\title{
OCEANOBS'19: AN OCEAN OF OPPORTUNITY. VOLUME I
}

EDITED BY: Tong Lee, Sabrina Speich, Laura Lorenzoni, Sanae Chiba, Frank E. Muller-Karger, Minhan Dai, Amos T. Kabo-Bah, John Siddorn, Justin Manley, Maria Snoussi and Fei Chai PUBLISHED IN: Frontiers in Marine Science

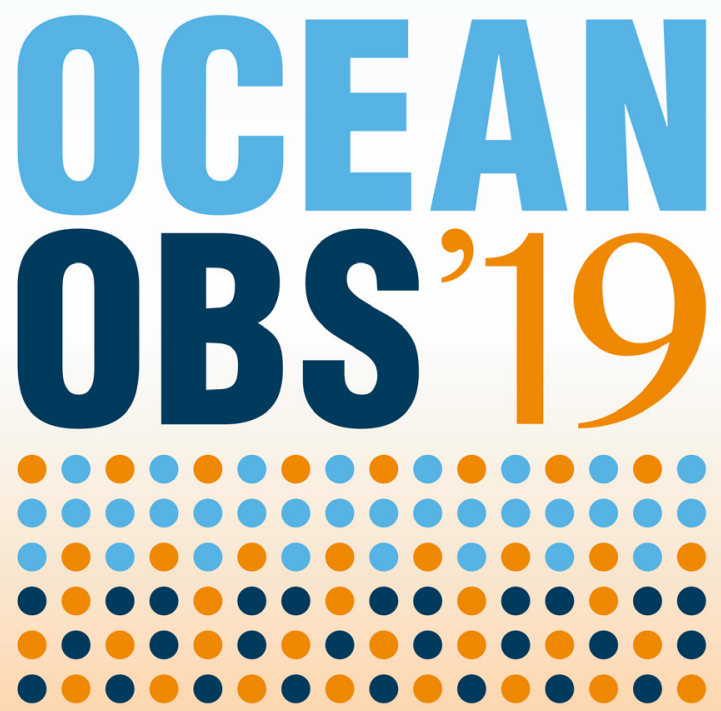

(1)

1000000

00000000000

10000000000000000

10000000000000000000000

10000000000000000000000000

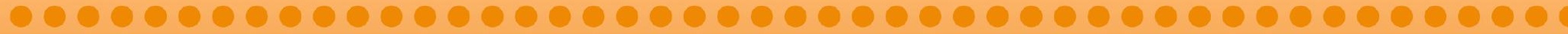
0000000000000000000000000000000000000000000000 10000000000000000000000000000000000000000000000 0000000000000000000000000000000000000000000000 


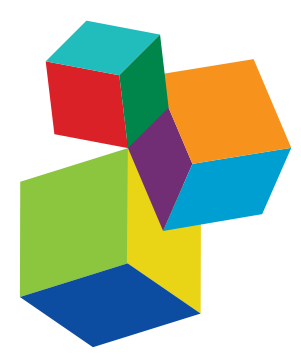

Frontiers eBook Copyright Statement

The copyright in the text of individual articles in this eBook is the property of their respective authors or their respective institutions or funders. The copyright in graphics and images within each article may be subject to copyright of other parties. In both cases this is subject to a license granted to Frontiers.

The compilation of articles constituting this eBook is the property of Frontiers.

Each article within this eBook, and the eBook itself, are published under the most recent version of the Creative Commons CC-BY licence.

The version current at the date of publication of this eBook is CC-BY 4.0. If the CC-BY licence is updated, the licence granted by Frontiers is automatically updated to the new version.

When exercising any right under the CC-BY licence, Frontiers must be attributed as the original publisher of the article or eBook, as applicable.

Authors have the responsibility of ensuring that any graphics or other materials which are the property of others may be included in the

CC-BY licence, but this should be checked before relying on the

CC-BY licence to reproduce those materials. Any copyright notices relating to those materials must be complied with.

Copyright and source acknowledgement notices may not be removed and must be displayed in any copy, derivative work or partial copy which includes the elements in question.

All copyright, and all rights therein, are protected by national and international copyright laws. The above represents a summary only.

For further information please read Frontiers' Conditions for Website

Use and Copyright Statement, and the applicable CC-BY licence.

ISSN 1664-8714

ISBN 978-2-88963-118-6 DOI 10.3389/978-2-88963-118-6

\section{About Frontiers}

Frontiers is more than just an open-access publisher of scholarly articles: it is a pioneering approach to the world of academia, radically improving the way scholarly research is managed. The grand vision of Frontiers is a world where all people have an equal opportunity to seek, share and generate knowledge. Frontiers provides immediate and permanent online open access to all its publications, but this alone is not enough to realize our grand goals.

\section{Frontiers Journal Series}

The Frontiers Journal Series is a multi-tier and interdisciplinary set of open-access, online journals, promising a paradigm shift from the current review, selection and dissemination processes in academic publishing. All Frontiers journals are driven by researchers for researchers; therefore, they constitute a service to the scholarly community. At the same time, the Frontiers Journal Series operates on a revolutionary invention, the tiered publishing system, initially addressing specific communities of scholars, and gradually climbing up to broader public understanding, thus serving the interests of the lay society, too.

\section{Dedication to Quality}

Each Frontiers article is a landmark of the highest quality, thanks to genuinely collaborative interactions between authors and review editors, who include some of the world's best academicians. Research must be certified by peers before entering a stream of knowledge that may eventually reach the public - and shape society; therefore, Frontiers only applies the most rigorous and unbiased reviews.

Frontiers revolutionizes research publishing by freely delivering the most outstanding research, evaluated with no bias from both the academic and social point of view. By applying the most advanced information technologies, Frontiers is catapulting scholarly publishing into a new generation.

\section{What are Frontiers Research Topics?}

Frontiers Research Topics are very popular trademarks of the Frontiers Journals Series: they are collections of at least ten articles, all centered on a particular subject. With their unique mix of varied contributions from Original Research to Review Articles, Frontiers Research Topics unify the most influential researchers, the latest key findings and historical advances in a hot research area! Find out more on how to host your own Frontiers Research Topic or contribute to one as an author by contacting the Frontiers Editorial Office: researchtopics@frontiersin.org 


\section{OCEANOBS'19: AN OCEAN OF OPPORTUNITY. VOLUME I}

Topic Editors:

Tong Lee, NASA Jet Propulsion Laboratory, United States

Sabrina Speich, Laboratoire de Météorologie Dynamique, ENS, France Laura Lorenzoni, NASA, United States and University of South Florida, United States

Sanae Chiba, Japan Agency for Marine-Earth Science and Technology, Japan Frank E. Muller-Karger, University of South Florida, United States

Minhan Dai, Xiamen University, China

Amos T. Kabo-Bah, University of Energy and Natural Resources, Ghana

John Siddorn, Met Office Exeter, United Kingdom

Justin Manley, Just Innovation Inc., United States

Maria Snoussi, Mohammed V University, Morocco

Fei Chai, Ministry of Natural Resources, China and University of Maine, United States

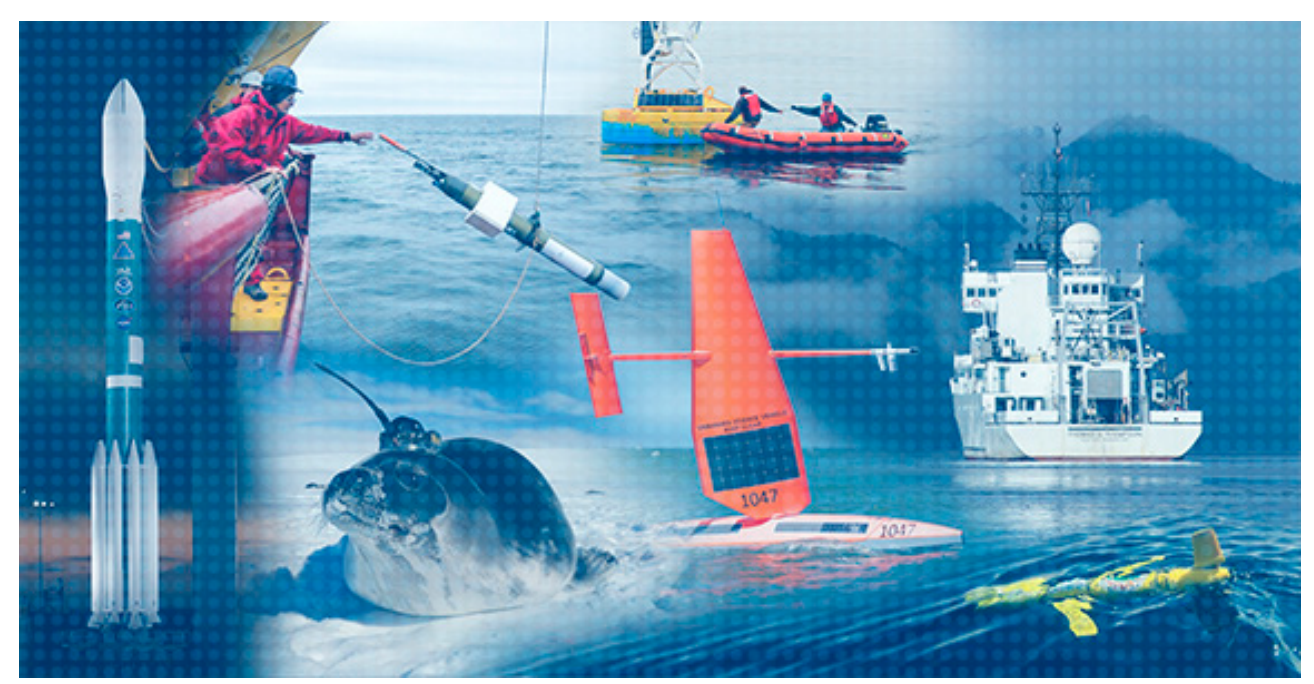

Cover and introductory image by Consortium for Ocean Leadership.

This eBook contains peer-reviewed community white papers (CWPs) as part of the community inputs to the OceanObs'19 Conference. The OceanObs conferences are held once every ten years for the scientific, technical, and operational communities involved in the planning, implementation, and use of ocean observing systems. The goal of the conferences is to communicate progress, promote plans, and to define advances to ocean observing system in response to societies' needs. Each conference provides a forum for the community to review the state of the ocean 
observing science and operations, and to define goals and plans to achieve over the next decade. The OceanObs'19 conference is the third in the series. It seeks to further align the science, technology, and human capacity of ocean observing to address growing and urgent societal needs.

CWPs have always been an integral part of the OceanObs conference series. The objective of this OceanObs'19 Research Topic in Frontiers in Marine Science is to provide a forum for community recommendations to inform the outcome of OceanObs'19 conference and to guide post-conference actions. The 140 CPWs collected under this Research Topic encompass perspectives from interested groups, including science, operational and commercial end-users, and stakeholders, on the needs and aspirations for the coming decade. Over 2500 authors from 79 countries contributed to the CWPs. These papers promote international collaboration, describe the status of a truly large-scale sustained ocean observing effort, and collectively help shape a vision for the future. They garner the collective knowledge of the community to evaluate and enhance the efficacy of our global and regional ocean observing networks.

The CWPs summarize key accomplishments in ocean observing, address gaps, and discuss the way forward. They specifically address improved connections between end users and providers of ocean observations, opportunities for integration of observing efforts and applications of information at the global and regional levels. Together, they contribute to a vision for ocean observing opportunities in the coming decade. For example, the importance of ocean observing as the key source of information on natural hazards (e.g., harmful algae and bacteria blooms, tsunamis, storm surges, marine heatwaves, and storms and other extreme weather events), ecosystem health and biodiversity (including shifting distributions of organisms and the increased risk of extinctions), ocean pollution (including acidification, de-oxygenation, and plastics), and sea level change are highlighted by various CWPs. They also identify substantial challenges that need to be overcome as a community, and offer suggestions for solutions. The needs for observations to support ecosystem-based management, marine and weather forecasting, climate predictions and projection, marine safety and navigation, decision support for climate adaptation, deep-ocean exploration, and seafloor mapping, among many other areas, are underscored. These issues are all at the core of a developing blue economy.

The papers address observing systems of various scales, including global ocean (e.g., Argo, GO-SHIP, Volunteer Observing Ships, and an active constellation of satellites), basin-scale (e.g., AtlantOS, Tropical Pacific Observing System 2020, Indian Ocean Observing System, Tropical Atlantic Observing System, Arctic Ocean and Southern Ocean observing systems, and a developing Deep Ocean Observing Strategy), regional (e.g., for boundary currents and inter-ocean exchanges), and coastal. They also address the goal of OceanObs'19 to further refine a governance framework that designates responsibility for product definition, production and timely delivery of fit-for-purpose information to serve user needs at the appropriate scales (global, basin, regional, national).

Taken together, the CWPs represent a call to governmental and non-governmental organizations, industries, scientists and technologists, stewards and citizens to work together to support furthering a coordinated development of the Global Ocean 
Observing System (GOOS) to ensure the delivery of information that will benefit human society over the long term. Together, the CWPs and OceanObs'19 will contribute to the development of a vibrant and blue economy that comprises many sectors, that supports policies that sustain development and conservation, and shape the next decade of ocean observing.

The organizers of the OceanObs'19 conference thank the authors that conceived and jointly crafted the CWPs for their tremendous efforts, extensive international collaborations, and community wisdom. The organizers also thank the hundreds of reviewers of the CWPs for their dedication, and the time invested in reviewing the papers. The organizers are also indebted to the entire team of Frontiers in Marine Science for their effort in handling the publications of the CWPs, and the compilation of the eBook.

The articles included in this version of the eBook include CWPs for the OceanObs'19 Research Topic published up to late July. Those published subsequently will be included in an updated version of the eBook.

Citation: Lee, T., Speich, S., Lorenzoni, L., Chiba, S., Muller-Karger, F. E., Dai, M., Kabo-Bah, A. T., Siddorn, J., Manley, J., Snoussi, M., Chai, F., eds. (2020).

Oceanobs'19: An Ocean of Opportunity. Volume I. Lausanne: Frontiers Media SA. doi: 10.3389/978-2-88963-118-6 


\section{Table of Contents}

13 Editorial: Oceanobs'19: An Ocean of Opportunity

Sabrina Speich, Tong Lee, Frank E. Muller-Karger, Laura Lorenzoni, Ananda Pascual, Di Jin, Eric Delory, Gilles Reverdin, John Siddorn, Marlon R. Lewis, Nuria Marba, Pier Luigi Buttigieg, Sanae Chiba, Justin Manley, Amos T. Kabo-Bah, Kruti Desai and Abby Ackerman

Marine Observing Applications Using AIS: Automatic Identification System

Darren Wright, Carol Janzen, Robert Bochenek, Jessica Austin and Edward Page

25 Global in situ Observations of Essential Climate and Ocean Variables at the Air-Sea Interface

Luca R. Centurioni, Jon Turton, Rick Lumpkin, Lancelot Braasch, Gary Brassington, Yi Chao, Etienne Charpentier, Zhaohui Chen, Gary Corlett, Kathleen Dohan, Craig Donlon, Champika Gallage, Verena Hormann, Alexander Ignatov, Bruce Ingleby, Robert Jensen, Boris A. Kelly-Gerreyn, Inga M. Koszalka, Xiaopei Lin, Eric Lindstrom, Nikolai Maximenko, Christopher J. Merchant, Peter Minnett, Anne O'Carroll, Theresa Paluszkiewicz, Paul Poli, Pierre-Marie Poulain, Gilles Reverdin, Xiujun Sun, Val Swail, Sidney Thurston, Lixin Wu, Lisan Yu, Bin Wang and Dongxiao Zhang

48 Adequacy of the Ocean Observation System for Quantifying Regional Heat and Freshwater Storage and Change

Matthew D. Palmer, Paul J. Durack, Maria Paz Chidichimo, John A. Church, Sophie Cravatte, Katy Hill, Johnny A. Johannessen, Johannes Karstensen, Tong Lee, David Legler, Matt Mazloff, Eitarou Oka, Sarah Purkey, Ben Rabe, Jean-Baptiste Sallée, Bernadette M. Sloyan, Sabrina Speich,

Karina von Schuckmann, Josh Willis and Susan Wijffels

74 Satellite Ocean Colour: Current Status and Future Perspective Steve Groom, Shubha Sathyendranath, Yai Ban, Stewart Bernard, Robert Brewin, Vanda Brotas, Carsten Brockmann, Prakash Chauhan, Jong-kuk Choi, Andrei Chuprin, Stefano Ciavatta, Paolo Cipollini, Craig Donlon, Bryan Franz, Xianqiang He, Takafumi Hirata, Tom Jackson, Milton Kampel, Hajo Krasemann, Samantha Lavender, Silvia Pardo-Martinez, Frédéric Mélin, Trevor Platt, Rosalia Santoleri, Jozef Skakala, Blake Schaeffer, Marie Smith, Francois Steinmetz, Andre Valente and Menghua Wang

104 ILTER - The International Long-Term Ecological Research Network as a Platform for Global Coastal and Ocean Observation

José H. Muelbert, Nicholas J. Nidzieko, Alicia T. R. Acosta, Stace E. Beaulieu, Angelo F. Bernardino, Elmira Boikova, Thomas G. Bornman, Bruno Cataletto, Klaas Deneudt, Erika Eliason, Alexandra Kraberg, Masahiro Nakaoka, Alessandra Pugnetti, Olivier Ragueneau, Mirco Scharfe, Thomas Soltwedel, Heidi M. Sosik, Angela Stanisci, Kremena Stefanova, Pierre Stéphan, Adrian Stier, Johan Wikner and Adriana Zingone 


\section{Toward the Integrated Marine Debris Observing System}

Nikolai Maximenko, Paolo Corradi, Kara Lavender Law, Erik Van Sebille, Shungudzemwoyo P. Garaba, Richard Stephen Lampitt, Francois Galgani, Victor Martinez-Vicente, Lonneke Goddijn-Murphy, Joana Mira Veiga, Richard C. Thompson, Christophe Maes, Delwyn Moller, Carolin Regina Löscher, Anna Maria Addamo, Megan R. Lamson, Luca R. Centurioni, Nicole R. Posth, Rick Lumpkin, Matteo Vinci, Ana Maria Martins, Catharina Diogo Pieper, Atsuhiko Isobe, Georg Hanke, Margo Edwards, Irina P. Chubarenko, Ernesto Rodriguez, Stefano Aliani, Manuel Arias, Gregory P. Asner, Alberto Brosich, James T. Carlton, Yi Chao, Anna-Marie Cook, Andrew B. Cundy, Tamara S. Galloway, Alessandra Giorgetti, Gustavo Jorge Goni, Yann Guichoux, Linsey E. Haram, Britta Denise Hardesty, Neil Holdsworth, Laurent Lebreton, Heather A. Leslie, Ilan Macadam-Somer, Thomas Mace, Mark Manuel, Robert Marsh, Elodie Martinez, Daniel J. Mayor, Morgan Le Moigne, Maria Eugenia Molina Jack, Matt Charles Mowlem, Rachel W. Obbard, Katsiaryna Pabortsava, Bill Robberson, Amelia-Elena Rotaru,

Gregory M. Ruiz, Maria Teresa Spedicato, Martin Thiel, Alexander Turra and Chris Wilcox

\section{Remotely Sensed Winds and Wind Stresses for Marine Forecasting and} Ocean Modeling

Mark A. Bourassa, Thomas Meissner, Ivana Cerovecki, Paul S. Chang, Xiaolong Dong, Giovanna De Chiara, Craig Donlon, Dmitry S. Dukhovskoy, Jocelyn Elya, Alexander Fore, Melanie R. Fewings, Ralph C. Foster, Sarah T. Gille, Brian K. Haus, Svetla Hristova-Veleva, Heather M. Holbach, Zorana Jelenak, John A. Knaff, Sven A. Kranz, Andrew Manaster, Matthew Mazloff, Carl Mears, Alexis Mouche, Marcos Portabella, Nicolas Reul, Lucrezia Ricciardulli, Ernesto Rodriguez, Charles Sampson, Daniel Solis, Ad Stoffelen, Michael R. Stukel, Bryan Stiles, David Weissman and Frank Wentz

\section{Observational Needs of Sea Surface Temperature}

Anne G. O'Carroll, Edward M. Armstrong, Helen M. Beggs, Marouan Bouali, Kenneth S. Casey, Gary K. Corlett, Prasanjit Dash, Craig J. Donlon, Chelle L. Gentemann, Jacob L. Høyer, Alexander Ignatov, Kamila Kabobah, Misako Kachi, Yukio Kurihara, Ioanna Karagali, Eileen Maturi, Christopher J. Merchant, Salvatore Marullo, Peter J. Minnett, Matthew Pennybacker, Balaji Ramakrishnan, RAAJ Ramsankaran, Rosalia Santoleri, Swathy Sunder, Stéphane Saux Picart, Jorge Vázquez-Cuervo and Werenfrid Wimmer

Measuring Global Ocean Heat Content to Estimate the Earth Energy Imbalance

Benoit Meyssignac, Tim Boyer, Zhongxiang Zhao, Maria Z. Hakuba, Felix W. Landerer, Detlef Stammer, Armin Köhl, Seiji Kato, Tristan L'Ecuyer, Michael Ablain, John Patrick Abraham, Alejandro Blazquez, Anny Cazenave, John A. Church, Rebecca Cowley, Lijing Cheng, Catia M. Domingues, Donata Giglio, Viktor Gouretski, Masayoshi Ishii, Gregory C. Johnson, Rachel E. Killick, David Legler, William Llovel, John Lyman, Matthew Dudley Palmer, Steve Piotrowicz, Sarah G. Purkey, Dean Roemmich, Rémy Roca, Abhishek Savita, Karina von Schuckmann, Sabrina Speich, Graeme Stephens, Gongjie Wang, Susan Elisabeth Wijffels and Nathalie Zilberman 
229 What we Have Learned From the Framework for Ocean Observing: Evolution of the Global Ocean Observing System

Toste Tanhua, Andrea McCurdy, Albert Fischer, Ward Appeltans, Nicholas Bax, Kim Currie, Brad DeYoung, Daniel Dunn, Emma Heslop, Linda K. Glover, John Gunn, Katherine Hill, Masao Ishii, David Legler, Eric Lindstrom, Patricia Miloslavich, Tim Moltmann, Glenn Nolan, Artur Palacz, Samantha Simmons, Bernadette Sloyan, Leslie M. Smith, Neville Smith, Maciej Telszewski, Martin Visbeck and John Wilkin

247 Ocean Climate Monitoring

Rick Cole, Jeff Kinder, Weidong Yu, Chun Lin Ning, Fujun Wang and Yang Chao

252 Public-Private Partnerships to Advance Regional Ocean-Observing Capabilities: A Saildrone and NOAA-PMEL Case Study and Future Considerations to Expand to Global Scale Observing

Christian Meinig, Eugene F. Burger, Nora Cohen, Edward D. Cokelet, Meghan F. Cronin, Jessica N. Cross, Sebastien de Halleux, Richard Jenkins, Andrew T. Jessup, Calvin W. Mordy, Noah Lawrence-Slavas, Adrienne J. Sutton, Dongxiao Zhang and Chidong Zhang

267 SEASTAR: A Mission to Study Ocean Submesoscale Dynamics and Small-Scale Atmosphere-Ocean Processes in Coastal, Shelf and Polar Seas

Christine Gommenginger, Bertrand Chapron, Andy Hogg,

Christian Buckingham, Baylor Fox-Kemper, Leif Eriksson, Francois Soulat, Clément Ubelmann, Francisco Ocampo-Torres, Bruno Buongiorno Nardelli, David Griffin, Paco Lopez-Dekker, Per Knudsen, Ole Andersen, Lars Stenseng, Neil Stapleton, William Perrie, Nelson Violante-Carvalho, Johannes Schulz-Stellenfleth, David Woolf, Jordi Isern-Fontanet, Fabrice Ardhuin, Patrice Klein, Alexis Mouche, Ananda Pascual, Xavier Capet, Daniele Hauser, Ad Stoffelen, Rosemary Morrow, Lotfi Aouf, Øyvind Breivik, Lee-Lueng Fu, Johnny A. Johannessen, Yevgeny Aksenov, Lucy Bricheno, Joel Hirschi, Adrien C. H. Martin, Adrian P. Martin, George Nurser, Jeff Polton, Judith Wolf, Harald Johnsen, Alexander Soloviev, Gregg A. Jacobs, Fabrice Collard, Steve Groom, Vladimir Kudryavtsev, John Wilkin, Victor Navarro, Alex Babanin, Matthew Martin, John Siddorn, Andrew Saulter, Tom Rippeth, Bill Emery, Nikolai Maximenko, Roland Romeiser, Hans Graber, Aida Alvera Azcarate, Chris W. Hughes, Doug Vandemark, Jose da Silva, Peter Jan Van Leeuwen, Alberto Naveira-Garabato, Johannes Gemmrich, Amala Mahadevan, Jose Marquez, Yvonne Munro, Sam Doody and Geoff Burbidge

274 The Development of a Canadian Integrated Ocean Observing System (CIOOS)

Andrew Stewart, Brad deYoung, Mike Smit, Karen Donaldson, Alexa Reedman, Andréane Bastien, Ray Brunsting, Bill Carter, Brad Covey, Richard Kelly, Eric Peterson, Benoît Pirenne, Ariane Plourde, Anne-Sophie Ste-Marie, Cristina Tollefsen, Douglas Wallace and Fred Whoriskey 
281 Ocean Observations to Improve Our Understanding, Modeling, and Forecasting of Subseasonal-to-Seasonal Variability

Aneesh C. Subramanian, Magdalena A. Balmaseda, Luca Centurioni, Rajib Chattopadhyay, Bruce D. Cornuelle, Charlotte DeMott, Maria Flatau, Yosuke Fujii, Donata Giglio, Sarah T. Gille, Thomas M. Hamill, Harry Hendon, Ibrahim Hoteit, Arun Kumar, Jae-Hak Lee, Andrew J. Lucas,

Amala Mahadevan, Mio Matsueda, SungHyun Nam, Shastri Paturi, Stephen G. Penny, Adam Rydbeck, Rui Sun, Yuhei Takaya, Amit Tandon, Robert E. Todd, Frederic Vitart, Dongliang Yuan and Chidong Zhang

A Framework for the Development, Design and Implementation of a Sustained Arctic Ocean Observing System

Craig M. Lee, Sandy Starkweather, Hajo Eicken, Mary-Louise Timmermans, Jeremy Wilkinson, Stein Sandven, Dmitry Dukhovskoy, Sebastian Gerland, Jacqueline Grebmeier, Janet M. Intrieri, Sung-Ho Kang, Molly McCammon, An T. Nguyen, Igor Polyakov, Benjamin Rabe, Hanne Sagen, Sophie Seeyave, Denis Volkov, Agnieszka Beszczynska-Möller, Léon Chafik, Matthew Dzieciuch, Gustavo Goni, Torill Hamre, Andrew Luke King, Are Olsen, Roshin P. Raj, Thomas Rossby, Øystein Skagseth, Henrik Søiland and Kai Sørensen

310 Delivering Sustained, Coordinated, and Integrated Observations of the Southern Ocean for Global Impact

Louise Newman, Petra Heil, Rowan Trebilco, Katsuro Katsumata, Andrew Constable, Esmee van Wijk, Karen Assmann, Joana Beja, Phillippa Bricher, Richard Coleman, Daniel Costa, Steve Diggs,

Riccardo Farneti, Sarah Fawcett, Sarah T. Gille, Katharine R. Hendry, Sian Henley, Eileen Hofmann, Ted Maksym, Matthew Mazloff, Andrew Meijers, Michael M. Meredith, Sebastien Moreau, Burcu Ozsoy, Robin Robertson, Irene Schloss, Oscar Schofield, Jiuxin Shi, Elisabeth Sikes, Inga J. Smith, Sebastiaan Swart, Anna Wahlin, Guy Williams, Michael J. M. Williams, Laura Herraiz-Borreguero, Stefan Kern, Jan Lieser, Robert A. Massom, Jessica Melbourne-Thomas, Patricia Miloslavich and Gunnar Spreen

341 Global Perspectives on Observing Ocean Boundary Current Systems Robert E. Todd, Francisco P. Chavez, Sophie Clayton, Sophie Cravatte, Marlos Goes, Michelle Graco, Xiaopei Lin, Janet Sprintall, Nathalie V. Zilberman, Matthew Archer, Javier Arístegui, Magdalena Balmaseda, John M. Bane, Molly O. Baringer, John A. Barth, Lisa M. Beal, Peter Brandt, Paulo H. R. Calil, Edmo Campos,

Luca R. Centurioni, Maria Paz Chidichimo, Mauro Cirano, Meghan F. Cronin, Enrique N. Curchitser, Russ E. Davis, Marcus Dengler, Brad deYoung, Shenfu Dong, Ruben Escribano, Andrea J. Fassbender, Sarah E. Fawcett, Ming Feng, Gustavo J. Goni, Alison R. Gray, Dimitri Gutiérrez, Dave Hebert, Rebecca Hummels, Shin-ichi Ito, Marjorlaine Krug, François Lacan, Lucas Laurindo, Alban Lazar, Craig M. Lee, Matthieu Lengaigne, Naomi M. Levine, John Middleton, Ivonne Montes, Mike Muglia, Takeyoshi Nagai, Hilary I. Palevsky, Jaime B. Palter, Helen E. Phillips, Alberto Piola, Albert J. Plueddemann, Bo Qiu, Regina R. Rodrigues, Moninya Roughan, Daniel L. Rudnick, Ryan R. Rykaczewski, Martin Saraceno, Harvey Seim, Alex Sen Gupta, Lynne Shannon, Bernadette M. Sloyan, Adrienne J. Sutton, LuAnne Thompson, Anja K. van der Plas, Denis Volkov, John Wilkin, Dongxiao Zhang and Linlin Zhang 
379 The Global Ocean Ship-Based Hydrographic Investigations Program (GO-SHIP): A Platform for Integrated Multidisciplinary Ocean Science

Bernadette M. Sloyan, Rik Wanninkhof, Martin Kramp, Gregory C. Johnson, Lynne D. Talley, Toste Tanhua, Elaine McDonagh, Caroline Cusack, Eleanor O'Rourke, Evin McGovern, Katsuro Katsumata, Steve Diggs, Julia Hummon, Masao Ishii, Kumiko Azetsu-Scott, Emmanuel Boss, Isabelle Ansorge, Fiz F. Perez, Herlé Mercier, Michael J. M. Williams, Leif Anderson, Jae Hak Lee, Akihiko Murata, Shinya Kouketsu, Emil Jeansson, Mario Hoppema and Edmo Campos

400 Ocean FAIR Data Services

Toste Tanhua, Sylvie Pouliquen, Jessica Hausman, Kevin O'Brien, Pip Bricher, Taco de Bruin, Justin J. H. Buck, Eugene F. Burger, Thierry Carval, Kenneth S. Casey, Steve Diggs, Alessandra Giorgetti, Helen Glaves, Valerie Harscoat, Danie Kinkade, Jose H. Muelbert, Antonio Novellino, Benjamin Pfeil, Peter L. Pulsifer, Anton Van de Putte, Erin Robinson, Dick Schaap, Alexander Smirnov, Neville Smith, Derrick Snowden, Tobias Spears, Shelley Stall, Marten Tacoma, Peter Thijsse, Stein Tronstad, Thomas Vandenberghe, Micah Wengren, Lesley Wyborn and Zhiming Zhao

417 Evolving the Physical Global Ocean Observing System for Research and Application Services Through International Coordination

Bernadette M. Sloyan, John Wilkin, Katherine Louise Hill, Maria Paz Chidichimo, Meghan F. Cronin, Johnny A. Johannessen, Johannes Karstensen, Marjolaine Krug, Tong Lee, Eitarou Oka, Matthew D. Palmer, Benjamin Rabe, Sabrina Speich,

Karina von Schuckmann, Robert A. Weller and Weidong Yu

430 Polar Ocean Observations: A Critical Gap in the Observing System and its Effect on Environmental Predictions From Hours to a Season

Gregory C. Smith, Richard Allard, Marcel Babin, Laurent Bertino, Matthieu Chevallier, Gary Corlett, Julia Crout, Fraser Davidson, Bruno Delille, Sarah T. Gille, David Hebert, Patrick Hyder, Janet Intrieri, José Lagunas, Gilles Larnicol, Thomas Kaminski, Belinda Kater, Frank Kauker, Claudie Marec, Matthew Mazloff, E. Joseph Metzger, Calvin Mordy, Anne O'Carroll, Steffen M. Olsen, Michael Phelps, Pamela Posey, Pierre Prandi, Eric Rehm, Phillip Reid, Ignatius Rigor, Stein Sandven, Matthew Shupe, Sebastiaan Swart, Ole Martin Smedstad, Amy Solomon, Andrea Storto, Pierre Thibaut, John Toole, Kevin Wood, Jiping Xie, Qinghua Yang and the WWRP PPP Steering Group

458 SMART Cables for Observing the Global Ocean: Science and Implementation

Bruce M. Howe, Brian K. Arbic, Jérome Aucan, Christopher R. Barnes, Nigel Bayliff, Nathan Becker, Rhett Butler, Laurie Doyle, Shane Elipot, Gregory C. Johnson, Felix Landerer, Stephen Lentz, Douglas S. Luther, Malte Müller, John Mariano, Kate Panayotou, Charlotte Rowe, Hiroshi Ota, Y. Tony Song, Maik Thomas, Preston N. Thomas, Philip Thompson, Frederik Tilmann, Tobias Weber and Stuart Weinstein on behalf of the Joint Task Force for SMART Cables 
Dean Roemmich, Matthew H. Alford, Hervé Claustre, Kenneth Johnson, Brian King, James Moum, Peter Oke, W. Brechner Owens, Sylvie Pouliquen, Sarah Purkey, Megan Scanderbeg, Toshio Suga, Susan Wijffels, Nathalie Zilberman, Dorothee Bakker, Molly Baringer, Mathieu Belbeoch, Henry C. Bittig, Emmanuel Boss, Paulo Calil, Fiona Carse, Thierry Carval, Fei Chai, Diarmuid Ó. Conchubhair, Fabrizio d'Ortenzio, Giorgio Dall'Olmo, Damien Desbruyeres, Katja Fennel, Ilker Fer, Raffaele Ferrari, Gael Forget, Howard Freeland, Tetsuichi Fujiki, Marion Gehlen, Blair Greenan, Robert Hallberg, Toshiyuki Hibiya, Shigeki Hosoda, Steven Jayne, Markus Jochum, Gregory C. Johnson, KiRyong Kang, Nicolas Kolodziejczyk, Arne Körtzinger, Pierre-Yves Le Traon, Yueng-Djern Lenn, Guillaume Maze, Kjell Arne Mork, Tamaryn Morris, Takeyoshi Nagai, Jonathan Nash, Alberto Naveira Garabato, Are Olsen, Rama Rao Pattabhi, Satya Prakash, Stephen Riser, Catherine Schmechtig, Claudia Schmid, Emily Shroyer, Andreas Sterl, Philip Sutton, Lynne Talley, Toste Tanhua, Virginie Thierry, Sandy Thomalla, John Toole, Ariel Troisi, Thomas W. Trull, Jon Turton, Pedro Joaquin Velez-Belchi, Waldemar Walczowski, Haili Wang,

Rik Wanninkhof, Amy F. Waterhouse, Stephanie Waterman, Andrew Watson, Cara Wilson, Annie P. S. Wong, Jianping Xu and Ichiro Yasuda

513 Lessons From the Pacific Ocean Portal: Building Pacific Island Capacity to Interpret, Apply, and Communicate Ocean Information

Molly Powers, Zulfikar Begg, Grant Smith and Elaine Miles

520 The Joint IOC (of UNESCO) and WMO Collaborative Effort for Met-Ocean Services

Nadia Pinardi, Johan Stander, David M. Legler, Kevin O'Brien, Tim Boyer, Tom Cuff, Pierre Bahurel, Mathieu Belbeoch, Sergey Belov, Shelby Brunner, Eugene Burger, Thierry Carval, Denis Chang-Seng, Etienne Charpentier, S. Ciliberti, Giovanni Coppini, Albert Fischer, Eric Freeman, Champika Gallage, Hernan Garcia, Lydia Gates, Zhiqiang Gong, Juliet Hermes, Emma Heslop, Sarah Grimes, Katherine Hill, Kevin Horsburgh, Athanasia Iona, Sebastien Mancini, Neal Moodie, Mathieu Ouellet, Peter Pissierssens, Paul Poli, Roger Proctor, Neville Smith, Charles Sun, Val Swail, Jonathan Turton and Yue Xinyang

543 Ship-Based Contributions to Global Ocean, Weather, and Climate Observing Systems

Shawn R. Smith, Gaël Alory, Axel Andersson, William Asher, Alex Baker, David I. Berry, Kyla Drushka, Darin Figurskey, Eric Freeman, Paul Holthus, Tim Jickells, Henry Kleta, Elizabeth C. Kent, Nicolas Kolodziejczyk, Martin Kramp, Zoe Loh, Paul Poli, Ute Schuster, Emma Steventon, Sebastiaan Swart, Oksana Tarasova, Loic Petit de la Villéon and Nadya Vinogradova-Shiffer

569 Ocean Reanalyses: Recent Advances and Unsolved Challenges Andrea Storto, Aida Alvera-Azcárate, Magdalena A. Balmaseda, Alexander Barth, Matthieu Chevallier, Francois Counillon, Catia M. Domingues, Marie Drevillon, Yann Drillet, Gaël Forget, Gilles Garric, Keith Haines, Fabrice Hernandez, Doroteaciro lovino, Laura C. Jackson, Jean-Michel Lellouche, Simona Masina, Michael Mayer, Peter R. Oke, Stephen G. Penny, K. Andrew Peterson, Chunxue Yang and Hao Zuo 
579 Ocean Climate Observing Requirements in Support of Climate Research and Climate Information

Detlef Stammer, Annalisa Bracco, Krishna AchutaRao, Lisa Beal, Nathaniel L. Bindoff, Pascale Braconnot, Wenju Cai, Dake Chen, Matthew Collins, Gokhan Danabasoglu, Boris Dewitte, Riccardo Farneti, Baylor Fox-Kemper, John Fyfe, Stephen M. Griffies, Steven R. Jayne, Alban Lazar, Matthieu Lengaigne, Xiaopei Lin, Simon Marsland, Shoshiro Minobe, Pedro M. S. Monteiro, Walter Robinson, Mathew Koll Roxy, Ryan R. Rykaczewski, Sabrina Speich, Inga J. Smith, Amy Solomon, Andrea Storto, Ken Takahashi, Thomas Toniazzo and Jerome Vialard

597 Air-Sea Fluxes With a Focus on Heat and Momentum

Meghan F. Cronin, Chelle L. Gentemann, James Edson, Iwao Ueki, Mark Bourassa, Shannon Brown, Carol Anne Clayson, Chris W. Fairall, J. Thomas Farrar, Sarah T. Gille, Sergey Gulev, Simon A. Josey, Seiji Kato, Masaki Katsumata, Elizabeth Kent, Marjolaine Krug, Peter J. Minnett, Rhys Parfitt, Rachel T. Pinker, Paul W. Stackhouse Jr., Sebastiaan Swart, Hiroyuki Tomita, Douglas Vandemark, Robert A.Weller, Kunio Yoneyama, Lisan Yu and Dongxiao Zhang

627 Constraining Southern Ocean Air-Sea-Ice Fluxes Through Enhanced Observations

Sebastiaan Swart, Sarah T. Gille, Bruno Delille, Simon Josey, Matthew Mazloff, Louise Newman, Andrew F. Thompson, Jim Thomson, Brian Ward, Marcel D. du Plessis, Elizabeth C. Kent, James Girton, Luke Gregor, Petra Heil, Patrick Hyder, Luciano Ponzi Pezzi, Ronald Buss de Souza, Veronica Tamsitt, Robert A. Weller and Christopher J. Zappa

637 The UN Decade of Ocean Science for Sustainable Development Vladimir Ryabinin, Julian Barbière, Peter Haugan, Gunnar Kullenberg, Neville Smith, Craig McLean, Ariel Troisi, Albert Fischer, Salvatore Aricò, Thorkild Aarup, Peter Pissierssens, Martin Visbeck, Henrik Oksfeldt Enevoldsen and Julie Rigaud

647 Observing Requirements for Long-Term Climate Records at the Ocean Surface

Elizabeth C. Kent, Nick A. Rayner, David I. Berry, Ryan Eastman, Vika G. Grigorieva, Boyin Huang, John J. Kennedy, Shawn R. Smith and Kate M. Willett

675 Ocean Observations in Support of Studies and Forecasts of Tropical and Extratropical Cyclones

Ricardo Domingues, Akira Kuwano-Yoshida, Patricia Chardon-Maldonado, Robert E. Todd, George Halliwell, Hyun-Sook Kim, I.-I. Lin, Katsufumi Sato, Tomoko Narazaki, Lynn K. Shay, Travis Miles, Scott Glenn, Jun A. Zhang, Steven R. Jayne, Luca Centurioni, Matthieu Le Hénaff, Gregory R. Foltz, Francis Bringas, M. M. Ali, Steven F. DiMarco, Shigeki Hosoda, Takuya Fukuoka, Benjamin LaCour, Avichal Mehra, Elizabeth R. Sanabia, John R. Gyakum, Jili Dong, John A. Knaff and Gustavo Goni 
698 Observing System Evaluation Based on Ocean Data Assimilation and Prediction Systems: On-Going Challenges and a Future Vision for Designing and Supporting Ocean Observational Networks

Yosuke Fujii, Elisabeth Rémy, Hao Zuo, Peter Oke, George Halliwell, Florent Gasparin, Mounir Benkiran, Nora Loose, James Cummings, Jiping Xie, Yan Xue, Shuhei Masuda, Gregory C. Smith, Magdalena Balmaseda, Cyril Germineaud, Daniel J. Lea, Gilles Larnicol, Laurent Bertino, Antonio Bonaduce, Pierre Brasseur, Craig Donlon, Patrick Heimbach, YoungHo Kim, Villy Kourafalou, Pierre-Yves Le Traon, Matthew Martin, Shastri Paturi, Benoit Tranchant and Norihisa Usui

723 An Integrated All-Atlantic Ocean Observing System in 2030

Brad deYoung, Martin Visbeck, Moacyr Cunha de Araujo Filho, Molly O'Neil Baringer, CarolAnne Black, Erik Buch, Gabrielle Canonico, Paulo Coelho, Janice T. Duha, Martin Edwards, Albert Fischer, Jan-Stefan Fritz, Sandra Ketelhake, Jose-Henrique Muelbert, Pedro Monteiro, Glenn Nolan, Eleanor O'Rourke, Michael Ott, Pierre Y. Le Traon, Sylvie Pouliquen, Isabel Sousa-Pinto, Toste Tanhua, Filomena V. Velho and Zdenka Willis

745 Observing the Oceans Acoustically Bruce M. Howe, Jennifer Miksis-Olds, Eric Rehm, Hanne Sagen, Peter F. Worcester and Georgios Haralabus

767 Data Interoperability Between Elements of the Global Ocean Observing System

Derrick Snowden, Vardis M. Tsontos, Nils Olav Handegard, Marcos Zarate, Kevin O' Brien, Kenneth S. Casey, Neville Smith, Helge Sagen, Kathleen Bailey, Mirtha N. Lewis and Sean C. Arms 


\section{Editorial: Oceanobs'19: An Ocean of Opportunity}

\author{
Sabrina Speich ${ }^{1 *}$, Tong Lee ${ }^{2}$, Frank Muller-Karger ${ }^{3}$, Laura Lorenzoni ${ }^{4}$, Ananda Pascual ${ }^{5}$, \\ Di Jin $^{6}$, Eric Delory ${ }^{7}$, Gilles Reverdin ${ }^{8}$, John Siddorn ${ }^{9}$, Marlon R. Lewis ${ }^{10}$, Nuria Marba ${ }^{5}$, \\ Pier Luigi Buttigieg ${ }^{11}$, Sanae Chiba ${ }^{12}$, Justin Manley ${ }^{13}$, Amos Tiereyangn Kabo-Bah ${ }^{14}$, \\ Kruti Desai ${ }^{15}$ and Abby Ackerman ${ }^{15}$ \\ 1 Laboratoire de Meteorologie Dynamique, UMR 8539 Ecole Polytechnique, ENS, CNRS, Paris, France, ${ }^{2}$ NASA Jet \\ Propulsion Laboratory, California Institute of Technology, Pasadena, CA, United States, ${ }^{3}$ College of Marine Science, \\ University of South Florida, St. Petersburg, FL, United States, ${ }^{4}$ NASA Headquarters, Science Mission Directorate, Earth \\ Science Division, Washington, DC, United States, ${ }^{5}$ Instituto Mediterráneo de Estudios Avanzados (CSIC-UIB), Esporles, \\ Spain, ${ }^{6}$ Marine Policy Center, Woods Hole Oceanographic Institution, Woods Hole, MA, United States, ${ }^{7}$ Oceanic Platform of \\ the Canary Islands, Telde, Spain, ${ }^{8}$ Laboratoire d'Océanographie et du Climat: Expérimentations et Approches Numériques, \\ Paris, France, ${ }^{9}$ Met Office, Exeter, United Kingdom, ${ }^{10}$ Department of Oceanography, Dalhousie University, Halifax, NS, \\ Canada, ${ }^{11}$ HGF MPG Joint Research Group for Deep-Sea Ecology and Technology, Max Planck Institute for Marine \\ Microbiology, Bremen, Germany, ${ }^{12}$ Japan Agency for Marine-Earth Science and Technology, Yokosuka, Japan, ${ }^{13}$ Just \\ Innovation, Inc., Duxbury, MA, United States, ${ }^{14}$ Department of Energy and Environmental Engineering, University of Energy \\ and Natural Resources, Sunyani, Ghana, ${ }^{15}$ Consortium for Ocean Leadership, Washington, DC, United States
}

Keywords: OceanObs'19, ocean observing, innovation, information, integration, interoperability, governance

\section{Editorial on the Research Topic}

\section{OPEN ACCESS}

Edited and reviewed by: Carlos M. Duarte,

King Abdullah University of Science and Technology, Saudi Arabia

${ }^{*}$ Correspondence: Sabrina Speich sabrina.speich@lmd.ens.fr

Specialty section:

This article was submitted to Ocean Observation,

a section of the journal Frontiers in Marine Science

Received: 23 August 2019 Accepted: 28 August 2019 Published: 06 September 2019

Citation: Speich S, Lee T, Muller-Karger F, Lorenzoni L, Pascual A, Jin D, Delory E, Reverdin G, Siddorn J, Lewis MR, Marba N, Buttigieg PL, Chiba S, Manley J, Kabo-Bah AT, Desai K and Ackerman A (2019) Editorial: Oceanobs'19: An Ocean of Opportunity. Front. Mar. Sci. 6:570.

doi: 10.3389/fmars.2019.00570
Oceanobs'19: An Ocean of Opportunity

\section{BRIEF HISTORY OF THE OCEANOBS DECADAL CONFERENCE SERIES}

The OceanObs conferences are held once every 10 years for the scientific, technical, and operational communities involved in the planning, implementation, and use of ocean observing systems. They serve to communicate progress, promote plans, and to define advances in ocean observing in response to societies' needs. Each conference provides a forum for the community to review the state of the ocean observing science and operations, and to define goals and plans to achieve over the next decade.

The First International Conference on the Ocean Observing System for Climate (OceanObs'99, Saint Raphaël, France, 17-22 Oct 1999) was jointly convened by the Ocean Observations Panel for Climate (OOPC) and the World Climate Research Program's Climate Variability and Predictability Programme (CLIVAR) Upper Ocean Panel (UOP). The main objective was to define the operational measurements needed by several related programs, specifically CLIVAR, the Global Ocean Observing System (GOOS), and the Global Climate Observing System (GCOS). OceanObs'99 brought together about 300 attendees. The conference addressed technical and scientific challenges of ocean observing systems, and also covered data analysis, modeling, and assimilation. This conference led to concrete actions. Most importantly, consensus was reached within the ocean observing community to establish and grow an internationally coordinated ocean observing system for global physical climate and ocean carbon. The details of this system were subsequently defined and presented in the first GCOS Implementation Plan. OceanObs'99 also emphasized amongst other networks the need to build an international autonomous float program. This opportunity was taken up by sponsors, and the Argo system now provides critical operational data supporting 
a diverse range of services through improved short-term to seasonal weather and marine forecasting, and a better understanding of climate change, among many other benefits.

OceanObs'09 (21-25 September 2009, Venice, Italy) celebrated a decade of progress in implementing the initial ocean observing system. The conference convened over 600 scientists from 36 countries who represented diverse ocean stakeholder communities. Among the successes of this conference was a revolution in how physical and chemical oceanographers agreed to share data to better understand the global ocean. The outcomes included benefits through better weather forecasting, quantifying the change in $\mathrm{pH}$ of the global ocean, better understanding the physical and biogeochemical interactions between the ocean and the atmosphere, and heightened awareness that improvements in the tracking of biological variables are required. Many of these efforts led to major advances in understanding and projecting climate change. The conference developed the Framework for Ocean Observing (FOO; Lindstrom et al., 2012), which provided a strategy for defining and implementing physical, biogeochemical, and biological observing needs. This conference demonstrated the scientific and societal benefits of a sustained ocean observing system.

Now, the OceanObs'19 conference (16-20 September, 2019) seeks to further align the science, technology, and human capacity of ocean observing to address growing and urgent societal needs. The 140 Community White Papers collected under this Frontiers in Marine Science OceanObs'19 Research Topic summarize the perspective from all interested groups, including science, operational and commercial end-users, on the needs and aspirations for the coming decade. The aspirations span a broad set of communities, such as addressing the requirements of fisheries and ecosystem-based management practitioners, the maritime sector, national and local authorities, search and rescue, public and private sector researchers, and the public in general. An important goal is to further refine a governance framework that designates responsibility for product definition, production and timely delivery of fit-for-purpose information to serve user needs at the appropriate scales (global, basin, regional, national).

To reach these ambitious goals, the OceanObs'19 conference is focused around seven vital themes: Ecosystem Health and Biodiversity, Climate Variability and Change, Water Food and Energy Security, Pollution and Human Health, Ocean Hazards and Maritime Safety, Discovery, and Blue Economy. Two cross-cutting topics, Data and Information Systems and Observing Technologies and Networks, connect and integrate these themes. An overarching subject is Observing System Governance, which focuses all actors on adopting best practices for observing Essential Ocean Variables. It establishes communications protocols between elements of the Global Ocean Observing System. In addition, it guides activities to address gaps in our capacity to observe life in the sea. Ultimately, governance coordinates advocacy and planning for the funding needed to sustain ocean observations. Much of this coordination falls on the shoulders of the GOOS, and OceanObs'19 calls for the global community to participate and support this enormous effort.

\section{OBJECTIVE OF THE OCEANOBS'19 RESEARCH TOPIC COLLECTION}

Community White Papers have been an integral part of the OceanObs conference series. The objective of the OceanObs'19 Research Topic in Frontiers in Marine Science is to provide a forum for community recommendations to inform the outcome of OceanObs'19 conference and to guide post-conference activities. The community responded to a call for input to OceanObs'19 with an overwhelming number of concepts and ideas, by submitting 430 abstracts as proposals for white papers. These abstracts were refined and combined into 140 Community White Papers (CWPs). These papers promote international collaboration, describe the status of a truly largescale sustained ocean observing effort, and collectively help shape a vision for the future. They garner the collective knowledge of the community to evaluate and enhance the efficacy of our global and regional ocean observing networks. Each CWP addresses one or more of the seven themes described above and the two crosscutting topics, and makes recommendations on ocean observing governance. The CWPs specifically address improved connections between end users and providers of ocean observations, opportunities for integration of observing efforts and applications of information at the global and regional levels, and contribute to a vision in terms of ocean observing opportunities in the coming decade. They also identify substantial challenges that we need to overcome as a community and offer suggestions for solutions.

\section{BROADER CONTEXT}

The ocean provides essential services to our society. Ocean life and water quality are at the heart of the tourism, fisheries, and industries that provide benefits to millions of people. The ocean provides renewable energy and is major sources of oil, gas, and other minerals. By absorbing $\mathrm{CO}_{2}$ emissions from the combustion of fossil fuels and land use changes, the ocean moderates global warming.

Ocean observing systems are now essential to monitoring and forecasting changes in Earth's weather (minutes to days) and climate (decades to centuries; IPCC, 2013; Gleckler et al., 2016; Cheng et al., 2019). The public increasingly recognizes the critical importance of information about current and future ocean conditions to support diverse user needs. This includes better observations and predictions of wave, current, sea level, water quality, the abundance marine living resources, as well as improving marine, weather, and climate forecasting services. Major international weather and climate forecasting groups, including the European Centre for Medium-Range Weather Forecasts (ECMWF, 2016), the NOAA National Weather Service, and the World Meteorological Organization, have requirements for ocean information to enable a resilient and sustainable blue economy (WMO, 2019¹).

\footnotetext{
${ }^{1}$ https://en.unesco.org/news/new-agreement-bolsters-work-ocean-observationsresearch-and-services; https://public.wmo.int/en/media/news/wmo-hostsoceans-meetings
} 
Today, over $40 \%$ of the global human population lives within $200 \mathrm{~km}$ of the ocean. Intense urban and industrial development are exerting increasing pressures on the ocean, especially on marine ecosystems on which all human societies depend. Climate change, resource extraction, pollution from our activities on land and in the ocean, and habitat degradation due to other human activities threaten the sustained productivity and health of the ocean. The fundamental link between the ocean and human livelihood and health has led to a global social outcry over the irresponsible use of marine ecosystems. This, among other global challenges, has led the United Nations to formulate a set of Sustainable Development Goals (SDG's; 2030 Agenda for Sustainable Development). The 2030 Agenda recognizes the connection between the ocean and human well-being under SDG14 and many other interlinked SDG's (Visbeck et al., 2014; United Nations General Assembly, 2015). With this momentum, the first-ever UN Ocean conference (United Nations, 2017) was convened to support the implementation of the SDG's. Subsequently, the UN proclaimed a Decade of Ocean Science for Sustainable Development (2021-2030). The Decade of Ocean Science seeks to mobilize the scientific community, policymakers, business, and civil society around a program of joint research and technological innovation (UNESCO, 2017).

The outcomes of the OceanObs'19 conference represent a critical benchmark on how we address the challenge of sustainable development, and will significantly contribute to the Decade of Ocean Science. It is becoming more important than ever to integrate ocean observation and research agendas to meet societal needs (Visbeck, 2018). This opens opportunities for all nations to more actively engage in capacity development and in wise resource management. It provides opportunities for coastal and small island states to establish effective collaborations in multi-stakeholder processes to advance maritime activities, marine spatial planning, and effective ocean governance. It also helps to develop innovative strategies to adapt to climate change and to mitigate its impacts.

\section{DEMOGRAPHICS OF COMMUNITY WHITE PAPER PARTICIPATION}

The CWPs were contributed by more than 2,500 authors from over 79 countries. Figure 1 illustrates the geographic distributions of the authors. The authors represent organizations from various sectors, including governmental organizations (including civil and military), non-governmental organizations, non-profit organizations, industry, academic institutions, and private citizens. The number of authors, and the countries and sectors they represent, reflect the overwhelming interest and active involvement of the community. The community has high expectations for OceanObs'19 to shape the next decade of ocean observing.

\section{TOPICS OF THE COMMUNITY WHITE PAPERS IN THE COLLECTION}

The OceanObs'19 CWPs provide important contributions to the overall conference objectives (http://www.oceanobs19.net/ mission-and-vision/). The recommendations included in these papers contributed to the outcome of the conference by addressing the strategies and actions in four broad topics:

- Information to meet future user needs;

- Innovation to spur observing technologies, products, and user services;

- Integration to balance user and operator needs, capabilities, and knowledge worldwide;

- Interoperability to better communicate among observing systems, and to deliver products for users, following usability and other best practices across the globe.

The CWPs summarize key accomplishments in ocean observing, address gaps, and discuss the way forward. They emphasize the importance of ocean observations as the key source of information on natural hazards (e.g., harmful algae and bacteria blooms, tsunamis, storm surges, marine heatwaves, and storms and other extreme weather events), ecosystem health and biodiversity (including shifting distributions of organisms and the increased risk of extinctions), ocean pollution (including acidification, de-oxygenation, and plastics), and sea level change. They highlight the need for observations to support ecosystem-based management, marine and weather forecasting, climate predictions and projection, marine safety and navigation, decision support for climate adaptation, deep-ocean exploration, and seafloor mapping, among many other areas. The papers address observing systems of various scales, from the global ocean (e.g., Argo, GO-SHIP, Volunteer Observing Ships, and an active constellation of satellites), basin-scale (e.g., AtlantOS, Tropical Pacific Observing System 2020, Indian Ocean Observing System, Tropical Atlantic Observing System, Arctic Ocean and Southern Ocean observing systems, and a developing Deep Ocean Observing Strategy), regional scale (e.g., for boundary currents and inter-ocean exchanges), to various coastal observing systems. These issues and systems are all at the core of a developing blue economy.

Taken together, the CWPs represent a call to governmental and non-governmental organizations, industries, scientists and technologists, stewards and citizens to work together to support global ocean observing. They stress the need of furthering a coordinated development of the Global Ocean Observing System (GOOS) to ensure the delivery of information that will benefit human society over the long term.

Some of the strategies and actions the CWPs addressed included:

- Sustain the observation of Essential Ocean Variables (EOVs) for operational applications, including conservation and sustainable development, scientific research, and the assembling of climate data records;

- Reduce capability gaps, including the improvements of coverage and sampling in space and in time, measurement accuracies, and measurements of critical but under-measured observables (e.g., air-sea fluxes and ocean surface currents, ecosystem health and biodiversity in deep ocean as well as coastal areas).

- Develop cost-effective strategies and accelerate the infusion of technology to advance sensors, platforms, and networks; 


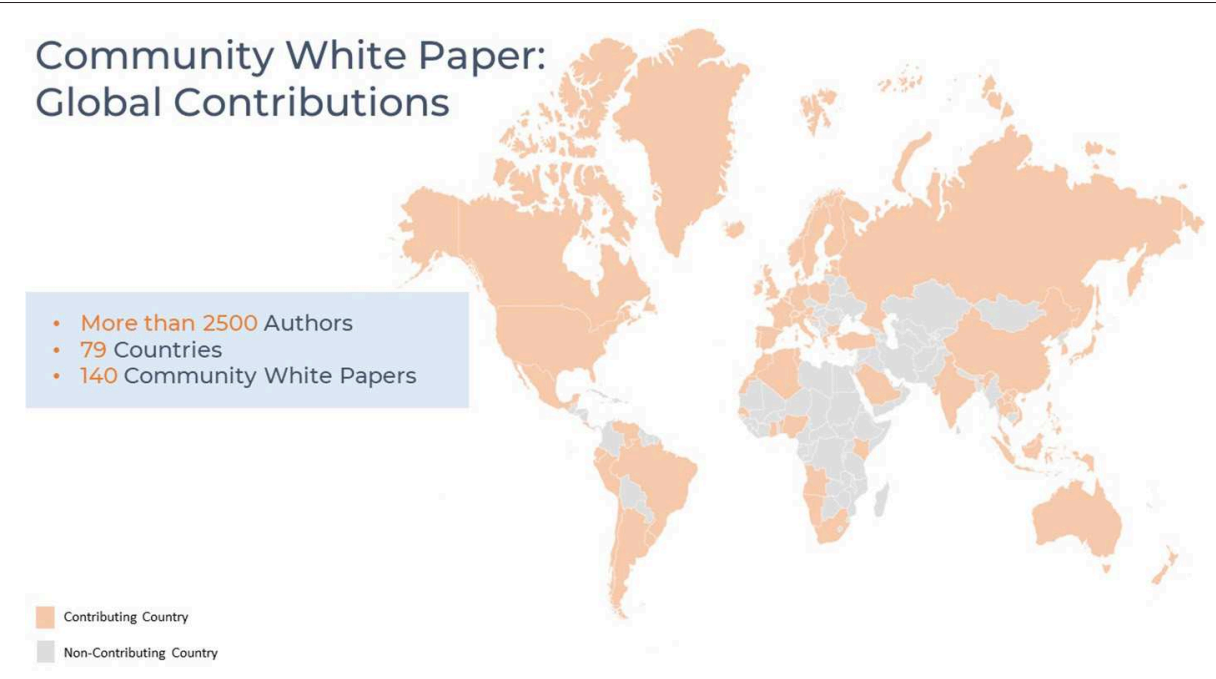

FIGURE 1 | Geographic distribution of authors contributing to the OceanObs'19 Community White Papers.

- Facilitate a governance approach that integrates observational capabilities under the framework of GOOS (e.g., Moltmann et al., 2019) across (1) different scales in space and time (global, basin-scale, regional, to coastal, and routinely, frequently, and over the long-term) (2) various platforms (in-situ, suborbital, and satellite), (3) disciplines (physical, biological, biogeochemical, and geological, and improved linkages to the social sciences), and (4) observations and forecasts (including improvements of models, assimilation, and initialization of forecasts);

- Improve the link between users and observing systems, including the private sector and bodies such as the World Meteorological Organization, the Food and Agricultural Organization, and many other government and academic entities. This includes re-enforcing the feedback loop between users and observing systems, so that system design and implementation ensure a fit-for-purpose enterprise as described in the Framework for Ocean Observing (FOO);

- Innovate data and information services to support diverse user needs across different regions and sectors, and to meet the challenges of Big Data and distributed data systems;

- Enhance and refine best practices in sensor and observing system development as well as in data and information curation and delivery, to build an international, collaborative, and multidisciplinary community of practice that jointly collects and shares global observations on EOVs for measuring change accurately and reliably;

- Strengthen capacity building and knowledge integration across the ocean observing value chain, from data collection to the generation of information and its application;

- Foster international collaborations and coordination, including free and public data access (real-time or near real-time where applicable), sharing of resources, coordination of education and outreach, and management of a shared and responsible observing system governance that provides funds for these activities.

The OceanObs'19 conference has taken the recommendations from these CPWs and many other sources and voices of the community to inform plenary presentations, panel discussion, breakout sessions, and conference posters. This input is critical for the success of the OceanObs'19 conference, and will help define the outcome of the conference and the post-conference actions. Together, the CWP and OceanObs'19 will contribute to the development of a vibrant and blue economy that comprises many sectors, that supports policies that sustain development and conservation, and shape the next decade of ocean observing.

\section{AUTHOR CONTRIBUTIONS}

SS, TL, FM-K, and LL drafted the content of this document and coordinated other author contributions. AP, DJ, ED, GR, JS, $\mathrm{ML}, \mathrm{NM}, \mathrm{PB}, \mathrm{SC}, \mathrm{JM}$, and AK-B provided edits. KD and AA contributed figures and final editing.

\section{ACKNOWLEDGMENTS}

The organizers of the OceanObs'19 conference thank the authors that conceived and jointly crafted the Community White Papers for their tremendous efforts, extensive international collaborations, and community wisdom. The organizers also thank the hundreds of reviewers of the CWPs for their dedication, and the time invested in reviewing the papers. The organizers of the OceanObs'19 conference wish to thank the chief editor of the journal, Dr. Carlos Duarte, and the Frontiers Marine Science staff, for their professionalism and support in this process. 


\section{REFERENCES}

Cheng, L., Abraham, J., Hausfather, Z., and Trenberth, K. E. (2019). How fast are the oceans warming? Science 363, 128-129. doi: 10.1126/science. aav7619

ECMWF (2016). ECMWF Strategy 2016-2025: The Strength of a Common Goal. ECMWF Tech. Rep., 27. Available online at: www.ecmwf.int/sites/default/files/ ECMWF_Strategy_2016-2025.pdf

Gleckler, P. J., Durack, P. J., Stouffer, R. J., Johnson, G. C., and Forest, C. E. (2016). Industrial-era global ocean heat uptake doubles in recent decades. Nature Clim. Change 6, 394-398. doi: 10.1038/nclimate2915

IPCC (2013). Climate Change 2013: The Physical Science Basis. Contribution of Working Group I to the Fifth Assessment Report of the Intergovernmental Panel on Climate Change, eds T. F. Stocker, D. Qin, G.-K. Plattner, M. Tignor, S. K. Allen, J. Boschung, A. Nauels, Y. Xia, V. Bex, and P. M. Midgley (Cambridge; New York, NY: Cambridge University Press), 1535. doi: 10.1017/CBO9781107415324

Lindstrom, E., Gunn, J., Fischer, A., McCurdy, A., and Glover, L. (2012). A Framework for Ocean Observing: A Report by the Task Team for an Integrated Framework for Sustained Ocean Observing. Paris: UNESCO.

Moltmann, T., Turton, J., Zhang, H-M., Nolan, G., Gouldman, C., Griesbauer, L., et al. (2019). A Global Ocean Observing System (GOOS), delivered through enhanced collaboration across regions, communities, and new technologies. Front. Mar. Sci. 6:291. doi: 10.3389/fmars.2019.00291

UNESCO (2017). United Nations Decade of Ocean Science for Sustainable Development (2021-2030). UNESCO Press Release. Available online at: https:// en.unesco.org/ocean-decade
United Nations (2017). UN Ocean Conference 2017. Available online at: https:// oceanconference.un.org/

United Nations General Assembly (2015). Transforming Our World: The 2030 Agenda for Sustainable Development. A/RES/70/1 Available online at: http:// www.un.org/sustainabledevelopment/development-agenda/

Visbeck, M. (2018). Ocean science research is key for a sustainable future. Nat. Commun. 9:690. doi: 10.1038/s41467-018-03158-3

Visbeck, M., Kronfeld-Goharani, U., Neumann, B., Rickels, W., Schmidt, J., Van Doorn, E., et al. (2014). Securing blue wealth: the need for a special sustainable development goal for the ocean and coasts. Mar. Policy 48, 184-191. doi: 10.1016/j.marpol.2014.03.005

Conflict of Interest Statement: The authors declare that the research was conducted in the absence of any commercial or financial relationships that could be construed as a potential conflict of interest.

The handling editor declared past co-authorship with one of the authors NM.

Copyright (C) 2019 Speich, Lee, Muller-Karger, Lorenzoni, Pascual, Jin, Delory, Reverdin, Siddorn, Lewis, Marba, Buttigieg, Chiba, Manley, Kabo-Bah, Desai and Ackerman. This is an open-access article distributed under the terms of the Creative Commons Attribution License (CC BY). The use, distribution or reproduction in other forums is permitted, provided the original author(s) and the copyright owner(s) are credited and that the original publication in this journal is cited, in accordance with accepted academic practice. No use, distribution or reproduction is permitted which does not comply with these terms. 


\section{OPEN ACCESS}

Edited by:

Laura Lorenzoni,

University of South Florida,

United States

Reviewed by:

Vicki Ferrini,

Lamont Doherty Earth Observatory

(LDEO), United States

Elizabeth Nyman,

Texas A\&M University at Galveston,

United States

Kurt Schwehr

Google, United States

${ }^{*}$ Correspondence:

Darren Wright

darren.wright@noaa.gov

Specialty section:

This article was submitted to

Ocean Observation,

a section of the journal

Frontiers in Marine Science

Received: 31 October 2018

Accepted: 15 August 2019

Published: 30 August 2019

Citation:

Wright $D$, Janzen $C$, Bochenek $R$, Austin J and Page E (2019) Marine Observing Applications Using AIS:

Automatic Identification System.

Front. Mar. Sci. 6:537.

doi: 10.3389/fmars.2019.00537

\section{Marine Observing Applications Using AIS: Automatic Identification System}

\author{
Darren Wright ${ }^{1 *}$, Carol Janzen ${ }^{2}$, Robert Bochenek ${ }^{3}$, Jessica Austin ${ }^{3}$ and Edward Page ${ }^{4}$ \\ ${ }^{1}$ National Marine Program, National Oceanic and Atmospheric Administration, National Weather Service, Silver Spring, MD, \\ United States, ${ }^{2}$ Alaska Ocean Observing System, Anchorage, AK, United States, ${ }^{3}$ Axiom Data Science, LLC, Anchorage, \\ AK, United States, ${ }^{4}$ Marine Exchange of Alaska, Juneau, AK, United States
}

The Automatic Identification System (AIS) is a real-time network of transmitters and receivers that allow vessel movements to be broadcast, tracked, and recorded. Though traditionally used for real-time maritime applications related to keeping track of vessel traffic for collision avoidance, there is increasing interest in using AIS data and the AIS platform for maritime safety planning, resource management, and weather forecasting. AIS data are being made tractable for alternative non-real-time applications like determining trends and patterns in vessel traffic and helping to prioritize where modern bathymetric surveys are needed to ensure safe maritime transit. The AIS is also being used for widespread transmission of critical environmental conditions information, such as sea state and weather, to mariners, forecasters, and emergency response providers. Several pilot projects are underway that demonstrate the capacity and promise of AIS data and the AIS platform to serve multiple purposes, providing overall maritime domain awareness while maintaining its most important objective of tracking vessels to aid safe, secure, efficient and environmentally sound maritime operations.

Keywords: automatic identification system, vessel tracking, weather observations, nautical charting, oil spill risk, subsistence use, hydrography, maritime domain awareness

\section{INTRODUCTION}

The Automatic Identification System (AIS) is a real-time network of transmitters and receivers that allow vessel movements to be broadcasted, tracked and recorded. The international maritime organization (IMO) international convention for the safety of life at sea (SOLAS) requires AIS to be operational aboard international voyaging ships of 300 gross tonnage or larger. It is also required on all passenger vessels with capacity of 12 or more passengers. The original purpose of the AIS was collision avoidance, and knowing the location of ships in real-time supports vessel traffic control, search and rescue operations, and enforcement of fisheries regulations (Natale et al., 2015; DeSouza et al., 2016). As maritime shipping increases worldwide, and ships are transiting new regions, knowing the location of vessels in time, and space becomes even more critical.

In addition to vessel information (e.g., type of vessel, dimensions, destination, position, course, and speed), three other types of data (environmental, area notice, and waterways management) can be incorporated into the AIS message, known as application specific messages (ASMs)(IMO Safety of Navigation Circular 289, 2010). This makes the AIS a valuable platform for the exchange of critical information such as weather, waves, water level, and current data from nearby observation installations. Examples in this paper describe how AIS is currently being tested and utilized to co-host local weather 
observations from both shore and ship. Emerging roles for the historical, as well as real-time, AIS data and AIS platform are presented in several real-world examples as well.

The focus of this paper is to highlight multiple ways the AIS can be utilized. The examples below highlight three manners of utilizing this system to perform vessel movement analysis and improve weather awareness and forecasting. The three techniques discussed include the use of AIS heat maps, use of application specific messages (ASM), and use of real-time AIS information to determine vessel operator awareness.

\section{ALTERNATIVE APPLICATIONS OF AIS}

\section{AIS Heat Maps}

AIS heat maps utilize historic AIS information to determine where vessels typically travel. This information can help determine where to focus resources for identifying where to carry out hydrographic surveys and update outdated bathymetry among other applications. AIS heatmaps can also be used to determine areas where there is no marine traffic, for projects such as wind farm development.

\section{Use of AIS Application Specific Messages}

Application specific messages, or ASMs, are IMO approved binary sections of the AIS message that can be used for environmental, area notice, and waterways management information. This document only focuses on examples that use the environmental ASM for transmission of environmental information.

\section{Use of Real-Time AIS Data}

Real-time use of AIS data using a holistic approach can help determine ship operator behavior. The example below proposed by the NOAA National Weather Service (NWS) plans to utilize real-time AIS data to determine if vessels are avoiding major storm events to see if they are properly communicating storm warnings. Historic AIS data can also be post processed to look back at historic storms to evaluate vessel operator behavior.

\section{DEMONSTRATION OF PROJECTS USING ALTERNATIVE APPLICATIONS OF AIS}

\section{AIS PAC: AIS for Prioritizing Arctic Charting}

Sea ice coverage has decreased to the point that existing northern shipping lanes around the world are open for longer periods of time and are projected to experience a continued increase in marine vessel traffic (Arctic Council, 2009). An accident in this region could be catastrophic to communities, and difficult to respond to because of the Arctic's remoteness, harsh environmental conditions, and lack of sufficient infrastructure for support. The grounding of the tanker CHAMPION EBONY in the summer of 2016 near Nunivak Island, Alaska, occurred in an area with outdated bathymetry that falsely reflected adequate water depth for safe passage. This demonstrates the importance of identifying areas where vessels are currently operating and ensuring that the availability of critical navigational charts and bathymetric survey information meet current standards.

In a collaborative effort funded by the Arctic Domain Awareness Center (ADAC) at the University of Alaska, Anchorage, investigators from the Marine Exchange of Alaska (MXAK), Axiom Data Science (Axiom), and the Alaska Ocean Observing System (AOOS) created AIS data products that inform decision-making about vessel traffic and safety in a rapidly changing Arctic environment ${ }^{1}$. The research effort helped develop and improve the capability to significantly reduce the execution time for handling and analyzing extremely large collections of AIS vessel tracking data, and produced a variety of AIS-derived data products formatted for use in the National Oceanic and Atmospheric Administration (NOAA) Office of Coast Survey (OCS) Hydrographic Health Model (Keown et al., 2016). NOAA uses this model to identify vessel safety risk areas, such as those with outdated bathymetry or insufficient coastal detail for safe passage of vessel traffic.

A pipeline workflow was developed and enhanced during this project in which AIS data are operated on over several stages rather than as a single phase process. The workflow stages take raw AIS messages, pull all valid position reports, create vessel voyages, and finally generate vessel traffic "heat maps." Heat maps are raster data that are downloadable as GeoTIFF (images) or NetCDF (files) in various resolutions and projections to allow visual and computational means for identifying high concentrations of ship traffic in the areas summarized. A pipeline workflow makes it so the entire process does not have to be rerun if there is a need to redo the analysis at any stage in the processing. The workflow stages also produce individual data products. To illustrate, if a new vessel type query is desired that differs from previous queries, it is easy to simply create new voyages for the new category, rather than rerunning the entire process. This saves time, as it might take $24 \mathrm{~h}$ to parse and clean the raw messages for a single dataset, but only take minutes to hours to generate voyages and minutes to generate a heat map from those voyages. The high-compute cluster workflow optimized during this effort was able to rapidly transform a 5-year historical record of terrestrial Arctic AIS data from MXAK for U.S. Exclusive Economic Zone (EEZ) waters into vessel density heat maps. It has since been used on U.S. Coast Guard Terrestrial and Satellite records, as well as Marine Cadastre Terrestrial and Danish Terrestrial AIS records. More details on the processing methods and access to a variety of processed AIS data products are publicly available on the Axiom AIS vessel traffic data products website ${ }^{2}$.

Stakeholder feedback guided the development of a specialized project portal hosting over 30 data layers for comparison and analysis against this 5-year AIS data record, hosted by the AOOS website ${ }^{3}$ and data portal ${ }^{4}$. Data tools continue to advance

\footnotetext{
${ }^{1}$ https://www.aoos.org/ais-4-bathy/

${ }^{2}$ http://ais.axds.co/

${ }^{3}$ https://aoos.org/

${ }^{4}$ https://pac.portal.aoos.org/
} 
end-user access to and utility of these datasets as illustrated in Figure 1. AOOS and the Arctic Domain Awareness Center (ADAC) continue to work together to provide annual updates to the AIS PAC data portal with the next update including 2018 data, and will continue to develop user-driven tools that will improve how stakeholders can interact with, visualize, and analyze these data.

\section{Synthesizing AIS, Oil Spill Model, and Subsistence Use Information for Planning}

AIS information is finding use in socioeconomic planning projects, including a recent effort in the Beaufort Sea region funded by the National Academies of Science. This project integrated data products describing vessel traffic patterns, estimated oil spill impacts, and subsistence use data from the communities of Barrow (Utqiagvik), Nuiqsut, and Kaktovik along the Beaufort Sea coast, and incorporated them into the advanced visualization tools provided by the AOOS data portal for planners and subsistence community members. Spatial heat maps of vessel traffic density were developed by analyzing 2017 AIS data from both the MXAK and NOAA in order to identify the marine vessel corridors in the Beaufort Sea experiencing the heaviest traffic. Investigators from Axiom ran several hundred oil spill trajectory scenarios powered by ocean circulation and atmospheric wind models initialized at randomized locations and times coinciding with vessel traffic hotspots.

Spill trajectory results were then summarized to produce a spill impact density map. Subsistence use patterns were produced from aggregating individual subsistence mapping, harvest, and other socio-economic surveys conducted by Stephen R. Braund and Associates from the Alaska Beaufort Sea communities above. These datasets had been previously unavailable and include species descriptions, timing and area for targeted (hunted) species, and other traditional ecological variables from each community. The subsistence mapping and species distribution data can now be plotted with and compared to the Spill Impact Density maps to understand how different subsistence activities and their relative timing could be impacted in the event of a spill.

All data gathered and synthesized throughout this project are now publicly accessible through a project specific, interactive web-based data portal equipped with mapping tools operated through the AOOS data system. The portal and associated tools provide a mechanism for increasing public, private sector, government, and community knowledge about possible spill effects to subsistence uses in the U.S. Arctic. Results are expected to inform government agency and community emergency response planning and decision-making. The Arctic Oil Spill Risk Assessment Portal $^{5}$ provides examples of how to visualize

\footnotetext{
${ }^{5}$ https://aoos.org/ais-oil-spill-support/
}

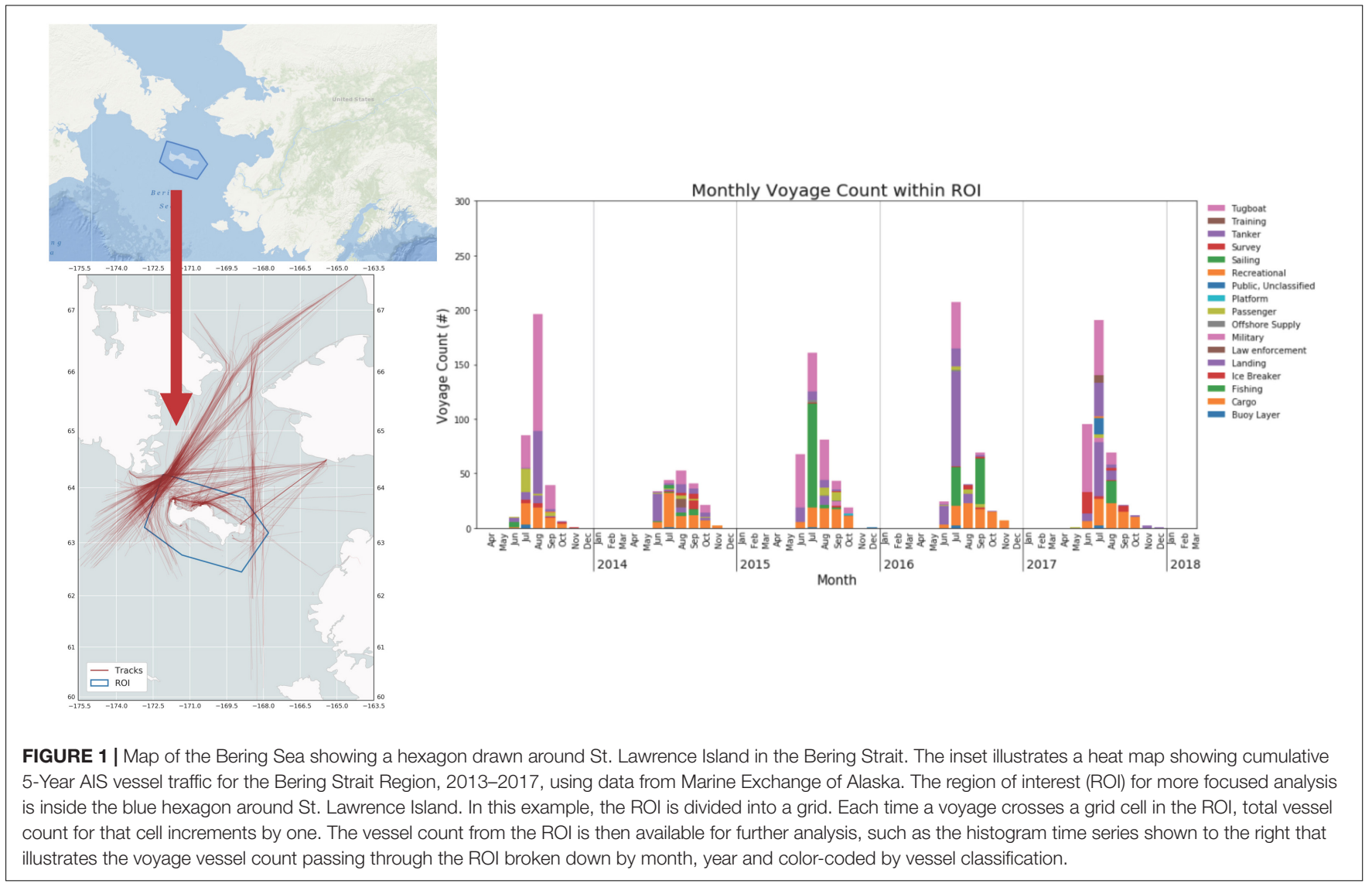


data, a tutorial on getting started with the data products and mapping tools offered on the portal, and access to project reports that offer more detailed information on the data sets and methodologies used for the project. Methods developed during this project can be applied to other regions where oil spill risks may impact at-risk resources, such as a fishery or sensitive habitat area.

\section{Reporting Weather Observations in Alaska}

An extensive AIS network has been established in Alaska with over 120 AIS receiving stations in place throughout the State. The Marine Exchange of Alaska (MXAK) builds and operates the only terrestrial AIS network in Alaska that is serving as the primary component of the U.S. Coast Guard's and State of Alaska's maritime domain awareness network. The MXAK has worked with the U.S. Coast Guard (USCG) in a cooperative research and development agreement (CRADA) to develop the Arctic next generation navigational safety information system that utilizes AIS transmitters to send environmental and safety information to mariners to aid safe and environmentally sound maritime operations.

Partnering with AOOS, the MXAK tested and developed the technology to collect weather observations and transmit this information to mariners using the environmental ASM portion of the AIS message and the internet accessible by smartphones and computers. This project enhances maritime safety and environmental protection in Alaska by providing real-time, environmental information to vessels over the AIS.

By establishing joint weather/AIS stations at existing AIS locations, and installing new stations in remote areas, real-time weather information can be presented on a vessel's AIS display in a more reliable and clear manner and at greater distances than the method in which weather information is presently disseminated. With most commercial vessels required to be equipped with AIS by the IMO treaties, AIS is one of the most reliable means of communicating weather and other environmental information (e.g., weather and ice reports) to vessels operating in coastal waters out to 50 miles offshore.

Up to 29 of the Alaska AIS stations now have co-located weather sensors that, along with an additional 20 standalone weather stations operated by the MXAK, are providing local observations through the AIS. Data are displayed and made publicly available by the MXAK weather and tracking website and phone application product ${ }^{6}$, AOOS on their realtime sensor map, and can also be accessed through the National Data Buoy Data Center (NDBC). Information is also provided directly to the NWS to aid forecasting and the dissemination of real-time weather information via their very high frequency (VHF) broadcast and web notification system. The success of the AIS weather project in Alaska has led to other environmental and maritime safety information being shared across the AIS, including wave and current

\footnotetext{
${ }^{6}$ http://www.mxak.org/mobile/index.html
}

observations from the Coastal Data Information Program (CDIP) (https://cdip.ucsd.edu/m/) buoy deployed off the Port of Nome, Alaska.

\section{Transmitting Weather Observations From Ships}

In 2018, the USCG, United States Army Corps of Engineers (USACE), Maritime Administration (MARAD), and the NWS partnered on an effort to develop an automated shipboard weather observation system that will format weather observations into an environmental ASM for transmission as part of an AIS message to the USCG and other vessels, and to the NWS for use in its marine weather forecast models. The NWS currently receives many thousands of weather observations terrestrially on an hourly basis or less, but observations from the open ocean are much more sparse. The NWS has to rely on satellite information to help fill large gaps for offshore conditions, but satellites do not provide full coverage and, as a result, large gaps remain (e.g., Figure 2; Smith et al., 2019).

By working with these agencies and manufacturers of both weather and AIS systems, the NWS hopes to significantly increase the number of observations it receives from ships, and at the same time, reduce workloads of mates on ships that would otherwise have to manually take and report out weather observations. Conditions like cloud cover and in situ wave height, along with a number of essential climate variables, may still need to be reported manually, but information such as air temperature, barometric pressure, sea surface temperature, and wind speed and direction can easily be recorded directly from sensors and formatted for transmission. The increase of observations reported out from ships will help improve forecast models and ultimately will assist the NWS with providing better forecasts and warnings over the open ocean, important for the safety of life at sea.

\section{Transmission of Environmental Information for Safe Maritime Navigation}

NOAA's National Ocean Service (NOS) has been working with the USCG to transmit data from its physical oceanographic real-time system (PORTS ${ }^{\circledR}$ ) over AIS to ships, providing critical environmental information to aid ships in navigating safely. PORTS $^{\circledR}$ is a decision support tool for mariners that improves the safety and efficiency of maritime commerce and coastal resource management through the integration of real-time environmental observations, forecasts and other geospatial information. PORTS ${ }^{\circledR}$ measures and disseminates observations and predictions of water levels, currents, salinity, waves and meteorological parameters (e.g., winds, visibility, atmospheric pressure, air, and water temperatures) that mariners need to navigate safely.

By transmitting this information over AIS, manufacturers of ship navigation display systems will be able to integrate this environmental information into one display, so multiple screens or devices to acquire the information onboard are not needed. The NOS and the USCG conducted two demonstration projects in Tampa Bay, Florida and on the Columbia River in Oregon. 


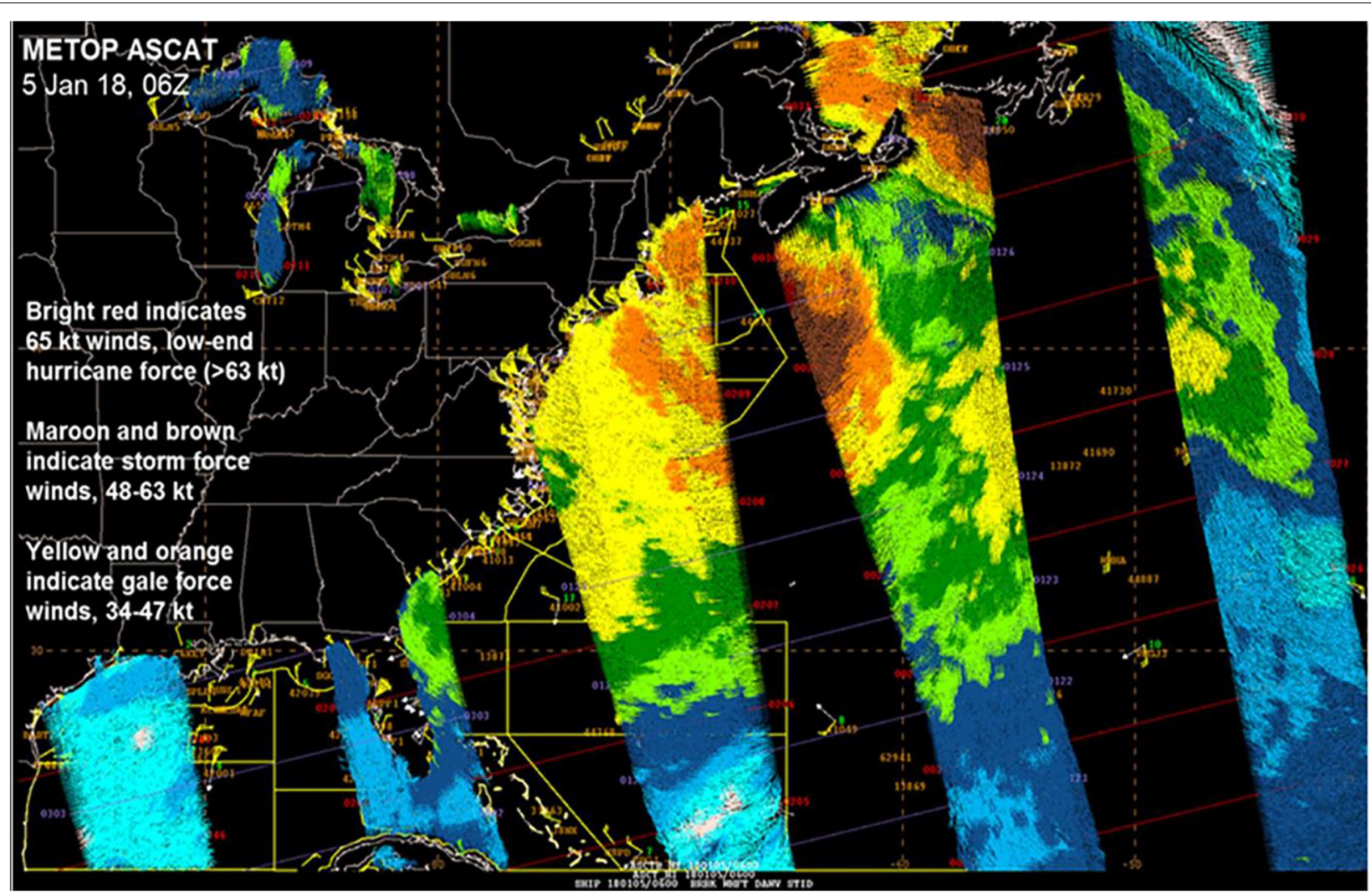

FIGURE 2 | EUMETSAT MetOp Advanced Scatterometer data (colored swaths), with available ship observations (using standard synoptic station notation), from 0600 UTC on January 5, 2018. Courtesy of National Oceanic and Atmospheric Administration, NWS, Ocean Prediction Center.

Both trials demonstrated this as a viable method of transmitting environmental information. The USCG is now working to make this transmission capability operational over its Nationwide AIS Network, which has full coastline coverage in the continental U.S. In the meantime, the Columbia River Pilots, working with U.S. Department of Transportation (USDOT) Volpe Center, have made the transmission of PORTS ${ }^{\circledR}$ data operational on the Columbia River and have integrated PORTS ${ }^{\circledR}$ data into their portable pilot units, which are devices used by the Pilots to assist in navigating ships to port.

With the transmission of real-time and forecast information over AIS, like water levels, one can envision navigational software providing dynamic bathymetry on electronic nautical charts and dynamic ship channel displays, notifying captains and pilots if they are passable, based on the ship's under keel clearance.

\section{Monitoring Ship Responses to Major Storm Events and Warnings}

The NWS is investigating the use of real-time ship locations to see how vessels are responding to storm/hurricane warnings. The NWS, working with the USCG, intends to get access to a real-time AIS data feed, download the information into a database and convert the information into a format that its advanced weather interactive processing system (AWIPS) can read and display. Once ingested, the AIS data can be overlayed on top of weather maps to see if ships are avoiding dangerous weather areas. A plot of ship locations from AIS data during Hurricane Florence in September 2018 shows a zone (red shaded region) of essentially complete ship avoidance in an area where a hurricane warning was in effect (Figure 3). The red arrow within this warning zone represents the hurricane's predicted path. From this image, it is clear that ships (represented by the small triangles) successfully practiced avoidance of the hurricane.

\section{CONCLUSION}

AIS was designed and developed as a method of tracking real-time vessel movements for both security and safety of navigation through traffic awareness. There are a variety of other applications in which this technology can be utilized.

Companies and government agencies will continue to use AIS information in new and different ways, like the examples presented in this paper. Some other examples include the USACE transmission of both environmental and lock information on inland rivers via its River Information Service, transmission of weather information all along the St. Lawrence Seaway in the Great Lakes, which has been done for years, and the Columbia River Pilots, who are receiving water level 


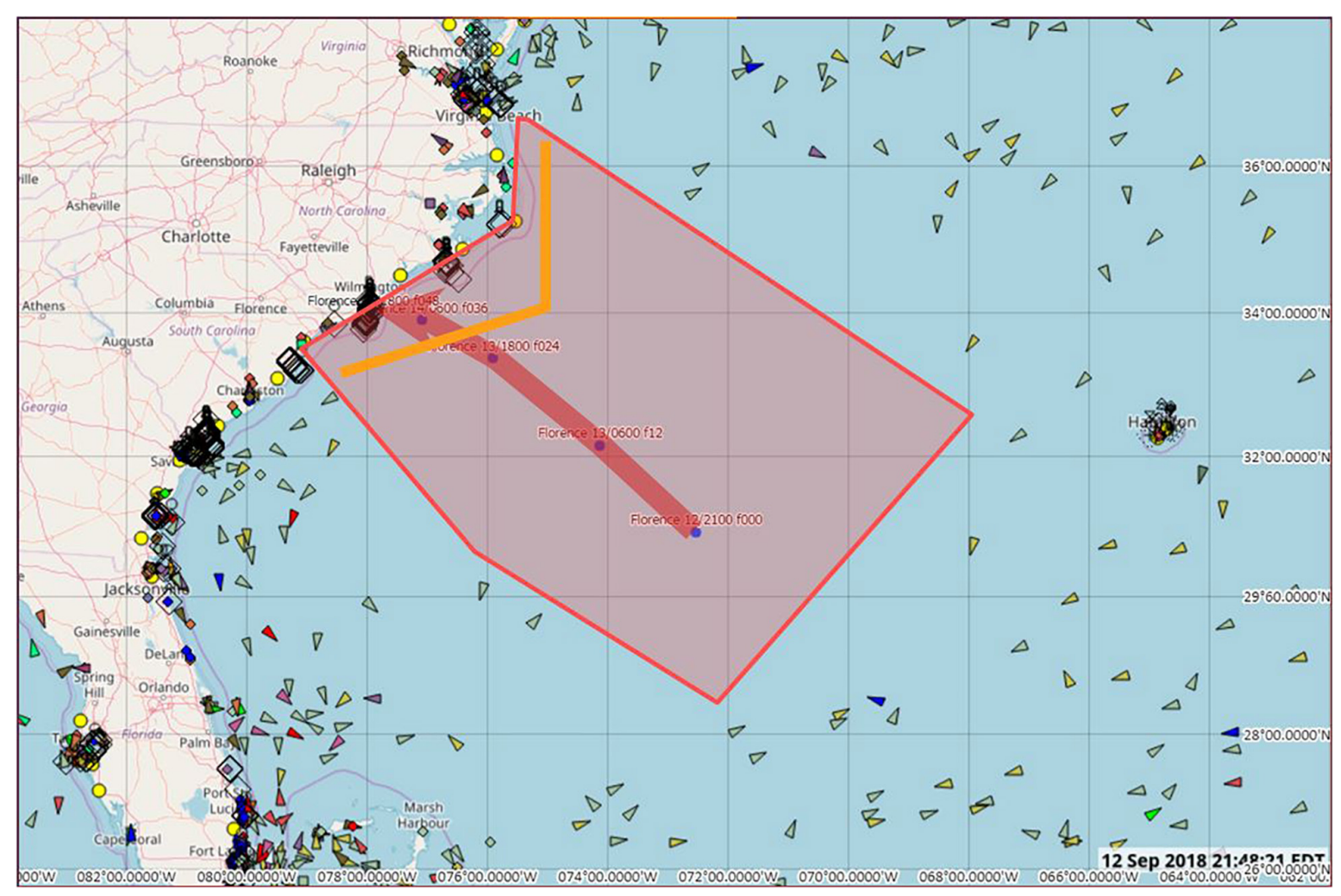

FIGURE 3 | AIS data plot shows ships (small triangles) avoiding Hurricane Florence, 2018. The NWS plans to use this tool to help ensure vessels are receiving the proper warnings and are avoiding hazardous seas, part of a decision support framework in support of a Weather Ready Nation.

data over AIS through their portable pilot units to assist in navigation. The USCG, in its Maritime Commerce Strategic Outlook document (U.S. Coast Guard, 2018), stated “The Coast Guard will bolster engagement with NOAA to integrate realtime information to broadcast hydrographic and weather data through the AIS, and continue to seek additional partnerships and information sources." This initiative, along with many others, paints a bright picture for the expanded use of AIS in the future.

\section{AUTHOR CONTRIBUTIONS}

DW is lead author of the manuscript, co-authored the "Abstract," "Introduction," and "Conclusion" sections, and was lead author for "Demonstration of Projects Using Alternative Applications of AIS" sections: "Transmitting Weather Observations From Ships," "Transmission of Environmental Information for Safe Maritime Navigation," and "Monitoring Ship Responses to Major Storm Events and Warnings." CJ co-authored the "Abstract," "Introduction," and "Conclusion" sections and was lead author on "Alternative Applications of AIS" section and "Demonstration of Projects Using Alternative Applications of AIS" sections: "AIS PAC: AIS for Prioritizing Arctic Charting," "Synthesizing AIS, Oil Spill Model, and Subsistence Use Information for Planning," and "Reporting Weather Observations in Alaska." RB and JA were responsible for developing the data processing protocols for handling large AIS records, for data analysis and performing oil spill modeling scenarios seeded using AIS information, and co-authored the discussions on methods described in "AIS PAC: AIS for Prioritizing Arctic Charting" and "Synthesizing AIS, Oil Spill Model, and Subsistence Use Information for Planning" sections. EP is the Executive Director and founder of the Marine Exchange of Alaska and supported the Alaska projects described in this manuscript by acquiring and providing $100 \%$ of quality controlled AIS data, also co-authored the section "Reporting Weather Observations in Alaska."

\section{FUNDING}

Primary funding for AOOS and associated projects comes from NOAA NOS grant-NA16NOS0120027. Support for the AIS PAC project was provided by the Arctic Domain Awareness Center (ADAC), a U.S. Department of Homeland Security (DHS) Center of Excellence in Maritime Research, hosted by the University of Alaska, where associated activities were supported by DHS under the grant award number 2014-ST-061-ML0002. The Gulf Research Program of the National Academies of Sciences, Engineering, and Medicine project assimilating oil spill, AIS, and subsistence harvest information was supported under the grant award number 2000007630 . 


\section{ACKNOWLEDGMENTS}

The authors would like to recognize the U.S. Coast Guard, the U.S. Army Corps of Engineers (USACE), the Maritime Administration (MARAD), the NOAA Office of Coast Survey (OCS), the NOAA National Weather Service (NWS), the

\section{REFERENCES}

Arctic Council (2009). Arctic Marine Shipping Assessment 2009 Report, Second Printing. Available at: https://www.pmel.noaa.gov/arctic-zone/detect/ documents/AMSA_2009_Report_2nd_print.pdf

DeSouza, E. N., Boerder, K., Matwin, S., and Worm, B. (2016). Improving fishing pattern detection from satellite AIS using data mining and machine learning. PLoS One 11:e0158248. doi: 10.1371/journal.pone. 0158248

IMO Safety of Navigation Circular 289 (2010). Guidance on the Use of Ais Application-Specific Messages. Available at: https://www.navcen.uscg.gov/pdf/ IMO_SN1_Circ289_Guidance_on_use_of_AIS_ASM.pdf

Keown, P., Gonsalves, M., Allen, C., Fandel, C., Gallagher, B., and Hick, L. (2016). "A risk-based approach to determine hydrographic survey priorities using GIS,” in Proceedings: 2016 ESRI Ocean GIS, (Redlands, CA: Forum).

Natale, F., Gibin, G., Alessandrini, A., Vespe, M., and Paulrud, A. (2015). Mapping fishing effort through AIS data. PLoS One 10:e130746. doi: 10.137/journal. pone. 0130746

Smith, S., Alory, G., Andersson, A., Asher, W., Baker, A., Berry, D., et al. (2019). Ship-based contributions to global ocean, weather, and climate observing systems. Front. Mar. Sci. 6:434. doi: 10.3389/fmars.2019. 00434
Integrated Ocean Observing System (IOOS), the Marine Exchange of Alaska, the Arctic Domain Awareness Center (ADAC) at the University of Alaska, the Department of Homeland Security, and the National Academy of Sciences for their role in the development of AIS applications for marine observing.

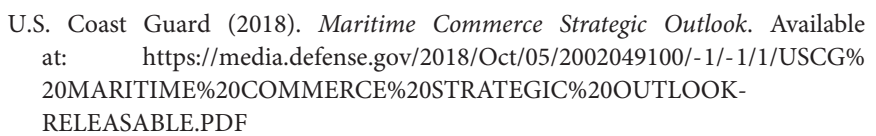

Disclaimer: The content is solely the responsibility of the authors and does not necessarily represent the official views of the Gulf Research Program or the National Academies of Sciences, Engineering, and Medicine.

Conflict of Interest Statement: RB and JA are employed by Axiom Data Science, which provides data management support for AOOS, IOOS, SECOORA, and CenCOOS.

The remaining authors declare that the research was conducted in the absence of any commercial or financial relationships that could be construed as a potential conflict of interest.

Copyright (c) 2019 Wright, Janzen, Bochenek, Austin and Page. This is an open-access article distributed under the terms of the Creative Commons Attribution License (CC BY). The use, distribution or reproduction in other forums is permitted, provided the original author(s) and the copyright owner(s) are credited and that the original publication in this journal is cited, in accordance with accepted academic practice. No use, distribution or reproduction is permitted which does not comply with these terms. 


\section{OPEN ACCESS}

Edited by:

Minhan Dai,

Xiamen University, China

Reviewed by:

Scott Doney,

University of Virginia, United States

Nadia Pinardi,

University of Bologna, Italy

*Correspondence:

Luca R. Centurioni

Icenturioni@ucsd.edu

${ }^{\dagger}$ Present address:

Theresa Paluszkiewicz,

Octopus Ocean Consulting LLC,

Oak Hill, VA, United States

Specialty section:

This article was submitted to

Ocean Observation,

a section of the journal

Frontiers in Marine Science

Received: 30 October 2018

Accepted: 05 July 2019

Published: 30 August 2019

Citation:

Centurioni $L R$, Turton J,

Lumpkin R, Braasch L,

Brassington G, Chao Y, Charpentier E,

Chen Z, Corlett G, Dohan K,

Donlon C, Gallage C, Hormann V,

Ignatov $A$, Ingleby $B$, Jensen $R$,

Kelly-Gerreyn BA, Koszalka IM, Lin X,

Lindstrom E, Maximenko $N$, Merchant CJ, Minnett P, O'Carroll A, Paluszkiewicz T, Poli P, Poulain P-M,

Reverdin G, Sun X, Swail V,

Thurston S, Wu L, YU L, Wang $B$ and

Zhang D (2019) Global in situ

Observations of Essential Climate

and Ocean Variables at the Air-Sea

Interface. Front. Mar. Sci. 6:419.

doi: 10.3389/fmars.2019.00419

\section{Global in situ Observations of Essential Climate and Ocean Variables at the Air-Sea Interface}

Luca R. Centurioni1*, Jon Turton'2, Rick Lumpkin ${ }^{3}$, Lancelot Braasch', Gary Brassington 4 , Yi Chao ${ }^{5}$, Etienne Charpentier 6 , Zhaohui Chen ${ }^{7}$, Gary Corlett ${ }^{8}$, Kathleen Dohan ${ }^{9}$, Craig Donlon ${ }^{10}$, Champika Gallage ${ }^{6}$, Verena Hormann', Alexander Ignatov ${ }^{11}$, Bruce Ingleby ${ }^{12}$, Robert Jensen ${ }^{13}$, Boris A. Kelly-Gerreyn ${ }^{4}$, Inga M. Koszalka' ${ }^{14}$, Xiaopei Lin ${ }^{7}$, Eric Lindstrom ${ }^{15}$, Nikolai Maximenko ${ }^{16}$, Christopher J. Merchant ${ }^{17}$, Peter Minnett ${ }^{18}$, Anne O'Carroll19, Theresa Paluszkiewicz ${ }^{20+}$, Paul Poli21, Pierre-Marie Poulain'22, Gilles Reverdin'23, Xiujun Sun', Val Swail24, Sidney Thurston ${ }^{25}$, Lixin $\mathrm{Wu}^{7}$, Lisan $\mathrm{Yu}^{26}$, Bin Wang ${ }^{27}$ and Dongxiao Zhang ${ }^{28}$

${ }^{1}$ Scripps Institution of Oceanography, University of California, San Diego, San Diego, CA, United States, ${ }^{2}$ Met Office, Exeter, United Kingdom, ${ }^{3}$ NOAA's Atlantic Oceanographic and Meteorological Laboratory, Miami, FL, United States, ${ }^{4}$ Bureau of Meteorology, Melbourne, VIC, Australia, ${ }^{5}$ Remote Sensing Solutions, Barnstable, MA, United States, ${ }^{6}$ World Meteorological Organization, Geneva, Switzerland, ${ }^{7}$ Ocean University of China, Qingdao, China, ${ }^{8}$ EUMETSAT, Darmstadt, Germany, ${ }^{9}$ Earth and Space Research, Seattle, WA, United States, ${ }^{10}$ European Space Agency, Paris, France, ${ }^{11}$ Center for Satellite Applications and Research (STAR), NOAA, College Park, MD, United States, ${ }^{12}$ European Centre for Medium-Range Weather Forecasts, Reading, United Kingdom, ${ }^{13}$ Engineer Research and Development Center, Vicksburg, MS, United States, ${ }^{14}$ Department of Meteorology, Stockholm University (MISU), Stockholm University Baltic Sea Centre, Stockholm, Sweden, ${ }^{15}$ National Aeronautics and Space Administration, Washington, DC, United States,

${ }^{16}$ International Pacific Research Center, School of Ocean and Earth Science and Technology, University of Hawai'i, Honolulu, HI, United States, ${ }^{17}$ Department of Meteorology, University of Reading, Reading, United Kingdom, ${ }^{18}$ Rosenstiel School of Marine and Atmospheric Science, University of Miami, Miami, FL, United States, ${ }^{19}$ European Organization for the Exploitation of Meteorological Satellites, Darmstadt, Germany, ${ }^{20}$ Office of Naval Research, Arlington, VA, United States, ${ }^{21}$ Météo-France, Toulouse, France, ${ }^{22}$ Istituto Nazionale di Oceanografia e di Geofisica Sperimentale, Trieste, Italy, ${ }^{23}$ UMR 7159 Laboratoire d'Océanographie et du Climat: Expérimentations et Approches Numériques, Paris, France, ${ }^{24}$ Environment and Climate Change Canada, Gatineau, QC, Canada, ${ }^{25}$ National Oceanic and Atmospheric Administration, Washington, DC, United States, ${ }^{26}$ Woods Hole Oceanographic Institution, Woods Hole, MA, United States, ${ }^{27}$ National Marine Technology Center, Tianjin, China, ${ }^{28}$ Pacific Marine Environmental Laboratory (NOAA), Seattle, WA, United States

The air-sea interface is a key gateway in the Earth system. It is where the atmosphere sets the ocean in motion, climate/weather-relevant air-sea processes occur, and pollutants (i.e., plastic, anthropogenic carbon dioxide, radioactive/chemical waste) enter the sea. Hence, accurate estimates and forecasts of physical and biogeochemical processes at this interface are critical for sustainable blue economy planning, growth, and disaster mitigation. Such estimates and forecasts rely on accurate and integrated in situ and satellite surface observations. High-impact uses of ocean surface observations of essential ocean/climate variables (EOVs/ECVs) include (1) assimilation into/validation of weather, ocean, and climate forecast models to improve their skill, impact, and value; (2) ocean physics studies (i.e., heat, momentum, freshwater, and biogeochemical air-sea fluxes) to further our understanding and parameterization of air-sea processes; and (3) calibration and validation of satellite ocean products (i.e., currents, temperature, salinity, sea level, ocean color, wind, and waves). We review strengths and limitations, impacts, and sustainability of in situ ocean surface observations of several ECVs and EOVs. We draw a 10-year vision 
of the global ocean surface observing network for improved synergy and integration with other observing systems (e.g., satellites), for modeling/forecast efforts, and for a better ocean observing governance. The context is both the applications listed above and the guidelines of frameworks such as the Global Ocean Observing System (GOOS) and Global Climate Observing System (GCOS) (both co-sponsored by the Intergovernmental Oceanographic Commission of UNESCO, IOC-UNESCO; the World Meteorological Organization, WMO; the United Nations Environment Programme, UNEP; and the International Science Council, ISC). Networks of multiparametric platforms, such as the global drifter array, offer opportunities for new and improved in situ observations. Advances in sensor technology (e.g., low-cost wave sensors), high-throughput communications, evolving cyberinfrastructures, and data information systems with potential to improve the scope, efficiency, integration, and sustainability of the ocean surface observing system are explored.

Keywords: global in situ observations, air-sea interface, essential climate and ocean variables, climate variability and change, weather forecasting, SVP drifters

\section{INTRODUCTION}

The air-sea interface is a key gateway in the Earth system. It is where the atmosphere sets the ocean in motion, climate and weather-relevant air-sea forcing and feedback processes occur, and pollutants such as plastic, anthropogenic carbon dioxide, and some radioactive and chemical waste enter the sea. Hence, improving the accuracy of estimates and forecasts of physical and biogeochemical processes at this interface has direct impacts on society and is essential for a sustainable blue economy planning, growth, and disaster mitigation. Such estimates and forecasts rely on accurate and integrated in situ and satellite surface observations of essential climate and ocean variables (ECVs and EOVs) at the ocean-atmosphere interface and in the respective boundary layers.

Satellite observations offer near-global coverage at useful spatial and temporal resolution that make them well suited for assimilation, alongside in situ observations, into forecasting models and for estimating the state of the ocean/atmosphere system. Both active and passive remote sensing techniques are used in a wide spectral interval spanning visible, infrared, and microwave parts of the spectrum, to measure many of the ECVs and EOVs also sampled by the in situ network. In situ sensors also provide the data against which satellite retrievals are calibrated, for example, ocean color, and validated, for example, ocean color, sea surface temperature (SST), and surface winds (Kilpatrick et al., 2001, 2015; Xu and Ignatov, 2010; Xu and Ignatov, 2014; Ignatov et al., 2016; Loew et al., 2017). However, some variables such as sea level pressure (SLP) cannot be determined from space with current technology, and thus, their acquisition relies solely on in situ platforms. A comprehensive description of the satellite systems used for remote sensing of the air-sea interface is beyond the scope of this review but can be found in Ardhuin et al. (2019a,b), Bourassa et al. (2019), Cronin et al. (2019), Gommenginger et al. (2019), Kent et al. (2019), O'Carroll et al. (2019), Smith et al. (2019), Swart et al. (2019), Villas Bôas et al. (2019), Wanninkhof et al. (2019), and Weller et al. (2019).
In situ observations at the air-sea interface are currently obtained from several programs such as the global surface drifter array (GSDA), the Voluntary Observing Ship (VOS) scheme, the tropical moored buoy array (TMBA), OceanSITES, and various national moored buoy networks. The Argo program, mainly aimed at studying the depth of the ocean from the surface to $2 \mathrm{~km}$ with profiling floats (Roemmich et al., 2009; Riser et al., 2016), also contributes, although to a lesser extent, to characterizing the air-sea interface.

The focus of many of the sustained observational programs operating at the interface between the ocean and the atmosphere is to measure key ECVs that are defined under the global climate observing system (GCOS) framework. Established in 1992 and co-sponsored by the World Meteorological Organization (WMO), the Intergovernmental Oceanographic Commission (IOC) of the United Nations Educational, Scientific and Cultural Organization (UNESCO), the United Nations Environment Program (UNEP), and the International Science Council (ISC), GCOS provides a list of 54 ECVs (including near-surface currents, SST, and SLP) needed to support intergovernmental and national oceanographic, climate, and weather services. The WMO Integrated Global Observing System (WIGOS) provides an overarching framework for integrating the various sources of observations, including the networks mentioned above, contributing to WMO applications. The framework includes relevant tools, standards, guidance, and WMO regulatory material. WIGOS also provisions for observing network design and observing system evolution through the WMO's Rolling Review of Requirements (RRR), where observational user requirements are compared with observing systems capabilities ${ }^{1}$ together with impact studies for the identification of observational gaps, leading to prioritization for the evolution of global observing systems and key recommended actions to WMO members and other significant programs to address such gaps. One component of WIGOS, the Global

\footnotetext{
${ }^{1}$ https://oscar.wmo.int/surface//index.html\#/
} 
Observing System (GOS), provides for national meteorological services (NMSs) and other partners' contributions to the Global Ocean Observing System (GOOS) and GCOS (meteorological satellites, drifters, moored buoys, and VOS). See Moltmann et al. (2019) for more details on WIGOS.

Many of these global observing networks are made of collections of national scientific and operational programs designed and implemented in support of a wide range of multidisciplinary scientific research and operational applications. Often, data from multiple programs and networks are consolidated to facilitate their accessibility and use. One example is the International Comprehensive Ocean-Atmosphere Data Set (ICOADS) project, which attempts to bring together all available data of the ocean-atmosphere interface (Freeman et al., 2016). Likewise, satellite communities, including the Group for High-Resolution SST (GHRSST; Donlon et al., 2009), consolidate and uniformly quality control the in situ data for the satellite era for use in their calibration and validation systems, standardize the matchup criteria, and unify and consolidate the comparison metrics (O'Carroll et al., 2019). The European Copernicus Marine Environment Monitoring Service (CMEMS) also relies on data from in situ monitoring networks to provide robust integrated information and to calibrate and validate the data from satellites.

The purpose of this article is to review strengths, weaknesses, impacts, and sustainability of in situ global observing networks measuring target ECVs and EOVs at the air-sea interface and to provide a 10-year vision for improved synergy and integration with other observing systems (e.g., satellites), for modeling/forecast efforts, and for a better ocean observing governance. We review new emerging technologies, sensors, newly developed platforms, as well as information technology advances that have the potential to improve the impact and efficiency of the existing ocean surface observing networks or the creation of new ones. The context is both the applications listed above and the guidelines of frameworks such as IOC-WMO-UNEP, WMO-IOC Joint Technical Commission for Oceanography and Marine Meteorology (JCOMM), ISC, GCOS, GOOS, and WIGOS (Moltmann et al., 2019).

\section{STATE OF THE ART: EXISTING GLOBAL NETWORKS FOR OBSERVATIONS AT THE AIR-SEA INTERFACE. CONFIGURATION AND IMPACTS}

\section{The Global Surface Drifter Array Background and Objectives}

The large-scale deployment of Lagrangian (i.e., water following) surface drifters drogued at a depth of $15 \mathrm{~m}$ and designed according to the specifications of the Surface Velocity Program (SVP; Niiler et al., 1995; Niiler, 2001; Centurioni, 2018; see also Figure 3 for schematics of several SVP drifter configurations) began in the tropical Pacific Ocean in 1988 (Hansen and Poulain, 1996). The World Ocean Circulation Experiment (WOCE) requirements were instrumental in laying the foundations of the United States NOAA (National Oceanic and Atmospheric Administration)-funded Global Drifter Program (GDP; Niiler, 2001; Maximenko et al., 2013), which represents along with the SVP the basis of the GSDA. Sustained deployments of drifters quickly grew into a global array with contributions from over 25 countries (see Figure 1) delivering primarily near real-time observations of SST and geolocation. The collaboration between the GSDA and NMSs in the 1990s facilitated the development, testing, and adoption of the barometer drifter (SVP-B; see Figure 3) as a reliable source of SLP data to support weather forecasting with the deployment of such SVP-B drifters in large numbers since then. The GSDA reached its full implementation when drifter number 1,250 was deployed off Halifax, Nova Scotia, Canada, on September 12, 2005, by Distinguished Professor Dr. Peter Niiler of the Scripps Institution of Oceanography (SIO) and Dr. Michael Johnson of NOAA, becoming the first fully implemented component of GOOS. The GDP, a branch of NOAA's GOOS, is a scientific program that also has substantial impacts on operational activities, including satellite SST validation and numerical weather prediction (NWP) and constitutes the principal component of the GSDA. The GSDA is coordinated internationally through the Data Buoy Cooperation Panel (DBCP), an international body coordinating the use of both drifting and moored data buoys to observe atmospheric and oceanographic conditions over the ocean, including regions where few other measurements are taken, such as the Southern Ocean. The DBCP was created in 1985 as a joint body of WMO and UNESCO's IOC. The DBCP constitutes the data buoy component of WMO-IOC JCOMM (Pinardi et al., 2019).

The main objectives of the GDP and GSDA are very closely aligned and can be summarized as follows:

- Maintain a global $5^{\circ} \times 5^{\circ}$ array of satellite-tracked SVP drifters (excluding marginal seas and latitudes higher than $60^{\circ} \mathrm{N} / \mathrm{S}$ ) to meet the need for an accurate and globally dense set of in situ observations of near-surface currents, SST, and SLP.

- Provide a data processing system to deliver the data to operational and research users via the WMO Global Telecommunication System (GTS) and via qualitycontrolled (QC), delayed mode products. The release of QC data products is led by the United States GDP component at the Atlantic Oceanographic and Meteorological Laboratory (AOML) of NOAA.

- Under the lead of the United States GDP component at SIO, the Lagrangian Drifter Laboratory (LDL), to innovate the drifter technology and expand the scope of the international program by transitioning new technologies and sensors into operation. Examples of recently developed technologies include water-following drifters measuring wind, solar radiation, and directional wave spectra (see section "Emerging Technologies").

The GCOS and GOOS guidelines specify an array of at least 1,250 Lagrangian SVP drifting buoys, as needed to achieve a $5^{\circ} \times 5^{\circ}$ global coverage. More details on this can be found in Lumpkin et al. (2016), who also discuss the modern 


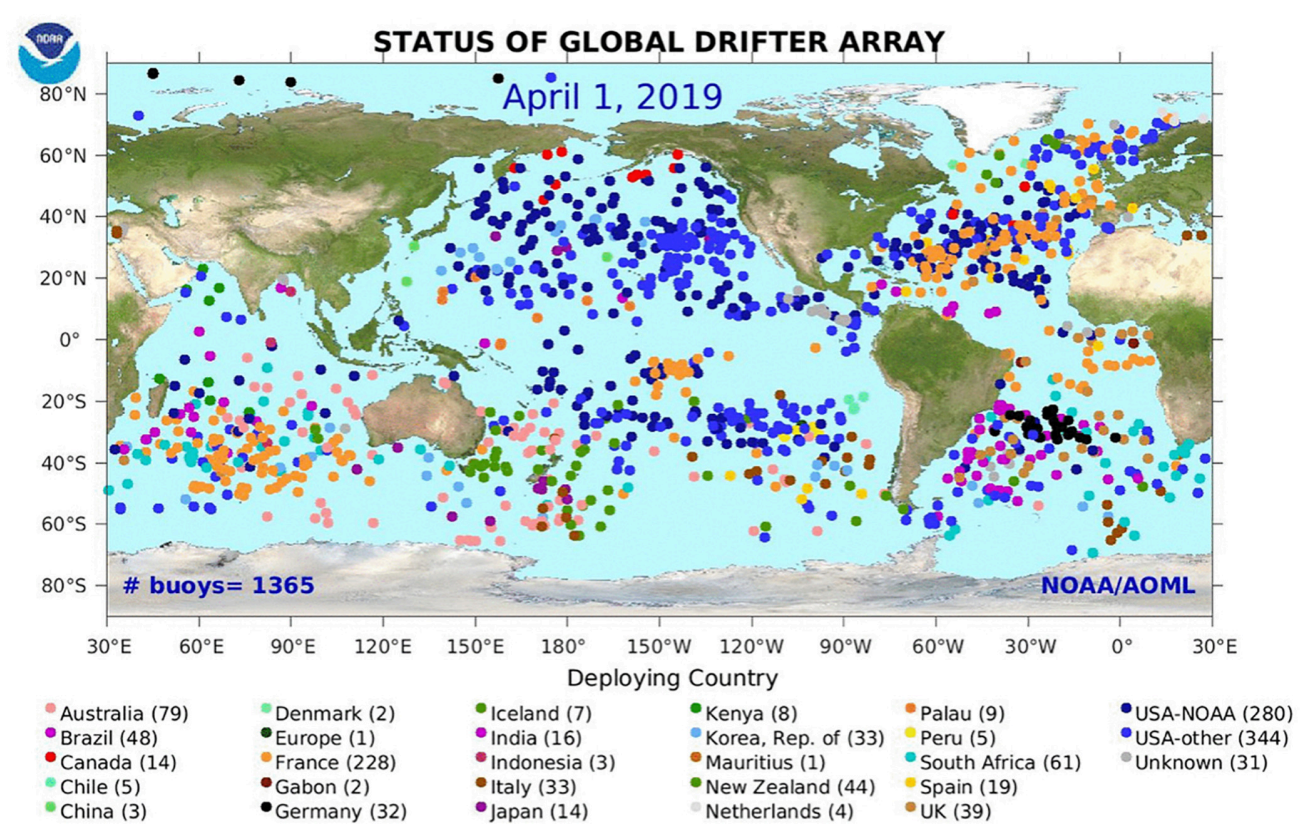

FIGURE 1 | Location of SVP drifters forming the GSDA array, color coded by deploying country.

implementation strategy of the GDP and GSDA. A crucial aspect of the data delivery is that the time interval between data collection and delivery to the GTS needs to be as short as possible, typically $15 \mathrm{~min}$ or less, in order to meet operational forecasting needs. The Iridium satellite system currently satisfies this requirement, and, at the time of writing, around $80 \%$ of the GSDA now transmits its data via Iridium telemetry. It is anticipated that by the end of 2019, all drifters in the GSDA will be using Iridium modems.

\section{Horizontal Near-Surface Current Observations From SVP Drifters}

The historical drifter data archive maintained and served by the GDP's Data Assembly Center (DAC) at NOAA/AOML dates back to 1979 and consists of observations from more than 22,000 Lagrangian SVP drifters. Near-surface, 15-m deep, ocean currents from SVP drifters are computed from satellite-derived time series of geolocation obtained with the Argos satellite system for older drifters and now with Global Positioning System (GPS) satellites. The accuracy of GPS geolocation is one to two orders of magnitude better than Argos. Consequently, GPS drifters provide more accurate ocean current data. Standardized QC techniques and interpolating procedures (Hansen and Poulain, 1996) onto regularly spaced 6-h time series are described, for example, in Niiler (2001) and Maximenko et al. (2013). QC and interpolation of recent data to hourly intervals are described in Elipot et al. (2016). The temporal extension of the GSDA dataset testifies to its value as a reference series relevant for climate studies and supports a detailed description of most oceanic mesoscale and submesoscale features and the major persistent current systems of the World Ocean.
The NOAA-funded GDP releases QC ocean current data that have supported more than 1,000 peer-reviewed scientific publications (see http://www.aoml.noaa.gov/phod/ dac/gdp_biblio.php for a partial list). The quality of the data collected with Lagrangian SVP drifters is discussed, for example, in Niiler (2001), Pazan and Niiler (2001), Lumpkin and Pazos (2007), and Maximenko et al. (2013). Ocean current data are available in delayed-time mode because the strain gauge sensor used to detect the drogue presence requires extensive operator interpretation (Lumpkin et al., 2012), historically with a 3-month delay; automatic drogue loss detection using strain gauge and GPS "time-to-first-fix," implemented in 2018 by AOML's DAC, has decreased this delay by a factor of three.

Surface current velocity observations derived from SVP drifters are directly used to validate the ocean velocity products of global monitoring and forecasting systems at Mercator Ocean (Lellouche et al., 2013, 2018), including the state-of-the-art Global Ocean $1 / 12^{\circ}$ Physics Analysis and Forecast. Surface velocities are also assimilated into regional ocean model forecast systems, leading to substantial improvement in the mesoscale Eulerian and Lagrangian forecast skills (e.g., Muscarella et al., 2015; Carrier et al., 2016; Phillipson and Toumi, 2017).

A good synopsis of recent surface ocean circulation studies based on drifter observations can be found in Maximenko et al. (2013), and state-of-the-art climatologies produced at AOML/GDP are discussed by Lumpkin and Johnson (2013) as well as Laurindo et al. (2017). These climatologies are used as benchmarks to validate the mean surface currents in the monitoring and forecasting systems at Mercator Ocean (Gasparin et al., 2018). Regional-scale SVP drifter climatologies have been used to study the seasonal and interannual variabilities 
of the ocean circulation where the data coverage is sufficient (e.g., Andersson et al., 2011; Poulain et al., 2013; Peng et al., 2015). Methods to remove the biases resulting from the irregular spatial and temporal sampling of the GSDA and to reconstruct the time-dependent geostrophic and Ekman velocities (Gill, 1982) consist in combining satellite altimetry, wind reanalysis products, and, sometimes, the large-scale geoid (Centurioni et al., 2008, 2009; Maximenko et al., 2009; Niiler et al., 2003a,b) (see Figure $\mathbf{2 b}$ as an example of such a synthesis). The GDP surface drifter dataset also enabled global mapping of the mean circulation (Figure 2a) and of characteristics such as cyclonic and anticyclonic motions from global SVP drifter observations for scales from large eddies to submesoscales (Griffa et al., 2008). Further, high-frequency (super-inertial) drifter velocity observations have been used to map the amplitude and phase of the baroclinic and barotropic tidal currents (Poulain and Centurioni, 2015). Since 2005, multisatellite processing of drifters transmitting through the Argos satellite system and the increasing use of GPS on some Argos and all Iridium drifters have allowed interpolation down to hourly intervals (Elipot et al., 2016) to support not only studies of global tidal currents but also of near- and super-inertial motions. When a wind stress is applied to the ocean surface, such as by strong storms or tropical cyclones, the transient ocean response in the mixed layer consists in the generation of cyclonically rotating near-surface currents that arise due to the Coriolis effect. These are commonly referred to as (near-)inertial oscillations, and their frequency is given by the Coriolis parameter. The overall current experienced by a Lagrangian SVP drifter results from the superposition of geostrophic currents and preexisting and wind-generated inertial currents, which often manifest in cycloidal drifter trajectories; analysis of drifter observations

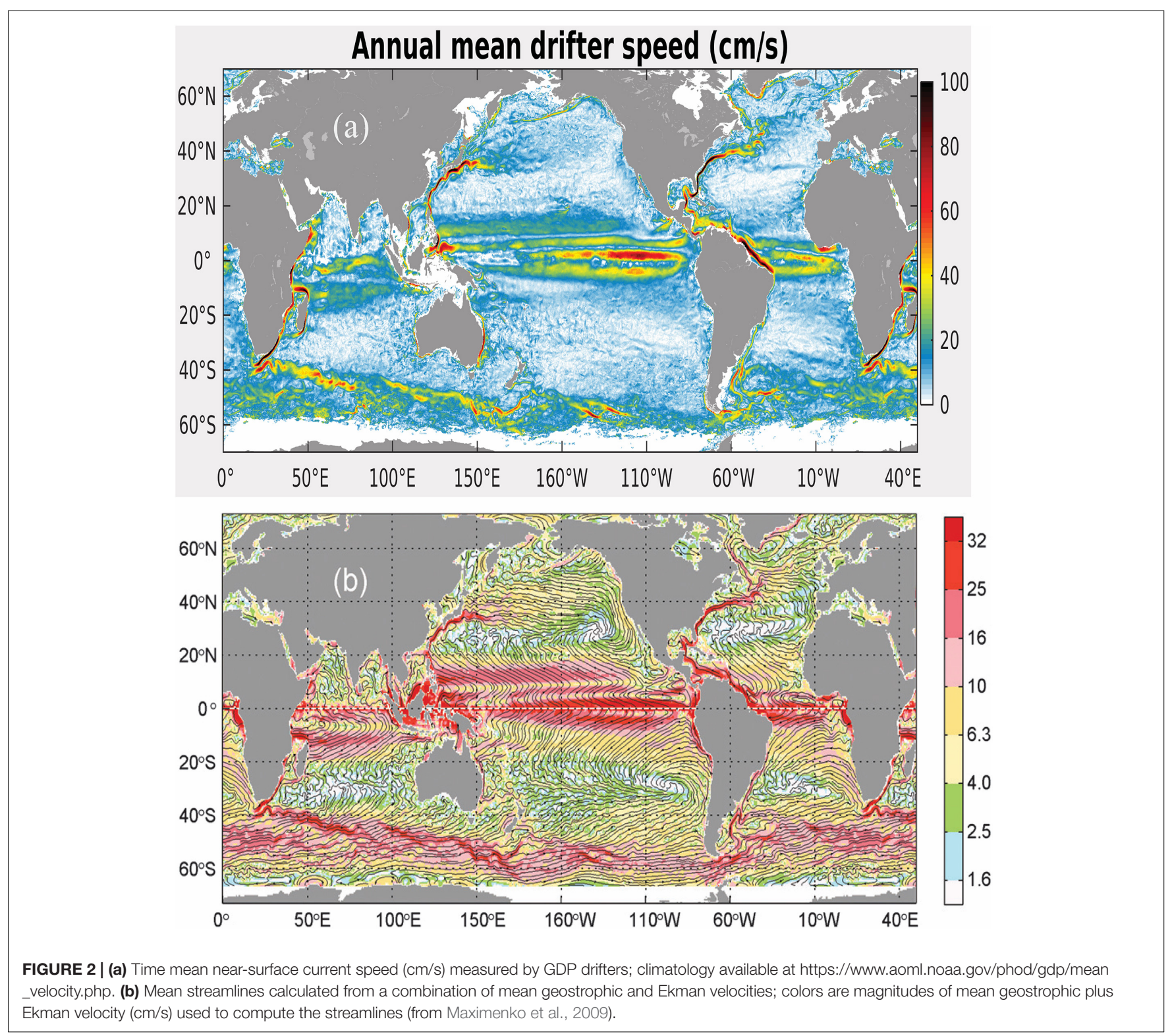


demonstrates that the period of these oscillations is also modified by the vorticity of the background eddy field and has been used to quantify the decay time scales of the oscillations (Elipot et al., 2010; Sykulski et al., 2016) in agreement with theory (e.g., Kunze, 1985). These inertial oscillations are believed to decay by propagating to greater depth as internal waves and typically subside over a few days to several weeks (e.g., Pollard and Millard, 1970; Price, 1983; D’Asaro et al., 1995; Hormann et al., 2014).

Because of the unmatched temporal and spatial resolution and their ability to accurately measure horizontal ocean currents, the observations from SVP drifters have been used in studies of lateral dispersion and mixing due to mesoscale and submesoscale circulations, providing estimates of mixing rates (e.g., Koszalka et al., 2011; Zhurbas et al., 2014; Roach et al., 2018), regional turbulent transport regimes, and time scale dependence of mixing (e.g., Lacorata et al., 2001; Koszalka et al., 2009; Lumpkin and Elipot, 2010). The drifter-derived mixing rates are used to evaluate regional ocean models with implications for eddy parameterizations (e.g., Haza et al., 2007; Döös et al., 2011; Rühs et al., 2018). Turbulent parameters estimated from drifters are used to build stochastic Lagrangian models (e.g., Griffa et al., 1995; Sykulski et al., 2016) that are further used to study signal propagation of hydrographic anomalies (e.g., Koszalka et al., 2013) and dispersion of floating debris (e.g., Cozar et al., 2017; McAdam and van Sebille, 2018).

A further description of ocean current observations in the upper-ocean mixed layer from Lagrangian drifters is given by Lumpkin et al. (2017).

\section{SST Observations From SVP Drifters}

Through the GTS, the drifter SSTs contribute to the majority of reanalysis and validation datasets (e.g., OSTIA: Donlon et al., 2012; Coriolis CORA; the CMEMS drifter validation product: Etienne, 2018; EUMETSAT OSI SAF). Today, SVP drifters provide more SST observations than any other source of in situ data, including ships (factor of about 4), coastal moorings (factor of about 4), tropical moorings (factor of about 50), or Argo floats (factor of about 100) (Xu and Ignatov, 2014). Together with tropical moorings, they cover most of the global oceans, producing highly accurate data with near-uniform quality across multiple temperature sensors ( $\mathrm{Xu}$ and Ignatov, 2016). Argo floats currently provide more uniform global coverage than SVP drifters and tropical moorings and have a lower measurement uncertainty. However, their normal 10-day operating cycle reduces the number of surface ${ }^{2}$ measurements by two orders of magnitude, leading to more limited validation statistics (e.g., Ignatov et al., 2016; Xu and Ignatov, 2016).

SVP drifters and tropical moorings are currently the main data sources for validation of satellite SST retrievals and are used by many groups to characterize the satellite data (e.g., Kilpatrick et al., 2001, 2015; Minnett and Barton, 2010; Dash et al., 2010; Ignatov et al., 2016), including the retrieval uncertainty (e.g., Bulgin et al., 2016) and the long-term stability of the satellitederived SSTs (e.g., Kilpatrick et al., 2015; Xu and Ignatov, 2016;

\footnotetext{
${ }^{2}$ Here, we refer to the Argo measurement taken nearest to the surface in either pumped or unpumped operation.
}

Berry et al., 2018). Three-way error analysis methods attempt to decouple errors in the three datasets being compared (e.g., O'Carroll et al., 2008; Xu and Ignatov, 2010, 2016; Lean and Saunders, 2013; Gentemann, 2014). The nominal accuracy and digitization of the temperature probes used to measure SST from SVP drifters deployed before 2014 were $00.1^{\circ} \mathrm{C}$. The use of more accurate temperature sensors, with smaller drift, improved accuracy $\left(O 0.05^{\circ} \mathrm{C}\right)$ and digitization $\left(O 0.01^{\circ} \mathrm{C}\right)$, and more accurate geolocation using GPS, is becoming the new standard. The triple-collocation studies (e.g., O'Carroll et al., 2008; Xu and Ignatov, 2010, 2016; Lean and Saunders, 2013), reviewed and summarized in Kennedy (2014), estimate the root-mean-square error (rmse; which equates to a standard uncertainty) in drifter SSTs in open ocean conditions to be in the range of $0.15-0.25^{\circ} \mathrm{C}$, higher than the expected range of $0.05-0.1^{\circ} \mathrm{C}$, and the exact cause of this difference is not fully understood.

Such estimates of SVP drifter SST uncertainty try to exclude values affected by "gross errors" (i.e., instrument malfunctioning), but the details of any QC used can significantly affect the uncertainty estimate (Kennedy, 2014). Other subtle issues may play a role in the observed discrepancy between the nominal and inferred quality of old-generation drifters, and these include the stability of the analog/electronic components of the temperature sensors and variations in the drifters' sampling methodology through the years. The contribution of real geophysical variability between point-wise in situ observations and space-averaged satellite observations that sense SST over about $1 \mathrm{~km}$ or larger pixels was first estimated by Minnett (1991) as $0.2^{\circ} \mathrm{C}$ for AVHRR and, more recently, by Castro et al. (2017) as $0.1^{\circ} \mathrm{C}$ for MODIS. New methods for satellite SST validation explicitly include geophysical effects (including depth and time differences as well as point in space) as summarized in Corlett et al. (2014). A benefit of the method detailed in Corlett et al. (2014) is the ability to validate the satellite SST uncertainty (Lean and Saunders, 2013; Bulgin et al., 2016; Nielsen-Englyst et al., 2018). Here, the uncertainty of the SVP drifter data is vital and ideally should be lower than that of the satellite data itself, which for three-channel retrievals from dual-view satellite radiometers is $<0.1^{\circ} \mathrm{C}$ (Embury and Merchant, 2012). This requirement for SVP drifters with lower SST uncertainty has led to the development of and subsequent deployment by the United States GDP/LDL of SIO of hundreds of Iridium/GPS drifters with carefully calibrated temperature sensors, providing an accuracy better than $0.05^{\circ} \mathrm{C}$ (Centurioni, 2018) and reporting temperature data with higher resolution compared to the historical drifters. Between the DBCP and GHRSST, it has also led to the development of other versions of drifters with a target measurement uncertainty of $0.05^{\circ} \mathrm{C}$, which are currently being evaluated (Poli et al., 2019).

\section{Sea Level Atmospheric Pressure Observations From SVP Drifters}

Since the 19th century, atmospheric pressure sensors have been relatively well distributed over land and have become increasingly common over the ocean, mainly due to the GSDA, the VOS scheme, and the moored buoy networks. While 
the GSDA represents a relatively short time series from a climate perspective, it represents the only source of in situ SLP data in many ocean regions. SLP observations from SVP-B drifters (Figure 3) are more accurate than those from ships because buoy barometers are located much closer to the sea level, whereas ship measurements require extrapolation from deck-level measurements (so-called reduction to sea level and subject to human errors), generating additional uncertainty, of the order of $0.8-0.9 \mathrm{hPa}$. SLP observations from ship-automated systems are free from human error and are of the order of $0.5 \mathrm{hPa}$ (Poli et al., 2017). By the same diagnostics, SLP data from SVP-B drifters are characterized by 0.3 to $0.4 \mathrm{hPa}$ rmse, which is consistent with the nominal accuracy of the sensor installed on the drifters (Centurioni, 2018). This also suggests that the sampling methodology of the SVP-B drifters, which are affected by surface waves that can be several meters in height (it should be recalled here that SLP can only be measured when the barometer port is exposed to air and that, because of their large drogues, drifters tend to float in the troughs and be submerged at crests in high seas), is not introducing a bias.

The spatial distribution of SLP data over the ocean, if SVP-B drifters were removed, would be affected by large gaps that exist between commercial shipping lanes, especially in the Southern Ocean and in the Arctic region (Woodruff et al., 2011). Even on trafficked shipping lanes, drifters are often the only source of SLP data when severe weather conditions occur and ships move out of the impacted areas.

SLP observations from SVP-B drifters contribute, directly or indirectly, to many reanalysis products used for climate assessments. Climate-related changes of the mean atmospheric load, or SLP, correspond to about $1-\mathrm{cm}$ change of sea level for a $100-\mathrm{hPa}$ difference, the so-called inverse barometer effect (Wunsch and Stammer, 1997). Although a study of the impact of SLP drifter data on the correction of sea-level satellite altimetry has never been performed, drifter-derived SLP data are likely to provide an important contribution to correct altimetry-derived sea level for atmospheric effects, especially in areas where drifters are the main or unique source of SLP ocean data.

Sea level pressure data from SVP-B drifters are used in the computation of trends to diagnose climate models and to construct climate indices. For example, the tropical Pacific SLP field is used to define the Multivariate El Niño-Southern Oscillation (ENSO) Index (MEI; Wolter and Timlin, 2011). The Southern Annular Mode (SAM) index is defined as the
A
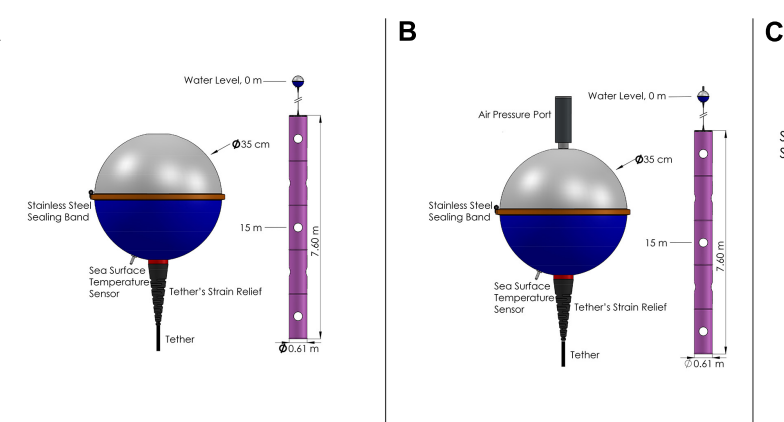

C

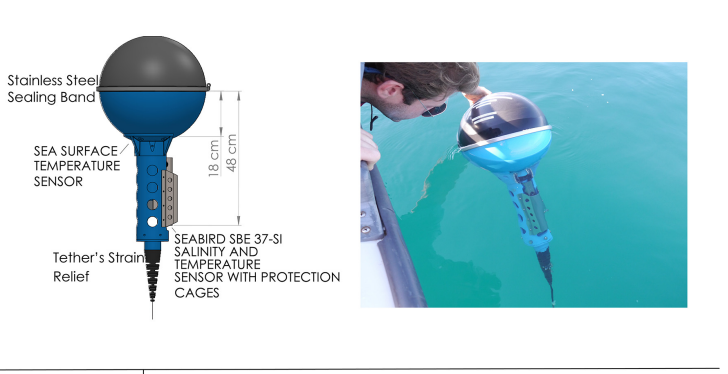

$\mathbf{F}$

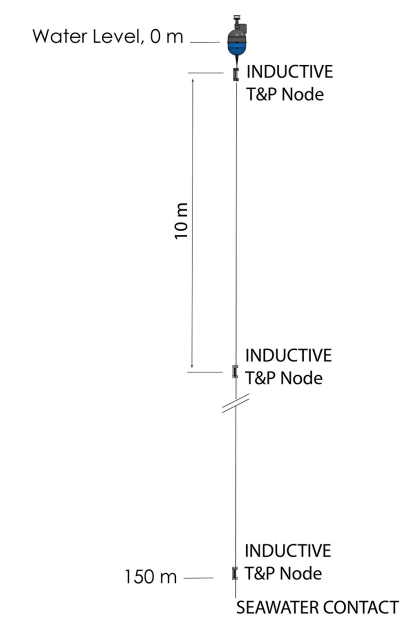

FIGURE 3 | (A) Schematic of the LDL SVP drifter (SST, 15-m depth currents). (B) Schematic of the LDL SVP-B drifter (SST, SLP, 15-m depth currents). (C) Schematic and picture of the LDL SVP-S drifter (SST, SSS, SLP optional, 15-m depth currents). (D) Schematic of the surface buoy (horizontal wind, SLP, SST) used for the LDL Minimet and ADOS drifters. (E) Schematic of the Minimet drifter (SST, SLP, horizontal wind, and 15-m depth currents). (F) Schematic of the ADOS drifter (SST, SLP, horizontal wind, subsurface temperature and pressure, up to $200 \mathrm{~m}$ with 10-m resolution). The ADOS can also be configured with ADCPs and conductivity sensors. All sensors use inductive communication modules to relay the data in real time through the Iridium satellite system. 
SLP difference between 40 and $65^{\circ} \mathrm{S}$ (Gong and Wang, 1999), and the North Atlantic Oscillation (NAO) index is based on the SLP difference between the subtropical (Azores) high and the subpolar low. SLP data from SVP-B drifters are also included in the Hadley Centre's monthly historical mean SLP dataset (HadSLP2) and the ICOADS v.2.5 (ranging from 1662 to 2007), and subsequent updates are based exclusively on observations available on the GTS (Woodruff et al., 2011). Substantial differences between reanalysis products are observed in regions where observations are sparse, including the Southern Ocean and southeastern Pacific (Allan and Ansell, 2006). Other climate products that use the drifter data (i.e., SST and SLP) are global reanalyses, such as the Climate Forecast System Reanalysis (CFSR) of the National Centers for Environmental Prediction (NCEP; Saha et al., 2010), the European Centre for Medium-Range Weather Forecasts (ECMWF) Interim reanalysis (ERA-Interim; Dee et al., 2011), the United States National Aeronautics and Space Administration (NASA) Modern-Era Retrospective analysis for Research and Applications, Version 2 (MERRA-2; Gelaro et al., 2017), the 55-year Japanese Reanalysis (JRA-55; Kobayashi et al., 2015), the 20th Century Reanalysis (20CR; Compo et al., 2011), and the ECMWF 20th century reanalysis (Poli et al., 2016). As reanalysis systems are evolving to describe the Earth system, they move toward ocean-atmosphere coupling, as noted at the 5th International Conference on Reanalyses (Buizza et al., 2018); to support advances in atmosphere-ocean coupling, the subsequent demand for air-sea interface observations is expected to grow.

SLP observations are used to assess changes in the frequency of occurrence and intensity of extratropical storms, to monitor and predict monsoon variabilities as well as trends in extreme weather. However, recent Intergovernmental Panel on Climate Change (IPCC) reports indicate that the confidence in some of these evaluations is low due to inconsistencies between reanalysis products that use data from different years (e.g., early 1900s) and of different quality as well as the lack of long-term data, particularly in the southern hemisphere (IPCC, 2013 report, pg. 2-62). Ensuring the continuity of in situ SLP observations coming from a variety of sources, including the GSDA, should be considered a priority.

A particularly significant impact of the GSDA is the beneficial influence of drifter SLP data for NWP. Ingleby (2010) provides an overview of in situ ocean surface observations from a NWP perspective. Recent studies have quantified the implications of the non-availability of these data (Centurioni et al., 2017b). Observing system experiments (OSEs), as discussed in Centurioni et al. (2017b) and Horányi et al. (2017), were performed with the objective to evaluate associated NWP errors against reanalyses when only drifter SLP data were removed. The main conclusions of these studies are that the in situ drifter SLP data reduce the forecast error up to 5 days ahead near the surface and higher in the troposphere, up to $250 \mathrm{hPa}$. The largest error reductions were observed for the mean SLP and wind field forecasts. Such studies also suggest that the expansion of the SVP-B array to the tropics should be considered. Forecast sensitivity observation impact (FSOI) studies were also used to quantify the impact on the principal components of GCOS.
Such assessments are often run operationally by ECMWF, the United Kingdom Met Office, and the Global Modeling and Assimilation Office of NASA. When the impact per observation or the fraction of beneficial observations is computed, the in situ drifter SLP data provide some of the largest values among the main components of GOS (Centurioni et al., 2017b; Horányi et al., 2017). In addition, Ingleby and Isaksen (2018) assessed the impact of removing half of the drifter SLP data from the assimilation; they concluded that the observing system was not yet saturated, and there was much room for improvement with additional SLP drifter data.

\section{Other Observations From SVP Drifters}

Lagrangian drifters based on the SVP design can be configured with a variety of meteorological and oceanographic sensors (Figure 3), including Gill's Windsonic anemometers, conductivity sensors to measure sea surface salinity (SSS), radiometers and pyranometers, hydrophones, acoustic Doppler current profilers (ADCPs), and subsurface temperature and conductivity sensors (see Centurioni, 2018 for a review of the current technology). A large number of salinity drifters were deployed in recent years in support of the two "Salinity Processes in the Upper-ocean Regional Study" (i.e., SPURS-1 and SPURS-2) campaigns (Centurioni et al., 2015; Hormann et al., 2015; Reverdin et al., 2015; Schmitt et al., 2015), and in the Bay of Bengal during the Office of Naval Research (ONR) Departmental Research Initiative (DRI) "Air-Sea Interactions in the Northern Indian Ocean-Regional Initiative" (ASIRI; Hormann et al., 2016). Salinity drifters can provide SSS observations with an accuracy of about 0.01 psu for 1 year (Hormann et al., 2015).

\section{Voluntary Observing Ship Scheme}

International coordination of observing the weather from ships started as long ago as 1853 and has led to the WMO VOS scheme, which is an international program in which ships are recruited by NMSs to make meteorological observations at sea. Participation in the VOS scheme is encouraged in the International Maritime Organization (IMO) Safety of Life at Sea (SOLAS) convention. The VOS scheme is an observing program of the Ship Observations Team (SOT), coordinated by the WMO-IOC JCOMM and described by Smith et al. (2019), including the contribution of oceanographic research vessels to observations at the air-sea interface. VOS report air and sea variables that are assimilated in forecasting models and used for reanalysis products, similarly to those from drifters described above. In addition, VOS generally also report critical variables such as air temperature as well as relative humidity and wind, which are also assimilated in operational weather models and global reanalyses. Furthermore, when observers are present, observations of clouds and sea state are also collected. Figure 4 shows the locations of observations from VOS for a selected time period. This map indicates that the concentration of VOS is greater near economic centers of activity and shipping corridors or along coastlines. A few research vessels are further equipped with sensors to measure salinity, $\mathrm{pH}$, fluorescence, and currents, though such information is generally not included in the VOS reports, but only available 


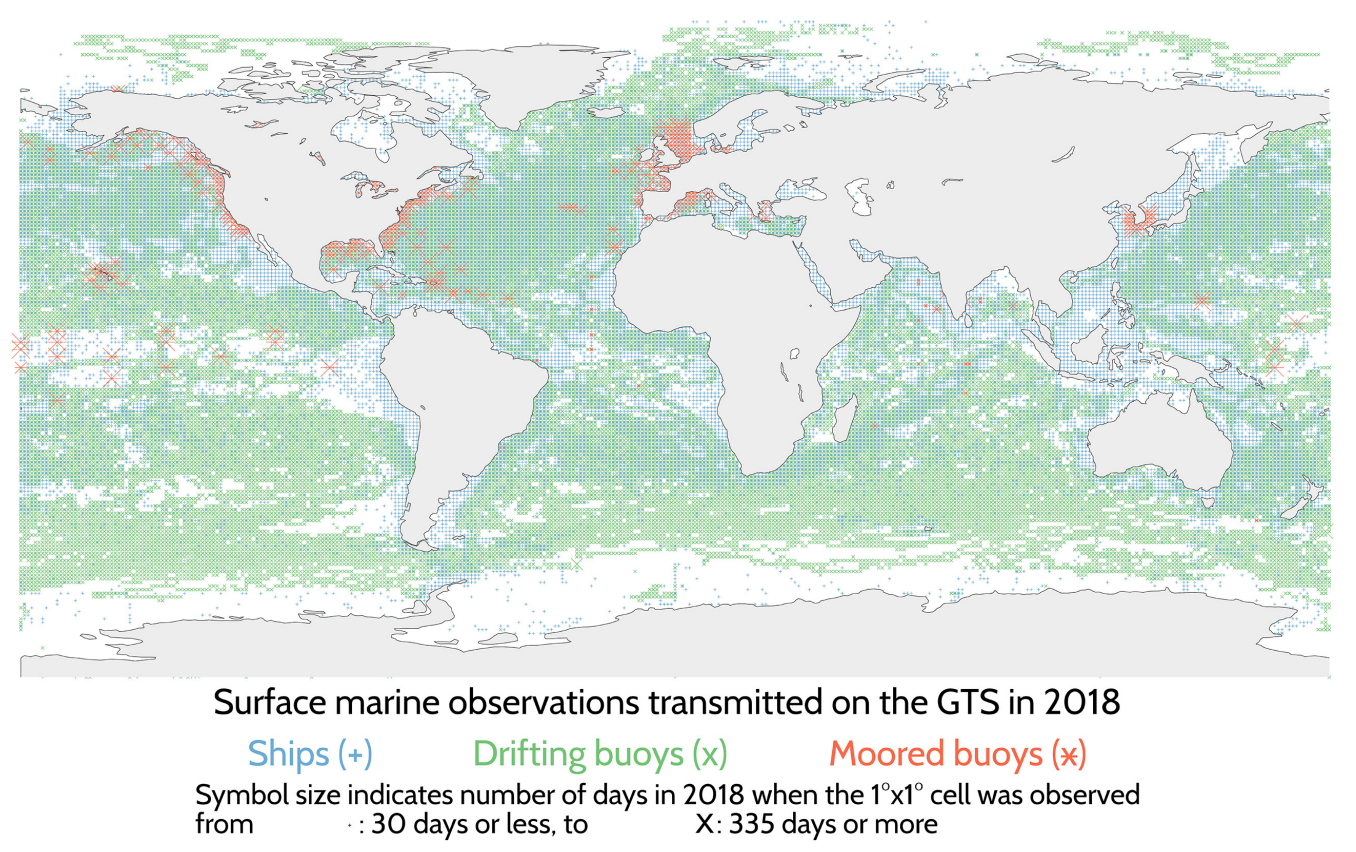

FIGURE 4 | Data coverage by the three major components of GOS (i.e., ships, drifting buoys, and moored buoys) based on information received by Météo-France through the GTS in 2018 (see legend for symbol details).

in delayed mode and for research. Currently, only few or no automated stations with broadcasting to the GTS report parameters such as $\mathrm{pH}$, fluorescence, clouds, sea state, and currents. However, notable efforts are underway for $\mathrm{pH}$ and fluorescence (e.g., Choquer et al., 2013), for waves (Christensen et al., 2013) as well as for currents from position, compass direction, and ship speed transmitted through the Automatic Identification System (AIS; Guichoux et al., 2016). In recent decades, there has been a slow decline in the number of ships in the traditional VOS scheme, partly offset by an increase in automated (usually hourly) ship reports. There has also been an increase in average ship size. About half of the ship measurements are relatively close to the coast (see Ingleby, 2010 and references therein).

Compared to drifters, the more complete set of variables from VOS enables the computation of air-sea fluxes from bulk parametrizations. A notable example is the surface flux and meteorological dataset of the National Oceanography Centre (NOC) version 2.0 (Berry and Kent, 2009), where air and sea temperatures observed by ships, along with observations of humidity, wind speed, and cloud cover, are used to estimate various components of heat fluxes (i.e., sensible, latent, short-, and longwave radiation). Such estimates are then useful benchmarks for the performance of weather and climate models and lead advances toward ocean-atmosphere coupling in models and data assimilation (Freeman et al., 2019).

Finally, research vessels can be equipped to directly measure some components of the air-sea fluxes (e.g., outgoing longwave radiation). This information is readily comparable to satellite measurements, enabling a better understanding of the uncertainties caused by atmospheric absorption in the retrievals.
Such data serve to develop fiducial reference measurements (FRM) of SST from ships (Theocharous et al., 2016).

For further information, we refer the reader to the review by Smith et al. (2019) on the role of ship-based observations, including but not limited to VOS, in support of the physical, biological, and carbon dioxide $\left(\mathrm{CO}_{2}\right)$ communities.

\section{Moored Buoys}

As noted earlier, the DBCP also coordinates observations from the TMBA and the various national (mainly coastal) meteorological and oceanographic moored buoy networks (including directional wave buoys). Figure 4 shows that the coverage achieved by moored buoys, especially the TMBA, complements the surface drifter array, which tends to be advected away from the equatorial band by the ocean circulation. The TMBA spans the Pacific (TAO/TRITON), Atlantic (PIRATA), and Indian (RAMA) ocean basins. It consists primarily of T-FLEX moorings developed at NOAA/Pacific Marine Environmental Laboratory (PMEL), and their configurations are determined by climate and regional processes of interest in the different basins. Reevaluations, such as the so-called TPOS 2020 effort (Cravatte et al., 2016), aim to maintain or increase the value of the arrays while responding to changes in partner commitments and funding pressures as well as other constraints (e.g., vandalism on data buoys). Numerous regional partners work together to maintain the three arrays, which require significant investment of ship time. TMBA moorings measure wind speed and direction, air temperature, humidity and downward shortwave radiation, and many also measure SLP. Ocean parameters measured include temperature and salinity in the upper $500 \mathrm{~m}$ of the ocean, and most also measure ocean 
currents at a depth of about $10 \mathrm{~m}$ using point acoustic current meters. The TMBA additionally includes a small number of ADCP moorings providing vertical profiles of ocean currents. Some TMBA as well as OceanSITES moorings have added biogeochemical observations such as oxygen, $\mathrm{CO}_{2}$ fugacity, and acoustic monitoring.

Over the last 25 years or so, national moored buoy networks have been developed by many countries as part of their operational observing capabilities. As such, there is no single global network of moored buoys but rather a "network of networks," capable of measuring a wide range of ECVs and EOVs from marine meteorology and waves to subsurface oceanographic measurements that complement other sources of synoptic observations in coastal seas and the open ocean. They provide real-time data for operational services such as marine weather and wave forecasts (both for assimilation into and validation of forecast models), maritime safety information and warnings to end users, information for marine disaster and accident response, calibration/validation of satellite-derived observations, and data for research purposes. Many of these networks have been in place for at least a couple of decades, some for as long as 40 years, and so provide valuable time series for marine climate studies, in particular for wave climate analyses. Advances in sensor technology, moorings, best practices, and sharing of experience and expertise are coordinated through the $\mathrm{DBCP}$ and have helped improve the performance and reliability of these systems at sea.

Similar to drifters and ships, moored buoy data also assist in the development of ocean-atmosphere coupling in numerical models. For example, the June 2018 upgrade of the ECMWF operational system to coupling was validated using moored buoys measuring SLP, air and sea temperatures as well as wind speed (Mogensen et al., 2018).

\section{Other Impacts of the Existing Air-Sea Networks}

Besides the demonstrated beneficial impact of several air-sea in situ observations for NWP as well as operational ocean forecasting and their importance for assembling climate datasets used for research and assessments discussed in the previous paragraphs, data relayed from the existing air-sea in situ observational networks are commonly supporting a variety of other applications with direct relevance to society and the development of blue economy.

One example of the latter is the exploitation of the Lagrangian properties of drogued SVP drifters to study the biological dispersal (e.g., Carlton et al., 2017; Miller, 2018) and recruitment of fish larvae to support stock management (e.g., Hare and Walsh, 2007; Booker et al., 2008).

Also, surface currents transport oil spills and marine debris. Generally, every type of pollution moves differently from each other as well as from the water parcels. Modeling this drift remains challenging because it is a result of the rectification of high-frequency motions and a function of the near-surface vertical structure of the currents, neither of which are resolved by the present observing system. Maximenko et al. (2018) demonstrated that data from Lagrangian SVP drifters were critical for adequate modeling of debris drift from the 2011 tsunami in Japan to North American and Hawaiian shorelines. Drifters are expected to play an important role in the future Integrated Marine Debris Observing System (IMDOS; De Dominicis et al., 2012; Maximenko et al., 2019).

It should also be noted that some observing programs, driven by a scientific rather than fully operational rational, are capable of addressing fast response needs to emergencies or can provide critical data for such applications in an opportunistic way. For example, the GDP can quickly air-deploy special drifters designed to measure wind speed and direction, SLP, SST, subsurface temperature, and directional wave spectra ahead of tropical storms and provide real-time data through the GTS and web server interfaces to forecasters and for post-storm season assessments. These buoys are deployed from C-130J aircrafts by the United States Air Force Reserve's 53rd Weather Reconnaissance Squadron "Hurricane Hunters." The latest deployment, at the time of writing occurred ahead of Hurricane Michael in the Gulf of Mexico on October 8, 2018, when drifters sampled storm conditions at the sea surface for several hours ahead of the storm, inside the eye, and in the wake of the storm (Figure 5). It should be noted that the drifter technology has matured to a level of sophistication that allows such instruments to go through tropical cyclones unscathed (e.g., D’Asaro et al., 2013; Mrvaljevic et al., 2013; Hormann et al., 2014; Centurioni, 2018).

Furthermore, the GSDA is maintained with a buoy density that makes encounters of drifters with tropical cyclones a rather common occurrence, thus providing valuable SST and SLP observations for assimilation into forecasting models and to support forecast analysis. In the North Atlantic alone and for the January 2013-September 2018 period, a total of 160 SVP and SVP-B drifters were within 30 nautical miles $(\mathrm{nm})$ of the tracks of systems that eventually developed into hurricanes (Figure 6).

\section{GAPS AND LIMITATIONS OF THE EXISTING AIR-SEA IN SITU NETWORK}

It is fair to say that the current air-sea in situ observing system is covering the basic needs for SST, SLP, and mixed-layer current observations. Notable lack of observed ECVs/EOVs at a spatial scale and temporal resolution compared to that provided by the GSDA include SSS, surface wind, directional wave spectra, air temperature, and relative humidity. For many (but not all) of these variables, the VOS and the moored buoy networks are the only sources of data, thus leaving many critical regions undersampled or not sampled at all. In the following, we review gaps and limitations of each observing system component.

\section{The Global Surface Drifter Array}

In its present configuration, the GSDA is sustainable with respect to global SST and near-surface currents, but gaps in the drifter distribution remain. Notable areas include some parts of the western Indian Ocean, stretching into the Arabian Sea, where sustained deployments in partnership with the shipping industry 

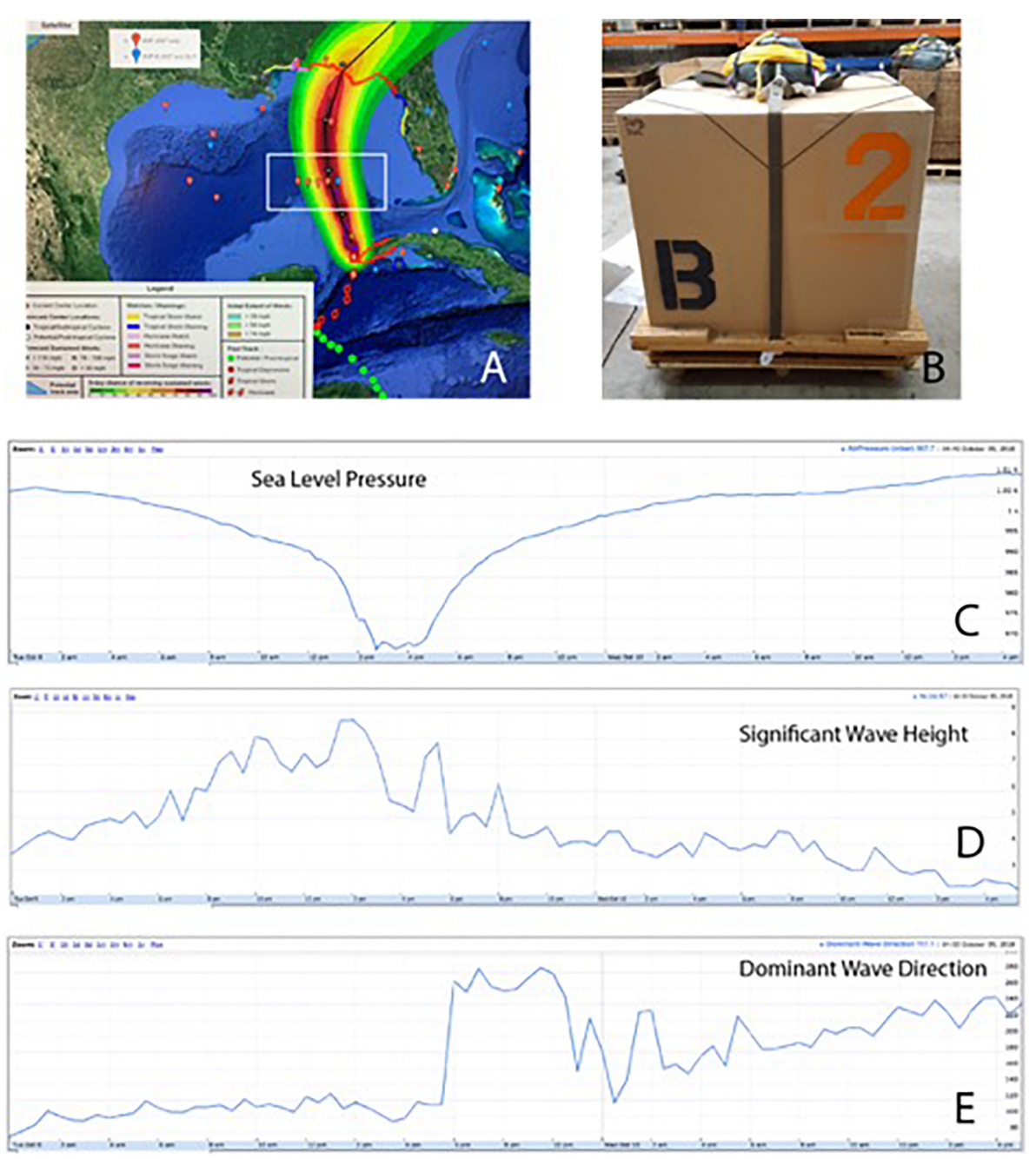

FIGURE 5 | (A) Deployment location of 10 drifters ahead of Hurricane Michael (white box). The drifters were deployed on October 8, 2018, from a C-130J aircraft by the United States Air Force Reserve's 53rd Weather Reconnaissance Squadron "Hurricane Hunters." The transect was located approximately $24 \mathrm{~h}$ ahead of the predicted track of the storm, which is shown by green and red symbols. The forecast for the wind intensity is overlaid as a contour plot (see legend in panel A). The storm data were provided by NOAA's National Hurricane Center (NHC). The 10 drifters were air-deployed at five stations using the deployment package shown in panel (B). Each box contained one SVP-B drifter and one directional wave spectra (DWS) drifter. One SVP-B drifter measured a minimum air pressure of $967.7 \mathrm{hPa}$ inside the eye of the hurricane (C). The DWS drifter deployed at the same location measured a maximum significant wave height of $8.7 \mathrm{~m}$ (D). Note the sharp change of the dominant wave direction due to the hurricane moving over the DWS drifter (E) and the rapid decay of the wave field in the wake of the storm.

still present logistical challenges (Centurioni et al., 2017a). Furthermore, drifting buoys are largely absent in the Arctic, notably during summer months when the basin becomes largely ice free, with the exception of ice buoys deployed as part of the Arctic Buoy Program and the Year of Polar Prediction (YOPP) targeting important SLP observations.

The demonstrated beneficial impact of drifter SLP data in climate research and NWP is sufficient to justify the installation of barometers on all of the GSDA drifters, but as of now, only about $50 \%$ of the drifters carry barometers, mainly due to the additional cost (approximately 1,100 US\$ per unit) and funding limitations. One of the most difficult tasks is to sustain a global drifter distribution; crucial to achieving this goal is the collaboration of the GDP, which evolved with a strong oceanographic connotation, with various NMSs from several countries. These include NOAA's National Weather Services, Members of the European Meteorological Services Network (EUMETNET), Canada, Australia, South Africa, New Zealand, India, and many other partners who are interested in maintaining a global network of barometers at the sea surface. Such collaborations are coordinated by the DBCP and implemented through both the direct purchase of barometer drifters and by means of the GDP barometer drifter upgrade program. Therefore, the collaboration with the meteorological services has the effect to provide a significant number (i.e., 25-30\%) of additional drifter deployments.

During the last decade, the drifter technology has evolved to measure other ECVs/EOVs. Drifters designed at SIO can measure 

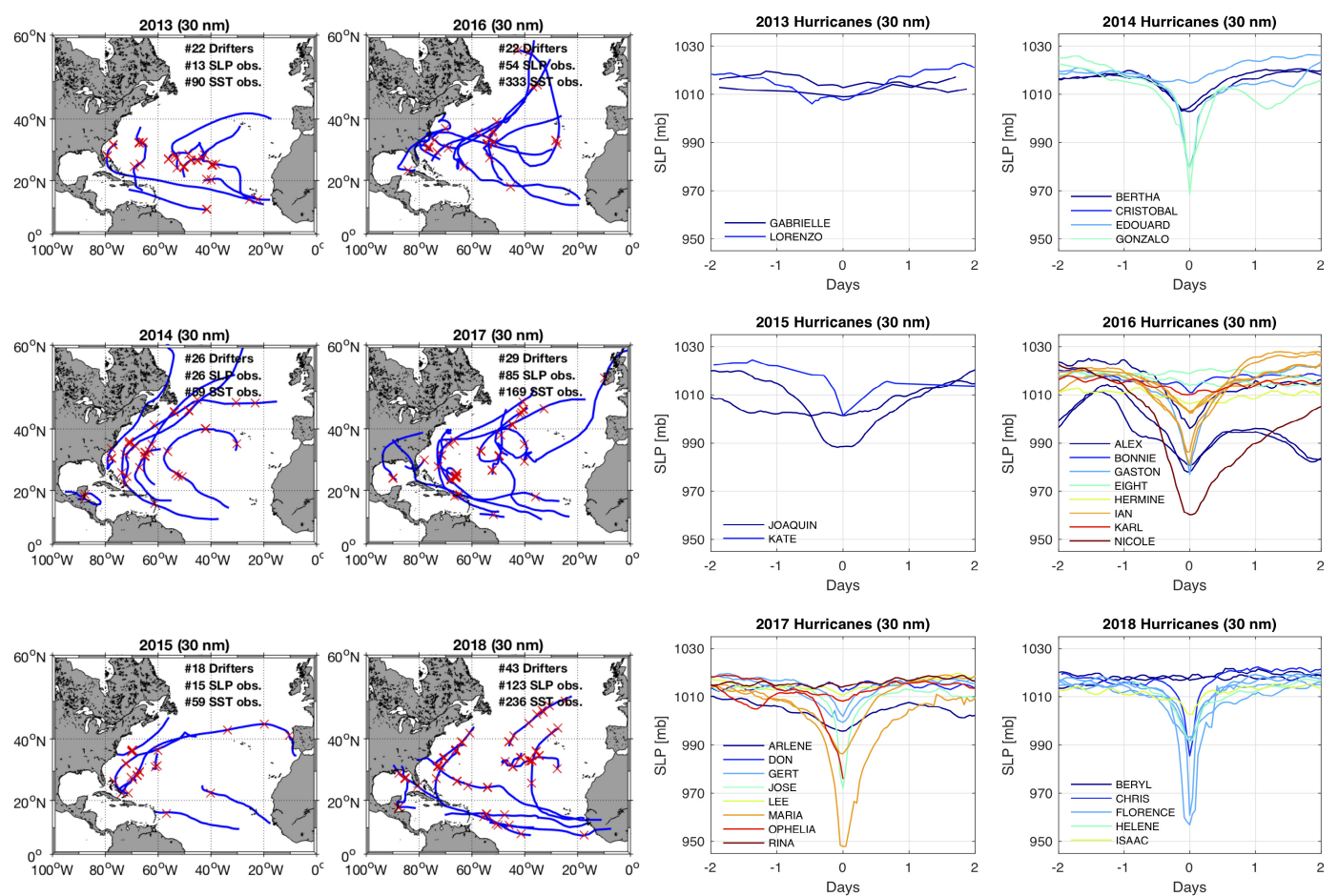

FIGURE 6 | Drifter/hurricane encounters (June 2013-September 2018). All drifter observations are within 60 min of the track location. Matches within 30 nm (left) are marked by red crosses. The hurricane tracks are shown in blue. For each hurricane season, the numbers of drifter/hurricane encounters are reported for each matching criterion as well as the total number of SST and SLP observations collected. Time series of SLP for drifter/hurricane matches selected using the 30-nm proximity criteria, color coded by storm name, are also shown. The time series are 4 days long and are centered around the minimum SLP pressure observed by the drifters.

SSS, sea-level wind velocity (Centurioni, 2018), upper-ocean temperature profiles, and surface radiation. Such technologies could be deployed for sustained operations, but funding limitations prevent the implementation on a global scale. Other observational gaps that could be addressed with surface drifters include air temperature, water vapor, sea state, and velocity shear in the upper ocean.

\section{Voluntary Observing Ship Scheme}

While ships can host a wide variety of sensors, placement of sensors is a primary issue for all variables. For SLP, the barometer can be up to several tens of meters above the surface, which introduces considerable additional errors when adjusting the measurements to the sea surface, especially when the proper barometer height is not used or misused (e.g., height depends on ship's load, which is variable and often not reported). For temperature, the ship structures are known to affect the measurements, and daytime measurements are most affected by biases (Berry et al., 2004). For wind, in spite of general placement at the highest point (to reduce circulation effects on the ship structures), it should also be noted that the ship structure can sometimes disturb the air flow (Popinet et al., 2004). For some of these challenges, there are possible ways forward. For example, reduction in cost of multifrequency, multisystem Global Navigation Satellite System (GNSS) technology may permit dynamic estimation of the barometer height, along with the sea level, to report accurate SLP measurements. For wind, accounting for airflow distortion can help to reconcile air-flux measurements (using corrected winds) with direct flux measurements (Landwehr et al., 2015).

SST is most often measured at the engine intake or using hull contact sensors, but on a small number of commercial vessels as well as research ships, infrared radiometers can be deployed to provide skin SST measurements of high accuracy. Two such instruments, the ISAR (Infrared Sea Surface Temperature Autonomous Radiometer), a filter radiometer with internal calibration (Donlon et al., 2008), and the M-AERI (Marine-Atmospheric Emitted Radiance Interferometer), a Fourier-transform infrared spectroradiometer, also have internal blackbodies for at-sea calibration (Minnett et al., 2001). These radiometers were specifically developed to take measurements suitable for the validation of satellite-derived SSTs (Corlett et al., 2014), and both types have been deployed on commercial ships for this purpose (Minnett, 2010; Wimmer et al., 2012; Donlon et al., 2014a,b) as well as on research vessels. A major advantage of using a M-AERI-type instrument is that besides measuring the skin SST, a near-surface air temperature can be derived (Minnett et al., 2005) and, hence, an accurate determination of the air-sea temperature difference. In addition, the analysis of the infrared emission spectra of the sea surface can lead to an estimation of the temperature profile through the ocean skin layer (Wong and Minnett, 2016a,b) and the response 
of the skin layer to infrared radiative forcing (Wong and Minnett, 2018), which are not accessible by in situ methods.

\section{Moored Buoys}

Moored buoys are generally regarded as providing the highest-quality observations of a wide range of marine meteorological variables and, as noted earlier, are used to provide "ground truth" observations for satellite calibration/validation as well as for estimates of surface fluxes (e.g., Bourras, 2006) for which wind speed is an essential parameter. However, due to the costs and logistics in operating moored buoys, they cannot provide global spatial coverage as drifters do since they are primarily located in the tropics and adjacent to coastlines (mainly in the northern hemisphere) to meet national operational requirements. Deployment and servicing of moored buoys require dedicated ship time, which is becoming increasingly costly. Typically, servicing intervals can be as long as 2 years, during which time the sensors, which are often exposed to severe weather conditions, may drift or fail. On some moored buoy networks, this can be partly mitigated by having dual sensor systems to provide increased resilience. In addition, the mooring lines generally need replacing every 2 to 4 years, and these can be expensive for deep ocean moorings that can be several thousand meters long. Vandalism is also a major problem, particularly for moored buoys that are deployed in equatorial regions near areas of intense fishing activity. This can include moored buoys being dragged off station, mooring lines being cut by fishing vessels so that the buoy goes adrift and has to be recovered, sensors and satellite transmitters being removed and stolen, and deliberate or accidental (e.g., collision with a ship) damage. The DBCP is trying to mitigate the problem of vandalism through an outreach strategy that aims to inform the fishing communities that the buoys are operated to provide data that are to their benefit (i.e., improved weather and wave predictions, and tsunami warnings).

\section{Gaps Across Programs and Platforms}

Users exploiting past observational data always wish to know the actual sensing equipment used and the direct sensing environment (e.g., platform type, sensor placement on the platform). Accurate and exhaustive metadata are the basis for the correct utilization of the data and to maximize their impact. While this information is always recorded locally, there is very little coordination among individual projects regarding the content and format of the metadata. This lack of coordination constitutes a potential limitation to the full exploitation of the observations. Under WMO guidance, metadata are only recently started being collected internationally. We also identify this effort as a way forward to improve the current situation, and we refer the reader to the guide on WIGOS (https://library.wmo. int/doc_num.php?explnum_id=6010) which explains how the international community has now arranged for a comprehensive collection of metadata, and for a process to consolidate realtime and delayed-mode data repositories in the Marine Climate Data System (MCDS; Pinardi et al., 2019). Here, we just wish to point out the importance that individual programs invest adequate resources to endorse and facilitate the harmonization of their metadata.

\section{EMERGING TECHNOLOGIES}

In this section, we describe some of the emerging technologies that are either mature for operations and large-scale deployments or have the potential to address some of the gaps and shortcomings described in the previous section.

\section{Directional Wave Spectra (DWS) Drifters}

Developed by the LDL at SIO, the DWS drifter (Centurioni et al., 2016 and Figure 7) is a very-low-cost and accurate wave rider based on the GPS technology that returns in real time the directional wave spectra of surface gravity waves and GHRSST-compliant SST. The DWS drifter is capable of a 1-year-long mission with 6-h measurements. The power spectral density, co-spectra, and quadrature-spectra parameters are derived by a Fourier transform of the correlation functions related to each pair of the three-dimensional wave orbital velocities, giving the "First-5" independent Fourier coefficients $\left(a_{0}, a_{1}, a_{2}, b_{1}, b_{2}\right)$ and, thus, the wave spectra for each 6-h (but as often as half-hourly) sea state. For each measured sea state, the three velocity components, the computed "First-5" Fourier coefficients, and the main wave data parameters can be stored onboard an optional data logger while platform information (timestamp, latitude, longitude, battery voltage, internal pressure, temperature, and humidity) at start of data collection, computed wave parameters $\left(\mathrm{H}_{\mathrm{mo}}, \mathrm{T}_{\mathrm{p}}, \mathrm{T}_{\mathrm{a}}\right.$, and $\left.\mathrm{D}_{\mathrm{p}}\right)$, and, optionally, "First-5" coefficients in the $0.031-0.496 \mathrm{~Hz}$ frequency range with $1 / 256$ $\mathrm{Hz}$ bandwidth are transmitted to shore in real time through the Iridium satellite system. The interval between measurements is programmable over the air, and spectral wave data can be retrieved as often as every $30 \mathrm{~min}$. At the time of writing, a GTS format for the DWS drifter has been approved by WMO, with the objective to facilitate the distribution of these data on the GTS and the use of in situ spectral wave data with wave forecasting models and to assist operational forecasting centers. The DWS drifter technology has a technology readiness level (TRL) of 9 , which has been demonstrated through the operational deployment from ships of 62 units in the Global Ocean and with the air deployment of 7 units in the Bay of Bengal and the Atlantic Ocean.

\section{Drifting Air-Sea Interface Buoy (DrlB)}

A newly designed non-Lagrangian drifter, called the drifting air-sea interface buoy (DrIB), was recently tested in the western Pacific. The DrIB is intended for atmospheric observations of surface wind, air temperature, air pressure, and relative humidity at a height of $3 \mathrm{~m}$ as well as for measuring SST (Figure 8). The data are transmitted by the Iridium or Beidou satellite systems.

The total weight of the DrIB is about $50 \mathrm{~kg}$, and the maximum diameter is $0.54 \mathrm{~m}$. An Airmar ultrasonic weather station is located at the top of the $3-\mathrm{m}$ mast to measure wind velocity and atmospheric pressure. The NOTC SHT16-1 sensor is used to measure air temperature and relative humidity, and the NOTC MT15 is used to measure SST. Both sensors are manufactured by the National Ocean Technology Center of China and tested and calibrated by the National Center of Ocean Standard and Metrology of China. 

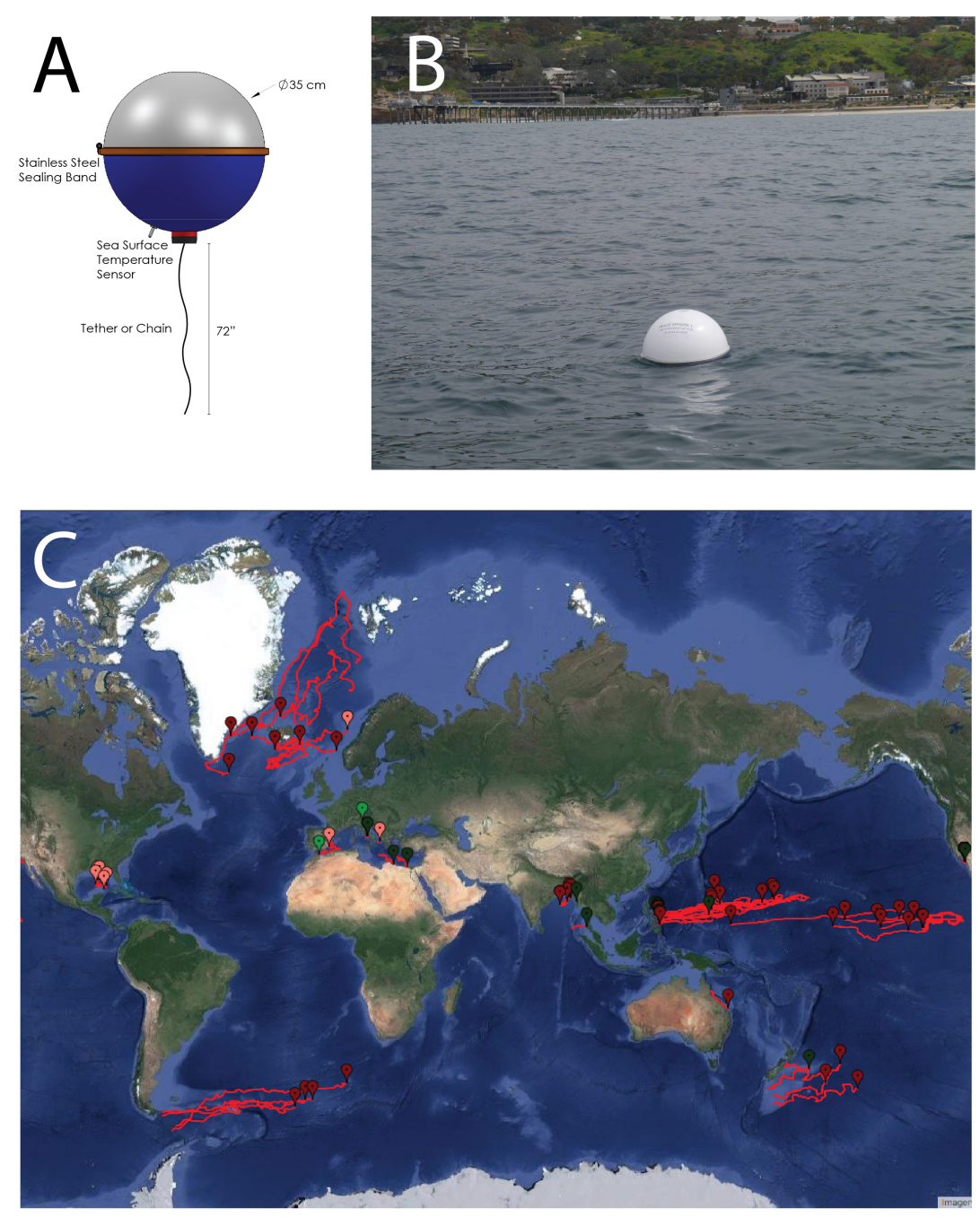

FIGURE 7 | Schematic of the DWS drifter (A). A LDL DWS drifter test deployment off the SIO pier (B). Tracks of the 69 DWS drifters deployed to date by the LDL (C).

The data communication, processing, and control systems have already been built, and two DrIBs have been used operationally by the State Oceanic Administration of China in 2018.

The DrIB was tested in the Kuroshio Extension region, and observations were compared with those obtained from the Kuroshio Extension Observatory (KEO). In 2019, over 40 DrIBs are planned to be deployed in the South China Sea and western Pacific, forming a mini-network for related studies on air-sea interaction and climate change. This will be beneficial to further validate/optimize forecast models.

\section{Unmanned Surface Vehicles}

In recent years, the use of autonomous vehicles, sometimes referred to as "gliders," for ocean data collection has increased. These include unmanned (or autonomous) surface and underwater vehicles (USVs), where the former have potential for making measurements at the air-sea interface and, in the absence of an international community of practice, fall under the ambit of the DBCP. A number of commercially available USVs have been developed (e.g., Wave Glider, Saildrone, Sailbuoy, AutoNaut, and C-Enduro) that are becoming more widely used by the research community and industry. The various USVs typically have the following characteristics: long endurance at sea (e.g., by harnessing wave or wind energy for propulsion), ability to carry a range of meteorological and/or oceanographic sensors and payloads, possibility to post-calibrate sensors on recovery, and two-way communication, allowing them to be piloted remotely (e.g., to make measurements along a preset route or to operate around a fixed station) and to transmit data in real time.

Possible sensors that can be integrated in USVs include meteorological sensors for wind, air temperature, air pressure as well as directional wave sensors, and oceanographic sensors such as ADCPs, conductivity/salinity, temperature, and dissolved oxygen probes. Wave Gliders are capable of shortterm to seasonal targeted sustained observations in data-void 

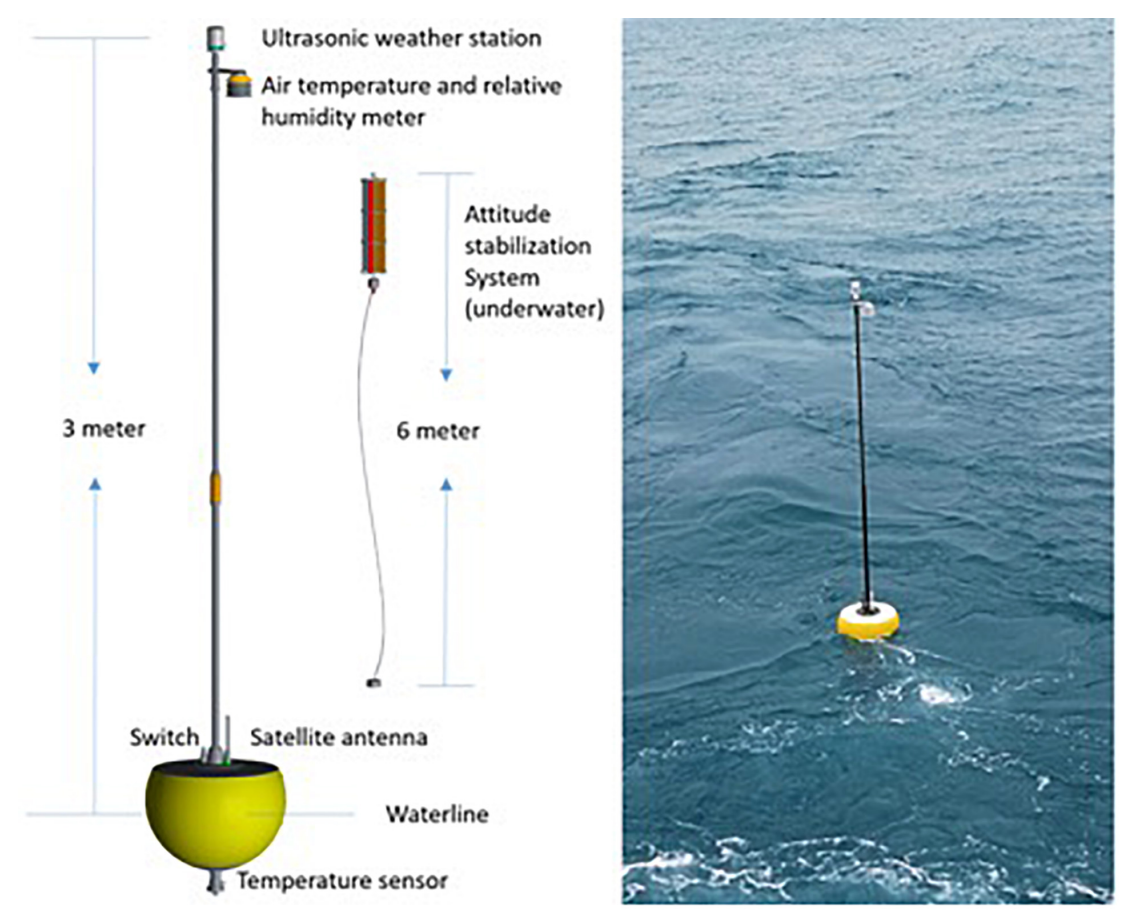

FIGURE 8 | Schematics and picture of the DrIB.

regions and, although slow moving, in tropical cyclones (Mitarai and McWilliams, 2016; Schmidt et al., 2017). Saildrones are larger in size and also capable of long-range missions in harsh conditions (Zhang et al., 2017). In 2015, NOAA/PMEL, the University of Washington, and Saildrone Inc., ran Saildrone missions in the Bering Sea and Norton Bay (Crance et al., 2017) traveling more than 4,100 nm. In 2017, two Saildrones were also used in the intensive SPURS-2 field campaign and in the pilot study of TPOS 2020 and are currently under evaluation (e.g., Meinig et al., 2019). A potential limiting factor on expanding the use of the Saildrones and potentially other emerging USV platforms is that some companies use a for-profit business model in which they maintain ownership of the USV and/or of the environmental and engineering data collected. This approach may ultimately increase the cost and decrease the effectiveness and rate of uptake of these platforms in the observing system.

Over the coming years, it is the intention of the DBCP community to evaluate USVs, which have the potential to complement and enhance the ocean observing system, as observations from USVs can complement those from fixed platforms such as moored buoys. In particular, USVs could also be used to provide observational backup during periods when moored buoys are out of service or even provide, in some cases, a cost-effective alternative to operating moored buoys. However, this will require a detailed examination of the quality of the data, the reliability of the USV platforms over extended deployments at sea, their ability to operate in and withstand severe weather conditions, and their operating costs. At this time, the use of USV for long-term operational data collection has still to be fully demonstrated, even though they have been more widely used in shorter-period research campaigns.

\section{New Opportunities for Data Relay, Archiving, and Metadata}

Collecting observations is not limited to operating the in situ infrastructure. In order to maximize the impact of the observations to the benefit of the scientific and operational communities and to support the development of the blue economy, the data and accompanying metadata need to be archived in an easily accessible way and in a variety of formats. Data collection, archiving, distribution, and the creation of QC products are an integral part of GCOS and should be harmonized and coordinated across the various subnetworks.

For example, observations from the air-sea observing system are currently underutilized for multidisciplinary scientific research, which often requires access to multiple datasets for interdisciplinary oceanographic and meteorological studies and implies that datasets from multiple sources need to be merged for subsequent analysis.

The WMO Information System (WIS; Pinardi et al., 2019) provides the global infrastructure for the exchange of data and information between all NMSs and incorporates the long-established WMO GTS for the delivery of real-time observational data (and increasingly those metadata needed to make best use of the real-time data) needed for their operational requirements. While the GTS remains the standard method of global data exchange between NMS and fulfills their operational requirements and applications, the academic community and the 
public have a clear need for a more streamlined and consolidated data management architecture, which should provide access to data and, equally important, to metadata in a common format.

Metadata, providing information such as sensor manufacturer, model, and accuracy, are a crucial component of any observing system because they allow to track changes in the technology that inevitably occur over the life of the programs, and their importance cannot be understated. For example, the GSDA contains data from drifters deployed in the late 1970, when the technology and the accuracy of the sensors were widely different from today's drifters. The metadata need to be standardized to ensure that they contain a comprehensive set of information suitable to support multiple applications, and such formats need to evolve with the technology. Most importantly, metadata must feed into a searchable database that provides key information that can be quickly extracted, cross-correlated, or correlated with environmental data to investigate biases and ultimately increase our confidence, for example, in the computation of trends associated with a changing climate. Further, as issues in batch manufacturing are discovered, instrument metadata can be easily identified and updated to address anomalous measurements or behaviors. Such discoveries are critical in understanding differences in QC data and their unfiltered counterparts (see also Pearlman et al., 2019).

Managing metadata effectively within the current framework is a very difficult task. Current issues include not only the lack of standardization of the digital information and accessibility but also the very real possibility of human errors when the metadata are transferred from the fabricator to the operators of the observing system networks. While metadata systems are being developed now, they are still based on manual operator declarations, and the trend is to minimize human interventions using machine-to-machine protocols. More interestingly, technology develops in parallel, with Sensor Model Language (SensorML), which allows the observing platforms to broadcast sensor information. We believe that a metadata relay framework, in which every instrument reports its identification at regular intervals, will greatly simplify operations, enabling the traceability of the metadata contents to the original fabricator of the observing hardware. It is conceivable that metadata originating from the SensorML methodology could be distributed using a system analogous to the GTS or integrated within.

The evolving computer technology is quickly shifting the paradigm from specialized data centers with racks of physical servers, which require constant modernization and substantial maintenance efforts, to cloud-based services and interfaces that are increasingly platform independent and widely accessible.

We anticipate that the following features will shape the way we access data in the next decade. A network with service cloud computing availability, which can be compliant with various government regulations, can now provide multiple and robust backups to traditional and expensive physical data servers. Physical data servers require sophisticated data centerlevel monitoring to continuously house, climate control, and provide electricity and connections to the World Wide Web. Further, as hardware fatigues, interfaces rendered obsolete, the hardware requires careful maintenance or refreshes on the order of every 5-10 years. Conversely, multiple virtual servers can be run in parallel and spun-up as user load and privacy requirements dictate, thus reducing downtime in data distribution and ancillary costs during surges of end-user requirements. Otherwise, complex hardware upgrades, including optimizations for increased disk input/output, random-access memory (RAM), or central processing unit (CPU) throughput are reduced to simple graphical user interfaces, enabling endusers to customize their computing platforms with an operating system reboot. Utilizing such a network infrastructure enables the end-user to accomplish otherwise complex, costly, and time-consuming tasks such as relocating and/or replicating the geographical location of the server instance from one region to another with a few menu selections in the user's dashboard interface.

\section{SUSTAINABILITY OF THE IN SITU OBSERVING NETWORKS}

Key to sustaining large observational programs is the fulfillment of at least three main requirements. The first is the demonstration of positive impacts of the data in the context of WMO's and IOC's missions and programs and to their members'/member states' scientific, operational, and economic interests. The second is the ability of each program to adjust to evolving requirements, innovate, and expand its scope as new emerging technologies allow. A third, very practical, requirement is the cost-effectiveness of the programs relative to the scientific and operational needs.

Major changes to the design of the existing air-sea in situ observing networks can have significant implications for their sustainability, for example, by hampering and/or dissipating scientific and technical knowledge and supporting infrastructures. However, there is an intrinsic need for all observing programs to evolve to better meet existing and new requirements. This may include altering the number and types of variables measured, their geographical distribution, and the frequency of observations. Such actions should be supported by tools designed to quantify the implications of such changes, which include OSE, observing system simulation experiments (OSSE), and FSOI studies. New technologies through which the cost-effectiveness of the observing networks can be improved should be tested with dedicated pilot experiments, and be complemented, whenever possible, with preliminary impact studies and validation of numerical models. The cost-effectiveness criterion is undeniably difficult to evaluate because it cannot be separated from the impact of the observations. Simple metrics such as the cost of equally accurate and comparable observations are, nevertheless, very valuable and need to be considered in the context of their scientific and operational impact.

Some established networks, such as the GSDA, have operated for a long time on specific design prerequisites and metrics that are, in some cases, several decades old. Modifications of the GSDA design and its performance metrics are discussed and reviewed each year by the DBCP, which is a particularly good example of an environment where scientific discussion, 
operational needs, and WMO guidance are integrated into planning the activities of the upcoming year and are instrumental in shaping the long-term vision of the buoy networks. Similarly, metrics have been, or are being, developed for the other components of the ocean observing system under the guidance of the JCOMM Observations Coordination Group (OCG).

\section{GOVERNANCE AND COORDINATION OF THE IN SITU AIR-SEA OBSERVATIONAL NETWORKS}

At present, the various global ocean observing networks under the JCOMM Observations Programme Area (OPA) are coordinated by the OCG, which includes representatives of all the individual observing networks such as the DBCP, SOT, and Argo. OCG is looking to build on synergies, develop best practices across the networks, and looking toward a more optimal multiplatform observing system design based on EOV/ECV requirements (see also Pinardi et al., 2019). In general, GCOS has very little observational redundancy, but given the complexity of the air-sea in situ observing system and the complementarity with observations from space, there is room for optimization. Program operators need a forum to discuss cross-network synergies, technical developments, and future directions. Benefits from developing a cross-network expert team tasked to oversee and optimize the observing capabilities for global observations at the air-sea interface and to advise the panels coordinating the contributing networks (i.e., DBCP and SOT) and OCG will include a more widespread awareness of the scope and capabilities of the various contributing programs, better planning, and more efficient management of the resources dedicated to the implementation, research, and development, ultimately resulting in faster and more efficient adaptation of GCOS to WMO's RRR.

\section{THE NEXT DECADE}

Based on the discussion of emerging technologies presented in the section "Sustainability of the in situ Observing Networks", it is anticipated that in the next 10 years, the in situ observational network located at the interface between the ocean and the atmosphere will evolve to address many of the weaknesses and limitations identified in the section "Gaps and Limitations of the Existing Air-Sea in situ Network".

Accurate directional wave observations are crucial for operational, engineering, and scientific applications, which include safety at sea; public safety warnings resulting from extreme events such as storm surges, winter storms, and hurricanes; the design of offshore structures; and, from a scientific research standpoint, improving our understanding of the physics of wave/wave interactions, wave/current interactions, mixed-layer depth models, to name a few. Accurate and standardized wave observations are also important for climate studies and for increasing resilience of coastal communities to the consequences of sea-level rise and severe storms.
In situ wave measurements are difficult in nature and are often plagued by biases that are highly dependent on the instrument type, configuration, and sampling methodology. The DBCP Task Team on Wave Measurements (TT-WM) aims to evaluate different wave measurement techniques, ensuing biases and standardization of wave measurement methods.

We are quickly reaching a stage at which we can fill the gap in offshore wave measurements using a sustainable methodology that consists of an array of expendable, low-cost, and yet accurate drifting wave riders, similar to the DWS drifter technology described above, thus providing fundamental and unique directional observations of the offshore wave field. Such observations will be crucial to validate existing wave forecasting models, better assess the severity of seasonal and extreme storms (e.g., hurricanes), and improve intraseasonal weather forecasting. They will also complement the existing wave climate dataset and will advance the science of physical oceanography. In situ offshore directional wave observations will play a critical role in validating and possibly calibrating satellite wave measurements, and we anticipate that such measurements will soon be assimilated in wave forecasting models. Altimetry satellites and the European Space Agency (ESA) Sentinel missions are already able to measure the significant wave height globally, but in situ reference directional wave data are substantially missing in offshore regions. The Surface Waves Investigation and Monitoring (SWIM) radar on the Chinese-French Oceanography Satellite (CFOSAT) is the first space radar designed to measure ocean directional wave spectra and, in conjunction with a global array of directional wave buoys, such as the DWS drifter described in the section "Directional Wave Spectra (DWS) Drifters", may herald a new era in determining surface wave spectra, linking the spatially separated in situ measurements.

Recent research has also shown the importance of measuring SSS to gain insight into the accelerating global water cycle (e.g., Durack, 2015). Lagrangian platforms are an ideal observational tool to investigate the modulation of SSS by upper-ocean processes, an essential ingredient to quantify such changes. Furthermore, there are regions, such as the Bay of Bengal, where rapidly evolving shallow layers of freshwater and strong lateral salinity gradients at the surface modify the physics of air-sea interactions and affect the evolution of the Indian monsoon, which impact the welfare of a large fraction of the world's population (e.g., Mahadevan et al., 2016). As ocean-atmosphere coupled models are increasingly used for intraseasonal and climate prediction, the need for in situ SSS observations for direct assimilation and/or for the validation of satellite products will increase. The development of low-cost and expendable conductivity sensors will pave the way for the large-scale deployment of salinity drifters (SVP-S) and will complement the observations from the Argo float array, which is designed to address the evolution of the salinity field of the ocean interior at a temporal resolution more relevant for climate studies.

SLP, wind, and other meteorological in situ observations over the World Ocean should be expanded. The Task Team on High-Resolution Marine Meteorology (TT-HRMM) is focused on improving VOS data. 
Undeniably, enabling a stream of real-time, 15-m depth ocean currents from the GSDA would be very useful for the evaluation and future assimilation in ocean forecasting models. The widespread use of the GPS technology to obtain the location of the drifters should offer a solution for the near real-time drogue presence detection problem through the use of the GPS "time-to-first-fix" parameter, which is generally longer when the drogue is present. It is anticipated that, 10 years from now, currents from Lagrangian surface drifters will be used operationally.

More funds should be made available to sustain the in situ air-sea observing system, and as new technologies are transitioned to operations, there will be opportunities to augment the number of observed meteorological ECVs from expendable buoys and USVs to include surface winds, air temperature, and air humidity. To this end, the backbone of the in situ observing system array for air-sea observations (i.e., GSDA, moorings, and VOS) could be complemented with a sustainable and integrated use of USVs.

\section{SUMMARY OF RECOMMENDATIONS}

- Given the large positive impact of SLP observations from SVP drifters on the accuracy of global NWP and the potential for improving our understanding and forecasting of other important atmospheric phenomena (e.g., atmospheric rivers and fast developing storms), it is recommended that all drifters in the GSDA carry a barometer. This recommendation should be considered a high priority.

- The evaluation of real-time drogue detection for SVP drifters should become fully operational to facilitate the use of real-time near-surface current observations, with the goal to validate ocean circulation models. Efforts to assimilate drifter velocity data in ocean, and ocean-atmosphere coupled models should also be prioritized. The GSDA should be expanded to sustain deployments in undersampled regions, including, but not limited to, the Arctic, the Arabian Sea, and the southern Indian Ocean.

- SST observations from SVP drifters are the benchmark for the calibration and validation of satellite SST products. The DBCP, in full synergy with network operators, should ensure that the accuracy of SST observations from SVP drifters is maintained below $0.05^{\circ} \mathrm{C}$. Efforts to understand and fully quantify other sources of error and biases of in situ SST measurements from drifters should be prioritized. Sustainable and cost-effective technical solutions to improve the quality of in situ SST observations from SVP drifters should be supported.

- Expendable, low-cost drifters designed to measure the directional spectra of surface waves should be utilized to implement a wave observing network in the open ocean. The impact of global wave observations in improving forecasts and our scientific understanding of upperocean mixed-layer processes (i.e., mixed-layer depth, wave/current interaction) should be assessed, for example, through OSEs. The findings will assist with the design of a global array. Other metrics pertaining to the validation of the forecast from wave models, useful to assess the impact of offshore wave observations, should be developed.

- Extending the range of meteorological and oceanographic observations from SVP drifters should be given full consideration and support. Critical in situ observations that should be considered include air temperature and relative humidity (a high priority, especially for tropical cyclone intensification), surface radiation, SSS, surface wind, and ocean color as technical developments permit.

- The VOS scheme is a very valuable air-sea observing network that allows unique measurements that cannot be obtained from SVP drifters, moorings, and USVs. The use of automated weather stations with dynamic estimation of barometer height should be prioritized to reduce the uncertainty of SLP observations. The expanded use of ISAR and M-AERI radiometers should be supported.

- Observations collected by the air-sea observing network form a record of climate relevance. The content of the metadata should comply with WIGOS requirements and should also contain all key, program-specific information that will form a permanent record. The overall objective is not only to facilitate the use of the data for scientific research and operations but also to allow a reassessment of the quality of the observations and subsequent reanalyses for years to come. Methods of metadata dissemination should favor machine-to-machine transfer to remove the human error component and to achieve full consistency and accuracy of the same records when they are stored at different locations. Cloud computing resources should be used alongside physical servers to improve the efficiency of data and metadata storage and distribution. Metadata self-reporting by the observing platforms should be considered as it will provide another means to improve the consistency of the data and metadata records.

- Governance bodies should be comprehensive and facilitate the exchange of scientific, operational, and technical topics across the operators and stakeholders of all in situ observing networks. Governance bodies should always include both the network operators and users and should address the synergy with other stakeholders, including the satellite observing community.

- The use of USVs to complement the existing air-sea observing network should be fully evaluated in the context of increasing capabilities, and their long-term endurance and operating costs should be assessed with respect to sustainability and quantifiable impacts.

\section{AUTHOR CONTRIBUTIONS}

LC conceived the review manuscript and wrote the manuscript with many of the coauthors. All authors contributed to discussions of the review and the final manuscript. 


\section{FUNDING}

LC, LB, and VH were supported by NOAA grant NA15OAR4320071 and ONR grant N00014-17-1-2517. RL was supported by NOAA/AOML and NOAA's Ocean Observation and Monitoring Division. NM was partly supported by NASA

\section{REFERENCES}

Allan, R., and Ansell, T. (2006). A new complete monthly historical gridded mean sea level pressure dataset (HadSLP2): 1850-2004. J. Clim. 19, 5816-5842. doi: 10.1175/JCLI3937.1

Andersson, M., LaCasce, J. H., Koszalka, I., Orvik, K. A., and Mauritzen, C. (2011). Variability of the Norwegian Atlantic current and associated eddy field from surface drifters. J. Geophys. Res. 116:C08032. doi: 10.1029/2011JC0 07078

Ardhuin, F., Stopa, J. E., Chapron, B., Collard, F., Husson, R., Jensen, R. E., et al. (2019a). Observing sea states. Front. Mar. Sci. 6:124. doi: 10.3389/fmars.2019. 00124

Ardhuin, F., Brandt, P., Gaultier, L., Donlon, C., Battaglia, A., Boy, F., et al. (2019b). SKIM, a candidate satellite mission exploring global ocean currents and waves. Front. Mar. Sci. 6:209. doi: 10.3389/fmars.2019.00209

Berry, D. I., and Kent, E. C. (2009). A new air-sea interaction gridded dataset from ICOADS with uncertainty estimates. Bull. Am. Meteorol. Soc. 90, 645-656. doi: 10.1175/2008BAMS2639.1

Berry, D. I., Corlett, G. K., Embury, O., and Merchant, C. J. (2018). Stability assessment of the (A)ATSR sea surface temperature climate dataset from the European Space Agency climate change initiative. Remote Sens. 10:126. doi: $10.3390 /$ rs10010126

Berry, D. I., Kent, E. C., and Taylor, P. K. (2004). An analytical model of heating errors in marine air temperatures from ships. J. Atmos. Ocean. Technol. 21, 1198-1215. doi: 10.1175/1520-0426(2004)021<1198:aamohe>2.0.co;2

Booker, D. J., Wells, N. C., and Smith, I. P. (2008). Modelling the trajectories of migrating Atlantic salmon (Salmo salar). Can. J. Fish. Aquat. Sci. 65, 352-361. doi: 10.1139/f07-173

Bourassa, M. A., Meissner, T., Cerovecki, I., Chang, P., Dong, X., De Chiara, G., et al. (2019). Remotely sensed winds and wind stresses for marine forecasting and ocean modeling. Front. Mar. Sci. doi: 10.3389/fmars.2019.00443

Bourras, D. (2006). Comparison of five satellite-derived latent heat flux products to moored buoy data. J. Clim. 19, 6291-6313. doi: 10.1175/JCLI3977.1

Buizza, R., Poli, P., Rixen, M., Alonso-Balmaseda, M., Bosilovich, M. G., Brönnimann, S., et al. (2018). Advancing global and regional reanalyses. Bull. Am. Meteorol. Soc. 99, ES139-ES144. doi: 10.1175/BAMS-D-17-0312.1

Bulgin, C. E., Embury, O., Corlett, G., and Merchant, C. J. (2016). Independent uncertainty estimates for coefficient based sea surface temperature retrieval from the Along-Track Scanning Radiometer instruments. Remote Sens. Environ. 178, 213-222. doi: 10.1016/j.rse.2016.02.022

Carlton, J. T., Chapman, J. W., Geller, J. G., Miller, J. A., Carlton, D. A., McCuller, M. I., et al. (2017). Tsunami-driven rafting: transoceanic species dispersal and implications for marine biogeography. Science 357, 1402-1406. doi: 10.1126/ science.aao 1498

Carrier, M., Ngodock, H., Muscarella, P., and Smith, S. (2016). Impact of assimilating surface velocity observations on the model sea surface height using the NCOM-4DVAR. Mon. Weather Rev. 144, 1051-1068. doi: 10.1175/mwr-d14-00285.1

Castro, S. L., Emery, W., Wick, G. A., and Tandy, W. (2017). Submesoscale sea surface temperature variability from UAV and satellite measurements. Remote Sens. 9:1089. doi: 10.3390/rs9111089

Centurioni, L. R. (2018). "Drifter technology and impacts for sea surface temperature, sea-level pressure, and ocean circulation studies," in Observing the Oceans in Real Time, eds R. Venkatesan, A. Tandon, E. D'Asaro, and M. A. Atmanand (Cham: Springer International Publishing), doi: 10.1007/978-3-31966493-4_3

Centurioni, L. R., Braasch, L., Di Lauro, E., Contestabile, P., De Leo, F., Casotti, R., et al. (2016). A new strategic wave measurement station off Naples port main breakwater. Coast. Eng. Proc. 1:36. doi: 10.9753/icce.v35.waves.36 grant NNX17AH43G. IK was supported by the Nordic Seas Eddy Exchanges (NorSEE) funded by the Norwegian Research Council (Grant 221780). DZ was partly funded by the Joint Institute for the Study of the Atmosphere and Ocean (JISAO) under NOAA Cooperative Agreement NA15OAR4320063. RJ was supported by the USACE's Civil Works $096 \times 3123$.

Centurioni, L. R., Hormann, V., Chao, Y., Reverdin, G., Font, J., and Lee, D.K. (2015). Sea surface salinity observations with Lagrangian drifters in the tropical north Atlantic during SPURS: circulation, fluxes, and comparisons with remotely sensed salinity from Aquarius. Oceanography 28, 96-105. doi: 10.5670/oceanog.2015.08

Centurioni, L. R., Hormann, V., Talley, L. D., Arzeno, I., Beal, L., Caruso, M., et al. (2017a). Northern Arabian Sea Circulation-Autonomous Research (NASCar): a research initiative based on autonomous sensors. Oceanography 30, 74-87. doi: 10.5670/oceanog.2017.224

Centurioni, L. R., Hornayi, A., Cardinali, C., Charpentier, E., and Lumpkin, R. (2017b). A global observing system for measuring sea level atmospheric pressure: effects and impacts on numerical weather prediction. Bull. Am. Meteorol. Soc 98, 231-238. doi: 10.1175/BAMS-D-15-00080.1

Centurioni, L. R., Niiler, P. N., and Lee, D.-K. (2009). Near-surface circulation in the South China Sea during the winter monsoon. Geophys. Res. Lett. 36:L06605. doi: 10.1029/2008GL037076

Centurioni, L. R., Ohlmann, J. C., and Niiler, P. P. (2008). Permanent meanders in the California Current System. J. Phys. Oceanogr. 38, 1690-1710. doi: 10.1175/ 2008JPO3746.1

Choquer, M., Blouch, P., Gaillard, F., Reynaud, T., Reverdin, G., Metzl, N., et al. (2013). The OceanoScientific Programme: Scientific Data Acquisition by Sailing Ships. Available at: https://www.wmo.int/pages/prog/www/ IMOP/publications/IOM-116_TECO-2014/Session\%201/O1_12_Choquer_ OceanoScientific.pdf (accessed October, 2018).

Christensen, K. H., Röhrs, J., Ward, B., Fer, I., Broström, G., Saetra, Ø, et al. (2013). Surface wave measurements using a ship-mounted ultrasonic altimeter. Methods Oceanogr 6, 1-15. doi: 10.1016/j.mio.2013.07.002

Compo, G. P., Whitaker, J. S., Sardeshmukh, P. D., Matsui, N., Allan, R. J., Yin, X., et al. (2011). The twentieth century reanalysis project. Q. J. Roy. Meteor. Soc. 137, 1-28. doi: 10.1002/qj.776

Corlett, G. K., Merchant, C. J., Minnett, P. J., and Donlon, C. J. (2014). “Assessment of long-term satellite derived sea surface temperature records," in Experimental Methods in the Physical Sciences, Optical Radiometry for Ocean Climate Measurements, Vol. 47, eds G. Zibordi, C. J. Donlon, and A. C. Parr (Cambridge, MA: Academic Press), 639-677. doi: 10.1016/b978-0-12-417011-7.00021-0

Cozar, A., Marti, E., Duarte, C. M., Garcia-de-Lomas, J., van Sebille, E., Ballatore, T. J., et al. (2017). The Arctic Ocean as a dead end for floating plastics in the North Atlantic branch of the Thermohaline circulation. Sci. Adv. 3:e1600582. doi: $10.1126 /$ sciadv. 1600582

Crance, J. L., Berchock, C. L., and Tabisola, H. (2017). Saildrone Summer 2016 Bering Sea Mission-Final Report. Available at: https://www.mmc.gov/wpcontent/uploads/Saildrone-2017-MMC-Final-Report.pdf (accessed October, 2018).

Cravatte, S., Kessler, W. S., Smith, N., Wijffels, S. E., Yu, L., Ando, K., et al. (2016). First Report of TPOS 2020. Available at: http://tpos2020.org/first-report/ (accessed October, 2018).

Cronin, M. F., Gentemann, C. L., Edson, J., Ueki, I., Bourassa, M., Brown, S., et al. (2019). Air-sea fluxes with a focus on heat and momentum. Front. Mar. Sci. 6:430. doi: 10.3389/fmars.2019.00430

D’Asaro, E. A., Black, P. G., Centurioni, L. R., Chang, Y. T., Chen, S. S., Foster, R. C., et al. (2013). 'Impact of typhoons on the ocean in the Pacific'. Bull. Am. Meteorol. Soc. 95, 1405-1418.

D’Asaro, E. A., Eriksen, C. C., Levine, M. D., Paulson, C. A., Niiler, P., and Van Meurs, P. (1995). Upper-ocean inertial currents forced by a strong storm. Part I: data and comparisons with linear theory. J. Phys. Oceanogr. 25, 2909-2936. doi: 10.1175/1520-0485(1995)025<2909:uoicfb > 2.0.co;2

Dash, P., Ignatov, A., Kihai, Y., and Sapper, J. (2010). The SST Quality Monitor (SQAM). J. Atmos. Ocean. Technol. 27, 1899-1917. doi: 10.1175/ 2010JTECHO756.1 
De Dominicis, M., Leuzzi, G., Monti, P., Pinardi, N., and Poulain, P.-M. (2012). Eddy diffusivity derived from drifter data for dispersion model applications. Ocean Dynam. 62, 1381-1398. doi: 10.1007/s10236-012-0564-2

Dee, D. P., Uppala, S. M., Simmons, A. J., Berrisford, P., Poli, P., Kobayashi, S., et al. (2011). The ERA-Interim reanalysis: configuration and performance of the data assimilation system. Q. J. Roy. Meteor. Soc. 137, 553-597. doi: 10.1002/ qj. 828

Donlon, C. J., Casey, K. S., Robinson, I. S., Gentemann, C. L., Reynolds, R. W., Barton, I., et al. (2009). The GODAE high-resolution sea surface temperature pilot project. Oceanography 22, 34-45. doi: 10.5670/oceanog.2009.64

Donlon, C. J., Martin, M., Stark, J., Roberts-Jones, J., Fiedler, E., and Wimmer, W. (2012). The Operational sea surface temperature and sea ice analysis (OSTIA) system. Remote Sens. Environ. 116, 140-158. doi: 10.1016/j.rse.2010. 10.017

Donlon, C. J., Minnett, P. J., Fox, N., and Wimmer, W. (2014a). “Strategies for the laboratory and field deployment of ship-borne fiducial reference thermal infrared radiometers in support of satellite-derived sea surface temperature climate data records," in Experimental Methods in the Physical Sciences, Optical Radiometry for Ocean Climate Measurements, Vol. 47, eds G. Zibordi, C. J. Donlon, and A. C. Parr (Cambridge, MA: Academic Press), 557-603. doi: 10.1016/b978-0-12-417011-7.00018-0

Donlon, C. J., Minnett, P. J., Jessup, A., Barton, I., Emery, W., Hook, S., et al. (2014b). "Ship-borne thermal infrared radiometer systems," in Experimental Methods in the Physical Sciences, Optical Radiometry for Ocean Climate Measurements, Vol. 47, eds G. Zibordi, C. J. Donlon, and A. C. Parr (Cambridge, MA: Academic Press), 305-404. doi: 10.1016/b978-0-12-417011-7.00 011-8

Donlon, C., Robinson, I. S., Reynolds, M., Wimmer, W., Fisher, G., Edwards, R., et al. (2008). An Infrared Sea Surface Temperature Autonomous Radiometer (ISAR) for deployment aboard volunteer observing ships (VOS). J. Atmos. Ocean. Technol. 25, 93-113. doi: 10.1175/2007jtecho505.1

Döös, K., Rupolo, V., and Brodeau, L. (2011). Dispersion of surface drifters and model-simulated trajectories. Ocean Model. 39, 301-310. doi: 10.1016/j. ocemod.2011.05.005

Durack, P. J. (2015). Ocean salinity and the global water cycle. Oceanography 28, 20-31. doi: 10.5670/oceanog.2015.03

Elipot, S., Lumpkin, R., and Prieto, G. (2010). Modification of inertial oscillations by the mesoscale eddy field. J. Geophys. Res. Oceans 115:20. doi: 10.1029/ 2009JC005679

Elipot, S., Lumpkin, R., Perez, R., Lilly, J., Early, J., and Sykulski, A. (2016). A global surface drifter data set at hourly resolution. J. Geophys. Res. Oceans 121, 2937-2966. doi: 10.1002/2016JC011716

Embury, O., and Merchant, C. J. (2012). A reprocessing for climate of sea surface temperature from the Along-Track Scanning Radiometers: a new retrieval scheme. Remote Sens. Environ. 116, 47-61. doi: 10.1016/j.rse.2010. 11.020

Etienne, H. (2018). Product User Manual for Global Ocean Delayed-Mode in-situ Observations Of Ocean Surface Currents and Temperature from Drifters. Available at: http://resources.marine.copernicus.eu/documents/PUM/ CMEMS-INS-PUM-013-044.pdf (accessed May, 2019).

Freeman, E., Kent, E. C., Brohan, P., Cram, T., Gates, L., Huang, B., et al. (2019). The International comprehensive ocean-atmosphere data set - meeting users needs and future priorities. Front. Mar. Sci. 6:435. doi: 10.3389/fmars.2019. 00435

Freeman, E., Woodruff, S. D., Worley, S. J., Lubker, S. J., Kent, E. C., Angel, W. E., et al. (2016). ICOADS Release 3.0: a major update to the historical marine climate record. Int. J. Climatol. 37, 2211-2232. doi: 10.1002/joc.4775

Gasparin, F., Greiner, E., Lellouche, J.-M., Legalloudec, O., Garric, G., Drillet, Y., et al. (2018). A large-scale view of oceanic variability from 2007 to 2015 in the global high resolution monitoring and forecasting system at Mercator Océan. J. Mar. Syst. 187, 260-276. doi: 10.1016/j.jmarsys.2018.06.015

Gelaro, R., McCarty, W., Suarez, M. J., Todling, R., Molod, A., Takacs, L., et al. (2017). The modern-era retrospective analysis for research and applications, Version 2 (MERRA-2). J. Clim. 30, 5419-5454. doi: 10.1175/JCLI-D-16-0758.1

Gentemann, C. L. (2014). Three way validation of MODIS and AMSR-E sea surface temperatures. J. Geophys. Res. Oceans 119, 2583-2598. doi: 10.1002/ 2013)C009716

Gill, A. (1982). Atmosphere-Ocean Dynamics. Amsterdam: Elsevier, 662.
Gommenginger, C., Chapron, B., Hogg, A., Buckingham, C., Fox-Kemper, B., Eriksson, L., et al. (2019). SEASTAR: a mission to study ocean submesoscale dynamics and small-scale atmosphere-ocean processes in coastal, shelf and polar seas. Front. Mar. Sci. 6:457. doi: 10.3389/fmars.2019.00457

Gong, D. Y., and Wang, S. W. (1999). Definition of Antarctic oscillation index. Geophys. Res. Lett. 26, 459-462. doi: 10.1029/1999GL900003

Griffa, A., Lumpkin, R., and Veneziani, M. (2008). Cyclonic and anticyclonic motion in the upper ocean. Geophys. Res. Lett. 35:L01608. doi: 10.1029/ 2007GL032100

Griffa, A., Owens, K., Piterbarg, L., and Rozovskii, B. (1995). Estimates of turbulence parameters from Lagrangian data using a stochastic particle model. J. Mar. Res. 53, 371-401. doi: 10.1357/0022240953213151

Guichoux, Y., Lennon, M., and Thomas, N. (2016). "Sea surface currents calculation using vessel tracking data," in Proceedings of the Maritime Knowledge Discovery and Anomaly Detection Workshop, (Ispra: JRC Science Hub), 5-6.

Hansen, D. V., and Poulain, P.-M. (1996). Quality control and interpolations of WOCE-TOGA drifter data. J. Atmos. Ocean. Technol. 13, 900-909. doi: 10.1175/1520-0426(1996)013<0900:qcaiow>2.0.co;2

Hare, J. A., and Walsh, H. J. (2007). Planktonic linkages among marine protected areas on the south Florida and southeast United States continental shelves. Can. J. Fish. Aquat. Sci. 64, 1234-1247. doi: 10.1139/f07-089

Haza, A. C., Piterbarg, L. I., Martin, P., Ozgokmen, T. M., and Griffa, A. (2007). Lagrangian subgridscale model for particle transport improvement and application in the Adriatic Sea using the navy coastal ocean model. Ocean Model. 17, 68-91. doi: 10.1016/j.ocemod.2006. 10.004

Horányi, A., Cardinali, C., and Centurioni, L. (2017). The global numerical weather prediction impact of mean-sea-level pressure observations from drifting buoys. Q. J. Roy. Meteor. Soc. 143, 974-985. doi: 10.1002/qj.2981

Hormann, V., Centurioni, L. R., and Reverdin, G. (2015). Evaluation of drifter salinities in the subtropical North Atlantic. J. Atmos. Ocean. Technol. 32, 185-192. doi: 10.1175/JTECH-D-14-00179.1

Hormann, V., Centurioni, L. R., Mahadevan, A., Essink, S., D’Asaro, E. A., and Kumar, B. P. (2016). Variability of near-surface circulation and sea surface salinity observed from Lagrangian drifters in the Northern Bay of Bengal during the waning 2015 southwest monsoon. Oceanography 29, 124-133. doi: 10.5670/ oceanog.2016.45

Hormann, V., Centurioni, L. R., Rainville, L., Lee, C. M., and Braasch, L. J. (2014). Response of upper ocean currents to Typhoon Fanapi. Geophys. Res. Lett 41, 3995-4003. doi: 10.1002/2014GL060317

Ignatov, A., Zhou, X., Petrenko, B., Liang, X., Kihai, Y., Dash, P., et al. (2016). AVHRR GAC SST reanalysis version 1 (RAN1). Remote Sens. 8:315. doi: 10. $3390 /$ rs8040315

Ingleby, B. (2010). Factors affecting ship and buoy data quality: a data assimilation perspective. J. Atmos. Ocean. Technol. 27, 1476-1489. doi: 10.1175/ 2010JTECHA1421.1

Ingleby, B., and Isaksen, L. (2018). Drifting buoy pressures: impact on NWP. Atmos. Sci. Lett. 19:e822. doi: 10.1002/asl.822

IPCC (2013). "Climate Change 2013: The physical science basis," in Contribution of Working Group I to the Fifth Assessment Report of the Intergovernmental Panel on Climate Change, eds T. F. Stocker, D. Qin, G.-K. Plattner, M. Tignor, S. K. Allen, J. Boschung, et al. (Cambridge: Cambridge University Press), 1535.

Kennedy, J. (2014). A review of uncertainty in in-situ measurements and data sets of sea surface temperature. Rev. Geophys. 52, 1-32. doi: 10.1002/2013RG000434

Kent, E. C., Rayner, N. A., Berry, D. I., Eastman, R., Grigorieva, V. G., Huang, B., et al. (2019). Observing requirements for long-term climate records at the ocean surface. Front. Mar. Sci. 6:441. doi: 10.3389/fmars.2019.00441

Kilpatrick, K. A., Podestá, G. P., and Evans, R. H. (2001). Overview of the NOAA/NASA Pathfinder algorithm for sea surface temperature and associated Matchup Database. J. Geophys. Res. 106, 9179-9198.

Kilpatrick, K. A., Podestá, G., Walsh, S., Williams, E., Halliwell, V., Szczodrak, M., et al. (2015). A decade of sea surface temperature from MODIS. Remote Sens. Environ. 165, 27-41. doi: 10.1016/j.rse.2015.04.023

Kobayashi, S., Ota, Y., Harada, Y., Ebita, A., Moriya, M., Onoda, H., et al. (2015). The JRA-55 reanalysis: general specifications and basic characteristics. J. Meteorol. Soc. Japan 93, 5-48. doi: 10.2151/jmsj.2015-001

Koszalka, I., LaCasce, J. H., and Orvik, K. A. (2009). Relative dispersion in the Nordic Seas. J. Mar. Res. 67, 411-433. doi: 10.1357/002224009790741102 
Koszalka, I., LaCasce, J. H., Andersson, M., Orvik, K. A., and Mauritzen, C. (2011). Surface circulation in the Nordic Seas from clustered drifters. Deep Sea Res. Part I Oceanogr. Res. Pap. 58, 468-485. doi: 10.1016/j.dsr.2011.01.007

Koszalka, I., LaCasce, J. H., and Mauritzen, C. (2013). In pursuit of anomaliesAnalyzing the poleward transport of Atlantic Water with surface drifters. Deep Sea Res. II 85, 96-108. doi: 10.1016/j.dsr2.2012.07.035

Kunze, E. (1985). Near-inertial wave propagation in geostrophic shear. J. Phys. Oceanogr. 15, 544-565. doi: 10.1175/1520-0485(1985)015<0544:niwpig $>2.0$. $\mathrm{co} ; 2$

Lacorata, G., Aurell, E., and Vulpiani, A. (2001). Drifter dispersion in the Adriatic Sea: Lagrangian data and chaotic model. Ann. Geophys. 19, 121-129. doi: 10. 5194/angeo-19-121-2001

Landwehr, S., O’Sullivan, N., and Ward, B. (2015). Direct flux measurements from mobile platforms at sea: motion and airflow distortion corrections revisited. J. Atmos. Ocean. Technol. 32, 1163-1178. doi: 10.1175/JTECH-D-14-00137.1

Laurindo, L. C., Mariano, A., and Lumpkin, R. (2017). An improved surface velocity climatology for the global ocean from drifter observations. Deep Sea Res. Part I Oceanogr. Res. Pap. 124, 73-92. doi: 10.1016/j.dsr.2017.04.009

Lean, K., and Saunders, R. W. (2013). Validation of the ATSR Reprocessing for Climate (ARC) dataset using data from drifting buoys and a three-way error analysis. J. Clim. 26, 4758-4772. doi: 10.1175/JCLI-D-12-00206.1

Lellouche, J.-M., Greiner, E., Le Galloudec, O., Garric, G., Regnier, C., Drevillon, M., et al. (2018). Recent updates on the Copernicus Marine Service global ocean monitoring and forecasting real-time $1 / 12^{\circ}$ high resolution system. Ocean Sci. 14, 1093-1126. doi: 10.5194/os-14-1093-2018

Lellouche, J.-M., Le Galloudec, O., Drevillon, M., Regnier, C., Greiner, G., Ferry, N., et al. (2013). Evaluation of global monitoring and forecasting systems at Mercator Ocean. Ocean Sci. 9, 57-81. doi: 10.5194/os-9-57-2013

Loew, A., Bell, W., Brocca, L., Bulgin, C. E., Burdanowitz, J., Calbet, X., et al. (2017). Validation practices for satellite-based Earth observation data across communities. Rev. Geophys. 55, 779-817. doi: 10.1002/2017RG000562

Lumpkin, R., and Elipot, S. (2010). Surface drifter pair spreading in the North Atlantic. J. Geophys. Res. 115:C12017. doi: 10.1029/2010JC006338

Lumpkin, R., and Johnson, G. (2013). Global ocean surface velocities from drifters: mean, variance, ENSO response, and seasonal cycle. J. Geophys. Res. Oceans 118, 2992-3006. doi: 10.1002/jgrc.20210

Lumpkin, R., and Pazos, M. (2007). "Measuring surface currents with Surface Velocity Program drifters: the instrument, its data and some recent results," in Lagrangian Analysis and Prediction of Coastal and Ocean Dynamics, Chap. 2, ed. A. Griffa (Cambridge: Cambridge University Press), 39-67. doi: 10.1017/ cbo9780511535901.003

Lumpkin, R., Centurioni, L., and Perez, R. C. (2016). Fulfilling observing system implementation requirements with the global drifter array. J. Atmos. Ocean. Technol. 33, 685-695. doi: 10.1175/JTECH-D-15-0255.1

Lumpkin, R., Maximenko, N., and Pazos, M. (2012). Evaluating where and why drifters die. J. Atmos. Ocean. Technol. 29, 300-308. doi: 10.1175/JTECH-D-1100100.1

Lumpkin, R., Ozgokmen, T., and Centurioni, L. (2017). Advances in the applications of surface drifters. Annu. Rev. Mar. Sci. 9, 59-81. doi: 10.1146/ annurev-marine-010816-060641

Mahadevan, A., Paluszkiewicz, T., Ravichandran, M., Sengupta, D., and Tandon, A. (2016). Introduction to the special issue on the Bay of Bengal: from monsoons to mixing. Oceanography 29, 14-17. doi: 10.5670/oceanog.2016.34

Maximenko, N. A., Hafner, J., Kamachi, M., and MacFadyen, A. (2018). Numerical simulations of debris drift from the 2011 tsunami in Japan, verified with boat reports. Mar. Pollut. Bull. 132, 5-25. doi: 10.1016/j.marpolbul.2018. 03.056

Maximenko, N., Corradi, P., Law, K. L., Van Sebille, E., Garaba, S. P., Lampitt, R. S., et al. (2019). Towards the Integrated Marine Debris Observing System. Front. Mar. Sci. doi: 10.3389/fmars.2019.00447

Maximenko, N., Lumpkin, R., and Centurioni, L. (2013). "Ocean surface circulation," in Ocean Circulation and Climate: A 21st Century Perspective, eds G. Siedler, S. M. Griffies, J. Gould, and J. A. Church (Cambridge, MA: Academic Press), 283-304. doi: 10.1016/b978-0-12-391851-2.00012-x

Maximenko, N., Niiler, P., Rio, M.-H., Melnichenko, O., Centurioni, L., Chambers, D., et al. (2009). Mean dynamic topography of the ocean derived from satellite and drifting buoy data using three different techniques. J. Atmos. Ocean. Technol. 26, 1910-1919. doi: 10.1175/2009JTECHO672.1
McAdam, R., and van Sebille, E. (2018). Surface connectivity and interocean exchanges from drifter-based transition matrices. J. Geophys. Res. Oceans 123, 514-532. doi: 10.1002/2017JC013363

Meinig, C., Burger, E. F., Cohen, N., Cokelet, E. D., Cronin, M. F., Cross, J. N., et al. (2019). Public-private partnerships to advance regional ocean-observing capabilities: a saildrone and NOAA-PMEL case study and future considerations to expand to global scale observing. Front. Mar. Sci. 6:448. doi: 10.3389/fmars. 2019.00448

Miller, J. (2018). Antarctica is not protected from an influx of new species. Phys. Today 71, 14-16. doi: 10.1063/pt.3.4012

Minnett, P. J. (1991). Consequences of sea surface temperature variability on the validation and applications of satellite measurements. J. Geophys. Res. 96, $18475-18489$.

Minnett, P. J., and Barton, I. J. (2010). "Remote sensing of the Earth's surface temperature," in Radiometric Temperature Measurements and Applications, eds Z. M. Zhang, B. K. Tsai, and G. Machin (Cambridge, MA: Academic Press), 333-391. doi: 10.1016/S1079-4042(09)04306-9

Minnett, P. J., Knuteson, R. O., Best, F. A., Osborne, B. J., Hanafin, J. A., and Brown, O. B. (2001). The Marine-Atmospheric Emitted Radiance Interferometer (MAERI), a high-accuracy, sea-going infrared spectroradiometer. J. Atmos. Ocean. Technol. 18, 994-1013. doi: 10.1175/1520-0426(2001)018<0994:tmaeri $>2.0$. $\operatorname{co} ; 2$

Minnett, P. J., Maillet, K. A., Hanafin, J. A., and Osborne, B. J. (2005). Infrared interferometric measurements of the near surface air temperature over the oceans. J. Atmos. Ocean. Technol. 22, 1016-1029.

Minnett, P. J. (2010). "The validation of sea surface temperature retrievals from spaceborne infrared radiometers," in Oceanography from Space, eds V. Barale, J. Gower, and L. Alberotanza (Dordrecht: Springer).

Mitarai, S., and McWilliams, J. C. (2016). Wave glider observations of surface winds and currents in the core of Typhoon Danas. Geophys. Res. Lett. 43, 11312-11319. doi: 10.1002/2016GL071115

Mogensen, K. S., Hewson, T., Keeley, S., and Magnusson, L. (2018). Effects of Ocean Coupling on Weather Forecasts. Available at: https://www.ecmwf.int/ en/newsletter/156/news/effects-ocean-coupling-weather-forecasts (accessed October, 2018).

Moltmann, T., Turton, J., Zhang, H. M., Nolan, G., Gouldman, C., Griesbauer, L., et al. (2019). A Global Ocean Observing System (GOOS), delivered through enhanced collaboration across regions, communities, and new technologies. Front. Mar. Sci. 6:291. doi: 10.3389/fmars.2019. 00291

Mrvaljevic, R. K., Black, P. G., Centurioni, L. R., Chang, Y.-T., D’Asaro, E. A., Jayne, S. R., et al. (2013). 'Observations of the cold wake of Typhoon Fanapi (2010)'. Geophys. Res. Lett. 40, 316-321. doi: 10.1029/2012gl054282

Muscarella, P., Carrier, M. J., Ngodock, H., Smith, S., Lipphardt, B. L., Kirwan, A. D., et al. (2015). Do assimilated drifter velocities improve Lagrangian predictability in an operational ocean model? Mon. Weather Rev. 143, 1822-1832. doi: 10.1175/mwr-d-14-00164.1

Nielsen-Englyst, P., Høyer, J. L., Toudal Pedersen, L., Gentemann, C. L., Alerskans, E., Block, T., et al. (2018). Optimal estimation of sea surface temperature from AMSR-E. Remote Sens. 10:229. doi: 10.3390/rs10020229

Niiler, P. P. (2001). “The world ocean surface circulation," in Ocean Circulation and Climate, International Geophysics Series, Vol. 77, eds G. Siedler, J. Church, and J. Gould (New York, NY: Academic Press), 193-204. doi: 10.1016/s0074-6142(01) 80119-4

Niiler, P. P., Maximenko, N. A., and McWilliams, J. C. (2003a). Dynamically balanced absolute sea level of the global ocean derived from near-surface velocity observations. Geophys. Res. Lett. 30:2164. doi: 10.1029/2003GL018628

Niiler, P. P., Maximenko, N. A., Panteleev, G. G., Yamagata, T., and Olson, D. B. (2003b). Near-surface structure of the Kuroshio extension. J. Geophys. Res. Oceans 108:3193. doi: 10.1029/2002JC0 01461

Niiler, P. P., Sybrandy, A. S., Bi, K., Poulain, P. M., and Bitterman, D. (1995). Measurements of the water-following capability of holey-sock TRISTAR drifters. Deep Sea Res. Part I Oceanogr. Res. Pap. 42, 1951-1964. doi: 10.1016/ 0967-0637(95)00076-3

O’Carroll, A. G., Armstrong, E. M., Beggs, H., Bouali, M., Casey, K. S., Corlett, G. K., et al. (2019). Observational needs of sea surface temperature. Front. Mar. Sci. 6:420. doi: 10.3389/fmars.2019.00420 
O'Carroll, A. G., Eyre, J. R., and Saunders, R. W. (2008). Three-way error analysis between AATSR, AMSR-E, and in-situ sea surface temperature observations. J. Atmos. Ocean. Technol. 25, 1197-1207. doi: 10.1175/2007JTECHO542.1

Pazan, S. E., and Niiler, P. P. (2001). Recovery of near-surface velocity from undrogued drifters. J. Atmos. Ocean. Technol. 18, 476-489. doi: 10.1175/15200426(2001)018<0476:ronsvf>2.0.co;2

Pearlman, J., Bushnell, M., Coppola, L., Karstensen, J., Buttigieg, P. L., Pearlman, F., et al. (2019). Evolving and sustaining ocean best practices and standards for the next decade. Front. Mar. Sci. 6:277. doi: 10.3389/fmars.2019.00277

Peng, S., Qian, Y.-K., Lumpkin, R., Du, Y., Wang, D., and Li, P. (2015). Characteristics of the near-surface currents in the Indian Ocean as deduced from satellite-tracked surface drifters. Part I: Pseudo-Eulerian statistics. J. Phys. Oceanogr. 45, 441-458. doi: 10.1175/jpo-d-140050.1

Phillipson, L., and Toumi, R. (2017). Impact of data assimilation on ocean current forecasts in the Angola Basin. Ocean Model. 114, 45-58. doi: 10.1016/j.ocemod. 2017.04.006

Pinardi, N., Stander, J., Legler, D. M., O’Brien, K., Boyer, T., Cuff, T., et al. (2019). The joint IOC (of UNESCO) and WMO collaborative effort for met-ocean services. Front. Mar. Sci. 6:410. doi: 10.3389/fmars.2019.00410

Poli, P., Cohuet, J.-B., Kleta, H., and Verboom, H. (2017). E-SURFMAR Operational Service VOS Activities. London: Ninth Session of the JCOMM Ship Observations Team.

Poli, P., Hersbach, H., Dee, D. P., Berrisford, P., Simmons, A. J., Vitart, F., et al. (2016). ERA-20C: an atmospheric reanalysis of the twentieth century. J. Clim. 29, 4083-4097. doi: 10.1175/JCLI-D-15-0556.1

Poli, P., Lucas, M., O'Carroll, A., Le Menn, M., David, A., Corlett, G. K., et al. (2019). The Copernicus surface velocity platform drifter with barometer and reference sensor for temperature (SVP-BRST): genesis, design, and initial results. Ocean Sci. 15, 199-214. doi: 10.5194/os-15-1992019

Pollard, R. T., and Millard, R. C. (1970). Comparison between observed and simulated wind-generated inertial oscillations. Deep Sea Res. Oceanogr. Abstr. 17, 813-821. doi: 10.1016/0011-7471(70)90043-4

Popinet, S., Smith, M., and Stevens, C. (2004). Experimental and numerical study of the turbulence characteristics of airflow around a research vessel. J. Atmos. Ocean. Technol. 21, 1575-1589. doi: 10.1175/1520-0426(2004) $021<1575$ :eansot $>2.0$.co; 2

Poulain, P.-M., and Centurioni, L. (2015). Direct measurements of world ocean tidal currents with surface drifters. J. Geophys. Res. Oceans 120, 6986-7003. doi: 10.1002/2015JC01081

Poulain, P.-M., Bussani, A., Gerin, R., Jungwirth, R., Mauri, E., Menna, M., et al. (2013). Mediterranean surface currents measured with drifters: from basin to subinertial scales. Oceanography 26, 38-47. doi: 10.5670/oceanog. 2013.03

Price, J. F. (1983). Internal wave wake of a moving storm. Part I: scales, energy budget and observations. J. Phys. Oceanogr. 13, 949-965. doi: 10.1175/15200485(1983)013<0949:iwwoam>2.0.co;2

Reverdin, G., Morisset, S., Marie, L., Bourras, D., Sutherland, G., Ward, B., et al. (2015). Surface salinity in the North Atlantic subtropical gyre during the STRASSE/SPURS summer 2012 cruise. Oceanography 28, 114-123. doi: 10. 5670/oceanog.2015.09

Riser, S., Freeland, H. J., Roemmich, D., Wijffels, S., Troisi, A., Belbéoch, M., et al. (2016). Fifteen years of ocean observations with the global Argo array. Nat. Clim. Change 6, 145-153. doi: 10.1038/NCLIMA TE2872

Roach, C. J., Balwada, D., and Speer, K. (2018). Global observations of horizontal mixing from Argo float and surface drifter trajectories. J. Geophys. Res. Oceans 123, 4560-4575. doi: 10.1029/2018JC013750

Roemmich, D., Johnson, G., Riser, S., Davis, R., Gilson, J., Owens, B., et al. (2009). The Argo Program: observing global ocean with profiling floats. Oceanography 22, 34-43. doi: 10.1029/2018JC014059

Rühs, S., Zhurbas, V., Koszalka, I. M., Durgadoo, J. V., and Biastoch, A. (2018). Eddy diffusivity estimates from Lagrangian trajectories simulated with ocean models and surface drifter data-a case study for the Greater Agulhas System. J. Phys. Oceanogr. 48, 175-196. doi: 10.1175/JPO-D-170048.1
Saha, S., Moorthi, S., Pan, H.-L., Wu, X., Wang, J., Nadiga, S., et al. (2010). The NCEP climate forecast system reanalysis. Bull. Am. Meteorol. Soc 91, 1015-1057. doi: 10.1175/2010BAMS3001.1

Schmidt, K. M., Swart, S., Reason, C., and Nicholson, S.-A. (2017). Evaluation of satellite and reanalysis wind products with in-situ Wave Glider wind observations in the Southern Ocean. J. Atmos. Ocean. Technol. 34, 2551-2568. doi: 10.1175/JTECH-D-17-0079.1

Schmitt, R. W., Asher, W., Bingham, F., Carton, J., Centurioni, L., Farrar, T., et al. (2015). From salty to fresh-Salinity processes in the upper-ocean regional study-2 (SPURS-2): diagnosing the physics of a rainfall-dominated salinity minimum. Oceanography 28, 150-159. doi: 10.5670/oceanog. 2015.15

Smith, S. R., Alory, G., Andersson, A., Asher, W., Baker, A., Berry, D. I., et al. (2019). Ship-based contributions to global ocean, weather, and climate observing systems. Front. Mar. Sci. 6:434. doi: 10.3389/fmars.2019.00434

Swart, S., Gille, S. T., Delille, B., Josey, S., Mazloff, M., Newman, L., et al. (2019). Constraining Southern Ocean air-sea-ice fluxes through enhanced observations. Front. Mar. Sci. 6:421. doi: 10.3389/fmars.2019.00421

Sykulski, A. M., Olhede, S. C., Lilly, J. M., and Danioux, E. (2016). Lagrangian time series models for ocean surface drifter trajectories. J. R. Stat. Soc. Ser. C 65, 29-50. doi: 10.1111/rssc. 12112

Theocharous, E., Fox, N., Göttsche, F., Høyer, J. L., Wimmer, W., et al. (2016). Fiducial Reference Measurements for Validation of Surface Temperature from Satellites (FRM4STS) Technical Report 1: Procedures and Protocols for the Verification of TIR FRM Field Radiometers and Reference Blackbody Calibrators. ESA Contract No. 4000113848_15I-LG. Teddington: National Physical Laboratory.

Villas Bôas, A. B., Ardhuin, F., Ayet, A., Bourassa, M. A., Brandt, P., Chapron, B., et al. (2019). Integrated Observations of Global Surface Winds, Currents, and Waves: Requirements and Challenges for the Next Decade. Front. Mar. Sci. 6:425. doi: 10.3389/fmars.2019.00425

Wanninkhof, R., Pickers, P. A., Omar, A. M., Sutton, A., Murata, A., Olsen, A., et al. (2019). A surface ocean $\mathrm{CO}_{2}$ reference network, SOCONET and associated marine boundary layer $\mathrm{CO}_{2}$ measurements. Front. Mar. Sci. 6:400. doi: 10.3389/ fmars.2019.00400

Weller, R. A., Baker, D. J., Glackin, M. M., Roberts, S. J., Schmitt, R. W., Twigg, E. S., et al. (2019). The challenge of sustaining ocean observations. Front. Mar. Sci. 6:105. doi: 10.3389/fmars.2019.00105

Wimmer, W., Robinson, I. S., and Donlon, C. J. (2012). Long-term validation of AATSR SST data products using shipborne radiometry in the Bay of Biscay and English Channel. Remote Sens. Environ. 116, 17-31. doi: 10.1016/j.rse.2011. 03.022

Wolter, K., and Timlin, M. S. (2011). El Nino/Southern Oscillation behaviour since 1871 as diagnosed in an extended multivariate ENSO index (MEI.ext). Int. J. Climatol. 31, 1074-1087. doi: 10.1002/joc. 2336

Wong, E. W., and Minnett, P. J. (2016a). Retrieval of the ocean skin temperature profiles from measurements of infrared hyperspectral radiometers-part I: derivation of an algorithm. IEEE Trans. Geosci. Remote Sens. 54, 1879-1890. doi: 10.1109/TGRS.2015.2483746

Wong, E. W., and Minnett, P. J. (2016b). Retrieval of the ocean skin temperature profiles from measurements of infrared hyperspectral radiometers-part II: field data analysis. IEEE Trans. Geosci. Remote Sens. 54, 1891-1904. doi: 10. 1109/TGRS.2015.2501425

Wong, E. W., and Minnett, P. J. (2018). The response of the ocean thermal skin layer to variations in incident infrared radiation. J. Geophys. Res. Oceans 123:19. doi: 10.1002/2017JC013351

Woodruff, S. D., Worley, S. J., Lubker, S. J., Ji, Z., Freeman, J. E., Berry, D. I., et al. (2011). ICOADS release 2.5: extensions and enhancements to the surface marine meteorological archive. Int. J. Climatol. 31, 951-967. doi: 10.1002/joc. 2103

Wunsch, C., and Stammer, D. (1997). Atmospheric loading and the oceanic “inverted barometer" effect. Rev. Geophys. 35, 79-107. doi: 10.1029/96RG0 3037

Xu, F., and Ignatov, A. (2010). Evaluation of in-situ sea surface temperatures for use in the calibration and validation of satellite retrievals. J. Geophys. Res. Oceans 115:C09022. doi: 10.1029/2010JC006129 
Xu, F., and Ignatov, A. (2014). In-situ SST quality monitor. J. Atmos. Ocean. Technol. 31, 164-180. doi: 10.1175/JTECH-D-13-00121.1

$\mathrm{Xu}, \mathrm{F}$., and Ignatov, A. (2016). Error characterization in iQuam SSTs using triple collocations with satellite measurements. Geophys. Res. Lett. 43, 10826-10834. doi: 10.1002/2016GL070287

Zhang, D., Cronin, M. F., Lin, X., Inoue, R., Fassbender, A., Bishop, S., et al. (2017). Observing air-sea interaction in the western boundary currents and their extension regions: considerations for OceanObs 2019. CLIVAR Variat. 15, 23-30.

Zhurbas, V., Lyzhkov, D., and Kuzmina, N. (2014). Drifter-derived estimates of lateral eddy diffusivity in the world ocean with emphasis on the Indian Ocean and problems of parametrization. Deep Sea Res. Part I Oceanogr. Res. Pap. 83, 1-11. doi: 10.1016/j.dsr.2013. 09.001
Conflict of Interest Statement: The authors declare that the research was conducted in the absence of any commercial or financial relationships that could be construed as a potential conflict of interest.

Copyright (๑) 2019 Centurioni, Turton, Lumpkin, Braasch, Brassington, Chao, Charpentier, Chen, Corlett, Dohan, Donlon, Gallage, Hormann, Ignatov, Ingleby, Jensen, Kelly-Gerreyn, Koszalka, Lin, Lindstrom, Maximenko, Merchant, Minnett, O'Carroll, Paluszkiewicz, Poli, Poulain, Reverdin, Sun, Swail, Thurston, Wu, Yu, Wang and Zhang. This is an open-access article distributed under the terms of the Creative Commons Attribution License (CC BY). The use, distribution or reproduction in other forums is permitted, provided the original author(s) and the copyright owner(s) are credited and that the original publication in this journal is cited, in accordance with accepted academic practice. No use, distribution or reproduction is permitted which does not comply with these terms. 
OPEN ACCESS

Edited by:

Eric Delory,

Oceanic Platform of the Canary

Islands, Spain

Reviewed by:

Oscar Schofield,

Rutgers University, The State

University of New Jersey,

United States

Shinya Kouketsu,

Japan Agency for Marine-Earth

Science and Technology, Japan

*Correspondence:

Matthew D. Palmer

matthew.palmer@metoffice.gov.uk

Specialty section:

This article was submitted to

Ocean Observation,

a section of the journal

Frontiers in Marine Science

Received: 23 October 2018

Accepted: 05 July 2019

Published: 29 August 2019

Citation:

Palmer MD, Durack PJ,

Chidichimo MP, Church JA,

Cravatte S, Hill K, Johannessen JA,

Karstensen J, Lee T, Legler D,

Mazloff M, Oka E, Purkey S, Rabe B,

Sallée J-B, Sloyan BM, Speich S,

von Schuckmann K, Willis J and

Wijffels S (2019) Adequacy of the

Ocean Observation System

for Quantifying Regional Heat

and Freshwater Storage and Change.

Front. Mar. Sci. 6:416.

doi: 10.3389/fmars.2019.00416

\section{Adequacy of the Ocean Observation System for Quantifying Regional Heat and Freshwater Storage and Change}

\author{
Matthew D. Palmeri*, Paul J. Durack², Maria Paz Chidichimo³, John A. Church", \\ Sophie Cravatte ${ }^{5}$, Katy Hill', Johnny A. Johannessen ${ }^{7}$, Johannes Karstensen ${ }^{8}$, \\ Tong Lee ${ }^{9}$, David Legler ${ }^{10}$, Matt Mazloff'1, Eitarou Oka ${ }^{12}$, Sarah Purkey ${ }^{11}$, Ben Rabe ${ }^{13}$, \\ Jean-Baptiste Sallée ${ }^{14}$, Bernadette M. Sloyan ${ }^{15}$, Sabrina Speich ${ }^{16}$, \\ Karina von Schuckmann ${ }^{17}$, Josh Willis ${ }^{9}$ and Susan Wijffels ${ }^{18}$
}

\begin{abstract}
${ }^{1}$ Met Office Hadley Centre, Exeter, United Kingdom, ${ }^{2}$ Lawrence Livermore National Laboratory, United States Department of Energy (DOE), Livermore, CA, United States, ${ }^{3}$ National Scientific and Technical Research Council (CONICET), Buenos Aires, Argentina, ${ }^{4}$ Climate Change Research Centre, University of New South Wales, Sydney, NSW, Australia, ${ }^{5}$ Institut de Recherche pour le Développement, Noumea, New Caledonia, ${ }^{6}$ World Meteorological Organization, Geneva, Switzerland, ${ }^{7}$ Nansen Environmental and Remote Sensing Center, Bergen, Norway, ${ }^{8}$ GEOMAR Helmholtz Centre for Ocean Research Kiel, Kiel, Germany, ${ }^{9}$ NASA Jet Propulsion Laboratory, California Institute of Technology, Pasadena, CA, United States, ${ }^{10}$ Ocean Observing and Monitoring Division, Climate Program Office (NOAA), Silver Spring, MD, United States, ${ }^{11}$ Scripps Institution of Oceanography, University of California, San Diego, San Diego, CA, United States, ${ }^{12}$ Atmosphere and Ocean Research Institute, The University of Tokyo, Kashiwa, Japan, ${ }^{13}$ Alfred Wegener Institute, Helmholtz Centre for Polar and Marine Research, Bremerhaven, Germany, ${ }^{14}$ UMR 7159, Laboratoire d'Océanographie et du Climat: Expérimentations et Approches Numériques (LOCEAN), Paris, France, ${ }^{15}$ CSIRO, Oceans and Atmosphere, Hobart, TAS, Australia,

${ }^{16}$ Laboratoire de Météorologie Dynamique (LMD) UMR8539, École Normale Supérieure, Paris, France, ${ }^{17}$ Mercator Ocean, Ramonville-Saint-Agne, France, ${ }^{18}$ Woods Hole Oceanographic Institution, Woods Hole, MA, United States
\end{abstract}

Considerable advances in the global ocean observing system over the last two decades offers an opportunity to provide more quantitative information on changes in heat and freshwater storage. Variations in these storage terms can arise through internal variability and also the response of the ocean to anthropogenic climate change. Disentangling these competing influences on the regional patterns of change and elucidating their governing processes remains an outstanding scientific challenge. This challenge is compounded by instrumental and sampling uncertainties. The combined use of ocean observations and model simulations is the most viable method to assess the forced signal from noise and ascertain the primary drivers of variability and change. Moreover, this approach offers the potential for improved seasonal-to-decadal predictions and the possibility to develop powerful multi-variate constraints on climate model future projections. Regional heat storage changes dominate the steric contribution to sea level rise over most of the ocean and are vital to understanding both global and regional heat budgets. Variations in regional freshwater storage are particularly relevant to our understanding of changes in the hydrological cycle and can potentially be used to verify local ocean mass addition from terrestrial and cryospheric systems associated with contemporary sea level rise. This White Paper will examine the ability of the current ocean observing system to quantify changes in regional heat and freshwater storage. In particular we will seek to answer the question: What time and space scales are currently 
resolved in different regions of the global oceans? In light of some of the key scientific questions, we will discuss the requirements for measurement accuracy, sampling, and coverage as well as the synergies that can be leveraged by more comprehensively analyzing the multi-variable arrays provided by the integrated observing system.

Keywords: heat content, freshwater content, salinity, temperature, ocean observing system, climate change, climate variability, observing system design

\section{INTRODUCTION}

The global ocean plays a fundamental role in the climate system through its ability to store and redistribute large quantities of heat and freshwater. The IPCC Fifth Assessment Report of Working Group I (hereafter "IPCC AR5") has highlighted the critical role played by the ocean in both the long-term response of the Earth system under global warming and also nearer-term prediction (IPCC, 2013). Variations in heat content (HC) and freshwater content (FWC) can give rise to predictability of societally relevant information on seasonal-to-decadal timescales, such as El Niño Southern Oscillation (ENSO) (Chen et al., 2004) and the North Atlantic Oscillation (Scaife et al., 2014). More than $90 \%$ of the planetary heating associated with anthropogenic climate change is manifested in warming of the global oceans (Rhein et al., 2013). Climate model simulations show that global ocean HC change becomes the dominant term in the planetary heat budget on a timescale of several months and provides a more reliable indication of Earth's net radiative forcing than changes in global surface temperature (Palmer et al., 2011; Palmer and McNeall, 2014; von Schuckmann et al., 2016).

The thermal expansion associated with global ocean warming accounts for about $30-40 \%$ of the observed global sea level rise (Church et al., 2011, 2013b; WCRP Global Sea Level Budget Group, 2018) and is expected to make a substantial contribution in future projections for the 21st century (Church et al., 2013a). The spatial pattern of ocean $\mathrm{HC}$ change exerts a strong influence on local sea level change and remains a key uncertainty in regional projections of sea level rise (e.g., Slangen et al., 2014; Cannaby et al., 2016; Carson et al., 2016). In addition, we are beginning to understand the importance of the spatial pattern of ocean warming on the strength of climate feedbacks and climate sensitivity (Rose et al., 2014; Rose and Rayborn, 2016), which determines the amount of surface warming we will see for a given amount of greenhouse gas emissions. Understanding, and accurately monitoring the patterns of $\mathrm{HC}$ change from global to regional scales are therefore of central importance to the development of robust information on future climate change.

Alterations to the global water cycle, such as water availability, droughts and floods are a major concern as Earth's climate changes. The ocean is the largest water store in Earth's hydrosphere, accounting for $97 \%$ by storage across all water reservoirs, and $80 \%$ of Earth's surface freshwater fluxes occur at the ocean-atmosphere interface (Durack, 2015). Accurate monitoring of ocean salinity variability and change can provide important insights into broad-scale changes to the global water cycle. Oceanic waters contain simple salts (e.g., Pawlowicz et al., 2016), and through the process of evaporation, these salts remain, ensuring that as water is cycled from ocean to atmosphere to land and back again, salinity anomalies persist and accumulate, providing a salty or fresh marker of water cycle variability and change.

Observed changes in HC and FWC are computed based upon the available in situ subsurface temperature and salinity measurements. While the earliest trans-global survey of subsurface temperature dates back to the H.M.S Challenger expedition in the late 19th century (Roemmich et al., 2012), it was not until the late 1960s that widespread upper ocean temperature measurements became available (Abraham et al., 2013). The historical ocean observations over the latter half of the 20th century have a strong bias toward the Northern Hemisphere, as these measurements are associated predominantly with research vessels and shipping activity. However, it is only since the mid-2000s with the advent of the Argo array of autonomous profiling floats that we have regular, quasi-global sampling of the upper ocean (0-2000 m) (Roemmich et al., 2012, 2019; Riser et al., 2016). Argo also delivers co-located observations of salinity, from which changes in ocean FWC can be derived. These co-located temperature and salinity observations allow us to compute the density field and its influence on regional sea level change (Willis et al., 2008), related changes in the geostrophic flow field (Gray and Riser, 2014) and mechanistic insights to the observed changes through water mass analysis (Desbruyères et al., 2017). The discussion of the global in situ ocean observing system presented here focuses on this post-Argo era.

The advent of remote sensing in 1978 with the Seasat Earth-orbiting satellite heralded a new era in global ocean investigations, with the first of these remote platforms including a radar altimeter to measure satellite height above the ocean surface, a microwave scatterometer to measure wind speed and direction, microwave radiometer to measure sea surface temperature and visible and infrared radiometers to identify clouds, land and water features. The utility of these space-borne remote platforms was proven for sea surface temperature in the early 1980s (e.g., McConaghy, 1980). In the early 1990s the integrated quantity of sea surface height (SSH) was robustly captured (e.g., Le Traon et al., 1998; Ducet et al., 2000), and the first of the sea surface salinity satellites was launched in November 2009 (ESAs Soil Moisture and Ocean Salinity satellite) with a number of other missions launching soon after (e.g., Berger et al., 2002; Lagerloef et al., 2008; Fore et al., 2016; Vinogradova et al., 2019). Satellite based observations provide an exceptional high-resolution view on the surface ocean manifestation of (sub)mesoscale dynamics in SST (Group for High Resolution 
SST; GHRSST), sea level anomaly (Jason \& Sentinel - see https:// sentinel.esa.int/web/sentinel/missions) and surface salinity fields (SMOS and SMAP). These satellite observations are highly complementary to the in situ observing array and can provide, for example, propagation of information from single profile observations (e.g., Argo) over wider spatial domains.

Satellite and in situ measurements are complementary. Satellite measurements help resolve the scales or monitor regions not adequately sampled or covered by in situ observations. Examples include variability in coastal oceans and marginal seas such as those associated with river plumes that influence regional FWC (e.g., Fournier et al., 2016). In situ measurements are generally much more accurate, thereby providing ground truth for the calibration and validation of satellite data. Synthesized satellite/in situ products (e.g., Reynolds et al., 2007; Guinehut et al., 2012; Xie et al., 2014) combine strengths of the satellite and in situ observing systems to facilitate studies of regional HC and FWC changes.

The multi-decadal three-dimensional response of the ocean temperature and salinity fields offers the potential for more powerful means to quantify the influence of different climate forcings than using surface temperature alone. In particular, the systematic horizontal and vertical re-arrangement of heat in the ocean through ocean circulation and processes such as ENSO variability (Roemmich and Gilson, 2011; England et al., 2014; Roemmich et al., 2015) poses a challenge for interpretation for both regional and global changes in surface temperature. However, this "noise" arising from internal climate variability is also an important source of decadal predictability (e.g., Smith et al., 2007; Meehl et al., 2014), which is an important element in the development of climate services for societal benefit. Advancing the science of the impacts of $\mathrm{HC}$ and FWC variability and change requires us to move beyond qualitative descriptions of past changes afforded by the 20th century ocean observing. This White Paper reviews the capabilities of the contemporary ocean observing array for quantifying regional HC and FWC changes and presents recommendations for the future development of the ocean observing system.

The remaining sections of the paper are organized as follows. In section "Observed Changes in Ocean Heat and Freshwater Content," we present a discussion of the observed trends and variability of regional $\mathrm{HC}$ and FWC, with linkages to predictability and forecast initialization. In section "Projected Changes in Ocean Heat and Freshwater Content," we present a synopsis of what is known about the emergent patterns of $\mathrm{HC}$ and FWC change from climate model simulations. We then present a number of regional case studies in subsequent sections, focused on the Atlantic Ocean, the tropical Pacific, the Arctic Ocean and the Southern Ocean. These geographic regions are selected based on their importance in the global climate system and each case study is focused on the key scientific questions and the current observing system capabilities in each of these regions respectively. In the "Discussion", we cover some of the overarching issues, including the challenge of sustaining the long-term observations that are needed to monitor regional $\mathrm{HC}$ and $\mathrm{FWC}$ variability and change.
Finally, we conclude with the recommendations in which we present a series of goals for future development of the ocean observing system.

\section{OBSERVED CHANGES IN OCEAN HEAT AND FRESHWATER CONTENT}

Prior to the comprehensive data coverage of Argo, much of the assessment of global ocean variability was limited to climatological annual and seasonal cycles (e.g., Levitus, 1984, 1986, 1989; Boyer and Levitus, 2002; Kara et al., 2003; de Boyer Montegut et al., 2004), or pentadal temporal epochs for the deeper ocean (e.g., Levitus, 1989). With the explosion of ocean measurements thanks to the international Argo Program, much more comprehensive investigations into modern-era ocean variability have been made possible. Most recently, with the so-called surface warming "hiatus" (e.g., Meehl et al., 2011), numerous studies have focused on the role of the ocean during the Earth surface warming slow down. Many of these studies have highlighted the importance of ocean HC redistribution, either basin to basin (e.g., Lee et al., 2015; Nieves et al., 2015) or vertically (Drijfhout et al., 2014; e.g., Chen and Tung, 2014; England et al., 2014), in explaining the reduced rate of surface warming during this period. Model simulations show that "hiatus" events can arise purely through internal climate variability (e.g., Easterling and Wehner, 2009; Meehl et al., 2011; Risbey et al., 2014; Roberts et al., 2016) but there is evidence that both anthropogenic and natural external forcings can also play an important role (e.g., Maher et al., 2014; Smith et al., 2016).

The upper ocean $(0-700 \mathrm{~m})$ is where the bulk of historical measurements exist, and consequently where our knowledge of long-term change is most robust (Abraham et al., 2013). On multi-decadal timescales, a consistent picture of forced ocean change has been clearly evident in ocean observations, since the first assessments of ocean warming became available (e.g., Levitus et al., 2000). Subsequently a clearer picture of change has emerged, with a near-global, upper-ocean warming evident from 1971 to 2010 at a mean rate of 107 TW (74-137 TW for the range of 5 independent estimates), and a weaker warming signal over 1870-1971 (Rhein et al., 2013), broadly consistent with our understanding of changes in Earth's radiative forcing (e.g., Myhre et al., 2013). While measurement coverage reduces in the intermediate depths (700-2000 m) prior to Argo, pentadal (5-year) estimates have been calculated that extend back to 1957 (Levitus et al., 2012). These too show marked warming over the observed record, but at a slower rate compared to the upper-ocean. While all available analyses show a marked historical warming, the patterns and rates of these diverge due to measurement coverage limitations, and the different methods used to reconstruct global changes from sparse observations (e.g., Boyer et al., 2016; Palmer et al., 2017). This issue largely disappears for the upper and intermediate ocean during the Argo period (Roemmich et al., 2015, 2019).

Along with ocean warming, coherent salinity changes have also been observed for both the near-surface global ocean and the ocean interior (Boyer et al., 2005; Hosoda et al., 2009; 

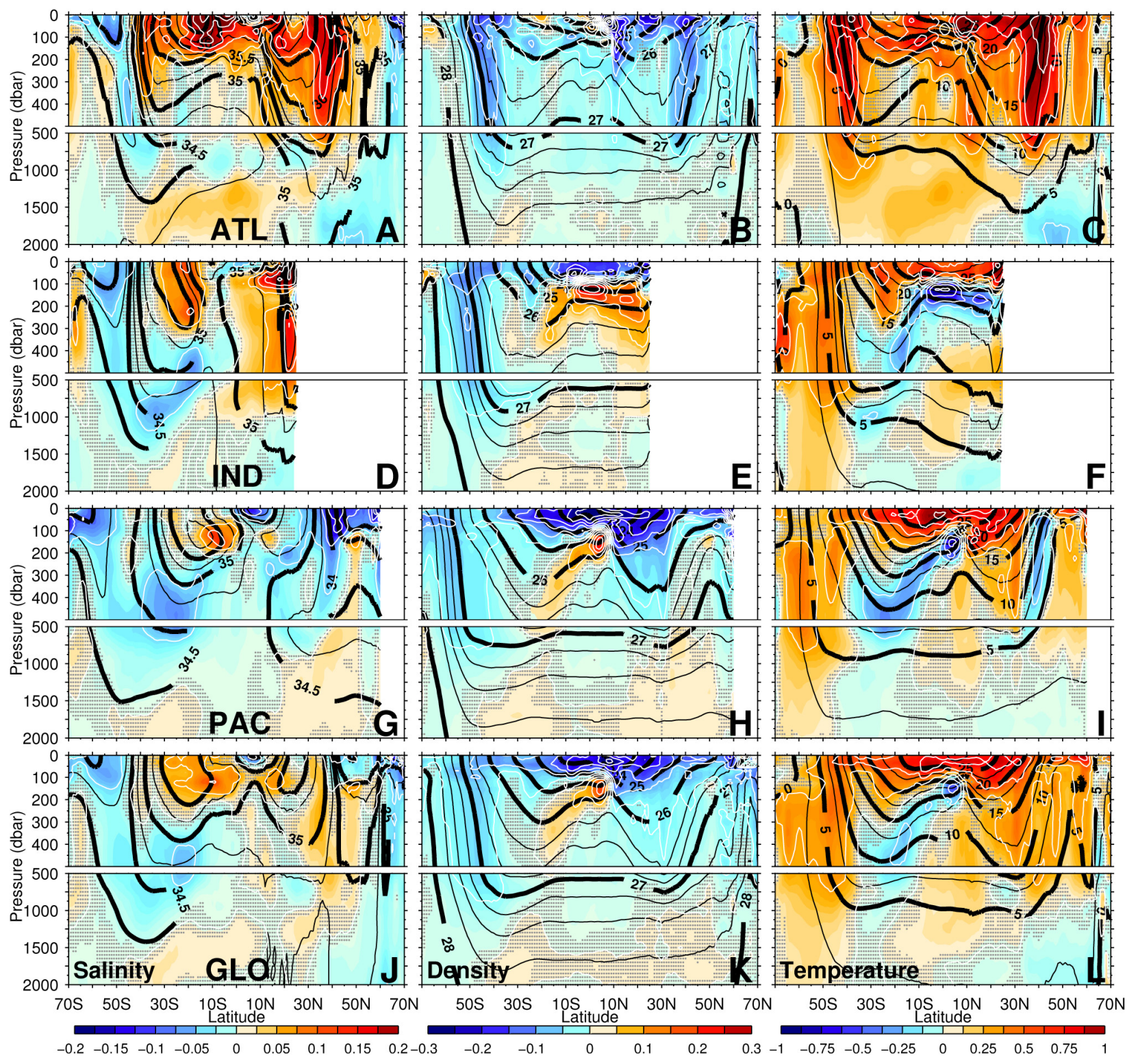

FIGURE 1 | Upper 2000 dbar (pressure) zonally averaged linear trend (1950-2000) (colors with white contours) of salinity changes (column 1, PSS-78 per 50 year; white contours: 0.05 PSS-78, -0.5 to 0.5 ), neutral density changes (column $2, \mathrm{~kg} \mathrm{~m}^{-3}$ per 50 year; white contours: $0.05 \mathrm{~kg} \mathrm{~m}^{-3},-0.3$ to 0.3 ), and potential temperature changes (column $3,{ }^{\circ} \mathrm{C}$ per 50 year; white contours: $0.25^{\circ} \mathrm{C},-1$ to 1 ), for the Atlantic Ocean (ATL) in row 1 (A-C), Indian Ocean (IND) in row 2 (D-F), Pacific Ocean (PAC) in row 3 (G-I), and global ocean (GLO) in row 4 (J-L). Mean fields are shown as black lines (Salinity: thick black contours 0.5 PSS-78, thin contours 0.25 PSS-78; Neutral density: thick black contours $1.0 \mathrm{~kg} \mathrm{~m}^{-3}$, thin contours $0.25 \mathrm{~kg} \mathrm{~m}^{-3}$; Potential temperature: thick black contours $5.0^{\circ} \mathrm{C}$, thin contours $2.5^{\circ} \mathrm{C}$ ). Trends are calculated on pressure surfaces. Regions where the resolved linear trend is not significant at the $90 \%$ confidence level are stippled in gray. Reproduced from IPCC AR5 (Rhein et al., 2013, Figure 3.9).

Durack and Wijffels, 2010; Helm et al., 2010; Mulet et al., 2018). Surface ocean salinity patterns mirror the climatological mean pattern evaporation and precipitation fluxes at the oceanatmosphere interface, such that high evaporation regions express high climatological salinities (the subtropical convergence zones) and high precipitation regions express low climatological salinities (tropical and sub-polar regions). The broad-scale patterns of salinity change reflect an enhancement to these mean patterns, both for the surface and subsurface ocean, and agree well with those from ocean model simulations driven by enhanced surface evaporation and precipitation patterns (e.g., Williams et al., 2007; Durack et al., 2012; Lago et al., 2016), suggesting that, as with temperature, anthropogenically forced changes are already underway (Figure 1).

While deep $(>2000 \mathrm{~m})$ ocean measurements are sparse, these are captured by dedicated research cruises using highly specialized, calibrated measurement platforms under the auspices of GO-SHIP (Sloyan et al., 2019) and represent the "gold 
standard" for subsurface observations. Regions of the deep ocean have also undergone statistically significant warming and freshening since the 1990s with large regional variability (Purkey and Johnson, 2010, 2013). Monitoring the deep ocean is currently limited to ship-based, full-depth hydrographic sections based on a sparse network of ship tracks that are typically repeated every few years, and from purposely designed transbasin Atlantic Meridional Overturning Circulation (AMOC) and boundary currents arrays (Frajka-Williams et al., 2019). Based on the available observations it has been estimated that the deep (below $2000 \mathrm{~m}$ ) and abyssal (below $4000 \mathrm{~m}$ ) oceans have accumulated heat at a rate of $22.3 \pm 23.7 \mathrm{TW}$ and $10.7 \pm 3.4 \mathrm{TW}$, respectively; mostly owing to the deep Southern Ocean which has warmed 10 times faster than the deep basins to the north (Purkey and Johnson, 2010; Desbruyères et al., 2016, 2017). In addition, the abyssal Indian and Pacific sectors of the Southern Ocean have freshened at a rate equivalent to a freshwater flux of $73 \pm 26$ Gtonne/year (Purkey and Johnson, 2012). As a third decade of repeat hydrography becomes available, there has been some evidence that the warming is slowing in the South Atlantic and accelerating in the Pacific, but variability in local trends are not statistically significant owing to limited data (Sloyan et al., 2019). To fully resolve deep ocean $\mathrm{HC}$ and FWC changes on local to global scales, a continuously, global, full depth monitoring system is needed. Plans are underway for a $5^{\circ} \times 5^{\circ} \times 15$-day cycle Deep Argo array capable of sampling to 4000-6000 m (Le Reste et al., 2016; Roemmich et al., 2019). This new array will be able to resolve local trends above $0.001^{\circ} \mathrm{C} /$ decade in the North Pacific and above $0.026^{\circ} \mathrm{C} /$ decade in the noisier Southern Ocean based on current observed local variance (Johnson et al., 2015).

Ocean and climate models represent critical tools to advance our understanding of observed changes and their causes. In addition to improvements in observations, ocean models have consistently improved in the last decade, with the latest eddypermitting ( $\sim 1 / 10$ th degree) high-resolution simulations capable of reproducing the variability represented in modern satellite altimetry measurements of SSH, both spatially and in magnitude (Griffies et al., 2015), and even the coarser resolution CMIPgrade simulations have utility in capturing the spatial gradients in SSH variability (Landerer et al., 2014). A number of studies have attributed observed temperature (Levitus et al., 2001; Domingues et al., 2008; Palmer et al., 2009; Gleckler et al., 2012; Pierce et al., 2012; Weller et al., 2016) and salinity changes (Stott et al., 2008; Pierce et al., 2012; Terray et al., 2012) to anthropogenic forcing. In addition, ensemble approaches in ocean modeling have been used to disentangle the forced response from chaotic ocean variability (e.g., Sérazin et al., 2017). Sub-sampling of spatially complete model fields has provided insights into our ability to constrain observed climate variability and change based on the sparse historical coverage of ocean profile measurements (Gregory et al., 2004; AchutaRao et al., 2006, 2007; Good, 2017; Allison et al., 2019). Studies using both models and observations have provided provocative perspectives that question the observationonly estimates of historical changes, noting that potentially large underestimates of historical changes may have occurred due to poor Southern Hemisphere sampling (e.g., Durack et al., 2014), and that due to poor spatial coverage, particularly in the deeper ocean, the rate of deep ocean warming may have been underestimated (Gleckler et al., 2016; Allison et al., 2019). In particular, these studies have highlighted the propagation of the climate change signal into the deeper ocean over time (Figure 2; Cheng et al., 2016; Gleckler et al., 2016). Ocean reanalyses and ocean state estimates, where data assimilation schemes are used to fuse observations with numerical model simulations, are increasingly being used to gain insights into past ocean variability and change (Balmaseda et al., 2015; Palmer et al., 2017).

Ocean and climate variability is an important source of predictability on seasonal-to-decadal timescales. The best-known example is probably that associated with seasonal forecasts of ENSO (e.g., Chen et al., 2004; Barnston et al., 2012), with large global impacts on patterns of temperature and precipitation (McPhaden et al., 2006) and sea level in the Pacific (Widlansky et al., 2017). Skill in ENSO prediction arises from well-understood and conceptually simple ocean dynamics (e.g., Roberts et al., 2016). The most skilful seasonal forecasts tend to be those that incorporate subsurface ocean initial conditions and include an active dynamical ocean as part of a coupled model prediction system (e.g., Doblas-Reyes et al., 2013). On longer timescales, one of the main sources of predictability arises from initialization of the subsurface ocean (e.g., Smith et al., 2007; Meehl et al., 2014). Therefore, a major challenge in the development of decadal prediction systems is the lack of historical temperature and salinity observations that are required to: (i) assess the representation of variability in climate model simulations; (ii) generate initial conditions for hindcast simulations; and (iii) provide skill assessment of prediction systems based on hindcast sets. Idealized predictability studies and assessment of internal variability in CMIP5 models both suggest that improved forecast skill should be possible with more complete sampling of the ocean temperature and salinity fields (Dunstone and Smith, 2010; Palmer et al., 2011; Palmer and McNeall, 2014).

\section{PROJECTED CHANGES IN OCEAN HEAT AND FRESHWATER CONTENT}

The ocean response under anthropogenic greenhouse gas forcing will be a critical determinant of both the magnitude and regional impacts of future climate change. We have a high degree of scientific confidence in some of the systematic changes that will occur in the climate system under anthropogenic warming. These changes include: intensification of the hydrological cycle (with corresponding amplification of the spatial variations in surface and subsurface salinity); continued warming of the global oceans and sea level rise; a weakening of the AMOC (and associated northward heat transport); intensification of the Southern Ocean winds; and increased freshwater input to the high-latitudes (from both moisture transport and melting ice). However, the magnitude of these changes and the associated spatial patterns generally show a large degree of uncertainty in climate model simulations (e.g., Collins et al., 2013; Gregory et al., 2016).

Global warming gives rise to increased global precipitation and atmospheric moisture transports, due to the capacity for a 


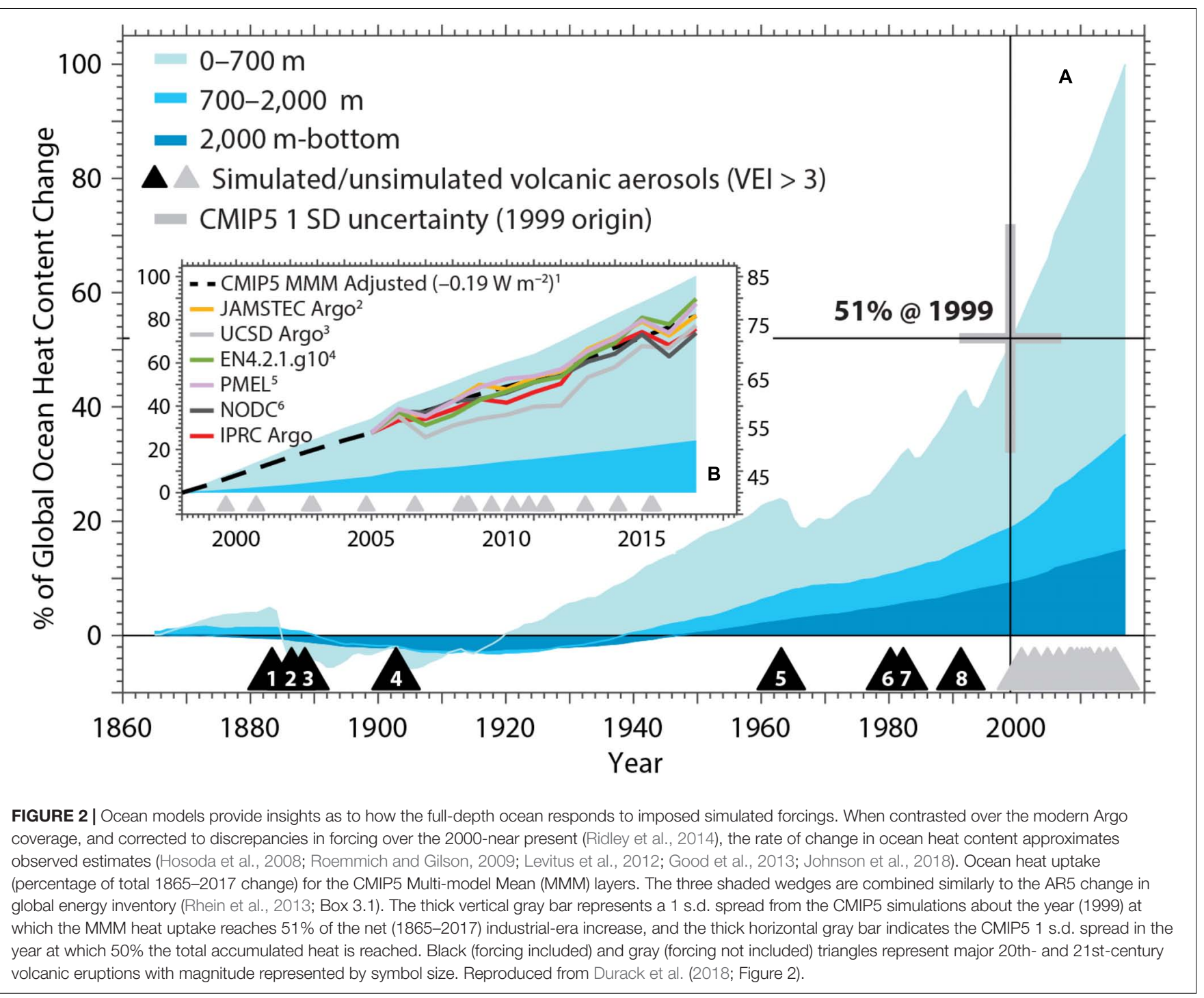

warmer atmosphere to hold more moisture (Held and Soden, 2006). CMIP5 climate model simulations suggest that the rate of global precipitation will increase with global surface temperature change, at rate between 0.5 and $3 \%$ per $\mathrm{C}$ depending on the climate change scenario (Collins et al., 2013). The first-order picture that has often been presented for precipitation is "the richer get richer and the poor get poorer" in reference to an intensification of the hydrological cycle, with corresponding amplification of rainfall extremes and drought events (Trenberth, 2011). However, more recent studies have challenged this viewpoint as too simplistic (Collins et al., 2013; e.g., Chadwick et al., 2013) and there remains a large degree of uncertainty in the spatial patterns of precipitation changes among climate models in general (Collins et al., 2013). Given the challenges of measuring changes in global precipitation and evaporation, using observations of sea surface salinity may be the most tractable approach to monitoring long-term changes in the global hydrological cycle (e.g., Durack and Wijffels, 2010; Durack et al., 2012; Li et al., 2016a,b; Liu et al., 2018).
Global ocean heat uptake acts to delay the surface temperature rise and mitigates the worst impacts of future global warming. The spatial pattern of heat uptake affects the evolution of sea surface temperature and climate feedbacks, with important implications for the magnitude of global surface temperature change (e.g., Rose et al., 2014; Rose and Rayborn, 2016). This spatial pattern of warming also has important implications for both global and regional sea level rise (e.g., Kuhlbrodt and Gregory, 2012); and may also directly influence the future ice mass input from the West Antarctic Ice Sheet (e.g., Levermann et al., 2014; Golledge et al., 2015). Global ocean $\mathrm{HC}$ change is an important indicator of the rate of global warming and provides more reliable information on annual-todecadal timescales than changes in global surface temperature (Roemmich et al., 2015; von Schuckmann et al., 2016). The spatial pattern of ocean heat uptake under climate change therefore has important implications for the design of the future observing array if we are to accurately monitor the rate of anthropogenic global change. 
The IPCC AR5 (Rhein et al., 2013) provided a good overview of the historical observed spatial trends in upper ocean $\mathrm{HC}$ and FWC/salinity (see section "Observed Changes in Ocean Heat and Freshwater Content”). However, relatively little information was provided on the spatial patterns of $\mathrm{HC}$ and FWC under climate change. The surface temperature response under greenhouse gas forcing varies considerably among CMIP5 models (Collins et al., 2013), both in terms of the overall magnitude and the spatial pattern of warming. In general, the response of any given climate model is associated with relatively large-scale patterns and key features typically extend over several degrees of longitude/latitude or more. The CMIP5 multi-model mean suggests the largest surface temperature response in the Arctic and the least warming in areas of the North Atlantic, South Pacific, and Southern Ocean (Collins et al., 2013).

CMIP5, and previous climate model simulations, exhibit widespread warming of the global ocean under all RCP climate change scenarios. The spatial pattern is broadly characterized by a surface intensified warming, with the majority of the warming below $2000 \mathrm{~m}$ occurring in the Southern Ocean (Figure 3; Collins et al., 2013). Model simulations suggest that the climate change signal steadily propagates into the deeper ocean over time (Collins et al., 2013; Gleckler et al., 2016).

The connection between air-sea heat fluxes and the deep ocean is strongest in regions of deep and bottom formation. Therefore, in addition to the surface-intensified warming, climate model simulations also show a bottom-intensified warming, particularly for the Southern Ocean sector (Figure 4; Heuzé et al., 2015). This aspect of the change signal is also in broad agreement with observational estimates of abyssal ocean warming and consistent with theoretical understanding of water mass formation and ocean circulation. While the signals for bottom warming are fairly consistent among CMIP5 models this is less true for projections of changes in bottom salinity. Details of the climate change response vary substantially across CMIP5 models and are linked to changes in the mean transports of key water masses (Heuzé et al., 2015). Uncertainty in the spatial pattern of the ocean response is also reflected in depth-integrated properties, such as HC and dynamic sea level (Kuhlbrodt and Gregory, 2012; Gregory et al., 2016). The largest ocean heat uptake is seen in the Southern Ocean and Atlantic sectors (Kuhlbrodt and Gregory, 2012; Exarchou et al., 2014; Garry, 2017) with ocean circulation playing a substantial role in the associated spatial pattern through both advection of, and response to, the warming signal (Marshall et al., 2015; Gregory et al., 2016).

The various aspects of climate change discussed above are all either directly, or indirectly, associated with patterns of regional $\mathrm{HC}$ and FWC, through changes in air-sea fluxes and/or horizontal ocean transports. Changes in storage act to integrate out atmospheric/weather "noise" that is inherent to air-sea fluxes and provide better signal-to-noise ratios than attempting to monitor the fluxes directly. While changes in upper ocean HC feedback on air-sea fluxes via the surface temperature field (e.g., Gregory et al., 2016), salinity behaves more like a passive tracer. Therefore, there is a high degree of complementarity between $\mathrm{HC}$ and FWC changes in water mass frameworks and the influence of both on density offers insights into changes in the geostrophic flow field and the steric component of sea level. Accurately monitoring regional HC and FWC changes offers the potential for improved understanding of the emergent patterns of climate change and developing multi-variate observational constraints for climate model projections (e.g., Cox et al., 2018) across a wide range of societal-relevant metrics. While we must be cognizant of limitations and known biases in climate model simulations, they can provide useful information to help inform development of the future ocean observing array. We can have most confidence in projections of future ocean climate changes where there is a consistent picture between theory, observations and the model simulations of future climate change. Comparison across these three elements may provide a useful framework for assessing and communicating uncertainty in future projections of climate change.

\section{CASE STUDY: ATLANTIC OCEAN}

The Atlantic Ocean plays a unique role in Earth's climate as the sole basin connected to both the Arctic and Southern Oceans. The current systems of the Atlantic carry and redistribute $\mathrm{HC}$ and FWC not only poleward but there is a net heat transport from the southern to the northern hemisphere. This cross hemispheric transport is carried by the AMOC (Frajka-Williams et al., 2019), and the HC and FWC pattern reflect this close link and show significant variability in both, thermocline waters and in the deep ocean below $2000 \mathrm{~m}$.

As for other basins, thermocline waters affecting $\mathrm{HC}$ changes are essentially mode and intermediate waters, formed in both the subpolar and subtropical regions, and penetrating the subtropical gyres of both hemispheres at depth (Arbic and Owens, 2001; Häkkinen et al., 2015; Desbruyères et al., 2017). Recent estimates show that during the period 1950s to 2012, the upper $2000 \mathrm{~m}$ of the North Atlantic Ocean have gained $5 \times 10^{22} \mathrm{~J}, 30 \%$ of the global ocean warming over this period (Häkkinen et al., 2015) and are accompanied by a downward displacement of midthermocline isopycnals (Arbic and Owens, 2001; Häkkinen et al., 2015; Desbruyères et al., 2017).

The water mass transformation processes that create North Atlantic Deep Water (NADW) have two drivers: (i) buoyancy fluxes at the air/sea interface, creating dense mode water in the subpolar gyre (densest one is Labrador Sea Water; LSW); and (ii) entrainment processes at the Greenland-Scotland Ridges, where dense water masses that enter from the Nordic Seas are transformed into Denmark Strait Overflow Water (densest part of the NADW) and the Iceland Scotland Overflow Water. The hydrographic variability of subpolar Mode Water masses is well documented and also correlated with air/sea buoyancy fluxes (Yashayaev and Loder, 2017). Variability of the hydrography of the overflow waters is more complex because of the entrainment of ambient waters adding twice the volume to the overflow (Jochumsen et al., 2015). The northward spreading of Antarctic Bottom Water (AABW) in the Atlantic Ocean is supplied by dense water formed around Antarctica, in particular from the 


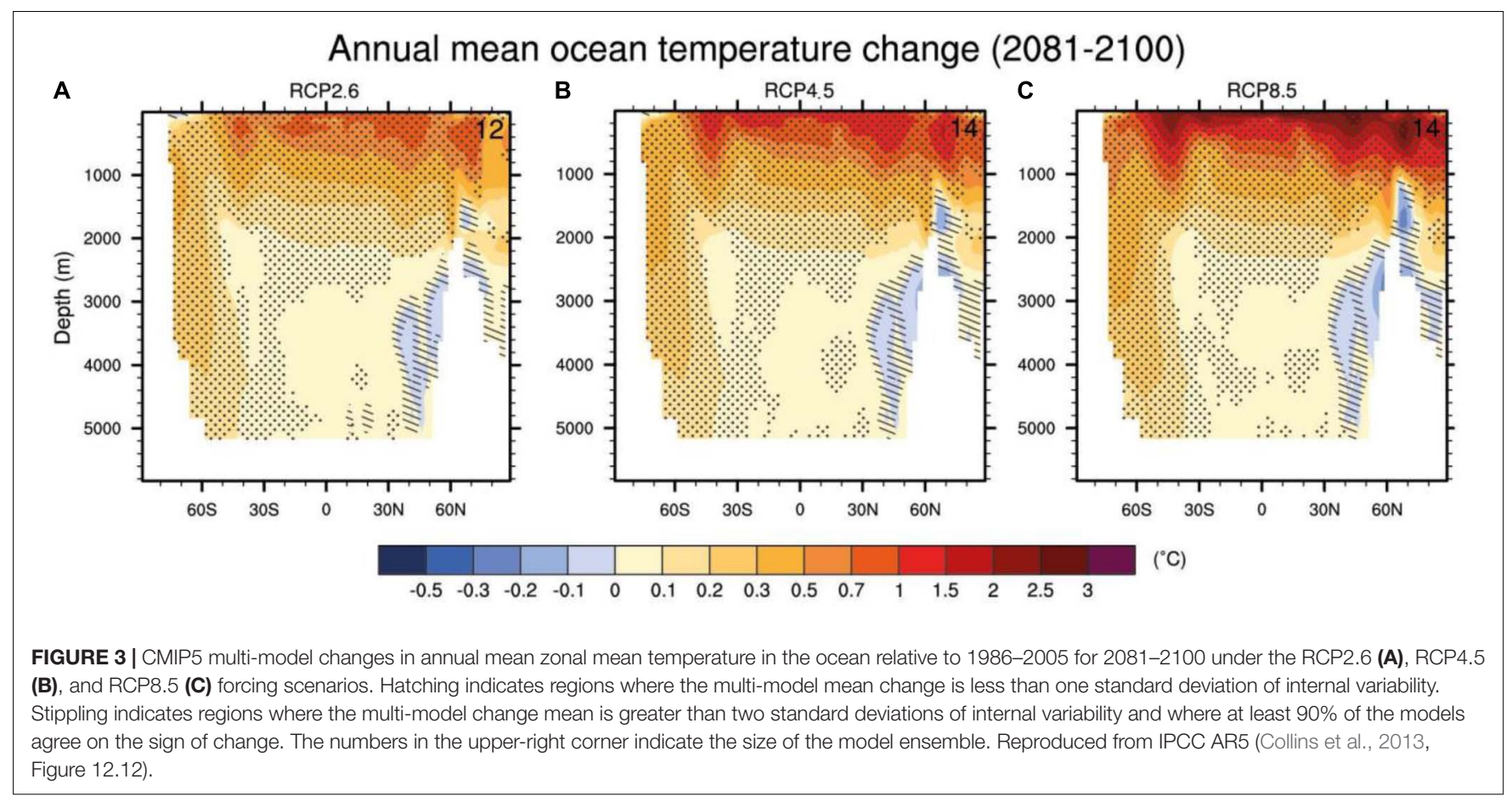

Weddell Sea. The northward penetration is limited by the topographic structure of the Atlantic basin and almost all AABW has to cross through the Vema Channel $\left(28^{\circ} \mathrm{S} / 38^{\circ} \mathrm{W}\right)$ where a steady increase in AABW temperature (about $2.8 \mathrm{mK} /$ year; Zenk and Morozov, 2007) has been reported.

Adequately monitoring the $\mathrm{HC}$ in the Atlantic Ocean is important in terms of planetary energy budget but also in relation to ocean-atmosphere heat fluxes (Rhines et al., 2008), seasonalto-decadal climate prediction (Czaja and Frankignoul, 2002), to constrain future projections of atmospheric storminess and Arctic changes (Straneo and Heimbach, 2013; Jung et al., 2017). The Atlantic HC in the upper 0-2000 m exhibits strong decadal variability (e.g., Cheng et al., 2017), which is likely linked with decadal changes in the AMOC, which has been shown to increase its total overturning transport up to the mid-1990s followed by a decrease from 2000 onward (e.g., Danabasoglu et al., 2015; Jackson et al., 2016).

Analysis of the strong weakening of the AMOC in 2009/2010 (McCarthy et al., 2012) suggest that we are able to present a consistent picture between changes in horizontal transports, heat storage and air-sea fluxes at least for basin-scale changes (Cunningham et al., 2013; Bryden et al., 2014). Since 2005, a large part of the North Atlantic evidenced a shift from warming to cooling (Robson et al., 2012a, 2016), which led to decreasing regional HC. The regional North Atlantic cooling is likely linked to changes in the circulation such as anomalously weak LSW deep convection (Thornalley et al., 2018) and/or long-term AMOC weakening (e.g., Caesar et al., 2018; Smeed et al., 2018) and to changes in the air-sea fluxes (e.g., Robson et al., 2014; Dubois et al., 2018; Josey et al., 2018). These results suggest that longterm changes in the AMOC will have a first-order impact on the future HC (and FWC) changes in the North Atlantic.
The deep reaching overturning circulation in the North Atlantic and the proximity to large freshwater reservoirs (Arctic, Greenland) means that surface freshwater anomalies can be efficiently introduced into deep waters and spread southward (Curry and Mauritzen, 2005). One such example is the Great Salinity Anomalies observed during the 1970s, 1980s, and 1990s (Belkin, 2004). Examination of a range of salinity products collectively suggests widespread freshening of the subpolar North Atlantic from the mid-2000s to the present (Tesdal et al., 2018). In the western subpolar gyre, a negative correlation between near-surface salinity and the circulation strength of the subpolar gyre suggests that negative salinity anomalies are connected to an intensification of the subpolar gyre, which is causing increased flux of freshwater from the East Greenland Current and subsequent transport into the Labrador Sea during the melting season. A freshening has been also observed in the Antarctic Intermediate Water (AAIW) layer in the South Atlantic during the period 2005-2014 (Yao et al., 2017) and it has been suggested that it is linked to increased southern hemisphere subpolar precipitation and a decrease in the Agulhas leakage. Concurrently to the so called "North Atlantic cold event," distinct freshening of the upper $1000 \mathrm{~m}$ layer has been reported in the subpolar North Atlantic since 2005 (Dubois et al., 2018). A schematic of the observed heat and salinity changes is presented in Figure 5.

Monitoring the Atlantic Ocean FWC budget is relevant for understanding and monitoring the global hydrological cycle with a clear connection to seasonal-to-decadal predictability (Schlundt et al., 2014; Fu et al., 2018). It is also an important element in the discussion around potential abrupt changes of the AMOC (Clark et al., 2002; de Vries and Weber, 2005; Liu et al., 2017).

In the Atlantic Ocean the different pathways of freshwater transport, the variability of air/sea fluxes and internal water 


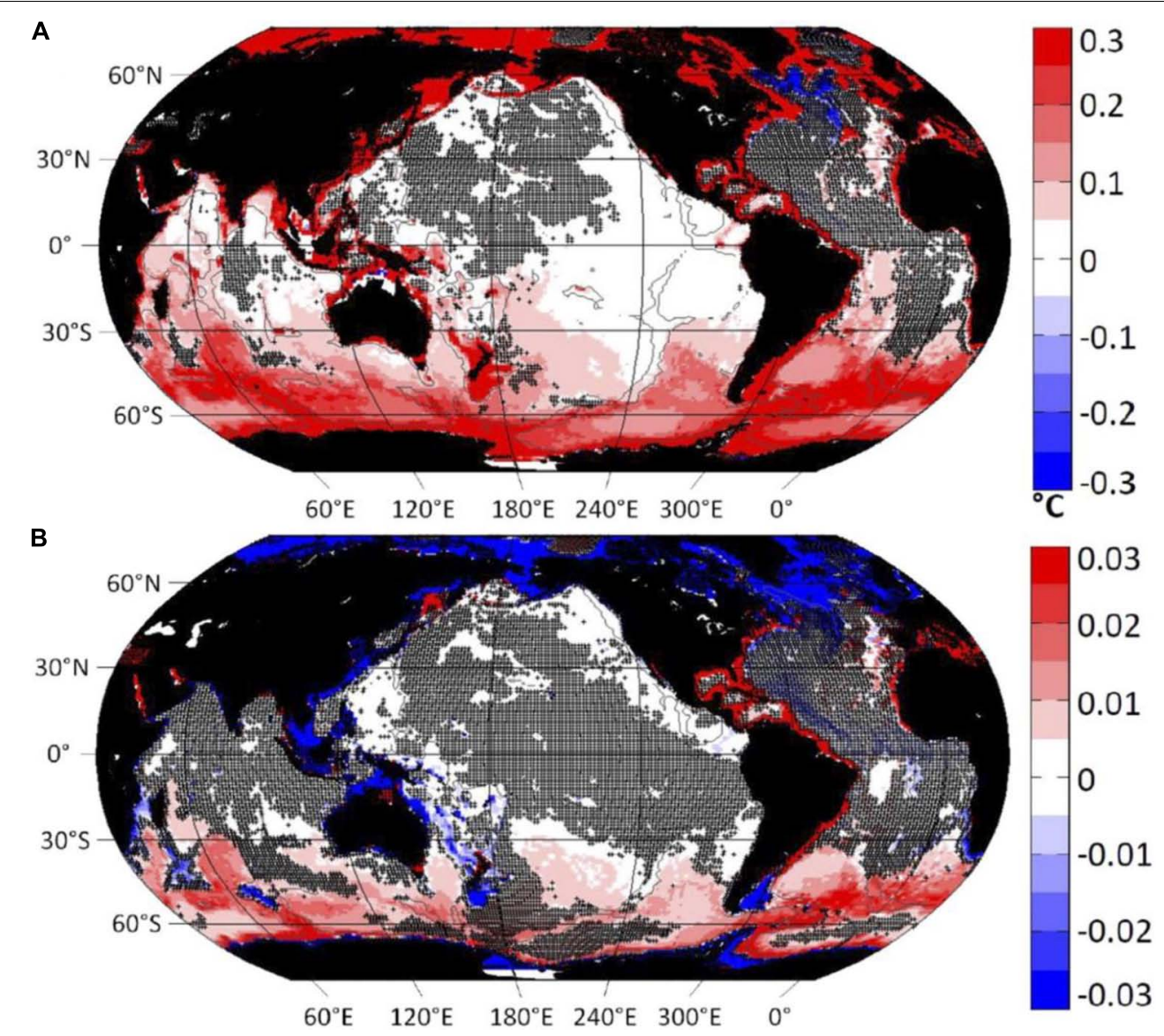

FIGURE 4 | RCP8.5 multimodel mean change (2081-2100 minus 1986-2005) in (A) bottom temperature, and (B) bottom salinity. Control drift has been removed. Black stippling indicates areas where fewer than 16 models agree on the sign of the change. Gray contour indicates the 3000-m isobath. Figure adapted from Heuzé et al. (2015).(CAmerican Meteorological Society. Used with permission.

mass transformation in particular at the overflow sills create highly variable $\mathrm{HC}$ and FWC storage throughout the water column. This sets very demanding observational requirements for monitoring the variations of these ocean properties in this basin. However, the characteristic timescales of $\mathrm{HC}$ and FWC anomalies differ by the depth of the water mass and corresponding ventilation regions and water transformation processes. Indeed, the upper to lower thermocline layers are highly affected by processes at intraseasonal to interdecadal timescales, due to the strong influence of air-sea fluxes, mesoscale variability and lower frequency natural variability. Changes in the deeper layers are characterized by interannual to centennial timescales (Park and Latif, 2008; McCarthy et al., 2012; Xu et al., 2014; Gastineau et al., 2018). In order to constrain variability and changes in the global energy and freshwater budgets and initialize seasonal-to-decadal forecasts, monitoring at monthly timescales is required for the upper $(0-2000 \mathrm{~m})$ layers and annual timescales for the deeper layers as only these observations provide a strong constraint on ocean temperatures beneath the mixed layer and have been shown to have a very significant impact on such forecasts (Dunstone and Smith, 2010; Robson et al., 2012b; Dunstone, 2014). Such timescales are defined by the time variability scales of the upper ocean (essentially mesoscale) and longer for deeper layers.

For the monthly monitoring of the upper layers, the current observing system provision of $\mathrm{T}$ and $\mathrm{S}$ vertical profiles at 5-10 days timescales at a $3^{\circ} \times 3^{\circ}$ resolution in the open ocean, should be adequate to characterize month-tomonth variations in $\mathrm{HC}$ and FWC. Higher spatio-temporal sampling is needed at choke points along the continental slopes and shelf areas in order to accurately monitor boundary currents, ocean-atmosphere buoyancy exchanges hot-spots and the freshwater inflow from continental sources (rivers, sea-ice and icebergs/continental ice-cap interaction along Greenland and the Labrador Sea) and exchanges with other oceans and marginal seas (Indian and Southern Ocean in the Southern Hemisphere, the Mediterranean and Caribbean seas and the Arctic in the Northern Hemisphere). For deeper layers, a yearly to interannual timescale of full depth $\mathrm{T}$ and $\mathrm{S}$ vertical profile should be sufficient for decadal prediction and longer-term climate change detection.

The combined elements of the current Atlantic observing system described above, allow good broadscale characterization of $\mathrm{HC}$ and FWC changes in the upper (especially 0-700 m 

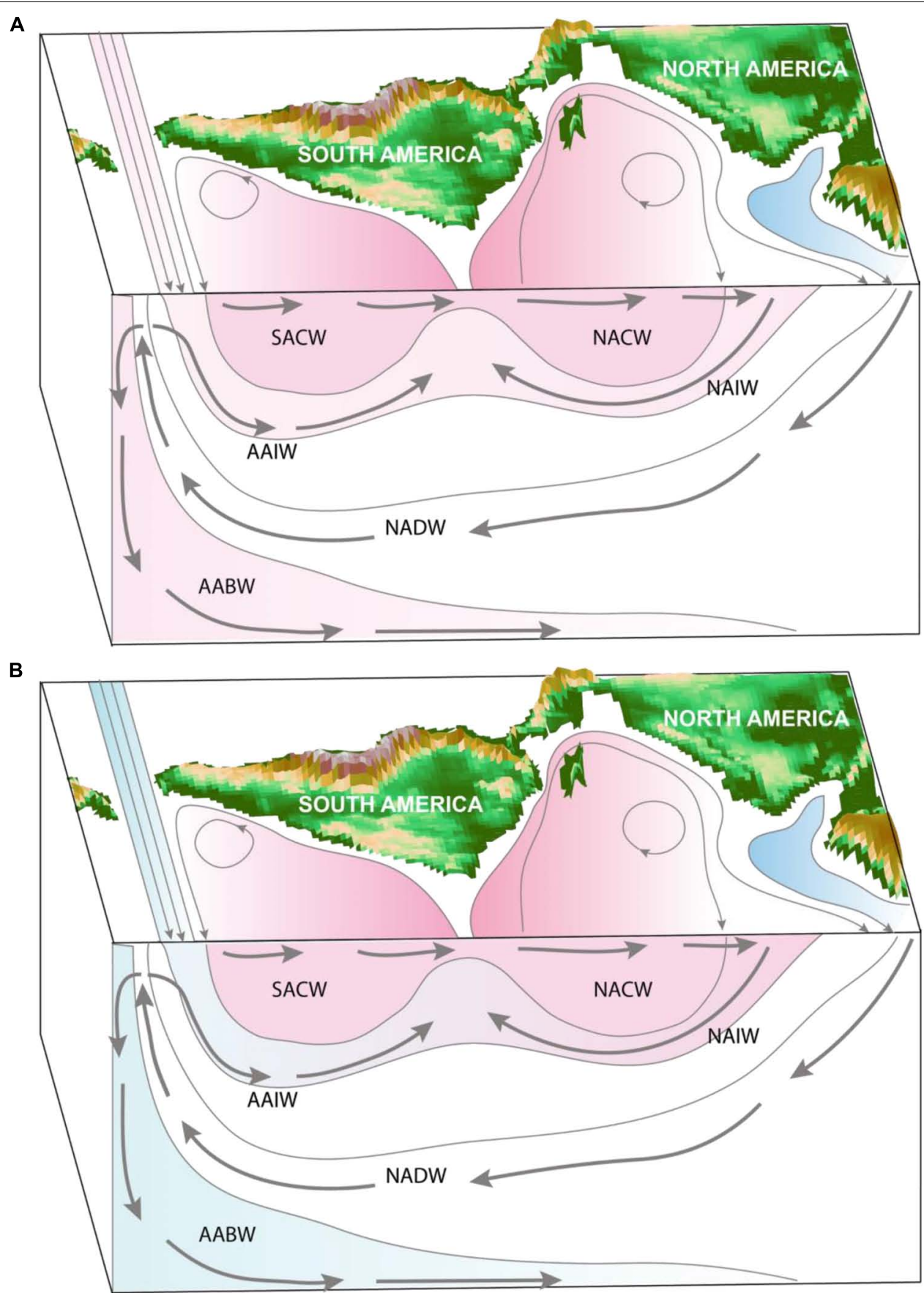

FIGURE 5 | Schematic showing temperature (A) and salinity (B) trends in different layers of the Atlantic Ocean. The layers are defined as main water-masses of the Atlantic Ocean. From the upper layers down to the ocean bottom: South Atlantic Central Water (SACW), North Atlantic Central Water (NACW), Antarctic Intermediate Water (AAIW), North Atlantic Intermediate Water (NACW), North Atlantic Deep Water (NADW), and Antarctic Bottom Water (AABW). Gray arrows show the main overturning pathways in the basin, and the black contours show a vertical slice of the vertical and meridional water masses distribution in terms of potential density. The color shading corresponds to: warming/saltier in red, cooling/freshening in blue, and white where no clear trends are documented.

with XBTs for OHC and down to $2000 \mathrm{~m}$ with Argo for both, HC and FWC) open ocean. However, the upper $1500 \mathrm{~m}$ of continental slopes around the Atlantic basin are undersampled since Argo floats tend to be advected parallel to, or away from, these regions by fast-moving boundary currents.
For the deep layers (below $2000 \mathrm{~m}$ ), the AMOC boundary arrays, boundary current arrays (Todd et al., 2019), choke point OceanSITES moorings (e.g., Vema Channel) and the oceanographic research cruises (incl. GO-SHIP, Sloyan et al., 2019) are, for the time being, the only source of deep $T$ 
and $\mathrm{S}$ data. However, it must be said that, in the Atlantic Ocean, due to the AMOC dedicated mooring arrays and the fact that these moorings need to be serviced, the amount of yearly data at depth exceed the number of data available for other ocean basins.

\section{CASE STUDY: TROPICAL PACIFIC}

The Tropical Pacific Ocean is the global "hot spot" for upper-ocean $\mathrm{HC}$ variability on interannual-to-decadal timescales (e.g., Chen and Tung, 2014). During ENSO events, OHC is redistributed across the basin and between the surface and subsurface layers: the upper 0-100 m layer warms while the 100$500 \mathrm{~m}$ layer cools during an El Nino, and vice versa during La Nina (Roemmich and Gilson, 2011; Roemmich et al., 2015; Johnson and Birnbaum, 2017). Deeper, the short observational time series suggest a weak warming in the intermediate layers (Piecuch and Quinn, 2016; Johnson and Birnbaum, 2017). Figure 6 illustrates schematically the fundamental processes of the coupled ocean-atmosphere system in the Tropical Pacific that links upper-ocean $\mathrm{HC}$ to climate variability and change from interannual-to-decadal and longer time scales.

During El Nino, trade winds weaken or reverse, leading to the deepening of the thermocline in the central and eastern Pacific, to a weakening/suppression of the upwelling, and to an eastward displacement of the warm waters and atmospheric convection. These changes induce a zonal redistribution of the upper oceanic HC from west to east. Schematic arrows indicate the recharge/discharge from the low-latitude western boundary currents (WBCs) and the interior ocean, forced by wind stress curl anomalies, both locally and through Kelvin and Rossby waves propagations.

ENSO-related $\mathrm{HC}$ variability in the $0-100 \mathrm{~m}$ layer is largely compensated by opposing changes in the 100-500 m layer, with generally small changes in the vertically integrated change. Several studies have suggested that the global ocean loses heat during El Nino events and gains heat during La Nina events (Roemmich and Gilson, 2011; Abraham et al., 2013; Rhein et al., 2013; Roemmich et al., 2015). However, a recent study by Piecuch and Quinn (2016) and satellitebased estimates of changes in Earth's energy storage lead to opposite conclusions (Wong et al., 2006; Johnson and Birnbaum, 2017). This emphasizes the need to continue accurate monitoring of the temperature anomalies in sub-thermocline layers in the Tropical Pacific, at least to the depth extent of ENSO-related variations, for constraining the global ocean HC. Such redistributions are also seen at decadal timescales, linked to the phase of the PDO (Pacific Decadal Oscillation) and IPO (interdecadal Pacific Oscillation; Corre et al., 2012; Nieves et al., 2015).

The tropical Pacific is a region where the long-term trend in ocean HC is masked by large interannual-to-decadal variability, and thus long continuous records are needed to separate out the forced response from climate variability. HC trends estimates are therefore subject to large uncertainties, and depend greatly on the time period, and on the depth of the layer considered (e.g., Wang et al., 2018). Recent estimates indicate a weak warming of the upper ocean (0-700 m) during 19712010 (Rhein et al., 2013) and the 1993-2016 (Johnson et al., 2016). Deeper, historical observations are too sparse to establish the relative importance of multi-decadal versus interannual variability. Argo observations of the 700-2000 m layer exhibit a steadier warming than seen in the upper layers (Roemmich et al., 2015), consistent with expectations based on climate model simulations (Palmer and McNeall, 2014).

Adequate monitoring $\mathrm{HC}$ in the Tropical Pacific is fundamental to better constraining variability in the planetary energy budget and for accurate prediction of ENSO events (e.g., Jin, 1997). The "Warm Water Volume" (WWV), (i.e the equatorial Pacific volume warmer than $20^{\circ} \mathrm{C}$; Meinen and McPhaden, 2000), is a useful precursor to El Nino events. The WWV is used in simple statistical ENSO forecasts (McPhaden et al., 2006), forecast center expert assessments, and is a key evaluation metric for coupled forecast systems (e.g., Balmaseda et al., 2014).

The Tropical Pacific also shows substantial interannual-todecadal variability in 0-100 m FWC (Delcroix et al., 2007; Wang et al., 2017). Recently unprecedented FWC anomalies were observed during the 2015 El Nino event (Gasparin and Roemmich, 2016; von Schuckmann et al., 2016), arising from both changes in surface freshwater fluxes and oceanic dynamics. Longer-term freshening of the Tropical Pacific has been attributed to anthropogenic forcing and is related to an intensification of the hydrological cycle (Cravatte et al., 2009; Durack and Wijffels, 2010; Durack et al., 2012; Terray et al., 2012; Rhein et al., 2013). Freshwater changes in the Tropical Pacific have important impacts on local seawater density and ENSO dynamics and contribute to both regional and global sea level change (Wang et al., 2017).

In the tropics, the observational requirements for monitoring $\mathrm{HC}$ and FWC anomalies are less stringent than in other regions: the drivers are mostly deterministic (Sérazin et al., 2017) and the signals generally have long zonal length scales compared to the mid-latitudes (Kessler et al., 1996). These requirements have been discussed in the context of the Tropical Pacific Observing System 2020 (TPOS 2020; Cravatte et al., 2016; Smith et al., 2019). Monitoring of the upper ocean $\mathrm{HC}$ and FWC $(0-300 \mathrm{~m})$ requires temperature and salinity profiles at 5 to 10 -days timescales, $500 \mathrm{~km}$ in the zonal and $200 \mathrm{~km}$ in the meridional directions, with a higher $100 \mathrm{~km}$ meridional resolution in the equatorial band to allow direct estimation of $\mathrm{HC}$ changes and to provide adequate constraints for ocean data assimilation products (Fujii et al., 2015). T and S profiles to $1000 \mathrm{~m}$ at monthly timescales and full-depth measurements at yearly or longer timescales to support decadal prediction and climate monitoring, respectively.

The current Tropical Pacific Observing System (TPOS) capability for measuring $\mathrm{HC}$ and FWC changes has been discussed by Roemmich et al. (2014) and Cravatte et al. (2016). The in situ elements of the TPOS include the Tropical Moored Array (TMA; McPhaden et al., 1998) since the 1980s, the Argo array since the 2000s, XBT lines and some repeat hydrographic measurements. The historical TMA TAO/TRITON array consists of around 70 moorings located at 11 longitudes across the Pacific 


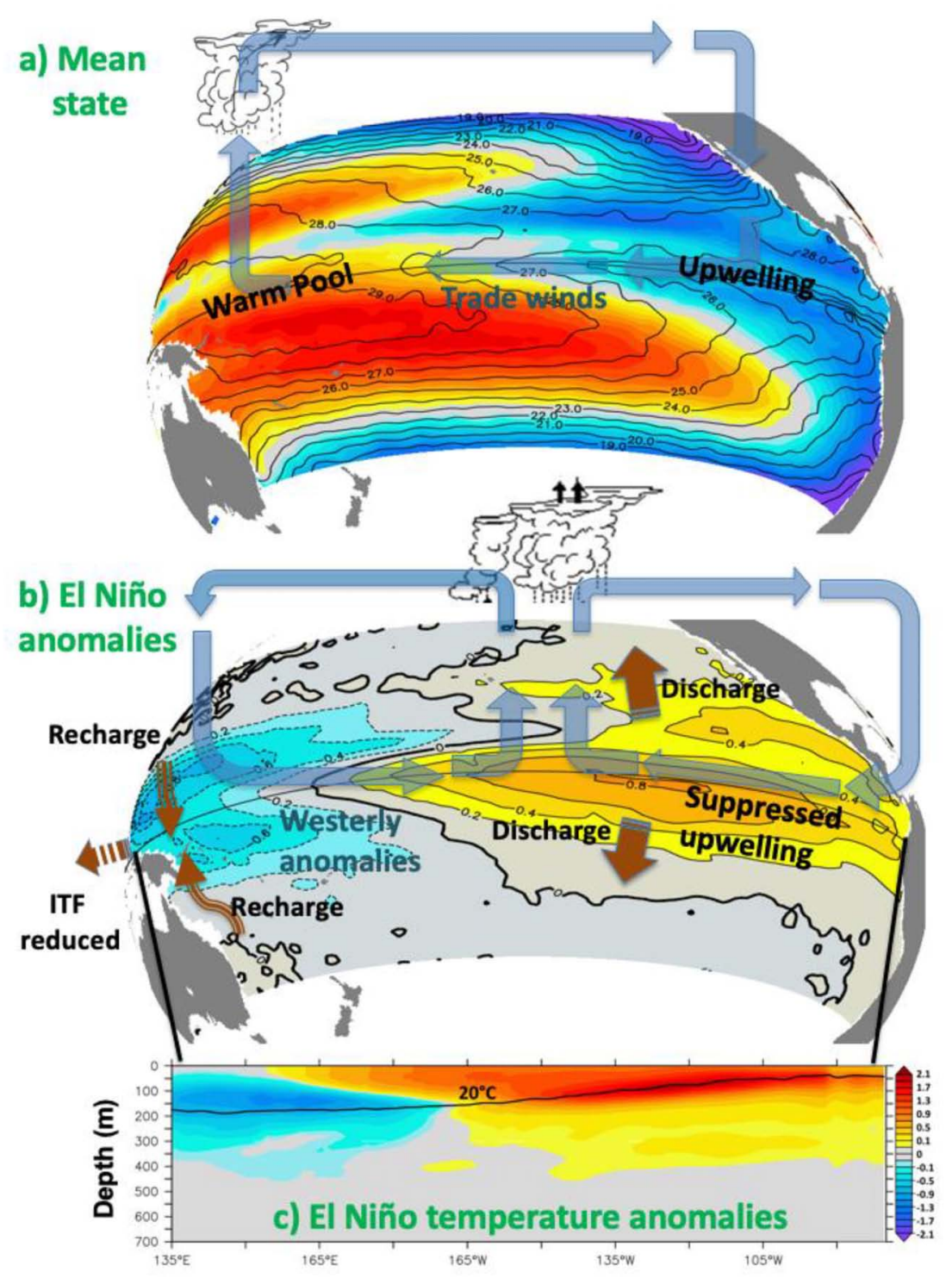

FIGURE 6 | Schematics of the oceanic and atmospheric processes in the mean state (a) and El Nino state (b,c). (a) Mean 0-300 m heat content from Argo gridded product (colors), and mean SST (contours). In the mean state, easterly trade winds produce eastern upwelling, fostering higher sea surface temperatures and atmospheric convection in the west. That in turn strengthens the easterly surface winds in a positive feedback. Upper oceanic heat content is larger in the southwestern and northwestern parts of the basin. (b,c) 0-300 m temperature anomalies regressed onto Nino3.4 index during the $2004-2016$ period, both for the upper 0-300 m layer (b), and for the vertical equatorial section to $700 \mathrm{~m}$ depth (c). The mean position of the $20^{\circ} \mathrm{C}$ isotherm is also shown. ITF $=$ Indonesian Throughflow.

from $137^{\circ} \mathrm{E}$ to $95^{\circ} \mathrm{W}$ and nominally at latitudes of $\pm 8^{\circ}, \pm 5^{\circ}$, $\pm 2^{\circ}$, and the equator. They measure temperature generally at 11 or 12 depths from the surface down to $500 \mathrm{~m}$, or to $700 \mathrm{~m}$ in the west, with coarser resolution below the thermocline. Subsurface salinity is measured from the surface to $500 \mathrm{~m}$ at the same depths in the western Pacific on TRITON moorings, and from the surface to $120 \mathrm{~m}$ at many TAO equatorial moorings. The divergence of Argo floats around the equatorial central and eastern Pacific has been an issue, but the new Iridium floats partly overcome this issue. Other regions where the Argo coverage is limited are the western and eastern boundary regions (Roemmich et al., 2014).

Hydrographic stations have been collected regularly along the TAO longitudes in the eastern equatorial Pacific since 1979, and since the mid 1980s for basin-wide sections, nominally to $1000 \mathrm{~m}$. Unfortunately, this profiles timeseries was interrupted since 2007 due to resourcing issues. Additional full-depth repeat hydrographic stations have been collected along $137^{\circ} \mathrm{E}, 149^{\circ} \mathrm{E}$, $165^{\circ} \mathrm{E}, 170^{\circ} \mathrm{W}$ (in the southern hemisphere only), $150^{\circ} \mathrm{W}$, and $110^{\circ} \mathrm{W}$ at roughly decadal intervals since the 1990s (WOCE 
and GO-SHIP programs). These data are precious to document long-term trends for the deep tropical Pacific HC and FWC.

The $0-300 \mathrm{~m}$ equatorial $\mathrm{HC}$ is well captured since the advent of the moored array in the mid-1980s, when these observations are combined with other hydrographic data (Smith, 1995) ${ }^{1}$. The spread among ocean reanalysis has been drastically reduced (Xue et al., 2017). The development of the Argo array has significantly enhanced the capability to estimate tropical Pacific FWC and to reduce uncertainties in HC. Due to the dominance of thermosteric contribution to sea level variability in the tropical Pacific, altimeter-derived sea level anomalies have also been used to derive proxy estimate of tropical Pacific upper-ocean $\mathrm{HC}$ (Willis et al., 2004; Bunge and Clarke, 2014; Lyman and Johnson, 2014). The combination of XBT, TMA, Argo, and altimetry has enabled longer time series and more accurate estimates of tropical Pacific upper-ocean HC over time. The complementarity of arrays plays an essential role in resolving the required scales effectively (Gasparin et al., 2015; Xue et al., 2017).

Current shortcomings in the TPOS are the limited meridional resolution and poor sampling in the vicinity of the equator, especially in the eastern Pacific where the meridional scales are smaller, the influence of Tropical Instability Waves are important, and where the HC variability in the upper layer is largest. Near the boundaries Argo floats are sparsely distributed, and the estimates of $\mathrm{HC}$ and FWC are subject to larger uncertainties. Altimetry data, especially those from the upcoming SWOT mission, can provide proxy estimates of upper-ocean HC, but cannot distinguish thermosteric from halosteric contributions without the help from in situ measurements. An additional challenge is to understand the tropical Pacific Ocean volume, heat and freshwater budgets, referred to as the "Wyrtki Challenge" (Wyrtki, 1981; Cravatte et al., 2016). The ability of the observing system to characterize the horizontal convergences, vertical fluxes and surface fluxes is yet to be established. The analysis of highresolution ocean or coupled ocean-atmosphere models and data assimilation products can help shed light on the requirements.

\section{CASE STUDY: ARCTIC OCEAN}

The HC and FWC transiting the Arctic Ocean is of prime importance not only for region but also for the global ocean and climate. The large amount of river input $(11 \%$ of the world's continental runoff; Fichot et al., 2013), low-salinity water from the Pacific and ice-ocean coupled processes lead to a very strong halocline overlying the relatively warm waters of Atlantic origin below. This practically isolates most of that heat from the surface and therefore prevents heat release to the atmosphere and melting of sea-ice (e.g., Rudels, 2009). Only in the inflow regions from the Atlantic and Pacific sides, through the Barents Sea and Fram Strait as well as through the Bering Strait, do the relatively warm water masses near the surface interact with sea-ice and release heat to the atmosphere. On a larger scale, the large amounts of FWC passing the Arctic lead to a large reservoir in the Beaufort Gyre but also across much of the

${ }^{1}$ https://www.pmel.noaa.gov/elnino/upper-ocean-heat-content-and-enso central Arctic. The release of this FWC to the North Atlantic is mostly driven by changes in atmospheric pressure leading to varying surface stress on the ocean and resulting Ekman transports/pumping. FWC input from the Arctic to the North Atlantic has the potential to strongly influence the subpolar gyre circulation (Brauch and Gerdes, 2005) and deep convection (e.g., Koenigk et al., 2007). On the other hand, FWC variability in the Arctic largely controls changes in the steric SSH leading to variability in the upper ocean circulation. A recent study shows that the FWC in the Arctic Ocean is characterized by a remarkable increase since the mid 1990s strongly linked to sea ice volume variability (Garric et al., 2018). Finally, the warm waters of Atlantic origin are slowly modified in passing the Arctic Ocean, with a potential to influence the overflow across the Denmark Strait sill into the NADW (Karcher et al., 2011). Changes in basin-wide ocean HC during the past two decades show considerable increase, but estimates have still large uncertainties due to insufficient sampling (Mayer et al., 2016; von Schuckmann et al., 2018).

Observations of salinity in the upper few $100 \mathrm{~m}$ of the Arctic Ocean basins by various platforms since the early 1990s and intensified use of autonomous ice-tethered systems (ITP) since about 2004 have allowed to robustly quantify the liquid FWC variability on decadal timescales (Rabe et al., 2014). In addition, year-round observations across the basins now allow studying winter ice formation and convection processes in relation to vertical heat fluxes from the warm water of Atlantic origin to the surface (Polyakov et al., 2013). However, ITP still leave temporary gaps in different parts of the Arctic. This is due to the deployments upstream in the Transpolar Drift, north of the Siberian shelves, being tied to icebreaker expeditions in summer/early autumn. These ITP then drift to north of $85^{\circ}$ $\mathrm{N}$ by April and leave a gap until late summer to the south. In addition, buoys only drift to the region north of Greenland by chance. Hence, it remains an unsolved challenge to reliably quantify a full seasonal cycle and interannual variability of the Arctic basin-wide liquid FWC. To tackle this issue, it is necessary to bring additional, newly innovated technology into the Arctic. The future use of Argo-style drifting profilers (floats) as well as advanced seafloor-moored systems, measuring from the warm Atlantic Water layer across the whole halocline and well into the mixed-layer should cover many of these spatial and temporal gaps. This could include floats operating a reliable ice detection sensor and/or used with an appropriate algorithm, supported by underwater sound sources (positions), as well as mooring systems with winches for year-round upper ocean measurements. The continuation of observing programs such as the Beaufort Gyre Project/Ice-tethered profiler (United States) ${ }^{2}$, FRontiers in Arctic-marine Monitoring (FRAM; Germany ${ }^{3}$ ) alongside the introduction of new technology are vital to sustain and enhance Arctic upper ocean observations of $\mathrm{HC}$ and FWC in future years. Furthermore, coordinated, extended observations on the shelves, near the continental slope and across the gateways

\footnotetext{
${ }^{2}$ http://www.whoi.edu/itp/

${ }^{3}$ https://www.awi.de/en/science/biosciences/deep-sea-ecology-and-technology/ observatories/fram-ocean-observing-system.html
} 
are vital to determine transports in-/out of the Arctic Ocean basins and to close the budget, as current surface flux products are highly uncertain (Haine et al., 2015). In addition, the continued use of research icebreakers to obtain high-quality, full-depth profiles of temperature and salinity across the Arctic Ocean basins is paramount to measure variability in the deep ocean and to ensure adequate calibration/quality-control of the autonomous systems. Estimating sea-ice volume, representing the solid part of liquid FWC, has been facilitated since about 2011 by various satellite missions (e.g., Cryosat-2), that allow the determination of seasonal near-surface changes (largely icemelt/freeze) and interannual variability (Armitage et al., 2016; Ricker et al., 2016). The differences between SSH from altimetry and ocean bottom pressure (OBP) from gravimetry, i.e., depthintegrated steric height that is dominated by FWC changes in the Arctic Ocean, have provided the capability to monitor broadscale FWC changes in the Arctic Ocean (e.g., Morison et al., 2012). SSS from the L-band SMOS and SMAP satellites, with better spatiotemporal sampling then the satellite-derived SSHOBP, have the potential to complement satellite SSH-OBP and in situ measurements to study Arctic FWC changes (e.g., Tang et al., 2018). However, improvement in retrievals and innovation in technology (e.g., multi-frequency sensors as opposed to be L-band only sensors) are necessary to reduce the uncertainty of satellite SSS in the Arctic Ocean to facilitate the studies of Arctic Ocean FWC changes.

One of the most important consequences of human-caused climate warming is rising sea levels, and although thermal expansion is a significant component of sea level rise the largest potential sea level threat comes from ice lost from the Greenland and Antarctic Ice Sheet. Greenland alone contains enough ice to raise sea levels by more than $7 \mathrm{~m}$, and recent work has shown that warmer waters and higher $\mathrm{HC}$ on the continental shelves surrounding Greenland may drive enhanced ice loss at the margins (Holland et al., 2008; Straneo and Heimbach, 2013; Rignot et al., 2016; Truffer and Motyka, 2016).

On the continental shelf, a shallow layer of fresh, cold water of Polar origin to overlay a layer of warm, salty Atlantic Water (Figure 7). At depths greater than about $150 \mathrm{~m}$, and with temperatures ranging from 1 to $6^{\circ} \mathrm{C}$, Atlantic Water can interact directly with glaciers that terminate in waters deeper than about $200 \mathrm{~m}$. The presence of this water enhances melt at the glacier face, which can increase calving and result in retreat and acceleration of glacier mass loss, driving sea levels higher. Because the Atlantic water usually sits more than $100 \mathrm{~m}$ beneath the ocean surface, remote sensing techniques are not effective in detecting it, and in situ observations are required to monitor Atlantic Water on the shelf.

Despite its importance, there is no planned or existing observing system to measure the volume and extent of warm, salty Atlantic Water on the shelves surrounding Greenland. As a result, ocean forcing of ice loss from Greenland's more than 200 marine terminating glaciers may go unmeasured in the decades to come. NASA has undertaken a 5-year airborne campaign called Oceans Melting Greenland (OMG) that is designed to fill this gap from 2016 to 2020 (Fenty et al., 2016). Once per year surveys of temperature and salinity profiles with approximately $50 \mathrm{~km}$

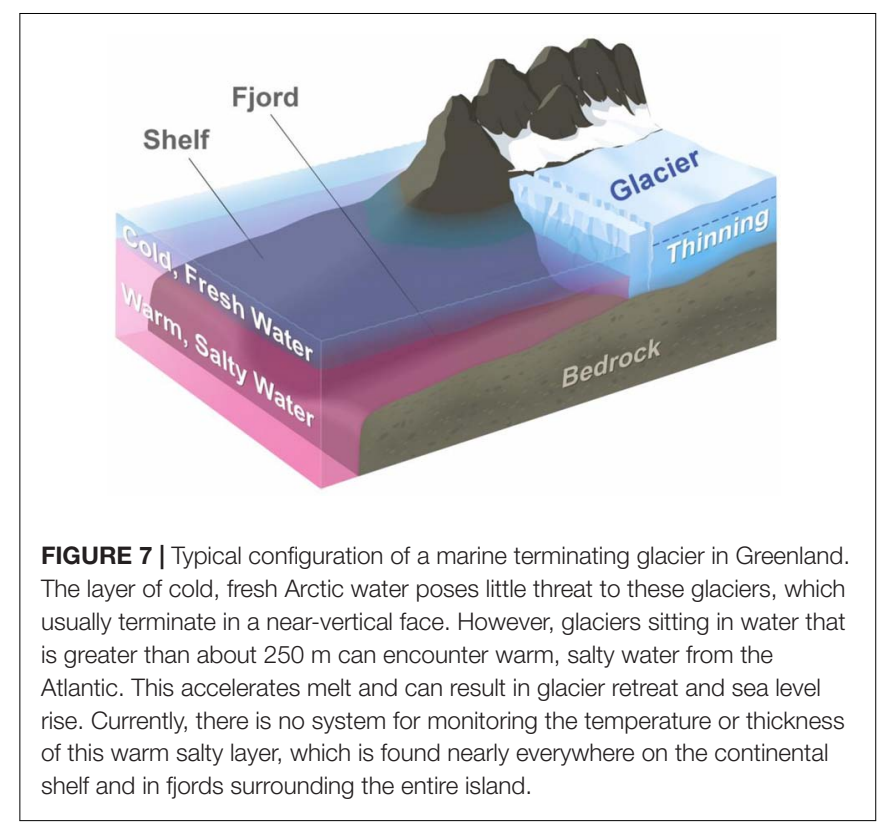

spatial resolution are carried out as a part of OMG, with enhanced sampling in deep troughs and depressions on the shelf. While short-term variability clearly exists in many places on the shelves and in glacial fjords, interannual increases in the amount and temperature of warm water on the shelf have been suggested as a driver of enhanced ice loss from the glaciers (Holland et al., 2008; Straneo and Heimbach, 2013).

This suggests that a broad-scale survey of ocean temperatures and salinities on the continental shelf may be necessary, at least in key regions like southeast and northwest Greenland where oceanice interactions are thought to be most important. Such a survey would have to augment existing efforts to monitor key glaciers and straights with moorings and other in situ instruments. A combination of ship-surveys, gliders and autonomous floats (such as the air-deployable Alamo floats) may be necessary to cover key regions effectively.

\section{CASE STUDY: SOUTHERN OCEAN}

The Southern Ocean is an essential piece of the global ocean heat uptake, Earth Energy Imbalance and global warming, and is associated with some of the world's ocean largest deep-reaching freshwater storage changes (e.g., Durack and Wijffels, 2010; Durack et al., 2012; Rhein et al., 2013; Roemmich et al., 2015). Its complex circulation connecting most of the world's ocean deep water-masses to the sea surface makes it a unique place on Earth for the transport and storage of heat and freshwater anomalies to great depths where such anomalies are then stored for decades to millennia (DeVries et al., 2011).

In stark contrast with its pivotal role for the global $\mathrm{HC}$ and FWC (and other tracers such as carbon) budgets, the Southern Ocean has remained poorly observed compared to other ocean basins (Sallée, 2018). Specific challenges keep the observation system from growing as fast as in other basins: the difficulty 
of access to these regions, the vastness of the ocean basin, and presence of sea-ice in large part of the domain, which makes ship-based observation complex and expensive, prevents satellite-based observation of the ocean surface, as well as satellite communication with autonomous platforms. Ship-based observations of heat and salt remain infrequent and seasonally biased, being largely limited to quasi-decadal summer repeats of a few repeat hydrographic sections. Nevertheless, the development of the international Argo Program has revolutionized the sampling of the upper $2000 \mathrm{~m}$ of the Southern Ocean. Argo observations have no seasonal biases, but they are still sparse in the region poleward of the Antarctic Circumpolar Current, where sea-ice cover is a challenge for their survival and for data recovery. Moreover, Argo coverage does not yet include the deep seas below $2000 \mathrm{~m}$. The current development of networks of ice-capable Argo floats (Klatt et al., 2007), and Deep-Argo floats promises great advances in the sampling of the Southern Ocean in the coming decades. In addition, the development of observations from animal-born sensors have considerably increased the sampling of the upper ocean (upper $500 \mathrm{~m}$ ) under sea-ice over the last decade (Treasure et al., 2017), but the accuracy of such observations is still limiting their usefulness in studies seeking to detect subtle long-term change.

Despite these sampling challenges, the current observation system has allowed documenting clear and significant changes $\mathrm{HC}$ and FWC changes in the Southern Ocean. It is estimated that the Southern Ocean has accounted for $30-50 \%$ of the 0-700 m ocean HC change since 1970 (Smith and Murphy, 2007; Domingues et al., 2008; Ishii and Kimoto, 2009), while the Southern Ocean only represents $30 \%$ of the world's ocean surface. In addition, recent work suggests that the Southern Ocean heat storage in these estimates might be biased low as a result of the sparse sampling of the Southern Hemisphere, and it is hypothesized it may instead represent around $60 \%$ of the global ocean HC trend since 1970 (Durack et al., 2014). Estimates in the most recent period, which is better observed by autonomous profiling floats, suggest that the Southern Ocean contribution to the global $0-2000 \mathrm{~m}$ HC climbs to $67-98 \%$ for the period 2006-2013, with a clear peak in the latitude band of the Antarctic Circumpolar Current or north of it $\left(30-50^{\circ} \mathrm{S}\right.$; Figure 3C; Roemmich et al., 2015; Llovel and Terray, 2016; Durack et al., 2018; Sallée, 2018).

The significant HC of the Southern Ocean is, however, spatially inhomogeneous (Sallée, 2018). In particular, the surface layer of subpolar seas stands out as a region where temperatures are relatively stable, or even slightly cooling (e.g., Armour et al., 2016; Sallée, 2018; Figure 8), and associated with a marked freshening (Durack and Wijffels, 2010; Rhein et al., 2013; de Lavergne et al., 2014). The freshening trend might be associated to a regime shift in sea-ice growth/melt (Haumann et al., 2016), and accelerated melt of the Antarctic ice-sheet (Schmidtko et al., 2014), leading to an increase of the stratification, isolating surface waters from the relatively warmer underlying waters (Sallée, 2018). This increased stratification may possibly lead to an increase HC accumulation in the Circumpolar Deep Water layer, directly below the surface layer in the subpolar seas (Lecomte et al., 2017). However, the processes associated with temperature and salinity changes in the subpolar seas remains difficult to disentangle given the short and incomplete nature of the observed time-series.

The delayed warming of the subpolar seas creates a disequilibrium with the warming atmosphere, which tends to make the subpolar seas a region of large HC uptake (Frölicher et al., 2015). The heat taken up in the subpolar seas is then transported northward and accumulate within and north of the Antarctic Circumpolar Current, in the first $1 \mathrm{~km}$ of the water column which is well ventilated (Mode and Intermediate Waters; Figure 8). Strong warming at a rate of $\sim 0.2^{\circ} \mathrm{C} /$ decade is observed in this region of the Southern Ocean (Böning et al., 2008; Gille, 2008; Giglio and Johnson, 2017). Similarly, the freshening trend observed in the subpolar seas, propagates with the northward and downward circulation, within the Antarctic Circumpolar Current, in the AAIWs ventilated in the first $1 \mathrm{~km}$ of the water column (Figure 8; Durack and Wijffels, 2010).

The abysses of the Southern Ocean are also associated with significant warming and freshening trends of order of $0.05^{\circ} \mathrm{C} /$ decade, and 0.001-0.005 PSS-78/decade (Purkey and Johnson, 2012, 2013; Shimada et al., 2012; Jullion et al., 2013; van Wijk and Rintoul, 2014). However, these regions are the least well observed, and it remains hard to distinguished between natural and forced variability. It is however likely that these trends are explained by changes of ocean properties in the Antarctic coastal region, where bottom water forms (Jacobs and Giulivi, 2010; Masuda et al., 2010; Johnson et al., 2014; van Wijk and Rintoul, 2014).

Potential change of the circulation might also impact Southern Ocean HC and FWC. Several studies have discussed the possibility of an acceleration of the upper meridional overturning circulation as a results of increase winds (Southern Annular Mode), with increased volume of mid-depth waters (CDW) upwelled south of the Antarctic Circumpolar Current, and increased volume of water subducted north of the Antarctic Circumpolar Current (Le Quéré et al., 2009; Waugh et al., 2013; DeVries et al., 2017). If such change of the circulation is still debated, it would increase even more Southern Ocean HC and FWC accumulation in the upper ventilated layers (mode and intermediate waters; Figure 8). Other studies have discussed the possibility of a change in the ventilation of the abysses, caused by increased stratification due to freshening of the surface ocean in the subpolar seas. While still unclear, oxygen observations tend to argue for a persisting ventilation of the abysses (van Wijk and Rintoul, 2014). However, the short and incomplete nature of existing time series still makes the causes and consequences of the observed HC and FWC changes difficult to assess.

The deployment of the Southern Ocean Argo array in 2004 revolutionized Southern Ocean research. This array has revealed the changes discussed above, which are occurring across all distinct dynamical regimes of the Southern Ocean. Yet challenges attributing the causes of these changes remain. Remoteness and ice-cover have resulted in significant observational gaps in the subpolar regions. Meanwhile strong interannual variability combined with high-levels of internal variability occurring on short spatial scales reduce the confidence in some diagnosed trends. Continued and expanded coverage over the coming 


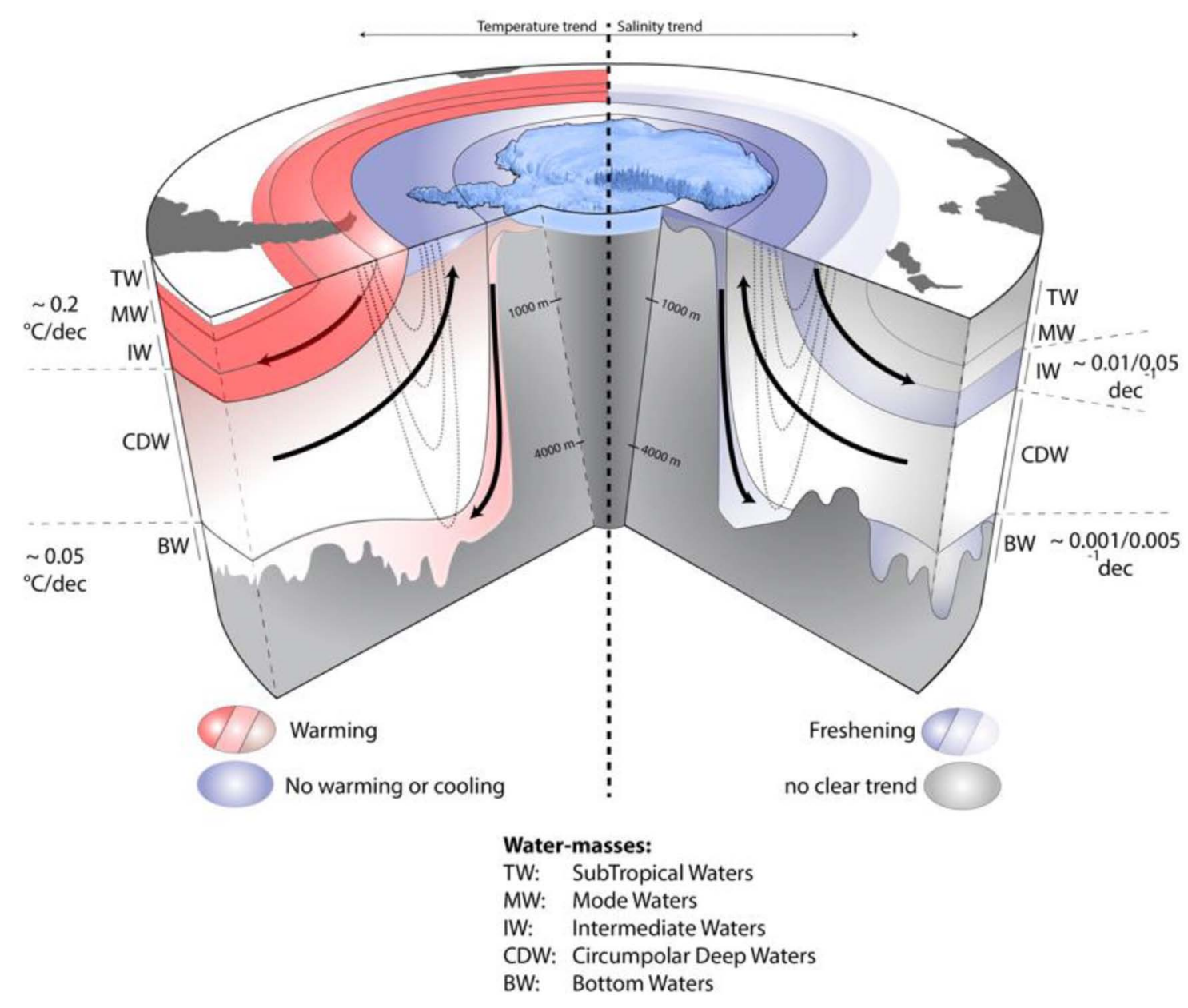

FIGURE 8 | Schematic showing temperature and salinity trends in different layers of the Southern Ocean. The layers are defined as main water-masses of the Southern Ocean: subtropical water (TW), mode water (MW), intermediate water (IW), circumpolar deep water (CDW), and bottom water (BW). Black arrows show the main overturning pathways in the basin, and the dashed black contours show a vertical slice of the deep-reaching Antarctic Circumpolar Current circulating clockwise around the Antarctic continent. Adapted from Sallée (2018, Figure 1).

decades will allow better quantification of the trends and attribution to their causes, which is a priority for documenting and understanding global ocean HC and FWC.

\section{DISCUSSION}

Evaluation of the potential spatiotemporal characteristics of the emergent climate change signals is a key element in the assessment of the adequacy of the ocean observing system. While the emergent patterns of climate change are uncertain (both in terms of spatial pattern and magnitude), they are associated with a large-scale coherent ocean responses and features that typically extend over several degrees of longitude/latitude or more (see section "Projected Changes in Ocean Heat and Freshwater Content"). In this regard, the nominal $3^{\circ} \times 3^{\circ}$ sampling of Argo should be sufficient to capture these emergent signals in the upper $2000 \mathrm{~m}$ ocean. In addition to the surface-intensified upper ocean warming, model simulations, observations and theory all support the expectation of a deep and bottom-intensified signature of ocean climate change associated with deep and bottom water formation and transport processes. A recent observing design study for a deep Argo array (based on high quality repeat ship sections from the GO-SHIP observing network) to monitor basin-scale deep ocean temperature trends suggests that a $5^{\circ} \times 5^{\circ}$ spatial sampling and 15 -day repeat cycle should be sufficient (Johnson et al., 2015). Likewise, observational data of adequate quality can be acquired with moored instrumentation (OceanSITES observing network). For the deep ocean, given the absence of a large amplitude seasonal cycle and wave signals (White, 1995), a spatially homogenous grid is not mandatory and a multiplatform observing approach is feasible.

In addition to the persistent anthropogenic greenhouse gas forcing, we know from historical observations that the total radiative forcing has been punctuated by the short-lived cooling effects of major volcanic eruptions (Figure 9). Future volcanic eruptions will no doubt play an important role in the future climate change and quantifying their effects on the Earth system 


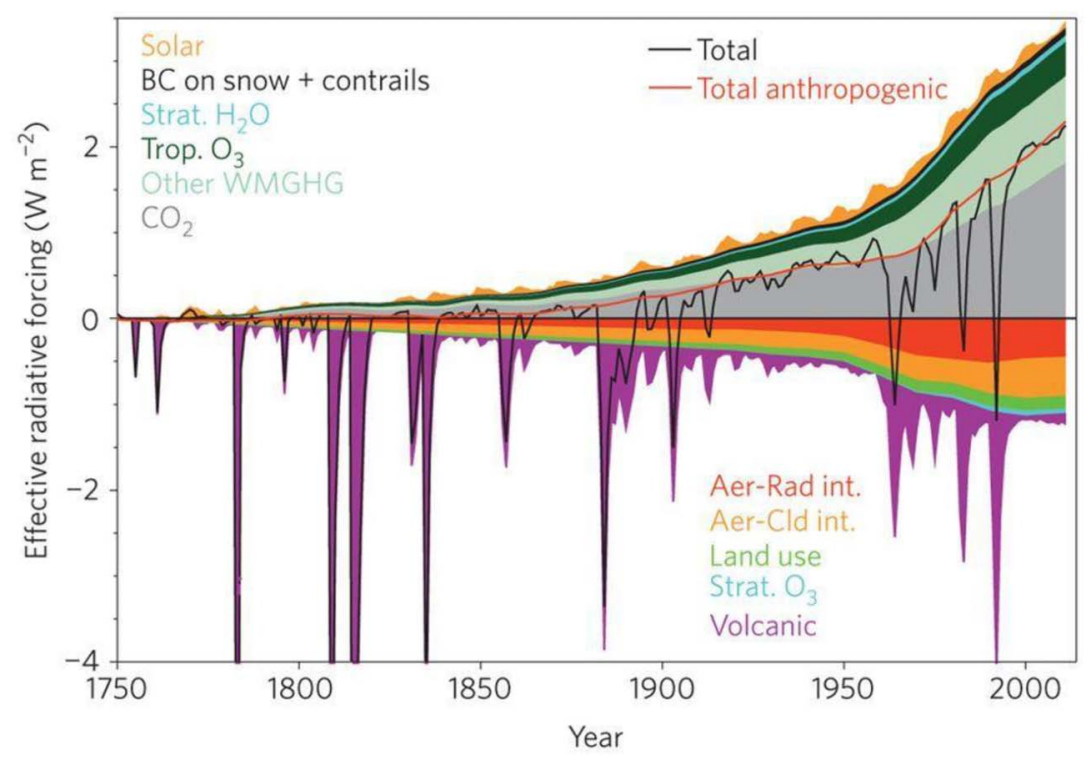

FIGURE 9 | An estimate of the radiative forcing of the climate system since 1750, including both natural (solar and volcanic) and anthropogenic climate forcings. Aer-Rad int. = aerosol-radiation interaction; Aer-Cld int. = aerosol-cloud interaction; BC = black carbon; Strat. = stratospheric; Trop. = tropospheric;

WMGHG = well-mixed greenhouse gases. Reproduced from von Schuckmann et al. (2016, Figure 1).

is an important element of our understanding of radiative forcing. The strong negative radiative forcing of a major volcanic eruption has an associated timescale of 1-2 years and has a distinct signature in ocean warming (e.g., Gleckler et al., 2016). While satellite-based sensors are best placed to capture the magnitude of volcanic radiative forcings, quantifying the ocean threedimensional response requires resolving the spatial patterns of regional ocean $\mathrm{HC}$ and FWC on sub-annual timescales.

The operational oceanography community has been particularly active in assessing the utility of the contemporary ocean observing system using a variety of ocean modeling and data assimilation systems (e.g., Oke et al., 2015; Gasparin et al., 2019). However, these efforts are often focused on short time-horizons (e.g., 1-2 years) and therefore can provide limited insights into the observing system capability for constraining longer-term climate variability and change. Climate and ocean model simulations have the potential to offer insights into observing system capability on longer time horizons. One such approach is by extracting "synthetic profiles" from these model simulations, i.e., model data with the same sampling characteristics as the real observations, in order to assess our ability to constrain both integrated quantities (such as global HC) and spatial patterns of change (e.g., Allison et al., 2019). The main innovation in this approach compared to previous efforts (e.g., Gregory et al., 2004; AchutaRao et al., 2006, 2007) is that the synthetic data can be ingested directly into the same mapping methods as used on the real observations and assessed by comparison with model "truth" fields. This type of approach may add substantial value to observation-based observing system assessments, particularly when considering future observing strategies in the presence of climate variability and change. Coordinated multi-system experiments are required to promote robust findings and used alongside analyses of observations and theoretical considerations, with full cognizance of any model biases limitations of model physics.

The challenge of funding and maintaining the ocean observing system has been discussed for decades, with mixed progress in establishing persistent funding sources and assigning custodial responsibilities (e.g., Baker et al., 2007; Wunsch et al., 2013; Wunsch, 2016; National Academies of Sciences Engineering and Medicine [NASEM], 2017; Weller et al., 2019). It is a human imperative, that these observing networks are protected, maintained and augmented, with increasing pressures accumulating on their continuation (e.g., Durack et al., 2016).

Sparsity of measurement coverage poses a primary limitation to improving our observed understanding, and validating and improving model representations of the physical world. Observational coverage becomes more valuable the longer, more complete, and more accurate the measurements become, with multi-platform, overlapping and complementary coverage the ultimate aim for any observing array. The Argo program has provided a step change that has turbo-charged progress and considerably improved our ability to monitor, understand, simulate and validate the physical world in respect to ocean heat and freshwater variability in the upper $2000 \mathrm{~m}$.

While great progress in physical ocean observing have been made in the last decades, sponsor agency priorities and funding pressures ensure that the observing array remains on tenterhooks. The success of the core physical Argo Program (see Roemmich et al., 2019), and the 30 contributing countries, has led to additional programs such as biogeochemical Argo which was initiated in 2012, however for the most part the funding sources of these networks primarily originate from identical agencies leading to competition for maintenance and 


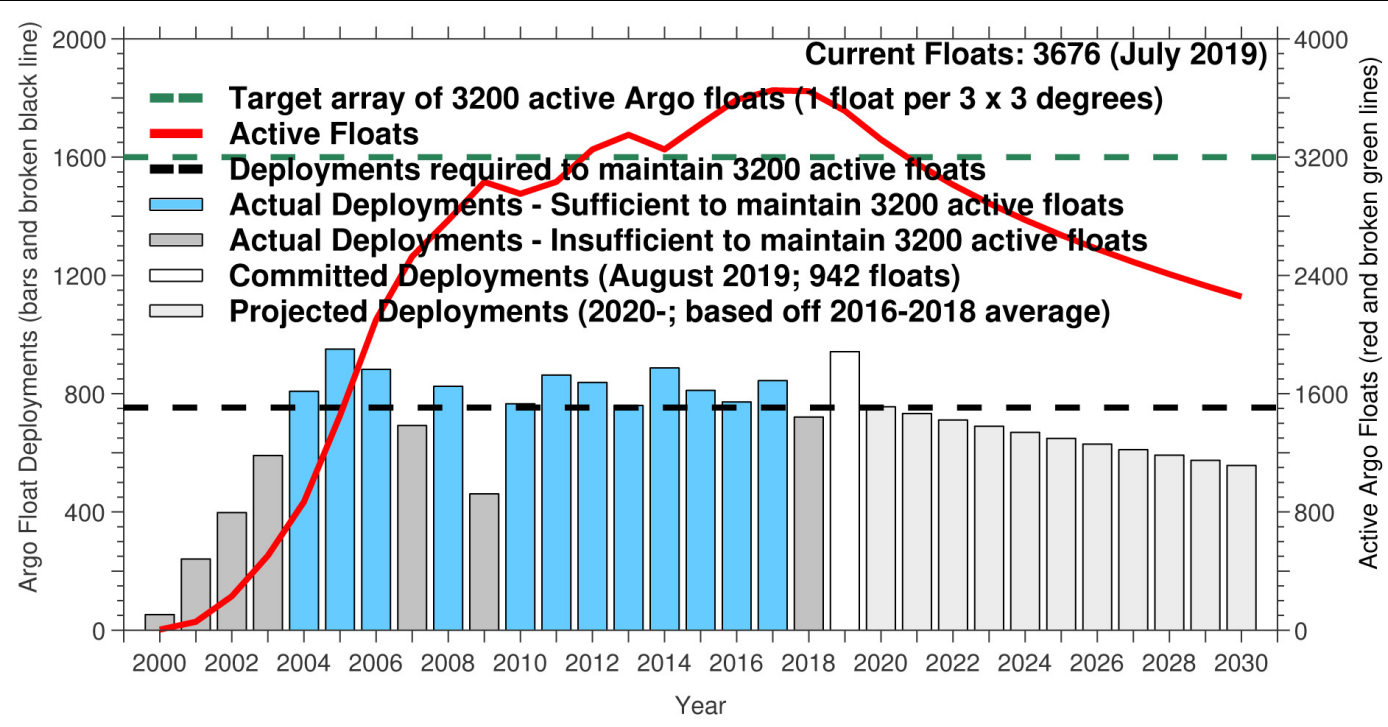

FIGURE 10 | The time history of Argo Programme deployments since 2000, and float coverage projections into the future assuming a business as usual funding scenario across the 30 partner countries. The broken green line shows the target 3200 active Argo float (right $y$-axis), since 2010 this target was met, but deployments dropped below this value in 2018. The broken black lines show the $\sim 800$ float annual deployments required (left $y$-axis) to maintain the 3200 -strong array. If additional deployments are not prioritized, the array will start falling beneath the deployments required to maintain the 3200 -strong global target array in 2021, with a continuing degradation of the array from 2022 onward (based off the average deployments from the last 3 years 2016-2018; updated from Durack et al., 2016). The expansion of the program to include BioArgo and Deep Argo needs to be prioritized in a way that preserves the physical observing capability of the core array.

augmentation of core Argo. There is a growing acknowledgment that the marine biological community needs to pay attention to the physical drivers of the system (e.g., Bates et al., 2018), however funding challenges remain while agency and country budgets are constrained, and the coordination of the physical and biological communities takes considerable work. In addition, most funding for the physical Argo network comes from fluctuating and competitive research-based grants, which are difficult to predict and maintain year-to-year, and consequently the near-term outlook for Argo core coverage is a significant cause for concern (see Figure 10).

The situation is similar with GO-SHIP, the ship-based observing network. Currently 10 nations share responsibility for the 62 repeat transects covering the global ocean (Sloyan et al., 2019). GO-SHIP is focused on increasing the number of participating nations to maintain the program due to the continued pressure by the realignment of national research priorities of the partner nations. While the current decadal survey is planned up to 2023, fewer cruises are committed when contrasted with previous decades. This is particularly concerning as Deep Argo spins up, because GO-SHIP measurements are the only source of the key deep observations that allow calibration drift of the remote sensors to be evaluated. Without GO-SHIP measurements, it will not be clear whether a large change in deep ocean temperature and/or salinity recorded by a remote Argo float is real or whether a problem with the developing equipment, with cross-platform consistency checks an imperative to ensure accuracy and utility of the observing network.

Stresses on marine ecosystems are likely to increase into the future, with a recent works suggesting that marine heatwaves are becoming more prevalent and intense and this trend is expected to continue into the future (e.g., Frolicher et al., 2018) alongside their terrestrial counterparts (e.g., Perkins, 2015). For this reason, our future observing array must be capable of addressing relevant questions now, as well as those questions which may be posed into the future.

\section{RECOMMENDATIONS}

- It is essential that the core physical (temperature and salinity) Argo array of autonomous profiling floats be maintained at current levels to preserve the $3 \times 3$ degree near-global coverage and monitor heat and freshwater changes in the 0-2000 m open ocean. Current evidence suggests this sampling density provides nearglobal coverage at the monthly frequency which will capture emergent climate change signals and provide initial conditions for seasonal-to-decadal prediction systems. Any expansion of Argo to observe deep, biogeochemical or other ocean properties must occur whilst preserving this core physical array.

- It is essential that the full-depth hydrographic sections sampled by GO-SHIP are maintained and augmented, enabling a linkage between physical ocean variables to biogeochemical and ecosystem changes, and provide key calibration data for emerging deep autonomous observations.

- It is essential that mooring arrays such as the AMOC, and the tropical Pacific TAO-TRITON as well as moorings 
that provide global ocean circulation "choke point" measurements (e.g., Indonesian Throughflow/ITF, Arctic gateways) be preserved to provide insights on interbasin transports, along with estimates of heat and freshwater variability and the ability to close regional and global budgets.

- Satellite measurements of sea-surface height, ocean mass, sea surface temperature and sea surface salinity must be maintained in order to monitor and understand the origins of regional sea level variability and change driven by changing ocean heat and freshwater.

- In order to promote combined observational and model analysis, the international research community should continue to improve data interoperability through implementing data standards (e.g., CF conventions), and supporting current and future host platforms such as the Earth System Grid Federation (ESGF $\left.{ }^{4}\right)$ and the observations for Model Intercomparisons Project (obs $4 \mathrm{MIPs}^{5}$ ). In addition, data streams should be implemented to be near real-time (similar to Argo) to facilitate data usage by seasonal and decadal forecasting activities.

- It is essential that the Deep Argo Program (>2000 m) be developed and implemented in coordination with core physical Argo, and GO-SHIP (which provides deep measurement calibration). The Southern and Atlantic Oceans are highlighted in model and observational analyses, as the regions where signals will first emerge and so deployments should be prioritized for these regions.

- Coordinated efforts are required to sample regional seas, continental shelves, near-coastal and subpolar regions to elucidate terrestrial-ocean interactions and allow global budget closure for heat and freshwater. In particular a focus on the upper $1500 \mathrm{~m}$ of continental slopes is required to constrain AMOC estimates, and better categorizing WBCs $\mathrm{HC}$ and variability, as well as the large air-sea interactions that occur in these regions. To progress this agenda we call for a dedicated working group to be formed to facilitate developed and developing nations to achieve these goals in their corresponding regions.

- Targeted observational campaigns are needed to promote understanding of the ocean forcing of ice mass loss from the polar ice sheets (Greenland and Antarctica), which represent a key uncertainty in projections of future global and regional sea level rise.

- Understanding ocean forcing of Greenland and Antarctica requires additional surveys of temperature and salinity on the continental shelves. A combination of airborne and ship-based surveys, gliders and autonomous floats (such as the air-deployable Alamo floats) may be necessary to cover key regions effectively.

- In the Arctic Ocean, additional observations are required to improve monitoring of the seasonal cycle and interannual variability in liquid FWC. These observations

${ }^{4}$ https://esgf.llnl.gov/

${ }^{5}$ https://esgf-node.llnl.gov/projects/obs4mips/ should exploit emerging technologies, such as ITP, drifting profilers and seafloor-moored systems. The conditions under which each of these systems can operate dictate the use of different modern instrumentation in conjunction with traditional CTD surveys carried out by research icebreakers.

- Improvements in satellite retrieval algorithms and innovation in technology (e.g., multi-frequency sensors as opposed to be L-band only sensors) are necessary to reduce the uncertainty of satellite sea surface salinity in the Arctic Ocean to facilitate the studies of Arctic Ocean freshwater changes.

- Ocean and climate model simulations provide an opportunity to test and prototype proposed changes to the observing system, and to set priorities for future network development. The use of model-derived "synthetic observations" is a promising approach that should be further exploited.

\section{AUTHOR CONTRIBUTIONS}

MP and PD led the writing of the manuscript. $\mathrm{KH}$ led the coordination of group discussions and teleconferences during the writing process. All authors made contributions via discussions or by writing text and figures.

\section{FUNDING}

MP was supported by the Met Office Hadley Centre Climate Programme funded by the BEIS and Defra, and the European Union's Horizon 2020 Research and Innovation Program under grant Agreement No. 633211 (AtlantOS). The work of PD was prepared the by Lawrence Livermore National Laboratory (LLNL) under Contract DE-AC52-07NA27344 and is a contribution to the U.S. Department of Energy, Office of Science, Climate and Environmental Sciences Division, Regional and Global Modeling and Analysis Program. LLNL Release number: LLNL-JRNL-761158. BS and JC was partially supported by the Centre for Southern Hemisphere Oceans Research, a joint research center between the QNLM and the CSIRO. BS was also supported by the Australian Government Department of the Environment and CSIRO through the National Environmental Science Program. SC was supported by the IRD and by the French national program LEFE/INSU. SC thanks W. Kessler for suggestions concerning Figure 6. BR was supported by the German Alfred-Wegener-Institut Helmholtz-Zentrum für Polar-und Meeresforschung (AWI). J-BS was supported by the CNRS/INSU and the Horizon 2020 Research and Innovation Program under Grant Agreement 637770. SS was supported by the French Institutions ENS, LMD, IPSL, and CNRS/INSU. The work of JW was performed in part at the Jet Propulsion Laboratory, California Institute of Technology, under contract with the National Aeronautics and Space Administration. 


\section{ACKNOWLEDGMENTS}

This manuscript contributes to an ongoing GCOS-GOOSWCRP Ocean Observations Physics and Climate Panel led review of the Observing System for tracking Ocean Heat and Freshwater Content in support of the Global Climate Observing System (GCOS) and the Global Ocean

\section{REFERENCES}

Abraham, J., Baringer, P. M., Bindoff, N. L., Boyer, T., Cheng, L. J., Church, J. A., et al. (2013). A review of global ocean temperature observations: implications for ocean heat content estimates and climate change. Rev. Geophys. 51, 450-483. doi: $10.1002 / \operatorname{rog} .20022$

AchutaRao, K. M., Santer, B. D., Gleckler, P. J., Taylor, K. E., Pierce, D. W., Barnett, T. P., et al. (2006). Variability of ocean heat uptake: reconciling observations and models. J. Geophys. Res. 111 doi: 10.1029/2005JC003136

AchutaRao, K. M., Ishii, M., Santer, B. D., Gleckler, P. J., Taylor, K. E., Barnett, T. P., et al. (2007). Simulated and observed variability in ocean temperature and heat content. Proc. Natl. Acad. Sci. U.S.A. 104, 10768-10773. doi: 10.1073/pnas. 0611375104

Allison, L. C., Roberts, C. D., Palmer, M. D., Killick, R., Hermanson, L., and Rayner, N. A. (2019). Towards quantifying uncertainty in ocean heat content changes using synthetic profiles. Env. Res. Lett. 14:8. doi: 10.1088/1748-9326/ab2b0b

Arbic, B. K., and Owens, W. B. (2001). Climatic warming of Atlantic intermediate waters. J. Clim. 14, 4091-4108. doi: 10.1175/1520-0442(2001)014<4091: cwoaiw $>2.0 . \mathrm{co} ; 2$

Armitage, T. W. K., Bacon, S., Ridout, A. L., Thomas, S. F., Aksenov, Y., and Wingham, D. J., (2016). Arctic sea surface height variability and change from satellite radar altimetry and GRACE, 2003-2014. J. Geophys. Res. Oceans 121, 4303-4322. doi: 10.1002/2015JC011579

Armour, K. C, Marshall, J., Scott, J., Donohoe, A., and Newsom, E. R. (2016). Southern ocean warming delayed by circumpolar upwelling and equatorward transport. Nat. Geosci. 9, 549-554. doi: 10.1038/ngeo2731

Balmaseda, M. A., Kumar, A., Andersson, E., Takaya, Y., Anderson, D., Janssen, P., et al. (2014). "Operational forecasting systems," in Proceedings of the Report of the Tropical Pacific Observing System 2020 Workshop (TPOS 2020), Vol. II. (San Diego, CA: Scripps Institution of Oceanography), 64-101.

Balmaseda, M. A., Hernandez, F., Storto, A., Palmer, M. D., Alves, O., Shi, L., et al. (2015). The ocean reanalyses intercomparison project (ORA-IP). J. Oper. Oceanogr. 7, 81-99

Baker, D. J., Schmitt, R. W., and Wunsch, C. (2007). Endowments and new institutions for long-term observation. Oceanography 20, 10-14. doi: 10.5670/ oceanog.2007.19

Barnston, A. G., Tippett, M. K., L’Heureux, M. L., Li, S., and Dewitt, D. G. (2012). Skill of real-time seasonal ENSO model predictions during 2002-11: is our capability increasing? Bull. Am. Meteorol. Soc. 93, 631-651. doi: 10.1175/ BAMS-D-11-00111.1

Bates, A. E., Helmuth, B., Burrows, M. T., Duncan, M. I., Garrabou, J., Guy-Haim, T., et al. (2018). Biologists ignore ocean weather at their peril. Nature 560, 299-301. doi: 10.1038/d41586-018-05869-5

Belkin, I. M. (2004). Propagation of the "great salinity anomaly" of the 1990s around the northern North Atlantic. Geophys. Res. Lett. 31:L08306. doi: 10. 1029/2003GL019334

Berger, M., Camps, A., Font, J., Kerr, Y., Miller, J., Johannessen, J. A., et al. (2002). Measuring ocean salinity with ESA's SMOS mission - advancing the science. ESA Bull. 111, 113-121.

Böning, C. W., Dispert, A., Visbeck, M., Rintoul, S. R., and Schwarzkopf, F. U. (2008). The response of the antarctic circumpolar current to recent climate change. Nat. Geosci. 1, 864-869. doi: 10.1038/ngeo362

Boyer, T. P., and Levitus, S. (2002). Harmonic analysis of climatological sea surface salinity. J. Geophys. Res. 107, 7-14. doi: 10.1029/2001JC000829

Boyer, T. P., Levitus, S., Antonov, J. I., Locarnini, R. A., and Garcia, H. E. (2005). Linear trends in salinity for the world ocean, 1955-1998. Geophys. Res. Lett. 32:L01604. doi: 10.1029/2004GL021791
Observing System (GOOS). The work presented here draws on community efforts of the International Quality Controlled Ocean Database initiative (IQuOD; www.iquod.org), which is sponsored by the Scientific Committee on Oceanic Research and the International Oceanographic Data and Information Exchange program of the Intergovernmental Oceanographic Commission (IOC).

Boyer, T. P., Domingues, C. M., Good, S. A., Johnson, G. C., Lyman, J. M., Ishii, M., et al. (2016). Sensitivity of global upper-ocean heat content estimates to mapping methods, XBT bias corrections, and baseline climatologies. J. Clim. 29, 4817-4842. doi: 10.1175/JCLI-D-15-0801.1

Brauch, J. P., and Gerdes, R. (2005). Reaction of the northern North Atlantic and arctic oceans to a sudden change of the NAO. J. Geophys. Res. 110:C11018. doi: 10.1029/2004JC002436

Bryden, H. L., King, B. A., McCarthy, G. D., and McDonagh, E. L. (2014). Impact of a 30\% reduction in Atlantic meridional overturning during 2009-2010. Ocean Sci. 10, 683-691. doi: 10.5194/os-10-683-2014

Bunge, L., and Clarke, A. J. (2014). On the warm water volume and its changing relationship with ENSO. J. Phys. Oceanogr. 44, 1372-1385. doi: 10.1175/JPOD-13-062.1

Clark, P. U., Pisias, N. G., Stocker, T. F., and Weaver, A. J. (2002). The role of the thermohaline circulation in abrupt climate change. Nature 415, 863-869. doi: $10.1038 / 415863$ a

Caesar, L., Rahmstorf, S., Robinson, A., Feulner, G., and Saba, V. (2018.) Observed fingerprint of a weakening Atlantic ocean overturning circulation. Nature 556, 191-196. doi: 10.1038/s41586-018-0006-5

Cannaby, H., Palmer, M. D., Howard, T., Bricheno, L., Calvert, D., Krijnen, J. et al. (2016)Projected sea level rise and changes in extreme storm surge and wave events during the 21 st century in the region of singapore. Ocean Sci. 12, 613-632. doi: 10.5194/os-12-613-2016

Carson, M., Köhl, A., Stammer, D., Slangen, A. A. B., Katsman, C. A., van de Wal, R. S. W., et al. (2016). Coastal sea level changes, observed and projected during the 20th and 21st century. Clim. Chang. 134, 269-281. doi: 10.1007/s10584-0151520-1

Chadwick, R., Boutle, I., and Martin, G. (2013). Spatial patterns of precipitation change in CMIP5: why the rich do not get richer in the tropics. J. Clim. 26, 3803-3822. doi: 10.1175/JCLI-D-12-00543.1

Chen, D., Cane, M. A., Kaplan, A., Zebiak, S. E., and Huang, D. (2004). Predictability of El Niño over the past 148 years. Nature 428, 733-736. doi: 10.1038 /nature02439

Chen, X., and Tung, K.-K. (2014). Varying planetary heat sink led to globalwarming slowdown and acceleration. Science 345, 897-903. doi: 10.1126/ science. 1254937

Cheng, L., Trenberth, K. E., Palmer, M. D., Zhu, J., and Abraham, J. P. (2016). Observed and simulated full-depth ocean heat-content changes for 1970-2005. Ocean Sci. 12, 925-935. doi: 10.5194/os-12-925-2016

Cheng, L., Trenberth, K. E., Fasullo, J., Boyer, T., Abraham, J., and Zhu, J. (2017). Improved estimates of ocean heat content from 1960 to 2015. Sci. Adv. 3:e1601545. doi: 10.1126/sciadv.e1601545

Church, J. A., White, N. J., Konikow, L. F., Domingues, C. M., Cogley, J. G., Rignot, E., et al. (2011). Revisiting the Earth's sea-level and energy budgets for 1961 to 2008. Geophys. Res. Lett. 38:L18601. doi: 10.1029/2011GL048794

Church, J. A., Clark, P. U., Cazenave, A., Gregory, J. M., Jevrejeva, S., Levermann, A., et al. (2013a). "sea level change," in Climate Change 2013: The Physical Science Basis. Contribution of Working Group I to the Fifth Assessment Report of the Intergovernmental Panel on Climate Change Stocker, eds T. F. D. Qin, G.K. Plattner, M. Tignor, S. K. J. Allen, A. Boschung, Y. Nauels, et al. (New York, NY: Cambridge University Press), 1137-1216.

Church, J. A., White, N. J., Konikow, L. F., Domingues, C. M., Cogley, J. G., Rignot, E., et al. (2013b). Correction to revisiting the earth's sea-level and energy budgets for 1961 to 2008. Geophys. Res. Lett. 40:4066. doi: 10.1002/grl. 50752

Collins, M., Knutti, R., Arblaster, J., Dufresne, J.-L., Fichefet, T., Friedlingstein, P., et al. (2013). "Long-term Climate Change: Projections, Commitments and 
Irreversibility," in Climate Change 2013: The Physical Science Basis. Contribution of Working Group I to the Fifth Assessment Report of the Intergovernmental Panel on Climate Change Stocker, eds T. F. D. Qin, G.-K. Plattner, M. Tignor, S.K. Allen, J. Boschung, A. Nauels, et al. (New York, NY: Cambridge University Press).

Corre, L., Terray, L., Balmaseda, M., Ribes, A., and Weaver, A. (2012). Can oceanic reanalyses be used to assess recent anthropogenic changes and low-frequency internal variability of upper ocean temperature? Clim. Dyn. 38, 877-896. doi: $10.1007 / \mathrm{s} 00382-010-0950-8$

Cox, P. M., Huntingford, C., and Williamson, M. S. (2018). Emergent constraint on equilibrium climate sensitivity from global temperature variability. Nature 553, 319-322. doi: 10.1038/nature25450

Cravatte, S., Delcroix, T., Zhang, D., McPhaden, M., and Leloup, J. (2009). Observed freshening and warming of the western pacific warm pool. Clim Dyn 33, 565-589. doi: 10.1007/s00382-009-0526-7

Cravatte, S., Kessler, W. S., Smith, N., Wijffels, S. E., Yu, L., Ando, K., et al. (2016). First Report of TPOS 2020. Salisbury: TPOS, 200.

Cunningham, S. A., Roberts, C. D., Frajka-Williams, E., Johns, W. E., Hobbs, W., Palmer, M. D., et al. (2013). Atlantic meridional overturning circulation slowdown cooled the subtropical ocean. Geophys. Res. Lett. 40, 6202-6207. doi: 10.1002/2013GL05846

Curry, R., and Mauritzen, C. (2005). Dilution of the northern North Atlantic ocean in recent decades. Science 308, 1772-1774. doi: 10.1126/science.1109477

Czaja, A., and Frankignoul, C. (2002). Observed impact of atlantic SST Anomalies on the North Atlantic Oscillation. J. Clim. 15, 606-623. doi: 10.1175/15200442(2002)015<0606:oioasa>2.0.co;2

Danabasoglu, G., Yeager, S. G., Kim, W. M., Behrens, E., Bentsen, M., Daohua, B., et al. (2015). North Atlantic simulations in coordinated ocean-ice reference experiments phase II (CORE-II). Part II: inter-annual to decadal variability. Ocean Mod. 97, 65-90. doi: 10.1016/j.ocemod.2015.11.007

de Boyer Montegut, C., Madec, G., Fischer, A. S., Lazar, A., and Ludicone, D. (2004). Mixed layer depth over the global ocean: an examination of profile data and a profile-based climatology. J. Geophys. Res. 109:20. doi: 10.1029/2004JC002378

de Lavergne, C., Palter, J. B., Galbraith, E. D., Bernardello, R., and Marinov, I. (2014). Cessation of deep convection in the open Southern Ocean under anthropogenic climate change. Nat. Clim. Chang 4, 278-282. doi: 10.1038/ nclimate2132

de Vries, P., and Weber, S. L. (2005). The Atlantic freshwater budget as a diagnostic for the existence of a stable shut down of the meridional overturning circulation. Geophys. Res. Lett. 32 doi: 10.1029/2004GL021450

Delcroix, T., Cravatte, S., and McPhaden, M. J. (2007). Decadal variations and trends in tropical pacific sea surface salinity since 1970. J. Geophys. Res. 112:C03012. doi: 10.1029/2006JC003801

Desbruyères, D. G., Purkey, S. G., McDonagh, E. L., Johnson, G. C., and King, B. A. (2016). Deep and abyssal ocean warming from 35 years of repeat hydrography. Geophys. Res. Lett. 43, 10356-10365. doi: 10.1002/2016GL070413

Desbruyères, D., McDonagh, E. L., King, B. A., and Thierry, V. (2017). Global and full-depth ocean temperature trends during the early twenty-first century from argo and repeat hydrography. J. Clim. 30, 1985-1997. doi: 10.1175/JCLI-D-160396.1

DeVries, T., Primeau, F., DeVries, T., and Primeau, F. (2011). Dynamically and observationally constrained estimates of water-mass distributions and ages in the global ocean. J. Phys. Oceanogr. 41, 2381-2401. doi: 10.1175/JPO-D-1005011.1

DeVries, T., Holzer, M., and Primeau, F. (2017). Recent increase in oceanic carbon uptake driven by weaker upper-ocean overturning. Nature 542, 215-218. doi: 10.1038 /nature21068

Doblas-Reyes, F. J., García-Serrano, J., Lienert, F., Biescas, A. P., and Rodrigues, L. R. (2013), Seasonal climate predictability and forecasting: status and prospects. WIRES Clim. Chang. 4, 245-268. doi: 10.1002/wcc.217

Domingues, C. M., Church, J. A., White, N. J., Gleckler, P. J., Wijffels, S. E., Barker, P. M., et al. (2008). Improved estimates of upper-ocean warming and multi-decadal sea-level rise. Nature 453, 1090-1093. doi: 10.1038/nature 07080

Drijfhout, S. S., Blaker, A. T., Josey, S. A., Nurser, A. J., Sinha, G. B., and Balmaseda, M. A. (2014). Surface warming hiatus caused by increased heat uptake across multiple ocean basins. Geophys. Res. Lett. 41, 7868-7874. doi: 10.1002/2014GL061456
Dubois, C., von Schuckmann, K., Josey, S., and Ceschin, A., (2018). "Changes in the North Atlantic," in Copernicus Marine Service Ocean State Report, Issue 2: Journal of Operational Oceanography, vol. 11, eds K. von Schuckmann, P.-Y. Le Traon, N. Smith, A. Pascual, P. Brasseur, K. Fennel, et al. (Ditcot: Taylor \& Francis), s66. doi: 10.1080/1755876X.2018.1489208

Ducet, N., Le Traon, P. Y., and Reverdin, G. (2000). Global high-resolution mapping of ocean circulation from TOPEX/poseidon and ERS-1 and -2 . J. Geophys. Res. 105, 19477-19498. doi: 10.1029/2000JC900063

Dunstone, N. J. (2014). A perspective on sustained marine observations for climate modelling and prediction. Philos. Trans. A Math. Phys. Eng. Sci. 37:20130340. doi: 10.1098/rsta.2013.0340

Dunstone, N. J., and Smith, D. M. (2010). Impact of atmosphere and sub-surface ocean data on decadal climate prediction. Geophys. Res. Lett. 37. doi: 10.1029/ 2009GL041609

Durack, P. J., and Wijffels, S. E. (2010). Fifty-year trends in global ocean salinities and their relationship to broad-scale warming. J. Clim. 23, 4342-4362. doi: 10.1175/2010JCLI3377.1

Durack, P. J., Wijffels, S. E., and Matear, R. J. (2012). Ocean salinities reveal strong global water cycle intensification during 1950 to 2000. Science 336, 455-458. doi: $10.1126 /$ science. 1212222

Durack, P. J., Gleckler, P. J., Landerer, F. W., and Taylor, K. E. (2014). Quantifying underestimates of long-term upper-ocean warming. Nat. Clim. Chang. 4, 9991005. doi: $10.1038 /$ nclimate2389

Durack, P. J., Gleckler, P. J., Purkey, S. G., Johnson, G. C., Lyman, J. M., and Boyer, T. P. (2018). Ocean warming: from the surface to the deep in observations and models. Oceanography 31, 41-51. doi: 10.5670/oceanog.2018.227

Durack, P. J. (2015). Ocean salinity and the global water cycle. Oceanography 28 , 20-31. doi: 10.5670/oceanog.2015.03

Durack, P. J., Lee, T., Vinogradova, N. T., and Stammer, D. (2016). Keeping the lights on for global ocean salinity observation. Nat. Clim. Chang. 6, 228-231. doi: $10.1038 /$ nclimate2946

Easterling, D. R., and Wehner, M. F. (2009). Is the climate warming or cooling? Geophys. Res. Lett. 36:L08706. doi: 10.1029/2009GL037810

England, M. H., McGregor, S., Spence, P., Meehl, G. A., Timmermann, A., and Cai, W., (2014). Recent intensification of wind-driven circulation in the pacific and the ongoing warming hiatus. Nat. Clim. Chang. 4, 222-227. doi: 10.1038/ nclimate2106

Exarchou, E., Kuhlbrodt, T., Gregory, J. M., and Smith, R. S. (2014). Ocean heat uptake processes: a model intercomparison. J. Clim. 28, 887-908. doi: 10.1175/ jcli-d-14-00235.1

Frölicher, T. L., Sarmiento, J. L., Paynter, D. J., Dunne, J. P., Krasting, J. P., Winton, M., et al. (2015). Dominance of the southern ocean in anthropogenic carbon and heat uptake in CMIP5 models. J. Clim. 28, 862-886. doi: 10.1175/JCLI-D14-00117.1

Fenty, I., Willis, J. K., Khazendar, A., Dinardo, S., Forsberg, R., Fukumori, I., et al. (2016). Oceans melting greenland: early results from nasa's ocean-ice mission in greenland. Oceanography 29, 72-83. doi: 10.5670/oceanog.2016.100

Fichot, C. G., Kaiser, K., Hooker, S. B., Amon, R. M. W., Babin, M., Belanger, S., et al. (2013), Pan-Arctic distributions of continental runoff in the arctic ocean. Sci. Rep. 3:1053

Fore, A. G., Yueh, S. H., Tang, W., Stiles, B. W., and Hayashi, A. K. (2016). Combined active/passive retrievals of ocean vector wind and sea surface salinity with SMAP. IEEE Trans. Geosci. Remote Sens. 54, 7396-7404. doi: 10.1109/ TGRS.2016.2601486

Fournier, S., Lee, T., and Gierach, M. M. (2016). Seasonal and interannual variations of sea surface salinity associated with the Mississippi River plume observed by SMOS and aquarius. Remote Sens. Env. 180, 431-439. doi: 10.1016/ j.rse.2016.02.050

Frajka-Williams, E., Ansorge, I. J., Baehr, J., Bryden, H. L., Chidichimo, M. P., Cunningham, S. A., et al. (2019). Atlantic meridional overturning circulation: observed transport and variability. Front. Mar. Sci. 6:260. doi: 10.3389/fmars. 2019.00260

Frolicher, T. L., Fischer, E. M., and Gruber, N. (2018). Marine heatwaves under global warming. Nature 560, 360-364. doi: 10.1038/s41586-018-0383-9

Fu, Y., Karstensen, J., and Brandt, P. (2018). Atlantic Meridional Overturning Circulation at $14.5^{\circ} \mathrm{N}$ in 1989 and 2013 and $24.5^{\circ} \mathrm{N}$ in 1992 and 2015: volume, heat, and freshwater transports. Ocean Sci. 14, 589-616. doi: 10.5194/os-14589-2018 
Fujii, Y., James, C., Yan, X., Andreas, S., Tong, L., Magdalena, A. B., et al. (2015). Evaluation of the tropical pacific observing system from the ocean data assimilation perspective. Q. J. R. Meteorol. Soc. 141, 2481-2496. doi: 10.1002/ qj.2579

Garric, G., Hernandez, O., Bricaud, C., Storto, A., Peterson, K. A., and Zuo, H., (2018). “Arctic ocean freshwater content," in Copernicus Marine Service Ocean State Report, Issue 2: Journal of Operational Oceanography, vol. 11, eds K. von Schuckmann, P.-Y. Le Traon, N. Smith, A. Pascual, P. Brasseur, K. Fennel, et al. (Ditcot: Taylor \& Francis), s70. doi: 10.1080/1755876X.2018.1489208

Garry, F. (2017). Deep Ocean Heat Content Observing Strategy: Evaluating the Past and Preparing for the Future. Ph.D thesis, University of Southampton, Southampton.

Gasparin, F., Roemmich, D., Gilson, J., and Cornuelle, B. (2015). Assessment of the upper-ocean observing system in the equatorial pacific: the role of argo in resolving intraseasonal to interannual variability. J. Atmos. Oceanic. Technol. 32, 1668-1688. doi: 10.1175/jtech-d-14-00218.1

Gasparin, F., and Roemmich, D. (2016). The strong freshwater anomaly during the onset of the 2015/2016 El Niño. Geophys. Res. Lett. 43, 6452-6460. doi: 10.1002/2016GL069542

Gasparin, F., Guinehut, S., Mao, C., Mirouze, I., Rémy, E., King, R. R., et al. (2019). Requirements for an integrated in situ atlantic ocean observing system from coordinated observing system simulation experiments. Front. Mar. Sci. 6:83. doi: 10.3389/fmars.2019.00083

Gastineau, G., Mignot, J., Arzel, O., and Huck, T. (2018). North Atlantic ocean internal decadal variability: role of the mean state and ocean-atmosphere coupling. J. Geophys. Res. Oceans 123, 5949-5970. doi: 10.1029/2018JC014074

Giglio, D., and Johnson, G. C. (2017). Middepth decadal warming and freshening in the South Atlantic. J. Geophys. Res. Oceans 122, 973-979. doi: 10.1002/ 2016)C012246

Gille, S. T. (2008). Decadal-scale temperature trends in the southern hemisphere ocean. J. Clim. 21, 4749-4765. doi: 10.1175/2008JCLI2131.1

Gleckler, P. J., Durack, P. J., Stouffer, R. J., Johnson, G. C., and Forest, C. E. (2016) Industrial-era global ocean heat uptake doubles in recent decades. Nat. Clim. Chang. 6, 394-398. doi: 10.1038/nclimate2915

Gleckler, P. J., Santer, B. D., Domingues, C. M., Pierce, D. W., Barnett, T. P., Church, J. A., et al. (2012). Human-induced global ocean warming on multidecadal timescales. Nat. Clim. Chang. 2, 524-529. doi: 10.1038/ nclimate1553

Golledge, N. R., Kowalewski, D. E., Naish, T. R., Levy, R. H., Fogwill, C. J., and Gasson, E. G. W. (2015). The multi-millennial Antarctic commitment to future sea-level rise. Nature 526, 421-425. doi: 10.1038/nature15706

Good, S. A. (2017). The impact of observational sampling on time series of global 0 700 m ocean average temperature: a case study. Int. J. Climatol. 37, 2260-2268. doi: $10.1002 /$ joc. 4654

Good, S. A., Martin, M. J., and Rayner, N. A. (2013). EN4: quality controlled ocean temperature and salinity profiles and monthly objective analyses with uncertainty estimates. J. Geophys. Res. 118, 6704-6716. doi: 10.1002/ 2013JC009067

Gray, A. R., and Riser, S. C. (2014). A global analysis of sverdrup balance using absolute geostrophic velocities from argo. J. Phys. Oceanogr. 44, 1213-1229. doi: 10.1175/JPO-D-12-0206.1

Gregory, J. M., Banks, H. T., Stott, P. A., Lowe, J. A., and Palmer, M. D. (2004), Simulated and observed decadal variability in ocean heat content. Geophys. Res. Lett. 31:L15312. doi: 10.1029/2004GL020258

Gregory, J. M., Bouttes, N., Griffies, S. M., Haak, H., Hurlin, W. J., Jungclaus, J., et al. (2016). The flux-anomaly-forced model intercomparison project (FAFMIP) contribution to CMIP6: investigation of sea-level and ocean climate change in response to CO2 forcing. Geosci. Mod. Dev. 9, 3993-4017. doi: 10. 5194/gmd-9-3993-2016

Griffies, S. M., Winton, M., Anderson, W. G., Benson, R., Delworth, T. L., Dufour, C. O., et al. (2015). Impacts on ocean heat from transient mesoscale eddies in a hierarchy of climate models. J. Clim. 28, 952-977. doi: 10.1175/JCLI-D-1400353.1

Guinehut, S., Dhomps, A.-L., Larnicol, G., and Le Traon, P.-Y., (2012). High resolution $3 \mathrm{D}$ temperature and salinity fields derived from in situ and satellite observations. Ocean Sci. 8, 845-857. doi: 10.5194/os-8-845-2012

Haine, T. W. N., Beth, C., Rüdiger, G., Edmond, H., Michael, J. K., Craig, L., et al. (2015). Arctic freshwater export: status, mechanisms, and prospects. Global. Planet. Chang. 125, 13-35. doi: 10.1016/j.gloplacha.2014 11.013

Häkkinen, S., Rhines, P. B., and Worthen, D. L. (2015). Heat content variability in the North Atlantic Ocean in ocean reanalyses. Geophys. Res. Lett. 42, 2901-2909. doi: 10.1002/2015GL063299

Haumann, F. A., Gruber, N., Münnich, M., Frenger, I., and Kern, S. (2016). Seaice transport driving Southern Ocean salinity and its recent trends. Nature 537, 89-92. doi: 10.1038/nature19101

Held, I. M., and Soden, B. J. (2006). Robust responses of the hydrological cycle to global warming. J. Clim. 19, 5686-5699. doi: 10.1175/JCLI3990.1

Helm, K. P., Bindoff, N. L., and Church, J. A. (2010). Changes in the global hydrological-cycle inferred from ocean salinity. Geophys. Res. Lett. 37. doi: 10.1029/2010GL044222

Heuzé, C., Heywood, K. J., Stevens, D. P., and Ridley, J. K. (2015). Changes in global ocean bottom properties and volume transports in CMIP5 models under climate change scenarios. J. Clim. 28, 2917-2944. doi: 10.1175/JCLI-D-1400381.1

Holland, D. M., Thomas, R. H., De Young, B., Ribergaard, M. H., and Lyberth, B. (2008). Acceleration of Jakobshavn Isbrae triggered by warm subsurface ocean waters. Nat. Geosci. 1, 659-664. doi: 10.1038/ngeo316

Hosoda, S., Ohira, T., and Nakamura, T., (2008). A monthly mean dataset of global oceanic temperature and salinity derived from Argo float observations. JAMSTEC Rep. Res. Dev. 8, 47-59. doi: 10.5918/Jamstecr.8.47

Hosoda, S., Suga, T., Shikama, N., and Mizuno, K. (2009). Global surface layer salinity change detected by Argo and its implication for hydrological cycle intensification. J. Oceanogr. 65, 579-586. doi: 10.1007/s10872-009-0049-1

IPCC, (2013). "Climate Change 2013: The Physical Science Basis," in Contribution of Working Group I to the Fifth Assessment Report of the Intergovernmental Panel on Climate Change Stocker, eds T. F. D. Qin, G.-K. Plattner, M. Tignor, S.K. Allen, J. Boschung, A. Nauels, et al. (New York, NY: Cambridge University Press), 1535.

Ishii, M., and Kimoto, M. (2009). Reevaluation of historical ocean heat content variations with time-varying XBT and MBT depth bias corrections. J. Oceanogr. 65, 287-299. doi: 10.1007/s10872-009-0027-7

Jacobs, S. S., and Giulivi, C. F. (2010). large multidecadal salinity trends near the pacific-antarctic continental margin. J. Clim. 23, 4508-4524 doi: 10.1175/ 2010jcli3284.1

Jackson, L. C., Peterson, K. A., Roberts, C. D., and Wood, R. A. (2016). Recent slowing of Atlantic overturning circulation as a recovery from earlier strengthening. Nat. Geosci. 9, 518-522. doi: 10.1038/ngeo2715

Jin, F. F. (1997). An equatorial ocean recharge paradigm for ENSO. Part I conceptual model. J. Atmos. Sci. 54, 811-829. doi: 10.1175/1520-0469(1997) 054<0811:aeorpf > 2.0.co;2

Johnson, G. C., and Birnbaum, A. N. (2017), As El Niño builds, Pacific Warm Pool expands, ocean gains more heat. Geophys. Res. Lett. 44, 438-445. doi: 10.1002/2016GL071767

Jochumsen, K., Köllner, M., Quadfasel, D., Dye, S., Rudels, B., and Valdimarsson, H. (2015). On the origin and propagation of denmark strait overflow water anomalies in the irminger basin. J. Geophys. Res. Oceans 120, 1841-1855. doi: 10.1002/2014JC010397

Johnson, G. C., Lyman, J. M., Boyer, T., Cheng, L., Domingues, C. M., Gilson, J., et al. (2018). Global oceans: ocean heat content. state of the climate in 2017. Bull. Am. Meteorol. Soc. 99, S72-S77.

Johnson, G. C., McTaggart, K. E., and Wanninkhof, R. (2014), Antarctic bottom water temperature changes in the western South Atlantic from 1989 to 2014. J. Geophys. Res. Oceans 119, 8567-8577. doi: 10.1002/2014JC010367

Johnson, G. C., Lyman, J. M., and Purkey, S. G. (2015). Informing deep argo array design using argo and full-depth hydrographic section data. J. Atmos. Ocean. Technol. 32, 2187-2198. doi: 10.1175/JTECH-D-15-0139.1

Johnson, G. C., Lyman, J. M., and Loeb, N. G. (2016). Improving estimates of Earth's energy imbalance. Nat. Clim. Chang. 6, 639-640. doi: 10.1038/nclimate 3043

Josey, S. A., Hirschi, J. J., Sinha, B., Duchez, A., Grist, J. P., and Marsh, R. (2018). The recent atlantic cold anomaly: causes, consequences, and related phenomena. Annu. Rev. Mar. Sci. 10, 475-501. doi: 10.1146/annurev-marine12191-2

Jullion, L., Naveira Garabato, A. C., Meredith, M. P., Holland, P. R., Courtois, P., and King, B. A. (2013). Decadal freshening of the antarctic bottom water 
exported from the weddell sea. J. Clim. 26, 8111-8125. doi: 10.1175/JCLI-D12-00765.1

Jung, O., Sung, M. K., Sato, K., Lim, Y. K., Kim, S. J., Baek, E. H., et al. (2017). How does the SST variability over the western North atlantic ocean control arctic warming over the barents-kara seas? Environ. Res. Lett. 12:034021. doi: 10.1088/1748-9326/aa5f3b

Kara, A., Rochford, P. A., and Hurlburt, H. E. (2003). Mixed layer depth variability over the global ocean. J. Geophys. Res. Oceans 108:3079

Karcher, M., Beszczynska-Möller, A., Kauker, F., Gerdes, R., Heyen, S., Rudels, B., et al. (2011), Arctic Ocean warming and its consequences for the Denmark Strait overflow. J. Geophys. Res. 116:C02037. doi: 10.1029/2010JC006265

Kessler, W. S., Spillane, M. C., McPhaden, M. J., and Harrison, D. E. (1996). Scales of variability in the equatorial pacific inferred from the TAO buoy array. J. Clim. 9, 2999-3024. doi: 10.1175/1520-0442(1996)009<2999:sovite>2.0.co;2

Klatt, O., Boebel, O., and Fahrbach, E., (2007). A profiling float's sense of ice. J. Atmos. Ocean. Technol. 24, 1301-1308. doi: 10.1175/JTECH2026.1

Koenigk, T., Mikolajewicz, U., Haak, H., and Jungclaus, J. (2007). Arctic freshwater export in the 20th and 21st centuries. J. Geophys. Res. 112:G04S41. doi: 10.1029/ 2006JG000274

Kuhlbrodt, T., and Gregory, J. M. (2012), Ocean heat uptake and its consequences for the magnitude of sea level rise and climate change. Geophys. Res. Lett. 39:L18608. doi: 10.1029/2012GL052952

Lagerloef, G., Colomb, F. R., Le Vine, D., Wentz, F., Yueh, S., Ruf, C., et al. (2008). The Aquarius/SAC-D mission: designed to meet the salinity remote-sensing challenge. Oceanograph 21, 68-81. doi: 10.5670/oceanog.2008.68

Lago, V., Wijffels, S. E., Durack, P. J., Church, J. A., Bindoff, N. L., and Marsland, S. J. (2016). Simulating the role of surface forcing on observed multidecadal upper-ocean salinity changes. J. Clim. 29, 5575-5588. doi: 10.1175/JCLI-D-150519.1

Landerer, F. W., Gleckler, P. J., and Lee, T. (2014). Evaluation of CMIP5 dynamic sea surface height multi-model simulations against satellite observations. Clim. Dyn. 43, 1271-1283. doi: 10.1007/s00382-013-1939-x

Lecomte, O., Goosse, H., Fichefet, T., de Lavergne, C., Barthélemy, A., and Zunz, V. (2017). Vertical ocean heat redistribution sustaining sea-ice concentration trends in the ross sea. Nat. Commun. 8:258. doi: 10.1038/s41467-017-00347-4

Le Traon, P. Y., Nadal, F., and Ducet, N. (1998). an improved mapping method of multisatellite altimeter data. J. Atmos. Ocean. Technol. 15, 522-534. doi: 10.1175/1520-04261998015<0522:AIMMOM<2.0.CO;2

Lee, S.-K., Park, W., Baringer, M. O., Gordon, A. L., Huber, B., and Liu, Y. (2015). Pacific origin of the abrupt increase in Indian Ocean heat content during the warming hiatus. Nat. Geosci. 8, 445-449. doi: 10.1038/ngeo2438

Levitus, S. (1984). Annual cycle of temperature and heat storage in the world ocean. J. Phys. Oceanogr. 14, 727-746. doi: 10.1175/1520-04851984014<0727: ACOTAH $<2.0 . \mathrm{CO} ; 2$

Le Quéré, C., Raupach, M. R., Canadell, J. G., Marland, G., Le Quéré, C., Raupach, M. R., et al. (2009). Trends in the sources and sinks of carbon dioxide. Nat. Geosci. 2, 831-836. doi: 10.1038/ngeo689

Le Reste, S., Dutreuil, V., André, X., Thierry, V., Renaut, C., Le Traon, P., et al. (2016). "Deep-Arvor": a new profiling float to extend the argo observations down to 4000-m depth. J. Atmos. Oceanic Technol. 33, 1039-1055. doi: 10.1175/ JTECH-D-15-0214.1

Levermann, A., Winkelmann, R., Nowicki, S., Fastook, J. L., Frieler, K., Greve, R., et al. (2014). Projecting Antarctic ice discharge using response functions from SeaRISE ice-sheet models. Earth Syst. Dyn. 5, 271-293. doi: 10.5194/esd-5-2712014

Levitus, S. (1986). Annual cycle of salinity and salt storage in the world ocean. J. Phys. Oceanogr. 16, 322-343. doi: 10.1175/1520-0485(1986)016<0322: acosas $>2.0 . \operatorname{co} ; 2$

Levitus, S. (1989). Interpentadal variability of temperature and salinity at intermediate depths of the north Atlantic Ocean, 1970-74 versus 1955-1959. J. Geophys. Res. 94, 6091-6131. doi: 10.1029/JC094iC05p 06091

Levitus, S., Antonov, J. I., Boyer, T. P., and Stephens, C. (2000). Warming of the World Ocean. Science 287, 2225-2229. doi: 10.1126/science.287.5461. 2225

Levitus, S., Antonov, J. I., Wang, J., Delworth, T. L., Dixon, K. W., and Broccoli, A. J. (2001). Anthropogenic warming of the earth's climate system. Science 292, 267-270. doi: 10.1126/science. 1058154
Levitus, S., Antonov, J. I., Boyer, T. P., Baranova, O. K., Garcia, H. E., Locarnini, R. A., et al. (2012). World ocean heat content and thermosteric sea level change (0 - 2000 m), 1955-2010. Geophys. Res. Lett. 39:L10603. doi: 10.1029/ 2012GL051106

Li, L., Schmitt, R. W., Ummenhofer, C. C., and Karnauskas, K. B. (2016a). North Atlantic salinity as a predictor of Sahel rainfall. Sci. Adv. 2:e1501588. doi: 10. 1126/sciadv. 1501588

Li, L., Schmitt, R. W., Ummenhofer, C. C., and Karnauskas, K. B. (2016b). Implications of North Atlantic Sea surface salinity for summer precipitation over the U.S. midwest: mechanisms and predictive value. J. Clim. 29, 3143-3159. doi: 10.1175/JCLI-D-15-0520.1

Liu, X., Köhl, A., and Stammer, D. (2017). Dynamical ocean response to projected changes of the global water cycle. J. Geophys. Res. Oceans 122, 6512-6532. doi: 10.1002/2017JC013061

Liu, T., Schmitt, R. W., and Li, L. (2018). Global search for autumn-lead sea surface salinity predictors of winter precipitation in Southwestern United States. Geophys. Res. Lett. Banner 45, 8445-8454. doi: 10.1029/2018GL079293

Lyman, J. M, and Johnson, G. C. (2014). Estimating global ocean heat content changes in the upper $1800 \mathrm{~m}$ since 1950 and the influence of climatology choice. J. Clim. 27, 1945-1957. doi: 10.1175/JCLI-D-12-00752.1

Maher, N., Sen Gupta, A., and England, M. H. (2014). Drivers of decadal hiatus periods in the 20th and 21st centuries. Geophys. Res. Lett. 41, 5978-5986. doi: 10.1002/2014GL060527

Marshall, J., Scott, J. R., Armour, K. C., Campin, J. M., Kelley, M., and Romanou, A. (2015). The ocean's role in the transient response of climate to abrupt greenhouse gas forcing. Clim. Dyn. 44, 2287-2299. doi: 10.1007/s00382-0142308-0

Masuda, S., Awaji, T., Sugiura, N., Matthews, J. P., Toyoda, T., Kawai, Y., et al. (2010). Simulated rapid warming of abyssal North Pacific waters. Science 329, 319-322. doi: 10.1126/science. 1188703

Mayer, M., Fasullo, J. T., Trenberth, K. E., and Haimberger, L. (2016). ENSO-driven energy budget perturbations in observations and CMIP models. Clim. Dyn. 4, 4009-4029. doi: 10.1007/s00382-016-3057-z

McCarthy, G., Frajka-Williams, E., Johns, W. E., Baringer, M. O., Meinen, C. S., and Bryden, H. L., (2012). Observed interannual variability of the Atlantic meridional overturning circulation at $26.5^{\circ} \mathrm{N}$. Geophysi. Res. Lett. 39:L19609.

McConaghy, D. C. (1980). Measuring sea surface temperature from satellites: a ground truth approach. Remote Sens. Environ. 10, 307-310. doi: 10.1016/00344257(80)90090-5

McPhaden, M. J., Zebiak, S. E., and Glantz, M. H. (2006). ENSO as an integrating concept in earth science. Science 314, 1740-1745. doi: 10.1126/science.1132588

McPhaden, M. J., Antonio, J. B., Robert, C., Jean-René, D., Kenneth, S. G., David, H., et al. (1998). The tropical ocean-global atmosphere observing system: a decade of progress. J. Geophys. Res. 103, 14169-14240

Meehl, G. A., Arblaster, J. M., Fasullo, J. T., Hu, A., and Trenberth, K. E. (2011). Model-based evidence of deep-ocean heat uptake during surface-temperature hiatus periods. Nat. Clim. Chang. 1, 360-364. doi: 10.1038/nclimate 1229

Meehl, G. A., Goddard, L., Boer, G., Burgman, R., Branstator, G., Cassou, C., et al. (2014). Decadal climate prediction an update from the trenches. Bull. Am. Meteorol. Soc. 95, 243-267. doi: 10.1175/BAMS-D-12-00241.1

Meinen, C. S., and McPhaden, M. J. (2000), Observations of warm water volume changes in the equatorial pacific and their relationship to El niño and la niña. J. Clim. 13, 3551-3559 doi: 10.1175/1520-0442(2000)013<3551:oowwvc $>2.0$. $\mathrm{co} ; 2$

Morison, J., Kwok, R., Peralta-Ferriz, C., Alkire, M., Rigor, I., Andersen, R., et al. (2012). Changing arctic ocean freshwater pathways. Nature 481, 66-70. doi: 10.1038 /nature 10705

Mulet, S., Buongiorno Nardelli, B., Good, S., Pisan, A., Greiner, E., Monier, M., et al. (2018). "Ocean temperature and salinity," in Copernicus Marine Service Ocean State Report, Issue 2: Journal of Operational Oceanography, vol. 11, eds K. von Schuckmann, P.-Y. Le Traon, N. Smith, A. Pascual, P. Brasseur, K. Fennel, et al. (Ditcot: Taylor \& Francis), s5. doi: 10.1080/1755876X.2018.1489208

Myhre, G., Shindell, D., Bréon, F.-M., Collins, W., Fuglestvedt, J., Huang, J., Koch, D., et al. (2013). "Anthropogenic and Natural Radiative Forcing" in Climate Change 2013: The Physical Science Basis. Contribution of Working Group I to 
the Fifth Assessment Report of the Intergovernmental Panel on Climate Change Stocker, eds T. F. D. Qin, G.-K. Plattner, M. Tignor, S. K. Allen, J. Boschung, A. Nauels, et al. (New York, NY: Cambridge University Press).

National Academies of Sciences, Engineering, and Medicine [NASEM], (2017). Sustaining Ocean Observations to Understand Future Changes in Earth's Climate. Washington, DC: The National Academies Press.

Nieves, V., Willis, J. K., and Patzert, W. C. (2015). Recent hiatus caused by decadal shift in indo-pacific heating. Science 349, 532-535. doi: 10.1126/science. aaa 4521

Oke, P. R., Larnicol, G., Fujii, Y., Smith, G. C., Lea, D. J., Guinehut, S., et al. (2015). Assessing the impact of observations on ocean forecasts and reanalyses: part 1, global studies. J. Oper. Oceanogr. 8, s49-s62. doi: 10.1080/1755876x.2015. 1022067

Palmer, M. D., Good, S. A., Haines, K., Rayner, N. A., and Stott, P. A. (2009). A new perspective on warming of the global oceans. Geophys. Res. Lett. 36:5. doi: 10.1029/2009GL039491

Palmer, M. D., McNeall, D. J., and Dunstone, N. J. (2011). Importance of the deep ocean for estimating decadal changes in earth's radiation balance. Geophys. Res. Lett. 38:L13707. doi: 10.1029/2011GL047835

Palmer, M. D., and McNeall, D. J. (2014). Internal variability of Earth's energy budget simulated by CMIP5 climate models. Environ. Res. Lett. 9:34016.

Palmer, M. D., Roberts, C. D., Balmaseda, M., Chang, Y-S., Chepurin, G., Ferry, N., et al. (2017). Ocean heat content variability and change in an ensemble of ocean reanalyses. Clim. Dyn. 49, 909-930. doi: 10.1007/s00382-015-2801-0

Park, W, and Latif, M. (2008). Multidecadal and multicentennial variability of the meridional overturning circulation. Geophys. Res. Lett. 35:L22703

Pawlowicz, R., Feistel, R., McDougall, T. J., Ridout, P., Seitz, S., and Wolf, H. (2016). Metrological challenges for measurements of key climatological observables Part 2: oceanic salinity. Metrologia 53, R1-R11. doi: 10.1088/0026-1394/53 /1/R12

Perkins, S. E. (2015). A review on the scientific understanding of heatwaves - their measurement, driving mechanisms, and changes at the global scale. Atmos. Res. 164-165, 242-267. doi: 10.1016/j.atmosres.2015.05.014

Piecuch, C. G., and Quinn, K. J. (2016), El Niño, La Niña, and the global sea level budget. Ocean Sci. 12, 1165-1177.

Pierce, D. W., Gleckler, P. J., Barnett, T. P., Santer, B. D., and Durack, P. J. (2012). The fingerprint of human-induced changes in the ocean's salinity and temperature fields. Geophys. Res. Lett. 39:6. doi: 10.1029/2012GL053389

Polyakov, I. V., Pnyushkov, A. V., Rember, R., Padman, L., Carmack, E. C., and Jackson, J. M. (2013). Winter convection transports atlantic water heat to the surface layer in the eastern arctic ocean. J. Phys. Oceanogr. 43, 2142-2155. doi: 10.1175/JPO-D-12-0169.1

Purkey, S. G., and Johnson, G. C. (2010). Warming of global abyssal and deep southern ocean waters between the 1990s and 2000s: contributions to global heat and sea level rise budgets. J. Clim. 23, 6336-6351. doi: 10.1175/ 2010JCLI3682.1

Purkey, S. G., and Johnson, G. C. (2012). Global contraction of antarctic bottom water between the 1980s and 2000s. J. Clim. 25, 5830-5844. doi: 10.1175/JCLID-11-00612.1

Purkey, S. G., and Johnson, G. C. (2013). Antarctic bottom water warming and freshening: contributions to sea level rise, ocean freshwater budgets, and global heat gain. J. Clim. 26, 6105-6122. doi: 10.1175/JCLI-D-12-00834.1

Rabe, B., Karcher, M., Kauker, F., Schauer, U., Toole, J. M., Krishfield, R. A., et al. (2014). Arctic ocean basin liquid freshwater storage trend 1992-2012. Geophys. Res. Lett. 41, 961-968. doi: 10.1002/2013GL058121

Reynolds, R. W., Smith, T. M., Liu, C., Chelton, D. B., Casey, K., and Schlax, G. (2007). Daily high-resolution-blended analyses for sea surface temperature. J. Clim. 20, 5473-5496. doi: 10.1175/2007jcli1824.1

Rhein, M., Rintoul, S. R., Aoki, S., Campos, E., Chambers, D., Feely, R. A., et al. (2013). "Observations: Ocean," in Climate Change 2013: The Physical Science Basis. Contribution of Working Group I to the Fifth Assessment Report of the Intergovernmental Panel on Climate Change, ed. T. F Stockercpesnm, (New York, NY: Cambridge University Press, Cambridge) 255-315.

Rhines, P., Häkkinen, S., and Josey, S. A. (2008). "Is oceanic heat transport significant in the climate system?", in Arctic-Subarctic Ocean Fluxes eds R. R. Dickson, J. Meincke, P. Rhinescpesnm, (Dordrecht: Springer). doi: 10.1007/ 978-1-4020-6774-7-5
Ricker, R., Hendricks, S., and Beckers, J. F. (2016), The impact of geophysical corrections on sea-ice freeboard retrieved from satellite altimetry. Remote Sens. 8, 1415-1425. doi: 10.3390/rs8040317

Ridley, D. A., Solomon, S., Barnes, J. E., Burlakov, V. D., Deshler, T., Dolgii, S. I., et al. (2014). Total volcanic stratospheric aerosol optical depths and implications for global climate change. Geophys. Res. Lett. 41. 7763-7769. doi: 10.1002/2014GL061541

Rignot, E., Xu, Y., Menemenlis, D., Mouginot, J., Scheuchl, B., Li, X., et al. (2016). Modeling of ocean-induced ice melt rates of five west Greenland glaciers over the past two decades. Geophys. Res. Lett. 43, 6374-6382. doi: 10.1002/ 2016GL068784

Risbey, J. S., Lewandowsky, S., Langlais, C., Monselesan, D. P., O’Kane, T. J., and Oreskes, N. (2014). Well-estimated global surface warming in climate projections selected for ENSO phase. Nat. Clim. Chang. 4, 835-840. doi: 10. 1038/nclimate2310

Riser, S. C., Freeland, H. J., Roemmich, D., Wijffels, S., Troisi, A., Belbéoch, M., et al. (2016). Fifteen years of ocean observations with the global argo array. Nat. Clim. Chang. 6, 145-153. doi: 10.1038/nclimate2872

Roberts, C. D., Calvert, D., Dunstone, N., Hermanson, L., Palmer, M. D., and Smith, D. (2016). On the drivers and predictability of seasonal-to-interannual variations in regional sea level. J. Clim. 29, 7565-7585. doi: 10.1175/JCLI-D-150886.1

Robson, J., Sutton, R., Lohmann, K., Smith, D., and Palmer, M. D. (2012a). Causes of the rapid warming of the north atlantic ocean in the mid-1990s. J. Clim. 25, 4116-4134. doi: 10.1175/JCLI-D-11-00443.1

Robson, J., Sutton, R., and Smith, D. (2012b). Initialized decadal predictions of the rapid warming of the north Atlantic ocean in the mid 1990s. Geophys. Res. Lett. 39:L19713. doi: 10.1029/2012GL053370

Robson, J., Sutton, R., and Smith, D. (2014). Decadal predictions of the cooling and freshening of the North Atlantic in 1960s and the role of ocean circulation. Clim. Dyn. 42, 2353-2365. doi: 10.1007/s00382-014-2115-7

Robson, J., Ortega, P., and Sutton, R. (2016). A reversal of climatic trends in the North Atlantic since 2005. Nat. Geosci. 9, 513-517. doi: 10.1038/ngeo2727

Roemmich, D., Alford, M. H., Claustre, H., Johnson, K., King, B., Moum, J., (2019). On the future of argo: a global, full-depth, multi-disciplinary array. Front. Mar. Sci. 6:439. doi: 10.3389/fmars.2019.00439

Roemmich, D., and Gilson, J. (2009). The 2004-2008 mean and annual cycle of temperature, salinity, and steric height in the global ocean from the Argo Program. Prog. Oceanogr. 82, 81-100. doi: 10.1016/j.pocean.2009.03.004

Roemmich, D., and Gilson, J. (2011). The global ocean imprint of ENSO. Geophys. Res. Lett. 38:L13606. doi: 10.1029/2011GL047992

Roemmich, D., John Gould, W., and Gilson, J. (2012). 135 years of global ocean warming between the challenger expedition and the argo programme. Nat. Clim. Chang. 2, 425-428. doi: 10.1038/nclimate1461

Roemmich, D., Cravatte, S., Delcroix, T., Gasparin, F., Hu, D., Johnson, G. C., et al. (2014). "White paper \#10-In situ temperature, salinity, and velocity observations," in Proceedings of the Report of the Tropical Pacific Observing System 2020 Workshop (TPOS 2020), Vol. II, (San Diego, CA: Scripps Institution of Oceanography), 27-30

Roemmich, D., Church, J., Gilson, J., Monselesan, D., Sutton, P., and Wijffels, S. (2015). Unabated planetary warming and its ocean structure since 2006. Nat. Clim. Chang. 5, 240-245. doi: 10.1038/nclimate2513

Rose, B. E. J., Armour, K. C., Battisti, D. S., Feldl, N., and Koll, D. D. B. (2014). The dependence of transient climate sensitivity and radiative feedbacks on the spatial pattern of ocean heat uptake. Geophys. Res. Lett. 41, 1071-1078. doi: 10.1002/2013GL058955

Rose, B. E. J., and Rayborn, L. (2016). The effects of ocean heat uptake on transient climate sensitivity. Curr. Clim. Chang. Rep. 2, 190-201. doi: 10.1007/s40641$016-0048-4$

Rudels, B. (2009). "Arctic ocean circulation," in Encyclopedia of Ocean Sciences, 2 Edn, eds J. H. Steele, K. K. Turekian, S. A. Thorpecpesnm, (Oxford: Academic Press), 211-225 doi: 10.1016/b978-012374473-9.00601-9

Sallée, J. B. (2018). Southern Ocean warming. Oceanography 31, 52-62. doi: 10. 5670/oceanog.2018.215

Scaife, A. A., Arribas, A., Blockley, E., Brookshaw, A., Clark, R. T., Dunstone, N., et al. (2014). Skillful long-range prediction of European and North American winters. Geophys. Res. Lett. 41, 2514-2519. doi: 10.1002/2014GL059637 
Schlundt, M., Brandt, P., Dengler, M., Hummels, R., Fischer, T., Bumke, K., et al. (2014). Mixed layer heat and salinity budgets during the onset of the 2011 Atlantic cold tongue. J. Geophys. Res. Oceans 119, 7882-7910. doi: 10.1002/ 2014JC010021

Schmidtko, S., Heywood, K. J., Thompson, A. F., and Aoki, S. (2014). Multidecadal warming of antarctic waters. Science 346, 1227-1231. doi: 10.1126/science. 1256117

Sérazin, G., Jaymond, A., Leroux, S., Penduff, T., Bessières, L., Llovel, W., et al. (2017). A global probabilistic study of the ocean heat content low-frequency variability: atmospheric forcing versus oceanic chaos. Geophys. Res. Lett. 44, 5580-5589. doi: 10.1002/2017GL073026

Shimada, K., Aoki, S., Ohshima, K. I., and Rintoul, S. R. (2012). Influence of ross sea bottom water changes on the warming and freshening of the antarctic bottom water in the australian-antarctic basin. Ocean Sci. 8, 419-432. doi: 10.5194/os8-419-2012

Slangen, A. B. A., Carson, M., Katsman, C. A., van de Wal, R. S. W., Koehl, A., Vermeersen, L. L. A., et al. (2014). Projecting twenty-first century regional sea-level changes. Clim. Chang. 124, 317-332. doi: 10.1007/s10584-014-1080-9

Sloyan, B. M., Wanninkhof, R., Kramp, M., Johnson, G. C., Talley, L. D., Tanhua, T., et al. (2019). The global ocean ship-based hydrographic investigations program (GO-SHIP): a platform for integrated multidisciplinary ocean science. Front. Mar. Sci. 6:445. doi: 10.3389/fmars.2019.00445

Smith, D. M., Cusack, S., Colman, A. W., Folland, C. K., Harris, G. R., and Murphy, J. M. (2007). Improved surface temperature prediction for the coming decade from a global climate model. Science 317, 796-799. doi: 10.1126/science. 1139540

Smith, D. M., and Murphy, J. M. (2007). An objective ocean temperature and salinity analysis using covariances from a global climate model. J. Geophys. Res. 112:C02022. doi: 10.1029/2005JC003172

Smith, D. M., Booth, B. B. B., Dunstone, N. J., Eade, R., Hermanson, L., Jones, G. S., et al. (2016). Role of volcanic and anthropogenic aerosols in the recent global surface warming?slowdown. Nat. Clim. Chang. 6, 936-940. doi: 10.1038/ nclimate3058

Smith, N., Kessler, W. S., Cravatte, S., Sprintall, J., Wijffels, S., Cronin, M. F., et al. (2019). Tropical pacific observing system. Front. Mar. Sci. 6:31. doi: 10.3389/ fmars.2019.00031

Smith, N. R. (1995). An improved system for tropical ocean sub-surface temperature analyses. J. Atmos. Oceanic Technol. 12, 850-870. doi: 10.1175/ 1520-0426(1995)012<0850:aisfto > 2.0.co;2

Smeed, D. A., Josey, S. A., Beaulieu, C., Johns, W. E., Moat, B. I., and FrajkaWilliams, E., et al. (2018). The north atlantic ocean is in a state of reduced overturning. Geophys. Res. Lett. 45, 1527-1533. doi: 10.1002/2017GL076350

Stott, P. A., Sutton, R. T., and Smith, D. M. (2008). Detection and attribution of Atlantic salinity changes. Geophys. Res. Lett. 35:L21702. doi: 10.1029/ 2008GL035874

Straneo, F., and Heimbach, P. (2013). North Atlantic warming and the retreat of Greenland's outlet glaciers. Nature 504, 36-43. doi: 10.1038/nature12854

Tang, W., Yueh, S., Yang, D., Fore, A., Hayashi, A., Lee, T., et al. (2018). The potential and challenges of using soil moisture active passive (SMAP) sea surface salinity to monitor arctic ocean freshwater changes. Remote Sens. 10:869. doi: 10.3390/rs10060869

Terray, L., Corre, L., Cravatte, S., Delcroix, T., Reverdin, G., and Ribes, A. (2012). Near-surface salinity as nature's rain gauge to detect human influence on the tropical water cycle. J. Clim. 25, 958-977. doi: 10.1175/JCLI-D-1005025.1

Llovel, W., and Terray, L. (2016). Observed southern upper-ocean warming over 2005-2014 and associated mechanisms. Environ. Res. Lett. 11:124023. doi: 10. 1088/1748-9326/11/12/124023

Tesdal, J., Abernathey, R. P., Goes, J. I., Gordon, A. L., and Haine, T. W. (2018). Salinity trends within the upper layers of the subpolar north atlantic. J. Clim. 31, 2675-2698. doi: 10.1175/JCLI-D-17-0532.1

Thornalley, D. J. R., Oppo, D. W, Ortega, P., Robson, J. I., Brierley, C. M., Davis, R. et al. (2018). Anomalously weak Labrador Sea convection and Atlantic overturning during the past 150 years. Nature 556, 227-230. doi: 10.1038/ s41586-018-0007-4

Todd, R. E., Chavez, F. P., Clayton, S., Cravatte, S. E., Goes, M. P., Graco, M. I., et al. (2019). Global perspectives on observing ocean boundary current systems. Front. Mar. Sci. 6:423.
Treasure, A. M., Roquet, F., Ansorge, I. J., Bester, M. N., Boehme, L., Bornemann, H. et al. (2017). Marine mammals exploring the oceans pole to pole: a review of the MEOP consortium. Oceanography 30, 132-138. doi: 10.5670/oceanog.2017. 234

Trenberth, K. E. (2011). Changes in precipitation with climate change. Clim. Res. 47, 123-138. doi: 10.3354/cr00953

Truffer, M., and Motyka, R. J. (2016), Where glaciers meet water: subaqueous melt and its relevance to glaciers in various settings. Rev. Geophys. 54, 220-239. doi: 10.1002/2015RG000494

van Wijk, E. M., and Rintoul, S. R. (2014). Freshening drives contraction of antarctic bottom water in the australian antarctic basin. Geophys. Res. Lett. 41, 1657-1664. doi: 10.1002/2013GL058921

Vinogradova, N., Lee, T., Boutin, J., Drushka, K., Fournier, S., Sabia, R., et al. (2019) Satellite salinity observing system: recent discoveries and the way forward. Front. Mar. Sci. 6:243. doi: 10.3389/fmars.2019.00243

von Schuckmann, K., Palmer, M. D., Trenberth, K. E., Cazenave, A., Chambers, D., Champollion, N., et al. (2016). An imperative to monitor Earth's energy imbalance. Nat. Clim. Chang. 6, 138-144. doi: 10.1038/nclimate2876

von Schuckmann, K., Storto, A., Simoncelli, S., Raj, R. P., Samuelsen, A., de Pascual Collar, A. et al. (2018). "Ocean heat content," in Copernicus marine Service Ocean state Report, Issue 2: Journal of Operational Oceanography, vol. 11, eds K. von Schuckmann, P.-Y. Le Traon, N. Smith, A. Pascual, P. Brasseur, K. Fennel, et al. (Ditcot: Taylor \& Francis), s41. doi: 10.1080/1755876X.2018.1489208

Wang, G., Cheng, L., Boyer, T., and Li, C. (2017). Halosteric sea level changes during the argo Era. Water 9:484. doi: 10.3390/w9070484

Wang, G., Cheng, L., Abraham, J., and Li, C. (2018). Consensuses and discrepancies of basin-scale ocean heat content changes in different ocean analyses. Clim. Dyn. 50:2471. doi: 10.1007/s00382-017-3751-5

Waugh, D. W., Primeau, F., DeVries, T., and Holzer, M. (2013). Recent changes in the ventilation of the southern oceans. Science 339, 568-570. doi: 10.1126/ science. 1225411

WCRP Global Sea Level Budget Group, (2018). Global sea-level budget 1993 present. Earth Syst. Sci. Data 10, 1551-1590. doi: 10.5194/essd-10-1551-2018

Weller, E., Min, S.-K., Palmer, M. D., Lee, D., Yim, B. Y., and Yeh, S.-W. (2016). Multi-model attribution of upper-ocean temperature changes using an isothermal approach. Sci. Rep. 6:26926. doi: 10.1038/srep26926

Weller, R. A., Baker, D. J., Glackin, M. M., Roberts, S. J., Schmitt, R. W., Twigg, E. S. et al. (2019) The Challenge of Sustaining Ocean Observations. Front. Mar. Sci. 6:105. doi: 10.3389/fmars.2019.00105

White, W. (1995). Design of a global observing system for gyre-scale upper ocean temperature variability. Prog. Oceanogr. 36, 169-217. doi: 10.1016/00796611(95)00017-8

Widlansky, M. J., Marra, J. J., Chowdhury, M. R., Stephens, S. A., Miles, E. R., Fauchereau, N., et al. (2017). Multimodel ensemble sea level forecasts for tropical pacific islands. J. Appl. Meteorol. Climatol. 56, 849-862. doi: 10.1175/ jamc-d-16-0284.1

Williams, P. D., Guilyardi, E., Sutton, R., Gregory, J., and Madec, G. (2007). A new feedback on climate change from the hydrological cycle. Geophys. Res. Lett. 34:L08706. doi: 10.1029/2007GL029275

Willis, J. K., Roemmich, D., and Cornuelle, B. (2004), Interannual variability in upper ocean heat content, temperature, and thermosteric expansion on global scales. J. Geophys. Res. 109:C12036. doi: 10.1029/2003JC0 02260

Willis, J. K., Chambers, D. P., and Nerem, R. S. (2008). Assessing the globally averaged sea level budget on seasonal to interannual timescales. J. Geophys. Res. Oceans 113:C06015. doi: 10.1029/2007JC004517

Wong, T., Wielicki, B. A., Lee, R. B., Smith, G. L., Bush, K. A., and Willis, J. K. (2006). Reexamination of the observed decadal variability of the earth radiation budget using altitude-corrected ERBE/ERBS nonscanner WFOV data. J. Clim. 19, 4028-4040. doi: 10.1175/jcli3838.1

Wunsch, C. (2016). Global ocean integrals and means, with trend implications. Annu. Rev. Mar. Sci. 8, 1-33. doi: 10.1146/annurev-marine-122414-034040

Wunsch, C., Schmitt, R. W., and Baker, D. J. (2013). Climate change as an intergenerational problem. Proc. Natl. Acad. Sci. U.S.A. 110, 4435-4436. doi: 10.1073/pnas.1302536110

Wyrtki, K. (1981). An estimate of equatorial upwelling in the pacific. J. Phys. Oceanogr. 11, 1205-1214. doi: 10.1175/1520-04851981011<1205:AEOEUI<2.0. $\mathrm{CO} ; 2$ 
Xie, P., Boyer, T., Bayler, E., Xue, Y., Byrne, D., Reagan, J., et al. (2014). An in situsatellite blended analysis of global sea surface salinity. J. Geophys. Res. Oceans 119, 6140-6160. doi: 10.1002/2014JC010046

Xu, X., Chassignet, E. P., Johns, W. E., Schmitz, W. J. Jr., and Metzger, E. J. (2014), Intraseasonal to interannual variability of the Atlantic meridional overturning circulation from eddy-resolving simulations and observations. J. Geophys. Res. Oceans 119, 5140-5159. doi: 10.1002/2014JC009994

Xue, Y., Wen, C., Kumar, A., Balmaseda, M., Fujii, Y., Alves, O., et al. (2017). A real-time ocean reanalyses intercomparison project in the context of tropical pacific observing system and ENSO monitoring. Clim Dyn. 49:3647. doi: 10. 1007/s00382-017-3535-y

Yao, W., Shi, J., and Zhao, X. (2017). Freshening of antarctic intermediate water in the south atlantic ocean in 2005-2014. Ocean Sci. 13, 521-530. doi: 10.5194/os13-521-7

Yashayaev, I. M., and Loder, J. W. (2017). Further intensification of deep convection in the labrador sea in 2016. Geophys. Res. Lett. 44, 1429-1438. doi: 10.1098/rsta. 2016.0321
Zenk, W., and Morozov, E. (2007). Decadal warming of the coldest antarctic bottom water flow through the vema channel. Geophys. Res. Lett. 34:L14607. doi: $10.1029 / 2007$ GL030340

Conflict of Interest Statement: The authors declare that the research was conducted in the absence of any commercial or financial relationships that could be construed as a potential conflict of interest.

At least a portion of this work is authored by Paul J. Durack on behalf of the U.S. Government and, as regards Dr. Durack and the U.S. Government, is not subject to copyright protection in the United States. Foreign and other copyrights may apply. This is an open-access article distributed under the terms of the Creative Commons Attribution License (CC BY). The use, distribution or reproduction in other forums is permitted, provided the original author(s) and the copyright owner(s) are credited and that the original publication in this journal is cited, in accordance with accepted academic practice. No use, distribution or reproduction is permitted which does not comply with these terms. 


\section{Satellite Ocean Colour: Current Status and Future Perspective}

OPEN ACCESS

Edited by:

Frank Edgar Muller-Karger, University of South Florida,

United States

Reviewed by:

James Yoder,

Woods Hole Oceanographic Institution, United States Robert J. Frouin,

University of California, San Diego, United States

*Correspondence: Steve Groom sbg@pml.ac.uk

Specialty section: This article was submitted to Ocean Observation,

a section of the journal Frontiers in Marine Science

Received: 05 December 2018

Accepted: 18 July 2019

Published: 29 August 2019

Citation:

Groom S, Sathyendranath S, Ban Y, Bernard S, Brewin R, Brotas V, Brockmann C, Chauhan P, Choi J-k,

Chuprin A, Ciavatta S, Cipollini P,

Donlon C, Franz B, He X, Hirata T, Jackson T, Kampel M, Krasemann $H$,

Lavender S, Pardo-Martinez S, Mélin F, Platt T, Santoleri R, Skakala J,

Schaeffer B, Smith M, Steinmetz F,

Valente $A$ and Wang M (2019) Satellite Ocean Colour: Current Status and Future Perspective.

Front. Mar. Sci. 6:485.

doi: 10.3389/fmars.2019.00485

\begin{abstract}
Steve Groom ${ }^{1,2 *}$, Shubha Sathyendranath ${ }^{1,2}$, Yai Ban $^{3}$, Stewart Bernard ${ }^{4}$, Robert Brewin ${ }^{1,2}$, Vanda Brotas ${ }^{5}$, Carsten Brockmann ${ }^{6}$, Prakash Chauhan ${ }^{7}$, Jong-kuk Choi ${ }^{8}$, Andrei Chuprin ${ }^{1}$, Stefano Ciavatta ${ }^{1,2}$, Paolo Cipollini $^{9}$, Craig Donlon ${ }^{10}$, Bryan Franz ${ }^{11}$, Xianqiang He ${ }^{3}$, Takafumi Hirata ${ }^{12}$, Tom Jackson ${ }^{1}$, Milton Kampel13, Hajo Krasemann ${ }^{14}$, Samantha Lavender ${ }^{15}$, Silvia Pardo-Martinez ${ }^{1}$, Frédéric Mélin ${ }^{16}$, Trevor Platt', Rosalia Santoleri'17, Jozef Skakala ${ }^{1,2}$, Blake Schaeffer ${ }^{18}$, Marie Smith ${ }^{4}$, Francois Steinmetz ${ }^{19}$, Andre Valente ${ }^{5}$ and Menghua Wang ${ }^{20}$
\end{abstract}

${ }^{1}$ Plymouth Marine Laboratory, Plymouth, United Kingdom, ${ }^{2}$ National Centre for Earth Observation, Plymouth Marine Laboratory, Plymouth, United Kingdom, ${ }^{3}$ State Key Laboratory of Satellite Ocean, Environment Dynamics, Second Institute of Oceanography, Ministry of Natural Resources, Hangzhou, China, ${ }^{4}$ CSIR Earth Systems Earth Observation, CSIR - NRE, Cape Town, South Africa, ${ }^{5}$ MARE, Faculdade de Ciências, Universidade de Lisboa, Lisbon, Portugal, ${ }^{6}$ Brockmann Consult, Geesthacht, Germany, ${ }^{7}$ Indian Institute of Remote Sensing, Dehradun, India, ${ }^{8}$ KIOST-PML Science Lab, Korea Institute of Ocean Science and Technology, Plymouth, United Kingdom, ${ }^{9}$ Telespazio VEGA UK Ltd. for ESA Climate Office, European Centre for Space Applications and Telecommunications, European Space Agency, Didcot, United Kingdom, ${ }^{10}$ European Space Research and Technology Centre, European Space Agency, Noordwijk, Netherlands, " ${ }^{11}$ Goddard Space Flight Center, NASA, Greenbelt, MD, United States, ${ }^{12}$ Arctic Research Center, Hokkaido University, Sapporo, Japan, ${ }^{13}$ Instituto Nacional de Pesquisas Espaciais São Jose dos Campos, São Paulo, Brazil, ${ }^{14} \mathrm{Helmholtz-Zentrum} \mathrm{Geesthacht} \mathrm{-} \mathrm{Zentrum} \mathrm{für} \mathrm{Material-}$ und Küstenforschung GmbH, Geesthacht, Germany, ${ }^{15}$ Pixalytics Ltd., Plymouth, United Kingdom, ${ }^{16}$ European Commission, Joint Research Centre (JRC), Ispra, Italy, ${ }^{17}$ Consiglio Nazionale delle Ricerche, Rome, Italy, ${ }^{18}$ Office of Research and Development, United States Environmental Protection Agency, Research Triangle, NC, United States, ${ }^{19} \mathrm{HYGEOS}$, Lille, France, ${ }^{20}$ Marine Ecosystems and Climate Branch, NOAA NESDIS STAR, College Park, MD, United States

Spectrally resolved water-leaving radiances (ocean colour) and inferred chlorophyll concentration are key to studying phytoplankton dynamics at seasonal and interannual scales, for a better understanding of the role of phytoplankton in marine biogeochemistry; the global carbon cycle; and the response of marine ecosystems to climate variability, change and feedback processes. Ocean colour data also have a critical role in operational observation systems monitoring coastal eutrophication, harmful algal blooms, and sediment plumes. The contiguous ocean-colour record reached 21 years in 2018; however, it is comprised of a number of one-off missions such that creating a consistent time-series of ocean-colour data requires merging of the individual sensors (including MERIS, Aqua-MODIS, SeaWiFS, VIIRS, and OLCI) with differing sensor characteristics, without introducing artefacts. By contrast, the next decade will see consistent observations from operational ocean colour series with sensors of similar design and with a replacement strategy. Also, by 2029 the record will start to be of sufficient duration to discriminate climate change impacts from natural variability, at least in some regions. This paper describes the current status and future prospects in the field of ocean colour focusing on large to medium resolution observations of oceans and coastal seas. It reviews the user requirements in terms of products and uncertainty characteristics and then describes features of current and future satellite ocean-colour sensors, both operational and innovative. The key role of in situ validation and calibration is highlighted as are ground segments that process the data received from the ocean-colour sensors and deliver analysis-ready products 
to end-users. Example applications of the ocean-colour data are presented, focusing on the climate data record and operational applications including water quality and assimilation into numerical models. Current capacity building and training activities pertinent to ocean colour are described and finally a summary of future perspectives is provided.

Keywords: ocean colour, phytoplankton, ground-segment, climate data records, water-quality, capacity building

\section{INTRODUCTION}

Satellite observation of ocean-colour radiometry involves detection of spectral variations in the water-leaving radiance (or reflectance), which is the sunlight backscattered out of the ocean after interaction with water and its constituents. In the open ocean the signal is primarily dependent on phytoplankton which contain photosynthetic pigments, primarily chlorophyll-a (chl-a) and an assemblage of other pigments, and which coexist together with associated detrital and coloured dissolved organic matter (CDOM) that are related to the phytoplankton. Coastal waters are more complex optically on account of the additional influences of re-suspended particulates, or river run-off which could contain terrestrial suspended particulates or CDOM which are independent of the phytoplankton assemblage (International Ocean Colour Coordinating Group [IOCCG], 2000).

To infer the concentration of chl-a or other optically active constituents from ocean-colour data, algorithms have been constructed relating characteristics of the water signal to the property of interest. These may be empirical algorithms (e.g., O'Reilly et al., 1998) or more complex semi-analytical approaches (e.g., Lee et al., 2002). However, because of the addition of light scattered by the atmosphere into the satellite view, the ocean colour signal is a relatively small part $(<10 \%)$ of the satellite-detected top-of-atmosphere (TOA) radiance; so highly accurate atmospheric correction schemes are needed to retrieve the water signal (Wang et al., 2009). Furthermore, because the water signal is needed to within 5\% (GCOS, 2016), the spaceborne sensor needs to retrieve TOA signal to $0.5 \%$ (International Ocean Colour Coordinating Group [IOCCG], 2012) and, hence, be well calibrated to avoid residual errors propagating to the water signal. This is accomplished through a combination of preflight calibration, post-launch onboard calibration of observation of external targets (such as the sun, moon or on the Earth) and a 'system vicarious calibration' undertaken to compare the expected TOA signal based on the water signal and atmospheric components with that measured by the sensor (see section 'Ocean Colour EO Sensors and in situ Observations'). The system vicarious calibration is considered essential to reach the $0.5 \%$ requirement (International Ocean Colour Coordinating Group [IOCCG], 2012).

The first ocean colour sensor was the proof-of-concept Coastal Zone Color Scanner (CZCS: Gordon et al., 1980; Hovis et al., 1980) launched in 1978 and operated until 1986. After this there was a gap in the record until the launch of the Japanese Ocean Color Temperature Scanner (OCTS) in 1996 and the United States Sea-viewing Wide field-of-view Sensor (SeaWiFS) in 1997, which marked the start of the contiguous ocean colour era. Since then there have been further missions, typically of oneoff instruments. This situation is, however, changing with the recent launches of operational ocean-colour sensor series (NOAA JPSS VIIRS) and ESA Sentinel 3 OLCI (see section 'Ocean Colour EO Sensors and in situ Observations').

The International Ocean Colour Coordinating Group $\left(\mathrm{IOCCG}^{1}\right.$ ) has been, and continues to be, the principal forum for space agencies, domain experts and user representatives to discuss coordination and integration of the ocean-colour field. Key activities include formation of scientific working groups and the resulting reports and protocols that describe requirements and developments in the field. Importantly, these reports are freely available and so accessible to scientists in developing countries who may not have journal access. IOCCG report 8 'Why ocean colour' (International Ocean Colour Coordinating Group [IOCCG], 2008) gave an overview of the varied applications of ocean colour for academic and societal applications, while other reports have focused more on specific issues including, inter alia: mission requirements for sensors (International Ocean Colour Coordinating Group [IOCCG], 2012), atmospheric correction (International Ocean Colour Coordinating Group [IOCCG], 2010), fisheries applications (International Ocean Colour Coordinating Group [IOCCG], 2009), phytoplankton functional types (International Ocean Colour Coordinating Group [IOCCG], 2014), and the most recent in the series focused on inland and coastal water quality (International Ocean Colour Coordinating Group [IOCCG], 2018). IOCCG initiated the biennial International Ocean Colour Coordinating Group [IOCCG], 2013 with an aim of bringing together the ocean colour community; the fourth meeting was in Korea in 2019.

This review starts with a summary of the user community requirements, then discusses current and upcoming satellite EO missions and in situ data, including citizen science/crowdsourcing, that support the ocean-colour sensors. It then describes ground segments, which take the raw ocean-colour data to generate intermediary or end products for users and provide data delivery and access mechanisms. Next, it discusses climate data records and presents example operational applications, considering how current and future developments in EO are likely to make an impact. The review discusses ongoing activities focused on ocean-colour relevant capacity building and training. Finally, a summary is given on perspectives for ocean colour in integrated, sustained observations for

\footnotetext{
${ }^{1}$ www.ioccg.org
} 
ocean, shelf and off-shore coastal applications but not on near-coastal, estuarine or inland water observation using high-resolution sensors. Furthermore, it does not cover polarimetry or LiDAR observations that are both described in Jamet et al. (2019).

\section{USER REQUIREMENTS}

In considering user requirements it is instructive to review how community white papers from OceanObs'09 articulated the requirements for ocean-colour observations from a variety of perspectives and how these have led to progress in the subsequent decade. Bonekamp et al. (2010) discussed how the operational requirements of satellite observations in general, including ocean colour, differed from research requirements, and how science-driven single missions were transitioning to multi-mission, sustained ocean observations to meet a variety of operational requirements. The paper by Yoder et al. (2010) led to the development of an oceancolour radiometry virtual constellation (OCR-VC) by the Committee on Earth Observation Satellites $\left(\mathrm{CEOS}^{2}\right)$ to ensure sustainability of ocean colour observations through the coordinated efforts of the individual national space agencies. Yoder et al. (2010) also identified a number of operational applications for ocean colour, for health (identification of pollution, eutrophication, and harmful algal blooms; impacts of aerosols on health); climate (contribution to carbon observations in the oceans); marine ecosystems; fisheries; and aquaculture. They showed how the ocean-colour capabilities were beginning to meet the requirements identified by the various tasks of the Group on Earth Observations (GEO). The community white paper by Forget et al. (2010) presented the ocean-colour requirements from a fisheries and aquaculture perspective; and that by Sathyendranath et al. (2010) highlighted the need to integrate satellite with in situ observations to address the requirements for monitoring marine ecosystems. Le Quéré et al. (2010) placed the needs for ocean colour in the broader context of observational requirements for validating dynamic green-ocean models; and Claustre et al. (2010) articulated how ocean colour fitted into the need for an integrated sustained observation system for marine ecosystems and biogeochemistry. Finally, Drinkwater et al. (2010) in their discussion of the role of space observations in operational oceanography, drew attention to the importance of ocean colour for marine ecology and applications thereof.

The decade since OceanObs'09 has seen many reports that have refined and elaborated the user requirements for oceancolour data and introduced additional requirements. They emerge from a variety of needs, including to understand the structure and function of the marine ecosystem and how it might be affected by climate variability and change, or by various types of pollution and their societal implications.

\footnotetext{
${ }^{2}$ http://ceos.org/ourwork/virtual-constellations/ocr/
}

The areas of studies reported to benefit from sustained oceancolour observations include:

- The global carbon cycle and its fluctuations at many time scales;

- Ocean acidification;

- Marine biodiversity and function;

- Validation and improvement of Earth System and ocean biogeochemical models;

- Data assimilation to improve model performance;

- Data for assessing impact and adaptation of marine ecosystem to climate change;

- Bio-feedback mechanisms, understanding Earth System;

- Flow of material through the marine food webs, implications for marine resources;

- Marine pollution; and

- The need to inform debates about bioengineering.

Notably, the IPCC Impacts and Adaptation Assessments (WGII) AR5 WGII Summary for Policymakers noted: 'Openocean net primary production is projected to redistribute and, by 2100 , fall globally under all RCP scenarios. Climate change adds to the threats of over-fishing and other non-climatic stressors, thus complicating marine management regimes (high confidence).'

The Global Climate Observing System (GCOS) defines a climate data record (CDR) as 'a time series of measurements of sufficient length, consistency and continuity to determine climate variability and change' and essential climate variables (ECVs) as measurements that contribute to characterisation of the Earth's climate, for which a CDR may be created. Ocean colour is an ECV with two specific products: spectrally resolved remote-sensing reflectances or (normalised) waterleaving radiances and chlorophyll concentration. GCOS specifies requirements in terms of spatial resolution and uncertainties $^{3}$ (Table 1).

The ESA Climate Change Initiative (CCI - see section 'Time Series for Climate') has been working toward producing ECVs that meet the GCOS requirements, and as part of the process has undertaken extensive user consultations. For ocean colour CCI the User Requirement Documents gave detailed requirements from a climate perspective $e^{4}$ revealing that in addition to the GCOS ocean colour ECV products, the user community has additional requirements for products that include carbon products and phytoplankton types. Improvements to coastal algorithms are also a requirement. The theme of carbon products from space was picked up by the CEOS 'Carbon from Space' Report (CEOS, 2014), which in turn was a response to the GEO Carbon Strategy Report. The CEOS report clearly recognises the central role played by ocean colour in generating carbon products (both pools and fluxes of carbon) in the aquatic environment (including marine and freshwater). It recognises the importance of integration at various levels: that of satellite observations

\footnotetext{
${ }^{3}$ https://www.ncdc.noaa.gov/gosic/gcos-essential-climate-variable-ecv-dataaccess-matrix/gcos-ocean-biogeochemistry-ecv-ocean-color

${ }^{4}$ http://www.esa-oceancolour-cci.org/?q?webfm_send/582
} 
TABLE 1 | The Global Climate Observing System uncertainty requirements for the ocean colour ECV.

\begin{tabular}{|c|c|c|c|c|}
\hline Products & Frequency & Spatial resolution & Required measurement uncertainty & Stability/decade \\
\hline Water-leaving radiance & Daily & $4 \mathrm{~km}$ & $5 \%$ in blue and green wavelengths & $0.5 \%$ \\
\hline Chl-a concentration & Weekly averages & $4 \mathrm{~km}$ & $30 \%$ & $3 \%$ \\
\hline
\end{tabular}

with in situ observations and modelling; that across domains (land, water and air) given the importance of interfaces and cross-domain fluxes; the need to account for the role of coasts as the interface between land and ocean; and threeway coupling and feedbacks across domains. Another report worth mentioning in this context is the white paper on 'Oceans and society: Blue Planet,' which is a GEO initiative ${ }^{5}$ that amalgamates the marine tasks within GEO. Many of the more recent reports have placed increasing emphasis on data harmonisation, uncertainty characterisation, traceability and transparency.

Considerations for identifying key variables have differed from report to report, and included factors such as the important environmental issues to be addressed, key questions to be answered, feasibility, cost, technology available for detection, platforms available for deployment, impact, and spatial and temporal scales of interest. However, the requirements for ocean-colour observations for operational oceanography and for societal applications have remained a common factor since POGO (Partnership for Observation of the Global Oceans) and CoML (Census of Marine Life) commissioned a report on 'Biological observations of the global ocean: requirements and how to meet them' in 2001. However, the products required from ocean-colour observations have grown over the years.

In addition to the products required from ocean colour, the uncertainty requirements merit consideration. For climate purposes, GCOS specifies (Table 1) 5\% for water-leaving radiance and $30 \%$ for chl-a, taken to mean relative error related to bias (see section 'Time Series for Climate' and Figure 3). The OC CCI user consultation also sought input on uncertainties and responses were received from EO scientists engaged in global and regional analyses, trend analysis, primary production studies, fisheries, phenology and other applications, together with modellers working on global and regional models, validation/skill assessment, model development and data assimilation. A clear message from respondents (100\% of modellers and $95 \%$ of EO scientists) is that product uncertainty estimates should be made available along with the product values and that uncertainties should be referenced to in situ data. Responses showed threshold (minimum) accuracy and precision requirements for chl-a of 10-25\% (modellers) and 25-50\% (EO scientists) with a goal of $<10 \%$. This goal was considered to be equivalent to the uncertainty in in situ observation (CCI URD, 2014). However, within the EO and end-user communities the commonly quoted uncertainty requirement for chl-a is 35\%. This is probably because the community is aware of the current

\footnotetext{
${ }^{5}$ https://geoblueplanet.org/
}

capabilities of satellite EO observations where uncertainties of products are dependent on the uncertainties of retrieval algorithms, which in turn are based on linking in situ radiances and chl-a. each of which has its intrinsic uncertainties: this is discussed further in section 'In situ Observations and Algorithms.'

Ocean colour is also a cross cutting Essential Ocean Variable (EOV) as defined by the Global Ocean Observing System $\left(\mathrm{GOOS}^{6}\right)$, and EOVs are identified by GOOS Expert Panels on the basis of relevance, feasibility and cost effectiveness. Satellite ocean-colour measurements contribute to observations of a number of other EOVs including, inter alia: phytoplankton biomass and diversity, inorganic carbon, and dissolved organic carbon. Furthermore, analysis of ocean colour data can contribute understanding of other EOVs, such as frontal locations in relation to fish distributions. Hence, ocean colour is a vital component in the GOOS 'Framework for Ocean Observing' concept and, hence, to the aims of OceanObs19.

Ocean colour is relevant to the GEO Biodiversity Observation Network (GEO-BON) that has defined Essential Biodiversity Variables (e.g., Muller-Karger et al., 2018) as 'the derived measurements required to study, report, and manage biodiversity change, focusing on status and trend in elements of biodiversity ${ }^{7}$.' There are six EBV classes and 21 EBV 'candidates': the latter are linked to targets for the United Nations (UN) Convention on Biological Diversity $\left(\mathrm{CBD}^{8}\right)$ as well as the UN Sustainable Development Goals (SDGs - see below). Ocean colour is of relevance to EBV class 'Species traits' candidate 'phenology' to observe global seasonal and interannual changes including bloom timing and duration, 'Ecosystem function' candidate 'Net primary productivity' and 'Ecosystem structure' candidate 'Ecosystem composition by functional type.'

Finally, the UN SDGs provide a major global challenge ${ }^{9}$ over the next decade. The SDGs have the aim of, inter alia, action to end poverty and economic inequality, address climate change, and sustainable consumption. There are 17 goals and the most relevant in the context of this review are Goal 14: 'Life Below Water', while inland and coastal water colour and water quality is particularly relevant to Goal 6: 'Clean water and sanitation.' The SDGs are sometimes considered only with regard to developing countries, but whilst issues in many developing countries are acute, marine pollution, sustainable management of marine ecosystem,

\footnotetext{
${ }^{6}$ http://www.goosocean.org

${ }^{7}$ https://geobon.org/ebvs/what-are-ebvs/

${ }^{8}$ https://www.un.org/en/events/biodiversityday/convention.shtml

${ }^{9}$ http://www.undp.org/content/undp/en/home/sustainable-development-goals. html
} 
ocean acidification and increasing scientific knowledge are relevant to communities worldwide. Some training activities focusing on SDGs are presented in section 'Training and Capacity Building.'

\section{OCEAN COLOUR EO SENSORS AND IN SITU OBSERVATIONS}

This section provides an overview of current and future elements of ocean colour sensors together with relevant in situ data gathering to support ocean colour.

\section{EO Sensors With Ocean-Colour Capability \\ Polar-Orbiting Sensors}

The first 15 years of the contiguous ocean colour record was characterised by one-off sensors or systems, like SeaWiFS, MERIS and MODIS (albeit with instruments on two spacecraft). By contrast, the next decade will see the availability of consistent observations from a series of missions supporting long-time series CDRs and operational monitoring applications, with processing by operational agencies such as NOAA in the United States and EUMETSAT in Europe. Complementing these systems are a number of current or planned missions that provide new capabilities (such as hyperspectral observations), geostationary platforms and systems that add to the CEOS ocean colour virtual constellation. These are all summarised in Table 2 and described below.

- In the United States:

- The NASA Aqua MODIS instrument, launched in 2002, has provided a backbone for ocean colour observations over the past 16 years. With 9 bands in the visible/NIR and wide swath it provides coverage every day. Aqua-MODIS was planned to operate in constellation with Terra-MODIS launched in 1999 but sensor calibration of the latter limits its use in ocean colour applications (Franz et al., 2008; Kwiatkowska et al., 2008) and the long-term trend of TerraMODIS has been linked to that of SeaWiFS and AquaMODIS and so is not recommended for use in CDRs.

- The Joint Polar Satellite System (JPSS) programme comprises a series of launches of the Visible Infrared Imaging Radiometer Suite (VIIRS) instrument: the first on Suomi-NPP in October 2011 and the second on NOAA20 in November 2017. Subsequent launches are planned on JPSS-2, 3 and 4 in 2021, 2026 and 2031, respectively ${ }^{10}$, all with the same instrument as SNPP and NOAA-20. VIIRS does not have some of the wavebands recommended by the IOCCG for ocean-colour sensors (International Ocean Colour Coordinating Group [IOCCG], 2012) but data have been shown to be of sufficient quality for addition to CDRs such the European Ocean Colour Climate Change Initiative products (see section 'Time Series for Climate').
In addition, VIIRS has three SWIR bands recommended by International Ocean Colour Coordinating Group [IOCCG] (2012) for deriving ocean colour products over turbid coastal and inland waters.

- The NASA Plankton, Aerosol, Cloud, ocean Ecosystem (PACE) mission, currently scheduled for launch in late 2022, is planned to carry the Ocean Color Instrument (OCI) that will provide hyperspectral radiometric measurements between 340- and 890-nm at 5-nm spectral resolution and at seven discrete bands in the short-wave infrared (940, $1038,1250,1378,1615,2130$, and 2160-nm), with 1-day global coverage (2663-km swath) at $1-\mathrm{km}$ resolution (nadir view). OCI is designed to support heritage and advanced ocean-colour applications, as well as atmosphere and cloud science. PACE is also expected to carry two polarimetres that measure polarised light at multiple view angles: the Hyperangular Rainbow Polarimetre 2 (HARP2), and the Spectro-polarimetre for Planetary Exploration (SPEXone). HARP2 is a wide-swath polarimetre $(1556-\mathrm{km})$ that provide measurements at four discrete wavelengths in the visible to near infrared, and at 20-60 along-track viewing angles. SPEXone is a narrow-swath (106-km at nadir) hyperspectral polarimetre measuring from 385 to $770 \mathrm{~nm}$ with $2-4 \mathrm{~nm}$ spectral sampling and 5 along-track viewing angles. In addition to supporting cloud science and detailed aerosol property retrievals, the combined capabilities of the PACE polarimetres ${ }^{11}$ is expected to contribute to improvements in atmospheric correction for OCI ocean-colour retrievals.

- In Europe, the Copernicus programme plans four Sentinel 3 spacecraft carrying the Ocean and Land Colour Imager (OLCI: Donlon et al., 2012) with Sentinel 3A and 3B launched, respectively in 2016 and 2018. The motivation for Copernicus is to ensure continuity and consistency of observations enabling construction of services utilising these EO data. OLCI has 21 spectral bands from 400 to $1020 \mathrm{~nm}$ in the SWIR. Copernicus has an open data policy and supports upstream data processing; for OLCI processing of data from L1 to L2 will be undertaken by EUMETSAT and data are available via ftp and satellite digital broadcast (Eumetcast) making the data readily available even in developing countries with poor internet connections. Level 3 and regional (European) products are provided through the Copernicus Marine Environment Monitoring Service while the CDRs are available from the Copernicus Climate Change Service.

- In India, ocean-colour missions started with the launch of Oceansat-1 (on IRS-P4) with the Ocean Colour Monitor (OCM) in 1999 followed by Oceansat-2 in 2009 carrying a modified OCM-2 sensor. OCM is in operation and providing data with a spatial resolution of $360 \mathrm{~m}$ and global data at $1 \mathrm{~km}$ spatial resolution and temporal resolution of every 2-3 days. The OCM sensor has been used extensively for studying ocean phytoplankton, suspended particulate matter (SPM), and aerosol optical depth over ocean and data are operationally used for services like

\footnotetext{
${ }^{10} \mathrm{https} / / /$ www.jpss.noaa.gov
}

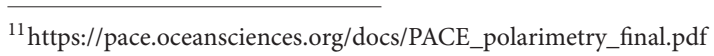


TABLE 2 | Recent, existing, and near-future satellite sensor systems of relevance for ocean colour (revised and updated after International Ocean Colour Coordinating Group [IOCCG], 2018).

\begin{tabular}{|c|c|c|c|c|c|c|c|c|c|c|c|c|c|}
\hline \multirow[t]{2}{*}{ Status } & \multirow[t]{2}{*}{ Sensor type } & \multirow[t]{2}{*}{ Platform } & \multirow[t]{2}{*}{ Sensor } & \multirow{2}{*}{$\begin{array}{c}\text { Spatial } \\
\text { Resolution= } \\
\text { Pixel size }\end{array}$} & \multirow{2}{*}{$\begin{array}{l}\text { Spectral bands } \\
(400-1000 \mathrm{~nm})\end{array}$} & \multirow{2}{*}{$\begin{array}{l}\text { Revisit frequency } \\
\text { (at equator) }\end{array}$} & \multirow[t]{2}{*}{ Launch (end) } & \multicolumn{6}{|c|}{ Water quality variables } \\
\hline & & & & & & & & Chl & CYP & TSM & CDOM & Kd & Turb/ SD \\
\hline \multirow[t]{2}{*}{ Defunct } & Sun-sync & SeaStar & SeaWiFS & 4 and $1 \mathrm{~km}$ & 8 & 2 days & 1997-(2010) & & S & & & & \\
\hline & & Envisat & MERIS & $\begin{array}{l}1.2 \mathrm{~km} \text { and } \\
0.3 \mathrm{~km}\end{array}$ & 15 & 2 days & 2002-(2012) & & & & & & \\
\hline \multirow[t]{7}{*}{ Current } & Sun-sync & Terra/Aqua & MODIS-A\&T & $1 \mathrm{~km}$ & 9 & daily & $1999 / 2000$ & & S & & & & \\
\hline & & Oceansat 2 & OCM-2 & $300 \mathrm{~m}$ & 8 & 2-3 days & 2009 & & & & & & \\
\hline & & Suomi/NOAA-20 & VIIRS & $750 \mathrm{~m}$ & 7 & daily & $2011 / 2017$ & & S & & & & \\
\hline & & Sentinel 3 A/B & OLCl & $300 \mathrm{~m}$ & 21 & $\begin{array}{l}\text { Daily (with } 2 \\
\text { satellites) }\end{array}$ & 2016/18 & & & & & & \\
\hline & & GCOM-C & SGLI-2 & $250 \mathrm{~m}$ & 9 & 2-4 days & 2017 & & S & & & & \\
\hline & GEO & KOMPSAT & $\mathrm{GOCl}$ & $500 \mathrm{~m}$ & 8 & Half hourly & 2010 & & & & & & \\
\hline & & $\begin{array}{l}\text { Himawari-8\&9, } \\
\text { GOES-R }\end{array}$ & $\mathrm{AHI}$ & $500 \mathrm{~m}-2 \mathrm{~km}$ & 4 & $10 \mathrm{~min}$ & 2014 & & & & & & \\
\hline \multirow[t]{6}{*}{ Future } & Sun-sync & JPSS-2, JPSS-3 & VIIRS. & $750 \mathrm{~m}$ & 7 & daily & 2017, 2022, & & $S$ & & & & \\
\hline & & Sentinel 3 C/D & OLCl & $300 \mathrm{~m}$ & 21 & $\begin{array}{l}\text { Daily (with } 2 \\
\text { satellites) }\end{array}$ & $>2022$ & & & & & & \\
\hline & & Oceansat-3 & OCM-3 & $300 \mathrm{~m}$ & 13 & 2-3 days & 2020 & & & & & & \\
\hline & & Sabia-MAR & MUS & $200 / 800 \mathrm{~m}$ & 13 & $1-2$ days global & & & & & & & \\
\hline & & PACE & $\mathrm{OCl}$ & $1 \mathrm{~km}$ & $\begin{array}{c}\text { Hyperspectral; } \\
5 \mathrm{~nm}(340-890 \mathrm{~nm})\end{array}$ & Daily & 2022 & & & & & & \\
\hline & GEO & KOMPSAT-3B & $\mathrm{GOCl}$ & $500 \mathrm{~m}$ & $8 s$ & Half hourly & 2019 & & & & & & \\
\hline
\end{tabular}

: highly suitable, ㅁ: suitable, ㅁ: potential, $\mathbf{\square}:$ not suitable, Chl, Chlorophyll; CYP, cyanobacterial pigments (S denotes surface blooms); TSM, total suspended matter; CDOM, coloured dissolved organic matter; Kd, diffuse attenuation coefficient (or attenuation coefficient of diffuse light); Turb, Turbidity / SD, Secchi Disk Depth. 
potential fishing zone selection (see section 'Operational Ocean-Colour Applications'). OCM-3 is due for launch on Oceansat-3 in 2020.

- In Japan, the Japan Aerospace eXploration Agency (JAXA) launched the polar-orbiting Global Change Observation Mission - Climate (GCOM -C) satellite in December 2017 carrying the Second-generation GLobal Imager (SGLI), a successor of the Global Imager (GLI) on-board the ADEOS II satellite. SGLI observes in 11 nadir channels in the visible - near-IR, with spatial resolution of $250 \mathrm{~m}$, including the first observations at $380 \mathrm{~nm}$, together with two polarisation forward/back along-track slant views channels at 673.5 and $865.5 \mathrm{~nm}$ with $1-\mathrm{km}$ resolution. The initial plan for GCOM-C included the intermittent launch of 3 identical or similar satellite sensors over 15 years for a continuous monitoring of ocean colour.

- InChina, the civil space infrastructure development plan (2015-2025) foresees the launch of more than 15 ocean satellites, which includes ocean-colour satellites (HY-1 series), ocean-dynamics satellites (HY-2 series), and high spatial or temporal resolution monitoring satellites (HY-3 series). Three ocean-colour satellites (HY-1A, HY-1B, and HY-1C) have been launched, the last in 2018, and HY-1D will be launched in 2019. HY-1C and HY-1D, both with global ocean-colour observing capability, will be combined as a constellation observing in the morning (10:30 AM) and afternoon (1:30 PM), respectively. In addition to the sensors on HY-1B (Chinese Ocean Colour and Temperature Scanner and Coastal Zone Imager), HY-1C also has a new ultraviolet imaging sensor on-board, with 2 bands at 345-365 nm and 375-395 nm (550 m pixel resolution), which will be of benefit for atmospheric correction over turbid waters and for monitoring of CDOM. Ocean-colour satellites HY-1E and HY-1F, planned to be launched in 2021, will carry ocean-colour sensors similar to MERIS and OLCI, with high signal-to-noise ratios. The Moderate-resolution Wide-wavelengths Imager (MWI) on-board the Chinese Tiangong-2 Space Lab was launched in 2016, as an experimental precursor to HY-1E and HY-1F. With $100 \mathrm{~m}$ spatial resolution and 18 bands in the visible light and infrared wavelengths, MWI has proven the high quality of its ocean-colour observations, especially over coastal and inland waters (He et al., 2017).

- In Argentina, the Satélites Argentino-Brasileño para Información Ambiental del Mar (Sabia-MAR) mission is planned for launch in 2022 carrying optical sensors in the VIS/NIR/SWIR with $200 \mathrm{~m}$ resolution (regional) and $800 \mathrm{~m}$ (global). It was originally part of a two-satellite mission but the Brazilian spacecraft is unlikely to proceed, according to current status.

\section{Geostationary Sensors}

- Geostationary (GEO) ocean colour sensors offer the potential to observe variations in water optical properties over a day or a tidal cycle as well as mitigating the effects of clouds through repeated observations (see review by Ruddick et al., 2014). However, to date only the Korea Institute of Ocean Science and Technology (KIOST) Geostationary Ocean Color Imager (GOCI) instrument launched in June 2010 with 8 VIS/NIR bands at $500 \mathrm{~m}$ resolution has provided GEO ocean colour capabilities (Choi et al., 2012, 2014a). Neither of the proposed European GeOCAPI or US GEO-CAPE missions have progressed beyond initial planning. GOCI-II is scheduled for launch in 2019 on NEO-KOMPSAT-2B, possesses 13 VNIR bands at $300 \mathrm{~m}$ resolution and has Full Disk mode (providing one image per day) in addition to the Local Area mode of GOCI. China plans to launch a GEO ocean colour satellite, HY-3C, in 2022 with the prototype GEO ocean-colour sensor with 10 bands from 412 to $1640 \mathrm{~nm}$ (8 VIS/NIR; 2 SWIR), signal-to-noise ratio higher than $600,250 \mathrm{~m}$ spatial resolution for VIS/NIR and $1 \mathrm{~km}$ for SWIR, and hourly observation over $2500 \mathrm{~km} \times 2500 \mathrm{~km}$ (25 slots, each slot $512 \mathrm{~km} \times 512 \mathrm{~km}$ ). He et al. (2018) have developed a vector radiation transmission model for a coupled oceanatmosphere system that takes into account Earth's curvature (PCOART-SA), which can be applied to the high solar zenith angle satellite data from geostationary satellites (e.g., dawn and dusk observations).

- Geostationary meteorological sensors: the European Meteosat Second Generation SEVIRI was designed for meteorological applications, but with a single visible band has demonstrated capability to observe SPM or turbidity dynamics (Alvera-Azcarate et al., 2015; Kwiatkowska et al., 2016; Ody et al., 2016) and has been used in synergy with polar-orbiting ocean colour sensor observations (Vanhellemont et al., 2014). The Japanese Himawari 8 has 6 vis/NIR spectral bands including blue, green and red channels and can retrieve chl-a if data are averaged over $1 \mathrm{~h}$ (Murakami, 2016). Likewise, the forthcoming Meteosat Third Generation meteorological-focused Flexible Combined Imager has three visible bands and simulations have shown chl-a retrieval capability if data are averaged in space and time (Lavigne and Ruddick, 2018).

\section{Novel Observation Platforms}

- The European TROPOspheric Monitoring Instrument (TROPOMI) is carried on board the Copernicus Sentinel-5 Precursor satellite, the first of the atmospheric composition Sentinels, launched in October 2017. TROPOMI, similar to SCIAMACHY on the ESA ENVISAT platform (2002-2012), has ocean-colour capability, notably of phytoplankton functional types, through processing of the hyperspectral top-of-atmosphere radiance (e.g., Losa et al., 2017). Similarly, hyperspectral data from the Global Ozone Monitoring Experiment-2 (GOME-2) instrument carried on EUMETSAT's MetOp series has been used to retrieve solar induced fluorescence (SIF) from chl-a (e.g., Joiner et al., 2016).

- NASA's Earth Polychromatic Imaging Camera (EPIC) onboard the Deep Space Climate Observatory (DSCOVR) satellite, launched in 2015 and located $1.510^{6} \mathrm{~km}$ above the Earth's surface orbiting the Lagrange point 1, provides 
repeated colour images of the Earth's surface (its position is fixed with respect to the Earth and Sun). It possesses 10 narrow bands including three of ocean-colour relevance, but the pixel size at nadir is $8-\mathrm{km}$ and the sensor signalto-noise is only $200: 1$, with the consequence that it does not meet requirements for most ocean-colour applications. Nevertheless, Gao et al. (2019) produced atmospherecorrected reflectances over various inland and ocean sites and described requirements for an ocean-colour capable sensor located at Lagrange 1.

- EUMETSAT's MetOp second generation A missions will carry the Multi-Viewing Multi-Channel Multi-Polarisation Imaging (3MI) instrument with launches planned in 2022, 2029 , and 2036. This mission complements the NOAA JPSS described above. $3 \mathrm{MI}$ has a secondary objective of oceancolour observations, though it does not possess an on-board calibration facility and has relatively low SNR in its 12 VIS/NIR/SWIR spectral bands.

\section{Higher Resolution Sensors}

- Higher-resolution sensors are summarised in Table 3. Some, such as the United States Geological Survey Landsat 8 OLI and Copernicus Sentinel 2 MSI at 10-60 m resolutions were designed for terrestrial applications but have inland-water colour (e.g., Bresciani et al., 2018) or ocean-colour capabilities such as for monitoring suspended particulates (e.g., Vanhellemont and Ruddick, 2014; Vanhellemont and Ruddick, 2015) or coastal floating vegetation (Dogliotti et al., 2018). Very high-resolution sensors, some commercial, have been exploited for coastal monitoring of underwater vegetation, coral reefs, etc.

- Nano-satellites (or cube-sats) offer potential to create relatively low-cost ocean-colour constellations, and the proof-of-concept HawkEye instrument launched in December 2018 is the first of two sensors ${ }^{12}$ offering multispectral ocean colour observations in 8 bands with a sensitivity similar to SeaWiFS. The $120 \mathrm{~m}$ resolution and baseline of 15 scenes per day (each $200 \mathrm{~km} \times 600 \mathrm{~km}$ ) leads to a coastal or inland water observing paradigm, but global observing capability could be provided with a swarm of instruments.

- Finally, High Altitude Pseudo-Satellites (or High Altitude Platforms: HAP) are aircraft flying $\sim 20 \mathrm{~km}$ high in the stratosphere, and may be balloons, airships, gliders or powered 'planes, either manned or unmanned (d'oliveira et al., 2016). At such heights HAPs provide repeated observations similar to GEO spacecraft, but over a smaller range and at a much lower cost. A particular advantage is that missions would be typically of shorter duration and so HAP-borne instruments could be re-calibrated regularly. A number of concepts are under investigation (d'oliveira et al., 2016): disaster management or emergency response, or marine traffic situational awareness, form typical proposed environmental monitoring applications. In the ocean-colour field, monitoring for eutrophication

\footnotetext{
${ }^{12} \mathrm{https} / /$ uncw.edu/socon/mission.html
}

or harmful algal blooms could be potential applications in areas of dense aquaculture or in response to a specific pollution event.

These sensors and applications, however, deserve a dedicated review paper, and so for brevity are not discussed further herein.

\section{Adequacy of Ocean-Colour Missions}

It is clear from the sections above that there are many oceancolour missions in existence or in planning phase. This section reviews the adequacy of the missions for different scientific and operational needs. However, adequacy depends significantly on the nature of the application.

Polar-orbiting missions provide the mainstay for observational needs for scientists, for operational applications and monitoring purposes. The virtual constellation of two operational Sentinel 3 OLCI and two S-NPP/JPSS VIIRS together provide two observations per day at slightly different overpass times, albeit reduced by cloud cover. These are complemented by older sensors such as Aqua-MODIS or newer missions like GCOM-C.

For climate applications the key requirements are longevity and consistency of the ocean-colour record, with biases between the sensors needed to provide a record of sufficient duration, adequately characterised - this is discussed further in section 'Time Series for Climate.'

Operational applications, such as monitoring for harmful algal blooms, are also served by the virtual constellations, with the multiple sensors providing gap-filling capabilities. It is important to note that the development of operational applications, notably by commercial companies, relies on the continuity provided in operational missions: that is to say, it would be difficult to justify commercial investment based on a single mission that may not be replaced. It is also worth noting that some applications do not need highly accurate ocean-colour estimates but instead use boundaries or gradients in water masses [e.g., see section 'Use of Ocean Colour Data for Location of Potential Fishing Zones (PFZ)'].

Regarding spatial resolution, OLCI provides full oceancolour capability to $300 \mathrm{~m}$ but higher resolution (of order $10-100 \mathrm{~m}$ ) to detect in bays or estuaries, where natural or farmed aquaculture is often located, or the observation of colour in inland waters, are not adequately supported, since missions providing such data are focused on land applications (e.g., Sentinel 2 or Landsat 8). However, as noted above, sensors on these missions have limited capabilities for retrieval of SPM concentrations, and in some cases of chl-a concentrations. Mission requirements to meet these needs were presented in International Ocean Colour Coordinating Group [IOCCG] (2018). Improvements to the capability of Sentinel 2 or Landsat 8 sensors could partly meet these requirements, along with HAP or nanosatellites, assuming that issues of calibration are addressed.

Applications requiring high temporal frequency, such as to observe $\mathrm{HAB}$ species that migrate to surface waters during the day, require GEO ocean-colour sensors that 
TABLE 3 | Recent, existing, and near-future satellite sensor systems of relevance for inland and near-coastal water quality (revised and updated after International Ocean Colour Coordinating Group [IOCCG], 2018).

\begin{tabular}{|c|c|c|c|c|c|c|c|c|c|c|c|c|c|}
\hline \multirow[t]{2}{*}{ Status } & \multirow[t]{2}{*}{ Sensor type } & \multirow[t]{2}{*}{ Platform/Sensor } & \multirow{2}{*}{$\begin{array}{l}\text { Spatial } \\
\text { Resolution = Pixel } \\
\text { size }\end{array}$} & \multirow{2}{*}{$\begin{array}{l}\text { Spectral bands } \\
(400-1000 \mathrm{~nm})\end{array}$} & \multirow{2}{*}{$\begin{array}{l}\text { Revisit } \\
\text { frequency (at } \\
\text { equator) }\end{array}$} & \multirow{2}{*}{$\begin{array}{l}\text { Raw Data Cost } \\
\text { per km² [USD*] }\end{array}$} & \multirow[t]{2}{*}{ Launch (End) } & \multicolumn{6}{|c|}{ Water quality variables } \\
\hline & & & & & & & & Chl & CYP & TSM & CDOM & Kd & Turb/ SD \\
\hline \multirow[t]{3}{*}{ Defunct } & $\begin{array}{l}\text { Mid-spatial } \\
\text { resolution }\end{array}$ & LANDSAT 1 to 7 & $30 \mathrm{~m}$ & 4 & 16 days & Free & Various & & S & & & & \\
\hline & Hyperspectral & Hyperion & $30 \mathrm{~m}$ & 60 & 60 & Free & $2000(2017)$ & & & & & & \\
\hline & Hyperspectral & ISS/HICO & $90 \mathrm{~m}$ & 100 & $\begin{array}{l}\text { Orbit } 51 \mathrm{~N} \text { and } \\
51 \mathrm{~S}=>3 \text { to } \\
5 \text { days cadence }\end{array}$ & Free & 2009 (2014) & & & & & & \\
\hline \multirow[t]{9}{*}{ Current } & $\begin{array}{l}\text { Mid-spatial } \\
\text { resolution }\end{array}$ & $\begin{array}{l}\text { Terra /Aqua } \\
\text { MODIS }\end{array}$ & 500 m & 2 & Daily & Free & 1999/ 2002 & & & & & & \\
\hline & & “ & $250 \mathrm{~m}$ & 2 & Daily & Free & & & & & & & \\
\hline & & $\begin{array}{l}\text { Suomi/NOAA20 } \\
\text { VIIRS }\end{array}$ & 375 m & 3 & Daily & Free & $2011 / 2017$ & & & & & & \\
\hline & $\begin{array}{l}\text { Mid-to high } \\
\text { spatial resolution }\end{array}$ & LANDSAT 8 -OLI & $30 \mathrm{~m}$ & 5 & 16 days & Free & 2013 & & S & & & & \\
\hline & & SENTINEL-2 MSI & $\begin{array}{l}10 \mathrm{~m} \text { to } 60 \mathrm{~m} \\
\text { bands }\end{array}$ & 10 & $\begin{array}{l}10 \text { days per } \\
\text { sensor; } 5 \text { days } \\
\text { with two S-2's }\end{array}$ & Free & 2015 & & S & & & & \\
\hline & $\begin{array}{l}\text { High-spatial } \\
\text { resolution }\end{array}$ & $\begin{array}{l}\text { QuickBird, SPOT- } \\
6 \text { GeoEye, etc. }\end{array}$ & $2-4$ m & 3 to 4 & $\begin{array}{l}\text { Programmable - } \\
60 \text { to } 2-3 \text { days }\end{array}$ & 5 to 15 & 1999 and onward & & S & & & & \\
\hline & & RapidEye & $6.5 \mathrm{~m}$ & 5 & Daily & 1.5 & 2008 & & S & & & & \\
\hline & & WORLDVIEW-2 & $\begin{array}{l}2 \mathrm{~m} \text { spectral - } \\
0.5 \mathrm{~m} \text { B\&W }\end{array}$ & 8 & $\begin{array}{l}\text { Programmable - } \\
60 \text { to } 1 \text { day }\end{array}$ & 30 & 2009 & & & & & & \\
\hline & & WORLDVIEW-3 & $\begin{array}{l}1.24 \mathrm{~m} \text { spectral - } \\
0.50 \mathrm{~m} \mathrm{B \& W}\end{array}$ & 8 & $\begin{array}{l}\text { Programmable - } \\
60 \text { to } 1 \text { days }\end{array}$ & 30 & 2014 & & & & & & \\
\hline \multirow[t]{5}{*}{ Future } & Hyperspectral & EnMap & $30 \mathrm{~m}$ & 90 & $\begin{array}{l}\text { Programmable } \\
\text { (once per } 4 \text { days) }\end{array}$ & Free(?) & 2020 & & & & & & \\
\hline & & ISS/DESIS & $30 \mathrm{~m}$ & 235 & $\begin{array}{l}\text { Orbit } 51 \mathrm{~N} \text { and } \\
51 \mathrm{~S}=>3 \text { to } \\
5 \text { days cadence }\end{array}$ & Free(?) & ???? & & & & & & \\
\hline & & ISS/ HISUI-hyper & $30 \mathrm{~m}$ & 60 & $\begin{array}{l}\text { Orbit } 51 \mathrm{~N} \text { and } \\
51 \mathrm{~S}=>3 \text { to } \\
5 \text { days cadence }\end{array}$ & Free(?) & 2018 & & & & & & \\
\hline & & PRISMA & $\begin{array}{l}20 \mathrm{~m} \\
\text { spectral }-2.5 \mathrm{~m} \\
\text { B\&W }\end{array}$ & 66 & $\begin{array}{l}25 \text { days/pointing } \\
7 \text { days }\end{array}$ & Free(?) & ???? & & & & & & \\
\hline & & $\begin{array}{l}\text { Surface Biology } \\
\text { Geology = HySpIRI }\end{array}$ & 30 & 60 & 16 & Free & 2022 & & & & & & \\
\hline
\end{tabular}

口: highly suitable, I: suitable, $\mathbf{\square}:$ potential, $\mathbf{\square}$ : not suitable. CHL, Chlorophyll; CYP, cyanobacterial pigments; TSM, total suspended matter; CDOM, coloured dissolved organic matter; Kd, diffuse attenuation coefficient (or attenuation coefficient of diffuse light); Turb, Turbidity; SD, Secchi Disk Depth. Free, publicly available; Free(?), available upon request for research. 
currently are only available over east Asia (with GOCI see section 'Geostationary Sensor Monitoring of a Harmful Algal Bloom'). Conversely, commercial EO companies are addressing the need for frequent observations using constellations of satellites (Muller-Karger et al., 2018); however, these authors describe the stringent requirements for such a constellation designed for ocean colour observations, in terms of radiometric quality (high signalto-noise) and spectral resolution as well as spatial and temporal resolution.

Applications requiring hyperspectral observations are also not served by operational missions, though the launch of NASA PACE will support this area.

\section{In situ Observations and Algorithms}

To infer in-water properties such as chl-a from satellite observations of ocean colour requires retrieval of the waterleaving radiance that, as noted above, may be less than $10 \%$ of the top of atmosphere detected signal and, hence, requires high precision in the satellite sensor calibration. Then, the retrieved water-leaving radiances are linked to inwater properties using models or algorithms (harnessing new capabilities when available, e.g., hyperspectral measurements). Radiometric calibration of spaceborne sensors was addressed by IOCCG report 14 (International Ocean Colour Coordinating Group [IOCCG], 2013) and various recommendations were made, including pre-flight characterisation of sensors; calibration of reflectance instead of radiance; vicarious calibration to meet accuracy requirements (see below); the location of calibration sites, i.e., spatially homogenous oceanic regions sufficiently far from land; consistency in approaches between space agencies; and easy availability of calibration data to the ocean colour community.

Regarding in situ data for algorithm development or calibration/validation of satellite-retrieved geophysical products, the in situ measurements must meet strict criteria, including being taken using instruments with documented SI traceability based on metrology standards and an associated uncertainty budget, collected following community-agreed protocols and procedures, and freely available to other researchers for independent verification. Such data have been termed Fiducial Reference Measurements (FRM). Recommendations on the need for in situ measurements for satellite calibration and validation of long-term CDRs were documented in a white paper by the International Network for Sensor Inter-comparison and Uncertainty assessment for Ocean Color Radiometry (INSITU-OCR) initiative $^{13}$ and are updated as part of the on-going activities of the Ocean Colour Radiometry - Implementation Team (OCR-IT). Protocols for in situ observations were produced during the SeaWiFS era and recent efforts and discussions at meetings such as the IOCS, have been made to update these protocols, taking into account developments in sensors and sampling platforms; draught and final texts are available at

\footnotetext{
${ }^{13} \mathrm{http}: / /$ ioccg.org/wp-content/uploads/2016/02/INSITU-OCR-white-paper.pdf
}

http://ioccg.org/what-we-do/ioccg-publications/ocean-opticsprotocols-satellite-ocean-colour-sensor-validation/.

\section{Algorithms}

Algorithms link the satellite observations of water-leaving radiance (retrieved after atmospheric correction) and the inwater quantities of interest such as chl-a. Many algorithms have been proposed for chl-a, for example, and this field has been the subject of many review papers and is only covered briefly herein: readers can consult International Ocean Colour Coordinating Group [IOCCG] (2018) and forthcoming reports (such as the GEO AquaWatch review) for further details.

For ocean and shelf waters the most commonly used algorithms are band ratios of blue green wavelengths (e.g., O'Reilly et al., 1998) constructed by regression of in situ observations. A common feature of such algorithms, even in socalled 'case 1' waters where the optical properties are driven by phytoplankton and co-varying optically active substances, is the considerable variability around the regression. This is probably due to the diversity of water types encountered and a result of the uncertainties in the in situ measurements (e.g., radiances and chl-a) used in construction of algorithms. Furthermore, an in situ chl-a sample may be from $1 \mathrm{~L}$ of water and is compared with in situ upwelling radiance observations representing a signal from a much larger mass of water.

A summary of studies comparing chl-a algorithms applied to single sensor or multi-sensor merged satellite records with global in situ datasets showed average root mean square error of 0.337 $\log _{10}$ chl-a (Brewin et al., 2016). A further issue is that global analyses are usually based on measurements from independent investigators meaning that methodological differences between laboratories can introduce additional uncertainties. Sørensen et al. (2007) compared measurements of chl-a by high performance liquid chromatography (HPLC - considered the reference technique for chl-a) by 11 teams involved in the validation of the ESA MERIS sensor. Measurements showed wide variations amongst teams: in the first intercomparison using algal cultures the coefficient of variation (CV) spanned 10-25\%; in a second intercomparison based on natural waters, CV was 10$16 \%$ in 'case 2' waters and $7-40 \%$ for case 1 waters. These results were after removal of 'outliers' which, in the field, may not be recognised as such. They attributed much of the difference to extraction methods since an intercomparison of chl-a extracts showed CV of 8-15\%. Another intercomparison (Claustre et al., 2004) of four HPLC laboratories found a lower uncertainty (expressed as absolute percentage difference) of 7\% for total chla across a wide trophic range. Furthermore, by applying further quality assurance procedures the uncertainty was reduced to $\sim 5.5 \%$ for total chl-a. Both these studies reinforce the need to follow internationally agreed protocols as described above.

Another issue in the validation of satellite algorithms (and manifested in the results of a comparison of satellite retrievals with in situ observations) is the differences in scales of observations between satellite and in situ data. Applying an algorithm to average upwelling radiance observed over a pixel $\sim 1$ $\mathrm{km}^{2}$ with a nominal penetration depth of $\sim 10 \mathrm{~m}$, and comparing the result with an in situ observation based on $1 \mathrm{~L}$ of water 
represents a scale difference of $1: 10^{-10}$. This raises concerns on how representative an in situ sample of a $1 \mathrm{~km}^{2}$ body of water is. This issue was investigated by Brewin et al. (2016) who used measurements from two Atlantic Meridional Transect (AMT) cruises between $50^{\circ} \mathrm{N}$ and $50^{\circ} \mathrm{S}$ of along-track particulate absorption calibrated against HPLC-chl-a to obtain multiple in situ measurements within a satellite 'pixel.' With this approach they found that the uncertainty in satellite retrievals reduced to $0.157 \log _{10}$ chl-a on average, which is less than half that reported from previous global studies. They suggested that the better performance may be due to the timing of the AMT cruises (in October-November 2009 and 2012) or the restriction to just the Atlantic (limiting the variety of waters encountered), but it could also reflect HPLC analysis at one laboratory (avoiding interinvestigator differences) using an accurate automated system that minimises human error, and also taking multiple measurements within an individual satellite pixel to better characterise the sub-pixel variability.

Rather than applying algorithms globally, another approach is to first classify individual pixels optically on the basis of their reflectance spectra (Moore et al., 2009) and then use the bestperforming algorithm selected for each water class (Moore et al., 2014; Jackson et al., 2017). This approach is described in more detail in section 'Time Series for Climate' along with a view on the ability of algorithms to meet requirements.

\section{In situ Observations to Calibrate Ocean-Colour Sensors and Evaluate Products \\ Radiometric calibration}

As noted by International Ocean Colour Coordinating Group [IOCCG] (2013) 'uncertainty requirements for scientific applications, e.g., $5 \%$ absolute in the blue and $1 \%$ relative (i.e., band to band), or $30 \%$ on chl-a concentration, radiometric calibration should be accurate to a fraction of $1 \%$.' However, despite careful pre-flight and post-launch calibration efforts such as periodically viewing on-board calibration sources or external targets such as the sun and moon (International Ocean Colour Coordinating Group [IOCCG], 2013; Zibordi et al., 2015) it has not been possible to retrieve radiances of sufficient accuracy. Hence, System Vicarious Calibration (SVC) of spaceborne sensors is undertaken whereby the retrieved satellite top of atmosphere radiance is compared with that expected from transmission of measured water-leaving radiance to the top of the atmosphere, using the same atmospheric model used for the in-water radiance retrieval. It is described as a system calibration since it incorporates the effects of the entire processing chain and is therefore specific to individual atmospheric correction procedures. The NOAA operated Marine Optical BuoY (MOBY: Clark et al., 1997) system located off Hawaii in very low chl-a water is used for SVC, along with the French Boussole system in the Mediterranean, though SVC can also utilise other systems (such as AERONET-OC). Maintenance of MOBY and other SVC systems is a key continuing requirement: in addition to continued support of MOBY by NOAA in the United States, EUMETSAT is conducting projects to develop SVC capability in Europe.

Ongoing high-quality in situ radiometric measurements are also needed to validate new sensors in a satellite constellation as well as to test continually the performance of instruments that have been in orbit for many years. This is important since the premature addition of a new sensor to a CDR before intersensor biases are accounted for, or failure or degradation of an older instrument, may introduce spurious trends into the record (e.g., Brewin et al., 2016). Likewise, for operational applications a new sensor with a bias could potentially lead to spurious alerts for events such as eutrophication. The AERONET-OC initiative provides quality-controlled multispectral data for a number of fixed locations worldwide (Zibordi et al., 2009), which is likely to be complemented by a hyperspectral observation network, for example, the recently started European Hypernet project ${ }^{14}$. Biooptical instruments such as above-water radiometers, and flowthrough absorption/attenuation instruments, are increasingly being installed on moving platforms such as ships of opportunity, research vessels, or even yachts of environmentally aware philanthropists. Quality control of along-track data is vital (see IOCCG page on protocols) but such systems have shown considerable potential to increase significantly the number of satellite validation matchups (Brewin et al., 2016; Dall'Olmo et al., 2017).

\section{In situ matchup database}

For satellite validation and SVC it is important to have access to the various sources of data in one place. The ESA Ocean Colour CCI project has constructed such a database with global distribution that includes data acquired from several sources: a version was published as a paper/dataset in Valente et al. (2016) and is also available at PANGAEA ${ }^{15}$. The latest version (v4) comprises data from 1997 to late 2017 on spectral remotesensing reflectances, concentrations of chl-a, spectral inherent optical properties, spectral diffuse attenuation coefficients and total suspended matter.

\section{The argo float network}

The argo float network comprises 4000 floats worldwide measuring vertical ocean physical structure. The network of Biogeochemical-Argo (BGC-Argo ${ }^{16}$ ) floats is smaller, comprising hundreds of floats, carrying a variety sensors to measure oceanic biogeochemical properties such as chl-a fluorescence, oxygen pH or nitrate (e.g., Dall'Olmo and Mork, 2014; Dall'Olmo et al., 2016). In principle, BGC-Argo-measured chl-a could provide the depth dimension not sensed by oceancolour satellites but this is complicated by chl-a fluorescence quenching near the surface, though Xing et al. (2011) have proposed correction of fluorescence using irradiance sensors on the float. There are also various physiological processes that contribute to complex relationships between chl-a fluorescence and concentration, such that interpretation of the fluorescence signal as concentration is not straightforward. BGC-Argo data on spectral diffuse attenuation of downward irradiance, fluorescent dissolved organic matter concentrations, and particulate optical backscattering have been compared with satellite retrievals (Organelli et al., 2017). Upwelling radiance sensors on BGC

\footnotetext{
${ }^{14}$ http://www.hypernets.eu/from_cms/objectives

${ }^{15}$ https://www.pangaea.de

${ }^{16}$ http://biogeochemical-argo.org/
} 
Argo floats (Leymarie et al., 2018; Wojtasiewicz et al., 2018) offer potential for validation of EO sensors, though long-term calibration and bio-fouling may impact upon the performance.

\section{Citizen Science}

Citizen science or crowdsourced environmental observations is an area that has grown rapidly and is likely to grow further over the next decade as the worldwide usage of smart phones increases. Citizen science may provide useful contributions to ocean-colour observations, albeit primarily in coastal and inland waters as well as raising environmental awareness. Examples of ocean-colour relevant citizen science projects include:

- The Secchi Disk Project ${ }^{17}$ is the largest worldwide citizen science study collecting measurements of ocean and coastal transparency with the Secchi disk (Lavender et al., 2017). A phone app is used to geo-locate and record the Secchi disk measurements, which are subsequently transmitted to a data repository.

- Citizen science measurements using a 3D-printed mini Secchi disk (Brewin et al., 2019) are contributing to a research project monitoring water quality (visibility and colour) of waters of Lake Vembanad in India, for comparison with satellite products of the region, contributing to a United Kingdom-India jointly funded research project.

- The EyeOnWater ${ }^{18}$ mobile phone app was originally developed in a European citizen science project (Citclops) that enables the user to classify a water body using the Forel-Ule 21 water-type colour scale that is displayed on the smartphone screen. Data are then uploaded to a central repository.

- The BloomWatch app ${ }^{19}$ documents the location and frequency of potentially harmful cyanobacteria blooms. The user is prompted through a series of screens to provide site information and photos.

\section{GROUND SEGMENT AND DATA PROCESSING}

\section{Ground Segments}

The term ground segment is used loosely herein to define the systems that include reception of EO data via satellite downlink and lower-level processing for calibration and geolocation, followed by atmospheric correction and production of analysis-ready data and end-user focused services; it also includes timely and appropriate delivery mechanisms (International Ocean Colour Coordinating Group [IOCCG], 2018). The ground segment is an area that has, arguably, improved considerably over the past 10 years and, indeed, the success of an EO mission, once launched, is ultimately determined by the use of the data, information and services by stakeholders for applications. Below we describe example ground segments.

\footnotetext{
${ }^{17}$ http://www.secchidisk.org/

${ }^{18} \mathrm{http}: / /$ www.eyeonwater.org/

${ }^{19}$ https://cyanos.org/
}

\section{Copernicus}

Copernicus is a flagship European programme that incorporates Earth Observations encompassing satellite constellations (the Sentinel series, notably Sentinel 3 - see section 'Ocean Colour EO Sensors and in situ Observations'); ground-segments producing operational products and CDRs; and training and outreach activities. Copernicus has an open data policy whereby data can be used free of charge for any purpose, academic or commercial. For ocean-colour data from the OLCI sensor, the basic data processing from L0 to L1 and L2 is undertaken operationally by EUMETSAT. Users can also obtain the L1 and L2 data and process using tools such as the Sentinel Application Platform $\left(\mathrm{SNAP}^{20}\right)$, provided through the ESA which offer additional capabilities, such as different atmospheric correction procedures. Copernicus supports a marine-focused processing service, the Copernicus Marine Environment Monitoring Service (CMEMS). The CMEMS ocean-colour team uses OLCI, VIIRS and AquaMODIS data to produce near-real time and reprocessed time series of global mapped products, and regional products with bespoke, regionally tuned algorithms, such as for the Mediterranean Sea (Volpe et al., 2007). CMEMS produces ocean monitoring indicators (OMIs) and publishes annual ocean state reports which include ocean-colour results (von Schuckmann et al., 2016, 2018 see below). Copernicus envisages that 'downstream' services addressing individual end-user or application sectors are produced by national agencies or private companies building on the CMEMS 'upstream' products. An ocean-colour CDR is produced by the Copernicus Climate Change Service (C3S) and brokered to CMEMS. Although Copernicus services are operational and do not explicitly support $\mathrm{R} \& \mathrm{D}$, the products are maintained at the state-of-theart and so incorporate the latest R\&D: in the case of C3S the CDR benefits from R\&D in the ESA CCI project (see section 'Time Series for Climate'). Finally, training in oceancolour data is undertaken through, inter alia, the EUMETSAT marine training programme that includes European and international elements, the latter primarily, but not exclusively, in developing countries.

\section{NASA OBPG}

The NASA GSFC Ocean Biology Processing Group (OBPG) produces ocean colour data from NASA sensors such as CZCS, SeaWiFS and MODIS, as well as third-party missions including NOAA's VIIRS, ESA's MERIS (both reduced and full resolution, and operated 2002-2012) and the Korean GOCI. All source data and derived data products are freely distributed through the Ocean Biology Distributed Active Archive Center (OB.DAAC) and directly accessible via the Ocean Color Web portal ${ }^{21}$. Data are provided at Level-1: raw or calibrated and geolocated observations at native resolution; Level-2: derived geophysical variables at native resolution; and Level-3: derived geophysical variables that have been aggregated/projected onto a well-defined spatial grid over a given time period, including daily, weekly, monthly, seasonal and annual composites at 4.6 or $9.2-\mathrm{km}$

\footnotetext{
${ }^{20} \mathrm{http}: / /$ step.esa.int/main/download/

${ }^{21}$ https://oceancolor.gsfc.nasa.gov/
} 
spatial resolution (depending on sensor). Individual images can be downloaded as PNG or NetCDF files, and orders can be placed for bulk downloads with subsetting options. For the global missions supported by the OBPG, a primary focus is on the use of consistent algorithms and calibration approaches to produce a continuous time-series of standardised ocean colour products for global change research and Earth system science applications.

\section{NOAA}

Data from both VIIRS sensors are processed by NOAA to produce global maps ${ }^{22}$ and merged VIIRS products are under development. Data are also produced in near-real time for different user regions-of-interest via CoastWatch ${ }^{23}$. In addition, near-real time ocean-colour data compared with in situ data from MOBY and several AERONET-OC sites are available through the NOAA website. All VIIRS global ocean-colour data are freely available through NOAA CoastWatch website.

\section{Data Delivery}

- Traditional methods such as ftp and web-downloads, e.g., via the NASA browser noted above or the ESA Ocean Colour Climate Change Initiative $\operatorname{site}^{24}$, will continue to be exploited by many users.

- GEONETCast is a distribution system based on Digital Video Broadcast technology with multicasting of environmental data via commercial satellites. It is particularly valuable for institutes or universities in developing countries with poor internet access due to their location, such as marine institutes located at the coast, or due to limited national infrastructure. For Africa, two European Commission funded projects installed low-cost GEONETCast receivers at sites across the continent. The systems had mixed success, not least due to the exposed locations at the coast. The Monitoring of Environment and Security in Africa programme (MESA ${ }^{25}$ : completed in 2017) installed further reception capacity based around Regional Implementation Centers. Ocean colour data are still transmitted via the Eumetcast and data provision is being maintained in the 'GMES and Africa' project.

- Mobile applications. According to the World Bank in 2016 there were more mobile cellular subscriptions than people in the world $^{26}$ while by 2019 it is forecast that $63 \%$ of the population will have mobile technology with internet connectivity ${ }^{27}$. Indeed, in Africa, mobile coverage has enabled '...Africans to skip the landline stage of development and jump right to the digital age' (Pew Research Centre, April 2015). Mobiles offer great potential for dissemination of oceancolour data for a variety of applications. It obviously opens up two-way conversation, encouraging citizen scientists (see section 'Citizen Science'). Example mobile apps focusing on ocean and inland colour applications include:

\footnotetext{
${ }^{22}$ https://www.star.nesdis.noaa.gov/sod/mecb/color/

${ }^{23}$ https://coastwatch.noaa.gov/

${ }^{24}$ www.oceancolour.org

${ }^{25} \mathrm{http}: / /$ moi.govmu.org/mesa/

${ }^{26} \mathrm{https}$ //data.worldbank.org/indicator/IT.CEL.SETS

${ }^{27}$ www.statista.com
}

- The CyAN mobile app. Most water quality managers and local interest groups do not have time for extensive training in building a technical background for deriving and processing satellite derived water quality data and information. Therefore, a passive software system may benefit end-users that limit this technical burden and time commitment. Schaeffer et al. (2018) demonstrated an operational mobile application (see Figure 1) that allows passive reception of Copernicus Sentinel-3 OLCI data instead of active acquisition minimising the barrier to receiving actionable information (Schaeffer et al., 2013). The app functionality was successfully replicated across 25 individual state health advisories in 2017, proving it could provide a user-friendly platform reducing complexity traditionally associated with using satellite data (Schaeffer et al., 2018). Currently, a number of state environmental and health departments, non-government organisations, and some local interest groups are using this application across large lakes and coastal regions.

- The ABALOBI mobile $a p p^{28}$ is planned to be used by the GMES and Africa southern Africa centre to deliver Potential fishing zone products. PFZ information is already supplied to Indian fishers through a mobile from INCOIS (see section 'Operational Ocean-Colour Applications').

- Web-based analysis tools. For exploitation of ocean-colour data by non-specialist end-users it is important to provide easy-to-use tools, ideally web-based, that support simple analysis of datasets provided by the ground segments. This ensures that EO data have an impact beyond specialist academic communities. Such a system should be built ideally on recognised standards and allow sharing of data or visualisation of data from multiple providers. The Open Geospatial Consortium (OGC) specifies standards for accessing data via the web including Web Mapping Service (WMS) serving images (such as GIF), Web Coverage Service (WCS) which serves arrays of data and Web Feature Service (WFS) that specifies point or other vector data such as shiptracks or region of interest boundaries. In principle, webbased portals using these standards can request and visualise data from other portals. Many portals have built-in analysis capabilities, such as time-series plotting; this is also efficient since only delivery of the results is required, not the full dataset from which it was derived and which may comprise many gigabytes or terabytes of data. An example of a web-vis system is the ocean colour CCI portal ${ }^{29}$ (see Figure 2A) which allows selection of regions of interest (e.g., via hand-drawn polygons or uploaded shapefiles) then produces time series, Hovmöller plots, or time animations of these data. Figure 2B shows an extracted time series of chl-a from a user region of interest. The portal also provides additional analyses such as scatterplots between two variables, and data extraction in time and space, from an uploaded location/date file, such as along a ship track. Hence, it can be used to for satellite matchup

\footnotetext{
${ }^{28}$ http://abalobi.info/

${ }^{29}$ www.oceancolour.org/portal
} 
analysis. Another important ocean-colour tool is GIOVANNI as provided by NASA ${ }^{30}$.

\section{Cloud Platforms}

The increase of data volumes resulting from new higher resolution or hyperspectral sensors and the growing length of the ocean-colour record is creating challenges for data processing, and even more so for accessing the data. Bandwidth can inhibit systematic near-real time processing at a global scale, or analysis of a complete archive. In recent years, there has been a paradigm shift from downloading and processing data locally, to moving the processing software at the data source, together with provision of data processing resources. This is an ongoing process and most users still download data and process in the traditional way. This may be attributed to the fact that specialised systems, i.e., hardware and software with cost implications, are required to support the new way of processing EO data close to the data source as well as conservative practice. The Copernicus Data and Information Access Services (DIAS) are planned to facilitate access to data and information from the Copernicus services. By providing data and information access alongside processing resources, tools and other relevant data, this initiative is expected to boost user uptake, stimulate innovation and create new business models based on EO data and information. More information can be found $\mathrm{at}^{31}$.

Accessing_Copernicus_data_made_easier. ESA's Thematic Exploitation Platforms (TEPs) concept aims to provide a working environment where users can access algorithms and data remotely, providing them with computing resources and tools that they might not otherwise have, and avoiding the need to download and store large volumes of data. Currently, there is a coastal TEP focusing on coastal ocean-colour applications. Finally, various commercial suppliers like Amazon Web Services offer cloud computing with EO data series.

\section{TIME SERIES FOR CLIMATE}

Ocean-colour CDRs of sufficient duration to quantify natural variability and to detect climate change impacts on the marine ecosystem are the key requirement emerging from user consultations. Henson et al. (2010) showed that a contiguous recorded in excess of 40 years is needed to distinguish a climate-related trend in chl-a and primary production from natural variability, though only $>20-30$ years is needed for chl-a in some regions such as equatorial waters. Furthermore, the minimum length of time series required increases in the event of gaps in the record. The uninterrupted ocean-colour record started in 1997 with SeaWiFS and so in 2018 is only 21 years; no individual sensor covers all of this period and in any case most sensors have finite lifespans, and older sensors exhibit calibration degradation. So, to create a continuous time series requires merging (International Ocean Colour Coordinating Group [IOCCG], 2007) data from individual

\footnotetext{
${ }^{30}$ https://giovanni.gsfc.nasa.gov/giovanni/

${ }^{31}$ https://www.esa.int/Our_Activities/Observing_the_Earth/Copernicus/
}

sensors without introducing biases, artifacts or discontinuities (Hammond et al., 2017). However, the sensors launched over this period each have different characteristics in terms of orbits (overpass in the morning, mid-day or afternoon), different swaths (and, hence, re-visit times), spatial resolution $(4 \mathrm{~km}$ to $300 \mathrm{~m}$ ), and a different number and location of spectral wavebands. So construction of a multi-sensor merged time-series is not a trivial task.

ESA's Climate Change Initiative (CCI; Hollmann et al., 2013) has been supporting the research to develop and validate algorithms, initially for 13 Essential Climate Variables (ECVs) including ocean colour, to produce consistent, stable, errorcharacterised global satellite data products from multi-sensor data archives, that meet the GCOS requirements (Table 1). Since 2010 the Ocean Colour CCI team has been working with support and help from NOAA and NASA to perform research and development, and undertake regular re-processing to extend the time series implementing the R\&D (Brewin et al., 2015; Muller et al., 2015a,b; Sathyendranath et al., 2017). The team includes representatives of the global climate research community, including marine ecosystem modellers and remote sensing scientists, who provide ongoing feedback. Community consultation and participation have been cornerstones of this initiative.

To produce the ocean-colour CDR, MERIS, Aqua-MODIS, SeaWiFS, and SNPP-VIIRS remote sensing reflectances $\left(\mathrm{R}_{\mathrm{rs}}\right)$ are merged following band-shifting (Melin and Sclep, 2015) to a reference sensor (SeaWiFS) and pixel-specific inter-sensor bias correction. Bias climatologies between a sensor and the reference (e.g., MERIS and SeaWiFS) are computed using 5 years (20032007) of overlapping data, taking into account seasonal and regional variations (Bias correction of VIIRS uses a two-step process of shifting Aqua-MODIS to SeaWiFS and then VIIRS to Aqua-MODIS). The project used a novel atmospheric correction approach (Steinmetz et al., 2011) to better deal with areas of sunglint and haze: this is important since some areas such as the Arabian Sea (Al-Naimi et al., 2017) or Red Sea (Racault et al., 2015) previously had long periods of no coverage in some precursor multi-sensor merged or single sensor data sets. Such gaps also confound elucidation of long-term trends. Sensor-specific $\mathrm{R}_{\mathrm{rs}}$ are then merged using simple averaging of bias-corrected, band shifted data.

To compute per-pixel uncertainty metrics (bias and rootmean-square difference, RMSD) and also to select the in-water retrieval algorithm, an optical water-type classification approach is used to compute pixel water type (Moore et al., 2014; Jackson et al., 2017). Satellite-derived radiances are used to construct a set of optical water types (OWT); then uncertainty metrics are computed per OWT through matchups between satellite $\mathrm{R}_{\mathrm{rs}}$ and in-water constituent values from the OC CCI database (Valente et al., 2016). For each pair of satellite $\mathrm{R}_{\mathrm{rs}}$ and in situ water constituent the pixel water class memberships (from the $\mathrm{R}_{\mathrm{rs}}$ ) are used to weight the differences in computation of RMSD and bias. This approach was followed since the OC-CCI user consultation indicated a clear preference for uncertainties based on validation of the products against in situ data. Finally, for each pixel, uncertainty is computed from the per-class 


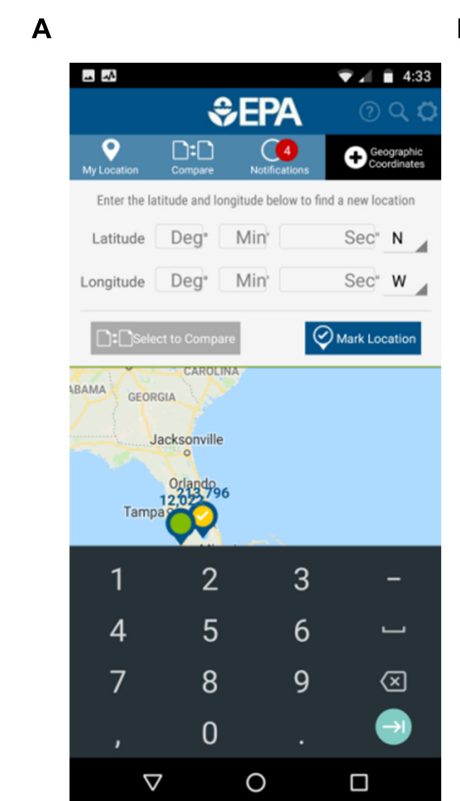

B

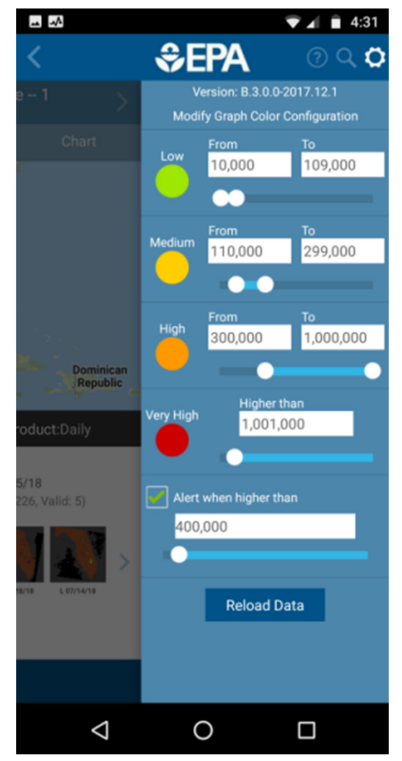

C
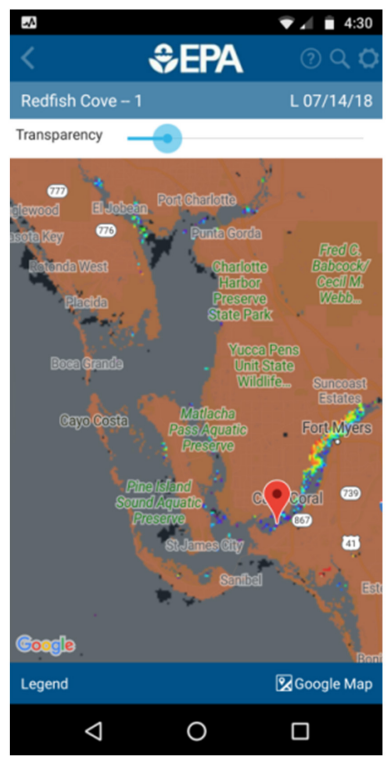

D

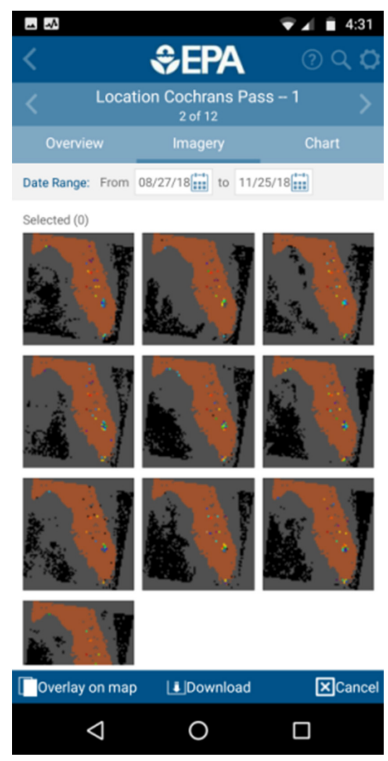

FIGURE 1 | Screenshots of CyAN mobile app (Schaeffer et al., 2018): (A) The main page of the app allows for dropping locations of interest pins using latitude and longitude coordinates or by responsive touch on the screen. (B) The user is also able to set pin colour thresholds and an alert icon setting with the cogwheel based on their own criteria. (C) selecting a pin allows the user to visualise the complete satellite tile for the location of interest for spatial patterns, such as demonstrated with a coastal estuary on the west Florida shelf where Google maps is a transparent layer for quick geographic reference. (D) The user can view a temporal series of images related to their pin location and visualise temporal trends amongst different locations, where each line graph is a single pixel (not shown).

uncertainties using the pixel class membership (Jackson et al., 2017). Chl-a and other in-water variables are computed using class membership and the optimal algorithm per water class. Chlorophyll algorithms were selected from amongst OC4/OCI (Hu et al., 2012) and OC5 (Gohin et al., 2002) through round robin assessment utilising a scoring system similar to that of Brewin et al. (2015).

Assessment of the OC CCI products has included suitability for use as a CDR (Melin et al., 2017); the impact of discontinuities on computing trends (Hammond et al., 2017); and comparison of OC CCI data with precursor datasets (Couto et al., 2016). Example results from OC-CCI version 3.1 (available at www.oceancolour.org) are shown in Figures 3A-C including a chl-a product, RMSD and bias expressed as log $10 \mathrm{chl}$ a. RMSD and bias vary with water class, with typically lower RMSD in water classes representing clearer open-ocean waters (Jackson et al., 2017).

OC CCI v3.1 data have been compared with the GCOS requirements for uncertainty (Table 1) by computing absolute per-pixel relative error $\left(\in=\left|100\left[1-\left(1 / 10^{\mathrm{m}}\right)\right]\right|\right)$ from bias (m) e.g., for chl-a, $m$ is in units of $\log _{10}(\mathrm{chl})$. In general, OC CCI products meet the GCOS requirements for uncertainty (see Figures 3D,E) though long-term stability is still under investigation.

Although the time series is too short to unambiguously detect climate change impacts (Henson et al., 2010), the $\mathrm{CDR}$ can be compared to climate indicators. Figure $4 \mathrm{~A}$ from von Schuckmann et al. (2016) shows interannual variations in chl-a concentration in the Pacific Ocean and close correspondence with the ENSO index. This regional response to climate variability gives important clues on how phytoplankton might respond to long-term climate changes. The 2015-2016 ENSO event was the strongest observed since 1997. The OC CCI reflectance data are used in the CMEMS project with regionally tuned algorithms to provide optimal ocean-colour time series for European seas. Figure $4 \mathrm{~B}$ from von Schuckmann et al. (2018) depicts the general trend in chl-a in the northeast Atlantic from 1997 to 2016 where the red line shows a simple linear fit to the data: no correction was made for outliers nor was any seasonal signal removed. The trend shows a steady increase until 2014-2016. An anomaly map (with respect to the 1997-2014 period) for 2016 (Figure 4C) for the northeast Atlantic shows positive anomalies except for European shelf waters, which show mostly negative anomalies.

Sathyendranath et al. (2017) described the characteristics of algorithms and methods needed for an ocean colour CDR. Amongst the 20 requirements are included: use of retrieval algorithms robust to changes in the environment; methods or algorithms should perform routinely and globally and should minimise gaps in data; inter-sensor bias needs to be corrected before multi-sensor merging; and algorithms should retrieve products with minimal uncertainties. Striving to meet these requirements needs additional research and development such as approaches to minimise gaps in the data, and improved coverage is expected through constellations of EO sensors. The next decade will see the time series extending to 30 years, which could allow investigation of climate impacts on the 

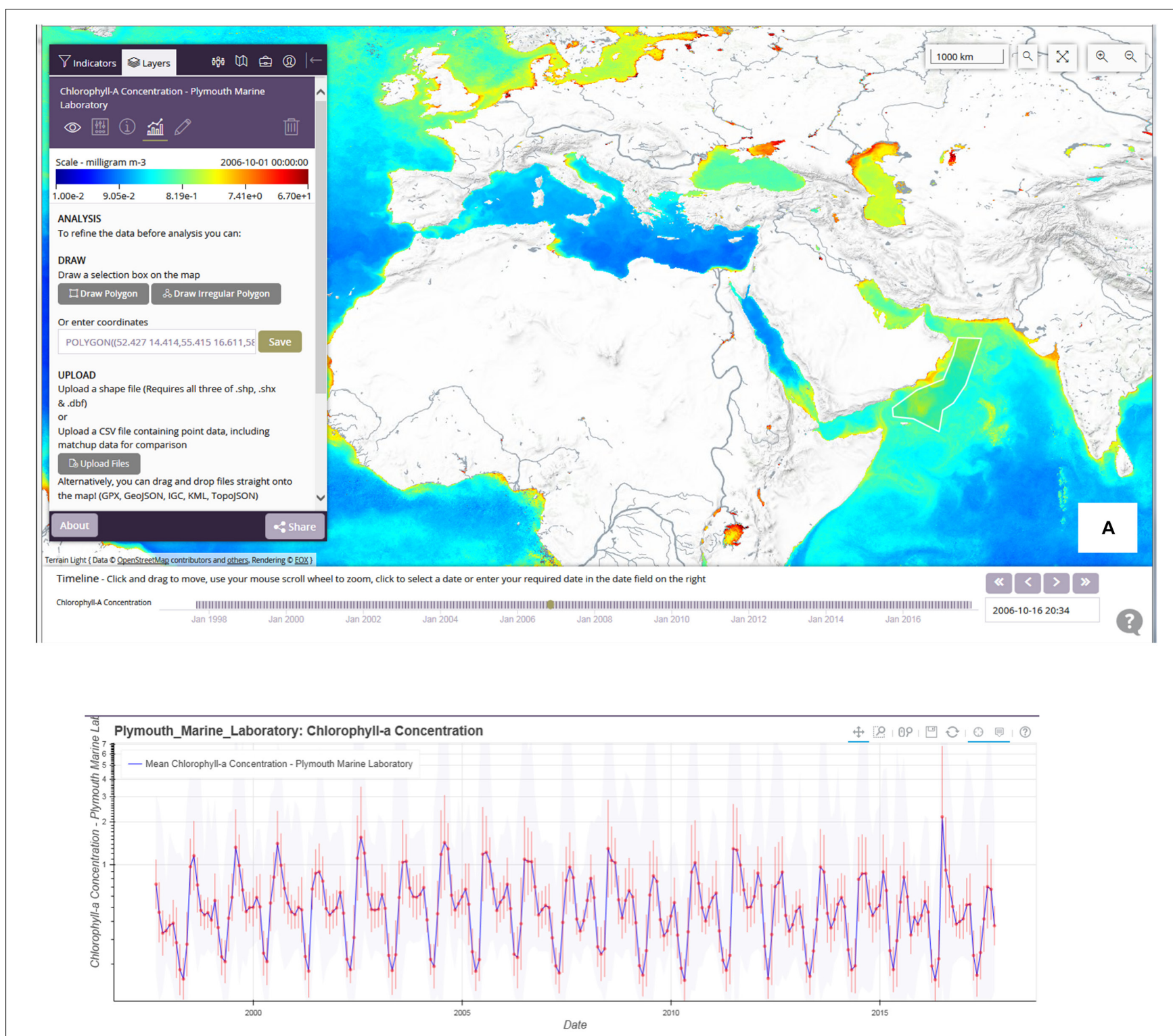

1) Chlorophyll-a Concentration - Plymouth Marine Laboratory

Region: undefined

Confidence: undefined

Provider: Plymouth_Marine_Laboratory

Interval: Monthly

Bounding Box: POLYGON(I52.427 14.414,55.415 16.611,58.667 17.93,59.897 20.918,60.425 23.027,.63.325 22.939,62.622 20.83,61.392 17.227,59.897 15.029,58.579 14.15,58.14 11.514,54.976 13.096,.52.515 13.359,52.427 14.414))

Download

FIGURE 2 | Screenshots for ESA ocean colour CCI OGC-compliant visualisation/analysis portal: (A) monthly version 3.1 global chl-a map (comprising merged bias-corrected SeaWiFS, Aqua-MODIS, MERIS and VIIRS) with uploaded shapefile for image data extraction. (B) Time series plot for region of interest; vertical bars show standard deviation and background grey shows range.

ocean ecosystems, for some regions, albeit with caveats on gaps and discontinuities (Henson et al., 2010). The similarity in sensor characteristics and orbits of the operational systems (Sentinel 3 OLCI and NOAA VIIRS) at least within each constellation should minimise discontinuities in the CDR caused by the introduction or removal of an individual sensor, whilst regularity in sensor launches should remove the issue of gaps, at least through lack of sensors, even if problems with extended cloud-cover may continue to pose problems. It is important that the Sentinel-3 OLCI-like series be continued well beyond the planned 20 years, to provide a baseline for long-term climate data records.

A further development will be the integration of the oceancolour CDR with in situ data from Argo and BGC-Argo programmes, as well as other EO data in synergy, to characterise the $3 \mathrm{D}$ structure of the ocean. In particular, the BGC-Argo 


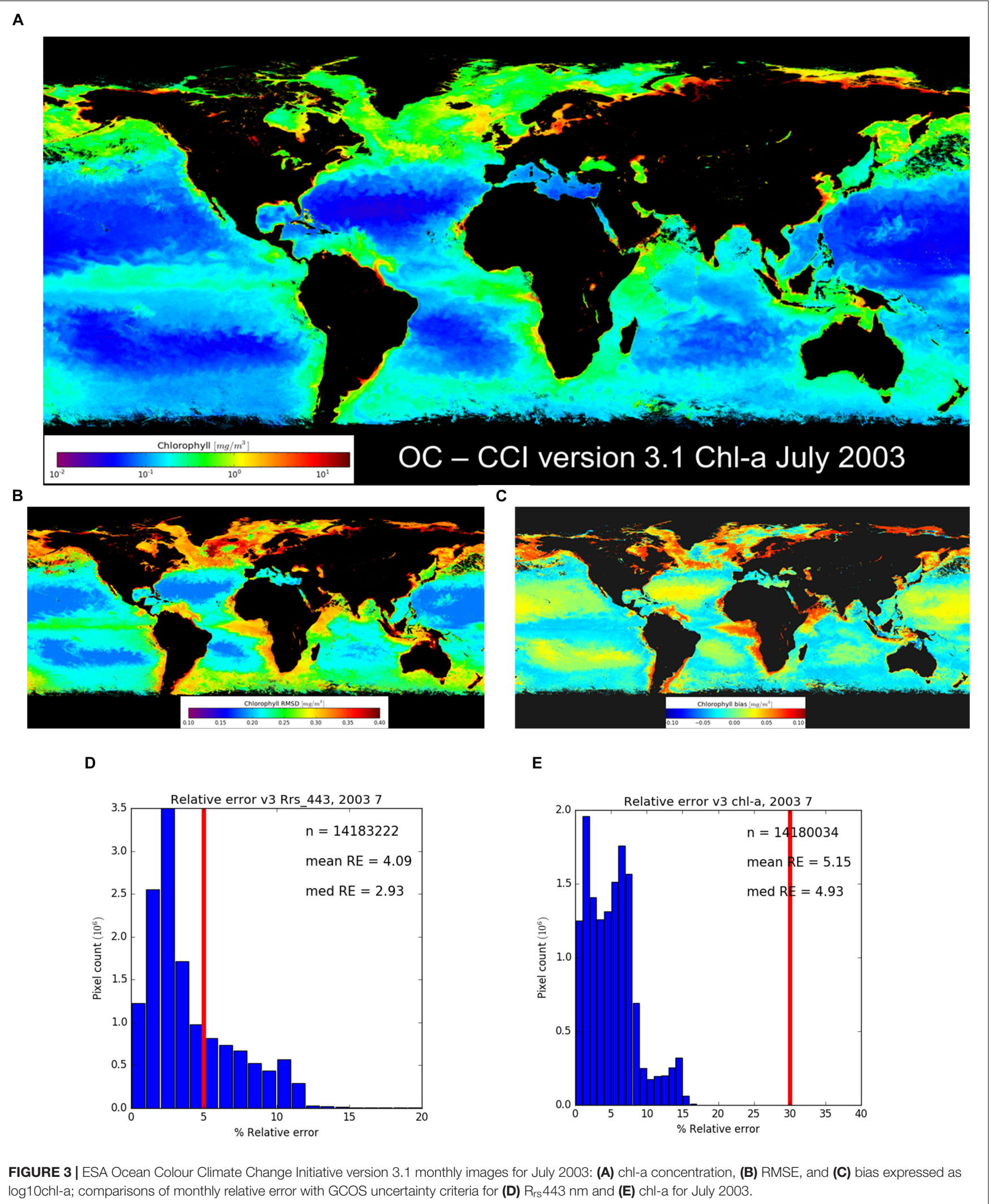




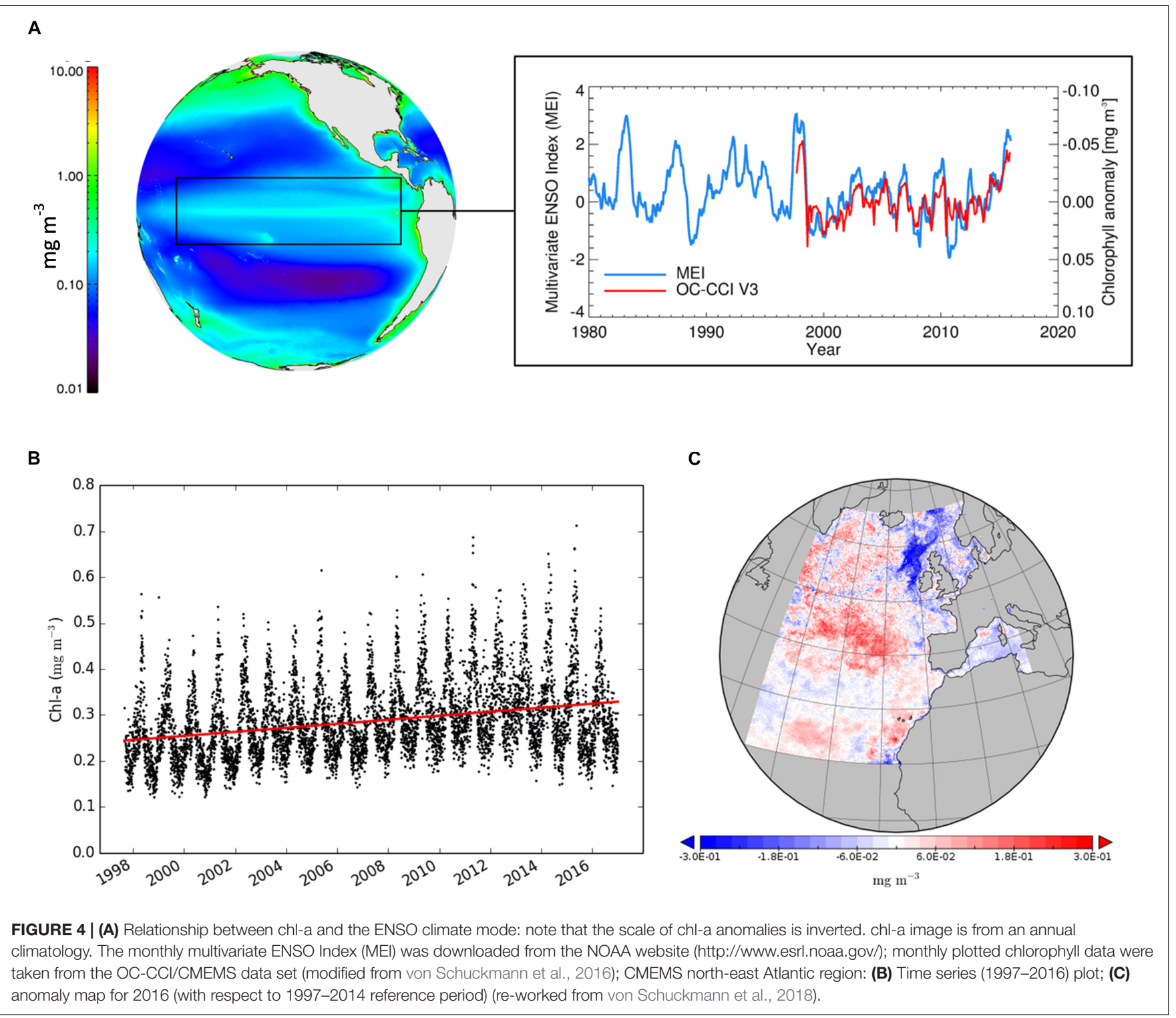

fleet is expected to expand, albeit not to the density of Argo, but with backscattering sensors (Dall'Olmo and Mork, 2014; Dall'Olmo et al., 2016; Organelli et al., 2017) and radiance sensors (Leymarie et al., 2018; Wojtasiewicz et al., 2018), which will enable greater understanding of the biogeochemical structure and possibly additional data for sensor validation. Furthermore, additional sensors could provide observations on biological diversity (Boss et al., 2018).

\section{OPERATIONAL OCEAN-COLOUR APPLICATIONS}

The operational applications of ocean-colour data are described in International Ocean Colour Coordinating Group [IOCCG] (2008) and include inter alia: natural fisheries; marine protected area selection and monitoring; ecosystem model data assimilation; aquaculture site selection and monitoring; water quality and eutrophication; hazard monitoring such as nuisance or harmful algal blooms (HAB); dredging/dumping; coastal erosion; or sediment plumes.

This section presents a few examples of ocean colour applications; it is not intended to be exhaustive, and it is recognised that there are very many operational or preoperational services and applications ongoing or planned. Instead, it is meant to give a flavour of current activities and future developments, with an emphasis on activities in developing countries.

\section{Harmful Algal Blooms and Aquaculture in South Africa}

Aquaculture is a growing industry in South Africa, with the majority of the marine sector focusing on the in-water culture of the mussel Mytilus galloprovincialis and the Pacific oyster 
Crassostrea gigas, as well as the land-based farming of the abalone Haliotis midae (daff, 2016). All of these industries are to some extent susceptible to the negative impacts of HABs, whether directly through the assimilation of toxins, mechanical damage, or indirectly through anoxia from bloom-collapse (Pitcher and Calder, 2000). As a result, HABs have the potential to cause devastating economic losses to the aquaculture industry.

An example of such an event occurred in January 2017 in the vicinity of Walker Bay, Hermanus where a yessotoxin-producing dinoflagellate bloom (Pitcher et al., 2019) led to the mortalities of approximately 250 tonnes of farmed abalone, with an estimated consumer value of $\$ 33$ million. The spatial and temporal extent of the bloom, captured by S3A-OLCI, is depicted in Figure 5: this used a regional switching algorithm for optimised chl-a estimates in high biomass bloom water types (Smith et al., 2018).

Partially in response to this devastating event, the National Oceans and Coastal Information Management System $\left(\mathrm{OCIMS}^{32}\right)$ of South Africa released an alpha version of a HAB decision support tool in March 2017. While OCIMS supports the general socio-economic potential and ecological conservation of South Africa's oceans and coasts, the HAB decision support tool specifically provides information to the aquaculture industry and environmental managers, allowing the timely implementation of appropriate mitigation steps.

Looking to the future, operational HAB monitoring services based on EO are likely to significantly expand as the industry develops worldwide, and notably in Africa: aquaculture contributes $\sim 50 \%$ to global fish supply, but $<1 \%$ of South Africa's fish supply. The availability of regular satellitebased hyperspectral data may assist in discrimination of certain HAB species (Kurekin et al., 2014) but only if there are distinct spectral features (e.g., as found in Mesodinium rubrum).

\section{Use of Ocean Colour Data for Location of Potential Fishing Zones (PFZ)}

Commercial exploitation of natural fishery resources can be made more efficient through use of information on fish location and abundance. Since phytoplankton forms the base of the food chain, ocean colour information indicates productive regions of the marine environment. Complementing ocean colour images of chl-a, sea surface temperature (SST) images provide information on oceanographic processes such as upwelling, convective mixing, etc., important for enrichment of nutrients and increasing productivity. An integrated approach using ocean colour from the Indian OCM sensor and SST from NOAA AVHRR data was developed (Figure 6A) and made operational for potential fishing zone (PFZ) forecasts (Dwivedi et al., 2005). Currently, ocean colour and SST data are used by the Indian National Centre of Ocean Information System (INCOIS) for locating PFZ based on oceanographic features including gradients in SST and colour, fronts, eddies, rings, meanders, and upwelling regions. PFZ advisories (Figure 6B) are generated by INCOIS routinely using OCM-2 data for dissemination to fishers (Chauhan and Raman, 2017) across the entire Indian coast. The bulletins are widely disseminated as maps and text, via fax, phone,

\footnotetext{
${ }^{32}$ https://eo.meraka.csir.co.za/hab/app/
}

newspapers, internet, e-mail, electronic display boards, radio and TV broadcasts, and information kiosks. A cost-benefit analysis to assess the impact of using satellite PFZ forecasts on fish catch showed that the value of the fish catch was higher than the cost of generating PFZ maps and the fishing effort, indicating the value of satellite data in improving the economics of fish catch (Nayak et al., 2003). The bulletins also show restricted zones based on information such as habitats of species including whale sharks off the Gujarat coast during winter monsoon (Kumari and Raman, 2010) or turtle habitats [based on the impact of longline fisheries on turtle bycatch (Bhatpuria et al., 2015)]. Such 'No Fishing Zones' can be selected by environmental managers and policy makers to reduce environmental impacts from fishing. Finally, PFZ maps are not produced during periods when fishing is banned, creating an incentive for fishers to observe the ban. The goal of PFZ advisories is to improve catch per fishing effort and not to encourage overfishing.

\section{Geostationary Sensor Monitoring of a Harmful Algal Bloom}

Since 1995 blooms of the harmful dinoflagellate Cochlodinium polykrikoides have frequently appeared and caused damage to aquaculture in Korean coastal waters. Choi et al. (2014b) used the Korean GOCI geostationary sensor to monitor diurnal variations in a bloom of C. polykrikoides identified through in situ measurements. Eight hourly water-leaving radiance composite images and corresponding chl-a images, on August 13, 2013, enabled monitoring of diurnal variations in spatial structure and concentration. HAB species are known to vertically migrate finding optimal conditions, in terms of light, temperature and nutrients (Schofield et al., 2006): cells of C. polykrikoides along the Korean coast begin to ascend before sunrise and begin to descend at about 16:00 (Kim et al., 2010). Figures 7A,B clearly show the temporal variations in GOCI-derived chl-a, particularly in the area where C. polykrikoides cell abundance is high (area 1 and area 2) with highest chl-a at 14:25. Chl-a profiles extracted from the GOCI data (Figure 7C) show the gradual increase in chl-a until 14:25 in the HAB area. It is worth noting that a sunsynchronous sensor over-passing earlier in the day would detect much lower concentrations in the bloom.

Over the next decade diurnally resolved observations from GEO satellites in far east Asian waters will be possible from Korea's GOCI-II and China's HY-3C due for launch in 2019 and 2023, respectively. Although no sensors are planned for other regions, nano-satellite constellations or high-altitude pseudosatellites may allow similar diurnal observations, albeit regionally. Furthermore, analysis of such diurnal observations should give insights into satellite data merging techniques that composite over 1 day from sensors with differing overpass times.

\section{ECOSYSTEM MODELLING}

Marine ecosystem models are being used increasingly to investigate ecosystem functioning, predict marine changes in response to different climate change scenarios or for supporting ecosystem management in relation to, e.g., eutrophication, HABs 

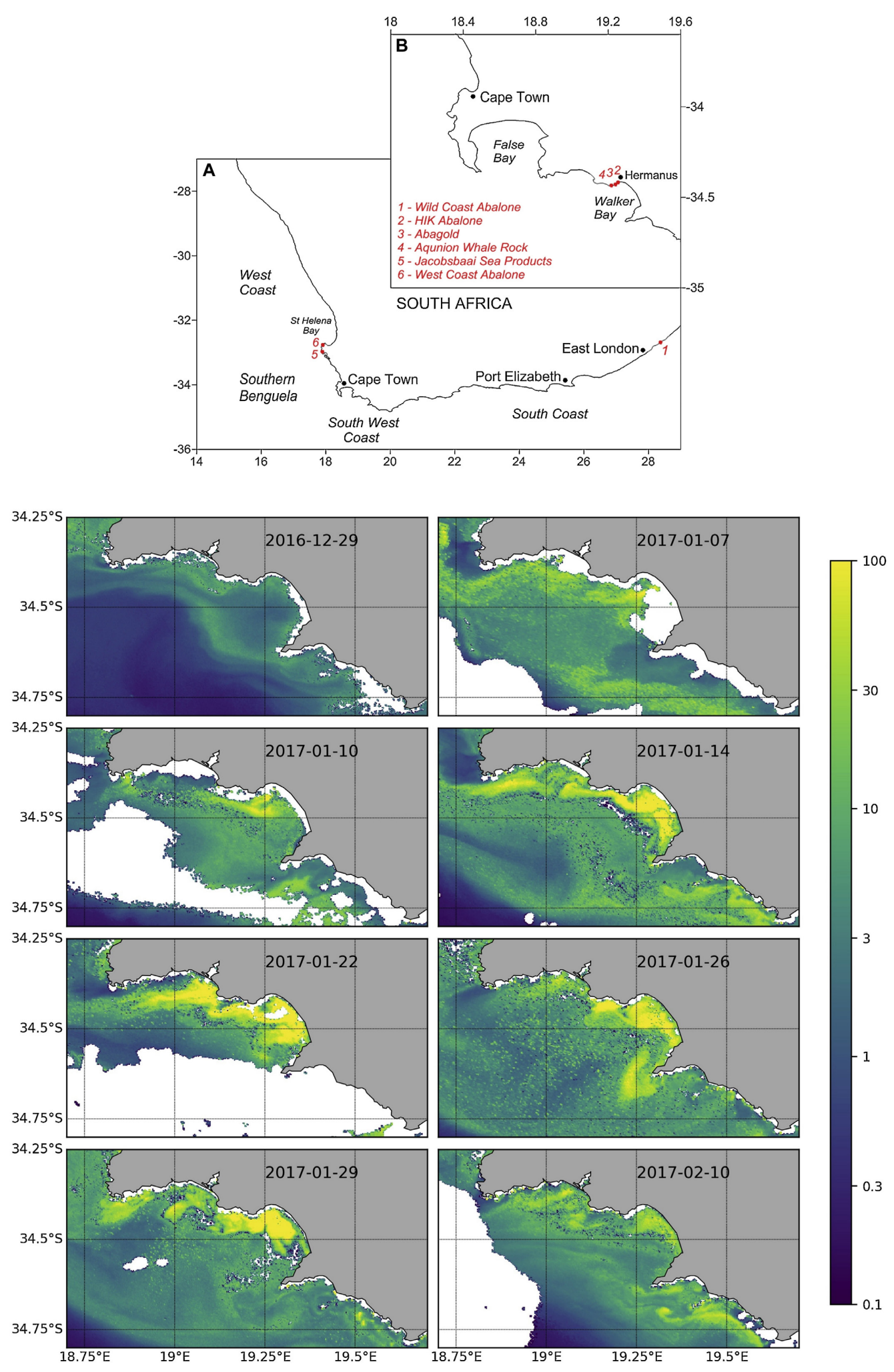

FIGURE 5 | (A,B) Map depicting the location of abalone farms on the South African coastline; lower panel shows satellite-derived chl-a concentration (in mg m-3) from the OLCl sensor for the Walker Bay area between the 29th of December 2016 and the 10th of February 2017. White areas represent either cloud or chl-a algorithm failure (Pitcher et al., 2019). 
A

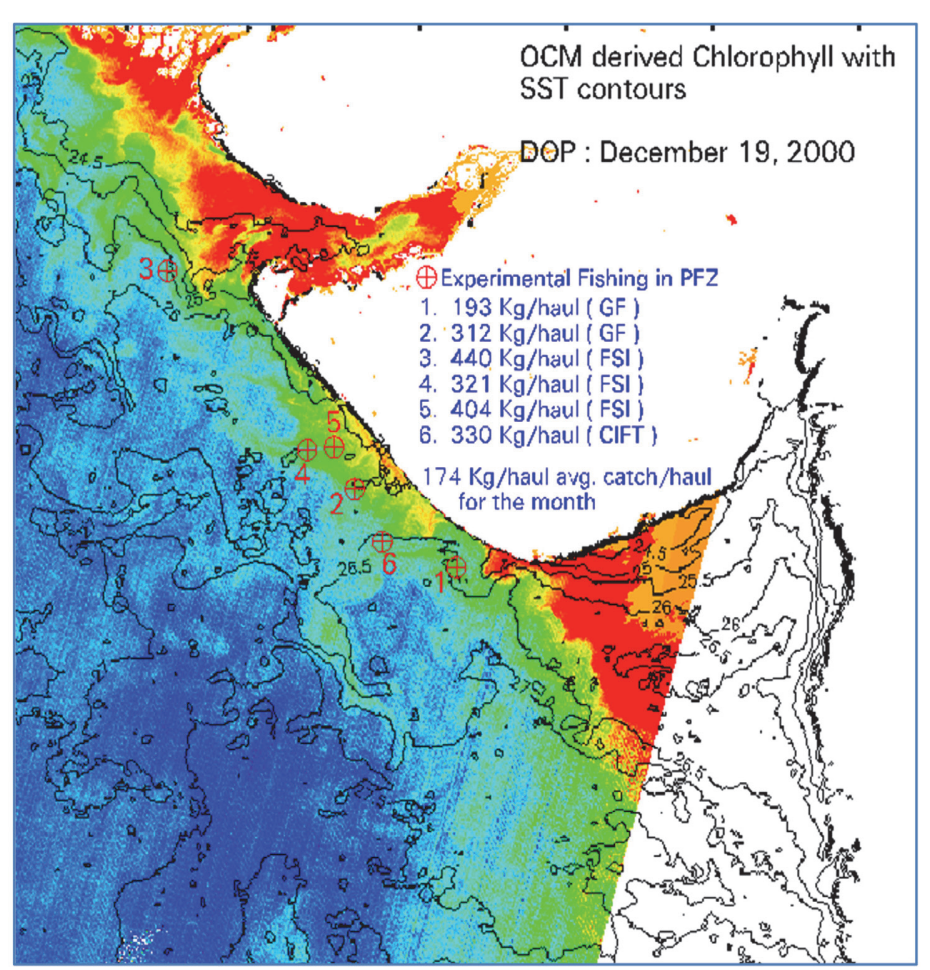

B

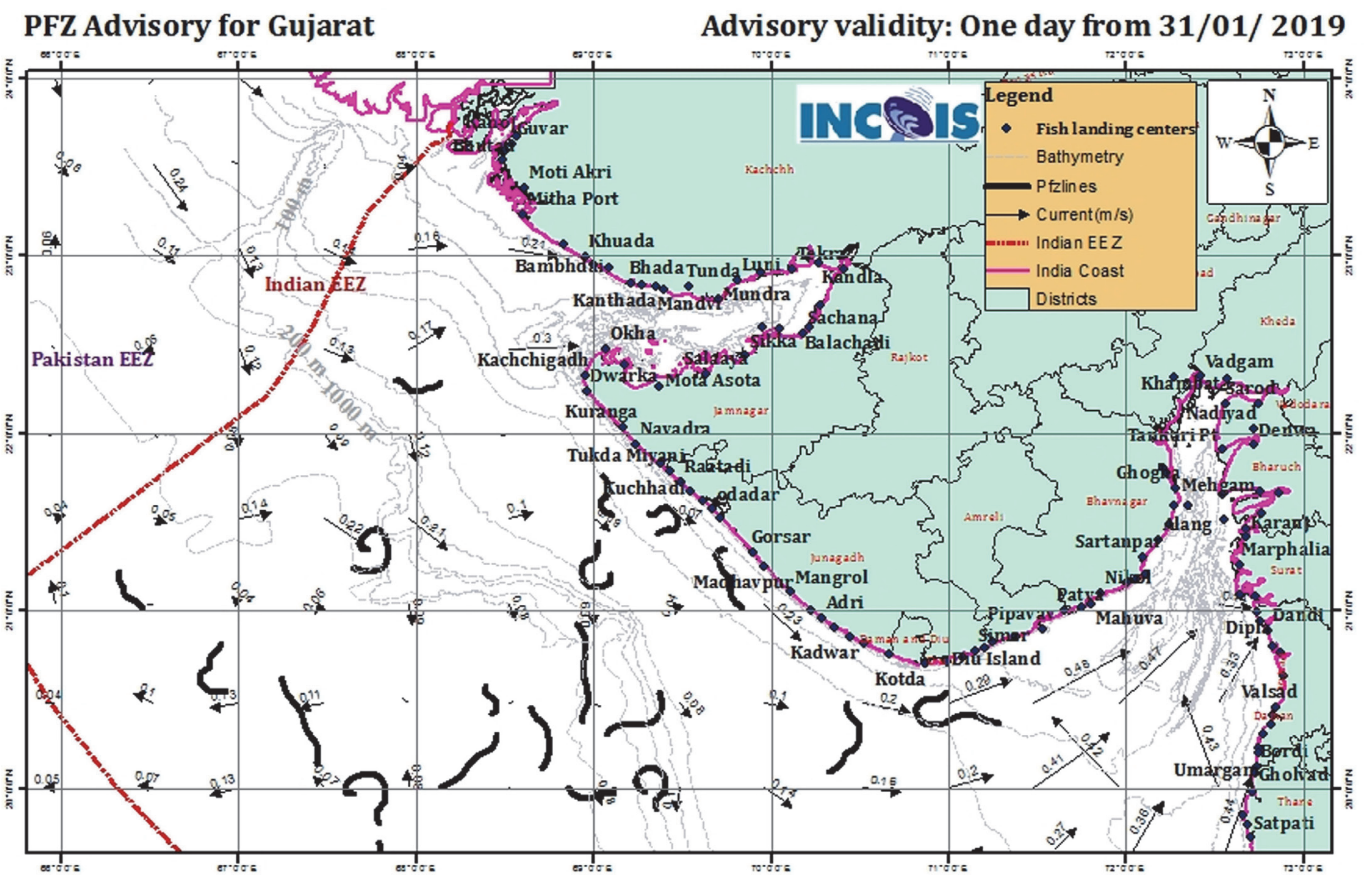

Please provide your valuable feedback: Director, Indian National Centre for Ocean Information Services (INCOIS), MoE S, Govt. of India, Ocean Valley, Pragathi Nagar (B.O), Nizampet (S.O), Hyderabad - 500090.

E-mail:pfz@incois.gov.in/ pfzmission@gmail.com, website: www.incois.gov.in, Phone No: 040-23895013, 040-23886031(ext) Fax No: 040-23895014

FIGURE 6 | (A) Ocean Colour Monitor-derived chl-a concentration with overlaid SST contours used to find PFZ regions. (B) Example PFZ advisory issued by INCOIS, Hyderabad on a routine basis for the Orissa coast of India showing both potential fishing zones (noted as 'Pfzlines') as well as restricted fishing areas ('Restricted_zones'). Such advisories are provided for the entire Indian coastline (Credit: INCOIS, Hyderabad; see https://incois.gov.in/MarineFisheries/PfzAdvisory). 
A
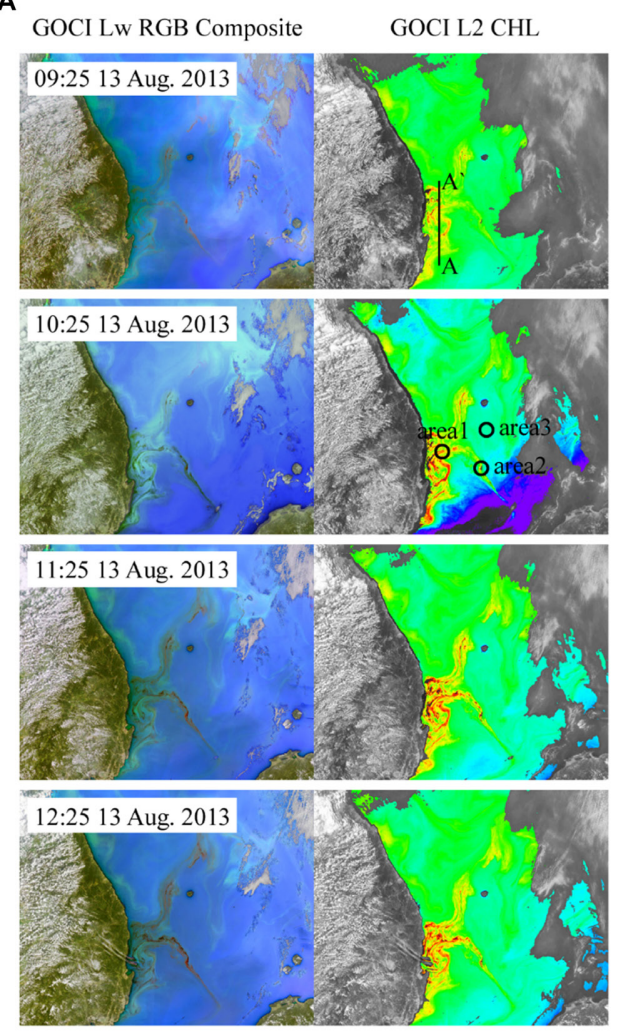

B

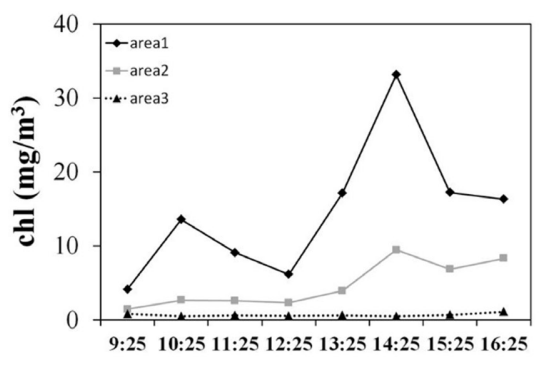

Observation time (h)
GOCI Lw RGB Composite GOCI L2 CHL
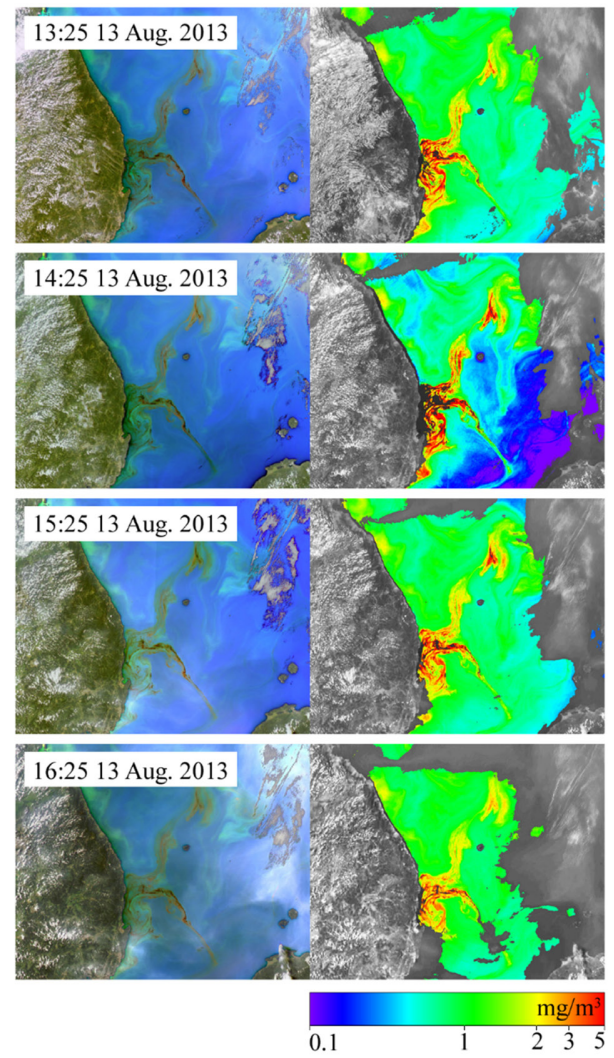

C

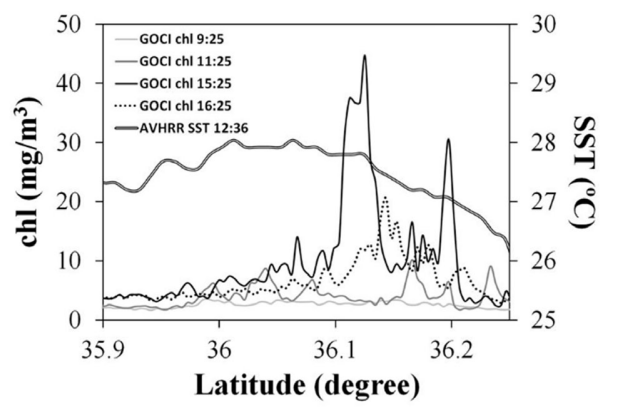

FIGURE 7 | (A) Geostationary Ocean Colour Imager radiance composite images and corresponding chl-a images from 09:25 to 16:25 on 13 August 2013: the chl-a profile line $\left(\mathrm{AA}^{\prime}\right)$ and the hourly chl-a investigation points (1-3) are marked; (B) Hourly GOCl chl-a values at points 1-3; and (C) chl-a values along line $A A^{\prime}$ at 09:25, $11: 25,15: 25$, and 16:25 local time.

and hypoxia (see the reviews by Fennel et al., 2019). Oceancolour data are being used for assessing the skill of model simulations of chl-a fields (see, e.g., the review by Gregg et al. (2009) or phytoplankton community structure (Hirata et al., 2013), and increasingly to improve simulations by means of data assimilation (see the review by Ford et al., 2018). Data assimilation (DA) algorithms correct simulations toward Earth observations (both satellite and in situ), to provide better estimates of the 'true' state of the ecosystem, taking account of the uncertainties in the models and in the data. In practice, DA is operated either to estimate parameter values in a model (e.g.,
Roy et al., 2012; Hoshiba et al., 2018) or to directly adjust the model state-variable simulation with the data (e.g., Tsiaras et al., 2017), or for simultaneous state and parameter estimation (e.g., Gharamti et al., 2017).

Data assimilation of ocean colour is, arguably, not as widespread as assimilation of physical variables: for example, in the European Copernicus Marine Environment Monitoring Service, ocean-colour assimilation is run for operational reanalysis and forecasts in the Mediterranean Sea (Teruzzi et al., 2018) and for operational reanalysis in the North West European Shelf, while the implementation for short-term forecasting is 


\section{A - Analysis (PFT fractions) \\ B - Observations (PFT fractions) \\ C - Forecast error improvements [\%]}
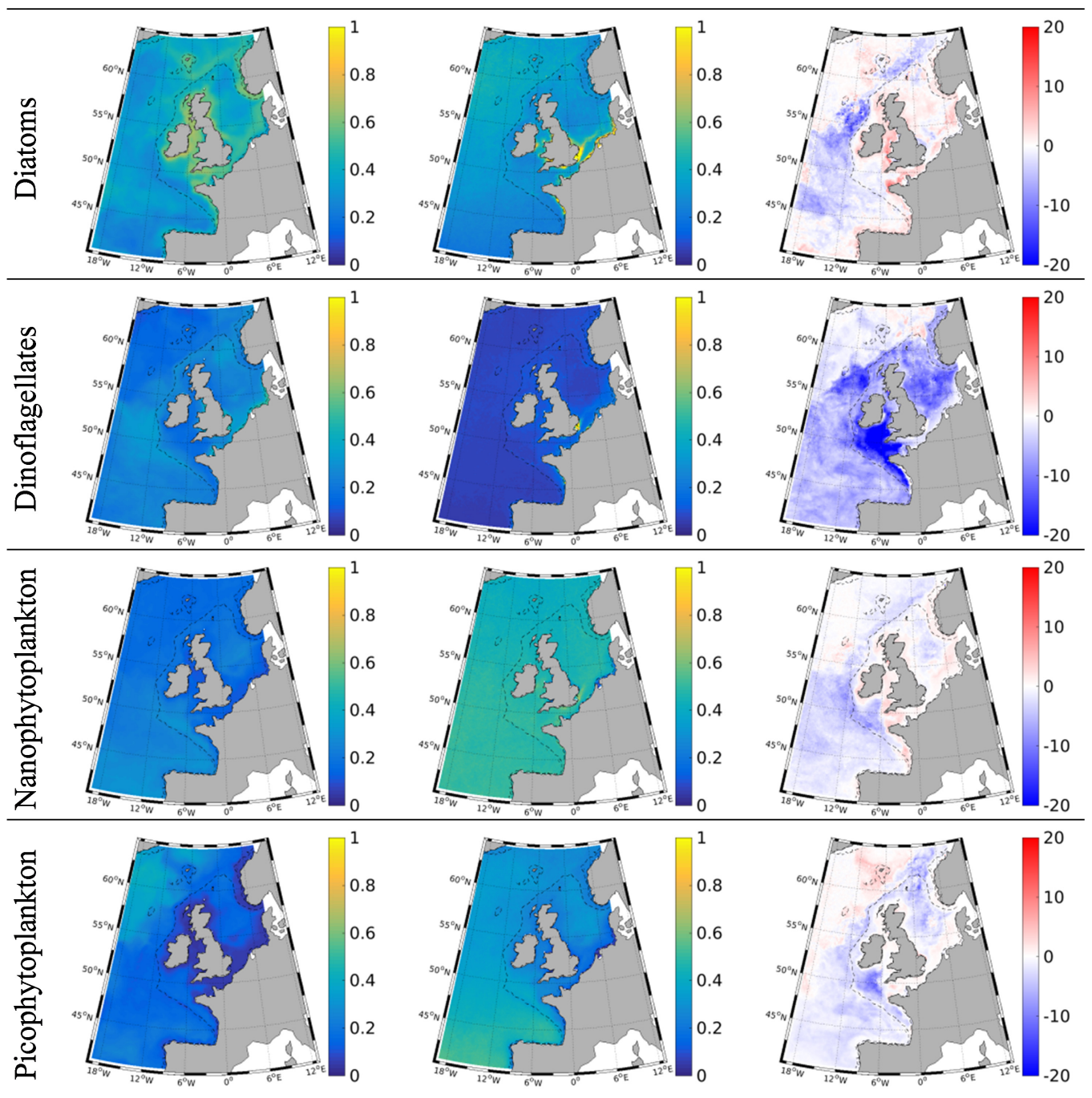

FIGURE 8 | Improved simulation of the plankton community structure by assimilating ocean-colour plankton functional type (PFT) products into a marine ecosystem model of the North East Atlantic. Column (A) PFTs fractions from the assimilative simulation (i.e., ratio of the PFT chlorophyll to the total chlorophyll); (B) PFTs fractions from ocean-colour data; and (C) percentage difference between the RMSD of the PFT assimilation (1-month forecasts) and of the reference model simulation without assimilation; RMSD is the root-mean-square-deviation between grid-point time series of model output and ocean-colour data. Figure elaborated from Figure 8 in Ciavatta et al. (2018), delivered by the Copernicus Marine Environment Monitoring Service TOSCA project.

ongoing (Skakala et al., 2018). The seminal work using CZCS ocean-colour for data assimilation involved 'nudging' a simple model to the chl-a observed value to evaluate ocean productivity (Armstrong et al., 1995). The most recent applications use an expanded suite of ocean-colour products with multivariate DA techniques that can correct the whole set of ocean variables in complex, highly non-linear models. For example, the chl-a time series from ESA's CCI Ocean Colour (see section 'Time Series for Climate') has been assimilated in applications ranging from mapping the risk of oxygen deficiency in shelf-bottom waters (Ciavatta et al., 2016) to improving global estimates of carbon cycle variables (Ford and Barciela, 2017). DA of new oceancolour products, such as phytoplankton functional types (PFTs), outperformed total chlorophyll DA in improving the reanalysis of the plankton community structure (Figure 8; Ciavatta et al., 2018) and the pre-operational prediction of some biogeochemical indicators (e.g., CO2 fugacity, Skakala et al., 2018).

Moving forward, the development of bio-optical modules in marine ecosystem models is opening new frontiers for ocean-colour DA (see e.g., the model by Dutkiewicz et al., 2015). Assimilation of ocean-colour phytoplankton absorption (Shulman et al., 2013), spectral diffuse attenuation coefficient 
(Ciavatta et al., 2014), remote sensing reflectance (Jones et al., 2016), absorption of CDOM and detritus (Gregg and Rousseaux, 2017) have been shown to enhance the estimation of the biooptically active compounds, including in case II waters. This approach is tackling the issue of possible mismatches between the variables that are simulated by models and those retrieved from ocean colour (e.g., observed 'chlorophyll' concentration might include simulated suspended solids in coastal areas, Jones et al., 2016). Finally, simulating optical properties can allow one to use ecosystem models for design and analysis of future satellite missions, e.g., for the selection of bands in hyper-spectral remote sensors (Gregg and Rousseaux, 2017).

\section{TRAINING AND CAPACITY BUILDING}

Capacity building and training, notably in developing countries, is recognised as an on-going need to make best use of the ocean colour resources and data sets available to the user community. The IOCCG has supported regular lecture series at the Laboratoire d'Océanographie de Villefranche (LOV) in Villefranche-sur-Mer: the lectures and other materials are available on the IOCCG web site, and in the past, has supported training courses in various locations around the world. EUMETSAT undertakes marine training courses in Europe and around the world. The Partnership for Observation of the Global Ocean (POGO) offers a number of training, education and fellowship opportunities. Below are described some ocean-colour relevant initiatives contributing to community and capacity building and training.

\section{Group on Earth Observations EO4SDG}

EO4SDG is a GEO initiative on promoting EO for Sustainable Development Goals ${ }^{33}$. It has three goals '(1) Demonstrate how Earth observations, geospatial information, and socio-economic and other data contribute in novel and practical ways to support achievement of the SDGs. (2) Increase skills and capabilities in uses of Earth observations for SDG activities and their broader benefits. (3) Broaden interest and awareness of Earth observations support to the SDGs and contributions to social, environmental, and economic benefits' (EO4SDG Implementation Plan: GEO Initiative 18: Earth Observations in Service of the 2030 Agenda for Sustainable Development, available at eo4sdg.org).

\section{The Chlorophyll Globally Integrated Network (ChloroGIN)}

ChloroGIN $^{34}$ (Sathyendranath et al., 2010) was an early attempt to create a network of regional ocean-colour data providers and users. Mapped level 2 satellite ocean-colour products were made available to the users through a single portal. The Africa data production has been maintained with complete coverage of African waters at 1-km since 2010. Data have been used in a number of capacity building projects in Africa such as the

${ }^{33}$ http://eo4sdg.org

${ }^{34}$ www.chlorogin.org
Monitoring for Environment and Security in Africa (MESA) with data available via the web site and also via Eumetcast. The data continue to be used in the follow-up GMES and Africa project and production is transitioning to Sentinel 3 OLCI. ChloroGIN has had a strong capacity building goal, and though there has been little resource of late for global coordination of this activity, the regional nodes within ChloroGIN have remained active, and become more self-sustained (see for example, the case of GMES Africa and Antares, the Latin American networks detailed below). But the benefits from enhanced global coordination have not yet been fully realised, because of lack of funding.

\section{ANTARES Latin and South American Network}

The Antares network was created in July 2003 under the auspices of the IOCCG, Partnership for Observation of the Global Ocean (POGO), Nippon Foundation and Inter-American Institute for Global Change Research (IAI). Antares served as a seed for ChloroGIN. Antares aims to monitor and detect long-term changes in coastal waters around Latin-America with the main approach of developing time-series of oceanographic observations (physical, chemical and biological) integrated with remote sensing data, products and modelling. Capacity building as well as scientific and technical collaboration are key for Antares network. Current participating countries are Argentina, Brazil, Chile, Colombia, Ecuador, Mexico, Peru, United States, and Venezuela.

The Antares stations are located in a region with scarce in situ observations. They are distributed along the Pacific, Caribbean and Atlantic coasts of Latin America, covering about 70 degrees of latitude from $31^{\circ} \mathrm{N}$ (Ensenada, Mexico) up to $38^{\circ} \mathrm{S}$ (EPEA, Argentina). The time-series observations carried out at these sites register valuable ecological information in different areas with different oceanographic characteristics. Among the Pacific stations, one is located in the northern hemisphere (Ensenada, Mexico) and another in the southern hemisphere (IMARPE, Peru). The Caribbean station (CARIACO, Venezuela) is no longer operational and was located on a basin more than $1000 \mathrm{~m}$ deep. On the South Atlantic side, Ubatuba (Brazil) located in a Subtropical regime and EPEA (Argentina) on a temperate regime. Data will be made available via the InterAmerican Institute for Global Change Research (IAI) Open Data Portal ${ }^{35}$.

Through an IAI project, Antares is studying trends in phytoplankton and its associated ecosystem services in a context of climate variability. It aims at making more 'visible' the link between phytoplankton and the concentration of $\mathrm{CO}_{2}$, a key variable for the biogeochemistry of the planet and the organisation of human society.

Satellite ocean colour imagery is available via the SigmaAntares tool, at antares2.cptec.inpe.br for the entire coast of Latin and South America.

\footnotetext{
${ }^{35} \mathrm{http} / /$ www.iai.int/en/post/detail/open-dataopen-science
} 


\section{ESA Earth Observation for Sustainable Development}

ESA's $\mathrm{EO}_{4} \mathrm{SD}^{36}$ is a major project promoting use and uptake of $\mathrm{EO}$ data (e.g., Anderson et al., 2017) to support the UN Sustainable Development Goals by working with International Funding Institutes like the World Bank or East Asian Development Bank and their individual client states. The marine and coastal project has just started (mid 2018) and is one of eight including, inter alia, water resource management, disaster risk reduction and climate resilience. The marine and coastal project aims at supporting a number of services, including water quality monitoring, aquaculture site selection, invasive species monitoring together with near coastal and high-resolution services, coastal and benthic habitat status and MPA management. The project aims to address five regions worldwide: west Africa, western Indian Ocean, Caribbean, east Asia and Pacific Islands.

\section{Global Monitoring for Environment and Security and Africa (GMES and Africa)}

Global Monitoring for Environment and Security and Africa is a pan-continental project using EO data, technologies and services in support of African sustainable development; it is, arguably, an exemplar of construction of an integrated ocean and coastal motoring system in developing countries. As a joint initiative between the African and European Unions, there is strong emphasis on realising value from the data, information, expertise and know-how offered by the Copernicus programme. In the marine and coastal domains, four consortia around the continent are developing and implementing a variety of services, including the provision of oceanographic products, fisheries and aquaculture support, and coastal ecosystem monitoring and assessment. Private companies are also encouraged to participate in the services with $20 \%$ of the regional budget reserved for the sector. From an ocean-colour perspective, Sentinel 2 and Sentinel 3 in particular will provide substantial value to these services, using a variety of products and approaches.

In South and East Africa under the 'MarCoSouth' consortium, application services will provide additional value to ocean colour science products, with user co-design a major aspect of the service development. Standard Copernicus reduced resolution $(1-\mathrm{km})$ Sentinel $3 \mathrm{~A}$ and $3 \mathrm{~B}$ OLCI products will provide base ocean biogeochemistry products for the African shelf. In addition to standard chl-a and Total Suspended Matter products, regional switching algorithms will be used for optimised chl-a estimates in high biomass bloom water types. OLCI Full Resolution data at $300 \mathrm{~m}$ resolution, and Sentinel 2A/SB MSI derived products will be provided for specific coastal sites, prioritised for aquaculture, desalination, estuarine conservation and fisheries, effluent discharge and other applications. Three application services will provide additional value to these ocean colour science products:

- Aquaculture support will focus primarily on determining $\mathrm{HAB}$ risk, gross water quality monitoring and temperature

\footnotetext{
${ }^{36} \mathrm{http}: / /$ eo4sd.esa.int/
}

range analysis for a range of target organisms, both in nearreal time and for historical analysis. A range of sectors will be targeted, including the relatively high-technology and high-investment abalone farms in South Africa (see section 'Harmful Algal Blooms and Aquaculture in South Africa'), bivalve operations in Namibia and South Africa, industrial and artisanal finfish farming regionally, and artisanal seaweed farming in Tanzania and Kenya.

- Fisheries support is already well established for coastal fisheries in West Africa and large pelagics in the Western Indian ocean. In Southern Africa, new services will focus on the provision of ocean colour and SST frontal maps primarily for pelagic fisheries such as sardine and tuna across the shelf and in Lake Victoria. PFZ products will also be provided through mobile platforms (see section 'Ground Segment and Data Processing'). Longer term development will focus on integration of catch data, refinement of PFZ products through chl-a/SST heat maps, and provision of longer-term phenologies and time series analyses to national and regional resource management agencies.

- The ecosystem monitoring service will focus primarily on reef and mangrove mapping and assessment from a habitat perspective, with additional risk monitoring capabilities at prioritised sites for high-resolution water quality and Virtual Buoy oceanographic monitoring. Routine near-real time products from Sentinel 2 and 3 will play a major role here, monitoring for effluent discharge and turbidity, eutrophication and temperature anomalies.

\section{Partnership for Observation of the Global Ocean (POGO)}

Partnership for Observation of the Global Ocean ${ }^{37}$ offers various ocean-colour relevant training education and fellowship opportunities including, inter alia, the Nippon Foundation (NF)-POGO sponsored Centre of Excellence for training in observational oceanography hosted currently at the Alfred Wegener Institute, Germany and regional training courses; a fellowship training programme in collaboration with the Scientific Committee on Oceanic Research (SCOR) to support young professionals from developing countries to spend up to 3 months at a major oceanographic institution; and shipboard training.

\section{OCEAN COLOUR WITHIN OCEAN OBSERVING SYSTEMS}

Developments since the last OceanObs conference in 2009 have rapidly expanded the applications that make use of satellite ocean-colour data. The record has extended to 22 years (in 2019); a virtual constellation of operational missions is now in orbit, with replacements planned; routine global observations are available at $300 \mathrm{~m}$; production of ocean-colour observations has developed significantly with operational production of L1, L2 and L3 data by Copernicus in Europe, and NOAA and NASA in the

\footnotetext{
${ }^{37}$ www.ocean-partners.org/
} 
United States (for example); and access to data is now possible through a variety of methods including $\mathrm{ftp}$, OPeNDAP and web-GIS, while cloud platforms offer greater flexibility for data analysis (see section 'Ground Segment and Data Processing'). Areas still to be addressed include higher spatial resolution, for observation of near-coastal or inland waters, and diurnal observations through the day (which are indispensable for the meteorological community) with geostationary missions and only east Asia has such a mission in place. Since this paper is associated with OceanObs 2019 it is instructive to consider how ocean colour will contribute to the aims of the conference to improve response to scientific and societal needs of a fit-for-purpose integrated ocean observing system, for better understanding the environment of the Earth, monitoring climate, and informing adaptation strategies as well as the sustainable use of ocean resources ${ }^{38}$.'

Ocean colour is central to ocean observing systems, providing the only direct global observations of phytoplankton, the base of the ocean ecosystem. Such observing systems are driven by the United Nations Sustainable Development Goals (SDGs) and the Convention on Biological Diversity (CBD). Ocean colour is noted as a cross-cutting Essential Ocean Variable (EOV) by GOOS, is implicated in other EOVs and is relevant to a number of GEO-BON Essential Biodiversity Variables (Muller-Karger et al., 2018). Observing systems operate at a number of spatial and temporal scales reflecting the range of applications from climate observation (global, long-term) to operational (usually regional or local, short-term); nevertheless, some commonalities can be drawn.

Ocean colour is fundamental to observing the ocean ecosystem and carbon system including primary production, particulate organic or inorganic carbon and dissolved organic carbon; these also require inputs from multiple disciplines such as sea-surface temperature or solar irradiance, encouraging collaboration between these communities. Extending the primarily surface observations to the ocean interior will need synergy with in situ data, notably from BGC-Argo floats in oceans, instrumented gliders or moorings in shelf-seas and novel in situ plankton monitoring systems (Lombard et al., 2019). Likewise, stepping from observations to forecasts implies data assimilation into models or validation of models using the ocean-colour record (see section 'Ecosystem Modeling' and Fennel et al., 2019). All these considerations lead to the need to sustain ocean-colour observations in operational mode; further improve ocean-colour observations and the derived products (including data uncertainty characteristics) by improving the constituent retrieval algorithms, through strict adherence to international protocols and thorough uncertainty characterisation of instrumentation for in situ data gathering (see section 'Ocean Colour EO Sensors and in situ Observations'); improve the methods to remove atmospheric contamination and combine multiple sensors (e.g., see section 'Time Series for Climate'); and improve access to ocean-colour data, following international standards and conventions for data storage (like netCDF) or data

${ }^{38} \mathrm{http}: / /$ www.oceanobs19.net/ serving (like OGC compliant web servers). There is also an obvious need for greater training opportunities for specialists and non-specialists alike, in both developed countries and developing countries, to make use of the wider range of products, where issues of impact of climate change, of food security, of water availability and biodiversity loss are particularly acute.

\section{CONCLUSION AND FORWARD LOOK}

- The conclusions in this section focus on ocean, shelf and off-shore coastal applications: the reader is reminded that near-coastal, estuarine and inland water observations are not considered herein.

- Operational satellite sensor constellations, such as Copernicus Sentinel 3 OLCI and NOAA JPSS VIIRS, will ensure provision of ocean-colour data as elements of integrated, sustained observing systems. This ensures that commercial and governmental operators who invest in ocean-colour-related services can do so with the confidence that continuity of data is guaranteed. The operational missions will be complemented by new capability missions such as NASA PACE.

- Over the next decade the contiguous ocean-colour record will cover 30 years, reaching the minimum length of time series essential for discrimination of climate change impacts from shorter-scale natural variability, at least for some regions.

- The higher spatial resolution available from Sentinel 3 OLCI, $300 \mathrm{~m}$ globally with full ocean colour capability, will support applications closer to the coast as well as in estuaries and inland waters.

- Although not considered explicitly herein there is a gap in high-resolution $(\sim<10 \mathrm{~m})$ observations of ocean colour (or inland water colour) and it is recommended that space agencies consider this as a future development. Sentinel 2 MSI and Landsat 8, although not optimised for aquatic applications, can provide observations of features such as sediment plumes, floating vegetation and phytoplankton information in some cases, at the $10-100 \mathrm{~m}$ resolution required for coastal and fresh-water systems.

- Significant steps are being taken to provide analysis-ready data in a variety of formats, with increasingly faster turnaround from data capture to product delivery to the enduser, which will support applications that need a rapid response such as alerts for nuisance or harmful algal bloom in the vicinity of aquaculture farms and beaches.

- There is a continuing need to improve retrieval algorithms, as well as the methods to establish the quality of their performance: hence, it is recommended that strict protocols for in situ data gathering be followed, with full system uncertainty characterisation, to obtain Fiducial Reference Measurements. This will help ensure that combined datasets used for algorithm construction and satellite product validation and product evaluation have minimal inter-investigator differences. 
- As hyperspectral satellite sensors are about to become a reality, it is important to explore novel algorithms to reduce uncertainties in products by exploiting the new capability.

- Continuity in campaigns to obtain Fiducial Reference Measurements supporting system vicarious calibration and validation of sensors is key to the success of oceancolour missions, both when they join the ocean-colour constellation and as they extend the duration of their operation in space. This is an area that needs to be funded continuously by space agencies.

- Novel instrumentation, including autonomous or flowthrough systems installed on research vessels or ships of opportunity, have the potential to greatly increase the number of satellite 'matchup' data but they need to follow strict protocols with respect to appropriate system characterisation, calibration, and maintenance including treatment of bio-fouling. Such systems will also help characterise the variability within a satellite pixel and hence reduce the retrieval uncertainties. Furthermore, additional sensors could provide observations on biological diversity (Boss et al., 2018).

- EO data will be increasingly combined with operational in situ sensors to provide integrated observations with the temporal or depth capability of in situ data complementing the synoptic spatial scale of satellite EO data.

- Given the value and cost of in situ data it is important that international or de facto standards are followed that allow linking or interoperability when data are held in multiple databases.

- Ocean colour data will be increasingly used for initialisation and updating of short-term numerical forecast models as well as validating hindcast climate model runs.

- Mobile communication is expected to grow worldwide, especially in developing countries, supporting citizen science and communication of EO-based information to citizens: this two-way communication will both aid the ocean-colour field and increase scientific awareness.

- Capacity building and training will remain important to fully utilise satellite observation and integrated observing systems for societal benefit worldwide, but notably in developing countries.

- Finally, the UN Sustainable Development Goals aiming for the implementation of the 2030 Agenda for Sustainable

\section{REFERENCES}

Al-Naimi, N., Raitsos, D. E., Ben-Hamadou, R., and Soliman, Y. (2017). Evaluation of satellite retrievals of chlorophyll-a in the Arabian Gulf. Remote Sens. 9:301.

Alvera-Azcarate, A., Vanhellemont, Q., Ruddick, K., Barth, A., and Beckers, J. M. (2015). Analysis of high frequency geostationary ocean color data using DINEOF. Estuar. Coast. Shelf Sci. 159, 28-36.

Anderson, K., Ryan, B., Sonntag, W., Kavvada, A., and Friedl, L. (2017). Earth observation in service of the 2030 Agenda for sustainable development. Geo Spat. Inform. Sci. 20, 77-96.

Armstrong, R. A., Sarmiento, J. L., and Slater, R. D. (1995). "Monitoring ocean productivity by assimilating satellite chlorophyll into ecosystem models," in
Development will set a wide context for developments in the ocean colour field for the next decade.

\section{AUTHOR CONTRIBUTIONS}

All authors listed have made a substantial, direct and intellectual contribution to the work, and approved it for publication.

\section{FUNDING}

We acknowledge funding from the Natural Environment Research Council, National Centre for Earth Observation; the NERC EO Data Acquisition and Analysis Service; the European Space Agency Climate Change Initiative; the European Commission; the CMEMS SE OPTIMA project; NASA ROSES 17-TASNPP17-0065; Global Change Observation Mission-Climate (GCOM-C) from JAXA; Inter-American Institute for Global Changes Research (IAI/US-NSF GEO1128040 grant); NASA Ocean Biology and Biogeochemistry Programme/Applied Sciences Programme (proposal 14SMDUNSOL14-0001); United States Environmental Protection Agency, National Oceanic and Atmospheric Administration; United States Geological Survey Toxic Substances Hydrology Program; the Korean Ministry of Oceans and Fisheries (PM61110); and a sabbatical grant from FCT, Portugal, SFRH/BSAB/142981/2018. This work was supported by the Simons Collaboration on Computational Biogeochemical Modelling of Marine Ecosystems/CBIOMES (Grant ID: 549947, SS). This article has been reviewed by the National Exposure Research Laboratory and Office of Water and approved for publication. Mention of trade names or commercial products does not constitute endorsement or recommendation for use by the United States Government. The views expressed in this article are those of the authors and do not necessarily reflect the views or policies of the United States Environmental Protection Agency. This work was also supported by funding from the European Union's Horizon 2020 Research and Innovation Programme grant agreement $\mathrm{N}^{\circ}$ 810139: Project Portugal Twinning for Innovation and Excellence in Marine Science and Earth Observation - PORTWIMS.

Ecological Time Series, eds T. M. Powellcpesnm, and J. H. Steelecpesnm, (Boston, MA: Springer), 371-390.

Bhatpuria, D., Solanki, H. U., Varghese, S., and Chauhan, P. (2015). Applications of satellite derived meso-scale features and in-situ bycatch to understand sea turtle habitats along the Indian Coast. Curr. Sci. 108, 326-329.

Bonekamp, H., Parisot, F., Wilson, S., Miller, L., Donlon, C., Drinkwater, M., et al. (2010). "Transition towards operational space-based ocean observations: from single research missions into series and constellations," in Proceedings of OceanObs'09: Sustained Ocean Observations and Information for Society, Vol. 1, eds J. Hall, D. E. Harrison, and D. Stammercpesnm, (Venice: ESA Publication WPP-306).

Boss, E., Waite, A., Muller-Karger, F., Yamazaki, H., Wanninkhof, R., Uitz, J., et al. (2018). Beyond chlorophyll fluorescence: the time is right to expand biological 
measurements in ocean observing programs. Limnol. Oceanogr. Bull. 27, 89-90.

Bresciani, M., Cazzaniga, I., Austoni, M., Sforzi, T., Buzzi, F., Morabito, G., et al. (2018). Mapping phytoplankton blooms in deep subalpine lakes from Sentinel-2A and Landsat-8. Hydrobiologia 824, 197-214.

Brewin, R. J. W., Brewin, T. G., Phillips, J., Rose, S., Abdulaziz, A., Wimmer, W., et al. (2019). A printable device for measuring clarity and color in lake and nearshore waters. Sensors 19:936. doi: 10.3390/s19040936

Brewin, R. J. W., Dall'olmo, G., Pardo, S., Van Dongen-Vogels, V., and Boss, E. S. (2016). Underway spectrophotometry along the Atlantic meridional transect reveals high performance in satellite chlorophyll retrievals. Remote Sens. Environ. 183, 82-97.

Brewin, R. J. W., Sathyendranath, S., Muller, D., Brockrnann, C., Deschamps, P. Y., Devred, E., et al. (2015). The ocean color climate change initiative: III. A roundrobin comparison on in-water bio-optical algorithms. Remote Sens. Environ. 162, 271-294. doi: 10.1371/journal.pone.0168440

CEOS, (2014). CEOS Strategy for Carbon Observations From Space: The Committee on Earth Observation Satellites (CEOS) Response to the Group on Earth Observations (GEO) Carbon Strategy. Japan: JAXA and I\&A Corporation. Available at: http://ceos.org/document_management/ Publications/WGClimate_CEOS-Strategy-for-Carbon-Observations-fromSpace_Apr2014.pdf

Chauhan, P., and Raman, M. (2017). Satellite remote sensing for ocean biology: an indian perspective. Proc. Natl. Acad. Sci. India Sec. Phys. Sci. 87, 629-640.

Choi, J. K., Park, Y. J., Ahn, J. H., Lim, H. S., Eom, J., and Ryu, J. H. (2012). GOCI, the world's first geostationary ocean color observation satellite, for the monitoring of temporal variability in coastal water turbidity. J. Geophys. Res. Oceans 117:c09004.

Choi, J.-K., Park, Y. J., Lee, B. R., Eom, J., Moon, J.-E., and Ryu, J.-H. (2014a). Application of the geostationary ocean color imager (GOCI) to mapping the temporal dynamics of coastal water turbidity. Remote Sens. Environ. 146, 24-35. doi: $10.1016 /$ j.rse.2013.05.032

Choi, J.-K., Min, J.-E., Noh, J. H., Han, T.-H., Yoon, S., Park, Y. J., et al. (2014b). Harmful algal bloom (HAB) in the East Sea identified by the Geostationary Ocean Color Imager (GOCI). Harmful Algae 39, 295-302. doi: 10.1016/j.hal. 2014.08.010

Ciavatta, S., Brewin, R. J. W., Skakala, J., Polimene, L., De Mora, L., Artioli, Y., et al. (2018). Assimilation of ocean-color plankton functional types to improve marine ecosystem simulations. J. Geophys. Res. Oceans 123, 834-854.

Ciavatta, S., Kay, S., Saux-Picart, S., Butenschon, M., and Allen, J. I. (2016). Decadal reanalysis of biogeochemical indicators and fluxes in the North West European shelf-sea ecosystem. J. Geophys. Res. Oceans 121, 1824-1845.

Ciavatta, S., Torres, R., Martinez-Vicente, V., Smyth, T., Dall'olmo, G., Polimene, L., et al. (2014). Assimilation of remotely-sensed optical properties to improve marine biogeochemistry modelling. Prog. Oceanogr. 127, 74-95.

Clark, D. K., Gordon, H. R., Voss, K. J., Ge, Y., Broenkow, W., and Trees, C. (1997). Validation of atmospheric correction over the oceans. J. Geophys. Res. Atmos. 102, 17209-17217.

Claustre, H., Antoine, D., Boehme, L., Boss, E., D’ortenzio, F., Fanton D’andon, O., et al. (2010). "Guidelines towards an integrated ocean observation system for ecosystems and biogeochemical cycles," in Proceedings of OceanObs'09: Sustained Ocean Observations and Information for Society, Vol. 1, eds J. Hall, D. E. Harrison, and D. Stammercpesnm, (Venice: ESA Publication WPP-306), 21-25.

Claustre, H., Hooker, S. B., Van Heukelem, L., Berthon, J.-F., Barlow, R., Ras, J., et al. (2004). An intercomparison of HPLC phytoplankton pigment methods using in situ samples: application to remote sensing and database activities. Mar. Chem. 85, 41-61. doi: 10.1016/j.marchem.2003.09.002

Couto, A. B., Brotas, V., Melin, F., Groom, S., and Sathyendranath, S. (2016). Intercomparison of OC-CCI chlorophyll-a estimates with precursor data sets. Int. J. Remote Sens. 37, 4337-4355.

daff (2016). Aquaculture Yearbook 2015. Cape Town: Department of Agriculture, Forestry and Fisheries.

Dall'Olmo, G., Brewin, R. J. W., Nencioli, F., Organelli, E., Lefering, I., Mckee, D., et al. (2017). Determination of the absorption coefficient of chromophoric dissolved organic matter from underway spectrophotometry. Opt. Express 25, A1079-A1095. doi: 10.1364/OE.25.0A1079

Dall'Olmo, G., Dingle, J., Polimene, L., Brewin, R. J. W., and Claustre, H. (2016). Substantial energy input to the mesopelagic ecosystem from the seasonal mixed-layer pump. Nat. Geosci. 9, 820-823.
Dall'Olmo, G., and Mork, K. A. (2014). Carbon export by small particles in the Norwegian Sea. Geophys. Res. Lett. 41, 2921-2927.

Dogliotti, A. I., Gossn, J. I., Vanhellemont, Q., and Ruddick, K. G. (2018). Detecting and quantifying a massive invasion of floating aquatic plants in the Rio de la Plata turbid waters using high spatial resolution ocean color imagery. Remote Sens. 10:1140.

d'oliveira, F. A., De Melo, F. C. L., and Devezas, T. C. (2016). High-altitude platforms - present situation and technology trends. J. Aerosp. Technol. Manag. $8,249-262$.

Donlon, C., Berruti, B., Buongiorno, A., Ferreira, M. H., Femenias, P., Frerick, J., et al. (2012). The global monitoring for environment and security (GMES) Sentinel-3 mission. Remote Sens. Environ. 120, 37-57. doi: 10.1364/AO.52. 002125

Drinkwater, M., Bonekamp, H., Bontempi, P., Chapron, B., Donlon, C., Fellous, J. L., et al. (2010). "Status and outlook for the space component of an integrated ocean observing system," in Proceedings of OceanObs'09: Sustained Ocean Observations and Information for Society, Vol. 1, eds J. Hall, D. E. Harrison, and D. Stammercpesnm, (Venice: ESA Publication WPP-306), 21-25.

Dutkiewicz, S., Hickman, A. E., Jahn, O., Gregg, W. W., Mouw, C. B., and Follows, M. J. (2015). Capturing optically important constituents and properties in a marine biogeochemical and ecosystem model. Biogeosciences 12, 4447-4481.

Dwivedi, R. M., Solanki, H. U., Nayak, S. R., Gulati, D., and Somvanshi, V. S. (2005). Exploration of fishery resources through integration of ocean color with sea surface temperature: Indian experience. Indian J. Mar. Sci. 34, 430-440.

Fennel, K., Gehlen, M., Brasseur, P., Brown, C. W., Ciavatta, S., Cossarini, G., et al. (2019). Advancing marine biogeochemical and ecosystem reanalyses and forecasts as tools for monitoring and managing ecosystem health. Front. Mar. Sci. 6:89. doi: 10.3389/fmars.2019.00089

Ford, D., and Barciela, R. (2017). Global marine biogeochemical reanalyses assimilating two different sets of merged ocean color products. Remote Sens. Environ. 203, 40-54.

Ford, D., Kay, S., Mcewan, R., and Totterdell, I. A. (2018). "Marine biogeochemical modelling and data assimilation for operational forecasting, reanalysis, and climate research," in New Frontiers in Operational Oceanography, eds E. Chassignet, A. Pascual, J. Tintoré, and J. Verroncpesnm, (Tallahassee, FL: Florida State University), 625-652.

Forget, M.-H., Platt, T., Sathyendranath, S., Stuart, V., and Delaney, L. (2010). "Societal applications in fisheries and aquaculture using remotely-sensed imagery - the SAFARI project," in Proceedings of OceanObs'09: Sustained Ocean Observations and Information for Society, Vol. 2, eds J. Hall, D. E. Harrison, and D. Stammercpesnm, (Venice: ESA Publication WPP-306).

Franz, B. A., Kwiatkowska, E. J., Meister, G., and Mcclain, C. R. (2008). Moderate resolution imaging spectroradiometer on Terra: limitations for ocean color applications. J. Appl. Remote Sens. 2:023525.

Gao, B. C., Li, R. R., and Yang, Y. K. (2019). Remote sensing of daytime water leaving reflectances of oceans and large inland lakes from EPIC onboard the DSCOVR spacecraft at lagrange-1 point. Sensors 19:E1243. doi: 10.3390/ s19051243

GCOS, (2016). The Global Observing System for Climate: Implementation Needs GCOS200. Bonn: United Nations Framework Convention on Climate Change (UNFCCC).

Gharamti, M. E., Tjiputra, J., Bethke, I., Samuelsen, A., Skjelvan, I., Bentsen, M., et al. (2017). Ensemble data assimilation for ocean biogeochemical state and parameter estimation at different sites. Ocean Model. 112, 65-89.

Gohin, F., Druon, J. N., and Lampert, L. (2002). A five channel chlorophyll concentration algorithm applied to SeaWiFS data processed by SeaDAS in coastal waters. Int. J. Remote Sens. 23, 1639-1661.

Gordon, H. R., Clark, D. K., Mueller, J. L., and Hovis, W. A. (1980). Phytoplankton pigments from the Nimbus-7 coastal zone color scanner - comparisons with surface measurements. Science 210, 63-66.

Gregg, W. W., Friedrichs, M. A. M., Robinson, A. R., Rose, K. A., Schlitzer, R., Thompson, K. R., et al. (2009). Skill assessment in ocean biological data assimilation. J. Mar. Syst. 76, 16-33.

Gregg, W. W., and Rousseaux, C. S. (2017). Simulating PACE global ocean radiances. Front. Mar. Sci. 4:60. doi: 10.3389/fmars.2017.00060

Hammond, M. L., Beaulieu, C., Sahu, S. K., and Henson, S. A. (2017). Assessing trends and uncertainties in satellite-era ocean chlorophyll using space-time modeling. Glob. Biogeochem. Cycles 31, 1103-1117.

He, X. Q., Bai, Y., Wei, J., Ding, J., Shanmugam, P., Wang, D. F., et al. (2017). Ocean color retrieval from MWI onboard the Tiangong-2 space 
lab: preliminary results. Opt. Express 25, 23955-23973. doi: 10.1364/OE.25. 023955

He, X. Q., Stamnes, K., Bai, Y., Li, W., and Wang, D. F. (2018). Effects of earth curvature on atmospheric correction for ocean color remote sensing. Remote Sens. Environ. 209, 118-133.

Henson, S. A., Sarmiento, J. L., Dunne, J. P., Bopp, L., Lima, I., Doney, S. C., et al. (2010). Detection of anthropogenic climate change in satellite records of ocean chlorophyll and productivity. Biogeosciences 7, 621-640.

Hirata, T., Saux-Picart, S., Hashioka, T., Aita-Noguchi, M., Sumata, H., Shigemitsu, M., et al. (2013). A comparison between phytoplankton community structures derived from a global 3D ecosystem model and satellite observation. J. Mar. Syst. 109, 129-137.

Hollmann, R., Merchant, C. J., Saunders, R., Downy, C., Buchwitz, M., Cazenave, A., et al. (2013). THE ESA climate change initiative satellite data records for essential climate variables. Bull. Am. Meteorol. Soc. 94, 1541-1552.

Hoshiba, Y., Hirata, T., Shigemitsu, M., Nakano, H., Hashioka, T., Masuda, Y., et al. (2018). Biological data assimilation for parameter estimation of a phytoplankton functional type model for the western North Pacific. Ocean Sci. 14, 371-386.

Hovis, W. A., Clark, D. K., Anderson, F., Austin, R. W., Wilson, W. H., Baker, E. T., et al. (1980). NIMBUS-7 coastal zone color scanner - system description and initial imagery. Science 210, 60-63.

Hu, C., Lee, Z., and Franz, B. (2012). Chlorophyll a algorithms for oligotrophic oceans: a novel approach based on three-band reflectance difference. J. Geophys. Res. Oceans 117:C01011.

International Ocean Colour Coordinating Group [IOCCG] (2000). Remote Sensing of Ocean Color in Coastal and Other Optically-Complex Waters. Dartmouth, NS: Bedford Institute of Oceanography.

International Ocean Colour Coordinating Group [IOCCG] (2007). Ocean-Color Data Merging. Dartmouth, NS: IOCCG.

International Ocean Colour Coordinating Group [IOCCG] (2008). Why Ocean Color? The Societal Benefits of Ocean-Color Technology. Dartmouth, NS: IOCCG.

International Ocean Colour Coordinating Group [IOCCG] (2009). Remote Sensing in Fisheries and Aquaculture. Dartmouth, NS: IOCCG.

International Ocean Colour Coordinating Group [IOCCG] (2010). Atmospheric Correction for Remotely-Sensed Ocean-Color Products. Dartmouth, NS: IOCCG.

International Ocean Colour Coordinating Group [IOCCG] (2012). Mission Requirements for Future Ocean-Color Sensors. Dartmouth, NS: IOCCG.

International Ocean Colour Coordinating Group [IOCCG] (2013). In-Flight Calibration of Satellite Ocean-Color Sensors. Dartmouth, NS: IOCCG.

International Ocean Colour Coordinating Group [IOCCG] (2014). Phytoplankton Functional Types from Space. Dartmouth, NS: IOCCG.

International Ocean Colour Coordinating Group [IOCCG] (2018). Earth Observations in Support of Global Water Quality Monitoring, IOCCG Report Series No. 17. Dartmouth, NS: IOCCG.

Jackson, T., Sathyendranath, S., and Melin, F. (2017). An improved optical classification scheme for the ocean color essential climate variable and its applications. Remote Sens. Environ. 203, 152-161.

Jamet, C., Ibrahim, A., Ahmad, Z., Angelini, F., Babin, M., Behrenfeld, M. J., et al. (2019). Going beyond standard ocean color observations: lidar and polarimetry. Front. Mar. Sci. 6:251. doi: 10.3389/fmars.2019.00251

Joiner, J., Yoshida, Y., Guanter, L., and Middleton, E. M. (2016). New methods for the retrieval of chlorophyll red fluorescence from hyperspectral satellite instruments: simulations and application to GOME-2 and SCIAMACHY. Atmos. Meas. Techniq. 9, 3939-3967.

Jones, E. M., Baird, M. E., Mongin, M., Parslow, J., Skerratt, J., Lovell, J., et al. (2016). Use of remote-sensing reflectance to constrain a data assimilating marine biogeochemical model of the Great Barrier Reef. Biogeosciences 13, 6441-6469.

Kim, Y. S., Jeong, C. S., Seong, G. T., Han, I. S., and Lee, Y. S. (2010). Diurnal vertical migration of Cochlodinium polykrikoides during the red tide in Korean coastal sea waters. J. Environ. Biol. 31, 687-693.

Kumari, B., and Raman, M. (2010). Whale shark habitat assessments in the northeastern Arabian Sea using satellite remote sensing. Int. J. Remote Sens. 31, 379-389.

Kurekin, A. A., Miller, P. I., and Van Der Woerd, H. J. (2014). Satellite discrimination of Karenia mikimotoi and Phaeocystis harmful algal blooms in
European coastal waters: merged classification of ocean color data. Harmful Algae 31, 163-176.

Kwiatkowska, E. J., Franz, B. A., Meister, G., Mcclain, C. R., and Xiong, X. X. (2008). Cross calibration of ocean-color bands from moderate resolution imaging spectroradiometer on Terra platform. Appl. Opt. 47, 6796-6810.

Kwiatkowska, E. J., Ruddick, K., Ramon, D., Vanhellemont, Q., Brockmann, C., Lebreton, C., et al. (2016). Ocean color opportunities from meteosat second and third generation geostationary platforms. Ocean Sci. 12, 703-713.

Lavender, S., Beaugrand, G., Outram, N., Barlow, N., Crotty, D., Evans, J., et al. (2017). Seafarer citizen scientist ocean transparency data as a resource for phytoplankton and climate research. PLoS One 12:e0186092. doi: 10.1371/ journal.pone.0186092

Lavigne, H., and Ruddick, K. (2018). The potential use of geostationary MTG/FCI to retrieve chlorophyll-a concentration at high temporal resolution for the open oceans. Int. J. Remote Sens. 39, 2399-2420.

Le Quéré, C., Sathyendranath, S., Bopp, L., Buitenhuis, E., Doney, S., Dutkiewicz, S., et al. (2010). "Observational needs of dynamic green ocean models," in Proceedings of OceanObs'09: Sustained Ocean Observations and Information for Society, Vol. 2, eds J. Hall, D. E. Harrison, and D. Stammercpesnm, (Venice: ESA Publication WPP-306).

Lee, Z. P., Carder, K. L., and Arnone, R. A. (2002). Deriving inherent optical properties from water color: a multiband quasi-analytical algorithm for optically deep waters. Appl. Opt. 41, 5755-5772.

Leymarie, E., Penkerc'h, C., Vellucci, V., Lerebourg, C., Antoine, D., Boss, E., et al. (2018). ProVal: a new autonomous profiling float for high quality radiometric measurements. Front. Mar. Sci. 5:437. doi: 10.3389/fmars.2018. 00437

Lombard, F., Boss, E., Waite, A. M., Vogt, M., Uitz, J., Stemmann, L., et al. (2019). Globally consistent quantitative observations of planktonic ecosystems. Front. Mar. Sci. 6:196. doi: 10.3389/fmars.2019.00196

Losa, S. N., Soppa, M. A., Dinter, T., Wolanin, A., Brewin, R. J. W., Bricaud, A., et al. (2017). Synergistic exploitation of hyper- and multi-spectral precursor sentinel measurements to determine phytoplankton functional types (SynSenPFT). Front. Mar. Sci. 4:22. doi: 10.3389/fmars.2017.00203

Melin, F., and Sclep, G. (2015). Band shifting for ocean color multi-spectral reflectance data. Opt. Express 23, 2262-2279. doi: 10.1364/OE.23.002262

Melin, F., Vantrepotte, V., Chuprin, A., Grant, M., Jackson, T., and Sathyendranath, S. (2017). Assessing the fitness-for-purpose of satellite multi-mission ocean color climate data records: a protocol applied to OC-CCI chlorophyll-a data. Remote Sens. Environ. 203, 139-151. doi: 10.1016/j.rse.2017.03.039

Moore, T. S., Campbell, J. W., and Dowell, M. D. (2009). A class-based approach to characterizing and mapping the uncertainty of the MODIS ocean chlorophyll product. Remote Sens. Environ. 113, 2424-2430.

Moore, T. S., Dowell, M. D., Bradt, S., and Ruiz Verdu, A. (2014). An optical water type framework for selecting and blending retrievals from bio-optical algorithms in lakes and coastal waters. Remote Sens. Environ. 143, 97-111.

Muller, D., Krasemann, H., Brewin, R. J. W., Brockmann, C., Deschamps, P. Y., Doerffer, R., et al. (2015a). The ocean color climate change initiative: I. A methodology for assessing atmospheric correction processors based on in-situ measurements. Remote Sens. Environ. 162, 242-256.

Muller, D., Krasemann, H., Brewin, R. J. W., Brockmann, C., Deschamps, P. Y., Doerffer, R., et al. (2015b). The ocean color climate change initiative: II. Spatial and temporal homogeneity of satellite data retrieval due to systematic effects in atmospheric correction processors. Remote Sens. Environ. 162, 257-270. doi: 10.1371/journal.pone.0168440

Muller-Karger, F. E., Hestir, E., Ade, C., Turpie, K., Roberts, D. A., Siegel, D., et al. (2018). Satellite sensor requirements for monitoring essential biodiversity variables of coastal ecosystems. Ecol. Appl. 28, 749-760. doi: 10.1002/eap.1682

Murakami, H. (2016). "Ocean color estimation by Himawari-8/AHI," in Remote Sensing of the Oceans and Inland Waters: Techniques, Applications, and Challenges, eds R. J. Frouin, S. C. Shenoi, and K. H. Raocpesnm, (New Delhi: SPIE).

Nayak, S., Solanki, H., and Dwivedi, R. (2003). Utilization of IRS P4 ocean color data for potential fishing zone-a cost benefit analysis. Indian J. Mar. Sci. 32, $244-248$.

Ody, A., Doxaran, D., Vanhellemont, Q., Nechad, B., Novoa, S., Many, G., et al. (2016). Potential of high spatial and temporal ocean color satellite data to study the dynamics of suspended particles in a micro-tidal River Plume. Remote Sens. $8: 245$. 
O’Reilly, J. E., Maritorena, S., Mitchell, B. G., Siegel, D. A., Carder, K. L., Garver, S. A., et al. (1998). Ocean color chlorophyll algorithms for SeaWiFS. J. Geophys. Res. Oceans 103, 24937-24953.

Organelli, E., Barbieux, M., Claustre, H., Schmechtig, C., Poteau, A., Bricaud, A., et al. (2017). Two databases derived from BGC-Argo float measurements for marine biogeochemical and bio-optical applications. Earth Syst. Sci. Data 9, 861-880.

Pitcher, G. C., and Calder, D. (2000). Harmful algal blooms of the southern Benguela current: a review and appraisal of monitoring from 1989 to 1997. South Afr. J. Mar. Sci. 22, 255-271.

Pitcher, G. C., Foord, C. J., Macey, B. M., Mansfield, L., Mouton, A., Smith, M. E., et al. (2019). Devastating farmed abalone mortalities attributed to yessotoxinproducing dinoflagellates. Harmful Algae 81, 30-41. doi: 10.1016/j.hal.2018.11. 006

Racault, M. F., Raitsos, D. E., Berumen, M. L., Brewin, R. J. W., Platt, T., Sathyendranath, S., et al. (2015). Phytoplankton phenology indices in coral reef ecosystems: application to ocean-color observations in the Red Sea. Remote Sens. Environ. 160, 222-234.

Roy, S., Broomhead, D. S., Platt, T., Sathyendranath, S., and Ciavatta, S. (2012). Sequential variations of phytoplankton growth and mortality in an NPZ model: a remote-sensing-based assessment. J. Mar. Syst. 92, 16-29.

Ruddick, K., Neukermans, G., Vanhellemont, Q., and Jolivet, D. (2014). Challenges and opportunities for geostationary ocean color remote sensing of regional seas: a review of recent results. Remote Sens. Environ. 146, 63-76.

Sathyendranath, S., Ahanhanzo, J., Bernard, S., Byfield, V. L. D., Dowell, M., Field, J., et al. (2010). "ChloroGIN: use of satellite and in situ data in support of ecosystem-based management of marine resources," in Proceedings of OceanObs'09: Sustained Ocean Observations and Information for Society, Vol. 2, eds J. Hall, D. E. Harrison, and D. Stammercpesnm, (Venice: ESA Publication WPP-306).

Sathyendranath, S., Brewin, R. J. W., Jackson, T., Melin, F., and Platt, T. (2017). Ocean-color products for climate-change studies: what are their ideal characteristics? Remote Sens. Environ. 203, 125-138.

Schaeffer, B. A., Bailey, S. W., Conmy, R. N., Galvin, M., Ignatius, A. R., Johnston, J. M., et al. (2018). Mobile device application for monitoring cyanobacteria harmful algal blooms using Sentinel-3 satellite ocean and land color instruments. Environ. Model. Softw. 109, 93-103.

Schaeffer, B. A., Schaeffer, K. G., Keith, D., Lunetta, R. S., Conmy, R., and Gould, R. W. (2013). Barriers to adopting satellite remote sensing for water quality management. Int. J. Remote Sens. 34, 7534-7544.

Schofield, O., Kerfoot, J., Mahoney, K., Moline, M., Oliver, M., Lohrenz, S., et al. (2006). Vertical migration of the toxic dinoflagellate Karenia brevis and the impact on ocean optical properties. J. Geophys. Res. Oceans 111:C06009.

Shulman, I., Frolov, S., Anderson, S., Penta, B., Gould, R., Sakalaukus, P., et al. (2013). Impact of bio-optical data assimilation on short-term coupled physical, bio-optical model predictions. J. Geophys. Res. Oceans 118, 2215-2230.

Skakala, J., Ford, D., Brewin, R. J. W., Mcewan, R., Kay, S., Taylor, B., et al. (2018). The assimilation of phytoplankton functional types for operational forecasting in the Northwest European Shelf. J. Geophys. Res. Oceans 123, 5230-5247.

Smith, M. E., Lain, L. R., and Bernard, S. (2018). An optimized chlorophyll a switching algorithm for MERIS and OLCI in phytoplankton-dominated waters. Remote Sens. Environ. 215, 217-227.

Sørensen, K., Grung, M., and Röttgers, R. (2007). An intercomparison of in vitro chlorophyll $\alpha$ determinations for MERIS level 2 data validation. Int. J. Remote Sens. 28, 537-554.

Steinmetz, F., Deschamps, P.-Y., and Ramon, D. (2011). Atmospheric correction in presence of sun glint: application to MERIS. Opt. Express 19, 9783-9800. doi: 10.1364/OE.19.009783

Teruzzi, A., Bolzon, G., Salon, S., Lazzari, P., Solidoro, C., and Cossarini, G. (2018). Assimilation of coastal and open sea biogeochemical data to improve phytoplankton simulation in the Mediterranean Sea. Ocean Model. 132, 46-60.

Tsiaras, K. P., Hoteit, I., Kalaroni, S., Petihakis, G., and Triantafyllou, G. (2017). A hybrid ensemble-OI Kalman filter for efficient data assimilation into a 3-D biogeochemical model of the Mediterranean. Ocean Dyn. 67, 673-690.

Valente, A., Sathyendranath, S., Brotas, V., Groom, S., Grant, M., Taberner, M., et al. (2016). A compilation of global bio-optical in situ data for ocean-color satellite applications. Earth Syst. Sci. Data 8, 235-252.
Vanhellemont, Q., Neukermans, G., and Ruddick, K. (2014). Synergy between polar-orbiting and geostationary sensors: remote sensing of the ocean at high spatial and high temporal resolution. Remote Sens. Environ. 146, 49-62.

Vanhellemont, Q., and Ruddick, K. (2014). Turbid wakes associated with offshore wind turbines observed with Landsat 8. Remote Sens. Environ. 145, $105-115$.

Vanhellemont, Q., and Ruddick, K. (2015). Advantages of high quality SWIR bands for ocean color processing: examples from Landsat-8. Remote Sens. Environ. 161, 89-106.

Volpe, G., Santoleri, R., Vellucci, V., D’alcala, M. R., Marullo, S., and D’ortenzio, F. (2007). The color of the Mediterranean Sea: global versus regional biooptical algorithms evaluation and implication for satellite chlorophyll estimates. Remote Sens. Environ. 107, 625-638.

von Schuckmann, K., Le Traon, P. Y., Alvarez-Fanjul, E., Axell, L., Balmaseda, M., Breivik, L. A., et al. (2016). The copernicus marine environment monitoring service ocean state report. J. Operat. Oceanogr. 9, s235-s320.

von Schuckmann, K., Le Traon, P.-Y., Smith, N., Pascual, A., Brasseur, P., Fennel, K., et al. (2018). Copernicus marine service ocean state report. J. Operat. Oceanogr. 11, S1-S142.

Wang, M. H., Son, S., and Shi, W. (2009). Evaluation of MODIS SWIR and NIRSWIR atmospheric correction algorithms using SeaBASS data. Remote Sens. Environ. 113, 635-644.

Wojtasiewicz, B., Hardman-Mountford, N. J., Antoine, D., Dufois, F., Slawinski, D., and Trull, T. W. (2018). Use of bio-optical profiling float data in validation of ocean color satellite products in a remote ocean region. Remote Sens. Environ. 209, 275-290.

Xing, X. G., Morel, A., Claustre, H., Antoine, D., D’ortenzio, F., Poteau, A., et al. (2011). Combined processing and mutual interpretation of radiometry and fluorimetry from autonomous profiling Bio-Argo floats: chlorophyll a retrieval. J. Geophys. Res. Oceans 116:C06020.

Yoder, J., Dowell, M., Hoepffner, N., Murakami, H., and Stuart, V. (2010). "The ocean color radiance virtual constellation (OCR)," in Proceedings of OceanObs'09: Sustained Ocean Observations and Information for Society, Vol. 2, eds J. Hall, D. E. Harrison, and D. Stammercpesnm, (Venice: ESA Publication WPP-306).

Zibordi, G., Holben, B., Slutsker, I., Giles, D., D’alimonte, D., Melin, F., et al. (2009). AERONET-OC: a network for the validation of ocean color primary products. J. Atmos. Ocean. Technol. 26, 1634-1651.

Zibordi, G., Melin, F., Voss, K. J., Johnson, B. C., Franz, B. A., Kwiatkowska, E., et al. (2015). System vicarious calibration for ocean color climate change applications: requirements for in situ data. Remote Sens. Environ. 159, 361-369.

Disclaimer: The views, opinions, and findings contained in this paper are those of the authors and should not be construed as an official NOAA or United States Government position, policy, or decision. Mention of trade names or commercial products does not constitute endorsement or recommendation for use by the United States Government.

Conflict of Interest Statement: SL was employed by Pixalytics Ltd. CB was employed by Brockmann Consult GmbH. FS was employed by HYGEOS. PCi was employed by Telespazio VEGA UK.

The remaining authors declare that the research was conducted in the absence of any commercial or financial relationships that could be construed as a potential conflict of interest.

Copyright (C) 2019 Groom, Sathyendranath, Ban, Bernard, Brewin, Brotas, Brockmann, Chauhan, Choi, Chuprin, Ciavatta, Cipollini, Donlon, Franz, He, Hirata, Jackson, Kampel, Krasemann, Lavender, Pardo-Martinez, Mélin, Platt, Santoleri, Skakala, Schaeffer, Smith, Steinmetz, Valente and Wang. This is an openaccess article distributed under the terms of the Creative Commons Attribution License (CC BY). The use, distribution or reproduction in other forums is permitted, provided the original author(s) and the copyright owner(s) are credited and that the original publication in this journal is cited, in accordance with accepted academic practice. No use, distribution or reproduction is permitted which does not comply with these terms. 


\section{OPEN ACCESS}

Edited by:

Laura Lorenzoni,

University of South Florida,

United States

Reviewed by:

Craig E. Nelson,

University of Hawai'i at Mānoa

United States

Maria Kavanaugh,

Oregon State University,

United States

*Correspondence:

José H. Muelbert

jmuelbert@furg.br

Specialty section:

This article was submitted to

Ocean Observation,

a section of the journal

Frontiers in Marine Science

Received: 13 November 2018 Accepted: 12 August 2019

Published: 28 August 2019

Citation:

Muelbert JH, Nidzieko NJ,

Acosta ATR, Beaulieu SE,

Bernardino AF, Boikova $E$,

Bornman TG, Cataletto B, Deneudt K

Eliason E, Kraberg A, Nakaoka M,

Pugnetti A, Ragueneau O, Scharfe M,

Soltwedel T, Sosik HM, Stanisci A,

Stefanova K, Stéphan P, Stier A,

Wikner $J$ and Zingone A (2019) ILTER

- The International Long-Term

Ecological Research Network as

a Platform for Global Coastal

and Ocean Observation.

Front. Mar. Sci. 6:527.

doi: 10.3389/fmars.2019.00527

\section{ILTER - The International Long-Term Ecological Research Network as a Platform for Global Coastal and Ocean Observation}

\begin{abstract}
José H. Muelbert ${ }^{1,2 *}$, Nicholas J. Nidzieko ${ }^{3}$, Alicia T. R. Acosta ${ }^{4}$, Stace E. Beaulieu ${ }^{5}$, Angelo F. Bernardino ${ }^{6}$, Elmira Boikova ${ }^{7}$, Thomas G. Bornman ${ }^{8,9}$, Bruno Cataletto $^{10}$, Klaas Deneudt'11, Erika Eliason ${ }^{3}$, Alexandra Kraberg ${ }^{12}$, Masahiro Nakaoka ${ }^{13}$, Alessandra Pugnetti ${ }^{14}$, Olivier Ragueneau ${ }^{15}$, Mirco Scharfe ${ }^{16}$, Thomas Soltwedel ${ }^{12}$, Heidi M. Sosik ${ }^{5}$, Angela Stanisci ${ }^{17}$, Kremena Stefanova ${ }^{18}$, Pierre Stéphan ${ }^{19}$, Adrian Stier ${ }^{3}$, Johan Wikner ${ }^{20}$ and Adriana Zingone ${ }^{21}$
\end{abstract}

1 Instituto de Oceanografia, Universidade Federal do Rio Grande, Rio Grande, Brazil, ${ }^{2}$ Institute for Marine Sciences and Antarctic Studies, University of Tasmania, Hobart, TAS, Australia, ${ }^{3}$ Department of Geography and Marine Science Institute, University of California, Santa Barbara, Santa Barbara, CA, United States, ${ }^{4}$ Department of Sciences, Roma Tre University, Rome, Italy, ${ }^{5}$ Biology Department, Woods Hole Oceanographic Institution, Woods Hole, MA, United States, ${ }^{6}$ Grupo de Ecologia Bêntica, Departamento de Oceanografia, Universidade Federal do Espirito Santo, Vitoria, Brazil, ${ }^{7}$ Marine Ecology Laboratory, Institute of Biology, University of Latvia, Riga, Latvia, ${ }^{8}$ South African Environmental Observation Network, Elwandle Coastal Node, Port Elizabeth, South Africa, ${ }^{9}$ Coastal and Marine Research Institute, Nelson Mandela University, Port Elizabeth, South Africa, ${ }^{10}$ Istituto Nazionale di Oceanografia e di Geofisica Sperimentale, Trieste, Italy, ${ }^{11}$ Flanders Marine Institute, Ostend, Belgium, ${ }^{12}$ Alfred Wegener Institute, Helmholtz Center for Polar and Marine Research, Bremerhaven, Germany, ${ }^{13}$ Akkeshi Marine Station, Field Science Center for Northern Biosphere, Hokkaido University, Sapporo, Japan, ${ }^{14}$ National Research Council - Institute of Marine Sciences, Venice, Italy, ${ }^{15}$ LTSER "Zone Atelier Brest-Iroise", Laboratoire des Sciences de l'Environnement Marin, Institut Universitaire Européen de la Mer, Plouzané, France, ${ }^{16}$ Biologische Anstalt Helgoland, Alfred Wegener Institute, Helmholtz Center for Polar and Marine Research, Bremerhaven, Germany, ${ }^{17}$ Dipartimento di Bioscienze e Territorio, Università del Molise, Termoli, Italy, ${ }^{18}$ Institute of Oceanology, Bulgarian Academy of Sciences, Varna, Bulgaria, ${ }^{19}$ LTSER "Zone Atelier Brest-Iroise", Centre National de la Recherche Scientifique, Laboratoire LETG, Institut Universitaire Européen de la Mer, Plouzané, France, ${ }^{20}$ Umeå Marine Sciences Center, Umeå University, Umeå, Sweden, ${ }^{21}$ Stazione Zoologica Anton Dohrn, Naples, Italy

Understanding the threats to global biodiversity and ecosystem services posed by human impacts on coastal and marine environments requires the establishment and maintenance of ecological observatories that integrate the biological, physical, geological, and biogeochemical aspects of ecosystems. This is crucial to provide scientists and stakeholders with the support and knowledge necessary to quantify environmental change and its impact on the sustainable use of the seas and coasts. In this paper, we explore the potential for the coastal and marine components of the International Long-Term Ecological Research Network (ILTER) to fill this need for integrated global observation, and highlight how ecological observations are necessary to address the challenges posed by climate change and evolving human needs and stressors within the coastal zone. The ILTER is a global network encompassing 44 countries and 700 research sites in a variety of ecosystems across the planet, more than 100 of which are located in coastal and marine environments (ILTER-CMS). While most of the ILTER-CMS were established after the year 2000, in some cases they date back to the early 1900s. At ILTER sites, a broad variety of abiotic and biotic variables 
are measured, which may feed into other global initiatives. The ILTER community has produced tools to harmonize and compare measurements and methods, allowing for data integration workflows and analyses between and within individual ILTER sites. After a brief historical overview of ILTER, with emphasis on the marine component, we analyze the potential contribution of the ILTER-CMS to global coastal and ocean observation, adopting the "Strength, Weakness, Opportunity and Threats (SWOT)" approach. We also identify ways in which the in situ parameters collected at ILTER sites currently fit within the Essential Ocean Variables framework (as proposed by the Framework for Ocean Observation recommendations) and provide insights on the use of new technology in long-term studies. Final recommendations point at the need to further develop observational activities at LTER sites and improve coordination among them and with external related initiatives in order to maximize their exploitation and address present and future challenges in ocean observations.

Keywords: climate change, marine ecosystems, ecology, EOVs, SWOT, DEIMS

\section{INTRODUCTION}

Human activities threaten both the natural functioning of coastal and marine ecosystems and their sustainable use by present and future generations (Worm et al., 2006; Defeo et al., 2009; Halpern et al., 2012; Howes et al., 2015; Drius et al., 2016; Malavasi et al., 2018). Developing and delivering the ecological knowledge necessary to quantify how threats to coastal ecosystems impact national and international economies, policies and the sustainable use of the sea poses a significant challenge. Gaining such ecological knowledge on a local scale requires long-term observations of both environmental and biological variables, such as temperature and species richness. Effective assessment, management and prediction at the global scale, however, requires infrastructure and ecological observatories capable of systematically integrating from a long-term, large-scale, and whole-system perspective. Developing new observing systems and strengthening existing initiatives in a coordinated, standardized, global effort is essential to address these challenges.

Marine observatories provide the infrastructure and logistical support necessary to acquire data and knowledge for these purposes. The Global Ocean Observing System $\left(\mathrm{GOOS}^{1}\right)$ was established to provide a sustained, collaborative system for ocean observation that brings together in situ networks, remote sensing systems, government stakeholders, UN agencies and individual scientists. An important outcome of OceanObs'09 was the recognition of an imbalance between physical and biogeochemical/biological/ecological observations within most observing systems and the recommendation that GOOS consider how to expand the scope of observations to best address socially pressing global issues, including food security, harmful algal blooms, the spread of dead zones, and biodiversity conservation (Lindstrom et al., 2012). To that end, GOOS has established Biology and Ecosystem Essential Ocean Variables (EOVs ${ }^{2}$,

${ }^{1}$ http://goosocean.org/

${ }^{2}$ http://www.goosocean.org/eov
Miloslavich et al., 2018). The International Long-Term Ecological Research Network (ILTER ${ }^{3}$ ) is uniquely poised to contribute to the need for integrated, global observation of biological and ecological aspects of coastal ecosystems. Focal areas of the ILTER coastal and marine sites (henceforth ILTER-CMS) include: the consequences of biodiversity alteration for ecosystem functioning and services, documenting productivity changes, and understanding the cumulative impacts of multiple stressors including overfishing and ocean acidification. In this paper we explore the coastal and marine component of ILTER as a platform for global coastal and ocean observation, highlighting the role of ecological observations to address the challenges posed by climate change and evolving human needs and stressors within the coastal zone. To begin, we provide a brief historical overview of ILTER and describe its organization, with emphasis on the marine component. Next, we analyze the potential contribution of the ILTER marine component to global coastal and ocean observation adopting the "Strength, Weakness, Opportunity and Threats (SWOT)" approach. From this analysis, we elaborate on two specific opportunities for ILTER-CMS to contribute to global ocean observing. One, we identify ways that the in situ parameters collected at ILTER sites fit within the EOVs framework, as proposed by the "Framework for Ocean Observation" (FOO, Lindstrom et al., 2012) recommendations, and, two, provide insights on the use of new technology in long-term studies. Finally, we recommend paths forward to address future challenges.

\section{HISTORICAL PERSPECTIVE AND OVERVIEW OF ILTER}

The ILTER was established in 1993, 13 years after the launch of the Long-Term Ecological Research program by the National Science Foundation (NSF) of the United States, as a means of coordinating synthesis between LTER sites. Fully

${ }^{3} \mathrm{https} / /$ www.ilter.network/ 
supported by the NSF until 2003, ILTER gradually became a self-reliant network, growing from three founding members to 34 organizations by 2006 , when a 10 -year strategic plan was ratified (ILTER, 2006). At that time, ILTER transitioned to a broader disciplinary approach that included researchers, managers and stakeholders (ILTER, 2006). In 2007, the ILTER Association was founded in Costa Rica, making the ILTER a legal entity with its own governance structure, unifying strategy, operational goals and by-laws (Mirtl et al., 2018). Formally, member networks join the ILTER Association.

The ILTER Network provides a globally distributed network of long-term research sites for multiple purposes and uses in the fields of ecosystem, biodiversity, critical zone, and socioecological research (Mirtl et al., 2018). It currently consists of 44 national networks with robust governance structures, managing more than 700 sites worldwide, with a systematic coverage of terrestrial, freshwater, and marine environments (Haase et al., 2018; Mirtl et al., 2018). This site-based research network measures a broad variety of abiotic and biotic environmental variables, which may feed into other global initiatives. LTER national networks have mainly been developed from the bottom-up and LTER sites were established for different research and monitoring purposes. The ILTER community has produced tools to harmonize and compare measurements and methods, allowing for data integration workflows and analyses between and within individual LTER sites, to ensure the highest quality interoperable services in close interaction with related regional and global research infrastructures and networks (Haase et al., 2018; Mirtl et al., 2018). Long Term Socioeconomic and Ecosystem Research (LTSER) platforms emerged as initiatives aimed at enhancing the capacity of ecological knowledge combined with social science to produce useful knowledge for facing global environmental challenges (Mauz et al., 2012). This emphasis in the ILTER network reflects the desire to produce knowledge particularly useful for addressing complex environmental challenges emerging from nature-society interactions (Dick et al., 2018).

One of the goals of ILTER is to improve the comparability of site metadata and of long-term ecological data, facilitating their exchange and preservation around the world. ILTER member networks are committed to free and open data sharing (Vanderbilt et al., 2010; Vanderbilt and Gaiser, 2017), in agreement with the F.A.I.R (Findable, Accessible, Interoperable, and Reusable) principles for data management and Open Science (European Commission, 2016 'FAIR'; Mirtl et al., 2018; Tanhua et al., 2019). The Dynamic Ecological Information Management System Site and Dataset Registry (DEIMS-SDR ${ }^{4}$ ) provides a common and standardized metadata catalog for the distinct identification of observation facilities (e.g., sites, stations, sensors, datasets, persons) used by ILTER members. DEIMS-SDR also provides a web-based service to document and share scientific datasets, implements the ILTER community profile (Kliment and Oggioni, 2011), and allows the export to different XML formats (e.g., EML 2.1.1, BDP, ISO19115, INSPIRE). ILTER identified DataONE as the main facility to share and distribute ILTER data,

\footnotetext{
${ }^{4}$ http://data.lter-europe.net/deims/
}

but it also shares data through the GEOSS (Group on Earth Observation System of Systems ${ }^{5}$ ) Data Portal. ILTER agrees with the open data principles at the global scale in principle, but putting them into practice is still a challenging issue in most member networks and at the site level.

\section{THE COASTAL AND MARINE ILTER SITES (ILTER-CMS)}

There are 70 coastal and 60 marine sites in the ILTER (Figure 1 and Supplementary Table S1). Based on classifications in the ILTER's DEIMS-SDR, coastal sites include sand dunes and beaches, lagoons, estuaries, river deltas, fjords, salt marshes and mangroves, while marine sites are located on continental shelves and oceanic islands (Figure 2). Nearly half of the CMS include data records that precede the formal establishment of the ILTER (Figure 3). For example, the "Dutch Wadden Sea Area" in the Netherlands has records dating to 1872. Observations began in the Western Gulf of Finland in 1902; the Mar Piccolo of Taranto, Italy in 1914; and Shirahama, Japan in 1922. The length of these observations enhances the opportunities for ILTER-CMS to contribute to documenting global change.

The ILTER-CMS are distributed from tropical-equatorial to polar regions in what can be considered a global observing system (Figure 1). There is a large concentration of sites in LTER-Europe, with broad distribution along most of the European Seas. The European LTER sites are predominantly characterized by coastal and transitional waters, such as lagoons, river deltas, estuaries, and fjords. In the Atlantic, sites are located predominantly along the United States, Caribbean, and Brazilian coasts. There is a lack of coverage for the equatorial Atlantic and the African coast, where site distribution is restricted to South Africa. The Indian Ocean has only one oceanic site located at Reunion Island. There are some sites in the South and East China Sea and a good concentration of sites around Japan. In the South Pacific, a coastal site is located in Australia and one oceanic site in Tahiti. In the East Pacific, there are no sites along South America, but only along the Mexican and North American coasts.

The main focus of the ILTER-CMS is on the primary role of ecosystem structure, function, and services in response to a wide range of environmental forcing factors, using long-term, site-based research. Consistent with the general ILTER mission, the coastal and marine sites have been established to contribute to a global, multi-disciplinary community of ecosystem observation and research capable of delivering socially relevant information on sustainable use of natural resources. To that end, ILTER-CMS represents a strong component in a global ocean observation system linking ILTER more strongly into the GOOS framework (Figure 4). ILTER-CMS and GOOS can mutually benefit from setting similar requirements and deciding on what to measure, monitor Essential Ocean Variables, and interact through the instrumentation deployment and

${ }^{5}$ http://www.geoportal.org/ 


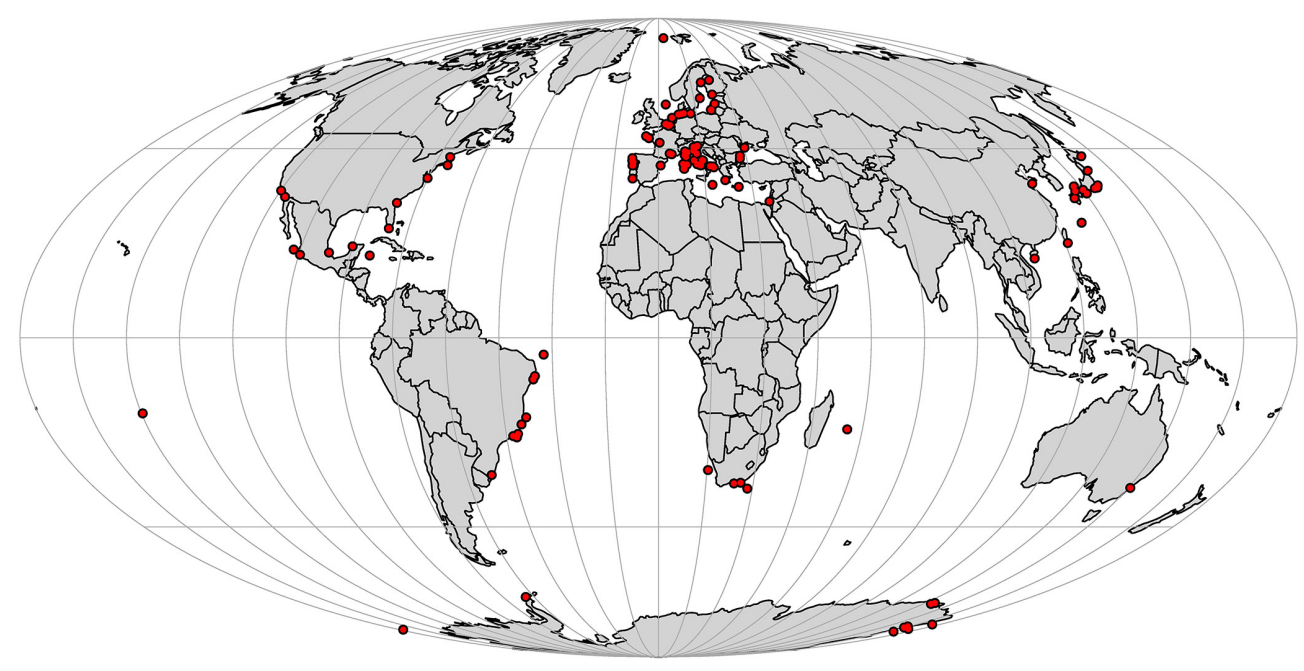

FIGURE 1 | Worldwide distribution of coastal and marine International Long-Term Ecological Research (ILTER) sites. Based on DEIMS status as of 21 st July, 2018.

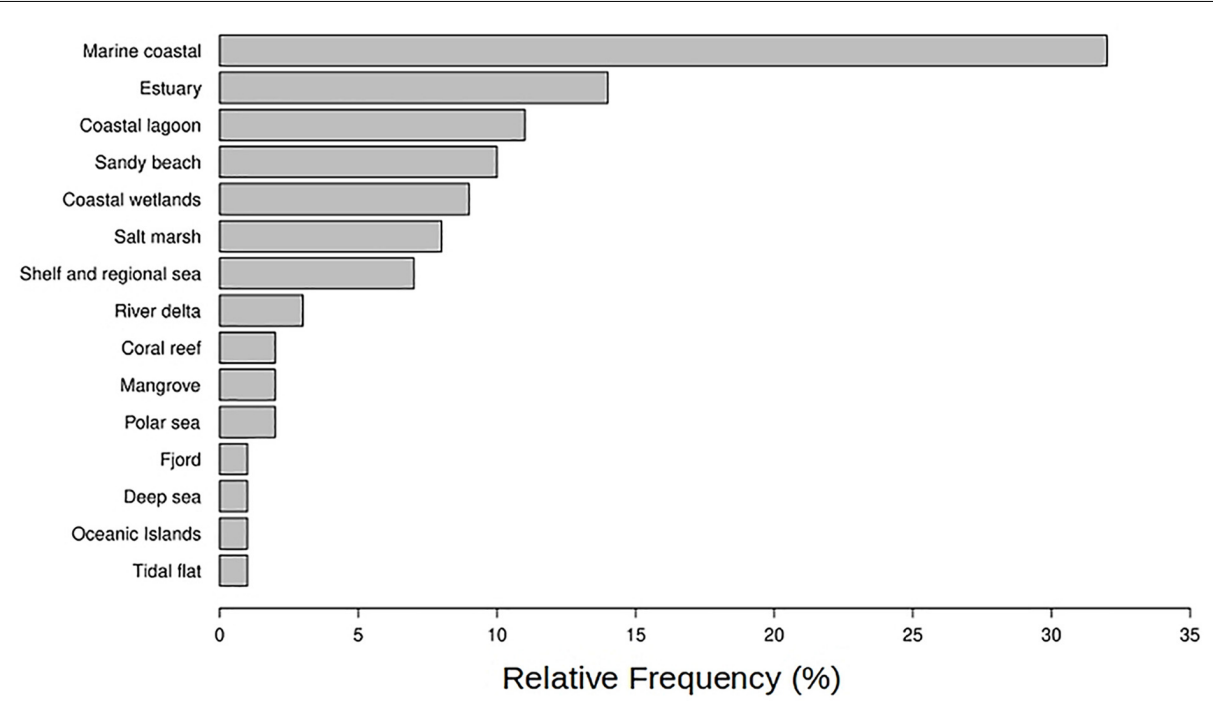

FIGURE 2 | The diversity of habitats represented at coastal and marine International Long-Term Ecological Research sites.

maintenance. ILTER-CMS monitors many biological variables by classical methods, providing added value to the sensor-based measurements in the GOOS program. The LTSER with social science competence can contribute to define issues and priorities and assess the impact of observations on society (Figure 4). In the following paragraphs we will briefly analyze the potential for ILTER-CMS to contribute to global coastal and marine observations by means of a "Strengths, Weaknesses, Opportunities and Threats" (SWOT) approach (Table $\mathbf{1}$ ).

\section{Strengths}

- ILTER coastal and marine sites are uniquely poised to contribute to biological and ecosystem EOVs by virtue of the existing ILTER governance, infrastructure, and research resources (both human and technological).

- The long time span of many ILTER-CMS members enables the identification of global trends (e.g., warming) and local pressures (e.g., nutrient loads) against the background of natural variation. Recent evidence of global changes and impacts from marine organisms and ecosystems are mainly derived from global databases of in situ observations (Poloczanska et al., 2016), stressing the value of global networks such as ILTER-CMS.

- A marked diversity and wide range of partners and institutions across the globe characterize ILTERCMS, guaranteeing multi-disciplinary data acquisition, analysis, integration and synthesis, and cost-effective sustainable observations. 


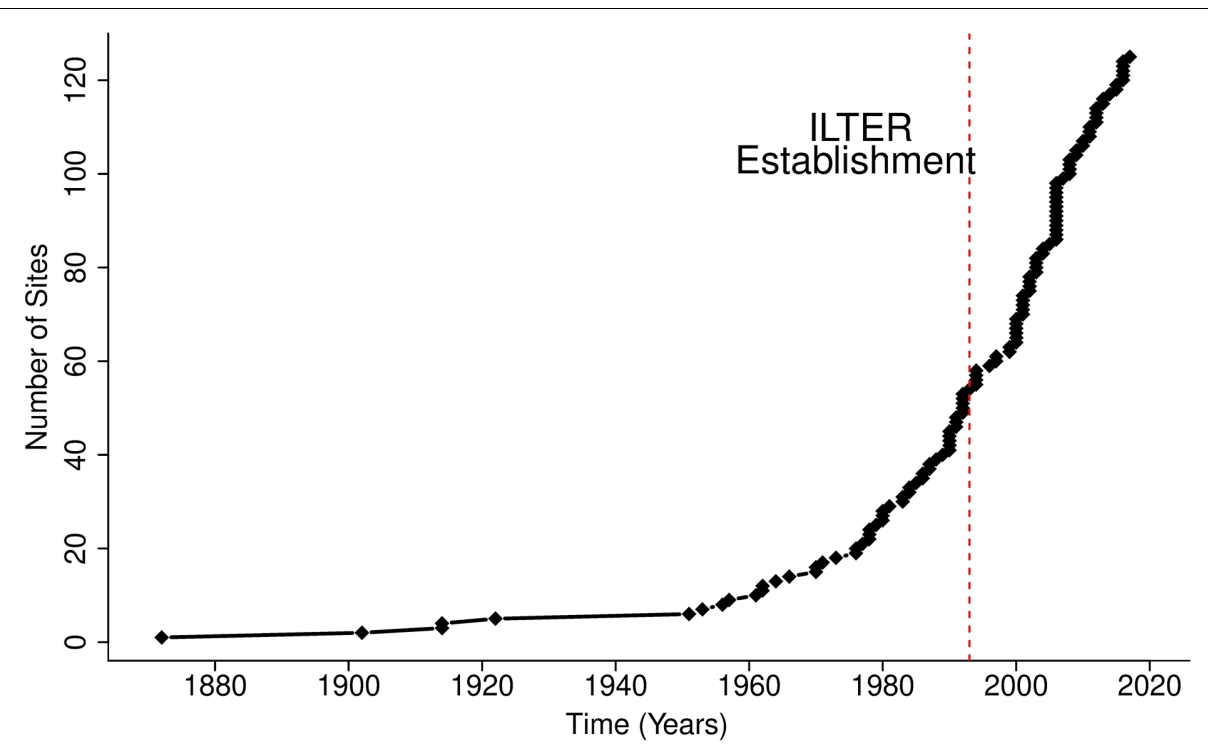

FIGURE 3 | Timeline of the establishment of long-term coastal and marine observation sites and the implementation of the International Long-Term Ecological Research (ILTER) Program.

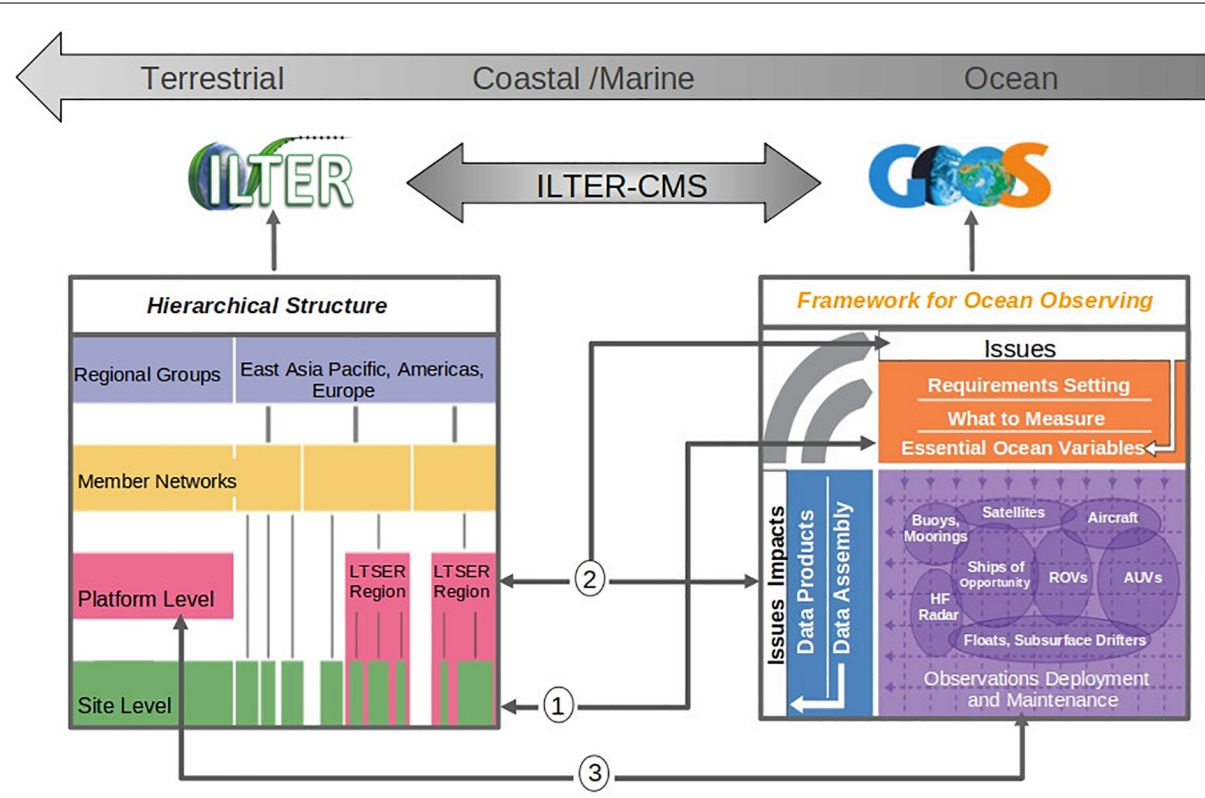

FIGURE 4 | ILTER coastal and marine sites can provide several interaction points between the ILTER structure and the GOOS Framework. ILTER-CMS and GOOS can mutually benefit from setting similar requirements and deciding on what to measure. (1) ILTER sites can provide GOOS with monitoring of Essential Ocean Variables, including many biological variables by classical methods, providing added value to the sensor-based measurements in the GOOS program. (2) The LTSER with social science competence can contribute to define issues and priorities and assess the impact of observations on society. (3) At the platform level the interaction can take place through instrumentation deployment and maintenance.

- The ILTER Network provides a platform for discussion among experts and stakeholders on key oceanographic and ecological themes, optimizing active links or developing new ones with a diverse array of global and regional processes and initiatives. Indeed, since its onset, ILTER had adopted a more interdisciplinary approach to research and monitoring, recognizing that in dealing with environmental problems, natural and human systems cannot be considered as separate entities (Dick et al., 2018). ILTER-CMS provides and fosters the use of standard protocols and open access data. This is critical for the study of climate change and its effect on biota and ecosystems. Additionally, ILTER-CMS already monitors Physical, Biochemical, and Biological/Ecological EOVs. 


\section{Weaknesses}

- Most LTER sites and national networks have been developed from the bottom-up. The different research and monitoring aims, some of which may have changed over time, as well as the wide variety of ecosystem types, infrastructure, instrumentation and technological development may all hinder comparisons within and across networks, sites and scales.

- ILTER-CMS is a relatively new network, with $50 \%$ of the sites being established after the year 2000. Environmental and socio-ecological issues are often regional and not yet clearly defined at the global scale.

- Despite recent efforts (Haase et al., 2018) and progress with the development of the DEIMS-SDR, the harmonization of data and metadata for CMS is still far from complete. Standardized/transparent data management procedures cannot easily be implemented in a number of locations and datasets are often not readily available. The obstacles include inadequate funding, a lack of training opportunities, and/or hesitancy to submit data to internationally accepted repositories.

- ILTER-CMS was not designed to be an operational monitoring system but to study ecosystem and biodiversity. Consequently, there is not final consensus about the variables to be measured

TABLE 1 | The strengths, weakness, opportunities, and threats presented by the ILTER-CMS.

\begin{tabular}{ll}
\hline Strengths & Weakness \\
\hline - Multi- and inter-disciplinary & - ILTER is mostly terrestrial, \\
- Links with a wide range of & overarching strategy and \\
global/regional processes and & conceptual framework are broad \\
initiatives & and not specific for coastal and \\
- Platform for discussion among & marine environments \\
experts on oceanographical and & Variables to be measured, \\
ecological themes & methodologies, technological \\
- Multi-institutional cooperation & development and sampling \\
- Metadata organized and updated & schemes are not homogeneous \\
in DEIMS-SDR & among sites \\
- Socio-ecological aspects & The socio-ecological aspects are \\
developed in the ILTER mission & not yet fully developed \\
- ILTER-CMS monitor Physical, & Harmonization of data and \\
Biochemical, and & metadata for coastal and marine \\
Biological/Ecological EOVs & environments is still incomplete \\
- Potential for large syntheses and & The geographic location of time \\
detection of long-term trends & series has notable gaps \\
across sites, spatial and temporal & - The standardization of variables \\
scales & gathered has not been \\
- Potential for developing and & accomplished, and EEVs or EOVs \\
testing concepts and theoretical & coverage is inconsistent \\
frameworks & Intercalibration of approaches and \\
- A consolidated data policy and & methodologies is lacking \\
information availability system & The data management is relatively \\
- Quick response to & poor at several sites \\
methodological/technological & Some data linked to ecological \\
advances, standardization and & research activities not immediately \\
implementation of these & available \\
technologies on a large spatial & \\
scale and link to existing time & \\
series and spatial data & \\
\hline
\end{tabular}

(Continued)
TABLE 1 | Continued

\begin{tabular}{ll}
\hline Opportunities & Threats \\
\hline - Optimal sites for experiments on & - Missing link with society, \\
observation and pilot integrated & hampering the identification of \\
biological observatories & questions with societal relevance \\
- Promote the use of new & Reduction in focus on in situ \\
technologies for ocean observation & sampling as a consequence of \\
and compare the information that & linking with more technological or \\
technologies make available & model-centric networks \\
- Merging frameworks from different & $\begin{array}{l}\text { Inadequate training of new } \\
\text { global research and monitoring }\end{array}$ \\
initiatives, producing guidelines for & generation of researchers with \\
future site-based long-term & relevant skills set (e.g., taxonomy, \\
research and monitoring marine & data science, database \\
and coastal ecosystems & management), able to recognize \\
- Support the use of costly & the relevance of these kinds of \\
infrastructure, fostering & activities and maintain LTER in the \\
cross-initiative collaborative & future \\
research & Reduction of ILTER activities at \\
- Monitoring EOVs at a global scale & some sites leading to temporal \\
at 130 discrete sites & and spatial gaps \\
- Improvement of models and & \\
predictions of possible future & \\
developments & \\
glatform for citizen science & \\
the transfer of knowledge & \\
\hline
\end{tabular}

and the methodologies and sampling schemes that should be adopted.

\section{Opportunities}

- ILTER-CMS has the governance structure to coordinate with management and policy programs, interact and link with other large-scale initiatives, create interfaces between the different approaches from the various communities, and establish a co-located network of sites within similar ecosystem typologies, with shared research and monitoring tasks for multi-purpose uses (Haase et al., 2018). The ILTERCMS can contribute substantially to merging frameworks that are behind different global research and monitoring initiatives, producing guidelines for future site-based longterm research and monitoring. The site network could generate connected ecosystem monitoring methodologies and datasets, supporting, as well, synergies in the use of costly infrastructures, through cross-initiative collaborative research.

- Stakeholder interactions play a key role in implementing ILTER outcomes for sustainable regional and local development in light of global trends, and for true integration of the sites into local/regional innovation systems, where the societal and human dimension is considered together with the scientific one. Long-term ecosystem studies, in comparison to the public funding they need, possess a disproportionately high capacity to inform policymakers about relevant environmental issues (Hughes et al., 2017). The ILTER-CMS represent ideal places for establishing pilot integrated biological observatories where the use of new technologies for ocean observation can also be promoted 
and where the information that technologies make available can be tested and compared to develop standardized approaches for their use (e.g., high throughput molecular or imaging techniques). With enhanced standardization and increased adoption across coastal and marine sites, the ILTER offers the opportunity to monitor EOVs at a global scale.

- The interaction between modelers and observers provides the opportunity to better design and plan new long-term observation initiatives. Several sites record periods of decades and thus a large number of different past conditions, which can be used to calibrate and validate various kinds of models. Model-generated environmental parameters can help to reduce temporal and spatial observation gaps. Advanced models are vital to create reliable (future) scenarios that facilitate the understanding of ecosystem functioning and evolution and are necessary to improve links between science and policy makers. Observations can be used to generate operational models needed to sustainably manage and protect marine and coastal ecosystems (ODS 14.2) by indicating areas of adverse impacts, habitat loss, and changes in ocean state that are relevant for ecosystems services. ILTER can strengthen capacity building by providing site access and training on advanced ecosystem monitoring and management. This is an invaluable opportunity to attract young scientists from around the globe and to teach a new generation of scientists innovative ways to use resources, recognize the relevance of these kinds of activities, and maintain and develop the network with a long-term perspective.

- ILTER-CMS could be an ideal context for the development of citizen science (CS) activities (Irwin, 1995; Bonney et al., 2009). CS can (i) improve the frequency and geographical coverage of observations, and (ii) improve pedagogy and communication at the science/society/policy interface by involving citizens, managers or different stakeholders in a research program. Hands-on involvement of people in research and monitoring activities is more effective than communicating about science and its benefits. Increasingly apps can be used to assist the citizen scientists to report data in a standardized manner (e.g., the Secchi disk project ${ }^{6}$ or beach observer). CS programs can yield significant results (see, e.g., Abbott et al., 2018) and could be applied, for example, in programs with divers, sailors, and beach-goers looking at biodiversity in coastal zones and collecting data of great interest to ILTER and contributing to GOOS.

- ILTER Network may provide improved data management at site-level and enhanced data flow toward global and European marine data infrastructure like the Ocean Biogeographic Information System (OBIS) and the European Marine Observation and Data Network (EMODnet). ILTER may make use as well of the standardization tools provided by World Register of Marine Species (WoRMS) and the improved analytical capacity provided by marine virtual research environments constructed under the European Research

\footnotetext{
${ }^{6} \mathrm{http}: / /$ www.secchidisk.org/
}

Infrastructure (ESFRI) by LifeWatch-ERIC and the European Open Science Cloud.

- The concepts of EOVs and Essential Biodiversity variables (EBVs) could facilitate the task of identifying key variables for LTER coastal and marine biological observations (Miloslavich et al., 2018; Navarro et al., 2018). ILTER-CMS needs to assess the readiness levels of the EOVs to assure implementation of an operational system. These concepts could be merged with the Ecosystem Integrity framework (Müller, 2005), at the moment adopted by ILTER, based on a comprehensive set of abiotic variables for identifying drivers of biodiversity changes within the context of ecosystem structures and processes (Haase et al., 2018).

\section{Threats}

- Long-term initiatives are difficult to maintain, and they need to be cost-effective and sustainable at the country level. Reduction of ILTER-CMS activities at some sites could produce temporal and spatial gaps that would hamper the functionality and relevance of the network.

- A failure in engaging users and stakeholders could limit the full exploitation of ILTER-CMS services and products. The lack of transfer of knowledge and understanding to policy makers and society would prevent informed decision making regarding the long-term safeguarding and effective management of marine ecosystem services.

- ILTER Network has historically been more oriented toward "terrestrial," inland ecosystems: conceptual frameworks, harmonization, and data models not well-suited for the marine component may discourage future ocean initiatives.

- Future ILTER-CMS activities must be sustained by young scientists. A failure to make ILTER-CMS attractive and meaningful for early career researchers (and the next generation of stakeholders) could be a major threat for the long-term sustainability of integrated coastal and marine ecological observatories.

In the following two sections, we elaborate on two specific opportunities for ILTER-CMS in contributing to global ocean observing: Essential Ocean Variables and Emerging Technology.

\section{Essential Ocean Variables and the ILTER Contribution to Observation, Science and Management Programs}

Many programs and initiatives at the regional and global level are dedicated to the study of coastal and marine environments and they express a common need to harmonize and coordinate observations to allow comparison and synthesis across ecosystems and scales. In this respect, efforts need to be dedicated to enable interoperability and to create interfaces among the different initiatives. In particular, consolidated global networks, such as GOOS and ILTER, should be leaders in proposing and demonstrating how interoperability and linking of conceptual frameworks could be tackled.

We argue that one way to achieve this integration is through the use of Essential Ecosystem Variables (EEVs) or EOVs. One of the outputs of the OceanObs'09 conference was the 
adoption of FOO (Lindstrom et al., 2012). The FOO proposed the use of routine and sustained observations of physical, biogeochemical and biological EOVs. GOOS has adopted the FOO recommendations, and recently the Biology and Ecosystem Panel has approved a set of EOVs for global sustained observations of biodiversity and ecosystem change (Miloslavich et al., 2018). This is also in line with a recent joint proposition of ILTER and the Group on Earth Observations Biodiversity Observation Network (GEOBON) to merge ecosystem integrity and EBVs to serve as an improved guideline for future long-term environmental research and monitoring (Haase et al., 2018).

As a first step, we conducted a survey involving ILTER-CMS site managers in order to record how many sites already monitor the EOVs proposed by the GOOS. The GOOS Panels have identified 11 Physical, 9 Biogeochemical, and 11 Biological/Ecosystem essential variables. The results of the survey were quite encouraging (Figure 5). Eleven EOVs are observed routinely in more than $50 \%$ of the surveyed sites. Surface and sub-surface temperature and salinity are among the most observed physical EOVs, but a good number of sites also measure sea state (42\%), surface (42\%) and subsurface currents (42\%) and sea surface height (38\%). The most common physical EOVs within ILTER-CMS sites (Figure 5) are already in a mature readiness level. For the biogeochemical EOVs, nutrients, oxygen and particulate matter are measured in more than $50 \%$ of the sites, while inorganic carbon (40\%), dissolved organic carbon (40\%) and ocean color (32\%) are also well-represented. Nutrients and oxygen are at a mature level while particular matter can vary from concept to mature depending on the environment. Phytoplankton biomass and diversity, the abundance and distribution of benthic invertebrates, fish and zooplankton are the biological/ecosystem EOVs measured at $50 \%$ or more sites. These EOVs have a varying degree of readiness level, ranging from concept to mature. Other biological/ecosystem EOVs are site specific and reflect habitat specificity. Therefore, it is expected that seagrass, hard coral, and mangrove covers would only be measured at a few sites.

ILTER coastal and marine sites also measure several other variables that are relevant for coastal and marine ecosystems, but are not indicated as EOVs. Among them: physical (e.g., incident wave height and cumulative wave energy, and currents along the entire water column parameters), water quality (e.g., transparency, turbidity, sediment concentration, and composition) and biogeochemical and biological parameters (redox potential and water $\mathrm{pH}, \mathrm{CO}_{2}$ fluxes to and from the atmosphere, primary productivity, toxic phytoplankton composition, and production of toxins).

These results show that a comprehensive coastal and marine ecosystem monitoring system could benefit from collaboration and synergy with the ILTER network. At this time, when GOOS has just defined biological/ecological EOVs and is working on implementation strategies and coordination of observations, the ILTER network already provides infrastructure and logistical support to conduct monitoring of coastal and marine ecosystems around the world.
Another possible contribution of the ILTER-CMS observations is the establishment of integrated supersites. Supersites are focal points for instrument intensive research, ideally suited for co-location with small-scale experiments and specialized observations, and situated in areas that will provide important information on environmental change, i.e., critical zones. Co-locating ILTER-CMS supersites with GOOS-proposed Sentinel sites (PICO-I, 2012) would link the mechanistic ecological and biophysical understanding from the ILTER with an ocean observatory framework that explicitly seeks to understand the influence of anthropogenic pressures and the roles of ecosystem services in that location. ILTER has sites in most Sentinel Sites including some of the most stressed ones: Greenland-North-Baltic Seas/Bay of Biscay; Indonesian Archipelago-South China Sea; and, East China/Yellow Seas. Thus, the ILTER-CMS is poised to provide observations of scientific and societal benefit immediately to vulnerable regions.

Long-term ecological time series are crucial for setting realistic baselines and limits in the classification systems used for assessing ecosystem environmental status. The 130 globally-distributed coastal and marine sites of the ILTER provide an exceptional observation platform for the GOOS-defined EOVs and invaluable information for several regional and global programs. This integration could benefit the European Water Framework Directive (WFD) and the EU Marine Strategy Framework Directive (MSFD), the accomplishment of the Aichi Targets of the Convention for Biological Diversity (CBD), the Intergovernmental Panel for Climate Change (IPCC), the Intergovernmental SciencePolicy Platform on Biodiversity and Ecosystem Services (IPBES) and the United Nations World Ocean Assessment. Information on coastal and marine ecosystems is urgently required to address the UN Sustainable Development Goal (SDG) 14.

ILTER coastal and marine sites can also provide information for science programs such as Future Earth Coasts, formerly LOICZ (Land-Ocean Interactions in the Coastal Zone) programme. Future Earth Coasts was launched by IGBP (International Geosphere-Biosphere Programme) and IHDP (International Human Dimensions Programme) as an international research project and global expert network exploring the pressures and social-environmental impacts of global environmental change in coastal zones. ILTER-CMS can also contribute to the Marine Biodiversity Observation Network $(\mathrm{MBON})$, a thematic component of GEO BON, that aims to coordinate, promote and augment the capabilities of present and future national and international observing systems to characterize and monitor diversity of marine life at the genetic, species, and ecosystem levels using a broad array of in situ and remote sensing observations (Duffy et al., 2013; Muller-Karger et al., 2018). ILTER-CMS sites are located in 20 of the 66 Large Marine Ecosystem (LME, Sherman, 1991) around the world and can contribute to identify areas of the oceans for conservation purposes and enable ecosystem-based management to provide a collaborative approach to management of resources within ecologically-bounded transnational areas. Data collected at 


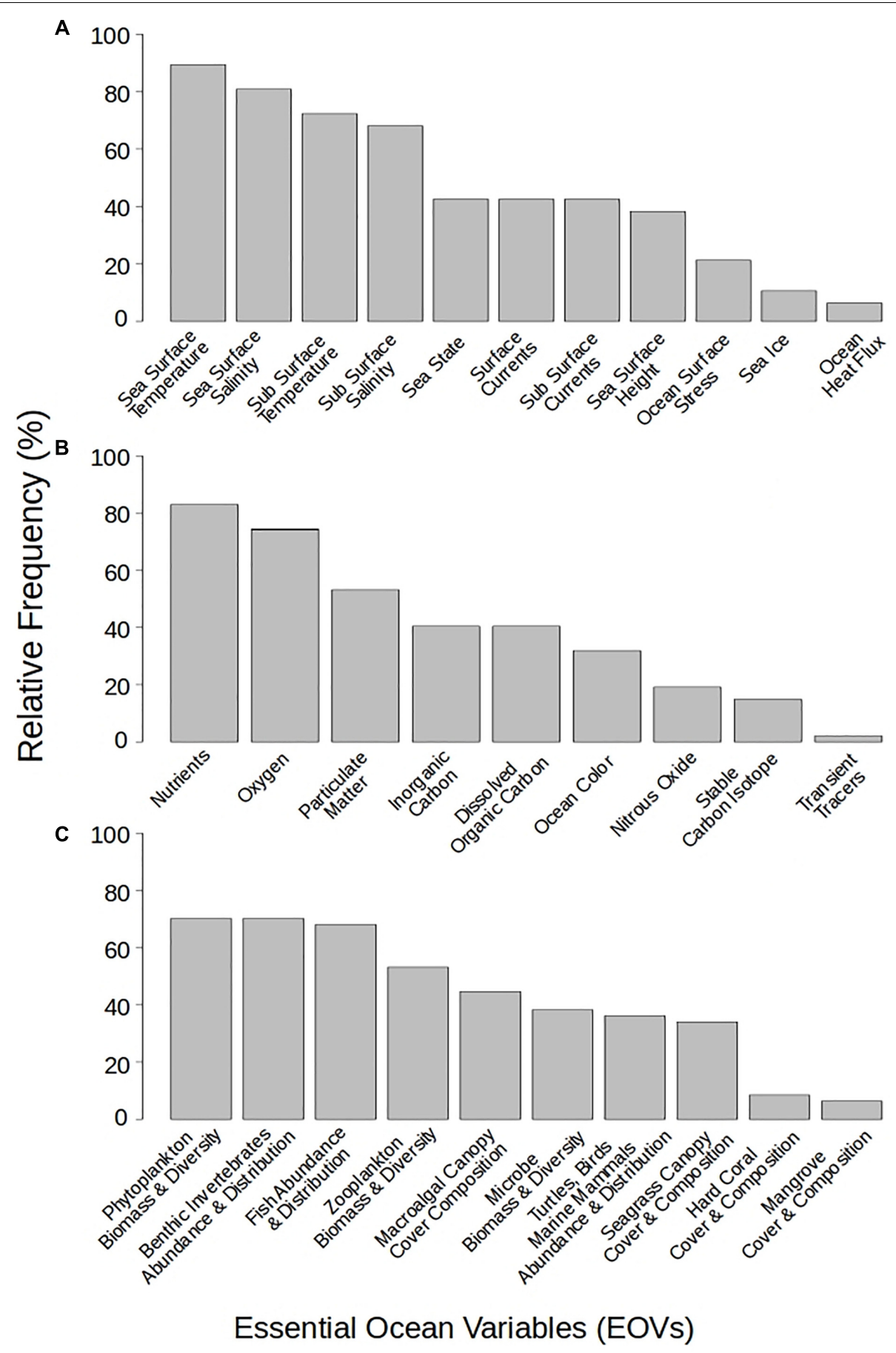

FIGURE 5 | Frequency of coastal and marine International Long-Term Ecological Research (ILTER) sites that observe Essential Ocean Variables recommended by the GOOS Biology and Ecosystem Panel. Physical variables (A), biogeochemical variables (B), and biological/ecosystem variables (C).

ILTER-CMS could be streamed into the OBIS and contribute to increasingly free and open access biodiversity and biogeographic data and information on marine life. ILTER can harbor and link with many other long-term coastal and ocean ecosystem monitoring programs, which may be explicitly reported also on DEIMS - SDR, in each site description. Furthermore, there is a well-established bottom-up procedure from the request of a site to become an LTER site, to the assessment of its suitability, i.e., of the possession of specific requisites fulfilling the LTER network requirements, which occurs at the national level, to the admittance to the national network and hence to ILTER. 


\section{NEW TECHNOLOGIES FOR ILTER}

One aspect determining the future success of the ILTER will be its ability to adopt and leverage emerging technologies at all levels of organization. Many of these technological developments represent opportunities to catalyze education of and collaboration between young scientists across LTER sites, strengthening the network and thus mitigate identified threats in the SWOT-analysis (Table 1). Mature technologies that are economic to implement can directly contribute to counteract currently identified weaknesses by better geographical coverage and harmonized data quality. This spans innovative in situ technologies developed at individual sites to global data networks that enable novel syntheses via increasingly powerful computational technologies or leverage citizen science. Here we consider some of these technologies, building up through the hierarchical organization of ILTER (see Figure 4 in Mirtl et al., 2018).

\section{Researcher Level}

International Long-Term Ecological Research Network sites are ideal incubators for the development and/or deployment of emerging technologies. As noted in the introduction, the general sampling rate of eco/bio/geo/chemical phenomena has long lagged behind the rapid data collection possible by physical oceanographers. In the last two decades, however, there has been a rapid development of novel high-throughput technologies. These include chemical, imaging, acoustic and molecular sensor systems that have enabled continuous and long-term measurement of new parameters that are of great interest (Johnson et al., 2007). Examples include nitrate (Johnson and Coletti, 2002), ammonium (Plant et al., 2009), and pH (Seidel et al., 2008; Martz et al., 2010) sensors. Similarly, advances in rapid image collection and processing have enabled cataloging and enumeration of phytoplankton and zooplankton biodiversity at tidal to interannual scales (Olson and Sosik, 2007; Sosik and Olson, 2007; Faillettaz et al., 2016; Hunter-Cevera et al., 2016), while high-throughput molecular analyses facilitate biodiversity assessments of unprecedented taxonomic resolution, facilitating the regular monitoring of taxa that were not reliably identifiable previously (Stern et al., 2018). This, in turn, provides the potential for unique insights into the diversity and function of marine foodwebs (e.g., Leray et al., 2015).

One major trend that will shape the future of ILTER research is the continued miniaturization of electronics and improvements to memory and batteries. For example, the Imaging FlowCytobot is now half as large, weighs half as much, and consumes one-third of the power of the original (Olson et al., 2017). This technology has now been commercialized (McLane Research Laboratories, Inc.), is in routine use at the Northeast U.S. Shelf (NES) LTER site and other time series locations in the U.S., and has a growing user base around the world. Continued advances along this trajectory will enable novel biological and ecological studies. For example, embedded sensors within organisms have enabled metabolic and physiological studies (McGaw et al., 2018); underwater tracking technology has revealed ecological interactions between predator, prey and the environment (Osterback et al., 2013). The rich contextual data at the ILTER sites provide an ideal framework to develop this new technology and interpret the data it produces.

In addition to developments of in situ technologies, ILTER sites are also promoting advances in remote sensing technologies that are critical for characterizing ecosystems at large scales. For example, in coastal dune systems the use of high-resolution remote sensed imagery (LiDAR - Light Detection and Ranging - and orthophotos) is helping to explain the invasion success of some alien species and the roles of propagule pressure, abiotic, and biotic factors in coastal landscapes (Bazzichetto et al., 2018). The use of remotely sensed data may make it possible to model the invader-landscape relationship over a large geographic extent and to highlight the coastal sectors that are most likely to be invaded in the future.

\section{Site Level}

Across individual ILTER sites (which may comprise a number of individual locations), autonomous systems, robotics, machine learning, and advances in -omics technologies will drive discovery in the coming decades.

The maturation and miniaturization of robotic platforms (taken here to be all manner of autonomous mobile platforms, including drifters, profiling floats, buoyancy gliders, propeller-driven autonomous underwater vehicles, and unmanned aerial systems, cf. Nidzieko et al., 2018) will gradually enable their incorporation into a broader range of research. There are three barriers to more widespread adoption at the moment. One, the maturation of suitable sensors, as mentioned above, is necessary prior to incorporation onto mobile platforms. Two, most platforms are still not "turnkey" devices and thus require specialized operator knowledge. And three, costs are still prohibitive for acquisition of more mature technologies. The popular trend of the DIY/makerspace ethos may result in less costly, yet still capable platforms that can be readily employed in coastal and marine research. Once deployed, however, the ability of robots to do "dull, dirty, dangerous" tasks will improve both the temporal and spatial measurements collected across an LTER site. For example, the time-consuming task of manually counting kelp forest biomass is only conducted monthly across all 10 of the individual sites within the Santa Barbara Coastal LTER and measurements are done on subsampled transects within a kelp forest. Underwater and/or aerial platforms could be used to survey the entire $70 \mathrm{~km}$ coastline on a weekly basis, enabling better understanding spatial patterns in synchrony and disturbance (Bell et al., 2015).

Machine learning will enable the deployment of technologies that have heretofore been too data-rich to make significant impacts to understanding biology and ecology within LTER sites. We give two examples: passive acoustics and flow cytometers. The use of passive acoustics across a broad sound spectrum has not been feasible due to limitations in memory, battery power, and processing capabilities. With the advances mentioned above and the maturation of machine learning techniques in parallel with increasingly powerful computers (at both the personal and cloud/cluster level), tackling the processing requirements of such large datasets in now feasible (Mooney et al., 2017). We envision that monitoring the soundscape within ILTER-CMS will become 
one of the most widely adopted technologies in the coming decade. Flow cytometry techniques similarly produce massive amounts of data and have traditionally required extensive manual efforts to classify cytometric and image data into quantified products; the potential for automation of these tasks across ILTER sites could revolutionize researcher's understanding of variations in plankton biodiversity.

Finally, at the site level, emerging molecular methods will bring major insights by identifying important microbial actors, determining their interactions, and providing deeper insights into biogeochemical processes at ILTER sites. One example of this technology is the MBARI Environmental Sample Processor (Scholin et al., 2009), which has been used to autonomously collect and preserve microbial samples (Ottesen et al., 2011), revealing complex shifts in microbial communities over very short time scales (Needham et al., 2018). See McQuillan and Robidart (2017) for a comprehensive review of recent advances. Importantly, these technological advances are multiplicative: not only are the measurement techniques novel and advanced, but these measurements can also be conducted adaptively in response to the feature or event of interest (Harvey et al., 2012). Furthermore, these technologies can present advantages in accuracy, efficiency, and cost (Danovaro et al., 2016). It is important though that all new observing technologies pass through the four stages of evolution of a sustained Ocean Observing system (Nowlin et al., 2001).

\section{Regional/Global Levels}

While many of the roles of technology at the researcher and site levels will provide fundamental disciplinary discoveries and enable the establishment of new time series for incorporation into ILTER core products, several aspects of technology will drive the interdisciplinary discoveries that integrate multiple sites at regional and/or global levels. First, common metadata and easy, rapid access to data assets are essential to synthesis efforts. Improvements to cloud storage and data discovery tools will be critical to this end (Buck et al., 2019). As these tools mature, and perhaps converge from bespoke applications into more generic platforms, ILTER data will become an integral component of the broader suite of data products provided from regional observing systems, operational (and data assimilative) forecasts, numerical hindcasts, and weather data that researchers draw upon.

The major challenge to delivering the sound ecological knowledge necessary to address human impacts will require incorporating patterns/trends from site-level records into observed and predicted global climate models. This is not a trivial task, because it requires both scaling up local measurements to discover and explain emergent patterns (that might only be detectable within the distributed network of the ILTER) while also scaling down from climate predictions to expectations/hypotheses of what might be observed at individual site. Synthesis using cross ILTER site have contributed to the knowledge of ecosystem spatial and temporal variability (e.g., Bestelmeyer et al., 2011), and serve as example that this challenge can be addressed.
Advances in computational capabilities will certainly be an asset to this end, but ultimately the larger hurdle may be not be technological be rather operational, as described in the threats above.

\section{RECOMMENDATIONS AND CHALLENGES}

ILTER coastal and marine sites has great potential to contribute to global coastal and ocean observation. Here we provide recommendations to improve the opportunities for ILTER-CMS to enhance collaboration among researchers, institutions and governments and funding agencies in support of long-term ocean observing initiatives.

(1) Recommendations to the network members and related organizations:

- Regional nodes should promote the expansion of coastal and marine sites by strengthening networks of marine scientists.

- Financial and educational support necessary to minimize the coverage gaps of ILTER-CMS (Figure 1), especially in developing countries should be addressed.

- Members should provide a quality-assured web-based data archiving, effective data retrieval and relevant data products within the network; promote the use of bestavailable statistical tools for data analysis and synthesis; and build up their respective infrastructures for sharing data and data handling, analysis and visualization.

- The ILTER-CMS need to harmonize and coordinate longterm environmental (both biotic and abiotic) observations in line with the EOVs to the extent possible, to allow comparisons within and across networks, sites and scales. These efforts should consider socio-ecological aspects, as well.

- Launch or improve coordination and integration of observations across scales (e.g., from coast to open sea) in concert with other international observing networks.

- Develop standard operating procedures, adopt guidelines for measurement program design, and establish routines for recurrent intercalibration exercises for all subject fields in line with the OceanBestPractices $\left(\mathrm{OBP}^{7}\right)$. Such activities may include increasing the use of shared infrastructure, protocols and data platforms; and developing metadata and dataset harmonization/interoperability necessary to foster and facilitate sharing and open access.

- Define a consistent overarching research and monitoring framework, taking into account the wide range of ILTERCMS typologies. LTER has historically been more oriented toward terrestrial, inland ecosystems, therefore coastal and marine conceptual frameworks, harmonization and data models still need improvement and should be strengthened and better integrated.

\footnotetext{
${ }^{7}$ https://www.oceanbestpractices.net/
} 
- Strengthen the communication with relevant stakeholders to improve the role of ILTER-CMS network as a data and knowledge provider for society.

- Promote citizen science initiatives to raise awareness about the broad importance of ILTER-CMS research activities. A collaboration with existing citizen science networks, e.g., ECSA (European Citizen Science Association) and other observation networks should be encouraged.

- Seek consensus for the adoption of parameters and monitoring methods in order to overcome fragmentation between sites, improve interfaces among networks (e.g., GOOS) and promote cooperation on shared environmental issues and targets.

- Further develop existing biological observations through the implementation of new technological and -omic approaches and improve their integration with physical and chemical observing systems and modeling initiatives.

- Provide the knowledge needed by policy makers and society for informed decision-making regarding the long-term safeguarding and effective management and sustainability of coastal and marine ecosystem services.

(2) General recommendations to government, funding agencies, and other organizations:

- Partners should address the need to support and protect existing LTER sites, recruit technical workforce, allow for their development and improvement, and increase their number for better spatial coverage, while recognizing funding limitations.

- Support formation of expert working groups providing urgent scientific environmental knowledge for society, capacity strengthening, education, and training.

- Support technological innovation to implement in situ observing systems, develop smart technologies for costeffective automated monitoring of biological variables, and transition from research to operational status.

- Engage society in the definition of relevant research questions to strengthen the science/society/policy interface.

- Engage governments in prioritizing and sustaining ILTERCMS activities as well as engage users, stakeholders, and

\section{REFERENCES}

Abbott, B. W., Moatar, F., Gauthier, O., Fovet, O., Antoine, V., and Ragueneau, O. (2018). Trends and seasonality of river nutrients in agricultural catchments: 18 years of weekly citizen science in France. Sci. Total Environ. 624, 845-858. doi: 10.1016/j.scitotenv.2017.12.176

Bazzichetto, M., Malavasi, M., Barták, V., Acosta, A. T. R., Moudrý, V., and Carranza, M. L. (2018). Modeling plant invasion on Mediterranean coastal landscapes: an integrative approach using remotely sensed data. Landsc. Urban Plan. 171, 98-106. doi: 10.1016/j.landurbplan.2017.11.006

Bell, T. W., Cavanaugh, K. C., Reed, D. C., and Siegel, D. A. (2015). Geographical variability in the controls of giant kelp biomass dynamics. J. Biogeogr. 42, 2010-2021. doi: 10.1111/jbi.12550

Bestelmeyer, B. T., Ellison, A. M., Fraser, W. R., Gorman, K. B., Holbrook, S. J., Laney, C. M., et al. (2011). Analysis of abrupt transitions in ecological systems. Ecosphere 2:129. doi: 10.1890/ES11-00216.1 other existing observing networks to fully exploit ILTER services and products, and demonstrate the impact on science and society.

\section{AUTHOR CONTRIBUTIONS}

$\mathrm{JM}, \mathrm{NN}, \mathrm{AA}, \mathrm{AB}, \mathrm{AK}, \mathrm{AP}$, and $\mathrm{AZ}$ helped to conceive the manuscript, coordinated the author contributions, wrote and edited the manuscript, and contributed to tables and figures. $\mathrm{SB}, \mathrm{EB}, \mathrm{TB}, \mathrm{BC}, \mathrm{KD}, \mathrm{EE}, \mathrm{MN}, \mathrm{OR}$, MS, TS, HS, AdS, KS, PS, AnS, and JW contributed to manuscript ideas and text.

\section{FUNDING}

JM was supported by a CNPq fellowship (Grant No. 310047/2016-1) and by PELD Estuário da Lagoa dos Patos e Costa Adjacente (CNPq/CAPES/FAPERGS). SB was supported by US NSF (Grant \#OCE-1655686). AB was supported by CAPES/CNPq/FAPES grant no. 441243/2016-9 to PELD Coastal Habitats of Espírito Santo as part of the Brazilian LTER program. HS was supported by US NSF (Grant \#CCF-1539256 and \#OCE-1655686), Simons Foundation (Grant \#561126) and US NOAA/CINAR (Cooperative Agreement NA14OAR4320158).

\section{ACKNOWLEDGMENTS}

The authors acknowledge the contribution of all ILTER-CMS that have provided updated information to the Dynamic Ecological Information Management System Site and Dataset Registry (DEIMS-SDR) and that responded the EOV questionnaire.

\section{SUPPLEMENTARY MATERIAL}

The Supplementary Material for this article can be found online at: https://www.frontiersin.org/articles/10.3389/fmars. 2019.00527/full\#supplementary-material

Bonney, R., Ballard, H., Jordan, R., Mc Callie, E., Phillips, T., Shirk, J., et al. (2009). CAISE Report on Public Participation in Scientific Research. A CAISE Inquiry Group Report. Washington, DC: Center for Advancement of Informal Science Education.

Buck, J. J. H., Bainbridge, S. J., Burger, E. F., Kraberg, A. C., Casari, M., Casey, K. S., et al. (2019). Ocean data product integration through innovation-the next level of data interoperability. Front. Mar. Sci. 6:32. doi: 10.3389/fmars.2019.00032

Danovaro, R., Carugati, L., Berzano, M., Cahill, A., Carvalho, S., Chenuil, A., et al. (2016). Implementing and innovating marine monitoring approaches for assessing marine environmental status. Front. Mar. Sci. 3:213. doi: 10.3389/ fmars.2016.00213

Defeo, O., McLachlan, A., Schoeman, D. S., Schlacher, T. A., Dugan, J., Jones, A., et al. (2009). Threats to sandy beach ecosystems: a review. Estuar. Coast. Shelf Sci. 81, 1-12. doi: 10.1016/j.ecss.2008.09.022

Dick, J., Orenstein, D. E., Holzer, J. M., Wohner, C., Achard, A.-L., Andrews, C., et al. (2018). What is socio-ecological research delivering? A literature survey 
across 25 international LTSER platforms. Sci. Total Environ. 62, 1225-1240. doi: 10.1016/j.scitotenv.2017.11.324

Drius, M., Carranza, M. L., Stanisci, A., and Jones, L. (2016). The role of Italian coastal dunes as carbon sinks and diversity sources. A multi-service perspective. Appl. Geogr. 75, 127-136. doi: 10.1016/j.apgeog.2016.08.007

Duffy, J. E., Amaral-Zettler, L. A., Fautin, D. G., Paulay, G., Rynearson, T. A., Sosik, H. M., et al. (2013). Envisioning a national marine biodiversity observation network. Bioscience 63, 350-361. doi: 10.1525/bio.2013.63.5.8

European Commission (2016). H2020 Programme - Guidelines on FAIR Data Management in Horizon 2020. Available at: http://ec.europa.eu/research/ participants/data/ref/h2020/grants_manual/hi/oa_pilot/h2020-hi-oa-datamgt_en.pdf (accessed August 13, 2019).

Faillettaz, R., Picheral, M., Luo, J. Y., Guigand, C., Cowen, R. K., and Irisson, J.-O. (2016). Imperfect automatic image classification successfully describes plankton distribution patterns. Methods Oceanogr. 15, 60-77. doi: 10.1016/j.mio.2016. 04.003

Haase, P., Tonkin, J. D., Stoll, S., Burkhard, B., Frenzel, M., Geijzendorffer, I. R., et al. (2018). The next generation of site-based long-term ecological monitoring: linking essential biodiversity variables and ecosystem integrity. Sci. Total Environ. 613-614, 1376-1384. doi: 10.1016/j.scitotenv.2017.08.111

Halpern, B. S., Frazier, M., Potapenko, J., Casey, K. S., Koenig, K., Longo, C., et al. (2012). Spatial and temporal changes in cumulative human impacts on the world's ocean. Nat. Comm. 6:7615. doi: 10.1038/nccomms8615

Harvey, J. B. J., Ryan, J. P., Marin, R., Preston, C. M., Alvarado, N., Scholin, C. A., et al. (2012). Robotic sampling, in situ monitoring and molecular detection of marine zooplankton. J. Exp. Mar. Biol. Ecol. 413, 60-70. doi: 10.1016/j.jembe. 2011.11.022

Howes, E. L., Joos, F., Eakin, C. M., and Gattuso, J.-P. (2015). An updated synthesis of the observed and projected impacts of climate change on the chemical, physical and biological processes in the oceans. Front. Mar. Sci. 2:36. doi: $10.3389 /$ fmars. 2015.00036

Hughes, B. R., Beas-Luna, R., Barner, A. K., Brewitt, K., Brumbaugh, D. R., CernyChipman, E. B., et al. (2017). Long-Term studies contribute disproportionately to ecology and policy. Bioscience 67, 271-281. doi: 10.1093/biosci/biw185

Hunter-Cevera, K. R., Neubert, M. G., Olson, R. J., Solow, A. R., Shalapyonok, A., and Sosik, H. M. (2016). Physiological and ecological drivers of early spring blooms of a coastal phytoplankter. Science 354, 326-329. doi: 10.1126/science. aaf8536

ILTER (2006). ILTER Strategic Plan: International long-term ecological research network - Strategic Plan. Available at: http://www.lter-europe.net/documentarchive/central/ECOLEC-D-08-00262.pdf (accessed August 13, 2019).

Irwin, A. (1995). Citizen Science: A Study of People, Expertise and Sustainable Development. New York, NY: Routledge.

Johnson, K. S., and Coletti, L. J. (2002). In situ ultraviolet spectrophotometry for high resolution and long-term monitoring of nitrate, bromide and bisulfide in the ocean. Deep Sea Res. I 49, 1291-1305. doi: 10.1016/s0967-0637(02) 00020-1

Johnson, K. S., Needoba, J. A., Riser, S. C., and Showers, W. J. (2007). Chemical sensor networks for the aquatic environment. Chem. Rev. 107, 623-640. doi: $10.1021 / \mathrm{cr} 050354 \mathrm{e}$

Kliment, T., and Oggioni, A. (2011). Metadatabase: EnvEurope Metadata specification for Dataset Level. EnvEurope (LIFE08 ENV/IT/000339) Project Report PD.A1.1.4. Available at: http://www.enveurope.eu/misc/PD_1_1_4_ Kliment_Metadatabase_201112_final_v1.0.pdf (accessed August 13, 2019).

Leray, M., Meyer, C. P., and Mills, S. C. (2015). Metabarcoding dietary analysis of coral dwelling predatory fish demonstrates the minor contribution of coral mutualists to their highly partitioned, generalist diet. PeerJ 3:e1047. doi: 10 . 7717/peerj.1047

Lindstrom, E., Gunn, J., Fischer, A., McCurdy, A., Glover, L., Alverson, K., et al. (2012). A Framework for Ocean Observing. By the Task Team for an Integrated Framework for Sustained Ocean Observing, UNESCO 2012, IOC/INF1284. Available at: http://www.oceanobs09.net/foo/FOO_Report.pdf (accessed August 13, 2019).

Malavasi, M., Acosta, A. T. R., Carranza, M. L., Bartolozzi, L., Basset, A., Bassignana, M., et al. (2018). Plant invasions in Italy: an integrative approach using the european lifewatch infrastructure database. Ecol. Indic. 91, 182-188. doi: 10.1016/j.ecolind.2018.03.038
Martz, T. R., Connery, J. G., and Johnson, K. S. (2010). Testing the honeywell durafet $B$ for seawater ph applications. Limnol. Oceanogr. Methods 8, 172-184. doi: 10.4319/lom.2010.8.172

Mauz, I., Peltola, T., Granjou, C., van Bommel, S., and Buijs, A. (2012). How scientific visions matter: insights from three long-term socio-ecological research (LTSER) platforms under construction in Europe. Environ. Sci. Pol. 19, 90-99. doi: 10.1016/j.envsci.2012.02.005

McGaw, I. J., Steell, S. C., Van Leeuwen, T. E., Eliason, E. J., and Cooke, S. J. (2018). Application of miniature heart rate data loggers for use in large free-moving decapod crustaceans: method development and validation. Physiol. Biochem. Zool. 91, 731-739. doi: 10.1086/695839

McQuillan, J. S., and Robidart, J. C. (2017). Molecular-biological sensing in aquatic environments: recent developments and emerging capabilities. Curr. Opin. Biotechnol. 45, 43-50. doi: 10.1016/j.copbio.2016.11.022

Miloslavich, P., Bax, N. J., Simmons, S. E., Klein, E., Appeltans, W., AburtoOropeza, O., et al. (2018). Essential ocean variables for global sustained observations of biodiversity and ecosystem changes. Glob. Change Biol. 24, 2416-2433. doi: 10.1111/gcb.14108

Mirtl, M., Borer, E. T., Djukic, I., Forsius, M., Haubold, H., Hugo, W., et al. (2018). Genesis, goals and achievements of long-term ecological research at the global scale: a critical review of ILTER and future directions. Sci. Total Environ. 626, 1439-1462. doi: 10.1016/j.scitotenv.2017.12.001

Mooney, T. A., Lillis, A., Kaplan, M. B., Suca, J., and Lammers, M. (2017). Variability in coral reef soundscapes, spatiotemporal differences, biophysical and behavioral drivers, and associations with local biota. J. Acoust. Soc. Am. 141, 3939-3939. doi: 10.1121/1.4988920

Muller-Karger, F. E., Miloslavich, P., Bax, N. J., Simmons, S., Costello, M. J., Sousa Pinto, I., et al. (2018). Advancing marine biological observations and data requirements of the complementary essential ocean variables (EOVs) and essential biodiversity variables (EBVs) frameworks. Front. Mar. Sci. 5:211. doi: 10.3389/fmars.2018.00211

Müller, F. (2005). Indicating ecosystem and landscape organisation. Ecol. Indic. 5, 280-294. doi: 10.1016/j.ecolind.2005.03.017

Navarro, L., Fernández, N., Guerra, C., Guralnick, R., Kissling, W. D., Londoño, M. C., et al. (2018). Monitoring biodiversity change through effective global coordination. Curr. Opin. Environ. Sust. 29, 158-169. doi: 10.1016/j.cosust. 2018.02.005

Needham, D. M., Fichot, E. B., Wang, E., Berdjeb, L., Cram, J. A., Fichot, C. G., et al. (2018). Dynamics and interactions of highly resolved marine plankton via automated high-frequency sampling. ISME J. 12, 2417-2432. doi: 10.1038/ s41396-018-0169-y

Nidzieko, N. J., Todd, R. E., and Edwards, C. R. (2018). "ALPS in coastal oceanography," in ALPS II - Autonomous and Lagrangian Platforms and Sensors. A Report of the ALPS II Workshop, eds D. Rudnick, D. Costa, K. Johnson, C. Lee, and M.-L. Timmermans (La Jolla, CA: National Oceanographic Partnership Program).

Nowlin, W. D. Jr., Briscoe, M., Smith, N., McPhaden, M., Roemmich, D., Chapman, P., et al. (2001). Evolution of a sustained ocean observing system. Bull. Am. Met. Soc. 82, 1369-1376.

Olson, R. J., Shalapyonok, A., Kalb, D. J., Graves, S. W., and Sosik, H. M. (2017). Imaging FlowCytobot modified for high throughput by in-line acoustic focusing of sample particles. Limnol. Oceanogr. Methods 15, 867-874. doi: 10.1002/lom3.10205

Olson, R. J., and Sosik, H. M. (2007). A submersible imaging-in-flow instrument to analyze nano-and microplankton: imaging flowcytobot. Limnol. Oceanogr. Methods 5, 195-203. doi: 10.4319/lom.2007.5.195

Osterback, A.-M. K., Frechette, D. M., Shelton, A. O., Hayes, S. A., Bond, M. H., Shaffer, S. A., et al. (2013). High predation on small populations: avian predation on imperiled salmonids. Ecosphere 4:116. doi: 10.1890/ES13-0 0100.1

Ottesen, E. A., Marin, R., Preston, C. M., Young, C. R., Ryan, J. P., Scholin, C. A., et al. (2011). Metatranscriptomic analysis of autonomously collected and preserved marine bacterioplankton. ISME J. 5, 1881-1895. doi: 10.1038/ismej. 2011.70

PICO-I (2012). Requirements for Global Implementation of the Strategic Plan for Coastal GOOS. Available at: http://goosocean.org/index.php?option=com_oe\& task=viewDocumentRecord\&docID=7702 (accessed August 13, 2019). 
Plant, J., Johnson, K., Needoba, J., and Coletti, L. (2009). NH4-Digiscan: an in situ and laboratory ammonium analyzer for estuarine, coastal and shelf waters. Limnol. Oceanogr. Methods 7, 144-156. doi: 10.4319/lom.2009. 7.144

Poloczanska, E. S., Burrows, M. T., Brown, C. J., García Molinos, J., Halpern, B. S., Hoegh-Guldberg, O., et al. (2016). Responses of marine organisms to climate change across oceans. Front. Mar. Sci. 3:62. doi: 10.3389/fmars.2016. 00062

Scholin, C., Doucette, G., Jensen, S., Roman, B., Pargett, D., Marin, R. III, et al. (2009). Remote detection of marine microbes, small invertebrates, harmful algae, and biotoxins using the environmental sample processor (ESP). Oceanography 22, 158-167. doi: 10.5670/oceanog. 2009.46

Seidel, M. P., DeGrandpre, M. D., and Dickson, A. G. (2008). A sensor for in situ indicator-based measurements of seawater pH. Mar. Chem. 109, 18-28. doi: 10.1016/j.marchem.2007.11.013

Sherman, K. (1991). The large marine ecosystem concept: research and management strategy for living marine resources. Ecol. Appl. 1, 349-360. doi: $10.2307 / 1941896$

Sosik, H. M., and Olson, R. J. (2007). Automated taxonomic classification of phytoplankton sampled with imaging-in-flow cytometry. Limnol. Oceanogr. Methods 5, 204-216. doi: 10.4319/lom.2007. 5.204

Stern, R., Kraberg, A., Bresnan, E., Kooistra, W. H. C. F., Lovejoy, C., Montresor, M., et al. (2018). Molecular analyses of protists in long-term observation programmes-current status and future perspectives. J. Plankton Res. 40, 519-536. doi: 10.1093/plankt/fby035
Tanhua, T., Pouliquen, S., Hausman, J., O’Brien, K., Bricher, P., de Bruin, T., et al. (2019). Ocean FAIR data services. Front. Mar. Sci. 6:440. doi: 10.3389/fmars. 2019.00440

Vanderbilt, K. L., Blankman, D., Guo, X., He, H., Lin, C.-C., Lu, S.-S., et al. (2010). A multilingual metadata catalog for the ILTER: issues and approaches. Ecol. Inform. 5, 187-193. doi: 10.1016/j.ecoinf.2010. 02.002

Vanderbilt, K. L., and Gaiser, E. (2017). The international long term ecological research network: a platform for collaboration. Ecosphere 8:e01697. doi: 10. 1002/ecs 2.1697

Worm, B., Barbier, E. B., Beaumont, N., Duffy, J. E., Folke, C., Halpern, B. S., et al. (2006). Impacts of biodiverstiy loss on ocean ecosystem services. Science 314, 787-790.

Conflict of Interest Statement: The authors declare that the research was conducted in the absence of any commercial or financial relationships that could be construed as a potential conflict of interest.

Copyright (c) 2019 Muelbert, Nidzieko, Acosta, Beaulieu, Bernardino, Boikova, Bornman, Cataletto, Deneudt, Eliason, Kraberg, Nakaoka, Pugnetti, Ragueneau, Scharfe, Soltwedel, Sosik, Stanisci, Stefanova, Stéphan, Stier, Wikner and Zingone. This is an open-access article distributed under the terms of the Creative Commons Attribution License (CC BY). The use, distribution or reproduction in other forums is permitted, provided the original author(s) and the copyright owner(s) are credited and that the original publication in this journal is cited, in accordance with accepted academic practice. No use, distribution or reproduction is permitted which does not comply with these terms. 


\section{OPEN ACCESS}

Edited by:

Sanae Chiba,

Japan Agency for Marine-Earth Science and Technology, Japan

Reviewed by: Hans-Peter Plag,

Old Dominion University, United States

Rene Garello,

IMT Atlantique Bretagne-Pays

de la Loire, France

*Correspondence:

Nikolai Maximenko

maximenk@hawaii.edu

Specialty section:

This article was submitted to

Ocean Observation,

a section of the journal

Frontiers in Marine Science

Received: 21 November 2018

Accepted: 05 July 2019

Published: 28 August 2019

Citation:

Maximenko N, Corradi P, Law KL, Van

Sebille E, Garaba SP, Lampitt RS,

Galgani F, Martinez-Vicente V,

Goddijn-Murphy L, Veiga JM,

Thompson RC, Maes C, Moller D,

Löscher CR, Addamo AM,

Lamson MR, Centurioni LR,

Posth NR, Lumpkin R, Vinci $M$,

Martins AM, Pieper CD, Isobe A,

Hanke G, Edwards M, Chubarenko IP,

Rodriguez E, Aliani S, Arias M,

Asner GP, Brosich A, Carlton JT,

Chao $Y$, Cook $A-M$, Cundy $A B$,

Galloway TS, Giorgetti A, Goni GJ, Guichoux Y, Haram LE, Hardesty BD, Holdsworth N, Lebreton L, Leslie HA,

Macadam-Somer I, Mace T,

Manuel M, Marsh R, Martinez E,

Mayor DJ, Le Moigne M, Molina Jack ME, Mowlem MC, Obbard RW

Pabortsava K, Robberson B, Rotaru A-E, Ruiz GM, Spedicato MT Thiel M, Turra A and Wilcox C (2019)

Toward the Integrated Marine Debris Observing System

Front. Mar. Sci. 6:447. doi: 10.3389/fmars.2019.00447

\section{Toward the Integrated Marine Debris Observing System}

\author{
Nikolai Maximenko ${ }^{1 *}$, Paolo Corradi ${ }^{2}$, Kara Lavender Law ${ }^{3}$, Erik Van Sebille ${ }^{4}$, \\ Shungudzemwoyo P. Garaba ${ }^{5}$, Richard Stephen Lampitt ${ }^{6}$, Francois Galgani ${ }^{7}$, \\ Victor Martinez-Vicente ${ }^{8}$, Lonneke Goddijn-Murphy ${ }^{9}$, Joana Mira Veiga ${ }^{10}$, \\ Richard C. Thompson ${ }^{11}$, Christophe Maes ${ }^{12}$, Delwyn Moller ${ }^{13}$, Carolin Regina Löscher ${ }^{14}$, \\ Anna Maria Addamo ${ }^{15}$, Megan R. Lamson ${ }^{16}$, Luca R. Centurioni ${ }^{17}$, Nicole R. Posth ${ }^{18}$, \\ Rick Lumpkin ${ }^{19}$, Matteo Vinci ${ }^{20}$, Ana Maria Martins ${ }^{21}$, Catharina Diogo Pieper ${ }^{21}$, \\ Atsuhiko Isobe ${ }^{22}$, Georg Hanke ${ }^{15}$, Margo Edwards ${ }^{23}$, Irina P. Chubarenko ${ }^{24}$, \\ Ernesto Rodriguez ${ }^{25}$, Stefano Aliani ${ }^{26}$, Manuel Arias ${ }^{27}$, Gregory P. Asner ${ }^{28}$, \\ Alberto Brosich ${ }^{20}$, James T. Carlton ${ }^{29}$, Yi Chao ${ }^{13}$, Anna-Marie Cook ${ }^{30}$, Andrew B. Cundy ${ }^{31}$, \\ Tamara S. Galloway ${ }^{32}$, Alessandra Giorgetti ${ }^{20}$, Gustavo Jorge Goni ${ }^{19}$, Yann Guichoux ${ }^{33}$, \\ Linsey E. Haram ${ }^{34}$, Britta Denise Hardesty ${ }^{35}$, Neil Holdsworth ${ }^{36}$, Laurent Lebreton ${ }^{37}$, \\ Heather A. Leslie ${ }^{38}$, Ilan Macadam-Somer ${ }^{39}$, Thomas Mace ${ }^{40}$, Mark Manuel ${ }^{41,42}$, \\ Robert Marsh ${ }^{31}$, Elodie Martinez ${ }^{12}$, Daniel J. Mayor ${ }^{6}$, Morgan Le Moigne ${ }^{7}$, \\ Maria Eugenia Molina Jack ${ }^{20}$, Matt Charles Mowlem ${ }^{6}$, Rachel W. Obbard ${ }^{43}$, \\ Katsiaryna Pabortsava ${ }^{6}$, Bill Robberson ${ }^{30}$, Amelia-Elena Rotaru ${ }^{14}$, Gregory M. Ruiz ${ }^{34}$, \\ Maria Teresa Spedicato ${ }^{44}$, Martin Thiel ${ }^{45}$, Alexander Turra ${ }^{46}$ and Chris Wilcox ${ }^{35}$
}

${ }^{1}$ International Pacific Research Center, School of Ocean and Earth Science and Technology, University of Hawaii, Honolulu, HI, United States, ${ }^{2}$ European Space Research and Technology Centre, European Space Agency, Noordwijk, Netherlands, ${ }^{3}$ Sea Education Association, Falmouth, MA, United States, ${ }^{4}$ Institute for Marine and Atmospheric Research, Utrecht University, Utrecht, Netherlands, ${ }^{5}$ Marine Sensor Systems, Institute for Chemistry and Biology of the Marine Environment, University of Oldenburg, Oldenburg, Germany, ${ }^{6}$ National Oceanography Centre, Southampton, United Kingdom, ${ }^{7}$ Département Océanographie et Dynamique des Écosystémes, Institut Français de Recherche pour l'Exploitation de la Mer, Bastia, France, ${ }^{8}$ Plymouth Marine Laboratory, Plymouth, United Kingdom, ${ }^{9}$ Environmental Research Institute, University of the Highlands and Islands, Thurso, United Kingdom, ${ }^{10}$ Deltares, Delft, Netherlands, ${ }^{11}$ University of Plymouth, Plymouth, United Kingdom, ${ }^{12}$ Laboratoire d'Océanographie Physique et Spatiale, Institute of Research for Development, Brest, France, ${ }^{13}$ Remote Sensing Solutions, Los Angeles, CA, United States, ${ }^{14}$ Department of Biology, Faculty of Science, University of Southern Denmark, Odense, Denmark, ${ }^{15}$ European Commission, Joint Research Centre (JRC), Ispra, Italy, ${ }^{16}$ Hawai'i Wildlife Fund, Paia, HI, United States, ${ }^{17}$ Scripps Institution of Oceanography, University of California, San Diego, La Jolla, CA, United States, ${ }^{18}$ Department of Geosciences and Natural Resource Management (IGN), University of Copenhagen, Copenhagen, Denmark, ${ }^{19}$ Atlantic Oceanographic and Meteorological Laboratory (NOAA), Miami, FL, United States, ${ }^{20}$ Sezione di Oceanografia, Istituto Nazionale di Oceanografia e di Geofisica Sperimentale, Trieste, Italy, ${ }^{21}$ Department of Oceanography and Fisheries, University of the Azores, Ponta Delgada, Portugal, ${ }^{22}$ Research Institute for Applied Mechanics, Kyushu University, Fukuoka, Japan, ${ }^{23}$ Applied Research Laboratory, Hawaii Institute of Geophysics and Planetology, University of Hawaii, Honolulu, HI, United States, ${ }^{24}$ P.P. Shirshov Institute of Oceanology, Russian Academy of Sciences, Moscow, Russia, ${ }^{25}$ NASA Jet Propulsion Laboratory, California Institute of Technology, Pasadena, CA, United States, ${ }^{26}$ CNR Institute of Marine Sciences, Lerici, Italy, ${ }^{27}$ Argans, Plymouth, United Kingdom, ${ }^{28}$ Center for Global Discovery and Conservation Science, Arizona State University, Tempe, AZ, United States, ${ }^{29}$ Mystic Seaport Program, Williams College, Mystic, CT, United States, ${ }^{30}$ Environmental Protection Agency (EPA), San Francisco, CA, United States, ${ }^{31}$ School of Ocean and Earth Science, National Oceanography Centre, University of Southampton, Southampton, United Kingdom, ${ }^{32}$ College of Life and Environmental Science, University of Exeter, Exeter, United Kingdom, ${ }^{33}$ eOdyn, Plouzané, France, ${ }^{34}$ Smithsonian Environmental Research Center, Edgewater, MD, United States, ${ }^{35}$ CSIRO Oceans and Atmosphere, Hobart, TAS, Australia,

${ }^{36}$ International Council for the Exploration of the Sea, Copenhagen, Denmark, ${ }^{37}$ The Ocean Cleanup, Delft, Netherlands,

${ }^{38}$ Department of Environment and Health, Vrije Universiteit Amsterdam, Amsterdam, Netherlands, ${ }^{39}$ Algalita Marine Research and Education, Long Beach, CA, United States, ${ }^{40}$ Mace Geospatial, LLC, Menasha, WI, United States, ${ }^{41}$ Freestone Environmental Services, Richland, WA, United States, ${ }^{42}$ National Oceanic and Atmospheric Administration, Seattle, WA, United States, ${ }^{43}$ Carl Sagan Center, SETI Institute, Mountain View, CA, United States, ${ }^{44}$ COISPA Tecnologia and Ricerca, Bari, Italy, ${ }^{45}$ Facultad Ciencias del Mar, Universidad Católica del Norte, Coquimbo, Chile, ${ }^{46}$ Oceanographic Institute, Universidade de São Paulo, São Paulo, Brazil 
Plastics and other artificial materials pose new risks to the health of the ocean. Anthropogenic debris travels across large distances and is ubiquitous in the water and on shorelines, yet, observations of its sources, composition, pathways, and distributions in the ocean are very sparse and inaccurate. Total amounts of plastics and other man-made debris in the ocean and on the shore, temporal trends in these amounts under exponentially increasing production, as well as degradation processes, vertical fluxes, and time scales are largely unknown. Present ocean circulation models are not able to accurately simulate drift of debris because of its complex hydrodynamics. In this paper we discuss the structure of the future integrated marine debris observing system (IMDOS) that is required to provide long-term monitoring of the state of this anthropogenic pollution and support operational activities to mitigate impacts on the ecosystem and on the safety of maritime activity. The proposed observing system integrates remote sensing and in situ observations. Also, models are used to optimize the design of the system and, in turn, they will be gradually improved using the products of the system. Remote sensing technologies will provide spatially coherent coverage and consistent surveying time series at local to global scale. Optical sensors, including high-resolution imaging, multi- and hyperspectral, fluorescence, and Raman technologies, as well as SAR will be used to measure different types of debris. They will be implemented in a variety of platforms, from hand-held tools to ship-, buoy-, aircraft-, and satellite-based sensors. A network of in situ observations, including reports from volunteers, citizen scientists and ships of opportunity, will be developed to provide data for calibration/validation of remote sensors and to monitor the spread of plastic pollution and other marine debris. IMDOS will interact with other observing systems monitoring physical, chemical, and biological processes in the ocean and on shorelines as well as the state of the ecosystem, maritime activities and safety, drift of sea ice, etc. The synthesized data will support innovative multi-disciplinary research and serve a diverse community of users.

Keywords: plastics, marine debris, sensor development, observing network, ecosystem stressors, maritime safety

\section{INTRODUCTION}

In this paper, serving as a Community White Paper contributed to the OceanObs19 conference $^{1}$, we present the concept of an Integrated Marine Debris Observing System (IMDOS) that will provide global coverage and accuracy, required for understanding the dynamics of marine debris and monitoring of changes in its distribution and effectiveness of mitigation of its impacts. IMDOS builds on previous initiatives (e.g., MSFD, 2013; GESAMP, 2019) to include into consideration a broad variety of debris and its complete life cycle in the marine environment, and aims to stimulate the establishment of best practices as well as optimization and expansion of the existing observational networks. We review the properties and impacts of different types of marine debris, as well as observation techniques and technologies that are used or could potentially be used in the next decade and beyond, and we share our vision of how direct observation, remote sensing, and numerical modeling can be integrated to compose a global observing system.

${ }^{1}$ http://www.oceanobs19.net/
The World Ocean plays an integral role in connecting remote areas by transporting substances and materials over large distances and in between continents. This is not limited to transport of heat and chemical compounds that shape the climate of the planet, but also includes solid objects and particulate matter floating on or near the ocean surface or suspended in the water column, including dispersal of many forms of living organisms. This way, over millions of years, floating seeds have populated new volcanic islands, and kelp species have spread over large areas. Land-derived plant material, entering the ocean, provides a substrate and food for marine life, and sinking particles in turn support the diverse ecosystems on the ocean floor (Dupont et al., 2009). Introduction of durable synthetic materials, especially plastics, has dramatically increased the amount of marine debris. This increase has cooccurred with other natural and anthropogenic changes in the ocean, such as warming, sea level rise, acidification, and loss of biodiversity; and plastic pollution is one particular, and very visible, example of the lack of sustainability in our actions.

The advent of commercial mass production of plastics in the mid-twentieth-century, and the subsequent exponential rise 
in consumption to an estimated 407 million metric tons in 2015 (Geyer et al., 2017), resulted in 4.8-12.7 million metric tons of plastic waste entering the oceans in 2010 from land and an estimated 120 million tons cumulative total (Jambeck et al., 2015). Plastics comprise the majority of marine debris and represent its most durable and persistent part (e.g., 88\% of samples analyzed by Cózar et al., 2014 were composed of plastics). In the absence of comprehensive international law regulating marine pollution, MARPOL Annex $\mathrm{V}^{2}$ was introduced in 1988 and has been signed by more than 150 countries to prevent ocean pollution with garbage from ships. However, it does not extend to land-based sources.

Now, as part of the Decade of Ocean Science for Sustainable Development (2021-2030) $)^{3}$, proclaimed by the United Nations, the Sustainable Development Goal (SDG) Target $14.1^{4}$ aims to prevent and significantly reduce marine pollution of all kinds by 2025, including marine debris (or marine litter). Marine litter is also addressed in the voluntary Global Programme of Action (GPA) through the Global Partnership on Marine Litter $^{5}$ (GPML), promoting the implementation of the Honolulu Strategy $^{6}$ from 2011, and through the UN Environment Clean Seas ${ }^{7}$ campaign launched in 2017. These initiatives engage governments, the general public, civil society and the private sector to transform habits, practices, standards and policies in the fight against marine plastic litter. Sessions and resolutions of the United Nations Environment Assembly (UNEA, which encompass GPA) highlighted marine plastic debris and microplastics amongst the issues of global importance and moved to act toward the negotiation of a new international legally binding instrument.

The Implementation of the G7 Action Plan on Marine Litter $^{8}$ mainstreams the work of the Regional Seas Programmes and includes capacity building and sharing of best practices, ongoing coordination with European Regional Seas conventions, strengthening the collaboration with GPA and GPML, and a contribution to the G20 Action Plan on Marine Litter ${ }^{9}$. The latter will be implemented through a voluntary Global Network of the Committed (GNC). The actions focus on the promotion of the socio-economic benefits of establishing policies on marine litter prevention, waste reduction and resource efficiency, waste/stormwater management, public awareness, education and research, remediation action and the promotion of stakeholder engagement. Under the presidency of Canada in 2018, the G7 countries agreed on a short list of seven main challenges in the areas of health and environment, and committed to move toward a more resource-efficient and

\footnotetext{
${ }^{2}$ http://www.imo.org/en/OurWork/Environment/PollutionPrevention/Garbage/ Pages/Default.aspx

${ }^{3}$ https://en.unesco.org/ocean-decade

${ }^{4}$ https://sustainabledevelopment.un.org/sdg14

${ }^{5}$ https://www.unenvironment.org/explore-topics/oceans-seas/what-we-do/ addressing-land-based-pollution/global-partnership-marine

${ }^{6}$ https://marinedebris.noaa.gov/sites/default/files/publications-files/

Honolulu_Strategy.pdf

${ }^{7}$ http://www.cleanseas.org/

${ }^{8}$ http://www.g8.utoronto.ca/science/G7_Science_2015-en.pdf

${ }^{9}$ https://ieep.eu/news/g20-adopts- $\mathrm{t} 20$-recommendations-on-plastics-andmarine-litter
}

sustainable approach to the management of marine plastic litter, working with industries toward $100 \%$ reusable, recycled, or recovered plastics by 2040. In support of these plans, the International Association for the Physical Sciences of the Oceans (IAPSO), the International Council for Science (ICSU), the International Union of Geodesy and Geophysics (IUGG), and the Scientific Committee on Oceanic Research (SCOR) published a document, providing a scientific perspective on these issues (Thompson and Maximenko, 2016).

To be effective, new regulations must be based on solid knowledge, and changes resulting from their implementation must be monitored comprehensively, accurately, and in a standardized way. Decades of scientific research have addressed many important aspects of the abundance, composition, and dynamics of marine debris, and plastic pollution in particular. The earliest scientific reports documented a variety of items in many marine habitats: industrial resin pellets and millimetersized fragments (Carpenter and Smith, 1972) and plastic bottles (Venrick et al., 1973) floating at the sea surface in the open ocean; synthetic fibers derived from marine netting and rope in filtered coastal water column samples (Buchanan, 1971); and items derived from food and beverage consumption, and fishing and boating activities on a beach near recreational waters (Cundell, 1973). A systematic chronology of marine debris research was well-described by Ryan (2015).

At the same time many questions remain unanswered. There are many reasons why measuring and understanding marine debris is difficult, including: (i) variety of object sizes (from tens of meters to microns) and shapes (e.g., spherical pellets, packaging films, fibers, and composite objects), (ii) complexity of chemical composition (e.g., different polymers, metals, glass, and organic materials), (iii) unknown sources and sinks, as well as (iv) pathways and (v) decay processes. Also, because different laboratories and groups use different methodologies to study marine litter, local observations are often hard to generalize into a global picture. For example, estimates of the amount of microplastic floating at the sea surface vary between 6,350 and 236,000 metric tons (Cózar et al., 2014; Eriksen et al., 2014; van Sebille et al., 2015). Note that even the highest estimate constitutes only $0.1 \%$ of the estimated total amount of plastic added to the ocean from land-based sources. Inclusion of estimates of other debris types (184,000 metric tons of larger plastic debris floating on the ocean surface; Eriksen et al., 2014), sinks (8,000 metric tons removed annually from the shorelines by the cleanup groups), and reservoirs (seabed, buried in beaches, water column, ingested by marine life, etc.) still leaves the mass balance of plastic debris open, with the fate of at least $90 \%$ of it unknown.

\section{IMPACTS OF MARINE DEBRIS AND IMPORTANCE OF THEIR GLOBAL MONITORING}

Marine debris has numerous impacts on the environment and society. Those influenced by these impacts, managing them professionally, or responding voluntarily are the potential users 
of the IMDOS. The global extent and growing magnitude of the impacts justifies the importance and urgency of the global observing system. It is also expected that increased knowledge of marine debris will reveal new impacts that are currently unrecognized. Most impacts are specific to the type of marine debris. People's activities increase the amount of natural material entering the ocean (such as driftwood from logging) and also introduce artificial materials that pose new threats to the environment.

\section{Microplastics and Biological Interactions}

Consumption of oil grows exponentially, with about 10\% used to produce plastic, a large fraction of which is intended for single-use applications and/or packaging. Jambeck et al. (2015) estimated that trends in plastic input entering the ocean from land-based sources follow the trends in oil consumption. These human-made polymers can last in the marine environment for a significant time; during their lifespan plastic items can travel over large distances and accumulate in some areas in high concentrations. Weakened by ultraviolet light and broken apart by storms, larger objects gradually fragment into smaller and smaller pieces (Figure 1) that become increasingly accessible to different marine species. Pre-production pellets and microbeads are deliberately manufactured in small sizes. The abundance and impacts of the smallest plastic particles, nanoplastics, are not well-studied, but their demonstrated ability to penetrate cell membranes and accumulate in organisms raises great concern (c.f., Koelmans et al., 2015). Fish, seabirds, turtles, and other marine animals, mistaking microplastics for food, ingest them, which may cause physical injury, starvation and even death. After entering the food web, plastics may travel to upper trophic levels, resulting in bioaccumulation and biomagnification, but such trophic transfer is not yet well-studied (c.f., Provencher et al., 2018).

Additives incorporated during production (such as colorants, plasticizers, stabilizers, antioxidants, flame retardants, UV absorbers, antistatic agents, etc.) make some plastics toxic; if ingested by organisms, these toxins may enter the food chain, posing a threat to ecosystem structure and function and possibly even to human health. Leaching of additives to the seawater may also pose ecotoxicological threats to the marine biodiversity. Toxins can be also adsorbed by the plastic when it moves through areas contaminated with industrial pollution or via natural events (e.g., such as "red tides" ${ }^{10}$ ). Because of their large relative surface area, nanoplastics may be most efficient transporters of these added or adsorbed chemicals (c.f., Galloway, 2015).

\section{Derelict Fishing Gear}

One of most abundant and conspicuous types of marine debris is derelict fishing gear. Used in large numbers, fishing nets are often lost during storms. According to Lebreton et al. (2018), they comprise $46 \%$ of the plastic mass floating in the upper ocean in the North Pacific Subtropical Gyre. The lifespan of these "ghost nets" is not known but as long as they float they continue killing not only fish, but also sea turtles, seabirds and

\footnotetext{
$\overline{{ }^{10} \mathrm{https} / / / \text { oceanservice.noaa.gov/facts/redtide.html }}$
}

other marine animals (Figure 2A). Washed ashore, partly buried in sand or stuck on rocks, nets are difficult to remove. Every year, fishing nets damage large areas of coral reef ecosystems: the Northwestern Hawaiian Islands alone collect on average an estimated 52 tons per year ${ }^{11}$, with 80 tons removed in $2018^{12}$

While concentration of heavy floating fishing nets tend to be higher in the subtropical "garbage patches" or in other frontal convergence regions of the ocean, light floats or buoys separating from these nets or lost during other fishing or aquaculture activities are blown by the wind onto selected shorelines. For example, plastic and styrofoam buoys, used for fishing and in aquafarms in the western Pacific and Chinese seas do not only pollute Asian shoreline (Lee et al., 2013) but reach in large numbers the coast of Alaska (Figures 2B,C), where inaccessible terrain, sparse populations, short summers, and dangerous wildlife make cleanup very difficult. Winter storms break debris into tiny pieces and blow them inland, contaminating large areas of land. Similarly, crab and lobster tags arrive from North America on the coasts of Europe, and Styrofoam is one of major types of plastic debris in coastal waters of Chile (Hinojosa and Thiel, 2009).

\section{Large Debris and Maritime Safety}

In addition to small items that sneak through our waste management systems, marine debris also includes large items that carry a threat to maritime activity. The "All is Lost" movie that starts with a sailboat collision with a marine container is not a completely unrealistic story. The World Shipping Council estimated that in 2008-2016 there were on average $568^{13}$ containers lost at sea each year during normal operational activity (Figure 3A). This is a tiny fraction of millions of containers delivered safely to sustain the World's economy, but even one 40 -foot object drifting without control can be tremendously dangerous. Its path and associated risks depend on the cargo inside the container, making predictions virtually impossible.

Smaller objects, including large natural debris, are hard to document. For example, driftwood is abundant on many shores, but extensive data on its amounts in the open ocean are lacking. However, individual observations (Figure 3B) and collision reports ${ }^{14}$ indicate that the risk is real. In some regions encounters with fishing nets and entanglement of ship propellers also poses significant threat to navigation (Hong et al., 2017).

\section{Debris on the Ocean Floor}

The ocean floor is one of the main destinations of marine debris. Many materials are denser than water and sink immediately after entering the ocean. For example, the seabed near big cities, ports, and river mouths are often densely covered with whole PET bottles and other presumably recent debris (Figure 4) of local

\footnotetext{
${ }^{11} \mathrm{https} / /$ www.fisheries.noaa.gov/feature-story/removal-and-research-marinedebris-team-strikes-again

${ }^{12}$ https://response.restoration.noaa.gov/over-80-tons- marine-debris-removednorthwestern-hawaiian-islands

${ }^{13}$ http://www.worldshipping.org/industry-issues/safety/

Containers_Lost_at_Sea_-_2017_Update_FINAL_July_10.pdf

${ }^{14}$ https://2019.transpacyc.com/race-archives/news-article/2013/2013-honolulu/

dismastings-debris-dazzling-speed-in-transpac
} 


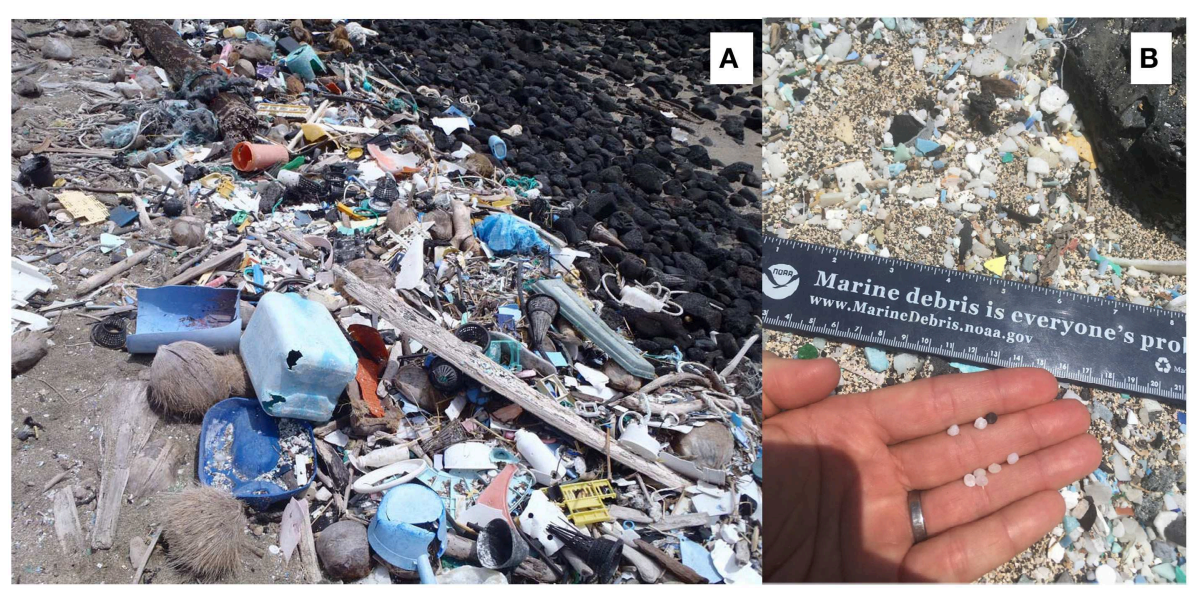

FIGURE 1 | Plastic debris accumulation at Kamilo Point, Island of Hawai'i. Large items (A) are often still identifiable, but gradually break into small fragments (microplastics, B). Photos courtesy of the 2011 IPRC project (A) and Hawai' i Wildlife Fund (B) (Maximenko holds copyright on 1A and Lamson holds copyright on 1B).

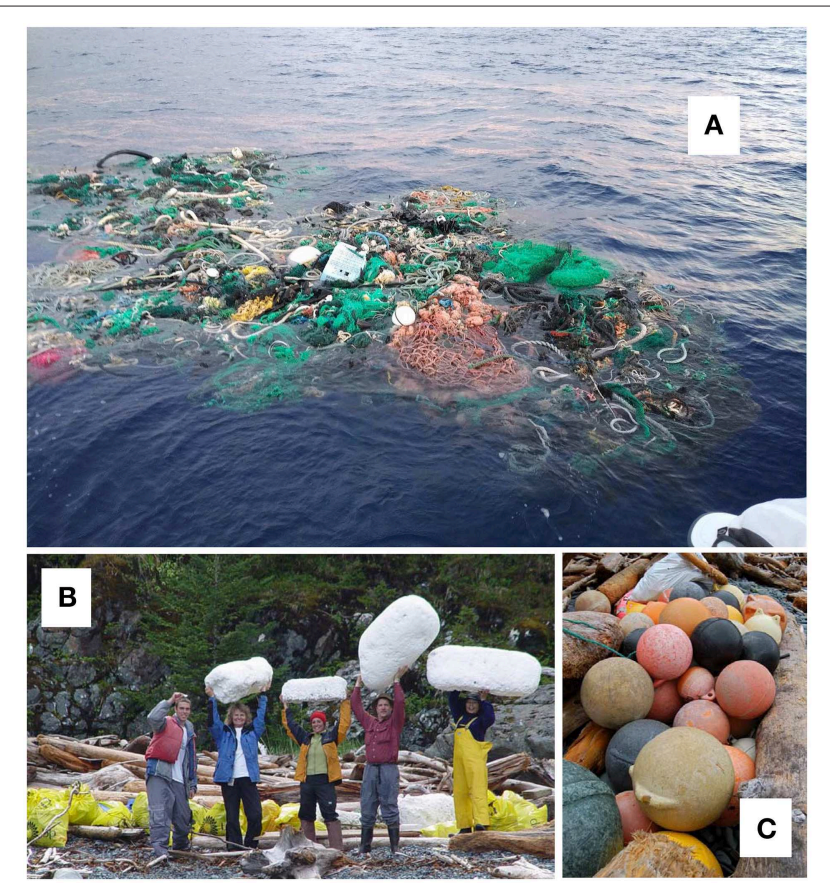

FIGURE 2 | Derelict fishing nets (A) floating in the eastern North Pacific. Photo courtesy of Raymond McCormack, who tagged the net with a satellite tracker during the Ocean Voyages Institute's projects. Styrofoam (B) and plastic (C) fishing buoys, removed from the shoreline of Alaska. Photos courtesy of Christopher Pallister, Gulf of Alaska Keeper ${ }^{15}$ (Maximenko has permissions from McCormack and Pallister to use the photos).

origin. Other types of debris or their parts float initially, but gradually lose their buoyancy due to biofouling and degradation, processes that may remove debris from the sea surface. Wooden debris, by being exposed to water may saturate and increase its density and sink. The ocean floor is difficult to study (section

\footnotetext{
${ }^{15} \mathrm{http}: / /$ www.goak.org/
}

Direct Observations of Marine Debris provides more information about benthic observations). However, because the benthic zone is a very important and vulnerable part of the marine ecosystem, it is exposed to critical impacts of natural and artificial marine debris (c.f., Galgani et al., 2015).

Over centuries, a large number of shipwrecks, lost in accidents and combat or sunk intentionally, as well as dumped munitions and containers with chemicals, including toxic and radioactive materials $^{16}$ have been also deposited on the ocean floor. These wrecks and dumpsites will sooner or later start leaking contaminants into the ocean; their retrieval or conservation and close monitoring are essential for the health of the deep ocean.

\section{Biological Dispersal}

"Floating macroalgae, wood and volcanic pumice have been part of the natural flotsam assemblage of the oceans for millions of years" (Kiessling et al., 2015). They provide not only a substratum for the pelagic ocean, but also facilitate the spread of new life to volcanic islands. Together with fouled ship hulls and ballast water, artificial marine debris opens new opportunities for long-distance travel and elevates risks of invasions of alien species, which are considered a major threat to coastal ecosystems (Molnar et al., 2008). Additionally, changing climate and ocean currents open up novel pathways for rafters (Miller, 2018).

\section{Catastrophic Events}

Natural disasters such as hurricanes, floods, and tsunamis, along with accidents created by human activity, can greatly increase inputs of all kinds of natural and artificial debris overviewed above. For example, when catastrophic events are considered, the number of marine containers lost at sea in 2008-2016 increased three times to 1,582 per year on average. According to the Japan Ministry of the Environment. (2012), the 2011 tsunami in Japan washed about 5 million tons of debris into the ocean within hours. Of this amount, 3.5 million tons sank on the shelf, severely damaging the benthic ecosystem and, together

\footnotetext{
${ }^{16}$ https://www.epa.gov/ocean-dumping/learn-about-ocean-dumping\#Before
} 

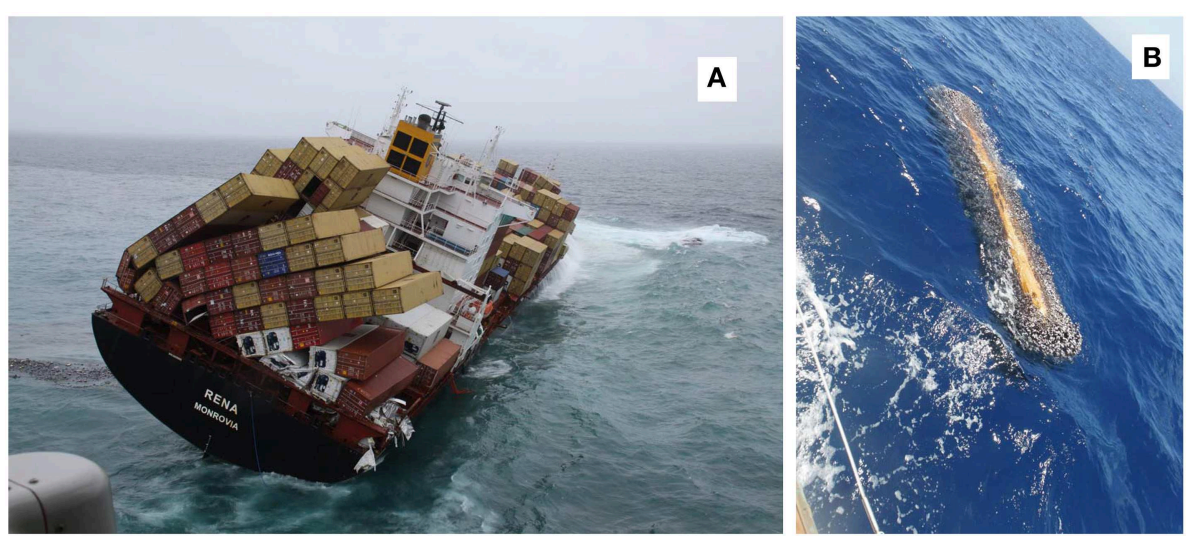

FIGURE 3 | (A) The MV Rena lost an estimated 900 containers when it ran aground and broke up off the coast of New Zealand in October 2011 (Photo courtesy of Maritime New Zealand). (B) Heavily fouled driftwood, floating in the North Pacific (courtesy of Randal Reeves) (Maximenko has permissions from Maritime New Zealand and Reeves to use the photos).

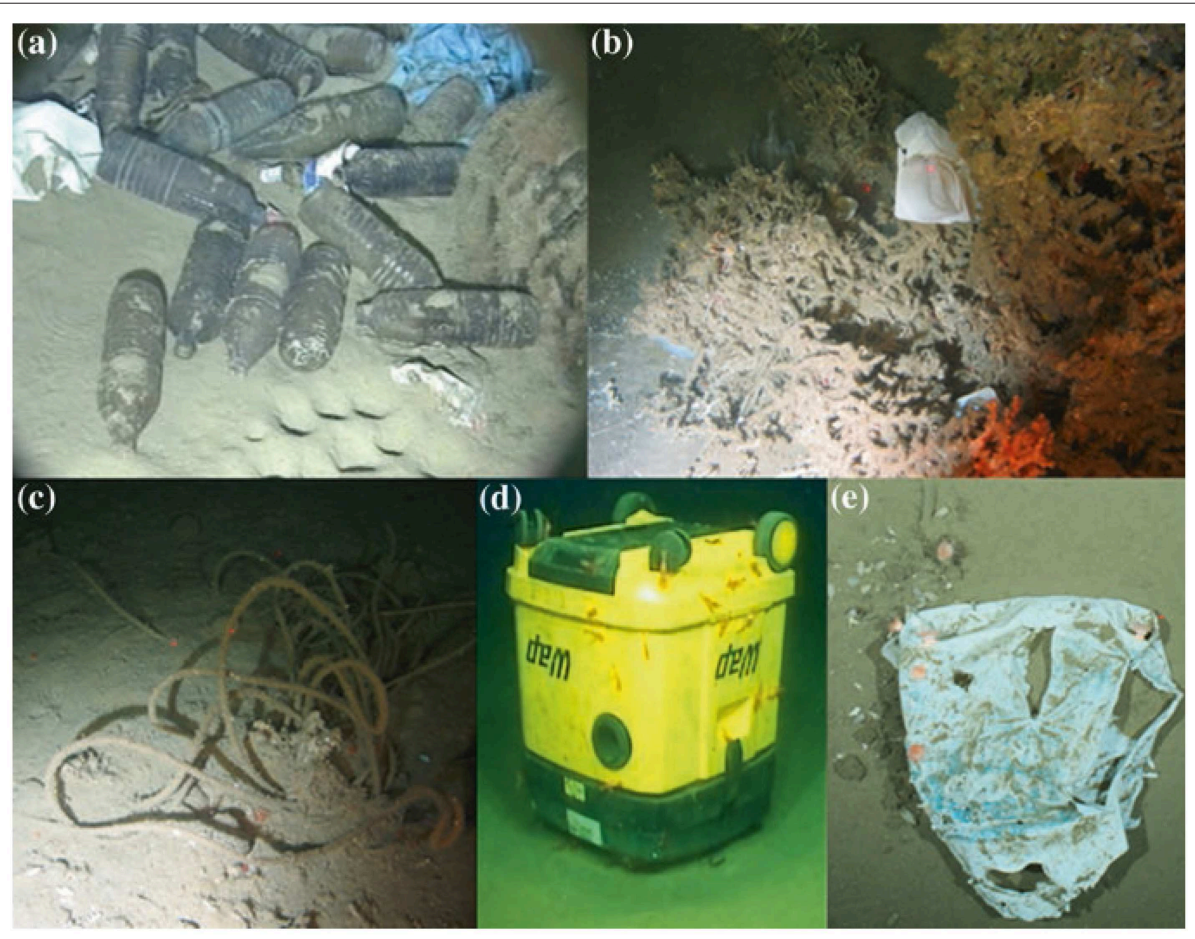

FIGURE 4 | Litter on the deep seafloor. (a) Plastic bags and bottles dumped $20 \mathrm{~km}$ off the French Mediterranean coast at 1,000 m in close vicinity to burrow holes; (b) food package entrapped at 1,058 m in deep-water coral colony; (c) rope at 1,041 m depth, both from Darwin Mounds; (d) waste disposal bin or a vacuum cleaner with prawns on the seafloor off Mauritania at 1,312 m depth; (e) plastic carrier bag found at 2,500 m depth at the HAUSGARTEN observatory (Arctic) colonized by hormathiid anemones and surrounded by dead tests of irregular sea urchins (adopted from Galgani et al., 2015 Galgani holds copyright of this figure).

with the radioactive spill from the Fukushima nuclear plant, badly affecting the local fishing industry. The remaining 1.5 million tons (an amount close to a full-year input of land-based plastic debris for the entire North Pacific) became flotsam and a fraction of this drifted to North America and Hawai'i. Four 20-meter floating docks, about 1,000 vessels (Maximenko et al., 2018), and other large objects posed threats to navigation and remote coastlines. At least 289 documented Japanese species
(Carlton et al., 2017) were also transported over large distances due to this event, some of which are known as past invaders. Many near-shore mariculture farms were set afloat and produced large numbers of floats. The composition of tsunami debris was very complex: according to Murray et al. (2018), counts of all categories of debris, monitored on Washington state beaches, increased in 2012 by a factor of 10 compared to pretsunami levels. 
Disasters and accidents often occur under conditions when limited resources, difficult access and emergency priorities do not allow for accurate documentation of the amounts of marine debris generated. For example, little is known about flotsam from the 2004 tsunami in Indonesia that caused 20 times more casualties than the tsunami in Japan. On a similar note, the Malaysian Airlines Flight 370 that disappeared in 2014 is still missing even though satellite engineers determined the approximate location of the crash, verified fragments were found on Reunion Island, Madagascar, and in Africa, their drift was studied in research projects (Trinanes et al., 2016) and 100 million Australian dollars were invested in the search.

\section{Economics}

Plastic plays an important role in the economy: every member of society benefits from this inexpensive material. Yet, the market price of the virgin plastics is only based on the low cost of plastic production and does not take into account the potentially much higher cost of its end-of-life processing and mitigation of its leakage into the environment. The consequences are often hard to estimate because they are coupled with other factors, and impacts are indirect, unproven, or even unknown. Newman et al. (2015) overviewed published studies that revealed high or significant costs of marine litter impacts on tourism and recreation, shipping and yachting, fisheries, and aquaculture. Generally, the data available for consolidation are rare. In the UK, the cost of professional litter removal is estimated at $€ 7,000$ per $\mathrm{km}$ per year (Mouat et al., 2010). Jang et al. (2014) linked more than 20 million dollars lost revenue in 2011 (compared to 2010) due to increased litter on Goeje Island beaches. Leggett et al. (2014) estimated that a $75 \%$ reduction of marine debris at six popular beaches of Orange County, California generated 40 million dollars benefit in just 3 months. Fisheries, shipping industries, navy, and aquaculture lose time and money by cleaning marine debris from their nets, ship propellers, cooling intakes, and farms.

Much higher risks and expenses are associated with potential future degradation of ecosystems, reduction of food production, and human health issues. The role of marine debris in these impacts is yet to be quantified. IMDOS will provide such data, without which such quantification is impossible. A recent study by Royer et al. (2018) also suggests that aging plastics release trace gases that contribute to the greenhouse effect. This impact may be significant because of its delayed effect and because it can be localized to areas (such as the seabed) where other sources are weak. Plastic contamination is one of the multiple stressors on the ocean that is projected to increase as the human population grows ${ }^{17}$.

\section{IMDOS STAKEHOLDERS}

IMDOS will provide data to individuals, organizations, and governments dealing with the surveillance, impact management

\footnotetext{
${ }^{17}$ https://en.unesco.org/ocean-decade
}

and mitigation of marine debris, including but not limited to the following groups:

- Policy and decision makers. The long-term resolution of the problems of anthropogenic debris will likely be achieved through integrated local, national, and international laws, balancing rewards and enforcement. IMDOS will provide knowledge for their development and tools to monitor their implementation. Such a system will have a strategic role in supporting the international agenda on the oceans, including reporting to the SDG 14.1 at different but complimentary scales.

- Management of relevant marine environments. Local agencies and companies will have first-hand information on risks, "hot spots" and "extreme events" associated with marine debris spills, improving strategies to locate and remove litter from shorelines, diving sites and Marine Protected Areas.

- First responders. IMDOS will inform about abnormal amounts of marine debris generated by disasters and accidents, and determine risks and influenced areas. It will also track large objects and ensembles of debris that will help with search and rescue operations and prevention of subsequent collisions.

- Scientists. With the current general understanding of sources, pathways, processes, and impacts related to marine debris, quantitative description is very poor; amounts, fluxes, and densities, estimated in different studies, often disagree by orders of magnitude and fate of as much as $90 \%$ of plastic debris is not known. Marine physicists, biologists, ecologists, chemists, and climate scientists will use the IMDOS data in their research, to reduce these uncertainties to both understand marine litter dynamics and its interaction with other oceanic processes.

- Businesses. Industries affected by marine debris (such as fisheries, tourism, etc.) will use IMDOS to plan preparedness and mitigation of potential impacts. The marine debris problem also opens new opportunities for inventions and new technology development. Given the co-responsibility of the private sector in generating marine litter (e.g., plastic pellets), they will also be able to inspect the effectiveness of implemented mitigation measures (e.g., Operational Clean Sweep, Circular economy). IMDOS will help high-tech companies to use marine debris information as a resource and to monitor impacts of new technologies on the environment.

- Environmental activists and citizen scientists. Plastic pollution has mobilized millions of concerned citizens, who are working to raise awareness, push legislation and, when possible, to clean the most polluted areas. Using IMDOS will strengthen their arguments, avoid biases and misunderstandings, and optimize their field work. In addition, IMDOS will offer a platform to consolidate and share validated marine litter data produced by citizen scientists.

- Educators. IMDOS will help to promote a more holistic view of our planet by raising population awareness of the consequences of our actions as a civilization and our responsibilities for personal and collective decisions. 


\section{STRUCTURE OF THE INTEGRATED MARINE DEBRIS OBSERVING SYSTEM}

An effective global IMDOS will make use of a variety of components, collecting in situ observations and sensing the environment remotely. It will be augmented by numerical models and theoretical analyses.

The structure of the IMDOS will be designed to provide best possible accurate estimates of variables required by the users, such as the SDG 14.1 indicators and subindicators under development at UN Environment (GESAMP, 2019). The list of variables includes, but is not limited to: concentration, composition, origin and pathways of marine debris. Concentration (abundance and mass) data will highlight patterns of debris distribution, identify areas of accumulation, and monitor temporal variability and trends. IMDOS will help identify main sources (i.e., varying both geographically and possibly in terms of human activities) and quantify inputs and ways debris enters the marine environment, estimate total amounts residing in different reservoirs [such as upper ocean, water column, seabed, shoreline, etc. (Hardesty et al., 2017; GESAMP, 2019)], close the gaps in the budget of marine litter as a system, and characterize connectivity between different regions. Distinct types of debris come from different sources, are transported along different paths and have different impacts on the environment and people's activity (e.g., Pieper et al., 2019). Data on the composition of debris will help scientists choose the right set of instruments for its monitoring, optimized for a given region.

A key goal of IMDOS is to monitor and assess the risk posed by marine debris. This risk can be distilled as a superposition of: (1) exposure or concentration; and (2) vulnerability ofor harms to-the system. Large knowledge gaps exist for both of these risk-determining variables. For example, in terms of exposure, it is still not clear among the scientific community how precise a measurement should be such as to accurately estimate the amount of debris in a region, and to calculate the fluxes of debris in and out of that region. All of these demand more scientific studies, but once risks of environmental impact of marine debris can be computed in IMDOS, these combined with data from other observing systems (e.g., systems monitoring the ecosystem, biodiversity, climate and/or maritime activity, and safety), can allow for the diagnosis of extreme situations, can support the mitigation and/or response to these risks, and can help to assess the effectiveness of those responses.

Direct observations of marine debris will provide "groundtruth" information and will be the most critical component of IMDOS. Different instruments will be used to measure distinct variables and characteristics of debris types. Abundance of debris will be monitored in all parts of the Earth hydrological system, including surface, water-column and seafloor compartments of the open ocean, coastal areas, shoreline, sea ice, lakes, rivers, streams and watersheds. To monitor changes in debris density, spatial patterns, and composition, IMDOS will include a set of permanent sites and repeat sections in the main areas of debris accumulation. IMDOS will have a global spatial coverage and resolution sufficient to describe large-scale patterns of debris distribution. While one-time observational and regional campaigns may also provide important insights, the core of IMDOS will be designed for multi-year functionality, with gradual enhancement driven by national monitoring capacities, developing technologies, gained knowledge and changes in the ocean circulation, climate, and ecosystem. To provide data for calibration/validation of satellite sensors, a part of in situ observations will be located along satellite tracks and maintained for the lifetime of these satellite missions. The regional structure of in situ observations will be adjusted to best cover regional sources of debris as well as the areas most affected by local and remote marine debris. To increase the volume of highquality observational data, new instruments and techniques will be developed and used in collaboration with governmental organizations, citizen scientists, volunteers, and ships and aircraft of opportunity. Crowdsourcing will be used to establish better connections to various groups worldwide and to collect reports on extreme events, such as accidental spills or unusual floating objects. Techniques will be developed to validate and synthesize low-level data (such as photographs or even verbal reports) with products of IMDOS. This in situ observational component of IMDOS is discussed in more detail in section Direct Observations of Marine Debris.

Remote sensing will fill gaps between sparse in situ observations (an example of the present coverage is shown in Figure 5A) and provide nearly uniform, nearly global coverage over long time scales. Spatiotemporal scales, resolved by IMDOS, will include scales of important sources and accumulation zones of marine debris and their variability as well as scales of physical processes controlling the dynamics of debris (such as oceanic gyres, fronts, eddies, seasonal cycle, El Nino-Southern Oscillation, Pacific Decadal Oscillation, etc.). Remote sensing will extend into such remote, unpopulated areas as the Arctic and Southern Oceans, as well as coastlines of uninhabited islands (Lavers and Bond, 2017). Calibration/validation of remote sensors will be conducted through comparison with data from a network of dedicated in situ observations and specialized models. To enhance regional monitoring, with higher spatiotemporal resolution, remote technologies will be implemented on suborbital missions using drones and small aircraft. Portable, robust, automated instruments, using remote sensors, will be used on ships and airplanes of opportunity as well as by citizen scientists and volunteers to sustain a cost-effective web of additional observations. The remote sensing component of IMDOS is discussed in more detail in section Remote Sensing of Marine Debris.

Like any effective observing system, scientific analysis and numerical modeling will play important roles in all stages of IMDOS design, implementation, and data analysis. Among the advantages of numerical modeling is that dynamical models can provide a global perspective on extremely sparse observations and fill the regions between the observations with an "educated guess" (e.g., Figures 5B-D; also, Hardesty et al., 2017). This extrapolation will be especially relevant in areas where direct 

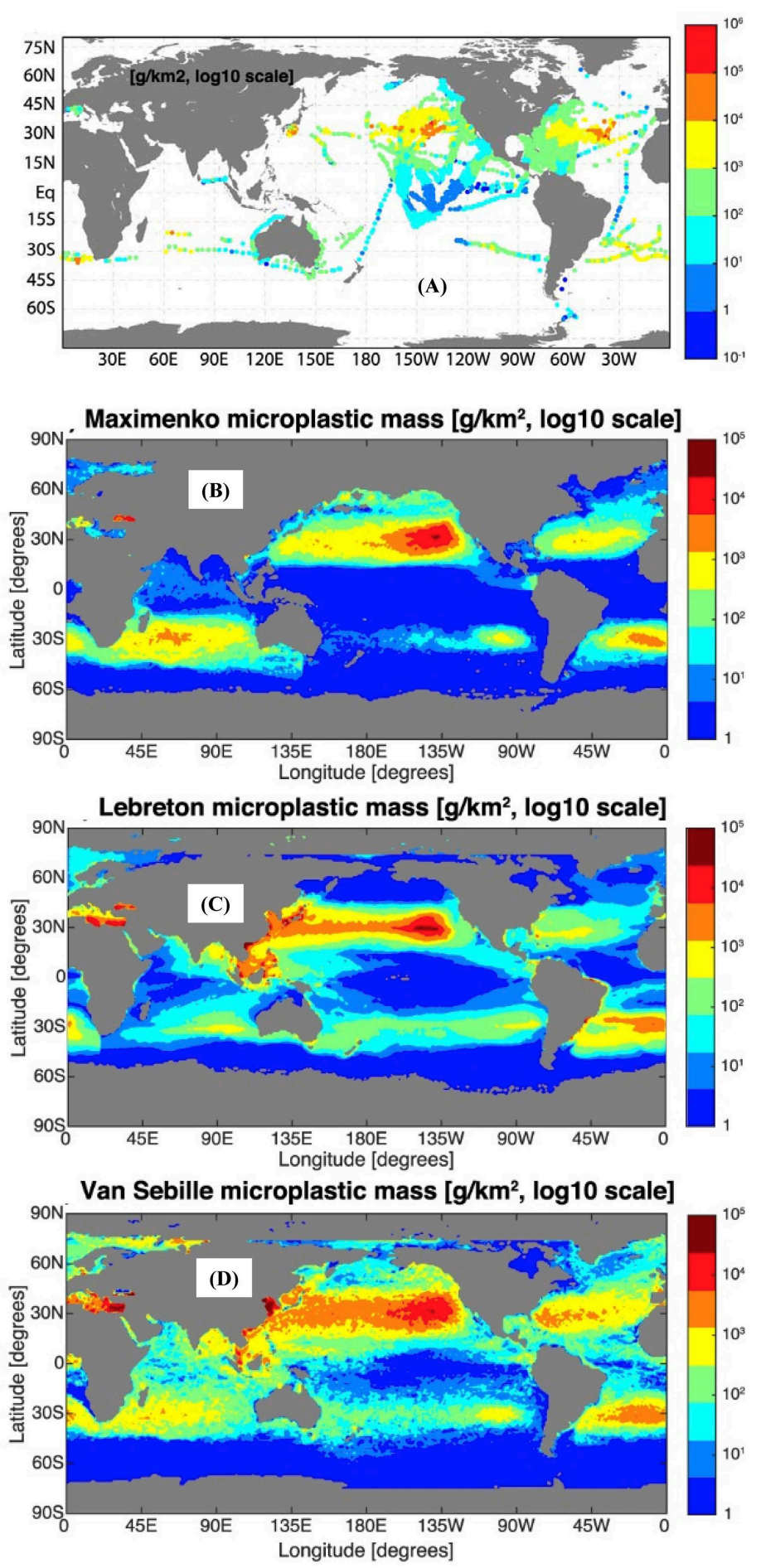

FIGURE 5 | (A) Density of microplastics near the ocean surface in the global dataset and (B-D) three model simulations [adopted from (van Sebille et al., 2015)] (Van Sebille holds copyright on this figure).

observations are difficult to make, such as the high Arctic region (Cózar et al., 2017; Peeken et al., 2018).

Moreover, dynamical models can be used to better understand the processes that affect transport, fate and distribution of marine debris. For example, the hypothesized sinking and rising of plastic particles due to biofouling and possible subsequent remineralization at depth (Kooi et al., 2017) will be extremely difficult to observe, but its large-scale effect on the horizontal distribution of marine plastic can be modeled. 
A possibly even more important task for modeling is to underpin Observing System Simulation Experiments (OSSEs), where the design of the large-scale structure of the IMDOS can be fine-tuned with the aid of simulations. OSSEs are a mature and proven methodology for effective design of observing systems given a realistic "nature run," but in the case of marine debris the difficulty will be that the simulated distribution of debris can be orders of magnitude different between models (van Sebille et al., 2015). How to deal with this large inter-model uncertainty in the scope of OSSEs will require further research.

Over the last decade or so, a number of different models for marine debris have been developed. These range from statistical observation-driven Markov models (Maximenko et al., 2012; van Sebille et al., 2012), to highly idealized mathematical models (e.g., Koelmans et al., 2017), to full-blown two- and three-dimensional particle tracking models (Lebreton et al., 2012; Robinson et al., 2017).

While designing an IMDOS with uncertain models will be challenging, the fact that these models are so poorly constrained is a primary motivation for the IMDOS in itself. The high sensitivity to unknown (or vaguely known) parameters in sets of model experiments will be used as guidance for upgrading the initial IMDOS with additional, targeted observations. Examples of these include fragmentation, biofouling, and sinking rates of marine plastic particles.

Once operational, the models will then be used to estimate the overall performance and efficiency of the observing system as well as to indicate the degree to which the whole dynamics of marine debris are understood and monitored. As such, the planning and operation of the IMDOS go hand in hand with the development of the models.

Specialized studies may be necessary to understand poorlyknown processes responsible for changes in debris properties, degradation, and interaction with the ecosystem, economics, and other factors. These studies will be designed to improve the products but will not become a permanent part of the IMDOS.

Some model uncertainties reflect gaps in the current state of knowledge or in critical ocean observations. For example, surface currents are one of the most complex components of the ocean dynamics, presently not measured by satellites directly, but derived from other remotely sensed variables (sea level, winds, temperature, etc.), using either relatively coarse ocean global circulation models (OGCMs) or simplified mixed layer models ${ }^{18}$. The development of IMDOS, with its emphasis on debris drift on or near the ocean surface, will motivate development and improvement of technologies of observations of ocean currents, waves and surface winds and their effects on movement of debris (e.g., Fraser et al., 2018; Putman et al., 2018), while objects of dimensions upwards of a few $\mathrm{cm}$ are subject to inertial forces that scale with mass of the object (e.g., Brooks et al., 2019).

The overall structure and operation of IMDOS will be intrinsically based on the scientific analysis of existing observations and models. The efficiency of IMDOS will be achieved by focusing on variables demanded by end-users of the data products. Because (based on our experience) the majority of

\footnotetext{
${ }^{18}$ http://www.globcurrent.org/
}

users have local and regional interests, the observing system will have an important focus on regional products while maintaining global coverage.

IMDOS will build on the positive experience of such successful programs as the Global Ocean Observing System $(\text { GOOS })^{19}$ and, after a setup period, may become a part of the GOOS. International groups of experts will maintain the list of Essential Ocean Variables and discuss the effective methodologies. Data centers will be set up for producing, managing, and distributing quality-controlled data and near real-time products. These data centers will work together to develop and implement observational methods and techniques, providing uniform format and accuracy of observations. They will also work with satellite mission science teams and modelers to fully use the wealth of information into gridded products useful for various applications. Synthesis of heterogeneous data (such as counts, images, and coarse satellite indices) will require development of new advanced methods such as those using machine learning. Interaction with users will be improved by involving them in the voluntary data collection process as boats of opportunity or coastal observers.

\section{DIRECT OBSERVATIONS OF MARINE DEBRIS}

In order to characterize the abundance of different types of marine debris, a variety of observing platforms are required. Each of these have advantages and disadvantages in terms of the level of temporal and spatial resolution they can provide, the sensors and samplers they can carry, debris types and size ranges they can cover, and the physical parts of the marine environment from which they acquire data. Until appropriate sensors become available and provide sufficient temporal and spatial coverage, samplers will be broadly used for "point" observations. At the same time, only samplers provide "ground truthing" in its entirety and complexity. It was the collection of samples that raised concern for the trends of plastic debris in the marine environment. Samples are also irreplaceable in process studies investigating biological interactions, chemical degradation, and changes in the chemical composition of debris over time.

\section{Platforms}

The following platforms are used for marine debris observations (Figure 6).

- Satellites, Aircraft, and Drones. Development of advanced sensors makes the boundary between "direct" and "remote" observations somewhat fuzzy. Presently, aerial surveys and remote technologies (described in section Remote Sensing of Marine Debris) provide rich information on debris at the ocean or shoreline surface (e.g., Lecke-Mitchell and Mullin, 1992). As the major part of marine debris is submerged in the ocean or buried in marine sediments (e.g., van Sebille et al., 2015; Koelmans et al., 2017; Chubarenko et al., 2018), direct measurements remain critical for comprehensive monitoring.

${ }^{19}$ http://www.goosocean.org/ 


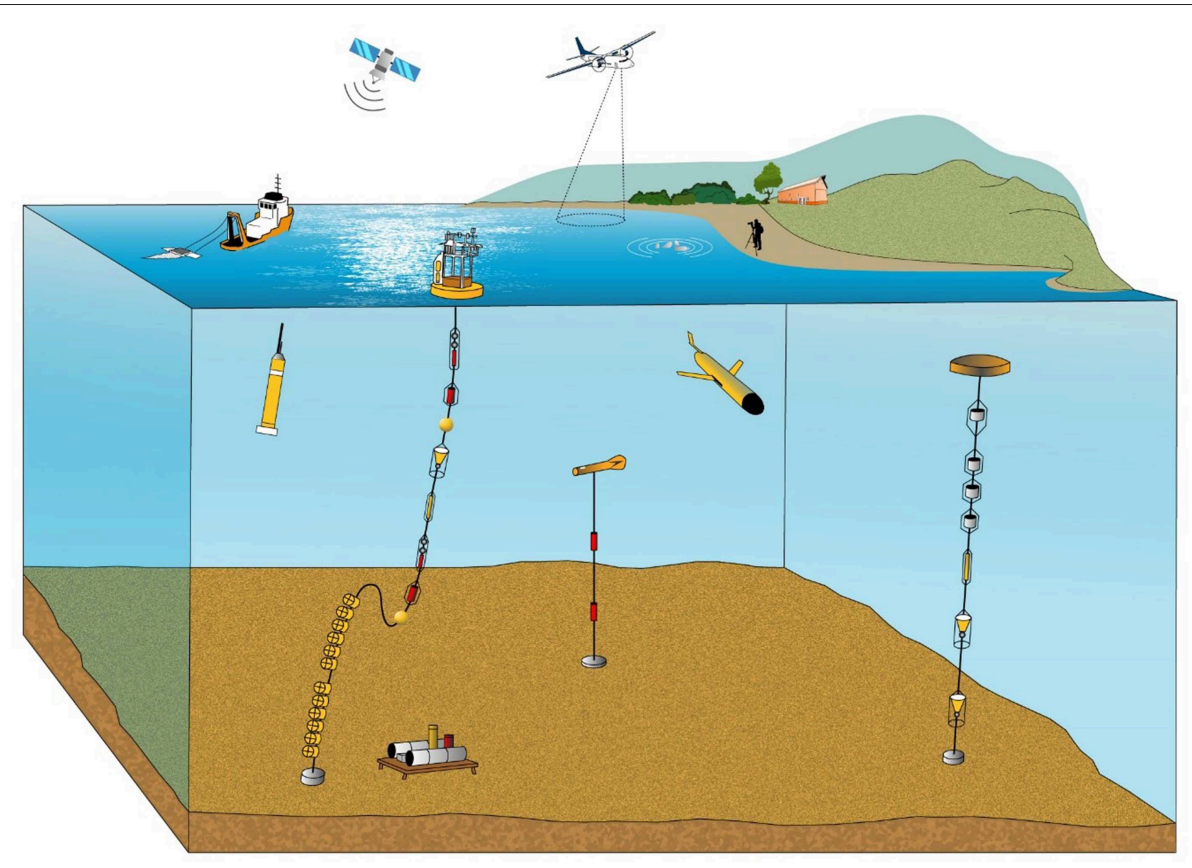

FIGURE 6 | Schematic of the various observation platforms currently in use and on which a variety of sensors and samplers can be carried (Courtesy Kate Davis) (Lampitt has permission to use this figure).

In a comprehensive discussion of sensors applicable in monitoring of hazardous materials and organisms in the marine environment Zielinski et al. (2009) present how different measurement platforms can contribute to the depth of knowledge at varying geo-spatial and temporal scales.

- Ships. Ships are traditionally used to collect data on marine debris floating on or near the surface. Ship observations are sparse, vulnerable to the weather conditions, and often sensitive to the type of ship and expertise of the operator. Nevertheless, they provide a platform from which a broad variety of sensors and samplers can be used for comprehensive study of the entire water column, from seabed to the surface. With the small number of research vessels, ships of opportunity have a great potential to greatly improve coverage by increasing the number of visual observations and using ship-borne autonomous systems (e.g., Ferrybox) ${ }^{20}$.

- Autonomous platforms. Floats, gliders (both seagliders and wavegliders), and Autonomous Surface and Underwater Vehicles (ASVs and AUVs), equipped with adequate sensors can provide unique measurements in hard-to-reach parts of the ocean. As a rule these platforms have insufficient space for samplers and are often not recovered. The expanding Animal Telemetry Network ${ }^{21}$ can be also used for marine debris data collection as well as to monitor the interaction of debris with marine life. Lagrangian platforms may play an important role in tracking marine debris and understanding its pathways. The Global Drifter Program ${ }^{22}$ (Niiler, 2001) maintains a network of $\sim 1,400$ active satellite-tracked drifting buoys reporting hourly (Centurioni, 2018), and an archive of more than 30,000 drifter-years of historical trajectories, used in many studies (e.g., Lumpkin et al., 2012; Maximenko et al., 2012; van Sebille et al., 2012). Satellite trackers, attached to large debris (such as fishing net in Figure 2A) facilitate their retrieval from the ocean and reduction of associated hazards for maritime activities and coastal businesses. Specialized Lagrangian tools are developed to study drift of debris and other pollutions in focused regional projects. For example, Meyerjürgens et al. (2019) built compact surface drifters and used them on the southern North Sea shelf to study the transport patterns in the nearshore zones and the beaching-refloating dynamics.

- Fixed Point Observatories. These are important and efficient platforms to monitor temporal variability and, in particular, long-term trends of the problem. Moored platforms have the great advantage of being able to carry sensors and samplers. At present, there are about 120 open ocean observatories (OceanSITES) ${ }^{23}$ and even higher numbers of coastal and shelf observatories that can be used for marine debris observations. Some of the observatories are cabled ${ }^{24}$ and can produce a large volume of real-time data. Choice of locations of sites optimal for marine debris trend observations as well as interpretation of the time series, contaminated with the spatial

\footnotetext{
${ }^{20}$ https://www.ferrybox.com/

${ }^{21}$ https://ioos.noaa.gov/project/atn/
}

\footnotetext{
${ }^{22}$ http://www.aoml.noaa.gov/phod/gdp/index.php

${ }^{23} \mathrm{http}: / /$ www.oceansites.org/

${ }^{24} \mathrm{http}$ ://aco-ssds.soest.hawaii.edu/
} 
signal will require coordination with satellite observations and numerical models.

- Benthic landers and crawlers. A range of devices have been developed which remain on the seabed for protracted periods of time some of which photograph the seabed repeatedly (e.g., Lampitt and Burnham, 1983; Lampitt, 1985) or can take time series sediment samples when crawling over the seabed (Purser et al., 2013). These provide unique information about the arrival of debris on the seabed and the interaction with this rather different benthic ecological community.

- Shoreline monitoring and Beachcombing. The highest known concentrations of marine debris are reported from selected shorelines, including both anthropogenic debris ${ }^{25}$ (Figure 1) and natural materials such as driftwood (e.g., in the background of Figure 2B). Societal concern has promoted a massive beachcombing activity. In 2017, the International Coastal Cleanup ${ }^{26}$, including more than 0.5 million people worldwide, removed more than 8,000 tons of artificial debris. Despite patchy geographical distribution and sparse timetables, cleanups have great potential of crowdsourcing qualitative and quantitative marine debris data through coordinated surveys using approved protocols [e.g., NOAA's Marine Debris Tracker $^{27}$, Litterati ${ }^{28}$, and JRC Floating Litter App (González-Fernández and Hanke, 2017)]. Easy-to-access shorelines facilitate citizen science initiatives (GESAMP, 2019), can be used for sampling and monitored with beach cameras (Kako et al., 2010).

\section{Sensors and Samplers}

A combination of samplers and sensors is used for direct observations of marine debris.

\section{Sensors}

While items at the sea and land surface can be detected and classified using remote sensing (as discussed in section Remote Sensing of Marine Debris), the development of autonomous and field deployable sensors for marine debris including microplastics remains a significant research challenge. Some field-deployed deep capable systems carry sensors and combine them with sampling and post-retrieval analysis (Wang and Wang, 2018): the sensors in this case are only used to trigger sampling and to provide environmental context (Edson and Patterson, 2015). Other imaging systems developed for macrofauna or microorganism studies can also be used to study plastic debris. For example, a towed camera and human assisted semi-automated image analysis (BIIGLE-Bio-Image Indexing and Graphical Labeling Environment-database) has been used to track increases in debris 2004-2014 in the eastern Fram Strait (Bergmann and Klages, 2012; Tekman et al., 2017). For microplastics, many of the techniques used in the lab (e.g., see Wang and Wang, 2018) are being semi-automated and can

\footnotetext{
${ }^{25}$ https://www.axios.com/dominican-republic-garbage-plastic-waste-coverbeach-c16f8637-6002-476c-bcf5-1964fe38abc1.html

${ }^{26}$ https://oceanconservancy.org/wp-content/uploads/2017/06/InternationalCoastal-Cleanup_2017-Report.pdf

${ }^{27}$ https://marinedebris.noaa.gov/partnerships/marine-debris-tracker

${ }^{28}$ https://www.litterati.org/
}

be applied in situ after developing the necessary engineering / instrumentation. For example, staining with the dye Nile Red (Shim et al., 2016) results in discrimination of plastics from other particles using fluorescence excitation and emission in the visible range (Maes et al., 2017): a spectral range that can be implemented in situ. Whilst chitin and some other organic matter is also stained, these particles may be discriminated using other means, e.g., by density (Maes et al., 2017) or digestion (Erni-Cassola et al., 2017). This technique has been semi-automated using image analysis software (Erni-Cassola et al., 2017). Full automation will require in situ filtration and image capture and likely separation or digestions steps which though onerous are not beyond the capabilities of in situ instrumentation (e.g., Scholin et al., 2017). Popular and powerful direct hyperspectral/FT-IR techniques used on filtered samples (e.g., Karlsson et al., 2016) and being increasingly automated may be problematic in situ due to the optical absorption of water in preferred spectral ranges (e.g., 1,000-2,500 nm, Karlsson et al., 2016). However, Raman spectroscopy has previously been applied in situ for other applications (Kirkwood et al., 2013; Peltzer et al., 2016; Guo et al., 2017; Jing et al., 2018; Li et al., 2018), and for microplastics can be operated in a spectral range with low absorption in water (e.g., $785 \mathrm{~nm}$ laser, Frere et al., 2016). Imaging (Nelson et al., 2018) and flow cytometry (Sgier et al., 2016; Long et al., 2017) can also be used, currently in the lab to analyze small plastic particles. Indeed cytometry is calibrated using plastic microbeads. In situ flow and imaging cytometers have been deployed (Dubelaar and Jonker, 2000; Dubelaar and Geerders, 2004; Lambert et al., 2017; Olson et al., 2017; Sosik, 2017), and an intriguing possibility is the reduction in size and power of deployed systems using microcytometers (Benazzi et al., 2007; Barat et al., 2012; Spencer et al., 2014, 2016; McGrath et al., 2017). Focused and significant effort is required to turn these opportunities into mature sensor technologies that can address operational metrology of marine debris across a wide size range in the marine environment.

\section{Samplers and Subsequent Analytics}

Beach litter surveys, using standardized protocols, are the simplest sampling technique, in which all analytical methods can be applied in order to deduce debris characteristics, including composition and possibly even origin. For smaller particles of debris found in the water, plankton nets of various types have frequently been used, some focusing on the surface layer (Neuston nets, Eriksen et al., 2018), and all usually with mesh sizes $>250-300 \mu \mathrm{m}$. These techniques are relatively inexpensive and easy to operate but they may be missing a significant mass fraction of plastic debris associated with smaller particles. Direct measurements are needed to derive the size distribution to assess the missing mass. Presently, smaller particles have been sampled using in situ and deck-mounted filters with pore sizes down to $0.5 \mu \mathrm{m}$, which addresses the smaller size classes very effectively. However, such techniques are expensive demanding specialist equipment and operators.

Depending on their composition and degree of biofouling, many common microplastics are less dense than seawater so tend to float at or near the ocean surface, and as such may exhibit 
high concentrations in the upper ocean relative to deeper water. Manta trawls are used near the surface and a number of methods are available for sampling in the $1-1,000 \mu \mathrm{m}$ surface microlayer, including rapid and facile glass plate sampling methods, which collect samples that contain relatively small amounts of sediment and biogenic material, facilitating subsequent microplastics identification (Anderson et al., 2018).

Sediments are typically sampled using corers such as the multicorer, which retains the sediment/water interface (Barnett et al., 1984). The separation of anthropogenic debris from naturally occurring material is a challenging task and significant research is still required to perfect techniques, particularly for micro-debris.

Biota is an additional and relevant source of information to estimate litter distribution and exposure to biodiversity and humans (GESAMP, 2019). It may also be used to validate models since the greater amount of micro and nano-particles in the tissues reflects a more chronic instead of episodic contamination in the surrounding environment. Particles in the digestive tract are also highly informative on the risks of trophic transfer and impacts in the ecosystem. However, as sediment samples, biotic sample processing and analysis still demand improved intercalibration between laboratories.

\section{Sample Contamination and Intercompatibility}

In terms of sample analyses, a range of techniques has been used often with unknown precision and specificity, which frequently leads to data of unknown quality. A key aspiration for the coming decade is that rigorous and standardized analytical techniques are developed so that data are intercomparable between laboratories and in order to determine temporal and spatial variability.

Sampling of the smaller size categories of debris and particularly nanoplastics and fibers (due to the relative ubiquity of microparticles in clothing, buildings, etc.), has to date been frequently characterized by poor protocols which will have generated large numbers of contaminated samples. In order to obtain adequate data on which to base decisions, the coming decade must be characterized by high principles of "best practice."

\section{REMOTE SENSING OF MARINE DEBRIS}

The remote sensing of marine debris, in particular plastic pollution, is in its infancy (Maximenko et al., 2016a,b; Garaba et al., 2018). Despite encouraging results of first experiments with detection of large floating items (e.g., Acuña-Ruz et al., 2018; Garaba et al., 2018; Topouzelis et al., 2019) overall marine debris monitoring represents a significant technological challenge. Successful remote sensing can fill gaps between sparse in situ observations and provide uniform coverage over large areas and long time periods. Quantifying how much and where debris enters the marine environment over time is critical to formulate and evaluate proper responses but on a global scale has, so far, only been roughly estimated (Jambeck et al., 2015). Global coverage is particularly important because some artificial marine debris such as plastic can travel over long distances and accumulate over time. Satellites can also help to survey remote, hard-to-reach areas, in which direct observations are sparse and difficult. Because of the huge diversity of chemistry and geometry of different types of debris, no single sensor can see it all. A list of instruments is presented to highlight those currently available on in situ, air- and space-borne remote sensing platforms that have potential applications relevant to the aims of IMDOS (Table 1). A number of these sensors have already been tested in past missions but each sensor has observational limitations related to spectral resolution, spectral range, sensitivity, revisit time, geospatial resolution and coverage. To this end, an integrated sensor system combining sensors of different capabilities on different platforms is needed to advance future operational remote sensing efforts of marine debris especially in remote areas of the global oceans (Zielinski et al., 2009; Garaba and Dierssen, 2018).

\section{High Spatial Resolution Imaging}

Washed ashore, floating and slightly submerged marine debris has been monitored using high-resolution cameras on fixed platforms (Kako et al., 2018), shipborne (Hanke and GonzalezFernandez, 2014), airborne (e.g., Veenstra and Churnside, 2012; Kataoka et al., 2017; Garaba et al., 2018; Martin et al., 2018; Moy et al., 2018) and satellites (e.g., Matthews et al., 2017; Topouzelis et al., 2019). Generally this technique is applied to the visible spectrum (400-700 $\mathrm{nm}$ ) by making true color RGB composite images. Visible images have been used, for example, to study the dynamics of rafts of marine debris generated by the March 11, 2011 tsunami that devastated the coastline of eastern Japan (Figure 7, Matthews et al., 2017). The key requirement for the imaging technique is high spatial resolution sufficient to not only detect flotsam but desirably also identify it as a particular type of debris or specific object. While military satellite technology is capable of providing higher resolution, commercial products are limited to $25-50 \mathrm{~cm}$, restricting their utility to several-meterssized objects. Identification of smaller objects is feasible in suborbital missions.

Analysis of visible images requires advanced interpretation techniques to eliminate environmental perturbations from ocean bright targets (breaking waves, white caps, sea foam, surface reflected glint), clouds and cloud shadow (Matthews et al., 2017; Garaba and Dierssen, 2018). The unwanted effects can be mitigated by capturing a series of images with intervals of several seconds and at an optimal viewing geometry. True color RGB images provide crucial complementary information in monitoring marine debris about the apparent color and shape of litter that can be used to, for example, discriminate man-made objects from marine organisms, such as kelp or whales. However, the RGB images do not provide information on the physical and chemical composition of the litter. The potential of new "machine learning" techniques, combined with hyperspectral information, demonstrated recently by Acuña-Ruz et al. (2018) in their pilot study on the shoreline, will increase the value of high-resolution visible imaging in the future. Costs related to managing "big data" might be an operational challenge for such very high temporal and geo-spatial resolution RGB imagery. Efficiency of visual observations can be optimized through interaction with other components of IMDOS, used to identify "hot spots," and 
TABLE 1 | Examples of actual sensors already used for detection and/or polymer identification of plastic debris.

\begin{tabular}{|c|c|c|c|c|c|}
\hline $\begin{array}{l}\text { Satellite/Sensor } \\
\text { name }\end{array}$ & $\begin{array}{l}\text { Spatial resolution } \\
(\mathrm{HR}>10 \mathrm{~m} \text {; } \\
\text { VHR }<10 \mathrm{~m})\end{array}$ & $\begin{array}{l}\text { Spectral } \\
\text { resolution }\end{array}$ & $\begin{array}{l}\text { Wavelength } \\
\text { range [nm] }\end{array}$ & Platform & Instrument \\
\hline Sentinel-2/ MSI & $\mathrm{HR}$ & Multispectral & $450-1,400$ & Satellite & \\
\hline TanDEM-X & $\mathrm{HR}$ & & & Satellite & SAR \\
\hline WorldView3 & VHR & Multispectral & $400-2,365$ & Satellite & \\
\hline PlanetScope & VHR & Multispectral & $455-860$ & Cubesat/Satellite & \\
\hline SASI & VHR & Hyper & $950-2,450$ & Airborne imager & \\
\hline APEX & VHR & Hyper & $372-2,540$ & Airborne imager & \\
\hline AVIRIS-NG & VHR & Hyper & $380-2,510$ & Airborne imager & \\
\hline $\begin{array}{l}\text { ASD FieldSpec } \\
\text { Pro }\end{array}$ & VHR & Hyper & $350-2,500$ & Handheld & \\
\hline $\begin{array}{l}\text { Spectra vista } \\
\text { corporation }\end{array}$ & VHR & Hyper & $350-2,500$ & Handheld & \\
\hline Spectral evolution & VHR & Hyper & $350-2,500$ & Handheld & \\
\hline
\end{tabular}

in concert with other observing ocean systems, such as weather forecast and disaster/accident warning systems.

\section{Optical Spectro-Radiometric Techniques}

Spectro-radiometric analysis from the ultraviolet to the far infrared spectrum has opened new avenues for detection and characterization of plastic and other types of marine debris. Garaba and Dierssen (2018) demonstrated the existence of unique spectral absorption features in the near infrared (NIR) and short-wave infrared (SWIR) spectrum using washed ashore macroplastics harvested from the west coast of the USA (Figure 8). These absorption features were found to be insensitive to the object size (even in the marine-harvested microplastics from the North Atlantic and Pacific waters), apparent color or polymer type of plastic particles, suggesting that these features have potential applications in remote detection of ocean plastics under various backgrounds, including vegetation. Spectral information from multi- and hyperspectral optical sensors can be used to infer the abundance of plastic objects of a subpixel size. In the infrared spectrum plastics have identifying spectral signatures with a higher reflectance signal when compared to the ocean, which is a dark target with a very low nearly flat reflectance. However, this approach should take into account recent substantial increases in the quantity of pelagic Sargassum (e.g., since 2011 in the tropical Atlantic, Gower et al., 2013) also characterized by high reflectance in the NIR. It is therefore possible to detect the reflectance of floating ocean plastics, but this will depend on sensor capability as even shallowly submerged objects might be completely masked by water, a strong absorber of light in the SWIR spectrum. Although the relationship between degree of submersion and reflectance properties of plastics has not been fully investigated, it has been shown spectral reflectance shape of the samples was retained but the magnitude of the reflectance was lower for wet samples compared to dry samples (Garaba and Dierssen, 2018). A combination of drones and satellite imagery was recently experimented with to detect typical household items as floating plastic targets. Topouzelis et al. (2019) confirmed that floating plastics are seen from space as bright objects and demonstrated the benefits of using very high $(\sim 0.02 \mathrm{~m})$ geo-spatial resolution imagery from drones to improve geo-referencing of Sentinel 2 data, resampled to $10 \mathrm{~m}$ resolution.

Spectral observations provide a wealth of information that can be applied to algorithms that are key to inferring abundance of marine debris, polymer types and degree of weathering or degradation relating this to the possible age of debris. To become useful, large spectral libraries of different types of debris as well as inorganic substrates and biological environment need to be established (e.g., Garaba and Dierssen, 2017; Kokaly et al., 2017). Because marine debris attracts all kind of organisms, from bacteria and algae to fishes, separation of the signals may be challenging but, if successful, will provide a very important characterization of the impact on the ecosystem. Additionally, atmospheric correctional approaches (such as the "black pixel assumption" approach which assumes that in the open ocean the signal in the NIR is negligible since the ocean is a dark target) for satellite and airborne observations need to be revised to account for the contribution of floating plastics to the bulk water leaving signal. More recently, the use of SWIR at-sensor radiance was presented showing promising results in the airborne detection, quantification and characterization of floating ocean plastics (Garaba et al., 2018). Goddijn-Murphy and Dufaur (2018) and Goddijn-Murphy et al. (2018) developed and verified an optical model of sunlight hitting a water surface, littered with floating plastic, that describes how inherent optical properties of plastic items (such as transparency) affect reflectance in the visible to SWIR spectrum.

A prospective technique to observe submerged debris is active remote sensing using a light detection and ranging system (LIDAR) that can measure the onboard laser lights backscattered from the ocean. Alternative approaches with potential application are based on fluorescence and Raman spectroscopy although the latter operates with a low signal that is presently challenging to detect from current satellites missions. 


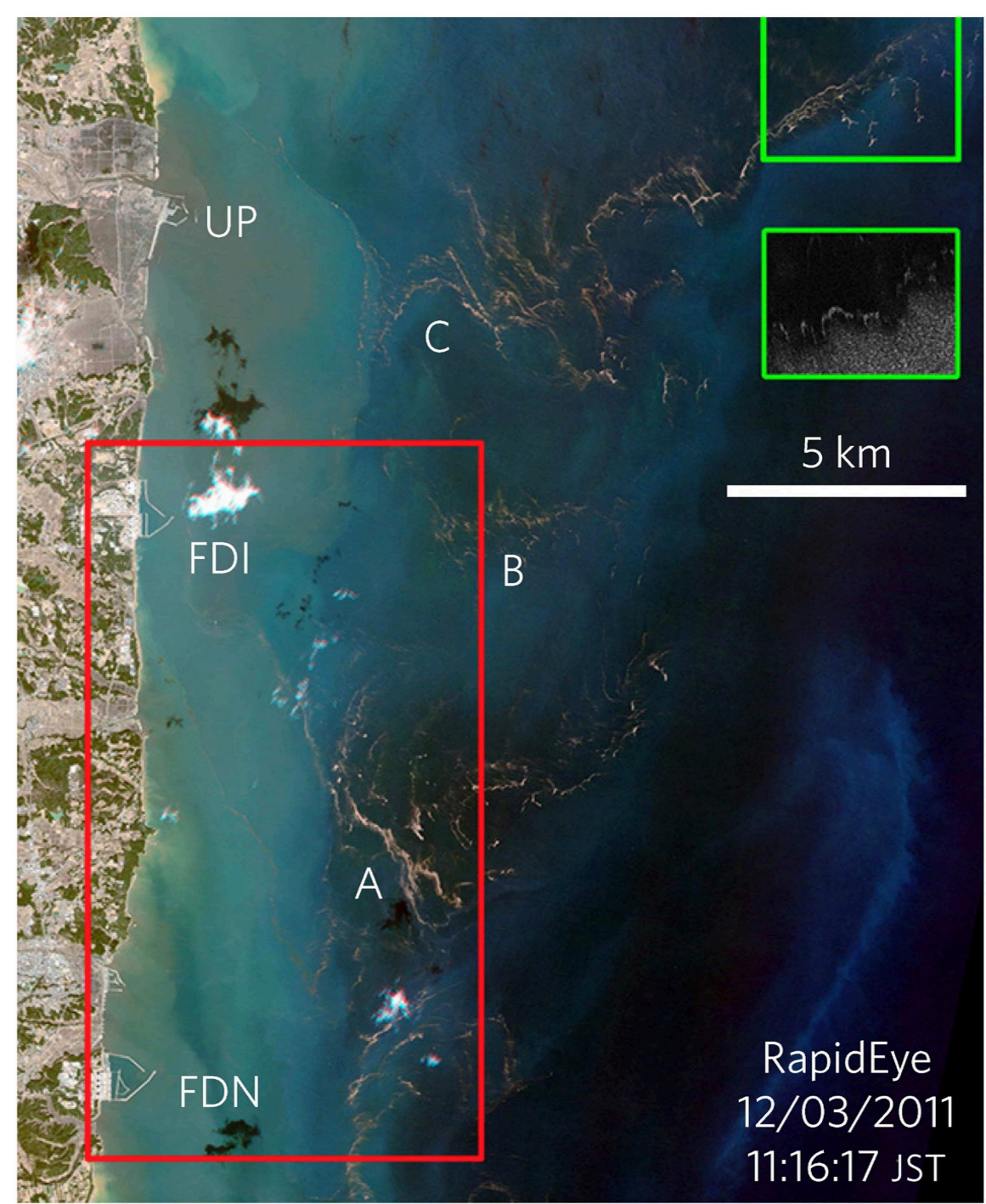

FIGURE 7 | RapidEye image of debris, floating east of Japan 1 day after the March 11, 2011 tsunami showing Ukedo Port (Port), Fukushima Daiichi (FDI) nuclear power station and Fukushima Daini (FDN), floating litter A-C visible on 12 March 2011 [Adopted from (Matthews et al., 2017)] (Maximenko has permission from Nature Geosciences to use this figure).

\section{Radar Sensors}

Radars are active sensors that transmit an electromagnetic pulse and measure the signal as scattered by the scene, in this case the water surface. Radar sensors are broadly used to measure such essential ocean variables as sea surface topography (and thereby geostrophic currents), wind-speed and direction, waves as well as whitecaps and sea ice coverage and for oceanic surveillance.

With respect to marine debris, one of most promising radar technologies is synthetic aperture radar (SAR), whereby the forward movement of the platform (aircraft or satellite) is exploited to synthesize a large aperture and thereby greatly increase the spatial resolution, possibly even to sub-meter resolution depending on sensor design and scene properties. Figure 9 shows an example of SAR image exhibiting many oceanic features. These data were collected from the NASA
JPL AirSAR system and employed a technique called alongtrack interferometry to enable measurement of the ocean radial surface velocity (indicated by the color) in addition to traditional backscatter contrast (indicated by brightness). Employing various techniques and capabilities one can exploit interferometry, frequency and polarization diversity to measure not only the signal reflected from marine debris but also its profile above the sea surface, the wake generated by its interaction with ambient surface waves, and velocity of its drift relative to the surrounding area. Combined with remotely sensed currents and wind, SAR has the potential to provide a comprehensive description of the dynamics of the floating object.

Other radars presently used in satellite missions, such as coarse-resolution altimeters and scatterometers, provide 


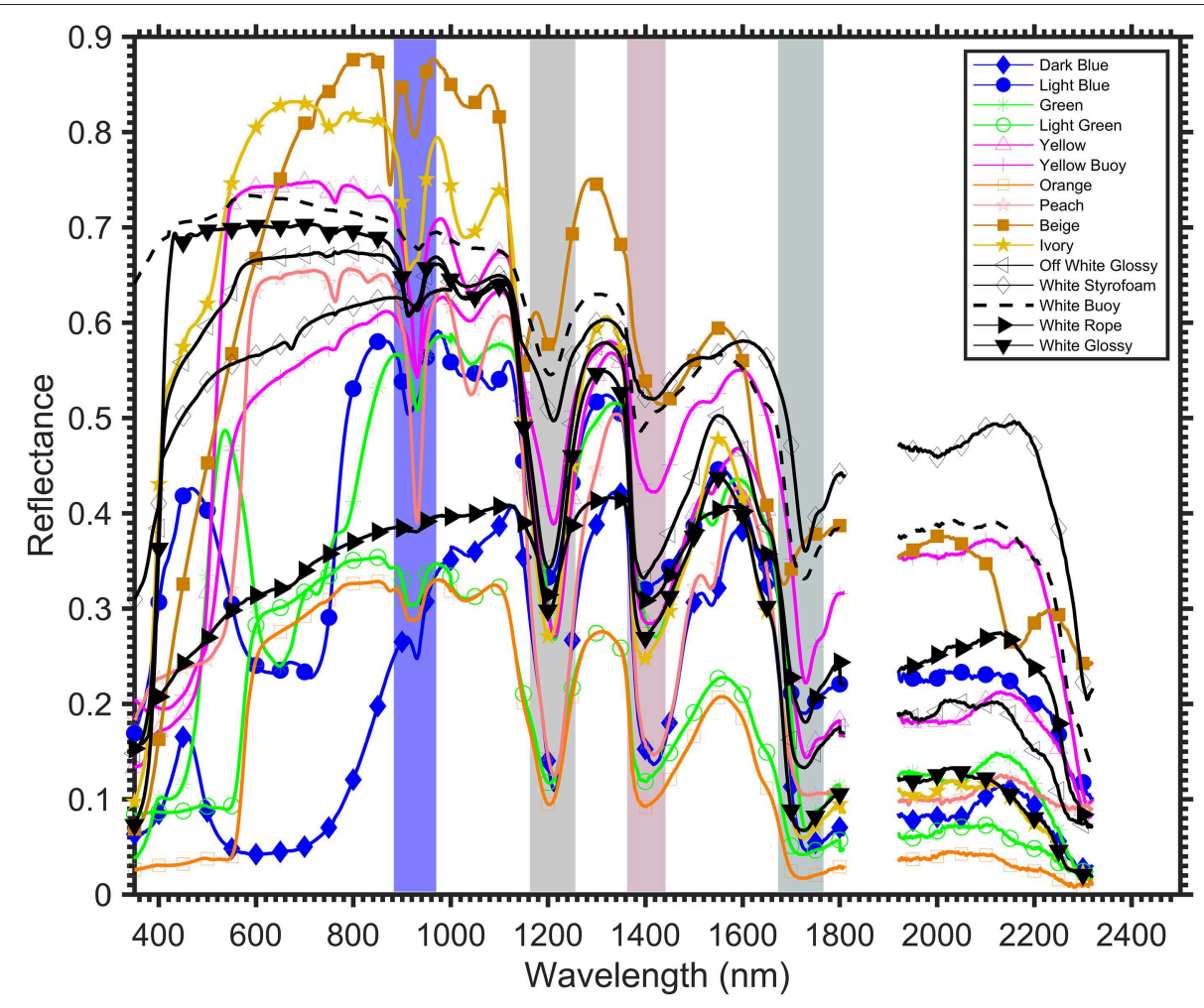

FIGURE 8 | Reflectance of washed ashore macroplastics from beaches along the west coast of the USA reveals spectral absorption features at 931, 1,215, 1,417, and $1,732 \mathrm{~nm}$ (shaded) that are fairly consistent across the variety of plastic objects (adopted from Garaba and Dierssen, 2018) (Garaba holds copyright on this figure).

data that are used to derive mesoscale surface currents ${ }^{29}, 30$ (Maximenko and Hafner, 2010) that play an important role in the transport of marine debris, other pollution (such as oil spills), as well as biological rafting and climate systems. At the same time, model studies (McWilliams, 2016), supported by high-resolution images of the sea surface temperature and oil spills suggest that the strongest convergences on the ocean surface occur on the submesoscale $(1-10 \mathrm{~km})$. These local convergences, whose signatures are seen in Figure 9, play a very important role in the dynamics of floating marine debris. Such missions as SKIM $^{31}$ (Sea Surface KInematics Multiscale monitoring satellite mission; Ardhuin et al., 2018, 2019), SWOT $^{32}$ (Surface Water and Ocean Topography), SEASTAR ${ }^{33}$, and $\mathrm{WACM}^{34}$ (Wind And Currents Mission) are expected to give insight into submesoscale dynamics. SAR observations, embedded in these missions, or used as complementing projects, will significantly enrich our knowledge of marine debris sources, sinks, patterns, and pathways. Passive microwave radiometers,

\footnotetext{
${ }^{29}$ https://www.esr.org/research/oscar/oscar-surface-currents/

${ }^{30} \mathrm{http}: / /$ marine.copernicus.eu/services- portfolio/access- to-products/

${ }^{31}$ https://www.umr-lops.fr/en/Projects/Active-projects/SKIM

${ }^{32}$ https://swot.jpl.nasa.gov/

${ }^{33}$ https://www.researchgate.net/publication/323629810_SEASTAR_A_mission_ to_study_ocean_submesoscale_dynamics_and_small-scale_atmosphere-ocean_ processes_in_coastal_shelf_and_polar_seas

${ }^{34}$ https://mdc.coaps.fsu.edu/scatterometry/meeting/docs/2016/Tue_PM/ B_winds_update.pdf
}

broadly used in satellite missions, may also be helpful in tracking marine debris, however, their capability is yet to be demonstrated.

As a part of IMDOS, remote sensing will require adequate calibration and validation, based on an observational in situ component, described in section Direct Observations of Marine Debris. Analysis of the influence of subsurface ocean processes (such as the vertical shear of currents) on debris drift will also require close interaction with other components of the Global Ocean Observing System (see Centurioni et al., 2019).

While satellites are important to cover the largest scales, the same or similar remote sensing technologies will be used from suborbital platforms [High Altitude Pseudo Satellites (HAPS), aircraft, drones] ships and as portable devices to provide focus on important scales and priority regions as required by the users.

\section{Using Existing and Planned Satellite Missions to Measure Marine Debris}

While remote sensing is needed to provide a global view of the marine litter concentration in the oceans, dedicated satellite missions are expensive, no single sensor responds to all needs of IMDOS, and none of the currently orbiting instruments were specifically designed to detect plastic marine litter. Neither are "marine debris" satellites in the short-term plans of leading space agencies (e.g., recommendation on the Decadal Strategy for Earth Observation from Space from the US National Academies, 


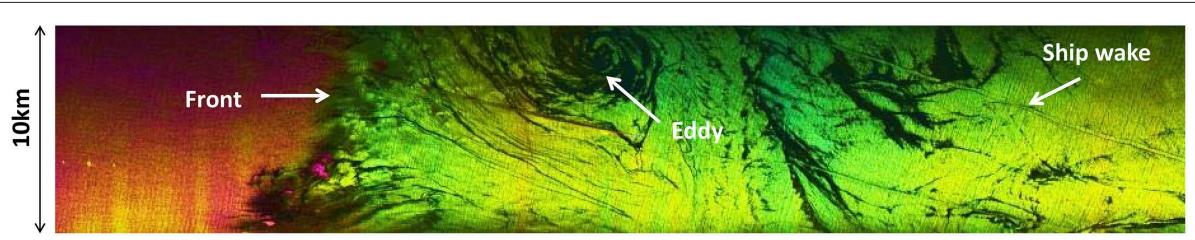

FIGURE 9 | High-resolution sea surface map off Santa Barbara, California, derived from suborbital C-band SAR (Moller, 2016$)^{35}$.

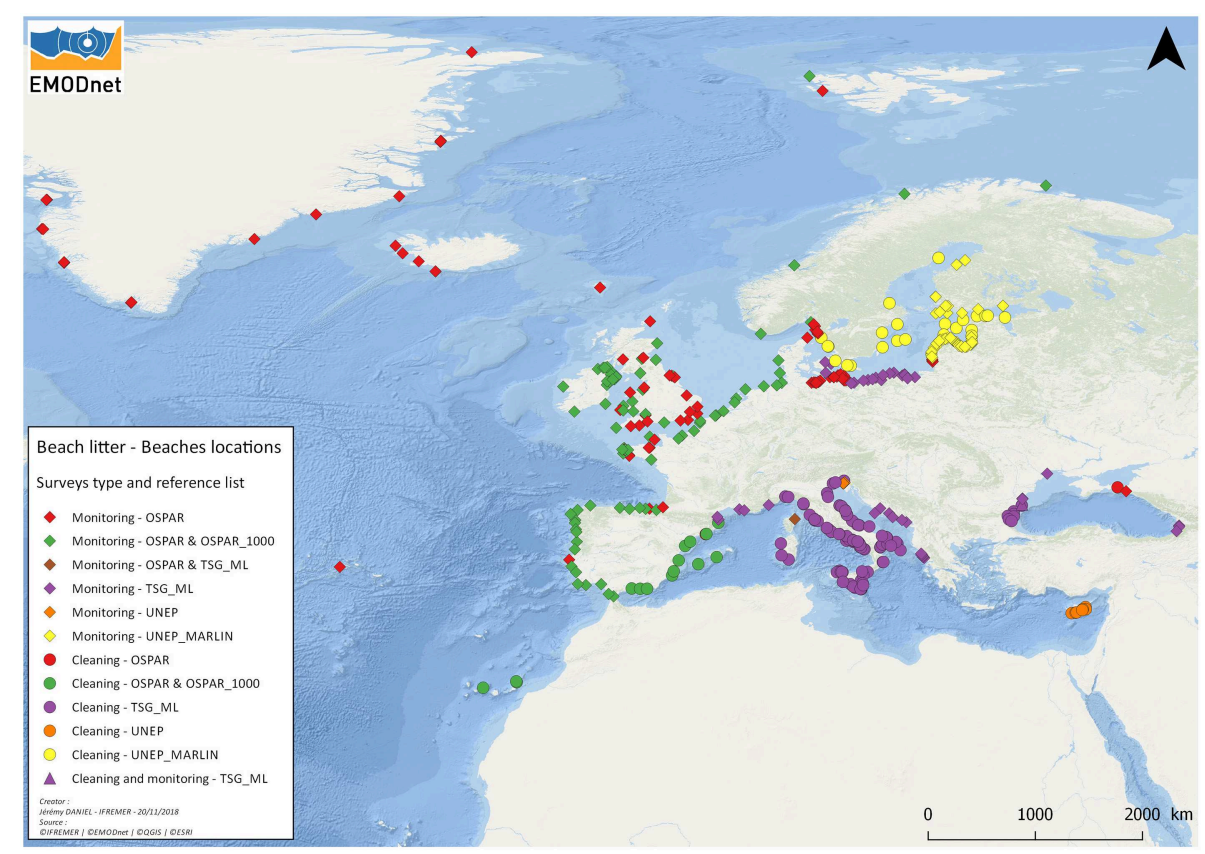

FIGURE 10 | Spatial distribution of surveyed beaches, and reference lists used for litter items in EMODnet and cleaning/monitoring sites (The EMODnet Chemistry project, whose Pls are among the authors of this paper, holds the copyright on this figure).

ESAS2017 $7^{36}$ ). At the same time, the scope and capabilities of some flying or coming soon missions do overlap with the properties and dynamics of marine debris ${ }^{37}$. With the significant increase of the number of satellite launches foreseen, including commercial launches (Euroconsult, 2017), this provides new opportunities for remote sensing of marine debris. For example, the ESAS2017 list of designated (top priority) targeted variables includes Surface Biology and Geology, studied with hyperspectral imagery in the visible and SWIR, multi- or hyperspectral imagery in the thermal IR. As described in previous sections, the same sensors can be used for monitoring marine debris. Similarly, other missions will provide data on submesoscale currents that will advance models, simulating marine debris drift.

\footnotetext{
${ }^{35} \mathrm{http}: / /$ iprc.soest.hawaii.edu/NASA_WS_MD2016/agenda.php

${ }^{36} \mathrm{https} / / / \mathrm{www}$.nap.edu/catalog/24938/thriving- on- our-changing-planet-adecadal-strategy-for-earth

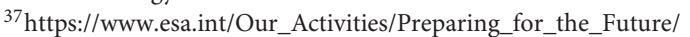

Discovery_and_Preparation/Cleaning_up_our_oceans;

https://www.esa.int/Our_Activities/Space_Engineering_Technology/

Seeking_innovative_ideas_space_for_the_oceans
}

The PRISMA satellite, carrying a hyperspectral instrument operating in the $400-2500 \mathrm{~nm}$ range, with spectral and spatial resolution of $12 \mathrm{~nm}$ and $30 \mathrm{~m}$, respectively, has been launched in March 2019 by Italian Space Agency (ASI). The Plankton, Aerosol, Cloud, ocean Ecosystem mission (PACE, NASA), expected to be launched late 2022, will include a hyperspectral Ocean Color Instrument (OCI), and Environmental Mapping and Analysis Program (EnMAP, DLR) will carry a hyperspectral "pushbroom" imager with high spectral $(6.5-10 \mathrm{~nm})$ and spatial $(30 \mathrm{~m})$ resolutions. More examples of future missions with varying spectral and spatial resolution capabilities relevant to marine debris remote sensing are summarized below and in Table 2. To identify characteristics (spectral bands, spectral resolution, signal-to-noise ratio, minimum and maximum ground sampling distance, etc.) of orbiting optical sensors optimal for detection of plastic marine debris, the European Space Agency (ESA) sponsored two parallel ongoing projects (OPTIMAL and RESMALI) focusing on passive optical spectroradiometric remote sensing.

Expansion of the satellite mission tasks to include marine debris will require collaborative work of marine debris scientists, 


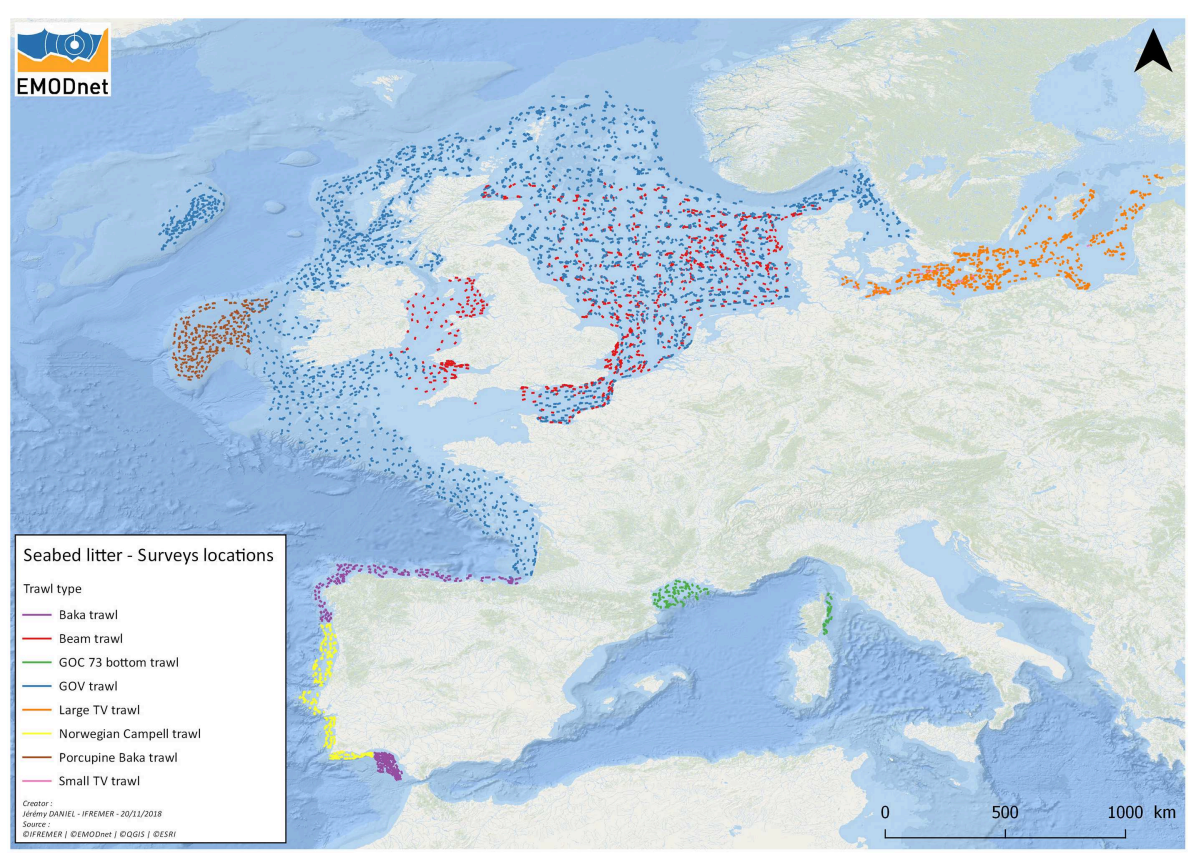

FIGURE 11 | Spatial distribution of seafloor litter survey transects and gear types used for sampling (the EMODnet Chemistry project, whose Pls are among the authors of this paper, holds the copyright on this figure).

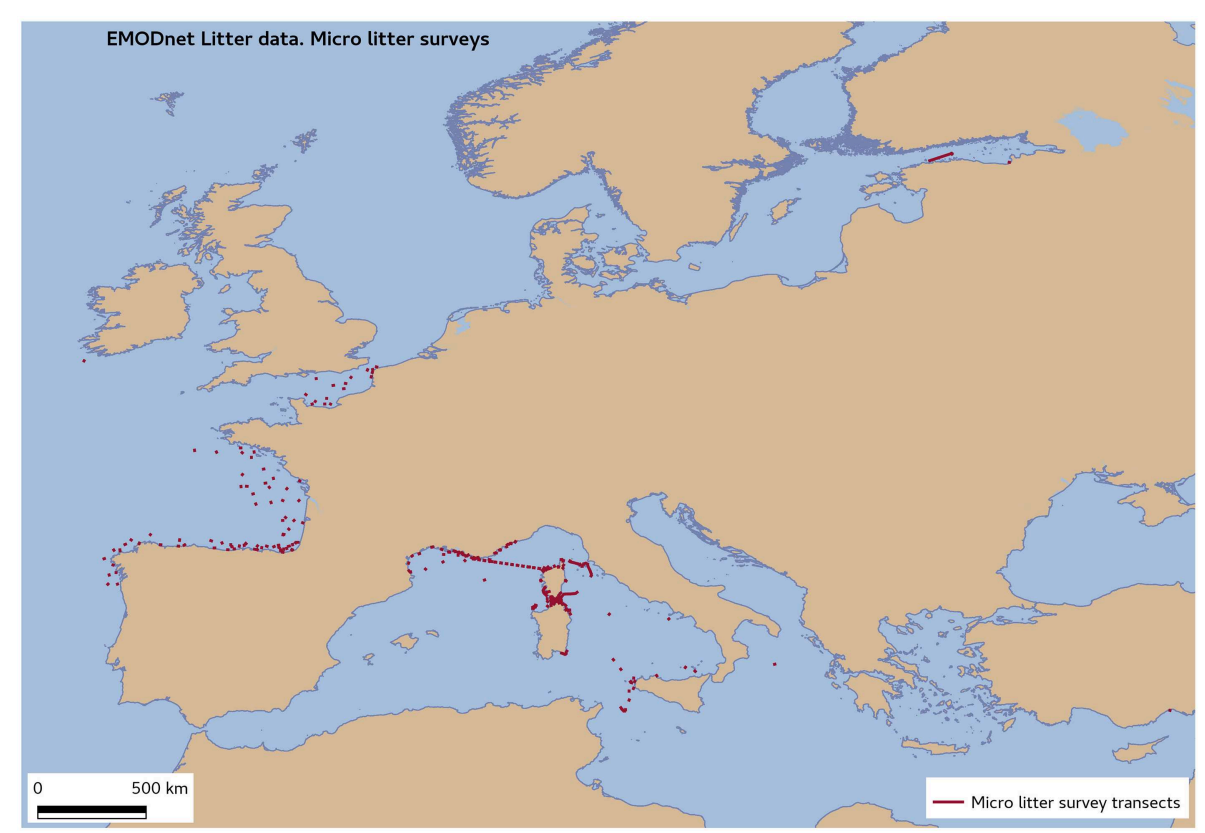

FIGURE 12 | Spatial distribution of micro-litter survey transects in EMODnet (The EMODnet Chemistry project, whose Pls are among the authors of this paper, holds the copyright on this figure).

data users, and mission teams to demonstrate the presence of significant debris signal in the sensor data and develop methodologies for its extraction and calibration-validation efforts. In some cases, this may require modification of standard data processing procedures or adjustment of the sensor parameters (e.g., Garaba and Dierssen, 2018). It is critical that researchers, focusing on the dynamics and impacts of marine debris, penetrate mission science teams and gain influence on the team decisions. Backed by the growing interest of society in the marine debris problem, the activity of these scientists can accelerate development of new sensors or modification of sensors on some planned missions and inclusion of marine 


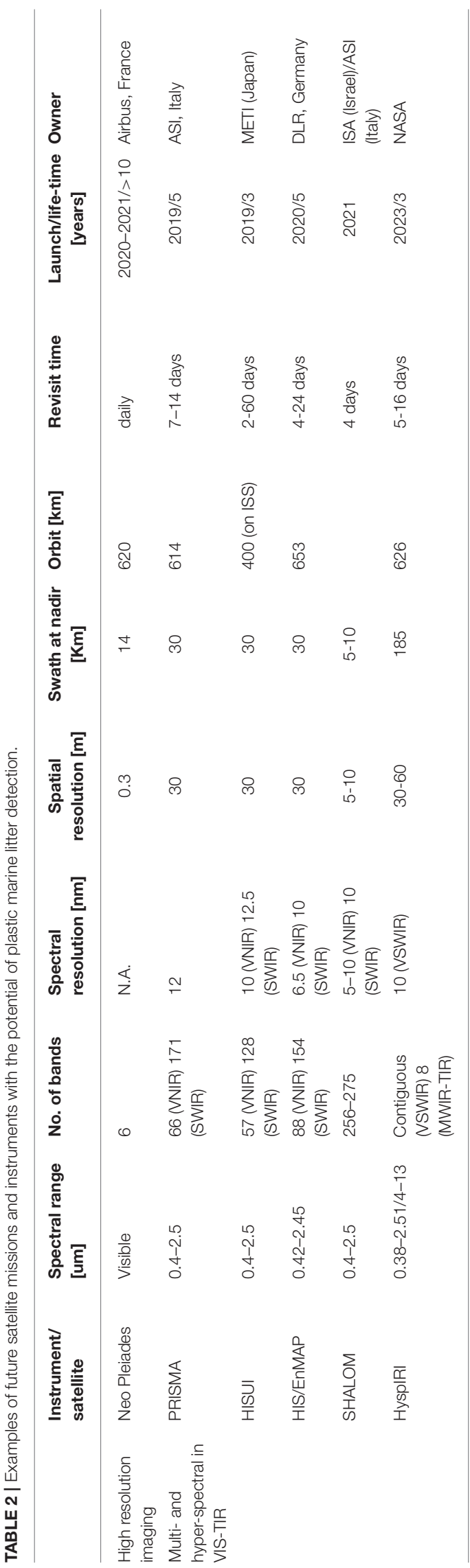

debris applications in the mission goals. Furthermore, small satellites (such as Cubesat) ${ }^{38}$ offer an affordable solution for deployment of experimental sensors or their constellations but require development of compact space systems. Monitoring marine pollution is addressed as a potential application in several small-satellite initiatives proposed recently.

\section{NETWORKING}

Consolidation of distributed platforms and their groups into an integrated system requires active networking. Presently, there are several platforms that monitor marine litter on a large scale by assembling local observations (GESAMP, 2019). These platforms include national (run by such governmental agencies as NOAA, CSIRO, SOA, etc.) and regional (such as OSPAR ${ }^{39}$ in the Northeast Atlantic or UNEP/MED ${ }^{40}$ in the Mediterranean Sea) programs as well as such coordinated efforts as MSFD (European Parliament and Council of the European Union, 2008; European Commission, 2017). Some of the contributing observations are collected on a regular basis while some other are opportunistic or acquired in the course of short-time projects, experiments, or initiatives. Several data-collecting systems, often managed by non-governmental organizations (NGOs), rely on coordinated crowdsourcing (e.g., The Trawlshare Program ${ }^{41}$, The International Coastal Cleanup ${ }^{42}$ ).

With the fast-growing number of local initiatives and datasets, their assembling into larger products and databases for joint analyses requires unified definitions, standards and formats, complemented by well-developed infrastructure for data flow and storage. For example, division on such categories as mega-, meso-, macro-, micro-, and nano-plastics should be based on clear size ranges (e.g., Frias and Nash, 2019; GESAMP, 2019), and accepted by all contributors and users of the observing system. While precise boundaries of the observed range of marine debris are hard to enforce with respect to observational tools and methods that are currently used, ambiguity of the analysis should be avoided by carefully following unified methodologies. This also includes harmonization of indicators and sampling strategies, as recently proposed by GESAMP (2019).

A good example of successful regional partnership is the European Marine Observation and Data Network (EMODnet). In Europe, the Marine Strategy Framework Directive (MSFD) establishes principles and rules for a monitoring network of environmental data and marine litter (Galgani et al., 2013). This monitoring is based on the measurements of stranded litter, litter at sea, microplastics, and litter ingested by animals (like birds or sea turtles). Also, in some cases, entanglement of marine organisms is rated on a regular basis. The data acquired by EU Member States are used for harmonized assessments and aims at maintaining or progressing toward Good

\footnotetext{
${ }^{38}$ http://www.cubesat.org/

${ }^{39}$ https://www.ospar.org/

${ }^{40}$ http://web.unep.org/unepmap/

${ }^{41}$ https://www.5gyres.org/trawlshare-application

${ }^{42}$ https://oceanconservancy.org/trash-free-seas/international-coastal-cleanup/ annual-data-release/
} 
Environmental Status. The MSFD Technical Group on Marine Litter $^{43}$ is working toward consolidated approaches in order to derive compatible data (see also GESAMP, 2019). EMODnet was launched by the European Commission to promote and support the large-scale collection and harmonization of environmental data in European Seas, including (as defined by the UNEP) the North Atlantic Ocean (OSPAR) and Baltic $(\mathrm{HELCOM})^{44}$, Mediterranean (UNEP MAP), and Black (Black Sea Commission $)^{45}$ seas that are currently at different degrees of maturity. The aim is to provide reliable information to set targets and baselines for policy decisions (Addamo et al., 2018).

The EMODnet Chemistry ${ }^{46}$ represents one of the seven thematic data portals, each being related to a different area of expertise. It was created in 2009 by a wide consortium of European research institutes and environmental agencies involved in the collection, standardization, aggregation, and sharing of data related to eutrophication (oxygen, chlorophyll, phosphate, nitrogen and silicate compounds), and contaminants (hydrocarbons, metals, pesticides, radionuclides). The scope has been recently extended to marine debris with specific foci on beach litter, seafloor litter (collected by fish trawl surveys) and micro-litter (microplastics).

The overall structure of the management of litter information in Europe is heterogeneous. There are different levels of development and harmonization of formats and protocols for different compartments (beach and seafloor), particle size (macro and micro-litter) and among geographic scales (regional, national, etc.). The EMODnet Chemistry plans to deal with this heterogeneity by adopting consolidated data formats (when available) and adapting them as needed (Galgani et al., 2017, 2018). Specific approaches have been proposed, based on the best available reference documents, to address the task at European scale.

The management of beach litter is based on OSPAR, with the possibility to report data using OSPAR, MSFD, UNEP/MAP, or UNEP-Marlin protocols. The EMODnet Chemistry beach litter format is designed as an Excel spreadsheet with four worksheets that handle separately individual topics: beach metadata, survey metadata, animals and litter data.

The first pan-European beach litter database has been populated with all relevant datasets available in the European seas. It has been developed in synergy with the European Commission Joint Research Center (JRC), OSPAR, HELCOM, and the MSFD Technical Group on Marine Litter. Presently, 518 beaches and 4,772 surveys from 29 countries have been imported in this database (Addamo et al., 2018). Figures 1012 show distribution of data of beach litter, seafloor litter and micro-litter, respectively.

For the bottom trawls litter, the database used the experience of ICES DATRAS ${ }^{47}$ but added reports from some of the

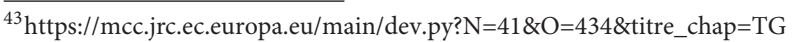
$\% 2520$ Marine\%2520Litter

${ }^{44}$ http://www.helcom.fi/

${ }^{45} \mathrm{http}: / /$ www.blacksea-commission.org/

${ }^{46}$ http://www.emodnet-chemistry.eu/welcome

${ }^{47}$ http://www.ices.dk/marine-data/data-portals/Pages/DATRAS.aspx
}

International Bottom Trawl Surveys in the Mediterranean (MEDITS $)^{48}$ as well as general data submitted according to the Marine Strategy Framework Directive $\left(\mathrm{MSFD}^{49}\right.$.)

For floating marine microplastics, the SeaDataNet ${ }^{50}$ metadata and data formats have been adopted after some adaptation to address the diversity of information from other European sources. With the development of autonomous instruments and sensors, the observing network system will also benefit from the use of platforms of opportunity, such as ships, airplanes, and coastal structures, from which they can be operated either remotely or with minimum intervention of operators.

Data of beach, floating micro and (soon) also seafloor litter can be accessed through the dedicated discovery and access service in the EMODnet Chemistry portal ${ }^{51}$.

Depending on the interests and agenda of the international community, the EMODnet could serve as a template for networks developing in other regions and/or expand beyond the European Seas. In any case, priorities of marine litter indicators and associated environmental variables may differ among regions and space scales and the work successfully performed in Europe needs to be redone elsewhere, with economical, political, ecological, and cultural differences accounted for.

The global governance scheme for marine debris data acquisition, streaming, quality control, and distribution to users is still to be designed and built. This infrastructure is critical for IMDOS and it will develop in collaboration with UN Environment, assuming the coordinating role in international harmonization, and all other stakeholders, described in section IMDOS Stakeholders.

\section{BUILDING IMDOS}

The implementation of IMDOS is difficult to plan. The marine debris community is still under construction, observations are sparse, many impacts of marine debris are poorly documented or unstudied, numerical models do not capture all relevant processes, different groups follow different methodologies, international regulations are vague, national plans are incomplete and often missing, and overall funding to develop the observing system is not secured.

This is exactly why we believe that this paper, presenting the concept of the "ideal" observing system and identifying gaps and problems as current and future opportunities, is very timely. The concept of IMDOS aligns efforts of very different entities (from international to individuals) in a coherent way, maximizing the efficacy of use of available resources. This includes geographical expansion of the experience of leading countries and groups, exemplified in previous sections, and interaction with other observing systems. For example, just as neuston nets are used to sample microplastics and plankton, some other instruments can collect both debris and non-debris data simultaneously, so that adding debris

\footnotetext{
${ }^{48} \mathrm{http} / / /$ www.sibm.it/SITO\%20MEDITS/principaleprogramme.htm

${ }^{49}$ https://eur-lex.europa.eu/legal-content/EN/TXT/?uri=CELEX:32008L0056

${ }^{50}$ https://www.seadatanet.org/

${ }^{51} \mathrm{http}: / /$ emodnet-chemistry.maris2.nl/v_cdi_v3/search.asp
} 
measurements to existing protocols will be beneficial to both observing systems.

With the growing international concern and momentum toward resolution of the problem of artificial pollutions, the present states of local, regional and national marine debris programs are very heterogeneous. Generally, developed countries have resources and information systems to better address the problem but this leaves large geographical areas unattended, preventing a global action. In the next decade we expect increased activity on all scales that will lead to development of new national initiatives, unification and/or harmonization of approaches, standards, instruments and formats and consolidation of individual components into an integrated observing system. Exchange of the data, their processing, archiving and serving to the users will require construction of efficient global informational infrastructure. This is a tremendously difficult task but examples of such European programs as EMODnet and Copernicus Marine Environment Monitoring Service (MEMS ${ }^{52}$ ) are inspiring.

On a global scale, GOOS exemplifies the evolution of ocean science and technology in recent decades from exploration and understanding the basic dynamics to monitoring and practical applications. The governance structure of GOOS as a "collaborative system of ocean observations, encompassing in situ networks, satellite systems, governments, UN agencies and individual scientists," combining sustainability with evolution, can be adopted when constructing IMDOS. The problem of marine debris is interdisciplinary by its nature and close collaboration between IMDOS and GOOS and other global observing systems as well as the diversity of actors and institutions, monitoring the environment and society, is imperative for its effective control and successful resolution.

${ }^{52} \mathrm{http}: / /$ marine.copernicus.eu/

\section{AUTHOR CONTRIBUTIONS}

This review paper is produced by the members of five working groups that proposed Community White Papers relevant to the dynamics and impacts of marine debris and merged on advice of the OceanObs19 organizers. NM developed the structure of the paper and coordinated work on individual sections. KL, RT, and NM drafted section Introduction. NM and KL drafted section Impacts and CM, CL, AA, MRL, NP, MV, GH, ME, JC, and A-ER provided critical revision of this section. EV and NM drafted section Structure of IMDOS and JV, CM, and MA provided critical revision. Section Direct Measurements was drafted by RSL, KP, DM, MEM, AC, RT, and TG and critically revised by KL, CL, LC, AM, RL, CP, AI, GH, ME, IC, SA, JC, GR, LH, A-MC, TG, GG, BH, LL, HL, RM, EM, RO, BR, MT, AT, and

\section{REFERENCES}

Acuña-Ruz, T., Uribe, D.,Taylor, R., Amézquita, L., Guzmán, M. C., Merrill, J., et al. (2018). Anthropogenic marine debris over beaches: spectral characterization for remote sensing applications. Remote Sens. Environ. 217, 309-322. doi: $10.1016 /$ j.rse.2018.08.008
CW. PC, VM-V, SG, and NM drafted section Remote Sensing and LG-M, DM, ER, GA, YC, YG, and TM provided critical revision of the section. MV, FG, AG, MJ, NH, AB, AA, GH, MS, and MLM drafted section Networking and PC, RSL, MRL, IM-S, MM, and AT provided critical revision. Section Interactions was drafted by PC, NM, and SG, and critically revised by $\mathrm{BH}, \mathrm{EV}$, and CW. Every part of the paper went through the full-team discussion. All authors approve the version to be published and agree to be accountable for all aspects of the work in ensuring that questions related to the accuracy or integrity of any part of the work are appropriately investigated and resolved.

\section{FUNDING}

NM, LC, GR, LH, and JC were partly funded by NASA Interdisciplinary Science through Grant 80NSSC17K0559 (FloatEco project). The SCOR WG 153 partners were partly supported by the national committees of the Scientific Committee on Oceanic Research (SCOR) and Grant OCE-1546580 to SCOR from the U.S. National Science Foundation. EMODnet Chemistry was supported by DG MARE under the Service Contract number EASME/EMFF/2016/1.3.1.2-Lot4/SI2.749773. SG was supported by Deutsche Forschungsgemeinschaft (DFG, German Research Foundation)-Projektnummer 417276871. CP was funded through an FCT fellowship (SFRH/BD/111757/2015). AM was supported through the EAPA_165/2016: iFADO: Innovation in the Framework of the Atlantic Deep Ocean, financed by the INTERREG | Atlantic Area. EV was supported through funding from the European Research Council (ERC) under the European Union's Horizon 2020 research and innovation programme (Grant Agreement No. 715386). IC was supported by Russian Foundation for Basic Research, grant number 18-55-76001. $\mathrm{VM}-\mathrm{V}$ was supported through funding from the European Space Agency (ESA) (project No. 4000120879/17/NL/PS). NP was supported by the VILLUM FONDEN (grant number: 15397). RL was supported by NOAA/AOML and NOAA's Ocean Observation and Monitoring Division. AT was supported by the Chili National Council for Scientific and Technological Development (CNPq) through research grant 309697/2015-8. AI was supported by the Environmental Research and Technology Development Fund (SII-2) of the Japan Ministry of the Environment. TG was partly supported through UK NERC grant NE/S003975/1.

\section{ACKNOWLEDGMENTS}

The authors would like to thank the editor, reviewers, and Frontiers Production staff, whose excellent help allowed to significantly improve the paper. et al. (2018). Marine Litter Database: Lessons Learned in Compiling the First PanEuropean Beach Litter Database, EUR 29469 EN. Luxembourg: Publications Office of the European Union.

Anderson, Z. T., Cundy, A. B., Croudace, I. W., Warwick, P. E., Celis-Hernandez, O., and Stead, J. L. (2018). A rapid method for assessing the accumulation of 
microplastics in the sea surface microlayer (SML) of estuarine systems. Sci. Reports 8:9428. doi: 10.1038/s41598-018-27612-W

Ardhuin, F., Aksenov, Y., Benetazzo, A., Bertino, L., Brandt, P., Caubet, E., et al. (2018). Measuring currents, ice drift, and waves from space: the sea surface kinematics multiscale monitoring (SKIM) concept. Ocean Sci. 14, 337-354. doi: 10.5194/os-14-337-2018

Ardhuin, F.,Brandt, P., Gaultier, L., Donlon, C., Battaglia, A., Boy, F., et al. (2019). SKIM, a candidate satellite mission exploring global ocean currents and waves. Front. Mar. Sci. 6:209. doi: 10.3389/fmars.201 9.00209

Barat, D., Spencer, D., Benazzi, G., Mowlem, M. C., and Morgan, H. (2012). Simultaneous high speed optical and impedance analysis of single particles with a microfluidic cytometer. Lab Chip 12, 118-126. doi: 10.1039/C1LC2 0785G

Barnett, P. R. O., Watson, J., and Connelly, D. (1984). The multiple corer for taking virtually undisturbed samples from shelf, bathyal and abyssal sediments. Oceanol. Acta. 7, 399-408.

Benazzi, G., Holmes, D., Sun, T., Mowlem, M. C., and Morgan, H. (2007). Discrimination and analysis of phytoplankton using a microfluidic cytometer. Nanobiotechnology 1, 94-101. doi: 10.1049/iet-nbt:20070020

Bergmann, M., and Klages, M. (2012). Increase of litter at the Arctic deep-sea observatory Hausgarten. Mar. Poll. Bull. 64, 2734-2741. doi: 10.1016/j.marpolbul.2012.09.018

Brooks, M. T., Coles, V. J., and Coles, W. C. (2019). Inertia influences pelagic sargassum advection and distribution. Geophys. Res. Lett. 46, 2610-2618. doi: 10.1029/2018GL081489

Buchanan, J. B. (1971). Pollution by synthetic fibres. Marine Pollution Bulletin. 2:23. doi: 10.1016/0025-326X(71)90136-6

Carlton, J. T., Chapman, J. W., Geller, J. B., Miller, J. A., Carlton, D. A., McCuller, M. I., et al. (2017). Tsunami-driven rafting, transoceanic species dispersal and implications for marine biogeography. Science 357, 1402-1406. doi: 10.1126/science.aao 1498

Carpenter, E. J., and Smith, K. L. Jr. (1972). Plastics on the Sargasso Sea surface. Science 175, 1240-1241. doi: 10.1126/science.175.4027.1240

Centurioni, L., Chen, Z., Lumpkin, R., Braasch, L., Brassington, G., Chao, Y., et al. (2019). Multidisciplinary global in-situ observations of essential climate and ocean variables at the air-sea interface in support of climate variability and change studies and to improve weather forecasting, pollution, hazard and maritime safety assessments. Front. Mar. Sci.

Centurioni, L. R. (2018). "Drifter technology and impacts for sea surface temperature, sea-level pressure, and ocean circulation studies," in Observing the Oceans in Real Time, eds. R. Venkatesan, A. Tandon, E. D'Asaro, and M. A. Atmanand. 37-57. doi: 10.1007/978-3-319-66493-4_3

Chubarenko, I. P., Esiukova, E. E., Bagaev, A. V., Bagaeva, M. A., and Grave, A. N. (2018). Three-dimensional distribution of anthropogenic microparticles in the body of sandy beaches. Sci. Total Environ. 628-629, 1340-1351. doi: 10.1016/j.scitotenv.2018.02.167

Cózar, A., Echevarría, F., González-Gordillo, J. I., Irigoien, X., Úbeda, B., Hernández-León, S., et al. (2014). Plastic debris in the open ocean. Proc. Natl. Acad. Sci. U.S.A. 111, 10238-10244. doi: 10.1073/pnas.1314705111

Cózar, A., Martí, E., Duarte, J. C. M., García-de-Lomas, E. J., van Sebille, E., Ballatore, T. J., et al. (2017). The Arctic Ocean as a dead end for floating plastics in the North Atlantic branch of the thermohaline circulation. Sci. Adv. 3:e1600582. doi: 10.1126/sciadv.1600582

Cundell, A. M. (1973). Plastic materials accumulating in Narragansett Bay. Mar. Poll. Bull. 4, 187-188. doi: 10.1016/0025-326X(73)90226-9

Dubelaar, G. B. J., and Geerders, P. J. F. (2004). Innovative technologies to monitor plankton dynamics - Scanning flow cytometry, a new dimension in real-time, in-situ water quality monitoring. Sea Technol. 45, 15-21.

Dubelaar, G. B. J., and Jonker, R. R. (2000). Flow cytometry as a tool for the study of phytoplankton. Sci. Mar. 64, 135-156. doi: 10.3989/scimar.2000.64n2135

Dupont, J., Magnin, S., Rousseau, F., Zbinden, M., Frebourg, G., Samadi, S., et al. (2009). Molecular and ultrastructural characterization of two ascomycetes found on sunken wood off Vanuatu Islands in the deep Pacific Ocean. Mycol. Res. 113, 1351-1364. doi: 10.1016/j.mycres.2009.08.015

Edson, E. C., and Patterson, M. R. (2015). "MantaRay: a novel autonomous sampling instrument for in situ measurements of environmental microplastic particle concentrations," in Oceans 2015 Conference (Washington, DC).
Eriksen, M., Lebreton, L., Carson, H., Thiel, M., Moore, C., Borerro, J., et al. (2014). Plastic pollution in the World's oceans: more than 5 trillion plastic pieces weighing over 250,000 Tons Afloat at sea. PLoS ONE 9:e111913. doi: 10.1371/journal.pone.0111913

Eriksen, M., Liboiron, M., Kiessling, T., Charron, L., Alling, A., Lebreton, L., et al. (2018). Microplastic sampling with the AVANI trawl compared to two neuston trawls in the Bay of Bengal and South Pacific. Environ. Poll. 232, 430-439. doi: 10.1016/j.envpol.2017.09.058

Erni-Cassola, G., Gibson, M. I.,Thompson, R. C., and Christie-Oleza, J. A. (2017). Lost, but found with nile red: a novel method for detecting and quantifying small microplastics ( $1 \mathrm{~mm}$ to $20 \mathrm{mu} \mathrm{m}$ ) in environmental samples. Environ. Sci. Technol. 51, 13641-13648. doi: 10.1021/acs.est.7b04512

Euroconsult (2017). Satellites to be Built and Launched by 2026. Analysis and Report. Available online at: http://www.euroconsult-ec.com/research/ satellites-built-launched-by-2026-brochure.pdf (accessed August 16, 2019).

European Commission (2017). Laying Down Criteria and Methodological Standards on Good Environmental Status of Marine Waters and Specifications and Standardized Methods for Monitoring and Assessment, and Repealing Decision 2010/477/EU COMMISSION DECISION (EU) 2017/848. Journal of the European Union. Available online at: https://eur-lex.europa.eu/legal-content/ EN/TXT/PDF/?uri=CELEX:32017D0848\&from=EN

European Parliament and Council of the European Union (2008). Marine strategy framework directive, directive 2008/56/EC of the European parliament and of the council. J. Eur. Union 164, 19-40.

Fraser, C. I., Morrison, A. K., Hogg, A., Mc, C., Macaya, E. C., Van Sebille, E., et al. (2018). Antarctica's ecological isolation will be broken by storm-driven dispersal and warming. Nat. Clim. Change 8, 704-708. doi: 10.1038/s41558-018-0209-7

Frere, L., Paul-Pont, I., Moreau, J., Soudant, P., Lambert, C., Huvet, A., et al. (2016). A semi-automated Raman micro-spectroscopy method for morphological and chemical characterizations of microplastic litter. Mar. Poll. Bull. 113, 461-468. doi: 10.1016/j.marpolbul.2016.10.051

Frias, J. P. G. L., and Nash, R. (2019). Microplastics: finding a consensus on the definition. Mar. Poll. Bull. 138, 145-147. doi: 10.1016/j.marpolbul.2018.11.022

Galgani, F., Giorgetti, A., Le Moigne, M., Brosich, A., Vinci, M., Lipizer, M., et al. (2018). Guidelines and Forms for Gathering Marine Litter Data. European scale. 58. doi: 10.6092/8ce4e8b7-f42c-4683-9ece-c32559606dbd

Galgani, F., Giorgetti, A., Vinci, M., Le Moigne, M., Moncoiffe, G., Brosich, A., et al. (2017). Proposal for Gathering and Managing Data Sets on Marine Micro-Litter on a European Scale. 35.

Galgani, F., Hanke, G., and Maes, T. (2015). "Global distribution, composition and abundance of marine litter,"in Marine Anthropogenic Litter, eds M. Bergmann, L. Gutow, and M. Klages (Cham: Springer Open; Elsevier), 29-56.

Galgani, F., Hanke, G., Werner, S., and De Vrees, L. (2013). Marine litter within the european marine strategy framework directive. Ices J. Mar. Sci. 70, 1055-1064. doi: $10.1093 /$ icesjms/fst122

Galloway, T. S. (2015). "Micro- and nano-plastics and human health" in Marine Anthropogenic Litter, eds M. Bergmann, L. Gutow, and M. Klages (Cham: Springer Open; Elsevier), 343-366. doi: 10.1007/978-3-319-16510-3_13

Garaba, S. P., Aitken, J., Slat, B., Dierssen, H. M., Lebreton, L., Zielinski, O., et al. (2018). Sensing ocean plastics with an airborne hyperspectral shortwave infrared imager. Environ. Sci. Technol. 52, 11699-11707. doi: 10.1021/acs.est.8b02855

Garaba, S. P., and Dierssen, H. M. (2017). Spectral Reference Library of 11 Types of Virgin Plastic Pellets Common in Marine Plastic Debris. The Ecological Spectral Information System (EcoSIS). Available online at: http://dx.doi.org/10.21232/ C27H34 (accessed July 25, 2019).

Garaba, S. P., and Dierssen, H. M. (2018). An airborne remote sensing case study of synthetic hydrocarbon detection using short wave infrared absorption features identified from marine harvested macro- and microplastics. Rem. Sens. Environ. 205, 224-235. doi: 10.1016/j.rse.2017.11.023

GESAMP (2019). Guidelines or the Monitoring and Assessment of Plastic Litter and Microplastics in the Ocean, eds P. J. Kershaw, A. Turra, and F. Galgani, Rep. Stud. GESAMP No. 99 (IMO/FAO/UNESCOIOC/UNIDO/WMO/IAEA/UN/UNEP/UNDP/ISA Joint Group of Experts on the Scientific Aspects of Marine Environmental Protection).

Geyer, R., Jambeck, J. R., and Law, K. L. (2017). Production, use, and fate of all plastics ever made. Sci. Adv. 3:e1700782. doi: 10.1126/sciadv.1700782 
Goddijn-Murphy, L., Peters, S., Van Sebille, E., James, N. A., and Gibb, S. (2018). Concept for a hyperspectral remote sensing algorithm for floating macro plastics. Mar. Poll. Bull. 126:11. doi: 10.1016/j.marpolbul.2017.11.011

Goddijn-Murphy, L. M., and Dufaur, J. (2018). Proof of concept for a model of light reflectance of plastics floating on natural waters. Mar. Poll. Bull. 135, 1145-1157. doi: 10.1016/j.marpolbul.2018.08.044

González-Fernández, D., and Hanke, G. (2017). Toward a harmonized approach for monitoring of riverine floating macro litter inputs to the marine environment. Front. Mar. Sci. 4:86. doi: 10.3389/fmars.2017.00086

Gower, J., Young, E., and King, S. (2013), Satellite images suggest a new Sargassum source region in 2011. Remote Sens. Lett. 4, 764-773. doi: 10.1080/2150704X.2013.796433

Guo, J. J., Zhang, F., Liu, C. H., Li, Y., and Zheng, R. E. (2017). Raman-fluorescence spectroscopy for underwater in-situ application. Spectrosc. Spectr. Anal. 37, 3099-3102.

Hanke, G., and Gonzalez-Fernandez, D. (2014). "Long term deployment of the JRC Sealittercam on the Western Mediterranean Sea," in Perseus 2nd Scientific Workshop. Marrakesh. Book of Abstracts. PERSEUS Project. Available online at: http://www.perseus-net.eu/assets/media/PDF/GA\%20Marrakesh/3408.pdf

Hardesty, B. D., Harari, J., Isobe, A., Lebreton, L., Maximenko, N., Potemra, J., et al. (2017). Using numerical model simulations to improve the understanding of microplastic distribution and pathways in the marine environment. Front. Mar. Sci. 30, 1-9. doi: 10.3389/fmars.2017.00030

Hinojosa, I. A., and Thiel, M. (2009). Floating marine debris in fjords, gulfs and channels of southern Chile. Mar. Poll. Bull. 58, 341-350. doi: 10.1016/j.marpolbul.2008.10.020

Hong, S., Lee, J., and Lim, S. (2017). Navigational threats by derelict fishing gear to navy ships in the Korean seas. Mar. Poll. Bull. 119, 100-105 doi: 10.1016/j.marpolbul.2017.04.006

Jambeck, J. R., Geyer, R., Wilcox, C., Siegler, T. R., Perryman, M., Andrady, A., et al. (2015). Plastic waste inputs from land into the ocean. Science 347, 768-771. doi: $10.1126 /$ science. 1260352

Jang, Y. C., Hong, S., Lee, J., Lee, M. J., and Shim, W. J. (2014). Estimation of lost tourism revenue in Geoje Island from the 2011 marine debris pollution event in South Korea. Mar. Poll. Bull. 81, 49-54. doi: 10.1016/j.marpolbul.2014.02.021

Japan Ministry of the Environment. (2012). Estimated Total Amount of Debris Washed out by the Great East Japan Earthquake. Available online at: http:// www.env.go.jp/en/focus/docs/files/20120901-57.pdf (accessed August 10).

Jing, X., Gou, H., Gong, Y., Su, X., Xu, L., Ji, Y., et al. (2018). Raman-activated cell sorting and metagenomic sequencing revealing carbon-fixing bacteria in the ocean. Environ. Microbiol. 20, 2241-2255. doi: 10.1111/1462-2920.14268

Kako, S., Isobe, A., Kataoka, T., Yufu, K., Sugizono, S., Plybon, C., et al. (2018). Sequential webcam monitoring and modeling of marine debris abundance. Mar. Poll. Bull. 132, 33-43. doi: 10.1016/j.marpolbul.2018.04.075

Kako, S. I., Isobe, A., and Magome, S. (2010). Sequential monitoring of beach litter using webcams. Mar. Poll. Bull. 60, 775-779. doi: 10.1016/j.marpolbul.2010.03.009

Karlsson, T. M., Grahn, H., van Bavel, B., and Geladi, P. (2016). Hyperspectral imaging and data analysis for detecting and determining plastic contamination in seawater filtrates. J. Infr. Spectros. 24, 141-149. doi: 10.1255/jnirs. 1212

Kataoka, T., Murray, C. C., and Isobe, A. (2017). Quantification of marine macrodebris abundance around Vancouver Island, Canada, based on archived aerial photographs processed by projective transformation. Mar. Poll. Bull. 132, 44-51. doi: 10.1016/j.marpolbul.2017.08.060

Kiessling, T., Gutow, L., and Thiel, M. (2015). "Marine litter as habitat and dispersal vector," in Marine Anthropogenic Litter, eds. M. Bergmann, L. Gutow, and M. Klages (Cham: Springer Open; Elsevier), 141-181. doi: 10.1007/978-3-319-16510-3_6

Kirkwood, W. J., Peltzer, E., and Brewer, P. G. (2013). Development and deployment of a deep-sea Raman instrumentation for in situ analysis. Abstr. Am. Chem. Soc. 245.

Koelmans, A. A., Besseling, E., and Shim, W. J. (2015). "Nanoplastics in the aquatic environment. Critical review," in Marine Anthropogenic Litter, eds. M. Bergmann, L. Gutow, and M. Klages (Cham: Springer Open; Elsevier), 325-340. doi: 10.1007/978-3-319-16510-3_12

Koelmans, A. A., Kooi, M., Law, K. L., and van Sebille, E. (2017). All is not lost, deriving a top-down mass budget of plastic at sea. Environ. Res. Lett. 12:500. doi: $10.1088 / 1748-9326 /$ aa9500
Kokaly, R. F., Clark, R. N., Swayze, G. A., Livo, K. E., Hoefen, T. M., Pearson, N. C., et al. (2017). USGS spectral library version 7, US. Geolog. Surv. Data Ser. 61, 1035. doi: $10.3133 /$ ds 1035

Kooi, M., Nes, E. H. V., Scheffer, M., and Koelmans, A. A. (2017). Ups and downs in the ocean, effects on biofouling on vertical transport of microplastics. Environ. Sci. Technol. 51, 7963-7971. doi: 10.1021/acs.est.6b04702

Lambert, B. S., Olson, R. J., and Sosik, H. M. (2017). A fluorescence-activated cell sorting subsystem for the imaging flowcytobot. Limnol. Oceanogr. Methods 15:145. doi: 10.1002/lom3.10145

Lampitt, R. S. (1985). Evidence for the seasonal deposition of detritus to the deep-sea floor and its subsequent resuspension. Deep Sea Res. 32, 885-897. doi: 10.1016/0198-0149(85)90034-2

Lampitt, R. S., and Burnham, M. P. (1983). A free fall time lapse camera and current meter system "Bathysnap" with notes on the foraging behaviour of a bathyal decapod shrimp. Deep Sea Res. 30. 1009-1017. doi: 10.1016/0198-0149(83)90055-9

Lavers, J. L., and Bond, A. L. (2017). Exceptional and rapid accumulation of anthropogenic debris on one of the world's most remote and pristine islands, Proc. Natl. Acad. Sci. U.S.A. 114, 6052-6055. doi: 10.1073/pnas.1619818114

Lebreton, L., Slat, B., Ferrari, F., Sainte-Rose, B., Aitken, J., Marthouse, R., et al. (2018). Evidence that the Great Pacific Garbage Patch is rapidly accumulating plastic. Sci. Reports. 8:4666. doi: 10.1038/s41598-018-22939-w

Lebreton, L. C.-M., Greer, S. D., and Borrero, J. C. (2012). Numerical modelling of floating debris in the world's oceans. Mar. Poll. Bull. 64, 653-661. doi: 10.1016/j.marpolbul.2011.10.027

Lecke-Mitchell, K. M., and Mullin, K. (1992). Distribution and abundance of large floating plastic in the north-central Gulf of Mexico. Mar. Poll. Bull. 24, 598-601. doi: 10.1016/0025-326X(92)90279-F

Lee, J., Hong, S., Song, Y. K., Hong, S. H., Jang, Y. C., Jang, M.,., et al. (2013). Relationships among the abundances of plastic debris in different size classes on beaches in South Korea. Mar. Poll. Bull. 77, 349-354. doi: 10.1016/j.marpolbul.2013.08.013

Leggett, C., Scherer, N., Curry, M., and Bailey, R. (2014). Assessing the Economic Benefits of Reductions in Marine Debris, A Pilot Study of Beach Recreation in Orange County, California. Final report, June 15, 2014, National Oceanic and Atmospheric Administration. Cambridge, MA. Available online at: http:// marinedebris.noaa.gov/sites/default/files/MarineDebrisEconomicStudy.pdf

Li, L. F., Zhang, X., Luan, Z. D., Du, Z. F., Xi, S. C., Wang, B., et al. (2018). In situ quantitative raman detection of dissolved carbon dioxide and sulfate in deepsea high-temperature hydrothermal vent fluids. Geochem. Geophys. Geosyst. 19:7445. doi: 10.1029/2018GC007445

Long, M., Paul-Pont, I., Hegaret, H., Moriceau, B., Lambert, C., Huvet, A., et al. (2017). Interactions between polystyrene microplastics and marine phytoplankton lead to species-specific hetero-aggregation. Environ. Poll. 2017:228. doi: 10.1016/j.envpol.2017.05.047

Lumpkin, R., Maximenko, N., and Pazos, M. (2012). Evaluating where and why drifters die. J. Atmos. Ocean. Techn. 29, 300-308. doi: 10.1175/JTECH-D-11-00100.1

Maes, T., Jessop, R., Wellner, N., Haupt, K., and Mayes, A. G. (2017). A rapidscreening approach to detect and quantify microplastics based on fluorescent tagging with Nile Red. Sci. Reports 2017:7. doi: 10.1038/srep44501

Martin, C., Parkes, S., Zhang, Q., Zhang, X., McCabe, M. F., and Duarte, C. M. (2018). Use of unmanned aerial vehicles for efficient beach litter monitoring. Mar. Poll. Bull. (2018) 131, 662-673. doi: 10.1016/j.marpolbul.2018. 04.045

Matthews, J. P., Ostrovsky, L., Yoshikawa, Y., Komori, S., and Tamura, H. (2017). Dynamics and early post-tsunami evolution of floating marine debris near Fukushima Daiichi. Nat. Geosci. 10, 598-605. doi: 10.1038/ngeo2975

Maximenko, N., Arvesen, J., Asner, G., Carlton, J., Castrence, M., Centurioni, L., et al. (2016a) "Remote sensing of marine debris to study dynamics, balances and trends," in NASA Workshop on Mission Concepts for Marine Debris Sensing, 2016. (Hawai'i: University of Hawai'i at Manoa).

Maximenko, N., Arvesen, J., Asner, G., Carlton, J., Castrence, M., Centurioni, L., et al. (2016b). Remote Sensing of Marine Debris to Study Dynamics, Balances and Trends. White Paper, Decadal Survey for Earth Science and Applications from Space, 22.

Maximenko, N., and Hafner, J. (2010). SCUD, Surface Currents From Diagnostic Model, IPRC Technical Note No. 5. Available online at: http://iprc.soest.hawaii. 
edu/publications/tech_notes/SCUD_manual_02_17.pdf (accessed on February $16,2010)$

Maximenko, N., Hafner, J., and Niiler, P. (2012). Pathways of marine debris derived from trajectories of Lagrangian drifters. Mar. Poll. Bull. 65, 51-62. doi: 10.1016/j.marpolbul.2011.04.016

Maximenko, N. A., Hafner, J., Kamachi, M., and MacFadyen, A. (2018). Numerical simulations of debris drift from the 2011 tsunami in Japan, verified with boat reports. Mar. Pollut. Bull. 132, 5-25. doi: 10.1016/j.marpolbul.2018.03.056

McGrath, J. S., Honrado, C., Spencer, D., Horton, B., Bridle, H. L., and Morgan, H. (2017). Analysis of parasitic protozoa at the single-cell level using microfluidic impedance cytometry. Sci. Reports 2017:7. doi: 10.1038/s41598-017-02715-y

McWilliams, J. C. (2016). Submesoscale currents in the ocean. Proc. R. Soc. A 472:20160117. doi: 10.1098/rspa.2016.0117

Meyerjürgens, J., Badewien, T. H., Garaba, S. P., Wolff, J. O., and Zielinski, O. (2019). A state-of-the-art compact surface drifter reveals pathways of floating marine litter in the German bight. Front. Marine Sci. 6:58. doi: $10.3389 /$ fmars.2019.00058

Miller, J. (2018). Antarctica is not protected from an influx of new species. Phys. Today 71, 14-16. doi: 10.1063/PT.3.4012

Moller, D. (2016). "SAR and IfSAR observational concepts for marine debris mapping and oceanic monitoring," in Presentation at Workshop on Mission Concepts for Marine Debris Sensing (Honolulu, HI). Available online at: http://iprc.soest.hawaii.edu/NASA_WS_MD2016/pdf/Moller2016. pdf (accessed August 10, 2019).

Molnar, J. L., Gamboa, R. L., Revenga, C., and Spalding, M. D. (2008). Assessing the global threat of invasive species to marine biodiversity. Front. Ecol. Environ. 6, 485-492. doi: 10.1890/070064

Mouat, J., Lozano, R. L., and Bateson, H. (2010). Economic Impacts of Marine Litter. KIMO International. Retrieved from: http://www.kimointernational. org/wp/wp-content/uploads/2017/09/KIMO_Economic-Impacts-of-MarineLitter.pdf (accessed on November 29, 2013).

Moy, K., Neilson, B., Chung, A., Meadows, A., Castrence, M., Ambagis, S., et al. (2018). Mapping coastal marine debris using aerial imagery and spatial analysis. Mar. Poll. Bull. 132, 52-59. doi: 10.1016/j.marpolbul.201 7.11 .045

MSFD (2013). Marine Strategy Framework Directive GES Technical Group on Marine Litter. Guidance on Monitoring of Marine Litter in European Seas, Luxembourg. Available online at: https://mcc.jrc.ec.europa.eu/documents/ 201702074014.pdf

Murray, C. C., Maximenko, N., and Lippiatt, S. (2018). The influx of marine debris from the Great Japan Tsunami of 2011 to North American shorelines. Mar. Pollut. Bull. 132, 26-32. doi: 10.1016/j.marpolbul.2018.01.004

Nelson, H., Woods, M., Lorenz, C., Gerdts, G., Fields, D., Matrai, P., et al. (2018). "Use of Imaging Flow Cytometry (FlowCam) in the Study of Microplastics," in Ocean Sciences Meeting. (Portland, OR: Oregon Convention Center).

Newman, S., Watkins, E., Farmer, A., ten Brink, P. J., and Schweitzer, P. (2015). "The economics of marine litter," in Marine Anthropogenic Litter, eds. M. Bergmann, L. Gutow, and M. Klages (Cham: Springer Open; Elsevier), 367-394. doi: 10.1007/978-3-319-16510-3_14

Niiler, P. P. (2001). "The world ocean surface circulation," in Ocean Circulation and Climate. International Geophysics Series, Vol. 77, eds G. Siedler, J. Church, and J. Gould (New York, NY: Academic Press), 193-204. doi: 10.1016/S0074-6142(01)80119-4

Olson, R. J., Shalapyonok, A., Kalb, D. J., Graves, S. W., and Sosik, H. M. (2017). Imaging flowcytobot modified for high throughput by in-line acoustic focusing of sample particles. Limnol. Oceanogr. Methods 15:10. doi: 10.1002/lom3.10205

Peeken, I., Primpke, S., Beyer, B., Gütermann, J., Katlein, C., Krumpen, T., et al. (2018). Arctic sea ice is an important temporal sink and means of transport for microplastic. Nat Commun. 9:1505. doi: 10.1038/s41467-01803825-5

Peltzer, E. T., Zhang, X., Walz, P. M., Luna, M., and Brewer, P. G. (2016). In situ Raman measurement of HS- and H2S in sediment pore waters and use of the HS-:H2S ratio as an indicator of pore water pH. Mar. Chem. 2016:184. doi: 10.1016/j.marchem.2016.05.006

Pieper, C., Amaral-Zettler, L., Law, K. L., Loureiro, C. M., and Martins, A. (2019). Application of matrix scoring techniques to evaluate marine debris sources in the remote islands of the azores archipelago. Environ. Poll. 249, 666-675. doi: 10.1016/j.envpol.2019.03.084
Provencher, J. F., Ammendolia, J., Rochman, C. M., and Mallory, M. L. (2018). Assessing plastic debris in aquatic food webs, what we know and don't know about uptake and trophic transfer. Environ. Rev. doi: 10.1139/er-20180079

Purser, A., Thomsen, L., Barnes, C., Best, M., Chapman, R., Hofbauer, M., et al. (2013). Temporal and spatial benthic data collection via an internet operated deep sea crawler. Methods Oceanogr. 5, 1-18. doi: 10.1016/j.mio.2013. 07.001

Putman, N. F., Goni, G. J., Gramer, L. J., Hu, C., Johns, E. M., Trinanes, J., et al. Wang (2018). Simulating transport pathways of pelagic sargassum from the Equatorial Atlantic into the Caribbean Sea. Progr. Oceanogr. 165, 205-214. doi: 10.1016/j.pocean.2018.06.009

Robinson, J., New, A. L., Popova, E. E., Srokosz, M. A., and Yool, A. (2017). Farfield connectivity of the UK's four largest marine protected areas, Four of a kind? Earth Fut. 5, 475-494. doi: 10.1002/2016EF000516

Royer, S. J., Ferrón, S., Wilson, S. T., and Karl, D. M. (2018). Production of methane and ethylene from plastic in the environment. PLoS ONE 13:e0200574. doi: 10.1371/journal.pone.0200574

Ryan, P. G. (2015). "A brief history of marine litter research," in Marine Anthropogenic Litter, eds. M. Bergmann, L. Gutow, and M. Klages (Cham: Springer Open; Elsevier), 1-25. doi: 10.1007/978-3-31916510-3_1

Scholin, C. A., Birch, J., Jensen, S., Marin, R., Massion, E., Pargett, D., et al. (2017).the quest to develop ecogenomic sensors a 25 -year history of the environmental sample processor (esp) as a case study. Oceanography 30:427. doi: 10.5670/oceanog.2017.427

Sgier, L., Freimann, R., Zupanic, A., and Kroll, A. (2016). Flow cytometry combined with viSNE for the analysis of microbial biofilms and detection of microplastics. Nat. Commun. 7:11587. doi: 10.1038/ ncomms 11587

Shim, W. J., Song, Y. K., Hong, S. H., and Jang, M. (2016). Identification and quantification of microplastics using Nile Red staining. Mar. Pollu. Bull. 113:49. doi: 10.1016/j.marpolbul.2016.10.049

Sosik, H. M. (2017). Life in the plankton, stories from automated submersible microscopy and flow cytometry. Integr. Compar. Biol. 57. Available online at: http://www.sicb.org/meetings/2017/schedule/abstractdetails.php?id=1946

Spencer, D., Caselli, F., Bisegna, P., and Morgan, H. (2016). High accuracy particle analysis using sheathless microfluidic impedance cytometry. Lab Chip. 16:13. doi: 10.1039/C6LC00339G

Spencer, D., Elliott, G., and Morgan, H. (2014). A sheath-less combined optical and impedance micro-cytometer. Lab Chip 14, 3064-3073. doi: 10.1039/C4LC00224E

Tekman, M. B., Krumpen, T., and Bergmann, M. (2017). Marine litter on deep Arctic seafloor continues to increase and spreads to the North at the HAUSGARTEN observatory. Deep Sea Res. I Oceanogr. Res. Papers 120:11. doi: 10.1016/j.dsr.2016.12.011

Thompson, R. C., and Maximenko, N. (2016). "Plastic pollution in the marine environment," in Future of the Ocean and Its Seas, a Non-Governmental Scientific Perspective on Seven Marine Research Issues of G7 Interest, eds. P. Williamson, D. Smythe-Wright, and P. Burkill (Paris: ICSU-IAPSO-IUGGSCOR), 12-18.

Topouzelis, K., Papakonstantinou, A., and Garaba, S. P. (2019). Detection of floating plastics from satellite and unmanned aerial systems (plastic litter project 2018). Int. J. Appl. Earth Observ. Geoinform. 79, 175-183.doi: 10.1016/j.jag.2019. 03.011

Trinanes, J. A., Olascoaga, M. J., Goni, G. J., Maximenko, N. A., Griffin, D. A., and Hafner, J. (2016). Analysis of potential MH370 debris trajectories using ocean observations and numerical model results. J. Oper. Oceanogr. 9, 126-138. doi: 10.1080/1755876X.2016.1248149

van Sebille, E., England, M., H., and Froyland, G. (2012). Origin, dynamics and evolution of ocean garbage patches from observed surface drifters. Environ. Res. Lett. 7:044040. doi: 10.1088/1748-9326/7/4/ 044040

van Sebille, E., Wilcox, C., Lebreton, L., Maximenko, N. A., Hardesty, B. D., van Franeker, J. A., et al. (2015). A global inventory of small floating plastic debris. Environ. Res. Lett. 10:124006. doi: 10.1088/1748-9326/10/12/1 24006 
Veenstra, T. S., and Churnside, J. H. (2012). Airborne sensors for detecting large marine debris at sea. Mar. Pollut. Bull. 65, 1-3. doi: 10.1016/j.marpolbul.2010.11.018

Venrick, E. L., Backman, T., Bartram, W. C., Platt, C. J., Thornhill, M. S., and Yates, R. E. (1973). Man-made objects on the surface of the Central North Pacific ocean. Nature 241, 271-271. doi: 10.1038/241271a0

Wang, W., and Wang, J. (2018). Investigation of microplastics in aquatic environments, An overview of the methods used, from field sampling to laboratory analysis. Trends Anal. Chem. 108:26. doi: 10.1016/j.trac.2018.08.026

Zielinski, O., Busch, J., Cembella, A., Daly, K., Engelbrektsson, J., Hannides, A., et al. (2009). Detecting marine hazardous substances and organisms, sensors for pollutants, toxins, and pathogens. Ocean Sci. 5, 329-349. doi: 10.5194/os-5-329-2009

Disclaimer: The information in this paper reflects the views of the authors, and does not necessarily reflect the official positions or policies of NOAA, EPA, or Smithsonian Institution.
Conflict of Interest Statement: MA was employed by Argans. YG was employed by eOdyn. TM was employed by Mace Geospatial, LLC.

The remaining authors declare that the research was conducted in the absence of any commercial or financial relationships that could be construed as a potential conflict of interest.

At least a portion of this work is authored by Rick Lumpkin, Anna-Marie Cook, Gustavo Goni, Bill Robberson, and Gregory Ruiz on behalf of the U.S. Government and, as regards Dr. Lumpkin, Mrs. Cook, Dr. Goni, Mr. Robberson, Dr. Ruiz, and the U.S. Government, is not subject to copyright protection in the United States. Foreign and other copyrights may apply. This is an open-access article distributed under the terms of the Creative Commons Attribution License (CC BY). The use, distribution or reproduction in other forums is permitted, provided the original author(s) and the copyright owner(s) are credited and that the original publication in this journal is cited, in accordance with accepted academic practice. No use, distribution or reproduction is permitted which does not comply with these terms. 


\section{OPEN ACCESS}

Edited by:

Tong Lee,

NASA Jet Propulsion Laboratory

(JPL), United States

Reviewed by:

Amanda Plagge,

Global Science and Technology, Inc.

United States

Aaron Christopher Paget,

Concord University, United States

*Correspondence:

Mark A. Bourassa

mbourassa@fsu.edu

Specialty section:

This article was submitted to

Ocean Observation,

a section of the journal

Frontiers in Marine Science

Received: 16 November 2018

Accepted: 05 July 2019

Published: 23 August 2019

Citation:

Bourassa MA, Meissner T, Cerovecki I, Chang PS, Dong X, De Chiara G, Donlon C, Dukhovskoy DS, Elya J,

Fore A, Fewings MR, Foster RC,

Gille ST, Haus BK, Hristova-Veleva S, Holbach HM, Jelenak Z, Knaff JA, Kranz SA, Manaster A, Mazloff M, Mears C, Mouche A, Portabella M, Reul N, Ricciardulli L, Rodriguez E, Sampson C, Solis D, Stoffelen A, Stukel MR, Stiles B, Weissman D and Wentz F (2019) Remotely Sensed

Winds and Wind Stresses for Marine

Forecasting and Ocean Modeling.

Front. Mar. Sci. 6:443.

doi: 10.3389/fmars.2019.00443

\section{Remotely Sensed Winds and Wind Stresses for Marine Forecasting and Ocean Modeling}

\author{
Mark A. Bourassa ${ }^{1,2 *}$, Thomas Meissner ${ }^{3}$, Ivana Cerovecki ${ }^{4}$, Paul S. Chang ${ }^{5}$, \\ Xiaolong Dong ${ }^{6}$, Giovanna De Chiara ${ }^{7}$, Craig Donlon ${ }^{8}$, Dmitry S. Dukhovskoy ${ }^{2}$, \\ Jocelyn Elya ${ }^{2}$, Alexander Fore ${ }^{9}$, Melanie R. Fewings ${ }^{10}$, Ralph C. Foster ${ }^{11}$, Sarah T. Gille ${ }^{4}$, \\ Brian K. Haus ${ }^{12}$, Svetla Hristova-Veleva ${ }^{9}$, Heather M. Holbach ${ }^{13}$, Zorana Jelenak ${ }^{14}$, \\ John A. Knaff ${ }^{15}$, Sven A. Kranz ${ }^{1}$, Andrew Manaster ${ }^{3}$, Matthew Mazloff ${ }^{4}$, Carl Mears ${ }^{3}$, \\ Alexis Mouche ${ }^{16}$, Marcos Portabella ${ }^{17}$, Nicolas Reul ${ }^{16}$, Lucrezia Ricciardulli ${ }^{3}$, \\ Ernesto Rodriguez ${ }^{9}$, Charles Sampson ${ }^{18}$, Daniel Solis ${ }^{19}$, Ad Stoffelen ${ }^{20}$, \\ Michael R. Stukel ${ }^{1}$, Bryan Stiles ${ }^{9}$, David Weissman ${ }^{21}$ and Frank Wentz ${ }^{3}$ \\ ${ }^{1}$ EOAS, Florida State University, Tallahassee, FL, United States, ${ }^{2}$ COAPS, Florida State University, Tallahassee, FL, \\ United States, ${ }^{3}$ Remote Sensing Systems, Santa Rosa, CA, United States, ${ }^{4}$ Scripps Institution of Oceanography, University \\ of California, San Diego, San Diego, CA, United States, ${ }^{5}$ NOAA/NESDIS/Center for Satellite Applications and Research, \\ Silver Spring, MD, United States, ${ }^{6}$ The Key Laboratory of Microwave Remote Sensing, NSSC, CAS, Beijing, China, \\ ${ }^{7}$ European Centre for Medium-Range Weather Forecasts, Reading, United Kingdom, ${ }^{8}$ Mission Science Division, Earth \\ Observation Programme Directorate, ESTEC, European Space Agency, Noordwijk, Netherlands, ${ }^{9}$ Jet Propulsion Laboratory \\ and California Institute of Technology, Pasadena, CA, United States, ${ }^{10}$ College of Earth, Ocean, and Atmospheric Sciences, \\ Oregon State University, Corvallis, OR, United States, ${ }^{11}$ Applied Physics Laboratory, University of Washington, Seattle, WA, \\ United States, ${ }^{12}$ Department of Ocean Sciences - Rosenstiel School of Marine and Atmospheric Science, University of \\ Miami, Miami, FL, United States, ${ }^{13}$ NOAA/AOML Hurricane Research Division, Northern Gulf Institute, Florida State \\ University, Miami, FL, United States, ${ }^{14}$ NOAA/NESDIS/STAR-UCAR, Washington, DC, United States, ${ }^{15}$ NOAA Center for \\ Satellite Applications and Research, Fort Collins, CO, United States, ${ }^{16}$ Laboratoire d'Océanographie Physique et Spatiale, \\ Institut Français de Recherche et d'Exploitation de la Mer, Brest, France, ${ }^{17}$ Barcelona Expert Centre, Institute of Marine \\ Sciences (ICM-CSIC), Barcelona, Spain, ${ }^{18}$ U.S. Naval Research Laboratory, Monterey, CA, United States, ${ }^{19}$ Florida \\ Agricultural and Mechanical University, Tallahassee, FL, United States, ${ }^{20}$ RDSW, Royal Netherlands Meteorological Institute, \\ De Bilt, Netherlands, ${ }^{21}$ School of Engineering and Applied Science, Hofstra University, Hempstead, NY, United States
}

Strengths and weakness of remotely sensed winds are discussed, along with the current capabilities for remotely sensing winds and stress. Future missions are briefly mentioned. The observational needs for a wide range of wind and stress applications are provided. These needs strongly support a short list of desired capabilities of future missions and constellations.

Keywords: satellite, wind, stress, ocean, requirements

\section{INTRODUCTION}

This paper is focused on remotely sensed surface winds (scalar winds and vector winds) with related material on surface stress, air-sea heat fluxes, currents, sea state, and precipitation. It begins with definitions used in the description of remotely sensed winds followed by a description of wind sensing techniques including strengths, weaknesses, spatial coverage, and resolution (section Observation Technologies and Networks). Planned and suggested improvements to these observations are also discussed. Three of the largest concerns with modern observations are rain contamination (section Rain Contamination), calibration at very high wind speeds (section High Wind Speed retrieval) and the lack of observations near land and ice. Section Multi-Satellite Wind Products is a brief description of merged satellite products that are produced on a regular grid. 
The connections between observing system governance (section Observing System Governance), science teams and users, and data centers (section Data and Information Systems) are explained. This is followed by sections on applications, with the greatest detail provided on operations (section Hazards and Marine Safety) and discovery (section Discovery), and the requirements for these applications. The last section is a summary of key issues and requirements.

\section{OBSERVATION TECHNOLOGIES AND NETWORKS}

\section{Scalar Speed Sensors}

Passive microwave sensors (radiometers) measure the wind speed over the ocean by receiving and analyzing the power spectrum (brightness temperatures) of the electromagnetic radiation that is emitted by the wind roughened water surface, largely from foam when foam is present. The retrieval of ocean surface wind speed from microwave radiometers is based on a physical radiative transfer model that calculates the microwave emission from flat and rough ocean surfaces and the absorption and emission by the Earth's atmosphere (Wentz, 1997; Meissner and Wentz, 2012).

Past and currently operating sensors that can measure ocean surface wind speed include the Special Sensor Microwave Imager (SSM/I; Hollinger et al., 1990; Wentz, 1997) and the Special Sensor Microwave Imager Sounder (SSMIS), the Tropical Rainfall Mission Microwave Imager (TMI; Wentz, 2015), the Global Precipitation Mission (GMI; Draper et al., 2015; Wentz and Draper, 2016), the Advanced Microwave Scanning Radiometers (AMSR-E and AMSR2; Imaoka et al., 2010), and the Micro-Wave Radiation Imager (MWRI) on the Feng-Yun FY-3 platforms (Yang et al., 2012). All of these instruments are conically scanning. For measuring scalar ocean surface wind speeds, they utilize the microwave frequency range between $6 \mathrm{GHz}$ (C-band) and $37 \mathrm{GHz}$ (Ka-band) at two polarizations (vertical $\mathrm{V}$ and horizontal $\mathrm{H}$ ). The spatial resolution of the wind speeds obtained from these instruments is about $20-35 \mathrm{~km}$. The multi-frequency and dual polarization channel configuration of these passive sensors makes it possible to remove the effects of atmospheric attenuation in non-precipitating scenes accurately enough to measure wind speeds at the ocean surface.

The accuracy of the scalar ocean wind speeds from microwave radiometers has been assessed through comparisons with buoys (Mears et al., 2001; Wentz, 2015; Wentz et al., 2017) and numerical weather prediction models (NWP, Meissner et al., 2001). In rain-free scenes, the accuracy matches that of an active instrument ( $<1 \mathrm{~m} / \mathrm{s}$; Wentz, 2015; Wentz et al., 2017). In precipitating scenes, the ability of these passive microwave sensors to measure ocean wind speeds degrades quickly with significant errors starting to appear in light rain (surface rain rates $>1 \mathrm{~mm} / \mathrm{h}$, Meissner and Wentz, 2009). This is mainly due to: (1) the attenuation of the surface signal by rain drops, and (2) the fact that the attenuation is difficult to simulate using radiative transfer models, in particular at the higher frequencies when scattering by rain drops occurs. However, because of their sensitivity to rain, the passive sensors are able to deliver an accurate measurement of the surface rain rate (Wentz and Spencer, 1998; Hilburn and Wentz, 2008). This provides valuable information for flagging wind speed retrievals in rain.

Since 1996, there have been 3-6 radiometers operating in polar and/or equatorial orbits, resulting in good spatial and temporal sampling and covering $95 \%$ of Earth's ocean surface in a given day. This temporal and spatial coverage makes it possible to integrate scalar wind speeds from satellite microwave radiometers into a long-term climate data record (Wentz et al., 2017).

Scalar surface wind data sets from these sensors are produced and distributed by several institutions (www.remss.com, https:// gportal.jaxa.jp/gpr, http://satellite.nsmc.org.cn/PortalSite/ Default.aspx).

\section{Vector Wind Sensors}

Vector winds are useful for a wider range of applications than scalar winds. The observational needs are most consistent with scatterometers (section Scatterometers), with existing applications discussed in sections Hazards and Marine Safety and Discovery, and goals discussed immediately below in section Scatterometers and in the summary. Passive polarimetric observations (section Polarimetric Radiometers) provide an alternative with a different set of strengths and weaknesses, while synthetic aperture radar [SAR; section Synthetic Aperture Radar (SAR)] is more capable when used very close to land and where finer resolution is required.

\section{Scatterometers}

There is a long history of scatterometer observations, which are based on active microwave radars: Seasat (Johnson et al., 1980), ERS1 and ERS2 (Attema, 1991; Lin et al., 2017), NSCAT (Naderi et al., 1991; Tsai et al., 1999; Wentz and Smith, 1999), SeaWinds instruments on QuikSCAT (Spencer et al., 2000), and ADEOS2, ASCAT (Figa-Saldaña et al., 2002) on METOP$\mathrm{A},-\mathrm{B}$, and $-\mathrm{C}$, the RapidScat (Durden and Perkovic-Martin, 2017) on the International Space Station as well as OSCAT on OceanSat2 (Kumar et al., 2013), ScatSat-1, and HY-2A. These instruments provide very accurate winds in rain-free conditions and in some raining conditions (Stiles and Dunbar, 2010; Lin et al., 2015a; Wentz et al., 2017; Priftis et al., 2018), with the longer wavelength $\mathrm{C}$-band instruments performing better in rain than the Ku-band instruments. The ASCAT products are more consistent and provide better directional accuracy across the measurement swath due to their constant azimuth viewing angles but at the expense of a large nadir gap in coverage. A new scatterometer with a rotating fan-beam began operating on CFOSAT in October 2018. It will provide an opportunity to evaluate coverage and directional performance near nadir due to its increased number of views (Li et al., 2019). Combined C and $\mathrm{Ku}$-band measurements have also been demonstrated by using data collected nearly simultaneously by ASCAT and ScatSat-1 (Fore et al., 2018).

In-swath grid spacing has improved from 25 to $12.5 \mathrm{~km}$ for standard products (Fore et al., 2014), however, spectral analysis shows little improvement in resolution in products with finer grid spacing than $25 \mathrm{~km}$. There is a special 
coastal ASCAT product with $5.6 \mathrm{~km}$ grid spacing (and $<20 \mathrm{~km}$ resolution; Vogelzang and Stoffelen, 2017) as well as gridspaced ultra high resolution products from Brigham Young University (Lindsley and Long, 2016), which have grid spacing as fine as $2.5 \mathrm{~km}$ with resolution slightly better than $20 \mathrm{~km}$. The latest JPL QuikSCAT winds $12.5-\mathrm{km}$ product (Level 2B Version 4.0) has also been optimized to acquire winds close to the coast $(\sim 10 \mathrm{~km})$, at the price of reduced accuracy (Figure 1). Temporal sampling is a function of the orbit and the swath width. A single SeaWinds instrument has sufficient sampling for synoptic scale weather forcing, with substantial information on the mesoscale. A combined C- and Ku-band scatterometer is also planned by CMA FY-3 series satellite, which is expected to improve the performance for rain and higher wind speed conditions and to help evaluate calibration differences between $\mathrm{Ku}-$ and $\mathrm{C}$-band instruments for these conditions and to better characterize differences in dependence on sea surface temperature (SST; Wang et al., 2017). Moreover, an additional cross-polarized beam will fly on the scatterometers (SCA) onboard the Metop Second Generation (Metop-SG) series as of 2023, providing enhanced extreme-wind retrieval capabilities.

The main weaknesses of scatterometers are rain contamination for some rain conditions (far more so for Ku-band than C-band), a lack of data near land (typically within $15 \mathrm{~km}$ ), and temporal sampling. Satellites in low Earth orbit take roughly $100 \mathrm{~min}$ to orbit around the world. Consecutive sunsynchronous orbits have gaps between them and thus only cover portions of the globe in 1 day (Figure 2). Multiple scatterometers greatly improve the temporal sampling (Trindade et al., 2017; Stoffelen et al., 2019). However, the number of satellites is not a good metric for coverage, as shown in 6-hourly images of coverage from RapidScat, ASCAT-A, and ASCAT-B (Figure 3).

The World Meteorological Organization specifies $25 \mathrm{~km}$ resolution and sampling every $6 \mathrm{~h}$ (ideally from three satellites) as the operational goals for scatterometers. While existing instruments achieve this spatial resolution goal, a chart of the existing and projected sensors (Figure 2) shows that the target temporal resolution cannot be achieved without more international collaboration on the timing of orbits (Stoffelen et al., 2019). Instruments in more equatorial orbits, such as RapidSCAT, considerably improve tropical coverage and allow for very fast and good intercalibration with instruments in sun-synchronous orbits (Ricciardulli et al., 2015; Wang et al., 2017; Wentz et al., 2017). Stoffelen et al. (2013) found that two scatterometers (OSCAT and ASCAT) separated by $2.5 \mathrm{~h}$ in local overpass time generally doubled the contribution to the reduction of wind forecast error with respect to one scatterometer overpass time (ASCAT). This indicates that sampling every $2.5 \mathrm{~h}$ results in very little over-sampling. Consequently, the International Ocean Vector Winds Science Team (IOVWST) recommends at least one instrument in a more tropical orbit be combined with at least three satellites in coordinated sun-synchronous orbits. The planned HY-2 constellation will fill current gaps in coverage with sun-synchronous orbits: HY-2C (to be launched in 2019) and HY-2D (planned for launch in 2020 or 2021) will be flown with two more equatorial orbits, in addition to HY-2B, which will be flown in a sun-synchronous orbit. The next ASCAT mission and the second generation of European instruments will contribute to this coverage, while upcoming missions from India will fill in the gaps at midnight and midday.

Planned advances include the use of Doppler information to improve accuracy in future Indian instruments and upgraded antenna technology for modest improvements of resolution and polarization in the follow-on to the ASCAT series of instruments (Lin et al., 2017). One goal of the remote sensing community is to add Doppler capability to future scatterometers (Rodriguez, 2018; Rodriguez et al., 2019, this issue) and altimeters (Ardhuin et al. this issue) in a manner that allows for measurements of surface currents. Traditionally, surface currents are poorly defined, thought of as currents at the surface or in the upper few meters, and more often as currents in the mixed layer (GCOS, 2015; Belward et al., 2016). Thus, very few observations are made in the upper few meters where there is great deal of variation related to winds and waves. These variations impact the calibration of winds and stress and applications that depend on stress retrievals. Simultaneous ocean motion Doppler and backscatter measurements will allow the retrieval of both winds and currents (Mouche et al., 2012; Rodriguez et al., 2018; Rodriguez et al. this issue) and improve directional accuracy. The recent National Academies of Science Earth Science and Applications from Space Decadal Survey (National Academies of Sciences, 2018) recognized the value of this combination of observations and the need to use higher frequencies to improve resolution of a SeaWinds-like instrument (Rodriguez et al., 2018). It appears that studying mesoscale air-sea interaction requires much finer scale observations, with grid spacing of roughly $5 \mathrm{~km}$ or finer (Villa Boas et al. this issue). Observations on these scales, with sufficient averaging in space and time, would allow investigation of most mesoscale features.

\section{Polarimetric Radiometers}

Polarimetric radiometers (passive instruments) include the $\pm 45^{\circ}$ polarized and left/right circular polarized channels in addition to the vertical $(\mathrm{V})$ and horizontal $(\mathrm{H})$ polarizations. Thus, these sensors contain complete information about the state of polarization (4 Stokes parameters) of the electromagnetic radiation that is emitted from the ocean surface. The signal in the 4 Stokes parameters of the passive microwave emission from a rough ocean surface contains a small part that depends on wind direction relative to look direction (Yueh et al., 1999). This allows the determination of the ocean vector winds, i.e., both scalar wind speed and wind direction. Wind speed accuracies are similar for passive polarimetric radiometers and for scatterometers. However, the wind directions from passive systems become less accurate as wind speeds fall below $8 \mathrm{~m} \mathrm{~s}^{-1}$ (Ricciardulli et al., 2012).

The WindSat sensor is a conically scanning polarimetric radiometer operated by the US Navy and flown on the Coriolis platform (Gaiser et al., 2004). It is fully polarimetric 

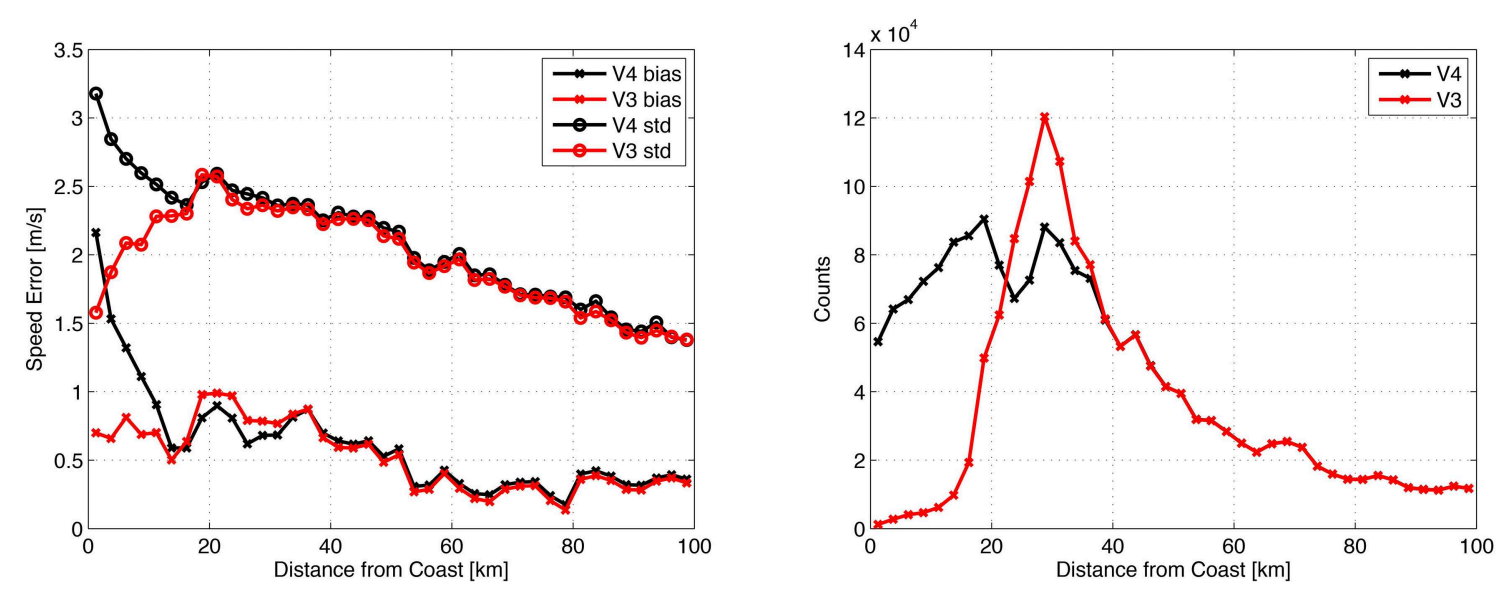

FIGURE 1 | QuikSCAT Coastal performance (left) for versions 3 (red) and 4 (black) showing biases (crosses) and standard deviations (circles) relative to buoy observations. Version 4 has at least 10 time as many buoy hits within $20 \mathrm{~km}$ of the coast as version 3 (right). All available NDBC buoys were utilized in this assessment. This may tend to overemphasize near-coastal errors, because some coastal buoys are known to be non-representative of the surrounding wind field. Two trends are apparent. Differences from buoys decrease out to $100-\mathrm{km}$ possibly due to more spatial variance closer to shore. There is a steeper trend from 0 to $20-\mathrm{km}$ perhaps due to residual errors in coastal processing. Agreement 10-km from coast is only marginally worse than 30-40-km. Agreement within 5-km from the coast shows significant degradation. Distance from the coast is included in the product and can be used as a quality flag.
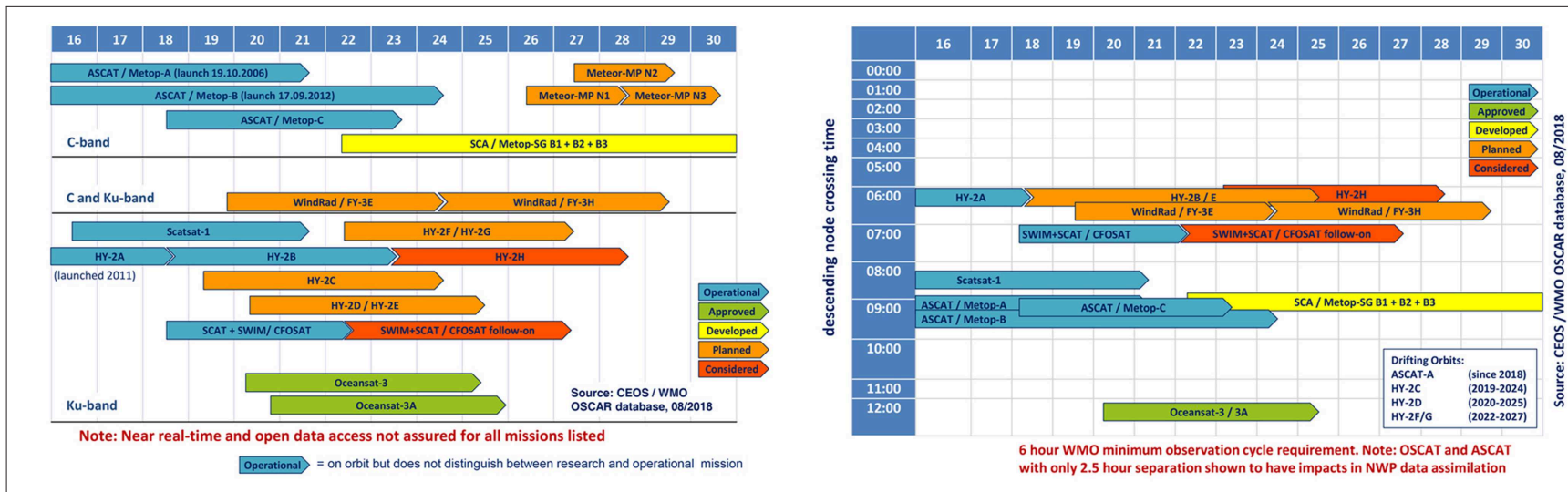

FIGURE 2 | Projection of existing and future scatterometers (left) and sampling through the diurnal cycle (right). While the World Meteorological Organization's requirement for the number of satellites should be met, the sampling through the diurnal cycle is insufficient. Graphic courtesy of Stefanie Linow.

(measuring all 4 Stokes parameters) at 10.7 (X-band), 18.7 (Ku-band) and 37.0 (Ka-band) $\mathrm{GHz}$ and dual polarized ( $\mathrm{V}$ and $\mathrm{H}$ ) at 6.8 (C-band) and $23.8 \mathrm{GHz}$ (K-band). The instrument has been operating since 2003, with just a few brief interruptions, and has been providing the oceanographic communities with high-quality ocean vector wind data (Bettenhausen et al., 2006; Meissner and Wentz, 2006).

At wind speeds below $6 \mathrm{~m} \mathrm{~s}^{-1}$, the passive wind direction signal is small in all polarizations and because of that the wind direction measurements are noisy at these wind speeds. The accuracy of the WindSat wind direction increases with increasing wind speed, because the wind direction signal increases. At $6 \mathrm{~m} \mathrm{~s}^{-1}$, the uncertainty of with WindSat wind direction is $20^{\circ}$ (Hilburn et al., 2016), and it becomes usable for most oceanographic and meteorological applications. Above $8 \mathrm{~m} \mathrm{~s}^{-1}$, the uncertainty of the WindSat wind direction measurements $\left(10^{\circ}-15^{\circ}\right)$ is similar to that of scatterometers (Ricciardulli et al., 2012; Hilburn et al., 2016). At high winds (above $10 \mathrm{~m} \mathrm{~s}^{-1}$ ) the passive wind direction signal is strong in all 4 Stokes parameters. Because the wind direction dependence differs for the 4 Stokes parameters it is possible to resolve the vector wind ambiguities without the use of an ancillary numerical weather prediction field. WindSat takes observations in both the forward (fore) and backward (aft) look. Using both look directions in the WindSat vector wind retrieval helps to improve its performance (Hilburn et al., 2016).

Like other passive microwave sensors, WindSat is strongly affected by rain; its ability to measure wind speed in the presence of rain is significantly diminished. However, this effect has been converted into a useful tool for estimating rain rates (and other atmospheric products) on a global 

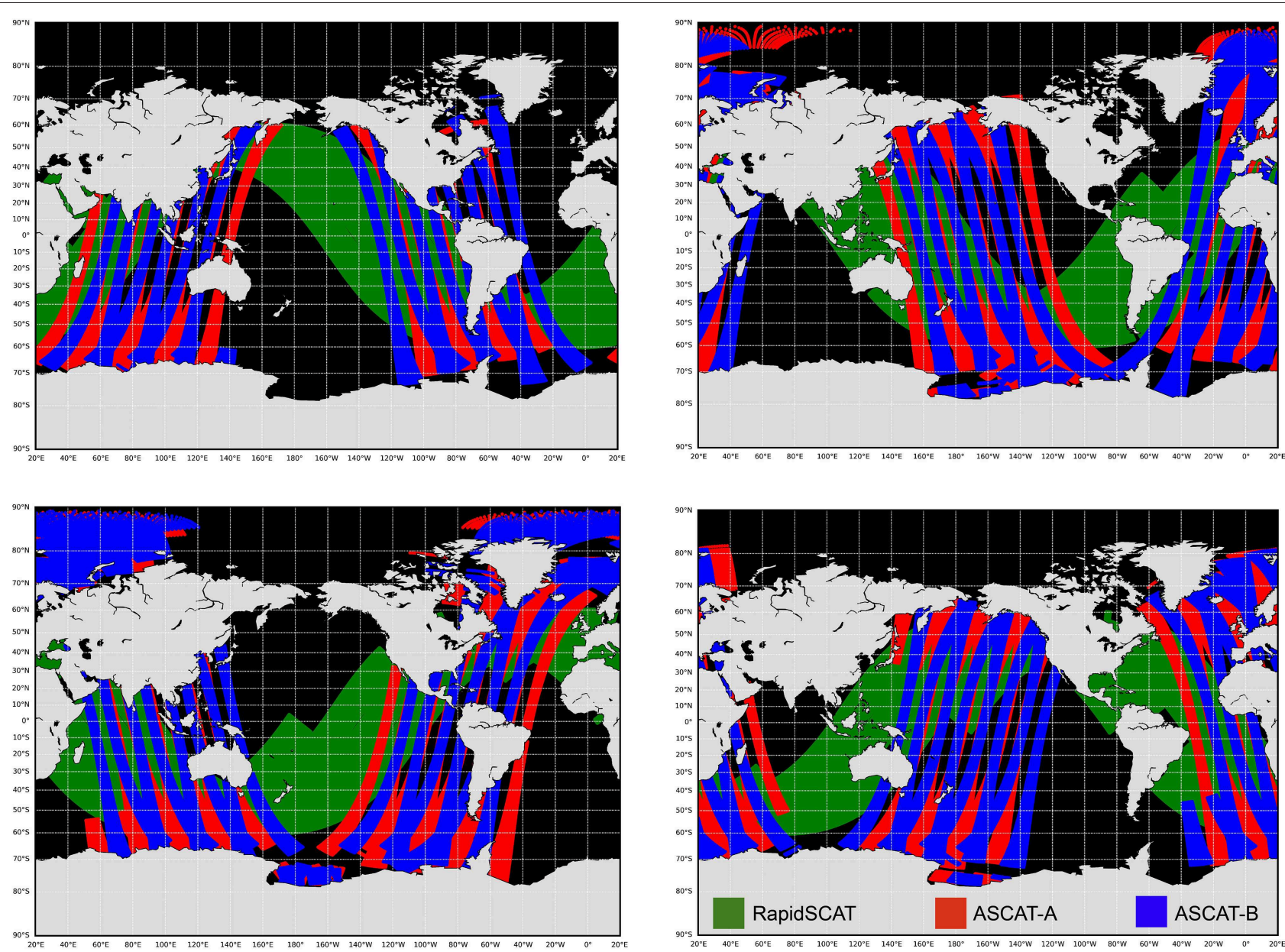

FIGURE 3 | Examples of coverage from 0 to $6 Z$ (upper left), 6 to $12 Z$ (upper right), 12 to $18 Z$ (lower left), and 18 to $24 Z$ (lower right) based on one equatorial orbiting instrument (RapidSCAT; green) and two instruments (ASCAT-A in red and ASCAT-B in blue) in sun synchronous orbits. While this configuration has considerable advantages for intercalibration and studies of convection, and has the World Meteorological Organization recommended number of satellites, the temporal sampling is clearly insufficient.

scale. WindSat ocean vector winds (and other products) are produced by the Naval Research Laboratory ${ }^{1}$ and Remote Sensing Systems ${ }^{2}$.

Current plans call for the Compact Ocean Wind Vector Radiometer (COWVR) developed by the NASA Jet Propulsion Laboratory (JPL) to be launched and mounted at the International Space Station within the next couple years (Brown et al., 2017). Like WindSat, it scans conically and takes observations over the full scan both in the fore and aft look direction. It has fully polarimetric channels (18.7, 23.8, and $33.9 \mathrm{GHz}$ ) and lower frequency channels can be easily added. COWVR's performance to measure ocean vector winds is expected to be similar to WindSat, but it will demonstrate new technology for polarimetric radiometers. Its calibration is done internally and, unlike the previously discussed passive spaceborne sensors, only its reflector antenna rotates while

${ }^{1}$ https://www.nrl.navy.mil/WindSat/

${ }^{2}$ www.remss.com/missions/windsat its receiver is fixed to the spacecraft. This is a more costeffective design and may pave the way to future polarimetric microwave radiometers.

The Weather System Follow-on Microwave (WSF-M) mission is currently being developed by Ball Aerospace for the U.S. Department of Defense as a continuation for the SSMIS and WindSat sensors. It will have a microwave imager with a configuration and polarimetric capabilities similar to WindSat, and is expected to be able to measure ocean surface wind vectors with an accuracy comparable to WindSat. The first of two planned sensors will launch in 2023.

Studies are underway at the European Space Agency using the Copernicus Imaging Microwave Radiometer (CIMR; see Donlon, 2018), a conically scanning imager that includes channels between 1.4 and $36.5 \mathrm{GHz}$, in a polar orbit, to provide SST, wind speed, sea surface salinity, thin sea ice thickness, and sea ice concentration with increased accuracy and spatial resolution. The initial design includes low noise dual polarization receivers 
and a large $7 \mathrm{~m}$ rotating mesh antenna. Low-noise receivers at $1.4,6.9,10.65,18.7$, and $36.5 \mathrm{GHz}$ (L-, C-, X-, Ku-, and $\mathrm{Ka}-$ band) and at spatial resolutions of $5-15 \mathrm{~km}$ (55 km at L-band) are foreseen over a swath of $\sim 1,920 \mathrm{~km}$. CIMR is designed to address the Integrated European Union Policy for the Arctic providing a follow up for the current L-band missions-Soil Moisture and Ocean Salinity (SMOS) and Soil Moisture Active Passive (SMAP) - and the continuity of AMSR/2 low frequency channels. It is expected to have excellent capabilities for ocean wind measurements under all conditions. The CIMR mission is currently in a Phase A/B1 study that is expected to move into full implementation for a launch in the 2025+ timeframe.

\section{Synthetic Aperture Radar (SAR)}

SAR sensors measure the NRCS, similar to scatterometers. Therefore, geophysical model functions derived from abundant wind scatterometer measurements may be used to infer wind information from SAR (Portabella et al., 2002), but they cannot simultaneously retrieve both wind speed and direction. Since SARs provide a single azimuth view, one cannot infer whether backscatter variations are due to wind speed (along-view wind vector component) or wind direction (across-view wind vector component) variation. Retrieval methodology is reviewed in Beal (2005) and the references therein. Young et al. (2007) developed both manual and semi-automated methods to correct for interactions between errors in wind speed and direction. Horstmann et al. (2000) described how wind direction on the kilometer-scale can be obtained at moderate winds by evaluating wind streaks in SAR images. Mouche et al. (2012) used approach to convert SAR's backscatter and Doppler measurements of radial wind speed into a wind vector. Gade and Stoffelen (2019) described the characteristics of widely used global ocean satellite wind measurement systems, where SARs are particularly unique in their spatial resolution but lack absolute calibration, temporal sampling, and complete information for wind vector retrieval.

SAR has a unique place in satellite wind observations, having the ability to image the sea surface on a relatively finer scale, $O(10 \mathrm{~m})$. However, in order to remove speckle noise and to filter ocean surface waves and other non-wind-induced features, wind retrieval is usually performed at spatial scales of $0.5-1 \mathrm{~km}$. C-band and L-band SAR systems have been used to retrieve surface winds on ERS-1,-2 Envisat, RADARSAT-1,-2 ALOS, and Sentinel-1A,B. Also, X-band SAR algorithms are being developed to retrieve winds on COSMO-SkyMed and TerraSAR-X.

The surface signature of marine atmospheric boundary layer organized roll vortices (Brown, 1980; Hein and Brown, 1988) has been detected in SAR imagery as far back as the Seasat L-band SAR (Fu and Holt, 1982). These rolls are kilometer-scale overturning coherent structures embedded in the marine atmospheric boundary layer turbulent flow that are approximately aligned with the surface wind direction. They induce a periodic enhancement and reduction of the surface wind speed that alternately enhances and reduces the backscatter. Beginning with Gerling (1986), this signature has been used to find the wind direction within a $180^{\circ}$ ambiguity (Horstmann et al., 2002; Horstmann and Koch, 2005, Wackerman et al., 2006; Horstmann et al., 2013). This ambiguity can be resolved by external, larger-scale flow maps or by flow features such as wind shadows within the SAR image.

\section{Coastal High Frequency Radar}

Coastal high frequency (HF) radars operate in frequency ranges from $\sim 5$ to $30 \mathrm{MHz}$ and at grazing angles. Their backscattered Doppler spectra are dominated by Bragg scattering off ocean waves ranging in length from roughly 50 to $10 \mathrm{~m}$. The Doppler spectra consistently reveal peaks corresponding to waves both advancing toward and receding from the transmit/receive direction at the Doppler-shifted Bragg frequency (known as the first order peaks). The ratio of the advancing and receding peaks is related to the wind direction component directed either toward or away from the transmitted signal (Long and Trizna, 1973), however there remains an ambiguity about the look direction. By looking at the same patch of the ocean from different directions using a monostatic HF radar system this ambiguity can be resolved. Wind direction has been proven to be a robustly derived parameter from monostatic shore based radars over long ranges because of the signal-to-noise ratio of the 1st order returns (e.g., Heron and Rose, 1986). The extension of this technique to bistatic transmit receive configurations (Huang et al., 2012) as well as shipboard observations (Xie et al., 2018) is under development.

Extraction of the wind speed from HF radars is more difficult because it has typically relied on the signal level of the 2nd order returns about the 1 st order peak, which are related to a longer wave modulation of the Bragg waves. These 2nd order returns are used to derive a wave spectral estimate that can then be related to the wind speed (Wyatt et al., 2006). One disadvantage of this approach is that the 2 nd order returns have a lower signalto-noise ratio than the 1st order peaks and are thus available at shorter ranges. Shen et al. (2012) developed a technique to extract the wind speed from the strength of the 1st order returns using neural networks that has the advantage of longer-range observation, but the range of wind speeds that can be observed from $\sim 4$ to $11 \mathrm{~m} / \mathrm{s}$ is limited due to saturation of the Bragg wave returns.

The measurement characteristics of the HF radar winds are related to the frequency of the radar as well as the receiver antenna technology used. The spatial resolution of HF radar winds can range from $\mathrm{O}(1-10 \mathrm{~km})$ in range and a few degrees in azimuth for phased-array systems, which can provide maps of the winds at 30-min intervals. The resolution of these approaches was reported by Shen et al. (2012), resulting in roughly $20^{\circ} \mathrm{rms}$ directional errors and $\sim 1.5 \mathrm{~m} / \mathrm{s}$ rms speed errors, limited to tens of $\mathrm{km}$ to roughly $79 \mathrm{~km}$ offshore.

The main contributions of HF radars within the context of ocean observing are their complementary capabilities to satellite based observations. In the United States the NOAA HF Radar National server presently provides continuous coastal ocean surface winds and currents at distributed sites. Expanding the data available from these systems would provide high temporal ( $\sim$ hourly) and spatial (a few $\mathrm{km}$ ) resolution winds in regions that are challenging for other types of remote sensors. In particular the capability of HF radars to provide wind information within $5 \mathrm{~km}$ of the coastline, where there are many critical applications such 
as search and rescue and oil-spill response can help to address observing system gaps as addressed below.

\section{Observing System Challenges}

There are two challenges related to communications. Satellite "winds" are estimates of stress tuned to be like winds (section Interpretation of Satellite Winds). Second, the radiometry community has done a poor job orally communicating the impressive capability at high wind speeds (section Tropical Cyclone Forecasting). With current instrument capabilities and ignoring very low wind speeds $\left(<2 \mathrm{~ms}^{-1}\right)$ where the variability in wind direction overwhelms the mean signal on the time scale of satellite observations, there are two main areas of ongoing concern. The first is degradation of the retrievals in rain and the other is retrievals at high wind speeds, which in tropical and sub-tropical latitudes often occur with rain. Moreover, in situ measurement systems disagree on the strength of wind speeds above $15 \mathrm{~m} / \mathrm{s}$ and an absolute wind reference needs to be established for calibrating the microwave satellite winds (e.g., Vandemark et al., 2018). Another issue is making observations closer to the coastline, which is challenging because the side lobes in the antenna pattern (areas outside the areas normally considered the footprint) extend roughly $60 \%$ of the footprint width beyond the edge of the traditional footprint, and because the signal from land and ice is much stronger than the signal from water. The single most effective solution to contamination from land and ice is to use finer resolution radar footprints, which is important for many applications mentioned later in this document.

Fast routines are being developed to allow on-the-fly processing of the radar spatial response functions on the ocean surface. This is useful for retrievals near land and ice because knowledge of the antenna pattern (i.e., the surface pattern including side lobes) can be used to mask backscatter observations that are contaminated by land and ice. This masking allows for retrievals to be made closer to the land or ice, albeit without all the information from an open ocean retrieval. This approach has been used to create special coastal scatterometer wind products with $5.6 \mathrm{~km}$ grid spacing and $<20 \mathrm{~km}$ resolution (Vogelzang and Stoffelen, 2017). Similarly, by taking account of the estimated coastal sea and land/ice backscatter relative to the total backscatter, more winds near the coast are being processed (Stiles et al., 2018).

\section{Interpretation of Satellite Winds}

As noted above, radiometers and scatterometers respond to ocean characteristics that are driven more by stress than by wind. Satellite-derived wind speed (also called equivalent neutral wind speed; Ross et al., 1985; Liu and Tang, 1996; Kara et al., 2008a) was developed to consider the influences of atmospheric stability in the conversion from "wind" to stress. It is a wind speed for which a neutral transfer coefficient can be used to convert a satellite's equivalent neutral winds to a kinematic stress. An updated definition of equivalent neutral winds (Bourassa et al., 2009) is consistent with scatterometry, which responds to surface stress (Bourassa and Hughes, 2018). It is also considered to be applicable to SAR (Takeyama et al., 2013) and radiometric
(Meissner and Wentz, 2012) wind speeds. De Kloe et al. (2017) further corrected for atmospheric mass density variations in what they called stress-equivalent winds. Unfortunately, accurate in situ measurements of near-surface stress over water are extremely sparse relative to wind speed, while surface layer models and auxiliary information used to convert winds to stress are not accurate enough (Portabella and Stoffelen, 2009); therefore, winds have historically been calibrated to equivalent neutral winds (or stress equivalent winds) rather than wind stress (Ross et al., 1985; Kara et al., 2008a,b; Stoffelen et al., 2017a). In other words, satellite instruments respond to ocean characteristics that are driven by stress rather than by wind; however, the products are "winds" that have been modified to be consistent with stress.

Another issue to consider is that satellite winds are relative to currents (Cornillon and Park, 2001; Kelly et al., 2001; Plagge et al., 2012) and thus likely to be further modified by surface wave characteristics via their dependence on stress (Quilfen et al., 2004; Bourassa, 2006). Still, some studies have not identified statistically significant differences (Portabella and Stoffelen, 2009). Improvements in our understanding of remotely sensed winds made in the last decade are expected to improve ocean forcing in the coming decade. Simultaneous observations of winds and currents (e.g., Rodriguez et al., 2019, this issue) will likely make a large contribution to better understanding observations related to air-sea coupling.

\section{Rain Contamination}

For scatterometers, there are two principal bodies of knowledge associated with the subject of rain contamination. The first is expanding the physical basis and modeling of the ocean surface spectrum (especially the relevant Bragg scattering elements) due to rain impacts as a function of rain intensity wind speed (Stiles and Yueh, 2002; Draper and Long, 2004; Weissman and Bourassa, 2008, 2011; Weissman et al., 2012; Ulaby and Long, 2014). Rain contamination is most pronounced at shorter wavelengths (Ku-band, Ka-band). At Ku-band, the primary effect of rain is increased backscatter from the rain column and raininduced roughening of the surface, resulting in positive speed biases in wind retrievals. Only for higher wind regimes $(>20$ $\mathrm{m} / \mathrm{s}$ ) does attenuation dominate, resulting in negative biases. Numerous algorithmic methods have been shown to reduce errors in Ku-band winds due to rain contamination (Draper and Long, 2004; Hilburn et al., 2006; Stiles and Dunbar, 2010; Weissman et al., 2012; Stiles and co-authors, 2014). Several of these algorithms are utilized in the latest version of JPL QuikSCAT data product. These are accompanied by physical measurements and their interpretation. Situations involving tropical cyclones and hurricanes are not very common but they are of special interest when accompanied by near simultaneous wind and rain measurements (Weissman and Bourassa, 2011). Early studies of the L-band capability for estimating winds in hurricanes did not lead to extensive applications because the sensitivity was not as strong as Ku-band. However, there were a few studies based on SEASAT technologies that entered the literature in the 1970s and 1980s. One experimental program for hurricane Gloria in 1976 involved three aircraft collecting extensive physical and L-band backscatter data. This included 
high winds and rainfall (Weissman et al., 1979). Also notable in the results was that heavy rainfall can suppress the L-band surface scattering coefficient. This is relevant for the new CYGNSS cyclone observation program, which depends on the reflected Lband signals from GPS satellites. This earlier work emphasizes the need to consider radar responses to modification of the rain-impacted surface for all wavelengths. Alpers et al. (2016) elaborated this issue using C-band SAR data.

The second body of knowledge focuses on rain screening. Several rain flags and quality control metrics have been proposed over the past two decades. These are mainly based on the inversion residual (Portabella and Stoffelen, 2001; Portabella et al., 2012), the singularity analysis or local wind field decorrelation estimation (Lin et al., 2014; Lin and Portabella, 2017), and/or a combination of different scatterometer-derived parameters which are found to be sensitive to rain (Huddleston and Stiles, 2000; Stiles and Yueh, 2002; Lin et al., 2014). It is found that for C-band systems, the impact of rain on the retrieved wind quality is lower than for Ku-band systems. For example, while only a small fraction (about $0.5-1 \%$ ) of ASCAT global winds are rejected by QC (Lin et al., 2015b), a much larger fraction (5-7\%) of Rapidscat winds are rejected using a similar QC algorithm (Lin and Portabella, 2017). However, thanks to the inclined orbit of Rapidscat, very tight collocations (within a few minutes distance in time) with ASCAT are available, which show that Rapidscat QC is far too conservative, i.e., it rejects a substantial amount of Rapidscat winds which are in good agreement with ASCAT winds (Lin and Portabella, 2017). As such, improved Ku-band QC is required in order to keep valuable $\mathrm{Ku}$-band derived winds in the vicinity of (thus not contaminated by) rain.

\section{High Wind Speed Retrievals}

It has been a long-standing challenge to accurately measure high wind speeds, such as those as found in tropical or extratropical cyclones. This is because: (1) in most sensors, the signal saturates when wind speed reaches $\sim 35 \mathrm{~m} / \mathrm{s}$ (the strength of category-1 hurricanes); (2) the signal is attenuated by heavy rainfall (where we include snow and mixed phase precipitation) that typically accompanies the majority of high wind events; (3) the resolution of the sensors is too coarse to resolve high-gradient regions that typically accompany high wind speeds; and (4) there is insufficient comparison data for validation, and some concern about the accuracy of these comparison data such as dropsondes and SFMR (Uhlhorn and Black, 2003; Uhlhorn et al., 2007; Klotz and Uhlhorn, 2014). All of these factors can result in large errors at very high wind speeds. Some next-generation scatterometers will use cross-polarization and enhanced spatial resolution to mitigate the reduced sensitivity and further improve hurricaneforce retrievals (van Zadelhoff et al., 2014; Lin et al., 2017).

Scatterometers, such as ASCAT or QuikSCAT, are able to reliably measure wind speeds up to $\sim 35 \mathrm{~m} / \mathrm{s}$, at which point they lose sensitivity and the signal begins to saturate (Donelan et al., 2004; Hwang et al., 2013; Hwang and Fois, 2015; Sapp et al., 2016). C-band scatterometers perform better in rainfall than Ku-band scatterometers, but existing C-band scatterometers sacrifice spatial coverage to do so. Most scatterometers have spatial resolutions of roughly $25 \mathrm{~km}$, despite $12.5 \mathrm{~km}$ grid spacing in products. The combination of spatial resolution and reduced sensitivity at extreme wind speeds prevents them from resolving winds in the eyewall of a hurricane or similar resolution high wind features in extratropical cyclones. However, extratropical cyclones have large areas of high winds behind the trailing cold front, and these are easily resolved. Scatterometers are used frequently in operational applications related to tropical and extratropical cyclones. Another major benefit of scatterometers is that they provide a wind vector, not just the scalar wind speed.

The new capabilities of recent Radarsat-2 and Sentinel-1 Cband SAR missions with both co- and cross-polarized channels demonstrated the Complementarity of these two polarizations for ocean surface wind measurements. In particular, compared to co-polarized signal, the cross-polarized backscattering has a very weak incidence and azimuth angle dependency with no indication of saturation for the strongest wind speeds (up to $75 \mathrm{~m} / \mathrm{s}$ ). Following the pioneering work from Fu and Holt (1982) or Katsaros et al. (2000) on hurricane observations with SAR, these capacities have opened new perspectives for ocean surface wind speed measurements in Tropical Cyclones (e.g., Zhang and Perrie, 2012; Horstmann et al., 2013; Hwang et al., 2015; Mouche et al., 2017), including the Tropical Cyclone inner core. Indeed, while there are a variety of sources for TC outer-core wind data, the only inner-core wind data that are routinely available at present are collected by airplanes and limited to the Atlantic and East pacific hurricane seasons. CBand SAR is the only space-borne instrument able to probe at very high resolution under extreme conditions. Moreover, dedicated acquisition strategy based on hurricane forecast tracks such as the Hurricane Watch program in Canada (Banal et al., 2007) or SHOC in Europe (Mouche et al., 2017) have proven to be efficient to mitigate the time sampling limitations (due to duty cycle) and provide comprehensive dataset over hurricane eyes ( $\mathrm{Li}$ et al., 2013; Mouche et al., 2017). To note, the potential of both cross and co-polarization yielded to several proposals of concept missions, including the scatterometer onboard the Metop-SG series (Stoffelen et al., 2017b). C-band scatterometer cross-pol measurements obtained from hurricane flights with the NOAA WP-3D aircraft have also shown the sensitivity of the cross-polarized response to extreme winds (Sapp et al., 2016, 2018).

Passive microwave radiometers, such as WindSat, overcome some of the precipitation attenuation issues by obtaining measurements at multiple channels that respond differently to precipitation, although the attenuation at high winds does negatively impact the direction retrievals (Meissner et al., 2011; Zabolotskikh et al., 2015). Microwave radiometers also tend to have slightly coarser spatial resolution than scatterometers, and hence cannot retrieve winds as close to the coast. However, the passive signal does not have the reduced sensitivity issues of scatterometers, allowing for wind speed retrievals at higher wind speeds in principle. The use of radiometer wind speeds to support operational weather applications is increasing and was recently included in the Automated Tropical Cyclone Forecasting Systems (Sampson and Schrader, 2000).

The recent availability of space-borne L-band radiometers, which operate in the low range microwave frequencies (1-2 
$\mathrm{GHz}$ ), also helps overcome the signal saturation and precipitation attenuation shortcomings of scatterometers. L-band radiometers are minimally affected by rain or frozen precipitation (Wentz, 2005; Reul et al., 2012). The signal they receive remains sensitive to increasing wind even in wind speeds up to $70 \mathrm{~m} / \mathrm{s}$ (Reul et al., 2012, 2016; Yueh et al., 2013; Meissner et al., 2014, 2017; Fore et al., 2016), which is the strength of category- 5 tropical cyclones. Both the European Space Agency Soil Moisture and Ocean Salinity (SMOS) and the NASA Soil Moisture Active Passive (SMAP) L-band radiometers are able to provide useful estimates of extreme ocean wind speeds at a spatial resolution of $40 \mathrm{~km}$ (Fore et al., 2016, 2018; Reul et al., 2016, 2017; Meissner et al., 2017). However, the high-gradient regions, such as the eye and eyewall of hurricanes, are still unable to be resolved with a spatial resolution of $40 \mathrm{~km}$.

SMOS (Kerr et al., 2010; Mecklenburg et al., 2012) is a passive interferometer that measures effectively the spatial Fourier transform of the emitted brightness temperature. SMAP (Entekhabi et al., 2010, 2014) has a real aperture consisting of a spinning mesh antenna $6 \mathrm{~m}$ in diameter. Achieving the desired spatial resolution of $40 \mathrm{~km}$ with the low L-band frequency requires either a large antenna or the utilization of the synthetic aperture technique. Wind speeds are processed and distributed for both sensors (www.smosstorm.org, ftp://podaac.jpl.nasa.gov/ allData/smap/L3/JPL/V4/, www.remss.com/missions/smap).

For wind speeds below $15 \mathrm{~m} / \mathrm{s}$, scatterometers (QuikSCAT, ASCAT, RapidScat, OceanSat, ScatSat) outperform L-band and polarimetric microwave radiometers in the measurement of scalar wind. However, at high wind speeds, above 25 $\mathrm{m} / \mathrm{s}$, L-band and polarimetric microwave radiometers have a distinct advantages (sensitivity) and disadvantages (lower spatial resolution and inability to be used close to the coast) compared to current scatterometers. All of these instruments could be further improved with better resolution. This applies, in particular, to areas of strong wind gradients and heavy precipitation, as found in tropical cyclones and strong cold fronts. Therefore, a combination of polarimetric microwave radiometers with multiple frequencies, scatterometers, and L-band radiometers provides a complementary set of instruments for measuring ocean surface wind speeds that can be made even better if the resolution is further improved.

\section{MULTI-SATELLITE WIND PRODUCTS}

Multi-satellite wind products provide vector winds on a regularly spaced temporal/spatial grid. They attempt to preserve the satellite wind information characteristics and facilitate many science and operational applications. Single satellite sampling view the Earth usually at a single local time (both AM and $\mathrm{PM}$ ) and are sometimes sensitive to precipitation or have no directional information. In contrast, much of the satellite wind information is generally ignored by design in global NWP models, which generally lack deterministic mesoscale structure. Multi-satellite wind products attempt to bridge the gap between single satellite products and NWP models. They resample or interpolate the satellite wind observations to regular (typically 6-h) time intervals and allow diurnal information into the processing stream of the winds over the world's oceans.

These products have several limitations for ocean vector winds including the lack of directional information in some satellite retrievals and inhomogeneous sampling caused by regions and times where there are no satellite observations or when satellite observations are affected by rain. In many blended products, a background field (usually obtained from NWP output) is used to fill regions with no observations. The background has different spatial characteristics to satellite measurements (Vogelzang et al., 2015) and may contain significant regional or global biases relative to satellites. For rainy regions, moist convection is generally poorly represented in global NWP (Lin et al., 2015b) except where observations are available for assimilation. The large mean and variance spatial biases in ERA-Interim may be corrected by computing and subtracting local mean and variable adaptations to ERA over a few days (Trindade et al., 2017; Stoffelen et al., 2019). Note that satellite winds are relative to ocean currents rather than relative to a fixed Earth surface, in contrast to both conventional observations and NWP data used as a background in these products. This difference likely leads to biases in regions with high velocity currents (McGregor et al., 2017).

Another limitation of the use of multi-satellite products is the difficulty in maintaining the subtle decadal wind trends that are contained in the stable satellite observations (Belmonte et al., 2017). These trends can be small $(0.1 \mathrm{~m} / \mathrm{s} / \mathrm{decade})$ and they can be distorted by the background field and by changing sampling. One remedy for this is to use the satellite record to monitor and correct for these types of systematic errors (Verhoef et al., 2017).

The Cross-Calibrated Multi-Platform (CCMP) ocean vector wind analysis (Atlas et al., 2011) is a quarter-degree, 6-hourly, near-global Level 4 vector wind product. Two similar products are OAFLUX ( $\mathrm{Yu}$ et al., 2008) and Bentamy et al. (2003), which are both created using a variational analysis method that combines wind measurements from satellite retrievals and in situ measurements with a background field from a weather model. For example, CCMP version 2 (V2.0) uses wind retrievals from imaging microwave radiometers (SSM/I, SSMIS, TMI, AMSRE, AMSR2, GMI, and WindSat) and microwave scatterometers (QuikSCAT and ASCAT). Measurements from moored buoys (NBDC, TAO-TRITON, PIRATA) are directly included in CCMP, whereas they are used to bias adjust the background field in the AOFLUX product. The CCMP V2.0 background field is the 6-hourly ERA-Interim reanalysis. The background for OAFLUX is the bias adjusted relative to OceanSITES buoys. Each of these products extends more than 30 years, from mid1987 to the present time, and will be further extended as buoy data and reanalysis fields become available. McGregor et al. (2017) recently evaluated CCMP V2.0 in the Tropical Pacific and identified several issues, including regional biases correlated with currents and distortion of derivative fields near the locations of moored buoys. We recommend a similar analysis of other gridded products. 


\section{OBSERVING SYSTEM GOVERNANCE}

There are three distinct satellite research communities (scatterometry, radiometry, and SAR) and a coastal HF radar community. The satellite communities interact at a very high level through the Committee on Earth Orbiting Satellites and the Coordination Group for Meteorological Satellites. Satellite data are widely used by national weather forecasting agencies that have international obligations to provide weather warning and forecasting information within their areas of responsibility under the umbrella of the World Meteorological Organization. Satellite ocean surface vector wind (OSVW) data are utilized in the daily operations of all national weather service offices with marine warning and forecasting responsibilities. These data are used to aid decisions to initiate, continue and terminate marine warnings, including advisories for tropical cyclones; adjust shortterm marine forecasts for the intensity and geographic coverage of winds; identify swell generation areas for longer-term wave forecasts; identify lows, highs, fronts, and convergence zones and examine their intensity and trends; and verify in real-time the NWP analyses of winds, waves, and feature intensity.

Militaries also provide marine weather forecasting and warning services to support military operations. At a science and applications level, the scatterometry community interacts with the wind vector portion of the radiometry community and a portion of the radiometric wind speed community through the IOVWST. There is overlap with the SAR research and applications community, with common definitions and model functions for retrieval. European and U. S. scientists contributed to the $\mathrm{cal} / \mathrm{val}$ of all scatterometers and started cal/val collaboration with India and more recently China.

Satellite wind products are made available by the producers and often through national laboratories. Specialized products are available from research centers and private companies. All scatterometry data are made publicly and freely available. Some SAR data are only available commercially. Scatterometer and radiometer data are very well-intercalibrated (Verhoef et al., 2017; Wentz et al., 2017) except for wind speeds $>20 \mathrm{~ms}^{-1}$, for which there is a $3 \mathrm{~ms}^{-1}$ bias between the most popular products (Vandemark et al., 2018) due to the absence of an absolute and a consistent in situ wind calibration reference.

The coastal HF radar community has high level governance from the Global Ocean Observing System (GOOS), with regional and a few national groups providing more localized governance. While there has been intercomparison with satellite data, much of the data from coastal HF radar have been too close to land for comparison to satellite date. The differences in spatial and temporal sampling between satellites and radars also contributes to differences in application and hence differences in user communities.

\section{DATA AND INFORMATION SYSTEMS}

The models for distribution of scatterometer data can be classified as either near-real time or delayed mode. NOAA and the Royal Netherlands Meteorological Institute (KNMI) produce near real-time scatterometer data for operational use that are subsequently archived. The Eumetsat Ocean \& Sea Ice Satellite
Application Facility (OSI SAF) operational product (developed by KNMI) is considered a science quality product, but to make it more homogeneous old data is reprocessed to the highest and most recent standards (Verhoef et al., 2017). Also, other science quality products are produced in a delayed mode. This is the norm for scatterometer products from JPL and Remote Sensing Systems. The speed of processing these science quality products had dramatically increased over the last decade. SAR wind data (section Synthetic Aperture Radar) are slower in production and cover only a small part of the ocean surface. All these products are made available through national laboratories or private companies. There are differences in formatting and metadata from group to group, which has resulted in a great deal of confusion in the user community. Similar to the Copernicus Marine Environment Monitoring Service (CMEMS), the Center for Ocean-Atmospheric Prediction Studies at Florida State University recently produced scatterometer data in a common format with common metadata, retaining the key information from the original data provider and stored in daily files rather than orbits. Thus, one code could be used to read data from several different scatterometers. One key shortcoming that must be addressed in future products is a rain impact flag that has a similar interpretation from product to product. The discussion in section Interpretation of Satellite Winds clearly indicates that while longer wavelengths can be used to greatly reduce problems with attenuation and backscatter associated with rain, that the rain-induced modification of the surface will impact retrievals. Therefore, we recommend that approaches such as Stiles and Dunbar (2010), with a rain impact flag that is similar to a rain-induced uncertainty in the vector wind components. Collocations of RapidScat with ASCAT and ASCAT with ScatSat will help to align Ku- ( $\sim 5 \%$ of data) and C-band $(\sim 0.5-1 \%$ of data) quality assessments (e.g., Lin and Portabella, 2017).

\section{HAZARDS AND MARINE SAFETY}

The primary operational benefit of satellite OSVW observations is the improvement of weather forecasting and warnings. In addition, knowledge of the winds and wind generated waves over the ocean is indispensable for the maritime transportation, fishing, and oil production industries, as well as for search and rescue efforts and the accurate tracking and management of marine hazards such as oil spills. It is also essential for determining the ocean forcing, wind induced mixing, currents (Kelly et al., 2004), and air/sea $\mathrm{CO}_{2}$ fluxes. Satellite OSVW data is now routinely utilized by operational weather centers around the world. The number of open ocean buoy and ship reports available at any given time is very limited, so the systematic mapping of the global ocean wind field delivered by satellite remote sensing instruments provides valuable situational awareness of conditions at the ocean surface.

\section{Impact on Numerical Weather Prediction Winds}

Currently, we can identify the deterministic scale for wind data assimilation, which is the scale supported by observations in both space and time to deterministically initialize the smallest 
(short-lived and small-amplitude) evolving scales in weather models. These scales will remain larger than $100 \mathrm{~km}$ over sea due to a persistent lack of temporal wind observation coverage. An effective dynamical model resolution can be defined, which is 5-10 times the grid length and generally smaller than the aforementioned deterministic scale. The ongoing challenge is to maximize the benefit from higher resolution observations to help support the evolution of the fast small-scale atmospheric structures, whilst the temporal coverage over sea remains limited (Stoffelen et al., 2019). This means that model scales smaller than the deterministic scale, essentially weather model noise, need to be accounted through data assimilation (Marseille and Stoffelen, 2017). For regional NWP, very high horizontal resolution models with grid lengths of $300 \mathrm{~m}$ or less are anticipated at the Met Office and elsewhere. For fine-scale model grid lengths, it will be important to consider the scatterometer footprint to reduce the representativeness errors and account for the deterministic scales, as mentioned above. With increased supercomputer resources, we can expect mesoscale model improvements from the use of rapidly updating $4 \mathrm{D}$-Var assimilation schemes, resolving the temporal and spatial scales of convection. Further research is needed to develop more realistic, situation-dependent models and observation error schemes [based, for example, on the wind variability within the resolution cell (Lin et al., 2016)] that can improve the balance in the weight given to observations and background, and provide the optimal spatial filtering in the analysis.

Although advancing meteorological applications to resolve smaller scales offers obvious benefits, measuring mesoscale, and convective-scale wind structures poses daunting challenges (Stoffelen et al., 2019):

1. Spatial resolution and coverage must be sufficient to capture the small atmospheric scales globally;

2. Accuracy must be sufficiently high to measure the gradients on the small scales;

3. Temporal sampling must be enhanced to match convective time scales ( $30 \mathrm{~min})$; and

4. Observation timeliness must match this time scale as well as the warning horizon needed to take action to avoid societal impacts.

NWP data assimilation is based on the best linear unbiased estimator (BLUE) and generally bias correction schemes are used to remove observation minus model biases. Figure 4 shows NWP model biases against scatterometers. Since typical random scatterometer errors on global model scales $(150 \mathrm{~km})$ are about $1.5 \mathrm{~m} / \mathrm{s}$ (Stoffelen et al., 2017b), many locations exist where monthly biases are larger and the BLUE paradigm is violated. We note that the largest differences between scatterometer and the European Centre for Medium-Range Weather Forecasting (ECMWF) model winds appear in regions near the Inter Tropical Convergence Zone, regions with ocean currents, and near land masses or sea ice. These areas correspond to areas with large atmospheric dynamics and extensive moist convection (Belmonte and Stoffelen, 2019). In addition to considerable speed biases, substantial systematic wind direction biases also exist in NWP (Sandu et al., 2011). NWP biases occur in all NWP models and are due to fast or mesoscale processes resolved by the scatterometer but not by models, such as (moist) convection (Lin et al., 2016), atmospheric turbulence (King et al., 2015), gravity waves and systematic errors in boundary layer parameterizations, or the lack of ocean currents. These biases tend to persist over time (not shown). The occurrence of these spatial biases is, however, ignored in data assimilation and their existence prevents the correct assimilation of observed dynamical weather features, following BLUE; thus, it could be worthwhile to develop local bias reduction schemes for scatterometers to further optimize their impact (Trindade et al., 2017).

Accurate strong winds are important in NWP in order to get an accurate analysis of high wind speed, thus improving forecasts of severe weather events and tracking of tropical and extra-tropical storms. Strong winds are relatively rare but their impact can be very large. The current generation of scatterometer instruments is still lacking in terms of analyzing high wind speeds, which are not sufficiently accurate above $20 \mathrm{~m} / \mathrm{s}$. Other surface wind measurements that provide strong wind observations do not provide wind vectors. Therefore, the idea of using cross-polarization measurements (which will be used on the next scatterometer generation on board the METOP-SG missions) to extend the useful range of wind speed to $40 \mathrm{~m} / \mathrm{s}$ or higher, is very promising. Alternatively, the Ka-band has greater sensitivity at high wind speeds and could provide a small enough footprint to see between rain bands (overcoming the issue of seeing through rain bands and dealing with rain modification of the surface).

Many NWP centers are moving toward coupled oceanatmosphere global models and coupled data assimilation systems. This is expected to allow a better use of near-surface observations, which could help to provide a more consistent analysis of both atmosphere and ocean variables. Recent experiments in the ECMWF CERA-SAT coupled assimilation system proved that the assimilation of surface winds, similar to scatterometer observations, has a substantial impact on the ocean parameters (e.g., temperature, salinity, and currents). The impact on the ocean variables is not only confined to the ocean surface but propagated down into the ocean column. Scatterometer observations provide information on the windinduced surface roughness (and therefore on the ocean mixing). Their assimilation in coupled systems is particularly important in areas where the detection of wind-induced ocean cooling is essential to correctly predict the intensification of the storm (e.g., tropical cyclones).

\section{Impact of Satellite Winds on Wave Model Development, Applications, and Forecasting}

Satellite winds offer a unique way to describe ocean surface conditions and the momentum transfer to the water, hence they support the modeling and forecasting of ocean wave generation and storm surges. Scatterometers respond to $\mathrm{cm}$-waves on the ocean, which generally do not appear to be affected by sea state. However, wind scatterometers may be sensitive to parameters 


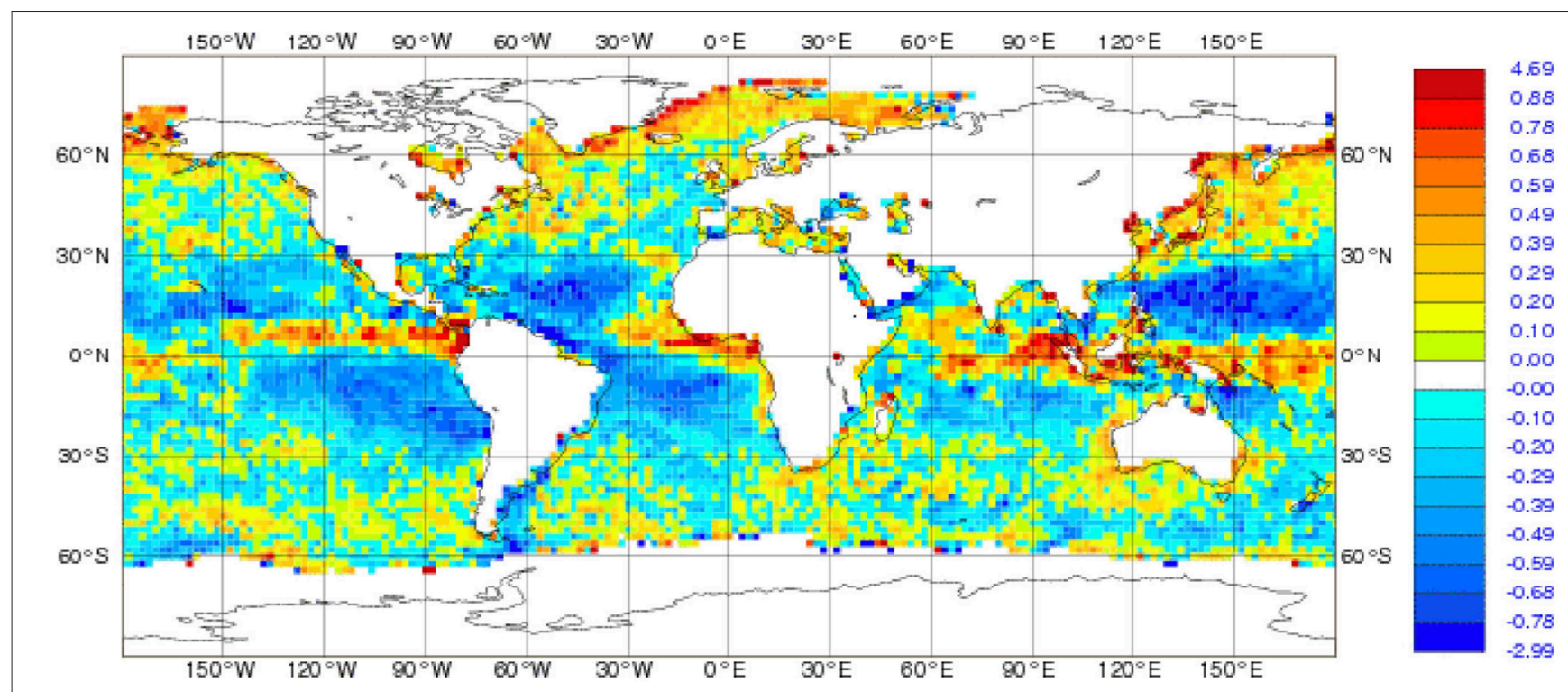

FIGURE 4 | Accumulated statistics for 10-m equivalent-neutral mean wind speed differences in ms ${ }^{-1}$ between ASCAT-A and the European Centre for Medium-Range Weather Forecasting (ECMWF) first guess (ASCAT-A minus ECMWF) for the period from 21:00 UTC on 31 October 2015 until 9 UTC on 5 December 2015 in boxes of two by two degrees. ${ }^{\circledR}$ EUMETSAT NWP SAF.

other than those that relate (correlate) with stress-equivalent winds, $\mathbf{U}_{10 S}$ (de Kloe et al., 2017). Variable effects such as rain (mainly for Ku-band scatterometers), extreme wind variability, complex sea states, SST, and currents can also affect geophysical model function accuracy, but are well-controlled.

\section{Impact on Storm Surge Forecasting}

Surface wind fields are the primary source of information for storm surge forecasting (Welander, 1961). Storm surges are mainly driven by momentum imparted by wind stress. Another important source of momentum for storm surges is large-scale coastally-trapped waves that transport energy over a distance impacting surge magnitude (Morey et al., 2006). Many storm surge forecast models do not directly include waves and may not include effects of tides, river runoff, and inverse barometer, implicitly adding these effects to the storm surge prediction (e.g., Jelesnianski et al., 1992; Weisberg and Zheng, 2008; Dukhovskoy and Morey, 2011). A basic system of linear storm surge prediction equations that has been used in many forecasting models is (Murty, 1984) dependent on the depthintegrated velocity components (transport), sea surface elevation, the wind stress vector, the bottom stress vector, the bottom depth, and atmospheric pressure. The evolution of a storm surge is controlled by balance between the pressure gradient force (due to the sea surface elevation), surface and bottom stresses, and the atmospheric pressure gradient. Calculation of the surface wind stress relies on the knowledge of the storm wind fields, which are very complex and vary greatly among the storms. The typical wind field of a storm is characterized by the radius of maximum sustained wind and the radius of the eyewall. However, storm wind fields are not symmetric, especially when the storm approaches land. Thus, high-resolution surface winds data are essential for accurate storm surge prediction. Temporal resolution is also important, especially for fast-moving storms, to force surge models.

Outer wind fields are also essential for storm surge forecasting. Many storm surges are preceded by forerunner waves (Kennedy et al., 2011) or strengthened by remotely generated shelf waves (Morey et al., 2006; Dukhovskoy and Morey, 2011). In both of these cases, outer wind fields determine the formation of these long waves propagating as coastal-trapped waves over large distances. Prediction of these waves is crucial for evacuation planning.

Observing the location and direction of stronger winds is key, with the more extreme winds in the core of a storm being much less important, as they have a smaller impact on the storm surge. Six-hourly sampling or better is desired.

\section{Marine Nowcasting}

Perhaps the most significant impact of satellite OSVW observations on national weather service operations has been the ability to routinely and consistently observe winds of hurricane force intensity in extratropical cyclones. This new capability, which started with the QuikSCAT mission, allowed forecasters to delineate and introduce (in December 2000) a new wind warning category of hurricane force intensity for extratropical cyclones. Prior to then, only two warning categories existed for extratropical wind sources-gale (33-47 knots) and storm (48 knots and higher) - with the most severe storms included in the rather common storm warning category, making it difficult to highlight and adequately warn for their severity. Forecasters had long been uncomfortable with this two-tier warning system, but a consistent observing capability was needed to divide the common occurrence of winds of 
48-63 knots and the less common and more dangerous winds in excess of 63 knots. QuikSCAT wind observations provided that capability.

The observational requirements for marine nowcasting are similar to those for storm surge forecasting except that measurements of extreme winds are required and near-shore winds are particularly useful.

\section{Tropical Cyclone Forecasting}

Over most of the global tropical cyclone formation basins, remotely sensed winds are the primary source of ocean surface wind information available to tropical cyclone forecasters and forecast models. The North Atlantic basin has the advantage of routine aircraft reconnaissance, but only when tropical cyclones are close enough to land for the aircraft to reach them. That said, there are too few non-satellite (in situ and aircraft) extreme wind observations $\left(>30 \mathrm{~ms}^{-1}\right)$ to calibrate satellite observations. Therefore, calibrations from sufficient observed conditions are extrapolated to higher wind speeds.

For forecasters, ocean surface wind vectors from scatterometers are an essential tool used for identifying when a tropical disturbance has obtained a closed circulation and tropical storm force winds, thus becoming a tropical cyclone. Surface wind information from scatterometers and radiometers can also be used to identify when a tropical cyclone has reached hurricane/typhoon strength and to identify important distances from the storm center (called wind radii) at which wind exceed operationally developed thresholds (Bender et al., 2017). Obtaining information on the wind radii of tropical cyclones is necessary for forecasters to be able to issue watches and warnings that enable advance preparations to reduce the loss of property and life (Knaff et al., 2018). In addition, the surface wind information provided by scatterometers and radiometers also allows the forecast models to initialize tropical cyclones and their environment more accurately, which leads to more accurate model forecasts (Isaksen and Stoffelen, 2000; Leidner et al., 2003; Stoffelen et al., 2013). Without remotely sensed surface winds, the accuracy of tropical cyclone forecasts would be degraded.

Meaningful comparisons between satellite-derived intensities and the operational 1-min sustained winds from the Best Track ${ }^{3}$ (BT) data set require that the BT data be scaled down to 10 min sustained winds. A scale factor of 0.93 has been applied to 1-min winds as recommended by the World Meteorological Organization (Harper et al., 2010). Powell et al. (2010) used 0.91 and others have used as much as 0.87 . The L-band radiometers (SMOS, SMAP) produced reliable estimates of the TC intensity when spatially averaged over the radiometer footprint. Examples are shown in Figures 5-7. The footprint averaged winds reached $60 \mathrm{~m} / \mathrm{s}$ in very intense TC. This calibration has been demonstrated by comparing the L-band radiometer wind speeds with spatially averaged wind speeds from the Stepped Frequency Microwave Radiometer (Reul et al., 2016; Meissner et al., 2017; Fore et al., 2018). The advantage of L-band radiometry is that the L-band emission from whitecaps keeps increasing approximately linearly with wind speed (Nordberg

$\overline{{ }^{3} \text { https://www.nhc.noaa.gov/ and http://www.metoc.navy.mil/jtwc }}$ et al., 1971; Monahan and O'Muircheartaigh, 1980; Reul and Chapron, 2003; Anguelova and Webster, 2006). Currently, the major limiting factor of the L-band radiometers to measure winds in tropical cyclones is their spatial resolution $(\sim 40 \mathrm{~km})$. In many cases, this does not allow the structure around the eye of the tropical cyclone to be resolved and also it puts a limitation on how close to the coast accurate ocean wind speed measurements can be performed. Another concern is the influence of heavy rain on whitecapping. Early analysis indicated there is some sensitivity to heavy rain, but this is small compared to the influence or rain (Draper and Long, 2004) on higher frequency radiometers and scatterometers. Finer spatial resolution is needed to further such studies.

Ocean surface winds also provide critical information to improve our still lacking understanding of the physical processes that determine hurricane genesis and evolution. Of particular interest to the community is improved understanding of what leads to hurricane rapid intensification (RI) and how we can better predict it. A very important aspect of the RI process is regarding the location of the convective activity with respect to the vortex structure as depicted by the Radius of Maximum Wind (RMW). Rogers et al. (2013) found that for intensifying hurricanes the peak in the distribution of deep convective clouds (CBs) was preferentially located inside the RMW, whereas for steadystate hurricanes the CBs were primarily located outside the RMW. Such a difference in the radial distribution of CBs was deemed important based on balance arguments (e.g., Shapiro and Willoughby, 1982; Vigh and Schubert, 2009). Near-simultaneous satellite observations of precipitation and ocean surface winds (e.g., Figure 8) allows investigation of the distribution of precipitation with respect to the RMW. Recent observational (e.g., Hristova-Veleva et al., 2016) and modeling (e.g., Hristova-Veleva et al., 2018; Saiprasanth et al., 2018) studies provide strong indications for the predictive capability of this approach.

An important use of SAR wind vector retrievals is the application to tropical cyclones (Li, 2017). While many wind sensors can retrieve wind speeds at or above the hurricane threshold $\left(\sim 33 \mathrm{~m} \mathrm{~s}^{-1}\right)$, currently only SAR can provide the kilometer-scale resolution needed to capture the extreme radial shear in the surface wind field. Gall et al. (2013) suggested that high resolution information, especially data associated with the inner core and eyewall region, is likely to be critical for improving hurricane intensity forecasts.

The Canadian Space Agency runs a Hurricane Watch program and the European Space Agency started the Satellite Hurricane Observations Campaign based on the Sentinel1A,B SAR missions. Tropical cyclone track forecasts are used to predict possible overlaps with the satellite image track and targeting decisions are made with relatively short lead times. Because the tropical cyclone boundary layer is an ideal environment for roll formation (Foster, 2005, 2013; Morrison et al., 2005) wind direction retrieval using the maximum gradient method of Koch (2004) or the maximum contrast method of Wackerman et al. (2006) work well (Horstmann et al., 2002, 2013; Horstmann and 

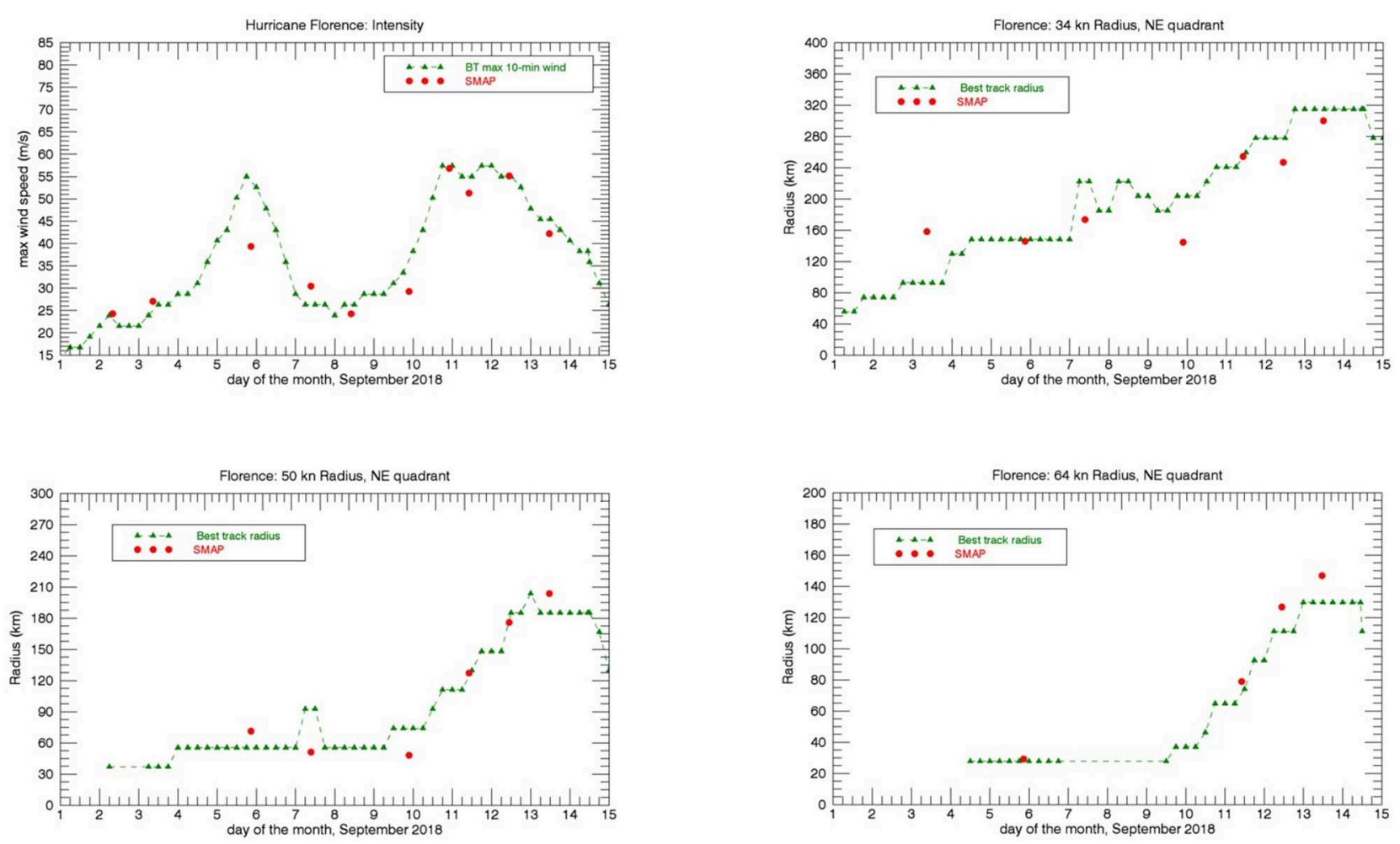

FIGURE 5 | Time series of intensity, 34-kt (gale force), 50-kt (storm force), and 64-kt (hurricane force) wind radii of Hurricane FLORENCE in September 2018. The red circles are the values from SMAP (40 km spatial resolution). The green dashed line shows the Best Track (BT) estimates from the U.S. National Hurricane Center. The BT intensity values have been scaled to 10-min sustained winds (Harper et al., 2010). The radii are shown for the NE sector.

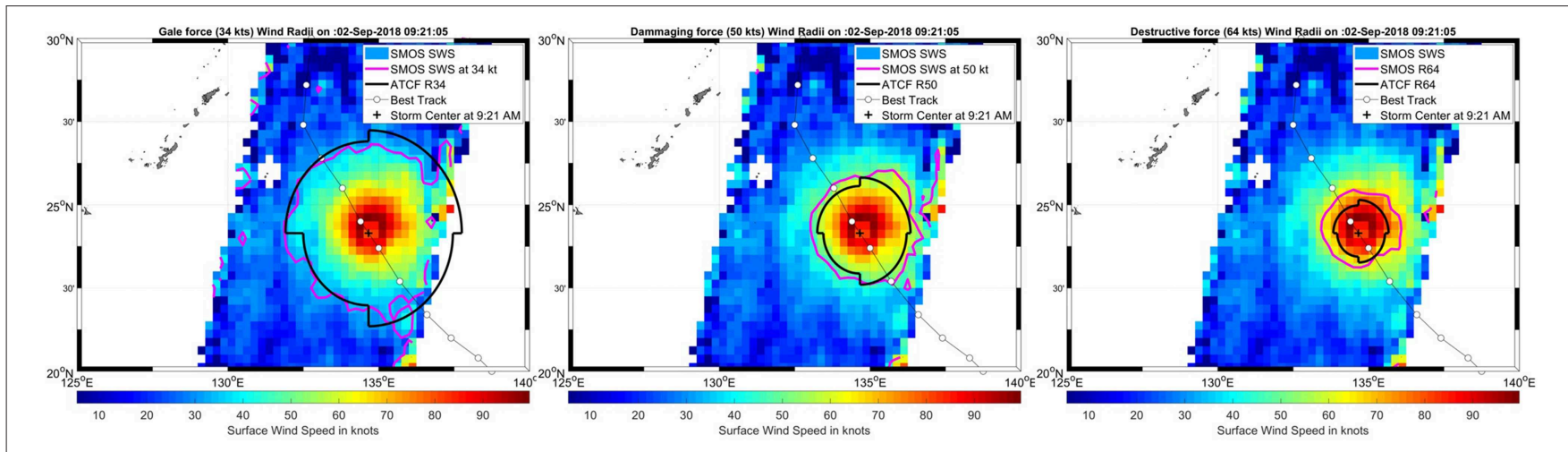

FIGURE 6 | Surface wind speed retrieved from SMOS data as the satellite swath intercepted Super-Typhoon JEBI on 02 SEP 2018. The wind speed contours from SMOS wind (pink) at 34 (left), 50 (middle), and 64 kt (right) are superimposed with Automated Tropical Cyclone Forecasting wind-radii forecasts (thick black). The direction of motion of the storm in the Best Track is from SE to NW.

Koch, 2005). Research has shown that cross-polarization SAR imagery has greater sensitivity at high winds than single polarization ( $\mathrm{HH}$ or $\mathrm{VV})$. Furthermore, its sensitivity to wind direction is relatively small. Very simple geophysical model functions have been constructed (Horstmann et al., 2013; Hwang et al., 2015). Sensors such as Sentinel-1 that can retrieve simultaneous co-polarization and crosspolarization imagery have the potential for improved wind vector retrievals.

\section{Ocean Forcing, Ecosystem Health, and Biodiversity}

Wind information is not only essential to depict convection in the tropics, but it also provides the Ekman ocean currents due to wind stress and an accurate view of the tropical circulation in the atmosphere, which is otherwise lacking. Observation requirements vary greatly depending on the spatial and temporal scales of phenomena that need to be captured in a model. To a rough approximation, the accuracy 

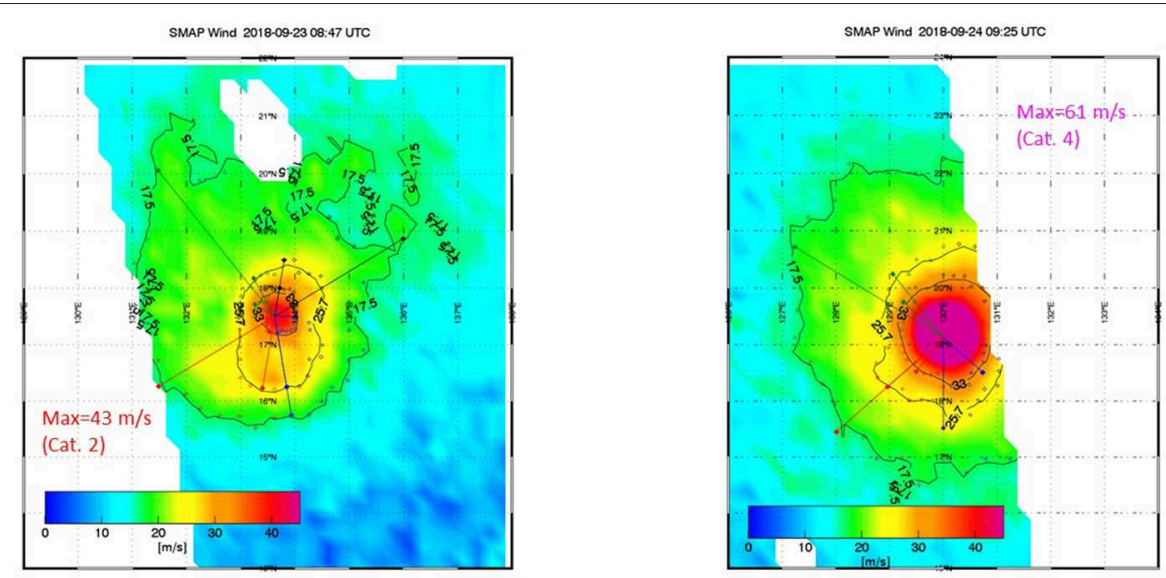

FIGURE 7 | Two snapshots of Typhoon TRAMl observed by SMAP (40-km spatial resolution). The observed intensity changed from $43 \mathrm{~ms}^{-1}$ (89 kts, category 2 ) on 2018-09-23 08:47 to $61 \mathrm{~ms}^{-1}$ (119 kts, category 4) on 2018-09-24 09:25 within <25 h. The lines show the 34, 50, and 64-kt wind radii in the various sectors.

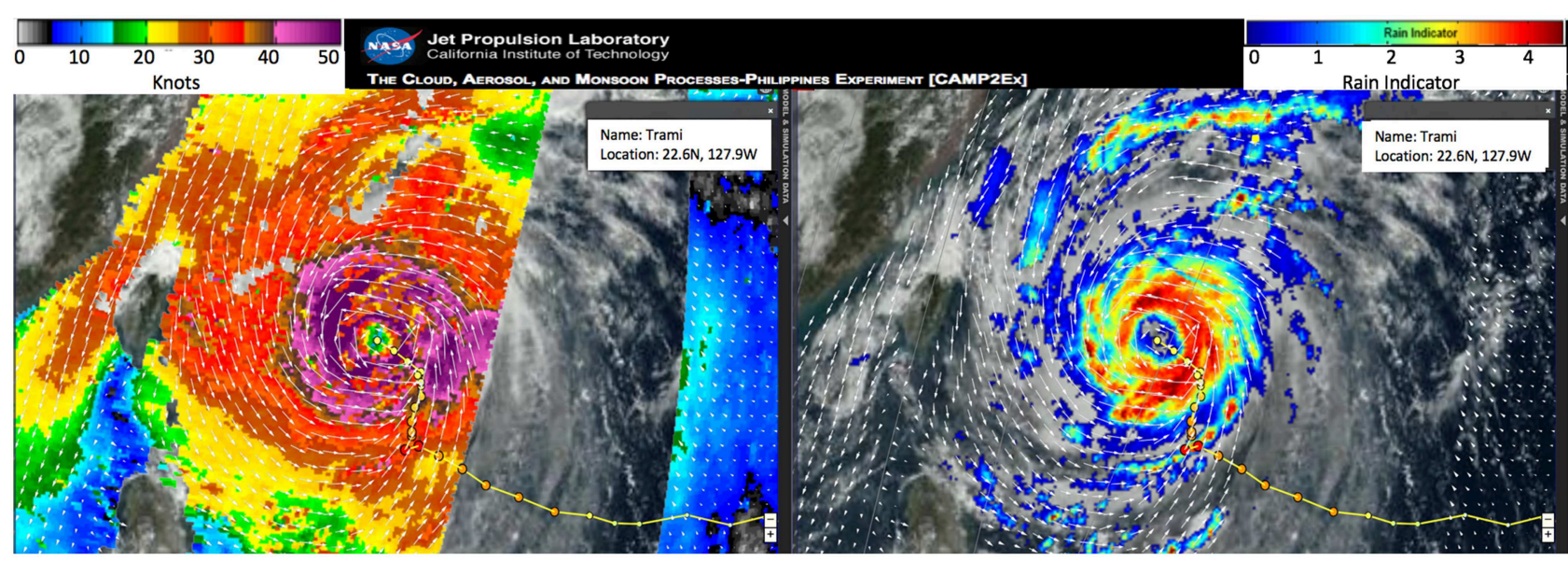

FIGURE 8 | Retrievals of the ocean surface winds from the Ku-band ScatSat-1 scatterometer as it flew over typhoon TRAMl on 28th September 2018 at 012 (left). Almost coincident depiction of the precipitation structure as observed by the passive microwave radiometer GMI on the GPM-core satellite, overlaid with the wind vectors retrieved from Scat-Sat1 (right). The background in both panels shows the cloud field as depicted by the visible channels of the Himawari geostationary satellite. Such multi-parameter and multi-instrument data can be visualized interactively in the CAMP2Ex and the North Atlantic Hurricane Watch portals that are part of the JPL Tropical Cyclone Information System.

requirement is 10 to $20 \%$ of the variability associated with the phenomena. Similarly, sampling requirements in space and time are driven by the need to capture the variability, and perhaps further constrained by the needs to reduce the random error in the observations. These requirements are estimated in Figure 9.

The need for these products in the ocean community is immense, as they provide spatial information on air-sea interaction and thus exchanges of heat, momentum, water vapor, gasses, and aerosols through processes of mixing, upwelling, and downwelling in both the atmosphere and the ocean. Moreover, this highly dynamic interface covers about $70 \%$ of the Earth's surface and, therefore, determines climate dynamics to a large degree.

Satellite vector wind products are essential for determining the large-scale ocean circulation and transport. Vector winds are also needed to estimate the ageostrophic (Ekman) component of ocean currents, and consequently are linked to coastal upwelling, primary productivity, cross shelf transport, ice transport, mixed layer evolution, and deepwater formation. Moreover, accurate wind speeds are essential for reliable computations of air/sea heat fluxes (sensible and latent heat fluxes) as well as mass fluxes (e.g., $\mathrm{CO}_{2}$ and $\mathrm{H}_{2} \mathrm{O}$ ), making surface winds critically important for budgeting energy, moisture and carbon, and for studies of ocean acidification and fish stocks. OSVW and surface stress are linked to ocean, atmospheric, cryospheric, and terrestrial climate change and listed as essential climate variables.

Marine pelagic communities are fueled by the productivity of tiny plankton that float with the currents and are supported by upwelling of deepwater nutrients. Surface wind stress, which simultaneously drives surface currents and introduces energy for turbulent mixing, is thus a primary driver of spatial and 
temporal variability in communities ranging from phytoplankton to fish. Accurate, high-resolution, remote measurements of wind stress have multiple potential utilities for biological and chemical oceanographers. Surface wind stress can be used to diagnose horizontal currents in the ocean, which are important for interpreting the past history of water parcels sampled during field campaigns. Surface wind stress (and the surface current velocities that must be determined as part of this measurement) can also be useful for diagnosing fronts, eddies, and other meso- and submesoscale features in the ocean. These regions are important habitats for many organisms for a variety of reasons. For example, increased turbulence in frontal regions can stimulate upwelling and vertical mixing, while cold-core eddies continually introduce nutrients to the surface ocean (McGillicuddy et al., 2007; Li et al., 2013). Frontal regions also serve as areas where different water parcels (with potentially different limiting nutrients) can mix, thus alleviating nutrient stress for phytoplankton. Fronts, eddies, and jets can also aggregate organisms in convergent zones and transport organisms long distances (Hofmann et al., 1991; Bakun, 2006). Consequently, these features have been found to be sites of increased primary productivity (Franks, 1992; Benitez-Nelson et al., 2007), higher mesozooplankton and fish biomass (LaraLopez et al., 2012; Ohman et al., 2012; Laiz-Carrión et al., 2015), and increased carbon export to the ocean interior (Omand et al., 2015; Stukel et al., 2017). High-resolution wind and current data are essential for interpreting the physical dynamics driving such patterns at the spatial scales sampled during field campaigns or using autonomous floats and gliders.

Surface wind stress is also crucial in mediating air-sea interactions. Air-sea fluxes of gases such as $\mathrm{O}_{2}$ and $\mathrm{CO}_{2}$, dimethyl sulfide, methane, and halocarbons are commonly estimated using a gas transfer velocity $(\mathrm{k})$ and the concentration gradient of the gasses between the oceanic mixed layer and the atmosphere (as measured from ships research cruises or automated ferry box systems). A necessity for an accurate estimate of those fluxes is a well-parameterized wind speed over the residence time of the gas within the water column (Wanninkhof, 1992). By using reanalyzed winds from e.g., the National Centers for Environmental Prediction (NCEP) and the ECMWF, changes in wind speeds prior to collection can be considered and a weighted average gas transfer velocity can be calculated (Jonsson et al., 2013). These data provide basin-wide reanalysis of wind speed data, however uncertainties in gas fluxes are large in oceanic regions where mesoscale events prevail (fronts, eddies, and coastal upwelling zone). High-resolution data can account for this variability and hence provide more precise estimates of gas transfer velocity.

While some regional measurements of wind speed are already in higher resolution (NCEP North American Regional Reanalysis: NARR), those data are lacking on a global scale. As shown in Bender et al. (2011), the standard deviation of differences between ship and reanalyzed winds is about $40 \%$ in the Atlantic and can lead to an even higher uncertainty as the gas transfer velocity scales with the square of wind speed.

Data products that would benefit from enhanced resolution include estimates of $\mathrm{CO}_{2}$ fluxes between the ocean and the atmosphere, estimates of net community production (the biological productivity in the mixed layer and hence the biological carbon pump), and estimates of other climate relevant gas fluxes mentioned above.

\section{Currents}

Currents are responsible for transporting debris, microorganisms, microplastics, and surface slicks (Maximenko et al., 2019). They are used in search and rescue and ship routing. Large-scale currents are mostly forced by surface winds and the rotation of the Earth. Deepwater currents are related to surface wind stress through Sverdrup transport, and surface currents related to wind and density-driven dynamic layer thicknesses plus Ekman currents (which are modeled by wind stress and frictional mixing). Thus, winds play a very important role in many types of currents, including wave-induced Stokes drift (Mouche et al., 2012).

The winds needed for large-scale currents are relatively easily met. Of greater interest is the episodic forcing from atmospheric storms and fronts, as well as mesoscale and smaller currents. Episodic currents have been shown to be responsible for greatly increasing across-shelf transport (Morey et al., 2009). Therefore, the observations must capture such features. Hurricane winds have been shown to produce $25 \%$ of the bioproductivity in a regional sea (Chu et al., 2000; Lin et al., 2003), therefore sampling and resolution should be sufficient to capture the winds around a hurricane. Through the assimilation of wind data in numerical weather models, an interpolation of winds between satellite overpasses may be performed on the larger scales $(>150 \mathrm{~km})$, but the small-scale variability measured by scatterometers will generally be lost in NWP fields (Belmonte and Stoffelen, 2019; Marseille and Stoffelen. Furthermore, four dimensional data assimilation does not work well with $25 \mathrm{~km}$-scale winds and observations only a few times a day.

Greater constraints come from the mesoscale interaction of winds and currents (Gaube et al., 2015; Shi, 2017). Currents modify surface roughness associated with waves. These changes in roughness and the currents influence the horizontal shear of wind and stress, causing very strong curl of stress and divergence of winds (Shi, 2017), which greatly increases vertical motion and vertical mixing, modifying the local energy budgets and surface temperature. Modeling studies indicate that changes in SST and winds alter air-sea fluxes and the moisture in the atmospheric column and hence the radiative fluxes. Observation of this kind of coupling requires winds on a $5 \mathrm{~km}$ scale and ideally every $3 \mathrm{~h}$, although the temporal resolution is likely to be achieved through data assimilation for coupled weather models. Traditional accuracies appear to be sufficient, albeit on a much smaller spatial scale. Doppler scatterometry has been suggested as an approach to measure coincident surface vector winds and currents (Rodriguez et al. this issue).

\section{Water, Food, and Energy Security}

Monsoon winds are critical to many agricultural regions, as they are essential for the supply of water, hydroelectric power, and 
agriculture. The Indian Monsoon is the best know example, supplying the water needs for a populous nation with agriculture as its leading economic activity. Monsoons also bring much needed rains to Eastern Asia, Australia, Central America, and the south central United States. In general, winds are a key part of the water cycle, crucial for evaporation ( $85 \%$ of rain comes from the ocean) and for transport of moisture. This transport is dominated by large-scale wind and pressure patterns and by atmospheric rivers. Many coastal regions benefit from rain associated with a sea breeze. Wind-driven upwelling supplies nutrients for fisheries. Wind also drive the currents (surface and deeper currents) that transport larva, thus influencing multiple aspects of the life cycle of finfish. In short, winds make enormous contributions to the availability of food from land and sea, and fresh water on land, which is often critical for the generation of energy.

The observational needs for the monsoon-related variability are mostly large-scale in both space and time, and hence sampling needs are well achieved with the existing system. Wind direction is important. However, changes in the winds on weekly to 20-day scales are associated with variability in monsoons (Roman-Stork, 2018). The observational needs for upwelling are much finer observations in space and time, requiring six hourly sampling or better, winds near the coast, and accurate directions. The coastal requirement is more easily met with highresolution winds, which better resolve upwelling and data closer to the coast.

Marine wind climatologies are also useful for estimating wind resources for power generation. However, sampling must be greatly increased for observations alone to be sufficient. Siting for wind power is often in near coastal regions, where NWP is poor and there are few observations from satellite. Thus, greater temporal and spatial resolution are highly desirable.

\section{Pollution and Human Health}

Marine pollution is transported by wind-driven currents and wind-driven waves, resulting in the collection of debris in surface convergence zones as elaborated in Maximenko et al. (2019). A large faction of protein consumed by humans now comes from the ocean, where some of this debris is consumed by marine creatures and then by humans (Cooley et al., 2009).

The other notable contribution of winds to the transport of pollution is associated with land-sea breezes. Cities can emit pollutants into the atmospheric boundary-layer and have them moved offshore by a land breeze only to be returned later by a land breeze. This return of pollution has been noted to substantially impact cities along the United States' Great Lakes (Walter, 1973). The observational requirements for this application are higher resolution winds $(5-10 \mathrm{~km})$ and frequent sampling that can be effectively assimilated into a coupled weather model.

\section{The Blue Economy}

Oceans are an intrinsic part of the global economy. Commercial fisheries add $\$ 270$ billion annually to the global gross domestic product, maritime and coastal tourism and recreation is a $\$ 7.2$ trillion industry, and $80 \%$ of international goods are transported by sea (World Bank, 2017). Other important ocean industries include offshore renewable energy, seabed extractive activities, and marine biotechnology and bioprospecting (UNEP, 2015). However, as a public good, oceans are overused, abused and/or degraded. Pendleton et al. (2012) argued that anthropogenic activities are the main reason for the significant deterioration in ocean, wetlands and coral reefs health. About $80 \%$ of the pollution and litter in oceans come from land-based human activities (Galgani et al., 2015). To avoid irreversible degradation and encourages better stewardship of natural resources, including oceans, the United Nations adopted the Sustainable Development Goals in 2015. Within the Sustainable Development Goals, the blue economy is an international agreement that seeks to promote economic growth while encouraging better manage the many aspects of oceanic sustainability (World Bank, 2017).

Other important developments include the fact that commercial ships have doubled in size, waterborne commerce has tripled, and the number of small boats and recreational water crafts has increased during the last 50 years. To keep ships on schedule and safe from dangerous ocean storms, the $\$ 200$ billion global marine shipping industry increasingly relies on accurate marine warnings and forecasts (Kite-Powell and Colgan, 2001; Kite-Powell, 2011). Accurate wind and wave information helps marine traffic avoid hazardous weather and keeps the costs of goods down, thus making products more affordable. Maritime commerce results in a contribution of $\$ 78.6$ billion annually and generates nearly 16 million jobs; one out of six jobs in the U.S. is marine-related (NOAA, 1998). As a result of QuikSCAT winds, marine warning and forecast services out to $48 \mathrm{~h}$ (for hurricane force cyclones) saves an estimated $\$ 135$ million annually in the North Pacific and North Atlantic dry bulk and container shipping industry alone by minimizing storm exposure (Kite-Powell et al., 2008; Kite-Powell, 2009, 2011).

The key observing requirements for the blue economy are sampling at 6-hourly intervals and accurate retrievals for extreme winds. Approaches to achieving this accuracy are discussed in section Near Coastal Processes.

\section{DISCOVERY}

\section{Air/Sea Surface Fluxes}

Estimation of air-sea exchanges of thermal energy (sensible and latent heat fluxes in this context), momentum, and gasses all require surface wind or surface stress. Cronin et al. (this issue) provide detailed accuracy requirements for heat exchange and stress needed for the study of a wide range of processes. In almost all applications, the bias in wind must be small to achieve the required accuracy in stress, latent heat, and sensible heat. The accuracy requirement for uncertainty (random errors) in wind is roughly 18 times the stress requirement, which is achievable given appropriate sampling and resolution of the variability discussed below. The sampling requirement cannot be met without sufficient coverage of diurnal and inertial variability, and the resolution requirement appears to be roughly $5 \mathrm{~km}$ assuming that sub-mesoscale variability makes only a minor contribution to fluxes. 
One challenge to determine air-sea fluxes stems from the use of transfer coefficients for momentum, sensible heat, and latent heat fluxes, particularly in cases when the fluxes are derived from in situ observations where currents were not measured. Satellite winds are current-relative, whereas in situ winds are Earth relative. We typically assume that eddy covariance methods provide unbiased in situ turbulent fluxes are measured without bias through an eddy covariance method and that in situ winds are measured without bias. In reality, turbulent fluxes depend on the vector difference of wind and current, rather than simply the Earth relative wind. The transfer coefficient is related to a ratio of the observed stress to the wind speed, where that wind speed should be current relative. If winds and currents tend to be in the same direction, the use of Earth-relative winds systematically underestimate the transfer coefficient and satellitederived fluxes. Measurements of surface currents coincident with surface winds would largely solve this problem and lead to improved flux models.

\section{Coupling Winds With SSTs and Currents}

Air-sea fluxes are also modulated by small-scale variability in winds associated with gradients in SSTs (Chelton et al., 2007; O'Neill, 2012) and currents (Gaube et al., 2015; Shi, 2017). A science challenge is to determine the relative importance of wind, boundary-layer stratification and SST in this modulation. Both wind and SST gradients modify the atmospheric boundary-layer in a manner that is correlated with air-sea differences associated with heat and momentum fluxes, leading to small-scale variability in heat fluxes and stress. Furthermore, these processes are nonlinear, meaning that the net changes because of this variability are non-zero. Net radiative fluxes are also modified because of changes in SST (modifying long-wave emission) and whitecap fraction (modifying longwave emission and shortwave albedo).

Observational studies of coupling between wind, currents, and SST have been largely based on satellite (Chelton et al., 2007) and in situ (O'Neill, 2012) measurements applicable on scales of $\geq 25 \mathrm{~km}$, with a few model-based studies (Zheng et al., 2013; Gaube et al., 2015; Ardhuin et al., 2017; Shi, 2017). Modeling research by Shi (2017) used two-way coupling for ocean, waves, and the atmosphere, with ocean-forced changes to the atmosphere feeding back to the ocean. Results showed much stronger coupling in the areas of strong current gradients than seen in studies that did not include waves and currents. These occurred because the ocean-induced changes in stress greatly modified the stress gradients and the curl of the stress, substantially enhancing vertical transport. This finding differed from observational studies, in part because the model had $3 \mathrm{~km}$ grid spacing and consequently could capture changes in stress on smaller spatial scales that favor stronger curl. Shi and Bourassa (2019) showed that the current gradients had a large impact on SST gradients and wind gradients. Furthermore, highresolution mesoscale ocean modeling studies found that strong gradients in currents occur over much of the ocean (McWilliams, 2016, others), suggesting that that the related signals in winds, turbulent fluxes, and upwelling have been missed in lowerresolution observations and models.
The oceanic vertical motion induced by winds and currents is important to biology and biogeochemistry. Vertical motions can transport nutrients and sub-surface water to the surface, with the nutrients causing greater bioproductivity and the exposure of sub-surface water enhancing gas exchanges that are exposurelimited. This motion also likely modifies vertical heat transport, suggesting that there should be greater vertical fluxes of heat along ocean fronts. The observational requirements to examine or better model these processes are $5 \mathrm{~km}$ resolution of winds and currents (and ideally SST and ocean color), where the winds and currents are coincident. The accuracy of wind vector components (speed alone is insufficient) is roughly the existing uncertainty for scatterometers (Chelton et al., 2018; Rodriguez et al., 2019) and about $0.5 \mathrm{~m} \mathrm{~s}^{-1}$ for currents, but at the $5 \mathrm{~km}$ scale. There must also be sufficient sampling (about 1.5 times per day, on average) to allow averaging to reduce the uncertainty in currents. Observations on these scales would be a tremendous breakthrough for understanding air-sea coupling processes and consequently for Earth systems modeling.

\section{High Winds}

More accurate observations of high surface winds from scatterometers, radiometers, and other spaceborne remote sensing instruments could lead to new discoveries about the magnitude of ocean surface mixing, air-sea fluxes, ocean currents, and the frequency of storm- and gale-force wind events. A better understanding of extreme ocean surface mixing, air-sea fluxes, and ocean currents from high winds may have implications on heat transfer within the ocean mixed layer, vertical nutrient transport, SST, and atmospheric stability, and these impacts can transfer all the way up to the atmospheric synoptic scale and the energy budget of the earth-atmosphere system.

Improved detection of storm- and gale-force wind events has applications in hazard avoidance for ships and extreme weather event impacts on humans, as well as the previously discussed implications on ocean surface mixing, air-sea fluxes, and ocean currents. If the frequency of storm- and gale-force wind events changes there will be implications on the climate scale.

Additionally, improved accuracy of remotely sensed surface winds will improve tropical cyclone detection and forecasting. In basins that rely on remotely sensed winds, being able to resolve high winds in tropical cyclones will provide forecasters with the necessary information to provide better analysis and forecast of surface winds to the public.

\section{Diurnal Variability}

Daily variations in solar radiative forcing result in diurnal wind fluctuations (e.g., Simpson, 1994). Near coastlines, differential heating of the land and ocean drives a land breeze/sea breeze circulation, most prominently in the summertime (e.g., Gille et al., 2003; Hyder et al., 2011; Dorman et al., 2013), but diurnal winds are not confined only to the coast. In the open ocean, nearly everywhere equatorward of $30^{\circ}$ latitude, statistically significant diurnal winds are observable (Dai and Deser, 1999; Gille et al., 2003, 2005). Diurnal winds can be comparatively small in magnitude relative to wind variability associated with synoptic storms, but their persistence makes them dynamically important 
and also a challenge for satellite retrievals. Quantifying diurnal wind variability is essential because diurnal winds can interact with diurnal heat fluxes and thus have a net (or "rectified") impact on upper ocean processes, influencing air-sea heat and gas exchange, mixed-layer depth, SST, and the timing of storms and coastal storm surge.

Full sampling of a diurnal cycle requires a minimum of four measurements, well separated in time, over the course of the 24-h cycle. A single sun-synchronous satellite (or a series of sun-synchronous satellites that all use the same equator crossing time) can only obtain measurements at two times of day, and thus samples at the Nyquist frequency of the diurnal cycle, which precludes determination of the amplitude and phase of the diurnal cycle. Two sun-synchronous satellites with different equator crossing times will sample different phases of the diurnal wind. If the satellites are well calibrated, their measurements can be combined to infer the structure of the diurnal wind, as was done for the QuikSCAT and ADEOS-2 tandem mission (e.g., Gille et al., 2005; Wood et al., 2009; Gille and Llewellyn Smith, 2014). Conversely, if the diurnal cycle were to be overlooked in cross-calibrating the satellites, then there would be a risk of misinterpreting diurnal wind fluctuations as satellite bias. Bias errors could, in principle, be minimized by using prior knowledge of the diurnal cycle to help interpret differences between measurements collected at different times of day. However, prior knowledge of the diurnal cycle is not trivially inferred from sparse observations; Giglio et al. (2018) highlighted the fact that the diurnal cycle has a non-stationary amplitude that varies throughout the year. Annual (and interannual) modulations of the diurnal cycle should be taken into account in evaluating diurnal wind variability. For instance, RapidScat, which flew on a non-sun-synchronous orbit, provided coincident measurements with multiple satellites, offering one strategy for intercalibrating different wind satellites. Diurnal variability and seasonal modulation of the diurnal cycle could be inferred with reasonable skill if the constellation of scatterometers met the IOVWST recommendation (summarized in Scatterometers) for three scatterometers, with two in sun-synchronous orbits at different times of day and a third in a non-sun-synchronous intercalibration orbit.

\section{Near Coastal Processes}

Winds in the region within $\sim 25 \mathrm{~km}$ of the coast are not wellresolved by currently operating satellite missions. Continentalscale reanalyses also have difficulty in this region as well because the model grids are too coarse to resolve the topography and the relevant dynamical scales (Kara et al., 2008b). However, sparse measurements from buoys, aircraft, and SAR, as well as high-resolution models with smaller domains, all show smallscale wind features generated by coastal topography. Hydraulic expansion fans in the marine boundary layer near capes and points (Winant et al., 1988; Rahn et al., 2013; Parish et al., 2016), coastally-trapped wind reversals (Nuss et al., 2000), and along-shore wind jets confined by coastal mountains can have cross-coast scales of $5-10 \mathrm{~km}$ or smaller. We only have limited knowledge of the climatology of these features. The associated wind stress divergence and curl (Dorman et al., 2013; Juliano et al., 2017) generate circulations in the ocean (Pringle and Dever, 2009; Perlin et al., 2011) that affect coastal fisheries and other ecosystems by changing transport patterns of fish and invertebrate larvae or harmful algal blooms; upwelling nutrients into the euphotic zone; and transporting carbon offshore. Surface stresses in coastal regions can also be larger than expected for the same wind and wave conditions in the open ocean, and directions of the stress and wind velocity can differ (Fisher et al., 2015; Ortiz-Suslow et al., 2015) due to the effects of short fetch or waves interacting with the bottom. Satellite ocean vector wind data have enabled recent progress in understanding coastal wind fluctuations in the region $>25 \mathrm{~km}$ from the coast (Fewings et al., 2016; Fewings, 2017) and their effects on ocean conditions (Flynn et al., 2017), but much remains to be learned about the area within $25 \mathrm{~km}$ of the coast.

Satellite wind measurements with $\sim 5 \mathrm{~km}$ resolution and coverage to within a few kilometers of the coast worldwide would advance our understanding of physical controls on coastal ecosystems, such as wind forcing of the water temperature and circulation variations that affect kelp forests and marine protected areas (Fewings et al., 2015; Aristizábal et al., 2016, 2017). Improved satellite-based coastal winds would also help marine weather forecasting (Samelson, 2019) for applications including search and rescue, oil spill and harmful algal bloom tracking, storm surge prediction, and wind energy production. Many of these applications do not require climate data record levels of accuracy in winds, because the variability at weatherband frequencies is strong-the wind velocity signals of interest are $O\left(\right.$ several $\left.\mathrm{m} \mathrm{s}^{-1}\right)$ or greater. Improvements in spatial resolution, spatial coverage within $25 \mathrm{~km}$ of the coast, and temporal sampling (ideally, at least four times daily) would advance our understanding of coastal processes, often more so than improving the accuracy of the wind measurements. To obtain the highest possible spatial resolution and finest coastal coverage, the Coastal Scatterometry Working Group of the International Ocean Vector Winds Science Team recommends that future satellite missions be designed to have as little onboard data averaging as possible. This will permit higher resolution reprocessing of the data beyond the standard products, such as the Ultra-High Resolution and coastal QuikSCAT products discussed in section Scatterometers (e.g., Plagge et al., 2009).

\section{High Latitude Processes and Water Mass Formation}

Vertical mixing resulting from air-sea fluxes of heat and momentum influence the rate of property exchange between the atmosphere and deeper ocean, and thus govern the ocean's capacity to store heat, carbon, and nutrients. Thus, even relatively small changes in wind stress can lead to significant changes in mixing and transport of these tracers into ocean interior (Klocker, 2018). Accurate representation of global water mass distribution and its recent trends crucially depends on skilled estimates of surface winds (Klocker, 2018).

The strongest trends of water mass properties in recent decades have been observed in the Southern Ocean (Roemmich et al., 2015; Haumann et al., 2016). The intensification and 
poleward shift of the westerly winds in the Southern Ocean in recent decades, enhanced upwelling of deep waters south of the Antarctic Circumpolar Current (ACC) and increased formation of mode and intermediate waters north of the ACC, causing large scale coherent changes in Southern Ocean circulation (Waugh et al., 2013). Much of the increase in global upper ocean heat content observed in recent decades has been attributed to this wind-forced volume changes of mode and intermediate waters (Gao et al., 2018).

Along with warming, Southern Ocean freshening is also a prominent climate change signal (Durack et al., 2012). Haumann et al. (2016) attributed freshening to an increase in wind-driven northward freshwater transport by Antarctic sea ice. Changing wind patterns lead to increased sea ice formation near Antarctica in winter, increasing transformation of deep water into denser bottom water due to brine rejection. After the sea ice is transported equatorward it melts, increasing the buoyancy of lighter variety of deep water, mode and intermediate waters (Abernathey et al., 2016; Haumann et al., 2016; Pellichero et al., 2018). This way wind-driven northward freshwater transport by Antarctic sea ice represents a dominant mechanism destroying the old, deep waters that upwell in the Southern Ocean driven by strong westerly winds (Abernathey et al., 2016).

The large-scale heat, freshwater, carbon, and nutrient content of the deep ocean is set by water masses that form via mechanisms acting on short time- and space scales (Li and Lee, 2017). Therefore, diagnosing, modeling, and predicting the highlatitude water mass changes requires accurate wind observations at high resolution in both time and space.

\section{Seasonal to Interannual Variability, Decadal Variability, Climate Variability, and Change}

Changes in large-scale wind patterns are associated with changes in surface pressure patterns, cloud cover, rainfall, ocean upwelling (Rodriguez et al., 2019, this issue), surface fluxes (Cronin et al., 2019), and temperature. Due to the large number of satellite marine wind observations, it is much easier to observe the changes in surface wind patterns than the changes in marine pressure patterns (Belmonte and Stoffelen, 2019). Decadal variability in winds is related to ocean biology and productivity (section High Latitude Processes and Water

\section{Wind \& Stress Accuracies and Processes}

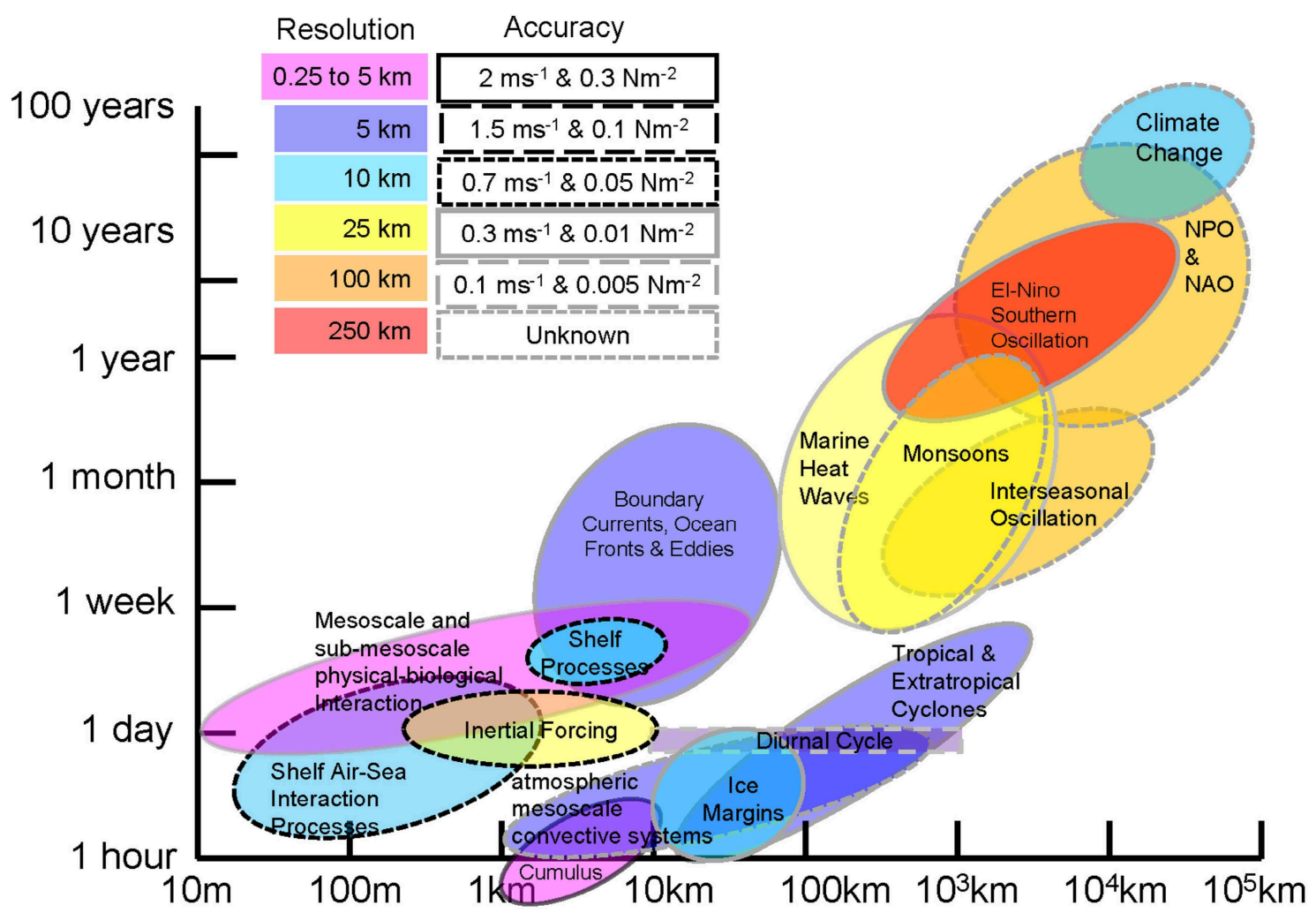

FIGURE 9 | Summary of observing system requirements for accuracy and resolution. For the larger-scale processes ( $\geq 100 \mathrm{~km}$ ), there will be sufficient sampling to reduce random errors to the point where they are a small concern compared to systematic errors in calibration. Currently, both bias and systematic errors are important at $25 \mathrm{~km}$ and at finer scales random errors will dominate on shorter timescales. Some of the smaller-scale processes (e.g., physical-biological interactions) are influenced more by horizontal shear than by the magnitude of the wind or stress, and therefore have very little sensitivity to systematic errors, but require finer spatial resolution to determine these spatial derivatives. 
Mass Formation). At one time, there was a concern about different satellites having different global trends. However, it was later found that these variations were due to the different spatial sampling of each satellite and that there was excellent agreement on local trends. Decadal changes in wind patterns appear to be far larger than observational limits (e.g., Yang et al., 2007; Zheng et al., 2017), making the restrictive factor the length of the observational record (e.g., Han et al., 2014).

Monitoring, understanding, and predicting oceanic variations associated with natural climate variability and human-induced changes is challenging (Stephens et al., 2018). For example, global change detection of $0.1 \mathrm{~m} / \mathrm{s}$ per decade is required, which corresponds to a calibration accuracy of a scatterometer of about $0.1 \mathrm{~dB}$ over 10 years. However, new methods provide precise instrument monitoring and potentially instrument intercalibration to within a few hundreds of $\mathrm{dB}$ (Belmonte et al., 2017). That said, current satellite data records and, as a result, multi-satellite gridded products do not yet achieve such precision and accurate intercalibration. As a result, the diverse instrument climate data records remain essential to monitor trends in climate, be it locally or globally (Verhoef et al., 2017; Wentz et al., 2017).

\section{SUMMARY OF OBSERVATIONAL NEEDS}

There is broad agreement across many oceanographic communities on the improvements needed for future wind sensors. These recommendations include:

1. Enhanced temporal coverage through an international virtual constellation to follow the fast, small transient mesoscales (Stoffelen et al., 2019);

2. Improved spatial resolution to better address mesoscale variability, improve coastal sampling, and make visible the areas between rain bands in tropical cyclones;

3. Coincident observations of surface vector winds and currents, to better understand the coupling of winds and currents and to improve surface flux parameterizations;

4. Instrument intercalibration (e.g., cone metrics) for all conditions;

5. Improved calibration for extreme winds (strong and weak);

6. In situ wind references (including surface currents) to calibrate and intercalibrate satellite winds and surface currents. This goal includes improved understanding of in situ and SFMR calibration at extreme winds to be used in the above calibration;

7. Consider new strategies to observe the TC inner-core and environment with high spatial and temporal resolution from the upper ocean (including pre-and post-storm) to the lower stratosphere. The observations should be used to diagnose physical processes, improve initialization through data assimilation, and evaluate and improve NWP models.

A. Prioritizes access to non-commercial C-Band SAR data collection (e.g., from Sentinel-1A and 1B satellites, NISAR, and PalSAR-2 and SAOCOM) in wide swath mode with dual-polarization over global tropical cyclones. Such observations will be useful for wind speed estimates (including RMW) for operational (and other) uses;

B. Set-up an internationally coordinated framework for targeting SAR acquisitions on TCs; and

8. Local bias correction in NWP to follow the BLUE paradigm and improve gridded products;

The accuracy requirements for surface winds and stress are highly dependent on the phenomena and the spatial/temporal scales of the variability associated with the phenomena. These complications are summarized in Figure 9, and underscore the needs listed above. The resolution needs are roughly 10 to $20 \%$ of the spatial extent of the phenomena except when spatial derivatives are critical, where the resolution need is increased by a factor of three. The temporal sampling requirement is roughly $10 \%$ of the timescale of the phenomena. The accuracy requirements are 10 to $20 \%$ of the typical dynamic range of the phenomena.

\section{AUTHOR CONTRIBUTIONS}

MB, TM, IC, PC, XD, GD, CD, DD, JE, AF, MF, RF, SG, BH, SH-V, HH, ZJ, JK, SK, AMa, MM, CM, AMo, MP, NR, LR, ER, CS, DS, AS, MS, BS, DW, and FW contributed to the text. JE developed an original figure.

\section{FUNDING}

This work was funded in part by the Ocean Observing and Monitoring Division, Climate Program Office (FundRef number 100007298), National Oceanic and Atmospheric Administration, U.S. Department of Commerce, through NGI grant 18-NGI342; NASA Physical Oceanography (Ocean Vector Wind Science Team grants NNH14CM09C NNX14AM69G, NNX14A078G, 80NSSC17K00537, 80NSSC18K1611, and 1419699 and 1531731 via JPL), NASA grant NNX17AH17G and NNX14AM69G, SMAP Science Utilization (contracts NNH17CA04C), the Office of Naval Research, Program Elements (0602435N), National Key Research \& Development Program of China (Contract No. 2016YFC1401002 and 2017YB0502800), the Spanish Research and Development Plan under Project L-BAND (ESP2017-89463C3-1-R), and NESDIS Base Research and Technology Funding (NESDIS Base Funding).

\section{ACKNOWLEDGMENTS}

The authors thank Roger Samelson, Dudley Chelton, Ted Strub, Eric Skyllingstad, and Larry O'Neill (all at Oregon State University), David Long (Brigham Young University), Timothy Lang (NASA Marshall Space Flight Center), and Jur Vogelzang (Royal Netherlands Meteorological Institute, KNMI) for helpful comments and suggestions, and Stefanie Linow (EUMETSAT) for the development of a figure. The views, opinions, and findings contained in this report are those of the authors and should not be construed as an official National Oceanic and Atmospheric Administration or U.S. Government position, policy, or decision. 


\section{REFERENCES}

Abernathey, R. P., Cerovecki, I., Holland, P. R., Newsom, E., Mazloff, M., and Talley, L. D. (2016). Water-mass transformation by sea ice in the upper branch of the Southern Ocean overturning. Nat. Geosci. 9:596. doi: 10.1038/ngeo2749

Alpers, W., Biao, Z., Alexis, M., Kan, Z., and Pak Wai, C. (2016). Rain footprints on C-band synthetic aperture radar images of the ocean - revisited. Remote Sens. Environ. 187, 169-185. doi: 10.1016/j.rse.2016.10.015

Anguelova, M., and Webster, F. (2006). Whitecap coverage from satellite measurements: a first step toward modeling the variability of oceanic whitecaps, 2008. J. Geophys. Res. 111. doi: 10.1029/2005JC003158

Ardhuin, F., Gille, S. T., Menemenlis, D., Rocha, C. B., Rascle, N., Chapron, B., et al. (2017). Small-scale open ocean currents have large effects on wind wave heights. J. Geophys. Res. Oceans 122, 4500-4517. doi: 10.1002/2016JC012413

Aristizábal, M. F., Fewings, M. R., and Washburn, L. (2016). Contrasting spatial patterns in the diurnal and semidiurnal temperature variability in the Santa Barbara Channel, California. J. Geophys. Res. Oceans 121, 427-440. doi: 10.1002/2015JC011239

Aristizábal, M. F., Fewings, M. R., and Washburn, L. (2017). Effects of the relaxation of upwelling-favorable winds on the diurnal and semidiurnal water temperature fluctuations in the Santa Barbara Channel, California. J. Geophys. Res. Oceans 122, 7958-7977. doi: 10.1002/2017JC013199

Atlas, R. M., Hoffman, R. N., Ardizzone, J., Leidner, S. M., Jusem, J. C., Smith, D. K., et al. (2011). A cross-calibrated, multi-platform ocean surface wind velocity product for meteorological and oceanographic applications. Bull. Am. Meteorol. Soc. 92, 157-174. doi: 10.1175/2010BAMS2946.1

Attema, E. P. (1991). The active microwave instrument on-board the ERS-1 satellite. Proc. IEEE 79, 791-799. doi: 10.1109/5.90158

Bakun, A. (2006). Fronts and eddies as key structures in the habitat of marine fish larvae: opportunity, adaptive response and competitive advantage. Sci. Mar. 70, 105-122. doi: 10.3989/scimar.2006.70s2105

Banal, S., Iris, S., and Saint-Jean, R. (2007). "Canadian space agency hurricane watch program: archive contents, data access and improved planning strategies," in Proceeding of IEEE International Geoscience and Remote Sensing Symposium (Barcelona), 3494-3497.

Beal, R. S. (ed.). (2005). High Resolution Wind Monitoring With Wide Swath SAR: A Users Guide. U.S. Washington, DC: Department of Commerce; National Oceanic and Atmospheric Administration; National Environmental Satellite, Data, and Information Serve; Office of Research and Applications.

Belmonte, R. M., and Stoffelen, A. (2019). Characterizing ERA-interim and ERA5 surface wind biases using ASCAT. Ocean Sci. Discuss. 15, 831-852. doi: 10.5194/os-15-831-2019

Belmonte, R. M., Stoffelen, A., Verspeek, J., Verhoef, A., Neyt, X., and Anderson, C. (2017). Cone metrics: a new tool for the intercomparison of scatterometer records. IEEE J. Select. Topics Appl. Earth Observ. Remote Sens. 10, 2195-2204. doi: 10.1109/JSTARS.2017.2647842

Belward, A., Bourassa, M., Dowell, M., Briggs, S., Dolman, H., Holmlund, K., et al. (2016). The Global Observing System for Climate: Implementation Needs. GCOS-200, 342.

Bender, M. A., Marchok, T. P., Sampson, C. R., Knaff, J. A., and Morin, M. J. (2017). Impact of storm size on prediction of storm track and intensity using the 2016 operational GFDL Hurricane Model. Wea Forecast. 32, 1491-1508. doi: 10.1175/WAF-D-16-0220.1

Bender, M. L., Kinter, S., Cassar, N., and Wanninkhof, R. (2011), Evaluating gas transfer velocity parameterizations using upper ocean radon distributions. J. Geophys. Res. 116:C02010. doi: 10.1029/2009JC005805

Benitez-Nelson, C. R., Bidigare, R. R., Dickey, T. D., Landry, M. R., Leonard, C. L., Brown, S. L., et al. (2007). Mesoscale eddies drive increased silica export in the subtropical Pacific Ocean. Science 316, 1017-1021. doi: $10.1126 /$ science. 1136221

Bentamy, A., Katsaros, K. B., Mestas-Nuñez, A. M., Drennan, W. M., Forde, E. B., and Roquet, H. (2003). Satellite estimates of wind speed and latent heat flux over the global oceans. J. Clim. 16, 637-656. doi: 10.1175/1520-0442(2003)016<0637:SEOWSA $>2.0 . C O ; 2$

Bettenhausen, M. H., Smith, C. K., Bevilacqua, R. M., Nai-Yu, W., Gaiser, P. W., and Cox, S. (2006). A nonlinear optimization algorithm for WindSat wind vector retrievals. IEEE Trans. Geosci. Remote Sens. 44, 597-610. doi: 10.1109/TGRS.2005.862504
Bourassa, M. A. (2006). "Satellite-based observations of surface turbulent stress during severe weather," in Atmosphere - Ocean Interactions, Vol. 2, ed W. Perrie (Torquay: Wessex Institute of Technology Press), 35-52.

Bourassa, M. A., and Hughes, P. J. (2018). "Turbulent heat fluxes and wind remote sensing," in New Frontiers in Operational Oceanography, eds E. P. Chassignet, A. Pascual, J. Tintoré, and J. Verron (Tallahassee, FL: GODAE OceanView), 245-270.

Bourassa, M. A., Rodriguez, E., and Gaston, R. (2009). Summary of the 2008 NASA ocean vector winds science team meeting. Bull. Am. Meteorol. Soc. 91, 925-928. doi: 10.1175/2010BAMS2880.1

Brown, R. A. (1980). Longitudinal instabilities and secondary flows in the planetary boundary layer: a review. Rev. Geophys. 18, 683-697. doi: 10.1029/RG018i003p00683

Brown, S., Paolo, F., Amarit, K., Frank, M., Lance, M., Oliver, M., et al. (2017). “The COWVR mission: demonstrating the capability of a new generation of small satellite weather sensors," in IEEE Aerospace Conference (Big Sky, MT), 1-7.

Chelton, D. B., Schlax, M. G., and Samelson, R. M. (2007). Summertime coupling between sea surface temperature and wind stress in the California current system. J. Phys. Oceanogr. 37, 495-517. doi: 10.1175/JPO 3025.1

Chelton, D. B., Schlax, M. G., Samelson, R. M., Farrar, J. T., Molemaker, M. J., McWilliams, J. C., et al. (2018). Prospects for future satellite estimation of smallscale variability of ocean surface velocity and vorticity. Prog. Oceanogr. 173, 256-350. doi: 10.1016/j.pocean.2018.10.012

Chu, P. C., Veneziano, J. M., Fan, C., Carron, M. J., and Liu, W. T. (2000). Response of the South China Sea to tropical cyclone Ernie 1996. J. Geophys. Res. Oceans 105, 13991-14009. doi: 10.1029/2000JC900035

Cooley, S. R., Kite-Powell, H. L., and Doney, S. C. (2009). Ocean acidification's potential to alter global marine ecosystem services. Oceanography 22, 172-181. doi: 10.5670/oceanog.2009.106

Cornillon, P., and Park, K. A. (2001), Warm core ring velocities inferred from NSCAT. Geophys. Res. Lett. 28, 575-578. doi: 10.1029/2000GL0 11487

Cronin, M. F., Gentemann, C. L., Edson, J., Ueki, I., Bourassa, M., Brown, S., et al. (2019). Air-sea fluxes with a focus on heat and momentum. Front. Mar. Sci. 6:430. doi: $10.3389 /$ fmars.2019.00430

Dai, A., and Deser, C. (1999). Diurnal and semidiurnal variations in global surface wind and divergence fields. J. Geophys. Res. 104, 31109-25. doi: 10.1029/1999JD900927

Donelan, M., Haus, B., Reul, N., Plant, W., Stiassnie, M., Graber, H., et al. (2004). On the limiting aerodynamic roughness of the ocean in very strong winds Geophys. Res. Lett. 31. doi: 10.1029/2004GL019460

Donlon, C. J. (2018). Copernicus Imaging Microwave Radiometer (CIMR) Mission Requirements Document. Norrdwijk: European Space Agency. Available online at: https://cimr.eu/documents/

Dorman, C. E., Mejia, J. F., and Koračin, D. (2013). Impact of U.S. west coastline inhomogeneity and synoptic forcing on winds, wind stress, and wind stress curl during upwelling season. J. Geophys. Res. Ocean 118, 4036-4051. doi: 10.1002 /jgrc. 20282

Draper, D. W., and Long, D. G. (2004). Evaluating the effect of rain on SeaWinds scatterometer measurements. J. Geophys. Res. 109. doi: 10.1029/2002JC0 01741

Draper, D. W., Newell, D. A., Wentz, F. J., Krimchansky, S., and SkofronickJackson, G. M. (2015). The global precipitation measurement (GPM) microwave imager (GMI): instrument overview and early on-orbit performance. IEEE J. Select. Topics Appl. Earth Observ. Remote Sens. 8, 3452-3462. doi: 10.1109/JSTARS.2015.2403303

Dukhovskoy, D. S., and Morey, S. L. (2011). Simulation of the Hurricane Dennis storm surge and considerations for vertical resolution. Nat. Hazards J. 58, 511-540. doi: 10.1007/s11069-010-9684-5

Durack, P. J., Wiiffels, S. E., and Matear, R. J. (2012). Ocean salinities reveal strong global water cycle intensification during 1950 to 2000. Science 336, 455-458. doi: $10.1126 /$ science. 1212222

Durden, S., and Perkovic-Martin, D. (2017). "The RapidScat ocean winds scatterometer," in IEEE Geoscience and Remote Sensing Magazine.

Entekhabi, D., Njoku, E. G., O’Neill, P. E., Kellogg, K. H., Crow, W. T., Edelstein, W. N., et al. (2010). The soil moisture active passive (SMAP) mission. Proc. IEEE 98, 704-716. doi: 10.1109/JPROC.2010.2043918 
Entekhabi, D., Yueh, S., O’Neill, P. E., Kellogg, K. H., Allen, A., Bindlish, R., et al. (2014). SMAP Handbook-Soil Moisture Active Passive: Mapping Soil Moisture and Freeze/Thaw from Space. Pasadena, CA: JPL Publication.

Fewings, M. R. (2017). Large-scale structure in wind forcing over the california current system in summer. Mon. Wea. Rev. 145, 4227-4247. doi: 10.1175/MWR-D-17-0106.1

Fewings, M. R., Washburn, L., and Carter Ohlmann, J. (2015). Coastal water circulation patterns around the Northern Channel Islands and Point Conception, California. Prog. Oceanogr. 138, 283-304. doi: 10.1016/j.pocean.2015.10.001

Fewings, M. R., Washburn, L., Dorman, C. E., Gotschalk, C., and Lombardo, K. (2016). Synoptic forcing of wind relaxations at Pt. Conception, California. J. Geophys. Res. Oceans 121, 5711-5730. doi: 10.1002/2016JC011699

Figa-Saldaña, J., Wilson, J. J. W., Attema, E., Gelsthorpe, R., Drinkwater, M. R., and Stoffelen, A. (2002). The advanced scatterometer (ASCAT) on the meteorological operational (MetOp) platform: a follow on for European wind scatterometers. Can. J. Remote Sens. 28, 404-412. doi: 10.5589/m02-035

Fisher, A. W., Sanford, L. P., and Suttles, S. E. (2015). Wind stress dynamics in Chesapeake Bay: Spatiotemporal variability and wave dependence in a fetch-limited en- vironment. J. Phys. Oceanogr. 45, 2679-2696. doi: 10.1175/JPO-D-15-0004.1

Flynn, K. R., Fewings, M. R., Gotschalk, C., and Lombardo, K. (2017), Large-scale anomalies in sea-surface temperature and air-sea fluxes during wind relaxation events off the United States West Coast in summer. J. Geophys. Res. Oceans 122, 2574-2594, doi: 10.1002/2016JC012613

Fore, A. G., Stiles, B. W., Chau, A. H., Williams, B. A., Dunbar, R. S., and Rodríguez, E. (2014). Point-wise wind retrieval and ambiguity removal improvements for the QuikSCAT climatological data set. IEEE Trans. Geosci. Remote Sens. 52, 51-59. doi: 10.1109/TGRS.2012.2235843

Fore, A. G., Yueh, S. H., Stiles, B. W., Tang, W., and Hayashi, A. K. (2018). SMAP radiometer-only tropical cyclone intensity and size validation. IEEE Geosci. Remote Sens. Lett. 15, 1480-1484. doi: 10.1109/LGRS.2018.2849649

Fore, A. G., Yueh, S. H., Tang, W., Stiles, B. W., and Hayashi, A. K. (2016). Combined active/passive retrievals of ocean vector wind and sea surface salinity with SMAP. IEEE Trans. Geosci. Remote Sens. 54, 7396-7404. doi: 10.1109/TGRS.2016.2601486

Foster, R. C. (2005). Why rolls are prevalent in the hurricane boundary layer. J. Atmos. Sci. 62, 2647-2661. doi: 10.1175/JAS3475.1

Foster, R. C. (2013). Signature of large aspect ratio roll vortices in SAR images of tropical cyclones. Oceanography 26, 58-67. doi: 10.5670/oceanog.2013.31

Franks, P. J. (1992). Phytoplankton blooms at fronts: patterns, scales, and physical forcing mechanisms. Rev. Aquatic Sci. 6, 121-137.

Fu, L.-L., and Holt, B. (1982). Seasat Views Oceans and Sea Ice With SyntheticAperture Radar. NASA; Jet Propulsion Laboratory Publication (81-120), 200.

Gade, M., and Stoffelen, A. (2019). "An introduction to microwave remote sensing of the Asian Seas," in Remote Sensing of the Asian Seas, 1st Edn., eds V. Barale and M. Gade (Cham: Springer), XXXV; 526; 81-101.

Gaiser, P., St Germain, K. M., Twarog, E. M., Poe, G. A., Purdy, W., Richardson, D., et al. (2004). The WindSat space borne polarimetric microwave radiometer: sensor description and early orbit performance. IEEE Trans. Geosci. Remote Sens. 42, 2347-2361. doi: 10.1109/TGRS.2004.836867

Galgani, F., Hanke, G., and Maes, T. (2015). "Global distribution, composition and abundance of marine litter," in Marine Anthropogenic Litter eds M. Bergmann, L. Gutow, and M. Klages (Cham, Heidelberg, New York, NY, Dordrecht, London: Springer International Publishing), 29-56. Available online at: https:// www.amazon.com/Marine-Anthropogenic-Litter-Melanie-Bergmann-ebook/ $\mathrm{dp} /$ B00YOFPEGC/ref=sr_1_fkmr0_1?keywords=Marine+anthropogenic + litter\&qid $=1564883584 \& s=$ books\&sr $=8-1$ - fkmr0\#reader_B00YOFPEGC

Gall, R., Franklin, J., Marks, F., Rappaport, E. N., and Toepfer, F. (2013). The hurricane forecast improvement project. Bull. Am. Meteorol. Soc. 94, 329-343. doi: 10.1175/BAMS-D-12-00071.1

Gao, L., Rintoul, S. R., and Yu, W. (2018). Recent wind-driven change in Subantarctic Mode Water and its impact on ocean heat storage. Nat. Clim. Change 8, 58-63. doi: 10.1038/s41558-017-0022-8

Gaube, P., Chelton, D. B., Samelson, R. M., Schlax, M. G., and O'Neill, L. W. (2015). Satellite observations of mesoscale eddy-induced Ekman pumping. J. Phys. Oceanogr. 45, 104-132. doi: 10.1175/JPO-D-14-0032.1

GCOS (2015). Status of the Global Observing System for Climate. GCOS.
Gerling, T. W. (1986). Structure of the surface wind field from the Seasat SAR. J. Geophys. Res. 91, 2308-2320. doi: 10.1029/JC091iC02p 02308

Giglio, D., Cornuelle, B. D., Northcott, D. M., and Gille, S. T. (2018). Modulation of Diurnal Winds in the Tropical Oceans. Barcelona: International Ocean Vector Wind Science Team. Available online at: https://mdc.coaps.fsu.edu/ scatterometry/meeting/docs/2018/docs/ThursdayApril26/Thursday_morning/ Giglio_OVWST2018.pdf

Gille, S. T., and Llewellyn Smith, S. G. (2014). When land breezes collide: converging diurnal winds over small bodies of water. Q. J. R. Meteorol. Soc. 140, 2573-2581. doi: 10.1002/qj.2322

Gille, S. T., Llewellyn Smith, S. G., and Lee, S. M. (2003). Measuring the sea breeze from QuikSCAT scatterometry. Geophys. Res. Lett. 30. doi: 10.1029/2002GL016230

Gille, S. T., Llewellyn Smith, S. G., and Statom, N. M. (2005). Global observations of the land breeze. Geophys. Res. Lett. 32. doi: 10.1029/2004GL022139

Han, W., Vialard, J., McPhaden, M. J., Lee, T., Masumoto, Y., Feng, M., et al. (2014). Indian ocean decedal variability. Bull. Am. Meteorol. Soc. 95, 1679-1703. doi: 10.1175/BAMS-D-13-00028.1

Harper, B., Kepert, J., and Ginger, J. (2010). Guidelines for Converting Between Various Wind Averaging Periods in Tropical Cyclone Conditions. World Metrological Organization. Available online at: https:/www.wmo.int/pages/ prog/www/tcp/documents/WMO_TD_1555_en.pdf

Haumann, F. A., Gruber, N., Münnich, M., Frenger, I., and Kern, S. (2016). Sea ice transport driving Southern Ocean salinity and its recent trends. Nature 537, 89-92. doi: 10.1038/nature19101

Hein, P. F., and Brown, R. A. (1988). Observations of longitudinal roll vortices during arctic cold air outbreaks over open water. Bound. Layer Meteorol. 45, 177-199. doi: 10.1007/BF00120822

Heron, M., and Rose, R. (1986). On the application of HF ocean radar to the observation of temporal and spatial changes in wind direction. IEEE J. Oceanic Eng. 11, 210-218. doi: 10.1109/JOE.1986.1145173

Hilburn, K., and Wentz, F. (2008). Intercalibrated passive microwave rain products from the unified microwave ocean retrieval algorithm (UMORA). J. Appl. Meteor. Climatol. 47, 778-794. doi: 10.1175/2007JAMC1635.1

Hilburn, K. A., Meissner, T., Wentz, F. J., and Brown, S. T. (2016). Ocean vector winds from WindSat two-look polarimetric radiances. IEEE Trans. Geosci. Remote Sens. 54, 918-931. doi: 10.1109/TGRS.2015.2469633

Hilburn, K. A., Wentz, F. J., Smith, D. K., and Ashcroft, P. D. (2006). Correcting active scatterometer data for the effects of rain using passive microwave data. J. Appl. Meteorol. Climatol. 45, 382-398. doi: 10.1175/JAM2357.1

Hofmann, E. E., Hedstrom, K. S., Moisan, J. R., Haidvogel, D. B., and Mackas, D. L. (1991). Use of simulated drifter tracks to investigate general transport patterns and residence times in the coastal transition zone. J. Geophys. Res. Oceans 96, 15041-15052. doi: 10.1029/91JC00832

Hollinger, J. P., Peirce, J. L., and Poe, G. A. (1990). SSM/I instrument evaluation. IEEE Trans. Geosci. Remote Sens. 28,781-790. doi: 10.1109/36.58964

Horstmann, J., Christopher, W., Silvia, F., and Salvatore, M. (2013). Tropical cyclone winds retrieved from synthetic aperture radars. Oceanography 26, 46-57.

Horstmann, J., and Koch, W. (2005). Measurement of sea surface winds using synthetic aperture radars IEEE Trans. Geosci. Remote Sens. 30, 508-515 doi: 10.1109/JOE.2005.857514

Horstmann, J., Koch, W., Lehner, S., and Tonboe, R. (2002). Ocean winds from RADARSAT-1 ScanSAR. Can. J. Remote Sens. 28, 524-533. doi: $10.5589 / \mathrm{m} 02-043$

Horstmann, J., Koch,W., Lehner, S., and Tonboe, R. (2000). Wind retrieval over the ocean using synthetic aperture radar with C-band $\mathrm{HH}$ polarization. IEEE Trans. Geosci. Remote Sens. 38, 2122-2131. doi: 10.1109/36.868871

Hristova-Veleva, S. M., Chen, H., Gopalakrishnan, S. G., and Haddad, Z. S. (2018). "Low-wave number analysis of observations and ensemble forecasts to develop metrics for the selection of most realistic members to study multiscale interactions between the environment and the convective organization of hurricanes: focus on rapid intensification," in 98th AMS Annual Meeting; $22^{n d}$ Conference on Integrated Observing and Assimilation Systems for the Atmosphere, Oceans, and Land Surface (IOAS-AOLS) (Austin, TX). Available online at: https://ams.confex.com/ams/98Annual/webprogram/Paper333206. html 
Hristova-Veleva, S. M., Haddad, Z. S., Stiles, B. W., Shen, T. P. J., Niamsuwan, N., Turk, F. J., et al. (2016). "Possible predictors for the rapid intensification and evolution of hurricanes from near-coincident satellite observations of the structure of precipitation and surface winds: Hurricane Joaquin," in $32^{\text {nd }}$ AMS Conference on Hurricanes and Tropical Meteorology (San Juan, PR). Available online at: https://ams.confex.com/ams/32Hurr/webprogram/ Paper293955.html

Huang, W., Gill, E., Wu, X., and Li, L. (2012). Measurement of sea surface wind direction using bistatic high-frequency radar. IEEE Trans. Geosci. Remote Sens. 50, 4117-4122. doi: 10.1109/TGRS.2012.2188298

Huddleston, J. N., and Stiles, B. W. (2000). "A multi-dimensional histogram technique for flagging rain contamination on QuikSCAT," in Proceedings of IEEE International Geoscience and Remote Sensing Symposium, Vol. 3 (Honolulu, HI), 1232-1234.

Hwang, P., Burrage, D., Wang, D., and Wesson, J. (2013). Ocean surface roughness spectrum in high wind condition for microwave backscatter and emission computations. J. Atmos. Ocean. Technol. 30, 2168-2188. doi: 10.1175/JTECH-D-12-00239.1

Hwang, P., and Fois, F. (2015). Surface roughness and breaking wave properties from polarimetric microwave radar backscattering. J. Geophys. Res. 120, 3640-3657. doi: 10.1002/2015JC010782

Hwang, P., Stoffelen, A., van Zadelhoff, G.-J., Perrie, W., Zhang, B., Li, H., et al. (2015). Cross-polarization geophysical model function for c-band radar backscattering from the ocean surface and wind speed retrieval. J. Geophys. Res. Oceans 120, 893-909. doi: 10.1002/2014JC010439

Hyder, P., Simpson, J. H., Xing, J., and Gille, S. T. (2011). Observations over an annual cycle and simulations of wind-forced oscillations near the critical latitude for diurnal-inertial resonance. Continent. Shelf Res. 31, 1576-1591. doi: 10.1016/j.csr.2011.06.001

Imaoka, K., Kachi, M., Kasahara, M., Ito, N., Nakagawa, K., and Oki, T. (2010). Instrument performance and calibration of AMSR-E and AMSR2. in International Archives of the Photogrammetry, Remote Sensing and Spatial Information Science. 38, 13-18. Available online at: www.isprs.org/ proceedings/XXXVIII/part8/pdf/JTS13_20100322190615.pdf

Isaksen, L., and Stoffelen, A. C. M. (2000). ERS scatterometer wind data impact on ECMWF's tropical cyclone forecasts. IEEE Trans. Geosci. Remote Sens. 38, 1885-1892. doi: $10.1109 / 36.851771$

Jelesnianski, C. P., Chen, J., and Shaffer, W. A. (1992). SLOSH: Sea, Lake, and Overland Surges From Hurricanes. Silver Spring: Department of Commerce.

Johnson, J., Williams, L., Bracalente, E., Beck, F., and Grantham, W. (1980). SEASAT-A satellite scatterometer instrument evaluation. IEEE J. Ocean. Eng. OE-5, 138-144. doi: 10.1109/JOE.1980.1145461

Jonsson, B. F., Doney, S. C., Dunne, J., and Bender, M. (2013). Evaluation of the Southern Ocean $\mathrm{O}_{2}$ /Ar-based NCP estimates in a model framework. J. Geophys. Res. Biogeosci. 118, 385-399. doi: 10.1002/jgrg.20032

Juliano, T. W., Parish, T. R., Rahn, D. A., and Leon, D. C. (2017). An atmospheric hydraulic jump in the Santa Barbara channel. J. Appl. Meteor. Climatol. 56, 2981-2998. doi: 10.1175/JAMC-D-16-0396.1

Kara, A. B., Wallcraft, A. J., Barron, C. N., Metzger, E. J., Hurlburt, H. E., and Bourassa, M. A. (2008b). Accuracy of $10 \mathrm{~m}$ Wind Speeds from Satellites and NWP Products Near Land-Sea Boundaries. J. Geophys. Res. 113:C04009. doi: 10.1029/2007JC004516

Kara, A. B., Wallcraft., A. J., and Bourassa, M. A. (2008a). Air-sea stability effects on the $10 \mathrm{~m}$ winds over the global ocean: evaluations of air-sea flux algorithms. J. Geophys. Res. Oceans 113. doi: 10.1029/2007JC004324

Katsaros, K., Vachon, P., Black, P., Dodge, P., and Ulhorn, E. (2000). Wind fields from SAR: could they improve our understanding of storm dynamics? Johns Hopkins APL Tech. Dig. 21, 86-93. doi: 10.4095/219617

Kelly, K. A., Dickinson, S., Chuan Li, J., Johnson, G. C., and Thompson, L. (2004). "Impact of ocean currents on scatterometer winds in the tropical Pacific Ocean," IGARSS 2004. 2004 IEEE International Geoscience and Remote Sensing Symposium (Anchorage, AK), 799.

Kelly, K. A., Dickinson, S., McPhaden, M. J., and Johnson, G. C. (2001). Ocean currents evident in satellite wind data. Geophys. Res. Lett. 28, 2469-2472. doi: 10.1029/2000GL012610

Kennedy, A. B., Gravois, U., Zachry, B. C., Westerink, J. J., Hope, M. E., Dietrich, J. C., et al. (2011). Origin of the Hurricane Ike forerunner surge. Geophys. Res. Lett. 38. doi: 10.1029/2011GL047090
Kerr, Y., Waldteufel, P., Wigneron, J.-P., Delwart, S., Cabot, F., Boutin, J., et al. (2010). The SMOS mission: new tool for monitoring key elements of the global water cycle. Proc. IEEE 98, 666-687. doi: 10.1109/JPROC.2010.2043032

King, G. P., Vogelzang, J., and Stoffelen, A. (2015). Upscale and downscale energy transfer over the tropical Pacific revealed by scatterometer winds. J. Geophys. Res. Oceans 120, 346-361. doi: 10.1002/2014JC009993

Kite-Powell, H., Colgan, C., and Weiher, R. (2008). Estimating the economic benefits of regional ocean observing systems. Coastal Manage. 36, 125-145. doi: 10.1080/08920750701868002

Kite-Powell, H. L. (2009). Economic considerations in the design of ocean observing systems. Oceanography 22, 44-49. doi: 10.5670/oceanog.2009.37

Kite-Powell, H. L. (2011). The value of ocean surface wind information for maritime commerce. Mar. Technol. Soc. J. 45, 75-84. doi: 10.4031/MTSJ. 45.1.13

Kite-Powell, H. L., and Colgan, C. S. (2001). The Potential Economic Benefits of Coastal Ocean Observing Systems, the Gulf of Maine. Washington, DC: US Department of Commerce; National Oceanic and Atmospheric Administratin; Office of Program Planning and Integration.

Klocker, A. (2018). Opening the window to the Southern Ocean: the role of jet dynamics. Sci. Adv. 4:eaao4719. doi: 10.1126/sciadv.aao4719

Kloe, J., de, Stoffelen, A., and Verhoef, A. (2017). Improved use of scatterometer measurements by using stress-equivalent reference winds. IEEE J. Select. Topics Appl. Earth Observ. Remote Sens. 10, 2340-2347. doi: 10.1109/JSTARS.2017.2685242

Klotz, B., and Uhlhorn, E. (2014). Improved stepped frequency microwave radiometer tropical cyclone surface winds in heavy precipitation. J. Atmos. Ocean. Technol. 31, 2392-2408. doi: 10.1175/JTECH-D-14-00028.1

Knaff, J. A., Sampson, C. R., and Musgrave, K. D. (2018). Statistical tropical cyclone wind radii prediction using climatology and persistence: Updates for the western North Pacific. Wea Forecast. 1093-1098. doi: 10.1175/WAF-D-18-0027.1

Koch, W. (2004). Directional analysis of SAR images aiming at wind direction. IEEE Trans. Geosci. Remote Sens. 42, 702-710. doi: 10.1109/TGRS.2003. 818811

Kumar, R., Chakraborty, A., Parekh, A., Sikhakolli, R., Gohil, B. S., and Kiran Kumar, A. S. (2013). Evaluation of Oceansat-2-derived ocean surface winds using observations from global buoys and other scatterometers. IEEE Trans. Geosci. Remote Sens. 51, 2571-2576. doi: 10.1109/TGRS.2012.2214785

Laiz-Carrión, R., Gerard, T., Uriarte, A., Malca, E., Quintanilla, J., Muhling, B., et al. (2015). Larval bluefin tuna (Thunnus thynnus) trophodynamics from Balearic Sea (WM) and Gulf of Mexico spawning ecosystems by stable isotope. Collect. Vol. Sci. Pap. ICCAT 71, 1354-1365.

Lara-Lopez, A. L., Davison, P., and Koslow, J. A. (2012). Abundance and community composition of micronekton across a front off Southern California. J. Plankton Res. 34, 828-848. doi: 10.1093/plankt/fbs016

Leidner, S. M., Isaksen, L., and Hoffman, R. N. (2003). Impact of NSCAT winds on tropical cyclones in the ECMWF $4 \mathrm{D}$-Var assimilation system. Mon. Weather Rev. 131, 3-26. doi: 10.1175/1520-0493(2003)131<0003:IONWOT>2.0.CO;2

Li, Q., and Lee, S. (2017). A mechanism of mixed-layer formation in the Indo-western Pacific Southern Ocean: preconditioning by an eddydriven jet-scale overturning circulation. J. Phys. Oceanogr. 47, 2755-2772. doi: 10.1175/JPO-D-17-0006.1

Li, X. (ed.). (2017). Hurricane Monitoring with Spaceborne Synthetic Aperture Radar. Singapore: Springer Natural Hazards, 398. doi: 10.1007/978-981-10-2893-9

Li, X., Zhang, J. A., Yang, X., Pichel, W. G., DeMaria, M., Long, D., et al. (2013). Tropical cyclone morphology from spaceborne synthetic aperture radar. Bull. Amer. Meteorol. Soc. 94, 215-230. doi: 10.1175/BAMS-D-11-00211.1

Li, Z., Stoffelen, A., and Verhoef, A. (2019). A generalized simulation capability for rotating- beam scatterometers. Atmos. Meas. Tech. 12, 3573-3594. doi: 10.5194/amt-12-3573-2019

Lin, C., Lengert, W., and Attema, E. (2017). Three generations of C-band wind scatterometer systems from ERS-1/2 to MetOp/ASCAT, and MetOp second generation. IEEE J. Select. Topics Appl. Earth Observ. Remote Sens. 10, 2098-2122. doi: 10.1109/JSTARS.2016.2616166

Lin, I., Liu, W. T., Wu, C. C., Wong, G. T., Hu, C., Chen, Z., et al. (2003). New evidence for enhanced ocean primary production triggered by tropical cyclone. Geophys. Res. Lett. 30. doi: 10.1029/2003GL017141 
Lin, W., and Portabella, M. (2017). Towards an improved wind quality control for RapidScat. IEEE Trans. Geosci. Remote Sens. 55, 3922-3930. doi: 10.1109/TGRS.2017.2683720

Lin, W., Portabella, M., Stoffelen, A., Turiel, A., and Verhoef, A. (2014). Rain identification in ASCAT winds using singularity analysis. IEEE Geosci. Remote Sens. Lett. 11, 1519-1523. doi: 10.1109/LGRS.2014.2298095

Lin, W., Portabella, M., Stoffelen, A., Verhoef, A., and Turiel, A. (2015b). ASCAT wind quality control near rain. IEEE Trans. Geosci. Remote Sens. 53, 4165-4177. doi: $10.1109 /$ TGRS.2015.2392372

Lin, W., Portabella, M., Stoffelen, A., Vogelzang, J., and Verhoef, A. (2015a). ASCAT wind quality under high subcell wind variability conditions. J. Geophys. Res. 120, 5804-5819. doi: 10.1002/2015JC010861

Lin, W., Portabella, M., Stoffelen, A., Vogelzang, J., and Verhoef, A. (2016). On mesoscale analysis and ASCAT ambiguity removal. Q. J. R. Meteorol. Soc. 142, 1745-1756. doi: 10.1002/qj.2770

Lindsley, R. D., and Long, D. G. (2016). Enhanced-resolution reconstruction of ASCAT backscatter measurements. IEEE Trans. Geosci. Remote Sens. 54, 2589-2601. doi: 10.1109/TGRS.2015.2503762

Liu, W. T., and Tang, W. (1996). Equivalent Neutral Wind. Pasadena, CA: JPL Publication 96-17; Jet Propulsion Laboratory.

Long, A., and Trizna, D. (1973). Mapping of North Atlantic winds by HF radar sea backscatter interpretation. IEEE Trans. Antennas Propagat. 21, 680-685.

Marseille, G. J., and Stoffelen, A. (2017). Toward scatterometer winds assimilation in the mesoscale HARMONIE Model. IEEE J. Select. Topics Appl. Earth Observ. Remote Sens. 10, 2383-2393. doi: 10.1109/JSTARS.2016.2640339

Maximenko, N., Corradi, P., Law, K. L., Van Sebille, E., Garaba, S. P., Lampitt, R. S., et al. (2019). Towards the integrated marine debris observing system. Front. Mar. Sci. 6:447. doi: 10.3389/fmars.2019.00447

McGillicuddy, D. J., Anderson, L. A., Bates, N. R., Bibby, T., Buesseler, K. O., Carlson, C. A., et al. (2007). Eddy/wind interactions stimulate extraordinary mid-ocean plankton blooms. Science 316, 1021-1026. doi: 10.1126/science.1136256

McGregor, S., Gupta, A. S., Dommenget, D., Lee, T., McPhaden, M. J., Kessler, W., et al. (2017). Factors influencing the skill of synthesized satellite wind products in the tropical Pacific. J. Geophys. Res. Oceans 18. doi: 10.1002/2016JC012340

McWilliams, J. C. (2016). Submesoscale currents in the ocean. Proc. R. Soc. A 472:20160117. doi: 10.1098/rspa.2016.0117

Mears, C. A., Smith, D. K., and Wentz, F. J. (2001). Comparison of special sensor microwave imager and buoy-measured wind speeds from 1987 - 1997. J. Geophys. Res. 106, 11719-11729. doi: 10.1029/1999JC0 00097

Mecklenburg, S., Drusch, M., Kerr, Y. H., Font, J., Martin-Neira, M., Delwart, S., et al. (2012). ESA's soil moisture and ocean salinity mission: mission performance and operations. IEEE Trans. Geosci. Remote Sens. 50, 1354-1366. doi: $10.1109 /$ TGRS.2012.2187666

Meissner, T., Ricciardulli, L., and Wentz, F. (2011). "All-weather wind vector measurements from intercalibrated active and passive microwave satellite sensors," Proceedings of the 2011 IEEE International Geoscience and Remote Sensing Symposium (IGARSS) (Vancouver, BC:IEEE).

Meissner, T., Ricciardulli, L., and Wentz, F. J. (2017). Capability of the SMAP mission to measure ocean surface winds in storms. Bull. Am. Meteorol. Soc. 98, 1660-1677. doi: 10.1175/BAMS-D-16-0052.1

Meissner, T., Smith, D., and Wentz, F. (2001). A 10-year intercomparison between collocated special sensor microwave imager oceanic surface wind speed retrievals and global analyses. J. Geophys. Res. 106, 11731-11742. doi: 10.1029/1999JC000098

Meissner, T., and Wentz, F. (2009). Wind vector retrievals under rain with passive satellite micro-wave radiometers. IEEE Trans. Geosci. Remote Sens. 47, 3065-3083. doi: 10.1109/TGRS.2009.20 27012

Meissner, T., and Wentz, F. (2012). The emissivity of the ocean surface between 6 - $90 \mathrm{GHz}$ over a large range of wind speeds and Earth incidence angles. IEEE Trans. Geosci. Remote Sens. 50, 3004-3026. doi: 10.1109/TGRS.2011.2179662

Meissner, T., Wentz, F., and Ricciardulli, L. (2014). The emission and scattering of L-band microwave radiation from rough ocean surfaces and wind speed measurements from the Aquarius sensor. J. Geophys. Res. 119, 6499-6522. doi: 10.1002/2014JC009837
Meissner, T., and Wentz, F. J. (2006). "Ocean retrievals for WindSat: radiative transfer model, algorithm, validation," in 9th Specialist Meeting on Microwave Radiometry and Remote Sensing Applications (San Juan, PR).

Monahan, E., and O'Muircheartaigh, I. (1980). Optimal power-law description of oceanic white-cap coverage dependence on wind speed. J. Phys. Oceanogr. 10, 2094-2099. doi: 10.1175/1520-0485(1980)010\&lt;2094:OPLDOO\&gt;2.0.CO;2

Morey, S. L., Baig, S., Bourassa, M. A., Dukhovskoy, D. S., and O'Brien, J. J. (2006). Remote forcing contribution to storm-induced sea level rise during Hurricane Dennis. Geophys. Res. Lett. 33, L19603-19607. doi: 10.1029/2006GL027021

Morey, S. L., Dukhovskoy, D. S., and Bourassa, M. A. (2009). Connectivity between variability of the Apalachicola River flow and the bio-optical oceanic properties of the northern West Florida Shelf. Cont. Shelf Res. 29, 1264-1275. doi: 10.1016/j.csr.2009.02.003

Morrison, I., Businger, S., Marks, F., Dodge, P., and Businger, J. A. (2005). An observational case for the prevalence of roll vortices in the hurricane boundary layer. J Atmos. Sci. 62, 2662-2673. doi: 10.1175/JAS3508.1

Mouche, A., Chapron, B., Zhang, B., and Husson, R. (2017). Combined co- and cross- polarized SAR measurements under extreme wind conditions. IEEE Trans. Geosci. Remote Sens. 55, 6476-6467. doi: 10.1109/TGRS.2017.2732508

Mouche, A. A., Collard, F., Chapron, B., Dagestad, K.-F., Guitton, G., Johannessen, J. A., et al. (2012). On the use of doppler shift for sea surface wind retrieval from SAR. IEEE Trans. Geosci. Remote Sens. 50, 2901-2909. doi: 10.1109/TGRS.2011.2174998

Murty, T. S. (1984). Storm Surges-Meteorological Ocean Tides. Altona, MB: Friesen Printers $L t d$.

Naderi, F., Freilich, M. H., and Long, D. G. (1991). Spaceborne radar measurement of wind velocity over the ocean: an overview of the NSCAT scatterometer system. Proc. IEEE 79, 850-866. doi: 10.1109/5.90163

National Academies of Sciences, Engineering, and Medicine (2018). Thriving on Our Changing Planet: A Decadal Strategy for Earth Observation From Space. Washington, DC: The National Academies Press.

NOAA (1998). Year of the Ocean Discussion Papers, Office of the Chief Scientist. Available online at: https://nepis.epa.gov/Exe/tiff2png.cgi/200051DL.PNG?-r+ 85+-g+15+- $\mathrm{h}+2,15,5,5,44,13 \$+\$ \mathrm{D} \% 3 \mathrm{~A} \%$ 5CZYFILES\%5CINDEX\%20DATA \%5C95THRU99\%5CTIFF\%5C00001056\%5C200051DL.TIF

Nordberg, W., Conaway, J., Ross, D., and Wilheit, T. (1971). Measurement of microwave emission from a foam covered wind driven sea. J. Atmos. Sci. 38, 429-433. doi: 10.1175/1520-0469(1971)028<0429:MOMEFA $>2.0 . C O ; 2$

Nuss, W. A., Bane, J. M., Thompson, W. T., Holt, T., Dorman, C. E., Ralph, F. M., et al. (2000). Coastally trapped wind reversals: progress toward understanding. Bull. Am. Meteorol. Soc. 81, 719-744. doi: 10.1175/1520-0477(2000)081<0719:CTWRPT>2.3.CO;2

Ohman, M. D., Powell, J. R., Picheral, M., and Jensen, D. W. (2012). Mesozooplankton and particulate matter responses to a deep-water frontal system in the southern California Current System. J. Plankton Res. 34, 815-827. doi: 10.1093/plankt/fbs028

Omand, M. M., D’asaro, E. A., Lee, C. M., Perry, M. J., Briggs, N., Cetinić, I., et al. (2015). Eddy-driven subduction exports particulate organic carbon from the spring bloom. Science 348, 222-225. doi: 10.1126/science.1260062

O'Neill, L. W. (2012). Wind speed and stability effects on the coupling between surface wind stress and SST observed from buoys and satellite. J. Climate 26, 1544-1569. doi: 10.1175/JCLI-D-11-00121.1

Ortiz-Suslow, D., Haus, B., Williams, N., Laxague, N., Reniers, A., and Graber, H. (2015). The spatial-temporal variability of air-sea momentum fluxes observed at a tidal inlet. J. Geophys. Res. 120, 660-676. doi: 10.1002/2014JC010412

Parish, T. R., Rahn, D. A., and Leon, D. C. (2016). Aircraft measurements and numerical simulations of an expansion fan off the California coast. J. Appl. Meteor. Climatol. 55, 2053-2062. doi: 10.1175/JAMC-D-16-0101.1

Pellichero, V., Sallée, J. B., Chapman, C. C., and Downes, S. M. (2018). The southern ocean meridional overturning in the sea-ice sector is driven by freshwater fluxes. Nat. Commun. 9:1789. doi: 10.1038/s41467-018-04101-2

Pendleton, L., Donato, D. C., Murray, B. C., Crooks, S., Jenkins, W. A., Sifleet, S., et al. (2012). Estimating global "blue carbon" emissions from conversion and degradation of vegetated coastal ecosystems. PLOS ONE 7:e43542. doi: 10.1371/journal.pone.0043542

Perlin, N., Skyllingstad, E. D., and Samelson, R. M. (2011). Coastal atmospheric circulation around an idealized cape during wind-driven upwelling studied 
from a coupled ocean-atmosphere model. Mon. Wea. Rev. 139, 809-829. doi: 10.1175/2010MWR3372.1

Plagge, A. M., Vandemark, D., and Chapron, B. (2012). Examining the impact of surface currents on satellite scatterometer and altimeter ocean winds. J. Atmos. Ocean. Technol. 29, 1776-1793. doi: 10.1175/JTECH-D-12-00017.1

Plagge, A. M., Vandemark, D. C., and Long, D. G. (2009). Coastal validation of ultra-high resolution wind vector retrieval from QuikSCAT in the Gulf of Maine. IEEE Geosci. Remote Sens. Lett. 6, 413-417. doi: 10.1109/LGRS.2009.2014852

Portabella, M., and Stoffelen, A. (2001). Rain detection and quality control of SeaWinds. J. Atmos. Ocean. Technol. 18, 1171-1183. doi: 10.1175/1520-0426(2001)018\&lt;1171:RDAQCO\&gt;2.0.CO;2

Portabella, M., and Stoffelen, A. (2009). On scatterometer ocean stress. J. Atmos. Ocean. Technol. 26, 368-382. doi: 10.1175/2008JTECHO578.1

Portabella, M., Stoffelen, A., and Johannessen, J. A. (2002). Toward an optimal inversion method for SAR wind retrieval. J. Geophys. Res. 107, 1-13. doi: 10.1029/2001JC000925

Portabella, M., Stoffelen, A., Verhoef, A., and Verspeek, J. (2012). A new method for improving scatterometer wind quality control. IEEE Geosci. Remote Sens. Lett. 9, 579-583. doi: 10.1109/LGRS.2011.2175435

Powell, M. D., Murillo, S., Dodge, P., Uhlhorn, E., Gamache, J., Cardone, V., et al. (2010). Reconstruction of Hurricane Katrina's wind fields for storm surge and wave hindcasting. Ocean Eng. 37, 26-36. doi: 10.1016/j.oceaneng.2009.08.014

Priftis, G., Garg, P., Lang, T., Chronis, T., Nesbitt, S., and Lindsley, R. (2018). "Examining convective signatures in scatterometer data," International Ocean Vector Winds Science Team IOVWST (Barcelona). Available online at: https:// mdc.coaps.fsu.edu/scatterometry/meeting/docs/2018/docs/TuesdayApril24/ TuesdayAfternoon/iovwst_presentation_lang.pptx

Pringle, J. M., and Dever, E. P. (2009). Dynamics of wind-driven upwelling and relaxation between Monterey Bay and Point Arena: Local-, regional-, and gyre-scale controls, J. Geophys. Res. 114:C07003. doi: 10.1029/2008JC005016

Quilfen, Y., Chapron, B., Collard, F., and Vandemark, D. (2004). Relationship between ERS scatterometer measurement and integrated wind and wave parameters. J. Atmos. Ocean. Technol. 21, 368-373. doi: 10.1175/1520-0426(2004)021<0368:RBESMA >2.0.CO;2

Rahn, D. A., Parish, T. R., and Leon, D. (2013). Airborne measurements of coastal jet transition around point conception, California. Mon. Weather Rev. 141, 3827-3839. doi: 10.1175/MWR-D-13-00030.1

Reul, N., and Chapron, B. (2003). A model of sea-foam thickness distribution for passive micro-wave remote sensing applications. J. Geophys. Res. 108. doi: 10.1029/2003JC001887

Reul, N., Chapron, B., Zabolotskikh, E., Donlon, C., Mouche, A., Tenerelli, J., et al. (2017). A new generation of tropical cyclone size measurements from space. Bull. Am. Meteorol. Soc. 98, 2367-2385. doi: 10.1175/BAMS-D-1500291.1

Reul, N., Chapron, B., Zabolotskikh, E., Donlon, C., Quilfen, Y., Guimbard, S., et al. (2016). A revised L-band radio-brightness sensitivity to extreme winds under Tropical Cy-clones: the five year SMOS-storm database. Remote Sens. Environ. 180, 274-291. doi: 10.1016/j.rse.2016.03.011

Reul, N., Tenerelli, J., Chapron, B., Vandemark, D., Quilfen, Y., and Kerr, Y. (2012). SMOS satellite L-band radiometer: a new capability for ocean surface remote sensing in hurricanes. J. Geophys. Res. 117:C02006. doi: 10.1029/2011JC007474

Ricciardulli, L., Meissner, T., and Wentz, F. (2012). "Towards a climate data record of satellite ocean vector winds," Proceedings of the 2012 IEEE International Geoscience and Remote Sensing Symposium (Munich: IEEE).

Ricciardulli, L., Wentz, F., and Meissner, T. (2015). "Bringing consistency among scatterometer winds using radiometer observations," IOVWST Meeting 2015 (Portland). Available online at: https://mdc.coaps.fsu.edu/scatterometry/ meeting/docs/2015/ClimateDataRecordDevelopmentAndAnalysis/ Ricciardulli_ovwst_2015.pdf

Rodriguez, E. (2018). On the optimal design of doppler scatterometers. Remote Sens. 10:1765. doi: 10.3390/rs10111765

Rodriguez, E., Bourassa, M., Chelton, D., Farrar, J. T., Long, D. D., PerkovicMartin, D., et al. (2019). The winds and currents mission concept. Front. Mar. Sci. 6, 1-8. doi: 10.3389/fmars.2019.00438

Rodriguez, E., Wineteer, A., Perkovic-Martin, D., Gál, T., Stiles, B. W., Niamsuwan, N., et al. (2018). Estimating ocean vector winds and currents using a Ka-band pencil-beam doppler scatterometer. Remote Sens. 10:576. doi: $10.3390 /$ rs 10040576

Roemmich, D., Church, J., Gilson, J., Monselesan, D., Sutton, P., and Wijffels, S., (2015): Unabated planetary warming and its ocean structure since 2006. Nat. Clim. Change. 5, 240-245. doi: 10.1038/nclimate2513

Rogers, R., Reasor, P., and Lorsolo, S. (2013). Airborne Doppler observations of the inner-core structural differences between intensifying and steady-state tropical cyclones. Mon. Wea. Rev. 141, 2970-91.

Roman-Stork (2018). Analysis of the 10-20-Day intraseasonal oscillation in the indian ocean using surface winds from composite satellite data (MS Thesis). Florida State University.

Ross, D. B., Cardone, V. J., Overland, J., McPherson, R. D., Pierson, W. J. Jr., and Yu, T. (1985). Oceanic surface winds. Adv. Geophys. 27, 101-138. doi: 10.1016/S0065-2687(08)60404-5

Saiprasanth, B., Haddad, Z. S., Hristova-Veleva, S., and Marks, F. D. Jr. (2018). "A low-wavenumber analysis of the environmental and vortex-scale variables responsible for rapid intensity changes in landfalling tropical cyclones," in Remote Sensing and Modeling of the Atmosphere, Oceans, and Interactions VII, Vol. 10782 (International Society for Optics and Photonics), 1078208. Available online at: https://www.spiedigitallibrary.org/conference-proceedings-of-spie/ 10782/1078208/A-low-wavenumber-analysis- of-the-environmental-andvortex-scale/10.1117/12.2500290.short?SSO=1

Samelson, R. M. (2019). Challenges and Opportunities in Coastal Prediction. Earth \& Space Science News, 100

Sampson, C., and Schrader, A. (2000). The automated tropical cyclone forecasting system (version 3.2). Bull. Am. Meteorol. Soc. 81, 1231-1240. doi: 10.1175/1520-0477(2000)081<1231:TATCFS $>2.3 . C O ; 2$

Sandu, I., Beljaars, A., Bechtold, P., Mauritsen, T., and Balsamo, G. (2011). Why is it so difficult to represent stably stratified conditions in numerical weather prediction (NWP) models? J. Adv. Model. Earth Syst. 5, 117-133. doi: $10.1002 /$ jame. 20013

Sapp, J., Alsweiss, S., Jelenak, Z., Chang, P., Frasier, S., and Carswell, J. (2016). Airborne co-polarization observations of the ocean-surface NRCS at C-band. IEEE Trans. Geosci. Remote Sens. 54, 5975-5992. doi: 10.1109/TGRS.2016.2578048

Sapp, J., Jelenak, Z., Chang, P., and Frasier, S. (2018). "C-band cross-polarization ocean surface observations in Hurricane Matthew," in IGARSS. (Valencia).

Shapiro, L. J., and Willoughby, H. E. (1982). The response of balanced hurricanes to local sources of heat and momentum. J. Atmos. Sci. 39, 378-394. doi: 10.1175/1520-0469(1982)039\&1t;0378:TROBHT\&gt;2.0.CO;2

Shen, W., Gurgel, K.-W., Voulgaris, G., Schlick, T., and Stammer, D. (2012). Windspeed inversion from HF radar first-order backscatter signal. Ocean Dyn. 62, 105-121. doi: 10.1007/s10236-011-0465-9

Shi, Q. (2017). Coupling ocean currents and waves with wind stress over the Gulf Stream (Ph.D. dissertation). Tallahassee, FL, USA: Florida State University.

Shi, Q., and Bourassa, M. A. (2019). Coupling ocean currents and waves with wind stress over the gulf stream. Remote Sens. 11:1476. doi: 10.3390/rs11121476

Simpson, J. E. (1994). Sea Breeze and Local Wind. Cambridge: Cambridge University Press.

Spencer, M. W., Wu, C., and Long, D. G. (2000). Improved resolution backscatter measurements with the SeaWinds pencil-beam scatterometer. IEEE Trans. Geosci. Remote Sens. 38, 89-104. doi: 10.1109/36.823904

Stephens, G. L., Hakuba,M. Z., Webb,M. J., Lebsock, M., Yue, Q., Kahn, B. H., et al. (2018). Regional intensification of the tropical hydrological cycle during ENSO. Geophys. Res. Lett. 45, 4361-4370. doi: 10.1029/2018GL077598

Stiles, B., Fore, A., Ricciardulli, L., Wineteer, A. G., Hristova-Veleva, S., Rodriguez, E., et al. (2018). "Imporvements in the version 4.0 QuikSCAT ocean vector winds," in International Ocean Vector Wind Science Team (Barcelona). Available online at: https://mdc.coaps.fsu.edu/scatterometry/meeting/docs/2018/docs/ TuesdayApril24/TuesdayAfternoon/Stiles_QSCATV4_slides.pptx

Stiles, B.W., and co-authors (2014). Optimized tropical cyclone winds from QuikSCAT: a neural network approach. IEEE Trans. Geosci. Remote Sens. 52, 7418-7434. doi: 10.1109/TGRS.2014.2312333

Stiles, B. W., and Dunbar, R. S. (2010). A neural network technique for improving the accuracy of scatterometer winds in rainy conditions. IEEE Trans. Geosci. Remote Sens. 48, 3114-3122. doi: 10.1109/TGRS.2010. 2049362 
Stiles, B. W., and Yueh, S. H. (2002). Impact of rain on spaceborne Ku-band wind scatterometer data. IEEE Trans. Geosci. Remote Sens. 40, 1973-1983. doi: $10.1109 /$ TGRS.2002.803846

Stoffelen, A., Aaboe, S., Calvet, J.-C., Cotton, J., De Chiara, G., Saldana, J., et al. (2017b). Scientific Developments and the EPS-SG Scatterometer. IEEE J. Select. Topics Appl. Earth Observ. Remote Sens. 10, 2086-2097. doi: 10.1109/JSTARS.2017.2696424

Stoffelen, A., Anton, V., Jeroen, V., Jur, V., Gert-Jan, M., Driesenaar, T., et al. (2013). Research and Development in Europe on Global Application of the OceanSat-2 Scatterometer Winds. Final Report of OceanSat-2 Cal/Val AO project, NWPSAF-KN-TR-022, SAF/OSI/CDOP2/KNMI/TEC/RP/196

Stoffelen, A., Kumar, R., Zou, J., Karaev, V., Chang, P. S., and Rodriguez, E. (2019). "Ocean surface vector wind observations," In Remote Sensing of the Asian Seas (Cham: Springer), 429-447.

Stoffelen, A., Verspeek, J. A., Vogelzang, J., and Verhoef, A. (2017a). The CMOD7 geophysical model function for ASCAT and ERS wind retrievals. IEEE J. Select. Topics Appl. Earth Observ. Remote Sens. 10, 2123-2134. doi: 10.1109/JSTARS.2017.2681806

Stukel, M. R., Aluwihare, L. I., Barbeau, K. A., Chekalyuk, A. M., Goericke, R., Miller, A. J., et al. (2017). Mesoscale ocean fronts enhance carbon export due to gravitational sinking and subduction. Proc. Natl. Acad Sci. U.S.A. 114, 1252-1257. doi: 10.1073/pnas.1609435114

Takeyama, Y., Ohsawa, T., Kozai, K., Hasager, C. B., and Badger, M. (2013). Comparison of geophysical model functions for SAR wind speed retrieval in Japanese coastal waters. Remote Sens. 5, 1956-1973. doi: 10.3390/rs5041956

Trindade, A., Portabella, M., Lin, W., and Stoffelen, A. (2017). "On the development of a scatterometer-based correction for NWP wind forcing systematic errors: impact of satellite sampling," in Proceedings of the International Geoscience and Remote Sensing Symposium (IGARSS) (Fort Worth, TX).

Tsai, W.-Y., Graf, J. E., Winn, C., Huddleston, J. N., Dunbar, S., Freilich, M. H., et al. (1999). Postlaunch sensor verification and calibration of the NASA scatterometer. IEEE Trans. Geosci. Remote Sens. 37, 1517-1542. doi: $10.1109 / 36.763264$

Uhlhorn, E., and Black, P. (2003). Verification of remotely sensed sea surface winds in hurricanes. J. Atmos. Ocean. Technol. 20, 99-116. doi: 10.1175/1520-0426(2003)020<0099:VORSSS $>2.0 . C O ; 2$

Uhlhorn, E., Franklin, J., Goodberlet, M., Carswell, J., and Goldstein, A. (2007). Hurricane surface wind measurements from an operational stepped frequency microwave radiometer. Mon. Wea. Rev. 135, 3070-3085. doi: 10.1175/MWR3454.1

Ulaby, F., and Long, D. (2014). Microwave Radar and Radiometric Remote Sensing. Ann Arbor: The University of Michigan Press.

UNEP. (2015). Blue Economy-Sharing Success Stories to Inspire Change. UNEP Regional Seas Report and Studies No. 195, Nairobi.

van Zadelhoff, G. J., Stoffelen, A., Vachon, P. W., Wolfe, J., Horstmann, J., Belmonte-Rivasm, M. (2014). Retrieving hurricane wind speeds using cross-polarization C-band measurements. Atmos. Measure. Tech. 7, 437-449. doi: 10.5194/amt-7-437-2014

Vandemark, D., James, E., and Marc, E. (2018). "Evaluating several key issues in satellite wind stress validation," in International Ocean Vector Winds Science Team IOVWST (Barcelona). Available online at: https://mdc. coaps.fsu.edu/scatterometry/meeting/docs/2018/docs/WednesdayApril25/ WednesdayMorning/vandemark_edson_OVW2018_talk.pptx

Verhoef, A., Vogelzang, J., Verspeek, J., and Stoffelen, A. (2017). Longterm scatterometer wind climate data records. IEEE J. Select. Topics Appl. Earth Observ. Remote Sens. 10, 2186-2194. doi: 10.1109/JSTARS.2016.26 15873

Vigh, J. L., and Schubert, W. H. (2009) Rapid development of the tropical cyclone warm core. J. Atmos. Sci. 66, 3335-3350. doi: 10.1175/2009JAS3092.1

Vogelzang, J., King, G. P., and Stoffelen, A. (2015). Spatial variances of wind fields and their relation to second-order structure functions and spectra. J. Geophys. Res. Oceans 120, 1048-1064. doi: 10.1002/2014JC0 10239

Vogelzang, J., and Stoffelen, A. (2017). ASCAT ultrahigh-resolution wind products on optimized grids. IEEE J. Select. Topics Appl. Earth Observ. Remote Sens. 10, 2332-2339. doi: 10.1109/JSTARS.2016.2623861
Wackerman, C. C., Pichel, W., Li, X., Clemente-Colòn, P. (2006). "Estimation of surface winds from SAR using a projection algorithm," in Proceedings of the 14th Conference on Satellite Meteorology and Oceanography (Atlanta, GA).

Walter, A. (1973). Detailed mesometeorological studies of air pollution dispersion in the Chicago Lake Breeze. Monthly Weather Rev. 101:387. doi: 10.1175/1520-0493(1973)101<0387:DMSOAP>2.3.CO;2

Wang,Z., Stoffelen, A., Fois, F., Verhoef, A., Zhao, C., Lin, M., et al. (2017). SST Dependence of Ku- and C-band backscatter measurements. IEEE J. Select. Topics Appl. Earth Observ. Remote Sens. 10, 2135-2145. doi: 10.1109/JSTARS.2016.2600749

Wanninkhof, R. (1992). Relationship between wind-speed and gas-exchange over the ocean. J. Geophys. Res. Oceans 97, 7373-7382. doi: 10.1029/92JC00188

Waugh, D. W., Primeau, F., DeVries, T., and Holzer, M. (2013). Recent changes in the ventilation of the Southern Oceans. Science 339, 568-570. doi: $10.1126 /$ science. 1225411

Weisberg, R. H., and Zheng, L. (2008). Hurricane storm surge simulations comparing three-dimensional with two-dimensional formulations based on an Ivan-like storm over the Tampa Bay, Florida region. J. Geophys. Res. Oceans 113. doi: $10.1029 / 2008$ JC005115

Weissman, D., King, D., and Thompson, T. W. (1979). Relationship between hurricane surface winds and L-band radar backscatter from the sea surface. Appl. Meteorol. J. 18, 1023-1034. doi: 10.1175/1520-0450(1979)018<1023:RBHSWA $>2.0 . C O ; 2$

Weissman, D. E., and Bourassa, M. A. (2008). Measurements of the effect of rain-induced sea surface roughness on the QuikSCAT scatterometer radar cross section. IEEE Trans. Geosci. Remote Sens. 46, 2882-2894. doi: 10.1109/TGRS.2008.2001032

Weissman, D. E., and Bourassa, M. A. (2011). The influence of rainfall on scatterometer backscatter within tropical cyclone environments - implications of parameterization of sea surface stress. IEEE Trans. Geosci. Remote Sens. 49, 4805-4814. doi: 10.1109/TGRS.2011.2170842

Weissman, D. E., Stiles, B., Hristova-Veleva, S., Long, D., Smith, D., Hilburn, K., et al. (2012). Challenges to satellite sensors of ocean winds; addressing precipitation effect. Atmos. J. Oceanic Technol. 29, 356-374. doi: 10.1175/JTECH-D-11-00054.1

Welander, P. (1961). Numerical prediction of storm surges. Adv. Geophys. 8, 315-379. doi: 10.1016/S0065-2687(08)60343-X

Wentz, F. (1997). A well calibrated ocean algorithm for special sensor microwave/imager. J. Geophys. Res. 102, 8703-8718. doi: 10.1029/96JC01751

Wentz, F. (2005). The Effects of Cloud and Rain on the Aquarius Salinity Retrieval. Algorithm Theoretical Basis Document, RSS Technical Report 3031805, Remote Sensing Systems (Santa Rosa, CA). Available online at: www.remss. com/papers/aquarius/rain_effect_on_salinity.pdf

Wentz, F. (2015). A 17-year climate record of environmental parameters derived from the Tropical Rainfall Measuring Mission (TRMM) Microwave Imager. J. Clim. 28, 6882-6902. doi: 10.1175/JCLI-D-15-0155.1

Wentz, F., Ricciardulli, L., Rodriguez, E., Stiles, B., Bourassa, M., Long, D., et al. (2017). Evaluating and extending the ocean winds data climate record. J. Select. Topics Appl. Earth Observ. Remote Sens. 99, 2165-2185. doi: $10.1109 /$ JSTARS.2016.2643641

Wentz, F., and Spencer, R. (1998). SSM/I rain retrievals within a unified all-weather ocean algorithm. J. Atmos. Sci. 55, 1613-1627. doi: 10.1175/1520-0469(1998)055<1613:SIRRWA >2.0.CO;2

Wentz, F. J., and Draper, D. (2016). On-orbit absolute calibration of the global precipitation measurement microwave imager. J. Atmos. Ocean. Technol. 33, 1393-1412. doi: 10.1175/JTECH-D-15-0212.1

Wentz, F. J., and Smith, D. K. (1999). A model function for the ocean normalized radar cross-section at $14 \mathrm{GHz}$ derived from NSCAT observations. J. Geophys. Res. 104, 11499-11524. doi: 10.1029/98JC02148

Winant, C. D., Dorman, C. E., Friehe, C. A., and Beardsley, R. C. (1988). The marine layer off northern California: an example of supercritical channel flow. J. Atmos. Sci. 45, 3588-3605. doi: 10.1175/1520-0469(1988)045<3588:TMLONC >2.0.CO;2

Wood, R., Koehler, M., Bennartz, R., and O’Dell, C. (2009). The diurnal cycle of surface divergence over the global oceans. Q. J. R. Meteorol. Soc. 135, 1484-1493. doi: 10.1002/qj.451 
World Bank (2017). The Potential of the Blue Economy: Increasing Long-Term Benefits of the Sustainable Use of Marine Resources for Small Island Developing States and Coastal Least Developed Countries. Washington, DC: World Bank.

Wyatt, L., Green, J., Middleditch, A., Moorhead, M., Howarth, J., Hol, M., et al. (2006). Operational wave, current, and wind measurements with the pisces HF radar. IEEE J. Ocean. Eng. 31, 819-834. doi: 10.1109/JOE.2006.888378

Xie, J., Yao, G., Sun, M., and Ji, Z. (2018). Measuring ocean surface wind field using shipborne high-frequency surface wave radar. IEEE Trans. Geosci. Remote Sens. 56, 3383-3397. doi: 10.1109/TGRS.2018.2799002

Yang, X.,-Y., and Huang, R. X., Wang, D. X. (2007). Decadal changes of wind stress over the southern ocean associated with Antarctic Ozone depletion. Clim. J. 20, 3395-3410. doi: 10.1175/JCLI4195.1

Yang, Z., Lu, N., Shi, J., Zhang, P., Dong, C., and Yang, J. (2012). Overview of FY-3 Payload and ground application system. IEEE Trans. Geosci. Remote Sens. 50, 4846-4853. doi: 10.1109/TGRS.2012.2197826

Young, G. S., Sikora, T. D., and Winstead, N. S. (2007). Manual and semiautomated wind direction editing for use in the generation of synthetic aperture radar wind speed imagery. J. Appl. Meteorol. Clim. 46, 776-790. doi: 10.1175/JAM 2507.1

Yu, L., Jin, X., and Weller, R. A. (2008). Multidecade Global Flux Datasets From the Objectively Analyzed Air-Sea Fluxes (OAFlux) Project: Latent and Sensible Heat Fluxes, Ocean Evaporation, and Related Surface Meteorological Variables. OAFlux Project Technical Report OA-2008-01.

Yueh, S., Tang, W., Fore, A., Neumann, G., Hayashi, A., Freedman, A., et al. (2013). L-band passive and active microwave geophysical model functions of ocean surface winds and applications to Aquarius retrieval. IEEE Trans. Geosci. Remote Sens. 51, 4619-4662 doi: 10.1109/TGRS.2013.2266915

Yueh, S. H., Wilson, W. J., Dinardo, S. J., and Li, F. K. (1999). Polarimetric microwave brightness signatures of ocean wind directions. IEEE Trans. Geosci. Remote Sens. 37, 949-959. doi: 10.1109/36.752213

Zabolotskikh, E., Mitnik, L., Reul, N., and Chapron, B. (2015). New possibilities for geophysical parameter retrievals opened by GCOM-W1 AMSR2.
IEEE J. Select. Topics Appl. Earth Observ. Remote Sens. 8, 4248-4261. doi: 10.1109/JSTARS.2015.2416514

Zhang, B., and Perrie, W. (2012). Cross-polarized synthetic aperture radar: a new potential measurement technique for Hurricanes. Bull. Am. Meteorol. Soc. 93, 531-541. doi: 10.1175/BAMS-D-11-00001.1

Zheng, C. W., Li, C. Y., and Li, X. (2017). Recent decadal trend in the North Atlantic wind energy resources. Adv. Meteorol. 2017, 1-8. doi: 10.1155/2017/7257492

Zheng, Y., Bourassa, M. A., and Hughes, P. J. (2013). Influences of sea surface temperature gradients and surface roughness changes on the motion of surface oil: a simple idealized study. J. Appl. Meteorol. Clim. 52, 1561-1575. doi: 10.1175/JAMC-D-12-0211.1

Conflict of Interest Statement: TM, AMa, CM, LR, and FW were employed by the company Remote Sensing Systems.

The remaining authors declare that the research was conducted in the absence of any commercial or financial relationships that could be construed as a potential conflict of interest.

The handling editor declared a shared affiliation, though no other collaboration with several of the authors AF, SH-V, ER, BS at time of review.

Copyright (c) 2019 Bourassa, Meissner, Cerovecki, Chang, Dong, De Chiara, Donlon, Dukhovskoy, Elya, Fore, Fewings, Foster, Gille, Haus, Hristova-Veleva, Holbach, Jelenak, Knaff, Kranz, Manaster, Mazloff, Mears, Mouche, Portabella, Reul, Ricciardulli, Rodriguez, Sampson, Solis, Stoffelen, Stukel, Stiles, Weissman and Wentz. This is an open-access article distributed under the terms of the Creative Commons Attribution License (CC BY). The use, distribution or reproduction in other forums is permitted, provided the original author(s) and the copyright owner(s) are credited and that the original publication in this journal is cited, in accordance with accepted academic practice. No use, distribution or reproduction is permitted which does not comply with these terms. 


\section{OPEN ACCESS}

Edited by:

Sabrina Speich,

École Normale Supérieure, France

Reviewed by:

Gilles Reverdin,

Centre National de la Recherche Scientifique (CNRS), France

Alberto Ricardo Piola,

Naval Hydrographic Service,

Argentina

*Correspondence:

Anne G.O'Carroll

anne.ocarrol/@eumetsat.int

Specialty section:

This article was submitted to

Ocean Observation,

a section of the journal

Frontiers in Marine Science

Received: 31 October 2018

Accepted: 05 July 2019

Published: 20 August 2019

Citation:

O'Carroll AG, Armstrong EM, Beggs HM, Bouali M, Casey KS,

Corlett GK, Dash P, Donlon CJ, Gentemann CL, Høyer JL, Ignatov A, Kabobah K, Kachi M, Kurihara Y, Karagali I, Maturi E, Merchant CJ, Marullo S, Minnett PJ,

Pennybacker M, Ramakrishnan B,

Ramsankaran $R$, Santoleri $R$,

Sunder $S$, Saux Picart $S$,

Vázquez-Cuervo J and Wimmer $W$ (2019) Observational Needs of Sea

Surface Temperature.

Front. Mar. Sci. 6:420.

doi: 10.3389/fmars.2019.00420

\section{Observational Needs of Sea Surface Temperature}

\begin{abstract}
Anne G. O'Carroll1*, Edward M. Armstrong', Helen M. Beggs ${ }^{3}$, Marouan Bouali4, Kenneth S. Casey ${ }^{5}$, Gary K. Corlett' ${ }^{1}$, Prasanjit Dash', Craig J. Donlon ${ }^{7}$, Chelle L. Gentemann ${ }^{8}$, Jacob L. Høyer ${ }^{9}$, Alexander Ignatov ${ }^{6}, K^{2}$ amila Kabobah ${ }^{10}$, Misako Kachi'1, Yukio Kurihara ${ }^{11}$, loanna Karagali'12, Eileen Maturi6, Christopher J. Merchant ${ }^{13}$, Salvatore Marullo ${ }^{14}$, Peter J. Minnett ${ }^{15}$, Matthew Pennybacker ${ }^{6}$, Balaji Ramakrishnan ${ }^{16}$, RAAJ Ramsankaran ${ }^{16}$, Rosalia Santoleri15, Swathy Sunder ${ }^{16}$, Stéphane Saux Picart ${ }^{17}$, Jorge Vázquez-Cuervo ${ }^{2}$ and Werenfrid Wimmer ${ }^{18}$

${ }^{1}$ EUMETSAT, Darmstadt, Germany, ${ }^{2}$ NASA Jet Propulsion Laboratory/California Institute of Technology, Pasadena, CA, United States, ${ }^{3}$ Bureau of Meteorology, Melbourne, VIC, Australia, ${ }^{4}$ Institute of Oceanography, Universidade de São Paulo, São Paulo, Brazil, ${ }^{5}$ NOAA NCEI, Silver Spring, MD, United States, ${ }^{6}$ NOAA STAR, College Park, MD, United States, ${ }^{7}$ ESA ESTEC, Noordwijk, Netherlands, ${ }^{8}$ Earth \& Space Research, Seattle, WA, United States, ${ }^{9}$ Danish Meteorological Institute, Copenhagen, Denmark, ${ }^{10}$ Earth Observation Research and Innovation Centre, University of Energy and Natural Resource, Sunyani, Ghana, ${ }^{11}$ Earth Observation Research Center, Japan Aerospace Exploration Agency, Chofu, Japan, ${ }^{12}$ Department of Wind Energy, Technical University of Denmark, Roskilde, Denmark, ${ }^{13}$ Department of Meteorology, University of Reading, Reading, United Kingdom, ${ }^{14}$ ENEA, ISAC-CNR, Frascati, Italy, ${ }^{15}$ Rosenstiel School of Marine and Atmospheric Science, University of Miami, Miami, FL, United States, ${ }^{16}$ Department of Civil Engineering, Indian Institute of Technology Bombay, Mumbai, India, ${ }^{17}$ CNRM, Université de Toulouse, Météo-France, CNRS, Lannion, France, ${ }^{18}$ Ocean and Earth Science, University of Southampton, Southampton, United Kingdom
\end{abstract}

Sea surface temperature (SST) is a fundamental physical variable for understanding, quantifying and predicting complex interactions between the ocean and the atmosphere. Such processes determine how heat from the sun is redistributed across the global oceans, directly impacting large- and small-scale weather and climate patterns. The provision of daily maps of global SST for operational systems, climate modeling and the broader scientific community is now a mature and sustained service coordinated by the Group for High Resolution Sea Surface Temperature (GHRSST) and the CEOS SST Virtual Constellation (CEOS SST-VC). Data streams are shared, indexed, processed, quality controlled, analyzed, and documented within a Regional/Global Task Sharing (R/GTS) framework, which is implemented internationally in a distributed manner. Products rely on a combination of low-Earth orbit infrared and microwave satellite imagery, geostationary orbit infrared satellite imagery, and in situ data from moored and drifting buoys, Argo floats, and a suite of independent, fully characterized and traceable in situ measurements for product validation (Fiducial Reference Measurements, FRM). Research and development continues to tackle problems such as instrument calibration, algorithm development, diurnal variability, derivation of high-quality skin and depth temperatures, and areas of specific interest such as the high latitudes and coastal areas. In this white paper, we review progress versus the challenges we set out 10 years ago in a previous paper, highlight remaining and new research and development challenges for the next 10 years 
(such as the need for sustained continuity of passive microwave SST using a $6.9 \mathrm{GHz}$ channel), and conclude with needs to achieve an integrated global high-resolution SST observing system, with focus on satellite observations exploited in conjunction with in situ SSTs. The paper directly relates to the theme of Data Information Systems and also contributes to Ocean Observing Governance and Ocean Technology and Networks within the OceanObs2019 objectives. Applications of SST contribute to all the seven societal benefits, covering Discovery; Ecosystem Health \& Biodiversity; Climate Variability \& Change; Water, Food, \& Energy Security; Pollution \& Human Health; Hazards and Maritime Safety; and the Blue Economy.

Keywords: sea surface temperature, observations, GHRSST, satellite, in situ, Fiducial Reference Measurements, operational oceanography, climate data records

\section{INTRODUCTION}

Sea surface temperature (SST) is a fundamental variable for understanding, monitoring and predicting fluxes of heat, momentum and gases at a variety of scales that determine complex interactions between the atmosphere and ocean. SST at the ocean-atmosphere interface has a significant societal impact, through, e.g., large ocean gyres and atmospheric circulation cells influencing weather and climate, weather systems and severe storms and local scale phenomena, such as the generation of sea breezes and convective clouds.

Mapping SST is now the responsibility of operational monitoring and forecasting agencies (Robinson et al., 2012) as accurate knowledge of global SST distribution and temporal variation is a key input to forecasting and prediction systems. SST fields constrain upper-ocean circulation and thermal structure on daily, seasonal, decadal and climatic timescales, for the exchange of energy between the ocean and atmosphere in coupled oceanatmosphere models, and as boundary conditions for ocean, weather and seasonal forecasting models. Other applications include maritime safety, military operations, ecosystem assessment, supporting fisheries and tourism, transport and energy, human health, food security and environmental policy. Well-defined and error-quantified measurements of SST are also required in the form of climate data records (CDRs) to reveal the role of the ocean in short and long term climate variability.

A sustained operational stream of satellite SST measurements has been available since 1981, which together with in situ measurements collectively form the modern-era SST observing system. By 1998, SST data were a mature component of the observing system with demonstrated capability and data products. However, SST product availability was limited to a few data sets that were large, scientific in format and difficult to exchange in near real time (NRT). Furthermore, product accuracy was insufficient for the emerging ocean modeling and data assimilation communities, while at the same time the number of applications requiring accurate spatial high-resolution SST was growing.

To address these issues, the Global Ocean Data Assimilation Experiment (GODAE; Bell et al., 2009) defined a minimum data specification for use in operational ocean models, requiring SST observations with global coverage, a spatial resolution of $10 \mathrm{~km}$ or higher, an accuracy of $0.2^{\circ} \mathrm{C}$ or better, and updated every $6 \mathrm{~h}$ (Smith and Koblinsky, 2001). The establishment of the Group for High Resolution Sea Surface Temperature, GHRSST (previously, GODAE High-Resolution Sea Surface Temperature Pilot Project) addressed these specifications (Donlon et al., 2009). GHRSST is a self-organizing group of researchers and operational practitioners working together to improve the accuracy and availability of SST fields for all applications.

To achieve the GODAE requirement, GHRSST devised an integrated approach to develop an SST observing system comprising: (1) improved SST data assembly/delivery; (2) quantified SST product accuracy; (3) improved consistency of SST products; and (4) demonstrated benefits for the improved observing system. Over the past 10 years, GHRSST has continued to support the delivery of operational SST data to the user community $^{1}$ and confirmed that the requirements of GODAE can be met (with the exception of accuracy). Much progress has been made on the inter-comparison of SST products, through activities such as the SST Quality Monitor (SQUAM ${ }^{2}$; Dash et al., 2010), in situ SST quality monitor (iQuam ${ }^{3}$; Xu and Ignatov, 2014), ACSPO Regional Monitor for SST (ARMS ${ }^{4}$ ) and Felyx ${ }^{5}$. Progress has been also made on SST applications, including assimilation and demonstrating the benefit of the improved observing system. Many users now assimilate SST in the global and regional models to provide continuous data and information on the physical state, variability and dynamics of the global ocean and marine ecosystems.

This paper focuses mainly on the progress and challenges for satellite SSTs over the past decade and continuing into the next decade. Fiducial Reference Measurements (FRM) and in situ data are described with respect to satellite validation activities and ensuring traceability for climate records. Centurioni et al. (2019), Kent et al. (2019), and Smith et al. (2019) address these surface-based observations in more detail. We conclude with a community-consensus vision for the next decade and beyond as a set of recommendations.

\footnotetext{
${ }^{1}$ https://www.ghrsst.org/quick-start-guide/

${ }^{2}$ http://www.star.nesdis.noaa.gov/sod/sst/squam

${ }^{3}$ http://www.star.nesdis.noaa.gov/sod/sst/iquam

${ }^{4}$ http://www.star.nesdis.noaa.gov/sod/sst/arms/

${ }^{5}$ http://hrdds.ifremer.fr/
} 


\section{SST'S ROLE IN AN INTEGRATED OCEAN OBSERVING SYSTEM}

Understanding oceanic and atmospheric processes requires knowledge of the temperature at which these processes occur. Processes at the ocean surface include radiative emission peaking in the infrared (IR), solubility of gasses (including $\mathrm{CO}_{2}$ and therefore ocean-atmosphere gas fluxes), evaporation (the latent heat flux), and sensible heat flux associated with the air-sea temperature difference (which also influences the momentum fluxes from atmosphere to ocean). The atmospheric and oceanic vertical temperature profile near the air-sea interface affect the stability and effectiveness of turbulent exchanges, and thus they influence the magnitudes of air-sea fluxes (Lindzen and Nigam, 1987; Fairall et al., 2003; Kara et al., 2005). Profiles of temperature throughout the ocean depth have indicated a warming of the oceans over the past six decades, the period for which such measurements are available (Levitus et al., 2012).

Satellite remote sensing has provided a mechanism for deriving SST on global scales over decades and needs to be sustained, improved, reconciled and extended into the next decade and beyond. The uninterrupted time-series of global, high resolution $(\sim 4-10 \mathrm{~km})$ SSTs retrieved from satellite IR radiometers extends back to the early 1980s (Kilpatrick et al., 2001), and SSTs from passive microwave (PMW) radiometers back to late 1997 in the tropics, and 2002 globally. In the framework of an integrated ocean observing system, satellitederived SST fields provide information on upper ocean variability and processes (e.g., Legeckis, 1977; Cornillon, 1986; Gentemann et al., 2008; Liu and Minnett, 2015) and on air-sea interactions (e.g., Chelton et al., 2001; O’Neill et al., 2012). The current constellation of satellites produces global measurements of SST at accuracies useful for many scientific, operational and climate purposes.

\section{THE SST MEASUREMENT CONSTELLATION}

Measurements of SST are made in situ with contact thermometers and retrieved from IR and PMW radiometers on satellites (for example, see Walton et al., 1998; Merchant et al., 2009b; Gentemann et al., 2010; Koner and Harris, 2016). IR- and PMWderived SSTs have different and complementary characteristics. IR emission at the ocean surface originates in a very thin electromagnetic skin layer of $<0.1 \mathrm{~mm}$ thickness, whereas PMW emission comes from an electromagnetic skin layer which is several millimeters thick. The SST as derived from IR radiometers is cooler than the water beneath, on average, by $\sim 0.17 \mathrm{~K}$, but can be several tenths of a degree cooler at low wind speeds (Donlon et al., 2002; Minnett et al., 2011). The spatial resolution of IR-derived SSTs from modern satellite instruments is typically 1-4 $\mathrm{km}$ at nadir, whereas the resolution of microwave (MW)derived SSTs is typically 50-75 km. PMW SSTs are not normally derived within $\sim 100 \mathrm{~km}$ of land or ice. A major advantage of PMW-derived SSTs over those from the IR is that the propagation of MW radiation at $6-10 \mathrm{GHz}$ which is largely insensitive to the presence of clouds, except where there is heavy rainfall. In contrast, satellite IR measurements are strongly influenced by scattering and emission from clouds, with the consequence that only measurements taken through clear atmospheres can be used to derive $\mathrm{SST}_{\text {skin. }}$. Effective "cloud-screening" algorithms are needed to ensure derived SST $_{\text {skin values are not tainted }}$ (Kilpatrick et al., 2001; Merchant et al., 2005). Merging SST retrievals from IR and MW sensors together with in situ data on any given day is a widely accepted approach to derive global fields (e.g., Chin et al., 2017).

\section{An Increasing Number and Diversity of Satellite Observations}

Since satellite SST observations began in the early 1980s, the number and diversity of sensors has increased dramatically, and is ever evolving. A combination of IR (onboard both low-Earth orbiters, LEO, and geostationary, GEO, platforms) and PMW (LEO only) radiometers provide a comprehensive global SST constellation, capable of meeting users' needs across a wide range of scales by exploiting their different capabilities and strengths. Figure 1 indicates the main satellite missions contributing to the current and recent SST constellation. Continuity and redundancy is ensured primarily by meteorological agencies' space programs, as well as initiatives such as Copernicus. It is vital to continue innovation to improve the resolution and accuracy of the SST fields. Table 1 lists the satellite SST measurement constellation at the present time, noting that further changes and developments are taking place to the constellation over the next decade and are summarized in the following sections.

\section{Polar-Orbit IR SST Capability}

IR radiometers on LEO satellites provide high spatial resolution SST with global coverage of the Earth up to twice daily at the equator, and more frequently in the higher latitudes. Current retrieval methods and sensor technologies enable highly accurate IR SST retrievals, yielding global monthly differences around $0.2^{\circ} \mathrm{C}$ with standard deviations around $0.4^{\circ} \mathrm{C}$ during night-time (e.g., Petrenko et al., 2014; Wang et al., 2016). Progress in IR SST also significantly reduced regional and seasonal differences inherent with non-linear SST (NLSST) retrieval algorithms (e.g., Merchant et al., 2009a; Marsouin et al., 2015). SST products cover Level 2 (original swath projection, with native spatial resolution) to regional and global Level 3 products, remapped and composited over areas and times defined by user needs.

Recently, AVHRR (Cracknell, 1997; Albiñana et al., 2007) and MODIS (Esaias et al., 1998; Kilpatrick et al., 2015) have been joined by newer generation sensors. The newest National Oceanographic and Atmospheric Administration (NOAA) JPSS satellites are now equipped with VIIRS, which first flew on the Suomi-NPP launched in October 2011, and has a wide range of IR channels including 3.70, 4.05, and $8.55 \mu \mathrm{m}$ as well as two traditional "split-window" 11-12 $\mu \mathrm{m}$ channels. Currently, two Copernicus Sentinel-3 satellites are flying, carrying an SLSTR (Donlon et al., 2012), which is a dual-view radiometer akin to the Along Track Scanning Radiometer (ATSR) series (Llewellyn-Jones and Remedios, 2012). The future European 


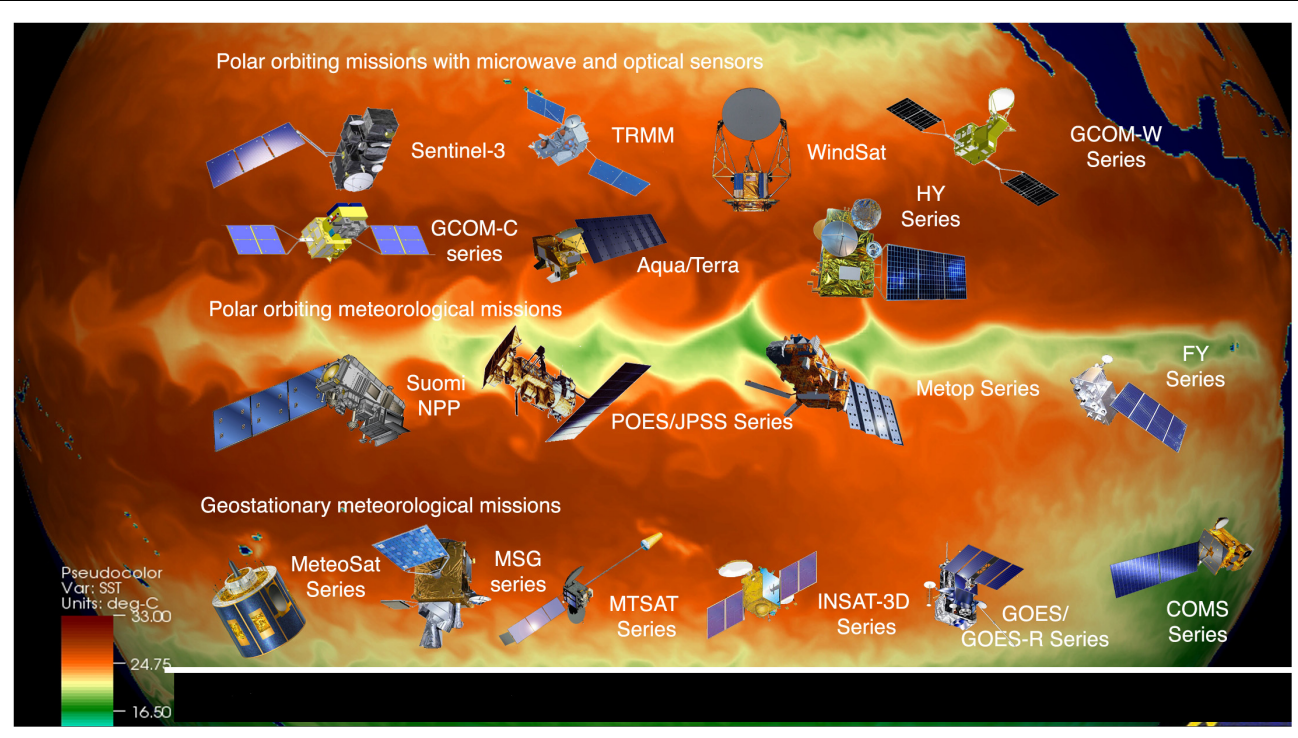

FIGURE 1 | The main satellite missions contributing to the current SST constellation. (Reproduced by permission under CC BY 4.0 from http://doi.org/10.6084/m9. figshare.7291694.

Organisation for the Exploitation of Meteorological Satellites (EUMETSAT) Polar System (EPS) Meteorological Operational Second Generation (Metop-SG) program will include the visible and infrared imager (METimage, Wallner et al., 2017), which will have 20 spectral bands channels, at $500 \mathrm{~m}$ resolution at nadir. In December 2017, Japan Aerospace Exploration Agency (JAXA) launched the Global Change Observation Mission - Climate (GCOM-C or "Shikisai") satellite carrying the Second-generation Global Imager (SGLI) with 19 spectral bands, and switchable resolution from 250 to 1,000 $\mathrm{m}$ at nadir (Urabe et al., 2018). Other instruments enabling SST retrieval include the Visible and InfraRed Radiometer (VIRR) on board the Chinese Feng-Yun satellites (Dong et al., 2009; Yang A. et al., 2017).

\section{Geostationary-Orbit Infrared SST Capability}

In contrast to LEO, GEO satellites provide many images each day over a fixed geographic region. New-generation GEO sensors include: the Advanced Baseline Imager (ABI; Schmit et al., 2005, 2017) onboard NOAA GOES-16 and -17 (launched in November 2016 and March 2017, respectively, and designated as NOAA operational GOES-East and -West satellites in December 2018 and February 2019, respectively); the Advanced Himawari Imager (AHI, a twin to ABI; Bessho et al., 2016), onboard Himawari-8 and -9 (launched in October 2014 and November 2016, respectively); the Advanced Meteorological Imager (AMI, another ABI twin; Choi and Ho, 2015) onboard Geo-Kompsat-2A (launched December 2018; Kim et al., 2015); the Advanced Geosynchronous Radiation Imager (AGRI; Yang J. et al., 2017) onboard the FY-4 series (FY-4A, launched in November 2016, will be followed by FY4B \& C in 2019 and 2021, respectively); and the Flexible Combined Imager (FCI; Durand et al., 2015) to be launched on EUMETSAT Meteosat Third Generation Imaging satellites (MTG-I) beginning in 2021.
All these new-generation sensors capture IR images every 10-15 min with $2 \mathrm{~km}$ nadir resolution (except FY-4, which takes images every $30 \mathrm{~min}$ with $4 \mathrm{~km}$ resolution at nadir) and have multiple spectral bands, sampling in the atmospheric windows around $3.7,8.6$, and $10-12 \mu \mathrm{m}$ suitable for SST retrievals. Other currently available GEO sensors with SST capability are the Spinning Enhanced Visible and Infrared Imager (SEVIRI) on Meteosat Second Generation (Meteosat-8, -9, -10, and -11), and the Multi-Spectral Imager onboard the Insat3D/3DR. The full constellation of geostationary satellites provides nearly global coverage, missing only high latitudes. There should always be a constellation of 5-6 geostationary platforms with SST-capable sensors.

An important feature of new generation GEO sensors is their capability to take measurements at high temporal resolution to improve clear-sky masking (e.g., using temporal information to separate the effects of faster moving clouds and other atmospheric features from the slower evolving SST fields). This approach can significantly improve coverage by using SSTs from successive clear-sky data allowing better characterization of the SST diurnal cycle (Gladkova et al., in review). Moreover, data volumes can be significantly reduced by providing a product at a reduced temporal rate (e.g., hourly) but still sufficient to resolve the diurnal cycle. Also, the sensitivity of the retrieval algorithm to real changes in temperature (Merchant et al., 2009a) is critical to accurately measure the temporal variability in geostationary SSTs, and in particular, to correctly estimate the amplitude of the SST diurnal cycle. Additional applications benefiting from highfrequency SST variability include semi-diurnal tides and their impact on shelf sea fronts. An illustration of the footprint and constellation of the global geostationary satellites can be found from the World Meteorological Organization (WMO) ${ }^{6}$.

\footnotetext{
${ }^{6}$ http://www.wmo.int/pages/prog/sat/globalplanning_en.php
} 
TABLE 1 | Current platform and sensor characteristics with capability for sea surface temperature, specifying whether in GEO or LEO; IR or PMW; daily coverage; spatial resolution at nadir; position or orbit and equator crossing time; agency and the expected lifetime till.

\begin{tabular}{|c|c|c|c|c|c|c|c|c|}
\hline Platform & Sensor & GEO/LEO & IR/PMW & Coverage & $\begin{array}{l}\text { Spatial resolution at } \\
\text { nadir (SST channels) }\end{array}$ & $\begin{array}{c}\text { Position/orbit } \\
\text { (equator crossing time) }\end{array}$ & Agency & $\begin{array}{l}\text { Expected } \\
\text { lifetime till }\end{array}$ \\
\hline Meteosat $^{1}$ & SEVIRI & GEO & $\mathbb{R}$ & Full disk 15 min & $3 \mathrm{~km}$ & $0^{\circ}$ & EUMETSAT & 2024 \\
\hline GOES-R (S/T/U) & $\mathrm{ABI}$ & GEO & $\mathbb{R}$ & Full disk $10 \mathrm{~min}$ & $2 \mathrm{~km}$ & $\mathrm{E}: 72.5^{\circ} \mathrm{W} W: 137.2^{\circ} \mathrm{E}$ & NOAA & 2036 \\
\hline Himawari & $\mathrm{AHI}$ & GEO & $\mathbb{R}$ & Full disk 10 min & $2 \mathrm{~km}$ & $140.7^{\circ} \mathrm{E}$ & JMA & 2031 \\
\hline $\mathrm{FY}-2$ & S-VISSR & GEO & $\mathbb{R}$ & Full disk 30 min & $5 \mathrm{~km}$ & $86.5^{\circ} \mathrm{E}$ & CMA & 2022 \\
\hline $\mathrm{FY}-4$ & AGRI & GEO & $\mathrm{IR}$ & Full disk 15 min & $4 \mathrm{~km}$ & $105^{\circ} \mathrm{E}$ & CMA & 2040 \\
\hline Electro-L & MSU-GS & GEO & IR & Full disk 15-30 min & $4 \mathrm{~km}$ & $76^{\circ} \mathrm{E}$ & RosHydroMet & 2039 \\
\hline GEO-KOMPSAT & AMl & GEO & $\mathbb{R}$ & Full disk <10 min & $2 \mathrm{~km}$ & $128.2^{\circ} \mathrm{E}$ & KMA & 2031 \\
\hline INSAT-3D & Imager & GEO & $\mathrm{IR}$ & Full disk 30 min & $4 \mathrm{~km}$ & $82^{\circ} \mathrm{E}$ & ISRO & 2029 \\
\hline Terra & MODIS & LEO & $\mathbb{R}$ & Global 2 days & $1 \mathrm{~km}$ & Sun-synchronous 10:30 & NASA & 2022 \\
\hline Aqua & MODIS & LEO & IR & Global 2 days & $1 \mathrm{~km}$ & Sun-synchronous 13:30 & NASA & 2025 \\
\hline Sentinel-3 & SLSTR & LEO & $\mathbb{R}$ & Global $1-2$ days & $1 \mathrm{~km}$ & Sun-synchronous 10:00 & EU & 2031 \\
\hline EPS $^{2}$ & AVHRR/3 IASI & LEO & $\mathbb{R}$ & $\begin{array}{l}\text { Global twice per day/near } \\
\text { global twice per day }\end{array}$ & $1.1 \mathrm{~km} / 12 \mathrm{~km}$ IFOV & Sun-synchronous09:30 & EUMETSAT & 2024 \\
\hline S-NPP & VIIRS & LEO & $\mathbb{R}$ & Global twice per day & $750 \mathrm{~m}$ & Sun-synchronous13:30 & NASA \& NOAA & 2025 \\
\hline $\begin{array}{l}\text { JPSS (NPP/N20 } \\
\text { and J2/3/4) }\end{array}$ & VIIRS & LEO & $\mathbb{R}$ & Global twice per day & $750 \mathrm{~m}$ & Sun-synchronous13:25 & NOAA & 2038 \\
\hline Meteor-M & MSU-MR & LEO & $\mathbb{R}$ & Global twice per day & $1 \mathrm{~km}$ & Sun-synchronous15:09 & RosHydroMet & 2030 \\
\hline GCOM-C & SGLI & LEO & $\mathbb{R}$ & Global in 3 days (day-light) & $250 \mathrm{~m} / 1 \mathrm{~km}$ & Sun-synchronous10:30 & JAXA & 2022 \\
\hline $\mathrm{HY}-2$ & MWI & LEO & PMW & Daily global & $80 \times 120 \mathrm{~km} \mathrm{IFOV}$ at $6.6 \mathrm{GHz}$ & Sun-synchronous06:00 & NSOAS & 2023 \\
\hline GPM-Core & GMI & LEO & PMW & $\begin{array}{l}\text { Near-global in } 2 \text { days } \\
<70^{\circ} \text { latitude }\end{array}$ & $19 \times 32 \mathrm{~km} \mathrm{IFOV}$ at $10.65 \mathrm{GHz}$ & Drifting, $65^{\circ}$ inclination & NASA/JAXA & 2019 \\
\hline GCOM-W & AMSR-2 & LEO & PMW & Global once per day & $35 \times 62 \mathrm{~km} \mathrm{IFOV}$ at $6.9 \mathrm{GHz}$ & Sun-synchronous13:30 & JAXA & 2019 \\
\hline Coriolis & WindSat & LEO & PMW & Global in 1.5 days & $39 \times 71 \mathrm{~km}$ IFOV at $6.8 \mathrm{GHz}$ & Sun-synchronous06:00 & US/NASA & 2019 \\
\hline $\mathrm{FY}-3$ & VIRR/MERSI-2/MWRI & LEO & IR/PMW & Global twice per day & $250 \mathrm{~m} / 51 \times 85 \mathrm{~km} \mathrm{IFOV}$ at $10.65 \mathrm{GHz}$ & Sun-synchronous06:00/14:00 & CMA & 2029 \\
\hline
\end{tabular}

${ }^{1}$ Meteosat Third Generation (MTG) will follow-on from MSG until 2038. 2EUMETSAT Polar System-Second Generation (EPS-SG) will follow-on from EPS until 2043. 


\section{Passive MW Polar-Orbiters and Low Inclination Orbiting SST Capability}

PMW SST retrievals from LEO satellites (in both sunsynchronous and non-sun-synchronous orbits) have been collected for approximately 20 years, starting with the joint USJapan Tropical Rainfall Measuring Mission, launched in 1997, which carried the TRMM Microwave Imager (TMI; Wentz et al., 2000). TRMM was in a low inclination orbit so measurements were limited to within about $40^{\circ}$ of the Equator. To sample the diurnal patterns in rainfall, TRMM was put in a sunasynchronous drifting orbit. The NASA satellite, Aqua, launched in 2002 carried the Advanced Microwave Scanning Radiometer for EOS (AMSR-E; Kawanishi et al., 2003). Aqua is in a sunsynchronous, polar orbit. The first PMW producing global SSTs started in 1978 with SMMR on SeaSat and Nimbus-7. A great advantage of the PMW SSTs is in the cloud penetrating capability of low frequency microwaves to capture SST under most clouds and through atmospheric aerosols. These capabilities make PMW SSTs an essential data source for producing multi-satellite merged SST products on a daily basis, though their spatial resolution is much coarser than IR SSTs. SST retrievals using PMW imagers are based on low-frequency channels of 6.9 and $10.65 \mathrm{GHz}$ that are sensitive to water temperature variations, but the SST sensitivity of the $10.65 \mathrm{GHz}$ emission decreases below SSTs colder than $13^{\circ} \mathrm{C}$ (Gentemann et al., 2010).

The spatial resolution of PMW SSTs depends on the antenna size and satellite altitudes. Larger antennas and lower satellite altitude result in finer spatial resolution, with the $10.65 \mathrm{GHz}$ measurements having finer footprints than at $6.9 \mathrm{GHz}$. At present, the finest spatial resolution of $6.9 \mathrm{GHz}$ SST is about $35 \times 62 \mathrm{~km}$ by AMSR2 on the Japanese GCOM-W satellite launched in 2012, having a $2.0 \mathrm{~m}$ diameter antenna and $699 \mathrm{~km}$ altitude. The finest spatial resolution of $10.65 \mathrm{GHz}$ SSTs is about $19 \times 32 \mathrm{~km}$ provided by Global Precipitation Measurement (GPM) Microwave Imager (GMI) onboard the US-Japan joint GPM Core Observatory (launched in 2014 into a low inclination orbit) with a $1.2 \mathrm{~m}$ diameter antenna and $402.5 \mathrm{~km}$ altitude. Recent validation indicates the accuracy of PMW SSTs to be around $0.5^{\circ} \mathrm{C}$ for $6.9 \mathrm{GHz}$, and $0.6^{\circ} \mathrm{C}$ for $10.65 \mathrm{GHz}$ SSTs by AMSR2 (JAXA, 2017). More research and international collaboration is recommended on harmonizing and improving the calibration of low-level PMW observations across all available missions. Further fundamental observation of and research into the dependence of sea-surface emissivity at SST-relevant frequencies on sea-state and wind speed will be crucial to driving future improvements in uncertainty in PMW SST.

\section{The Evolving in situ SST Observing System}

Measurements of SST have been made for more than 200 years for a wide variety of purposes. The earliest measurements of SST in the eighteenth century were taken as an aid to navigation. Over a time and after the connection between SST and ocean currents was strengthened, large numbers of measurements were made for the construction of navigational charts (Kennedy et al., 2012). Gradually, data needs for weather and oceanographic forecasting systems, as well as understanding a changing climate, became the main drivers for collecting SST data. This evolution reflected a change in the method of measurements from mainly human operators on voluntary observing ships (VOS) to fully automated measurements from buoys (and satellites). Satellite measurements rely on in situ data for retrieval and validation (see section "Improved Methods of Validation") and both sources of SST are used in many applications, such as climate and weather model boundary conditions (Folland, 2005; Kennedy et al., 2012) and SST analysis products (e.g., Roberts-Jones et al., 2012). Figure 2 shows the changes over time of geographical coverage and method for in situ measurements, illustrating the constraint of major shipping lanes have on the data collection and the under-sampling of low and high latitudes.

The change in observation method not only expanded the geographical distribution of the in situ SST measurements but also their quality. The changing requirements led to international efforts to define a common set of requirements and standards with the implementation of the climate observing system that is overseen and coordinated by the Global Climate Observing System (GCOS) providing advice and guidance at national and international levels (e.g., GCOS, 2009, 2010). Coordination of specific activities is through the GCOS-affiliated expert bodies and panels, such as the WMO/Intergovernmental Oceanographic Commission (IOC) Joint Technical Commission for Oceanography and Marine Meteorology (JCOMM), Ship Observations Team (SOT), and the Data Buoy Cooperation Panel (DBCP) to name a few (Berry and Kent, 2017). Different applications of SST fields have varying accuracy requirements, with those for CDR being the most stringent and difficult to meet. Continuation of the Global Tropical Moored Buoy Array (GTMBA; McPhaden et al., 2010) is essential, as it has a major impact on methods to assess the stability of long-term SST data records (e.g., see for example Merchant et al., 2012).

Sea surface temperature is identified by the WMO as an Essential Climate Variable $\left(\mathrm{ECV}^{7}\right)$, among other parameters (Bojinski, 2014), and a rigorous approach to data quality assessment must be followed, such as recommended by Barker et al. (2015). Most notably, this means that in situ SST measurements should be accompanied by estimates of their uncertainty, following formal metrological protocols (Joint Committee for Guides in Metrology (JCGM, 2008). These specifications should be followed for all SST products but are especially important if satellite-derived SSTs are to be used as a CDR (Minnett and Corlett, 2012). There are several approaches to estimate uncertainties of in situ measurements (e.g., Hirahara et al., 2014; Kennedy, 2014; Berry and Kent, 2017; Freeman et al., 2017). Broadly speaking, the uncertainties can be split into two groups: uncertainties due to random errors, such as incorrect readings or rounding errors; and uncertainties due to systematic errors, such as incorrect calibration or poor positioning of the measurement site. Although this is a convenient way to deal with the uncertainties, inaccuracies in SST measurements generally share characteristics (Kennedy, 2014). Most in situ SST datasets

\footnotetext{
${ }^{7}$ http://www.wmo.int/pages/prog/gcos/index.php?name= EssentialClimateVariables
} 


\section{A \\ World Ocean Database 2013 Decadal averaqe 1955 - 2012}

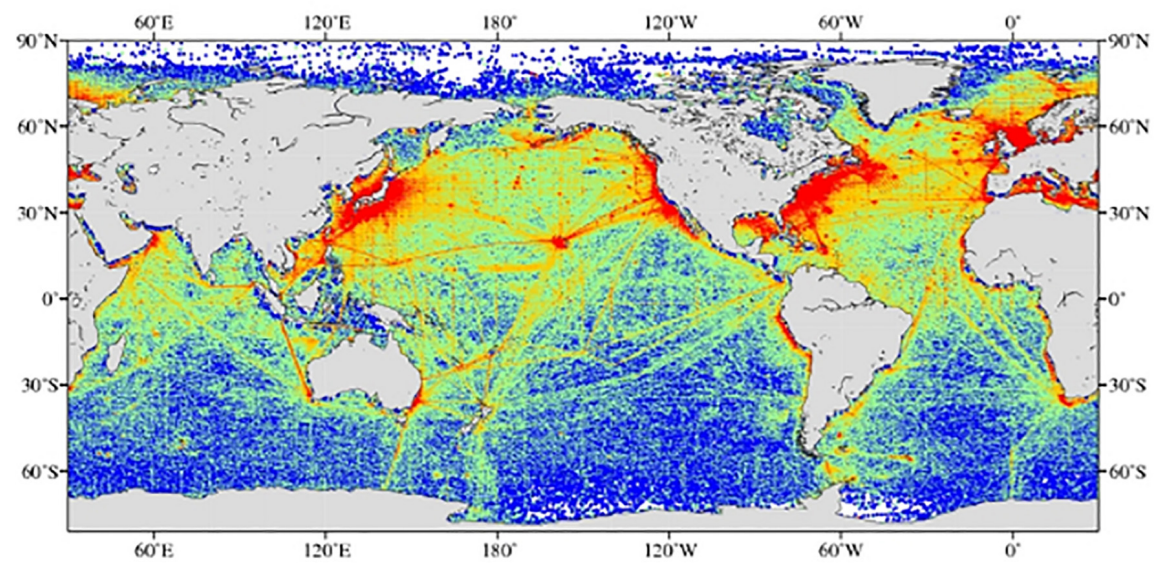

Annual temperature observations at surface (quarter-degree grid)

B
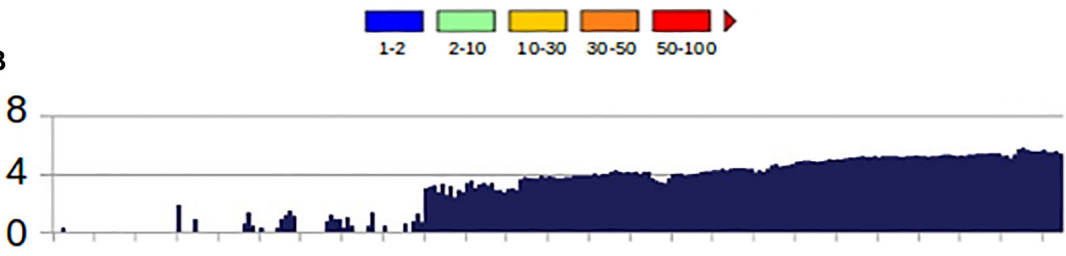

\section{Fraction of Measurements from each type in ICOADS}

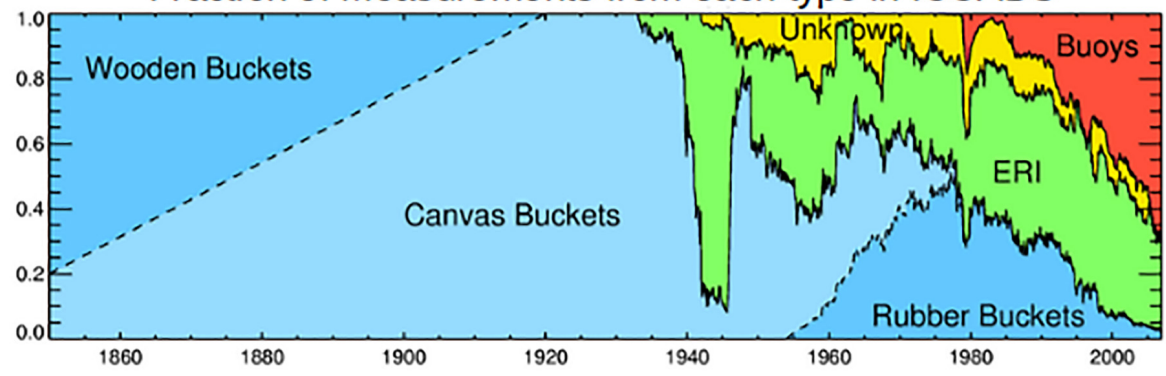

FIGURE 2 | Distribution of SST measurements and by method. (A) Shows the spatial distribution of SST measurements as included in the World Ocean Atlas in 2013. (B) Shows the temporal distribution of SST measurements in the World Ocean Atlas in 2013 (from Freeman et al., 2017). (C) How the measurement methods changed over time. ERI are Engine Room Intake measurements, Buoy measurements include drifting and moored buoys (from Rayner et al., 2018). Please note that the spatial and temporal time lines are different, 1955-2012 for the spatial distribution shown in plate (A) and 1770-2014 for the temporal distribution shown in plate (B).

(e.g., International Comprehensive Ocean-Atmosphere Data Set, ICOADS, and the Hadley Centre SST Dataset, HadSST3) include an estimate of a correction for the systematic component based on measurement type (e.g., bucket, engine room intake or buoy), which can reduce discontinuities in the long time records. Kennedy (2014) states that a typical SST measurement made by a ship has an uncertainty of around $1.0-1.5^{\circ} \mathrm{C}$ and a drifting buoy observation a typical uncertainty of around $0.1-0.7^{\circ} \mathrm{C}$. Vessels with well-calibrated and suitably located temperature sensors can provide SST measurements with similar uncertainties to currently deployed drifting buoys, and can therefore provide in situ SST for satellite SST validation in regions lacking in drifting or moored buoys, such as coastal regions and in the Australian region (Beggs et al., 2012).

While the effort to characterize uncertainties for in situ SST records is considerable not all of them are SI-traceable, in a metrological sense, which is needed for a CDR. Therefore, more recent efforts using ship-based infrared radiometers for satellite-derived SST validation have shown the value of SItraceable measurements, together with rigorous uncertainty budgets (Barton et al., 2004; Rice et al., 2004; Donlon et al., 2008; Minnett, 2010; Wimmer and Robinson, 2016) of $0.14^{\circ} \mathrm{C}$ 
(Theocharous et al., 2016), or better. However, the measurements are sparse compared to those of drifting buoys. Continued studies and activities are needed on the inter-comparison of FRM (Donlon et al., 2015) with data; the estimation and provision of the measurement uncertainties; and ensuring SI-traceability is established through international collaboration. In an effort to improve the sampling of SI-traceable in situ SSTs, a new generation of drifting buoys is being designed and tested through efforts of GHRSST, DBCP, European Union's Copernicus Programme and EUMETSAT (Poli et al., 2019). These efforts to improve in situ records are continuing and require considerable resources to improve the in situ SSTs not only as a standalone CDR but also as a verification tool for satellite-derived SST fields. It is important that improvements in correct metadata provision to in situ records continue to be made. Data from Argo profilers in the near surface layer are used for satellite SST validation, particularly those specifically designed to acquire SST close to the sea surface. These data could be further exploited to explore SST at depth and validate near-surface profiles. Additional in situ measurements, and ideally FRM, are required, especially in high-latitude and sea ice regions.

\section{Improving Current and Future SST Measurements for Users}

The increased number of SST products and large number of users makes user-driven developments within the current and future SST products essential. Comments and feedback are typically obtained through dedicated science and user meetings (such as the annual GHRSST meeting), which allow data producers to collect information to guide future product developments. This section presents and discusses necessary developments to provide improvements highlighted by users as the most important for their application, such as: cloud masking, aerosol impact assessment, uncertainty estimation, diurnal variability, and validation.

\section{Improved Cloud Masking of IR Imagery}

About $80-90 \%$ of pixels are fully or partially cloudy in any IR imagery of the ocean, and it is critically important to identify (and remove) these pixels. Clouds are colder and more reflective, more variable in space/time, and have a particular IR spectral emissivity, compared to clear ocean. Cloud masking algorithms for SST make use of the spectral, spatial, and temporal characteristics of the sensor brightness temperatures (BTs); solar reflectance bands may be additionally employed during daytime. Methods currently used are threshold-based (e.g., Saunders and Kriebel, 1988; Kilpatrick et al., 2001; Petrenko et al., 2010), probabilistic (based on blending physically and empirically derived joint probability distributions, in conjunction with Bayes' theorem; e.g., Merchant et al., 2005), or trained using artificial intelligence (AI) approaches (e.g., Kilpatrick et al., 2019).

For LEO sensors, an additional spatial pattern recognition approach has been found useful (Gladkova et al., 2015). Also, repeated sampling of the same area of the ocean, from different passes or satellites, may be utilized (Gladkova et al., 2019). For GEO, frequent sampling provides a valuable opportunity to dramatically improve cloud masking, by separating fast-moving atmospheric features from more slowly evolving ocean patterns (Gladkova et al., in review; see example in Figure 3). While not all clouds can be identified in this way, it offers a computationally effective improvement over using only static spatial information.

All current methods are formulated to deliver either a binary mask or a continuous score (e.g., probability of clear sky) that can be used to derive quality information. Albeit differently, all methods embed prior knowledge of the physical and radiative transfer properties of the surface, clouds and atmosphere. In dynamic and coastal regions of the ocean, and around ice, uncertainty in the prior SST is larger, and misclassification more likely. In general, dependence on prior information should be minimized wherever reliable classification can be made from the satellite observations themselves, which is aided by new SST sensors that provide measurements in more bands, and at higher spatial and temporal resolution.

\section{More Accurate SST Retrieval Through Aerosol-Laden Atmospheres}

Sea surface temperature retrievals from IR measurements are susceptible to forms of aerosol that absorb and scatter IR radiation, particularly mineral dust (such as lofted from the Sahara Desert) and stratospheric volcanic aerosol following major $\mathrm{SO}_{2}$-rich eruptions (the last being Mt. Pinatubo in 1991). Many SST algorithms have little robustness to such events (Blackmore et al., 2012) and will be subject to significant errors. Figure 4 indicates the influence of Saharan dust aerosol outflows on satellite SST retrievals, over the Atlantic Ocean and the benefits of a correction algorithm (Luo et al., 2019). Intermittent mineral-dust loadings cause variable SST errors, which have negative implications for prediction of important phenomena including monsoonal systems, as well as for other applications (Atkinson et al., 2013, 2015).

Dual-view SSTs from sensors such as SLSTR are less sensitive to mineral dust than single-view sensors (Merchant et al., 1999). The exploitation of non-traditional SST bands should also help reduce dust-related biases. Microwave SSTs are expected to be unaffected by mineral dust. Nonetheless, improvements in SST retrieval in mineral-dust conditions is possible and is needed, and should involve efforts both on single-sensor algorithms and optimum use of the constellation of sensors.

Some current (MODIS and VIIRS) and planned (METimage) sensors have more than one channel around $3.9 \mu \mathrm{m}$. With the information content of these channels, the potential for more accurate SST retrievals in the presence of aerosols arises (Merchant et al., 2006), but this is yet to be widely exploited. It is recommended that studies to address this are pursued to improve SST accuracy in areas of mineral dust aerosol and introduce resilience to major volcanic eruptions, which although rare will occur in future.

\section{Better Uncertainty and Error Estimation}

Users of SST increasingly demand uncertainty information, to give greater context for their applications and for quantitative use in systems such as data assimilation (ECMWF Workshop Report, 2018). Good-practice guidance for uncertainty provision 


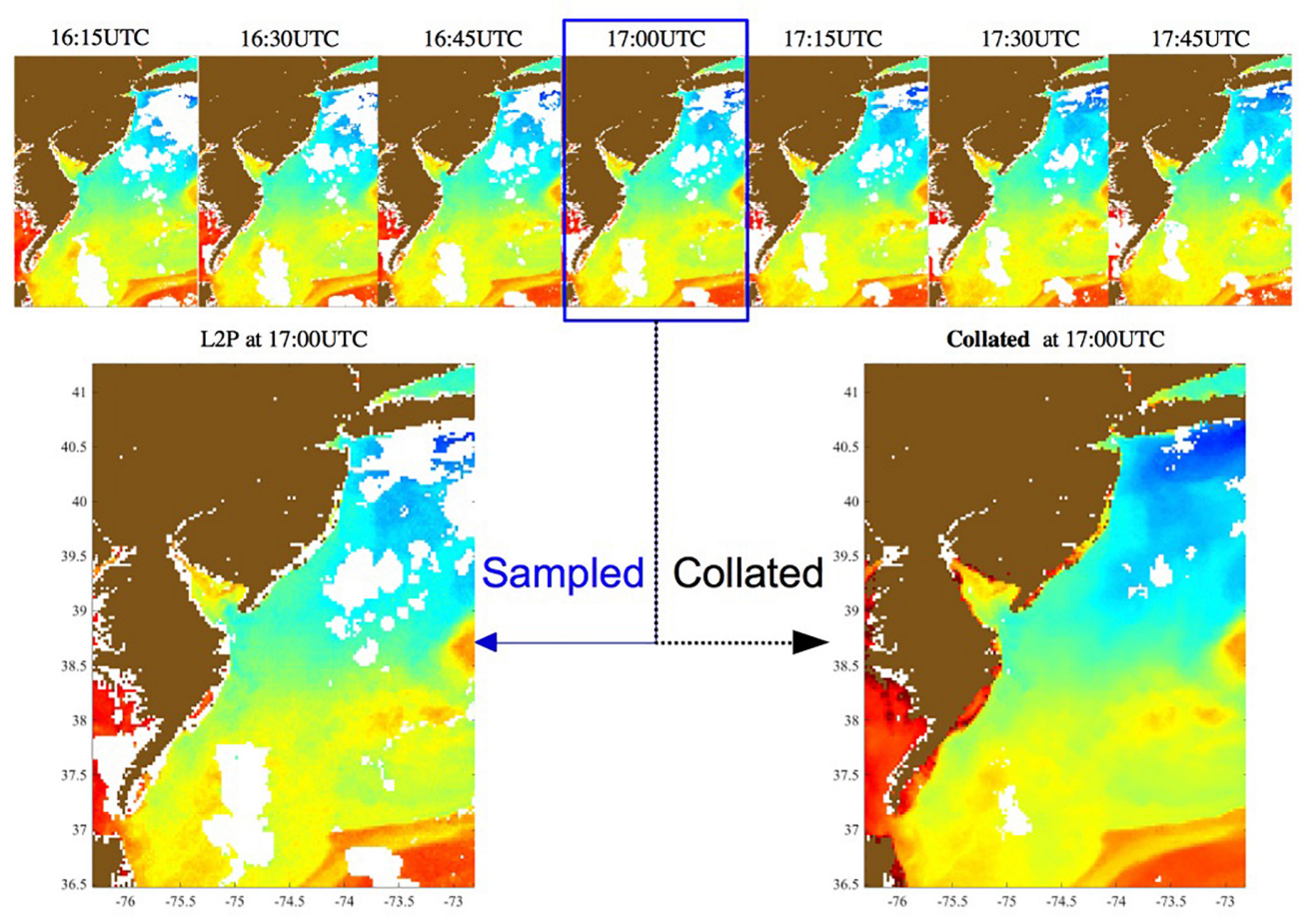

FIGURE 3 | An example of using the temporal context information available from the ABI on GOES-16. The image at lower left is the sampled instantaneous SST at 17UTC. At lower right is collated SST, produced using an algorithm described in Gladkova et al. (in review) that examines 6 h of temporal data to detect residual cloud from drop-outs in the SST time series, and to fill short temporal gaps by noise-tolerant curve fitting. The collated product significantly increases clear-sky coverage, and reduces cloud leakages and noise in the imagery. (Reproduced by permission under CC BY 4.0 from http://doi.org/10.6084/m9.figshare.7286267.v2).
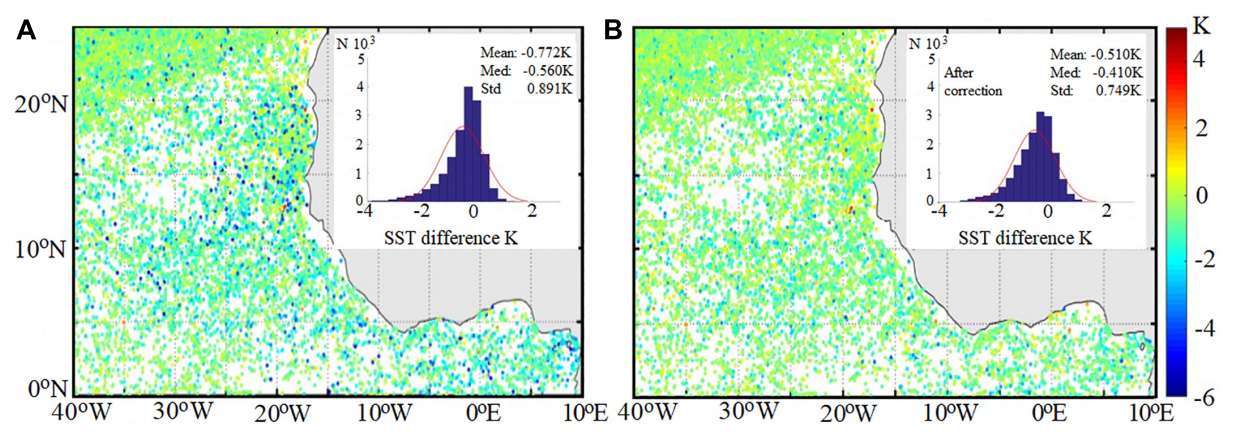

FIGURE 4 | Night-time differences between SST $_{\text {skin }}$ derived from MODIS on Aqua, and collocated, coincident subsurface temperatures from drifting buoys off West Africa, a region frequently subjected to the influence of Sahara dust aerosol outflows to the west and southwest, after Luo et al. (2019). The temperature differences

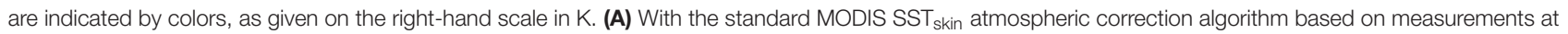
$\lambda=11$ and $12 \mu \mathrm{m}$ (Kilpatrick et al., 2015), indicating the predominantly negative differences in the dust outflow area. (B) As at panel (A) but after additional correction for aerosol effects with an algorithm that includes measurements at $\lambda=3.75$ and $8.96 \mu \mathrm{m}$.

for CDRs (Merchant et al., 2017) offers principles that should be applied to SST data products in general.

The error in a measured value of SST is the difference in that value from the unknown true value, and the uncertainty in SST represents the dispersion of error. Although the error in each point is unknown (otherwise we would correct for it), there are a variety of techniques for quantifying uncertainty, in a statistical way. These fall into two classes: empirical methods in which uncertainty is deduced from the distribution of differences between alternative measured values (such as satellites versus drifting buoys; e.g., Castro et al., 2008, 2012; O'Carroll et al., 2008; Petrenko et al., 2016; Xu and Ignatov, 2016); and uncertainty modeling, in which understanding of the instrumental uncertainty, cloud screening, retrieval process and representativity effects are quantified, propagated and combined to form an uncertainty estimate (e.g., Merchant et al., 2014).

To gain confidence of users in applying provided uncertainty information, we recommend that uncertainty estimates should 


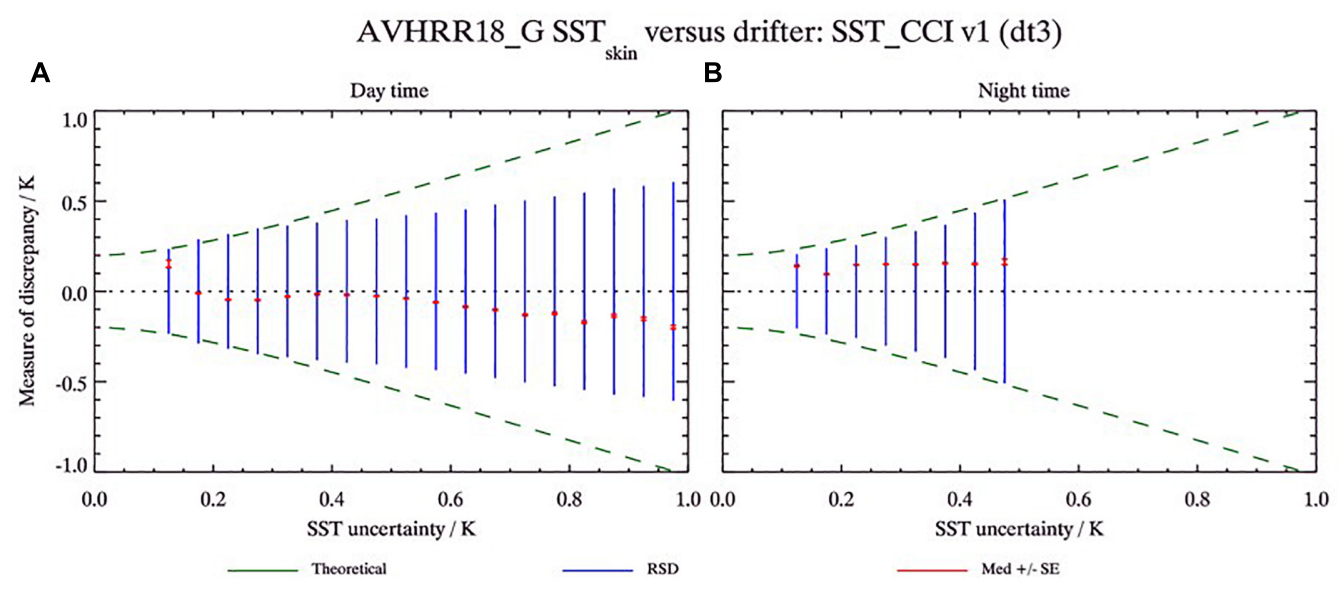

FIGURE 5 | Example of validation of an uncertainty model for an SST product. Here, SST uncertainty is defined per datum in an SST product, and varies over a range of 0.0-1.0 K. Matches to drifting buoys are binned in terms of the SST uncertainty, and the mean and standard deviation of the difference calculated per bin (indicated by the red and blue bars respectively). Accounting for an estimate of in situ uncertainty, the green dashed line is the envelope expected if the uncertainty estimates are accurate. As we can see from this example, the agreement at night time (A) is very good. However, for day time match-ups (B) the blue bars are within the theoretical envelope, indicating they are over estimated as the calculated standard deviation is smaller than the theoretical for much of the distribution.

(Reproduced by permission under CC BY 4.0 from https://doi.org/10.6084/m9.figshare.7286168.v2).

be developed using both uncertainty modeling and by empirical means. Provided a mature forward model exists, agreement of results between the two methods amounts to a convincing validation of the uncertainty model.

Approximate empirical methods of estimating uncertainty are presently the approach within GHRSST format SST products. These include Sensor Specific Error Statistics (SSES), comprising the mean and standard deviation of satellite SSTs differenced from a matched in situ temperature, such as measured from drifting buoys. Improved SSES require better quantification of in situ uncertainty (and preferably its reduction). It is recommended that understanding of drifting buoy uncertainty is improved in support of assessing satellite-derived SST accuracies. As uncertainty modeling methods are more widely developed for satellite SSTs over coming years, this will also enable more rigorous validation including validating uncertainties (see for example Figure 5 and section "Improved Methods of Validation").

\section{Better Practical Quantification of Diurnal Variability}

Progress in understanding and quantifying diurnal variability using hourly GEO IR SSTs over European seas and the Atlantic Ocean (Gentemann et al., 2008; Bellenger and Duvel, 2009; Marullo et al., 2010, 2016; Eastwood et al., 2011; Karagali et al., 2012; Karagali and Høyer, 2013), and the Tropical Warm Pool (Marullo et al., 2010; Zhang et al., 2016a,b), revealed consistent features regarding the frequency of all such events, their timing during the day and seasonal patterns. The difference between a daytime hourly SST value and the corresponding foundation temperature of the previous night is defined as diurnal warming (or anomaly), indicated by $\delta$ SST. Figure 6 shows the number of observations with $\delta S S T \geq 1 \mathrm{~K}$ estimated from hourly SEVIRI SST retrievals between 2006 and 2011. Areas with increased occurrences of $\delta$ SST exceeding $1 \mathrm{~K}$ include the Mediterranean, Red and Baltic Sea, the region of the Malvinas/Brazil currents (SW Atlantic), the Benguela Current offshore from Namibia and Angola (SE Atlantic) and the Mozambique Channel (Indian Ocean).

Different models of diurnal variability have been proposed (e.g., Filipiak et al., 2010) and evaluated (e.g., Karagali and Høyer, 2013, 2014; Karagali et al., 2017; While et al., 2017; Zhang et al., 2018). Diurnal SST variability has been quantified

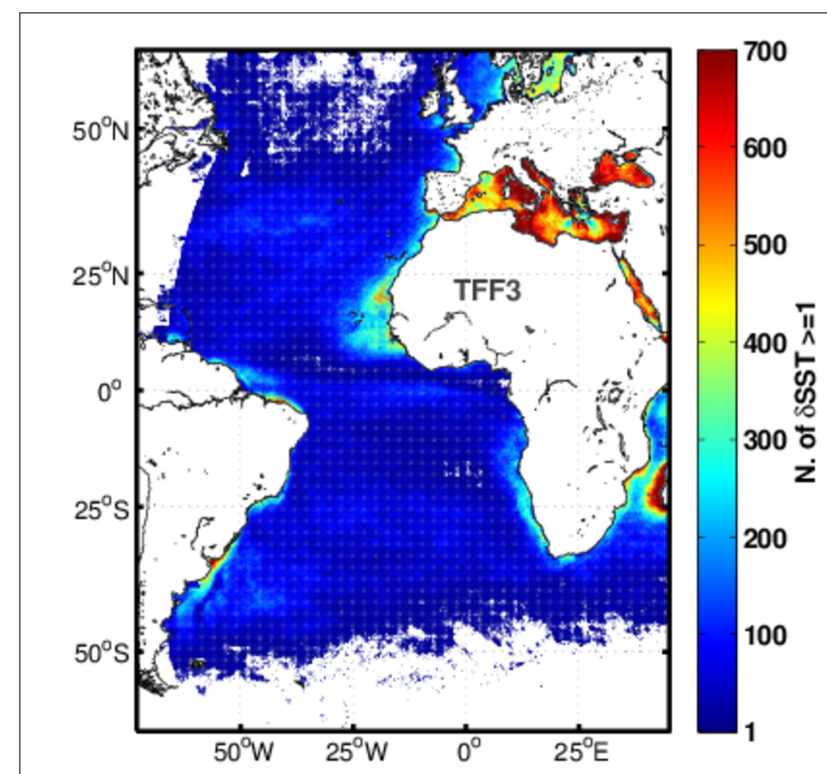

FIGURE 6 | Occurrences of diurnal warming of $1 \mathrm{~K}$ and larger, estimated from hourly SEVIRI SST retrievals between 2006 and 2011. (Reproduced by permission under CC BY 3.0 from https://doi.org/10.5194/os-10-745-2014). 

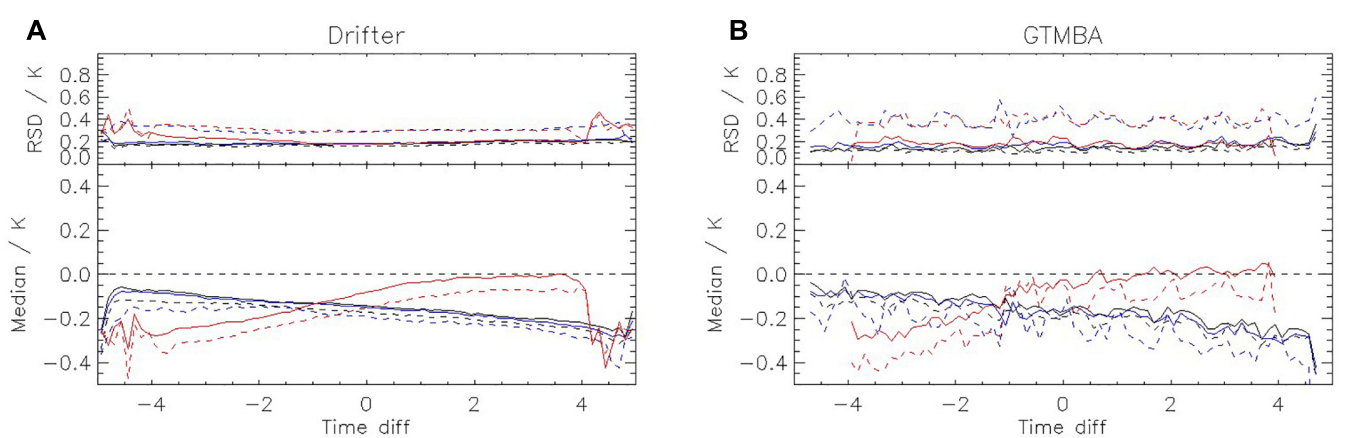

C

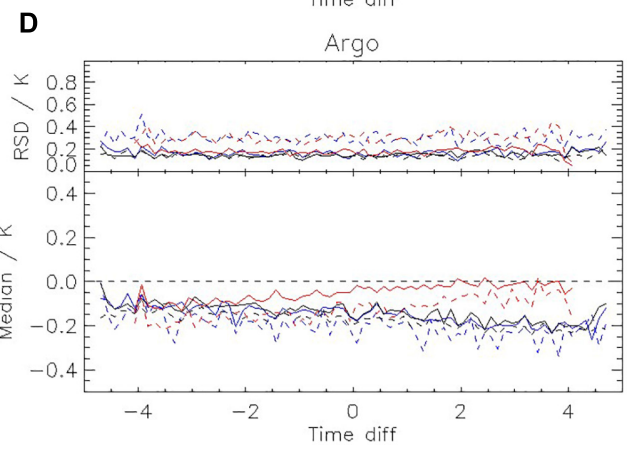

FIGURE 7 | Median (top) and robust standard deviation (bottom) for match-ups between AATSR and (A) drifters, (B) GTMBA, and (C,D) radiometers and Argo floats. Daytime results are shown in red, nighttime in blue and black; solid lines indicate AATSR dual-view retrievals, dashed lines indicate nadir-only. (Reproduced by permission under CC BY 4.0 from https://doi.org/10.6084/m9.figshare.7286216.v1).

by satellite (Gentemann et al., 2008) and in situ (MorakBozzo et al., 2016) observations, yet it is not fully resolved by forced ocean and coupled ocean-atmosphere models. Only some of the important diurnal variability and cool skin effects are parameterized or explicitly represented in models (e.g., Zeng and Beljaars, 2005; Fallmann et al., 2017). The diurnal cycle is represented in several operational GCMs that distribute hourly products. Here, the mean annual diurnal cycle is well reproduced but extreme events such as diurnal warming that exceed $1 \mathrm{~K}$ are always underestimated (Marullo et al., 2014). The implications associated with the lack of a properly resolved SST daily cycle in atmospheric, oceanic and climate models have been quantified in terms of heat budget errors in the Tropics (Clayson and Bogdanoff, 2012) and the Mediterranean Sea (Marullo et al., 2016). The community and users will benefit in the future if consistent methodology is developed, and a global study performed to cross-evaluate and validate against in situ data, the diurnal variability as seen in various geostationary products (SEVIRIs, GOES, etc.) over the longest period.

\section{Improved Methods of Validation}

The traditional approach to determine errors in satellite SSTs is comparison to in situ thermometers (e.g., Llewellyn-Jones et al., 1984; Strong and McClain, 1984; Kilpatrick et al., 2001; Reynolds et al., 2010; Gentemann and Hilburn, 2015) or shipboard radiometers (Kearns et al., 2000; Wimmer et al., 2012; Kilpatrick et al., 2015). GHRSST has supported efforts over the past 10 years to unify in situ data for satellite validation (e.g., in situ SST Quality Monitor, iQuam; Xu and Ignatov, 2014). However, the heritage approach to validation does not account for real physical differences between the two measurements and therefore may not correctly describe the error distribution in satellite SSTs. For example, Figure 7 shows the temporal dependence of differences between match-ups from AATSR and four different in situ datasets. The spread of values shown in Figure 7 contributes to the overall standard deviation of the match-ups if not minimized. Also, the uncertainty of the in situ measurement should be considered, which has been shown to be larger than expected for drifting buoys $(\sim 0.2 \mathrm{~K})$ from 3-way analysis methods (O'Carroll et al., 2008; Gentemann, 2014; $\mathrm{Xu}$ and Ignatov, 2016).

Corlett et al. (2014) proposed a new approach to satellite SST validation, by adapting the method of Wimmer et al. (2012) to cover all in situ measurement types and represent contributing terms as uncertainties rather than absolute errors. A benefit of the Corlett et al. (2014) approach is that it also offers validation of the satellite SST uncertainty (see section "Better Uncertainty and Error Estimation" and Figure 5; Lean and Saunders, 2013; Bulgin et al., 2016; Nielsen-Englyst et al., 2018). A continuing challenge is to understand the spatial variability within a satellite SST pixel (Castro et al., 2010) in order to justify assumptions in the treatment of spatial differences between the satellite and in situ data. Minnett (1991) estimated the variability within a pixel to be $\sim 0.2 \mathrm{~K}$ for AVHRR (which has $1.1 \mathrm{~km}$ spatial resolution at nadir) and more recently Castro et al. (2017) estimated a mean value of $O(0-0.1) \mathrm{K}$ for MODIS (which has $1 \mathrm{~km}$ spatial resolution in the thermal IR bands). 


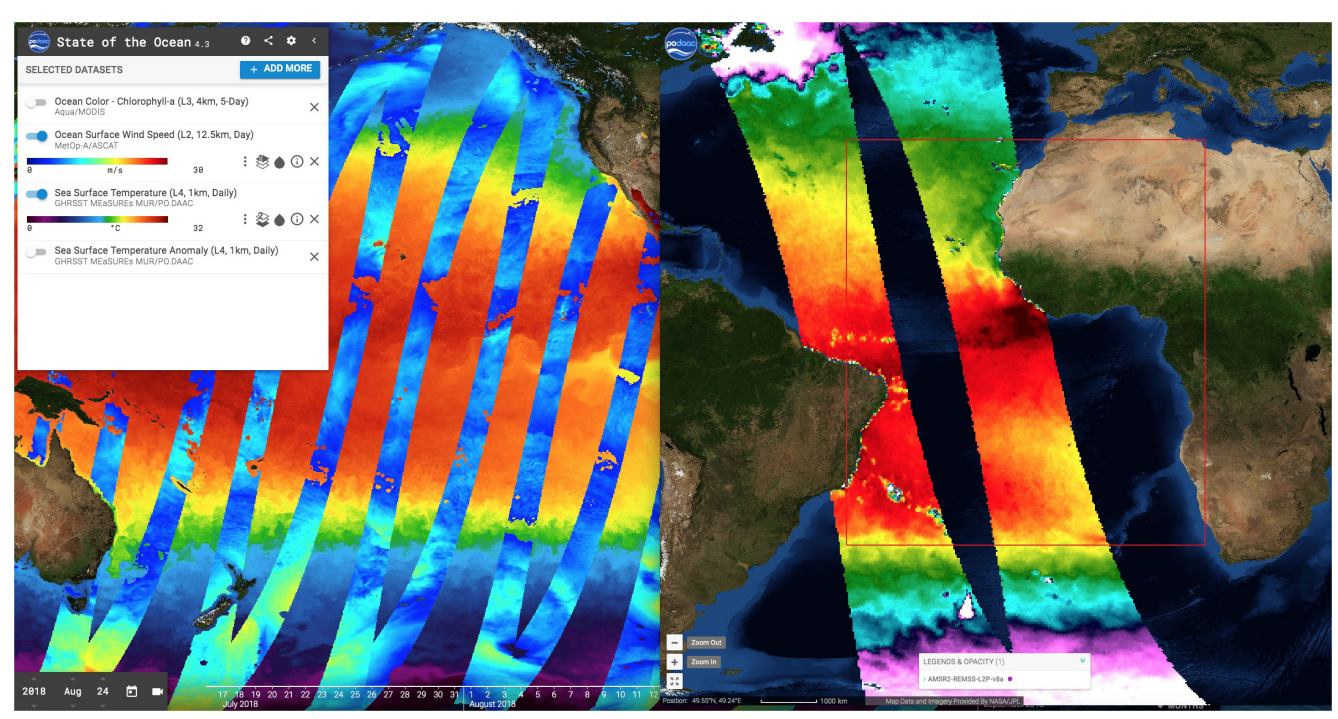

FIGURE 8 | The SOTO visualization tool (left) showing an overlay of ocean wind satellite tracks over a global Level 4 daily SST map juxtaposed with output from the HiTide Level 2 subsetter (right) showing two AMSR2 SST swaths overlapping a region of interest off Africa.

\section{EVOLVING THE INTERNATIONAL SST SERVICE TO USERS}

GHRSST began coordination of operational production and distribution of satellite SST datasets in 2005 governed by the foundational system of shared roles and responsibilities known as the Regional/Global Task Sharing (R/GTS). This framework has been highly successful and continues to operate today. However, the international GHRSST community, in response to ever-growing data volumes, improved national capabilities, and provider-specific data access policies, has established plans to evolve the R/GTS to become a more distributed and federated framework.

In the evolved R/GTS, multiple organizations around the globe will adhere to an agreed-upon set of data discovery, archive, and access standards, to enable the GHRSST Project Office to maintain a consolidated one-stop catalog and data discovery portal, while eliminating the need to physically consolidate the large and growing data volumes. In this new architecture existing systems will collaborate to migrate and evolve existing data, metadata and discovery systems toward this new, distributed system serving the entire community, and to ensure that current and future GHRSST format datasets maintain the required level of interoperability, discoverability, and metadata compliance.

Much of what this new architecture will require in terms of data cataloging, discovery, and access services had already been implemented using open source software and specifications. For example, the NASA Physical Oceanography Distributed Active Archive Center (PO.DAAC) and the NOAA National Centers for Environmental Information (NCEI) have implemented dataset and granule web services using the ESIP Opensearch specification for constructing web service discovery URLs. These services can be "chained" so that dataset to specific granule discoveries can be used as entry points to further data extraction, sub-setting, or visualization via additional web service calls. In addition, well established web services to access both gridded and non-gridded data via THREDDS and OPeNDAP have been implemented.

Users are also able to access a comprehensive tool set for GHRSST format data visualization, extraction and quality monitoring. For example, the PO.DAAC has developed the State Of The Ocean $\left(\mathrm{SOTO}^{8}\right)$ for rapid data visualization, overlay, and animation and the HiTide ${ }^{9}$ Level 2 subsetting service for extracting spatial and temporal subset of orbital swath data (see Figure 8). Examples of inter-comparison and data quality tools include SQUAM, iQuam, ARMS and Felyx, previously mentioned in this paper.

Looking forward to the next decade, given the ever-growing data volumes and need to utilize SST alongside other large volume datasets, the international SST service must also evolve to include cloud computing, storage and access capabilities as well. Users increasingly seek to bring their algorithms and applications to the data, rather than following the traditional model of downloading data and processing it locally. Numerous groups are actively experimenting with or deploying cloud-based SST services, which are expected to become the new normal over the course of the coming decade.

\section{APPLICATIONS OF SST}

Satellite derived SST products are used in applications encompassing a wide range of temporal (diurnal to decadal) and spatial (sub-km to global) scales and are required by many user communities with an interest in ocean processes. Daily global high-resolution $(<10 \mathrm{~km})$ products are now easily accessible in support of operational forecast systems (Donlon et al., 2009).

\footnotetext{
${ }^{8}$ http://podaac-tools.jpl.nasa.gov/soto

${ }^{9}$ http://podaac-tools.jpl.nasa.gov/hitide
} 
The ability to resolve mesoscale and submesoscale features is facilitating new applications in coastal regions and frontal detection (e.g., García-Morales et al., 2017; Vazquez-Cuervo et al., 2017). As a result of their rapid sampling, data from GEO satellites facilitates detecting temperature changes on sub-daily timescales (Wick et al., 2002). Long-term SST records (some now approaching 40-years in duration) can be used to observe interannual to decadal scale variability (e.g., Liu and Minnett, 2015; Ferster et al., 2018).

A full review of all applications of SST would be a review paper itself. So, in this section we first focus on two of the more established application areas of SST, in support of (1) operational forecast systems and (2) understanding changes to the Earth's climate over time. Then, we look at three evolving user-driven application areas, which are driving much of the required SST research and development activities for the next 10 years and where SST will play an increasingly more important role.

\section{Application Example 1: Use of SST Observations in Operational Forecast Systems}

Understanding the three-dimensional structure of the oceans requires the combined use of satellite observations, in situ observations and ocean numerical models through assimilation techniques. Due to limited coverage of in situ measurements, and assumed systematic errors in numerical weather and seasonal prediction models, satellite observations are required to constrain SST. Together with near-surface wind vectors and ice cover observations, satellite SST can be used to model heat and momentum exchange to characterize the ocean surface and the energy fluxes through it.

Sea surface temperature observations provide boundary conditions for Numerical Weather Prediction (NWP) models, are assimilated into general circulation ocean models, and are used to initialize air-sea coupled models from short (days) to seasonal or multi-year scales. This section summarizes existing and emerging requirements for operational forecast systems for SST.

\section{Numerical Weather Prediction (NWP)}

Numerical Weather Prediction uses current conditions as input into mathematical models of the atmosphere to predict the weather. SST affects the behavior of the overlying atmosphere and vice versa and so daily analyses of SST are required by many operational NWP systems (Beggs, 2010). Historically, NWP systems have used gap-free Level 4 SSTs equivalent to ocean temperatures at around $10 \mathrm{~m}$ depth (e.g., Puri et al., 2013). This takes no account of the temperature gradient between the air-sea interface and in the upper $10 \mathrm{~m}$ and may introduce errors into forecasts (Beggs, 2010). New techniques apply cool-skin and warm-layer models on top of the standard ocean model configuration to predict the actual ocean skin temperature (Gentemann and Akella, 2018). Two-way airsea coupled weather prediction models have been developed, such as the ECMWF Integrated Forecasting System (IFS) ${ }^{10}$ and the Met Office Unified Model Global Coupled Model 2.0

\footnotetext{
${ }^{10}$ http://www.ecmwf.int
}

(Williams et al., 2015). Satellite SST data are assimilated into an ocean model in coupled systems, which then exchanges data with the atmospheric model, including the dynamic evolution of the SST. In the case of the ECMWF IFS system, the $5 \mathrm{~km}$ OSTIA SST analysis (Donlon et al., 2012) is used to initialize the coupled model.

Although the highest spatial resolution global SST analysis used as a boundary condition for NWP is $5 \mathrm{~km}$ OSTIA (Donlon et al., 2012), it's observation correlation length scale is $\sim 40 \mathrm{~km}$, and unable to resolve meso-scale coastal ocean features. Regional SST analyses have smaller observation correlation length scales (e.g., 12 km for RAMSSA; Beggs et al., 2011), but suffer from a lack of high resolution IR observations in cloudy regions. Impact studies are needed to show whether the prediction of extreme meteorological events over the ocean or land might be improved by using either SST analyses that resolve ocean features at $<20 \mathrm{~km}$ spatial scales to constrain high-resolution $(1-2 \mathrm{~km})$ NWP models, or coupled models at high resolution.

\section{Ocean Forecasting}

Marine forecasting is important for defense, public safety and transportation. National forecast centers and naval agencies use SST as input into their marine high seas models, providing forecasts of currents, temperature and salinity fields. These fields are then used for a variety of operational applications (Beggs, 2010). The ocean models range from regional high resolution systems that include tides, and may be updated as frequently as hourly, to global eddy-resolving systems that provide estimates of the ocean state, updated regularly (from daily to monthly), providing forecasts from a few days to one month in advance (Dombrowsky et al., 2009). An example of a forecast from an operational, eddy-resolving, ocean model is shown in Figure 9. For 2018, with respect to drifting buoy observations typical RMS errors of forecast SST from operational ocean models ranged from $0.45 \mathrm{~K}$ at forecast day 1 to $0.55 \mathrm{~K}$ at day $6^{11}$.

SST strongly co-varies with the ocean temperature over the ocean mixed layer depth, of the order 50-100 m, and complements altimetry data in multi-variate ocean analyses (Brassington, 2009). Short-range ocean forecast systems assimilate satellite SST data; high resolution coastal ocean models require geostationary data. Long time series satellite SST are also assimilated by global and regional forecasting systems to produce ocean re-analyses (Palmer et al., 2017) and to provide information on the state of marine environment for policy and decision-makers (von Schuckmann et al., 2016). The forecast performance of operational ocean models is now critically dependent on satellite-derived SST observations having excellent coverage, high accuracy and low latency. The reduction in errors following assimilating NPP VIIRS SST into ocean models has demonstrated the significance of accurate SST, and the dependence of ocean prediction on this instrument (Gary Brassington, pers. com.) Ocean forecasting will also benefit from improvements in satellite SST cloud clearing algorithms to preserve cool ocean features, such as coastal upwelling. Reducing the footprint from microwave instruments and

\footnotetext{
${ }^{11}$ http://130.56.244.252/monitoring/index.php?pg=class4_stats
} 


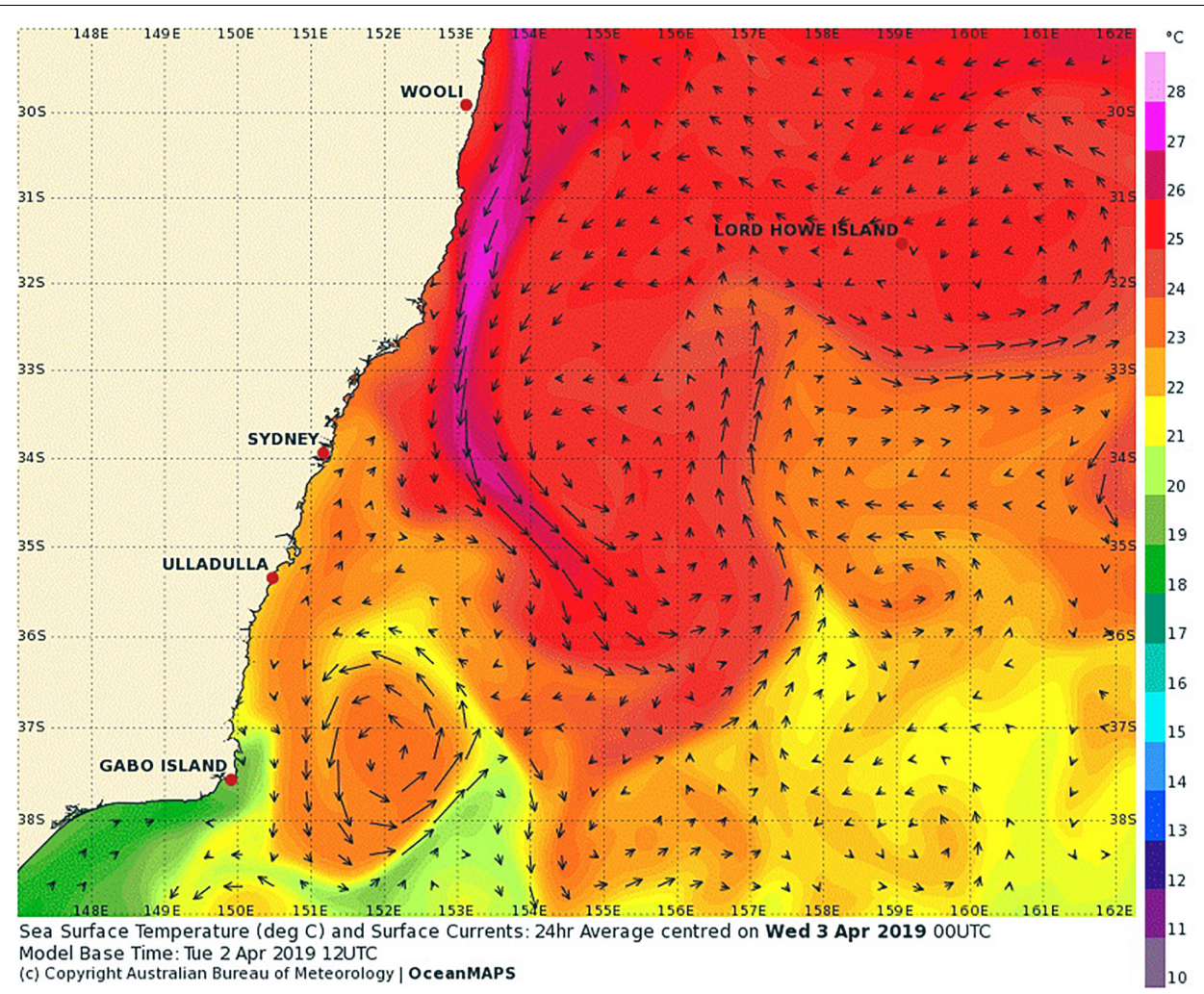

FIGURE 9 | Daily averaged forecast SST and surface currents in the East Australian Current, centered on April 03, 2019, obtained from the Bureau of Meteorology's operational OceanMAPS ocean model, with a model base time of April 02, 201912 UTC. (Sourced from http://www.bom.gov.au/oceanography/forecasts/, accessed on April 03, 2019; reproduced by permission of Bureau of Meteorology, (C) 2019 Commonwealth of Australia).

improving their SST accuracy will significantly benefit ocean forecasts, particularly in coastal and tropical regions. Improving temporal coverage and accuracy of SSTs from geostationary satellites will help to constrain diurnal processes in higher resolution ocean models.

\section{Seasonal and Inter-Annual Forecasting}

Operational centers also issue seasonal forecasts out to several weeks to months (Balmaseda et al., 2009). Most seasonal forecasting systems are based on coupled ocean-atmosphere general circulation models that predict SSTs and their impact on atmospheric circulation, and assimilate SST as part of their initial conditions. The aim of seasonal forecasts is to predict anomalies from the historical average for the forthcoming seasons (Balmaseda et al., 2009). The strongest relationship between SST patterns and seasonal weather trends are found in tropical regions (Beggs, 2010). Although most operational seasonal prediction models have horizontal spatial resolutions over the ocean of the order of 100 to $200 \mathrm{~km}$, recently, higher resolution coupled models have forecast the ocean state at weekly temporal resolution and $25 \mathrm{~km}$ spatial resolution (e.g., MacLachlan et al., 2014; Hudson et al., 2017). Seasonal forecasts from coupled ocean-atmosphere models can be used to predict anomalous SST several months in advance ${ }^{12}$.

\footnotetext{
${ }^{12}$ http://www.bom.gov.au/oceanography/oceantemp/sst-outlook-map.shtml
}

\section{Application Example 2: The Modern Era and Historical SST Climate Data Records}

Linking the satellite SST record to the historical in situ SST record is important to develop coherent CDRs. This is complicated by changes over time in both measurement systems. Early (e.g., 1800s) in situ measurements are from sailing ships and now the in situ system is comprised of measurements from ships, drifting and moored buoys, profiling floats and some other elements. Long-term satellite SST records are affected by constellation changes (sensors may be replaced, instrument calibration and satellite orbits may drift and instrument channels may fail). To assess any impact of variations between sensors on the overall long-term satellite record the GCOS recommends that "continuity of satellite measurements through appropriate launch and orbital strategies should be ensured" (GCOS, 2011) with at least 6-months overlap between successive sensors in order to develop homogeneity adjustments. Available satellite data holdings have not always met this minimum, and a full annual cycle of overlap would always be more robust. CDR requirements (Ohring et al., 2005) are very demanding to achieve and as is demonstrating whether the goals have been met (Merchant et al., 2017).

The long-term satellite SST record known as "Pathfinder" (Casey et al., 2010) utilizes empirical regression algorithms (Kilpatrick et al., 2001) tuned to drifting buoys to provide 


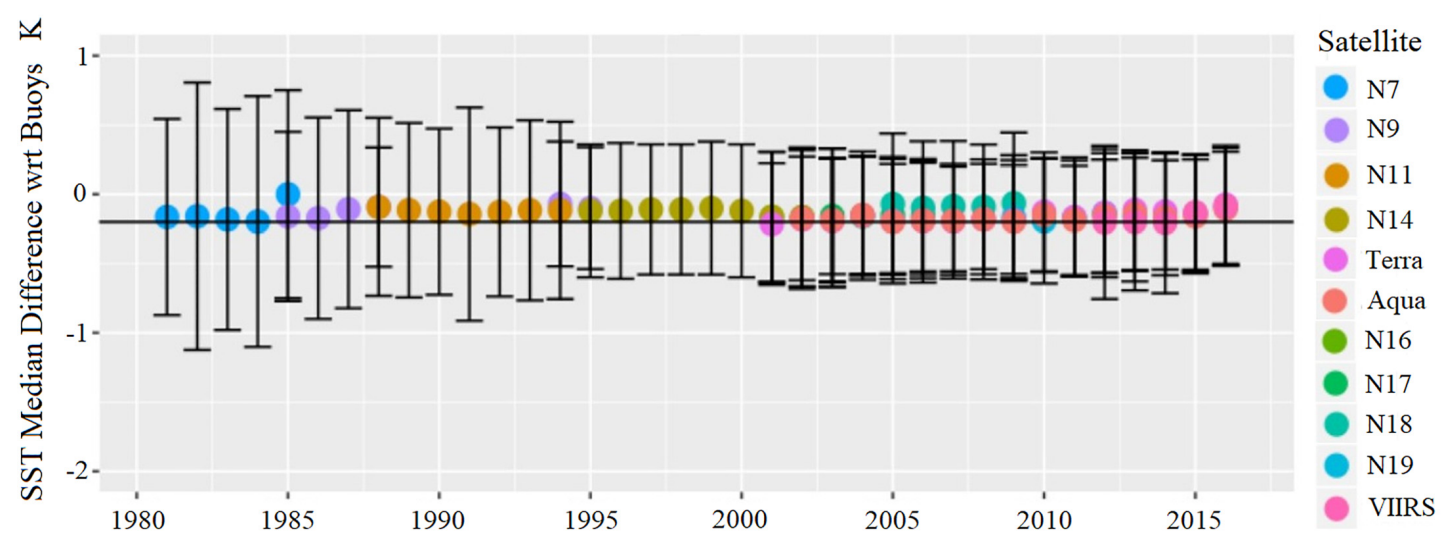

FIGURE 10 | Annual median and interquartile range of satellite SST skin versus SST $_{\text {depth }}$ from drifting buoys for a combined timeseries of AVHRR Pathfinder SSTs from NOAA-7 to -19, MODIS on Terra and Aqua, and S-NPP VIIRS.

a self-consistent approach across the series of AVHRRs from NOAA-7 onward. Although there have been some sensor overlaps in the series, Pathfinder is constructed from a single AVHRR sensor at any one time, and the tuning to in situ data is primarily to account for the many instrumental issues observed in the series, including errors in calibration (Mittaz et al., 2009). The latest version of Pathfinder is Version 5.3 and future developments of Pathfinder will be found in the version 6.0 dataset, including latitudinal banded coefficients to reduce latitudinal biases (Merchant et al., 2009a). In addition to Pathfinder, other AVHRR-only SST time-series include the NOAA science quality AVHRR GAC Reanalysis-1 (RAN1; Ignatov et al., 2016) and a regional Mediterranean product (Pisano et al., 2016).

The ATSR Reprocessing for Climate (ARC) project (Merchant et al., 2012) utilized the ATSR series of sensors, which were designed for providing SST suitable for climate studies. ARC differs from Pathfinder and AVHRR RAN in that it maintains independence from in situ measurements, has performed a careful harmonization of the BTs from successive sensors, and employs a physics-based retrieval of $\mathrm{SST}_{\text {skin }}$ using a Radiative Transfer Model (RTM) (Embury et al., 2012a,b). This then allows independent validation of the dataset using in situ measurements, accounting for depth and diurnal effects, both before (Embury et al., 2012b) and after harmonization of the ATSR BTs. Merchant et al. (2012) demonstrated how ARC is capable of quantifying inter-annual variability in SST and identifying major SST anomalies. However, the timespan of the ATSR series (1991-2012) compared to the AVHRRs is a limitation. The ESA SST_CCI project (Merchant et al., 2014) has pioneered using the ATSRs to reduce BT errors in the AVHRRs and in derived SSTs, providing a stabilized dataset of both ATSR and AVHRR for the period they co-exist (1991-2012). The next version product from SST CCI will extend backward and forward from the ATSR period using consistent physically based retrievals for all AVHRRs.

The possibilities for long-term satellite SST records will increase over the next 10 years, as new sensors such as VIIRS provide a capability to extend existing data records from the
MODIS series (Kilpatrick et al., 2015). These more recent satellite records can then be linked to the longer term records from AVHRR (Figure 10). In addition, new high spatio-temporal resolution climatologies are required in order to fully understand air-sea interactions across all relevant scales, for which an attractive approach will be to combine polar and geostationary observations consistently. While research will be required on newer sensors, the total length of record is a key parameter of CDR users, and can be maximized by work to extend the satellite SST record back to 1978 (the earliest feasible time). It is essential that this early record be addressed with wellcharacterized uncertainty and stability, taking advantage of advances in inter-satellite re-calibration techniques, the full SSTrelevant historic observing system and advances in radiative transfer and numerical weather reanalysis.

\section{User-Driven Priorities for SST Observations Over the Next Decade}

In this section, we summarize three application areas identified by SST users as being of high priority for SST developments in the next decade and focus on one specific priority in each area that requires research and development. The application areas are:

(1) Improving SST data quality in the Arctic: Arctic Amplification of the climate change signal requires understanding its cause and improving our ability to make more accurate predictions, including quantification of feedback processes operating in the Arctic Ocean. Included in the Arctic system is a temperature feedback (Pithan and Mauritsen, 2014), and better understanding of this requires more accurate SSTs. Coverage from IR sensors is poor mainly due to persistent cloud, so a priority is to improve PMW data coverage at high latitudes.

(2) Improving coastal SST data quality: Coastlines and coastal communities are under threat so improved understanding of coastal marine processes is of vital importance, and high resolution SST that can reveal 
mesoscale and sub-mesoscale features will make an increasingly important contribution. A priority is to use other satellite datasets (e.g., from ocean color or very high spatial resolution from Landsat) to help address limitations of SST products owing to severely limited in situ data.

(3) Improving SST feature resolution: Identification of ocean fronts for understanding air-sea exchanges (e.g., Cronin et al., 2019) requires better measurements, including high-resolution SST. Also, understanding changes in small scale ocean features such as coral reefs, requires improved high-resolution SSTs. The lack of high-resolution SST features is due to most SST analysis systems smoothing features as their original heritage is to support NWP, so a priority is to develop new methods to retain high resolution features in analyses.

\section{The Challenges of SST Provision in High Latitude Regions}

SST retrievals at high latitudes are difficult for a number of reasons. IR and PMW SSTs require in situ datasets for algorithm development, validation, and fine-tuning, and measurements of in situ SST are sparse at high latitudes, and often lack coincident atmospheric observations critical for algorithm development. Without adequate sampling of the variable atmospheric and oceanic conditions, IR and PMW algorithms rely on the data that is too sparse in time and space, creating unknown errors in conditions outside the relatively narrow range of existing observations. There are additional complications, for example, recent PMW missions lack a $6.9 \mathrm{GHz}$ channel that retains useful sensitivity below $13^{\circ} \mathrm{C}$, which includes most regions poleward of $60^{\circ}$ latitudes. Without a $6.9 \mathrm{GHz}$ channel, errors increase by $0.5^{\circ} \mathrm{C}$ (Gentemann et al., 2010). Additionally, accurate identification of sea ice can be difficult. Thin sea ice can form quickly, over large areas, and may not be accurately mapped by daily sea ice maps (Kwok et al., 2003). Identification of floating icebergs can also be difficult as some are sub-grid scale. Sea ice remains an issue for both IR and PMW data, with the PMW having the further complication of sidelobe contamination when sea ice is present near the observation footprint. A further complication comes from Arctic dynamics. River and sea-ice melt freshwater input to the Arctic can result in strong salinity gradients adding to errors in SST retrievals. The long high latitude night time can result in difficulty identifying cloud contaminated pixels and results in a seasonal dependence in the accuracy of IR retrievals. Figure 11 shows an example of the data coverage of all available infrared satellites during one day for the sea ice minimum in September 2012, together with simulated Copernicus Imaging Microwave Radiometry (CIMR) coverage for the same period.

\section{User case study 1: improved PMW observations at high latitudes}

Frequent observations of SST and other related variables in the Arctic and Antarctic Oceans are only practical via microwave imaging instruments. SST and other measurements are crucial to describe the seasonal and long-term variation of the polar sea ice caps. The long-term decline of sea ice has been monitored and quantified by satellite MW radiometer measurements over the past 30 years.

The continuation of PMW imagers that can be used to derive global SST is currently a major concern. SST under all weather conditions (except precipitation) can be derived from $6-10 \mathrm{GHz}$ channels. The challenge is to provide such measurements at higher spatial resolution and with high radiometric fidelity to serve modern operational needs. As of 2019, the Chinese Microwave Radiometer Imager (MWRI) onboard the HaiYang-2B (HY-2B) is the only approved future PMW imager that has a $6.9 \mathrm{GHz}$ channel. The Chinese FengYun3 series (FY-3D and FY-3F) also has a $10.65 \mathrm{GHz}$ channel. Those two missions may fill possible gaps in microwave SSTs, but their spatial resolutions are three times coarser than for AMSR2: $150 \times 90 \mathrm{~km}$ for HY-2B, and $51 \times 35 \mathrm{~km}$ for FY3. Another possibility to fill this gap is the JAXA GOSAT3 satellite which will carry the AMSR2 follow-on, AMSR3, and is currently a pre-project phase (Phase A) and expected to become "project" later in 2019. The orbit of GOSAT-3 satellite will be $666 \mathrm{~km}$ altitude (same as GOSAT-1) at 13:30 local time (same as GCOM-W). AMSR3 is almost equivalent to AMSR2 (antenna size, channels) with additional higher frequency channels of 166 and $183 \mathrm{GHz}$ for snowfall retrievals and water vapor analysis.

A new radiometer, CIMR, is currently being studied by the European Space Agency (ESA), responding to the need to expand the Copernicus satellite fleet driven by user requirements. CIMR addresses requirements to monitor the rapidly changing Arctic environment, providing evidence to underpin the Integrated European Union Policy for the Arctic. The CIMR mission would deploy a wide-swath $(>1900 \mathrm{~km})$ conically scanning multifrequency microwave radiometer. CIMR measurements will be made using a forward scan arc followed $\sim 260 \mathrm{~s}$ later by a second measurement of the same location using a backward scan arc. Full Stokes channels centered at 1.414, 6.925, 10.65, 18.7 and $36.5 \mathrm{GHz}$ are included in the mission design. The real-aperture resolution of the $6.925 / 10.65 \mathrm{GHz}$ channels is $<15 \mathrm{~km}, 5$ and $<5 \mathrm{~km}$ for the $18.7 / 36.5 \mathrm{GHz}$ channels respectively. The $1.414 \mathrm{GHz}$ channel will have a real-aperture resolution of $<60 \mathrm{~km}$ (fundamentally limited by the size of the $\sim 8 \mathrm{~m}$ deployable mesh reflector). However, channels will be oversampled allowing gridded products to be generated at much better spatial resolution. Channel NEdT is $0.2-0.8 \mathrm{~K}$ with an absolute radiometric accuracy goal of $\sim 0.5 \mathrm{~K}$. CIMR will fly in a 06:00/18:00 dawn-dusk orbit providing access to the foundation SST. With one satellite, $\sim 95 \%$ global coverage every day (except for rain conditions), better than daily coverage poleward of $55^{\circ} \mathrm{N}$ and $\mathrm{S}$, and no gap at the pole itself. The mission will operate in synergy with EPS-SG mission so that in regions $>65^{\circ} \mathrm{N}$ and $\mathrm{S}$, collocated and contemporaneous measurements between CIMR and complementary sensors on EPS-SG measurements will be available within $\pm 10 \mathrm{~min}$. The mission is currently completing Phase B1 and is expected to initiate Phase B2 in 2020 for a planned launch of the first of two satellites in $2026+$. 

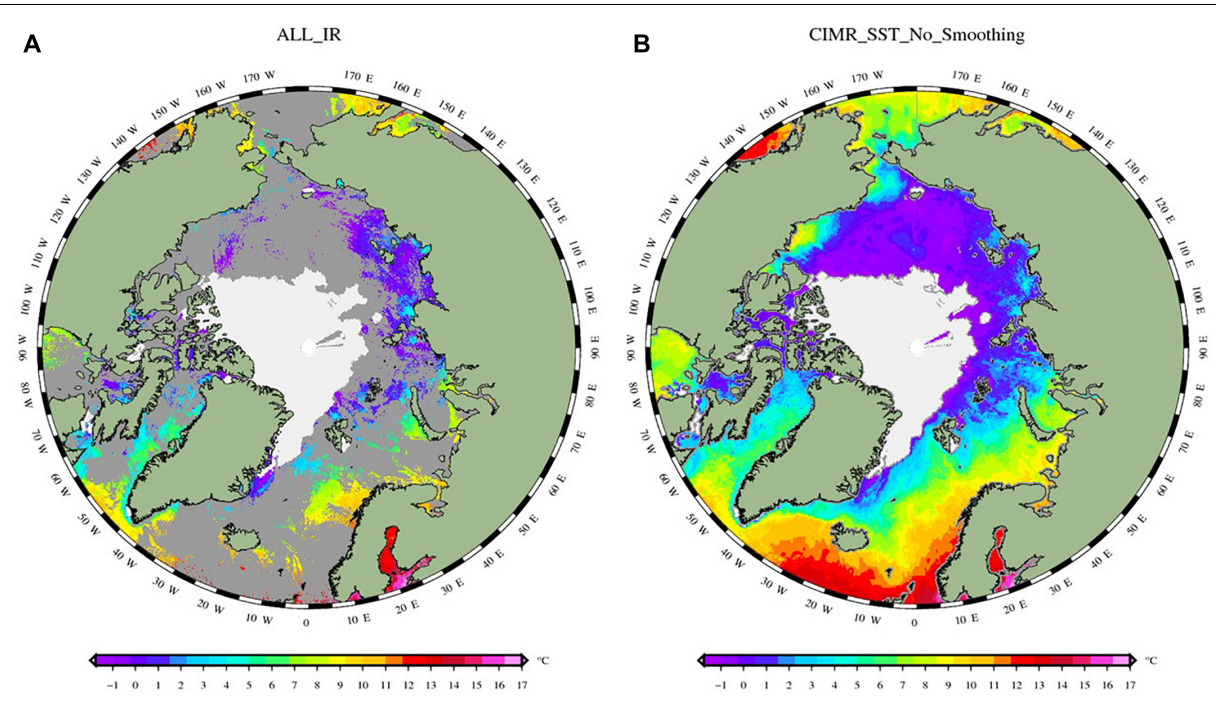

FIGURE 11 | Data coverage of (A) all available Infrared satellites during the sea ice minimum in September 2012 (taken from Copernicus Marine Environmental Services (CMEMS) data production). (B) Simulated CIMR coverage for the same period. (Reproduced by permission under CC-BY 4.0 from https://doi.org/10.6084/ m9.figshare.6969422.v1).

The combination of both the AMSR2 follow-on and CIMR missions are highly complementary and would provide an unprecedented coverage and revisit time of the global ocean.

\section{The Challenges of Systematic SST Observations Over Inland Seas, Lakes and in the Coastal Zones}

Challenges of systematic water temperature observations over the inland seas, lakes and in the ocean coastal zones include: greater variability in atmospheric water vapor, temperature and aerosol than over most of the ocean; avoiding (or accounting for) land contamination since most lakes and rivers are of scales not resolved or not well resolved by SST sensors; water surface contaminants and any modification of surface emissivity; and turbidity in interaction with cloud detection. Regarding the latter, products are prone to over-masking during crucial SpringSummer warming phase, and to screening of cold, clear water, leading to systematic observation bias (Crosman et al., 2017). Problems in satellite-based lake temperature products arise when applying "global" cloud detection and SST retrieval algorithms (optimized for the open ocean, where the atmosphere is usually close to equilibrium with the water surface). Inland water-specific methodologies are promising for derivation of Lake Surface Water Temperatures (LSWT) (e.g., MacCallum and Merchant, 2012; Crosman et al., 2017; Woolway and Merchant, 2018) although their application to rivers has been less well explored.

Satellite-derived LSWT can be assimilated into coupled models to produce more accurate local weather forecasts. The opportunity exists to evaluate LSWT products in regional models, which should enhance our ability to account for strong local effects exerted on the forecast. Possible ways are proposed to improve products, such as tailored QC filters to mitigate effects of increased retrieval error with a relaxed cloud mask. Such effects are likely to be regional and seasonal. Prospects for reducing error due to anomalous atmospheres (e.g., cool lake surrounded by warm land, or vice versa), emissivity differences, diurnal warming (water turbidity, wind speed, insolation) focus on retrievals informed by prior information used in a physical method, such as Modified Total Least Squares (MTLS; Koner et al., 2015) or optimal estimation (MacCallum and Merchant, 2012). These methods account for background variability, MTLS doing so via dynamic estimation of the regularization of the inversion. Both provide mechanisms for additional QC and pixellevel uncertainty estimation.

\section{User case study 2: the impact of pollution on sea surface temperature (SST) along the coast of the Gulf of Guinea of West Africa}

The Gulf of Guinea (GOG) is a maritime area located off Western Africa covering ten countries - Togo, Ghana, Benin, Nigeria, Cameroun, Gabon, Equatorial Guinea, Sao Tome and Principe, Congo and Angola. The area of the GOG is about 2.35 million $\mathrm{km}^{2}$ and has its major contributing freshwater inputs from the Volta and Niger rivers. The GOG is recognized for its economic importance. However, its vast resources, especially of the coastal ocean, have been heavily affected by rapid development of human activity (Scheren et al., 2002). Hazardous discharges of liquid and solid waste into the coastal oceans from urban expansion and developments, agriculture and sewage, oil exploration activities, dredging of channel, seismic surveys and pipelines (Spalding et al., 2010), have left the natural coastal zone of the GOG unsuitable to support its economic activities. This has resulted in significant eutrophication and heavy metal contamination. Polidoro et al. (2017) estimate that out of the 125 species of fish in the GOG, 33\% are affected by pollution, leading to a significant impact as significant as unsustainable fishing. Solid waste entering the GOG annually is estimated at 3.8 million T/year according to research by Scheren et al. (2002). 

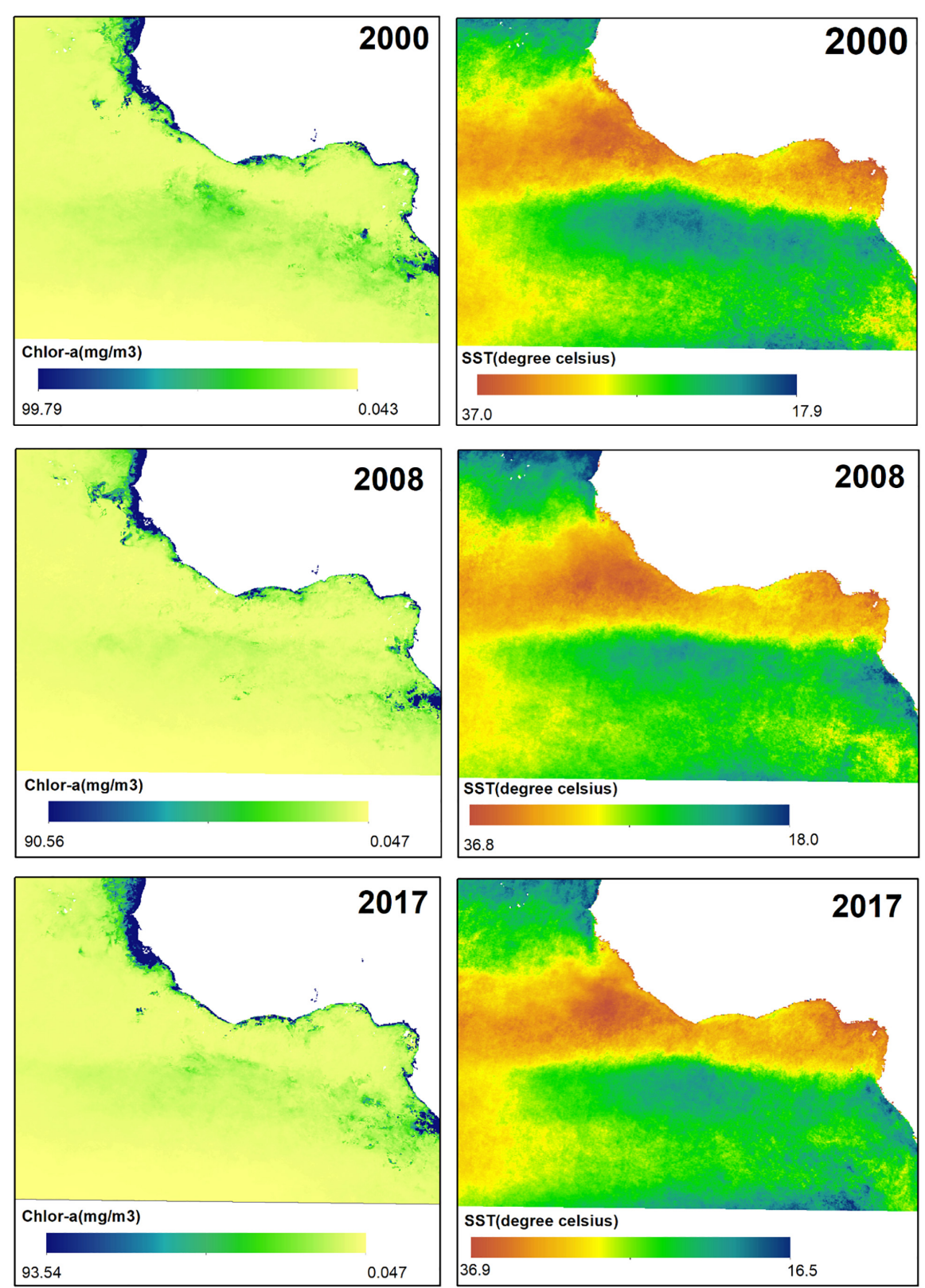

FIGURE 12 | Variations of Chl a and SST from 2000 to 2017.

The dominant items are plastics comprising about $62 \%$ of the waste related to fishing activities, including plastic carrier bags and packaging materials. About $80 \%$ of industrial effluents directly into the lagoon systems through pits, gutters and trenches (Scheren et al., 2002). Research conducted by Eriksen et al. (2014) has shown that plastic pollution is another key challenge for the oceans.

In order to properly model the distribution of ocean pollutants across the GOG and to be able to aid the identification and understanding of the evolution of such features, access to high spatial resolution satellite imagery is needed from multiple sources. It is expected that Chlorophyll-a will be a useful proxy in areas closer to the sources of pollutants and that SST will be a suitable proxy to track the evolution of the discharge across the GOG. Figure 12 shows the variation of both SST and Chlorophyll-a (Chl-a) variations over the period 2000-2017 from annual averages. Maximum values of Chl-a are located in coastal zones where concentrated discharges of pollutants occur. However, SST maps in the same region show significant variability across all years. Consequently, little correlation between the SST/Chl-a maps and the evolution of the plastics is found. Access to time-series of higher spatial resolution satellite imagery of these regions will help to better distinguish sub mesoscale ocean features and better understand 

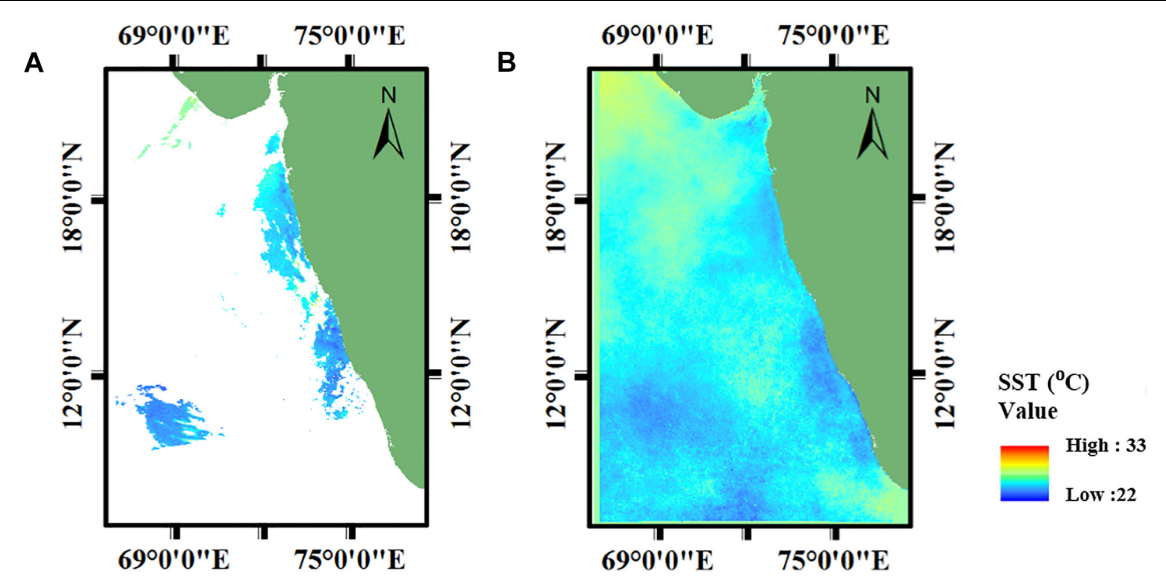

FIGURE 13 | |llustrative example for user case study 3 (A) The MODIS Aqua image with gaps. (B) Reconstructed SST image using DINEOF, both corresponding to the date November 30, 2015.

the limited correlations seen in the products as a way to track the evolution of pollutants.

\section{The Challenges of Providing Global High Resolution SSTs}

Increased modeling and observational efforts from the physical oceanography community have recently been dedicated to the study of ocean sub-mesoscale dynamics. This is due to the growing evidence that processes occurring at small spatial and temporal scales [i.e., $O(\sim 1 \mathrm{~km}$ and $\sim 1$ day $)$ ] are relevant for large scale ocean dynamics and thus affect weather and climate variability (Ferrari, 2011; Levy et al., 2012). This includes significant interest in the sub-mesoscale from potential biological and biogeochemical impacts on primary production, planktonic ecosystems and ocean carbon transport (Levy et al., 2012; Woodson and Litvin, 2015). Despite the major benefit of near global coverage, low spatial resolution SST fields derived from PMW instruments do not allow the observation of geometrical structures associated with sub-mesoscale processes. Such features are commonly seen in images acquired from GEO and LEO IR sensors provided clear-sky conditions are met. A spectral analysis of SST analyses is required to relate the true ocean variability, including methods to discriminate between signal and noise in these data with high spatio-temporal frequencies.

The impact of persistent clouds in IR SST datasets in SST analyses can be partly overcome through the additional ingestion of PMW SST (Donlon et al., 2011), multi-scale techniques (Buongiorno Nardelli et al., 2013) or the use of high-resolution data within a larger time window (Haines et al., 2007). However, the resulting multi-sensor gap free SST fields usually suffer from at least one of the following limitations: over-smoothed SST fields due to the spatio-temporal interpolation, with smoothing scales varying both in space and time (Lekouara, 2013); processing artifacts in the form of spatial patchiness due to imperfect bias correction, retrieval errors, or errors in masking erroneous retrievals (rain, clouds, sea ice or radio-frequency-interference). While these drawbacks do not directly affect the statistics of
SST, the analysis of SST gradients and the detection of fronts in optimally interpolated SST analyses is greatly affected.

\section{User case study 3: reconstruction of daily cloud free MODIS SST over South Eastern Arabian Sea}

High resolution SST products rely on IR sensors more than PMW due to the latter's larger footprint, relatively lower accuracy, and inability to derive SST close to coasts; IR sensors are themselves limited by cloud cover that results in significant data loss and poor data quality. The effect is largest in the tropics where cloud cover is more persistent even compared to higher latitude regions (NASA, 2018). Optimal Interpolation (OI) and data assimilation techniques are traditionally used to provide gap-free datasets. These approaches are often complex and based on a number of assumptions and parameterizations (Miles and He, 2010; Zhao and He, 2012; Fablet et al., 2018). An alternate approach is to use Data Interpolation Empirical Orthogonal functions (DINEOF)

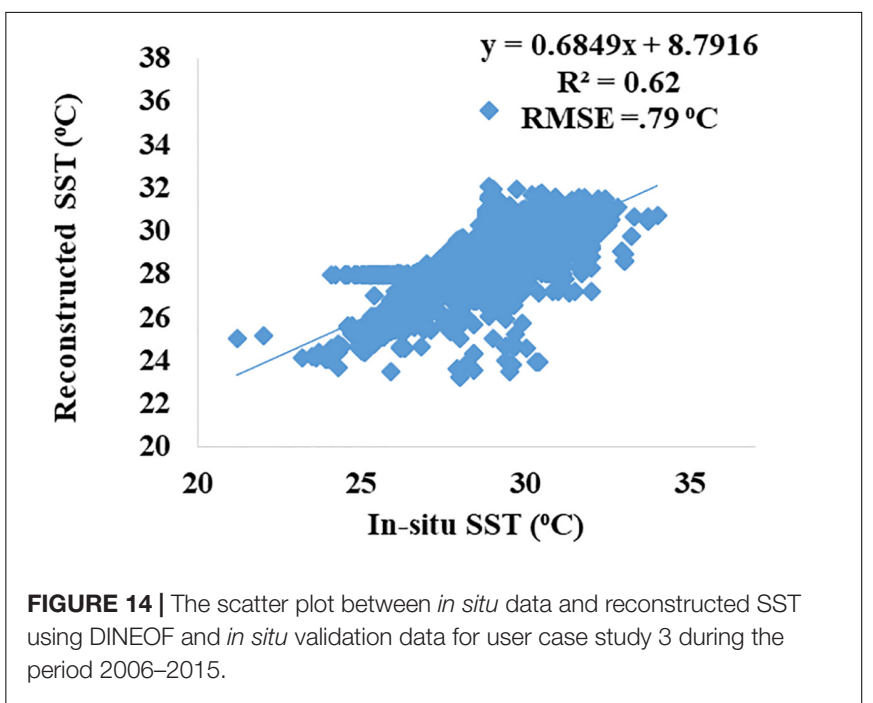


(Beckers and Rixen, 2003; Alvera-Azcárate et al., 2005, 2007), which can fill gaps in SST fields even if major portions of an image are cloudy. An example is shown for before and after the DINEOF in Figure 13, respectively, showing Aqua MODIS daytime SST data for the south eastern parts of Arabian Sea from 2006 to 2015. Following Alvera-Azcárate et al. (2007), the images with more than $95 \%$ cloud cover were discarded. For the entire period, $77.7 \%$ data was missing due to cloud.

Validation statistics of the reconstructed DINEOF cloud free images have a coefficient of determination is 0.62 and an RMSE of $0.79^{\circ} \mathrm{C}$ when compared to in situ data, as shown in Figure 14. No adjustments have been made to account for differences in the depth and time of the satellite and in situ SSTs, which will contribute to the observed differences, as well as differences due to the spatial offsets between the in situ data points and the footprints of the satellite image (Zhao and He, 2012). DINEOF based reconstructed SST datasets perform well and can be used to fill gaps in IR SST datasets due to persistent cloud. Further research is required to use this method as a pre-processing step into an SST analysis.

\section{CONCLUSION}

This full-review community white paper "Observational Needs of Sea Surface Temperature" provides a core contribution to OceanObs'19. It overviews the current SST observing systems, describes progress and challenges over the past decade and into the next, and overviews the forward-looking vision over the next decade and beyond. User requirements for SST products in the next decade need a complementary blend of satellites and in situ measurements. The establishment of a framework for the exchange and management of international SST data has been successfully implemented and is operating on a daily basis coordinated by GHRSST. A thriving user community has developed in which integrated SST data sets are being used at scientific institutions and operational agencies. Tools and data services have been developed and implemented to serve this user community. Through the activities of GHRSST, many lessons have been learned that provide the basis for an optimal configuration for the SST observing system in the next 10 years. Additional challenges and opportunities will emerge as datasets are migrated onto cloud processing environments. Open source software policies will help this process.

The following priority recommendations are made:

(a) Ensure continuity and redundancy of the multifrequency Passive Microwave (PMW) Radiometry constellation for SST including $6.9 \mathrm{GHz} \mathrm{V}$ \& $\mathrm{H}$ channel capability, with resilience to radio frequency interference.

(b) Make further developments and support projects toward provision of innovative FRM and in situ SST data, particularly at high-latitudes and in marginal ice zones.

(c) Enhance SST algorithms and improve SST products and data quality (including cloud screening, aerosol-screening and correction, retrievals, uncertainty assessment, analysis and inter-sensor harmonization), with focus on coastal, dynamic, polar and upwelling regions, and regions subject to persistent atmospheric aerosols.

(d) In climate applications, follow a rigorous approach to SST data quality, and the Fiducial Reference Measurement (FRM) and in situ SST data used in SST validation should have estimates of the uncertainty of those measurements.

(e) In response to ever-growing data volumes of SST data, improved regional and national capabilities, and provider-specific data access policies, establish plans to evolve the GHRSST Regional/Global Task Sharing framework to become a more distributed and federated system for data and information distribution and archiving.

Detailed recommendations are given following the themes of constellation, validation and FRM, algorithms and cloudscreening, climate, assimilation and merged products and user applications.

Constellation:

(1) For a range of applications including NWP, climate and research, it is imperative that satellite derived SST timeseries are improved and sustained into the next decade and beyond. This encompasses SST from polar-orbiting and geostationary, using both infrared and microwave sensors, with continued research vital to improve the resolution and accuracy of the SST fields.

(2) There is an increased need for SST sensors with high spatial resolution and high radiometric fidelity to serve modern operational services for sea-ice and SST analysis, and services for modeling and prediction of ocean and weather.

(3) Future satellite infrared radiometers targeting SST should include channels centered at $4.05,3.7 / 3.9$, and $8.6 \mu \mathrm{m}$, in addition to the window channels of $10.8 / 11$ and $12 \mu \mathrm{m}$, to improve clear-sky determination and SST uncertainty under aerosol. A day-night band (as on VIIRS) would be beneficial for night-time cloud detection.

(4) There should be continuation of dual-view infrared capability with redundancy, particularly to act as a satellite reference for the operational constellation, including resilience to extreme stratospheric aerosol events, through planned continuation of these missions in the long-term.

(5) There should be a constellation of at least six geostationary platforms (with operational redundancy) with high-quality sensors designed for SST retrieval.

(6) Improved accuracy and spatial resolution of SST observations derived from PMW radiometer data are needed, particularly for coastal and sea-ice regions.

(7) More research and international collaboration is recommended to improve and harmonize BT 
calibration across all available microwave missions, and to better constrain the quantification of sea surface emissivity under varying conditions over SST-relevant frequencies. This should include the potential for harmonization research for infrared BTs calibration using dual-view radiometers as a reference.

(8) The highly complementary CIMR and AMSR2 follow-on missions should be both pursued, to provide unprecedented coverage, redundancy and revisit of the global ocean and high latitude sea-ice conditions.

Validation and FRM:

(9) There should be increased further research and activities into estimating and documenting the uncertainties of SST FRM, including improved uncertainty models, data and research into understanding of the sampling errors introduced during validation inter-comparison exercises, to facilitate SI-traceability of satellite SSTs.

(10) Further developments of the drifting buoy network are needed to improve the calibration and resolution of SST measurements (achieving uncertainties better than $<0.05 \mathrm{~K}$ ), provide improved uncertainty information per drifting buoy, understand the depth of the sensor and how it behaves in the near-surface, and improved metadata, to aid satellite inter-comparisons, and progress toward SI-traceable drifting buoy SSTs to facilitate the SI-traceability of satellite SSTs in a way comparable to that currently done with ship radiometers with SI-traceable calibration.

(11) All FRM and/in situ SST data should be provided with complete metadata including depth of measurement. Efforts should be made to further expand the FRM available for satellite SST validation including those at high-latitudes and those capable of giving estimates of spatial variability such as Saildrone.

(12) Methodologies on the use of satellite-derived SST as a reference for performing automatic quality control of in situ observations should be extended and continued.

(13) The way uncertainties are communicated to users should be improved in response to the increasing number of users and data products, to address context sensitive uncertainties provided at the appropriate scale, ranging from noise at the pixel-level to correlated or systematic uncertainties per file. Documentation should be improved, including to define the traceability chain and efforts made for the uptake of this information by users.

(14) Uncertainty estimates of satellite SST should be developed using uncertainty modeling validated by empirical means against uncertainty-quantified in situ references, establishing that the joint satellite-in situ error budget explains observed discrepancies.

(15) Satellite SST uncertainty validation using in situ SST needs to consider the geophysical contribution to satellite-in situ collocated differences in addition to the uncertainty of the in situ data.

(16) Improved uncertainty models of SST data, and uncertainty models used for CDRs should be the basis for all satellite SST products in general. Uncertainty representations need to be developed that can supply uncertainty information across all scales of application, including providing observing-system stability estimates, which need a continuation of consistent in situ sources such as the GTMBA.

Algorithms and cloud-screening:

(17) Further studies and research are needed on cloudidentification at high-latitudes and sea-ice regions, such as the Marginal Ice Zone.

(18) New generation geostationary SST data should be further exploited and researched to understand SST diurnal variability characteristics and provide information to users on the SST diurnal cycle, SST spatial contrasts and temporal variability, with consistent methodologies for estimating the diurnal variability.

(19) There should be further research and development into new approaches for cloud detection methods, as a major contributor to uncertainties on the derived SST. These should fully exploit the new generation of sensors including those with more channels, improved calibration, lower uncertainties, and improved spatial and temporal resolution; assess the potential for pattern recognition and temporal collation techniques; and use the repeated sampling of all observations.

(20) There should be further research and studies toward ensuring robustness of SST algorithms to stratospheric volcanic aerosol conditions, and desert dust aerosol conditions, for all infrared satellite observations with the necessary spectral and/or dual-view channels, and particularly to fully exploit the approximate 3.9 and $8.7 \mu \mathrm{m}$ channel capability on VIIRS and eventually METimage.

(21) Further research is needed to improve operational Lake Surface Water Temperature products, addressing the additional challenges of cloud screening and surface temperature retrieval in the contexts of inland and coastal waters.

(22) To respond to the greater availability of high-resolution high-quality sensors, further research is needed on the potential for machine-learning techniques to improve the accuracy of SST algorithms.

\section{Climate:}

(23) Maximizing the benefit of the SST CDR involves increasing the length of the stable record, requiring ongoing focus on the early AVHRR record (19781995), exploiting recent improvements in inter-satellite re-calibration techniques, radiative transfer and earth observation metrology. 
(24) Climatologies with high spatial and temporal resolution are needed to fully understand the relevant scales and diurnal cycle on long-term CDRs by combining both polar orbiting and geostationary data from PMW and infrared radiometers.

(25) Ship-based infrared radiometer SST data are crucial for the assessment and qualification of a satellite SST CDR, as they provide a means for providing SI-traceable FRM, and should be extended and continued globally, with regular inter-comparison exercises.

(26) Further research into analyzing the different CDRs from IR and PMW and in harmonizing the CDRs from IR and PMW missions through the use of radiative transfer techniques to obtain more accurate and consistent CDRs.

Assimilation and merged products:

(27) The use of satellite SST is important for assimilation into global and regional ocean models to improve the estimation of the ocean state over the past decades and to aid the understanding of ocean variability and trends, and further effort should be made to ensure long-times series data are used and sustained.

(28) The power spectrum properties of SST analyses need to be understood in relation to the true geophysical spectrum from ocean variability, including methods to discriminate analysis noise and real signal at high spatio-temporal frequencies.

(29) Impact studies are needed to show whether the prediction of extreme meteorological events over the ocean or land, including coastal regions, might be improved by using either SST analyses that resolve ocean features at less than $20 \mathrm{~km}$ scales to constrain high resolution NWP models of less than $2 \mathrm{~km}$, or coupled models at high resolution.

User needs:

(30) To respond to the rapidly growing number and volume of GHRSST products there is a need for a comprehensive, one-stop catalog for data discovery and access now that data providers are distributing their own datasets.

(31) User tools for GHRSST data visualization, extraction and quality monitoring should continue to be supported, developed and expanded, for example: The SOTO; HiTide; satellite SST inter-comparison tools (such as NOAA SQUAM) and matchup datasets/tools to ocean-surface SST measurements (such as Felyx and NOAA iQuam).

\section{REFERENCES}

Albiñana, A. P., Battles, D., Monteiro, D., Lambeck, R. W., Alemán, R., and Jackson, C. (2007). "The performance of the AVHRR, HIRS, and AMSU-A instruments on board Metop-A," in Proceedings of the Optical Engineering + Applications, (Bellingham, WA: SPIE), 13. doi: 10.1117/12.730839

\section{AUTHOR CONTRIBUTIONS}

AO'C, GC, AI, and PM involved in overall editing and wrote the sections "Introduction," "SST's Role in an Integrated Ocean Observing System," and "Conclusion." SS wrote the section "Polar-Orbit IR SST Capability". MP and AI wrote the sections "Geostationary-Orbit Infrared SST Capability" and "Improved Cloud Masking of IR Imagery." WW wrote the section "The Evolving in situ SST Observing System." CM wrote the sections "More Accurate SST Retrieval Through Aerosol-Laden Atmospheres," "Better Uncertainty and Error Estimation," and "The Challenges of Systematic SST Observations Over Inland Seas, Lakes and in the Coastal Zones" along with EM. IK, RS, and SM wrote the section "Better Practical Quantification of Diurnal Variability." GC wrote the sections "Improved Methods of Validation" and "Application Example 2: The Modern Era and Historical SST Climate Data Records." JV-C, JH, and HB wrote the sections "Evolving the International SST Service to Users" and "Application Example 1: Use of SST Observations in Operational Forecast Systems." KC, PD, and EA wrote the section "Evolving the International SST Service to Users." CD, CG, MK, and YK wrote the sections "Passive MW Polar-Orbiters and Low Inclination Orbiting SST Capability" and "The Challenges of SST Provision in High Latitude Regions." MB wrote the section "The Challenges of Providing Global High Resolution SSTs.” KK wrote the section "User Case Study 2: The Impact of Pollution on Sea Surface Temperature (SST) Along the Coast of the Gulf of Guinea of West Africa." SS, BR, and RR wrote the section "User Case Study 3: Reconstruction of Daily Cloud Free MODIS SST Over South Eastern Arabian Sea." All authors contributed to the writing of section "Conclusion."

\section{FUNDING}

CG was supported in this work by the NASA Grant 80NSSC18K0837. All other authors were supported by their institutes in preparing this review article.

\section{ACKNOWLEDGMENTS}

SS, BR, and RR acknowledge the GHER Group at the University of Liège and the NASA OBPG. AI and MP acknowledge support from JPSS, GOES-R, and ORS Programs (their views expressed in this report are their own and should not be construed as an official NOAA or U.S. Government Position or Policy). JV-C and EA acknowledge support from NASA JPL.

Alvera-Azcárate, A., Barth, A., Beckers, J.-M., and Weisberg, R. H. (2007). Multivariate reconstruction of missing data in sea surface temperature, chlorophyll, and wind satellite fields. J. Geophys. Res. Oceans 112, 1-11.

Alvera-Azcárate, A., Barth, A., Rixen, M., and Beckers, J. M. (2005). Reconstruction of incomplete oceanographic data sets using empirical orthogonal functions: application to the Adriatic Sea surface temperature. Ocean Model. 9, 325-346. 
Atkinson, C., Kennedy, J., and Rayner, N. (2015). ESA SST CCI Phase 2 Climate Assessment Report. Available at: http://www.esa-sst-cci.org (accessed November, 2015).

Atkinson, C. P., Rayner, N. A., Roberts-Jones, J., and Smith, R. O. (2013). Assessing the quality of sea surface temperature observations from drifting buoys and ships on a platform-by-platform basis. J. Geophys. Res. Oceans 118, 3507-3529. doi: 10.1002 /jgrc. 20257

Balmaseda, M. A., Alves, O. J., Arribas, A., Awaji, T., Behringer, D. W., Ferry, N., et al. (2009). Ocean initialization for seasonal forecasts. Oceanography 22, 154-159. doi: 10.1002/2017MS001204

Barker, A., Banks, A., Bell, W., Dowell, M., Fox, N. P., Green, P., et al. (2015). "Metrology for climate," in Metrology Priorities for the Earth Observation and Climate Community, eds A. Barker, et al. (Brussels: European Commission), 36.

Barton, I. J., Minnett, P. J., Donlon, C. J., Hook, S. J., Jessup, A. T., Maillet, K. A., et al. (2004). The Miami2001 infrared radiometer calibration and intercomparison: 2. Ship comparisons. J. Atmos. Ocean. Technol. 21, 268-283. doi: 10.1175/1520-0426(2004)021<0268:tmirca>2.0.co;2

Beckers, J. M., and Rixen, M. (2003). EOF calculations and data filling from incomplete oceanographic datasets. J. Atmos. Ocean. Technol. 20, 1839-1856. doi: 10.1175/1520-0426(2003)020<1839:ecadff> 2.0.co;2

Beggs, H. (2010). "Use of TIR from Space in Operational Systems," in Oceanography from Space Revisited, eds V. Barale, J. F. R. Gower, and L. Alberotanza (Berlin: Springer), 249-271. doi: 10.1007/978-90-481-86 81-5_15

Beggs, H., Verein, R., Paltoglou, G., Kippo, H., and Underwood, M. (2012). Enhancing ship of opportunity sea surface temperature observations in the Australian region. J. Operat. Oceanogr. 5, 59-73. doi: 10.1080/1755876x.2012. 11020132

Beggs, H., Zhong, A., Warren, G., Alves, O., Brassington, G., and Pugh, T. (2011). RAMSSA - an operational, high-resolution, regional Australian multi-sensor sea surface temperature analysis over the Australian region. Aust. Meteorol. Oceanogr. J. 61, 1-22. doi: 10.22499/2.6101.001

Bell, M. J., Lefèbvre, M., le Traon, P.-Y., Smith, N., and Wilmer-Becker, K. (2009). GODAE the global ocean data assimilation experiment. Oceanography 22, 14-21. doi: 10.1016/j.marpolbul.2011.06.026

Bellenger, H., and Duvel, J.-P. (2009). An analysis of tropical ocean diurnal warm layers. J. Clim. 20, 3629-3646. doi: 10.1175/2008jcli2 598.1

Berry, D. I., and Kent, E. C. (2017). Assessing the health of the in situ global surface marine climate observing system. Int. J. Climatol. 37, 2248-2259. doi: $10.1002 /$ joc. 4914

Bessho, K., Date, K., Hayashi, M., Ikeda, A., Imai, T., Inoue, H., et al. (2016). An introductionIntroduction to Himawari-8/9 - Japan's- Japan's new-generation geostationary meteorological satellites. J. Meteorol. Soc. Jpn. 94, 151-183. doi: 10.2151/jmsj.2016-2019

Blackmore, T., O'Carroll, A. G., Fennig, K., and Saunders, R. W. (2012). Correction of AVHRR Pathfinder SST data for volcanic aerosol effects using ATSR SSTs and TOMS aerosol optical depth. Remote Sens. Environ. 116, 107-117. doi: 10.1016/j.rse.2011.04.040

Bojinski, S. (2014). The concept of essential climate variables in support of climate research, applications, and policy, BAMS, September 2014. Bull. Am. Meteorol. Soc. 95, 1431-1443. doi: 10.1175/BAMS-D-13-00047.1

Brassington, G. B. (2009). Ocean Prediction Issues Related to Weather and Climate Prediction, Pre-CAS Public Web Consultation: Vision Paper (Agenda Item 8.5). Seoul: WMO CAS.

Bulgin, C. E., Embury, O., Corlett, G., and Merchant, C. J. (2016). Independent uncertainty estimates for coefficient based sea surface temperature retrieval from the along-track scanning radiometer instruments. Remote Sens. Environ. 178, 213-222. doi: 10.1016/j.rse.2016.02.022

Buongiorno Nardelli, B., Tronconi, C., Pisano, A., and Santoleri, R. (2013). High and ultra-high resolution processing of satellite Sea Surface temperature data over Southern European Seas in the framework of MyOcean project. Remote Sens. Environ. 129, 1-16. doi: 10.1016/j.rse.2012.10.012

Casey, K. S., Brandon, T. B., Cornillon, P., and Evans, R. (2010). "The past, present, and future of the AVHRR pathfinder SST program," in Oceanography from Space, eds V. Barale, J. Gower, and L. Alberotanza (Dordrecht: Springer), 273-287. doi: 10.1007/978-90-481-8681-5_16
Castro, S., Wick, G., and Emery, W. (2012). Evaluation of the relative performance of SST measurements from different types of drifting and moored buoys using satellite-derived reference products. J. Geophys. Res. 117:C02029. doi: 10.1029/ 2011JC007472

Castro, S., Wick, G., Jackson, D., and Emery, W. (2008). Error characterization of infrared and microwave sea surface temperature products for merging and analysis. J. Geophys. Res. 113:C03010. doi: 10.1029/2006JC00 3829

Castro, S. L., Emery, W. J., Tandy, W. Jr., and Good, W. S. (2017). Submesoscale sea surface temperature variability from UAV and satellite measurements. Remote Sens. 9:1089. doi: 10.3390/rs9111089

Castro, S. L., Wick, G. A., Minnett, P. J., Jessup, A. T., and Emery, W. J. (2010). The impact of measurement uncertainty and spatial variability on the accuracy of skin and subsurface regression-based sea surface temperature algorithms. Remote Sens. Environ. 114, 2666-2678. doi: 10.1016/j.rse.2010.06.003

Centurioni, L. R., Turton, J. D., Lumpkin, R., Braasch, L., Brassington, G., Chao, Y., et al. (2019). Multidisciplinary global in-situ observations of essential climate and ocean variables at the air-sea interface in support of climate variability and change studies and to improve weather forecasting, pollution, hazard and maritime safety assessments. Front. Mar. Sci.

Chelton, D. B., Esbensen, S. K., Schlax, M. G., Thum, N., Freilich, M. H., Wentz, F., et al. (2001). Observations of coupling between surface wind stress and sea surface temperature in the eastern tropical pacific. J. Clim. 14, 1479-1498. doi: 10.1175/1520-0442(2001)014<1479:oocbsw >2.0.co;2

Chin, T. M., Vazquez-Cuervo, J., and Armstrong, E. M. (2017). A multi-scale highresolution analysis of global sea surface temperature. Remote Sens. Environ. 200, 154-169. doi: 10.1039/b911677j

Choi, Y.-S., and Ho, C.-H. (2015). Earth and environmental remote sensing community in South Korea: a review. Remote Sens. Appl. Soc. Environ. 2, 66-76. doi: 10.1016/j.rsase.2015.11.003

Clayson, C. A., and Bogdanoff, A. S. (2012). The effect of diurnal sea surface temperature warming on climatological air-sea fluxes. J. Clim. 26, 2546-2556. doi: 10.1175/jcli-d-12-00062.1

Corlett, G. K., Merchant, C. J., Minnett, P. J., and Donlon, C. J. (2014). "Assessment of long-term satellite derived sea surface temperature records," in Experimental Methods in the Physical Sciences Optical Radiometry for Ocean Climate Measurements, Vol. 47, eds G. Zibordi, C. J. Donlon, and A. C. Parr (Cambridge: Academic Press), 639-677. doi: 10.1016/b978-0-12-4170117.00021-0

Cornillon, P. (1986). The effect of the new england seamounts on gulf stream meandering as observed from satellite IR imagery. J. Phys. Oceanogr. 16, 386-389. doi: 10.1175/1520-0485(1986)016<0386:teotne >2.0.co;2

Cracknell, A. P. (1997). The Advanced Very High Resolution Radiometer. London: CRC Press.

Cronin, M. F., Gentemann, C. L., Edson, J., Ueki, I., Ando, K., Bourassa, M., et al. (2019). Air-sea fluxes with a focus on heat and momentum. Front. Mar. Sci. doi: 10.3389/fmars.2019.00430

Crosman, E., Vazquez-Cuervo, J., and Chin, T. M. (2017). Evaluation of the multiscale ultra-high resolution (MUR) analysis of lake surface temperature. Remote Sens. 9:723. doi: 10.3390/rs9070723

Dash, P., Ignatov, A., Kihai, Y., and Sapper, J. (2010). The SST quality monitor (SQUAM). J. Atmos. Ocean. Technol. 27, 1899-1917. doi: 10.1175/ 2010JTECHO756.1

Dombrowsky, E., Bertino, L., Brassington, G. B., Chassignet, E. P., Davidson, F., Hurlburt, H. E., et al. (2009). GODAE systems in operation. Oceanography 22, 80-95. doi: 10.5670/oceanog.2009.68

Dong, C., Yang, J., Zhang, W., Yang, Z., Lu, N., Shi, J., et al. (2009). An overview of a new chinese weather satellite FY-3A. Bull. Am. Meteorol. Soc. 90, 1531-1544. doi: $10.1175 / 2009$ bams 2798.1

Donlon, C., Berruti, B., Buongiorno, A., Ferreira, M. H., Féménias, P., Frerick, J., et al. (2012). The global monitoring for environment and security (GMES) sentinel-3 mission. Remote Sens. Environ. 120, 37-57. doi: 10.1016/j.rse.2011. 07.024

Donlon, C., Robinson, I. S., Reynolds, M., Wimmer, W., Fisher, G., Edwards, R., et al. (2008). An infrared sea surface temperature autonomous radiometer (ISAR) for deployment aboard volunteer observing ships (VOS). J. Atmos. Ocean. Technol. 25, 93-113. doi: 10.1175/2007jtecho505.1 
Donlon, C. J., Casey, K. S., Robinson, I. S., Gentemann, C. L., Reynolds, R. W., Barton, I., et al. (2009). The GODAE high-resolution sea surface temperature pilot project. Oceanography 22, 34-45. doi: 10.5670/oceanog.2009.64

Donlon, C. J., Martin, M., Stark, J. D., Roberts-Jones, J., Fiedler, E., and Wimmer, W. (2011). The operational sea surface temperature and sea ice analysis (OSTIA). Remote Sens. Environ. 116, 140-158. doi: 10.1016/j.rse.2010.10.017

Donlon, C. J., Minnett, P. J., Fox, N., and Wimmer, W. (2015). "Strategies for the Laboratory and Field Deployment of Ship-Borne Fiducial Reference Thermal Infrared Radiometers in Support of Satellite-Derived Sea Surface Temperature Climate Data Records," in Optical Radiometry for Oceans Climate Measurements:Experimental Methods in Sciences, eds G. Zibordi, C. Donlon, and A. Parr (Amsterdam: Elsivier).

Donlon, C. J., Minnett, P. J., Gentemann, C., Nightingale, T. J., Barton, I. J., Ward, B., et al. (2002). Toward improved validation of satellite sea surface skin temperature measurements for climate research. J. Clim. 15, 353-369. doi: 10.1175/1520-0442(2002)015<0353:tivoss>2.0.co;2

Durand, Y., Hallibert, P., Wilson, M., Lekouara, M., Grabarnik, S., Aminou, D., et al. (2015). "The flexible combined imager onboard MTG: from design to calibration," in Proceedings of the SPIE Remote Sensing, (Bellingham, WA: SPIE), doi: 10.1117/12.2196644

Eastwood, S., Le Borgne, P., Préré, S., and Poulter, D. (2011). Diurnal variability in sea surface in the Arctic. Remote Sens. Environ. 115, 2594-2602. doi: 10.1016/j. rse.2011.05.015

ECMWF Workshop Report (2018). Observations and Analysis of Sea-Surface Temperature and Sea-ice for Numerical Weather Prediction and Climate Applications. Available at: https://www.ecmwf.int/sites/default/files/elibrary/ 2018/18157-observations-and-analysis-sea-surface-temperature- and-sea-icenwp-and-climate-applications-ws.pdf (accessed January, 2018).

Embury, O., Merchant, C. J., and Filipiak, M. J. (2012a). A reprocessing for climate of sea surface temperature from the along-track scanning radiometers: basis in radiative transfer. Remote Sens. Environ. 116, 32-46. doi: 10.1016/j.rse.2010. 10.016

Embury, O., Merchant, C. J., and Corlett, G. K. (2012b). A reprocessing for climate of sea surface temperature from the along-track scanning radiometers: initial validation, accounting for skin and diurnal variability effects. Remote Sens. Environ. 116, 62-78. doi: 10.1016/j.rse.2011.02.028

Eriksen, M., Lebreton, L. C. M., Carson, H. S., Theil, M., Moore, C. J., Borerro, J. C., et al. (2014). Plastic pollution in the World's oceans: more than 5 trillion plastic pieces weighing over 250,000 tons afloat at Sea. PLoS One 9:e111913. doi: 10.1371/journal.pone.0111913

Esaias, W. E., Abbott, M. R., Barton, I., Brown, O. B., Campbell, J. W., Carder, K. L., et al. (1998). An overview of MODIS capabilities for ocean science observations. IEEE Trans. Geosci. Remote Sens. 36, 1250-1265. doi: 10.1109/36.701076

Fablet, R., Phi, V., Redouane, L., Pierre-Henri, H., and Bertrand, C. (2018). Spatiotemporal interpolation of cloudy SST fields using conditional analog data assimilation. Remote Sens. 10:310. doi: 10.3390/rs10020310

Fairall, C. W., Bradley, E. F., Hare, J. E., Grachev, A. A., and Edson, J. B. (2003). Bulk parameterization of air-sea fluxes: updates and verification for the COARE algorithm. J. Clim. 16, 571-591. doi: 10.1175/1520-0442(2003) $016<0571$ :bpoasf $>2.0$. co; 2

Fallmann, J., Lewis, H., Castillo, J. M., Arnold, A., and Ramsdale, S. (2017). Impact of sea surface temperature on stratiform cloud formation over the North Sea. Geophys. Res. Lett. 44, 4296-4303. doi: 10.1002/2017gl073105

Ferrari, R. (2011). A frontal challenge for climate models. Science 332, 316-317. doi: $10.1126 /$ science. 1203632

Ferster, B. S., Subrahmanyam, B., and Macdonald, A. M. (2018). Confirmation of ENSO-southern ocean teleconnections using Satellite-Derived SST. Remote Sens. 10:331. doi: 10.3390/rs10020331

Filipiak, M. J., Merchant, C. J., Kettle, H., and Le Borgne, P. (2010). A statistical model for sea surface diurnal warming driven by numerical weather prediction fluxes and winds. Ocean Sci. Discuss. 7, 1497-1532. doi: 10.5194/osd-7-14972010

Folland, C. (2005). Assessing bias corrections in historical sea surface temperature using a climate model. Int. J. Climatol. 25, 895-911. doi: 10.1002/joc.1171

Freeman, E., Woodruff, S. D., Worley, S. J., Lubker, S. J., Kent, E. C., Angel, W. E., et al. (2017). ICOADS Release 3.0: a major update to the historical marine climate record. Int. J. Climatol. 37, 2211-2232. doi: 10.1002/joc.4775
García-Morales, R., López-Martínez, J., Valdez-Holguin, J. E., Herrera-Cervantes, H., and Espinosa-Chaurana, L. D. (2017). Environmental variability and oceanographic dynamics of the central and southern coastal zone of sonora in the Gulf of California. Remote Sens. 9:925. doi: 10.3390/rs9090925

GCOS (2009). Guideline for the Generation of Satellite-based Datasets and Products Meeting GCOS Requirements. Paris: GCOS.

GCOS (2010). Guideline for the Generation of Datasets and Products Meeting GCOS Requirements. Paris: GCOS.

GCOS (2011). Systematic Observation Requirements for Satellite-Based Products for Climate. Supplemental Details to the Satellite-Based Component of the Implementation Plan for the Global Observing System for Climate in Support of the UNFCC. Geneva: UNFCC.

Gentemann, C. L. (2014). Three way validation of MODIS and AMSR-E sea surface temperatures. J. Geophys. Res. Oceans 119, 2583-2598. doi: 10.1002/ 2013jc009716

Gentemann, C. L., and Akella, S. (2018). Evaluation of NASA GEOS-ADAS modeled diurnal warming through comparisons to SEVIRI and AMSR2 SST observations. J. Geophys. Res. Oceans 123, 1364-1375. doi: 10.1002/ 2017 jc013186

Gentemann, C. L., and Hilburn, K. A. (2015). In situ validation of sea surface temperatures from the GCOM-W1 AMSR2 RSS calibrated brightness temperatures. J. Geophys. Res. Oceans 120, 3567-3585. doi: 10.1002/ $2014 \mathrm{jc} 010574$

Gentemann, C. L., Meissner, T., and Wentz, F. J. (2010). Accuracy of satellite sea surface temperatures at 7 and $11 \mathrm{GHz}$. IEEE Trans. Geosci. Remote Sens. 48, 1009-1018. doi: 10.1109/tgrs.2009.2030322

Gentemann, C. L., Minnett, P. J., LeBorgne, P., and Merchant, C. J. (2008). Multisatellite measurements of large diurnal warming events. Geophys. Res. Lett. 35:L22602.

Gladkova, I., Ignatov, A., Pennybacker, M., and Kihai, Y. (2019). "Towards highresolution multi-sensor gridded ACSPO SST Product," in Proceedings of the SPIE, Ocean Sensing and Monitoring XI, Bellingham.

Gladkova, I., Kihai, Y., Ignatov, A., Shahriar, F., and Petrenko, B. (2015). SST pattern test in ACSPO clear-sky mask for VIIRS. Remote Sens. Environ. 160, 87-98. doi: 10.1016/j.rse.2015.01.003

Haines, S. L., Jedlovec, G. J., and Lazarus, S. M. (2007). A MODIS sea surface temperature composite for regional applications. IEEE Trans. Geosci. Remote Sens. 45, 2919-2927. doi: 10.1109/tgrs.2007.898274

Hirahara, S., Ishii, M., and Fukuda, Y. (2014). Centennial-Scale sea surface temperature analysis and its uncertainty. J. Clim. 27, 57-75. doi: 10.1175/jclid- $12-00837.1$

Hudson, D., Alves, O., Hendon, H. H., Lim, E., Liu, G., Luo, J.-J., et al. (2017). ACCESS-S1: the new bureau of meteorology multi-week to seasonal prediction system. J. South. Hemisphere Earth Syst. Sci. 67, 132-159. doi: 10.22499/3. 6703.001

Ignatov, A., Zhou, X., Petrenko, B., Liang, X., Kihai, Y., Dash, P., et al. (2016). AVHRR GAC SST reanalysis version 1 (RAN1). Remote Sens. 8:315. doi: 10. $3390 / \mathrm{rs} 8040315$

JAXA (2017). AMSR2 Products Update. Available at: https://suzaku.eorc.jaxa.jp/ GCOM_W/materials/product/170222_Ver3.0_release_e.pdf (accessed March, 2017).

JCGM (2008). Evaluation of Measurement Data - Guide to the Expression of Uncertainty in Measurement. Available at: https://www.bipm.org/utils/ common/documents/jcgm/JCGM_100_2008_E.pdf (accessed September, 2008).

Kara, A. B., Hurlburt, H. E., and Wallcraft, A. J. (2005). Stability-Dependent exchange coefficients for Air-Sea fluxes. J. Atmos. Ocean. Technol. 22, 10801094. doi: $10.1175 /$ jtech 1747.1

Karagali, I., and Høyer, J. L. (2013). Observations and modeling of the diurnal SST cycle in the North and Baltic Seas. J. Geophys. Res. Oceans 118:4488. doi: 10.1002/jgrc.20320

Karagali, I., and Høyer, J. L. (2014). Characterisation and quantification of regional diurnal SST cycles from SEVIRI. Ocean Sci. Discuss. 11, 1093-1128. doi: 10. 5194/osd-11-1093-2014

Karagali, I., Høyer, J. L., and Donlon, C. J. (2017). Using a 1-D model to reproduce the diurnal variability of SST. J. Geophys. Res. Oceans 122, 2945-2959. doi: $10.1002 / 2016 j \mathrm{c} 012542$ 
Karagali, I., Høyer, J. L., and Hasager, C. B. (2012). SST diurnal variability in the North and the Baltic Sea. Rem. Sens. Envinron. 121, 159-170. doi: 10.1016/j.rse. 2012.01.016

Kawanishi, T., Sezai, T., Ito, Y., Imaoka, K., Takeshima, T., Ishido, Y., et al. (2003). The advanced microwave scanning radiometer for the earth observing system (AMSR-E), NASDA's contribution to the EOS for global energy and water cycle studies. IEEE Trans. Geosci. Remote Sens. 41, 184-194. doi: 10.1109/tgrs.2002. 808331

Kearns, E. J., Hanafin, J. A., Evans, R. H., Minnett, P. J., and Brown, O. B. (2000). An independent assessment of Pathfinder AVHRR sea surface temperature accuracy using the Marine-Atmosphere emitted radiance interferometer (MAERI). Bull. Am. Meteorol. Soc. 81, 1525-1536. doi: 10.1175/1520-0477(2000) $081<1525$ :aiaopa $>2.3 . c 0 ; 2$

Kennedy, J. J. (2014). A review of uncertainty in in situ measurements and data sets of sea surface temperature. Rev. Geophys. 52, 1-32. doi: 10.1002/2013RG000434

Kennedy, J. J., Smith, R. O., and Rayner, N. A. (2012). Using AATSR data to assess the quality of in situ sea-surface temperature observations for climate studies. Remote Sens. Environ. 116, 79-92. doi: 10.1016/j.rse.2010.11.021

Kent, E. C., Rayner, N. A., Berry, D. I., Huang, B., Kennedy, J. J., and Wilkinson, C. (2019). Observing requirements for long-term climate records at the ocean surface. Front. Mar. Sci. 6:441, doi: 10.3389/fmars.2019.00441

Kilpatrick, K. A., Podestá, G., Walsh, S., Williams, E., Halliwell, V., Szczodrak, M., et al. (2015). A decade of sea surface temperature from MODIS. Remote Sens. Environ. 165, 27-41. doi: 10.1016/j.rse.2015.04.023

Kilpatrick, K. A., Podestá, G., Williams, E., Walsh, S., and Minnett, P. J. (2019). Alternating decision trees for cloud masking MODIS and VIIRS NASA SST products. J. Atmos. Ocean. Technol. 36, 387-407. doi: 10.1175/jtech-d-180103.1

Kilpatrick, K. A., Podestá, G. P., and Evans, R. H. (2001). Overview of the NOAA/NASA pathfinder algorithm for sea surface temperature and associated matchup database. J. Geophys. Res. 106, 9179-9198.

Kim, H., Kim, H., Lim, H., and Choi, H. (2015). Space-Based earth observation activities in south korea. IEEE Geosci. Remote Sens. Mag. 3, 34-39. doi: 10.1109/ MGRS.2014.2382652

Koner, P., and Harris, A. (2016). Improved quality of MODIS sea surface temperature retrieval and data coverage using physical deterministic methods. Remote Sens. 8:454. doi: 10.3390/rs8060454

Koner, P. K., Harris, A. R., and Maturi, E. M. (2015). A physical deterministic inverse method for operational satellite remote sensing: an application for sea surface temperature retrievals. IEEE Trans. Geosci. Rem. Sens. 53, 5872-5888. doi: $10.1109 /$ tgrs.2015.2424219

Kwok, R., Cunningham, G. F., and Hibler, W. D. I. I. I. (2003). Sub-daily sea ice motion and deformation from RADARSAT observations. Geophys. Res. Lett. 30:2218. doi: 10.1029/2003GL018723

Lean, K., and Saunders, R. (2013). Validation of the ATSR reprocessing for climate (ARC) dataset using data from drifting buoys and a three-way error analysis. J. Clim. 26, 4758-4772. doi: 10.1175/JCLI-D-12-00206.1

Legeckis, R. (1977). Long waves in the eastern equatorial pacific ocean: a view from a geostationary satellite. Science 197, 1179-1181. doi: 10.1126/science.197.4309. 1179

Lekouara, M. (2013). Exploring Frontogenesis Processes in New Satellite Sea Surface Temperature Data Sets. Doctoral thesis, University of Southampton, Southampton.

Levitus, S., Antonov, J. I., Boyer, T. P., Baranova, O. K., Garcia, H. E., Locarnini, R. A., et al. (2012). World ocean heat content and thermosteric sea level change (0-2000 m), 1955-2010. Geophys. Res. Lett. 39:L10603. doi: 10.1029/ 2012 GL051106

Levy, M., Raffaele, F., Franks, P. J. S., Martin, A. P., and Pascal, R. (2012). Bringing physics to life at the submesoscale. Geophys. Res. Lett. 39:L14602.

Lindzen, R. S., and Nigam, S. (1987). On the role of sea surface temperature gradients in forcing low-level winds and convergence in the tropics. J. Atmos. Sci. 44, 2418-2436. doi: 10.1175/1520-0469(1987)044<2418:otross >2.0.co;2

Liu, Y., and Minnett, P. J. (2015). Evidence linking satellite-derived seasurface temperature signals to changes in the Atlantic meridional overturning circulation. Remote Sens. Environ. 169, 150-162. doi: 10.1016/j.rse.2015. 08.014

Llewellyn-Jones, D., Minnett, P., Saunders, R., and Zavody, A. (1984). Satellite multi-channel infrared measurements of sea surface temperature of the N.E.
Atlantic Ocean using AVHRR/2. Q. J. R. Meteorol. Sci. 110, 613-631. doi: 10.1256/smsqj. 46503

Llewellyn-Jones, D., and Remedios, J. (2012). The advanced along track scanning radiometer (AATSR) and its predecessors ATSR-1 and ATSR-2: an introduction to the special issue. Remote Sens. Environ. 116, 1-3. doi: 10.1016/j.rse.2011. 06.002

Luo, B., Minnett, P. J., Gentemann, C., and Szczodrak, G. (2019). Improving satellite retrieved night-time infrared sea surface temperatures in aerosol contaminated regions. Remote Sens. Environ. 223, 8-20. doi: 10.1016/j.rse.2019. 01.009

MacCallum, S. N., and Merchant, C. J. (2012). Surface water temperature observations of large lakes by optimal estimation. Can. J. Remote Sens. 38, 25-45. doi: 10.5589/m12-010

MacLachlan, C., Arribas, A., Peterson, K. A., Maidens, A., Fereday, D., Scaife, A. A., et al. (2014). Global Seasonal forecast system version 5 (GloSea5): a highresolution seasonal forecast system. Q. J. R. Met. Soc. 141:689. doi: 10.1002/qj. 2396

Marsouin, A., Le Borgne, P., Legendre, G., Péré, S., and Roquet, H. (2015). Six years of OSI-SAF METOP-A AVHRR sea surface temperature. Remote Sens. Environ. 159, 288-306. doi: 10.1016/j.rse.2014.12.018

Marullo, S., Minnett, P. J., Santoleri, R., and Tonani, M. (2016). The diurnal cycle of sea-surface temperature and estimation of the heat budget of the Mediterranean Sea. J. Geophys. Res. Oceans. 121, 8351-8367. doi: 10.1002/2016JC012192

Marullo, S., Santoleri, R., Banzon, V., Evans, R. H., and Guarracino, M. (2010). A diurnal-cycle resolving sea surface temperature product for the tropical Atlantic. J. Geophys. Res. Oceans 115:C05011. doi: 10.1029/2009JC005466

Marullo, S., Santoleri, R., Ciani, D., Le Borgne, P., Pere, S., Pinardi, N., et al. (2014). Combining model and geostationary satellite data to reconstruct hourly SST field over the Mediterranean Sea. Remote Sens. Environ. 146, 11-23. doi: 10.1016/j.rse.2013.11.001

McPhaden, M. J., Ando, K., Bourlès, B., Freitag, H. P., Lumpkin, R., Masumoto, Y., et al. (2010). "The global tropical moored buoy array," in Proceedings of the OceanObs'09:Sustained Ocean Observations and Information for Society Conference, Vol. 2, eds J. Hall, D. E. Harrison, and D. Stammer (Venice: ESA Publication WPP-306).

Merchant, C. J., Embury, O., Le Borgne, P., and Bellec, B. (2006). Saharan dust in night-time thermal imagery: detection and reduction of related biases in retrieved sea surface temperature. Remote Sens. Environ. 104, 15-30. doi: 10. 1016/j.rse.2006.03.007

Merchant, C. J., Embury, O., Rayner, N. A., Berry, D. I., Corlett, G., Lean, K., et al. (2012). A twenty-year independent record of sea surface temperature for climate from along-track scanning radiometers. J. Geophys. Res. 117:C12013. doi: 10.1029/2012JC008400

Merchant, C. J., Embury, O., Roberts-Jones, J., Fiedler, E., Bulgin, C. E., Corlett, G. K., et al. (2014). Sea surface temperature datasets for climate applications from Phase 1 of the european space agency climate change initiative (SST CCI). Geosci. Data J. 1, 179-191. doi: 10.1002/gdj3.20

Merchant, C. J., Harris, A. R., Maturi, E., and MacCallum, S. (2005). Probabilistic physically based cloud screening of satellite infrared imagery for operational sea surface temperature retrieval. Q. J. R. Meteorol. Soc. 131, 2735-2755. doi: 10.1256/qj.05.15

Merchant, C. J., Harris, A. R., Murray, M. J., and Zavody, A. M. (1999). Toward the elimination of bias in satellite retrievals of skin sea surface temperature 1. Theory, modeling and inter-algorithm comparison. J. Geophys. Res. 104, 23565-23578. doi: 10.1029/1999JC900105

Merchant, C. J., Harris, A. R., Roquet, H., and Le Borgne, P. (2009a). Retrieval characteristics of non-linear sea surface temperature from the advanced very high resolution radiometer. Geophys. Res. Lett. 36:L17604. doi: 10.1029/ 2009GL039843

Merchant, C. J., Le Borgne, P., Roquet, H., and Marsouin, A. (2009b). Sea surface temperature from a geostationary satellite by optimal estimation. Remote Sens. Environ. 113, 445-457. doi: 10.1016/j.rse.2008.10.012

Merchant, C. J., Paul, F., Popp, T., Ablain, M., Bontemps, S., Defourny, P., et al. (2017). Uncertainty information in climate data records from Earth observation. Earth Syst. Sci. Data 9, 511-527. doi: 10.5194/essd-9-511-2017

Miles, T. N., and He, R. (2010). Temporal and spatial variability of Chl-a and SST on the south atlantic bight: revisiting with cloud-free reconstructions of MODIS satellite imagery. Cont. Shelf Res. 30, 1951-1962. doi: 10.1016/j.csr.2010.08.016 
Minnett, P. J. (1991). Consequences of sea surface temperature variability on the validation and applications of satellite measurements. J. Geophys. Res. 96, 18475-18489.

Minnett, P. J. (2010). "The validation of sea surface temperature retrievals from spaceborne infrared radiometers," in Oceanography from Space, eds V. Barale, J. F. R. Gower, and L. Alberotanza (Berlin: Springer), 273-295.

Minnett, P. J., and Corlett, G. K. (2012). A pathway to generating climate data records of sea-surface temperature from satellite measurements. Deep Sea Res. Part II Top. Stud. Oceanogr. 77-80, 44-51. doi: 10.1016/j.dsr2.2012. 04.003

Minnett, P. J., Smith, M., and Ward, B. (2011). Measurements of the oceanic thermal skin effect. Deep Sea Res. Part II Top. Stud. Oceanogr. 58, 861-868. doi: 10.1016/j.dsr2.2010.10.024

Mittaz, J. P. D., Harris, A. R., and Sullivan, J. T. (2009). A physical method for the calibration of the AVHRR/3 thermal IR Channels 1: the prelaunch calibration data. J. Atmos. Ocean. Technol. 26:996. doi: 10.1175/2008JTECHO636.1

Morak-Bozzo, S., Merchant, C. J., Kent, E. C., Berry, D. I., and Carella, G. (2016). Climatological diurnal variability in sea surface temperature characterized from drifting buoy data. Geosci. Data J. 3, 20-28. doi: 10.1002/gdj3.35

NASA (2018). Cloud Climatology, Global Distribution and Character of Clouds. Available at: https://www.giss.nasa.gov/research/briefs/rossow_01/distrib.html (accessed April, 2018).

Nielsen-Englyst, P., Høyer, J. L., Toudal Pedersen, L., Gentemann, C., Alerskans, E., Block, T., et al. (2018). Optimal estimation of sea surface temperature from AMSR-E. Remote Sens. 10:229. doi: 10.3390/rs10020229

O'Carroll, A. G., Eyre, J. R., and Saunders, R. W. (2008). Three-Way error analysis between AATSR, AMSR-E, and in situ sea surface temperature observations. J. Atmos. Oceanic Technol. 25, 1197-1207. doi: 10.1175/2007jtecho542.1

Ohring, G., Wielicki, B., Spencer, R., Emery, B., and Datla, R. (2005). Satellite instrument calibration for measuring global climate change: report of a workshop. Bull. Am. Meteorol. Soc. 86, 1303-1313.

O'Neill, L. W., Chelton, D. B., and Esbensen, S. K. (2012). Covariability of surface wind and stress responses to sea surface temperature fronts. J. Clim. 25, 5916-5942. doi: 10.1175/jcli-d-11-00230.1

Palmer, M. D., Roberts, C. D., Balmaseda, M., Chang, Y.-S., Gennady, A., Chepurin, N. F., et al. (2017). Ocean heat content variability and change in an ensemble of ocean reanalyses. Clim. Dyn. 49, 909-930. doi: 10.1007/s00382-015-28012800

Petrenko, B., Ignatov, A., Kihai, Y., and Dash, P. (2016). Sensor-specific error statistics for SST in the advanced clear-sky processor for ocean. J. Atmos. Ocean. Tech. 27, 345-359. doi: 10.1175/JTECH-D-15-0166.1

Petrenko, B., Ignatov, A., Kihai, Y., and Heidinger, A. (2010). Clear-Sky mask for the advanced clear-sky processor for ocean. J. Atmos. Ocean. Tech. 27, 1609-1623. doi: 10.1175/2010JTECHA1413.1

Petrenko, B., Ignatov, A., Kihai, Y., Stroup, J., and Dash, P. (2014). Evaluation and selection of SST regression algorithms for JPSS VIIRS. J. Geophys. Res. 119, 4580-4599. doi: 10.1002/2013JD020637

Pisano, A., Buongiorno Nardelliab, B., Tronconia, C., and Santoleri, R. (2016). The new mediterranean optimally interpolated pathfinder AVHRR SST Dataset (1982-2012). Remote Sens. Environ. 176, 107-116. doi: 10.1016/j.rse.2016. 01.019

Pithan, F., and Mauritsen, T. (2014). Arctic amplification dominated by temperature feedbacks in contemporary climate models. Nat. Geosci. 7:181. doi: $10.1038 /$ ngeo2071

Poli, P., Lucas, M., O’Carroll, A., Le Menn, M., David, A., Corlett, G. K., et al. (2019). The copernicus surface velocity platform drifter with barometer and reference sensor for temperature (SVP-BRST): genesis, design, and initial results. Ocean Sci. 15, 199-214. doi: 10.5194/os-15-199-2019

Polidoro, B., Ralph, G., Strongin, K., Harvey, M., Carpenter, K. E., Arnold, R., et al. (2017). The status of marine biodiversity in the eastern central atlantic (West and Central Africa). Aquat. Conserv. 27, 1021-1034. doi: 10.1002/aqc.2744

Puri, K., Dietachmayer, G., Steinle, P., Dix, M., Rikus, L., Logan, L., et al. (2013). Implementation of the initial ACCESS numerical weather prediction system. Aust. Meteorol. Oceanogr. J. 63, 265-284.

Rayner, N., Kennedy, J., Titchner, H., and Kent, E. (2018). Observations of seaSurface Temperature Made in Situ: Evolution, Uncertainties and Considerations on Their Use. Available at: https://www.ecmwf.int/en/elibrary/17967observations-sea-surface-temperature-made-situ-evolution- uncertaintiesand (accessed January, 2018).
Reynolds, R. W., Gentemann, C. L., and Corlett, G. K. (2010). Evaluation of AATSR and TMI Satellite SST Data. J. Clim. 23, 152-165. doi: 10.1175/2009jcli3252.1

Rice, J. P., Butler, J. J., Johnson, B. C., Minnett, P. J., Maillet, K. A., Nightingale, T. J., et al. (2004). The Miami2001 infrared radiometer calibration and intercomparison: 1. Laboratory characterization of blackbody targets. J. Atmos. Ocean. Technol. 21, 258-267. doi: 10.1175/1520-0426(2004)021<0258:tmirca> 2.0.co;2

Roberts-Jones, J., Fiedler, E. K., and Martin, M. J. (2012). Daily, global, highresolution SST and sea ice reanalysis for 1985-2007 Using the OSTIA system. J. Clim. 25, 6215-6232. doi: 10.1175/JCLI-D-11-00648.1

Robinson, I., Piolle, J.-F., Le Borgne, P., Poulter, D., Donlon, C., and Arino, O. (2012). Widening the application of AATSR SST data to operational tasks through the medspiration service. Remote Sens. Environ. 116, 126-139. doi: 10.1016/j.rse.2010.12.019

Saunders, R. W., and Kriebel, K. T. (1988). An improved method for detecting clear sky and cloudy radiances from AVHRR data. Int. J. Remote Sens. 9, 123-150. doi: 10.1080/01431168808954841

Scheren, P., Ibe, A. C., Jansenn, F. J., and Lemmens, A. M. (2002). Environmental pollution in the Gulf of Guinea - A regional approach. Mar. Pollut. Bull. 44, 633-641. doi: 10.1016/s0025-326x(01)00305-8

Schmit, T. J., Griffith, P., Gunshor, M. M., Daniels, J. M., Goodman, S. J., and Lebair, W. J. (2017). A closer look at the ABI on the GOES-R Series. Bull. Am. Meteorol. Soc. 98, 681-698. doi: 10.1175/bams-d-15-00230.1

Schmit, T. J., Gunshor, M. M., Menzel, P. W., Gurka, J. J., Li, J., and Bachmeier, A. S. (2005). Introducing the next-generation advanced baseline imager on GOES-R. Bull. Am. Meteorol. Soc. 86, 1079-1097. doi: 10.1175/BAMS-86-8-1079

Smith, G. C., Allard, R., Babin, M., Bertino, L., Chevallier, M., Corlett, G. K., et al. (2019). Polar ocean observations: a critical gap in the observing system and its effect on environmental prediction. Front. Mar. Sci.

Smith, N. R., and Koblinsky, C. J. (2001). "The ocean observing system for the 21st Century: a consensus statement," in Observing the Oceans in the 21st Century, eds C. Koblinsky and N. R. Smith (Melbourne: Bureau of Meteorology).

Spalding, M., Kainuma, M., and Collins, L. (2010). World Atlas of Mangroves. London: Earthscan.

Strong, A., and McClain, P. (1984). Improved ocean surface temperatures from space - comparisons with drifting buoys. Bull. Am. Met. Soc 65, 138-142. doi: 10.1175/1520-0477(1984)065<0138:iostfs > 2.0.co;2

Theocharous, E., Fox, N. P., Barker-Snook, I., Niclos, R., Garcia Santos, V., Minnett, P. J., et al. (2016). The 2016 CEOS infrared radiometer comparison: Part II: laboratory comparison of radiation thermometers. J. Atmos. Ocean. Technol. 36:6.

Urabe, T., Okamura, Y., Tanaka, K., and Mokuno, M. (2018). "In-orbit commissioning activities results of GCOM-C/SGLI," in Proceedings of the SPIE Remote Sensing, (Bellingham, WA: SPIE), doi: 10.1117/12.2325572

Vazquez-Cuervo, J., Torres, H. S., Menemenlis, D., Chin, T. M., and Armstrong, E. M. (2017). Relationship between SST gradients and upwelling off Peru and Chile: model/satellite data analysis. Int. J. Remote Sens. 38, 6599-6622. doi: 10.1080/01431161.2017.1362130

von Schuckmann, K., Palmer, M. D., Trenberth, K. E., Cazenave, A., Chambers, D., Champollion, N., et al. (2016). An imperative to monitor Earth's energy imbalance. Nat. Clim. Change 6, 138-144. doi: 10.1038/nclimate2876

Wallner, O., Reinert, T., and Straif, C. (2017). "METIMAGE: a spectro-radiometer for the VII mission onboard METOP-SG," in Proceedings of International Conference on Space Optics - ICSO 2016, eds B. Cugny, N. Karafolas, and Z. Sodnik (Biarritz: SPIE), doi: 10.1117/12.2296103

Walton, C. C., Pichel, W. G., Sapper, J. F., and May, D. A. (1998). The development and operational application of nonlinear algorithms for the measurement of sea surface temperatures with the NOAA polar-orbiting environmental satellites. J. Geophys. Res. 103, 27999-28012. doi: 10.1029/98jc02370

Wang, H., Guan, L., and Chen, G. (2016). Evaluation of Sea surface temperature from FY-3C VIRR Data in the Arctic. IEEE Geosci. Remote Sens. Lett. 13, 292-296. doi: 10.1109/LGRS.2015.2511184

Wentz, F. J., Gentemann, C., Smith, D., and Chelton, D. (2000). Satellite measurements of sea surface temperature through clouds. Science 288, 847-850. doi: 10.1126/science.288.5467.847

While, J., Mao, C., Martin, M. J., Roberts-Jones, J., Sykes, P. A., Good, S. A., et al. (2017). An operational analysis system for the global diurnal cycle of sea surface temperature: implementation and validation. Q. J. R. Meteorol. Soc. 143, 1787-1803. doi: 10.1002/qj.3036 
Wick, G. A., Bates, J. J., and Scott, D. J. (2002). Satellite and skin-layer effects on the accuracy of sea surface temperature measurements from the GOES satellites. J. Atmos. Ocean. Technol. 19, 1834-1848. doi: 10.1175/1520-0426(2002) $019<1834$ :sasleo $>2.0$. co; 2

Williams, K. D., Harris, C. M., Bodas-Salcedo, A., Camp, J., Comer, R. E., Copsey, D., et al. (2015). The met office global coupled model 2.0 (GC2) configuration. Geosci. Model. Dev. 8, 1509-1524. doi: 10.5194/gmd-8-1509-2015

Wimmer, W., and Robinson, I. S. (2016). The ISAR instrument uncertainty model. J. Atmos. Ocean. Technol. 33, 2415-2433. doi: 10.1175/JTECH-D-16-0096.1

Wimmer, W., Robinson, I. S., and Donlon, C. J. (2012). Long-term validation of AATSR SST data products using shipborne radiometry in the Bay of Biscay and English Channel. Remote Sens. Environ. 116, 17-31. doi: 10.1016/j.rse.2011. 03.022

Woodson, C. B., and Litvin, S. Y. (2015). Ocean fronts drive marine fishery production and biogeochemical cycling. Proc. Natl. Acad. Sci. U.S.A. 112, 1710-1715. doi: 10.1073/pnas. 1417143112

Woolway, R. I., and Merchant, C. J. (2018). Intralake heterogeneity of thermal responses to climate change: a study of large northern hemisphere lakes. J. Geophys. Res. Atmos. 123, 3087-3098. doi: 10.1002/2017JD027661

$\mathrm{Xu}, \mathrm{F}$., and Ignatov, A. (2014). In situ SST Quality Monitor (iQuam). J. Atmos. Ocean. Tech. 31, 164-180. doi: 10.1175/JTECH-D-13-00121.1

$\mathrm{Xu}, \mathrm{F}$., and Ignatov, A. (2016). Error characterization in $i$ Quam SSTs using triple collocations with satellite measurements. Geophys. Res. Lett. 43, 10826-10834. doi: 10.1002/2016GL070287

Yang, A., Zhong, B., Wu, S., and Liu, Q. (2017). Evaluation on radiometric capability of chinese optical satellite sensors. Sensors 17:204. doi: 10.3390/ s17010204

Yang, J., Zhang, Z., Wei, C., Lu, F., and Guo, Q. (2017). Introducing the new generation of chinese geostationary weather satellites, Fengyun-4. BAMS. Bull. Am. Meteorol. Soc. 98, 1637-1658. doi: 10.1175/BAMS-Dbams-d-160065.1
Zeng, X., and Beljaars, A. (2005). A prognostic scheme of sea surface skin temperature for modeling and data assimilation. Geophys. Res. Lett. 32:L14605. doi: 10.1029/2005GL023030

Zhang, H., Beggs, H., Majewski, L., Wang, X. H., and Kiss, A. (2016a). Investigating sea surface temperature diurnal variation over the tropical warm pool using MTSAT-1R data. Remote Sens. Environ. 183, 1-12. doi: 10.1016/j.rse.2016. 05.002

Zhang, H., Beggs, H., Wang, X. H., Kiss, A. E., and Griffin, C. (2016b). Seasonal patterns of SST diurnal variation over the Tropical Warm Pool region. J. Geophys. Res. Oceans 121, 8077-8094. doi: 10.1002/2016jc 012210

Zhang, H., Beggs, H., Merchant, C. J., Wang, X. H., Majewski, L., Kiss, A. E., et al. (2018). Comparison of SST diurnal variation models over the tropical warm pool region. J. Geophys. Res. Oceans 123, 3467-3488. doi: 10.1029/2017jc013517

Zhao, Y., and He, R. (2012). Cloud-Free sea surface temperature and colour reconstruction for the gulf of Mexico: 2003-2009. Remote Sens. Lett. 3, 697-706. doi: 10.1080/01431161.2012.666638

Conflict of Interest Statement: The authors declare that the research was conducted in the absence of any commercial or financial relationships that could be construed as a potential conflict of interest.

Copyright (C) 2019 O'Carroll, Armstrong, Beggs, Bouali, Casey, Corlett, Dash, Donlon, Gentemann, Høyer, Ignatov, Kabobah, Kachi, Kurihara, Karagali, Maturi, Merchant, Marullo, Minnett, Pennybacker, Ramakrishnan, Ramsankaran, Santoleri, Sunder, Saux Picart, Vázquez-Cuervo and Wimmer. This is an open-access article distributed under the terms of the Creative Commons Attribution License (CC BY). The use, distribution or reproduction in other forums is permitted, provided the original author(s) and the copyright owner(s) are credited and that the original publication in this journal is cited, in accordance with accepted academic practice. No use, distribution or reproduction is permitted which does not comply with these terms. 


\section{OPEN ACCESS}

Edited by:

Maria Snoussi,

Mohammed V University, Morocco

Reviewed by:

Patrick Heimbach,

University of Texas at Austin,

United States

Ru Chen,

University of California, Los Angeles,

United States

*Correspondence: Benoit Meyssignac benoit.meyssignac@

legos.obs-mip.fr

Specialty section:

This article was submitted to

Ocean Observation,

a section of the journal

Frontiers in Marine Science

Received: 08 November 2018

Accepted: 05 July 2019

Published: 20 August 2019

Citation:

Meyssignac B, Boyer T, Zhao Z,

Hakuba MZ, Landerer FW,

Stammer D, Köhl A, Kato S,

L'Ecuyer T, Ablain M, Abraham JP,

Blazquez A, Cazenave A, Church JA,

Cowley R, Cheng L, Domingues CM,

Giglio D, Gouretski V, Ishii M,

Johnson GC, Killick RE, Legler D,

Llovel W, Lyman J, Palmer MD,

Piotrowicz S, Purkey SG,

Roemmich D, Roca R, Savita A,

von Schuckmann K, Speich S,

Stephens G, Wang G, Wijfels SE and Zilberman N (2019) Measuring Global

Ocean Heat Content to Estimate the Earth Energy Imbalance.

Front. Mar. Sci. 6:432.

doi: 10.3389/fmars.2019.00432

\section{Measuring Global Ocean Heat Content to Estimate the Earth Energy Imbalance}

Benoit Meyssignac ${ }^{1 *}$, Tim Boyer2, Zhongxiang Zhao ${ }^{3}$, Maria Z. Hakuba ${ }^{4,5}$, Felix W. Landerer ${ }^{4}$, Detlef Stammer ${ }^{6}$, Armin Köh/6, Seiji Kato ${ }^{7}$, Tristan L'Ecuyer ${ }^{8}$, Michael Ablain ${ }^{9}$, John Patrick Abraham ${ }^{10}$, Alejandro Blazquez ${ }^{1}$, Anny Cazenave', John A. Church ${ }^{11}$, Rebecca Cowley ${ }^{12}$, Lijing Cheng ${ }^{13}$, Catia M. Domingues ${ }^{14,15,16}$, Donata Giglio ${ }^{17}$, Viktor Gouretski ${ }^{18}$, Masayoshi Ishii ${ }^{19}$, Gregory C. Johnson ${ }^{20}$, Rachel E. Killick ${ }^{21}$, David Legler ${ }^{22}$, William Llovel', John Lyman ${ }^{20,23,}$ Matthew Dudley Palmer ${ }^{21}$, Steve Piotrowicz ${ }^{22}$, Sarah G. Purkey ${ }^{24}$, Dean Roemmich ${ }^{17}$, Rémy Roca', Abhishek Savita 14,16, Karina von Schuckmann ${ }^{25}$, Sabrina Speich ${ }^{26}$, Graeme Stephens ${ }^{4}$, Gongjie Wang ${ }^{27}$, Susan Elisabeth Wijffels ${ }^{28}$ and Nathalie Zilberman ${ }^{17}$

${ }^{1}$ LEGOS, CNES, CNRS, UPS, IRD, Université de Toulouse, Toulouse, France, ${ }^{2}$ NOAA National Centers for Environmental Information, Silver Spring, MD, United States, ${ }^{3}$ Applied Physics Laboratory, University of Washington, Seattle, WA, United States, ${ }^{4}$ Jet Propulsion Laboratory, California Institute of Technology, Pasadena, CA, United States, ${ }^{5}$ Department of Atmospheric Science, Colorado State University, Fort Collins, CO, United States, ${ }^{6}$ Centrum für Erdsystemforschung und Nachhaltigkeit, Universität Hamburg, Hamburg, Germany, ${ }^{7}$ NASA Langley Research Center, Hampton, VA, United States, ${ }^{8}$ Department of Atmospheric and Oceanic Sciences, University of Wisconsin-Madison, Madison, WI, United States, ${ }^{9}$ Collecte Localisation Satellite, Ramonville-Saint-Agne, France, ${ }^{10}$ University of St. Thomas, St. Paul, MN, United States, ${ }^{11}$ Climate Change Research Centre, University of New South Wales, Sydney, NSW, Australia, ${ }^{12}$ Climate Science Centre, Commonwealth Scientific and Industrial Research Organisation, Hobart, TAS, Australia, ${ }^{13}$ International Center for Climate and Environment Sciences, Institute of Atmospheric Physics, Chinese Academy of Sciences, Beijing, China, ${ }^{14}$ Institute for Marine and Antarctic Studies, University of Tasmania, Hobart, TAS, Australia, ${ }^{15}$ Antarctic Climate and Ecosystems Cooperative Research Centre, Hobart, TAS, Australia, ${ }^{16}$ Centre of Excellence for Climate System Science, Australian Research Council, Hobart, TAS, Australia, ${ }^{17}$ Department of Atmospheric and Oceanic Sciences, University of Colorado Boulder, Boulder, CO, United States, ${ }^{18}$ Center for Earth System Research and Sustainability, CliSAP, Integrated Climate Data Center, University of Hamburg, Hamburg, Germany, ${ }^{19}$ Meteorological Research Institute, Japan Meteorological Agency, Tsukuba, Japan, ${ }^{20}$ NOAA Pacific Marine Environmental Laboratory, Seattle, WA, United States, ${ }^{21}$ Met Office Hadley Centre, Exeter, United Kingdom, ${ }^{22}$ NOAA Climate Program Office, Silver Spring, MD, United States, ${ }^{23}$ Joint Institute for Marine and Atmospheric Research, University of Hawai'i at Mānoa, Honolulu, HI, United States, ${ }^{24}$ Scripps Institution of Oceanography, University of California, San Diego, La Jolla, CA, United States, ${ }^{25}$ Mercator Ocean International, Ramonville-Saint-Agne, France, ${ }^{26}$ Laboratoire de Météorologie Dynamique, Ecole Normale Supérieure, Paris, France, ${ }^{27}$ College of Meteorology and Oceanography, National University of Defense Technology, Nanjing, China, ${ }^{28}$ Woods Hole Oceanographic Institution, Woods Hole, MA, United States

The energy radiated by the Earth toward space does not compensate the incoming radiation from the Sun leading to a small positive energy imbalance at the top of the atmosphere $\left(0.4-1 \mathrm{Wm}^{-2}\right)$. This imbalance is coined Earth's Energy Imbalance (EEI). It is mostly caused by anthropogenic greenhouse gas emissions and is driving the current warming of the planet. Precise monitoring of $\mathrm{EEl}$ is critical to assess the current status of climate change and the future evolution of climate. But the monitoring of EEl is challenging as EEI is two orders of magnitude smaller than the radiation fluxes in and out of the Earth system. Over 93\% of the excess energy that is gained by the Earth in response to the positive EEl accumulates into the ocean in the form of heat. This accumulation of heat can be tracked with the ocean observing system such that today, the monitoring of Ocean Heat Content $(\mathrm{OHC})$ and its long-term change provide the most 
efficient approach to estimate EEI. In this community paper we review the current four state-of-the-art methods to estimate global $\mathrm{OHC}$ changes and evaluate their relevance to derive EEl estimates on different time scales. These four methods make use of: (1) direct observations of in situ temperature; (2) satellite-based measurements of the ocean surface net heat fluxes; (3) satellite-based estimates of the thermal expansion of the ocean and (4) ocean reanalyses that assimilate observations from both satellite and in situ instruments. For each method we review the potential and the uncertainty of the method to estimate global $\mathrm{OHC}$ changes. We also analyze gaps in the current capability of each method and identify ways of progress for the future to fulfill the requirements of EEl monitoring. Achieving the observation of EEI with sufficient accuracy will depend on merging the remote sensing techniques with in situ measurements of key variables as an integral part of the Ocean Observing System.

Keywords: ocean heat content, sea level, ocean mass, ocean surface fluxes, ARGO, altimetry, GRACE, Earth Energy Imbalance

\section{INTRODUCTION}

Estimating and analyzing the Earth Energy Imbalance (EEI) is essential for understanding the evolution of the Earth's climate. This is possible only through a careful computation and monitoring of the climate energy budget. The climate system exchanges energy with outer space at the top of the atmosphere (TOA) (through radiation) and with solid Earth at the Earth crust surface (essentially through geothermal flux). If the climate system were free from external perturbations and internal variability during millennia, then the climate energy budget would be in a steady state in which the net TOA radiation budget compensates the geothermal flux of $+0.08 \mathrm{Wm}^{-2}$ (Davies and Davies, 2010). But the climate system is not free from external perturbations and from internal variability. Although the geothermal flux does not generate any perturbations at interannual to millenia time scales (because it varies only at geological time scales), other external forcing from natural origin (such as the sun radiation, the volcanic activity) or anthropogenic origin (such as Greenhouse Gas emissions -GHG-) perturb the system. These perturbations generate anomalies in the net TOA radiation budget. In response, the climate system adjusts toward a new steady state with zero anomalies in the net TOA radiation budget. The time of adjustment depends on the type of perturbation and on the internal climate feedbacks that the perturbation triggers. It can last from a few days (fast feedbacks such as atmospheric temperature, clouds and moisture feedback) to several tens of thousands of years (slow feedback such as ice sheet and vegetation feedback).

At daily to multicentennial time scales, the climate system is constantly excited by internal variability and external forcing such that it actually never reaches any steady state with zero anomalies in the net TOA radiation budget. Thus, at each moment, there is an imbalance at TOA between the anomaly in incoming solar radiation and the anomaly in outgoing long wave radiation. This imbalance is called the EEI. EEI characterizes the energy state of the climate system. It results from the integrated response of the climate system to past and present internal and external perturbations.

From days to interannual time scales, EEI variations are dominated by the effects of internal climate modes of variability such as the El Niño Southern Oscillation (Loeb et al., 2018a). Primary causes for variability on decadal and longer time scales are changes in solar irradiance, large volcanic eruptions and natural variations in GHG concentrations (Hansen et al., 2011; von Schuckmann et al., 2016). Since the beginning of the industrial era, human activities caused GHG and aerosol emissions as well as land use changes that perturb EEI on decadal to millennial time scales (Hartmann et al., 2013).

Integrated over time EEI provides an estimate of the energy that is stored or released to space by the climate system in its effort to relax toward the TOA steady state. Because anthropogenic activities have been the dominant cause for a positive EEI (0.4$1 \mathrm{Wm}^{-2}$ ) over the last decades (Hansen et al., 2011; Trenberth et al., 2014), EEI represents a measure of the excess of energy that is stored in the climate system as a response to anthropogenic forcing (Trenberth et al., 2014; von Schuckmann et al., 2016). As such, measuring EEI provides a mean to monitor and understand the anthropogenic perturbation of the energy flows (and water flows) in the climate system.

Measuring EEI is difficult because EEI is a globally integrated variable whose magnitude and variations are small (of the order of $1 \mathrm{Wm}^{-2}$, von Schuckmann et al., 2016) compared to the amount of energy entering and leaving the climate system (e.g., $\sim 340 \mathrm{Wm}^{-2}$ for solar irradiance, L'Ecuyer et al., 2015). Separating EEI variations generated by anthropogenic GHG emissions from other sources of EEI variations is even more difficult because the EEI response to GHG emissions is a small long term variation (of a few tenth of $\mathrm{Wm}^{-2}$ over decades to centuries) buried in the monthly to interannual noise generated by the natural variability. The typical amplitude of EEI variations at monthly to interannual time scales generated by the natural variability is on the order of $\pm 2 \mathrm{Wm}^{-2}$ (Loeb et al., 2018b). Recent estimates of EEI on decadal time scales suggest that the EEI response to anthropogenic GHG and aerosol emissions is 
0.4-1 Wm $\mathrm{Wm}^{-2}$ (Llovel et al., 2014; Trenberth et al., 2014; Wild et al., 2014; Smith et al., 2015; von Schuckmann et al., 2016). It implies that an accuracy of $<0.3 \mathrm{Wm}^{-2}$ at decadal time scales is necessary to evaluate the long term mean EEI associated with anthropogenic forcing. Ideally an accuracy of $<0.1 \mathrm{Wm}^{-2}$ at decadal time scales is desirable if we want to monitor future changes in EEI associated with GHG mitigation policies (see for example the difference in 21st century EEI between the 1.5 and $2^{\circ} \mathrm{C}$ scenario from Rogelj et al., 2018). A similar level of accuracy of $<0.1 \mathrm{Wm}^{-2}$ at interannual time scales would also help in analyzing and understanding the response of EEI to phenomena such as the so-called "climate change hiatus" (Allan et al., 2014; Hedemann et al., 2017).

To date there are four approaches to estimate EEI. First, EEI can be directly measured by estimating the global budget of incoming and outgoing radiation at TOA. The current implementation of this method with the Clouds and the Earth's Radiant Energy System (CERES) instruments allows accurate determination of the time variations of EEI (with an uncertainty of $\pm 0.17 \mathrm{Wm}^{-2}$ at monthly time scales, Loeb et al., 2018a). But the accuracy on the absolute global mean value of EEI is limited within $\pm 4 \mathrm{Wm}^{-2}$ mainly due to instrument calibration uncertainty (Loeb et al., 2018a). Second, EEI can be indirectly measured by estimating the surface energy budget (on both land and ocean). The current implementation of this method using surface energy fluxes from either observations or reanalyses has large uncertainties. The surface energy budget can be closed with an uncertainty of up to $\pm 15 \mathrm{Wm}^{-2}$ at the global scale (e.g., L'Ecuyer et al., 2015). Third, EEI can be estimated with climate models by calculating the net radiation budget at TOA due to different radiative forcing and the associated radiative responses of the climate system. Differences among climate model estimates do not allow calculation of EEI with an uncertainty below $\pm 0.21 \mathrm{Wm}^{-2}$ at decadal time scales (5-95\% CL from Smith et al., 2015). This is a lower bound estimated from the spread among climate model simulations. It does not take into account any known systematic biases in climate model simulations.

The fourth approach to estimate EEI is indirect as well and consists of taking an inventory of the energy stored in different climate system reservoirs and estimating their changes with time. To date, this is the most accurate method and yields a global mean EEI at 0.4-1 $\mathrm{Wm}^{-2}$ over 2005-2015 (e.g., Johnson et al., 2016; von Schuckmann et al., 2016; Hakuba et al., 2018). There are four reservoirs of energy in the climate system: the atmosphere, the land, the cryosphere and the ocean. In each of these reservoirs the stored energy takes different forms: internal and latent heat energy, potential energy and kinetic energy. At large scales, variations in internal and latent heat energy dominate largely over the variations in other forms of energy (Trenberth et al., 2002; Trenberth and Stepaniak, 2003), such that EEI can be estimated by an inventory of heat content changes in the different reservoirs. Among all reservoirs, the ocean concentrates the vast majority of energy uptake $(\sim 93 \%)$ associated with EEI (Trenberth and Fasullo, 2016). For this reason the global Ocean Heat Content $(\mathrm{OHC})$ places a strong constraint on the absolute magnitude of EEI and its uncertainty. Likewise, the accuracy of the EEI estimate through the inventory method essentially relies on the accuracy of the estimated change in global mean OHC.

This paper is a community effort that is made in the framework of the Oceanobs' 19 initiative. It reviews the potential of the current ocean observing system to monitor EEI, identifies gaps in the observing systems' capabilities and proposes ways forward to improve the observation of EEI in the future. We mainly consider the inventory method because it is by far the most accurate method to estimate EEI, and we focus on estimates of global OHC, because the oceans represent the main sink for heat uptake. This paper does not address any scientific questions associated with $\mathrm{OHC}$ other than the estimation of EEI. Other scientific questions associated with $\mathrm{OHC}$ are addressed by the Oceanobs'19 community white paper from Palmer et al. (2019).

In total, we identify four methods to estimate global $\mathrm{OHC}$ changes that make use of: (1) direct measurement of in situ temperature (2) the measurement of the net ocean surface heat fluxes from space (3) the measurement of the thermal expansion of the ocean from space and (4) ocean reanalyses that assimilate observations from both satellite and in situ instruments. We review the potential and the uncertainty of each method to estimate global OHC changes and EEI within required accuracy (see "Estimating the Ocean Temperature from in situ Observations," "Estimating the Ocean Surface Net Flux From Space Observations," Estimating the Ocean Thermal Expansion From Space Observations," and "Estimating the Global OHC From Ocean Reanalyses"); and suggest ways of progress to fulfill the requirements on the EEI observation (minimum accuracy of $\pm 0.3 \mathrm{Wm}^{-2}$ and desired accuracy of $\pm 0.1 \mathrm{Wm}^{-2}$, see Comparison of Global Mean Sea Level Budget, Ocean Heat). Based on this analysis we define a set of priorities for the development of an optimal and integrated (satellite and in situ) ocean observing system for EEI monitoring today and in the future (see Conclusion, Synthesis and Perspective).

Here all estimates of the $\mathrm{OHC}$ changes, are given in $\mathrm{Wm}^{-2}$ relative to the total area of the Earth at the top of the atmosphere, unless stated otherwise. All uncertainties are given at the 5-95\% confidence level (CL) unless stated otherwise.

\section{PAST AND CONTEMPORARY OBSERVING SYSTEMS FOR GLOBAL OHC}

Past and contemporary observing systems for the evaluation of global OHC can be separated into three periods (Figure 1). The first is linked to historical shipboard in situ ocean temperature measurements with sampling biased to the northern hemisphere, coastal regions and hemispheric summer, particularly in high latitudes (e.g., Abraham et al., 2013). In situ ocean measurements are available from the early 19th century, but larger scale sampling of the upper 300 and $700 \mathrm{~m}$ only started around 1960 and 1970 respectively, although with noticeable spatio-temporal data gaps and instrumental biases (Lyman and Johnson, 2008, 2014; Cowley et al., 2013; Rhein et al., 2013; Boyer et al., 2016; Cheng et al., 2016a). 


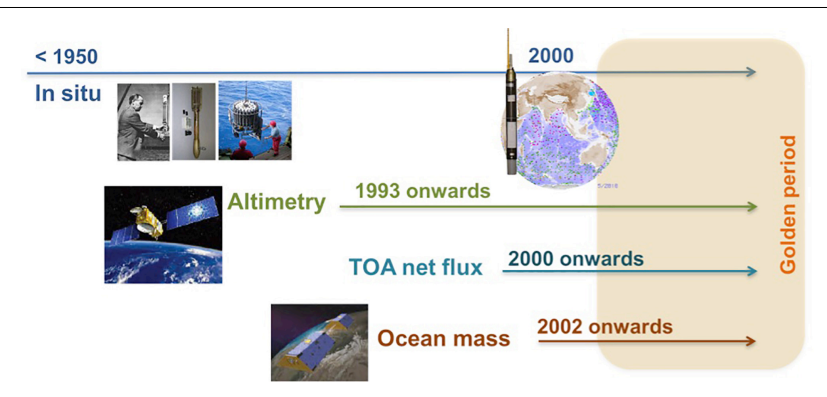

FIGURE 1 | Schematic representation of the evolution of in situ and remote sensing observing systems for the evaluation of global ocean heat content. The shaded area indicates the so-called "golden period" of Earth system measurements for global ocean heat content estimates, which starts circa 2005 and is characterized by initially sparse but steadily improving global coverage of in situ temperature measurements through the Argo program.

The second period, which starts with satellite altimetry in 1993, includes more complementary observing systems, from remote sensing techniques, fixed stations, modern shipboard measurements and autonomous in situ platforms ${ }^{1}$. This era also saw the development of reanalysis systems, which assimilate in situ and satellite observations into numerical models to provide a four-dimensional perspective of the global ocean (Balmaseda et al., 2013; Palmer et al., 2017; von Schuckmann et al., 2018). Storto et al. (2019) outline advances and current challenges for ocean reanalyses.

The third period, an ongoing "golden era" for $\mathrm{OHC}$, is characterized by a surge in temperature measurements with near global ocean data coverage for the upper $2000 \mathrm{~m}$, mainly from Argo profiling floats (Riser et al., 2016), and the availability of information for Earth energy/sea level budget constraint evaluations (Loeb et al., 2012; Llovel et al., 2014; Trenberth and Fasullo, 2016; von Schuckmann et al., 2016; Chambers et al., 2017; Dieng et al., 2017).

\section{ESTIMATING THE OCEAN TEMPERATURE FROM IN SITU OBSERVATIONS}

\section{The in situ Observing System}

Accurate reconstruction of $\mathrm{OHC}$ requires subsurface measurements that are sustained over time (decades and longer) and sufficiently widespread to adequately capture spatio-temporal changes. Evolving changes to instrumentation, geographic range and depth coverage (Figure 2) can introduce uncertainty into the determination of long-term global trends and regional patterns (Wunsch, 2016).

The primary modern instruments comprising the $\mathrm{OHC}$ observing system since the 1940s (Figure 2) are Mechanical Bathythermographs (MBTs), Expendable Bathythermographs (XBTs), Nansen/Nisken bottles, and Conductivity-TemperatureDepth (CTD) instruments. Argo floats, gliders, ice-drifters,

${ }^{1}$ http://www.goosocean.org instrumented pinnipeds, and moored buoys often carry CTDs. Nansen or Niskin bottle hydrocasts with attached reversing thermometers and the CTD casts represent together an important portion of the global archive as they are superior in precision and provide more full-depth temperature profiles compared to other instrumentation types. Several old expeditions provided observations suitable for the estimation of long-term temperature changes along specific tracks (Roemmich et al., 2012; Gouretski et al., 2013) relative to the contemporary ocean thermal state.

Together, MBT and XBT data contribute $36 \%$ of the total ocean temperature profile data available to 2013 ; there are $\sim 2.4$ million MBT (1931-2004) and 2.5 million XBT profiles (1960present) (Boyer et al., 2013). MBTs typically go down to $\sim 125-$ $250 \mathrm{~m}$ and were widely deployed from 1938 to the early 1960s (Figure 2). Shallow XBTs (e.g., T4/T6) reach $450 \mathrm{~m}$, and were widely deployed during the 1970s 1980s (Figure 2). On the other hand, deep XBTs (e.g., T7/DB) provide data to $800 \mathrm{~m}$, and were widely used during the 1990s and early 2000s (Figure 2). These devices have typically been deployed from naval and research vessels and, more recently, from merchant ships of opportunity for XBTs.

The Argo Program, designed in 1998 (Argo Science Team, 1999), was transformational for OHC estimation because it enabled high-quality profile CTD data to be obtained nearly anywhere in the ocean without a human monitor present, thus reducing or eliminating coverage biases of ship-based systems. Argo first achieved its initial goal of 3000 profiling floats in November 2007. Its present coverage of about 3800 floats (Figure 3A) is close to the target of 4000, and is beginning to move into marginal seas, seasonally ice-covered regions, and increasing float density in critical areas (Jayne et al., 2017). The data coverage is $>80 \%$ of the global ocean area ( 3 by 3 degree box) after 2007 from depth $0-1200 \mathrm{~m}$ and $>70 \%$ for $1200-2000 \mathrm{~m}$ (Figure 2). Advances in profiling float technology, including bidirectional communications, have increased float lifetime and improved coverage. Argo's near-global uniform coverage has resulted in a dramatic reduction of the uncertainty of global $\mathrm{OHC}$ changes and related ocean thermal expansion estimates (e.g., Domingues et al., 2008; Lyman and Johnson, 2014; Boyer et al., 2016; Cheng et al., 2016b; Johnson et al., 2018; The WCRP Global sea level budget group, 2018).

Other instrument platforms have contributed to temperature profile data used to calculate OHC. The tropical moored buoy array, as well as moored buoys represented by OceanSITES (Figure 3B), have provided temperature measurements at specific depths across the global tropical latitudes and as point sources elsewhere. Gliders, autonomous vehicles more directly controllable than Argo floats with shorter deployment periods, have become a valuable source for CTD data with programs focused on United States coastal areas, Australian waters, and the European Union areas of interest. These platforms have the potential to contribute in a more coordinated fashion, including measurements across boundary currents. Instrumented pinnipeds may be a valuable source of CTD data from seasonal ice-bound waters and elsewhere. 

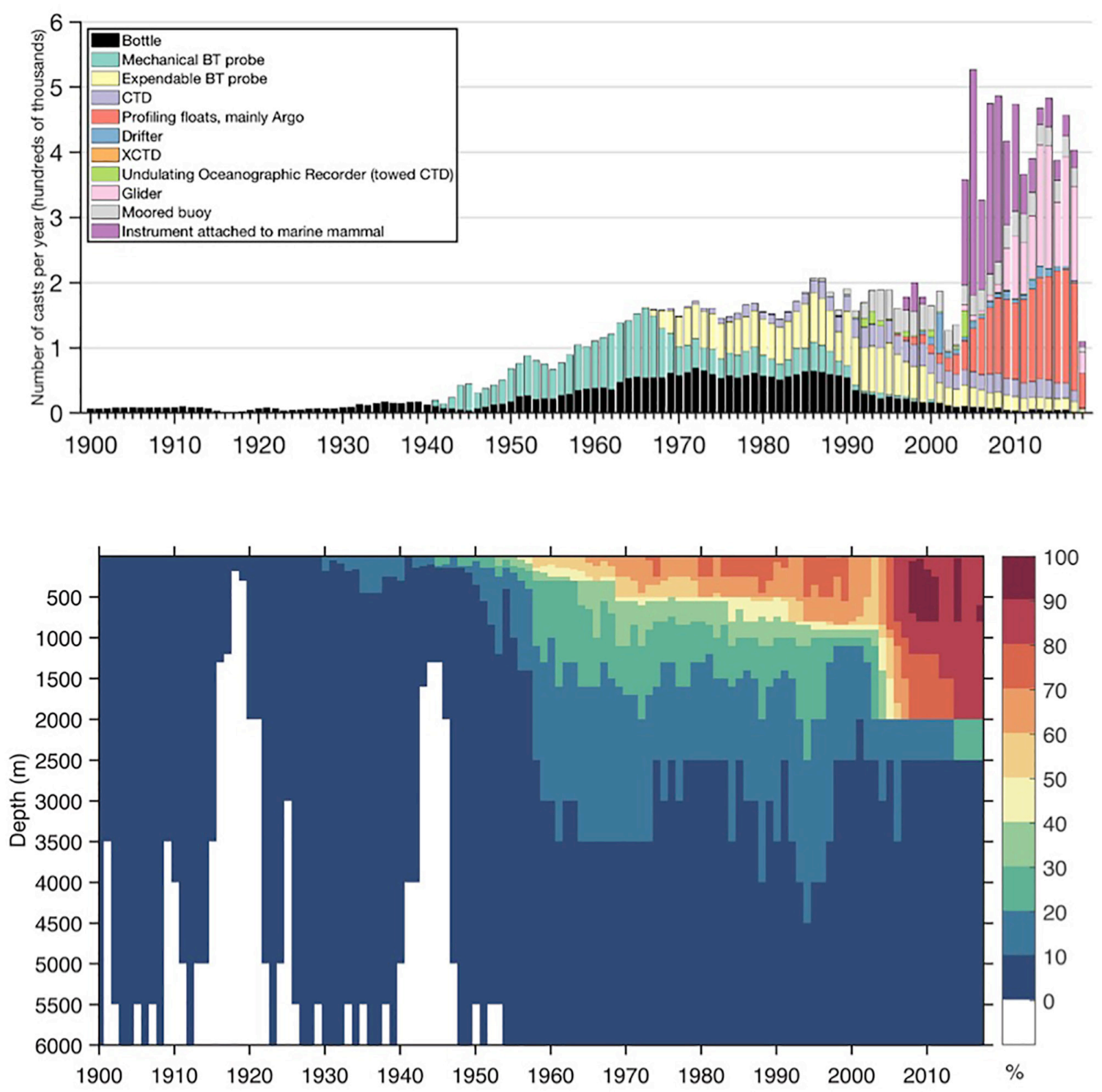

FIGURE 2 | (Upper) Number of subsurface ocean temperature profiles per year by instrument type 1900-2017. [BT, Bathythermograph; CTD, ConductivityTemperature-Depth; XCTD, Expendable CTD]. (Lower) Percentage (\%) of data coverage for $3 \times 3$ boxes over the global ocean area from 5 to $6000 \mathrm{~m}$.

\section{Importance of Data Management}

Effective management of subsurface ocean temperature information is the basis for the dissemination and reproducibility of accurate scientific knowledge of ocean warming and its causes. Effective management is also needed for timely and user-friendly access to data products and services to various community sectors (including scientists, industry, government etc.). Data management starts at the time of data collection and persists throughout the data lifecycle.

Consistent synthesis of the various data sources is crucial to ensure optimal $\mathrm{OHC}$ changes estimates. The optimization process includes quality control $(\mathrm{QC})$ of the available data within the individual expert communities (e.g., the Global Temperature and Salinity Profile [GTSPP], for XBTs) and collectively, as well as pre-processing with any bias corrections that are necessary.

Historical ocean temperature profiles, particularly those outside expert community control such as the Argo Data Management System (ADMS) or outside highly controlled programs such as the World Ocean Circulation Experiment (WOCE) can suffer from inconsistencies in QC that can impact OHC estimation. The International Quality Controlled Oceanographic Database (IQuOD) program (Domingues and Palmer, 2015) ${ }^{2}$ is filling this gap by developing internationally coordinated delayed QC standards which will be implemented in a homogeneous, structured, and fully documented form.

${ }^{2}$ www.iquod.org 


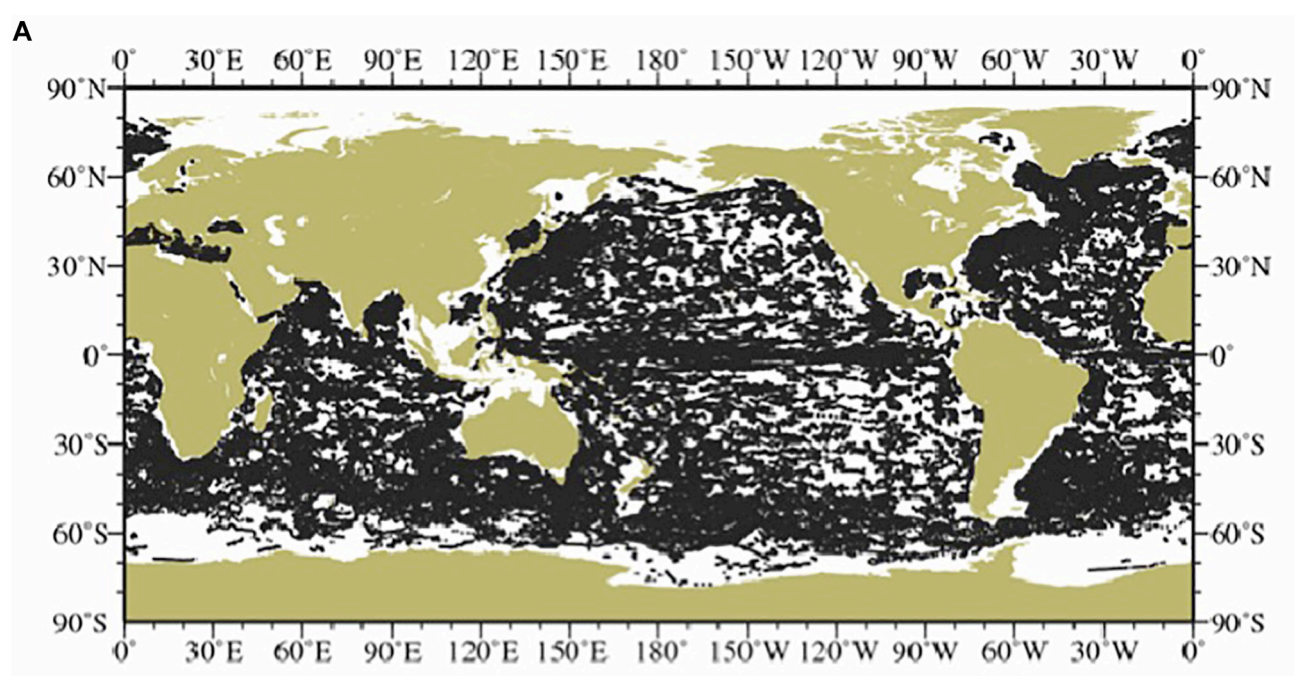

B

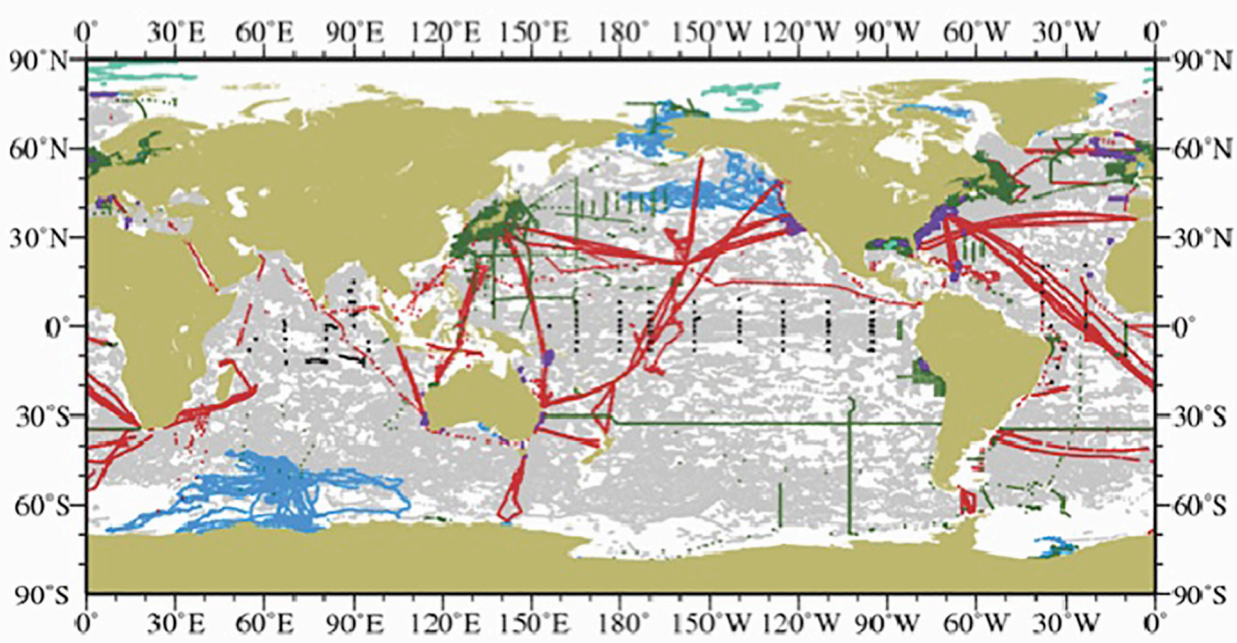

FIGURE 3 | Temperature profile locations in 2017 from (A) Argo floats and (B) ship-based bottle/Conductivity-Temperature-Depth (CTD) casts (dark green), Expendable Bathythermographs (XBT) drops (red), tropical moored buoy daily means (black), glider cycles (purple), instrumented pinniped dives (blue) and ice-tethered profilers (light green) [Argo cycles in background (gray) for comparison].

\section{Calculating OHC Changes From in situ Temperature Profiles and Sources of Uncertainty}

For a given depth layer, $\mathrm{OHC}$ is defined as the volume integral of temperature multiplied by seawater density and the specific heat capacity, with units of Joules:

$$
\mathrm{OHC}=\iiint_{h 2}^{h 1} p C_{p} \Theta d x d y d z
$$

Here, (following TEOS-10; IOC et al.,, 2010) $\rho$ is seawater density, $C_{p}$ is the (constant) specific heat capacity, $\Theta$ is conservative temperature (derived from in situ temperature, absolute salinity, and pressure), and $h_{1}$ and $h_{2}$ are the depth range over which the heat content is computed.

The traditional approach to estimate OHC from ocean temperature profiles involves gridding the available observations and interpolating across data gaps using a statistical mapping method (e.g., Abraham et al., 2013). Prior to the gridding of data, a seasonal climatology is usually subtracted from each profile to convert the observations into temperature anomalies with the annual cycle removed. Temperature anomalies have larger de-correlation length scales than the full temperature field and therefore provide a more useful basis for mapping and interpolation. A reliable mapping method should provide a good estimate of signal and error while minimizing sources of uncertainty.

Ocean heat content trends are sensitive to the choice of statistical model for the mapping, which may include both a least squares fit (e.g., to estimate the annual climatology to be removed from the data) and objective mapping of residuals. For the Argo period, including a climatological trend in the least squares fit results in smaller biases and larger long-term changes in $\mathrm{OHC}$ (Domingues et al., 2008). Objective mapping is the most common 
statistical approach used to map the residuals, although details on how it is implemented differ among groups (e.g., Levitus et al., 2000, 2012; Willis, 2004; Ishii and Kimoto, 2009; Good et al., 2013; Cheng et al., 2014, 2017; Ishii et al., 2017; Kuusela and Stein, 2018). The approach can evolve over time within the same group (e.g., Lyman and Johnson, 2008; Lyman et al., 2010). Modeling the time dimension in objective mapping yields smoother monthto-month transitions and smaller overall uncertainty in $\mathrm{OHC}$ changes. Estimating space-varying decorrelation scales from observations is key to quantifying uncertainty (Kuusela and Stein, 2018). Other approaches to mapping include simple grid box averaging (e.g., Palmer et al., 2007; von Schuckmann and LeTraon, 2011; Gouretski, 2012, 2018) or reduced-space optimal interpolation (Domingues et al., 2008; Church et al., 2011). Boyer et al. (2016) estimates an uncertainty of $\pm 1 \mathrm{Wm}^{-2}$ annually for 1970-2008 due to mapping method differences. Lyman and Johnson (2008), Cheng and Zhu (2014a), and Durack et al. (2014) noted that estimates of OHC trends from many mapping methods are biased, because the mapping methods tend to relax toward the climatological values in the data gaps. As data increase with time, the uncertainty due to mapping is reduced and OHC estimates from different groups show more consistency (Johnson et al., 2018). Nevertheless, understanding of the performance of the mapping methods and improving them are the major step forward to reduce the uncertainty in OHC estimate.

Lyman and Johnson (2014) shows that the observed temperature profiles can be integrated in depth first for $\mathrm{OHC}$ calculation, which reduces the dimensionality of the (mapping) problem, as well as reducing uncertainty due to modeling the vertical dimension. Cheng and Zhu (2014b) show that using different interpolation schemes can lead to small differences in $\mathrm{OHC}$ calculation, because of the insufficient vertical resolution in the old observation records.

In 2007, it was discovered that systematic errors in XBT and MBT data significantly impacted the accuracy of OHC changes (Gouretski and Koltermann, 2007), creating a spurious "hump" in the OHC record during the late 1970 s to the early 1980 s (Bindoff et al., 2013). Since then, multiple methods have been proposed to correct biases in XBT data (Cheng et al., 2016a, 2018). By applying six correction schemes for OHC calculation separately and after calculating the standard deviation among the obtained OHC time series, Boyer et al. (2016) found that the uncertainty in $\mathrm{OHC}$ due to XBT error is $0.5-1.1 \mathrm{Wm}^{-2}$ annually for 1970-2008 and $0.7-1.4 \mathrm{Wm}^{-2}$ annually for 1993-2008, depending on the mapping method. Since 2014, the community has recommended the Cheng et al. (2014) method as the most complete correction for XBT data for calculating $\mathrm{OHC}$ changes; it accounts for all of the known factors that influence XBT error. Consequently, the uncertainty in $\mathrm{OHC}$ changes due to XBT error is expected to be smaller than that shown in Boyer et al. (2016). For example, the mean standard deviation of the best two schemes (Levitus et al., 2009; Cheng et al., 2014) identified in Cheng et al. (2018) is only $0.2 \mathrm{Wm}^{-2}$ annually for the 1970-2004 period (Cheng et al., 2018). XBTs are now a much smaller part of the overall observing system than in the pre-Argo time period, with corresponding smaller uncertainty contribution.
As stated above, in general, OHC changes are computed by using ocean temperature anomalies (residuals) relative to a baseline climatology. In some cases, the selected climatology affects $\mathrm{OHC}$ changes estimates and quality control results (Ishii and Kimoto, 2009; Lyman and Johnson, 2014; Cheng and Zhu, 2015; Boyer et al., 2016; Gouretski, 2018). Several centers adopt objective analysis as a global mapping method, and some types of this method yield temperature values close to the climatology particularly in data-sparse regions. Uncertainty due to baseline climatology ranges $0.2-0.9 \mathrm{Wm}^{-2}$ for the six mapping methods in Boyer et al. (2016). The quality of climatology is not uniform in space because of the spatio-temporal data sampling density and observational biases like those in XBT observations. Temperature biases for XBTs tend to be larger around the thermocline and at greater depths (Gouretski and Reseghetti, 2010; Cheng et al., 2018). Furthermore, the climatological baseline of ocean temperatures has temporally been changing due to global warming. To obtain reliable $\mathrm{OHC}$ changes over 60 years or more is equivalent to understanding acceptable climatological mean fields of ocean temperature before the Argo era. Reconstructing high-quality ocean observations is key to solving this.

In summary, each of the available observational OHC changes estimates is affected to different extents by uncertainties due to specific systematic instrumental adjustments, to baseline climatology from which the anomaly is calculated, and data distribution irregularity (mapping). These errors are not independent, therefore, it is still difficult to fully isolate them and quantify their contributions separately. Further actions and novel methods are needed to tackle this problem.

\section{Present and Future Observational Coverage}

A key to reducing uncertainties in $\mathrm{OHC}$ changes is the flow of high quality observations to researchers making the calculations. Many elements of the Global Ocean Observing System routinely take ocean temperature profiles (Figure 3), including the Argo profiling float program, the XBT network, GO-SHIP, OceanSITES, regular national hydrographic surveys, and the activities of short-term research campaigns (crosslink to Palmer et al., 2010). These observations vary in accuracy and many have been northern-hemisphere focused. A stepchange in our ability to monitor the upper $\mathrm{OHC}$ came with the implementation of the global Argo program (Figure 2). Delivering a profile nominally every $3^{\circ}$ lat. $\times 3^{\circ}$ long every 10 days (Jayne et al., 2017), the nearly global reach and high quality of Argo temperature and pressure observations allow mapping heat content patterns on roughly seasonal and $1000 \mathrm{~km}$ scales in the ice-free open ocean.

Argo's revolutionary impacts on basic research, climate assessment, ocean reanalysis and forecasting, and education are widely recognized. Nevertheless, Argo's future includes major organizational and technical challenges. Major enhancements to Argo, including Deep and Biogeochemical Argo must be implemented with new resources and without eroding Core Argo. The successful ADMS must continue responding to new requirements in ways that do not overwhelm data 
managers. International protocols for floats drifting into Exclusive Economic Zones (EEZs) must be broadened to simplify float deployment in these regions. The supply chain for Argo floats and sensors must be made robust against sole-source failures. The mean lifetime of Argo floats, presently $>4$ years, should be extended further through design improvements, analysis of long-term failure modes, and adoption of improved battery technologies. Argo's leadership model must prove capable of spanning scientific generations while preserving its focus, originality, and collaborative nature. Finally, Argo's role as an inter-dependent element of the integrated observing system requires that all elements thrive together.

Diversity in sensors and platforms are essential to help build confidence in the OHC record, particularly for tracking the small but persistent global ocean warming signals. With its present dependence on one sensor manufacturer, Argo is highly vulnerable to manufacturing errors. GO-SHIP and OceanSITE records, which are post calibrated and of high quality, are essential points of cross-reference for Argo. Satellite altimeter data are also used to identify and remove suspect Argo data. XBT lines give an insight into scales of variability not resolved by Argo, particularly near the margins of the open oceans. In this way, a robust $\mathrm{OHC}$ observing system involves a mixture of platforms to ensure robustness and confirmation of signals observed across networks.

Boundary currents (eastern and western as well as northern and southern currents in closed basin) are not fully represented by Argo as the core floats have a parking depth of $1000 \mathrm{~m}$ and therefore they do not sample waters located in the upper $1500 \mathrm{~m}$ of every continental slope (that represent an important fraction of boundary currents). Also, Argo floats swiftly pass through the energetic regions. Ocean analyses at present have limited capability to identify the mesoscale variability among boundary current (WBC) and Antarctic Circumpolar Current (ACC) regions which could induce an inverse cascade of kinetic energy and affect the large scale low-frequency variability (Penduff et al., 2018). Inter-comparison among three available ocean analyses (EN4, Ishii and IAP dataset) revealed a large spread of OHC change in the $\mathrm{WBC}$ and $\mathrm{ACC}$ regions: $>10 \times 10^{8}$ $\mathrm{J} \mathrm{m}^{-2}$ even during the Argo era (2005-2012), 2-10 times larger than the open ocean regions (Wang et al., 2018). Removal of WBC and ACC regions reduces the spread of global integrated $\mathrm{OHC}$ change estimates in the upper $1500 \mathrm{~m}$ by $13 \%$ during 1976-2012, despite these regions small $(\sim 6 \%)$ portion of the global ocean. Within these regions, the rootmean-squared error (RMSE) of OHC tendency could be larger than $100 \mathrm{Wm}^{-2}$ locally (i.e., with respect to the local surface and not with respect to the global surface at TOA, Wang et al., 2018), which has the same magnitude as the climatology mean net air-sea heat flux (Liang and $\mathrm{Yu}, 2016$ ). The large error among these regions is partially because the calculation of tendency from a noisy time series exacerbates the noise. Therefore, advanced ocean observing systems in the WBC and ACC regions are required to better resolve mesoscale and sub-mesoscale variability and aid in higher resolution ocean analysis. Complementary to Argo, high-resolution XBT casts, gliders under pre-set routines, as well as mooring networks such as the North Pacific Ocean Circulation Experiment (NPOCE) (Wang and $\mathrm{Hu}, 2010$ ) could reduce the uncertainty of $\mathrm{OHC}$ change estimates.

It has always been difficult to obtain subsurface ocean temperature measurements in the Arctic, leading to a dearth of historic and recent data (Zweng et al., 2018). In 2017, there were subsurface temperature data from only three research cruises north of $66^{\circ} \mathrm{N}$ generally available through the World Ocean Database, down from 12 in 2016. There are some Argo floats at high northern latitudes, mainly in the GreenlandIceland-Norwegian seas (GIN) area. Argo floats are more prevalent at southern high latitudes, including some with ice-sensing technology (Riser et al., 2018). The only regular subsurface temperature measurements presently gathered in the high Arctic $\left(>80^{\circ}\right)$ are from the Ice Tethered Profiler (ITP) program (Toole et al., 2011). A rough estimate of OHC difference between the 1955-1964 and 2005-2012 periods using decadal mean temperature fields from the World Ocean Atlas (Locarnini et al., 2013) shows $\sim 4 \%$ of global $\mathrm{OHC}$ change occurred in the Arctic (including GIN Seas and Baffin Bay). Coverage in the Russian Arctic and high Arctic was actually better in the 1955-1964 period. Sustaining the ITP program, purposeful planning of Arctic cruises, better global data exchange, and extending Argo can close the data gaps in the Arctic. Sustaining Southern Ocean Argo, increasing deployment of under-ice Argo floats, and utilization of quality pinniped mounted sensors can help close the data gaps in the Southern Ocean.

It is also difficult to obtain subsurface ocean temperature measurements in EEZs. A $\sim 0.1 \mathrm{Wm}^{-2}$ increase between 19551964 and 2005-2012 (calculated as above for the Arctic) is found for the Tropical Asian Archipelago (TAA) and the Andaman Sea, or slightly less than $2 \%$ of the total $+6.5 \mathrm{Wm}^{-2}$ increase calculated from the same mean fields. A similar rough estimate for non-TAA continental shelf/coastal areas adds another $\sim 1.5 \%$ $\mathrm{OHC}$ change in shallow areas not presently well sampled by Argo. While shelf $\mathrm{OHC}$ changes can be important regionally (e.g., Forsyth et al., 2015; Turner et al., 2017) they constitute only a small percentage of global change. Some semi-enclosed ocean areas such as the Mediterranean and the Gulf of Mexico are sampled by Argo and other systems. Others, such as the Sea of Okhotsk have almost no available data for the last 10 years. Argo extensions and the systematic deployment of gliders can add reliable data collection in marginal seas.

The ocean below $2000 \mathrm{~m}$ has warmed significantly since the 1990s, accounting for $\sim 10 \%$ of the total ocean heat uptake (Purkey and Johnson, 2010; Rhein et al., 2013; Desbruyères et al., 2016). Recent estimates of the deep OHC change are based on decadal repeats of coarse hydrographic sections (Talley et al., 2016) and the only statistically robust deep OHC trends are basin-wide, decadal averages owing to limited data (Figure 2). Nonetheless, a spatially coherent global picture has emerged of an intensified deep warming, originating from deep water formation sites in the Southern Ocean and propagating through the Meridional Overturning Circulation, with a statistically significant contribution to the global ocean heat uptake. 
The global deep (below $2000 \mathrm{~m}$ ) and abyssal (below $4000 \mathrm{~m}$ ) $\mathrm{OHC}$ accumulation rates between the 1992 and 2009 are 0.04 $( \pm 0.05) \mathrm{Wm}^{-2}$ and $0.02( \pm 0.01) \mathrm{Wm}^{-2}$, respectively, and local estimates show large spatial variability (Figure 4; updated from Purkey and Johnson, 2010). The Southern Ocean below $1000 \mathrm{~m}$ has warmed 10 times faster than elsewhere in the deep ocean. While there has been some regional variability, the total deep-ocean warming rate has not changed outside the error bars over the past 3 decades (Lyman and Johnson, 2014; Desbruyères et al., 2016).

Continuous, global, top-to-bottom ocean temperature data are needed to monitor the deep OHC change. Plans are underway to make it possible through the implementation of the new Deep Argo array, capable of sampling the water column down to $6000 \mathrm{~m}$ (Roemmich et al., 2019). The envisioned $5^{\circ}$ lat. $\times 5^{\circ}$ long. $\times 15$-day Deep Argo array with highly accurate temperature and pressure standards $\left(0.001^{\circ} \mathrm{C}\right.$ and 3 dbar, respectively) would decrease errors in decadal deep OHC trends to $\pm 0.006 \mathrm{Wm}^{-2}$, compared to $\pm 0.04 \mathrm{Wm}^{-2}$ uncertainty based on present observing systems (Johnson et al., 2015). Deep Argo will complement the ongoing decadal repeat hydrography (Sloyan et al., 2019) and existing deep moorings (Cronin et al., 2012). In addition, new technologies, including deep-gliders that operate to 6000-m depth, are under development to bridge gaps in deep-ocean temperature observations in boundary regions (Eriksen, 2017).

\section{Time Scale of OHC Estimates}

When estimating the uncertainty in OHC changes on decadal time scales, ideally all sources of uncertainty explained above should be taken into account and combined. This is difficult because the in situ observing system and therefore the different sources of uncertainty change with time and space. Some sources of uncertainty may include spatial and temporal correlation adding to the complexity of the calculation. Different groups have elaborated different strategies to calculate $\mathrm{OHC}$ trend uncertainties. In Table $\mathbf{1}$ we show the most recent estimate of the uncertainty in OHC trends over the last two decades from in situ data and recall the different sources of uncertainty they take into account. Because different groups account for different sources of uncertainty, their total uncertainty estimates differ substantially. However, in general, for uncertainty estimates over recent periods (estimates starting in 1993 or in 2005), the time and space error correlation and the error due to the data distribution in time and space appear to be the most important terms (see Table 1). Here after (e.g., Table 3), we consider the uncertainty estimate based on work by Johnson et al. (2018) for the in situ based estimates of $\mathrm{OHC}$ trends, because it is the most comprehensive estimate considering OHC over the full 0-2000 m ocean column and covering both the altimetry period (1993 onwards) and the Argo period (2005 onwards, see Table 1). We apply a least square method to the average of the four time series used in Johnson et al. (2018) weighted by the square sum of the four associated standard errors in order to estimate the trend. In the trend uncertainty calculation, degrees of freedom are adjusted taking into account the temporal correlation of the residuals following Johnson et al. (2018). This yields an uncertainty of $\pm 0.11 \mathrm{Wm}^{-2}$ for the OHC changes over 2006-2015. The result is given in Table 3.This uncertainty does not take into account the uncertainty due to data distribution, which may amount to $\sim 0.1 \mathrm{Wm}^{-2}$ (see Table 1 ).

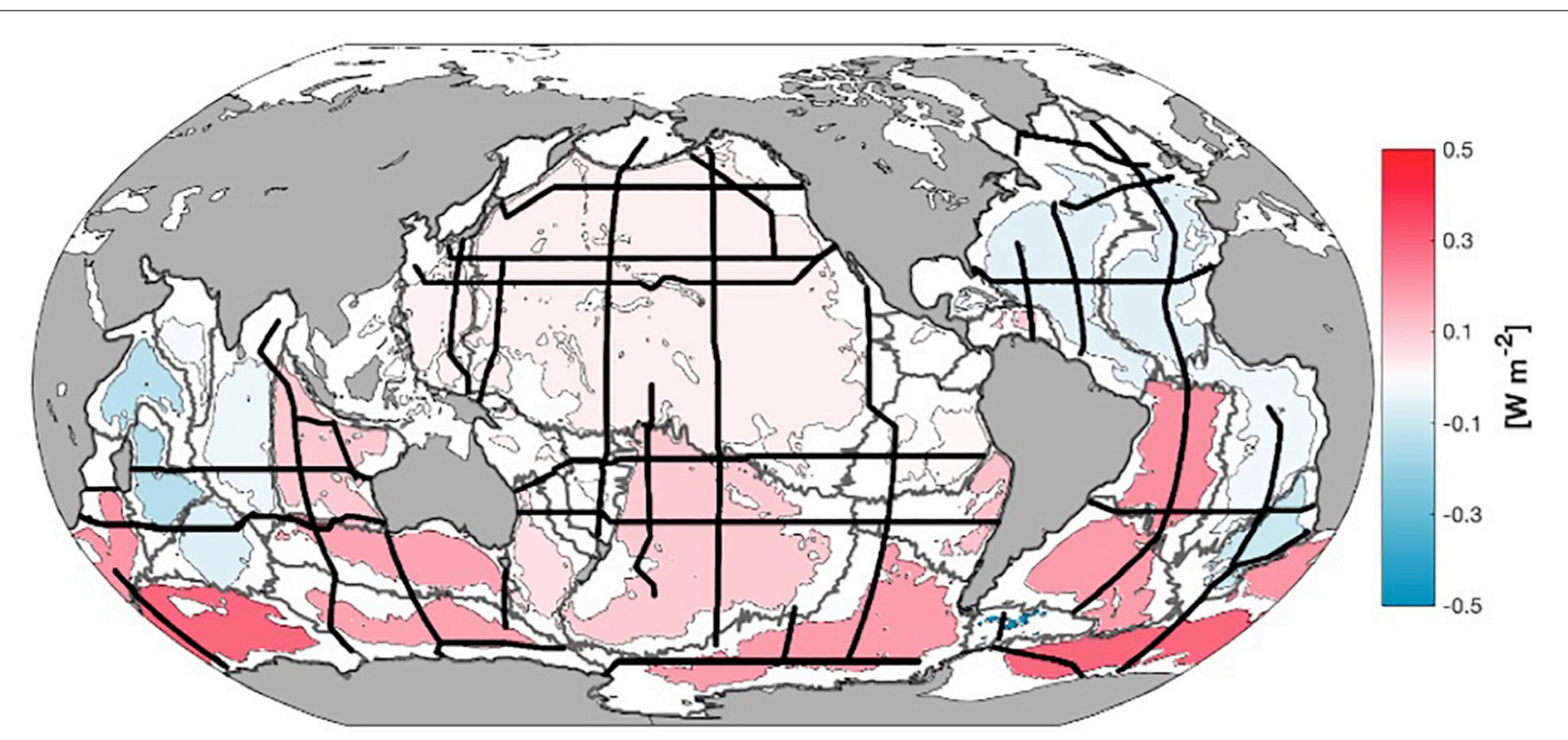

FIGURE 4 | Heat flux (colors) through the $4000 \mathrm{~m}$ isobath (thin gray lines) needed to account for the mean local basin (thick gray lines) abyssal ocean warming estimated from GO-SHIP full depth hydrography sections occupied two or more times (black) between 1981 and 2018. Methods follow Purkey and Johnson (2010) with warming rates updated through 2018. 
TABLE 1 | Ocean heat content change in $\mathrm{Wm}^{-2}$ and associated uncertainties from recent studies based on in situ data. Column 2-8 indicate the different sources of uncertainty accounted for. Column 9 indicates the period of computation. Column 10 indicates the region that is considered.

\begin{tabular}{|c|c|c|c|c|c|c|c|c|c|c|c|}
\hline & $\begin{array}{l}\text { Uncertainty } \\
\text { due to quality } \\
\text { control }\end{array}$ & $\begin{array}{l}\text { Uncertainty } \\
\text { due to errors } \\
\text { in the bias } \\
\text { corrections }\end{array}$ & $\begin{array}{l}\text { Uncertainty } \\
\text { due to the } \\
\text { choice of } \\
\text { climatology }\end{array}$ & $\begin{array}{l}\text { Uncertainty } \\
\text { due to the } \\
\text { mapping } \\
\text { method }\end{array}$ & $\begin{array}{l}\text { Uncertainty due } \\
\text { to the time } \\
\text { correlation of the } \\
\text { measurements }\end{array}$ & $\begin{array}{c}\text { Uncertainty due } \\
\text { to data } \\
\text { distribution }\end{array}$ & $\begin{array}{l}\text { Uncertainty due } \\
\text { to the formal } \\
\text { error of the } \\
\text { optimal } \\
\text { procedure (e.g., } \\
\text { least square) }\end{array}$ & $\begin{array}{l}\text { Time Period } \\
\text { (years) }\end{array}$ & $\begin{array}{l}\text { Depth range } \\
\text { (m) }\end{array}$ & Trend $\mathrm{Wm}^{-2}$ & $\begin{array}{l}\text { Uncertainty } \\
\mathrm{Wm}^{-2}\end{array}$ \\
\hline $\begin{array}{l}\text { Cheng et al. } \\
\text { (2019) }\end{array}$ & No & No & No & No & $\begin{array}{l}\text { Yes but the method } \\
\text { shows problems } \\
\text { when applied over } \\
\text { the period } \\
\text { 2005-2017. } \\
\text { This method is not } \\
\text { mature yet and is } \\
\text { subject to further } \\
\text { adjustments. }\end{array}$ & No & $\begin{array}{l}\text { Yes (ordinary least } \\
\text { square) }\end{array}$ & 2005-2017 & 0-2000 & 0.54 & \pm 0.02 \\
\hline \multirow[t]{3}{*}{$\begin{array}{l}\text { Cheng et al., } \\
2017\end{array}$} & No & No & No & No & No & No & $\begin{array}{l}\text { Yes (ordinary least } \\
\text { square) }\end{array}$ & 1992-2015 & $0-700$ & 0.38 & \pm 0.03 \\
\hline & & & & & & & & & $700-2000$ & 0.23 & \pm 0.02 \\
\hline & & & & & & & & & 0-2000 & $0.61^{b}$ & $\pm 0.036^{\mathrm{c}}$ \\
\hline \multirow[t]{3}{*}{$\begin{array}{l}\text { Johnson et al., } \\
2018\end{array}$} & $\begin{array}{l}\text { No (except in } \\
\text { range of } \\
\text { estimates) }\end{array}$ & $\begin{array}{l}\text { No (except in } \\
\text { range of } \\
\text { estimates) }\end{array}$ & $\begin{array}{l}\text { No (except in } \\
\text { range of } \\
\text { estimates) }\end{array}$ & $\begin{array}{l}\text { No (except in } \\
\text { range of } \\
\text { estimates) }\end{array}$ & $\begin{array}{l}\text { Yes (autocorrelation } \\
\text { analysis) }\end{array}$ & No & $\begin{array}{l}\text { Yes (ordinary least } \\
\text { square) }\end{array}$ & 1993-2017 & $0-700$ & $0.36-0.40$ & $\pm 0.06- \pm 0.18$ \\
\hline & & & & & & & & & $700-2000$ & $0.19-0.35$ & $\pm 0.01- \pm 0.07$ \\
\hline & & & & & & & & & 0-2000 & $0.48-0.70^{b}$ & $\pm 0.06- \pm 0.16^{\mathrm{c}}$ \\
\hline $\begin{array}{l}\text { Lyman and } \\
\text { Johnson, } 2008\end{array}$ & No & No & No & No & No & Yes & No & 2000-2005 & $0-750$ & $\mathrm{n} / \mathrm{a}$ & \pm 0.06 to $\pm 0.18^{\circ}$ \\
\hline $\begin{array}{l}\text { Lyman et al., } \\
2010\end{array}$ & Yes & Yes & Yes & Yes & $\begin{array}{l}\text { Yes (autocorrelation } \\
\text { analysis) }\end{array}$ & No & $\begin{array}{l}\text { Yes (weighted least } \\
\text { square) }\end{array}$ & 1993-2008 & $0-700$ & 0.64 & \pm 0.11 \\
\hline $\begin{array}{l}\text { Johnson et al., } \\
2016\end{array}$ & No & No & No & No & $\begin{array}{l}\text { Yes (autocorrelation } \\
\text { analysis) }\end{array}$ & No & $\begin{array}{l}\text { Yes (ordinary least } \\
\text { squares) }\end{array}$ & 2005-2015 & 0-bottom & 0.68 & \pm 0.10 \\
\hline $\begin{array}{l}\text { Levitus et al., } \\
2009\end{array}$ & No & No & Yes & Yes & No & No & Yes & 2005-2017 & 0-2000 & 0.51 & \pm 0.15 \\
\hline $\begin{array}{l}\text { Palmer et al., } \\
\text { 2007; Palmer } \\
\text { and Brohan, } \\
2011\end{array}$ & No & $\begin{array}{l}\text { No (except in } \\
\text { range of } \\
\text { estimates) }\end{array}$ & $\begin{array}{l}\text { No (except in } \\
\text { range of } \\
\text { estimates) }\end{array}$ & Yes & No & No & No & 1993-2017 & $0-700$ & 0.40 & \pm 0.18 \\
\hline $\begin{array}{l}\text { Domingues } \\
\text { et al., } 2008\end{array}$ & Yes & No & $\begin{array}{l}\text { No but remove } \\
\text { a trend to } \\
\text { reduce biases } \\
\text { from historical } \\
\text { climatologies } \\
\text { (space, time, } \\
\text { location data) }\end{array}$ & Yes & No & No & Yes & 1993-2018 & $0-700$ & 0.64 & \pm 0.03 \\
\hline
\end{tabular}




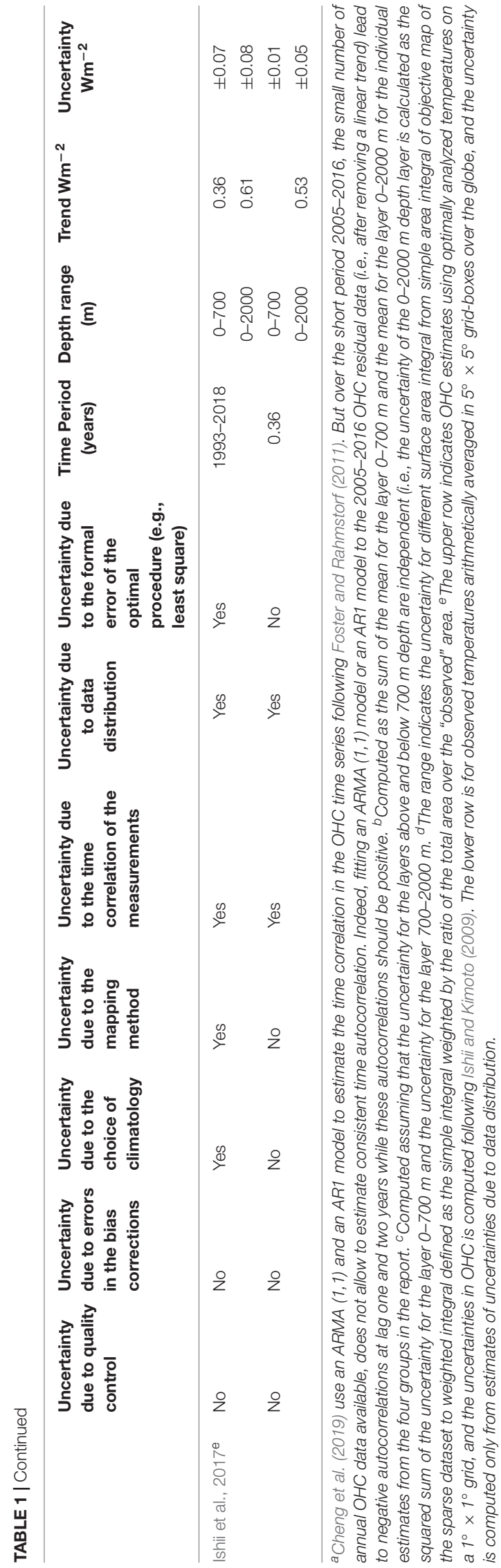

Estimating the uncertainty in $\mathrm{OHC}$ changes at interannual time scales is more challenging because of insufficient spatiotemporal coverage. So far, few studies have provided estimates. Estimates of annual OHC changes for 0-2000 $\mathrm{m}$ have standard errors of $0.3-0.6 \mathrm{Wm}^{-2}$ over the Argo era, and those errors increase substantially for the pre-Argo time period (Johnson et al., 2018, their Figure 2). Thus, while year-to-year variations in global $\mathrm{OHC}$ change during the Argo time period may be well correlated with El Niño indices and TOA radiative imbalance variability, the interannual signal does not quite rise above the uncertainties in the estimates, and monthly estimates from in situ ocean observations alone are much noisier (Johnson and Birnbaum, 2017).

Monthly estimates of global OHC for 0-2000 m exhibit variability several times that of TOA satellite estimates (Johnson and Birnbaum, 2017, their Figure 1B) and are not yet useful for the study of EEI when made from ocean temperature observations only (Trenberth and Fasullo, 2016). Nonetheless, the seasonal cycle is well resolved by observations at monthly time-scales when using data from several years (Roemmich and Gilson, 2009), and even more so now with more than a decade of Argo data ${ }^{3}$.

\section{A New Technique to Monitor Global OHC Changes: Internal Tide Oceanic Tomography}

Review of the Concept, Advantages, and Challenges

A new concept of internal tide oceanic tomography (ITOT) was recently proposed to monitor global OHC changes (Zhao, 2016). ITOT detects OHC changes by measuring travel time changes of long-range internal tides. The underlying principle is that upper ocean warming strengthens ocean stratification and thus increases the propagation speed of internal tides. ITOT is similar to ocean acoustic tomography but that the work waves are internal tidal waves. Acoustic tomography was brought up about 40 years ago to detect ocean temperature changes from travel time changes of acoustic waves (Munk and Worcester, 1976; Munk et al., 1995; Dushaw, 2018; Howe et al., 2019). The two tomographic techniques have the same advantages: they suppress the temperature perturbations caused by mesoscale processes (major error sources in field measurements) and measure basinscale OHC changes (compared to station-wise measurements). Therefore, the tomographic techniques may complement the currently existing in situ ocean profile technique described above (Dushaw, 2018).

ITOT monitors $\mathrm{OHC}$ changes by tracking long-range propagating internal tides. Internal tides are generated in tide-bottom interactions over topographic features. Low-mode internal tides may travel hundreds to thousands of kilometers. Internal tides have $\mathrm{cm}$-scale sea surface height (SSH) fluctuations, which can be detected by satellite altimetry. Figure 5 shows the global mode-1 M2 internal tides from 20 years of satellite altimeter data. The internal tide field has been separated into northbound and southbound components. The separation makes

\footnotetext{
${ }^{3}$ http://sio-argo.ucsd.edu/RG_Climatology.html
} 


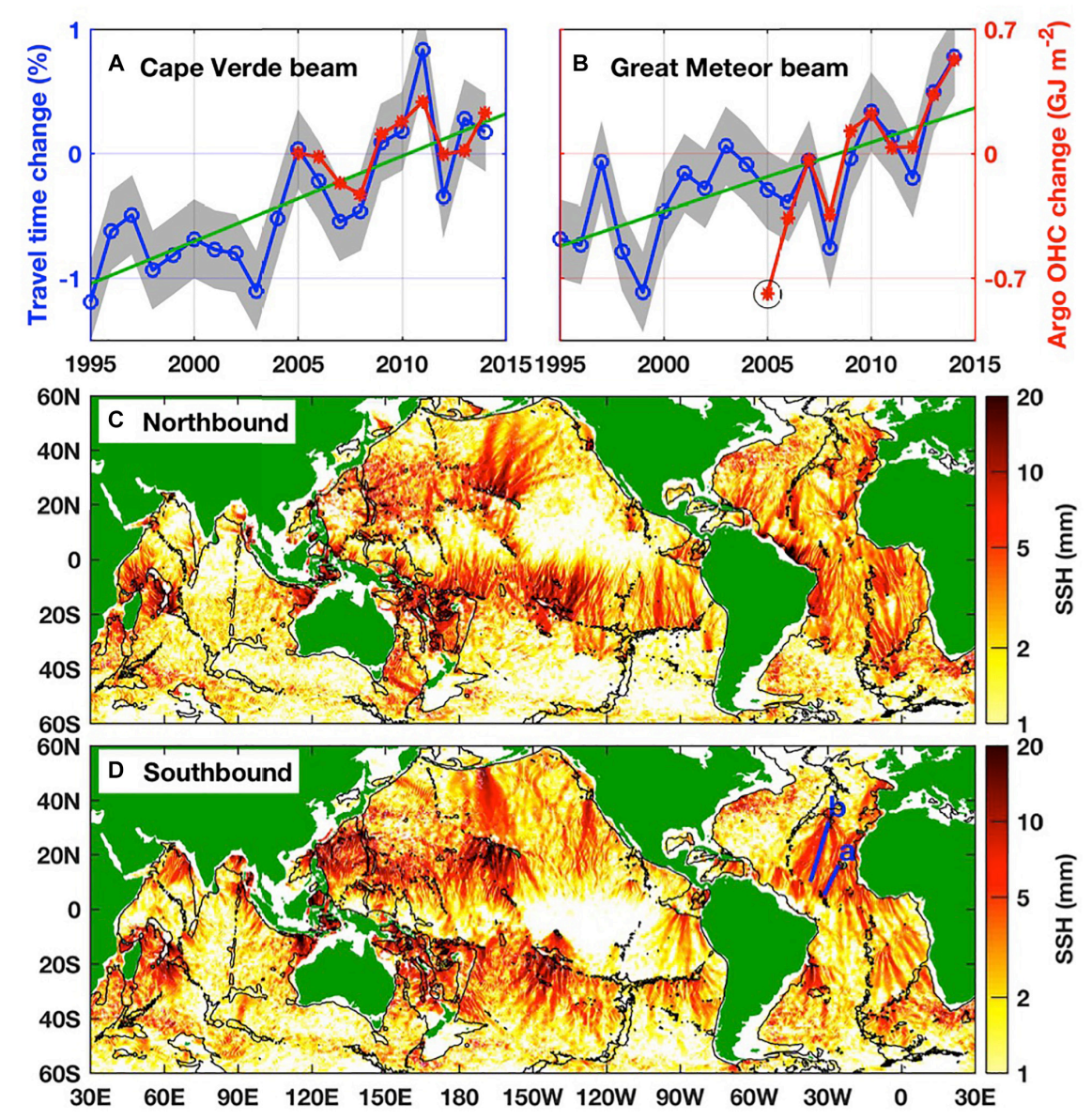

FIGURE 5 | Internal tide oceanic tomography. (A,B) Travel time changes in percentage during 1995-2014 along mode-1 M2 internal tidal beams from (A) Cape Verde Islands and (B) Great Meteor Seamount. See panel (D) for beam locations. Both beams reveal significant interannual variations and bidecadal trends in the travel time change. Argo-measured $\mathrm{OHC}$ changes are overlapped with a conversion rate of $1 \%$ versus 0.7 GJ/m2 as explained in the text. (C,D) Global mode-1 M2 internal tides from 20 years of satellite altimeter data. The northbound (propagation direction ranging $0^{\circ}-180^{\circ}$ ) and southbound $\left(180^{\circ}-360^{\circ}\right.$ ) components have been separated. $\mathrm{OHC}$ changes can be monitored by tracking long-range internal tidal beams.

it possible to track the long-range propagation of each internal tidal beam and estimate OHC changes. Figure 5 shows that long-range internal tidal beams are widespread in the Pacific, Atlantic, and Indian Oceans. ITOT can track M2, S2, O1 and K1 internal tides, and for each tidal constituent both mode-1 and -2 waves. The multi-constituent, multi-mode method has a much better spatial coverage of the global ocean. Internal tides are powered by the astronomical tidal potential, and thus no cost is needed to maintain their radiation sources. ITOT has a relatively low temporal resolution, due to long repeat cycles of satellite altimetry. Therefore, ITOT may offer a long-term low-cost observing network.

Much work is needed to develop ITOT from a proof-ofconcept level to a mature level. There are two major challenges. The first challenge is how to precisely measure the phase of internal tides by satellite altimetry. It stems from the complex nature of the internal tide field and the low spatio-temporal sampling rates of altimeter satellites. The current-generation nadir-looking satellite altimetry samples the ocean along sparse ground tracks with time intervals of $\mathrm{O}(10)$ days. To resolve spatio-temporal variations of internal tides, time series of 1 year or longer are needed, depending on the number of satellites in the constellation. Uncertainties in the speed changes may be caused by background currents and salinity anomalies. They can be evaluated using overlapping internal tidal beams and/or in situ measurements. The second challenge is to derive $\mathrm{OHC}$ changes from the speed changes of internal tides. ITOT itself cannot distinguish the upper- and lower-layer contributions. The ambiguity requires constraints from in situ measurements. Heat enters the ocean from the sea surface and is redistributed in the ocean interior. Ocean warming can be approximated using Argo and shipboard measurements. It generally follows a baroclinic profile-more heat is stored in the upper layer (Levitus et al., 2012). Assuming that ocean warming follows a normalized profile, its magnitude can be computed from travel time changes of internal tides. In the North Atlantic, about $0.7 \mathrm{GJm}^{-2}$ is required to increase the internal tide's phase speed by $1 \%$. The spatially varying conversion rate 
can be determined using available in situ measurements and internal tide dynamics.

The propagation of two southbound mode-1 M2 internal tidal beams in the North Atlantic has been studied (Figure 5). For each beam, the annual travel times are calculated from the phase increase along the beam. The travel time change rates (in percentage) demonstrate significant inter-annual variability, compared to its 5-95\% confidence intervals (shading). Bidecadal trends are obtained by linear fit (green lines). Both beams reveal that their travel times decreased by about $1 \%$ over the past two decades. The propagation is about $1-2 \mathrm{~h}$ faster, suggesting that this part of the North Atlantic is getting warmer. Argo measured along-beam-mean $\mathrm{OHC}$ changes are calculated and overlapped in Figure 5. The ITOT estimates and Argo measurements agree well for both interannual variations and bidecadal trends. This example confirms that ITOT is feasible and reasonable.

\section{Gap Analysis of the Current Measurement Capacity and Ways for Improvement}

Currently there are about 25 years of satellite altimeter data since 1993 made by a series of altimeter missions. The dataset is long enough to study interannual variations and bidecadal trends in the global OHC. ITOT can be used to analyze 3 years of GeoSat data from 1986-1989 to retrieve OHC changes in the 1980's. In the next 5 years, there will be a few new altimeter missions in operation including Jason classes, Sentinal3 series, HY-2 series (Haiyang-2, Dong et al., 2004), and GFO-2 (Geosat Follow-On-2, Benveniste, 2011). The combination of these satellites will maintain the measurement capability of ITOT at the present level.

In the next 5-10 years, the two major challenges of ITOT may be addressed, leading to improvements of ITOT's measurement capability. First, the next-generation wide-swath altimetry (such as SWOT -Surface Water and Ocean Topography, Morrow et al., 2019- and COMPIRA -Coastal and Ocean Measurement mission with Precise and Innovative Radar Altimeter, Uematsu et al., 2013) will measure high-resolution SSH in the real twodimensional ocean. In contrast, the conventional nadir-looking altimetry has low spatial resolution. Wide-swath altimetry will greatly improve our capability of mapping internal tides and their propagation speed. Second, the ITOT derived OHC changes will be calibrated against other $\mathrm{OHC}$ estimate techniques. In addition, the ECCO2 state estimate (Estimating the Circulation and Climate of the Ocean, Phase II, Menemenlis et al., 2005) can be used to directly calculate the internal tide's speed and OHC changes, so that ECCO products can be used to assess the accuracy of ITOT. In particular, acoustic tomography has been conducted in a series of field experiments (Dushaw et al., 2009). It will be very useful to compare the $\mathrm{OHC}$ changes estimated by the two tomographic techniques.

\section{ESTIMATING THE OCEAN SURFACE NET FLUX FROM SPACE OBSERVATIONS}

Because energy storage in the atmosphere over time scales longer than a year is two order of magnitude smaller than heating rate of the ocean (Church et al., 2011), the global annual mean net surface flux is in principle nearly equal to the TOA irradiance. Thus TOA irradiance can in principle be used to estimate the ocean heating rate. The flux components needed to compute the net surface flux are radiative flux, turbulent flux, and flux associated with mass transfer. In the following sections, we provide brief descriptions of algorithms to estimate surface fluxes and uncertainties in the fluxes derived from the algorithms.

\section{Radiative Flux}

Radiative fluxes (irradiances) at the ocean and atmosphere boundary are estimated by radiative transfer models. These models are based on radiative transfer theory and solve an integro-differential equation of radiative transfer typically with a two- or a four-stream approximation. Polarization state is neglected. Primary inputs to the radiative transfer model are height dependent atmospheric temperature and water vapor mixing ratio, cloud and aerosol properties, ocean surface albedo and emissivity. Satellite observation-based estimates generally take temperature and humidity from reanalysis data products. Physical and optical properties of clouds and aerosols are estimated from satellite observations either passive sensors (e.g., imagers like Moderate Resolution Imaging Spectroradiometer (MODIS), Visible Infrared Imaging Radiometer Suite (VIIRS), and imagers on geostationary satellites) or active sensors [e.g., Cloud-Aerosol Light Detection and Ranging (LIDAR) with Orthogonal Polarization and Cloud Profiling Radar on CloudSat]. Cloud properties include cloud top and base heights, optical thickness, and particle size and water phase. When passive sensors are used to retrieve cloud properties, the cloud top height is estimated from the effective cloud top temperature combined with the vertical temperature profile. Passive sensors cannot observe the cloud base height directly. It is usually estimated empirically with the combination of the cloud optical thickness and cloud top height. Active sensors can directly detect cloud top and base heights. In additions, the vertical profile of particle size and phase can be derived from their observations. Once particle size and phase are derived, wavelength dependent extinction coefficient, single scattering albedo and asymmetry parameter are determined theoretically by assuming cloud particle shape. Spherical particles are assumed for warm clouds and their optical properties are computed with Mie theory. Various shapes are used for ice clouds. However, as long as the same ice crystal shape is used in both the cloud retrieval algorithm and surface irradiance computations, the error is generally small (Loeb et al., 2018b).

Similar to cloud properties, aerosol optical properties are determined from observations. Aerosol optical thickness can be derived from passive sensors and active sensors. Passive sensors can provide optical thickness at various wavelengths. Particle size is estimated from the wavelength dependent optical thickness. LIDAR that measure the backscatter extinction need to assume the ratio of the backscatter extinction and extinction coefficient to derive aerosol optical thickness. The wavelength dependent aerosol optical thickness (as well as depolarization ratio for the case of LIDAR) combined with geolocation is used to determine aerosol types. Once aerosol type 
is determined, wavelength dependent optical thickness, single scattering albedo, and asymmetry parameters are determined by assuming particle shape and size distribution. Wavelength dependent refractive indices of pure substances or those based on laboratory measurements are used to compute aerosol optical properties.

Surface albedos and emissivity also depend on wavelength. Ocean surface albedo (Cox and Munk, 1954; Jin et al., 2004) and emissivity (e.g., Sidran, 1981; Masuda et al., 1988) can be derived from models with relatively small uncertainty.

All inputs discussed above are used in a radiative transfer model to compute radiative flux. Diurnal cycle of temperature and humidity and cloud and aerosol properties need to be known to compute diurnally averaged radiative fluxes. Reanalysis data products provide the diurnal cycles of temperature and humidity. The diurnal cycle of clouds can be derived from geostationary satellites. An aerosol transport model (e.g., Collins et al., 2001) can be used for estimating the diurnal cycle of aerosols.

All assumptions and approximations made in deriving input variables and in the radiative transfer model introduce errors in irradiances. The uncertainty is reduced when TOA irradiances derived from observations are used to constrain the surface irradiance. Shortwave irradiances can be well constrained while constraint on longwave irradiance is somewhat weaker (Ellingson, 1995; Kato et al., 2018).

Radiative fluxes observed at limited ocean and land sites are used to evaluate computed radiative fluxes. Comparisons reported by Kato et al. (2018) show that surface monthly mean downward fluxes agree with observations to within $5 \mathrm{Wm}^{-2}$ for shortwave fluxes and $2 \mathrm{Wm}^{-2}$ for longwave fluxes when the differences are averaged over 46 ocean sites. In addition, the correlation coefficient of deseasonalized anomalies of computed and observed monthly mean regional fluxes (with the annual cycle removed) is greater than 0.94 over ocean for both shortwave and longwave radiative fluxes (Kato et al., 2018). These comparison results are used to determine the uncertainty in the net radiative flux over ocean. The uncertainty in the annual mean irradiance over the global ocean is significantly smaller than the uncertainty in the regional mean irradiance because of partial cancelation of spatially random errors. Once errors in all radiative flux components are assumed to be independent, the uncertainty in the global annual mean radiative flux over the ocean is $8.7 \mathrm{Wm}^{-2}$. Similarly, with the assumption of independent errors among all surface irradiance components, the uncertainty estimated by L'Ecuyer et al. (2015) for land and ocean combined leads to the global mean surface net irradiance uncertainty of $8.3 \mathrm{Wm}^{-2}$. The same assumption applied to the uncertainty estimated by Wild et al. (2014) leads to $7.0 \mathrm{Wm}^{-2}$ uncertainty in the global annual mean irradiance over the ocean. Uncertainties in the surface irradiances at different temporal and spatial scales are given in Kato et al. (2018).

\section{Turbulent Fluxes}

With the assumption of no Coriolis force and no adiabatic heating by radiation, the sensible heat, latent heat, and momentum vertical fluxes that are assumed to be uniform within the lowest atmospheric layer can be expressed as a function of temperature, water vapor mixing ratio, and wind speed scaling parameters (Monin and Obukhov, 1954). The scaling parameters, which are independent of height, are a product of a height dependent non-dimensional number that is a property of medium and height dependent temperature, mixing ratio, and wind speed. Based on this theory, turbulent fluxes are estimated using parameterized form of vertical energy transfer. One of popular algorithms is the Coupled Ocean-Atmosphere Response Experiment (COARE) algorithm (Fairall et al., 1996, 2003). The COARE bulk parameterization expresses the sensible and latent heat fluxes as a product of the density of dry air, transfer coefficient, wind speed relative to the sea surface, temperature or water vapor mixing ratio difference, and thermodynamic constant. The difference is expressed the value at the surface and at the reference height. The transfer coefficient is a product of the non-dimensional numbers that are used to express the scaling parameters.

Turbulent fluxes in the algorithm are composed of various terms that are needed to account for corrections. Although the inclusion of the sensible heat flux associated with mass transfer depending on the data product, as discussed in Fairall et al. (1996), the sensible heat flux associated with precipitation and water vapor evaporating from the ocean surface is computed by the COARE algorithm. The reference temperature used for these flux estimates is the sea surface skin temperature. In addition, an additional correction is applied to the latent heat flux estimate to account for the upward mass flow associated with non-negligible mean vertical velocity (Webb et al., 1980).

Uncertainty in turbulent fluxes are caused by the uncertainty in the transfer coefficient and in the temperature, water vapor mixing ratio, and wind speed used for the input to the algorithm. Approximately, a 10\% uncertainty in the transfer coefficient used in the bulk formula results in a $10 \mathrm{Wm}^{-2}$ uncertainty in the latent heat flux under a tropical condition (Fairall et al., 1996). The overall error in the flux estimated by the COARE algorithm for wind speeds $<10 \mathrm{~ms}^{-1}$ is less than $5 \%$ and for wind speeds between $10 \mathrm{~ms}^{-1}$ to $20 \mathrm{~ms}^{-1}$ is less than $10 \%$ (Fairall et al., 2003). In addition, Andreas (1992) shows that sea spray, which is generally not considered in turbulent flux algorithms, contributes the sensible and latent heat fluxes over ocean, especially when the wind speed exceeds $10 \mathrm{~ms}^{-1}$. The uncertainty in the global annual mean fluxes (land and ocean combined) estimated by L'Ecuyer et al. (2015) is $7 \mathrm{Wm}^{-2}$ for the latent heat flux and $5 \mathrm{Wm}^{-2}$ for sensible heat flux. The uncertainty in the global annual mean latent and sensible heat fluxes over the ocean estimated by Wild et al. (2014) is, respectively, $15 \mathrm{Wm}^{-2}$ and $7 \mathrm{Wm}^{-2}$.

\section{Role of the Surface Net Flux Approach}

If we simply average uncertainties in the global annual mean irradiance discussed above, the result is an $8 \mathrm{Wm}^{-2}$ uncertainty. Similarly, averaging the sensible and latent heat uncertainty of Wild et al. (2014) and L'Ecuyer et al. (2015) discussed above leads to an uncertainty of $11 \mathrm{Wm}^{-2}$ in the latent heat flux and $6 \mathrm{Wm}^{-2}$ in the sensible heat flux. If errors in these flux components are independent, the uncertainty in the global annual mean net surface flux over the ocean is $15 \mathrm{Wm}^{-2}$. The uncertainty in the 
surface fluxes is, therefore, approximately three times larger than the uncertainty in the TOA net irradiance derived from CERES observations (see the introduction and Loeb et al., 2009). In addition, when the global annual mean surface energy budget is computed from satellite-based data products, there is a significant residual of 10-15 $\mathrm{Wm}^{-2}$ (Kato et al., 2011; Stephens et al., 2012; Loeb et al., 2014; L'Ecuyer et al., 2015), which is about the same magnitude as the uncertainty in the global annual mean net surface flux. The accuracy of inter-annual variability of the net surface flux computed by this approach, however, needs to be investigated. While this approach has a significant disadvantage compared to the TOA approach in estimating EEI, this approach provides the spatial distribution of net surface energy. The net surface energy flux is the energy input to the regional ocean. The heating rate of the ocean column is balanced by the net surface energy flux. This flux includes input from the surface boundary of the ocean water column as well as horizontal energy transport by ocean dynamics through lateral boundaries. In addition, internal energy transport by river runoff needs to be considered for coastal regions (e.g., Rodell et al., 2015). Therefore, if the uncertainty in the net surface energy flux is sufficiently small, observationally derived net surface energy flux can constrain energy transport by ocean dynamics (e.g., Trenberth and Stepaniak, 2004), provided regional ocean heating is known by, for example, in situ ocean temperature measurements.

Two different approaches are currently available to estimate regional surface energy budget. The first approach is to use satellite-based surface radiative flux, and turbulent fluxes. The uncertainty in these fluxes were discussed earlier. The second approach is to use TOA radiative fluxes derived from satellite observations and energy divergence and tendencies derived from an atmospheric reanalysis data product (Fasullo and Trenberth, 2008; Trenberth and Fasullo, 2013; Liu et al., 2017; Mayer et al., 2018). Both approaches have advantages and disadvantages. The first approach provides all components of surface fluxes, which is an advantage. As a consequence, the error in the net regional flux can be computed based on the error in each component so the error is traceable. As mentioned earlier, the disadvantage of this approach is that the net flux computed by summing up all components over the ocean differs significantly from EEI. How the residual is distributed among regions is not known but the spatial distribution affects the regional energy balance. The regional net flux can be estimated in an objective way, as demonstrated by L'Ecuyer et al. (2015). The advantage of the second approach is that a relatively long time series of surface net flux can be estimated (Allan et al., 2014). The disadvantage of the second approach is that the error estimate in the regional surface net flux is difficult. The uncertainty in the regional dry static energy and kinetic energy divergence estimated in Kato et al. (2016) is about 15\%. However, the error in the total energy divergence (i.e., moist energy plus kinetic energy divergence) may be smaller than $15 \%$ because of partial cancelation.

Regardless of the approach taken, the reason for the residual of global and regional surface energy balance needs to be understood in order to use the ocean surface flux method to constrain ocean dynamics.

\section{ESTIMATING THE OCEAN THERMAL EXPANSION FROM SPACE OBSERVATIONS}

As the oceans warm, the sea water expands and sea level rises. This physical relationship allows to estimate OHC change from observed sea level change, provided the mass component of sea level change is known and accounted for. Changes in ocean mass occur through the transfer of water between continents, the cryosphere and the ocean (the atmosphere plays a negligible role on all time scales due to its negligible water holding capacity). When corrected for ocean mass variability, the so-called steric component of sea level change provides an estimate of the thermal expansion of the ocean. The relationship between sea level change $\left(\Delta \mathrm{SL}_{\text {total }}\right)$, ocean mass change $\left(\Delta \mathrm{SL}_{\text {mass }}\right)$ and ocean thermal expansion change $\left(\Delta \mathrm{SL}_{\text {thermo }}\right)$ is expressed by the sea level budget equation (see Equation 1). Variability in ocean salinity yields sea level changes as well, but at the global scale this effect is practically zero (Lowe and Gregory, 2006; Gregory et al., 2019). Since we focus here on the global scale, salinity changes are excluded (it would include a spurious global mean halosteric sea level change in the calculation owing to the heterogeneous spatial coverage of salinity measurements).

$$
\Delta \mathrm{SL}_{\text {total }}=\Delta \mathrm{SL}_{\text {mass }}+\Delta \mathrm{SL}_{\text {thermo }}
$$

Once the ocean thermal expansion is retrieved, OHC changes can be derived by dividing the thermal expansion changes by the expansion efficiency of heat $\left(\varepsilon, \mathrm{mYJ}^{-1}\right)$ as in Equation 2.

$$
\Delta \mathrm{OHC}_{\text {alti-GRACE }}=\frac{\Delta \mathrm{SL}_{\text {thermo }}}{\varepsilon}
$$

Sea level change is observed from space with radar altimetry missions (see Sea Level). Ocean mass change is observed from space with the gravimetry missions GRACE and GRACE-FO (see Ocean Mass).

\section{Sea Level}

Since October 1992 and the launch of TOPEX/Poseidon (T/P), twelve satellite altimeters have been launched providing high precision ( $\pm 1.6 \mathrm{~cm}, 5-95 \% \mathrm{CL}$, Ablain et al., 2015) and highresolution (every $7 \mathrm{~km}$ ) measurements of the ocean surface topography. In total, satellite altimeters have retrieved more than 26 years of high accuracy sea level measurement with a quasi-global coverage and a revisit time between 9.9 and 35 days.

Satellite altimeters carry onboard an instrument, which emits microwave radiation impulses in the nadir direction. Part of the radiation impulses reflects off the sea surface back to the altimeter. The measurement of the round-trip travel time of the radiation impulses is used to estimate the distance between the satellite and the sea surface (this distance is called the altimeter range). Satellite altimeters also carry onboard tracking instruments (Global Positioning System-GPS-, Doppler Orbitography and Radiopositioning Integrated by SatelliteDORIS-, and Satellite Laser Ranging system -SLR-) that estimate the height of the altimeter with respect to the center of the Earth in the International Terrestrial Reference Frame (ITRF). The 
difference between the altimeter height and the altimeter range gives the sea level. To get accurate sea level estimates the altimeter range must be corrected for delays in the travel of the microwave impulse through the atmosphere. It must be also corrected for biases due to the scattering of the impulse at the sea surface and for the aliasing of various geophysical signals (see for example Chelton et al., 2001 for more details).

When all corrections are applied, the error on each single sea level measurement is $\pm 3.5 \mathrm{~cm}$ (5-95\% CL, Ablain et al., 2015). To estimate the global mean sea level, all single measurements of a given satellite altimeter are averaged over an orbit cycle (see Figure 6). In this process, the instrument's random errors average out leading to an uncertainty in global mean sea level over an orbit cycle of $\pm 3 \mathrm{~mm}$ (5-95\% CL, Ablain et al., 2015).

In the sea level budget approach, the estimate of the $\mathrm{OHC}$ trend is derived from the trend in global mean sea level corrected for the mass contribution. The trend in global mean sea level over the last 25 years is of $3.2 \mathrm{mmyr}^{-1}$ (e.g., The WCRP Global sea level budget group, 2018) Estimating such a small trend over multi-decadal time scales requires both high accuracy (of a few tenth of $\mathrm{mmyr}^{-1}$ ) and high stability in the measurement system over decades. The high stability requirement has been achieved with the series of altimeters T/P, Jason 1 Jason 2 and Jason 3 through dedicated inter-calibration phases where a satellite altimeter and it's successor fly on the same orbit, a few seconds apart. These inter-calibration phases allow for the comparison of precise measurements of the same sea surface topography by different satellite altimeters (Legeais et al., 2018).
Six different groups provide estimates of the trend in global mean sea level (GMSL) from T/P and Jason 1-2-3 (see Figure 6). Over the period 2002-2017 (when GRACE is available to calculate the mass budget) the different groups indicate a sea level rise of $3.3 \pm 0.1 \mathrm{mmyr}^{-1}$ (1.65 sigma, The WCRP Global sea level budget group, 2018). The spread of $\pm 0.1 \mathrm{mmyr}^{-1}$ (1.65 sigma) across these estimates is due to the use of different retracking techniques, different orbit solutions, different corrections and different interpolation methods applied by the different groups (Masters et al., 2012; Henry et al., 2014). This spread is smaller than the uncertainty in the sea level trend because all groups use the same (or similar) methods and corrections to process the altimeter data leading to some potential systematic uncertainty that is not accounted for in the spread.

Two different approaches have been developed to estimate the uncertainty in the trend in sea level so far. The first approach is an error budget approach, which consists of estimating all the possible sources of uncertainty in the satellite measurement system that affect the estimate of the trend in global mean sea level. A careful analysis of all subsystem errors (Ablain et al., 2009,2015 ) indicates that the main source of error comes from the correction of the delay in the radar impulse round-trip travel caused by the water content in the atmospheric column (called hereafter the "wet tropospheric correction," see Table 2). This correction is based on the measurement of a radiometer on board the altimeter that tends to drift with time between two calibrations. This drift causes a spurious drift in the sea level estimate that generates an error of up to $\pm 0.2 \mathrm{mmyr}^{-1}$

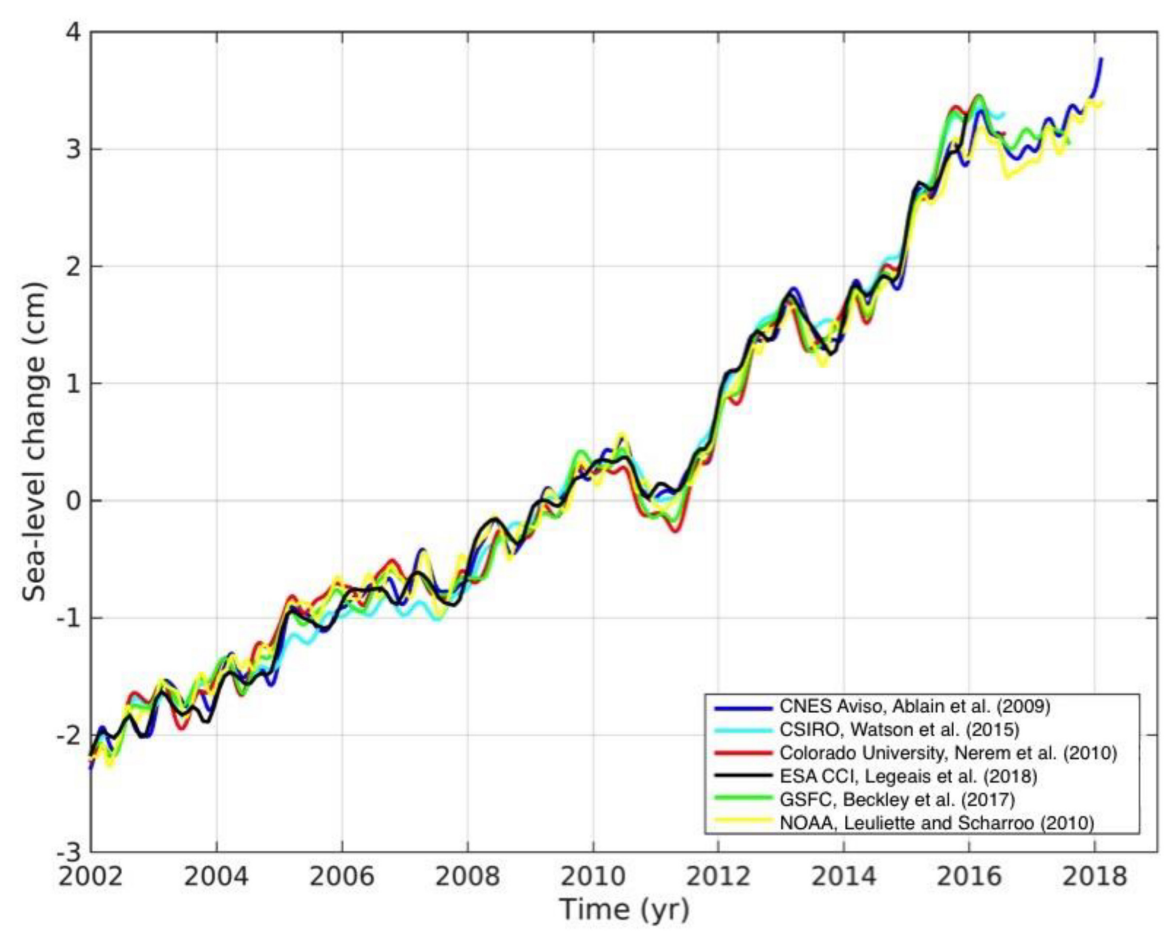

FIGURE 6 | Global mean sea level changes estimated from satellite altimetry by CNES Aviso, CSIRO, Colorado University, Copernicus Climate service, ESA CCI, GSFC, and NOAA. 
TABLE 2 | GMSL trend uncertainties (in $\mathrm{mmyr}^{-1}$ ) over 2002-2017 estimated from the error budget approach and from the comparison with tide gauge records.

\begin{tabular}{lc}
\hline Source & $\begin{array}{c}\text { Trend uncertainty over } \\
\text { 2002-2017 (mmyr }\end{array}$ \\
\hline $\begin{array}{l}\text {-1) } \\
\text { Orbit (Beckley et al., 2007; Couhert et al., 2015; }\end{array}$ & 0.2 \\
Ablain et al., 2019) & 0.17 \\
Wet atmos. (TMR/JMR drift) (Ablain et al., 2019) & 0.1 \\
Intercalibration (Ablain et al., 2019) & 0.1 \\
Dry atmos. (pressure fields) (Ablain et al., 2015) & 0.1 \\
Sea state bias (Ablain et al., 2015) & 0.33 \\
Quadratic sum (including the main sources & \\
cited above plus other minor sources) & 0.4 \\
Tide gauge comparison (Mitchum et al., 2010; & \\
Valladeau et al., 2012; Watson et al., 2015) & \\
\hline
\end{tabular}

(5-95\% CL) on trends computed over periods less than 10 years (e.g., Legeais et al., 2014; Thao et al., 2014; Fernandes et al., 2015). For decadal and longer trends the error gets smaller because the wet tropospheric signal decorrelates at decadal and longer time scales. The second largest source of error comes from the orbit correction. The errors in the time variable gravity field retrieved with SLR and GRACE and the errors in the realization of the ITRF lead each to an uncertainty of $\pm 0.1 \mathrm{mmyr}^{-1}$ in the GMSL trend on annual to multi-decadal time scales (e.g., Couhert et al., 2015). The inter-calibration between satellite altimeters is also a source of uncertainty. The inter-calibration phases allow for the correction of biases between altimeters within an uncertainty of $\pm 0.5 \mathrm{~mm}$ for Jason1-2-3 and $\pm 2 \mathrm{~mm}$ for T/P in terms of GMSL (Zawadzki and Ablain, 2016; Ablain et al., 2019). This bias uncertainty leads to an uncertainty in the GMSL trend of up to a few tenth of mmyr $^{-1}$ for decadal trends (see Figure 7 and Ablain et al., 2019). To a lesser extent the uncertainty in geophysical corrections also lead to some uncertainty in the GMSL trend. In total, the error budget approach indicates an error in the GMSL trend of $\pm 0.5 \mathrm{mmyr}^{-1}$ (5-95\% CL) on decadal trends, down to $\pm 0.33 \mathrm{mmyr}^{-1}$ on 15 -year trends (5-95\% CL, see Figure 7 and Ablain et al., 2019).

The second approach to estimate the error in the GMSL trend is to compare sea level estimates from satellite altimeters with independent estimates from tide gauge records. A careful comparison between altimeters and tide gauges at hundreds of tide gauge sites (Valladeau et al., 2012; Watson et al., 2015) indicates no significant bias between altimeters and tide gauge records at global scale with a RMSE of $\pm 0.4 \mathrm{mmyr}^{-1}$ (5-95\% CL) over 2002-2017. This uncertainty confirms the results from the error budget approach. Table 2 summarizes the uncertainty estimate over the period of interest here 2002-2017.

There are some limitations in the satellite measurement of sea level. Satellite altimeters do not cover the polar regions (the series $\mathrm{T} / \mathrm{P}$ and Jason do not reach regions above $66^{\circ}$ latitude, the other altimeters reach latitudes up to $82.5^{\circ}$ but there are issues in retrieving $\mathrm{SSH}$ under sea ice and the sea level estimate in sea ice covered regions is not as accurate). Satellite altimeters do not cover the coastal ocean within $20 \mathrm{~km}$ of the coast either (because many geophysical corrections are not valid close to the coast). In addition, satellite altimetry can be affected by systematic drifts that have been accounted for only partially in the error estimate, such as drifts in the ITRF realization or in the glacial isostatic adjustment (GIA) estimate. In the future, progress in interferometry synthetic aperture radar altimetry, which can measure sea level in the leads within sea ice and also close to the coast, should lead to improvements in GMSL trend estimates. Progress in the ITRF realization using assimilation techniques to combine all available geodetic techniques should also lead to improvements (D. Coulot and l'équipe du projet Geodesie, 2017).

For the time-being the only available approach to estimate the error associated with these limitations is to simulate them with models. Several studies (Prandi et al., 2012; Couhert et al., 2015) showed that the associated error on the GMSL trend is likely small $\left(<0.05 \mathrm{mmyr}^{-1}\right.$ for the limitation in coverage and $<0.06 \mathrm{mmyr}^{-1}$ for the limitation due to the ITRF) compared to the total error of $\pm 0.33 \mathrm{mmyr}^{-1}$.

\section{Ocean Mass}

The ocean mass component of sea level changes is a significant contributor to global mean sea level rise over the last 1020 years. About $2 / 3$ of the observed sea level change is attributed to mass gain, mostly related to land ice melt on decadal and longer time-scales (The WCRP Global sea level budget group, 2018). On inter-annual timescales, ocean mass changes are modulated by terrestrial water storage changes in response to large-scale precipitation/evaporation variability (i.e., through ENSO). Accurately measuring mass changes on a global scale (both land, ice and oceans) can only be done from space. The GRACE (Gravity Recovery And Climate Experiment) mission, launched in 04/2002 and in operation through 06/2017, provided month-to-month estimates of mass changes, at a spatial resolution of about $300 \mathrm{~km}$ (e.g., Wouters et al., 2014). In 05/2018, the GRACE Follow-On mission was launched to continue the GRACE data record.

The measurement system of both missions is similar: two identical satellites orbit the Earth at $\sim 490 \mathrm{~km}$, and a microwave ranging system tracks minute variations (order if micro-meters) of the separation distance, which is about $220 \mathrm{~km}$. Mass variations at or near the Earth's surface alter the Earth's gravity field, which in turn impacts the relative distance between the twin gravimetry satellites. The observations are used to calculate mass changes to an accuracy of $\sim 1 \mathrm{~cm}$ over an area of $300 \times 300 \mathrm{~km}$. Averaged over the global oceans, the measurement uncertainty on a monthly time scale is on the order of 1$2 \mathrm{~mm}$. However, as GRACE(-FO) cannot distinguish between the source of the observed gravity change, the isolation of ocean mass from the observations requires several corrections, each of which have different error characteristics. The following corrections are necessary: (1) removing atmospheric mass effects, typically achieved by subtracting the mean atmospheric mass over the oceans from a reanalysis data set (e.g., ERA-Interim), (2) removing long-term trends associated with GIA, typically achieved by subtracting a data-constrained model estimate of the GIA-related gravity signals, and (3) adjusting for signal leakage across the land-ocean boundary due to the limited spatial resolution. While atmospheric mass corrections for ocean mass are considered to be well accounted for, the GIA correction, 


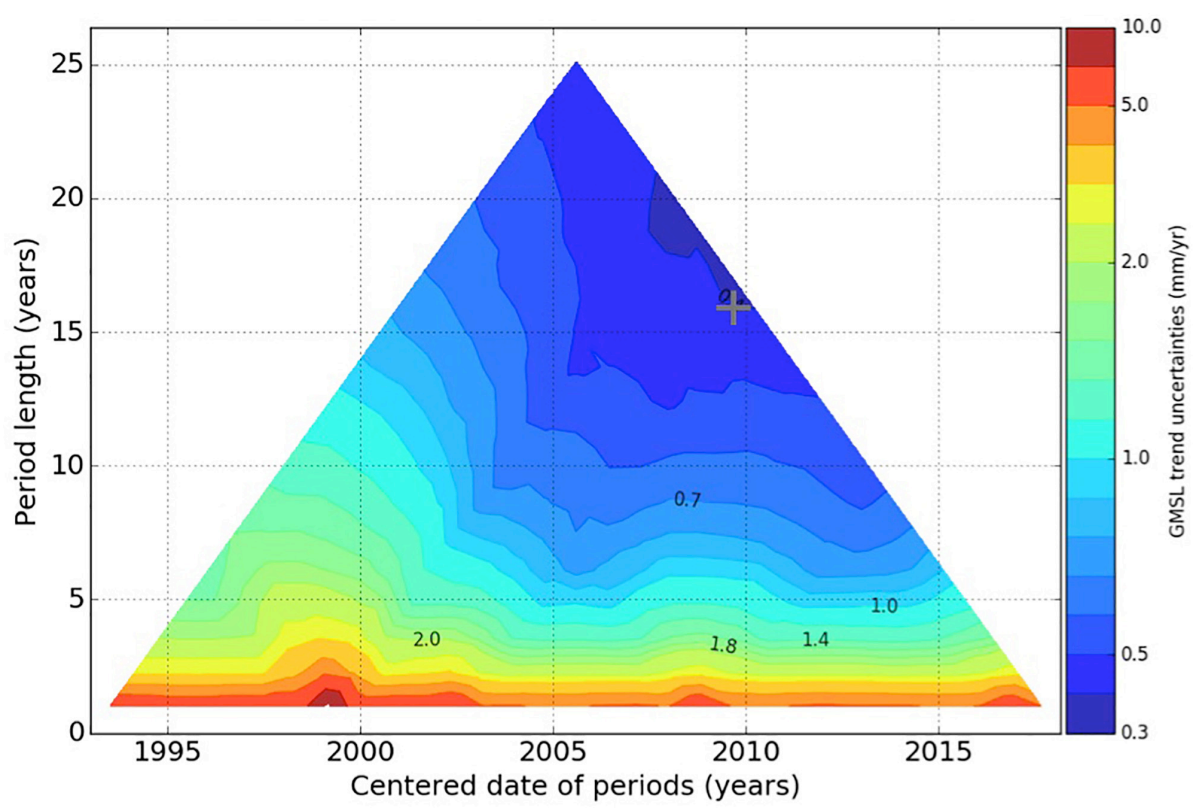

FIGURE 7 | GMSL trend uncertainties (mmyr ${ }^{-1}$ ) estimated for any altimeter period between 1993 and 2017. The $Y$-axis represents the length of the period over which the trend is computed (in years). The $X$-axis represents the central date of the period over which the trend is computed (in years). The colorbar indicates the uncertainty associated to trend in sea level. The confidence level is 5-95\% (1.65-sigma because we assume a Gaussian distribution). The gray cross indicates the value taken for filling Table 2. Figure updated from Ablain et al. (2019).

especially for global ocean mass, is one of the leading error terms. Different GIA corrections can introduce ocean mass trend differences of $0.5 \mathrm{mmyr}^{-1}$, mainly due to GIA uncertainty in Antarctica (Blazquez et al., 2018; The WCRP Global sea level budget group, 2018). Signal leakage between the land-ocean boundary, which tends to bias ocean mass changes low, has been addressed in different ways: (1) to obtain unbiased ocean mass directly from conventional GRACE(-FO) observations (socalled spherical harmonic solutions), ocean grid points closer than $300 \mathrm{~km}$ from land can be discarded (often referred to as 'buffer'); (2) so-called mascon solutions (e.g., Watkins et al., 2015) intrinsically address signal leakage via a priori constraints and generally agree with the 'buffer' approach; (3) inversion approaches seek to combine GRACE(-FO) observations with other observations (e.g., altimetry, land ice changes, vertical deformation) to obtain an indirect ocean mass estimates (for more details, see for example Wiese et al., 2016b; The WCRP Global sea level budget group, 2018; Uebbing et al., 2019). When compatible correction models (such as GIA) are used, direct and indirect ocean mass estimates agree.

In addition to these corrections, GRACE(-FO) observations need to be augmented with a geocenter offset estimate to take into account center-of-mass to center-of-figure effects on surface mass. Different geocenter estimates exist (using different geodetic data sources and methods), and also contribute to monthly as well as trend uncertainty, on the order of $\pm 0.2 \mathrm{mmyr}^{-1}$ (5-95\% CL, Blazquez et al., 2018).

The measurement error of GRACE(-FO) is a function of the instrument accuracies, as well as the science data system background models that need to be employed to account for aliasing effects (e.g., tides, sub-monthly ocean and atmosphere changes). Background model uncertainty is somewhat larger than the instrument errors, but on interannual and longer timescales, the corrections from GIA and geocenter trend dominate the error budget (Chen et al., 2013; Blazquez et al., 2018). Over the period 2002-2017 this leads to an uncertainty of $\pm 0.5 \mathrm{mmyr}^{-1}$ in the estimation of the ocean mass change (Wiese et al., 2016a).

\section{Sea Level Budget}

Following equation (1), the residual of remotely sensed $\Delta \mathrm{SL}_{\text {total }}$ and $\Delta \mathrm{SL}_{\text {mass }}$ provides an estimate of $\Delta \mathrm{SL}_{\text {thermo. We compare }}$ this $\Delta \mathrm{SL}_{\text {thermo }}$ estimate to estimates derived from in situ measurements (see Figure 2) over the period 2006 to 2015. The in situ measurements of ocean temperature are available for limited ocean depths. With the onset of Argo measurements in 2005 , the majority of temperature profiles are available down to $\sim 2000 \mathrm{~m}$ depth (see the section "Past and Contemporary Observing Systems for Global OHC”). Technically, the altiGRACE residual approach provides an independent estimate of $\Delta \mathrm{SL}_{\text {thermo }}$ representative of the full ocean column and therefore the additional deep ocean contribution $(>2000 \mathrm{~m})$ to sea level change that is otherwise estimated by analysis of hydrographic section measurements at about $0.1 \pm 0.08 \mathrm{mmyr}^{-1}$ (5-95\% CL, Purkey and Johnson, 2010; Desbruyères et al., 2016) which correspond to $0.04 \pm 0.04 \mathrm{Wm}^{-2}$. However, presently the magnitude of measurement uncertainty associated with $\Delta \mathrm{SL}_{\text {thermo }}\left( \pm 0.5 \mathrm{mmyr}^{-1}\right.$, see below) does not allow for the closure of the sea level budget accurately enough to estimate the deep ocean contribution on decadal time scales (The WCRP Global sea level budget group, 2018). 


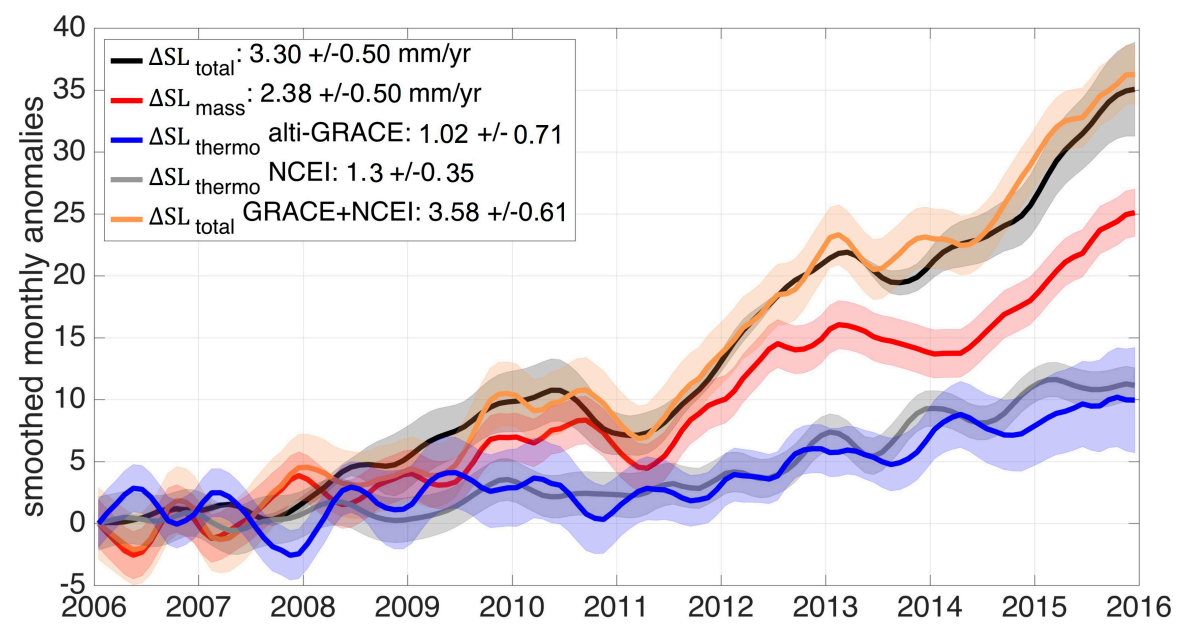

FIGURE 8 | Global mean anomalies of global mean sea level in mm (monthly anomalies, 5-months running means) derived from six altimetry data sets presented in section "Sea Level" ( $\Delta S L_{\text {total }}$, black), ocean mass change from four GRACE products ( $\Delta S L_{\text {mass }}$, red), thermosteric sea level change as the residual of $\Delta S L_{\text {thermo }}=\Delta S L_{\text {total }}-\Delta S L_{\text {mass }}$ (blue), $\Delta \mathrm{SL}_{\text {thermo }}$ from NOAA NCEl (gray) based on interpolated seasonal anomalies, and $\Delta S L_{\text {total }}$ as the sum of $\Delta S L_{\text {thermo }}$ from $\mathrm{NCEl}$ and $\Delta S L_{\text {mass }}$ (orange). The shadings indicate the measurement uncertainty (5-95\% CL); the 5-95\% CL trend uncertainties in the legend are provided by the data centers and are detailed in the main text.

From $\Delta \mathrm{SL}_{\text {thermo }}$ and with knowledge of the ocean's expansion efficiency of heat, $\varepsilon, \Delta \mathrm{OHC}$ can be derived (see Equation 2). The period over which we present the published datasets, represents the Argo-era (2006-2015). The global mean $\Delta \mathrm{SL}_{\text {total }}$ shown in Figure 8 (black line) is a multi-product average based on the six time series presented in section "Sea Level." The shading indicates the monthly measurement error derived using the error budget approach (see section "Sea Level") that considers all possible sources of error in the measurement system. We calculate the $\Delta \mathrm{SL}_{\text {total }}$ trend using weighted least squares regression. The calculated trend is $3.3 \pm 0.5 \mathrm{mmyr}^{-1}$ (5-95\% CL) largely in line with earlier estimates, by Dieng et al. (2017) and The WCRP Global sea level budget group (2018) over similar time period. The time series represent monthly anomalies (mean annual cycle removed) that have been smoothed by applying a 5 -month running average filter. The calculation of decadal trend uncertainty is detailed in section "Sea Level."

$\Delta S L_{\text {mass }}$ is a multi-product average (Figure 8, red line) derived from four GRACE-based datasets provided by the Center for Space Research (CSR), the Helmholtz Centre Potsdam, German Research Centre for Geosciences (GFZ), and the Jet Propulsion Laboratory (JPL). The global mean series together with standard errors after Johnson and Chambers (2013) and Chambers et al. (2017) is based on the three institutes' spherical harmonics products as well as the global mean series from Wiese et al. (2016a) that is based on the JPL mascon product (Watkins et al., 2015). The trend in $\Delta \mathrm{SL}_{\text {mass }}$ at $2.39 \pm 0.5 \mathrm{mmyr}^{-1}$ (5-95\% CL) is in line with previous estimates from Chambers et al. (2017), Blazquez et al. (2018), and The WCRP Global sea level budget group (2018). The trend uncertainty (5-95\% CL) calculation after Wiese et al. (2016a) considers the propagation of monthly uncertainties into the trend, assumes uncorrelated observations, and includes GIA uncertainty according to Chambers et al. (2017). It is important to note that the altimetry and GRACE products do not share the same spatial footprint. This might introduce systematic errors and requires evaluation.

The $\Delta \mathrm{SL}_{\text {total }}-\Delta \mathrm{SL}_{\text {mass }}$ residual timeseries is shown in blue on Figure 8 and represents the alti-GRACE time series of $\Delta \mathrm{SL}_{\text {thermo. }}$. The trend in $\mathrm{SL}_{\text {thermo }}$ amounts to $1.02 \pm 0.71 \mathrm{mmyr}^{-1}$ over 2006-2015 when the mass estimate from GRACE is corrected for the elastic response of the solid Earth (Frederikse et al., 2017; Lickley et al., 2018). The trend uncertainty is calculated assuming the trend uncertainties in $\Delta \mathrm{SL}_{\text {total }}$ and $\Delta \mathrm{SL}_{\text {mass }}$ are independent. The in situ estimate of global mean $\Delta \mathrm{SL}_{\text {thermo }}$ and its standard error (gray) originate from the NOAA National Centers for Environmental Information (NCEI) and represent the thermal expansion of the upper $2000 \mathrm{~m}$ ocean column (Levitus et al., 2012). The trend in $\mathrm{SL}_{\text {thermo }}$ from NCEI at $1.29 \pm 0.35 \mathrm{mmyr}^{-1}$ is slightly larger than the $\mathrm{SL}_{\text {thermo }}$ trend obtained from the Alti-GRACE residual approach, but in line with estimates over 2005-2016 by von Schuckmann et al. (2018). The associated uncertainty is smaller than the uncertainty in $\mathrm{SL}_{\text {thermo }}$ derived from GRACE and altimetry but it is likely biased low as it does not take into account the bias correction errors, the error temporal correlations (see Table 1) and the sampling error.

The orange line represents the $\Delta \mathrm{SL}_{\text {total }}$ as the sum of in situ $\Delta \mathrm{SL}_{\text {thermo }}$ and $\Delta \mathrm{SL}_{\text {mass }}$ and is associated with a trend at $3.58 \pm 0.61 \mathrm{mmyr}^{-1}$. Accounting for the uncertainties in both $\Delta \mathrm{SL}_{\text {total }}$ estimates, the sea level budget is considered to be closed. This is in accordance with various studies conducted over the altimetry/GRACE era (e.g., Leuliette and Willis, 2011; Church et al., 2013; Llovel et al., 2014; Feng and Zhong, 2015; Chambers et al., 2017; Dieng et al., 2017). The closure of the sea level budget demonstrate the capability of the sea level budget approach to estimate the ocean thermal expansion. The accuracy of the closure represents the accuracy of the sea level budget approach in estimating the thermal expansion of the ocean. This accuracy 
is $\pm 0.7 \mathrm{mmyr}^{-1}$ (5-95\% CL) over 2006-2015. It corresponds to an uncertainty in $\mathrm{OHC}$ changes of $\pm 0.37 \mathrm{Wm}^{-2}$ (see below).

\section{Estimating the $\mathrm{OHC}$ Change From the Ocean Thermal Expansion Change}

From the alti-GRACE estimates of $\Delta \mathrm{SL}_{\text {thermo, }}$, we calculate global mean $\mathrm{OHC}$ anomalies by dividing $\Delta \mathrm{SL}_{\text {thermo }}$ by the expansion efficiency of heat $\left(\mathrm{mYJ}^{-1}\right)$ (following Equation 2).

For the expansion efficiency of heat, we adopt here literature values by Kuhlbrodt and Gregory (2012), who estimate $\varepsilon$ from in situ observations (Levitus et al., 2012) at $0.12 \pm 0.01 \mathrm{mYJ}^{-1}$ (equivalent to $0.52 \mathrm{Wm}^{-2} / \mathrm{mmyr}^{-1}$ ) representative of the 0-2000 m ocean column over 1955-2010. Alternate observational estimates by Church et al. (2011) for the full ocean depth over 1972-2008 suggest $\varepsilon=0.15 \pm 0.03 \mathrm{mYJ}^{-1}$, which is not significantly different. The conversion performed is a one-time adjustment, though $\varepsilon$ is known to vary spatially and over time and ocean depth. However changes in the global thermal expansion efficiency of heat are likely negligible on decadal time scales because the warming pattern of the ocean is likely the same on decadal time scales (as suggested by climate models, see Kuhlbrodt and Gregory, 2012).

The trend in the OHC series amounts to $0.53 \pm 0.38 \mathrm{Wm}^{-2}$ over 2006-2015 using $\varepsilon=0.12 \pm 0.03 \mathrm{mYJ}^{-1}$. The associated uncertainty is translated from the trend uncertainty in $\Delta \mathrm{SL}_{\text {thermo }}$ $\left( \pm 0.37 \mathrm{Wm}^{-2}\right)$ and we conservatively add the uncertainty in $\varepsilon$ at $\pm 0.03 \mathrm{mYJ}^{-1}$ taken from Church et al. (2011) which adds an extra $\pm 0.1 \mathrm{Wm}^{-2}$ of uncertainty. The choice of $\varepsilon$ plays an important role in modulating the OHC trend estimate from alti-GRACE. Consensus neither exists about the magnitude and uncertainty of $\Delta \mathrm{SL}_{\text {thermo }}$ from alti-GRACE nor about $\varepsilon$. For example, $\mathrm{Fu}(2016)$ found $\Delta \mathrm{SL}_{\text {thermo }}$ trend at $0.88 \mathrm{mmyr}^{-1}(2003-2014)$ that is similar to the estimate presented here, but derived a trend in OHC of $0.66 \mathrm{Wm}^{-2}$ using $\varepsilon=0.13 \mathrm{mYJ}^{-1}$ after Wunsch and Heimbach (2014). Assuming $\varepsilon=0.12 \pm 0.03 \mathrm{mYJ}^{-1}$ yields a change in $\mathrm{OHC}$ at $0.52 \mathrm{Wm}^{-2}$ per $1 \mathrm{mmyr}^{-1}$ change in sea level, which is consistent with Trenberth and Fasullo (2016). Based on this value Dieng et al. (2017) derived an OHC trend from alti-GRACE over 2003-2013 at $0.65 \pm 0.1 \mathrm{Wm}^{-2}$ (uncertainty represents trend uncertainty only). To avoid systematic errors and to decrease the uncertainty in OHC requires more detailed assessment of $\varepsilon$ and ideally the production of $\varepsilon$ datasets for the $\Delta \mathrm{SL}_{\text {thermo }}$ conversion to OHC. These datasets potentially can be derived from observational datasets or ocean reanalysis/models that capture the entire ocean column globally.

\section{ESTIMATING THE GLOBAL OHC FROM OCEAN REANALYSES}

Ocean reanalyses (ORAs) represent an important tool to create a complete picture of ocean variability and climate change based on sparse observations. Typically, ORAs employ an ocean general circulation model (OGCM) and data assimilation schemes to synthesize diverse network of ocean observations ranging from in situ networks to remote sensing systems. An overview of assimilation methods and approaches to ORA is given by Stammer et al. (2016). As summarized there some assimilation approaches are dynamically self-consistent conserving the dynamics embedded into model equations, while other methods violate conservation principles and may be considered as interpolation approaches to ocean observations. Early estimate of the ocean's role in modulating global energy budget on interannual to decadal timescales (Domingues et al., 2008; Levitus et al., 2009; Ishii and Kimoto, 2009) were only based only on observations and statistical information while during the recent decade more and more products appeared that make use of dynamical information to fill the gaps in the observations.

As part of the international ORA intercomparison project (ORA-IP, Balmaseda et al., 2015), Palmer et al. (2017) used a total of 19 ORAs to intercompare their estimates of various aspects of OHC changes. While the majority of products included a dynamic OGCM, three of the products are still based on statistical analysis of the observations and do not include a dynamic model component. Results indicate that estimates of regional $\mathrm{OHC}$ of the top $300 \mathrm{~m}$ agree between ORAs over large regions of the Pacific and Indian Oceans over the period 1993-2009. However, at deeper levels, the ORAs are less well-constrained by existing observations leading to substantial differences across the ensemble of existing ORAs, especially in areas of high eddy kinetic energy.

Several studies build on global OHC estimates from ORAs to study the ocean's role in the Earth's energy budget and transient climate sensitivity (Balmaseda et al., 2013; Trenberth and Fasullo, 2016; see also von Schuckmann et al., 2018). However, the spread of global OHC estimates of an ensemble of ORAs increase considerably with depth. ORA time series of OHC change in various depth ranges vary in a number of aspects, including: interannual variations; the estimated response to the major volcanic eruptions in 1963,1982, and 1991 and decadal and multi-decadal trends (Figure 9). The decrease of in situ data coverage available for assimilation is the main factor for large differences at depth layers below $700 \mathrm{~m}$ depth (Storto et al., 2015; Palmer et al., 2017). The range of trends and spread among the analyses for the global ocean is similar to the statistical products presented in the Intergovernmental Panel on Climate Change (IPCC) 5th Assessment report (Rhein et al., 2013). A number of products show large initialization or spin-up 'shock'-i.e., an initial and rapid change in $\mathrm{OHC}$ in the first few years of the time series. Separating the time series by hemisphere illustrates the larger spread in the Southern Hemisphere- consistent with the lack of observations over this domain.

Estimated spatial patterns of $\mathrm{OHC}$ change for the period 1970-2009 show good agreement in the upper $300 \mathrm{~m}$ and are characterized by a strong dipole pattern in the Pacific Ocean (Figure 10). There is less agreement in the patterns of change at deeper levels, potentially linked to differences in the representation of ocean dynamics, such as water mass formation processes. Nevertheless the Atlantic and Southern Oceans are regions in which many ORAs show widespread warming below $700 \mathrm{~m}$ over the period 1997-2009.

ORA-based estimates of the past OHC are fundamentally limited by the availability of historical ocean profiles. This holds 

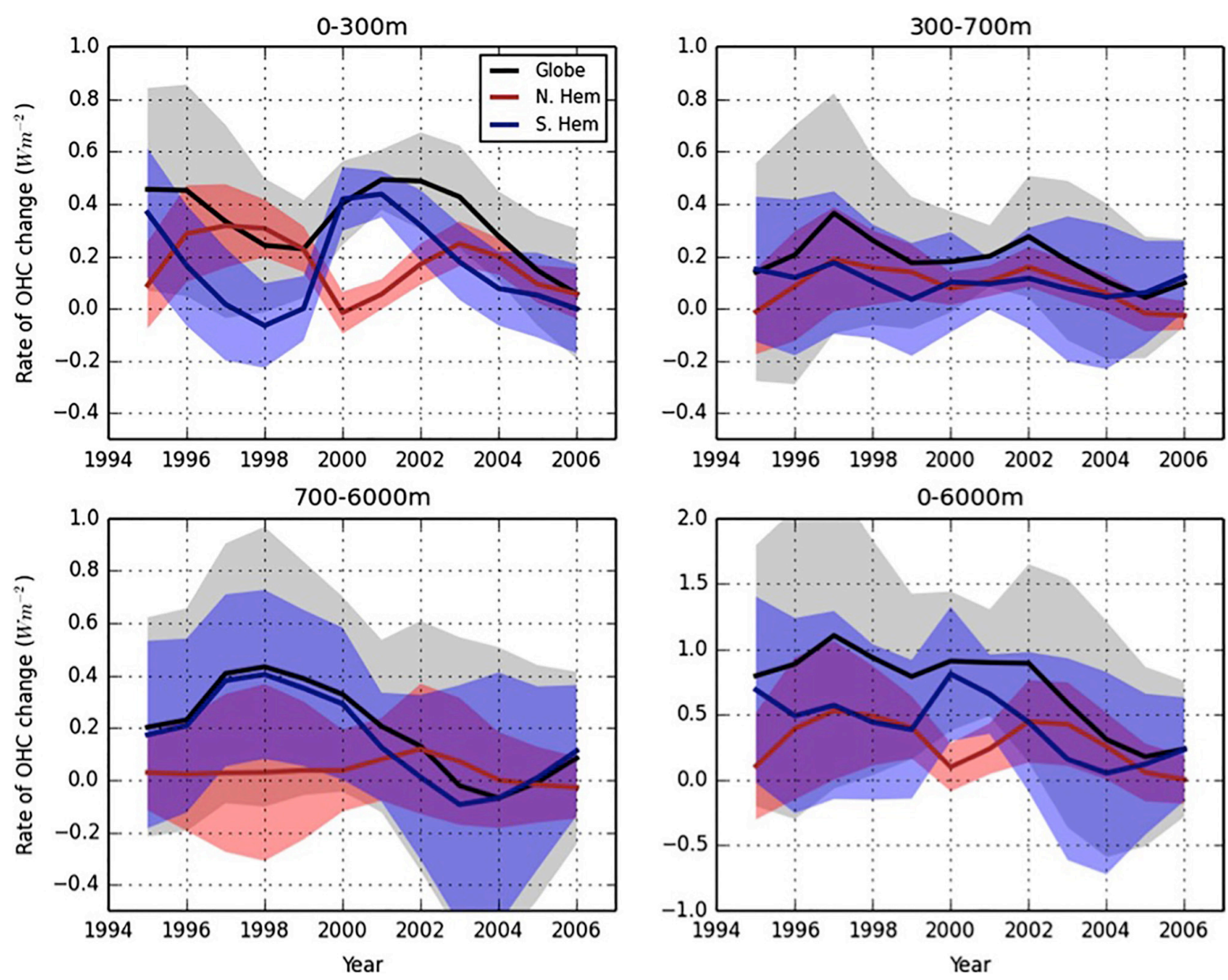

FIGURE 9 | Five-year rolling trends of ocean heat content change over various depth layers for: the Globe (black); Northern Hemisphere (red); and Southern Hemisphere (b/ue). The solid lines show the ensemble mean with shaded regions indicating \pm 1 standard deviation. Changes are expressed as equivalent heating rate, in $\mathrm{W} \mathrm{m}^{-2}$, relative to Earth's surface area. Trends plotted relative to the mid-point of each 5-year period. From Palmer et al. (2017).

especially at depths which are considerably under sampled, historically. The intercomparison of ORAs included a reanalysis based on a coupled model (CFSR). In the future more and more of the ORA will be replaced by coupled reanalyses (Penny and Hamill, 2017), in which the combined observations of the atmospheric and ocean compartment can provide a more complete and more consistent picture of the global energy balance of the Earth. Most current ORAs are based on rather coarse resolution models and a further necessary development is the inclusion of the meso-scale in the underlying ocean models due to the importance of upward eddy heat transports for the distribution of heat (Griffies et al., 2015).

\section{COMPARISON OF GLOBAL MEAN SEA LEVEL BUDGET, OCEAN HEAT CONTENT AND EEI ESTIMATION}

In this section, we compare the $\mathrm{OHC}$ estimates obtained from the different approaches described above, namely the remote sensing technique (altimetry and GRACE), the analysis of in situ observations of temperature as well as ocean reanalysis. We furthermore compare time series of Ocean Heat Storage (OHS), the time derivative of $\mathrm{OHC}$, to EEI as measured at TOA from CERES (EBAF, Loeb et al., 2017) dataset. The CERES data is known to be temporally stable $\left(<0.17 \mathrm{Wm}^{-2}\right.$ at interannual time scales, Loeb et al., 2018a), but biased in the long-term global mean (and therefore adjusted to agree with long-term OHC trends based on Argo measurements, Loeb et al., 2009). The goal of this comparison is to gain insight into the capabilities and weaknesses of the individual approaches, and to formulate recommendations that lead to improvements in retrieval and the complementary use of the datasets.

\section{Ocean Heat Content}

The OHC time series resulting from the sea level budget approach (see Estimating the OHC Change From the Ocean Thermal Expansion Change) is shown in Figure 11 (black line) together with the measurement uncertainty (shading) translated from $\Delta \mathrm{SL}_{\text {thermo }}$ uncertainty (see Estimating the OHC Change From 


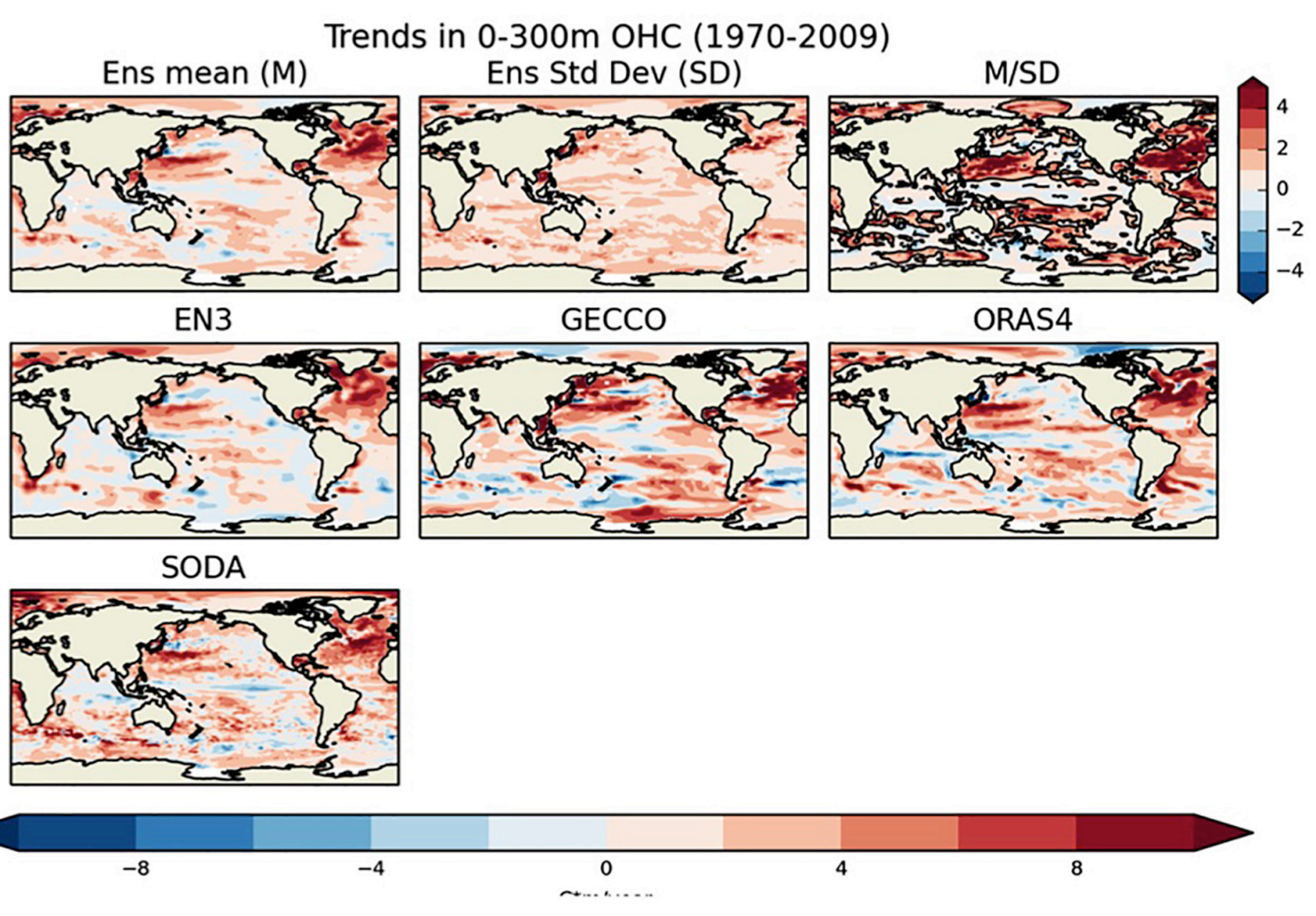

FIGURE 10 | Trends in 0-300 m vertically integrated temperature (Celsius meters per year) for the period 1970-2009. White areas indicate where no data are available. Also shown is the ensemble mean trend (M), the standard deviation of ensemble trends (SD), and the ratio of the two (M/SD). The ensemble values (top row) are computed for each grid box based on all available data (from Palmer et al., 2017).

the Ocean Thermal Expansion Change). For comparison, we show $\mathrm{OHC}$ series based on in situ data $\left(\mathrm{OHC}_{\text {in situ }}\right)$ and ocean reanalysis $\left(\mathrm{OHC}_{\text {rean }}\right)$ in Figure 11. The $\mathrm{OHC}_{\text {in situ }}$ representative of the 0-2000 m ocean column, is a multi-product time series calculated as the average of four products (PML/JPL/JIMAR, MRI/JMA, NCEI, IAP/CAS) detailed in Johnson et al. (2018) by summing the individual detrended anomalies and trends separately. The series of $\mathrm{OHC}_{\text {in situ }}$ representative of the $0-$ $700 \mathrm{~m}$ column additionally includes series from CSIRO/ACE and the Met Office Hadley Centre. The $\mathrm{OHC}_{\text {rean }}$ series for the $0-700 \mathrm{~m}$ and $0-2000 \mathrm{~m}$ columns are representative of the global oceans between $60^{\circ} \mathrm{S}$ and $60^{\circ} \mathrm{N}$ and are based on three reanalysis, GLORYS, C-GLORS, and ORAS5, described in von Schuckmann et al. (2018). Both $\mathrm{OHC}_{\text {in situ }}$ and $\mathrm{OHC}_{\text {rean }}$ are consistent in that they agree on the absolute change in $\mathrm{OHC}$ over the period 2006-2015 within given error bars and on the increase in OHC going from the upper 0-700 $\mathrm{m}$ to the $0-2000 \mathrm{~m}$ column by about $0.3 \mathrm{Wm}^{-2}(\sim 40 \%$ of $0-2000 \mathrm{~m})$.

However, overall, the $\mathrm{OHC}_{\text {rean }}$ trend at $0.71 \mathrm{Wm}^{-2}\left(0_{-}\right.$ $2000 \mathrm{~m}$ ) is larger by $0.1 \mathrm{Wm}^{-2}$ than the $\mathrm{OHC}_{\text {in situ }}$ trend $\left(0.61 \mathrm{Wm}^{-2}\right)$, which is of the order of the trend uncertainty. A slight systematic difference is plausible for different reasons. For example, some of the $\mathrm{OHC}_{\text {in situ }}$ series are not entirely representative of the global oceans (i.e., PML/JPL/JIMAR) and even if global coverage is provided by including all available ocean profiling data, such as originating from XBT or mooring (see Past and Contemporary Observing Systems for Global $\mathrm{OHC}$ ), observational sparsity can bias the global reconstructions. $\mathrm{OHC}_{\text {in situ }}$ based on Argo data only do not fully cover the Arctic and marginal seas. Missing the latter two regions has been shown to reduce the trend in $\Delta \mathrm{SL}_{\text {thermo }}$ by about $0.25 \mathrm{mmyr}^{-1}$ (or $0.13 \mathrm{Wm}^{-2}$ ) as compared to the ocean reanalysis system ORAS4 (Desbruyères et al., 2014; Dieng et al., 2015). On the other hand, $\mathrm{OHC}_{\text {rean }}$ exhibits a larger spread especially at deeper levels, linked to differences in the representation of ocean dynamics (Palmer et al., 2017) and the unavailability of in situ data for assimilation (see Estimating the Global OHC From Ocean Reanalyses).

The most recent estimate of EEI at $0.71 \pm 0.11 \mathrm{Wm}^{-2}$ (OHU over 2006-2015 at $0.68 \pm 0.10 \mathrm{Wm}^{-2}+$ other forms of heat uptake; Johnson et al., 2016) that is used to constrain spaceborn radiometric EEI observations (from CERES) is well met by all approaches within their range of uncertainty. In Table 3 we summarize the different $\mathrm{OHC}$ trend estimates and find good agreement over 2006-2015 at the 5-95\% CL. However some approaches provide more accurate estimates than others. The uncertainty of EEI derived from the Earth surface net heat flux is about 2 orders of magnitude more uncertain than the other estimates hence does not provide any relevant information on the global mean EEI compared to other approaches. The OHC trend based on in situ data is the most accurate of all, with an 


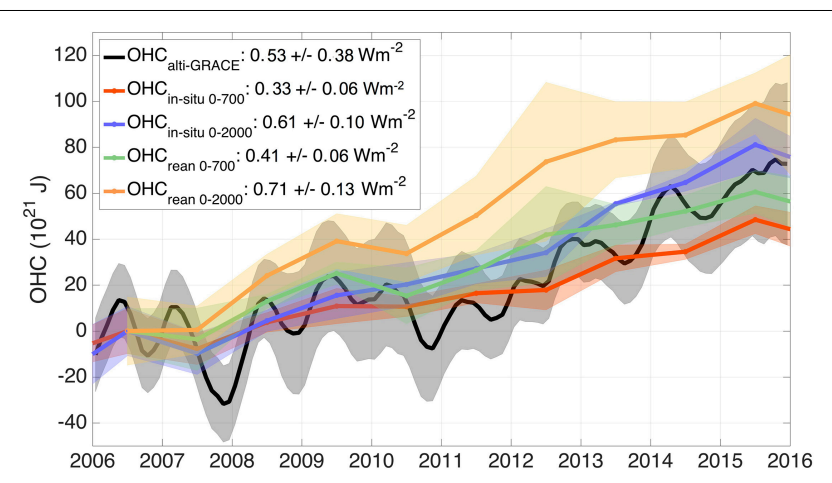

FIGURE 11 | Global mean smoothed (5-month running average) monthly anomalies of $\mathrm{OHC}$ derived from alti-GRACE $\Delta \mathrm{SL}_{\text {thermo }}$ (black,

$\mathrm{OHC}$ alti-GRACE), annual mean $\mathrm{OHC}$ over the 0-700 $\mathrm{m}$ ocean column based on six in situ datasets $\left(\mathrm{OHC}_{\text {in situ }}\right.$, red), annual mean $\mathrm{OHC}$ over the 0-2000 m ocean column based on four in situ datasets $\left(\mathrm{OHC}_{\text {in situ }}\right.$, blue), annual mean $\mathrm{OHC}$ over the 0-700 $\mathrm{m}$ ocean column based on three ocean reanalyses ( $\mathrm{OHC}_{\text {rean }}$, green), annual mean $\mathrm{OHC}$ over the 0-2000 m ocean column based on three ocean reanalyses $\left(\mathrm{OHC}_{\text {rean }}\right.$, orange). For $\mathrm{OHC}_{\text {rean }}$ and $\mathrm{OHC}_{\text {in situ }}$, the shading indicates the spread between timeseries (1.65-sigma). The trend uncertainties represent 5-95\% CL from weighted least square regression and adjusted degrees of freedom. For $\mathrm{OHC}_{\text {rean }}$, the trend is adapted from von Schuckmann et al. (2018). The trend 5-95\% uncertainty in $\mathrm{OHC}_{\text {alti-GRACE }}$ is translated from the trend uncertainty in $S L A_{\text {thermo }}$ and uncertainty in $\varepsilon$ (see text in section "Estimating the $\mathrm{OHC}$ Change From the Ocean Thermal Expansion Change" and Table 3 for details).

uncertainty of $\pm 0.11 \mathrm{Wm}^{-2}$. This uncertainty may be biased low by as much as $\sim 0.1 \mathrm{Wm}^{-2}$ because it does not take into account the sources of uncertainty due to data distribution (see Table 1 and Lyman and Johnson, 2008). Even after correction of this bias, the in situ approach meets the required accuracy for EEI of $\pm 0.3 \mathrm{Wm}^{-2}$ as proposed in the introduction but it does not meet the desired accuracy of $\pm 0.1 \mathrm{Wm}^{-2}$. More research is needed to estimate the uncertainty in the in situ estimate of $\mathrm{OHC}$ trend, in particular due to data distribution. The ocean reanalyses based estimate of the OHC trend shows a spread of $\pm 0.13 \mathrm{Wm}^{-2}$ over 2006-2015 down to $2000 \mathrm{~m}$ depth. This spread, which is a lower bound of the uncertainty in the reanalysis estimates, does not decrease the uncertainty compared to the in situ approach. Below $2000 \mathrm{~m}$ depth, where very few in situ data are available to constrain the reanalyses, the reanalyses estimates diverge quickly (Palmer et al., 2017) with a spread up to $\pm 0.5 \mathrm{Wm}-2$ (see Figure 9). Thus, ocean reanalyses does not provide any significant additional constraints on the $\mathrm{OHC}$ trend estimates (this is also true for the deep ocean). More research is needed to determine the causes for the spread and the ways of improvement. The remote sensing estimate of $\mathrm{OHC}$ trends based on satellite altimetry and GRACE shows an uncertainty of $\pm 0.38 \mathrm{Wm}^{-2}$ over 2006-2015 for the global ocean (this estimate includes the uncertainty due to data distribution). This is slightly higher than the required uncertainty of $\pm 0.3 \mathrm{Wm}^{-2}$ as proposed in the introduction and further research is needed to improve this uncertainty if this approach is to provide constraints on the EEI estimate.
However, it is important to note that a significant part of the uncertainty associated with the remote sensing technique is actually coming from the temporal correlation in errors (in particular in altimetry, see section "Estimating the Ocean Thermal Expansion From Space Observations" and Ablain et al., 2019). This source of uncertainty naturally decays as the period of analysis increases above the error correlation time scales. As a result, the uncertainty in EEI associated with the remote sensing approach is actually expected to reduce below the $\pm 0.3 \mathrm{Wm}^{-2}$ limit as the record period becomes longer than $\sim 15$ years. Over the period 2002-2016, the uncertainty associated with the remote sensing approach is already down to $\pm 0.29 \mathrm{Wm}^{-2}$ (see Table 3) because of this effect of reduced uncertainty due to temporal correlations in errors.

\section{Interannual Variability in Ocean Heat Uptake and EEI}

In the global annual mean, the amplitude and phase in EEI and OHS are expected to be equal, since all other forms of heat uptake are an order of magnitude smaller. Direct measurements of EEI from CERES EBAF are known for their high temporal stability $\left( \pm 0.17 \mathrm{Wm}^{-2}\right.$ at interannual time scales, Loeb et al., 2018a), reflecting essentially internal climate variability, such as induced by ENSO (Loeb et al., 2017). In Figure 12, we compare the interannual variability of CERES EBAF EEI with OHS, derived from the temporal gradient in $\mathrm{OHC}(\mathrm{dOHC} / \mathrm{dt})$, using annual anomalies (long-term mean subtracted) of $\mathrm{OHC}_{\text {rean }}(0-$ $2000 \mathrm{~m}$ ) and $\mathrm{OHC}_{\text {in situ }}(0-2000)$, and annual as well as monthly anomalies (with annual signal removed) for $\mathrm{OHC}_{\mathrm{alti}-\text { grace }}$. Pearson correlation coefficients ( $\mathrm{R}$ ) as well as residual root mean square error (RMSE) of the EEI minus each individual OHS time series are provided in Table 3. These measures illustrate the degree of agreement in the phase (R) and in the amplitude (RMSE) of the time series. Although $\mathrm{OHS}_{\text {alti-grace }}$ (yellow line) underestimates the amplitude of EEI (red line) (see Figure 12), the series are in phase with a high correlation at $R=0.89$, which is in line with the good agreement found by Dieng et al. (2017). Preceding the phase of EEI by 1 year, OHS rean (blue line) tracks the phase and amplitude of the EEI time series reasonably well $(R=0.50, \mathrm{RMSE}=0.41)$. For $\mathrm{OHS}_{\text {in situ }}$ (gray line), both the amplitude and phase are captured well until $2012(R=0.85)$, but including the latter 3 years reduces the $\mathrm{R}$ to 0.44 . Comparisons with a different subset of $\mathrm{OHS}_{\text {in situ }}$ by Johnson et al. (2016) yields better correlation at $R=0.78$ over 2005-2015. These results strengthen the confidence in the $\mathrm{OHS}_{\text {in situ }}, \mathrm{OHS}_{\mathrm{alti}-\text { grace }}$ and CERES measurement systems. When going to shorter time scales and conducting comparisons using monthly anomalies as done with $\mathrm{OHS}_{\mathrm{alti}-\text { grace }}$ (green line), we expect the agreement to decline. Temporal variability in Argoderived $\mathrm{OHS}_{\text {in situ }}$ beyond the decadal and year-to-year scale is overpowered by substantial noise (see Past and Contemporary Observing Systems for Global OHC), owing to measurement errors and spatial coverage deficiencies (e.g., Dieng et al., 2015; Trenberth and Fasullo, 2016). For the monthly anomalies series, the correlation of $\mathrm{OHS}_{\text {alti-grace }}$ with CERES EBAF is $\mathrm{R}=0.42$ and the $\mathrm{OHS}_{\text {alti-grace }}$ precedes the phase of CERES by 2 months. 
TABLE 3 | Ocean heat uptake and associated uncertainty as estimated with the different methods listed in this paper. The correlation and the RMSE with the TOA radiative budget estimate of the EEl from CERES EBAF is given in column 6 and 7 . CERES EBAF values taken from Johnson et al. (2016). All with respect to global surface.

\begin{tabular}{|c|c|c|c|c|c|c|}
\hline $\begin{array}{l}\text { Ocean heat } \\
\text { uptake }\end{array}$ & Time period & $\begin{array}{l}\text { Spatial coverage and/or } \\
\text { depth range }\end{array}$ & mean in $\mathrm{Wm}^{-2}$ & $\begin{array}{l}\text { Uncertainty in } \mathrm{Wm}^{-2} \text { at } \\
\text { the } 5-95 \% \mathrm{CL}\end{array}$ & $\begin{array}{c}\text { Correlation } \\
\text { with CERES } \\
\text { EBAF EEI }\end{array}$ & $\begin{array}{l}\text { RMSE with } \\
\text { CERES EBAF } \\
\text { EEI Wm }{ }^{-2}\end{array}$ \\
\hline \multirow[t]{6}{*}{$\begin{array}{l}\text { From in situ } \\
\text { observations }\end{array}$} & 2006-2015 & 0-2000 m & $\begin{array}{l}0.61 \text { (update of Johnson } \\
\text { et al., 2018) }\end{array}$ & $\pm 0.1^{b}$ & 0.44 & 0.40 \\
\hline & & deep ocean contribution & $\begin{array}{l}0.04 \text { (update of Purkey and } \\
\text { Johnson, 2010). }\end{array}$ & $\begin{array}{l} \pm 0.04 \text { (update of Purkey } \\
\text { and Johnson, 2010); }\end{array}$ & & \\
\hline & & 0-bottom & $0.65^{\mathrm{C}}$ & $\pm 0.11^{d}$ & & \\
\hline & 1993-2017 & $\begin{array}{l}\text { 0-2000 m (no marginal } \\
\text { seas, no ice covered areas) }\end{array}$ & $\begin{array}{l}0.62 \text { (update of Johnson } \\
\text { et al., 2018) }\end{array}$ & $\pm 0.22^{\mathrm{e}}$ & & \\
\hline & & $\begin{array}{l}\text { deep ocean contribution } \\
\text { (below } 2000 \text { m, no ice } \\
\text { covered areas) }\end{array}$ & $\begin{array}{l}0.04 \text { (update of Purkey and } \\
\text { Johnson, 2010); }\end{array}$ & $\begin{array}{l} \pm 0.04 \text { (update of Purkey } \\
\text { and Johnson, 2010); }\end{array}$ & & \\
\hline & & 0-bottom & $0.66^{c}$ & $\pm 0.22^{d}$ & & \\
\hline $\begin{array}{l}\text { From surface net } \\
\text { heat flux }\end{array}$ & 2006-2015 & Net ocean surface heat flux & 10 to $15^{f}$ & $\pm 15^{9}$ & & \\
\hline \multirow{2}{*}{$\begin{array}{l}\text { From satellite } \\
\text { altimetry and } \\
\text { GRACE }\end{array}$} & 2006-2015 & $\begin{array}{l}\text { 0-bottom (no sea ice } \\
\text { covered areas above } 82^{\circ} \mathrm{N} \text { ) }\end{array}$ & $0.53^{h}$ & $\pm 0.38^{i}$ & 0.89 & 0.26 \\
\hline & 2002-2016 & $\begin{array}{l}\text { 0-bottom (no sea ice } \\
\text { covered areas above } 82^{\circ} \mathrm{N} \text { ) }\end{array}$ & $0.57^{j}$ & $\pm 0.29^{k}$ & & \\
\hline \multirow[t]{4}{*}{$\begin{array}{l}\text { From ocean } \\
\text { reanalyses }\end{array}$} & 2006-2015 & 0-2000 m & $\begin{array}{l}0.7 \text { (update of von } \\
\text { Schuckmann et al., 2018)' }\end{array}$ & $\begin{array}{l} \pm 0.13 \text { (update of von } \\
\text { Schuckmann et al., 2018) }\end{array}$ & 0.50 & 0.41 \\
\hline & & $\begin{array}{l}\text { deep ocean contribution } \\
\text { (below 2000m, no ice } \\
\text { covered areas) }\end{array}$ & $\begin{array}{l}0.04 \text { (update of Purkey and } \\
\text { Johnson, 2010). }\end{array}$ & $\begin{array}{l} \pm 0.04 \text { (update of Purkey } \\
\text { and Johnson, 2010); }\end{array}$ & & \\
\hline & & 0-bottom & $0.74^{\mathrm{C}}$ & $\pm 0.14^{d}$ & & \\
\hline & 1993-2008 & 0-bottom & $\begin{array}{l}0.71 \text { (from Palmer et al., } \\
2017 \text { ) }\end{array}$ & $\begin{array}{l} \pm 0.7 \text { (spread across } 15 \\
\text { ocean reanalyses from } \\
\text { Palmer et al., 2017) }\end{array}$ & & \\
\hline $\begin{array}{l}\text { From CMIP5 } \\
\text { climate model } \\
\text { simulations }\end{array}$ & 2000-2010 & 0-bottom & $\begin{array}{l}0.73 \text { (from Smith et al., } \\
2015 \text { ) }\end{array}$ & $\begin{array}{l} \pm 0.21 \text { (spread across } 21 \\
\text { CMIP5 climate model } \\
\text { simulations, from Smith } \\
\text { et al., 2015) }\end{array}$ & & \\
\hline
\end{tabular}

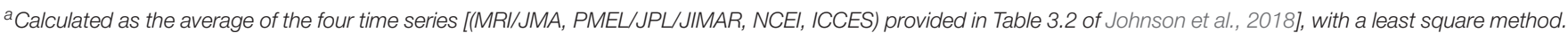

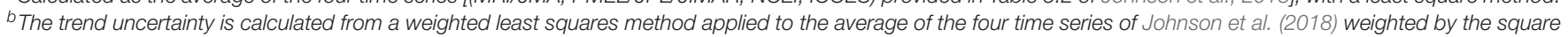

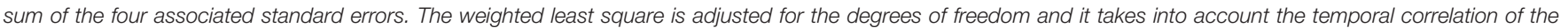

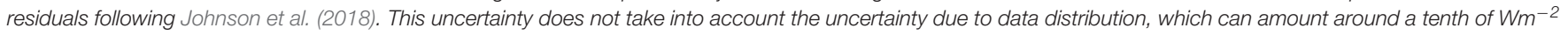

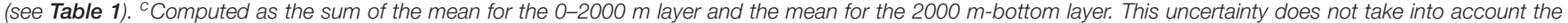

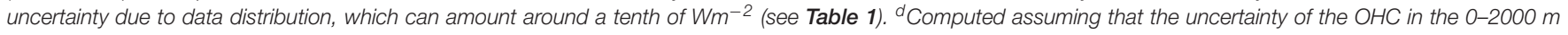

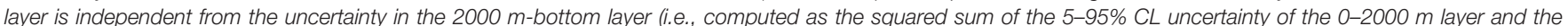

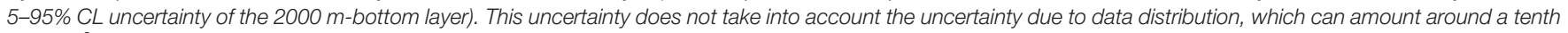

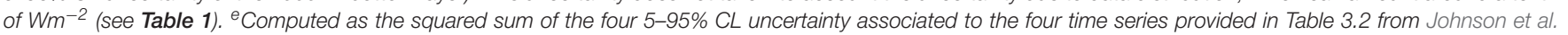

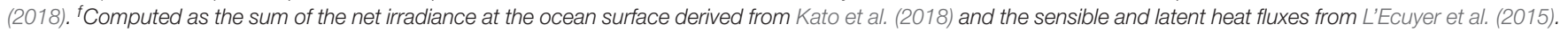

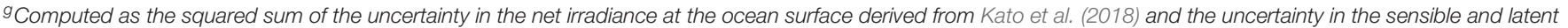

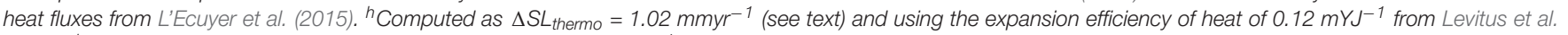

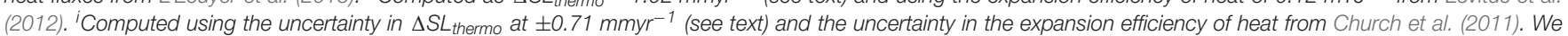

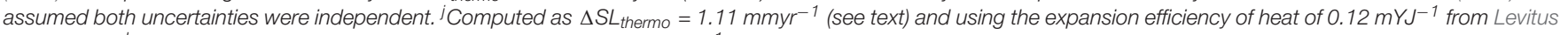

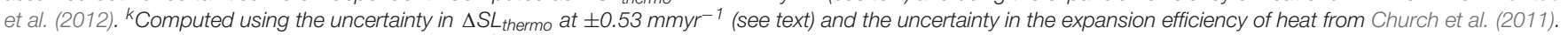

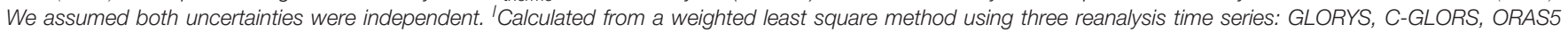

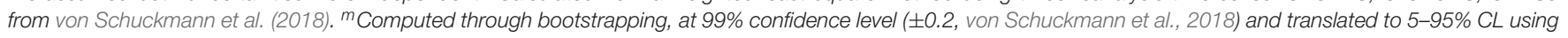
t-test table.

While the question of the phase has to be resolved, the significant positive correlation shows some capacity of the Alti-GRACE approach to reproduce the monthly anomalies in EEI. When comparing annual means, as opposed to the monthly anomaly series, all the approaches agree reasonably well in their depiction of interannual variability.

\section{CONCLUSION, SYNTHESIS AND PERSPECTIVE}

The correlation coefficients and RMSE are provided in Table 3 together with the long-term EEI and OHC trend estimates and their uncertainties derived above. In section 


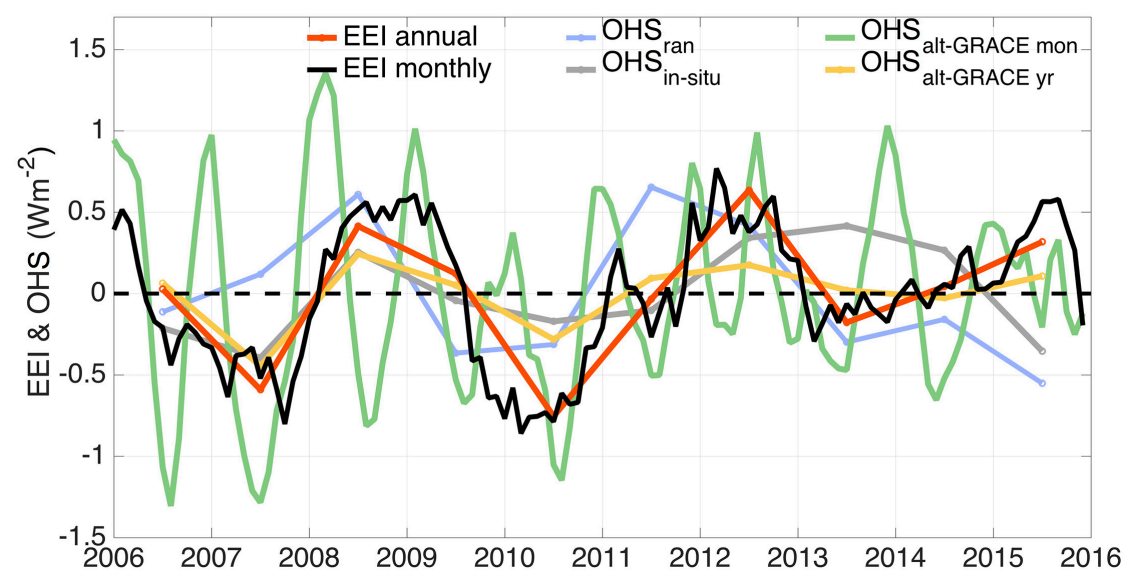

FIGURE 12 | EEI (CERES EBAF) and OHS (dOHC/dt) timeseries from ocean reanalysis (OHS ran), in situ estimation (OHS in situ), and alti-GRACE (OHS alti-GRACE), We show annual timeseries for all parameters (with long-term mean removed) and deseasonalized monthly anomalies for OHS alti-GRACE and EEI that have been smoothed applying a 5-months running average filter.

"Comparison of Global Mean Sea Level Budget, Ocean Heat Content and EEI Estimation" we paid little attention to the surface net heat flux as a way to approximate global mean EEI, owing to biases and measurement errors two orders of magnitude larger than in the other approaches. In Table 3, we include the global long-term mean (10$\left.15 \mathrm{Wm}^{-2}\right)$ and its uncertainty $\left(15 \mathrm{Wm}^{-2}\right)$ as presented in section "Estimating the Ocean Surface Net Flux From Space Observations" (e.g., L'Ecuyer et al., 2015). From this comparison it is obvious that a reliable estimate for long-term global mean EEI from surface heat flux is not possible, as there is both substantial overestimation and random error. The limitations of current data products of surface heat flux are outlined in section "Estimating the Ocean Surface Net Flux From Space Observations." The character of these datasets is complementary to the other approaches, in that they provide constraints at the ocean surface, allow for studies of the separate contributions of ocean and atmospheric energy variability (Liu et al., 2017), and allow regional analysis that can provide information on the magnitude and direction of lateral heat fluxes (e.g., L'Ecuyer et al., 2015). The emerging ITOT method (see Estimating the Ocean Temperature From in situ Observations) is also absent from our comparison because it is an emerging method that is not operational yet and needs further improvements. But we identify it as a promising technique for the future.

The approaches to estimate global mean EEI via the estimation of OHC from either in situ, reanalysis or combined remote sensing techniques, are more reliable and complement each other. The most direct way to estimate $\mathrm{OHC}$ is from in situ temperature profiles and represents so far the most accurate method to estimate long-term ocean heat storage. In addition it delivers unprecedented information on how the ocean heat is distributed vertically. Making use of comprehensive ocean models, reanalysis augments the spatial and temporal coverage of OHC physically consistent with dynamical information and provides the means to study sensitivities and uncertainties systemically. But an improvement of $\mathrm{OHC}$ trend uncertainty as compared to the in situ approach is not achievable yet. The alti-GRACE sea level budget approach provides a full depth estimate of $\mathrm{OHC}$ but is associated with uncertainties that are slightly larger than what is needed to pin down the global mean value of EEI within needed accuracy levels (e.g., Table 3 and von Schuckmann et al., 2016; Hakuba et al., 2018). The alti-Grace approach is promising as it provides consistent spatial and temporal sampling, is representative of the entire global oceans, and estimates OHC over the ocean's entire depth. A number of issues need to be addressed to improve such datasets, comprising improved estimates of heat expansion efficiencies needed to translate thermosteric sea level change to $\mathrm{OHC}$ changes (which relies on in situ measurements of temperature and salinity), analysis of footprint discrepancies and their impact, the retrieval of thermosteric sea level at regional scale including the varying role of halosteric effects, as well as a range of technical challenges in the retrieval of both $\Delta \mathrm{SL}_{\text {mass }}$ and $\Delta \mathrm{SL}_{\text {total }}$, such as inter-calibration, orbit and geocenter corrections, and improved GIA and background models (see Estimating the Ocean Thermal Expansion From Space Observations). To date, only the method employing ocean in situ data (and potentially also the method based on reanalyses, but a robust and comprehensive uncertainty estimate is not yet available) enables to estimate the EEI with the required accuracy of $\pm 0.3 \mathrm{Wm}^{-2}$ on decadal time scale. The method based on the altimetry minus GRACE sea level budget reach the same level of accuracy on 15 years time scales. However none of the methods quite reach the desired accuracy of $\pm 0.1 \mathrm{Wm}^{-2}$ necessary to analyze hiatus periods or to monitor the EEI response to GHG mitigation policies in the future. Significant improvements in the observing system are necessary in the coming decade if we want to achieve this target. We summarize here the recommendations on the 
observing system that would enable to reach the accuracy target for EEI estimation.

Recommendation \#1 - Sustained observations: The top priority for the observing system from the perspective of EEI estimates is to maintain support for core Argo and to maintain observations of sea level and all contributions to sea level. The sustained observations must be accompanied with research and development activities to improve the climate records (including the record from old satellite missions such as Topex or from old in situ instruments such as XBT) when errors or biases are identified.

Recommendation \#2 - Full spatial coverage: Full spatial and temporal implementation of Deep Argo can reduce decadal uncertainties in the deep ocean from \pm 0.04 to about $\pm 0.006 \mathrm{Wm}^{-2}$. A concerted international effort should be made to set up observational campaigns to measure subsurface temperature measurements in areas not presently well covered. These areas include marginal seas, high latitude seasonal ice zones, boundary currents, and shelf regions. Achieving adequate sampling will require an observing system design based on a mixture of observing technologies adopted to the different operating environments. Full spatial coverage including the polar regions of satellite Altimetry will reduce as well the uncertainty in EEI estimate through the alti-grace approach. It is possible to retrieve sea level in the leads over sea ice covered area using new retracking techniques in traditional altimetry (low resolution mode) or using new altimetry techniques like synthetic aperture radar altimetry. The objective is to provide sea level estimates up to $82^{\circ} \mathrm{N}$ over the last 2 decades with old missions (like ERS 1 and 2 and ENVISAT) and to provide sea level estimates up to $88^{\circ} \mathrm{N}$ over the last 7 years with recent polar missions such as CRYOSAT.

Recommendation \#3 - Uncertainties: Over the last decade progress has been made in quantifying uncertainties in estimates of in situ temperatures, sea level, ocean mass and its contributors. But it is still necessary to improve the uncertainty estimates. Uncertainties should be validated against other independent observations when possible. This is essential to increase the confidence in the EEI estimate. There are several directions of improvements:

(1) The first direction is to improve the uncertainty in in situ temperature. This is possible by developing/maintaining multiple platform observations for cross-validation and calibration purposes for current Argo measurements in the upper $2000 \mathrm{~m}$ depth, as well as for new extensions such as deep Argo.

(2) The second direction is to get a better confidence in GRACE uncertainty of ocean mass estimates by validation against independent data sources. Independent estimates of the ocean mass can be obtained through the ocean freshwater budget (Munk, 2003). This implies to improve the salinity record from Argo and the sea ice thickness estimate from altimetry.
(3) The third direction is to improve altimetry uncertainty by reducing the uncertainty in orbit determination (which depends on the accuracy of the gravity field prior of GRACE and the accuracy of the geocenter motion estimate) and by revisiting the uncertainty in the wet tropospheric correction (which can show biases with respect to independent estimates from SSMI satellites). To get higher confidence in altimetry uncertainty the comparison with tide gauge records should also be improved. This is possible by equipping more tide gauge records with global positioning system in order to improve tide gauge measurement of the absolute sea level and improve the comparison with satellite altimetry at both global and regional scale.

\section{AUTHOR CONTRIBUTIONS}

This manuscript resulted from the merger, suggested by OceanObs'19 organizers, of three different abstracts led by BM, TB, and ZZ. BM, TB, and ZZ led and designed the manuscript. All authors participated in the discussions and writing that led to the final contents of the manuscript.

\section{FUNDING}

GJ was supported by the NOAA Research. MP and RK were supported by the Met Office Hadley Centre Climate Programme funded by BEIS and Defra. JC was partially supported by the Centre for Southern Hemisphere Oceans Research, a joint research centre between QNLM and CSIRO. CD and AS were funded by the Australian Research Council (FT130101532 and DP160103130) and its Centre of Excellence for Climate Extremes (CLEX). IQuOD team members (TB, RC, LC, CD, VG, MI, MP, and SW) were supported by the Scientific Committee on Oceanic Research (SCOR) Working Group 148, funded by the National SCOR Committees and a grant to SCOR from the U.S. National Science Foundation (Grant OCE-1546580), as well as the Intergovernmental Oceanographic Commission of UNESCO/International Oceanographic Data and Information Exchange (IOC/IODE) IQuOD Steering Group. ZZ was supported by the National Aeronautics and Space Administration (NNX17AH14G). LC was supported by the National Key Research and Development Program of China (2017YFA0603200 and 2016YFC1401800).

\section{ACKNOWLEDGMENTS}

We thank the CNES AVISO project and the ESA CCI project for making their sea level data freely available. PMEL Contribution Number 4865. 


\section{REFERENCES}

Ablain, M., Cazenave, A., Larnicol, G., Balmaseda, M., Cipollini, P., Faugère, Y., et al. (2015). Improved sea level record over the Satellite Altimetry Era (19932010) from the climate change initiative project. Ocean Sci. 11, 67-82. doi: 10.5194/os-11-67-2015

Ablain, M., Cazenave, A., Valladeau, G., and Guinehut, S. (2009). A new assessment of the error budget of global mean sea level rate estimated by satellite altimetry over 1993-2008. Ocean Sci. 5, 193-201. doi: 10.5194/os-5-193-2009

Ablain, M., Meyssignac, B., Zawadzki, L., Jugier, R., Ribes, A., Cazenave, A., et al. (2019). Uncertainty in satellite estimate of global mean sea level changes, trend and acceleration. Earth Syst. Sci. Data Discuss. 1-26. doi: 10.5194/essd2019-10

Abraham, J. P., Baringer, M., Bindoff, N. L., Boyer, T., Cheng, L. J., Church, J. A., et al. (2013). A review of global ocean temperature observations: implications for ocean heat content estimates and climate change. Rev. Geophys. 51, 450-483. doi: 10.1002/rog.20022

Allan, R. P., Liu, C., Loeb, N. G., Palmer, M. D., Roberts, M., Smith, D., et al. (2014). Changes in global net radiative imbalance 1985-2012. Geophys. Res. Lett. 41, 5588-5597. doi: 10.1002/2014GL060962

Andreas, E. L. (1992). Sea spray and the surbulent air-sea heat fluxes. J. Geophys. Res. 97, 11429-11441. doi: 10.1029/92jc00876

Argo Science Team (1999). Report of the Argo Science Team Meeting (Argo-1). Melbourne: GODAE International Project Office, 27.

Balmaseda, M. A., Hernandez, F., Storto, A., Palmer, M. D., Alves, O., Shi, L., et al. (2015). The ocean reanalyses intercomparison project (ORA-IP). J. Operat. Oceanogr. 8(Suppl. 1), s80-s97. doi: 10.1080/1755876X.2015.1022329

Balmaseda, M. A., Trenberth, K. E., and Källén, E. (2013). Distinctive climate signals in reanalysis of global ocean heat content. Geophys. Res. Lett. 40, 1754-1759. doi: 10.1002/grl.50382

Beckley, B. D., Callahan, P. S., Hancock, D. W., Mitchum, G. T., and Ray, R. D. (2017). On the 'Cal-Mode' correction to TOPEX satellite altimetry and its effect on the global mean sea level time series. J. Geophys. Res. Oceans 122, 8371-8384. doi: $10.1002 / 2017 \mathrm{jc} 013090$

Beckley, B. D., Lemoine, F. G., Luthcke, S. B., Ray, R. D., and Zelensky, N. P. (2007). A reassessment of global and regional mean sea level trends from TOPEX and Jason-1 altimetry based on revised reference frame and orbits. Geophys. Res. Lett. 34:L14608. doi: 10.1029/2007GL030002

Benveniste, J. (2011). "Radar altimetry: past, present and future," in Coastal Altimetry, eds S. Vignudelli, A. Kostianoy, P. Cipollini, and J. Benveniste (Berlin: Springer), 1-17. doi: 10.1007/978-3-642-12796-0_1

Bindoff, N. L., Stott, P. A., AchutaRao, K. M., Allen, M. R., Gillett, N., Gutzler, D., et al. (2013). "Detection and attribution of climate change: from global to regional," in Climate Change 2013: The Physical Science Basis. Contribution of Working Group I to the Fifth Assessment Report of the Intergovernmental Panel on Climate Change, eds T. F. Stocker, D. Qin, G.-K. Plattner, M. Tignor, S. K. Allen, J. Boschung, et al.. (Cambridg: Cambridge University Press).

Blazquez, A., Meyssignac, B., Lemoine, J. M., Berthier, E., Ribes, A., and Cazenave, A. (2018). Exploring the uncertainty in GRACE estimates of the mass redistributions at the Earth surface: implications for the global water and sea level budgets. Geophys. J. Int. 215, 415-430. doi: 10.1093/gji/ ggy 293

Boyer, T., Domingues, C. M., Good, S. A., Johnson, G. C., Lyman, J. M., Ishii, M., et al. (2016). Sensitivity of global upper ocean heat content estimates to mapping methods, XBT bias corrections, and baseline climatologies. J. Clim. 29, 4817-4842. doi: 10.1175/JCLI-D-15-0801.1

Boyer, T. P., Antonov, J. I., Baranova, O. K., Coleman, C., Garcia, H. E., Grodsky, A., et al. (2013). World Ocean Database 2013. Silver Spring, MD: NOAA Printing Officce, 208

Chambers, D. P., Cazenave, A., Champollion, N., Dieng, H., Llovel, W., Forsberg, R., et al. (2017). Evaluation of the global mean sea level budget between 1993 and 2014. Surv. Geophys. 38:309. doi: 10.1007/s10712-016-9381-3

Chelton, D. B., Ries, J. C., Haines, B. J., Fu, L.-L., and Callahan, P. S. (2001). "Satellite altimetry," in Satellite Altimetry and Earth Sciences, Vol. 69, eds L. Fu and A. Cazenave (New York, NY: Elsevier), 1-131.

Chen, J. L., Wilson, C. R., and Tapley, B. D. (2013). Contribution of ice sheet and mountain glacier melt to recent sea level rise. Nat. Geosci. 9, 549-552. doi: $10.1038 /$ NGEO1829
Cheng, L., Abraham, J., Goni, G., Boyer, T., Wijffels, S., Cowley, R., et al. (2016a). XBT science: assessment of instrumental biases and errors. Bull. Am. Meterol. Soc. 96, 924-933. doi: 10.1175/BAMS-D-15-00031.1

Cheng, L., Abraham, J., Hausfather, Z., and Trenberth, K. E. (2019). How fast are the oceans warming? Science 363, 128-129. doi: 10.1126/science.aav 7619

Cheng, L., Luo, H., Boyer, T., Cowley, R., Abraham, J., Gouretski, V., et al. (2018). How well can we correct systematic errors in historical XBT data? J. Atmos. Oceanic Technol. 35, 1103-1125. doi: 10.1175/JTECH-D-17-0122.1

Cheng, L., Trenberth, K. E., Boyer, T., Fasullo, J., Zhu, L., and Abraham, J. (2017). Improved estimates of ocean heat content from 1960-2015. Sci. Adv. 3:e1601545. doi: 10.1126/sciadv.1601545

Cheng, L., Trenberth, K. E., Palmer, M. D., Zhu, J., and Abraham, J. P. (2016b). Observed and simulated full-depth ocean heat-content changes for 1970-2005. Ocean Sci. 12, 925-935. doi: 10.5194/os-12-925-2016

Cheng, L., and Zhu, J. (2014a). Artifact in variations of ocean heat content induced by the observation system changes. Geophys. Res. Lett. 41, 7276-7283. doi: 10.1002/2014GL061881

Cheng, L., and Zhu, J. (2014b). Uncertainties of the ocean heat content estimation induced by insufficient vertical resolution of historical ocean subsurface observations. J. Atmos. Oceanic Technol. 31, 1383-1396. doi: 10.1175/JTECHD-13-00220.1

Cheng, L., and Zhu, J. (2015). Influences of the choice of climatology on ocean heat content estimation. J. Atmos. Oceanic Technol. 32, 388-394. doi: 10.1175/ JTECH-D-14-00169.1

Cheng, L., Zhu, J., Cowley, R., Boyer, T., and Wijffels, S. (2014). Time, probe type and temperature variable bias corrections to historical expendable bathythermograph observations. J. Atmos. Oceanic Technol. 31, 1793-1825. doi: 10.1175/JTECH-D-13-00197.1

Church, J. A., Clark, P. U., Cazenave, A., Gregory, J. M., Jevrejeva, S., Levermann, A., et al. (2013). "Sea level change," in Climate Change 2013: The Physical Science Basis. Contribution of Working Group I to the Fifth Assessment Report of the Intergovernmental Panel on Climate Change, eds T. F. Stocker, D. Qin, G.-K. Plattner, M. Tignor, S. K. Allen, J. Boschung, et al. (Cambridge: Cambridge University Press), 1137-1216.

Church, J. A., White, N. J., Konikow, L. F., Domingues, C. M., Graham Cogley, J., Rignot, E., et al. (2011). Revisiting the Earth's sea-level and energy budget from 1961 to 2008. Geophys. Res. Lett. 38:L18601. doi: 10.1029/2011GL048794

Collins, W. D., Rasch, P. J., Eaton, B. E., Khattatov, B. V., Lamarque, J.-F., and Zender, C. S. (2001). Simulating aerosols using a chemical transport model with assimilation of satellite aerosol retrievals: methodology for INDOEX. J. Geophys. Res. 106, 7313-7336. doi: 10.1029/2000JD900507

Couhert, A., Cerri, L., Legeais, J. F., Ablain, M., Zelensky, N. P., Haines, B. J., et al. (2015). Towards the $1 \mathrm{~mm} / \mathrm{y}$ stability of the radial orbit error at regional scales. Adv. Space Res. 55, 2-23. doi: 10.1016/j.asr.2014.06.041

Cowley, R., Wijffels, S., Cheng, L., Boyer, T., and Kizu, S. (2013). Biases in expendable bathythermograph data: a new view based on historical side-by-side comparisons. J. Atmos. Ocean. Technol. 30, 1195-1225. doi: 10.1175/JTECH-D12-00127.1

Cox, C., and Munk, W. (1954). Measurement of the roughness of the sea surface from photographs of the Sun's glitter. J. Opt. Soc. Am. 44, 838-850.

Cronin, M. F., Weller, R. A., Lampitt, R. S., and Send, U. (2012). "Ocean reference stations," in Earth Observation, eds R. B. Rustamov and S. E. Salahova (London: IntechOpen). Available at: https://www.intechopen.com/ books/earth-observation/ocean-reference-stations

D. Coulot and l'équipe du projet Geodesie (2017). “Assimilation de données géodésiques et estimation de références pour l'étude du changement climatique," in Paper Présented at the Projet of ANR GEODESIE. XYZ, (SaintMandé: Association française de topographie (AFT)), 39-46.

Davies, J. H., and Davies, D. R. (2010). Earth's surface heat flux. Solid Earth 1, 5-24. doi: 10.5194/se-1-5-2010

Desbruyères, D. G., McDonagh, E. L., King, B. A., Garry, F. K., Blaker, A. T., Moat, B. I, et al. (2014). Full-depth temperature trends in the northeastern Atlantic through the early 21st century. Geophys. Res. Lett. 41, 7971-7979. doi: 10.1002/(ISSN)1944-8007

Desbruyères, D. G., Purkey, S. G., McDonagh, E. L., Johnson, G. C., and King, B. A. (2016). Deep and abyssal ocean warming from 35 years of repeat hydrography. Geophys. Res. Lett. 43, 356-310. doi: 10.1002/2016GL070413 
Dieng, H. B., Cazenave, A., Meyssignac, B., von Schuckmann, K., and Palanisamy, H. (2017). Sea and land surface temperatures, ocean heat content, Earth's energy imbalance and net radiative forcing over the recent years. Int. J. Climatol. 37, 218-229. doi: 10.1002/joc.4996

Dieng, H. B., Cazenave, A., von Schuckmann, K., Ablain, M., and Meyssignac, B. (2015). Sea level budget over(2005)-2013: missing contributions and data errors. Ocean Sci. 11, 789-802. doi: 10.5194/os-11-789-2015

Domingues, C., and Palmer, M. (2015). The IQuOD initiative: towards an international quality controlled ocean database. CLIVAR Exchanges 67, $38-40$.

Domingues, C. M., Church, J. A., White, N. J., Gleckler, P. J., Wijffels, S. E., Barker, P. M., et al. (2008). Improved estimates of upper-ocean warming and multi-decadal sea-level rise. Nature 453, 1090-1093. doi: 10.1038/nature07080

Dong, X., Xu, K., Liu, H., and Jiang, J. (2004). "The radar altimeter and scatterometer of China's HY-2 satellite," in Proceedings of the IEEE International Geoscience and Remote Sensing Symposium 2004, Vol. 13, (Anchorage, AK), 1703-1706. doi: 10.1109/IGARSS.2004.1370659

Durack, P. J., Gleckler, P. J., Landerer, F. W., and Taylor, K. E. (2014). Quantifying underestimates of long-term upper-ocean warming. Nat. Clim. Change 4, 9991005. doi: 10.1038/nclimate2389

Dushaw, B., Au, W., Beszczynska-Moller, A., Brainard, D., Bruce, D. C., Duda, T., et al. (2009). "A Global Ocean Acoustic Observing Network," in Proceedings of OceanObs'09: Sustained Ocean Observations and Information for Society, Vol. 2. Venice: European Space Agency, 279-291. doi: 10.5270/OceanObs09.cwp.25

Dushaw, B. D. (2018). Ocean acoustic tomography in the North Atlantic. J. Atmos. Oceanic Technol. 36, 183-202. doi: 10.1175/JTECH-D-18-0082.1

Ellingson, R. G. (1995). Surface longwave fluxes from satellite observations: a critical review. Remote Sens. Environ. 51, 89-97. doi: 10.1016/0034-4257(94) 00067-W

Eriksen, C. C. (2017). Deep gliders for observing circulation and climate. US CLIVAR Variat. 2, 34-38.

Fairall, C. W., Bradley, E. F., Hare, J. E., Grachev, A. A., and Bdson, J. B. (2003). Bulk parameterization of air-sea fluxes: update and verification for the COARE algorithm. J. Clim. 16, 571-591. doi: 10.1175/1520-0442(2003) $016<0571$ :bpoasf $>2.0$. co; 2

Fairall, C. W., Bradley, E. F., Rogers, D. P., Edson, J. B., and Young, G. S. (1996). Bulk parameterization of air-sea flux for tropical ocean-global atmosphere coupled-ocean atmosphere response experiment. J. Geophys. Res. 101, 37473746.

Fasullo, J. T., and Trenberth, K. E. (2008). The annual cycle of the energy budget Part I: global mean and land-ocean exchange. J. Clim. 21, 2297-2312. doi: 10.1175/2007JCLI11935.1

Feng, W., and Zhong, M. (2015). Global sea level variations from altimetry, GRACE and Argo data over 2005-2014. Geod. Geodyn. 6:4. doi: 10.1016/j.geog.2015. 07.001

Fernandes, M. J., Lázaro, C., Ablain, M., and Pires, N. (2015). Improved wet path delays for all ESA and reference altimetric missions. Remote Sens. Environ. 169, 50-74. doi: 10.1016/j.rse.2015.07.023

Forsyth, J. S. T., Andres, M., and Gawarkiewicz, G. G. (2015). Recent accelerated warming of the continental shelf off New Jersey: observations from the CMV Oleander expendable bathythermograph line. J. Geophys. Res. Oceans 120, 2370-2384. doi: 10.1002/2014JC010516

Foster, G., and Rahmstorf, S. (2011). Global temperature evolution 1979-2010. Environ. Res. Lett. 6:044022. doi: 10.1088/1748-9326/6/4/044022

Frederikse, T., Riva, R. E. M., and King, M. A. (2017). Ocean bottom deformation due to present-day mass redistribution and its impact on sea level observations. Geophys. Res. Lett. 44, 306-312. doi: 10.1002/2017GL075419

Fu, L.-L. (2016). On the decadal trend of global mean sea level and its implication on ocean heat content change. Front. Mar. Sci. 3:37. doi: 10.3389/fmars.2016. 00037

Good, S. A., Martin, M. J., and Rayner, N. A. (2013). EN4: quality controlled ocean temperature and salinity profiles and monthly objective analyses with uncertainty estimates. J. Geophys. Res. Oceans 118, 6704-6716. doi: 10.1002/ 2013JC009067

Gouretski, V. (2012). Using GEBCO digital bathymetry to infer depth biases in the XBT data. Deep-Sea Res. I 62, 40-52. doi: 10.1016/j.dsr.2011.12.012
Gouretski, V. (2018). World ocean circulation experiment - argo global hydrographic climatology. Ocean Sci. 14, 1127-1146. doi: 10.5194/os-14-11272018

Gouretski, V., Jungclaus, J., and Haack, H. (2013). Revisiting the Meteor 19251927 hydrographic dataset reveals centennial full-depth changes in the Atlantic Ocean. Geophys. Res. Lett. 40, 2236-2224. doi: 10.1002/grl.50503

Gouretski, V., and Koltermann, K. P. (2007). How much is the ocean really warming? Geophys. Res. Lett. 34:L01610. doi: 10.1029/2006GL027834

Gouretski, V., and Reseghetti, V. (2010). On depth and temperature biases in bathythermograph data: development of a new correction scheme based on analysis of a global ocean database. Deep Sea Res. Part I Oceanogr. Res. Pap. 57, 812-833. doi: 10.1016/j.dsr.2010.03.011

Gregory, J. M., Griffies, S. M., Hughes, C. W., Lowe, J. A., Church, J. A., Fukimori, I., et al. (2019). Concepts and terminology for sea level: mean, variability and change, both local and global. Surv. Geophys. 1-39. doi: 10.1007/s10712-01909525-Z

Griffies, S. M., Winton, M., Anderson, W. G., Benson, R., Delworth, T. L., Dufour, C. O., et al. (2015). Impacts on ocean heat from transient mesoscale eddies in a hierarchy of climate models. J. Clim. 28, 952-977. doi: 10.1175/jcli-d-1400353.1

Hakuba, M. Z., Stephens, G. L., Bruno, C., Nash, A. N., Foulon, B., Bettadpur, S. V., et al. (2018). Earth's energy imbalance measured from space. IEEE Trans. Geosci. Remote Sens. 57, 32-45. doi: 10.1109/TGRS.2018.2851976

Hansen, J., Sato, M., Kharecha, P., and von Schuckmann, K. (2011). Earth's energy imbalance and implications. Atmos. Chem. Phys. 11, 13421-13449. doi: 10. 5194/acp-11-13421-2011

Hartmann, D. L., Klein Tank, A. M. G., Rusticucci, M., Alexander, L. V., Brönnimann, S., Rahman Charabi, Y. A., et al. (2013). "Observations: atmosphere and surface, climate change 2013: the physical science basis," in Proceedings of the Working Group I Contribution to the Fifth Assessment Report of the Intergovernmental Panel on Climate Change, (Cambridge: Cambridge University Press), 159-254. doi: 10.1017/cbo9781107415324.008

Hedemann, C., Mauritsen, T., Jungclaus, J., and Marotzke, J. (2017). The subtle origins of surface-warming hiatuses. Nat. Clim. Change 7, 336-339. doi: 10. 1038/nclimate 3274

Henry, O., Ablain, M., Meyssignac, B., Cazenave, A., Masters, D., Nerem, S., et al. (2014). Investigating and reducing differences between the satellite altimetrybased global mean sea level time series provided by different processing groups. J. Geod. 88, 351-361. doi: 10.1007/s00190-013-0687-3

Howe, B., Worcester, P., Sagen, H., et al. (2019). The roles of underwater acoustics in observing the global ocean. Front. Mar. Sci.

IOC, SCOR, and IAPSO (2010). The International Thermodynamic Equation of Seawater - 2010: Calculation and Use of Thermodynamic Properties. Intergovernmental Oceanographic Commission, Manuals and Guides No. 56. Paris: UNESCO, 196.

Ishii, M., Fakuda, Y., Hirahara, S., Yasui, S., Suzuki, T., and Sato, K. (2017). Accuracy of global upper ocean heat content estimation expected from present observational data sets. SOLA 13, 163-167. doi: 10.2151/sola.2017-030

Ishii, M., and Kimoto, M. (2009). Reevaluation of historical ocean heat content variations with time-varying XBT and MBT depth bias corrections. J. Oceanogr. 65, 287-299. doi: 10.1007/s10872-009-0027-7

Jayne, S. R., Roemmich, D., Zilberman, N., Riser, S. C., Johnson, K. S., Johnson, G. C., et al. (2017). The argo program present and future. Oceanography. 30, 18-28. doi: 10.5670/oceanog.2017.213

Jin, Z., Charlock, T. P., Smith, W. L. Jr., and Rutledge, K. (2004). A parameterization ocean surface albedo. Geophys. Res. Lett. 31:L22301. doi: 10. 1029/2004GL021180

Johnson, G. C., and Birnbaum, A. N. (2017). As El Nino builds, Pacific Warm Pool expands, ocean gains more heat. Geophys. Res. Lett. 44, 438-445. doi: 10.1002/2016gl071767

Johnson, G. C., and Chambers, D. (2013). Ocean bottom pressure seasonal cycles and decadal trends from GRACE Release-05: ocean circulation implications. J. Geophys. Res. Oceans 118, 4228-4240. doi: 10.1002/jgrc.20307

Johnson, G. C., Lyman, J. M., Boyer, T., Cheng, L., Domingues, C. M., Gilson, J., et al. (2018). Ocean heat content. In State of the Climate in 2017. Bull. Am. Meteorol. Soc. 99, S72-S77. doi: 10.1175/2018BAMSStateoftheClimate.1 
Johnson, G. C., Lyman, J. M., and Loeb, N. G. (2016). CORRESPONDENCE: improving estimates of Earth's energy imbalance. Nat. Clim. Change 6, 639-640. doi: $10.1038 /$ nclimate 3043

Johnson, G. C., Lyman, J. M., and Purkey, S. G. (2015). Informing deep argo array design using argo and full- depth hydrographic section data. J. Atmos. Oceanic Technol. 32, 2187-2198. doi: 10.1175/JTECH-D-15-0139.1

Kato, S., Rose, F. G., Rutan, D. A., Thorsen, T. J., Loeb, N. G., Doelling, D. R., et al. (2018). Surface irradiances of edition 4.0 clouds and the earth's radiant energy system (CERES) energy balanced and filled (EBAF) data product. J. Clim. 31, 4501-4527. doi: 10.1175/JCLI-D-17-0523.1

Kato, S., Rose, F. G., Sun-Mack, S., Miller, W. F., Chen, Y. R., David, A. S., et al. (2011). Improvements of top-of-atmosphere and surface irradiance computations with CALIPSO-, CloudSat-, and MODIS-derived cloud and aerosol properties. J. Geophys. Res. 116:D19209. doi: 10.1029/2011JD016050

Kato, S., Xu, K.-M., Wong, T., Loeb, N. G., Rose, F. G., Trenberth, K. E., et al. (2016). Investigation of the bias in column integrated atmospheric energy balance using cloud objects. J. Clim. 29, 7435-7452. doi: 10.1175/JCLI-D-150782.1

Kuhlbrodt, T., and Gregory, J. M. (2012). Ocean heat uptake and its consequences for the magnitude of sea level rise and climate change. Geophys. Res. Lett. 39:L18608. doi: 10.1029/2012GL052952

Kuusela, M., and Stein, M. L. (2018). Locally stationary spatio-temporal interpolation of Argo profiling float data. Proc. R. Soc. A 474:20180400. doi: 10.1098/rspa.2018.0400

L'Ecuyer, T., Beaudoing, H. K., Rodell, M., Olson, W., Lin, B., and Kato, S. (2015). The observed state of the energy budget in the early 21 st century. J Clim. 28, 8319-8346. doi: 10.1175/JCLI-D-14-00556.1

Legeais, J.-F., Ablain, M., and Thao, S. (2014). Evaluation of wet troposphere path delays from atmospheric reanalyses and radiometers and their impact on the altimeter sea level. Ocean Sci. 10, 893-905. doi: 10.5194/os-10-8932014

Legeais, J.-F., Ablain, M., Zawadzki, L., Zuo, H., Johannessen, J. A., Scharffenberg, M. G., et al. (2018). An accurate and homogeneous altimeter sea level record from the ESA climate change initiative. Earth Syst. Sci. Data Discuss. 10, 1-35. doi: 10.5194/essd-2017-116

Leuliette, E. W., and Scharroo, R. (2010). Integrating Jason-2 into a multiplealtimeter climate data record. Mar. Geod. 33(Suppl. 1), 504-517. doi: 10.1080/ 01490419.2010.487795

Leuliette, E. W., and Willis, J. K. (2011). Balancing the sea level budget. Oceanography 24, 122-129. doi: 10.5670/oceanog. 2011.32

Levitus, S., Antonov, J., Boyer, T. P., and Stephens, C. (2000). Warming of the world ocean. Science 287, 2225-2229. doi: 10.1126/science.287.5461.2225

Levitus, S., Antonov, J. I., Boyer, T. P., Baranova, O. K., Garcia, H. E., Locarnini, R. A., et al. (2012). World ocean heat content and thermosteric sea level change (0-2000 m), 1955-2010. Geophys. Res. Lett. 39:L10603. doi: 10.1029/ 2012GL051106

Levitus, S., Antonov, J. I., Boyer, T. P., Locarnini, R. A., Garcia, H. E., and Mishonov, A. V. (2009). Global ocean heat content 1955-2008 in light of recently revealed instrumentation problems. Geophys. Res. Lett. 36:L07608. doi: 10.1029/2008GL037155

Liang, X., and Yu, L. (2016). Variations of the global net air-sea heat flux during the "Hiatus" Period (2001-10). J. Clim. 29, 3647-3660. doi: 10.1175/JCLI-D15-0626.1

Lickley, M. J., Hay, C. C., Tamisiea, M. E., and Mitrovica, J. X. (2018). Bias in estimates of global mean sea level change inferred from satellite altimetry. J. Clim. 31, 5263-5271. doi: 10.1175/JCLI-D-18-0024.1

Liu, C., Allan, R. P., Mayer, M., Hyder, P., Loeb, N. G., Roberts, C. D., et al. (2017). Evaluation of satellite and reanalysis-based global net surface energy flux and uncertainty estimates. J. Geophys. Res. Atmos. 122, 6250-6272. doi: 10.1002/2017JD026616

Llovel, W., Willis, J. K., Landerer, F. W., and Fukumori, I. (2014). Deep-ocean contribution to sea level and budget not detectable over the past decade. Nat. Clim. Change 4, 1031-1035. doi: 10.1038/NCLIMATE2387

Locarnini, R. A., Mishonov, A. V., Antonov, J. I., Boyer, T. P., Garcia, H. E., and Baranova, O. K. E. T. A. L. (2013). "World ocean atlas 2013, volume 1: temperature," in Proceedings of the NOAA Atlas NESDIS 73, ed. S. Levitus (Silver Spring, MD: NOAA), 40. doi: 10.1073/pnas.1303904110
Loeb, N. G., Doelling, D. R., Wang, H., Su, W., Nguyen, C., Corbett, J. G., et al. (2017). Clouds and the earth's radiant energy system (CERES) energy balanced and filled (EBAF) Top-of-atmosphere (TOA) edition 4.0 data product. J. Clim. 31, 895-918. doi: 10.1175/JCLI-D-17-0208.1

Loeb, N. G., Lyman, J. M., Johnson, G. C., Allan, R. P., Doelling, D. R., Wong, T., et al. (2012). Observed changes in top-of-the-atmosphere radiation and upper-ocean heating consistent within uncertainty. Nat. Geosci. 5, 110-113. doi: 10.1038/NGEO1375

Loeb, N. G., Rutan, D. A., Kato, S., and Wang, W. (2014). Observing interannual variations in Hadley circulation atmospheric diabatic heating and circulation strength. J. Clim. 27, 4139-4158. doi: 10.1175/JCLI-D-13-00656.1

Loeb, N. G., Thorsen, T. J., Norris, J. R., Wang, H., and Su, W. (2018a). Changes in earth's energy budget during and after the "pause" in global warming: an observational perspective. Climate 6:62. doi: 10.3390/cli603 0062

Loeb, N. G., Yang, P., Rose, F. G., Hong, G., Sun-Mack, S., Minnis, P., et al. (2018b). Impact of ice cloud microphysics on satellite cloud retrievals and broadband flux radiative transfer model calculations. J. Clim. 31, 1851-1864. doi: 10.1175/JCLI-D-17-0426.1

Loeb, N. G., Wielicki, B. A., Doelling, D. R., Smith, G. L., Keyes, D. F., Kato, S., et al. (2009). Toward optimal closure of the earth's top-of-atmosphere radiation budget. J. Clim. 22, 748-766. doi: 10.1175/2008jcli2637.1

Lowe, J. A., and Gregory, J. M. (2006). Understanding projections of sea level rise in a Hadley Centre coupled climate model. J. Geophys. Res. 111:C11014. doi: 10.1029/2005JC003421

Lyman, J. M., Good, S. A., Gouretski, V., Ishii, M., Johnson, G. C., and Palmer, M. D. (2010). Robust warming of the global upper ocean. Nature 465, 334-337. doi: 10.1038 /nature 09043

Lyman, J. M., and Johnson, G. C. (2008). Estimating annual global upper-ocean heat content anomalies despite irregular in situ ocean sampling. J. Clim. 21, 5629-5641. doi: 10.1175/2008JCLI2259.1

Lyman, J. M., and Johnson, G. C. (2014). Estimating global ocean heat content changes in the upper $1800 \mathrm{~m}$ since 1950 and the influence of climatology choice. J. Clim. 27, 1945-1957. doi: 10.1175/jcli-d-12-00752.1

Masters, D., Nerem, R. S., Choe, C., Leuliette, E., Beckley, B., White, N., et al. (2012). Comparison of global mean sea level time series from TOPEX/Poseidon, Jason1, and Jason-2. Mar. Geod. 35(Suppl. 1), 20-41. doi: 10.1080/01490419.2012. 717862

Masuda, K., Takashima, T., and Takayama, Y. (1988). Emissivity of pure and sea waters for the model sea surface in the infrared window regions. Remote Sens. Environ. 24, 313-329. doi: 10.1016/0034-4257(88)90032-6

Mayer, M., Haimberger, L., Edwards, J. M., and Hyder, P. (2018). Toward consistent diagnostics of the coupled atmosphere and ocean energy budget. J. Clim. 30, 9225-9246. doi: 10.1175/JCLI-D-17-0137.1

Menemenlis, D., Hill, C., Adcroft, A., Campin, J.-M., Cheng, B., Ciotti, B., et al. (2005). NASA supercomputer improves prospects for ocean climate research. EOS Trans. AGU 86, 89-96. doi: 10.1029/2005EO090002

Mitchum, G. T., Nerem, R. S., Merrifield, M. A., and Gehrels, R. (2010). "20th Century sea level change estimates from tide gauges and altimeters," in Understanding Sea-level Rise and Variability, eds J. A. Church, P. L. Woodworth, T. Aarup, and W. S. Wilson (London: Blackwells Publishing).

Monin, S. A., and Obukhov, A. M. (1954). Basic laws of turbulent mixing in the surface layer of the atmosphere. Contrib. Geophys. Inst. Acad. Sci. 24, 163-187.

Morrow, R., Fu, L.-L., Ardhuin, F., Benkiran, M., Chapron, B., Cosme, E., et al. (2019). Global observations of fine-scale ocean surface topography with the Surface Water and Ocean Topography (SWOT) Mission. Front. Mar. Sci. 6:232. doi: $10.3389 /$ fmars. 2019.00232

Munk, W. (2003). Ocean freshening, sea level rising. Science 300, 2041-2043. doi: $10.1126 /$ science. 1085534

Munk, W., and Worcester, P. F. (1976). "Monitoring the ocean acoustically," in Science, Technology, and the Modern Navy, Thirtieth Anniversary, 1946-1976, ed. E. I. Salkovitz (Arlington, VA: Office of Naval Research), 497-508.

Munk, W. H., Worcester, P. F., and Wunsch, C. (1995). Ocean Acoustic Tomography. New York, NY: Cambridge University Press.

Nerem, R. S., Chambers, D. P., Choe, C., and Mitchum, G. T. (2010). Estimating mean sea level change from the TOPEX and Jason altimeter missions. Mar. Geod. 33(Suppl. 1), 435-446. doi: 10.1080/01490419.2010.491031 
Palmer, M., Antonov, J., Barker, P., Bindoff, N., Boyer, T., Carson, M., et al. (2010). "Future observations for monitoring global ocean heat content," in Proceedings of OceanObs'09: Sustained Ocean Observations and Information for Society, Vol. 2, eds J. Hall, D. E. Harrison, and D. Stammer (Venice: ESA Publication).

Palmer, M. D., and Brohan, P. (2011). Estimating sampling uncertainty in fixeddepth and fixed-isotherm estimates of ocean warming. Int. J. Climatol. 31, 980-986. doi: 10.1002/joc.2224

Palmer, M. D., Durack, P., Chidichimo, M. P., Church, J., Cravatte, S. E., Hill, K. L., et al. (2019). Adequacy of the ocean observation system for quantifying regional heat and freshwater storage and change. Front. Mar. Sci. 6:416. doi: 10.3389/fmars.2019.00416

Palmer, M. D., Haines, K., Tett, S. F. B., and Ansell, T. J. (2007). Isolating the signal of global ocean warming. Geophys. Res. Lett. 34:L23610. doi: 10.1029/ 2007GL031712

Palmer, M. D., Roberts, C. D., Balmaseda, M., Chang, Y.-S., Chepurin, G., Ferry, N., et al. (2017). Ocean heat content variability and change in an ensemble of ocean reanalyses. Clim. Dyn. 49, 909-930. doi: 10.1007/s00382-015-2801-0

Penduff, T., Sérazin, G., Leroux, S., Close, S., Molines, J. M., Barnier, B., et al. (2018). Chaotic variability of ocean: heat content climate-relevant features and observational implications. Oceanography 31, 63-71. doi: 10.5670/oceanog. 2018.210

Penny, S. G., and Hamill, T. M. (2017). Coupled data assimilation for integrated earth system analysis and prediction. Bull. Am. Meteorol. Soc. 97, ES169-ES172.

Prandi, P., Ablain, M., Cazenave, A., and Picot, N. (2012). A new estimation of mean sea level in the arctic ocean from satellite altimetry. Mar. Geod. 35(Suppl. 1), 61-81. doi: 10.1080/01490419.2012.718222

Purkey, S. G., and Johnson, G. C. (2010). Warming of global abyssal and deep southern ocean waters between the 1990s and 2000s: contributions to global heat and sea level rise budgets. J. Clim. 23, 6336-6351. doi: 10.1175/ 2010JCLI3682.1

Rhein, M., Rintoul, S. R., Aoki, S., Campos, E., Chambers, D., Feely, R. A., et al. (eds) (2013). "Observations: ocean. in: climate change 2013: the physical science basis," in Proceedings of the Contribution of Working Group I to the Fifth Assessment Report of the Intergovernmental Panel on Climate Change, (Cambridge: Cambridge University Press), 255-316. doi: 10.1017/ CBO9781107415324.010

Riser, S. C., Howard, J. F., Dean, R., Susan, W., Ariel, T., Mathieu, B., et al. (2016). Fifteen years of ocean observations with the global Argo array. Nat. Clim. Change 6, 145-153. doi: 10.1038/NCLIMATE2872

Riser, S. C., Swift, D., and Drucker, R. (2018). Profiling floats in SOCCOM: technical capabilities for studying the Southern Ocean. J. Geophys. Res. Oceans 123, 4055-4073. doi: 10.1002/2017JC013419

Rodell, M., Beaudoing, H. K., L’Ecuyer, T. S., Olson, W. S., Famiglietti, J. S., Houser, P. R., et al. (2015). The observed state of the water cycle in the early twenty-first century. J. Clim. 28, 8289-8318. doi: 10.1175/JCLI-D-14-00555.1

Roemmich, D., Alford, M. H., Claustre, H., Johnson, K., King, B., Moum, J., et al. (2019). On the future of argo: a global, full-depth, multi-disciplinary array. Front. Mar. Sci. 6:439. doi: 10.3389/fmars.2019.00439

Roemmich, D., and Gilson, J. (2009). The 2004-2008 mean and annual cycle of temperature, salinity, and steric height in the global ocean from the Argo Program. Prog. Oceanogr. 82, 81-100. doi: 10.1016/j.pocean.2009. 03.004

Roemmich, D., Gould, J., and Gilson, J. (2012). 135 years of global warming between the challenger expedition and the argo programme. Nat. Clim. Change 2, 425-428. doi: 10.1038/nclimate1461

Rogelj, J., Popp, A., Calvin, K. V., Luderer, G., Emmerling, J., Gernaat, D., et al. (2018). Scenarios towards limiting global mean temperature increase below $1.5^{\circ} \mathrm{C}$. Nature Climate Change 8, 325-332. doi: 10.1038/s41558-0180091-3

Sidran, M. (1981). Broadband reflectance and emissivity of specular and rough water surfaces. Appl. Opt. 20, 3176-3183. doi: 10.1364/AO.20.003176

Sloyan, B. M., Wanninkhof, R., Kramp, M., Johnson, G. C., Talley, L. D., Tanhua, T., et al. (2019). The global ocean ship-based hydrographic investigations program (GO-SHIP): a platform for integrated multidisciplinary ocean science. Front. Mar. Sci. 6:445. doi: 10.3389/fmars.2019.00445

Smith, D. M., Allan, R. P., Coward, A. C., Eade, R. O., Hyder, P., Liu, C., et al. (2015). Earth's energy imbalance since 1960 in observations and CMIP5 models. Geophys. Res. Lett. 42, 1205-1213. doi: 10.1002/2014gl062669
Stammer, D., Balmaseda, M., Heimbach, P., Köhl, A., and Weaver, A. (2016). Ocean data assimilation in support of climate applications - status and perspectives. Ann. Rev. Mar. Sci. 8, 491-518. doi: 10.1146/annurev-marine-122414034113

Stephens, G. L., Li, J.-L., Wild, M., Anne Clayson, C., Loeb, N. G., Kato, S., et al. (2012). An update on Earth's energy balance in light of the latest global observations. Nat. Geosci. 5, 691-696. doi: 10.1038/ngeo1580

Storto, A., Alvera-Azcárate, A., Balmaseda, M. A., Barth, A., Chevallier, M., Counillon, F., et al. (2019). Ocean reanalyses: recent advances and unsolved challenges. Front. Mar. Sci. 6:418. doi: 10.3389/fmars.2019.00418

Storto, A., Masina, S., Balmaseda, M., Guinehut, S., Xue, Y., Szekely, T., et al. (2015). Steric sea level variability (1993-2010) in an ensemble of ocean reanalyses and objective analyses. Clim. Dyn. 49, 1-21. doi: 10.1007/s00382-015-2554-9

Talley, L. D., Feely, R. A., Sloyan, B. M., Wanninkhof, R., Baringer, M. O., Bullister, J. L., et al. (2016). Changes in ocean heat, carbon content, and ventilation: a review of the first decade of GO-SHIP global repeat hydrography. Annu. Rev. Mar. Sci. 8, 185-215. doi: 10.1146/annurev-marine-052915-100829

Thao, S., Eymard, L., Obligis, E., and Picard, B. (2014). Trend and variability of the atmospheric water vapor: a mean sea level issue. J. Atmos. Oceanic Technol. 31, 1881-1901. doi: 10.1175/JTECH-D-13-00157.1

The WCRP Global sea level budget group (2018). Global sea-level budget 1993present. Earth Syst. Sci. Data 10, 1551-1590. doi: 10.5194/essd-10-1551-2018

Toole, J. M., Krishfield, R. A., Timmermans, M.-L., and Proshutinsky, A. (2011). The ice-tethered profiler: argo of the arctic. Oceanography 24, 126-135. doi: 10.5670/oceanog.2011.64

Trenberth, K., and Fasullo, J. (2016). Insights into earth's energy imbalance from multiple sources. J. Clim. 29, 7495-7505. doi: 10.1175/JCLI-D-16-0339.1

Trenberth, K. E., and Fasullo, J. T. (2013). Regional energy and water cycle: transports from ocean to Land. J. Clim. 26, 7837-7851. doi: 10.1175/JCLI-D13-00008.1

Trenberth, K. E., Fasullo, J. T., and Balmaseda, M. A. (2014). Earth's energy imbalance. J. Clim. 27, 3129-3144.

Trenberth, K. E., and Stepaniak, D. P. (2003). Co-variability of components of poleward atmospheric energy transports on seasonal and interannual timescales. J. Clim. 16, 3691-3705. doi: 10.1175/1520-0442(2003)016<3691: cocopa $>2.0$. co; 2

Trenberth, K. E., and Stepaniak, D. P. (2004). The flow of energy trough the earth's climate system. Q. J. R. Meteorol. Soc. 130, 2677-2701.

Trenberth, K. E., Stepaniak, D. P., and Caron, J. M. (2002). Accuracy of atmospheric energy budgets from analyses. J. Clim. 15, 3343-3360. doi: 10.3390/ rs10081175

Turner, R. E., Rabalais, N. N., and Justiæ, D. (2017). Trends in summer bottom-water temperatures on the northern Gulf of Mexico continental shelf from 1985 to 2015. PLoS One 12:e0184350. doi: 10.1371/journal.pone.01 84350

Uebbing, B., Kusche, J., Rietbroek, R., and Landerer, F. W. (2019). Processing choices affect ocean mass estimates from GRACE. J. Geophys. Res. Oceans 124, 1029-1044. doi: 10.1029/2018JC014341

Uematsu, A., Nakamura, R., Nakajima, Y., Yajima, Y., and the Jaxa Compira team. (2013). "X-band interferometric SAR sensor for the Japanese altimetry mission, COMPIRA," in Proceedings of the IGARSS, (Melbourne, VIC).

Valladeau, G., Legeais, J. F., Ablain, M., Guinehut, S., and Picot, N. (2012). Comparing altimetry with tide gauges and argo profiling floats for data quality assessment and mean sea level studies. Mar. Geod. 35(Suppl. 1), 42-60. doi: 10.1080/01490419.2012.718226

von Schuckmann, K., and LeTraon, P.-Y. (2011). How well can we derive global ocean indicators from Argo data? Ocean Sci. 7, 783-791. doi: 10.5194/os-7-7832011

von Schuckmann, K., Palmer, M. D., Trenberth, K. E., Cazenave, A., Chambers, D., Champollion, N., et al. (2016). An imperative to monitor Earth's energy imbalance. Nat. Clim. Change 6, 138-144. doi: 10.1038/NCLIMATE 2876

von Schuckmann, K., Pierre-Yves, L. T., Neville, S., Ananda, P., Pierre, B., Katja, F., et al. (2018). Copernicus marine service ocean state report. J. Operat. Oceanogr. 11(Suppl. 1), S1-S142. doi: 10.1080/1755876X.2018.148 9208

Wang, F., and Hu, D. (2010). Introduction to international NPOCE program. Chin. J. Oceanol. Limnol. 28, 953-953. doi: 10.1007/s00343-010-0953-7 
Wang, G., Cheng, L., Abraham, J., and Li, C. (2018). Consensuses and discrepancies of basin-scale ocean heat content changes in different ocean analyses. Clim. Dyn. 50, 2471-2487. doi: 10.1007/s00382-017-3751-5

Watkins, M. M., Wiese, D. N., Yuan, D.-N., Boening, C., and Landerer, F. W. (2015). Improved methods for observing Earth's time variable mass distribution with GRACE using spherical cap mascons. J. Geophys. Res. Solid Earth 120, 2648-2671. doi: 10.1002/2014JB011547

Watson, C. S., White, N. J., Church, J. A., King, M. A., Burgette, R. J., and Legresy, B. (2015). Unabated global mean sea-level rise over the satellite altimeter Era. Nat. Clim. Change 5, 565-568. doi: 10.1038/nclimate2635

Webb, E. K., Pearman, G. I, and Leuning, R. (1980). Correction of the flux measurements for density effects due to heat and water vapour transfer. Quart. J. R. Meteorol. Soc. 106, 85-100. doi: 10.1256/smsqj.44706

Wiese, D. N., Yuan, D.-N., Boening, C., Landerer, F. W., and Watkins, M. M. (2016b). JPL GRACE Mascon Ocean, Ice, and Hydrology Equivalent Water Height RL05M.1 CRI Filtered Version 2., Ver. 2. California, CA: PODAAC, doi: 10.5067/TEMSC-2LCR5

Wiese, D. N., Landerer, F. W., and Watkins, M. M. (2016a). Quantifying and reducing leakage errors in the JPL RL05M GRACE mascon solution. Water Resour. Res. 52, 7490-7502. doi: 10.1002/2016WR019344

Wild, M., Folini, D., Hakuba, M. Z., Schär, C., Seneviratne, S. I., Kato, S., et al. (2014). The energy balance over land and oceans: and assessment based on direct observations and CMIP5 climate models. Clim. Dyn. 44, 3393-3429. doi: $10.1007 / \mathrm{s} 00382-014-2430-\mathrm{Z}$

Willis, J. K. (2004). Interannual variability in upper ocean heat content, temperature, and thermosteric expansion on global scales. J. Oceanogr. 109:C12036. doi: 10.1029/2003JC002260

Wouters, B., Bonin, J. A., Chambers, D. P., Riva, R. E. M., Sasgen, I., and Wahr, J. (2014). GRACE, time-varying gravity, earth system dynamics and climate change. Rep. Prog. Phys. 77:116801. doi: 10.1088/0034-4885/77/11/116801
Wunsch, C. (2016). Global ocean integrals and means, with trend implications. Annu. Rev. Mar. Sci. 8, 1-33. doi: 10.1146/annurev-marine-122414-0 34040

Wunsch, C., and Heimbach, P. (2014). Bidecadal thermal changes in the abyssal ocean. J. Phys. Oceanogr. 44, 2013-2030. doi: 10.1175/JPO-D-13-096.1

Zawadzki, L., and Ablain, M. (2016). Accuracy of the mean sea level continuous record with future altimetric missions: Jason-3 vs. Sentinel-3a. Ocean Sci. 12, 9-18. doi: 10.5194/os-12-9-2016

Zhao, Z. (2016). Internal tide oceanic tomography. Geophys. Res. Lett. 43, 91579164. doi: 10.1002/2016GL070567

Zweng, M. M., Boyer, T. P., Baranova, O. K., Reagan, J. R., Seidov, D., and Smolyar, I. V. (2018). An inventory of arctic ocean data in the world ocean database. Earth Syst. Sci. Data 10, 677-687. doi: 10.5194/essd-10-6772018

Conflict of Interest Statement: The authors declare that the research was conducted in the absence of any commercial or financial relationships that could be construed as a potential conflict of interest.

Copyright (c) 2019 Meyssignac, Boyer, Zhao, Hakuba, Landerer, Stammer, Köhl, Kato, L'Ecuyer, Ablain, Abraham, Blazquez, Cazenave, Church, Cowley, Cheng, Domingues, Giglio, Gouretski, Ishii, Johnson, Killick, Legler, Llovel, Lyman, Palmer, Piotrowicz, Purkey, Roemmich, Roca, Savita, von Schuckmann, Speich, Stephens, Wang, Wijffels and Zilberman. This is an open-access article distributed under the terms of the Creative Commons Attribution License (CC BY). The use, distribution or reproduction in other forums is permitted, provided the original author(s) and the copyright owner(s) are credited and that the original publication in this journal is cited, in accordance with accepted academic practice. No use, distribution or reproduction is permitted which does not comply with these terms. 
OPEN ACCESS

Edited by:

Sanae Chiba,

Japan Agency for Marine-Earth

Science and Technology, Japan

Reviewed by:

Robert Andrew Weller,

Woods Hole Oceanographic

Institution, United States

Detlef Stammer,

Universität Hamburg, Germany

*Correspondence:

Toste Tanhua

ttanhua@geomar.de

Specialty section:

This article was submitted to

Ocean Observation,

a section of the journal

Frontiers in Marine Science

Received: 15 November 2018

Accepted: 12 July 2019

Published: 20 August 2019

Citation:

Tanhua T, McCurdy A, Fischer A,

Appeltans W, Bax N, Currie K,

DeYoung B, Dunn D, Heslop E,

Glover LK, Gunn J, Hill K, Ishii M,

Legler D, Lindstrom E, Miloslavich P,

Moltmann T, Nolan G, Palacz A,

Simmons S, Sloyan B, Smith LM,

Smith N, Telszewski M, Visbeck M

and Wilkin J (2019) What We Have

Learned From the Framework for

Ocean Observing: Evolution of the

Global Ocean Observing System.

Front. Mar. Sci. 6:471.

doi: 10.3389/fmars.2019.00471

\section{What We Have Learned From the Framework for Ocean Observing: Evolution of the Global Ocean Observing System}

\author{
Toste Tanhua ${ }^{*}$, Andrea McCurdy ${ }^{2}$, Albert Fischer ${ }^{3}$, Ward Appeltans ${ }^{4}$, Nicholas Bax ${ }^{5,6}$, \\ Kim Currie ${ }^{7}$, Brad DeYoung ${ }^{8}$, Daniel Dunn 9,10, Emma Heslop $^{3}$, Linda K. Glover ${ }^{11}$, \\ John Gunn ${ }^{6}$, Katherine Hill'2, Masao Ishii ${ }^{13}$, David Legler ${ }^{14}$, Eric Lindstrom ${ }^{15}$, \\ Patricia Miloslavich ${ }^{6,16}$, Tim Moltmann ${ }^{6}$, Glenn Nolan ${ }^{17}$, Artur Palacz ${ }^{18}$, \\ Samantha Simmons ${ }^{19}$, Bernadette Sloyan ${ }^{5}$, Leslie M. Smith ${ }^{20}$, Neville Smith ${ }^{21}$, \\ Maciej Telszewski ${ }^{17}$, Martin Visbeck ${ }^{1}$ and John Wilkin ${ }^{22}$
}

${ }^{1}$ GEOMAR Helmholtz Centre for Ocean Research Kiel, Marine Biogeochemistry, Kiel, Germany, ${ }^{2}$ Cooperative Programs for the Advancement of Earth System Science, University Corporation for Atmospheric Research, Boulder, CO, United States, ${ }^{3}$ Global Ocean Observing System, Paris, France, ${ }^{4}$ Intergovernmental Oceanographic Commission of UNESCO, Oostende, Belgium, ${ }^{5}$ CSIRO and University of Tasmania, Hobart, TAS, Australia, ${ }^{6}$ Institute for Marine and Antarctic Studies, University of Tasmania, Hobart, TAS, Australia, ${ }^{7}$ National Institute for Water and Atmospheric Research, Dunedin, New Zealand, ${ }^{8}$ Department of Physics and Physical Oceanography, Memorial University, St. John's, NL, Canada, ${ }^{9}$ Nicholas School of the Environment, Duke University, Beaufort, NC, United States, ${ }^{10}$ School of Earth and Environmental Sciences, University of Queensland, Brisbane, QLD, Australia, ${ }^{11}$ Glover Works Consulting, Alexandria, VA, United States, ${ }^{12}$ World Meteorological Organization, Geneva, Switzerland, ${ }^{13}$ Meteorological Research Institute, Ibaraki, Japan, ${ }^{14}$ National Oceanic and Atmospheric Administration, Climate Program Office, Silver Spring, MD, United States, ${ }^{15}$ National Aeronautics and Space Administration, Washington, DC, United States, ${ }^{16}$ Departamento de Estudios Ambientales, Universidad Simón Bolivar, Caracas, Venezuela, ${ }^{17}$ European Global Ocean Observing System, Brussels, Belgium, ${ }^{18}$ International Ocean Carbon Coordination Project, Institute of Oceanology, Polish Academy of Sciences, Sopot, Poland, ${ }^{19}$ Marine Mammal Commission, Bethesda, MD, United States, ${ }^{20}$ Your Ocean Consulting, LLC, Knoxville, TN, United States, ${ }^{21}$ GODAE Ocean Services, Melbourne, VIC, Australia, ${ }^{22}$ Department of Marine and Coastal Sciences, Rutgers University, New Brunswick, NJ, United States

The Global Ocean Observing System (GOOS) and its partners have worked together over the past decade to break down barriers between open-ocean and coastal observing, between scientific disciplines, and between operational and research institutions. Here we discuss some GOOS successes and challenges from the past decade, and present ideas for moving forward, including highlights of the GOOS 2030 Strategy, published in 2019. The OceanObs'09 meeting in Venice in 2009 resulted in a remarkable consensus on the need for a common set of guidelines for the global ocean observing community. Work following the meeting led to development of the Framework for Ocean Observing (FOO) published in 2012 and adopted by GOOS as a foundational document that same year. The FOO provides guidelines for the setting of requirements, assessing technology readiness, and assessing the usefulness of data and products for users. Here we evaluate successes and challenges in FOO implementation and consider ways to ensure broader use of the FOO principles. The proliferation of ocean observing activities around the world is extremely diverse and not managed, or even overseen by, any one entity. The lack of coherent governance has resulted in duplication and varying degrees of clarity, responsibility, coordination and data sharing. GOOS has had considerable success over the past decade in encouraging voluntary collaboration across much of this broad community, including increased use 
of the FOO guidelines and partly effective governance, but much remains to be done. Here we outline and discuss several approaches for GOOS to deliver more effective governance to achieve our collective vision of fully meeting society's needs. What would a more effective and well-structured governance arrangement look like? Can the existing system be modified? Do we need to rebuild it from scratch? We consider the case for evolution versus revolution. Community-wide consideration of these governance issues will be timely and important before, during and following the OceanObs'19 meeting in September 2019.

Keywords: ocean observing, governance, framework for ocean observing, sustainable development, multidisciplinary, international

\section{THE MANDATE FOR SUSTAINED OCEAN OBSERVING}

The ocean affects humans in many ways, regardless of where we live. It continues to produce most of the oxygen we breathe and is the primary controller of the global climate that makes this planet habitable. It provides humans with food, materials, energy, transportation, and recreation.

However, the ocean is also the source of many hazards, both natural and anthropogenic induced including increasingly strong hurricanes and severe coastal flooding, tsunamis, storm surges, sea level rise, toxic algal blooms and other pollution. An ability to observe and forecast the ocean and its links to weather, climate and biogeochemical phenomena are required to mitigate risks via improved early warning systems.

Assessing progress of the United Nations (UN) Agenda 2030 and its Sustainable Development Goal 14 to, "conserve and sustainably use the oceans, seas and marine resources for sustainable development," and many of the other 17 Sustainable Development Goals will require sustained ocean data. There will be a profound need for essential ocean information to guide policy and progress toward both local public safety needs as well as the range of internationally-agreed upon goals.

The international community has identified global goals related to sustainable development, climate change, and disaster risk reduction that all require systematic ocean observations:

- The UN Sustainable Development Goals: At the June 2017 UN Ocean Conference, governments called for more resources for sustained ocean and coastal observation, "in order to increase our knowledge of the ocean, to better understand the relationship between climate and the health and productivity of the ocean, to strengthen the development of coordinated early warning systems on extreme weather events and phenomena, and to promote decision-making based on the best available science."

- Improved monitoring of marine ecosystems also supports global goals under the Convention for Biodiversity, regional frameworks such as Europe's Marine Strategy Framework Directive, and assessments like those produced by the Intergovernmental Science-Policy Platform for Biodiversity and Ecosystem Services (IPBES) and the World Ocean Assessment.
- The UN Framework Convention on Climate Change (UNFCCC) and the Paris Agreement (2015) note the importance of ensuring the integrity of all ecosystems, including those in the ocean, and call on countries to strengthen, "systematic observation of the climate system and early warning systems, in a manner that informs climate services and supports decision-making." Ocean observations are also essential to the validation of climate projections assessed by the Intergovernmental Panel for Climate Change.

Governments and policymakers are facing complex decisions that require information from sustained ocean observations. We lack both the observations and integration necessary to fully meet these needs. In many areas, sustained ocean observations are simply too infrequent, sparse, inadequate, or imprecise. A step-change is required in worldwide investment in order to take advantage of the changes made possible by increasing requirements and the expansion of technological developments and the adoption of open data policies. The improved understanding of the ocean based on decades of scientific effort, in concert with a recognized need for continued coordination of efforts to observe, analyze, understand and predict the ocean will assist in the realization of a return on this important societal investment.

\section{THE GLOBAL OCEAN OBSERVING SYSTEM (GOOS) AND THE FRAMEWORK FOR OCEAN OBSERVING (FOO)}

GOOS was established in 1991 by the Member States of the Intergovernmental Oceanographic Commission (IOC) of the United Nations Educational, Scientific and Cultural Organization (UNESCO), with the World Meteorological Organization (WMO), UN Environment, and the International Science Council (ISC) later joining as sponsors.

Over the past quarter-century, the GOOS community and partners have worked well in coordinating global ocean climate observing and information products and in supporting observations for operational forecast systems. More recently, GOOS has had a growing focus on an integrated global observing 
system including a wider range of data types and serving a broader range of users, consisted with the Framework of Ocean Observing (FOO, Lindstrom et al., 2012).

In 2012, the IOC General Assembly unanimously endorsed all the FOO recommendations. A new GOOS Steering Committee was established to replace the IOC Intergovernmental Committee on GOOS and its supporting GOOS Scientific Steering Committee. Three new recommended expert panels were formed, and the GRA Council was reinvigorated.

\section{Origin and Early Accomplishments of the FOO}

The OceanObs'09 Conference ${ }^{1}$ in Venice, Italy achieved broad agreement on the need for interdisciplinary, internationally integrated ocean observations. Based on general consensus at the meeting, its 18 sponsors commissioned a working group of international program representatives to create a systematic approach for defining requirements for ocean observations, deciding appropriate technology for measurements, and assessing data standards and dissemination. The resulting Framework for Ocean Observing has been widely endorsed by the ocean observing community, and adopted formally by GOOS as a guiding document.

In addition to its extensive recommendations on the design of an enhanced ocean observing system, the FOO made two recommendations on governance:

- To simplify and strengthen the high-level governance of GOOS, establish a single, expertise-based Steering Committee reporting directly to the IOC officers and members; and,

- Establish two new GOOS Panels - for Biogeochemistry, and for Biology and Ecosystems, to complement the existing Observations of Ocean Physics and Climate Panel.

The FOO argues that it is essential that governance of the global ocean observing system reflect the needs and contributions of both the broad ocean observing system community (scientists, institutions, observing system managers), and the IOC member states who should represent their national and collectively the international community's interests, and users of ocean information. The changes to the GOOS Program governance made in 2012 were a step-change toward providing a balance between the interests of these two communities. However, since OceanObs'09 and the FOO the proliferation of consortia/organizations (the "acronym soup") that now share the broad ocean observing mission makes the governance challenge even more complex.

It is important to note that the 2012 IOC endorsement of the expanded GOOS focus has not resulted in increased IOC budgetary support. Indeed, in real terms core budgetary support for GOOS has declined. Operations of the significantly expanded work plans for GOOS have been funded through short term grants and financial support from various institutions and regional funding programs. This has driven a significant decentralization of GOOS efforts and weakens sustainability.

\footnotetext{
${ }^{1}$ http://www.oceanobs09.net/index.php
}

\section{Elements of the Framework for Ocean Observing}

The FOO provides a structure that allows ocean observing providers and users to engage in the system at various points. It traces the path from Inputs (e.g., essential ocean variables) to Processes (observations and maintenance), to Outputs (data and products). It has helped form an understanding of the elements of the system as a whole and has facilitated the activities of GOOS in many areas (Figure 1).

The common language and system design principles introduced by the FOO are:

- Essential Ocean Variables (EOVs)

- Requirements

- Observing system elements

- Data management and information products

- Readiness levels (for requirements, observations, and data and information)

- Incorporation of both coastal and open ocean observations

- Feedback loops addressing science challenges and social needs

\section{Elements of the GOOS}

The organization of GOOS is comprised of several key elements (see Figure 2).

\section{GOOS Steering Committee}

GOOS is guided by a Steering Committee, with 10 expert members appointed by the IOC Executive Secretary in consultation with sponsors, and five members selected by IOC regional electoral groups. The Steering Committee reports to the IOC Assembly and other sponsors, defines the GOOS work plan, and manages the structures that report to it.

\section{GoOS Office}

The GOOS Office, headquartered at the IOC, consists of a small core team with in-kind contributions from several supporting agencies. The Office supports the work and actions of the Steering Committee, panels, and implementation structures of GOOS, serving as a hub of communication, and point of contact for partners.

\section{Expert Panels}

Three panels for global ocean observing are focused on developing essential ocean variables (EOVs), evaluating success of the system, and synthesizing across the climate, operational services and ocean ecosystem health requirements. The three panels are: Physics (The Ocean Observations Physics and Climate panel, co-sponsored with the Global Climate Observing System, GCOS), Biogeochemistry (the International Ocean Carbon Coordination Project, IOCCP), and the Biology and Ecosystems Panel.

\section{The Observations Coordination Group}

The IOC/WMO Joint Technical Commission for Oceanography and Marine Meteorology (JCOMM) has an Observations Coordination Group (OCG) charged with reviewing, advising on, 


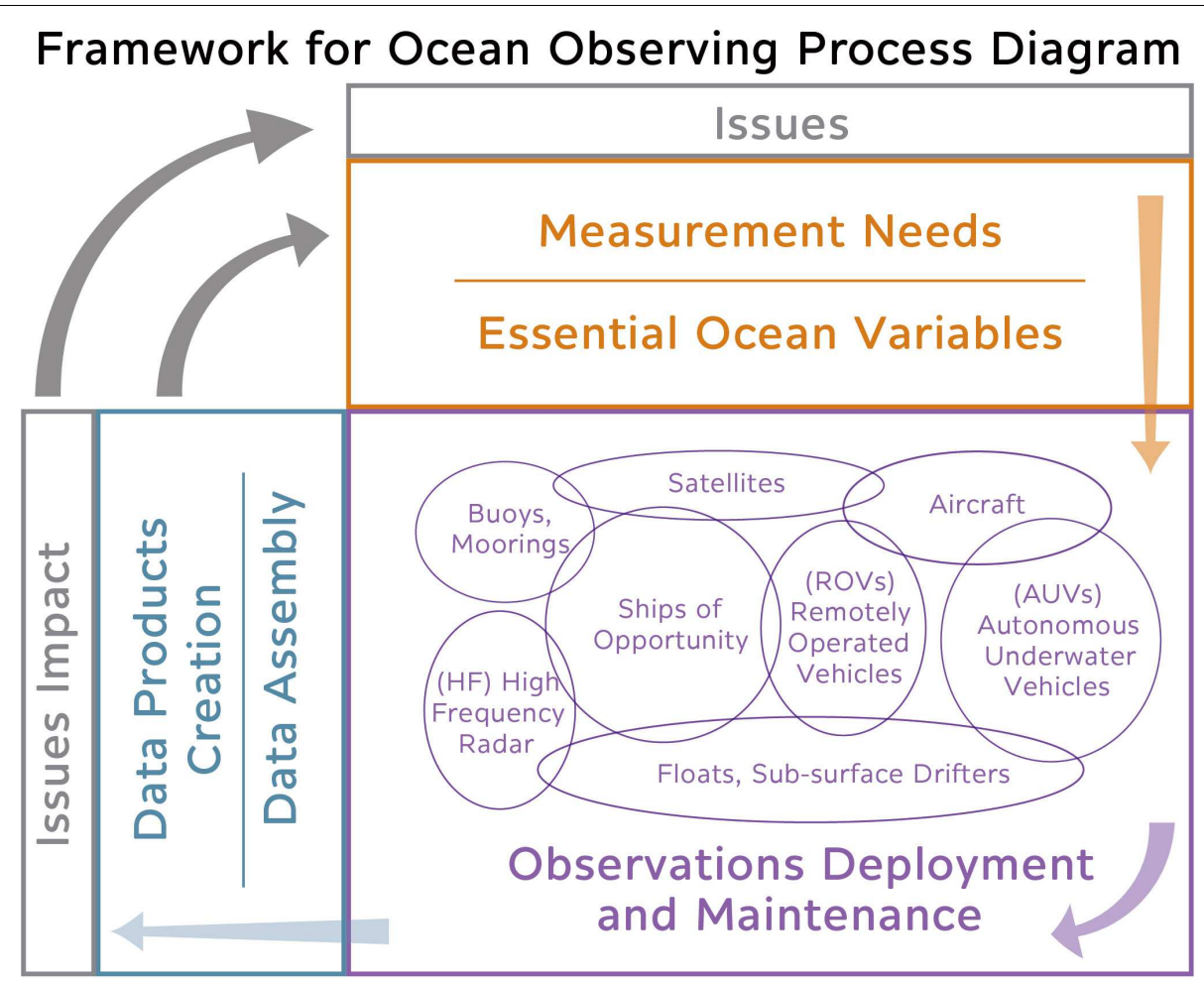

FIGURE 1 | Overview of the key elements in the Framework of Ocean Observing (FOO).

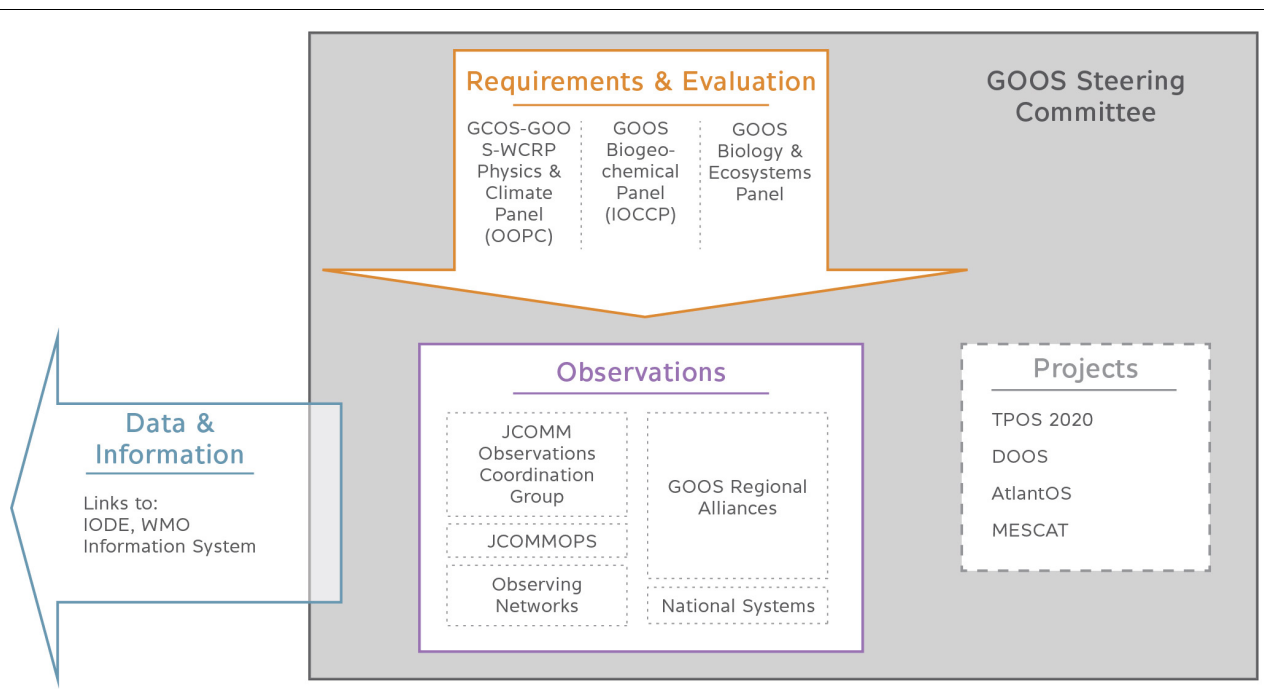

FIGURE 2 | Primary areas of activity and influence for key GOOS elements and linkages in 2018.

and coordinating the effective operation of the ocean and marine observing networks and related activities. Notable progress has been made in several areas: engaging networks to address new requirements; developing metrics to assess observing system performance; advancing the exchange of international data and metadata, encouraging system-wide standards and best practices, data management standards and integration pilot projects.
The OCG monitors and reports on progress of, and risks to, the ocean observing networks. Increasing the use of metrics throughout the ocean observing value chain allows for more robust evaluations of the system, and eventually will enable monitoring of its performance and provision of feedback into improved requirements and value. The OCG monitors and coordinates testing and assessments of ocean 


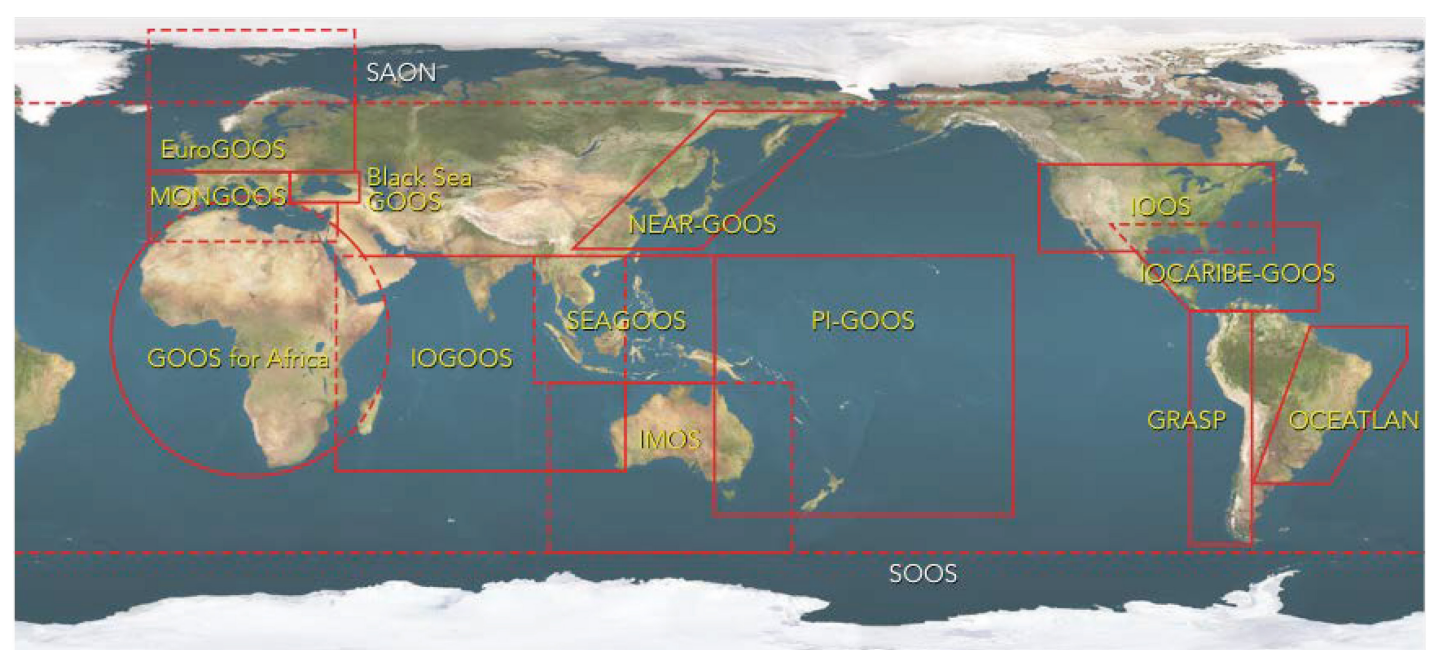

\section{GOOS Regional Alliances (GRAs)}

Black Sea GOOS

EuroGOOS

GOOS-Africa

GRASP = GOOS Regional Alliance of the SE Pacific

IOCARIBE GOOS = Wider Caribbean GOOS

IMOS = Integrated Marine Observing System (Australia)

lOGOOS = Indian Ocean GOOS

MONGOOS = Mediterranean Operational Network for GOOS
NEAR-GOOS - NE Asia Regional GOOS

OCEATLAN = Upper SW \& Tropical Pacific

PIGOOS - Pacific Islands GOOS

SEAGOOS = SE Asia GOOS

US IOOS = US Integrated Ocean Observing System

Not GRAs, but related programs

SAON = Sustained Arctic Observing Network

SOOS = Southern Ocean Observing System

FIGURE 3 | GOOS Regional Alliances (GRAs).

observing technologies as they mature and approach readiness for sustained operation, and it will support assessments that consider the mix of platforms and /or technologies to best meet requirements.

Note that the Joint WMO-IOC Collaborative Board, a high-level coordination mechanism with broader engagement of the key relevant bodies of the WMO and IOC, which will be established and, subsequently, JCOMM will phase out as a decision during the WMO Congress-18. The congress also decided to incorporate appropriate JCOMM functions and activities on observation and operational ocean forecasting systems into GOOS, with functional connections to the Commission for Observation, Infrastructure and Information Systems.

\section{GOOS Regional Alliances}

Thirteen GOOS Regional Alliances (GRAs) that have organized themselves over the past two decades covering most regions of the globe (Figure 3). GRAs enable regional cooperation in ocean observing and in some cases in ocean forecasting and services. There is great variability among the GRAs in terms of governance, scope and maturity of activity. GRA attitudes to data sharing also vary widely, from full open access in some regions to restrictions on data sharing and use in others. The regional level of governance of GOOS is therefore ripe for evolution and adaptation, a process that will need to take stock of the regional structures that organize both science and policy.

\section{GOOS Projects}

GOOS Projects inform the community on how to develop, and/or mature technologies and programs, and provide architectural patterns or best practices. These Projects are finite-term endeavors focused on common challenges that span scientific or geographic boundaries.

A key benefit to the adoption of system engineering and architecture practices is the reuse of knowledge. By taking advantage of what is known or has worked successfully in the past and making required adjustments, a Project can be a mechanism for demonstrating and/or bringing best practices into the mainstream.

The ongoing GOOS Projects also actively use the FOO processes. The Tropical Pacific Observing System 2020 (TPOS 2020, Smith et al., 2019) focused on an ocean region of high importance to global seasonal climate variability, the Deep Ocean Observing Strategy (DOOS, Levin et al., 2019) is designing and implementing an observing approach for the very under-sampled areas of the deep sea. The AtlantOS program, that arose from a similarly named European project, aims to engage a larger set of actors around the Atlantic Ocean to organize ocean observing on an ocean basin-wide level ${ }^{2}$. These projects cut across GOOS requirements, panels and observing systems, and provide insight into observing system development and best practices for future efforts (more detailed coverage of TPOS 2020 and case studies of the use of FOO are in section Case Studies).

\footnotetext{
${ }^{2}$ http://atlantos-ocean.org/
} 


\section{GOOS Partners}

In response to the requirements of a wide range of users, GOOS has developed a strategy to drive and guide implementation of a global ocean observing system. Meeting these challenges will require concerted efforts to strengthen a suite of committed and funded international partnerships.

A generalized list of partnerships that must be formed or strengthened includes:

- Organizations within the UN system, such as between IOC/UNESCO and WMO whose members increasingly recognize the importance of ocean observations for weeklyto-seasonal weather prediction, and the UN Environment Program (UNEP) with their strong mandate to monitor ecosystem health and pollution.

- Executive bodies for international conventions and agreements that require ocean information to assess progress toward their agreed objectives.

- Groups in the international marine science, management, and policy communities, such as those in climate, operational oceanography, ocean carbon, and marine ecosystems and fisheries, marine ecosystem health assessment, as well as national and regional bodies charged with evaluation of risk and management of marine systems.

- Industries and manufacturers that develop and provide instruments, platforms, and communications technologies for global sustained networks, observatories, and operational infrastructures.

- National and regional agencies responsible for funding and running ocean observing systems, many of whom are research-based, rather than operational.

- Industry sector users for whom ocean data and information are critical for sustainable, efficient and safe operations, such as shipping, tourism, offshore oil and gas, offshore wind power, seabed mining, fisheries, ocean services.

- Marine/ocean research and development sector, such as the Partnership for Observations of the Global Ocean (POGO), who often provide the innovation engine for advancing ocean observations, the proof-of-concept processes for many new observation programs, and many of the ongoing ocean observation programs.

- The data innovation and technical services sector, including the International Oceanographic Data and Information Exchange (IODE), the national ocean data centers, and other ocean data centers and data integrators.

- Non-governmental bodies that speak directly to societal concerns such as the World Wildlife Fund (WWF), Ocean Conservancy and Greenpeace.

- Finally, funders and educational partners that enable development of technical capacity in ocean observation across the globe.

\section{GOOS/FOO SUCCESSES}

Over the past decade, GOOS has shown some success in organizing and expanding the global observing system.
The FOO has provided a rigorous, standardized way for the ocean observing enterprise to be understood and advanced. It provides a framework of processes, best practices for requirements-setting based on societal needs, identification of common EOVs to be observed, technology readiness assessments (Figure 4), data sharing, product development and information delivery. As the global ocean observing system is a complex and highly connected system, addressing these information needs is an enormous challenge that has benefited from the engineering approach of the FOO.

\section{An Expanding User Base}

Over the past 5 years, GOOS has expanded to include expertise related to ocean observations across physical, chemical, biological and ecological properties. This supports not just the Intergovernmental Panel on Climate Change (IPCC) and GCOS, but also the IPBES, the Convention for Biological Diversity (CBD), the UN Environment Program, the Committee on Fisheries of the Food and Agriculture Organization (FAO), the International Council for the Exploration of the Sea (ICES), and the Regional Fisheries Management Organizations (RFMOs), among others. GOOS has been delivering relevant ocean data for various applications, often including coupling between the ocean, atmosphere and terrestrial systems. For instance, interior ocean data is increasingly used for weather forecasts, and ocean carbon fluxes are used to constrain the global carbon budget.

\section{Increased Cooperation Across Elements of the Observing Community}

GOOS has long emphasized the link between observations and end-user products in its system design and implementation. It has also encouraged GRAs and IOC member nations to make observations to support marine ecosystem health and climate issues where the link to end-users is often less obvious. This expansion of focus has encouraged the transfer of know-how among the physics, biology and biogeochemistry domains which has been a welcome development at the regional level.

GOOS has demonstrated some success in facilitating closer collaboration between the in situ and satellite observing communities, and the ocean modeling and forecasting communities. In EuroGOOS, for instance, the recent establishment of a cross-cutting coastal working group that considers the link between satellite, in situ and modeling data across scientific domains and different user groups is an impressive result of GOOS influence. Similarly, GOOS provides the ocean component of the GCOS which in turn provides a mechanism for coordination with the terrestrial, atmospheric and cryospheric observing systems.

\section{Essential Ocean Variables (EOVs)}

Much of the implementation effort to date has focused on EOVs and requirements, and it is here that we can most clearly see the demonstrated value of the FOO. The new GOOS 


\section{FOO Readiness Levels and Activities Matrix}

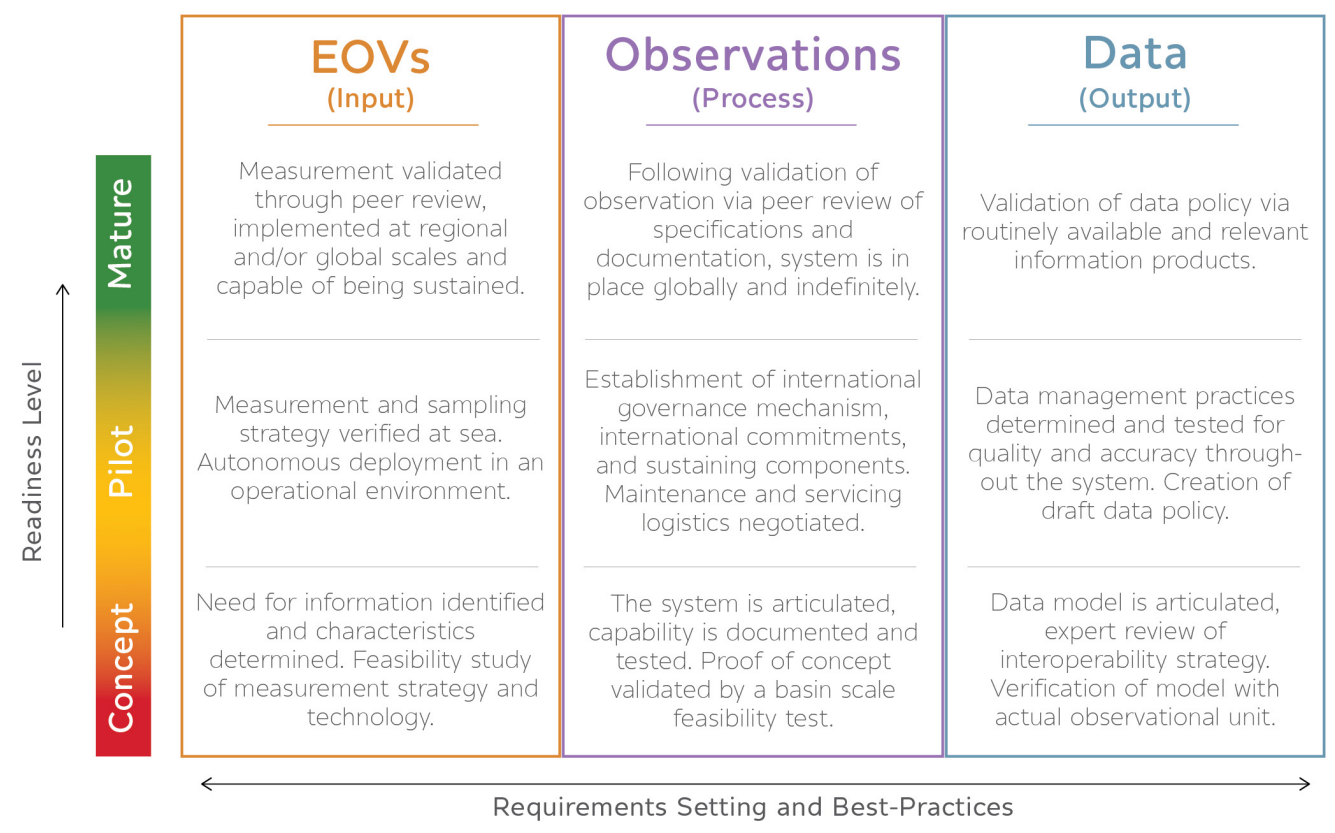

FIGURE 4 | Matrix of FOO-element attributes at increasing readiness levels.

Biology and Ecosystems Panel was able to start its requirementssetting process from the outset using FOO principles, as well as a thorough analysis using the Driver Pressure State Impact Response framework commonly used in ecosystem management (Miloslavich et al., 2018b). The panel developed a list of new, priority EOVs (Table 1), with clear societal benefit for developed and developing nations. Implementation planning is now underway.

The GOOS Biogeochemistry Panel used the FOO to evolve from its singular focus on carbon under the IOCCP to identify a new, broader set of priority EOVs (Table 2), with relevance to the United Nation's Sustainable Development Goals (SDGs). The goal is for some of these EOV observations to be established

TABLE 1 | GOOS Biology and Ecosystems Panel supported EOVs (October 2018).

\author{
Biology and ecosystem EOVs \\ Phytoplankton biomass and diversity \\ Zooplankton biomass and diversity \\ Fish abundance and distribution \\ Marine turtles, birds, mammals abundance, and distribution \\ Hard coral cover and composition \\ Seagrass cover and composition \\ Macroalgal canopy cover and composition \\ Mangrove cover and composition \\ Ocean Sound \\ Microbe biomass and diversity $\left({ }^{*}\right.$ emerging) \\ Benthic invertebrate abundance and distribution (*emerging)
}

as "indicators" that can be used internationally as monitors of progress toward the SDG goals, and those of related intergovernmental conventions.

The FOO has also influenced priorities under the most recent review of GCOS, enabling better linkages across ocean physics, biogeochemistry, and biology and ecosystems. It has enabled the GOOS Physics Panel, whose EOVs are shown in Table 3, to begin responding to requirements in continental shelf and coastal systems through a focus on boundary currents.

A number of the EOVs identified by the three panels are clearly interdisciplinary, such as ocean sound, which is physical measurement but is often measured to assess its effects on ocean mammals and fish. Lead responsibility for these cross-disciplinary EOVs has been assigned to the Panel which is deemed most in need of the data. The EOVs identified by the three panels are continuously evaluated and

TABLE 2 | GOOS Biogeochemistry Panel supported EOVs (October 2018).

Biogechemistry EOVs
Oxygen
Nutrients
Inorganic carbon
Transient tracers
Particulate matter
Nitrous oxide
Stable carbon isotopes
Dissolved organic carbon
Ocean color

\section{Biogechemistry EOVs}

xygen

Inorganic carbon

Transient tracers

Particulate matter

Nitrous oxide

Dissolved organic carbon

Ocean color 
TABLE 3 | GOOS Physics Panel supported EOVs (October 2018).

\section{Physics EOVs \\ Sea state \\ Ocean surface stress \\ Sea ice \\ Sea surface height \\ Sea surface temperature \\ Subsurface temperature \\ Surface currents \\ Subsurface currents \\ Sea surface salinity \\ Subsurface salinity \\ Ocean surface heat flux}

evolved by interaction with their scientific and operational user communities.

\section{Best Practices}

The use of best practices fostered by the FOO has supported many positive outcomes for the ocean observing system (Pearlman et al., 2019):

- Identified (minimal) system attributes for multiple system components, such as sensor performance, observing, data models, data quality, and data flow

- Encouraged more complete capture of metadata, important for data quality

- Enabled more rapid capacity development through sharing of knowledge

- Encouraged more contributions of usable data and better data quality

- Enabled system-wide integration across networks around EOVs.

\section{User Feedback}

Use of the FOO has also addressed the need to involve the end user in assessing and achieving the full societal benefit of sustained ocean observing by:

- Encouraging the practice of establishing user-driven requirements around EOVs

- Requiring assessment and feedback of the effectiveness of the observing system in addressing these requirements/needs

- Encouraging and assessing synthesis-based products based on EOV observations

- Recognizing and advancing these synthesized EOV Products (e.g., Sea Surface Temperature, ocean currents, global sea level rise estimates, wave field) as a critical bridge between raw observations and user-driven needs.

\section{USING THE FRAMEWORK}

As GOOS responds to new requirements for measuring additional EOVs in coastal and open ocean environments, it must also include new observing system elements and networks. In many instances, GRAs are already operating observing networks that are potentially fit for these purposes. Examples include high frequency radar, ocean glider, and animal tracking networks. Here we have seen the networks and GRAs, and large Projects come together as ocean observing communities to propose expansion of GOOS in line with the FOO (while not a GOOS Project the Southern Ocean Observing System generated its own version of ecosystem EOVs or eEOVs). The need to address requirements, measure priority EOVs, and provide data and information products is accepted by these communities. This indicates that the usefulness of guidance provided by the FOO is also being recognized by many from the "bottom up."

Argo provides a good example of how the FOO can be used to evolve an existing observing system element in response to new requirements. The Argo profiling float network, which is at a mature level of readiness for its core variables and spatial coverage, is now challenged to mature technologies and data delivery for floats measuring additional EOVs. Biogeochemical and bio-optical Argo floats (Bio-Argo) are now at a pilot level of readiness and are being trialed in the Southern Ocean and other locations. Deep Argo is at a proof-of-concept level of readiness, also within FOO guidelines, with several experiments underway.

\section{Case Studies}

Several case studies presented in the text boxes below address both successes and challenges in real-world application of the FOO guidelines.

\section{A national case study - the Integrated Marine Observing System (IMOS)}

The Integrated Marine Observing System (IMOS) is a national collaborative research infrastructure funded by the Australian Government, doing systematic and sustained observing of Australia's vast ocean territory, and making all its data openly accessible for science, research, and other uses. IMOS is integrated across scales (open ocean, continental shelf, and coast), and across disciplines (physics, biogeochemistry, and biology and ecosystems). Established in 2007, it has been expanded, consolidated, and sustained over the past decade.

This period overlapped with development and dissemination of the FOO, and IMOS has used the common language and system design principles of the FOO in numerous ways.

IMOS requirements were initially set through national science planning, subject to international peer review. Within a socio-economic context, major research themes and science questions were identified (requirements), leading to the prioritization of variables to be measured at relevant time and space scales (EOVs), along with platforms and sensors to be utilized (observing system elements). Direct investment in information management infrastructure was a design feature from the outset.

IMOS has also used the FOO concept of readiness to assess technology investments over time. Investing mostly in mature technologies to ensure delivery of quality data for its missions, IMOS has also run pilot projects of some newer technologies, maturing them if successful, or discontinuing them if not.

Now funded to 2023, with strong prospects out to 2029, IMOS is looking to strengthen its use of FOO elements that have served it well. Based on the 
expectations of Australian Government, requirements will be more clearly defined based on social, environmental and economic drivers. The strategy is to move from use and impact being something that emerges from what we do, to something that is explicitly planned for and measured. Direct investment will also be made in new technology assessment, with more rigorous selection and evaluation of pilot projects. There will also be emphasis on areas where the FOO has been less influential to date: increasing effectiveness and efficiency, greater integration across EOVs, and more investment in value-added information products.

A regional case study - the European Ocean Observing System (EOOS)

European stakeholders in ocean observation are working together in the European Ocean Observing System (EOOS) under the guidance of EuroGOOS and the European Marine Board. Stakeholder events and consultations have been held to gather perspectives on how the current system can be broadened to include marine ecosystem health, climate observations and applications, as well as the traditional data collection that supports real time oceanographic services for the user community. EOOS aims to establish a mechanism for a wide range of users to formulate and convey their needs to ocean observation system implementers, where they can be transformed into data requirements and the most appropriate measurement strategies can be identified. EOOS will also provide a mechanism to track and assess the implementation of solutions to meet user needs.

The requirements feedback loop advocated in the FOO has provided a globally-adopted context for this cycle of user requirements, implementation and tracking of observing system implementation for EOOS. In the future EOOS will look to a more mature FOO to help address the effectiveness of various mechanisms for gathering user feedback to inform future advances in ocean observing system design.

\section{A basin-scale case study - TPOS 2020 \\ The TPOS 2020 Project is evaluating all elements that contribute to ocean observing in this area, based on a modern understanding of the science and the capabilities of new sensing technologies, and recommending a redesign that will deliver enhanced effectiveness for all stakeholders, including operational climate prediction systems (Cravatte et al., 2016; Smith et al., 2019). In the context of FOO it is a regional Project, owned by regional stakeholders, but otherwise well aligned with the basic concepts of the FOO. The First Report of TPOS 2020 is structured according to the FOO in the following ways:}

- User requirements are expressed in terms of EOVs and characteristic scales and quality

- Generic, platform-agnostic recommendations are high-level responses to those requirements, which manifest as requirements on the various platforms and networks

- Possible platform and network solutions take account of the complementary capabilities of different approaches.

Differentiating these distinct levels of requirements was a constant source of debate, with the ever-present temptation to immediately focus on the technology solutions. It is critical these steps are considered independently to avoid conflicts of interest. Research and societal needs must be considered together. The First Report further refined the meaning of "essential" and differentiated between experimental and sustained measurements. Process experiments and pilot studies were managed somewhat differently from FOO, but their important role was fully recognized (Smith et al., 2019).

TPOS 2020 is moving toward a regional governance model involving key stakeholders and partners; again, this differs somewhat from FOO which emphasizes global aspects. This does require further elaboration - FOO is a top-down construct but allows for bottom-up development and direction.

\section{GOOS CHALLENGES}

Requirements for the ocean observing system are expanding. GOOS faces many challenges to its further success in addressing the entire value chain (Figure 5). These range from an increased urgency to articulate EOV requirements for an ever more complex and demanding user community, to the engagement with industry. Technologies need to mature to meet expanding needs along with the markets for these technologies such that they are both reliable and affordable. In addition, better systems for standardizing instruments, including quality assurance and telecommunications methodologies, and for encouraging a stable but diverse community of technology providers, is needed. There is also a need for engagement of data and user communities to ensure the uptake and expanded utility of data and information products. In addition, a global and inclusive governance architecture is needed to enable direction setting and coordination of ocean observing (see section Improving Governance of the Ocean Observing System: Revolution OR Evolution?).

\section{New Technology}

While observing technology (including sensor systems, platforms, data transmission, archival systems, data analysis software, and user product) evolves rapidly, the sustained observing system must balance responsiveness and continuity. It is challenging to determine where and why investment should be made for maximum utility. This applies both to new sensors, such as those for measuring biogeochemical properties, and to more well-established systems such as instruments for measuring sea level. It is a significant challenge to balance the incorporation of new technologies while sustaining the appropriate legacy components of the system, while ensuring the necessary calibration, verification and integration of all data sets, models, and end-user products, (see for instance the GCOS monitoring principles ${ }^{3}$ ).

\section{Gaps in the Observing System}

As the system grows to serve a broader suite of users across operational services and marine ecosystem health, encompassing both open ocean and coastal applications, the complexity of the environment is increasing. There are profound gaps in ocean observing coverage. For instance, the ocean observing capacity to monitor human impacts on the global ocean and climate must be engaged and improved.

\section{Best Practices}

GOOS's observations come from many heterogeneous observing elements funded by different nations and agencies and run by different groups, which can all benefit from increased sharing of best practices and integration.

Some observing networks, such as Argo or HF Radar, that focus on particular technologies are quite effective at developing and sharing best practices. Given the wide range of observing systems and end-users around the globe, however, we have

\footnotetext{
${ }^{3} \mathrm{https} / /$ gcos.wmo.int/en/essential-climate-variables/gcos-monitoring-principles
} 


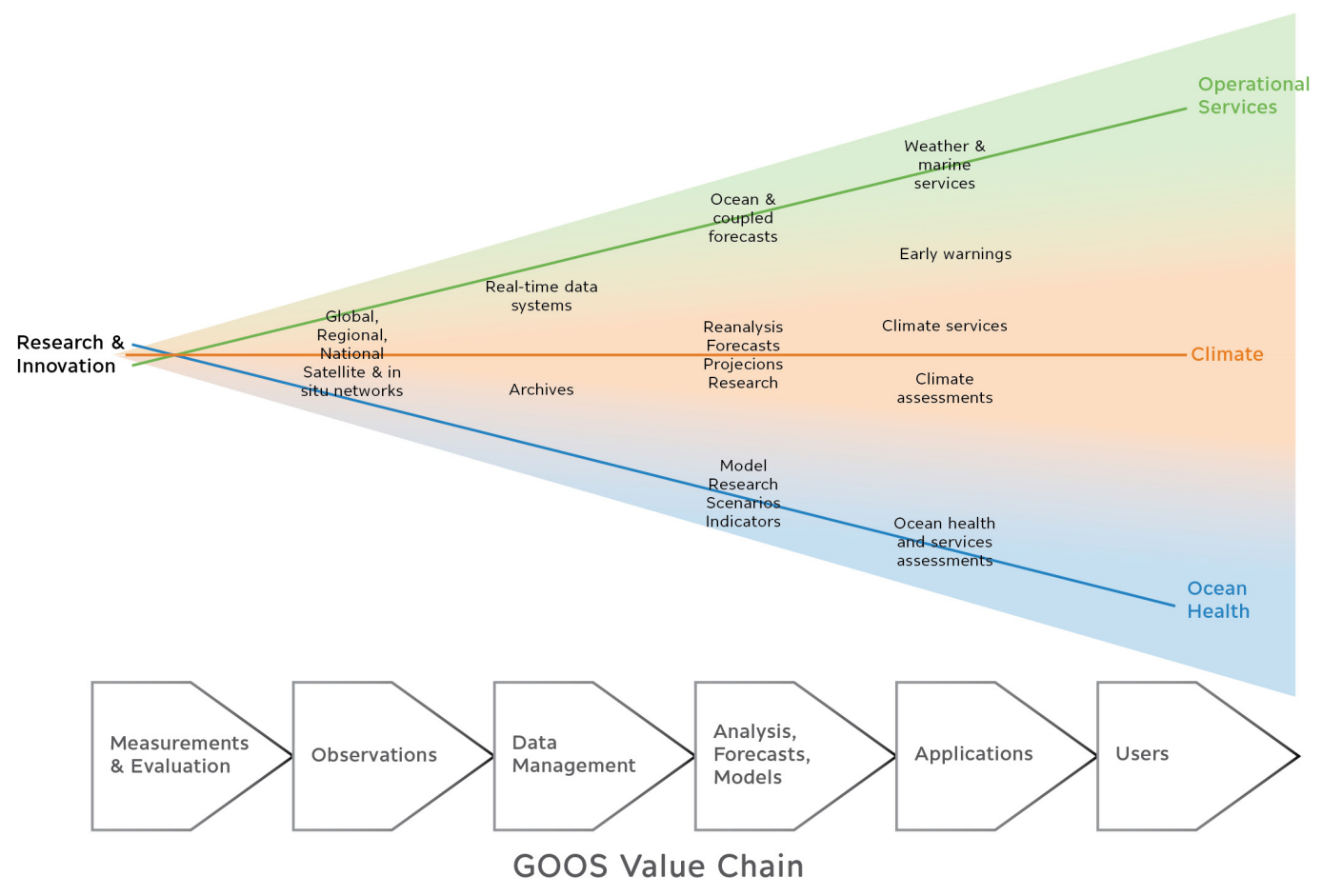

FIGURE 5 | GOOS value chain and associated activities and outcomes.

learned that relying on informal processes to share best practices is inadequate. There is a critical need to increase emphasis on identifying, sharing and following lessons learned and best practices across the GOOS enterprise. Important aspects of this work are being addressed by the Best Practices Working Group (Pearlman et al., 2019). Once these best practices are better socialized, it is expected user group communities will form to mature these practices and create a better understanding of the need for more formal interfaces and associated governance or institutional support.

\section{Integration and Feedback}

Given the evolution of user needs, the observing system and protocols for the analysis of observations, it is important that there be regular periodic reviews of the value chain to ensure that it continues to meet both scientific and societal needs. Currently feedback from end-users to the observing system is largely ad hoc. Development of a robust assessment component for GOOS is needed, with a wide range of questions addressed: How well are requirements being met? Do the requirements need revising? Is the mix of observing elements optimal? Are data quality and attributes acceptable? Do the products contain useful information? How well are user needs being met? The FOO identifies the need for regular cycles of evaluation to ensure the data products meet designated requirements and to ensure the information generated is having impact on the societal issues the observing system is designed to address. However, there is a fundamental lack of connection across the value chain from observations to end use, and in the ability to ensure fit-for-purpose delivery of information. Feedback and assessments should also track progress over time. Metrics for measuring GOOS system performance are under discussion and must be developed.

\section{Funding the Observing System}

Although we have grown the capability and associated funding for the ocean observing system (all elements) enormously over the last 25 years, particularly from satellites and modeling, the current findings of the in-situ observing system rely on a mixed funding model, with proposal-driven sources continuing to provide the bulk of the capability. New requirements, such as those from ocean health and sustainable development of the marine environment remain largely unfunded. Many of the ocean observing networks are largely dependent on short-term research projects. Nations have traditionally funded observing networks in their own waters to support marine transportation and public safety needs. Expansion of the scope and requirements into ocean health and environmental concerns has greatly increased the demand for observations without a commensurate increase in funding, which is putting enormous pressure on the system. Building the expanded system needed will require more cooperation and more funding across the ocean research, operations and policy communities worldwide. Ocean observing must be moved further up the political agenda internationally, and there is a fundamental need for long-term funding mechanisms to support ocean observing. Except for weather forecast systems, there is no collective knowledge base for assessing the value of ocean data products and services. 


\section{Capacity Development}

Building an operational system that is truly global requires expanding participation to include a far broader representation of developing and less-resourced countries. Significant global efforts to support capacity-building have been sponsored through a variety of organizations including the IOC, but the truth is these have not been sufficiently effective. To succeed, capacity development strategy must be sustained, and stronger partnerships, new funding models, innovative technologies, and new training approaches will be required (Bax et al., 2018; Miloslavich et al., 2018a). The goal is to have more countries actively participating in GOOS observing and benefiting from its information products.

\section{Engaging the Private Sector and New Partners}

There is a growing need to address the expanding role played by volunteer and chartered vessels hired to conduct observational efforts. In order to deliver ocean information to the end-user it is important to engage partners through the whole value chain. This includes a stronger engagement with private sector partners, both as a delivery of observations, or as users of ocean information. For example, in 2017, 27\% of the total CMEMS (Copernicus Marine Environmental Monitoring Service; a large operational GOOS user) download volume (around 45 Terabyte) was for private/commercial applications. On the observation side, private sector vessels (volunteer, charter) are widely used (e.g., volunteer observing vessels; maintenance of the tropical moored buoy array; tsunami moorings). As noted above, GOOS needs to better engage instrument and communication system manufacturers in setting and providing standardized platform operations and in evolving the cadre of data collection providers from the private sector.

\section{Data Sharing}

The ocean data system architecture is incomplete and fragmented, and the revolution of free and open data sharing achieved for most open-ocean physical variables is not universally inclusive of the biogeochemical and biological variables, or to certain other ocean areas. GOOS is a strong supporter of the principle "measure once/use many times." As an IOC program, GOOS adheres to the IOC oceanographic data exchange policy (Resolution IOC-XXII-6, 2003), which stipulates that Member States shall provide timely, free and unrestricted access to all data, associated metadata and products generated under the auspices of IOC programs ${ }^{4}$.

On 21 March 2018, Nature published the results of a large survey on the practical challenges of scientific data sharing. It showed that $76 \%$ of respondents highly rated the importance of data being discoverable, but the main challenge to data sharing is organizing data in a presentable and useful way (46\%), followed by confusion around copyright (37\%) and not knowing where to share data (33\%). This confirms that there is still a strong need

\footnotetext{
${ }^{4}$ www.iode.org/policy

${ }^{5} \mathrm{http}: / /$ go.nature.com/ResearchDataWhitepaper
}

to improve capacity in data management, promote best practices in global common data standards, data exchange protocols, and expert-controlled vocabularies to ensure interoperability between datasets.

The FAIR (Findable, Accessible, Interoperable, and Reusable) Guiding Principles (Wilkinson et al., 2016; Tanhua et al., 2019) now enjoy broad recognition through the data community. Several oceanographic organizations/projects, such as the AtlantOS Blueprint - Data flow (de Young et al., 2019), are already embracing the FAIR Principles alongside the consideration of EOVs and requirements, so it makes sense to bring them into GOOS.

\section{FOO COMMUNITY REVIEW}

A community-wide review of the FOO's usefulness was launched in August 2017. Twenty-one extensive interviews were conducted with representatives from federal agencies, research institution, academia, and the private sector. These discussions focused on three broad categories: technology and implementation, data and analysis, and management and governance.

This effort resulted in several key findings that will guide changes to use and implementation of the FOO. Recommended changes so far are that there should be an increased emphasis on the multi-scale (coastal, open-ocean, local, regional, national, global) aspects of the observing system. Also, there must be improvements in assessment methods to clarify the path to maturity across the system.

A brief summary of early results from the FOO Community Review:

- The FOO has been helpful in the establishment of EOVs, however, the observing community could benefit from an ongoing review of the EOV setting process and its outcomes.

- While alignment with FOO did facilitate the dialog around what should be measured, it was not as useful in negotiations of what sensors should be deployed on observing platforms, or in the design or redesign of observing networks or arrays.

- There is a greater need for interaction among data managers and integrators within the system. The implementation and data management teams are often overlooked in conversations when calling for enhanced relationships with users, and the needs of these users are often not sufficiently funded or managed in a sustained manner.

- There is a need for greater awareness of the role of GOOS and other groups functioning in the international coordination arena.

- Improved understanding and strengthened partnerships can assist the international community in addressing the entire value chain of the ocean observation system.

- In addition to the expanded emphasis on building an observation and data and information infrastructure that is based on the scientific understanding generated by the EOV setting process, practitioners are also challenged to 
develop the more direct or concerted relationships required to develop the system's feedback loops further addressing science and societal needs.

\section{THE WAY AHEAD}

GOOS is looking forward to the coming decade by assessing its unique role in ocean observing to date, reviewing remaining challenges, and considering how it can best improve its contributions in the future. The GOOS 2030 vision (IOC, 2019) was published in April 2019 (pending approval of IOC General Assembly); the mission, goals, and strategic objectives are presented in this paper. Responses before and during the OceanObs'19 Conference in September 2019 (Honolulu, United States) will inform and refine how GOOS will implement the strategy during the next decade.

\section{The GoOS Vision}

\section{A Truly Integrated Global Ocean Observing System That Delivers the Essential Information Needed for Our Sustainable Development, Safety, Wellbeing and Prosperity}

Compared with GOOS at its creation in 1991, GOOS now has a very broad, societally-driven perspective, responding as required to all relevant SDGs and to the many other users of, and stakeholders in, the GOOS. This in turn mandates GOOS to provide a systematic, integrated and effective response to this complex set of information requirements.

\section{The GOOS Mission}

To Lead the Ocean Observing Community and Create the Partnerships to Grow an Integrated, Responsive, and Sustained Observing System

A fully implemented global ocean observing system will provide the critical ocean information needed to address climate change, generate forecasts, and protect ocean health. By 2030, GOOS will engage a greatly expanded level of partnership and participation from more countries, other observing organizations, and users of data and products.

\section{GOOS Goals and Strategic Objectives}

GOOS will work with its partners over the next decade to address ocean information issues and achieve its vision through 11 Strategic Objectives, grouped under Three Overarching Goals shown in Figure 6.

- Goal 1: Deepening Engagement and Impact

Deepen engagement and partnership from observations to end users to advance the use and impact of the observations and demonstrate its benefits

(1) Strengthen partnerships to improve delivery of forecasts, services, and scientific assessments.

(2) Build advocacy and visibility with stakeholders through communicating with key users and national funders.

(3) Regularly evaluate system impact to assess fit for purpose.
(4) Strengthen knowledge and exchange around services and products, to boost local uptake.

- Goal 2: Supporting Integration and Delivery

Deliver an integrated, "fit for purpose" observing system built on the systems approach outlined in the Framework for Ocean Observing

(5) Provide authoritative guidance on integrated observing system design, synthesizing across evolving requirements and identifying gaps.

(6) Sustain, strengthen and expand observing system implementation through GOOS and partner communities, promoting standards and best practice, and developing metrics to measure success.

(7) Ensure GOOS ocean observing data and information are findable, accessible, interoperable, and reusable, with appropriate quality and latency.

- Goal 3: Building for the Future

Building for the future through innovation, capacity development, and evolving good governance

(8) Support innovation in observing technologies and networks.

(9) Develop capacity to ensure a broader range of beneficial stakeholder participation.

(10) Extend systematic observations to understand human impacts on the ocean.

(11) Champion effective governance for global in situ and satellite observing, together with partners and stakeholders.

\section{IMPROVING GOVERNANCE OF THE OCEAN OBSERVING SYSTEM: REVOLUTION OR EVOLUTION?}

The world at large is recognizing the magnitude of the ocean's impact on global, regional and local lives and livelihoods. These include the ocean's impacts on regulating climate and the tendency toward local extreme storm and flooding events; global sea level rise; and the growing problems of ocean warming, acidification, plastics and other forms of pollution. Many nations are calling for improved public safety forecasts and warnings; international conventions and regional agreements are calling for more ocean observations to address their various concerns; and ocean scientists urgently require more ocean observations to support these many needs. At the same time, there is a growing "blue economy" with many innovations in marine transportation, search/salvage, food production, underwater mining, recreational boating, and many other maritime industries. The requirements for increased ocean observations to address all of these issues, and to provide products to support them is growing in number.

There is a wide and growing range of participants worldwide in ocean observing with different scopes, aims and ambitions and different geographical, thematic and technical foci. Significant 


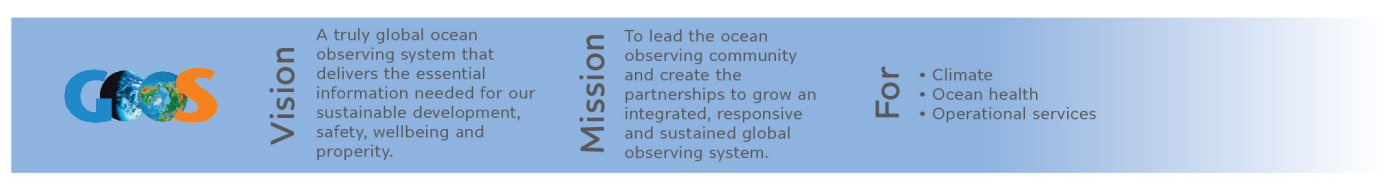

Strategic Goals \& Objectives

\begin{tabular}{|c|c|c|c|c|c|c|c|}
\hline 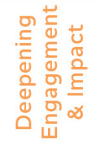 & 1 & $\begin{array}{l}\text { Strengthen partnerships, } \\
\text { to improve delivery of } \\
\text { forecasts, services, and } \\
\text { scientific assessments. }\end{array}$ & 2 & $\begin{array}{l}\text { Build advocacy and } \\
\text { visibility with stakehold- } \\
\text { ers through communicat- } \\
\text { ing with key users and } \\
\text { national funders. }\end{array}$ & 3 & $\begin{array}{l}\text { Regularly evaluate } \\
\text { system impact to } \\
\text { assess fit for } \\
\text { purpose. }\end{array}$ & $\begin{array}{l}\text { Strengthen knowledge and } \\
\text { exchange around services and } \\
\text { products, to boost local } \\
\text { uptake. }\end{array}$ \\
\hline
\end{tabular}

\begin{tabular}{|c|c|c|c|c|c|c|}
\hline 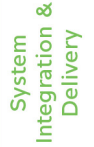 & 5 & $\begin{array}{l}\text { Provide authoritative } \\
\text { guidance on integrated } \\
\text { observing system design, } \\
\text { synthesizing across } \\
\text { evolving requirements } \\
\text { and indentifying gaps. }\end{array}$ & 6 & $\begin{array}{l}\text { Sustain, strengthen and expand } \\
\text { observing system implementa- } \\
\text { tion through GOOS and partner } \\
\text { communities, promoting } \\
\text { standards and best practice, and } \\
\text { developing metrics to measure } \\
\text { success. }\end{array}$ & 7 & $\begin{array}{l}\text { Ensure GOOS ocean } \\
\text { observing data and } \\
\text { information are findable, } \\
\text { accessible, interoperable, } \\
\text { and reusable, with } \\
\text { appropriate quality and } \\
\text { latency. }\end{array}$ \\
\hline
\end{tabular}

\begin{tabular}{|c|c|c|c|c|c|c|c|c|}
\hline 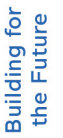 & 8 & $\begin{array}{l}\text { Support innovation in } \\
\text { observing technolo- } \\
\text { gies and networks. }\end{array}$ & 9 & $\begin{array}{l}\text { Develop capacity to ensure } \\
\text { a broader range of } \\
\text { beneficial stakeholder } \\
\text { participation. }\end{array}$ & 10 & $\begin{array}{l}\text { Extend systematic } \\
\text { observations to understand } \\
\text { human impacts on the } \\
\text { ocean. }\end{array}$ & 11 & $\begin{array}{l}\text { Champion effective } \\
\text { governance for global in } \\
\text { situ and satellite observing, } \\
\text { together with partners and } \\
\text { stakeholders. }\end{array}$ \\
\hline
\end{tabular}

FIGURE 6 | GOOS 2030 Strategy; vision and mission statements with strategic goals and correlating objectives.

expansion of new observing efforts has recently increased the community's intellectual and operational capability, which is a great outcome. However, there is unbridled growth in the number of groups taking on responsibilities without appropriate coordination: the system has ineffective systemlevel management, lack of planning coordination across the system, and sub-optimal financial and management support levels for many of the efforts. This lack of awareness of, and/or coordination with, already existing observing systems in some areas has resulted in less positive outcomes, including duplication of observing efforts, use of less-than-optimal observing technology, and limitations in data standards and data sharing. The rapidly increasing requirements, the growing landscape of actors and activities in ocean observing, and the constrained resources, require that some form of improved ocean observing governance evolve that can effectively and efficiently address the growing needs of the many stakeholders.

Most of the funding for ocean observation infrastructure continues to come from national governmental funding sources, so the strongest level of governance around planning, commitments and implementation continues to be at the national level. Improved governance must demonstrate to the national players how engagement across regional and global levels can bring advantages, for example from cooperation and coordination and leveraging the best practices of others. Furthermore, engagement at the global level will give all participants in ocean observing more access and input to the development and implementation of intergovernmental conventions that require ocean observations.

Improvement of the governance system must focus foremost on finding common requirements and building strong partnerships across the expanding observing system.
It is necessary to define roles and responsibilities and agree on goals and strategies including processes for setting system requirements, assessing technology choices, setting standards for data management and sharing, coordinating the suite of public products, and cooperating in global capacity development on all levels.

It is important to understand where commitments take place for the observing system, and how governance can be more effective and efficient internally, while at the same time recognizing and working more closely with other partners to build the case for additional investment in the observing system.

\section{Dimensions of Ocean Observing Governance Structures Today}

This diversity, energy and activity in the global ocean observing community can be seen both as a strength and a weakness of the current ocean observing system. The global ocean observing community is multi-faceted, loosely organized, and growing more so every year. We operate now with an historical accretion of organizations and networks working at different scales and focused on different parts of the value chain from observations to end users.

Structurally the community can be looked at as being aligned around three dimensions:

\section{- A first dimension is that of platform-based observing.}

A core component of the observing system has long been the observing networks organized around particular observing platforms. Good examples are profiling floats (Argo), moored time series (OceanSites), large scale hydrography (Global Ocean Ship-Based Hydrographic Investigations Program). These networks operate under well-defined criteria and shared best 
practices. They have their own governance systems, are global in ambition, promote free and open data sharing and aspire to be sustained. These networks tend to be focused on specific scientific and/or societal user needs but are not well integrated with other observing systems even when in the same area.

\section{- A second dimension is that of ocean observing requirement themes.}

Good examples are the newly developed Global Ocean Acidification Observation Network (GOA-ON) and the Group on Earth Observations Biodiversity Observation Networks (GEO BON) and the Deep Ocean Observing Strategy (DOOS) Project. Thematic based collaborations allow for broad, and as appropriate, multi-disciplinary engagement across geographic areas. Currently there are efforts underway to more systematically align these thematic networks within the broader GOOS structure and encourage wider use of the FOO principles and processes.

- The third dimension is that of scale.

Governance challenge varies as we move from national and regional scales, to basin and global scales. The bulk of the funding for ocean observations comes from the national level where the strongest governance also exists (for example, accountability for public funds), although governance arrangements differ from nation to nation, and there is no standard or organized coordination across nations. The nations mostly oversee their own local observing networks, although in some nations there are so many that they are not really well coordinated, and in other nations, outside organizations sometimes take the lead without coordinating strongly with national agencies.

The regional level has both geopolitical and natural ocean drivers and motivation for cooperation, like regional current systems, pollution, fisheries, and other issues of resource management and protection. Yet today there is no single governance solution for regional efforts, not just for ocean observations, but also for the broader issues of transboundary problems and regional politics. The regional scale of governance for both science and policy is ripe for improvement.

A recent positive development has been the emergence of coordination at the scale of complete ocean basins. Good examples include the Southern Ocean Observing System (SOOS), AtlantOS for the Atlantic, the Indian Ocean Observing System and TPOS2020 focusing on the tropical Pacific. The basin-scale focus provides a new and effective vehicle for collaboration on ocean observation requirements, observing strategies, data sharing, capacity building, and resourcing, though still with some of the challenges identified for the regional scale. The European Commission has pursued this scale of cooperation for ocean science with the Galway Statement (2013) and the Beléem Statement (2017), covering North and South Atlantic Ocean research and observing cooperation, respectively.

GOOS governance focuses on the global scale, but also embraces elements of regional and basin-scale approaches.

\section{Voluntary Contributions}

Perhaps most importantly, there is currently no mechanism in place to assess the effectiveness of the total contribution of ocean observations and data streams from the variety of networks and components toward the goals of the scientific and societal benefit areas recognized as the overarching purpose for the observing system. This speaks to both one of the greatest achievements of the observing system in the previous decade - the more widespread adoption of open data policies but also to one of its greatest challenges for the next decade: the lack of an appropriate governance structure for the vast resources of scientists, technology developers, and data experts that involved. The governance is under tremendous pressure to both respond as well as lead to resolve the issues related to associated requirements development and communications gaps.

The current governance system is loosely coordinated based on voluntary commitments with little of the rule and control characteristics usually associated with more formalized governance structures. The variety of differently focused observing systems makes it challenging to find a governance model that works; the current model is clearly not adequate to accommodate, oversee, guide, or support all systems. Additionally, governance of ocean observing is not well supported; both in terms of investment and commitment. One possible reason for this is the inherently unclear and complex structures, roles and responsibilities of the current governance arrangements.

\section{Attributes and Objectives of a Good Governance System}

All indications are that the diversity of expertise, interests, and support for the fragmented global ocean observing community will continue to expand in the coming decade. The "next step" for high-level governance of the global ocean observing system must be attractive in order to involve more representatives from the community, including some who are currently working in isolation. The FOO principles will be a valuable resource toward establishing guidelines, standards and procedures for moving forward.

In a recent assessment of over 100 international agreements comprising the global ocean governance architecture for fisheries, pollution, biodiversity and climate change, Mahon et al. (2014) found two emerging network structures. The first were "global-regional, issue-based networks" building from the siloed global agreements touching fisheries, pollution, biodiversity and climate change, which they suggested should be better integrated. At the regional level, they found 16 crosscutting regional clusters of networks, where regional agreements for several issues coincide spatially. They suggested these clusters provide the opportunity for integration, focusing broadly on ecosystem-based management of the ocean, and improving regional implementation of global agreements. Sustained ocean observations are a necessary input for all of these initiatives, as is scientific input for setting requirements and policy and in monitoring outcomes, but Mahon et al. (2014) found that many of these mechanisms to incorporate science into policy 
were weak. This study makes clear the requirements and the opportunities for strengthened governance at both the global and regional levels.

Based on experience with GOOS and similar systems, and on initial stakeholder consultation, we propose the following principles for an observing system governance structure:

- Responsiveness: Governance must respond to the needs of stakeholders and participants, from local, regional to global, across all relevant sectors, and include governmental and non-governmental aspects.

- Purposeful: Governance must demonstrate purposefulness for, and on behalf of, the community.

- Clear objectives: Good governance relies on clear and relevant objectives and strategy.

- Transparency: Transparency and openness must be a priority, to ensure broad and public access to and benefit from the system. While some private (closed) networks may be warranted on the grounds of security or because they address a narrow target audience, in general information should be public and governance arranged accordingly.

- Efficiency and Effectiveness: Governance must ensure that maximum value is derived from invested resources and must have sufficient flexibility and nimbleness to ensure that both decisions and/or guidance are provided in a timely way.

- Adaptive: Governance must support innovation and an openness to change, in order to ensure that benefits accrue for new solutions and improved best practices.

- Sustainability: Governance must have a long-term orientation, taking account of the broad range of existing and likely future drivers, and the need for dependability and robustness.

- Authoritative: The individuals and teams contributing to governance must have the appropriate capability, skills, and respect of the community to act on their behalf.

- Performance and accountability: The governance processes must include monitoring and feedback to measures of success and performance.

An additional principle when dealing with multiple levels of governance is that a central authority should have a subsidiary function, performing only those tasks which cannot be performed at a more local level. This subsidiarity principle establishes a link of co-responsibility among different levels of governance. The central idea is that local governance will be the most responsive to local needs, increasing quality and effectiveness and managing risk at the appropriate level (Jachtenfuchs and Krisch, 2016).

The above principles are intended to provide a firm foundation for building a new governance system and are not prescriptive. If after further consultation with stakeholders these principles are judged to capture the needed objectives and attributes, then the details can follow.

It is important to recognize the nesting from national to regional or basin-scale to global efforts; the needs and contributions of each level; and how a governance system must work with and help to coordinate the efforts of all of these levels to achieve the best system of systems for all users. We need to invest in projects to test and demonstrate the linking of the various governance levels.

\section{Scenarios for Improved Ocean Observing Governance}

The ultimate objective is a better-structured, efficient system-ofsystems, or enterprise with clear roles and responsibilities and a sense of ownership among all members in the overall system, where all individual parts work in concert, with observations, standards, data sharing and data product needs met in the most efficient and cost-effective ways.

How do we get there? We offer several scenarios for improving governance of the overall ocean observing community.

\section{Starting Over - A Revolution}

If we could start over and build the ideal governance structure from scratch, what could it look like?

There would be a strong, single international organization with a clear mandate and adequate funding to direct, coordinate, integrate, monitor and assess ocean observations, data and products worldwide, and to provide and coordinate robust capacity building for nations in need of it. It would direct and oversee expanded use of the FOO, including requirement setting organized around EOVs, observing technology and design readiness assessments, increasing user feedback, and assessing the adequacy of the system in meeting societal benefits. It would establish and support strong channels for two-way communications and mechanisms for the input of ideas and leadership from national and regional levels.

This organization would adhere to all the principles, objectives, and attributes of governance outlined above. All other intergovernmental organizations needing ocean information would be incentivized to coordinate their needs and efforts with the lead organization.

Every nation with an ocean coastline would have an "ocean ministry," responsible for coordination of ocean science and observations. In nations with multiple ocean agencies, one would be clearly designated as the lead for ocean observations. These national ocean ministries would coordinate their ocean observing efforts with the lead international organization. Regional observing efforts would have clear and shared common governance arrangements and would be required to coordinate with and take direction from the lead international organization. Both national and regional observing efforts would work closely together by participating in established mechanisms for their input and leadership of various aspects of the global effort. All the geographical scales of ocean observing would be linked, both "upward" and "downward" to assure engagement, ownership, consistency and cost-effectiveness of the overall system.

Clearly, starting over is not a realistic option. There is no identified mechanism to make that level of change achievable in a system with so many players and, even if possible, it would cause too much disruption to important ongoing and emerging observing efforts. Perhaps this look at an idealized model will inform the goals and attributes of the evolutionary approaches outlined below. 


\section{Top-Down Model}

A top-down governance system led from some part of the UN system has some strengths: it is rooted in Member State governments, it is consensual and inclusive, and conditioned to treat capacity development as a priority to bring the community of nations up to a common level of development. That leads to some of its disadvantages: a consensual drive can lead to lowest-common denominator responses to innovation.

A good example of a top-down model is the rather strongly regulated framework around meteorological observations, recognized many decades ago as vital to public safety (weather and storm forecasts), business interests (agriculture, aviation forecasts), and military needs. Governed by the WMO under the WMO convention, members have a strong say in formulating regulations, but once promulgated the WMO regulations are largely complied with by most nations, and each nation has a designated responsible agency to ensure compliance.

Without such a top-down instrument like the WMO Convention, the current ocean observing governance system under IOC/UNESCO has become fractured and chronically underfunded. User needs are represented within different UN agencies, ranging from operational users (for example, WMO), scientific users (IOC/UNESCO and International Science Council), and policy/regulatory users (e.g., UN Environment, International Maritime Organization, among others), requiring strong partnerships that are sometimes difficult to establish across agencies.

\section{Bottom-Up Model}

A strength of the bottom-up, community-based self-organizing model is that the governance energy is naturally concentrated in the elements that see the greatest advantage in collaboration. This approach harnesses the energy, enthusiasm, and funding of self-organizing efforts. In this bottom-up model, governance of the global ocean observing system is left to the observing communities to self-organize around their own objectives and goals, but sometimes without the guidance of broader international knowledge, experience and goals. Many of the existing networks, organized around a particular technology for example, were organized by scientists in a bottom-up manner.

This approach could be organized around voluntary participation in an overall governing body financed by membership fees from organizations that would then have a seat at the table of their governance structure. This model could be structured as an independent legal entity and could potentially be inclusive and recognize all participants in ocean observing. An example of this approach is EuroGOOS, the European GOOS Regional Alliance (GRA), that is funded and governed by membership organizations to form a strong regional ocean observing body with well-defined mission and goals.

This bottom-up approach is, to a large extent, already happening with many organizations and structures developing around emerging ocean observing/application themes, networks, and systems. Drawbacks of this approach are lack of coordination and difficulties in access to new technology assessments, best practices, and data sharing globally; the difficulty of accessing advantages provided in the UN system, such as global targets and global/regional development funds; the difficulty of influencing development and implementation of the intergovernmental conventions.

\section{Loosely Coupled Hybrid Model, "Business as Usual”}

The current governance system can be described as a weak "hybrid model" with governance provided by GOOS within the UN system and working as much as possible with partners at all geographic scales. GOOS provides credibility for national and regional observing efforts through its presence within the UN system and by providing member states a voice into the intergovernmental processes.

This loosely coupled model requires stability in order to remain credible and thus effective. Stability and sustainability require long-term commitment to objectives and observing capability, and adequate and appropriate resourcing for the governance model. Resources are currently obtained from funders outside of the UN system for components of the governance system, such as the GOOS panels and most of the network structures. Although this provision of funds from partners outside of the UN system is currently essential to governance operations, it leads to a loosely coordinated, difficult to manage system.

\section{Tightly Coupled Hybrid Model}

This approach is similar to the "business as usual" model, but with a much stronger link for governance partners of the observing system. This model builds on a strong UN presence but with official membership status of partners that work in concert, a hybrid of top-down and bottom-up approaches. Outside of the core activities of coordinating and implementing sustained ocean observing activity, this puts emphasis on building partnerships for delivery, advocacy and visibility with stakeholders, supporting innovation, and developing capacity. A governance approach where partners from the observing system are members and directly participate in governance would facilitate engagement, foster common solutions, and encourage sharing of best practices and data.

How would such an approach look in practice? Perhaps a lead UN agency clearly designated to provide the top-down coordination, plus an office of the lead UN agency placed outside of the UN system as a legal entity that engages the national, regional, scientific, and industry observing partners as members, working in concert with the UN system. It would empower partners to participate and facilitate co-design and management of the observing system.

This starts to define a global common observing governance infrastructure. Though still a small fraction of the investment in observing systems by nations, this approach would give more voice at the intergovernmental level to the national efforts. The benefits of such a global common coordinated infrastructure arguably flow to all nations, but the capacity of many countries to use data for their local purposes must be further developed. The creation of a G7 "Future of Oceans and Seas" working group has led to ongoing discussions of the 
establishment of a G7 sustained ocean observing coordination center, linked to GOOS. This, or a somewhat larger grouping of countries, might form part of a tightly coupled hybrid model for governing GOOS.

\section{Polycentric Multi-Level Governance Systems}

This section seeks to describe the current situation in a framework of multiple centers of responsibility and can be applied to some of the models described above. The situation of multiple centers of responsibility and the lack of an entity or mechanisms with responsibility for overall coordination of the system is common in the world of natural resources governance. Polycentrism is the term given to such system (Ostrom, 2010); they are defined by the existence of multiple centers of influence or decision-making that address a particular problem area. A fully polycentric governance system exists at one end of a "governance modality spectrum" with a central authority system at the other end (Mahon, personal communication). Fully functional polycentric governance systems have some theoretical advantages, including adaptive capacity, fit to institutions, and risk mitigation. Some potential disadvantages are the transactional cost of coordination across structures, and the dispersion of accountability.

Polycentric systems are characterized by having overarching rules, mutual adjustment of activities (collaborate and avoid conflict), a willingness to experiment, trust among members (domains), and are supported by local action of members. Ostrom (2010) considers the benefits of polycentric governance arrangements; "An important lesson is that simply recommending a single governance unit to solve global collective-action problems - because of global impacts - needs to be seriously rethought."

Clearly, the current GOOS governance system is polycentric; there are multiple centers of responsibility for GOOS components, there is a lack of an entity or mechanisms with responsibility for overall for coordination of the system, despite this there are attempts to coordinate and facilitate interaction of existing structures. Significant complications also arise as the GOOS polycentric system is also multilevel, see section Dimensions of Ocean Observing Governance Structures Today.

There are several examples of effective multilevel polycentric governance systems, for instance in the wider Caribbean region (Mahon et al., 2014). There are lessons to be learned from these systems across the observing system. A realization by all actors, that GOOS is a polycentric governance system seems to be an important first step toward moving GOOS toward a co-designed polycentric system. GOOS needs to start with mapping of existing structures, including roles and responsibilities, and then analyze areas of weakness and point to where a focus on improved use of polycentric governance principles is desired. GOOS should use the principles and experience of polycentric multilevel governance systems to evolve toward a more effective system than we have at present.

\section{NEXT STEPS IN OCEAN OBSERVING GOVERNANCE}

GOOS is an organization that includes involvement with in situ networks, satellite systems, governments, UN agencies, research organizations, individual scientists, design engineers, and data managers. GOOS adopted and oversees the FOO guidelines which have been widely embraced and used throughout the ocean observing community. Through the FOO, GOOS is coordinating the assessment of ocean observing requirements, observing system implementation, and innovation through GOOS Projects. Sitting within the UN structure allows GOOS to enable a 2way interaction with nations through many forums. By building community consensus, GOOS enables stakeholders to engage with the system as a whole.

Since its establishment the GOOS mandate has grown in size to include multiple scientific disciplines, responding to a growing range of societal and policy drivers, and operating in an increasingly crowded governance landscape. In 2019, the "global ocean observing system" brings together individuals and organizations from multiple inter-governmental organizations (e.g., UN Conference of the parties to the Framework Convention on Climate Change, UNESCO-IOC, WMO, UN Environment, European Community), and national and academic sectors from more than one hundred countries.

The authors conclude that a polycentric governance model is appropriate for GOOS, and that a set of Principles should be agreed to ensure the basis for this model aligns with stakeholder needs and expectations. We further think that the model would include governance action at the global, basin, regional and local/national levels, coordinated according to polycentric methods; not all nations, regions or basins need be explicitly represented, but their needs must be. GOOS governance should be recognized within the UN and intergovernmental system, but may need some separation to fulfill ideals of responsiveness and adaptability.

\section{Call to Action}

- We invite all participants across the ocean observing community to consider and comment on the governance ideas laid out in this paper. Leading up to the OceanObs19 conference a concerted effort will be conducted to collect input on the governance recommendations from across the community. This process will be designed to make feedback into the process as seamless as possible while providing a forum for ongoing discussion and comment.

- Further, as these governance discussions will be an important part of the agenda at OceanObs19 in September 2019 , to ensure the community is ready to make meaningful progress on this issue at those meetings, a series of workshops will be held beforehand (in early to mid2019) with invitations to representatives of all the varied parts of the observing community. The workshop agenda will address various ways to improve communication, coordination, partnership and governance across the global ocean observing enterprise. 


\section{AUTHOR CONTRIBUTIONS}

TT, AM, and AF helped to conceive the study, coordinated the author contributions, wrote and edited the manuscript, and contributed to tables and figures. LG, LS, and AM coordinated the submission of manuscript, completion of tables and figures, and final report editing. WA, NB, KC, BD, DD, EH, JG, KH, MI, DL, EL, PM, TM, GN, AP, SS, BS, NS, MT, MV, and JW contributed to the manuscript ideas and text.

\section{FUNDING}

Preparation of this manuscript was supported by 80NSSC17M0007 (NASA) University Corporation for Atmospheric Research (UCAR). Some recommendations from this work resulted from PEGASuS 2: Ocean Sustainability, a partnership between Future Earth, the National Center

\section{REFERENCES}

Bax, N. J., Appeltans, W., Brainard, R., Duffy, J. E., Dunstan, P., Hanich, Q., et al. (2018). Linking capacity development to GOOS monitoring networks to achieve sustained ocean observation. Front. Mar. Sci. 5:346. doi: 10.3389/fmars. 2018.00346

Cravatte, S., Kessler, W. S., Smith, N., and Wijffels, S. E. (2016). First Report of TPOS 2020. GOOS-215, 200. Available at: http://tpos2020.org/first-report/

de Young, B., Visbeck, M., de Araujo Filho, M. C., Baringer, M. O., Black, C. A., Buch, E. et al. (2019). An integrated all-atlantic ocean observing system in 2030. Front. Mar. Sci. 26. doi: 10.3389/fmars.2019.00428

IOC (2019). The Global Ocean Observing System 2030 Strategy. Paris: IOC. 2019.

Jachtenfuchs, M., and Krisch, N. (2016). Subsidiarity in global governance. Law Contemp. Probl. 79, 1-26.

Levin, L. A., Bett, B. J., Gates, A. R., Heimbach, P., Howe, B. M., Janssen, F., et al. (2019). Global observing needs in the deep ocean. Front. Mar. Sci. 6:241. doi: 10.3389/fmars.2019.00241

Lindstrom, E., Gunn, J., Fischer, A., McCurdy, A., Glover, L., Alverson, K., et al. (2012). A Framework for Ocean Observing. By the Task Team for an Integrated Framework for Sustained Ocean Observing. Paris: UNESCO. doi: 10.5270/ OceanObs09-FOO

Mahon, R., Fanning, L., Gjerde, K., Young, O., Reid, M. R., and Douglas, S. (2014). "Governance," in The Open Ocean: Status and Trends, eds UNESCO, IOC, and UNEP (Nairobi: United Nations Environmental Program), 21-45.

Miloslavich, P., Bax, N. J., Simmons, S. E., Klein, E., Appeltans, W., AburtoOropeza, O., et al. (2018a). Essential ocean variables for global sustained observations of biodiversity and ecosystem changes. Glob. Change Biol. 24, 2416-2433. doi: 10.1111/gcb.14108

Miloslavich, P., Seeyave, S., Muller-Karger, F., Bax, N., Ali, E., Delgado, C., et al. (2018b). Challenges for global ocean observation: the need for increased human capacity. J. Operat. Oceanogr. 11, 1-20. doi: 10.1080/1755876X.2018.15 26463 for Ecological Analysis and Synthesis (NCEAS), and Global Biodiversity Center at Colorado State University. TT and AP acknowledges support from EU's Horizon 2020 Research and Innovation Program under grant agreement 63321 (AtlantOS). MT acknowledges support from the US National Science Foundation grant OCE-1840868 to the Scientific Committee on Oceanic Research (SCOR, US).

\section{ACKNOWLEDGMENTS}

We thank many people who contributed to the preparation of the 2012 Framework for Ocean Observing, the GOOS 2030 Strategic Plan, and the multitude of Project Reports, Annual Reports, Conference Proceedings, and Blueprint documents referenced in this White Paper. All of their efforts and energy contributed to the ideas and expertise herein.

Ostrom, E. (2010). Polycentric systems for coping with collective action and global environmental change. Glob. Environ. Change 20, 550-557. doi: 10.1016/j. gloenvcha.2010.07.004

Pearlman, J., Bushnell, M., Coppola, L., Karstensen, J., Buttigieg, P. L., Pearlman, F., et al. (2019). Evolving and sustaining ocean best practices and standards for the next decade. Front. Mar. Sci. 6:277. doi: 10.3389/fmars.2019. 00277

Smith, N., Kessler, W. S., Cravatte, S., Sprintall, J., Wijffels, S., Cronin, M. F., et al. (2019). Tropical pacific observing system. Front. Mar. Sci. 6:31. doi: 10.3389/ fmars.2019.00031

Tanhua, T., Pouliquen, S., Hausman, J., O’brien, K., Bricher, P., Bruin, T. D., et al. (2019). Ocean FAIR data services. Front. Mar. Sci. (in press).

Wilkinson, M. D., Dumontier, M., Aalbersberg, I. J., Appleton, G., Axton, M., Baak, A., et al. (2016). The FAIR guiding principles for scientific data management and stewardship. Sci. Data 3:160018. doi: 10.1038/sdata.2016.18

Conflict of Interest Statement: LS was employed by company Your Ocean Consulting, LLC, and LG was employed by company Glover Works Consulting.

The remaining authors declare that the research was conducted in the absence of any commercial or financial relationships that could be construed as a potential conflict of interest.

Copyright (C) 2019 Tanhua, McCurdy, Fischer, Appeltans, Bax, Currie, DeYoung, Dunn, Heslop, Glover, Gunn, Hill, Ishii, Legler, Lindstrom, Miloslavich, Moltmann, Nolan, Palacz, Simmons, Sloyan, Smith, Smith, Telszewski, Visbeck and Wilkin. This is an open-access article distributed under the terms of the Creative Commons Attribution License (CC BY). The use, distribution or reproduction in other forums is permitted, provided the original author(s) and the copyright owner(s) are credited and that the original publication in this journal is cited, in accordance with accepted academic practice. No use, distribution or reproduction is permitted which does not comply with these terms. 


\section{Ocean Climate Monitoring}

\author{
Rick Cole ${ }^{1 *}$, Jeff Kinder ${ }^{2}$, Weidong Yu ${ }^{3}$, Chun Lin Ning ${ }^{3}$, Fujun Wang ${ }^{4}$ and Yang Chao ${ }^{5}$ \\ ${ }^{1}$ RDSEA International, Inc., St. Pete Beach, FL, United States, ${ }^{2}$ Down East Instrumentation, LLC, Parish, FL, United States, \\ ${ }^{3}$ State Oceanic Administration, First Institute of Oceanography, Qingdao, China, ${ }^{4}$ Institute of Oceanology, Chinese Academy \\ of Sciences, Qingdao, China, ${ }^{5}$ Proteus Solutions, Beijing, China
}

Measuring ocean physics and atmospheric conditions at the sea-surface has been taking place for decades in our world's oceans. Enhancing R\&D technologies developed in Federal and academic institutions and laboratories such as WHOl's Vector Averaging Current Meter (VACM, 1970s) and NOAA - PMEL's: Autonomous Temperature Line Acquisition System (ATLAS, 1980s) as example, in situ ocean measurements and realtime telemetry for data processing and dissemination from remote areas of oceans and seas are now common place. A transition of this "ocean monitoring" technology has occurred with additional support from individual and group innovative efforts in the field of ocean instrumentation. As a result, long-term monitoring of ocean processes and changes has become more accessible to the research community at large. Here; we discuss a "Hybrid" air-sea interaction deep-sea monitoring system that has been developed in the private sector to mirror ocean-climate community data streams and has been successfully deployed on three basin-scaled programs in the Indian Ocean (RAMA, First Institute of Oceanography, FIO, China), the Andaman Sea (MOMSEI, Monsoon Onset Monitoring, FIO) and the Pacific Ocean (China's Institute of Oceanology, Academy of Sciences (IOCAS) research in the western tropical Pacific). This application is a base to build upon as new sensors are developed and increased sampling at higher resolutions is required. Surface vehicles measure the surface, with some profiling available. Water column density sampling is still a much-needed measurement within the Ocean Climate Monitoring community. The "Hybrid" is a multidisciplinary tool to integrate new biological and biogeochemical sensors for continued interaction studies of the physical processes of our oceans. This application can also be used at FLUX sites to enhance the Argo Program, telemetry applications and docking stations for autonomous vehicles such as sail-drones, gliders and wave riders for enhancement and contribution to the Global Tropical Moored Buoy Array (GTMBA), Global Ocean Observing System (GOOS), Global Climate Observing System (GCOS), and the Global Earth Observing System of Systems (GEOS).

Keywords: density, $\mathrm{CO}_{2}$, ocean temperature, ocean currents measurement, ocean physics

\section{INTRODUCTION}

With the Ocean Climate Monitoring community, worldwide, now settled into a "relaxed funding stage," the future of ocean observing is taking shape and continues to evolve. Technology driven change is occurring, with a mutual goal: continue and increase gathering critical information on how the world's oceans interact with each other along with peripheral terrestrial ecosystems. Oceanographers and Atmospheric scientists must engage in long-term ocean measurements to 
piece together the physical structure of the surface and subsurface below. Understanding physical processes such as air-sea interaction, heat and momentum exchange, freshwater movement, changes in temperature and salinity (density), velocity and direction of major current flow and their spin off eddies along with ocean acidification characteristics are considered the basics. The tools and capabilities for measuring these parameters are forever evolving. New Satellite systems with higher resolutions, automation, micro-electrical-mechanical (MEMS) sensors and even artificial intelligence (AI) are becoming standard and set to be used at length in the coming decades of ocean observation.

Three large multinational programs have set the tone for basin-scale ocean observing systems including; "The Tropical Atmosphere Ocean/Triangle Trans-Ocean Buoy Network" (TAO/TRITON, Pacific), "The Prediction and Research Moored Array in the Tropical Atlantic" (PIRATA), and "The Research Moored Array for the African-Asian-Australian Monsoon Analysis and Prediction Program" (RAMA; McPhaden et al., 2009) a subcomponent of the "Indian Ocean Observing System" (IndOOS). All are components of the "Global Tropical Moored Buoy Array" (GTMBA) and systems within a system; the "Global Ocean Observing System" (GOOS).

Tropical Atmosphere Ocean, formerly the "Tropical Ocean Global Atmosphere" program (TOGA) and the "Equatorial Pacific Ocean Climate Studies" (EPOCS) of the 1970s and $80 \mathrm{~s}$, is now a well-established operational program. PIRATA, still in research mode, covers the tropical region of the Atlantic Ocean. RAMA is the youngest of the arrays with emphasis on the role of the ocean for prediction of the Asian Monsoon, Indian Ocean Dipole (IOD) and El Niño/La Niña-Southern Oscillation (ENSO), all contributing factors to global climate. Buoy monitoring systems deployed in these regions take a "snap shot" of ocean climate as it changes over time creating basin-wide time series. Smaller spin-off programs collecting high resolution data, in the coastal-boundary regions have added additional data sets for model integration. Two key-words above; "Buoy monitoring" are a focus of a transition to less fixed-point assets on programs (i.e., anchored buoy systems) to a more automated configuration, taking advantage of new technologies, both satellite and in situ, with the goal of increased efficiency yet maintaining data quality.

The IndOOS and RAMA Programs now close the longstanding gap in data between the three major Oceans. This research also benefits many outside the Indian Ocean (IO) region due to the atmospheric connections that influence surrounding nations. Additionally, these data will contribute to improve forecasting of tropical cyclones and storm surge. Continued science and technology transfer to the IO-Rim countries helps establish observing capabilities in the region and to promote programs to better understand their role in the global climate system. China's State Oceanic Administration (SOA), First Institute of Oceanography (FIO) has engaged in field operations and data support to the RAMA array, occupying two locations in the eastern region of the tropical IO. These sites help capture the first signal from the western Pacific via the "Indonesian Throughflow" into the IO monitoring array.

First Institute of Oceanography is also deployed on the Monsoon Onset Monitoring and its Social and Ecosystem Impact (SEAGOOS-MOMSEI), a program set to improve the understanding and forecasting of the Asia monsoon and its multi-scaled variability within the Andaman Sea, west of Thailand, a common boundary region in the eastern IO and Bay of Bengal (monsoon behavior and coral reef bleaching). The Institute of Oceanology, Chinese Academy of Sciences (IOCAS, Qingdao, China) has expanded their monitoring program in the north to the tropical western Pacific with increased data collection throughout the region. With the loss of sites in the south (i.e., funding), the Japan Agency for Marine-Earth Science and Technology (JAMSTEC) Triangle Trans Ocean Buoy Network (TRITON) data streams have decreased, leaving gaps in model input. JAMSTEC collaborates with NOAA-PMEL on the TAO/TRITION array. IOCAS hopes to regain some of these data sets in the future by re-populating critical locations along this Equatorial region.

RDSEA International (St Pete Beach, FL, United States) and Down East Instrumentation (Parish, FL, United States), CoAuthors on this writing, have a vast background in oceanobserving, systems integration and data dissemination on U.S. Federal and State programs over many decades, some mentioned above. One of their goals as a team was to develop and mirror a deep-water monitoring buoy and mooring system such as the U.S. National Oceanographic and Atmospheric Administration (NOAA), Pacific Marine Environment Laboratory (PMEL), Automated Temperature Line Acquisition System (ATLAS; Milburn and McLain, 1986), with available, present day, Original Equipment Manufacturer (OEM, off the shelf) components. This goal was achieved with the collaborative efforts of the FIO and IOCAS in Qingdao, China, and Proteus Solutions, Hong Kong, in the Indian and Pacific Oceans. This support has contributed successful technology transfer to our colleagues in China and Southeast Asia (Indonesia and Thailand) with data streaming now for nearly a decade. New laboratories have been populated to receive, process, and disseminate data expanding needed input to the ever-challenging task of monitoring the changing dynamics of the tropical ocean.

\section{SYSTEM BACKGROUND}

In the early 1980s, the National Oceanic and Atmospheric Administration's (NOAA) Pacific Marine Environmental Laboratory (PMEL) began development of an inexpensive buoy and mooring system called the "Autonomous Temperature Line Acquisition System” (ATLAS; Milburn and McLain, 1986). These efforts paralleled one of the most significant ENSO events ever to take place in the Pacific (El Niño, 1982-83). ATLAS measures basic parameters at the sea surface including wind speed and direction, air and sea surface temperature. Ten subsurface locations record temperature at various depths down to 500 meters with redundant pressure recorders at the bottom of the 
string. These data are then sent to shore using the ARGOS telemetry system based on NOAA polar-orbiting satellites (data are also recorded onboard in memory).

The ATLAS system was based on earlier designs used at PMEL on current meter projects (McPhaden, 1995) and would be used to enhance studies on the thermal structure of the upper water column, wind-fields and the transport of heat in the tropical regions of the Pacific Ocean (i.e., airsea interaction). From the few initial deployments, ATLAS was quickly incorporated into the "Tropical Ocean Global Atmosphere" program (TOGA, Hayes et al., 1991) which was initiated to better understand ENSO phenomenon via realtime oceanographic measurements. TOGA laid the foundation for what is in place today; The Tropical Atmosphere Ocean (TAO) program, an array of 70-plus buoys and moorings spanning the equatorial region of the Pacific between $8^{\circ} \mathrm{N}$ latitude and $8^{\circ} \mathrm{S}$ latitude. The Japan Agency for Marine-EarthScience and Technology (JAMSTEC) maintains and manages the western component of the array with their TRITON (similar to ATLAS) buoy systems.

Autonomous Temperature Line Acquisition System electronics (designed and manufactured at PMEL) were completely re-engineered in the early to mid-1990s with new sensors added (relative humidity, radiation, precipitation at the surface and conductivity at depth) (Milburn et al., 1996). The initial systems design of bringing sub-surface temperature data up to the buoy used a thermistor string with temperature pod breakouts at the following depths: 20,40, 60, 80, 100, $120,140,180,300$, and 500 meters in the eastern Pacific and $25,50,75,100,125,150,200,250,300$, and 500 meters at western locations. Pressure is also measured at the bottom of each string at the 300 - and 500-meter levels. These data are used to monitor mooring motion and allow for depth corrections in post processing of temperature data. Subsurface data telemetry to the buoy was replaced by the process of "induction" allowing for simple attachment of sensors using inductively coupled components to the main mooring line and sending a signal (data) up to the buoy controller. Wind speed and direction are measured at 4 meters above the sea surface at $2 \mathrm{~Hz}$ along with a thermistor mounted in a radiation shield to reduce the effects of solar heating for air temperature records. Sea-surface temperature is also measured at a depth of 1 meter.

RDSEA, in partnership with Down East Instrumentation, began a system's engineering approach using the TAO Legacy ATLAS System among other coastal buoy system designs as a baseline for hardware, instrumentation and sample rates. Having been involved in similar design concepts with NOAA and academia using "off-the-shelf" sensors and equipment, we believed it was possible to mirror TAO type buoys and their data streams using existing OEM instrumentation and components.

\section{THE BUOY SYSTEM}

Requested from colleagues at FIO and IOCAS was delivery of real-time data acquisition buoy and mooring systems consisting of a full suite of meteorology (MET) at the sea surface, conductivity, temperature and pressure (CTD) throughout the water column to a depth of 700 meters along with current velocity and direction in the upper $100 \mathrm{~m}$. Project goals were to provide complete turn-key oceanographic buoy and mooring systems for deployment on RAMA. The design would be consistent with ongoing mooredbuoy programs within the GTMBA (Thurston, 2019). All instrumentation and electronic components used are standard "off-the-shelf" ocean instruments proven in the field to provide scientific quality data with long-term durability. All data, surface and subsurface, are transmitted in near realtime and follow standard software and data collection format configurations. A "taut-line" mooring was chosen due to its' proven success throughout decades of robust, long-term use in the deep ocean.

\section{THE BUOY}

A wave following hull design (double chine) of thick walled, closed-cell structure ionomer foam (Softlite Surlyn ${ }^{\circledR}$ ) was chosen for all applications. Many research groups have achieved success with this product on both blue water and coastal programs. For our deployments in over 5000 meters depth a 2.3-meter diameter hull with a nominal density of 8,580 lbs. (buoyancy) was developed.

\section{THE MOORING}

The "taut-line" mooring design dates' back to the early configurations used at the Woods Hole Oceanographic Institution (WHOI) on buoy projects and is the mooring of choice on programs throughout the GTMBA. This design is well proven, relatively easy to deploy and recover with the proper deck gear aboard ship and may be redeployed again on the same research cruise. The philosophy behind a taut-line mooring is to cut the nylon shorter than the deployment site depth (0.985 scope) allowing the elongation factor of the nylon to keep the mooring "taut." All instruments then take measurements at their actual deployed depths (i.e., locations along the mooring line). The buoy system is designed to lift the mooring and anchor during events of heavy seas once maximum stretch is achieved in the nylon, preventing the buoy from sinking. A back up positioning beacon is installed separately (position only) to monitor this buoy movement across the sea-surface.

\section{THE “INDUCTION" PROCESS}

Until recently, sub-surface ocean instrumentation had to be directly cabled to the surface for the transfer of data (as in the ATLAS Legacy System). This is a weak-link in mooring ocean instrumentation and usually the first line of data loss due to cable fatigue and failure. As technology advanced, the use of 
cabled sub-surface sensors at depth on moorings has diminished. Using the jacketed wire rope as a conduit for data transfer, an inductive modem mounted internally on instruments attached to the mooring line transfers signals to a buoy controller at the surface (McPhaden et al., 2016). These instruments include an integrated coupler (magnet) made up of two halves attached externally, allowing them to simply clamp around the mooring. Swaged fittings at both ends of the mooring cable form the seawater-ground needed to complete the inductive loop through the cable. This induces a signal in the single-turn secondary winding formed by the mooring cable passing through the coupler. Data is received from depth via an inductive cable coupler (ICC) that clamps onto the wire rope just below the buoy and mooring attachment (clevis) and is then cabled up to the buoy controller where the sub-surface signal (data) from each instrument is received, decoded and processed.

\section{SYSTEM PAYLOAD}

The buoy system maintains a suite of atmospheric sensors: air temperature, wind (speed and direction), barometric pressure, relative humidity, precipitation, short and long wave solar radiation. Additionally, the system collects near real-time data inductively from sub-surface instruments: SBE37 MicroCats measuring temperature and conductivity, SBE-39 temperature recorders measuring temperature including pressure sensors, and single point Doppler Volume Samplers (DVS, current speed and velocity). A GPS receiver is used to track the position of the buoy and sync the data logger/controller clock with Universal Coordinated Time (UTC). A redundant satellite communications system (dual modems) is used for near real-time telemetry of all data collected via the Iridium satellite constellation. The controller is responsible for scheduling when the sensors will begin sampling, calculating averages, storing information from the sensors in data files and outputting the data records to the satellite modem. When the controller is not performing any of these functions, it reverts to a low power sleep state until the next scheduled sample is to be taken. This is done to conserve systems power as much as possible. The system is designed to be a turn-key, self-contained control and logging application (Figure 1). The Supplementary Data Sheet S1 displays the entire system design.

Additional sensors are presently being added to monitoring arrays and data streams from our world's oceans and the large basin-scale programs. Understanding the partial pressure of $\mathrm{CO}_{2}\left(\mathrm{pCO}_{2}\right)$ at the sea-surface is critical for monitoring ocean acidification levels and the effects and consequences $\mathrm{CO}_{2}$ emissions have on offshore and terrestrial ecosystems. Incorporating these sensors and beginning time-series along with measuring $\mathrm{pH}$, dissolved oxygen, turbidity and chlorophyll will help the ocean sciences better understand the changes in sea-water chemistry as it occurs. In the spring of $2011 \mathrm{CO}_{2}$ measurements were added to the FIO RAMA buoy at a depth

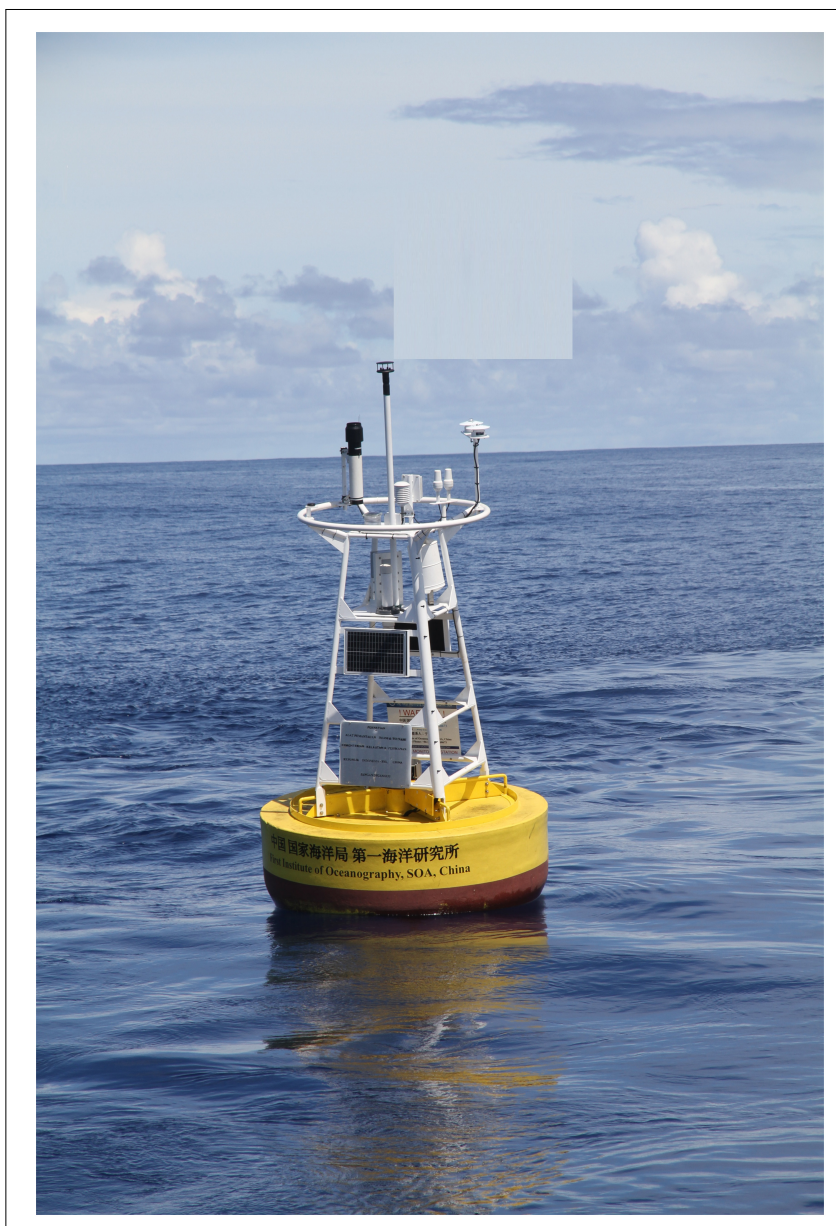

FIGURE 1 | Deployed system, RAMA - Indian Ocean.

of one meter (sea-surface) and on the tower (atmospheric), the first on RAMA.

\section{CONCLUSION}

The development of an "off-the-shelf" deep water buoy system, mimicking the NOAA-TAO-ATLAS design of the 80s and 90s has been tested and deployed. The framework to enhance upon this design has been laid with new ideas in motion for the future longevity of ocean climate monitoring sites. Fixed monitoring sites are still needed on the GTMBA programs, especially for collecting critical wind and density data at FLUX locations. A multinational redesign is well underway (Tropical Pacific Observing System, TPOS-2020; Cravatte et al., 2016), taking advantage of new ocean observing technologies to maintain and extend the tropical ocean climate record. A combination of buoys and moorings plus automated in situ devices such as; gliders, water column samplers (ARGO Program), surface wave riders and sail-drones enhance the needs of the community. RDSEA and Down East Instrumentation 
will continue to support these efforts, buoy system assets and programs and continue to work at sustaining the data flow from present and future locations. Plans for adding additional sensors such as dissolved oxygen and fluorescence are in discussion with hopes of integration and deployment in the very near future.

\section{AUTHOR CONTRIBUTIONS}

All authors listed have made a substantial, direct and intellectual contribution to the work, and approved it for publication.

\section{FUNDING}

This work was supported by the First Institute of Oceanography, State Oceanic Administration and Institute of Oceanology Chinese Academy of Sciences (IOCAS).

\section{REFERENCES}

Cravatte, S., Kessler, W. S., Smith, N., Wijffels, S. E., Ando, K., and Cronin, M. (2016). First Report of TPOS 2020. GOOS-215-200. Available at: http://tpos2020. org/first-report (accessed October, 2018).

Hayes, S. P., Mangum, L. J., Picaut, J., Sumi, A., and Takeuchi, K. (1991). TOGATAO: a moored array for real-time measurements in the tropical Pacific Ocean. Bull. Am. Meteorol. Soc. 72, 339-347. doi: 10.1175/1520-0477(1991)072<0339: ttamaf $>2.0 . \mathrm{co} ; 2$

McPhaden, M. J. (1995). The tropical atmosphere ocean array is completed. Bull. Am. Meteorol. Soc. 76, 739-741.

McPhaden, M. J., Ando, K., Bourlès, K. B., Freitag, H. P., Lumpkin, R., Masumoto, Y., et al. (2016). Real-Time Oceanography with Inductive Moorings and the Inductive Modem Module, (Application Note 92). Bellevue, WA: Sea-Bird Electronics, Inc.

McPhaden, M. J., Meyers, G., Ando, K., Masumoto, Y., Murty, V. S. N., Ravichandran, M., et al. (2009). RAMA - the research moored array for African-Asian-Australian monsoon analysis and prediction. Bull. Am. Meteorol. Soc. 90, 459-480. doi: 10.1175/2008bams 2608.1

Milburn, H. B., and McLain, P. D. (1986). "ATLAS-A low cost satellite data telemetry mooring developed for NOAA's Climate Research Mission," in Proceedings from MTS Marine Data Systems, New Orleans, LA.

\section{ACKNOWLEDGMENTS}

The authors would like to thank the individual and group efforts and contributions at all levels that have made possible the multiple successes of collaborative efforts over the past decade, including FIO and IOCAS staff, scientists, engineers, technicians and students, manufactures, and federal agencies from China, Indonesia and the U.S. Mooring Systems, Inc., of Cape Cod, MA, United States played a huge role in the success of these projects.

\section{SUPPLEMENTARY MATERIAL}

The Supplementary Material for this article can be found online at: https://www.frontiersin.org/articles/10.3389/fmars. 2019.00503/full\#supplementary-material

DATA SHEET S1 | Hybrid-ocean climate monitoring system mooring design.

Milburn, H. B., McLain, P. D., and Meinig, C. (1996). “ATLAS buoy-reengineered for the next decade," in Proceedings of IEEE/MTS Oceans '96 (Fort Lauderdale, FL: NOAA, Pacific Marine Environmental Laboratory), 698-702.

Thurston, S. (2019). The Tropical Atmosphere Ocean (TAO) Array Expands into Indian Ocean. Seattle, WA: NOAA Office of Climate Observation.

Conflict of Interest Statement: RC (RDSEA International) and JK (Down East Instrumentation) were employed/contracted by Chinese Academic Institutions: First Institute of Oceanography (FIO) and the Institute of Oceanology and Academy of Sciences (IOCAS) for this work. YC of Proteus Solutions, Beijing, China is the agent representing all foreign entities on all FIO and IOCAS projects via China and United States representatives.

The remaining authors declare that the research was conducted in the absence of any commercial or financial relationships that could be construed as a potential conflict of interest.

Copyright (c) 2019 Cole, Kinder, Yu, Ning, Wang and Chao. This is an open-access article distributed under the terms of the Creative Commons Attribution License (CC BY). The use, distribution or reproduction in other forums is permitted, provided the original author(s) and the copyright owner(s) are credited and that the original publication in this journal is cited, in accordance with accepted academic practice. No use, distribution or reproduction is permitted which does not comply with these terms. 
OPEN ACCESS

Edited by:

Fei Chai,

State Oceanic Administration, China

Reviewed by:

Wayne Homer Slade,

Sequoia Scientific, United States

lan Walsh,

Sea-Bird Scientific, United States

*Correspondence:

Christian Meinig

Christian.Meinig@noaa.gov

Specialty section: This article was submitted to

Ocean Solutions,

a section of the journal

Frontiers in Marine Science

Received: 30 November 2018

Accepted: 05 July 2019

Published: 13 August 2019

Citation:

Meinig C, Burger EF, Cohen N, Cokelet ED, Cronin MF, Cross JN, de Halleux $S$, Jenkins $R$, Jessup AT, Mordy CW, Lawrence-Slavas N,

Sutton $A J$, Zhang $D$ and Zhang $C$

(2019) Public-Private Partnerships

to Advance Regional

Ocean-Observing Capabilities:

A Saildrone and NOAA-PMEL Case

Study and Future Considerations

to Expand to Global Scale Observing.

Front. Mar. Sci. 6:448.

doi: 10.3389/fmars.2019.00448
Public-Private Partnerships to

Advance Regional Ocean-Observing

Capabilities: A Saildrone and

NOAA-PMEL Case Study and Future

Considerations to Expand to Global Scale Observing

Christian Meinig ${ }^{1 *}$, Eugene F. Burger ${ }^{1}$, Nora Cohen ${ }^{2}$, Edward D. Cokelet',

Meghan F. Cronin', Jessica N. Cross', Sebastien de Halleux², Richard Jenkins'2, Andrew T. Jessup ${ }^{3}$, Calvin W. Mordy ${ }^{1,4}$, Noah Lawrence-Slavas', Adrienne J. Sutton', Dongxiao Zhang ${ }^{1,4}$ and Chidong Zhang ${ }^{1}$

\footnotetext{
National Oceanic and Atmospheric Administration (NOAA) Pacific Marine Environmental Laboratory (PMEL), Seattle, WA, United States, ${ }^{2}$ Saildrone, Alameda, CA, United States, ${ }^{3}$ Applied Physics Laboratory, University of Washington, Seattle, WA, United States, ${ }^{4}$ Joint Institute for the Study of the Atmosphere and Ocean, University of Washington, Seattle, WA, United States
}

Partnership between the private sector and the ocean-observing community brings exciting opportunities to address observing challenges through leveraging the unique strengths of each sector. Here, we discuss a case study of a successful relationship between the National Oceanic and Atmospheric Administration (NOAA) Pacific Marine Environmental Laboratory (PMEL) and Saildrone to instrument an unmanned surface vehicle (USV) in order to serve shared goals. This case study demonstrates that a private company working with a federal laboratory has provided innovative oceanobserving solutions deployed at regional scale in only a few years, and we project that this model will be sustainable over the long term. An alignment of long-term goals with practical deliverables during the development process and integrating group cultures were key to success. To date, this effort has expanded NOAA's interdisciplinary observing capabilities, improved public access to ocean data, and paved the way for a growing range of USV applications in every ocean. By emphasizing shared needs, complementary strengths, and a clear vision for a sustainable future observing system, we believe that this case study can serve as a blueprint for public and private partners who wish to improve observational capacity. We recommend that the international scientific community continues to foster collaborations between the private sector and regional ocean-observing networks. This effort could include regional workshops that build community confidence through independent oversight of data quality. We also recommend that an international framework should be created to organize public and private partners in the atmospheric and oceanographic fields. This body would coordinate development of observational technologies that adhere to best practices and standards for sensor integration, verification, data quality control and delivery, 
and provide guidance for unmanned vehicle providers. Last, we also recommend building bridges between the private sector, the ocean-observing community, and the operational forecast community to consider the future of this new private sector, with goals to determine targeted ocean-observing needs; assess the appropriateness of USVs as science platforms, sensors, and data format standards; and establish usage and data quality control and distribution protocols for ocean observing and operational forecasting.

Keywords: unmanned surface vehicle, Saildrone, National Oceanic and Atmospheric Administration, Pacific Marine Environmental Laboratory, surface essential ocean variables, essential climate variables, ocean observations, public-private partnership

\section{INTRODUCTION}

In 2009, the previous OceanObs conference ended with a call to action to significantly enhance global ocean observing. With over 600 participants across 36 countries and more than 100 papers submitted, the community put forward many recommendations. The key, consensus outcome of the global ocean science community represented by OceanObs'09 was the development of the Framework for Ocean Observing (FOO) (Lindstrom et al., 2012). The vision was aspirational: sustained, high-resolution observing networks that openly share data in near real time, distributed and quality controlled according to best practices and international standards, that support society's needs for understanding and forecasting marine variability on a variety of scales. In order to achieve this vision, it was clear that development of new technologies would be essential, although the pathway forward was largely unclear.

In the intervening decade, technical progress has been made that has rapidly scaled the global ocean-observing system, sometimes through important partnerships with the private sector. Many industries, from insurance to agriculture, feel illequipped to plan for rapid environmental changes. As a result, private capital has been increasingly motivated to support the global science community to enhance earth observations.

Given these shared goals, public science entities and private partners can work together to make significant, high-quality, and rapid advances to the observing system by harnessing the strengths of each partner. Through the public sector, the scientific community provides guidance and shares expertise to ensure that any new assets or protocols meet data quality and access standards and target the public mission goals. The private sector can quickly harness capital and build assets, shouldering a significant amount of development and management risk. Together, these partners can quickly build, deploy, and apply high-quality scientific tools that both serve the public good and meet private sector needs.

Here, we present a public-private partnership (PPP) case study and model that has delivered innovative solutions to technological gaps and addressed requirements necessary for integration into sustained observing systems. For the purposes of this discussion, we define a PPP as an agreement between the U.S. government and a private corporate entity, each bearing the strengths and responsibilities suggested above. In 2014, a PPP was established between the U.S. National
Oceanic and Atmospheric Administration's (NOAA) Pacific Marine Environmental Laboratory (PMEL) and Saildrone. This partnership resulted in the successful development of the Saildrone USV $^{1}$, an unmanned surface vehicle (USV) for ocean research, and has provided 4 years of validated data for demonstration missions that have been conducted on regional scales.

Based on these early successes, it seems clear that USVs could substantially expand the global ocean-observing network. One vision is to leverage private industry investment in the development of a variety of USVs coordinated in regional fleets. These focused networks would be adaptable to specific observing requirements and fill targeted regional observing gaps. To fulfill that vision, we recommend that the international community continue to foster close relationships between the private sector, the regional ocean-observing community, and the operational forecast community to determine these observing needs; assess the appropriateness of existing USVs, sensors, sampling schemes, and data format standards for achieving these needs; and establish usage and data distribution protocols for data generated by USVs at these scales. Establishing community confidence, transparency, and independent oversight of data quality will be critical to building on these early successes and achieving a robust role for USVs in the global observing system.

\section{Public Infrastructure}

At NOAA-PMEL, large-scale global observatories have traditionally evolved to address specific scientific needs such as providing time series data and forecasts for high-impact phenomenon like El Niño Southern Oscillation (ENSO), tsunamis, and carbon flux. Research programs are developed around each specific science requirement and encompass the entire observational effort from engineering development, sensor and platform procurement, data quality control (QC), and assurance, to ingestion of data into research models and products. If the research program's data and products prove to be of significantly high impact, then the program is eventually transferred from the public research laboratory to a public operational entity. An example is the decade-long investment of PMEL research in developing an effective tsunami monitoring system that now delivers global data into NOAA National

\footnotetext{
${ }^{1}$ In this publication, "Saildrone" refers to the company name, and "Saildrone USV" refers to company's unmanned surface vehicle.
} 
Weather Service forecast systems in real time to save lives and property (Bernard and Meinig, 2011).

This well-proven public observational data model has several benefits. Observational data generated by scientists through a rigorous scientific process are of high quality, and the model closely couples data users with data gatherers during the research development phase. Unfortunately, this public observational model also has several key weaknesses that negatively affect the scalability of the public observational arrays. When the research array transitions into operations, there is a considerable loss in flexibility and adaptability, and data users and data gatherers become decoupled. Implementing and maintaining the array come at considerable cost due to the need for skilled instrument technicians, seagoing personnel, and staff to manage the logistical and data pipelines. While this model generates isolated areas of specific expertise, such arrays are not easily ported, adopted, or adapted by other organizations that may have different cultures and funding priorities. Finally, arrays built in isolation from the private sector may be slow to develop and implement and, therefore, not well suited to the urgency of a rapidly changing world.

\section{Private Capacity}

The PPP with Saildrone offered the ability to leverage private capital and invest in complex, multiyear efforts. Saildrone possessed the capacity to scale up rapidly, and this streamlined purchasing and hiring. In return, NOAA received a large quantity of high-quality standardized observations of essential climate and ocean variables (ECVs and EOVs). From Saildrone's perspective, NOAA provided expertise on the collection of observational data that were based on prior community development, including sensors, standards, and data quality management. Through the PPP, NOAA also serves as a potential customer for observational data.

The vision set forth in this paper is for distributed regional fleets of USVs that fill spatiotemporal data gaps in observing arrays, are adaptive to specific observing requirements, and serve as an end-to-end tool for the larger oceanographic community. Under the model presented here, a primary concern is the use of a private entity as a data provider. High-quality data provide the basis for decisions that affect weather, marine environment, fish stocks, climate, commerce, and security. For these reasons, a main driving force behind this PPP is the shared goal of making "fit for purpose" observations that have known and transparent QCs and, therefore, are trusted by the research community and, ultimately, by the public. To implement this vision, the roles of each partner must be defined, and the community must provide standards for data management and verification that are transparent and standardized. A cautionary lesson in the proper setting of data quality acceptance standards, availability, and archiving of contracted data is highlighted in an example from the ground-based Global Navigation Satellite System (GNSS) data (Serra et al., 2018). Because exact specifications for the data were not written into the vendor contract, the U.S. government must now pay for data produced by the private entity that it cannot use and cannot change. It is also imperative for the government to maintain in-house expertise to continually evaluate these data and act as a repository of expertise, should any commercial venture fail.

\section{PPP: SAILDRONE AS A CASE STUDY}

\section{Public Interest}

NOAA-PMEL has a long history of bringing innovations to ocean observations primarily through global-scale buoy arrays and sensor developments (McPhaden et al., 2010; Bernard and Meinig, 2011; Sutton et al., 2014). Saildrone's founder spent $\sim 10$ years perfecting highly innovative and robust wind-powered platforms. These innovative cultures mesh well, with each bringing unique strengths and shared goals to ocean-observing development. The team built momentum around shared values of "learning by doing" and, in the first year, tested prototypes with several integrated sensors and extended missions off central California, Gulf of Mexico, and the Bering Sea (Cokelet et al., 2015; Meinig et al., 2015).

Broader high-level impacts of interest to both parties for developing a scientific USV include the opportunity to address mission goals for oceanographic observing, as well as the opportunity to improve infrastructure and approach important scientific questions. NOAA's mission is to understand and predict changes in climate, weather, oceans, and coasts and to share that information in support of the blue economy, environmental sustainability, and coastal management. The cutting-edge measurements that Saildrone USVs can provide may result in model and forecasting improvements that are central to this mission.

\section{Saildrone USV}

A goal of Saildrone is to address the scarcity of in situ ocean data by augmenting ship and buoy infrastructure with flexible and efficient autonomous technology. The core components of this network are the Saildrone USVs, which combine windpowered vehicle technology with solar-powered meteorological and oceanographic sensors for long-range data collection missions in the toughest of ocean environments.

The Saildrone USV is an autonomous ocean-going data collection platform designed for long-range, long-duration missions of up to 12 months. The Saildrone USVs operate solely on renewable energy, using wind power for propulsion and solar power to run a suite of science-grade sensors. Each vehicle consists of a $7 \mathrm{~m}$ long narrow hull, a $5 \mathrm{~m}$ tall hard wing, and a keel with a $2.5 \mathrm{~m}$ draft. Each weighs approximately $750 \mathrm{~kg}$ and can be launched and recovered from a dock.

The Saildrone USV's propulsion system is the result of a 10 years research effort in high-performance land sailing and consists of the tall hard wing, a longitudinal spar, and a vertical tail. The wing angle to the wind is adjusted by a trim-tab on the tail, similar to the way an elevator trim tab controls the pitch of the aircraft. Each vehicle travels at an average speed of $1.5-2.5 \mathrm{~m} / \mathrm{s}$ and can reach top speeds above $4 \mathrm{~m} / \mathrm{s}$, enabling these USVs to cover large survey areas and reach most ocean locations within 30 days from the nearest shore. 
The sensor payload consists of $\sim 20$ sensors that measure key atmospheric and oceanographic environmental variables, with the goal of making climate quality measurements. These include solar irradiance, longwave radiation, atmospheric pressure, air temperature and humidity, wind speed and direction, ocean skin temperature, bulk water temperature, salinity, ocean color (Chl-a, CDOM), atmospheric and seawater partial pressure of $\mathrm{CO}_{2}\left(p \mathrm{CO}_{2}\right)$, dissolved oxygen, and $\mathrm{pH}$. The vehicles have also been equipped with acoustic doppler current profilers (ADCP), scientific echosounders for biomass assessment and bathymetry surveys, and passive acoustic recorders for marine mammal studies (Mordy et al., 2017). The sensors are connected to onboard computers and transmit data in real time via satellite communications to shore-based data centers.

The Saildrone USVs transmit their position and health status via satellite, and the entire fleet is under the constant supervision of a centralized mission control, staffed by human pilots aided by sophisticated automation tools. The vehicles navigate autonomously from prescribed waypoint to waypoint, accounting for wind and currents, while staying within a userdefined safety corridor. To further ensure safe operation, each USV is equipped with an Automated Identification System (AIS) transceiver, navigation lights, radar reflector, high visibility wing colors, and four onboard cameras. Together, these measures have resulted in a strong track record of safe operations.

\section{Saildrone Business Model}

The Saildrone USV is just a small component of this new data collection infrastructure, and as such, the vehicles themselves are not for sale. Instead, Saildrone offers the data collected by its vehicles on a data-as-a-service basis, for a flat daily rate, without any upfront investment required from the user, while Saildrone takes care of all operational mission execution. This rate covers the lease of the vehicles and all of their core sensors, as well as integration, calibration, and testing; it also includes vehicle deployment and transit to the area of operation, 24/7 navigation, and piloting; data retrieval via satellite link, and its visualization via a robust data portal; and the delivery of high-resolution data in $\mathrm{NetCDF}^{2}$ format (minute-level subsampled data in near real-time and raw data at mission end for full transparency and further QC).

The private sector has, so far, provided all upfront capital expenses (over $\$ 100 \mathrm{M}$ has been contributed to date), which ensures that this new infrastructure can become operational without the delays associated with large and complex public investment and also ensures that each user's budget is fully directed toward its specific data collection efforts while still leveraging the full power of the overall infrastructure.

Furthermore, in cases where multiple stakeholders with overlapping data interests can be served by the same regional USV fleet, operating expenses can be distributed among the group, further increasing cost-efficiencies for all. This mode of operation is analogous to a satellite array managed by a command-and-control center and serviced by a global operations team of engineers serving

${ }^{2}$ https://www.unidata.ucar.edu/software/netcdf/ a global user base. The long-term nature of observations ensures the financial sustainability of the infrastructure over a long horizon.

\section{Forming the PPP}

The PMEL and Saildrone partnership started in 2014 with an exploration of what value a long-endurance USV outfitted with numerous scientific sensors could bring to PMEL mission areas from the Arctic to the tropics. After a prototype Saildrone USV (without sensors) proved it could cross the Pacific from San Francisco, CA, to Honolulu, HI, the teams met several times to discuss forming a partnership to integrate PMEL sensors and leverage experience from decades of operating global buoy arrays. Common organizational goals were (1) high-quality surface and subsurface ocean observations in near real time; (2) highly reliable, long-endurance USVs that can operate globally; (3) elimination of shiptime for operations; and (4) a large, multidisciplinary payload capacity.

Based on common interest in developing a scientific USV, the teams established a Cooperative Research and Development Agreement (CRADA). A CRADA is an agreement under which a U.S. government laboratory contributes personnel, services, facilities, equipment, or other resources - but not funding toward the conduct of specified research or development efforts. The CRADA business partner contributes any necessary funding to the project as well as personnel, services, facilities, equipment, or other resources. A CRADA is intended to speed the transfer and commercialization of technology by optimizing resources and protecting the private company's intellectual property (IP) rights. Additionally, the CRADA contains high-level goals for the partnership and lays out the basic framework for the development effort.

Once benchmark standards of data quality have been assured, the PPP between NOAA and Saildrone has the potential to improve efficiency and cost-effectiveness as follows: (1) private investment is able to capitalize on cost savings through economy of scale, (2) consolidation of expertise by the private sector allows scientific investigators to focus on data ingestion and results, and (3) easily accessible data ensure that the data products generated are provided to multiple investigators, maximizing impact.

\section{Challenges Met by This PPP}

In a resource-limited environment, the FOO (Lindstrom et al., 2012) encouraged increased coordination and leveraging of existing observing assets, but a pathway to the development of the necessary technological advancements through increasing readiness levels (RL) was a slow, multiyear endeavor. However, our PPP was able to significantly accelerate this process.

At the start of the PPP, the Saildrone USV as an ocean science platform had an RL of 3 as defined by Lindstrom et al. (2012). Four years later, the Saildrone USV (Figure 1) has 20 sensor packages integrated into the platform, with near real-time data delivery (Figure 2) and an RL range of 7-9. The system, including all subsystem components and processes, was successfully tested as part of this PPP. Saildrone has completed over 200,000 nautical miles of 


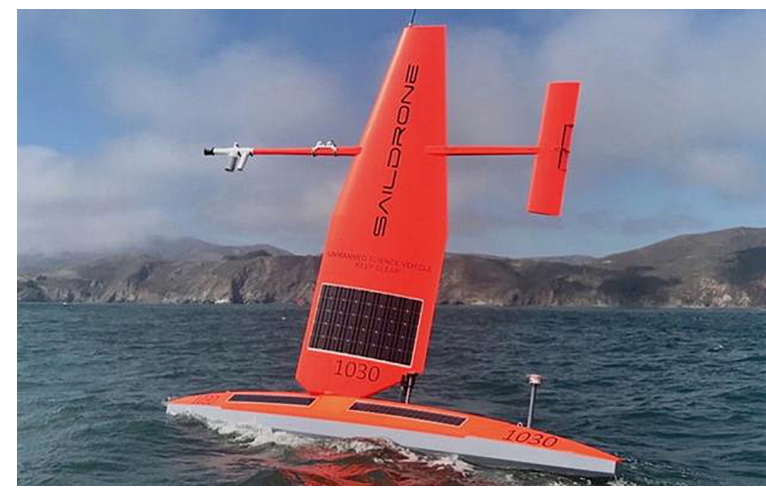

FIGURE 1 | Saildrone USV off the coast of California.

missions and continues to work closely with NOAA-PMEL (Figure 3) and other entities to demonstrate that the mission data are of high quality and can meet the requirements of the community.

These successes would not have been possible without acknowledging and carefully planning to face several important challenges. From the beginning, both parties shared a vision for a large fleet and an entirely new scale of data production. We also noted that these new platform and survey capabilities would likely lead to a demand for new sensor development in the future, although a relatively low demand has historically limited this market. Each of these issues on their own had historically caused many headaches for the scientific community. Here, we share some of our insights based on these three difficult issues and how they informed our planning and the vision for a large-scale Saildrone fleet.

\section{Observing Network}

A Saildrone USV network can provide data at an entirely new scale, including an unprecedented new flexibility. However, this scale requires operating a large fleet of data collection platforms. Historically, stitching together disparate data sets from individual campaigns has been a major challenge. Many programs rely on postcorrecting and synthesizing data to generate global data sets and climatological products [e.g., Surface Ocean $\mathrm{CO}_{2}$ Atlas (SOCAT) Program; Lauvset et al., 2018].

When envisioning the activity of a Saildrone USV fleet, we did our best to consider the scientific community's long experience with these challenges by thinking through how to plan surveys that would be easily intercomparable. Part of this is an innovative survey design that not only includes calibration and intercomparison opportunities and overlapping tracks but also suggests that the fleet should carry standard sensors, use consistent calibration methods, and set clear QC metrics.

The fleet refresh rate was also a critical point to consider: because of issues like biofouling and recalibration, deployments would likely be limited to approximately 1 year. This could be less in some regions: for instance, as a sailing vehicle that depends on sunlight to power sensors, in high latitudes,
Saildrone USVs have an operational limit based on solar availability, freezing sea spray, and ice cover. However, with a scaled fleet, year-round persistence outside of extreme latitudes will be possible by swapping vehicles on location periodically.

\section{Data}

Multidisciplinary observational platforms like the Saildrone USV generate huge amounts of data, especially when integrated with complex motion-corrected instrumentation such as echosounders, ADCPs, and three-dimensional (3D) wind measurements. After the first scientific mission in 2015 generated several terabytes of scientific data in 3 months, PMEL and Saildrone formed a data management working group and began to develop a data management and delivery pathway. This effort focused on the automated delivery of a climate and forecast (CF)-compliant, self-documented, NetCDF files with goals of making data readily available to the broad oceanographic community and enabling researchers to focus directly on data analysis and investigation results.

In the days of Amazon, Apple, Facebook, and Google-size data sets, tools exist to manage data at scale. However, this scale is new for in situ oceanographic data and means that Saildrone must rely on modern big data methodologies as data sets grow exponentially. Saildrone is working not just to implement largescale storage and computation but also to manage data quality and metadata at very large scale. The science-driven technology development PPP method laid out in this paper provides an outline for addressing the very important concerns of data quality and chain of custody when a private company is involved in the collection and processing of data sets.

\section{New Sensor Development}

Oceanographic sensor development has been slow because the market size for such sensors is small when compared to sensor markets such as laboratory, industrial, and commercial monitoring, measurement, and testing equipment. Additionally, the oceanographic sensor market is dispersed over both a wide range and number of public, academic, and private institutions with stringent and often unique sensor requirements. Building an array of hundreds of USV vehicles equipped with standardized sensors has the potential to contribute significantly to market share and generate enough focused demand to drive the commercial development of new specialized sensors.

One example of new sensor development is the SaildronePMEL autonomous surface vehicle $\mathrm{pCO}_{2}\left(\mathrm{ASVCO}_{2}\right)$ system. This system is based on the $\mathrm{MApCO}_{2}$ technology (Sutton et al., 2014), which was transitioned from PMEL to Battelle Memorial Institute and sold commercially as the Seaology $\mathrm{pCO}_{2}$ monitoring system. Over the last decade, Battelle sold $\sim 120$ systems worldwide. This lack of demand relative to the commercial demand of other Battelle products has resulted in the stagnation of technical development, obsolescence issues, and, ultimately, the abandonment of this sensor as a commercial product. Despite these commercial challenges, there is a significant scientific demand for a $\mathrm{pCO}_{2}$ sensor based on ocean-observing needs. Targeting key gaps in the Surface Ocean $\mathrm{CO}_{2}$ NETwork 


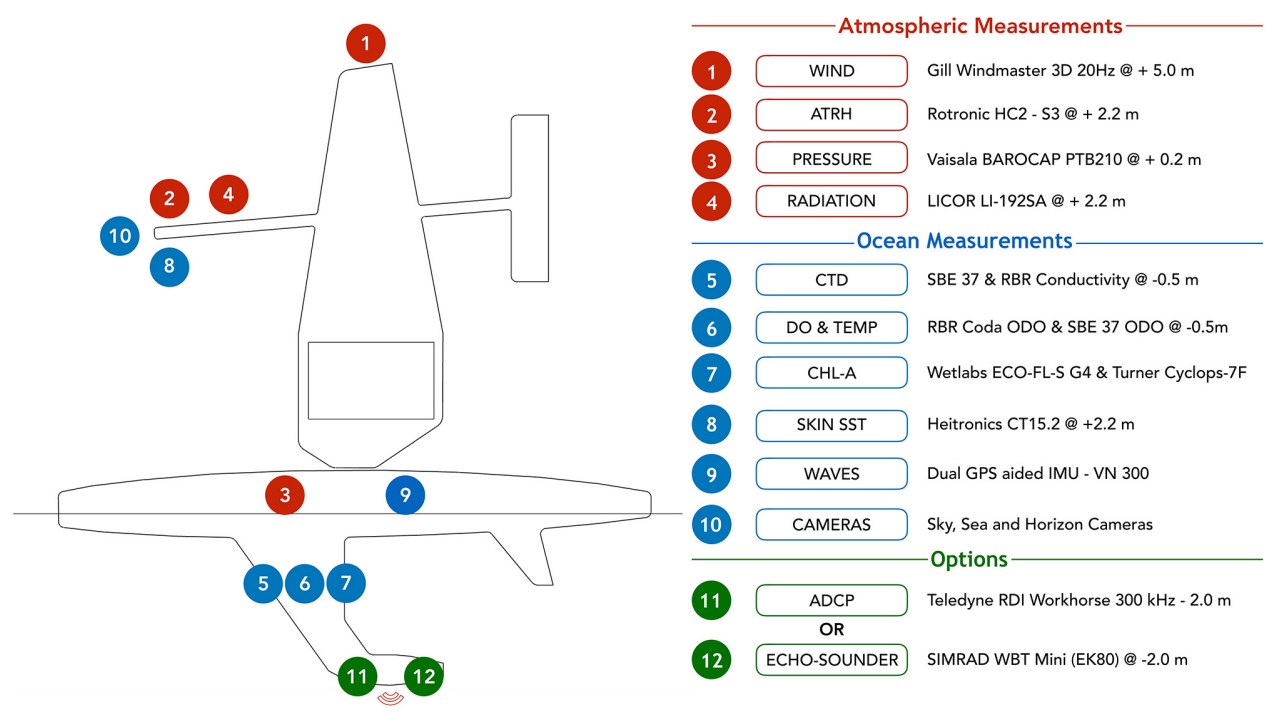

FIGURE 2 | Saildrone USV surface and subsurface core sensor suite. Additional application-specific sensors have routinely been added to the core suite to measure parameters such as atmospheric \& dissolved $\mathrm{pCO}_{2}$, short and long wave solar radiation, passive acoustic recordings, and other specialized measurements.

(SOCONET) array could reduce uncertainties in the ocean sink of anthropogenic $\mathrm{CO}_{2}$ (Wanninkhof et al., 2019). If the PMELSaildrone partnership can build and deliver codeveloped $p \mathrm{CO}_{2}$ systems that collect climate-quality surface ocean $p \mathrm{CO}_{2}$ data $(<2 \mu \mathrm{atm})$, this opens a new market to fill in major observing gaps in SOCONET.

\section{Development of a Drone Into a Science Tool}

Considering these questions helped inform the design of a large-scale USV fleet and what the key operational design challenges would be. However, we first needed to develop the Saildrone USV into a scientific tool. In order to make highquality measurements of ECVs and EOVs, many factors need to be considered, including details about the sensor's initial quality, drift, calibration, and platform effects. Engineers and scientists need to build and maintain tight feedback loops so that each sensor and essential variable is properly understood and calibration and validation is tracked during hardware and software design cycles. Critically, the overall development needs to include not only laboratory testing but also at-sea comparisons. These experiments need to be properly designed with detailed knowledge of temporal and spatial variabilities of each essential variable in the operating environment so that new sensors and platforms such as the Saildrone USV can be properly compared with established standards, which are usually ships or buoys.

\section{Sensor Integration}

The team collaboratively chose an incremental sensor integration strategy to gain some early "easy" successes based on science requirements before proceeding to more difficult sensors. Figure 4 shows a progression of testing and validation for several sensors suites. In general, the team progressed from basic atmospheric (solar irradiance, wind, barometric pressure, air temperature, and relative humidity) and ocean physics measurements (water temperature and salinity) to biogeochemical measurements (ocean color, dissolved oxygen, $\mathrm{pH}$, and $\mathrm{pCO}_{2}$ ) to complex active acoustics (fisheries, ADCP, charting) and broadband passive acoustics (marine mammals, subsea volcanoes).

This incremental approach divided the integration effort into discrete, year-long, sensor suite developments, which contained the following steps:

(1) Determine variables to measure based on ECVs, EOVs, and NOAA research priorities.

(2) Identify sensors to integrate and sensor sampling protocols based on science specifications.

(3) Integrate identified sensors onto the Saildrone USV platform with the goal of producing high-quality data.

(4) Validate the suite of instrumentation through carefully designed sensor intercomparisons.

\section{Codevelopment Working Groups}

To ensure the delivery of high-quality data, the PMEL-Saildrone effort was structured around joint science-engineering working groups. Within these groups the PMEL engineers, who were embedded with the scientists at the research laboratory, facilitated the flow of information and communicated science and engineering requirements/realities across the culture gap that existed between the government scientists and the private company engineers. This communication structure has proven very successful in both development and validation. Because of the multidisciplinary nature of the Saildrone USV platform, these working groups shared a common core of PMEL and Saildrone engineers. Working groups varied in size and scope from large groups containing scientific expertise from all of PMEL's major 

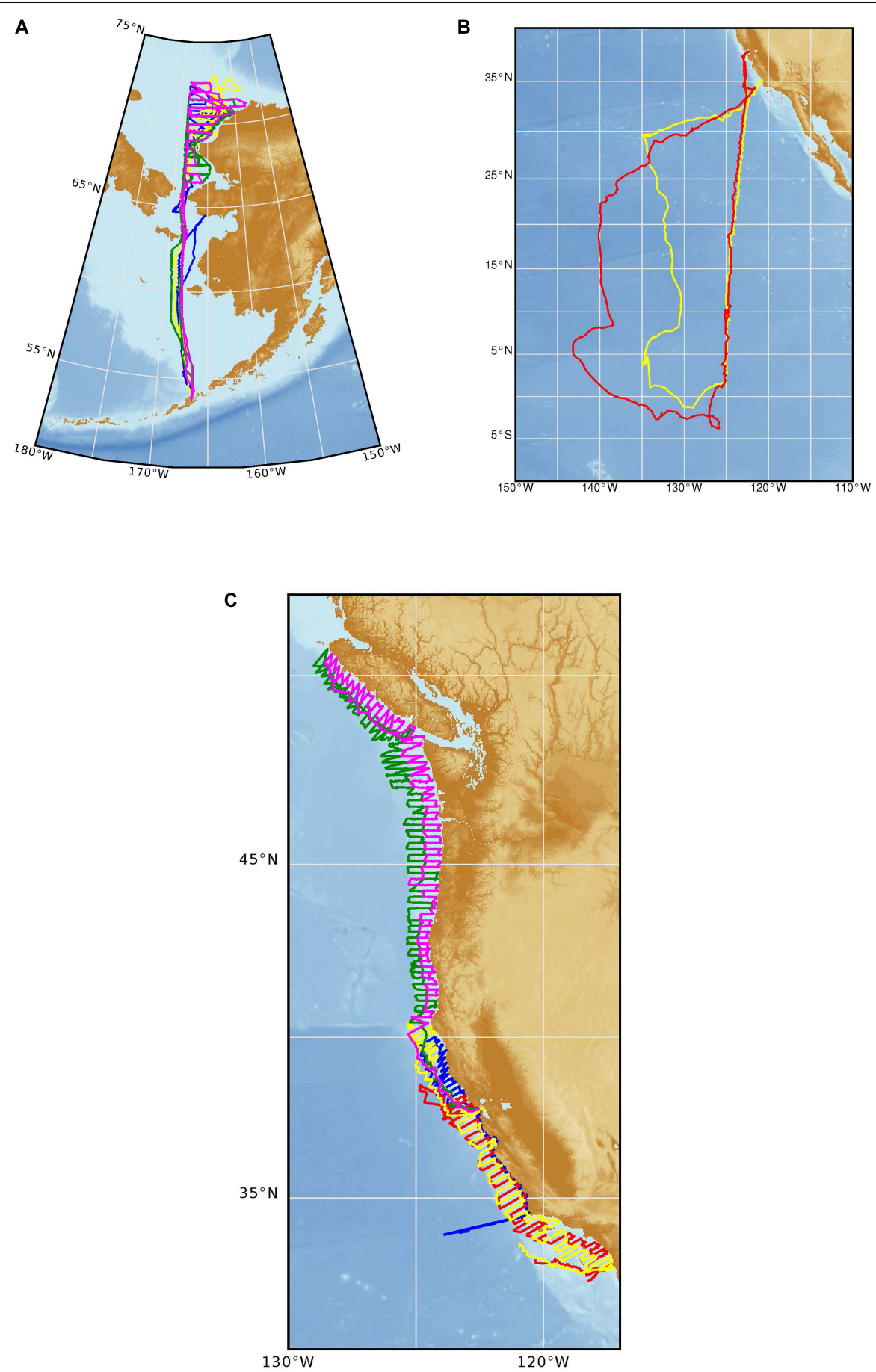

FIGURE 3 | Saildrone USV tracks from (A) four vehicles during a four-month mission in the Bering and Chukchi Seas in 2018, (B) two vehicles during a nine-month equatorial mission in 2017-2018, and (C) five vehicles during a four-month fisheries mission on the North American West Coast.

research programs, to small and highly focused groups of measurement and data specialists. An important aspect of these working groups was their flexibility. As new sensors were integrated and data processes developed, new working groups were formed or the focus of existing groups was modified.
In order to establish scientific confidence in observations outside of PMEL's expertise, the PMEL-Saildrone team sought out and developed external partnerships with established experts in the fields of fisheries acoustics, 3D wind flux, and wave measurement. These relationships varied in scope and included research partnerships that resulted in multidisciplinary scientific 


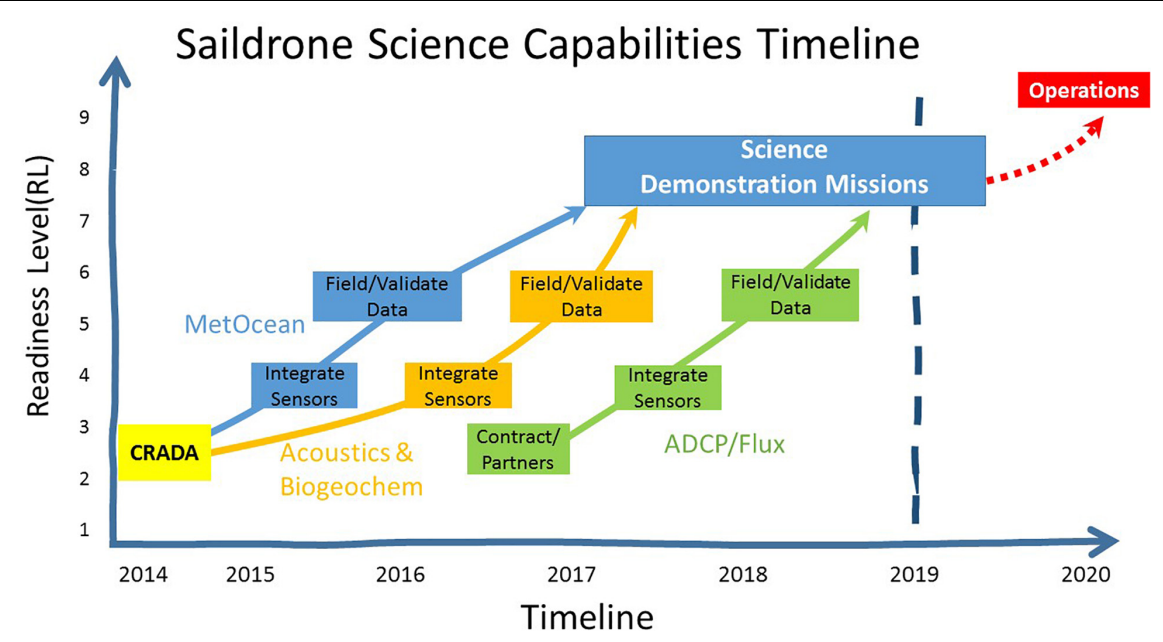

FIGURE 4 | Timeline of Readiness Level for Saildrone USV sensor suites.

USV missions, joint peer-reviewed journal publications on observational methods, contracts for specific sensor development, and informal review and advice on sensor integration and validation methodologies.

Working groups for bulk surface measurements and specialized sensor measurement groups for ADCP, 3D wind flux, and echosounder established scientific confidence in the Saildrone USV sensor measurements and sampling protocols. Additional working groups addressing pre- and postmission sensor calibration requirements, CF compliant data formats, and a drone-to-desktop data delivery pathway ensure data quality and chain of custody.

\section{Validation of Observations}

Detailed comparisons have been run in several oceans for EOVs and ECVs to understand the effects and corrections of a moving Saildrone USV in a variety of sea states. Saildrone and PMEL engineers and scientists collaboratively design the validation experiments and view the data in real time to catch biases and work out difficult sensing problems. As an example, underway ADCP measurements require extremely accurate heading information and precise sensor installation geometry so that ping-by-ping corrections can be made without introducing bias. These types of comparisons are typically intensive during the early development cycles but continue as spot checks for all missions. To date, EOV comparisons have been done with (1) Saildrone USV ADCP and an upward-looking moored ADCP off California deployed specifically for the intercomparison purpose; (2) Saildrone USV surface air and sea measurements against shipboard and surface moored buoys during science missions in the Arctic/Bering Sea, the tropical Pacific, the U.S. West Coast, and the Tasman Sea; and (3) Saildrone USV and shipboard fisheries acoustic sounders. Figure $\mathbf{5}$ is an example of wind speed validation between Saildrone USVs, ship, and buoy in the Bering Sea.

To further establish community-wide confidence in the validity of the Saildrone USV data, the results of these validation studies are in the process of being published in peer-reviewed journals. Currently, there are papers in early stages of development addressing 3D wind flux, USV carbon measurements, bulk surface met and ocean measurements, and fisheries acoustics (De Robertis et al., 2019; Zhang et al., 2019).

\section{Data Delivery}

Saildrone assembles data into two delivery modes, real time and postmission. The real-time datastream delivers averaged data samples during the mission to the mission owner via the Saildrone mission portal and as data files. Meteorological and oceanographic parameters are averaged to $1 \mathrm{~min}$ samples and delivered every $2 \mathrm{~h}$ to the mission operator as self-documenting, CF metadata-compliant, NetCDF Discrete Sampling Geometry (DSG) files that meet the underway observation feature type. For the postmission data, a NetCDF template is defined for the delivery of a larger selection of scientific and engineering data. These data are averaged to 1 and $10 \mathrm{~Hz}$ and delivered as a single file per day. Under this scheme, the data volume is $\sim 6 \mathrm{~GB}$ per month per vehicle. These data are not quality controlled at present.

\section{Data Acceptance and Reprocessing}

The objective of the data acceptance workflow is rapid data ingestion so that the data are available to scientists within 5 min after delivery to the mission operator (PMEL). As Saildrone delivers data in fully documented NetCDF files, minor data reformatting is required to make these data available through interoperable data services. Data are transferred to PMEL by FTP, where timed processes move the data from the FTP server to an internal server (Figure 6). Derived parameters (e.g., wind speed, direction, and USV separation distances) are computed and added to the NetCDF files.

Operational use of measurements from Saildrone USVs requires these data to be available to weather forecast centers via the World Meteorological Organization (WMO) Global 


\section{A Wind Speed from Saildrones $s d-126$ and $s d-128$ 24-MAY-2016 to 03-SEP-2016}
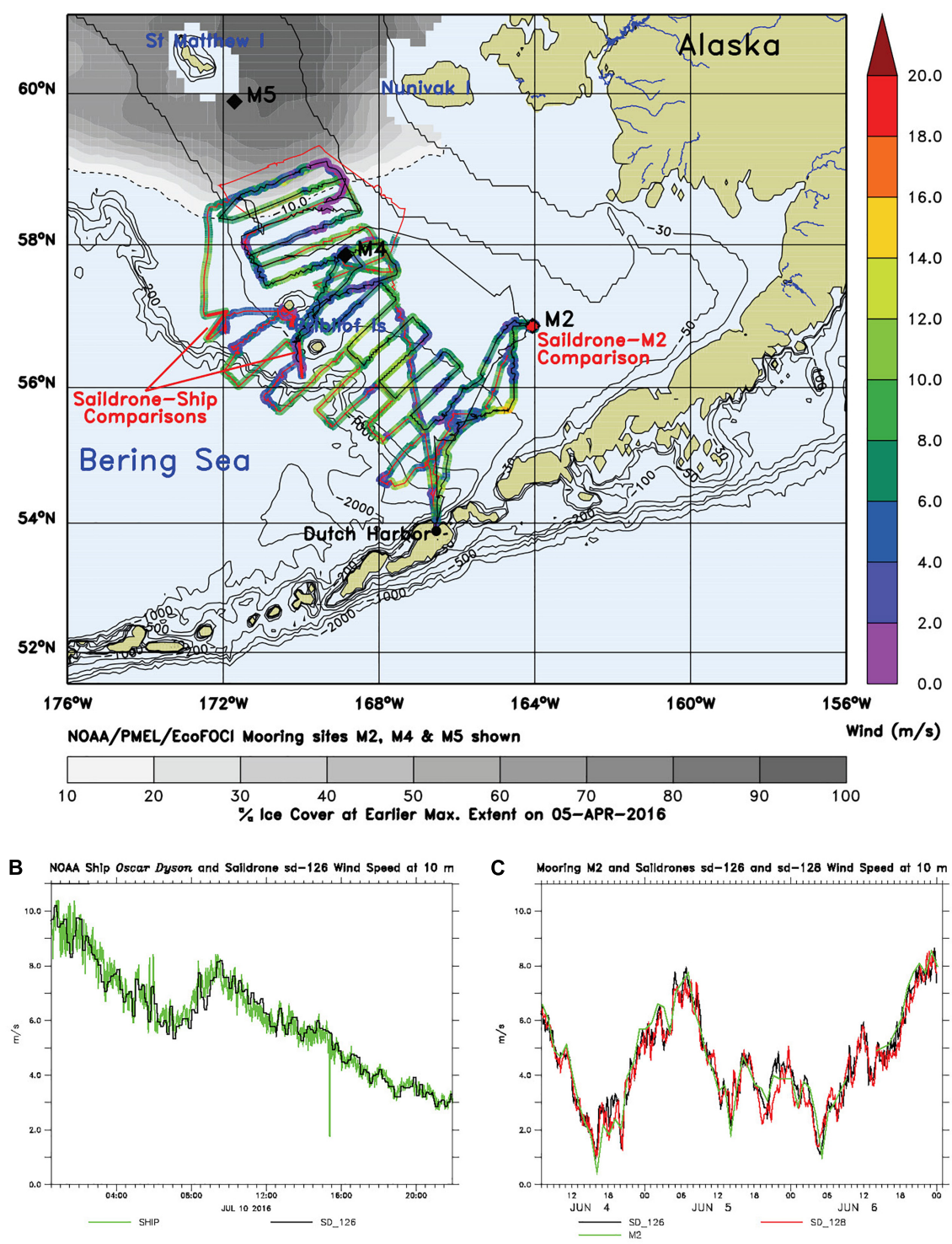

FIGURE 5 | (A) Map of the wind speed (m/s) along the tracks of two Saildrone USVs in the Bering Sea, 24 May-3 September 2016. Saildrone USV sd-126's track line is black, and sd-128's is red. During this 103-day mission, the mean wind speed was $5.7 \mathrm{~m} / \mathrm{s}$ with peak gusts to $23 \mathrm{~m} / \mathrm{s}$. Also shown are the sites of the comparisons between the Saildrone USVs and NOAA Ship Oscar Dyson and NOAA/PMEL research mooring M2. (B) The wind speed as measured by the ship (green) and Saildrone USV sd-126 (black). The comparison is good with an rms difference of $0.44 \mathrm{~m} / \mathrm{s}$. (C) The wind speed as measured by mooring M2 (green) and Saildrone USVs sd-126 (black) and sd-128 (red). The rms differences between the M2 and Saildrone USV winds were 0.49 and 0.54 m/s, respectively.

Telecommunications System (GTS). The GTS is a system of dedicated and shared network connections organized to distribute meteorological and oceanographic data to operational forecast centers.

By leveraging a pilot project undertaken by PMEL, the OpenGTS project ${ }^{3}$, some variables from Saildrone USV platforms are placed on to the GTS every $10 \mathrm{~min}$ in highly structured formats that require a minimum amount of

${ }^{3}$ http://www.opengts.org/ metadata. A Binary Universal Form for the Representation of meteorological data ${ }^{4}$ (BUFR) template specific to unmanned surface platforms, such as Saildrone USV, is now being defined.

QC is essential to the long-term viability of this effort, and groups 1 and 2 QC will be applied to these data using methods developed for the Integrated Ocean Observing System

\footnotetext{
${ }^{4}$ A Guide to the Code Form FM-94 BUFR (see https://www.wmo.int/pages/prog/ www/WDM/Guides/Guide-binary-1A.html).
} 


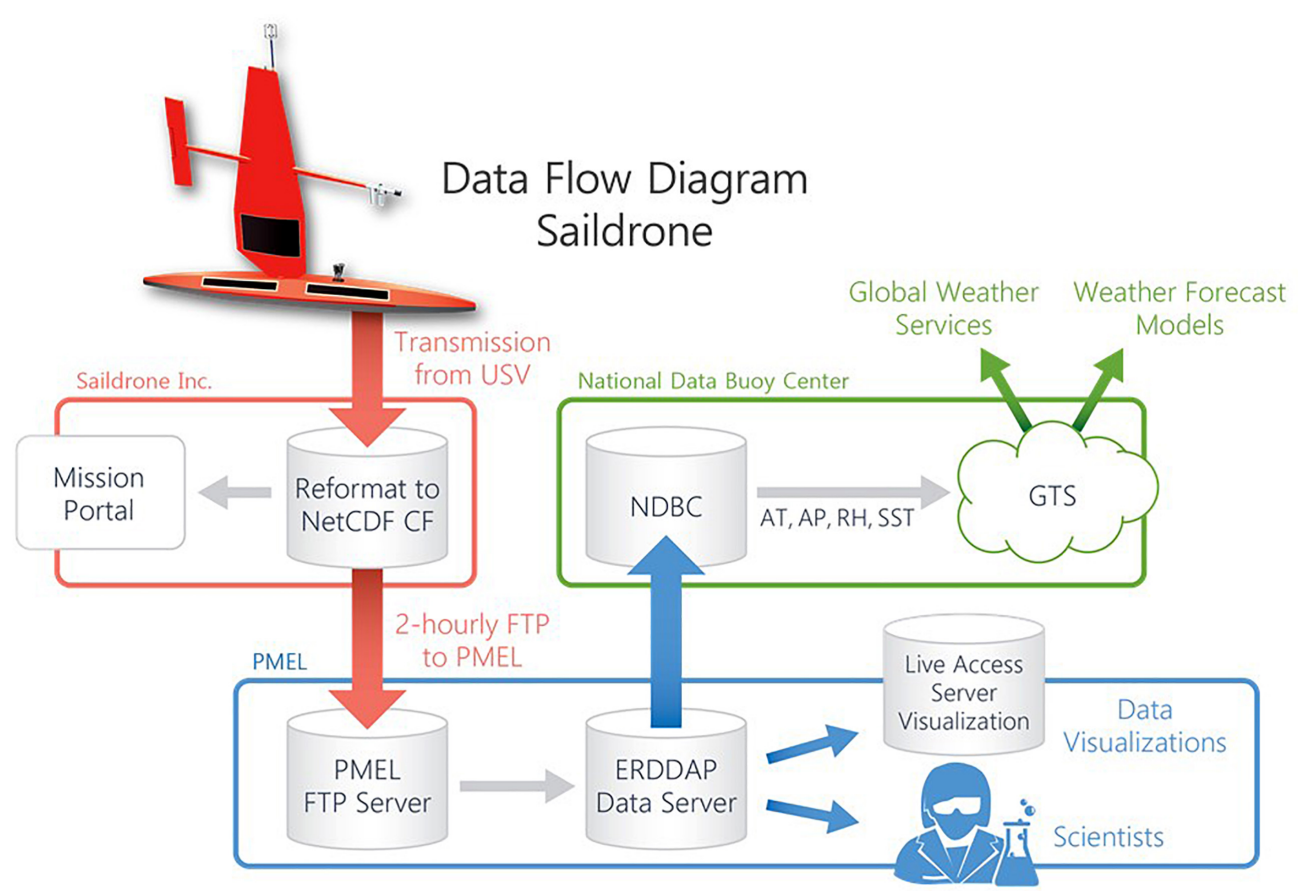

FIGURE 6 | Near real-time data workflow between Saildrone Inc., PMEL, and operational data dissemination network (GTS).

(IOOS) Quality Assurance for Real Time Oceanographic Data (QARTOD) as the guidance (Willis et al., 2015).

\section{Data Interoperability}

The Environmental Research Division Data Access Platform ${ }^{5}$ (ERDDAP) data server is used to provide interoperable access to the data. ERDDAP ingests data in many formats and outputs aggregated data sets in more than 30 formats via a graphical user interface and through a machine-to-machine Representational State Transfer (REST) interface. This REST interface to ERDDAP's rich functionality can be leveraged by other applications to provide the user with specialized services, such as visualizations or data analysis tools.

ERDDAP's machine-to-machine services provide users with powerful data access functions with applications that use ERDDAP as a middle-tier data broker. In general, data access is increasingly steering away from graphically rich "data portals" to data platforms that provide a broad set of services through which data can be accessed by other applications to serve a particular science community.

\section{Current Regional Saildrone Projects}

The current fleet of $\sim 70$ Saildrone USVs is composed of a collection of regional fleets, with each fleet ultimately deploying between 20 and 50 vehicles from a local operating hub (e.g., Dutch Harbor, AK, for U.S. Arctic operations). Each section below includes a brief summary of work to date that has been completed in each region.

\footnotetext{
${ }^{5}$ https://coastwatch.pfeg.noaa.gov/erddapinfo/index.html.
}

\section{Arctic Fleet}

Since 2015, a Saildrone USV fleet has been conducting annual surveys of the U.S. Arctic (Bering and Chukchi Seas) (Cokelet et al., 2015; Mordy et al., 2017). These surveys have focused on the following activities: meteorological observations to improve forecasting, multisensor improved SST (MISST) for calibration of remotely sensed data, surveys of carbon flux using $\mathrm{ASVCO}_{2}$ systems, ADCP surveys of ocean circulation to understand basin-basin and open ocean-shelf exchange of water masses and nutrients, acoustic surveys of Arctic cod (Boreogadus saida) and walleye pollock (Gadus chalcogrammus), bathymetric data, focal follows of tagged northern fur seals (Callorhinus ursinus), passive acoustics, and measurements focused on the marginal ice zone (MIZ).

\section{Tropical Pacific Fleet}

Missions to the tropical Pacific began in 2017 to test the Saildrone USV's ability to make climate-quality meteorological, oceanic, and biogeochemical observations as part of Tropical Pacific Observing System (TPOS) 2020 project (Cravatte et al., 2016; Smith et al., 2019). These pilot study surveys (Zhang et al., 2019) have included field intercomparisons of a full suite of meteorological, biogeochemical, and oceanic sensors used to estimate the wind stress and the air-sea exchanges of heat and $\mathrm{CO}_{2}$. These pilot study surveys have also tested Saildrone USV performance under challenging tropical Pacific conditions, including low wind, strong currents, and strong air-sea interaction at fronts. The ability to adaptively sample and measure multiscale variability make the Saildrone USV a powerful tool for monitoring evolving 
ENSO. A scientific focus of these missions has been to resolve mesoscale air-sea interactions because of their potential importance in ENSO development and rectification into largescale air-sea interaction and teleconnections. These smallscale processes are neither adequately observed by the current TPOS nor are they resolved or correctly simulated in state-ofscience coupled models.

\section{Southern Ocean Fleet}

Following initial work conducted in the Southern Ocean based out of Hobart, Australia, in partnership with the Commonwealth Scientific and Industrial Research Organisation (CSIRO), an inaugural Saildrone USV fleet has launched to circumnavigate Antarctica. The Southern Ocean fleet is optimized for the high wind and heavy sea state prevalent in the Southern Ocean and is equipped to measure $\mathrm{CO}_{2}$ flux using $\mathrm{ASVCO}_{2}$ systems, conduct acoustic krill surveys using science-grade echosounders, track tagged animal migrations, conduct marine mammal studies using passive acoustic recorders, and measure currents (ADCP).

\section{Coastal Fleet}

Saildrone USVs are highly capable of detailed coastal surveys. In 2018, Saildrone completed a full survey of the North American West Coast from Vancouver Island, Canada, to San Diego, CA, using five Saildrone USVs to complete a grid of 40 nautical mile transects spaced 10 nautical miles apart over 100 days (Figure 3C). Coastal surveys can significantly augment existing assets such as ships and moorings, and Saildrone USV fleets have been deployed for acoustic fish stock and bathymetry surveys, maritime domain awareness (AIS and smart cameras), meteorological data for improved weather forecasting, environmental monitoring (surface hydrocarbon detection and MIZ studies), and methane and $\mathrm{CO}_{2}$ seep detection near carbon sequestration sites.

Saildrone is committed to long-term PPPs to ensure this technology meets the scientific community's needs at scale. By marrying the best science from the ocean-observing community with the private sector's capability to invest in next-generation infrastructure, new high-accuracy and large-scale data sets can be obtained in the very short term to help address the current scarcity of in situ ocean observations. This partnership works primarily due to a shared commitment to operational excellence and high data quality standards. As this fleet of USVs expands, it is critical to expand engagement with the broader community around needs and requirements.

\section{VISION}

The promise of autonomous technology has always been to lower the data acquisition cost by an order of magnitude, something modeled by the Argo float network and expanded on by unmanned technologies such as Saildrone USVs. In turn, this reduction in cost enables very large-scale geospatial coverage for persistent or adaptive observations.
The main obstacle for implementing such networks has traditionally been the high level of upfront capital expenditures required. Some, such as Argo, successfully rallied national funding agencies to make large-scale investments, while others, such as underwater gliders, have built more localized networks.

As funding for infrastructure has become more limited, a PPP may serve as a pipeline to ensure continuity and serve new demands in ocean observations. The private sector is now taking a more involved role in setting up this type of infrastructure, in large part because it is a key beneficiary of the insights obtained and because the present era of evolving global weather and climate patterns has a substantial impact on the private sector. This creates an unprecedented alignment of global observation needs, technology advancement and cost-effectiveness, and private sector investment capacity and interest. However, to reap the scientific benefits of such efforts, communication and involvement of the regional-based and observation-based communities, beyond government agencies, are key requirements.

Observations are about "Systems of Systems," and success depends on community involvement to ensure the right mix of technologies for each domain while not stifling innovation. USVs would not meet the community's needs in isolation, but instead community input can ensure that the unique strengths of different technology solutions are leveraged to enable the System of Systems to have the maximum impact. For example, when unmanned systems augment ship-based surveys, they may provide reconnaissance, allow for adaptive sampling, and enable the ship to focus on high-value use cases, such as on-board science. USVs also can augment buoys by providing measurements in areas that are impractical for buoy deployment, provide in situ validation to enhance the value of remote sensing data sets, and measure air-sea interactions to augment subsurface (e.g., the Argo network) and atmospheric measurements (e.g., remote sensing and moored fixed-point time series measurements).

Optimizing the effectiveness of this type of System of Systems requires design input from the community to ensure that the network of USVs can address the most pressing questions at scale as it continues to extend its operating area to cover the world's oceans.

\section{Vision Structure and Framework}

This case study has demonstrated that USVs can provide high-quality atmospheric and oceanographic data for individual projects on a regional scale. Specifically, the committed and cooperative working relationship between NOAA and Saildrone has led to scientific confidence in the quality and accuracy of valuable measurements. Similar outcomes have been achieved by partnerships with other national and international agencies through regional deployments distributed in a variety of ocean basins. The experience, to date, compels us to consider the potential for a coordinated international effort to establish a global network of USVs that ultimately could contribute to the Global Ocean Observing System (GOOS) and the Global Climate Observing System (GCOS). 
The evolution and ongoing success of the Argo float program is a natural place to begin a discussion of how a global USV system might be achieved. While there are similarities with Argo, such as the concept of global network of autonomous platforms, several important differences need to be considered. Operational Argo floats were developed from community experience with the ALACE floats used for the 1990-1997 World Ocean Circulation Experiment (WOCE). In contrast, the existing USVs have been developed largely by private industry. Argo floats are designed to be expendable, while most USVs are designed to be retrieved and redeployed multiple times. Other technological contrasts include the added complexity of making measurements at the ocean surface as well as the increased number and variety of potential sensors. A complete treatment of the issues relevant to designing and implementing a global USV observing system is beyond the scope of this paper. Here, we seek to highlight some of the important issues and to provide a broad outline of the steps that would be necessary to evaluate the possibility of implementing a global network of USVs.

The history of how the Argo program evolved can provide valuable guidance for evaluating the need and practicality of a global USV observing network. The major components that led to the development of Argo were outlined in a presentation at the 13th meeting of the Argo Steering Committee in 2012 (Roemmich, 2012). These elements included the following:

- A statement of requirements

- Appropriate, cost-effective technology

- Consensus among user groups on its value

- Entrainment of agency sponsors and collaborators

- International scientific collaboration

- Intergovernmental coordination

- Commercial partnerships

Argo is an independent and voluntary entity administered by the Argo Steering Committee and is a major contributor to GOOS, which is under the Intergovernmental Oceanographic Commission (IOC) of UNESCO. As noted above, the idea of a global array of ocean profiling floats was an outcome of WOCE. Community consensus included endorsement by the Global Ocean Data Assimilation Experiment and the CLIVAR Upper Ocean Panel. Following these endorsements, a formal statement of requirements was developed at an international workshop. U.S. agency participation followed and was led by NOAA, with Argo seen as a global compliment to the Tropical Atmosphere Ocean array. A pilot project through the National Ocean Partnership Program, sponsored by NOAA and the Office of Naval Research, led to the multi-institution U.S. Argo Float Consortium. International partners (Japan, India, United Kingdom, France, Australia, and SOPAC) were engaged through contacts at the agency level. In addition to the Steering Committee, Argo includes a Data Management Team and runs science, technical, and training workshops. Argo is managed by the Argo Project Office, with each participating country responsible for funding and determining priorities for their own deployments.
Over the past several years, the potential for USVs to contribute to the global ocean-observing network has gained significant interest. For example, the Data Buoy Cooperation Panel (DBCP), under the Joint Technical Commission for Oceanography and Marine Meteorology (JCOMM), has an ongoing effort to evaluate USVs as a compliment, or even a cost-effective alternative, to moored buoys. DBCP states that such an evaluation will require detailed examination of data quality, reliability over extend deployments, and operating costs. Our experience and other reports using a variety of highendurance ( $>6$ months) surface platforms have demonstrated the endurance and versatility of currently available USVs. These can be categorized by their means of propulsion as either wave or wind driven. In addition to Saildrone USVs, currently available wind-propelled USVs include the C-Enduro, Sailbuoy (Ghani et al., 2014), Ocean Aero, and Harborwing. Wave-propelled USVs include the Wave Glider (Hine et al., 2009) and the Autonaut (Johnston and Poole, 2017).

In addition to providing many of the functions of surface moorings, the ability of USVs to be remotely piloted allows them to operate as a movable array, alternating between station keeping and transect survey mode. Thus, USVs can provide an extension and enhancement of fixed arrays through sampling on spatial and temporal scales that are not currently captured. The flexibility and independence from ship operations for deployment and recovery provide the ability for USVs to fill gaps in observing system arrays, such as the existing need for moored observations in the western equatorial Pacific or other emerging needs, such as air-sea flux measurements to capture ENSO precursors. The TPOS 2020 project, tasked with evaluating all elements that contribute to the existing TPOS, considers USVs to be at the pilot project RL and is evaluating their potential contributions. There are also several international efforts to evaluate USVs for extended observations such as the flux glider project sponsored by JAMSTEC and the United Kingdom-based Marine Autonomous Systems in Support of Marine Observations (MASSMO) through the National Oceanography Centre (NOC).

In summary, experience over the past few years has demonstrated pilot level technology for individual projects at the regional scale. Private industry investment in the development of a variety of USVs has allowed this new technology to evolve much more rapidly than would be possible through government-funded research alone. The scientific and operational communities need to decide how to take advantage of this unprecedented opportunity for expansion of the ocean-observing network, potentially to the global scale. For example, Cronin et al. (2019) have proposed deploying a global fleet of USVs to produce an in situ air-sea flux product with a $10^{\circ} \times 10^{\circ}$ resolution. The community needs to determine gaps in the existing network that USV can address, prioritize resources, and establish sensor and data standards perhaps through existing organizations such as the DBCP. For companies with mission-as-service business models, the community needs to explore how national and international agency sponsorship can be engaged since, in general, there is no precedent for this type of PPPs at the global scale. A plan should be developed to establish community confidence in the capability of USVs to 
provide accurate measurements, and an organizational structure should be established to ensure ongoing QC for data that would be widely distributed. A starting point would be to convene an international workshop to address these questions by involving current users, potential beneficiaries, manufacturers, and governing agencies.

\section{Vision - Saildrone Fleets}

The current fleet of 70 Saildrone USV s is composed of a collection of regional fleets, with each fleet ultimately deploying between 20 and 50 vehicles from a local operating hub. Saildrone is planning a fleet expansion of 200 USVs by the end of 2019 divided into regional fleets. In the coming years, Saildrone's target is to deploy $\sim 1,000$ vehicles, capable of real-time data collection across all oceans at a target resolution of $6^{\circ}$ by $6^{\circ}$ and operated from $\sim 20$ regional hubs. The data generated from this large set of sensors (20 per USV or $\sim 20,000$ total) will continue to be subject to a strict QC framework developed and managed on an ongoing basis in close partnership with the global science community, following the successful blueprint of established systems such as the Argo network.

\section{Vision - Data Delivery}

In a rapidly changing climate, the efficient delivery of in situ ocean observations at a high spatial and temporal resolution will become increasingly important. Data handling services should allow data from USVs to be delivered via cloud computing to mission operators in near real time for immediate interoperable access (Buck et al., 2019). Ease of access will need to be balanced with IT system protections to ensure data integrity. As illustrated by the cases described in this paper, it is imperative that the scientific community work closely with commercial cloud providers and platform operators to ensure services are in line with scientific best practices (Pearlman et al., 2019; Vance et al., 2019).

\section{Quality Control}

An important distinction in the case of the Saildrone USV is that the platform cannot be purchased, deployed, or operated by the end user of the data. Saildrone's business model is based on a feefor-service structure in which the vendor provides and maintains both the platform and all instrumentation, including calibration, data QC, and data distribution. A major advantage of this model, as noted above, is that infrastructure costs are provided through investment by private industry. From a data quality point of view, this model has worked well for the individual regionally focused projects to date since the end users have been intimately involved. When considering extension to a global network with open data access, a natural concern is how to establish community confidence in the data quality.

This observing platform model will only succeed if confidence and trust are established in the quality and veracity of the data. To establish such community data confidence, the private industry and the science community should jointly develop data quality verification processes that are transparent and standardized. We recommend the formation of a stakeholder group, derived from the meteorological and oceanographic communities, to provide an international framework for developing USV observation guidelines and standards. This could be a working group of the DBCP, a joint body of the WMO and IOC. Focus areas should include existing best practices and standards for sensor integration, validation, QC, and data delivery. Guidance documents from this group should be reviewed by subject matter experts to establish best practices documents.

The QC processes for USV data should take into account the unique characteristics of each platform. For example, acoustic backscatter measurements are affected by platform orientation. Community-wide distributed QC as a Service (QCaaS) capability should be established, where centers of excellence address the QC requirements for targeted parameters. Collectively, a wealth of QC capability could be leveraged to benefit a much broader community.

\section{Discovery and Access}

Observational data should be made available in an easily digestible format within minutes of the observation to fully utilize a USV's capability to respond to changing conditions in real time. Interoperable access would allow the users to access the data in a format that best suits their needs. Focus should be on a servicesbased approach that will empower communities to establish data portals that cater to their requirements.

Observational data should be discoverable and formatted for assimilation by modeling centers. This would require cooperation and would advance the scientific objectives of both observationalists and modelers. Information on data access points should be published at internationallevel discovery portals provided by the IOC and WMO. Data discovery functionality should be independent of the data hosting platform. New data discovery technologies and capabilities are becoming available, and leveraging tools such as Google's Dataset Search and the NOAA ERDDAP broker can assist the oceanographic community, and USV operators, to improve data discovery and data access services.

\section{ROADMAP}

Vision Statement \#1:

Continue fostering close collaboration between the private sector and the regional ocean-observing community to leverage strengths of each sector to incorporate USVs to fill regional observing and forecast gaps. Additional PPPs will form interdisciplinary teams that will define the focus, drive innovation, and result in rapid deployments to fill observing system gaps.

Recommendation \# 1 :

Convene regional workshops that include the ocean-observing community and private sector engineers and scientists to establish regional teams that focus on all aspects of observing from development to data delivery. To build community confidence, PPPs must have transparent processes for continued verification. An independent oversight of data quality is necessary 
for confidence in the business model for "Mission as Service." Communicate early successes through public engagement and communication.

Key Results \# 1 :

Convene five regional workshops by 2022; establish oversight and data quality criteria on at least $10 \mathrm{ECV}$ s and EOVs delivered through "Mission as Service" from industry, and attract $>\$ 300$ million in private capital to form PPPs to address regional overserving gaps.

Vision Statement \#2:

Provide an international framework for coordinating and developing observation technologies from USVs within the meteorological and oceanographic communities.

Recommendation \#2:

Form a USV working group of the long-established DBCP. Focus areas could include best practices and standards for sensor integration, verification and data delivery, and guidance for unmanned vehicle data providers.

Key Results \#2:

Expand the terms of reference of the DBCP to include other met-ocean observation platforms and establish a USV task team by 2021 .

Vision Statement 3:

Over the next decade, USVs will be incorporated into the ocean-observing system on a global scale for both the research and operational forecast community, providing new insight into phenomena at finer spatial and temporal scales than currently possible.

Recommendation 3:

We recommend that an international workshop be convened to establish community consensus on the needs and priorities for USVs in the global ocean-observing network and their contributions to forecast models. Important questions include the appropriateness of existing USVs for ocean-observing, including their complexity and cost-effectiveness and how to best ingest data from these moving platforms. Various models for PPPs should be explored. The workshop should address the issues of sensor and data format standards as well as an open access data distribution and access infrastructure. Calibration and validation methodologies should be developed to ensure community confidence in the data quality.

Key Results \#3:

Convene three international workshops by 2025; establish oversight and data quality criteria; determine model forecast value on at least five ECVs and EOVs in coordination with GCOS and GOOS; and attract $>\$ 1$ billion in private capital in additional USV PPP's.

\section{CONCLUSION}

As new technologies continue to rapidly increase observing assets and expand the international ocean-observing network, protecting the integrity of the global array is essential. In our view, the most efficient way to ensure rapid development that meets global standards is by encouraging close collaborations between the ocean-observing community and private developers. Private capacity can streamline capitalization and hiring, allowing development to scale up rapidly. Public involvement in private development can ensure that data quality, delivery, and access meet important community benchmarks and target key parts of the global observing array. According to our experience working together, NOAA and Saildrone suggest that the success of these relationships is based on close alignment of long-term goals that focus on practical, short-term deliverables. However, integrating workflows alone is insufficient: an essential element for achieving these successes is harmonizing group cultures. Good collaborations are based on relationships where both sectors understand and value their respective strengths and share goals that easily dovetail. By describing this case study in closedetail here, we hope to provide the international community with a blueprint for collaborating successfully with private industry partners.

We see the potential for the rapid expansion of private participation in regional and global ocean observing, particularly with USVs. While this will be dependent, in part, on individual teams working together, it is our view that the future of the industry could greatly benefit from a coordinating infrastructure on the international scale. This body could help provide oversight of this community by determining best practices and transparent data quality standards for using USVs and other new platforms for ocean observing. This would provide crucial guidance to private participants and build community confidence in these new assets. This body also has a role to play in driving the community forward by bringing the regional ocean-observing community together with operational forecasters to determine observing requirements, assessing the appropriateness of existing USVs, sensors, sampling schemes, QC, and assurance and data format standards for achieving these needs, and establish usage and data distribution protocols for data generated by USVs at these scales. Including private partners in this body will also help them efficiently identify and target community demand for new assets and development.

In this case study, we suggest one vision that would leverage private industry investment in the development of a variety of USV s coordinated in regional fleets, adapted to specific observing requirements, and targeted to fill regional observing gaps. We are confident that the international community will be able to use the information we have provided here to suggest a variety of other operating modes. We look forward to avid discussion with our colleagues and partners about the far-reaching opportunities that PPPs provide for the global ocean-observing community.

\section{AUTHOR CONTRIBUTIONS}

CM provided the overall guidance and executive editing for the manuscript, and wrote the sections "Introduction," "Public Infrastructure," "Private Capacity," "Forming the PPP," and "Roadmap." All authors provided text input and editing. RJ, SdH, and NC provided Figures 1, 2; EFB, Figures 3, 6; CM, Figure 4; and EDC, Figure 5. 


\section{FUNDING}

All authors gratefully acknowledge the support of their home institutions. CM, EFB, EDC, MFC, JNC, NL-S, AJS, and CZ are supported by PMEL and NOAA's Office of Oceanic and Atmospheric Research. ATJ was supported by APL-UW. CWM and DZ were partially funded by the Joint Institute for the Study of the Atmosphere and Ocean (JISAO) under NOAA Cooperative Agreement NA15OAR4320063.

\section{REFERENCES}

Bernard, E., and Meinig, C. (2011). "History and future of deep-ocean tsunami measurements". in Proceedings of Oceans '11 MTS/IEEE, Kona, (Piscataway, NJ: IEEE).

Buck, J. J. H., Bainbridge, S. J., Burger, E. F., Kraberg, A. C., Casari, M., Casey, K. S., et al. (2019). Ocean data product integration through innovation-the next level of data interoperability. Front. Mar. Sci. 6:32 doi: 10.3389/fmars.2019.00032.

Cokelet, E. D., Jenkins, R., Meinig, C., Lawrence-Slavas, N., Mordy, C. W., Stabeno, P. J., et al. (2015). "The use of saildrones to examine spring conditions in the bering sea: instrument comparisons, sea ice meltwater and yukon river plume studies. in Proceedings of the Oceans'15 MTS/IEEE, Marine Technology Society and Institute of Electrical and Electronics Engineers, Washington, DC, 19-22.

Cravatte, S., Kessler, W. S., Smith, N., Wijffels, S., Ando, K., Cronin, M., et al. (2016). First Report of TPOS 2020. GOOS-215. Available at: http://TPOS2020. org/first-report/ (accessed April 15, 2017).

Cronin, M. F., Gentemann, C. L., Edson, J., Ueki, I., Bourassa, M., Brown, S., et al. (2019). Air-sea fluxes with a focus on heat and momentum. OceanObs'19. Front. Mar. Sci 6:430, doi: 10.3389/fmars.2019.00430

De Robertis, A., Lawrence-Slavas, N., Jenkins, R., Wangen, I., Mordy, C. W., Meinig, C., et al. (2019). Long-term measurements of fish backscatter from Saildrone unmanned surface vehicles and comparison with observations from a noise-reduced research vessel. ICES J. Mar. Sci. fsz124, doi: 10.1093/icesjms/ fsz 124

Ghani, M. H., Hole, L. R., Fer, I., Kourafalou, V. H., Wienders, N., Kang, H., et al. (2014). The SailBuoy remotely controlled unmanned vessel: measurements of near surface temperature, salinity and oxygen concentration in the Northern gulf of mexico. Methods Oceanogr. 10, 104-121. doi: 10.1016/j.mio.2014.08.001

Hine, R., Willcox, S., Hine, G., and Richardson, T. (2009). "The Wave glider: a wave-powered autonomous marine vehicle". in Proceedings of the OCEANS '09, (Biloxi, MS: IEEE). doi: 10.23919/OCEANS.2009.5422129

Johnston, P., and Poole, M. (2017). "Marine surveillance capabilities of the autonaut wave-propelled unmanned surface vessel (USV)".in Proceedings of the OCEANS. (Aberdeen: IEEE). doi: 10.1109/OCEANSE.2017.80 84782

Lauvset, S. K., Olsen, A., Gehlen, M., and Sommer, A. (2018). Surface Carbon EOV Report. AtlantOS Deliverable, D7.10. (Kiel: AtlantOS). doi: 10.3289/AtlantOSD7.10

Lindstrom, E., Gunn, J., Fischer, A., McCurdy, A., and Glover, L. K. (2012). "A framework for ocean observing," in By the Task Team for an Integrated Framework for Sustained Ocean Observing. (Paris: UNESCO).

McPhaden, M. J., Ando, K., Bourlès, B., Freitag, H. P., Lumpkin, R., Masumoto, Y., et al. (2010). The global tropical moored buoy array. in Proceedings of the "OceanObs'09: Sustained Ocean Observations and Information for Society Vol. 2. eds J. Hall, D. E., Harrison, D. Stammer, (Venice: ESA Publication).

Meinig, C., Jenkins, R., Lawrence-Slavas, N., and Tabisola, H. (2015). “The use of Saildrones to examine spring conditions in the Bering Sea: vehicle specification and mission performance," in Oceans'15 MTS/IEEE, Marine Technology Society and Institute of Electrical and Electronics Engineers (Washington, DC).

Mordy, C. W., Cokelet, E. D., DeRobertis, A., Jenkins, R., Kuhn, C. E., LawrenceSlavas, N., et al. (2017). Advances in ecosystem research: saildrone surveys of

\section{ACKNOWLEDGMENTS}

The success of this PMEL-Saildrone PPP case study involved a large team of dedicated technicians, engineers, and scientists including R. Bott, C. Berchok, M. Casari, J. Crance, A. DeRobertis, J. Keene, C. Kuhn, S. Maenner, H. Tabisola, K. O’Brien, D. Peacock, J. Shanley, T. Walton, I. Wangen, and C. Wilson. This study is a PMEL contribution 4898 and JISAO contribution 2018-0180.

oceanography, fish, and marine mammals in the Bering Sea. Oceanography 30, 113-115. doi: 10.5670/oceanog.2017.230

Pearlman, J., Bushnell, M., Coppola, L., Karstensen, J., Buttigieg, P. L., Pearlman, F., et al. (2019). Evolving and sustaining ocean best practices and standards for the next decade. Front. Mar. Sci. 6:277. doi: 10.3389/fmars.2019.00277

Roemmich, D. (2012). "On the beginnings of Argo: ingredients of an ocean observing system", in Proceedings of the 13th Meeting of the Argo Steering Committee, Paris.

Serra, Y. L., Haase, J. S., Adams, D. K., Fu, Q., Ackerman, T. P., Alexander, M. J., et al. (2018). The risks of contracting the acquisition and processing of the nation's weather and climate data to the private sector. Bull. Am. Meteorol. Soc. 99, 869-870. doi: 10.1175/BAMS-D-180034.1

Smith, N., Kessler, W. S., Cravatte, S., Sprintall, J., Wijffels, S., Cronin, M. F., et al. (2019). Tropical Pacific observing system. Front. Mar. Sci. 6:31. doi: 10.3389/ fmars.2019.00031

Sutton, A. J., Sabine, C. L., Maenner-Jones, S., Lawrence-Slavas, N., Meinig, C., Feely, R. A., et al. (2014). A high-frequency atmospheric and seawater pCO2 data set from 14 open ocean sites using a moored autonomous system. Earth Syst. Sci. Data 6, 353-366. doi: 10.5194/essd-6-353-2014

Vance, T. C., Wengren, M., Burger, E. F., Hernandez, D., Kearns, T., Merati, N., et al. (2019). From the oceans to the cloud: opportunities and challenges for data, models, computation and workflows. Front. Mar. Sci. 6:211.doi: 10.3389/ fmars.2019.00211

Wanninkhof, R., Pickers, P. A., Omar, A. M., Sutton, A., Murata, A., Olsen, A., et al. (2019). A surface ocean $\mathrm{CO}_{2}$ reference network, SOCONET and associated marine boundary layer $\mathrm{CO}_{2}$ measurements. Front. Mar. Sci. 6:400. doi: 10.3389/fmars.2019.00400

Willis, Z., Swaykos, J., and Paternostro, C. (2015). Manual for the Real Time Quality Control of In-Situ Current Observations. (Silver Spring, MD: IOOS).

Zhang, D., Cronin, M. F., Meinig, C., Farrar, J. T., Jenkins, R., Peacock, D., et al. (2019). Comparing air-sea flux measurements from a new unmanned surface vehicle and proven platforms during the SPURS-2 field campaign. Oceanography. 32, 122-133. doi: 10.5670/oceanog.2019.220

Conflict of Interest Statement: NOAA-PMEL and Saildrone have a CRADA since 2014 that covers joint development work and in-kind contributions from Saildrone, in the form of USV testing days. PMEL researchers (CM, EFB, EDC, MFC, JNC, NL-S, AJS, and CZ) have benefitted from additional vehicle days-at-sea, and Saildrone (RJ, SdH, and NC) has benefitted from the sensor development and data validation. University of Washington JISAO researchers (CWM and DZ) have benefitted from the additional data collected during Arctic and Tropical Pacific missions. ATJ has no formal relationship with either NOAA-PMEL or Saildrone.

Copyright (C) 2019 Meinig, Burger, Cohen, Cokelet, Cronin, Cross, de Halleux, Jenkins, Jessup, Mordy, Lawrence-Slavas, Sutton, Zhang and Zhang. This is an open-access article distributed under the terms of the Creative Commons Attribution License (CC BY). The use, distribution or reproduction in other forums is permitted, provided the original author(s) and the copyright owner(s) are credited and that the original publication in this journal is cited, in accordance with accepted academic practice. No use, distribution or reproduction is permitted which does not comply with these terms. 


\section{OPEN ACCESS}

Edited by:

Eric Delory,

Oceanic Platform of the Canary

Islands, Spain

Reviewed by:

Werner R. Alpers,

Universität Hamburg, Germany

Samantha Jane Lavender,

Pixalytics Ltd., United Kingdom

${ }^{*}$ Correspondence:

Christine Gommenginger cg1@noc.ac.uk

Specialty section:

This article was submitted to

Ocean Observation,

a section of the journal

Frontiers in Marine Science

Received: 06 December 2018

Accepted: 09 July 2019

Published: 13 August 2019

\section{SEASTAR: A Mission to Study Ocean Submesoscale Dynamics and Small-Scale Atmosphere-Ocean Processes in Coastal, Shelf and Polar Seas}

Christine Gommenginger ${ }^{1 *}$, Bertrand Chapron², Andy Hogg ${ }^{3}$, Christian Buckingham ${ }^{4}$, Baylor Fox-Kemper ${ }^{5}$, Leif Eriksson ${ }^{6}$, Francois Soulat ${ }^{7}$, Clément Ubelmann ${ }^{7}$, Francisco Ocampo-Torres ${ }^{8}$, Bruno Buongiorno Nardelli ${ }^{9}$, David Griffin ${ }^{10}$, Paco Lopez-Dekker ${ }^{11}$, Per Knudsen ${ }^{12}$, Ole Andersen ${ }^{12}$, Lars Stenseng ${ }^{13}$, Neil Stapleton ${ }^{14}$, William Perrie ${ }^{15}$, Nelson Violante-Carvalho ${ }^{16}$, Johannes Schulz-Stellenfleth ${ }^{17}$, David Woolf ${ }^{18}$, Jordi Isern-Fontanet ${ }^{19}$, Fabrice Ardhuin ${ }^{2}$, Patrice Klein ${ }^{2}$, Alexis Mouche ${ }^{2}$, Ananda Pascual ${ }^{20}$, Xavier Capet ${ }^{21}$, Daniele Hauser ${ }^{22}$, Ad Stoffelen ${ }^{23}$, Rosemary Morrow ${ }^{24}$, Lotfi Aouf25, Øyvind Breivik ${ }^{26,27}$, Lee-Lueng Fu' ${ }^{28,}$ Johnny A. Johannessen ${ }^{29}$, Yevgeny Aksenov ${ }^{1}$, Lucy Bricheno ${ }^{30}$, Joel Hirschi', Adrien C. H. Martin ${ }^{1}$, Adrian P. Martin ${ }^{1}$, George Nurser ${ }^{1}$, Jeff Polton ${ }^{30}$, Judith Wolf ${ }^{30}$, Harald Johnsen ${ }^{31}$, Alexander Soloviev'32, Gregg A. Jacobs ${ }^{33}$, Fabrice Collard ${ }^{34}$, Steve Groom ${ }^{35}$, Vladimir Kudryavtsev ${ }^{36}$, John Wilkin ${ }^{37}$, Victor Navarro ${ }^{38}$, Alex Babanin ${ }^{39}$, Matthew Martin ${ }^{40}$, John Siddorn ${ }^{40}$, Andrew Saulter ${ }^{40}$, Tom Rippeth ${ }^{41}$, Bill Emery ${ }^{42}$, Nikolai Maximenko ${ }^{43}$, Roland Romeiser ${ }^{44}$, Hans Graber ${ }^{44}$, Aida Alvera Azcarate ${ }^{45}$, Chris W. Hughes ${ }^{30,46}$, Doug Vandemark ${ }^{47}$, Jose da Silva ${ }^{48}$, Peter Jan Van Leeuwen ${ }^{49,50,}$ Alberto Naveira-Garabato ${ }^{51}$, Johannes Gemmrich ${ }^{52}$, Amala Mahadevan ${ }^{53}$, Jose Marquez ${ }^{54}$, Yvonne Munro ${ }^{54}$, Sam Doody ${ }^{54}$ and Geoff Burbidge ${ }^{54}$

\footnotetext{
${ }^{1}$ National Oceanography Centre, Southampton, United Kingdom, ${ }^{2}$ Institut Français de Recherche pour l'Exploitation de la Mer, Brest, France, ${ }^{3}$ Research School of Earth Sciences, Australian National University, Canberra, ACT, Australia, ${ }^{4}$ Institut Universitaire Européen de la Mer, Brest, France, ${ }^{5}$ Department of Earth, Environmental, and Planetary Sciences, Brown University, Providence, RI, United States, ${ }^{6}$ Department of Space Earth and Environment, Chalmers University of Technology, Gothenburg, Sweden, ${ }^{7}$ Collecte Localisation Satellites, Toulouse, France, ${ }^{8}$ Centro de Investigación Científica y de Educación Superior de Ensenada, Ensenada, Mexico, ${ }^{9}$ Consiglio Nazionale delle Ricerche, Bologna, Italy, ${ }^{10}$ Commonwealth Scientific and Industrial Research Organisation, Canberra, ACT, Australia, ${ }^{11}$ Department of Geoscience and Remote Sensing, Delft University of Technology, Delft, Netherlands, ${ }^{12}$ Department of Geodesy, DTU Space, Technical University of Denmark, Kongens Lyngby, Denmark, ${ }^{13}$ Department of Planning, Aalborg University, Aalborg, Denmark, ${ }^{14}$ Defence Science and Technology Laboratory, Salisbury, United Kingdom, ${ }^{15}$ Bedford Institute of Oceanography, Fisheries and Oceans Canada, Dartmouth, NS, Canada, ${ }^{16}$ Program of Ocean Engineering, Federal University of Rio de Janeiro, Rio de Janeiro, Brazil, ${ }^{17}$ Institute of Coastal Research, Helmholtz-Zentrum Geesthacht - Centre for Materials and Coastal Research, Geesthacht, Germany, ${ }^{18}$ School of Energy, Geoscience, Infrastructure and Society, Heriot-Watt University, Edinburgh, United Kingdom, ${ }^{19}$ Institut de Ciencies del Mar, Barcelona, Spain, ${ }^{20}$ Institut Mediterrani d'Estudis Avançats, Esporles, Spain, ${ }^{21}$ Institut Pierre Simon Laplace, Laboratoire d'Océanographie et du Climat Expérimentations et Approches Numériques, Paris, France, ${ }^{22}$ Laboratoire Atmosphères, Milieux, Observations Spatiales, Guyancourt, France, ${ }^{23}$ Koninklijk Nederlands Meteorologisch Instituut, De Bilt, Netherlands, ${ }^{24}$ Laboratoire d'Etudes en Géophysique et Océanographie Spatiales, Toulouse, France, ${ }^{25}$ Météo-France, Toulouse, France, ${ }^{26}$ Norwegian Meteorological Institute, Oslo, Norway, ${ }^{27}$ Geophysical Institute, University of Bergen, Bergen, Norway, ${ }^{28}$ NASA Jet Propulsion Laboratory, La Cañada Flintridge, CA, United States, ${ }^{29}$ Nansen Environmental and Remote Sensing Center, Bergen, Norway, ${ }^{30}$ National Oceanography Centre, University of Liverpool, Liverpool, United Kingdom, ${ }^{31}$ Northern Research Institute, Tromsø, Norway, ${ }^{32}$ Halmos College of Natural Sciences and Oceanography, Nova Southeastern University, Fort Lauderdale, FL, United States, ${ }^{3}$ United States Naval Research Laboratory, Washington, DC, United States, ${ }^{34}$ OceanDataLab, Brest, France, ${ }^{35}$ Plymouth Marine Laboratory,
} 
Plymouth, United Kingdom, ${ }^{36}$ Satellite Oceanography Laboratory, Russian State Hydrometeorological University, Saint Petersburg, Russia, ${ }^{37}$ Department of Marine and Coastal Sciences, Rutgers University, New Brunswick, NJ, United States, ${ }^{38}$ Starlab, Barcelona, Spain, ${ }^{39}$ Department of Infrastructure Engineering, The University of Melbourne, Parkville, VIC, Australia, ${ }^{40}$ Met Office, Exeter, United Kingdom, ${ }^{41}$ School of Ocean Sciences, Bangor University, Bangor, United Kingdom, ${ }^{42}$ Colorado Center for Astrodynamics Research, University of Colorado Boulder, Boulder, CO, United States, ${ }^{43}$ School of Ocean and Earth Science an Technology, International Pacific Research Center, University of Hawai'i at Mānoa, Honolulu, HI, United States, ${ }^{44}$ Department of Ocean Sciences, Rosenstiel School of Marine and Atmospheric Science, University of Miami, Coral Gables, FL, United States, ${ }^{45}$ GeoHydrodynamics and Environment Research, University of Liège, Liège, Belgium, ${ }^{46}$ Department of Earth, Ocean and Ecological Sciences, University of Liverpool, Liverpool, United Kingdom, ${ }^{47}$ College of Engineering and Physical Sciences, University of New Hampshire, Durham, NH, United States, ${ }^{48}$ Departamento de Geociências, Ambiente e Ordenamento do Território, University of Porto, Porto, Portugal, ${ }^{49}$ Department of Meteorology, University of Reading, Reading, United Kingdom, ${ }^{50}$ Department of Meteorology, Colorado State University, Fort Collins, CO, United States, ${ }^{51}$ Ocean and Earth Science, University of Southampton, Southampton, United Kingdom, ${ }^{52}$ Department of Physics and Astronomy, School of Earth and Ocean Science, University of Victoria, Victoria, BC, Canada, ${ }^{53}$ Woods Hole Oceanographic Institution, Woods Hole, MA, United States, ${ }^{54}$ Airbus Defence and Space Ltd., Portsmouth, United Kingdom

High-resolution satellite images of ocean color and sea surface temperature reveal an abundance of ocean fronts, vortices and filaments at scales below $10 \mathrm{~km}$ but measurements of ocean surface dynamics at these scales are rare. There is increasing recognition of the role played by small scale ocean processes in ocean-atmosphere coupling, upper-ocean mixing and ocean vertical transports, with advanced numerical models and in situ observations highlighting fundamental changes in dynamics when scales reach $1 \mathrm{~km}$. Numerous scientific publications highlight the global impact of small oceanic scales on marine ecosystems, operational forecasts and long-term climate projections through strong ageostrophic circulations, large vertical ocean velocities and mixed layer re-stratification. Small-scale processes particularly dominate in coastal, shelf and polar seas where they mediate important exchanges between land, ocean, atmosphere and the cryosphere, e.g., freshwater, pollutants. As numerical models continue to evolve toward finer spatial resolution and increasingly complex coupled atmosphere-wave-ice-ocean systems, modern observing capability lags behind, unable to deliver the high-resolution synoptic measurements of total currents, wind vectors and waves needed to advance understanding, develop better parameterizations and improve model validations, forecasts and projections. SEASTAR is a satellite mission concept that proposes to directly address this critical observational gap with synoptic two-dimensional imaging of total ocean surface current vectors and wind vectors at $1 \mathrm{~km}$ resolution and coincident directional wave spectra. Based on major recent advances in squinted along-track Synthetic Aperture Radar interferometry, SEASTAR is an innovative, mature concept with unique demonstrated capabilities, seeking to proceed toward spaceborne implementation within Europe and beyond.

Keywords: satellite, air sea interactions, upper ocean dynamics, submesoscale, coastal, marginal ice zone, radar, along-track interferometry

\section{THE NEED FOR SYNOPTIC HIGH-RESOLUTION OCEAN CURRENT, WIND AND WAVE MEASUREMENTS}

Processes at the ocean-atmosphere interface are fundamental regulators of the Earth System, impacting a multitude of phenomena on global to local scales. This section highlights prevailing scientific questions that call for new high-resolution observations of ocean currents, winds and waves. Interested readers are referred to Villas Bôas et al. (2018) for a comprehensive review of relevant phenomena and of presentday observational gaps for ocean surface currents, winds and waves.

\section{Understanding the Ocean Submesoscale, Upper Ocean Dynamics and Vertical Exchanges}

High-resolution satellite images of the ocean reveal that, far from being quiescent and uniform, the ocean is teeming with dynamic structures at different scales. Together with the jets and eddies 
of the energetic mesoscale (scales between 10 and $100 \mathrm{~km}$ ) and internal waves, the ocean displays intense variability at scales between 0.1 and $10 \mathrm{~km}$ known as the submesoscale. These small scale features were ignored until relatively recently but a growing body of research now indicates that the interactions of these small features with the larger ocean mesoscale and the atmosphere make these key drivers of upper ocean mixing, horizontal and vertical transport, air-sea exchanges and marine ecosystem response (Lapeyre and Klein, 2006; Ferrari and Wunsch, 2009; D’Asaro et al., 2011; McWilliams, 2016; Lévy et al., 2018).

High-resolution numerical model simulations were among the first to reveal the role of the submesoscale for ocean stratification, large-scale circulation and climate (Capet et al., 2008; Lévy et al., 2010). Many studies have confirmed the associated high vertical ocean velocities and the subsequent impact on phytoplankton and biological productivity (Lapeyre and Klein, 2006; McGillicuddy et al., 2007; Mahadevan et al., 2008; Lévy et al., 2012; Woodson and Litvin, 2015). At the surface, these processes strongly affect the dispersion of floating materials (e.g., oil, plastics), which accumulate in high concentrations in convergence zones associated with density fronts and cyclonic vortices (Maximenko et al., 2017; D'Asaro et al., 2018). Below the surface, large vertical velocities several orders of magnitude greater than average penetrate to several hundred meters depth, enabling rapid exchange of properties (e.g., heat, $\mathrm{CO}_{2}$ ) between the turbulent surface boundary layer and the ocean interior (Lévy et al., 2012; Callies et al., 2015; Balwada et al., 2018).

Present-day knowledge identifies the critical role for upper ocean mixing of stirring by submesoscale eddies at the km-scale. Capet et al. (2008) was first to identify a clear transition in the eddy field variability as the horizontal grid scale of models reaches $\mathrm{O}(1) \mathrm{km}$. Multiple studies since highlighted the need for new synoptic observations of the two-dimensional horizontal structure of the mesoscale flow field. Poje et al. (2014) studied the role of submesoscale processes in the dispersion of oil contamination from the Deepwater Horizon spill in the Gulf of Mexico using data from an unprecedented simultaneous release of about 300 surface drifters, concluding that the experiment allowed "quantification of the submesoscale-driven dispersion [that is] missing in current operational circulation models and satellite altimeter-derived velocity fields" and that "Fundamental questions concerning the structure of the velocity field at the submesoscales (100 $\mathrm{m}$ to tens of kilometers, hours to days) remain unresolved due to a lack of synoptic measurements at these scales."

There is growing awareness also in the climate community that the restratification of the mixed layer by the submesoscale is a leading order process on longer scales (Fox-Kemper and Ferrari, 2008; Flato et al., 2013). As climate models remain too coarse to resolve submesoscales explicitly, models have to rely on parameterizations. The parameterization of Fox-Kemper et al. (2011) was used in some CMIP5 models included in IPCC AR5, but further developments are needed, most notably regarding front-wind and front-wave interactions, for which synoptic two-dimensional observations of currents, winds and waves are essential.

\section{Observing Small Scale Processes in Coastal and Continental Shelf Seas}

Coastal and shelf seas provide vital resources and services to society including food, energy, transport and recreation, but also mediate the transfer of terrestrial material from land to the open ocean (freshwater, carbon, nitrogen, plastics, and other pollutants). At the same time, the coastal zone presents mankind with some of the most urgent and challenging environmental hazards, including sea level change, coastal erosion and coastal flooding. Dynamic processes in coastal and shelf seas are more complex and occur on shorter spatial and temporal scales than in the open ocean (Schulz-Stellenfleth and Stanev, 2016; Cavaleri et al., 2018). Major differences from the open ocean include stronger tidal flows, rapidly changing bathymetry, spatially varying ocean waves and sharp water density fronts associated with freshwater river plumes or upwelling. Atmospheric circulation and air-sea interactions are affected too, with surface winds presenting much greater heterogeneity due to coastlines, the orography of nearby land and land/sea surface temperature contrasts (Bricheno et al., 2013; Müller et al., 2013).

Today, the representation of coastal and shelf seas in global ocean models remains inadequate due to the coarse spatial resolution and poor representation of relevant processes in global models. Current state-of-the-art $1 / 12^{\circ}$ global ocean models ( $\sim 9 \mathrm{~km}$ resolution away from poles) only resolve fine scale processes with sufficient resolution for $\sim 20 \%$ of coastal and shelf seas. It is estimated that to represent them globally would require substantially finer resolution of the order of $1.5 \mathrm{~km}$, which "would be routinely practical in about a decade given substantial effort on numerical and computational development" (Holt et al., 2017). State-of-the-art regional coastal and shelf seas models already operate at hourly and kmscale resolutions, but progress is hampered by the scarcity of in situ and remote sensing observations available for validation, assimilation and development. Paradoxically given their proximity and relevance to humans, simultaneous synoptic measurements of currents, winds and waves in coastal and shelf seas remain elusive, whether from spaceborne observatories or by other means.

\section{Atmosphere-Wave-Ice-Ocean Interactions in Polar Seas}

The rapid decline in Arctic sea ice over the past decades has stimulated much interest in the mechanisms contributing to sea ice breakup, highlighting in particular the role of surface waves, winds and currents in determining the size distribution of ice floes and the dynamics of ice growth and decay. Sea ice extent is a key climate change indicator, responsible for major climate feedbacks through its impact on Earth surface albedo and airsea heat fluxes. Polar seas are also the sites of globally important water mass transformation and are famously supporting intense primary production and marine life. Finally, there are important strategic and economic considerations associated with the navigability and accessibility of the Arctic with less or no ice in summer (Stephenson et al., 2011; Aksenov et al., 2017). 
Currents associated with eddies at $5-20 \mathrm{~km}$ scales play an important role in horizontal and vertical fluxes of heat, mass, momentum and tracers (Horvat et al., 2016) but evidence also points to complicated submesoscale structures at scales of $1 \mathrm{~km}$ or less (Carmack et al., 2015; Manucharyan and Thompson, 2017). Upwelling at the sea ice edge generates eddies and ice edge oceanic jets (Bulczak et al., 2015; Rynders, 2017). These interact with surface waves through complex coupling mechanisms with major effects on the near-surface mixing, heat balance and momentum transfer between the atmosphere, sea ice and the ocean (Giles et al., 2012; Thomson et al., 2018). Studies about the future state of the Arctic indicate the need for high-resolution model projections that account not only for changes in sea ice but also changes in ocean circulation, waves and wind (e.g., Aksenov et al., 2017). At present, no parameterization of these interactions is used in any Earth System Model, even though changes affecting the Marginal Ice Zone (MIZ) tend to bear directly on reanalysis discrepancies and climate challenges, e.g., predicted rates of Arctic sea ice loss (Chevallier et al., 2017). Here too, new observations have a crucial role to play to improve understanding and parameterizations of these critical processes in these extremely challenging environments.

\section{THE SEASTAR MISSION CONCEPT}

The clearly articulated needs identified in previous sections for simultaneous two-dimensional high-resolution measurements of current vectors, wind vectors and directional wave spectra cannot be addressed by the present-day ocean observing system. Detailed discussions of the observational gaps for currents, winds and waves are beyond this mini-review but can be found elsewhere in this issue (Villas Bôas et al., 2018; Rodriguez et al., 2018; Ardhuin et al., 2019a,b) and in the full SEASTAR mission proposal (Gommenginger et al., 2018).

\section{Objectives of the Mission}

The prime objective of SEASTAR is to address the observational gap for synoptic measurements of ocean surface currents and winds at the critical $1 \mathrm{~km}$ scale that are required to understand, model and forecast ocean submesoscale dynamics, air-sea interactions and small-scale processes in coastal, shelf and polar seas. Based on innovative interferometric technology, SEASTAR represents a major step forward from existing ground-based and spaceborne observing systems, offering two-dimensional imaging of total ocean surface current vectors and wind vectors at $1 \mathrm{~km}$ resolution with unprecedented accuracy, and coincident directional swell spectra. SEASTAR thus directly addresses the challenging but well-articulated multidisciplinary needs of the ocean, air-sea interactions, forecasting and climate communities for new measurements of:

- total surface currents (including ageostrophic currents)

- total Surface Current Vectors (TSCV; measuring two orthogonal vectorial components simultaneously)

- high-accuracy current data at $1 \mathrm{~km}$ resolution
- synoptic two-dimensional current field maps (to provide the wider dynamical context)

- TSCV collocated with high-resolution wind vectors and directional wave spectra.

The associated scientific objectives of SEASTAR are:

- to characterize, for the first time, total ocean surface current vectors and wind vectors at $1 \mathrm{~km}$ resolution, globally in open waters, coastal, shelf seas and the MIZ, to describe their nature, magnitude, spatial and seasonal variabilities

- to use current and wind derivative products (vorticity, divergence...) to study the relations between horizontal ocean surface circulation, air-sea exchanges and vertical ocean transports

- to use improved observations in the MIZ to better understand the role of surface winds, ocean waves and currents in sea ice dynamics, thermal evolution and break-up

- to exploit synergy with $1 \mathrm{~km}$ products from other satellite missions to study the impact of small scale ocean dynamics and air-sea exchanges on vertical transport, heat fluxes and marine biological productivity

- to support the validation of high-resolution models and the development of improved assimilation and parameterizations of submesoscale dynamics and small scale atmosphere-wave-ocean interactions for inclusion in multi-disciplinary Earth System climate models.

\section{Technical Concept}

SEASTAR consists of a single active microwave instrument on a single satellite flying in sun-synchronous Low-Earth orbit. The payload is a squinted Synthetic Aperture Radar along-track interferometer (SAR ATI) with two pairs of beams pointing fore and aft of the satellite at $\pm 45^{\circ}$ in azimuth, plus a standard SAR beam pointing broadside (Figure 1). The basic measuring principle of SEASTAR relies on Along-Track Interferometry (ATI), whereby the line-of-sight motion of the ocean surface is measured from the Doppler shift between two SAR images acquired within a few milliseconds of each other in a single satellite overpass. The use of beams squinting fore and aft of the satellite is a highly innovative solution that makes it possible to retrieve both components of the ocean surface motion vector in a single pass. SEASTAR is the first mission to propose squinted ATI from space.

The SEASTAR instrument operates at $\mathrm{Ku}$-band and produces a single-sided $170 \mathrm{~km}$ swath covered in 3 sub-swaths in ScanSAR mode. The inherent spatial resolution of SAR and InSAR images is $30 \times 150 \mathrm{~m}$ (range $\times$ azimuth), which are processed to Level 2 ocean surface current vectors and wind vectors at $1 \mathrm{~km}$ resolution over the full swath. The accuracy requirements for current vectors at $1 \mathrm{~km}$ resolution are $0.1 \mathrm{~m} / \mathrm{s}$ and $20^{\circ}$, and are achievable using the Level 2 products and instrument specifications. The two squinted beams operate in VV polarization whereas the broadside beam provides dual-polarization capability and additional azimuth diversity to retrieve unambiguous current and wind vectors at Level 2. 
The satellite flies in a sun-synchronous orbit (SSO) around $550 \mathrm{~km}$ altitude, which gives the option of alternating shortrevisit (1-2 day repeat) and medium-revisit (7-30 days) orbital phases. SEASTAR does not depend on data from other satellites but important scientific benefits can be expected from synergy with Sentinel-3 (particularly the high-resolution Sea Surface Temperature and Ocean Color images and high-resolution SAR altimetry), Sentinel-1 (Radial Velocity and Backscatter Coefficient data), Sentinel-2 (ultra-high resolution images in nearshore regions) and Jason-CS/Sentinel-6 (high-resolution SAR altimetry). A high level of scientific complementarity exists also with the Chinese-French Oceanography Satellite (CFOSat) and the Surface Water and Ocean Topography (SWOT) mission, as well as with the proposed SKIM and WACM mission concepts (Rodriguez et al., 2018; Ardhuin et al., 2019b).

\section{Retrieval Performance Assessment}

The SEASTAR inversion algorithm seeks to retrieve wind and current vectors from the SEASTAR data alone without reliance on ancillary information from models. Martin et al. (2018) propose a Bayesian approach derived from scatterometry to perform inversion and evaluation retrieval performance for currents and winds. The method accounts for the wind relative to the surface current and the effects of the Wind-wave Artifact Surface Velocity (WASV), a bias in all microwave Doppler data linked to the motion of ocean waves on the sea surface (Martin et al., 2016). Figure 2 shows the root-mean-square error (RMSE) of the retrieved current and wind estimated by Monte-Carlo simulations for the proposed instrument specifications, revealing excellent retrieval performance for both currents (better than $0.1 \mathrm{~m} / \mathrm{s}$ and $10^{\circ}$ ) and winds (better than $0.5 \mathrm{~m} / \mathrm{s}$ and $10^{\circ}$ ). Performance degrades slightly when wind direction is aligned with either squinted look directions but errors for currents typically remain below $0.1 \mathrm{~m} / \mathrm{s}$ across the $170 \mathrm{~km}$ swath. Overall, SEASTAR proposes to deliver observations of complementary ocean dynamic properties that have never before been measured simultaneously from space at this level of resolution and accuracy, and which far outstrip the capabilities of existing and proposed satellite ocean surface current missions.

\section{Airborne Demonstration and Validation Against HF Radar Data}

The measuring principle of SEASTAR was successfully demonstrated experimentally and scientifically with data from the Wavemill airborne demonstrator proof-of-concept campaign over the Irish Sea (Martin et al., 2016). Level-0 data were successfully processed to produce Level-1 interferograms and Level 2 ocean surface current vectors maps corrected for aircraft attitude fluctuations and wind-wave induced biases. Surface current vectors were retrieved at $100 \mathrm{~m}$ resolution and subsequently averaged for validation against coastal $\mathrm{HF}$ radar data (Martin and Gommenginger, 2017). At $1.5 \mathrm{~km}$ resolution, current RMS errors against HF radar were typically below $0.1 \mathrm{~m} / \mathrm{s}$ and $10^{\circ}$. The airborne system also detected sharp current jets over known deep bathymetry channels, giving confidence that the observing principle remains valid in
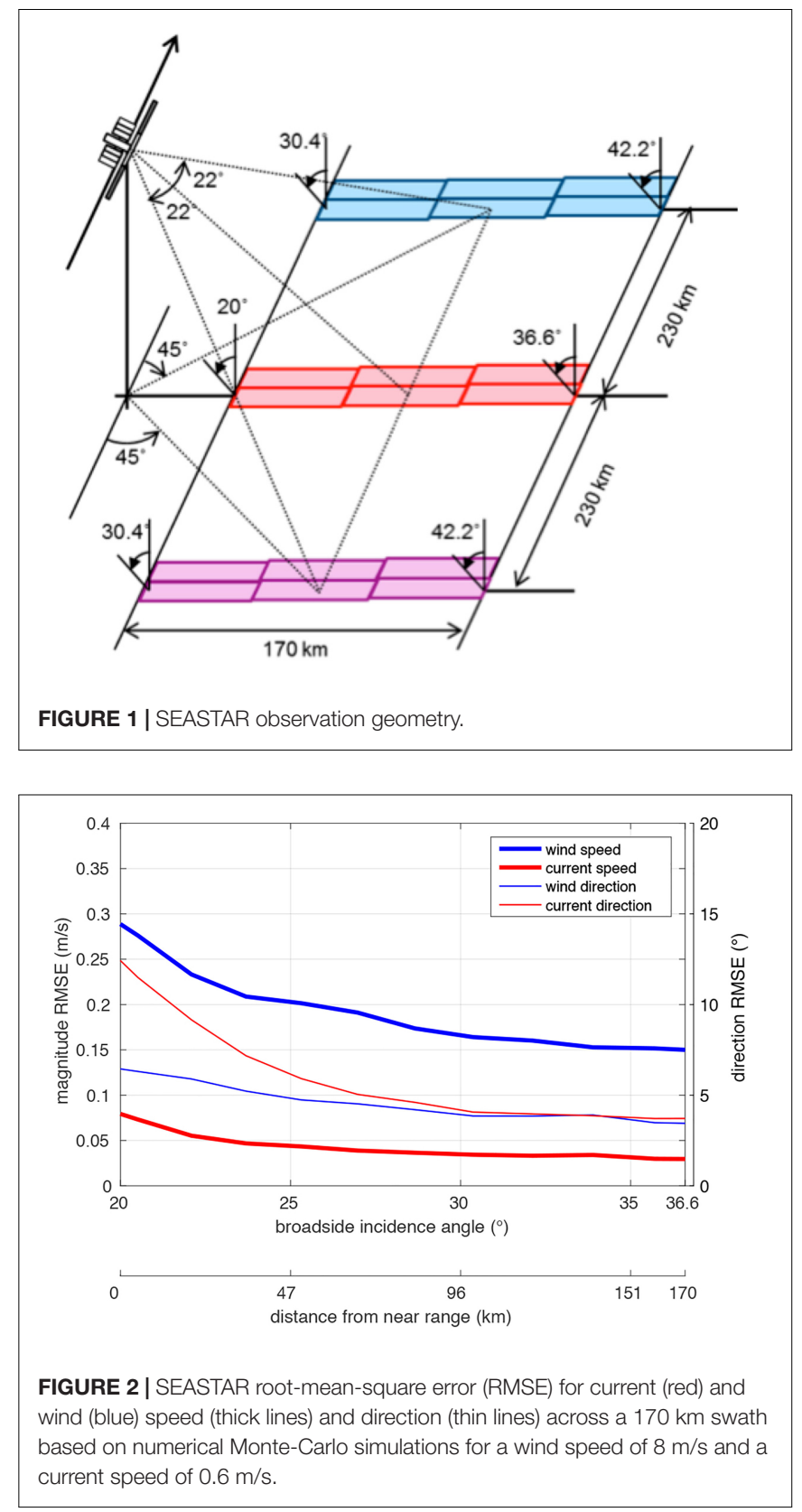

complex coastal environments. A new airborne instrument called OSCAR is currently under development for ESA to demonstrate the three-look SEASTAR configuration with airborne trials planned in July 2019.

\section{CONCLUSION AND RECOMMENDATIONS}

High-resolution ocean color and sea surface temperature images reveal an abundance of fronts, swirls, vortices and filaments at scales below $10 \mathrm{~km}$ but measurements of ocean surface dynamics at these scales are rare. 
Large numbers of numerical and experimental studies highlight the global role played by small ocean features in air-sea interactions, upper-ocean mixing, ocean vertical transports and marine ecosystem response.

Small-scale processes also visibly dominate ocean dynamics in coastal, shelf seas and polar seas, where they mediate important exchanges between land, the ocean, the atmosphere and the cryosphere (e.g., freshwater, nutrients, pollutants).

Advanced numerical models and in situ observations highlight the need for synoptic two-dimensional imaging of ocean surface properties at $1 \mathrm{~km}$ resolution where fundamental changes in dynamics are known to occur.

Present-day observing systems do not deliver the synoptic high-resolution measurements of current and wind vectors and directional wave spectra needed to observe small scale processes and improve their representation through parameterizations in multi-disciplinary Earth System models used for forecasting and climate projections.

SEASTAR is a new satellite mission concept to address the observational gap for synoptic measurements of ocean surface currents and winds at the critical $1 \mathrm{~km}$ scale that are required to understand, model, validate and forecast ocean submesoscale dynamics, air-sea interactions and small-scale processes in coastal, shelf and polar seas.

Based on innovative interferometric technology, SEASTAR represents a major step forward from existing ground-based

\section{REFERENCES}

Aksenov, Y., Popova, E. E., Yool, A., Nurser, A. G., Williams, T. D., Bertino, L., et al. (2017). On the future navigability of arctic sea routes: high-resolution projections of the arctic ocean and sea ice. Mar. Policy 75, 300-317. doi: 10.1016/j.marpol.2015.12.027

Ardhuin, F., Stopa, J. E., Chapron, B., Collard, F., Husson, R., Jensen, R. E., et al. (2019a). Observing sea states. Front. Mar. Sci. 6. doi: 10.3389/fmars.2019.00124

Ardhuin, F., Brandt, P., Gaultier, L., Donlon, C., Battaglia, A., Boy, F., et al. (2019b). SKIM, a candidate satellite mission exploring global ocean currents and waves. Front. Mar. Sci. 6. doi: 10.3389/fmars.2019.00209

Balwada, D., Smith, K. S., and Abernathey, R. (2018). Submesoscale vertical velocities enhance tracer subduction in an idealized antarctic circumpolar current. Geophys. Res. Lett. 45, 9790-9802. doi: 10.1029/2018gl079244

Bricheno, L. M., Soret, A., Wolf, J., Jorba, O., and Baldasano, J. M. (2013). Effect of high-resolution meteorological forcing on nearshore wave and current model performance. J. Atmos. Ocean. Technol. 30, 1021-1037. doi: 10.1175/jtech-d12-00087.1

Bulczak, A. I., Bacon, S., Garabato, A. C. N., Ridout, A., Sonnewald, M. J., and Laxon, S. W. (2015). Seasonal variability of sea surface height in the coastal waters and deep basins of the nordic seas. Geophys. Res. Lett. 42, 113-120. doi: $10.1002 / 2014$ gl061796

Callies, J., Ferrari, R., Klymak, J. M., and Gula, J. (2015). Seasonality in submesoscale turbulence. Nat. Commun. 6:6862. doi: 10.1038/ncomms7862

Capet, X., McWilliams, J. C., Molemaker, M. J., and Shchepetkin, A. (2008). Mesoscale to submesoscale transition in the california current system. part i: flow structure, eddy flux, and observational tests. J. Phys. Oceanogr. 38, 29-43. doi: 10.1175/2007jpo3671.1

Carmack, E., Polyakov, I., Padman, L., Fer, I., Hunke, E., Hutchings, J., et al. (2015). Toward quantifying the increasing role of oceanic heat in sea ice loss in the new Arctic. Bull. Am. Meteorol. Soc. 96, 2079-2105. doi: 10.1175/bams-d-1300177.1

Cavaleri, L., Abdalla, S., Benetazzo, A., Bertotti, L., Bidlot, J. R., Breivik, $\varnothing$, et al. (2018). Wave modelling in coastal and inner seas. Prog. Oceanogr. 167, $164-233$. and spaceborne observing systems, offering new capability and unprecedented accuracy, and addressing the challenging but well-articulated multidisciplinary needs of the ocean, airsea interactions, coastal processes, cryosphere, forecasting and climate communities.

SEASTAR is an innovative, mature and demonstrated mission concept looking for opportunities to proceed toward spaceborne implementation within Europe and beyond.

\section{AUTHOR CONTRIBUTIONS}

CG drafted and revised the manuscript. All authors reviewed the manuscript and approved the submitted version of the manuscript.

\section{FUNDING}

CG and AM received funding from the United Kingdom Centre for Earth Observation Instrumentation SEASTAR+ project (Contract No. RP10G0435A02). PVL was supported by the European Research Council (ERC) CUNDA project 694509 under the European Union Horizon 2020 Research and Innovation Program.

Chevallier, M., Smith, G. C., Dupont, F., Lemieux, J. F., Forget, G., Fujii, Y., et al. (2017). Intercomparison of the arctic sea ice cover in global ocean-sea ice reanalyses from the ora-ip project. Clim. Dyn. 49, 1107-1136. doi: 10.1007/ s00382-016-2985-y

D’Asaro, E., Lee, C., Rainville, L., Harcourt, R., and Thomas, L. (2011). Enhanced turbulence and energy dissipation at ocean fronts. Science 332, 318-322. doi: 10.1126/science. 1201515

D’Asaro, E. A., Shcherbina, A. Y., Klymak, J. M., Molemaker, J., Novelli, G., Guigand, C. M., et al. (2018). Ocean convergence and the dispersion of flotsam. Proc. Natl. Acad. Sci. U.S.A. 115, 1162-1167. doi: 10.1073/pnas.1718453115

Ferrari, R., and Wunsch, C. (2009). Ocean circulation kinetic energy: reservoirs, sources, and sinks. Annu. Rev. Fluid Mech. 41, 253-282. doi: 10.1146/annurev. fluid.40.111406.102139

Flato, G., Marotzke, J., Abiodun, B., Braconnot, P., Chou, S. C., Collins, W. J., et al. (2013). "Climate change 2013: the physical science basis. contribution of working group i to the fifth assessment report of the intergovernmental panel on climate change," in Evaluation of Climate Models, eds T. F. Stocker, D. Qin, G.K. Plattner, M. Tignor, S. K. Allen, J. Boschung, et al. (Cambridge: Cambridge University Press).

Fox-Kemper, B., Danabasoglu, G., Ferrari, R., Griffies, S., Hallberg, R., Holland, M., et al. (2011). Parameterization of mixed layer eddies. III: implementation and impact in global ocean climate simulations. Ocean Model. 39, 61-78. doi: 10.1016/j.ocemod.2010.09.002

Fox-Kemper, B., and Ferrari, R. (2008). Parameterization of mixed layer eddies. Part II: prognosis and impact. J. Phys. Oceanogr. 38, 1166-1179. doi: 10.1175/ 2007jpo3788.1

Giles, K. A., Laxon, S. W., Ridout, A. L., Wingham, D. J., and Bacon, S. (2012). Western arctic ocean freshwater storage increased by wind-driven spin-up of the beaufort gyre. Nat. Geosci. 5, 194-197. doi: 10.1038/ngeo 1379

Gommenginger, C. P., Chapron, B., Marquez, J., Fox-Kemper, B., Eriksson, L., Lopez-Dekker, P., et al. (2018). "SEASTAR: a mission to study ocean submesoscale dynamics and small-scale atmosphere-ocean processes in coastal, shelf and polar seas," in Proceedings of the EUSAR 2018 - 12th European Conference on Synthetic Aperture Radar, (Piscataway, NJ). 
Holt, J., Hyder, P., Ashworth, M., Harle, J., Hewitt, H. T., Liu, H., et al. (2017). Prospects for improving the representation of coastal and shelf seas in global ocean models. Geosci. Model Dev. 10, 499-523. doi: 10.5194/gmd-10-4992017

Horvat, C., Tziperman, E., and Campin, J. M. (2016). Interaction of sea ice floe size, ocean eddies, and sea ice melting. Geophys. Res. Lett. 43, 8083-8090. doi: $10.1002 / 2016$ gl069742

Lapeyre, G., and Klein, P. (2006). Impact of the small-scale elongated filaments on the oceanic vertical pump. J. Mar. Res. 64, 835-851. doi: 10.1357/ 002224006779698369

Lévy, M., Ferrari, R., Franks, P. J., Martin, A. P., and Rivière, P. (2012). Bringing physics to life at the submesoscale. Geophys. Res. Lett. 39:L14602.

Lévy, M., Franks, P. J., and Smith, K. S. (2018). The role of submesoscale currents in structuring marine ecosystems. Nat. Commun. 9:4758. doi: 10.1038/s41467018-07059-3

Lévy, M., Klein, P., Tréguier, A. M., Iovino, D., Madec, G., Masson, S., et al. (2010). Modifications of gyre circulation by sub-mesoscale physics. Ocean Model. 34, 1-15. doi: 10.1016/j.ocemod.2010.04.001

Mahadevan, A., Thomas, L. N., and Tandon, A. (2008). Comment on "Eddy/wind interactions stimulate extraordinary mid-ocean plankton blooms". Science 320:448. doi: 10.1126/science.1152111

Manucharyan, G. E., and Thompson, A. F. (2017). Submesoscale sea ice-ocean interactions in marginal ice zones. J. Geophys. Res. Oceans 122, 9455-9475. doi: $10.1002 / 2017 \mathrm{jc} 012895$

Martin, A. C. H., and Gommenginger, C. P. (2017). Towards wide-swath highresolution mapping of total ocean surface current vectors from space: airborne proof-of-concept and validation.”. Remote Sens. Environ. 197, 58-71. doi: 10. 1016/j.rse.2017.05.020

Martin, A. C. H., Gommenginger, C. P., Marquez, J., Doody, S., Navarro, V., and Buck, C. (2016). Wind-wave-induced velocity in ATI SAR ocean surface currents: first experimental evidence from an airborne campaign. Geophys. J. Res. Oceans 121, 1640-1653. doi: 10.1002/2015JC011459

Martin, A. C. H., Gommenginger, C. P., and Quilfen, Y. (2018). Current and wind vectors simultaneous inversion with a dual-beam ATI SAR: strategy and evaluation. Remote Sensing of Environment 216, 798-808.

Maximenko, N., Arvesen, J., Asner, G., Carlton, J., Castrence, M., Centurioni, L., et al. (2017). Remote Sensing of Marine Debris to Study Dynamics, Balances and Trends. Available at: https:/ecocast.arc.nasa.gov/las/Reports\% 20and\%20Papers/Marine-Debris-Workshop-2017.pdf (accessed 30 July, 2019).

McGillicuddy, D. J., Anderson, L. A., Bates, N. R., Bibby, T., Buesseler, K. O., Carlson, C. A., et al. (2007). Eddy/wind interactions stimulate extraordinary mid-ocean plankton blooms. Science 316, 1021-1026. doi: 10.1126/science. 1136256

McWilliams, J. C. (2016). Submesoscale currents in the ocean. Proc. Math. Phys. Eng. Sci. 472:20160117. doi: 10.1098/rspa.2016.0117

Müller, S., Stanev, E. V., Schulz-Stellenfleth, J., Staneva, J., and Koch, W. (2013). Atmospheric boundary layer rolls: quantification of their effect on the hydrodynamics in the german bight. J. Geophys. Res. Oceans 118, 5036-5053. doi: 10.1002/jgrc.20388

Poje, A. C., Özgökmen, T. M., Lipphardt, B. L., Haus, B. K., Ryan, E. H., Haza, A. C., et al. (2014). Submesoscale dispersion in the vicinity of the deepwater horizon spill. Proc. Natl. Acad. Sci. U.S.A. 111, 12693-12698. doi: 10.1073/pnas. 1402452111

Rodríguez, E., Bourassa, M., Chelton, D., Farrar, J. T., Long, D., PerkovicMartin, D., et al. (2019). The winds and currents mission concept. Front. Mar. Sci. 6. doi: 10.3389/fmars.2019.00438

Rynders, S. (2017). Impact of Waves on Sea Ice and Oceans in the Marginal Ice Zone. Southampton: University of Southampton. PhD Thesis.
Schulz-Stellenfleth, J., and Stanev, E. (2016). Analysis of the upscaling problema case study for the barotropic dynamics in the north sea and the german bight. Ocean Model. 100, 109-124. doi: 10.1016/j.ocemod.2016. 02.002

Stephenson, S. R., Smith, L. C., and Agnew, J. A. (2011). Divergent long-term trajectories of human access to the Arctic. Nat. Clim. Chang. 1:156. doi: 10. 1038/nclimate1120

Thomson, J., Ackley, S., Girard-Ardhuin, F., Ardhuin, F., Babanin, A., Boutin, G., et al. (2018). Overview of the arctic sea state and boundary layer physics program. J. Geophys. Res. Oceans 123, 8674-8687.

Villas Bôas, A. B., Ardhuin, F., Ayet, A., Bourassa, M. A., Brandt, P., Chapron, B., et al. (2019). Integrated observations of global surface winds, currents, and waves: requirements and challenges for the next decade. Front. Mar. Sci. 6. doi: 10.3389/fmars.2019.00425

Woodson, C. B., and Litvin, S. Y. (2015). Ocean fronts drive marine fishery production and biogeochemical cycling. Proc. Natl. Acad. Sci. 112, 1710-1715. doi: $10.1073 /$ pnas. 1417143112

Conflict of Interest Statement: The authors declare that the research was conducted in the absence of any commercial or financial relationships that could be construed as a potential conflict of interest.

The reviewer SL declares an ongoing collaboration on a project with the author SG, as a contribution to the OceanObs collaboration, a decadal conference series on ocean observation. The peer review was handled under the close supervision of the Chief Editors to ensure an objective process.

Citation: Gommenginger C, Chapron B, Hogg A, Buckingham C, Fox-Kemper B, Eriksson L, Soulat F, Ubelmann C, Ocampo-Torres F, Buongiorno Nardelli B, Griffin D, Lopez-Dekker P, Knudsen P, Andersen O, Stenseng L, Stapleton N, Perrie W, Violante-Carvalho N, Schulz-Stellenfleth J, Woolf D, Isern-Fontanet J, Ardhuin F, Klein P, Mouche A, Pascual A, Capet X, Hauser D, Stoffelen A, Morrow R, Aouf L, Breivik Ø, Fu L-L, Johannessen JA, Aksenov Y, Bricheno L, Hirschi J, Martin ACH, Martin AP, Nurser G, Polton J, Wolf J, Johnsen H, Soloviev A, Jacobs GA, Collard F, Groom S, Kudryavtsev V, Wilkin J, Navarro V, Babanin A, Martin M, Siddorn J, Saulter A, Rippeth T, Emery B, Maximenko N, Romeiser R, Graber H, Alvera Azcarate A, Hughes CW, Vandemark D, da Silva J, Van Leeuwen PJ, Naveira-Garabato A, Gemmrich J, Mahadevan A, Marquez J, Munro Y, Doody S and Burbidge G (2019) SEASTAR: A Mission to Study Ocean Submesoscale Dynamics and Small-Scale Atmosphere-Ocean Processes in Coastal, Shelf and Polar Seas. Front. Mar. Sci. 6:457. doi: 10.3389/fmars.2019.00457

Copyright (c) 2019 Gommenginger, Chapron, Hogg, Buckingham, Fox-Kemper, Eriksson, Soulat, Ubelmann, Ocampo-Torres, Buongiorno Nardelli, Griffin, LopezDekker, Knudsen, Andersen, Stenseng, Stapleton, Perrie, Violante-Carvalho, SchulzStellenfleth, Woolf, Isern-Fontanet, Ardhuin, Klein, Mouche, Pascual, Capet, Hauser, Stoffelen, Morrow, Aouf, Breivik, Fu, Johannessen, Aksenov, Bricheno, Hirschi, Martin, Martin, Nurser, Polton, Wolf, Johnsen, Soloviev, Jacobs, Collard, Groom, Kudryavtsev, Wilkin, Navarro, Babanin, Martin, Siddorn, Saulter, Rippeth, Emery, Maximenko, Romeiser, Graber, Alvera Azcarate, Hughes, Vandemark, da Silva, Van Leeuwen, Naveira-Garabato, Gemmrich, Mahadevan, Marquez, Munro, Doody and Burbidge. This is an open-access article distributed under the terms of the Creative Commons Attribution License (CC BY). The use, distribution or reproduction in other forums is permitted, provided the original author(s) and the copyright owner(s) are credited and that the original publication in this journal is cited, in accordance with accepted academic practice. No use, distribution or reproduction is permitted which does not comply with these terms. 


\title{
The Development of a Canadian Integrated Ocean Observing System (CIOOS)
}

\author{
Andrew Stewart ${ }^{1 *}$, Brad deYoung ${ }^{2}$, Mike Smit ${ }^{3}$, Karen Donaldson', Alexa Reedman4, \\ Andréane Bastien ${ }^{5}$, Ray Brunsting ${ }^{6}$, Bill Carter ${ }^{7}$, Brad Covey ${ }^{5}$, Richard Kelly ${ }^{7}$, \\ Eric Peterson ${ }^{6}$, Benoît Pirenne ${ }^{8}$, Ariane Plourde ${ }^{9}$, Anne-Sophie Ste-Marie ${ }^{5}$, \\ Cristina Tollefsen ${ }^{10}$, Douglas Wallace ${ }^{4}$ and Fred Whoriskey ${ }^{11}$

\begin{abstract}
${ }^{1}$ Fisheries and Oceans Canada, Ottawa, ON, Canada, ${ }^{2}$ Department of Physics and Physical Oceanography, Faculty of Science, Memorial University, St. John's, NL, Canada, ${ }^{3}$ School of Information Management, Faculty of Management, Dalhousie University, Halifax, NS, Canada, ${ }^{4}$ Marine Environmental Observation Prediction and Response Network, Halifax, NS, Canada, ${ }^{5}$ St. Lawrence Global Observatory, Rimouski, QC, Canada, ${ }^{6}$ Tula Foundation, Heriot Bay, BC, Canada, ${ }^{7}$ Fisheries and Marine Institute of Memorial, University of Newfoundland, St. John's, NL, Canada, ${ }^{8}$ Ocean Networks Canada, Victoria, BC, Canada, ${ }^{9}$ Institut des Sciences de la Mer de Rimouski (ISMER), Université du Québec à Rimouski, Rimouski, QC, Canada, ${ }^{10}$ Defence Research and Development Canada, Halifax, NS, Canada, ${ }^{11}$ Ocean Tracking Network, Dalhousie University, Halifax, NS, Canada
\end{abstract}

OPEN ACCESS

Edited by:

Justin Manley,

Just Innovation, Inc., United States

Reviewed by:

Christoph Waldmann,

University of Bremen, Germany

William Douglas Wilson,

Independent Researcher,

United States

*Correspondence:

Andrew Stewart

Andrew.stewart@dfo-mpo.gc.ca

Specialty section:

This article was submitted to

Ocean Observation,

a section of the journal

Frontiers in Marine Science

Received: 07 November 2018

Accepted: 05 July 2019

Published: 09 August 2019

Citation:

Stewart A, deYoung B, Smit M, Donaldson K, Reedman A, Bastien A, Brunsting $R$, Carter $B$, Covey $B$, Kelly $R$, Peterson E, Pirenne $B$, Plourde A, Ste-Marie A-S, Tollefsen C, Wallace D and Whoriskey F (2019) The Development of a Canadian Integrated Ocean Observing System (CIOOS). Front. Mar. Sci. 6:431. doi: 10.3389/fmars.2019.00431
Ocean observation is fundamental to Canada's ocean science community. The federal government, academia, small businesses, not-for-profit organizations, and other research partners, collect and synthesize physical, chemical and biological observations for research purposes, to model ocean changes, to support resource management decision-making, and to establish baseline data for long-term monitoring. Aside from building comprehensive ocean observatories (Fisheries and Oceans Canada, 2010), there is no easy mechanism to integrate the large amounts of data from the various sources or to explore interrelationships among variables, and no coordination and collaboration mechanism for the ocean community as a whole to generate an efficient system Ocean Science and Technology Partnership (OSTP), for Fisheries and Oceans Canada (2011). Consequently, we observe fragmented and isolated data that is only discoverable by a limited range of end users. Canada's ocean science community (Wallace et al., 2014), led and supported by Fisheries and Oceans Canada (DFO), is developing a Canadian Integrated Ocean Observation System (ClOOS) that brings together and leverages existing Canadian and international ocean observation data into a federated data system. This system (Wilson et al., 2016) will improve coordination and collaboration among diverse data producers, improve access to information for decision making, and enable discovery and access to data to support a wide variety of applied and theoretical research efforts to better understand, monitor, and manage activities in Canada's oceans. Canada is implementing a ClOOS test-phase, which will eventually lead to the development of a robust and integrated observing system, improving connections between end users and providers of ocean observations. The improved coordination of regional and national efforts within ClOOS will contribute to global ocean observing, maximizing the overall benefit of integrated observing.

Keywords: ocean, observations, ocean observing systems, Canada, marine, data, data management, governance 


\section{INTRODUCTION}

The world's oceans are a critical component of the Earth system. Sound knowledge and understanding of the ocean is essential to mitigate human impacts on the global environment and to promote the ocean's sustainable use. Effective ocean management depends on observations of the ocean, which are generated by existing national or regional ocean observing systems and networks. Such data are also increasingly relevant to a broad array of stakeholders, with a recent report suggesting that the industry sector engaged with ocean observation had revenues of over $\$ 7$ billion in the United States alone, driven in part by their national ocean observing system (National Oceanic and Atmospheric Administration [NOAA], 2016).

Canada has the largest coastline of any nation in the world $(244,000 \mathrm{~km})$ along three ocean basins - Pacific, Arctic, and Atlantic. It has two major inland Seas, the Gulf of St. Lawrence, Hudson Bay, and the Laurentian Great Lakes. These waters are important to Canadians, serving as the backbone for the socio-economic well-being of Canada's coastal communities. The scale of our ocean resource poses many real challenges to developing a national observation system. The needs for ocean data vary significantly among regions, with conditions varying from extreme tidal dynamics in the Bay of Fundy, to the iceberg infested Grand Banks of Newfoundland, to the iced passages of the Arctic regions of Canada, and the coastal waters of the Salish Sea inside Vancouver Island. These regions support many different types of coastal communities with varying relationships with the ocean-from small fishing communities, to major ports, to First Nations, Métis, and Inuit communities distributed along the coasts up into the Arctic and Inuit Nunangat. Any nationally coordinated ocean observing system must, therefore, support ocean observing and share data to meet the needs of diverse groups of people countrywide, in addition to contributing to global efforts to address shared ocean issues.

Ocean science and observing in Canada are carried out by government, academia, non-governmental organizations, the private sector, and the general public (e.g., through citizen science). Many different sectors contribute, including coastal communities, Indigenous partners, fisheries, transportation, and tourism sectors. Canada's international memberships such as in the Intergovernmental Oceanographic Commission and the Arctic Council positions it well to contribute to global monitoring. Given the scale of the observational challenge, we must pay close attention to efficiency, necessary for longterm sustainability.

Fisheries and Oceans Canada (DFO) has the greatest investment and capacity for ocean science and serves as the lead agency for Canada’s cooperative international ocean observation efforts and has a mandate to pursue fundamental science to support and enhance fisheries and oceans management. Canada is an innovator in the development of specialized ocean observing capabilities. For example, ONC and Smart Ocean ${ }^{\mathrm{TM}}$ Systems offer continuous, near real-time monitoring and measurements throughout the year, and ONC has been a global leader in the design, operation and maintenance of cabled oceanographic systems (Heesemann et al., 2014). The SmartAtlantic Alliance supports operational efficiency, situational awareness, and safety in the marine environment, and the Ocean Tracking Network (OTN) is a global monitoring and internationally certified data system for tracking the movements of aquatic animals to provide the scientific foundation for sustainable oceans management (Hussey et al., 2015). SLGO promotes and facilitates access to and dissemination of data from the St. Lawrence ecosystem in support of public safety, climate change, transportation and resource management. The Ocean Frontier Institute is a transnational hub for ocean research, bringing together researchers from across the North Atlantic. Industry, Indigenous communities, NGOs, and the public also undertake ocean research, sometimes tied to the specific interests or values of their organization(s). There are good opportunities for private-public partnerships building first upon open and transparent data-sharing to address shared environmental concerns.

Until now Canada has lacked a national vision and strategy for coordinated ocean observation, resulting in overlaps and gaps in our observation activities, from collection to sharing and interpreting data. Canada needs an integrated ocean observing system to effectively and efficiently observe the ocean, and to gain the greatest value from its efforts.

Fisheries and Oceans Canada, in partnership with the Marine Environmental Observation Prediction and Response Network (MEOPAR), is spearheading the development of a Canadian Integrated Ocean Observation System (CIOOS) that brings together and leverages existing Canadian and international ocean observation data into a national data system. This national System (Wilson et al., 2016) will improve coordination and collaboration among data producers, ensure data and metadata interoperability, improve access to existing information, and enable discovery and reuse of data. These activities will support a wide variety of ocean science efforts to better understand, monitor, and manage activities in Canada's oceans leading to improved decision making. CIOOS will also provide a single access point for data of potential commercial or community value and serve as the foundation for the development of future ocean observation in the country. The formation of CIOOS is a step toward addressing the concerns highlighted by the Council of Canadian Academies [CCA] (2013), Expert Panel on Canadian Ocean Science 2013 report that identified poor coordination as a current fundamental weakness of Canadian marine science and the need for national governance structures to integrate existing regional efforts.

In moving to create its national ocean observing system, Canada benefits from the experiences and lessons learned by similar efforts in other countries. Many other countries recognize the need for national integration (e.g., O'Callaghan et al., 2019). Two models of particular interest are the U.S. Integrated Ocean Observing System (U.S. IOOS ${ }^{1}$ ) and Australia’s Integrated Marine Observing System (IMOS; Hill et al., 2010). Both systems were built by incorporating and scaling-up existing ocean observing capabilities. Such a process may be perceived as threatening for existing observation platforms or regional bodies. To garner community support, careful attention was paid during the

\footnotetext{
${ }^{1}$ https://ioos.noaa.gov/about/ioos-history/
} 
development of both systems to the governance structure that gives the community confidence that the new system would operate openly and transparently. National integration also enables communities to obtain significant new funding and recognition (e.g., the National Integrated Coastal and Ocean Observing Act of 2007 in the United States; the Australian Government's National Collaborative Research Infrastructure Strategy (NCRIS) support for IMOS) that flowed to the regions/platforms, an attractive carrot for those trying to sustain costly infrastructures. CIOOS governance is being crafted in a similar manner, and while the pathway to future funding is less clear at present, the existence of a valued national system will be instrumental in enabling new funding opportunities.

The United States and Australian experience also demonstrated the importance of inclusion. U.S. IOOS was initially focused on practical services to society (weather forecasts, flood control, advice to government), whereas IMOS was more academically focused. To prosper, both systems evolved to embrace the broadest possible array of stakeholders, meeting a wide variety of needs ${ }^{2}$ (Hill et al., 2010; Roughan et al., 2010; National Ocean Council, 2015). CIOOS from its start will engage with the broadest possible community. The importance of diversity, in all forms, is recognized in Canada. One important aspect of diversity, strongly supported, is partnership with Indigenous communities across the country. Their ties with the ocean span generations and they have rights, land claims, treaties, and governance structures over oceans and waterways, which ensure their role in ocean governance and management. Recognizing, respecting and including the values, expertise and traditional knowledge and Indigenous sciences of Indigenous peoples are essential to CIOOS.

\section{STRATEGY AND APPROACH TO THE DEVELOPMENT OF CIOOS}

The development of CIOOS has included several years of discussion, community meetings, white papers, and reports (Wallace et al., 2014; Smit et al., 2017; Tremblay et al., 2017; Whoriskey et al., 2017). This planning process for CIOOS has helped to unify the Canadian ocean observation community. The first step was to define key principles that help to unify the different actors who can contribute to the system, which should:

- Build on existing strengths;

- Include a wide variety of stakeholders, including Indigenous rightsholders;

- Have sustainability as a core feature;

- Support a culture of open data sharing;

- Be forward-looking and work to bring stakeholders and partners together; and

- Develop as an integrated system of regional nodes with many partners from different sectors.

Representatives from the federal government, academia, and non-governmental organizations worked together to develop

${ }^{2}$ https://ioos.noaa.gov/about/ioos-by-the-numbers/ the scope for a CIOOS. Based on recommendations derived from consultations with the ocean observing community, the pilot phase of CIOOS is underway and consists of three Regional Associations (RAs), a national web presence, and a data stewardship node. CIOOS will be led primarily by the RAs. In addition to DFO and MEOPAR, these RAs include universities (Memorial University and the Marine Institute, Dalhousie University, and the University of Victoria) plus existing networks such as SLGO, ONC, OTN, and the Hakai Institute, with the expectation that more partners will join in the future. The first steps will be to create user engagement plans, gather data from multiple providers within a data system, and demonstrate dissemination and visualization for an initial subset of Essential Ocean Variables (EOVs). CIOOS has been developed in line with the Global Ocean Observing System (GOOS) framework, in particular the use of EOVs as discussed in the Framework on Ocean Observing (Lindstrom et al., 2012) and with an eye to future inclusion of the currently developing Ecosystem and Biodiversity EOVs (Miloslavich et al., 2017; Muller-Karger et al., 2018).

A national web presence will provide core branding, an asset map and metadata catalog, and a data stewardship node will facilitate the development of data standards with partners. The core branding, a common look and feel across CIOOS, will be important to ensure partner organizations are recognized as an integral part of the community, and with the support of clear and consistent messaging, can demonstrate the benefits of CIOOS to new and future stakeholders (Mackenzie et al., 2019). The importance of branding and consistent messaging was one of the lessons learned from consultations with U.S. IOOS.

An effective national strategy and governance structure will help maximize the benefits of investments in the system, system capabilities, and national ocean science expertise through national coordination, leadership, and accountability (Fisheries and Oceans Canada, 2011). The multilevel governance model for CIOOS will allow for strong representation and mobility at the regional level, while ensuring a consistent national approach. An Executive Committee, with representatives from the regions and across stakeholder and rightsholder groups, ensures a clear and transparent decision-making process, integration across the whole of CIOOS, oversight for a common vision and implementation plan, and enables the sharing of best practices. At the regional level, a Board of Directors for each RA will gather feedback and ensure regional adaptability for their unique challenges and opportunities. Committees, with representatives from across the regions, will address more specialized issues and report to the Executive Committee (see Figure 1).

Already, there are strong networks of ocean observation in the Canadian Pacific, St. Lawrence, and Atlantic regions (Figure 2) monitoring a variety of ocean variables. The initial RAs to launch CIOOS are located in these areas. The System will grow to additional areas, e.g., the Arctic/Inuit Nunangat and the Great Lakes. Northern expansion is particularly important given the recent designation of an Arctic ocean region by DFO that is in partnership with and under the leadership of Inuit Nunangat.

The pilot phase focuses on the development of data infrastructure, including the data system architecture, the 


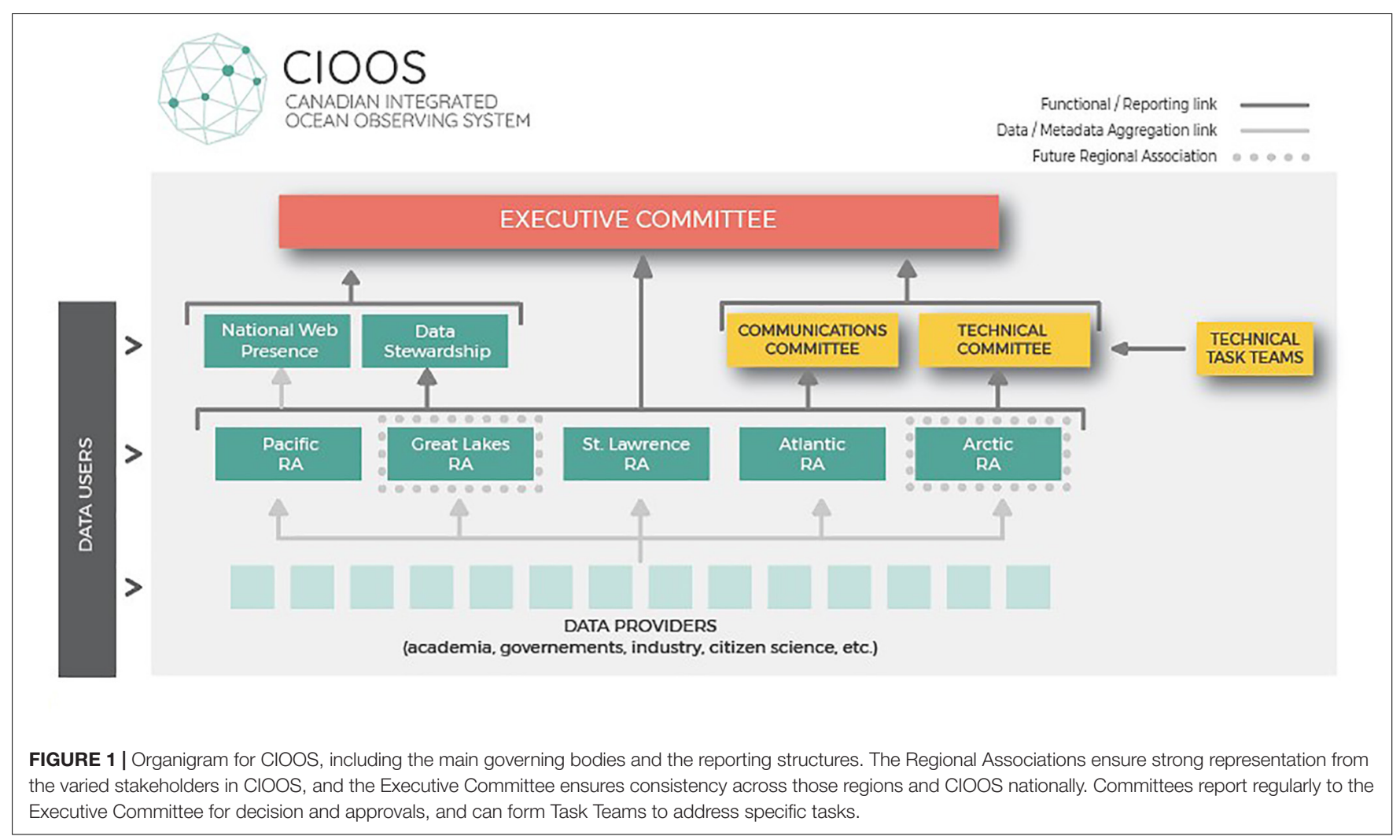

management structure and visualization tools, and begins by treating existing key data streams. By concentrating on a selection drawn from an initial subset of 11 EOVs, CIOOS will focus on development of a robust system that is scalable to new data types and variables, ensuring that the System is adaptable to new technology developments and societal changes. The initial 11 EOVs were agreed upon by the ocean observing community, based on quality and availability of data (Figure 2), and the ability of those variables to demonstrate the value of an integrated system by addressing national and international DFO mandates and ocean science community needs (Whoriskey et al., 2017).

The adoption of a common approach to metadata, software architecture, and data visualization is another critical piece of CIOOS. Within the ocean data management sector, there are a wide variety of competing standards, which were reviewed with recommendations during the design stage of CIOOS (Smit et al., 2017; Tremblay et al., 2017). It is important that good choices are made during the planning phases of the project. Due to the scope of work involved in making these decisions effectively, a technical decision-making structure is a part of the CIOOS project. A national Technical Committee, with representation from all regions oversees technical aspects of CIOOS, including metadata standards and practices, solution architecture and visualization tools. The Technical Committee initiates Task Teams to research and recommends to the Executive Committee on standards, frameworks, tools, methods and best practices. Task Teams include representation from all regions, in addition to external experts and collaborators. The Technical Committee helps to create and sustain collaborative working relationships between groups directly involved with developing CIOOS and national and international partners (Figure 1).

User engagement is critical to CIOOS, including training and capacity development. These activities will initially target a predominantly Canadian audience, focusing on the needs of regional data providers and users, as well as building awareness across the nation. The system will begin with a focus on meeting the needs of the initial partners, expanding to meet the wider needs of other groups as CIOOS matures. While CIOOS is currently focusing on a national approach, future phases of CIOOS could consider the basin-scale approach. Canada's coastlines are spread across three oceans, and so, in addition to Canadian partners, it will be important to work with international partners to develop basin strategies, such as the developing Atlantic Ocean Observing Blueprint (deYoung et al., 2019), and future initiatives such as an Arctic OOS.

\section{HOW CIOOS WILL BENEFIT CANADIANS}

Canadian Integrated Ocean Observation System will address immediate needs regarding quality and interoperability of ocean data, and will enable Canada to better adapt to changing requirements and a changing environment. Provision of open oceanographic data will help meet the societal needs for access to ocean information, benefit the country by assisting in the development of ground-breaking research, contribute to the global ocean observation efforts and provide 


\section{CORE VARIABLE READINESS BY REGION}

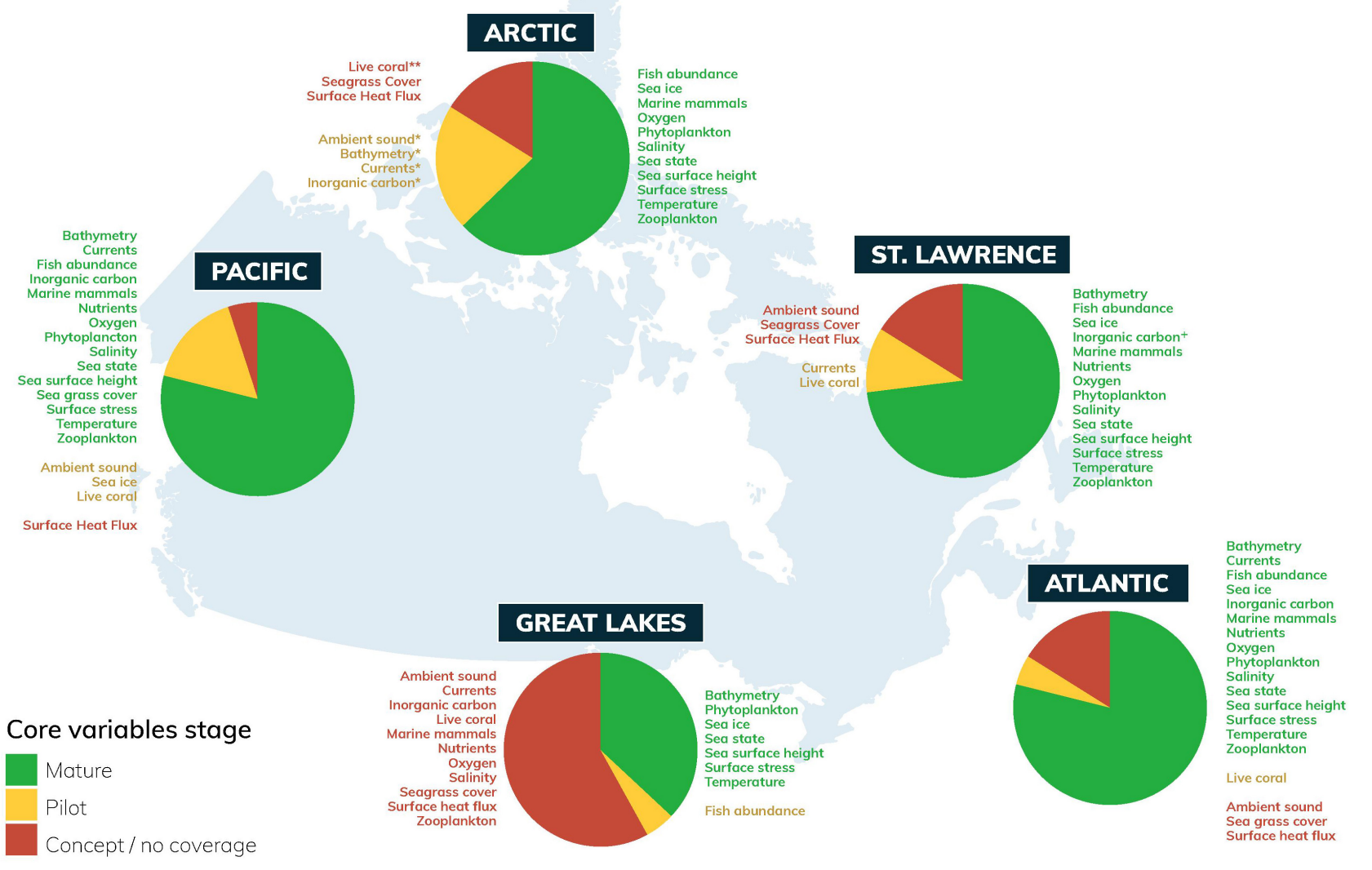

FIGURE 2 | Core variables readiness for GOOS compliance (level of maturity in terms of requirement process, coordination of observation elements, and data management and information product) according to GOOS three levels: Mature (green) -"products are well understood, documented, consistently available, and of societal benefit," Pilot (yellow) -"planning, negotiating, testing and approval within appropriate local, regional, global arenas," and Concept or have No Coverage (red) -"peer review of ideas and studies at science, engineering, and data management community level." * Mature technology, but low coverage. **Pilot, but low coverage. +Mature technology, but inconsistent sampling. Credit: St. Lawrence Global Observatory (SLGO) with data from Whoriskey et al. (2017).

opportunities for Canada to become a global leader in multidisciplinary ocean science.

The system's multi-level governance structure provides an opportunity to bring in new partners, both nationally and internationally, as emerging partners or observers. International involvement is important to ensure that as CIOOS grows, it enables Canada's engagement on the international ocean observing landscape (e.g., Barth et al., 2019), for example through the GOOS and the GOOS Regional Alliances (GRAs) and the proposed All Atlantic Ocean Observing System AtlantOS (deYoung et al., 2019). CIOOS will enhance ocean observing internationally, by ensuring the data are easily accessible, following the FAIR (Findable, Accessible, Interoperable, Reusable) principles of data management (Tanhua et al., 2019) and working with other countries to share innovations and value-added products. Many of the partner organizations contributing to the development of CIOOS are already involved in international projects. CIOOS can augment their international collaborations by providing new avenues and forums for partnerships.
CIOOS will help build ocean observing capacity across Canada, by reaching out to involve smaller organizations and communities, bringing together existing groups, and through enhanced collaboration and coordination strengthen existing activities. Many partners involved in CIOOS are world leaders in ocean observing and in the dissemination of ocean observing data, as noted above. The goal is for the national program to be stronger than the sum of all these components.

Ocean observing systems support many different human concerns about the ocean, from operational services, to ocean health, to climate. These concerns were behind the creation of GOOS. CIOOS will span these three key aspects of ocean observing by bringing together stakeholders from the ocean community to provide an integrated approach. Central to this approach is the partnership between the federal government and the academic community. This partnership will ensure that the strengths of each are mobilized to build the most flexible and dynamic system possible, and to ensure sustainable operations. This will require a focus on innovation to improve the efficiency and effectiveness of the System. The partnership also includes 
the private sector, to directly support the blue economy, the growth of which is rapidly accelerating, bringing both benefits but also challenges. CIOOS will regularly review the observing system activities to ensure that they are fit-for-purpose in the socio-economic sense to meet the needs of Canadians.

\section{LOOKING FORWARD}

Canada faces increasing demand for oceanographic data to support sustainable growth and address issues stemming from a changing ocean and needs a national data system to meet that demand. We currently face numerous environmental issues, including pollution, ocean acidification, sea-level rise, and managing sustainable fisheries. The challenges in addressing such issues include:

- Need for coordinated, global, sustained (long-term) data collection;

- Demand for timely access to data of known quality, information and knowledge, in user-friendly formats;

- Increasing data rates and volumes;

- Greater diversity of data types and observing instruments; and

- Limited collaboration mechanisms/structures to foster data standardization, integration, discovery, and visualization.

Only a national, integrated ocean observing system can address these challenges. We require a transparent process for decision making that will enable us to address key gaps in our observing activities. A system that encourages stakeholder engagement will help with system sustainability as it will be clearly seen to be meeting stakeholder needs which will encourage participatory support of the System. CIOOS will help to create

\section{REFERENCES}

Barth, J. A., Allen, S. E., Dever, E. P., Dewey, R. K., Evans, W., Feely, R. A., et al. (2019). Better regional ocean observing through cross-national cooperation: a case study from the Northeast Pacific. Front. Mar. Sci. 6:93. doi: 10.3389/fmars. 2019.00093

Council of Canadian Academies [CCA] (2013). Expert Panel on Canadian Ocean Science. Ocean Science in Canada: Meeting the Challenge, Seizing the Opportunity. Available at: http://scienceadvice.ca/reports/ocean-sciencein-canada-meeting-the-challenge-seizing-the-opportunity/ (accessed July 11, 2019).

deYoung, B., Visbeck, M., Araujo Filho, M. C., Baringer, M. O., Black, C.-A., Buch, E., et al. (2019). An integrated all-atlantic ocean observing system in 2030 . Front. Mar. Sci. 6:428. doi: 10.3389/fmars.2019.00428

Fisheries and Oceans Canada (2010). Ocean Science and Technology Partnership, With the Financial Support of the Canadian Space Agency. Canadian Survey of Atlantic, Pacific, Arctic and Great Lakes Observing Systems. Ottawa: Fisheries and Oceans Canada .

Fisheries and Oceans Canada (2011). Ocean Science and Technology Partnership. Lessons Learned from OOS in Canada: Preliminary Assessment of OOS Value. Available at: http://www.qc.dfo-mpo.gc.ca/publications/science/ documents/Preliminary\%20OOS\%20value\%20assessment_e.pdf (accessed July 11, 2019).

Heesemann, M., Insua, T. L., Scherwath, M., Juniper, S. K., and Moran, K. (2014). Ocean networks Canada: from geohazards research laboratories to smart Ocean Systems. Oceanography 27, 151-153. doi: 10.5670/oceanog.2014.50 the environment, and will encourage greater investment by the federal and provincial governments as well as by other partners who will contribute to and benefit from the observations.

Canadian Integrated Ocean Observation System will enable Canada to play a global leadership role in multidisciplinary ocean science and will provide access to valuable data currently not discoverable, especially within the extensive holdings of the federal government. This ocean observing system will help coordinate the collection of ocean data on a national scale, and critically, will be adaptable in the face of growing needs and a changing environment.

\section{VISION}

To develop an integrated ocean observation system that meets the needs of Canadians and contributes to global ocean observing.

\section{AUTHOR CONTRIBUTIONS}

All authors listed have made a substantial, direct and intellectual contribution to the work, and approved it for publication.

\section{ACKNOWLEDGMENTS}

We would like to thank the Canadian and the international ocean observing community for the support and input we have received and continue to receive in the development of CIOOS. We would also like to thank Fisheries and Oceans Canada and the Marine Environmental Observation, Prediction, and Response Network for funding this initiative.

Hill, K., Moltmann, T., Proctor, R., and Allen, S. (2010). The Australian integrated marine observing system: delivering data streams to address national and international research priorities. Mar. Technol. Soci. J. 44, 65-72. doi: 10.4031/ MTSJ.44.6.13

Hussey, N. E., Kessel, S. T., Aarestrup, K., Cooke, S. J., Cowley, P. D., Fisk, A. T., et al. (2015). Aquatic animal telemetry: a panoramic window into the underwater world. Science 348:1255642. doi: 10.1126/science.12 55642

Lindstrom, E., Gunn, J., Fischer, A., McCurdy, A., and Glover, L. K. (2012). A Framework for Ocean Observing. By the Task Team for an Integrated Framework for Sustained Ocean Observing, UNESCO 2012, IOC/INF-1284. Available at: http://unesdoc.unesco.org/images/0021/002112/211260e.pdf (accessed July 11, 2019).

National Oceanic and Atmospheric Administration [NOAA] (2016). The Ocean Enterprise: A study of US Business Activity in Ocean Measurement, Observation and Forecasting. Available at: http://www.ioos.noaa.gov/ioos_in_action/ocean_ enterprise_study.html (accessed July 11, 2019).

Mackenzie, B., Celliers, L., de Freitas Assad, L. P., Heymans, J. J., and Rome, N. (2019). The role of stakeholders and actors in creating societal value from coastal and ocean observations. Front. Mar. Sci. 6:137.

Miloslavich, P., Bax, N. J., Simmons, S. E., Klein, E., Appeltans, W., AburtoOropeza, O., et al. (2017). Essential ocean variables for global sustained observations of biodiversity and ecosystem changes. Glob. Change Biol. 24, 2416-2433. doi: 10.1111/gcb.14108

Muller-Karger, F. E., Miloslavich, P., Bax, N. J., Simmons, S., Costello, M. J., Sousa Pinto, I., et al. (2018). Advancing marine biological observations and 
data requirements of the complementary essential ocean variables (EOVs) and essential biodiversity variables (EBVs) frameworks. Front. Mar. Sci. 5:211. doi: $10.3389 /$ fmars.2018.00211

National Ocean Council (2015). US Integrated Ocean Observing System (U.S.IOOS $\left.{ }^{\circledR}\right) 2015$ Report to Congress. Available at: https://cdn.ioos.noaa.gov/ media/2017/12/ioos_report_congress2015.pdf (accessed July 11, 2019).

O'Callaghan, J., Stevens, C., Roughan, M., Cornelisen, C., Sutton, P., Garrett, S., et al. (2019). Developing an integrated ocean observing system for New Zealand. Front. Mar. Sci. 6:143. doi: 10.3389/fmars.2019.00143

Roughan, M., Morris, B. D., and Suthers, I. M. (2010). NSW-IMOS: an integrated marine observing system for Southeastern Australia. IOP Conf. Ser. Earth Environ. Sci. 11:012030. doi: 10.1088/1755-1315/11/1/012030

Smit, M., Kelly, R., Fitzsimmons, S., Bruce, S., Bulger, C., Covey, B., et al. (2017). Canadian Integrated Ocean Observing System: Cyberinfrastructure Investigative Evaluation. Halifax, NS: Dalhousie University.

Tanhua, T., Pouliquen, S., Hausman, J., O’brien, K., Bricher, P., Bruin, T. D., et al. (2019). Ocean FAIR data services. Front. Mar. Sci. (in press). doi: 10.3389/fmars. 2019.00440

Tremblay, C., Desjardins, A. B., Dewey, R., Guillemot, E., Lapointe, S., Owens, D., et al. (2017). Canadian Integrated Ocean Observing System: Visualization Investigative Evaluation. Available at: https://ogsl.ca/commons/doc/cioos_ie_ vis.pdf

Wallace, D. R., de Young, B., Iverson, S., Laroche, J., Whoriskey, F., Archambault, P., et al. (2014). "A Canadian contribution to an integrated atlantic ocean observing system (IAOOS)," in Proceedings of the Presented at the IEEE/OES Marine Technology Society OCEANS 2014, (St. John's, NL: IEEE).

Whoriskey, F. G., Morton, K., Bajona, L., Beck, M., Dewey, R., Guillemot, E., et al. (2017). Tremblay. Canadian Integrated Ocean Observing System Investigative Evaluations: Observation and Data. Available at: http://meopar.ca/uploads/IE_Report_-_Observations_and_Data.pdf (accessed July 11, 2019).

Wilson, L., Smit, M., and Wallace, D. W. R. (2016). "Towards a unified vision for ocean data management in canada: results of an expert forum)," in Proceedings of the Advancing the Frontiers of Data in Research from SciDataCon 2016, Denver, CO.

Conflict of Interest Statement: The authors declare that the research was conducted in the absence of any commercial or financial relationships that could be construed as a potential conflict of interest.

Copyright $(2019$ Stewart, deYoung, Smit, Donaldson, Reedman, Bastien, Brunsting, Carter, Covey, Kelly, Peterson, Pirenne, Plourde, Ste-Marie, Tollefsen, Wallace and Whoriskey. This is an open-access article distributed under the terms of the Creative Commons Attribution License (CC BY). The use, distribution or reproduction in other forums is permitted, provided the original author(s) and the copyright owner(s) are credited and that the original publication in this journal is cited, in accordance with accepted academic practice. No use, distribution or reproduction is permitted which does not comply with these terms. 


\section{Ocean Observations to Improve Our Understanding, Modeling, and Forecasting of Subseasonal-to-Seasonal Variability}

\section{OPEN ACCESS}

Edited by:

Amos Tiereyangn Kabo-Bah,

University of Energy and Natural

Resources, Ghana

Reviewed by:

Eric Hackert,

National Aeronautics and Space Administration (NASA), United States

Richard James Matear

Commonwealth Scientific and Industrial Research Organisation (CSIRO), Australia

${ }^{*}$ Correspondence:

Aneesh C. Subramanian aneeshcs@colorado.edu;

acsubram@ucsd.edu

Specialty section: This article was submitted to

Ocean Observation,

a section of the journal

Frontiers in Marine Science

Received: 01 November 2018

Accepted: 05 July 2019

Published: 08 August 2019

Citation:

Subramanian AC, Balmaseda MA, Centurioni L, Chattopadhyay $R$, Cornuelle BD, DeMott C, Flatau M, Fujii Y, Giglio D, Gille ST, Hamill TM, Hendon H, Hoteit I, Kumar A,

Lee J-H, Lucas AJ, Mahadevan A, Matsueda M, Nam S, Paturi S,

Penny SG, Rydbeck A, Sun $R$,

Takaya Y, Tandon A, Todd RE, Vitart $F$, Yuan D and Zhang C (2019) Ocean

Observations to Improve Our Understanding, Modeling, and Forecasting

of Subseasonal-to-Seasonal Variability. Front. Mar. Sci. 6:427. doi: 10.3389/fmars.2019.00427
Aneesh C. Subramanian ${ }^{*}$, Magdalena A. Balmaseda ${ }^{2}$, Luca Centurioni ${ }^{3}$, Rajib Chattopadhyay ${ }^{4}$, Bruce D. Cornuelle ${ }^{3}$, Charlotte DeMott ${ }^{5}$, Maria Flatau, Yosuke Fujii ${ }^{7}$, Donata Giglio ${ }^{1}$, Sarah T. Gille ${ }^{3}$, Thomas M. Hamill ${ }^{8}$, Harry Hendon ${ }^{9}$, Ibrahim Hoteit ${ }^{10}$, Arun Kumar'11, Jae-Hak Lee ${ }^{12}$, Andrew J. Lucas ${ }^{3}$, Amala Mahadevan ${ }^{13}$, Mio Matsueda ${ }^{14}$, SungHyun Nam ${ }^{15}$, Shastri Paturi" ${ }^{16}$, Stephen G. Penny ${ }^{17}$, Adam Rydbeck ${ }^{18}$, Rui Sun ${ }^{3}$, Yuhei Takaya ${ }^{7}$, Amit Tandon ${ }^{19}$, Robert E. Todd ${ }^{13}$, Frederic Vitart ${ }^{2}$, Dongliang Yuan ${ }^{20}$ and Chidong Zhang ${ }^{21}$

${ }^{1}$ Atmospheric and Oceanic Sciences, University of Colorado Boulder, Boulder, CO, United States, ${ }^{2}$ ECMWF, Reading, United Kingdom, ${ }^{3}$ Scripps Institution of Oceanography, University of California, San Diego, San Diego, CA, United States, ${ }^{4}$ Indian Institute of Tropical Meteorology, Pune, India, ${ }^{5}$ Department of Atmospheric Science, Colorado State University, Fort Collins, CO, United States, ${ }^{6}$ United States Naval Research Laboratory, Monterey, CA, United States, ${ }^{7}$ Meteorological Research Institute, Japan Meteorological Agency, Tsukuba, Japan, ${ }^{8}$ National Oceanic and Atmospheric Administration, Earth System Research Laboratory, Physical Sciences Division, Boulder, CO, United States, ${ }^{9}$ Bureau of Meteorology, Melbourne, VIC, Australia, ${ }^{10}$ Earth Science and Engineering, King Abdullah University of Science and Technology, Thuwal, Saudi Arabia, " National Centers for Environmental Prediction, Climate Prediction Center, College Park, MD, United States, ${ }^{12}$ Korea Institute of Ocean Science and Technology, Busan, South Korea, ${ }^{13}$ Woods Hole Oceanographic Institution, Woods Hole, MA, United States, ${ }^{14}$ Center for Computational Sciences, University of Tsukuba, Tsukuba, Japan, ${ }^{15}$ School of Earth and Environmental Sciences/Research Institute of Oceanography, Seoul National University, Seoul, South Korea, ${ }^{16}$ I.M. Systems Group (IMSG), National Oceanic and Atmospheric Administration, Environmental Modeling Center, College Park, MD, United States, ${ }^{17}$ Department of Atmospheric and Oceanic Science, University of Maryland, College Park, College Park, MA, United States, ${ }^{18}$ United States Naval Research Laboratory, Stennis Space Center, Hancock, MS, United States, ${ }^{19}$ Mechanical Engineering, University of Massachusetts Dartmouth, Dartmouth, MA, United States, ${ }^{20}$ Institute of Oceanology, Chinese Academy of Sciences, Qingdao, China, ${ }^{21}$ National Oceanic and Atmospheric Administration, Pacific Marine Environmental Laboratory, Seattle, WA, United States

Subseasonal-to-seasonal (S2S) forecasts have the potential to provide advance information about weather and climate events. The high heat capacity of water means that the subsurface ocean stores and re-releases heat (and other properties) and is an important source of information for S2S forecasts. However, the subsurface ocean is challenging to observe, because it cannot be measured by satellite. Subsurface ocean observing systems relevant for understanding, modeling, and forecasting on S2S timescales will continue to evolve with the improvement in technological capabilities. The community must focus on designing and implementing low-cost, high-value surface and subsurface ocean observations, and developing forecasting system capable of extracting their observation potential in forecast applications. S2S forecasts will benefit significantly from higher spatio-temporal resolution data in regions that are sources of predictability on these timescales (coastal, tropical, and polar regions). While ENSO has been a driving force for the design of the current observing system, the subseasonal time scales present new observational requirements. Advanced observation technologies such as autonomous surface and subsurface profiling devices as well as satellites that observe the ocean-atmosphere interface simultaneously can lead to breakthroughs in coupled data assimilation (CDA) and coupled initialization for S2S forecasts. 
These observational platforms should also be tested and evaluated in ocean observation sensitivity experiments with current and future generation CDA and S2S prediction systems. Investments in the new ocean observations as well as model and DA system developments can lead to substantial returns on cost savings from disaster mitigation as well as socio-economic decisions that use S2S forecast information.

Keywords: subseasonal, seasonal, predictions, air-sea interaction, satellite, Argo, gliders, drifters

\section{INTRODUCTION}

Operational centers are undertaking a seamless extension of weather forecasts to Subseasonal-to-Seasonal (S2S) forecasts. There is a growing consensus that coupled atmosphere-ocean modeling will be essential to forecasts on S2S time scales (Penny and Hamill, 2017; Penny et al., 2019). The role of oceanatmosphere coupling in S2S variability and its representation in models is one of the research foci of the World Meteorological Organization (WMO) S2S Prediction Project. In particular, the initialization and configuration of the ocean and sea ice are key modeling research issues ${ }^{1}$.

The role of ocean-atmosphere coupling in S2S variability must be well understood not only for improving atmospheric predictions but also for improving predictions relevant to the entire gamut of socio-economic scenarios encompassing everything from disaster mitigation to marine ecosystems and coastal management. Detailed studies to help improve strategies for coupled initialization are underway at various global modeling centers (e.g., ECMWF, NCEP, NRL; Lea et al., 2015; Frolov et al., 2016; Laloyaux et al., 2016).

Innovative observing technology in the subsurface ocean and at the air-sea interface will help us understand air-sea interaction and its role in S2S variability and prediction. S2S forecasts for high- and mid-latitudes can be improved with better observations of sea-ice thickness and concentration and ocean basin boundary currents (current intensity and stratification). Evaluating impacts of new ocean observing technology on ocean analyses and S2S forecasts will contribute to the design and the evolution of oceanic observing systems, such as the Tropical Pacific Observing System (TPOS-2020; Smith et al., 2019), the Atlantic Ocean Observing System (AtlantOS; Foltz et al., 2019), and regional boundary current observing systems (Todd et al., 2019).

Multi-system evaluation of observation impacts based on international collaboration is effective to deliver fair and robust information to society and observational agencies. Coordination by international groups such as CLIVAR and GODAE OceanView aims to support these efforts to make significant progress (see also Fujii et al., 2019). Here, we provide scientific rationales for ocean observations being important for S2S predictions, discuss gaps in observations, and recommend designs of observational and modeling experiments to evaluate the impact of ocean observations on S2S forecasts.

${ }^{1}$ http://s2sprediction.net/file/documents_reports/S2S_Implem_plan_en.pdf

\section{NEED FOR OCEAN OBSERVATIONS FOR S2S PREDICTIONS}

The top $100 \mathrm{~m}$ of the ocean have 40 times the heat capacity of the entire atmosphere. This makes the upper ocean a major reservoir of heat energy for the weather/climate system and a critical component of S2S prediction. In addition to the ocean surface temperatures, several other potential sources of S2S predictability have been identified such as: tropical intraseasonal oscillation (namely, the Madden-Julian Oscillation, MJO; Vitart et al., 2012), subsurface ocean heat anomalies (Alexander et al., 1999; Vimont et al., 2001), and sea ice (Vitart et al., 2012). These phenomena serve as sources of S2S predictability because of their relative persistence or known oscillatory behaviors. Some of them are directly related to ocean conditions. In addition, there are requirements to correctly represent coastal SST, ocean boundary currents, ocean mixed-layer structures, and other oceanic fields relevant to teleconnections and regional variability on S2S timescales.

ENSO is the most dominant mode of climate variability, with a significant influence on S2S variability through global teleconnections (Yeh et al., 2018). The recharge and discharge of warm water volume (WWV) in the Tropical Pacific is closely related to ENSO variability (Jin, 1997; Meinen and McPhaden, 2000). Hence, accurate observations of the evolution of Tropical Pacific WWV can help improve ENSO process understanding and predictions on seasonal timescales. Accurate analysis and initialization of ocean sub-surface states in the equatorial Pacific is of great importance for S2S prediction (Fujii et al., 2015).

Air-sea coupling has been considered a key process that needs to be accurately represented in models for MJO and Monsoon IntraSeasonal Oscillation (MISO) prediction (DeMott et al., 2015 and references therein). MJO prediction skill is higher in coupled models compared to uncoupled atmosphere-only models (e.g., Woolnough et al., 2007; Subramanian et al., 2017). The biases in sea surface temperature in operational forecast models are known to impact the operational predictability of MISOs over Indian region (Sahai et al., 2019). Yet, our understanding of the exact processes of air-sea coupling that are crucial to MJO and MISO prediction is incomplete. The diurnal cycle in air-sea coupling associated with the MJO (Seo et al., 2014) and MISO needs to be observed and understood further. The myriad phenomena that govern the S2S variability in the Tropical region (e.g., Tropical Pacific) are depicted in the schematic shown in Figure 1. Comprehensive observations of these processes will help improve their representation and prediction in the S2S forecasting systems. 


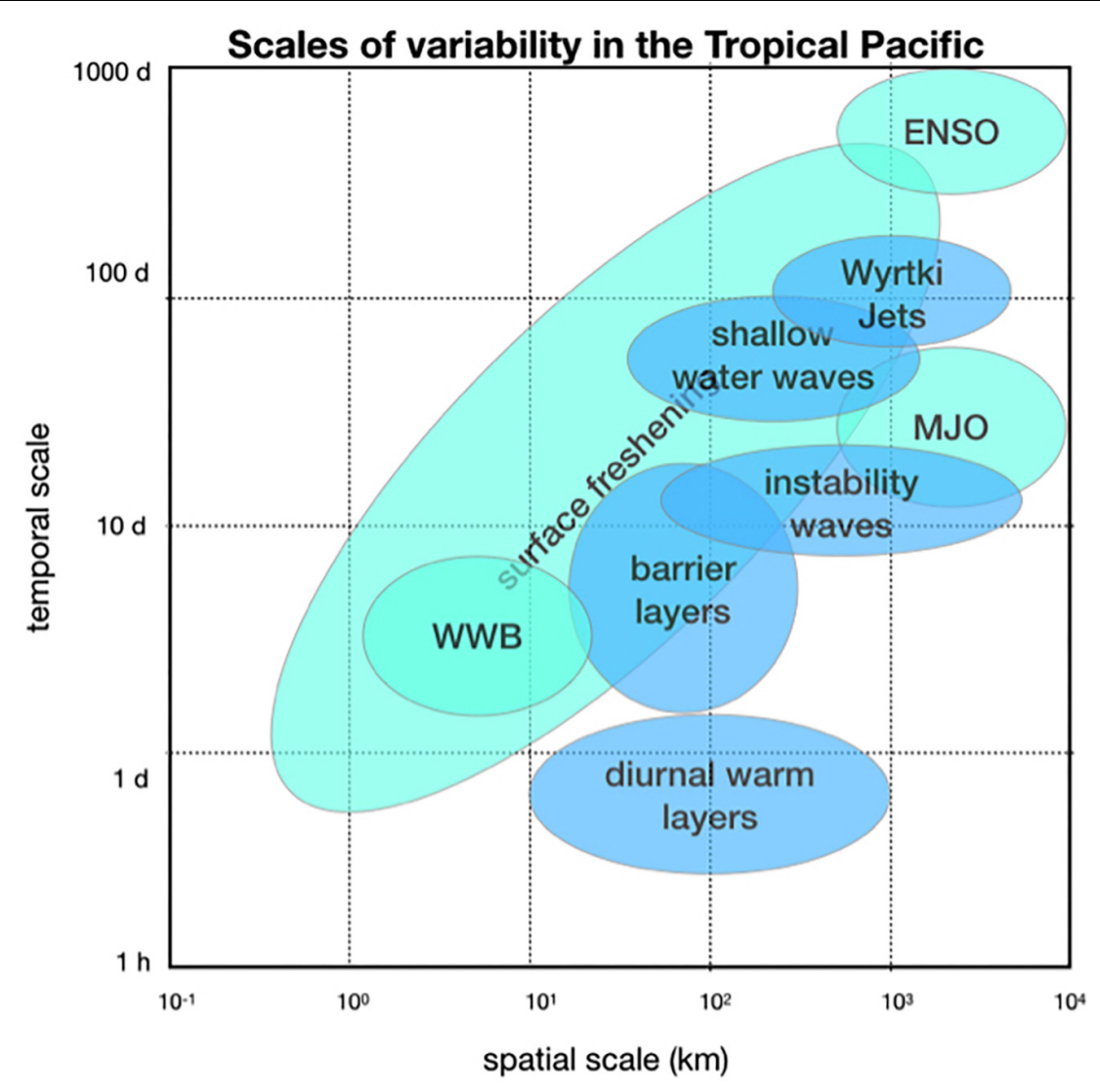

FIGURE 1 | Schematic depicting spatial and temporal scales of phenomena in the ocean-atmosphere coupled system that govern the variability in the Tropical Pacific. Due to limitations in space, we have depicted only a few of the key processes. The blue shaded ovals represent ocean processes and green ovals represent atmospheric processes.

Another outstanding issue related to air-sea coupling is the MJO propagation over the Indo-Pacific Maritime Continent (MC), where some MJO events terminate as consequences of the "barrier effect" of the MC (Zhang and Ling, 2017). This barrier effect is often exaggerated in numerical models, which limits MJO prediction skill. Air-sea interaction in the MC seas plays a key role in determining the MJO propagation through the region. In situ ocean observations are needed for understanding air-sea interaction in the region and their representation in S2S prediction models. The international program Years of the Maritime Continent (YMC, 2017-2020) includes several simultaneous measurements of the upper ocean, atmosphere, and their interactions. These observations provide sorely needed in situ observations from the region.

Predicting sea ice at S2S timescales has also been challenging, but progress is being made (Zampieri et al., 2018). Sea-ice prediction at S2S time scales is challenging in part due to a lack of in situ observations to develop adequate sea-ice models and initialization techniques (Day et al., 2014; Guemas et al., 2014, 2016). The unseasonably early and rapid sea-ice retreat in the Bering Sea in spring 2018 and unprecedented springtime retreat of Antarctic sea ice in 2016 (e.g., Turner et al., 2017) highlight priorities that should be accurately captured by S2S prediction models. Sea-ice-related predictions will not be possible without relevant observations that provide needed initial conditions in terms of upper ocean current and temperature, surface radiation and heat fluxes, surface wind, and wave height in and around the marginal ice zone (MIZ) and underneath sea ice during the sea-ice growing and retreating seasons.

Mid-latitude SST anomalies are a source of predictability on S2S time scales (McKinnon et al., 2016; Saravanan and Chang, 2019 and references therein). Regional warm SST anomalies that persist for 5 days or longer, known as the marine heat waves (Frölicher and Laufkötter, 2018), are being studied as a potential source and target of S2S predictability. Recently, a marine heat wave near the west coast of North America between 2013 and 2015 (Bond et al., 2015; Di Lorenzo and Mantua, 2016; Zaba and Rudnick, 2016) including the 2015-2016 El Niño event persisted for over a season and is being studied as a cause of several regional anomalies in both atmospheric and land conditions. The majority of marine heat waves, however, are not covered by in situ observations. This makes it difficult to understand the physical processes responsible for the formation and termination of the heat waves, let alone their prediction. Representation of marine heat waves requires good observations of air-sea fluxes, as well as the ocean mixed layer. 


\section{NEED FOR NEW OBSERVING TECHNOLOGY FOR S2S PREDICTIONS}

The current global ocean observing system comprises a range of in situ and satellite based observing networks, including moored buoys, Argo profiling floats (Roemmich et al., 2019), surface drifters, satellites, ship-based measurement, and gliders (Testor et al., 2019). In addition, emerging observing technology offers the promise of innovative devices [e.g., Autonomous Surface Vehicles (ASVs)] that can supplement the conventional platforms to fill gaps in ocean observations for S2S prediction.

Moored buoys provide high-frequency sampling to resolve the surface diurnal cycle. Argo profiling floats measure oceanic temperature and salinity profiles throughout the top $2000 \mathrm{~m}$ of the ocean with near global coverage. Surface drifters (Centurioni, 2018) measure mixed-layer currents while also recording sea surface temperature (SST) and sea-level atmospheric pressure (SLP) globally. These different platforms provide near-real time observations through the Global Telecommunication System for initialization of global prediction models. The SLP data from drifters are known to have a significant and positive impact on numerical weather prediction (Centurioni et al., 2017; Horányi et al., 2017). They are extremely valuable for the global in situ ocean observation network that supports the S2S forecasting systems for initialization and verification. Skillful prediction on S2S timescales demands high temporal (hourly) and vertical resolution $(<1 \mathrm{~m})$ observations in the upper ocean (Bernie et al., 2008; Seo et al., 2014).

Current satellites do not provide measurements for all surface variables critical to air-sea fluxes (e.g., surface air temperature, humidity, and downwelling radiation), nor can they measure within the ocean interior. China-France Oceanography SATellite (CFOSAT, launched in 2018) and proposed satellite projects including the European Sea surface KInematics Multiscale monitoring (SKIM) mission for waves and surface currents, as well as a United States concept for a Wind and Currents Mission (WaCM) offer the prospect of enhancing the range of information available from space. One challenge for satellite observations is that most Earth-observing satellites use sunsynchronous orbits, which means that they sample at the same local time (e.g., 6 am and $6 \mathrm{pm}$ ) everywhere, and thus multiple satellites with similar objectives but different local sampling times may be needed to capture diurnal variability or the scales of fast-moving synoptic storms.

Autonomous Surface Vehicles have been developed over the last decade to measure air-sea surface fields. Advanced ASVs can measure surface radiation, air pressure, upper-ocean currents, in addition to the standard surface variables. These are variables important to initialize coupled S2S prediction. Collocated ocean-atmosphere observations using ASV are essential for strongly coupled data assimilation (CDA) (Penny et al., 2019). The ASVs can be used to adaptively target special events (e.g., El Niño development) to supplement existing sources of ocean observations. As another example, ASVs are particularly appropriate for observing the diurnal and subsurface structure of marine heat waves. Hence, the advent of the ASVs to gather collocated ocean-atmosphere boundary layer observations, especially in locations where moored buoys are impractical (e.g., near seasonally migrating sea-ice edge) could be an irreplaceable source of information for both improving the models as well as for coupled assimilation for S2S prediction.

Autonomous underwater gliders (Rudnick, 2016; Testor et al., 2019) provide high-resolution, particularly within boundary current systems (Rudnick et al., 2017; Todd et al., 2019). Glider observations resolve the strong gradients that typify coastal and boundary current systems and have demonstrated impact on operational forecast models (e.g., Todd and Locke-Wynn, 2017). Increased utilization of gliders in boundary and coastal regions with strong gradients can help improve our ability to initialize S2S forecasts that are sensitive to upper-ocean gradients.

Some aspects of the MJO genesis and large-scale evolution are captured by the RAMA moored buoy array in the Indian Ocean and by the TAO array in the tropical Pacific, where MJO events interact with and affect ENSO evolution. However, these arrays do not resolve the gradients of temperature and salinity fields or upper ocean currents that potentially influence S2S predictions (Moum et al., 2016). Another example is the eastern edge of the western Pacific warm pool, where strong zonal gradients of temperature with a sharp salinity front at and near the surface migrate in longitude on the S2S timescales. In these two cases, sustained observation platforms complemented with ASVs are needed to cover the gradients in between fixed moorings. Observations in the atmospheric boundary layer are also required as the zonal migration of the salinity front and SST gradient are driven by intraseasonal fluxes of buoyancy, radiation, water vapor, and stress. The deliberate discussion that is taking place as part of TPOS 2020 on the future of the tropical Pacific observing system is essential for improving the skill of S2S predictions.

Western boundary current regions are areas of strong currents and hence are a challenge for Argo and drifting floats to make sustained observations. Moorings, though challenging to maintain in strong current regions, can still act as an important source of measurements of surface fluxes that are useful for initializing S2S forecasts as well as verifying the forecasts (Cronin et al., 2008; Weller et al., 2012; Bigorre et al., 2013). Gliders are able to deliver the data from these moorings via real-time acoustic telemetry to monitoring and assimilation networks (Send et al., 2013). These should ideally be complemented by more spatially integrated transport and surface flux measurements in these regions for forecast validation. Observational efforts should go hand in hand with modeling and data assimilation methods to help improve predictions in these regions.

Although assimilation of sea-ice concentration data into S2S prediction models has started in several operational centers, assimilation of sea-ice thickness is rarely performed due to the limitations of ice thickness data. Sea-ice thickness, while difficult to measure via remote sensing, is an important variable in sea-ice prediction (Blockley and Peterson, 2018). Water and air properties surrounding sea-ice are needed for seaice prediction. Currently, there are no routine observations taken near the edge of sea-ice nor in the transition marginal ice zone (MIZ) between sea ice and open water (Lee et al., 2017; Nguyen et al., 2017). Permanent moored subsurface buoys and ice-tethered profilers could be a key source of time-series 
data in these data sparse regions. However, mobile vehicles (ASVs, and subsurface gliders) are dynamic platforms that can monitor surface and subsurface conditions following the seasonal migration of sea ice. The value of data collected by these mobile vehicles in polar regions to S2S prediction needs to be explored.

\section{OBSERVING SYSTEM EXPERIMENTS TO EVALUATE OBSERVATION NETWORKS AND S2S MODELS}

Ocean observing system experiments are conducted at ECMWF and other global forecasting centers to evaluate the use of ocean observations in ocean reanalyses (Balmaseda et al., 2007; Fujii et al., 2015; Xue et al., 2017). In these ocean reanalyses produced at ECMWF, specific observing systems are withdrawn in a controlled manner. We call these experiments OSEORAs (Observing System Experiments - Ocean ReAnalyses). Differences between OSE-ORAs give information on the possible impact of a given observation type on the mean and variability of the reanalyses. The impact of observations on forecasts could in principle be further quantified by using these OSE-ORAs to initialize coupled forecast, although this assessment may be hindered by low statistical significance and issues related to model biases. This is the approach followed in previous studies targeting seasonal time scales
(Balmaseda and Anderson, 2009; Balmaseda et al., 2010; Fujii et al., 2011).

It is important to use a metric with significant level of skill and clearly influenced by the ocean state. Experiments have been conducted to quantify the impact of altimeter-derived sea level anomaly on sub-seasonal forecasts of tropical cyclone activity. The impact is measured by the differences between two sets of sub-seasonal reforecasts spanning the period 1994-2016, which differ only in the ocean initial conditions. The reference experiment uses the operational ocean reanalyses ORAS5 (Zuo et al., 2019), where all the ocean observing systems are used. The second experiment is initialized using a variant of ORAS5 where the altimeter sea level data are withdrawn from the assimilation, keeping everything else the same as in ORAS5. Figure 2 shows that by withdrawing the altimeter sea-level from the ocean initial conditions, the forecast of tropical cyclones is degraded in the 16-45-day time range. Correlations between observed and predicted Accumulated Cyclone Energy (ACE) during the monthly period are lower over the North Atlantic, the eastern North Pacific, the western north Pacific and the South Pacific in the experiment initialized with the assimilation without altimeter data than in the control experiment. The assessment of the adequacy of the current ocean observing system for the subseasonal range will be a focus activity of the WWRP/WCRP subseasonal to seasonal prediction project (S2S) in the next few years. Hence, the engagement of the ocean observations community with this WMO activity would be very beneficial for all involved.

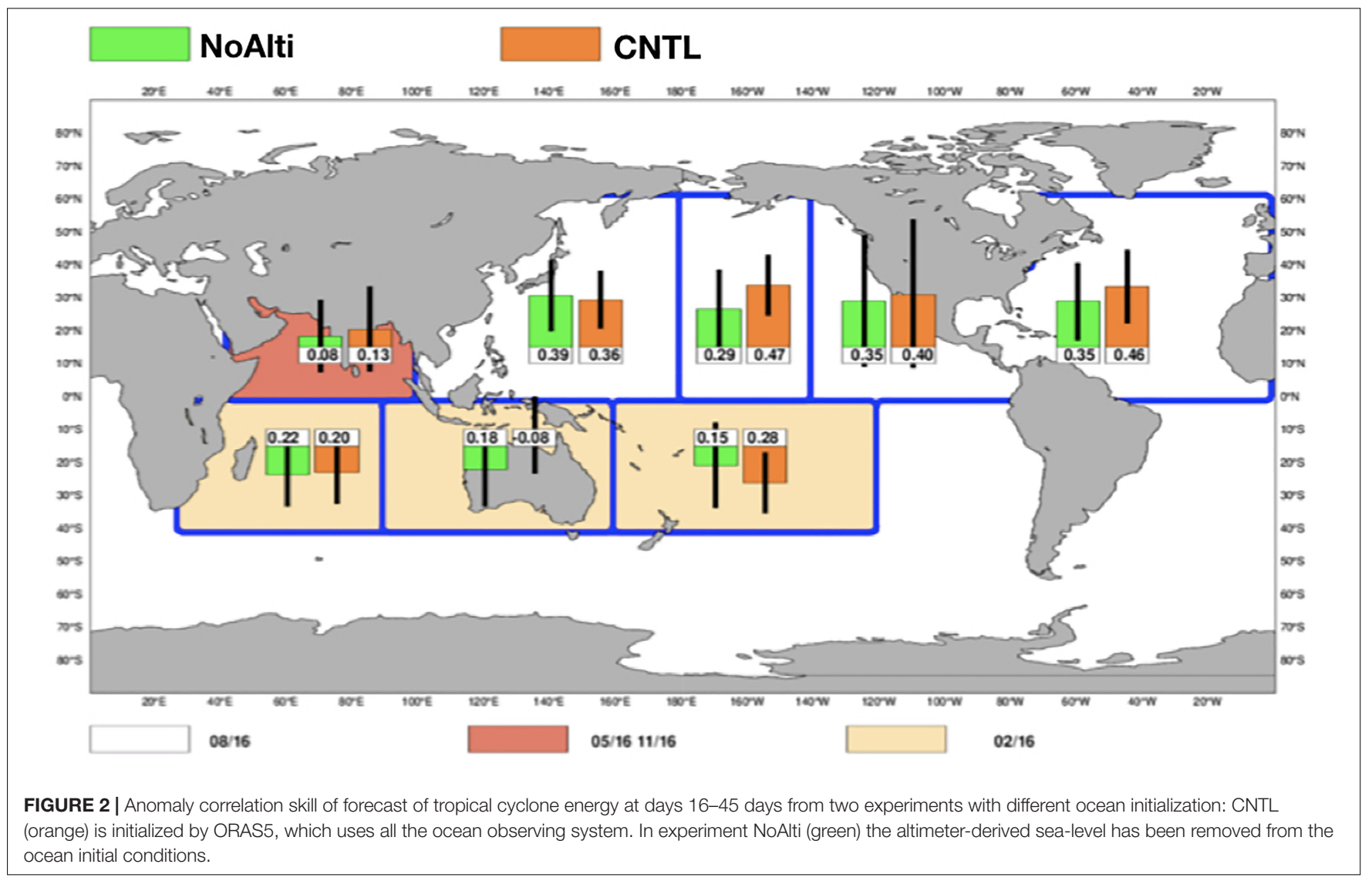




\section{CONCLUSION}

Global S2S forecasting systems are evolving rapidly in centers around the world. Their success critically depends on the development of a CDA capability that demands adequate observations of the upper ocean and ocean-atmosphere interface.

The ocean is under-observed relative to the number of routine observations collected in the atmosphere over land. The subsurface ocean and many variables at the ocean surface are hard, if possible, to observe with satellite remote sensing technologies. The surface and upper-ocean observing systems relevant for understanding, modeling and forecasting on S2S timescales will continue to evolve with the improvement in technological capabilities. Current and future challenges in ocean observing system designs and implementations are complicated by the desire to balance resources for routine ocean observation systems (e.g., moorings, Argo, and satellites) with new and more sophisticated technologies (e.g., ASVs). Hence, the community must focus on designing and implementing cost-effective surface and subsurface ocean observations with a combination of various technologies that provide needed data for initialization and validation of S2S prediction systems as well as advancing understanding of ocean-atmosphere coupling on S2S timescales.

The community can advance the skill of S2S forecasts significantly by focusing next generation ocean observations on higher temporal and spatial resolution observations in the ocean-atmosphere boundary layers in key regions hosting known sources of S2S predictability (Tropics, coastal regions and polar regions) including the diurnal cycle of air-sea coupling and mixed-layer structure as highlighted by studies referenced in the above sections.

Breakthroughs in the accuracy of S2S predictions are finally within reach with the current generation coupled forecasting systems. New tools from CDA and advanced satellite and autonomous technology to observe the oceanatmosphere boundary layers will make it possible to integrate global observations and local high-resolution simulations in S2S forecasting systems. Scientific, computational, and observation

\section{REFERENCES}

Alexander, M. A., Deser, C., and Timlin, M. S. (1999). The reemergence of SST anomalies in the North Pacific Ocean. J. Clim. 12, 2419-2433. doi: 10.1175/ 1520-0442(1999)012<2419:trosai $>2.0$. co;2

Balmaseda, M., Alves, O., Awaji, T., Behringer, D., Ferry, N., Fujii, Y., et al. (2010). "Initialization for seasonal and decadal forecasts," in OceanObs 09 (Paris: European Space Agency), 19-26.

Balmaseda, M., and Anderson, D. (2009). Impact of initialization strategies and observations on seasonal forecast skill. Geophys. Res. Lett. 36:L01701. doi: 10. 1029/2008GL035561

Balmaseda, M., Anderson, D., and Vidard, A. (2007). Impact of Argo on analyses of the global ocean. Geophy. Res. Lett. 34:L16605.

Bernie, D. J., Guilyardi, E., Madec, G., Slingo, J. M., Woolnough, S. J., and Cole, J. (2008). Impact of resolving the diurnal cycle in an ocean-atmosphere GCM. Part 2: A diurnally coupled CGCM. Clim. Dyn. 31, 909-925. doi: 10.1007/ s00382-008-0429-z

Bigorre, S. P., Weller, R. A., Edson, J. B., and Ware, J. D. (2013). A surface mooring for air-sea interaction research in the Gulf Stream. Part II: analysis of technology challenges need to be addressed to accomplish these required advances in the coming decade. While these challenges are substantial, the revolution in autonomous observing systems and computational breakthroughs focused on addressing high priority S2S prediction problems can help make key progress possible. This will not only benefit the scientific community but also society as a whole by providing climate information that can change the world of climate-data driven decision-making.

\section{AUTHOR CONTRIBUTIONS}

AS led the writing of the manuscript. FV and MB contributed to the analysis and creation of Figure 2. CD and AS contributed to creation of Figure 1. DG contributed to the text of the manuscript. All authors contributed to the text of the manuscript.

\section{FUNDING}

AS was funded by NOAA Climate Variability and Prediction Program (NA14OAR4310276) and the NSF Earth System Modeling Program (OCE1419306). CD was funded by NA16OAR4310094. SG and DG were funded by NASA awards NNX14AO78G and 80NSSC19K0059. DY was supported by NSFC (91858204, 41720104008, and 41421005).

\section{ACKNOWLEDGMENTS}

The authors would like to thank support by a grant from NOAA Climate Variability and Prediction Program (NA14OAR4310276) and the NSF Earth System Modeling Program (OCE1419306). PMEL contribution number 4888. CD was funded by NA16OAR4310094. SG and DG were funded by NASA awards NNX14AO78G and 80NSSC19K0059. DY was supported by NSFC (91858204, 41720104008, and 41421005).

the observations and their accuracies. J. Atmos. Oceanic Technol. 30, 450-469. doi: 10.1175/jtech-d-12-00078.1

Blockley, E. W., and Peterson, K. A. (2018). improving met office seasonal predictions of Arctic sea ice using assimilation of CryoSat-2 thickness. Cryosphere 12, 3419-3438. doi: 10.5194/tc-12-3419-2018

Bond, N. A., Cronin, M. F., Freeland, H., and Mantua, N. J. (2015). Causes and impacts of the 2014 warm anomaly in the NE Pacific. Geophy. Res. Lett. 42, 3414-3420. doi: 10.1002/2015GL063306

Centurioni, L., Horányi, A., Cardinali, C., Charpentier, E., and Lumpkin, R. (2017). A global ocean observing system for measuring sea level atmospheric pressure: effects and impacts on numerical weather prediction. Bull. Am. Meteorol. Soc. 98, 231-238. doi: 10.1175/bams-d-1500080.1

Centurioni, L. R. (2018). "Drifter technology and impacts for sea surface temperature, sea-level pressure, and ocean circulation studies," in Observing the Oceans in Real Time, eds R. Venkatesan, A. Tandon, E. D'Asaro, and M. A. Atmanand (Cham: Springer International Publishing).

Cronin, M. F., Meinig, C., Sabine, C. L., Ichikawa, H., and Tomita, H. (2008). Surface mooring network in the Kuroshio Extension. IEEE Syst. J. 2, 424-430. doi: 10.1109 /jsyst.2008.925982 
Day, J. J., Hawkins, E., and Tietsche, S. (2014). Will Arctic sea ice thickness initialization improve seasonal forecast skill? Geophy. Res. Lett. 41, 7566-7575. doi: $10.1002 / 2014$ gl061694

DeMott, C. A., Klingaman, N. P., and Woolnough, S. J. (2015). Atmosphereocean coupled processes in the Madden-Julian oscillation. Rev. Geophys. 53, 1099-1154. doi: 10.1002/2014rg000478

Di Lorenzo, E., and Mantua, N. (2016). Multi-year persistence of the 2014/15 North Pacific marine heatwave. Nat. Clim. Change 11, 1042-1047. doi: 10.1038/ nclimate 3082

Foltz, G. R., Brandt, P., Richter, I., Rodríguez-Fonseca, B., Hernandez, F., Dengler, M., et al. (2019). The tropical Atlantic observing system. textitFront. Mar. Sci. 6:206. doi: 10.3389/fmars.2019.00206

Frölicher, T. L., and Laufkötter, C. (2018). Emerging risks from marine heat waves. Nat. Commu. 9:650. doi: 10.1038/s41467-018-03163-6

Frolov, S., Bishop, C. H., Holt, T., Cummings, J., and Kuhl, D. (2016). Facilitating strongly coupled ocean-atmosphere data assimilation with an interface solver. Mon. Wea. Rev. 144, 3-20. doi: 10.1175/MWR-D-150041.1

Fujii, Y., Cummings, J., Xue, Y., Schiller, A., Lee, T., and Balmaseda, M. A. (2015). Evaluation of the Tropical Pacific Observing System from the ocean data assimilation perspective. Quart. J. Roy. Meteor. Soc. 141, 2481-2496. doi: 10.1002/qj.2579

Fujii, Y., Kamachi, M., Nakaegawa, T., Yasuda, T., Yamanaka, G., Toyoda, T., et al. (2011). "Assimilating ocean observation data for ENSO monitoring and forecasting," in Climate Variability - Some Aspects, Challenges and Prospects, ed. A. Hannachi (Rijeka: InTech Open), 75-98. doi: 10.5772/30330

Fujii, Y., Rémy, E., Zuo, H., Oke, P., Halliwell, G., Gasparin, F., et al. (2019). Observing system evaluationbased on ocean data assimilation and prediction systems: on-going challenges and a future vision for designing and supporting ocean observational networks. Front. Mar. Sci. 6:417. doi: 10.3389/fmars.2019. 00417

Guemas, V., Blanchard-Wrigglesworth, E., Chevallier, M., Day, J. J., Déqué, M., Doblas-Reyes, F. J., et al. (2016). A review on Arctic sea-ice predictability and prediction on seasonal to decadal time-scales. Q. J. R. Meteorol. Soc. 142, 546-561. doi: 10.1002/qj.2401

Guemas, V., Doblas-Reyes, F. J., Mogensen, K., Keeley, S., and Tang, Y. (2014). Ensemble of sea ice initial conditions for interannual climate predictions. Clim. Dyn. 43, 2813-2829. doi: 10.1007/s00382-0142095-7

Horányi, A., Cardinali, C., and Centurioni, L. (2017). The global numerical weather prediction impact of mean-sea-level pressure observations from drifting buoys. Q. J. R. Meteorol. Soc. 143, 974-985. doi: 10.1002/qj.2981

Jin, F. F. (1997). An equatorial ocean recharge paradigm for ENSO. Part I: conceptual model. J. Atmos. Sci. 54, 811-829. doi: 10.1175/1520-0469(1997) 054<0811:aeorpf $>2.0 . \mathrm{co} ; 2$

Laloyaux, P., Balmaseda, M., Dee, D., Mogensen, K., and Janssen, P. (2016). A coupled data assimilation system for climate reanalysis. Q. J. Roy. Meteor. Soc. 142, 65-78. doi: 10.1002/qj.2629

Lea, D. J., Mirouze, I., Martin, M. J., King, R. R., Hines, A., Walters, D., et al. (2015). Assessing a new coupled data assimilation system based on the met office coupled atmosphere-land-ocean-sea ice model. Mon. Wea. Rev. 143, 4678-4694. doi: 10.1175/mwr-d-15-0174.1

Lee, C. M., Thomson, J., and The Marginal Ice Zone Team, and Arctic Sea State Teams (2017). An autonomous approach to observing the seasonal ice zone in the western Arctic. Oceanography 30, 56-68. doi: 10.5670/oceanog.2017.222

McKinnon, K. A., Rhines, A., Tingley, M. P., and Huybers, P. (2016). Longlead predictions of eastern United States hot days from Pacific sea surface temperatures. Nat. Geosci. 9:389. doi: 10.1038/ngeo2687

Meinen, C. S., and McPhaden, M. J. (2000). Observations of warm water volume changes in the equatorial Pacific and their relationship to El Niño and La Niña. J. Clim. 13, 3551-3559. doi: 10.1175/1520-0442(2000)013<3551:oowwvc $>$ 2.0.co; 2

Moum, J. N., Pujiana, K., Lien, R. C., and Smyth, W. D. (2016). Ocean feedback to pulses of the Madden-Julian oscillation in the equatorial Indian Ocean. Nat. Commun. 7:13203. doi: 10.1038/ncomms13203

Nguyen, A. T., Ocaña, V., Garg, V., Heimbach, P., Toole, J. M., Krishfield, R. A., et al. (2017). On the benefit of current and future ALPS data for improving
Arctic coupled ocean-sea-ice state estimation. Oceanography 30, 69-73. doi: 10.5670/oceanog.2017.223

Penny, S. G., Akella, S., Balmaseda, M. A., Browne, P., Carton, J. A., Chevallier, M., et al. (2019). Observational needs for improving ocean and coupled reanalysis, S2S prediction, and decadal prediction. Front. Mar. Sci. 6:391. doi: 10.3389/ fmars.2019.00391

Penny, S. G., and Hamill, T. M. (2017). Coupled data assimilation for integrated earth system analysis and prediction. Bull. Am. Meteorol. Soc. 97, ES169-ES172.

Roemmich, D., Alford, M. H., Claustre, H., Johnson, K. S., King, B., Moum, J., et al. (2019). On the future of Argo: a global, full-depth, multi-disciplinary array. Front. Mar. Sci. doi: 10.3389/fmars.2019.00439

Rudnick, D. L. (2016). Ocean research enabled by underwater gliders. Ann. Rev. Marine Sci. 8, 519-541. doi: 10.1146/annurev-marine-122414033913

Rudnick, D. L., Zaba, K. D., Todd, R. E., and Davis, R. E. (2017). A climatology of the California current system from a network of underwater gliders. Progr. Oceanogr. 154, 64-106. doi: 10.1016/j.pocean.2017. 03.002

Sahai, A. K., Chattopadhyay, R., Susmitha, J., Phani, M. K., Pattanaik, D. R., and Abhilash, S. (2019). "Chapter 20 - seamless prediction of monsoon onset and active/break phases," in Sub-Seasonal to Seasonal Prediction, eds A. W. Robertson, F. Vitart, and A. Robertson (Amsterdam: Elsevier), 421-438.

Saravanan, R., and Chang, P. (2019). "Midlatitude mesoscale oceanatmosphere interaction and its relevance to S2S prediction," in Sub-Seasonal to Seasonal Prediction, eds A. W. Robertson and V. Frédéric (Amsterdam: Elsevier), 183-200. doi: 10.1016/b978-0-12-811714-9. 00009-7

Send, U., Regier, L., and Jones, B. (2013). Use of underwater gliders for acoustic data retrieval from subsurface oceanographic instrumentation and bidirectional communication in the deep ocean. J. Atmos. Oceanic Technol. 30, 984-998. doi: 10.1175/JTECH-D-11-00169.1

Seo, H., Subramanian, A. C., Miller, A. J., and Cavanaugh, N. R. (2014). Coupled impacts of the diurnal cycle of sea surface temperature on the Madden-Julian oscillation. J. Clim. 27, 8422-8443. doi: 10.1175/jcli-d-1400141.1

Smith, N., Kessler, W. S., Cravatte, S., Sprintall, J., Wijffels, S., Cronin, M. F., et al. (2019). Tropical Pacific observing system. Front. Mar. Sci. 6:31. doi: 10.3389/ fmars.2019.00031

Subramanian, A. C., Vitart, F., Zhang, C., Kumar, A., and Balmaseda, M. A. (2017). Ocean observations for operational subseasonal and seasonal forecasts. (in press).

Testor, P., DeYoung, B., Rudnick, D. L., Glenn, S., Hayes, D., Lee, C., et al. (2019). OceanGliders: A component of the integrated GOOS. Front. Marine Sci. doi: 10.3389/fmars.2019.00422

Todd, R. E., Chavez, F. P., Clayton, S., Cravatte, S. E., Goes, M. P., Graco, M. I., et al. (2019). Global perspectives on observing ocean boundary current systems. Front. Marine Sci. doi: 10.3389/fmars.2019.00423

Todd, R. E., and Locke-Wynn, L. (2017). Underwater glider observations and the representation of western boundary currents in numerical models. Oceanography 30, 88-89. doi: 10.5670/oceanog. 2017.225

Turner, J., Phillips, T., Marshall, G. J., Hosking, J. S., Pope, J. O., Bracegirdle, T. J., et al. (2017). Unprecedented springtime retreat of Antarctic sea-ice in 2016. Geophys. Res. Lett. 44, 6868-6875. doi: 10.1002/2017GL073656

Vimont, D. J., Battisti, D. S., and Hirst, A. C. (2001). Footprinting: a seasonal connection between the tropics and mid-latitudes. Geophys. Res. Lett. 28, 3923-3926. doi: 10.1029/2001gl013435

Vitart, F., Robertson, A. W., and Anderson, D. L. (2012). Subseasonal to Seasonal Prediction Project: bridging the gap between weather and climate. WMO Bull. 61, 23-28.

Weller, R. A., Bigorre, S. P., Lord, J., Ware, J. D., and Edson, J. B. (2012). A surface mooring for air-sea interaction research in the Gulf Stream. Part I: Mooring design and instrumentation. J. Atmos. Oceanic Technol. 29, 1363-1376. doi: 10.1175/jtech-d-12-00060.1

Woolnough, S. J., Vitart, F., and Balmaseda, M. A. (2007). The role of the ocean in the Madden-Julian Oscillation: implications for MJO prediction. Q. J. R. Meteorol. Soc. 133, 117-128. doi: 10.1002/qj.4 
Xue, Y., Wen, C., Yang, X., Behringer, D., Kumar, A., Vecchi, G., et al. (2017). Evaluation of tropical Pacific observing systems using NCEP and GFDL ocean data assimilation systems. Clim. Dyn. 49, 843-868. doi: 10.1007/s00382-0152743-6

Yeh, S.-W., Cai, W., Min, S.-K., McPhaden, M. J., Dommenget, D., and Dewitte, B. (2018). ENSO atmospheric teleconnections and their response to greenhouse gas forcing. Rev. Geophy. 56, 185-206. doi: 10.1002/2017RG00 0568

Zaba, K. D., and Rudnick, D. L. (2016). The 2014-2015 warming anomaly in the Southern California current system observed by underwater gliders. J. Geophy. Res.: Oceans 2016, 1241-1248. doi: 10.1002/2015gl06 7550

Zampieri, L., Goessling, H. F., and Jung, T. (2018). Bright prospects for arctic sea ice prediction on subseasonal time scales. Geophys. Res. Lett. 45, 9731-9738. doi: $10.1029 / 2018 \mathrm{gl} 079394$

Zhang, C., and Ling, J. (2017). Barrier effect of the Indo-Pacific maritime continent on the MJO: perspectives from tracking MJO precipitation. J. Clim. 30, 34393459. doi: 10.1175/jcli-d-16-0614.1
Zuo, H., Balmaseda, M. A., Tietsche, S., Mogensen, K., and Mayer, M. (2019) The ECMWF operational ensemble reanalysis-analysis system for ocean and sea ice: a description of the system and assessment. Ocean Sci. 15, 779-808. doi: 10.5194/os-15-779-2019

Conflict of Interest Statement: The authors declare that the research was conducted in the absence of any commercial or financial relationships that could be construed as a potential conflict of interest.

Copyright (c) 2019 Subramanian, Balmaseda, Centurioni, Chattopadhyay, Cornuelle, DeMott, Flatau, Fujii, Giglio, Gille, Hamill, Hendon, Hoteit, Kumar, Lee, Lucas, Mahadevan, Matsueda, Nam, Paturi, Penny, Rydbeck, Sun, Takaya, Tandon, Todd, Vitart, Yuan and Zhang. This is an open-access article distributed under the terms of the Creative Commons Attribution License (CC BY). The use, distribution or reproduction in other forums is permitted, provided the original author(s) and the copyright owner(s) are credited and that the original publication in this journal is cited, in accordance with accepted academic practice. No use, distribution or reproduction is permitted which does not comply with these terms. 


\section{A Framework for the Development, Design and Implementation of a Sustained Arctic Ocean Observing System}

Craig M. Lee ${ }^{1 *}$, Sandy Starkweather 2,3 , Hajo Eicken ${ }^{4}$, Mary-Louise Timmermans ${ }^{5}$, Jeremy Wilkinson ${ }^{6}$, Stein Sandven ${ }^{7}$, Dmitry Dukhovskoy ${ }^{8}$, Sebastian Gerland ${ }^{9}$, Jacqueline Grebmeier ${ }^{10}$, Janet M. Intrieri ${ }^{3}$, Sung-Ho Kang ${ }^{11}$, Molly McCammon ${ }^{12}$, An T. Nguyen ${ }^{13}$, Igor Polyakov ${ }^{4}$, Benjamin Rabe ${ }^{14}$, Hanne Sagen ${ }^{7}$, Sophie Seeyave ${ }^{15}$,

OPEN ACCESS

Edited by:

Tong Lee,

NASA Jet Propulsion Laboratory

(JPL), United States

Reviewed by:

Patrick Heimbach,

The University of Texas at Austin,

United States

Ananda Pascual,

Instituto Mediterráneo de Estudios

Avanzados (IMEDEA), Spain

${ }^{*}$ Correspondence:

Craig M. Lee

craiglee@uw.edu

Specialty section:

This article was submitted to

Physical Oceanography,

a section of the journal

Frontiers in Marine Science

Received: 12 February 2019

Accepted: 05 July 2019

Published: 08 August 2019

Citation:

Lee CM, Starkweather S,

Eicken $H$, Timmermans $M-L$,

Wilkinson J, Sandven S,

Dukhovskoy D, Gerland $S$,

Grebmeier J, Intrieri JM, Kang S-H,

McCammon M, Nguyen AT,

Polyakov I, Rabe B, Sagen H,

Seeyave $S$, Volkov $D$,

Beszczynska-Möller A, Chafik L,

Dzieciuch M, Goni G, Hamre T,

King AL, Olsen A, Raj RP, Rossby T,

Skagseth Ø, Søiland $H$ and

Sørensen K (2019) A Framework

for the Development, Design and Implementation of a Sustained

Arctic Ocean Observing System.

Front. Mar. Sci. 6:451.

doi: 10.3389/fmars.2019.00451
Denis Volkov 16,17, Agnieszka Beszczynska-Möller ${ }^{18}$, Léon Chafik ${ }^{19}$, Matthew Dzieciuch ${ }^{20}$, Gustavo Goni" ${ }^{16}$, Torill Hamre7, Andrew Luke King ${ }^{21}$, Are Olsen ${ }^{22}$, Roshin P. Raj', Thomas Rossby ${ }^{23}$, Øystein Skagseth ${ }^{24}$, Henrik Søiland ${ }^{24}$ and Kai Sørensen ${ }^{21}$

${ }^{1}$ Applied Physics Laboratory, University of Washington, Seattle, WA, United States, ${ }^{2}$ Cooperative Institute for Research in Environmental Sciences, University of Colorado, Boulder, Boulder, CO, United States, ${ }^{3}$ Earth Systems Research Laboratory, National Oceanographic and Atmospheric Administration, Boulder, CO, United States, ${ }^{4}$ International Arctic Research Center, University of Alaska Fairbanks, Fairbanks, AK, United States, ${ }^{5}$ Department of Geology and Geophysics, Yale University, New Haven, CT, United States, ${ }^{6}$ British Antarctic Survey, Cambridge, United Kingdom, ${ }^{7}$ Nansen Environmental and Remote Sensing Center, Bergen, Norway, ${ }^{8}$ Center for Ocean-Atmospheric Prediction Studies, Florida State University, Tallahassee, FL, United States, ${ }^{9}$ Norwegian Polar Institute, Fram Centre, Tromsø, Norway, ${ }^{10}$ Center for Environmental Science, University of Maryland, Solomons, MD, United States, ${ }^{11}$ Korean Polar Research Institute, Incheon, South Korea, ${ }^{12}$ Alaska Ocean Observing System, Anchorage, AK, United States, ${ }^{13}$ Oden Institute, The University of Texas at Austin, Austin, TX, United States, ${ }^{14}$ Alfred-Wegener-Institut Helmholtz-Zentrum für Polar- und Meeresforschung, Bremerhaven, Germany, ${ }^{15}$ Partnership for Observation of the Global Oceans, Plymouth Marine Laboratory, Plymouth, United Kingdom, ${ }^{16}$ Physical Oceanography Division, Atlantic Oceanographic and Meteorological Laboratory, National Oceanic and Atmospheric Administration, Miami, FL, United States, ${ }^{17}$ Cooperative Institute for Marine and Atmospheric Studies, University of Miami, Miami, FL, United States, ${ }^{18}$ Institute of Oceanology, Polish Academy of Sciences, Sopot, Poland, ${ }^{19}$ Department of Meteorology and Bolin Centre for Climate Research, Stockholm University, Stockholm, Sweden,

${ }^{20}$ Institute of Geophysics and Planetary Physics, Scripps Institution of Oceanography, La Jolla, CA, United States, ${ }^{21}$ Marine Biogeochemistry and Oceanography, Norwegian Institute for Water Research, Oslo, Norway, ${ }^{22}$ Bjerknes Centre for Climate Research, University of Bergen, Bergen, Norway, ${ }^{23}$ Graduate School of Oceanography, The University of Rhode Island, Narragansett, RI, United States, ${ }^{24}$ Oceanography and Climate, Institute of Marine Research, Bergen, Norway

Rapid Arctic warming drives profound change in the marine environment that have significant socio-economic impacts within the Arctic and beyond, including climate and weather hazards, food security, transportation, infrastructure planning and resource extraction. These concerns drive efforts to understand and predict Arctic environmental change and motivate development of an Arctic Region Component of the Global Ocean Observing System (ARCGOOS) capable of collecting the broad, sustained observations needed to support these endeavors. This paper provides a roadmap for establishing the ARCGOOS. ARCGOOS development must be underpinned by a broadly endorsed framework grounded in high-level policy drivers and the scientific and operational objectives that stem from them. This should be guided by a transparent, internationally accepted governance structure with recognized authority and organizational relationships with the national agencies that ultimately execute network plans. A governance model for ARCGOOS must guide selection of objectives, assess performance and fitness-to-purpose, and advocate for resources. A requirements-based framework for an ARCGOOS begins with the Societal Benefit 
Areas (SBAs) that underpin the system. SBAs motivate investments and define the system's science and operational objectives. Objectives can then be used to identify key observables and their scope. The domains of planning/policy, strategy, and tactics define scope ranging from decades and basins to focused observing with near real time data delivery. Patterns emerge when this analysis is integrated across an appropriate set of SBAs and science/operational objectives, identifying impactful variables and the scope of the measurements. When weighted for technological readiness and logistical feasibility, this can be used to select Essential ARCGOOS Variables, analogous to Essential Ocean Variables of the Global Ocean Observing System. The Arctic presents distinct needs and challenges, demanding novel observing strategies. Cost, traceability and ability to integrate region-specific knowledge have to be balanced, in an approach that builds on existing and new observing infrastructure. ARCGOOS should benefit from established data infrastructures following the Findable, Accessible, Interoperable, Reuseable Principles to ensure preservation and sharing of data and derived products. Linking to the Sustaining Arctic Observing Networks (SAON) process and involving Arctic stakeholders, for example through liaison with the International Arctic Science Committee (IASC), can help ensure success.

Keywords: Arctic, observing system, Essential Ocean Variable, autonomous platforms, observing system design, societal benefit areas, Sustaining Arctic Observing Networks, Global Ocean Observing System

\section{INTRODUCTION}

\section{Motivation}

Nowhere on Earth are the effects of climate change more apparent than in the Arctic. Long-term temperature records clearly show that the region is warming at more than double the global average (Richter-Menge et al., 2018; Box et al., 2019), an effect known as Arctic amplification. The rapid warming, identified in early climate models (e.g., Manabe and Stouffer, 1980), impacts all areas of the Arctic, from the tundra to the

Abbreviations: AMAP, Arctic Monitoring and Assessment Programme; ARCGOOS, Arctic Region Component of the Global Ocean Observing System; CAFF, Conservation of Flora and Fauna; CBMP, Circumpolar Biodiversity Monitoring Program; DA, Data Assimilation; DBO, Distributed Biological Observatory; EAV, Essential ARCGOOS Variable; ECV, Essential Climate Variable; EOV, Essential Ocean Variable; FOO, Framework for Ocean Observing; FRAM, Frontiers in Arctic Marine Monitoring; GOOS, Global Ocean Observing System; GEO, Group on Earth Observations; GEOBON, GEO Biodiversity Observation Network; GEOCRI, GEO Cold Regions Initiative; GEOSS, Global Earth Observation System of Systems; GRA, GOOS Regional Alliance; IAOAF, International Arctic Observing Assessment Framework; IASC, International Arctic Science Committee; IMOBAR, Impact Assessment on a Long-Term Investment on Arctic Observations; INTAROS, Integrated Arctic Observation System; IOC, Intergovernmental Oceanographic Commission; IOOS-AOOS, US Integrated Ocean Observing System - Alaska Ocean Observing System; IPY, International Polar Year; NABOS, Nansen and Amundsen Basins Observational System; NR, Nature Run; OED, Optimal Experimental Design; OSE, Observing System Experiment; OSSE, Observing System Simulation Experiment; PAG, Pacific Arctic Group; PAME, Protection of the Arctic Marine Environment; POGO, Partnership for Observation of the Global Ocean; SAON, Sustaining Arctic Observing Networks; SBA, Societal Benefit Area; SCAR, Scientific Committee on Antarctic Research; SCOR, Scientific Committee on Oceanic Research; SDM, Structured Decision-Making; SDWG, Sustainable Development Working Group; SOOS, Southern Ocean Observing System; STPI, Science and Technology Policy Institute; UNFCCC, United Nations Framework Convention on Climate Change; WMO, World Meteorological Organization. highest mountains, from to the deepest part of the Arctic Ocean through to the upper levels of the atmosphere (AMAP, 2017). The speed of these changes is particularly worrying, as most have occurred within a generation, thus making adaptation for the people, animals and plants that live in the region particularly challenging (Crépin et al., 2017).

It is fair to say that the Arctic marine environment has borne the brunt of these changes. For example, a key indicator of climate change, summertime sea ice extent, has declined substantially. Satellite measurements have revealed that sea ice extent has declined by roughly a half, due to melting and export, since the late 1970s; with the 12 lowest minimum sea ice extents all occurring in the last 12 years (Stroeve and Notz, 2018). The remaining ice is thinner (Lindsay and Schweiger, 2015) and more mobile (e.g., Rampal et al., 2011), and the area of thick multiyear ice has significantly reduced (Kwok, 2018). The Arctic is rapidly moving toward a seasonal ice cover.

Regionally, a warmer Arctic will accelerate sea ice loss, increase coastal erosion, alter ecosystem dynamics, increase glacial runoff and enhance permafrost decay, thus impacting the people (and infrastructure) that rely on the Arctic marine environment for their livelihood (AMAP, 2017). These changes are setting off complex chain reactions that have far-reaching environmental, social and economic impacts extending far beyond the region. A recent socio-economic analysis, performed within the EU funded ICE-ARC (Ice, Climate, Economics- Arctic Research on Change) program, calculated that compared with previous estimates, acceleration of climate change driven by thawing Arctic land permafrost and melting sea ice and land snow could cause up to USD $\$ 70$ trillion worth of extra economic losses globally over the next three centuries under the levels of 
mitigation ambition consistent with the current national pledges, known as Nationally Determined Contributions (NDCs) (total climate change cost of this scenario: $\$ 1400$ trillion). Importantly, their analysis revealed that if global warming is limited to $1.5^{\circ} \mathrm{C}$ in 2100 in line with the Paris Climate Agreement, the additional cost will be reduced to $\$ 25$ trillion (total climate change cost of this scenario: \$600 trillion) (Yumashev et al., 2019).

However, because our projected greenhouse gas emissions path is not clear, the extent and impact of future changes in the Arctic marine environment remain uncertain. At present the most likely scenario is projected to cause a global average warming of $3.0^{\circ} \mathrm{C}$ above pre-industrial temperatures by 2100 , and very likely an ice-free summer Arctic before the mid-century (Sigmond et al., 2018). If global average temperatures stabilize at $1.5^{\circ} \mathrm{C}$ above the pre-industrial levels, as stipulated in the Paris Agreement, the chances for an icefree summer are predicted to be quite low, about 2.5\% (Jahn, 2018). It is important to realize that even under a $1.5^{\circ} \mathrm{C}$ warmer world the Arctic temperatures will rise up to $5^{\circ} \mathrm{C}$ (Schlosser et al., 2016).

Understanding the full extent of changes underway, predicting their impacts, and positioning to exploit new opportunities requires a sustained effort to collect and interpret a great volume of highly diverse information. This paper aims to provide a roadmap for the complex and daunting task of establishing the ARCGOOS. Efforts to define and develop an inclusive ARCGOOS have been ongoing. In 2006, a report from the US National Academy of Sciences' Polar Research Board entitled, 'Toward an Integrated Arctic Observing Network', (National Research Council [NRC], 2006) recommended that an Arctic observing network should be initiated, for which a sustained observing system for the Arctic Ocean would fulfill the critical marine component of the envisioned linked system of observing systems. Since then the concept has been refined through the bi-yearly Arctic Observing Summit (AOS) workshops and reports (Murray et al., 2018). The AOS is an important planning activity that complements ongoing efforts of the SAON process, a multi-governmental body that has a joint mandate from the Arctic Council and the IASC. The need for an internationally coordinated observing system in the Arctic Ocean was stressed at the highest level in 2016 and 2018 at the first and second "Arctic Science Ministerials" respectively (German Arctic Office., 2019). The output from these high level discussions focused in part on the grand challenge of coordinating and advancing Arctic observing, with a recognition of the role SAON might play as a regional facilitator.

In summary, a successful ARCGOOS needs the cooperation of a broad range of experts including different scientific disciplines, economic sectors and society, and indigenous peoples. An ARCGOOS that is co-designed with multiple partners and user needs in mind will have the greatest likelihood of long-term sustainability, usability, and relevance. A recent collaborative effort, the IAOAF (Science, and Technology Policy Institute [STPI], and Sustaining Arctic Observing Networks [SAON], 2017) provides an effective means to comprehensively assess the impact of an ARCGOOS across users from an Arctic-specific set of SBAs. Areas include regionally relevant considerations like "Accessible, Available, and Sustainable Food," "Disaster Mitigation," and "Maintain areas of cultural significance in the Arctic." This multi-faceted cooperation is essential to ensure ARCGOOS plays a crucial role in the sustainable development of the Arctic in accordance with the United Nations 17 Sustainable Development Goals (SDGs), and importantly to inform scientific, economic, environmental and societal policies. An ARCGOOS must be flexible to respond to the changing needs and conditions of the region and provide the needed information for Arctic peoples and wider society, science, the private sector and decision makers. Whilst the development of an ARCGOOS is moving forward, a financial commitment remains elusive.

\section{Objectives}

This paper advocates a requirements-based approach for developing an ARCGOOS. Successful development and sustained operation of an ARCGOOS requires a broadly supported framework within which essential observational targets and approaches for observing them will be identified, the appropriate system metrics are monitored and integrated data delivery systems developed. Through observational targets, like the EOVs of the FOO (Lindstrom et al., 2012), relevant observing actions from contributing entities can be aligned, leveraged and made accessible. Observing targets and approaches are both intimately tied to the intended benefits of such an observing system. Motivated by the need to understand ecosystem response to sea ice retreat and other Arctic environmental change, and the potential implications for food security, the PAG (see section "Examples") coordinates the DBO (Moore and Grebmeier, 2018) and Pacific Arctic Climate Ecosystem Observatory. These two systems provide contemporary examples of sustained international observing in which a diverse, lightly coordinated consortium aligns around the consistent collection of a suite of essential measurements at mutually agreed-upon locations. The governance and operating structures employed by the consortium enables broad international collaboration capable of sustaining measurements over extended, decadal timescales and can be readily scaled to tackle new regions and measurements.

Design of an ARCGOOS can be optimized through application of quantitative approaches - defined as mathematically rigorous assessments of impact, redundancy, representativeness, and efficiency of existing, planned or hypothetical observational networks using numerical or statistical models. These quantitative approaches will help in determining observational sites and strategies (where, when, and for how long to conduct measurements, what variables are the most critical/influential/sensitive to observe, etc.). In environments undergoing rapid change, or for more complicated predictand variables, for example parameters integrating biophysical measures of food security, quantitative design approaches likely have to be refined through expert input.

The design and implementation tradeoffs of the ARCGOOS must be systematically evaluated in a transparent and broadly acceptable manner. It is thus critical to have an accepted benefit structure and quantitative framework for evaluating ARCGOOS impacts that incorporates a broad range of expertise, 
including science, decision and policy makers. The IAOAF (Science, and Technology Policy Institute [STPI], and Sustaining Arctic Observing Networks [SAON], 2017), which has identified 12 Arctic-specific SBAs supported (Figure 1) by $160+\mathrm{Key}$ Objectives, provides a valuable starting point for an ARCGOOS framework and assessment system, and it will elucidate variables of highest common dependence. The range of interests so identified will impact how those variables are observed and data are shared.

A successful framework for ARCGOOS needs to be led by an authoritative, international body or consortium of bodies with the mandate to coordinate observations at the intersection of Arctic and oceanic systems. Several bodies partially address such a mandate. For oceanic observing, the GOOS and the POGO each have meaningful roles to play. GOOS is a program executed by the IOC of the United Nations Educational, Scientific and Cultural Organization (UNESCO) with the mandate to sustain a coordinated set of ocean observations globally (Lindstrom et al., 2012). The European component of GOOS, EuroGOOS, founded in 1994, is an international association of 44 insitutitions from 18 countries coordinating ocean observations in five regional seas, including the European sector of the Arctic. POGO was founded in 1999 by directors of oceanographic institutions around the world as a forum to promote and advance the observation of the global ocean. POGO's membership includes most of the world's leading ocean science and technology institutions, whose expertise, experience and infrastructure provide the unique and long term capability to design, build, operate and innovate the GOOS. For Arctic observing, SAON has the joint mandate of Arctic Council and the IASC. SAON has been invited by the GRAs Council to participate as the Arctic representative and has been recognized as a Community Activity "Arctic GEOSS" under the GEO. As with all ocean/ice observing, transnational partnership and transnational access are critical. The 2017 Arctic Council "Agreement on Enhancing International Arctic Scientific Cooperation" should prove useful.

\section{Scope}

The SBAs dictate diverse, overlapping needs for a sustained Arctic observing system, including documenting Arctic environmental change, understanding the Arctic Ocean's role in climate, supporting planning and decision making, and providing near real-time support for operators in the Arctic (Figure 1, middle column). These needs strongly constrain system design by defining spatial and temporal scope and resolution, and by dictating the speed at which data and products must be delivered.

A first step toward organizing these requirements comes in recognizing that there are three overlapping domains of information needs: 1) long-range planning and policy; 2) strategy; and 3) tactics (Figure 1, left column). Long-range planning concerns the use of science to inform governance and management of the natural and built environment and human communities on decadal timescales, and thus focuses on long-term sustainability through planning for infrastructure, activity, regulation and environmental protection. Observations for policy must characterize environmental change and support model-based prediction. These needs prioritize measurements with large geographic scope (national to pan-Arctic) sustained over the long, decadal timescales needed to resolve climate variability. Scientific rigor, accuracy and precision are important considerations due to the subtle nature of some climate signals. Successful collection of sustained, climate-quality measurements requires long-term planning (National Academies of Sciences Engineering Medicine [NASEM], 2017). The distributed nature of these observations will place many sites far from areas of human activity, presenting logistical challenges that will limit the range of variables that can be observed. Policy frameworks respond slowly to new information, making data updates at annual or longer timeframes sufficient. Long records hold great value in the policy domain, prioritizing collection of decadal time series of consistent, high-quality measurements with broad geographic distribution.

Strategy involves the use of environmental data to support seasonal to decadal planning by governments, industry, science and communities. Here, an Arctic observing system might support activities such as decision-making surrounding transArctic shipping, resource extraction or the design of new vessels. Observations focus on regions of planned activity, and are thus limited in geographic coverage, but might still require data at regional or pan-Arctic scales to support numerical prediction. Data from these systems would also contribute to numerical prediction efforts. An understanding of synoptic-scale events can be important for robust decision making. For example, knowing both the statistics of internal waves and eddy variability and the physics that govern their generation and propagation can be important for planning resource extraction and designing the necessary offshore structures. The need for robust statistics makes long, sustained records valuable, while also driving the requirement for regional measurements that resolve synoptic scales of a few days and kilometers. Strategic decisions can unfold more rapidly than those in the policy domain, and thus require more rapid access to data, with updates at daily to annual intervals.

Tactics encompasses environmental/situational awareness and data collection in support of operational forecasting, informing decisions surrounding day-to-day activity. How far out onto the ice can I venture? What's the most cost or time efficient route for my vessel? Data needs for any given application will be narrow in geographic and temporal coverage, focused on the time and place where activity unfolds. Rapid and robust data delivery and analysis is critical, as tactical information loses value quickly with the passage of time. All data and analysis products should be provided with ample metadata, including estimates of uncertainty, and documentation of processing and quality control.

This paper focuses on frameworks for systems aimed at "Planning/Policy" and "Strategy," thus emphasizing the challenges associated with sustained observations over the larger temporal and spatial scales (years to decades, regional to panArctic). The paper considers how such systems might be optimized to provide tactical information when feasible, and how frameworks might be engineered to more readily integrate these needs into the design process. It further recognizes that a focus 


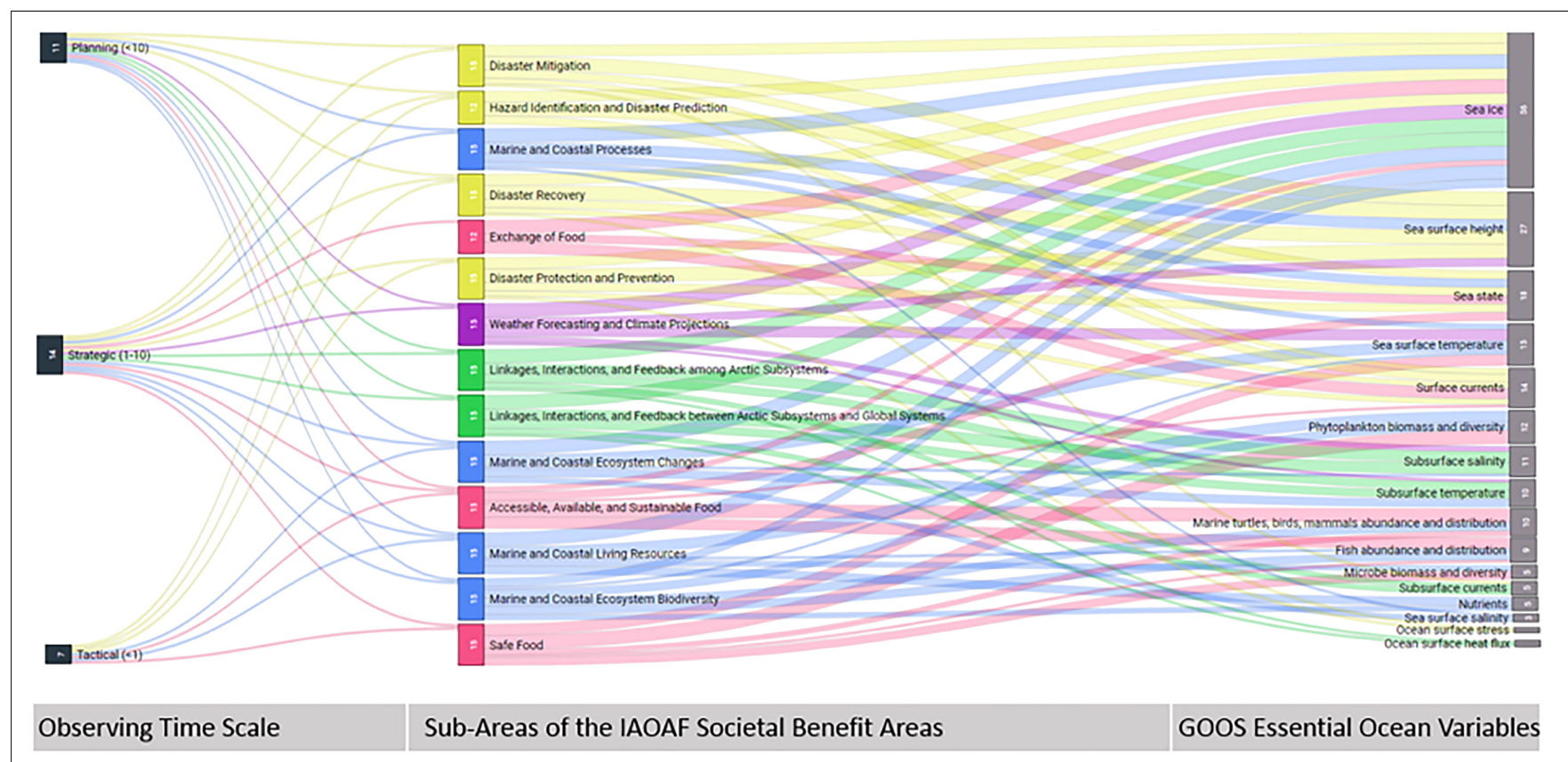

Observing Time Scale

Sub-Areas of the IAOAF Societal Benefit Areas

GOOS Essential Ocean Variables

Food Security

Disaster

Preparedness
Fundamental
Understanding
Marine,

Coastal

Weather and

Climate

\section{KEY: Arctic-Marine Relevant Subset of the 12 Societal Benefit Areas Identified by the IAOAF}

FIGURE 1 | In this example, temporal scales for observing information (left) are related to IAOAF Societal Benefit Sub-Areas of 5 ocean-relevant SBA's (center) and in turn linked to GOOS EOV's of highest impact to that topic. Through such relational mapping, the IAOAF can support the design of ARCGOOS through: (1) clarifying the user base for the observing system; (2) demonstrating variables of highest common dependence; (3) identifying the range of interests that will impact how those variables are observed and data is shared.

on longer-term understanding will ultimately support the most effective use of tactical information.

\section{A Path to an Arctic Ocean Observing System}

The following sections describe a process (Figure 1) that begins with selecting a subset of SBAs (Figure 1, denoted by color) that require understanding and information to support longterm planning, policy making and strategy (section "Framework and Governance"). These requirements drive the selection of research questions and operational objectives (Figure 1, middle column), from which flow identification of EAVs (Figure 1, right column), along with a broad sense of the scope (Figure 1, left column) of the necessary observing effort (section "System Design"). Subsequent design efforts (section "System Optimization") focus on understanding and optimizing the scope and scales of the observations needed to deliver the required information within specified uncertainties and representativeness. Implementation (see section "System Implementation") considers the technologies and approaches that could be employed to deliver the measurements specified in the design phase.

The implementation of an ARCGOOS that covers the breadth of these three application domains amounts to more than mere incremental technological and methodological innovation, cross-linkage of system components, and response to new scientific discoveries. Rather, it represents a challenge that requires transformative approaches, integrating physical and biological marine sciences, engineering, computational, social and behavioral sciences, and other knowledge systems (traditional). Convergence of these methods (McNutt, 2017) has not been achieved yet at this scale, but could be possible in the Arctic, where connections between different disciplines, sectors and knowledge systems are possibly tighter than elsewhere on the globe (Young, 2009). However, such transformational, convergent approaches require highly collaborative efforts from the regional to the international level, sustained by resourcing mechanisms that transcend challenges at the national scale. These approaches have to inform scope, design and implementation of the ARCGOOS - kernels of which are apparent in some of the examples listed in section "Examples." 


\section{FRAMEWORK AND GOVERNANCE}

\section{Introduction}

A sustainable ARCGOOS capable of advancing system-wide, collaborative sampling of the Arctic Ocean must be underpinned by a broadly endorsed framework grounded in: (i) high-level policy drivers (articulated as SBAs), and (ii) scientific and operational objectives that stem from these drivers. A framework provides a systems-level view of observing requirements, best practices and data delivery plans toward applications for which the observing system is intended. Requirements and best practices for system design, including platforms and instrumentation, data processing, quality control and long term preservation, as well as for dissemination strategies, derive from the scientific and operational objectives. This will provide a welldefined pathway toward implementation. Given the complexity of what a framework should accomplish, considerations for developing a successful observing framework for ARCGOOS will be discussed in the following.

First, the ARCGOOS requires an effective, internationally accepted governance structure with recognized authority building on the work of national agencies and marine institutions that ultimately execute observing network plans. Second, the ARCGOOS requires a clear framework for defining the scientific and operational objectives that support the selected SBAs. This requires a systems-level perspective to identify the necessary measurements (variables, scale and scope), and a methodology for prioritizing those measurements as a function of impact across the suite of objectives and SBAs. Given the vast number of potential scientific objectives and areas of interest in the Arctic marine system, the system-level view of the ARCGOOS framework should include a mechanism for weighing trade-offs based on importance, urgency and technical maturity. The framework should include a strategy to allow for the collaborative development of the most urgent set of requirements, while outlining a development pathway for less urgent requirements. It should include an evaluation process which allows the system to mature and change over time. Most urgently, the need for both governance and a framework need to be recognized and initiated by those in the best position to do so. This section explores each of these general aspects in greater detail, with specific recommendations for the ARCGOOS.

\section{Governance and the SBA-Driven ARCGOOS}

Governance guides the broad objectives of the ARCGOOS by identifying the most relevant policy mandates, as embodied by the SBAs, conducts ongoing evaluations of performance and fitness-to-purpose, and advocates for resources to support design and implementation. Given the fundamental importance of selecting SBAs, the ARCGOOS governance structure will need to identify and engage the bodies that have the most relevant policy mandate to lead efforts topically and regionally. It should further consider how these bodies are connected to the partners and resources needed to succeed with transnational implementation. Governance arrangements have implications for the observing framework that a network will use (e.g., extend an existing framework or create its own); the policy drivers that will guide observing priorities (e.g., climate-oriented versus operational); and the resource sharing schemes, including transnational access, data and cyberinfrastructure. Lastly, the governance structure must define clear lines of responsibility and processes for decision-making.

ARCGOOS sits at the confluence of many relevant governance schemes, including those of global actors (e.g., GOOS, POGO, GEO, WMO), thematic observing efforts (e.g., SCOR, Global Cryosphere Watch (GCW), GEO-CRI, Climate and Cryosphere (CliC), IASC), and plural regional, indigenous and national organizations. Achieving alignment between national, regional, thematic and existing global governance schemes is critical for all, and will influence the selection of policy drivers for the observing system. An example is whether the ARCGOOS should follow the SBAs outlined by GEO or refer to something more regionally specific like the IAOAF SBAs (Dobricic et al., 2018).

As the global coordinating body for ocean observing GOOS provides the mandate and framework under which ARCGOOS should operate. GOOS fosters the development of regional GOOS systems through GRAs. EuroGOOS, the European component of GOOS, contributes observations in the European Arctic with its Arctic Regional Ocean Observing System. POGO is an international non-governmental organization supporting the development of GOOS through innovation, advocacy and capacity development. POGO has recently been focusing on the challenges facing sustained Arctic observations, but needs to liaise with organizations and programs already active in the region to ensure that its activities are complementary.

The Global Climate Observing System (GCOS) is a United Nations-ratified program which regularly assesses the status of global climate observations and produces guidance for its improvement. GCOS is co-sponsored by the WMO, the Intergovernmental Oceanographic Commission of the United Nations Educational, Scientific and Cultural Organization (IOCUNESCO), the United Nations Environment Programme (UN Environment), and the International Science Council (ISC). ARCGOOS will contribute to GCOS through its climate related objectives.

GEO is an intergovernmental organization that works to actively improve and coordinate global earth observing systems, and their connection to users, promoting broad, open data sharing to the benefit of society. ARCGOOS can connect to GEO through its initiatives such as the Cold Regions Initiative (GEO-CRI), Biodiversity Observations Network (GEOBON) and Blue Planet Initiative. ARCGOOS would clearly benefit from reviewing the overlapping policy drivers of these organizations, such as the UNFCCC and its Paris Agreement, the Sendai Framework for Disaster Reduction and the United Nations SDGs. It would also benefit from working with some or all to take advantage of their strong agreements for transnational cooperation and data sharing.

A number of existing regional and topical observing systems serve specific SBAs, providing examples of governance structures that should be accounted for in the development of the ARCGOOS. Examples of such systems include the International 
Arctic Buoy Program (IABP, perhaps the oldest international Arctic observing program), the FRAM, the Svalbard Integrated arctic Earth Observation System (SIOS), the PAG, the CBMP and the IOOS-AOOS. The Arctic Regional Ocean Observing System (Arctic ROOS ${ }^{1}$ ), under EuroGOOS, provides an example of formal collaboration between European marine institutions. These systems are well established and will provide building blocks for ARCGOOS.

Since the IPY, SAON has emerged with a governance mandate to coordinate Arctic observing. It was jointly initiated and later formalized by the Arctic Council and IASC through the 2011 Nuuk Declaration (Arctic Council, 2011). This mandate has been renewed and reinforced through the Joint Statement of the 2016 and the 2018 Arctic Science Ministerials ${ }^{2}$ yet SAON's still-maturing strategy and lack of resources have limited its ability to develop clear direction for regional networks. SAON's policy mandate comes from the Arctic Council, which has working groups focussing on climate and contaminants (AMAP), ecosystems and biodiversity (CAFF), marine activity (PAME), sustainability (SDWG) and more.

At the intersection of GOOS, GEO, POGO and SAON lies the strengths of long-standing intergovernmental agreements to collaboratively observe the global ocean and related systems, a consortium of ocean observing expertise, and strong regional policy drivers and engagement with indigenous communities with sovereign rights in the Arctic. In the same manner as the SOOS was developed jointly through the work of SCOR, the SCAR and POGO, ARCGOOS development should proceed under a consortium governance approach.

\section{Framework Design}

A framework for the ARCGOOS must support a system-level view of policy drivers, the resulting scientific challenges, essential variables and the interrelationships that bind them. SBAs are central to the ARCGOOS framework, motivating investment in an ARCGOOS and associated scientific efforts. The results of the "Impact assessment study on societal benefits of Arctic observing systems" (IMOBAR) project has demonstrated the economic value that accrues from Arctic observations in support of the IAOAF SBAs (Dobricic et al., 2018). Science objectives derive from the information needs of the selected SBAs. An effective ARCGOOS must be firmly grounded in science, with system design tailored to the identified science questions. EAVs can be identified as those that offer maximum impact, as measured by the number or urgency of science objectives and SBAs served, and feasibility, as measured by technological maturity and logistical constraints. System scope and temporal and spatial resolution requirements flow from design studies aimed at achieving efficient delivery of the required data. Data delivery systems should support the range of objectives that the framework identifies. The ARCGOOS framework should also provide processes for continued system evaluation, and evolution in response to changing science needs and introduction of new technologies.

${ }^{1}$ https://arctic-roos.org/

${ }^{2}$ https://www.arcticscienceministerial.org/en/conclusions- $1740 . \mathrm{html}$
A system-level view overcomes the difficulty of looking at pieces of the Arctic marine system in isolation, while not getting overwhelmed by the complexity of their relationships. This view should also be a starting point for identifying which aspects of the observing system have the greatest impact on the policy drivers. The SBA frameworks provide a mechanism to systematically assess the impact of different observing targets and develop a hierarchy of key observables - or essential variables - around which to organize.

Even as the ARCGOOS seeks alignment of relevant governance schemes, it should also strive to align with relevant global observing frameworks. From a practical standpoint, global frameworks have made tremendous progress and many national funding programs have developed to support them. The ARCGOOS could leverage this progress, along with existing international agreements for collaborative sampling and data dissemination. For example, the GOOS FOO is organized around EOVs. EOVs support the scientific objectives of the FOO while providing a systematic means to align and coordinate observing strategies. EOVs allow discrete aspects of the observing system to develop independently of one another, as they reach sufficient maturity. Other global essential variable systems like the GCOS ECVs, the GEOBON Essential Biodiversity Variables, or the WMO's GCW observing specifications should be taken into consideration, leveraged and extended where possible.

If ARCGOOS is to follow the pathway of merging and extending existing essential variable frameworks, it would need to attend to two important considerations. First, global EOVs were identified and prioritized under globally relevant policy mandates. ARCGOOS should review and refine these policy mandates for Arctic relevance. As a result of the first Arctic Science Ministerial, SAON, in partnership with the STPI, jointly developed a key collaboration tool through the IAOAF. The IAOAF was developed through SAON partners, national, indigenous and non-governmental organizations, and articulated 12 Arctic-specific SBAs, supported by more than 160 key objectives for regional observing. While broad, it provides a critical starting point for bridging between global and regionally significant observing targets.

Second, Arctic regional climate and ecosystems themselves are unique and require specific observing strategies. Ice cover impedes satellite services for geopositioning and communication of underwater platforms, and threatens instruments at or near the ice-ocean interface. The Argo program (Riser et al., 2016), which revolutionized climate-scale observing in the ice-free oceans with an array of roughly 4000 profiling floats, has not yet gained traction in the Arctic because of its reliance on satellite services for data transfer and geolocation. Polar night limits the seasonal range of visible satellite imagery. Both sea and land ice play important roles in dynamic coastal ecosystems. The demanding environment, remoteness and operational challenges make logistics a stronger constraint than they might be in lower latitude oceans. This has produced a culture of shared logistics that can encourage interdisciplinary research and, in more recent years, has motivated accelerated efforts to employ autonomous platforms. 


\section{Strategy for Initiating a Framework}

At the second Arctic Science Ministerial in Berlin (German Arctic Office., 2019), ministers from 24 countries emphasized the urgency of implementing sustained Arctic observing and pointed to SAON to demonstrate the necessary leadership. SAON's Committee on Observing Networks (CON) is initiating a new task force to advance the application of IAOAF SBAs toward framework design. Recently, SAON was invited to participate in the GOOS GRAs, and POGO has become more engaged with SAON. As a follow up we recommend that SAON invites GOOS GRAs, GEOSS, WMO, and POGO to form an exploratory task team to make recommendations for Arctic Ocean Observing System. ARCGOOS could serve as an excellent focal point to follow up the implementation of the task team recommendations.

\section{Requirements-Driven Identification of Essential ARCGOOS Variables}

Applied within the context of the broad Arctic observing domains described above, and governed by the processes discussed in section "Framework and Governance," the GOOS EOV approach provides a framework for guiding the selection of observables and scales to be addressed by the various components of an ARCGOOS. EAVs are selected for relevance to climate, operational services and ocean health, modulated by feasibility and cost effectiveness. Unique aspects of the Arctic Ocean and its role in global climate imprint on the shape of the ARCGOOS, while practical considerations, including logistics, resource availability, and sustainability place strong constraints on scope. While the ultimate selection and definition of EAVs, and the scales at which they must be measured, should flow from the identification of SBAs and the science questions they engender (section "Framework and Governance"), the GOOS EOVs provide a starting point for considering some of the ARCGOOS baseline variables and how they might be shaped by challenges and concerns particular to the Arctic.

To illustrate the flow that might lead from SBAs to the identification of EAVs, consider the example of Arctic heat and freshwater budgets. Several SBAs, including Fundamental Understanding of Arctic Systems, Environmental Quality, Food Security, Weather and Climate (all linked to the sustainable development of the region), motivate the need to understand and document the fate of Arctic Ocean heat and freshwater. The Arctic freshwater cycle plays an important role in modulating the Arctic energy balance and in global climate (e.g., Prowse et al., 2015; Carmack et al., 2016). Upper ocean freshwater modulates sea ice formation and melt by insulating ice from warmer waters below (e.g., Aagaard and Coachman, 1975) and, through this, impacts coupling between the upper ocean and local atmospheric forcing. Sea ice growth and melt are also fundamental components of the Arctic heat balance, which plays an important role in constraining meridional atmospheric energy transport (Nakamura and Oort, 1988). Seasonal changes in sea ice timing and extent directly influence marine ecosystems both through loss of habitat for ice-obligate species such as polar cod and ice seals, but also via changes in the timing and amount of primary productivity (e.g., Arrigo et al., 2008; Macintyre et al., 2015). Arctic residents in turn have a subsistence reliance on local food sources (Eicken et al., 2009; Nilsson et al., 2013), the shifts in which impact both their food security and culture. Globally, the fisheries of the Bering and Barents Seas are highly productive and vulnerable to system-wide shifts (Hunt and Megrey, 2005; Wassmann, 2015). Measurements of ocean temperature, salinity and mass are fundamental to understanding in these areas, and thus have the broad impact that suggest them as EAVs.

Science and operational needs, derived from the SBAs, identify EAVs (Figure 1) and, in a broad sense, the scope and scale at which they must be measured. Continuing the example, documenting the fate of freshwater and heat will require maps of temperature and salinity suitable for quantifying storage and fluxes. Surrounded by land, the Arctic Ocean communicates with the Pacific and Atlantic Oceans through a handful of gateways, with additional inputs from net precipitation and riverine inflow. Estimates of heat and freshwater storage will require measurements suitable for mapping the interior of the ocean under the drifting sea ice, along with observations over the shallow shelves that account for over a third of the Arctic Ocean surface area and are the focus of most human activity. Technological and political challenges have limited access to some Arctic shelf systems, creating a critical gap in the observing system. Flux estimates will require sustained observations of temperature, salinity and velocity at the main ocean gateways (Bering, Fram and Davis Straits and the Barents Sea Opening), and in-situ recording of discharge rates and temperatures for the major rivers (Mackenzie, Yukon, Ob, Yenisey and Lena rivers), as well as quantification of sea ice-associated export fluxes in the North American and Eurasian Arctic and broad estimates of net precipitation. For this example, the SBAs point to science for long-term planning, policy and strategy, and thus longer timescales and sustained measurements over years to decades.

Applying a similar analysis systematically across multiple SBAs identifies a suite of EAVs along with usage patterns that can be interpreted as a measure of impact (Figure 1, right column). For this example, SBAs yield science and operational objectives, each of which points to a maximum of five dependent variables, specified in priority order. Impact can be gauged by the number of objectives served weighted by priority within each objective, with high-impact variables being candidates for EAVs. While illustrative of the process, this simplified evaluation does not capture some important factors that must be accounted for in a more thorough EAV analysis. Interdependencies can elevate the impact of some variables. For example, although sea ice ranks among the most impactful EAVs, understanding and predicting sea ice evolution requires a host of other measurements. These are termed 'supporting variables' in the GOOS EOV framework, and include subsurface temperature and salinity, radiative surface fluxes, surface air temperature, precipitation and wind velocity. EAV definitions would build on those created for GOOS EOVs, incorporating refinements specific to the needs of Arctic observing. Scope (Figure 1, left column) is not reflected in this analysis. The challenges associated with Arctic observing make weighting EAV candidates for technological readiness and logistical feasibility particularly important. 
Taking into account the caveats articulated above, the simplified example plotted in Figure 1 identifies potential highimpact EAVs that include sea ice, sea surface height, sea state, phytoplankton, surface and subsurface temperature, salinity and ocean velocity. Although this subset is clearly incomplete, the example serves to illustrate how a requirements-driven framework might be used to trace and understand the impact of observations across multiple scientific and operational endeavors. This knowledge then informs planning and investment toward developing the ARCGOOS.

\section{Overview of Arctic Marine System Observables \\ Ocean}

The energy imbalance in Earth's climate system is caused by rising concentrations of heat-trapping gases. About 93\% of the energy imbalance accumulates in the ocean as increased ocean heat content (Cheng et al., 2019). Heat introduced by summertime solar warming, and through Pacific and Atlantic inflows, plays an increasingly important role in the extent and timing of sea ice growth and retreat (e.g., Carmack et al., 2015), while freshwater (salinity) forms a cold halocline that modulates contact between sea ice and the warmer, subsurface waters below (Aagaard and Coachman, 1975). To document changes in upper ocean heat content, stratification and the resulting impact on sea ice, water-column measurements must include in situ temperature and salinity profiles from the ocean surface through the warm core of the Atlantic Water Layer (at least to $500 \mathrm{~m}$ depth) with sufficient vertical resolution to quantify mixed-layer depth and the strength of the strong halocline stratification at the mixed layer base. Waters deeper than this change on timescales longer than interannual and deep measurements every several years will suffice.

Other high-priority water column observations might include dissolved oxygen, velocity, nutrients, bio-optical measurements (sensor-based proxies for phytoplankton) and seawater inorganic carbon chemistry (at least two of the four variables: dissolved inorganic carbon, total alkalinity, $\mathrm{pCO} 2$, and $\mathrm{pH}$ ). Geochemical parameters (trace elements and isotopes - TEI) including chlorofluorocarbons (CFCs), sulfur hexafluoride (SF6), tritium, helium isotopes, stable isotopes of water and ${ }^{14} \mathrm{C}$ integrate over large spatial and temporal scales, and can thus be useful for understanding water mass formation and movement in the Arctic Ocean. These TEI have been successfully used with the US GEOTRACES study and other studies to investigate water mass structure, pathways, and residence times. Recently, 'ocean sound' was approved as an EOV by GOOS. This is a highly relevant EOV to monitor for the Arctic region due to the increased human influence on the ocean acoustic environment.

High-priority sea surface measurements include sea state and sea level. Sea state observations might include remotely sensed and in situ measurement of surface waves and in situ observations of upper ocean velocity and turbulence. Sea level monitoring relies on a combination of remote sensing (satellite altimetry and gravimetry measurements; e.g., Armitage et al., 2016), bottom pressure and coastal tide gauges (Proshutinsky et al., 2004;
Volkov and Pujol, 2012; Calafat et al., 2013; Peralta-Ferriz and Morison, 2014). Measurements of sea level provide information on ocean dynamics and thermohaline properties at weekly to interannual timescales as well as longer-term land ice (and snow) melt.

\section{Sea Ice}

Sea ice plays a central role in Arctic marine environments. Its presence constrains atmosphere-ocean interaction (e.g., Rösel et al., 2018), structures marine ecosystems (e.g., Nicolaus et al., 2012; Wassmann, 2015), and greatly affects human activities (Eicken et al., 2009; Andrews et al., 2017). Sea ice loss is both an indicator of broader, system-wide transformations (Polyakov et al., 2017; Wilkinson and Julienne, 2018) as well as a driver of change in many of the components of the Arctic system. In this context, concentration, thickness, roughness and mechanical characteristics, in addition to snow cover, optical and biogeochemical properties of the Arctic ice pack have been identified as high-priority variables. Reductions in sea ice extent (the 12 lowest minimum sea ice extents all occurred in the last 12 years, Stroeve and Notz, 2018) and thickness (Lindsay and Schweiger, 2015; Kwok, 2018) and the underlying changes in the heat and momentum budget call for detailed observations of ice thickness and snow depth at the basin-scale, but with a resolution sufficient to capture key processes, e.g., in the context of ice deformation or its evolution as a critical habitat (Post et al., 2013).

Recent advances in remote sensing, in particular in terms of radar and laser altimetry, and synthetic aperture radar (e.g., Markus et al., 2017; Kwok, 2018) have the potential to greatly improve our understanding of the transformation of the Arctic Ocean from a perennially to a seasonally ice-covered body of water. However, remote sensing, modeling, and prediction system development all require in situ observations for constraint and validation. This problem is exacerbated by the fact that our research understanding and model development are built on an Arctic formerly dominated by multi-year ice. Near-term correction of this paradigm is needed if we are to provide more accurate predictions on sea ice behavior and evolution on planning/policy to strategic to tactical timescales. For improving understanding and to be able to predict changes, the new conditions need more in situ and remote sensing observations, especially for the winter season from which relatively little data exist (Webster et al., 2018; Gerland et al., 2019). Satellite remote sensing provides good spatial and temporal coverage of the Arctic Ocean through long-term programs such as the Defense Meteorological Satellite Program (DMSP), the RADARSAT Constellation Mission and the Copernicus Sentinel program. Copernicus is the most ambitious satellite program today, with more than 10 satellites providing near real time data for environmental and climate monitoring, in particular ocean and sea ice observations. However, there are substantial gaps in in situ observations of Arctic sea ice variables such as sea ice thickness and snow cover, both in terms of coverage and longevity (Sandven et al., 2018).

The increase in human activity and potential for maritime disasters in the presence of ice (e.g., Barber et al., 2018), as 
well as the need to better understand potential restructuring of food webs as a result of sea ice changes, call for a new suite of sustained observations targeting biogeochemical variables and the coastal zone where much of human activity is concentrated and risks are most pronounced (Eicken and Mahoney, 2015). Much of the demand for these types of observations can be met through a combination of newly developed autonomous sensing systems (Whitlock, 2018) and community-based observations (Johnson et al., 2015).

\section{Atmosphere}

The atmosphere links all components of the Arctic climate system (ocean, ice, land, biology), driving changes within each of them, responding to these changes, and extending their influence across spatial areas as extensive as hemispheric. Understanding the complex, interconnected processes and exchanges of energy between the atmosphere and the underlying ocean, sea ice, and land surfaces plays a central role in the modulation of weather, the seasonal growth and decay of sea ice, and climate. Within the atmospheric column, transport of heat, humidity, aerosols and momentum are all important contributors in the generation of clouds with potential impacts on global circulation patterns governing regional climate.

Essential atmospheric parameters that need to be monitored to quantify the surface energy balance are atmosphere-ocean (-ice and -land) radiative and turbulent fluxes; these govern ocean and sea-ice dynamics and thermodynamics. At the same time, changes in ocean heat content and sea ice conditions alter the heat fluxes to the atmosphere. Atmosphere-sea ice-ocean fluxes of momentum and freshwater must also be quantified. For example, winds play a central role in driving oceanic mixing from above through momentum exchange. The role of sea ice in this exchange remains an open question, with changes to ice concentration and roughness likely to alter the efficiency of momentum transfer from atmosphere to ocean and the extent to which wind stress can impact the mixed layer through waves and other mixing processes. Additionally, wind speed, in conjunction with the temperature contrast between the ocean and atmosphere dictate the magnitude of turbulent heat exchange between these bodies, elevating the importance of wind orientation and localized flow and the need to understand lower tropospheric dynamics. Salinity controls upper ocean stratification in the Arctic. Freshwater inputs thus modulate mixing and the distribution of momentum and heat within the upper ocean.

Although the Arctic represents one of the most challenging environments, year-round measurements are needed to capture the annual cycle of energy exchanges over sea ice, open ocean, and land, and to constrain global atmospheric reanalyses, which can be problematic in data-sparse regions such as the Arctic (e.g., Lindsay et al., 2014). These measurements would include long and shortwave radiation, wind, air temperature and relative humidity profiles (within the atmospheric boundary layer), and turbulent fluxes of heat and momentum. The next level of priorities might include aerosols, clouds, precipitation, atmospheric chemistry and ocean-atmosphere $\mathrm{CO}_{2}$ fluxes. Seasonal and interannual changes of atmospheric $\mathrm{CO}_{2}$ are poorly resolved over the Arctic and the role of the Arctic Ocean as a $\mathrm{CO}_{2}$ sink is yet to be determined. Remote sensing can provide measurements of near-surface winds over open water, but other measurements must be made using in situ.

\section{SYSTEM DESIGN}

Key guidance and constraints on the design of an ARCGOOS derive from the consensus that the system has to cover a range of purposes and applications National Research Council [NRC] (2006), Lee et al. (2015), Science, and Technology Policy Institute [STPI], and Sustaining Arctic Observing Networks [SAON] (2017). Consequently, it is helpful to consider the expected benefits, requirements, and demands on system components by different user communities in the context of planning and policy, strategy, and tactics - as laid out in section "Introduction." From these three aspects of system characteristics, a range of observing system design criteria and approaches can be derived, augmented by the constraints placed upon the system at the implementation stage (Table 1, see also section "System Implementation" and Lee et al., 2015 for further background). A distinguishing characteristic of the Arctic region compared to other marine environments is the tight coupling between the different components of the extant social-environmental systems, corresponding to the need for similar fit between different disciplines, sectors and knowledge systems (e.g., Young, 2009). These circumstances place major demands on the process of observing system design. Below, we consider how such transformational challenges can be addressed through a portfolio of different design approaches and briefly discuss their advantages and drawbacks, drawing on specific examples from the Arctic where possible.

Past assessments and surveys conducted by SAON, the AOSs (Murray et al., 2018; Sandven et al., 2018), and related efforts e.g., INTAROS (Ludvigsen et al., 2018) demonstrate that an integrated Arctic observing system will not be designed in its entirety from the ground up. Rather, with a range of observing activities, from numerous research projects (such as those highlighted in section "Examples"), agency-led programs at the national and international level (e.g., the International Arctic Buoy Program ${ }^{3}$ ), and community-based monitoring efforts (Johnson et al., 2015; Williams et al., 2018). ARCGOOS design and implementation needs to leverage from a range of existing observing efforts operating at different scales with different objectives and missions. In fact, the sparsity of observations and resources in Arctic marine settings have resulted in operators and planners having to disproportionately rely on data from research observing networks (Lee et al., 2015; Eicken et al., 2016; Ludvigsen et al., 2018). These challenges need to be addressed through development of a framework that allows for the design of new components and optimization, modification, and integration of existing pieces to go hand in hand (AOS Executive Organizing Committee, 2018). Global observing frameworks

\footnotetext{
${ }^{3}$ http://iabp.apl.washington.edu/
} 


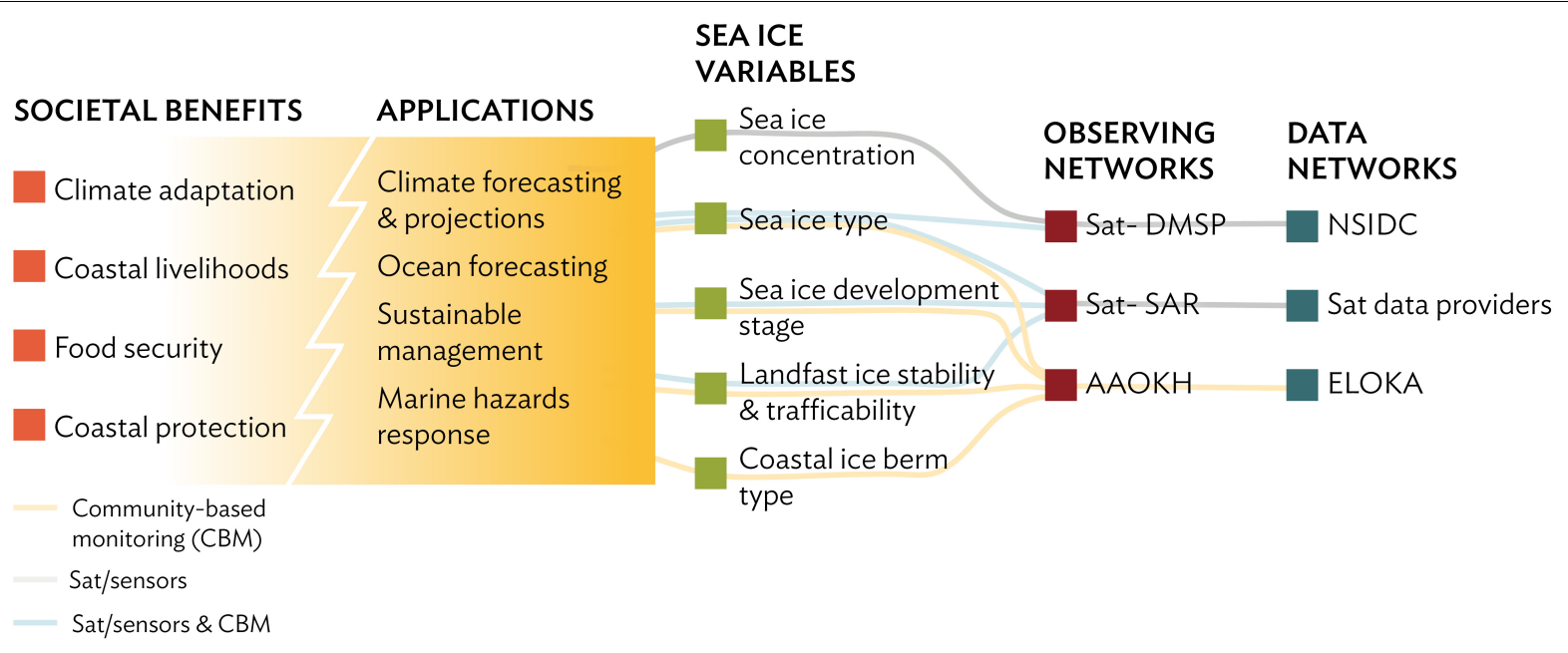

FIGURE 2 | (Adopted from Lindstrom et al., 2012) Example of observing network design and implementation as seen through the lens of a top-down approach based on Starkweather et al. (2018). Shown is the link between societal benefit areas, EOVs, and observing and data management systems for the GOOS FOO (Lindstrom et al., 2012) as applied to Arctic sea-ice observations. Abbreviations: DMSP, Defense Meteorological Satellite Program; SAR, Synthetic Aperture Radar; NSIDC, National Snow and lce Data Center.

have grappled with this issue, though maybe not to the extent as required in the Arctic.

While a number of observing frameworks exist, over the past decade or so approaches to derive specific requirements from mission and SBAs have been converging. The GOOS FOO (Lindstrom et al., 2012; Figure 2) is particularly well suited in the Arctic since it aligns with other relevant efforts under GEO and GCOS. Further guidance on the combination of observing system design and optimization can be drawn from the Ten GCOS Climate Monitoring Principles ${ }^{4}$, four of which explicitly address key aspects of integrating new observations into nascent, loosely assembled networks:

(1) The impact of new systems or changes to existing systems should be assessed prior to implementation.

(2) Consideration of the needs for environmental and climate-monitoring products and assessments, such as IPCC assessments, should be integrated into national, regional and global observing priorities.

(3) High priority for additional observations should be focused on regions sensitive to change, data-poor regions, poorly observed parameters, and key measurements with inadequate temporal resolution.

(4) The conversion of research observing systems to long-term operations in a carefully planned manner should be promoted.

The three different user-base contexts and associated information needs (augmented by information guiding system implementation) can be linked to different observing system design and optimization methods appropriate for the particular application (Table 1; Lee et al., 2015). Progressively finer detail needs to be resolved in moving from the policy to the

${ }^{4}$ https://gcos.wmo.int/en/essential-climate-variables/gcos-monitoring-principles (retrieved January 24, 2018). tactics and implementation scale. At the policy and planning level, information needs and observing program design focus on broad societal benefits and applications. To be sure, such demands on the system still require identification of EOVs, but as demonstrated in the value tree analysis (VTA) carried out by the STPI and SAON in 2017, siting of observations and temporal resolution are not as much of a concern in demonstrating value to policy makers and funding organizations. For example, the European Union's Horizon 2020 Program supported a high level study that focused mainly on demonstrating economic value of a portfolio of observations in broadly defined sectors, without delving into the detail that would be part of tactics or even strategy-focused observations (Dobricic et al., 2018).

A key challenge at the policy and planning scale is the identification of suitable target variables. Here, the use of participatory scenarios has proven valuable in having observing system users or stakeholders identify indicator variables (Flynn et al., 2018; Preston and Lovecraft, 2018) that can then be translated into or linked to EOVs. Even at this level of data use, it is in principle possible to derive more specific guidance on the siting of specific observations. For example, Vargas-Moreno et al. (2016) were able to use geospatially explicit scenarios to identify broad target regions for monitoring linked to energy and resource development in coastal and offshore Alaska. Along the same lines, institutional analysis, with a focus on formal and informal regulations governing specific ecosystem services or societal benefits, can provide some guidance on the locus of observations (Lovecraft et al., 2013). While studies such as Science, and Technology Policy Institute [STPI], and Sustaining Arctic Observing Networks [SAON] (2017) or Dobricic et al. (2018) can help address the due diligence required by policymakers and funding agencies in terms of guiding sustained observations, there is comparatively little reliance on the approaches outlined in Table 1 to date. 


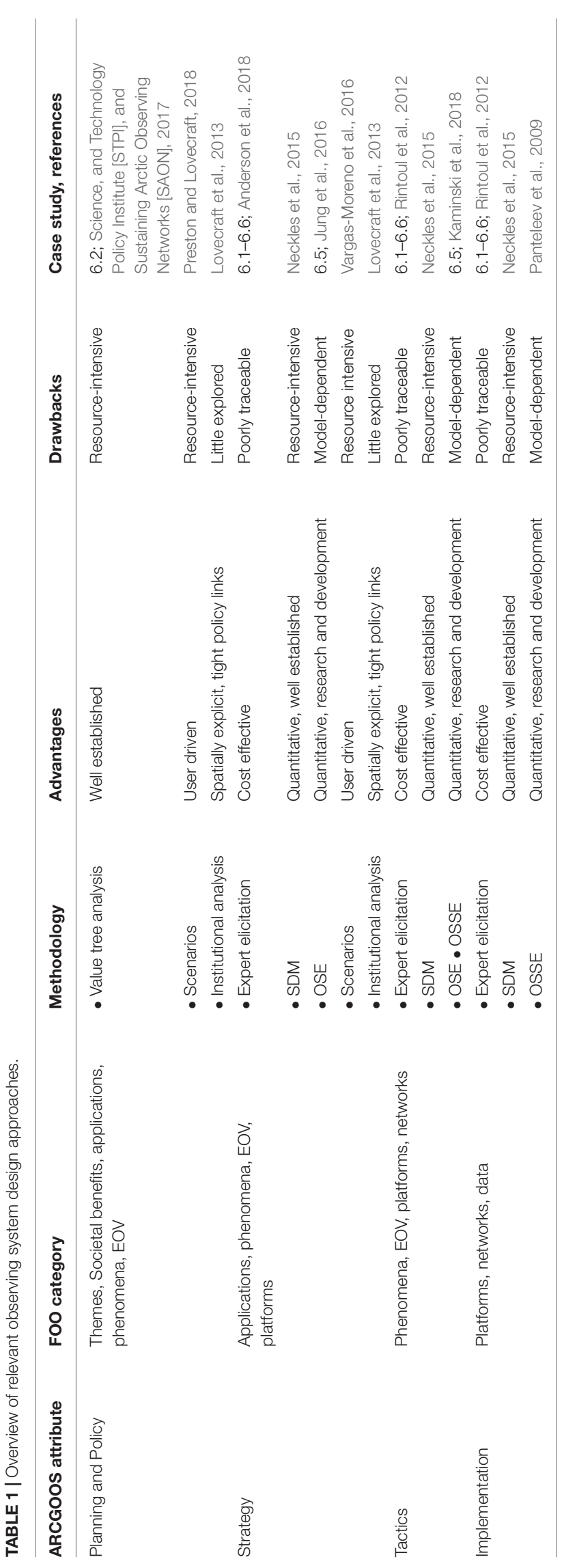

At the Strategy and even more so at the tactics and system implementation level, more detail is required in terms of the specifications as well as the observing assets and networks and data management aspects of the monitoring (Table 1 and Figure 2). The necessary detail can be obtained through a combination of different methods, with heuristic input from experts being the most common. In fact, all of the examples listed in section "Examples" draw most heavily on input by experts, typically researchers or agency personnel who are conducting the actual measurements. While this group often has extensive knowledge of both the underlying science and the technology details that are relevant for system design, such input is typically poorly traceable and may not meet requirements, e.g., by regulatory agencies where such decisions may have to hold up in court.

\section{SYSTEM OPTIMIZATION}

Design of ARCGOOS should be dictated by cost-efficiency and representativeness of sampled EOVs. Quantitative methods, including OSEs, OSSEs, or adjoint-based optimal experimental design can assist the design of ARCGOOS. Below we briefly describe and discuss each of these methods.

Observing System Experiments involve analyses designed to withhold or aggregate certain subsets of data to quantitatively assess the impact of different observational platforms within already implemented observing systems (Oke et al., 2015). Impacts are assessed versus observations withheld (or aggregated) from assimilation or from state-of-the-art analysis products derived from observations. One disadvantage of OSEs is that the reference experiment that includes all data might not represent the true state, thus the assessed impacts of the withheld data relative to this reference experiment might not fully capture the true impacts. An additional disadvantage is that OSEs can only assess impacts of existing but not of future observing systems.

Observing System Simulation Experiments follow the same procedures as OSEs, but assimilate synthetic observations instead of existing real-world observations. OSSE systems (Atlas, 1997; Hoffman and Atlas, 2015) facilitate quantitative impact assessment on analyses and strategies of planned observing systems, and of different deployment strategies for existing systems. An OSSE system consists of three components: (a) NR, which is a free-running model, that represents the groundtruth, i.e., assumed to be representative of the true ocean; b) data-assimilative ocean forecast system that couples different ocean models (the Forecast Model, or FM) to an ocean DA scheme; and c) simulations of ocean observations within the NR containing realistic or expected observational errors for each observing platform being assessed for assimilation into the forecast system. Ocean profiles (e.g., temperature, salinity) and other observations are generally re-mapped prior to assimilation (Halliwell et al., 2014). Impacts can then be assessed by comparing experimental analyses to high-resolution three-dimensional representations of the "groundtruth" ocean provided by the NR. Therefore, OSSE systems can not only 
assess new observing systems and strategies, but also assess impacts of existing observing systems more thoroughly than it is possible with OSEs. OSSEs also have an advantage over linearized procedures to assess impact, such as adjoint methods, because the influence of non-linear processes on observing system impact can be documented. The disadvantage of OSSEs is that the NR might not fully capture the realworld ocean dynamics (Hoffman and Atlas, 2015). In addition, the impact assessment can be dependent on the modelsystem being used.

In contrast to both OSEs and OSSEs, where statistical approach is an important component of the DA step, adjointbased methods utilize the dynamical information in the tangent linear and adjoint models of the underlying general circulation model (GCM). Through the equations which capture conservation and constitutive laws, propagation of information up- and down-stream of any quantity of interest (QoI) is used to (a) assess impactful regions where new observations can be potentially deployed (Marotzke et al., 1999; Zanna et al., 2010; Heimbach et al., 2011; Nguyen et al., 2017; Stammer et al., 2018); (b) assess the redundancy of existing observing networks (Köhl and Stammer, 2004; Moore et al., 2017b); (c) quantify the impacts of selected existing/new observational networks on reducing posterior uncertainties of the GCM control parameters and/or potential unobserved remote QoI (Moore et al., 2011, 2017a; Bui-Thanh et al., 2012; Kalmikov and Heimbach, 2014, 2018; Kaminski et al., 2015, 2018); (d) find an optimal observing network through Hessian-based OED that minimizes the posterior uncertainties as a function of the control parameters and/or targeted QoI (Alexanderian et al., 2016; Loose, 2019). The advantage of the adjoint-based methods is not only the quantification of uncertainty reduction of the GCM control parameters and/or any specific QoI to the observing network but also the identification of dynamical connection and causal relationship between them. The disadvantage of these methods is the prohibitive computational cost of calculating the full Hessian and its inverse. Recent advances in the field of computational sciences (e.g., Flath et al., 2011; Bui-Thanh et al., 2012; Loose, 2019) have helped reduce the computational cost and thus can make adjoint-based OEDs tractable for oceanographic applications.

To date, OSE, OSSE, and adjoint-based OED have not been fully utilized for optimization of observational work due to the disadvantages associated with each of the methods as discussed above. As a result, there are only a few examples of OSEs employed to provide quantitative, traceable guidance on key aspects of observing system design (e.g., Panteleev et al., 2009; Jung et al., 2016; Kaminski et al., 2018; Stammer et al., 2018). An argument put forward against over-reliance on OSEs and OSSEs for system design is that measurements in a rapidly changing Arctic are typically meant to provide information that can help anticipate or prepare for major changes and transformations. Hence, the siting and overall sampling strategy may not account for the impacts of rapidly changing conditions not captured or predicted by the model. This is particularly true if statistical models are relied upon. For example, in the
Arctic Ocean the loss of perennial sea ice has impacts on many other components of the marine system that are still poorly captured by models and in particular statistics of past behavior (e.g., Lindsay and Zhang, 2006).

However, it should be noted that comprehensive observations covering both the spatial and temporal scales required to fully measure and understand the rapidly changing Arctic are also not available. In addition, ad hoc estimates of spatial/temporal correlation scales are often utilized to interpolate data within the existing observational systems without full knowledge of error covariance (Wunsch, 2006). Thus, a careful synthesis of different bodies of knowledge is needed to guide system design. In other words, an approach where the imperfect tools available within OSE, OSSE, and adjoint-based OED should be employed in iterative steps with deployments of observations to push forward development and improvements for ocean applications, similar to the steady progress achieved in DA and models used for NWP applications (e.g., Bauer et al., 2015).

Advances in forward modeling and 4D-var DA have facilitated model guidance that is more tightly linked to, e.g., sensor and observing technology constraints such that OS(S)Es and OEDs can potentially help support the design of the sensor systems themselves (Kaminski et al., 2018). It should be expected that, due to the potentially limited and serious deficiencies, an optimal network based on these quantitative tools might not capture the dynamics when compared to field observations and other political/economic constraints. One example is the case at the Bering Strait where, based on inverse modeling and drawing on a large dataset for the region, Panteleev et al. (2009) determined that optimal observations of Bering Strait throughflow should include two moorings located within the Strait and two at some distance further south. In practice, due to economical and political constraints, and in combination with knowledge gained from the existing observations at the strait, the array has been reduced to three moorings, two in the eastern channel and next to the Alaskan Coast, and one located north of the strait (Woodgate, 2018). Such discrepancies should be anticipated in the iterative process, and should be used to facilitate further communication and system co-design by modelers and observationalists. Important QoI should be identified, e.g., monitoring ocean dynamics, detecting temporal variability on a range of scales, capturing air-sea interaction processes, or detecting sea level changes. Proposed observing systems based on different OS(S)Es and OEDs should be weighed against each other and used judiciously, as with any decision-making.

\section{SYSTEM IMPLEMENTATION}

Arctic Region Component of the Global Ocean Observing System implementation will involve a complex network of national and international partners, addressing a myriad of scientific and operational objectives, under significant and varied logistical challenges. In practice, implementation of a sustained ARCGOOS will mean adapting and integrating existing 
observing activities alongside the development of new systems deliberately designed to meet identified ARCGOOS needs. Although the choice of specific operational approaches and technologies clearly depends on the requirements and constraints of the specific system, some considerations that apply more broadly are discussed here.

An ARCGOOS capable of spanning diverse demands will need to rely on a heterogeneous mix of reference frameworks and approaches. The needs of SBAs that require data in the domains of planning/policy and strategy skew toward measurements at the basin and pan-Arctic scales. At present, such observations are often collected by stochastic sampling using large arrays of drifting instruments such as Argo (e.g., Argo Steering Team, 1998; Riser et al., 2016), the Global Drifter Program (e.g., Lumpkin and Pazos, 2007; Centurioni et al., 2017), IABP ${ }^{5}$ and the Ice-Tethered Profiler program (Krishfield et al., 2008). Instruments are deployed in sufficient numbers to achieve a target density (e.g., one float per $300 \mathrm{~km}$ for Argo), with the system relying on drift to maintain distribution over the target region. Although collected by drifting instruments, measurements are interpreted in an Eulerian (geographically fixed) frame. Though powerful, this approach has limitations. The same currents that distribute platforms also create areas of convergence and divergence, which concentrate or exclude instruments from those regions. Instruments rapidly flush from areas of strong flow, limiting measurements in many regions that are of particular importance. Eulerian measurements e.g., moorings, sea floor installations, tied to key locations, such as across straits and boundary currents, address these weaknesses. Fixed installations complement the broad stochastic array by ensuring consistent data return at specific temporal and spatial resolution. Lastly, measurements collected in the Lagrangian frame, drifting with a water parcel or ice floe, can be employed to isolate time evolution driven by internal processes from advective effects. For instance, estimate the difference between observing the time-evolution of a single ice floe and recording variability in sea ice due to different ice floes passing through the domain.

Approaches that incorporate small, long-endurance autonomous platforms, including ice-based instruments, surface drifters, profiling floats, underwater gliders and autonomous surface vessels, offer capabilities that address many of the challenges confronting ARCGOOS implementation. Operational constraints have historically restricted measurements to the times and locations accessible by ships and aircraft, thus biasing observations toward summertime regions lacking thick, multiyear sea ice. Some autonomous platforms, such as ice-based observatories, profiling floats and gliders, offer endurance that exceeds a year, thus providing persistent sampling through the full annual cycle. Flexible deployment logistics afforded by small size, coupled with endurance and mobility (self-propelled, or drifting in water or sea ice) allows access to remote regions. Relatively low unit cost provides the potential to scale to large numbers, both for achieving large scope and for creating robust systems that are resilient to instrument losses. Systems of autonomous platforms (e.g.,

${ }^{5}$ http://iabp.apl.washington.edu
Lee et al., 2017) can provide a flexible observing capability that can be readily reconfigured in response to changing needs and objectives. Lastly, relatively light logistical requirements and low cost help make sustained operation over many-year timescales more tractable.

Autonomous approaches also face challenges. Sensor technologies currently limit autonomous platforms to a modest suite of variables, with clear needs for advances in biological, biogeochemical and atmospheric sensors. Sustained collection of critical in situ observations, for measurements that cannot be made autonomously and for referencing autonomous sensors, will require periodic ship-based sampling, such as that planned by the Synoptic Arctic Survey (Anderson et al., 2018). When operating beneath ice, autonomous platforms cannot access satellite services (GPS and Iridium), and must instead rely on acoustic networks (Lee and Gobat, 2006; ANCHOR Working Group, 2008; Mikhalevsky et al., 2015) for underwater geopositioning (UW-GPS) and telemetry. Acoustic infrastructure might thus be required to maximize the utility of autonomous approaches. Acoustic networks augmented with hydrophones and oceanographic instruments could provide a scalable multipurpose ocean observing system including UW-GPS, acoustic thermometry along fixed sections, passive acoustics, and oceanographic point measurements (e.g., Mikhalevsky et al., 2015).

A critical and growing component of the ARCGOOS is satellite remote sensing. Satellite mounted sensors provide a pan-Arctic observing capability. They are a powerful tool to put land/marine/ice based in-situ measurements in a large-scale context. There are numerous satellite mounted sensors, from different space agencies, that have significant relevance for the Arctic region. With respect to sea ice, passive microwave satellite measurements (which can see through clouds and the polar night) can be used to obtain sea ice characteristics such as sea ice concentration and extent (Martin and Cavalieri, 1989; Bareiss and Goergen, 2005). Passive microwave measurements have been made from US satellites since the late 1970s, thus providing a continuous record of sea ice conditions for over 40 years. PanArctic sea ice thickness can be calculated from radar (ESA) and, with the 2018 launch of ICESat-2, laser (NASA) altimetry by accurately measuring the height of the ice (and snow) above the sea surface (its freeboard) and converting this information into an ice thickness (e.g., Kwok and Cunningham, 2008; Laxon et al., 2013). Scatterometer-derived information can be used for identification of the sea ice edge (Long and Hicks, 2005) as well as to provide high-resolution information on ice type (Kwok, 2004). The most widely used satellite sensor for operational human activities in ice covered seas is Synthetic Aperture Radar (SAR). SAR images, which also see through clouds and the polar night, are used routinely for ship navigation of ice-covered waters through to inferring sea ice characteristics (Willmes et al., 2010), sea ice drift (e.g., Spreen et al., 2011) and deformation (e.g., Bouillon and Rampal, 2015).

Many recent advances in physical oceanography have become possible due to the advent of altimetry and gravimetry satellites that observe the total and ocean mass components of sea level change. Radar altimetry cannot measure sea level over sea ice, 
but significant progress has been made in data retrieval over leads and polynyas (Laxon, 1994; Peacock and Laxon, 2004; Farrell et al., 2012; Kwok and Morison, 2016; Anderson et al., 2018). Satellite altimetry measurements have proven to be efficient for monitoring the large-scale variability of ocean circulation in the Arctic (e.g., Armitage et al., 2017).

While the spatial coverage of satellite altimetry data is limited to leads and open ocean, the Gravity Recovery and Climate Experiment (GRACE) and its follow-on (GRACE-FO) missions provide basin-wide observations of monthly ocean mass variations that also reveal changes in Arctic Ocean circulation patterns (Volkov and Landerer, 2013; Peralta-Ferriz and Morison, 2014). Subtracting GRACE records from altimetric sea level yields estimates of the steric sea level variability (due to density changes), which are indicative of changes in the Arctic Ocean freshwater content (Häkkinen and Proshutinsky, 2004; Giles et al., 2012; Morison et al., 2012). The combined use of satellite derived geostrophic surface currents and subsurface velocities derived from hydrography data makes it possible to routinely monitor the volume transport of the warm and saline Atlantic Water in the Nordic Seas (Chafik et al., 2015; Raj et al., 2018). In the near-future, progress can be made with wide-swath Surface Water and Ocean Topography (SWOT) interferometric altimetry mission, scheduled for launch in 2021. With a spatial resolution of about $10 \mathrm{~km}$ this mission will further enhance our knowledge about mesoscale and coastal zone dynamics (e.g., Fu and Ferrari, 2008). High-resolution surface velocity data from Synthetic Aperture Radar data (SAR) is another potential source of information about ocean circulation (Chapron et al., 2005; Johannessen et al., 2008; Hansen et al., 2012). Other satellite sensors provide information on, for example, phytoplankton blooms, sea surface temperature and salinity, albedo, and surface wind speeds.

In addition to identification of SBAs and the resulting ARCGOOS objectives, the engagement of local communities is also critical to implementation. Many communities already maintain a persistent presence in key areas for the observing system, and could thus inform and support the sustained collection of high-quality in situ measurements. Furthermore, the efficient operation of autonomous platforms depends highly on local logistics, such that a sustained measurement program would benefit greatly from partnerships with communities near the target region. By nature, community-based monitoring programs tend to focus on those issues of greatest concern to local stakeholders, thus having considerable potential to influence on-the-ground management activities e.g., acoustic pollution from seismic surveys. It is found that CBM programs inform decisions at local, regional and national levels and often provide insight into processes and changes not captured in agency or research-driven monitoring programs. An evaluation of the capabilities, good practice and challenges of CBM programs is provided by Danielsen et al. (2018). Engagement surrounding implementation might also establish relationships that guide analyses and enhance uptake of the resulting products.

Although not a direct component of the sustained observing system, focused, limited-duration process studies will continue to be crucial for advancing understanding of the marine system and informing the evolution of ARCGOOS. Examples include recent international programs focused on the Marginal Ice Zone during melt (Lee et al., 2017), Arctic Sea state and boundary layer physics during freeze up (Thomson et al., 2018) and the large Multidisciplinary drifting Observatory for the Study of Arctic Climate (MOSAiC), which will conduct intensive measurements of that atmosphere-ice-ocean system using the German icebreaker Polarstern as a drifting platform for a year.

The changing Arctic presents new challenges and opportunities to ARCGOOS implementation. Steady decline in sea ice extent, accompanied by changes in thickness distribution and character, make landing aircraft on the ice, and thus deploying instruments, increasingly difficult. The growing dominance of first year ice makes it more challenging to deploy ice-based platforms onto floes that are likely to survive beyond a year, motivating new platform designs that can withstand the rigors of melt-out, open water drift and freezein. In contrast, growing open water extent in summer eases the operation of autonomous platforms sampling within the water column, as they have increasing access to satellite services. Important opportunities for the ARCGOOS include exploiting the current generation of autonomous platforms and accelerated development of sensors for water column biology and biogeochemistry and for fully autonomous, untended atmospheric measurements including radiative fluxes, winds, aerosols and clouds.

\section{EXAMPLES}

There are many broad and important efforts underway regionally and topically. These include, for example, observing systems characterizing Arctic gateways (Fram Strait, e.g., Soltwedel et al., 2013; Hansen et al., 2013; Rabe et al., 2013; Sagen et al., 2016; de Steur et al., 2018; Bering Strait, e.g., Woodgate,. 2018; Davis Strait, e.g., Curry et al., 2014), a network of distributed, drifting Ice Tethered Profilers (e.g., Toole et al., 2011) and observing systems under the PAG, such as the DBO (see Moore and Grebmeier, 2018). Basin-wide Arctic Ocean observations are particularly challenging, but efforts such as the NABOS (e.g., Polyakov et al., 2017) and the INTAROS are leading the way. These examples demonstrate that international collaboration with common scientific and logistical work components is crucial for meeting the challenges of the complexity of natural systems, demanding specific tailored observing systems suitable for Arctic conditions. They offer successful models for operating and sustaining systems driven by science choices, motivated by immediate SBAs.

Fisheries potential and sound management in the Pacific sector of the Arctic Ocean requires understanding how the marine ecosystem will respond to seasonal shifts in the timing of spring sea ice retreat and/or delays in fall sea ice formation. To more systematically track the broad biological response to sea ice retreat and associated environmental change, an international consortium of scientists implemented a coordinated DBO that includes selected biological measurements at multiple trophic levels (Moore and Grebmeier, 2018). These measurements are being made simultaneously with hydrographic surveys and satellite observations. The DBO focuses on biological "hotspot" 
locations in the Bering and Chukchi Seas. From European side intensive environmental and ecosystem monitoring programs are carried out in the Barents Sea used for resource management in this important region for fisheries in Norway and Russia.

The internationally coordinated NABOS has focused on the detection of Arctic Ocean change in the Eurasian Basin. The program's primary monitoring tool is a series of moorings and quasi-synoptic oceanographic surveys to quantify circulation, water mass transformations, and key mechanisms of variability in the Arctic Ocean, including links to lower-latitude processes. The observing array captured several warming events in the Atlantic Water layer, and a general weakening of the stratification in this sector of the Arctic (Carmack et al., 2015). As successful as these programs are, they are still reliant on the good will of independent Arctic nations to allow for international monitoring efforts within their territorial seas or exclusive economic zones.

Integrated Arctic Observation System involves 48 partners from 20 different countries to contribute to the development of an INTAROS addressing ocean, atmosphere and land. With a focus on coordination, observing system assessments and data integration for user applications. INTAROS aims to produce a roadmap for a sustained Arctic observing that will encompass coordination, data collection, data handling, and services benefiting specific stakeholders.

These regional and topical efforts are not presently framed within a unified strategy for synthesis (e.g., Behrendt et al., 2018) or network development. Beyond advancing more coordination of existing efforts (capabilities-based approaches), we recommend requirements-driven approaches, which require a shared assessment system to identify and weigh priorities.

\section{VISION}

The different assessments, planning processes, and systems referenced above provide a foundation for a multi-function ARCGOOS. The functions associated with an ARCGOOS (Figure 1) for a subset of all SBAs, serve broad and diverse end user and scientific communities. The considerations in this review paper strongly support the establishment of ARCGOOS with the following benefits:

- The scientific community has at its disposal decadal scale observations of a broad array of variables that describe the state and dynamics of core Arctic socialenvironmental systems in order to track and anticipate major changes, inform model development, and support validation of remote sensing data and model output, and advance our understanding of an environment undergoing transformative, globally relevant change;

- Arctic residents and communities can rely on a combination of community-based monitoring and sustained pan-Arctic sensor-based observations to help them understand and respond to a rapidly changing Arctic, preparing for rapid- and slow-onset hazards and supporting sustainable development;
- National, local, and Indigenous governments are receiving focused, meaningful information about long term change and variability from an observing system that supports planning and decision-making for community health and sustainability;

- The global community of Arctic and non-Arctic countries is furnished with data from Arctic observing networks that guides long-term planning to minimize the impacts of environmental hazards associated with a rapidly changing Arctic;

- Resource management agencies access reliable long-term records available to inform prudent management action and policy;

- Emergency response organizations draw on a suite of near real-time environmental observations that guide emergency response and hazard mitigation efforts;

- The global community is able to trace the impacts and determine the efficacy of international treaty systems and mitigative measures meant to address the negative impacts of climate change by drawing on an observing system with the requisite sensitivity and resolution.

With this review paper a foundation has been laid for the design and optimization of ARCGOOS leveraging existing system components at the international level. SAON in particular has emerged as the entity that is both tasked and capable with additional resources emerging following the second Arctic Science Ministerial in October 2018 - to address this challenge. Collaboration and coordination with national, regional, and local efforts, and global observing programs such as GEOSS, GCOS, GOOS, and others is essential. A further critical component to observing system success is to ensure relevance of the system to the people of the Arctic. The latter can only be achieved through co-design and co-management of such a system and the data it produces. Recent assessments by Johnson et al. (2015) and Williams et al. (2018) as well as a survey of best practices by Fidel et al. (2017) and Danielsen et al., 2018 point the way on how to achieve these outcomes through integration of communitybased observations.

We conclude with a few specific recommendations drawn from this review article and some of the documents and initiativesthis work draws on.

Recommendation 1: While progress has been made in several disciplines or sectors, what is lacking is a framework that facilitates and supports coordinated implementation and integration of observing system components that fill critical thematic, regional, or trans-sectoral gaps. We recognize that an Arctic Ocean observing system will require such a framework that allows for the optimization, modification, and integration of existing observing programs and networks to go hand in hand with design and implementation of new observing components. Quantitative, simulation-based frameworks for optimal observing network design (including, OSE, OSSE, OED) should be advanced, matured and be made available as a tool for supporting ARCGOOS efforts. Such an approach 
should build on capacity and leadership by SAON, partnering with global observing programs and systems (in particular those called out above) where appropriate. Private-public partnerships and the approach taken by the Copernicus, a European Program for the establishment of capacity for earth observation, may serve as models and incentives for increased attention needed at the level of potential public and private sector funders of such activities.

Recommendation 2: An emerging governance structure under SAON will help minimize duplication, channel resources, and support shared benefits. What is urgently needed as a first step is international collaboration on developing a roadmap for an integrated observing system that meets user requirements as well as putting in place components that draw on existing networks and help maximize shared benefits in the near- and mid-term. The AOS and venues such as OceanObs need to be drawn upon as platforms to advance exchange and define specific action. $\mathrm{AOS}^{6}$ in particular provides a forum for different user groups, engineers and sensor network designers, and the broader research community to come together and make progress with some of the thornier issues, that require intense, focused input and reconciliation of different approaches. The Arctic-themed Community White Papers submitted to OceanObs'19 illustrate both the breadth of contributions and the potential for developing closer links and optimization along regional or sectoral interfaces. Beyond these surveys, a commitment toward parsing and consolidation of planning efforts is needed.

Recommendation 3: An efficient ARCGOOS that provides information across the range of scales demanded by the SBAs and missions laid out above, needs to focus attention on the integration of remote sensing and in-situ observations. Robustness and reliable data delivery play a major role in the design of observing systems, as well as multipurpose use of autonomous observing platforms and infrastructure. Low-cost, long-endurance autonomous platforms offer promising new approaches for large-scale, sustained observing. Development of small, low-power sensors suitable for deployment on these platforms requires further support and attention by both the engineering and research community. Development and implementation of systems for geopositioning and telemetry is needed to make underwater autonomous observing systems fully operational.

Recommendation 4: To maximize the uptake of observations and derived products, all data must be secured in long-term storage in an established data repository adhering to best practices for scientific data management. This includes standardized metadata describing the content, processing and quality control procedures applied, and assignment of unique persistent identifiers (such as DOIs). Datasets must be searchable

${ }^{6} \mathrm{http}: / /$ www.arcticobservingsummit.org/ and accessible through standard protocols, enabling a wide range of software clients to utilize them, and must be accompanied by a data license. The license will define acceptable use of the data, and include a citation statement linking back to the data providers giving them credit for their datasets. There is a need for a governance structure to coordinate the many ongoing initiatives in Spatial Data Infrastructures (SDIs) for Arctic data to ensure all future ARCGOOS data follow the FAIR Principles. SAON, through its Arctic Data Committee, should take the lead in establishing a pan-Arctic SDI in collaboration with the major players in Arctic data management.

Recommendation 5: To ensure societal relevance, address critical capacity issues, and acknowledge relevant expertise and authority, Indigenous peoples of the Arctic and Arctic residents need to be involved in the definition, design, and implementation of an Arctic Ocean observing system; co-design of the system, co-management of resulting data products, and integration of communitybased observations into the observing system fabric best serve this goal.

\section{AUTHOR CONTRIBUTIONS}

CL, SSt, HE, M-LT, JW, and SSa contributed to the overall concept, writing of the initial draft, and revision of all sections. DD, SG, JG, JI, S-HK, MM, IP, BR, SSe, DV, AN, and TH contributed writing to individual sections. AB-M, MD, GG, $\mathrm{AK}, \mathrm{AO}, \mathrm{TR}, \mathrm{HS}, \emptyset \mathrm{S}, \mathrm{HS}, \mathrm{LC}, \mathrm{RR}$, and $\mathrm{KS}$ contributed ideas and material for individual sections. All authors have read and approved the submitted version.

\section{FUNDING}

CL was supported by the US Office of Naval Research under the grant N00014-16-1-2377. SSt was supported by the US National Oceanic and Atmospheric Administration - Arctic Research Program. DD was supported by HYCOM NOPP (Award No. N00014-15-1-2594). SSa, TH, HS, AK, KS, and AB-M were supported by INTAROS project. This project has received funding from the European Union's Horizon 2020 Research and Innovation Programme under the grant agreement number 727890. BR strongly benefited from the work within the German Helmholtz Association strategic investment Frontiers in Arctic Marine Monitoring (FRAM). DV was supported by the Atlantic Oceanographic and Meteorological Laboratory under the auspices of the Cooperative Institute for Marine and Atmospheric Studies of the University of Miami and NOAA (cooperative agreement NA10OAR4320143). JG was supported by the US National Science Foundation Arctic Observing Network grant \#1702456 and National Oceanic and Atmospheric Administration grant \#CINAR 22309.02. SG was supported in part by the Norwegian Polar Institute's project "The Fram Strait Arctic Outflow Observatory." LC was supported by the Swedish National Space Agency (Dnr:133/17). 


\section{REFERENCES}

Aagaard, K., and Coachman, L. (1975). Toward an ice-free Arctic ocean. Eos Trans. AGU 56, 484-486. doi: 10.1029/EO056i007p00484

Alexanderian, A., Petra, N., Stadler, G., and Ghattas, O. (2016). A fast and scalable method for a-optimal design of experiments for infinite-dimensional bayesian non-linear inverse problems. SIAM J. Sci. Comput. 38, A243-A272.

AMAP (2017). Snow, Water, Ice and Permafrost in the Arctic (SWIPA). Oslo: Arctic Monitoring and Assessment Programme (AMAP), 269.

Anchor Working Group. (2008). Acoustic Navigation and Communication for High-Latitude Ocean Research. Seattle, WA: University of Washington, 54.

Anderson, L. G., Ashjian, C., Azetsu-Scott, K., Carmack, E., Chierici, M., Cho, K.H., et al. (2018). Synoptic Arctic Survey - a Pan-Arctic Research Program. Science and Implementation Plan. 40. Available at: http://www.synopticarcticsurvey. info/ (accessed January 25, 2019).

Andrews, J., Babb, D., and Barber, D. G. (2017). Climate change and sea ice: shipping accessibility on the marine transportation corridor through Hudson Bay and Hudson Strait (1980-2014). Elem. Sci. Anth. 5:15. doi: 10.1525/ elementa. 130

AOS Executive Organizing Committee [AOS EOC] (2018). Report of the 4th Arctic Observing Summit: AOS 2018, Davos Switzerland, 24-26 June 2018. Calgary, AB: International Study of Arctic Change (ISAC) Program Office.

Arctic Council (2011). Nuuk Declaration. Canada, Denmark, Faroe Islands, Finland, Greenland, Iceland, Norway, Russian Federation, Sweden, United States of America. Available at: https://oaarchive.arctic-council.org/handle/11374/92 (accessed May 12, 2011).

Argo Steering Team (1998). On the Design and Implementation of Argo: An Initial Plan for a Global Array of Profiling Floats. International CLIVAR Project Office Report 21, GODAE Report 5. Melbourne, VIC: GODAE International Project Office, 32 .

Armitage, T. W. K., Bacon, S., Ridout, A. L., Petty, A. A., Wolbach, S., and Tsamados, M. (2017). Arctic Ocean surface geostrophic circulation 2003-2014. Cryosphere 11, 1767-1780. doi: 10.5194/tc-11-1767-2017

Armitage, T. W. K., Bacon, S., Ridout, A. L., Thomas, S. F., Aksenov, Y., and Wingham, D. J. (2016). Arctic sea surface height variability and change from satellite radar altimetry and GRACE, 2003-2014. J. Geophys. Res. Oceans 121, 4303-4322. doi: 10.1002/2015JC011579

Arrigo, K. R., van Dijken, G., and Pabi, S. (2008). Impact of a shrinking Arctic ice cover on marine primary production. Geophys. Res. Lett. 35:L19603. doi: 10.1029/2008GL035028

Atlas, R. (1997). Atmospheric observations and experiments to assess their usefulness in data assimilation. J. Meteor. Soc. Japan 75, 111-130. doi: 10.2151/ jmsj1965.75.1b_111

Barber, D. G., Babb, D. G., Ehn, J. K., Chan, W., Matthes, L., Dalman, L. A., et al. (2018). Increasing mobility of high Arctic Sea ice increases marine hazards off the east coast of Newfoundland. Geophys. Res. Lett. 45, 2370-2379. doi: $10.1002 / 2017 \mathrm{gl} 076587$

Bareiss, J., and Goergen, K. (2005). Spatial and temporal variability of sea ice in the Laptev sea: analysis and review of satellite passive-microwave data and model results, 1997 to 2002. Glob. Plan. Change 48, 28-54. doi: 10.1016/j.gloplacha. 2004.12.004

Bauer, P., Thorpe, A., and Brunet, G. (2015). The quiet revolution of numerical weather prediction. Nature 525, 47-55. doi: 10.1038/nature14956

Behrendt, A., Sumata, H., Rabe, B., and Schauer, U. (2018). UDASH - unified database for Arctic and Subarctic hydrography. Earth Syst. Sci. Data 10, 11191138. doi: 10.5194/essd-10-1119-2018

Bouillon, S., and Rampal, P. (2015). On producing sea ice deformation data sets from SAR-derived sea ice motion. Cryosphere 9, 663-673. doi: 10.5194/tc-9663-2015

Box, J. E., Colgon, W. T., Christensen, T. R., Schmidt, N. M., Lund, M., and Parmentier, F. W. (2019). Key indicators of Arctic climate change: 1971-2017. Environ. Res. Lett. 14:045010.

Bui-Thanh, T., Burstedde, C., Ghattas, O., Martin, J., Stadler, G., and Wilcox, L. C. (2012). "Extreme-scale UQ for Bayesian Inverse Problems Governed by PDEs," in Proceedings of the International Conference on High Performance Computing, Networking, Storage and Analysis, SC '12, (Los Alamitos, CA: IEEE Computer Society Press), 3:1-3:11.
Calafat, F. M., Chambers, D. P., and Tsimplis, M. N. (2013). Interannual to decadal sea level variability in the coastal zones of the Norwegian and Siberian seas: the role of atmospheric forcing. J. Geophys. Res. 118, 1287-1301. doi: 10.1002/ JGRC.20106

Carmack, E., Polyakov, I., Padman, L., Fer, I., Hunke, E., Hutchings, J., et al. (2015). Toward quantifying the increasing role of oceanic heat in sea ice loss in the New Arctic. Bull. Amer. Meteor. Soc. 96, 2079-2105. doi: 10.1175/BAMS-D-1300177.1

Carmack, E. C., Yamamoto-Kawai, M., Haine, T. W. N., Bacon, S., Bluhm, B. A., Lique, C., et al. (2016). Freshwater and its role in the Arctic marine system: sources, disposition, storage, export, and physical and biogeochemical consequences in the Arctic and global oceans. J. Geophys. Res. Biogeosci. 121, 675-717. doi: 10.1002/2015JG003140

Centurioni, L., Horányi, A., Cardinali, C., Charpentier, E., and Lumpkin, R. (2017). A global ocean observing system for measuring sea level atmospheric pressure: effects and impacts on numerical weather prediction. Bull. Am. Meteorol. Soc. 98, 231-238. doi: 10.1175/bams-d-15-00080.1

Chafik, L., Nilsson, J., Skagseth, $\varnothing$, and Lundberg, P. (2015). On the flow of Atlantic water and temperature anomalies in the Nordic seas toward the Arctic ocean. J. Geophys. Res. Oceans 120, 7897-7918. doi: 10.1002/2015JC011012

Chapron, B., Collard, F., and Ardhuin, F. (2005). Direct measurements of ocean surface velocity from space: interpretation and validation. J. Geophys. Res. Oceans 110:7008.

Cheng, L., Abraham, J., Hausfather, Z., and Trenberth, K. E. (2019). How fast are the oceans warming? Science 363, 128-129. doi: 10.1126/science.aav7619

Crépin, A. S., Karcher, M., and Gascard, J. C. (2017). Arctic climate change, economy and society (ACCESS): integrated perspectives. Ambio 46(Suppl. 3), 341-354. doi: 10.1007/s13280-017-0953-3

Curry, B., Lee, C. M., Petrie, B., Moritz, R., and Kwok, R. (2014). Multi-year volume, liquid 1978 freshwater, and sea ice transports through Davis Strait, 2004-2010. J. Phys. Oceanogr. 44, 1244-1266. doi: 10.1175/JPO-D-13-0177.1

Danielsen, F., Fidel, M., Johnson, N., Poulsen, M. K., Eicken, H., Alba, A., et al. (2018). INTAROS D4.1. Community based Monitoring Programmes in the Arctic: Capabilities, Good Practice and Challenges. Research and Innovation Action under EC Horizon 2020 Grant Agreement no. 727890. Brussels: European Commission.

de Steur, L., Peralta-Ferriz, C., and Pavlova, O. (2018). Freshwater export in the East Greenland current freshens the North Atlantic. Geophys. Res. Lett. 45, 359-313. doi: 10.1029/2018GL080207

Dobricic, S., Monforti, F., Pozzoli, L., Wilson, J., Gambardella, A., and Tilche, A. (2018). Impact Assessment Study on Societal Benefits of Arctic Observing Systems IMOBAR. Luxembourg: European Union, doi: 10.2760/713084

Eicken, H., Lee, O. A., and Lovecraft, A. L. (2016). Evolving roles of Observing Systems and data Co-management in Arctic Ocean Governance, OCEANS 2016 MTS/IEEE Monterey. Monterey, CA: IEEE, 1-8. doi: 10.1109/OCEANS.2016. 7761298

Eicken, H., Lovecraft, A. L., and Druckenmiller, M. L. (2009). Sea-ice system services: a framework to help identify and meet information needs relevant for Arctic observing networks. Arctic 62, 119-136.

Eicken, H., and Mahoney, A. R. (2015). "Sea ice: Hazards, risks, and implications for disasters," in Coastal and Marine Hazards, Risks, and Disasters, eds J. Ellis and D. J. Sherman (Amsterdam: Elsevier), 381-401. doi: 10.1016/b978-0-12 396483-0.00013-3

Farrell, S. L., McAdoo, D. C., Laxon, S. W., Zwally, H. J., Li, D., Ridout, A., et al. (2012). Mean dynamic topography of the arctic ocean, Geophys. Res. Lett. 39:L01601. doi: 10.1029/2011GL050052

Fidel, M., Johnson, N., Danielsen, F., Eicken, H., Iversen, L., Lee, O., et al. (2017). INTAROS Community-based Monitoring Experience Exchange Workshop Report. Alaska, AK: Yukon River Inter-Tribal Watershed Council (YRITWC).

Flath, H., Wilcox, L., Akçelik, V., Hill, J., van Bloemen Waanders, B., and Ghattas, O. (2011). Fast algorithms for bayesian uncertainty quantification in largescale linear inverse problems based on low-rank partial hessian approximations. SIAM J. Sci. Comput. 33, 407-432. doi: 10.1137/090780717

Flynn, M., Ford, J. D., Pearce, T., Harper, S., and Ihacc Research Team. (2018). Participatory scenario planning and climate change impacts, adaptation and vulnerability research in the Arctic. Environ. Sci. Policy 79, 45-53. doi: 10.1016/ j.envsci.2017.10.012 
Fu, L.-L., and Ferrari, R. (2008). Observing oceanic submesoscale processes from space. Eos Trans. Am. Geophys. Union 89:488. doi: 10.1029/2008eo480003

Gerland, S., Barber, D., Meier, W., Mundy, C. J., Holland, M., Kern, S., et al. (2019). Essential gaps and uncertainties in the understanding of the roles and functions of Arctic sea ice. Environ. Res. Lett. 14:043002. doi: 10.1088/1748-9326/ab09b3

German Arctic Office. (2019). Co-Operation in Arctic Science - Challenges and Joint Actions. Report of the 2nd Arctic Science Ministerial. Bonn: Federal Ministry of Education and Research, 150.

Giles, K. A., Laxon, S. W., Ridout, A. L., Wingham, D. J., and Bacon, S. (2012). Western Arctic ocean freshwater storage increased by wind-driven spin-up of the Beaufort Gyre. Nat. Geosci. 5, 194-197. doi: 10.1038/NGEO1379

Häkkinen, S., and Proshutinsky, A. (2004). Freshwater content variability in the Arctic ocean. J. Geophys. Res. 109:C03051. doi: 10.1029/2003JC001940

Halliwell, G. R., Srinivasan, A., Kourafalou, V., Yang, H., Willey, D., Le Hénaff, M., et al. (2014). Rigorous evaluation of a fraternal twin ocean OSSE system in the open Gulf of Mexico. J. Atmos. Ocean. Technol. 31, 105-130. doi: 10.1175/ JTECH-D-13-00011.1

Hansen, E., Gerland, S., Granskog, M. A., Pavlova, O., Renner, A. H. H., Haapala, J., et al. (2013). Thinning of Arctic sea ice observed in Fram Strait: 1990-2011. J. Geophys. Res. 18, 5202-5221. doi: 10.1002/jgrc.20393

Hansen, M. W., Johannessen, J. A., and Raj, R. P. (2012). "Mapping the Nordic Seas surface velocity using Envisat ASAR," in Proceedings of the ESA SeaSAR 2012, Tromsø.

Heimbach, P., Wunsch, C., Ponte, R. M., Forget, G., Hill, C., and Utke, J. (2011). Timescales and regions of the sensitivity of Atlantic meridional volume and heat trans- port: toward observing system design. Deep Sea Res. Part 2 Topic. Stud. Oceanogr. 58, 1858-1879. doi: 10.1016/j.dsr2.2010.10.065

Hoffman, R. N., and Atlas, R. (2015). Future observing system simulation experiments. Bull. Am. Meteorol. Soc. 97, 1601-1616. doi: 10.1175/bams-d-1500200.1

Hunt, G. L. Jr., and Megrey, B. A. (2005). Comparison of the biophysical and trophic characteristics of the Bering and Barents Seas. ICES J. Mar. Sci. 62, 1245-1255. doi: 10.1016/j.icesjms.2005.04.008

Jahn, A. (2018). Reduced probability of ice-free summers for $1.5^{\circ} \mathrm{C}$ compared to $2^{\circ} \mathrm{C}$ warming. Nat. Clim. Change 8, 409-413. doi: 10.1038/s41558-018$0127-8$

Johannessen, J. A., Chapron, B., Collard, F., Kudryavtsev, V., Mouche, A., Akimov, D., et al. (2008). Direct ocean surface velocity measurements from Space: improved quantitative interpretation of Envisat ASAR observations. Geophys. Res. Lett. 35:L22608.

Johnson, N., Alessa, L., Behe, C., Danielsen, F., Gearheard, S., GofmanWallingford, V., et al. (2015). The contributions of community-based monitoring and traditional knowledge to Arctic observing networks: reflections on the state of the field. Arctic 68, 28-40.

Jung, T., Gordon, N. D., Bauer, P., Bromwich, D. H., Chevallier, M., Day, J. J., et al. (2016). Advancing polar prediction capabilities on daily to seasonal time scales. Bull. Am. Meteorol. Soc. 97, 1631-1647. doi: 10.1175/bams-d-14-00246.1

Kalmikov, A., and Heimbach, P. (2014). A hessian-based method for uncertainty quantification in global ocean state estimation. SIAM J. Sci. Comput. 36:S267S295.

Kalmikov, A. G., and Heimbach, P. (2018). On barotropic mechanisms of uncertainty propagation in estimation of drake passage transport. arXiv

Kaminski, T., Kauker, F., Eicken, H., and Karcher, M. (2015). Exploring the utility of quantitative network design in evaluating Arctic sea ice thickness sampling strategies. Cryosphere 9, 1721-1733. doi: 10.5194/tc-9-1721-2015

Kaminski, T., Kauker, F., Toudal Pedersen, L., Voßbeck, M., Haak, H., Niederdrenk, L., et al. (2018). Arctic mission benefit analysis: impact of sea ice thickness, freeboard, and snow depth products on sea ice forecast performance. Cryosphere 12, 2569-2594. doi: 10.5194/tc-12-2569-2018

Köhl, A., and Stammer, D. (2004). Optimal observations for variational data assimilation. J. Phys. Oceanogr. 34, 529-542. doi: 10.1175/2513.1

Krishfield, R., Toole, J., Proshutinsky, A., and Timmermans, M.-L. (2008). Automated ice-tethered profilers for seawater observations under pack ice in all seasons. J. Atmos. Oceanic Technol. 25, 2091-2105. doi: 10.1175/2008jtecho 587.1

Kwok, R. (2004). Annual cycles of multiyear sea ice coverage of the Arctic Ocean: 1999-2003. J. Geophys. Res. 109:C11004. doi: 10.1029/2003JC002238
Kwok, R. (2018). Arctic sea ice thickness, volume, and multiyear ice coverage: losses and coupled variability (1958-2018). Environ. Res. Lett. 13:105005. doi: 10.1088/1748-9326/aae3ec

Kwok, R., and Cunningham, G. F. (2008). ICESat over Arctic sea ice: estimation of snow depth and ice thickness. J. Geophys. Res. 113:C08010. doi: 10.1029/ 2008JC004753

Kwok, R., and Morison, J. (2016). Sea surface height and dynamic topography of the ice-covered oceans from CryoSat-2: 2011-2014. J. Geophys. Res. Oceans 121, 674-692. doi: 10.1002/2015JC011357

Laxon, S. (1994). Sea ice altimeter processing scheme at the EODC. Int. J. Remote Sens. 15, 915-924. doi: 10.1080/01431169408954124

Laxon, S., Giles, K. A., Ridout, A., Wingham, D. J., Willatt, R. C., Cullen, R., et al. (2013). CryoSat-2 estimates of Arctic sea ice thickness and volume. Geophys. Res. Lett 40, 732-737. doi: 10.1098/rsta.2014.0157

Lee, C. M., and Gobat, J. I. (2006). Acoustic navigation and communication for high-latitude ocean research workshop. EOSTrans. AGU 87:268. doi: 10.1029/ 2006 eo270004

Lee, C. M., and Thomson, J., and The Marginal Ice Zone and Arctic Sea State Teams (2017). An autonomous approach to observing the seasonal ice zone in the western Arctic. Oceanography 30, 56-68. doi: 10.5670/oceanog.2017.222

Lee, O., Eicken, H., Kling, G., and Lee, C. (2015). A framework for prioritization, design and coordination of arctic long-term observing networks: a perspective from the US SEARCH program. Arctic 68, 76-88.

Lindsay, R., and Schweiger, A. (2015). Arctic sea ice thickness loss determined using subsurface, aircraft, and satellite observations. Cryosphere 9, 269-283. doi: 10.5194/tc-9-269-2015

Lindsay, R., Wensnahan, M., Schweiger, A., and Zhang, J. (2014). Evaluation of seven different atmospheric reanalysis products in the Arctic. J. Clim. 27, 2588-2606. doi: 10.1175/jcli-d-13-00014.1

Lindsay, R. W., and Zhang, J. (2006). Arctic ocean ice thickness: modes of variability and the best locations from which to monitor them. J. Phys. Oceanogr. 36, 496-506. doi: 10.1175/jpo2861.1

Lindstrom, E., Gunn, J., Fischer, A., McCurdy, A., and Glover, L. (2012). A Framework for Ocean Observing. By the Task Team for an Integrated Framework for Sustained Ocean Observing. Paris: UNESCO, doi: 10.5270/OceanObs09FOO

Long, D. G., and Hicks, B. R. (2005). Standard BYU QuikSCAT/SeaWinds Land/Ice Image Products, Report. Provo, UT: Brigham Young University.

Loose, N. (2019). Adjoint Modeling and Observing System Design in the Subpolar North Atlantic. Ph.D. dissertation, University of Bergen, Bergen.

Lovecraft, A. L., Meek, C. L., and Eicken, H. (2013). Connecting scientific observations to stakeholder needs in sea ice social-environmental systems: the institutional geography of northern Alaska. Polar Geogr. 36, 105-125. doi: 10.1080/1088937x.2012.733893

Ludvigsen, C. A., Pirazzini, R., Sagen, H., Hamre, T., Sandven, S., Stette, M., et al. (2018). INTAROS Deliverable 2.1. Report on Present Observing Capacities and Gaps: Ocean and Sea Ice Observing System. Research and Innovation Action under EC Horizon 2020 Grant Agreement no. 727890. Brussels: European Commission.

Lumpkin, R., and Pazos, M. (2007). Measuring surface currents with Surface Velocity Program drifters: the instrument, its data, and some recent results. Chapter two of Lagrangian Analysis and Prediction of Coastal and Ocean Dynamics (LAPCOD), eds A. Griffa, A. D. Kirwan, A. J. Mariano, T. Ozgokmen, and T. Rossby (New York, NY: Cambridge University Press).

MacIntyre, K. Q., Stafford, K. M., Conn, P. B., Laidre, K. L., and Boveng, P. L. (2015). The relationship between sea ice concentration and the spatio-temporal distribution of vocalizing bearded seals Erignathus barbatus in the Bering, Chukchi, and Beaufort Seas from 2008 to 2011. Prog. Ocean. 136, 241-249. doi: $10.1016 /$ j.pocean.2015.05.008

Manabe, S., and Stouffer, R. J. (1980). Sensitivity of the global climate model to an increase of $\mathrm{CO} 2$ in the atmosphere. J. Geophys. Res. 85, 5529-5554.

Markus, T., Neumann, T., Martino, A., Abdalati, W., Brunt, K., Csatho, B., et al. (2017). The ice, cloud, and land elevation satellite-2 (ICESat-2): science requirements, concept, and implementation. Remote Sens. Environ. 190, 260273. doi: 10.1016/j.rse.2016.12.029

Marotzke, J., Giering, R., Zhang, K. Q., Stammer, D., Hill, C., and Lee, T. (1999). Construction of the adjoint MIT ocean general circulation model and 
application to Atlantic heat transport sensitivity. J. Geophys. Res. Oceans 104, 29529-29547. doi: 10.1029/1999jc900236

Martin, S., and Cavalieri, D. J. (1989). Contributions of the Siberian shelf polynyas to the Arctic ocean intermediate and deep water. J. Geophys. Res 94, 12725 12738. doi: 10.1029/JC094iC09p12725

McNutt, M. K. (2017). Convergence in the Geosciences. GeoHealth 1, 2-3. doi: 10.1002/2017GH000068

Mikhalevsky, P. N., Sagen, H., Worcester, P. F., Baggeroer, A. B., Orcutt, J., Moore, S. E., et al. (2015). Multipurpose acoustic networks in the integrated Arctic ocean observing system. Arctic 68, 1-17.

Moore, A. M., Arango, H. G., Broquet, G., Edwards, C., Veneziani, M., Powell, B., et al. (2011). The regional ocean modeling system (ROMS) 4-dimensional variational data assimilation systems: part ii - perfor- mance and application to the California current system. Progr. Oceanogr. 91, 50-73. doi: 10.1016/j. pocean.2011.05.003

Moore, A. M., Arango, H. G., and Edwards, C. A. (2017a). Reduced-rank array modes of the California current observing system. J. Geophys. Res. Oceans 123, 452-465. doi: 10.1002/2017jc013172

Moore, A. M., Jacox, M. G., Crawford, W. J., Laughlin, B., Edwards, C. A., and Fiechter, J. (2017b). The impact of the ocean observing system on estimates of the California current circulation spanning three decades. Progr. Oceanogr. 156, 41-60. doi: 10.1016/j.pocean.2017.05.009

Moore, S. E., and Grebmeier, J. M. (2018). The distributed biological observatory: linking physics to biology in the Pacific Arctic region. Arctic 71(Suppl. 1), 1-7. doi: 10.14430/arctic4606

Morison, J., Kwok, R., Peralta-Ferriz, C., Alkire, M., Rigor, I., Andersen, R., et al. (2012). Changing Arctic Ocean freshwater pathways. Nature 481, 66-70. doi: 10.1038/nature 10705

Murray, M. S., Sankar, R. D., and Ibarguchi, G. (2018). The Arctic Observing Summit, Background and Synthesis of Outcomes 2013-2016. Calgary, AB: International Study of Arctic Change (ISAC) Program Office.

Nakamura, N., and Oort, A. H. (1988). Atmospheric heat budgets of the polar regions. J. Geophys. Res. 93, 9510-9524. doi: 10.1029/JD093iD08p09510

National Academies of Sciences, Engineering, and Medicine (2017). Sustaining Ocean Observations to Understand Future Changes in Earth's Climate. Washington, DC: The National Academies Press, doi: 10.17226/ 24919

National Research Council (2006). Toward an Integrated Arctic Observing Network. Washington, DC: The National Academies Press, doi: 10.17226/ 11607

National Research Council (NRC) Committee on Designing an Arctic Observing Network (2006). Toward an integrated Arctic Observing Network. Washington, DC: National Academies Press, 1-182.

Neckles, H. A., Lyons, J. E., Guntenspergen, G. R., Shriver, W. G., and Adamowicz, S. C. (2015). Use of structured decision making to identify monitoring variables and management priorities for salt marsh ecosystems. Estuaries Coasts 38, 1215-1232. doi: 10.1007/s12237-014-9822-5

Nguyen, A., Ocaña, V., Garg, V., Heimbach, P., Toole, J., Krishfield, R., et al. (2017). On the benefit of current and future ALPS data for improving Arctic coupled ocean-sea ice state estimation. Oceanography 30, 69-73. doi: 10.5670/oceanog. 2017.223

Nicolaus, M., Katlein, C., Maslanik, J., and Hendricks, S. (2012). Changes in Arctic sea ice result in increasing light transmittance and absorption. Geophys. Res. Lett. 39:L24501. doi: 10.1029/2012GL053738

Nilsson, L. M., Destouni, G., Berner, J., Dudarev, A. A., Mulvad, G., Odland, J. Ø, et al. (2013). A call for urgent monitoring of food and water security based on relevant indicators for the Arctic. Ambio 42, 816-822. doi: 10.1007/s13280-0130427-1

Oke, P. R., Larnicol, G., Fujii, Y., Smith, G. C., Lea, D. J., Guinehut, S., et al. (2015). Assessing the impact of observations on ocean forecasts and reanalyses: Part 1, Global studies. J. Oper. Oceanogr. 8, s49-s62. doi: 10.1080/1755876X.2015. 1022067

Panteleev, G., Yaremchuk, M., and Nechaev, D. (2009). Optimization of mooring observations in Northern Bering Sea. Dynam. Atmos. Oceans 48, 143-154. doi: 10.1016/j.dynatmoce.2008.11.004

Peacock, N. R., and Laxon, S. W. (2004). Sea surface height determination in the Arctic Ocean from ERS altimetry. J. Geophys. Res. 109:C07001. doi: 10.1029/ 2001JC001026
Peralta-Ferriz, C., and Morison, J. H. (2014). Arctic ocean circulation patterns revealed by GRACE. J. Clim. 27, 1445-1468. doi: 10.1175/JCLI-D-13-00013.1

Polyakov, I. V., Pnyushkov, A. V., Alkire, M. B., Ashik, I. M., Baumann, T. M., Carmack, E. C., et al. (2017). Greater role for Atlantic inflows on sea-ice loss in the Eurasian Basin of the Arctic Ocean. Science 356, 285-291. doi: 10.1126/ science.aai8204

Post, E., Bhatt, U. S., Bitz, C. M., Brodie, J. F., Fulton, T. L., Hebblewhite, M., et al. (2013). Ecological consequences of sea-ice decline. Science 341, 519-524. doi: $10.1126 /$ science. 1235225

Preston, B., and Lovecraft, A. L. (2018). "Chapter 8 Scenarios thinking for the Bering-Chukchi-Beaufort Region," in Adaptation Actions for a Changing Arctic - Perspectives from the Bering/Chukchi/Beaufort Region, ed. AMAP (Oslo: Arctic Monitoring and Assessment Programme (AMAP)), 209-230.

Proshutinsky, A., Ashik, I. M., Dvorkin, E. N., Hakkinen, S., Krishfield, R. A., and Peltier, W. R. (2004). Secular sea level change in the Russian sector of the Arctic Ocean. J. Geophys. Res. 109:C03042. doi: 10.1029/2003JC002007

Prowse, T., Bring, A., Mård, J., and Carmack, E. (2015). Arctic freshwater synthesis: introduction. J. Geophys. Res. Biogeosci. 120, 2121-2131. doi: 10.1002/ 2015JG003127

Rabe, B., Dodd, P. A., Hansen, E., Falck, E., Schauer, U., Mackensen, A., et al. (2013). Liquid export of Arctic freshwater components through the Fram Strait 1998-2011. Ocean Sci. 9, 91-109. doi: 10.5194/osd-9-91-2013

Raj, R. P., Nilsen, J. E. Ø, Johannessen, J. A., Furevik, T., Andersen, O. B., and Bertino, L. (2018). Quantifying Atlantic water transport to the Nordic seas by remote sensing. Remote Sens. Environ. 216, 758-769. doi: 10.1016/j.rse.2018.04. 055

Rampal, P., Weiss, J., Dubois, C., and Campin, J. M. (2011). IPCC climate models do not capture Arctic sea ice drift acceleration: consequences in terms of projected sea ice thinning and decline. J. Geophys. Research 116:C00D07. doi: 10.1029/2011JC007110

Richter-Menge, J., Jeffries, M. O., and Osborne, E. (eds). (2018). The Arctic in "State of the Climate in 2017". Bull. Amer. Meteor. Soc. 99, S143-S173. doi: 10.1175/2018BAMSStateoftheClimate.I

Rintoul, S. R., Sparrow, M., Meredith, M. P., Wadley, V., Speer, K., Hofmann, E., et al. (2012). The Southern Ocean Observing System: Initial Science and Implementation Strategy. Cambridge: Scientific Committee on Antarctic Research.

Riser, S. C., Freeland, H. J., Roemmich, D., Wijffels, S., Troisi, A., Belbéoch, M., et al. (2016). Fifteen years of ocean observations with the global Argo array. Nat. Clim. Change 5, 145-153. doi: 10.1038/nclimate2872

Rösel, A., Itkin, P., King, J., Divine, D., Wang, C., Granskog, M. A., et al. (2018). Thin sea ice, thick snow, and widespread negative freeboard observed during N-ICE2015 North of Svalbard. J. Geophys. Res. Oceans 123, 1156-1176. doi: 10.1002/2017JC012865

Sagen, H., Dushaw, B. D., Skarsoulis, E. K., Dumont, D., Dzieciuch, M. A., and Beszczynska-Möller, A. (2016). Time series of temperature in Fram Strait determined from the 2008-2009 DAMOCLES acoustic tomography measurements and an ocean model. J. Geophys. Res. 121, 4601-4617. doi: 10. 1002/2015JC011591

Sandven, S., Sagen, H., Buch, E., Pirazzini, R., Gustavson, D., BeszczynskaMöller, A., et al. (2018). The in situ component of Arctic observing systems opportunities and challenges in implementation of platforms and sensors. in Proceedings of the AOS 2018 Statement paper, (Calgary, AB: Arctic Observing Summit).

Schlosser, P., Pfirman, S. L., Pomerance, R., Williams, M., Ack, B., Duffy, P., et al. (2016). A $5^{\circ} \mathrm{C}$ Arctic in a $2^{\circ} \mathrm{C}$ World: Challenges and Recommendations for Immediate Action. New York, NY: Columbia University, 10.

Science, and Technology Policy Institute [STPI], and Sustaining Arctic Observing Networks [SAON] (2017). International Arctic Observations Assessment Framework. Washington, DC: IDA Science and Technology Policy Institute, 73.

Sigmond, M., Fyfe, J. C., and Swart, N. C. (2018). Ice-free Arctic projections under the Paris agreement. Nat. Clim. Change 8, 404-408. doi: 10.1038/s41558-0180124-y

Soltwedel, T., Schauer, U., Boebel, O., Nöthig, E.-M., Bracher, A., Metfies, K., et al. (2013). "FRAM - FRontiers in Arctic marine monitoring: Permanent observations in a gateway to the Arctic ocean," in Proceedings of the OCEANS Bergen, 2013 MTS/IEEE, (Bergen: IEEE). 
Spreen, G., Kwok, R., and Menemenlis, D. (2011). Trends in Arctic sea ice drift and role of wind forcing: 1992-2009. Geophys. Res. Lett. 38:L19501. doi: 10.1029/ 2011GL048970

Stammer, D., Lyu, G., Pirazzini, R., Naakka, T., Nygaård, T., Vihma, T., et al. (2018). INTAROS D2.12: Observational Gaps Revealed by Model Sensitivity to Observations. Research and Innovation Action under EC Horizon 2020 Grant Agreement no. 727890. Brussels: European Commission.

Starkweather, S., Intrieri, J., Solomon, A., Farrell, S., and Woods, J. (2018). Lessons from the Arctic Observing Framework for Sea Ice Forecasting-A Task of the US Arctic Observing Network (US AON). Washington, DC: AGU Fall Meeting Abstracts.

Stroeve, J., and Notz, D. (2018). Changing state of Arctic sea ice across all seasons. Environ. Res. Lett. 13:103001. doi: 10.1088/1748-9326/ aade56

Thomson, J., Ackley, S., Girard-Ardhuin, F., Ardhuin, F., Babanin, A., Boutin, G., et al. (2018). Overview of the Arctic Sea state and boundary layer physics program. J. Geophys. Res.: Oceans 123, 8674-8687. doi: 10.1002/2018JC01 3766

Toole, J. M., Krishfield, R. A., Timmermans, M.-L., and Proshutinsky, A. (2011). The ice-tethered profiler: argo of the Arctic. Oceanography 24, 126-135. doi: 10.5670/oceanog.2011.64

Vargas-Moreno, J. C., Fradkin, B., Emperador, S., and Lee, O. (2016). Prioritizing Science needs Through Participatory Scenarios for Energy and Resource Development on the North Slope and Adjacent Seas. Boston, MA: GeoAdaptive, LLC.

Volkov, D. L., and Landerer, F. W. (2013). Nonseasonal fluctuations of the Arctic Ocean mass observed by the GRACE satellites. J. Geophys. Res. Oceans 118, 6451-6460. doi: 10.1002/2013JC009341

Volkov, D. L., and Pujol, M.-I. (2012). Quality assessment of a satellite altimetry data product in the Nordic, Barents, and Kara seas. J. Geophys. Res. 117:C03025. doi: 10.1029/2011JC007557

Wassmann, P. (2015). Overarching perspectives of contemporary and future ecosystems in the Arctic Ocean. Progr. Oceanogr. 139, 1-12. doi: 10.1016/j. pocean.2015.08.004

Webster, M., Gerland, S., Holland, M., Hunke, E., Kwok, R., Lecomte, O., et al. (2018). Snow in the changing sea-ice systems. Nat. Clim. Change 8, 946-953. doi: 10.1038/s41558-018-0286-7

Whitlock, J. D. (2018). Autonomous Sea Ice Measurements for the Changing Arctic, Ph.D. dissertation, Dartmouth College, Hanover, NH.

Wilkinson, J., and Julienne, S. (2018). "Polar sea ice as a barometer and driver of change," in The Routledge Handbook of the Polar Regions London and New York, eds M. Nuttall, T. R. Christensen, and M. J. Siegert (New York, NY: Routledge), $176-184$.
Williams, P., Alessa, L., Abatzoglou, J. T., Kliskey, A., Witmer, F., Lee, O., et al. (2018). Community-based observing networks and systems in the Arctic: human perceptions of environmental change and instrument-derived data. Reg. Environ. Change 18, 547-559. doi: 10.1007/s10113-017-1220-7

Willmes, S., Krumpen, T., Adams, S., Rabenstein, L., Haas, C., Hoelemann, J., et al. (2010). Cross-validation of polynya monitoring methods from multisensor satellite and airborne data: a case study from the Laptev Sea. Can. J. Remote Sens. 36, S196-S210.

Woodgate, R. A. (2018). Increases in the Pacific inflow to the Arctic from 1990 to 2015, and insights into seasonal trends and driving mechanisms from yearround Bering Strait mooring data. Progr. Oceanogr. 160, 124-154. doi: 10.1016/ j.pocean.2017.12.007

Wunsch, C. (2006). Discrete Inverse and State Estimation Problems: With Geophysical Fluid Applications. Cambridge: Cambridge University Press, doi: 10.1017/CBO9780511535949

Young, O. R. (2009). The Arctic in play: Governance in a time of rapid change. Int. J. Mar. Coastal Law 24, 423-442. doi: 10.1163/157180809x421833

Yumashev, D., Hope, C., Schaefer, K., Riemann-Campe, K., Iglesias-Suarez, F., Jafarov, E., et al. (2019). Climate policy implications of nonlinear decline of Arctic land permafrost, snow and sea ice. Geophys. Res. Abstracts 21:EGU2019-15815.

Zanna, L., Heimbach, P., Moore, A. M., and Tziperman, E. (2010). Optimal excitation of interannual atlantic meridional overturning circulation variability. J. Clim. 24, 413-427. doi: 10.1175/2010jcli3610.1

Conflict of Interest Statement: The authors declare that the research was conducted in the absence of any commercial or financial relationships that could be construed as a potential conflict of interest.

The reviewer $\mathrm{PH}$ declared a shared affiliation and a past co-authorship, with one of the authors, AN, to the handling Editor at the time of review. The peer review was handled under the close supervision of the Chief Editors to ensure an objective process.

Copyright (c) 2019 Lee, Starkweather, Eicken, Timmermans, Wilkinson, Sandven, Dukhovskoy, Gerland, Grebmeier, Intrieri, Kang, McCammon, Nguyen, Polyakov, Rabe, Sagen, Seeyave, Volkov, Beszczynska-Möller, Chafik, Dzieciuch, Goni, Hamre, King, Olsen, Raj, Rossby, Skagseth, Søiland and Sørensen. This is an open-access article distributed under the terms of the Creative Commons Attribution License (CC BY). The use, distribution or reproduction in other forums is permitted, provided the original author(s) and the copyright owner(s) are credited and that the original publication in this journal is cited, in accordance with accepted academic practice. No use, distribution or reproduction is permitted which does not comply with these terms. 


\section{OPEN ACCESS}

\section{Edited by: \\ Laura Lorenzoni, \\ University of South Florida, Tampa, \\ United States \\ Reviewed by: \\ Frank Dehairs, \\ Vrije Universiteit Brussel, Belgium \\ Mathieu Ardyna, \\ Stanford University, United States \\ Matthew Long, \\ National Center for Atmospheric Research (UCAR), United States \\ *Correspondence: \\ Louise Newman \\ newman@soos.aq; \\ louise.newman@utas.edu.au \\ tThese authors have contributed equally to this work \\ Specialty section: \\ This article was submitted to \\ Ocean Observation \\ a section of the journal \\ Frontiers in Marine Science}

Received: 03 December 2018

Accepted: 05 July 2019

Published: 08 August 2019

Citation:

Newman L, Heil P, Trebilco R,

Katsumata K, Constable A, van Wijk E, Assmann K, Beja J, Bricher P, Coleman R, Costa $D$

Diggs $S$, Farneti $R$, Fawcett $S$,

Gille ST, Hendry KR, Henley S,

Hofmann E, Maksym T, Mazloff M,

Meijers A, Meredith MM, Moreau S,

Ozsoy B, Robertson R, Schloss I,

Schofield O, Shi J, Sikes E, Smith IJ,

Swart S, Wahlin A, Williams $G$,

Williams MJM, Herraiz-Borreguero L,

Kern S, Lieser J, Massom RA,

Melbourne-Thomas J, Miloslavich P

and Spreen G (2019) Delivering

Sustained, Coordinated,

and Integrated Observations of the

Southern Ocean for Global Impact.

Front. Mar. Sci. 6:433.

doi: 10.3389/fmars.2019.00433

\section{Delivering Sustained, Coordinated, and Integrated Observations of the Southern Ocean for Global Impact}

\author{
Louise Newman ${ }^{1 *}$, Petra Heil2,3†, Rowan Trebilco ${ }^{3,4+}$, Katsuro Katsumata ${ }^{5}$, \\ Andrew Constable ${ }^{2,3}$, Esmee van Wijk ${ }^{3,4}$, Karen Assmann ${ }^{6}$, Joana Beja ${ }^{7}$, \\ Phillippa Bricher ${ }^{1}$, Richard Coleman ${ }^{3,8}$, Daniel Costa ${ }^{9}$, Steve Diggs ${ }^{10}$, Riccardo Farneti ${ }^{11}$, \\ Sarah Fawcett ${ }^{12}$, Sarah T. Gille ${ }^{10}$, Katharine R. Hendry ${ }^{13}$, Sian Henley ${ }^{14}$, \\ Eileen Hofmann ${ }^{15}$, Ted Maksym ${ }^{16}$, Matthew Mazloff10, Andrew Meijers ${ }^{17}$, \\ Michael M. Meredith ${ }^{17}$, Sebastien Moreau ${ }^{18}$, Burcu Ozsoy ${ }^{19}$, Robin Robertson ${ }^{20}$, \\ Irene Schloss ${ }^{21,22,23}$, Oscar Schofield24, Jiuxin Shi25, Elisabeth Sikes ${ }^{24}$, Inga J. Smith ${ }^{26}$, \\ Sebastiaan Swart ${ }^{6,12}$, Anna Wahlin 6 , Guy Williams ${ }^{3,8}$, Michael J. M. Williams ${ }^{27}$, \\ Laura Herraiz-Borreguero ${ }^{4,28}$, Stefan Kern ${ }^{29}$, Jan Lieser ${ }^{3,8}$, Robert A. Massom ${ }^{2,3}$, \\ Jessica Melbourne-Thomas $2,3,4$, Patricia Miloslavich ${ }^{8,30}$ and Gunnar Spreen ${ }^{31}$
}

${ }^{1}$ Southern Ocean Observing System International Project Office, Institute for Marine and Antarctic Studies, College of Sciences and Engineering, University of Tasmania, Hobart, TAS, Australia, ${ }^{2}$ Australian Antarctic Division, Kingston, TAS, Australia, ${ }^{3}$ Antarctic Climate and Ecosystems Cooperative Research Centre, University of Tasmania, Hobart, TAS, Australia, ${ }^{4}$ Commonwealth Scientific and Industrial Research Organisation, Oceans and Atmosphere, Hobart, ACT, Australia, ${ }^{5}$ Research and Development Center for Global Change, Japan Agency for Marine-Earth Science and Technology, Yokosuka, Japan, ${ }^{6}$ Department of Marine Sciences, University of Gothenburg, Gothenburg, Sweden, ${ }^{7}$ British Oceanographic Data Centre, Liverpool, United Kingdom, ${ }^{8}$ Institute for Marine and Antarctic Studies, College of Sciences and Engineering, University of Tasmania, Hobart, TAS, Australia, ${ }^{9}$ Department of Ecology \& Evolutionary Biology, University of California, Santa Cruz, Santa Cruz, CA, United States, ${ }^{10}$ Scripps Institution of Oceanography, University of California, San Diego, La Jolla, CA, United States, ${ }^{11}$ Earth System Physics Section, The Abdus Salam International Centre for Theoretical Physics, Trieste, Italy, ${ }^{12}$ Department of Oceanography, Faculty of Science, University of Cape Town, Cape Town, South Africa, ${ }^{13}$ School of Earth Sciences, University of Bristol, Bristol, United Kingdom, ${ }^{14}$ School of Geosciences, University of Edinburgh, Edinburgh, United Kingdom, ${ }^{15}$ Center for Coastal Physical Oceanography, Old Dominion University, Norfolk, VA, United States, ${ }^{16}$ Woods Hole Oceanographic Institution, Woods Hole, MA, United States, ${ }^{17}$ British Antarctic Survey, Cambridge, United Kingdom, ${ }^{18}$ Norwegian Polar Institute, Tromsø, Norway, ${ }^{19}$ Polar Research Center, Istanbul Technical University, Istanbul, Turkey, ${ }^{20}$ China-ASEAN College of Marine Sciences (CAMS), Xiamen University Malaysia, Sepang, Malaysia, ${ }^{21}$ Instituto Antártico Argentino, Buenos Aires, Argentina, ${ }^{22}$ CONICET Centro Austral de Investigaciones Científicas (CADIC), Ushuaia, Argentina, ${ }^{23}$ Universidad Nacional de Tierra del Fuego, Ushuaia, Argentina, ${ }^{24}$ Department of Marine and Coastal Sciences, School of Environmental and Biological Sciences, Rutgers University, New Brunswick, NJ, United States, ${ }^{25}$ Key Laboratory of Physical Oceanography, Ocean University of China, Qingdao, China, ${ }^{26}$ Department of Physics, University of Otago, Dunedin, New Zealand, ${ }^{27}$ National Institute of Water and Atmospheric Research, Wellington, New Zealand, ${ }^{28}$ Centre for Southern Hemisphere Oceans Research, Hobart, TAS, Australia, ${ }^{29}$ Integrated Climate Data Center, Center for Earth System Research and Sustainability, University of Hamburg, Hamburg, Germany, ${ }^{30}$ Departamento de Estudios Ambientales, Universidad Simón Bolivar, Caracas, Venezuela, ${ }^{31}$ Institute of Environmental Physics, University of Bremen, Bremen, Germany

The Southern Ocean is disproportionately important in its effect on the Earth system, impacting climatic, biogeochemical, and ecological systems, which makes recent observed changes to this system cause for global concern. The enhanced understanding and improvements in predictive skill needed for understanding and projecting future states of the Southern Ocean require sustained observations. Over the last decade, the Southern Ocean Observing System (SOOS) has established networks for enhancing regional coordination and research community groups to advance development of observing system capabilities. These networks support delivery of the SOOS 20-year vision, which is to develop a circumpolar system that ensures 
time series of key variables, and delivers the greatest impact from data to all key end-users. Although the Southern Ocean remains one of the least-observed ocean regions, enhanced international coordination and advances in autonomous platforms have resulted in progress toward sustained observations of this region. Since 2009, the Southern Ocean community has deployed over 5700 observational platforms south of $40^{\circ} \mathrm{S}$. Large-scale, multi-year or sustained, multidisciplinary efforts have been supported and are now delivering observations of essential variables at space and time scales that enable assessment of changes being observed in Southern Ocean systems. The improved observational coverage, however, is predominantly for the open ocean, encompasses the summer, consists of primarily physical oceanographic variables, and covers surface to $2000 \mathrm{~m}$. Significant gaps remain in observations of the ice-impacted ocean, the sea ice, depths $>2000 \mathrm{~m}$, the air-ocean-ice interface, biogeochemical and biological variables, and for seasons other than summer. Addressing these data gaps in a sustained way requires parallel advances in coordination networks, cyberinfrastructure and data management tools, observational platform and sensor technology, twoway platform interrogation and data-transmission technologies, modeling frameworks, intercalibration experiments, and development of internationally agreed sampling standards and requirements of key variables. This paper presents a community statement on the major scientific and observational progress of the last decade, and importantly, an assessment of key priorities for the coming decade, toward achieving the SOOS vision and delivering essential data to all end-users.

Keywords: Southern Ocean, observations, modeling, ocean-climate interactions, ecosystem-based management, long-term monitoring, international coordination

\section{IMPETUS AND PROGRESS TOWARD DEVELOPING A SOUTHERN OCEAN OBSERVING SYSTEM}

The Southern Ocean has a profound influence on the Earth system (e.g., IPCC, 2013). It has wide-ranging climatic, ecological, and human impacts via its key role in the global circulation of heat, freshwater and nutrients, biogeochemical and carbon cycles, ocean productivity, and sea-level rise, as well as providing intrinsic value for conservation and management. Yet despite its importance, the Southern Ocean remains one of the most under-observed regions on Earth, leading to significant uncertainties in estimates of the future state of Southern Ocean processes and the consequences for the globe and its inhabitants (IPCC, 2013).

The Southern Ocean Observing System (SOOS) was established in 2011 as a partnership between the Scientific Committee on Antarctic Research (SCAR) and the Scientific Committee on Oceanic Research (SCOR) to enhance delivery of the data required to reduce these uncertainties. This collaboration followed years of international discussions that were distilled and published in the OceanObs'09 White Papers (Rintoul et al., 2010), and then more comprehensively in the Initial Science and Implementation Strategy (Rintoul et al., 2012). Broad international involvement and enhanced coordination and collaboration were highlighted as key mechanisms to deliver integrated and sustained observations to all end-users. These initial documents provided community-defined scientific drivers for SOOS and six Science Themes that are used to prioritize all subsequent $\mathrm{SOOS}$ efforts:

(1) The role of the Southern Ocean in the planet's heat and freshwater balance.

(2) The stability of the Southern Ocean overturning circulation.

(3) The role of the ocean in the stability of the Antarctic Ice Sheet and its future contribution to sea-level rise.

(4) The future and consequences of Southern Ocean carbon uptake.

(5) The future of Antarctic sea ice.

(6) Impacts of global change on Southern Ocean ecosystems.

\section{The Vision}

Southern Ocean Observing System involves many nations, significant resources, and multiple end-users, which makes its 20 -year vision critical for enabling long-term strategic planning and preventing mission drift (Meredith et al., 2013). Although ambitious, the SOOS vision identifies the required networks and infrastructure to deliver a backbone of sustained, fundamental observations for use by a breadth of stakeholders across scientific, policy, and educational communities. It allows for inclusion of global initiatives that are advancing observing and data-acquisition technologies (e.g., the Digital Ocean initiative; Gysin, 2017). The long-term vision for SOOS (Figure 1) is 


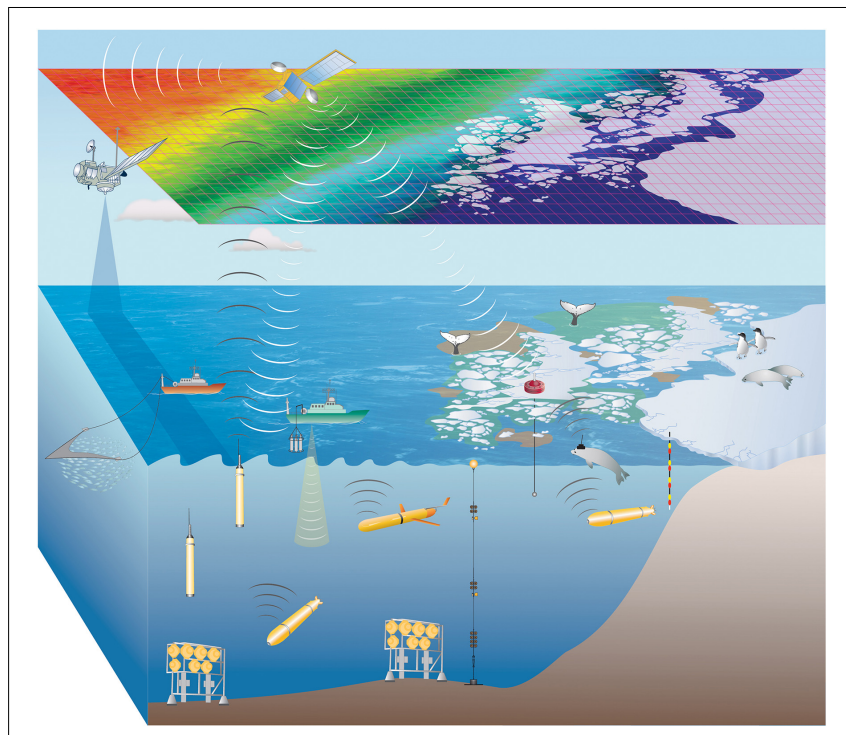

FIGURE 1 | Schematic of a cyberinfrastructure-based vision for SOOS (Meredith et al., 2013; reproduced with permission). Combined with satellite remote sensing, data from both autonomous and non-autonomous platforms would be relayed to ground stations in real time. Assimilating ocean models would produce near-real time state estimates of each of the parameters in the system and associated error fields would then be used to re-task the autonomous platforms in real time. This will maximize the spatial-temporal coverage of each of the parameters being measured, without specific need for human intervention.

to have a cyberinfrastructure-based program with increasing reliance on autonomous platforms as technology develops, as a means of readily delivering "essential" data to stakeholders, including in real time.

Achieving such a vision requires parallel advances in coordination networks, cyberinfrastructure and data management tools, observational platform and sensor technology, platform interrogation and data-transmission technologies, modeling frameworks, and internationally agreed sampling requirements of key variables. Toward this end, SOOS is working with the broader community to: develop regional networks for integration of international observational field activities (Figure 2); enhance observing capabilities (e.g., technology, methods) ${ }^{1}$; build a robust data network that encompasses oceanographic and Antarctic expertise; deliver data discovery (Figure 3) and field coordination tools (Figure 4); facilitate advances in observing system design (section "Essential Ocean Variables and Observing System Design"); and collaborate with the global community to identify Essential Ocean Variables (EOVs) for the Southern Ocean, following the Framework for Ocean Observing (Lindstrom et al., 2012).

\section{A Decade of Progress}

Since 2009, the number of Southern Ocean observations collected has increased considerably, enabled by rapid progress

${ }^{1}$ More information on SOOS Capability Working Groups at http://soos.aq/ activities/capability-wgs

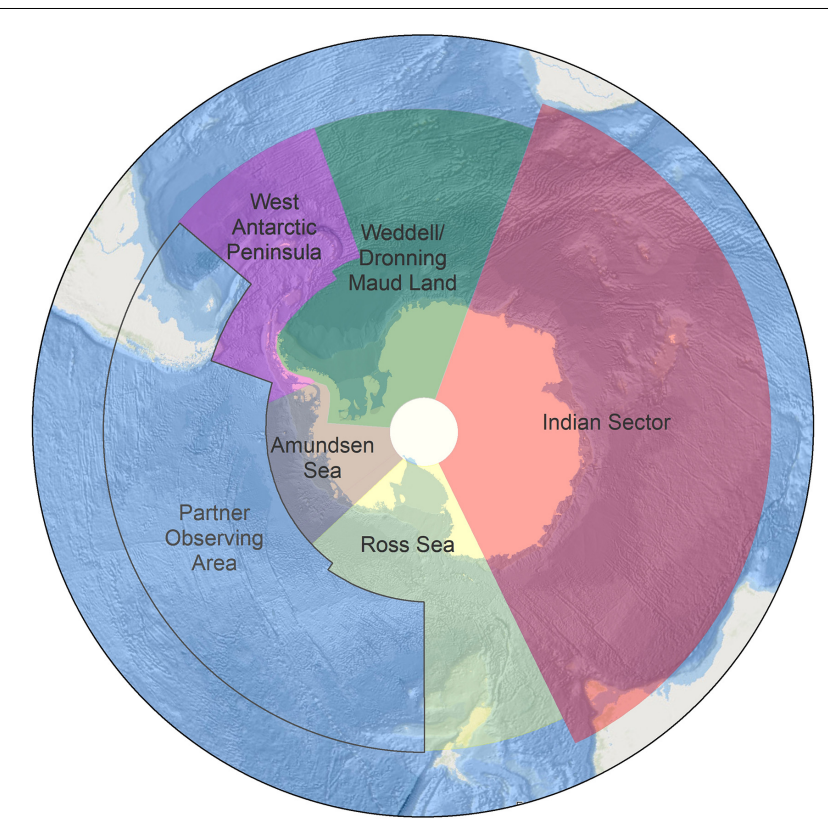

FIGURE 2 | Map of the five Regional Working Groups developed by SOOS to integrate the existing observational efforts in a region and facilitate efforts to address key gaps in observational coverage. The regions are based on the natural areas of focus for nations working in the Southern Ocean and facilitate regional coordination in (1) scientific information exchange, (2) technology transfer/collaboration, (3) standardization of measurements, and (4) sharing of data. The Partner Observing Area is a region not presently covered by a working group. In the interim, the SOOS Scientific Steering Committee will maintain oversight of the observational coverage of this region to ensure requirements are met. Base map data from ESRI, GARMIN, GEBCO, NOAA NGDC, and others.

in the development, capability, and use of autonomous and robotic systems. SOOS has supported this progress by defining and promoting the vision around the systems that are needed, and also by endorsing and supporting the funding acquisition by various nations' investigators [e.g., Research of Oceanice BOundry InTeraction and Change around Antarctica $(\text { ROBOTICA })^{2}$, Japan; Southern Ocean Carbon and Climate Observations and Modeling (SOCCOM $)^{3}$, United States; Ocean Regulation of Climate by Heat and Carbon Sequestration and Transports (ORCHESTRA) ${ }^{4}$, United Kingdom; Research Center Dynamics of High Latitude Marine Ecosystems (IDEAL) ${ }^{5}$, Chile; Southern Ocean Seasonal Cycle Experiment $(\operatorname{SOSCEx})^{6}$, South Africa; Marine Observatory in the Ross Sea $(\mathrm{MORSea})^{7}$, Italy; Kerguelen Axis $(\mathrm{K}-\mathrm{Axis})^{8}$, Australia; Water-mass transformation and Pathways In The Weddell Sea

\footnotetext{
${ }^{2}$ http://soos.aq/images/soos/downloads/endorsement/robotica-web.pdf

${ }^{3} \mathrm{https} / / /$ soccom.princeton.edu/

${ }^{4}$ https://www.bas.ac.uk/project/orchestra/

${ }^{5}$ http://www.centroideal.cl/antarctica/

${ }^{6}$ http://socco.org.za/research/

${ }^{7}$ http://morsea.uniparthenope.it/

${ }^{8}$ http://www.antarctica.gov.au/magazine/2011-2015/issue-29-december-2015/ science/spotlight-on-the-k-axis
} 


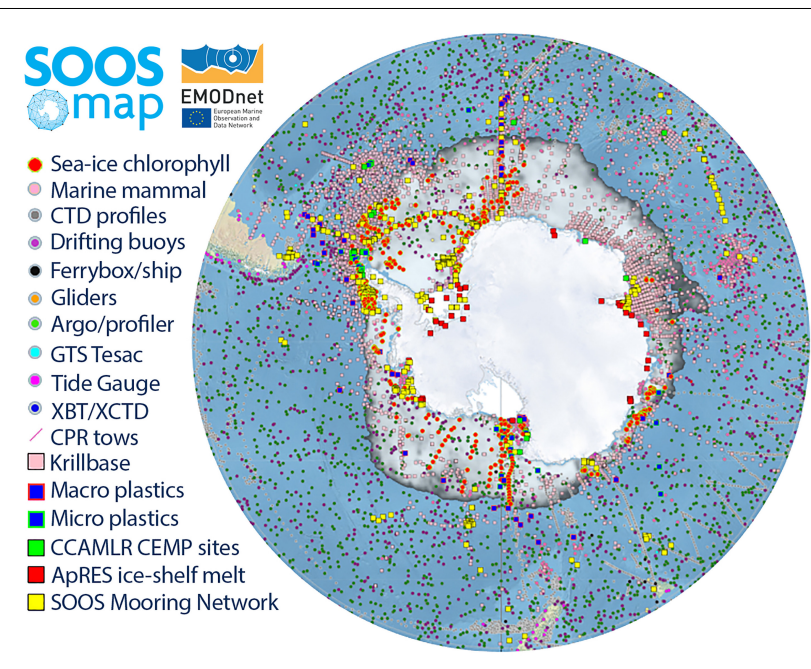

FIGURE 3 | SOOSmap is an interactive webmap that allows users to explore circumpolar datasets. It was developed for SOOS by EMODnet Physics and enables users to search within different spatial and temporal filters, and to select different platforms, variables, and data layers. SOOSmap is an open access and provides direct data downloads where possible (www.soos.aq/soosmap).

(WAPITI) $^{9}$, France; Polynyas, Ice Production, and seasonal Evolution in the Ross Sea (PIPERS) ${ }^{10}$, United States; Amundsen Sea Program ${ }^{11}$, Korea; Dynamics of the Orkney Passage Outflow (DynOPO) ${ }^{12}$, United Kingdom; Robotic Observations and Modelling in the Marginal Ice Zone (ROAM-MIZ) ${ }^{13}$, Sweden; and Investigation of Bottom Water Formation in Prydz Bay ${ }^{14}$, China]. In addition to these initiatives, sustained time-series programs are vital for revealing the ongoing change on decadal timescales; SOOS has supported the continuation of many of these, such as Rothera Time Series (RaTS, United Kingdom) ${ }^{15}$, Palmer Station Long-Term Ecological Research (LTER), United States ${ }^{16}$, Australia's Integrated Marine Observing System Southern Ocean Time Series (IMOS SOTS) ${ }^{17}$, and Potter Cove Time Series (Argentina/Germany) ${ }^{18}$, as well as internationally coordinated observing programs, such as Network for the Collection of Knowledge on Melt of Antarctic Ice Shelves: (UK-led international Southern Ocean program) (NECKLACE) ${ }^{19}$, OceanSITES ${ }^{20}$, Argo ${ }^{21}$, Southern

\footnotetext{
${ }^{9}$ http://wapiti-project.com/

${ }^{10} \mathrm{http} / /$ www.utsa.edu/signl/pipers/index.html

${ }^{11} \mathrm{http}: / /$ soos.aq/news/current-news/188-kopriamundsenproject

${ }^{12} \mathrm{https}$ ///www.bas.ac.uk/project/dynamics-of-the-orkney-passage-outflow/

${ }^{13} \mathrm{http}: / /$ soos.aq/images/soos/downloads/endorsement/floating-glaciers.pdf

${ }^{14}$ https://www.ldeo.columbia.edu/ xyuan/Prydz/index.html

${ }^{15} \mathrm{https} / / /$ www.bas.ac.uk/project/rats/

${ }^{16} \mathrm{http}: / /$ pal.lternet.edu/

${ }^{17} \mathrm{http} / /$ imos.org.au/facilities/deepwatermoorings/sots/

${ }^{18}$ https://www.st.nmfs.noaa.gov/copepod/time-series/de-30401/

${ }^{19} \mathrm{http}: / /$ www.soos.aq/activities/soos-at-sea/necklace

${ }^{20} \mathrm{~A}$ worldwide system of deepwater reference stations, http://www.oceansites.org/ index.html

${ }^{21}$ A global array of free-drifting profiling floats, http://www.argo.ucsd.edu/
}

Ocean Continuous Plankton Recorder program (SO-CPR) ${ }^{22}$, the Global Ocean Ship-Based Hydrographic Investigations Program (GO-SHIP) ${ }^{23}$, International Program for Antarctic Buoys (IPAB $)^{24}$ and Data Buoy Cooperation Panel (DBCP) $)^{25}$, and Marine Mammals Exploring the Oceans Pole to Pole (MEOP) ${ }^{26}$. Through the above-mentioned programs and many others, the international community has deployed more than 5700 observational platforms since 2009 (Figure 3; compared to approx. 3000 for the previous decade), significantly enhancing our understanding of the Southern Ocean and delivering essential data to core stakeholders.

The implementation of these programs over the last decade has enabled the community to make significant statements on the state of the Southern Ocean and how it is changing.

\section{Themes 1 and 2: The Role of the Southern Ocean in the Planet's Heat and Freshwater Balance; And the Stability of the Southern Ocean Overturning Circulation}

The Southern Ocean is warming faster than any other sector of the global ocean (Roemmich et al., 2015), accounting for $60-90 \%$ of the total modeled anthropogenic ocean heat uptake since the start of the industrial period (Frölicher et al., 2015). This warming is concentrated in the upper $2000 \mathrm{~m}$ north of the Sub-Antarctic Front (SAF) and is largely due to ongoing wind-driven thickening of the intermediate and mode-water layers and consequent deepening of isopycnals and consequent thickening of the intermediate and mode water layers (Desbruyeres et al., 2017; Gao et al., 2018). Observations and modeling efforts indicate that the prevailing westerly winds over the Southern Ocean have shifted poleward and strengthened, due to ozone depletion (Polvani et al., 2011) and $\mathrm{CO}_{2}$ forcing (Bracegirdle et al., 2013). Despite this, no changes in the Antarctic Circumpolar Current (ACC) density structure (Böning et al., 2008) and lateral transport (Gille, 2014; Shao et al., 2015) have been definitively detected.

Increases in the westerly winds overlying the ACC are hypothesized to result in an increased net meridional overturning circulation (MOC) associated with enhanced poleward transport of heat by mesoscale eddies and a stronger equatorward return Ekman transport at the surface (Meredith et al., 2012; Gent, 2016). However, existing observations have not shown changes in the MOC, which is hard to explicitly observe due to the difficulty in resolving the small-scale but widely distributed contribution of eddies. Yet, satellite observations suggest that mesoscale eddy activity has increased with winds over recent decades (Hogg et al., 2015). Consistent with this increase, recent methods using indirect metrics, such as water mass age (Waugh et al., 2013; Waugh, 2014; Ting and Holzer, 2017), warming (Gille, 2008; Meijers et al., 2011), and circulation obtained using inverse methods (DeVries et al., 2017), are consistent with an increase in upwelling and overturning, although uncertainty is high.

\footnotetext{
${ }^{22}$ https://data.aad.gov.au/aadc/cpr/

${ }^{23}$ http://www.go-ship.org/

${ }^{24}$ http://www.ipab.aq/

${ }^{25}$ http://www.jcommops.org/dbcp/

${ }^{26}$ http://www.meop.net/
} 


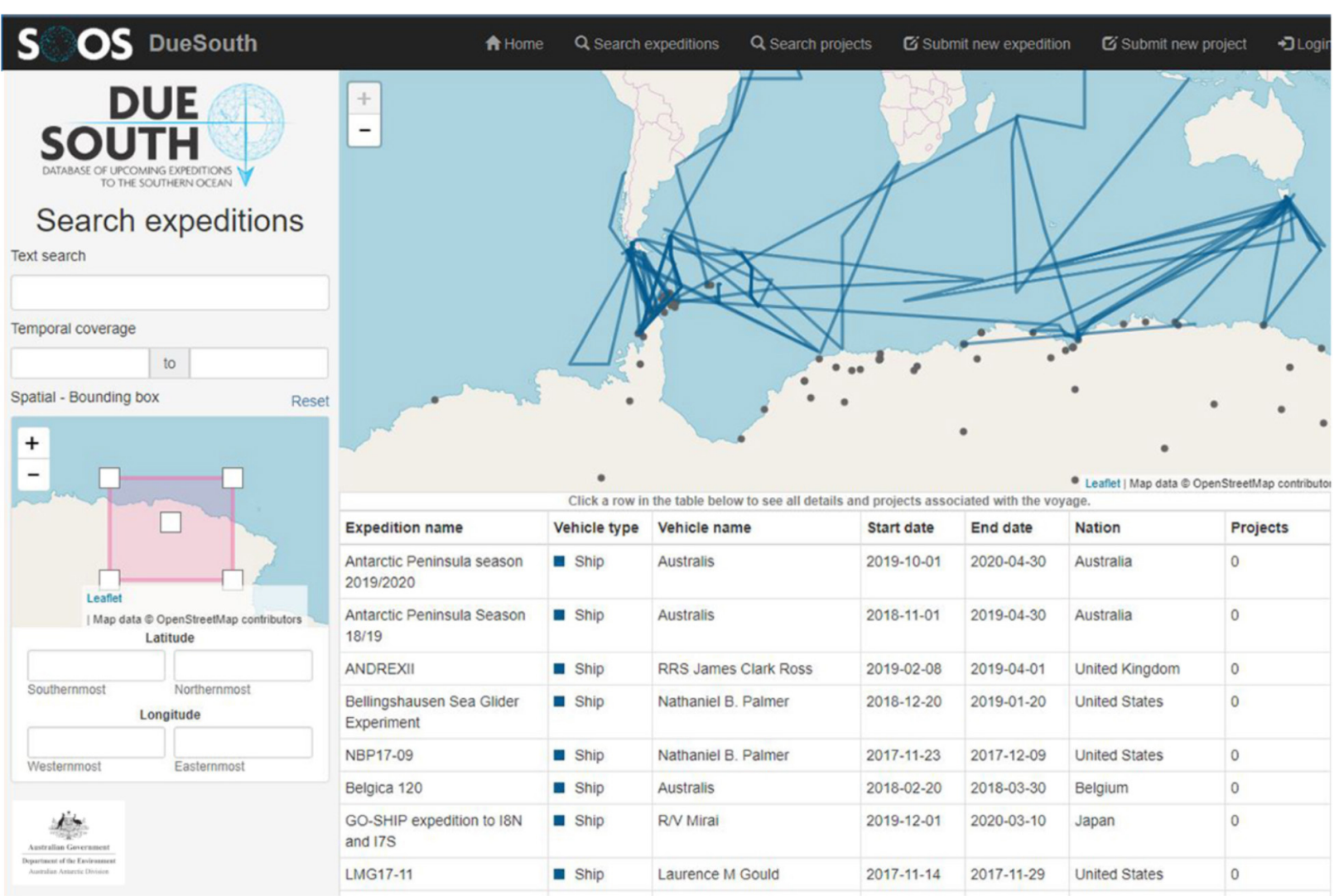

FIGURE 4 | DueSouth is the Database of Upcoming Expeditions to the Southern Ocean and is a coordination tool that enables users to find out which expeditions (e.g., voyages, flights, traverses) are planned for future field seasons, and what observational projects are funded to take place on each expedition. It is developed and maintained for SOOS by the Australian Antarctic Data Centre.

While the waters north of the SAF have warmed significantly, the sea-surface waters south of the ACC core and Polar Front have changed less than the global sea-surface temperature trend $\left(0.02^{\circ} \mathrm{C} /\right.$ decade cf. $0.08^{\circ} \mathrm{C} /$ decade since 1950$)$. This northsouth temperature trend contrast reflects the continual renewal of the surface waters south of the Polar Front from below by old Circumpolar Deep Water (CDW) that has yet been largely unmodified by anthropogenic influences (Armour et al., 2016). This demonstrates that Southern Ocean circulation has a major impact on the distribution of climate change signals. Warming closer to the continent, notably in the AmundsenBellingshausen seas, has been observed on decadal timescales (Schmidtko et al., 2014) and has been linked to wind-driven increases in warm CDW upwelling onto the continental shelf (Jenkins et al., 2010). This has significant consequences for sea-level rise via accelerated ice-shelf melt but remains poorly observed and understood.

The Southern Ocean is also freshening (Durack and Wijffels, 2010), particularly in the upper layers and near Antarctica itself (Jacobs and Giulivi, 2010; Schmidtko et al., 2014). This has been attributed to an increase in precipitation (Helm et al., 2010), increased basal melting of ice shelves (Paolo et al., 2015), and enhanced sea-ice melt and export (Haumann et al., 2016), though the relative contribution of these processes is highly uncertain. The bottom waters that are formed in coastal regions and exported to the global ocean are becoming warmer (Purkey and Johnson, 2010, 2013), fresher (Rintoul, 2007; Jullion et al., 2013; Menezes et al., 2017), and decreasing in volume (Purkey and Johnson, 2012, 2013), although these bottom waters remain sparsely sampled. Recent model experiments suggest that the observed warming and freshening are inconsistent with natural variability, and require increased surface fluxes of heat and freshwater that are driven by greenhouse gas forcing, and to a lesser extent, ozone depletion (Swart et al., 2018).

\section{Theme 3: The Role of the Ocean in the Stability of the Antarctic Ice Sheet and Its Future Contribution to Sea-Level Rise}

The Antarctic Ice Sheet is the greatest source of uncertainty in projections of future sea level (IPCC, 2013), with estimates ranging from zero to a catastrophic $5 \mathrm{~cm} /$ year (IPCC, 2013; Bakker et al., 2017; Shepherd et al., 2018). Observations and modeling during the last decade indicate increased ice-shelf basal melt through contact with warm, salty deep water (Rignot et al., 2013; Paolo et al., 2015). This thinning is accelerating (Paolo et al., 2015), threatening the West Antarctic Ice Shelf (WAIS) in particular, although recent research suggests more of the East Antarctic Ice Sheet is marine-based (i.e., its base lies below 
sea level) than previously thought, earning itself the title of a "sleeping giant" in future global sea-level projections (Gulick et al., 2017; Rintoul et al., 2018). A full collapse of the WAIS would result in 3-4 $\mathrm{m}$ of sea-level rise over timescales estimated to be from many decades to centuries (DeConto and Pollard, 2016).

Although the largest ice shelves (Ross, Filchner-Ronne, and Amery) have remained within their observed historical ranges, many smaller ice shelves have thinned, decreased in extent or disintegrated, leading to increased rates of discharge of grounded inland ice to the ocean. Large changes have occurred in the Amundsen Sea Embayment, where warm salty deep water intrudes on the continental shelf (Pritchard et al., 2012; Shepherd et al., 2018). The five largest ice shelves in this region have lost between 10 and 18\% of their volume (Paolo et al., 2015). The basal melt appears to co-oscillate with ENSO (Paolo et al., 2018). Ice shelves also decline through calving-front retreat, especially on the West Antarctic Peninsula (WAP) (Shepherd et al., 2010), where a number of ice shelves have disintegrated including Larsen-A and -B (Scambos et al., 2004) and Wilkins (Humbert and Braun, 2008).

Recent observations indicate that other parts of the Antarctic Ice Sheet are equally vulnerable, such as the Filchner-Ronne Ice Shelf (Hellmer et al., 2012; Darelius et al., 2016). In East Antarctica, warm water has been found beneath the Amery Ice Shelf (Herraiz-Borreguero et al., 2015), and on the shelf that accesses the ice-shelf cavity of the rapidly melting Totten Glacier (Greenbaum et al., 2015; Rintoul et al., 2016).

\section{Theme 4: The Future and Consequences of Southern Ocean Carbon Uptake}

The Southern Ocean is Earth's largest $\mathrm{CO}_{2}$ sink (Sabine et al., 2004; Sallée et al., 2012); however, the magnitude of air-sea exchange appears extremely variable on short timescales. Data and model simulations suggest that the Southern Ocean $\mathrm{CO}_{2}$ sink weakened between 1980 and the early 2000s, possibly due to changes in the Southern Hemisphere westerlies driving enhanced upwelling of $\mathrm{CO}_{2}$-rich waters (Le Quéré et al., 2007). Analysis of multidecadal sea-surface $\mathrm{pCO}_{2}$ [Surface Ocean $\mathrm{CO}_{2}$ Atlas (SOCAT); Bakker et al., 2014] showed a reversal of this trend in 2002, with the Southern Ocean $\mathrm{CO}_{2}$ sink apparently regaining its expected strength by 2012 (Landschützer et al., 2015).

Much uncertainty remains regarding the Southern Ocean's role in global carbon cycling due largely to data gaps, particularly in non-summer seasons and coastal regions (Takahashi et al., 2009). For example, recent profiling float measurements suggest significantly higher wintertime outgassing of $\mathrm{CO}_{2}$ in the Antarctic Zone than previously estimated (Gray et al., 2018), which, if confirmed, imply a significantly weaker Southern Ocean $\mathrm{CO}_{2}$ sink. Sea ice has been suggested to affect the Southern Ocean $\mathrm{CO}_{2}$ sink (Delille et al., 2014), yet its role in air-sea $\mathrm{CO}_{2}$ fluxes is not clear. Likewise, the role of (sub)mesoscale features (e.g., eddies) is under debate (Dufour et al., 2015; Li et al., 2017; Moreau et al., 2017). Further, CMIP5 Earth system models do not agree on the amplitude or sign of the Southern Ocean air-sea $\mathrm{CO}_{2}$ fluxes (Anav et al., 2013).

Estimating Southern Ocean annual net community production (ANCP) is important for understanding the role of the biological pump in sequestering anthropogenic $\mathrm{CO}_{2}$ (Nevison et al., 2012). Previous ANCP estimates (Lee, 2001; MacCready and Quay, 2001; Dunne et al., 2007) provide no information on seasonal or interannual variability, yielding an incomplete picture of Southern Ocean's $\mathrm{CO}_{2}$ drawdown. Estimates of ANCP from profiling floats ${ }^{27}$ agree well with existing estimates for all biogeochemical zones of the Southern Ocean (Johnson et al., 2017b), and indicate that ANCP south of the "biogeochemical divide" ( $\left.50^{\circ} \mathrm{S}\right)$ (Marinov et al., 2006) removes dissolved inorganic carbon (DIC) from surface water to the deep ocean where it remains sequestered for long periods of time; changes in ANCP in this region can thus drive significant changes in atmospheric $\mathrm{CO}_{2}$ concentrations.

Antarctic coastal regions, such as the WAP, are highly productive yet spatially and temporally variable. Long-term, multi-national observations have revealed the interconnectivity of climate oscillations and physical forcing mechanisms (windstress, temperature) in controlling WAP seasonal sea-ice coverage, seasonality, and properties (Stammerjohn et al., 2008; Meredith et al., 2017), which influence primary production, macronutrients, trace-metal cycling, and carbonate chemistry across the shelf (Venables et al., 2013; Hauri et al., 2015; Henley et al., 2017). More localized processes also impact nutrient and carbon cycling, including channeling of nutrientrich deep-water by glacial troughs (Schofield et al., 2013), glacial meltwater influence on productivity (Kim et al., 2018), oceanic stratification and mixing (Cassarino et al., 2016), and exchange with shallow marine sediments (Henley et al., 2018; Sherrell et al., 2018). Sustained and multi-disciplinary research along the WAP and in other high-productivity coastal regions is necessary to fully understand the processes driving biogeochemical "patchiness" and the impact of longer-term climate forcing. Such research should include process-based and experimental studies for a better mechanistic understanding of nutrient cycling, e.g., the role of sediment fluxes and seaice formation.

Considerable progress has been made since Rintoul et al. (2012) outlined the need for new technologies for biogeochemistry and biology. Biogeochemical sensors deployed on SOCCOM and Southern Ocean and Climate (SOCLIM) ${ }^{28}$ floats are beginning to address this need, measuring chlorophylla, nitrate, oxygen, light, optical properties, and $\mathrm{pH}$. Air-sea $\mathrm{CO}_{2}$ fluxes, ANCP, mesopelagic respiration, and particulate organic carbon export can now be estimated on seasonal to interannual scales, and around the Antarctic (Hennon et al., 2016; Johnson et al., 2017b; Poteau et al., 2017; Williams et al., 2017; Llort et al., 2018). Gliders are yielding seasonal, sub-seasonal, and sub-mesoscale information (Kaufman et al., 2014), and icecapable bio-Argo floats can monitor biogeochemical cycles during ice-covered periods, increasing the temporal and spatial range of the available data (Briggs et al., 2017). Additionally, phytoplankton fluorescence sensors deployed on marine mammals provide winter data from otherwise-inaccessible coastal regions (Labrousse et al., 2018).

\footnotetext{
${ }^{27}$ SOCCOM Project: https://soccom.princeton.edu/

${ }^{28}$ SOCLIM field studies with innovative tools: http://soclim.com/index.php
} 


\section{Theme 5: The Future of Antarctic Sea Ice}

Sea ice influences surface albedo and oceanic overturning circulation; affects stratification and properties of upper ocean; regulates heat, momentum, and gas transfer between the ocean and atmosphere; protects and stabilizes certain ice shelves (Massom et al., 2018); provides an important habitat for ice-associated species (Thomas, 2017); and has a major impact on safe shipping and operations. Antarctic sea ice exhibits substantial spatio-temporal variability (Stammerjohn and Maksym, 2016), principally driven by wind and modulated by ocean feedbacks (e.g., Goosse and Zunz, 2014; Holland et al., 2014). Between 1979 and 2014, the circum-Antarctic annual maximum sea-ice extent increased slightly, as a result of larger and opposing regional trends (Hobbs et al., 2016; Stammerjohn and Maksym, 2016). While sea-ice variability is strongly linked to large-scale modes of atmospheric variability, these links vary regionally and seasonally, and do not fully explain observed trends (Hobbs et al., 2016; National Academies of Sciences, Engineering, and Medicine, 2017). Moreover, Antarctic sea ice has undergone recent strong variability that is poorly understood (Turner and Comiso, 2017) - fluctuating from record maxima in overall extent in 2012-2014, to unprecedented minima from 2016 until present (early 2019).

While sea-ice extent and concentration have been quantified to reasonable accuracy from space since 1979, more robust statements on the nature of sea-ice changes and their drivers (including their regional and seasonal dependence) are impeded by a lack of observations and process understanding. Atmosphere and ocean forcing are poorly observed and understood; sea-ice thickness (and volume) are poorly known, as is the distribution of snow-cover depth (Sturm and Massom, 2017; Webster et al., 2018); and surface flooding and resultant snow-ice formation are poorly quantified (Maksym et al., 2012). Sea-ice observations from ship-based underway observations ${ }^{29}$ have provided a climatology for ice thickness and snow depth (Worby et al., 2008), but are biased to thinner sea ice (Williams et al., 2015) and are limited in space and time. Space-borne laser and radar altimetry are the key to large-scale monitoring of sea-ice thickness (Zwally et al., 2008; Kurtz and Markus, 2012; Ozsoy-Cicek et al., 2013; Kern et al., 2016), yet uncertainties (chiefly in snow depth and ice- and snow densities) are currently too large to derive reliable circum-Antarctic sea-ice thickness estimates (Giles et al., 2008; Schwegmann et al., 2016; Paul et al., 2018). Sea-ice motion and deformation determine ice production, thickness, and the transport of freshwater, but satellite products have insufficient accuracy, consistency, duration and/or spatial and temporal resolution to provide accurate long-term drift trends or largescale ice deformation fields (Schwegmann et al., 2011; Holland and Kwok, 2012; Haumann et al., 2016; Spreen et al., 2017). Drifting sea-ice buoys can provide key information on both the seasonal evolution of the ice and snow-cover thicknesses (e.g., Maksym et al., 2012) and high-resolution observations of ice drift and deformation (e.g., Heil et al., 2008, 2016), but deployments are limited. Coastal polynyas play an important though regionally

${ }^{29}$ Coordinated through the SCAR program "Antarctic Sea Ice Processes and Climate," http://aspect.antarctica.gov.au/ dependent role in Antarctic sea-ice formation (Drucker et al., 2011; Tamura et al., 2016) and are closely coupled to fast ice (Nihashi and Ohshima, 2015).

\section{Theme 6: Impacts of Global Change on Southern Ocean Ecosystems}

The Southern Ocean, with its sea-ice cover, modulates nearly all ecosystems and life in Antarctica. While birds and some mammals breed on land, they all feed in the ocean. Changing air temperatures, wind patterns, sea ice, ocean frontal areas, and water-mass circulation therefore have profound and regionally specific effects on biota, impacting the adaptation capacity and survival of individual species, and promoting cascading effects throughout the ecosystem (Murphy et al., 2012; Constable et al., 2014, 2016; Gutt et al., 2015, 2017).

At the base of the food web, trends in phytoplankton differ among areas and latitudes. Kim et al. (2018) showed that while phytoplankton increased in the northern WAP, around Carlini Station (Potter Cove) in the South Shetlands (Schloss et al., 2014) and near Palmer Station, they decreased further south due to the differential impact of ENSO and the Southern Annular Mode on spring phytoplankton. This suggests important localized forcings. Similar comparative studies are not available for other areas of the Southern Ocean, which are differentially affected by sea ice, glacial meltwater (Hernando et al., 2015), iron availability, and other stressors such as $\mathrm{CO}_{2}$ increase and the consequent ocean acidification (Hoppe et al., 2013; Trimborn et al., 2013, 2017; Hancock et al., 2018). Other than phytoplankton, bulk community composition and a deeper understanding of the microbial realm, including bacteria, archaea, and viruses, and the role of recalcitrant dissolved organic matter (microbial carbon pump, Jiao et al., 2010) are essential to the food-web carbon flux as well as to the ocean-carbon pump and $\mathrm{CO}_{2}$ sequestration.

For first-order phytoplankton consumers, zooplankton, and micronekton (those organisms in the size range between zooplankton and higher predators - notably krill, and small midwater fishes and squids), accurate biomass estimations remain a major challenge. A recent modeling effort (Klein et al., 2018) forecasted that a reduction in krill growth rate due to warming will propagate to the dependent predators (penguins, seals, whales, and fishes). The biomass of mid-water (mesopelagic) fishes and squids, how they will respond to climate change, and implications for higher trophic levels are increasingly recognized as gaps in our understanding of ocean ecosystems globally (St. John et al., 2016), and the Southern Ocean is no exception (Murphy et al., 2012).

Top predators are excellent sentinels of change, as their species composition, demography, behavior, and diet reflect the community structure (Constable et al., 2014, 2016). Climatelinked oceanographic features determine the distribution and abundance of their prey (reviewed in De Broyer et al., 2014), which affects foraging success, juvenile recruitment, breeding phenology, growth rates, and population stability (e.g., Ducklow et al., 2013; Hinke et al., 2017b). As ecosystems change, there will be both winners and losers (Costa et al., 2010; MelbourneThomas et al., 2013; Clucas et al., 2014; Constable et al., 2014). For example, while some Adélie and chinstrap penguin 
populations have plummeted, gentoo penguin colonies have increased (Trivelpiece et al., 2011; Ducklow et al., 2013; Hinke et al., 2017b). Similarly, while Weddell seals near Palmer Station have declined, Antarctic fur seal and southern elephant seals have increased (Siniff et al., 2008). The increased intensity of winds has allowed wandering albatross to forage farther and faster, making their foraging trips shorter and increasing their foraging efficiency and breeding success (Weimerskirch et al., 2012). The foraging behavior and demographics of Antarctic fur seals and penguins have been monitored by the Convention for the Conservation of Antarctic Marine Living Resources (CCAMLR) as part of the management of krill fisheries. CCAMLR have produced long-term time series that can be used as an important baseline for future comparison (Trivelpiece et al., 2011; Ducklow et al., 2013; Hinke et al., 2017a,b).

The combined implications of changes across multiple trophic levels for the structure and function of Southern Ocean ecosystems are not well understood, and will require coordinated observations and modeling to resolve (see the sections "Assessing Status and Trends of Key Southern Ocean Taxa" and "Southern Ocean Modelling progress and priorities").

\section{DRIVERS OF THE FUTURE DEVELOPMENT OF SOOS}

The next 10 years will see a step-change in the delivery of integrated, multidisciplinary Southern Ocean data. The SOOS Regional Working Groups and other networks developed over the last few years will become well-established, and will work with the community to design regional observing systems to collect sustained data and deliver them to all end-users. Importantly, the systems designed will need to be flexible, to take advantage of sensor and platform developments, new networks, funding or infrastructures, and to ensure continued impact of the data in addressing the most pressing issues for society. Toward this end, regular community review and updating of the scientific drivers of the observing system will be essential.

\section{Southern Ocean Observations: Future Priorities}

The SOOS Science Themes have provided clear focus for SOOS's activities over the last decade, and in many instances, remain priorities for the coming decade of observations. Taking into account the progress of the community in delivering knowledge relevant to those themes (sections "Themes 1 and 2: The Role of the Southern Ocean in the Planet's Heat and Freshwater Balance; and the Stability of the Southern Ocean Overturning Circulation," "Theme 3: The Role of the Ocean in the Stability of the Antarctic Ice Sheet and Its Future Contribution to Sea-Level Rise," "Theme 4: The Future and Consequences of Southern Ocean Carbon Uptake," "Theme 5: The Future of Antarctic Sea Ice," and "Theme 6: Impacts of Global Change on Southern Ocean Ecosystems"), the Southern Ocean community has identified eight key issues of focus for the coming decade. These issues are identified as major data bottlenecks in addressing the six themes, and are introduced in the following sections.

\section{Observing Antarctic Bottom Water Production Processes}

Conspicuous signals of climate change have been observed in Antarctic Bottom Water (AABW) including warming (Purkey and Johnson, 2010; Couldrey et al., 2013), freshening (Purkey and Johnson, 2013), and a reduction in volume (Purkey and Johnson, 2012). It is tempting to associate these changes with the observed freshening and warming over the continental shelves surrounding Antarctica (Schmidtko et al., 2014); however, our knowledge of the physical processes linking these changes is limited (e.g., van Wijk and Rintoul, 2014). In situ observations to describe and understand the processes involving forcings from the atmosphere and ice (both land-based and floating) and AABW are needed. New technologies are improving our knowledge of such physical processes, including animalborne sensors, under-ice autonomous floats, microscale sensors attached to Conductivity, Temperature, Depth profiler (CTD), and more are becoming available such as under-ice autonomous underwater vehicles (AUV), remotely operated vehicles (ROVs), and gliders. Figure 5 shows the observing system required to observe these production processes.

These processes involve large-scale physics to meso- and submeso-scale physics (e.g., Stewart and Thompson, 2014) with complicating factors such as the Antarctic Slope Current, patchy bathymetric data coverage, tides, and sea-ice variability. Diapycnal mixing is a key quantity, but knowledge of its spatial and temporal distribution is lacking, except for a limited number of field campaigns (Gille et al., 2007; Meyer et al., 2015). Observed mixing is yet to be attributed to generation mechanisms (e.g., current/topography interactions; Nikurashin and Ferrari, 2011). Earth system models poorly resolve Southern Ocean water-mass behaviors, including AABW formation, often leading to unphysical deep open-ocean convection (Heuzé et al., 2013) or large spread in an ensemble (Meijers et al., 2012). Observations of AABW, along with meso- and submeso-scale processes, are needed for better parameterization of watermass transformation (see the section "Ecosystem Modeling Efforts"). The observation should cover air-sea and sea-ice fluxes for buoyancy transformation driving AABW formation (see the next section).

\section{Reducing Uncertainties in Air-Sea and Air-Ocean-Ice Fluxes of Heat, Momentum, Freshwater, and Carbon}

Southern Ocean in situ air-sea and air-sea-ice flux observations are rare (Bourassa et al., 2013), with almost no observations in autumn or winter (Gille et al., 2016; Swart et al., 2019) or in the sea-ice zone. Reanalyses and satellite-derived surface flux products therefore have major errors and vary considerably (Josey et al., 2013; Bentamy et al., 2017; Schmidt et al., 2017).

In 2015 , the SOOS community identified fluxes as a priority observation gap, resulting in the development of the SOOS Southern Ocean Air-Sea Flux (SOFLUX ${ }^{30}$ ) Working Group. SOFLUX has identified priorities for the coming decade, which are articulated in Swart et al. (2019). Specifically, SOFLUX noted that the climate-scale net air-sea heat flux of $0.6 \mathrm{~W} \mathrm{~m}^{-2}$ is

\footnotetext{
${ }^{30}$ http://soos.aq/activities/capability-wgs/soflux
} 


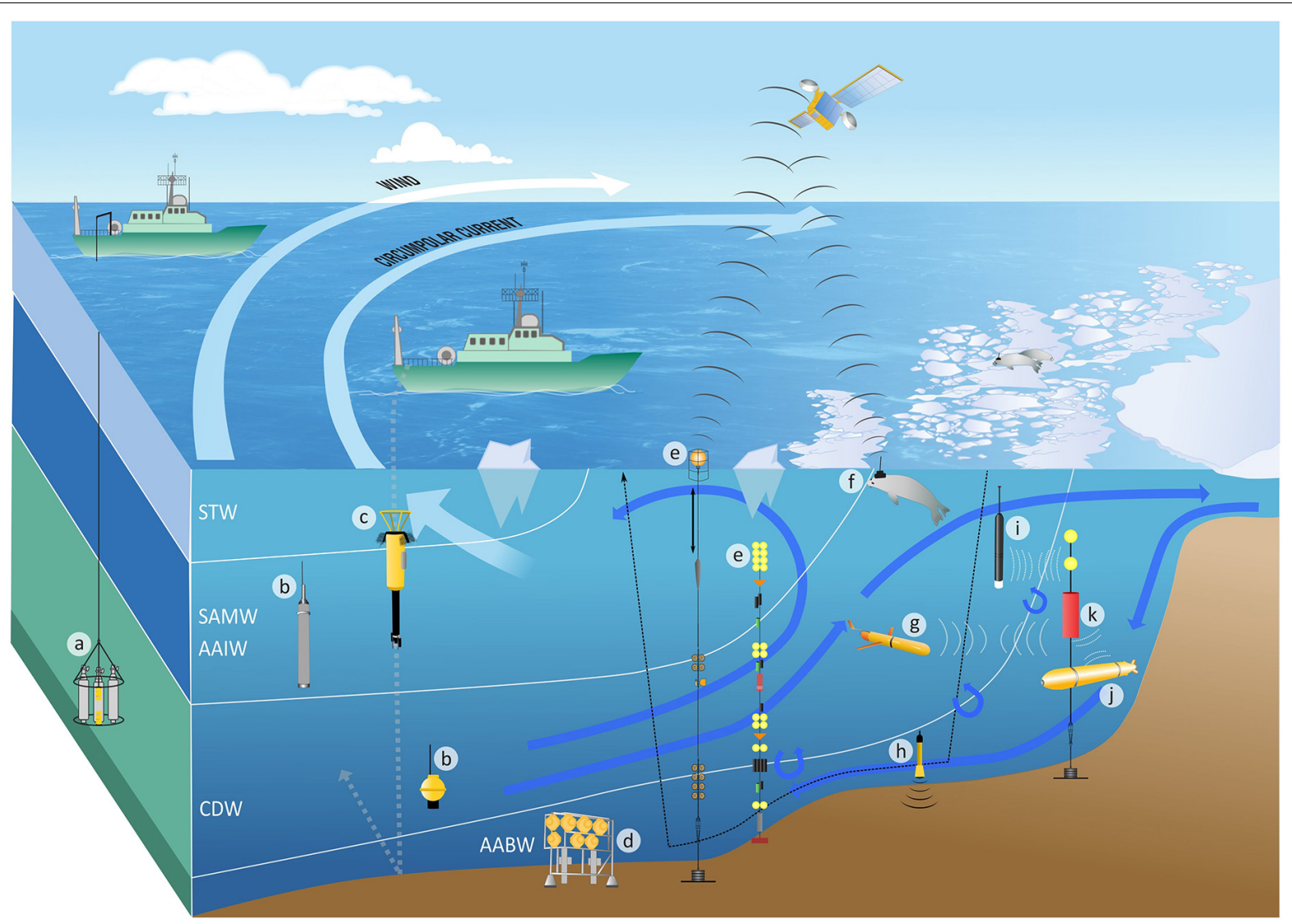

FIGURE 5 | A schematic of the observational platforms required to observe the AABW formation. (a) Conductivity-temperature-depth profiler often equipped with additional sensors such as dissolved oxygen, horizontal velocity (lowered ADCP - yellow cylinders), and temperature turbulence; (b) Argo profiler, both on core mission (depth < $2000 \mathrm{~m}$ ) and deep mission (deeper); (c) microstructure profiler measuring energy and material dissipation rate; (d) bottom lander with a suite of instruments including water sample; (e) mooring system with physical (temperature, salinity, velocity) and biogeochemical (sediment trap, etc.) measurements, some with an underwater winch-driven surface buoy for ice avoidance; (f) animal tagged profiler; (g) glider; (h) bottom drifter, a float specially targeting AABW; (i) under-ice Argo profiler with acoustic locating capability; (j) autonomous underwater vehicle; and (k) a sound source for acoustic float locating. Modified from Meredith et al. (2013).

determined from Argo float measurements of ocean-heat content and not from direct flux measurements, and while the Argobased level of accuracy is not expected, direct observations will provide validation for products based on remote sensing and reanalyses. Additionally, direct flux measurements provide process-level understanding, particularly intraseasonal variations associated with oceanic eddies and fronts or with the passage of atmospheric storms. This justifies targeted process studies aimed at measuring flux differences over the scale of the first baroclinic Rossby radius (e.g., $50 \mathrm{~km}$ or less).

The key priority for the coming decade is to obtain more in situ observations, particularly in winter. A significant increase in observations would occur if all Southern Ocean vessels were instrumented with a standard meteorological package (e.g., IMET system), and a thermosalinograph to provide the key variables needed for air-sea flux bulk formulae (i.e., air-sea temperature differences, wind speed, and humidity). Vessel flowdistortion calculations done prior to installation are needed for optimizing sensor locations to minimize airflow disruption, or to account for these errors. Wave gliders, saildrones, and surface-flux moorings offer the possibility of year-round air-sea flux observations, but can be prone to potentially greater logistical and/or power challenges. Moored buoy flux observations are valuable for investigating temporal variability in air-sea fluxes, but they have only been deployed in two locations in the Southern Ocean (Schulz et al., 2012; Ogle et al., 2018). Figure 6 represents this proposed optimal observing system, and further recommendations on priority flux observations can be found in Swart et al. (2019).

\section{Understanding the Contribution of Oceanic Heat to Ice-Shelf Basal Melt}

Drivers of ice-shelf melt are poorly understood (Kennicutt et al., 2014a). Transport of warm oceanic water to the ice-shelf front and base, and the importance of different processes and pathways (i.e., eddies, tides, winds, variability, and intensity of the Antarctic Slope Current and topography) need to be evaluated. Long timeseries ( $>10$ years) of temperature, salinity, and current velocities are required in key locations near ice shelves, such as those shown in Figure 7. A number of locations in the Weddell and Amundsen 


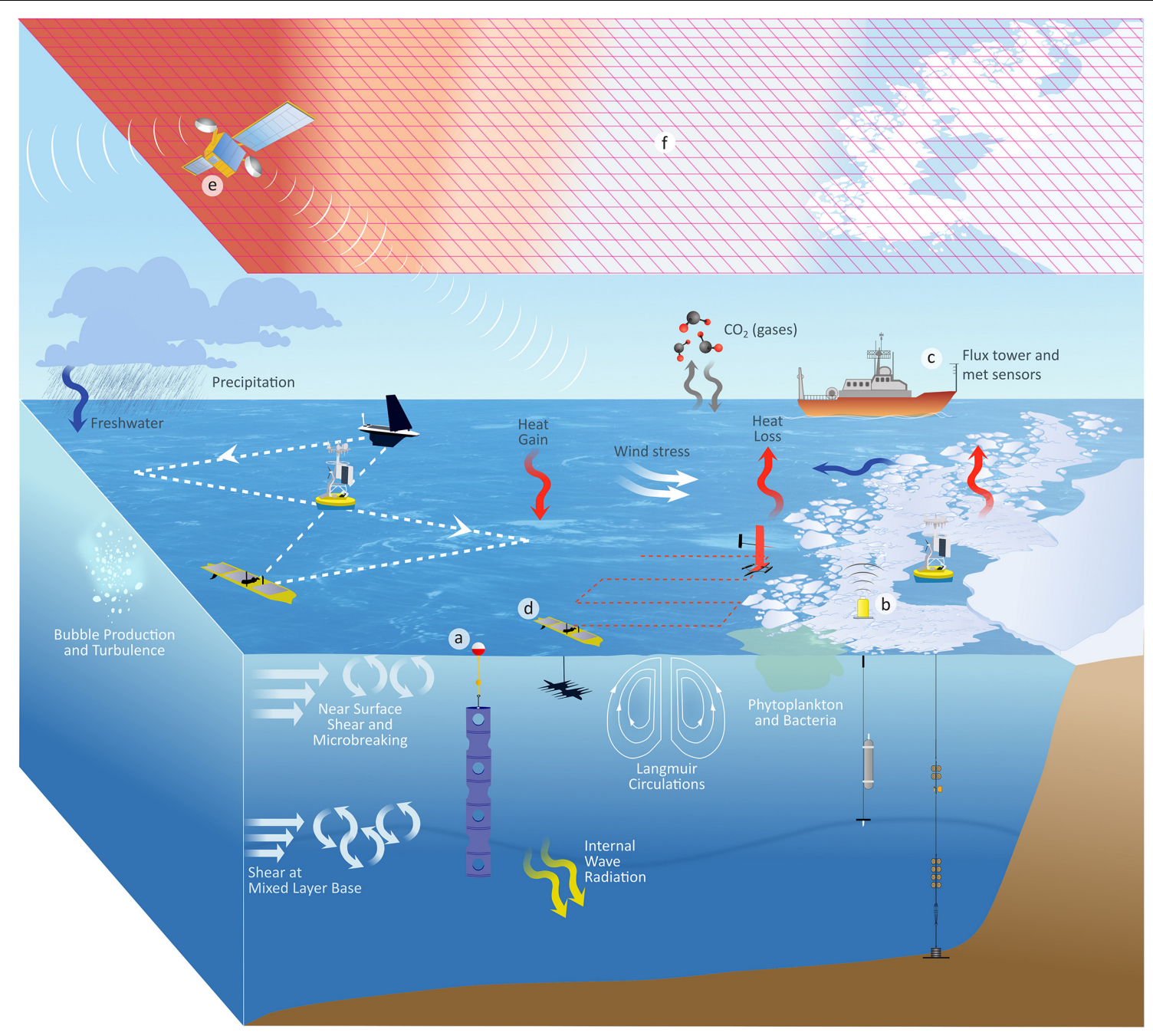

FIGURE 6 | Schematic of the key elements of a Southern Ocean air-sea flux observing system in order to reduce uncertainties in air-sea and air-sea-ice fluxes of heat, momentum, freshwater, and carbon (section "Reducing Uncertainties in Air-Sea and Air-Sea-Ice Fluxes of Heat, Momentum, Freshwater, and Carbon"). The optimal observing system would include buoys (a) in the open ocean, and ice-tethered profilers (b) in the marginal ice zone, as well as ships instrumented with flux covariance systems (c). Autonomous surface systems (d) such as wave gliders and saildrones are proposed to provide a more extensive spatial network of flux sensors than enabled by ships and buoys alone and also for targeted process studies aimed at understanding air-sea flux variability across fronts or eddies, possibly surveying in the vicinity of a buoy. Satellite measurements (e) of surface wind, SST, and near surface variables provide global measurements that can fill spatial gaps between in situ observations. The grid overlaying the entire domain (f) is intended to indicate the role of assimilating models, such as reanalyses, in ingesting all available observations to provide a consistent gridded flux product. Modified from Meredith et al. (2013).

Seas and off East Antarctica have maintained mooring sites on the open shelf and through the ice shelf that have the potential to achieve important, long time series if they are sustained over the next decade.

Increased use of Autonomous Phase Sensitive Radars (ApRES; Nicholls et al., 2015) through the NECKLACE initiative will provide direct observations of the basal melt rate and help validate basal melt rates inferred from satellite observations. Gliders and floats with under-ice capabilities, together with AUV missions, have enabled measures of sub-ice shelf ocean circulation, processes controlling the ocean interaction with the ice shelf base, and exchange processes across the ice-shelf front that we are currently lacking (e.g., Miles et al., 2015). Improvements in under-ice navigation/geolocation will lead to a rapid transition from pilot projects to large-scale arrays and longer duration missions.

To constrain numerical ocean and ice-sheet models, high resolution bathymetry on the continental shelves and within iceshelf cavities, observations of ice-shelf draft, basal topography and roughness, and ocean observations that resolve the seasonal and intra-annual water mass and current variability are required. Increased use of autonomous technologies, long-range missions, improved under-ice navigation, and satellite transmission of the data will decrease the effort and cost involved in monitoring the transport of ocean heat toward the ice shelf bases. 


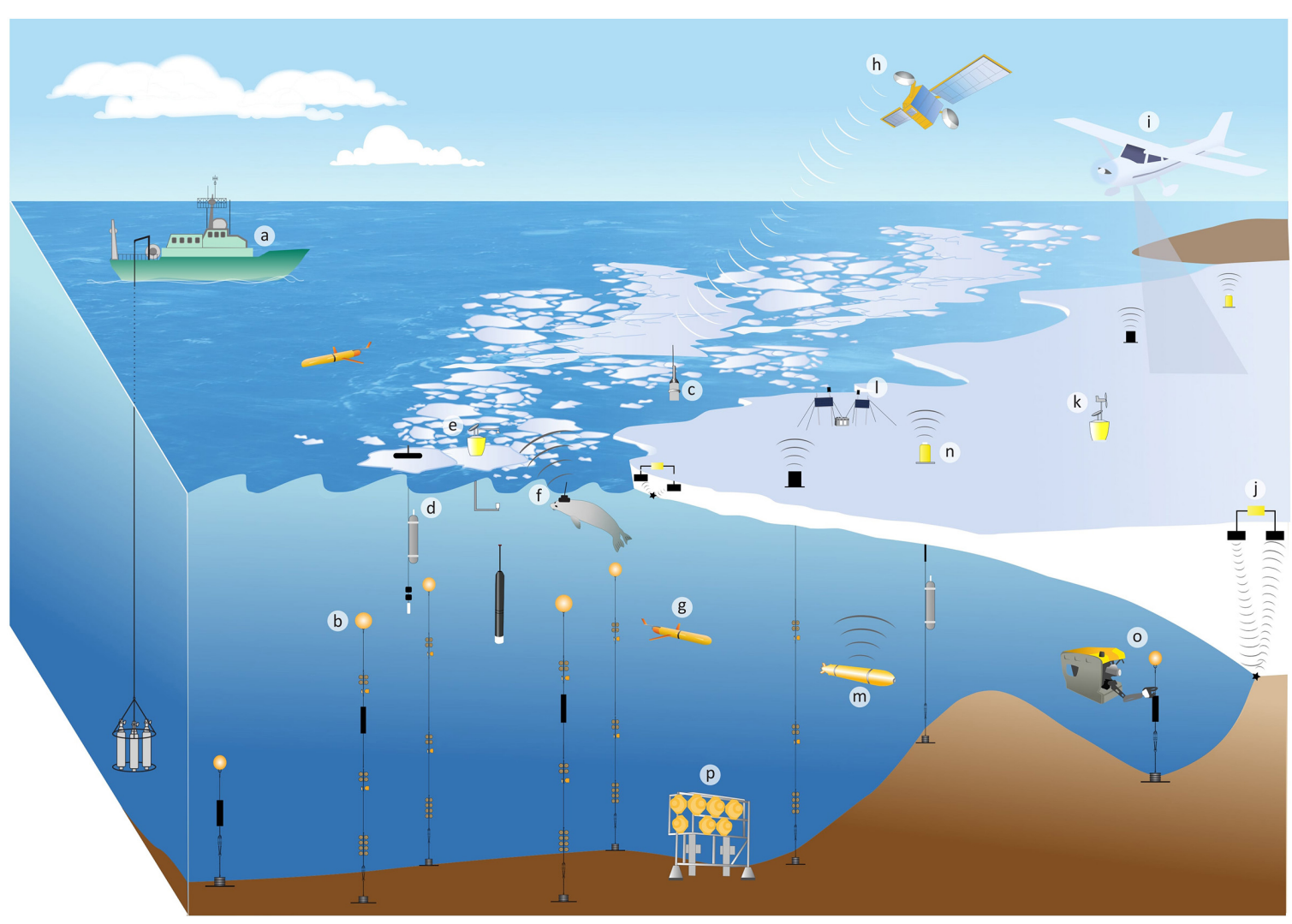

FIGURE 7 | A schematic of the integrated system of observational platforms required to determine the contribution of oceanic heat to ice-shelf melt for a generic ice-shelf configuration. An integrated system to observe the processes important for grounding line retreat and basal melt consists of three main components. Firstly, observing how ocean currents approach and exit the ice shelf cavity, achieved by a combination of ship-based observations, such as hydrographic sections (a); moored instrumentation (b) deployed in key locations across the front of the ice shelf (e.g., polynyas, dense overflows); ice-capable profiling floats (c); ice-tethered profilers (d) and ice-mass buoys (e) particularly in fast- or multi-year ice; seal tags (f); and gliders (g) for high-resolution transects on the shelf and slope. Secondly, determining changes in ice-shelf thickness, basal melt-rate, and grounding-line retreat, achieved by deployment of satellite (h) and airborne (i) remote sensing for ice shelf properties; ApRES radars (j) for direct basal-melt rates; automatic weather stations (k), GPS, boreholes, and other sensors (l) for other properties, such as snow accumulation. Finally, observation of processes within the ice-shelf cavity itself requires AUVs (m) for observations of water properties, as well as swath mapping of sea floor and sub-ice shelf topography; sensors/moorings deployed through boreholes ( $\mathbf{n}$ ) for sub-ice shelf properties, circulation, and direct basal melt data, including mooring with ITPs and telemetry; moorings deployed by ROVs in ice shelf cavity (o), which is not currently implemented but is a promising technological advance in the coming decade; and bottom landers (p) out the front of the ice shelf for a multitude of roles, such as communication gateways, imaging, water sampling, etc. This figure was modified from Meredith et al. (2013) and Rintoul et al. (2014) "seeing below the ice" 2014 strategy available at http://soos.aq/resources/science-strategies?view=product\&pid=26.

Figure 7 schematically represents the integrated observing system required to understand and constrain ice-shelf basal melt.

The Partnership for Observation of the Global Oceans (POGO) has supported a SOOS working group Observing and Understanding the ocean below Antarctic Sea Ice and Ice Shelves $\left(\right.$ OASIIS $^{31}$ ) to focus the international effort to enhance sustained observations of the ocean near and under major ice shelves. This group will make an important contribution to addressing the above-mentioned data gaps.

\section{Toward a Better Understanding of Processes Controlling Antarctic Sea-Ice Variability and Change}

Although climate models include sea-ice components, they exhibit low skill in simulating observed Antarctic sea-ice

\footnotetext{
${ }^{31}$ http://soos.aq/activities/capability-wgs/oasiis
}

properties and their spatio-temporal variability, i.e., seasonality, extent, concentration, and thickness (Shu et al., 2015; National Academies of Sciences, Engineering, and Medicine, 2017; Roach et al., 2018). This is due to a combination of poor process understanding and sub-optimal parameterization of the iceand snow physics and embedded ecosystems, for example, the representation of ocean-ice-atmosphere interactions and feedbacks (Stammerjohn et al., 2012; Goosse and Zunz, 2014; Holland et al., 2014, 2017; National Academies of Sciences, Engineering, and Medicine, 2017). In turn, this severely undermines confidence in model projections of future sea-ice cover and conditions around Antarctica (IPCC, 2013). Key observations and processes requiring attention include coupled ice-ocean processes driving short-term and seasonal advance and retreat (including wave-ice interaction in the marginal ice zone); the relative role of dynamic and thermodynamic processes in 
driving the ice-thickness evolution; the thickness distribution of Antarctic sea ice and its snow cover; effect of snow processes on sea-ice properties and ice-mass balance; the role of polynyas as regional sea-ice "factories"; and pack-ice - fast-ice - ice-sheet interactions (Figure 8).

Of high priority are gap-filling, multi-disciplinary in situ experiments [e.g., in style of Antarctic Remote Ice Sensing Experiment (ARISE) $)^{32}$, Ice Station POLarstern (ISPOL) ${ }^{33}$, PIPERS (see text footnote 10)] in combination with welldistributed but sustained monitoring programs [e.g., Antarctic Fast-Ice Network (AFIN) $)^{34}$ ] to capture and resolve seaice characteristics, processes, and interactions at sufficient spatio-temporal scales to enable comparison with (and thus improvement of) model parameterizations, as well as

${ }^{32}$ http://www.antarctica.gov.au/science/cool-science/2007/antarctic-remote-icesensing-experiment

${ }^{33}$ https://www.awi.de/ueber-uns/service/presse-detailansicht/presse/ forschungseisbrecher-polarstern-driftet-im-antarktischen-eis.html

${ }^{34}$ seaice.acecrc.org.au/afin/ satellite-derived estimates. Such an approach is required to improve quantification and understanding of, for example, ice deformation and ice production; snow accumulation and distribution; autumn-winter ice formation and ice-edge advance, and spring-summer ice break-up and ice-edge retreat; and largescale characterization of the sea ice. Autonomous technologies [e.g., long-range AUV and Unmanned Aerial Vehicles (UAVs)] have a crucial role to play in bridging the scale gap between floes [of the order of $(1-100) \mathrm{m}$ ] and regional-scale satellite products and models [typical resolution (1-25) km], supplemented by (i) sophisticated mass-balance buoys that measure the evolution and drift of the coupled ocean-ice-snow-atmosphere system, and (ii) routine underway observations from instrumented icebreakers to measure snow- and ice thickness, floe-size distribution, and more. Around coastal Antarctica, the more stable fastice environment lends itself to sustained and internationally coordinated long-term observations at AFIN stations (Heil et al., 2011), which, together with supporting atmospheric and oceanographic data, may contribute to the WMO-endorsed

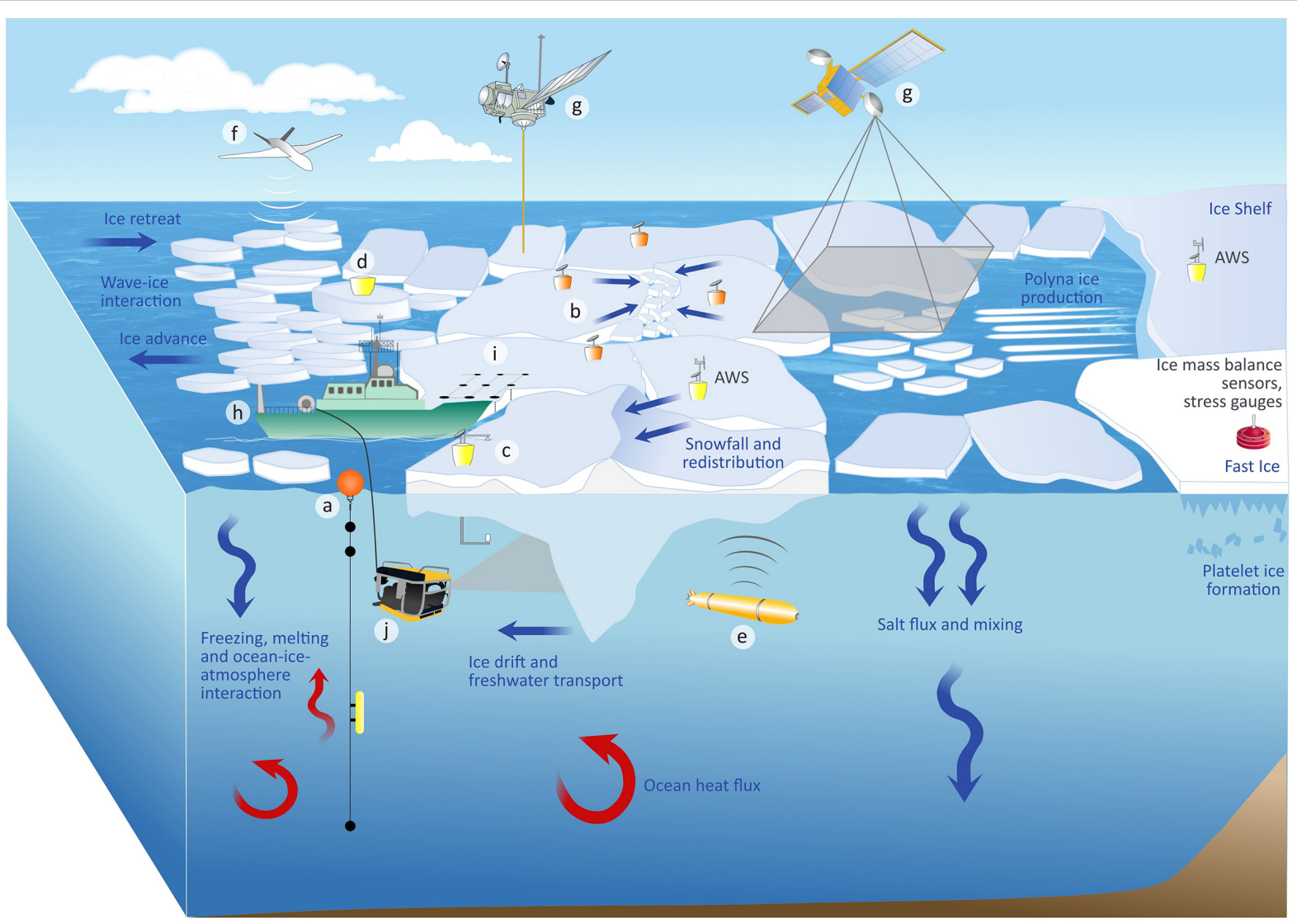

FIGURE 8 | Schematic of the platforms required to observe key sea-ice processes toward capturing circumpolar sea-ice variability (section "Toward a Better Understanding of Processes Controlling Antarctic Sea-Ice Variability and Change") and deriving sea-ice thickness and volume (section "Observing Sea-Ice Thickness and Volume"). Due to the remoteness, vastness, and hostile conditions of the sea-ice zone, autonomous platforms will be crucial. They include high-resolution in situ sensors such as drifting buoys (a); ice-deformation arrays (b), mass-balance- and snow-buoys (c), wave buoys (d), and autonomous underwater (e) and airborne vehicles (f). Observations of snow depth, ice thickness, ice concentration, drift and properties from satellite altimeters, and radars and radiometers (g) are vital to sustain for long-term time series. Manned observation platforms include vessels (h) [for underway and in situ sea-ice sampling (i) including ROV deployments (j)], aircraft (e.g., EMBird and NASA's Operation IceBridge), and near-coastal research stations (for fast-ice and sea-ice/ice-shelf studies). Modified from Meredith et al. (2013). 
CryoNet initiative (Smith et al., 2018). Such long-term and internally consistent datasets not only provide key information on variability/change and processes responsible; they also enable calibration and validation of satellite-derived sea-ice thickness, as well as model verification.

Satellite remote sensing - calibrated and validated by targeted in situ observations (see above) - has a key role in both extending in situ observations in space and time, and in providing unique large-scale information on the Antarctic sea-ice environment (Lubin and Massom, 2006). Yet, important challenges remain, not only in improving satellite-derived products and quantifying uncertainties (see the section "Theme 5: The Future of Antarctic Sea Ice"), but also with respect to satellite data availability around Antarctica. For example, continuation of the crucial satellite passive microwave sea-ice concentration record (dating back to 1978) is not assured. Moreover, high resolution data required for sea-ice deformation, as well as polynya, fast-ice, and marginal-ice zone studies (e.g., synthetic aperture radar and high-resolution visible-thermal infrared data) are sparse over the Antarctic sea-ice zone. These, and other issues relating to data calibration/validation requirements over the next decade, are discussed in detail by Pope et al. (2017).

\section{Observing Sea-Ice Thickness and Volume}

The two most important reasons for not being able to obtain accurate estimates of circum-Antarctic sea-ice thickness (Giles et al., 2008; Schwegmann et al., 2016; Paul et al., 2018) are the lack of reliable snow depth and ice/snowdensity data over broad scales, which are required for the sea-ice thickness retrieval, and the lack of sufficient in situ, UAV-, and air-borne sea-ice, and snow thickness measurements required for the training and evaluation of algorithms to derive sea-ice and snow thickness. The recent advent of ICESat-2, plus increased EMBird deployments and ongoing in situ observations of fast and pack ice (through the "AFIN" and "Antarctic Sea Ice Processes and Climate program," respectively), provide a renewed impetus to tackle this in the near term.

Improved observations of snow-depth distribution will require sustained and much broader deployment of snowdepth and ice mass balance stations/buoys (IMBs) that capture sufficient spatial and temporal variability to evaluate satellite and modeled snow-depth products. Increased use of airborne, and particularly unmanned aerial systems (UASs), are needed to provide broader-scale snow distributions and to extend radar-derived snow-thickness estimates from NASA's Operation IceBridge mission to other regions and seasons (Webster et al., 2018).

To develop and validate ice-thickness products (e.g., from ICESat-2 and CryoSat-2), long-range, long-endurance AUVs (section "Priorities for Future Observational Technologies") or long-range aerial sampling (i.e., EMBird) will also be needed. Traditional aerial platforms, UASs, and AUVs are critical to both extend traditional in situ point measurements to satellite and model resolution scales and mitigate the logistical challenges of ship-based sea-ice measurement campaigns.
Additionally, wider networks of IMBs are required to provide crucial data for evaluation of satellite-derived ice-thickness products. More extensive drifting buoy networks are also critical in the sea-ice zone to evaluate and improve reanalysis products (which are key to understanding the drivers of sea-ice variability) and are much more sparsely deployed than in the Arctic. Ice-tethered and under-ice floats (SOCCOM and ice-capable Argo) can help determine coupling between ice production and upper-ocean properties. At present, few floats are deployed under sea ice, and almost no ice-tethered sensors have yet been deployed. Lastly, drifting buoy networks provide highresolution deformation data. This is essential to evaluating satellite-based deformation products that have more limited temporal resolution.

\section{Constraining the Seasonal Carbon Cycle}

To constrain the Southern Ocean's biogeochemical cycles, observations of oxygen, nutrients, dissolved inorganic carbon (DIC), dissolved organic carbon (DOC), transient tracers (e.g., CFCs), particulate organic carbon, and ocean-color measurements are a priority. Stable isotope measurements of nitrate, as well as DIC and silicic acid, are becoming powerful tools for constraining past and present biogeochemical processes and ocean circulation, and substantial datasets are accumulating across the Southern Ocean over the annual cycle (e.g., Smart et al., 2015; Henley et al., 2018; Kemeny et al., 2018; Fripiat et al., 2019). Carbon isotopes and elements such as neodymium are also becoming increasingly important for paleo-circulation reconstructions (Basak et al., 2018), but modern measurements are scarce, such that there is a need to include these parameters in future observing efforts. In addition to traditional oceanographic sampling approaches, such as repeat hydrographic sections, moorings, underway measurements, and continuous plankton recorders, future efforts should include more under-sampled EOVs such as DIC and DOC, supplemented by data from bio-Argo floats, tagged marine mammals, and gliders (Figure 9; Roquet et al., 2014; Erickson et al., 2016; Johnson et al., 2017a).

Despite significant progress in new technologies for biogeochemical observations, recent studies indicate that at least 100 Biogeochemical-Argo floats (BGC Argo) are needed to detect climate-scale changes in air-sea $\mathrm{CO}_{2}$ fluxes and Southern Ocean carbon and heat content (Mazloff et al., 2018). Even then, this will not cover coastal regions and may not resolve seasonal carbon dynamics well enough to reduce $\mathrm{CO}_{2}$ flux uncertainty to the levels required to reveal climatic trends (Monteiro et al., 2015). In the next decade, a new generation of bio-Argo floats may help to fill these data gaps. For example, Air Launched Autonomous Micro Observer (ALAMO) floats could be launched from air into polynyas or open water adjacent to sea-ice zones, and ArvorC floats are designed to operate in coastal environments. Improved battery capacity will increase sampling frequency, allowing for the study of blooms or sub-mesoscale dynamics and permitting the installation of new and/or additional sensors. Floats capable of profiling below $2000 \mathrm{~m}$ will help to constrain deep heat and carbon storage as well as deep 


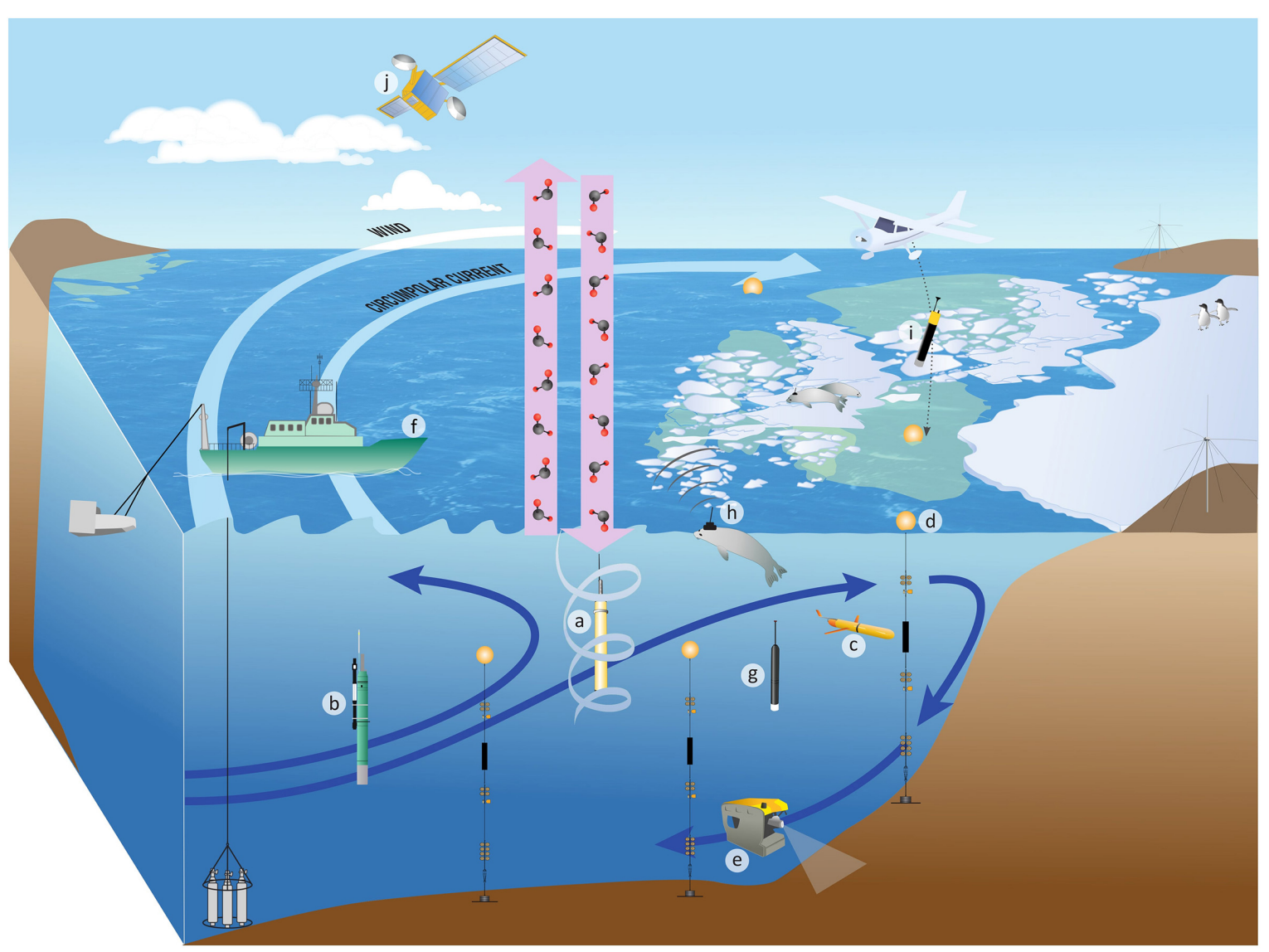

FIGURE 9 | Components of an optimal observing system to constrain and quantify biogeochemical cycling processes in the Southern Ocean. New methodologies such as biogeochemical sensors deployed on SOCCOM (a) and SOCLIM (b) floats, gliders (c), and moorings (d), and sampling from ROVs (e) must be integrated with traditional ship- (f) and station-based approaches. Ice-capable bio-Argo floats (g) and biogeochemical sensors deployed on marine mammals (h) are needed to extend the observational range in time and space. Airborne deployment of ALAMO floats (i) could increase spatial coverage substantially. All of these in situ approaches should be combined with existing and improved satellite-based measurements (j) of sea surface height, sea ice, sea surface temperature, and ocean color to develop an integrated biogeochemical observing system for the Southern Ocean. Modified from Meredith et al. (2013).

oceanic circulation. Observations from aircraft-mounted devices also have the potential to increase the coverage of high-resolution air-sea $\mathrm{CO}_{2}$ and $\mathrm{O}_{2}$ exchange measurements, together with ocean color and atmospheric data, e.g., the $\mathrm{O}_{2} / \mathrm{N}_{2}$ ration and $\mathrm{CO}_{2}$ Airborne Southern Ocean (ORCAS) study (Stephens et al., 2018).

Central to the Southern Ocean carbon cycle are the trace metals required for biological activity, with the most important being iron (e.g., Tagliabue et al., 2012). The GEOTRACES program $^{35}$ has vastly increased the number of available observations; nevertheless, data coverage remains poor compared to other ocean regions. Early trace-metal studies focused largely on the open Southern Ocean, but coastal areas such as the Ross and Amundsen Seas where ice shelves are melting are now receiving increased attention (Alderkamp et al., 2012; Mack et al., 2017).

\footnotetext{
${ }^{35}$ Twenty-four GEOTRACES cruises and process studies occurring between 2006 and 2018 across all sectors of the Southern Ocean (Atlantic: 8; Indian: 12; Pacific: 7) (http://www.geotraces.org/cruises/cruise-summary)
}

\section{Constraining Biological Energy Pathways}

An ongoing challenge in observing ecosystems is that key processes and major components are difficult or impossible to observe directly, and uncertainties around indirect observation methods are not well characterized. This is true for important processes and rates - such as predator-prey interactions, consumption and energy use, and growth rates - and also for estimating abundance and biomass of many key taxa (see section "Assessing Status and Trends of Key Southern Ocean Taxa"). Ecosystem EOVs (eEOVs) provide a mechanism to address these challenges (section "Conclusion and Recommendations") and models are also important in integrating, contextualizing, and optimizing observations (section "Southern Ocean Modelling").

Improved data streams that constrain biological energy pathways by better characterizing the transfer of mass through food webs, into carbon export, and into fish stocks are a priority to inform Southern Ocean ecosystem modeling, assessment, and management (Constable et al., 2017; Figure 10). Potential consequences of shifts in energy pathways in the next 50100 years include: (i) declines in krill that may negatively impact 


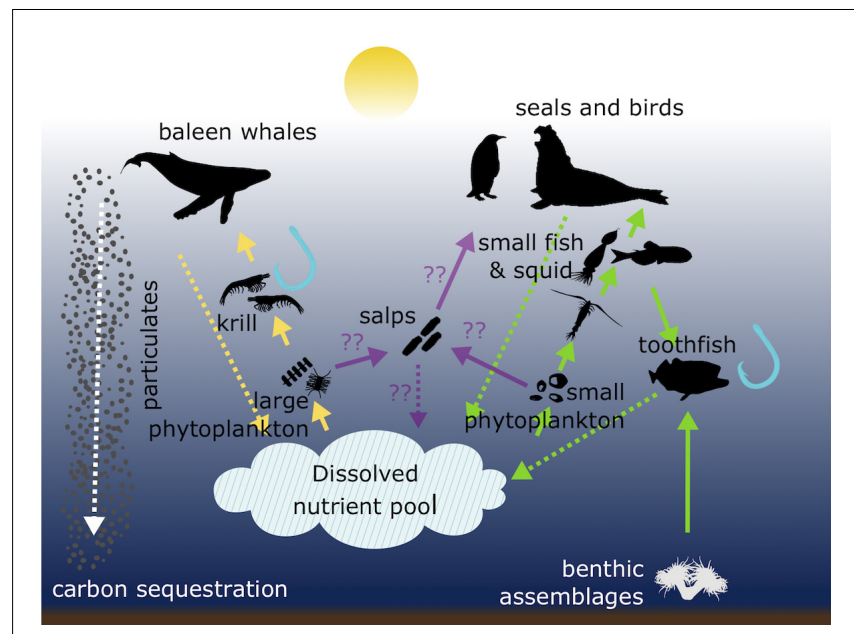

FIGURE 10 | Major biological energy pathways by which energy moves from primary producers to higher trophic levels in Southern Ocean ecosystems (integrated across seasons and across open water and sea-ice environments). These pathways include the well-studied Krill food-chain (yellow), as well as less well-studied pathways via small fish and squid (green), and potentially salps (purple). The relative dominance of these pathways underpins ecosystem structure and function and the provision of services such as fisheries (blue hooks) and carbon sequestration (white dotted arrow). However, these pathways and the factors that determine their relative dominance in space and time are not well resolved. Targeted observing effort will be required to fill this knowledge gap.

baleen whales, seals, seabirds, and krill fishery production; (ii) increases in copepods may result in increased toothfish catch; and/or (iii) increases in gelatinous salps may increase carbon sequestration and food for benthic communities (Constable et al., 2017). Observations that can resolve the likelihood of these outcomes include diet data (section 3.8) and biochemical tracers (isotopes, fatty acids, DNA, trace metals; see Pethybridge et al., 2017) together with targeted process studies that can inform estimates of the magnitude of biological fluxes at large scales.

\section{Assessing Status and Trends of Key Southern Ocean Taxa}

Rapid development of telemetry and remote-sensing technologies are yielding increasingly rich observational datasets for taxa at the bottom and top of Southern Ocean food webs (i.e., phytoplankton and predators). Mid-trophic level groups, however, represent a major data gap due to a lower observing effort and uncertainties around primary observing methods: nets and acoustic backscatter (Davison et al., 2015; Proud et al., 2018). For net data, catch depends upon the sampling method as much as what is in the water column, and relative catchability of different taxa (and size classes within taxa) is difficult to quantify but hugely influential on catch composition (Kaartvedt et al., 2012). Similarly, there are large uncertainties in converting acoustic backscatter information to estimates of community composition (biomass and abundance of different taxa) in the water column. Although initiatives like MESOPP ${ }^{36}$ are working

\footnotetext{
${ }^{36}$ www.mesopp.eu
}

to improve the models used to convert acoustic backscatter data to estimates of community composition, this remains a key challenge in ecosystem observing (Proud et al., 2018).

Identifying the drivers of changes in abundance requires information on foraging behavior and tactics, which need to be integrated with data on the relevant biophysical factors. Changes in diet inferred from stomach flushing (Miller and Trivelpiece, 2007, 2008), from tissue stable isotope analysis (Newsome et al., 2010; Ben-David and Flaherty, 2012), or from dietary DNA (Deagle et al., 2007) can be integrated with measurements of foraging behavior such as trip duration, movement patterns, and diving patterns using electronic tags (Miller et al., 2009; Hückstädt et al., 2012b; Kowalczyk et al., 2014). When coupled with demographic data, these provide a mechanistic understanding of how and why populations are changing and whether they can persist under climate change (Hückstädt et al., 2012a; Hinke et al., 2017b).

Other important gaps for biological observations that represent opportunities where the "value" of observation effort can be maximized include:

- Observations from under sea ice and fast ice (especially measures of under-ice production and habitat characteristics);

- Data on winter ecology or year-round studies;

- Co-located (and co-incident) sampling of multiple ecosystem components (e.g., net sampling, acoustics, profiles, predator observations); and

- System-level structural knowledge (i.e., measures of relative biomass of key taxa, links, and flux rates; see the section “Constraining Biological Energy Pathways").

Figure 11 represents the observational capabilities and data required to address these gaps in knowledge.

\section{Southern Ocean Modeling: Progress and Priorities}

The ocean and climate modeling community has achieved great progress in simulating the Southern Ocean during the last 10 years (see Stammer et al., 2019); however, many processes remain poorly represented. The Southern Ocean is not only one of the harshest environments to observe, but also one of the most challenging to simulate, because of complex interactions between climate system components (ocean, atmosphere, sea ice, ice sheets), and the fundamental role of mesoscale and submesoscale eddy fluxes in determining Southern Ocean circulation and its response to variable forcing. Realistic simulation of all dynamical interactions between components, delivering an explicit representation of eddy fluxes and isopycnal/diapycnal mixing, as well as ensuring that models can robustly represent the response of the overturning circulation to variation in wind and buoyancy forcing, constitute the major challenges for the next decade of physical oceanographic modeling of the Southern Ocean. All of the above can only be achieved with a hierarchy of model complexities and continued efforts toward the improvement of parameterizations of eddy-driven mixing. 


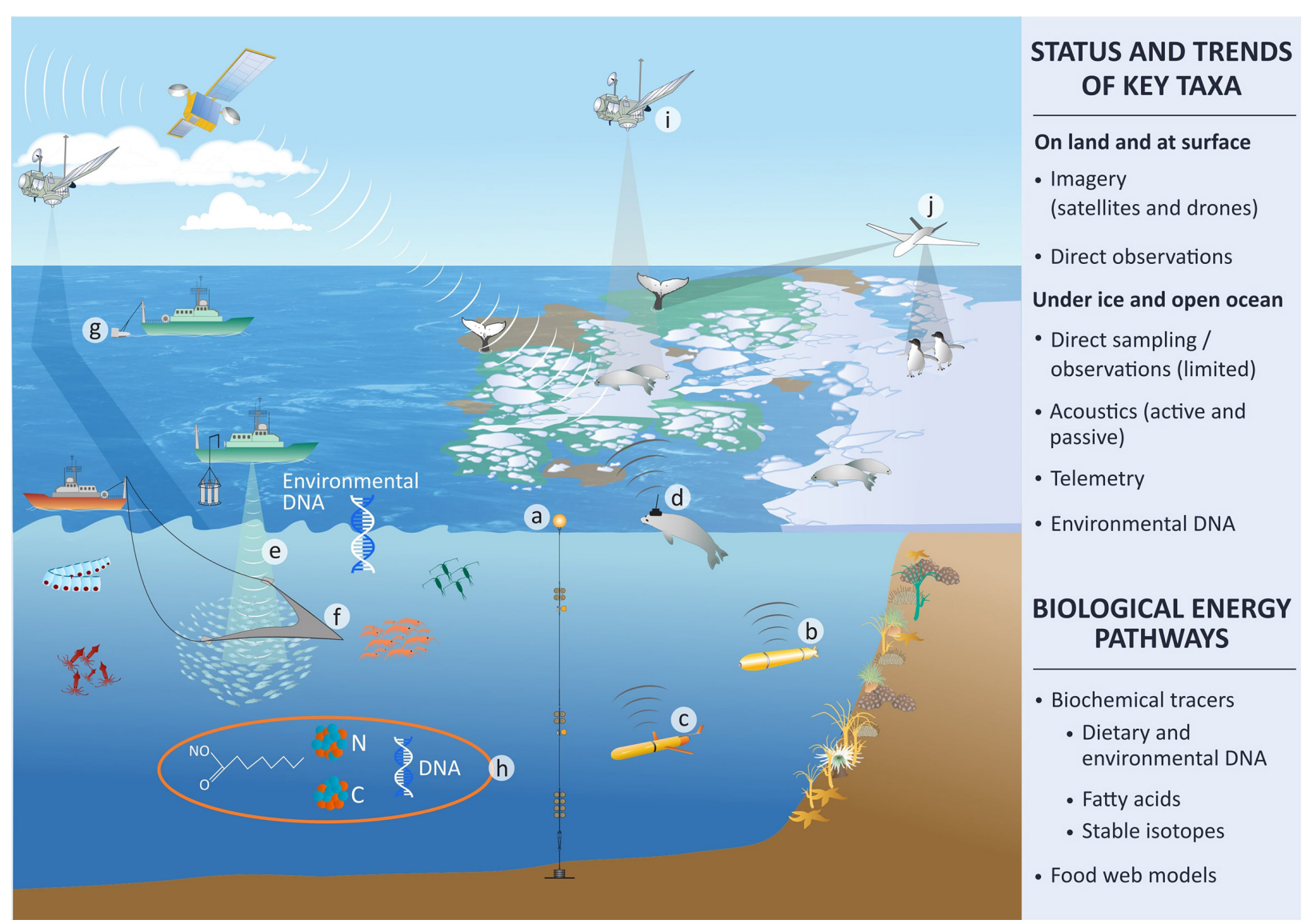

FIGURE 11 | Schematic illustration of the observation and models required to resolve key uncertainties regarding the impacts of global change on Southern Ocean ecosystems (adapted from Meredith et al., 2013). Mid-water taxa and food webs (particularly under seasonal ice and during the winter) are a key gap. Observations from platforms such as moorings (a), AUVs (b), gliders (c), and animal-borne instruments (d), along with improvements in acoustics (e) (including acoustic observation models) will help resolve status and trends of taxa, complementing traditional methods such as net sampling (f) and CPR (g). Integration of biochemical tracer data (such as dietary and environmental DNA, fatty acids, stable isotopes, $\mathbf{h}$ ) and observations into food web models will be required to constrain biological energy pathways. Imagery from satellites (i) and drones (j) will greatly improve knowledge of marine mammals, penguins, and other seabirds in difficult to observe areas such as pack ice.

Past and present freshwater input from ice-shelf melt is not well-constrained by observations, leading to a wide range of possible values used in global climate model studies of impacts on sea ice, water-mass formation, and overturning circulation (e.g., Bintanja et al., 2013, 2015; Swart and Fyfe, 2013; Abernathey et al., 2016; Pauling et al., 2016, 2017). Similarly, the factors controlling the seasonality, spatial distribution, and volume of Antarctic sea ice are still uncertain, as are present day effects and implications of future changes in sea ice on other parts of the Earth system, such as atmospheric and ocean circulation (Kennicutt et al., 2014a). In fact, none of the Coupled Model Intercomparison Project Phase 5 (CMIP5) models can reproduce the satellite record of Antarctic sea-ice extent (Shu et al., 2015). An increased observational coverage, in both space and time, can guide and support improved simulations of Southern Ocean dynamics, near- to seasonal-term forecasting of sea-ice distribution, dynamics and thickness, upper ocean heat content, and detection and attribution analyses of observed changes. Furthermore, sustained and integrated observations can better constrain and promote Southern Ocean state estimates, which are now starting to provide coupled biogeochemical-sea ice-ocean state information (Verdy and Mazloff, 2017).

\section{Required Ocean Modeling Efforts}

Recent modeling intercomparison studies have highlighted the pervasive biases and uncertainties in Southern Ocean dynamics and future projections (e.g., Meijers et al., 2012; Sallée et al., 2013; Downes et al., 2015; Farneti et al., 2015). Reliable simulations together with more confidence in future projections would have benefits beyond the Southern Ocean due to the fundamental role of the Southern Ocean in water-mass ventilation and carbon and heat sequestration. Polar climate change is largely dependent on surface fluxes, but their magnitude and variability are poorly constrained (Bourassa et al., 2013) due to spatial and temporal observational gaps, resulting in large uncertainties that limit our ability to assess the performance of our coupled climate models, to reduce model uncertainties and biases, and to guide model development. 
Russell et al. (2018) stressed the importance of the westerlies in shaping the circulation, and heat and carbon fluxes in the Southern Ocean. However, the correct simulation of easterlies and katabatic winds is equally important in simulating sea-ice extent and advective freshwater fluxes, particularly for latent heat polynyas where a large fraction of AABW precursors are produced.

Heat and freshwater fluxes are responsible for the formation of Antarctic Intermediate Water (AAIW) and Sub-Antarctic Mode Water (SAMW), constituting the upper limb of the MOC (Sallée et al., 2010), and have a crucial role in deep and bottom-water formation. Limited understanding of these fluxes is a significant problem in the present generation of coupled climate models (Sallée et al., 2013). This is partly due to poorly constrained surface and sea-ice fluxes, with CMIP5 models showing large inter-model spread in both present-day simulations and future projections (Russell et al., 2018). Also, sea-ice freshwater flux is recognized as playing a major role in water-mass formation and transformation (Abernathey et al., 2016; Pellichero et al., 2018), sustaining a large part of the overturning circulation. Unprecedented observations in seasonally covered regions of the Southern Ocean should be used to evaluate model results. This is also true in the context of heat and carbon sequestration, given the availability of biogeochemical profiling floats (Gray et al., 2018). Recent estimates of meridional transports of mass, heat, and salt along the Atlantic sector, as well as new observational estimates of ACC transport across Drake Passage (Donohue et al., 2016), provide a new opportunity for model evaluation, particularly as observations are sustained and enhanced over the next decade.

Southern Ocean circulation, and heat and carbon uptake, are not only dependent on wind and buoyancy forcing, but also on isopycnal and diapycnal mixing, eddies, and topographic features. Mesoscale mixing is ubiquitous in the ocean, and plays a fundamental role in setting the overturning circulation, watermass formation, and transport of tracers in the Southern Ocean. An accurate parameterization of mesoscale eddy mixing is a pressing challenge for the global ocean modeling community, and much work has been devoted to improving its representation in coarse-resolution models. For example, the eddy diffusivity has been related to the eddy kinetic energy derived through satellite altimetry. However, not only an estimate for the mean value is needed, but also its sensitivity to changes in forcing and interannual variability is crucial for properly representing mesoscale mixing processes and their effects on circulation and transport of tracers (e.g., Busecke and Abernathey, 2019). Mixing is critical for AABW formation, ice shelf and seaice melt, nutrient distribution, and other processes. The major Southern Ocean mixing mechanisms are winds, tides, interaction of currents and topography, and deep convection cells associated with polynyas. The influence of winds is limited to the upper ocean (Robertson et al., 1995) while the other mechanisms dominate deeper mixing. Tidal mixing plays a significant role in melting near the front of ice shelves (Robertson, 2013; Padman et al., 2018) and sea ice (Koentopp et al., 2005).

Presently, vertical mixing for all these mechanisms is parameterized in ocean and climate models. Some of the parameterizations reproduce the wind-generated upper mixedlayer depth well, whereas others overmix the water column (Robertson and Hartlipp, 2017). Likewise, with a resolution of $1 \mathrm{~km}$ or finer, internal tides and tidal mixing can be replicated by some parameterizations (Robertson, 2006). However, there is room for improvement in the vertical mixing schemes and their parameterization in the global ocean and climate models. In an effort to address this mixing issue, national and international groups in the United States, China, France, Japan, Netherlands, and other nations have been formed and are collaborating. Progress in improving the vertical mixing would improve forecasts of future changes. Ice shelf-ocean interactions remain a challenge for observationalists and a source of errors and uncertainties in the production of bottom waters in models. Improvements will require a better representation of continental shelf ocean circulation, sea ice, winds, and higher resolution topography. All of which remain daunting tasks for the present generation of climate models.

\section{Ecosystem Modeling Efforts}

Ecosystem models are essential for integrating and contextualizing ecosystem observations, identifying how changes will affect ecosystems and the services they provide (Addison et al., 2017; Melbourne-Thomas et al., 2017), and for optimizing observing strategies (Constable et al., 2016). Ecosystem modeling efforts develop representations of the food web in relation to key biophysical drivers, and over the last decade have largely focused on sub-systems, such as open ocean, coastal and sea-ice areas, or particular regions (Hill et al., 2006; Murphy et al., 2012; Cordone et al., 2018; Marina et al., 2018a,b). Much of this work has been qualitative, with quantitative analyses of the structure of parts of the food web mostly limited to high data-density regions (e.g., WAP, Ross Sea, Potter Cove).

Common frameworks for ecosystem modeling include:

(i) Population, growth, and habitat models for individual species (autecological models), focused on understanding how focal species respond to environmental conditions and external drivers such as harvest and bycatch (e.g., stock assessment models).

(ii) Nutrient-phytoplankton-zooplankton-detritus models focused on understanding lower trophic level dynamics in response to physical drivers;

(iii) Network models, representing linkages among components of ecosystems (synecological models) such as parts of the food web (functional groups and/or species), physical ecosystem components, and human activities.

International coordination of Southern Ocean ecosystem modeling is achieved through the Integrating Climate and Ecosystem Dynamics (ICED) subprogram of Integrating Marine Biosphere Research (IMBeR; Murphy et al., 2008). A longstanding goal of ICED is the development of "end-to-end" models that encompass the entire food web, coupled to key biological and physical forcings (Murphy et al., 2008). Simple end-toend models using mass-balance approaches (Ecopath) have been implemented in West Antarctica (Hill et al., 2012; Ballerini et al., 2014; Gurney et al., 2014; Surma et al., 2014; Klein et al., 2018), 
the Ross Sea (Pinkerton et al., 2010), and are in development for East Antarctica (McCormack et al., 2017), along with a more comprehensive end-to-end model in the "Atlantis" framework, which is targeted to inform management and policy (Fulton et al., 2011; Melbourne-Thomas et al., 2017).

Southern Ocean food-web models are currently oriented around krill (Ballerini et al., 2014; Gurney et al., 2014; Surma et al., 2014; Klein et al., 2018). These have been valuable in understanding the implications of climate change and changing patterns of krill fishing on krill and their major predators, yet are unlikely to represent non-krill dominated ecosystems. The sensitivity of krill dominance to structural uncertainty in these models has not been explored, but are potentially problematic (Hill et al., 2012). "Ensembles" or "toolboxes" of multiple structurally different ecosystem models will reduce this uncertainty and enhance understanding of patterns and drivers of ecosystem structure and function (Murphy et al., 2012, 2016; Gregr and Chan, 2014). Integrated modeling across multiple scales and dimensions (i.e., individual to global scales, and physical, ecological, and socio-ecological dimensions) will also be important (Melbourne-Thomas et al., 2017).

Ecosystem modeling capacity is currently limited by a lack of fit-for-purpose models and methods to evaluate the representativeness of models under development (i.e., model skill). This is due to difficulties in assembling data for difficultto-observe parts of the ecosystem (section "Assessing Status and Trends of Key Southern Ocean Taxa") and for trophic relations and rate parameters (section "Constraining Biological Energy Pathways"). Consolidating and comparing diets are a priority (Murphy et al., 2012) and efforts such as the SCAR Southern Ocean Diet and Energetics Database ${ }^{37}$ improve the availability of trophic data, allowing comparisons of regional variation in foodweb characteristics. Combining traditional dietary information from stomach contents with information from stable isotopes and dietary DNA will identify "missing links" in food-web models and thus reduce structural uncertainty (i.e., unknown and missed trophic links and/or important groups; Gregr and Chan, 2014). Additional priorities include improved understanding of the dependence of food-web structure and function on habitat structure and biogeochemical cycling through targeted process studies and data syntheses.

\section{Essential Ocean Variables and Observing System Design}

A core objective of SOOS is to ensure sustained delivery of the fundamental observations that meet the majority of stakeholder requirements. In the same way that the global network of meteorological stations provides end-users with a foundation of standardized observations around which to build specific requirements, SOOS will work with the broader Southern Ocean community to develop regionally defined, quantified sampling targets defining which observations are needed, where, how, and when. This will not only ensure base-level time series of key variables, but will support funders in identifying the observations that will deliver the greatest impact to the highest

\footnotetext{
${ }^{37}$ https://data.aad.gov.au/trophic/
}

number of end-users. In combination with parallel efforts within the international community (e.g., through the Global Ocean Observing System ${ }^{38}$; GOOS), SOOS has identified candidate EOVs for the Southern Ocean ${ }^{39}$, which are the most feasible (possible to observe in a sustained way) and highest impact (those variables that address the highest priority issues) variables that deliver observations to address the SOOS Science Themes. In the coming decade, the community must continue to prioritize candidate EOVs and develop the required documentation for determination of sampling requirements.

Ecosystem EOVs are the least developed in any observing system. Significant progress has been made in defining the process and criteria for determining eEOVs for the Southern Ocean (Constable et al., 2016). Sixty-four candidate eEOVs have been nominated from this process. They include variables for benthic species (11), pelagic and sea-ice taxa (17), marine mammals and birds (27), and direct human pressures (9). Nine of these are regarded as mature, and relate to fisheries catch and zooplankton from the SO-CPR Survey. The remainder are currently under review. Although specific to the Southern Ocean, these eEOVs have synergies with the GOOS biological EOVs ${ }^{40}$ (Miloslavich et al., 2018), ensuring harmony in statements on global priorities.

The Southern Ocean Observing System has developed an Observing System Design Working Group to advance the knowledge and tools used in designing optimal observing systems. These tools, known as Observing System Simulation Experiments (OSSEs), are used to estimate the value of ocean observations, with respect to how well they constrain the goals of the observing system. Observing system design evaluation entails subsampling a realistic model solution and determining the ability to then reconstruct this model solution (e.g., Forget et al., 2008; Kurahashi-Nakamura et al., 2014; Kamenkovich et al., 2017). The realistic model solution being sampled is known as the "nature run." The realism of this model run is the most important aspect of the endeavor, as it determines the partitioning between errors of commission, which arise from inability to reconstruct the nature run from the subsampling, versus errors of omission. These refer to phenomenon not in the nature run, and thus omitted from evaluation with regards to the observing system. For example, most models fail to capture the full spectrum of internal wave energy in the ocean, meaning that if these are used for the nature run, this source of variability is omitted with regards to assessment of observing system design.

Having a nature run with minimal omission errors allows accurate assessment of the observing system design and mapping method. It builds confidence in estimates of the observing system design and its skill in meeting the goals. Global ocean models are now being run with $2 \mathrm{~km}$ resolution in the Southern Ocean and appear to capture the internal wave spectrum (Rocha et al., 2016). A primary challenge with respect to observing system

\footnotetext{
${ }^{38} \mathrm{http}: / /$ www.goosocean.org/index.php?option=com_content\&view=article\&id= $14 \&$ Itemid $=114$

${ }^{39} \mathrm{http}: / /$ soos.aq/activities/system-design

${ }^{40}$ Figure showing links between SOOS and GOOS eEOVs, http://soos.aq/activities/ system-design
} 
design over the next decade is to validate these models. Do they fill in the spectrum for the correct reason? What physics (e.g., surface-gravity waves or ice shelves) are they omitting and what needs to be included? What aspects of ecosystem dynamics are omitted?

Tools exist to design and prioritize the observing system. These are primarily based on model statistics (e.g., Mazloff et al., 2018). By utilizing these tools, the observing system will provide estimates of quantities established by stakeholder needs at a minimal cost and with associated uncertainty estimates. However, adequate process studies are necessary to validate state-of-the-art numerical model statistics used in these OSSEs. Confidence in Southern Ocean science and policy relies on having simulations that capture all the biological, chemical, and physical dynamics of the region.

\section{Priorities for Future Observational Technologies}

Given the size, complexity, operational cost, and harsh conditions, a broad suite of technologies is required to observe the Southern Ocean. The biggest driver of technology development is the urgent need to address the spatio-temporal data gaps in traditional ship-based sampling, and the last decade has seen significant progress on this front. Augmenting ship-based observations with satellite-sensor technology, autonomous platforms, and fixed assets such as moorings, has made a step-change in our observational capabilities (Schofield and Kohut, 2018).

Autonomous underwater vehicles have experienced strong growth in Antarctic science applications over the last two decades, operating under ice shelves and sea ice to collect otherwise inaccessible ocean and ice data. The UK's National Oceanographic Centre's Autosub group was the first to conduct AUV missions beneath sea ice (Brierley et al., 2002, 2003; Hayes et al., 2007) and is exploring the cavities of major Antarctic ice shelves (Nicholls et al., 2006, 2008; McPhail, 2009; Jenkins et al., 2010; Graham et al., 2013). Other countries (Australia, Sweden, and Japan) are now developing their own polar AUV capabilities, with other nations certain to follow.

In situ sea-ice observations that are scalable to remote sensing and modeling products are a priority. New floe-scale measurements of sea-ice draft and morphology are now being delivered by the dual-hulled SeaBED-class of AUV that are reconfigured to face the bottom of the sea ice (Williams et al., 2015). New longer range AUVs will be able to address these observation gaps on a larger scale $(100+\mathrm{km})$. Better understanding of key processes such as the role of ice-wave interaction in sea-ice advance and retreat (Kohout et al., 2014, 2016), and the role of fast ice and polynyas in interactions with ice shelves (e.g., Massom et al., 2010, 2018; Silvano et al., 2018) will require coordinated in situ and autonomous observations (buoys and moorings) with high-resolution satellite-data products. Aerial electromagnetic induction techniques that measure seaice thickness will be important for validation of remote-sensing products (Price et al., 2015). Despite these new technologies, observing seasonal dynamics and snow thickness will still require ice-mass balance buoys, which are rapidly evolving a range of new sensors (fluorometers, bio-optical sensors) (Maksym et al., 2012).

Profiling floats have revolutionized sampling of the Southern Ocean (coordinated through the global Argo program), but the coverage of 1 float per 3 degrees is well behind the global average, with large gaps particularly south of $60^{\circ} \mathrm{S}$ and for $>2000 \mathrm{~m}$ depth everywhere. In the coming decade, we expect the underice domains to be addressed but technological challenges related to fixing positioning and communication under the ice will need to improve, for example, through expansion of sound sources such as RAFOS. Deployment of deep Argo floats will close the data gap for depths $>2000 \mathrm{~m}$, and new "bottom drifters" (Figure 7), currently being trialed by Sorbonne University and IFREMER through the WAPITI program (see footnote 9), will further support this.

Tagged marine animals, namely seals, have provided significant complementary datasets to the Argo program for temperature-salinity, and most recently fluorescence, from around the Antarctic margin and seasonal sea-ice zone. This technology has provided crucial winter and under-ice observations that could not be otherwise collected (Costa et al., 2010; Roquet et al., 2013; Heywood et al., 2016; Williams et al., 2016b; Treasure et al., 2017). Areas for improvement include sensor accuracy and rates, which have so far impacted data quality, as well as increasing sensor types for broader application, such as biogeochemical and acoustic sensors (Roquet et al., 2011; Frazer et al., 2018).

Underwater gliders now provide significant observations for the upper $1000 \mathrm{~m}$ at high spatio-temporal resolution and for multiple months at a time. They have been deployed over all parts of the Southern Ocean, including the ACC (Swart et al., 2015; Du Plessis et al., 2017; Viglione et al., 2018) and continental shelves (Kahl et al., 2010; Schofield et al., 2013; Thompson et al., 2014; Carvalho et al., 2016, 2017; Couto et al., 2017), as well as near ice shelves (Miles et al., 2015; Schofield et al., 2015; Naveira Garabato et al., 2017). They have a growing sensor suite capable of measuring nutrients, $\mathrm{pH}$, acoustics, and $\mathrm{CO}_{2}$. As battery technology and robustness of such platforms increase, autumn to winter observations will become ubiquitous (Du Plessis et al., 2019). Furthermore, autonomous surface vehicles (e.g., windand wave-powered and Lagrangian platforms) are also filling a growing need for surface-based observations. Recent studies assessing physical air-sea fluxes (Schmidt et al., 2017; Thomson and Girton, 2017) or gas fluxes, notably $\mathrm{CO}_{2}$ (Monteiro et al., 2015), have demonstrated that surface vehicles are able to collect data for many months.

The use of UAS/drones for atmospheric (Cassano et al., 2010; Knuth et al., 2013), sea ice (Williams et al., 2016a), and ecological studies (Borowicz et al., 2018) is also expanding around Antarctica. They provide the ability to map at ecologically relevant scales and are proving effective tools to observe higher trophic levels. A growing suite of scientific sensors are becoming available for UAS applications, rapidly increasing the number of countries using these platforms. A major barrier for UAS is the significant regulatory issues involved. These technologies complement traditional satellite and airborne remote-sensing 
approaches that continue to be the backbone of broad seasonal scale sampling of the Southern Ocean.

Advances in moored platforms have been less broadly cited, but promising developments include bottom landers, such as the cheap, soccer-ball sized, ice-resistant, anchor-weighted " $\mathrm{T}$ pop" buoys deployed to depths of $6000 \mathrm{~m}$. Dropped from a ship, ice, or helicopter, they measure temperature and pressure for several years before surfacing and relaying the data via satellite. T-pops were successfully deployed by Sweden in the Amundsen Sea in 2016. Further, the newly developed ApRES instruments (coordinated through the NECKLACE program) collect time series of melt rates from point locations and complement the satellite-derived maps of melt rates that are now becoming available.

In addition to new platforms, a wide range of physical, chemical, and biological sensors are rapidly maturing. Advances in bio-acoustic technologies - both on ships and other platforms - will be critical to resolving uncertainties around the abundance and biomass of key mid-trophiclevel taxa (Handegard et al., 2012). Parallel development of observation models will be required to support use of complex data streams (e.g., Proud et al., 2018). Advances in technologies used to quantify biochemical tracers from biological samples will also be invaluable in resolving biological energy pathways and supporting the development of ecosystem models (Pethybridge et al., 2017).

Along with new technologies, observational coverage can be enhanced using existing technology through more efficient use of resources. Although industry-based shipping in the Southern Ocean is marginal, fishing and tourism are growing industries with regular trips and, in many cases, the capability and interest to support observational activities. Additionally, station resupply voyages rarely operate underway observations without specific research requests, which, if coordinated, could provide a step-wise enhancement of observational coverage. The installation of sensors such as flux towers, multibeam echo sounders, standardized camera systems, snow radar, and electromagnetic induction sensors are important systems that could be installed on all vessels to greatly enhance spatial coverage. The GOOS Ship Of Opportunity Program (SOOP) ${ }^{41}$ facilitates such coordination globally, but the Southern Ocean community is yet to take full advantage of these opportunities. Further, new initiatives (see SCOR Working Group 154: Integration of Plankton-Observing Sensor Systems to Existing Global Sampling Programs (P-OBS) ${ }^{42}$; Lombard et al., 2019) are developing ways to integrate biological observing sensor systems into existing physical oceanographic networks, such as GO-SHIP and OceanSITES, to enhance biological observation coverage with minimal additional resourcing. Leveraging efforts in this way is critical to achieving multidisciplinary observational coverage. Another important consideration is the continued development of inexpensive systems capable of sustained broadscale deployments (e.g., Expendable Bathythermograph (XBT), sea-ice mass balance buoys, ocean- and sea-ice drifters) by

\footnotetext{
${ }^{41}$ http://www.wmo.int/pages/prog/amp/mmop/JCOMM/OPA/SOT/soop.html

${ }^{42}$ https://scor-int.org/group/154/
}

ship or aircraft. Further, year-round coastal Antarctic stations have under-utilized potential for circumpolar field work stations to monitor seasonal and long-term evolution of, for instance, sea-ice algal biomass and production. Observing the scale and complexity of the Southern Ocean will require a full portfoli o of these technologies.

\section{Connecting and Delivering Southern Ocean Data}

The international data-science landscape has changed significantly over the last decade, with the development of new data tools and an unprecedented increase in both the volume and diversity of data; presenting both opportunity and challenges. A particular challenge for SOOS is to link the science and data communities associated with National Antarctic Programs (NAPs) to the broader oceanographic community. The SOOS data system must leverage existing efforts, thus SOOS is working with other data initiatives to develop tools that bring together these efforts, to serve this thematically broad but regionally focused community.

Enhanced observations of the Southern Ocean will be of limited value if the data (in situ, satellite-derived, and model outputs) are not easily "Findable, Accessible, Interoperable, and Reusable" (the FAIR Principles; Wilkinson et al., 2016; Tanhua et al., 2019). Data complexity, lack of data- and metadataexchange standards, and difficulty finding relevant extant datasets are major challenges for researchers (Lucas, 2017). Addressing these challenges is the core objective of the SOOS data strategy, and a "one size fits most" approach to data management will not solve these problems; therefore, we envisage several interlocking components.

The SOOS data ecosystem (Figure 12) provides multiple pathways to access data (Figure 12a), based on the geometry of the datasets and the communities that produce them. A core focus for SOOS to date has been the development of SOOSmap by the European Marine and Observation Data Network Physics group (EMODnet Physics) (Figure 12c) to deliver standardized and aggregated in situ observational data in both real-time and delayed modes. A central task for SOOS this decade will be to populate SOOSmap with key observational datasets that allow researchers to explore temporal and spatial patterns in parameters. To create these datasets, SOOS is encouraging scientists and data managers to mine historical data to generate aggregations of similar datasets that have undergone standardized quality control. Examples of such projects include the aggregation of chlorophyll-a data from fastice cores (Meiners et al., 2018), the KRILLBASE database for krill and salp observations (Atkinson et al., 2017), and the SCAR Retrospective Analysis of Antarctic Tracking Data. Similarly, groups such as SCORs ECV-Ice group ${ }^{43}$ are promoting the intercalibration of in situ measurement methods, a vital step for integrating observations from different research groups.

Southern Ocean Observing System is partnering with Antarctic data management organizations to deliver relevant gridded datasets (Figure 12d), and while satellite imagery is vital

\footnotetext{
${ }^{43}$ https://scor-int.org/group/152/
} 


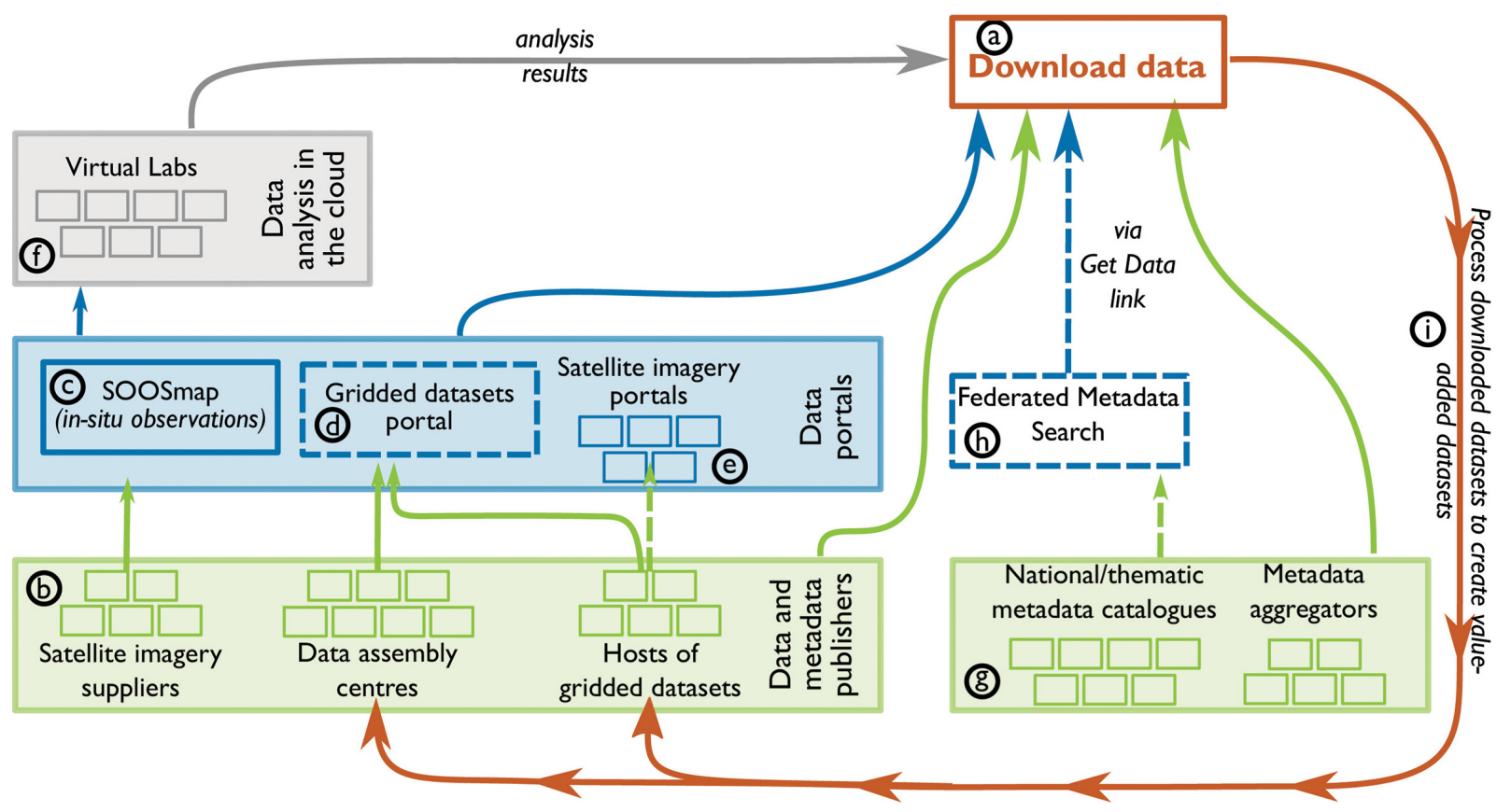

FIGURE 12 | A SOOS-centric view of the data ecosystem. Components that do not exist yet are denoted with dashed lines. (a) Downloading data is possible through multiple paths in this ecosystem; (b) existing data curation services, divided by data geometry; (c) SOOS' data aggregation portal, SOOSmap, for discovery of in situ observation data; (d) a proposed portal for aggregating gridded datasets for Antarctic regions; (e) aggregation portals for satellite imagery discovery (e.g., NSIDC); (f) virtual laboratories to allow analysis in the cloud rather than creating a local copy of the input data; (g) existing metadata discovery services; (h) a proposed tool to federate searching across all relevant metadata discovery services; (i) in an enclosed data ecosystem, downloaded data can be aggregated, quality-controlled, and converted in value-added datasets that can in turn be fed into the data system.

for SOOS, its data are globally managed (Figure 12e). These portals are likely to develop connections with cloud analytics services (Figure 12f).

Standardized datasets, such as those delivered through SOOSmap, require significant effort and for many data types the only practicable way to provide access is through comprehensive metadata discovery tools. At present, there are more than 70 metadata catalogs that contain SOOS-relevant data records (Figure 12g). Thus, SOOS and other data management initiatives are working to develop a federated metadata search tool for single-window searches of SOOS-relevant metadata repositories (Figure 12h) and exploring opportunities for structured schemas to improve metadata search (Beck et al., 2019).

This suite of tools will support the Southern Ocean community to add value to raw datasets (Figure 12i), producing aggregated datasets that can be republished through the data centers, iteratively improving the data available to the SOOS community.

All aspects of this data strategy require adequate and sustained funding, and given recent issues with sustained funding of data products, SOOS has identified core values for how community tools should be developed, including using free and open source software where available; not duplicating efforts; and creating a sense of community ownership so that the tools can survive changes in funding environments. Collectively, the SOOS ecosystem enables sharing and accessing data while being adaptable to the needs, varying levels of standardization, and patterns of IT use among the disciplines that comprise SOOS. As the global data management community further develops tools to make data more FAIR, the mix of tools in the SOOS data vision will allow its community to adapt to these changes.

\section{International Coordination and End-User Engagement}

The success of SOOS will ultimately be determined by the delivery of sustained observations to end-users, which include scientists, policy makers, and industry. This requires significant efforts to ensure an integrated and coordinated approach.

Unique to the Southern Ocean, the Antarctic Treaty provides a framework within which the use of Antarctic resources and services can be monitored and managed. Because of this, all users of the Southern Ocean (south of $60^{\circ} \mathrm{S}$ ) are obligated to record their activities and monitor their impact. This requires sustained observation of environmental variables and transfer of data into knowledge and statements of impact or change. This requirement has supported the development of coordinating bodies who manage specific aspects of program monitoring requirements.

Ecosystem-based management approaches to regulate fisheries activity, for example, are managed through CCAMLR. This requires a range of sustained environmental, biological, and ecosystem-level observations. CCAMLR takes an ecosystem approach to management, and this require access to data sets that contribute to understanding the whole ecosystem, the 
physical environment within which it sits, and the rates of change in both of these systems. Specific areas where accessible, sustained, and integrated decisions can contribute include the management of the marine protected areas, which have been established by CCAMLR. Additionally, the Antarctic Treaty System's “Committee for Environmental Protection" provides recommendations to manage the impact of other human activities in Antarctica, including climate change, and as such requires sustained observations of environmental conditions. It is expected to benefit from improved observations that enhance our understanding of environmental change, including the implications this will have for logistical and scientific operations.

The network of 53 NAPs has a broad remit to support strategic science, logistics, and their national commitments to Antarctic Treaty obligations. Further, their operations require observationally based services, such as sea ice and weather forecasts that are coordinated through programs such as the International Ice Chart Working Group (IICWG), and the World Meteorological Organization (WMO). Industry-based activities such as fisheries and tourism (coordinated through International Association of Antarctic Tourism Operators, IAATO) also require observationally based environmental information, and can additionally enhance opportunistic ship-based collection of data, as a strong statement of commitment to Treaty obligations toward environmental protection.

The influence of SOOS on policy is through the mechanisms described above for the Antarctic Treaty System, and also in terms of inputs to the Intergovernmental Panel for Climate Change (IPCC) assessment process. SOOS-supported datasets informed the last major assessment (AR5 in 2013) and are underpinning much of the Southern Ocean material in the ongoing Special Report on Oceans and Cryosphere in a Changing Climate (scheduled for release in 2019). Such data are also used to test and challenge climate projection models used by the IPCC and are critical to assessments of oceanic influence on the Antarctic Ice Sheet and hence global sea-level rise. The IPCC process includes direct connection with policy makers and outlining of response options, enabling the use and recognition of SOOS-supported observations.

Fundamentally for SOOS, the primary end-users are the scientists and research communities that collect and bring together the diverse observations to answer societies evolving questions on how the Southern Ocean is changing, and what the ramifications are for humanity. The Southern Ocean scientific community, through the NAPs, SCAR, SCOR, the World Climate Research Program (WCRP), and the IMBeR project, provides the scientific knowledge and recommendations that underpin the management and policy decisions.

Delivering the breadth of observations required by these endusers is an enormous task, and is greater than can be provided within each forum or by any one nation alone - a key driver for the development of SOOS in 2011. Over the last 7 years, SOOS has worked with all stakeholders to build networks and connections around the identified data priorities. With these structures now in place, the next decade sees a strong lean toward designing a network of regional systems that incorporate all overlapping enduser requirements where possible. This will not only facilitate the delivery of data to end-users, but also enable funders to quantify and broaden their impact and up-take. Working with end-users and organizations such as the Committee of Managers of National Antarctic Programs (COMNAP), the SOOS Regional Working Groups will be central to developing and implementing these observing systems in an integrated and flexible way.

\section{CONCLUSION AND RECOMMENDATIONS}

Significant investment in data collection over the last decade has enhanced knowledge of Southern Ocean systems and their impact on the Earth system. Yet, the Southern Ocean remains poorly observed leading to uncertainty in estimates of future states of Southern Ocean processes and the consequences for the Earth system.

This community paper assesses decades of research and observational efforts in the Southern Ocean to provide forwardlooking priorities that require sustained observations over the coming decade. Distilled into eight key issues, these are identified as the major data bottlenecks in addressing the SOOS Science Themes that have underpinned the focus of SOOS networks and efforts thus far. For Themes 1 and 2, the role of the Southern Ocean in the planet's heat and freshwater balance, and the stability of the Southern Ocean overturning circulation, respectively, observations of bottom water production processes are critical, and should be prioritized in the coming decade (Key Issue 1). Key Issue 2 on "reducing uncertainties in air-sea and air-sea-ice fluxes of heat, momentum, freshwater, and carbon" is a cross-cutting issue that delivers into each of the six Science Themes, and is fundamental in modeling efforts to project future states of Southern Ocean systems. Theme 3 on "The role of the ocean in the stability of the Antarctic Ice Sheet" is imperative to better constrain uncertainties in the contribution of the Antarctic Ice Sheet to future sea level. Key issue 3 supports this by prioritizing efforts to observe the contribution of oceanic heat to ice-shelf basal melt. The "future and consequences of Southern Ocean carbon uptake" is Theme 4 , and fundamental to this are observations that enable constraining of the seasonal carbon cycle (Key issue 4). Theme 5 on "The future of Antarctic sea ice" is a broad challenge underpinned by two Key Issues "Integrating sea-ice satellite and in situ observations with modeling efforts to constrain circumpolar sea-ice variability" and "observing sea-ice thickness and volume." The final Theme 6, "Impacts of global change on Southern Ocean ecosystems" is so broad as to be difficult to define with respect to observational priorities. Now, two Key Issues have been defined "constraining biological energy pathways" and "Assessing status and trends of key Southern Ocean taxa." A focus of observational efforts on these Key Issues will thus deliver benefits across the SOOS Themes and directly to the networks and end-users of the data.

Further, the above disciplinary priorities share common needs for observation systems priorities:

- Enhanced observations of most variables from nonsummer seasons are needed. Year-round use of autonomous vehicles year-round where possible, 
deployment of sustained moorings, and other time-series platforms, as well as focused autumn/winter observational programs are needed to address this critical data gap.

- A strong relationship between implementation of new technologies and development of internationally agreed standards for collection, quality control, and management of the new data, delivered where possible in alignment with the FAIR data principals is a critical need. This requires appropriate funding, and will ensure that new data streams are high-quality, openly available, integrated data sets following the example of Argo and other successful ocean observing programs.

- Related to this, standardization and aggregation of similar observations are vital. This requires concerted, long-term data management efforts to discover, quality control, standardize, and publish aggregated datasets for both historic and future observations.

- Requirements for the full suite of observations needed by the broad end-user community must be quantified and underpinned by robust observing system design efforts. The end-user community includes numerical modelers and scientists from other disciplines. The requirements need to be delivered across disciplinary barriers, through existing or new forums. In this way, the combined community can advocate for sustained efforts that deliver across user needs.

- Further, models should be better incorporated into the observing system design and evaluation. As the datastream grows, these models can be used to semi-automate data quality control. Moreover, data assimilation can be exploited to extract maximum value from the observations and provide gridded products for baseline assessments of the Southern Ocean state.

- Remote-sensing data are vital for achieving observational coverage across large spatio-temporal scales for all disciplines. The Southern Ocean community must identify mechanisms to better articulate and advocate their requirements to Space Agencies.

Emerging direct human pressures will also require monitoring in the coming decade. Global issues such as organic pollutants, ocean plastics, and over-fishing are gaining attention. Although mechanisms exist for the reporting of pollutant and waste spills, plastics and fishing catch south of $60^{\circ} \mathrm{S}$ (e.g., through COMNAP, CEP, and CCAMLR), the impacts on the Antarctic ecosystems are not yet routinely observed and monitored. Some efforts are being made for single-species monitoring [e.g., Southern Ocean Persistent Organic Pollutants Program (SOPOPP $)^{44}$ ], and monitoring efforts for micro- and macro-plastics are gaining traction internationally. Future recommendations to SOOS for observing needs will come both directly from the stakeholder communities and through science organizations such as SCAR and SCOR. Another emerging issue is the impact of ocean acidification on the Antarctic ecosystem, which is encompassed in Science Theme 4, but is not yet addressed by SOOS. It is an increasing focus of international

\footnotetext{
${ }^{44}$ https://www.griffith.edu.au/griffith-sciences/southern-ocean-persistentorganic-pollutants-program/projects-and-collaboration
}

research and this will stimulate the development of observational requirements. These emerging issues highlight the need for any observing system developed for the Southern Ocean to be flexible, and responsive to scientific advice from the broader community.

In the past decade, SOOS and the Southern Ocean community have made considerable progress toward designing a comprehensive and sustainable observing system by integrating existing efforts. In the next decade, the focus will turn to addressing the gaps in the system through a combination of technical innovation and greater coordination and sharing of data and logistics.

\section{AUTHOR CONTRIBUTIONS}

LN and EH: section "Abstract." AC, MMM, and LN: sections "Impetus and Progress Toward Developing a Southern Ocean Observing System" and "The Vision." LN: sections "A Decade of Progress," "Drivers of the Future Development of SOOS," and "Southern Ocean Observations: Future Priorities." AM and JS: section "Themes 1 and 2: The Role of the Southern Ocean in the Planet's Heat and Freshwater Balance; and the Stability of the Southern Ocean Overturning Circulation.” AW and RC: section "Theme 3: The Role of the Ocean in the Stability of the Antarctic Ice Sheet and Its Future Contribution to SeaLevel Rise." SH and KH: section "Theme 4: The Future and Consequences of Southern Ocean Carbon Uptake.” PH, TM, RM, and BO: section "Theme 5: The Future of Antarctic Sea Ice." DC and IS: section "Theme 6: Impacts of Global Change on Southern Ocean Ecosystems." KK and AM: section "Observing Antarctic Bottom Water Production Processes." SS and SG: section "Reducing Uncertainties in Air-Sea and AirSea-Ice Fluxes of Heat, Momentum, Freshwater, and Carbon." $\mathrm{KA}$ and EvW: "Understanding the Contribution of Oceanic Heat to Ice-Shelf Basal Melt." TM, RM, and PH: "Toward a Better Understanding of Processes Controlling Antarctic Sea-Ice Variability and Change." IJS, RM, and PH: section "Observing Sea-Ice Thickness and Volume." SM, SF, and ES: "Constraining the Seasonal Carbon Cycle." RT: sections "Constraining Biological Energy Pathways," "Assessing Status and Trends of Key Southern Ocean Taxa," and "Southern Ocean Modelling Progress and Priorities." RF: sections "Southern Ocean Modeling: Progress and Priorities," "Required Ocean Modeling Efforts," and "Ecosystem Modeling Efforts." MM and LN: "Essential Ocean Variables and Observing System Design." GW, OS, LN, PB, JB, RM, and SD: section "Priorities for Future Observational Technologies.” AC, SS, EH, MW, and LN: section "International Coordination and End-User Engagement." LN, MW, EH, and PB: section "Conclusion and Recommendations."

\section{FUNDING}

PH was supported by the Australian Government's Cooperative Research Centers Program through the Antarctica Climate and 
Ecosystems Cooperative Research Centre, and the International Space Science Institute's team grant \#406. This work contributes to the Australian Antarctica Science projects 4301 and 4390.

\section{ACKNOWLEDGMENTS}

This paper is a joint contribution of the SCAR-SCOR Southern Ocean Observing System, and the CLIVARCliC-SCAR Southern Ocean Regional Panel (SORP), in addition to many authors from the broader Southern Ocean community. The authors thank Stacey McCormack

\section{REFERENCES}

Abernathey, R. P., Cerovecki, I., Holland, P. R., Newsom, E., Matzloff, M., and Talley, L. D. (2016). Water-mass transformation by sea ice in the upper branch of the Southern Ocean overturning. Nat. Geosci. 9, 596-601. doi: 10.1038/ ngeo 2749

Addison, P. F. E., Collins, D. J., Trebilco, R., Howe, S., Bax, N., Hedge, P., et al. (2017). A new wave of marine evidence-based management: emerging challenges and solutions to transform monitoring, evaluating, and reporting. ICES J. Mar. Sci. 75, 941-952. doi: 10.1093/icesjms/fsx216

Alderkamp, A.-C., Mills, M. M., van Dijken, G. L., Laan, P., Thuróczy, C.-E., Gerringa, L. J. A., et al. (2012). Iron from melting glaciers fuels phytoplankton blooms in the Amundsen Sea (Southern Ocean): phytoplankton characteristics and productivity. Deep Sea Res. II Top. Stud. Oceanogr. 71-76, 32-48. doi: 10.1016/j.dsr2.2012.03.005

Anav, A., Friedlingstein, P., Kidston, M., Bopp, L., Ciais, P., Cox, P., et al. (2013). Evaluating the land and ocean components of the global carbon cycle in the CMIP5 earth system models. J. Clim. 26, 6801-6843. doi: 10.1175/JCLI-D-1200417.1

Armour, K. C., Marshall, J., Scott, J. R., Donohoe, A., and Newsom, E. R. (2016). Southern Ocean warming delayed by circumpolar upwelling and equatorward transport. Nat. Geosci. 9, 549-554. doi: 10.1038/ngeo2731

Atkinson, A., Hill, S. L., Pakhomov, E., Siegel, V., Anadon, R., Chiba, S., et al. (2017). KRILLBASE: a circumpolar database of Antarctic krill and salp numerical densities, 1926-2016. Earth Syst. Sci. Data 9, 193-210. doi: 10.5194/ essd-9-193-2017

Bakker, A. M. R., Wong, T. E., Rukkert, K. L., and Keller, K. (2017). Sealevel projections representing the deeply uncertain contribution of the West Antarctic ice sheet. Sci. Rep. 7:3880. doi: 10.1038/s41598-017-04134-5

Bakker, D. C. E., Pfeil, B., Smith, K., Hankin, S., Olsen, A., Alin, S. R., et al. (2014). An update to the Surface Ocean CO2 Atlas (SOCAT version 2). Earth Syst. Sci. Data 6, 69-90. doi: 10.5194/essd-6-69-2014

Ballerini, T., Hofmann, E. E., Ainley, D. G., Daly, K., Marrari, M., Ribic, C. A., et al. (2014). Productivity and linkages of the food web of the southern region of the western Antarctic Peninsula continental shelf. Prog. Oceanogr. 122, 10-29. doi: 10.1016/j.pocean.2013.11.007

Basak, C., Fröllje, H., Lamy, F., Gersonde, R., Benz, V., Anderson, R. F., et al. (2018). Breakup of last glacial deep stratification in the South Pacific. Science 359:900. doi: 10.1126/science.aao2473

Beck, J. J. H., Bainbridge, S. J., Burger, E., Kraberg, A., Casari, M., Casey, K. S., et al. (2019). Ocean data product integration through innovation-The next level of data interoperability. Front. Mar. Sci 6:32. doi: 10.3389/fmars.2019.00032

Ben-David, M., and Flaherty, E. A. (2012). Stable isotopes in mammalian research: a beginner's guide. J. Mammal. 93, 312-328. doi: 10.1644/11-mamm-s-166.1

Bentamy, A., Piolle, J. F., Grouazel, A., Danielson, R., Gulev, S., Paul, F., et al. (2017). Review and assessment of latent and sensible heat flux accuracy over the global oceans. Remote Sens. Environ. 201, 196-218. doi: 10.1016/j.rse.2017. 08.016

Bintanja, R., van Oldenborgh, G. J., Drijfhout, S. S., Wouters, B., and Katsman, C. A. (2013). Important role for ocean warming and increased ice-shelf melt in Antarctic sea ice expansion. Nat. Geo. 6, 376-379. doi: 10.1038/ngeo1767 for development of Figures 5-11. SOOS thanks SCAR and SCOR, as well as the sponsors of the SOOS International Project Office for their support; the Australian Research Council's Special Research Initiative for Antarctic Gateway Partnership (Project ID SRI40300001); the Institute for Marine and Antarctic Studies, University of Tasmania; Gothenburg University, Sweden; the State Oceanic Administration of China; the Australian Antarctic Division; and Antarctica New Zealand. SORP thank their sponsors; the WCRP programs "Climate and Ocean Variability, Predictability and Change (CLIVAR)" and "Climate and Cryosphere (CliC)" and SCAR.

Bintanja, R., van Oldenbrough, G. J., and Katsman, C. A. (2015). The effect of increased freshwater from Antarctic ice shelves on future trends in Antarctic sea ice. Ann. Glaciol. 56, 120-126. doi: 10.3189/2015AoG69A001

Böning, C. W., Dispert, A., Visbeck, M., Rintoul, S. R., and Schwarzkopf, F. U. (2008). The response of the antarctic circumpolar current to recent climate change. Nat. Geosci. 1, 864-869. doi: 10.1038/ngeo362

Borowicz, A., McDowall, P., Youngflesh, C., Sayre-McCord, T., Clucas, G., Herman, R., et al. (2018). Multi-modal survey of Adélie penguin mega-colonies reveals the Danger Islands as a seabird hotspot. Sci. Rep. 8:3926. doi: 10.1038/ s41598-018-22313-w

Bourassa, M. A., Gille, S. T., Bitz, C., Carlson, D., Cerovecki, I., Clayson, C. A., et al. (2013). High-latitude ocean and sea ice surface fluxes: challenges for climate research. Bull. Am. Meteor. Soc. 94, 403-423. doi: 10.1175/BAMS-D-1100244.1

Bracegirdle, T. J., Shuckburgh, E., Sallee, J.-B., Wang, Z., Meijers, A. J. S., Bruneau, N., et al. (2013). Assessment of surface winds over the Atlantic, Indian, and Pacific Ocean sectors of the Southern Ocean in CMIP5 models: historical bias, forcing response, and state dependence. J. Geophys. Res. Atmos. 118, 547-562. doi: 10.1002/jgrd.50153

Brierley, A. S., Fernandes, P. G., Brandon, M. A., Armstrong, F., Millard, N. W., McPhail, S. D., et al. (2002). Antarctic krill under sea ice: elevated abundance in a narrow band just south of the ice edge. Science 295, 1890-1892. doi: $10.1126 /$ science. 1068574

Brierley, A. S., Fernandes, P. G., Brandon, M. A., Armstrong, F., Millard, N. W., McPhail, S. D., et al. (2003). An investigation of avoidance by Antarctic krill of RRS James Clark Ross using the Autosub-2 autonomous underwater vehicle. Fish. Res. 12, 569-576. doi: 10.1016/S0165-7836(02)00144-3

Briggs, E. M., Martz, T. R., Talley, L. D., Mazloff, M. R., and Johnson, K. S. (2017). Physical and biological drivers of biogeochemical tracers within the seasonal sea ice zone of the southern ocean from profiling floats. J. Geophys. Res. 123, 746-758. doi: 10.1002/2017JC012846

Busecke, J., and Abernathey, R. P. (2019). Ocean mesoscale mixing linked to climate variability. Sci. Adv. 5:eaav5014. doi: 10.1126/sciadv.aav5014

Carvalho, F., Kohut, J., Oliver, M. J., and Schofield, O. (2017). Estimating ecological relevant mixed layer depths in Antarctica's coastal seas. Geophys. Res. Lett. 44:L071205. doi: 10.1002/2016GL071205

Carvalho, F., Kohut, J., Oliver, M. J., Sherrell, R. M., and Schofield, O. (2016). Mixing and phytoplankton dynamics in a submarine canyon in the West Antarctic Peninsula. J. Geophys. Res. 121, 5069-5083. doi: 10.1002/ 2016JC011650

Cassano, J. J., Maslanik, J. A., Zappa, C. J., Gordon, A. L., Cullather, R. I., and Knuth, S. L. (2010). Wintertime observations of an Antarctic polynya with unmanned aerial systems. EOS 91, 245-246. doi: 10.1029/2010EO 280001

Cassarino, L., Hendry, K. R., Meredith, M. P., Venables, H. J., and Christina, L. (2016). Silicon isotope and silicic acid uptake in surface waters of Marguerite Bay, West Antarctic Peninsula. Deep Sea Res. II Top. Stud. Oceanogr. 139, 143-150. doi: 10.1016/j.dsr2.2016.11.002

Clucas, G. V., Dunn, M. J., Dyke, G., Emslie, S. D., Levy, H., Naveen, R., et al. (2014). A reversal of fortunes: climate change 'winners and losers' in Antarctic Peninsula penguins. Sci. Rep. 4:5024. doi: 10.1038/srep05024 
Constable, A. J., Costa, D. P., Schofield, O., Newman, L., Urban, E. R., Fulton, E. A., et al. (2016). Developing priority variables ("ecosystem Essential Ocean Variables" - eEOVs) for observing dynamics and change in Southern Ocean ecosystems. J. Mar. Syst. 161, 26-41. doi: 10.1016/j.jmarsys.2016. 05.003

Constable, A. J., Melbourne-Thomas, J., Corney, S. P., Arrigo, K. R., Barbraud, C., Barnes, D. K., et al. (2014). Climate change and Southern Ocean ecosystems I: how changes in physical habitats directly affect marine biota. Glob. Chang Biol. 20, 3004-3025. doi: 10.1111/gcb.12623

Constable, A. J., Melbourne-Thomas, J., Trebilco, R., Press, A. J., and Haward, M. (2017). ACE CRC Position Analysis: Managing Change in Southern Ocean Ecosystems. Hobart, TAS: Antarctic Climate and Ecosystems Cooperative Research Centre.

Cordone, G., Marina, T. I., Salinas, V., Doyle, S. R., Saravia, L. A., and Momo, F. R. (2018). Effects of macroalgae loss in an Antarctic marine food web: applying extinction thresholds to food web studies. PeerJ 6:e5531. doi: 10.7717/peerj. 5531

Costa, D. P., Huckstadt, L. A., Crocker, D. E., McDonald, B. I., Goebel, M. E., and Fedak, M. A. (2010). Approaches to studying climatic change and its role on the habitat selection of Antarctic pinnipeds. Integr. Comp. Biol. 50, 1018-1030. doi: 10.1093/icb/icq054

Couldrey, M. P., Jullion, L., Naveira Garabato, A. C., Rye, C., Herráiz-Borreguero, L., Brown, P. J., et al. (2013). Remotely induced warming of antarctic bottom water in the eastern Weddell gyre. Geophys. Res. Lett. 40, 2755-2760. doi: 10.1002/grl.50526

Couto, N., Martinson, D., Kohut, J., and Schofield, O. (2017). Distribution of upper circumpolar deep water on the warming continental shelf of the West Antarctic Peninsula. J. Geophys. Res. 122, 5306-5315. doi: 10.1002/2017JC012840

Darelius, E., Fer, I., and Nicholls, K. W. (2016). Observed vulnerability of filchnerronne ice shelf to wind-driven inflow of warm deep water. Nat. Commun. 5:12300. doi: 10.1038/ncomms 12300

Davison, P. C., Koslow, J. A., and Kloser, R. J. (2015). Acoustic biomass estimation of mesopelagic fish: backscattering from individuals, populations, and communities. ICES J. Mar. Sci. 72, 1413-1424. doi: 10.1093/icesjms/fsv023

De Broyer, E. B. C., Koubbi, P., Griffiths, H. J., Raymond, B., dAcoz, U. C., Van de Putte, A. P., et al. (2014). Biogeographic Atlas of the Southern Ocean. Cambridge: Scientific Committee on Antarctic Research.

Deagle, B. E., Gales, N. J., Evans, K., Jarman, S. N., Robinson, S., Trebilco, R., et al. (2007). Studying seabird diet through genetic analysis of faeces: a case study on macaroni penguins (Eudyptes chrysolophus). PLoS One 2:e831. doi: 10.1371/journal.pone.0000831

DeConto, R. M., and Pollard, D. (2016). Contribution of Antarctica to past and future sea-level rise. Nature 531, 591-597. doi: 10.1038/nature17145

Delille, B., Vancoppenolle, M., Geilfus, N.-X., Tilbrook, B., Lannuzel, D., Schoemann, V., et al. (2014). Southern ocean CO2 sink: the contribution of sea ice. J. Geophys. Res. Oceans 119, 6340-6355. doi: 10.1002/2014JC009941

Desbruyeres, D., McDonagh, E. L., King, B. A., and Thierry, V. (2017). Global and full-depth ocean temperature trends during the early twenty-first century from argo and repeat hydrography. J. Clim. 30, 1985-1997. doi: 10.1175/JCLI-D-160396.1

DeVries, T., Holzer, M., and Primeau, F. (2017). Recent increase in oceanic carbon uptake driven by weaker upper-ocean overturning. Nature 542:215. doi: 10 . 1038/nature21068

Donohue, K. A., Tracey, K. L., Watts, D. R., Chidichimo, M. P., and Chereskin, T. K. (2016). Mean antarctic circumpolar current transport measured in drake passage. Geophys. Res. Lett. 43, 11760-11767. doi: 10.1002/2016GL070319

Downes, S., Farneti, R., Uotila, P., Griffies, S. M., Marsland, S. J., Bailey, D., et al. (2015). An assessment of Southern Ocean water masses and sea-ice during 1988-2007 in a suite of interannual CORE-II simulations. Ocean Model. 94, 67-94. doi: 10.1016/j.ocemod.2015.07.022

Drucker, R., Martin, S., and Kwok, R. (2011). Sea ice production and export from coastal polynyas in the Weddell and Ross Seas. Geophys. Res. Lett. 38:L17502. doi: 10.1029/2011GL048668

Du Plessis, M. D., Swart, S., Ansorge, I. J., and Mahadevan, A. (2017). Submesoscale processes accelerate seasonal restratification in the Subantarctic Ocean. J. Geophys. Res. 122, 2960-2975. doi: 10.1002/2016JC012494
Du Plessis, M. D., Swart, S., Ansorge, I. J., Mahadevan, A., and Thompson, A. F. (2019). Southern Ocean seasonal restratification delayed by submesoscale windfront interactions. J. Phys. Oceanogr. 49, 1035-1053. doi: 10.1175/JPO-D-180136.1

Ducklow, H. W., Fraser, W. R., Meredith, M. P., Stammerjohn, S. E., Doney, S. C., Martinson, D. G., et al. (2013). West antarctic peninsula: an ice-dependent coastal marine ecosystem in transition. Oceanography 26, 190-203. doi: 10. 5670/oceanog.2013.62

Dufour, C. O., Griffies, S. M., de Souza, G. F., Frenger, I., Morrison, A. K., Palter, J. B., et al. (2015). Role of mesoscale eddies in cross-frontal transport of heat and biogeochemical tracers in the southern ocean. J. Phys. Ocean 45, 3057-3081. doi: 10.1175/jpo-d-14-0240.1

Dunne, J. P., Sarmiento, J. L., and Gnanadesikan, A. (2007). A synthesis of global particle export from the surface ocean and cycling through the ocean interior and on the seafloor. Glob. Biogeochem. Cycles 21:GB4006. doi: 10.1029/ 2006GB002907

Durack, P. J., and Wijffels, S. E. (2010). Fifty-year trends in global ocean salinities and their relationship to broad-scale warming. J. Clim. 23, 4342-4362. doi: 10.1175/2010JCLI3377.1

Erickson, Z. K., Thompson, A. F., Cassar, N., Sprintall, J., and Mazloff, M. R. (2016). An advective mechanism for deep chlorophyll maxima formation in southern Drake Passage. Geophys. Res. Lett. 43, 10846-10855. doi: 10.1002/ 2016GL070565

Farneti, R., Downes, S. M., Griffies, S. M., Marsland, S. J., Behrens, E., and Bensten, M. (2015). An assessment of antarctic circumpolar current and southern ocean meridional overturning circulation during 1958-2007 in a suite of interannual CORE-II simulations. Ocean Model. 93, 84-120. doi: 10.1016/j.ocemod.2015.07.009

Forget, G., Ferron, B., and Mercier, H. (2008). Combining Argo profiles with a general circulation model in the North Atlantic. Part 1: estimation of hydrographic and circulation anomalies from synthetic profiles, over a year. Ocean Model. 20, 1-16. doi: 10.1016/j.ocemod.2007.06.001

Frazer, E. K., Langhorne, P. J., Williams, M. J. M., Goetz, K. T., and Costa, D. P. (2018). A method for correcting seal-borne oceanographic data and application to the estimation of regional sea ice thickness. J. Mar. Syst. 187, 250-259. doi: 10.1016/j.jmarsys.2018.08.002

Fripiat, F., Martinez-Garcia, A., Fawcett, S. E., Kemeny, P. C., Studer, A. S., Smart, S. M., et al. (2019). The isotopic effect of nitrate assimilation in the Antarctic Zone: improved estimates and plaeoceanographic implications. Geochim. Cosmochim. Acta 247, 261-279. doi: 10.1016/j.gca.2018.12.003

Frölicher, T. L., Sarmiento, J. L., Paynter, D. J., Dunne, J. P., Krasting, J. P., and Winton, M. (2015). Dominance of the southern ocean in anthropogenic carbon and heat uptake in CMIP5 models. J. Clim. 28, 862-886. doi: 10.1175/JCLI-D14-00117.1

Fulton, E. A., Link, J. S., Kaplan, I. C., Savina-Rolland, M., Johnson, P., Ainsworth, C., et al. (2011). Lessons in modelling and management of marine ecosystems: the Atlantis experience. Fish Fish. 12, 171-188. doi: 10.1111/j.1467-2979.2011. 00412.x

Gao, L., Rintoul, S. R., and Yu, W. (2018). Recent wind-driven change in subantarctic mode water and its impact on ocean heat storage. Nat. Clim. Chem. 8:58. doi: 10.1038/s41558-017-0022-8

Gent, P. R. (2016). Effects of southern hemisphere wind changes on the meridional overturning circulation in ocean models. Ann. Rev. Mar. Sci. 8, 79-94. doi: 10.1146/annurev-marine-122414-033929

Giles, K. A., Laxon, S. W., and Worby, A. P. (2008). Antarctic sea ice elevation from satellite radar altimetry. Geophys. Res. Lett. 35:L03503. doi: 10.1029/ 2007GL031572

Gille, S., Josey, S., and Swart, S. (2016). New approaches for air-sea fluxes in the Southern Ocean. EOS 97, doi: 10.1029/2016EO052243 (accessed May 13, 2016).

Gille, S. T. (2008). Decadal-scale temperature trends in the Southern Hemisphere ocean. J. Clim. 21, 4749-4765. doi: 10.1175/2008JCLI2131.1

Gille, S. T. (2014). Meridional displacement of the Antarctic circumpolar current. Philos. Trans. R. Soc. A 372:20130273. doi: 10.1098/rsta.2013.0273

Gille, S. T., Speer, K., Ledwell, J. R., and Naveira Garabato, A. C. (2007). Mixing and stirring in the Southern Ocean. EOS Trans. Am. Geophys. Union 88, 382-383. doi: 10.1029/2007EO390002 
Goosse, H., and Zunz, V. (2014). Decadal trends in the Antarctic sea ice extent ultimately controlled by ice-ocean feedback. Cryosphere 8, 453-470. doi: 10. 5194/tc-8-453-2014

Graham, A. G. C., Dutrieux, P., Vaughan, D. G., Nitsche, F. O., Gyllencreutz, R., Greenwood, S. L., et al. (2013). Seabed corrugations beneath an Antarctic ice shelf revealed by autonomous underwater vehicle survey: origin and implications for the history of Pine Island Glacier. J. Geophys. Res. 118, 13561366. doi: 10.1002/jgrf.20087

Gray, A., Johnson, K. S., Bushinsky, S. M., Riser, S. C., Russell, J. L., Talley, L. D., et al. (2018). Autonomous biogeochemical floats detect significant carbon dioxide outgassing in the high-latitude Southern Ocean. Geophys. Res. Lett. 45, 9049-9057. doi: 10.1029/2018GL078013

Greenbaum, J., Blankenship, D. D., Young, D. A., Richter, T. G., Roberts, J. L., Aitken, A. R. A., et al. (2015). Ocean access to a cavity beneath totten glacier in east antarctica. Nat. Geosci. 8, 294-298. doi: 10.1038/ngeo2388

Gregr, E. J., and Chan, K. M. A. (2014). Leaps of faith: how implicit assumptions compromise the utility of ecosystem models for decision-making. Bioscience 65, 43-54. doi: 10.1093/biosci/biu185

Gulick, S. P. S., Shevenell, A. E., Montelli, A., Fernandez, R., Smith, C., Warny, S., et al. (2017). Initiation and long-term instability of the East Antarctic Ice Sheet. Nature 552, 225-229. doi: 10.1038/nature25026

Gurney, L. J., Pakhomov, E. A., and Christensen, V. (2014). An ecosystem model of the prince edward island archipelago. Ecol. Model. 294, 117-136. doi: 10.1016/j. ecolmodel.2014.09.008

Gutt, J., Bertler, N., Bracegirdle, T. J., Buschmann, A., Comiso, J., Hosie, G., et al. (2015). The southern ocean ecosystem under multiple climate change stresses - an integrated circumpolar assessment. Glob. Change Biol. 21, 1434-1453. doi: $10.1111 /$ gcb.12794

Gutt, J., Isla, E., Bertler, A. N., Bodeker, G. E., Bracegirdle, T. J., Cavanagh, R. D., et al. (2017). Cross-disciplinarity in the advance of Antarctic ecosystem research. Ma. Genomics. 37, 1-17. doi: 10.1016/j.margen.2017.09.006

Gysin, G. (2017). The Digital Ocean: Our Next Information Frontier. Scientific American. Available at: https://blogs.scientificamerican.com/guest-blog/thedigital-ocean-our-next-information-frontier1/ (accessed July 2018).

Hancock, A. M., Davidson, A. T., McKinlay, J., McMinn, A., Schulz, K. G., and Enden, R. L. (2018). Ocean acidification changes the structure of an Antarctic coastal protistan community. Biogeoscience 15, 2393-2410. doi: 10.5194/bg-152393-2018

Handegard, N. O., Buisson, L. D., Brehmer, P., Chalmers, S. J., De Robertis, A., Huse, G., et al. (2012). Towards an acoustic-based coupled observation and modelling system for monitoring and predicting ecosystem dynamics of the open ocean. Fish Fish. 14, 605-615. doi: 10.1111/j.1467-2979.2012.00480.x

Haumann, F. A., Gruber, N., Münnich, M., Frengerm, I., and Kern, S. (2016). Seaice transports driving Southern Ocean salinity and its recent trends. Nature 537, 89-92. doi: 10.1038/nature19101

Hauri, C., Doney, S. C., Takahashi, T., Erickson, M., Jiang, G., and Ducklow, H. W. (2015). Two decades of inorganic carbon dynamics along the West Antarctic Peninsula. Biogeoscience 12, 6761-6779. doi: 10.5194/bg-12-6761-2015

Hayes, D. R., Jenkins, A., and McPhail, S. D. (2007). Autonomous underwater vehicle measurements of surface wave decay and directional spectra in the marginal sea ice zone. J. Phys. Oceanogr. 37, 71-83. doi: 10.1175/JPO2979.1

Heil, P., Gerland, S., and Granskog, M. A. (2011). An antarctic monitoring initiative for fast ice and comparison with the Arctic. Cryos. Disc. 5, 2437-2463. doi: 10.5194/tcd-5-2437-2011

Heil, P., Hutchings, J. K., Worby, A. P., Johansson, M., Launiainen, J., Haas, C., et al. (2008). Tidal forcing on sea-ice drift and deformation in the western Weddell Sea in early austral summer, 2004. Deep Sea Res. II Top. Stud. Oceanogr. 55, 943-962. doi: 10.1016/j.dsr2.2007.12.026

Heil, P., Stammerjohn, S., Reid, P., Massom, R. A., and Hutchings, J. K. (2016). SIPEX 2012: extreme sea-ice and atmospheric conditions off East Antarctica. Deep Sea Res. II Top. Stud. Oceanogr. 131, 7-21. doi: 10.1016/j.dsr2.2016. 06.015

Hellmer, H. H., Kauker, F., Timmermann, R., Determann, J., and Rae, J. (2012). Twenty-first-century warming of a large Antarctic ice-shelf cavity by a redirected coastal current. Nature 485, 225-228. doi: 10.1038/nature 1064

Helm, K. P., Bindoff, N. L., and Church, J. A. (2010). Changes in the global hydrological-cycle inferred from ocean salinity. Geophys. Res. Lett. 37:L18701. doi: 10.1029/2010GL044222
Henley, S. F., Jones, E. M., Venables, H. J., Meredith, M. P., Firing, Y. L., Dittrich, R., et al. (2018). Macronutrient and carbon supply, uptake and cycling across the Antarctic Peninsula shelf during summer. Philos. Trans. R. Soc. A. 376:20170168. doi: 10.1098/rsta.2017.0168

Henley, S. F., Tuerena, R. E., Annett, A. L., Fallick, A. E., Meredith, M. P., Venables, H. J., et al. (2017). Macronutrient supply, uptake and recycling in the coastal ocean of the west Antarctic Peninsula. Deep Sea Res. II Top. Stud. Oceanogr. 139, 58-76. doi: 10.1016/j.dsr2.2016.10.003

Hennon, T. D., Riser, S. C., and Mecking, S. (2016). Profiling float-based observations of net respiration beneath the mixed layer. Glob. Biogeochem. Cycles 30, 920-932. doi: 10.1002/2016GB005380

Hernando, M. P., Schloss, I. R., Malanga, G. F., Almandoz, G. O., Ferreyra, G. A., Aguiar, M. B., et al. (2015). Effects of salinity changes on coastal antarctic phytoplankton physiology and assemblage composition. J. Exp. Mar. Biol. Ecol. 466, 110-119. doi: 10.1016/j.jembe.2015.02.012

Herraiz-Borreguero, L., Coleman, R., Allison, I., Rintoul, S. R., Craven, M., and Williams, G. D. (2015). Circulation of modified circumpolar deep water and basal melt beneath the amery ice shelf, East Antarctica. J. Geophys. Res. Oceans 120, 3098-3112. doi: 10.1002/2015jc010697

Heuzé, C., Heywood, K. J., Stevens, D. P., and Ridley, J. K. (2013). Southern Ocean bottom water characteristics in CMIP5 models. Geophys. Res. Lett. 40, 1409-1414. doi: 10.1002/grl.50287

Heywood, K., Biddle, L. C., Boehme, L., Dutrieux, P., Fedak, M., Jenkins, A., et al. (2016). Between the devil and the deep blue sea: the role of the amundsen sea continental shelf in exchanges between ocean and ice shelves. Oceanography 29, 118-129. doi: 10.5670/oceanog.2016.104

Hill, S. L., Keeble, K., Atkinson, A., and Murphy, E. J. (2012). A foodweb model to explore uncertainties in the South Georgia shelf pelagic ecosystem. Deep Sea Res. II Top. Stud. Oceanogr. 59-60, 237-252. doi: 10.1016/j.dsr2.2011. 09.001

Hill, S. L., Murphy, E. J., Reid, K., Trathan, P. N., and Constable, A. J. (2006). Modelling Southern Ocean ecosystems: krill, the food-web, and the impacts of harvesting. Biol. Rev. 81, 581-608. doi: 10.1111/j.1469-185x.2006.tb00219.x

Hinke, J. T., Cossio, A. M., Goebel, M. E., Reiss, C. S., Trivelpiece, W. Z., and Watters, G. M. (2017a). Identifying risk: concurrent overlap of the antarctic krill fishery with krill-dependent predators in the Scotia Sea. PLoS One 12:e170132. doi: 10.1371/journal.pone.0170132

Hinke, J. T., Trivelpiece, S. G., and Trivelpiece, W. Z. (2017b). Variable vital rates and the risk of population declines in Adelie penguins from the Antarctic Peninsula region. Ecosphere 8:e01666. doi: 10.1002/ecs2.1666

Hobbs, W. R., Massom, R., Stammerjohn, S., Reid, P., Williams, G., and Meier, W. (2016). A review of recent changes in Southern Ocean sea ice, their drivers and forcings. Glob. Planet. Change 143, 228-250. doi: 10.1016/j.gloplacha.2016. 06.008

Hogg, A. M., Meredith, M. P., Chambers, D. P., Abrahamsen, E. P., Hughes, C. W., and Morrison, A. K. (2015). Recent trends in the Southern Ocean eddy field. J. Geophys. Res. Oceans 120, 257-267. doi: 10.1002/2014JC0 10470

Holland, M. M., Landrum, L., Raphael, M., and Stammerjohn, S. E. (2017). Springtime winds drive Ross Sea ice variability and change in the following autumn. Nat. Commun. 8:731. doi: 10.1038/s41467-017-00820-820

Holland, P. R., Bruneau, N., Enright, C., Losch, M., Kurtz, N. T., and Kwok, R. (2014). Modeled trends in Antarctic sea ice thickness. J. Clim. 27, 3784-3801. doi: 10.1175/JCLI-D-13-00301.1

Holland, P. R., and Kwok, R. (2012). Wind-driven trends in Antarctic sea-ice drift. Nat. Geosci. 5, 872-875. doi: 10.1038/ngeo1627

Hoppe, C. J. M., Hassler, C. S., Payne, C. D., Tortell, P. D., Rost, B., and Trimborn, S. (2013). Iron limitation modulates ocean acidification effects on southern ocean phytoplankton communities. PLoS One 8:e79890. doi: 10.1371/journal.pone. 0079890

Hückstädt, L. A., Burns, J. M., Koch, P. L., McDonald, B. I., Crocker, D. E., and Costa, D. P. (2012a). Diet of a specialist in a changing environment: the crabeater seal along the western Antarctic Peninsula. Mar. Ecol. Prog. Ser. 455, 287-301. doi: 10.3354/meps09601

Hückstädt, L. A., Koch, P. L., McDonald, B. I., Goebel, M. E., Crocker, D. E., and Costa, D. P. (2012b). Stable isotope analyses reveal individual variability in the trophic ecology of a top marine predator, the southern elephant seal. Oecologia 169, 395-406. doi: 10.1007/s00442-011-2202-y 
Humbert, A., and Braun, M. (2008). The wilkins ice shelf, Antarctica: break-up along failure zones. J. Glaciol. 54, 943-944. doi: 10.3189/002214308787780012

IPCC (2013). "Climate change 2013: the physical science basis," in Contribution of Working Group I to the Fifth Assessment Report of the Intergovernmental Panel on Climate Change, eds T. F. Stocker, et al. (Cambridge: Cambridge University Press), doi: 10.1017/CBO9781107415324

Jacobs, S. S., and Giulivi, C. F. (2010). Large multidecadal salinity trends near the Pacific-Antarctic continental margin. J. Clim. 23, 4508-4524. doi: 10.1175/ 2010JCLI3284.1

Jenkins, A., Dutrieux, P., Jacobs, S. S., McPhail, S. D., Perrett, J. R., Webb, A. T., et al. (2010). Observations beneath pine island glacier in West Antarctica and implications for its retreat. Nat. Geosci. 3, 468-472. doi: 10.1038/ngeo890

Jiao, N., Herndl, G. J., Hansell, D. A., Benner, R., Kattner, G., Wilhelm, S. W., et al. (2010). Microbial production of recalcitrant dissolved organic matter: long-term carbon storage in the global ocean. Nat. Rev. Microbiol. 8, 593-599. doi: $10.1038 /$ nrmicro2386

Johnson, K. S., Plant, J. N., Coletti, L. J., Jannasch, H. W., Sakamoto, C. M., Riser, S. C., et al. (2017a). Biogeochemical sensor performance in the SOCCOM profiling float array. J. Geophys. Res. Oceans 122, 6416-6436. doi: 10.1002/ 2017JC012838

Johnson, K. S., Plant, J. N., Dunne, J. P., Talley, L. D., and Sarmiento, J. L. (2017b). Annual nitrate drawdown observed by SOCCOM profiling floats and the relationship to annual net community production. J. Geophys. Res. 122, 6668-6683. doi: 10.1002/2017JC012839

Josey, S. A., Gulev, S., and Yu, L. (2013). "Exchanges through the ocean surface," in Ocean Circulation and Climate: A 21st Century Perspective, eds G. Siedler, J. Church, W. J. Gould, and S. Griffies (Oxford: Academic Press), 115-140. doi: 10.1016/b978-0-12-391851-2.00005-2

Jullion, L., Naveira Garabato, A. C., Meredith, M. P., Holland, P. R., Courtois, P., and King, B. A. (2013). Decadal freshening of the antarctic bottom water exported from the weddell sea. J. Clim. 26, 8111-8125. doi: 10.1175/JCLI-D12-00765.1

Kaartvedt, S., Staby, A., and Aksnes, D. L. (2012). Efficient trawl avoidance by mesopelagic fishes causes large underestimation of their biomass. Mar. Ecol. Prog. Ser. 456, 1-6. doi: 10.3354/meps09785

Kahl, A., Fraser, W., and Schofield, O. (2010). Autonomous gliders reveal water column features associated with Adélie penguin foraging. Int. Comp. Biol. 50, 1041-1050. doi: 10.1093/icb/icq098

Kamenkovich, I., Haza, A., Gray, A. R., Dufour, C. O., and Garraffo, Z. (2017). Observing system simulation experiments for an array of autonomous biogeochemical profiling floats in the Southern Ocean. J. Geophys. Res. Oceans 122, 7595-7611. doi: 10.1002/2017JC012819

Kaufman, D. E., Friedrichs, M. A. M., Smith, W. O., Queste, B. Y., and Heywood, K. J. (2014). Biogeochemical variability in the southern Ross Sea as observed by a glider deployment. Deep Sea Res. I Oceanogr. Res. Pap. 92, 93-106. doi: 10.1016/j.dsr.2014.06.011

Kemeny, P. C., Kast, E. R., Hain, M. P., Fawcett, S. E., Fripiat, F., Studer, A. S., et al. (2018). A seasonal model of nitrogen isotopes in the ice age Antarctic zone: support for weakening of the Southern Ocean upper overturning cell. Paleoceanogr. Paleoclimatol. 33, 1453-1471. doi: 10.1029/2018PA00 3478

Kennicutt, M. C. II, Chown, S. L., Cassano, J. J., Liggett, D., Massom, R., Peck, L. S., et al. (2014a). Six priorities for Antarctic science. Nature 512, 23-25. doi: $10.1038 / 512023 a$

Kern, S., Ozsoy-Cicek, B., and Worby, A. P. (2016). Antarctic sea-ice thickness retrieval from ICESat: inter-comparison of different approaches. Remote Sens. 8:538. doi: 10.3390/rs8070538

Kim, H., Ducklow, H. W., Abele, D., Barlett, E. M. R., Buma, A. G., Meredith, M. P., et al. (2018). Inter-decadal variability of phytoplankton biomass along the coastal West Antarctic Peninsula. Philos. Trans. R. Soc. A. 376:20170174. doi: 10.1098/rsta.2017.0174

Klein, E. S., Hill, S. L., Hinke, J. T., Phillips, T., and Watters, G. M. (2018). Impacts of rising sea temperature on krill increase risks for predators in the Scotia Sea. PLoS One 13:e191011-21. doi: 10.1371/journal.pone.0191011

Knuth, S. L., Cassano, J. J., Maslanik, J. A., Herrmann, P. D., Kernebone, P. A., Crocker, R. I., et al. (2013). Unmanned aircraft system measurements of the atmospheric boundary layer over Terra Nova Bay, Antarctica. Earth Syst. Sci. Data 5, 57-69. doi: 10.5194/essd-5-57-2013
Koentopp, M., Eisen, O., Kottmeier, C., Padman, L., and Lemke, P. (2005). Influence of tides on sea ice in the Weddell Sea: investigations with a high-resolution dynamic-thermodynamic sea ice model. J. Geophys. Res. 110:C02014.

Kohout, A. L., Williams, M. J. M., Dean, S. M., and Meylan, M. H. (2014). Storm-induced sea-ice breakup and the implications for ice extent. Nature 509, 604-607. doi: 10.1038/nature13262

Kohout, A. L., Williams, M. J. M., Toyota, T., Lieser, J., and Hutchings, J. (2016). In situ observations of wave-induced sea ice breakup. Deep Sea Res. II Top. Stud. Oceanogr. 131, 22-27. doi: 10.1016/j.dsr2.2015.06.010

Kowalczyk, N. D., Chiaradia, A., Preston, T. J., and Reina, R. D. (2014). Linking dietary shifts and reproductive failure in seabirds: a stable isotope approach. Funct. Ecol. 28, 755-765. doi: 10.1111/1365-2435.12216

Kurahashi-Nakamura, T., Losch, M., and Paul, A. (2014). Can sparse proxy data constrain the strength of the Atlantic meridional overturning circulation? Geosci. Model Dev. 7, 419-432. doi: 10.5194/gmd-7-419-2014

Kurtz, N. T., and Markus, T. (2012). Satellite observations of Antarctic sea ice thickness and volume. J. Geophys. Res. 117:C08025. doi: 10.1029/2012JC008141

Labrousse, S., Williams, G., Tamura, T., Bestley, S., Sallée, J.-B., Fraser, A. D., et al. (2018). Coastal polynyas: winter oases for subadult southern elephant seals in East Antarctica. Sci. Rep. 8:3183. doi: 10.1038/s41598-018-2138821389

Landschützer, P., Gruber, N., Haumann, F. A., Rödenbeck, C., Bakker, D. C. E., van Heuven, S., et al. (2015). The reinvigoration of the Southern Ocean carbon sink. Science 349, 1221-1224. doi: 10.1126/science.aab2620

Le Quéré, C., Rödenbeck, C., Buitenhuis, E., Conway, T., Langenfelds, R., Gomex, A., et al. (2007). Saturation of the Southern Ocean CO2 sink due to recent climate change. Science 316, 1735-1738. doi: 10.1126/science/1136188

Lee, K. (2001). Global net community production estimated from the annual cycle of surface water total dissolved inorganic carbon. Limnol. Oceanogr. 46, 1287-1297. doi: 10.4319/lo.2001.46.6.1287

Li, Y., McGillicuddy, D. J., Dinniman, M. S., and Klinck, J. M. (2017). Processes influencing formation of low-salinity high-biomass lenses near the edge of the Ross Ice Shelf. J. Mar. Syst. 166, 108-119. doi: 10.1016/j.jmarsys.2016. 07.002

Lindstrom, E., Gunn, J., Fischer, A., McCurdy, A., and Glover, L. K. (2012). A Framework for Ocean Observing. By the Task Team for an Integrated Framework for Sustained Ocean Observing. Paris: UNESCO, doi: 10.25607/OBP-9

Llort, J., Langlais, C., Matear, R., Moreau, S., Lenton, A., and Strutton, P. (2018). Evaluating southern ocean carbon eddy-pump from biogeochemicalargo floats. J. Geophys. Res. Oceans 123, 971-984. doi: 10.1002/2017JC012861

Lombard, F., Boss, E., Waite, A. M., Vogt, M., Uitz, J., Stemmann, L., et al. (2019). Globally consisten quantitative observations of planktonic ecosystems. Front. Mar. Sci. 6:196. doi: 10.3389/fmars.2019.00196

Lubin, D., and Massom, R. A. (2006). Polar Remote Sensing. Volume 1: Atmosphere and Polar Oceans. Chichester: Springer.

Lucas, V. (2017). Skills Gap Analysis e-Infrastructures and Data Management in Global Change Research, Belmont Forum. Available at: http://www.bfeinf.org/sites/default/files/doc-repository/BF-Skills-Gap_Analysis-2017_0.pdf (accessed July 2018).

MacCready, P., and Quay, P. (2001). Biological export flux in the Southern Ocean estimated from a climatological nitrate budget. Deep Sea Res. II Top. Stud. Oceanogr. 48, 4299-4322. doi: 10.1016/S0967-0645(01) 00090-X

Mack, S. L., Dinniman, M. S., McGillicuddy, D. J., Sedwick, P. N., and Klinck, J. M. (2017). Dissolved iron transport pathways in the Ross Sea: influence of tides and horizontal resolution in a regional ocean model. J. Mar. Syst. 166, 73-86. doi: 10.1016/j.jmarsys.2016.10.008

Maksym, T., Stammerjohn, S. E., Ackley, S., and Massom, R. (2012). Antarctic sea ice - A polar opposite?. Oceanography 25, 140-151. doi: 10.5670/oceanog. 2012.88

Marina, T. I., Salinas, V., Cordone, G., Campana, G., Moreira, M. E., Deregibus, D., et al. (2018a). The food web of Potter Cove (Antarctica): complexity, structure and function. Estuar. Coast. Shelf Sci. 200, 141-151. doi: 10.1016/j.ecss.2017. 10.015

Marina, T. I., Saravia, L. A., Cordone, G., Salinas, V., Doyle, S., and Momo, F. R. (2018b). Architecture of marine food webs: to be or not be a 'small-world'. PLoS One 13:e198217. doi: 10.1371/journal.pone.0198217 
Marinov, A., Gnanadesikan, A., Toggweiler, J. R., and Sarmiento, J. L. (2006). The Southern Ocean biogeochemical divide. Nature 441, 964-967. doi: 10.1038/ nature 04883

Massom, R. A., Giles, A. B., Fricker, H. A., Warner, R. C., Legresy, B., Hyland, G., et al. (2010). Examining the interaction between multi-year landfast sea ice and the Mertz Glacier Tongue, East Antarctica: another factor in ice sheet stability? J. Geophys. Res. 115:C12027. doi: 10.1029/2009JC006083

Massom, R. A., Scambos, T. A., Bennetts, L. G., Reid, P., Squire, V. A., and Stammerjohn, S. E. (2018). Antarctic ice shelf disintegration triggered by sea ice loss and ocean swell. Nature 558, 383-389. doi: 10.1038/s41586-018-0212-1DO

Mazloff, M. R., Cornuelle, B. D., Gille, S. T., and Verdy, A. (2018). Correlation lengths for estimating the large-scale carbon and heat content of the southern ocean. J. Geophys. Res. Oceans 123, 883-901. doi: 10.1002/2017JC013408

McCormack, S., Melbourne-Thomas, J., Trebilco, R., Blanchard, J., and Constable, A. (2017). "Simplification of complex ecological networks - species aggregation in Antarctic food web models," in Proceedings of the 22nd International Congress on Modelling and Simulation, Hobart.

McPhail, S. D. (2009). Autosub6000: a deep diving long range AUV. J. Bionic Eng. 6, 55-62. doi: 10.1016/s1672-6529(08)60095-5

Meijers, A. J. S., Bindoff, N. L., and Rintoul, S. R. (2011). Frontal movements and property fluxes: contributions to heat and freshwater trends in the Southern Ocean. J. Geophys. Res. Oceans 116:C08024. doi: 10.1029/2010JC006832

Meijers, A. J. S., Shuckburgh, E., Bruneau, N., Sallee, J.-B., Bracegirdle, T. J., and Wang, Z. (2012). Representation of the antarctic circumpolar current in the CMIP5 climate models and future changes under warming scenarios. J. Geophys. Res. 117:C12008. doi: 10.1029/2012JC008412

Meiners, K. M., Vancoppenolle, M., Carnat, G., Castellani, G., Delille, B., Delille, D., et al. (2018). Chlorophyll-a in antarctic landfast sea ice: a first synthesis of historical ice core data. J. Geophys. Res. Oceans 123, 8444-8459. doi: 10.1029/ 2018JC014245

Melbourne-Thomas, J., Constable, A., Wotherspoon, S., and Raymond, B. (2013). Testing paradigms of ecosystem change under climate warming in Antarctica. PLoS One 8:e55093. doi: 10.1371/journal.pone.0055093.s004

Melbourne-Thomas, J., Constable, A. J., Fulton, E. A., Corney, S. P., Trebilco, R., Hobday, A. J., et al. (2017). Integrated modelling to support decisionmaking for marine social-ecological systems in Australia. ICES J. Mar. Sci. 74, 2298-2308.

Menezes, V. V., Macdonald, A. M., and Schatzman, C. (2017). Accelerated freshening of antarctic bottom water over the last decade in the Southern Indian Ocean. Sci. Adv. 3:e1601426. doi: 10.1126/sciadv.1601426

Meredith, M. P., Naveira Garabato, A. C., Hogg, A. M., and Farneti, R. (2012). Sensitivity of the overturning circulation in the southern ocean to decadal changes in wind forcing. J. Clim. 25, 99-110. doi: 10.1175/2011JCLI4204.1

Meredith, M. P., Schofield, O., Newman, L., Urban, E., and Sparrow, M. D. (2013). The vision for a southern ocean observing system. Curr. Opin. Environ. Sustain. 5, 306-313. doi: 10.1016/j.cosust.2013.03.002

Meredith, M. P., Stammerjohn, S. E., Venables, H. J., Ducklow, H. W., Martinson, D. G., Iannuzzi, R. A., et al. (2017). Changing distributions of sea ice melt and meteoric water west of the Antarctic Peninsula. Deep Sea Res. II Top. Stud. Oceanogr. 139, 40-57. doi: 10.1016/j.dsr2.2016.04.019

Meyer, A., Sloyan, B. M., Polzin, K. L., Phillips, H. E., and Bindoff, N. L. (2015). Mixing Variability in the Southern Ocean. J. Phys. Oceanogr. 45, 966-987. doi: 10.1175/JPO-D-14-0110.1

Miles, T. N., Lee, S. H., Wahlin, A., Ha, H. K., Assman, K., and Schofield, O. (2015). Glider observations of the dotson ice shelf outflow. Deep Sea Res. II Top. Stud. Oceanogr. 123, 16-29. doi: 10.1016/j.dsr2.2015.08.008

Miller, A. K., Karnovsky, N. J., and Trivelpiece, W. Z. (2009). Flexible foraging strategies of gentoo penguins Pygoscelis papua over 5 years in the South Shetland Islands, Antarctica. Mar. Biol. 156, 2527-2537. doi: 10.1007/s00227009-1277-z

Miller, A. K., and Trivelpiece, W. Z. (2007). Cycles of Euphausia superba recruitment evident in the diet of Pygoscelid penguins and net trawls in the South Shetland Islands. Antarctica. Polar Biol. 30, 1615-1623. doi: 10.1007/ s00300-007-0326-7

Miller, A. K., and Trivelpiece, W. Z. (2008). Chinstrap penguins alter foraging and diving behavior in response to the size of their principle prey, Antarctic krill. Mar. Biol. 154, 201-208. doi: 10.1007/s00227-0080909-z
Miloslavich, P., Bax, N. J., Simmons, S. E., Klein, E., Appeltans, W., AburtoOropeza, O., et al. (2018). Essential ocean variables for global sustained observations of biodiversity and ecosystem changes. Glob. Change Biol. 24, 2416-2433. doi: 10.1111/gcb.14108

Monteiro, P. M. S., Gregor, L., Lévy, M., Maenner, S., Sabine, C. L., and Swart, S. (2015). Intraseasonal variability linked to sampling alias in air-sea CO2 fluxes in the Southern Ocean. Geophys. Res. Lett. 42, 8507-8514. doi: 10.1002/ 2015GL066009

Moreau, S., Penna, A. D., Llort, J., Pastel, R., Langlai, C., Boyd, P. W., et al. (2017). Eddy-induced carbon transport across the antarctic circumpolar current. Glob. Biogeochem. Cycles 31, 1368-1386. doi: 10.1002/2017GB00 5669

Murphy, E. J., Cavanagh, R. D., Drinkwater, K. F., Grant, S. M., Heymans, J. J., Hofmann, E. E., et al. (2016). Understand the structure and functioning of polar pelagic ecosystems to predict the impacts of change. Proc. Biol. Sci. R. Soc. 283, doi: $10.1098 / \mathrm{rspb} .2016 .1646$

Murphy, E. J., Cavanagh, R. D., Hofmann, E. E., Hill, S. L., Constable, A. J., Costa, D. P., et al. (2012). Developing integrated models of Southern Ocean food webs: including ecological complexity, accounting for uncertainty and the importance of scale. Prog. Oceanogr. 102, 74-92. doi: 10.1016/j.pocean.2012.03.006

Murphy, E. J., Cavanagh, R. D., Johnston, N. M., Reid, K., and Hofmann, E. E. (2008). Integrating Climate and Ecosystem Dynamics in the Southern Ocean: A Circumpolar Ecosystem Program: Science Plan and Implementation Strategy. GLOBEC Report No. 26, IMBER Report No. 2. Plymouth: GLOBEC.

National Academies of Sciences, Engineering, and Medicine (2017). Antarctic Sea Ice Variability in the Southern Ocean-Climate System. Washington, DC: The National Academies Press, doi: 10.17226/24696

Naveira Garabato, A. C., Forryan, A., Dutrieux, P., Brannigan, L., Biddle, L. C., Heywood, K., et al. (2017). Vigorous lateral export of the meltwater outflow from beneath an Antarctic ice shelf. Nature 542:219. doi: 10.1038/nature20825

Nevison, C. D., Keeling, R. F., Kahru, M., Manizza, M., Mitchell, B. G., and Cassar, N. (2012). Estimating net community production in the Southern Ocean based on atmospheric potential oxygen and satellite ocean color data. Glob. Biogeochem. Cycles 26:GB1020. doi: 10.1029/2011GB0 04040

Newsome, S. D., Clementz, M. T., and Koch, P. L. (2010). Using stable isotope biogeochemistry to study marine mammal ecology. Mar. Mamm. Sci. 26, 509-572.

Nicholls, K. W., Abrahamsen, E. P., Buck, J. J. H., Dodd, P. A., Goldblatt, C., Griffiths, G., et al. (2006). Measurements beneath an Antarctic ice shelf using an autonomous underwater vehicle. Geophys. Res. Lett. 33:L08612. doi: 10.1029/ 2006GL025998

Nicholls, K. W., Abrahamsen, E. P., Heywood, K. J., Stansfield, K., and Østerhus, S. (2008). High-latitude oceanography using the Autosub autonomous underwater vehicle. Limnol. Oceanogr. 53(5 Part 2), 2309-2330.

Nicholls, K. W., Corr, H. F. J., Stewart, C. L., Lok, L. B., Brennan, P. V., and Vaughan, D. G. (2015). A ground-based radar for measuring vertical strain rates and time-varying basal melt rates in ice sheets and shelves. J. Glaciol. 61, 1079-1087. doi: 10.3189/2015JoG15J073

Nihashi, S., and Ohshima, K. I. (2015). Circumpolar mapping of Antarctic coastal polynyas and landfast sea ice: relationship and variability. J. Clim. 28, 36503670. doi: $10.1175 /$ jcli-d-14-00369.1

Nikurashin, M., and Ferrari, R. (2011). Global energy conversion rate from geostrophic flows into internal lee waves in the deep ocean. Geophys. Res. Lett. 38:L08610. doi: 10.1029/2011GL046576

Ogle, S. E., Tamsitt, V., Josey, S. A., Gille, S. T., Cerovecki, I., Talley, L. D., et al. (2018). Episodic Southern Ocean heat loss and its mixed layer impacts revealed by the furthest south multi-year surface flux mooring. Geophys. Res. Lett. 45, 5002-5010. doi: 10.1029/2017GL076909

Ozsoy-Cicek, B., Ackley, S., Xie, H., Yi, D., and Zwally, J. (2013). Sea ice thickness retrieval algorithms based on in situ surface elevation and thickness values for application to altimetry. J. Geophys. Res. Oceans 118, 3807-3822. doi: 10.1002/ jgrc.20252

Padman, L., Siegfried, M. R., and Fricker, H. (2018). Ocean tide influences on the Antarctic and Greenland ice sheets. Rev. Geophys. 56, 142-184. doi: 10.1002/ 2016RG000546

Paolo, F. S., Fricker, H. A., and Padman, L. (2015). Volume loss from Antarctic ice shelves is accelerating. Science 348:327. doi: 10.1126/science.aaa0940 
Paolo, F. S., Padman, L., Fricker, H. A., Adusumilli, S., Howard, S., and Siegfried, M. R. (2018). Response of Pacific-sector Antarctic ice shelves to the El Niño/Southern Oscillation. Nat. Geosci. 11, 121-126. doi: 10.1038/s41561-0170033-0

Paul, S., Hendricks, S., Ricker, R., Kern, S., and Rinne, E. (2018). Empirical parametrization of Envisat freeboard retrieval of Arctic and Antarctic sea ice based on CryoSat-2: progress in the ESA climate change initiative. Cryosphere 12, 2437-2460. doi: 10.5194/tc-12-2437-2018

Pauling, A. G., Bitz, C. M., Smith, I. J., and Langhorne, P. J. (2016). The response of the southern ocean and antarctic sea ice to fresh water from ice shelves in an earth system model. J. Clim. 29, 1655-1672. doi: 10.1175/JCLI-D-15-0501.1

Pauling, A. G., Smith, I. J., Langhorne, P. J., and Bitz, C. M. (2017). Time-dependent freshwater input from ice shelves: impacts on antarctic sea ice and the southern ocean in an earth system model. Geophys. Res. Lett. 44, 10454-10461. doi: 10.1002/2017GL075017

Pellichero, V., Sallee, J.-B., Chapman, C. C., and Downes, S. R. (2018). The southern ocean meridional overturning in the sea-ice sector is driven by freshwater fluxes. Nat. Commun. 9:1789. doi: 10.1038/s41467-018-04101-2

Pethybridge, H. R., Choy, C. A., Polovina, J. J., and Fulton, A. E. (2017). Improving marine ecosystem models with biochemical tracers. Annu. Rev. Mar. Sci. 10, 199-288. doi: 10.1146/annurev-marine-121916-063256

Pinkerton, M. H., Bradford-Grieve, J. M., and Hanchet, S. M. (2010). A balanced model of the food web of the Ross Sea, Antarctica. CCAMLR Science 17, 1-31.

Polvani, L. M., Waugh, D. W., Correa, G. J., and Son, S. (2011). Stratospheric ozone depletion: the main driver of twentieth-century atmospheric circulation changes in the southern hemisphere. J. Clim. 24, 795-812. doi: 10.1175/ 2010JCLI3772.1

Pope, A., Wagner, P., Johnson, R., Shutler, J. D., Baeseman, J., and Newman, L. (2017). Community review of Southern Ocean satellite data needs. Antarctic Sci. 29, 97-138. doi: 10.1017/S0954102016000390

Poteau, A., Boss, E., and Claustre, H. (2017). Particulate concentration and seasonal dynamics in the mesopelagic ocean based on the backscattering coefficient measured with Biogeochemical-Argo floats. Geophys. Res. Lett. 44, 6933-6939. doi: 10.1002/2017GL073949

Price, D., Beckers, J., Ricker, R., Kurtz, N., Rack, W., Haas, C., et al. (2015). Evaluation of CryoSat-2 derived sea-ice freeboard over fast ice in McMurdo Sound, Antarctica. J. Glaciol. 61, 285-300. doi: 10.3189/2015JoG14J157

Pritchard, H. D., Lightenberg, S. R. M., Fricker, H. A., Vaughan, D. G., van den Broeke, M. R., and Padman, L. (2012). Antarctic ice-sheet loss driven by basal melting of ice shelves. Nature 484, 502-505. doi: 10.1038/nature10968

Proud, R., Handegard, N. O., Kloser, R. J., Cox, M. J., and Brierley, A. S. (2018). From siphonophores to deep scattering layers: uncertainty ranges for the estimation of global mesopelagic fish biomass. ICES J. Mar. Scil 3, 1-16. doi: 10.1093/icesjms/fsy037

Purkey, S. G., and Johnson, G. C. (2010). Warming of global abyssal and deep southern ocean waters between the 1990s and 2000s: contributions to global heat and sea level rise budgets. J. Clim. 23, 6336-6351. doi: 10.1175/ 2010JCLI3682.1

Purkey, S. G., and Johnson, G. C. (2012). Global contraction of antarctic bottom water between the 1980 s and 2000s. J. Clim. 25, 5830-5844. doi: 10.1175/JCLID-11-00612.1

Purkey, S. G., and Johnson, G. C. (2013). Antarctic bottom water warming and freshening: contributions to sea level rise, ocean freshwater budgets, and global heat gain. J. Clim. 26, 6105-6122. doi: 10.1175/JCLI-D-12-00834

Rignot, E., Jacobs, S., Mouginot, J., and Scheuchl, B. (2013). Ice shelf melting around Antarctica. Science 341, 266-270. doi: 10.1126/1235798

Rintoul, S., van Wijk, E., Wahlin, A., and Boehme, L. (2014). Seeing Below the Ice: A Strategy for Observing the Ocean Beneath Antarctic Sea Ice and Ice Shelves. Hobart, TAS: Southern Ocean Observing System.

Rintoul, S. R. (2007). Rapid freshening of antarctic bottom water formed in the indian and pacific oceans. Geophys. Res. Lett. 34:L06606. doi: 10.1029/ 2006GL028550

Rintoul, S. R., Chown, S. L., DeConto, R. M., England, M. H., Fricker, H. A., Masson-Delmotte, V., et al. (2018). Choosing the future of Antarctica. Nature 558, 233-241. doi: 10.1038/s41586-018-0173-4

Rintoul, S. R., Silvano, A., Pena-Molino, B., van Wijk, E., Rosenberg, M., Stevens, J., et al. (2016). Ocean heat drives rapid basal melt of the Totten Ice Shelf. Sci. Adv. 2:e1601610. doi: 10.1126/sciadv.1601610
Rintoul, S. R., Sparrow, M., Meredith, M. P., Wadley, V., Speer, K., Hofmann, E. E., et al. (2012). The Southern Ocean Observing System: Initial Science and Implementation Strategy. Cambridge: Scientific Committee on Antarctic Research.

Rintoul, S. R., Speer, K., Sparrow, M., Meredith, M. P., Hofmann, E., Fahrbach, E., et al. (2010). "Southern ocean observing system (SOOS): rationale and strategy for sustained observations of the southern ocean," in Proceedings of OceanObs'09: Sustained Ocean Observations and Information for Society, Venice, Vol. 2, eds J. Hall, D. E. Harrison, and D. Stammer (France: ESA Publication), doi: 10.5270/OceanObs09.cwp.74

Roach, L. A., Horvat, C., Dean, S. M., and Bitz, C. M. (2018). An emergent sea ice floe size distribution in a global coupled ocean-sea ice model. J. Geophys. Res. Oceans 123, 4322-4337. doi: 10.1029/2017JC013692

Robertson, R. (2006). Modeling internal tides over fieberling guyot: resolution, parameterization, performance. Ocean Dyn. 56, 430-444. doi: 10.1007/s10236006-0062-65

Robertson, R. (2013). Tidally induced increases in melting of Amundsen Sea ice shelves. J. Geophys. Res. Oceans 118, 3138-3145. doi: 10.1002/jgrc. 20236

Robertson, R., and Hartlipp, P. (2017). Surface wind mixing in the regional ocean modeling system (ROMS). Geosci. Lett. 4:24. doi: 10.1186/s40562-017-0090-97

Robertson, R., Padman, L., and Levine, M. D. (1995). Fine structure, microstructure, and vertical mixing processes in the upper ocean in the western Weddell Sea. J. Geophys. Res. Oceans 100, 18517-18535.

Rocha, C. B., Chereskin, T. K., Gille, S. T., and Menemenlis, D. (2016). Mesoscale to submesoscale wavenumber spectra in Drake Passage. J. Phys. Ocean 46, 601-620. doi: 10.1175/jpo-d-15-0087.1

Roemmich, D., Church, J., Gilson, J., Monselesan, D., Sutton, S., and Wijffels, S. (2015). Unabated planetary warming and its ocean structure since 2006. Nat. Clim. Change 5, 240-245. doi: 10.1038/nclimate2513

Roquet, F., Charrassin, J. B., Marchand, S., Boehme, L., Fedak, M., Reverdin, G., et al. (2011). Delayed-mode calibration of hydrographic data obtained from animal-borne satellite relay data loggers. J. Atmos. Ocean Tech. 28, 787-801. doi: 10.1175/2010jtecho801.1

Roquet, F., Williams, G., Hindell, M. A., Harcourt, R., McMahon, C., and Guinet, G. (2014). A southern indian ocean database of hydrographic profiles obtained with instrumented elephant seals. Nat. Sci. Data 1:140028. doi: 10.1038/sdata. 2014.28

Roquet, F., Wunsch, C., Forget, G., Heimbach, P., Guinet, C., Reverdin, G., et al. (2013). Estimates of the Southern Ocean general circulation improved by animal-borne instruments. Geophys. Res. Lett. 40, 6176-6180. doi: 10.1002/ 2013gl058304

Russell, J. L., Kamenkovich, I., Bitz, C., Ferrari, R., Gille, S. T., Goodman, P. J., et al. (2018). Metrics for the evaluation of the southern ocean in coupled climate models and earth system models. J. Geophys. Res. Oceans 123, 3120-3143. doi: 10.1002/2017JC013461

Sabine, C. L., Feely, R. A., Gruber, N., Key, R. M., Lee, K., Bullister, J. L., et al. (2004). The Oceanic sink for anthropogenic CO2. Science 305, 367-371. doi: 10.1126/science.1097403

Sallée, J.-B., Matear, R. J., Rintoul, S. R., and Lenton, A. (2012). Localized subduction of anthropogenic carbon dioxide in the Southern Hemisphere oceans. Nat. Geosci. 5, 579-584. doi: 10.1038/ngeo1523

Sallée, J.-B., Shuckburgh, E., Bruneau, N., Meijers, A. J. S., Bracegirdle, T. J., Wang, Z., et al. (2013). Assessment of southern ocean water mass circulation and characteristics in CMIP5 models: historical bias and forcing response. J. Geophys. Res. Oceans 118, 1830-1844. doi: 10.1002/jgrc. 20135

Sallée, J.-B., Speer, K., Rintoul, S. R., and Wijffels, S. (2010). Southern Ocean thermocline ventilation. J. Phys. Oceanogr. 40, 509-529. doi: 10.1175/ 2009jpo4291.1

Scambos, T. A., Bohlander, J. A., Shuman, C. A., and Skvarca, P. (2004). Glacier acceleration and thinning after ice shelf collapse in the Larsen B embayment, Antarctica. Geophys. Res. Lett. 31:L18402.

Schloss, I. R., Wasilowska, A., Dumont, D., Almandoz, G. O., Hernando, M. P., Michaud-Tremblay, C.-A., et al. (2014). On the phytoplankton bloom in coastal waters of southern King George Island (Antarctica) in January 2010: an exceptional feature? Limnol. Oceanogr. 59, 195-210. doi: 10.4319/lo.2014.59.1. 0195 
Schmidt, K. M., Swart, S., Reason, C., and Nicholson, S. (2017). Evaluation of satellite and reanalysis wind products with in situ wave glider wind observations in the Southern Ocean. J. Atmos. Oceanic Tech. 34, 2551-2568. doi: 10.1175/ jtech-d-170079.1

Schmidtko, S., Heywood, K. J., Thompson, A. F., and Aoki, S. (2014). Multidecadal warming of Antarctic waters. Science 346, 1227-1231. doi: 10.1126/science. 1256117

Schofield, O., Ducklow, H., Bernard, K., Doney, S., Patterson-Fraser, D., Gorman, K., et al. (2013). Penguin biogeography along the west antarctic peninsula: testing the canyon hypothesis with Palmer LTER observations. Oceanography 26, 204-206. doi: 10.5670/oceanog.2013.63

Schofield, O., and Kohut, J. (2018). Sampling the southern ocean: technology for observing the marine system. Antarctic Environ. Portal

Schofield, O., Miles, T., Aldercamp, A.-C., Lee, S. H., Haskin, C., Roaglsky, E., et al. (2015). In situ phytoplankton distributions in the Amundsen Sea polynya measured by autonomous gliders. Elem. Sci. Anth. 3:000073. doi: 10.12952/ journal.elementa.000073

Schulz, E. W., Josey, S. A., and Verein, R. (2012). First air-sea flux mooring measurements in the Southern Ocean. Geophys. Res. Let. 39:L16606.

Schwegmann, S., Haas, C., Fowler, C., and Gerdes, R. (2011). A comparison of satellite-derived sea-ice motion with drifting buoy data in the Weddell Sea, Antarctica. Ann. Glaciol. 52, 103-110. doi: 10.3189/17275641179593 1813

Schwegmann, S., Rinne, E., Ricker, R., Hendricks, S., and Helm, V. (2016). About the consistency between Envisat and CryoSat-2 radar freeboard retrieval over Antarctic sea ice. Cryosphere 10, 1415-1425. doi: 10.5194/tc-10-1415-2016

Shao, A. E., Gille, S. T., Mecking, S., and Thompson, L. (2015). Properties of the subantarctic front and polar front from the skewness of sea level anomaly. J. Geophys. Res. Oceans 120, 5179-5193. doi: 10.1002/2015JC010723

Shepherd, A., Fricker, H. A., and Farrell, S. L. (2018). Trends and connections across the Antarctic cryosphere. Nature 558, 223-232. doi: 10.1038/s41586-0180171-6

Shepherd, A., Wingham, D., Wallis, D., Giles, K., Laxon, S., and Venke Sundal, A. (2010). Recent loss of floating ice and the consequent sea level contribution. Geophys. Res. Lett. 37:L13503. doi: 10.1029/2010GL042496

Sherrell, R. M., Annett, A. L., Fitzsimmons, J. N., Roccanova, V. J., and Meredith, M. P. (2018). A 'shallow bathtub ring' of local sedimentary iron input maintains the Palmer Deep biological hotspot on the West Antarctic Peninsula shelf. Philos. Trans. R. Soc. A. 376:20170171. doi: 10.1098/rsta.2017. 0171

Shu, Q., Song, Z., and Qiao, F. (2015). Assessment of sea ice simulations in the CMIP5 models. Cryosphere 9, 399-409. doi: 10.5194/tc-9-399-2015

Silvano, A., Rintoul, S. R., Pena-Molina, B., Hobbs, W. R., van Wijk, E., Aoki, S., et al. (2018). Freshening by glacial meltwater enhances melting of ice shelves and reduces formation of Antarctic Bottom Water. Sci. Adv. 4:eaa9467. doi: 10.1126/sciadv.aap9467

Siniff, D. B., Garrott, R. A., Rotella, J. J., Fraser, W. R., and Ainley, D. G. (2008). Opinion: projecting the effects of environmental change on Antarctic seals. Antarctic Sci. 20, 425-435. doi: 10.1017/S0954102008001351

Smart, S. M., Fawcett, S. E., Thomalla, S. J., Weigand, M. A., Reason, C. J. C., and Sigman, D. M. (2015). Isotopic evidence for nitrification in the Antarctic winter mixed layer. Glob. Biogeochem. Cycles 29, 427-445. doi: 10.1002/2014GB0 05013

Smith, C. D., Thorteinsson, T., Heil, P., Fierz, C., Nitu, R., Buisan, S., et al. (2018). "A new WMO Guide for the measurement of cryospheric variables," in Proceedings of the WMO Technical Conference on Meteorological and Environmental Instruments and Methods of Observation (CIMO TECO 2018), Geneva.

Spreen, G., Kwok, R., Menemelis, D., and Nguyen, A. T. (2017). Sea-ice deformation in a coupled ocean-sea-ice model and in satellite remote sensing data. Cryosphere 11, 1553-1573. doi: 10.5194/tc-11-1553-2017

St. John, M. A., Borja, A., Chust, G., Heath, M., Grigorov, I., Mariani, P., et al. (2016). A dark hole in our understanding of marine ecosystems and their services: perspectives from the mesopelagic community. Front. Mar. Sci. 3:31. doi: $10.3389 /$ fmars.2016.00031

Stammer, D., Bracco, A., AchutaRao, K., Beal, L., Bindoff, N., Braconnot, P., et al. (2019). Ocean climate observing requirements in support of Climate Research and Climate Information. Front. Mar. Sci. doi: 10.3389/fmars.2019.00444
Stammerjohn, S., and Maksym, T. (2016). “Gaining (and losing) Antarctic sea ice: variability, trends and mechanisms," in Sea Ice, ed. D. N. Thomas (Chichester: John Wiley \& Sons, Ltd), doi: 10.1002/9781118778371.ch10

Stammerjohn, S., Massom, R., Rind, D., and Martinson, D. (2012). Regions of rapid sea ice change: ant inter-hemispheric comparison. Geophys. Res. Lett. 39:L06501. doi: 10.1029/2012GL050874

Stammerjohn, S. E., Martinson, D. G., Smith, R. C., and Iannuzzi, R. A. (2008). Sea ice in the western Antarctic Peninsula region: spatio-temporal variability from ecological and climate change perspectives. Deep Sea Res II Top. Stud. Oceanogr. 55, 2041-2058. doi: 10.1016/j.dsr2.2008.04.026

Stephens, B. B., Long, M., Keeling, R. F., Kort, E. A., Sweeney, C., Apel, E. C., et al. (2018). The $\mathrm{O}_{2} / \mathrm{N}_{2}$ ratio and $\mathrm{CO}_{2}$ airborne Southern Ocean Study. Bull. Am. Meteorol. Soc. 99, 381-402. doi: 10.1175/BAMS-D-16-0206.1

Stewart, A. L., and Thompson, A. F. (2014). Eddy-mediated transport of warm circumpolar deep water across the antarctic shelf break. Geophys. Res. Lett. 42, 432-440. doi: 10.1002/2014GL062281

Sturm, M., and Massom, R. A. (2017). "Snow in the sea ice system: friend or foe?," in Sea Ice, 3rd Edn, ed. D. Thomas (New York, NY: Wiley-Blackwell).

Surma, S., Pakhomov, E. A., and Pitcher, T. J. (2014). Effects of whaling on the structure of the southern ocean food web: insights on the "Krill Surplus" from ecosystem modelling. PLoS One 9:e114978. doi: 10.1371/journal.pone.011 4978

Swart, N. C., and Fyfe, J. C. (2013). The influence of recent Antarctic ice sheet retreat on simulated sea ice area trends. Geophys. Res. Lett. 40, 4328-4332. doi: $10.1002 /$ grl.50820

Swart, N. C., Gille, S. T., Fyfe, J. C., and Gillett, N. P. (2018). Recent Southern Ocean warming and freshening driven by greenhouse gas emissions and ozone depletion. Nat. Geosci. 11, 836-841. doi: 10.1038/s41561-018-0226-221

Swart, S., Gille, S. T., Delille, B., Josey, S., Mazloff, M., Newman, L., et al. (2019). Constraining Southern Ocean air-sea-ice fluxes through enhanced observations. Front. Mar. Sci.

Swart, S., Thomalla, S. J., and Monteiro, P. M. S. (2015). The seasonal cycle of mixed layer characteristics and phytoplankton biomass in the Sub-Antarctic Zone: a high-resolution glider experiment. J. Mar. Syst. 147, 103-115. doi: 10.1016/j.jmarsys.2014.06.002

Tagliabue, A., Mtshali, T., Aumont, O., Bowie, A. R., Klunder, M. B., Roychoudhury, A. N., et al. (2012). A global compilation of dissolved iron measurements: focus on distributions and processes in the Southern Ocean. Biogeosciences 9, 2333-2349. doi: 10.5194/bg-9-2333-2012

Takahashi, T., Sutherland, S. C., Wanninkhof, R., Sweeney, C., Feely, R. A., Chipman, D. W., et al. (2009). Climatological mean and decadal change in surface ocean pCO2, and net sea-air CO2 flux over the global oceans. Deep Sea Res. II Top. Stud. Oceanogr. 56, 554-577. doi: 10.1016/j.dsr2.2008. 12.009

Tamura, T., Ohshima, K. I., Fraser, A. D., and Williams, G. D. (2016). Sea ice production variability in Antarctic coastal polynyas. J. Geophys. Res. Oceans 121, 2967-2979. doi: 10.1002/2015JC011537

Tanhua, T., Pouliquen, S., Hausman, J., O’Brien, K., Bricher, P., de Bruin, T., et al. (2019). Ocean FAIR data services. Front. Mar. Sci.

Thomas, D. N. (2017). Sea Ice, 3rd Edn. Hoboken, NJ: Wiley-Blackwell.

Thompson, A. F., Heywood, K. J., Schmidtko, S., and Stewart, A. L. (2014). Eddy transport as a key component of the Antarctic overturning circulation. Nat. Geosci. 7, 879-884. doi: 10.1038/ngeo2289

Thomson, J., and Girton, J. (2017). Sustained measurements of Southern Ocean air-sea coupling from a wave glider autonomous surface vehicle. Oceanography 30, 104-109. doi: 10.5670/oceanog.2017.228

Ting, Y. H., and Holzer, M. (2017). Decadal changes in Southern Ocean ventilation inferred from deconvolutions of repeat hydrographies. Geophys. Res. Lett. 44, 5655-5664. doi: 10.1002/2017GL073788

Treasure, A. M., Roquet, F., Ansorge, I. J., Bester, M. N., Boehme, L., Bornemann, H., et al. (2017). Marine mammals exploring the oceans pole to pole a review of the MEOP consortium. Oceanography 30, 132-138. doi: 10.5670/oceanog. 2017.234

Trimborn, S., Brenneis, T., Sweet, E., and Rost, B. (2013). Sensitivity of antarctic phytoplankton species to ocean acidification: growth, carbon acquisition, and species interaction. Limnol. Oceanogr. 58, 997-1007. doi: 10.4319/lo.2013.58.3. 0997 
Trimborn, S., Thoms, S., Brenneis, T., Heiden, J. P., Beszteri, S., and Bischof, K. (2017). Two southern ocean diatoms are more sensitive to ocean acidification and changes in irradiance than the prymnesiophyte Phaeocystis Antarctica. Physiol. Plant 160, 155-170. doi: 10.1111/ppl.12539

Trivelpiece, W. Z., Hinke, J. T., Miller, A. K., Reiss, C. S., Trivelpiece, S. G., and Watters, G. M. (2011). Variability in krill biomass links harvesting and climate warming to penguin population changes in Antarctica. Proc. Natl. Acad. Sci. U.S.A. 108, 7625-7628. doi: 10.1073/pnas.1016560108

Turner, J., and Comiso, J. (2017). Solve Antarctica's sea-ice puzzle. Comment Nat. 547, 19-21. doi: 10.1038/547019a

van Wijk, E., and Rintoul, S. R. (2014). Freshening drives contraction of Antarctic Bottom water in the australian antarctic basin. Geophys. Res. Lett. 41, $1657-$ 1664. doi: 10.1002/2013GL058921

Venables, H. J., Clarke, A., and Meredith, M. P. (2013). Wintertime controls on summer stratification and productivity at the western Antarctic Peninsula. Limnol. Oceanogr. 58, 1035-1047. doi: 10.4319/lo.2013.58.3.1035

Verdy, A., and Mazloff, M. R. (2017). A data assimilating model for estimating Southern Ocean biogeochemistry. J. Geophys. Res. Oceans 122, doi: 10.1002/ 2016JC012650

Viglione, G. A., Thompson, A. F., Flexas, M. M., Sprintall, J., and Swart, S. (2018). Abrupt transitions in submesoscale structure in southern Drake Passage: glider observations and GCM results. J. Phys. Oceanogr. 48, 2011-2027. doi: 10.1175/ jpo-d-17-0192.1

Waugh, D. W. (2014). Changes in the ventilation of the southern oceans. Philos. Trans. A Math. Phys. Eng. Sci. 372:20130269. doi: 10.1098/rsta.2013.0269

Waugh, D. W., Primeau, F., DeVries, T., and Holzer, M. (2013). Recent changes in the ventilation of the southern oceans. Science 339, 568-570. doi: 10.1126/ science. 1225411

Webster, M., Gerland, S., Holland, M., Hunke, E., Kwok, R., Lecomte, O., et al. (2018). Snow in the changing sea-ice systems. Nat. Clim. Change 8, 946-953. doi: 10.1038/s41558-018-0286-287

Weimerskirch, H., Louzao, M., de Grissac, S., and Delord, K. (2012). Changes in wind pattern alter albatross distribution and life-history traits. Science 335, 211-214. doi: 10.1126/science.1210270

Wilkinson, M. D., Dumontier, M., Aalbersberg, I. J. J., Appleton, G., Axton, M., Baak, A., et al. (2016). The fair guiding principles for scientific data management and stewardship. Sci. Data 3:160018. doi: 10.1038/sdata.2016.18
Williams, G. D., Fraser, A., Lucieer, A., Turner, D., Cougnon, E., Kimball, P., et al. (2016a). Drones in a cold climate. EOS Trans. 97 (accessed January 19, 2016).

Williams, G. D., Herraiz-Borreguero, L., Roquet, F., Tamura, T., Ohshima, K. I., Fukamachi, Y., et al. (2016b). The suppression of Antarctic bottom water formation by melting ice shelves in Prydz Bay. Nat. Commun. 7:12577. doi: $10.1038 /$ ncomms 12577

Williams, G. D., Maksym, T., Wilkinson, J., Kunz, C., Murphy, C., Kimball, P., et al. (2015). Thick and deformed Antarctic sea ice mapped with autonomous underwater vehicles. Nat. Geosci. 8, 61-67. doi: 10.1038/ngeo2299

Williams, N. L., Juranek, L. W., Feely, R. A., Johnson, K. S., Sarmiento, J. L., Talley, L. D., et al. (2017). Calculating surface ocean pCO2 from biogeochemical Argo floats equipped with $\mathrm{pH}$ : an uncertainty analysis. Glob. Biogeochem. Cycles 31, 591-604. doi: 10.1002/2016GB0 05541

Worby, A. P., Geiger, C. A., Paget, M. J., Van Woert, M. L., Ackley, S. F., and DeLiberty, T. L. (2008). Thickness distribution of Antarctic sea ice. J. Geophys. Res. 113:C05S92. doi: 10.1029/2007JC00 4254

Zwally, H. J., Yi, D., Kwok, R., and Zhao, Y. (2008). ICESat measurements of sea ice freeboard and estimates of sea-ice thickness in the Weddell Sea. J. Geophys. Res. 113:C02S15.

Conflict of Interest Statement: The authors declare that the research was conducted in the absence of any commercial or financial relationships that could be construed as a potential conflict of interest.

Copyright (c) 2019 Newman, Heil, Trebilco, Katsumata, Constable, van Wijk Assmann, Beja, Bricher, Coleman, Costa, Diggs, Farneti, Fawcett, Gille, Hendry, Henley, Hofmann, Maksym, Mazloff, Meijers, Meredith, Moreau, Ozsoy, Robertson, Schloss, Schofield, Shi, Sikes, Smith, Swart, Wahlin, Williams, Williams, HerraizBorreguero, Kern, Lieser, Massom, Melbourne-Thomas, Miloslavich and Spreen. This is an open-access article distributed under the terms of the Creative Commons Attribution License (CC BY). The use, distribution or reproduction in other forums is permitted, provided the original author(s) and the copyright owner(s) are credited and that the original publication in this journal is cited, in accordance with accepted academic practice. No use, distribution or reproduction is permitted which does not comply with these terms. 


\section{Global Perspectives on Observing Ocean Boundary Current Systems}

\section{OPEN ACCESS}

Edited by:

Sabrina Speich,

École Normale Supérieure, France

Reviewed by:

Moacyr Cunha de Araujo Filho, Federal Rural University

of Pernambuco, Brazil

Eitarou Oka

The University of Tokyo, Japan

*Correspondence:

Robert E. Todd

rtodd@whoi.edu

Specialty section

This article was submitted to

Ocean Observation,

a section of the journal

Frontiers in Marine Science

Received: 31 October 2018

Accepted: 05 July 2019

Published: 08 August 2019

Citation:

Todd RE, Chavez FP, Clayton S,

Cravatte $S$, Goes $M$, Graco $M$, Lin X, Sprintall J, Zilberman NV, Archer M, Arístegui J, Balmaseda M, Bane JM,

Baringer MO, Barth JA, Beal LM, Brandt P, Calil PHR, Campos E

Centurioni LR, Chidichimo MP

Cirano M, Cronin MF, Curchitser EN,

Davis RE, Dengler M, deYoung $B$,

Dong S, Escribano R, Fassbender AJ,

Fawcett SE, Feng M, Goni GJ,

Gray $A R$, Gutiérrez $D$, Hebert $D$,

Hummels $R$, Ito $S$-i, Krug M, Lacan F,

Laurindo L, Lazar A, Lee CM,

Lengaigne $M$, Levine NM,

Middleton J, Montes I, Muglia M,

Nagai T, Palevsky HI, Palter JB,

Phillips HE, Piola A, Plueddemann AJ,

Qiu B, Rodrigues RR, Roughan M,

Rudnick DL, Rykaczewski RR,

Saraceno M, Seim H, Sen Gupta A,

Shannon L, Sloyan BM, Sutton AJ,

Thompson L, van der Plas AK,

Volkov $D$, Wilkin J, Zhang $D$ and

Zhang L (2019) Global Perspectives

on Observing Ocean Boundary

Current Systems.

Front. Mar. Sci. 6:423.

doi: 10.3389/fmars.2019.00423
Robert E. Todd ${ }^{*}$, Francisco P. Chavez ${ }^{2}$, Sophie Clayton ${ }^{3}$, Sophie Cravatte Marlos Goes ${ }^{5,6}$, Michelle Graco ${ }^{7}$, Xiaopei Lin ${ }^{8}$, Janet Sprintall', Nathalie V. Zilberman ${ }^{9}$, Matthew Archer ${ }^{10}$, Javier Arístegui ${ }^{11}$, Magdalena Balmaseda ${ }^{12}$, John M. Bane ${ }^{13}$, Molly O. Baringer ${ }^{5}$, John A. Barth ${ }^{14}$, Lisa M. Beal ${ }^{6}$, Peter Brandt ${ }^{15,16}$, Paulo H. R. Calil17, Edmo Campos $^{18}$, Luca R. Centurioni ${ }^{9}$, Maria Paz Chidichimo ${ }^{19}$, Mauro Cirano ${ }^{20}$, Meghan F. Cronin ${ }^{21}$, Enrique N. Curchitser ${ }^{22}$, Russ E. Davis ${ }^{9}$, Marcus Dengler ${ }^{15}$, Brad deYoung ${ }^{23}$, Shenfu Dong ${ }^{5}$, Ruben Escribano ${ }^{24}$, Andrea J. Fassbender ${ }^{2}$, Sarah E. Fawcett ${ }^{25}$, Ming Feng ${ }^{26}$, Gustavo J. Goni ${ }^{5}$, Alison R. Gray ${ }^{27}$, Dimitri Gutiérrez ${ }^{7}$, Dave Hebert ${ }^{28}$, Rebecca Hummels ${ }^{15}$, Shin-ichi Ito ${ }^{29}$, Marjorlaine Krug $^{30}$, François Lacan ${ }^{4,31}$, Lucas Laurindo ${ }^{6}$, Alban Lazar ${ }^{32}$, Craig M. Lee ${ }^{33}$, Matthieu Lengaigne ${ }^{32}$, Naomi M. Levine ${ }^{34}$, John Middleton ${ }^{35}$, Ivonne Montes ${ }^{36}$, Mike Muglia ${ }^{13,37}$, Takeyoshi Nagai ${ }^{38}$, Hilary I. Palevsky ${ }^{39}$, Jaime B. Palter ${ }^{40}$, Helen E. Phillips ${ }^{41}$, Alberto Piola19,42, Albert J. Plueddemann11, Bo Qiu'43, Regina R. Rodrigues ${ }^{44}$, Moninya Roughan ${ }^{45}$, Daniel L. Rudnick ${ }^{9}$, Ryan R. Rykaczewski ${ }^{46}$, Martin Saraceno ${ }^{42,47}$, Harvey Seim ${ }^{13}$, Alex Sen Gupta ${ }^{45}$, Lynne Shannon ${ }^{48}$, Bernadette M. Sloyan ${ }^{49}$, Adrienne J. Sutton ${ }^{21}$, LuAnne Thompson ${ }^{27}$, Anja K. van der Plas ${ }^{50}$, Denis Volkov, ${ }^{5,6}$, John Wilkin ${ }^{51}$, Dongxiao Zhang ${ }^{21,52}$ and Linlin Zhang ${ }^{53}$

${ }^{1}$ Woods Hole Oceanographic Institution, Woods Hole, MA, United States, ${ }^{2}$ Monterey Bay Aquarium Research Institute, Moss Landing, CA, United States, ${ }^{3}$ Department of Ocean, Earth, and Atmospheric Sciences, Old Dominion University, Norfolk, VA, United States, ${ }^{4}$ LEGOS, IRD, CNES, CNRS, UPS, Universite de Toulouse, Toulouse, France, ${ }^{5}$ NOAA's Atlantic Oceanographic and Meteorological Laboratory, Miami, FL, United States, ${ }^{6}$ Rosenstiel School of Marine and Atmospheric Science, University of Miami, Miami, FL, United States, ${ }^{7}$ Instituto del Mar del Peru, Lima, Peru, ${ }^{8}$ Qingdao National Laboratory for Marine Science and Technology, Ocean University of China, Qingdao, China, ${ }^{9}$ Scripps Institution of Oceanography, University of California, San Diego, La Jolla, CA, United States, ${ }^{10}$ Jet Propulsion Laboratory, California Institute of Technology, Pasadena, CA, United States, ${ }^{11}$ Instituto de Oceanografía y Cambio Global, Universidad de Las Palmas de Gran Canaria, Las Palmas, Spain, ${ }^{12}$ European Centre for Medium-Range Weather Forecasts, Reading, United Kingdom, ${ }^{13}$ Department of Marine Sciences, The University of North Carolina at Chapel Hill, Chapel Hill, NC, United States, ${ }^{14}$ College of Earth, Ocean, and Atmospheric Sciences, Oregon State University, Corvallis, OR, United States, ${ }^{15}$ GEOMAR Helmholtz Centre for Ocean Research Kiel, Kiel, Germany, ${ }^{16}$ Faculty of Mathematics and Natural Sciences, Kiel University, Kiel, Germany, ${ }^{17}$ Institute of Coastal Research, Helmholtz-Zentrum Geesthacht, Geesthacht, Germany, ${ }^{18}$ Departamento de Oceanografia Física, Química e Geológica University of São Paulo, São Paulo, Brazil, ${ }^{19}$ Consejo Nacional de Investigaciones Cientificas y Técnicas, Servicio de Hidrografía Naval, Buenos Aires, Argentina, ${ }^{20}$ Department of Meteorology, Institute of Geosciences, Federal University of Rio de Janeiro (UFRJ), Rio de Janeiro, Brazil, ${ }^{21}$ NOAA Pacific Marine Environmental Laboratory, Seattle, WA, United States, ${ }^{22}$ Deptartment of Environmental Science, Rutgers University, New Brunswick, NJ, United States, ${ }^{23}$ Department of Physics and Physical Oceanography, Memorial University of Newfoundland, St. John's, NL, Canada, ${ }^{24}$ Instituto Milenio de Oceanografía, Universidad de Concepción, Concepción, Chile, ${ }^{25}$ Department of Oceanography, University of Cape Town, Cape Town, South Africa, ${ }^{26}$ CSIRO Oceans and Atmosphere, Crawley, WA, Australia, ${ }^{27}$ School of Oceanography, University of Washington, Seattle, WA, United States, ${ }^{28}$ Fisheries and Oceans Canada, Dartmouth, NS, Canada, ${ }^{29}$ Atmosphere and Ocean Research Institute, The University of Tokyo, Kashiwa, Japan, ${ }^{30}$ Council for Scientific and Industrial Research, Cape Town, South Africa, ${ }^{31}$ LEGOS, CNRS, CNES, UPS, University of Toulouse, Toulouse, France, ${ }^{32}$ LOCEAN-IPSL, Sorbonne Université, Paris, France, ${ }^{33}$ Applied Physics Laboratory, University of Washington, Seattle, WA, United States, ${ }^{34}$ Department of Biological Sciences, University of Southern California, Los Angeles, CA, United States, ${ }^{35}$ South Australian Research and Development Institute (Aquatic Sciences), West Beach, SA, Australia, ${ }^{36}$ Instituto Geofísico del Perú, Lima, Peru, ${ }^{37}$ UNC Coastal Studies Institute, Wanchese, NC, United States, ${ }^{38}$ Tokyo University of Marine Science and Technology, Tokyo, Japan, ${ }^{39}$ Wellesley College, Wellesley, MA, United States, ${ }^{40}$ Graduate School of Oceanography, University of Rhode Island, Narragansett, RI, United States, ${ }^{41}$ Institute for Marine and Antarctic Studies, University of Tasmania, Hobart, TAS, Australia, ${ }^{42}$ Departamento de Ciencias de la Atmósfera y los Oceanos, University of Buenos Aires, Buenos Aires, Argentina, ${ }^{43}$ School of Ocean and Earth Science and Technology, University of Hawai'i at Mānoa, Honolulu, HI, United States, ${ }^{44}$ Departamento de Oceanografia, Universidade Federal de Santa Catarina, Florianópolis, Brazil, ${ }^{45}$ School of Mathematics and Statistics, University of New South Wales, Sydney, NSW, Australia, ${ }^{46}$ Department of Biological Sciences, University of South Carolina, Columbia, SC, United States, ${ }^{47}$ Consejo Nacional de Investigaciones Cientificas y Técnicas, Buenos Aires, Argentina, 


\begin{abstract}
${ }^{48}$ Department of Biological Sciences, University of Cape Town, Cape Town, South Africa, ${ }^{49}$ CSIRO Oceans and Atmosphere, Hobart, TAS, Australia, ${ }^{50}$ Ministry of Fisheries and Marine Resources, Swakopmund, Namibia, ${ }^{51}$ Department of Marine and Coastal Sciences, Rutgers University, New Brunswick, NJ, United States, ${ }^{52}$ Joint Institute for the Study of the Atmosphere and Ocean, University of Washington, Seattle, WA, United States, ${ }^{53}$ Institute of Oceanology, Chinese Academy of Sciences, Qingdao, China
\end{abstract}

Ocean boundary current systems are key components of the climate system, are home to highly productive ecosystems, and have numerous societal impacts. Establishment of a global network of boundary current observing systems is a critical part of ongoing development of the Global Ocean Observing System. The characteristics of boundary current systems are reviewed, focusing on scientific and societal motivations for sustained observing. Techniques currently used to observe boundary current systems are reviewed, followed by a census of the current state of boundary current observing systems globally. The next steps in the development of boundary current observing systems are considered, leading to several specific recommendations.

Keywords: western boundary current systems, eastern boundary current systems, ocean observing systems, time series, autonomous underwater gliders, drifters, remote sensing, moorings

\section{INTRODUCTION}

Ocean boundary current systems are where society most frequently interacts with the ocean through fisheries, maritime transportation, oil and gas extraction, and recreation. These systems are home to intense and highly variable oceanic currents that redistribute mass, heat, salt, biogeochemical constituents, plankton, and pollution. Circulation patterns also influence the life history, foraging behavior, and abundance of many marine species (e.g., Mansfield et al., 2017). The coastal and open oceans are linked through boundary current systems where events such as coastal upwelling, sea level anomalies, primary productivity, fisheries, and weather are propagated between domains by various processes (e.g., eddies, Rossby waves, and advection). Boundary currents may be broadly categorized as either western boundary currents (WBCs; Imawaki et al., 2013) or eastern boundary currents (EBCs) based on their governing dynamics. In each ocean basin, WBCs play a prominent role in the climate system by redistributing heat from the equator toward the poles, while EBCs are some of the most biologically productive regions in the world and respond dramatically to climate variability (Chavez et al., 2008; Chavez and Messié, 2009).

In our changing climate, shifting hydrological cycles and weather patterns are expected to strongly impact oceanic boundary current processes. Observational evidence for such shifts is beginning to appear. Wu et al. (2012) noted enhanced warming of subtropical WBCs and their extensions during the 20th century, possibly linked to their poleward shift or intensification. Changes in the stability of WBCs have also been noted, with instabilities in the Gulf Stream shifting westward (Andres, 2016), increasing influence of warm core rings on shelf circulation (Gawarkiewicz et al., 2018), and a trend toward greater instability in the East Australian and Agulhas Currents (Sloyan and O'Kane, 2015; Beal and Elipot, 2016).

Oceanic ecosystems are being exposed to increasing pressure from major stressors including warming, deoxygenation, fishing, and acidification. EBCs in particular are projected to be strongly impacted by these stressors (Bakun et al., 2015). For instance, the Peru-Chile (Humboldt) Current system (see the section Peru-Chile Current System), a highly productive EBC and a regional source of greenhouse gases, is naturally affected by upwelling of offshore waters with low oxygen and $\mathrm{pH}$ onto the continental shelf (Helly and Levin, 2004) and by periodic El Niño Southern Oscillation (ENSO) events that change the water mass distributions, oxygenation, and productivity (Chavez et al., 2008; Gutiérrez, 2016; Graco et al., 2017); further, stress could have significant consequences for the regional ecosystem. Similarly, changes in the Gulf Stream under global warming are predicted to negatively impact fisheries in the Gulf of Maine and on the New England Shelf (Saba et al., 2016; Claret et al., 2018).

Sustained, interdisciplinary observations in boundary current regions are required for a comprehensive ocean observing system. For OceanObs'09, Send et al. (2010) proposed a global network of sustained monitoring arrays as part of the Global Ocean Observing System (GOOS). Send et al. (2010) broadly defined the properties to be observed as (1) the transports of mass, heat, and freshwater needed for monitoring the global climate in conjunction with basin-scale measurements and (2) local boundary-specific properties including eddy activity, changes in potential vorticity, air-sea interactions (Cronin et al., 2019), ecosystem dynamics, and biogeochemistry. More recently, the 2017 GOOS workshop on "Implementation of Multi-Disciplinary Sustained Ocean Observations" (IMSOO; Palacz et al., 2017) focused, in part, on how to proceed with the development of a truly multidisciplinary boundary current observing system, building upon the more physical and climatefocused plans of Send et al. (2010). In particular, it was noted that observations that resolve along-boundary variability are needed in order to understand climate impacts on various societally relevant uses of boundary current systems (e.g., fisheries). The need to maintain a global perspective that targets all boundary current systems has been repeatedly recognized (Send et al., 2010; Palacz et al., 2017), particularly in developing nations where fisheries can be centrally important (Palacz et al., 2017). To that end, IMSOO planned to review established observing systems in 
the California Current System and East Australian Current in order to develop a blueprint for an adaptive, multidisciplinary observing system with relocatable subsystems to capture finer scales (Palacz et al., 2017).

Oceanic boundaries present a variety of challenges for sustained observing systems (Send et al., 2010). With strong flows in relatively shallow areas, spatial scales of $\mathrm{O}(1)-\mathrm{O}(10) \mathrm{km}$, and temporal scales often shorter than a few days (e.g., He et al., 2010; Todd et al., 2013; Rudnick et al., 2017), the broad-scale (i.e., Argo and gridded satellite altimetry) and long-duration (e.g., HOTS, BATS, Station P, and CARIACO) measurements that constitute the observing system for the ocean interior are insufficient for boundary current systems. Multiple observing strategies are needed to measure the essential ocean variables (EOVs; Table 1) that can be used to understand and track the physical and biogeochemical processes of interest within boundary currents (Lindstrom et al., 2012). The optimal combination of observing methods will depend upon characteristics unique to each region. Send et al. (2010) noted that an additional challenge in observing boundary current systems is that there is no well-defined offshore "end" of a boundary current but rather a temporally and spatially variable transition to the interior. At the same time, oceanic boundaries generally lie within exclusive economic zones (EEZs), so the implementation of observing systems requires significant international cooperation.

The overarching purpose of this review is to examine the current state of the boundary current system component of GOOS, updating and building upon the OceanObs'09 review of Send et al. (2010). The section Scientific and Societal Needs considers the scientific and societal needs that comprehensive boundary current observing systems must fulfill. The section Observing Techniques reviews how various observing techniques are employed in boundary currents, highlighting key scientific advances from each platform. The section Current Status of Regional Boundary Current Observing Systems surveys the current state of boundary current observing systems globally. Table 2 provides a comprehensive collection of publications and datasets from the past decade, organized by region and platform. The section Future Outlook then considers the future development of boundary current observing systems. The section Summary Recommendations concludes with specific recommendations to promote development of a comprehensive global network of boundary current observing systems.

\section{SCIENTIFIC AND SOCIETAL NEEDS}

The Framework for Ocean Observing (Lindstrom et al., 2012), developed after OceanObs'09, recommended that ocean observing systems (1) be "fit for purpose" and driven by "scientific inquiry and societal needs"; (2) include physical, biogeochemical, and biological observations; (3) operate collaboratively based on established best practices; (4) balance innovation with stability; (5) promote alignment of independent user groups; (6) build on existing infrastructure as much as possible; and (7) provide maximum benefit to all users from each observation. Here, we present the scientific and societal needs that that should be met by comprehensive observing of oceanic boundary current systems, focusing on three broad categories: ecosystems and biogeochemistry (see the section Ecosystems and Biogeochemistry), weather and climate (see the section Climate and Weather), and connections between the shelves and deep ocean (see the section Shelf-Deep Ocean Connections).

\section{Ecosystems and Biogeochemistry}

Boundary current systems play an important role in carbon cycling through the physical and biological carbon pumps. WBCs are major sites of air-sea $\mathrm{CO}_{2}$ exchange (e.g., Rodgers et al., 2008; Gorgues et al., 2010; Nakano et al., 2011) and have been shown to exhibit enhanced contemporary carbon uptake from the atmosphere (Takahashi et al., 2009; Landschützer et al., 2014). $\mathrm{WBC} \mathrm{CO}_{2}$ uptake is driven by a large $\mathrm{pCO}_{2}$ disequilibrium with the overlying mid-latitude atmosphere, which is due to the rapid cooling of low Revelle factor waters advected from the tropics to midlatitudes. Since thick subtropical mode waters form during wintertime convection on the equatorward edges of the WBC extensions, the mode waters are key carbon sinks (e.g., Bates et al., 2002; Gruber et al., 2002; Ito and Follows, 2003; Levine et al., 2011; DeVries, 2014; Iudicone et al., 2016) and have been the target of detailed observational carbon studies (Andersson et al., 2013; Palevsky and Quay, 2017). However, it is still unclear how variability in the rate of mode water formation might impact ocean carbon uptake in these regions and what impacts these changes might have on the biological pump and higher trophic levels (e.g., fisheries). In the Kuroshio Extension region, there is evidence that the majority of carbon exported from the surface ocean during the spring and summer productive season is subsequently respired in the seasonal thermocline and ventilated back to the atmosphere during wintertime mode water formation (Palevsky et al., 2016; Fassbender et al., 2017b; Palevsky and Quay, 2017; Bushinsky and Emerson, 2018). The Southern Hemisphere WBCs are chronically undersampled, particularly during winter, leading to significant uncertainty in their contribution to the global ocean carbon sink.

Boundary current systems are highly productive regions (Chavez et al., 2008). The mechanisms of nutrient supply to surface waters that drive increased primary productivity differ among EBC and WBC systems, but their global contributions are similar (Chavez and Toggweiler, 1995). In EBC systems, the dominant source of nutrients is coastal upwelling (Chavez and Messié, 2009), while in WBC systems, geostrophic- and eddydriven upwelling predominates (Pelegrí and Csanady, 1991). Nutrient streams are important in the Gulf Stream (Pelegrí and Csanady, 1991; Pelegrí et al., 1996; Williams et al., 2006, 2011; Palter and Lozier, 2008) and the Kuroshio (Guo et al., 2012, 2013), transporting subsurface positive nitrate anomalies, which are delivered to the photic zone primarily by mesoscale and submesoscale processes (Nagai and Clayton, 2017; Honda et al., 2018; Yamamoto et al., 2018; Zhang et al., 2018). Nutrient cycles and drivers have not yet been studied in WBC systems of the Southern Hemisphere.

Western boundary currents are also enriched in micronutrients (e.g., Fe, Zn, Cd, Co, and Ni) from land-sea exchanges. They ultimately feed open ocean surface waters and, at lower 
TABLE 1 | List of essential ocean variables from www.goosocean.org/eov with indications of which observing platforms are able to sample each variable.

\begin{tabular}{|c|c|c|c|c|c|c|c|c|c|c|c|c|}
\hline & & $\begin{array}{c}\text { Autonomous } \\
\text { Underwater } \\
\text { Gliders }\end{array}$ & Drifters & Moorings & $\begin{array}{l}\text { Profiling } \\
\text { Floats }\end{array}$ & $\begin{array}{l}\text { Satellite } \\
\text { Remote } \\
\text { Sensing }\end{array}$ & $\begin{array}{l}\text { High- } \\
\text { Frequency } \\
\text { Radar }\end{array}$ & $\begin{array}{c}\text { Ships of } \\
\text { Opportunity }\end{array}$ & $\begin{array}{l}\text { Research } \\
\text { Ships }\end{array}$ & $\begin{array}{c}\text { Expendable } \\
\text { Bathythermographs }\end{array}$ & $\begin{array}{l}\text { Inverted } \\
\text { Echo } \\
\text { Sounders }\end{array}$ & $\begin{array}{c}\text { Submarine } \\
\text { Cables }\end{array}$ \\
\hline \multirow[t]{11}{*}{ Physics } & Sea state & & $x$ & $x$ & & $x$ & & $x$ & $x$ & & & \\
\hline & Ocean surface stress & & & $x$ & & $x$ & & & $x$ & & & \\
\hline & Sea ice & & & $x$ & & $x$ & & & $x$ & & & \\
\hline & Sea surface height & & & & & $x$ & & & & & & \\
\hline & Sea surface temperature & $x$ & $x$ & $x$ & $x$ & $x$ & & $x$ & $x$ & $x$ & & \\
\hline & Subsurface temperature & $x$ & & $x$ & $x$ & & & & $x$ & $x$ & $x$ & \\
\hline & Surface currents & $x$ & $x$ & $x$ & $x$ & $x$ & $x$ & $x$ & $x$ & & & \\
\hline & Subsurface currents & $x$ & & $x$ & $x$ & & & $x$ & $x$ & & $x$ & $x$ \\
\hline & Sea surface salinity & $x$ & & $x$ & $x$ & $x$ & & $x$ & $x$ & & & \\
\hline & Subsurface salinity & $x$ & & $x$ & $x$ & & & & $x$ & & & \\
\hline & Ocean surface heat flux & & & $x$ & & $x$ & & & $x$ & & & \\
\hline \multirow[t]{9}{*}{ Biogeochemistry } & Oxygen & $x$ & & $x$ & $x$ & & & $x$ & $x$ & & & \\
\hline & Nutrients & $x$ & & $x$ & & & & $x$ & $x$ & & & \\
\hline & Inorganic carbon & & & $x$ & & & & $x$ & $x$ & & & \\
\hline & Transient tracers & & & $x$ & & & & & $x$ & & & \\
\hline & Particulate matter & $x$ & & $x$ & & & & $x$ & $x$ & & & \\
\hline & Nitrous oxide & & & $x$ & & & & & $x$ & & & \\
\hline & Stable carbon isotopes & & & & & & & $x$ & $x$ & & & \\
\hline & Dissolved organic carbon & & & & & & & & $x$ & & & \\
\hline & Ocean color & & & & & $x$ & & & & & & \\
\hline \multirow[t]{11}{*}{$\begin{array}{l}\text { Biology and } \\
\text { Ecosystems }\end{array}$} & $\begin{array}{l}\text { Phytoplankton biomass } \\
\text { and diversity }\end{array}$ & $x$ & $x$ & $x$ & $x$ & $x$ & & $x$ & $x$ & & & \\
\hline & $\begin{array}{l}\text { Zooplankton biomass and } \\
\text { diversity }\end{array}$ & $x$ & & $x$ & & & & & $x$ & & & \\
\hline & $\begin{array}{l}\text { Fish abundance and } \\
\text { distribution }\end{array}$ & $x$ & & $x$ & & & & & $x$ & & & \\
\hline & $\begin{array}{l}\text { Marine turtles, birds, and } \\
\text { mammals abundance and } \\
\text { distribution }\end{array}$ & & & $x$ & & & & & $x$ & & & \\
\hline & $\begin{array}{l}\text { Hard coral cover and } \\
\text { composition }\end{array}$ & & & & & & & & $x$ & & & \\
\hline & Seagrass cover & & & & & & & & $x$ & & & \\
\hline & Macroalgal canopy cover & & & & & & & & $x$ & & & \\
\hline & Mangrove cover & & & & & & & & $x$ & & & \\
\hline & Ocean sound & $x$ & & $x$ & & & & & $x$ & & & \\
\hline & $\begin{array}{l}\text { Microbe biomass and } \\
\text { diversity (*emerging) }\end{array}$ & & & & & & & & $x$ & & & \\
\hline & $\begin{array}{l}\text { Benthic invertebrate } \\
\text { abundance and distribution } \\
\left({ }^{*} \text { emerging) }\right.\end{array}$ & & & & & & & & $x$ & & & \\
\hline
\end{tabular}


TABLE 2 | Examples of sustained boundary current observing efforts since 2009

\begin{tabular}{|c|c|c|}
\hline Region & Platform & References \\
\hline Agulhas & Gliders & Krug et al., 2017 \\
\hline Agulhas & Moorings & $\begin{array}{l}\text { Beal et al., 2015; Elipot and Beal, 2015, 2018; Beal and } \\
\text { Elipot, 2016; Kersalé et al., } 2018\end{array}$ \\
\hline Agulhas & XBT & \\
\hline Angola Current & Moorings & Kopte et al., 2017, 2018; Tchipalanga et al., 2018 \\
\hline Benguela Current & Argo & Pegliasco et al., 2015; Majumder and Schmid, 2018 \\
\hline Benguela Current & Research Vessels & \\
\hline Benguela Current & Moorings & Junker et al., 2017a, 2019 \\
\hline Brazil Current & Drifters & Oliveira et al., 2009 \\
\hline Brazil Current & Moorings & Meinen et al., 2017, 2018 \\
\hline Brazil Current & Research Vessels & Valla et al., 2018 \\
\hline Brazil Current & XBT & $\begin{array}{l}\text { Garzoli et al., 2012; Mata et al., 2012; Lima et al., 2016; } \\
\text { Majumder et al., } 2019\end{array}$ \\
\hline Brazil Current & Argo & Schmid and Majumder, 2018 \\
\hline North Brazil Undercurrent & Moorings & Hummels et al., 2015 \\
\hline California Current System & Drifters & \\
\hline California Current System & Gliders & $\begin{array}{l}\text { Todd et al., 2011a,b, 2012; Pelland et al., 2013; Mazzini } \\
\text { et al., 2014; Johnston and Rudnick, 2015; Adams et al., } \\
\text { 2016; Zaba and Rudnick, 2016; Rudnick et al., 2017; } \\
\text { Henderikx Freitas et al., } 2018\end{array}$ \\
\hline California Current System & High-Frequency Radar & Kim, 2010; Kim et al., 2011; Kim and Kosro, 2013; \\
\hline California Current System & Moorings & $\begin{array}{l}\text { Nam et al., 2011; Harris et al., 2013; Ohman et al., 2013; } \\
\text { Sutton et al., 2014, 2016; Fassbender et al., 2016, 2017a, } \\
\text { 2018; Siedlecki et al., } 2016\end{array}$ \\
\hline California Current System & Research Vessels & $\begin{array}{l}\text { Juranek et al., 2009; Fassbender et al., 2011, 2017a, 2018; } \\
\text { Alin et al., 2012; Bednaršek et al., 2014, 2017, 2018; } \\
\text { McClatchie, 2014; Reum et al., 2014, 2016; Feely et al., } \\
\text { 2016, 2018; McClatchie et al., } 2016\end{array}$ \\
\hline California Current System & Ship of Opportunity & Fassbender et al., 2018 \\
\hline California Current System & XBT & Douglass et al., 2010; Auad et al., 2011 \\
\hline California Current System & Argo & Pegliasco et al., 2015 \\
\hline East Auckland Current & XBT & Bowen et al., 2017; Fernandez et al., 2018 \\
\hline
\end{tabular}

Data source

http://www.aoml.noaa.gov/phod/research/moc/samoc/sam/;

http://www.aoml.noaa.gov/phod/hdenxbt/index.php;

http://www-hrx.ucsd.edu

https://doi.org/10.1594/PANGAEA.868684;

https://doi.org/10.1594/PANGAEA.886492

http://www.argodatamgt.org

http://www.mfmr.gov.na/;

http://data.ocean.gov.za/pub/DATA

https://doi.org/10.1594/PANGAEA.871251 (Junker et al., 2017b); https://doi.org/10.1594/PANGAEA.871253 (Junker et al., 2017c); https://doi.org/10.1594/PANGAEA.872098 (Junker et al., 2017d); https://doi.org/10.1594/PANGAEA.872099 (Junker et al., 2017e);

https://www.ocims.gov.za

http://www.aoml.noaa.gov/phod/gdp/index.php

http://www.aoml.noaa.gov/phod/SAMOC_international/samoc_data.php

http://www.aoml.noaa.gov/phod/hdenxbt/index.php

http://argodatamgt.org

https://doi.org/10.1594/PANGAEA.886415;

https://doi.org/10.1594/PANGAEA.886420;

https://doi.org/10.1594/PANGAEA.886426;

https://doi.org/10.1594/PANGAEA.886428

http://www.aoml.noaa.gov/phod/gdp/index.php

https://spraydata.ucsd.edu/projects/CUGN (Rudnick, 2016a);

https://spraydata.ucsd.edu/projects/CORC (Send, 2018);

http://www.oceanobservatories.org/

http://www.oceanobservatories.org/;

https://www.nodc.noaa.gov/ocads/oceans/Coastal/north_america_west.html;

$\mathrm{ftp}: / /$ data.ndbc.noaa.gov/data/oceansites/

http://www.calcofi.org;

https://www.nodc.noaa.gov/ocads/oceans/Coastal/north_america_west.html

http://www-hrx.ucsd.edu

http://www.argodatamgt.org

http://www-hrx.ucsd.edu 


\begin{tabular}{|c|c|c|c|}
\hline Region & Platform & References & Data source \\
\hline East Australian Current & Argo & Zilberman et al., 2014, 2018 & $\begin{array}{l}\text { https://portal.aodn.org.au; } \\
\text { http://www.argodatamgt.org }\end{array}$ \\
\hline East Australian Current & Drifters & Brassington, 2010; Brassington et al., 2011 & http://www.aoml.noaa.gov/phod/gdp/index.php \\
\hline East Australian Current & Gliders & $\begin{array}{l}\text { Roughan et al., 2015; Schaeffer and Roughan, 2015; } \\
\text { Schaeffer A. et al., 2016; Schaeffer A. M. et al., } 2016\end{array}$ & $\begin{array}{l}\text { https://portal.aodn.org.au; } \\
\text { http://imos.org.au/facilities/aodn/ }\end{array}$ \\
\hline East Australian Current & High-Frequency Radar & $\begin{array}{l}\text { Roughan et al., 2015; Archer et al., 2017a, 2018; Mantovanelli } \\
\text { et al., 2017; Schaeffer et al., 2017; Wyatt et al., } 2018\end{array}$ & $\begin{array}{l}\text { www.oceanography.unsw.edu.au/radar.html } \\
\text { https://portal.aodn.org.au }\end{array}$ \\
\hline East Australian Current & Moorings & $\begin{array}{l}\text { Roughan et al., 2013, 2015; Schaeffer et al., 2013, 2014; Lynch } \\
\text { et al., 2014; Sloyan et al., 2016; Schaeffer and Roughan, 2017; } \\
\text { Alford et al., } 2017\end{array}$ & https://portal.aodn.org.au \\
\hline East Australian Current & XBT & $\begin{array}{l}\text { Hill et al., 2011; Suthers et al., 2011; Sloyan and O'Kane, 2015; } \\
\text { Zilberman et al., 2018; }\end{array}$ & $\begin{array}{l}\text { https://portal.aodn.org.au; } \\
\text { http://www-hrx.ucsd.edu }\end{array}$ \\
\hline Gulf Stream & Gliders & $\begin{array}{l}\text { Todd et al., 2016, 2018b; Todd, 2017; Todd and Locke-Wynn, } \\
\text { 2017; Gula et al., } 2019\end{array}$ & https://spraydata.ucsd.edu/projects/GS (Todd and Owens, 2016) \\
\hline Gulf Stream & High-Frequency Radar & Parks et al., 2009; Archer et al., 2015, 2017b; Haines et al., 2017 & http://cordc.ucsd.edu/projects/mapping/maps/ \\
\hline Gulf Stream & Moorings & $\begin{array}{l}\text { Weller et al., 2012; Bigorre et al., 2013; Bane et al., 2017; } \\
\text { Lowcher et al., } 2017\end{array}$ & http://www.whoi.edu/science/PO/linew/ \\
\hline Gulf Stream & Research Vessels & Meinen et al., 2010 & $\begin{array}{l}\text { http://www.aoml.noaa.gov/phod/floridacurrent/; } \\
\text { http://www.whoi.edu/science/PO/linew/ }\end{array}$ \\
\hline Gulf Stream & Ship of Opportunity & Rossby et al., 2010; Wang et al., 2010 & http://oleander.bios.edu/ \\
\hline Gulf Stream & XBT & Domingues et al., 2018 & http://www.aoml.noaa.gov/phod/hdenxbt/index.php \\
\hline Gulf Stream & Submarine Cable & Meinen et al., 2010 & http://www.aoml.noaa.gov/phod/floridacurrent/ \\
\hline Kuroshio & Argo & $\begin{array}{l}\text { Sugimoto and Hanawa, 2014; Oka et al., 2015; Bushinsky et al., } \\
\text { 2016; Inoue et al., 2016a,b; Fassbender et al., 2017b; Bushinsky } \\
\text { and Emerson, } 2018\end{array}$ & http://www.argodatamgt.org \\
\hline Kuroshio & HF Radar & Yang et al., 2015 & \\
\hline Kuroshio & Drifters & Vélez-Belchí et al., 2013; Gordon et al., 2014; Andres et al., 2015 & http://www.aoml.noaa.gov/phod/gdp/index.php \\
\hline Kuroshio & Gliders & $\begin{array}{l}\text { Rudnick et al., 2011, 2013; Johnston et al., 2013; Rainville et al., } \\
\text { 2013; Lien et al., 2014, } 2015\end{array}$ & \\
\hline Kuroshio & Moorings & $\begin{array}{l}\text { Bond et al., 2011; Cronin et al., 2013, 2015; Hu et al., 2013; } \\
\text { Wada et al., 2013; Lien et al., 2014, 2015; Sutton et al., 2014, } \\
\text { 2016, 2017; Zhou et al., 2014; Chen et al., 2015; Yang et al., } \\
\text { 2015; Zhang et al., 2015; Fassbender et al., 2017b; Honda et al., } \\
\text { 2018 }\end{array}$ & $\begin{array}{l}\text { https://www.nodc.noaa.gov/ocads/oceans/Moorings/Pacific.html; } \\
\text { ftp://data.ndbc.noaa.gov/data/oceansites }\end{array}$ \\
\hline Kuroshio & Research Vessels & $\begin{array}{l}\text { Yasunaka et al., 2013, 2014; Sugimoto and Hanawa, 2014; } \\
\text { Nakano et al., 2015; Yang et al., 2015; Oka et al., } 2018\end{array}$ & \\
\hline Kuroshio & Ship of Opportunity & Palevsky et al., 2016; Palevsky and Quay, 2017 & \\
\hline Kuroshio & XBT & Nagano et al., 2016 & http://www-hrx.ucsd.edu \\
\hline Labrador Current & Gliders & deYoung et al., 2018; Howatt et al., 2018 & \\
\hline
\end{tabular}




\begin{tabular}{|c|c|c|c|}
\hline Region & Platform & References & Data source \\
\hline Labrador Current & Moorings & deYoung et al., 2018 & \\
\hline Leeuwin Current & Gliders & Pattiaratchi et al., 2011 & \\
\hline Leeuwin Current & Argo & Furue et al., 2017 & http://www.argodatamgt.org \\
\hline Leeuwin Current & High-Frequency Radar & Mihanoviæ et al., 2016 & \\
\hline Leeuwin Current & Moorings & Lynch et al., 2014; & \\
\hline Loop Current (Gulf of Mexico) & Gliders & Gopalakrishnan et al., 2013; Rudnick et al., 2015a; Todd et al., 2016 & https://spraydata.ucsd.edu/projects/GoM (Rudnick, 2017) \\
\hline Malvinas Current & Argo & Artana et al., 2016, 2018b & http://www.argodatamgt.org \\
\hline Malvinas Current & Moorings & $\begin{array}{l}\text { Valla and Piola, 2015; Ferrari et al., 2017; Artana et al., 2018a; Paniagua } \\
\text { et al., } 2018\end{array}$ & $\begin{array}{l}\text { https://doi.org/10.17882/51492 (Saraceno et al., 2017); } \\
\text { https://www.seanoe.org/data/00403/51479/; } \\
\text { https://www.seanoe.org/data/00403/51492/ }\end{array}$ \\
\hline Mediterranean & Gliders & Heslop et al., 2012 & \\
\hline Middle Atlantic Bight & Gliders & $\begin{array}{l}\text { Castelao et al., 2010; Todd et al., 2013; Zhang and Gawarkiewicz, } \\
\text { 2015; Dever et al., 2016; Gawarkiewicz et al., } 2018\end{array}$ & http://www.oceanobservatories.org/ \\
\hline Middle Atlantic Bight & Moorings & Chen et al., 2018; Gawarkiewicz et al., 2018; Zhang and Partida, 2018 & http://www.oceanobservatories.org/ \\
\hline Mindanao & Gliders & Schönau et al., 2015; Schönau and Rudnick, 2017 & \\
\hline Mindanao & Moorings & Zhang et al., 2014; Hu et al., 2016; Wang et al., 2017 & \\
\hline NE Atlantic (Subpolar) & Gliders & Houpert et al., 2018 & \\
\hline Canary Current System & Drifters & Menna et al., 2016 & \\
\hline Canary Current System & Gliders & Karstensen et al., 2017; Kolodziejczyk et al., 2018 & \\
\hline Canary Current System & Mooring & Nowald et al., 2015 & http://www.fixo3.eu \\
\hline Canary Current System & Research Vessels & $\begin{array}{l}\text { Steinfeldt et al., 2015; Capet et al., 2017; Klenz et al., 2018; Machu } \\
\text { et al., 2019; Thomsen et al., } 2019\end{array}$ & \\
\hline Canary Current System & Argo & Pegliasco et al., 2015 & http://www.argodatamgt.org \\
\hline $\begin{array}{l}\text { NW Atlantic Deep Western } \\
\text { Boundary Current }\end{array}$ & Moorings & $\begin{array}{l}\text { Fischer et al., 2004, 2010; Dengler et al., 2006; Johns et al., 2008, 2011; } \\
\text { Bacon and Saunders, 2010; Toole et al., 2017; Zantopp et al., } 2017\end{array}$ & $\begin{array}{l}\text { http://www.whoi.edu/science/PO/linew/; } \\
\text { www.oceansites.org }\end{array}$ \\
\hline $\begin{array}{l}\text { NW Atlantic Deep Western } \\
\text { Boundary Current }\end{array}$ & Research Vessels & van Sebille et al., 2011 & http://www.whoi.edu/science/PO/linew/ \\
\hline Oyashio & Research Vessels & Kuroda et al., 2015, 2017 & http://tnfri.fra.affrc.go.jp/seika/a-line/a-line_index2.html \\
\hline Peru-Chile Current System & Gliders & Pietri et al., 2013, 2014; Pizarro et al., 2016 & \\
\hline Peru-Chile Current System & Argo & Pegliasco et al., 2015 & http://www.argodatamgt.org \\
\hline Peru-Chile Current System & Research Vessels & Espinoza et al., 2017; Graco et al., 2017; Grados et al., 2018 & \\
\hline Peru-Chile Current System & Research Vessels & Escribano and Morales, 2012; Schneider et al., 2016 & http://www.antares.ws \\
\hline Solomon Sea & Argo & Zilberman et al., 2013 & http://www.argodatamgt.org \\
\hline Solomon Sea & Gliders & Davis et al., 2012 & https://spraydata.ucsd.edu/projects/Solomon (Davis, 2016) \\
\hline Solomon Sea & Moorings & Ganachaud et al., 2014, 2017; Alberty, 2018 & $\begin{array}{l}\text { http://www.solomonseaoceanography.org/; } \\
\text { https://doi.org/10.6075/J09W0CS2 (Cravatte et al., 2019); } \\
\text { https://doi.org/10.6075/J0639N12 (Alberty et al., 2019) }\end{array}$ \\
\hline Solomon Sea & XBT & Zilberman et al., 2013 & http://www-hrx.ucsd.edu \\
\hline Somali Current & Drifters & Beal et al., 2013; Centurioni et al., 2017 & http://www.aoml.noaa.gov/phod/gdp/index.php \\
\hline South China Sea & Drifters & Centurioni et al., 2009 & http://www.aoml.noaa.gov/phod/gdp/index.php \\
\hline
\end{tabular}

Included are in situ and land-based observing efforts extending longer than 1 year in the period 2009-2018. Key references since 2009 and sources for publicly available data are included. 
latitudes, the equatorial undercurrent, where these micronutrients are critical in maintaining high levels of productivity. For instance, iron transported by boundary currents in the western Pacific feeds into the Pacific Equatorial Undercurrent, which then supplies iron to the eastern equatorial Pacific (e.g., Mackey et al., 2002; Ryan et al., 2006). In the North Atlantic, Gulf Stream rings supply iron to the subtropical gyre (e.g., Conway et al., 2018). Subpolar WBCs such as the Oyashio and Malvinas Currents are also likely to transport waters enriched in nutrients; wind-driven and shelfbreak upwelling then supplies nutrients to the euphotic layers, enhancing biological productivity (Matano and Palma, 2008; Ito et al., 2010; Valla and Piola, 2015). Locations at which subtropical and subpolar WBCs meet provide ideal environments for biological production, as warm subtropical waters converge with nutrient-rich subpolar waters (Brandini et al., 2000).

The upwelling of deep, poorly ventilated water masses rich in inorganic nutrients and $\mathrm{CO}_{2}$ and low in $\mathrm{O}_{2}$ make EBCs areas of high air-sea fluxes, and the sensitivity of the upwelling process to climate variability contributes to large interannual and decadal scale changes in the magnitude of these fluxes (Friederich et al., 2002; Brady et al., 2019). EBCs also exhibit strong cross-shore gradients in fluxes; narrow strips of the nearshore ocean act as intense sources of $\mathrm{CO}_{2}$ to the atmosphere, while the abundance of nutrients in these upwelled waters facilitates primary production that results in net uptake of $\mathrm{CO}_{2}$ (Hales et al., 2005). The supply of poorly ventilated waters combined with high levels of organic-matter remineralization resulting from intense primary production in surface waters can trigger periods of anoxia and low pH in shelf waters (Feely et al., 2008; Zhang et al., 2010) with severe consequences for demersal and pelagic ecosystems (Chan et al., 2008; Monteiro et al., 2008; Bertrand et al., 2011).

Boundary currents play an important role in ocean ecosystems across all trophic levels. The intense levels of primary production associated with EBCs support rich ecosystems with relatively short food chains, and these systems provide at least $20 \%$ of the world's wild-caught fish despite covering less than $1 \%$ of the global ocean (Chavez and Messié, 2009). WBCs and EBCs are also oceanic regions where coastal and open ocean ecosystems are brought together and interact. Modeling studies have suggested that boundary currents are hotspots of microbial biodiversity (Barton et al., 2010; Clayton et al., 2013). This has been supported in the Kuroshio Extension by some in situ surveys (Clayton et al., 2014, 2017). At the other end of the trophic spectrum, recent work combining tag data and satellite altimetry data has shown that white sharks (Carcharodon carcharias) actively occupy warm-core anticyclonic eddies in the Gulf Stream (Gaube et al., 2018). The warmer waters in these mesoscale features allow the sharks to reduce the physiological costs of thermoregulation in cold water, thereby making prey more accessible and energetically more profitable. Similarly, the location of the Kuroshio axis and associated changes in water temperature have been shown to influence the behavior of juvenile Pacific bluefin tuna (Thunnus orientalis; Fujioka et al., 2018). In the Southern Benguela EBC upwelling system, the coastal, wind-driven upwelling along the southwest African coast supports planktonic food supplies for young pelagic fish, while the temperate Agulhas
Bank shelf region provides suitable spawning habitat for large communities of fish including in particular anchovy and sardine (Hutchings et al., 2009a). Likewise, southern elephant seals feed along the intense fronts and eddies in the Brazil/Malvinas Confluence (Campagna et al., 2006). WBCs are also known to play an important role in the migration of other coastal and pelagic organisms, such as eels (Shinoda et al., 2011; Rypina et al., 2014) and salmon (Wagawa et al., 2016).

Marine heat waves (MHWs) are strongly linked with boundary current systems. For instance, the exceptional and devastating MHW event off Western Australia during summer of 2010/2011 was caused by a strengthening of the Leeuwin Current associated with La Niña conditions (Pearce and Feng, 2013; Feng et al., 2015), a 2014-2015 MHW had unprecedented impacts on the California Current System (Di Lorenzo and Mantua, 2016; Zaba and Rudnick, 2016), and an MHW in 2015-2016 impacted the Tasman Sea (Oliver et al., 2017). These discrete, prolonged periods of anomalously warm waters at particular locations (Hobday et al., 2016) can stress ecosystems, leading to increased mortality of marine species, closing of commercial and recreational fisheries, and coral bleaching (Cavole et al., 2016; Stuart-Smith et al., 2018). The addition of other stressors such as ocean acidification and deoxygenation, which are projected to increase in future warming scenarios, could amplify the ecosystem impacts of MHWs. Sustained physical and biogeochemical observations are necessary to improve forecasts of the frequency and magnitude of MHWs, as well as to assess the risk and vulnerability of marine ecosystems to extreme climate events (Frölicher and Laufkötter, 2018).

\section{Climate and Weather}

Boundary currents are an integral part of the global climate system as they redistribute heat and facilitate carbon uptake from the atmosphere (see the section Ecosystems and Biogeochemistry). In the Atlantic, boundary currents are key components of the Atlantic Meridional Overturning Circulation (AMOC; FrajkaWilliams et al., 2019). Low-latitude WBCs that connect the subtropics to the equator at thermocline and intermediate levels are important contributors to the mass and heat budgets of the equatorial oceans, which influence climate modes such as ENSO (Lengaigne et al., 2012). Low-latitude WBCs are also suspected to contribute to the decadal modulation of the equatorial thermocline background state (e.g., Lee and Fukumori, 2003). Sustained monitoring of WBC transports would be particularly useful for climate and seasonal-to-decadal forecast centers (see Smith et al., 2019).

As climate change progresses, boundary current systems are likely to undergo further significant changes. Subtropical WBCs and their extensions are the fastest warming regions of the world ocean (Wu et al., 2012; Yang et al., 2016). Climate model simulations have suggested that western boundary current extensions may move poleward under climate change (Saba et al., 2016). This poleward expansion of energetic WBCs may impact extreme temperatures and marine species migration (Johnson et al., 2011), as well as enhance eddy activity regionally (e.g., Oliver et al., 2015). While low-resolution climate models suggest strengthening and poleward migration of several of these currents 
under climate change, particularly in the Southern Hemisphere (Sen Gupta et al., 2012; Hu et al., 2015; Pontes et al., 2016), studies leveraging in situ velocity and satellite data suggest no significant increase in their transports since the early 1990s (Rossby et al., 2014; Beal and Elipot, 2016). This discrepancy motivates the collection of long-term measurements of baroclinic changes in boundary currents (i.e., subsurface temperature and salinity properties), as well as the vertical structure of the velocity, in order to understand and predict future changes.

In addition, ocean warming and a magnified hydrological cycle could drive significant changes in shelf ocean stratification, while changes to wind forcing will directly alter rates of upwelling. These ocean circulation processes, and meteorological forcing at the scales that impact upwelling, are poorly represented in climate models (Richter, 2015; Zuidema et al., 2016). Thus, we have little capability to predict how upwelling, winds, and other physical drivers of ocean property exchanges at the coastal/open ocean boundary will change in the future. The impact these changes will have on coastal ecosystems is simply unknown.

Detection and attribution of global sea level variability has improved considerably in the last decade (Cazenave et al., 2014; Marzeion et al., 2014). The location and strength of WBCs considerably influence the mean local sea level (Domingues et al., 2016; Archer et al., 2017b), possibly accounting for part of the mismatch between forecasts and observations of sea level at the coast (Ezer, 2015). Relationships between large-scale wind anomalies, basin-wide sea surface height (SSH), and WBCs (e.g., Boening et al., 2012; Volkov et al., 2019) suggest that observations of current strength and oceanic teleconnections can be used to improve seasonal to decadal coastal sea level forecasts, leading to improved assessments of impacts on infrastructure and groundwater quality (Slangen et al., 2014; Park and Sweet, 2015).

Boundary current systems influence synoptic and longer scale weather patterns. Large upper ocean heat content within WBCs can fuel development and intensification of tropical cyclones (Bright et al., 2002; Wu et al., 2008; Nguyen and Molinari, 2012; Galarneau et al., 2013). Strong sea surface temperature (SST) gradients across WBCs, particularly during winter months, destabilize the atmospheric boundary layer, fueling the midlatitude storm tracks and atmospheric blocking frequency, which in turn impact regional climate (Kelly et al., 2010; Nakamura, 2012; O’Reilly and Czaja, 2015; O’Reilly et al., 2016; Révelard et al., 2016; Ma et al., 2017). For instance, a weaker Gulf Stream SST front leads to a decrease in cold and dry spells over Europe (O'Reilly et al., 2016), while a sharper SST front in the Kuroshio Extension increases cyclogenesis and shifts the storm track northward, causing warming over eastern Asia and the western United States that can reduce snow cover by $4-6 \%$ (O'Reilly and Czaja, 2015; Révelard et al., 2016). Variability in the warm waters of the Agulhas influences summer rainfall over parts of South Africa (Jury et al., 1993; Nkwinkwa Njouodo et al., 2018). In EBC systems, SST minima are collocated with maxima in sea level pressure that are in turn associated with alongshore wind stress, wind stress curl, and cloud cover along the boundary (Sun et al., 2018), suggesting coupling with the full HadleyWalker tropical atmospheric circulation, though the details of such coupling remain an open question.
Accurate weather and climate forecasting thus requires accurate representation of boundary current systems. However, most of the current ocean reanalyses used to initialize the monthly, seasonal, and decadal forecasts exhibit large errors in the boundary currents (Rouault et al., 2003; Valdivieso et al., 2017), hampering forecast performance. Coupled climate models, such as those used in the Intergovernmental Panel on Climate Change reports, also exhibit large deficiencies in boundary current regions (e.g., Siqueira and Kirtman, 2016; Zuidema et al., 2016), including warm SST biases in EBCs (e.g., Large and Danabasoglu, 2006). Current modeling and data assimilation capabilities are insufficient to fully represent boundary currents at the small spatial scales needed for forecasting. Subramanian et al. (2019) further consider how observing efforts, including within boundary currents, can contribute to improved subseasonal-toseasonal forecasting.

\section{Shelf-Deep Ocean Connections}

The coastal ocean and nearshore zones support a broad range of human activities in maritime industries and resource extraction, and the environmental health and productivity of these regions deliver important ecosystem services. As already noted, the proximity of energetic boundary currents in deep water adjacent to continental shelves mediates shelf-sea/deep-ocean exchange of properties. Along many coasts, this forcing can match or exceed local drivers of circulation such as tides, wind, and river inflows. Coastal ocean and shelf edge dynamics have immediate impacts on ecosystem function and productivity on weekly to seasonal timescales but can also drive multi-decadal changes in ecosystem structure through effects on habitat ranges and biodiversity, not only in coastal zones but also at basin scales.

While we have a broad understanding of the dynamics of upwelling in both WBC and EBC regimes, quantitative estimates of net shelf-sea/deep-ocean exchanges of freshwater and tracers integrated over extended along-shelf distances are few. Quantifying these exchanges is challenging where shelf-edge flow-bathymetry interactions foster variability at short length and timescales. Similarly, exchange flows are not always readily observable at the sea surface from satellite or shore-based remote sensing technologies (see the section Remote Sensing) because they are associated with bottom boundary layer flow driven by the boundary current encountering the seafloor or subduction at the sea surface due to boundary current detachment and mixing. Two efforts along the U.S. East Coast are striving to make such measurements using multi-platform observing arrays: the Processes driving Exchange At Cape Hatteras (PEACH) program and the Ocean Observatories Initiative (OOI; Smith et al., 2018; Trowbridge et al., 2019) Pioneer Array (see the section Northwestern Atlantic). Similarly, in situ and satellite remote sensing observations combined with high-resolution numerical simulations have provided insights into the shelfsea/deep-ocean exchanges near the confluence of the Brazil and Malvinas Currents (Guerrero et al., 2014; Matano et al., 2014; Strub et al., 2015).

On narrow continental shelves adjacent to intense boundary currents, the impact of deep-ocean circulation on the shelf system is immediate, driving significant fluxes across the continental 
shelf edge through mesoscale and boundary layer dynamics. For example, mesoscale and submesoscale meandering of the Agulhas jet leads to strong episodic exchanges with shelf waters (Krug et al., 2017; Leber et al., 2017) that support high productivity over the eastern Agulhas Bank (Probyn et al., 1994) and may influence the well-known sardine run (Fréon et al., 2010). On broad continental shelves, bathymetric constraints on cross-isobath flow can hamper exchange at the shelf edge, trapping terrestrial inflows and establishing appreciable crossshelf buoyancy gradients that in turn sustain shelf-edge fronts (Fratantoni and Pickart, 2007; Howatt et al., 2018).

With changing climate, ocean warming and changes to the hydrological cycle could drive changes in vertical thermal stratification and across-shelf salinity stratification, altering ocean conditions at the inshore edge of boundary current systems (e.g., Gawarkiewicz et al., 2018) and potentially impacting across-shelf fluxes of nutrients and micro-nutrients that are important to sustaining coastal productivity (Fennel et al., 2006). Changes in watershed land use and global weather will alter the volume and characteristics of river flows discharged into the coastal zone. At continental shelf scales, key areas of uncertainty in the oceanographic response to climate variability and change include submesoscale processes and open oceanshelf exchange. Sustained observing efforts are needed that more fully capture the influence of boundary currents on exchanges with the coastal zone. Designing and deploying boundary current observing systems capable of operating across shelf and deep ocean regimes to deliver coherent views of the shelf-edge exchange is challenging.

\section{OBSERVING TECHNIQUES}

The highly variable and multi-scale characteristics of boundary currents necessitate an integrated observing system approach, in which high-resolution observations are nested within a backbone of observations over a broad area. Under the Framework for Ocean Observing (Lindstrom et al., 2012), design and implementation of ocean observing systems are focused around a set of EOVs that include physical, biogeochemical, and ecosystem parameters (Table 1) ${ }^{1}$. Design of an observing system for a particular region (e.g., a specific boundary current system) should proceed through a series of "readiness levels." In the concept phase, initial feasibility studies and peer review of proposed plans take place. Then, in the pilot phase, smallscale deployments are used to test and validate the proposed approach. Once the observing system reaches the mature phase, it is part of the sustained Global Ocean Observing System. No single observing platform can provide all of the necessary measurements (Table 1), so an optimal mix of observing platforms is needed. Determination of this mix of platforms will be specific to a particular boundary current system, taking into consideration the unique processes and scales at play in that system. Here, we briefly review how various observing platforms are currently being used in boundary current systems; Table 2

${ }^{1}$ http://www.goosocean.org/eov refers to many other examples of these observing techniques being applied to boundary current systems.

\section{Time Series}

Time series measured from platforms fixed to the seafloor have long been and continue to be central to observing system design and implementation since they uniquely enable collection of long-term measurements at high temporal resolution (minutes to hours) at key locations. Traditional tall moorings (e.g., Johns et al., 2005) typically carry instruments on the mooring wire, within subsurface floats, and on surface buoys, if present; instruments are available to measure most physical EOVs and a growing number of biogeochemical and ecosystem EOVs (Table 1). Moored surface buoys additionally carry suites of meteorological sensors on the buoy tower and sensors for biogeochemical and physical EOVs on the buoy bridle and mooring line just below the sea surface; these air- and seasurface measurements can be combined to estimate the air-sea exchanges of heat, moisture, $\mathrm{CO}_{2}$, and momentum (Cronin et al., 2019). Inverted echo sounders (IESs) measure the time for sound pulses to travel from the bottom-mounted IES to the surface and back, which, in regions with good databases of hydrographic measurements, can provide full water column estimates of temperature, salinity, and density using the gravest empirical mode technique (Meinen and Watts, 2000). In the Florida Strait, a unique time series of volume transport has resulted from measuring the voltage induced in a submarine cable by seawater moving through the Earth's magnetic field (Larsen and Sanford, 1985; Baringer and Larsen, 2001; Meinen et al., 2010).

Dense, moored arrays of instruments remain the most effective way to return volume and property transport measurements with high temporal resolution. Subsurface moorings are more typical in WBCs due to the strong surface currents, although surface moorings have also been successfully deployed in the Gulf Stream (Weller et al., 2012) and Kuroshio Extension (Cronin et al., 2013). Arrays of IESs can be used to infer geostrophic shear profiles and, with the addition of bottom pressure sensors (PIES) and near-bottom current measurements (CPIES), can provide estimates of the absolute geostrophic current (Donohue et al., 2010; Meinen et al., 2018). However, the high costs of building, deploying, and turning around such arrays makes them feasible only at a few key locations. Other observing assets are needed to provide spatially broad measurements.

\section{Ship-Based Measurements}

Measurements from both dedicated research vessels and ships of opportunity have been central to observing boundary current systems for decades. Research vessels can measure nearly every EOV (Table 1) through the full depth of the water column and are uniquely capable of collecting many types of samples (e.g., net tows and large-volume water samples). Ongoing sustained research vessel surveys of ocean boundary currents include the global GO-SHIP transects at 25- to $50-\mathrm{km}$ resolution (Talley et al., 2016) and the California Cooperative Oceanic Fisheries Investigations (CalCOFI) surveys (McClatchie, 2014) in the California Current System (see the section California Current System). The servicing of boundary current mooring arrays, 
generally undertaken from research vessels, provides unique opportunities to undertake intensive process studies targeting key scientific questions. The primary limitations on research vessels' contribution to sustained boundary current observing are their high costs of operation (typically tens of thousands of dollars per day, excluding science personnel) and the infrequency of cruises.

The World Meteorological Organization (WMO) Voluntary Observing Ship (VOS) Program and Ship of Opportunity Program (SOOP) both make use of non-research vessels to collect observations globally, substantially augmenting the amount of ship-based observing. Both programs collect meteorological measurements with real-time observations benefiting weather forecasting, while SOOP additionally uses commercial ships to collect oceanographic measurements along frequently occupied trade routes in the global ocean. Oceanic measurements from SOOP include temperature profiles from expendable bathythermographs (XBTs) at $10-$ to $25-\mathrm{km}$ resolution in boundary currents (Goni et al., 2019), surface temperature, salinity, plankton, and $\mathrm{pCO}_{2}$ from flow-through systems, and, on specially equipped vessels, velocity profiles from hull-mounted ADCPs (e.g., M/V Oleander; Rossby et al., 2010). Several repeat transects across boundary currents have been maintained for multiple decades and so represent some of the longer datasets available (see the section Current Status of Regional Boundary Current Observing Systems). Fast-moving ships are often able to occupy transects directly across strong boundary currents in short periods of time, a feat not yet possible with other sampling platforms. However, subsurface measurements of variables other than temperature and velocity have remained elusive from ships of opportunity, and recovery of instruments deployed over the side is not practical on cargo vessels.

\section{Autonomous Underwater Gliders}

Autonomous underwater gliders (Rudnick, 2016b; Testor et al., 2019) routinely collect long-duration, high-resolution observations in a variety of boundary current systems globally (Todd et al., 2018b; Table 2). Gliders typically profile from the surface to 500-1,000 m, taking 3-6 h to complete a cycle from the surface to depth and back while covering 3-6 km horizontally through the water at a speed of about $0.25 \mathrm{~m} \mathrm{~s}^{-1}$. During a mission lasting 3-6 months, a glider's survey track extends well over $2,000 \mathrm{~km}$. Owing to the relatively slow speed of gliders, care must be taken when interpreting the observations, which contain both spatial and temporal variability (Rudnick and Cole, 2011). Sustained deployment of networks of gliders can provide observations with both high spatial resolution and year-round coverage (e.g., Figures 1A,B).

Realizable glider-based sampling plans in boundary currents vary primarily due to the strength of currents relative to a glider's speed. In EBCs and other boundary currents with relatively weak depth-average currents, gliders can occupy repeat survey lines. The California Underwater Glider Network (CUGN; Figure 1, left), which consists of three cross-shore transects off southern and central California that have been continuously occupied for more than a decade (Rudnick et al., 2017), exemplifies sustained glider observations in an EBC. In WBCs and other boundary currents where depth-average currents are significantly faster than a glider's speed through the water, gliders can be navigated so as to cross the observed flow as they are advected downstream, returning oblique transects. For example, multi-year surveys of the Gulf Stream (Figure 1, right; Todd et al., 2016, 2018a; Todd, 2017; Todd and Locke-Wynn, 2017) have now returned over 150 high-resolution transects across the WBC of the North Atlantic. Testor et al. (2019) further discuss efforts associated with the OceanGliders Boundary Ocean Observing Network (BOON).

Gliders can carry a variety of sensors (e.g., Figures 1C-F). Measurements of pressure, temperature (Figure 1D), conductivity, and depth-average currents are standard, enabling estimates of absolute geostrophic transport and other physical parameters at relevant scales in boundary currents. Measurements of biooptical (e.g., Niewiadomska et al., 2008; Henderikx Freitas et al., 2016) and bio-acoustic properties (e.g., Baumgartner and Fratantoni, 2008; Van Uffelen et al., 2017), dissolved oxygen (e.g., Figure 1E; Perry et al., 2008), nitrate, turbulent microstructure (St. Laurent and Merrifield, 2017), and velocity profiles (Figure 1F; Todd et al., 2017) are becoming increasingly common. The main constraints on sensors for gliders are the requirements for small-size, low-power consumption and multimonth stability. As sensor technology continues to mature, gliders will be well suited to carry sensors for additional EOVs, such as $\mathrm{pH}$, in boundary currents.

\section{Drifters}

Surface Velocity Program (SVP) drifters drogued at 15-m depth (Niiler et al., 1995; Niiler, 2001; Centurioni, 2018) deployed as part of the Global Drifter Program (GDP) and the Global Surface Drifter Array (GSDA) are also important for understanding the structure and variability of boundary current systems. The GSDA archive dates back to February 1979 and includes over 32 million records of geographical location, 15-m depth velocity, and SST at 6-h resolution (e.g., Hansen and Poulain, 1996; Lumpkin and Pazos, 2007).

Drifter observations have been widely used in both EBCs and WBCs (see Table 2). Recently improved analysis techniques (e.g., Lumpkin, 2003; LaCasce, 2008; Koszalka and LaCasce, 2010; Laurindo et al., 2017) and expansion of the Lagrangian drifter array have allowed gridded, Eulerian statistics of nearsurface velocity to be produced at higher resolution, resulting in improved estimates of near-surface flow in boundary currents (e.g., Figure 2) at seasonal to interannual timescales (e.g., Niiler et al., 2003; Lumpkin and Johnson, 2013). Drifter observations in boundary currents offer opportunities for new analyses of long-term variability and trends (e.g., Johnson, 2001; Lumpkin and Johnson, 2013) and the dispersion of tracers and marine debris in the upper ocean (Lumpkin et al., 2012; van Sebille et al., 2015), which is driven by turbulence at scales from surface waves through the submesoscale to large-scale geostrophic eddies (Lumpkin et al., 2017; Lund et al., 2018).

\section{Argo Floats}

Over the past two decades, autonomous profiling Argo floats have become cost-effective and robust platforms. Over 3,700 active Argo floats provide global measurements of temperature, salinity, and pressure in the upper 2,000 $\mathrm{m}$ of the ocean, and some are also equipped with sensors measuring biogeochemical properties (Riser et al., 2016; Jayne et al., 2017; Roemmich et al., 2019). 


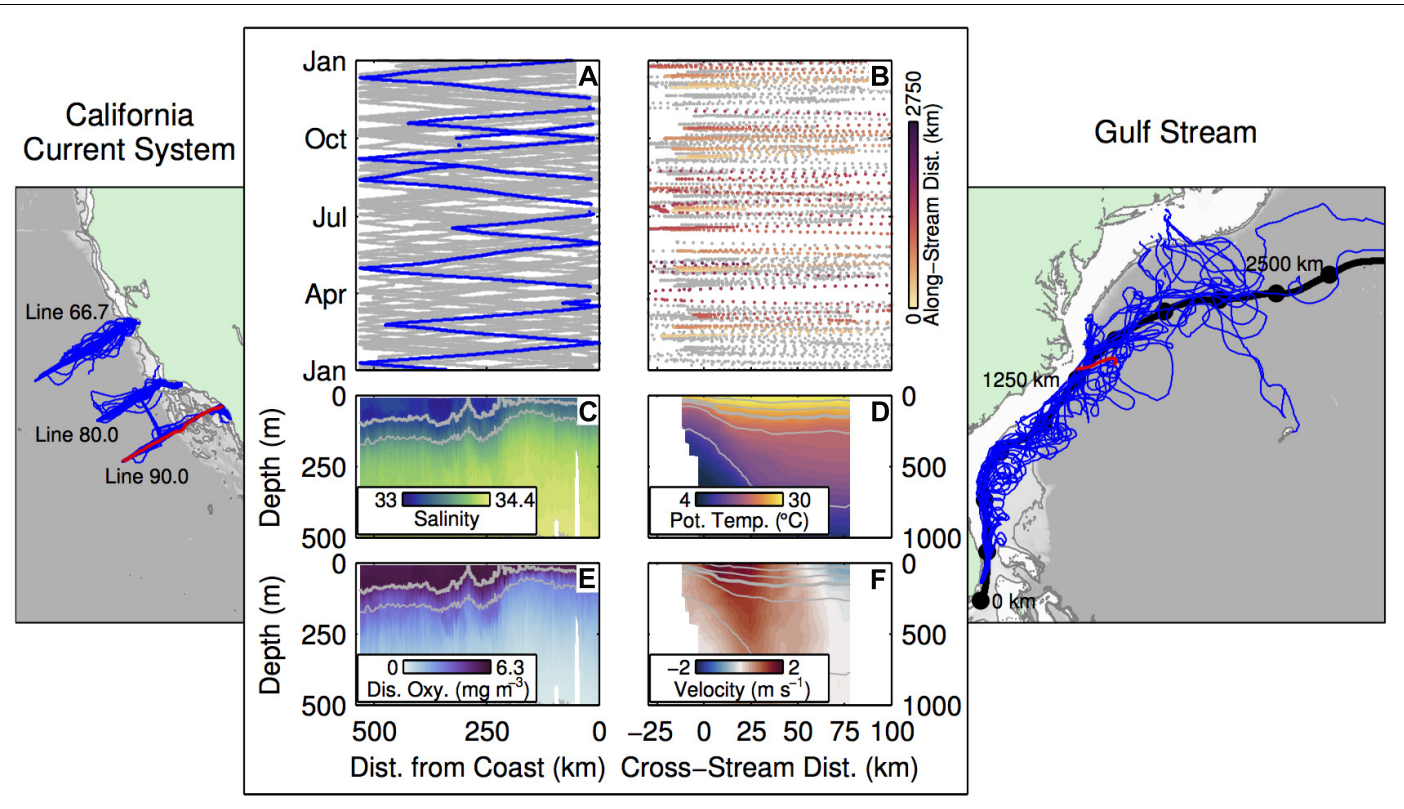

FIGURE 1 | Examples of multi-year, glider-based sampling in (left) an eastern boundary current system and (right) a western boundary current. Trajectories of all Spray gliders surveying the California Current System along CalCOFI lines 66.7, 80.0, and 90.0 (Rudnick et al., 2017 and references therein) and the Gulf Stream along the U.S. East Coast (Todd, 2017; Todd and Locke-Wynn, 2017; Todd et al., 2018a) are shown on the background map. (A,B) Glider sampling as a function of month and cross-shore or cross-stream distance with sampling in all years in gray and calendar year 2017 in color; Gulf Stream sampling in 2017 is colored by along-stream distance from $25^{\circ} \mathrm{N}$ following the mean 40-cm SSH contour (black trajectory on map with dots every $\left.250 \mathrm{~km}\right)$. (C-F) Example transects of salinity and dissolved oxygen along CalCOFI line 90.0 off Southern California in May 2017 and of potential temperature and velocity toward $50^{\circ}$ across the Gulf Stream near Cape Hatteras in August 2017 (red transects on map).

Though the Argo network was not designed to capture the details of boundary currents and lacks the resolution necessary to resolve narrow boundary currents, Argo data have nevertheless been used extensively in both WBCs and EBCs (see Table 2). Argo complements other boundary current observing efforts by providing collocated temperature and salinity measurements that are used to infer geostrophic shear from XBT temperature profiles, extending geostrophic shear from XBT and ocean glider data to 2,000 $\mathrm{m}$, measuring reference velocities at parking depth (typically 1,000 m), and linking transport measurements of boundary currents to the ocean interior through basinwide integration (e.g., Zilberman et al., 2018). Following recommendations at OceanObs'09 (Roemmich et al., 2010), the Argo program is currently moving to double float density in WBC regions (Jayne et al., 2017). The Kuroshio (Figure 3A) and Gulf Stream have historically been among the more densely populated sectors in the Argo array, while other boundary current regions (e.g., the Peru-Chile system, Figure 3B) lack the desired coverage.

\section{Remote Sensing}

Among the many oceanic variables that are routinely measured from satellites (Table 1), SSH, SST, and ocean color have been most used to study boundary current systems. Satellite measurements typically have resolutions of $\mathrm{O}(1)-\mathrm{O}(10) \mathrm{km}$ along the satellite track, with repeated measurements on daily to 10-day timescales at a given location. Boundary currents often have strong signatures in both SSH and SST, so satellite-derived gradients in these properties can approximate the strength and/or position of these currents (e.g., Imawaki et al., 2001), including variability on longer timescales (e.g., Qiu and Chen, 2005; Qiu et al., 2014; Andres, 2016). Synergy between dynamic height derived from temperature and salinity profiles and SSH can be exploited to produce synthetic reconstructions of boundary currents (van Sebille et al., 2010; Beal and Elipot, 2016; Majumder and Schmid, 2018; Zilberman et al., 2018), although these reconstructions crucially depend on assumptions about the nonsteric (barotropic and mass) variability. Weaknesses of SSH for observing boundary currents include reduced measurement quality within $40 \mathrm{~km}$ of the coast due to large uncertainties in the wet-tropospheric correction, unfiltered tides, and a lack of sufficient temporal and spatial resolution to capture the full spectrum of near-surface current variability observed by drifters (Poulain and Niiler, 1989; Centurioni and Niiler, 2003; Fratantoni and Richardson, 2006; Centurioni et al., 2008, 2009; Maximenko et al., 2009). Products that combine SSH and drifter measurements have improved eddy kinetic energy and dynamic topography estimates (Maximenko et al., 2009; Lumpkin and Garzoli, 2011; Rio et al., 2014; Rio and Santoleri, 2018). Estimates of chlorophyll derived from satellite ocean color measurements provide information on biological productivity in boundary current systems worldwide (e.g., Messié and Chavez, 2015; Gómez-Letona et al., 2017). Because ocean color observations have higher resolution $(\mathrm{O}(1) \mathrm{km})$ than satellite altimetry measurements, they potentially provide insight into the rich fields of submesoscale instabilities that exist within 


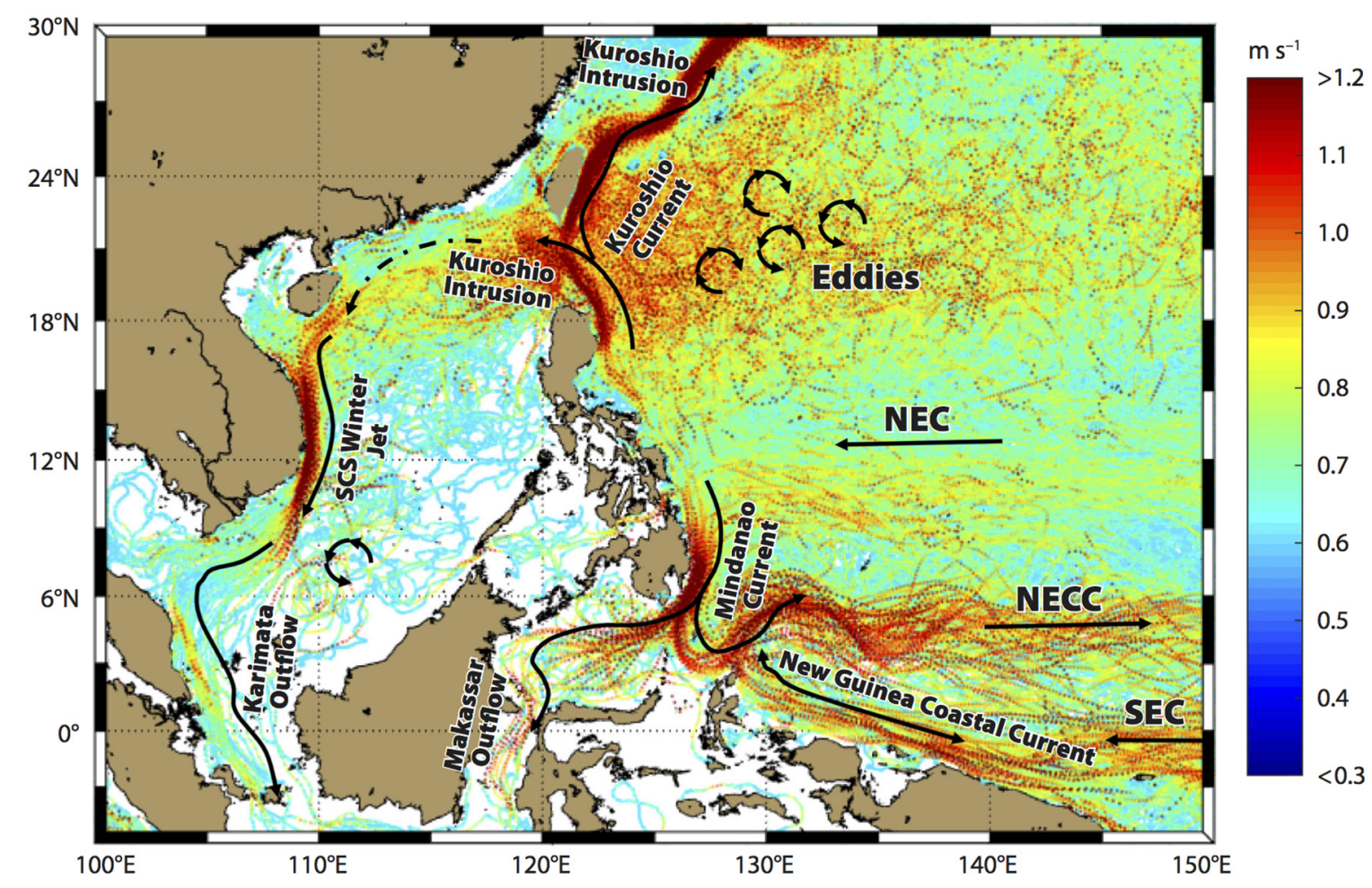

FIGURE 2 | Trajectories and near-surface velocity estimates from Global Drifter Program drifters in the western Pacific and marginal seas. Over 1.2 million discrete measurements from 1982 to 2014 are included. Paths of various boundary currents are clearly visible, as is the rich eddy field in the region of the Subtropical Countercurrent around $18-24^{\circ} \mathrm{N}$. NEC $=$ North Equatorial Current, NECC $=$ North Equatorial Counter Current, SEC $=$ South Equatorial Current, SCS $=$ South China Sea (Figure from Todd et al., 2018b).
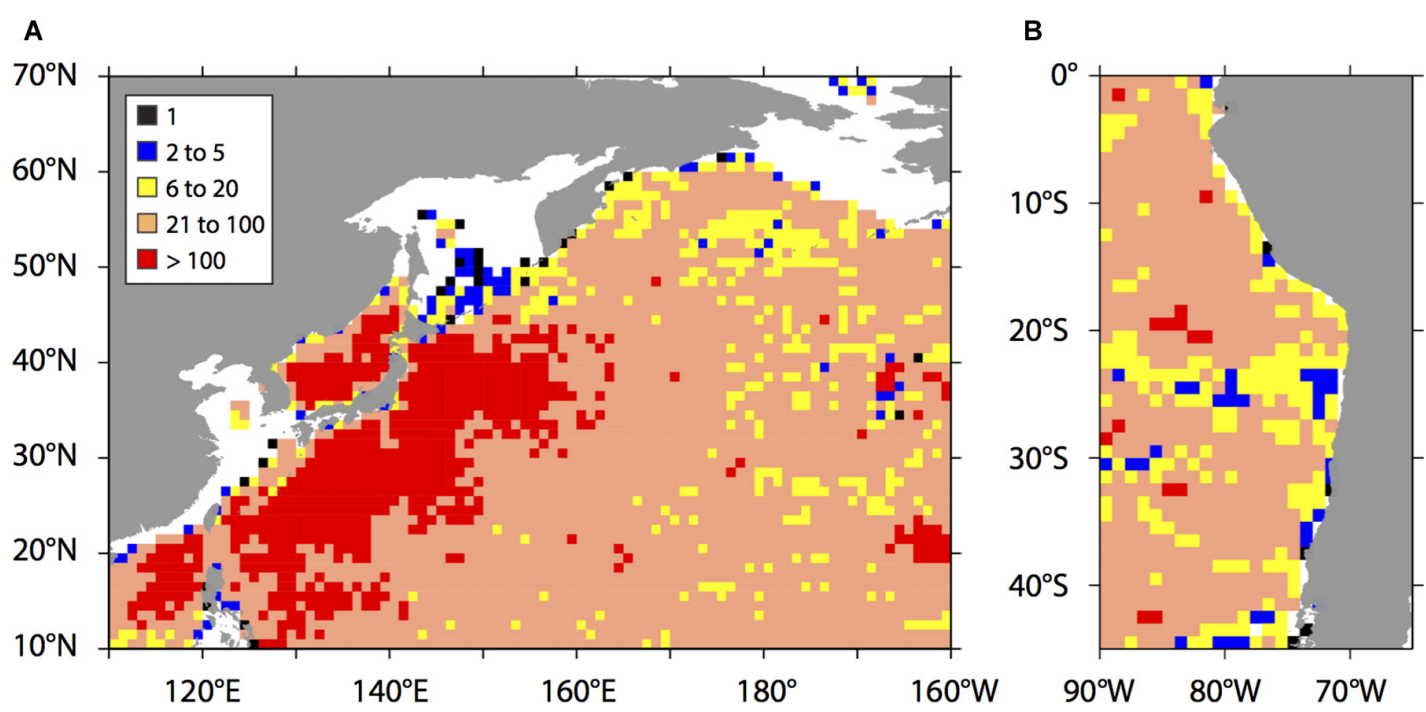

FIGURE 3 | Sampling density of Argo float (including Core Argo and BGC Argo) profiles per $1^{\circ}$ latitude $\times 1^{\circ}$ longitude bin, collected between January 2009 and September 2018, in the Kuroshio region (A), and the Peru-Chile Current region (B)

boundary current systems (Figure 4; Everett et al., 2014; Lee and Kim, 2018).

High-frequency (HF) radars (Paduan and Washburn, 2013) have been used effectively to monitor surface current variability of boundary currents (e.g., Kim et al., 2011; Archer et al., 2018). They directly map the total surface current within $\mathrm{O}(100) \mathrm{km}$ of the coast at high resolution in time $(\sim 1 \mathrm{~h})$ and space $(\sim 1 \mathrm{~km})$ during long-term deployments ( $\sim 10$ years). 


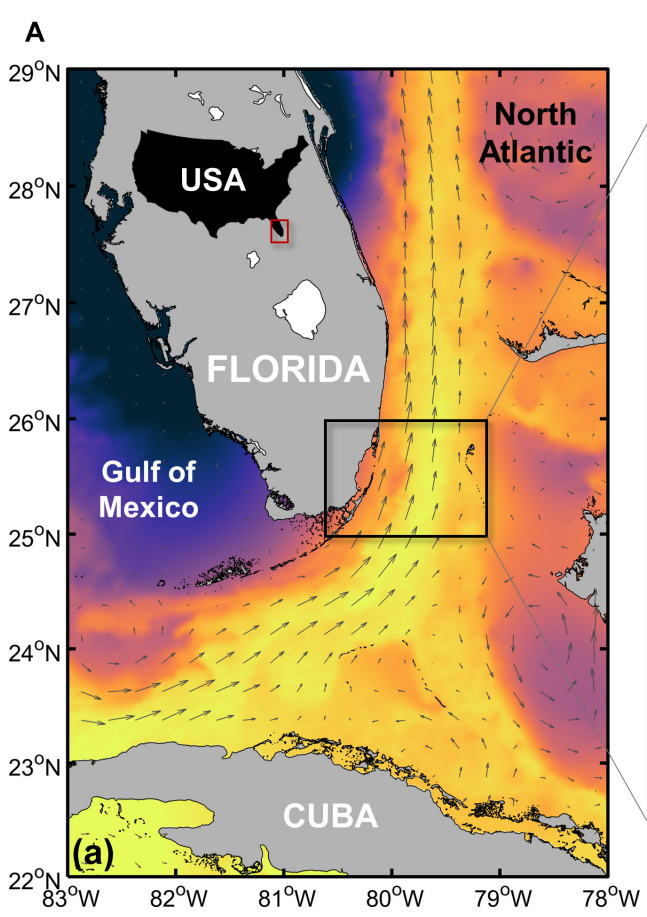

B

\section{FLORIDA CURRENT}

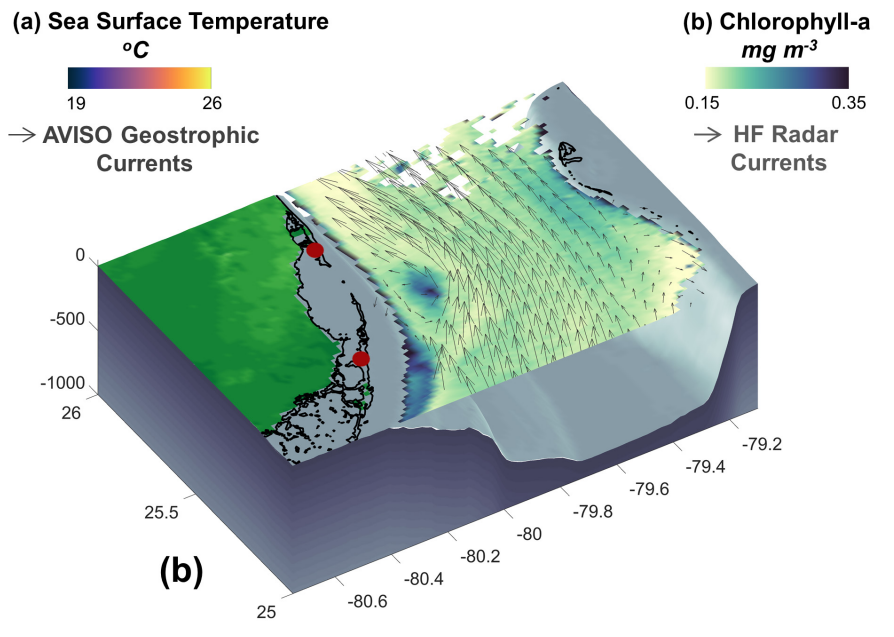

FIGURE 4 | Example of combined satellite- and land-based remote sensing of the Florida Current. (A) SST from GHRSST and surface geostrophic currents from AVISO. (B) Chlorophyll from MODIS AQUA and surface currents from HF radars (HF radar data from Archer et al., 2017b).

HF radar observations have proven useful for investigating both the mean surface velocity structure of boundary currents and associated submesoscale features that develop as boundary currents meander and shed eddies (Archer et al., 2018; Soh and Kim, 2018). Combining HF radar velocity estimates with satellitebased measurements of SST and ocean color (e.g., Figure 4) can provide a multidisciplinary view of surface circulation features at O(1)-km scales (e.g., Schaeffer et al., 2017). Some radar sites have been in continuous operation for more than a decade, offering opportunities to examine interannual to decadal variability of surface circulation. New radar sites can be installed and daisychained with existing sites, providing measurements of the alongshore evolution of boundary currents, as has been achieved along the West Coast of the United States (Kim et al., 2011).

\section{CURRENT STATUS OF REGIONAL BOUNDARY CURRENT OBSERVING SYSTEMS}

Existing observing systems for particular boundary currents are in various stages of development. Here, we review the current status of the observing systems currently operating in several EBCs and WBCs globally. The California Current System (see the section California Current System) is arguably the most well sampled boundary current in the world, offering hope that a fully integrated physical and biogeochemical system is achievable. Other boundary current systems, particularly in the Southern Hemisphere, are much less sampled. As was the case a decade ago
(Send et al., 2010), biogeochemical and ecosystem EOVs (Table 1) remain much less well sampled than physical EOVs. Table 2 provides a more comprehensive collection of recent scientific results for each boundary current system as well as sources of publicly available observations.

\section{Eastern Boundary Current Systems California Current System}

The California Current System is the EBC system of the subtropical North Pacific (Checkley and Barth, 2009). The equatorward flowing California Current carries relatively cool and fresh waters of subpolar origin, while the poleward California Undercurrent (Gay and Chereskin, 2009; Todd et al., 2011b) transports warmer saltier waters from the tropics along the continental margin. The California Current System is strongly influenced by the predominantly upwelling-favorable winds along the West Coast of North America.

Owing to the need to understand the collapse of the regional sardine fishery in the 1940s, there is a well-developed multidisciplinary observing system in the California Current System with a decades-long history of routine observations by the CalCOFI program (McClatchie, 2014, and references therein). Since 1949, CalCOFI has made regular (currently quarterly) measurements of physical, biological, and chemical properties at fixed stations along survey lines oriented perpendicular to the coast from research vessels (Figure 5). The establishment of the California Current Ecosystem Long Term Ecological Research program in 2004 brought further ship-based surveys and longterm moorings (Figure 5) focused on non-linear transitions in 


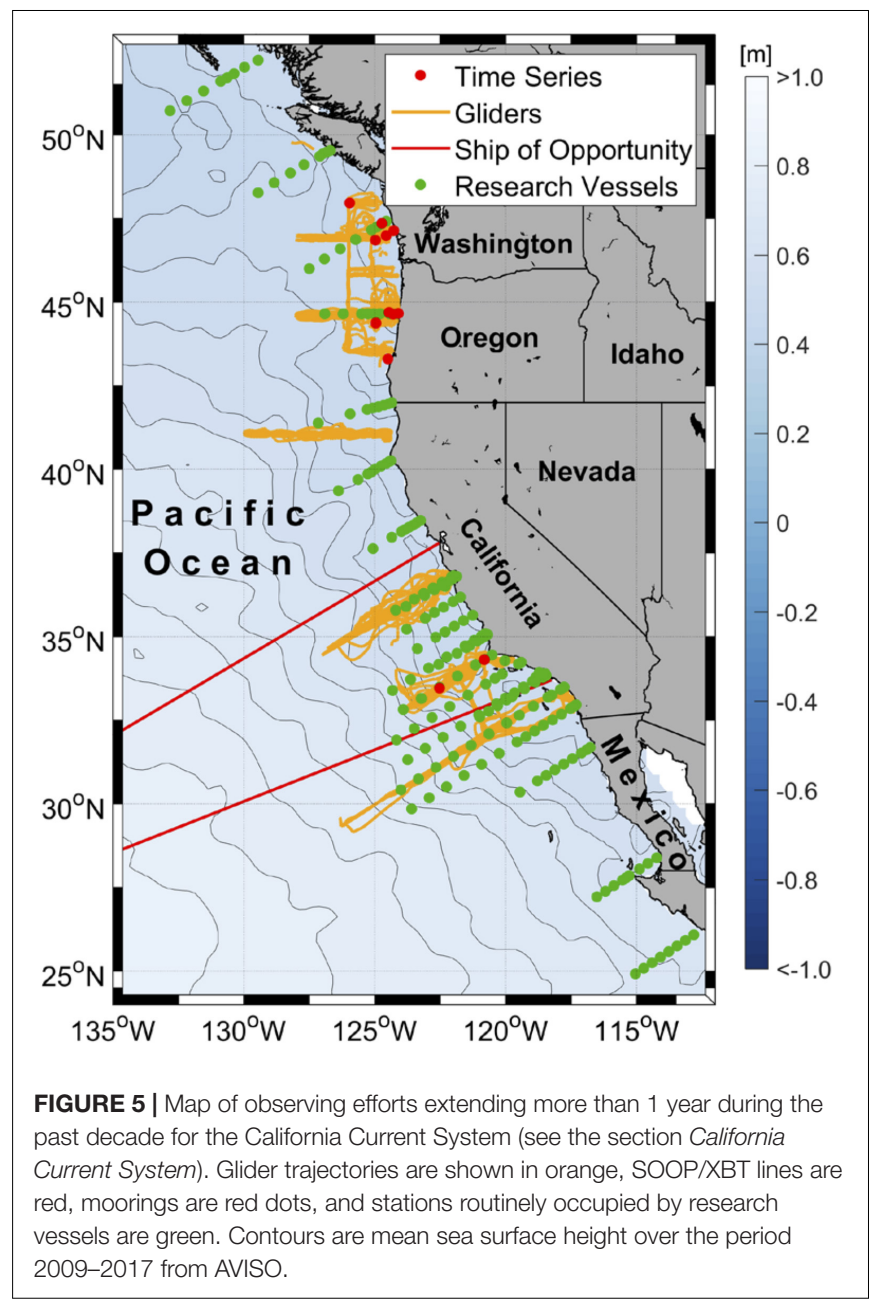

the pelagic ecosystem in response to ENSO, the Pacific Decadal Oscillation, and secular trends. In the Northern California Current, the Newport Hydrographic Line $\left(44^{\circ} 39.1^{\prime} \mathrm{N}\right)$ has been continuously occupied since 1961 (Huyer et al., 2007). Since 2005, autonomous underwater gliders have continuously surveyed along three of the CalCOFI lines as part of the CUGN (see the section Autonomous Underwater Gliders; Figures 1, 5) as well as along cross-shore transects as far north as the Washington Coast (Figure 5), returning measurements of physical properties and some biological proxies; the gliders complement the ship-based surveys by providing observations at higher spatial and temporal resolutions (e.g., Rudnick et al., 2017), albeit of a more limited set of properties. An array of PIES with end-point moorings off of Southern California monitors full-depth geostrophic transport; gliders routinely retrieve data from the PIES and transmit them to shore (Send et al., 2013). Since 2007, NOAA has led largescale coastal surveys along the U.S. West Coast every 2-4 years to determine the spatial distributions of carbon, oxygen, nutrient, biological, and hydrographic parameters (Feely et al., 2008, 2018). Starting in 2010, moored platforms throughout the California Current System established high-frequency time series of physical and biogeochemical parameters (Nam et al., 2011;
Harris et al., 2013; Sutton et al., 2016). More recently, the OOI Endurance Array (Smith et al., 2018; Trowbridge et al., 2019) has been deployed in the northern California Current System (Figure 5); moorings on the shelf and continental slope provide high-resolution time series, while gliders provide high-spatialresolution observations between the mooring sites. A network of shore-based HF radars provides real-time surface currents within about $150 \mathrm{~km}$ of the coast along nearly the entire U.S. West Coast (Kim et al., 2011).

\section{Peru-Chile Current System}

The Peru-Chile Current System (or Humboldt Current System) is the EBC system of the subtropical South Pacific, extending from the equator to southern Chile $\left(\sim 45^{\circ} \mathrm{S}\right)$. It is characterized by a persistent stratus cloud deck, equatorward surface currents, strong wind-driven coastal upwelling, poleward undercurrents, and filaments and eddies that develop along the coasts of Peru and Chile (see Colas et al., 2012 and references therein). A subsurface oxygen minimum zone (e.g., Paulmier and RuizPino, 2009) results in upwelled waters being nutrient rich but low in oxygen (e.g., Silva et al., 2009; Pizarro et al., 2016). Due to its proximity to the equator, the Peru-Chile Current System is strongly influenced by equatorial variability through propagation of Kelvin and coastal trapped waves (Dewitte et al., 2012; Mosquera-Vásquez et al., 2013) and anomalous advection during strong El Niños (e.g., Colas et al., 2008).

The dramatic impacts of El Niño events on both weather and fisheries have driven monitoring of oceanographic properties and fish stock assessments along the Peruvian coast since the 1960s (Figure 6; Grados et al., 2018). Over the past decade, these surveys have taken place monthly along the northern Peruvian coast and at least twice per year along the entire Peruvian coast; shipboard ADCP surveys are conducted at least seasonally. Biweekly time series along the $100-\mathrm{m}$ isobath between Paita $\left(5^{\circ} \mathrm{S}\right)$ and Ilo $\left(17^{\circ} \mathrm{S}\right)$, coastal tide-gauge stations, daily SST measurements at coastal laboratories, and a nearshore thermistor chain and bottom-mounted ADCP at $4^{\circ} 30^{\prime}$ S (Figure 6) allow monitoring of temperature and sea level anomalies and associated fluctuations in thermocline, oxycline, and nutricline depths. Measurements from the TAO/TRITON moored array and the Argo program (Figure 3B) provide key broad-scale context to these coastal observations. Efforts are underway to improve monitoring of the Peru-Chile Current System. For instance, sustained glider surveys across the frontal region off northern Peru, where El Niño impacts are large, are planned to begin by 2020 .

\section{Leeuwin Current System and South Australian Current System}

The boundary currents along the western and southern coasts of Australia have some unique features. The Leeuwin Current, which is the subtropical EBC of the southeastern Indian Ocean, is unusual in that it flows poleward along an eastern boundary, transporting warm, fresh tropical waters southward due to forcing by the Indonesian Throughflow and ocean atmosphere interactions in the Indian Ocean (Godfrey and Weaver, 1991); it is important for the upper ocean heat balance in the southeast 


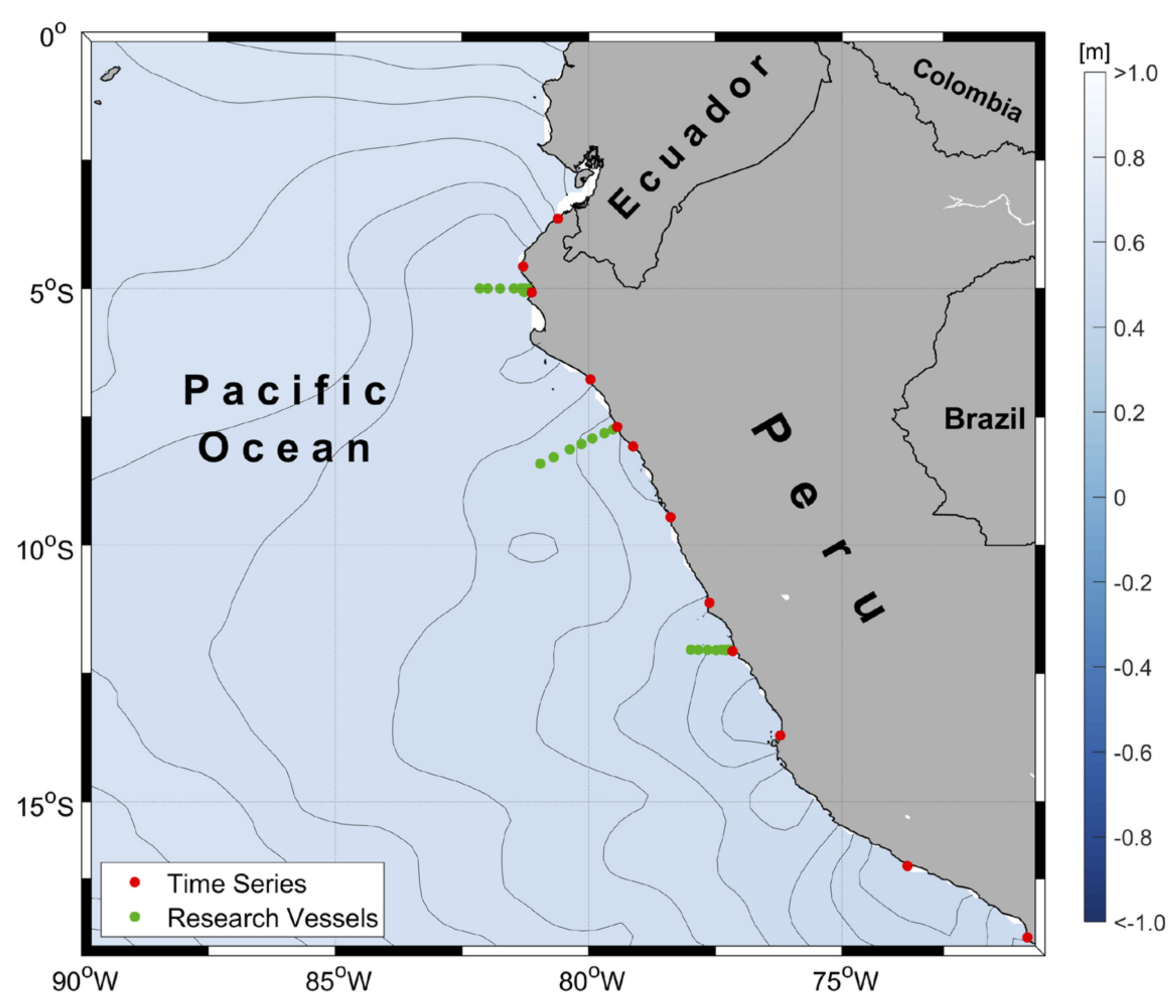

FIGURE 6 | Map of the boundary current observing effort for the Peru-Chile Current System (see the section Peru-Chile Current System) with details as in Figure $\mathbf{5}$.

Indian Ocean (Domingues et al., 2006). The Leeuwin Current hosts broad-scale downwelling (Furue et al., 2017; Liang et al., 2017) where eastward surface currents merge with the Leeuwin Current and then downwell into the Leeuwin Undercurrent at depths of 200-1,000 m. The equatorward Leeuwin Undercurrent carries waters of subantarctic origin along the western Australian coast (Woo and Pattiaratchi, 2008), leaving the coast near $22^{\circ} \mathrm{S}$ to contribute to the lower limb of a zonal overturning (Furue et al., 2017) and the subtropical gyre (Schott et al., 2009). In winter, the Leeuwin Current merges with the southwestward-flowing Holloway Current off the northwest coast of Australia, the eastward-flowing South Australian Current off the south coast, and the southward-flowing Zeehan Current off the west coast of Tasmania to form the longest shelfbreak boundary current system in the world (Ridgway and Condie, 2004; D'Adamo et al., 2009; Ridgway and Godfrey, 2015). Along the continental slope south of Australia, the westward flowing Flinders Current results from the collision of the equatorward deep ocean Sverdrup transport with the deep shelf slope of the Great Australian Bight (Middleton and Cirano, 2002; Middleton and Bye, 2007) and is a unique northern boundary current.

Coastal sea level observations at Fremantle have long been used as a proxy for the strength of the Leeuwin Current (Feng et al., 2003). Since 2008, the Australian Integrated Marine Observing System (IMOS; Hill et al., 2010) has been monitoring the shelf component of the Leeuwin Current near $32^{\circ} \mathrm{S}$ using shelf moorings (Feng et al., 2013), gliders, and HF radars (Figure 7). Short-term deployments (2012-2014) have also been carried out off the northwest coast of Australia (Ridgway and Godfrey, 2015). XBT surveys from ships of opportunity in and out of Fremantle, though not targeted for the Leeuwin Current, have taken place since the mid-1980s (Wijffels et al., 2008). IMOS makes ongoing observations of the South Australian Current system with dedicated moorings and glider missions monitoring the Flinders Current (Figure 7).

\section{Benguela Current System}

The Benguela Current Large Marine Ecosystem is the eastern boundary upwelling system of the South Atlantic. The equatorward Benguela Current is unique in that it is bounded by warm currents at its northern and southern edges, the Angola Current to the north and the Agulhas Current (see the section Agulhas Current) to the south. Coastal upwelling is linked to the seasonal position of the South Atlantic high pressure system, resulting in a number of upwelling cells along the southern African coast with divergent seasonality; the strongest year-round upwelling occurs off Lüderitz $\left(\sim 26^{\circ} S\right)$, effectively dividing the Benguela Current System into northern and southern subsystems. The northern Benguela upwelling system is highly productive (e.g., Louw et al., 2016) but also prone to hypoxia over the continental shelf that is modulated by a seasonal poleward undercurrent bringing low-oxygen waters to the shelf in summer and fall and an equatorward undercurrent that brings oxygenated water in winter and spring (Duncombe Rae, 2005; Mohrholz et al., 2008; Monteiro et al., 2008). The southern Benguela upwelling system experiences 


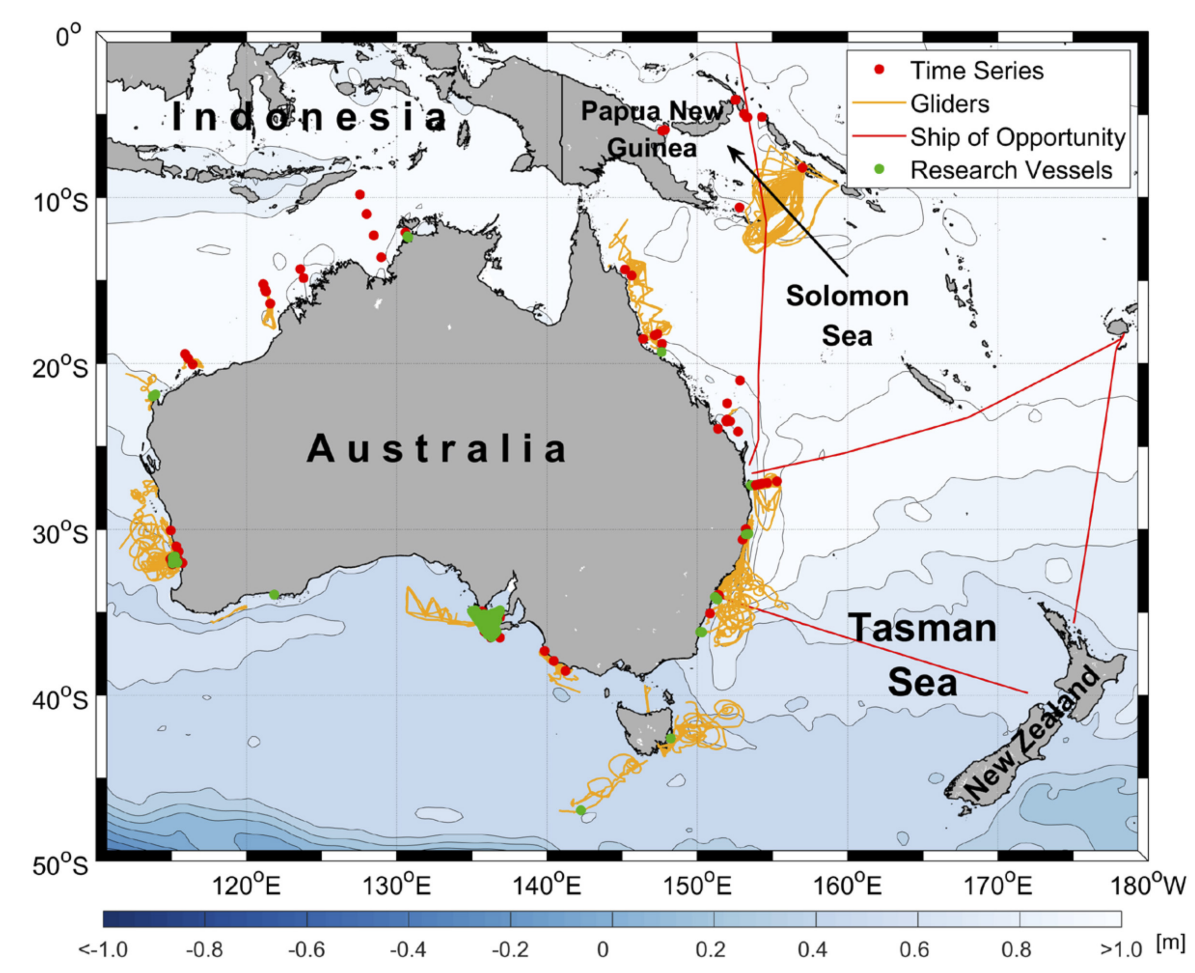

FIGURE 7 | Map of the boundary current observing efforts for the Leeuwin and South Australian Current Systems (see the section Leeuwin Current System and South Australian Current System) and the Southwestern Pacific (see the section Southwestern Pacific) with details as in Figure $\mathbf{5}$.

intense, pulsed upwelling in summer and quiescence in winter (Shannon and Nelson, 1996; Weeks et al., 2006; Hutchings et al., 2009c), although the direction of net Ekman transport appears to be offshore year-round (Carr and Kearns, 2003). This region also experiences hypoxia (and occasionally anoxia) in inshore waters, particularly in the region of St. Helena Bay (Pitcher and Probyn, 2011; Pitcher et al., 2014), but low-oxygen events are driven solely by bacterial respiration of organic matter from surface waters (Monteiro and van der Plas, 2006) and can result in mass mortalities of commercial fish stocks and rock lobster (e.g., Cockcroft et al., 2000, 2008; Van der Lingen et al., 2006).

In the southern Benguela Current System, monthly ship-based sampling of fisheries-relevant parameters took place through the 1950s and 1960s and then intermittently until 1988, after which surveys of fisheries, hydrographic, and chemical properties have been conducted two to three times per year (Figure 8; Moloney et al., 2004). Since 2012, quarterly surveys as part of the Integrated Ecosystem Program have additionally monitored the carbonate system. Various multifunctional moorings have been deployed over the years, including a buoy for oxygen and temperature and a harmful algal bloom detection system in the vicinity of St. Helena Bay (see Hutchings et al., 2009b). The Namibian Ministry of Fisheries and Marine Resources conducts regular monitoring of hydrographic conditions and commercial fish resources in Namibian waters of the northern Benguela (Figure 8); regular shipboard oceanographic monitoring began in 1999 with sampling frequency varying from two to eight occupations annually along most lines and up to twice per month off Lüderitz during the lobster fishing season. Long-term, though intermittent, moored observations have been collected at $23^{\circ} \mathrm{S}$, $14^{\prime} 03^{\circ} \mathrm{E}$, and coastal stations are maintained along the Namibian coast (Figure 8).

\section{Canary Current System}

The Canary Current Large Marine Ecosystem extends from the northern tip of the Iberian Peninsula $\left(43^{\circ} \mathrm{N}\right)$ to south of Senegal $\left(12^{\circ} \mathrm{N}\right)$, corresponding to the extent of the northeasterly trade winds in the northeastern Atlantic. Upwelling occurs yearround with meridional shifts in the trade winds leading to seasonality in the latitudinal range of upwelling, particularly in the south (Benazzouz et al., 2014; Faye et al., 2015), where strong intraseasonal to longer timescale variability is driven by internal or remotely forced pulsations of the trade winds, passages of African easterly waves, and oceanic coastally trapped waves (Polo et al., 2008; Diakhaté et al., 2016; Oettli et al., 2016). The ecosystem is broadly divided by the Strait of Gibraltar into the Iberian and the Northwest African areas, though strong subregional differences are observed due to variability in factors including coastal configuration, oxygen concentration, nutrient fertilization, and productivity (Arístegui et al., 2009). The continental shelf in the Canary Current System is the most extensive of any EBC, and persistent circulation features are associated with the topography of the shelf. Large filaments of coastal upwelled water stretch offshore from the numerous capes and promontories (e.g., Cape Guir and Cape Blanc), transporting waters rich in organic matter into the oligotrophic 


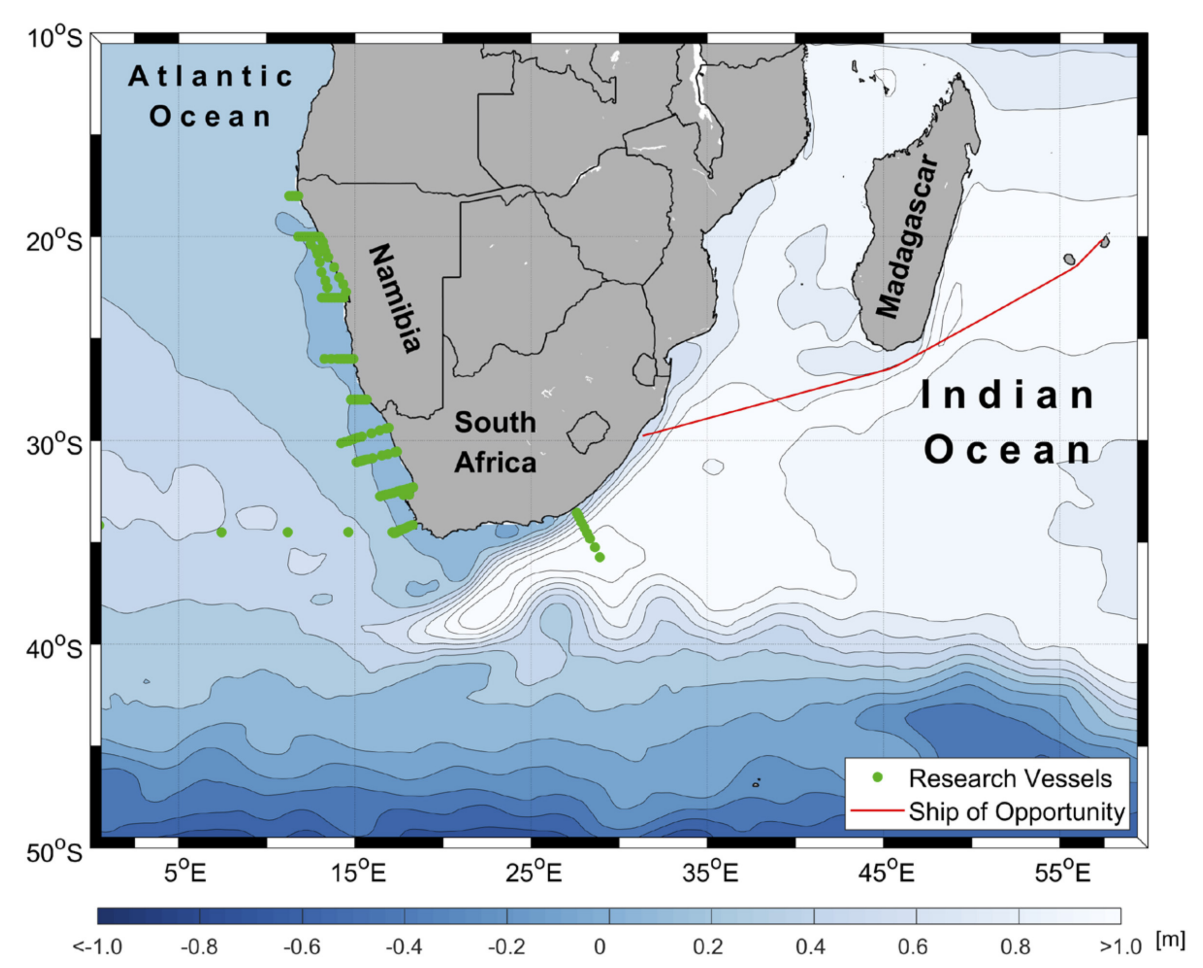

FIGURE 8 | Map of the boundary current observing effort for the Bengula Current System (see the section Benguela Current System) and the Agulhas Current (see the section Agulhas Current) with details as in Figure $\mathbf{5}$.

subtropical gyre (Álvarez-Salgado et al., 2007; Lovechio et al., 2018). The Canary Archipelago interrupts the equatorward flow of the Canary Current, leading to strong mesoscale variability downstream of the islands (Arístegui et al., 1994). Island eddies and upwelling filaments interact to exchange water properties, resulting in an efficient route for transporting organic matter to the open ocean (Arístegui et al., 1997; Barton et al., 1998). As a major upwelling area, the Canary Current System is highly productive and the focus of intensive fisheries. Interannual and decadal variability in fisheries landings and distributions of small pelagic fishes has been related to environmental changes associated with the North Atlantic Oscillation and, to a lesser extent, ENSO in the southern part of the region (see reviews in Arístegui et al., 2006; Arístegui et al., 2009).

There have been numerous process-oriented field programs in Canary Current System in the past 20 years, including the Coastal Transition Zone (CTZ; Barton and Arístegui, 2004) and Canary Islands Azores Gibraltar Observations (CANIGO; Parrilla et al., 2002) programs. However, physical, biogeochemical, and ecosystem monitoring efforts have been less routine than have other EBCs (Figure 9). The European Station for Time series in the Ocean Canary Islands (ESTOC) has completed over 20 years of continuous meteorological and surface and mid-water physical and biogeochemical monitoring. The Cape Verde Ocean and Atmospheric Observatories (CVOO/CVAO) are a deep water mooring and an atmospheric station that have been deployed since 2006 in a region critical for climate and greenhouse gas studies and for investigating dust impacts on marine ecosystems.
Both ESTOC and CVOO/CVAO are part of the European open ocean fixed point observatories (FixO3). An additional mooring has been recording oceanographic properties and particle fluxes with sediment traps off Cape Blanc continuously since 2003 (Nowald et al., 2015). Long-term measurements of coastal oceanic and atmospheric properties from buoys off Morocco and Senegal have begun during the last 4 years. Ship-based hydrographic and biogeochemical sampling has taken place twice per year since 2006 at the latitude of the Canary Archipelago as part of the RAPROCAN program (Figure 9), which aims to monitor the Canary Current and maintain the ESTOC mooring. Gliders have periodically surveyed between the African coast and the Cape Verde Islands (Figure 9; Karstensen et al., 2017; Kolodziejczyk et al., 2018).

\section{Western Boundary Current Systems Northwestern Atlantic}

The Gulf Stream comprises the upper limb of the AMOC in the North Atlantic subtropical gyre, carrying warm, saline waters from the tropics to higher latitudes. It flows along the eastern seaboard of the United States before separating from the continental margin near Cape Hatteras. The Labrador Current is the WBC of the subpolar gyre. The North Atlantic Deep Western Boundary Current is a deep limb of the AMOC that carries cold water masses from the tail of the Grand Banks of Newfoundland equatorward (Pickart and Watts, 1990). It encounters the Gulf Stream at the tail of the Grand Banks and again at Cape Hatteras, where a portion is entrained into the abyssal interior 


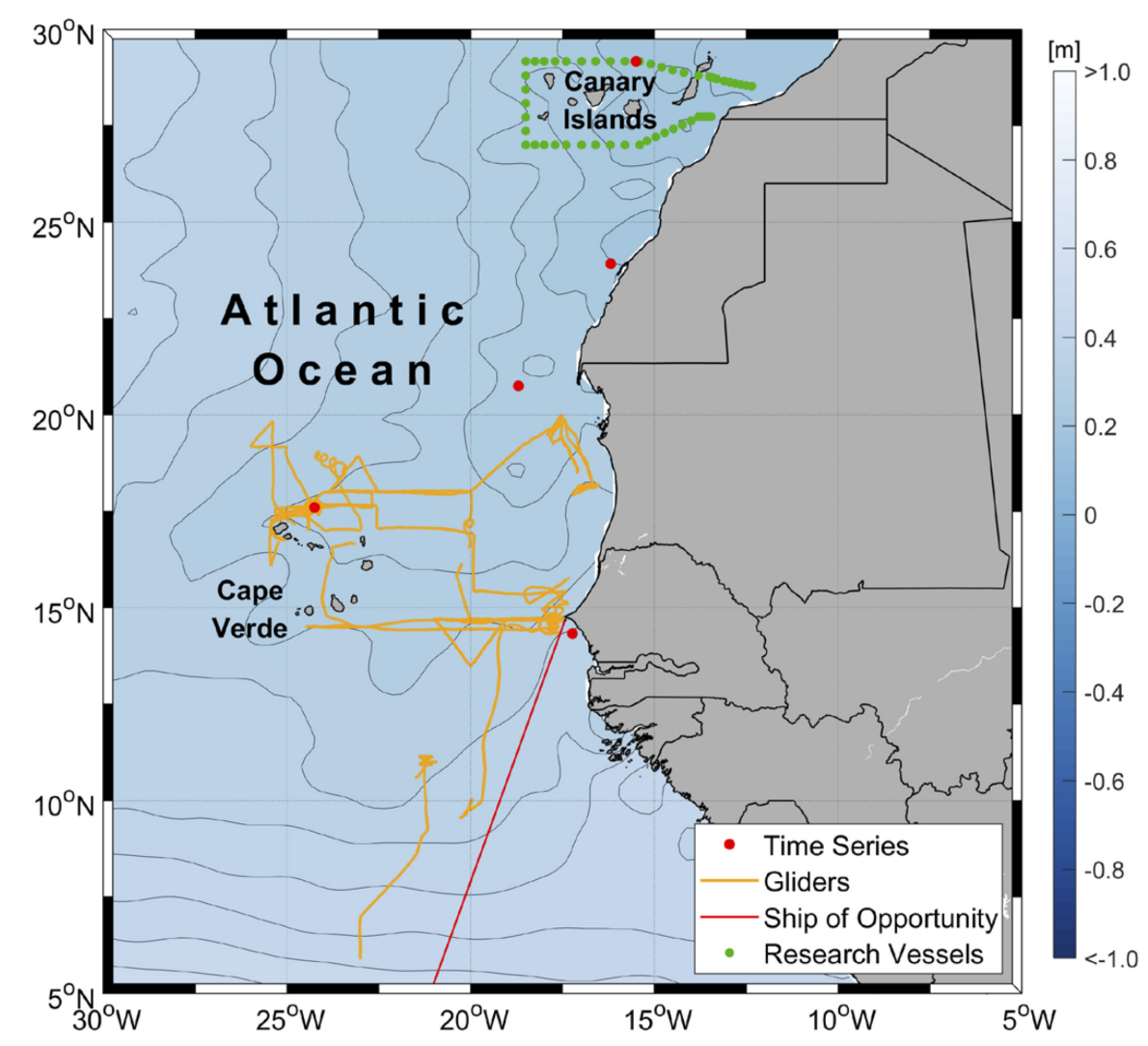

FIGURE 9 | Map of the boundary current observing effort for the Canary Current System (see the section Canary Current System) with details as in Figure 5.

(Pickart and Smethie, 1993; Bower and Hunt, 2000a,b) while the rest continues to flow equatorward along the western boundary and into the Southern Hemisphere (Southwestern Atlantic). The strength of the Deep Western Boundary Current may influence the latitude at which the Gulf Stream detaches from the continental margin (Thompson and Schmitz, 1989). Along the edge of the adjacent Middle Atlantic Bight shelf, a persistent shelfbreak front and associated shelfbreak jet (Linder and Gawarkiewicz, 1998) transport waters equatorward with secondary frontal circulation leading to upwelling and elevated primary productivity (Marra et al., 1990). The shelfbreak jet continues southward until just north of Cape Hatteras, where it turns offshore as it encounters the much stronger Gulf Stream (Gawarkiewicz and Linder, 2006).

The boundary current observing system for the subtropical northwest Atlantic (Figure 10) is anchored by decades-long measurements at several fixed locations along the boundary. In the Florida Strait near $27^{\circ} \mathrm{N}$, cable-based measurements of Gulf Stream transport and quarterly to bi-monthly ship-based sampling have been ongoing since 1982 as part of the Western Boundary Time Series (WBTS; Baringer and Larsen, 2001; Meinen et al., 2010). Far to the northeast, where the Gulf Stream has separated from the continental margin, XBT, shipboard ADCP, and surface temperature and salinity measurements are obtained twice weekly from $M / V$ Oleander, a cargo ship running between New Jersey and Bermuda (Rossby et al., 2010, 2014;
Wang et al., 2010). The AX10 XBT line between New York and Puerto Rico crosses the Gulf Stream just upstream of the Oleander line and conducts high-resolution sampling within the boundary current (e.g., Domingues et al., 2018). Since 2015, gliders have been used to routinely survey across the Gulf Stream between Florida and Massachusetts (Figure 1; Todd, 2017; Todd and Locke-Wynn, 2017; Todd et al., 2018b), providing subsurface observations that fill the gap between the WBTS and Oleander and AX10 lines. Two moored arrays with complementary repeat hydrographic sampling have focused on the Deep Western Boundary Current for a decade or more. The RAPID-MOCHA array of subsurface moorings and PIES near $26.5^{\circ} \mathrm{N}$ has been in place since 2004 with hydrographic stations reoccupied about every 9 months (Meinen et al., 2013). Farther north, the Line W array of subsurface moorings was in place from 2004 to 2014 with repeat ship-based sampling every 6-12 months (Toole et al., 2017). The OOI Pioneer Array south of New England (Smith et al., 2018; Trowbridge et al., 2019) and the PEACH array near Cape Hatteras use a mixture of moorings, gliders (e.g., Gawarkiewicz et al., 2018), and land-based remote sensing (e.g., Haines et al., 2017) to characterize the dynamics of the shelfbreak jet and exchange between the shelf and deep ocean in the vicinity of the Gulf Stream and its eddies. In the subpolar northwestern Atlantic at $53^{\circ} \mathrm{N}$, transport of the Labrador Current has been monitored since 1997 using a combination of moored and shipboard observations (Zantopp et al., 2017). 


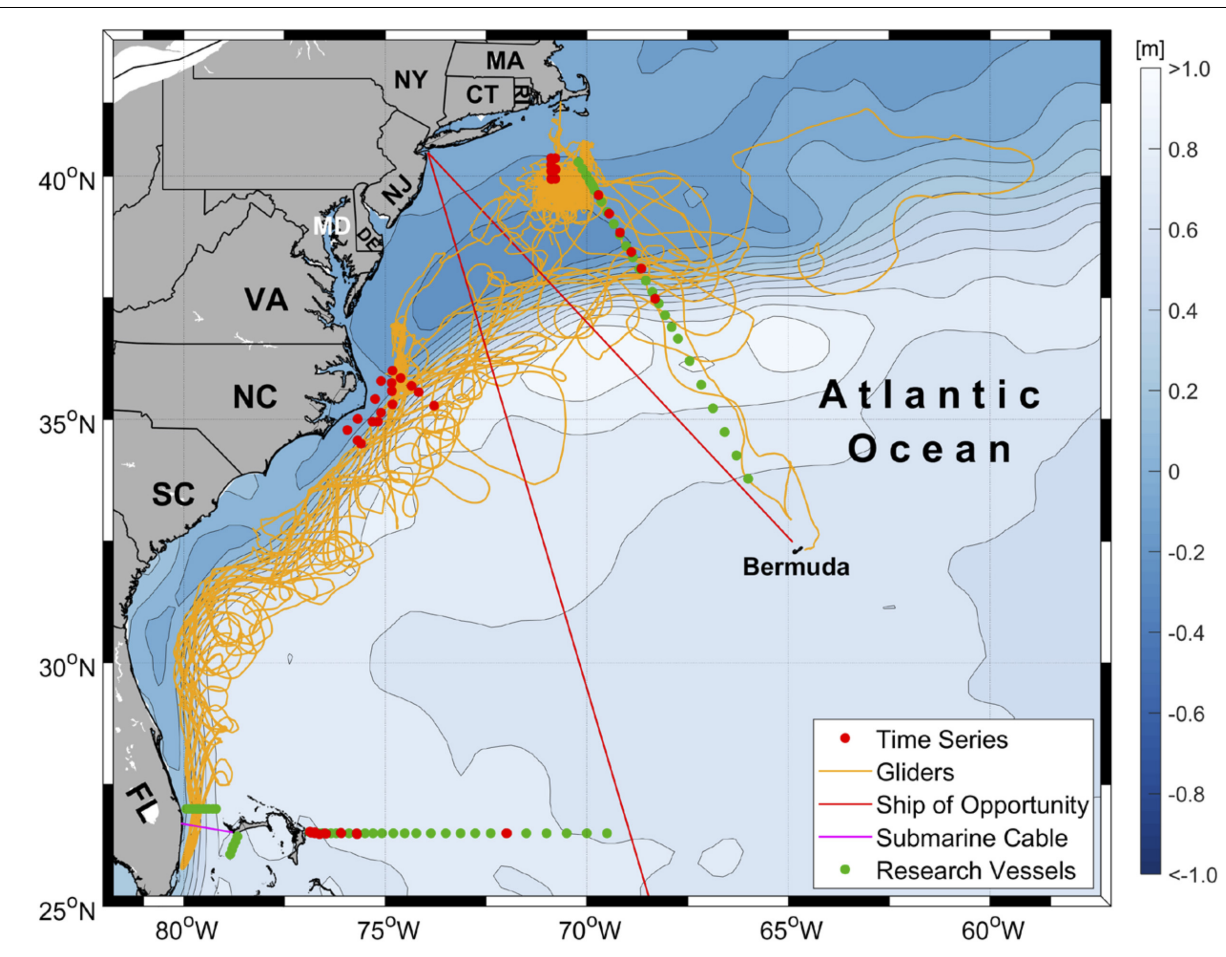

FIGURE 10 | Map of the boundary current observing efforts for the Northwestern Atlantic (see the section Northwestern Atlantic) with details as in Figure $\mathbf{5}$ and the addition of the submarine cable location in the Florida Strait.

\section{Northwestern Pacific}

In the Northwestern Pacific, bifurcation of the westward North Equatorial current between $11^{\circ}$ and $13^{\circ} \mathrm{N}$ along the Philippine coast (Qiu and Chen, 2010; Rudnick et al., 2015b) forms the poleward Kuroshio and the equatorward Mindanao Current. The Kuroshio becomes a more coherent jet as it flows along the Taiwanese coast (e.g., Centurioni et al., 2004), into the East China Sea, and along the southern Japanese coast before separating from the continental margin near $35^{\circ} \mathrm{N}$ to form the Kuroshio Extension, an eastward, meandering jet in the open North Pacific. The Mindanao carries waters from the North Pacific southward to feed tropical circulation in both the Pacific and Indian Oceans (Schönau et al., 2015). The Oyashio is the western boundary current of the North Pacific subpolar gyre and converges with the Kuroshio to the east of Japan. This convergence region has rich frontal structure as various water masses meet and are modified and is a key area for fisheries (Yasuda, 2003).

The Japan Meteorological Agency (JMA) has carried out repeat hydrographic surveys two to five times annually at the PN line in the East China Sea since 1971 (Aoyama et al., 2008; Figure 11) and at the TK line south of Kyushu since 1987 (Oka and Kawabe, 2003) to monitor physical and biogeochemical EOVs in the Kuroshio. JMA has also monitored the Ryukyu Current system (Ichikawa et al., 2004) flowing south of the Ryukyu Islands at the OK line southeast of Okinawa, which is connected to a zonal section along $24^{\circ} \mathrm{N}$. Furthermore, the JMA has maintained physical and biogeochemical surveys along $137^{\circ} \mathrm{E}$ across the western North Pacific to monitor major currents of the subtropical and tropical gyres including the Kuroshio (Nakano et al., 2015; Oka et al., 2018). Monthly fisheries surveys and hydrographic stations along the A-line off Hokkaido have been occupied since 1987 (Kuroda et al., 2015) with collocated long-term moorings (Kono and Kawasaki, 1997). JAMSTEC has sampled hydrographic stations $\mathrm{K} 2\left(47^{\circ} \mathrm{N}, 160^{\circ} \mathrm{E}\right)$ and KNOT $\left(44^{\circ} \mathrm{N}, 155^{\circ} \mathrm{E}\right)$ in the subpolar north Pacific at least annually since 1997 (Wakita et al., 2010). The Kuroshio Extension Observatory (KEO; Cronin et al., 2015) is a surface mooring that has been located in the subtropical recirculation gyre south of the Kuroshio Extension at $32.3^{\circ} \mathrm{N}, 144.6^{\circ} \mathrm{E}$ (Figure 11) since 2004. KEO monitors air-sea exchanges of heat, moisture, momentum, and $\mathrm{CO}_{2}$; sea surface temperature, salinity, and ocean acidification; and upper ocean temperature, salinity, and currents below the surface buoy. Since 2014, a sediment trap mooring has been located at KEO (Honda et al., 2018). More recently, the CLIVAR Northwestern Pacific Ocean Circulation and Climate Experiment (NPOCE) has deployed an array of subsurface moorings, some with real-time data transmission, across the western Pacific, South China Sea, and Indonesian seas (Figure 11) that cover the major currents in these regions (e.g., Hu et al., 2013, 2015, 2016; Zhang et al., 2014; Chen et al., 2015; Wang et al., 2017). Multiple XBT transects cross boundary currents within the region (see Goni et al., 2019). Gliders have been deployed for extended periods in the Kuroshio and Mindanao (Figure 11), generally sampling obliquely across the boundary currents as they were advected downstream (e.g., Rainville et al., 2013; Schönau and Rudnick, 2017). 


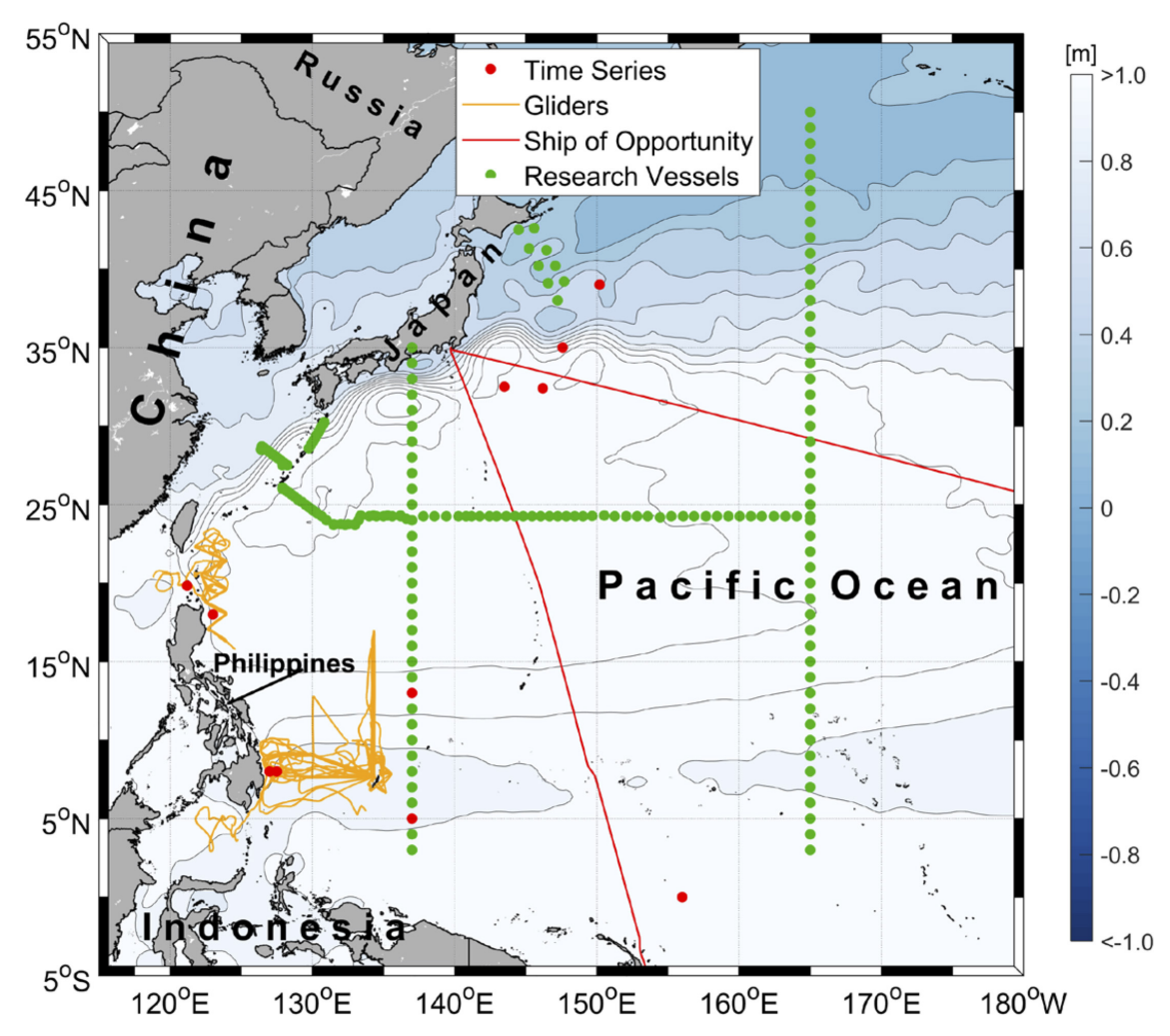

FIGURE 11 | Map of the boundary current observing effort for the Northwestern Pacific (see the section Northwestern Pacific) with details as in Figure $\mathbf{5}$.

\section{Southwestern Pacific}

The East Australian Current is the subtropical WBC of the South Pacific; it is a strong, meandering current with large poleward heat transport (Sloyan et al., 2016) that separates from the continental margin between $30^{\circ} \mathrm{S}$ and $32^{\circ} \mathrm{S}$ to join a dynamic eddy field (Cetina Heredia et al., 2014) in the Tasman Sea. The low-latitude WBC system of the South Pacific originates as the equatorward Gulf of Papua Current along the northeast coast of Australia, which then flows through the Solomon Sea as the New Guinea Coastal Undercurrent before feeding into the equatorial current system. This is a major contributor to the mass and heat budget of the tropical Pacific, acting as a conveyor belt for micronutrients from the western continental margins to the eastern Equatorial Pacific upwelling region. These low-latitude WBCs split into numerous branches around topographic obstacles and flow through narrow passages, presenting challenges for sustained observing.

The sustained observing system for the East Australian Current and its extension (Figure 7) currently consists of highdensity XBT transects (PX05, PX06, PX30, and PX34; Goni et al., 2019); Argo floats; a deep moored array at approximately $27^{\circ} \mathrm{S}$; HF radar sites near $32^{\circ} \mathrm{S}$ and $30^{\circ} \mathrm{S}$; a regional array of shelf moorings (including biogeochemical and biological sensors) at $30^{\circ} \mathrm{S}, 34^{\circ} \mathrm{S}$, and $36^{\circ} \mathrm{S}$; and numerous glider deployments from northern Australia $\left(11^{\circ} \mathrm{S}\right)$ to the Tasman Sea $\left(42^{\circ} \mathrm{S}\right)$ (Roughan and Morris, 2011; Roughan et al., 2013, 2015). These observational platforms complement each other well, providing a distributed boundary current observational system for the East Australian Current that has been shown to constrain ocean models well (Kerry et al., 2018). Additional sustained measurements are needed to characterize the seasonal changes in the transports of mass, heat, and freshwater in the East Australian Current and its eddy field. Effective monitoring strategies would be to deploy moored arrays in key regions; to increase Argo float and drifter density in the WBC region; and to implement glider sampling along existing high-density XBT lines within the East Australian Current, its eddy field, and recirculation.

In the low-latitude WBC system, long-term, sustained observations of the heat and mass transport through the southern entrance of the Solomon Sea have been provided by gliders since 2007 (Davis et al., 2012) and an array of PIES since 2012 (Figure 7). Concurrent, short-term process studies including mooring deployments have been conducted as part of the CLIVAR-SPICE program (Ganachaud et al., 2014). Future monitoring efforts should integrate measurements across platforms, with the existing measurements in the southern entrance complemented by observations at the northern exits of the Solomon Sea (e.g., moorings, HF radars, and glider transects) to resolve the partitioning of the flow joining the equator (see Smith et al., 2019).

\section{Agulhas Current}

The Agulhas Current is the poleward WBC of the subtropical South Indian Ocean (Lutjeharms, 2006). It flows as a fast 
$\left(>1.5 \mathrm{~m} \mathrm{~s}^{-1}\right)$, deep-reaching $(>3,000 \mathrm{~m})$ jet along the continental slope of southeast Africa (Beal and Bryden, 1999; Beal et al., 2015). Near $40^{\circ} \mathrm{S}$, the Agulhas flows into the open ocean, where it retroflects under the influence of the large-scale wind stress curl to flow eastward into the Indian Ocean as the Agulhas Return Current (de Ruijter et al., 1999). Leakage of warm, salty Agulhas waters into the South Atlantic by rings, eddies, and filaments (Boebel et al., 2003; Richardson, 2007) is thought to influence the AMOC on timescales from decades to millennia (Beal et al., 2011).

In 2010, the Agulhas Current Time-series experiment (ACT) established a moored array to measure the volume transport of the Agulhas Current along a satellite altimeter ground-track (\#96) near $34^{\circ} \mathrm{S}$ (Figure 8) for a period of 3 years. The array consisted of seven full-depth current meter moorings and four CPIES that captured the breadth and depth of the Agulhas jet, including during offshore meander events (Beal et al., 2015). Following ACT, a consortium of South African, U.S., and Dutch scientists deployed the Agulhas System Climate Array (ASCA) in 2016 for long-term monitoring of the Agulhas Current as part of GOOS. ASCA augmented the original ACT array design with conductivity-temperature recorders to measure the heat and freshwater fluxes. The long-term success of ASCA was dependent on an ambitious plan of capacity building and resource sharing among nations, and, owing to a number of challenges, this plan was not fulfilled, and the array was pulled out of the water in 2018, following a 2-year deployment. In 2015, the Shelf Agulhas Glider Experiment (SAGE) demonstrated the feasibility of operating autonomous robotic platforms to sample the shelf regions of the Agulhas Current (Krug et al., 2017). Since SAGE, growing regional interest in monitoring with autonomous platforms led to formation of a South African multi-institutional scientific consortium named Gliders in the Agulhas (GINA). GINA conducted two additional glider missions in 2017 and 2018 and is working toward the development of a sustained glider observing system for the Agulhas Current coastal and shelf regions. The influence of the Agulhas leakage on the AMOC has been monitored since 2013 by an array of CPIES and tall moorings as part of the SAMBA line at $34.5^{\circ} \mathrm{S}$ (Figure 8; Ansorge et al., 2014). Thus far, no sustained ecological or biogeochemical measurements have been made in the Agulhas, though the addition of oxygen sensors to SAMBA moorings is planned.

\section{Southwestern Atlantic}

In the South Atlantic, the WBC system consists of the poleward Brazil Current and the equatorward North Brazil Undercurrent, both of which originate from the bifurcation of the South Equatorial Current between $10^{\circ} \mathrm{S}$ and $20^{\circ} \mathrm{S}$ (e.g., da Silveira et al., 1994; Rodrigues et al., 2007), and the equatorward Malvinas current in the subpolar gyre. The Brazil Current and Malvinas Current both separate from the South American continental margin between $35^{\circ} \mathrm{S}$ and $40^{\circ} \mathrm{S}$ to flow eastward at the BrazilMalvinas confluence (Olson et al., 1988). The North Brazil Undercurrent constitutes a bottleneck for the interhemispheric mean flow of the upper limb of the AMOC as it transports warm waters of South Atlantic origin across the equator (e.g., Schott et al., 1998; Zhang et al., 2011; Rühs et al., 2015). The Deep
Western Boundary Current carries much of the lower limb of the AMOC off the coast of South America (Schott et al., 2005; Meinen et al., 2013).

For more than a decade, high-density XBT transects (Goni et al., 2019) have been occupied near $22^{\circ} \mathrm{S}$ and $34^{\circ} \mathrm{S}$ (AX97 and AX18) across South Atlantic WBCs (Figure 12; Dong et al., 2015; Lima et al., 2016). Near $11^{\circ} \mathrm{S}$, an array of four tall moorings and two PIES has measured transport of the North Brazil Current since 2013 (Figure 12; Hummels et al., 2015). At $34.5^{\circ} \mathrm{S}$ (Figure 12), an array of PIES, CPIES, and a bottom-mounted ADCP has monitored the Brazil Current and Deep Western Boundary Current (Meinen et al., 2013, 2017, 2018) in conjunction with periodic hydrographic surveys (Valla et al., 2018). A series of yearlong deployments of current meter arrays along $41^{\circ} \mathrm{S}$ since 1993 (Figure 12; Vivier and Provost, 1999; Spadone and Provost, 2009; Paniagua et al., 2018), in conjunction with satellite altimetry, has allowed for production of a 24-year transport time series for the Malvinas Current (Artana et al., 2018a).

\section{FUTURE OUTLOOK}

We recommend establishing and maintaining a global network of boundary current observing systems. Each distinct observing system will need to be tailored to the unique aspects of that particular boundary current system and also follow best practices established by the international community. Such a network of regional boundary current observing systems is a crucial element of GOOS. To date, boundary current observing systems in different regions and countries have developed largely independently. Development and maintenance of a global network of boundary current observing systems that is fit for purpose would benefit from the standards outlined in the Framework for Ocean Observing (Lindstrom et al., 2012). In particular, application of the Framework across boundary current observing systems should foster communication and data sharing; contribute to capacity building, particularly in developing countries; encourage confidence and support from funding agencies; and promote international collaboration and scientific and technological innovation.

Boundary currents typically lie within the EEZs of coastal states, so development and maintenance of boundary current observing systems will require the cooperation and support of appropriate governing authorities. Considering the difficulty of obtaining international funding for observations in national waters, there is a need for a community of regional boundary observers. Moreover, many boundary currents span multiple countries, so the observing system for a single boundary current system is likely to require collaboration and coordination between several countries. The advective nature of boundary currents may even require that mobile or drifting assets deployed within one country's EEZ be recovered within another EEZ. Sharing of measurements taken within EEZs, particularly those that have economic impacts such as some biogeochemical measurements, remains a challenge. By moving toward international collaboration in the design and implementation 


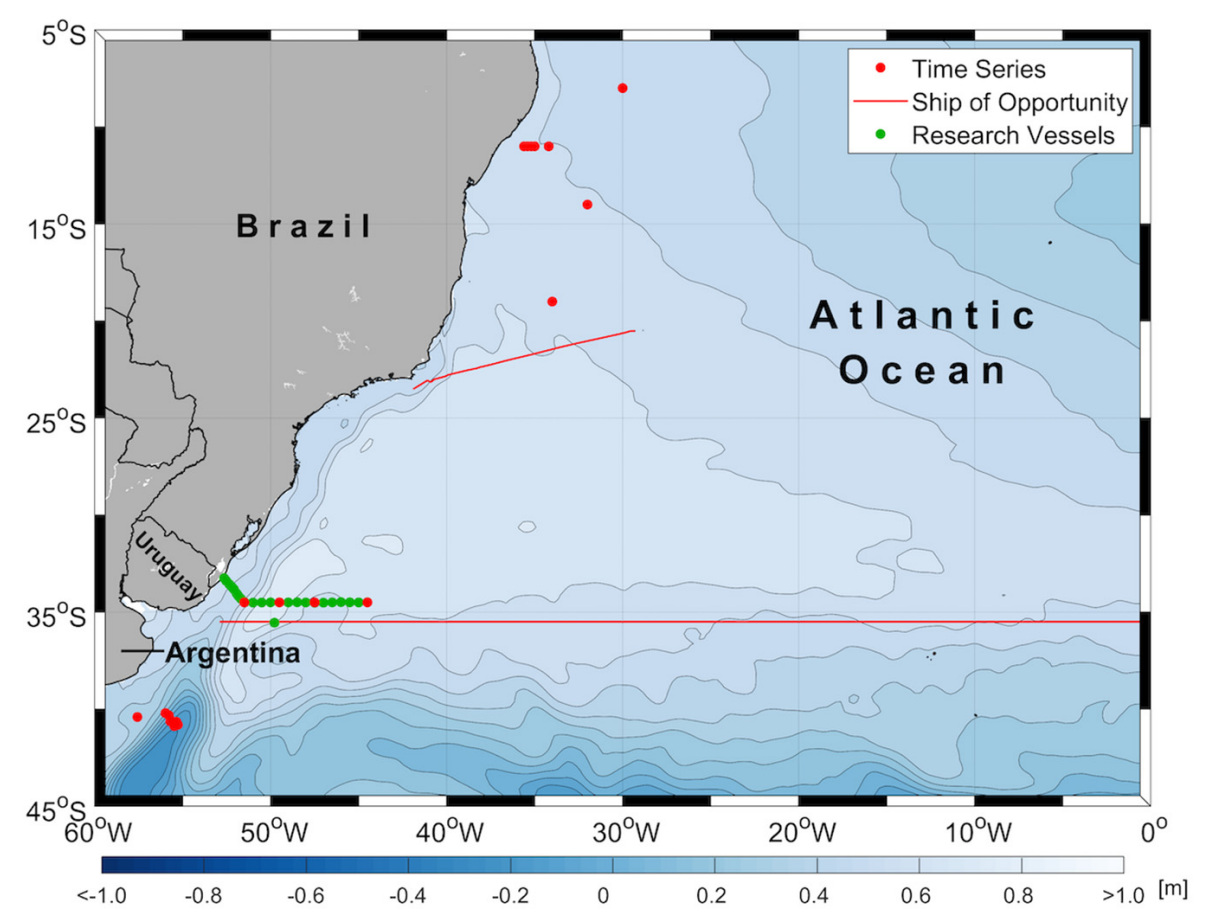

FIGURE 12 | Map of the boundary current observing system for the Southwestern Atlantic (see the section Southwestern Atlantic) with details as in Figure $\mathbf{5}$.

of boundary current systems as suggested by the Framework for Ocean Observing, there is hope for building the high-level governance structure needed to surmount the challenges posed by boundary currents falling within EEZs. The Large Marine Ecosystems effort has identified distinct boundary regions that cross international borders and has gained international traction through the Global Environment Facility and the International Union for Conservation of Nature; leveraging this effort to facilitate international cooperation and governance for sustained boundary current observations may be fruitful.

For any particular boundary current system, a complete observing system will require a combination of currently available observing platforms (see the section Observing Techniques), as well as future platforms, to optimally measure EOVs at necessary spatial and temporal resolutions to address relevant scientific and societal needs. Through the Framework process, specific observing platforms, sampling choices, and instruments would be matched to the relevant questions. Drifting and mobile assets that provide spatially resolved measurements at the expense of temporal resolution will need to be combined with moored assets that provide high-frequency measurements at key locations and land- or satellite-based remote sensing that provides spatially broad measurements of sea surface properties. Such integrated arrays, as are already in place in the California Current System, at the Ocean Observatories Initiative (OOI; Smith et al., 2018; Trowbridge et al., 2019) Endurance and Pioneer Arrays, and along the Australian coasts as part of the Integrated Marine Observing System, offer critical opportunities for intercalibration between instruments on fixed and mobile assets; such intercalibration is particularly important for biogeochemical sensors (e.g., Palevsky and Nicholson, 2018). Since similar needs arise in most boundary current systems, the Framework process should provide a means for determining the extent to which the same observing strategies should be applied to address similar needs in different systems. Additional studies that compare different sampling techniques in a given boundary current system could provide guidance on the strengths and limitations of each technique and how to better exploit their complementarity.

While the discussion of observing platforms in the section Observing Techniques focused on mature observing platforms with proven records of sustained operation in boundary currents, there is no doubt that recently developed observing platforms and sensing technology will become integral parts of future boundary current observing systems. For instance, more fast-moving autonomous underwater vehicles (AUVs) and autonomous surface vehicles (ASVs) will be deployed to conduct adaptive and targeted sampling in response to real-time needs. Propellerdriven AUVs have thus far seen limited use in boundary currents. Though able to carry large instrument payloads and move much faster $\left(1-2 \mathrm{~m} \mathrm{~s}^{-1}\right)$ than gliders, propeller-driven AUVs have been limited by battery endurance to missions typically lasting hours to days; improvements in battery technology and autonomous charging are expected to make propeller-driven vehicles capable of long-duration sampling in the near future. Fast-moving, longendurance ASVs (e.g., Saildrones and WaveGliders) are poised to become key platforms for making measurements near the air-sea interface, including meteorological measurements, $\mathrm{pCO}_{2}$, subsurface currents, and plankton biomass. Due to the use of renewable energy, these ASVs generally carry a larger number 
of sensors and have longer duration than other autonomous platforms (e.g., Zhang et al., 2017). Planned high-resolution, satellite-based altimetry measurements (e.g., SWOT), smaller and dramatically cheaper satellites (e.g., Cubesats), and, potentially, geostationary satellites positioned over boundary regions offer the prospect of dramatically increased spatial and temporal resolution of surface properties.

At some locations, boundary currents have been continuously observed for many years using various techniques. For instance, the CalCOFI program has maintained quarterly ship-based stations for more than 65 years (McClatchie, 2014), the WBTS has made cable- and ship-based measurements in the Florida Strait for more than 35 years (see the section Northwestern Atlantic), and hydrographic sampling has occurred monthly along the inside edge of the East Australian Current since the 1940s (Lynch et al., 2014) and is now an integral part of the East Australian Current observing system (Roughan and Morris, 2011). Long-term measurements like these are invaluable for capturing decadal variability and secular trends. Sites at which decades-long measurements exist should be maintained and serve as anchors for comprehensive boundary current observing systems. These long-term measurement sites at the boundaries also serve as points at which the boundary current observing systems are linked to the basin-scale ocean observing system. Since 2004, the WBTS has been integrated with the U.K.-U.S. RAPID-MOCHA program that measures meridional transport at $26.5^{\circ} \mathrm{N}$ in the North Atlantic, while several long-standing, crossPacific XBT transects intersect the U.S. West Coast within the CalCOFI domain (Goni et al., 2019).

Existing boundary current observing systems are largely focused on measuring physical processes, with biogeochemical and ecosystem processes only beginning to gain traction, largely due to the advent of new sensors. The California Current System (see the section California Current System) and Benguela (see the section Benguela Current System) are exceptions, having had sustained observations of EOVs relevant to physics, biogeochemistry, and biology and ecosystems for over 65 and 30 years, respectively. However, these ship-intensive models are unlikely to be suited to all boundary current systems due to a wide range of factors (e.g., cost, proximity to the coast, existing infrastructure, and available manpower). Although the methods for measuring many of the EOVs needed to monitor biogeochemical and, to a greater extent, ecosystem processes are time-intensive and require a platform for collecting water, new sensors are being developed to reliably measure a range of biogeochemical and biological EOVs. Many of these sensors have been successfully deployed on BGC-Argo floats as part of the SOCCOM project (Johnson et al., 2017). Increasing the measurements of biological and ecological EOVs should be prioritized if we are to understand, monitor, and predict the physical-biological connections and processes that support marine-based industries and activities and, importantly, seafood security.

Providing publicly available data in a timely manner is a key attribute of any ocean observing system. These observations should be provided in formats that are discoverable, accessible, and readily subset, following conventions agreed upon by the community (see Wilkinson et al. (2016) for a set of general principles for management of scientific data). Many platforms already provide observations in near real-time through a variety of services. Transmission of data through the Global Telecommunications System is particularly important if those observations are to be used in operational numerical modeling. Advances in real-time data collection from sub-surface moorings (e.g., Send et al., 2013) will be critical to providing boundary current observations in a timely manner for forecasting and prediction. Widespread dissemination of comprehensive boundary current observations can foster synergies with other disciplines, including the geophysics (tsunamis and earthquakes), physics, meteorological (e.g., tropical and extratropical cyclone forecasting; Domingues et al., 2019), and fisheries communities.

In addition to providing raw observations, there is a need for providing synthesized data products that are tailored to user needs. Integration of complementary data types can yield useful metrics. Further advances in data analysis techniques and statistical methods should aid in using multi-platform data to increase temporal and/or spatial resolution of metrics. The Southern California Temperature Index (Rudnick et al., 2017) is an example of such a data product.

Boundary current observations play a key role in constraining ocean models (e.g., Todd and Locke-Wynn, 2017), while models play a complementary role by filling gaps between sparse observations in a dynamically consistent manner (e.g., Todd et al., 2011b, 2012; Gopalakrishnan et al., 2013). Increased availability of boundary current observations, particularly in regions that are currently poorly sampled, should lead to continued improvements in regional models and predictive tools. At the same time, higher resolution climate models that can resolve boundary currents are becoming more plentiful and should begin to rely on high-resolution boundary current observations as constraints. One specific goal would be to reduce climate models' warm SST biases within EBCs; continuation and expansion of long-term measurements in EBCs as well as focused process studies to study upper ocean and atmospheric dynamics in EBCs would contribute to this goal. Observation impact studies derived from data assimilating models provide guidance on the value of a range of observation types in resolving boundary current transport, as well as for constraining the eddy field in ocean reanalyses (e.g., Kerry et al., 2016, 2018). It remains an open question how best to integrate models with interdisciplinary (e.g., biogeochemical) observations to study ecosystem dynamics, though advances are being made in the assimilation of biological parameters (e.g., Song et al., 2012). Observing System Simulation Experiments tailored for boundary current systems can also provide insight to the type, spatial distribution, and frequency of observations required to improve numerical simulations of boundary current dynamics (Hoffman and Atlas, 2016). Targeted observations can reduce biases in the initialization of models used to forecast extreme weather events and support local decision making (Halliwell et al., 2017).

Downscaling coarse resolution climate model predictions through the application of higher resolution regional and coastal models is now common and has shown promise but still faces research challenges. Furthermore, a significant amount 
of physical, biogeochemical, and biological response on the continental shelf is due to episodic oceanic and atmospheric events at timescales of variability that are absent from coarse models and cannot be recovered locally. To be valid globally, the veracity of downscaled models needs to be appraised by supporting observations of shelf edge fluxes in a diversity of circulation regimes.

Funding sustained observing efforts is a significant challenge. Portions of the observing system that have proven their readiness for long-term deployment have been discontinued after one or more short-term funding cycles. For instance, it is currently not clear how ship-time and funding challenges will be met for a reestablishment of ASCA (see the section Agulhas Current) in the future. In the typical 3-to-4-year cycles of scientific funding, early years (e.g., pilot phases) of observing efforts are readily fundable based on the promise of quick scientific results. Observing efforts that have endured for a decade or longer can leverage their long histories and clear relevance to decadal variability or secular trends to secure continued funding. The middle years, roughly years 4 through 10 as programs transition from pilot to mature components of the GOOS, are particularly difficult to fund.

The provision of robust three-dimensional and time-varying ocean circulation estimates in boundary current systems, resolving scales of a few kilometers, is seemingly within reach through advances in data-assimilative ocean models and rapid developments in observations platforms and sensors. However, the development of integrated observing systems that deliver the scope of observations required and the models capable of fully utilizing those observations is challenging. Success will require coordinated international collaborations, bringing together the expertise of the ocean modeling and observational communities. Establishment of an Ocean Boundary Task Team would provide a mechanism for the exchange of information regarding observing and model strategies, sensor developments, analysis techniques to combine data from the various observing platforms, and model development and application. The Task Team would also enable capacity building, encourage timely and appropriate transfer of knowledge, and provide a mechanism to instigate multinational observing systems with shared goals amongst participating nations. Endorsement of the Task Team by IOC/WMO or similar international organization is critical due to interests of multiple coastal state EEZs and the resulting complex governance needs.

\section{SUMMARY RECOMMENDATIONS}

The following actions are recommended to promote development of a comprehensive global network of boundary current observing systems in the next decade:

(1) Maintain existing long-term (i.e., multi-year) observational records;

(2) Expand the use of mobile, autonomous platforms (e.g., gliders, AUVs, and ASVs) to provide continuous, highresolution, broad-scale monitoring of EOVs;

(3) Deploy moored platforms at key locations to measure high-frequency variability;

(4) Continue and expand the provisioning of real-time observations and encourage post-processed data to be made publicly available as quickly as possible; data should be provided in readily discoverable formats that can easily be subset;

(5) Continue development and expand deployment of sensors for ecological and biogeochemical EOVs;

(6) Establish an Ocean Boundary Task Team to foster international community development and end-user engagement and to guide evolution of observing systems as user requirements change;

(7) Expand collaborations between observational efforts, modeling efforts, and societal users to meet stakeholder and end-user needs; and

(8) Increase focus on exchange between continental shelves and the deep ocean boundary currents to develop observing systems that span the continuum from the land to the deep ocean.

\section{AUTHOR CONTRIBUTIONS}

RT led the manuscript. Other lead authors (FC, SCl, SCr, MGo, MGr, XL, JS, and NZ) helped to conceive the manuscript and participated in all the stages of development. All authors provided input and/or edited the text.

\section{FUNDING}

RT was supported by The Andrew W. Mellon Foundation Endowed Fund for Innovative Research at WHOI. FC was supported by the David and Lucile Packard Foundation. MGo was funded by NSF and NOAA/AOML. XL was funded by China's National Key Research and Development Projects (2016YFA0601803), the National Natural Science Foundation of China (41490641, 41521091, and U1606402), and the Qingdao National Laboratory for Marine Science and Technology (2017ASKJ01). JS was supported by NOAA's Global Ocean Monitoring and Observing Program (Award NA15OAR4320071). DZ was partially funded by the Joint Institute for the Study of the Atmosphere and Ocean (JISAO) under NOAA Cooperative Agreement NA15OAR4320063. BS was supported by IMOS and CSIRO's Decadal Climate Forecasting Project. We gratefully acknowledge the wide range of funding sources from many nations that have enabled the observations and analyses reviewed here.

\section{ACKNOWLEDGMENTS}

We gratefully acknowledge the efforts of all parties involved in collecting, analyzing, and disseminating the wide-ranging observations discussed here. We thank the OCB Program, US CLIVAR, MBARI, and OMIX for supporting the Ocean Carbon Hot Spots Workshop, which facilitated the discussion of carbon cycle research in Western Boundary Current systems. We also gratefully acknowledge J. Hildebrandt (WHOI) for the assistance in editing the final manuscript. This is PMEL contribution \# 4890. 


\section{REFERENCES}

Adams, K., Barth, J. A., and Shearman, R. K. (2016). Intraseasonal cross-shelf variability of hypoxia along the Newport, Oregon, hydrographic line. J. Phys. Oceanogr. 46, 2219-2238. doi: 10.1175/JPO-D-15-0119.1

Alberty, M. S. (2018). Water Mass Transport and Transformation in the Tropics and Arctic. San Diego, CA: University of California. Available at: https:// escholarship.org/uc/item/9rf158pq

Alberty, M., Sprintall, J., MacKinnon, J., Germineaud, C., Cravatte, S., and Ganachaud, A. (2019). "Data from: moored observations of transport in the Solomon Sea," in Solomon Sea SPICE Mooring Data, (La Jolla, CA: UC San Diego Library Digital Collections), doi: 10.6075/J0639N12

Alford, M. H., Sloyan, B. M., and Simmons, H. L. (2017). Internal waves in the East Australian Current. Geophys. Res. Lett. 44, 12280-12288. doi: 10.1002/ 2017GL075246

Alin, S. R., Feely, R. A., Dickson, A. G., Hernández-Ayón, J. M., Juranek, L. W., Ohman, M. D., et al. (2012). Robust empirical relationships for estimating the carbonate system in the southern California Current System and application to CalCOFI hydrographic cruise data (2005-2011). J. Geophys. Res. Oceans 117:C05033. doi: 10.1029/2011JC007511

Álvarez-Salgado, X. A., Arístegui, J., Barton, E. D., and Hansell, D. A. (2007). Contribution of upwelling filaments to offshore carbon export in the subtropical Northeast Atlantic Ocean. Limnol. Oceanogr. 52, 1287-1292. doi: 10.4319/lo. 2007.52.3.1287

Andersson, A. J., Krug, L. A., Bates, N. R., and Doney, S. C. (2013). Sea-air CO2 flux in the North Atlantic subtropical gyre: role and influence of sub-tropical mode water formation. Deep Sea Res. Part II Top. Stud. Oceanogr. 91, 57-70. doi: $10.1016 /$ j.dsr2.2013.02.022

Andres, M. (2016). On the recent destabilization of the Gulf Stream path downstream of Cape Hatteras. Geophys. Res. Lett. 43, 9836-9842. doi: 10.1002/ 2016GL069966

Andres, M., Jan, S., Sanford, T. B., Mensah, V., Centurioni, L. R., and Book, J. W. (2015). Mean structure and variability of the Kuroshio from northeastern Taiwan to southwestern Japan. Oceanography 28, 84-95. doi: 10.5670/oceanog. 2015.84

Ansorge, I. J., Baringer, M. O., Campos, E. J. D., Dong, S., Fine, R. A., Garzoli, S. L., et al. (2014). Basin-wide oceanographic array bridges the South Atlantic. EOS 95, 53-54. doi: 10.1002/2014eo060001

Aoyama, M., Goto, H., Kamiya, H., Kaneko, I., Kawae, S., Kodama, H., et al. (2008). Marine biogeochemical response to a rapid warming in the main stream of the Kuroshio in the western North Pacific. Fish. Oceanogr. 17, 206-218. doi: $10.1111 / j .1365-2419.2008 .00473 . x$

Archer, M. R., Keating, S. R., Roughan, M., Johns, W. E., Lumkpin, R., BeronVera, F., et al. (2018). The kinematic similarity of two western boundary currents revealed by sustained high-resolution observations. Geophys. Res. Lett. 45, 6176-6185. doi: 10.1029/2018GL078429

Archer, M. R., Roughan, M., Keating, S. R., and Schaeffer, A. (2017a). On the variability of the East Australian Current: jet structure, meandering, and influence on shelf circulation. J. Geophys. Res. Oceans 122, 8464-8481. doi: 10.1002/2017JC013097

Archer, M. R., Shay, L. K., and Johns, W. E. (2017b). The surface velocity structure of the Florida Current in a jet coordinate frame. J. Geophys. Res. Oceans 122, 9189-9208. doi: 10.1002/2017JC013286

Archer, M. R., Shay, L. K., Jaimes, B., and Martinez-Pedraja, J. (2015). "Observing frontal instabilities of the Florida Current using high frequency radar," in Coastal Ocean Observing Systems, eds Y. Liu, H. Kerkering, and R. H. Weisberg (Amsterdam: Elsevier, Inc.), 179-208. doi: 10.1016/B978-0-12802022-7.00011-0

Arístegui, J., Alvarez-Salgado, X. A., Barton, E. D., Figueiras, F. G., HernándezLeón, S., Roy, C., et al. (2006). "Oceanography and fisheries of the Canary Current/Iberian region of the eastern North Atlantic," in The Sea, Vol. 14, eds A. R. Robinson and K. H. Brink (Cambridge, MA: Harvard University Press), 877-931.

Arístegui, J., Barton, E. D., Alvarez Salgado, X. A., Santos, A. M. P., Figueiras, F. G., Kifani, S., et al. (2009). Sub-regional ecosystem variability in the Canary Current upwelling. Prog. Oceanogr. 83, 33-48. doi: 10.1016/j.pocean.2009.07.031

Arístegui, J., Sangrà, P., Hernández-León, S., Cantón, M., Hernández Guerra, A., and Kerling, J. L. (1994). Island-induced eddies in the Canary
Islands. Deep Sea Res. I 41, 1509-1525. doi: 10.1016/0967-0637(94)90 058-2

Arístegui, J., Tett, P., Hernández-Guerra, A., Basterretxea, G., Montero, M. F., Wild, K., et al. (1997). The influence of island-generated eddies on chlorophyll distribution: a study of mesoscale variation around Gran Canaria. Deep Sea Res. 44, 71-96. doi: 10.1016/s0967-0637(96)00093-3

Artana, C., Ferrari, R., Koenig, Z., Saraceno, M., Piola, A. R., and Provost, C. (2016). Malvinas Current variability from argo floats and satellite altimetry. J. Geophys. Res. Oceans 121, 4854-4872. doi: 10.1002/2016JC011889

Artana, C., Ferrari, R., Koenig, Z., Sennechael, N., Saraceno, M., Piola, A. R., et al. (2018a). Malvinas Current volume transport at $41^{\circ} \mathrm{S}$ : a 24 yearlong time series consistent with mooring data from 3 decades and satellite altimetry. J. Geophys. Res. Oceans 123, 378-398. doi: 10.1002/2017JC013600

Artana, C., Lellouche, J., Park, Y., Garric, G., Koenig, Z., Sennéchael, N., et al. (2018b). Fronts of the Malvinas Current System: surface and subsurface expressions revealed by satellite altimetry, Argo floats, and Mercator operational model outputs. J. Geophys. Res. Oceans 123, 5261-5285. doi: 10. 1029/2018JC013887

Auad, G., Roemmich, D., and Gilson, J. (2011). The California Current System in relation to the northeast Pacific ocean circulation. Prog. Oceanogr. 91, 576-592. doi: $10.1016 /$ j.pocean.2011.09.004

Bacon, S., and Saunders, P. M. (2010). The deep western boundary current at Cape Farewell: results from a moored current meter array. J. Phys. Oceanogr. 40, 815-829. doi: 10.1175/2009jpo4091.1

Bakun, A., Black, B. A., Bograd, S. J., Garcia-Reyes, M., Miller, A. J., Rykaczewski, R. R., et al. (2015). Anticipated effects of climate change on coastal upwelling ecosystems. Curr. Clim. Change Rep. 1, 85-93. doi: 10.1007/s40641-015-0008-4

Bane, J. M., He, R., Muglia, M., Lowcher, C. F., Gong, Y., and Haines, S. M. (2017). Marine hydrokinetic energy from western boundary currents. Annu. Rev. Mar. Sci. 9, 105-123. doi: 10.1146/annurev-marine-010816-060423

Baringer, M. O., and Larsen, J. (2001). Sixteen years of Florida Current transport at 27N. Geophys. Res. Lett. 28, 3179-3182. doi: 10.1029/2001GL013246

Barton, A. D., Dutkiewicz, S., Flierl, G., Bragg, J., and Follows, M. J. (2010). Patterns of diversity in marine phytoplankton. Science 327, 1509-1511. doi: 10.1126/ science. 1184961

Barton, E. D., and Arístegui, J. (2004). The Canary Islands transition-upwelling, eddies and filaments. Prog. Oceanogr. 62, 67-69. doi: 10.1016/j.pocean.2004. 08.003

Barton, E. D., Arístegui, J., Tett, P., Cantón, M., García-Braun, J., Hernández-León, S., et al. (1998). The transition zone of the Canary Current upwelling region. Prog. Oceanogr. 41, 455-504. doi: 10.1016/s0079-6611(98)00023-8

Bates, N., Pequignet, A. C., Johnson, R. J., and Gruber, N. (2002). A variable sink for atmospheric $\mathrm{CO}_{2}$ in subtropical mode water of the North Atlantic Ocean. Nature 420, 489-493. doi: 10.1038/nature01253

Baumgartner, M. F., and Fratantoni, D. M. (2008). Diel periodicity in both sei whale vocalization rates and the vertical migration of their copepod prey observed from ocean gliders. Limnol. Oceanogr. 53, 2197-2209. doi: 10.4319/lo.2008.53. 5_part_2.2197

Beal, L. M., and Bryden, H. L. (1999). The velocity and vorticity structure of the Agulhas Current at $32^{\circ}$ S. J. Geophys. Res. 104, 5151-5176. doi: 10.1029/ 1998JC900056

Beal, L. M., and Elipot, S. (2016). Broadening not strengthening of the Agulhas Current since the early 1990s. Nature 540, 570-573. doi: 10.1038/nature19853

Beal, L. M., De Ruijter, W. P. M., Biastoch, A., Zahn, R., Cronin, M., Hermes, J., et al. (2011). On the role of the Agulhas system in ocean circulation and climate. Nature 472, 429-436. doi: 10.1038/nature09983

Beal, L. M., Elipot, S., Houk, A., and Leber, G. M. (2015). Capturing the transport variability of a western boundary jet: results from the Agulhas Current TimeSeries Experiment (ACT). J. Phys. Oceanogr. 45, 1302-1324. doi: 10.1175/JPOD-14-0119.1

Beal, L. M., Hormann, V., Lumpkin, R., and Foltz, G. R. (2013). The response of the surface circulation of the Arabian Sea to monsoonal forcing. J. Phys. Oceanogr. 43, 2008-2022. doi: 10.1175/JPO-D-13-033.1

Bednaršek, N., Feely, R. A., Beck, M. W., Glippa, O., Kanerva, M., and EngströmÖst, J. (2018). El Niño-related thermal stress coupled with upwelling-related ocean acidification negatively impacts cellular to population-level responses in pteropods along the California Current System with implications for increased bioenergetic costs. Front. Mar. Sci. 4:486. doi: 10.3389/fmars.2018.00486 
Bednaršek, N., Feely, R. A., Reum, J. C. P., Peterson, B., Menkel, J., Alin, S. R., et al. (2014). Limacina helicina shell dissolution as an indicator of declining habitat suitability owing to ocean acidification in the California Current Ecosystem. Proc. Biol. Sci. 281:20140123. doi: 10.1098/rspb.2014. 0123

Bednaršek, N., Feely, R. A., Tolimieri, N., Hermann, A. J., Siedlecki, S. A., Waldbusser, G. G., et al. (2017). Exposure history determines pteropod vulnerability to ocean acidification along the US West Coast. Sci. Rep. 7:4526. doi: 10.1038/s41598-017-03934-z

Benazzouz, A., Mordane, S., Orbi, A., Chagdali, M., Hilmi, K., Atillah, A., et al. (2014). An improved coastal upwelling index from sea surface temperature using satellite-based approach-the case of the Canary Current upwelling system. Cont. Shelf Res. 81, 38-54. doi: 10.1016/j.csr.2014.03.012

Bertrand, A., Chaigneau, A., Peraltilla, S., Ledesma, J., Graco, M., Monetti, F., et al. (2011). Oxygen: a fundamental property regulating pelagic ecosystem structure in the coastal southeastern tropical Pacific. PLoS One 6:e29558. doi: 10.1371/journal.pone.0029558

Bigorre, S. P., Weller, R. A., Edson, J. B., and Ware, J. D. (2013). A surface mooring for air-sea interaction research in the Gulf Stream. Part II: analysis of the observations and their accuracies. J. Atmos. Ocean. Technol. 30, 450-469. doi: 10.1175/JTECH-D-12-00078.1

Boebel, O., Lutjeharms, J., Schmid, C., Zenk, W., Rossby, T., and Barron, C. (2003). The cape cauldron: a regime of turbulent inter-ocean exchange. Deep Sea Res. Part II 50, 57-86. doi: 10.1016/S0967-0645(02)00379-X

Boening, C., Willis, J. K., Landerer, F. W., Nerem, R. S., and Fasullo, J. (2012). The 2011 La Niña: so strong, the oceans fell. Geophys. Res. Lett. 39:L19602. doi: 10.1029/2012GL053055

Bond, N. A., Cronin, M. F., Sabine, C., Kawai, Y., Ichikawa, H., Freitag, P., et al. (2011). Upper ocean response to Typhoon Choi-wan as measured by the Kuroshio Extension Observatory mooring. J. Geophys. Res. 116:C02031. doi: 10.1029/2010JC006548

Bowen, M., Markham, J., Sutton, P., Zhang, X., Wu, Q., Shears, N. T., et al. (2017). Interannual variability of sea surface temperature in the southwest Pacific and the role of ocean dynamics. J. Clim. 30, 7481-7492. doi: 10.1175/JCLI-D-160852.1

Bower, A. S., and Hunt, H. D. (2000a). Lagrangian observations of the Deep Western Boundary Current in the North Atlantic Ocean. Part I: large-scale pathways and spreading rates. J. Phys. Oceanogr. 30, 764-783. doi: 10.1175/ 1520-0485(2000)030<0764:lootdw>2.0.co;2

Bower, A. S., and Hunt, H. D. (2000b). Lagrangian observations of the Deep Western Boundary Current in the North Atlantic Ocean. Part II: the Gulf Stream-Deep Western Boundary Current crossover. J. Phys. Oceanogr. 30, 784-804. doi: 10.1175/1520-0485(2000)030<0784:lootdw>2.0.co;2

Brady, R. X., Lovenduski, N. S., Alexander, M. A., Jacox, M., and Gruber, N. (2019). On the role of climate modes in modulating the air-sea $\mathrm{CO}_{2}$ fluxes in eastern boundary upwelling systems. Biogeosciences 16, 329-346. doi: 10.5194/bg-16329-2019

Brandini, F. P., Boltovskoy, D., Piola, A., Kocmur, S., Röttgers, R., Abreu, P. C., et al. (2000). Multiannual trends in fronts and distribution of nutrients and chlorophyll in the southwestern Atlantic (30-62 S). Deep Sea Res. Part I 47, 1015-1033. doi: 10.1016/s0967-0637(99)00075-8

Brassington, G. B. (2010). Estimating surface divergence of ocean eddies using observed trajectories from a surface drifting buoy. J. Atmos. Ocean. Technol. 27, 705-720. doi: 10.1175/2009jtecho651.1

Brassington, G. B., Summons, N., and Lumpkin, R. (2011). Observed and simulated Lagrangian and eddy characteristics of the East Australian Current and the Tasman Sea. Deep Sea Res. 58, 559-573. doi: 10.1016/j.dsr2.2010.10.001

Bright, R. J., Xie, L., and Pietrafesa, L. J. (2002). Evidence of the Gulf Stream's influence on tropical cyclone intensity. Geophys. Res. Lett. 29:1801. doi: 10.1029/ 2002GL014920

Bushinsky, S. M., and Emerson, S. R. (2018). Biological and physical controls on the oxygen cycle in the Kuroshio Extension from an array of profiling floats. Deep Sea Res. I 141, 51-70. doi: 10.1016/j.dsr.2018.09.005

Bushinsky, S. M., Emerson, S. R., Riser, S. C., and Swift, D. D. (2016). Accurate oxygen measurements on modified Argo floats using in situ air calibrations. Limnol. Oceanogr. Methods 14, 491-505. doi: 10.1002/lom3.10107

Campagna, C., Piola, A. R., Marin, M. R., Lewis, M., and Fernández, T. (2006). Southern elephant seal trajectories, ocean fronts and eddies in the
Brazil/Malvinas Confluence. Deep Sea Res. I 53, 1907-1924. doi: 10.1016/j.dsr. 2006.08.015

Capet, X., Estrade, P., Machu, É., Ndoye, S., Grelet, J., Lazar, A., et al. (2017). On the dynamics of the southern Senegal upwelling center: observed variability from synoptic to superinertial scales. J. Phys. Oceanogr. 47, 155-180. doi: 10.1175/ JPO-D-15-0247.1

Carr, M.-E., and Kearns, E. J. (2003). Production regimes in four eastern boundary current systems. Deep Sea Res. II 50, 3199-3221. doi: 10.1016/j.dsr2.2003. 07.015

Castelao, R., Glenn, S., and Schofield, O. (2010). Temperature, salinity, and density variability in the central Middle Atlantic Bight. J. Geophys. Res. 115:C10005. doi: 10.1029/2009JC006082

Cavole, L. M., Demko, A. M., Diner, R. E., Giddings, A., Koester, I., Pagniello, C. M. L. S., et al. (2016). Biological impacts of the 2013-2015 warm-water anomaly in the Northeast Pacific: winners, losers, and the future. Oceanography 29, 273-285. doi: 10.5670/oceanog.2016.32

Cazenave, A., Dieng, H.-B., Meyssignac, B., von Schuckmann, K., Decharme, B., and Berthier, E. (2014). The rate of sea-level rise. Nat. Clim. Change 4, 358-361. doi: $10.1038 /$ nclimate2159

Centurioni, L. R. (2018). "Drifter technology and impacts for sea surface temperature, sea-level pressure, and ocean circulation studies," in Observing the Oceans in Real Time, eds R. Venkatesan, A. Tandon, E. D'Asaro, and M. A. Atmanand (Cham: Springer International Publishing), 37-57. doi: 10.1007/ 978-3-319-66493-4_3

Centurioni, L. R., and Niiler, P. P. (2003). On the surface currents of the Caribbean Sea. Geophys. Res. Lett. 30:1279. doi: 10.1029/2002GL016231

Centurioni, L. R., Hormann, V., Talley, L. D., Arzeno, I., Beal, L., Caruso, M., et al. (2017). Northern Arabian Sea Circulation-autonomous research (NASCar). A research initiative based on autonomous sensors. Oceanography 30, 74-87. doi: 10.5670/oceanog.2017.224

Centurioni, L. R., Niiler, P. N., and Lee, D.-K. (2009). Near-surface circulation in the South China Sea during the winter monsoon. Geophys. Res. Lett. 36:L06605. doi: 10.1029/2008GL037076

Centurioni, L. R., Niiler, P. P., and Lee, D.-K. (2004). Observations of inflow of Philippine sea surface water into the South China Sea through the Luzon Strait. J. Phys. Oceanogr. 34, 113-121. doi: 10.1175/1520-0485(2004)034<0113: ooiops $>2.0 . \mathrm{co} ; 2$

Centurioni, L. R., Ohlmann, J. C., and Niiler, P. P. (2008). Permanent meanders in the California Current System. J. Phys. Oceanogr. 38, 1690-1710. doi: 10.1175/ 2008JPO3746.1

Cetina Heredia, P., Roughan, M., Van Sebille, E., and Coleman, M. A. (2014). Longterm trends in the East Australian Current separation latitude and eddy driven transport. J. Geophys. Res. 119, 4351-4366. doi: 10.1002/2014JC010071

Chan, H. Y., Xu, W. Z., Shin, P. K. S., and Cheung, S. G. (2008). Prolonged exposure to low dissolved oxygen affects early development and swimming behaviour in the gastropod Nassarius festivus (Nassariidae). Mar. Biol. 153, 735-743. doi: 10.1007/s00227-007-0850-6

Chavez, F. P., and Messié, M. (2009). A comparison of eastern boundary upwelling ecosystems. Prog. Oceanogr. 83, 80-96. doi: 10.1016/j.pocean.2009.07.032

Chavez, F. P., and Toggweiler, J. R. (1995). "Physical estimates of global new production: the upwelling contribution," in Upwelling in the Ocean: Modern Processes and Ancient Records, eds C. P. Summerhayes, et al. (Hoboken, NJ: John Wiley), 313-320.

Chavez, F. P., Bertrand, A., Guevara, R., Soler, P., and Csirke, J. (2008). The northern Humboldt Current System: brief history, present status and a view towards the future. Prog. Oceanogr. 79, 95-105. doi: 10.1016/j.pocean.2008. 10.012

Checkley, D., and Barth, J. A. (2009). Patterns and processes in the California Current System. Prog. Oceanogr. 83, 49-64. doi: 10.1016/j.pocean.2009.07.028

Chen, K., Gawarkiewicz, G., and Plueddemann, A. J. (2018). Atmospheric and offshore forcing of temperature variability at the shelf break: observations from the OOI Pioneer Array. Oceanography 31, 72-79. doi: 10.5670/oceanog. 2018.112

Chen, Z., Wu, L., Qiu, B., Li, L., Hu, D., Liu, C., et al. (2015). Strengthening Kuroshio observed at its origin during November 2010 to October 2012. Oceans 120, 2460-2470. doi: 10.1002/2014JC010590

Claret, M., Galbraith, E. D., Palter, J. B., Bianchi, D., Fennel, K., Gilbert, D., et al. (2018). Rapid coastal deoxygenation due to ocean circulation shift in the 
northwest Atlantic. Nat. Clim. Chang. 8, 868-872. doi: 10.1038/s41558-0180263-1

Clayton, S., Dutkiewicz, S., Jahn, O., and Follows, M. J. (2013). Dispersal, eddies, and the diversity of marine phytoplankton. Limnol. Oceanogr. 3, 182-197. doi: 10.1215/21573689-2373515

Clayton, S., Lin, Y. C., Follows, M. J., and Worden, A. Z. (2017). Co-existence of distinct Ostreococcus ecotypes at an oceanic front. Limnol. Oceanogr. 62, 75-88. doi: 10.1002/lno.10373

Clayton, S., Nagai, T., and Follows, M. J. (2014). Fine scale phytoplankton community structure across the Kuroshio Front. J. Plankton Res. 36, 1017-1030. doi: $10.1093 /$ plankt/fbu020

Cockcroft, A. C., Schoeman, D. S., Pitcher, G. C., Bailey, G. W., and van Zyl, D. L. (2000). "A mass standing, or "walkout," of west coast rock lobster Jasus lalandii in Elands Bay, South Africa: causes, results and implications," in The Biodiversity Crisis and Crustacea, Vol. 12, eds J. C. von Kaupel Klein and F. R. Schram (Rotterdam: A. A. Balkema), 673-688.

Cockcroft, A. C., van Zyl, D., and Hutchings, L. (2008). Large-scale changes in the spatial distribution of South African West Coast rock lobsters: an overview. Afr. J. Mar. Sci. 30, 149-159. doi: 10.2989/ajms.2008.30.1.15.465

Colas, F., Capet, X., McWilliams, J. C., and Shchepetkin, A. (2008). 1997-1998 El Niño off Peru: a numerical study. Prog. Oceanogr. 79, 138-155.

Colas, F., McWilliams, J. C., Capet, X., and Kurian, J. (2012). Heat balance and eddies in the Peru-Chile current system. Clim. Dyn. 39, 509-529. doi: 10.1007/ s00382-011-1170-6

Conway, T. M., Palter, J. B., and de Souza, G. F. (2018). Gulf Stream rings as a source of iron to the North Atlantic subtropical gyre. Nat. Geosci. 11, 594-598. doi: 10.1038/s41561-018-0162-0

Cravatte, S., Ganachaud, A., Sprintall, J., Alberty, M., Germineaud, C., Brachet, C., et al. (2019). Solomon Sea SPICE Mooring Data. La Jolla, CA: UC San Diego Library Digital Collections, doi: 10.6075/J09W0CS2

Cronin, M. F., Bond, N. A., Farrar, J. T., Ichikawa, H., Jayne, S. R., Kawai, Y., et al. (2013). Formation and erosion of the seasonal thermocline in the Kuroshio Extension recirculation gyre. Deep Sea Res. 85, 62-74. doi: 10.1016/j.dsr2.2012. 07.018

Cronin, M. F., Gentemann, C. L., Edson, J., Ueki, I., Bourassa, M., Brown, S., et al. (2019). Air-sea fluxes with a focus on heat and momentum. Front. Mar. Sci. 6:430. doi: 10.3389/fmars.2019.00430

Cronin, M. F., Pelland, N. A., Emerson, S. R., and Crawford, W. R. (2015). Estimating diffusivity from the mixed layer heat and salt balances in the North Pacific. J. Geophys. Res. Oceans 120, 7346-7362. doi: 10.1002/2015JC011010

D'Adamo, N., Fandry, C., Bubarton, S., and Domingues, C. (2009). Northern sources of the Leeuwin Current and the "Holloway Current" on the North West Shelf. J. R. Soc. West Aust. 92, 53-66.

da Silveira, I. C. A., deMiranda, L. B., and Brown, W. S. (1994). On the origins of the North Brazil Current. J. Geophys. Res. 99, 22501-22512. doi: 10.1029/ 94JC01776

Davis, R. (2016). Data From: Solomon Sea Ocean Transport From Gliders. La Jolla, CA: Scripps Institution of Oceanography, Instrument Development Group, doi: 10.21238/s8SPRAY2718

Davis, R. E., Kessler, W. S., and Sherman, J. T. (2012). Gliders measure western boundary current transport from the South Pacific to the Equator. J. Phys. Oceanogr. 42, 2001-2013. doi: 10.1175/JPO-D-12-022.1

de Ruijter, W. P. M., Biastoch, A., Drijfhout, S. S., Lutjeharms, J. R. E., Matano, R. P., Pichevin, T., et al. (1999). Indian-Atlantic interocean exchange: dynamics, estimation and impact. J. Geophys. Res. 104, 20885-20910. doi: 10.1029/ 1998JC900099

Dengler, M., Fischer, J., Schott, F. A., and Zantopp, R. (2006). Deep Labrador Current and its variability in 1996-2005. Geophys. Res. Lett. 33:L21S06. doi: 10.1029/2006GL026702

Dever, M., Hebert, D., Greenan, B. J. W., Sheng, J., and Smith, P. C. (2016). Hydrography and coastal circulation along the Halifax Line and the connections with the Gulf of St. Lawrence. Atmos. Ocean 54, 199-217. doi: 10.1080/ 07055900.2016.1189397

DeVries, T. (2014). The oceanic anthropogenic $\mathrm{CO}_{2}$ sink: storage, air-sea fluxes, and transports over the industrial era. Glob. Biogeochem. Cycles 28, 631-647. doi: 10.1002/2013GB004739

Dewitte, B., Vazquez-Cuervo, J., Goubanova, K., Illig, S., Takahashi, K., Cambon, G., et al. (2012). Change in El Niño flavours over 1958-2008: implications for the long-term trend of the upwelling off Peru. Deep Sea Res. Part II 77, $143-156$.
deYoung, B., von Oppeln-Bronikowski, N., Matthews, J. B. R., and Bachmayer, R. (2018). Glider operations in the Labrador Sea. J. Ocean Tech. 13, 108-120.

Di Lorenzo, E., and Mantua, N. (2016). Multi-year persistence of the 2014/15 North Pacific marine heatwave. Nat. Clim. Change 6, 1042-1047. doi: 10.1038/ nclimate 3082

Diakhaté, M., de Coëtlogon, G., Lazar, A., Wade, M., and Gaye, A. T. (2016). Intraseasonal variability of tropical Atlantic sea-surface temperature: air-sea interaction over upwelling fronts. Q. J. R. Meteorol. Soc. 142, 372-386. doi: 10.1002/qj.2657

Domingues, C. M., Wijffels, S. E., Maltrud, M. E., Church, J. A., and Tomczak, M. (2006). Role of eddies in cooling the Leeuwin Current. Geophys. Res. Lett. 33:L05603. doi: 10.1029/2005GL025216

Domingues, R., Baringer, M., and Goni, G. (2016). Remote sources for year-to-year changes in the seasonality of the Florida Current transport. J. Geophys. Res. 121, 7547-7559. doi: 10.1002/2016JC012070

Domingues, R., Goni, G., Baringer, M., and Volkov, D. L. (2018). What caused the accelerated sea level changes along the United States East Coast during 2010-2015? Geophys. Res. Lett. 45, 13367-13376. doi: 10.1029/2018GL081183

Domingues, R., Kuwano-Yoshida, A., Chardon-Maldonado, P., Todd, R. E., Halliwell, G. R., Kim, H.-S., et al. (2019). Ocean observations in support of studies and forecasts of tropical and extratropical cyclones. Front. Mar. Sci. 6:446. doi: 10.3389/fmars.2019.00446

Dong, S., Goni, G., and Bringas, F. (2015). Temporal variability of the south Atlantic Meridional Overturning Circulation between $20^{\circ} \mathrm{S}$ and $35^{\circ} \mathrm{S}$. Geophys. Res. Lett. 42, 7655-7662. doi: 10.1002/2015GL065603

Donohue, K. A., Watts, D. R., Tracey, K. L., Greene, A. D., and Kennelly, M. (2010). Mapping circulation in the Kuroshio Extension with an array of current and pressure recording inverted echo sounders. J. Atmos. Ocean. Technol. 27, 507-527. doi: 10.1175/2009JTECHO686.1

Douglass, E., Roemmich, D., and Stammer, D. (2010). Interannual variability in North Pacific heat and freshwater budgets. Deep Sea Res. Part II 57, 1127-1140. doi: 10.1016/j.dsr2.2010.01.001

Duncombe Rae, C. M. (2005). A demonstration of the hydrographic partition of the Benguela upwelling ecosystem at $26^{\prime} 40^{\circ}$ S. Afr. J. Mar. Sci. 27, 617-628. doi: $10.2989 / 18142320509504122$

Elipot, S., and Beal, L. M. (2015). Characteristics, energetics, and origins of Agulhas Current meanders and their limited influence on ring shedding. J. Phys. Oceanogr. 45, 2294-2314. doi: 10.1175/JPO-D-14-0254.1

Elipot, S., and Beal, L. M. (2018). Observed Agulhas Current sensitivity to interannual and long-term trend atmospheric forcings. J. Clim. 31, 3077-3098. doi: 10.1175/JCLI-D-17-0597.1

Escribano, R., and Morales, C. E. (2012). Spatial and temporal scales of variability in the coastal upwelling and coastal transition zone off central-southern Chile (35-40 S). Progr. Oceanogr. 92, 1-7. doi: 10.1016/j.pocean.2011.07.019

Espinoza, P., Lorrain, A., Ménard, F., Cherel, Y., Tremblay-Boyer, L., Argüelles, J., et al. (2017). Trophic structure in the northern Humboldt Current System: new perspectives from stable isotope analysis. Mar. Biol. 164:86. doi: 10.1007/ s00227-017-3119-8

Everett, J. D., Baird, M. E., Roughan, M., Suthers, I. M., and Doblin, M. A. (2014). Relative impact of seasonal and oceanographic drivers on surface chlorophyll a along a Western Boundary Current. Prog. Oceanogr. 120, 340-351. doi: 10. 1016/j.pocean.2013.10.016

Ezer, T. (2015). Detecting changes in the transport of the Gulf Stream and the Atlantic overturning circulation from coastal sea level data: the extreme decline in 2009-2010 and estimated variations for 1935-2012, Global Planet. Change 129, 23-36. doi: 10.1016/j.gloplacha.2015.03.002

Fassbender, A. J., Alin, S. R., Feely, R. A., Sutton, A. J., Newton, J. A., Krembs, C., et al. (2018). Seasonal carbonate chemistry variability in marine surface waters of the US Pacific Northwest. Earth Syst. Sci. Data 10, 1367-1401. doi: 10.5194/essd-10-1367-2018

Fassbender, A. J., Alin, S. R., Feely, R. A., Sutton, A. J., Newton, J. A., and Byrne, R. H. (2017a). Estimating total alkalinity in the Washington State coastal zone: complexities and surprising utility for ocean acidification research. Estuar. Coast. 40, 404-418. doi: 10.1007/s12237-016-0168-z

Fassbender, A. J., Sabine, C. L., Cronin, M. F., and Sutton, A. J. (2017b). Mixedlayer carbon cycling at the Kuroshio extension observatory. Glob. Biogeochem. Cycles 31, 272-288. doi: 10.1002/2016GB005547

Fassbender, A. J., Sabine, C. L., and Feifel, K. M. (2016). Consideration of coastal carbonate chemistry in understanding biological calcification. Geophys. Res. Lett. 43, 4467-4476. doi: 10.1002/2016gl068860 
Fassbender, A. J., Sabine, C. L., Feely, R. A., Langdon, C., and Mordy, C. W. (2011). Inorganic carbon dynamics during northern California coastal upwelling. Cont. Shelf Res. 31, 1180-1192. doi: 10.1016/j.csr.2011.04.006

Faye, S., Lazar, A., Sow, B. A., and Gaye, A. T. (2015). A model study of the seasonality of sea surface temperature and circulation in the Atlantic northeastern tropical upwelling system. Front. Phys. 3:76. doi: 10.3389/fphy.2015. 00076

Feely, R. A., Alin, S., Carter, B., Bednaršek, N., Hales, B., Chan, F., et al. (2016). Chemical and biological impacts of ocean acidification along the west coast of North America. Estuar. Coast. Shelf Sci. 183, 260-270. doi: 10.1016/j.ecss.2016. 08.043

Feely, R. A., Okazaki, R. R., Cai, W.-J., Bednaršek, N., Alin, S. R., Byrne, R. H., et al. (2018). The combined effects of acidification and hypoxia on $\mathrm{pH}$ and aragonite saturation in the coastal waters of the California Current Ecosystem and the northern Gulf of Mexico. Cont. Shelf Res. 152, 50-60. doi: 10.1016/j.csr.2017. 11.002

Feely, R. A., Sabine, C. L., Hernandez-Ayon, J. M., Ianson, D., and Hales, B. (2008). Evidence for upwelling of corrosive 'acidified' water onto the continental shelf. Science 320, 1490-1492. doi: 10.1126/science.1155676

Feng, M., Hendon, H. H., Xie, S. P., Marshall, A. G., Schiller, A., Kosaka, Y., et al. (2015). Decadal increase in Ningaloo Niño since the late 1990s. Geophys. Res. Lett. 42, 104-112. doi: 10.1002/2014GL062509

Feng, M., McPhaden, M. J., Xie, S. P., and Hafner, J. (2013). La Niña forces unprecedented Leeuwin Current warming in 2011. Sci. Rep. 3:1277. doi: 10. 1038/srep01277

Feng, M., Meyer, G., Pearce, A., and Wijffels, S. (2003). Annual and interannual variations of the Leeuwin Current at 32S. J. Geophys. Res. 108:3355. doi: 10. 1029/2002JC001763

Fennel, K., Wilkin, J., Levin, J., Moisan, J., O’Reilly, J., and Haidvogel, D. (2006). Nitrogen cycling in the middle atlantic bight: results from a threedimensional model and implications for the North Atlantic nitrogen budget. Glob. Biogeochem. Cycles 20:GB3007. doi: 10.1029/2005GB002456

Fernandez, D., Bowen, M., and Sutton, P. (2018). Variability, coherence and forcing mechanisms in the New Zealand ocean boundary currents. Prog. Oceanogr. 165, 1680-1688. doi: 10.1016/j.pocean.2018.06.002

Ferrari, R., Artana, C., Saraceno, M., Piola, A. R., and Provost, C. (2017). Satellite altimetry and current-meter velocities in the Malvinas Current at $41^{\circ} \mathrm{S}$ : comparisons and modes of variations. J. Geophys. Res. 122, 9572-9590. doi: 10.1002/2017JC013340

Fischer, J., Schott, F., and Dengler, M. (2004). Boundary circulation at the exit of the Labrador Sea. J. Phys. Oceanogr. 34, 1548-1570. doi: 10.1175/1520-0485(2004) $034<1548$ :bcateo $>2.0$. co; 2

Fischer, J., Visbeck, M., Zantopp, R., and Nunes, N. (2010). Interannual to decadal variability of outflow from the Labrador Sea. Geophys. Res. Lett. 37:L24610. doi: 10.1029/2010GL045321

Frajka-Williams, E., Ansorge, I. J., Baehr, J., Bryden, H. L., Chidichimo, M. P., Cunningham, S. A., et al. (2019). Atlantic Meridional Overturning Circulation: observed transport and variability. Front. Mar. Sci. 6:260. doi: 10.3389/fmars. 2019.00260

Fratantoni, D. M., and Richardson, P. L. (2006). The evolution and demise of North Brazil current rings. J. Phys. Oceanogr. 36, 1241-1264. doi: 10.1175/JPO 2907.1

Fratantoni, P. S., and Pickart, R. S. (2007). The western North Atlantic shelfbreak current system in summer. J. Phys. Oceanogr. 37, 2509-2533. doi: 10.1175/ JPO3123

Fréon, P., Coetzee, J. C., Van der Lingen, C. D., Connell, A. D., O’Donoghue, S. H., Roberts, M. J., et al. (2010). A review and tests of hypotheses about causes of the KwaZulu-Natal sardine run. Afr. J. Mar. Sci. 32, 449-479. doi: 10.2989/1814232X.2010.519451

Friederich, G. E., Walz, P. M., Burczynski, M. G., and Chavez, F. P. (2002). Inorganic carbon in the central California upwelling system during the 19971999 El Niño-La Niña event. Prog. Oceanogr. 54, 185-203. doi: 10.1016/S00796611(02)00049-6

Frölicher, T. L., and Laufkötter, C. (2018). Emerging risks from marine heat waves. Nat. Commun. 9:650. doi: 10.1038/s41467-018-03163-6

Fujioka, K., Fukuda, H., Furukawa, S., Tei, Y., Okamoto, S., and Ohshimo, S. (2018). Habitat use and movement patterns of small (age-0) juvenile Pacific bluefin tuna (Thunnus orientalis) relative to the Kuroshio. Fish. Oceanogr. 27, 185-198. doi: $10.1111 /$ fog. 12244
Furue, R., Guerreiro, K., Phillips, H. E., McCreary, J. P., and Bindoff, N. (2017). On the Leeuwin Current system and its linkage to zonal flows in the South Indian ocean as inferred from a gridded hydrography. J. Phys. Oceanogr. 47, 583-602. doi: 10.1175/JPO-D-16-0170.1

Galarneau, T. J. Jr., Davis, C. A., and Shapiro, M. A. (2013). Intensification of Hurricane Sandy (2012) through extratropical warm core seclusion. Mon. Weather Rev. 141, 4296-4321. doi: 10.1175/MWR-D-13-00181.1

Ganachaud, A., Cravatte, S., Melet, A., Schiller, A., Holbrook, N. J., Sloyan, B. M., et al. (2014). The Southwest Pacific Ocean Circulation and Climate Experiment (SPICE). J. Geophys. Res. Oceans 119, 7660-7686. doi: 10.1002/2013JC00967

Ganachaud, A., Cravatte, S., Sprintall, J., Germineaud, C., Alberty, M., Jeandel, C., et al. (2017). The Solomon Sea: its circulation, chemistry, geochemistry and biology explored during two oceanographic cruises. Elem. Sci. Anth. 5:33. doi: 10.1525/elementa.221

Garzoli, S. L., Baringer, M. O., Dong, S., Perez, R. C., and Yao, Q. (2012). South Atlantic meridional fluxes. Deep Sea Res. Part I 71, 21-32. doi: 10.1016/j.dsr. 2012.09.003

Gaube, P., Braun, C. D., Lawson, G. L., McGillicuddy, D. J., Della Penna, A., Skomal, G., et al. (2018). Mesoscale eddies influence the movements of mature female white sharks in the Gulf Stream and Sargasso Sea. Sci. Rep. 8:7363. doi: 10.1038/s41598-018-25565-8

Gawarkiewicz, G. G., and Linder, C. A. (2006). Lagrangian flow patterns north of Cape Hatteras using near-surface drifters. Prog. Oceanogr. 70, 181-195. doi: 10.1016/j.pocean.2006.03.020

Gawarkiewicz, G., Todd, R. E., Zhang, W., Partida, J., Gangopadhyay, A., Monim, M.-U.-H., et al. (2018). The changing nature of shelf-break exchange revealed by the OOI Pioneer Array. Oceanography 31, 60-70. doi: 10.5670/oceanog. 2018.110

Gay, P. S., and Chereskin, T. K. (2009). Mean structure and seasonal variability of the poleward undercurrent off southern California. J. Geophys. Res. 114:C02007. doi: 10.1029/2008JC004886

Godfrey, J. S., and Weaver, A. J. (1991). Is the Leeuwin current driven by Pacific heating and winds? Prog. Oceanogr. 27, 225-272. doi: 10.1016/0079-6611(91) 90026-I

Gómez-Letona, M., Ramos, A. G., Coca, J., and Arístegui, J. (2017). Trends in primary production in the Canary Current upwelling system-a regional perspective comparing remote sensing models. Front. Mar. Sci. 4:370. doi: 10.3389/fmars.2017.00370

Goni, G. J., Sprintall, J., Bringas, F., Cheng, L., Cirano, M., Dong, S., et al. (2019). More than 50 years of continuous temperature section measurements by the Global Expendable Bathythermograph (XBT) Network, its integrability, societal benefits, and future. Front. Mar. Sci. 6:452. doi: 10.3389/fmars.2019.00452

Gopalakrishnan, G., Cornuelle, B. D., Hoteit, I., Rudnick, D. L., and Owens, W. B. (2013). State estimates and forecasts of the Loop Current in the Gulf of Mexico using the MITgcm and its adjoint. J. Geophys. Res. 118, 3292-3314. doi: $10.1002 /$ jgrc.20239

Gordon, A. L., Flament, P., Villanoy, C., and Centurioni, L. (2014). The nascent Kuroshio of Lamon Bay. J. Geophys. Res. 119, 4251-4263. doi: 10.1002/ 2014JC009882

Gorgues, T., Aumont, O., and Rodgers, K. B. (2010). A mechanistic account of increasing seasonal variations in the rate of ocean uptake of anthropogenic carbon. Biogeosciences 7, 2581-2589. doi: 10.5194/bg-7-2581-2010

Graco, M., Purca, S., Dewitte, B., Castro, C., Morón, O., Ledesma, J., et al. (2017). The OMZ and nutrient features as a signature of interannual and low-frequency variability in the Peruvian upwelling system. Biogeosciences 14, 4601-4617. doi: 10.5194/bg-14-1-2017

Grados, C., Chaigneau, A., Echevin, V., and Dominguez, N. (2018). Upper ocean hydrology of the northern Humboldt Current System at seasonal, interannual and interdecadal scales. Prog. Oceanogr. 165, 123-144. doi: 10.1016/j.pocean. 2018.05.005

Gruber, N., Keeling, C. D., and Bates, N. R. (2002). Interannual variability in the North Atlantic Ocean carbon sink. Science 298, 2374-2378. doi: 10.1126/ science. 1077077

Guerrero, R. A., Piola, A. R., Fenco, H., Matano, R. P., Combes, V., Chao, Y., et al. (2014). The salinity signature of the cross-shelf exchanges in the Southwestern Atlantic Ocean: satellite observations. J. Geophys. Res. Oceans 119, 7794-7810. doi: 10.1002/2014JC010113

Gula, J., Blacic, T. M., and Todd, R. E. (2019). Submesoscale coherent vortices in the Gulf Stream. Geophys. Res. Lett. 46, 2704-2714. doi: 10.1029/2019GL081919 
Guo, X. Y., Zhu, X. H., Long, Y., and Huang, D. J. (2013). Spatial variations in the Kuroshio nutrient transport from the East China Sea to south of Japan. Biogeosciences 10, 6403-6417. doi: 10.5194/bg-10-6403-2013

Guo, X., Zhu, X. H., Wu, Q. S., and Huang, D. (2012). The Kuroshio nutrient stream and its temporal variation in the East China Sea. J. Geophys. Res. Oceans 117:C01026. doi: 10.1029/2011JC007292

Gutiérrez, D. (2016). Variabilidad climática, procesos oceanográficos y producción primaria frente al Perú. Boletín Técnico "Generación de información y monitoreo del Fenómeno El Niño.”. Instituto Geofísico del Perú 3, 4-8.

Haines, S., Seim, H., and Muglia, M. (2017). Implementing quality control of highfrequency radar estimates and application to Gulf Stream surface currents. J. Atmos. Ocean. Technol. 34, 1207-1224. doi: 10.1175/JTECH-D-16-0203.1

Hales, B., Takahashi, T., and Bandstra, L. (2005). Atmospheric $\mathrm{CO}_{2}$ uptake by a coastal upwelling system. Glob. Biogeochem. Cycles 19:GB1009. doi: 10.1029/ 2004GB002295

Halliwell, G. R., Mehari, M., Shay, L. K., Kourafalou, V. H., Kang, H., Kim, J.-S., et al. (2017). OSSE quantitative assessment of rapid-response prestorm ocean surveys to improve coupled tropical cyclone prediction. J. Geophys. Res. Oceans 122, 5729-5748. doi: 10.1002/2017JC012760

Hansen, D. V., and Poulain, P.-M. (1996). Quality control and interpolation of WOCE/TOGA drifter data. J. Atmos. Ocean. Technol. 13, 900-909. doi: 10. 1175/1520-0426(1996)013<0900:qcaiow >2.0.co;2

Harris, K. E., DeGrandpre, M. D., and Hales, B. (2013). Aragonite saturation states in a coastal upwelling zone. Geophys. Res. Lett. 40, 2720-2725. doi: 10.1002/grl. 50460

He, R., Chen, K., Moore, T., and Li, M. (2010). Mesoscale variations of sea surface temperature and ocean color patterns at the Mid-Atlantic Bight shelfbreak. Geophys. Res. Lett. 37:L09607. doi: 10.1029/2010GL042658

Helly, J. J., and Levin, L. A. (2004). Global distribution of naturally occurring marine hypoxia on continental margins. Deep Sea Res. I 51, 1159-1168. doi: 10.1016/j.dsr.2004.03.009

Henderikx Freitas, F., Saldiìas, G. S., Goñi, M., Shearman, R. K., and White, A. E. (2018). Temporal and spatial dynamics of physical and biological properties along the Endurance Array of the California Current Ecosystem. Oceanography 31, 80-89. doi: 10.5670/oceanog.2018.113

Henderikx Freitas, F., Siegel, D. A., Washburn, L., Halewood, S., and Stassinos, E. (2016). Assessing controls on cross-shelf phytoplankton and suspended particle distributions using repeated bio-optical glider surveys. Oceans 121, 7776-7794. doi: 10.1002/2016JC011781

Heslop, E. E., Ruiz, S., Allen, J., López-Jurado, J. L., Renault, L., and Tintoré, J. (2012). Autonomous underwater gliders monitoring variability at "choke points" in our ocean system: a case study in the Western Mediterranean Sea. Geophys. Res. Lett. 39:L20604.

Hill, K., Moltmann, T., Meyers, G., and Proctor, R. (2010). "The Australian integrated marine observing system (IMOS)," in Proceedings of OceanObs'09: Sustained Ocean Observations and Information for Society, eds J. Hall, D. E. Harrison, and D. Stammer (Venice: ESA Publication WPP-306), doi: 10.5270/ OceanObs09

Hill, K., Rintoul, S. R., Ridgway, K. R., and Oke, P. R. (2011). Decadal changes in the South Pacific western boundary current system revealed in observations and ocean state estimates. J. Geophys. Res. 116:C01009. doi: 10.1029/2009JC005926

Hobday, A. J., Alexander, L. V., Perkins, S. E., Smale, D. A., Straub, S. C., Oliver, E. C. J., et al. (2016). A hierarchical approach to defining marine heatwaves. Prog. Oceanogr. 141, 227-238. doi: 10.1016/j.pocean.2015.12.014

Hoffman, R. N., and Atlas, R. (2016). Future observing system simulation experiments. Bull. Am. Meteor. Soc. 97, 1601-1616. doi: 10.1175/BAMS-D-1500200.1

Honda, M. C., Sasai, Y., Siswanto, E., Kuwano-Yoshida, A., Aiki, H., and Cronin, M. F. (2018). Impact of cyclonic eddies and typhoons on biogeochemistry in the oligotrophic ocean based on biogeochemical/physical/meteorological timeseries at station KEO. Prog. Earth Planet. Sci. 5:42. doi: 10.1186/s40645-0180196-3

Houpert, L., Inall, M. E., Dumont, E., Gary, S., Johnson, C., Porter, M., et al. (2018). Structure and transport of the North Atlantic Current in the eastern subpolar gyre from sustained glider observations. J. Geophys. Res. Oceans 123, 6019-6038. doi: 10.1029/2018JC014162

Howatt, T., Palter, J. B., Matthews, J. B., deYoung, B., Bachmayer, R., and Claus, B. (2018). Ekman and eddy exchange of freshwater and oxygen across the
Labrador Shelf Break. J. Phys. Oceanogr. 48, 1015-1031. doi: 10.1175/JPO-D17-0148.1

Hu, D., Hu, S., Wu, L., Li, L., Zhang, L., Diao, X., et al. (2013). Direct measurements of the Luzon undercurrent. J. Phys. Oceanogr. 43, 1417-1425. doi: 10.1175/JPOD-12-0165.1

Hu, D., Wu, L., Cai, W., Sen Gupta, A., Ganachaud, A., Qiu, B., et al. (2015). Pacific western boundary currents and their roles in climate. Nature 522, 299-308. doi: 10.1038/nature14504

Hu, S., Hu, D., Guan, C., Wang, F., Zhang, L., Wang, F., et al. (2016). Interannual variability of the Mindanao Current/Undercurrent in direct observations and numerical simulations. J. Phys. Oceanogr. 46, 483-499. doi: 10.1175/JPO-D-150092.1

Hummels, R., Brandt, P., Dengler, M., Fischer, J., Araujo, M., Veleda, D., et al. (2015). Interannual to decadal changes in the western boundary circulation in the Atlantic at 11 degrees S. Geophys. Res. Lett. 42, 7615-7622. doi: 10.1002/ 2015 gl065254

Hutchings, L., Augustyn, C. J., Cockcroft, A., Van der Lingen, C., Coetzee, J., Leslie, R. W., et al. (2009a). Marine fisheries monitoring programmes in South Africa. S. Afr. J. Sci. 105, 182-192.

Hutchings, L., Roberts, M. J., and Verheye, H. M. (2009b). Marine environmental monitoring programmes in South Africa: a review. S. Afr. J. Sci. 105, 94-102.

Hutchings, L., van der Lingen, C., Shannon, L. J., Cawford, R. J. M., Verheye, H. M., Bartholomae, C. H., et al. (2009c). The Benguela Current: an ecosystem of four components. Progr. Oceanogr. 83, 15-32. doi: 10.1016/j.pocean.2009. 07.046

Huyer, A., Wheeler, P. A., Strub, P. T., Smith, R. L., Letelier, R., and Kosro, P. M. (2007). The Newport Line off Oregon-studies in the northeast Pacific. Prog. Oceanogr. 75, 126-160. doi: 10.1016/j.pocean.2007.08.003

Ichikawa, H., Nakamura, H., Nishina, A., and Higashi, M. (2004). Variability of northeastward current southeast of Northern Ryukyu Islands. J. Oceanogr. 60, 351-363. doi: 10.1023/B:JOCE.0000038341.27622.73

Imawaki, S., Bower, A., Beal, L., and Qiu, B. (2013). "Western boundary currents," in Ocean Circulation and Climate - a 21st Century Perspective, eds G. Siedler, S. M. Griffies, J. Gould, and J. A. Church (Amsterdam: Elsevier Academic Press), 305-338. doi: 10.1016/B978-0-12-391851-2.00013-1

Imawaki, S., Uchida, H., Ichikawa, H., Fuksawa, M., Umatani, S., and The Asuka group. (2001). Satellite altimeter monitoring the Kuroshio transport south of Japan. Geophys. Res. Lett. 28, 17-20. doi: 10.1029/2000GL011796

Inoue, R., Honda, M., Fujiki, T., Matsumoto, K., Kouketsu, S., Suga, T., et al. (2016a). Western North Pacific integrated physical-biogeochemical ocean observation experiment (INBOX): Part 2. Biogeochemical responses to eddies and typhoons revealed from the S1 mooring and shipboard measurements. J. Mar. Res. 74, 71-99. doi: 10.1357/002224016819257335

Inoue, R., Suga, T., Kouketsu, S., Kita, T., Hosoda, S., Kobayashi, T., et al. (2016b). Western north Pacific integrated physical-biogeochemical ocean observation experiment (INBOX): Part 1. Specifications and chronology of the S1-INBOX floats. J. Mar. Res. 74, 43-69. doi: 10.1357/002224016819257344

Ito, S., Yoshie, N., Okunishi, T., Ono, T., Okazaki, Y., Kuwataet, A., et al. (2010). Application of an automatic approach to calibrate the NEMURO nutrientphytoplankton-zooplankton food web model in the Oyashio region. Progr. Oceanogr. 87, 186-200. doi: 10.1016/j.pocean.2010.08.004

Ito, T., and Follows, M. J. (2003). Upper ocean control on the solubility pump of $\mathrm{CO}_{2}$. J. Mar. Res. 61, 465-489. doi: 10.1357/002224003322384898

Iudicone, D., Rodgers, K. B., Plancherel, Y., Aumont, O., Ito, T., Key, R. M., et al. (2016). The formation of the ocean's anthropogenic carbon reservoir. Sci. Rep. 6:35473. doi: $10.1038 /$ srep35473

Jayne, S. R., Roemmich, D., Zilberman, N., Riser, S. C., Johnson, K. S., Johnson, G. C., et al. (2017). The Argo program: present and future. Oceanography 30, 18-28. doi: 10.5670/oceanog.2017.213

Johns, W. E., Baringer, M. O., Beal, L. M., Cunningham, S. A., Kanzow, T., Bryden, H. L., et al. (2011). Continuous array-based estimates of Atlantic Ocean heat transport at $26.5^{\circ}$ N. J. Clim. 24, 2429-2449. doi: 10.1175/2010JCLI3997.1

Johns, W. E., Beal, L. M., Baringer, M. O., Molina, J. R., Cunningham, S. A., Kanzow, T., et al. (2008). Variability of shallow and deep western boundary currents off the Bahamas during 2004-2005: results from the $26^{\circ} \mathrm{N}$ RAPIDMOC array. J. Phys. Oceanogr. 38, 605-623. doi: 10.1175/2007JPO3791.1

Johns, W. E., Kanzow, T., and Zantopp, R. (2005). Estimating ocean transports with dynamic height moorings: an application in the Atlantic Deep Western 
Boundary Current at $26^{\circ}$ N. Deep Sea Res. Part I 52, 1542-1567. doi: 10.1016/j. dsr.2005.02.002

Johnson, C. R., Banks, S. C., Barrett, N. S., Cazassus, F., Dunstan, P. K., Edgar, G. J., et al. (2011). Climate change cascades: shifts in oceanography, species' ranges and subtidal marine community dynamics in eastern Tasmania. J. Exp. Mar. Biol. Ecol. 400, 17-32. doi: 10.1016/j.jembe.2011.02.032

Johnson, G. C. (2001). The Pacific Ocean subtropical cell surface limb. Geophys. Res. Lett. 28, 1771-1774. doi: 10.1029/2000gl012723

Johnson, K. S., Plant, J. N., Coletti, L. J., Jannasch, H. W., Sakamoto, C. M., Riser, S. C., et al. (2017). Biogeochemical sensor performance in the SOCCOM profiling float array. J. Geophys. Res. 122, 6416-6436. doi: 10.1002/ 2017JC012838

Johnston, T. M. S., and Rudnick, D. L. (2015). Mixing estimates in the California Current System from sustained observations by underwater gliders. Deep Sea Res. II 112, 61-78. doi: 10.1016/j.dsr2.2014.03.009

Johnston, T. M., Rudnick, D. L., Alford, M. H., Pickering, A., and Simmons, H. L. (2013). Internal tidal energy fluxes in the South China Sea from density and velocity measurements by gliders. J. Geophys. Res. Oceans 118, 3939-3949. doi: 10.1002 /jgrc.20311

Junker, T., Mohrholz, V., Schmidt, M., Siegfried, L., and van der Plas, A. (2019). Coastal trapped wave propagation along the southwest African shelf as revealed by moored observations. J. Phys. Oceanogr. 49, 851-866. doi: 10.1175/JPO-D18-0046

Junker, T., Mohrholz, V., Siegfried, L., and van der Plas, A. (2017a). Seasonal to interannual variability of water mass characteristics and currents on the Namibian shelf. J. Mar. Syst. 165, 36-46. doi: 10.1016/j.jmarsys.2016.09.003

Junker, T., Mohrholz, V., Siegfried, L., van der Plas, A., Heene, T., and Beier, S. (2017b). Daily Means of Bottom Temperature and Bottom Oxygen Measurements on the Namibian Shelf at $18^{\circ} \mathrm{S}$. Warnemünde: Leibniz Institute for Baltic Sea Research, doi: 10.1594/PANGAEA.871251

Junker, T., Mohrholz, V., Siegfried, L., van der Plas, A., Heene, T., and Beier, S. (2017c). Daily Means of Horizontal Currents Measurements on the Namibian Shelf at $18^{\circ} \mathrm{S}$. Warnemünde: Leibniz Institute for Baltic Sea Research, doi: 10.1594/PANGAEA.871253

Junker, T., Mohrholz, V., Siegfried, L., van der Plas, A., Heene, T., Beier, S., et al. (2017d). Daily Means of Horizontal Currents Measurements on the Namibian Shelf at $20^{\circ} \mathrm{S}$. Warnemünde: Leibniz Institute for Baltic Sea Research, doi: 10.1594/PANGAEA.872098

Junker, T., Mohrholz, V., Siegfried, L., van der Plas, A., Heene, T., and Breier, S. (2017e). Daily Means of Bottom Temperature and Bottom Oxygen Measurements on the Namibian Shelf at $20^{\circ} \mathrm{S}$. Warnemünde: Leibniz Institute for Baltic Sea Research, doi: 10.1594/PANGAEA.872099

Juranek, L. W., Feely, R. A., Peterson, W. T., Alin, S. R., Hales, B., Lee, K., et al. (2009). A novel method for determination of aragonite saturation state on the continental shelf of central Oregon using multi-parameter relationships with hydrographic data. Geophys. Res. Lett. 36:L24601. doi: 10.1029/2009GL04 0778

Jury, M. R., Valentine, H. R., and Lutjeharms, J. R. (1993). Influence of the Agulhas Current on summer rainfall along the southeast coast of South Africa. J. Appl. Meteorol. 32, 1282-1287. doi: 10.1175/1520-0450(1993)032<1282:iotaco > 2.0.co; 2

Karstensen, J., Schütte, F., Pietri, A., Krahmann, G., Fiedler, B., Grundle, D., et al. (2017). Upwelling and isolation in oxygen-depleted anticyclonic modewater eddies and implications for nitrate cycling. Biogeosciences 14, 2167-2181. doi: 10.5194/bg-14-2167-2017

Kelly, K. A., Small, R. J., Samelson, R. M., Qiu, B., Joyce, T. M., Kwon, Y. O., et al. (2010). Western boundary currents and frontal air-sea interaction: Gulf Stream and Kuroshio Extension. J. Clim. 23, 5644-5667. doi: 10.1175/2010JCLI3346.1

Kerry, C., Powell, B., Roughan, M., and Oke, P. (2016). Development and evaluation of a high-resolution reanalysis of the East Australian Current region using the regional ocean modelling system (ROMS 3.4) and incremental strongconstraint 4-Dimensional variational (IS4D-Var) data assimilation. Geosci. Model. Dev. 9, 3779-3801. doi: 10.5194/gmd-9-3779-2016

Kerry, C., Roughan, M., and Powell, B. (2018). Observation impact in a regional reanalysis of the East Australian Current System. J. Geophys. Res. 123, 75117528. doi: $10.1029 / 2017 \mathrm{JC} 013685$

Kersalé, M., Lamont, T., Speich, S., Terre, T., Laxenaire, R., Roberts, M. J., et al. (2018). Moored observations of mesoscale features in the Cape Basin: characteristics and local impacts on water mass distributions. Ocean Sci. 14, 923-945. doi: 10.5194/os-14-923-2018

Kim, S. Y. (2010). Observations of submesoscale eddies using high-frequency radar-derived kinematic and dynamic quantities. Cont. Shelf Res. 30, 16391655. doi: $10.1016 /$ j.csr.2010.06.011

Kim, S. Y., and Kosro, P. M. (2013). Observations of near-inertial surface currents off Oregon: decorrelation time and length scales. Oceans 118, 3723-3736. doi: $10.1002 /$ jgrc. 20235

Kim, S. Y., Terrill, E. J., Cornuelle, B. D., Jones, B., Washburn, L., Moline, M. A., et al. (2011). Mapping the U.S. West Coast surface circulation: a multiyear analysis of high-frequency radar observations. J. Geophys. Res. 116:C03011. doi: 10.1029/2010JC006669

Klenz, T., Dengler, M., and Brandt, P. (2018). Seasonal variability of the Mauritania Current and hydrography at $18^{\circ}$ N. J. Geophys. Res. 123, 8122-8137. doi: 10. 1029/2018JC014264

Kolodziejczyk, N., Testor, P., Lazar, A., Echevin, V., Krahmann, G., Chaigneau, A., et al. (2018). Subsurface fine-scale patterns in an anticyclonic eddy off CapVert peninsula observed from glider measurements. J. Geophys. Res. Oceans 123, 6312-6329. doi: 10.1029/2018JC014135

Kono, T., and Kawasaki, Y. (1997). Results of CTD and mooring observations southeast of Hokkaido. 1. Annual verocity and transport variations in the Oyashio. Bull. Hokkaido Natl. Fish. Res. Inst. 61, 65-81.

Kopte, R., Brandt, P., Claus, M., Greatbatch, R. J., and Dengler, M. (2018). Role of equatorial basin-mode resonance for the seasonal variability of the Angola Current at $11^{\circ}$ S. J. Phys. Oceanogr. 48, 261-281. doi: 10.1175/JPO-D-17-0111.1

Kopte, R., Brandt, P., Dengler, M., Tchipalanga, P. C. M., Macuéria, M., and Ostrowski, M. (2017). The Angola Current: flow and hydrographic characteristics as observed at $11^{\circ} \mathrm{S}$. J. Geophys. Res. Oceans 122, 1177-1189. doi: 10.1002/2016JC012374

Koszalka, I., and LaCasce, J. H. (2010). Lagrangian analysis by clustering. Ocean Dyn. 60, 957-972. doi: 10.1007/s10236-010-0306-2

Krug, M., Swart, S., and Gula, J. (2017). Submesoscale cyclones in the Agulhas Current. Geophys. Res. Lett. 44, 346-354. doi: 10.1002/2016GL071006

Kuroda, H., Wagawa, T., Kakehi, S., Shimizu, Y., Kusaka, A., Okunishi, T., et al. (2017). Long-term mean and seasonal variation of altimetry-derived Oyashio transport across the A-line off the southeastern coast of Hokkaido. Jpn. Deep Sea Res. Part I 121, 95-109. doi: 10.1016/j.dsr.2016.12.006

Kuroda, H., Wagawa, T., Shimizu, Y., Ito, S., Kakehi, S., Okunishi, T., et al. (2015). Interdecadal decrease of the Oyashio transport on the continental slope off the southeastern coast of Hokkaido, Japan. J. Geophys. Res. Oceans 120, 2504-2522. doi: 10.1002/2014JC010402

LaCasce, J. H. (2008). Statistics from Lagrangian observations. Prog. Oceanogr. 77, 1-29. doi: 10.1016/j.pocean.2008.02.002

Landschützer, P., Gruber, N., Bakker, D. C. E., and Schuster, U. (2014). Recent variability of the global ocean carbon sink. Glob. Biogeochem. Cycles 28, 927949. doi: $10.1002 / 2014 \mathrm{~GB} 004853$

Large, W. G., and Danabasoglu, G. (2006). Attribution and impacts of upper-ocean biases in CCSM3. J. Clim. 19, 2325-2346. doi: 10.1175/JCLI3740.1

Larsen, J. C., and Sanford, T. B. (1985). Florida Current volume transports from voltage measurements. Science 227, 302-304. doi: 10.1126/science.227. 4684.302

Laurindo, L. C., Mariano, A., and Lumpkin, R. (2017). An improved near-surface velocity climatology for the global ocean from drifter observations. Deep Sea Res. I 124, 73-92. doi: 10.1016/j.dsr.2017.04.009

Leber, G. M., Beal, L. M., and Elipot, S. (2017). Wind and current forcing combine to drive strong upwelling in the Agulhas Current. J. Phys. Oceanogr. 47, 123-134. doi: 10.1175/JPO-D-16-0079.1

Lee, E. A., and Kim, S. Y. (2018). Regional variability and turbulent characteristics of the satellite-sensed submesoscale surface chlorophyll concentrations. J. Geopyhs. Res. 123, 4250-4279. doi: 10.1029/2017JC013732

Lee, T., and Fukumori, I. (2003). Interannual-to-decadal variations of tropicalsubtropical exchange in the Pacific Ocean: boundary versus interior pycnocline transports. J. Clim. 16, 4022-4042. doi: 10.1175/1520-0442(2003)016<4022: ivotei>2.0.co;2

Lengaigne, M., Hausmann, U., Madec, G., Menkes, C. E., Vialard, J., and Molines, J. M. (2012). Mechanisms controlling warm water volume interannual variations in the equatorial Pacific: diabatic versus adiabatic processes. Clim. Dyn. 38, 1031-1046. doi: 10.1007/s00382-011-1051-z 
Levine, N. M., Doney, S. C., Lima, I., Wanninkhof, R., Bates, N. R., and Feely, R. A. (2011). The impact of the North Atlantic Oscillation on the uptake and accumulation of anthropogenic $\mathrm{CO}_{2}$ by North Atlantic Ocean mode waters. Glob. Biogeochem. Cycles 25:GB3022. doi: 10.1029/2010GB003892

Liang, X., Spall, M., and Wunsch, C. (2017). Global ocean vertical velocity from a dynamically consistent ocean state estimate. J. Geophys. Res. 122, 8208-8224. doi: 10.1002/2017JC012985

Lien, R.-C., Ma, B., Cheng, Y.-H., Ho, C.-R., Qiu, B., Lee, C. M., et al. (2014). Modulation of Kuroshio transport by mesoscale eddies at the Luzon Strait entrance. J. Geophys. Res. Oceans 119, 2129-2142. doi: 10.1002/2013JC009548

Lien, R.-C., Ma, B., Lee, C. M., Sanford, T. B., Mensah, V., Centurioni, L. R., et al. (2015). The Kuroshio and Luzon Undercurrent east of Luzon Island. Oceanography 28, 54-63. doi: 10.5670/oceanog.2015.81

Lima, M. O., Cirano, M., Mata, M., Goes, M., Goni, G., and Baringer, M. O. (2016). An assessment of the Brazil Current baroclinic structure and variability near $22^{\circ} \mathrm{S}$ in distinct ocean forecasting and analysis systems. Ocean Dyn. 66, 893-916. doi: 10.1007/s10236-016-0959-6

Linder, C. A., and Gawarkiewicz, G. G. (1998). A climatology of the shelfbreak front in the Middle Atlantic Bight. J. Geophys. Res. 103, 18405-18423. doi: 10.1029/98JC01438

Lindstrom, E., Gunn, J., Fischer, A., McCurdy, A., and Glover, L. K. (2012). A Framework for Ocean Observing. By the Task Team for an Integrated Framework for Sustained Ocean Observing. Paris: UNESCO, doi: 10.5270/Ocean Obs09-FOO

Louw, D., van der Plas, A., Mohrholz, V., Wasmund, N., Junker, T., and Eggert, A. (2016). Seasonal and interannual phytoplankton dynamics and forcing mechanisms in the northern Benguela upwelling system. J. Mar. Syst. 157, 124-134. doi: 10.1016/j.jmarsys.2016.01.009

Lovechio, E., Gruber, N., and Münnich, M. (2018). Mesoscale contribution to the long-range offshore transport of organic carbon from the Canary Upwelling system to the open North Atlantic. Biogeosciences 15, 5061-5091. doi: 10.5194/ bg-15-5061-2018

Lowcher, C. F., Muglia, M., Bane, J. M., He, R., Gong, Y., Haines, S. M., et al. (2017). "Marine hydrokinetic energy in the Gulf Stream off North Carolina: an assessment using observations and ocean circulation models," in Marine Renewable Energy, eds Z. Yang and A. Copping (Cham: Springer), doi: 10.1007/ 978-3-319-53536-4_10

Lumpkin, R. (2003). Decomposition of surface drifter observations in the Atlantic Ocean. Geophys. Res. Lett. 30:1753. doi: 10.1029/2003GL017519

Lumpkin, R., and Garzoli, S. L. (2011). Interannual to decadal changes in the western South Atlantic's surface circulation. J. Geophys. Res. 116:C01014. doi: 10.1029/2010JC006285

Lumpkin, R., and Johnson, G. (2013). Global ocean surface velocities from drifters: mean, variance, El Niño-Southern Oscillation response, and seasonal cycle. J. Geophys. Res. 118, 2992-3006. doi: 10.1002/jgrc.20210

Lumpkin, R., and Pazos, M. C. (2007). "Measuring surface currents with surface velocity program drifters: the instrument, its data, and some recent results," in Lagrangian Analysis and Prediction of Coastal and Ocean Dynamics, eds A. Griffa, A. D. Kirwan, A. J. Mariano, T. Ozgokmen, and H. T. Rossby (Cambridge: Cambridge University Press), 39-67. doi: 10.1017/ cbo9780511535901.003

Lumpkin, R., Maximenko, N., and Pazos, M. (2012). Evaluating where and why drifters die. J. Atmos. Ocean. Technol. 29, 300-308. doi: 10.1175/jtech-d-1100100.1

Lumpkin, R., Özgökmen, T., and Centurioni, L. (2017). Advances in the application of surface drifters. Annu. Rev. Mar. Sci. 9, 59-81. doi: 10.1146/annurev-marine010816-060641

Lund, B., Haus, B. K., Horstmann, J., Graber, H. C., Carrasco, R., Laxague, N. J., et al. (2018). Near-surface current mapping by shipboard marine X-band radar: a validation. J. Atmos. Oceanic Technol. 35, 1077-1090. doi: 10.1175/JTECH-D17-0154.1

Lutjeharms, J. R. (2006). The Agulhas Current. Berlin: Springer.

Lynch, T. P., Morello, E. B., Evans, K., Richardson, A., Rochester, W., Steinberg, C. R., et al. (2014). IMOS national reference stations: a continental scale physical, chemical, biological coastal observing system. PLoS One 9:e113652. doi: 10.1371/journal.pone.0113652

Ma, X., Chang, P., Saravanan, R., Montuoro, R., Nakamura, H., Wu, D., et al. (2017). Importance of resolving Kuroshio front and eddy influence in simulating the North Pacific storm track. J. Clim. 30, 1861-1880. doi: 10.1175/ JCLI-D-16-0154.1

Machu, E., Capet, X., Estrade, P. A., Ndoye, S., Brajard, J., Baurand, F., et al. (2019). First evidence of anoxia and nitrogen loss in the southern Canary upwelling system. Geophys. Res. Lett. 46, 2619-2627. doi: 10.1029/2018GL079622

Mackey, D. J., O'Sullivan, J. E., and Watson, R. J. (2002). Iron in the western Pacific: a riverine or hydrothermal source for iron in the equatorial undercurrent. Deep Sea. Res. I 49, 877-893. doi: 10.1016/S0967-0637(01)00075-9

Majumder, S., and Schmid, C. (2018). A study of the variability in the Benguela Current volume transport. Ocean Sci. 14, 273-283. doi: 10.5194/os-14-2732018

Majumder, S., Goes, M., Polito, P. S., Lumpkin, R., Schmid, C., and Lopez, H. (2019). Propagating modes of variability and their impact on the western boundary current in the South Atlantic. J. Geophys. Res. Oceans 124, 3168-3185. doi: 10.1029/2018JC014812

Mansfield, K. L., Mendilaharsu, M. L., Putman, N. F., dei Marcovaldi, M. A., Sacco, A. E., Lopez, G., et al. (2017). First satellite tracks of South Atlantic sea turtle 'lost years': seasonal variation in trans-equatorial movement. Proc. Biol. Sci. 284:20171730. doi: 10.1098/rspb.2017.1730

Mantovanelli, A., Keating, S., Wyatt, L., Roughan, M., and Schaeffer, A. (2017). Lagrangian and Eulerian characterization of two counter rotating submesoscale eddies in a western boundary current. J. Geophys. Res. Oceans 122, 4902-4921. doi: 10.1002/2016JC011968

Marra, J., Houghton, R. W., and Garside, C. (1990). Phytoplankton growth at the shelf-break front in the Middle Atlantic Bight. J. Mar. Res. 48, 851-868. doi: 10.1357/002224090784988665

Marzeion, B., Cogley, J. G., Richter, K., and Parkes, D. (2014). Attribution of global glacier mass loss to anthropogenic and natural causes. Science 345, 919-921. doi: $10.1126 /$ science. 1254702

Mata, M. M., Cirano, M., van Caspel, M. R., Fonteles, C. S., Goni, G., and Baringer, M. (2012). Observations of Brazil Current baroclinic transport near $22^{\circ} \mathrm{S}$ : variability from the AX97 XBT transect. CLIVAR Exchanges 58, 5-10. doi: 10.1007/s10236-016-0959-6

Matano, R. P., and Palma, E. D. (2008). On the upwelling of downwelling currents. J. Phys. Oceanogr. 38, 2482-2500. doi: 10.1175/2008jpo3783.1

Matano, R. P., Combes, V., Piola, A. R., Guerrero, R. A., Palma, E. D., Strub, P. T., et al. (2014). The salinity signature of the cross-shelf exchanges in the Southwestern Atlantic Ocean: numerical simulations. J. Geophys. Res. Oceans 119, 7949-7968. doi: 10.1002/2014JC010116

Maximenko, N., Niiler, P., Centurioni, L., Rio, M.-H., Melnichenko, O., Chambers, D., et al. (2009). Mean dynamic topography of the ocean derived from satellite and drifting buoy data using three different techniques. J. Atmos. Ocean. Technol. 26, 1910-1919. doi: 10.1175/2009JTECHO672.1

Mazzini, P. L. F., Barth, J. A., Shearman, R. K., and Erofeev, A. (2014). Buoyancydriven coastal currents off the Oregon coast during fall and winter. J. Phys. Oceanogr. 44, 2854-2876. doi: 10.1175/JPO-D-14-0012.1

McClatchie, S. (2014). Regional Fisheries Oceanography of the California Current System: The CalCOFI Program. Berlin: Springer, doi: 10.1007/978-94-0077223-6

McClatchie, S., Thompson, A. R., Alin, S. R., Siedlecki, S., Watson, W., and Bograd, S. J. (2016). The influence of Pacific Equatorial Water on fish diversity in the southern California Current System. J. Geophys. Res. Oceans 121, 6121-6136. doi: 10.1002/2016JC011672

Meinen, C. S., and Watts, D. R. (2000). Vertical structure and transport on a transect across the North Atlantic Current near $42^{\circ} \mathrm{N}$ : time series and mean. J. Geophys. Res. 105, 21869-21891. doi: 10.1029/2000JC900097

Meinen, C. S., Baringer, M. O., and Garcia, R. F. (2010). Florida Current transport variability: an analysis of annual and longer-period signals. Deep Sea Res. I 57, 835-846. doi: 10.1016/j.dsr.2010.04.001

Meinen, C. S., Garzoli, S. L., Perez, R. C., Campos, E., Piola, A. R., Chidichimo, M. P., et al. (2017). Characteristics and causes of Deep Western Boundary Current transport variability at $34.5^{\circ} \mathrm{S}$ during 2009-2014. Ocean Sci. 13, 175194. doi: 10.5194/os-13-175-2017

Meinen, C. S., Johns, W. E., Garzoli, S. L., van Sebille, E., Rayner, D., Kanzow, T., et al. (2013). Variability of the Deep Western Boundary Current at $26.5 \mathrm{~N}$ during 2004-2009. Deep Sea Res. II 85, 154-168. doi: 10.1016/j.dsr2.2012.07.036

Meinen, C. S., Speich, S., Piola, A. R., Ansorge, I., Campos, E., Kersale, M., et al. (2018). Meridional overturning circulation transport variability at $34.5^{\circ} \mathrm{S}$ 
during 2009-2017: baroclinic and barotropic flows and the dueling influence of the boundaries. Geophys. Res. Lett. 45, 4180-4188. doi: 10.1029/2018GL07 7408

Menna, M., Faye, S., Poulain, P.-M., Centurioni, L., Lazar, A., Gaye, A., et al. (2016). Upwelling features of the coast of north-western Africa in 2009-2013. Boll. Geofis. Teor. Appl. 57, 71-86. doi: 10.4430/bgta0164

Messié, M., and Chavez, F. P. (2015). Seasonal regulation of primary production in eastern boundary upwelling systems. Prog. Oceanogr. 134, 1-18. doi: 10.1016/j. pocean.2014.10.011

Middleton, J. F., and Bye, J. T. (2007). A review of the shelf-slope circulation along Australia's southern shelves: Cape Leeuwin to Portland. Prog. Oceanogr. 75, 1-41. doi: 10.1016/j.pocean.2007.07.001

Middleton, J. F., and Cirano, M. (2002). A boundary current along Australia's southern shelves: the Flinders Current. J. Geophys. Res. 107:3129. doi: 10.1029/ 2000JC000701

Mihanoviæ, H., Pattiaratchi, C., and Verspecht, F. (2016). Diurnal sea breezes force near-inertial waves along Rottnest continental shelf, southwestern Australia. J. Phys. Oceanogr. 46, 3487-3508. doi: 10.1175/JPO-D-16-0022.1

Mohrholz, V., Bartholomae, C. H., van der Plas, A. K., and Lass, H. U. (2008). The seasonal variability of the northern Benguela undercurrent and its relation to the oxygen budget on the shelf. Cont. Shelf Res. 28, 424-441. doi: 10.1016/j.csr. 2007.10.001

Moloney, C. L., Van Der Lingen, C. D., Hutchings, L., and Field, J. G. (2004). Contributions of the Benguela ecology programme to pelagic fisheries management in South Africa. Afr. J. Mar. Sci. 26, 37-51. doi: 10.2989/ 18142320409504048

Monteiro, P. M. S., and van der Plas, A. K. (2006). "Low oxygen water (LOW) variability in the Benguela system: key processes and forcing scales relevant to forecasting," in Benguela: Predicting a Large Marine Ecosystem, eds V. Shannon, et al. (Netherlands: ElsevierAmsterdam), 71-90. doi: 10.1016/s1570-0461(06) 80010-8

Monteiro, P. M. S., van der Plas, A. K., Mélice, J.-L., and Florenchie, P. (2008). Interannual hypoxia variability in a coastal upwelling system: ocean-shelf exchange, climate and ecosystem-state implications. Deep Sea Res. I 55, 435450. doi: 10.1016/j.dsr.2007.12.010

Mosquera-Vásquez, K., Dewitte, B., Illig, S., Takahashi, K., and Garric, G. (2013). The 2002/2003 El Niño: equatorial waves sequence and their impact on sea surface temperature. J. Geophys. Res. Oceans 118, 346-357.

Nagai, T., and Clayton, S. (2017). Nutrient interleaving below the mixed layer of the Kuroshio Extension front. Ocean Dyn. 67, 1027-1046. doi: 10.1007/s10236017-1070-3

Nagano, A., Kizu, S., Hanawa, K., and Roemmich, D. (2016). Heat transport variation due to change of North Pacific subtropical gyre interior flow during 1993-2012. Ocean Dyn. 66, 1637-1649. doi: 10.1007/s10236-016-1007-2

Nakamura, M. (2012). Impacts of SST anomalies in the Agulhas Current System on the regional climate variability. J. Clim. 25, 1213-1229. doi: 10.1175/JCLID-11-00088.1

Nakano, H., Tsujino, H., Yasuda, M., Hirabara, T., Motoi, T., Ishii, M., et al. (2011). Uptake mechanisms of anthropogenic $\mathrm{CO}_{2}$ in the Kuroshio Extension region in an ocean general circulation model. J. Oceanogr. 67, 765-783. doi: 10.1007/s10872-011-0075-7

Nakano, T., Kitamura, T., Sugimoto, S., Suga, T., and Kamachi, M. (2015). Long-term variations of North Pacific Tropical Water along the $137^{\circ} \mathrm{E}$ repeat hydrographic section. J. Oceanogr. 71, 229-238. doi: 10.1007/s10872-0150279-3

Nam, S., Kim, H. J., and Send, U. (2011). Amplification of hypoxic and acidic events by La Niña conditions on the continental shelf off California. Geophys. Res. Lett. 38:L22602. doi: 10.1029/2011g1049549

Nguyen, L. T., and Molinari, J. (2012). Rapid intensification of a sheared, fastmoving hurricane over the Gulf Stream. Mon. Weather Rev. 140, 3361-3378. doi: 10.1175/MWR-D-11-00293.1

Niewiadomska, K., Claustre, H., Prieur, L., and d'Ortenzio, F. (2008). Submesoscale physical-biogeochemical coupling across the Ligurian Current (northwestern Mediterranean) using a bio-optical glider. Limnol. Oceanogr. 53, 2210-2225. doi: 10.4319/lo.2008.53.5_part_2.2210

Niiler, P. P. (2001). "The world ocean surface circulation," in Ocean Circulation and Climate, eds G. Siedler, J. Church, and J. Gould (Cambridge, MA: Academic Press), 193-204. doi: 10.1016/s0074-6142(01)80119-4
Niiler, P. P., Maximenko, N. A., Panteleev, G. G., Yamagata, T., and Olson, D. B. (2003). Near-surface dynamical structure of the Kuroshio Extension. J. Geophys. Res. 108:3193. doi: 10.1029/2002JC001461

Niiler, P. P., Sybrandy, A., Bi, K., Poulain, P. M., and Bitterman, D. (1995). Measurements of the water-following capability of holey-sock and TRISTAR drifters. Deep Sea Res. I 42, 1951-1964. doi: 10.1016/0967-0637(95)00076-3

Nkwinkwa Njouodo, A. S., Koseki, S., Keenlyside, N., and Rouault, M. (2018). Atmospheric signature of the Agulhas Current. Geophys. Res. Lett. 45, 51855193. doi: 10.1029/2018GL077042

Nowald, N., Iversen, M. H., Fischer, G., Ratmeyer, V., and Wefer, G. (2015). Time series of in-situ particle properties and sediment trap fluxes in the coastal upwelling filament off Cape Blanc, Mauritania. Prog. Oceanogr. 137, 1-11. doi: 10.1016/j.pocean.2014.12.015

O'Reilly, C. H., and Czaja, A. (2015). The response of the Pacific storm track and atmospheric circulation to Kuroshio Extension variability. Q. J. R. Meteorol. Soc. 141, 52-66. doi: 10.1002/qj.2334

O'Reilly, C. H., Minobe, S., and Kuwano-Yoshida, A. (2016). The influence of the Gulf Stream on wintertime European blocking. Clim. Dyn. 47, 1545-1567. doi: 10.1007/s00382-015-2919-0

Oettli, P., Morioka, Y., and Yamagata, T. (2016). A regional climate mode discovered in the North Atlantic: Dakar Niño/Niña. Sci. Rep. 6:18782. doi: $10.1038 /$ srep 18782

Ohman, M. D., Rudnick, D. L., Chekalyuk, A., Davis, R. E., Feely, R. A., Kahru, M., et al. (2013). Autonomous ocean measurements in the California Current Ecosystem. Oceanography 26, 18-25. doi: 10.5670/oceanog.2013.41

Oka, E., and Kawabe, M. (2003). Dynamic structure of the Kuroshio south of Kyushu in relation to the kuroshio path variations. J. Oceanogr. 59, 595-608. doi: 10.1023/B:JOCE.0000009589.28241.93

Oka, E., Ishii, M., Nakano, T., Suga, T., Kouketsu, S., Miyamoto, M., et al. (2018). Fifty years of the 137E repeat hydrographic section in the western North Pacific Ocean. J. Oceanogr. 74, 115-145. doi: 10.1007/s10872-017-0461-x

Oka, E., Qiu, B., Takatani, Y., Enyo, K., Sasano, D., Kosugi, N., et al. (2015). Decadal variability of Subtropical Mode Water subduction and its impact on biogeochemistry. J. Oceanogr. 71, 389-400. doi: 10.1007/s10872-015-0300-x

Oliveira, L. R., Piola, A. R., Mata, M. M., and Soares, I. D. (2009). Brazil Current surface circulation and energetics observed from drifting buoys. J. Geophys. Res. 114:C10006. doi: 10.1029/2008JC004900

Oliver, E. C. J., O’Kane, T. J., and Holbrook, N. J. (2015). Projected changes to Tasman Sea eddies in a future climate. Oceans 120, 7150-7165. doi: 10.1002/ 2015JC010993

Oliver, E. C., Benthuysen, J. A., Bindoff, N. L., Hobday, A. J., Holbrook, N. J., Mundy, C. N., et al. (2017). The unprecedented 2015/16 Tasman Sea marine heatwave. Nat. Commun. 8:16101. doi: 10.1038/ncomms16101

Olson, D., Podesta, G. P., Evans, R. H., and Brown, O. (1988). Temporal variations in the separation of Brazil and Malvinas Currents. Deep Sea Res. 35, 1971-1990. doi: 10.1016/0198-0149(88)90120-3

Paduan, J. D., and Washburn, L. (2013). High-frequency radar observations of ocean surface currents. Annu. Rev. Mar. Sci. 5, 115-136. doi: 10.1146/annurevmarine-121211-172315

Palacz, A. P., Pearlman, J., Simmons, S., Hill, K., Miloslavich, P., Telszewski, M., et al. (2017). Report of the Workshop on the Implementation of Multidisciplinary Sustained Ocean Observations (IMSOO). Global Ocean Observing System (GOOS) Report No. 223. Available at: http://www.goosocean.org/imsooreport (accessed July 23, 2019).

Palevsky, H. I., and Nicholson, D. P. (2018). The North Atlantic biological pump: insights from the Ocean Observatories Initiative Irminger Sea array. Oceanography 31, 42-49. doi: 10.5670/oceanog.2018.108

Palevsky, H. I., and Quay, P. D. (2017). Influence of biological carbon export on ocean carbon uptake over the annual cycle across the North Pacific Ocean. Glob. Biogeochem. Cycles 31, 1-15. doi: 10.1002/2016GB005527

Palevsky, H. I., Quay, P. D., Lockwood, D. E., and Nicholson, D. P. (2016). The annual cycle of gross primary production, net community production, and export efficiency across the North Pacific Ocean. Glob. Biogeochem. Cycles 30, 361-380. doi: 10.1002/2015GB005318

Palter, J. B., and Lozier, M. S. (2008). On the source of Gulf Stream nutrients. J. Geophys. Res. 113:C06018. doi: 10.1029/2007jc004611

Paniagua, G. F., Saraceno, M., Piola, A. R., Guerrero, R., Provost, C., Ferrari, R., et al. (2018). Malvinas Current at $40^{\circ} \mathrm{S}-41^{\circ} \mathrm{S}$ : first assessment of temperature 
and salinity temporal variability. J. Geophys. Res. Oceans 123, 5323-5340. doi: 10.1029/2017JC013666

Park, J., and Sweet, W. (2015). Accelerated sea level rise and Florida Current transport. Ocean Sci. 11, 607-615. doi: 10.5194/os-11-607-2015

Parks, A. B., Shay, L. K., Johns, W. E., Martinez-Pedraja, J., and Gurgel, K. W. (2009). HF radar observations of small-scale surface current variability in the Straits of Florida. J. Geophys. Res. 114:C08002. doi: 10.1029/2008JC005025

Parrilla, G., Neuer, S., and Le Traon, P.-Y. (2002). Topical studies in oceanography: Canary Islands Azores Gibraltar Observations (CANIGO). Volume 1: studies in the northern Canary Islands basin. Deep Sea Res. II 49, 3409-3413. doi: 10.1016/s0967-0645(02)00104-2

Pattiaratchi, C., Hollings, B., Woo, M., and Welhena, T. (2011). Dense shelf water formation along the south-west Australian inner shelf. Geophys. Res. Lett. 38:L10609. doi: 10.1029/2011GL046816

Paulmier, A., and Ruiz-Pino, D. (2009). Oxygen minimum zones (OMZs) in the modern ocean. Prog. Oceanogr. 80, 113-128. doi: 10.1016/j.pocean.2008.08.001

Pearce, A., and Feng, M. (2013). The rise and fall of the "marine heat wave" off western Australia during the summer of 2010/11. J. Mar. Syst. 111-112, 139-156. doi: 10.1016/j.jmarsys.2012.10.009

Pegliasco, C., Chaigneau, A., and Morrow, R. (2015). Main eddy vertical structures observed in the four major eastern boundary upwelling systems. Oceans 120, 6008-6033. doi: 10.1002/2015JC010950

Pelegrí, J. L., and Csanady, G. T. (1991). Nutrient transport and mixing in the Gulf Stream. J. Geophys. Res. 96, 2577-2583. doi: 10.1029/90JC02535

Pelegrí, J. L., Csanady, G. T., and Martins, A. (1996). The North Atlantic nutrient stream. J. Oceanogr. 52, 275-299. doi: 10.1007/BF02235924

Pelland, N. A., Eriksen, C. C., and Lee, C. M. (2013). Subthermocline eddies over the Washington continental slope as observed by Seagliders, 2003-09. J. Phys. Oceanogr. 43, 2025-2053. doi: 10.1175/jpo-d-12-086.1

Perry, M. J., Sackmann, B. S., Eriksen, C. C., and Lee, C. M. (2008). Seaglider observations of blooms and subsurface chlorophyll maxima off the Washington coast. Limnol. Oceanogr. 53, 2169-2179. doi: 10.4319/lo.2008.53.5_part_2. 2169

Pickart, R. S., and Smethie, W. M. (1993). How does the Deep Western Boundary Current cross the Gulf Stream? J. Phys. Oceanogr. 23, 2602-2616. doi: 10.1038/ s41598-018-22758-z

Pickart, R. S., and Watts, D. R. (1990). Deep western boundary current variability at Cape Hatteras. J. Mar. Res. 48, 765-791. doi: 10.1357/002224090784988674

Pietri, A., Echevin, V., Testor, P., Chaigneau, A., Mortier, L., Grados, C., et al. (2014). Impact of a coastal-trapped wave on the near-coastal circulation of the Peru upwelling system from glider data. Oceans 119, 2109-2120. doi: 10.1002/ 2013JC009270

Pietri, A., Testor, P., Echevin, V., Chaigneau, A., Mortier, L., Eldin, G., et al. (2013). Finescale vertical structure of the upwelling system off southern Peru as observed from glider data. J. Phys. Oceanogr. 43, 631-646. doi: 10.1175/JPOD-12-035.1

Pitcher, G. C., and Probyn, T. A. (2011). Anoxia in southern Benguela during the autumn of 2009 and its linkage to a bloom of the dinoflagellate Ceratium balechii. Harmful Algae 11, 23-32. doi: 10.1016/j.hal.2011.07.001

Pitcher, G. C., Probyn, T. A., du Randt, A., Lucas, A. J., Bernard, S., Evers-King, H., et al. (2014). Dynamics of oxygen depletion in the nearshore of a coastal embayment of the southern Benguela upwelling system. J. Geophys. Res. Oceans 119, 2183-2200. doi: 10.1002/2013JC009443

Pizarro, O., Ramírez, N., Castillo, M. I., Cifuentes, U., Rojas, W., and Pizarro-Koch, M. (2016). Underwater glider observations in the oxygen minimum zone off central Chile. Bull. Am. Meteor. Soc. 97, 1783-1789. doi: 10.1175/BAMS-D-1400040.1

Polo, I., Lazar, A., Rodriguez-Fonseca, B., and Arnault, S. (2008). Oceanic Kelvin waves and tropical Atlantic intraseasonal variability: 1. Kelvin wave characterization. J. Geophys. Res. 113:C07009. doi: 10.1029/2007JC004495

Pontes, G. M., Sen Gupta, A., and Taschetto, A. S. (2016). Projected changes to South Atlantic boundary currents and confluence region in the CMIP5 models: the role of wind and deep ocean changes. Environ. Res. Lett. 11:094013. doi: 10.1088/1748-9326/11/9/094013

Poulain, P. M., and Niiler, P. P. (1989). Statistical-analysis of the surface circulation in the California Current System using satellite-tracked drifters. J. Phys. Oceanogr. 19, 1588-1603. doi: 10.1175/1520-0485(1989)019<1588:saotsc $>$ 2.0.co;2
Probyn, T. A., Mitchellinnes, B. A., Brown, P. C., Hutchings, L., and Carter, R. A. (1994). Review of primary production and related processes on the Agulhas Bank. S. Afr. J. Sci. 90, 166-173.

Qiu, B., and Chen, S. (2005). Variability of the kuroshio extension jet, recirculation gyre, and mesoscale eddies on decadal time scales. J. Phys. Oceanogr. 35, 2090-2103. doi: 10.1175/JPO2807.1

Qiu, B., and Chen, S. (2010). Interannual-to-decadal variability in the bifurcation of the North Equatorial Current off the Philippines. J. Phys. Oceanogr. 40, 2525-2538. doi: 10.1175/2010JPO4462.1

Qiu, B., Chen, S., Schneider, N., and Taguchi, B. (2014). A coupled decadal prediction of the dynamic state of the Kuroshio Extension system. J. Clim. 27, 1751-1764. doi: 10.1175/JCLI-D-13-00318.1

Rainville, L., Lee, C. M., Rudnick, D. L., and Yang, K.-C. (2013). Propagation of internal tides generated near Luzon Strait: observations from autonomous gliders. J. Geophys. Res. 118, 4125-4138. doi: 10.1002/jgrc.20293

Reum, J. C. P., Alin, S. R., Harvey, C. J., Bednaršek, N., Evans, W., Feely, R. A., et al. (2016). Interpretation and design of ocean acidification experiments in upwelling systems in the context of carbonate chemistry co-variation with temperature and oxygen. ICES J. Mar. Sci. 73, 582-595. doi: 10.1093/icesjms/ fsu231

Reum, J. C., Alin, S. R., Feely, R. A., Newton, J., Warner, M., and McElhany, P. (2014). Seasonal carbonate chemistry covariation with temperature, oxygen, and salinity in a fjord estuary: implications for the design of ocean acidification experiments. PLoS One 9:e89619. doi: 10.1371/journal.pone.0089619

Révelard, A., Frankignoul, C., Sennéchael, N., Kwon, Y. O., and Qiu, B. (2016). Influence of the decadal variability of the kuroshio extension on the atmospheric circulation in the cold season. J. Clim. 29, 2123-2144. doi: 10.1175/ JCLI-D-15-0511.1

Richardson, P. L. (2007). Agulhas leakage into the Atlantic estimated with subsurface floats and surface drifters. Deep Sea Res. I 54, 1361-1389. doi: 10. 1016/j.dsr.2007.04.010

Richter, I. (2015). Climate model biases in the eastern tropical oceans: causes, impacts and ways forward. WIREs Clim. Chang 6, 345-358. doi: 10.1002/ wcc. 338

Ridgway, K. R., and Condie, S. A. (2004). The 5500-km-long boundary flow off western and southern Australia. J. Geophys. Res. 109:C04017. doi: 10.1029/ 2003JC001921

Ridgway, K. R., and Godfrey, J. S. (2015). The source of the Leeuwin Current seasonality. J. Geophys. Res. 120, 6843-6864. doi: 10.1002/2015JC011049

Rio, M. H., and Santoleri, R. (2018). Improved global surface currents from the merging of altimetry and Sea surface temperature data. Remote Sens. Environ. 216, 770-785. doi: 10.1016/j.rse.2018.06.003

Rio, M.-H., Mulet, S., and Picot, N. (2014). Beyond GOCE for the ocean circulation estimate: synergetic use of altimetry, gravimetry, and in situ data provides new insight into geostrophic and Ekman currents. Geophys. Res. Lett. 41, 8918-8925. doi: 10.1002/2014GL061773

Riser, S. C., Freeland, H. J., Roemmich, D., Wijffels, S., Troisi, A., Belbéoch, M., et al. (2016). Fifteen years of ocean observations with the global Argo array. Nat. Clim. Change 6, 145-153. doi: 10.1038/nclimate2872

Rodgers, K. B., Sarmiento, J. L., Crevoisier, C., de Boyer Montegut, C., Metzl, N., and Aumont, O. (2008). A wintertime uptake window for anthropogenic $\mathrm{CO}_{2}$ in the North Pacific. Glob. Biogeochem. Cycles 22:GB2020. doi: 10.1029/ 2006GB002920

Rodrigues, R. R., Rothstein, L. M., and Wimbush, M. (2007). Seasonal variability of the South Equatorial Current bifurcation in the Atlantic Ocean: a numerical study. J. Phys. Oceanogr. 37, 16-30. doi: 10.1175/JPO2983.1

Roemmich, D., Alford, M. H., Claustre, H., Johnson, K. S, King, B., Moum, J., et al. (2019). On the future of Argo: A global, full-depth, multi-disciplinary array. Front. Mar. Sci. 6:439. doi: 10.3389/fmars.2019. 00439

Roemmich, D., Boehme, L., Claustre, H., Freeland, H., Fukasawa, M., Goni, G., et al. (2010). "Integrating the ocean observing system: mobile platforms," in Proceedings of OceanObs'09: Sustained Ocean Observations and Information for Society, eds J. Hall, D. E. Harrison, and D. Stammer (Venice: ESA Publication WPP-306), 21-25. doi: 10.5270/OceanObs09

Rossby, H. T., Flagg, C. N., and Donohue, K. (2010). On the variability of Gulf Stream transport from seasonal to decadal timescales. J. Mar. Res. 68, 503-522. doi: $10.1357 / 002224010794657128$ 
Rossby, T., Flagg, C. N., Donohue, K., Sanchez-Franks, A., and Lillibridge, J. (2014). On the long-term stability of Gulf Stream transport based on 20 years of direct measurements. Geophys. Res. Lett. 41, 114-120. doi: 10.1002/2013GL05 8636

Rouault, M., Reason, C. J. C., Lutjeharms, J. R. E., and Beljaars, A. C. M. (2003). Underestimation of latent and sensible heat fluxes above the Agulhas Current in NCEP and ECMWF analyses. J. Clim. 16, 776-782. doi: 10.1175/15200442(2003)016<0776:uolash>2.0.co;2

Roughan, M., and Morris, B. D. (2011). "Using high-resolution ocean timeseries data to give context to long term hydrographic sampling off Port Hacking, NSW, Australia," in Proceedings of the Oceans'11 MTS/IEEE Kona, Waikoloa, HI, doi: 10.23919/OCEANS.2011.6107032

Roughan, M., Schaeffer, A., and Kioroglou, S. (2013). "Assessing the design of the NSW-IMOS Moored Observation Array from 2008-2013: recommendations for the future," in Proceedinds of the 2013 OCEANS -San Diego, San Diego, CA, doi: 10.23919/OCEANS.2013.6741092

Roughan, M., Schaeffer, A., and Suthers, I. M. (2015). Sustained ocean observing along the coast of Southeastern Australia: NSW-IMOS 2007-2014. Coast. Ocean Observing Syst. 2015, 76-98. doi: 10.1016/B978-0-12-802022-7.00006-7

Rudnick, D. L. (2017). Data from: Spray Underwater Glider Campaign in Gulf of Mexico. La Jolla, CA: Instrument Development Group, Scripps Institution of Oceanography, doi: 10.21238/s8SPRAY0420

Rudnick, D. L. (2016a). Data from: California Underwater Glider Network. La Jolla, CA: Instrument Development Group, Scripps Institution of Oceanography, doi: 10.21238/s8SPRAY1618

Rudnick, D. L. (2016b). Ocean research enabled by underwater gliders. Annu. Rev. Mar. Sci. 8, 519-541. doi: 10.1146/annurev-marine-122414-033913

Rudnick, D. L., and Cole, S. T. (2011). On sampling the ocean using underwater gliders. J. Geophys. Res. 116:C08010. doi: 10.1029/2010JC006849

Rudnick, D. L., Gopalakrishnan, G., and Cornuelle, B. D. (2015a). Cyclonic eddies in the Gulf of Mexico: observations by underwater gliders and simulations by numerical model. J. Phys. Oceanogr. 45, 313-326. doi: 10.1175/JPO-D-140138.1

Rudnick, D. L., Jan, S., and Lee, C. M. (2015b). A new look at circulation in the western North Pacific. Oceanography 28, 16-23. doi: 10.5670/oceanog.2015.77

Rudnick, D. L., Jan, S., Centurioni, L., Lee, C., Lien, R.-C., Wang, J., et al. (2011). Seasonal and mesoscale variability of the Kuroshio near its origin. Oceanography 24, 52-63. doi: 10.5670/oceanog.2011.94

Rudnick, D. L., Johnston, T. M., and Sherman, J. T. (2013). High-frequency internal waves near the Luzon Strait observed by underwater gliders. J. Geophys. Res. Oceans 118, 774-784. doi: 10.1002/jgrc.20083

Rudnick, D. L., Zaba, K. D., Todd, R. E., and Davis, R. E. (2017). A climatology of the California Current System from a network of underwater gliders. Prog. Oceanogr. 154, 64-106. doi: 10.1016/j.pocean.2017.03.002

Rühs, S., Getzlaff, K., Durgadoo, J. V., Biastoch, A., and Böning, C. W. (2015). On the suitability of North Brazil current transport estimates for monitoring basin-scale AMOC changes. Geophys. Res. Lett. 42, 8072-8080. doi: 10.1002/ 2015GL065695

Ryan, J. P., Ueki, I., Chao, Y., Zhang, H., Polito, P. S., and Chavez, F. P. (2006). Western Pacific modulation of large phytoplankton blooms in the central and eastern equatorial Pacific. J. Geophys. Res. 111:G02013. doi: 10.1029/ 2005JG000084

Rypina, I. I., Llopiz, J. K., Pratt, L. M., and Lozier, M. S. (2014). Dispersal pathways of American eel larvae from the Sargasso Sea. Limnol. Oceanogr. 59, 1704-1714. doi: 10.4319/lo.2014.59.5.1704

Saba, V. S., Griffies, S. M., Anderson, W. G., Winton, M., Alexander, M. A., Delworth, T. L., et al. (2016). Enhanced warming of the Northwest Atlantic Ocean under climate change. J. Geophys. Res. 121, 118-132. doi: 10.1002/ 2015JC011346

Saraceno, M., Guerrero, R., Piola, A., Provost, C., Perault, F., Ferrari, R., et al. (2017). Malvinas Current 2014-2015: Mooring Velocities. France: SEANOE, doi: $10.17882 / 51492$

Schaeffer, A., Roughan, M., Jones, E. M., and White, D. (2016). Physical and biogeochemical spatial scales of variability in the East Australian Current separation from shelf glider measurements. Biogeosciences 13, 1967-1975. doi: 10.5194/bg-13-1967-2016

Schaeffer, A. M., Roughan, T., Austin, J. D., Everett, D., Griffin, B., Hollings, E., et al. (2016). Mean hydrography on the continental shelf from 26 repeat glider deployments along Southeastern Australia. Sci. Data 3:160070. doi: 10.1038/ sdata.2016.70

Schaeffer, A., and Roughan, M. (2015). Influence of a western boundary current on shelf dynamics and upwelling from repeat glider deployments. Geophys. Res. Lett. 42, 121-128. doi: 10.1002/2014GL062260

Schaeffer, A., and Roughan, M. (2017). Subsurface intensification of marine heatwaves off southeastern Australia: the role of stratification and local winds. Geophys. Res. Lett. 44, 5025-5033. doi: 10.1002/2017GL073714

Schaeffer, A., Gramoulle, A., Roughan, M., and Mantovanelli, A. (2017). Characterizing frontal eddies along the East Australian Current from HF radar observations. J. Geophys. Res. 122, 3964-3980. doi: 10.1002/2016JC012171

Schaeffer, A., Roughan, M., and Morris, B. (2013). Cross-shelf dynamics in a western boundary current. Implications for upwelling. J. Phys. Oceanogr. 43, 1042-1059. doi: 10.1175/JPO-D-12-0177.1

Schaeffer, A., Roughan, M., and Morris, B. (2014). Corrigendum. J. Phys. Oceanogr. 44, 2812-2813. doi: 10.1175/JPO-D- 14-0091.1

Schmid, C., and Majumder, S. (2018). Transport variability of the Brazil Current from observations and a data assimilation model. Ocean Sci. 14, 417-436. doi: 10.5194/os014-417-2018

Schneider, W., Donoso, D., Garcés-Vargas, J., and Escribano, R. (2016). Watercolumn cooling and sea surface salinity increase in the upwelling region off central-south Chile driven by a poleward displacement of the South Pacific. Prog. Oceanogr. 141, 38-58. doi: 10.1016/j.pocean.2016.11.004

Schönau, M. C., and Rudnick, D. L. (2017). Mindanao Current and Undercurrent: thermohaline structure and transport from repeat glider observations. J. Phys. Oceanogr. 47, 2055-2075. doi: 10.1175/JPO-D-16-0274.1

Schönau, M. C., Rudnick, D. L., Cerovecki, I., Gopalakrishnan, G., Cornuelle, B. D., McClean, J. L., et al. (2015). The Mindanao Current: mean structure and connectivity. Oceanography 28, 34-45. doi: 10.5670/oceanog.2015.79

Schott, F. A., Fischer, J., and Stramma, L. (1998). Transports and pathways of the upper-layer circulation in the western tropical Atlantic. J. Phys. Oceanogr. 28, 1904-1928. doi: 10.1175/1520-0485(1998)028<1904:tapotu >2.0.co;2

Schott, F., Dengler, M., Zantopp, R. J., Stramma, L., Fischer, J., and Brandt, P. (2005). The shallow and deep western boundary circulation of the South Atlantic at $5^{\circ}-11^{\circ}$ S. J. Phys. Oceanogr. 35, 2031-2053. doi: 10.1175/JPO 2813.1

Schott, F., Xie, S.-P., and McCreary, J. P. (2009). Indian Ocean circulation and climate variability. Rev. Geophys. 47:RG1002. doi: 10.1029/2007RG000245

Sen Gupta, A., Ganachaud, A., McGregor, S., Brown, J. N., and Muir, L. (2012). Drivers of the projected changes to the Pacific Ocean equatorial circulation. Geophys. Res. Lett. 39:L09605. doi: 10.1029/2012GL051447

Send, U. (2018). Data from: Spray Glider Data in Support of Mooring Observations from the CORC Project in the California Current Starting 2007. La Jolla, CA: Instrument Development Group, Scripps Institution of Oceanography, doi: 10.21238/s8SPRAY8857

Send, U., Davis, R. E., Fischer, J., Imawaki, S., Kessler, W., Meinen, C., et al. (2010). "A global boundary current circulation observing network," in Proceedings of OceanObs'09: Sustained Ocean Observations and Information for Society, Vol. 2, eds J. Hall, D. E. Harrison, and D. Stammer (Venice: ESA Publication WPP-306), doi: 10.5270/OceanObs09.cwp.78

Send, U., Regier, L., and Jones, B. (2013). Use of underwater gliders for acoustic data retrieval from subsurface oceanographic instrumentation and bidirectional communication in the deep ocean. J. Atmos. Ocean Technol. 30, 984-998. doi: 10.1175/JTECH-D-11-00169.1

Shannon, L. V., and Nelson, G. (1996). "The Benguela: large-scale features and processes and system variability," in The South Atlantic: Present and Past Circulation, eds G. Wefer, W. H. Berger, G. Seidler, and D. J. Webb (Berlin: Springer-Verlag), 163-210. doi: 10.1007/978-3-642-80353-6_9

Shinoda, A., Aoyama, J., Miller, M. J., Otake, T., Mochioka, N., Watanabe, S., et al. (2011). Evaluation of the larval distribution and migration of the Japanese eel in the western North Pacific. Rev. Fish. Biol. Fisher. 21, 591-611. doi: 10.1007/ s11160-010-9195-1

Siedlecki, S. A., Kaplan, I. C., Hermann, A. J., Nguyen, T. T., Bond, N. A., Newton, J. A., et al. (2016). Experiments with seasonal forecasts of ocean conditions for the Northern region of the California Current upwelling system. Sci. Rep. 6:27203. doi: 10.1038/srep27203

Silva, N., Rojas, N., and Fedele, A. (2009). Water masses in the Humboldt Current System: properties, distribution, and the nitrate deficit as a chemical water mass 
tracer for Equatorial Subsurface Water off Chile. Deep Sea Res. Part II 56, 1004-1020. doi: 10.1016/j.dsr2.2008.12.013

Siqueira, L., and Kirtman, B. P. (2016). Atlantic near-term climate variability and the role of a resolved Gulf Stream. Geophys. Res. Lett. 43, 3964-3972. doi: 10.1002/2016GL068694

Slangen, A. B. A., Carson, M., Katsman, C. A., van de Wal, R. S. W., Köhl, A., Vermeersen, L. L. A., et al. (2014). Projecting twenty-first century regional sea-level changes. Clim. Chang. 124, 317-332. doi: 10.1007/s10584-014-1080-9

Sloyan, B. M., and O'Kane, T. J. (2015). Drivers of decadal variability in the Tasman Sea. J. Geophys. Res. 120, 3193-3210. doi: 10.1002/2014JC010550

Sloyan, B. M., Ridgway, K., and Cowley, R. (2016). The East Australian Current and property transport at $27^{\circ} \mathrm{S}$ from 2012-2013. J. Phys. Oceanogr. 46, 993-1008. doi: 10.1175/JPO-D-15-0052.1

Smith, L. M., Barth, J. A., Kelley, D. S., Plueddemann, A., Rodero, I., Ulses, G. A., et al. (2018). The Ocean Observatories Initiative. Oceanography 31, 16-35. doi: 10.5670/oceanog.2018.105

Smith, N., Kessler, W. S., Cravatte, S. E., Sprintall, J., Wijffels, S. E., Cronin, M. F., et al. (2019). Tropical Pacific Observing System. Front. Mar. Sci. 6:31. doi: $10.3389 /$ fmars.2019.00031

Soh, H. S., and Kim, S. Y. (2018). Diagnostic characteristics of submesoscale coastal surface currents. J. Geophys. Res. 123, 1838-1859. doi: 10.1002/2017JC013428

Song, H., Edwards, C. A., Moore, A. M., and Fiechter, J. (2012). Incremental fourdimensional variational data assimilation of positive-definite oceanic variables using a logarithm transformation. Ocean Model. 54-55, 1-17. doi: 10.1016/j. ocemod.2012.06.001

Spadone, A., and Provost, C. (2009). Variations in the Malvinas Current volume transport since October 1992. J. Geophys. Res. 114:C02002. doi: 10.1029/ 2008JC004882

St. Laurent, L., and Merrifield, S. (2017). Measurements of near-surface turbulence and mixing from autonomous ocean gliders. Oceanography 30, 116-125. doi: 10.5670/oceanog.2017.231

Steinfeldt, R., Sültenfuß, J., Dengler, M., Fischer, T., and Rhein, M. (2015). Coastal upwelling off Peru and Mauritania inferred from helium isotope disequilibrium. Biogeosciences 12, 7519-7533. doi: 10.5194/bg-12-7519-2015

Strub, P. T., James, C., Combes, V., Matano, R., Piola, A., Palma, E., et al. (2015). Altimeter-derived seasonal circulation on the SW Atlantic Shelf: $27^{\circ}-43^{\circ} \mathrm{S}$. J. Geophys. Res. Oceans 120, 3391-3418. doi: 10.1002/2015JC010769

Stuart-Smith, R. D., Brown, C. J., Ceccarelli, D. M., and Edgar, G. J. (2018). Ecosystem restructuring along the Great Barrier Reef following mass coral bleaching. Nature 560, 92-96. doi: 10.1038/s41586-018-0359-9

Subramanian, A., Balmaseda, M. A., Chattopadhyay, R., Centurioni, L. R., Cornuelle, B. D., DeMott, C., et al. (2019). Ocean observations to improve our understanding, modeling, and forecasting of subseasonal-to-seasonal variability. Front. Mar. Sci. 6:427. doi: 10.3389/fmars.2019.00427

Sugimoto, S., and Hanawa, K. (2014). Influence of Kuroshio path variation south of Japan on formation of subtropical mode water. J. Phys. Oceanogr. 44, 1065-1077. doi: 10.1175/jpo-d-13-0114.1

Sun, X., Vizy, E. K., and Cook, K. H. (2018). Land-atmosphere-ocean interactions in the southeastern Atlantic: interannual variability. Clim. Dyn. 52:539. doi: 10.1007/s00382-018-4155-x

Suthers, I. M., Young, J. W., Baird, M. E., Roughan, M., Everett, J. E., Brassington, G. B., et al. (2011). The strengthening East Australian Current, its eddies and biological effects-an introduction and overview. Deep Sea Res. 58, 538-546. doi: 10.1016/j.dsr2.2010.09.029

Sutton, A. J., Sabine, C. L., Feely, R. A., Cai, W.-J., Cronin, M. F., McPhaden, M. J., et al. (2016). Using present-day observations to detect when anthropogenic change forces surface ocean carbonate chemistry outside preindustrial bounds. Biogeosciences 13, 5065-5083. doi: 10.5194/bg-2016-104

Sutton, A. J., Sabine, C. L., Maenner-Jones, S., Lawrence-Slavas, N., Meinig, C., Feely, R. A., et al. (2014). A high-frequency atmospheric and seawater $\mathrm{pCO}_{2}$ data set from 14 open-ocean sites using a moored autonomous system. Earth Syst. Sci. Data 6, 353-366. doi: 10.5194/essd-6-353-2014

Sutton, A. J., Wanninkhof, R., Sabine, C. L., Feely, R. A., Cronin, M. F., and Weller, R. A. (2017). Variability and trends in surface seawater $\mathrm{pCO}_{2}$ and $\mathrm{CO}_{2}$ flux in the Pacific Ocean. Geophys. Res. Lett. 44, 5627-5636. doi: 10.1002/ 2017GL073814

Takahashi, T., Sutherland, S. C., Wanninkhof, R., Sweeney, C., Feely, R. A., Chipman, D. W., et al. (2009). Climatological mean and decadal change in surface ocean $\mathrm{pCO}_{2}$, and net sea-air $\mathrm{CO}_{2}$ flux over the global oceans. Deep Sea Res. II 56, 554-577. doi: 10.1016/j.dsr2.2008.12.009

Talley, L. D., Feely, R. A., Sloyan, B. M., Wanninkhof, R., Baringer, M. O., Bulliser, J. L., et al. (2016). Changes in ocean heat, carbon content, and ventilation: a review of the first decade of GO-SHIP global repeat hydrography. Ann. Rev. Mar. Sci. 8, 185-215. doi: 10.1146/annurev-marine-052915100829

Tchipalanga, P., Dengler, M., Brandt, P., Kopte, R., Macuéria, M., Coelho, P., et al. (2018). Eastern boundary circulation and hydrography off Angola-building Angolan oceanographic capacities. Bull. Am. Meteor. Soc. 99, 1589-1605. doi: 10.1175/BAMS-D-17-0197.1

Testor, P., DeYoung, B., Rudnick, D. L., Glenn, S., Hayes, D., Lee, C., et al. (2019). OceanGliders: a component of the integrated GOOS. Front. Mar. Sci. 6:422. doi: $10.3389 /$ fmars.2019.00422

Thompson, J. D., and Schmitz, W. J. Jr. (1989). A limited-area model of the Gulf Stream: design, initial experiments, and model-data intercomparison. J. Phys. Oceanogr. 19, 791-814. doi: 10.1175/1520-0485(1989)019<0791:alamot> 2.0.co; 2

Thomsen, S., Karstensen, J., Kiko, R., Krahmann, G., Dengler, M., and Engel, A. (2019). Remote and local drivers of oxygen and nitrate variability in the shallow oxygen minimum zone off Mauritania in June 2014. Biogeosciences 16, 979-998. doi: 10.5194/bg-16-979-2019

Todd, R. E. (2017). High-frequency internal waves and thick bottom mixed layers observed by gliders in the Gulf Stream. Geophys. Res. Lett. 44, 6316-6325. doi: 10.1002/2017GL072580

Todd, R. E., and Locke-Wynn, L. (2017). Underwater glider observations and the representation of western boundary currents in numerical models. Oceanography 30, 88-89. doi: 10.5670/oceanog.2017.225

Todd, R. E., and Owens, B. (2016). Data from: Gliders in the Gulf Stream. La Jolla, CA: Instrument Development Group, Scripps Institution of Oceanography, doi: 10.21238/s8SPRAY2675

Todd, R. E., Asher, T. G., Heiderich, J., Bane, J. M., and Luettich, R. A. (2018a). Transient response of the Gulf Stream to multiple hurricanes in 2017. Geophys. Res. Lett. 45, 10509-10519. doi: 10.1029/2018GL079180

Todd, R. E., Rudnick, D. L., Centurioni, L. R., Jayne, S. R., and Lee, C. M. (2018b). "Boundary current observations with ALPS," in ALPS II-Autonomous and Lagrangian Platforms and Sensors. A Report of the ALPS II Workshop, eds D. Rudnick, D. Costa, C. Lee, and M.-L. Timmermans (Washington, DC: National Oceanographic Partnership Program).

Todd, R. E., Gawarkiewicz, G. G., and Owens, W. B. (2013). Horizontal scales of variability over the Middle Atlantic Bight shelf break and continental rise from finescale observations. J. Phys. Oceanogr. 43, 222-230. doi: 10.1175/JPO-D-12099.1

Todd, R. E., Owens, W. B., and Rudnick, D. L. (2016). Potential vorticity structure in the North Atlantic western boundary current from underwater glider observations. J. Phys. Oceanogr. 46, 327-348. doi: 10.1175/JPO-D-150112.1

Todd, R. E., Rudnick, D. L., Davis, R. E., and Ohman, M. D. (2011a). Underwater gliders reveal rapid arrival of El Niño effects off California's coast. Geophys. Res. Lett. 38:L03609. doi: 10.1029/2010GL046376

Todd, R. E., Rudnick, D. L., Mazloff, M. R., Davis, R. E., and Cornuelle, B. D. (2011b). Poleward flows in the southern California Current System: Glider observations and numerical simulation. J. Geophys. Res. 116:C02026. doi: 10. 1029/2010JC006536

Todd, R. E., Rudnick, D. L., Mazloff, M. R., Cornuelle, B. D., and Davis, R. E. (2012). Thermohaline structure in the California Current System: Observations and modeling of spice variance. J. Geophys. Res. 117:C02008. doi: 10.1029/ 2011JC007589

Todd, R. E., Rudnick, D. L., Sherman, J. T., Owens, W. B., and George, L. (2017). Absolute velocity estimates from autonomous underwater gliders equipped with Doppler current profilers. J. Atmos. Ocean. Technol. 34, 309-333. doi: 10.1175/JTECH-D-16-0156.1

Toole, J. M., Andres, M., Le Bras, I. A., Joyce, T. M., and McCartney, M. S. (2017). Moored observations of the deep western boundary current in the NW Atlantic: 2004-2014. Oceans 122, 7488-7505. doi: 10.1002/2017JC012984

Trowbridge, J., Weller, R., Kelley, D., Dever, E., Plueddemann, A., Barth, J. A., et al. (2019). The Ocean Observatories Initiative. Front. Mar. Sci. 6:74. doi: $10.3389 /$ fmars.2019.00074 
Valdivieso, M., Haines, K., Balmaseda, M., Chang, Y.-S., Drevillon, M., Ferry, N., et al. (2017). An assessment of air-sea heat fluxes from ocean and coupled reanalyses. Clim. Dyn. 49, 983-1008. doi: 10.1007/s00382-015-2843-3

Valla, D., and Piola, A. R. (2015). Evidence of upwelling events at the northern Patagonian shelf break. J. Geophys. Res. Oceans 120, 7635-7656. doi: 10.1002/ $2015 \mathrm{jc} 011002$

Valla, D., Piola, A. R., Meinen, C. S., and Campos, E. (2018). Strong mixing and recirculation in the northwestern Argentine Basin. J. Geophys. Res. Oceans 123, 4624-4648. doi: 10.1029/2018JC013907

Van der Lingen, C. D., Fréon, P., Hutchings, L., Roy, C., Bailey, G. W., Bartholomae, C., et al. (2006). "Forecasting shelf processes of relevance to living marine resources in the BCLME," in Benguela: Predicting a Large Marine Ecosystem, Large Mar. Ecosyst. Ser, Vol. 14, ed. V. Shannon (Amsterdam: Elsevier), 309347. doi: 10.1016/s1570-0461(06)80019-4

van Sebille, E., Baringer, M. O., Johns, W. E., Beal, L. M., de Jong, M. F., van Aken, H. M., et al. (2011). Propagation pathways of classical Labrador Sea Water from its source region to $26^{\circ}$ N. J. Geophys. Res. 116:C12027. doi: 10.1029/ 2011JC007171

van Sebille, E., Beal, L. M., and Biastoch, A. (2010). Sea surface slope as a proxy for Agulhas Current strength. Geophys. Res. Lett 37:L09610.

van Sebille, E., Wilcox, C., Lebreton, L., Maximenko, N., Hardesty, B. D., Van Franekar, J. A., et al. (2015). A global inventory of small floating plastic debris. Environ. Res. Lett 10:124006. doi: 10.1088/1748-9326/10/12/124006

Van Uffelen, L. J., Roth, E. H., Howe, B. M., Oleson, E. M., and Barkley, Y. (2017). A Seaglider-integrated digital monitor for bioacoustic sensing. IEEE J. Ocean. Eng. 42, 800-807. doi: 10.1109/JOE.2016.2637199

Vélez-Belchí, P., Centurioni, L. R., Lee, D.-K., Jan, S., and Niiler, P. P. (2013). Eddy induced Kuroshio intrusions onto the continental shelf of the East China Sea. J. Mar. Res. 71, 83-107. doi: 10.1357/002224013807343470

Vivier, F., and Provost, C. (1999). Direct velocity measurements in the malvinas current. J. Geophys. Res. 104, 21083-21103. doi: 10.1029/1999JC900163

Volkov, D. L., Baringer, M., Smeed, D., Johns, W., and Landerer, F. (2019). Teleconnection between the Atlantic meridional overturning circulation and sea level in the Mediterranean Sea. J. Clim. 32, 935-955. doi: 10.1175/JCLI-D18-0474.1

Wada, A., Cronin, M. F., Sutton, A. J., Kawai, Y., and Ishii, M. (2013). Numerical simulations of oceanic $\mathrm{pCO}_{2}$ variations and interactions between typhoon Choi-wan (0914) and the ocean. J. Geophys. Res. Oceans 118, 2667-2684. doi: 10.1002 /jgrc. 20203

Wagawa, T., Tamate, T., Kuroda, H., Ito, S., Kakehi, S., Yamanome, T., et al. (2016). Relationship between coastal water properties and adult return of chum salmon (Oncorhynchus keta) along the Sanriku coast, Japan. Fish. Oceangr. 25, 598-609. doi: 10.1111/fog. 12175

Wakita, M., Watanabe, S., Murata, A., Tsurushima, N., and Honda, M. (2010). Decadal change of dissolved inorganic carbon in the subarctic western North Pacific Ocean. Tellus B 62, 608-620. doi: 10.1111/j.1600-0889.2010.00476.x

Wang, D., Flagg, C. N., Donohue, K., and Rossby, H. T. (2010). Wavenumber spectrum in the Gulf Stream from shipboard ADCP observations and comparison with altimetry measurements. J. Phys. Oceanogr. 40, 840-844. doi: 10.1175/2009JPO4330.1

Wang, F., Zhang, L., Hu, D., Wang, Q., Zhai, F., and Hu, S. (2017). The vertical structure and variability of the western boundary currents east of the Philippines from direct observations. J. Oceanogr. 73, 743-758. doi: 10.1007/ s10872-017-0429-x

Weeks, S. J., Barlow, R., Roy, C., and Shillington, F. A. (2006). Remotely sensed variability of temperature and chlorophyll in the southern Benguela: upwelling frequency and phytoplankton response. Afr. J. Mar. Sci. 28, 493-509. doi: 10. 2989/18142320609504201

Weller, R. A., Bigorre, S. P., Lord, J., Ware, J. D., and Edson, J. B. (2012). A surface mooring for air-sea interaction research in the Gulf Stream. Part I: mooring design and instrumentation. J. Atmos. Ocean. Technol. 29, 1363-1376. doi: 10.1175/JTECH-D-12-00060.1

Wijffels, S. E., Meyers, G., and Godfrey, J. S. (2008). A 20-yr average of the Indonesian Throughflow: regional currents and the interbasin exchange. J. Phys. Oceanogr. 38, 1965-1978. doi: 10.1175/2008JPO3987.1

Wilkinson, M. D., Dumontier, M., Aalbersberg, I. J., Appleton, G., Axton, M., Baak, A., et al. (2016). The FAIR guiding principles for scientific data management and stewardship. Sci. Data 3:160018. doi: 10.1038/sdata.2016.18
Williams, R. G., McDonagh, E. L., Roussenov, V. M., Torres-Valdes, S., King, B., Sanders, R., et al. (2011). Nutrient streams in the North Atlantic: advective pathways of inorganic and organic nutrients. Glob. Biogeochem. Cycles 25:GB4008. doi: 10.1029/2010GB003853

Williams, R. G., Roussenov, V., and Follows, M. J. (2006). Nutrient streams and their induction into the mixed layer. Glob. Biogeochem. Cycles 20:GB1016. doi: 10.1029/2005gb002586

Woo, L. M., and Pattiaratchi, C. B. (2008). Hydrography and water masses off the western Australian coast. Deep Sea Res. I 55, 1090-1104. doi: 10.1016/j.dsr.2008. 05.005

Wu, C.-R., Chang, Y.-L., Oey, L.-Y., Chang, C.-W. J., and Hsin, Y.-C. (2008). Airsea interaction between tropical cyclone Nari and Kuroshio. Geophys. Res. Lett. 35:L12605. doi: 10.1029/2008GL033942

Wu, L., Cai, W., Zhang, L., Nakamura, H., Timmermann, A., Joyce, T., et al. (2012). Enhanced warming over the global subtropical western boundary currents. Nat. Clim. Chang. 2, 161-166. doi: 10.1038/nclimate1353

Wyatt, L. R., Mantovanelli, A., Heron, M. L., Roughan, M., and Steinberg, C. R. (2018). Assessment of surface currents measured with high-frequency phasedarray radars in two regions of complex circulation IEEE. J. Ocean. Eng. 43, 484-505. doi: 10.1109/JOE.2017.2704165

Yamamoto, A., Palter, J. B., Dufour, C. O., Griffies, S. M., Bianchi, D., Claret, M., et al. (2018). Roles of the ocean mesoscale in the horizontal supply of mass, heat, carbon and nutrients to the Northern Hemisphere subtropical gyres. J. Geophys. Res. 123, 7016-7036. doi: 10.1029/2018JC013969

Yang, H., Lohmann, G., Wei, W., Dima, M., Ionita, M., and Liu, J. (2016). Intensification and poleward shift of subtropical western boundary currents in a warming climate. Oceans 121, 4928-4945. doi: 10.1002/2015JC0 11513

Yang, Y. J., Jan, S., Chang, M.-H., Wang, J., Mensah, V., Kuo, T.-H., et al. (2015). Mean structure and fluctuations of the Kuroshio east of Taiwan from in situ and remote observations. Oceanography 28, 74-83. doi: 10.5670/oceanog. 2015.83

Yasuda, I. (2003). Hydrographic structure and variability in the KuroshioOyashio transition area. J. Oceanogr. 59, 389-402. doi: 10.1023/A:10255803 13836

Yasunaka, S., Nojiri, Y., Nakaoka, S., Ono, T., Mukai, H., and Usui, N. (2013). Monthly maps of sea surface dissolved inorganic carbon in the North Pacific: basin-wide distribution and seasonal variation. J. Geophys. Res. Oceans 118, 3843-3850. doi: 10.1002/jgrc.20279

Yasunaka, S., Nojiri, Y., Nakaoka, S.-I., Ono, T., Whitney, F. A., and Telszewski, M. (2014). Mapping of sea surface nutrients in the North Pacific: basin-wide distribution and seasonal to interannual variability. J. Geophys. Res. Oceans 119, 7756-7771. doi: 10.1002/2014jc010318

Zaba, K. D., and Rudnick, D. L. (2016). The 2014-2015 warming anomaly in the Southern California Current System observed by underwater gliders. Geophys. Res. Lett. 43, 1241-1248. doi: 10.1002/2015GL067550

Zantopp, R., Fischer, J., Visbeck, M., and Karstensen, J. (2017). From interannual to decadal: 17 years of boundary current transports at the exit of the Labrador Sea. J. Geophys. Res. Oceans 122, 1724-1748. doi: 10.1002/2016JC01 2271

Zhang, D., Cronin, M. F., Lin, X., Inoue, R., Fassbender, A. J., Bishop, S. P., et al. (2017). Observing air-sea interaction in western boundary currents and their extension regions: considerations for OceanObs' 19. CLIVAR Variations 15, 23-30. doi: 10.5065/D6SJ1JB2

Zhang, D., Msadek, R., McPhaden, M. J., and Delworth, T. (2011). Multidecadal variability of the North Brazil Current and its connection to the atlantic meridional overturning circulation. J. Geophys. Res. 116:C04012. doi: 10.1029/ 2010JC006812

Zhang, J., Gilbert, D., Gooday, A. J., Levin, L., Naqvi, S. W. A., Middelburg, J. J., et al. (2010). Natural and human-induced hypoxia and consequences for coastal areas: synthesis and future development. Biogeosciences 7, 1443-1467. doi: 10.5194/bg-7-1443-2010

Zhang, L., Hu, D., Hu, S., Wang, F., Wang, F., and Yuan, D. (2014). Mindanao current/undercurrent measured by a subsurface mooring. J. Geophys. Res. 119, 3617-3628. doi: 10.1002/2013JC009693

Zhang, S., Curchitser, E. N., Kang, D., Stock, C. A., and Dussin, R. (2018). Impacts of mesoscale eddies on the vertical nitrate flux in the Gulf stream region. J. Geophys. Res. 123, 497-513. doi: 10.1002/2017JC013402 
Zhang, W. G., and Gawarkiewicz, G. G. (2015). Dynamics of the direct intrusion of gulf stream ring water onto the Mid-Atlantic Bight shelf. Geophys. Res. Lett. 42, 7687-7695. doi: 10.1002/2015GL065530

Zhang, W. G., and Partida, J. (2018). Frontal subduction of the Mid-Atlantic Bight shelf water at the onshore edge of a warm-core ring. J. Geophys. Res. 123, 7795-7818. doi: 10.1029/2018JC013794

Zhang, Z., Zhao, W., Tian, J., Yang, Q., and Qu, T. (2015). Spatial structure and temporal variability of the zonal flow in the Luzon Strait. J. Geophys. Res. 120, 759-776. doi: 10.1002/2014JC010308

Zhou, C., Zhao, W., Tian, J., Yang, Q., and Qu, T. (2014). Variability of the deepwater overflow in the luzon strait. J. Phys. Oceanogr. 44, 2972-2986. doi: 10. 1175/JPO-D-14-0113.1

Zilberman, N. V., Roemmich, D. H., Gille, S. T., and Gilson, J. (2018). Estimating the velocity and transport of western boundary current systems: a case study of the East Australian Current near Brisbane. J. Atmos. Ocean. Technol. 35, 1313-1329. doi: 10.1175/JTECH-D-170153.1

Zilberman, N. V., Roemmich, D., and Gille, S. (2013). The mean and the time variability of the shallow meridional overturning circulation in the tropical South Pacific Ocean. J. Clim. 26, 4069-4087. doi: 10.1175/JCLI-D-1200120.1

Zilberman, N. V., Roemmich, D., and Gille, S. (2014). Meridional volume transport in the South Pacific: mean and SAM-related variability. J. Geophys. Res. Oceans. 119, 2658-2678. doi: 10.1002/2013JC009688
Zuidema, P., Chang, P., Medeiros, B., Kirtman, B. P., Mechoso, R., Schneider, E. K., et al. (2016). Challenges and prospects for reducing coupled climate model SST biases in the eastern tropical Atlantic and Pacific Oceans: the U.S. CLIVAR eastern tropical oceans synthesis working group. Bull. Am. Meteor. Soc. 97, 2305-2328. doi: 10.1175/BAMS-D-15-00274.1

Conflict of Interest Statement: The authors declare that the research was conducted in the absence of any commercial or financial relationships that could be construed as a potential conflict of interest.

Copyright (C) 2019 Todd, Chavez, Clayton, Cravatte, Goes, Graco, Lin, Sprintall, Zilberman, Archer, Arístegui, Balmaseda, Bane, Baringer, Barth, Beal, Brandt, Calil, Campos, Centurioni, Chidichimo, Cirano, Cronin, Curchitser, Davis, Dengler, deYoung, Dong, Escribano, Fassbender, Fawcett, Feng, Goni, Gray, Gutiérrez, Hebert, Hummels, Ito, Krug, Lacan, Laurindo, Lazar, Lee, Lengaigne, Levine, Middleton, Montes, Muglia, Nagai, Palevsky, Palter, Phillips, Piola, Plueddemann, Qiu, Rodrigues, Roughan, Rudnick, Rykaczewski, Saraceno, Seim, Sen Gupta, Shannon, Sloyan, Sutton, Thompson, van der Plas, Volkov, Wilkin, Zhang and Zhang. This is an open-access article distributed under the terms of the Creative Commons Attribution License (CC BY). The use, distribution or reproduction in other forums is permitted, provided the original author(s) and the copyright owner(s) are credited and that the original publication in this journal is cited, in accordance with accepted academic practice. No use, distribution or reproduction is permitted which does not comply with these terms. 


\section{OPEN ACCESS}

Edited by: Sabrina Speich,

École Normale Supérieure, France

Reviewed by:

Maciej Telszewski,

Institute of Oceanology (PAN), Poland

Maria Paz Chidichimo,

National Scientific and Technical

Research Council (CONICET),

Argentina

*Correspondence:

Bernadette M. Sloyan

Bernadette.Sloyan@csiro.au

Rik Wanninkhof

rik.wanninkhof@noaa.gov

Martin Kramp

mkramp@jcommops.org

Specialty section:

This article was submitted to

Ocean Observation,

a section of the journal

Frontiers in Marine Science

Received: 20 November 2018

Accepted: 05 July 2019

Published: 07 August 2019

Citation:

Sloyan BM, Wanninkhof $R$, Kramp M, Johnson GC, Talley LD, Tanhua T, McDonagh E, Cusack C,

O'Rourke E, McGovern E, Katsumata K, Diggs S, Hummon J,

Ishii M, Azetsu-Scott K, Boss E,

Ansorge I, Perez FF, Mercier H, Williams MJM, Anderson L, Lee JH, Murata A, Kouketsu S, Jeansson E, Hoppema M and Campos E (2019)

The Global Ocean Ship-Based Hydrographic Investigations Program (GO-SHIP): A Platform for Integrated

Multidisciplinary Ocean Science.

Front. Mar. Sci. 6:445.

doi: 10.3389/fmars.2019.00445

\section{The Global Ocean Ship-Based} Hydrographic Investigations Program (GO-SHIP): A Platform for Integrated Multidisciplinary Ocean Science

Bernadette M. Sloyan ${ }^{1 *}$, Rik Wanninkhof ${ }^{2 *}$, Martin Kramp ${ }^{3 *}$, Gregory C. Johnson ${ }^{4}$, Lynne D. Talley ${ }^{5}$, Toste Tanhua 6 , Elaine McDonagh ${ }^{7}$, Caroline Cusack ${ }^{8}$, Eleanor O'Rourke ${ }^{8}$, Evin McGovern ${ }^{8}$, Katsuro Katsumata ${ }^{9}$, Steve Diggs ${ }^{5}$, Julia Hummon ${ }^{10}$, Masao Ishii ${ }^{11}$, Kumiko Azetsu-Scott ${ }^{12}$, Emmanuel Boss ${ }^{13}$, Isabelle Ansorge ${ }^{14}$, Fiz F. Perez ${ }^{15}$, Herlé Mercier ${ }^{16}$, Michael J. M. Williams ${ }^{17}$, Leif Anderson ${ }^{18}$, Jae Hak Lee ${ }^{19}$, Akihiko Murata ${ }^{9}$, Shinya Kouketsu ${ }^{9}$, Emil Jeansson ${ }^{20}$, Mario Hoppema ${ }^{21}$ and Edmo Campos 22,23

${ }^{1}$ CSIRO, Oceans and Atmosphere, Hobart, TAS, Australia, ${ }^{2}$ NOAA's Atlantic Oceanographic and Meteorological Laboratory, Miami, FL, United States, ${ }^{3}$ JCOMMOPS, WMO/IOC-UNESCO, Brest, France, ${ }^{4}$ NOAA's Pacific Marine Environmental Laboratory, Seattle, WA, United States, ${ }^{5}$ Scripps Institution of Oceanography, University of California, San Diego, La Jolla, CA, United States, ${ }^{6}$ GEOMAR Helmholtz Centre for Ocean Research Kiel, Kiel, Germany, ${ }^{7}$ National Oceanography Centre, Southampton, United Kingdom, ${ }^{8}$ Marine Institute, Galway, Ireland, ${ }^{9} \mathrm{Global}$ Chemical and Physical Oceanography Group, JAMSTEC, Yokosuka, Japan, ${ }^{10}$ SOEST, University of Hawai'i, Honolulu, Honolulu, HI, United States, ${ }^{11}$ JMA, Meteorological Research Institute, Tsukuba, Japan, ${ }^{12}$ Bedford Institute of Oceanography, Dartmouth, NS, Canada, ${ }^{13}$ School of Marine Sciences, The University of Maine, Orono, ME, United States, ${ }^{14}$ Department of Oceanography, University of Cape Town, Cape Town, South Africa, ${ }^{15}$ Instituto de Investigaciones Marinas (IIM-CSIC), Vigo, Spain, ${ }^{16}$ CNRS, Ifremer Centre de Bretagne, University of Brest, Plouzané, France, ${ }^{17}$ National Institute of Water and Atmospheric Research, Wellington, New Zealand, ${ }^{18}$ Department of Marine Sciences, University of Gothenburg, Gothenburg, Sweden, ${ }^{19}$ Korea Institute of Ocean Science and Technology, Busan, South Korea, ${ }^{20}$ NORCE Norwegian Research Centre AS, Bjerknes Centre for Climate Research, Bergen, Norway, ${ }^{21}$ Alfred Wegener Institute, Helmholtz Centre for Polar and Marine Research, Bremerhaven, Germany, ${ }^{22}$ Oceanographic Institute, University of São Paulo, São Paulo, Brazil, ${ }^{23}$ Gulf Environments Research Institute, American University of Sharjah, Sharjah, United Arab Emirates

The Global Ocean Ship-Based Hydrographic Investigations Program (GO-SHIP) provides a globally coordinated network and oversight of 55 sustained decadal repeat hydrographic reference lines. GO-SHIP is part of the global ocean/climate observing systems (GOOS/GCOS) for study of physical oceanography, the ocean carbon, oxygen and nutrient cycles, and marine biogeochemistry. GO-SHIP enables assessment of the ocean sequestration of heat and carbon, changing ocean circulation and ventilation patterns, and their effects on ocean health and Earth's climate. Rapid quality control and open data release along with incorporation of the GO-SHIP effort in the Joint Technical Commission for Oceanography and Marine Meteorology (JCOMM) in situ Observing Programs Support Center (JCOMMOPS) have increased the profile of, and participation in, the program and led to increased data use for a range of efforts. In addition to scientific discovery, GO-SHIP provides climate quality observations for ongoing calibration of measurements from existing and new autonomous platforms. This includes biogeochemical observations for the nascent array of biogeochemical (BGC)-Argo floats; temperature and salinity for Deep Argo; and salinity for the core 
Argo array. GO-SHIP provides the relevant suite of global, full depth, high quality observations and co-located deployment opportunities that, for the foreseeable future, remain crucial to maintenance and evolution of Argo's unique contribution to climate science. The evolution of GO-SHIP from a program primarily focused on physical climate to increased emphasis on ocean health and sustainability has put an emphasis on the addition of essential ocean variables for biology and ecosystems in the program measurement suite. In conjunction with novel automated measurement systems, ocean color, particulate matter, and phytoplankton enumeration are being explored as GOSHIP variables. The addition of biological and ecosystem measurements will enable GO-SHIP to determine trends and variability in these key indicators of ocean health. The active and adaptive community has sustained the network, quality and relevance of the global repeat hydrography effort through societally important scientific results, increased exposure, and interoperability with new efforts and opportunities within the community. Here we provide key recommendations for the continuation and growth of GO-SHIP in the next decade.

Keywords: GO-SHIP, ship-based observations, multidisciplinary ocean research, contemporaneous ocean observations, global ocean change and variability, health, essential ocean variables, essential climate variables

\section{INTRODUCTION}

The Global Ocean Ship-based Hydrographic Investigations Program (GO-SHIP ${ }^{2}$ ), building on the successes of hydrographic surveys such as the international geophysical year, IGY (1957/58); the Indian Ocean expedition IIOE $(\approx 1959-1965)$; the Geochemical Sections Study, GEOSECS (1968-1978); the transient tracers in the ocean program, TTO (1979-1983); the World Ocean Circulation Experiment, World Hydrographic Survey /Joint Global Ocean Flux Study, WOCE/WHP/JGOFS (1986-1996); and the Climate Variability program (1995current, CLIVAR, 2018), maintains the global survey of select hydrographic sections. Hereafter, "GO-SHIP" refers to the global hydrographic observations with modern standards that began with WOCE in the 1990s.

Global Ocean Ship-Based Hydrographic Investigations Program, in combination with earlier programs, has led to improved understanding of the role of the ocean in climate variability and change, carbon cycling, and biogeochemical responses to climate change. GO-SHIP data have been, either exclusively or in combination with other data, used to document: (1) heat and freshwater storage and flux, (2) ocean circulation and circulation variability, (3) sequestration of anthropogenic carbon, (4) ventilation of deep and shallow water masses, and (5) changes in biogeochemical properties such as inorganic carbon, $\mathrm{pH}$, oxygen and nutrients. The data have also been extensively used for model initialisation, calibration and validation. Talley et al. (2016) provide a comprehensive review of the science achievements of GO-SHIP; here we summarize those achievements and their societal impacts.

\footnotetext{
${ }^{1}$ Significant ocean science programs and research activities are listed in Table $\mathbf{1}$. GO-SHIP data centers are identified in Table 2.

${ }^{2}$ http://www.go-ship.org
}

\section{Assessment of Ocean Change Heat and Fresh Water Changes}

Argo floats now routinely sample the upper ocean $(0-2000 \mathrm{~m})$ and provide monthly estimates of upper ocean heat and freshwater change, whereas the global ocean below $2000 \mathrm{~m}$ depth is currently primarily sampled through GO-SHIP (Talley et al., 2016). GO-SHIP data have revealed that the deep $(z>2000 \mathrm{~m})$ oceans have warmed from the 1990s to 2000s, storing some of the excess heat entering Earth's climate system and contributing to global sea level rise (SLR) through thermal expansion (Purkey and Johnson, 2010, 2013; Kouketsu et al., 2011; Desbruyères et al., 2016). The GO-SHIP global sampling has shown that the deep warming is significantly larger in regions of the global ocean ventilated largely by Antarctic Bottom Water (AABW) with the strongest warming in the Southern Ocean near AABW formation sites and decreasing to the north (Sloyan et al., 2013). The deep warming equates globally to a $0.07 \mathrm{~W} \mathrm{~m}^{-2}$ average flux applied over Earth's surface area (Purkey and Johnson, 2010; Rhein et al., 2013).

During the 2010s, GO-SHIP completed a reoccupation of the global hydrographic survey (Figure 1). The extended timeseries has shown rapid AABW changes, including accelerated freshening in the Australian-Antarctic Basin and accelerated warming in the Southwest Pacific Basin in the last decade (Menezes et al., 2017; Purkey et al., 2019). In contrast, Johnson et al. (2014) find, in the Southwest Atlantic, that near-bottom warming in the Argentine Basin was reduced or absent from 2005 to 2014 compared to between 1989 and 2005, although warming continued in the bottom Brazil Basin. The difference in deep heat uptake between regions suggests different dynamical controls and/or regional variability in forcing the deep ocean. Further investigations are required to thoroughly understand these mechanisms. GO-SHIP observations have clearly shown that the ocean below $2000 \mathrm{~m}$ is varying and 
plays a significant role in the Earth energy and freshwater balance (Palmer et al., 2019). The detection of deep ocean variability has highlighted the need for more deep ocean observations, and in-part has led to the development and beginning of implementation of deep Argo float deployments that cover the full water column (Johnson et al., 2015; Jayne et al., 2017).

TABLE 1 | Significant ocean science programs and research activities.

\begin{tabular}{|c|c|c|}
\hline Program/Research activity & Acronym & Link \\
\hline Argo & & www.argo.ucsd.edu \\
\hline Biogeochemical Argo & BGC Argo & $\begin{array}{l}\text { www.biogeochemical- } \\
\text { argo.org }\end{array}$ \\
\hline $\begin{array}{l}\text { Coriolis Operational } \\
\text { Oceanography }\end{array}$ & Coriolis & www.coriolis.eu.org \\
\hline $\begin{array}{l}\text { CLIVAR and Carbon } \\
\text { Hydrographical Data Office }\end{array}$ & $\mathrm{CCHDO}$ & https://cchdo.ucsd.edu/ \\
\hline $\begin{array}{l}\text { Data Publisher for Earth and } \\
\text { Environmental Science }\end{array}$ & PANGAEA & www.pangaea.de \\
\hline $\begin{array}{l}\text { Essential Climate Variable, } \\
\text { Essential Ocean Variable }\end{array}$ & ECV, EOV & www.goosocean.org \\
\hline $\begin{array}{l}\text { Global Ocean Acidification } \\
\text { Observing Network }\end{array}$ & GOA-ON & www.goa-on.org/ \\
\hline Global Ocean Data Analysis & GLODAP & www.glodap.info \\
\hline
\end{tabular}

Project

Global Climate Observing GCOS https:/public.wmo.int/en

System

Global Ocean Observing

System

Global Ocean Ship-based Hydrographic Program

International Oceanographic

Data and Information Exchange

International quality controlled

ocean database

National Centers for

Environmental Information

OceanSITES

Scientific Committee on

Oceanic Research

SCOR Working Group 147

Toward comparability of global

oceanic nutrient data

SCOR Working Group 154

Integration of

Plankton-Observing Sensor

Systems to Existing Global

Sampling Programs

Southern Ocean Carbon Cycle

Observation and Modeling

Surface Ocean $\mathrm{CO}_{2}$ Atlas

The International Association

for the Physical Sciences of the

Ocean

IAPSO Standard Seawater

World Ocean Database

WOD

\section{Circulation and Mixing}

Global Ocean Ship-Based Hydrographic Investigations Program coast-to-coast sections provide a unique data set to monitor and documents changes in the large-scale basin circulation of the global ocean (e.g., Ganachaud and Wunsch, 2000; Sloyan and Rintoul, 2001; Lumpkin and Speer, 2007; McDonagh et al., 2008; Katsumata et al., 2013; Talley, 2013; Mercier et al., 2015; Hernandez-Guerra and Talley, 2016). GO-SHIP data have been combined with other components of the observing system, including satellite altimetry sea surface height and Argo float velocity (e.g., Roemmich et al., 2007) or OceanSITES boundary current transport and midocean arrays (e.g., Carton and Hakkinen, 2011; Carton et al., 2014) to investigate key questions regarding basin circulation. In addition full-depth temperature, salinity and velocity profiles have been used to investigate the spatial and vertical distribution of ocean mixing (Kunze et al., 2006; Huussen et al., 2012; Talley et al., 2016).

\section{Transient Tracers}

Anthropogenic transient-tracers such as chlorofluorocarbons (CFCs) and sulfur hexafluoride $\left(\mathrm{SF}_{6}\right)$, collected as part of GOSHIP, enable the science community to investigate the pathways, rates, and temporal variations in the processes ventilating the ocean on decadal timescales (Johnson et al., 2008; Wanninkhof et al., 2013b; Purkey et al., 2018). In addition, CFC and other tracer data are used to reconstruct the spatial and temporal evolution of anthropogenic carbon in the global ocean (Sabine and Tanhua, 2010; Khatiwala et al., 2013; Wanninkhof et al., 2013a); to estimate apparent oxygen utilization rates (Jenkins, 1977; Stanley et al., 2012); and to separate observed changes in oxygen and nutrients into physical (e.g., solubility, circulation, and mixing) and biological processes (Jenkins, 1998; Emerson et al., 2001; Mecking et al., 2006; Sonnerup et al., 2013, 2015; de la Paz et al., 2017). Transient tracers observations have revealed decadal scale variability of ventilation (e.g., Waugh et al., 2013; Tanhua et al., 2017).

\section{Anthropogenic Carbon and Ocean Acidification}

The tracking of global ocean uptake of anthropogenic carbon since the 1990s has only been possible due to the sustained GOSHIP decadal survey. Changes in DIC concentration between repeat sections reflect both anthropogenic carbon uptake as well as natural variations in circulation and organic matter remineralization (Sabine and Tanhua, 2010).

Using GO-SHIP data a number of methods have been developed to estimate the ocean's anthropogenic carbon uptake and inventory (Gruber et al., 1996; Friis et al., 2005). Based on these methods, Kouketsu and Murata (2014) estimated an anthropogenic storage rate of $2.9( \pm 0.4) \mathrm{Pg} \mathrm{C}_{\mathrm{year}}{ }^{-1}$ for the most recent decade (Figure 2). Gruber et al. (2019) provide a comprehensive analyses of GO-SHIP, and other ocean interior data and determine an uptake of $2.6( \pm 0.3) \mathrm{Pg} \mathrm{C}$ year $^{-1}$ between 1994 and 2006. Data-constrained ocean models for the period 1994-2010 suggest an ocean mean annual uptake rate of approximately $2.3 \mathrm{Pg} \mathrm{C}$ year $^{-1}$. This equates to approximately $27 \%$ of the total anthropogenic carbon emissions absorbed by the ocean over this time period (Khatiwala et al., 2013). 
Takatani et al. (2014) used the GO-SHIP reference and highfrequency sections to show that the rate of anthropogenic carbon increase in the western North Pacific subtropical gyre over the past two decades is consistent with atmospheric $\mathrm{CO}_{2}$ increases. The mean rate of anthropogenic carbon inventory change over the past few decades varies from $0.22( \pm 0.05) \mathrm{mol} \mathrm{m}^{-2}$ year $^{-1}$ to $0.94( \pm 0.10) \mathrm{mol} \mathrm{m}^{-2}$ year $^{-1}$, depending on the depth to which the ocean is ventilated (Talley et al., 2016). There is still considerable debate on whether this uptake is stored locally or exported (Sabine et al., 2004; van Heuven et al., 2011) and what water masses are involved (e.g., Gruber et al., 1996; Sabine et al., 2004; van Heuven et al., 2011; Pardo et al., 2014; Groeskamp et al., 2016). Once the anthropogenic carbon enters the ocean it is redistributed by the ocean circulation. It has been found that the deep western boundary currents serve as important ventilation pathways and carry an appreciable amount of anthropogenic

TABLE 2 | Variables measured on Global Ocean Ship-Based Hydrographic Investigations Program (GO-SHIP) cruises ${ }^{1}$ with location of documentation, calibration, and data storage.

\begin{tabular}{|c|c|c|c|c|c|}
\hline Measurement & Level $^{1}$ & EOV $^{2}$ & Documentation $^{3}$ & Standards $^{4}$ & Data depository 5 \\
\hline \multicolumn{6}{|c|}{ Sensor-based: sensors on CTD frame } \\
\hline Temperature & 1 & $\mathrm{P}$ & Hydro man. & & $\mathrm{CCHDO}$ \\
\hline Salinity & 1 & $\mathrm{P}$ & Hydro man. & & $\mathrm{CCHDO}$ \\
\hline Oxygen & 1 & BGC & Hydro man. & & $\mathrm{CCHDO}$ \\
\hline L-ADCP & 1 & P & Hydro man. & & NCEI (United States) ${ }^{6}$, UH, BODC (United Kingdom) \\
\hline Transmissometer & 2 & $\mathrm{BE}$ & & & CCHDO, NCEI (United States) \\
\hline Chlorophyll & 3 (ocean color) & $\mathrm{BGC}$ & & & SeaBASS \\
\hline \multicolumn{6}{|c|}{ Underway: taken from uncontaminated seawater line or hull mounted sensor } \\
\hline $\mathrm{pCO}_{2}$ & 1 & BGC & Pierrot & & SOCAT \\
\hline Temperature & 1 & $\mathrm{P}$ & GOSUD & & GOSUD \\
\hline Salinity & 1 & $\mathrm{P}$ & GOSUD & & GOSUD \\
\hline S-ADCP & 1 & $P$ & Hydro Man. & & JASADCP/NCEI/UH ${ }^{7}$ \\
\hline Meteorological data & 1 & $P$ & & & SAMOS/COAPS/FSU/ICOADS ${ }^{8}$ \\
\hline Bathymetry & 1 & & & & $\mathrm{IHO}$ \\
\hline \multicolumn{5}{|c|}{ Bottles: samples taken from Niskin Samplers } & $\mathrm{CCHDO}$ \\
\hline Sampling protocols & & & Hydro man. & & \\
\hline Salinity & 1 & $\mathrm{P}$ & Hydro man. & IAPSO standard Seawater & $\mathrm{CCHDO}$ \\
\hline Oxygen & 1 & $\mathrm{BGC}$ & Hydro man. & lodate Std. & $\mathrm{CCHDO}$ \\
\hline CFC's incl. SF $_{6}$ & 1 & $\mathrm{BGC}$ & Hydro man. & & $\mathrm{CCHDO}$ \\
\hline Nitrous oxide & 3 & $B G C$ & Hydro man. & & $\mathrm{CCHDO}$ \\
\hline Inorganic nutrients & 1 & $\mathrm{BGC}$ & Hydro man. & Kanso Nutrient CRM & \\
\hline $\mathrm{NO}_{3} / \mathrm{NO}_{2}$ & 1 & & & & $\mathrm{CCHDO}$ \\
\hline $\mathrm{PO}_{4}$ & 1 & & & & $\mathrm{CCHDO}$ \\
\hline $\mathrm{SiO}_{3}$ & 1 & & & & $\mathrm{CCHDO}$ \\
\hline Inorganic carbon 9,10 & & BGC & Dickson- $\mathrm{CO}_{2}$ & & \\
\hline TAlk & 1 & & Dickson-SOP 3 & CRM-Dickson & $\mathrm{CCHDO}$ \\
\hline$D / C$ & 1 & & Dickson-SOP 2 & CRM-Dickson & $\mathrm{CCHDO}$ \\
\hline $\mathrm{pH}$ & 1 & & Dickson-SOP 6 & $N / A^{13}$ & $\mathrm{CCHDO}$ \\
\hline $\mathrm{pCO}_{2}$ & 2 & & Dickson-SOP 4 & & $\mathrm{CCHDO}$ \\
\hline DOC/DON & 2 & $B G C$ & Dickson-SOP 7 & CRM-Hansell & $\mathrm{CCHDO}$ \\
\hline $\mathrm{DIC}-{ }^{13} \mathrm{C},{ }^{14} \mathrm{C}$ & 2 & $B G C$ & Dickson-SOP 1 & & $\mathrm{CCHDO}$ \\
\hline POC/PON & 3 (Plankton) & $\mathrm{BE}$ & $\operatorname{socCOM}^{3,11}$ & $N / A^{13}$ & SeaBASS \\
\hline HPLC pigments & 3 (plankton/ocean color) & $\mathrm{BE} / \mathrm{BGC}$ & soccom $^{3,12}$ & $\mathrm{~N} / \mathrm{A}^{13}$ & SeaBASS \\
\hline
\end{tabular}

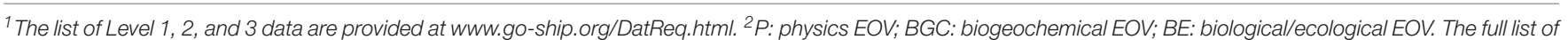

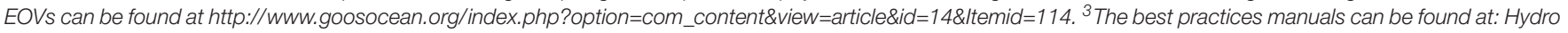

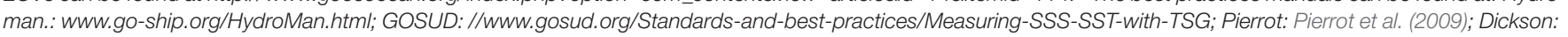

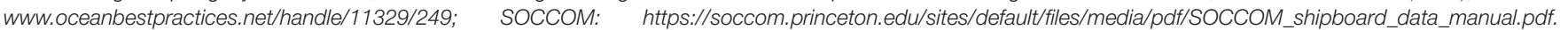

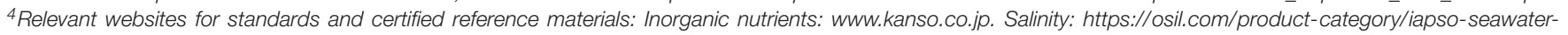

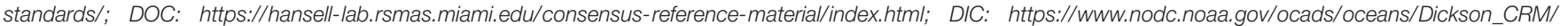

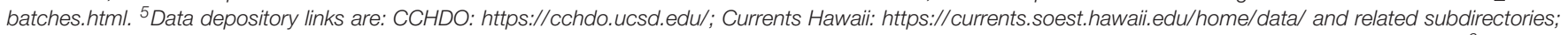

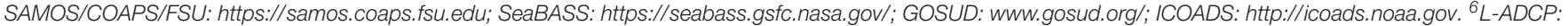

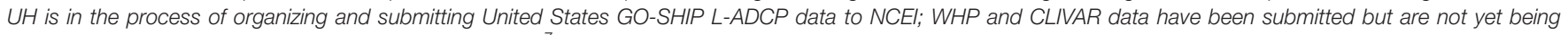

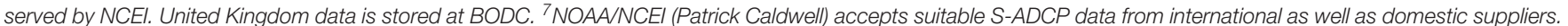

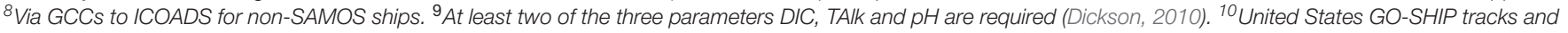
links to all of its component datasets: http://usgoship.ucsd.edu. ${ }^{11} \mathrm{Knap}$ et al. (1996). ${ }^{12}$ Hooker et al. (2005). ${ }^{13} \mathrm{~N} / \mathrm{A}$ : Currently not available but highly desired. 

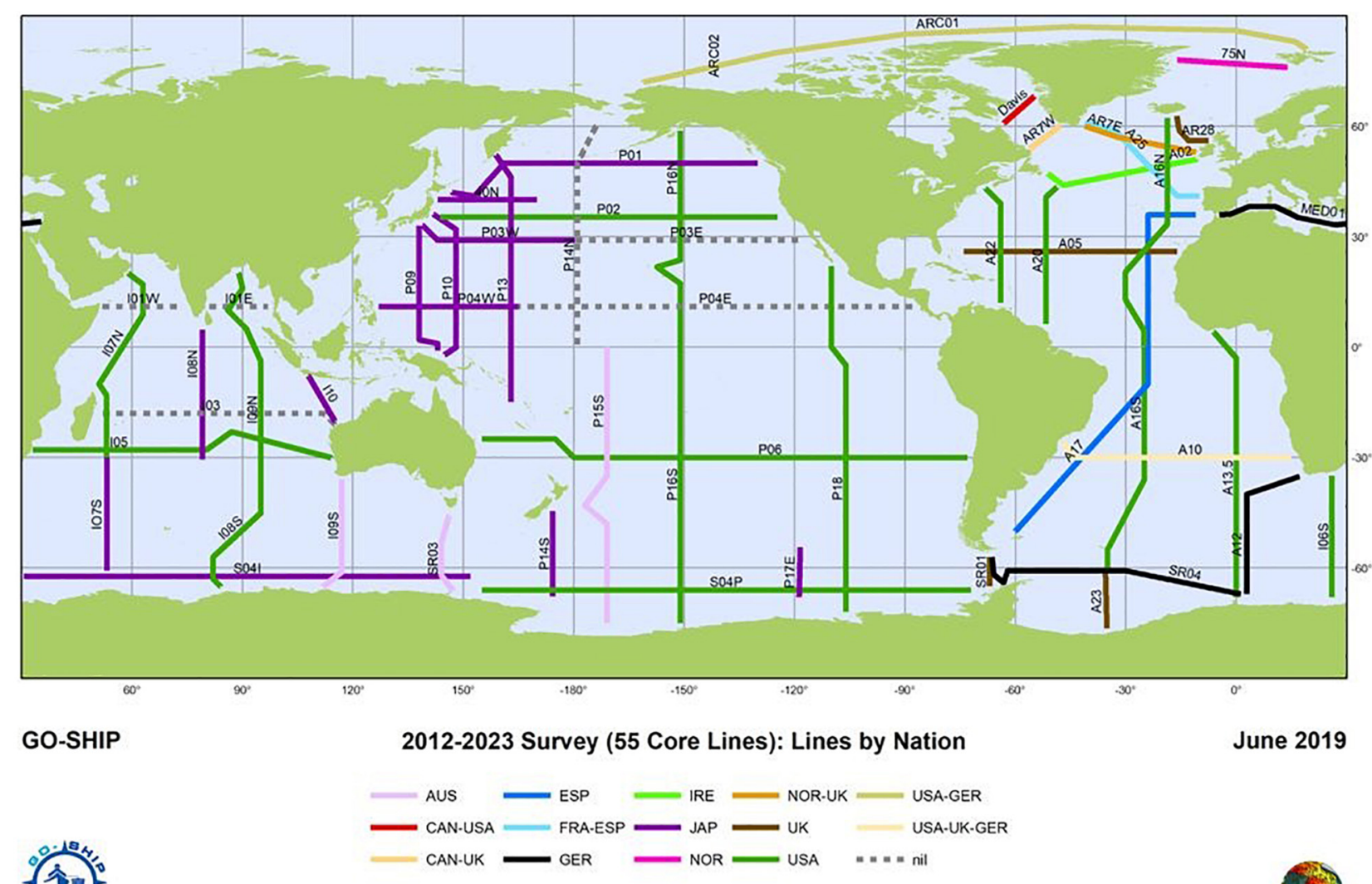

FIGURE 1 | Global Ocean Ship-Based Hydrographic Investigations Program (GO-SHIP) reference lines according the nations leading the effort (color coded).

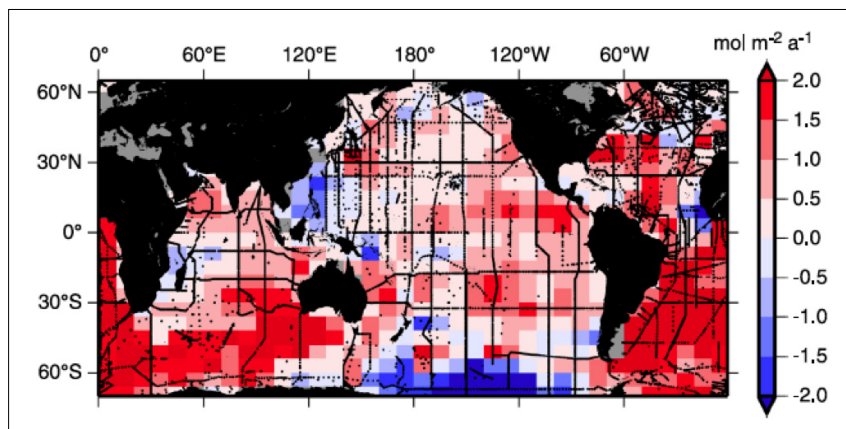

FIGURE 2 | Column inventory changes in anthropogenic carbon in the global ocean based on the CARINA and PACIFICA syntheses from Kouketsu and Murata (2014).

carbon into the interior (Körtzinger et al., 1999; Murata et al., 2010; Brown et al., 2010; Waters et al., 2011; Ríos et al., 2012; Sloyan et al., 2013; Guallart et al., 2015; Carter et al., 2019).

The increase in ocean carbon concentration due to anthropogenic carbon uptake and rising ocean temperature has also lead to ocean acidification (Feely et al., 2009; Doney et al., 2009; Jiang et al., 2015). Results from GO-SHIP and the earlier programs (Feely et al., 2004, 2009; Key et al., 2004; Sabine et al., 2004; García-Ibáñez et al., 2016), have shown a pH change in the surface water of approximately -0.002 year $^{-1}$ from 1991 to 2006 along sections in the North Pacific (for a summary, see Rhein et al., 2013). Based on frequently repeated GO-SHIP section in the North Atlantic it was shown how Meridional overturning circulation transports acidification signal to the deep waters (Perez et al., 2018).

\section{Oxygen and Deoxygenation}

Large and systematic changes in oxygen, mostly reductions, have been documented over the last two decades through GOSHIP and additional local time-series data (e.g., Keeling et al., 2010; Hahn et al., 2017). Based on modeling studies (Deutsch et al., 2005) and correlations with physical forcing (Johnson and Gruber, 2007), it is suggested that the oxygen changes are mainly driven by physical forcing changes. The deoxygenation in the open-ocean thermocline over the past two decades is consistent with the expectation that warmer waters will hold less dissolved oxygen (the solubility effect) and that warminginduced stratification leads to a decrease in the transport of dissolved oxygen from surface to subsurface waters (the stratification effect) (Matear and Hirst, 2003; Deutsch et al., 2005; Frölicher et al., 2009). 


\section{Nutrients}

Global Ocean Ship-Based Hydrographic Investigations Program provides high quality ocean nutrient data. The observed nutrient changes are due to a combination of both physical and land-use changes; physical stratification and wind changes and use of fertilizer in agriculture production leading to riverine discharge of nitrate to the ocean (Doney, 2010). A study by Kim et al. (2014), using derived excess nitrate, $\mathrm{N}^{*}$ measurements and ventilation rates from CFCs, determined that the atmospheric deposition of anthropogenic nitrate in the western North Pacific is leading to excess nitrate in the upper water column. Note, $\mathrm{N}^{*}=\mathrm{NO}_{3}-\mathrm{R}_{\mathrm{N}: \mathrm{P}}{ }^{*}$ $\mathrm{PO}_{4}$, where $\mathrm{NO}_{3}$ and $\mathrm{PO}_{4}$ are the measured nitrate and phosphate concentrations and $\mathrm{R}_{\mathrm{N}: \mathrm{P}}$ is the Redfield ratio of 16:1. The excess nitrate was highest close to Asia with rates decreasing eastward across the North Pacific and suggests implications for the ecosystem from nitrate to phosphate limitation (Kim et al., 2014).

\section{Multi-Variable Analyses}

The value of a suite of contemporaneous ocean variables in GOSHIP has been demonstrated in a number of studies. Oxygen data combined with transient-tracer data provides evidence regarding the timescales at which the ocean ventilation changes occur. The combination of oxygen data with carbon data (e.g., Sabine et al., 2008) and pH data (e.g., Byrne et al., 2010) has allowed separation of $\mathrm{DIC} / \mathrm{pH}$ changes along the GO-SHIP sections into anthropogenic and ventilation/remineralization components, including their respective effects on carbon storage and ocean acidification. Ko et al. (2018) estimated net community production $(\mathrm{NCP})$ in the $\mathrm{N}$-depleted oligotrophic oceans on the basis of the seasonal changes in surface water partial pressure of $\mathrm{CO}_{2}, \mathrm{pCO}_{2}$, and DIC in absence of nutrients, largely based on GO-SHIP cruises. The combinations of ocean tracers and carbon have been used to investigate air-sea gas exchange and reconstruct the spatial and temporal evolution of anthropogenic carbon in the global ocean. All of these biogeochemical variables rely on high quality temperature and/or salinity data for many of their analytical analysis techniques.

\section{GO-SHIP Measurement Attributes}

Global Ocean Ship-Based Hydrographic Investigations Program's unique capability of high-quality, contemporaneous ocean observations has and will continue to support physical, biogeochemical and importantly multidisciplinary ocean research. The provision of physics, biogeochemistry and increasingly biological/ecosystem ocean observations, combined with other observing systems (satellites and in situ platforms including increasing use of autonomous platforms) will support many of the user requirements of the observing system, such as climate monitoring and reporting and ocean health, in particular those identified by the UN Sustainable Development Goals ${ }^{3}$. GO-SHIP data are required to monitor the Earth's energy imbalance, and provide a significant contribution, and decadal

${ }^{3}$ https://www.undp.org/content/undp/en/home/sustainable-development-goals. $\mathrm{html}$ anchor point, to enabling tracking of the global carbon budget (Le Quéré et al., 2018). While the high quality data are generally provided in delayed-mode, the data are used in the calibration of near-real-time data that are heavily used by climate and ocean operational service providers.

\section{GO-SHIP: STRUCTURE AND OVERSIGHT ROLE}

As a component of the Global Climate Observing System (GCOS) and the Global Ocean Observing System (GOOS), it provides necessary international coordination and scientific oversight for repeat hydrography. The initial GO-SHIP Panel was established in 2007 to develop a strategy for a sustained global repeat hydrography program as a contribution to the OceanObs09 Conference (Hood et al., 2010).

As GO-SHIP established itself as an integral component of the Global Ocean Observing System (GOOS) network, the initial panel has evolved to a science committee with Executive Group and Steering Group Members committee representing all nations that execute GO-SHIP reference lines. The GOSHIP co-chairs serve on the Joint Technical Commission for Oceanography and Marine Meteorology Observation Coordination group (JCOMM OCG).

The main tasks of the national representatives and international GO-SHIP committees are to:

- Develop international agreements for a sustained international repeat ship-based hydrography program, including a strategy and program plan,

- Advocate for national contributions to this strategy and participation in the global program,

- Provide a central forum for communication, coordination, and best practices, and

- Partner in syntheses of hydrographic data, in collaboration with regional, national, international, and global research programs.

Ship-based hydrography remains the only method for obtaining coincident reference standard, high-quality, high lateral and vertical resolution measurements of a suite of physical, chemical, and biological parameters over the full water column. The GO-SHIP principal scientific objectives are: (1) understanding and documenting the large-scale ocean water property distributions, their decadal changes, and drivers of those changes; and (2) addressing questions of the large physical, biogeochemical, and biological changes anticipated for the future ocean. This includes increasing dissolved inorganic carbon (DIC), warming, acidification, deoxygenation, and increased stratification of the ocean due to atmospheric $\mathrm{CO}_{2}$ increases and associated warming caused by changes in the Earth's radiation balance. The observations will aid understanding of interaction of anthropogenic perturbations with natural cycles of water, including sea-ice, and with changes in circulation and ventilation processes. The addition of biological and ecosystem measurements will enable GO-SHIP to determine trends and variability in key indicators of ocean health. 
Currently 10 nations contribute to the GO-SHIP reference and high frequency lines (Figure 1) and GO-SHIP lines increasingly benefit from multi-national participation and support (see section "Multi-Nation Voyages"). Approximately a third are performed by the United States with support from the National Science Foundation (NSF) and the National Oceanic and Atmospheric Administration (NOAA); some Level 3 optical measurements (Table 2) are being supported by the National Aeronautics and Space Administration (NASA). Australia is a core contributor to observations in the Southern Ocean, relying on other nations for measurement of transient tracers (United States, Japan). Japan has been active in observing the Pacific; their large contributions extend to the Southern Ocean and the Indian Ocean. The United Kingdom has increased its participation in long line cruises with focus on Atlantic zonal sections and Atlantic sector Southern Ocean sections. Germany has had a long-standing effort in the Mediterranean Sea and in the Southern Ocean sector of the Atlantic, and recently completed a joint multi-nation effort with Brazil, Argentina, and South Africa that has aided the GO-SHIP capacity building activities. Spain continues its long-term commitment to Atlantic meridional and zonal sections, one of them with biennial occupancy. France and Spain contribute through a biennial line in the northern North Atlantic. Ireland led its first GO-SHIP cruise in the North Atlantic with participation from Germany, United States, United Kingdom, and Canada. Canada and Norway occupy annual lines at high northern latitudes in the Atlantic.

The GO-SHIP cruises are completed by highly qualified and experienced observing teams. The observations meet the highest standards necessary ("climate quality"), increasingly facilitated by use of certified reference materials (CRMs). The strict GOSHIP data release policy is grounded on the premise that the data are a shared community resource and should be publicly available as soon as possible. The use of high quality GOSHIP in situ data continues to grow with the expanding use of autonomous ocean observing platforms, now including those that measure biogeochemical parameters and small physical changes in the deep ocean. GO-SHIP's high quality global data are essential and irreplaceable for global climatologies that are used for calibration and reference standards for these autonomous sensors, particularly given decadal changes in ocean properties.

Global Ocean Ship-Based Hydrographic Investigations Program comprises sections in three different categories, and measurements in three priority levels. The sections are categorized as follows:

\section{Decadal Full GO-SHIP (Reference or Core Lines)}

These are a series of zonal and meridional lines that go coast to coast (or ice edge) and full depth of the ocean (Figure 1) at a target spacing of 30 nautical miles, sometimes less in regions of strong property gradients. Currently the reference network is 55 cruises sampling all major ocean basins. Level 1 data (see below) are collected at least once per decade on these sections.

\footnotetext{
${ }^{4}$ Climate quality is defined as measurements of quality sufficient to assess long term trends with a defined level of confidence.
}

\section{High Frequency GO-SHIP}

A subset of core GO-SHIP lines are occupied at higher frequencies (annual, biennial) typically with limited parameters, but with at least one decadal full GO-SHIP cruise with all Level 1 and many Level 2 and 3 measurements. High frequency cruises must (i) occupy the regular line end-to-end, (ii) cover full depth, and (iii) comply with the data policy.

\section{Associated GO-SHIP}

Repeat hydrographic sections that do not necessarily meet the sampling density and are not coast-to-coast/ice, which (i) deliver high quality data, (ii) establish full depth stations below $2000 \mathrm{~m}$ at least every $240 \mathrm{~nm}$, (iii) are repeated at least once per decade with sufficient Level 1 parameters to quantify decadal change in inorganic carbon and heat inventories, (iv) at a minimum resolution of $60 \mathrm{~nm}$, and (v) comply with the data policy.

\section{Measurement Levels}

Measurements in GO-SHIP are prioritized based on the overall goals of the program. GO-SHIP ranks the measurements as Level 1,2 , or 3. Level 1 "core" measurements are the highest priority for the scientific objectives; Level 2 are desirable and often include measurements of high interest for the particular region; and Level 3 are ancillary measurements. Level 3 measurements may include new instrumentation and experimental techniques that benefit from validation using the core measurements. The parameters for Level 1 and some Level 2 can be found in Table 2 with a full list at http://www.go-ship.org/DatReq.html.

The enduring legacy of GO-SHIP is dependent on ready access of the quality data. These yield the scientific and synthesis products, and discoveries. The value of easy access to a unified dataset for the global ocean, and recognition of the GO-SHIP contribution to these products, is important to sustain the GOSHIP effort.

\section{DATA PROCEDURES AND ACCESS}

\section{Data Acquisition: Best Practices, Standards, and Data Consistency}

The acquisition of GO-SHIP data involves several steps. GOSHIP data acquisition and laboratory analyses follow best practices, including providing standardized metadata and quality control. The quality-controlled (QC'd) data are submitted to central depositories in a timely fashion where they are checked and made available to the community at large. Coordination and assistance in these steps by the GO-SHIP science committee and JCOMMOPS coordinator are an important part of the program. Once collected and archived, GO-SHIP data form the basis for essential synthesis products and calibration of autonomous observations. The World Ocean Atlas (WOA) is a synthesized product based on the physical, oxygen, and nutrient data. For carbon and other biogeochemical analysis, the Global Ocean Data Analysis Project (GLODAP) is a major bottle data synthesis product involving extensive additional quality control in the form of cross-over adjustments. A tool to facilitate 
primary QC and coherent submission of bottle data was recently published $^{5}$. The most recent complete version is GLODAPv2 (Olsen et al., 2016) with an update in 2019, GLODAPv2.2019 (Olsen et al., 2019). Calibrated GO-SHIP CTD data are an important input for autonomous observations such as Argo floats. Climatologies of physical and biogeochemical parameters are produced by several national data centers, incorporating GOSHIP data.

The process of acquiring quality oceanographic data commences with cruise planning and follows a multi-step process to final product. Full documentation of these steps and easy access to the procedures at sites such as the online International Oceanographic Data and Information Exchange (IODE) best practices depository ${ }^{6}$ facilitate the process. This documentation is very useful to new participants in the program and when considering the inclusion of new measurements. The "how to" information is regularly reviewed to provide information and incorporate new procedures relevant to the planning and running of GO-SHIP sections.

Measurement techniques for all Level 1 and some Level 2 parameters are described in the GO-SHIP Repeat Hydrography Manual: A Collection of Expert Reports and Guidelines, often referred to as the GO-SHIP hydro manual (Hood et al., 2010). Chapters of the manual and other pertinent best practices related to GO-SHIP can be found at https://www.oceanbestpractices. net/handle/11329/371. As requirements change, parameters are included or deleted as Level 1 measurements. For example, currently the required transient tracer variables are under discussion. The CFCs, which are currently Level 1 , are analyzed by only a limited number of laboratories; moreover the atmospheric inputs have changed over time, with CFC-11, and CFC-12 concentrations leveling off in the atmosphere, reducing their usefulness as transient tracers. However, $\mathrm{SF}_{6}$, which is included in CFCs and measured from the same samples, continues to increase in the atmosphere and this variable is becoming more useful in meeting the GO-SHIP science objectives.

At present, GO-SHIP requires the following data-release timelines (Talley et al., 2016) ${ }^{7}$ :

- Preliminary datasets for all Level 1 and Level 2 ship-based measurements released within 5 weeks of the cruise

- Final datasets for all Level 1 and Level 2 ship-based measurements released within 6 months of the cruise

- Final datasets within 24 months for all Level 3 data

The relatively rapid release of data is motivated by their usefulness for climate studies and the observed rapid change in the ocean, which are of increasing societal importance. The GOSHIP data policy is stringent and geared toward rapid, open dissemination, with a clear structure for all data to undergo quality control, and to be sent to and available from recognized

\footnotetext{
${ }^{5}$ https://github.com/ocean-data-qc/ocean-data-qc Final datasets for all Level 1 and Level 2 measurements performed at a shore-based laboratory within 6 months of the shore based analysis (except for helium/tritium where the goal is 15-months after sample collection). Shore-side analyses in practice often do not meet these targets.

${ }^{6}$ https://www.oceanbestpractices.net/handle/11329/3

${ }^{7}$ http://www.go-ship.org/DatReq.html
}

data centers. All data collected as part of the program need to be submitted to a designated data management structure for quality control and dissemination for syntheses. As indicated in the recommendations, this is an ongoing effort.

The GO-SHIP suite of observations includes a significant number of Essential Ocean Variables (EOVs) and some are Essential Climate Variables (ECVs), reflecting their importance, spanning the physical, biogeochemical, and biological/ecosystem ocean (Table 2). These variables are collected by various national laboratories and universities, and rely on the international ocean research community to collaborate to collect and analyze samples following a suggested procedure and accuracy (Hood et al., 2010). Standardized collection and analysis procedures to ensure consistency of observations amongst the nations involved in the program are of utmost importance.

Global Ocean Ship-Based Hydrographic Investigations Program actively supports efforts to improve the standard and consistency amongst the research community involved in collection. In recent years, GO-SHIP has endorsed two Scientific Committee on Oceanic Research (SCOR) working groups: Toward comparability of global oceanic nutrient data (COMPONUT), and Integration of Plankton-Observing Sensor System to existing global sampling programs (P-OBS). These working groups bring together an international group of experts to develop agreed standards and procedures required to review and develop commonly agreed methodologies. GO-SHIP relies on these groups of experts to revise its hydrographic manual and update Best Practices. A significant outcome of the COMPONUT group has been the adoption of certified reference material and inter-laboratory calibration for nutrients that included 69 laboratories from 30 countries (Aoyama et al., 2012; Aoyama, 2018) and an updated chapter of Best Practices for nutrient analyses (Becker et al., 2019) ${ }^{8}$.

The production and increased use of reference materials and standards are critical for accurate measurements and their use is advocated in Best Practice procedures. Standard seawater has long been used for bottle salinity analyses, but other new reference materials have increased the quality of data. For example, use of reference material for inorganic carbon has led to a two to threefold increase in accuracy of the measurements of DIC and TAlk (Dickson et al., 2003). Reference materials come in different forms including primary standards (such a pure $\mathrm{CO}_{2}$ and compressed gas standards for $\mathrm{pCO}_{2}$ and $\mathrm{CFC}$ analyses), standard solutions for titrants (such as $\mathrm{KIO}_{3}$ for Winkler $\mathrm{O}_{2}$ and $\mathrm{HCl}$ for Alkalinity), and increasingly well-characterized seawater for the parameter of interest (for dissolved organic matter (DOM), DIC, Total Alkalinity (TAlk), and inorganic nutrients).

Reference materials are produced by manufacturers or by individual investigator's academic institutions. There can be a significant cost that, in some instances, leads to insufficient use, although costs may be subsidized by national funding agencies. Salinity, DIC, TAlk, DOM and nutrients references are prepared from natural seawater; oxygen standardization is based a calibrated iodate standard. Many of the references are "certified," that is tied to a primary standard, but nutrient

${ }^{8}$ http://www.ioccp.org/index.php/nutrients 
standards are consensus values (Aoyama et al., 2012; Aoyama, 2018). The reference materials are often used as calibrants and values measured are adjusted to the certified reference material values. For example CTD salinity (conductivity) and oxygen sensor values are adjusted to bottle salinity and oxygen measurements on GO-SHIP cruises. Bottle measurements have been declining significantly on high frequency and associate GO-SHIP cruises, and on non-GO-SHIP cruises, which typically have much less chemistry, but which all include CTD profiles. Column 5 of Table 2 indicates the priority variables in need of reference materials.

The research vessels used in GO-SHIP cruises are equipped with a number of (often semi-automatic) instruments. Seawater is pumped into the vessel through an intake, usually 1$8 \mathrm{~m}$ below the draft line. Temperature, salinity, and other parameters including dissolved oxygen and $\mathrm{pCO}_{2}$ in this intake water are continuously measured and reported. The intake temperature data have been used in the calibration of satellite SST (e.g., Reynolds, 1988). Sea surface salinities are obtained from thermosalinograph and stored in the GOSUD database. The underway $\mathrm{pCO}_{2}$ data are integral to the global surface ocean carbon data set (SOCAT) (Bakker et al., 2016), particularly in regions with little shipping traffic, that provides information necessary for air-sea carbon exchange and surface water ocean acidification monitoring.

\section{Data Repositories and Accessibility}

Global Ocean Ship-Based Hydrographic Investigations Program collects a large suite of measurements across multiple ocean disciplines. Thus there are multiple types of GO-SHIP datasets, including hydrographic, velocity, underway, and meteorological data, and the data therefore flow into different data centers and archives (Table 2). Development of a central data portal tracking and linking all component data sets is essential, including formal agreements on depositories, interoperability, and exchange, that extend beyond the vertical CTD and rosette-sample chemistry profile data for which there is a long-standing GO-SHIP archive. Several nations contributing to GO-SHIP provide concise links to all data types collected during each cruise (e.g., United States GO-SHIP's cruise data tracker), but there is no parallel international structure.

In addition to providing a centralized link to the component datasets from each GO-SHIP cruise, providing stable locations for submission and retrieval of some of the GO-SHIP data types is essential. Examples are the Level 1 shipboard acoustic Doppler profiler (S-ADCP) and lowered acoustic Doppler profiler (LADCP) velocity data, and underway data sets including Level 1 continuous sampling of temperature, salinity and $\mathrm{pCO}_{2}$ as well as Level 2 discrete surface chemistry collected in conjunction with the continuous underway sampling. There is increased recognition of the importance of data storage, and the need of exposure of the data and ease of retrieval in order to sustain and increase use of GO-SHIP data. However, funding for such activities has been lagging. Not having a clear unified strategy and resources to address data issues in global oceanography has hampered oceanographic data dissemination, in general.
That is, there is no dedicated data center or depository for all of the different types of international GO-SHIP data nor is there an international GO-SHIP requirement to submit data to a specific data center or link to a specific portal. However, GO-SHIP has identified and endorsed data centers for each type of GO-SHIP data (e.g., Table 2) and strongly recommends that data be sent to these data centers within the agreed data submission timelines (see section "Data Acquisition: Best Practices, Standards, and Data Consistency"). For several national efforts, the data submission requirements and timelines are mandated.

Full water column hydrographic profiles (CTD and discrete chemistry sampling) comprise the core data set for GO-SHIP. The most commonly used center for these data is the CLIVAR and Carbon Hydrographic Data Office (CCHDO). The mission of CCHDO is to deliver the highest possible quality global CTD and hydrographic data to users. These physical and biogeochemical data include observations carried out during numerous other past and ongoing oceanographic research programs. For GOSHIP, the CCHDO captures the Level 1 processed CTD data, all Level 1 bottle data, and as much Level 2 and 3 bottle data as possible. The GO-SHIP JCOMMOPS-based project officer and CCHDO have close interactions and exchange information regarding voyage status and data submission. These links need to be strengthened further to ensure that each GO-SHIP cruise data set is available to the community. A challenge is that there is currently no international requirement or structure to ensure that all of the completed data sets are available from a central source. The permanent repository for hydrographic data is at NOAA National Center of Environmental Information (NCEI) and routine connection between NCEI and CCHDO assure that the permanent archive is regularly updated.

CLIVAR and Carbon Hydrographic Data Office data managers work with GO-SHIP principal investigators and GO-SHIP committee members to ensure the open and timely availability of all the high quality repeat hydrography data to the international science community. GO-SHIP and CCHDO have established a strong working relationship. This is highlighted by the recent addition of an interactive map of GO-SHIP sections on the CCHDO website and direct links to data from the GO-SHIP web site. This has greatly enhanced data searching and access. GO-SHIP, JCOMMOPS and CCHDO have undertaken an audit of GO-SHIP Data Availability at CCHDO. This essential cooperative effort will be further enhanced through the JCOMMOPS Ship Coordinator, partly (but currently ad-hocly) funded by (multi) national GO-SHIP funding sources such as AtlantOS, CSIRO, and NOAA. GO-SHIP envisages that communication between the ship coordinator and CCHDO will allow for increased tracking of the international collection of CTD and bottle data and pursuit and submission of data to CCHDO.

While bottle and CTD data are well managed through CCHDO, data management efforts for several other GO-SHIP measurements as less advanced. Level 1 velocity data for GOSHIP are collected using S-ADCP and L-ADCP instrumentation. The S-ADCP and L-ADCP data acquired during GO-SHIP cruises can now be submitted to the Hawaii Currents group (Table 2). Establishing and maintaining a long-term data archive 
for all GO-SHIP velocity data, extending back through the CLIVAR and WOCE surveys that preceded it, is highly desirable, and a recommendation is to establish a routine data management system subscribed to by all GO-SHIP operators.

Underway data, predominantly $\mathrm{T}, \mathrm{S}$, and $\mathrm{pCO}_{2}$, do not have a single depository but are often included in parameter specific larger data collations. Much of the underway $\mathrm{pCO}_{2}$ data finds its way into SOCAT (Bakker et al., 2016) and some of the underway temperature and salinity are added to the GOSUD which is planned to become the general GO-SHIP archive for such data. In addition, there are efforts in the United States to place all data routinely acquired on research ships in a depository called rolling deck to depository, R2R'.

In addition to submission to centers that accept all specific GO-SHIP measurements, there are specific requirements of national funding agencies for submission to national data centers. The data centers, which are often part of world data centers under auspices of IODE or International Council for Science (ICSU), have mandates to store data in perpetuity. These include, for example, the British Oceanographic Data Centre, which is the United Kingdom facility for preserving and distributing marine data, the German data center PANGAEA, NCEI, which is the United States archive for hydrographic data, and JODC, which is the Japanese archive. These data centers often do not have an efficient way to retrieve data for programs such as GO-SHIP but rather are parameter focused. In the United States there are additional centers that house specific United States GO-SHIP datasets such as the SeaWiFS Bio-optical Archive and Storage System (SeaBASS), which is the publicly shared archive of in situ oceanographic and atmospheric data maintained by the NASA Ocean Biology Processing Group (OBPG), and the Biological and Chemical Oceanography Data Management Office (BCODMO) that serve data online from research projects funded by the oceanography programs at the United States National Science Foundation ${ }^{10}$.

A GO-SHIP Data Assembly Center (DAC) using modern data management and interoperability procedures would facilitate a means to submit and access data in the various repositories. The existing data assembly and archive centers need support to develop new tools and centers to manage the increasing variety of properties observed on hydrographic lines, to coordinate data management activities with programs such as Argo and OceanSITES, and to improve technology and data policies to release data in a timely manner. Linking datasets seamlessly through Global Data assembly centers (GDACs) such as proposed for marine biogeochemistry (GDAC-BGC) at the Bjerknes Climate Data Centre would aid access and discovery. GO-SHIP endorses a central portal and a logical query system where all the data obtained in GO-SHIP can be acquired.

Global Ocean Ship-Based Hydrographic Investigations Program will continue to focus on acquiring the highest quality, documented and referenced data. There is a need for clear documentation of practical information for cruise activities in an updated GO-SHIP manual and easily accessible voyage reports.

${ }^{9} \mathrm{http} / / / \mathrm{www}$. rvdata.us/

${ }^{10}$ www.bco-dmo.org/
This includes rosette sampling order of parameters, sample storage protocols, and cleaning and maintenance procedures for sampling bottles (Niskins). A protocol and recommendation for citation of GO-SHIP data should be developed and provided in prominent locations including all portals for GO-SHIP data, such that it is clearly referenced when used in publications or as reference data in calibration/validation of data from other observing systems such as Argo.

Systems for optimized monitoring of GO-SHIP from early cruise planning to the timely submission of data are needed. Emerging scientific products (including best practices, data synthesis and scientific publications) are presently implemented as coordinated efforts between data portals, data centers, and JCOMMOPS; coordination of these across all GO-SHIP contributors presents a major goal for the next decade.

\section{GO-SHIP EVOLVING TO MEET FUTURE OCEAN OBSERVATION DEMANDS}

\section{A Platform for Multidisciplinary Ocean Observations}

Global Ocean Ship-Based Hydrographic Investigations Program has advanced since the first global cycle during WOCE in the 1990s and is continuing to develop as requirements and technology are evolving. The evolution in large part consists of measurements of a broader range of variables, many of which are EOVs. These include physical, biogeochemical, and biological/ecosystem variables ${ }^{11}$. Open ocean research vessels are well suited to measure EOVs and there is a focus on including more EOVs in the suite of variables measured on the GO-SHIP reference sections. This has to be balanced by the feasibility of adding measurements along with the current measurement suite, considering factors such as costs, availability of channels on conducting cable, water requirements, number of berths available, station time and endurance.

Additional variables that are measured during a GO-SHIP cruise involve either Level 2 or Level 3 variables, some of which may be EOVs. The GO-SHIP community encourages and supports these additional observations to the extent possible, subject to limitations just mentioned. The GO-SHIP science committee, in consultation with the GO-SHIP community, shifts variables to a higher priority as feasibility and impact justify this. Conversely, some parameters can be relegated to a lower level when the need to observe them decreases, such as Helium/Tritium measurements given the decay of the atomic testing signal and completion of a global survey of the deeper signal. Decisions regarding additional parameters that should/could be measured on GO-SHIP cruises must include defining suitable observing strategies. This includes all aspects of observations, such as making sure that best practices (Pearlman et al., 2019) are developed and followed, that the data reporting is well defined, and that there is a data center able to accept, serve, and provide long term storage of the data and metadata (Tanhua et al., 2019).

\footnotetext{
${ }^{11} \mathrm{http} / / /$ goosocean.org/eov
} 
Sensing techniques have improved recently and can be used to determine diversity and biomass of zooplankton and phytoplankton, making these observations of biology and ecosystem EOVs on GO-SHIP cruises feasible. Techniques include: bio-optical sensors deployed on the rosette frame or in-line for underway sampling (e.g., measuring bulk absorption, attenuation, scattering and/or fluorescence at wavelengths useful to detect plankton pigments and/or their presence and size distribution); bio-acoustic techniques deployed on the rosette frame or on the hull of the research vessels for continuous operation. Imaging techniques such as the Underwater Video Profiler (UVP, Hydroptics) on the rosette, Imaging Flow Cyto-Bot (IFCB, McLane), and discrete samples for shore based analysis from rosette and/or underway surface samples. Observations include variables constraining bulk properties (e.g., POC, HPLC pigments) as well as species/group specific variables (e.g., genetics, flow-cytometry). Many of these observations (other than genetics and imaging) have long established protocols for operation and analysis. Imaging techniques have the advantage that they can be analyzed in the future through open source hosting. One example is Eco-Taxa, a web application dedicated to the visual exploration and the taxonomic annotation of images (Raes et al., 2018). The SCOR working group 154, P-OBS, is dedicated to the identification of plankton relevant technologies that could be incorporated into GO-SHIP (Boss et al., 2018; Lombard et al., 2019).

Additional areas and variables that are being considered to be elevated to Level 1 or 2 for GO-SHIP include: observations of air-sea fluxes and marine aerosols properties; additional biogeochemical EOVs such as $\mathrm{N}_{2} \mathrm{O}$ (currently a Level 2 measurement); NCP (e.g., using in-line $\mathrm{O}_{2}$-Ar Massspectroscopy), and observations of turbulence (currently a Level 3 measurement). Nitrous oxide $\left(\mathrm{N}_{2} \mathrm{O}\right)$ is routinely measured from bottle samples on United States GO-SHIP by the CFC groups using similar instrumentation and protocols as for CFC analyses.

There is also a need for near real-time data transmission of observations, in particular surface salinity and temperature from underway measurements and profile data from the CTD, for operational services. Currently, CTD profiles are transmitted from the United Kingdom GO-SHIP sections and retrieved by the United Kingdom MET service. The Coriolis data center is used by French and German ships for real-time CTD data transmission http://www.coriolis.eu.org/Data-Products/Submitdata. It is necessary to develop protocols to distinguish this nearreal time (NRT) data from the delayed-mode calibrated data used for the detection of decadal scale trends that is the core mission of GO-SHIP and the current procedures in Argo are being scrutinized as an effective example.

Global Ocean Ship-Based Hydrographic Investigations Program cruises are also useful for development and validation of new observing techniques. For example, GO-SHIP voyages have supported the validation of $\mathrm{pH}$ sensor measurements in underway systems (Takeshita et al., 2018), and turbulent mixing observations using CTD-chipods (Pickering et al., 2016). Given the range of variables observed on GO-SHIP, and the facilities on research vessels in comparison to autonomous platforms, or ships of opportunity, GO-SHIP cruises can be used to an even larger extent for validation and calibration of new sensors, sampling schemes, and analytical techniques.

\section{Autonomous Ocean Networks and Sensor Development}

Global Ocean Ship-Based Hydrographic Investigations Program contributes substantially to autonomous ocean observing networks in several ways. GO-SHIP (Talley et al., 2016) provides a variety of high-quality data required for calibration and validation of sensors on autonomous oceanographic platforms such as floats, gliders, and marine mammal tag CTDs. Importantly, GO-SHIP provides the highest-quality reference standard data of salinity for the core Argo array as well as those of oxygen, $\mathrm{pH}$, nitrate, and other biogeochemical data for emerging BGC Argo profiling float program. Now that Deep Argo (Johnson et al., 2015; Jayne et al., 2017) has begun sampling the full ocean depth, GO-SHIP data are crucial for ensuring the high precision and accuracy needed for deep ocean temperature and salinity analyses. GO-SHIP cruises also provide a platform for assessing the performance of new sensors. These sensors are sometimes added to the underwater package, and occasionally integrated into the CTD data-stream for direct comparisons.

\section{High Quality Reference Data for Sensor Calibration}

High quality salinity data are essential in understanding the variability and changes in ocean circulation and hydrological cycle. High quality and deep salinity profiles (reaching slightly below the 2000 dbar limit of standard Argo floats) from shipboard CTDs calibrated with bottle samples referenced to the International Association for the Physical Sciences of the Ocean (IAPSO) Standard Seawater are essential to delayedmode scientific quality control of Argo float salinity data (Wong et al., 2003).

Conductivity sensors on Argo float CTDs are calibrated at the manufacturer prior to deployment, but a variety of factors can introduce biases into the reported salinities once the floats is deployed. Float CTD conductivity cells are occasionally subject to fouling after calibration but prior to deployment, initially biasing reported salinities fresh of actual salinities. Float CTD conductivity cells can also foul owing to biological activity, and become fresh with time. Float CTD salinity data have also sometimes exhibited a salty drift (e.g., Figure 3), very recently hypothesized to result from the separation of the protective jackets from the exterior of the cells, increasing the conductivity measured.

CTD data that have been calibrated to bottle salinity data referenced to an international standard (IAPSO Standard Seawater) are essential for validating, and calibrating when necessary, Argo CTD salinity data. Profiles deep enough to sample water masses with relatively temporally stable and laterally homogeneous temperature-salinity relations (such as North Pacific and North Indian Deep Water, or Circumpolar Deep Water) are vital. Profiles contemporaneous and co-located with float deployments are also useful. As Deep Argo is coming on line, profiling to $6000 \mathrm{dbar}$, high accuracy GO-SHIP CTD data are essential to validate the Deep Argo CTD data. 


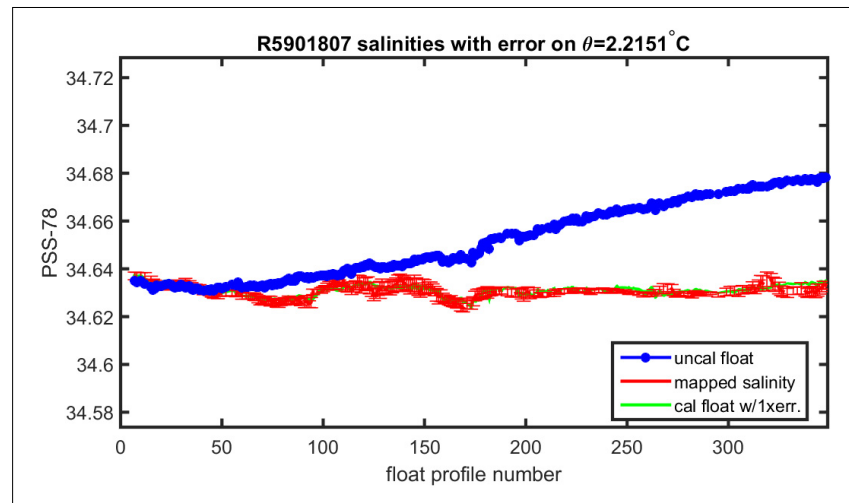

FIGURE 3 | Salinity on a deep potential isotherm plotted vs. profile number for Argo Float WMO ID 5901807 showing the uncalibrated float data (blue line), which drift salty, the climatological values mapped from GO-SHIP and other data (red lines with error bars), which are relatively constant, and the calibrated float data (green line), which has been adjusted to the climatology using a piecewise linear fit following Owens and Wong (2009). Profiles are taken every 10 days.

Global Ocean Ship-Based Hydrographic Investigations Program's decadal global monitoring of all components of the carbonate system is indispensable for being able to relate autonomous measurements from the newly expanding biogeochemical (BGC) Argo to the full carbon system. BGC Argo floats, including oxygen, $\mathrm{pH}$, nitrate, and other sensors, are powerful tools for dramatically better understanding the variability and changes in ocean biogeochemistry including biological production (Johnson et al., 2017a), deoxygenation (Stramma et al., 2008; Sasano et al., 2018), airsea $\mathrm{CO}_{2}$ fluxes (Gray et al., 2018), and ocean acidification. GO-SHIP plays two important roles in calibrating and validating these sensors. (1) The recently developed calibration methods for BGC Argo sensors (Johnson et al., 2017b) use the accurate and quality controlled Global Ocean Data Analysis Project (GLODAPv2) profile data set (Olsen et al., 2016), which is heavily based on GO-SHIP data. As the relationships between ocean properties evolve, for instance as acidification progresses and nutrient balances change, continued reference standard measurements collected in GO-SHIP, which are incorporated in the temporally evolving GLODAP synthesis, are essential for global implementation of sustained BGC Argo floats (Carter et al., 2017). (2) For validation of these $\mathrm{BGC}$ sensor calibrations in the large pilot Southern Ocean BGC float experiment as part of the Southern Ocean Carbon Cycle Observation and Modeling $\left(\mathrm{SOCCOM}^{12}\right)$ project, shipboard measurements of salinity, oxygen, nitrate, $\mathrm{pH}, \mathrm{DIC}$ and TAlk coincident with BGC Argo float deployments have been required (Williams et al., 2016). For the BGC-Argo bio-optical sensors, which are used to derive chlorophyll-a and POC, concurrent HPLC and POC shipboard sampling in the near-surface have been collected with all SOCCOM deployments. These are currently not GO-SHIP Level 1 measurements, but they

\footnotetext{
${ }^{12} \mathrm{https} / / /$ soccom.princeton.edu/
}

have been critical for validation of the NASA algorithms for converting satellite ocean color to in situ chlorophyll-a and particulate organic carbon (POC) (Haentjens et al., 2017). GO-SHIP cruises, and cruises that are modeled closely on GO-SHIP, are by far the most helpful and highest quality for these validations.

Measurements from marine mammals typically use the Conductivity-Temperature-Depth Satellite Relay Data Logger (CTD-SRDL), developed by the Sea Mammal Research Unit at the University of St Andrews. Marine mammals often sample remote, harsh environments, e.g., the sea-ice edge and the Antarctic continental shelf during winter. However, CTD-SRDL measurements have a random bias in the conductivity cell caused by the marine mammal interfering in the inductive field of the CTD-SRDL (Böhme et al., 2009). Several methods for correcting the individual offsets, which is assumed constant in space and time, have been developed (e.g., Böhme and Send, 2005; Roquet et al., 2011; Frazer et al., 2018). Most of these rely on either little variability in deep ocean measurements from GO-SHIP or delayed mode Argo data (corrected with GO-SHIP data), implicitly placing GO-SHIP as the underlying provider of reference quality data for CTD-SRDL.

Global Ocean Ship-Based Hydrographic Investigations Program provides a platform for assessing new sensors, including BGC sensors, mixing sensors, and biological sensors, inter alia. For instance, to determine their characteristics, oxygen-sensing optodes have been deployed alongside conventional electrode oxygen sensors, allowing both to be compared with bottle oxygen titration data (Sasano et al., 2011). In addition, float CTDs can be strapped onto the shipboard CTD package for in situ evaluation of their sensors relative to reference quality shipboard CTD sensors.

Given the importance of GO-SHIP as the provider of the high quality reference data needed by autonomous platforms and other components of the observing system, it is recommended that GOOS endorses GO-SHIP as the ocean reference data set, and that GO-SHIP data be given a unique identifier in the data system to enable users to identify it separate from other observing platforms or sensors.

\section{Deploying Autonomous Vehicles}

Global Ocean Ship-Based Hydrographic Investigations Program deploys many autonomous floats for Argo, Deep Argo, BGC Argo, and drifter programs. Deployment of gliders has started on a small scale. GO-SHIP cruises are desirable for autonomous instrument deployment because of the highquality calibration data they collect, because they are staffed by experienced scientists and technicians who can provide professional support and who recognize the value of the data collected from those vehicles, and because they reach remote locations where deployment opportunities for floats and gliders are limited. For instance, from 2002 to 2019 (Figure 4), GO-SHIP cruises deployed over 7\% of Argo floats, which included 70 of the 132 SOCCOM BGC Argo floats deployed up to 2019. 

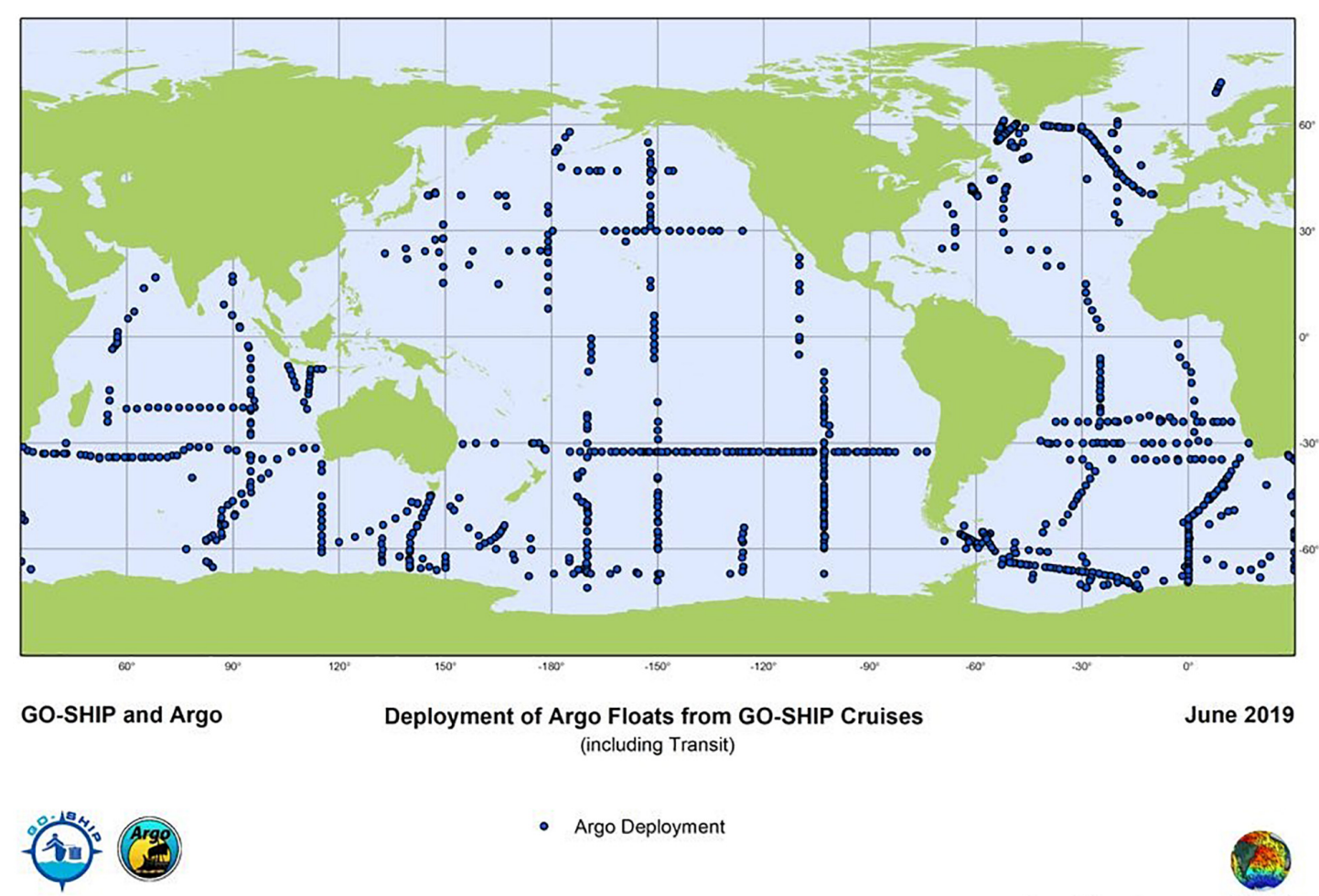

- Argo Deployment

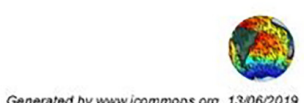

FIGURE 4 | Deployment locations of more than 1100 Argo floats from GO-SHIP cruises from 2002 to 2019 (www.jcommops.org). The 7\% deployment rate of Argo floats on GO-SHIP cruises during that time period is likely conservative, as required metadata for such an analysis are not always available Many GO-SHIP deployments are in remote locations outside commercial shipping lanes, making GO-SHIP cruises an important means to cover the global ocean.

\section{Data Synthesis, Reanalysis Products and GO-SHIP Contribution to Improved Climate Understanding}

Data syntheses are important products from GO-SHIP data (Figure 5). After the first decade of WOCE, a major synthesis effort resulted in a gridded data product, the Global Hydrographic Climatology (GHC) (Gouretski, 2018) ${ }^{13,14}$; print/online atlases for each of the ocean basins ${ }^{15}$; and synthesis in a digital atlas, $\operatorname{eWOCE}^{16}$ (Schlitzer, 2000). The latter utilized the widely used Ocean Data View (ODV) software package (Schlitzer, 2018). These products were focused primarily on the physical properties, oxygen, nutrients and transient tracers, although eWOCE incorporated all of the WOCE data, ranging beyond the hydrographic data sets.

Biogeochemical (BGC) data, with emphasis on the inorganic carbon parameters, were synthesized regionally for the Atlantic (CARINA) (Key et al., 2010) and Pacific (PACIFICA) (Suzuki et al., 2013). The overarching global synthesis of BGC data from $\approx 1980$ to 2000 was the GLODAP project (Key et al., 2004) ${ }^{17}$,

\footnotetext{
${ }^{13} \mathrm{https}: / /$ icdc.cen.uni-hamburg.de/daten/ocean/woce-climatology.html

${ }^{14} \mathrm{http} / / /$ icdc.cen.uni-hamburg.de/1/daten/ocean/waghc/

${ }^{15} \mathrm{http} / / /$ woceatlas.ucsd.edu

${ }^{16} \mathrm{http}: / /$ www.ewoce.org/

${ }^{17}$ www.glodap.info
}

involving extensive quality control and cross-over adjustments to yield a globally consistent data set. The recently completed GLODAPv2 2019 represents a major step forward for BGC data synthesis. It includes an iterative inversion process to optimize consistency. GLODAP products include both quality controlled profile data (Key et al., 2015; Olsen et al., 2016, 2019) and a gridded product (Lauvset et al., 2016). GLODAP will be updated bi-annually, using GLODAPv2 as a basis, with a full re-analysis including a new inversion every decade.

The surface water $\mathrm{CO}_{2}$ measurements performed on GOSHIP lines are incorporated in surface water $\mathrm{CO}_{2}$ collations such as the LDEO surface $\mathrm{CO}_{2}$ climatology (Takahashi et al., 2009) and the Surface Ocean Carbon Atlas (SOCAT) ${ }^{18}$ (Bakker et al., 2016). The GLODAPv2 and SOCAT efforts are focused on routine releases, documented submission, and QC procedures. These are labor intensive efforts, and increased automation is being implemented to facilitate uniformity and decrease the personnel time involved. GLODAP and SOCAT have working groups that are represented in the International Ocean Carbon Coordination Project (IOCCP) steering committee. A serious impediment for continuation is that they currently depend on project funds from individual countries that are proposal driven and of finite duration. Entrainment of new younger scientists in these efforts is also critical to maintain them.

\footnotetext{
${ }^{18}$ www.socat.info
} 


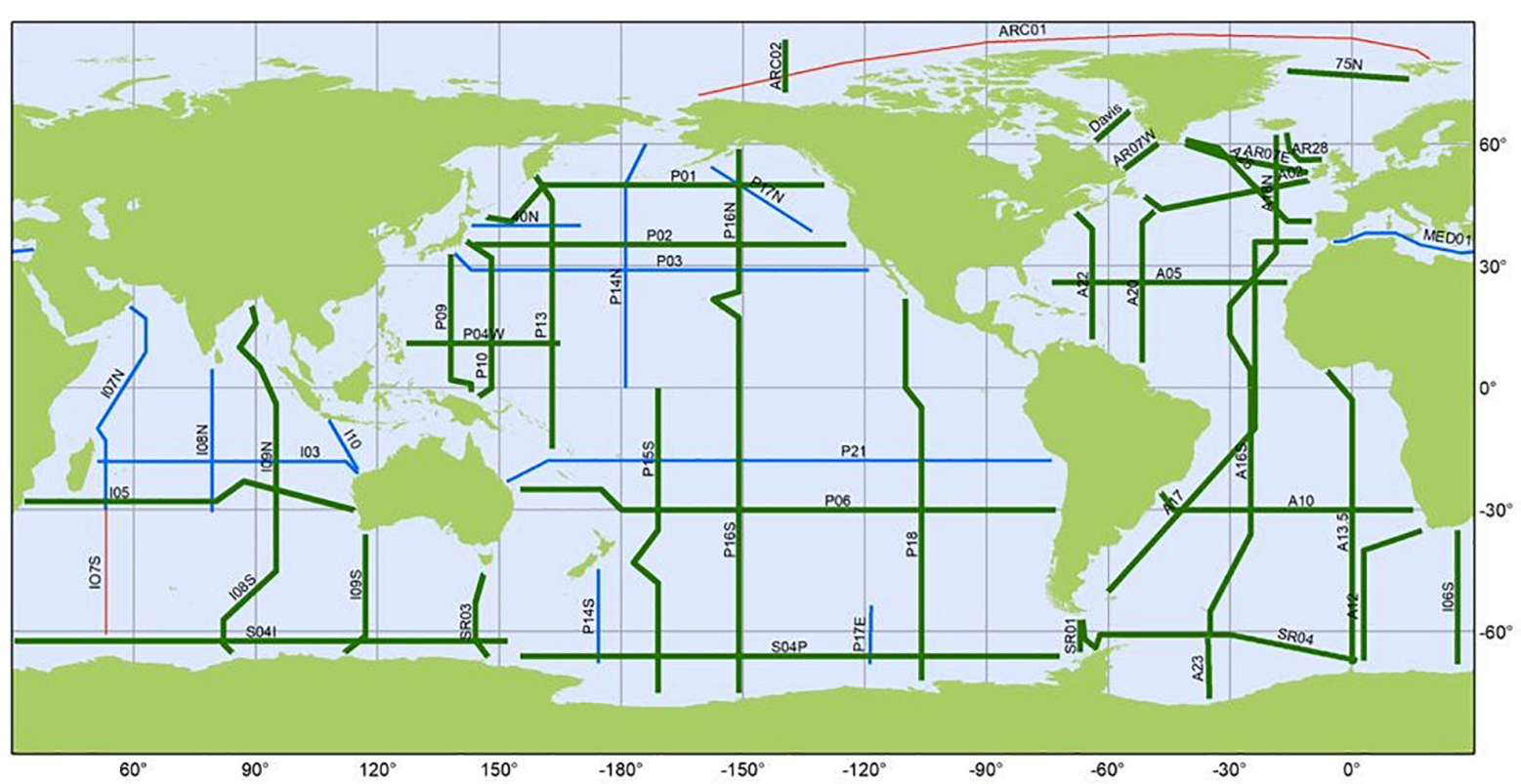

GO-SHIP

Three Decades of GO-SHIP: Time-Series

June 2019

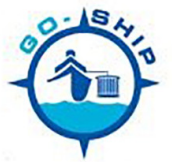

At least three occupations
Two occupations
Recently introduced new lines

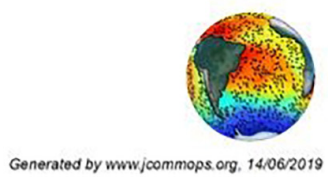

FIGURE 5 | Time-series of GO-SHIP sections, taking into account cruises which have already been confirmed for occupation in the future. Many of the decadal full GO-SHIP sections have now been occupied three or more times. Individual occupations of the sections can be accessed from CCHDO, however, as the number of section repeats increases improved data accessibility of the section time-series should be developed.

Global Ocean Ship-Based Hydrographic Investigations Program is developing the protocols and methods to generate a data product that concatenates all occupations of individual sections into a time-series (Figure 5). Initial efforts are focused on CTD (temperature, salinity, and dissolved oxygen) data, with the plan to include GLODAP quality-controlled bottle data in future versions in collaboration with GLODAP.

Global Ocean Ship-Based Hydrographic Investigations Program data held at $\mathrm{CCHDO}$ and national data repositories are included in the World Ocean Database (WOD; Boyer et al., 2013). Much of the nutrient, carbon and deep ( > 2000 dbar) physical data in this dataset originates from GO-SHIP cruises. The WOD is used by a large number of research institutions and meteorological and ocean operational centers for many purposes. In addition, the SCOR International quality controlled ocean database (IQuOD) working group aim is to add value to the WOD by providing a consistent quality controlled subset of data, that has comprehensive meta-data information for use by climate and ocean science researchers and operational services.

All gridded ocean climatology products [e.g., World Ocean Atlas (WOA), CSIRO Atlas of Regional Seas (CARS), and Global Hydrographic Climatology (WOCEGHC)] are derived from WOD (and other data sources). Such climatologies represent a significant route by which users access GO-SHIP data. The above products mostly grid temperature and salinity, with some including dissolved oxygen and nutrients.

Global Ocean Ship-Based Hydrographic Investigations Program data, largely via WOD and other databases, contributes to ocean state estimates and reanalysis products (Balmaseda et al., 2015 includes an extensive list of products). GO-SHIP hydrographic data are also used in datasets such as EN4 and Coriolis Ocean dataset for Reanalysis (CORA), which provide higher quality measurements at a global scale for numerical modeling, data assimilation, and scientific studies. Such data assimilation efforts are used for the initialization of the current climate state from which forecasts are run.

Products based on direct velocity measurements using S-ADCP and L-ADCP instrumentation are useful (e.g., Johnson et al., 2002), and more are desired. However, the primary data management challenge at this time for ADCP data is to process and archive all of it, including essential metadata, in a single data system. This includes preservation of the WOCE ADCP datasets. The community, particularly that involved with mixing and turbulence calculations (e.g., Kunze et al., 2006; Huussen et al., 2012; Waterhouse et al., 2014), has a clear need for easy access to these velocity profile data and all repeats (e.g., Kunze, 2017a,b whose analysis relied on hydrographic data alone to achieve global coverage). Synthesis products of mixing-related 
quantities based on these repeats would be a useful adjunct to hydrographic data syntheses.

Global Ocean Ship-Based Hydrographic Investigations Program must develop data assemblages by section and/or decade to increase the ease of access and use of all of its Level 1 observations. These data assemblages should follow standard data practices and be provided in a number of file formats that meet users' requirements. Funding of such activities should be a priority of the community. The use of GO-SHIP data in commonly used datasets enhances the use of the data. However, GO-SHIP should develop collaboration with the creators and users of ocean datasets to ensure attribution of the original data source, determine systematic biases in the data, and improve the quality of the observations.

\section{INTERNATIONAL COORDINATION}

Long-term funding commitments to sustain and enhance ocean observational efforts such as the activities carried out by the GO-SHIP network remain elusive. Current international efforts rely considerably on contributions from a limited number of countries and a small pool of dedicated scientists. Ship time is often secured 1 to 2 years in advance and is not always guaranteed. GO-SHIP activities are often funded by consecutive short-term research projects. Scientists sometimes struggle to cover the cost and must find multiple funding sources.

\section{Multi-Nation Voyages}

To maintain the GO-SHIP program and grow the number of participating nations, a modified operational and funding model should be developed to include multi-nation voyages. Active support and long-term investment from government decision makers and funders of multi-nation voyages has the potential to increase the number of participating countries, enhance capacity, accommodate new measurements of biological and ecosystem variables, and increase awareness of outputs in the form of decision support information products. Capacity building through training a new generation of observational oceanographers is also vital to ensure sustainability. Finally, greater coordination with other ocean observing networks could lead to cost sharing and increased impact within a more coordinated global ocean observing framework.

As the number of variables collected by the GO-SHIP community increases, the demand on human and financial resources becomes difficult to sustain. Multinational efforts offer the potential to share and distribute resource allocations to a wider community. This has occurred in the past. For example Australia has benefitted from United States and Japanese support to undertake tracer $\left(\mathrm{CFC} \mathrm{SF}_{6}\right)$ observations. GO-SHIP lines in the Atlantic enjoyed support from European laboratories for variables almost exclusively measured on GO-SHIP such as transient tracers. This approach also empowers new participant nations to take the lead while receiving support from experienced international collaborators. Of course, there are organizational challenges in this approach, but the benefits of shared cost and expertise will widen the pool of countries willing, and able, to lead
GO-SHIP expeditions. The opportunity to extend capabilities, facilitate knowledge exchange, build capacity and future scientific collaboration is huge.

\section{The Irish Example}

In 2016, Ireland, led by the Irish Marine Institute and National University of Ireland Galway, committed to complete the 2017 A02 GO-SHIP line on-board the RV Celtic Explorer. While a momentous task, considerable effort to showcase the importance of ocean observing to Irish decision makers and funders proved fruitful. The issue of ensuring the full complement of expertise on-board had to be addressed and the answer lay in an international cooperation. This was a great example of the Galway Statement (signed by United States, Canada and EU May 2013) in action with on-board teams from Canada, Germany, the United Kingdom, and the United States, and additional support from shore teams and experts in Denmark and France. Financial support for partner teams was found through the European Union Horizon 2020 AtlantOS and ATLAS projects, and the Canada Excellence Research Chair in Ocean Science and Technology. The international nature of the 2017 GO-SHIP A02 cruise offered an opportunity for cross-comparison of methods, data quality procedures, and exchange of technical expertise. For example, surface $\mathrm{pCO}_{2}$ was recorded along the track with the teams from Dalhousie University and the Marine Institute comparing different systems. By using independent systems with frequent multiple point calibration, the RV Celtic Explorer's General Oceanics equilibrator system and Canadian (Dalhousie) team's SubCTech permeable membrane system, there is added confidence in the data produced.

\section{Building Capacity and Training the Next Generation}

Global Ocean Ship-Based Hydrographic Investigations Program transects provide an ideal platform to train the next generation of observational oceanographers across the physical, chemical, biological and ecosystem disciplines. Transfer of knowledge and expertise and participation of students and early career scientists is highly encouraged. As commitment and support of international collaborations gain momentum, more opportunities will open to students from developing countries and to scientists in countries without basin and global-class research vessels.

A prime example is the South African floating university. Currently in South Africa, there is a lack of students proficient in mathematics, and by association physics, engineering, statistics, chemistry and computer science, who wish to major in the marine sciences. The majority of students who graduate from a marine science degree program have weak quantitative backgrounds and consequently, battle with understanding the core mathematical or physical dynamics behind open-ocean and coastal processes. "SEAmester" aims to mitigate this problem by introducing marine science as an applied and cross-disciplinary field to students who have shown an affinity for these core science disciplines. In doing so, a new generation of South African marine and atmospheric scientists with a far higher caliber will be trained. "SEAmester" combines traditional classroom lectures with hands-on ship-based deck activities, while providing 
students with an opportunity to support scientists specializing in a wide range of marine fields. The South African government's National Development Plan has identified education, training and innovation as being at the forefront of South Africa's longterm development, and specifically states: "Inadequate capacity will constrain knowledge production and innovation unless effectively addressed." The central strategy behind SEAmester is not only of a training program that aligns with South Africa's core Science and Technology objectives, but more importantly, a program designed to fill a growing gap in the capabilities of our current cohort of marine students. It aims to build critical mass within the marine sciences, so as to ensure sustained growth in this field within South Africa and beyond.

\section{Collaborating and Coordinating With Other Observing Networks}

Further efforts to consolidate surveys, for example between GOSHIP transects and deep water data buoy mooring deployment efforts, could lead to cost sharing. There are several examples of this type of coordination in the Prediction and Research Moored Array in the Tropical Atlantic (PIRATA) program and also in Australia. GO-SHIP is an active and dynamic community and keen to strengthen existing links, build new partnerships, and coordinate more closely with other observing networks particularly those focused on biological and ecosystem variables to monitor ocean health. An example to build on is the partnership with Global Ocean Acidification Observing Network (GOA-ON), whereby GO-SHIP is explicitly identified as providing key data for open ocean observing of physicochemical aspects of ocean acidification, and the active role of GO-SHIP in development of a marine biological and ecosystem observation methods and implementation (Boss et al., 2018). GO-SHIP is involved in the evolution of new regional scale coordinated observing systems, which will bring together the diverse communities of ocean observing networks. These initiatives include the Atlantic Ocean Blueprint, the Tropical Atlantic Observing system (TAOS) (Foltz et al., 2019), Tropical Pacific Observing System (TPOS) (Smith et al., 2019) and European Ocean Observing System (EOOS) together with existing programs such as the integrated ocean observing system (IOOS) and the integrated marine observing system (IMOS).

\section{GO-SHIP Project Office at JCOMMOPS}

The Observations Coordination Group (OCG) of JCOMM, part of the World Meteorological Organization (WMO), and UNESCO's Intergovernmental Oceanographic Commission (IOC), coordinates sustained ocean observing networks such as GO-SHIP, Argo, Data Buoy Coordination Panel (DBCP), OceanSITES, GLOSS and the Ship Observations Team (SOT) at the international level. The JCOMM in-situ Observations Programme Support Centre (JCOMMOPS), which is hosted by Ifremer in Brest (France), maintains an information system that allows for monitoring and implementation planning within and across these networks. The JCOMMOPS metadata tools help to set up multi-national and multi-institutional GO-SHIP cruises, and monitor the targets of an ocean observing program in terms of space, time, quality and availability of the emerging data. Scientific publications based on GO-SHIP data are captured in the GO-SHIP bibliography maintained by JCOMMOPS. Beyond core GO-SHIP activities, the JCOMMOPS system allows for identifying opportunities for other networks that rely on GOSHIP reference data (e.g., deployment of floats and drifters), or ship time in vicinity of a cruise (e.g., maintenance of a moored buoy). So far, JCOMMOPS monitoring capacity concerns cruise/platform metadata but it is planned to expand this to the observations made available in real-time and delayedmode. This would allow JCOMMOPS to track cruise progress in real-time and optimize data flow to users.

\section{Costing GO-SHIP, Determining Value, and Securing Sustainable Funding}

Efforts to cost ocean observing networks are at an early stage of development and a standard economic methodology has yet to be determined to assess the true cost of ocean observing efforts. As part of the European Horizon 2020 Optimizing and Enhancing the Integrated Atlantic Ocean Observing Systems project $\left(\right.$ AtlantOS $^{19}$ ) the cost of running the GO-SHIP network in the Atlantic for the period 2012-2023 was estimated at a total of about 100 million Euro. However, the report highlighted that this is a conservative number since many hidden costs exist such as costs of on-land laboratories and equipment required for pre- and post-QC parameter checks on methods. Determining the value of the GO-SHIP network has not been tackled in a quantitative manner due to the lack of economic and socioeconomic methods in this area. An important starting point for a clearer definition of the value of GO-SHIP is the definition of value chains describing how the data lead to meeting a variety of end user needs.

Currently, most GO-SHIP cruises are funded through short-term commitments of direct public sector government funding, together with time limited project based research funding. The questions of how to secure sustainable funding for GO-SHIP are echoed by many other ocean observing networks, and by the emerging coordinated observation systems concept. Is a new model of funding based on multinational voyages and/or increased participation of the private sector the path to sustainability? How can this be achieved? How would such funding models be governed to ensure that scientific independence, quality and open data access is enshrined? Could more coordination and integration of government funding be achieved with commitments that run over periods longer than national political cycles? These are questions to be addressed by GO-SHIP together with the ocean observation community as a whole, policy makers and national funders.

\section{SUMMARY AND RECOMMENDATIONS}

Global Ocean Ship-Based Hydrographic Investigations Program has successfully provided the necessary data for estimates of

\footnotetext{
${ }^{19}$ www.atlantos-h2020.eu
} 
decadal trends and changes of large-scale ocean circulation, deep heat uptake and carbon inventories. The contemporaneous collection of a suite of ocean variables has shown the value in simultaneously collecting physical, biogeochemical and biological observations if we wish to fully understand and track the impacts of ocean variability and change within the Earth's System. In the decades to come, GO-SHIP will continue to build the time-series of full-depth repeat ocean measurements capable of resolving decadal and longer time scale changes in the circulation and property storage (including heat, freshwater, oxygen, and carbon) of the global oceans. GO-SHIP will also continue to provide a means to access remote ocean areas for deployment of autonomous observing platforms, including Argo floats, surface-drifters and gliders. GO-SHIP will continue to evolve based on the findings of the previous work and emerging science requirements and technological developments. Future developments will include additional biogeochemistry and biology measurements to enable GO-SHIP to determine trends and variability in marine biogeochemistry, health, and ecosystems. These measurements will be incorporated into the sustained primary objectives of GO-SHIP. As the number of autonomous platforms increases and new sensors are developed, GOSHIP will be increasingly relied upon as the high quality ocean reference data set required to ensure the accuracy and consistency of a number of EOVs from the increasing diverse range platform/sensors comprising the ocean observing system. While the last decade has seen the establishment of GO-SHIP as a vital component of the global ocean observing system, there is still much work to do to ensure its sustainability and effectiveness. This paper identifies some key points that GO-SHIP needs to address and progress if it is to meet the needs and expectations of the increasingly diverse user community.

Key recommendations for GO-SHIP in the next decade are:

- GOOS should endorse GO-SHIP Level 1 data as a key "surface to bottom" ocean reference data set. GO-SHIP should continue to be the provider of the high quality ocean reference data needed by autonomous platforms and other components of the observing system. GO-SHIP data should be given a unique identifier in the data system to enable users to track and identify it from other shipbased and observing platforms or sensors, and its use in (gridded) products.

- Global Ocean Ship-Based Hydrographic Investigations Program must develop guidelines to evaluate the request of additional variables to the program in order to facilitate incorporation of new variables in the sampling suite. The documentation should include the feasibility, use and importance of the measurement. The logistical issues such as extra ship time, station time, personnel, ship laboratory space, and expertise will need to be considered, as well data management systems for storage and accessibility. Also, the cost needs to be considered for all cruises, including the availability of resources for all the GO-SHIP participating nations.
- Global Ocean Ship-Based Hydrographic Investigations Program needs to focus on establishing and seeking funds for an internationally agreed and adhered to data procedures and management pathways that is still somewhat ad hoc. There should be a single mandate/requirement for each GO-SHIP data type in terms of timing, posting, and archiving, and a central data portal. A central data portal tracking and linking all component data sets is essential, including formal agreements on depositories, interoperability, and exchange. This will require the inclusion of data management costs both at the national level and contribution to international coordination within national-led funding proposals.

- The protocol and recommendation for citation of GOSHIP data ${ }^{20}$ should be provided in prominent locations including all portals for GO-SHIP data, such that it is clearly referenced when used in publications or as reference data in calibration/validation of data from other observing systems such as Argo.

- Global Ocean Ship-Based Hydrographic Investigations Program must develop data assemblages (by section or decade) to increase the accessibility of the data to the wider community and use of all of its Level 1 observations. These data assemblages should follow standard data practices and be provided in a number of file formats that meet the users requirements.

- A modified operational and funding model should be developed to include multi-national voyages. Programs such as AtlantOS and intergovernmental agreements such as the Galway Statement have been successfully employed by GO-SHIP to increase national participation and share resources. GO-SHIP should work with national funding agencies to formalize these arrangements to increase national participation, capacity building and training of the next generation of skilled sea-going scientists and technicians.

- New models of funding based on multi-nation voyages and/or increased participation of the private sector need to be actively investigated as a means to sustainability. The questions of how to secure sustainable funding for GO-SHIP are echoed by many other ocean observing networks. This needs to be addressed by GO-SHIP together with the ocean observation community as a whole, policy makers and national funders to guarantee the existing ocean observing systems and provide investment to expand the ocean observing systems where required.

\section{AUTHOR CONTRIBUTIONS}

$\mathrm{BS}, \mathrm{RW}$, and MK led the writing and overall content of the manuscript. GJ, LT, TT, CC, EO'R, EMcG, EB, and KK led sections of the manuscript. All other authors contributed to different sections, to manuscript revision, read, and approved the submitted version.

\footnotetext{
${ }^{20} \mathrm{http}: / /$ www.go-ship.org/About.html
} 


\section{FUNDING}

BS was supported by the Australian Government Department of the Environment and CSIRO through the National Environmental Science Program and CSIROs Decadal Climate Forecasting Project. RW and GJ were supported by the NOAA office of Oceanic and Atmospheric Research, including the Ocean Observations and Monitoring Division (OOMD), funding

\section{REFERENCES}

Aoyama, M., Ota, H., Kimura, M., Kitao, T., Mitsuda, H., Murata, A., et al. (2012). Current status of homogeneity and stability of the reference materials for nutrients in seawater. Anal. Sci. 28, 1-6.

Aoyama, M. (2018). IOCCP-JAMSTEC 2018 Inter-Laboratory Calibration Exercise of a Certified Reference Material for Nutrients in Seawater. IOCCP report number 1/2018. Yokosuka: Japan Agency for Marine-Earth Science and Technology.

Bakker, D. C. E., Pfeil, B., Landa, C. S., Metzl, N., O’Brien, K. M., Olsen, A., et al. (2016). A multi-decade record of high-quality $\mathrm{fCO}_{2}$ data in version 3 of the Surface Ocean $\mathrm{CO}_{2}$ Atlas (SOCAT). Earth Syst. Sci. Data 8, 383-413, doi: 10.5194/essd-8-383-2016

Balmaseda, M. A., Hernandez, F., Storto, A., Palmer, M. D., Alves, O., et al. (2015). The ocean reanalyses intercomparison project (ORA-IP), J. Operat. Oceanogr. 8, s80-s97, doi: 10.1080/1755876X.2015.1022329

Becker, S., Aoyama, M., Woodward, E. M. S., Bakker, K., Coverly, S., Mahaffey, C., et al. (2019). GO-SHIP Repeat Hydrography Nutrient Manual, 2019: The Precise And Accurate Determination Of Dissolved Inorganic Nutrients In Seawater; Continuous Flow Analysis Methods And Laboratory Practices. Available at: http://www.ioccp.org/images/06Nutrients/GO-SHIPRepeatHydro graphyNutrientManual_October2018_Final-Draft.pdf (accessed July 10, 2019).

Böhme, L., Lovell, P., Biuw, M., Roquet, F., Nicholson, J., Thorpe, S. E., et al. (2009). Technical note: animal-borne CTD-satellite relay data loggers for realtime oceanographic data collection. Ocean Sci. 5, 685-695. doi: 10.5194/os-5685-2009

Böhme, L., and Send, U. (2005). Objective analyses of hydrographic data for referencing profiling float salinities in highly variable environments. Deep Sea Res. II 52, 651-664. doi: 10.1016/j.dsr2.2004.12.014

Boss, E., Waite, A., Muller-Karger, F., Yamazaki, H., Wanninkhof, R., Uitz, J., et al. (2018). Beyond chlorophyll fluorescence: the time in right to expand biological measurements in ocean observing programs. Limnol. Oceanogr. Bull. 27, 89-90 doi: 10.1002/lob.10243

Boyer, T. P., Antonov, J. I., Baranova, O. K., Coleman, C., Garcia, H. E., Grodsky, A., Johnson, D. R., et al. (2013). World Ocean Database 2013, eds S. Levitus, A. Mishonov (Silver Spring, MD: NOAA).

Brown, P. J., Bakker, D. C. E., Schuster, U., and Watson, A. J. (2010). Anthropogenic carbon accumulation in the subtropical North Atlantic. J. Geophys. Res. 115:C04016

Byrne, R. H., Mecking, S., Feely, R. A., and Liu, X. (2010). Direct observations of basin-wide acidification of the North Pacific. Geophys. Res. Lett. 37:L02601.

Carter, B. R., Feely, R. A., Williams, N. L., Dickson, A. G., Fong, M. B., and Takeshita, Y. (2017). Updated methods for globally locally-interpolated estimation of alkalinity, pH, and nitrate. Limnol. Oceanogr. Methods 16, 119131. doi: 10.1002/lom 3.10232

Carter, B. R., Feely, R. A., Wanninkhof, R., Kouketsu, S., Sonnerup, R. E., Pardo, P. C., et al. (2019). Pacific anthropogenic carbon between 1991 and 2017. Glob. Biogeochem. Cycles 33, 597-617, doi: 10.1029/2018GB00 6154

Carton, J. A., Cunningham, S. A., Frajka-Williams, E., Kwon, Y-O., Marshall, D. P., and Msadek, R. (2014). The Atlantic overturning circulation: more evidence of variability and links to climate. Bull. Am. Meteor. Soc. 95, ES163-ES166.

Carton, J. A., and Hakkinen, S. (2011). Introduction to: atlantic meridional overturning circulation (AMOC). Deep Sea Res. II 58, 1741-1743. reference \#100007298. PMEL Contribution Number 4864. LT was supported by the United States NSF Ocean Sciences OCE_1437015. TT and CC were supported by the EU Horizon 2020 grant agreement 633211, AtlantOS. ElM was supported by the United Kingdom NERC National Capability ORCHESTRA (NE/N018095/1) and CLASS (NE/R015953/1) projects. EO'R, $\mathrm{EMcG}$, and CC were supported under the Marine Research Programme of the Irish Government.

CLIVAR (2018). Climate and Ocean - Variability,Predictability and Change Science Plan and Implementation Strategy. Geneva: WCRP.

de la Paz, M., García-Ibáñez, M. I., Steinfeldt, R., Ríos, A. F., and Pérez, F. F. (2017). Ventilation versus biology: what is the controlling mechanism of nitrous oxide distribution in the North Atlantic. Glob. Biogeochem. Cycles 31, $1-16$.

Desbruyères, D. G., Purkey, S. G., McDonagh, E. L., Johnson, G. C., and King, B. A. (2016). Deep and abyssal ocean warming from 35 years of repeat hydrography. Geophys. Res. Lett. 43, 356-365. doi: 10.1002/2016GL070413

Deutsch, C., Emerson, S., and Thompson, L. (2005). Fingerprints of climate change in North Pacific oxygen. Geophys. Res. Lett. 32:L16604

Dickson, A. G., Afgan, J. D., and Anderson, G. C. (2003). Reference materials for oceanic $\mathrm{CO}_{2}$ analysis: a method for certification of total alkalinity. Mar. Chem. 80, 185-197. doi: 10.1016/s0304-4203(02)00133-0

Dickson, A. G. (2010). Standards for ocean measurements. Oceanography 23, 34-47. doi: 10.5670/oceanog.2010.22

Doney, S. C. (2010). The growing human footprint on coastal and open-ocean biogeochemistry. Science 328, 1512-1516. doi: 10.1126/science.1185198

Doney, S. C., Fabry, V. J., Feely, R. A., and Kleypas, J. A. (2009). Ocean Acidification: the other $\mathrm{CO}_{2}$ Problem. Ann. Rev. Mar. Sci. 1, 169-192. doi: 10.1146/annurev.marine.010908.163834

Emerson, S., Mecking, S., and Abell, J. (2001). The biological pump in the subtropical North Pacific Ocean: nutrient sources, redfield ratios, and recent changes. Glob. Biogeochem. Cycles 15, 535-554. doi: 10.1029/2000gb001320

Feely, R. A., Doney, S. C., and Cooley, S. R. (2009). Ocean acidification: present conditions and future changes in a high- $\mathrm{CO}_{2}$ world. Oceanography 22, 36-47. doi: $10.1098 /$ rstb.2012.0442

Feely, R. A., Sabine, C. L., Lee, K., Berelson, W., Kleypas, J., Fabry, V. J., Millero, F. J. (2004). Impact of anthropogenic $\mathrm{CO}_{2}$ on the $\mathrm{CaCO}_{3}$ system in the oceans. Science 305, 362-366. doi: 10.1126/science.1097329

Foltz, G., Brandt, P., Richter, I., Rodríguez-Fonseca, B., Hernandez, F., Dengler, M., et al. (2019). Tropical atlantic observing system. Front. Mar. Sci. 6:206. doi: 10.3389/fmars.2019.00206

Frazer, E. K., Langhorne, P. J., Williams, M. J. M., Goetz, K. T., and Costa, D. P. (2018). A method for correcting seal-borne oceanographic data and application to the estimation of regional sea ice thickness. J. Mar. Syst. 187, 250-259. doi: 10.1016/j.jmarsys.2018.08.002

Friis, K., Körtzinger, A., Pätsch, J., and Wallace, D. W. R. (2005). On the temporal increase of anthropogenic $\mathrm{CO}_{2}$ in the subpolar North Atlantic. Deep Sea Res. I 52, 681-698 doi: 10.1016/j.dsr.2004.11.017

Frölicher, T. L., Joos, F., Plattner, G. K., Steinacher, M., and Doney, S. C. (2009). Natural variability and anthropogenic trends in oceanic oxygen in a coupled carbon cycle-climate model ensemble. Glob. Biogeochem. Cycles 23:GB1003.

Ganachaud, A., and Wunsch, C. (2000). Improved estimates of global ocean circulation, heat transport and mixing from hydrographic data. Nature 408, 453-456.

García-Ibáñez, M. I., Zunino, P., Fröb, F., Carracedo, L. I., Ríos, A. F., Mercier, H., et al. (2016). Ocean acidification in the subpolar North Atlantic: rates and mechanisms controlling $\mathrm{pH}$ changes. Biogeosciences 13, 3701-3715, doi: 10.5194/bg-13-3701-2016

Gouretski, V. (2018). World ocean circulation experiment - argo global hydrographic climatology. Ocean Sci. 14, 1127-1146, doi: 10.5194/os-14-11272018

Gray, A. R., Johnson, K. S., Bushinsky, S. M., Riser, S. C., Russell, J. L., Talley, L. D., et al. (2018). Autonomous biogeochemical floats detect significant carbon 
dioxide outgassing in the high-latitude Southern Ocean. Geophys. Res. Let. 45, 9049-9057. doi: 10.1029/2018GL078013

Groeskamp, S., Lenton, A., Matear, R., Sloyan, B. M., and Langlais, C. (2016). Anthropogenic carbon surface to interior connections. Global Biogeochem. Cycles 30, 1682-1698. doi: 10.1002/2016gb005476

Gruber, N., Sarmiento, J. L., and Stocker, T. F. (1996). An improved method for detecting anthropogenic $\mathrm{CO}_{2}$ in the oceans. Global Biogeochem. Cycles 10, 809-837. doi: $10.1029 / 96 \mathrm{gb} 01608$

Gruber, N., Clement, D., Carter, B. R., Feely, R. A., van Heuven, S., Hoppema, M., et al. (2019). The oceanic sink for anthropogenic CO2 rom 1994 to 2007. Science 363, 1193-1199. doi: 10.1126/science.aau5153

Guallart, E. F., Schuster, U., Fajar, N. M., Legge, O., Brown, P., Pelejero, C., et al. (2015). Trends in anthropogenic CO2 in water masses of the Subtropical North Atlantic Ocean. Prog. Oceanogr. 131, 21-32. 10.1016/j.pocean.2014.11.006 doi: 10.1016/j.pocean.2014.11.006

Haentjens, N., Boss, E., and Talley, L. D. (2017). Revisiting ocean color algorithms for chlorophyll a and particulate organic carbon in the southern ocean using biogeochemical floats. J. Geophys. Res. Oceans 122, 6583-6593.

Hahn, J., Brandt, P., Schmidtko, S., and Krahmann, G. (2017). Decadal oxygen change in the eastern tropical North Atlantic. Ocean Sci. 13, 551-576 doi: 10.5194/os-13-551-2017

Hernandez-Guerra, A., and Talley, L. D. (2016). Meridional overturning transports at $30^{\circ} \mathrm{S}$ in the Indian and Pacific Oceans in 2002-2003 and 2009. Prog. Oceanogr. 146, 89-120. doi: 10.1016/j.pocean.2016.06.005

Hood, E. M., Sabine, C. L., and Sloyan, B. M. (Eds.) (2010). The GO-SHIP Repeat Hydrography Manual: A Collection of Expert Reports and Guidelines. IOCCP Report Number 14. Paris: SCOR.

Hooker, S. B., Van Heukelem, L., Thomas, C. S., Claustre, H., Ras, J., Schluter, L., et al. (2005). The Second SeaWiFS HPLC Analysis Round-Robin Experiment (SeaHARRE-2). Washington, DC: NASA Tech.

Huussen, T. N., Naveira Garabato, A. C., Bryden, H. L., and McDonagh, E. L. (2012). Is the deep Indian Ocean MOC sustained by breaking internal waves? J. Geophys. Res. Oceans 117:C080

Jayne, S. R., Roemmich, D., Zilberman, N., Riser, S. C., Johnson, K. S., Johnson, G. C., et al. (2017). The argo program present and future. Oceanography 30, 18-28. doi: 10.5670/oceanog.2017.213

Jenkins, W. J. (1977). Tritium-helium dating in the Sargasso Sea: a measurement of oxygen utilization rates. Science 196, 291-292 doi: 10.1126/science.196.4287. 291

Jenkins, W. J. (1998). Studying thermocline ventilation and circulation using tritium and 3He. J. Geophys. Res. 103, 15817-15831 doi: 10.1029/98ji 00141

Jiang, L.-Q., Feely, R. A., Carter, B. R., Greeley, D. J., Gledhill, D. K., and Arzayus, K. M. (2015). Climatological distribution of aragonite saturation state in the global oceans. Glob. Biogeochem. Cycles 29, 1656-1673 doi: 10.1002/ $2015 \mathrm{gb} 005198$

Johnson, G. C., and Gruber, N. (2007). Decadal water mass variations along $20^{\circ} \mathrm{W}$ in the northeastern Atlantic Ocean. Prog. Oceanogr. 73, 277-295, doi: 10.1016/ j.pocean.2006.03.022

Johnson, G. C., McTaggart, K. E., and Wanninkhof, R. (2014). Antarctic bottom water temperature changes in the western South Atlantic from 1989 to 2014. J. Geophys. Res. 119, 8567-8577. doi: 10.1002/2014jc010367

Johnson, G. C., Sloyan, B. M., Kessler, W. S., and McTaggart, K. E. (2002). Direct measurements of upper ocean currents and water properties across the tropical Pacific Ocean during the 1990's. Prog. Oceanogr. 52, 31-61, doi: 10.1016/S00796611(02)00021-6

Johnson, G. C., Lyman, J. M., and Purkey, S. G. (2015). Informing deep argo array design using argo and full- depth hydrographic section data. J. Atmos. Ocean. Technol. 32, 2187-2198. doi: 10.1175/jtech-d-15-0139.1

Johnson, K. S., Plant, J. N., Coletti, L. J., Jannasch, H. W., Sakamoto, C. M., Riser, S. C., et al. (2017a). Biogeochemical sensor performance in the SOCCOM profiling float array. J. Geophys. Res. 122, 6416-6436. doi: 10.1002/ 2017jc012838

Johnson, K. S., Plant, J. N., Dunne, J. P., Talley, L. D., and Sarmiento, J. L. (2017b). Annual nitrate drawdown observed by SOCCOM profiling floats and the relationship to annual net community production. J. Geophys. Res. 122, 6668-6683, doi: 10.1002/2017JC012839
Johnson, G. C., Purkey, S. G., and Toole, J. M. (2008). Reduced antarctic meridional overturning circulation reaches the North Atlantic Ocean. Geophys. Res. Lett. 35:L22601.

Katsumata, K., Sloyan, B. M., and Masuda, S. (2013). Diapycnal and isopycnal transports in the southern ocean estimated by a box inverse model. J. Phys. Oceanogr. 43, 2270-2287, doi: 10.1175/JPO-D-12-0210.1

Keeling, R. F., Körtzinger, A., and Gruber, N. (2010). Ocean deoxygenation in a warming world. Ann. Rev. Mar. Sci. 2, 199-229. doi: 10.1146/annurev.marine. 010908.163855

Key, R. M., Kozyr, A., Sabine, C. L., Lee, K., Wanninkhof, R., Bullister, J., et al. (2004). A global ocean carbon climatology: results from global data analysis project (GLODAP). Glob. Biogeochem. Cycles 19:GB4031

Key, R. M., Tanhua, T., Olsen, A., Hoppema, M., Jutterström, S., Schirnick, C., et al. (2010). The CARINA data synthesis project: introduction and overview. Earth Syst. Sci. Data 2, 105-121. doi: 10.5194/essd-2-105-2010

Key, R. M., Olsen, A., van Heuven, S., Lauvset, S. K., Velo, A., Lin, X., et al. (2015). Global Ocean Data Analysis Project, Version 2 (GLODAPv2). Oak Ridge, TN: US Department of Energy.

Khatiwala, S., Tanhua, T., Mikaloff Fletcher, S., Gerber, M., Doney, S. C., Graven, H. D., et al. (2013). Global ocean storage of anthropogenic carbon. Biogeosciences 10, 2169-2191.

Kim, I. N., Lee, K., Gruber, N., Karl, D. M., Bullister, J. L., Yang, S., et al. (2014). Increasing anthropogenic nitrogen in the North Pacific Ocean. Science 346, 1102-1106. doi: 10.1126/science. 1258396

Knap, A., Michaels, A., Close, A., Ducklow, H., and Dickson, A. (eds). (1996). Protocols for the Joint Global Ocean Flux Study (JGOFS) Core Measurements. Report Nr. 19, Paris: UNESCO.

Ko, Y. H., Lee, K., Takahashi, T., Karl, D. M., Kang, S-H., and Lee, E. (2018). Carbon-based estimate of nitrogen fixation-derived net community production in N-depleted ocean gyres. Glob. Biogeochem. Cycles 32, 1241-1252.

Körtzinger, A., Rhein, M., and Mintrop, L. (1999). Anthropogenic $\mathrm{CO}_{2}$ and CFCs in the North Atlantic Ocean-a comparison of man-made tracers. Geophys. Res. Lett. 26, 2065-2068. doi: 10.1029/1999g1900432

Kouketsu, S., Doi, T., Kawano, T., Masuda, S., Sugiura, N., et al. (2011). Deep ocean heat content changes estimated from observation and reanalysis product and their influence on sea level change. J. Geophys. Res. 116:C03012.

Kouketsu, S., and Murata, A. M. (2014). Detecting decadal scale increases in anthropogenic $\mathrm{CO}_{2}$ in the ocean. Geophys. Res. Lett. 41, 4594-4600. doi: 10. 1002/2014gl060516

Kunze, E. (2017a). Internal-wave-driven mixing: global geography and budgets. J. Phys. Oceanogr. 47, 1325-1345. doi: 10.1175/JPO-D-16-0141.1

Kunze, E. (2017b). The internal-wave-driven meridional overturning circulation. J. Phys. Oceanogr. 47, 2673-2689, doi: 10.1175/JPO-D-16-0142.1

Kunze, E., Firing, E., Hummon, J. M., Chereskin, T. K., and Thurnherr, A. M. (2006). Global abyssal mixing inferred from lowered ADCP shear and CTD strain profiles. J. Phys. Oceanogr. 36, 1553-1576, doi: 10.1175/JPO 2926.1

Lauvset, S. K., Key, R. M., Olsen, A., van Heuven, S., Velo, A., Lin, X., et al. (2016). A new global interior ocean mapped climatology: the $1^{\circ} \mathrm{x} 1^{\circ}$ GLODAP version 2. Earth Syst. Sci. Data 8, 325-340. doi: 10.5194/essd-8-3252016

Le Quéré, C., Andrew, R. M., Friedlingstein, P., Sitch, S., Pongratz, J., Manning, A. C., et al. (2018). Global carbon budget 2017. Earth Syst. Sci. Data 10, 405-448.

Lombard, F., Boss, E., Waite, A. M., Vogt, M., Uitz, J., Stemmann, L., et al. (2019). Globally consistent quantitative observations of planktonic ecosystems. Front. Mar. Sci. 6:196. doi: 10.3389/fmars.2019.00196

Lumpkin, R., and Speer, K. (2007). Global ocean meridional overturning. J. Phys. Oceanogr. 37, 2550-2562. doi: 10.1175/jpo3130.1

Matear, R. J., and Hirst, A. C. (2003). Long-term changes in dissolved oxygen concentrations in the ocean caused by protracted global warming. Glob. Biogeochem. Cycles 17:1125.

McDonagh, E. L., Bryden, H. L., King, B. A., and Sanders, R. J. (2008). The circulation of the Indian Ocean at $32^{\circ}$ S. Prog. Oceanogr. 79, 20-36.

Mecking, S., Warner, M. J., and Bullister, J. L. (2006). Temporal changes in pCFC-12 ages and AOU along two hydrographic sections in the eastern subtropical North Pacific. Deep Sea Res. I 53, 169-187. doi: 10.1016/j.dsr.2005. 06.018 
Menezes, V. V., Macdonald, A. M., and Schatzman, C. (2017). Accelerated freshening of antarctic bottom water over the last decade in the Southern Indian Ocean. Sci. Adv. 3:e1601426. doi: 10.1126/sciadv.1601426

Mercier, H., Lherminier, P., Sarafanov, A., Gaillard, F., Daniault, N., Desbruyères, D., et al. (2015). Variability of the meridional overturning circulation at the greenland-portugal ovide section from 1993 to 2010. Prog. Oceanogr. 132, 250-261. doi: 10.1016/j.pocean.2013.11.001

Murata, A., Kumamoto, Y., Sasaki, K., Watanabe, S., and Fukasawa, M. (2010). Decadal increases in anthropogenic $\mathrm{CO}_{2}$ along $20^{\circ} \mathrm{S}$ in the South Indian Ocean. J. Geophys. Res. 115:C12055.

Olsen, A., Key, R. M., van Heuven, S., Lauvset, S. K., Velo, A., Lin, X., et al. (2016). The global ocean data analysis project version 2 (GLODAPv2) - an internally consistent data product for the world ocean. Earth Syst. Sci. Data 8, 297-323. doi: 10.5194/essd-8-297-2016

Olsen, A., Lange, N., Key, R. M., Tanhua, T., Álvarez, M., Becker, S., et al. (2019). GLODAPv2.2019 - an update of GLODAPv2. Earth Syst. Sci. Data Discuss. 2019, 1-39. doi: 10.5194/essd-2019-66

Owens, W. B., and Wong, A. P. S. (2009). An improved calibration method for the drift of the conductivity sensor on autonomous CTD profiling floats by $\theta-S$ climatology. Deep Sea Res. I 56, 450-457. doi: 10.1016/j.dsr.2008.09.008

Palmer, M. D., Durack, P., Paz Chidichimo, M., Church, J., Cravatte, S. E., Hill, K. L., et al. (2019). Adequacy of the ocean observation system for quantifying regional heat and freshwater storage and change. Front. Mar. Sci. [in press]. doi: 10.3389/fmars.2019.00416

Pardo, P. C., Pérez, F. F., Khatiwala, S., and Ríos, A. F. (2014). Anthropogenic $\mathrm{CO}_{2}$ estimates in the Southern Ocean: storage partitioning in the different water masses. Prog. Oceanogr. 120, 230-242. doi: 10.1016/j.pocean.2013.09.005

Pearlman, J., Bushnell, M., Coppola, L., Karstensen, J., Buttigieg, P. L., Paerlman, F., et al. (2019). Evolving and sustaining ocean best practices and standards for the next decade. Front. Mar. Sci. 6:277. doi: 10.3389/fmars.2019.00277

Perez, F. F., Fontela, M., García-Ibáñez, M. I., Mercier, H., Velo, A., Lherminier, P., et al. (2018). Meridional overturning circulation conveys fast acidification to the deep Atlantic Ocean. Nature 554, 515-518. [in press]. doi: 10.1038/nature25493

Pickering, A., Nash, J. D., Moum, J. N., and MacKinnon, J. A., (2016). Global Patterns of Turbulence and Diapycnal Mixing from CTD-Chipods on the Global Repeat Hydrography Program. Washington, D.C: American Geophysical Union.

Pierrot, D., Neil, C., Sullivan, K., Castle, R., Wanninkhof, R., Lueger, H., et al. (2009). Recommendations for autonomous underway pCO2 measuring systems and data reduction routines. Deep Sea Res. II 56, 512-522. doi: 10.1016/j.dsr2. 2008.12.005

Purkey, S. G., Smethie, W. M., Gebbie, G., Gordon, A. L., Sonnerup, R. E., Warner, M. J., et al. (2018). A synoptic view of the ventilation and circulation of antarctic bottom water from chlorofluorocarbons and natural tracers. Ann. Rev. Mar. Sci. 10, 503-327. doi: 10.1146/annurev-marine-121916-063414

Purkey, S. G., and Johnson, G. C. (2010). Warming of global abyssal and deep Southern Ocean waters between the 1990s and 2000s: contributions to global heat and sea level rise budgets. J. Clim. 23, 6336-6351. doi: 10.1175/ 2010jcli3682.1

Purkey, S. G., and Johnson, G. C. (2013). Antarctic bottom water warming and freshening: contributions to sea level rise, ocean freshwater budgets, and gobal heat gain. J. Clim. 26, 6105-6122. doi: 10.1175/jcli-d-12-00834.1

Purkey, S. G., Johnson, G. C., Talley, L. D., Sloyan, B. M., Wijffels, S. E., Smethie, W., et al. (2019). Continued bottom water warming and freshening in the South Pacific Ocean. J. Geophys. Res. 124, 1778-1794. doi: 10.1029/2018JC014775

Raes, E. J., Bodrossy, L., van de Kamp, J., Bissett, A., Ostrowski, M., Brown, M. V., et al. (2018). Oceanographic biome boundaries constrain microbial richness in the South Pacific Ocean. Proc. Natl. Acad. Sci. U.S.A. 115, E8266-E8275, doi: $10.1073 /$ pnas.1719335115

Reynolds, R. W. (1988). A Real-time global sea surface temperature analysis. J. Climate 1, 75-86.

Rhein, M., Rintoul, S. R., Aoki, S., Campos, E., Chambers, D., Feely, R. A., et al. (2013). Observations: ocean. In Climate Change 2013: The Physical Science Basis. Contribution of Working Group I to the Fifth Assessment Report of the Intergovernmental Panel on Climate Change. eds T. F Stocker., D. Qin, G. -K. Plattner, M. Tignor, S. K Allen (Cambridge, MA: Cambridge University Press), 255-310.

Ríos, A. F., Velo, A., Pardo, P. C., Hoppema, M., and Pérez, F. F. (2012). An update of anthropogenic $\mathrm{CO}_{2}$ storage rates in the western South Atlantic basin and the role of Antarctic Bottom Water. J. Mar. Syst. 94, 197-203. doi: 10.1016/j. jmarsys.2011.11.023

Roemmich, D., Gilson, J., Davi, R., Sutton, P., Wijffels, S., and Riser, S. (2007). Decadal spinup of the South Pacific subtropical gyre. J. Phys. Oceanogr. 37, 162-173. doi: 10.1175/jpo3004.1

Roquet, F., Charrassin, J. -B., Marchand, S., Boehme, L., Fedak, M., Reverdin, G., et al. (2011). Delayed-mode calibration of hydrographic data obtained from animal-borne satellite relay data loggers. J. Atmos. Ocean. Technol. 28, 787-801. doi: 10.1175/2010jtecho801.1

Sabine, C. L., Feely, R. A., Gruber, N., Key, R. M., Lee, K., Bullister, J. L., et al. (2004). The oceanic sink for anthropogenic $\mathrm{CO}_{2}$. Science 305, 367-371. doi: 10.1126/science. 1097403

Sabine, C. L., Feely, R. A., Millero, F. J., Dickson, A. G., Langdon, C., et al. (2008). Decadal changes in pacific carbon. J. Geophys. Res. 113:C07021.

Sabine, C. L., and Tanhua, T. (2010). Estimation of anthropogenic $\mathrm{CO}_{2}$ inventories in the ocean. Ann. Rev. Mar. Sci. 2, 175-198.

Sasano, D., Takatani, Y., Kosugi, N., Nakano, T., Midorikawa, T., and Ishii, M. (2018). Decline and bidecadal oscillations of dissolved oxygen in the Oyashio region and their propagation to the western North Pacific. Glob. Biogeochem. Cycles 32, 909-931. doi: 10.1029/2017GB005876

Sasano, D., Ishii, M., Midorikawa, T., Nakano, T., Tokieda, T., and Uchida, H. (2011). Testing a new quick response oxygen sensor, "RINKO". Papers Meteorol. Geophys. 62, 63-73. doi: 10.2467/mripapers.62.63

Schlitzer, R. (2000). Electronic Atlas of WOCE Hydrographic and tracer data now. EOS Trans. AGU 81:45. doi: 10.1029/00eo00028

Schlitzer, R. (2018). Ocean Data View. odv.awi.de.

Sloyan, B. M., and Rintoul, S. R. (2001). The Southern Ocean limb of the global deep overturning circulation. J. Phys. Oceanogr. 31, 143-173. doi: 10.1175/ 1520-0485(2001)031<0143:tsolot $>2.0 . c 0 ; 2$

Sloyan, B. M., Wijffels, S. E., Tilbrook, B., Katsumata, K., Murata, A., and Macdonald, A. M. (2013). Deep ocean changes near the western boundary of the South Pacific Ocean. J. Phys. Oceanogr. 43, 2132-2141. doi: 10.1175/jpo-d12-0182.1

Smith, N., Kessler, W. S., Cravatte, S., Sprintall, J., Wijffels, S, Cronin, M. F., et al. (2019). Tropical pacific observing system. Front. Mar. Sci. 6:31. doi: 10.3389/ fmars.2019.00031

Sonnerup, R. E., Mecking, S., and Bullister, J. L. (2013). Transit time distributions and oxygen utilization rates in the Northeast Pacific Ocean from chlorofluorocarbons and sulfur hexafluoride. Deep Sea Res. I 72, 61-71.

Sonnerup, R. E., Mecking, S., Bullister, J. L., and Warner, M. J. (2015). Transit time distributions and oxygen utilization rates from chlorofluorocarbons and sulfur hexafluoride in the Southeast Pacific Ocean. J. Geophys. Res. 120, 3761-3776. doi: 10.1002/2015JC010781

Stanley, R. H. R., Doney, S. C., Jenkins, W. J., and Lott, D. E. I. (2012). Apparent oxygen utilization rates calculated from tritium and helium-3 profiles at the Bermuda Atlantic Time-series Study site. Biogeosciences 9, 1969-1983. doi: 10.5194/bg-9-1969-2012

Stramma, L., Johnson, G. C., Sprintall, J., and Mohrholz, V. (2008). Expanding oxygen-minimum zones in the tropical oceans. Science 320, 655-658. doi: 10. $1126 /$ science. 1153847

Suzuki, T., Ishii, M., Aoyama, M., Christian, J. R., Enyo, K., Kawano, T., et al. (2013). PACIFICA Data Synthesis Project. ORNL/CDIAC-159, NDP-092. Carbon Dioxide Information Analysis Center, Oak Ridge National Laboratory. Oak Ridg, TN: U.S. Department of Energy.

Takahashi, T., Sutherland, S. C., Wanninkhof, R., Sweeney, C., Feely, R. A., Chipman, D. W., et al. (2009). Climatological mean and decadal change in surface ocean $\mathrm{pCO} 2$, and net sea-air $\mathrm{CO} 2$ flux over the global oceans. Deep Sea Res. II 56, 554-577. doi: 10.1016/j.dsr2.2008.12.009

Takatani, Y., Kojima, A., Iida, Y., Nakano, T., Ishii, M., et al. (2014). Ocean acidification in the interior of the western North Pacific subtropical region, in Proceedings of the International Ocean Research. Conference, Spain, 17-21.

Takeshita, Y., Johnson, K. S., Martz, T. R., Plant, J. N., and Sarmiento, J. L. (2018). Assessment of autonomous $\mathrm{pH}$ measurements for determining surface seawater partial pressure of $\mathrm{CO}_{2}$. J. Geophys. Res. Oceans 123, 4003-4013. doi: 10.1029/ 2017JC013387

Talley, L. D., Feely, R. A., Sloyan, B. M., Wanninkhof, R., Baringer, M. O., and Bullister, J. L. (2016). Changes in ocean heat, carbon content, and ventilation: a review of the first decade of GO-SHIP global repeat hydrography. 
Ann. Rev. Mar. Sci. 8, 185-215. doi: 10.1146/annurev-marine-052915100829

Talley, L. D. (2013). Closure of the global overturning circulation through the Indian, Pacific and Southern Oceans: schematics and transports. Oceanography 26, 80-97. doi: 10.5670/oceanog.2013.07

Tanhua, T., Pouliquen, S., Hausman, J., O’Brien, K., Bricher, P., de Burin, T., et al. (2019). Ocean FAIR data services. Front. Mar. Sci. [in press]. doi: 10.3389/fmars. 2019.00440

Tanhua, T., Hoppema, M., Jones, E. M., Stöven, T., Hauck, J., Dávila, M. G., et al. (2017). Temporal changes in ventilation and the carbonate system in the Atlantic sector of the Southern Ocean. Deep Sea Res. Part II 138, 26-38. doi: 10.1016/j.dsr2.2016.10.004

van Heuven, S. M. A. C., Hoppema, M., Huhn, O., Slagter, H. A., and de Baar, H. J. W. (2011). Direct observation of increasing $\mathrm{CO}_{2}$ in the weddell gyre along the prime meridian during 1973-2008. Deep-Sea Res. II 58, 2613-2635. doi: 10.1016/j.dsr2.2011.08.007

Wanninkhof, R., Park, G. -H., Takahashi, T., Feely, R. A., Bullister, J. L., and Doney, S. C. (2013a). Changes in deep-water $\mathrm{CO}_{2}$ concentrations over the last several decades determined from discrete $\mathrm{pCO}_{2}$ measurements. Deep-Sea Res. I 74, 48-63. doi: 10.1016/j.dsr.2012.12.005

Wanninkhof, R., Park, G. -H., Takahashi, T., Sweeney, C., Feely, R., Nojiri, Y., et al. (2013b). Global ocean carbon uptake: magnitude, variability and trends. Biogeosciences 10, 1983-2000. doi: 10.1073/pnas.1900371116

Waterhouse, A. F., MacKinnon, J. A., Nash, J. D., Alford, M. H., Kunze, E., Simmons, H. L., et al. (2014). Global patterns of mixing from measurements of the turbulent dissipation rate. J. Phys. Oceanogr.. 44, 1854-1872. doi: 10.1175/ JPO-D-13-0104.1
Waters, J. F., Millero, F. J., and Sabine, C. L. (2011). Changes in South Pacific anthropogenic carbon. Glob. Biogeochem. Cycles 25:GB4011.

Waugh, D. W., Primeau, F., Devries, T., and Holzer, M. (2013). Recent changes in the ventilation of the southern oceans. Science 339, 568-570. doi: 10.1126/ science. 1225411

Williams, N. L., Juranek, L. W., Johnson, K. S., Feely, R. A., Riser, S. C., Talley, L. D., et al. (2016). Empirical algorithms to estimate water column $\mathrm{pH}$ in the Southern Ocean. Geophys. Res. Lett. 43, 3415-3422. doi: 10.1002/2016GL06 8539

Wong, A. P. S., Johnson, G. C., and Owens, W. B. (2003). Delayed-mode calibration of autonomous CTD profiling float salinity data by theta-S climatology. J. Atmos. Ocean. Technol. 20, 308-318. doi: 10.1175/15200426(2003)020<0308:dmcoac $>2.0 . c 0 ; 2$

Conflict of Interest Statement: The authors declare that the research was conducted in the absence of any commercial or financial relationships that could be construed as a potential conflict of interest.

Copyright (c) 2019 Sloyan, Wanninkhof, Kramp, Johnson, Talley, Tanhua, McDonagh, Cusack, O'Rourke, McGovern, Katsumata, Diggs, Hummon, Ishii, Azetsu-Scott, Boss, Ansorge, Perez, Mercier, Williams, Anderson, Lee, Murata, Kouketsu, Jeansson, Hoppema and Campos. This is an open-access article distributed under the terms of the Creative Commons Attribution License (CC BY). The use, distribution or reproduction in other forums is permitted, provided the original author(s) and the copyright owner(s) are credited and that the original publication in this journal is cited, in accordance with accepted academic practice. No use, distribution or reproduction is permitted which does not comply with these terms. 


\section{Ocean FAIR Data Services}

\section{OPEN ACCESS}

Edited by:

Amos Tiereyangn Kabo-bah, University of Energy and Natural Resources, Ghana

Reviewed by: Jan-Bart Calewaert, European Marine Observation and Data Network (EMODnet),

Belgium

Greg Zacharewicz,

Institut Mines-Télécom Mines Alès,

France

${ }^{*}$ Correspondence:

Toste Tanhua

ttanhua@geomar.de

Specialty section:

This article was submitted to

Ocean Observation,

a section of the journal

Frontiers in Marine Science

Received: 15 November 2018

Accepted: 05 July 2019

Published: 07 August 2019

Citation:

Tanhua T, Pouliquen $S$ Hausman J, O'Brien K, Bricher P, de Bruin T, Buck JJH, Burger EF,

Carval T, Casey KS, Diggs $S$,

Giorgetti A, Glaves $H$, Harscoat $V$, Kinkade D, Muelbert JH, Novellino A, Pfeil B, Pulsifer PL, Van de Putte $A$ Robinson E, Schaap D, Smirnov A,

Smith N, Snowden D, Spears T,

Stall S, Tacoma M, Thijsse $P$,

Tronstad S, Vandenberghe $T$, Wengren $M$, Wyborn $L$ and Zhao Z (2019) Ocean FAIR Data Services.

Front. Mar. Sci. 6:440.

doi: 10.3389/fmars.2019.00440
Toste Tanhua ${ }^{*}$, Sylvie Pouliquen², Jessica Hausman³ ${ }^{3}$ Kevin O’Brien ${ }^{4}$, Pip Bricher $^{5}$, Taco de Bruin', Justin J. H. Buck ${ }^{7}$ Eugene F. Burger ${ }^{8}$, Thierry Carval², Kenneth S. Casey ${ }^{9}$, Steve Diggs ${ }^{10}$, Alessandra Giorgetti11, Helen Glaves ${ }^{12}$, Valerie Harscoat ${ }^{2}$, Danie Kinkade ${ }^{13}$, Jose H. Muelbert ${ }^{14}$, Antonio Novellino ${ }^{15}$, Benjamin Pfeil ${ }^{16}$, Peter L. Pulsifer ${ }^{17}$, Anton Van de Putte ${ }^{18}$, Erin Robinson ${ }^{19}$, Dick Schaap ${ }^{20}$, Alexander Smirnov ${ }^{21}$, Neville Smith ${ }^{22}$, Derrick Snowden ${ }^{23}$, Tobias Spears ${ }^{24}$, Shelley Stall25, Marten Tacoma ${ }^{6}$, Peter Thijsse ${ }^{20}$, Stein Tronstad ${ }^{26}$, Thomas Vandenberghe ${ }^{18}$, Micah Wengren ${ }^{23}$, Lesley Wyborn ${ }^{27}$ and Zhiming Zhao ${ }^{28}$

1 GEOMAR Helmholtz Centre for Ocean Research Kiel, Kiel, Germany, ${ }^{2}$ IFREMER, Plouzané, France, ${ }^{3}$ Jet Propulsion Laboratory, California Institute of Technology, Pasadena, CA, United States, ${ }^{4}$ Joint Institute for the Study of the Atmosphere and Ocean, University of Washington, Seattle, WA, United States, ${ }^{5}$ Southern Ocean Observing System, University of Tasmania, Hobart, TAS, Australia, ${ }^{6}$ NIOZ Royal Netherlands Institute for Sea Research, and Utrecht University, Texel, Netherlands, ${ }^{7}$ National Oceanography Centre-British Oceanographic Data Centre, Liverpool, United Kingdom, ${ }^{8}$ NOAA Pacific Marine Environmental Laboratory, Seattle, WA, United States, ${ }^{9}$ NOAA National Centers for Environmental Information, Silver Spring, MD, United States, ${ }^{10}$ Scripps Institution of Oceanography, University of California, San Diego, La Jolla, CA, United States, ${ }^{11}$ Istituto Nazionale di Oceanografia e di Geofisica Sperimentale, Sgonico, Italy, ${ }^{12}$ British Geological Survey, Nottingham, United Kingdom, ${ }^{13}$ Woods Hole Oceanographic Institution, Woods Hole, MA, United States, ${ }^{14}$ Instituto de Oceanografia, Universidade Federal do Rio Grande, Rio Grande, Brazil, ${ }^{15}$ ETT, Genova, Italy, ${ }^{16}$ Bjerknes Centre for Climate Research, University of Bergen, Bergen, Norway, ${ }^{17}$ National Snow and Ice Data Center, University of Colorado Boulder, Boulder, CO, United States, ${ }^{18}$ Royal Belgian Institute for Natural Sciences, Brussels, Belgium, ${ }^{19}$ Earth Science Information Partners, Boulder, CO, United States, ${ }^{20}$ MARIS Mariene Informatie Service, Voorburg, Netherlands, ${ }^{21}$ Arctic Portal, Akureyri, Iceland, ${ }^{22}$ GODAE Ocean Services, Melbourne, VIC, Australia, ${ }^{23}$ U.S. Integrated Ocean Observing System, Silver Spring, MD, United States, ${ }^{24}$ Fisheries and Oceans, Science Branch, Maritimes Region Ocean Data and Information Section, Dartmouth, NS, Canada, ${ }^{25}$ American Geophysical Union, Washington, DC, United States, ${ }^{26}$ Norwegian Polar Institute, Tromsø, Norway, ${ }^{27}$ National Computational Infrastructure, Australian National University, Canberra, ACT, Australia, ${ }^{28}$ Informatics Institute, University of Amsterdam, Amsterdam, Netherlands

Well-founded data management systems are of vital importance for ocean observing systems as they ensure that essential data are not only collected but also retained and made accessible for analysis and application by current and future users. Effective data management requires collaboration across activities including observations, metadata and data assembly, quality assurance and control (QA/QC), and data publication that enables local and interoperable discovery and access and secures archiving that guarantees long-term preservation. To achieve this, data should be findable, accessible, interoperable, and reusable (FAIR). Here, we outline how these principles apply to ocean data and illustrate them with a few examples. In recent decades, ocean data managers, in close collaboration with international organizations, have played an active role in the improvement of environmental data standardization, accessibility, and interoperability through different projects, enhancing access to observation data at all stages of the data life cycle and fostering the development of integrated services targeted to research, regulatory, and operational users. As ocean observing systems evolve and an increasing number of autonomous platforms and sensors are deployed, the volume and variety of data increase dramatically. For instance, there are more than 70 data catalogs that contain metadata records for the polar oceans, a situation that makes comprehensive data discovery beyond the capacity of most researchers. To better serve research, operational, and commercial users, more efficient turnaround of quality data in known 
formats and made available through Web services is necessary. In particular, automation of data workflows will be critical to reduce friction throughout the data value chain. Adhering to the FAIR principles with free, timely, and unrestricted access to ocean observation data is beneficial for the originators, has obvious benefits for users, and is an essential foundation for the development of new services made possible with big data technologies.

Keywords: FAIR, ocean, data management, data services, ocean observing, standardization, interoperability

\section{INTRODUCTION}

Well-functioning and fit-for-purpose data management systems are essential to the sustained ocean observing system. This quote from the Intergovernmental Oceanographic Commission (IOC) of United Nations Educational, Scientific, and Cultural Organization (UNESCO) Oceanographic Data Exchange Policy articulates the high-level mandate for and the essence of the requirements for oceanographic data flow:

"The timely, free and unrestricted international exchange of oceanographic data is essential for the efficient acquisition, integration and use of ocean observations gathered by the countries of the world for a wide variety of purposes including the prediction of weather and climate, the operational forecasting of the marine environment, the preservation of life, the mitigation of human-induced changes in the marine and coastal environment, as well as for the advancement of scientific understanding that makes this possible."

It is worthwhile to note that the IOC member states, in this policy, agreed to "provide timely, free and unrestricted access to all data, associated metadata and products generated under the auspices of IOC programs," and encouraged the member states to do the same also for non-IOC programs. Although this is an excellent aspiration, in practice, this principle has been only loosely enforced by the IOC.

Fit-for-purpose data management systems are of vital importance as they ensure that essential data are not only collected but also retained and made accessible for analysis and application for current and future users. Data management systems that facilitate free and open access, use, and interpretation of data and products must be included as essential elements of the ocean observing system. Effective data management is based on collaboration across activities including observing, metadata and data assembly, quality assurance and control (QA/QC), and data publication. It enables local and interoperable discovery and access and secures archiving that guarantees long-term preservation.

As ocean observing systems evolve with an increasing number of autonomous platforms and sensors being deployed, measuring an increased range of essential ocean variables (EOVs), the volume and diversity of data are increasing dramatically. Automation of data workflows ${ }^{1}$ and effective standards will be critical to reduce data friction throughout the whole data life cycle (e.g., Taylor et al., 2006). This increased efficiency is relevant for all data types, from physical observations to biogeochemical

\footnotetext{
${ }^{1}$ See, for instance, https://www.wfmc.org/
}

observations and biological and ecosystem observations. With the development of information technologies, researchers expect easy access to a wide range of data and data products. As it becomes easier to aggregate huge amounts of data, the risk of mixing "apples with oranges" increases if the delivery services are not well designed and the data and data products are not clearly described using standardized schemas.

The challenge of enabling optimal use of research data and methods is complex with multiple stakeholders: researchers wanting to share their data and interpretations; professional data publishers offering their services; software and tool builders providing data analysis and processing services; funding agencies (private and public) increasingly concerned with proper data stewardship; and a data science community mining, integrating, and analyzing the output to advance discovery. Computational analysis to discover meaningful patterns in massive, interlinked datasets is rapidly becoming a routine research activity.

The global ocean data system should be designed as an interoperable system of systems that will allow data to be easily findable, accessible, interoperable, and allowing reusability through thematic integrated products and services. The longterm goal is to develop a data system of systems that allows the development of data services at different levels with a guarantee that the best version of the observed data is used at all levels. Data quality, interoperability, and good discoverability can only be assured with a standardized, traceable workflow throughout the lifetime of the datasets. This paper reviews recent developments in technical capacity and requirement setting of a data management system for the Global Ocean Observing System (GOOS). The focus is on EOVs, and the content reflects the increased attention to biogeochemical and biological ecosystem EOVs, building on successes of the physical data system that has evolved the fastest.

\section{THE CHALLENGES}

Over previous decades, the requirements for ocean information on an ever-increasingly diverse range of issues have increased. In the past, data management systems have largely developed in isolation and with different objectives to serve particular communities or funding routes. Here, we list some of the main challenges in moving ocean data management toward the FAIR principles of being findable, accessible, interoperable, and reusable (see next section for more details). 


\section{Wide Diversity}

The diversity of oceanographic data makes it difficult for the scientist or application developer to find, understand, and use data to optimal benefit. Significant time is invested in these activities before the actual research or data utilization can begin, while provenance and traceability are required for the sake of reproducibility. While automation can bring improved efficiencies to data management for some data types, there is highly variable uptake of these automation methods, and some disciplines will require considerable progress in standardizing observation methods and data management processes before they can take full advantage of these advances.

\section{Multitude of Disparate Data Management Structures}

The existence of a multitude of disparate data management infrastructures currently imposes problems for observing systems. These include delayed and duplicated data receipts, versioning issues, missing data and metadata, and undocumented data processing procedures. The interoperability issues resulting from the existence and use of various data management infrastructures are fundamental and wide ranging. Resources are often not available to resolve these issues by wholesale replacement of existing systems. For instance, there are more than 70 data catalouges for the Polar Ocean, see Box 1.

\section{Increased Volume of Data}

The past 10 years have seen the development of autonomous platforms able to acquire accurate measurements during yearslong deployments (e.g., Argo, glider, moorings, and ships-ofopportunity). These platforms are transmitting as much data in 1 year as has been acquired in the past century (Figure 1). This rapid increase in data volume puts high demands even on well-organized and interoperable data management systems. Not only is the real time (RT) in situ data increasing in volume, but also new variables, particularly for biogeochemistry and biology, are frequently being transmitted in RT. This has also resulted in a concomitant increase in the workload for delayed-mode data processing that corrects for biases that accumulate during time spent at sea.

\section{New Sensors Creating New Formats}

A major challenge is the management of novel data types produced from new sensors that require mapping to existing standards and conventions or the creation of new ones.

\section{Widely Used Formats Not Universally Applicable}

Implementation of widely used standards and formats can be beyond the capabilities of many scientific communities, even

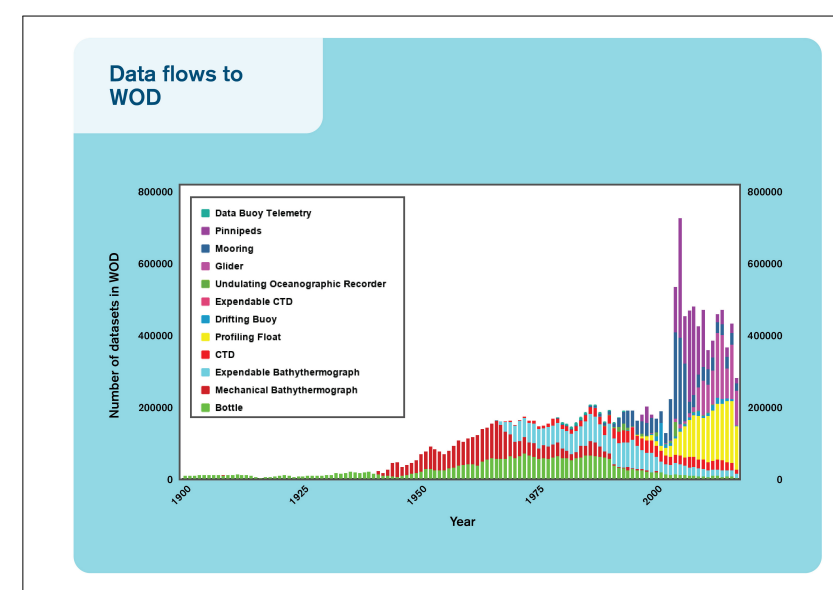

FIGURE 1 | The number of datasets flowing to the World Ocean Database over the past century.

BOX 1| Polar Oceans.

The Southern Ocean links the world's major ocean basins and the upper and lower levels of global ocean circulation (Meredith et al., 2013). Research in this region is largely conducted under the auspices of the Antarctic Treaty System (https://www.ats.aq/e/ats.htm). The polar oceans provide a particular example of the need for global cooperation, given the logistical challenges of working in high-latitude environments and the strategic and scientific importance to nations well beyond those that share geographic borders with the polar regions. The distances and logistical challenges in these oceans mean that much oceanographic research is conducted by national polar research programs rather than purely oceanographic programs. In data management terms, the data centers serving these communities must meet data and metadata standards for both terrestrial and national data communities, in addition to those agreed in the international oceanographic community. Serving 'many masters' as these polar data centers do highlights the need for oceanographic data managers to use agreed standards. More than 70 metadata catalogs hosting polar data have been identified, many of which are not part of metadata federations or aggregations and, hence, put comprehensive data discovery beyond the capacity of most researchers.

In order to achieve FAIR, the Southern Ocean Observing System (SOOS) explicitly accounts for differences in the workflows and levels of technological integration among its scientific communities and seeks to make data available through a variety of paths to "meet each scientist where they are." SOOS, along with the EMODnet Physics group, is making standardized and aggregated in situ datasets available for exploration and download through SOOSmap (http://www.soos.aq/news/current-news/362-explore-southern-ocean-soosmap). For the long tail of non-standardized data, SOOS is working with Arctic and Antarctic data management groups to investigate the best ways to achieve federated metadata search and to ensure that the EMODnet/SOOSmap infrastructure can be directly linked to virtual labs. SOOS is encouraging scientists in its community to use existing data discovery tools to identify key datasets that should be standardized and federated to publish through SOOSmap. Along this line, SOOS, the Standing Committee on Antarctic Data Management (SCADM), and the Arctic Data Committee (ADC) have founded the POLDER (http://www.soos.aq/data/federatedsearch) initiative (Polar Data Discovery Enhancement Research) to identify and advocate the needs of the polar data community in the development of federated metadata search for polar oceanographic and terrestrial data. The activities to date, described above, have largely focused on the findability and accessibility of polar ocean data, and it is likely that these will continue to be significant activities for the next decade. As new observing technologies develop, observing systems are encouraging researchers to standardize formats and QC processing, which should considerably improve on the interoperability and reusability of those datasets. 
when the benefits of using those formats are clear. While some progress has been made through tools like the National Oceanic and Atmospheric Administration (NOAA) National Centers for Environmental Information's Network Common Data Form (NetCDF) templates ${ }^{2}$, which help data producers across disciplines create Climate and Forecast (CF) and Attribute Conventions Dataset Discovery (ACDD)-compliant NetCDF, truly comprehensive adoption has not yet been achieved. IT activities are typically funded by science activities, so the requirements of particular science communities may be addressed effectively. However, this can be at the expense of universal interoperability.

\section{Gap Between Data-Producing Scientists and Downstream Users of the Data}

It is incumbent upon our community to develop or leverage existing tools that can bridge the gap between data-producing scientists and downstream users of the data, i.e., to remove barriers from "owning data" to "sharing data" for maximal community benefit would require cultural changes. Efficient (FAIR) sharing of data is a social responsibility of scientists, mostly funded by the society, to support the blue economy and ocean information building.

\section{Development of Common Protocols Takes Time}

As identified by de La Beaujardière et al. (2010) and Hankin et al. (2010), the development and adoption of common standards for data/metadata (Keeley et al., 2010) and sharing protocols (Pouliquen et al., 2010) take time, coordination, and careful testing.

\section{Best Practices Poorly Defined}

At present, best-practice data management (Pearlman et al., 2019) is often largely undefined and is generally left as a decision for the data curator and/or data publisher, although community standards for metadata, data formats, communication protocols, and data server software infrastructure are the foundation for interoperability. Data that are poorly documented can be considered lost and will have little or no value without access to the team that collected the data. Even the research team that collected the data will be challenged to remember details, or find notes, on how the data were collected if it is not properly curated at the time of collection and review.

\section{THE FAIR PRINCIPLES}

Open and free data policies are widely encouraged and increasingly required by many organizations, including the IOC and International Oceanographic Data and Information Exchange (IODE), the European Commission, and the Antarctic Treaty System, as well as many funding and operational agencies. Interoperability among data services has become a priority with the development of the FAIR principles ${ }^{3}$, a set of guiding principles to make data:

\section{- Findable}

Each dataset should be identified by a unique persistent identifier and described by rich, standardized metadata that clearly include the persistent identifier. The metadata record should be indexed in a catalog and carried with the data.

\section{- Accessible}

The dataset and its metadata record should be retrievable by using the persistent identifier and a standardized communications protocol. In turn, that protocol should allow for authentication and authorization, where necessary. All metadata records should remain accessible even when the datasets they describe are not easily accessible.

\section{- Interoperable}

Both metadata and datasets use formal, accessible, shared, and broadly applicable vocabularies and/or ontologies to describe themselves. They should also use vocabularies that follow FAIR principles and provide qualified references to other relevant metadata and data. Importantly, the data and metadata should be machine accessible and parsable.

\section{- Reusable}

To meet this principle, data must already be findable, accessible, and interoperable. Additionally, the data and metadata should be sufficiently richly described that it can be readily integrated with other data sources. Published data objects should contain enough information on their provenance to enable them to be properly cited and should meet domain-relevant community standards.

The FAIR data principles are gaining increasing traction across all scientific domains, triggered by an important publication that radically influenced data management and data sharing developments (Wilkinson et al., 2016). The FAIR principles specifically focus on machine use of data and metadata because this is more difficult than achieving FAIR data for human users, whose intuitive sense of semantics and ability to infer meaning from contextual cues assists them in navigating nonstandardized datasets and their metadata descriptions. While full implementation of the FAIR principles may rarely be achieved, these principles are designed to encourage data managers to take steps along a continuum from unstructured, undocumented data to fully FAIR data (Wilkinson et al., 2016). As ocean observing systems develop, they will need to account for the patchy legacy of data management approaches. Data management infrastructure-both technical and humanwill need to be flexible to assist those disciplines and nations without access to sophisticated data management systems to make their data FAIR, while also encouraging continued development toward the FAIR principles from those disciplines and nations with a strong history of data management.

Oceanographic data systems generally possess a high level of FAIRness compared to many other disciplines. The major, but certainly not only, hurdle today is machine-to-machine aspects

\footnotetext{
${ }^{2}$ https://www.nodc.noaa.gov/data/formats/netcdf
}

$\overline{{ }^{3} \text { https://www.force11.org/group/fairgroup/fairprinciples }}$ 
of interoperability. At the same time, we acknowledge that in some disciplines, it is important to the scientists who collect a dataset that they be given the opportunity to publish their findings before the data are made public. There is nothing in the FAIR principles that precludes data originators from embargoing their data for a limited period, even though the sustained ocean observing system heavily relies on timely availability of data. We believe that giving scientific data originators the option of embargo can be important to maintain confidence in the data management system.

\section{THE PAST DECADE}

The process of developing a multidisciplinary, integrated ocean observing system for operational uses, including sustained scientific research, follows the guidelines of the framework for ocean observing (Lindstrom et al., 2012). This framework was a major outcome of the OceanObs'09 conference and was developed through sponsorship of IOC, and the implementation is coordinated by the GOOS. Important aspects of the Framework of Ocean Observing (FOO) are the focus on EOVs and the expansion of GOOS to cover the biogeochemical and biological/ecological domains as well as physical variables. During the last few years, the ocean community has been working toward agreeing on a set of EOVs for physics, biogeochemistry, and biology/ecosystems. As a result, ocean data management systems have put emphasis on servicing the need to observe and report on EOVs for sustained ocean observing systems.

Both the means to acquire these data and the way they are used have evolved greatly in the past 10 years. In the past decade, new Global Data Assembly Centers (GDACs) were set up for some in situ networks, applying the OceanObs'09 recommendations (Pouliquen et al., 2010). In their contribution to OceanObs'09, (Hankin et al., 2010) recommended key areas in which oceanographic data managers should focus their attention during the decade that is now coming to a close. Their recommendations focused on what they considered pragmatic and realistic actions to improve the accessibility and interoperability of ocean-focused data. These included (1) working toward adopting common standards for data and metadata building on progress made in the past decade (Keeley et al., 2010); (2) establishment of a single entry point (GDAC) concept to network data or for aggregated products (Pouliquen et al., 2010); (3) the use of common standards that allow systems to interoperate; (4) leveraging the efforts of commercial search engines through the adoption of Web services with tools (Blower et al., 2010); (5) developing and adopting data models based on sampling geometry; (6) developing semantic Web tools to allow straightforward translations between metadata standards; and (7) specific recommendations for meteorological data, data archiving processes, biological data, satellite data, and software toolkits for systems developments. In particular, they advocated for all ocean observations to be made universally accessible through CF-compliant NetCDF files using common vocabularies served through Open Geospatial Consortium (OGC) Web services or commonly used tools such as Open-source Project for a Network Data Access Protocol (OPeNDAP) servers.
At the time of writing, these formats are already widely used for model outputs and satellite products. The last decade has seen the introduction of the discrete sampling geometries (DSG) into the CF standard as of version 1.6, released in December 2011. These geometries are designed to provide representations for in situ features such as time series, vertical profiles, and surface trajectories. More mature networks are currently implementing these features in their NetCDF data representations. Recently, these standards have also been embraced by the biogeochemical community, e.g., the Surface Ocean $\mathrm{CO}_{2}$ Atlas (SOCAT) project uses NetCDF, CF-compliant DSG as the backbone of its data ingest and QC system. The European SeaDataNet community, working on standards for validated, archived data, has adopted Ocean Data View (ODV) ASCII format as well as SeaDataNet NetCDF CF for the observation datasets (profiles, time series, and trajectories), and NetCDF CF for its data products such as climatologies. However, the biological communities have not embraced the CF conventions to describe their data but have converged on different standards; e.g., biological data standards are curated by the Biodiversity Information Standards (TDWG). The most popular standard for sharing biodiversity information is Darwin Core, which enables integration between the two largest communities, the Global Biodiversity Information Facility (GBIF) and Ocean Biogeographic Information System (OBIS). The difference between physical and biological data standards has likely to do with the requirement (RT vs. delayed-mode data delivery), the amount of data to be handled (physical data tend to have significant higher volume), and the methods of data collection.

In addition to developments within the oceanographic community, key interdisciplinary communities have emerged to address data informatics topics common to multiple communities. These include the American Geophysical Union (AGU) and European Geophysical Union (EGU) Earth Space and Science Informatics groups and the Research Data Alliance (RDA). Examples of efforts that have had links to these communities are the FAIR principles and developments in the use of Digital Object Identifiers (DOI).

Progress in information technologies over the past decade with an increase in data services available via the internet has led to the emergence of new computing paradigms and technologies such as high-performance and high-throughput computing; cloud, edge, and fog computing; big data analytics; machine learning; and virtual research environments. This opens up significant opportunities but requires FAIR data management practices to be implemented. One example demonstrating how embracing new technologies has improved data access for uses occurred when NOAA's Big Data Project (BDP) partnered with Amazon Web Services (AWS) to provide access to the complete historical archive of the Level-II Next Generation Weather Radar (NEXRAD). NEXRAD data are used for a variety of purposes, including weather forecasting, water management, etc. With these data available in the cloud, the data were accessed 2.3 times as often as the historical monthly access rates ${ }^{4}$, indicating it was much easier to find and use for data consumers. Similarly, within the European H2020 ENVRIplus project, a subscription

${ }^{4}$ https://www.ncei.noaa.gov/news/noaa-expands-big-data-access 
system was developed, allowing faster subsampling on the full Argo dataset, and semantic search based on FAIR Argo metadata system is under development using Elasticsearch and big data technologies.

Another example is SeaDataNet, which connects in excess of 110 data centers in Europe and gives harmonized discovery and access to a large volume of marine and ocean datasets. For this purpose, SeaDataNet dynamically maintains so-called data buffers for specific parameters. SeaDataNet is also performing data discovery and access as well as data buffer services for several European Marine Observation and Data Network (EMODnet) projects. Several data products are delivered with DOI, OGC Web services, NetCDF (CF), and other formats, depending on their user communities. In addition, all products carry SeaDataNet PIDs and related metadata for the used basis datasets for acknowledging data originators and following FAIRness principles. The experience is that these harmonized and validated data products are popular with users, encouraging more data centers to join the marine data infrastructures for standardized exchange. SeaDataNet is making good progress with developing a collaborative and high-performing cloud and virtual research environment (VRE), configured with tools and services for processing essential marine data. Using OGC, ISO, and $\mathrm{W} 3 \mathrm{C}$ standards and incorporating scientific expertise, dynamic workflows are configured for analyzing, processing, and combining subsets of data. The VRE and workflows will allow data product teams to work more efficiently for processing large amounts of input datasets and generating data products collaboratively, while also adopting innovations like machine learning for QA/QC of large data collections. This way, the production cycle for data products can be reduced in duration and higher-quality products can be achieved.

Hankin et al. (2010) provided a series of predictions and recommendations for how oceanographic data management systems would evolve over the past decade. Their prediction that data management would likely improve incrementally rather than in 'heroic leaps' has held true, though some of their other predictions have proved overly optimistic. Despite considerable progress and effort toward the goals outlined by Hankin et al. (2010), oceanographic data are generally not yet managed through independent and interoperable data management systems, forming a system of systems. Semantic interoperability tools are only patchily translating terminology, codes, conceptual models, and relationships across data and metadata standards. Progress has been made on all of these fronts, but true international interoperability seems only to have been achieved for a small fraction of the kinds of data being collected in the world's oceans. Excellent progress has been made when it comes to many physical and meteorological variables needed by the operational ocean community and on validated archives of marine data via metadata standards and semantics like the British Oceanographic Data Centre's (BODC) Natural Environment Research Council (NERC) Vocabulary Server. However, the biogeochemical (BGC) and biological data communities are still striving for improvement and need increased and sustained funding to meet observing systems' needs. There are multiple reasons for the slower progress of BGC and biological communities when it comes to data interoperability. These communities are largely operating more in "research mode," with low requirement for fast and interoperable data exchange and with a large and complex set of variables being measured. The definition of EOVs and wider acceptance of best practices will likely help remedy the situation.

\section{THE CURRENT SITUATION}

A great abundance of regularly acquired environmental data exist for a wide range of disciplines derived from both in situ and remote sensing observing platforms, available in real-time, near real-time, and delayed modes. These data are acquired within routine monitoring activities and scientific surveys by a few thousand institutes and agencies around the world. A number of projects have been working of improving data management practices for sustained ocean observing, for instance the AtlantOS project (see Box 2).

Increasingly, scientists directly consider societal needs and benefits, policy dimensions, environmental health, business needs ("blue economy"), and the operational utility of their research. The societal (both from the public and private sector actors) need of ocean information is increasing as society is relying more and more on the ocean for food, energy,

BOX 2 | AltantOS.

AltantOS is an EU-funded project with the aim of enhancing and optimizing the integrated Atlantic Ocean Observing System. The targeted European data system within the AtlantOS project enhances and integrates existing data systems to ingest and deliver more in situ data. The existing data systems are diverse, and integrators are charged with integrating the data streams. These integrated system are mature systems with long-term experience and established procedures for data collection and management, often agreed at an international level; trying to implement a sovereign and rigid set of rules would be highly challenging and not in the best interest of the observing system. By relying on sustained infrastructure, AtlantOS has furthered the implementation of the FAIR principles for Atlantic observations, achieved through a system of systems where ocean observations are made available to users on a free and unrestricted basis, ensuring timely, full, and open exchange of data, metadata, and products. This includes improving interfaces with observing networks, European monitoring centers, European nations' oceanographic data centers, and the way exiting integrators exchange data and products using FAIR principles. AtlantOS recommended to integrate existing standards and protocols, rather than reinvent the wheel, by first implementing a minimum set of mandatory information for metadata. Using agreed vocabularies in the data processing and distribution chain allows for traceability of the observations. AtlantOS encourages open and free data policy and focuses on data quality by implementing a set of common near real-time QC procedures for seven EOVs acquired in near real time. AtlantOS enhances access to network data by setting up a unique entry point to discover and download existing data, either by integrating the data in existing Global Data Centers or by setting up new ones, recognizing the importance of existing integrators. The enhancement of monitoring facilities offered by JCOMM in situ Observations Programme Support Centre (JCOMMOPS) associated with the documentation of existing services through a unique catalog is an important element for the development of integrator services. They also allow efficient connection to the Global Earth Observation System of Systems (GEOSS). 
natural materials, transport, etc. This results in integrative research that includes the social sciences and humanities as part of a much-needed holistic perspective on environmental change. It also introduces the need to ensure that open data policies consider ethical dimensions of such policies. While the default should be fully open data, in some cases (e.g., personally identifiable information, health data, sensitive species information, some indigenous knowledge, etc.), specific management and dissemination methods must be employed to avoid harm (i.e., aggregation, anonymization, etc.).

Data management practices across oceanographic sciences are highly variable in terms of their sophistication and the levels of support they offer to data providers to make their data available in a timely, free, and unrestricted manner. A good practice example is in the Argo program where observations from floats are uploaded by satellite link to national Data Assembly Centres (DACs) where homogeneous automated QC processes are applied, and the data made available rapidly through two Global Data Assembly Centres (GDACs) that synchronize their data holdings many times a day, see Box 3. All data from this program are open and free, in a highly standardized format, which allows data users to aggregate it, subset it, and manipulate it with comparative ease. Figure 2 illustrates typical interfaces between observing networks and data systems, and Figure 3 illustrates the need for data management systems to cover a variety of scales.

Tools, such as Environmental Research Division's Data Access Program (ERDDAP), that allow scientists to work in their format of choice, but make the data available through interoperable formats, such as NetCDF and Web services, without an added burden on the scientist, are crucial to increasing interoperability. Additionally, tools such as ODV, Thematic Realtime Environmental Distributed Data Services (THREDDS), and ERDDAP can reduce the technical barrier that NetCDF presents. For real-time data, the need for interoperable data streaming (for instance by piping different processes) has been partially borne out of the technological context (i.e., digital sensors) and of the impracticality of data transformations after the operational time of the sensor. Although real-time data exchange for many EOVs has been operational for decades, facilitated by World Meteorological Organization (WMO) standards, it is still difficult for non-operational users to get access to GTS data (see Box 4). In the European context, the EMODnet (Miguez et al., 2019) physics is trying to bridge the gap between realtime and delayed-mode data streams by linking existing data management systems developed in both communities, see Box 5. In addition, the Joint Technical Committee for Oceanography and Marine Meteorology (JCOMM) Observations Coordination Group (OCG) has led a successful pilot project to improve both distribution and access of real-time data from the Global

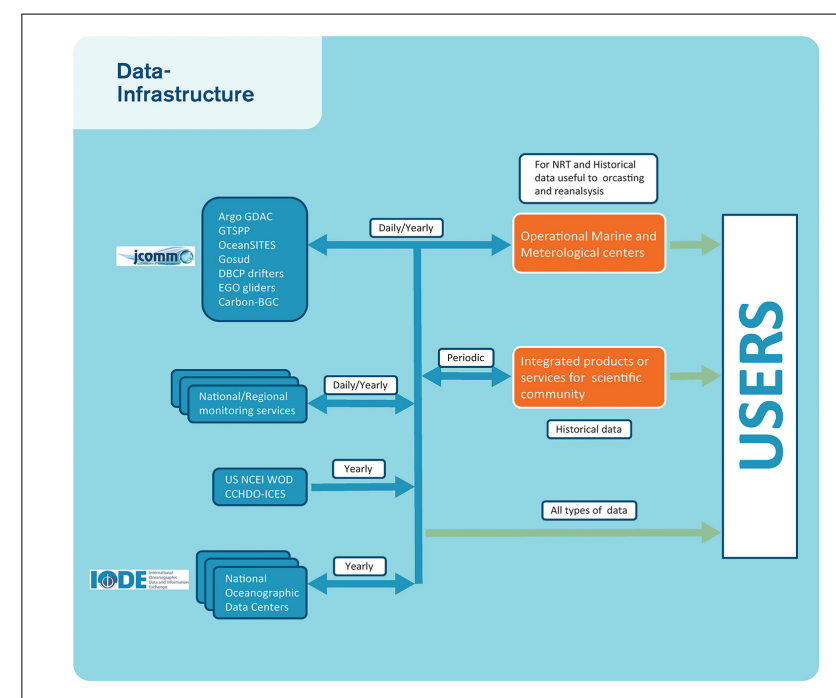

FIGURE 2 | An example of interfaces with observing networks, monitoring centers, and Nations Oceanographic data centers needed to build user products and how the integrators can collaborate together exchanging data and products using FAIR principles.

\section{BOX 3|Argo.}

Since its design in 2001, Argo data have become the dominant source of in situ data in the physical oceanographic community. The national DACs receiving data via satellite transmission decode and QC the data according to a set of RT automatic tests. Erroneous data are corrected or flagged and then sent to two GDACs and the GTS. The GDACs collect data from the 11 DACs, synchronize their databases daily, and serve the resulting data products on FTP sites

(http://www.argodatamgt.org/Access-to-data/Access-via-FTP-on-GDAC). The Argo Information Centre (AIC http://argo.jcommops.org/) monitors the status of the Argo program, including data distribution and metadata on float location, model, transmission system, owner, and other variables. The Argo Regional Centers (ARCs) perform a variety of tasks including coordinating float deployments, consistency checks on delayed-mode QC, finding additional reference data for delayed-mode work, adopting floats for delayed-mode QC, and producing Argo data products.

Argo supports FAIR principles with an open and free data policy both in real time and in delayed mode. The FAIR principles are also supported by the Argo team to enhance the interoperability of the Argo data system. Argo was the first network to apply a unique ID for each float in the program (unique WMO numbers) and has worked with the RDA to develop a strategy for managing DOls for continuously increasing datasets (DOI with a monthly tag for the GDAC monthly snapshots) (http://www.argodatamgt.org/Access-to-data/Argo-DOI-Digital-Object-Identifier). The metadata attached to a float, containing information such as serial number of the sensors and other technical information, support analysis from the Argo GDAC, a strategy that has shown its efficiency when anomalies were detected. QC of Argo data involves a complex sequence of both automatic and manual tests to produce delayed-mode data of high quality. With 400 profiles daily, the burden on human resources dedicated to $\mathrm{QC}$ is large and Argo is investigating machine learning solutions to improve the process.

While the complete data chain has been developed for the Argo core mission (P/T/S, 0-2000 dbar), the extension to Deep Argo and Biogeochemical (BGC) Argo is under development with a similar philosophy for the data system, realizing the increased need for resources to accommodate new data streams (Roemmich, 2019). Since Argo is often used in conjunction with data from other platforms, an important next step is enhancing the interoperability of Argo data with other data systems by implementing the FAIR principles. Improving the FAIRness of the Argo data system may require updates in data and metadata formats as standards and user requirements evolve. Serving high-quality data is a top priority for Argo, and comparison with other observing systems will help each system improve data quality and services. 


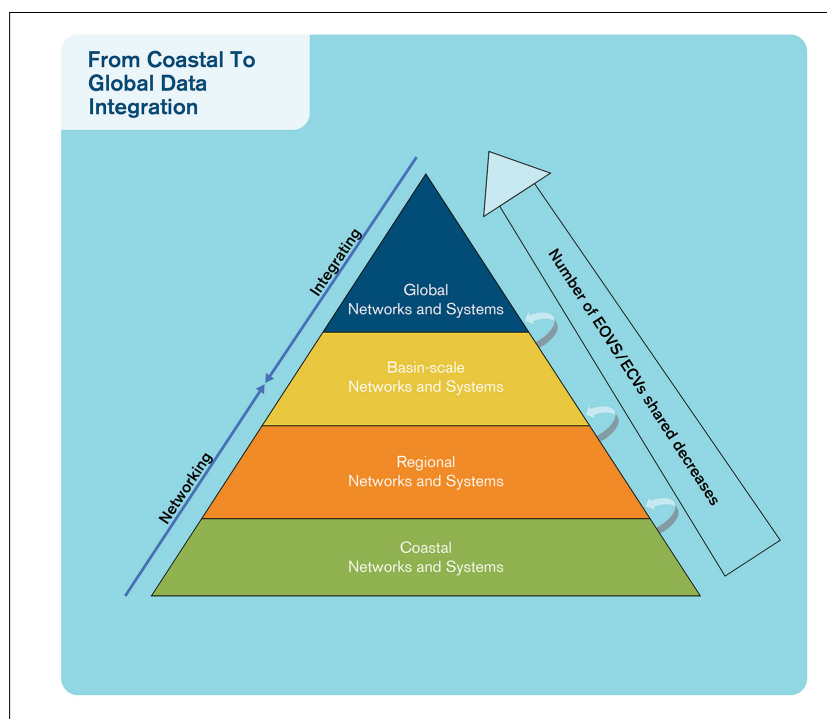

FIGURE 3 | A view of the data management systems ranging from coastal to global data integration.

Telecommunication System (GTS) Pinardi (2019). Leveraging and ensuring a sustainable and consistent implementation of such tools are also crucial in an era where funding is tight and where data management is often an afterthought. However, increasingly, management planning is obligatory, and funding and allocation of costs for data management and adopting FAIR principles are now encouraged. One example would be Argo (see text Box 3).

It is important to recognize that part of the failure to build fully interoperable data systems is that the use of a self-describing and highly effective data format such as NetCDF is beyond the technical reach of many science groups, even when they see the benefits of using the format.

\section{Data Management for Biology and Ecosystems}

In the biological and ecological disciplines, there is often a 5year lag in data publication so that the majority of effort is still allocated to data archeology rather than uploading more recent observations (Muller-Karger et al., 2018). Much of this delay is inevitable as the processing and identification of samples may take some time due to processing techniques and the consultation of taxonomic experts. Where the oceanographic community started working together in the 1980s on data archeology, standardization, and routine surveys, such cooperation has a much shorter history in many biological disciplines. In areas where this has happened, such as fisheries, the data are often not publicly available due to political and/or economic sensitivities. Development of systems that allow automated collection and/or analysis of samples is in its infancy. An exception may be in the field of aquatic telemetry, where a significant effort has been made to automate data acquisition and publication (Hussey et al., 2015; Treasure et al., 2017; Hoenner et al., 2018).

Continuous biological data acquisition is at an early stage, leading to little standardization and movement toward automated processes. A notable exception is the progress made by the Integrated Marine Observing System (IMOS) in Australia on timely distribution of non-physical data ${ }^{5}$. The vast majority of biodiversity research or monitoring happens by discrete sampling or human observation from a distance, each with their heterogeneous sampling protocols and statistical consequences. Within marine biology data management, the OBIS has contributed toward vocabulary and metadata-level

\footnotetext{
${ }^{5}$ https://portal.aodn.org.au/
}

BOX 4 | Evolution of the WMO Information System (WIS).

The GTS, implemented by the WMO, has been successful in meeting its primary objective-the cost-effective dissemination of meteorological information in near RT. However, the GTS was developed in a period when teletype communication was the norm and, as such, the capabilities of this system no longer meet the needs of the modern era. While the GTS is well managed, reliable, and effective, it is also limited in capability, expensive, complex, and with restricted access.

To address the shortcomings of the GTS and expanding the data services offered by the WMO, the WIS offers three services areas: (1) routine collection and dissemination service for time-critical and operation-critical data and products; (2) data discovery, access, and retrieval service; and (3) timely delivery service for data and products. WIS plans to expand the GTS offerings through the utilization of public networks, including the global Internet. This service area holds great promise to provide greater access to WMO data services ${ }^{5}$. The WIS does present a unique opportunity to increase the dissemination of marine observations and modeling data for use in operational forecasting. One such opportunity is for the WIS to adapt data transmission message standards to accommodate new platforms and current data conventions used in the oceanographic community. Through this evolution, the hope is that the WIS will provide not only greater access to near RT data but also streamlined procedures for data publishing without the procedural overhead now imposed by the GTS.

BOX 5 | European marine data initiatives. At the European scale, enhancing in situ data observation and product FAIRness has been identified as a priority, and collaboration between the different actors has been fostered through the EuroGOOS "DATA Management, Exchange and Quality" (DATAMEQ) working group. Within DATAMEQ, close collaboration is fostered between the observing network operators, the regional monitoring systems within EUROGOOS, and the main infrastructures dealing with in situ observations Copernicus Marine Service (CMEMS), SeaDataNet, and EMODnet. In particular, important are agreements on vocabularies, agreements on common QC automatic procedures for a selection of EOVs, improvements on interfaces between the different components to facilitate integrated product elaboration like those provided by CMEMS, EMODnet, or SeaDataNet. These three infrastructures have signed a memorandum of understanding (MoU) to strengthen and sustain their collaboration and move toward common products delivered by more than one infrastructure. The success came from a step-by-step approach, focusing first on physics and extending gradually to biogeochemistry, with biology being a new target. Another factor of success was a win-win relation established with in situ observing system operators that can use the European services for extending the use of their observations. Improving FAIRness of the European infrastructures is an important objective, and major steps should be achieved in the ENVRI-FAIR H2020 project in the next 4 years with experience that will be shared within EuroGOOS through the DATAMEQ working group within the EuroSea H2020 project. 
harmonization. For instance, the release of the Darwin Core Event Core in 2015 enables structured information on the sampling protocol and links a number of observations to a sampling event. This critical addition enables users to model population monitoring, simultaneous counting, and capturerecapture schemes-knowledge of which is essential to use such datasets for Essential Biodiversity Variable (EBV) products. For ingestion of data into OBIS, extensive semiautomated QC checks are employed, including completion of mandatory fields, correct typing and formatting of fields, and basic geographical checks, as well as taxonomical backbone mapping. The Darwin Core data standard means that there is little interest among biodiversity researchers in adopting formats and conventions from other disciplines, such as NetCDF and CF to primary occurrence data (e.g., presence/absence, abundance, and density measurements). To fully achieve interdisciplinary interoperability, current data catalog solutions will need additional interoperability layers, possibly based on those already mature in other domains, rather than building new services.

While some nations have considerable budgets for ocean observing and have significant resources to devote to data management, researchers in other nations have been left without professional data management support to aid them in publishing and curating their data (Parsons et al., 2011). Capacity development in data management and use is critical for a global reach and impact of ocean information. For instance, IOC/UNESCO's IODE are building capacities around the world via training and online learning materials ${ }^{6}$. In most countries, most of the time, data management is poorly funded compared to data acquisition, and therefore, the data are often not processed at a level suitable for true interoperability that would allow the full data life cycle to be documented. Internationally comparable numbers to assess this quantitatively could not be found, but personal experience of the authors demonstrates that all oceanographic disciplines and nations lack the sustained resources needed to fully underpin global and regional ocean observing systems.

\section{THE FAIR PRINCIPLES IN PRACTICE}

It has become clear that no single data access portal and application will ever fully satisfy the data access and requirements of all users. Rather, individual communities have very specific needs when it comes to how they access and use data, although cross-community data access is becoming more and more important. Rather than try to funnel users to an unfamiliar data portal, it is more valuable to focus on making data available through interoperable platforms. This can include direct access using protocols like the Data Access Protocol (DAP) Buck et al. (2019) or small, agile data portals that can potentially be easily and quickly built using the services provided by the data platform or be the result of thorough and consistent work over the years. A fundamental issue with data portals is their long-term maintenance, especially when they replicate the underlying data.

\footnotetext{
${ }^{6}$ For examble, Ocean Teacher, https://classroom.oceanteacher.org/
}

Data portals need to link to or regularly synchronize with the underlying data to avoid decoupled copies of datasets becoming increasingly different over time.

Professional data management is an essential element of the FAIRness of an observing system and should be designed and properly funded as part of the cost of collecting the observations. New data types, especially from autonomous observing platforms, will need to have professional data management streams developed for them. At the same time, the new focus on EOVs means that many older observing platforms will need to have new data management workflows developed and applied to the legacy data. Data management needs to be structured to work across EOVs and from local to global scales (Figure 3). As the volume and diversity of data increase, so does the need for professional data managers. The broader oceanographic community need to follow the path of physical oceanographers in terms of establishing DACs and GDACs to curate observations, along with the necessary standardized data management processes (Figure 4). This cannot be done without providing adequate and sustainable funding for the technical data management, as well as for the necessary coordination needed to define and agree on the best processes to be used.

Since one of the key tasks of data managers is to preserve data for the long term, it is imperative that new data management repositories or data assembly centers have sustainable, longterm funding, with the possible exception of targeted data rescue efforts. A common rule of thumb for scientific data management funding is that at least 5 to $10 \%$ of the funding for a science project should be committed to managing the resulting data. While it is difficult to accurately price the global cost of either oceanographic programs or data management efforts, the total cost of a single research voyage, for instance, is considerably higher than the cost of hiring a data manager for a year. For instance, a study by Shepherd (2018) indicate

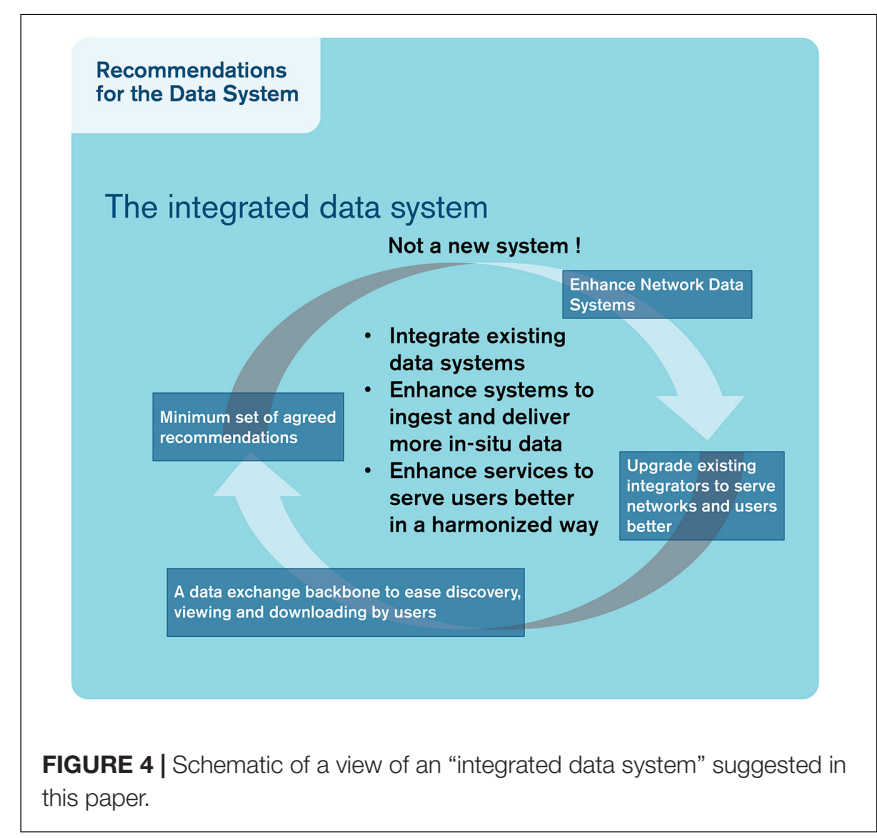




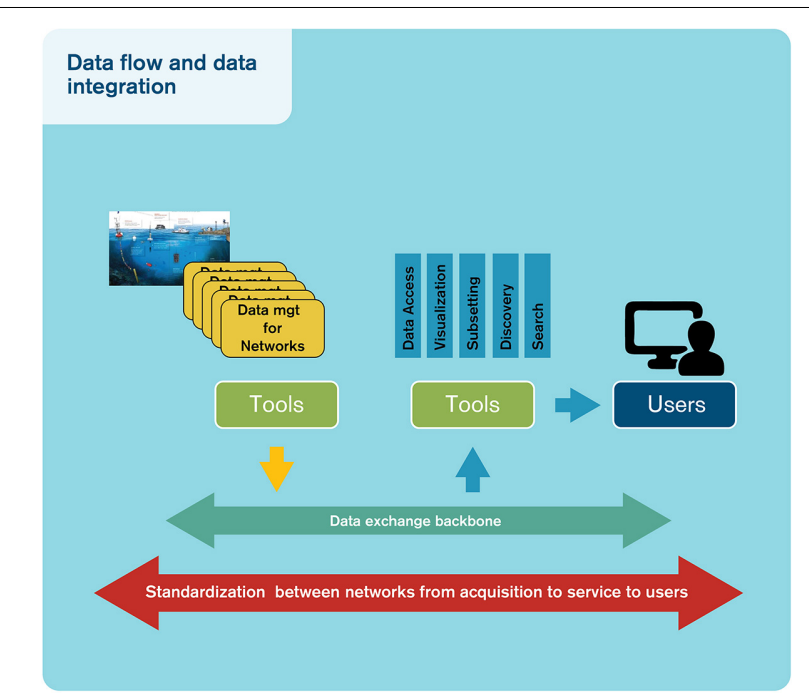

FIGURE 5 | Schematic representation of data flow from observing units through a data exchange backbone to, ultimately, users.

annual gains in the order of a billion euro within the EU of ocean/marine information being accessible. As an example of a comparatively well-funded data management activity, Australia's IMOS apportions $10 \%$ of its budget to building and maintaining the Australian Ocean Data Network (AODN), that is in addition to primary data management performed before the data reach the AODN (Lara-Lopez et al., 2016). Similarly, the TPOS 2020 2nd report recommend that $10 \%$ of observing effect should go toward data and information management ${ }^{7}$.

Figure 5 illustrates the principles of our suggestion, where data are delivered to the system from various networks or individual data providers through a data exchange backbone facilitated by appropriate tools and services to serve a wide data flow path from acquisition to user services.

\section{The FAIR Principles and the FOO}

In building any data system, a choice must be made between focusing on variables or on platforms/sampling events. It does not, in principle, matter from which platform observations of a variable originates, as long as the appropriate metadata are preserved, including estimation of the observation accuracy. This is in line with the focus on EOVs in the FOO. For RT data, it is essential to work with network operators to ensure complete data transmission and processing, appropriate QC steps, and labeling for practical/operational reasons. The integration of EOV data from different sensor, platforms, and networks require adequate characterization of accuracy and precision so a user can decide which data to use for a specific purpose. For delayedmode data, a first level of delayed-mode processing, correcting for offset or drift, must be performed at the platform level, as it requires solid technical knowledge on the measurement procedure. A second step must focus again on the data in an EOV perspective, allowing for evaluation of cross-platform coherency.

${ }^{7}$ http://tpos2020.org/project-reports/second-report/
This assures the most complete coverage by combining EOV data from multiple platforms and assures the highest possible quality. An interoperable data system should facilitate comparing the data on one EOV coming from different platforms with other products, for instance, surface ocean temperature from satellites or from Argo floats.

The FAIR principles now enjoy broad recognition through the data community, and increasingly in the ocean observing community (e.g., the GOOS 2030 Strategy $^{8}$ and the FOO). The FOO (Lindstrom et al., 2012) draw data and information systems, at the conceptual stage, in the discussion of maturity levels and in the discussion of processes of the Framework. However, it treats requirements, observations, data, and information as sequential, distinct parts of the framework and uniquely associates data and information with the outputs. In reality, those requirements will include data and information characteristics sitting alongside the EOVs. For example, an operational ocean prediction center that requires rapid access to physical data will have requirements in terms of quality, ease of access, and metadata. A climate assessment activity, on the other hand, will usually have a narrower requirement in terms of variables, but will emphasize quality, metadata, continuity, and comprehensive spatiotemporal coverage. The methods of data assembly and QC will be very important.

It is also clear that the requirements from stakeholders (providers and users) occur at both the input side and the output side according to the FOO framework. The observing systems themselves also have requirements; the effectiveness of data and information systems determines the impact of observing elements.

Several oceanographic organizations/projects are already embracing the FAIR principles alongside the consideration of EOVs and requirements, e.g., the Atlantic Blueprint vision document De Young et al. (2019); the latter conclude that "Following the FAIR principles must be a guiding principle for building and maintaining the Atlantic Ocean data management system." This should apply to GOOS and the FOO more generally so that data and information are recognized as essential alongside EOVs, with the variables replaced by a set of static attributes. Requirement setting for data and information systems should be performed alongside requirement setting for EOVs. The requirements will emanate from three levels: (a) from the data users who are specifying needed accuracy and sampling on variables, (b) from operators of observing elements, and (c) from the value chain of ocean observing where delivering fit-for-purpose data and products is paramount.

The essential elements of data and information might include requirements to follow FAIR principles. RT data submission should be as close to real time as possible but delayed enough to assure quality is fit for purpose, and the communication system should be open and accessible. Furthermore, QA/QC should be integrated with the instrument/platform and automated as far as possible. The FOO should also assess access and assembly of

\footnotetext{
${ }^{8}$ http://www.goosocean.org/index.php?option=com_oe\&task=
} viewDocumentRecord\&docID $=24590$ 
digital object identifiers and the use of long-term archives in the requirement setting.

The FAIR principles should not need to evolve with technological change, but the essential requirements for data and information almost certainly will. Just as new observing technologies, like gliders or the proposed Surface Water Ocean Topography (SWOT) satellite mission, open up new possibilities for data and products at different resolutions and quality, changing IT capabilities and emerging general standards will provide opportunities for improved solutions for data and information systems, encouraging stricter requirements.

\section{Testing FAIRness}

Some oceanographic data types already meet the FAIR principles. These are mostly those data for which international agreements already exist on the observation methods, dataset structures, and an infrastructure to coordinate data aggregation and QC processes. For most in situ oceanographic data types, however, the FAIR principles have only been partially implemented. Datasets that have been published through general purpose or national data centers are typically somewhat findable and accessible, though their findability is limited if the data center's metadata holdings are not connected to metadata aggregators or federations. Over the next decade, we anticipate general improvement in the implementation of the FAIR principles, leading to increased accessibility of data.

Many data systems are in the process of becoming FAIR; some even claim to be FAIR. However, it is important to utilize tools to measure FAIRness from a machine perspective. The GO FAIR office, active in the Netherlands, Germany, and France, has taken the initiative to develop metrics for testing FAIR readiness ${ }^{9}$. The metrics will assess the degree to which a data product meets the FAIR principles as accurately as possible. The aim is to give data holders the means to check where improvement is required (Wilkinson et al., 2018). For instance, SeaDataNet is a candidate implementation network, and the first FAIRness assessment has been made using the new metrics, providing a practical test ${ }^{10}$. SeaDataNet has made considerable progress in the last decade on metadata and data standardization on both syntax and semantics.

\footnotetext{
${ }^{9}$ https://www.go-fair.org/technology/go-fair-metrics/

${ }^{10}$ https://www.seadatanet.org/Data-Access
}

Focus has been given to the development of data access services and Web services to find and access the data.

\section{Integrated Data Services}

Integrated data services can be important tools in the FAIR process, facilitating uptake by users. DACs and Thematic Acquisitions Centers (TACs) assemble data from different providers to provide integrated products and services. While a DAC is linked to a network and the DAC process data from acquisition to data delivery, a TAC is a thematic center that aggregates data from other data centers to provide a service for a specific type of users.

The role of TACs is to collect, process, and QC upstream satellite and in situ data. The satellite TAC's main functions are to work on homogenization and intercalibration of data from multiple missions (so called L2P processing) and the development of higher-level data products. In situ TACs deal with the collection of data from a wide range of networks by the development of homogenized QC and validation procedures as well as high-quality data products. Such an approach can be used at global- and regional-level generating products in near real time as well as reprocessed data.

Such services have been developed in different continents, and collaboration exists between these initiatives through the Global Ocean Data Assimilation Experiment (GODAE) Ocean View for Operational services, RDA, and IODE. Past decades have seen the development of TACs [e.g., CMEMS, SeaDataNet, International Council for the Exploration of the Sea (ICES), or EMODnet] that developed cross-platform services targeted to a wider range of users. Other thematic centers have developed specific data products and services for specific EOVs [e.g., SOCAT for surface ocean $\mathrm{pCO}_{2}$ data, Global Ocean Data Analysis Project for Carbon (GLODAP) for interior ocean biogeochemistry (see Box 6), GEOTRACES for trace element chemistry, or OBIS for biodiversity and biogeography]. Setting up GDACs to provide an interoperable data platform for data access will allow for services on an EOV basis or a network basis. In turn, this will facilitate a rigorous and effective quality assessment service.

\section{Data and Information Products}

Data products are also useful ways of avoiding duplicate versions of the same dataset ingested in the analysis. Satellite data

BOX 6 | SOCAT and GLODAP.

The SOCAT and GLODAP are two complementary carbon-related data products. SOCAT is a QCed global ocean surface carbon dioxide (CO 2 ) dataset (Pfeil et al., 2013), and GLODAP is a QCed, internally consistent, global interior ocean data product on carbon-relevant variables.

SOCAT supports the FAIR data principles by leveraging current data standards, archiving the data and providing DOls as well as providing interoperable Web services to access the data. In addition, SOCAT has implemented a semiautomated data ingestion dashboard that allows the SOCAT data providers to submit data into the SOCAT QC system. Functionally, this means that scientists can work in their native data formats, and the ingestion system will manage the more technical tasks of creating standards-compliant files, associating the proper metadata with the files, providing submission to national data centers, and, finally, making the data available through high-level Web services. This automation of services has allowed SOCAT to evolve from a release every 4 years to annual releases. These annual releases serve to inform global products such as the Global Carbon Project's Annual Carbon Budget (http://www.globalcarbonproject.org/carbonbudget/). The automated system used by SOCAT demonstrates a method to efficiently manage the higher volumes of data expected with the future of new ocean observing efforts. The GLODAP (https://www.glodap.info/) product (Olsen et al., 2016) has evolved since its first release in 2004 (Key et al., 2004), with improved routines to retrieve data, for primary and secondary QC, and for archiving and viewing results from these processes. However, a large fraction of the work to produce the product is still manual, which places heavy demands on the community. The GLODAP community has begun to look at building SOCAT-like processes to handle future GLODAP releases in a more effective and efficient way. 
managers regularly produce higher-level data products that include quality flags and suggested editing features to fully applied QC and regular geolocated gridded data. NASA, NOAA, EUMETSAT, ESA, and other agencies use self-describing file formats [e.g., NetCDF, Hierarchical Data Format (HDF)] and CF metadata conventions. This makes indexing the data easier for the data centers' databases and makes sure that the quality flags are easily paired with their science variable for mid-level products so users can QC the data for their specific research needs. It lends itself to producing higher-level products, usually gridded, for data assimilation into models.

Web services are APIs, typically hosted by the data center. The Physical Ocean Distributed Active Archive Center (PO.DAAC) provides Web services to search on datasets and granule/file metadata and data. From there, the user can discover what files/granules fit their need, either by parameter, region, or time range. The user can then retrieve the entire dataset or subset it. This allows well-informed users to easily access data with their code as well as having smaller specialized data centers supplementing their community with satellite data without the need to archive themselves. As the data follow widely adopted conventions, specialized data readers are not required for the user to analyze the data.

Derived and model data outputs rely on knowing the quality of the data before assimilation by proper metadata. This provides traceability of the data assimilation and easier ingestion because the linkages between data and any quality flags are provided.

\section{Data Discovery for Non-EOVs}

Full data interoperability is the appropriate goal for data types that already have agreed standards and processes for collecting, documenting, and QCing the data. However, new variables will be observed and new observing methods are continuously being developed. It will always take time for new observation types to be validated and replicated. There are also historical datasets that gain new perceived value, making them "worth" aggregating in a standardized format (Griffin, 2015). It is important to provide data discovery tools, allowing researchers and data managers to find those datasets not yet entrained in sophisticated data management systems. For those datasets, it may not be possible to achieve all of the FAIR principles, but at least three of themfindability, accessibility, and reusability_can be applied to almost all ocean data.

To date, these non-standardized datasets are principally housed in nation- and discipline-specific data centers, which publish discovery metadata through in-house catalogs and through metadata aggregators. In the past, a common approach has been to ensure that a single master copy of data is preserved, but the accompanying metadata records have been translated among differing standards and republished through aggregating metadata catalogs to increase the visibility of the dataset. Combined with a lack of persistent identifiers on records, this has resulted in a legacy of partially duplicated metadata records across multiple catalogs, with limited capacity to keep those duplicates updated. These partially duplicated records provide a considerable challenge to data discovery, and we are not aware of any mature deduplication algorithms being applied in federated metadata discovery tools.

Increasingly, oceanographic metadata managers are investigating options to develop federated metadata search tools. Such tools will allow simultaneous searching of multiple metadata catalogs, thus considerably improving the findability and accessibility of non-standardized datasets while reducing opportunities for semi-duplicated metadata records to proliferate.

Federation of metadata search requires a brokering mechanism, which, in turn, requires well-understood crosswalks between the common metadata standards, vocabularies, and profiles in use by a community. This is done by having data that are in self-describing file formats (e.g., NetCDF or HDF) or well and regularly, easy-to-read file formats (e.g., ASCII). Following metadata standards is also a requirement as the brokering mechanisms can recognize the metadata and properly handle the data. The newly launched Google Dataset Search ${ }^{11}$ (currently as early release while gathering requirements for future iterations) relies on well-formatted metadata and tags following the schema.org definitions, where they see discipline-specific requirements on search and discovery as the responsibility of the discipline to implement. Smaller, more tightly focused federations will pose fewer challenges in terms of brokering these differences and will, hence, likely result in stronger search algorithms. In contrast, global federations will trade off searching power for larger holdings and greater economies of scale in terms of tool development. It is likely that the next decade will see a proliferation of metadata federations as communities balance the relative costs and benefits of small and large federations.

\section{Standards and Best Practices}

To achieve optimal use of research data and methods, we recognize the need to follow common community standards and best practices for data systems outlined by recognized international bodies such as IODE and GOOS. Implementing these practices requires that well-defined workflows are followed and that a sustained infrastructure is in place where the chain from data delivery by observing systems, initial QC/QA and feedback from the data center to the primary data producers, integration in data products, and arching in recognized data repositories are supported (Pearlman et al., 2019). Specialist support from data centers is a relatively small cost with potentially large positive gains in terms of timely data submission and quality. Careful metadata and quality flags are important attributes for ocean data, including information on the level of QC.

Best practices and standards for data management are increasingly being implemented by many observing networks and projects on national or continental scales. Successful cooperation among different operators is important for developing and promoting standards and best practices that facilitate the interoperability of systems.

\footnotetext{
${ }^{11}$ https://toolbox.google.com/datasetsearch
} 


\section{Credit Through Publication: Data Citation, Persistent Identifiers, Etc.}

Credit for developing new datasets, through attribution and citation, has several challenges as the supporting infrastructure needed for this is not fully developed. Journals increasingly require data that support an article to be in a FAIR-aligned repository that is open, uses persistent identifiers, and supports data citation (see the COPDESS Web site ${ }^{5}$ ).

We recognize that a significant fraction of the ocean data is collected "in research mode," i.e., from project-based science with limited-term funding agreements; in science, output is largely measured in citable publications rather than in published datasets (Mons et al., 2011). It is imperative that scientists are incentivized to make their data publicly available. Options include using data citation tools, such as DOIs, that make it easier to credit the originating scientist and publishing data reports as peerreviewed publications [e.g., Earth System Science Data (ESSD)]. Alternatively, a metadata record can contain fields identifying the data provider, giving recognition. To remedy this issue, the Coalition for Publishing Data in Earth and Space Sciences $(\text { COPDESS })^{12}$ is working with the scientific data facilities and scholarly publishers "to help translate the aspirations of open, available, and useful data from policy into practice."

Other efforts helping to automate data citations include a new task force by ORCID to work with repositories in capturing data creator ORCIDs and the necessary linking, and the MakeDataCount project ${ }^{13}$ defining consistent ways to count data usage and providing tools to repositories to consistently show these metrics.

Attribution and credit for data starts with the data repository and the registration of a persistent identifier associated with that data. Included in this metadata are the names and ORCIDs of the data creators. Through the registration process of the persistent identifier and services available through ORCID, a new dataset is made known in the research data infrastructure and linked with the repository and publication. For instance, publishers need to implement the full capability of the CrossRef Citation schema ${ }^{14}$ to include the portion about relationships. This identifies, in a machine-readable way, which citations are data and their association with research papers, which is particularly important for persistent identifiers that are not a DOI. Data facilities further need to capture the name of the data creator(s) along with their ORCID and provide that information when registering the persistent identifier for the dataset. This should also be provided to ORCID through the provided API that is created for repositories that have not implemented a globally unique persistent identifier. Similarly, researchers need to identify the best possible repository for their data, preferably one that is familiar with that type of data, providing curation services and is FAIR aligned.

Persistent Identifiers for Data and/or products (PID) is another important piece of metadata for traceability of processing (proper identification and versioning) on one side and traceability of use by end users and organizing feedback to

\footnotetext{
${ }^{12}$ COPDESS.org

${ }^{13} \mathrm{https} / / /$ makedatacount.org

${ }^{14} \mathrm{https} / / /$ support.crossref.org/hc/en- us/sections/202832803-Crossref-schema
}

providers. Such PIDs can be attached to the platform such as a WMO number for Argo, drifters, meteorological moorings, ICES code for vessels, etc. DOIs can also be attached to a version of a dataset, e.g., DOI on data from a research cruise, on one glider mission, on versions of aggregated products like for SOCAT, GLODAP, or the Coriolis Ocean database for ReAnalysis (CORA), or on a periodic snapshot of a GDAC. Different strategies have been developed in the past 10 years, and the PID technology has evolved to be able to manage network data that evolve continuously.

As datasets are aggregated into data products, it is not yet obvious how to give appropriate credit to all of the originating scientists who generated the data, with potentially thousands of scientists contributing to a single research article. Work is needed to develop ways to efficiently and effectively give credit to all those who have contributed. Similarly, data centers that curated datasets integrated into data products need to be efficiently credited as contributors, perhaps by ensuring that the full lineage of a metadata record is maintained through all levels of aggregation and federation, maybe by exploring the blockchain concept in marine data lineage. Certification of repositories (e.g., CoreTrustSeal ${ }^{15}$ ) plays a key role; there is strong alignment between the tenets of FAIR and the elements of certifications to help researchers and publishers make an informed decision on determining which repositories meet criteria and are FAIR aligned. Recognizing that data to support research is valuable as stand-alone products enables the community to work with institutions to enhance promotion and tenure criteria to publish data and data products. The more we cite data from repositories that support data citation, the more we can link that data back to cruises and other research objects from the same research effort and show a more complete view and demonstrate value.

Additionally, unambiguous citation of the underlying data used in the climate assessment is becoming a requirement for transparency. An example of such a policy emerged in the United States under Barack Obama's presidency with the open government initiative ${ }^{16}$ and open data policies on European Commission projects ${ }^{17}$.

\section{Implementing FAIR}

The Enabling FAIR Data project, which promotes mandatory exchanges across all journals to provide data creators the attribution and credit for the effort, building upon COPDESS through the commitment statement ${ }^{18}$ and author guidelines ${ }^{19}$, addresses this issue:

"Publication of scholarly articles in the Earth, Space, and Environmental science community is conditional upon the concurrent availability of the data underpinning the research finding, with only a few, standard, widely adopted exceptions, such as around privacy for human subjects or to protect heritage field samples. These data should, to the greatest extent possible, be

\footnotetext{
${ }^{15}$ https://www.coretrustseal.org/

${ }^{16}$ https://obamawhitehouse.archives.gov/open

${ }^{17}$ http://ec.europa.eu/research/participants/docs/h2020-funding-guide/crosscutting-issues/open-access-dissemination_en.htm

${ }^{18} \mathrm{http}$ ://www.copdess.org/enabling-fair-data-project/commitment-to-enablingfair-data-in-the-earth-space-and-environmental-sciences/

${ }^{19}$ https://zenodo.org/record/1447108\#.XUAR9EFS-Uk
} 
shared, open, and stored in community-approved FAIR-aligned repositories. Leading repositories provide additional quality checks around domain data and data services and facilitate discovery and reuse of data and other research outputs."

The recently started ENVRI-FAIR project aims to implement FAIR across the European research infrastructures, which include Argo, the European Multidisciplinary Seafloor and water column Observatory (EMSO), and the Integrated Carbon Observation System (ICOS). SeaDataNet is facilitating horizontal synergy between these research infrastructures (RIs) in the marine subdomain, but also toward RIs in the atmospheric, biological, and solid earth subdomains. The time of writing the project was at the stage of assessing FAIRness of the research infrastructures with a total project duration of 4 years.

\section{DATA SERVICES OF THE FUTURE}

Generating data that follow FAIR principles can be expensive upfront but generally pays big dividends in the long term. It is easier for the data producer to make data products according to their own needs, but that makes reuse much harder for other users. Those data products may not be formatted according to established standards, making it harder for users to read and access them. The quality of the data is not as obvious if it lacks quality flags or provenance information. This creates more work for the user as they try to understand the best way to read and use the data for their needs. When the data producer creates data that follow metadata standards and are well formatted, they facilitate access and use of the data. This implies some overhead as making the data products comply with standards is not a trivial task. It should, however, be noted that even if the workload for the data product producer is not trivial, we believe that it is a worthwhile endeavor when considering the amount of time that is saved for the users. Well-formatted data can almost act as their own service as there are many tools, software, and protocols that recognize NetCDF and HDF data that follow CF conventions, so less effort is needed by the user for finding, reading, and interpreting the data. Data that follow the FAIR principles also make it easier to attribute credit to the creators.

\section{Information and Communication Technology (ICT) and VRE}

One of the grand challenges of eScience is providing machinereadable data as the main substrate for knowledge discovery and to assure that these processes run smoothly and are sustainably.

At all levels of the value-adding chain (observation providers, products developer, and downstream services), we are facing an exponential increase in the volume of data acquired by different means of earth observing (in situ or satellites) or products made available to the community (in situ, satellite, and model outputs). Progress in the development of Internet and fast telecommunications services; big data capabilities, including machine learning and artificial intelligence; and standardized Web-services have and will continue to revolutionize the services available to users. VREs allowing users to remotely conduct processing not possible on desktop systems and to retrieve only the outputs (data/products) that are needed for their applications are being facilitated through cloud systems. These are providing important storage space and computing facilities through high-performance computers and are under development in most regions.

The addition of cloud and VRE as mainstream tools introduces opportunities and challenges. As a community, we must now consider "cloud-to-cloud" interoperability. This will build on standards and methods already in place and evolving for service-to-service interoperability. We must now consider how to ensure that data and application code (e.g., model code) developed on different platforms can be effectively integrated (e.g., Zacharewicz et al., 2017; Chang and Reinsch, 2018). Interoperability between the different elements of the Earth data management system is becoming a necessity driven by research and operational user requirements, and this will continue to evolve as cloud and VREs become more prominent and numerous. While big data and cloud tools will change the way services to users evolve in coming years, they will require sustainability in their development and in the funding scheme by member states.

\section{Web Services and Smart Sensors}

The acceleration of data production, diversity, and RT availability will increasingly demand machine-based processing of data flows. Data processing will require that machines (algorithms) be provided with properly structured metadata and data to discover and have access to services for catalogs, observations, and alerts on the Web. These Web services should be provided via uniform and compatible encodings, using community-adopted standards. Availability of open standards, supported by opensource software developers, and the advent of smart sensors, e.g., equipped with standard metadata on-board their communication interface and capable of data preprocessing, will support the FAIR principles for real-time data. Examples of such standards are the OGC sensor Web enablement (SWE) ${ }^{20}$ with the communityspecific marine SWE profile ${ }^{21}$ or the use of services such as data streaming 22 . While still on the path to maturity and with limited uptake in the marine community, these technologies should soon enable the transition to real-time machine-based processing and production of data products.

\section{SUMMARY AND RECOMMENDATIONS}

We have pointed out some challenges to support sustained and efficient ocean data management and pointed to the FAIR principles to guide future directions, and have shown some examples where the FAIR principles have started to demonstrate added value. Here, we try so summarize some of the main recommendations moving forward for the next decade, without going into technical details.

To progress toward increasing FAIRness of ocean data, there is a need to introduce the why and how of FAIR dataic to early career scientists in marine science so that adoption of

\footnotetext{
${ }^{20}$ http://www.opengeospatial.org/ogc/markets-technologies/swe

${ }^{21}$ https://odip.github.io/MarineProfilesForSWE/

${ }^{22}$ https://aws.amazon.com/streaming-data/
} 
these principles and this approach to data management becomes second nature and a natural part of doing ocean observations. We realize that future graduate students might not even go to sea but receive the data they need from autonomous platforms delivered through various integrated data services (Vance et al., 2019).

Hodson (2018) outline several recommendations to foster the FAIR principles in a recent EU report. For instance, they state that research facilities should be incentivized to follow FAIR principles by reviewing the FAIRness of data management processes. They also suggest that FAIR data practices should be included in the assessment of research contributions and career progression and that infrastructure and services that enable FAIR data must also be recognized and rewarded accordingly. A FAIR data policy is essential to enhance the accessibility of data, foster reuse of existing data, and turn observation data into information needed by end users. The FAIR principles should be embraced in assessing the technical readiness level for data and information. They should be an integral part of assessing requirements in the framework for ocean observing, and the principles should be backed up by appropriate investment in data and information management. Both pilot and mature systems should comply with the FAIR principles. Although there is great heterogeneity among oceanographic disciplines in terms of their progress toward the FAIR principles, considerable progress over the next decade can, and should, be made.

When data are not FAIR, access is limited to researchers in tight-knit networks. When data are FAIR, access is opened to people who are working outside their main discipline and outside the academy. The corollary of this is that there is an overhead in making data FAIR, which may be beyond the capacity of data creators working outside academia. It is therefore crucial that those organizations with the resources to develop data sharing tools do so in ways that are accessible to those outside their institutions.

Among the data lifetime models available, there are certain commonalities in the sense that they describe a series of steps in standardizing the handling of data from discovery to publication. These steps include (a) data acquisition, processing, and QA; (b) data description and representation; (c) data cataloging and dissemination; and d) repository services and preservation (Crowston and Qin, 2011). Modern data management infrastructures are needed to support the ocean observing system so that all these activities along the data flow pipeline are more automated and fault tolerant. Progressively, the systems should advance toward interoperability; this serves both the routine data exchanges within and between the observation networks, as well as user-friendly tools for data/products discovery viewing and access.

It is worth considering that the large majority of oceanographic ocean observations are funded by public funds; there are almost always requirements from the finding agency to make the data public, realizing that the exact requirements and the level to which they are imposed vary greatly from country to country. Submitting ocean data to a repository do not automatically mean that the data follow the FAIR principle. An important aspect is the latency of the process and the level of FAIRness of the data portal to which the data have been submitted. A FAIR data policy should be a priority for all marine datasets and should be supported by nations and stakeholders.

In chapter 2, we listed some challenges; here, we suggest actions to mitigate those.

\section{Large Diversity}

A high level of interoperability is required to harmonize these dispersed data systems to allow for easy access by users. Improving metadata services will be key at many steps in the data life cycle. For non-standardized datasets, better metadata search tools will be crucial. Leveraging the research infrastructures data systems to provide infrastructure for data of all EOVs at the same level of accessibility should be a priority. Data centers and scientific funding agencies should make international coordination an explicit part of data management staff's job description in order to achieve these outcomes.

\section{Multitude of Disparate Data Management Structures}

These existing systems need to be used as the 'building blocks' for an interoperable framework of data management systems. As an example on how this can work is the EMODnet effort in Europe, improving free, timely, and unrestricted access to interoperable European marine data, building on existing database, and open sharing infrastructures such as SeaDataNet, CMEMS, European Ocean Biogeographic Information System (EurOBIS), ICES, and EGDI (Shepherd, 2018). Similarly, open source data platform tools, such as ERDDAP, can reduce the burden of providing interoperable data services for users and improve data uptake by consumers.

\section{Increased Volume of Data}

As a consequence of increased data volume, it has become clear that it is beneficial to spread the data processing workload across many institutes and that harmonization of data processing and distribution is a priority. The data management systems must also be able to grow with the increasing volume of data expected in the future, leveraging on the IODE network of National Oceanographic Data Centres (NODCs), Associate Data Units (ADUs), online information sources, repositories, and learning objects. Based on robust FAIR data systems to deliver observation data and products, service development will drastically change in nature with the development of Big Data infrastructure and machine learning techniques that should foster reusability of existing observation and products exponentially.

\section{New Sensors Creating New Formats}

For all sensors, more complete metadata that are captured early in the data life cycle will improve the reusability of observations for many purposes. In communities that struggle to meet FAIR principles, leveraging existing tools can help those communities significantly increase their level of data interoperability with a minimum of resources. In addition, working more closely with sensor manufacturers to provide metadata directly from 
the sensors in community-accepted standards and conventions will make it much easier to properly document data further downstream in the data life cycle.

\section{Widely Used Formats Not Universally Applicable}

IT solutions need to meet the needs of particular science communities while at the same time facilitating the universal interoperability we desire. Both open source tools and services with community governance and commercial solutions are potentially possible.

\section{Gap Between Data-Producing Scientists and Downstream Users of the Data}

Modern data management architecture and infrastructures are needed so that all activities along the data pipeline are understood and efficient and progressively advanced toward interoperability. This serves the routine data exchanges within and between the observation networks, as well as user-centered tools for finding, accessing, analyzing, and (re)using data/products. Community standards for metadata, data formats, communication protocols, and data server software infrastructure are the foundation for interoperability. A key component is close monitoring and QC of data streams with communication between the observing system operators and data managers. Data centers need to give high priority to the use of modern information and communication technologies.

\section{Development of Common Protocols Takes Time}

These efforts, along with best-practice elaboration, should be organized and properly funded to develop new standards and enhance existing ones to meet the needs of the community in a reasonable timeframe.

\section{Best Practices Poorly Defined}

There is a growing need to identify the most efficient and systematic strategy for processing data all the way from the initial planning of data collection to the availability of the data products and their dissemination, i.e., development of a data management plan that evolves throughout the life cycle of the data. Data management for large, dispersed datasets benefits significantly from a well-established and standardized approach starting even before the data are collected. The FAIRness of the data system will rely on continuously updated standards and best practices for metadata and data harmonization as well as QA/QC common procedures; such activity should be properly funded at national, continent, and international level. This also includes support, training, and outreach to the teams that will develop data systems for networks or thematic services. Data can be more easily found and utilized if they are properly managed, follow best practices, are described with exhaustive and structured meta information, and are assigned persistent identifiers. These goals can be achieved by following internationally agreed standards and protocols for file formats, "content" (vocabularies/conventions), and "packaging" (metadata standards) and ensuring the data are preserved and curated in a sustained repository. Investing in the development and maintenance of freely available software utilities will pay dividends by assisting data producers in publishing data that meet community standards. Support for the implementation of standards and best practices by the research infrastructures is key to enhance interoperability and reusability of existing data and to avoid duplication of efforts.

Taking these steps will not only allow the scientist or developer to download the data and apply traditional data analysis techniques but will also enable the use of modern tools to transform, manipulate, visualize, and utilize the data in novel ways. These tools and platforms help ensure that true data interoperability is achieved, enabling interdisciplinary studies with a range of data from different domains. In addition, they promote reusability by making sure data can be understood by those who did not produce the data. These capacities are vital for international, interdisciplinary ocean observing systems.

\section{AUTHOR CONTRIBUTIONS}

TT, SP, JH, KO'B, and PB helped to conceive the study, coordinated the author contributions, wrote and edited the manuscript, and contributed to tables and figures. TdB, JB, EB, TC, KC, SD, AG, HG, VH, DK, JM, AN, BP, PP, AVdP, ER, DeS, AS, NS, DiS, TS, SS, MT, PT, ST, TV, MW, LW, and ZZ contributed to the manuscript ideas and text.

\section{FUNDING}

We thank the funding agencies and the data management projects that have made this work possible through dedicated funding for the data management activities and improvements. TT and JB acknowledge support from the EU Horizon 2020 project AtlantOS (grant agreement 633211). JM acknowledges support from the Integrated Oceanography and Multiple Uses of the Continental Shelf and the Adjacent Ocean Integrated Center of Oceanography (INCT-Mar COI, CNPq, Proc. 565062/2010-7). DS acknowledges support from the H2020 project SeaDataCloud (grant agreement 730960). SP acknowledges support from the EU Horizon 2020 project ENVRIplus (grant agreement 654182). AN acknowledges support from the EMODnet Physics (grant number EASME/EMFF/2016/1.3.1.2-Lot3/SI2.749411). HG acknowledges funding from the EU H2020 Ocean Data Interoperability Platform (ODIP) project (Grant No: 654310). JH acknowledges that funding came from the National Aeronautics and Space Agency as managed by the California Institute of Technology under task number 80NM0018F0848. AVdP acknowledges support from Belspo in the framework the EU Lifewatch ERIC (grant agreement FR/36/AN3). KO'B acknowledges that his publication is partially funded by the Joint Institute for the Study of the Atmosphere and Ocean (JISAO) under NOAA Cooperative Agreement NA15OAR4320063, Contribution No. 2018-0175. 


\section{REFERENCES}

Blower, J., Hankin, S. C., Keeley, R., Pouliquen, S., Beaujardire, J. D. L., Berghe, E. V., et al. (2010). "Ocean data dissemination: new challenges for data integration"," in Proceedings of OceanObs'09: Sustained Ocean Observations and Information for Society, eds J. Hall, D. E. Harrison, and D. Stammer (Paris: ESA Publication).

Buck, J. J. H., Bainbridge, S. J., Burger, E., Kraberg, A., Casari, M., Casey, K. S., et al. (2019). Ocean data product integration through innovation-the next level of data interoperability. Front. Mar. Sci. 6:32. doi: 10.3389/fmars.2019.00032

Chang, W. L., and Reinsch, R. (2018). DRAFT NIST Big Data Interoperability FrameworkAdoption and modernization.version 3. Gaithersburg, MD: National Institute of Standards and Technology

Crowston, K., and Qin, J. (2011). "A capability maturity model for scientific data management: evidence from the literature," in American Society for Information Science and Technology Annual Meeting, (Hoboken: Wiley).

de La Beaujardière, J., Beegle-Krause, C., Bermudez, L., Hankin, S., Hazard, L., Howlett, E., et al. (2010). "Ocean and coastal data management," in Proceedings of OceanObs'09: Sustained Ocean Observations and Information for Society, eds J. Hall, D. E. Harrison, and D. Stammer (Paris: ESA Publication).

De Young, B., Visbeck, M., De Araujo Filho, M. C., Baringer, M. O. N., Black, C. A., Buch, E., et al. (2019). An integrated all-atlantic ocean observing system in 2030. Front. Mar. Sci. 6:428. doi: 10.3389/fmars.2019. 00428

Griffin, E. R. (2015). When are old data new data? GeoResJ 6, 92-97. doi: 10.1016/ j.grj.2015.02.004

Hankin, S., Bermudez, L., Bowler, J. D., Blumenthal, B., Casey, K. S., Fornwall, M., et al. (2010). "Data management for the ocean sciences-perspectives for the next decade,", in Procedings of OceanObs'09: Sustained Ocean Observations and Information for Society, Vol. 1, eds J. Hall, D. E. Harrison, and D. Stammer (Venice: ESA Publication).

Hodson, J. (2018). FAIR Data Action Plan. Interim recommendations and actions from the European Commission Expert Group on FAIR data. Zenodo doi: 10.5281/zenodo. 1285290

Hoenner, X., Huveneers, C., Steckenreuter, A., Simpfendorfer, C., Tattersall, K., Jaine, F., et al. (2018). Australia's continental-scale acoustic tracking database and its automated quality control process. Sci. Data 5:170206. doi: 10.1038/ sdata.2017.206

Hussey, N. E., Kessel, S. T., Aarestrup, K., Cooke, S. J., Cowley, P. D., Fisk, A. T., et al. (2015). Aquatic animal telemetry: a panoramic window into the underwater world. Science 348, 1255642. doi: 10.1126/science.12 55642

Keeley, R., Woodruff, S., Pouliquen, S., Conkright-Gregg, M., and Reed, G. (2010). "The development of the data system and growth in data sharing," in Proceedings of OceanObs'09: Sustained Ocean Observations and Information for Society, eds J. Hall, D. E. Harrison, and D. Stammer (Paris: ESA Publication).

Key, R. M., Kozyr, A., Sabine, C. L., Lee, K., Wanninkhof, R., Bullister, J. L., et al. (2004). ). A global ocean carbon climatology: results from global data analysis project (GLODAP). Global Biogeochem. Cycles 18:GB4031.

Lara-Lopez, A., Moltmann, T., and Proctor, R. (2016). Australia’s Integrated marine observing system (imos): data impacts and lessons learned. Mar. Technol. Soc. J. 50, 22-33. doi: 10.4031/MTSJ.50.3.1

Lindstrom, E., Gunn, J., Fischer, A., Mccurdy, A., Glover, L., Alverson, K., et al. (2012). A Framework for Ocean observing. by the Task Team for an Integrated Framework for Sustained Ocean Observing. Paris: UNESCO.

Meredith, M. P., Schofield, O., Newman, L., Urban, E., and Sparrow, M. (2013). The vision for a southern ocean observing system. Curr. Opin. Environ. Sustain. 5, 306-313. doi: 10.1016/j.cosust.2013.03.002

Miguez, B. M., Novellino, A., Vinci, M., Claus, S., Calewaert, J., Vallius, H., et al. (2019). The european marine observation and data network (emodnet): visions and roles of the gateway to marine data in europe. Front. Mar. Sci. 6:313.

Mons, B., Van Haagen, H., Chichester, C., Hoen, P.-B. T., Den Dunnen, J. T., Van Ommen, G., et al. (2011). The value of data. Nat. Genet. 43:281. doi: 10.1038/ng0411-281

Muller-Karger, F. E., Miloslavich, P., Bax, N. J., Simmons, S., Costello, M. J., Sousa Pinto, I., et al. (2018). Advancing marine biological observations and data requirements of the complementary essential ocean variables (EOVs) and essential biodiversity variables (EBVs) frameworks. Front. Mar. Sci. 5:211. doi: $10.3389 /$ fmars.2018.00211

Olsen, A., Key, R. M., Van Heuven, S., Lauvset, S. K., Velo, A., Lin, X., et al. (2016). The Global ocean data analysis project version 2 (GLODAPv2) - an internally consistent data product for the world ocean. Earth Syst. Sci. Data 8, 297-323. doi: 10.5194/essd-8-297-2016

Parsons, M. A., de Bruin, T., Tomlinson, S., Campbell, H., Godøy, Ø, Leclert, J., et al. (2011). The state of polar data-the IPY experience. Edmonton: C. Press.

Pearlman, J., Bushnell, M., Coppola, L., Karstensen, J., Buttigieg, P. L., Pearlman, F., et al. (2019). Evolving and sustaining ocean best practices and standards for the next decade. Front. Mar. Sci. 6:277. doi: 10.3389/fmars.2019.00277

Pfeil, B., Olsen, A., Bakker, D. C. E., Hankin, S., Koyuk, H., Kozyr, A., et al. (2013). A uniform, quality controlled Surface Ocean CO2 Atlas (SOCAT). Earth Syst. Sci. Data 5, 125-143. doi: 10.5194/essd-5-1 25-2013

Pinardi, N. (2019). Marine monitoring to services: the IOC of UNESCO and WMO experience. Front. Mar. Sci. doi: 10.3389/fmars.2019.00410

Pouliquen, S., Hankin, S., Keeley, R., Blower, J., Donlon, C., Kozyr, A., et al. (2010). ")."The development of the data system and growth in data sharing"," in OceanObs'09: Sustained Ocean Observations and Information for Society, eds J. Hall, D. E. Harrison, and D. Stammer (Paris: ESA Publication).

Roemmich, D. (2019). On the future of Argo: a global, full-depth, multidisciplinary array. Front. Mar. Sci. doi: 10.3389/fmars.2019.00439

Shepherd, I. (2018). European efforts to make marine data more accessible. Ethics Sci. Environ. Polit. 18, 75-81. doi: 10.3354/esep00181

Taylor, I. J., Deelman, E., Gannon, D. B., and Shields, M. (2006). Workflows for e-Science: Scientific Workflows for Grids. Berlin: Springer-Verlag.

Treasure, A. M., Roquet, F., Ansorge, I. J., Bester, M. N., Boehme, L., Bornemann, H., et al. (2017). Marine mammals exploring the oceans pole to pole: a review of the MEOP consortium. Oceanography 30, 132-138. doi: 10.5670/oceanog.2017. 234

Vance, T. C., Wengren, M., Burger, E., Hernandez, D., Kearns, T., Medina-Lopez, E., et al. (2019). From the oceans to the cloud: opportunities and challenges for data, models, computation and workflows. Front. Mar. Sci. 6:211. doi: 10.3389/ fmars.2019.00211

Wilkinson, M. D., Dumontier, M., Aalbersberg, I. J., Appleton, G., Axton, M., Baak, A., et al. (2016). The FAIR guiding principles for scientific data management and stewardship. Sci. Data 3:160018. doi: 10.1038/sdata.2016.18

Wilkinson, M. D., Sansone, S.-A., Schultes, E., Doorn, P., Bonino da Silva Santos, L. O., and Dumontier, M. (2018). A design framework and exemplar metrics for FAIRness. Sci. Data 5:180118. doi: 10.1038/sdata.2018.118

Zacharewicz, G., Diallo, S., Ducq, Y., Agostinho, C., Jardim-Goncalves, R., Bazoun, H., et al. (2017). Model-based approaches for interoperability of next generation enterprise information systems: state of the art and future challenges. Inf. Syst. E-Bus. Manag. 15, 229-256. doi: 10.1007/s10257-0160317-8

Conflict of Interest Statement: AN was employed by the company ETT.

The remaining authors declare that the research was conducted in the absence of any commercial or financial relationships that could be construed as a potential conflict of interest.

The reviewer J-BC declares an ongoing collaboration on a project with the authors AN, AG, and DS as a contribution to the OceanObs collaboration, a decadal conference series on ocean observation. The peer review was handled under the close supervision of the Chief Editors to ensure an objective process.

Copyright () 2019 Tanhua, Pouliquen, Hausman, O’Brien, Bricher, de Bruin, Buck, Burger, Carval, Casey, Diggs, Giorgetti, Glaves, Harscoat, Kinkade, Muelbert, Novellino, Pfeil, Pulsifer, Van de Putte, Robinson, Schaap, Smirnov, Smith, Snowden, Spears, Stall, Tacoma, Thijsse, Tronstad, Vandenberghe, Wengren, Wyborn and Zhao. This is an open-access article distributed under the terms of the Creative Commons Attribution License (CC BY). The use, distribution or reproduction in other forums is permitted, provided the original author(s) and the copyright owner(s) are credited and that the original publication in this journal is cited, in accordance with accepted academic practice. No use, distribution or reproduction is permitted which does not comply with these terms. 


\section{Evolving the Physical Global Ocean Observing System for Research and Application Services Through International Coordination}

\section{OPEN ACCESS}

Edited by:

Marlon R. Lewis,

Dalhousie University, Canada

Reviewed by:

Stan Wilson,

Independent Researcher, Baltimore,

United States

A. J. Dolman,

Vrije Universiteit Amsterdam

Netherlands

Magdalena Alonso Balmaseda,

European Centre for Medium-Range Weather Forecasts, United Kingdom

Yuhei Takaya,

Meteorological Research Institute (MRI), Japan

*Correspondence:

Bernadette M. Sloyan

Bernadette.Sloyan@csiro.au

Specialty section:

This article was submitted to

Ocean Observation,

a section of the journal

Frontiers in Marine Science

Received: 12 December 2018

Accepted: 05 July 2019

Published: 06 August 2019

Citation:

Sloyan BM, Wilkin J, Hill KL,

Chidichimo MP, Cronin MF,

Johannessen JA, Karstensen J, Krug M, Lee T, Oka E, Palmer MD,

Rabe B, Speich S, von Schuckmann K, Weller RA and Yu W (2019) Evolving the Physical

Global Ocean Observing System for Research and Application Services

Through International Coordination.

Front. Mar. Sci. 6:449.

doi: 10.3389/fmars.2019.00449
Bernadette M. Sloyan ${ }^{*}$, John Wilkin², Katherine Louise Hill', Maria Paz Chidichimo4, Meghan F. Cronin ${ }^{5}$, Johnny A. Johannessen ${ }^{6}$, Johannes Karstensen ${ }^{7}$, Marjolaine Krug 8 , Tong Lee ${ }^{9}$, Eitarou Oka ${ }^{10}$, Matthew D. Palmer ${ }^{11}$, Benjamin Rabe ${ }^{12}$, Sabrina Speich ${ }^{13}$, Karina von Schuckmann ${ }^{14}$, Robert A. Weller ${ }^{15}$ and Weidong Yu ${ }^{16}$

${ }^{1}$ CSIRO Oceans and Atmosphere and Centre for Southern Hemisphere Oceans Research, Hobart, TAS, Australia, ${ }^{2}$ Rutgers, The State University of New Jersey, New Brunswick, NJ, United States, ${ }^{3}$ Global Ocean Observing System, Global Climate Observing System, World Meteorological Organization, Geneva, Switzerland, ${ }^{4}$ Consejo Nacional de Investigaciones Científicas y Técnicas (CONICET), Servicio de Hidrografía Naval and Instituto Franco-Argentino sobre Estudios de Clima y sus Impactos (UMI-IFAECI-CNRS), Buenos Aires, Argentina, ${ }^{5}$ National Oceanic and Atmospheric Administration (NOAA) Pacific Marine Environmental Laboratory, Seattle, WA, United States, ${ }^{6}$ Nansen Environmental and Remote Sensing Center and University of Bergen, Bergen, Norway, ${ }^{7}$ GEOMAR Helmholtz Centre for Ocean Research Kiel, Kiel, Germany, ${ }^{8}$ Council for Scientific and Industrial Research (CSIR), Cape Town, South Africa, ${ }^{9}$ National Aeronautics and Space Administration (NASA) Jet Propulsion Laboratory, Pasadena, CA, United States, ${ }^{10}$ Atmosphere and Ocean Research Institute, The University of Tokyo, Chiba, Japan, ${ }^{11}$ Met Office Hadley Centre, Exeter, United Kingdom, ${ }^{12}$ Alfred-Wegener-Institut Helmholtz-Zentrum für Polar- und Meeresforschung, Bremerhaven, Germany, ${ }^{13}$ École Normale Supérieure, Laboratoire de Météorologie Dynamique, Institut Pierre-Simon Laplace, Paris, France, ${ }^{14}$ Mercator Ocean International,

Ramonville-Saint-Agne, France, ${ }^{15}$ Woods Hole Oceanographic Institution, Woods Hole, MA, United States, ${ }^{16}$ National Marine Environmental Forecasting Center, Beijing, China

Climate change and variability are major societal challenges, and the ocean is an integral part of this complex and variable system. Key to the understanding of the ocean's role in the Earth's climate system is the study of ocean and sea-ice physical processes, including its interactions with the atmosphere, cryosphere, land, and biosphere. These processes include those linked to ocean circulation; the storage and redistribution of heat, carbon, salt and other water properties; and air-sea exchanges of heat, momentum, freshwater, carbon, and other gasses. Measurements of ocean physics variables are fundamental to reliable earth prediction systems for a range of applications and users. In addition, knowledge of the physical environment is fundamental to growing understanding of the ocean's biogeochemistry and biological/ecosystem variability and function. Through the progress from OceanObs'99 to OceanObs'09, the ocean observing system has evolved from a platform centric perspective to an integrated observing system. The challenge now is for the observing system to evolve to respond to an increasingly diverse end user group. The Ocean Observations Physics and Climate panel (OOPC), formed in 1995, has undertaken many activities that led to observing system-related agreements. Here, OOPC will explore the opportunities and challenges for the development of a fit-for-purpose, sustained and prioritized ocean observing system, focusing on physical variables that maximize support for fundamental research, climate monitoring, forecasting on different timescales, and society. OOPC recommendations are guided by the Framework for Ocean Observing which emphasizes identifying user requirements by considering time and space scales 
of the Essential Ocean Variables. This approach provides a framework for reviewing the adequacy of the observing system, looking for synergies in delivering an integrated observing system for a range of applications and focusing innovation in areas where existing technologies do not meet these requirements.

Keywords: observing system evaluation, observing system design, sustained observations, observing networks, observation platforms, climate, weather, operational services

\section{SOCIETAL REQUIREMENTS FOR OCEAN OBSERVATIONS}

That the ocean plays a central role in the Earth system is well known. Planetary scale transport of heat, freshwater and carbon by the ocean rivals that of the atmosphere, and the ocean's storage capacity for these quantities leads to slow modes of propagation that extend the predictability of climate conditions and have enabled the development of practical climate forecasting systems on seasonal to decadal time scales (e.g., Smith et al., 2012; DoblasReyes et al., 2013; Kirtman et al., 2013; Meehl et al., 2014). Much of the information that underpins these prediction systems comes from globally coordinated ocean basin scale observing systems.

For example, impacts of a predicted weakening of the Atlantic Meridional Overturning Circulation (AMOC) (e.g., Caesar et al., 2018) may already be evident in changing patterns of summer atmospheric circulation and heat waves in Europe (Duchez et al., 2016; Frajka-Williams et al., 2017) and in accelerating rates of sea level rise on the U.S. East Coast (e.g., Yin et al., 2009; Caesar et al., 2018). In the South Atlantic Ocean, numerical models have shown that low-frequency AMOC variations may influence decadal variability of global atmospheric circulation patterns (e.g., Lopez et al., 2016). The tropical Pacific's El Niño-Southern Oscillation (ENSO) dramatically affects global climate including regional temperature, precipitation, severe weather, marine and terrestrial ecosystems, fisheries and human activities (Timmermann et al., 2018; Smith et al., 2019). In Australia the severe droughts of 1982, 1994, 2002, and 2006

Abbreviations: AOPC, Atmopsheric Observations Panel for Climate; AMOC, Atlantic Meridional Overturning Circulation; CLIVAR, Climate and Ocean: Variability, Predictability and Change; CCCO, Committee on Climate Chagne and the Ocean; COES, Committee on Earth Observations Satellites; DOOS, Deep Ocean Observing Strategy; ENSO, El Niño-Southern Oscillation; ECVs, Essential Climate Variables; EOVs, Essential Ocean Variables; XBT, eXpendable Bathy Thermograph; FOO, Framework for Ocean Observing; GCOS, Global Climate Observing System; GODAE, Global Ocean Data Assimilation Experiment; GOOS, Global Ocean Observing System; GO-SHIP, Global Ocean Ship-based Hydrographic Program; GHRSST, Group for High Resolution Sea Surface Temperature; IOGOOS, Indian Ocean Global Ocean Observing System; IndOOS, Indian Ocean Observing System; IMBeR, Integrated Marine Biosphere Research; IOC, Intergovernmental Oceanographic Commission; ICSU, International Council for Science; IOCCP, International Ocean Carbon Coordination Project; IPCC, International Panel on Climate Change; IQuOD, International Quality Controlled Ocean Dsatabase; JCOMM, Joint WMO-IOC Technical Commission on Oceanography and Marine Meteorology; OCG, Ocean Coordination Group; OOPC, Ocean Observations Physics and Climate panel; OOSDP, Ocean Observing System Development Panel; SCOR, Scientific Committee on Oceanic Research; SOOP, Ship-of-Opportunity Programme; TOPC, Terrestrial Observation Panel for Climate; TOGA, Tropical Oceans Global Atmosphere; TAOS, Tropical Atlantic Observing System; TPOS, Tropical Pacific Observing System; WOCE, World Climate Circulation Experiment; WMO, World Meteorological Organization; WCRP, World Ocean Research Programme. were all associated with ENSO, while in South America reduced precipitation is observed in north/north-eastern South America and enhanced precipitation is found in south-eastern South America during ENSO events, with the opposite during La Niña events (Tedeschi et al., 2016).

At the coasts, rising sea level is increasing the probability that storm surge, or even the regular cycle of the tides, will produce sea level extremes that damage property and infrastructure, and present an immediate risk to human safety. This may be exacerbated by damage wrought by more frequent tropical storms of increasing duration and severity (Webster et al., 2005; Vecchi and Soden, 2007; IPCC, 2014). Coastal areas are densely populated, with about $27 \%$ of the global population living along the coastal fringes as of 2010 (Kummu et al., 2016). Many of these communities depend on the ocean for subsistence, livelihood, and general well-being through food provision, tourism, recreation, transportation services, water supply, and energy. In addition, for communities living by the sea, the ocean is also frequently a key aspect of their cultural identity (e.g., Marzeion and Levermann, 2014).

Ocean temperature extremes, commonly referred to as marine heatwaves (Hobday et al., 2018), impact marine habitats and ecosystems through thermal stress. There is a risk of increased frequency of marine heatwaves, which imperil coral reefs due to increased bleaching events. The marine heatwaves off Australia in 2016 has been linked to coral bleaching in the Great Barrier Reef (Hughes et al., 2018). In addition to natural variability and human-induced climate trends, ocean ecosystems are also vulnerable to direct anthropogenic forcing from overfishing, pollutant contamination, pathogens, coastal development, and maritime operations related to resource use and tourism.

In climate prediction (seasonal to decadal) and projection (centennial), demonstrating the veracity of coupled climate models with respect to future climate conditions relies on the ability to show meaningful skill in reproducing past climate variability by comparison to long observational time series. Seasonal and decadal prediction systems will rely principally on accurately forecasting the slow ocean modes and their role in modulating the fast atmosphere (Kirtman et al., 2013). In order to predict climate, models (coupled or ocean only) must be initialized with the ocean observations. Timely and sustained ocean observations, both satellite and in situ, will be crucial for the development of skillful climate predictions that meet societal expectations and needs (Smith et al., 2012).

The decades of oceanographic observation by research vessels, ships of opportunity, research and operational satellites, and experimental platforms and sensors, have contributed immeasurably to our knowledge of the ocean and our ability 
to respond usefully to calls for information regarding the important societal needs noted above. The ocean observing system remained relatively sparse and regionally-focused until the 1990s (Gould et al., 2013). These human pressures also need to be observed and monitored if their influence is to be placed in proper context compared to change driven by climate variability and change. In the decade since OceanObs'09, the use of autonomous in situ platforms has revolutionized the ocean observing system. Yet despite these successes, it remains that many of these data sets are brief relative to the time scales of natural variability (e.g., Jones et al., 2016) and trends or long-term cycles. Nevertheless, the data that the present observing system has delivered have been crucial in developing our understanding of the oceanic state, change and their underlying processes and their relation to observed climate variability to the extent that we can offer actionable guidance to users and stewards of the ocean environment. Sustained full-depth monitoring of ocean physical and biogeochemical parameters is required to deliver this information in order to determine our human influence upon the observed changes in the ocean.

It is anticipated that further technological advances in platforms and physical and biogeochemical sensors will continue to improve our ability to observe the ocean. Ensuring that the suite of ocean observations is adequate for the many uses to which they are put, and truly global in scope, demands a coordinated approach to using vessels, the deployment of instruments in the ocean, and satellite remote sensing in a manner that is complementary to their respective capabilities and meets agreed requirements for data quality, sampling frequency and geographic coverage. There are still many gaps and the observing system requires expansion into many regions that are poorly sampled or have simply never been visited (e.g., some shelf areas, marginal seas, sea ice covered oceans and the deep ocean below $2000 \mathrm{~m}$ ) to further improve assessments of the status of the global climate (e.g., von Schuckmann et al., 2016; Johnson et al., 2018).

Even for fundamental physical observations such as temperature and sea level, these requirements are difficult to quantify because of the diversity of potential users and observing technologies, and inevitable practical limitations on resources. As new observing technologies arise and analysis methods advance, the requirements for sustained observing should adapt and evolve if the system is to remain efficient and effective. Given the preponderance of pressing societal needs related to food supply, water quality and environmental health, coupled with emerging capabilities for observing the ocean's chemistry and key biological variables for ecosystems, it is becoming increasingly important to move beyond ocean physical variables to develop coordinated multidisciplinary observing technologies and systems to monitor and assess the status of marine ecosystems under different stressors. To date, this is only possible for a restricted number of variables and limited to regional scales (Miloslavich et al., 2018).

Given the increasing complex mix of observing platforms and sensor technologies, and the ever expanding users and their differing and sometimes divergent requirements, the Ocean Observations Physics and Climate panel (OOPC) faces new challenges. These challenges included the expanded role of OOPC to provide science-based recommendations for a fitfor-purpose, sustained and prioritized ocean observing system, that maximizes support for fundamental research, climate monitoring, forecasting on different timescales, and society. In this paper we take stock of progress to date; OOPC achievements and anticipated future challenges and opportunities to develop an integrated and consistent ocean observing for the future.

\section{OCEAN OBSERVATION COORDINATION EFFORTS}

The history of OOPC is linked to major milestones in the development of the sustained ocean observing system. The predecessor to the OOPC was the Ocean Observing System Development Panel (OOSDP), convened in 1990 under the Committee on Climate Change and the Ocean (CCCO) of the Scientific Committee on Oceanic Research-Intergovernmental Oceanographic Commission (SCOR-IOC) and the International Council for Science-World Meteorological Organization (ICSUWMO) Joint Scientific Council (JSC). Building upon the successful internationally coordinated World Climate Research Programme (WCRP)'s World Ocean Circulation Experiment (WOCE), and also motivated by the Tropical Oceans Global Atmosphere (TOGA) Experiment, OOSDP was established and charged with the design of an ocean observing system for climate.

While WOCE and TOGA advanced understanding of the ocean and ocean-atmosphere dynamics and the accuracies needed in the observing system, they also promoted mechanisms for the development of internationally coordinated globalscale ocean observations including standardized quality control procedures and open and timely data sharing protocols (Siedler et al., 2001). Building on this legacy, OOSDP met for 5 years and produced a series of in-depth background reports addressing the challenges and capabilities of different elements of the ocean climate system and of the role of models in a global ocean observing effort. The work of the OOSDP culminated in a realistic but aspirational plan for ocean observations, which was assessed on the basis of feasibility versus impact, a central theme of the Framework for Ocean Observing today. The OOSDP, as the name indicates, provided the required systems development plans and gave the foundation and terms of reference of a continuing ocean observations panel.

The OOPC met for the first time in 1996, as a panel of the Global Climate Observing System (GCOS), the Global Ocean Observing System (GOOS) and WCRP. OOPC took on the mantle from OOSDP to coordinate the respective capabilities of various observing approaches (National Research Council, 1997). Given the history of its development, the focus of OOPC within GOOS was the open ocean; other panels and groups were given charge of the enclosed and shelf seas and near-shore coastal seas. The initial tasks undertaken by OOPC were a number of reviews including: the global sea level observing system given the capability of satellite altimetry, and a review of the Ship-of-Opportunity Programme (SOOP) eXpendable BathyThermograph (XBT) program given plans 
for the International Argo program. These reviews were thus prompted by the advent of new technologies and observing capability. The panel was also involved in forming the Global Ocean Data Assimilation Experiment (GODAE; succeeded by GODAE OceanView), which placed new demands on the ocean observing system.

The panel's early work culminated in the OceanObs'99 conference, which cemented the foundations of what we know now as the sustained ocean observing system for climate. From OceanObs'99 there emerged a consensus within the ocean observing communities to undertake an internationally coordinated sustained global ocean observing effort for ocean physical and carbon variables with respect to climate applications, the details of which were subsequently agreed and presented in the first GCOS Implementation Plan.

During the 2000s OOPC, with other partners, supported the establishment of a number of sustained observing networks building on the OceanObs' 99 recommendations. These included the establishment of the OceanSITES moored time-series initiative in 1999, and development of the International Argo array of profiling floats in 2000. OOPC's involvement was critical to brokering data agreements and incorporating these new networks into existing programs such as SOOP and the global XBT network, and connecting to the expanding satellite missions (e.g., Sea Surface Temperature and Ocean Altimetry). In addition, OOPC co-sponsored a workshop on the Indian Ocean held in Perth, Australia, that led ultimately to the creation of a regional alliance to support GOOS in the Indian Ocean (IOGOOS). In 2001, OOPC conducted a review of the Tropical Moored Buoy Array. During this period OOPC established close and strong collaborations with WCRP including their Climate and Ocean: Variability, Predictability and Change (CLIVAR) project and other panels, ensuring both the development of a sustained ocean observing system and providing input to regional and basin process-studies. Building on the OOPC input to the establishment of GODAE, in 2002 OOPC's indirect sponsorship was vital to bringing climatechange consideration into the plan for the development of what is now the Group for High Resolution Sea Surface Temperature (GHRSST) project.

The consolidation of the OOPC activities and oversight of the ocean observing networks and sensor development culminated in the OceanObs'09 [OOPC co-led with CLIVAR and the Integrated Marine Biosphere Research (IMBeR)] conference. OceanObs' 09 recognized the utility of ocean observations beyond climate and the need to expand beyond physical variables to include biogeochemical and ecosystem variables within the ocean observing system. To this end there was strong engagement with the various ocean communities involved in ocean observations and end users. Leading up to OceanObs'09, OOPC in collaboration with the International Ocean Carbon Coordination Project (IOCCP) and CLIVAR developed a strategy for a sustained global repeat hydrography program as a contribution to the OceanObs09 Conference (Hood et al., 2010). Arising from this strategy, in 2009 the Global Ocean Shipbased Hydrographic Program (GO-SHIP) was established as a component of GOOS. A further outcome of the conference was the development of the Framework for Ocean Observing (FOO) (Lindstrom et al., 2012; Tanhua et al., 2019). The FOO provides guidelines for evolving the observing system in the service of a broad range of applications and users. To support expansion of the ocean observing system, GOOS expanded to include three disciplinary panels; OOPC became the physics and climate panel, IOCCP provides oversight of ocean biogeochemistry, and a new biology and ecosystems Panel (BioEco) was formed. OOPC retains, importantly, the dual roles as the ocean panel of GCOS and physics panel of GOOS. Delivery to GCOS requires OOPC to work across all components of GOOS.

OOPC has the demanding role of coordinating ocean input to GCOS and interacting with its sibling Terrestrial (TOPC) and Atmosphere (AOPC) GCOS panels, while also working with the other panels of GOOS. OOPC is the steward of the ocean Essential Climate Variables (ECV) of GCOS and the physical Essential Ocean Variables (EOVs) of GOOS, and hence must consider the requirements for observations across multiple time and space scales to meet users requirements from marine weather to climate, including both real-time and delayed mode data, while being mindful of complementing biogeochemistry and ecosystem observing at all scales. Since the OceanObs'09 Conference, GOOS has developed and articulated the need for ocean observation around application areas which cut across the disciplinary panels: climate, operational services, and ocean health. OOPC leads on delivery to Climate and Operational Services, and supports Ocean Health through articulating requirements for underpinning physical information. OOPC is also a panel of the WCRP, and provides scientific guidance and evaluation to observing networks through the Joint WMO IOC Technical Commission on Oceanography and Marine Meteorology (JCOMM) Observation Coordination Group.

These many responsibilities to GCOS, GOOS, WCRP, and JCOMM, spanning a broad spectrum of scales and including multiple disciplines, are demanding roles. Fundamental to delivering on these responsibilities, OOPC's, in conjunction with other programs, is undertaking regular assessments of elements of the observing system in order to provide objective guidance on the continued evolution of the system that will meet current and future user requirements.

We argue that the role of OOPC in the assessment and evolution of the observing system is becoming increasingly important given the expansion of ocean observing technologies, increased user dependency for sustained and timely ocean observations and expanding user groups. Given this, questions OOPC needs to consider are:

- How do we evolve the observing system to meet a broader range of applications, ranging from extreme events (e.g., cyclones, storms, marine heat waves, and coastal inundation forecasting) to climate monitoring and supporting ecosystem services?

- How do we maintain the interest and momentum for sustaining observations, when much of the funding is on short term cycles? 
- What actions do we need to take to keep exercising the system through reviews; engaging users, innovation, broadening participation?

- How do we continually evaluate and innovate the observing system to ensure it performs as an integrated system?

\section{DEFINING REQUIREMENTS FOR SUSTAINED OBSERVATIONS - THE ESSENTIAL OCEAN VARIABLES}

The challenge of measuring the ocean to meet the range of application needs discussed in section Societal Requirements for Ocean Observations may seem insurmountable. The Essential Ocean Variables are a tool to describe and communicate the "essential" requirements for sustained ocean observations and enable for evolution of the observing system with time. Essential Ocean Variables were inspired by the success of the Essential Climate Variables concept for articulating the critical variables that must be observed to meet user requirements (Bojinski et al., 2014).

\section{The Framework}

The Framework for Ocean Observing (Lindstrom et al., 2012) identifies lessons learned from the successes of previous and existing ocean observing efforts and provides an internationallyaccepted common language and guidance for expanded collaboration in sustained ocean observations. It is focused on:

- Delivering a system based on common requirements, coordinated ocean observing elements, and common data and information streams;

- Essential Ocean Variables (EOVs), a common focus for requirements defined based on feasibility and impact on societal and scientific drivers; and evaluation of readiness levels of sensors, platforms and data management procedures.

EOVs are identified by the GOOS Expert Panels, based on the following criteria:

Relevance: The variable is effective in addressing the overall GOOS Themes - Climate, Operational Ocean Services, and Ocean Health.

Feasibility: Observing or deriving the variable on a global scale is technically feasible using proven, scientifically understood methods.

Cost effectiveness: Generating and archiving data of the variable is affordable, mainly relying on coordinated observing systems using proven technology, taking advantage where possible of historical datasets.

When EOVs are identified, a series of recommendations are created and disseminated by the Expert Panels, including what measurements are to be made, various observing options, and data management practices. The adoption of EOVs is not intended to replace existing ocean observing networks and international coordination groups, but to provide a mechanism to bring them together to develop a holistic ocean observing system and strengthen the ocean observing communities ability to grow and best meet current and future user requirements.

\section{Key Ocean Phenomenon}

To help with the identification of scales (spatial and temporal, global, regional) and parameter spaces for a certain observing objective, OOPC has determined key or overarching ocean physical phenomena that the observing system should be able to resolve. In identifying these phenomena OOPC, considered the GOOS definition of a phenomenon as an observed process, event, or property, with characteristic spatial and timescales, measured or derived from one or a combination of EOVs, and needed to answer at least one of the scientific questions asked in order to address relevant societal need.

The list of phenomena, needed to be captured by EOVs, defined by OOPC (OOPC-19 Report) are:

- Heat storage;

- Salt storage;

- Water mass (i.e., Water properties and characteristics);

- Ocean Circulation;

- Sea level;

- Fronts and eddies;

- Stratification;

- Mixed layer;

- Sea ice;

- Air-sea fluxes;

- Upwelling;

- Coastal processes (encompassing a wide range of dynamics including, but not limited to, shelf-open ocean exchange of momentum, heat and salt);

- Tides;

- Riverine input;

- Surface waves;

- Near inertial oscillations;

- Extreme events (i.e., marine heatwaves, tsunamis, cyclones, storms).

Measurements of these key phenomena occupy a large spatial and temporal domain to meet the requirement of users (Figure 1). The phenomena span a spatial scale of 100's of meters to 1000's of kilometers and timescales of hours to centuries. These phenomena have different spatial and temporal measurement requirements from large basin-scale at annual resolution to coastal and weekly resolution. A diverse range of observing platforms are required to deliver this information including satellites, research and volunteer vessels, moorings, and surface and subsurface floats, and gliders. In addition, it is anticipated that as technologies (platform and sensors) advance, improved ocean observing techniques will be employed and these coupled with advances in telecommunications, will fill current gaps in the observing system and lead to improved impact of the observing system relative to investment (Sloyan et al., 2017). 


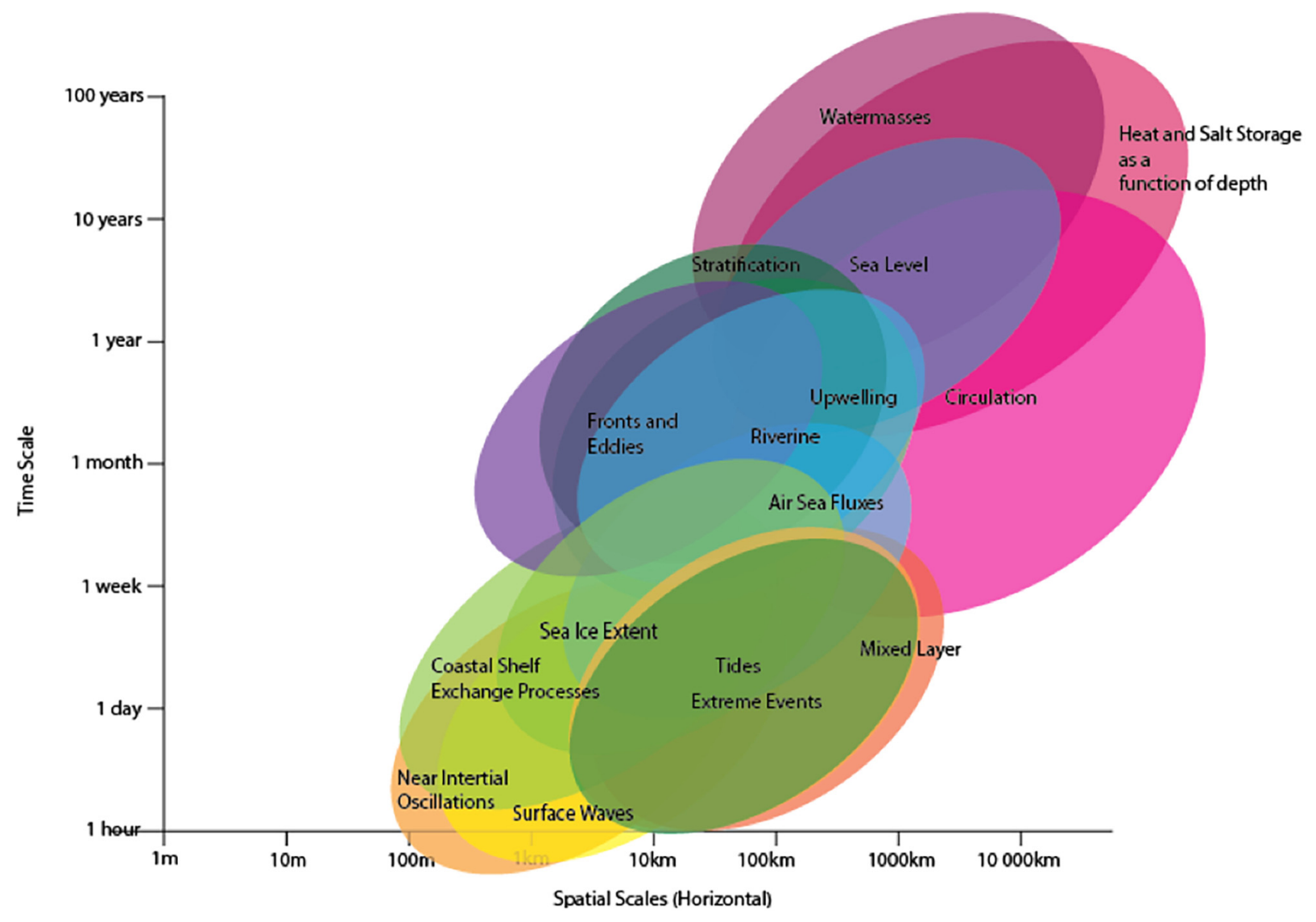

FIGURE 1 | Stommel diagram of key ocean physics phenomena that need to be derived or observed form ocean observations

\section{Essential Ocean Variables (EOVs)}

The set of key physical phenomena and processes are determined/estimated from observable ocean variables. OOPC, in conjunction with the GOOS Biogeochemistry and Biology and Ecosystem panels, motivated by the GCOS work on Essential Climate Variables (ECVs) and the strategic plan for integrated ocean observing "Framework for Ocean Observing" (FOO), have developed lists of Essential Ocean Variables (Table 1). The EOVs are judged to be the priority variables required to determine these key physical phenomena and meet most of the needs of the diverse user community. The EOVs also reflect the technical readiness to collect the observation. The selection of EOVs is based on key variables that are found to be required to describe the ocean state. They are also relevant in the context of UN Sustainable Development Goals, notably 13. Climate Action, 14. Life Below Water and 15. Life on Land. In general there are a number of observational platforms or methods used to measure an EOV. These platforms or methods may vary in their readiness level from concept to mature.

GOOS provides detailed information for each EOV including the spatial and temporal sampling requirements, identification of the key ocean processes and phenomena that require measurements of the variable, and characteristics of the observing platforms or programs that measure the variable (see www.goosocean.org for more information). The detailed EOV specification sheets demonstrate the interdependencies amongst EOVs and unique characteristics of observing platforms and programs to deliver the observations of the key ocean phenomena or process required by the diverse users of the ocean observing system. The selection of EOVs is based on their need to support improved scientific understanding and also societal needs; at the same time the mature status reflects technical readiness and feasibility of the observing methods. The importance of connecting the major drivers and themes with the societal benefits informed by scientific issues and applications, is moreover ensuring consistent identification of the EOVs and their corresponding observing element. In turn, strengths and deficiencies, synergies and interdependencies can be objectively tracked and evaluated.

The OOPC panel (and likewise the other GOOS panels) shall moderate such communication by informing the observing community. In that context, the panels have created EOVs specification documents that provide guidance on input variables, their definitions, observing platforms that sample the variables, information on accuracy, and examples for utilization of the data in the ocean observing value chain.

New scientific challenges may arise, as may societal needs. Observing technology will also evolve and improve. Thus, the 
EOVs specification sheets and EOVs will change with time to reflect need and readiness. At the same time, inclusion of the metric of readiness in the selection of mature EOVs, makes it clear that development of scientific understanding requires more progress in observation technology. For example, challenges of quantifying the large-scale heat budget of the Earth have been highlighted through the international WCRP/CLIVAR research focus "CONCEP-THEAT" (von Schuckmann et al., 2015). Quantifying the heat budget requires, for example, observation of the exchange of heat between the atmosphere and ocean at the sea surface. Ocean surface heat flux is also critically important for understanding and predicting climate variations on time scales of days to decades. The need for this observation was brought to the attention of the OOPC, and recently added ocean surface heat flux as an EOV. Ocean surface heat flux is one of the most complex EOV as it comprises both radiative and turbulent components. While ocean surface heat flux is considered an ocean ECV/EOV, its radiative components are considered atmospheric ECVs, with networks that extend over both land and ocean. Likewise, for many applications, the turbulent latent and sensible heat fluxes are estimated using a bulk algorithm applied to state variables, some or which are atmospheric ECVs, while others are oceanic ECVs. Ocean surface heat flux thus requires close coordination between all GCOS panels; Ocean, Atmosphere and Terrestrial. OOPC has thus initiated an ocean surface heat flux expert panel, with members from other GCOS panels, that will work to assess and improve capability and readiness. As one of the first activities of this group, Cronin et al. (2019) developed a strategy document for making breakthrough improvements to the gridded ocean surface heat fluxes. The ambitious strategy involves optimizing satellite-based observations for boundary layer measurement of humidity and air temperature, sea surface temperature and wind stress; and expansion of the in situ flux network. OOPC will continue to be active in evaluating this strategy and its roadmap.

CONCEPT-HEAT was also involved in highlighting the importance of observing the deep ocean. As they developed the Deep Ocean Observing Strategy under GOOS, they felt that additional observables should be considered as EOVs. To follow this up, three physical quantities were put forward to the OOPC by Deep Ocean Observing Strategy (DOOS) for consideration: notably bottom pressure, ocean mixing, and flux through the seafloor. OOPC assessed the requirements for each as well as the technical readiness and feasibility for observing on a global basis. As a result DOOS was encouraged to complete the documentation to advance bottom pressure as a potential EOV; ocean mixing and seafloor fluxes were judged to not yet be ready for identification as EOVs.

The expert panels for GOOS biogeochemistry (IOCCP) and biology/ecosystem (GOOS BioEco) follow a similar strategy to OOPC in providing structural elements as EOV and phenomena and estimating the relevant time/space and parameter space that is linked to a specific application. Ocean physics and its interaction with the marine ecosystem also plays a major role in understanding and predicting marine biology and biogeochemistry and in probably almost all of the ocean observing design for marine biology and biogeochemistry the physical processes (transport, thermodynamics) have to be considered as well. In other words, an apparently monodisciplinary experiment in marine biology or biogeochemistry is typical a multidisciplinary one involving ocean physics (and the associated sampling). Hence as we move toward an increasingly multidisciplinary observing system new challenges in a fully integrated system will need to be identified and eventually solved.

The EOV (and ECV) process very much focuses on observational quantities, meaning the sensor data and its combination with other variables. However, for the observing system and in particular for providing guidance on the individual observing networks it is mandatory to introduce a mechanism to trace each data point from the sensor mounted on an observing platform to a certain product that has been generated by the downstream services (e.g., a sea surface temperature map). Such an start-to-end evaluation is required if the complete value of the observations or observing system is required. This "value" assessment is further complicated by the interdependencies that exist in the observing system (e.g., GO-SHIP provides the highest quality observations of a suite of EOVs used by autonomous platforms for calibration). Thus assessment and attribution of value of the observing system is not a simple task.

As a common language for multi-platform global observing for the diverse user community, EOVs are fundamental to the implementation of FOO. In response to the advances in research and observing technology, OOPC will continually update specification sheets for each EOV and consider the addition of new EOVs. Moreover, increasing participation of private sectors in the ocean observing systems suggests the need to set target uncertainties for each EOV (Weller et al., 2019). The challenge that OOPC faces in keeping the EOV specifications relevant is formidable. EOV sampling requirements must acknowledge applications that span real-time weather and climate and maritime operations, fundamental ocean dynamical understanding to global climate, as well as requirements in support of ocean health applications. These are informed through strong liaison between GOOS, GCOS and JCOMM, and by the scientific and technical expertise and experience of the membership of the GOOS panels and steering committee.

\section{DATA INTEGRATION, DERIVED PRODUCTIONS AND INFORMATION DELIVERY}

Effective data management demands group collaboration across activities that include observation collection, metadata reporting and data assembly using community accepted standards, quality assurance and control (QA/QC), data publication that enables interoperable discovery and free, open access (both interactively and via machine to machine through standard protocols), and archiving that guarantees long-term preservation. Some sustained ocean observing networks are quite successful in accomplishing these data management functions, while others that are less centrally coordinated or are supported largely by research projects with short-term funding are challenged to 
TABLE 1 | GOOS essential ocean variables.

\begin{tabular}{|c|c|c|}
\hline Physics & Biogeochemistry & Biology and ecosystems \\
\hline Sea state & Oxygen & Phytoplankton biomass and diversity \\
\hline Ocean surface stress & Nutrients & Zooplankton biomass and diversity \\
\hline Sea ice & Inorganic carbon & Fish abundance and distribution \\
\hline Sea surface height & Transient tracers & Marine turtles, birds, mammals abundance and distribution \\
\hline Sea surface temperature & $\begin{array}{l}\text { Suspended } \\
\text { particles }\end{array}$ & Live coral \\
\hline Subsurface temperature & Nitrous oxide & Seagrass cover \\
\hline Surface currents & $\begin{array}{l}\text { Stable carbon } \\
\text { isotopes }\end{array}$ & Macroalgal canopy \\
\hline Subsurface currents & $\begin{array}{l}\text { Dissolved organic } \\
\text { carbon }\end{array}$ & Mangrove cover \\
\hline Sea surface salinity & Ocean color & Microbe biomass and diversity (*emerging) \\
\hline Subsurface salinity & & Benthic Invertebrate Biomass and Diversity. (*emerging). \\
\hline Ocean surface heat flux & & \\
\hline
\end{tabular}

OOPC is responsible for physics variables.

operate consistently. They may employ varying data policies and submission requirements, and may lack the resources to engage experienced staff to review and QA/QC data flowing from disparate providers or to offer cyberinfrastructure services that achieve the level of service to match more mature networks. Recent publication discuss many aspects of data management (e.g., Moltmann et al., 2019; Pinardi et al., 2019; Snowden et al., 2019); here we address the need for EOVs data collections.

Where EOV data are handled by a multitude of disparate data management infrastructures this imposes problems for the global observing system. These include, but are not limited to, delayed and duplicate data receipts, versioning issues, missing data and metadata, and undocumented data processing procedures, and data recovery. If data quality and supporting metadata are to carry over from observers to products then modern data management infrastructures are essential at every step along the data flow pipeline, from data recovery, collection through assembly and to preservation. The more automated and fault tolerant these steps are, the more they embrace community standard practices and the more efficiently and swiftly will systems advance toward the levels of reliability and interoperability that user communities seek. The Committee on Earth Observations Satellites (COES) and the International Quality controlled Ocean Database (IQuOD) are examples of groups that are focused on providing the community with high quality data that has a consistent and unified quality control standard applied. OOPC needs to support and promote these activities and products, respectively.

Interoperability serves both routine data flow within and amongst the networks, and user discovery and access. Community standards for metadata, data formats, communication protocols, and data server software infrastructure are the foundation for interoperability. These are not new considerations for the ocean data management community. The technical aspects have been demonstrated and successfully deployed in many regions and specific parts of the global networks. Expanding on these successes is important, and is being guided by various ocean observing programs led by individual national efforts, regional and international groupings, as well as by coordinating organizations independently and jointly in the WMO and IOC. The time is right to improve interoperability across the observing system networks and enable sustainable process that can create integrated datasets for the EOVs.

A data collection for EOVs must ensure the following:

- Data are quality assured and controlled according to agreed international standards;

- Feedback is given to the data sources as needed;

- Duplicates are identified and resolved;

- Metadata are complete and meet agreed best practices and existing standards;

- Data and metadata are discoverable and accessible through interoperable services;

- Data requests and searches from users can be reproduced;

- Data and metadata are electronically published with protocols for citation and acknowledgment;

- The complete provenance and lifecycle of data processing is traceable for each ECV/EOV dataset.

In addition to cataloging and preserving data and enabling effective delivery to users, these practices help ensure that observing programs/platforms and organizations get credit for data they make available and that users and reviewers can repeat precise requests for data referenced in scientific publications for the purposes of reproducing, verifying or expanding upon and enhancing prior work.

By providing interoperable access, and adhering to standards and conventions, this framework makes data synthesis efforts more efficient than with the less-integrated data management systems that exist in many quarters. It is such data synthesis efforts, founded on quality assured observational products, that deliver integrated assessments of regional and global ocean climate change and variability. 
We note that the periodical re-calculation of integrated gridded climatologies or model-data ocean and coupled atmosphere-ocean reanalysis products, such as is performed to inform State of the Ocean reports (e.g., von Schuckmann et al., 2018) and the IPCC process, are greatly facilitated by the existence of a stable, robust and interoperable data management and delivery system. Every effort should be made to sustain and enhance this data stream following the guiding principles, and for the reasons, noted above.

\section{PERIODIC REVIEWS OF THE OBSERVING SYSTEM}

From OOPC's perspective, a virtue of the EOV concept is that it helps guide global coordination of the ocean observing system through articulating requirements in a platform/sensor agnostic way. The EOV specification sheets emphasize requirements for accuracy, sampling resolution and frequency, and the timeliness of data delivery, without immediately calling out how these requirements might be met in terms of existing sensor types or scope of deployments. The actual deployments will always be the domain of the various expert observing networks (both in situ and satellite) and the national and international agencies that fund them. OOPC should advocate for national investment in the observing platforms.

Increasing use of the ocean observing system has driven OOPC to lead, support or provide substantive input to a number of systems-based-reviews of the observing system, some of which are developing into finite-lifetime development projects:

- 2011: The Deep Ocean Observing Strategy (DOOS) (Levin et al., 2019);

- 2014: The Tropical Pacific Observing System (TPOS) 2020 project (Smith et al., 2019);

- 2019: Ocean heat and freshwater review (Palmer et al., 2019);

- 2019: Air-Sea fluxes with focus on heat and momentum (Cronin et al., 2019);

- 2019: Boundary Currents (Todd et al., 2019);

- 2019: AtlantOS and the Atlantic blueprint (deYoung et al., 2019).

OOPC has also advised on two reviews led by the CLIVAR expert panels:

- 2019: Indian Ocean Observing System Review;

- 2019: Tropical Atlantic Observing System Review.

These system- or regional-based reviews are driven from the need to observe various targeted phenomenon, science questions and expanded user requirements. In this context an ongoing challenge for the stakeholders of the ocean observing system is designing, implementing and sustaining critical observations required by the diverse users groups. In addition, it is a significant challenge for OOPC to draw on these regional and thematic review and development activities to facilitate advancing the global observing effort in a consistent way.
To this end it would be valuable to turn to the community of global and regional modeling systems that formally meld data from the full suite of observing platforms with models using advanced data assimilation to derive ocean state estimates in support of reanalysis applications and weather-scale and subseasonal to seasonal and decadal prediction. For these groups, observations are vital to constraining the ocean state, and are the fundamental metric against which forecast skill is evaluated. Many such groups are also advanced service providers, and are important links in the value chain that translates observations into information products and OOPC needs to liaise with major institutions providing operational climate and environmental services. Where the observing network falls short in informing the analyses that underpin derived products generated by the service providers, it is important that this is communicated back to OOPC and GOOS to establish priorities for observing system enhancement.

The ocean modeling and analysis research communities and many operational centers have formulated and prototyped quantitative tools for rigorous assessment of the impact that individual observations or networks have on analysis and forecast skill. Though their use is not routine, such tools have the potential to systematically identify observational gaps and to evaluate new or revised deployment strategies or platforms to more effectively meet user requirements. More widespread analyses of observation gaps and EOV requirements by modeling groups would be a welcome addition to the OOPC-sponsored or coordinated network reviews. Modeling groups that emphasize product delivery to stakeholder communities are also well placed to advise on changing EOV requirements to inform new data uses, including beyond physics variables to multidisciplinary EOV.

Additionally, assimilation of similar data from multiple platforms, each with its estimate of accuracy, can help identify biases or inconsistencies in the data. Where models and data frequently disagree also identifies needs for improved model dynamics and parameterizations. By delivering formal expected errors of the merged analyses, these systems highlight where the observing network is sparse and in need of expansion. When executed with hypothetical data sets, these systems can offer rigorous assessments of observing system design and significantly inform and prioritize the need for new observations.

There remain many unanswered questions on the fundamental nature and drivers of the oceans on climate processes and feedbacks amongst the components of the Earth's climate system; atmosphere, terrestrial, cryosphere, oceans, and biosphere. Improved dynamical understanding of the ocean, land, ice and atmosphere, and the ocean-atmosphere-ice-land coupling and teleconnections will lead to more reliable and skillful multi-year to decadal climate forecasts. Key to improved knowledge are intensive process studies that integrate both observation and models and target specific key knowledge gaps. Leveraging existing components of the sustained observing system with short-period intensive process studies may prove an effective mechanism to achieve significant advancement of ocean and earth sciences. 
As we look to the next decade, OOPC must ensure that ocean observations are sustained and evolve to best meet the needs of our sponsoring programs GCOS-GOOS-WCRP and other user requirements. The assessment and recommendation of the evolution of the observing system should balance user requirements such as, providing improved knowledge of underpinning ocean dynamics, ocean state monitoring for assessment, and adaptation and mitigation, marine services operational weather and marine forecasting that encompass regional, basin and global scales and seasonal to decadal predictions systems. In addition, with the establishment of the biogeochemical and biological/ecosystem panels, GOOS has recognized that ocean observing in the service of existing and emerging users ultimately requires a multi-disciplinary approach. As such, the physics component will increasingly be integrated with biogeochemical and ecosystem observing sensors and platforms. This will require close collaboration amongst all GOOS panels.

We suggest that expanding model-based assessments of the accuracy and effective resolution of observing networks would be a valuable step in refining the guidance in the EOV specifications with respect to sampling resolution and frequency. There is an increasing need to assess the current and future observing system capabilities in light of the climate variability and potential climate change response that we can anticipate from both climate modeling systems and our existing knowledge and oceanographic theory. For example, closure of global energy and sea level budgets will increasingly demand improved sampling below $2000 \mathrm{~m}$ as the global warming signal propagates downward (Gleckler et al., 2016; von Schuckmann et al., 2016).

\section{AN INTEGRATED, SUSTAINED AND PRIORITIZED OCEAN OBSERVING SYSTEM FOR THE FUTURE}

Despite century-long efforts, observations are still limited for many parts of the global ocean. The ocean observing system must continue to evolve to maximize support for climate monitoring, process understanding, development of improved numerical models, and prediction of weather and climate on a range of timescales. Meeting societal needs will require more reliable ocean data for prediction of extreme events and their intensity and frequency distribution, monitoring of Earth's climate cycles (heat, water, and carbon) to inform discussion and development of mitigation and adaptation policy, marine forecasting, and weather to climate predictions and projections.

The OceanObs'09 Framework for Ocean Observing (Lindstrom et al., 2012) sets a foundation for how the GOOS a multiplatform, multidisciplinary and multifunctional ocean observing system - might evolve. However, it is recognized that the implementation of the observing system relies heavily on champions at the national level to bring the plan to reality. OOPC enables connections between the various observing systems/platforms and national implementers of the observing system to leverage the necessary funding from national agencies. OOPC's advocates for and endorsement of the ocean observing systems, through scientifically-informed assessments of stakeholder needs and observing system capabilities. Thus, OOPC's role is to provide objective advice, representing a wider community view of the observing system as a whole and acts as a broker between stakeholders, national agencies and intergovernmental constructs.

It is expected that through OceanObs'19, many communitydriven ideas will be voiced for improving the GOOS. Some of these recommendations may be readily implemented by new and current operators, while others may require trade-offs and/or international support and coordination. Transitioning to new observing system designs, for example, can be disruptive to long climate records that seek to minimize artifacts associated with changes in the measurement techniques and sampling characteristics that may mask the underlying climatic signals. Indeed, the next decade will present a number of opportunities that may enable genuine growth of the ocean observing system and the potential to provide a more secure funding mechanisms in future. The recent declaration of the United Nations Decade of Ocean Science for Sustainable Development (2021-2030) aims to provide ocean science, data and information to inform policy for a well-functioning ocean in support of all sustainable development goals of the Agenda 2030. This will generate scientific knowledge, underpinning infrastructures and partnerships and will require a cohesive GOOS across the coastal/shelf seas to the open ocean. OOPCs international coordination will be integral to building an integrated, sustained and prioritized ocean observing system and fostering cooperation with the GOOS biogeochemistry and biological/ecosystem expert panels, the JCOMM Observations Coordination Group, and GCOS.

\section{SUMMARY AND RECOMMENDATIONS}

OOPC, over the last 3 decades, has provided international guidance and a collaborative framework for the global ocean observing efforts that support fundamental research related to understanding and monitoring ocean physics and climate. More recently we have witnessed an acceleration in the technical capacity to deliver high quality observations in near-real time. This capacity for real-time data delivery has resulted in increasing demands for ocean observations in support of operational weather and maritime forecasting. Meteorological and ocean operational service providers have demonstrated how expanded ocean observations support the provision of improved forecasts and novel derived products of value to numerous user communities. OOPC needs to develop an inclusive partnership with these users to ensure support for the development and maintenance of the observing system.

Designing and operating a multidisciplinary observing system that goes beyond the physical ocean state into biogeochemistry and ecosystems will demand an even greater level of coordination and an expanded view of the phenomena that drive requirement specification. This will be accomplished by strong connections and collaborations between OOPC and the other GOOS panels, and it will fall on OOPC to provide informed consensus 
leadership on the expanding requirements for multidisciplinary ocean observing.

The heterogeneous nature of observing technology and the expanding sensors and platforms used, present great opportunities and challenges for the observing system. Coordination amongst disparate networks has the potential to offer efficiencies or enhanced complementarity of platforms. OOPC, with its responsibility to consider the observing system as a whole and its platform agnostic stewardship of the EOV/ECV requirements, is the natural forum to foster this coordinated evolution of the observing system. OOPC needs to ensure the observing system develops in a coordinated way and that data remain openly accessible in a timely manner. The synthesis and delivery of higher-level derived products based upon direct observations in support of research, operational and climate applications is a major scientific undertaking that demands significant added investment of both money and people. It is only through the coordinated synthesis of all observations for a given EOV that integrated observational datasets that meet user requirements and expand the use of the data will be achieved.

OOPC oversight and endorsement of ocean observing system reviews (e.g., TPOS2020, TAOS, IndOOS) and design studies for future systems (DOOS) is not currently undertaken in a consistent way. To ensure that these interconnected components of a global observing system are consistent, OOPC needs to provide a set of common guidelines to frame these review or design studies. Inevitably, there remain knowledge gaps in our understanding of how the ocean influences Earth's weather and climate across the full spectrum from daily to interannual and multidecadal time scales. Intensive short-term hypothesis-led observing studies targeted at specific processes are vital contributors to advancing our understanding of the oceans' influence on climate processes. Support for intensive ocean process studies will stand on their own intrinsic scientific merits, but may identify shortcomings in the global observing system, its design, its operation, or its fitness to deliver multidisciplinary objectives, OOPC should support the need for specific process studies wherever these emerge.

Key recommendations for OOPC to ensure that the ocean observing system delivers a globally integrated and consistent fit-for-purpose ocean observing system are:

1. OOPC should enhance links between observing networks and operational services that use data to generate higher level information products. Because of their dependency on these data, operational and modeling communities have a significant interest in supporting sustained ocean observing, and in offering guidance to ensure the system delivers data that meet evolving user needs.

2. OOPC should formulate a set of common principles for observing system evaluation and design. These will draw on the FOO and provide a consistent set of guidelines for the conduct of reviews, their terms of reference, and the reporting of their outcomes. It would be appropriate for OOPC to assume an oversight role in monitoring adherence to these principles to ensure that the guidelines are implemented consistently.

3. Under OOPC leadership, regular reviews of the EOV/ECV requirements should be undertaken to ensure they meet existing and emerging user needs. OOPC, with OCG, will work with observing networks to build unified data management practices to build consistent and findable data systems. OOPC must be a strong advocate that enables national implementers of the observing system to secure funding from national resources.

4. OOPC will support the coordination of EOV data collections, and production of synthesis and higher level products that users value. OOPC will publicize these products to increase data update and demonstrate to funders the value of these synthesis projects and need for ongoing resourcing of such efforts.

5. OOPC, in coordination with research partners (e.g., WRCP), will advocate for intensive process studies to address knowledge gaps.

\section{CONCLUSION}

The ever increasing complexity of the Ocean Observing System both in terms of the observation techniques and users requirement calls for a strong and robust management and evaluation process. OOPC provides an internationally recognized and supported mechanism for coordinating the review and evolution of existing observing systems that is essential to meeting the wide range of current and future user requirements.

Providing leadership on the continual assessment of the observing and data system, identifying gaps, risk and user needs is a central task of OOPC. We will continue these efforts reviewing existing observing systems and recommending unified data management and formats, all the while keeping in mind how their integration and expansion into multifunctional, multidisciplinary systems will meet an expanding range of user requirements. These review processes and the proposal of new observations require input and collaboration amongst the observing platforms/programs (satellite and in situ), research and user communities and insights from climate models and other modeling systems. Where reviews of regions or themes suggest continuation or expansion of observations, OOPC will be a strong advocate for continued support of the ocean observing system.

\section{AUTHOR CONTRIBUTIONS}

$\mathrm{KH}$, JW, and $\mathrm{BS}$ led the conception and writing of the manuscript. MPC, MFC, JJ, JK, MK, TL, EO, MP, BR, SS, KvS, RW, and WY contributed to the text.

\section{FUNDING}

BS received support from the Centre for Southern Hemisphere Oceans Research, a collaboration between the CSIRO and 
the Qingdao National Laboratory for Marine Science and Technology and the Australian Government Department of the Environment and CSIRO through the Australian Climate Change Science Programme and by the National Environmental Science Program. JK was supported by the European Union's Horizon 2020 Research and Innovation Programme under the grant agreement no. 633211 (AtlantOS). MP was supported by the Met Office Hadley Centre Climate Programme funded by the BEIS and Defra. SS was supported by the Ecole Normale Supérieure, CNRS, and Ifremer funded by the European Union's Horizon 2020 Research and Innovation Programme

\section{REFERENCES}

Bojinski, S., Verstraete, M., Peterson, T. C., Richter, C., Simmons, A., and Zemp, M. (2014). The concept of essential climate variables in support of climate research, applications, and policy. Bull. Amer. Meteor. Soc. 95, 1431-1443. doi: 10.1175/BAMS-D-13-00047.1

Caesar, L., Rahmstorf, S., Robinson, A., Feulner, G., and Saba, V. (2018). Observed fingerprint of weakening atlantic ocean overturning circulation. Nature 556, 191-196. doi: 10.1038/s41586-018-0006-5

Cronin, M. F., Gentemann, C. L., Edson, J., Ueki, I., Bourassa, M., Brown, S., et al. (2019). Air-sea fluxes with a focus on heat and momentum. Front. Mar. Sci. 6:430. doi: 10.3389/fmars.2019.00430

deYoung, B., Visbeck, M., de Araujo Filho, M. C., Baringer, M. O., Black, C.-A., Buch, E., et al. (2019). An integrated all-Atlantic Ocean observing system in 2030. Front. Mar. Sci. 6:428. doi: 10.3389/fmars.2019.00428

Doblas-Reyes, F. J., GarcíaSerrano, J., Lienert, F., Biescas, A. P., and Rodrigues, L. (2013). Seasonalclimate predictability and forecasting: status and prospects. WIREs Clim. Change 4, 4245-4268. doi: 10.1002/wcc.217

Duchez, A., Frajka-Williams, E., Josey, S. A., Evans, D. G., Grist, J. P., Marsh, R., et al. (2016). Drivers of exceptionally cold north atlantic ocean temperatures and their link to the 2015 European heat wave. Environ. Res. Lett. 11:074004. doi: 10.1088/1748-9326/11/7/074004

Frajka-Williams, E., Beaulieu, C., and Duchez, A. (2017). Emerging negative atlantic multidecadal oscillation index in spite of warm subtropics. Sci. Rep. 7, 11224. doi: 10.1038/s41598-017-11046-x

Gleckler, P. J., Durack, P. J., Stouffer, R. J., Johnson, G. C., and Forest, C. E. (2016). Industrial-era global ocean heat uptake doubles in recent decades. Nat. Clim. Chang. 6, 394-398. doi: 10.1038/nclimate2915

Gould, J., Sloyan, B. M., and Visbeck, M. (2013). "In-situ ocean observations: a brief history present status and future directions," in Ocean Circulation and Climate: A 21st Century Perspective, 2nd Edn eds G. Siedler, S. Griffies, J. Gould, and J. Church (Sydney: Academic Press), 59-79.

Hobday, A. J., Oliver, E. C. J., Sen Gupta, A., Benthuysen, J. A., Burrows, M. T., Donat, M. G., et al. (2018). Categorizing and naming marine heatwaves. Oceanography 31, 162-173. doi: 10.5670/oceanog.2018.205

Hood, E. M., Fukasawa, M., Gruber, N., Johnson, G. C., Körtzinger, A., Sabine, C., et al. (2010). "Ship-based repeat hydrography: a strategy for a sustained global survey," in Proceedings of OceanObs'09: Sustained Ocean Observations and Information for Society, Vol. 2 eds J. Hall, D. E. Harrison, and D. Stammer (Venice: ESA publication), 21-25.

Hughes, T. P., Kerry, J. T., Baird, A. H., Connolly, S. R., Dietzel, A., and Eakin, C. M. (2018). Global warming transforms coral reef assemblages. Nature 556:492. doi: 10.1038/s41586-018-0041-2

IPCC (2014). Climate Change (2014). Synthesis Report. Contribution of Working Groups I, II and III to the Fifth Assessment Report of the Intergovernmental Panel on Climate Change. Cambridge: Cambridge University Press.

Johnson, G. C., Lyman, J. M., Boyer, T., Cheng, L., Domingues, C. M., Gilson, J., et al. (2018). Ocean Heat Content. State of the Climate in 2017. Silver Spring, MA: NOAA.

Jones, J. M., Gille, S. T., Goosse, H., Abram, N. J., Canziani, P. O., Charman, D. J., et al. (2016). Assessing recent trends in high-latitude southern hemisphere surface climate. Nat. Clim. Chang. 6, 917-926. doi: 10.1038/nclimate 3103 under the grant agreement no. 633211 (AtlantOS), CNES, and ANR grants.

\section{ACKNOWLEDGMENTS}

We thank the scientific experts and observing programs who contributed to the development of the EOV specification sheets. We also thank David Legler for his valued contribution to OOPC activities. Comments from the reviewers improved the manuscript.

Kirtman, B., Stockdale, T., and Burgman, R. (2013). “The Ocean's role in modeling and predicting seasonal to-interannual climate variations," in Ocean Circulation and Climate: A 21st Century Perspective, 2nd Edn eds G. Siedler, S. Griffies, J. Gould, and J. Church (Sydney: Academic Press), 625-643. doi: 10.1016/b9780-12-391851-2.00024-6

Kummu, M., de Moel, H., Salvucci, G., Viviroli, D., Ward, P. J., Varis, O., et al. (2016). Over the hills and further away from coast: global geospatial patterns of human and environment over the 20th-21st centuries. Environ. Res. Lett. 11:034010. doi: 10.1088/1748-9326/11/3/03 4010

Levin, L. A., Bett, B. J., Gates, A. R., Heimbach, P., Howe, B. M., Janssen, F., et al. (2019). Global observing needs in the deep ocean. Front. Mar. Sci. 6:241. doi: 10.3389/fmars.2019.00241

Lindstrom, E., Gunn, J., Fischer, A., McCurdy, A., and Glover, L. K. (2012). “A Framework for Ocean Observing," in Proceedings of the Task Team for an Integrated Framework for Sustained Ocean Observing, UNESCO 2012 (revised in 2017), IOC/INF-1284 rev.2, Venice

Lopez, H., Dong, S., Lee, S.-K., and Goni, G. (2016). Decadal modulations of interhemispheric global atmospheric circulations and monsoons by the South Atlantic meridional overturning circulation. J. Clim. 29, 1831-1851. doi: 10. 1175/JCLI-D-15-0491.1

Marzeion, B., and Levermann, A. (2014). Loss of cultural world heritage and currently inhabited places to sea-level rise. Environ. Res. Lett. 9:34001.

Meehl, G. A., Goddard, L., Boer, G., Burgman, R., Branstator, G., Cassou, C., et al. (2014). Decadal climate prediction an update from the trenches. Bull. Am. Meteorol. Soc. 95, 243-267. doi: 10.1175/BAMS-D-12-00241.1

Miloslavich, P., Bax, N. J., Simmons, S. E., Klein, E., Appeltans, W., AburtoOropeza, O., et al. (2018). Essential ocean variables for global sustained observations of biodiversity and ecosystem changes. Glob. Change Biol. 2018, 2416-2433. doi: 10.1111/gcb.14108

Moltmann, T., Zhang, H.-M., Turton, J. D., Nolan, G., Gouldman, C. C., Griesbauer, L., et al. (2019). GOOS regional alliances enhance global ocean observing for regional and national benefit. Front. Mar. Sci. 6:291.

National Research Council (1997). The Global Ocean Observing System: Users, Benefits, and Priorities. Washington, DC: National Academies Press.

Palmer, M. D., Durack, P., Chidichimo, M. P., Church, J., Cravatte, S. E., Hill, K. L., et al. (2019). Adequacy of the ocean observation system for quantifying regional heat and freshwater storage and change. Front. Mar. Sci. doi: 10.3389/fmars. 2019.00416

Pinardi, N., Stander, J., Legler, D., O’Brien, K., Boyer, T., Cuff, T., et al. (2019). JCOMM: role and Vision. Front. Mar. Sci.

Siedler, G., Church, J., and Gould, J. (2001). Ocean Circulation and Climate: Observing and Modelling the Global Ocean. Cambridge, MA: Academic Press.

Sloyan, B., Craw, P., King, E., Neill, C., Kloser, R., and Bodrossy, L. (2017). "Future technologies," in Oceans: Science and solutions for Australia, Clayton South, ed. B. Mapstone (Australia: CSIRO Publishing), 179-186.

Smith, D. M., Scaife, A. A., and Kirtman, B. (2012). What is the current state of scientific knowledge with regard to seasonal and decadal forecasting. Environ. Res. Lett. 7:015602. doi: 10.1088/1748-9326/7/1/015602

Smith, N., Kessler, W. S., Cravatte, S., Sprintall, J., Wijffels, S., et al. (2019). Tropical pacific observing system. Front. Mar. Sci. 6:31. doi: 10.3389/fmars.2019. 00031 
Snowden, D., Belbeoch, M., Burnett, B., Carval, T., Graybeal, J., Habermann, T., et al. (2019). Framework for ocean observing: interoperability and vision. Front. Mar. Sci.

Tanhua, T., Fischer, A., and McCurdy, A. (2019). Successes and challenges in applying the framework for ocean observing. Front. Mar. Sci.

Tedeschi, R. G., Grimm, A. M., and Cavalcanti, I. F. A. (2016). Influence of central and east ENSO on precipitation and its extreme events in south america during austral autumn and winter. Int. J. Climatol. 36, 4797-4814. doi: 10.1002/joc. 4670

Timmermann, A., An, S. I, Kug, J. S., Jin, F. F., Cai, W., Capotondi, A., et al. (2018). El Niño-southern oscillation complexity. Nature 559, 535-545.

Todd, R., Chavez, F. P., Clayton, S., Cravatte, S., Goes, M., Graco, M., et al. (2019). Global perspectives on observing ocean boundary current systems. Front. Mar. Sci.

Vecchi, G. A., and Soden, B. J. (2007). Increased tropical Atlantic wind shear in model projections of global warming. Geophyis. Res. Lett. 34:L08702. doi: 10.1029/2006GL028905

von Schuckmann, K., Palmer, M. D., Trenberth, K. E., Cazenave, A., Chambers, D., Champollion, N., et al. (2015). A Prospectus for the CLIVAR Research Focus "Consistency Between Planetary Energy Balance and Ocean Heat Storage (CONCEPT-HEAT)". WCRP Report No. 14/2015. Geneva: WCRP.

von Schuckmann, K., Palmer, M. D., Trenberth, K. E., Cazenave, A., Chambers, D., Champollion, N., et al. (2016). An imperative to monitor Earth's energy imbalance. Nat. Clim. Chang. 6, 138-144. doi: 10.1038/nclimate 2876 von Schuckmann, K. P.-Y., Le Traon, N., Smith, A., Pascual, P., Brasseur, K., Fennel, S., et al. (2018). Copernicus marine service ocean state report. J. Operat. Oceanogr. 11, s1-s142.

Webster, P. J., Holland, G. J., Curry, J. A., and Chang, H. R. (2005). Changes in tropical cyclone number, duration, and intensity in a warming environment. Science 309, 1844-1846. doi: 10.1126/science.1116448

Weller, R. A., Baker, D. J., Glackin, M. M., Roberts, S. J., Schmitt, R. W., Twigg, E. S., et al. (2019). The challenge of sustaining ocean observations. Front. Mar. Sci. 6:105. doi: 10.3389/fmars.2019.00105

Yin, J., Schlesinger, M. E., and Stouffer, R. J. (2009). Model projections of rapid sealevel rise on the northeast coast of the United States. Nat. Geosci. 2, 262-266. doi: $10.1038 /$ ngeo462

Conflict of Interest Statement: The authors declare that the research was conducted in the absence of any commercial or financial relationships that could be construed as a potential conflict of interest.

Copyright (C) 2019 Sloyan, Wilkin, Hill, Chidichimo, Cronin, Johannessen, Karstensen, Krug, Lee, Oka, Palmer, Rabe, Speich, von Schuckmann, Weller and Yu. This is an open-access article distributed under the terms of the Creative Commons Attribution License (CC BY). The use, distribution or reproduction in other forums is permitted, provided the original author(s) and the copyright owner(s) are credited and that the original publication in this journal is cited, in accordance with accepted academic practice. No use, distribution or reproduction is permitted which does not comply with these terms. 


\section{Polar Ocean Observations: A Critical Gap in the Observing System and Its Effect on Environmental Predictions From Hours to a Season}

\section{OPEN ACCESS}

Edited by:

Tong Lee,

NASA Jet Propulsion Laboratory (JPL), United States

Reviewed by:

Agnieszka Beszczynska-Möller, Institute of Oceanology (PAN), Poland

Gunnar Spreen,

University of Bremen, Germany

*Correspondence:

Gregory C. Smith

Gregory.Smith2@canada.ca

Specialty section:

This article was submitted to

Ocean Observation,

a section of the journal

Frontiers in Marine Science

Received: 02 November 2018

Accepted: 05 July 2019

Published: 06 August 2019

Citation:

Smith GC, Allard R, Babin $M$,

Bertino L, Chevallier M, Corlett $G$,

Crout J, Davidson F, Delille B, Gille ST,

Hebert D, Hyder P, Intrieri J,

Lagunas J, Larnicol G, Kaminski T,

Kater B, Kauker F, Marec C,

Mazloff M, Metzger EJ, Mordy C,

O'Carroll A, Olsen SM, Phelps M,

Posey P, Prandi P, Rehm E, Reid P,

Rigor I, Sandven S, Shupe M,

Swart S, Smedstad OM, Solomon A, Storto A, Thibaut P, Toole J, Wood K,

Xie J, Yang $Q$ and

the WWRP PPP Steering Group (2019) Polar Ocean Observations:

A Critical Gap in the Observing

System and Its Effect on

Environmental Predictions From

Hours to a Season.

Front. Mar. Sci. 6:429.

doi: 10.3389/fmars.2019.00429
Gregory C. Smith ${ }^{1 *}$, Richard Allard ${ }^{2}$, Marcel Babin ${ }^{3}$, Laurent Bertino ${ }^{4}$,

Matthieu Chevallier 5,6, Gary Corlett ${ }^{7}$, Julia Crout ${ }^{8}$, Fraser Davidson ${ }^{9}$, Bruno Delille $^{10}$, Sarah T. Gille ${ }^{11}$, David Hebert², Patrick Hyder ${ }^{12}$, Janet Intrieri13, José Lagunas ${ }^{3}$, Gilles Larnicol'14, Thomas Kaminski ${ }^{15}$, Belinda Kater ${ }^{16}$, Frank Kauker ${ }^{17,18,}$ Claudie Marec ${ }^{3,19}$, Matthew Mazloff 11 , E. Joseph Metzger², Calvin Mordy ${ }^{20}$, Anne O'Carroll21, Steffen M. Olsen ${ }^{22}$, Michael Phelps ${ }^{8}$, Pamela Posey ${ }^{8}$, Pierre Prandi ${ }^{14}$, Eric Rehm ${ }^{3}$, Phillip Reid ${ }^{23}$, Ignatius Rigor ${ }^{24}$, Stein Sandven ${ }^{4}$, Matthew Shupe ${ }^{13,25}$, Sebastiaan Swart ${ }^{26,27}$, Ole Martin Smedstad ${ }^{8}$, Amy Solomon $^{28}$, Andrea Storto ${ }^{29}$, Pierre Thibaut ${ }^{14}$, John Toole ${ }^{30}$, Kevin Wood ${ }^{20}$, Jiping Xie ${ }^{4}$, Qinghua Yang ${ }^{31}$ and the WWRP PPP Steering Group ${ }^{32}$

${ }^{1}$ Environmental Numerical Prediction Research Section, Meteorological Research Division, Environment and Climate Change Canada, Dorval, QC, Canada, ${ }^{2}$ Stennis Space Center, U.S. Naval Research Laboratory, Bay St. Louis, MS, United States,

${ }^{3}$ Takuvik, UMI 3376, Université Laval-CNRS, Quebec City, QC, Canada, ${ }^{4}$ Nansen Environmental and Remote Sensing Center, Bergen, Norway, ${ }^{5}$ Division of Marine and Oceanography, Météo France, Toulouse, France, ${ }^{6}$ CNRM, Météo France, CNRS, Université de Toulouse, Toulouse, France, ${ }^{7}$ European Organisation for the Exploitation of Meteorological Satellites, Darmstadt, Germany, ${ }^{8}$ Perspecta, Inc., Stennis Space Center, Bay St. Louis, MS, United States, ${ }^{9}$ Northwest Atlantic Fisheries Centre, Fisheries and Oceans Canada, St. John's, NL, Canada, ${ }^{10}$ Chemical Oceanography Unit, Université de Liège, Liège, Belgium, ${ }^{11}$ Scripps Institution of Oceanography, University of California, San Diego, La Jolla, CA, United States, ${ }^{12}$ Met Office, Exeter, United Kingdom, ${ }^{13}$ Physical Sciences Division, NOAA Earth System Research Laboratory, Boulder, CO, United States, ${ }^{14}$ Collecte Localisation Satellites, Toulouse, France, ${ }^{15}$ The Inversion Lab, Hamburg, Germany, ${ }^{16}$ Arcadis Nederland B.V., Zwolle, Netherlands, ${ }^{17}$ Ocean Atmosphere Systems, Hamburg, Germany, ${ }^{18}$ Alfred Wegener Institute for Polar and Marine Research, Bremerhaven, Germany, ${ }^{19}$ Laboratoire d'Oceanographie Physique et Spatiale, UMR 6523, CNRS - IFREMER - IRD - UBO, Plouzané, France, ${ }^{20}$ Joint Institute for the Study of the Atmosphere and Oceans, University of Washington, Seattle, WA, United States, ${ }^{21}$ European Organisation for the Exploitation of Meteorological Satellites, Darmstadt, Germany, ${ }^{22}$ Danish Meteorological Institute, Copenhagen, Denmark, ${ }^{23}$ Bureau of Meteorology, Hobart, TAS, Australia, ${ }^{24}$ Polar Science Center, University of Washington, Seattle, WA, United States, ${ }^{25}$ Cooperative Institute for Research in Environmental Sciences, University of Colorado Boulder, Boulder, CO, United States, ${ }^{26}$ Department of Marine Sciences, University of Gothenburg, Gothenburg, Sweden, ${ }^{27}$ Department of Oceanography, University of Cape Town, Rondebosch, South Africa, ${ }^{28}$ Earth System Research Laboratory, National Oceanic and Atmospheric Administration, Boulder, CO, United States, ${ }^{29}$ Centre for Maritime Research and Experimentation, La Spezia, Italy, ${ }^{30}$ Woods Hole Oceanographic Institution, Woods Hole, MA, United States, ${ }^{31}$ Guangdong Province Key Laboratory for Climate Change and Natural Disaster Studies, School of Atmospheric Sciences, Sun Yat-sen University, Zhuhai, China, ${ }^{32}$ World Weather Research Programme (WWRP) Polar Prediction Project (PPP) Steering Group

There is a growing need for operational oceanographic predictions in both the Arctic and Antarctic polar regions. In the former, this is driven by a declining ice cover accompanied by an increase in maritime traffic and exploitation of marine resources. Oceanographic predictions in the Antarctic are also important, both to support Antarctic operations and also to help elucidate processes governing sea ice and ice shelf stability. However, a significant gap exists in the ocean observing system in polar regions, compared to most areas of the global ocean, hindering the reliability of ocean and sea ice forecasts. This gap can also be seen from the spread in ocean and sea ice reanalyses for polar regions which provide an estimate of their uncertainty. The reduced reliability of polar predictions may affect the quality of various applications 
including search and rescue, coupling with numerical weather and seasonal predictions, historical reconstructions (reanalysis), aquaculture and environmental management including environmental emergency response. Here, we outline the status of existing near-real time ocean observational efforts in polar regions, discuss gaps, and explore perspectives for the future. Specific recommendations include a renewed call for open access to data, especially real-time data, as a critical capability for improved sea ice and weather forecasting and other environmental prediction needs. Dedicated efforts are also needed to make use of additional observations made as part of the Year of Polar Prediction (YOPP; 2017-2019) to inform optimal observing system design. To provide a polar extension to the Argo network, it is recommended that a network of ice-borne sea ice and upper-ocean observing buoys be deployed and supported operationally in ice-covered areas together with autonomous profiling floats and gliders (potentially with ice detection capability) in seasonally ice covered seas. Finally, additional efforts to better measure and parameterize surface exchanges in polar regions are much needed to improve coupled environmental prediction.

Keywords: polar observations, operational oceanography, ocean data assimilation, ocean modeling, forecasting, sea ice, air-sea-ice fluxes, YOPP

\section{INTRODUCTION}

Over the last 10 years, there has been a significant maturing of ocean prediction systems, led by efforts such as GODAE OceanView (GOV; Bell et al., 2015; Davidson et al., 2019) and the European Union Copernicus Marine Environmental Monitoring Service (CMEMS; Le Traon et al., 2017). Numerous operational global and regional ocean analysis and forecast systems are now in place providing services for a range of applications including search and rescue, short- and long-range atmospheric and coupled prediction systems, aquaculture, energy sector activities and environmental management including environmental emergency response.

We have also seen the implementation of high-resolution operational ice-ocean prediction systems for polar regions providing forecasts on timescales of hours to days. These include the CMEMS Arctic Monitoring and Forecasting Centre (ARC MFC), the U.S. Navy Global Ocean Forecasting System, Environment and Climate Change Canada's Global and Regional Ice Ocean Prediction Systems among others (Carrieres et al., 2017). While these systems are intended to provide support for marine operations and related applications, there has been a growing acceptance of the importance of including coupled interactions across the marine surface in numerical weather prediction systems (Brassington et al., 2015). Indeed, operational medium-range weather forecasting systems in Canada and at the European Centre for Medium Range Forecasting (ECWMF) now include a dynamic coupling with ice-ocean models (Mogensen et al., 2017; Smith et al., 2018). As a result, polar ocean observations may now have impacts beyond the polar regions on mid-latitude weather predictions (Jung et al., 2015). Moreover, coupled atmosphere-ice-ocean models have been shown to provide skillful forecasts at monthly-to-seasonal time scales in the polar regions, especially for the Arctic sea ice cover (e.g., Guémas et al., 2016a; Sigmond et al., 2016). The importance of polar sea ice initial conditions in affecting skill of monthly-to-seasonal predictions at lower latitudes in the atmosphere has also been discussed (e.g., Guémas et al., 2016b). These advances are also fostering a coupled modeling approach in the context of Earth system reanalyses for climate monitoring (e.g., Buizza et al., 2018).

Environmental prediction systems rely heavily on ocean observations from a variety of platforms (both in situ and from remote sensing). Indeed, Observing System Experiments (OSEs) have shown the delicate balance and complementarity provided by the current basket of observations (Oke et al., 2015; Fujii et al., 2019). However, polar regions present a number of unique observing challenges (see Calder et al., 2010). For short leadtimes (hours to days), forecast skill depends strongly on initial conditions, which in turn depend on real-time observations ingested through data assimilation. The lack of real-time Argo profiling floats in polar regions due to the sea ice cover (Roemmich and Gilson, 2009) therefore creates a significant gap. This gap is compounded by additional errors in satellite products in polar regions, for example, due to difficulties in distinguishing snow and ice from clouds (Castro et al., 2016). The relative remoteness and harsh environmental conditions in polar regions further hinder efforts to provide in situ measurements. Moreover, the increasing use of coupled models noted above requires collocated observations of the atmosphere, ice and ocean, including flux estimates (Bourassa et al., 2013). This is all the more important because of the role of the Arctic region in particular in shaping the heat and freshwater transports at global scales, thus having a crucial remote impact on the midand low- latitude climate as well (Serreze and Barry, 2011; Jung et al., 2015).

Additionally, the rapidly receding summer ice cover in the Arctic is leading to both an increase in the demand for accurate ice-ocean and weather forecast products (Jung et al., 2016), as well as challenges in how to adapt prediction systems to 
accurately forecast conditions that may not have an existing analog in the historical record (e.g., the 2012 record low Arctic sea ice cover; Parkinson and Comiso, 2013). Similar issues apply in the Antarctic: rising temperatures and reports of both expanding and reduced sea ice have led to uncertainties about changes in the system (Maksym, 2019), while at the same time growing demands from Antarctic tourism and research logistics have driven a need for improved forecasts (Hoke et al., 2018).

Here, we outline the status of existing near real-time ocean observational efforts in polar regions, discuss gaps, and explore perspectives for the future. The first section is dedicated to in situ observations of temperature and salinity and several projects underway to further extend these efforts. Section "Satellite Observations" focuses on satellite observations of sea surface temperature (SST) and height (SSH), along with information on the sea ice cover. Section "Air-Sea Flux Measurements in Polar Oceans" discusses issues associated with surface flux measurements in polar regions. Section "Forecasting System Experiments" presents several impact studies in different prediction systems demonstrating the importance of ocean and sea ice observations to forecasting skill. Section "International Efforts to Address Gaps in Polar Ocean Observations" discusses several international projects working to address the gap in ocean observations in the polar regions. Finally, recommendations and an outlook for the future are presented in Sections "Recommendations" and "Outlook."

\section{IN SITU OBSERVATIONS OF TEMPERATURE AND SALINITY}

In the polar oceans there are several methods for collecting ocean and sea ice data and sending the data in near realtime via the Global Telecommunications System (GTS) or other transmission systems. In ice-free areas various methods are used, including ships, Argo floats, surface drifters, gliders, etc., while in ice-covered areas, only ice-borne instrument systems can operate throughout the year and transmit data in near real-time. Owing to their significant deployment and maintenance costs, the number of these platforms is very limited, resulting in large gaps in the data coverage in the ice-covered Arctic Ocean. Antarctic sea ice is largely single-year (or first-year) ice, which makes it illsuited for ice based measurement systems. A significant fraction of the ocean data from the Arctic, as well as under-ice Argo profiles from the Antarctic seasonal ice zone, are only available in delayed mode, because underwater platforms in ice-covered areas cannot transmit data via satellite communication. This limits the access to ocean data available in near real-time, which is required by monitoring and forecasting services.

In the following section, the most common observing systems for the Arctic Ocean and Antarctic marginal sea are described briefly. Sections "The WHOI Ice-Tethered Profiler," "ALAMO and Arctic Heat," "Deployment of Argo Floats in Canadian Marginal Ice Zone by CONCEPTS," "Under-ice BGC Argo Floats in the Canadian Arctic," and "Surface Drifters and IABP" detail several specific observing efforts as exemplars of each class of technology.

\section{Overview of Current Observing System Profiling Floats}

Argo floats are the backbone of the global ocean observing system developed over the last two decades with more than 3500 units currently in operation (e.g., Roemmich et al., 2009). In the north, Argo floats are used in ice-free areas of the North Atlantic/Nordic Seas, Baffin Bay and Bering Sea. In the North Atlantic/Nordic Seas about 10-20 Argo floats are deployed each year sustaining an operational array of about 40 - 50 Argo floats in these regions. These floats are provided mainly by national efforts (e.g., NorArgo) and coordinated at the European level by EuroArgo. The funding of these Argo floats is relatively secure, and provided for by the countries that participate in EuroArgo.

There is ongoing development to adapt Argo floats to operate under ice, using ice-sensing algorithms as well as acoustic and optical techniques. In the Southern Ocean, the Argo program has demonstrated success with under-ice profiling floats that delay data transmission until the float is in open water, typically in summer (e.g., projects SOCLIM, RemOcean, SOCCO Bioargo and ReMOCA). In particular, the Southern Ocean Carbon and Climate Observations and Modeling project (SOCCOM) has made an effort to deploy a significant number of underice Argo floats in the Southern Ocean. Moreover, RAFOS floats (Klatt et al., 2007; Reeve et al., 2016) provide the capability to triangulate their position using sound signals from nearby moorings for periods when they are not able to surface. In the Arctic, the ice-sensing algorithm is starting to become more mature and is being complemented with additional detection methods currently being tested by Takuvik (see Under-ice BGC Argo Floats in the Canadian Arctic).

\section{Ice-Borne Observing Systems}

Many types of ice-borne ocean measurement systems have been developed and fielded in the last 2-3 decades. Ice-based platforms are the only autonomous systems that can presently deliver near-real-time subsurface ocean observations year round from ice-covered areas. Early examples consisted of discrete sensors mounted on a floating mooring that drifts with the sea ice. More recent examples employ a drifting surface buoy deployed in the ice with a Conductivity-Temperature-Depth (CTD) sensor that is repeatedly transported along the mooring cable over the upper $800-1000 \mathrm{~m}$ of the water column. Ice-Tethered Profiler (ITP) systems from the Woods Hole Oceanographic Institution (WHOI, see The WHOI Ice-Tethered Profiler for details) have been fielded since 2004. Similarly, investigators from Laboratoire d'Océanographie et du Climat (LOCEAN) have developed and deployed IAOOS (Ice-Atmosphere-Arctic Ocean Observation System) buoys ${ }^{1}$. In addition to CTD profile observations, the IAOOS buoy includes ice mass balance measurements (snow and sea ice temperature and thickness), as well as a variety of meteorological sensors (Provost et al., 2015). Other similar platforms include the JAMSTEC Polar Ocean Profiling system (POPS; Kikuchi et al., 2007), the NPS Autonomous Ocean Flux

\footnotetext{
${ }^{1}$ See http://iaoos.ipev.fr/ for more details.
} 
Buoy $^{2}$ (AOFB), and the UpTempO ice-tethered buoy developed by APL-UW (Castro et al., 2016). The Arctic University of Norway has recently developed and field tested a new platform called the Ice-Tethered Platform Cluster for Optical, Physical, and Ecological Sensors (ICE-POPE) that will be deployed operationally in 2019 (Berge et al., 2016). The Polar Research Institute of China is also developing ice-tethered platforms, which are being tested during Arctic expeditions of the I/B Xuelong. In comparison to Argo-type profiling floats, icebased platforms are rather expensive (>100-300 k€ per unit). To date, funding to construct and field these systems and provide data to the community has derived from individual PI-led research projects. There are no operational programs to support the ice-tethered platforms at the moment, but there is a clear requirement from the Arctic Ocean modeling and data assimilation community that $10+$ such platforms need to be deployed every year and supported operationally.

It is widely known that sea ice in the Arctic is shrinking in areal coverage, thinning, and becoming more mobile. All of these changes present complications to an ice-based observing system. Although diminished, the sea ice will remain critically important to earth's climate and ecosystems as well as transportation and tourism, making ice-following observing platforms necessary into the future. However, future ice-borne instrument systems must be able to float and demonstrate resilience during fall freezeup. Thinner, more mobile ice can be more prone to ridging that can damage ice based buoys. It has not proven feasible to maintain the array of 20 ice-based observatory systems in the Arctic that was envisioned at the turn of the century. Nor have these technologies been used extensively in the seasonal ice zone surrounding Antarctica, where sea ice typically melts completely every summer. Nevertheless, ice-based observatories have and are continuing to return valuable year-round upper ocean data from the central Arctic. Buoy clusters sampling various elements of the atmosphere, sea ice and upper ocean have proven particularly valuable.

\section{Ice/Snow Surface Drifters}

A reasonable number of low-cost ice buoys operate in the Arctic, providing ice motion, air pressure and surface temperature data. At present, most of these buoys are drifting in the western part of the Arctic, whereas the eastern part, including the Eurasian Basin and the Russian shelves, has very few buoys. The drifter data are transmitted via the Argos or Iridium satellite systems then posted on the WMO/IOC GTS and provide baseline data for weather and ice forecasting in the Arctic (see Section Surface Drifters and IABP for more detail). Drifters are also routinely deployed throughout the Southern Ocean, providing important information (e.g., SST and sea level pressure) to forecast systems from extremely data sparse regions.

Sea ice mass balance buoys (IMBs) provide information on temperature within snow and ice as well as ice thickness and its motion. Development and use of these buoys have continued for several decades with more than 100 IMBs deployed

\footnotetext{
${ }^{2}$ See https://www.oc.nps.edu/ stanton/fluxbuoy/ for more details.
}

from 1957 to 2014. The data are transmitted in near realtime, but processing of the temperature profiles is mainly done manually. There are ongoing efforts to develop automated methods for retrieval of snow and ice thickness (Liao et al., 2018; Zuo et al., 2018). It is a challenge to develop robust algorithms that give accurate retrievals through the melting and freezing seasons.

Currently, three different systems are deployed in the Arctic including the CRREL-Dartmouth IMB (Richter-Menge et al., 2006), the SRSL Sea Ice Mass Balance Array (SIMBA; Jackson et al., 2013) and the TUT ice-tethered buoy developed by PRIC and TUC in China (Zuo et al., 2018). Some of the Ice-tethered platforms described in Section "Ice-Borne Observing Systems" are also equipped with SIMBA instruments.

\section{Other Systems}

(1) Ferrybox systems: The Norwegian Institute for Water Research (NIVA) operates a ferrybox between Tromsø and Longyearbyen/Ny Ålesund. Data are obtained every 2 weeks year-round. This system is fairly sustainable through support from a new infrastructure project led by NIVA.

(2) Ship-based CTD data during scientific cruises and fishery management cruises: Several research vessels in the North Atlantic/Nordic seas deliver CTD observations in near real-time. The data are provided more or less regularly, depending on the schedule of the research vessels.

(3) Profiling gliders: Several institutions conduct ocean glider experiments in the Arctic (e.g., Nordic Seas, David Strait, Fram Strait, north of Svalbard, Beaufort Sea) providing CTD data in near real-time. These experiments are mainly in the summer season, however, acoustically navigated ice-capable Seagliders (developed by APL-UW) were successfully used for the year-round missions in Davis Strait and in the Beaufort Sea including under ice measurements in winter (Curry et al., 2014; Lee et al., 2016). Glider experiments are also conducted with growing frequency in the Southern Ocean. These provide valuable data and upper ocean process understanding from winter through summer (e.g., Swart et al., 2015; Thomson and Girton, 2017; du Plessis et al., 2019 - in review).

(4) Autonomous surface vehicles: Wave Gliders have been deployed in both the Arctic and Southern Ocean, providing rare surface flux (heat, momentum and $\mathrm{CO}_{2}$ ) information over multiple months at a time and with high resolution (Monteiro et al., 2015; Schmidt et al., 2017; Thomson and Girton, 2017). Many of these platforms are reporting or being adapted to report real-time surface observational data.

(5) Sea-mammal borne instruments (CTD data) are becoming the most numerous in situ observations at high latitude in the Antarctic and also more recently in the Arctic, and may improve forecast skill and circulation patterns (Fedak, 2013; Roquet et al., 2013; Carse et al., 2015).

(6) Acoustic tomography: Acoustic sources and receivers have been deployed in the Arctic in the past (Mikhalevsky et al., 2015) and offer the unique capability to measure 
large-scale changes in temperature and heat content of the Arctic Ocean over long periods (Howe et al., 2019). New deployments of this technology were conducted under the CANAPE project (led by Scripps); CAATEx programs (led by NERSC) have been approved. While no observations are currently available in real-time, nor assimilated in delayed mode, acoustic tomography nonetheless presents an interesting possibility for constraining ocean stateestimate/prediction systems.

(7) Moored Arrays: Several long-term observatories have been running in both ice-covered and open-water areas with the capacity to provide real-time data. Examples include the German long-term observatory FRAM (Frontiers in Arctic Marine Monitoring; Soltwedel et al., 2013) and the Barrow Strait Observatory (Richards et al., 2017).

\section{The WHOI Ice-Tethered Profiler}

Historically, the Arctic Ocean has been poorly sampled relative to waters at low- and mid-latitudes, especially in winter time. To address this observing shortfall, the WHOI ITP was designed to sample the upper ocean below drifting sea ice throughout the year and to return data in near real time (Krishfield et al., 2008; Toole et al., 2011). As noted earlier, there are now a variety of systems with similar capability now being fielded; the ITP is detailed here as exemplar of the group. The expendable ITP consists of a surface buoy that supports a weighted wirerope tether extending through the ice and down to (at most) $800 \mathrm{~m}$ depth (Figures 1A,C,D). The heart of the ITP system is a cylindrical vehicle fitted with sensors (similar in size and shape to an Argo float) that travels up and down the tether. ITPs are equipped with CTD instruments for observing the ocean's thermohaline stratification and may also include sensors that sample, for example, dissolved oxygen (Timmermans et al., 2010), bio-optical properties (Laney et al., 2014), and ocean currents (Ice-Tethered Profiler with Velocity - ITV-V: Thwaites et al., 2011; Cole et al., 2015). In addition, instruments measuring temperature-conductivity, $\mathrm{pCO}_{2}$, dissolved $\mathrm{O}_{2}$ and $\mathrm{pH}$ have been affixed to the tether above the profiling interval (e.g., Islam et al., 2017). Deployments may be done from ice camps (supported by fixed-wing aircraft or helicopters) or ships. The majority of deployments have been through holes augured through ice floes but a handful of systems have been installed in open water (the buoy has sufficient buoyancy to support the system); most of those have survived fall freeze-up.

The basic ITP system was designed for an operational lifetime of more than 2 years assuming approximately $1500 \mathrm{~m}$ of profiling per day (e.g., 2 one-way profiles of $750 \mathrm{~m}$ span). Actual lifetimes of the full ITP system are often less than this, Figure 1B. There are two major failure modes of ITPs: crushing of the surface buoy and/or breaking of the tether in ice ridging events and dragging of the tether in shallow water. As is evident in Figure 1B, ITP surface buoys frequently transmit position data for an extended time after communication with the underwater unit is lost.

ITP data, available from the project website $^{3}$, the National Environmental Data Center and the Arctic Data Center, support a

\footnotetext{
${ }^{3}$ http://www.whoi.edu/itp
}

range of scientific investigations and student projects. The basinwide and year-round coverage facilitates studies of seasonal-tointerannual physical and biogeochemical processes (e.g., Rabe et al., 2011; McPhee, 2013; Laney et al., 2014, 2017; Islam et al., 2017) and basin-scale phenomena (e.g., Timmermans et al., 2014), as well as supporting the initialization (data assimilation) and/or validation of numerical models. Smaller-scale processes may also be investigated with ITP data, including meso- and submesoscale variability (e.g., Timmermans et al., 2011; Zhao et al., 2014, 2016), near-inertial internal waves (Cole et al., 2014; Dosser et al., 2014) and double diffusion (e.g., Timmermans et al., 2008; Shibley et al., 2017). Notably, the range of sensors able to be supported on ITPs provides a wide-ranging view of the evolving Arctic Ocean system.

\section{ALAMO and Arctic Heat}

Since 2014, NOAA Pacific Marine Environmental Laboratory (PMEL) and the University of Washington's Joint Institute for the Study of the Atmosphere and Ocean (JISAO) have been developing and field testing a series of autonomous marine systems designed for Arctic applications. This work has been carried out in collaboration with scientists, forecasters, and industry, primarily under the auspices of the Innovative Technologies for Arctic Exploration (ITAE ${ }^{4}$ ) program. Fifteen new types of technology have been developed or advanced for Arctic use under this program, including the Air-Launched Autonomous Micro-Observer (ALAMO), the Saildrone autonomous marine vehicle, and the Oculus Coastal Glider.

An example of a multi-platform experiment along these lines is the Arctic Heat Open Science Experiment (Wood et al., 2018). Here a NOAA Twin Otter research aircraft (NOAA-56) has been equipped with a range of weather and ocean-sensing instruments, which in 2018 included flight-level weather and radiometry, an A-size sonobuoy deployment tube used for a range of air-deployed probes - including AXBT/AXCTD/AXCP, atmospheric dropsondes, ALAMO autonomous profilers, and experimental UAS (Unmanned Aerial Systems) platforms LIDAR and thermal imaging camera. Arctic Heat deploys an array of ALAMO floats in the Chukchi Sea between May and September; floats deployed later in the season are active into winter (Figure 2), and are used to develop an experimental seasonal freeze-up projection, in combination with satellitederived SST and historical sea ice extent/concentration. In 2018 a combination of more than two dozen profiling floats of various types were deployed by three collaborating research groups in the Chukchi and Beaufort Seas ${ }^{5}$.

The dense array of floats in 2018 provides an opportunity to thoroughly investigate the potential gain in predictive skill provided by enhanced real-time ocean observations. Understanding how assimilating more observations will impact modeled analyses and short-term forecasts is of fundamental importance as new coupled models are developed. For example, sensitivity experiments with the NOAA-Earth System Research

\footnotetext{
${ }^{4}$ https://www.pmel.noaa.gov/itae/

${ }^{5}$ https://www.pmel.noaa.gov/arctic-heat/data
} 
A

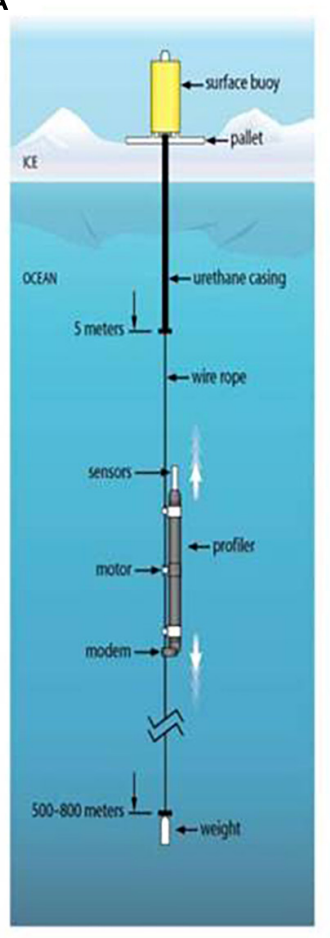

C

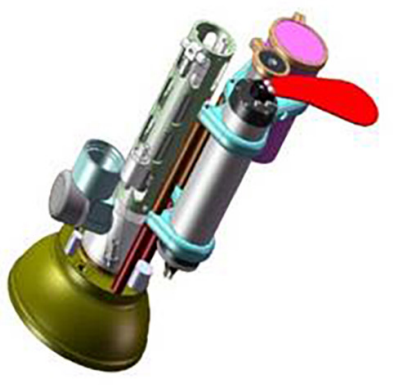

B

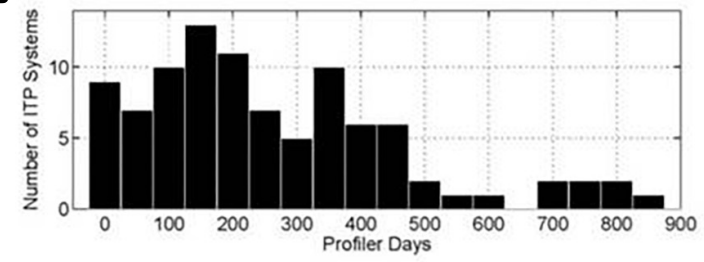

D

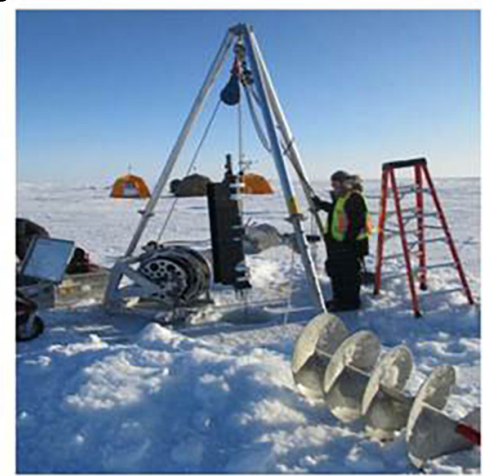

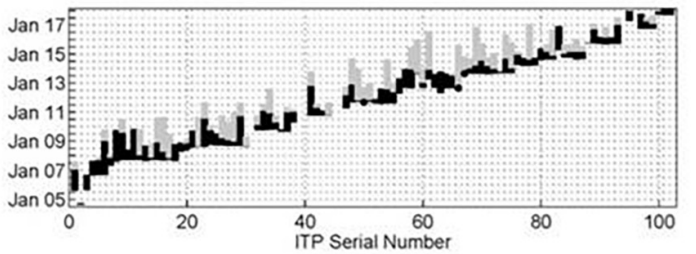

FIGURE 1 | (A) Schematic drawing of the WHOI lce-Tethered Profiler (ITP) system; (B) Histogram of ITP underwater vehicle lifetimes (top) and (bottom) the periods (shown as black vertical bars) over which telemetry was received from each ITP underwater unit and from each corresponding surface buoy (black plus gray bars). The history of ITP systems deployed in the Southern Ocean and in lakes are excluded from this plot. (C) Schematic drawing of the bio-optical ITP sensor suite with $\mathrm{CTD} / \mathrm{O}_{2}$, chlorophyll fluorescence, CDOM, optical backscatter and PAR (the latter suite housed under a retractable shutter), and (D) installation photograph of an ITP with a Modular Acoustic Velocity Sensor (ITP-V).

Laboratory Coupled Arctic Forecast System (CAFS) (see NOAAESRL/CIRES Coupled Arctic Forecast System) are being pursued.

\section{Deployment of Argo Floats in Canadian Marginal Ice Zone by CONCEPTS}

A Government of Canada initiative called CONCEPTS (Canadian Operational Network of Coupled Environmental Prediction Systems; Davidson et al., 2013) has implemented global and regional environmental prediction systems over the last few years. A particular need has been identified to complement the existing observing system with additional real-time observations to better constrain water mass properties in the ocean analyses, in particular in Canadian seasonally ice-infested seas. As a result, a project is underway to test the implementation of different observing technologies for their ability to provide reliable measurements of ocean temperature and salinity at a low cost. A first step was the deployment of a standard Argo float in the Gulf of St. Lawrence, configured with a shallow profiling and parking depth $(200 \mathrm{~m})$. The objective was to provide observations over the spring-to-fall ice-free period and to allow the float to remain subsurface in winter when ice forms. The use of existing technology (i.e., without any particular ice detection capability) reduced costs and increased feasibility. This initial effort was quite successful and provided an excellent complement to existing (and more costly) moorings deployed in the Gulf of St. Lawrence.

A second effort included the deployment of an Argo float on the Labrador shelf, again with a shallow profiling depth of $200 \mathrm{~m}$. This depth was greater than the local bathymetry allowing the float to rest on the sea floor and reduce lateral drift. Despite the presence of ice in winter, this float was able to breach the surface periodically (roughly every 10 days) and transmit ocean measurements. Additionally, the float observed temperature and salinity measurements during winter that deviated significantly from climatological conditions (Figure 3). As these climatological values are typically used by various applications in the absence of other data, the Argo float observations filled an important gap. Moreover, their availability in real time permits their use in data assimilation systems that can allow the detected anomaly in water mass conditions to be propagated over suitably correlated water masses (i.e., along the Labrador Shelf).

As part of the YOPP (see Year of Polar Prediction), this effort has been expanded to include the deployment of 7 Argo floats during the Arctic summer Special Observing Period (JulySeptember 2018). If successful, and found to provide adequate benefit for the cost, this effort could become part of the Canadian Argo Program, and also contribute to the newly forming 


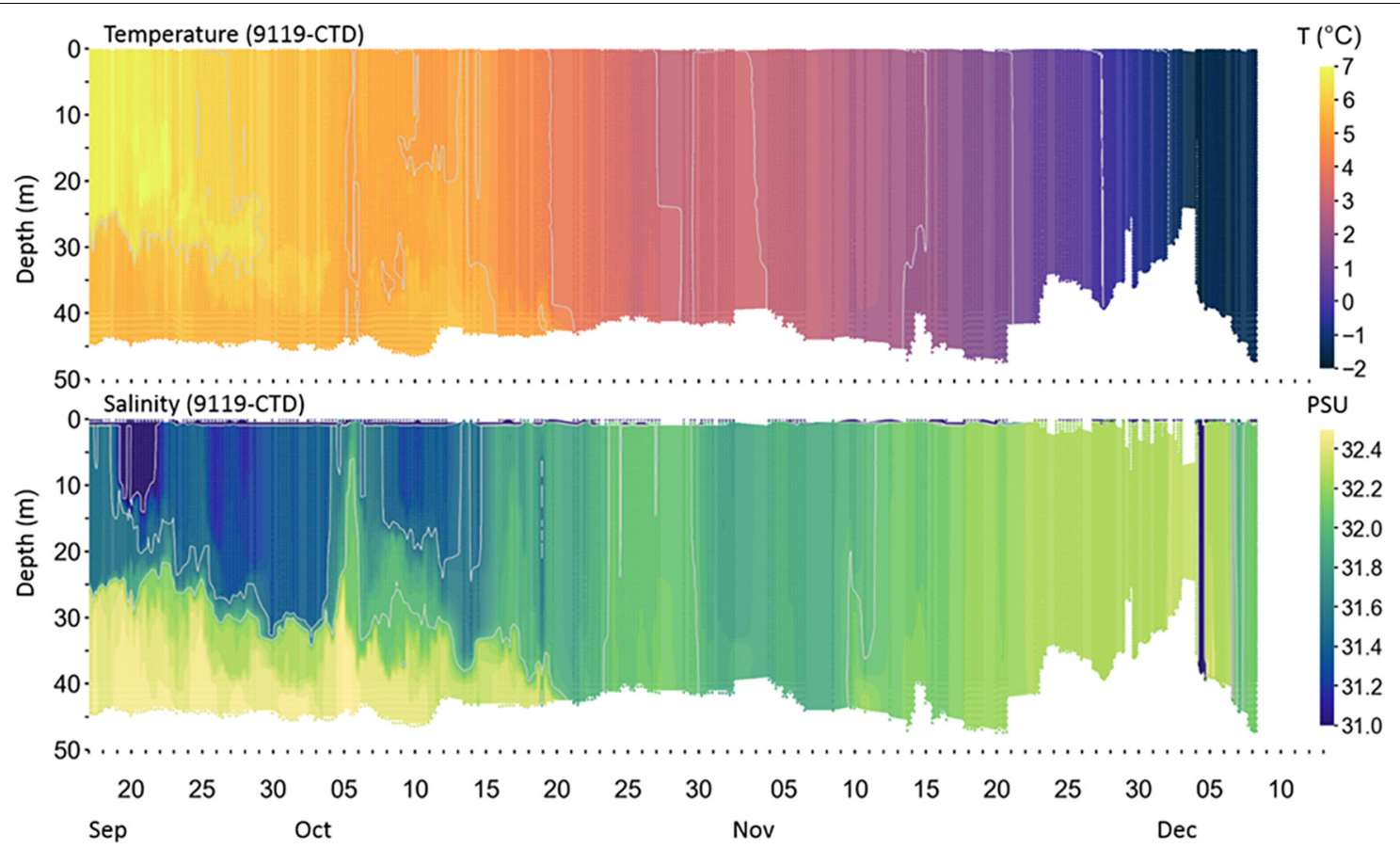

FIGURE 2 | Temperature and salinity profiles collected by ALAMO 9119-CTD from September 17 to December 8, 2017. The float began sampling near 167W, 70N and the last profile was near 165W, 72N. See: https://www.pmel.noaa.gov/arctic-heat/ for more information, including the float track.

Canadian Integrated Ocean Observing System (CIOOS). The use of air deployable ALAMO floats and floats with ice-detection capabilities is also being investigated.

\section{Under-Ice BGC Argo Floats in the Canadian Arctic}

As part of project Green Edge ${ }^{6}$, Takuvik deploys Biogeochemical Argo floats (BGC Argo; manufactured by $\mathrm{NKE}^{7}$ ) in the Arctic Ocean to study the dynamics of ice-edge phytoplankton spring blooms as controlled by sea ice dynamics, vertical mixing, light and nutrients. BGC Argo floats are more specialized profilers, equipped with sensors capable of sampling additional essential ocean variables (Claustre et al., 2010). The study focuses on Baffin Bay, which involves navigational challenges for floats in terms of bathymetry, ice coverage and circulation. Simulations of trajectories to choose the best dropping zones, combined with observations from ice charts (climatology and real-time charts) are necessary for safe deployments. An ice-covered ocean presents a real challenge for Argo floats that must surface for geo-localization and to use satellite networks for data transmission and command reception (Riser et al., 2016), see Figure 4. Therefore, some technical refinements have been needed to make the floats operational in the Arctic Ocean (Fennel and Greenan, 2017).

The development of a new generation of floats (PRO-ICE) to be operated under ice, was founded by the French project

\footnotetext{
${ }^{6}$ http://www.greenedgeproject.info/

${ }^{7}$ http://www.nke-instrumentation.com/
}

NAOS $^{8}$. If sea ice is present at the surface, Argo floats need to postpone surfacing. It will perform several consecutive profiles without sending stored data. PRO-ICE have additional nonvolatile memory and are able to record data from all profiles including those performed during wintertime. Because of the need to transmit a high volume of data, communications are performed via a two-way Iridium link, which is faster than the older ARGOS link. This minimizes time at the surface and allows instructions to be transmitted to the float. In addition, floats to be deployed in Arctic seas need to be able to deal with a wide range of seawater salinity/density. Some Argo floats, like PRO-ICE, do not require pre-ballasting as a function of mean seawater density. This is a huge advantage since they can surface easily in a low-density surface seawater layer (Riser et al., 2016).

The PRO-ICE floats use a combination of three technologies to detect ice: a reversed altimeter (active acoustics), an Ice Sensing Algorithm (Klatt et al., 2007) (ISA) based on seawater freezing temperature and an optical sensor. In the Arctic Ocean, the threshold of the ISA algorithm is difficult to determine because of the high levels of variation in salinity (seasonal and regional), compared to the homogeneous salinity present in Antarctica. Takuvik gathered a substantial data base of temperature and salinity data in Baffin Bay, linked to ice cover information in order to locally adapt the threshold of ISA.

Furthermore, the resolution provided by an altimeter is not sufficient to detect a thin-ice layer. For this, Takuvik has

\footnotetext{
${ }^{8}$ Naos, "Equipex Naos." http://www.naos-equipex.fr/.
} 


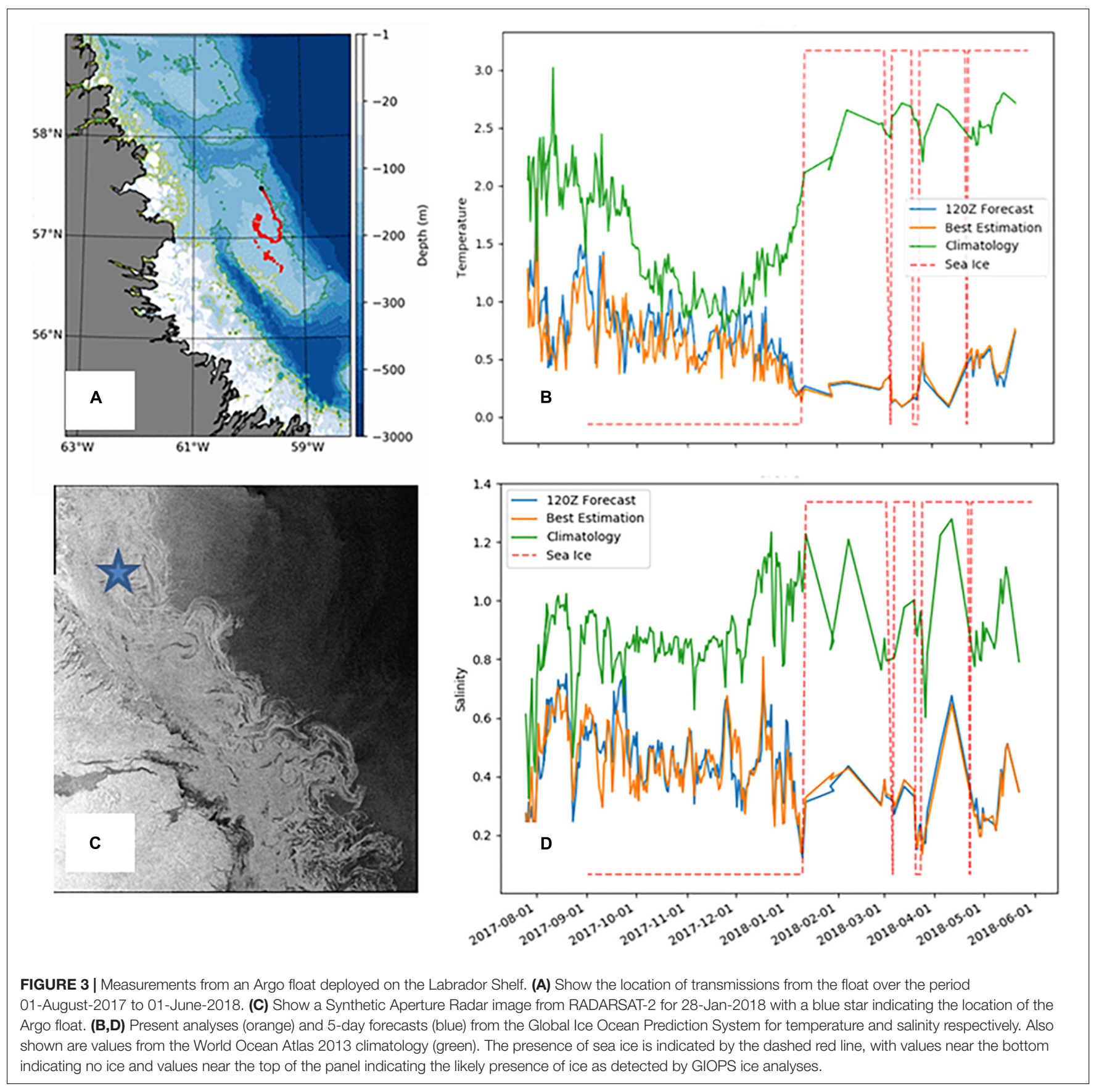

developed an ice-detection system based on laser polarimetry (Lagunas et al., 2018). Since sea-ice is a strong light depolarizer, this characteristic was used as an indicator of the presence or absence of ice. This ice-detection system has been installed on several PRO-ICE BGC-Argo floats deployed yearly in Baffin Bay since 2016.

A potential solution to data transmission issues caused by an inability to surface under the ice cover may be provided by underwater acoustic networks. Multipurpose underwater acoustic networks can provide under-ice positioning ("underwater GPS"; Lee and Gobat, 2008) and low bit-rate communication services (Freitag et al., 2015) to AUVs fitted with low-power acoustic receivers. Acoustic positioning systems have been deployed for under-ice navigation of Argo floats in the Weddell Sea (Klatt et al., 2007). Challenges for designing basin-scale acoustic systems include modeling the time-varying nature of the Arctic sound channel, for which measurements of annual variations of under-ice sound profiles and icebottom roughness are scarce (Rehm et al., 2018). The role of multipurpose acoustics networks as key components of polar observing systems is explored further in a complementary article (Howe et al., 2019). 


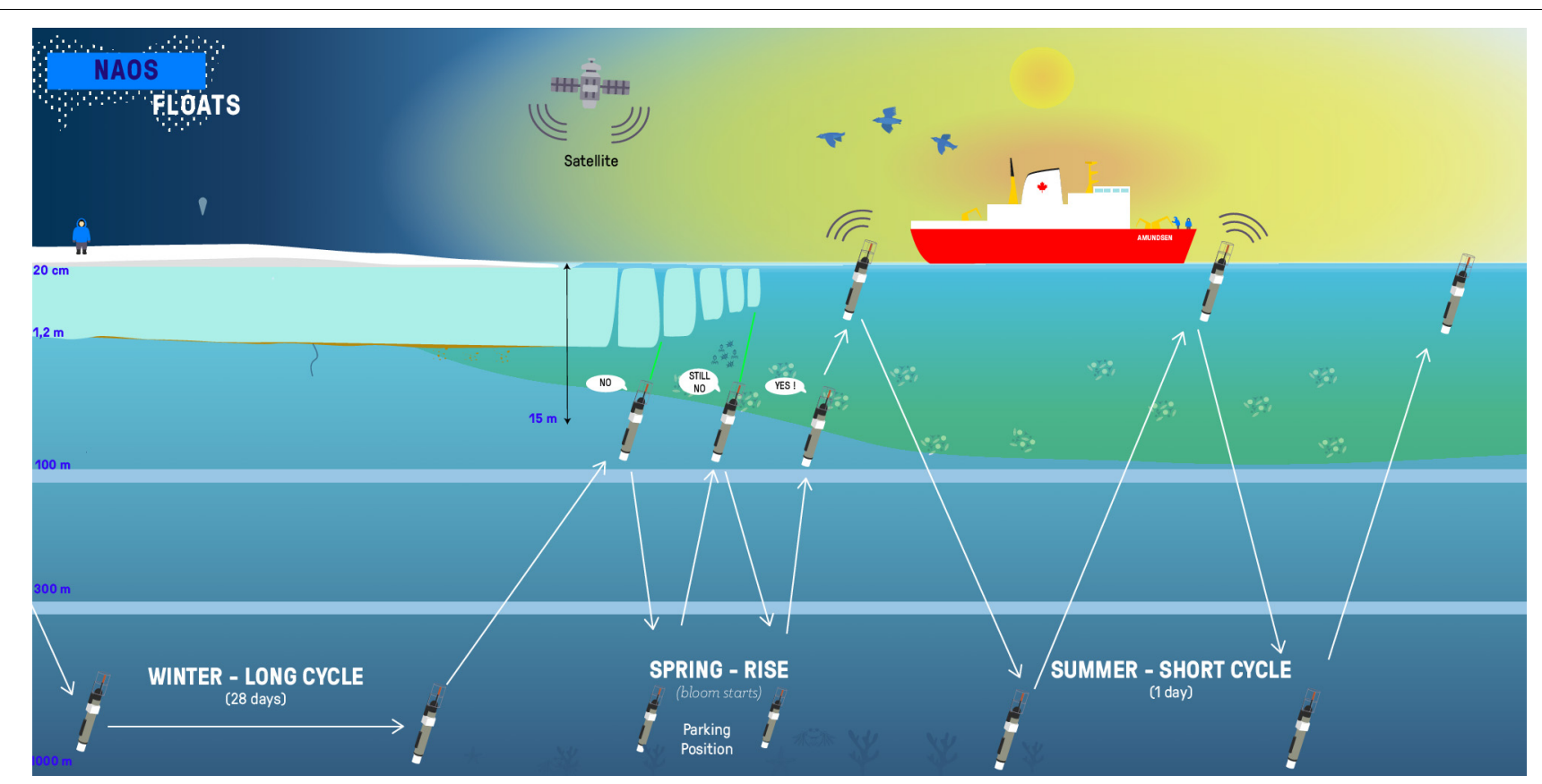

FIGURE 4 | A daily profile cycle of BGC-Argo floats deployed during the 2016 Green Edge scientific mission in Baffin Bay. The main goal is the understanding of the dynamics of the phytoplankton spring bloom and determine its role in the Arctic. During the spring-period the risk of colliding with sea-ice when emerging is a threat to the security of the floats. Moreover, during wintertime, geo-localization and the use satellite networks for data transmission and commands reception is not yet possible (Credit: J. Sansoulet, Takuvik).

Data acquired by Takuvik's PRO-ICE floats are available in the Argo database at the CORIOLIS Global Data Assembly Centre ${ }^{9}$ (GDAC). The data policy set for Argo mandates free access to the data for all interested scientists, research groups and operational agencies. The data management is designed to facilitate easy and immediate access to data not only in real time, but also in delayed mode.

\section{Surface Drifters and IABP}

Surface air pressure, temperature and ocean/ice circulation in the polar regions (Figure 5) are observed by a network of drifting buoys maintained by participants of the International Arctic Buoy Programme (IABP ${ }^{10}$, Figure 6) and the International Programme for Antarctic Buoys $\left(\mathrm{IPAB}^{11}\right)$ ). Over the Arctic Ocean and its peripheral seas (north of $60^{\circ} \mathrm{N}$ ), there were about 120 buoys reporting as of August 2018. Most of these buoys were located in the North American sector, with only a handful reporting in the Eurasian sector of the Arctic. This gap in the Arctic Observing Network creates a significant uncertainty in the analyzed fields of sea level pressure, temperature and winds (Inoue et al., 2009, Figure 7).

In the Southern Ocean (south of $40^{\circ} \mathrm{S}$ ) there were about 50 buoys as of August, 2018. Although over 100 buoys were deployed during the Austral summer near the coast of Antarctica, most of

${ }^{9}$ CORIOLIS, "Argo float data and metadata from Global Data Assembly Centre (Argo GDAC). Ifremer.” http://doi.org/10.17882/42182.

${ }^{10}$ http://IABP.apl.uw.edu

${ }^{11} \mathrm{http}: / /$ www.ipab.aq these buoys were destroyed by the sea ice during freeze up or have been blown north, away from the coast by the prevailing winds.

Maintaining the polar observing networks near the coasts around most of Antarctica, and in the Eurasian Arctic is an ongoing challenge, since the prevailing winds and ocean currents quickly transport the buoys away from the coast. Ideally, the IABP and IPAB networks should be reseeded during the winter, but it is difficult to deploy buoys from ships and aircraft during the polar night and extreme cold and harsh conditions of winter.

The participants of the IABP and IPAB strive to release their data onto the WMO/IOC GTS in near real-time by both the global research and operational weather and ice forecasting communities.

\section{SATELLITE OBSERVATIONS}

Satellite observations of polar oceans have been acquired for more than four decades by different measuring systems. The most prominent record comes from microwave radiometers that have been documenting the reduction of sea ice extent (Cavalieri and Parkinson, 2012; Osborne et al., 2018). Remote sensing systems also provide valuable information about other parameters of the sea ice (concentration and thickness) or about the underlying ocean (temperature and circulation). However, despite continuous technological advances and progress in data processing, several weaknesses can be pointed out and should be considered in the future for improving the observing system of polar regions. 


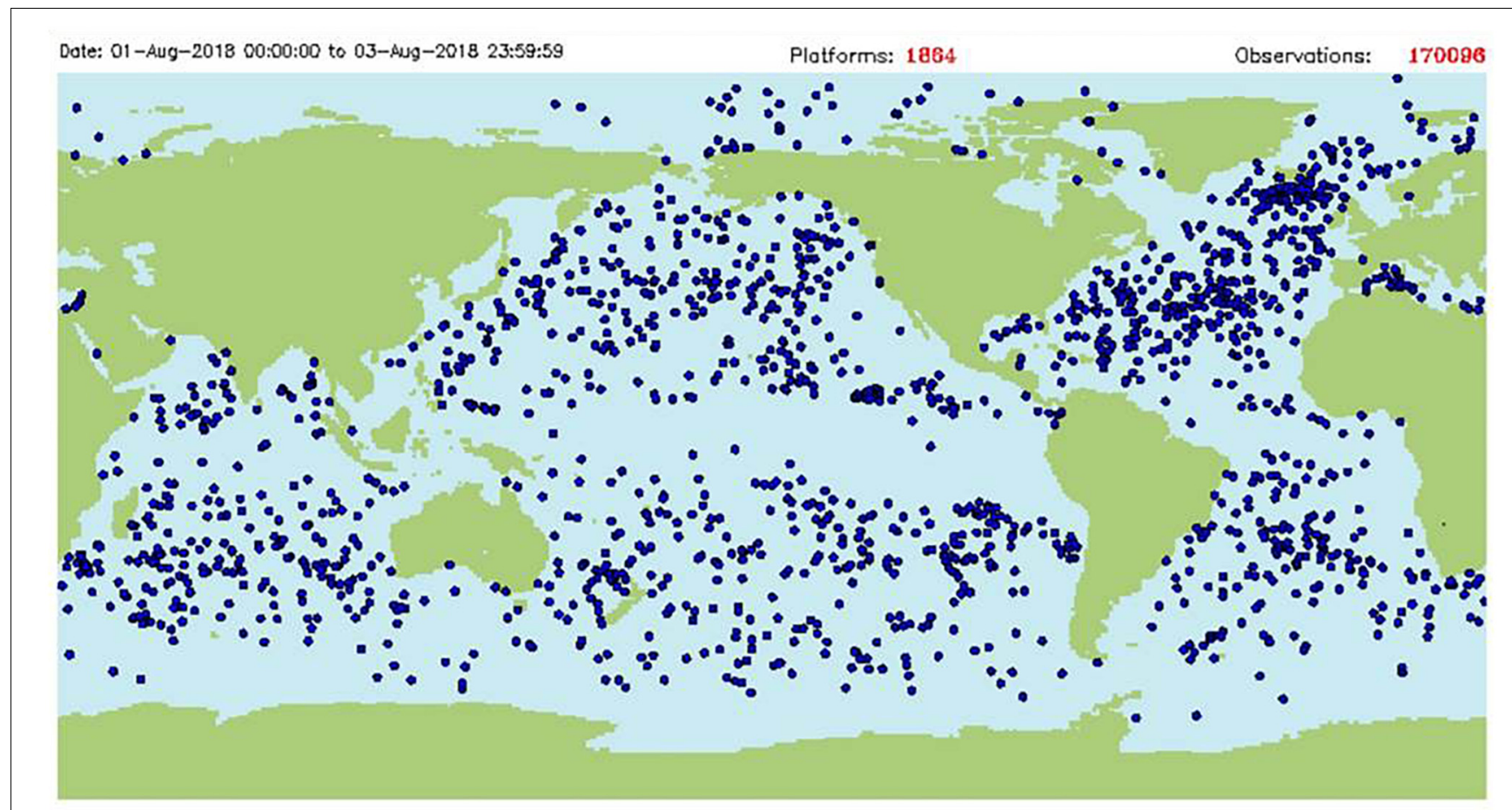

FIGURE 5 | Map of drifting buoys reporting on the WMO/IOC GTS in August 2018. Source: http://OSMC.NOAA.GOV.

\section{Sea Ice Concentration}

Satellite-based sea ice observations are required for assimilation to provide accurate polar environmental forecasts. These observations have been proven beneficial in improving prediction skill at different temporal scales when assimilated (e.g., Lisaeter et al., 2003; Lindsay and Zhang, 2006; Stark et al., 2008; Massonnet et al., 2013; Tietsche et al., 2013). In addition, it has also been shown that summer sea ice thickness (SIT) can be constrained to some extent by assimilating sea ice concentration (e.g., Yang et al., 2015). However, there is a significant spread in sea ice concentration products obtained through different retrieval algorithms (Ivanova et al., 2014), which affects the consistency of ocean-sea ice analyses that assimilate those products (Chevallier et al., 2016; Uotila et al., 2018), and the skill of seasonal predictions initialized from those reanalyses (e.g., Bunzel et al., 2016).

\section{Sea Ice Freeboard and Thickness}

Observing SIT from space is a challenge (Kwok and Sulsky, 2010), and gridded data are sparse. Recently SIT has been estimated using altimetry (CryoSat-2; Kwok and Cunningham, 2015), or the L-band radiometers of SMOS (Kaleschke et al., 2012; TianKunze et al., 2014) and NASA's SMAP (Paţilea et al., 2019) satellites, for thinner ice. The ICESat-2 laser altimeter satellite, which was launched in September 2018, is also starting to provide polar SIT estimates.

Sea ice thickness is typically derived from radar altimetry observations of freeboard using the hydro-static equilibrium assumption (e.g., Alexandrov et al., 2010). Both the freeboard retrieval from altimeter measurements (e.g., Ricker et al., 2014) and the freeboard to thickness conversion are active fields of research. The freeboard to thickness conversion is constrained by the limited availability of auxiliary information (sea ice type, density and snow parameters). Snow thickness on sea ice is still poorly known and is a major source of uncertainty. Snow depth models are not yet satisfactory, and alternative strategies will need to be defined to permit improving snow thickness estimation over sea ice. The joint use of $\mathrm{Ku}$ and $\mathrm{Ka}$ frequencies may facilitate estimates of the snow load above the ice pack. Studies based on AltiKa (Ka-band about $35.7 \mathrm{GHz}$ ) and CryoSat-2 (Ku-band about $13.5 \mathrm{GHz}$ ) satellite data have shown that differences in penetration of $\mathrm{Ka}$ - and $\mathrm{Ku}$-band are correlated with snow loading on sea ice (Armitage and Ridout, 2015; Guerreiro et al., 2016). Although AltiKa only provides measurements up to $81.5^{\circ} \mathrm{N}$ and thus cannot provide pan-Arctic data. The combination of laser (ICESat-2) and radar altimetry is also promising for this purpose. A better estimation of freeboard and then thickness would greatly benefit from such measurement complementarity. Data editing is also important on heterogeneous surfaces such as sea ice, where melt ponds act as bright targets in the radar footprint resulting in peaky waveforms that look very similar to returns from leads. As a result, radar altimeter ice thickness products based on CryoSat-2 (e.g., Hendricks et al., 2016) are not available in summer months.

Previous studies showed that assimilation of SMOS ice thickness significantly improves the first-year ice estimates (Yang et al., 2014; Xie et al., 2016). Furthermore, assimilating CryoSat2 and SMOS SIT leads to a reliable pan-Arctic SIT estimates (Mu et al., 2018; Xie et al., 2018), and also has the potential to 


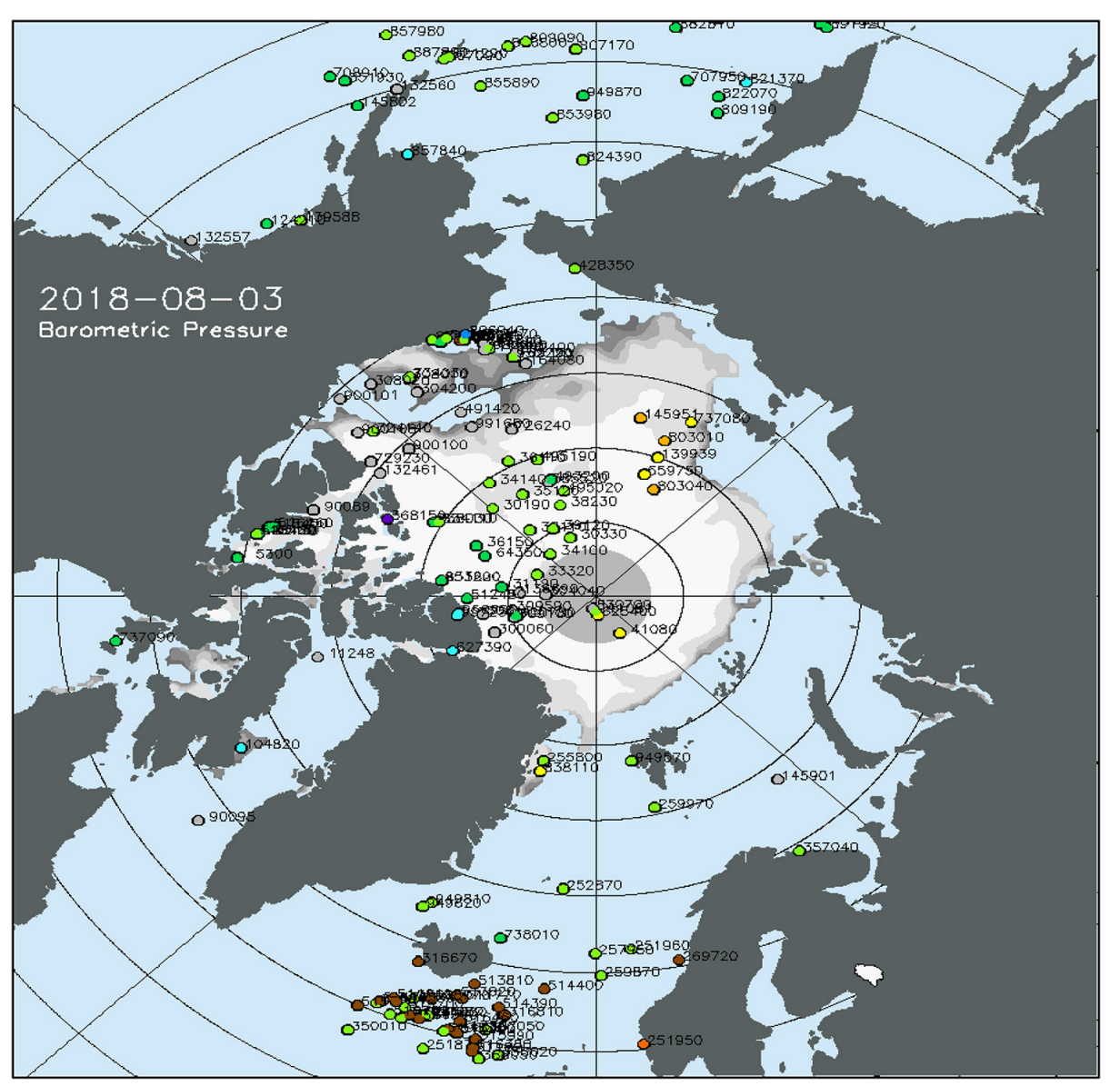

FIGURE 6 | Map of drifting buoys reporting in the Arctic on August 3, 2018. Source: http://IABP.apl.uw.edu.

improve seasonal forecasts of Arctic sea ice (Chen et al., 2017; Blockley and Peterson, 2018). The Cryorad mission has been proposed by Macelloni et al. (2017) as a microwave radiometer at very low frequency (down to $500 \mathrm{MHz}$, lower than SMOS) which longer wavelengths would penetrate through thicker SIT. A critical difficulty related to this mission is the contamination by Radio-Frequency Interference.

\section{Sea Surface Temperature}

Satellite SST retrievals in polar regions are challenging. SST is estimated from instruments operating in the infrared (IR) and microwave regions of the spectrum through the so-called atmospheric window regions. IR retrievals in polar regions are often limited by the observed abnormal (often very dry) atmospheric conditions (Vincent et al., 2008). In addition, there are issues in detecting clear-sky ocean conditions that are free from cloud and sea ice, issues which are compounded during polar nights and in areas of persistent cloud. Consequently, frequent measurements of SST at high latitudes rely on microwave imaging instruments. Although not impacted by cloud (unless precipitation), microwave retrievals are impacted by issues in detecting sea ice, especially at the edges of the instrument footprint.
Many SST retrieval algorithms for both IR and microwave rely on in situ data to account for both deficiencies in their calibration as well as correcting for atmospheric attenuation. The lack of in situ data should be addressed and new innovative approaches are needed (e.g., Castro et al., 2016). The Group for High Resolution Sea Surface Temperature (GHRSST) coordinates the production of multi-satellite merged SST products. However, the lack of accurate satellite and in situ data means there is little consistency between products, especially at high latitudes (Dash et al., 2010).

The current lack of continuity of microwave imagers that can be used to derive global SST is a major concern. For polar regions this requires the inclusion of a channel around $6.9 \mathrm{GHz}$ (Gentemann et al., 2010). Currently, the only approved future instrument with this capability is the Chinese Microwave Radiometer Imager (MWRI) onboard the HaiYang2B (HY-2B). A Copernicus Imaging Microwave Radiometry (CIMR) is currently being studied by the European Space agency (ESA) and JAXA is planning a follow-on to the Advanced Microwave Scanning Radiometer (AMSR2). The AMSR3 and CIMR missions are highly complementary and in combination would provide improved coverage and sampling in polar regions. 


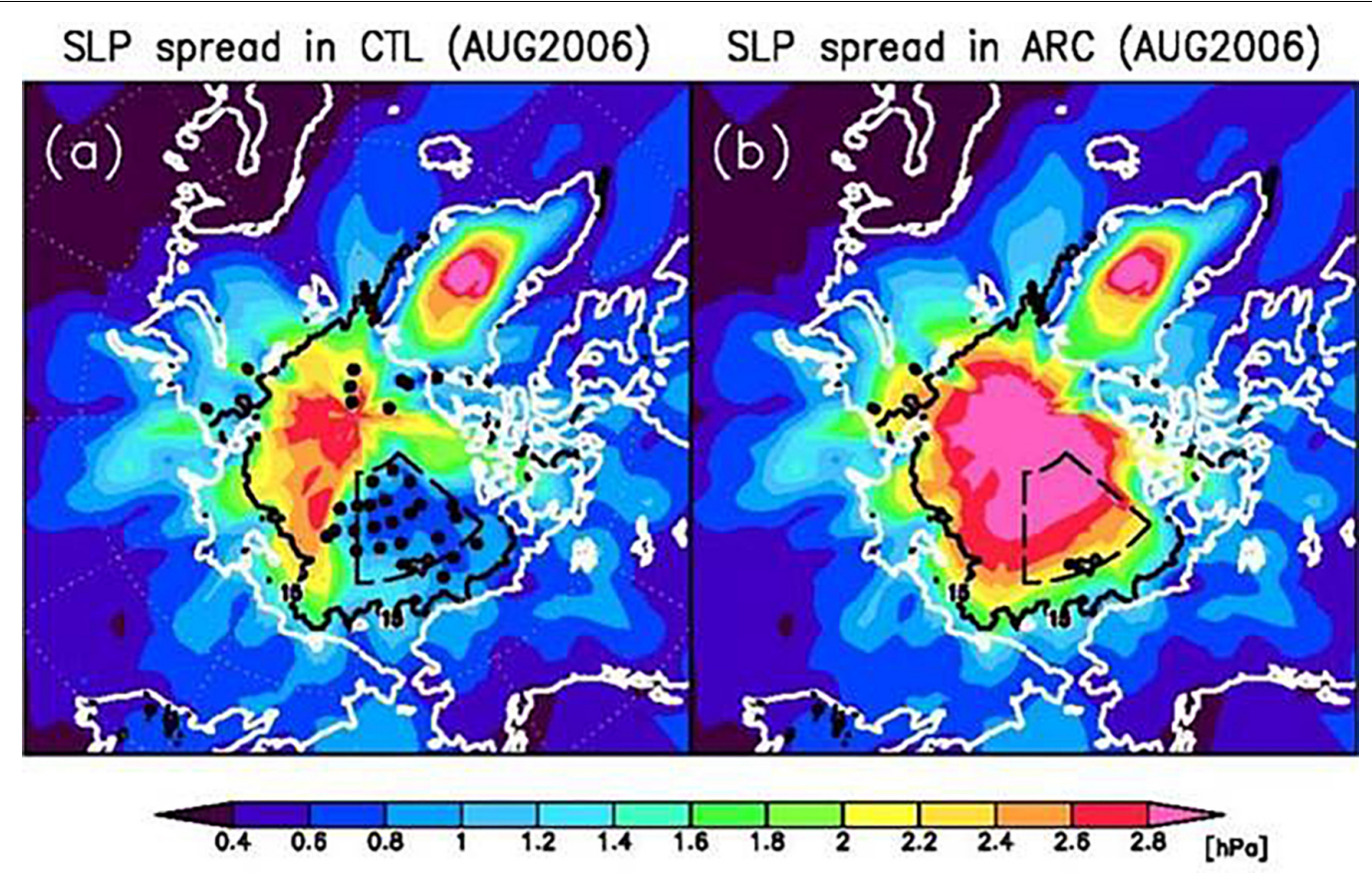

FIGURE 7 | Standard deviation (SD) of sea level pressure measurements from various atmospheric reanalyses. The SD is low in areas where there are buoy observations (A). The spread increases to cover the whole Arctic when the observations from the buoys are removed from the reanalyses (B) (Inoue et al., 2009).

\section{Sea Surface Height}

Satellite observations of sea level are required to constrain surface geostrophic currents in ocean forecasting systems, and several teams try to tackle the issue (Prandi et al., 2012; Andersen and Piccioni, 2016; Armitage et al., 2016). However, sea level observations from altimetry over polar regions suffer from three main issues:

1. First, the altimeter constellation has mainly been created to fulfill ocean requirements for the ice-free regions, in particular with regard to orbit coverage/inclination. With the exception of the CryoSat- 2 mission, which covers the Arctic Ocean up to $88^{\circ} \mathrm{N}$, altimetry missions do not cover poleward of $82^{\circ}$, leaving a vast region without any measurement.

2. Second, although significant progress has been made to distinguish whether measurements correspond to open ocean, ice floes or leads within the sea ice, further progress is still needed to unambiguously identify the different returns, in particular in complex mixed water/floe areas. Exploitation of close match-ups between SAR imagers and altimeter measurements as the potential for improving the identification of leads and the editing of ambiguous measurements coming from melt ponds or polynya in the melt season. An example of such collocation between a Sentinel-1 image and Sentinel-3 measurement is provided in Figure 8 (Longépé et al., 2019).

3. Lastly, the accurate retrieval of absolute SSHs, and therefore currents, in polar regions also suffers from degraded corrections. Tide models show higher

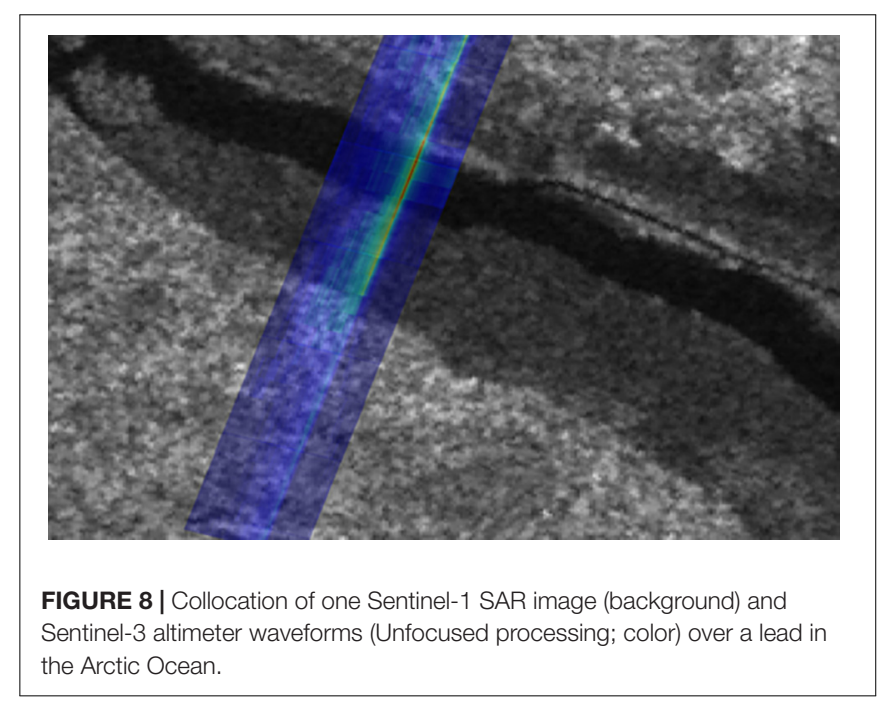

inter-model variability in polar regions than anywhere else, mean sea surfaces are not as accurate. An effort to refine the geophysical corrections applied to altimeter measurements is needed to improve polar SSH accuracy levels and make them useful for assimilation in operational oceanography systems.

As a result, there is currently no dedicated operational Arctic sea level product for assimilation in models. Efforts to provide Arctic sea level information in CMEMS, following the current SL-TAC products are ongoing. 
Other innovative concepts such as SKIM's (Sea surface KInematics Multiscale monitoring, Ardhuin et al., 2018) rotating radars at different angles offer opportunities to monitor waves, surface currents and possibly sea ice drift. The Surface Water Ocean Topography (SWOT) mission also has the potential to provide innovative new observations for sea level and sea ice cover, although its orbit is lower than 70 degrees.

\section{Sea-Ice Drift}

Sea ice drift data are now obtained all year round both in the Arctic and Antarctic by pattern cross-correlation of scatterometers and passive microwave images. For global information, Scanning Multichannel Microwave Radiometer (SMMR), the Special Sensor Microwave/Imager (SSM/I) and SSMI/Sounder (SSMIS), and the Advanced Very-HighResolution Radiometer (AVHRR) are used. Currently, daily products (e.g., Lavergne et al., 2010; Tschudi et al., 2019) typically use data acquired at Day D-1, with span of 24 or $48 \mathrm{~h}$. In addition, buoy observations of the International Arctic Buoy Program (IABP), and ice motion derived from NCEP/NCAR surface wind vectors can be used. Low resolution ice drift products are calculated daily from aggregate charts derived from radiometers (e.g., SSMIS, AMSR2) or scatterometers (e.g., ASCAT). The typical resolution of these input images is $12.5 \mathrm{~km}$. The large acquisitions, the repetition of the acquisitions, and their independence with respect to the weather conditions allow a daily polar coverage.

Sequences of SAR images can be used to derive higher resolution drift information. Algorithms have been developed to calculate ice drift from successive pairs of SAR images covering a common area. They are generally based on a spatial correlation calculation between these images, at several resolution scales (from the coarsest to the finest). In Europe, Sentinel-1 is used by DMI to produce an operational sea ice drift product as part of CMEMS (Pedersen et al., 2015). SAR data from Sentinel$1 \mathrm{~A} / \mathrm{B}$ constellation allow the derivation of daily fields of sea ice deformation at $2 \mathrm{~km}$ resolution (Korosov and Rampal, 2017). The algorithm developed by FMI has been operational in the Baltic Sea since early 2011 (Karvonen, 2012), using the wide Radarsat-1 ScanSAR mode and the wide swath ASAR Envisat mode data.

Revisit is the key here: higher revisit of SAR images is naturally required. Algorithms often use image tracking features between consecutive images. This type of algorithms perform better if the images are taken with the same frequency, short interval, and ideally same geometry. Joint acquisition of multifrequency SAR would enable accurate sea ice drift products, which is not possible with stand-alone current mono-frequency SAR missions. Cross-pol channel is often preferred as it is less sensitive to incidence angle variation (at least for C-band SAR). Drift cannot be calculated in areas without characteristics (i.e., so-called "level ice" and open water areas). These are of course only estimates of the integrated ice trajectories between the time instants corresponding to the SAR acquisition times. Lagrangian drift products are typically 2-day trajectories with coarse resolution $(62.5 \mathrm{~km})$ and are not often assimilated even though the accuracy is satisfactory ( $3 \mathrm{~km}$ for 2-days drift).
The TOPAZ4 system does assimilate these sea ice drift operationally, although the effect is relatively weak (Sakov et al., 2012). A different sea ice rheological model, more sensitive to winds, may be more adapted to assimilate this data (Rabatel et al., 2018). More precise (500 $\mathrm{m}$ for 1-day drift) and detailed (10 km resolution) sea ice drift products are now obtained year-round from Sentinel-1 SAR images, which cover about 70\% of the Arctic as of February 2019.

\section{AIR-SEA FLUX MEASUREMENTS IN POLAR OCEANS}

\section{The Need and Challenge for Air-Sea Flux Observations in Polar Regions}

Air-sea fluxes quantify the exchanges of heat, momentum, freshwater, gases and aerosols between different components of the polar climate system (i.e., atmosphere, water column, sea ice). Flux observations are essential for understanding the global energy budget (Trenberth and Fasullo, 2010), for evaluating forecasting and climate models, and for evaluating processes such as ocean heat uptake and mixed-layer temperature and SST variability (e.g., Dong et al., 2007). However, in situ air-sea or air-ice flux observations are extremely sparse in polar regions (e.g., Bourassa et al., 2013), with almost no winter observations in the Southern Ocean (e.g., Gille et al., 2016; Swart et al., 2019) or in ice-covered ocean domains in both the Arctic and Antarctic (e.g., Taylor et al., 2018). Quantifying air-sea exchange in regions with sea ice requires specialized approaches that must account for spatio-temporal heterogeneity. This has led to significant gaps in our knowledge of both air-sea and air-seaice exchanges.

Since there are few reliable near-surface atmospheric observations to serve as constraints, reanalyses and satellite derived surface flux products have major errors and vary considerably between products (Josey et al., 2013; Bentamy et al., 2017; Schmidt et al., 2017; Taylor et al., 2018). For example, in the Southern Ocean, atmospheric models (and reanalyses) have large air-sea heat flux biases, including substantial short-wave errors related to their inability to adequately represent super-cooled liquid cloud water (Bodas-Salcedo et al., 2014, 2016), and these errors appear to bias coupled model SST (Hyder et al., 2018), sea ice, and wind (Bracegirdle et al., 2018). Moored-buoy flux observations or year-round ice camps are required to evaluate and ultimately improve these products but to date buoys have been deployed in only two locations in the Southern Ocean (Schulz et al., 2012; Ogle et al., 2018) and in only a few instances in the Arctic, (Taylor et al., 2018). The Surface Heat Budget for the Arctic Ocean (SHEBA) program offered the only year-round ice camp in the Arctic (e.g., Persson et al., 2002), although Multidisciplinary drifting Observatory for the Study of Arctic Climate (MOSAiC; see MOSAiC) will soon extend this. The Southern Ocean Observing System (SOOS) working group on Southern Ocean air-sea fluxes (SOFLUX; Swart et al., 2019) has been working to coordinate observing system capabilities and requirements for high-latitude air-sea fluxes. 


\section{Impact of Flux Uncertainty on Forecasting/Prediction}

There are significant advantages to forecasting with a coupled atmosphere-ice-ocean system, especially on longer timescales. The intrinsic turbulence of the atmosphere limits predictive skill to timescales of days to weeks (Mariotti et al., 2018), whereas the large-scale ocean is predictable on monthly time scales. This provides a mechanism by which the predictability of atmosphere may be extended allowing skillful seasonal predictions.

A particular limitation in this regard is with respect to the significant uncertainty in the atmosphere-ocean boundary layer, made even more egregious when considering sea ice. Boundary layer dynamics involve vertical scales unresolved by coupled forecasting systems, which must be parameterized (e.g., Pullen et al., 2017). The exchanges between components are parameterized by so-called bulk formulae, which estimate airsea-ice exchanges based on near-surface large-scale properties. Currently, the uncertainties in bulk formulae are a primary bottleneck to seasonal prediction (Penny and Hamill, 2017). In other words, even if we had a perfect ocean model with perfect initial conditions, the information retained in the monthly ocean prediction would be degraded when propagated to the atmosphere due to uncertainty in estimating the true exchanges (Vecchi et al., 2014). Therefore, a priority in the coming decade must be to gather in situ estimates of air-sea-ice exchanges in the context of large-scale properties informing how to minimize errors in the bulk formulae parameterizations. Efforts such as YOPP, MOSAiC and ONR Marginal Ice Zone (MIZ) project are examples of projects aiming to address this gap.

We recommend further research to determine how best to represent these exchanges in a coupled forecasting system, with a focus on determining what aspects of boundary layer physics need to be resolved and what can be skillfully parameterized. For parameterized physics, we require that process studies be carried out to determine parameterizations and parameterization coefficients, including identifying the observations that should be sustained to validate estimated fluxes. Addressing these areas are primary goals to enable weekly-to-seasonal skillful predictions in the polar regions.

\section{FORECASTING SYSTEM EXPERIMENTS}

Understanding how assimilating more observations will impact modeled analyses and short-term forecasts is of fundamental importance as new coupled models are being developed. For instance, preliminary results using a regional coupled model [CAFS from the NOAA Earth System Research Laboratory (ESRL)] demonstrated that current ocean reanalyses do not include realistic representation of subsurface water masses relative to observations taken in 2015 and 2016 (including Arctic Heat data). Forecast experiments using a high-resolution fully coupled regional model show that these water masses impact sea ice evolution on synoptic time scales through upper-ocean mixing and heat flux at the ice-ocean interface. It is expected these effects will increase as sea ice continues to decline and surface heat flux processes increase. In addition, assimilating real-time ocean observations in the initial forecast conditions allows for the identification of biases in the coupled system, which are difficult to isolate when the ocean is allowed to drift away from the observed state. Some studies (e.g., Inoue et al., 2015; Lien et al., 2016; Xie et al., 2017) have begun to show the important potential impact of assimilating enhanced observations on model-based analyses and short-term sea ice forecasts. Additional focused research in this area would allow us to explore coupled model data assimilation issues, better understand physical processes, and assess model performance in comparison to noncoupled (atmospheric) model frameworks. In the next sections, we review a few results from operational centers.

\section{Impacts of Arctic and Antarctic Observations in U.S. Navy Coupled Ice-Ocean Models}

The ability to forecast sea ice conditions is of crucial importance for maritime operational planning (Greenert, 2014). The current U.S. Navy operational sea ice forecast system is the Global Ocean Forecast System (GOFS) version 3.1. GOFS utilizes the Los Alamos Community Ice CodE (CICE) version 4.0 (Hunke and Lipscomb, 2008) sea ice model, which is two-way coupled with the HYbrid Coordinate Model (HYCOM) (Metzger et al., 2014) ocean model. The grid resolution is $1 / 12^{\circ}$, with horizontal resolution approximately $3.5 \mathrm{~km}$ at the poles. Atmospheric forcing is provided by the NAVy Global Environment Model (NAVGEM; Hogan et al., 2014). The precursor to GOFS 3.1 was the Arctic Cap Nowcast/Forecast System (ACNFS; Posey et al., 2015). ACNFS is a coupled ice/ocean model similar to GOFS with two main differences: (1) ACNFS only covered areas north of $40^{\circ} \mathrm{N}$, and (2) in ACNFS HYCOM has 32 ocean layers compared to 41 in GOFS. An important part of both GOFS and ACNFS is the assimilation of observational data, which is accomplished using the Navy Coupled Ocean Data Assimilation (NCODA) system (Cummings and Smedstad, 2014).

Assimilation of observational data is performed to reduce errors in model forecasts that can result from many factors including non-linear processes that are not deterministic responses to atmospheric forcing, poorly parameterized physical processes, limitations in numerical algorithms, and limitations in model resolution. Polar observational data assimilation is an essential part of GOFS forecasts. Sea ice concentration observations are currently assimilated from the Defense Meteorological Satellite Program (DMSP) SSMIS and AMSR2. These observations are used in conjunction with the Interactive Multi-sensor Snow and Ice Mapping System produced by the U.S. National Ice Center (NIC; Helfrich et al., 2007). A full description of the assimilation process in GOFS and ACNFS can be found in Hebert et al. (2015).

The impact of data assimilation in the U.S. Navy models is significant. One measure that the U.S. Navy uses to determine the accuracy of the modeled sea ice edge (defined in Hebert et al., 2015) is the distance between it and the sea ice edge determined daily by analysts at NIC. The sea ice edge error over six regions in each hemisphere is shown in Figure 9 as well as the entire Arctic/Antarctic. GOFS was run for 1 year (2014) without data assimilation and compared to the current GOFS 

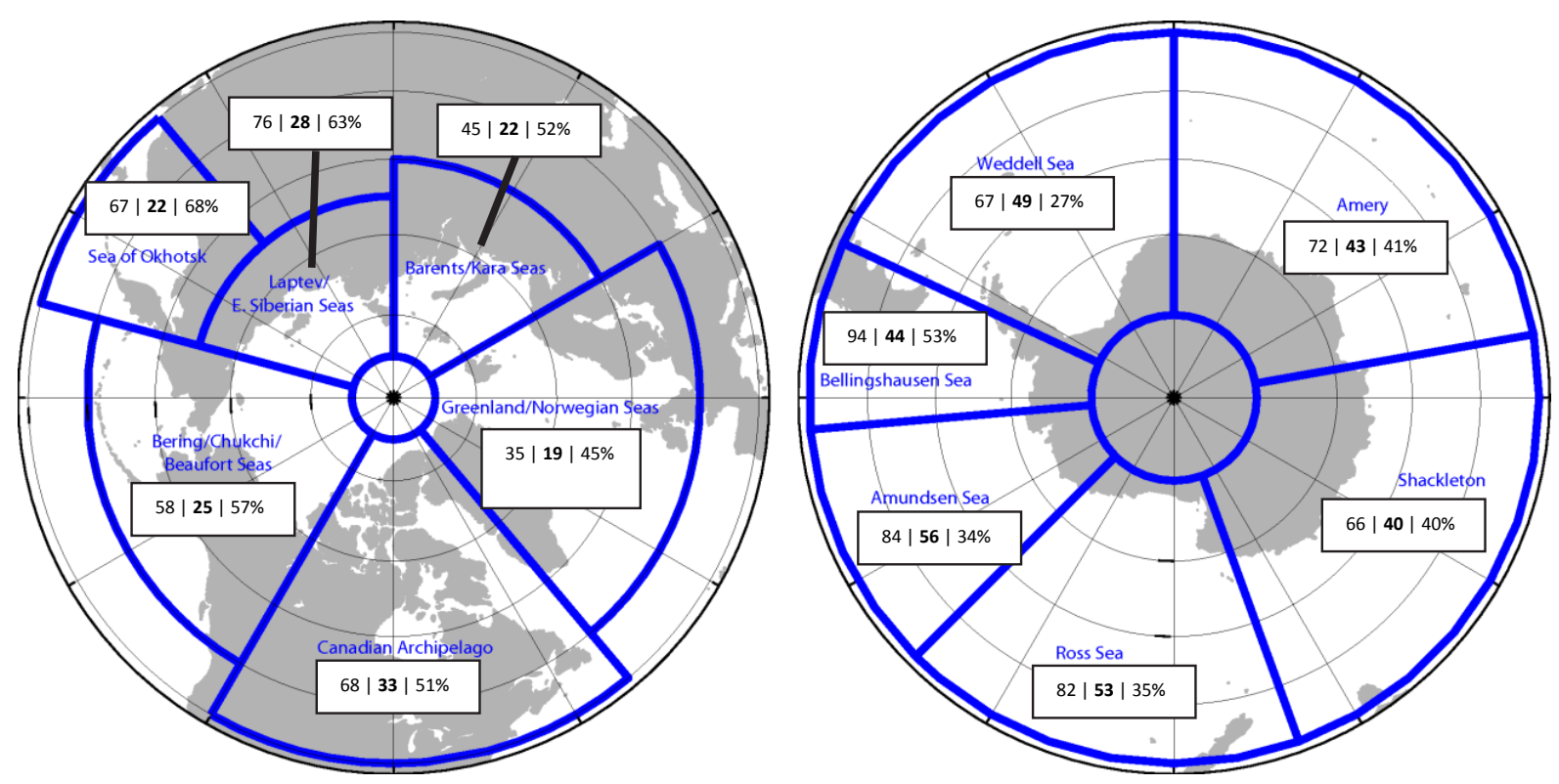

FIGURE 9 | lce edge error for individual regions ( $\mathrm{km}$ ). Each region contains three numbers. First number is ice edge error without assimilation. Second bold number is error with assimilation. Third number is percent improvement with assimilation. In the Arctic the overall reduction in ice edge error with observational data assimilation is $31 \mathrm{~km}$ (56\%); in the Antarctic the overall reduction is $28 \mathrm{~km}$ (37\%).

system with ocean and sea ice data assimilation, including sea ice concentration. The impact of assimilating these observations was to reduce the sea ice edge error in the Arctic by $56 \%(31 \mathrm{~km})$ and in the Antarctic by $37 \%(28 \mathrm{~km})$, with a reduction in sea ice edge error for each region ranging between 27 and $68 \%$.

As model resolution increases, so does the need for highresolution observations. SSMIS and AMSR2 are relatively coarse resolution (25 and $10 \mathrm{~km}$, respectively) compared that of GOFS. Higher resolution (less than $1 \mathrm{~km}$ ) sea ice concentration observations are available from the Suomi NPP Visible/Infrared (VIIRS). Although VIIRS observations can be obstructed by clouds, including high resolution VIIRS ice concentration into our data assimilation further reduced GOFS ice edge error by $19 \%$ $(5 \mathrm{~km})$ in the Arctic and $11 \%(4 \mathrm{~km})$ in the Antarctic. This result points to the need for higher resolution sea ice concentration observations to use in model applications.

In an earlier study using ACNFS to examine the impacts of sea ice concentration observations in ship routing and planning in boreal winter (January-March), assimilating satellite ice concentration observations reduced the projected track an ice breaker would take to a ship near the sea ice edge by an average of $150 \mathrm{~km}$ versus not assimilating sea ice concentration observations. This improved the time for planning operations by $12 \mathrm{~h}$ and reduced the distance a ship needs to prepare to encounter ice by $212 \mathrm{~km}$.

SIT observations are also important. Currently, pan-Arctic SIT observations on a daily basis are not available. Only limited satellite tracks per day are available that are aggregated on a monthly basis. In a recent study by Allard et al. (2018), ACNFS was re-initialized on March 15th using the March 2014 monthly CryoSat-2 thickness observations and integrated for 18 months.
It showed a reduction in SIT bias by $0.75-0.97 \mathrm{~m}$ compared to ACNFS SIT without CryoSat-2 initialization. The impact of this one-time re-initialization was significant and work is underway to assimilate daily satellite track SIT observations on a daily basis.

\section{Sensitivity of Sea Ice Forecasting Skill to Ocean Mixing Around Antarctica}

The rapid evolution of the sea ice cover can have important impacts on coupled environmental predictions through a variety of processes (Smith et al., 2013). These include the formation of leads and coastal polynyas, as well as changes in the ice cover along the marginal ice zone (MIZ). In these regions, the rapid formation, melt and advection of the sea ice cover can modify atmosphere-ocean fluxes on relatively short timescales. Interestingly, small-scale ocean variability has a role to play here as the timing and intensity of changes will be sensitive to the surface ocean mixing layer depth, water mass properties and mesoscale ocean circulation (e.g., Zhang et al., 1999).

As an illustration of the sensitivity of sea ice evolution to ocean mixing, an evaluation of the skill of two sets of sea ice forecasting experiments is shown in Figure 10. The first set uses the standard configuration of the Global Ice-Ocean Prediction System (GIOPS; Smith et al., 2016) running operationally at the Canadian Centre for Meteorological and Environmental Prediction. GIOPS combines the Système Assimilation Mercator (SAM2) ocean analysis system with a 3DVar ice analysis (Buehner et al., 2013) to produce daily 10-day forecasts using the NEMO ocean model at $1 / 4^{\circ}$ resolution coupled to the CICE ice model. The second set of experiments is identical to the first with the parameterization for surface wave breaking deactivated. Figure $\mathbf{1 0}$ shows the 7-day forecast skill evaluated 

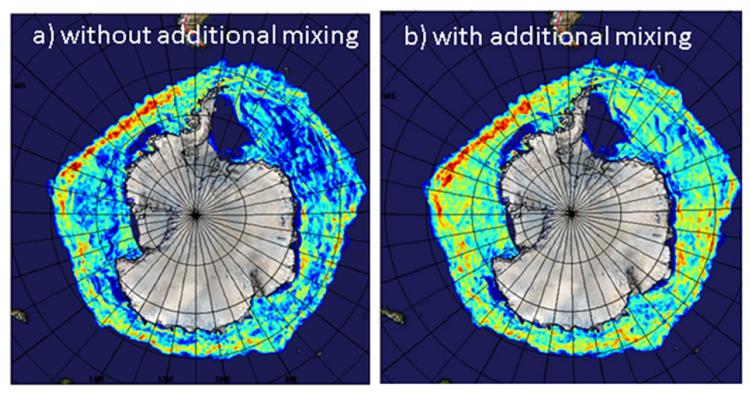

FIGURE 10 | Sensitivity of sea ice forecasting skill to ocean mixing around Antarctica. Weekly 7 days sea ice forecasts from the Global Ice-Ocean Prediction System (GIOPS) running operationally at the Canadian Centre for Meteorological and Environmental Prediction are evaluated against analyses over the year 2011. The evaluation of forecast skill is restricted to points where the analysis has changed by more than $10 \%$ over the forecast period (7 days). Warmer colors indicate larger root-mean squared error (maximum of 0.3 for dark red) with zero error shown as dark blue. Panels (a) and (b) show the forecast skill for experiments without and with additional ocean mixing respectively. Adapted from Smith et al. (2013).

against 3DVar ice analyses from weekly forecasts over 2011. The verification method used here (Lemieux et al., 2016) restricts the error evaluation to areas where the ice concentration analysis has changed by more than $10 \%$ over the forecast lead time (i.e., 7 days). This verification method has the advantage that it focuses the evaluation on 'hot spots' of activity, predominantly in the marginal ice zone.

From Figure 10 it can be seen that a small modification to the ocean vertical mixing can have a first order impact on the ice forecast errors. Interestingly, while the surface wave breaking parameterization degrades ice forecast skill, it does lead to an improvement in water mass properties over ice-free waters (as evaluated against Argo profiles; not shown). This is perhaps not surprising given that the mixing regime in polar regions is quite different from that at lower latitudes. This highlights the need for an expanded under-ice ocean monitoring program to be able to adequately model vertical mixing and constrain water mass properties and mixed layer depths.

\section{Impact of Temperature and Salinity Profiles in the CMEMS Arctic MFC}

There have been special observing periods of the Arctic and Antarctic in the past, in particular the successive International Polar Years (IPYs), the latest of which took place in 2007-2009 (with a gradual ramping up of ocean observing systems in the preceding years). Looking back at the impact of a recent IPY in a period with similar low-ice-coverage conditions in the Arctic, expanded sea ice in the Antarctic, and similar satellite coverage as today can provide another measure of the expected impact of the YOPP Special Observing Periods.

In the Arctic, the TOPAZ4 reanalysis is based on a regional configuration of the HYCOM model coupled to an early version of the CICE model at $12 \mathrm{~km}$ resolution. It assimilates both satellite and in situ observations using an Ensemble Kalman Filter (Sakov et al., 2012). As of 2018, the TOPAZ4 reanalysis system is almost identical to the real-time physical forecasting system used in the Copernicus Arctic MFC and operated by MET Norway (Melsom et al., 2015; Xie et al., 2017). It assimilates the same types of ocean and sea ice observations: along-track sea-level anomalies from altimeters, sea surface temperatures, sea ice concentrations and drift and in situ temperature and salinity profiles.

Ocean models have well-known limitations in simulating the advection of Atlantic Waters into the Arctic (Ilicak et al., 2016), which for a model like TOPAZ4 results in a typical cold bias of the 300-800 m water temperature by $0.5 \mathrm{~K}$ (see Figure 11, blue line) and regional differences by $1.5 \mathrm{~K}$ typically (green line). During the IPY time period, the assimilation of ITPs is able to constrain this bias down to $0.1 \mathrm{~K}$ and reduce the regional differences below $1 \mathrm{~K}$. The end of the IPY in 2009 relaxes the constraint and the bias and RMS errors return to larger values, although slightly lower than before the IPY (i.e., there is no "dynamical shock" after the IPY stops). Even though the quantitative impact on the TOPAZ4 system is dependent on the practical setup of the model and its assimilation scheme, the qualitative behavior may apply to other forecasting systems based on similar types of models and data assimilation schemes and indicates that a density of ITP profiles at least equal to that of the IPY should be sustained continuously to constrain efficiently the Atlantic Water properties in the Arctic.

\section{NOAA-ESRL/CIRES Coupled Arctic Forecast System}

NOAA ESRL has provided experimental, daily, 10-day forecasts of Arctic weather and sea ice evolution to stakeholders during freeze-up seasons since 2015 and daily forecasts year-round starting on February 14, 2018. CAFS produces high-resolution $(10 \mathrm{~km})$ regional coupled-model Arctic forecasts using global $0.5^{\circ}$ GFS forecast fields for lateral forcing. The current configuration of the model includes the POP2 dynamical ocean model (adapted from Maslowski et al., 2012), the CICE5 sea ice model (Hunke et al., 2015), the NCAR CLM4.5 land model, and the WRF3.6 ARW atmospheric model, coupled by the NCAR CPL7 coupler. The domain is the Arctic basin and surrounding coastal regions, including Bering Strait, to provide model guidance for the National Weather Service (NWS), and Fram Strait, to include the complete planned Multidisciplinary drifting Observatory for the Study of Arctic Climate campaign (MOSAiC) domain.

The CAFS forecasts are being used by NOAA ESRL to identify sources of skill on sub-seasonal time-scales due to coupled ocean-ice-atmosphere processes and by stakeholders as model guidance for sea ice forecasts. Real-time CAFS products are made available to the community ${ }^{12}$. Figures and animations from the 10-day forecasts are provided for sea ice, atmosphere, and ocean variability, as well as, an archive of model output for users to download. These forecasts are being used for model guidance by the NWS Alaska Sea Ice Program, the NOAA Arctic Testbed, the U.S. National Ice Center, and by the U.S. Navy and NOAA for operations during Arctic campaigns.

Coupled Arctic Forecast System forecasts use initial conditions that ingest SIT measurements from ESA's CryoSat-2 and SMOS satellites and the NASA Jet Propulsion Laboratory Multi-Scale Ultra-high Resolution Sea Surface Temperature

\footnotetext{
${ }^{12}$ https://www.esrl.noaa.gov/psd/forecasts/seaice
} 


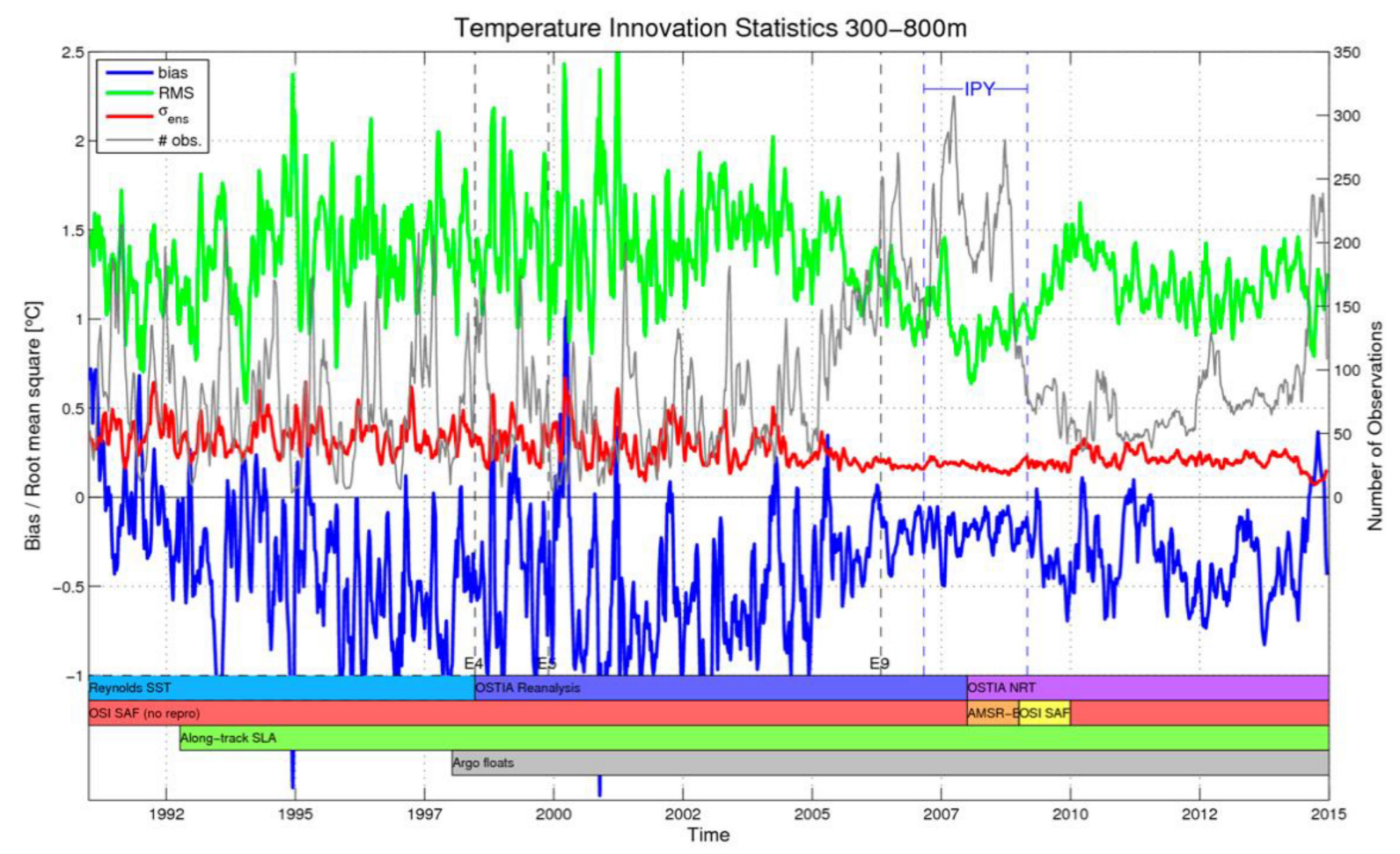

FIGURE 11 | Time series of TOPAZ4 data assimilation diagnostics across the 24-year reanalysis for all temperature profiles in the depths $300-800 \mathrm{~m}$ in the whole Arctic. The blue line is the bias, the green line is the related standard deviation (Root Mean Square), the red line is the ensemble spread, and the gray line the number of temperature observations, increasing during the IPY. The other vertical lines and the bottom bars indicate changes of the other observation data sources and modifications of the data assimilation system.

(MUR) SSTs and sea ice concentration. The MUR SSTs are used to initialize the ocean mixed layer with a mixed-layer depth diagnosed from the model. In order to identify whether using these satellite products in the initial conditions increases the skill of the 10-day forecasts, a series of 10-day hindcasts were performed for the time period of the ONR SeaState DRI, Oct. 1-Nov. 5 2015. The hindcasts are setup exactly like the real-time forecasts except the lateral boundary conditions are the GFS analyses instead of the GFS forecasts, in order to identify potential model biases. In addition, similar to the forecasts, hydrometeor mass and number are initialized with fields from the first day of the previous day's hindcast to reduce spin-up time of cloud fields.

Intensive measurements were taken of the ocean, surface, and atmospheric state during the SeaState campaign. This provides for a comprehensive observational database for model validation. Figure 12 shows the model error of the ocean-atmosphere state at the location of the R/V Sikuliaq over the 6-week campaign at lead times of 6 h, 1 day, and 5 days. At a 6-h lead time, there are equivalent errors in the ocean and atmosphere, less than $0.5^{\circ} \mathrm{C}$. Due to the initialization of the ocean mixed-layer with satellite SSTs, errors in the ocean grow slowly up to 5-day lead times. However, differently in the atmosphere, errors in the lowest $2 \mathrm{~km}$ grow rapidly with errors greater than $+1^{\circ} \mathrm{C}$ in the near-surface temperatures and errors greater than $-1^{\circ} \mathrm{C}$ at the top of the atmospheric boundary layer. This is an indication that the model is unable to maintain the observed boundary layer stratification and rapidly evolves into a less-stable state. It would not have been possible to identify this model bias without initialization of the ocean/ice state. Process studies are currently underway to identify if this bias is due to cloud processes or boundary layer parameterizations.

\section{Quantitative Network Design}

The Quantitative Network Design (QND) approach constitutes a computationally efficient alternative to Observing System Experiments (Fujii et al., 2019). The approach can be used to inform the design of observing networks or space missions. QND evaluates a set of observations (network) in terms of its constraint on a target quantity, i.e., a quantity of interest. This evaluation is performed in a modeling system that is capable of simulating counterparts of the observations and of the target quantity from a set of unknowns in the system (control variables). For a detailed description of the formalism we refer to Kaminski and Rayner (2017). Briefly, it proceeds in two steps: In the first step, the observational information is used to infer the uncertainty in the posterior control vector, $C(x)$, that is consistent with the observational uncertainty, $C(d)$, and the prior uncertainty of the control vector, $C\left(x_{0}\right)$ [Equation (1)]. In the second step the uncertainty in the control vector is mapped onto an uncertainty in the target quantity, $\sigma(y)$ [Equation (2)]. Both steps use appropriate sensitivities (linearizations/response 

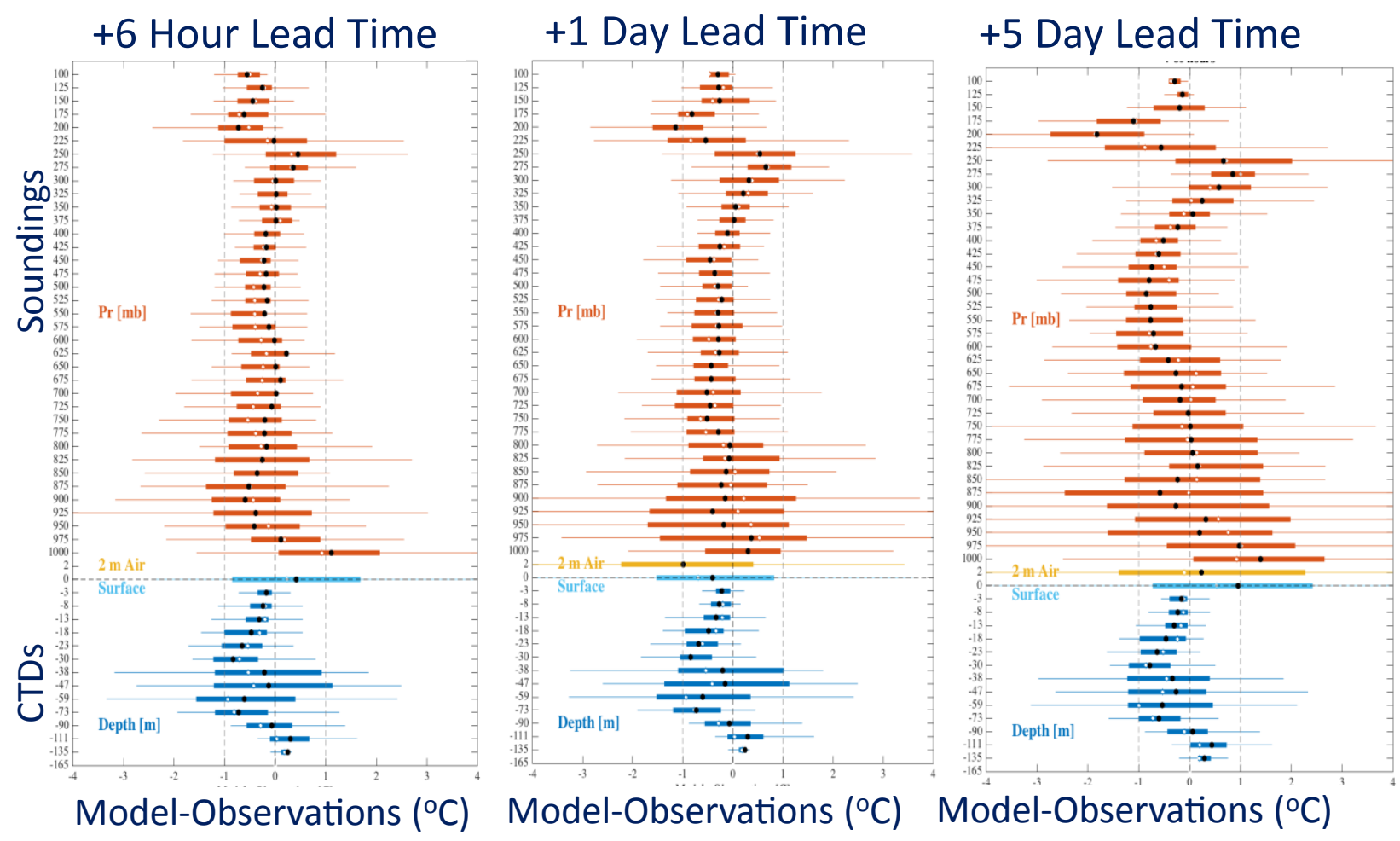

FIGURE 12 | Box and whisker plots of CAFS forecast temperature errors in the full atmosphere-ocean column at $6 \mathrm{~h}, 1$ day, and 5 days lead times compared to radiosondes (red) and CTDs (blue) from the RN Sikuliaq during the ONR SeaState campaign October 1 - November 52015 . Note, the vertical scale is model levels.

functions) of the modeling system. The first step, an inversion step, is formalized by

$$
C(x)^{-1}=M^{\prime T} C(d)^{-1} M^{\prime}+C\left(x_{0}\right)^{-1},
$$

and the second step by

$$
\sigma(y)^{2}=N^{\prime} C(x) N^{\prime T}+\sigma\left(y_{m o d}\right)^{2},
$$

where $\boldsymbol{M}^{\prime}\left(\boldsymbol{N}^{\prime}\right)$ denotes the sensitivity of the vector of observations (the target quantity) with respect to the control vector, as simulated by the modeling system, and $\sigma\left(y_{\mathrm{mod}}\right)$ is the residual uncertainty in the simulation of the target quantity that remains even for a perfect control vector. The approach represents a network through observational locations and times and the observational uncertainty, but does not require real observations. Consequently, it can evaluate hypothetical networks/space missions based on assumed instrumental specifications and space-time coverage. Observations can range from point-scale (in situ) to gridded data sets or level-1 satellite data, if appropriate forward models/observation operators mapping the model's state variables onto the respective data stream (Kaminski and Mathieu, 2017) are available.

Historically, QND was first applied by Hardt and Scherbaum (1994) to optimize the locations of a set of seismic sensors. Rayner et al. (1996) applied the approach to the in situ sampling network for atmospheric $\mathrm{CO}_{2}$. For the physical sea ice-ocean system in the Arctic domain, Kaminski et al. (2015) applied the approach to evaluate idealized flight transects derived from NASA's Operation IceBridge airborne altimeter ice surveys. Target quantities were 10-day to five-month forecasts of snow and ice volumes over areas relevant for maritime traffic (along the Alaskan coast) and offshore resource exploration (Chukchi Sea). The control vector was composed of physical constants in the model's process representations as well as initial and boundary conditions.

In an activity funded through ESA's Support to Science Element as a contribution to YOPP, Kaminski et al. (2017) constructed the Arctic Mission Benefit Analysis (ArcMBA) system. The system exploits the fact that model sensitivities at observational times and locations as well as the target quantities can be pre-computed, so that the actual assessment of a data set requires only matrix multiplications and inversions [Equation (1) and Equation (2)]. This means the assessment can be performed so fast that the ArcMBA system could be used as an interactive tool to assist decision makers, for example, in a meeting. Currently the system uses pre-computed model sensitivities $\boldsymbol{M}^{\prime}$ for observations of SIT, sea ice freeboard, radar freeboard, laser freeboard, and snow depth along with precomputed model sensitivities $\boldsymbol{N}^{\prime}$ for forecasts of snow and ice volumes for three regions along the Northern Sea Route (NorthEast Passage) respectively denoted by West Laptev Sea, Outer New Siberian Islands, and East Siberian Sea. These sensitivities were computed with the Max-Planck-Institute Ocean Model 
(MPIOM, Jungclaus et al., 2013) in a global configuration with high resolution over the Arctic (Niederdrenk, 2013). The control vector is composed of physical constants in the model's process representations as well as initial- and boundary conditions.

Kaminski et al. (2017) applied the system to evaluate real and hypothetical remote sensing products. The real products were monthly SIT, sea ice freeboard, and radar freeboard, all derived from CryoSat-2 by AWI. These real products were complemented by two hypothetical monthly laser freeboard products (2 and $20 \mathrm{~cm}$ accuracy, respectively), as well as two hypothetical monthly snow depth products ( 2 and $15 \mathrm{~cm}$ accuracy, respectively). Target quantities are 4-week forecasts of snow and ice volumes over three target regions along the Northern Sea Route.

As an example, Figure 13 shows the posterior uncertainty in snow and ice volumes over the three target regions, when the CryoSat-2 SIT product (Ricker et al., 2014) is used alone, in combination with the hypothetical snow depth product with $15 \mathrm{~cm}$ accuracy, or in combination with the hypothetical snow depth product with $2 \mathrm{~cm}$ accuracy. To sharpen the contrast between the observational scenarios, $\sigma\left(y_{\mathrm{mod}}\right)$ (which acts as an offset) is set to zero (perfect model scenario). Comparison of the top and middle panels shows the added value of the hypothetical snow product, not only for the snow volume forecast but also for the sea ice volume (SIV) forecast. SIV is sensitive not only to initial SIT but also to initial ice concentration and snow depth, which are both constrained by the snow depth product. SIV is also sensitive to some of the process parameters that are constrained by snow depth, notably the ice strength, see Kaminski et al. (2017) for details. Comparison of the middle and top panels shows the added value of a higher accuracy in the snow depth product. Increasing the accuracy from 15 to $2 \mathrm{~cm}$ results in a reduction in uncertainty of the SIV forecast for the East Siberian Sea target region from to 63 to $24 \mathrm{~km}^{3}$.

The ArcMBA can be extended to cover further Earth Observation (EO) products and further target variables. In the setup used here, the model can simulate a range of sea iceocean variables in addition to those considered in the present study (e.g., ice drift, mixed layer depth, freshwater/sea surface salinity, SST, circulation). Switching to a more comprehensive model configuration would enable the investigation of yet further variables. For example the model can be operated with its biogeochemistry module HAMOCC activated (Ilyina et al., 2013) or in a mode coupled to an atmospheric general circulation model and thus enable the analysis of biogeochemical products/target quantities or Arctic mid-latitude linkages. The extension of ArcMBA by a terrestrial biosphere component is planned, which will allow joint assessment of ocean and land observations.

\section{INTERNATIONAL EFFORTS TO ADDRESS GAPS IN POLAR OCEAN OBSERVATIONS}

Various international efforts contribute to coordinate and support the vast and complex polar observing networks. These networks are maintained by a collection of national and international efforts and scientific projects. These include

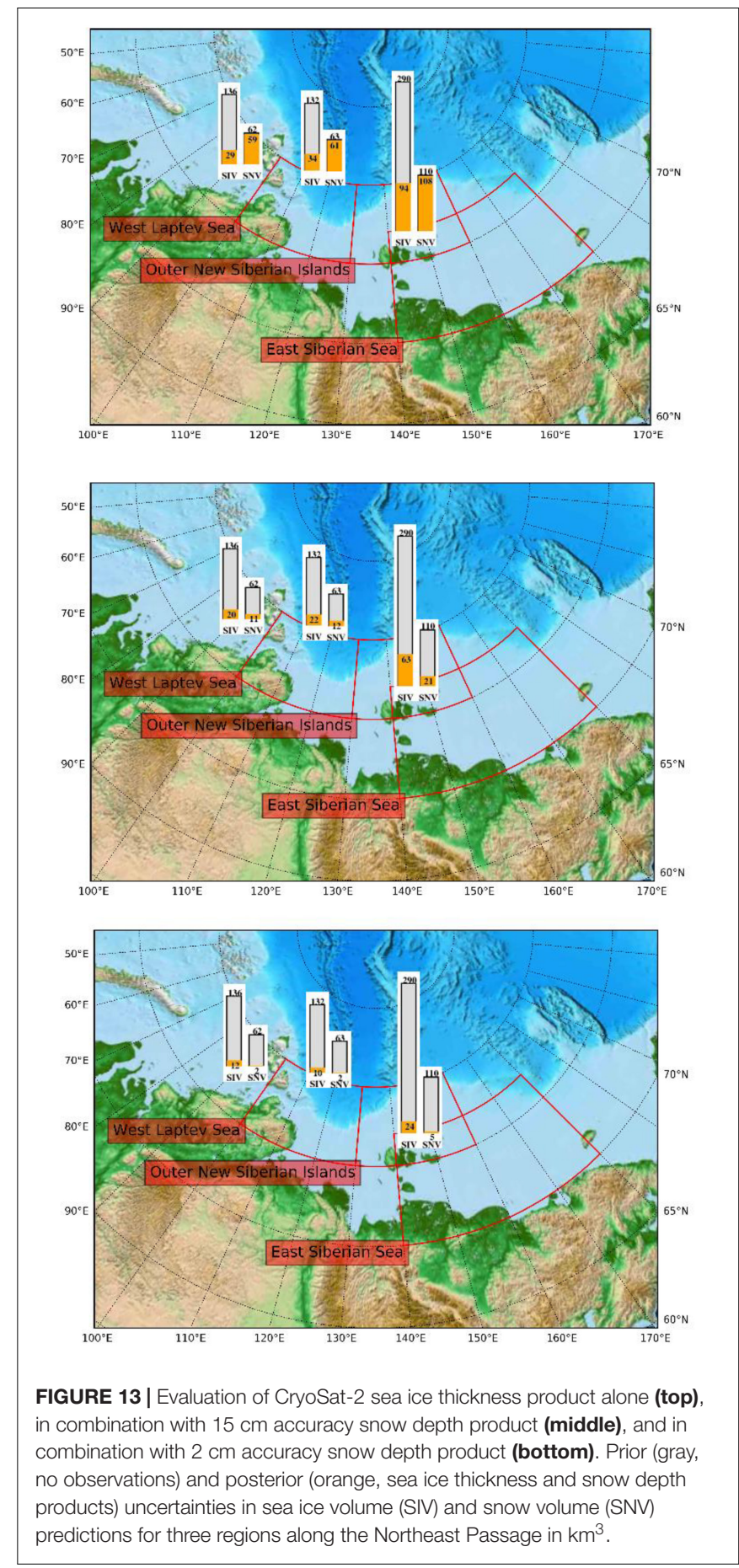

national efforts outlined above, such as IAOOS, FRAMs and NorArgo as well as diverse multi-platform observing projects such as the US SODA (Stratified Ocean Dynamics of the Arctic) program (Lee et al., 2016) that include many of the types of platforms described in Section "In situ Observations of Temperature and Salinity" (WHOI ITPs, ALAMO floats, drifters, gliders). In the south, these efforts are coordinated through the Southern Ocean Observing 
System (SOOS; Newman et al., 2019) supported by the Scientific Committee on Ocean Research (SCOR) and the Scientific Committee for Antarctic Research (SCAR).

A clear need for a coordinated approach for the Arctic has arisen following the International Polar Year (IPY) and is being developed through a series of bi-annual Arctic Observing Summits (Murray et al., 2018). These summits contribute to a broad initiative by Sustaining Arctic Observing Networks (SAON) that has arisen from the International Arctic Science Committee. Recently, a framework for the development of an Arctic Region Global Ocean Observing System (ARGOOS) has been proposed by Lee et al. (2019). Additionally, the CLIVAR/CliC Northern Oceans Panel serves as an international forum for coordinating and strategizing activities on the role of the Arctic Ocean in the context of the global climate system from a coupled perspective and facilitates progress in developing new tools and methods to observe the Arctic Ocean and neighboring seas.

While these efforts aim to address a broad range of societal needs, in this section we describe several particular initiatives that work to address specific gaps in the observing system important for environmental prediction.

\section{INTAROS (INTegrated ARctic Observation System)}

INTAROS is a research and innovation project under the European Union Horizon 2020 program, running from 2016 to 2021, with objective to build an efficient integrated Arctic Observation System in the Arctic. This requires collaborative efforts among many institutions to extend, improve and unify existing systems, which in many cases are designed and developed for specific scientific disciplines. INTAROS focuses on the in situ part of the observing systems, which represent the largest component of the integrated observing system. Satellite Earth Observation programs provide the most developed and operational components of the system, which are run by space agencies and satellite monitoring services such as Copernicus ${ }^{13}$. The satellite systems provide data for near real-time monitoring as well as for long-term climate observations. Validation of the satellite-derived variables is an important part of the operational services. In situ observations play an important role for this validation, but there is very limited access to such data from the Arctic Ocean. Furthermore, most of the ocean observations are only available in delayed mode, because they are provided by underwater moorings and seafloor observatories. Some platforms that operate at the surface can transmit data in near real-time and can therefore contribute to operational monitoring of sea ice and ocean variables.

INTAROS is multidisciplinary, implying that the observing systems encompass atmospheric, marine and terrestrial systems in the different regions of the Arctic. Marine observing systems are also divided into physical and biogeochemical components of the ocean surface (including sea ice), the water column and the seafloor. INTAROS contributes to all these components in collaboration with other observing programs and projects, by

\footnotetext{
${ }^{13} \mathrm{https}: / /$ www.copernicus.eu/en
}

deploying new sensors and platforms to enhance the observing capacity in different Arctic regions. The collection of new in situ data under INTAROS started in 2017 and will continue through 2020, coinciding with and contribute to the extensive data collection in the MOSAiC program (see MOSAiC). Examples of INTAROS supported platforms include: IMBs deployed in the Central Arctic Ocean by the Finnish Meteorological Institute during Chinese Arctic expeditions (Lei et al., 2018); a network of Argo floats in the Nordic Seas in ice-free areas; bio-Argo floats in Baffin Bay together with Takuvik; and the Ferry-box transect between Norway and Svalbard ${ }^{14}$. INTAROS also supports data collection from the cargo vessel NORBJØRN that runs between Troms $\varnothing$ and Longyearbyen throughout the year. Data can be transmitted to land in near realtime and is available for operational monitoring. Ship of Opportunity is promising method to collect oceanographic data in Arctic waters, since there is a growing number of ships operating in the Arctic, especially tourist ships.

Arctic observing systems will benefit greatly from collaboration with local communities (Johnson et al., 2018). INTAROS is therefore working with Community-Based Monitoring (CBM) systems which are under development in many places in North America, Greenland and Russia. CBMs can be supplementary to scientific observations when indigenous and local people collect scientifically relevant data and made them available via websites ${ }^{15}$. Community-based monitoring can also provide valuable data that cannot be obtained from normal research and monitoring programs. In the circumpolar Arctic region there are a number of observing programs addressing sea ice, oceanographic data and observations of marine mammals and fish which are very important for the communities.

\section{EMODNET}

Observations of the ocean are usually made for specific purposes. In order to save costs and improve marine knowledge, the European Union is now moving to a new paradigm where data are collected once and then used for many purposes. This paradigm is being implemented as part of the European Marine Observation and Data Network (EMODnet) consisting of a variety of organizations working together to assemble marine data and products, and to facilitate the dissemination of these resources to both public and private users. EMODnet is currently in its third development phase with the target to be fully deployed by 2020. Six Sea Basin Checkpoint programs were initiated as part of EMODnet, including the Sea Basin Checkpoint Arctic ${ }^{16}$, with the objective to examine the current status of Arctic data and to assess how fit-for-purpose the Arctic data are.

The project (2015-2018) aimed to identify problems and knowledge gaps and was organized in 10 challenges: wind farm sitting, marine protected areas, oil platform leak, climate change, coasts, fisheries management, fisheries impact, river input, bathymetry and alien species. Within the project, each dataset found (292 in total) was assessed on its spatial and temporal

\footnotetext{
${ }^{14}$ https://www.niva.no/en/projectweb/norsoop

${ }^{15} \mathrm{https} / / / \mathrm{mkp} 28$.wixsite.com/cbm-best-practice

${ }^{16}$ http://www.emodnet-arctic.eu
} 
coverage, its accessibility and cost to access, the responsiveness and the temporal and vertical resolution. Also, each assessment report using a dataset (840 in total) was assessed on its adequacy for the project. For the oil spill challenge an oil accident was simulated. This demonstrated the necessity for rapid acquisition and inspection of ocean current and wind data in order to provide a reliable response capacity.

\section{MOSAiC}

An important Arctic observing project called MOSAiC (Multidisciplinary Drifting Observatory for the Study of Arctic Climate; Shupe et al., 2018) is planned for 2019-2020. The German research icebreaker Polarstern will be frozen into the pack ice and over-winter in the Transpolar Drift to obtain measurements over a complete annual cycle. The MOSAiC sea ice platform provides an opportunity for greatly enhanced deployment of autonomous instrumentation and coordinated intensive field studies from research vessels, manned and unmanned aircraft, and distributed surface stations.

MOSAiC observations have been designed specifically to characterize the important coupled processes within the atmosphere-ice-ocean system that impact sea ice melting and freezing. These processes include heat, moisture, and momentum fluxes in the atmosphere and ocean, water vapor, clouds and aerosols, biogeochemical cycles in the ocean and ice, and many others.

The MOSAiC central observatory will be a manned, icebreaker ship-based ice camp with comprehensive instrumentation to measure coupled processes within the atmosphere, ice, and ocean. This intensive observatory will be embedded within a constellation of distributed measurements made by buoys, ice-tethered profilers, remote meteorology stations, underwater drifters, unmanned aerial systems, aircraft, additional ships, and satellites. These distributed observations will provide critical information on the spatial context and variability of key parameters, and allow for limited measurements in environments with sea ice of differing age, thickness, and concentration.

The additional observations from MOSAiC together with other sources during YOPP provide an ideal opportunity to assess the impact on forecast skill in environmental prediction systems through OSEs and process studies. Results will provide a quantitative baseline for use in decisions regarding how to configure a sustained Arctic observing system appropriate for the needs of environmental prediction.

\section{Year of Polar Prediction}

The need for improved environmental predictions (i.e., atmosphere, ice and ocean) has motivated an international effort called the Polar Prediction Project $\left(\mathrm{PPP}^{17}\right)$. The PPP was created under the auspices of the World Meteorological Organisation's World Weather Research Programme (WWRP) to "promote cooperative international research enabling development of improved weather and environmental prediction services for the polar regions, on time scales from hourly to seasonal." YOPP is a PPP flagship activity (Jung et al., 2016) with

\footnotetext{
${ }^{17}$ http://www.polarprediction.net
}

a core phase during 2017-2019, with the overarching goal to "Enable a significant improvement in environmental prediction capabilities for the polar regions and beyond, by coordinating a period of intensive observing, modeling, verification, userengagement and education activities." In particular, there are a series of Special Observing Periods for both the Arctic and Antarctic to improve the polar observing system to provide better coverage of high-quality observations in a cost effective manner, primarily by carrying out Observing System Experiments. The Southern Hemisphere SOP occurred from November 2018 to February 2019, and included deployment of extra radiosondes and drifting buoys, as well as the prolongation of the Ocean Observatories Initiative surface mooring at $55^{\circ} \mathrm{S}$. The Arctic SOPs were conducted for winter (February-March, 2018) and summer (July-September, 2018). A more comprehensive list of research activities is given by PPP Steering Group (2013, 2014). Additional observations gathered through field programs will also be used to improve our understanding of key polar processes relevant for improving prediction skill. A third Arctic SOP is planned for February-March 2020 as part of YOPP to capitalize on the MOSAiC observational effort.

The additional observations from MOSAiC together with other sources during YOPP provide an ideal opportunity to assess the impact on forecast skill in environmental prediction systems through OSEs and process studies. Results will provide a quantitative baseline for use in decisions regarding how to configure a sustained Arctic observing system appropriate for the needs of Environmental Prediction.

\section{RECOMMENDATIONS}

The scarcity of observations, the unique balance of physical processes, the key importance of sea ice, and the rapidly evolving climate of the Arctic, and the uncertainties in Antarctic sea ice trends and carbon uptake lead to a number of scientific challenges for observations in the context of a polar prediction system. Addressing these challenges motivates the following recommendations:

1. The presence of a seasonal ice cover limits the availability of real-time in situ data in polar regions to assist operational requirements. While several technologies have been developed (e.g., ITPs, gliders communicating via acoustic modems) a comprehensive real-time ocean observing network able to supplement Argo for polar regions has yet to be put in place, hindering progress toward coupled polar prediction. It is therefore recommended that a network of ice-borne measurement systems be deployed and supported operationally in ice-covered areas. These platforms represent well-proven technologies for year-round data collection and near-real time data transmission via satellites.

2. Antarctic measurements are also needed, in particular, to evaluate changes that could be harbingers of continental ice melt. Recent studies also highlight the importance of measuring and understanding the intra-hemispheric ocean interactions on numerical weather prediction to climate time-scales (Foppert et al., 2017). Ice-borne observing 
systems that have proven their utility in the Arctic should now be adapted and tested in the Antarctic marginal ice zone.

3. Conditions are changing rapidly with the loss of summer sea ice extent in the Arctic and changing ice-cover patterns in Antarctic marginal seas. Phenomena long considered negligible in the Arctic and Antarctic may be becoming important (e.g., ocean waves - Cavaleri et al., 2012; Massom et al., 2018). The observing system needs to be reevaluated with this in mind. The retreat of the Arctic ice cover increases the area where open-water (or seasonally ice free) observing systems (e.g., gliders, Argo) can operate providing the possibility in future to provide a substantial amount of near-real time data for polar prediction systems.

4. Ships of opportunity present a promising method to collect oceanographic data in polar waters, since there is a growing number of ships operating in these regions. Moreover, community-based monitoring can also provide valuable data that cannot be obtained from normal research and monitoring programs. These atypical observing methods should be encouraged and enhanced to provide a low-cost expansion to the in situ observing system.

5. In situ observations are routinely used for the calibration of remote sensing products over much of the globe, with fewer such calibrations made in polar regions due to lack of in situ observations. Studies have shown the large benefits that such calibrations can have (e.g., Castro et al., 2016; Kwok et al., 2017). The availability of nearreal time in situ measurements could be used to improve the quality of satellite products and thus on downstream environment predictions that assimilate them. Additional efforts involving multi-platform calibration are needed to improve the quality of remote sensing products.

6. The increasing maturity of satellite sea-ice thickness wintertime products merging several sensors (e.g., CryoSat-2 and SMOS) and its positive impact in preliminary assimilation experiments call for symmetrical efforts in the Antarctic ocean, where such products do not exist at the moment.

7. Polar surface properties are often dominated by various forms of ice that vary rapidly on small spatial scales. Some remote sensing methods of ice properties (ice cover, ice thickness, snow depth on ice, albedo, crystal structure) are not mature and offer little and/or coarseresolution information from within the ice, whereas in situ methods provide poor spatial coverage. Neither is currently able to address the need for high spatial and temporal resolution observations of sea ice deformation over large regions. Observations providing information regarding ice deformation and redistribution during ridging are also lacking. There is a need for highresolution (km-scale) remotely sensed snow and ice property data for both the Arctic and Southern Ocean with sufficient temporal resolution to address these relevant features.

8. The value of polar observations for multi-range environmental prediction emerged during the last decade from a variety of impact studies. The importance of SIT initialization for seasonal forecasting, the significance of sub-surface initialization in ice-covered areas, are non-exhaustive examples that call for coordinated efforts (including QND, OSEs and Observing System Simulation Studies) to enhance the Arctic and Southern Ocean observing networks.

9. To date, few data withholding experiments (OSEs) or observation design experiments (OSSEs) have been undertaken for polar regions using real-time prediction systems. Performing such experiments using additional observations available during YOPP (or other periods of additional observational coverage such as IPY) is suggested to provide valuable information to guide the design of a sustainable real-time observing system for polar regions suitable for environmental prediction. In particular, multisystem exercises shall be encouraged to gain robustness in the observation impact assessments.

10. Polar environmental prediction using coupled atmosphereice-ocean models is strongly sensitive to errors in fluxes across the surface interface and thus requires collocated information about the state of the atmosphere, sea ice and ocean, to be used for improving interface fluxes (i.e., coupled model validation) and eventually data assimilation. Direct flux covariance measurements, in particular, would be immensely valuable in constraining bulk parameterizations used to represent fluxes in models.

11. Open access to data, especially real-time data, is a critical capability for improved sea-ice and weather forecasting and other environmental prediction needs. The optimal observing system will no doubt include a suite of different instrument types, since no single platform can be optimized for the full range of observing needs (i.e., subsurface and lower atmosphere), and hence multi-platform coordination will be necessary, including coordination with local community-based observatories. This means that realtime dissemination of in situ observations in polar regions to global data assembly centers must be prioritized in order to make the observational efforts suitable for environmental prediction applications.

12. International collaboration will continue to be key for facilitating deployment of polar ocean instrument systems, including the fielding of drifting and anchored buoys, floats and gliders and free, rapid dissemination of the resulting data.

\section{OUTLOOK}

The relative remoteness and harsh environmental conditions over polar regions will always hinder efforts to provide adequate observations for polar prediction. Over recent years, we have seen improvements in observing technology and capabilities that create new possibilities for how to construct and maintain the polar ocean observing system. The technologies make an adequate polar ocean observing system feasible, but the question remains, is it worth the cost? YOPP aims 
to help address this question by coordinating international observing activities and fostering efforts to assess the impact of additional observations on environmental prediction skill, including impacts on downstream users and products. Following the YOPP core period (2017-2019), there will be a consolidation phase to assess these impacts and develop recommendations toward sustained polar observation. This effort will culminate in a YOPP Final Summit planned for summer 2022, providing an ideal opportunity for funding and implementation agencies to benefit from this community effort.

\section{AUTHOR CONTRIBUTIONS}

GS developed the structure of the manuscript and coordinated the writing team. GS and MC contributed to section "Introduction." SSa contributed to section "Overview of Current Observing System." JT contributed to section "The WHOI IceTethered Profiler." KW, JI, and CM contributed to section "ALAMO and Arctic Heat." GS and FD contributed to section "Deployment of Argo Floats in Canadian Marginal Ice Zone by CONCEPTS." CM, JL, ER, and MB contributed to section "Under-Ice BGC Argo Floats in the Canadian Arctic." IR contributed to section "Surface Drifters and IABP." GC and AS contributed to section "Sea Ice Concentration." QY, PPr, PT, and GL contributed to section "Sea Ice Freeboard and Thickness." PT, PPr, and GL contributed to section "Sea Surface Temperature." PT, PPr, and GL contributed to section "Sea Surface Height." PT, PPr, GL, LB, and JX contributed to section "Sea-Ice Drift." SSw, SG, PH, MM, and BD contributed to section "Air-Sea Flux Measurements in Polar Oceans." RA, JC, DH, EM, MP, PPo, and OS contributed to section "Impacts of Arctic and Antarctic Observations in U.S. Navy Coupled Ice-Ocean Models." GS contributed to section "Sensitivity of Sea Ice Forecasting Skill to Ocean Mixing Around Antarctica." LB and JX contributed to section "Impact of Temperature and Salinity Profiles in the CMEMS Arctic MFC." ASo contributed to section "NOAA-ESRL/CIRES Coupled Arctic Forecast System." TK and FK contributed to section "Quantitative Network Design." GC contributed to section "International Efforts to Address Gaps in Polar Ocean Observations." SSa contributed to section "INTAROS (INTegrated ARctic Observation System)." BK contributed to section "EMODNET." MS and JI contributed to section "MOSAiC." GS, MC, SO, PR, and WWRP PPP Steering Group contributed to section "YOPP." All coauthors contributed to section Recommendations. Revised manuscript were made by GS with particular contributions to overall editing by SG, SSw, ASt, JI, MC, and LB.

\section{REFERENCES}

Alexandrov, V., Sandven, S., Wahlin, J., and Johannessen, O. M. (2010). The relation between sea ice thickness and freeboard in the Arctic. Cryosphere 4, 373-380. doi: 10.5194/tc-4-373-2010

Allard, R. A., Farrell, S. L., Hebert, D. A., Johnston, W. F., Li, L., Kurtz, N. T., et al. (2018). Utilizing CryoSat-2 sea ice thickness to initialize a coupled ice-ocean modeling system. Adv. Space Res. 62, 1265-1280. doi: 10.1016/j.asr.2017.12.030

\section{FUNDING}

The development of the new generation of floats (PRO-ICE) to be operated under ice was funded by the French project NAOS. Twelve PRO-ICE were funded by NAOS and nine by the Canadian Foundation for Innovation (FCI-30124). The GreenEdge project is funded by the following French and Canadian programs and agencies: ANR (Contract \#111112), CNES (project \#131425), IPEV (project \#1164), CSA, Fondation Total, ArcticNet, LEFE and the French Arctic Initiative (GreenEdge project). The INTAROS project has received funding from the European Union's Horizon 2020 Research and Innovation Program under grant agreement No. 727890. The setup of the ArcMBA system and the experiment described in section "Quantitative Network Design" were funded by the European Space Agency through its support to science element (contract \#4000117710/16/I-NB). SSw was supported by a Wallenberg Academy Fellowship (WAF 2015.0186). The work at CLS (GL, PPr, and PT) has been funded by internal investment, in relation with on-going CNES and ESA funded studies making use of radar data over Polar regions. EMODNET $(\mathrm{BK})$ is funded by the European Commission. NRL Funding (for RA, JC, DH, EM, PPo, OS) provided by NRL Research Option "Determining the Impact of Sea Ice Thickness on the Arctic's Naturally Changing Environment (DISTANCE), ONR 6.2 Data Assimilation and under program element 0602435N (JC, RA, DH). JT's Arctic research activities are supported by the U.S. National Science Foundation and ONR. SG was funded by NSF grants/awards PLR-1425989 and OCE 1658001. IR is funded by contributors to the US IABP (including CG, DOE, NASA, NIC, NOAA, NSF, ONR). CAFS is supported by the NOAA ESRL Physical Sciences Division (AS and JI). LB and JX are funded by CMEMS. The WWRP PPP Steering Group is funded by a WMO trust fund with support from AWI for the ICO. The publication fee is provided by ECCC.

\section{ACKNOWLEDGMENTS}

We would like to acknowledge the valuable suggestions made by both reviewers that has improved the quality of this manuscript. Contributions to the PPP Trust Fund are acknowledged as well as AWI's financial contribution to hosting the ICO. For work done for section "Under-ice BGC Argo Floats in the Canadian Arctic," we thank E. Leymarie and C. Penkerc'h (LOV Laboratoire d'Océanographie de Villefranche sur Mer) for their contribution in the development of the PRO-ICE. We also thank officers and crew of CCGS Amundsen for their help during our deployments of floats.

Andersen, O. B., and Piccioni, G. (2016). Recent arctic sea level variations from satellites. Front. Mar. Sci. 3:76. doi: 10.3389/fmars.2016.00076

Ardhuin, F., Aksenov, Y., Benetazzo, A., Bertino, L., Brandt, P., Caubet, E., et al. (2018). Measuring currents, ice drift, and waves from space: the Sea surface KInematics Multiscale monitoring (SKIM) concept. Ocean Sci. 14, 337-354. doi: 10.5194/os-14-337-2018

Armitage, T. W. K., Bacon, S., Ridout, A. L., Thomas, S. F., Aksenov, A., and Wingham, D. J. (2016). Arctic sea surface height variability and change from 
satellite radar altimetry and GRACE, 2003-2013. J. Geophys. Res. Oceans 121, 4303-4322. doi: 10.1002/2015JC011579

Armitage, T. W. K., and Ridout, A. L. (2015). Arctic sea ice freeboard from AltiKa and comparison with Cryosat-2 and Operation IceBridge. Geophys. Res. Lett. 42, 6724-6731. doi: 10.1002/2015GL064823

Bell, M. J., Schiller, A., Le Traon, P.-Y., Smith, N. R., Dombrowsky, E., and WilmerBecker, K. (2015). An introduction to GODAE OceanView. J. Operat. Oceanogr. 8, s2-s11. doi: 10.1080/1755876X.2015.1022041

Bentamy, A., Piolle, J.-F., Grouazel, A., Danielson, R., Gulev, S., Paul, F., et al. (2017). Review and assessment of latent and sensible heat flux accuracy over the global oceans. Remote Sens. Environ. 201, 196-218. doi: 10.1016/j.rse.2017. 08.016

Berge, J., Geoffroy, M., Johnsen, G., Cottier, F., Bluhm, B., and Vogedes, D. (2016). Ice-tethered observational platforms in the Arctic Ocean pack ice. IFAC Pap. Online 49, 494-499. doi: 10.1016/j.ifacol.2016.10.484

Blockley, E. W., and Peterson, K. A. (2018). Improving Met Office seasonal predictions of Arctic sea ice using assimilation of CryoSat-2 thickness. Cryosphere 12, 3419-3438. doi: 10.5194/tc-12-3419-2018

Bodas-Salcedo, A., Hill, P. G., Furtado, K., Williams, K. D., Field, P. R., Manners, J. C., et al. (2016). Large contribution of supercooled liquid clouds to the solar radiation budget of the Southern Ocean. J. Clim. 29, 4213-4228. doi: 10.1175/ jcli-d-15-0564.1

Bodas-Salcedo, A., Williams, K. D., Ringer, M. A., Beau, I., Cole, J. N., Dufresne, J. L., et al. (2014). Origins of the solar radiation biases over the Southern Ocean in CFMIP2 models. J. Clim. 27, 41-56. doi: 10.1175/jcli-d-13-00169.1

Bourassa, M. A., Gille, S. T., Bitz, C., Carlson, D., Cerovecki, I., Clayson, C. A., et al. (2013). High-latitude ocean and sea ice surface fluxes: challenges for climate research. Bull. Am. Meteorol. Soc. 94, 403-423. doi: 10.1175/bams-d-11-00 244.1

Bracegirdle, T. J., Hyder, P., and Holmes, C. R. (2018). CMIP5 uncertainty in projected $21 \mathrm{C}$ change in southern westerlies significantly related to simulated historical sea ice area; strong link to strengthening and weak link to shift. J. Clim. 31, 195-211. doi: 10.1175/jcli-d-17-0320.1

Brassington, G. B., Martin, M. J., Tolman, H. L., Akella, S., Balmeseda, M., Chambers, C. R. S., et al. (2015). Progress and challenges in short- to mediumrange coupled prediction. J. Operat. Oceanogr. 8, s239-s258. doi: 10.1080/ 1755876x.2015.1049875

Buehner, M., Caya, A., Pogson, L., Carrieres, T., and Pestieau, P. (2013). A new Environment Canada regional ice analysis system. Atmos. Ocean 51, 18-34. doi: $10.1080 / 07055900.2012 .747171$

Buizza, R., Brönnimann, S., Haimberger, L., Laloyaux, P., Martin, M. J., Fuentes, M., et al. (2018). The EU-FP7 ERA-CLIM2 project contribution to advancing science and production of earth system climate reanalyses. Bull. Am. Meteorol. Soc. 99, 1003-1014. doi: 10.1175/bams-d-17-0199.1

Bunzel, F., Notz, D., Baehr, J., Müller, W. A., and Fröhlich, K. (2016). Seasonal climate forecasts significantly affected by observational uncertainty of Arctic sea ice concentration. Geophys. Res. Lett. 43, 852-859. doi: 10.1002/2015gl06 6928

Calder, J., Proshutinsky, A., Carmack, E., Ashik, I., Loeng, H., Key, J., et al. (2010). "Community white paper: an integrated international approach to arctic ocean observations for society (a legacy of the international polar year)," in Proceedings of OceanObs'09: Sustained Ocean Observations and Information for Society, Vol. 2, eds J. Hall, D. E. Harrison, and D. Stammer (Venice: ESA Publication), 306. doi: 10.5270/OceanObs09.cwp.14

Carrieres, T., Caya, A., Posey, P., Metzger, E. J., Bertino, L., Melsom, A., et al. (2017). Sea Ice Analysis and Forecasting: Towards an Increased Reliance on Automated Prediction Systems, eds T. Carrieres, M. Buehner, J.-F. Lemieux, and L. T. Pedersen (Cambridge: Cambridge University Press), 144-173. doi: $10.1017 / 9781108277600$

Carse, F., Martin, M. J., Sellar, A., and Blockley, E. W. (2015). Impact of assimilating temperature and salinity measurements by animal-borne sensors on FOAM ocean model fields. Q. J. R. Meteorol. Soc. 141, 2934-2943. doi: 10.1002/qj.2613

Castro, S. L., Wick, G. A., and Steele, M. (2016). Validation of satellite sea surface temperature analyses in the Beaufort Sea using UpTempO buoys. Remote Sens. Environ. 187, 458-475. doi: 10.1016/j.rse.2016.10.035

Cavaleri, L., Fox-Kemper, B., and Hemer, M. (2012). Wind waves in the coupled climate system. Bull. Am. Meteorol. Soc. 92, 1651-1661. doi: 10.1175/bams-d11-00170.1
Cavalieri, D. J., and Parkinson, C. L. (2012). Arctic sea ice variability and trends, 1979-2010. Cryosphere 6, 881-889. doi: 10.5194/tc-6-881-2012

Chen, Z., Liu, J., Song, M., Yang, Q., and Xu, S. (2017). Impacts of assimilating satellite sea ice concentration and thickness on Arctic sea ice prediction in the NCEP Climate Forecast System. J. Clim. 30, 8429-8446. doi: 10.1175/jcli-d-170093.1

Chevallier, M., Smith, G., Lemieux, J.-F., Dupont, F., Forget, G., Fujii, Y., et al. (2016). Intercomparison of the Arctic sea ice cover in global ocean-sea ice reanalyses from the ORA-IP project. Clim. Dyn. Spec. Issue Ocean Reanalysis 49, 1107-1136. doi: 10.1007/s00382-016-2985-y

Claustre, H., Bishop, J., Boss, E., Stewart, B., Berthon, J.-F., Coatanoan, C., et al. (2010). "Bio-optical profiling floats as new observational tools for biogeochemical and ecosystem studies: potential synergies with ocean color remote sensing," in Proceedings of OceanObs'09: Sustained Ocean Observations and Information for Society, Vol. 2, eds J. Hall, D. E. Harrison, and D. Stammer (Venice: ESA Publication), 306

Cole, S. T., Thwaites, F. T., Krishfield, R. A., and Toole, J. M. (2015). "Processing of velocity observations from ice-tethered profilers," in Proceedings of the Oceans 2015 MTS/IEEE, Washington, DC.

Cole, S. T., Timmermans, M. L., Toole, J. M., Krishfield, R. A., and Thwaites, F. T. (2014). Ekman veering, internal waves, and turbulence observed under Arctic sea ice. J. Phys. Oceanogr. 44, 1306-1328. doi: 10.1175/jpo-d-12-0191.1

Cummings, J. A., and Smedstad, O. M. (2014). Ocean data impacts in global HYCOM. J. Atmos. Ocean. Technol. 31, 1771-1791. doi: 10.1175/JTECH-D-1400011.1

Curry, B., Lee, C. M., Petrie, B., Moritz, R. E., and Kwok, R. (2014). Multiyear volume, liquid freshwater, and sea ice transports through Davis Strait, 2004-10. J. Phys. Oceanogr. 44, 1244-1266. doi: 10.1175/jpo-d-13-0177.1

Dash, P., Ignatov, A., Kihai, Y., and Sapper, J. (2010). The SST quality monitor (SQUAM). J. Atmos. Ocean. Technol. 27, 1899-1917. doi: 10.1175/ 2010JTECHO756.1

Davidson, F., Chassignet, E., Vinayachandran, P. N., Lu, Y., Smith, G. C., and Zhu, X. (2019). Synergies in operational oceanography: the intrinsic need for sustained ocean observations. Front. Mar. Sci. 6:450. doi: 10.3389/fmars.2019. 00450 doi: 10.1175/jtech-d-14-00011.1

Davidson, F., Smith, G. C., Lu, Y., and Woodbury, S. (2013). Operational atmosphere-ocean-ice prediction systems in Canada: providing decisionenabling marine environmental information to end users. Can. Ocean Sci. Newsl. 70, 2-5.

Dong, S., Gille, S. T., and Sprintall, J. (2007). An assessment of the Southern Ocean mixed-layer heat budget. J. Clim. 20, 4425-4442. doi: 10.1175/jcli4259.1

Dosser, H. V., Rainville, L., and Toole, J. M. (2014). Near-inertial internal wave field in the Canada basin from ice-tethered profilers. J. Phys. Oceanogr. 44, 413-426. doi: 10.1175/JPO-D-13-0117.1

du Plessis, M., Swart, S., Ansorge, I. J., Mahadevan, A., and Thompson, A. F. (2019). Southern Ocean seasonal stratification delayed by wind-front interaction at submesoscales. J. Phys. Oceanogr. 49, 1035-1053. doi: 10.1175/JPO-D-180136.1

Fedak, M. A. (2013). The impact of animal platforms on polar ocean observation. Deep Sea Res. Part II Top. Stud. Oceanogr. 88-89, 7-13. doi: 10.1016/j.dsr2. 2012.07.007

Fennel, K., and Greenan, B. (2017). Taking the Ocean's Pulse: A Vision for the Canadian Biogeochemical Argo Program. Available at: https://doi.org/10.13155/ 52451

Foppert, A., Donohue, K. A., Randolph Watts, D., and Tracey, K. L. (2017). Eddy heat flux across the Antarctic Circumpolar Current estimated from sea surface height standard deviation. J. Geophys. Res. Oceans 122, 6947-6964. doi: 10.1002/2017JC012837

Freitag, L., Ball, K., Partan, J., Koski, P., and Singh, S. (2015). "Long range acoustic communications and navigation in the Arctic," in Proceedings of the OCEANS'15 Conference and Exhibition, (Washington, DC: MTS), 1-5.

Fujii, Y., Remy, E., Zuo, H., Oke, P. R., Halliwell, G. R., Gasparin, F., et al. (2019). Observing system evaluation based on ocean data assimilation and prediction systems: on-going challenges and future vision for designing/supporting ocean observational networks. Front. Mar. Sci. 6:417. doi: 10.3389/fmars.2019.00417

Gentemann, C. L., Meissner, T., and Wentz, F. J. (2010). Accuracy of satellite sea surface temperatures at 7 and 11 GHz. IEEE Trans. Geosci. Remote Sens. 48, 1009-1018. doi: 10.1109/tgrs.2009.2030322 
Gille, S., Josey, S., and Swart, S. (2016). New approaches for air-sea fluxes in the Southern Ocean. Eos 97. doi: 10.1029/2016EO052243

Greenert, J. W. (2014). The United States Navy Arctic Roadmap for 2014 to 2030. Washington, DC: Office of the Chief of Naval Operations.

Guémas, V., Blanchard-Wrigglesworth, E., Chevallier, M., Day, J., Déqué, M., Doblas-Reyes, F., et al. (2016a). A review on Arctic sea ice predictability and prediction on seasonal-to-decadal timescales. Q. J. R. Meteorol. Soc. 142, 546-561. doi: 10.1002/qj.2401

Guémas, V., Chevallier, M., Déqué, M., Bellprat, O., and Doblas-Reyes, F. (2016b). Impact of sea ice initialization on sea ice and atmosphere prediction skill on seasonal timescales. Geophys. Res. Lett. 43, 3889-3896. doi: 10.1002/ 2015GL066626

Guerreiro, K., Fleury, S., Zakharova, E., Rémy, F., and Maisongrande, P. (2016). Potential for estimation of snow depth on Arctic sea ice from CryoSat-2 and SARAL/AltiKa missions. Remote Sens. Environ. 11, 2059-2073.

Hardt, M., and Scherbaum, F. (1994). The design of optimum networks for aftershock recordings. Geophys. J. Int. 117, 716-726. doi: 10.1111/j.1365-246x. 1994.tb02464.x

Hebert, D. A., Allard, R. A., Metzger, E. J., Posey, P. G., Preller, R. H., Wallcraft, A. J., et al. (2015). Short-term sea ice forecasting: an assessment of ice concentration and ice drift forecasts using the U.S. Navy's Arctic Cap Nowcast/Forecast System. J. Geophys. Res. Oceans 120, 8327-8345. doi: 10.1002/ 2015JC011283

Helfrich, S. R., McNamara, D., Ramsay, B. H., Baldwin, T., and Kasheta, T. (2007). Enhancements to, and forthcoming developments in the Interactive Multisensor Snow and Ice Mapping System (IMS). Hydrol. Process. 21, 15761586. doi: 10.1002/hyp.6720

Hendricks, S., Ricker, R., and Helm, V. (2016). User guide-AWI CryoSat-2 sea ice thickness data product (v1. 2). Alfred Wegener Institute Technical Report.

Hogan, T. F., Liu, M., Ridout, J. A., Peng, M. S., Whitcomb, T. R., Ruston, B. C., et al. (2014). The navy global environmental model. Oceanography 27, 116-125.

Hoke, W., Werner, K., Goessling, H., and Jung, T. (2018). "Engaging forecast users during the year of polar prediction," in Building Bridges at the ScienceStakeholder Interface, ed. G. Krause (Cham: Springer), 47-53. doi: 10.1007/9783-319-75919-7_7

Howe, B. M., Miksis-Olds, J., Rehm, E., Sagen, H., Worcester, P. F., and Haralabus, G. (2019). Observing the oceans acoustically. Front. Mar. Sci. 6:426. doi: 10. 3389/fmars.2019.00426

Hunke, E., and Lipscomb, W. (2008). "CICE: The Los Alamos Sea Ice Model documentation and software user's manual version 4.0," in Technical Report LACC-06-012, AWI CryoSat-2 Sea Ice Thickness Data Product (v1.2) User Guide, eds S. Hendricks, R. Ricker, and V. Helm (Los Alamos, NM: Los Alamos National Laboratory).

Hunke, E. C., Lipscomb, W. H., Turner, A. K., Jeffery, N., and Elliott, S. (2015). CICE: The Los Alamos Sea ice Model Documentation and Software User's Manual Version 5 (Tech. Rep. LA-CC-06-012). Los Alamos, NM: Los Alamos National Laboratory.

Hyder, P., Edwards, J. M., Allan, R. P., Hewitt, H. T., Bracegirdle, T. J., Gregory, J. M., et al. (2018). Critical Southern Ocean climate model biases traced to atmospheric model cloud errors. Nat. Commun. 9:3625. doi: 10.1038/s41467018-05634-2

Ilıcak, M., Drange, H., Wang, Q., Gerdes, R., Aksenov, Y., Bailey, D., et al. (2016). An assessment of the Arctic Ocean in a suite of interannual COREII simulations. Part III: hydrography and fluxes. Ocean Model. 100, 141-161. doi: 10.1016/j.ocemod.2016.02.004

Ilyina, T., Six, K. D., Segschneider, J., Maier-Reimer, E., Li, H., and NezRiboni, I. (2013). Global ocean biogeochemistry model HAMOCC: model architecture and performance as component of the MPI-Earth system model in different CMIP5 experimental realizations. J. Adv. Model. Earth Syst. 5, 287-315. doi: $10.1029 / 2012 \mathrm{MS} 000178$

Inoue, J., Enomoto, T., Miyoshi, T., and Yamane, S. (2009). Impact of observations from Arctic drifting buoys on the reanalysis of surface fields. Geophys. Res. Lett. 36:L08501.

Inoue, J., Yamazaki, A., Ono, J., Dethloff, K., Maturilli, M., Neuber, R., et al. (2015). Additional Arctic observations improve weather and sea-ice forecasts for the Northern Sea Route. Sci. Rep. 5:16868. doi: 10.1038/srep16868

Islam, F., DeGrandpre, M. D., Beatty, C. M., Timmermans, M. L., Krishfield, R. A., Toole, J. M., et al. (2017). Sea surface $\mathrm{pCO}_{2}$ and $\mathrm{O}_{2}$ dynamics in the partially ice-covered Arctic Ocean. J. Geophys. Res. 122, 1425-1438. doi: 10. 1002/2016JC012162

Ivanova, N., Johannessen, O. M., Pedersen, L. T., and Tonboe, R. T. (2014). Retrieval of Arctic sea ice parameters by satellite passive microwave sensors: a comparison of eleven sea ice concentration algorithms. IEEE Trans. Geosci. Remote Sens. 52, 7233-7246. doi: 10.1109/tgrs.2014.2310136

Jackson, K., Wilkinson, J., Maksym, T., Meldrum, D., Beckers, J., Haas, C., et al. (2013). A novel and low-cost sea ice mass balance buoy. J. Atmos. Ocean. Technol. 30, 2676-2688. doi: 10.1175/jtech-d-13-00058.1

Johnson, N., Fidel, M., Danielsen, F., Iversen, L., Poulsen, M. K., Hauser, D., et al. (eds). (2018). "INTAROS community-based monitoring experience exchange workshop report: Canada. Workshop organized as a contribution to INTAROS, December 11-12, 2017," in Exchange for Local Observations and Knowledge of the Arctic (ELOKA), Nordic Foundation for Development and Ecology (NORDECO), Yukon River Inter-Tribal Watershed Council (YRITWC), (Québec: International Arctic Research Center), 27.

Josey, S. A., Gulev, S., and Yu, L. (2013). "Exchanges through the ocean surface," in Ocean Circulation and Climate: A 21st Century Perspective, eds G. Siedler, J. Church, W. J. Gould, and S. Griffies (Cambridge, MA: Academic Press), 115-140. doi: 10.1016/b978-0-12-391851-2.00005-2

Jung, T., Doblas-Reyes, F., Goessling, H., Guemas, V., Bitz, C., Buontempo, C., et al. (2015). Polar lower-latitude linkages and their role in weather and climate prediction. Bull. Am. Meteorol. Soc. 96, ES197-ES200. doi: 10.1175/BAMS-D$15-00121.1$

Jung, T., Gordon, N. D., Bauer, P., Bromwich, D. H., Chevallier, M., Day, J. J., et al. (2016). Advancing polar prediction capabilities on daily to seasonal time scales. Bull. Am. Meteorol. Soc. 97, 1631-1647. doi: 10.1175/BAMS-D-1400246.1

Jungclaus, J. H., Fischer, N., Haak, H., Lohmann, K., Marotzke, J., Matei, D., et al. (2013). Characteristics of the ocean simulations in MPIOM, the ocean component of the MPI-Earth system model. J. Adv. Model. Earth Syst. 5, 422-446. doi: 10.1002/jame.20023

Kaleschke, L., Tian-Kunze, X., Maaß, N., Mäkynen, M., and Drusch, M. (2012). Sea ice thickness retrieval from SMOS brightness temperatures during the Arctic freeze-up period. Geophys. Res. Lett. 39:L05501.

Kaminski, T., Kauker, F., Eicken, H., and Karcher, M. (2015). Exploring the utility of quantitative network design in evaluating Arctic sea ice thickness sampling strategies. Cryosphere 9, 1721-1733. doi: 10.5194/tc-9-1721-2015

Kaminski, T., Kauker, F., Toudal Pedersen, L., Vobeck, M., Haak, H., Niederdrenk, L., et al. (2017). Arctic mission benefit analysis: impact of sea ice thickness, freeboard, and snow depth products on sea ice forecast performance. Cryosphere Discuss. 2017, 1-39. doi: 10.5194/tc-2017-249

Kaminski, T., and Mathieu, P.-P. (2017). Reviews and syntheses: flying the satellite into your model: on the role of observation operators in constraining models of the Earth system and the carbon cycle. Biogeosciences 14, 2343-2357. doi: 10.5194/bg-14-2343-2017

Kaminski, T., and Rayner, P. J. (2017). Assisting the evolution of the observing system for the carbon cycle through quantitative network design. Biogeosci. Discuss. 14, 1-22. doi: 10.5194/bg-2017-168

Karvonen, J. (2012). Operational SAR-based sea ice drift monitoring over the Baltic Sea. Ocean Sci. 8, 473-483. doi: 10.5194/os- 8-473-2012

Kikuchi, T., Inoue, J., and Langevin, D. (2007). Argo-type profiling float observations under the Arctic multiyear ice. Deep Sea Res. Part I Oceanogr. Res. Pap. 54, 1675-1686. doi: 10.1016/j.dsr.2007.05.011

Klatt, O., Boebel, O., and Fahrbach, E. (2007). A profiling float's sense of ice. J. Atmos. Ocean. Technol. 24, 1301-1308. doi: 10.1175/jtech2026.1

Korosov, A., and Rampal, P. (2017). A combination of feature tracking and pattern matching with optimal parametrization for sea ice drift retrieval from SAR data. Remote Sens. 9:258. doi: 10.3390/rs9030258

Krishfield, R., Toole, J., Proshutinsky, A., and Timmermans, M.-L. (2008), Automated Ice-Tethered Profilers for seawater observations under pack ice in all seasons. J. Atmos. Ocean. Technol. 25, 2091-2105. doi: 10.1175/ 2008jtecho587.1

Kwok, R., and Cunningham, G. F. (2015). Variability of Arctic sea ice thickness and volume from CryoSat-2. Philos. Trans. R. Soc. A Math. Phys. Eng. Sci. 373:20140157. doi: 10.1098/rsta.2014.0157

Kwok, R., Kurtz, N. T., Brucker, L., Ivanoff, A., Newman, T., Farrell, S. L., et al. (2017). Intercomparison of snow depth retrievals over Arctic sea ice from 
radar data acquired by Operation IceBridge. Cryosphere 11, 2571-2593. doi: 10.5194/tc-11-2571-2017

Kwok, R., and Sulsky, D. (2010). Arctic Ocean sea ice thickness and kinematics: satellite retrievals and modeling. Oceanography 23, 134-143. doi: 10.5670/ oceanog. 2010.11

Lagunas, J., Marec, C., Leymarie, É, Penkerc'h, C., Rehm, E., Desaulniers, P., et al. (2018). Sea-ice detection for autonomous underwater vehicles and oceanographic lagrangian platforms by continuous-wave laser polarimetry. Ocean Sens. Monitor. X 10631:106310W. doi: 10.1117/12.230 9571

Laney, S. R., Krishfield, R. A., and Toole, J. M. (2017). The euphotic zone under Arctic Ocean sea ice: vertical extents and seasonal trends. Limnol. Oceanogr. 62, 1910-1934. doi: 10.1002/lno.10543

Laney, S. R., Krishfield, R. A., Toole, J. M., Hammar, T. R., Ashjian, C. J., and Timmermans, M. L. (2014). Assessing algal biomass and bio-optical distributions in perennially ice-covered polar ocean ecosystems. Polar Sci. 8, 73-85. doi: 10.1016/j.polar.2013.12.003

Lavergne, T., Eastwood, S., Teffah, Z., Schyberg, H., and Breivik, L.-A. (2010). Sea ice motion from low-resolution satellite sensors: an alternative method and its validation in the Arctic. J. Geophys. Res. Atmos. 115:C10032. doi: 10.1029/ 2009JC005958

Le Traon, P. Y., Ali, A., Alvarez Fanjul, E., Aouf, L., Axell, L., and Aznar, R. (2017). The copernicus marine environmental monitoring service: main scientific achievements and future prospects. Spec. Issue Mercatorol. Ocean J. 56, $1-101$.

Lee, C. M., Cole, S., Doble, M., Guthrie, J. D., Harper, S., MacKinnon, J., et al. (2016). "Stratified ocean dynamics in the arctic: science and experiment plan. APL-UW TR 1601," in Technical Report, Applied Physics Laboratory, (Seattle, WA: University of Washington), 46.

Lee, C. M., and Gobat, J. (2008). Acoustic Navigation and Communications for High-Latitude Ocean Research. Seattle, WA: University of Washington.

Lee, C. M., Starkweather, S., Eicken, H., Timmermans, M.-L., Wilkinson, J., Sandven, S., et al. (2019). A framework for the development, design and implementation of a sustained arctic ocean observing system. Front. Mar. Sci. 6:451. doi: 10.3389/fmars.2019.00451

Lei, R., Cheng, B., Heil, P., Vihma, T., Wang, J., Ji, Q., et al. (2018). Seasonal and interannual variations of sea ice mass balance from the central arctic to the Greenland sea. J. Geophys. Res. Oceans 123, 2422-2439. doi: 10.1002/ 2017 jc013548

Lemieux, J. F., Beaudoin, C., Dupont, F., Roy, F., Smith, G. C., Shlyaeva, A., et al. (2016). The Regional Ice Prediction System (RIPS): verification of forecast sea ice concentration. Q. J. R. Meteorol. Soc. 142, 632-643. doi: 10.1002/qj.2526

Liao, Z., Cheng, B., Zhao, J., Vihma, T., Jackson, K., Yang, Q., et al. (2018). Snow depth and ice thickness derived from SIMBA ice mass balance buoy data using an automated algorithm. Int. J. Digit. Earth 12, 1-18. doi: 10.1080/17538947. 2018.1545877

Lien, V. S., Hjøllo, S. S., Skogen, M. D., Svendsen, E., Wehde, H., Bertino, L., et al. (2016). An assessment of the added value from data assimilation on modelled Nordic Seas hydrography and ocean transports. Ocean Model. 99, 43-59. doi: 10.1016/j.ocemod.2015.12.010

Lindsay, R. W., and Zhang, J. (2006). Assimilation of ice concentration in an ice-ocean model. J. Atmos. Ocean. Technol. 23, 742-749. doi: 10.1175/jtech1 871.1

Lisaeter, K. A., Rosanova, J., and Evensen, G. (2003). Assimilation of ice concentration in a coupled ice-ocean model, using the Ensemble Kalman filter. Ocean Dyn. 53, 368-388. doi: 10.1007/s10236-003-0049-4

Longépé, N., Thibaut, P., Vadaine, R., Poisson, J. C., Guillot, A., Boy, F., et al. (2019). Comparative evaluation of sea ice lead detection based on SAR imagery and altimeter data. IEEE Trans. Geosci. Remote Sens. 57, 4050-4061. doi: 10.1109/tgrs.2018.2889519

Macelloni, G., Brogioni, M., Montomoli, F., Leduc-Leballeur, M., De Carolis, G., Kaleschke, L., et al. (2017). "Preliminary study for a spaceborne ultrawideband microwave radiometer for the monitoring of cryosphere elements: the Cryorad project," in Proceedings of the 2017 IEEE International Geoscience and Remote Sensing Symposium (IGARSS), Fort Worth, TX, 1185-1188.

Maksym, T. (2019). Arctic and Antarctic Sea ice change: contrasts, commonalities, and causes. Annu. Rev. Mar. Sci. 11, 187-213. doi: 10.1146/annurev-marine010816-060610
Mariotti, A., Ruti, P. M., and Rixen, M. (2018). Progress in subseasonal to seasonal prediction through a joint weather and climate community effort. NPJ Clim. Atmos. Sci. 1:4.

Maslowski, W., Clement Kinney, J., Higgins, M., and Roberts, A. (2012). The future of Arctic sea ice. Annu. Rev. Earth Planet. Sci. 40, 625-654.

Massom, R. A., Scambos, T. A., Bennetts, L. G., Reid, P., Squire, V. A., and Stammerjohn, S. E. (2018). Antarctic Ice shelf disintegration triggered by sea ice loss and ocean swell. Nature 558, 383-389. doi: 10.1038/s41586-018-0212-1

Massonnet, F., Mathiot, P., Fichefet, T., Goosse, H., Beatty, C. K., Vancoppenolle, M., et al. (2013). A model reconstruction of the Antarctic sea ice thickness and volume changes over 1980-2008 using data assimilation. Ocean Model. 64, 67-75. doi: 10.1016/j.ocemod.2013.01.003

McPhee, M. G. (2013). Intensification of geostrophic currents in the Canada basin, Arctic ocean. J. Clim. 26, 3130-3138. doi: 10.1175/JCLI-D-12-00289.1

Melsom, A., Bertino, L., and Sutherland, G. (2015). Quality Information Document for the Arctic Ocean Physical Analysis and Forecast Product ARCTIC ANALYSIS FORECAST PHYS 002001 A. Available at: http://marine.copernicus.eu/ documents/QUID/CMEMS-ARC-QUID-002-001a.pdf

Metzger, E. J., Smedstad, O. M., Thoppil, P. G., Hurlburt, H. E., Cummings, J. A., Wallcraft, A. J., et al. (2014). US Navy operational global ocean and Arctic ice prediction systems. Oceanography 27, 32-43. doi: 10.5670/oceanog.2014.66

Mikhalevsky, P. N., Sagen, H., Worcester, P. F., Baggeroer, A. B., Orcutt, J., Moore, S. E., et al. (2015). Multipurpose acoustic networks in the integrated arctic ocean observing system. Arctic 68, 11-27.

Mogensen, K. S., Magnusson, L., and Bidlot, J. R. (2017). Tropical cyclone sensitivity to ocean coupling in the ECMWF coupled model. J. Geophys. Res. Oceans 122, 4392-4412. doi: 10.1002/2017jc012753

Monteiro, P. M. S., Gregor, L., Lévy, M., Maener, S., Sabine, C. L., and Swart, S. (2015). Seasonal-scale robotics experiment reveals the contribution made by fine scale dynamics to the uncertainties and biases in the mean seasonal $\mathrm{CO}_{2}$ flux in the Southern Ocean. Geophys. Res. Lett. 42, 8507-8514. doi: 10.1002/ 2015GL066009

Mu, L., Yang, Q., Losch, M., Losa, S. N., Ricker, R., Nerger, L., et al. (2018). Improving sea ice thickness estimates by assimilating CryoSat-2 and SMOS sea ice thickness data simultaneously. Q. J. R. Meteorol. Soc. 144, 529-538. doi: 10.1002/qj.3225

Murray, M. S., Sankar, R. D., and Ibarguchi, G. (2018). The Arctic Observing Summit, Background and Synthesis of Outcomes 2013-2016. Calgary: International Study of Arctic Change.

Newman, L., Heil, P., Trebilco, R., Katsumata, K., Constable, A. J., van Wijk, E., et al. (2019). Delivering sustained, coordinated and integrated observations of the southern ocean for global impact. Front. Mar. Sci. 6:433. doi: 10.3389/fmars. 2019.00433

Niederdrenk, L. (2013). The Arctic Hydrologic Cycle and its Variability in a Regional Coupled Climate Model. Ph.D. thesis, University of Hamburg, Hamburg, 1-186.

Ogle, S. E., Tamsitt, V., Josey, S. A., Gille, S. T., Ceroveèki, I., Talley, L. D., et al. (2018). Episodic Southern Ocean heat loss and its mixed layer impacts revealed by the furthest south multi-year surface flux mooring. Geophys. Res. Lett. 45, 5002-5010. doi: 10.1029/2017GL076909

Oke, P. R., Larnicol, G., Fujii, Y., Smith, G. C., Lea, D. J., Guinehut, S., et al. (2015). Assessing the impact of observations on ocean forecasts and reanalyses: part 1, Global studies. J. Operat. Oceanogr. 8, s49-s62. doi: 10.1080/1755876X.2015. 1022067

Osborne, E., Richter-Menge, J., and Jeffries, M. (eds). (2018). Arctic Report Card 2018. Available at: https://www.arctic.noaa.gov/Report-Card (accessed July 26, 2019).

Parkinson, C. L., and Comiso, J. C. (2013). On the 2012 record low Arctic sea ice cover: combined impact of preconditioning and an August storm. Geophys. Res. Lett. 40, 1356-1361. doi: 10.1002/grl.50349

Paţilea, C., Heygster, G., Huntemann, M., and Spreen, G. (2019). Combined SMAP-SMOS thin sea ice thickness retrieval. Cryosphere 13, 675-691. doi: 10.5194/tc-13-675-2019

Pedersen, L. T., Saldo, R., and Fenger-Nielsen, R. (2015). "Sentinel-1 results: sea ice operational monitoring," in Proceedings of the 2015 IEEE International Geoscience and Remote Sensing Symposium (IGARSS), Milan, 2828-2831. doi: 10.1109/IGARSS.2015.7326403

Penny, S. G., and Hamill, T. M. (2017). Coupled data assimilation for integrated earth system analysis and prediction. Bull. Am. Meteorol. Soc. 97, ES169-ES172. 
Persson, P. O. G., Fairall, C. W., Andreas, E. L., Guest, P. S., and Perovich, D. K. (2002). Measurements near the Atmospheric Surface Flux Group tower at SHEBA: near-surface conditions and surface energy budget. J. Geophys. Res. 107:8045.

Posey, P. G., Metzger, E. J., Wallcraft, A. J., Hebert, D. A., Allard, R. A., Smedstad, O. M., et al. (2015). Assimilating high horizontal resolution sea ice concentration data into the US Navy's ice forecast systems: arctic Cap Nowcast/Forecast System (ACNFS) and the Global Ocean Forecast System (GOFS 3.1). Cryosphere Discuss. 9, 2339-2365. doi: 10.5194/tcd-9-23392015

PPP Steering Group (2013). WWRP Polar Prediction Project Implementation Plan. WMO Report No. WWRP/PPP 2-2013, 72.

PPP Steering Group (2014). WWRP Polar Prediction Project Implementation Plan for the Year of Polar Prediction (YOPP). WMO Report No. WWRP/PPP 3-2014, 53.

Prandi, P., Ablain, M., Cazenave, A., and Picot, N. (2012). A new estimation of mean sea level in the Arctic Ocean from satellite altimetry. Mar. Geod. 35, 61-81. doi: 10.1080/01490419.2012.718222

Provost, C., Pelon, J., Sennéchael, N., Calzas, M., Blouzon, F., Desautez, A., et al. (2015). IAOOS (ice-atmosphere-arctic ocean observing system, 2011-2019). Mercator Ocean Q. Newsl. 51, 13-15.

Pullen, J., Allard, R., Seo, H., Miller, A. J., Chen, S., Pezzi, L. P., et al. (2017). Coupled ocean-atmosphere forecasting at short and medium time scales. J. Mar. Res. 75, 877-921. doi: 10.1357/002224017823523991

Rabatel, M., Rampal, P., Carrassi, A., Bertino, L., and Jones, C. K. (2018). Impact of rheology on probabilistic forecasts of sea ice trajectories: application for search and rescue operations in the Arctic. Cryosphere 12, 935-953. doi: 10.5194/tc12-935-2018

Rabe, B., Karcher, M., Schauer, U., Toole, J. M., Krishfield, R. A., Pisarev, S., et al. (2011). An assessment of pan-Arctic Ocean freshwater content changes from the 1990 s to the IPY period. Deep Sea Res. I 58, 173-185. doi: 10.1016/j.dsr. 2010.12.002

Rayner, P. J., Enting, I. G., and Trudinger, C. M. (1996). Optimizing the $\mathrm{CO}_{2}$ observing network for constraining sources and sinks. Tellus 48B, 433-444. doi: 10.3402/tellusb.v48i4.15924

Reeve, K., Boebel, O., Kanzow, T., Strass, V., Rohardt, G., and Fahrbach, E. (2016). A gridded data set of upper-ocean hydrographic properties in the Weddell Gyre obtained by objective mapping of Argo float measurements. Earth Syst. Sci. Data 8, 15-40. doi: 10.5194/essd-8-15-2016

Rehm, E., Dushaw, B. D., Freitag, L., Heaney, K. D., Carr, S., Dakin, T., et al. (2018). BBANC: A Baffin Bay Acoustic Navigation and Communication System Feasibility Study. Available at: https://gitlab.com/Takuvik/BBANC/wikis/home (accessed June 1, 2018).

Richards, C., Pittman, M., Phelan, K., Nudds, S., and Hamilton, J. (2017). “The barrow strait real time observatory: under-ice monitoring in the Canadian high arctic," in Proceedings of the International Conference on Underwater Networks \& Systems, (New York, NY: ACM), 8.

Richter-Menge, J. A., Perovich, D. K., Elder, B. C., Claffey, K., Rigor, I., and Ortmeyer, M. (2006). Ice mass-balance buoys: a tool for measuring and attributing changes in the thickness of the Arctic sea-ice cover. Ann. Glaciol. 44, 205-210. doi: 10.3189/172756406781811727

Ricker, R., Hendricks, S., Helm, V., Skourup, H., and Davidson, M. (2014). Sensitivity of CryoSat-2 Arctic sea-ice freeboard and thickness on radarwaveform interpretation. Cryosphere 8, 1607-1622. doi: 10.5194/tc-8-16072014

Riser, S. C., Freeland, H. J., Roemmich, D., Wijffels, S., Troisi, A., Belbéoch, M., et al. (2016). Fifteen years of ocean observations with the global Argo array. Nat. Clim. Change 6, 145-153.

Roemmich, D., and Gilson, J. (2009). The 2004-2008 mean and annual cycle of temperature, salinity, and steric height in the global ocean from the Argo Program. Prog. Oceanogr. 82, 81-100. doi: 10.1016/j.pocean.2009.03.004

Roemmich, D., Johnson, G. C., Riser, S., Davis, R., Gilson, J., Owens, W. B., et al. (2009). The Argo Program: observing the global ocean with profiling floats. Oceanography 22, 34-43. doi: 10.5670/oceanog.2009.36

Roquet, F., Wunsch, C., Forget, G., Heimbach, P., Guinet, C., Reverdin, G., et al. (2013). Estimates of the Southern Ocean general circulation improved by animal-borne instruments. Geophys. Res. Lett. 40, 6176-6180. doi: 10.1002/ 2013GL058304
Sakov, P., Counillon, F., Bertino, L., Lisaeter, K. A., Oke, P. R., and Korablev, A. (2012). TOPAZ4: an ocean-sea ice data assimilation system for the North Atlantic and Arctic. Ocean Sci. 8, 633-656. doi: 10.5194/os-8-633-2012

Schmidt, K., Swart, S., Reason, C., and Nicholson, S. (2017). Evaluation of satellite and reanalysis wind products with in situ Wave Glider wind observations in the Southern Ocean. J. Atmos. Ocean. Technol. 34, 2551-2568. doi: 10.1175/ JTECH-D-17-0079.1

Schulz, E. W., Josey, S. A., and Verein, R. (2012). First air-sea flux mooring measurements in the Southern Ocean. Geophys. Res. Lett. 39:L16606.

Serreze, M. C., and Barry, R. G. (2011). Processes and impacts of Arctic amplification: a research synthesis. Glob. Planet. Change 77, 85-96. doi: 10. 1016/j.gloplacha.2011.03.004

Shibley, N. C., Timmermans, M. L., Carpenter, J. R., and Toole, J. M. (2017). Spatial variability of the Arctic Ocean's double-diffusive staircase. J. Geophys. Res. Oceans 122, 980-994. doi: 10.1002/2016jc012419

Shupe, M., de Boer, G., Dethloff, K., Hunke, E., Maslowski, W., McComiskey, A., et al. (2018). The Multidisciplinary Drifting Observatory for the Study of Arctic Climate (MOSAIC) Atmosphere Science Plan. DOE Report No. DOE/SC-ARM18-005.

Sigmond, M., Reader, M. C., Flato, G. M., Merryfield, W. J., and Tivy, A. (2016). Skillful seasonal forecasts of Arctic sea ice retreat and advance dates in a dynamical forecast system. Geophys. Res. Lett. 43, 12457-12465.

Smith, G. C., Bélanger, J. M., Roy, F., Pellerin, P., Ritchie, H., Onu, K., et al. (2018). Impact of coupling with an ice-ocean model on global medium-range NWP forecast skill. Mon. Weather Rev. 146, 1157-1180. doi: 10.1175/MWR-D-170157.1

Smith, G. C., Roy, F., Belanger, J. M., Dupont, F., Lemieux, J. F., Beaudoin, C., et al. (2013). "Small-scale ice-ocean-wave processes and their impact on coupled environmental polar prediction," in Proceedings of the ECMWFWWRP/THORPEX Polar Prediction Workshop, 24-27 June 2013, (Reading: ECMWF).

Smith, G. C., Roy, F., Reszka, M., Surcel Colan, D., He, Z., Deacu, D., et al. (2016). Sea ice forecast verification in the Canadian global ice ocean prediction system. Q. J. R. Meteorol. Soc. 142, 659-671. doi: 10.1002/qj.2555

Soltwedel, T., Schauer, U., Boebel, O., Nöthig, E. M., Bracher, A., Metfies, K., et al. (2013). "FRAM - frontiers in arctic marine monitoring visions for permanent observations in a gateway to the arctic ocean," in Proceedings of the 2013 MTS/IEEE, Bergen. doi: 10.1109/OCEANS-Bergen.2013.660 8008

Stark, J. D., Ridley, J., Martin, M., and Hines, A. (2008). Sea ice concentration and motion assimilation in a sea ice- ocean model. J. Geophys. Res. Oceans 113:C05S91.

Swart, S., Gille, S. T., Delille, B., Josey, S., Mazloff, M., Newman, L., et al. (2019). Constraining Southern Ocean air-sea-ice fluxes through enhanced observations. Front. Mar. Sci. 6:421. doi: 10.3389/fmars.2019.00421

Swart, S., Thomalla, S. J., and Monteiro, P. M. S. (2015). The seasonal cycle of mixed layer dynamics and phytoplankton biomass in the Sub-Antarctic Zone: a highresolution glider experiment. J. Mar. Syst. 147, 103-115. doi: 10.1016/j.jmarsys. 2014.06.002

Taylor, P., Hegyi, B., Boeke, R., and Boisvert, L. (2018). On the increasing importance of air-sea exchanges in a thawing Arctic: a review. Atmosphere 9:41. doi: 10.3390/atmos9020041

Thomson, J., and Girton, J. (2017). Sustained measurements of Southern Ocean air-sea coupling from a wave glider autonomous surface vehicle. Oceanography 30, 104-109. doi: 10.5670/oceanog.2017.228

Thwaites, F. T., Krishfield, R., Timmermans, M. L., Toole, J. M., and Williams, A. J. (2011). "Flux measurements from an ice-tethered profiler: first look," in Proceedings of the Oceans 2011, 6-9 June 2011, IEEE/OES, (Santander: IEEE), 6.

Tian-Kunze, X., Kaleschke, L., Maaß, N., Mäkynen, M., Serra, N., Drusch, M., et al. (2014). SMOS-derived thin sea ice thickness: algorithm baseline, product specifications and initial verification. Cryosphere 8, 997-1018. doi: 10.5194/tc8-997-2014

Tietsche, S., Notz, D., Jungclaus, J. H., and Marotzke, J. (2013). Assimilation of sea-ice concentration in a global climate model-physical and statistical aspects. Ocean Sci. 9, 19-36. doi: 10.5194/os-9-19-2013

Timmermans, M.-L., Cole, S. T., and Toole, J. M. (2011). Horizontal density structure and restratification of the Arctic ocean surface layer. J. Phys. Oceanogr. 42, 659-668. doi: 10.1175/JPO-D-11-0125.1 
Timmermans, M. L., Krishfield, R., Laney, S., and Toole, J. (2010). Ice-Tethered Profiler measurements of dissolved oxygen under permanent ice cover in the Arctic Ocean. J. Atmos. Ocean. Technol. 27, 1936-1949. doi: 10.1175/ 2010JTECHO772.1

Timmermans, M. L., Proshutinsky, A., Golubeva, E., Jackson, J. M., Krishfield, R., McCall, M., et al. (2014). Mechanisms of pacific summer water variability in the Arctic's central Canada basin. J. Geophys. Res. 119, 7523-7548. doi: 10.1002/ 2014JC010273

Timmermans, M. L., Toole, J., Krishfield, R., and Winsor, P. (2008). IceTethered Profiler observations of the double-diffusive staircase in the Canada Basin thermocline. J. Geophys. Res. 113:C00A02. doi: 10.1029/2008JC00 4829

Toole, J. M., Krishfield, R. A., Timmermans, M.-L., and Proshutinsky, A. (2011). The ice-tethered profiler: Argo of the arctic. Oceanography 24, 126-135. doi: 10.5670/oceanog.2011.64

Trenberth, K. E., and Fasullo, J. T. (2010). Simulation of present day and twenty first century energy budgets of the Southern Oceans. J. Clim. 23, 440-454. doi: $10.1175 / 2009$ jcli3152.1

Tschudi, M., Meier, W. N., Stewart, J. S., Fowler, C., and Maslanik, J. (2019). Polar Pathfinder Daily 25 km EASE-Grid Sea Ice Motion Vectors, Version 4. Boulder, CO: NASA National Snow and Ice Data Center Distributed Active Archive Center.

Uotila, P., Goosse, H., Haines, K., Chevallier, M., Barthélémy, A., Bricaud, C., et al. (2018). An assessment of ten ocean reanalyses in the polar regions. Clim. Dyn. 52, 1613-1650. doi: 10.1007/s00382-018-4242-Z

Vecchi, G. A., Delworth, T., Gudgel, R., Kapnick, S., Rosati, A., Wittenberg, A. T., et al. (2014). On the seasonal forecasting of regional tropical cyclone activity. J. Clim. 27, 7994-8016.

Vincent, R. F., Marsden, R. F., Minnett, P. J., Creber, K. A. M., and Buckley, J. R. (2008). Arctic waters and marginal ice zones: a composite Arctic sea surface temperature algorithm using satellite thermal data. J. Geophys. Res. 113:C04021. doi: 10.1029/2007JC004353

Wood, K. R., Jayne, S. R., Mordy, C. W., Bond, N., Overland, J. E., Ladd, C., et al. (2018). Results of the first arctic heat open science experiment. Bull. Am. Meteorol. Soc. 99, 513-520. doi: 10.1093/aob/mcw237

Xie, J., Bertino, L., Counillon, F., Lisaeter, K. A., and Sakov, P. (2017). Quality assessment of the TOPAZ4 reanalysis in the Arctic over the period 1991-2013. Ocean Sci. 13, 123-144. doi: 10.5194/os-13-123-2017

Xie, J., Counillon, F., and Bertino, L. (2018). Impact of assimilating a merged sea-ice thickness from CryoSat-2 and SMOS in the Arctic reanalysis. Cryosphere 12, 3671-3691. doi: 10.5194/tc-12-36712018

Xie, J., Counillon, F., Bertino, L., Tian-Kunze, X., and Kaleschke, L. (2016). Benefits of assimilating thin sea ice thickness from SMOS into the TOPAZ system. Cryosphere 10, 2745-2761. doi: 10.5194/tc-10-2745-2016

Yang, Q., Losa, S. N., Losch, M., Liu, J., Zhang, Z., Nerger, L., et al. (2015). Assimilating summer sea-ice concentration into a coupled ice-ocean model using a LSEIK filter. Ann. Glaciol. 56, 38-44. doi: 10.3189/2015aog69a740

Yang, Q., Losa, S. N., Losch, M., Tian-Kunze, X., Nerger, L., Liu, J., et al. (2014). Assimilating SMOS sea ice thickness into a coupled ice-ocean model using a local SEIK filter. J. Geophys. Res. Oceans 119, 6680-6692. doi: 10.1002/ 2014jc009963

Zhang, Y., Maslowski, W., and Semtner, A. J. (1999). Impact of mesoscale ocean currents on sea ice in high-resolution Arctic ice and ocean simulations. J. Geophys. Res. 104, 18409-18429. doi: 10.1029/1999jc900158

Zhao, M., Timmermans, M. L., Cole, S., Krishfield, R., Proshutinsky, A., and Toole, J. (2014). Characterizing the eddy field in the Arctic Ocean halocline. J. Geophys. Res. 119, 8800-8817. doi: 10.1002/2014JC010488

Zhao, M., Timmermans, M. L., Cole, S., Krishfield, R., and Toole, J. (2016). Evolution of the eddy field in the Arctic ocean's Canada basin, 2005 - 2015. Geophys. Res. Lett. 43, 8106-8114. doi: 10.1002/2016FL069671

Zuo, G., Dou, Y., and Lei, R. (2018). Discrimination algorithm and procedure of snow depth and sea ice thickness determination using measurements of the vertical ice temperature profile by the ice-tethered buoys. Sensors 18:4162. doi: $10.3390 / \mathrm{s} 18124162$

Conflict of Interest Statement: The authors declare that the research was conducted in the absence of any commercial or financial relationships that could be construed as a potential conflict of interest.

Copyright (c) 2019 Smith, Allard, Babin, Bertino, Chevallier, Corlett, Crout, Davidson, Delille, Gille, Hebert, Hyder, Intrieri, Lagunas, Larnicol, Kaminski, Kater, Kauker, Marec, Mazloff, Metzger, Mordy, O'Carroll, Olsen, Phelps, Posey, Prandi, Rehm, Reid, Rigor, Sandven, Shupe, Swart, Smedstad, Solomon, Storto, Thibaut, Toole, Wood, Xie, Yang and the WWRP PPP Steering Group. This is an open-access article distributed under the terms of the Creative Commons Attribution License (CC BY). The use, distribution or reproduction in other forums is permitted, provided the original author(s) and the copyright owner(s) are credited and that the original publication in this journal is cited, in accordance with accepted academic practice. No use, distribution or reproduction is permitted which does not comply with these terms. 


\section{OPEN ACCESS}

Edited by:

John Siddorn,

Met Office, United Kingdom

Reviewed by:

Masanao Shinohara,

The University of Tokyo, Japan

Stanley Kim Juniper,

University of Victoria, Canada

*Correspondence:

Bruce M. Howe

bhowe@hawaii.edu

Specialty section:

This article was submitted to

Ocean Observation,

a section of the journal

Frontiers in Marine Science

Received: 31 October 2018

Accepted: 05 July 2019

Published: 02 August 2019

Citation:

Howe BM, Arbic BK, Aucan J, Barnes CR, Bayliff N, Becker N,

Butler R, Doyle L, Elipot $S$, Johnson GC, Landerer F, Lentz S,

Luther DS, Müller M, Mariano J,

Panayotou K, Rowe C, Ota H, Song YT, Thomas $M$, Thomas PN,

Thompson P, Tilmann F, Weber T and

Weinstein S (2019) SMART Cables for Observing the Global Ocean:

Science and Implementation.

Front. Mar. Sci. 6:424.

doi: 10.3389/fmars.2019.00424

\section{SMART Cables for Observing the Global Ocean: Science and Implementation}

\author{
Bruce M. Howe ${ }^{1 *}$, Brian K. Arbic ${ }^{2}$, Jérome Aucan ${ }^{3}$, Christopher R. Barnes ${ }^{4}$, \\ Nigel Bayliff5, Nathan Becker ${ }^{6}$, Rhett Butler ${ }^{7}$, Laurie Doyle ${ }^{8}$, Shane Elipot ${ }^{9}$, \\ Gregory C. Johnson ${ }^{10}$, Felix Landerer ${ }^{11}$, Stephen Lentz ${ }^{12}$, Douglas S. Luther ${ }^{13}$, \\ Malte Müller ${ }^{14}$, John Mariano ${ }^{15}$, Kate Panayotou ${ }^{16}$, Charlotte Rowe ${ }^{17}$, Hiroshi Ota ${ }^{18}$, \\ Y. Tony Song ${ }^{11}$, Maik Thomas ${ }^{19,20}$, Preston N. Thomas ${ }^{21}$, Philip Thompson ${ }^{22}$, \\ Frederik Tilmann 19,20, Tobias Weber ${ }^{19}$ and \\ Stuart Weinstein ${ }^{6}$ on behalf of the Joint Task Force for SMART Cables
}

${ }^{1}$ Ocean and Resources Engineering, School of Ocean and Earth Science and Technology, University of Hawai'i at Mānoa, Honolulu, HI, United States, ${ }^{2}$ Department of Earth and Environmental Sciences, University of Michigan, Ann Arbor, MI, United States, ${ }^{3}$ Laboratoire d'Études en Géophysique et Océanographie Spatiales (LEGOS), Toulouse, France, ${ }^{4}$ School of Earth and Ocean Sciences, University of Victoria, Victoria, BC, Canada, ${ }^{5}$ SIN Medida Limited, Newbury, United Kingdom, ${ }^{6}$ Pacific Tsunami Warning Center, National Oceanic and Atmospheric Administration, National Weather Service, Honolulu, HI, United States, ${ }^{7}$ Hawaii Institute of Geophysics and Planetology, School of Ocean and Earth Science and Technology, University of Hawai'i at Mānoa, Honolulu, HI, United States, ${ }^{8}$ Alcatel Submarine Networks, Hong Kong, Hong Kong, ${ }^{9}$ Rosenstiel School of Marine and Atmospheric Science, University of Miami, Miami, FL, United States, ${ }^{10}$ Pacific Marine Environmental Laboratory, National Oceanic and Atmospheric Administration, Seattle, WA, United States, ${ }^{11}$ Jet Propulsion Laboratory, California Institute of Technology, Pasadena, CA, United States, ${ }^{12}$ Ocean Specialists, Inc., Stuart, FL, United States, ${ }^{13}$ Department of Oceanography, Joint Institute for Marine and Atmospheric Research, School of Ocean and Earth Science and Technology, University of Hawai'i at Mānoa, Honolulu, HI, United States, ${ }^{14}$ Development Centre for Weather Forecasting, Nonwegian Meteorological Institute, Os/o, Norway, ${ }^{15}$ DRG Undersea Consulting, Inc., Morristown, NJ, United States, ${ }^{16}$ GHD Consulting, London, United Kingdom, ${ }^{17}$ Los Alamos National Laboratory, Los Alamos, NM, United States, ${ }^{18}$ International Telecommunications Union, United Nations, Geneva, Switzerland, ${ }^{19}$ Helmholtz Centre Potsdam, German Research Centre for Geosciences (GFZ), Potsdam, Germany, ${ }^{20}$ Freie Universität Berlin, Berlin, Germany, ${ }^{21}$ Thomas Strategies, Menlo Park, CA, United States, ${ }^{22}$ Sea Level Center, University of Hawai'i at Mānoa, Honolulu, HI, United States

The ocean is key to understanding societal threats including climate change, sea level rise, ocean warming, tsunamis, and earthquakes. Because the ocean is difficult and costly to monitor, we lack fundamental data needed to adequately model, understand, and address these threats. One solution is to integrate sensors into future undersea telecommunications cables. This is the mission of the SMART subsea cables initiative (Science Monitoring And Reliable Telecommunications). SMART sensors would "piggyback" on the power and communications infrastructure of a million kilometers of undersea fiber optic cable and thousands of repeaters, creating the potential for seafloor-based global ocean observing at a modest incremental cost. Initial sensors would measure temperature, pressure, and seismic acceleration. The resulting data would address two critical scientific and societal issues: the longterm need for sustained climate-quality data from the under-sampled ocean (e.g., deep ocean temperature, sea level, and circulation), and the near-term need for improvements to global tsunami warning networks. A Joint Task Force (JTF) led by three UN agencies (ITUMMO/UNESCO-IOC) is working to bring this initiative to fruition. This paper explores the ocean science and early warning improvements available 
from SMART cable data, and the societal, technological, and financial elements of realizing such a global network. Simulations show that deep ocean temperature and pressure measurements can improve estimates of ocean circulation and heat content, and cable-based pressure and seismic-acceleration sensors can improve tsunami warning times and earthquake parameters. The technology of integrating these sensors into fiber optic cables is discussed, addressing sea and land-based elements plus delivery of real-time open data products to end users. The science and business case for SMART cables is evaluated. SMART cables have been endorsed by major ocean science organizations, and JTF is working with cable suppliers and sponsors, multilateral development banks and end users to incorporate SMART capabilities into future cable projects. By investing now, we can build up a global ocean network of long-lived SMART cable sensors, creating a transformative addition to the Global Ocean Observing System.

Keywords: ocean circulation, ocean cabled observatories, submarine telecommunications cables, tsunami early warning, ocean observing, UN Joint Task Force, SMART subsea cables

\section{THE SMART CABLES CONCEPT}

Deploying oceanographic sensors on new undersea telecommunication cables is a promising solution for obtaining the extensive, longitudinal, real-time data that are critical for understanding and managing urgent environmental issues such as climate change and tsunami hazard mitigation. Such sensors can provide important environmental data from sites in the deep ocean that are otherwise difficult and expensive to obtain in real-time and over decadal time scales. Suitable sensors are already deployed on dedicated cabled ocean research observatories, and with modest non-recurring engineering expenses, such sensors can be integrated in future telecommunications cables to create SMART cable systems (Science Monitoring And Reliable Telecommunications; Figure 1).

The SMART cables concept originated decades ago and has been demonstrated on a small scale by placing a few sensors at the end of disused cables, such as off Japan in the 1990s for detecting earthquakes and tsunamis. Modern fiber-optic cables, capable of delivering power and high bandwidth, have been used as part of dedicated sustained cabled observatories to obtain data on complex ocean systems beyond what is available from conventional methods, such as research vessels and fixed buoys (Favali et al., 2010). The first such cabled observatories were the 2006 coastal VENUS system (Tunnicliffe et al., 2008) and the regional NEPTUNE observatory operational in 2009 (Barnes et al., 2015; Best et al., 2015), now within Ocean Networks Canada (ONC). Similar observatories, tailored to national, scientific, and geographical needs, have included Japan - DONET and S-net (Kawaguchi et al., 2015; Kanazawa et al., 2016), United States - Ocean Observatories Initiative (OOI) and others (Massion and Raybould, 2006; Kelley et al., 2014; Howe et al., 2015), China (Lu et al., 2015), and Europe (Best et al., 2014; Person et al., 2015). These developments have in turn fostered the evolution of progressively smaller, more precise and reliable sensors (Schaad, 2009; Paros et al., 2012; RBR, 2017; Delory and Pearlman, 2018).

Advocacy for the SMART cables concept began in earnest with a paper by You (2010). In 2012, following workshops in Rome (2011) and Paris (2012), three United Nations agencies established the Joint Task Force (JTF) to facilitate development of the concept (the International Telecommunication Union (ITU), the World Meteorological Organization (WMO), and the Intergovernmental Oceanographic Commission of the United Nations Educational, Scientific and Cultural Organization (UNESCO/IOC). The initial few years of development of JTF were described by Barnes (2018) and details of the various workshops and publications are provided on the JTF web site: https://www.itu.int/en/ITU-T/climatechange/task-force-sc/ Pages/default.aspx.

Science Monitoring And Reliable Telecommunications cables represent a potential major new element in the Global Ocean Observing System (GOOS), and JTF, as it develops SMART cables, is engaging closely with the GOOS Framework for Ocean Observing ( $\mathrm{FOO}^{1}$; Lindstrom et al., 2012). A core concept of GOOS FOO is "Essential Ocean Variables" (EOVs): high impact, discrete, feasibly monitored observable attributes of the global oceans. SMART cables, by their nature as extensive, deep-ocean, high-data-rate observatories, directly address several of the GOOS EOVs. For example, ocean bottom pressure (OBP) was recently accepted as an emerging EOV, and SMART cables are potentially the most extensive and costeffective source for such measurements. SMART cables also measure subsurface temperature, and the tsunami-measurement capabilities of SMART cables would address one aspect of the Sea Surface Height EOV. GOOS prescribes a phased approach for new ocean observing technologies, from concept to regional pilots through to global implementation. JTF is following this approach, taking steps to ensure that SMART cables and the

${ }^{1}$ http://www.oceanobs09.net/foo/ 


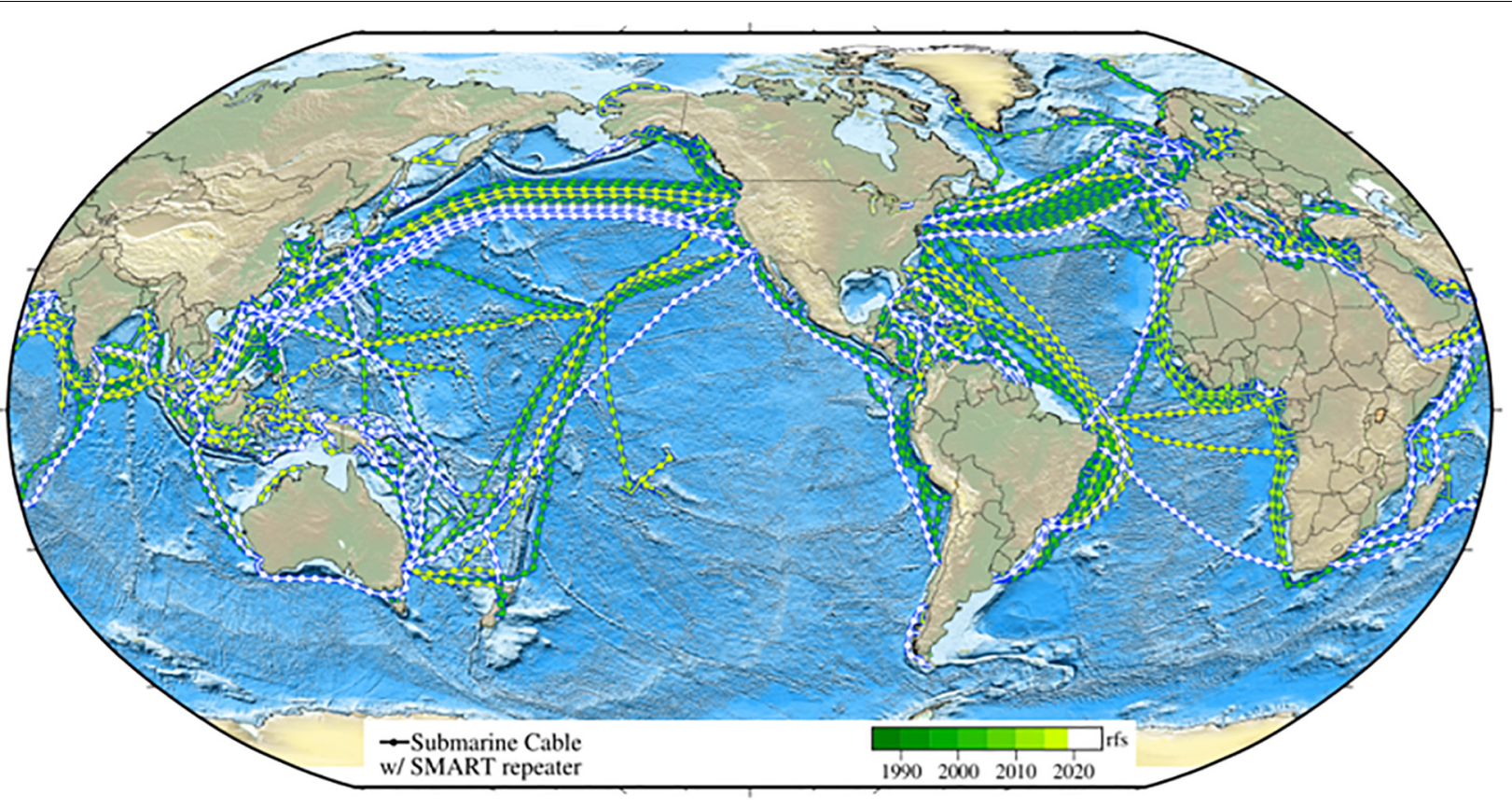

FIGURE 1 | Current and planned cables span the oceans, enabling the Internet and our society. As they are replaced and expanded over their 10-25 year refresh cycle, environmental sensors (pressure, temperature, acceleration) can be added to the cable repeaters every $\sim 100 \mathrm{~km}$, gradually obtaining real time global coverage (for clarity, repeaters are shown only every 300 km. rfs - year ready for service). Cable data: TeleGeography's Telecom Resources licensed under Creative Commons ShareAlike. Permission obtained for use of figure.

data derived from them can be seamlessly incorporated in GOOS as part of a comprehensive Deep Ocean Observing Strategy (DOOS; Levin et al., 2019).

A central feature of the SMART cables concept is that it brings together two key themes of the 21st century: the increasing pressure for global connectivity and the urgent need for coherent, concerted global effort on climate change and ocean management. The market-driven investment in information infrastructure can be harnessed to achieve tangible, social benefits in climate and ocean science. The relatively modest suite of proposed instruments will help address many of the basic science and societal needs, and will also facilitate the monitoring of the physical integrity of the cable itself. The importance of such synergy is reflected in the themes of OceanObs'19 (e.g., the "Blue Economy" and "Ocean Discovery," particularly in the deep oceans) and the United Nations' Sustainable Development Goals (SDG 13 - Climate and SDG 14 - Oceans).

Joint Task Force and its industry partners recognize the need for funding sources to bear the development costs of integrating sensors into existing submarine cable components and the incremental capital expenditures associated with adding SMART capabilities to a telecommunications cable system. JTF's next step is a wet demonstration/pilot project, in which sensor packages are included on a relatively short submarine cable using standard industry practices, with data retrieved in real-time over a minimum of 1 year. Multiple suitable cable projects are in the planning stages in the South Pacific, where JTF can validate not only the technical elements, but also the data management, regulatory clearances, and funding mechanisms (e.g., multilateral development banks).

This paper first explains how SMART cables can improve our understanding of myriad ocean and geophysical processes, including ocean temperature, circulation, sea level rise, tides and wind waves, as well as tsunami modeling and seismology (see section "Improvements in Ocean Observing with SMART Cables"). The paper then details the practical aspects of creating such a network: what sensors SMART repeaters will use and how will they integrate into subsea telecommunications cable systems (see section "Technical Approach"); how the resulting data will be managed, distributed, and used (see section "Data Management and Users"); how the international SMART cables program will be overseen (see section "Program Management"); what legal and permitting considerations are relevant to SMART cables (see section "Legal Outlook"); what the costs of a SMART cable system are and what sources of financing there are to meet those costs (see sections "Cost Estimate" and "Financing"). Recommendations for OceanObs'19 to consider are given in section "Summary." Cost elements and projections from year 1 through 25 are provided as a supplement.

\section{IMPROVEMENTS IN OCEAN OBSERVING WITH SMART CABLES}

The data collected by the SMART cables would greatly enhance and complement multiple observation networks already in place today. The variables measured by the SMART cable repeaters 
are temperature, pressure, and seafloor seismic acceleration. Importantly, as discussed in the previous section, the direct measurements and their derivatives respond directly to the GOOS need for greater attention to EOVs and the UN imperative to contribute to the SDGs. More broadly and in the future, the SMART cable infrastructure will provide a general interface into the deep ocean.

\section{Oceanography}

Oceans are currently monitored by in situ (ships, buoys, moorings, or floats) and remote sensing (satellite) techniques, yet the deep ocean and the important processes occurring remain undersampled and unobserved. Data from SMART cables would fill critical gaps in our existing monitoring systems, complement existing observations, increase our current level of understanding of the ocean, and improve our capability to predict its future evolution.

\section{Ocean Temperature}

Antarctic Bottom Waters, which fill much of the deep oceans (Johnson, 2008) are warming, absorbing substantial amounts of heat, and contributing to sea level rise (Figure 2; Purkey and Johnson, 2010). In addition, the Atlantic meridional overturning circulation (AMOC) is changing (Smeed et al., 2018), and those changes are associated with variations in ocean temperature, air-sea heat flux, and sea level, suggesting that this full-depth ocean circulation feature is important in modulating regional and global climate.

There are now several trans-basin moored arrays monitoring the AMOC at different latitudes (McCarthy et al., 2015; Lozier et al., 2017; Meinen et al., 2017), but outside of the Atlantic, oceanographers currently rely primarily on Global Ocean Ship
(GO-SHIP) repeated transoceanic hydrographic sections (Talley et al., 2016) to monitor the deep ocean water properties and circulation variations, along with a few time series stations (Lukas et al., 2001) and deep instruments on moorings both regional (McKee et al., 2011) and global (Send and Lankhorst, 2011) in scope. Deep Argo floats capable to a depth of $6,000 \mathrm{~m}$ have been developed, with a few regional pilot arrays already deployed or planned (Jayne et al., 2017), and a global network envisioned (Johnson et al., 2015). Changes in the deep ocean, however, remain undersampled (Johnson et al., 2015). SMART cables, with transoceanic sampling of temperature in the bottom boundary layer at roughly $50 \mathrm{~km}$ resolution, would complement other data sets that facilitate investigation into water temperature variability, trends, and circulation (together with SMART cable pressure sensors). SMART cable temperature sensors would provide much closer spatial sampling than either Deep Argo at $500 \mathrm{~km}$ nominal density (Johnson et al., 2015), transoceanic arrays moorings that are often much farther apart in the ocean interior (McCarthy et al., 2015; Lozier et al., 2017; Meinen et al., 2017), or the even sparser OceanSites moorings (Send and Lankhorst, 2011). They would provide much better temporal resolution than either the 10-15 day Deep Argo sampling or the decadal repeats of GO-SHIP transoceanic hydrographic sections (Talley et al., 2016).

\section{Ocean Circulation, Sea Level Rise, and Mass Distribution}

Since 2004, the RAPID/MOCHA array has been providing estimates of the AMOC at $26.5^{\circ} \mathrm{N}$ by estimating the pressure gradient between the western and eastern continental slopes (McCarthy et al., 2015). From the AMOC strength, the climate-relevant meridional heat transport and its variation can

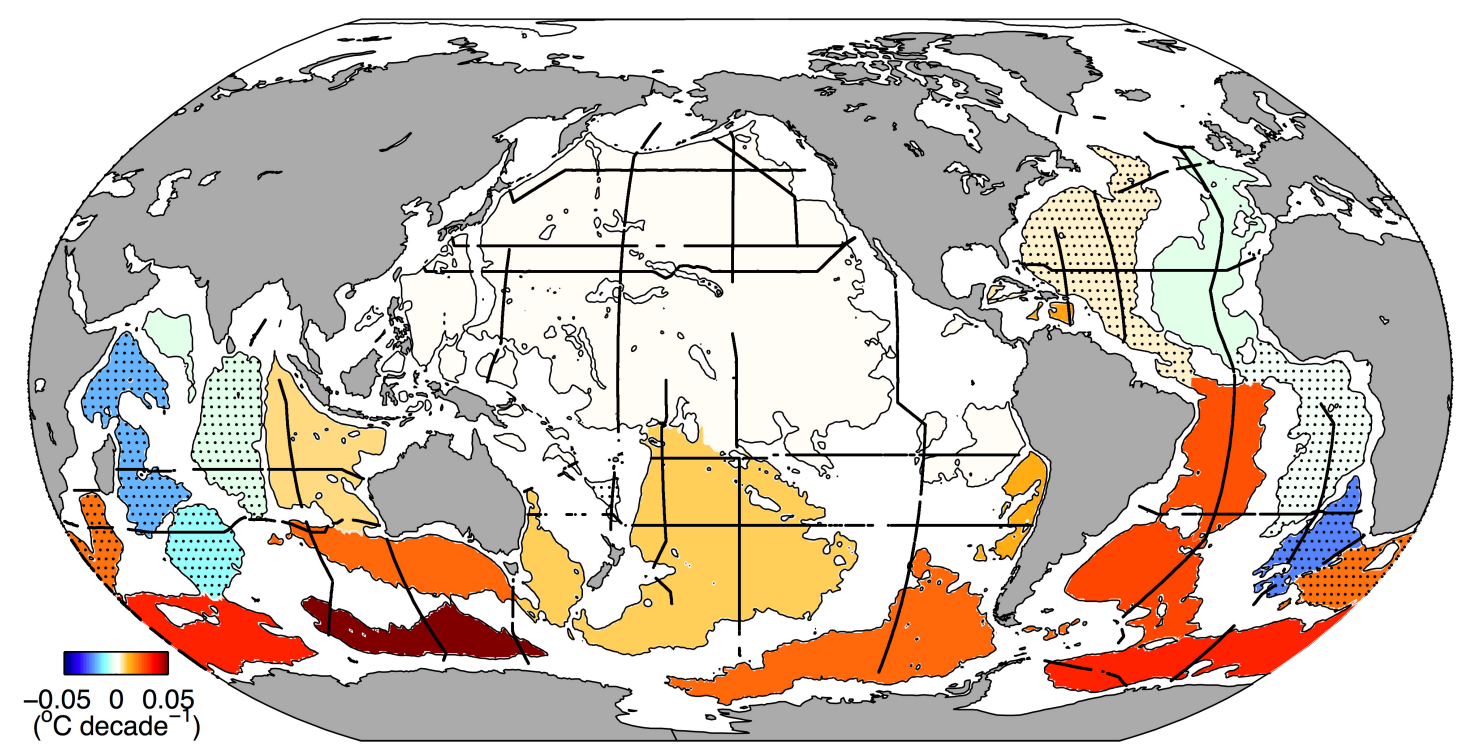

FIGURE 2 | Deep basin (thin solid lines) average warming rates below 4,000 $\mathrm{m}$ from the 1990 s to the 2000 s $\left({ }^{\circ} \mathrm{C}\right.$ decade ${ }^{-1}$, colorbar) based on data from Purkey and Johnson (2010). Estimates are based on data from decadal repeats of hydrographic sections (thick solid lines) first occupied during the World Ocean Circulation Experiment (WOCE) (King et al., 2001) and subsequently by the Global Ocean Ship-based Hydrographic Investigations Program (GO-SHIP) (Talley et al., 2016). Stippled basins have average warming rates that are not statistically significantly different from zero at $95 \%$ confidence. Permission obtained for use of figure. 
also be inferred, because a strong linear relationship exists between ocean volume and heat transports, at least at $26.5^{\circ} \mathrm{N}$ (Johns et al., 2011). SMART cables would complement and extend the existing AMOC estimates in two ways. First, OBP measurements on cables spanning an entire ocean basin could measure the pressure differences at many depths between the western and eastern boundaries of the basin. The pressure differences are directly related to the transports at those depths, as illustrated in Figure 3. Both the upper and lower limbs of the dominant geostrophic component of AMOC transport can be estimated in this way (Hughes et al., 2013, 2018; Elipot et al., 2014). Second, multiple cross-basin transects by SMART cables at different latitudes would allow a division of major ocean basins into boxes. Geostrophic transports across box boundaries could then be estimated from OBP observations as just described, allowing the mass balance of individual boxes to be calculated. These box models would finally allow a quantification of the long-term mass evolution in an ocean basin, based on unaliased measurements.

Global warming has caused global mean sea level to rise at a rate of $3.0 \pm 0.4 \mathrm{~mm} /$ year since 1992 (Figure 4), with an estimated current acceleration of $0.084 \pm 0.025 \mathrm{~mm} /$ year $^{2}$ (Nerem et al., 2018). This will lead to a sea level rise of approximately $65 \mathrm{~cm}$ by 2100 . Because individual contributions to sea level change, such as barystatic (mass changes, e.g., due to melting land ice) and steric (expansion of water, e.g., due to warming) effects in the ocean, as well as Earth-produced eustatic (changes in ocean volume) and isostatic (changes in height of land) effects, vary considerably across the oceans, sea level rise is not homogeneous. Sea level variability patterns can be determined by various measurement techniques and sensors, e.g., radar altimetry and tide gauges. The identification of individual contributing effects, however, requires complementary observation methods at each location. OBP observations provide the amount of local barystatic sea level change. SMART cables would provide a network of long-lasting, temporally unaliased OBP sensors that could be quite dense in some basins and unaliased along the cables. In conjunction with sea level observations by altimetry, or density field observations by Argo floats, for instance, the OBP measurements would enable

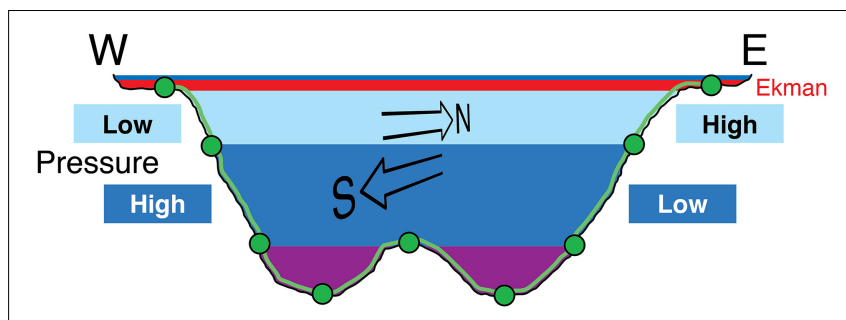

FIGURE 3 | Schematic of the geostrophic relationship between OBP (as measured by SMART pressure sensors, green dots/cables) at the western boundary of an ocean basin relative to the eastern boundary, given a net transport between the boundaries as indicated by the arrows. At the deepest depths, pressure differences on the sides of seafloor mountain ranges come into play and will also be captured by SMART cable OBP observations. Adapted from C. Hughes. Permission obtained for use of figure. separation of the steric and barystatic contributions to sea level change at a particular location, whose differentiation is required to understand the causes of sea level rise and thus for reliable sea level projections.

The satellite gravity missions - GRACE (Tapley et al., 2004) and its successor GRACE-FO (Flechtner et al., 2016) - provide measurements of the temporally varying gravity field, which can be interpreted as OBP and ocean mass. Such satellite data, however, are aliased by short-scale processes that cannot be resolved in a global gravity field due to the horizontally integrating nature of the space-based gravity measurement itself, and the characteristics of the satellite orbits, e.g., orbit period, repeat period, inclination, and ground distance between successive orbits. Hence, the data have to be "corrected" by a de-aliasing procedure, involving parameters for oceanic tides, Earth tides, short-term atmospheric pressure variability and short-period oceanic barotropic oscillations (Rietbroek et al., 2006). The quality of the resulting products is one of the main limiters of current and future gravity field missions (Panet et al., 2013). Local alterations of gravity are caused by changes in the vertically integrated mass distribution and a consequence of mass transport in the solid Earth, cryosphere, atmosphere, and ocean (Kelley et al., 2014; Dobslaw et al., 2017). Variations of oceanic mass are reflected in situ by OBP changes. Thus, OBP observations from SMART cables can significantly contribute to the improvement of the GRACE-derived oceanic products in two complementary ways.

First, the observed gravity signal is considerably "contaminated" by contributions from continental hydrology as far as $1,000 \mathrm{~km}$ from continental coasts. These contaminants are estimated and removed during post-processing by applying numerical models of the sub-systems with results that are useful, if not remarkable (Chambers and Schröter, 2011; Makowski et al., 2015). SMART cable observations from near-coastal areas are expected to substantially improve this signal separation by providing constraints for the determination of the oceanic contribution, especially over continental slopes where strong bottom pressure variations from boundary currents are common.

Second, SMART cable observations have the potential to improve simulations of OBP. Although observations by SMART cables are not yet available, observation system simulation experiments (OSSEs) can estimate these potential improvements. Assimilated synthetic OBP observations along possible SMART cable OBP routes from a global high-resolution version of the regional ocean modeling system (ROMS; Song and Hou, 2006) into a global coarse resolution version of the Max-Planck Institute-Ocean Model (MPIOM; Marsland et al., 2003) with an ensemble Kalman filter (Nerger et al., 2007). According to these model simulations, the root-mean-square difference between the OBP from ROMS and MPIOM is reduced by up to $40 \%$ in the Indian Ocean and up to $20 \%$ in the Pacific and Atlantic Oceans (Figure 5). The advantages of SMART cables compared to other observing systems considered in the study are the large number of instruments, the near-real-time availability, the high accuracy, and the global distribution of the network. The last is especially important for obtaining global improvements with the data assimilation approach. 


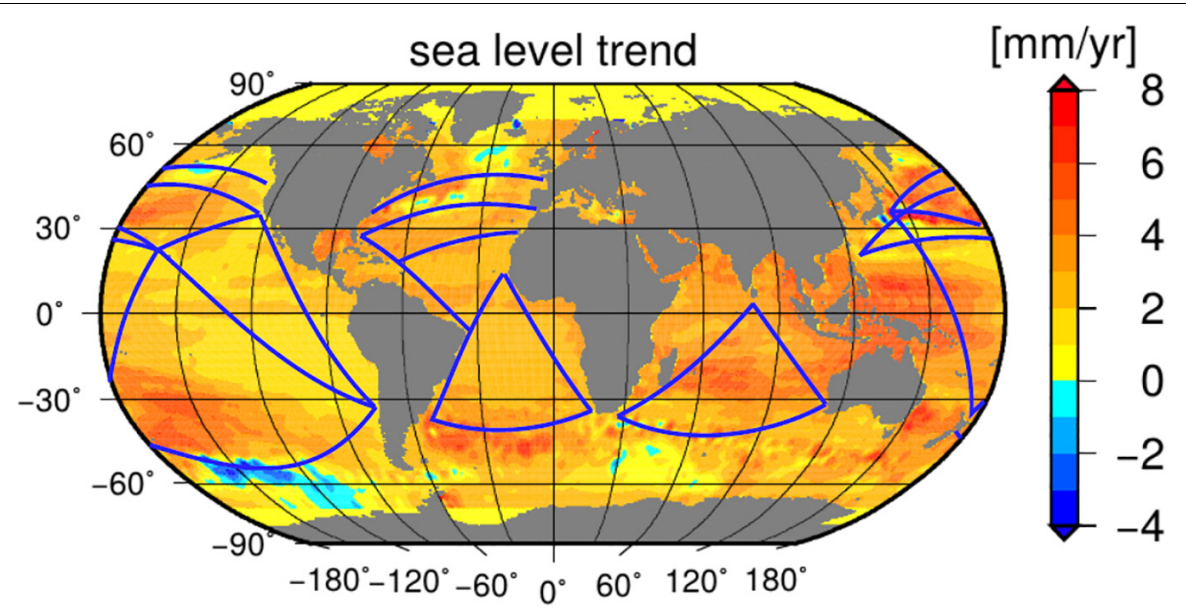

FIGURE 4 | Sea level trend in mm/year as derived from TOPEX, Jason-1, and Jason-2 for 1992-2018. Blue lines denote possible future SMART cable routes. Altimetry data is courtesy of NOAA/NESDIS Center for Satellite Applications and Research, and provided by the NOAA Laboratories for Satellite Altimetry (NOAA/NESDIS, 2018). Permission obtained for use of figure.

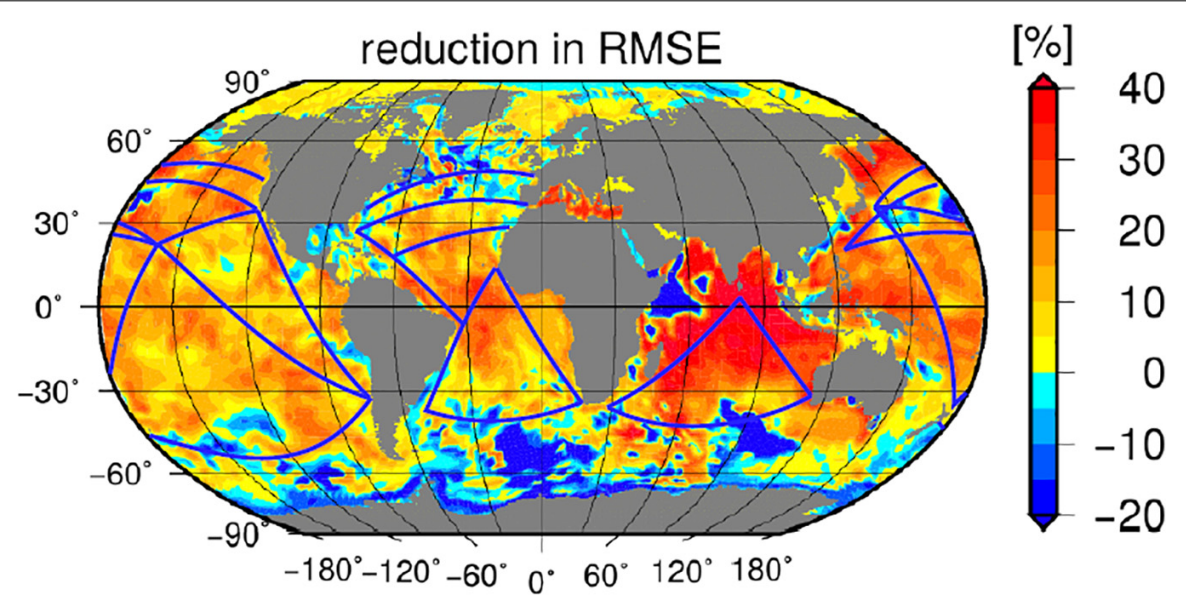

FIGURE 5 | Influence of data assimilation of synthetic SMART cable OBP observations. Reduction of root-mean-square difference between OBP simulated by ROMS and MPIOM for April 2014. Blue lines indicate possible future SMART cable transects. Permission obtained for use of figure.

\section{Ocean Surface (Barotropic) Tides}

Simulation of the gravitationally forced surface (barotropic) tides has now become quite accurate even without assimilation of satellite altimetric data. Forward tide models now routinely capture $90 \%$ or more of tidal sea surface height variance (Arbic et al., 2004; Egbert et al., 2004).

This success does not mean that further study of the tides is now of minimal value. On the contrary, there are still a number of poorly understood or poorly described tidal phenomena, such as the lesser tidal constituents, seasonal variability of all constituents, non-linear constituents, rapid variation of constituent structure in shallow water, and shifting sinks of energy as the global environment changes. Although these factors exhibit small amplitudes, their global distributions are sought due to their impacts on such phenomena as internal tide generation, deep ocean mixing, paleotide descriptions, and Earth structure, as well as due to their utility in defining the tidal "correction" that must be applied to satellite altimetry data to extract the subdiurnal variability of ocean circulation features as well as long period sea level rise.

Ocean bottom pressure observations provide one of the better tools for exploring the finer details of the barotropic tides, because the broad band, non-tidal "geophysical noise" within which the tides are embedded is much weaker at the seafloor than at the sea surface (Ray, 2013). The geophysical noise that limits the utility of satellite observations and in situ coastal sea level observations arises from surface-intensified processes such as internal waves, mixed-layer currents, and coastal-trapped edge waves.

Furthermore, although the astronomical gravitational potential that gives rise to the ocean tides is precisely known, tides are embedded in, and interact with, a three-dimensional ocean circulation that varies on seasonal and longer time scales, in ways that are not easily predicted. Recent results show that 
barotropic tides do vary on seasonal timescales (Müller et al., 2014), and even on secular timescales at the continental coasts (Ray, 2006; Müller et al., 2011). Detection of secular changes in the open ocean tides is less clear (Müller et al., 2011; Zaron and Jay, 2014; Schindelegger et al., 2018).

As an example of time-varying barotropic tide signals that could be seen by SMART cables, we can estimate tidal seasonal variability along the cable paths, using simulated hourly OBP samples from high-resolution global ocean models that are forced simultaneously by atmospheric fields and the astronomical tidal potential (Arbic et al., 2010, 2012, 2018; Müller et al., 2012, 2014; Rocha et al., 2016). The amplitude of the seasonal variability of the principal lunar semi-diurnal tide $\mathrm{M}_{2}$ from the Müller et al. (2014) simulation is given in Figure 6. The range is from 0 to $0.5 \mathrm{~cm}$, which is measurable by the OBP sensors envisioned within the SMART cable repeaters. There are currently no global-scale measurements for models of seasonal tidal variability. SMART cable measurements of OBP would allow unique, basin-scale quantification of barotropic tidal variability over a wide range of timescales, thus providing ground truth for secular and seasonal changes to tidal correction models used in altimetry and gravimetry.

\section{Wind Generated Waves, Microseisms, and Infragravity Waves}

Wind-generated waves at periods shorter than $30 \mathrm{~s}$ are ubiquitous at the ocean surface. In the open ocean, they play a crucial role in the exchange of heat and gases between the ocean and the atmosphere (Hasselmann, 1991), and they can be a major natural hazard at the coast (Hoeke et al., 2013). Because the wind-generated waves have wavelengths far shorter than the average ocean depth, these waves cannot be measured at the seafloor deeper than $1,000 \mathrm{~m}$ and satellites or floating buoys are used to measure them. The popularity of real-time wave observations from near-coast anchored buoys to mariners and surfers (such as the Coastal Data Information Program; Thomas et al., 2015) suggests that OBP observations on SMART cables on the continental shelves and upper slopes could provide useful information on wave amplitudes, periods, and even directions to the local coastal populace, especially in regions where wave buoys have not yet been deployed.

Microseisms constitute the principal seismic noise source on Earth. Wind waves breaking and interacting in the shallow waters of the continental shelves, as well as in the open ocean, generate seismic noise in the period band of the wind waves and at shorter periods (2-20 s). The larger, but termed "secondary," microseism generation mechanism (Longuet-Higgins, 1950) in the deep ocean is from opposing trains of ocean waves, which interact and generate a pressure signal at the seafloor with half the period of the interacting waves. This pressure signal generates seismic waves in the seafloor that can be observed and used for analysis even at the farthest reaches from the oceans in central Asia (Bromirski et al., 2005; Ardhuin et al., 2011; Chen et al., 2015; Butler and Aucan, 2018). These observations improve our understanding of both seismic waves in the seafloor and waves at the ocean surface.

Infragravity (IG) waves are surface waves with periods ranging from minutes to hours. Nonlinear interactions between wind

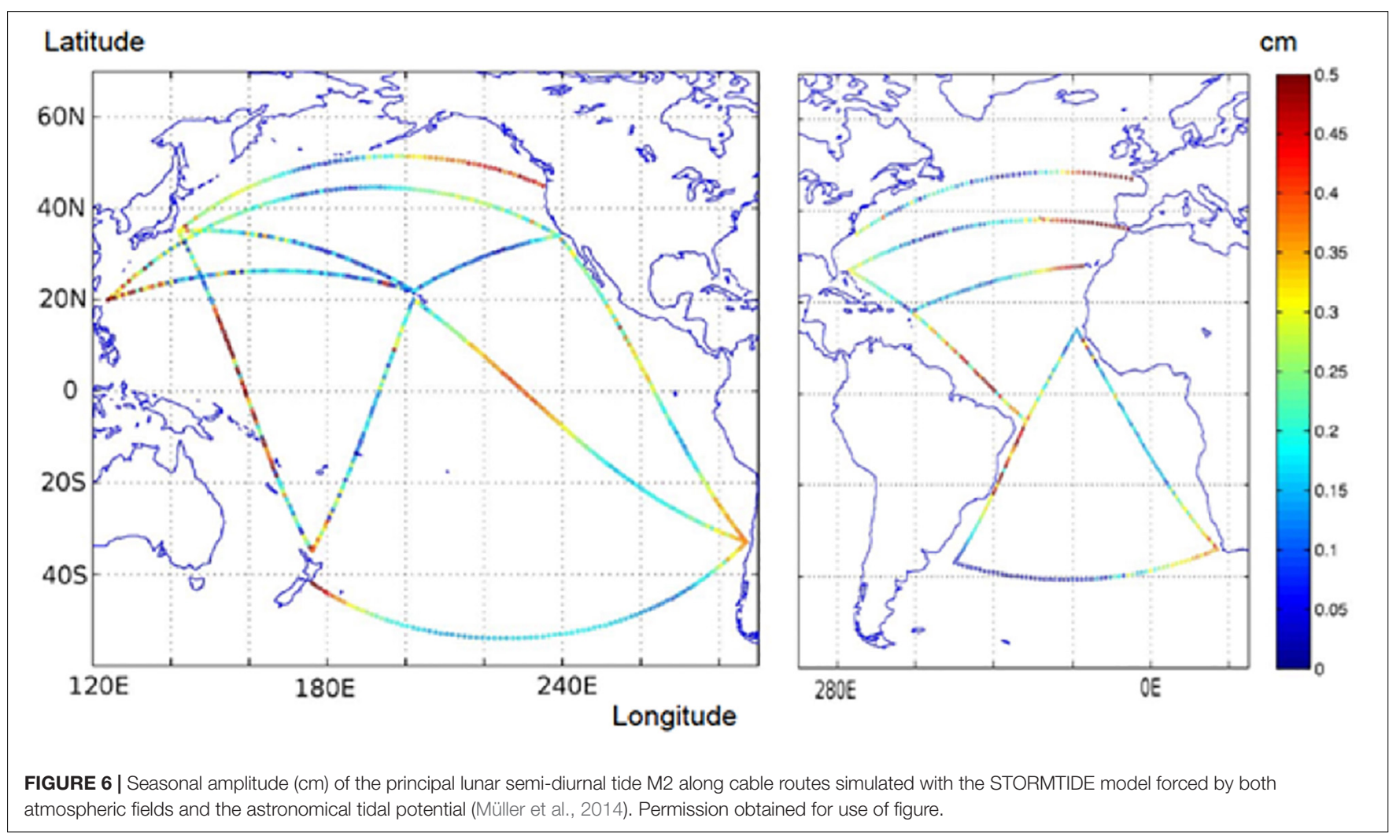


waves in the open ocean and at the coasts generate IG waves at periods from $0.5 \mathrm{~min}$ to many tens of minutes. IG waves appear as either "free" or "bound" waves (Herbers and Guza, 1994; Herbers et al., 1995), where the bound waves are tied to underlying groups of wind waves and become free at the shoreline where the short wind waves break (Bertin et al., 2018). A small fraction of the resultant free IG energy leaks into the open ocean where it can spread for thousands of kilometers, with horizontal wavelengths of up to $10 \mathrm{~s}$ of kilometers and heights of up to $10 \mathrm{~s}$ of centimeters with significant seasonal variability, as seen in Figure 7 (Aucan and Ardhuin, 2013). IG waves at longer periods up to hours have also been identified and appear to be forced by the surface barotropic tides and solar modes of oscillation (Chave et al., 2019). Given the size and wavelength of these IG waves, they are a source of alias noise in satellite measurements of sea surface elevation. Thus, a better understanding and modeling of the temporal and spatial variations of the IG waves could improve the processing of satellite altimetry data (Ardhuin et al., 2014).

Observations on wind-generated waves on the shelves, together with observation of IG waves and microseisms in the deep ocean on SMART cables have the potential to inform us about energy flow from the wind and tides into the deep ocean, as well as the source of Earth's "hum" at the same frequencies, a topic of considerable interest to seismologists using
Earth's vibrations to explore its structure (Nawa et al., 1998; Rhie and Romanowicz, 2004; Webb, 2008). OBP measurements on SMART cable repeaters would contribute significantly to this purpose since the number of ongoing OBP sensors is so small (currently tens of sensors versus the potential of many hundreds of sensors on SMART cables).

\section{Tsunami Monitoring and Warning}

One of the most pressing issues in the tsunami warning community concerns the ability to distinguish destructive tsunamis from those measurable on sea-level gauges but which do not pose a hazard along distant coastlines (Angove et al., 2019). Since the US tsunami warning system began in 1949, 75\% of the evacuations of Hawaii's coastlines have been unnecessary, with direct and indirect costs of millions or tens of millions of dollars per event. This is also true for other coastlines in the Pacific basin and elsewhere. In an attempt to alleviate this problem, NOAA's Pacific Marine Environmental Laboratory (PMEL) developed the Deep-Ocean Assessment and Reporting of Tsunamis (DART) system (Paros, 2011; Bernard and Titov, 2015) which consists of an OBP sensor that communicates via an acoustic modem with a surface buoy that in turn relays the pressure measurements through the IRIDIUM satellite constellation. Prior to the development of the DART system, warning systems had to rely on tide gauges and coastal

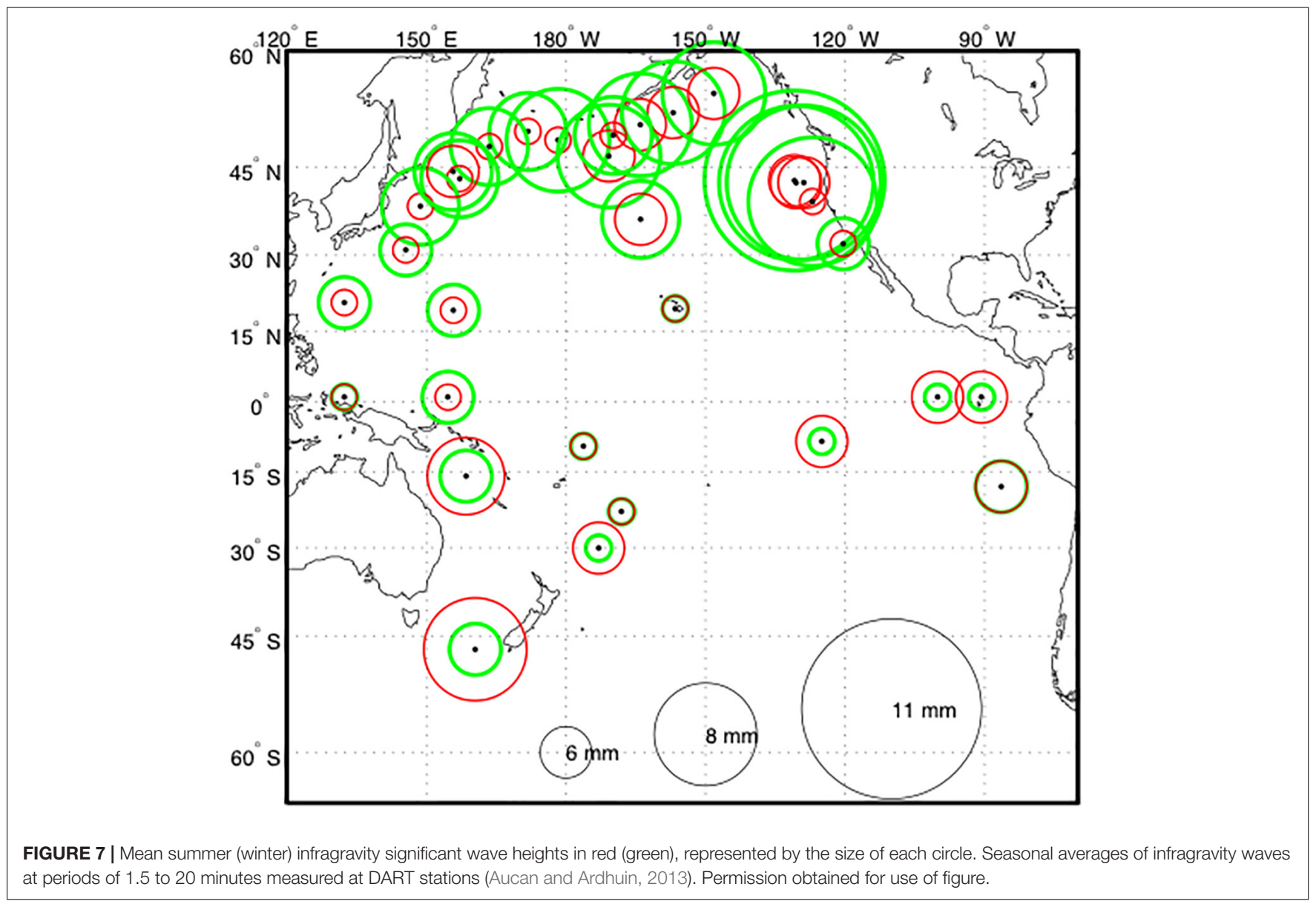


observations when evaluating the potential destructiveness of a tsunami on distant shores. As tsunami height can be strongly affected by nearshore bathymetry and harbor resonance, reliance on coastal observations coupled with the assumption of worstcase scenarios made unnecessary evacuations inevitable. The main limitations of the DARTs are that they require their own power source, need maintenance every 2-3 years, and their availability is reduced significantly by destructive weather and, in some regions, vandalism. Compared to DARTs, SMART cable-based pressure sensors offer much denser sampling and effectively zero maintenance costs after deployment.

An even greater challenge is warning in the near field, i.e., coastal areas directly adjacent to the earthquake rupture. The time between the occurrence of the earthquake and the arrival of the tsunami wave can be as short as $5 \mathrm{~min}$, and in many cases, the tsunami is exacerbated by a sudden co-seismic subsidence of the coast by up to 1-2 $\mathrm{m}$. In all recent catastrophic tsunamigenic earthquakes, fatalities due to the near field tsunami have dominated the overall death toll. Third generation and older DART buoys have to be placed too far from the coast to be helpful for near field warnings due to the necessity of separating in time the pressure fluctuations caused by the tsunami wave from the seismic disturbance caused by the seismic (seafloor) surface wave. SMART cables and fourth generation DART buoys offer higher sample rates, which can separate these two types of disturbances by frequency filtering.

Perhaps the largest uncertainty tsunami warning systems face is in the determination of the source. As some $72 \%$ of tsunamis in the historical record are generated by the static seafloor displacement associated with large submarine earthquakes (the remainder by landslides, volcanoes and others), tsunami warning systems have focused on real-time seismology to facilitate rapid tsunami hazard warnings. Until the last decade, the Pacific Tsunami Warning Center (PTWC) based such warnings purely on the earthquake's location and magnitude. However, these quantities alone are not sufficient to precisely assess the effects of a tsunami. As a result, over-warning or unnecessary warnings have been a flaw of whole-ocean warning systems since their inception. Recent advances in computational seismology, such as the ability to rapidly obtain the centroid moment tensor (CMT; Kanamori and Rivera, 2008; Duputel et al., 2012), have enabled the PTWC and other tsunami warning centers to generate whole-ocean forecasts with sufficient accuracy to be of service to emergency management systems. A robust CMT yields an authoritative assessment of the earthquake's magnitude and rupture orientation, subject to an inherent ambiguity between the fault and auxiliary plane (perpendicular to the fault plane and slip vector). It also helps fix the fault geometry for a finitefault model (Ammon et al., 2005; Weinstein and Lundgren, 2008) which gives the distribution of displacement on the fault plane. From this it is normally straightforward to calculate the sea-floor displacement, which is crucial for determining the overall size of the tsunami and the areas worst affected. A special case is represented by so-called tsunami earthquakes, which are earthquakes that produce tsunamis much larger than expected from their moment magnitude (Kanamori, 1972). Recent examples include the 2006 Java and the 2010 Mentawai earthquakes. Shaking in these earthquakes is strongly subdued, meaning that the tsunami hits an unprepared population if there is no systematic warning.

The tsunami excitation can be further exacerbated by displacement along splay faults and triggered submarine landslides (e.g., Tappin et al., 2014) which are very difficult to detect by seismology alone. SMART cables can help mitigate this issue by providing arrays of pressure sensors, which allow an accurate assessment of the tsunami wave field as it propagates. Currently there are only some 70 or so deep ocean sensors (e.g., DARTs and DONET, S-net, ONC and OOI cabled observatories) permanently in operation and the vast majority of them are in the Pacific Basin. Placing pressure sensors on SMART cables could increase that number well into the hundreds or thousands (Figure 8). This additional near-real-time information can be used to validate and/or revise forecasts making tsunami warnings for areas $>1,000 \mathrm{~km}$ from the earthquake more precise and greatly reducing the potential of unnecessary warnings and evacuations.

In addition to pressure sensors, SMART cables are envisioned to include strong-motion instruments (accelerometers), which measure the motion of the seafloor during an earthquake. With a few exceptions, currently all seismometers and strongmotion instruments are land-based, resulting in a one-sided view of subduction zone earthquakes. Accelerometers on SMART cables would fill in the gaps in the global seismic network (GSN) by acquiring data all along their routes, including in some cases, as they cross subduction zones, the source of the great earthquakes that generate ocean-crossing destructive tsunamis. Having a SMART cable with accelerometers near a submarine earthquake (i.e., having at least one sensor package within $100 \mathrm{~km}$, although more distant sensors also significantly contribute) would allow for faster, more accurate hypocenter locations, magnitude estimates, CMT calculations, and finitefault determinations. This additional information will speed up the process and improve the precision of tsunami wave height propagation models.

The PTWC has performed preliminary calculations as to how SMART cables can improve the tsunami warning system. Figure 9 shows the routes of five hypothetical cables that contain OBP sensor/seismometer packages and the division of the world's subduction zones into potential epicenters of great earthquakes. The routes chosen are hypothetical SMART cable routes and are not specifically based on any existing telecommunication cable route. The instrument packages are spaced $500 \mathrm{~km}$ apart and the calculations assume 688 potential earthquake centers located every $120 \mathrm{~km}$ on the world's subduction zones.

Pacific Tsunami Warning Center has estimated the potential impact of SMART cables on the speed with which an earthquake hypocenter might be determined. Five stations must detect the P-wave and the largest azimuth gap between any two (azimuthally) neighboring stations with respect to the hypocenter must be less than $180^{\circ}$. The calculation assumes an earthquake at each epicenter and then computes the minimum time required to meet the five station and azimuth gap requirement. The calculations are performed based on the seismic station distribution available to PTWC (as of 2016) with and without the 


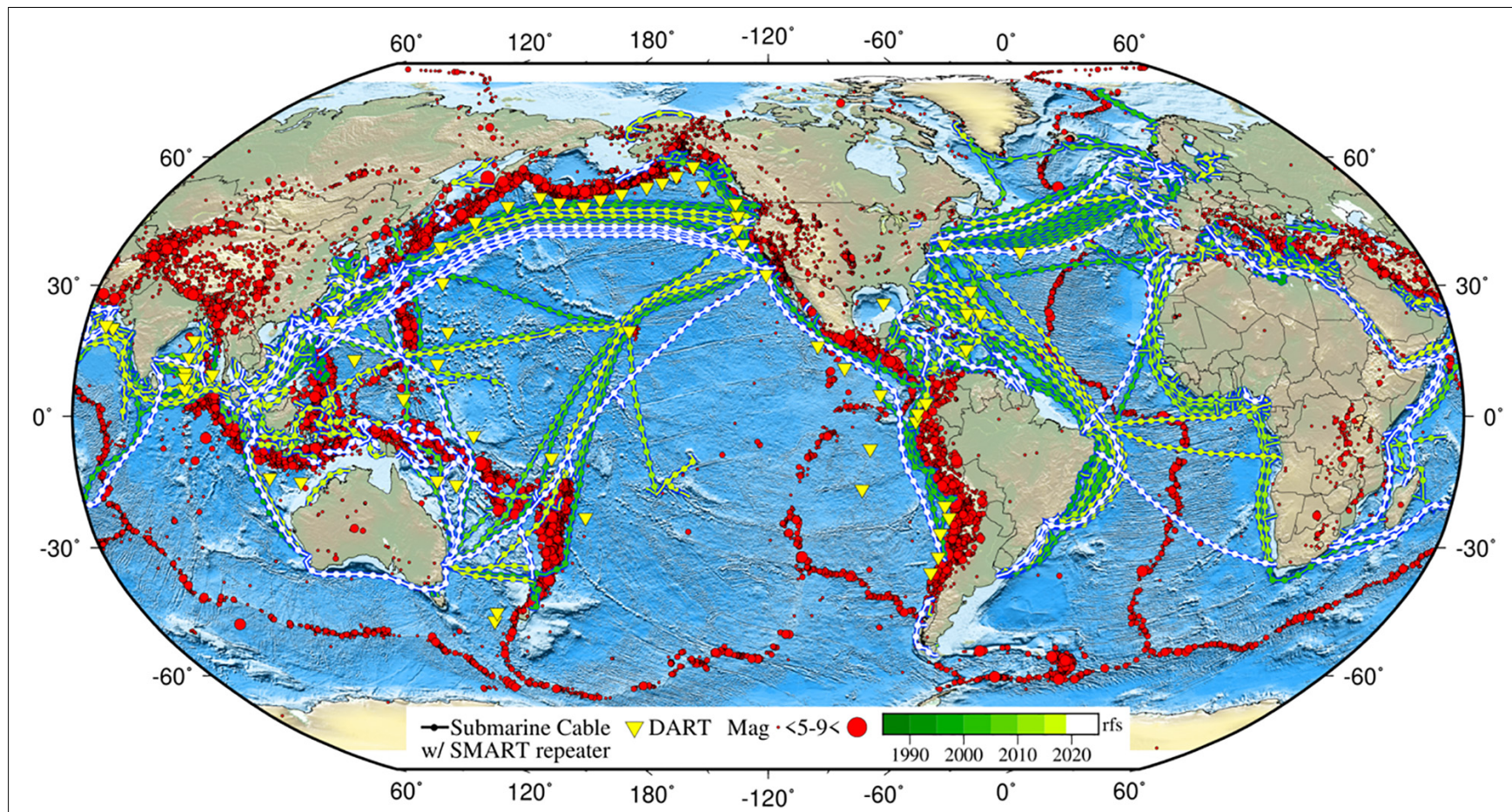

FIGURE 8 | Global map of $\sim 1$ million km of operational submarine telecommunications cables (green present, white in progress/planned; SMART repeaters shown every 300 km; rfs - year ready for service), historical earthquakes (red), and DART tsunami buoys (yellow triangles). Cable data: TeleGeography's Telecom Resources licensed under Creative Commons ShareAlike; DART Buoy locations: NOAA National Data Buoy Center; Seismic data: USGS Earthquake Catalog. Permission obtained for use of figure.

SMART cables in Figure 9. The resulting distribution of timeto-detection is shown in Figure 10 (left). The inclusion of just five SMART cables has the potential to speed up Pacific-wide earthquake epicenter determinations by an average of $~ 21 \%$.

Similarly, we calculated the reduction in latency in tsunami detection that is achievable with OBP sensors, following a tsunamigenic earthquake. Using the 688 epicenters in Figure 9, tsunami travel-times are computed from each epicenter to the set

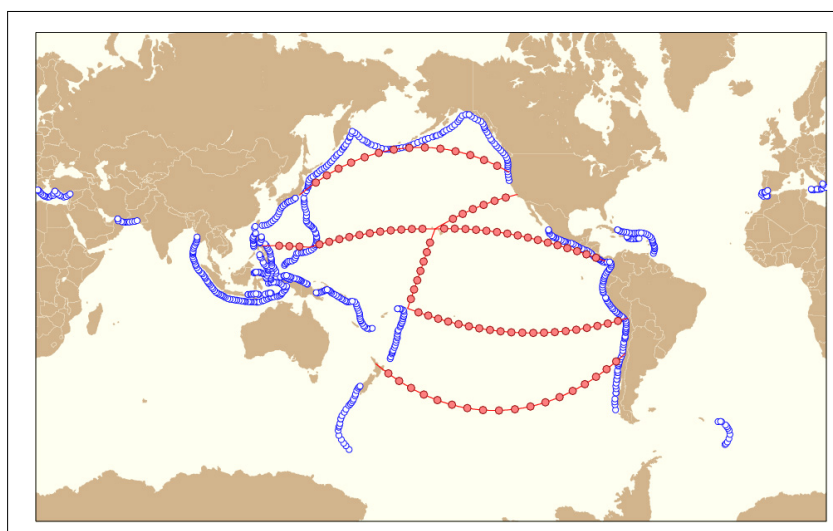

FIGURE 9 | Hypothetical cable routes and SMART repeater locations (red dots) used in tsunami simulations. Also shown are potential trench axis earthquake epicenters (blue circles). Permission obtained for use of figure. of OBP sensors received by PTWC with and without the OBP sensors associated with the SMART cables. With SMART cables in place, the time required to observe the tsunami arrival at three or more OBP sensors is reduced by $\sim 25 \%$ (Figure 10, right).

Specifically, the four charts in Figure 10 compare detection time for earthquakes (left) and tsunamis (right), first using only those sensors available in 2016 (top), and then using existing sensors augmented with simulated data from future SMART cable sensors (bottom). Detection of an earthquake - meaning reception on $\geq 5$ seismic instruments - is reduced from 2.4 to $1.9 \mathrm{~min}$, or from an average of $2 \mathrm{~min} 24 \mathrm{~s}$ down to $1 \mathrm{~min}$ $54 \mathrm{~s}$, which is a $21 \%$ reduction. Thirty seconds is an eternity in earthquake detection and warning.

Tsunami detection at $\geq 3$ pressure sensors showed a similar percentage improvement, dropping from 2.1 to $1.6 \mathrm{~h}$, for a $24 \%$ improvement. Although wide-area tsunami warnings are already helping save lives, unnecessary or overbroad warnings and evacuation orders have significant financial and safety costs. The reduction in detection time coupled with a better description of the wave field will help better characterize the source and improve forecasts in both the near and far field. This will help reduce unnecessary evacuations, which are a longstanding criticism of tsunami warning systems. Thus, SMART-cable augmented tsunami detection will help reduce the direct and indirect costs associated with tsunami warning and evacuation programs, as well as likely improving confidence in the warning system and compliance in the event of a necessary evacuation. 


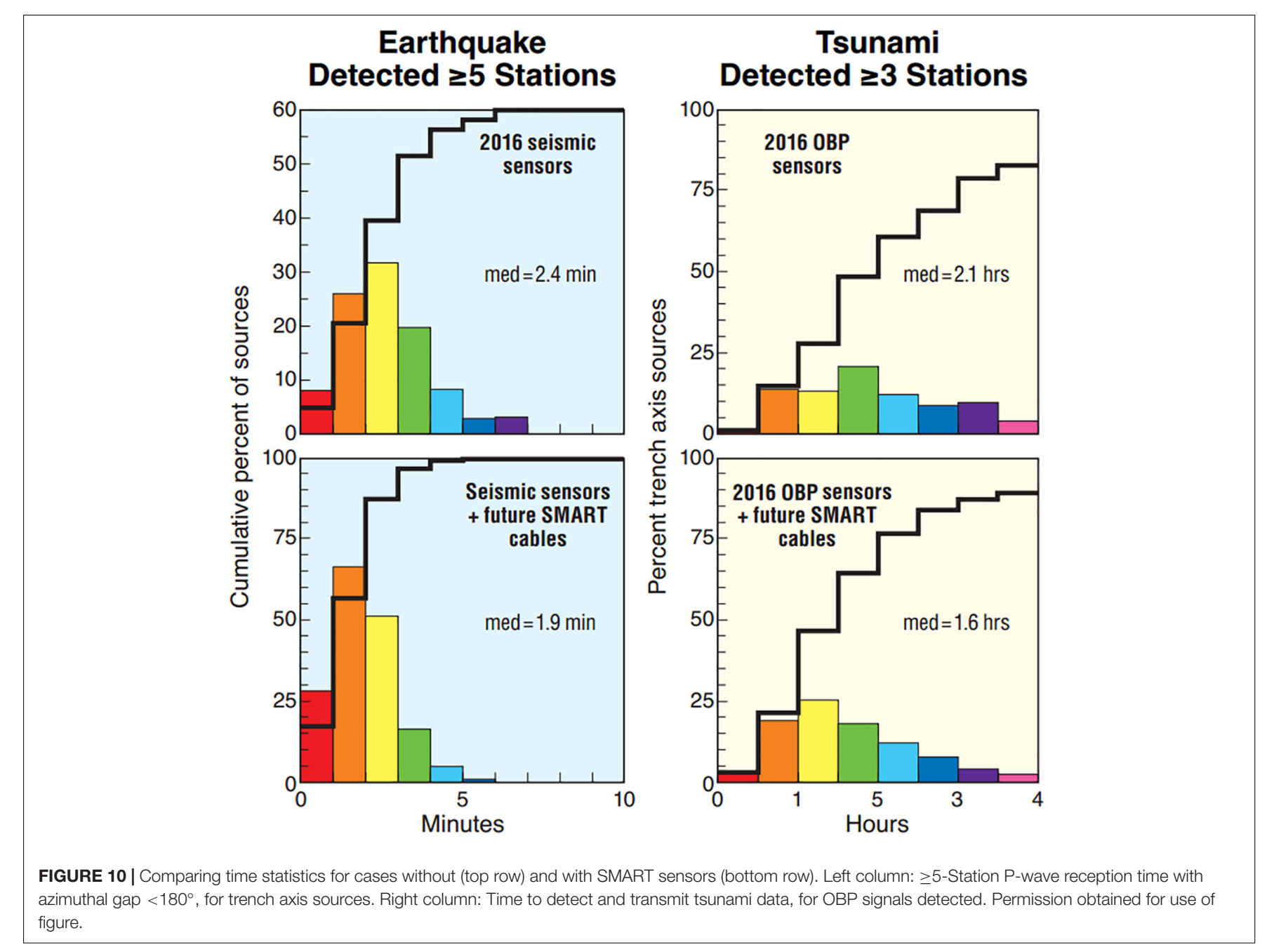

The minimum time required for earthquake location and time required to observe a tsunami at three or more pressure sensors would be further reduced if more repeaters were equipped with SMART capability, reducing the interval between sensors from 500 to $50 \mathrm{~km}$. To balance detection speed and cost, SMARTenabled repeaters could be deployed more densely near the coast and at greater intervals farther away.

Even larger gains, particularly for the near field, are possible for telecommunications cables running parallel to the coast, as they would provide several measurements from the near-source region, and immediately would constrain the lateral extent of any rupture. Even essential improvements in earthquake early warning, e.g., alerts ahead of strong shaking, might become feasible, depending on the exact position of the cable. The coast-parallel cable does not need to run in close proximity to the coast on the continental shelf but can be in deep water seaward of the trench.

To demonstrate the feasibility of SMART cables for tsunami early warning, the 2011 Tohoku-Oki earthquake and tsunami have been simulated with three hypothetical SMART cables from Tokyo to Oregon, Hawaii, and Auckland, respectively (Figure 11). We show that with these hypothetical cables, the
2011 Japanese tsunami could have been detected and its height confirmed within a few minutes after the earthquake and about 20 min earlier than was possible with the nearby DART buoy (Song et al., 2012). This would have provided enough time for warning the coastal communities. While the S-net early warning cable system mentioned above was specifically built to do this, this could apply just as well to other similar regions, such as the margin of South America.

\section{Seismology}

The inclusion of high-sensitivity accelerometers on SMART cables holds great potential for significant advances for the field of seismology by improving our capacity to detect and locate small earthquakes below the ocean floor by improving our ability to determine the rupture type and dynamics for larger offshore earthquakes and by enhancing our ability to image the interior of the Earth. Accelerometers are generally tuned to measure the strong ground motion occurring during large earthquakes, but modern instruments with exceptional sensitivity are available (Paros et al., 2012) that can capture the weak motions associated with distant or small earthquakes. Pressure sensors also provide very useful information to 

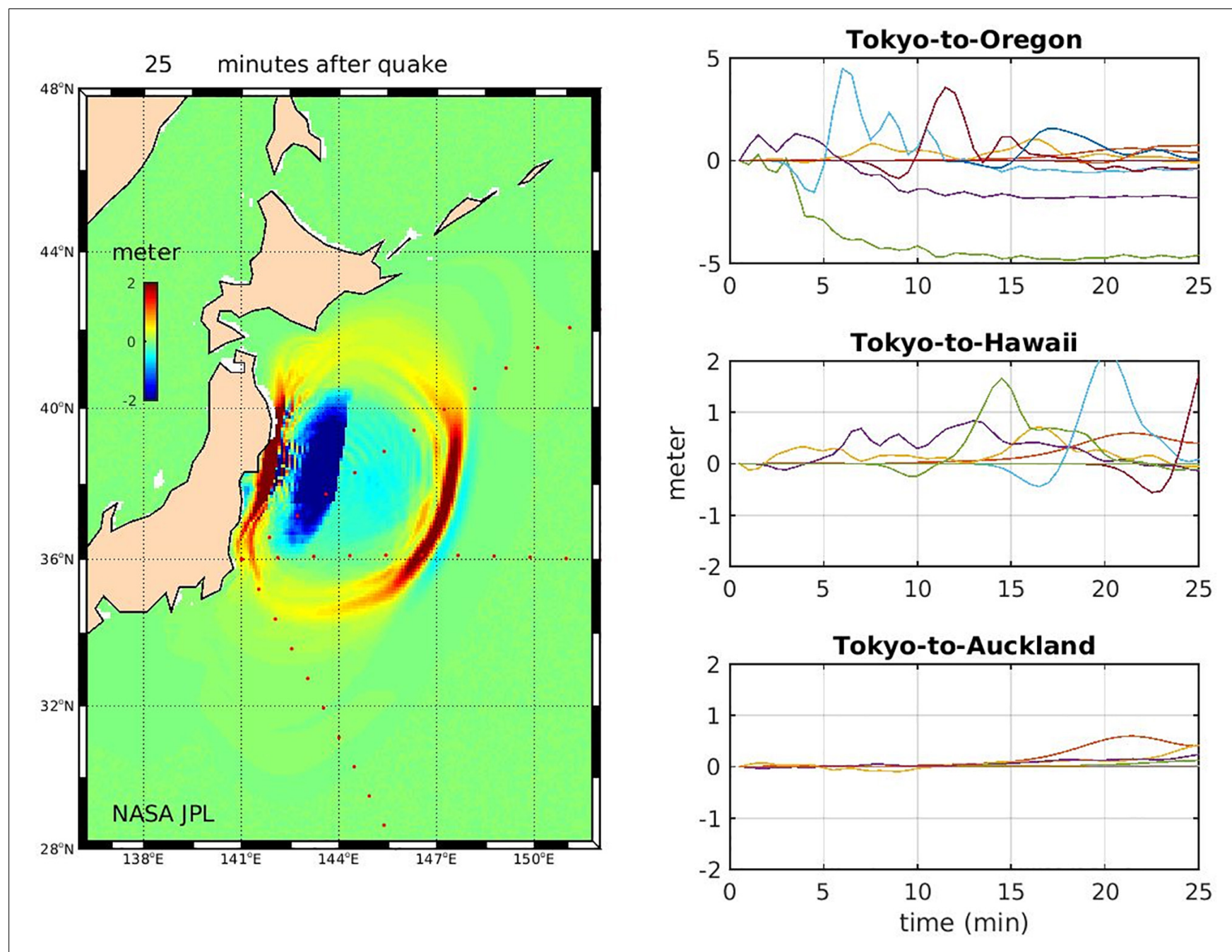

FIGURE 11 | Simulation of the 2011 Japanese tsunami with three hypothetical SMART cable routes: Tokyo to Oregon, Hawaii, and Auckland. Permission obtained for use of figure.

seismologists, as the elastic waves in the solid Earth easily couple into the water layer. They are particularly suitable for studying lithospheric structure and lithosphere-asthenosphere interactions with seismic surface waves.

As an example of the many possible applications, we focus on global body wave tomography as an example to illustrate the potential of data collected from SMART cables for improved sampling of parts of the Earth structure previously poorly illuminated due to the heterogeneous distribution of both earthquakes (largely at the margins of tectonic plates) and sensors (mostly on-land or near shore) (Figure 12A). Figure 12B depicts a slice through a $3 \mathrm{D}$ global model of seismic velocities, derived through tomographic inversion of travel times from the sources to the receivers illustrated in Figure 12A. Here, the intensity of color indicates a change in the velocity model, driven by the data, from the $1 \mathrm{D}$ radial ak135 reference model (Kennett et al., 1995). White regions indicate no change from the starting model. Because of reduced coverage below the oceans, it is difficult to say whether these are due to (expected) greater homogeneity below the ocean plates or because of lack of data in these places, hampering our ability to make robust geodynamic interpretation. The addition of SMART cable seismic sensors will significantly enhance the sampling of the Earth by augmenting existing ray paths of seismic propagation.

Figure 13 shows a likely sampling improvement for two example earthquakes, for which we performed raytracing to notional receivers along northern Pacific proposed SMART cable positions. We compare ray coverage for these sources using the existing GSN (left) with that possible with these notional SMART cables (right). Full global forward modeling using 20 years of earthquakes demonstrates over 300\% improvement in some suboceanic regions, where SMART cables would be deployed (Ranasinghe et al., 2018). This improved coverage will facilitate higher-fidelity 3D global models of both seismic wave speeds and attenuation, improving insight into the physical properties of the Earth mantle beneath the oceans and thus enabling better understanding of geodynamic processes. Improved models of the Earth's interior improve location estimates of seismic sources and 

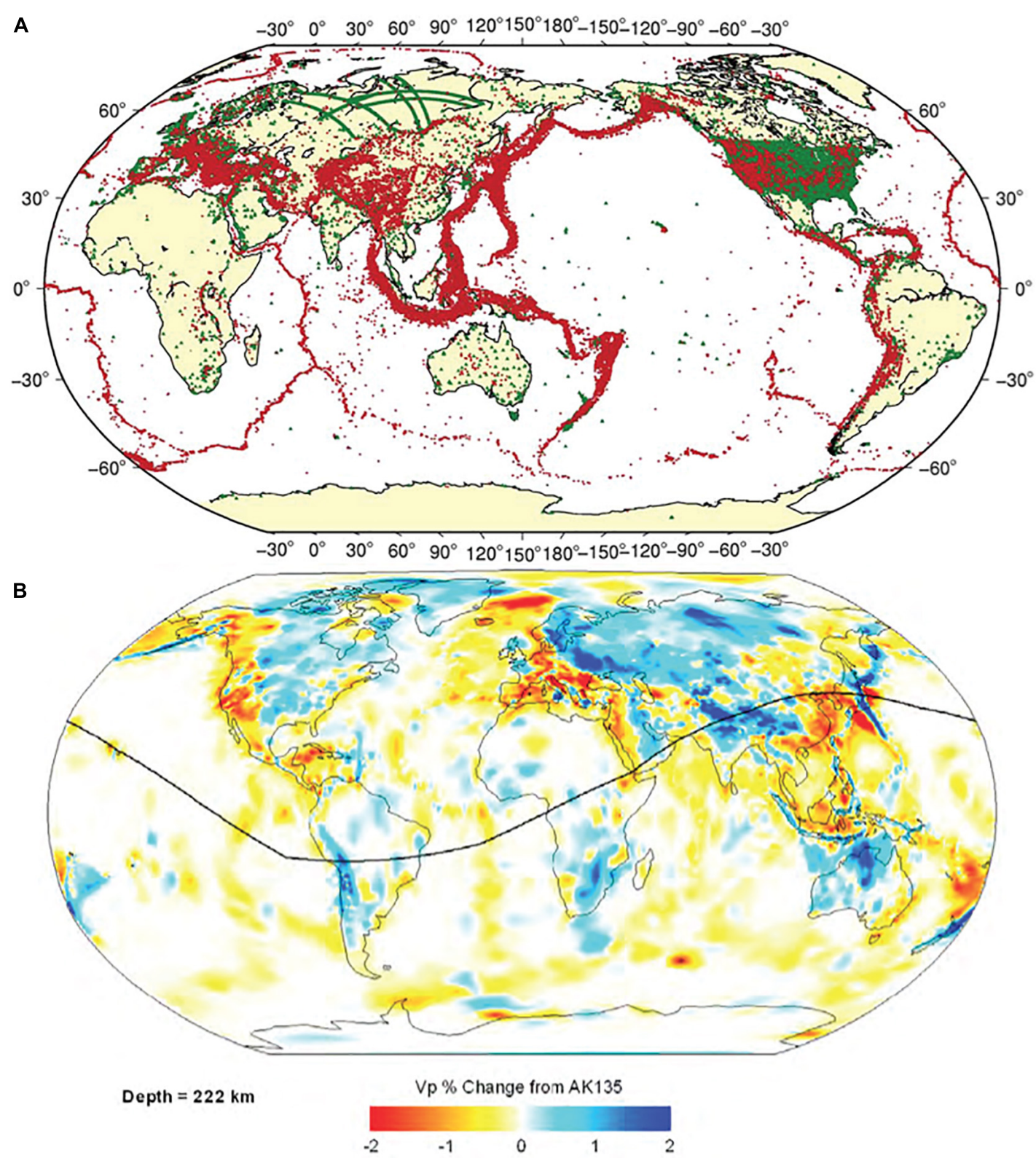

FIGURE 12 | (A) Distribution of global earthquakes (red) and existing seismic sensors (green) used in the derivation of the SALSA3D model. (B) Global seismic velocities at a $222 \mathrm{~km}$ depth in the 3D tomographic "SALSA3D" P-wave model shown as perturbation from the 1-D starting model (Begnaud et al., 2011; Ballard et al., 2016). Permission obtained for use of figure.

their sizes, which can retrospectively even benefit older events. The SMART cables will reduce detection thresholds not only for small events in proximity to the cables, but also for distant events whose signals can be enhanced by linear array methods using the new sensors.

Many large, destructive earthquakes occur in nearshore environments, where oceanic and continental lithosphere converge. Such plate interactions often occur offshore, hence on-shore seismic networks are some distance from the source, and provide a one-sided distribution of receivers, poorly constraining the location. If a nearby SMART cable exists, it would significantly enhance earthquake location estimates by reducing the azimuthal gap of sensors near the epicenter, as discussed in section "The Global Subsea Fiber Optic Network." SMART cable sensors may enhance early earthquake warning for offshore events via faster detection of first seismic waves at closer sensors. This could not only provide some seconds of additional seismic warning time for earthquakes occurring near a deployed SMART cable, but also the improved detection capability could be brought to bear on the analysis of moderate and small earthquakes occurring frequently at these margins. Based on their study we can determine the position of faults 


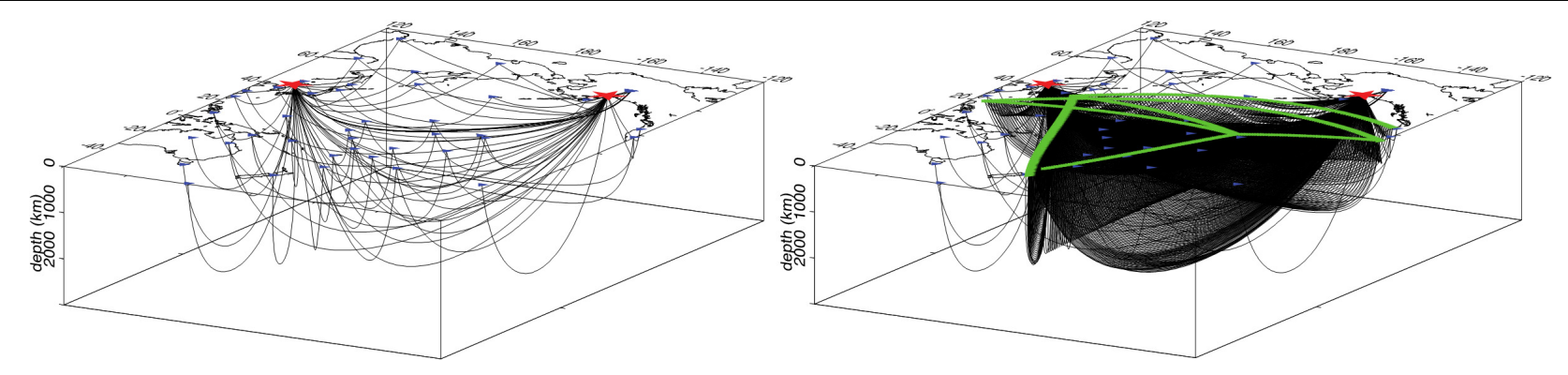

FIGURE 13 | Seismic ray coverage for two hypothetical sources in a block of the Pacific region. Coastlines are shown on the top. Sources are indicated as red stars in Cook Inlet, Alaska, and Korea. Rays, traced through the SALSA3D global P-wave model (Begnaud et al., 2011; Ballard et al., 2016) appear as curved lines from sources to receivers. Left: sampling of the Earth using today's GSN (blue circles). Right: greater sampling afforded by addition of notional SMART cables, with 75-km sensor spacing (green circles; Ranasinghe et al., 2018). Permission obtained for use of figure.

and then, by applying tomographic analysis at a much smaller scale than described above estimate the material properties in the forearc. In turn these can help us to enhance our understanding of the seismogenic potential of the margin.

\section{TECHNICAL APPROACH}

\section{SMART Cable Concept Requirements}

The fundamental premise of SMART cables is integrating environmental sensors into commercial submarine telecommunications cables. The crucial objectives are: (a) to obtain long term measurements of ocean bottom temperature, pressure, and three-axis seismic acceleration, (b) to have little or no impact on the operation of the telecommunications system that hosts the sensors, (c) to require no special handling or deployment methods, and (d) to be sufficiently reliable that $95 \%$ of all sensors operate for a minimum of 10 years.

\section{The Global Subsea Fiber Optic Network}

More than 1.1 million $\mathrm{km}$ of cable and 400 independent subsea cable systems are operated, maintained, and periodically renewed by the telecommunications industry. A full technical description of modern cable systems is given by Chesnoy (2016). Cables are installed across the North Atlantic, North Pacific, through the Mediterranean and Indian Oceans, through the Middle East, around South America and Africa, and throughout Oceania. Replacement or expansion cables are installed at intervals ranging from a few years on major routes to 10-15 years on minor routes. Thus, there is an opportunity on most or all cables to introduce sensor capabilities within the next 5-10 years.

\section{Subsea Cables}

A subsea fiber optic communications system comprises the cable (with varying layers of protection depending on water depth, seabed conditions, and potential risks), pressure housings containing erbium doped fiber amplifiers (EDFAs) (referred to as "repeaters") spaced at intervals from 60 to $150 \mathrm{~km}$, power feed equipment (PFE) that delivers a controlled direct current to the repeaters through the cable's single electrical conductor, and terminal station equipment (TSE) to transmit and receive signals on the optical fiber strands within the cable. Systems may include branching units (BUs) to provide connections to locations along the main cable route. Lengths range from less than $100 \mathrm{~km}$ to greater than $10,000 \mathrm{~km}$. Regional systems with lengths up to $2,500 \mathrm{~km}$ are ideal for the inclusion of sensor capabilities because these systems have sufficient design margin and can usually accommodate additional fibers to carry sensor data. Ocean spanning cables of $6,000 \mathrm{~km}$ or more are the most challenging. Successful operation of shorter SMART cables will be needed before sensor functions can be introduced into the longest cables.

The repeater is housed in a cylindrical pressure vessel typically $30 \mathrm{~cm}$ in diameter and $60-120 \mathrm{~cm}$ in length. Together with couplings and strain relief, the entire repeater assembly is 3$5 \mathrm{~m}$ long (Figure 14). The repeater houses EDFAs, which provide signal gain. The current generation of cable systems can have up to 16 fibers (eight bi-directional pairs) and work is underway to allow higher numbers of fibers.

\section{From Single-Purpose Cables to SMART Cables}

Functional elements to be added into the repeater include the sensors themselves, digital signal processing, an embedded processor, an Ethernet data switch, fiber optic transceivers, and power supplies. The most feasible way to transmit sensor data is to dedicate one fiber pair, although this reduces the overall capacity of the cable system. Options for embedding sensor data into the signal on a fiber pair dedicated to telecommunications are also under consideration.

To successfully incorporate sensors into a repeater, a number of engineering challenges must be addressed (Joint Task Force, 2015a; Lentz and Howe, 2018). Accelerometers will be mounted inside the repeater housing. The coupling characteristics between various types of seabed and the repeater housing are a subject for further study, although similar housings for ocean bottom seismometers are being used in systems such as Japan's S-net (Kanazawa et al., 2016). Temperature and pressure sensors must be placed outside the repeater housing, in contact with the environment, necessitating a penetration of the housing to connect these sensors to the internal circuitry (Figure 14). The 


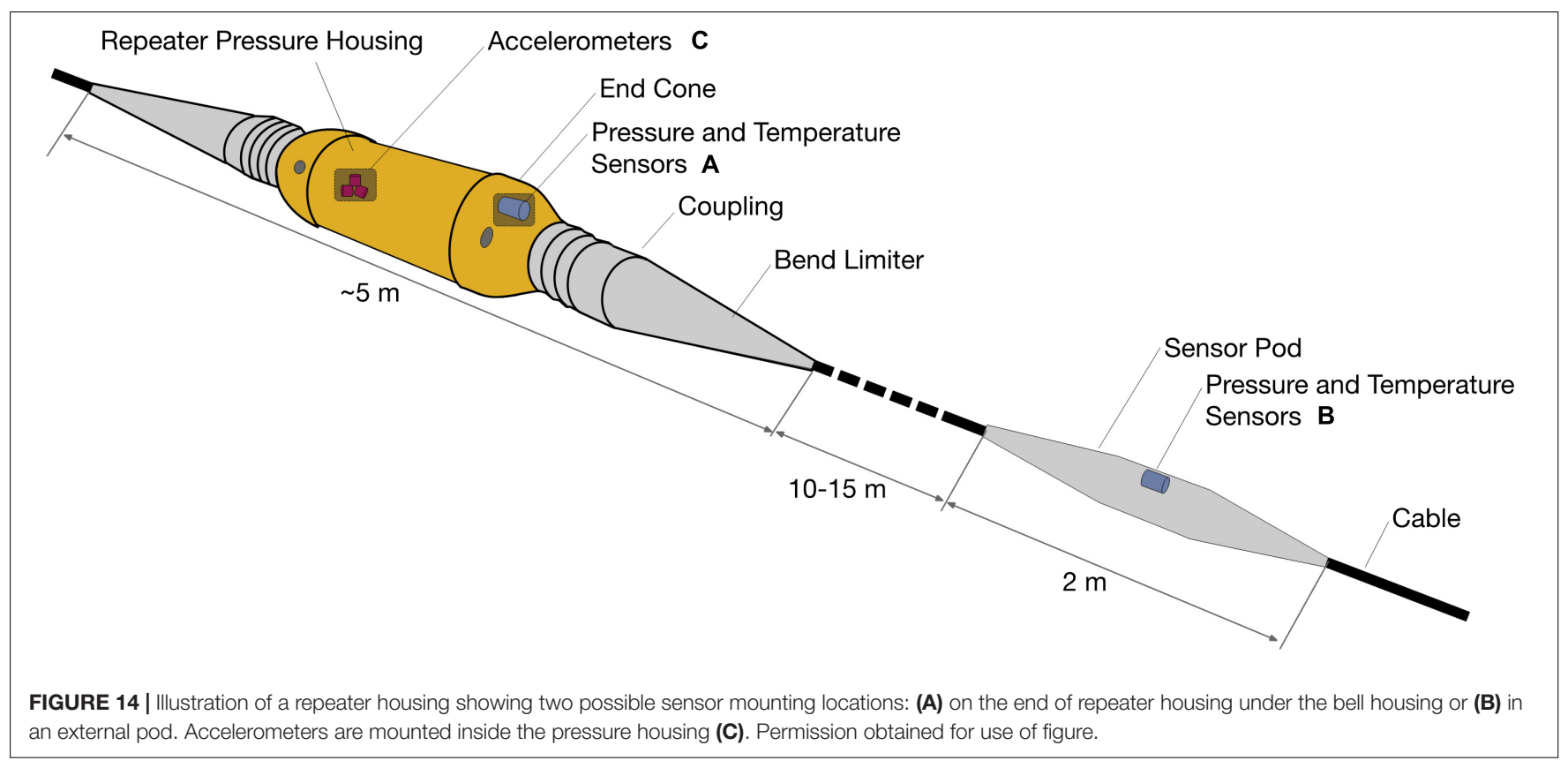

sensors must be isolated from high voltages present within the repeater and must be fail-safe so that the normal operation of the repeater cannot be impacted by faults in the external sensors. All of this must be done in a manner that is consistent with the 25-year expected operating life and 8,000 $\mathrm{m}$ deployment depth of a commercial repeater. Work is underway to identify and address these issues but will ultimately require the involvement of repeater manufacturers' development teams. Pressure sensor drift remains problematic and although a method of correction has been demonstrated (Wilcock et al., 2017), the resulting form factor is not yet suitable for incorporation into a repeater. The use of quartz pressure sensors without correction remains the best solution currently available.

An alternative, interim solution for adding SMART capabilities to commercial cable projects is to attach a set of SMART repeaters to a backbone cable by means of a BU. The backbone cable contains conventional telecommunications repeaters from which optical fibers and a power feed conductor "branch off" to a dedicated science cable that is independent of the telecommunications system. This arrangement has essentially no impact on the design of the backbone telecommunications system. The only non-standard component is the dual conductor cable required between the $\mathrm{BU}$ and shore station, although such a cable has been successfully deployed on multiple prior projects. The BU approach is attractive as an interim SMART cable demonstration because it avoids planning and technical complications of integrating SMART capabilities into tightly designed, standardized telecommunications repeaters. In a BU system, the power utilization, mechanical size, and reliability of the SMART sensors need not conform to the constraints of a fully functioning repeater. This flexibility can be used to prove SMART repeater designs, try other types of sensors, and demonstrate the data gathering and data management processes prior to approaching telecommunications and cable providers with the proposal for integration into the repeaters of the backbone cable. This interim variant is called a "wet demonstrator" (Joint Task Force, 2015b; Joint Task Force Engineering Team, 2016a,b). The chief drawback of this approach is the SMART functions are not hosted directly on the repeaters but rather are connected to a separate cable which does not provide telecommunications functions; while some costs can still be shared, this reduces the synergy between the telecommunications and science investment.

\section{Previous Work}

The earliest example of a submarine cable-based observatory is the Geophysical and Oceanographysical-Trans Ocean Cable (GeO-TOC), which was installed in 1997 midway between Guam and Japan using the retired TPC-1 communications cable (Kasahara et al., 1998). The GeO-TOC system anticipated the development of SMART cables by almost two decades yet included all essential SMART cable features: a three-axis accelerometer, pressure sensor, and precision thermometer. These were incorporated into an in-line pressure housing which was deployed from a cable ship in a conventional manner.

In the first decade of the 2000s, attention shifted to regional scale observatories such as NEPTUNE/ONC in Canada, DONET in Japan, and the OOI Regional Cabled Array (RCA) in the United States (Hazell et al., 2007; Kawaguchi et al., 2008; Consortium for Ocean Leadership, 2010; Barnes et al., 2015). Each of these employed telecommunications cable and repeaters; bespoke housings were developed for interconnection, power delivery, and communications. Sensors are installed on separate platforms outside the main pressure housings. These projects demonstrate the usefulness of commercial telecommunications technology in the realm of ocean observing but do not incorporate the close integration needed to create a true SMART cable. 
Following the Tôhoku earthquake and tsunami of 2011, Japan undertook rapid development of a large-scale network of subsea seismic and pressure sensors (Kanazawa, 2013; Kanazawa et al., 2016). The resulting S-net system incorporates many of the functions essential to a SMART cable. The overall deployment consists of 150 observation nodes along $5,700 \mathrm{~km}$ of cable divided into six independent subsystems. Each observation node consists of an underwater housing containing seismometers and pressure sensors connected in-line with a telecommunications cable. The result closely resembles a telecommunications repeater, however, as the rigid case has a length $1 \mathrm{~m}$ greater than that of a typical repeater, modifications to conventional cable laying equipment are required. Nevertheless, S-net provides evidence that SMART cables are close to being feasible using currently available technology.

Another in-line ocean bottom seismometer was developed by the University of Tokyo (Shinohara et al., 2014). This design is more compact than the S-net observatory at $50 \mathrm{~cm}$ long and $13 \mathrm{~cm}$ in diameter. A total of four units and $25 \mathrm{~km}$ of cable were deployed off the west coast of Awashima in 2012 using conventional cable laying equipment. Ethernet switches and optical transceivers are employed, an approach which could also be applied to SMART cables. In 2015, this was commercialized using an industry standard repeater housing and deployed off Sanriku with three nodes and a length of $105 \mathrm{~km}$. This design is commercially available and could be employed as an initial demonstration to build further confidence in the feasibility of SMART cables (Shinohara et al., 2016).

\section{Sensors}

The SMART cable sensor suite is comprised of just three sensors for temperature, pressure and acceleration, chosen based on the science described above and for engineering simplicity, especially important in facilitating the overall acceptance of the SMART concept by industry. These are well proven sensors, long used in oceanography and cabled observatories and early warning systems. Temperature is a local measurement while pressure and acceleration provide remote sensing of the entire water column and remote events and the intervening media. Detailed requirements for the SMART sensors are given in the several white papers (Lentz and Phibbs, 2012; Joint Task Force, 2015a).

Temperature sensors can meet the required initial accuracy of $1 \mathrm{mK}$ and stability of $2 \mathrm{mK} /$ year. They need to be mounted some distance from the repeater, a "heat island" dissipating $\sim 50 \mathrm{~W}$; this can be done in a sheath meters from the repeater (Figure 14).

Three-axis accelerometers, also called strong motion sensors or seismometers, reside inside the repeater housings; indeed, simple accelerometers are included in one supplier's repeaters for engineering purposes (Xtera, 2016). The key requirements for SMART accelerometers are a noise level less than $2 \mathrm{ng}$ at $1 \mathrm{~Hz}$ and a sample rate of $200 \mathrm{~Hz}$.

Pressure sensors also need to reside external to the repeater housing (see Figure 14) with access to local ambient pressure; there is typically a dedicated internal temperature sensor immediately next to the sensing element to account for temperature dependence. The main requirements are: depth rating to $7,000 \mathrm{~m}$ (with overpressure tolerance to $8,000 \mathrm{~m}$, the standard telecom rating); short term accuracy $1 \mathrm{~mm}$ water relative to recent measurements; $0.01 \%$ of full range absolute; and maximum allowable drift during a settling-in period of $20 \mathrm{~cm} /$ year.

Sensors meeting these requirements will be used for the first SMART systems. It is recognized though that sensors are continually improving, and different observables may be desired. In the first category, much effort has been devoted to removing long term drift from pressure sensor data with linear and exponential terms (Watts and Kontoyiannis, 1990; Polster et al., 2009). A new in situ calibration method has been devised that largely removes sensor calibration drift, reducing it from $\sim 10 \mathrm{~cm} /$ year (initial) to $\sim 1 \mathrm{~mm} /$ year, less than the nominal $3 \mathrm{~mm} /$ year sea level rise. In this "A-0-A" method, the pressure sensor is occasionally switched from Ambient water pressure to "0" internal case pressure, the latter nominally $1 \mathrm{~atm}$ measured with a barometer (Wilcock et al., 2017). While the current prototypes are too bulky, and the mechanical valving is cumbersome, it is worthwhile to monitor the development of the technology.

In the second category of other observables and sensors, many have been suggested, including hydrophones, conductivity sensors, inverted echosounders, acoustic modems, etc. Given that the SMART repeater will provide a general interface, it should be possible in principle to add these and others. In a different class is a new distributed sensing technology based on using optical fibers themselves as sensors. Any strain (stretch) in the fibers can be detected by Brillouin optical correlation domain reflectometry (BOCDR; currently to $50 \mathrm{~km}$; Galindez-Jamioy and LópezHiguera, 2012), Rayleigh backscatter interferometry (Lindsey et al., 2017), or a combination of forward transmission optical interferometry and absolute time measurement (Marra et al., 2018). The latter, based on connecting the world's ultra-stable optical clocks over all-optical networks, opens the possibility of passively using existing trans-ocean fibers as continuously distributed seismic sensors. We strongly emphasize that these additional sensor concepts are for the future. The first, essential step is to achieve successful deployments of the initial three chosen sensor types.

\section{Design and Development}

The design and development of SMART cables will require an unprecedented level of cooperation between scientific organizations, cable system suppliers, and cable system operators. The deployment of seismic observatories in Japan demonstrates that many of the necessary components and capabilities are already available. Achieving integration with telecommunications systems will require further refinement of the sensors, design and development of the signal processing and data transmission circuits, and mechanical integration into the repeaters. A full set of technical requirements is proposed in a whitepaper prepared by the Joint Task Force Engineering Team (2016a).

Integration of the SMART functions into cable systems requires a substantial investment on the part of the cable system suppliers. Despite similarities in function, each supplier has different mechanical arrangements and manufacturing processes. 
Development of a reference design and standard interfaces for the sensors, signal processing boards, and data communications should be pursued to lessen the burden on each potential supplier and to ensure the science objectives are met consistently. This reference design would incorporate, at a minimum, circuit diagrams and functional code; one or more working benchtop prototypes would be assembled. Individual suppliers could then use this to create functional circuit boards which are compatible with their repeater design.

Reliability is a chief concern for telecommunications cables. A rate of no more than one internal failure per 25 years in $5,000 \mathrm{~km}$ of cable is a typical objective for telecommunicationsonly cables. As a matter of principle, the sensor functions of a SMART cable must not reduce this reliability figure. Because the sensor functions are unlikely to achieve this same level of reliability, the integration must be designed to "fail safe" such that any sensor failures have no effect on the telecommunications function.

The initial design goal for the reliability of the sensor functions embedded within a SMART cable is to ensure 95\% of all sensors are still operable after 10 years. This goal is chosen because it is reasonably expected that a newer cable system would be installed alongside any existing cable within 10 years, providing a new source of data to first complement and then replace the original SMART cable. Initial review indicates this goal is achievable using currently available technology, with the main limitation being the reliability of laser diodes used to transmit the signals carrying the sensor data from each repeater. SMART cables are expected to use existing, off-the-shelf components, including laser diodes, which have been widely used in industry for decades and have well characterized reliability that meets or exceeds the 10-year design goal.

Commercial cable system operators must be persuaded to support SMART cables. Submarine cable systems represent a significant investment and a critical piece of strategic network infrastructure (Rauscher, 2010). Any interruption in operations has the potential to cause costly disruptions. For this reason, system owners are reluctant to accept anything new or unproven. Smaller projects, particularly those serving island nations that are most at risk from climate and sea level change and tsunamis, are expected to be initially most receptive to SMART cables. Regional systems are more likely to have surplus capacity or unused fiber pairs, thus eliminating the objection that adding SMART functions reduces the cable's overall capacity. Addressing the concerns of the telecommunications industry will require a series of projects that demonstrate that all technical issues have been fully addressed.

\section{DATA MANAGEMENT AND USERS}

Data from SMART cables is expected to be open and freely accessible. The overarching principle of data management for SMART cables is to leverage existing infrastructure in the oceanographic research and operations community rather than developing an independent data management system from scratch. As with any large-scale data stewardship program, findability, accessibility, interoperability, and reusability (the "FAIR" principles) are key (Tanhua et al., 2019a). Management of SMART cable data will require the ability to handle multiple variables at varying temporal resolutions and a system that can scale to handle the large volumes of data that will be generated by a mature cable network. In particular, SMART cables will resolve processes ranging in temporal scale from earthquake seismic signals and tsunamis to secular climate trends and will need to support both real-time and delayed-mode applications to facilitate hazard monitoring and research.

Data generated by SMART cable sensors will be transmitted along the underlying cable to a shore station where it may be stored in raw form, processed, and transmitted onward to data repositories, national agencies, and academic institutions (Figure 15). Seismic and pressure sensor data that will be used for early warning functions must be forwarded immediately, with minimal latency. Data will be processed and transmitted in recognized formats, such as SeedLink, to ensure compatibility with existing data processing and archival systems.

The Incorporated Research Institutions for Seismology (IRIS) has volunteered to receive, curate, and disseminate all of the data from SMART cable sensors through its well-developed data collection and dissemination process. Particularly valuable is IRIS' capability to ingest high-temporal resolution data, such as output from accelerometers at $100 \mathrm{~Hz}$. This early commitment by a world-leading data management cooperative ensures that SMART cable data products will have an immediate user, as well as being available to myriad other institutions that are not otherwise associated with SMART cable projects.

In addition to distribution through IRIS, SMART cables data products may be managed by a dedicated consortium. A close analog to such a network-specific data management consortium is the global tide gauge network. Like SMART cables, tide gauges are multi-purpose instruments that are also tasked with supporting both tsunami monitoring and climate research applications.

The global tide gauge network comprises approximately a thousand individual instruments organized within a multitude of small, regional networks maintained by independent international agencies in much the same way that SMART cables will be operated by an array of government and industry partners. To facilitate international coordination, the global tide gauge network is overseen by the Global Sea Level Observing System (GLOSS). While it is not possible for GLOSS to absorb SMART cables into its mission outright, there is a clear opportunity for partnership involving exchange of data management platforms and best practices, as well as leveraging existing GLOSS contacts within governmental hydrographic and oceanographic services.

The University of Hawaii Sea Level Center (UHSLC), a primary GLOSS datacenter, will foster collaboration between the SMART cables and GLOSS communities and will leverage existing resources to host first-generation versions of delayedmode datasets. This effort includes ensuring that these data are available to researchers via standard interfaces such as OPENDAP, ERRDAP, etc. The UHSLC will also work with the SMART cables community to establish standardized formatting 


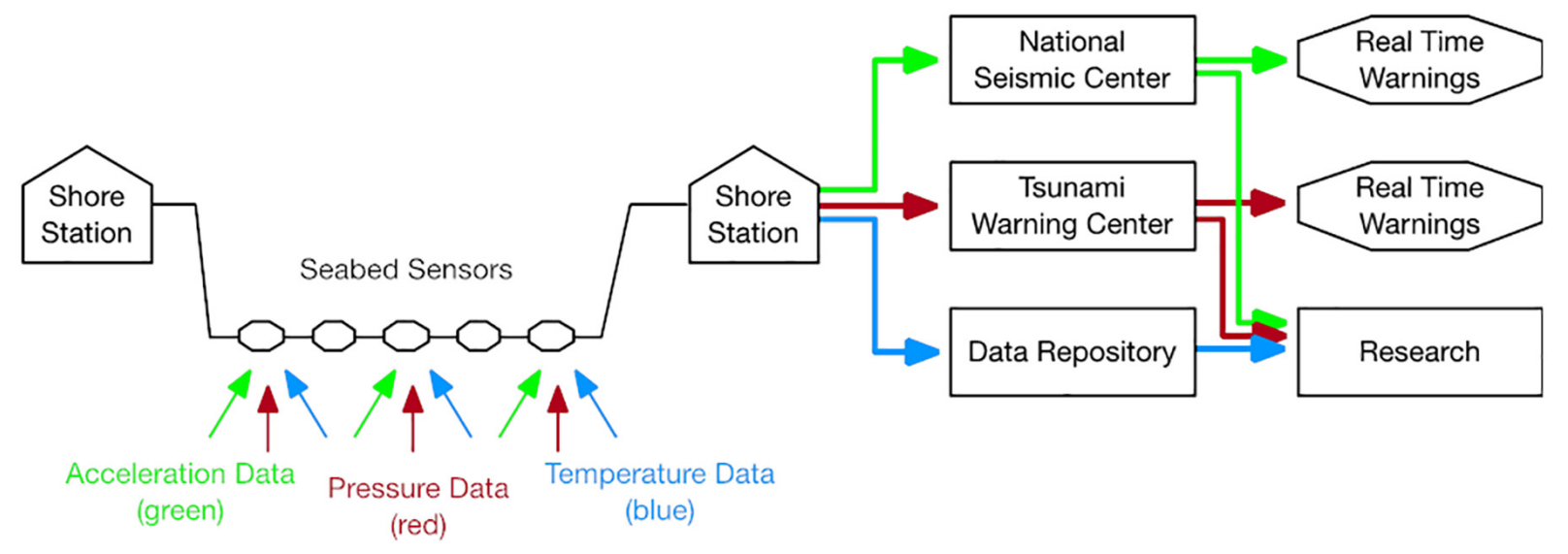

FIGURE 15 | Data flow from SMART cables to end users. Permission obtained for use of figure.

and transmission of real-time SMART cable data over the WMO Global Telecommunication System (GTS). Once on the GTS, tsunami and seismic monitoring facilities will have real-time access to the data and delayed-mode datacenters will be able to retrieve, process, and archive SMART cable data.

Researchers and institutions worldwide have already identified uses for the data products derived from SMART cables. As discussed in section "Technical Approach," the unprecedented volume of real-time, deep ocean data has the potential to advance oceanographic and seismic understanding across multiple and varied domains. The cable industry itself will be a user of the data, as cable systems necessarily evolve to smart infrastructure, monitoring for threats from trawling, anchors, submarine landslides, and earthquakes (Butler et al., 2014; Huchet and Brenne, 2018).

\section{PROGRAM MANAGEMENT}

Joint Task Force has about 130 members representing 80 organizations, almost entirely volunteer. It is sponsored by ITU, WMO, and IOC within the context of GOOS. ITU provides Secretariat support, and IOC provides modest support for reports (with some industry contributions), meetings, workshops, and travel. Major activities at this time include: building relationships with relevant stakeholders, presenting and obtaining support at meetings and conferences, working with multilateral development banks to secure funding, and interacting with candidate cable projects. JTF is not set up to directly participate in specific projects, but rather to facilitate the formation of the appropriate groups to accomplish the necessary tasks. Indeed, after a few fully operational systems are installed, JTF expects to transition into an international project office. The JTF terms of reference can be found on the web page: https://www.itu.int/en/ITU-T/climatechange/taskforce-sc/Pages/default.aspx.

For the management of the wet demonstration project (based on the scenario of adding a BU to a new system with SMART modules), it is expected that the management structure will be prescribed by the participants. One wet demo provider would be the recipient (after competition) of a subcontract with the main supplier of the cable system. A project management consultancy would participate. Part of this would include setting up mechanisms for data transfer, quality control, and use.

Following this phase, the first pilot systems with SMART repeaters in active telecom cables would be established. For each system, there would be a local/regional science/early warning working group representing the countries involved that would interface with the cable industry partners, arrange funding, provide oversight of the SMART component of the project (e.g., participation in relevant factory acceptance tests), and arrange for the data management and use. During this phase, it is expected that the provision of the SMART components is handled by the main telecom supplier. JTF would provide advice and facilitate as necessary.

After the first pilots are operating, an international project office will evolve, initially formed by the associated working groups and then expanding to provide oversight and an international and global perspective as additional pilots and then standard systems are installed and operated, following, for example, the International Argo project structure. The latter includes a Project Office with Director and Technical Coordinator, a Steering Team, a Data Management Team, and Information Center, and Oversight Committee. An important responsibility of the project office will be to coordinate education, outreach, and capacity building activities. This international project office would take over the activities of JTF, i.e., the ongoing implementation of the SMART concept within the context of GOOS and the FOO.

\section{LEGAL OUTLOOK}

Because SMART cables combine science and telecommunications into a single cable, they do not fit neatly into the international legal frameworks that developed while these were separate maritime activities. Fortunately, the current lag between international legal definitions and research innovation 
is unlikely to have a practical impact on near-term SMART cable projects, which will be carried out exclusively in the Exclusive Economic Zone ("EEZ") of cooperating nations and the high seas. As the dual-use cables concept turns from development to deployment, the collective international understanding of their legal status will be refined, and based on concrete examples, routes, and uses. The following discussion summarizes and updates JTF's comprehensive legal analysis conducted in 2012 (Bressie, 2012).

\section{The Legal Regime Governing Telecommunications Cables}

The laws regarding passage and usage of the seas are established by the United Nations Convention on the Law of the Sea (UNCLOS; United Nations, 1982), other treaties, and customary international law, which establish certain sovereign and jurisdictional rights for coastal states, depending on the distance from their coasts. These rights are generally most extensive in a nation's territorial waters, and diminish with distance through the contiguous zone, EEZ, continental shelf, and high seas.

International treaties dating back to 1884 guarantee unique freedoms to lay, maintain, and repair submarine cables not only on the high seas, but on the continental shelf and in the EEZ, making them among the most protected of marine activities (Convention, 1884; United Nations, 1958a,b). As the most comprehensive oceans-related treaty, UNCLOS is the applicable legal regime governing submarine cables and treated as customary international law, even by states that have not ratified them, including the United States (Presidential Proclamation No. 5030, 1983; Presidential Proclamation No. 7219, 1999). As a result, undersea telecommunications cables hold a "privileged place in international law, reflecting their status as an essential public good" (Davenport, 2013).

\section{The Legal Regime Governing Marine Data Collection}

By contrast, certain types of marine data collection are subject to varying levels of coastal state jurisdiction and regulation. There are several categories of marine data collection: (1) marine scientific research (MSR); (2) survey activities; (3) collection of marine meteorological data; (4) operational oceanography; and (5) exploration for and exploitation of natural resources (Roach, 2007). For SMART cables, the most relevant categories are MSR, marine meteorological data, and operational oceanography.

Importantly, UNCLOS does not define MSR. Its discussion in Article 243 is limited to references to scientists "studying the essence of phenomena and processes occurring in the marine environment and the interrelations between them" and in Article $246(3)$ to projects "exclusively for peaceful purposes and in order to increase scientific knowledge of the marine environment for the benefit of all mankind." Ultimately, UNCLOS Article 251 tasked signatories with defining MSR through practice, which has occasionally resulted in tension as different coastal states assert different definitions or scopes of MSR.

Article 87(1)(f) of UNCLOS recognizes MSR as one of the freedoms of the high seas and Article 245 categorizes it as subject to coastal State sovereignty in the territorial waters and EEZ. Article 246(1-2) set out that MSR in EEZ and continental shelf waters is in general subject to coastal state consent. Although the terms are not specified in UNCLOS, some states divide MSR into categories of "pure scientific research" (essentially activities encompassed by Article 246(3)) in which consent should in normal circumstances be given, and "applied scientific research" [those activities named in Article 246(5)], in which the coastal State may exercise their discretion to withhold consent. States wishing to conduct MSR (whether "pure" or "applied") in other States' EEZs or continental shelves are also subject to a number of duties, such as those laid out in Articles 248 and 249.

Other sub-divisions of MSR, which are also commonly used but do not appear in UNCLOS, are marine meteorology and operational oceanography. Marine meteorology generally refers to the collection of meteorological information from equipment such as voluntary observing ships, buoys, and other ocean platforms, and is treated by many coastal states as exempt from coastal State consent requirements applicable to MSR in the EEZ/continental shelf (Davenport, 2013). Operational oceanography is generally defined as an activity of systematic and long-term routine measurements of the seas and oceans and atmosphere, and their rapid interpretation and dissemination to assimilation centers (EuroGOOS, 2018). Some states view operational oceanography as exempt from the MSR regime, while other states view it as a type of MSR and subject to coastal state consent (Roach, 2007).

\section{A Pragmatic Approach: Dual-Use Cables in Cooperating Regions}

Thus, JTF, cable projects, and governments have noted the concern that combining science sensors and telecom cables could create uncertainty as to the legal status of such dual-purpose cables, potentially delaying cable deployment.

Fortunately, however, nothing in UNCLOS requires dualpurpose to be classified as MSR or operational oceanography, nor does customary international law reflect such a classification. Thus, individual coastal states have discretion regarding the treatment of dual-use cables, and their practice will form the precedent of how dual-use cables should be treated under international law. More importantly for practical purposes, any current uncertainty would not affect SMART cable projects operating in the high seas or within the EEZ of cooperating nations. Because all JTF projects are expected to be carried out in close cooperation with the national governments with coastal jurisdiction over the areas that the cables land on or transit through, JTF does not expect to encounter any well-founded legal objection.

In this regard, SMART cable projects for the foreseeable future should involve even less legal process than the Argo float system, which itself is well established among coastal states and the international legal community. The Argo system, consisting of nearly 4,000 ocean profiling floats, collects temperature and salinity data mostly in the upper 2,000 $\mathrm{m}$ of the ocean, and releases this data freely through the IOC and WMO. In recognition of the value of such measurements, as well as the 
absence of specific legal instruments relevant to the project, the IOC concluded that the Argo project must inform affected coastal states when Argo floats might drift into waters of their jurisdiction (i.e., within their EEZ) (International Oceanographic Commission, 2000).

Joint Task Force near term pilot projects are explicitly intended to validate the technology and business case for dualpurpose cables and create a climate where oceanographic sensorenabled telecommunications cables are a recognized part of maritime infrastructure. In doing so, they will also habituate the industry to such projects and reduce the perceived legal and business risk of this maturing concept.

The scientific community and its vendors can assist this process by developing specifications and standardized components to be included on telecommunications cables. This will provide all parties involved with a clear understanding of the capabilities of dual-purpose cables, thus reducing the potential for concerns by otherwise cooperative nations that such projects could stray from their stated scientific goals.

\section{COST ESTIMATE}

The anticipated costs to enable widespread use of SMART cables have been calculated based on known costs for submarine cable systems and sensor sets combined with estimates for development and operational costs. Assumptions regarding the number of cable systems and number of SMART enabled repeaters are a significant factor in establishing the overall costs. To achieve global coverage of 2,000 SMART repeaters, 200 repeaters must be deployed each year. We estimate it will take 10 years to achieve the widespread acceptance necessary to support this deployment rate. Full global deployment is projected to be achieved after 18 years at which time the rate of retirement will equal the rate of new deployments. Costs are separated into three broad categories: development, deployment, and operations.

Development costs include the cost of a demonstration system, the cost to develop a reference design, and non-recurring costs to support the first deliveries of SMART repeaters from cable system suppliers. The demonstration system consists of three prototype sensor sets and up to $300 \mathrm{~km}$ of cable. These sensor sets will be installed either as an independent system or as the branching unit off a commercial telecom cable. In either case, the incremental cost associated with the science functionality will be supported by science funding. The reference design is envisioned as an open-source project to develop circuit board schematics, digital signal processing methods, communications protocols and related micro-code which can be reused by each repeater supplier. Some professional input is expected to be required to complete this design, although a large portion of the work should be performed by academia. The final aspect of development costs are non-recurring costs incurred by the cable system suppliers. The first system delivered by each supplier will incur non-recurring development costs. We anticipate that funding will have to be allocated to the first three suppliers that participate in the SMART cable program for this purpose.
Any further participating suppliers will need to bear their own development costs. Development costs are the smallest portion of the overall budget and are incurred only during the first 5 years.

Deployment of fully developed SMART repeaters begins in year three. Deployment costs are calculated based on the number of SMART repeaters deployed times a unit cost, plus some costs for each cable system. We estimate a cost of US\$207,000 per repeater, which includes an allowance for additional fiber in the cable. The cost model assumes this target price will be achieved in the fifth year. For prior and subsequent years, a $10 \%$ inflation/deflation factor is applied on the basis that lower volumes will incur higher costs but as delivery volumes and experience increase, the cost will decline to $\$ 175,000$ per repeater. Deployment costs ramp from $\$ 6 \mathrm{M}$ in year three to a steady state of $\$ 36.5 \mathrm{M}$ in year nine. Deployment costs are the largest portion of the overall budget.

The final cost area is operational costs. Operations costs include program administration, data transmission, and data processing costs. Operational costs are about $10 \%$ of annual deployment costs but are an essential aspect of the overall program. Supplementary Table 1 shows the projected approximate system cost. Note the large initial development cost that decreases after approximately year 5, with deployment volume and costs increasing year over year until a steady state in approximately year 10 .

For comparison, the US NOAA DART program budget is $\$ 27 \mathrm{M} /$ year, comparable to the incremental cost for a SMART cable that spans the Pacific region where most of the US DART buoys are located. The Argo program, with 4,000 expendable floats, costs about $\$ 32 \mathrm{M}$ per year to maintain. The NSF funded OOI cost approximately $\$ 400 \mathrm{M}$ for the fabrication phase, with operating costs of approximately $\$ 44 \mathrm{M}$ annually. NOAA estimates it spends approximately $\$ 430 \mathrm{M}$ annually to operate and maintain its ocean, coastal, and Great Lakes observing systems.

\section{FINANCING}

Today's submarine cable projects fall into three major categories: consortium, private, and government. In consortium cables, a group of established carriers join to share costs on large projects. Consortium systems are difficult for SMART cables because of the need to get approval from many consortium members. Private cables are undertaken by a single developer using seed financing followed by full project financing with equity and debt. SMART cables in private systems are a reasonable option because the system developer is typically a small cadre of individuals who can be dealt with directly. Because these cables are focused on time to market and profits, however, introducing SMART technology must not cause undue delay or added costs to the owners. Government cables, or government-backed cables, seek to improve a country's link to the global network. With longer timelines and a broader range of goals, these cables represent a good opportunity for SMART cables. This section summarizes and extends a comprehensive funding study undertaken by JTF (OCI Group, 2015). 
Early engagement with potential projects is important for influencing the configuration to incorporate SMART requirements and to arrange funding. Because early awareness of a project is not always possible, the SMART efforts must be able to quickly react and introduce SMART requirements into the project technical requirements effort. Equally important at that time is that the SMART effort must clearly demonstrate funding is available for this integration.

Multiple funding options for SMART cables have been summarized in a previous ITU study (OCI Group, 2015). During the course of this investigation it was determined that the potential funders fell into the following categories:

- International Development Agencies

- Foundations

- Government Agencies

- Private Companies

International Development Agencies: Regional multilateral development banks have indicated their support for SMART capabilities in cable projects they are involved with. As of this writing, Asian Development Bank (ADB) supports including SMART capabilities on the Manatua cable in part because the landing point nations of Cook Islands, Niue, Samoa, and Tahiti are acutely exposed to tsunami and climate change risks. ADB expects science capabilities on the East Micronesia Cable for similar reasons. The InterAmerican Development Bank (IADB) is likewise interested, and the IOC Intergovernmental Coordination Group/Pacific Tsunami Warning System (ICP/PTWS) committee is preparing a report for the IADB to facilitate their participation. JTF is also in contact with the World Bank.

Foundations: Only large foundations can support grants of the size anticipated for SMART. JTF is in contact with these major foundations, including the Schmidt Ocean Institute, Schmidt Marine Technology Partners, Simons Foundation, Paul G. Allen Philanthropies, Moore Foundation, Packard Foundation, and Keck Foundation.

Government Agencies: All government agencies are bureaucratic and will require extensive effort to develop funding support, relationships, and consensus. In addition, all government agency funding will be contingent on appropriations and the government budget cycle.

There are a number of candidate government organizations that should be further investigated for funding or to facilitate funding, including in Canada the "Environment and Climate Change Canada" and "Natural Resources Canada"; in Europe the European Commission's Directorate for Research and Innovation-Research Infrastructures Unit, with the involvement of the Institute for Environment and Sustainability and the European Multidisciplinary Seafloor and Water Column Observatory (EMSO); in Japan the Japan Agency for Marine Earth Science and Technology (JAMSTEC); and in the United States several agencies including the Department of the Interior, the US Geological Survey, the Environmental Protection Agency, the Department of Defense, the Office of Naval Research, and the Department of Commerce, Economic
Development Administration and National Oceanic and Atmospheric Administration (NOAA). In the case of NOAA, funding may be facilitated by the Tsunami Warning, Education and Research Act 2017 (TWERA; Public Law 115-25) which gives NOAA the responsibility to consider "...integration of tsunami sensors into Federal and commercial submarine telecommunication cables."

Funding may most readily be obtained from a government that wishes to have its country connected with a new submarine cable and appreciates the value to its country of having SMART technologies as part of the solution. Further, developing countries may be able to arrange funding from the Ministry of Foreign Affairs, or other, of developed country partners for the purposes of community/international connectivity and climate and disaster monitoring.

Private Companies: The previous study effort concluded that commercial entities would be unlikely to allocate funding toward SMART technology because internal research and development dollars are a scarce resource and because such developments may indirectly assist a competitor's development efforts. However, JTF provides an avenue for commercial entities supportive of JTF's mission to provide in-kind support through engineering assistance, technical review, or establishing standards. As leading digital technology companies (e.g., Alibaba, Alphabet, Amazon, Apple, Facebook, Microsoft) become more involved with cable systems and more socially conscious, they may become more receptive to dual-purpose cables.

\section{IMPLEMENTATION PLAN}

The first deployment of SMART cables is anticipated to be a demonstration system that does not interface with the telecommunications portion of a cable, but instead focuses solely on sensor functionality (Joint Task Force Engineering Team, 2016b). Off-the-shelf components may be used to reduce development costs, even if these are physically larger than would be for a fully developed SMART cable. The demonstration system could be deployed as a branch of a commercial cable system, connected to an existing cabled observatory, or re-use a portion of an out-of-service cable. A minimum of three repeaters need to be included to fully demonstrate sensor functionality. It may be necessary to have more than one demonstration system, as the prototypes come closer to the final version, for instance to demonstrate the power isolation between the external sensors and the internal electronics. If the wet demonstration system was a branch off a commercial telecom system, it would demonstrate the coexistence of telecom and science with no interference.

Following the demonstration system, full development of SMART enabled repeaters must be undertaken by one or more system suppliers. The resulting repeater design will undergo qualification tests and sea trials after which it will be available for use in commercial telecom systems. To increase confidence, it may be necessary to deploy several fully developed repeaters as a further demonstration or in a situation that does not demand high reliability telecommunications services. At this stage of its 
development, the SMART repeater could also be used to create a dedicated earthquake/tsunami warning system.

After these confidence-building measures, deployment of the first true SMART cable system will take place. A regional cable, $1,500-2,500 \mathrm{~km}$ in length and containing 10-20 repeaters, is ideal as it is manageable both in scope and cost. Successful operation on this scale will provide a final demonstration of the value of SMART cables and ensure they have no impact on the telecommunications performance of the cable system. SMART cables could then be deployed with confidence on trans-Atlantic and trans-Pacific routes. Other cable routes, including the Indian Ocean, South Atlantic and South Pacific, South America, and the Arctic, will be addressed as opportunities arise. An achievable goal for SMART cables is to be generally accepted within 5-7 years and to serve all major ocean routes within the decade.

\section{RELATIONSHIP TO OTHER PROGRAMS}

There are natural scientific connections between the SMART program and many other ocean observing programs. There is an urgent need for deep ocean measurements of any type (Levin et al., 2019). Deep ocean temperatures intermittently and coarsely sampled in time and space by deep Argo and by the GO-SHIP program (Meyssignac et al., 2019; Sloyan et al., 2019) will benefit from cross comparison with the fixed high rate Eulerian cable-based measurements and those made by OceanSites moorings (Frajka-Williams et al., 2019), for improved estimates of heat content and deep ocean variability. SMART cable OBP observations will directly feed into the analysis of altimetry and gravity satellite data, as well as contribute to large scale ocean circulation and flux estimates, such as AMOC (Hughes et al., 2018). They are directly relevant to time and space dependent sea level rise estimates, measuring the component associated with increased water mass from melting land ice (Stammer et al., 2013; Ponte et al., 2019). The pressure data will feed directly into tsunami warning centers to improve early warning with better spatial and temporal sampling and array availability (Angove et al., 2019).

From the beginning, the SMART cables initiative has benefited for the sponsorship of its UN organizations - ITU, WMO, and UNESCO-IOC - with the associated imprimatur. With endorsements from the Joint Technical Commission for Oceanography and Marine Meteorology (JCOMM), Data Buoy Coordination Panel (DBCP), the ICG/PTWS, and more recently by the Partnership for Observing the Global Ocean (POGO), the SMART cable project is aligning itself within the FOO (Lindstrom et al., 2012; Snowden et al., 2019; Tanhua et al., 2019b).

\section{SUMMARY}

Science Monitoring And Reliable Telecommunications cables fill scientific and societal needs. The science described above and reviewed in multiple workshops - climate, ocean circulation and sea level rise, and tsunamis and earthquakes - requires the unique data that SMART cables can provide. SMART cables respond to the United Nations Sustainable Development Goals for Climate (SDG 13) and Oceans (SDG 14). Within the context of the OceanObs19 conference, SMART cables address observing technologies and networks, discovery, climate variability and change, and hazards and maritime safety. SMART Cables is an ocean observing technology that needs to be implemented during the UN Decade of Ocean Science for Sustainable Development (2021-2030).

Science Monitoring And Reliable Telecommunications cable systems will directly contribute to the measurement of the EOVs of subsurface temperature and OBP. OBP is an emerging EOV which, through the real-time, global scale sample enabled by SMART cables, can be elevated to full EOV status. Acceleration can be regarded as a supporting variable as it is needed to separate near field pressure and seismic signals for tsunami detection, as well as providing much seismic data, filling the gap of the $70 \%$ of the Earth's surface that is ocean. More modeling is required to quantify the benefits, not just for SMART cable scenarios but to the GOOS in general.

Science Monitoring And Reliable Telecommunications cable systems are technically achievable. There are no known technical obstacles, but clearly the engineering development must be performed and all related practical issues addressed. All of the essential components exist today in some form and projects such as DONET, S-net, and Sanriku, demonstrate the capability of the telecommunications industry to deliver ocean observing systems that meet nearly identical goals. Industry partnership and acceptance of the SMART functionality are commercial, rather than technical, challenges to be solved. A reference design that clearly separates the telecommunications and SMART functionality of a subsea cable system will help to address these concerns.

Science Monitoring And Reliable Telecommunications cable systems are allowable within legal and permitting frameworks. JTF is concentrating for the foreseeable future on cable systems in the EEZs of countries that support the SMART cable concept, where there will be strong legal support and few ongoing legal requirements.

Science Monitoring And Reliable Telecommunications cable systems are financially feasible. Cost estimates are similar to other observing system components. The best opportunities for funding appear to be in select development banks, government agencies, and private foundations. Discussions are underway with leading candidate entities, and JTF regularly briefs the others.

Initially, we are relying on development bank funds, with the business case based on combining community connectivity with climate monitoring and disaster mitigation, as encapsulated at the 2018 Global Infrastructure Forum, where "the leading multilateral development banks (MDBs)...expressed their condolences following the tragic loss of lives and livelihoods in Sulawesi, Indonesia and reaffirmed their commitment to 
work together to deliver infrastructure that is resilient, inclusive, and sustainable." (Asian Development Bank, 2018). Nonetheless, governments will have to supply funding as part of their ocean observing efforts. Smaller countries with short chains of command are preferable.

At this point in time, JTF SMART cables is in negotiations to include a wet demonstration component in a commercial system in the South Pacific, with the involvement of the ADB. Other cable systems in the same region are under consideration that include the World Bank. After the Sulawesi earthquake and tsunami of September 28, 2018, the Indonesian government recognizes the need for "cable-based tsunameters" (Kompass Press, 2018). We are engaging in smaller systems that have significant government and/or multilateral development bank involvement for initial wet demonstration and pilot systems. In addition, we are pursuing funding within Europe for the same. Once wet demonstrations and pilots are underway and a SMART repeater is offered "off-theshelf," then longer cable system crossing major ocean basins can be considered.

These findings lead to the following recommendations for OceanObs19 to include in the conference outcomes.

Recommendation 1: OceanObs19 recognize the utility of Science Monitoring And Reliable Telecommunications (SMART) subsea cable systems, both in terms of contribution to the observing system and efficiency of effort and investment, and encourages all stakeholders - sensor and system suppliers, system owners, multilateral development banks, other sponsors, and governments and their funding, permitting, early warning, and ocean observing agencies - to participate in the development of the SMART capability, so that it becomes a ubiquitous feature of future cable systems and an integral component of GOOS.

Recommendation 2: OceanObs19 and GOOS support the continuing evolution of OBP as an EOV.

Recommendation 3: GOOS develop a community capability to evaluate the current observing system and proposed additions thereto and apply the same to evaluating the benefits of data obtained using SMART subsea cable systems.

\section{JOINT TASK FORCE FOR SMART CABLES}

The Joint Task Force for SMART Cables is sponsored by the International Telecommunication Union (ITU), the World Meteorological Organization (WMO), and the Intergovernmental Oceanographic Commission of the United Nations Educational, Scientific and Cultural Organization (UNESCO/IOC), with members from academic, government, and industry institutions worldwide.

\section{AUTHOR CONTRIBUTIONS}

$\mathrm{BH}$ provided the overall guidance and executive editing for the manuscript, and wrote the sections "Sensors," "Program
Management," "Relationship to Other Programs," and the "Summary." PNT provided the detailed editing and organization, as well as writing the section "Legal Outlook." CB wrote the section "The SMART Cables Concept." JA led the writing of the section "Improvements in Ocean Observing with SMART Cables." Contributions to this latter section: GJ wrote the section "Ocean Temperature." DL, MT, and TW wrote the section "Ocean Circulation, Sea Level Rise, and Mass Distribution." TW contributed Figure 5. BA, DL, and MM contributed to the section "Ocean Surface (Barotropic) Tides." JA and DL contributed to the section "Wind Generated Waves, Microseisms, and Infragravity Waves." SW, NB, YS, and KP wrote the section "Tsunami Monitoring and Warning." NB contributed Figures 9, 10. YS contributed Figure 11. CR contributed to the section "Seismology." SL wrote the sections "Technical Approach," "Cost Estimate," and "Implementation Plan." PT and PNT wrote the section "Data Management and Users." JM and PNT wrote the section "Financing." RB, SE, and FT edited throughout. All authors provided input and edited the manuscript.

\section{FUNDING}

$\mathrm{BH}$ and PNT were supported by the NASA (via JPL) NNN13D462T for the writing of this manuscript. DL received support from NSF OCE-1460022. GJ is supported by the NOAA Research. PMEL Contribution Number 4854.

\section{ACKNOWLEDGMENTS}

D. Arcas, K. Bressie, C. Moore, T. Davenport, M. Gutscher, H. Kopp, D. Meldrum, N. Rakowsky, and D. Sieber all helped with various aspects of this paper. B. Bay and M. Izumi worked on illustrations and technical editing. Maps in Figures 1, 8 were provided by M. Chandler using GMT. Altimetry data were provided by NOAA Laboratory for Satellite Altimetry. Numerical simulations for section "Ocean Circulation, Sea Level Rise, and Mass Distribution" were carried out on the supercomputing system of the German Climate Computing Centre (DKRZ) in Hamburg, Germany. J. You is acknowledged for providing the initial vision for the SMART cables concept. Authors' and contributors' individual contributions and the support of their respective institutions is gratefully acknowledged. SOEST Contribution Number 10738. HIGP Number Contribution 2387. Los Alamos National Laboratory Publication LA-UR-18-31124.

\section{SUPPLEMENTARY MATERIAL}

The Supplementary Material for this article can be found online at: https://www.frontiersin.org/articles/10.3389/fmars. 2019.00424/full\#supplementary-material 


\section{REFERENCES}

Ammon, C. J., Ji, C., Thio, H.-K., Robinson, D., Ni, S., Hjorleifsdottir, V., et al. (2005). Rupture process of the 2004 sumatra-andaman earthquake. Science 308, 1133-1139. doi: 10.1126/science.111 2260

Angove, M., Arcas, D., Bailey, R., Carrasco, P., Coetzee, D., Fry, B., et al. (2019). Ocean Observations required to Minimize Uncertainty in global tsunami forecasts, warnings, and emergency response. Front. Mar. Sci. 6:350. doi: 10 . 3389/fmars.2019.00350

Arbic, B. K., Alford, M. H., Ansong, J., and Buijsman, M. C. (2018). “A primer on global internal tide and internal gravity wave continuum modeling in HYCOM and MITgcm," in New Frontiers in Operational Oceanography, eds E. Chassignet, A. Pascual, J. Tintore, and J. Verron (Scotts Valley, CA: CreateSpace).

Arbic, B. K., Garner, S. T., Hallberg, R. W., and Simmons, H. L. (2004). The accuracy of surface elevations in forward global barotropic and baroclinic tide models. Deep Sea Res. Part II Top. Stud. Oceanogr. 51, 3069-3101. doi: 10.1016/ j.dsr2.2004.09.014

Arbic, B. K., Richman, J. G., Shriver, J. F., Timko, P. G., Metzger, E. J., and Wallcraft, A. J. (2012). Global modeling of internal tides within an eddying ocean general circulation model. Oceanography 25, 20-29. doi: 10.5670/oceanog. 2012.38

Arbic, B. K., Wallcraft, A. J., and Metzger, E. M. (2010). Concurrent simulation of the eddying general circulation and tides in a global ocean model. Ocean Model. 32, 175-187. doi: 10.1016/j.ocemod.2010.01.007

Ardhuin, F., Rawat, A., and Aucan, J. (2014). A numerical model for free infragravity waves: Definition and validation at regional and globalscales. Ocean Model. 77, 20-32. doi: 10.1016/j.ocemod.2014.02.006

Ardhuin, F., Stutzmann, E., Schimmel, M., and Mangeney, A. (2011). Ocean wave sources of seismic noise. J. Geophys. Res. Oceans 116:C006952. doi: 10.1029/ 2011JC006952

Asian Development Bank (2018). Multilateral Banks Reaffirm Pledge to Support Resilient, Sustainable Infrastructure. Mandaluyong: Asian Development Bank.

Aucan, J., and Ardhuin, F. (2013). Infragravity waves in the deep ocean: An upward revision. Geophys. Res. Lett. 40, 1-5. doi: 10.1002/grl.50321

Ballard, S., Hipp, J. R., Begnaud, M. L., Young, C. J., Encarnacao, A. V., Chael, E. P., et al. (2016). SALSA3D: A tomographic model of compressional wave slowness in the Earth's mantle for improved travel-time prediction and traveltime prediction uncertainty. Bull. Seismological Soc. Am. 106, 2900-2916. doi: $10.1785 / 0120150271$

Barnes, C. R. (2018). "Quantum leap in platforms of opportunity: telecommunication cables," in Challenges and innovations in ocean in-situ sensors:Measuring Inner Ocean Processes and Health in the Digital Age, eds E. Delory and J. Pearlman (Amsterdam: Elsevier Science), 33-44.

Barnes, C. R., Best, M. M. R., Johnson, F. R., and Pirenne, B. (2015). "NEPTUNE Canada: Installation and initial operation of the world's first regional cabled ocean observatory," in Seafloor Observatories: A New Vision of Earth from the Abyss, eds A. De Santis, P. Favali, and L. Beranzoli (Berlin: Springer-Verlag Berlin Heidelberg), 415-438. doi: 10.1007/978-3-642-11374-1_16

Begnaud, M. L., Ballard, S., Young, C. J., Hipp, J. R., Chang, M. C., Encarnacao, A. V., et al. (2011). "Validating a Global 3D P-Velocity Model of the Earth's Crust and Mantle for Improved Event Location," in Proceedings of the 2012 Monitoring Research Review: Ground-Based Nuclear Explosion Monitoring Technologies, (Albuquerque, MN).

Bernard, E., and Titov, V. (2015). Evolution of tsunami warning systems and products. Philos. Trans. R. Soc. A 373:20140371. doi: 10.1098/rsta.2014.0371

Bertin, X., de Bakker, A., van Dongeren, A., Coco, G., André, G., Ardhuin, F., et al. (2018). Infragravity waves: From driving mechanisms to impacts. Earth Sci. Rev. 177, 774-799. doi: 10.1016/j.earscirev.2018.01.002

Best, M. M. R., Barnes, C. R., Bornhold, B. D., and Juniper, K. J. (2015). "Integrating continuous observatory data from the coast to the abyss: Assembling a multidisciplinary view of the ocean in four dimensions," in Seafloor Observatories: A New Vision of Earth from the Abyss, eds P. Favali, A. Santis, and L. Beranzoli (Berlin: Springer-Verlag Berlin Heidelberg), 5-21. doi: 10.1007/978-3-642-11374-1_2

Best, M. M. R., Favali, P., Beranzoli, L., Cannat, M., Çaðatay, N., and Dan̈obeitia, J. J. (2014). EMSO: A distributed infrastructure for addressing geohazards and global ocean change. Oceanography 27, 167-169. doi: 10.5670/oceanog 2014.52

Bressie, K. (2012). Using submarine cables for climate monitoring and disaster warning: Opportunities and legal challenges. Available at: https://www.itu. int/dms_pub/itu-t/opb/tut/T-TUT-ICT-2012-22-PDF-E.pdf (accessed July 15, 2019). doi: $10.5670 /$ oceanog.2014.52

Bromirski, P. D., Duennebier, F. K., and Stephen, R. A. (2005). Midocean microseisms. Geochem. Geophys. Geosyst. 6:Q04009. doi: 10.1029/ 2004GC000768

Butler, R., and Aucan, J. (2018). Multisensor, microseismic observations of a hurricane transit near the ALOHA cabled observatory. J. Geophys. Res. Solid Earth 123, 3027-3046. doi: 10.1002/2017JB014885

Butler, R., Cochran, E., Collins, J., Eblé, M., Evans, J., Favali, P., et al. (2014). The scientific and societal case for the integration of environmental sensors into new submarine telecommunication cables. Paris: UNESCO.

Chambers, D. P., and Schröter, J. (2011). Measuring ocean mass variability from satellite gravimetry. J. Geodyn. 52, 333-343. doi: 10.1016/j.jog.2011. 04.004

Chave, A. D., Luther, D. S., and Thomson, D. J. (2019). High-Q spectral peaks and nonstationarity in the deep ocean infragravity wave band: tidal harmonics and solar normal modes. J. Geophys. Res. Oceans 124, 2072-2087. doi: 10.1029/ 2018JC014586

Chen, B. R., Feng, X. T., Li, Q. P., Luo, R. Z., and Li, S. J. (2015). Rock burst intensity classification based on the radiated energy with damage intensity at Jinping II hydropower station, China. Rock Mech. Rock Eng. 48, 289-303. doi: 10.1007/s00603-013-0524-2

Chesnoy, J. (2016). Undersea Fiber Communication Systems, 2nd Edn. Amsterdam: Elsevier.

Consortium for Ocean Leadership (2010). Ocean Observatories Initiative Final Network Design. Washington DC: Consortium for Ocean Leadership.

Convention. (1884). Convention for the Protection of Submarine Telegraph Cables. Available at: https://www.loc.gov/law/help/us-treaties/bevans/m-ust0000010089.pdf

Davenport, T. (2013). "Submarine Communications Cables and Science: A New Frontier in Ocean Governance?," in Science, Technology, and New Challenges to Ocean Law, eds J. Kraska, M.-S. Kwon, and H. N. Scheiber (Berkely: Koninklijke Brill), 209-218.

Delory, E., and Pearlman, J. (eds) (2018). Challenges and innovations in ocean insitu sensors - Measuring Inner Ocean Processes and Health in the Digital Age. Amsterdam: Elsevier.

Dobslaw, H., Bergmann-Wolf, I., Dill, R., Poropat, L., Thomas, M., Dahle, C., et al. (2017). A new high-resolution model of non-tidal atmosphere and ocean mass variability for de-aliasing of satellite gravity observations: AOD1B RL06. Geophys. J. Int. 211, 263-269. doi: 10.1093/gji/ ggx302

Duputel, Z., Rivera, L., Kanamori, H., and Hayes, G. P. (2012). W phase source inversion for moderate to large earthquakes (1990-2010). Geophys. J. Int. 189, 1125-1147. doi: 10.1111/j.1365-246X.2012.05419.x

Egbert, G. D., Ray, R. D., and Bills, B. G. (2004). Numerical modeling of the global semidiurnal tide in the present day and in the last glacial maximum. J. Geophys. Res. 109:C03003. doi: 10.1029/2003JC001973

Elipot, S., Frajka-Williams, E., Hughes, C. W., and Willis, J. K. (2014). The observed north atlantic meridional overturning circulation: its meridional coherence and ocean bottom pressure. J. Phys. Oceanogr. 44, 517-537. doi: 10.1175/jpo-d-13026.1

EuroGOOS (2018). What is Operational Oceanography?. Available at: http://eurogoos.eu/about-eurogoos/what-is-operational-oceanography/ (accessed July 15, 2019).

Favali, P., Person, R., Barnes, C. R., Kaneda, Y., Delaney, J. R., and Hsu, S.-K. (2010). "Seafloor observatory science," in Proceedings of OceanObs'09: Sustained Ocean Observations and Information for Society, (venice: ESA publication).

Flechtner, F., Neumayer, K.-H., Dahle, C., Dobslaw, H., Fagiolini, E., Raimondo, J.-C., et al. (2016). "What Can be Expected from the GRACE-FO Laser Ranging Interferometer for Earth Science Applications?”, in Remote Sensing and Water Resources, eds A. Cazenave, N. Champollion, J. Benveniste, and J. Chen (Basel: Springer International Publishing), 263-280. doi: 10.1007/978-3-319-324 49-4_11 
Frajka-Williams, E., Ansorge, I. J., Baehr, J., Bryden, H. L., Chidichimo, M. P., Cunningham, S. A., et al. (2019). Atlantic meridional overturning circulation: observed transports and mechanisms. Front. Mar. Sci. 6:260. doi: 10.3389/ fmars.2019.00260

Galindez-Jamioy, C. A., and López-Higuera, J. M. (2012). Brillouin distributed fiber sensors: an overview and applications. J. Sens. 2012:204121. doi: 10.1155/2012/ 204121

Hasselmann, K. (1991). "Epilogue: waves, dreams, and visions," in Directional ocean wave spectra, ed. R. Beal (Baltimore, MD: Johns Hopkins University), 205-208.

Hazell, N., Lécroart, A., and Marcerou, J.-F. (2007). "Regional Cable Observatory Solutions," in proceedings of the IOCEANS '07 IEEE/MTS, (Vancouver, BC: IEEE).

Herbers, T. H. C., Elgar, S., and Guza, R. T. (1995). Generation and propagation of infragravity waves. J. Geophys. Res. 100, 24,863-24,872.

Herbers, T. H. C., and Guza, R. T. (1994). Nonlinear wave interactions and high-frequency seafloor pressure. J. Geophys. Res. 99, 35-48.

Hoeke, R. K., McInnes, K. L., Kruger, J. C., McNaught, R. J., Hunter, J. R., and Smithers, S. G. (2013). Widespread inundation of Pacific islands triggered by distant-source wind-waves. Glob. Planet. Change 108, 128-138. doi: 10.1016/j. gloplacha.2013.06.006

Howe, B. M., Duennebier, F. K., and Lukas, R. (2015). "The ALOHA cabled observatory," in Seafloor Observatories: A New Vision of Earth from the Abyss, eds A. De Santis, P. Favali, and L. Beranzoli (Berlin: Springer-Verlag Berlin Heidelberg), 439-464.

Huchet, G., and Brenne, J. K. (2018). Fiber Sensing is Everywhere. Sterling, VA: Subtel Forum Magazine.

Hughes, C. W., Elipot, S., Morales Maqueda, M. A., and Loder, J. (2013). Test of a method for monitoring the geostrophic meridional overturning circulation using only boundary measurements. J. Atmos. Ocean. Technol. 30, 789-809. doi: 10.1175/jtech-d-12-00149.1

Hughes, C. W., Williams, J., Blaker, A., Coward, A., and Stepanov, V. (2018). A window on the deep ocean: the special value of ocean bottom pressure for monitoring the large-scale, deep-ocean circulation. Prog. Oceanogr. 161, 19-46. doi: 10.1016/j.pocean.2018.01.011

International Oceanographic Commission (2000). IOC Assembly Resolution XX6. Available at http://www.wmo.int/pages/prog/amp/mmop/documents/JCLs_ JCOMM/JCOMM\%20JCL\%2000-2\%20Argo\%20Ann\%201.doc (accessed July 15, 2019)

Jayne, S. R., Roemmich, D., Zilberman, N., Riser, S. C., Johnson, K. S., Johnson, G. C., et al. (2017). The argo program present and future. Oceanography 30, 18-28. doi: 10.5670/oceanog.2017.213

Johns, W. E., Baringer, M. O., Beal, L. M., Cunningham, S. A., Kanzow, T., Bryden, H. L., et al. (2011). Continuous, array-based estimates of atlantic ocean heat transport at $26.5^{\circ} \mathrm{N}$. J. Clim. $24,2429-2449$. doi: $10.1175 / 2010$ JCLI 3997.1

Johnson, G. C. (2008). Quantifying antarctic bottom water and north atlantic deep water volumes. J. Geophys. Res. 113:C05027. doi: 10.1029/2007JC00 4477

Johnson, G. C., Lyman, J. M., and Purkey, S. G. (2015). Informing deep argo array design using argo and full- depth hydrographic section data. J Atmos. Ocean. Technol. 32, 2187-2198. doi: 10.1175/jtech-d-15-0139.1

Joint Task Force (2015a). Functional requirements of "green" submarine cable systems. ITU. Available at: https://www.itu.int/en/ITU-T/climatechange/taskforce-sc/Documents/Functional-requirements-2015-05.pdf (accessed July 15, 2019).

Joint Task Force (2015b). Wet Demonstrator: Scope Document and Budgetary Cost Estimate. Available at: https://www.itu.int/en/ITU-T/climatechange/taskforce-sc/Documents/Wet-demonstrator-requirements-2015-05.pdf (accessed July 15, 2019).

Joint Task Force Engineering Team (2016a). General Requirements for Sensor Enabled and Reliable Telecommunications (SMART) Cable Systems. Available at: https://www.itu.int/en/ITU-T/climatechange/task-force-sc/Documents/ General-Requirements-of-a-SMART-Cable-Issue-1.0.pdf (accessed June 2016).

Joint Task Force Engineering Team (2016b). Sensor Enabled Scientific Monitoring And Reliable Telecommunications (SMART) Cable Systems: Wet Demonstrator Project Description. Issue 1.0.. Available at: https://www.itu.int/en/ITU-T/
climatechange/task-force-sc/Documents/Wet-Demonstrator-Design-Issue-1.0. pdf (accessed July 2016).

Kanamori, H. (1972). Mechanism of tsunami earthquakes. Phys. Earth Planet. Int. 6, 346-359. doi: 10.1016/0031-9201(72)90058-1

Kanamori, H., and Rivera, L. (2008). Source inversion of WPhase: speeding up Seismic Tsunami Warning. Geophys. J. Int. 175, 222-238. doi: 10.1111/j.1365246x.2008.03887.x

Kanazawa, T. (2013). “Japan Trench earthquake and tsunami monitoring network of cable-linked ocean bottom observatories and its impact to earth disaster science," in Proceedings of the IEEE International Underwater Technology Symposium (UT), (Tokyo: IEEE).

Kanazawa, T., Uehira, K., Mochizuki, M., Takashi, S., Fujimoto, H., Noguchi, S. I., et al. (2016). S-net Project: Cabled Observation Network for Earthquakes and Tsunamis. Dubai: Suboptic.

Kasahara, J., Utada, H., Sato, T., and Kinoshita, H. (1998). Submarine cable OBS using a retired submarine telecommunication cable: GeO-TOC program. Phys. Earth Planet. Inter. 108, 113-127. doi: 10.1016/s0031-9201(98)00 090-9

Kawaguchi, K., Araki, E., Kaneko, S., and Kaneda, Y. (2008). Design of Deep Ocean Submarine Cable Observation Network for Earthquakes and Tsunamis. Kobe: IEEE

Kawaguchi, K., Kaneko, S., Nishida, T., and Komine, T. (2015). "Construction of the DONET real-time seafloor observatory for earthquakes and tsunami monitoring," in Seafloor Observatories: A New Vision of Earth from the Abyss, eds A. De Santis, P. Favali, and L. Beranzoli (Berlin: Springer-Verlag Berlin Heidelberg), 212-228.

Kelley, D. S., Delaney, J. R., and Juniper, S. K. (2014). Establishing a new era of submarine volcanic observatories: cabling axial seamount and the endeavour segment of the juan de fuca ridge. Mar. Geol. 352, 426-450. doi: 10.1016/j. margeo.2014.03.010

Kennett, B. L. N., Engdahl, E. R., and Buland, R. (1995). Constraints on seismic velocities in the Earth from traveltimes. Geophys. J. Int. 122, 108-124. doi: 10.1111/j.1365-246x.1995.tb03540.x

King, B. A., Firing, E., and Joyce, T. M. (2001). "Shipboard observations during WOCE," in Proceedings of the International Geophysics, (Amsterdam: Elsevier), 99-122. doi: 10.1016/s0074-6142(01)80114-5

Kompass Press (2018). "Technology Ready To Be Applied" (Original in Indonesian). Available at: https://kompas.id/baca/utama/2018/10/05/inovasiteknologi-kebencanaan-siap-diterapkan/ (accessed July 15, 2019).

Lentz, S., and Howe, B. (2018). Scientific Monitoring And Reliable Telecommunications (SMART) Cable Systems: Integration of Sensors into Telecommunications Repeaters. Kobe: IEEE.

Lentz, S., and Phibbs, P. (2012). Engineering feasibility study: Using submarine cables for climate monitoring and disaster warning. Joint Task Force. Geneva: ITU.

Levin, L., Betts, B. J., Gates, A. R., Heimbach, P., Howe, B. M., Janssen, F., et al. (2019). Global observational needs in the deep ocean. Front. Mar. Sci. 6:241. doi: $10.3389 /$ fmars.2019.00241

Lindsey, N. J., Martin, E. R., Dreger, D. S., Freifeld, B., Cole, S., James, S. R., et al. (2017). Fiber-optic network observations of earthquake wavefields. Geophys. Res. Lett. 44, 792-799. doi: 10.1002/2017GL075722

Lindstrom, E. J., Gunn, A., Fischer, A., and McCurdy, L. K. (2012). A Framework for Ocean Observing. By the Task Team for an Integrated Framework for Sustained Ocean Observing. Paris: UNESCO.

Longuet-Higgins, M. S. (1950). A theory of the origin of microseisms. Phil. Trans. R. Soc. Lond. A 243, 1-35. doi: 10.1098/rsta.1950.0012

Lozier, M. S., Bacon, S., Bower, A., and Zika, J. (2017). Overturning in the subpolar north atlantic program: a new international ocean observing system. Bull. Am. Meteorol. Soc. 98, 737-752.

Lu, F., Zhou, H., Peng, X., Yue, J., and Wang, P. (2015). “Technical preparation and prototype development for long-term cables seafloor observatories in Chinese marginal seas," in Seafloor Observatories: A New Vision of Earth from the Abyss, eds A. De Santis, P. Favali, and L. Beranzoli (Berlin: Springer-Verlag Berlin Heidelberg), 503-530.

Lukas, R., Santiago-Mandujano, F., Bingham, F., and Mantyla, A. (2001). Cold bottom water events observed in the Hawaii Ocean Time-series: implications for vertical mixing. Deep Sea Res. Part I Oceanogr. Res. Papers 48, 995-1021. doi: 10.1016/s0967-0637(00)00078-9 
Makowski, J. K., Chambers, D. P., and Bonin, J. A. (2015). Using ocean bottom pressure from the gravity recovery and climate experiment (GRACE) to estimate transport variability in the southern Indian Ocean. J. Geophys. Res. Oceans 120, 4245-4259. doi: 10.1002/2014JC010575

Marra, G., Clivati, C., Luckett, R., Tampellini, A., Kronjäger, J., Wright, L., et al. (2018). Ultrastable laser interferometry for earthquake detection with terrestrial and submarine cables. Science. 361, 486-490. doi: 10.1126/science.aat4458

Marsland, S. J., Haak, H., Jungclaus, J. H., Latif, M., and Röske, F. (2003). The Max-Planck-Institute global ocean/sea ice model with orthogonal curvilinear coordinates. Ocean Model 5, 91-127. doi: 10.1016/s1463-5003(02)00015-x

Massion, G., and Raybould, K. (2006). MARS: the monterey accelerated research system. Sea Technol. 47, 39-42.

McCarthy, G. D., Smeed, D. A., Johns, W. E., Frajka-Williams, E., Moat, B. I., Rayner, D., et al. (2015). Measuring the Atlantic Meridional Overturning Circulation at 26 degrees N. Prog. Oceanogr. 130, 91-111.

McKee, D. C., Yuan, X. J., Gordon, A. L., Huber, B. A., and Dong, Z. Q. (2011). Climate impact on interannual variability of Weddell Sea Bottom Water. J. Geophys. Res. Oceans 116:17. doi: 10.1016/b978-012472670-3/ 50005-7

Meinen, C. S., Garzoli, S. L., Perez, R. C., Campos, E., Piola, A. R., Paz Chidichimo, M., et al. (2017). Characteristics and causes of deep western boundary current transport variability at 34.5 degrees S during 2009-2014. Ocean Science 13, 175-194. doi: 10.5194/os-13-175-2017

Meyssignac, B., Boyer, T., Zhao, Z., Hakuba, M. Z., Landerer, F. W., Stammer, D., et al. (2019). Measuring global ocean heat content to estimate the earth energy imbalance. Front. Mar. Sci. doi: 10.3389/fmars.2019.00432

Müller, M., Arbic, B. K., and Mitrovica, J. X. (2011). Secular trends in ocean tides: Observations and model results. J. Geophys. Res. Oceans 116:C05013. doi: 10.1029/2010JC006387

Müller, M., Cherniawsky, J., Foreman, M., and von Storch, J.-S. (2012). Global map of M2 internal tide and its seasonal variability from high resolution ocean circulation and tide modelling. Geophys. Res. Lett. 39:L19607. doi: 10.1029/ 2012GL053320

Müller, M., Cherniawsky, J., Foreman, M., and von Storch, J. S. (2014). Seasonal variation of the M2 tide. Ocean Dyn. 64, 159-177. doi: 10.1007/s10236-0130679-0

Nawa, K., Suda, N., Fukao, Y., Sato, T., Aoyama, Y., and Shibuya, K. (1998). Incessant 698 excitation of the Earth's free oscillations. Earth Planets Space 50, 3-8. doi: 10.1186/bf03352080

Nerem, R. S., Beckley, B. D., Fasullo, J. T., Hamlington, B. D., Masters, D., and Mitchum, G. T. (2018). Climate-change-driven accelerated sea-level rise. Proc. Natl. Acad. Sci. 115, 2022-2025. doi: 10.1073/pnas.1717312115

Nerger, L., Danilov, S., Kivman, G., Hiller, W., and Schröter, J. (2007). Data assimilation with the ensemble kalman filter and the SEIK filter applied to a finite element model of the North Atlantic. J. Mar. Syst. 65, 288-298. doi: 10.1016/j.jmarsys.2005.06.009

NOAA/NESDIS (2018). Data from NOAA / NESDIS Center for Satellite Applications and Research. Available at: https://www.star.nesdis.noaa.gov/sod/ lsa/SeaLevelRise/LSA_SLR_maps.php (accessed September 14, 2018).

OCI Group (2015). Joint Task Force Green Cables Funding Study. Available at: https://www.itu.int/en/ITU-T/climatechange/task-force-sc/Documents/JTF\% 20Report\%20Green\%20Cable\%20Funding\%20Study.pdf (accessed July 1, 2015).

Panet, I., Flury, J., Biancale, R., Gruber, T., Johannessen, J., van den Broeke, M. R., et al. (2013). Earth system mass transport mission (e. motion): a concept for future earth gravity field measurements from space. Surv. Geophys. 34, 141-163. doi: 10.1007/s10712-012-9209-8

Paros, J. (2011). "Breakthrough underwater technology holds promise for improved local tsunami warnings," in Proceedings of the Symposium for Underwater Technology (UT), Workshop on Scientific Use of Submarine Cables and Related Technologies (SSC), (Tokyo: IEEE).

Paros, J., Migliacio, P., and Schaad, T. (2012). Nano-Resolution Sensors for Disaster Warning Systems. Yeosu: IEEE.

Person, R., Favali, P., Ruhl, H. A., Beranzoli, L., Rolin, J.-F., Waldmann, C. A., et al. (2015). "From ESONET multidisciplinary scientific community to EMSO novel European research infrastructure for ocean observatories," in Seafloor Observatories: A New Vision of Earth from the Abyss, eds A. De Santis, P. Favali, and L. Beranzoli (Berlin: Springer-Verlag Berlin Heidelberg), 531-564.
Polster, A., Fabian, M., and Villinger, H. (2009). Effective resolution and drift of Paroscientific pressure sensors derived from long-term seafloor measurements. Geochem. Geophys. Geosyst. 10:Q08008. doi: 10.1029/2009GC00 2532

Ponte, R. M., Carson, M., Cirano, M., Domingues, C., Jevrejeva, S., Marcos, M., et al. (2019). Towards comprehensive observing and modeling systems for monitoring and predicting regional to coastal sea level. Front. Mar. Sci. doi: 10.3389/fmars.2019.00437

Presidential Proclamation No. 5030 (1983). Proclamation No. 5030 Exclusive Economic Zone of the United States of America. Available at: https://www. archives.gov/federal-register/codification/proclamations/05030.html (accessde August 15, 2016).

Presidential Proclamation No. 7219 (1999). Proclamation No. 7219Contiguous Zone of the United States. Available at: https://www.govinfo.gov/content/pkg/ WCPD-1999-09-06/pdf/WCPD-1999-09-06-Pg1684.pdf (accessed July 15, 2019).

Purkey, S. G., and Johnson, G. C. (2010). Warming of global abyssal and deep southern ocean waters between the 1990s and 2000s: contributions to global heat and sea level rise budgets. J. Clim. 23, 6336-6351. doi: 10.1175/ 2010jcli3682.1

Ranasinghe, N., Rowe, C., Syracuse, E., Larmat, C., and Begnaud, M. (2018). Enhanced global seismic resolution using transoceanic SMART cables. Seismological Res. Lett. 89, 77-85. doi: 10.1785/0220170068

Rauscher, K. (2010). ROGUCCI, The Report, Issue 1, rev. 1. Available at: http: //www.ieee-rogucci.org/files/The\%20ROGUCCI\%20Report.pdf

Ray, R. D. (2006). Secular changes of the M2 tide in the Gulf of Maine. J. Cont. Shelf Res. 26, 422-427. doi: 10.1016/j.csr.2005.12.005

Ray, R. D. (2013). Precise comparisons of bottom-pressure and altimetric ocean tides. J. Geophys. Res. Oceans 118, 4570-4584. doi: 10.1002/jgrc. 20336

RBR (2017). Introducing Generation3. Available at: https://rbr-global.com/2017/ introducing-generation $\% \mathrm{C} 2 \% \mathrm{~B} 3$ (accessed November 16, 2017).

Rhie, J., and Romanowicz, B. (2004). Excitation of Earth's continuous free oscillations by atmosphere-ocean-seafloor coupling. Nature 431, 552-556. doi: 10.1038/nature02942

Rietbroek, R., LeGrand, P., Wouters, B., Lemoine, J.-M., Ramillien, G., and Hughes, C. W. (2006). Comparison of in situ bottom pressure data with GRACE gravimetry in the Crozet-Kerguelen region. Geophys. Res. Lett. 33:L21601. doi: 10.1029/2006GL027452

Roach, J. A. (2007). “Defining Scientific Research: Marine Data Collection," in Law, Science and Oceans Management, eds M. Nordquist, R. Long, T. Heidar, and J. N. Moore (Leiden: Martinus Nijhoff Publishers).

Rocha, C. B., Chereskin, T. K., Gille, S. T., and Menemenlis, D. (2016). Mesoscale to submesoscale wavenumber spectra in drake passage. J. Phys. Oceanogr. 46, 601-620. doi: 10.1175/JPO-D-15-0087.1

Schaad, T. (2009). Nano-Resolution: Oceanic, Atmospheric and Seismic Sensors with Parts-Per-Billion Resolution. Redmond, WA: Paroscientific, Inc.

Schindelegger, M., Green, J. A. M., Wilmes, S.-B., and Haigh, I. D. (2018). Can we model the effect of observed sea level rise on tides? J. Geophys. Res. Oceans 123, 4593-4609. doi: 10.1029/2018JC013959

Send, U., and Lankhorst, M. (2011). The global component of the US Ocean Observatories Initiative and the global OceanSITES project. Waikoloa, HI: IEEE.

Shinohara, M., Kanazawa, T., Yamada, T., Machida, Y., Shinbo, T., and Sakai, S.I. (2014). New compact ocean bottom cabled seismometer system deployed in the Japan Sea. Mar. Geophys. Res. 35, 231-242. doi: 10.1007/s11001-0139197-1

Shinohara, M., Yamada, T., Sakai, S., and Shiobara, H. (2016). Installation of new seafloor cabled seismic and tsunami observation system using ICT to off-Tohoku region, Japan. Dubai: Suboptic.

Sloyan, B., Wanninkhof, R., Kramp, M., Johnson, G. C., Talley, L., Tanhua, T., et al. (2019). The Global Ocean Ship-Based Hydrographic Investigations Program (GO-SHIP): A platform for integrated multidisciplinary ocean science. Front. Mar Sci. doi: 10.3389/fmars.2019.00445

Smeed, D. A., Josey, S. A., Beaulieu, C., Johns, W. E., Moat, B. I., Frajka-Williams, E., et al. (2018). The north atlantic ocean is in a state of reduced overturning. Geophys. Res. Lett. 45, 1527-1533. doi: 10.1002/2017gl076350

Snowden, D., Tsontos, V., Handegard, N. O., Zarate, M., O’Brien, K. M., Casey, K. S., et al. (2019). Data interoperability between elements of the global ocean observing system. Front. Mar. Sci. doi: 10.3389/fmars.2019.00442 
Song, Y. T., Fukumori, I., Shum, C. K., and Yi, Y. (2012). Merging tsunamis of the 2011 Tohoku-Oki earthquake detected over the open ocean. Geophys. Res. Lett. 39:L05606. doi: 10.1029/2011GL050767

Song, Y. T., and Hou, T. Y. (2006). Parametric vertical coordinate formulation for multiscale, Boussinesq, and non-Boussinesq ocean modeling. Ocean Model 11, 298-332. doi: 10.1016/j.ocemod.2005.01.001

Stammer, D., Cazenave, A., Ponte, R. M., and Tamisiea, M. E. (2013). Causes for Contemporary Regional Sea Level Changes. Ann. Rev. Mar. Sci. 5, 21-46. doi: 10.1146/annurev-marine-121211-172406

Talley, L. D., Feely, R. A., Sloyan, B. M., Wanninkhof, R., Baringer, M. O., Bullister, J. L., et al. (2016). Changes in ocean heat, carbon content, and ventilation: a review of the first decade of GO-SHIP global repeat hydrography. Ann. Rev. Mar. Sci. 8, 185-215. doi: 10.1146/annurev-marine-052915100829

Tanhua, T. S., Pouliquen, S., Hausman, J., O’Brien, K. M., Bricher, P., de Bruin, T., et al. (2019a). Ocean FAIR data services. Front. Mar. Sci. doi: 10.3389/fmars. 2019.00440

Tanhua, T., McCurdy, A., Fischer, A., Appeltans, W., Bax, N., Currie, K., et al. (2019b). What have we learned from the Framework for Ocean Observing: evolution of the Global Ocean Observing System. Front. Mar. Sci. doi: 10.3389/ fmars.2019.00471

Tapley, B. D., Bettadpur, S., Watkins, M., and Reigber, C. (2004). The gravity recovery and climate experiment: Mission overview and early results. Geophys. Res. Lett. 31, 1-4.

Tappin, D. R., Grilli, S. T., Harris, J. C., Geller, R. J., Masterlark, T., Kirby, J. T., et al. (2014). Did a submarine landslide contribute to the 2011 Tohoku tsunami? Mar. Geol. 357, 344-361. doi: 10.1016/j.margeo.2014.09.043

Thomas, J., Hazard, L., Jensen, R. E., Otero, M., Terrill, E., Keen, C., et al. (2015). "How High-Resolution Wave Observations and HF Radar-Derived Surface Currents are Critical to Decision-Making for Maritime Operations," in Coastal Ocean Observing Systems, eds Y. Liu, H. Kerkering, and R. H. Weisberg (Amsterdam: Elsevier).

Tunnicliffe, V. J., Barnes, C. R., and Dewey, R. (2008). "Major Advances in Cabled Ocean Observatories (VENUS and NEPTUNE Canada) in Shallow and Deep Sea Settings," in Proceedings of the US/EU-Baltic International Symposium, IEEE/OES, (Tallinn).

United Nations (1958a). Geneva Convention on the Continental Shelf. Available at: https://treaties.un.org/doc/Treaties/1964/06/19640610\%2002-10\%20AM/Ch_ XXI_01_2_3_4_5p.pdf (accessed January 2005).

United Nations (1958b). Geneva Convention on the High Seas. In 450 U.N.T.S. 82, arts. 2, 26(1), 26(3). New York, NY: United Nations Convention.

United Nations (1982). United Nations Law of the Sea Convention. 1833 U.N.T.S. 397 (entered into force on Nov. 16, 1994). New York, NY: United Nations Convention.
Watts, D. R., and Kontoyiannis, H. (1990). Deep-ocean bottom pressure measurement: drift removal and performance. J. Atmos. Ocean. Technol. 7, 296-306. doi: 10.1175/1520-0426(1990)007<0296:dobpmd> 2.0.co;2

Webb, S. C. (2008). The Earth's hum: the excitation of Earth normal modes by ocean waves. Geophys. J. Int. 174, 542-566. doi: 10.1111/j.1365-246x.2008. 03801.x

Weinstein, S. A., and Lundgren, P. R. (2008). "Finite Fault Modeling in a Tsunami Warning Center Context," in Earthquakes: Simulations, Sources and Tsunamis. Pageoph Topical Volumes, eds D. K. Weatherley, K. F. Tiampo, and S. A. Weinstein (Basel: Birkhäuser).

Wilcock, W. S. D., Manalang, D., Harrington, M., Cram, G., Tilley, J., Burnett, J., et al. (2017). A Seafloor Test of the A-0-A Approach to Calibrating Pressure Sensors for Vertical Geodesy. Washington, DC: American Geophysical Union.

Xtera (2016). Line monitoring and control in subsea networks. Available at: https://xtera.com/wp-content/uploads/2017/05/White-Paper-LineMonitoring-and-Control-in-Subsea-Networks-Xtera-August-2016.pdf

You, J. Y. (2010). Harnessing telecoms cables for science. Nature 466, 690-691. doi: $10.1038 / 466690 a$

Zaron, E. D., and Jay, D. A. (2014). An analysis of secular change in tides at openocean sites in the Pacific. J. Phys. Oceanogr. 44, 1704-1726. doi: 10.1175/jpo-d13-0266.1

Conflict of Interest Statement: NB is employed by SIN Medida Limited. LD is employed by Alcatel Networks. SL is employed Ocean Specialists, Inc. JM is employed by DRG Undersea Consulting, Inc. KP is employed by GHD Consulting. PNT was employed by Thomas Strategies.

The remaining authors declare that the research was conducted in the absence of any commercial or financial relationships that could be construed as a potential conflict of interest.

The reviewer SJ declared a shared affiliation, with no collaboration, with one of the authors, CB, to the handling Editor at the time of review.

Copyright (c) 2019 Howe, Arbic, Aucan, Barnes, Bayliff, Becker, Butler, Doyle, Elipot, Johnson, Landerer, Lentz, Luther, Müller, Mariano, Panayotou, Rowe, Ota, Song, Thomas, Thomas, Thompson, Tilmann, Weber and Weinstein. This is an open-access article distributed under the terms of the Creative Commons Attribution License (CC BY). The use, distribution or reproduction in other forums is permitted, provided the original author(s) and the copyright owner(s) are credited and that the original publication in this journal is cited, in accordance with accepted academic practice. No use, distribution or reproduction is permitted which does not comply with these terms. 


\section{On the Future of Argo: A Global, Full-Depth, Multi-Disciplinary Array}

\begin{abstract}
Dean Roemmich ${ }^{1 * t}$, Matthew H. Alford ${ }^{1 \dagger}$, Hervé Claustre ${ }^{2 \dagger}$, Kenneth Johnson ${ }^{3 \dagger}$, Brian King ${ }^{4 t}$, James Moum ${ }^{5 t}$, Peter Oke ${ }^{6 t}$, W. Brechner Owens ${ }^{7 t}$, Sylvie Pouliquen ${ }^{8 t}$, Sarah Purkey ${ }^{1 \dagger}$, Megan Scanderbeg ${ }^{1 \dagger}$, Toshio Suga ${ }^{9 \dagger}$, Susan Wijfels ${ }^{7 t}$, Nathalie Zilberman ${ }^{11}$, Dorothee Bakker ${ }^{10}$, Molly Baringer ${ }^{11}$, Mathieu Belbeoch ${ }^{12}$, Henry C. Bittig ${ }^{2}$, Emmanuel Bosss ${ }^{13}$, Paulo Calil ${ }^{14}$, Fiona Carse ${ }^{15}$, Thierry Carval ${ }^{8}$, Fei Chai ${ }^{16}$, Diarmuid Ó. Conchubhair ${ }^{17}$, Fabrizio d'Ortenzio ${ }^{2}$, Giorgio Dall'Olmo ${ }^{18}$, Damien Desbruyeres ${ }^{8}$, Katja Fennel ${ }^{19}$, Ilker Fer ${ }^{20}$, Raffaele Ferrari' ${ }^{21}$, Gael Forget ${ }^{21}$, Howard Freeland ${ }^{22}$, Tetsuichi Fujiki23, Marion Gehlen ${ }^{24}$, Blair Greenan ${ }^{25}$, Robert Hallberg' ${ }^{26}$, Toshiyuki Hibiya ${ }^{27}$, Shigeki Hosoda ${ }^{23}$, Steven Jayne', Markus Jochum ${ }^{28}$, Gregory C. Johnson ${ }^{29}$, KiRyong Kang ${ }^{30}$, Nicolas Kolodziejczyk ${ }^{31}$, Arne Körtzinger ${ }^{32}$, Pierre-Yves Le Traon ${ }^{33}$, Yueng-Djern Lenn ${ }^{34}$, Guillaume Maze ${ }^{8}$, Kjell Arne Mork ${ }^{35}$, Tamaryn Morris ${ }^{36}$, Takeyoshi Nagai ${ }^{37}$, Jonathan Nash ${ }^{5}$, Alberto Naveira Garabato ${ }^{4}$, Are Olsen ${ }^{20}$, Rama Rao Pattabhis8, Satya Prakash ${ }^{38}$, Stephen Riser ${ }^{39}$, Catherine Schmechtig ${ }^{40}$, Claudia Schmid ${ }^{11}$, Emily Shroyer ${ }^{5}$, Andreas Sterl ${ }^{41}$, Philip Sutton ${ }^{42}$, Lynne Talley ${ }^{1}$, Toste Tanhua ${ }^{32}$, Virginie Thierry ${ }^{8}$, Sandy Thomalla ${ }^{43}$, John Toole ${ }^{7}$, Ariel Troisi ${ }^{44}$, Thomas W. Trull 6 , Jon Turton ${ }^{15}$, Pedro Joaquin Velez-Belchi ${ }^{45}$, Waldemar Walczowski ${ }^{46}$, Haili Wang ${ }^{47}$, Rik Wanninkhof ${ }^{11}$, Amy F. Waterhouse ${ }^{1}$, Stephanie Waterman ${ }^{48}$, Andrew Watson ${ }^{49}$, Cara Wilson ${ }^{50}$, Annie P. S. Wong ${ }^{39}$, Jianping $\mathrm{Xu}^{16}$ and Ichiro Yasuda ${ }^{51}$
\end{abstract}

${ }^{1}$ Scripps Institution of Oceanography, La Jolla, CA, United States, ${ }^{2}$ French National Center for Scientific Research, Villefranche Oceanographic Laboratory, Sorbonne Université, Villefranche-sur-Mer, France, ${ }^{3}$ Monterey Bay Aquarium Research Institute, Moss Landing, CA, United States, ${ }^{4}$ National Oceanography Centre, Southampton, United Kingdom, ${ }^{5}$ College of Earth, Ocean, and Atmospheric Sciences, Oregon State University, Corvallis, OR, United States, ${ }^{6}$ Commonwealth Scientific and Industrial Research Organisation, Hobart, TAS, Australia, ${ }^{7}$ Woods Hole Oceanographic Institution, Woods Hole, MA, United States, ${ }^{8}$ Institut Français de Recherche pour l'Exploitation de la Mer, Brest, France, ${ }^{9}$ Department of Geophysics, Graduate School of Science, Tohoku University, Sendai, Japan, ${ }^{10}$ School of Environmental Sciences, Centre for Ocean and Atmospheric Sciences, University of East Anglia, Norwich, United Kingdom, ${ }^{11}$ Atlantic Oceanographic and Meteorological Laboratory, National Oceanic and Atmospheric Administration, Miami, FL, United States, ${ }^{12} \mathrm{JCOMMOPS}$, Brest, France, ${ }^{13}$ School of Marine Sciences, The University of Maine, Orono, ME, United States, ${ }^{14}$ Institute of Coastal Research, Helmholtz-Zentrum Geesthacht, Geesthacht, Germany, ${ }^{15}$ UK Met Office, Exeter, United Kingdom, ${ }^{16}$ State Key Laboratory of Satellite Ocean Environment Dynamics, Second Institute of Oceanography, Ministry of Natural Resources, Hangzhou, China, ${ }^{17}$ Irish Marine Institute, Galway, Ireland, ${ }^{18}$ Plymouth Marine Laboratory, Plymouth, United Kingdom, ${ }^{19}$ Department of Oceanography, Dalhousie University, Halifax, NS, Canada, ${ }^{20}$ Physical Oceanography, Geophysical Institute, University of Bergen, Bergen, Norway, ${ }^{21}$ Massachusetts Institute of Technology, Cambridge, MA, United States, ${ }^{22}$ Fisheries and Oceans Canada, Sidney, BC, Canada, ${ }^{23}$ Japan Agency for Marine-Earth Science and Technology, Yokosuka, Japan, ${ }^{24}$ Laboratoire des Sciences du Climat et de l'Environnement/Institut Pierre Simon Laplace, Gif-sur-Yvette, France, ${ }^{25}$ Bedford Institute of Oceanography and Fisheries and Oceans Canada, Dartmouth, NS, Canada, ${ }^{26}$ National Oceanic and Atmospheric Administration, Geophysical Fluid Dynamics Laboratory, Princeton, NJ, United States, ${ }^{27}$ Graduate School of Science, Department of Earth and Planetary Science, The University of Tokyo, Tokyo, Japan, ${ }^{28}$ Neils Bohr Institute, Copenhagen, Denmark, ${ }^{29}$ National Oceanic and Atmospheric Administration/Pacific Marine Environmental Laboratory, Seattle, WA, United States, ${ }^{30}$ Korea Meteorological Administration, Seoul, South Korea, ${ }^{31}$ CNRS-IRD-Ifremer, LOPS Laboratory, University of Brest, Brest, France, ${ }^{32}$ Helmholtz-Zentrum für Ozeanforschung Kiel, Kiel, Germany, ${ }^{33}$ Mercator-Ocean, Brest, France, ${ }^{34}$ School of Ocean Sciences, Bangor University, Bangor, United Kingdom, ${ }^{35}$ Institute of Marine Research, Bergen, Norway, ${ }^{36}$ Marine Research Unit, South African Weather Service, Cape Town, South Africa, ${ }^{37}$ Graduate School of Marine Science and Technology, Ocean Sciences, Tokyo University of Marine Science and Technology, Tokyo, Japan, ${ }^{38}$ Indian National Centre for Ocean Information Services, Hyderabad, India, ${ }^{39}$ School of Oceanography, College of the Environment, University of Washington, Seattle, WA, United States, ${ }^{40}$ French National Center for Scientific Research, OSU Ecce Terra, Sorbonne Université, Paris, France, ${ }^{41}$ Koninklijk Nederlands Meteorologisch Instituut, De Bilt, Netherlands, ${ }^{42}$ National Institute of Water and Atmospheric Research, Auckland, New Zealand, ${ }^{43}$ Southern Ocean Carbon \& Climate Observatory, Cape Town, South Africa, ${ }^{44}$ Servício de Hidrografia Naval, Buenos Aires, Argentina, ${ }^{45}$ Instituto Espanol de Oceanografia, Canary Islands, Spain, ${ }^{46}$ Institute of Oceanology Polish Academy of Sciences, Sopot, Poland, ${ }^{47}$ State Key Laboratory of Marine Environmental Science, Xiamen University, Xiamen, China, ${ }^{48}$ Department of Earth, 
Ocean and Atmospheric Sciences, The University of British Columbia, Vancouver, BC, Canada, ${ }^{49}$ Earth System Science Group, College of Life and Environmental Science, University of Exeter, Exeter, United Kingdom, ${ }^{50}$ National Oceanic and Atmospheric Administration - National Marine Fisheries Service, Pacific Grove, CA, United States, ${ }^{51}$ Atmosphere and Ocean Research Institute, The University of Tokyo, Tokyo, Japan

The Argo Program has been implemented and sustained for almost two decades, as a global array of about 4000 profiling floats. Argo provides continuous observations of ocean temperature and salinity versus pressure, from the sea surface to $2000 \mathrm{dbar}$. The successful installation of the Argo array and its innovative data management system arose opportunistically from the combination of great scientific need and technological innovation. Through the data system, Argo provides fundamental physical observations with broad societally-valuable applications, built on the cost-efficient and robust technologies of autonomous profiling floats. Following recent advances in platform and sensor technologies, even greater opportunity exists now than 20 years ago to (i) improve Argo's global coverage and value beyond the original design, (ii) extend Argo to span the full ocean depth, (iii) add biogeochemical sensors for improved understanding of oceanic cycles of carbon, nutrients, and ecosystems, and (iv) consider experimental sensors that might be included in the future, for example to document the spatial and temporal patterns of ocean mixing. For Core Argo and each of these enhancements, the past, present, and future progression along a path from experimental deployments to regional pilot arrays to global implementation is described. The objective is to create a fully global, top-to-bottom, dynamically complete, and multidisciplinary Argo Program that will integrate seamlessly with satellite and with other in situ elements of the Global Ocean Observing System (Legler et al., 2015). The integrated system will deliver operational reanalysis and forecasting capability, and assessment of the state and variability of the climate system with respect to physical, biogeochemical, and ecosystems parameters. It will enable basic research of unprecedented breadth and magnitude, and a wealth of ocean-education and outreach opportunities.

Keywords: Argo, floats, global, ocean, warming, circulation, temperature, salinity

\section{INTRODUCTION}

The Argo Program is a major component of both the Global Ocean Observing System (GOOS) and the Global Climate Observing System (GCOS), providing near-real time data for ocean and atmospheric services and high quality data for climate research. The Argo Program began its implementation in 1999 and has provided global coverage of the upper $2000 \mathrm{~m}$ of the oceans since 2006. By November 2018, Argo had provided $2,000,000$ profiles since the program began, and a comparable number of velocity drift estimates at $1000 \mathrm{~m}$ depth. Although originally designed to provide temperature and salinity profiles in the upper $2 \mathrm{~km}$ of the ice-free oceans, the array has been expanded into seasonal ice zones using floats equipped with ice avoidance algorithms. Argo profiling floats also are sampling in many marginal seas. In addition, ongoing regional pilot programs have demonstrated that Argo floats can now (1) measure biogeochemical parameters to address oceanic uptake of carbon, acidification and deoxygenation (Biogeochemical, BGC, Argo) and (2) make measurements throughout the water column to $6000 \mathrm{~m}$ depth (Deep Argo). Notification through the
Argo Information Center (AIC), following Intergovernmental Oceanographic Commission (IOC) guidelines to protect the rights of Coastal States, has enabled global coverage. Presently the number of functioning Argo floats remains steady, with total float count around 4000. This has been possible, despite relatively flat funding, through a collaboration of international partners and significant technological innovation. The Argo Data System provides real-time data within $24 \mathrm{~h}$ of collection through the Global Telecommunications System (GTS) and via the internet for use at global prediction centers. The Argo Data Management Team (ADMT) also oversees delayed-mode quality control of the data and the availability of Argo data at the Argo Global Data Assembly Centers.

To meet future needs, Argo should (1) support continuing innovation in float technology, (2) enhance coverage in critical regions such as the equatorial band, where higher temporal resolution is needed and the western boundary regions where mesoscale 'noise' is high, (3) implement Deep Argo and Biogeochemical Argo in the global array, (4) assess the technical readiness and scientific value of experimental measurements for possible future inclusion in Argo, for example those used to 
estimate small scale mixing, and (5) collaborate with our enduser community to improve the use of Argo data in prediction systems and services. This review lays out the motivation, development, and present status of the Argo Program, and addresses the five issues mentioned above. It is important to note that Core Argo has reached and maintained full implementation through innovation and broad community support. Only a small fraction of the funding needed to support the ambitious community requests for an expanded Argo Program can be identified at present. It is important for Argo to meet its future challenges as a single integrated program. The present elements of Argo - Core, Deep, and BGC - and of its data management system are not separable, and any other future enhancements will similarly be considered as contributions to the unified effort.

\section{MOTIVATION, DEVELOPMENT, TECHNOLOGY}

\section{Core Argo}

During the 1990s, the World Ocean Circulation Experiment brought increased understanding of important oceanic roles in climate variability and change (Siedler et al., 2001). The need to observe the global subsurface ocean, together with a fit-for-purpose revolutionary autonomous technology (Davis et al., 2001), led to a multinational proposal for a global subsurface ocean observing system (Argo Steering Team, 1998). The proposed 'Argo Program' would be comprised of over 3000 profiling floats, obtaining a snapshot of the physical state of the ocean from 0 to $2000 \mathrm{~m}$ every 10 days. All data would be freely shared in near-real time (NRT, within $24 \mathrm{~h}$ ) to support forecasting, and with a highly quality-controlled delayed-mode (DM) version delivered within 12 months for climate research and assessments. Argo floats were deployed in regional arrays beginning in 1999 and then globally from 2004 to the present. Argo has fulfilled its promise to complement and integrate across many satellite and in situ elements of the GOOS and across many regional observational networks (deYoung et al., 2019; Foltz et al., 2019; Hermes et al., 2019; Lee et al., 2019; Newman et al., 2019; Palazov et al., 2019; Smith et al., 2019; Todd et al., 2019).

A number of key elements that contributed to Argo's success over the past 20 years are evident. The underpinning profiling float technology is simple, robust, and cost-effective. A strong international consensus on the high value of Argo, by agencies and the science community, contributed to Argo's rapid roll-out. Once Argo was in place, a broad base of applications (see section "Core Argo" under the section "End User Engagement") including basic research, assessment of the state of the Earth's climate, tertiary and secondary education, and ocean modeling for reanalysis and operational prediction, drew strong community support. Effective partnerships developed between Argo teams and commercial suppliers, to exploit and improve float and sensor technologies. The IOC provided necessary protocols to facilitate the operation of Argo floats in national waters (Intergovernmental Oceanographic Commission
[IOC], 1999), while the $\mathrm{AIC}^{1}$ supplied the mechanisms for tracking and reporting to coastal states (Pinardi et al., 2019).

Technology advances have continued throughout Argo's 20year history. New generation profiling floats are smaller, lighter, and more energy efficient. A profoundly important transition from unidirectional to faster bidirectional communication (Iridium) improves vertical resolution and shortens surface times from $12 \mathrm{~h}$ to $20 \mathrm{~min}$, greatly reducing bio-fouling, array divergence due to surface drift, grounding, and other hazards. Ice-avoidance measures in float controllers (Klatt et al., 2007) have extended the range of Argo through the seasonal ice zones (Wong and Riser, 2011). Improved CTD sensors, as well as procedures for delayed-mode quality control (Owens and Wong, 2009) have increased the accuracy and consistency of the Argo dataset. Float lifetimes have increased, to 4-5 years for most Argo National Programs, reducing the cost per profile while extending reseeding intervals. All of these improvements are propagated across the Argo national programs through communication of Best Practices (Pearlman et al., 2019).

Argo's systematic and regular observation of the global subsurface ocean has transformed ocean observing. Northern hemisphere, near-coastal, and seasonal sampling biases of earlier eras are removed. The global Argo array has been sustained and improved for more than a decade, providing data for over 3000 research publications and becoming a mainstay of global ocean data assimilation, modeling, and prediction applications. The notable convergence, in the Argo era, of diverse estimates of historical global ocean heat content changes (e.g., Johnson et al., 2016) has increased the confidence that can be placed on the reliability of national and international assessments of climate change. International partners in Argo merge their efforts to produce a seamless global array, providing standardized observations, and delivering near realtime and research quality data with public access. Argo has led the way among ocean observing networks with regard to international cooperation, operations planning, Data Availability, and metadata quality.

\section{BGC-Argo}

The Biogeochemical (BGC)-Argo program began with the deployment of optical (Bishop et al., 2002; Mitchell, 2003; Boss et al., 2008) and oxygen (Körtzinger et al., 2004; Riser and Johnson, 2008) sensors on profiling floats between 2000 and 2003. The success of these efforts was highlighted at the Autonomous Platforms and Sensors meeting (Rudnick and Perry, 2003), which was the founding meeting for this community. It was followed by the development of a global vision for biogeochemical data acquisition through the inclusion of oxygen sensors on Argo platforms (Gruber et al., 2007), and the launch the same year of a working group of the International OceanColor Coordinating Group, "Bio-optical sensors on Argo floats" (IOCCG, 2011). In the meantime, both the oxygen and optical communities were promoting their vision for developing a global network of profiling floats carrying oxygen and optical sensors as part of the OceanObs09 conference (Claustre et al., 2010;

${ }^{1}$ http://argo.jcommops.org/ 
Gruber et al., 2010), following a meeting in Johnson et al. (2009) that addressed the development of a global observing system using both gliders and profiling floats.

In 2016, a meeting was held in Villefranche-sur-mer to develop an implementation plan for BGC-Argo. The subsequent report (Biogeochemical-Argo Planning Group, 2016) was the starting point of the BGC-Argo program. Observing system simulation experiments (OSSEs) performed for this meeting suggested that a 1000-float array would significantly constrain the processes that control global oxygen and carbon distributions, including air-sea fluxes and exports from the surface (Kamenkovich et al., 2017). Assuming a mean BGC float lifetime of 4 years, sustaining a 1000-float array requires 250 floats per year with an estimated annual cost near US\$25-M. Each of the floats would carry sensors for six core ocean variables measured with targeted accuracies ${ }^{2}$ : chlorophyll fluorescence (Chla), particle backscatter, oxygen, nitrate, $\mathrm{pH}$, and irradiance. The 1000-float array would provide observational data to transform ability to quantify: (i) air-sea carbon fluxes, (ii) ocean deoxygenation, oxygen minimum zones and related denitrification fluxes, (iii) ocean acidification, (iv) the biological carbon pump, and (v) phytoplankton communities. The observing system would improve management of living marine resources and carbon budget verification, both key societal goals. In 2017, a BGCArgo Scientific Steering Committee was formed (under the Argo Steering Team), to guide the development of the network and the implementation of the program objectives, and to continue developing a vision for the future. In 2018, during the Executive Council of IOC, unanimous support from Member States was given to the proposal to incorporate the six biogeochemical measurements in the Argo array. Additionally the Executive Council approved a framework for the future addition of new parameters to Argo.

The first BGC-Argo deployments consisted of a few floats at a time. These have evolved to regional scale projects such as remOcean (North Atlantic sub-polar Gyre, 20 floats) and NAOS (Mediterranean Sea, 30 floats), and to basin-scale projects such as SOCCOM (Southern Ocean Carbon and Climate Observations and Modeling) with more than 100 floats deployed thus far, toward a target of 200 . These pilots have showcased the potential of community-shared efforts to support better understanding of major biogeochemical processes at the global scale and to explore new research topics. The profiling float data sets have been vetted by research groups via publications approaching several 100 in total. BGC-Argo observations are open and free through the Argo data system both in NRT and DM.

These projects are transforming our understanding of variability in the ocean over time scales difficult to achieve with ship-based observations. A few of these achievements include characterization of ocean nitrate supply (Johnson et al., 2010; D'Ortenzio et al., 2014); observation of bloom dynamics beneath the surface (Boss and Behrenfeld, 2010; Mignot et al., 2018); novel carbon export mechanisms through a mixed-layer pump (Dall'Olmo and Mork, 2014; Dall'Olmo et al., 2016) or eddy subduction (Llort et al., 2018); oxygen

${ }^{2}$ http://biogeochemical-argo.org/measured-variables-general-context.php minimum zone processes (Whitmire et al., 2009; Prakash et al., 2012; Stanev et al., 2018); ocean net community production over complete annual cycles throughout the ocean (Riser and Johnson, 2008; Bushinsky and Emerson, 2015; Hennon et al., 2016; Plant et al., 2016); ocean ventilation (Körtzinger et al., 2004; Wolf et al., 2018); air-sea exchanges of $\mathrm{O}_{2}$ (Bushinsky et al., 2017) and $\mathrm{CO}_{2}$ (Williams et al., 2017; Bittig et al., 2018; Gray et al., 2018); and mesoscale/sub-mesoscale processes (Sukigara et al., 2011; Kouketsu et al., 2016). In a major advance, the data are now being assimilated into biogeochemical models to enable greater understanding and improved predictions (Verdy and Mazloff, 2017).

The development of BGC-Argo floats has been based on the standard Argo Pressure/Temperature/Salinity (P/T/S) platforms, integrating new sensors when their readiness level appeared compatible with long-term, operational use. Today, the community is operating three main BGC-Argo platforms (Figure 1): PROVOR, Navis, and APEX floats. While each of these platforms is capable of carrying the six core sensors outlined in the BGC-Argo implementation plan, due to present hardware limitations and the objectives of funded research programs, few have yet been deployed with all six. Deployments of floats carrying the six core variables are highly desirable as well as harmonizing of mission parameters with those of Core Argo.

\section{Deep Argo}

Deep Argo is motivated by the substantial oceanographic variability found in the $50 \%$ of ocean volume that lies below the 2000-dbar profiling target for conventional Argo floats. Development of floats and CTDs capable of accurate measurements to 6000 dbar makes global fulldepth Argo implementation feasible, including sampling of bottom-intensified ocean variability.

Antarctic Bottom Water, which fills much of the ocean below 2000 dbar (Johnson, 2008), has been warming and freshening during the past few decades, with these changes contributing to steric sea level rise (Purkey and Johnson, 2013). The rate of ocean heat-gain below 2000 dbar, of $0.065( \pm 0.04) \mathrm{W} \mathrm{m}^{-2}$ from 1991 to 2010 (Desbruyères et al., 2016) is about $10 \%$ of the $0.61( \pm 0.09) \mathrm{W} \mathrm{m}^{-2}$ from 2005 to 2015 in the upper 1800 dbar (Johnson et al., 2016). Deep ocean (>2000 dbar) heat content changes have been estimated over decadal intervals using a sparse network of repeat hydrographic sections that are sampled at quasi-decadal intervals (Talley et al., 2016), hence only decadal estimates are possible, and uncertainties due to the sparsity of observations are about $2 / 3$ the size of the signal. In contrast, Core Argo data enables decadal estimation of ocean heat uptake shallower than 2000 dbar with uncertainties only about $1 / 7$ the size of the signal. In addition, monthly global analyses of Core Argo data (Roemmich and Gilson, 20093) have provided a basis for investigation of seasonal-to-interannual variability (e.g., Johnson and Birnbaum, 2017). Deep Argo will similarly reduce the uncertainties in decadal deep ocean heat uptake estimates, while providing data for a broad range of scientific investigations of deep variability (Johnson et al., 2015).

\footnotetext{
${ }^{3}$ http://sio-argo.ucsd.edu/RG_Climatology.html
} 
A

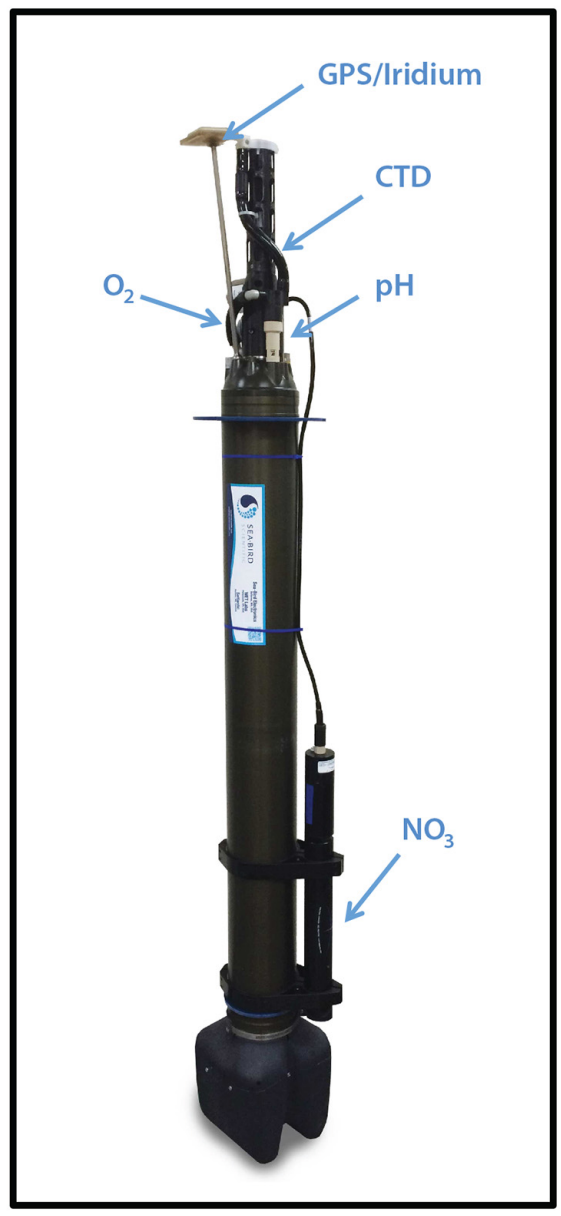

B

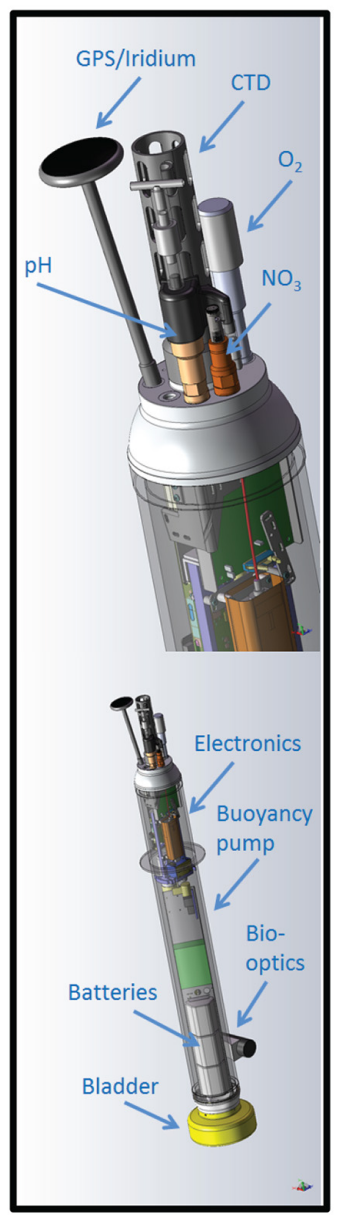

C

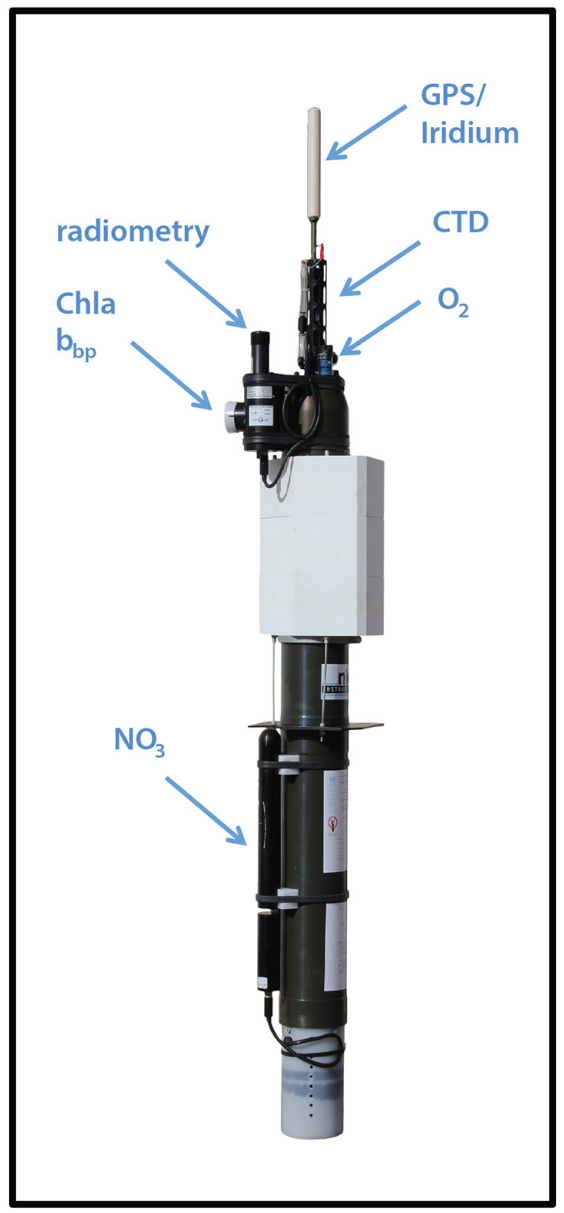

FIGURE 1 | The three main models of BGC-Argo floats presently in use include (A) Navis, (B) APEX, and (C) PROVOR.

The fact that abyssal trends and diffusivities both seem to be insufficiently constrained in ocean state estimates is further evidence that deep Argo is needed (Wunsch and Heimbach, 2014; Forget et al., 2015).

North Atlantic Deep Water is also changing, with the meridional overturning circulation decreasing measurably between 2004-2008 and 2008-2012 (Smeed et al., 2018). Deep ocean circulation variations have primarily been observed using transoceanic moored arrays, which are costly to maintain, and hence regionally limited (Lozier et al., 2017; Meinen et al., 2017; Smeed et al., 2018). Repeat hydrographic section data are also used for this purpose despite their sparse temporal sampling (Bryden et al., 2005; Kouketsu et al., 2011). Deep Argo would provide velocity and density information, complementing both the moored arrays and repeat hydrography, as well as facilitating decadal climate predictions and constraining full-depth ocean data assimilation (Robson et al., 2012; Yeager et al., 2012; Carrassi et al., 2016).

The value and technical feasibility of a Deep Argo Program were recognized at OceanObs'09 (Roemmich et al., 2010). Successful deployments of prototype deep floats and CTDs in
2012-2014 led to a Deep Argo Implementation Workshop in May 2015 (Zilberman and Maze, 2015), where a plan for Deep Argo's progression to a global $5^{\circ} \times 5^{\circ}$ array was endorsed. Regional pilot arrays have been established in the South Pacific, South Indian, and North Atlantic oceans, further demonstrating the feasibility of a global Deep Argo array.

\section{Argo Data Management}

The Argo data system was designed in 2001 at the $1^{\text {st }}$ Argo Data Management meeting in Brest, France and its main components remain in place and function well (Figure 2). The national Data Assembly Centres (DACs) receive data via satellite transmission, decode it, and apply quality control according to a set of agreed NRT tests. Erroneous data are corrected if possible, flagged accordingly and then sent to two Global Data Assembly Centres (GDACs) and the GTS. The GDACs collect the data from the 11 DACs, synchronize their databases daily and serve the data on FTP sites. The AIC monitors the status of the Argo Program, including data distribution, and meta data that incorporate float location, model, transmission system, owner, etc. In addition, the AIC gathers feedback on data quality from 


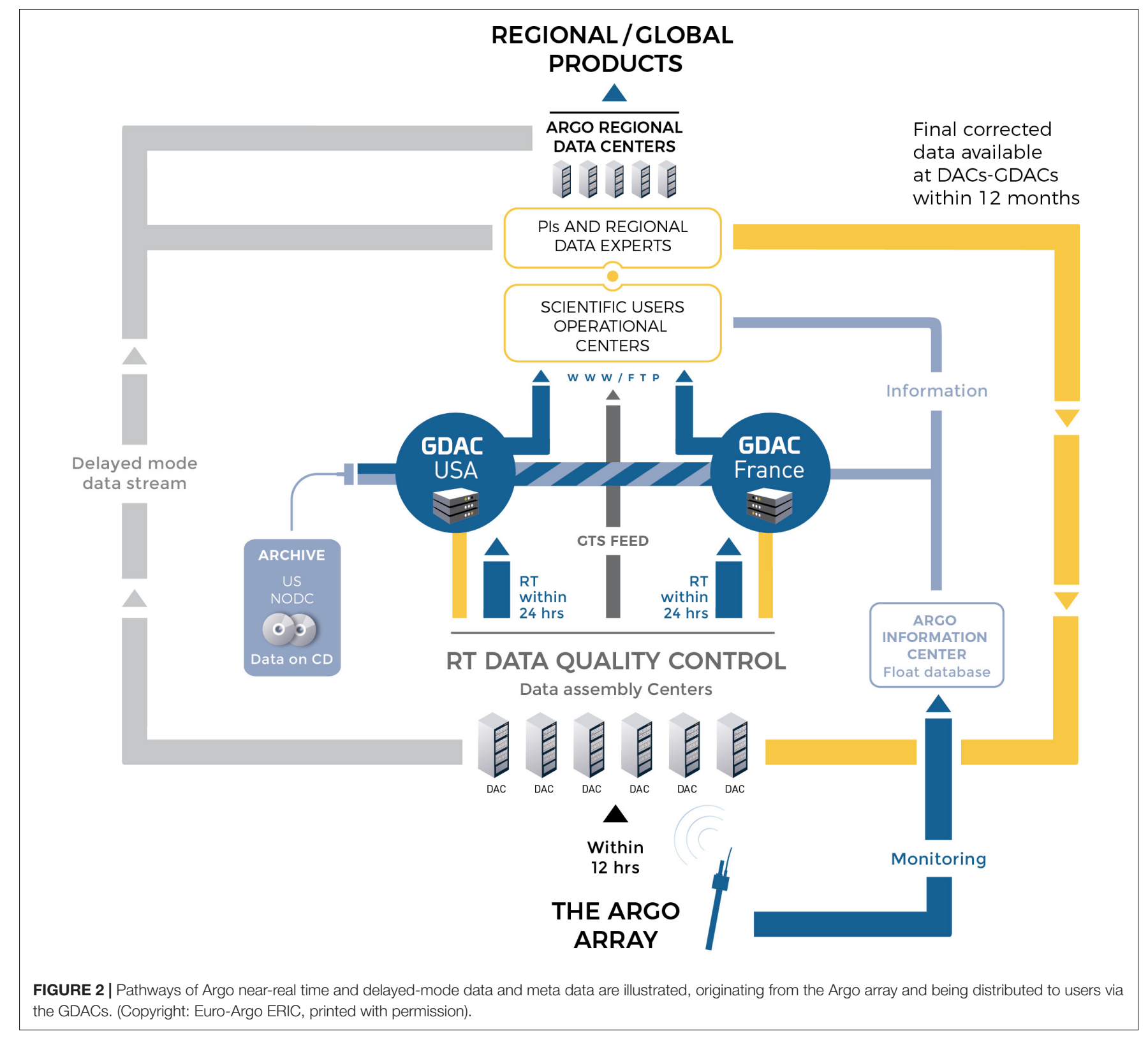

users and relays it to float owners and DACs. Argo's delayedmode data system for $\mathrm{P} / \mathrm{T} / \mathrm{S}$ variables relies on Argo data experts examining the data and reflagging where necessary, using a standard method (Owens and Wong, 2009) to estimate salinity drift, in addition to applying salinity thermal lag adjustments and pressure adjustments.

To improve the quality of $\mathrm{P} / \mathrm{T} / \mathrm{S}$ variables between NRT and DM versions, a few tests have been developed to run on a regular basis (e.g., monthly, quarterly, etc.) on the GDAC data holdings. One of these is an objective analysis run monthly by Coriolis, where profiles that are inconsistent with neighbors are identified for further examination. Another is a quarterly comparison with satellite altimetry performed by CLS/France. When suspect profiles are identified, float owners and DACs are notified to make changes to data QC flags as needed.
The Argo Regional Centers (ARCs) perform a variety of tasks including coordinating float deployments, consistency checks on delayed mode quality control, finding additional reference data for delayed mode work, adopting floats for delayed mode quality control, and producing Argo data products.

While the complete data management chain (Figure 2) has been developed for the core mission (P/T/S, 0-2000 dbar), the extensions to Deep Argo and BGC Argo are under development to form an integrated Argo Data Management System.

\section{ArgoMix}

Here a new enhancement is suggested for possible future inclusion in Argo, consisting of direct shear and scalar microstructure (turbulence) measurements for both the upper and deep ocean. The rationale is provided by recent scientific 
and technical developments. Microstructure measurements are not presently part of Argo but are presented as an example of how new experimental measurements on profiling floats can progress toward inclusion in the global array. This process, begins with limited deployment outside of Argo to demonstrate value and capabilities.

Turbulent mixing in the ocean is important because (1) it contributes to the transport and distributions of heat, freshwater, dissolved gasses, and pollutants, (2) it impacts biological processes by determining the flow field for the smallest plankton and setting large-scale gradients of nutrient availability, and (3) societally-valuable predictions from numerical models of quantities such as temperature and sea level depend sensitively on the geography of ocean mixing rates. Despite its importance, observations of ocean turbulence are extremely sparse, particularly in the deep ocean. Imprints of turbulent transport processes on the large scale ocean state, as readily observed by Core Argo, allow for inversion of diffusivity maps but direct mixing measurements are needed to further constrain inverse estimates (Forget et al., 2015).

Ocean turbulence is of leading order importance in determining the variability of many of the 11 physics-based GCOS "essential ocean variables" (EOVs). For example, turbulence determines the divergence of surface buoyancy fluxes across the surface mixed layer. On larger scales the flux of constituents (e.g., heat or salt) enhanced by turbulence are critical to understanding weather and climate. At present, physics-based EOVs include the surface and subsurface values of ocean currents $(u, v)$, temperature $(T)$ and salinity $(S)$. At any subsurface location, these three EOVs vary in accordance to the Navier-Stokes equations, but thermodynamically they vary solely with the vertical (or diapycnal) divergences of turbulence-enhanced fluxes of momentum, heat, and salt. The physics-based EOVs also include the fluxes of momentum (wind stress) and heat at the sea surface. These fluxes provide surface inputs but do not quantify internal redistributions and so cannot define changes in $u$ or $T$. However, fluxes of heat and momentum can now be reliably assessed using direct shear and scalar microstructure turbulence measurements (Pujiana et al., 2018) as witnessed by flux measurements that asymptote to and vary with independently-measured surface values (Figure 3 ).

Recent work suggests considerable temporal and spatial inhomogeneity in near-surface and deep-ocean mixing. Moored mixing time series in the upper few $100 \mathrm{~m}$ (Moum et al., 2009, 2013) have, for example, identified the importance of subsurface turbulence fluxes in setting the annual cycle of tropical sea surface temperature (SST), and shown variability on long time scales that include ENSO. Furthermore, mixing measurements in the deep ocean (Alford et al., 2011) show intense intermittency, indicating a need for sustained global measurements through the full water column.

The importance of small scale mixing to prediction of global scale circulation, including how this might be quantified, was a focus of the recent Climate Process Team on Ocean Mixing (MacKinnon et al., 2017). In particular, global distributions of mixing (Whalen et al., 2012, 2015; Waterhouse et al., 2014) based on indirect fine-scale parameterizations and internal wave

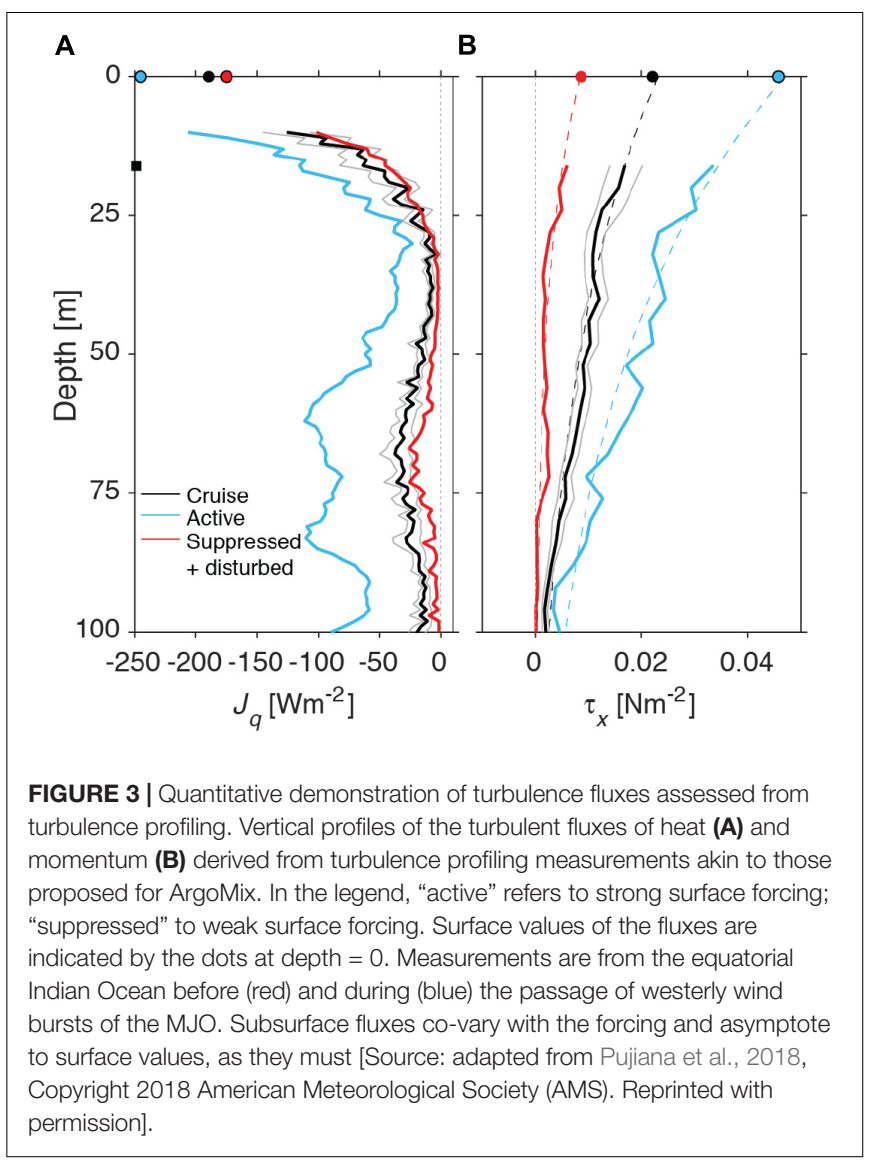

models (Gregg, 1989; Olbers and Eden, 2013; Polzin et al., 2014; Pollman et al., 2017) represent a new paradigm against which to test theory and models, and to target new process experiments. However, the underlying assumptions of these methods are uncertain and may be most suspect where the inferred turbulence is greatest. While the fine-scale parameterizations tend to agree with direct measurements to within a factor of two in the ocean interior, a significantly higher standard is required to understand turbulent flux divergences that govern evolution of EOVs. The assumptions in these methods are known to be violated near boundaries, with differences between parameterizations and direct measurements of up to a factor of 10 (Waterman et al., 2014). In addition to the diapycnal fluxes, direct microstructure measurements can also allow assessment of isopycnal stirring and its underpinning dynamics (Ferrari and Polzin, 2005; Naveira Garabato et al., 2015).

Direct measurements on both Argo and Deep Argo floats are now feasible, owing to recent advances in turbulence sensing technology that have reduced the cost, communications bandwidth, and power requirements. Recent technological advances in inexpensive and low-power circuitry, increased battery capacity and data storage density have led to an ability to make long-term continuous measurements of mixing from oceanographic moorings (Moum and Nash, 2009) and profiling instruments (Sherman and Davis, 1995; Nagai et al., 2015; Goto et al., 2016, 2018; Lucas et al., 2016; Shroyer et al., 2016). 
These systems have been tested quantitatively via inter-platform comparisons (Perlin and Moum, 2012; Pujiana et al., 2018) and schemes to compress data for satellite transmission have been developed (Becherer and Moum, 2017). These are key technical ingredients for successful implementation of mixing measurements on the Argo fleet.

Roughly a dozen moored mixing measurements are now distributed around ocean basins, permitting important but highly limited geographic comparisons. While this effort has been an exciting development, these measurements cannot address problems associated with widespread geographical variations in mixing, deep thermocline and abyssal mixing, or variability away from the moorings. Existing monitoring efforts specifically do not address mixing variability at Argo resolution, as seen in sparse but highly variable deep microstructure observations (Figure 4; Waterhouse et al., 2014).

\section{THE PRESENT STATUS, PROBLEMS, AND STRENGTHS}

\section{Core Argo}

The number of floats reporting Core Argo parameters increased rapidly from 1999 until it reached 3000 during 2007, and has increased slowly over the last 10 years to its present number of 4000 (Figure 5). Recent increases reflect floats deployed in previously unsampled regions and regions of higher than standard sampling density. More than $90 \%$ of measurements made on Argo floats are available within Argo's $24 \mathrm{~h}$ target (see section "Argo Data Management" under the section "The Present Status, Problems, and Strengths"), and all are freely distributed.

Core Argo has a mature leadership model, with Steering and Data Management Teams that meet annually in plenary, and with vigorous inter-sessional activity. These meetings are supplemented with workshops on technical aspects as required, such as hardware engineering, sensor development, and data quality control. Argo leadership has a strong corporate memory, which ensures the stability of the program. Argo regularly audits the performance of the array so that systematic problems can be identified before they seriously degrade the data.

Argo data acquisition is distributed across 25 national programs (Figure 5). The procedures for delivery of data in NRT and DM (see section "Argo Data Management" under the section "The Present Status, Problems, and Strengths") are comprehensively described in public documents. This ensures a global dataset of consistent quality and uniform format ready for public use. Reliability of data does not depend on the originating national program or investigator.

Since 2013, Argo has undertaken a major revision of its data format in order to incorporate data from biogeochemical sensors and other auxiliary measurements. This includes improving the quality of meta data to enable more detailed array audits. This additional complexity (see section "Argo Data Management" under the section "The Present Status, Problems, and Strengths"), while essential, has placed a burden on the data system and has diverted effort from DMQC of the core physical parameters.

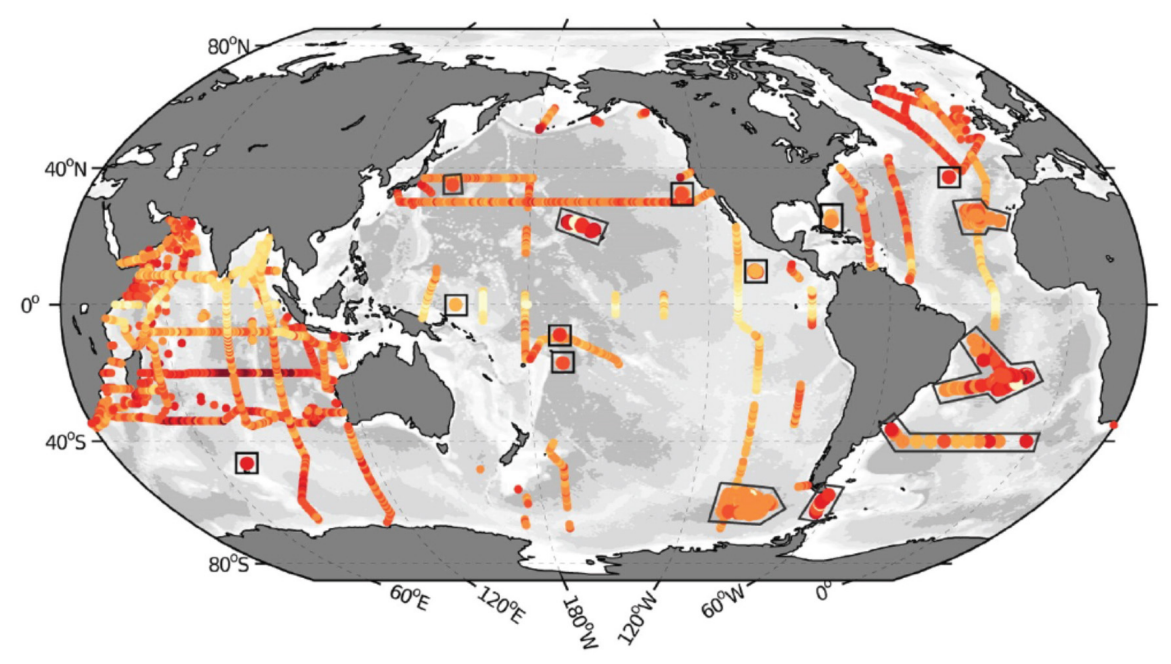

Full depth $\log _{10} K$

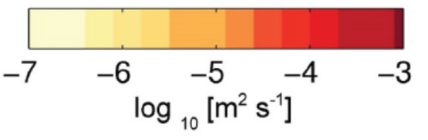

FIGURE 4 | Depth-averaged diffusivity from the surface to the bottom from all existing full-depth microstructure observations as of Waterhouse et al. (2014). [Source: Waterhouse et al., 2014, Copyright 2014 American Meteorological Society (AMS). Reprinted with permission]. 


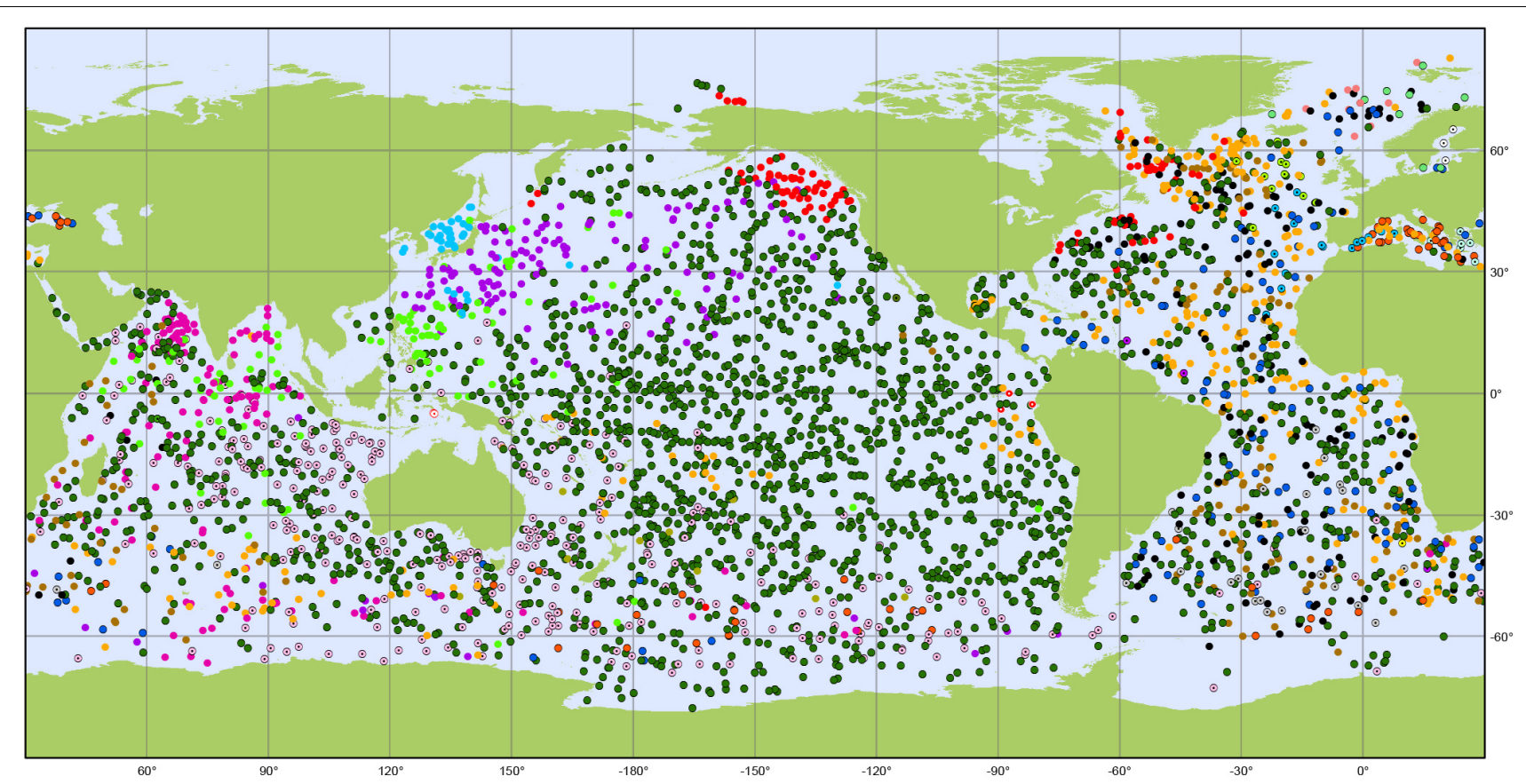

Argo

National contributions - 3983 Operational Floats

September 2018

Latest location of operational floats (data distributed within the last 30 days)

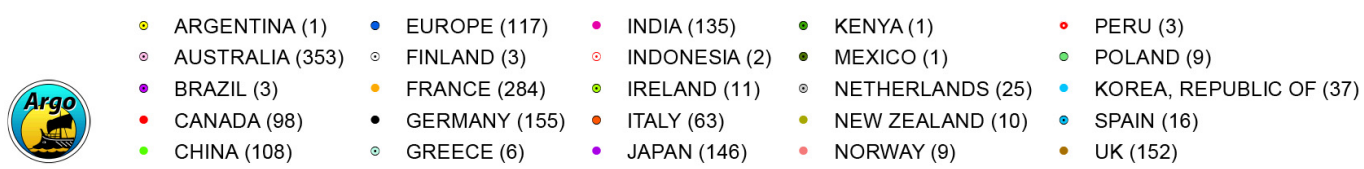

- USA (2234)

- AUSTRALIA (353) $\odot$ FINLAND (3) $\odot$ INDONESIA (2) $\bullet \operatorname{MEXICO}(1) \quad \circ$ POLAND (9)

- CANADA (98)

- GERMANY (155)

- JAPAN (146)

- $\operatorname{NORWAY}(9)$

- UK (152)

Generated by www.jcommops.org, 03/10/2018

FIGURE 5 | Global map of operational Core Argo floats, by nation, in September 2018 (Source: JCOMMOPS).

The greatest challenge facing Core Argo is simply to sustain the global array, especially in remote southern hemisphere regions, in the face of limited budgets. In order to maximize the number of active floats for a given rate of deployment, vigorous attention is being given to improving float and sensor lifetimes and identifying deployment opportunities. A float technical workshop in 2017 identified differences of float longevity between groups using the same hardware. Differences were noted in 'acceptance testing' when floats are delivered by manufacturers and 'final pre-deployment testing' at port of departure. Post-delivery test protocols are being devised to minimize the number of floats and sensors that fail before battery energy is fully utilized.

Technical issues presently under scrutiny include management of lithium batteries for efficient energy utilization, and stability of sensors, especially conductivity (and hence salinity) and pressure. Recent analysis has shown batches of floats in which the salinity reported by the float drifts toward higher than true values. The bias can exceed 0.03 in salinity. Salinity drift is estimated in DM by comparison with nearby measurements. The cause and mitigation of this new salinity drift is still under study. This issue highlights the need to exercise care in the use of NRT data, and the requirements for reference data from GO-SHIP and other programs, to ensure the long-term consistency of the Argo dataset.

While there have been many different designs of the hull and buoyancy engines on which sensors are deployed, Core Argo has depended almost exclusively on the SBE41 and SBE41CP CTDs by SeaBird Electronics (SBE). This has led to good consistency of data, and implementation of uniform procedures for data handling across the national programs, but exposes the program to the risk of single points of failure. Twice during Argo there has been a major problem with the supply of pressure sensors to SBE for use in float CTDs, resulting in lost deployment opportunities and impacting Argo coverage. To reduce this risk, the Steering Team welcomes initiatives to establish alternative sensor payloads. The Steering Team has set up a rigorous process of trial and evaluation to ensure that the Argo dataset remains of consistent quality if and when alternative CTD sensors are introduced.

\section{BGC Argo}

The BGC-Argo network presently relies on research-based projects operated by individuals or institutions. Globally, these regional pilot programs operate as a collective resource of 335 floats carrying BGC sensors that can be accessed through the 


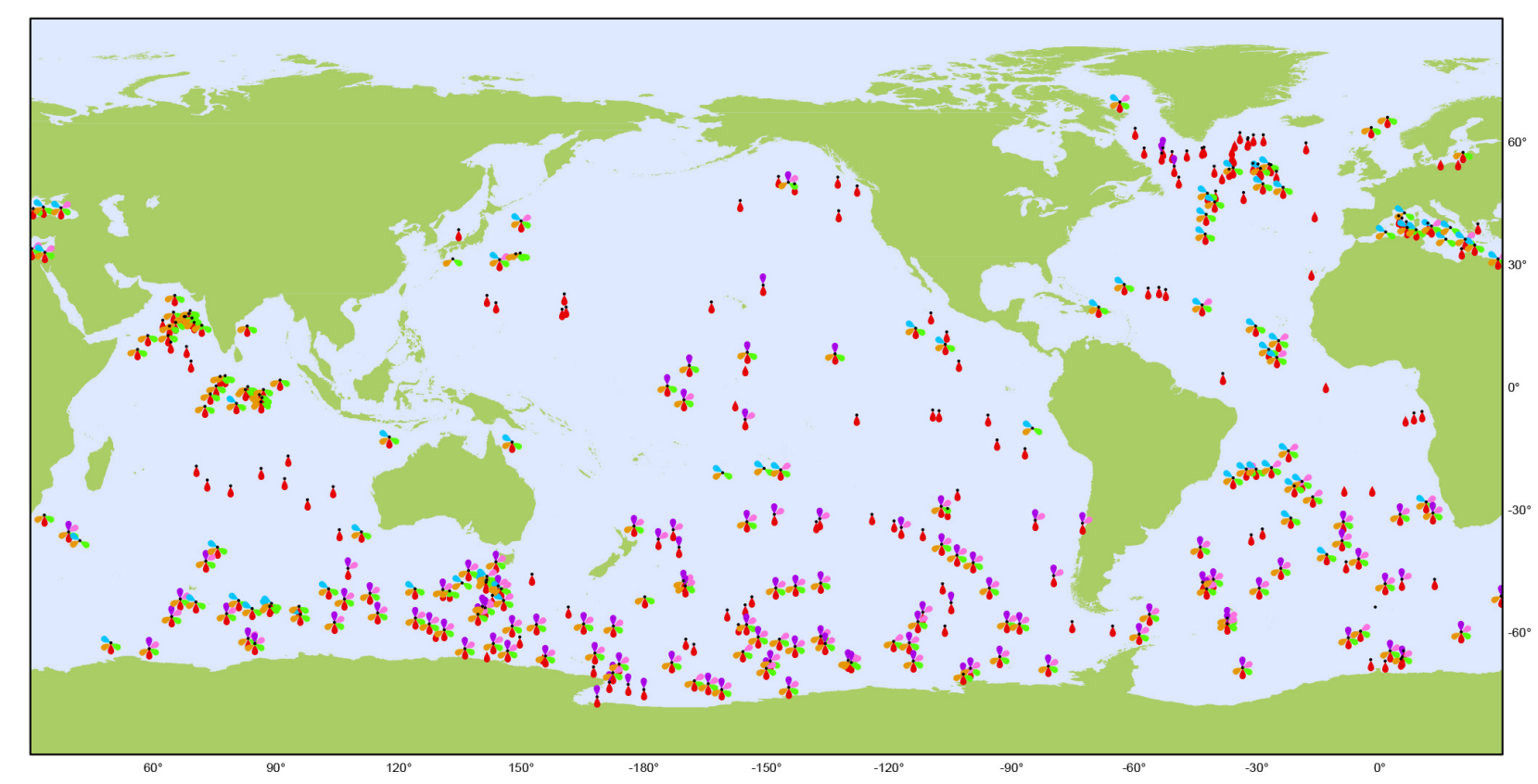

Biogeochemical Argo

Sensor Types

September 2018

Latest location of operational floats (data distributed within the last 30 days)

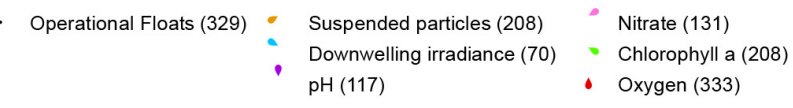

Generated by www.jcommops. org, 03/10/2018

FIGURE 6 | Global map of BGC-Argo floats, indicating sensor types on each float, as of September 2018 (Source: JCOMMOPS).

Argo data system (Figure 6). With a goal of 1000 floats equipped with the six core BGC sensors, system status ranges from nearly $30 \%$ complete for oxygen to only $8 \%$ complete for $\mathrm{pH}$ and irradiance (Figure 6). About 10\% of the floats in the BGC array carry 5 of the six core sensors, but few research programs have merged all six on one float. Moving from a 30\% complete oxygen array to a $30 \%$ complete array with all six sensors will be a critical step in BGC-Argo development, as key synergies arise when all six core sensors are deployed on a single float.

BGC-Argo is the first program with the capability to monitor biogeochemical processes globally, under seasonal seaice, throughout the year, and across all weather conditions. This allows BGC-Argo to access processes and timescales that cannot be observed from oceanographic cruises. It will revolutionize understanding of ocean biogeochemistry and marine resources, including fisheries, providing the foundation for informed ocean management. This strength results in particular from the costeffectiveness of such robotic measurements, which lower the cost of a vertical profile by more than an order of magnitude compared to classical techniques of data acquisition. The consistency of the data collected and processed in this manner enable global assessments based on the entire data set. In some cases, analyses of these consistent datasets (Poteau et al., 2017) have identified sensor calibration issues at the manufacturer level (Barnard and Koegle, 2018). The accessibility of BGC-Argo global datasets has allowed the measurements of Chla (Roesler et al., 2017) and optical backscatter (Poteau et al., 2017) to be reprocessed, producing an even more valuable product.

\section{Deep Argo}

Four models of Deep Argo floats have been developed and are currently in use. The Deep Arvor (Le Reste et al., 2016) and the Deep NINJA (Kobayashi, 2013) floats measure P/T/S, from the surface to $4000 \mathrm{dbar}$. The Deep SOLO and the Deep APEX record $\mathrm{P} / \mathrm{T} / \mathrm{S}$ to $6000 \mathrm{dbar}$ (Zilberman, 2017). Two CTD models developed by Sea-Bird, the $6000 \mathrm{dbar}$ SBE-61 CTD and the 4000 dbar SBE-41 CTD, were implemented on Deep Argo floats, and a 6000 dbar CTD from RBR is currently being tested. In addition, dissolved oxygen (DO) sensors have been mounted on most 4000 dbar floats (Figure 7). All Deep Argo floats have the ability to provide NRT P/T/S measurements during the float ascent to the surface. Deep Argo floats were designed originally to achieve 180 cycles to $6000 \mathrm{dbar}$; the migration to hybrid lithium batteries increases the Deep SOLO battery lifetime substantially above 200 cycles.

The trajectory uncertainty at parking depth, resulting from the vertical shear during the float ascent and descent, is higher for Deep Argo dives than for Core Argo. As for Core 


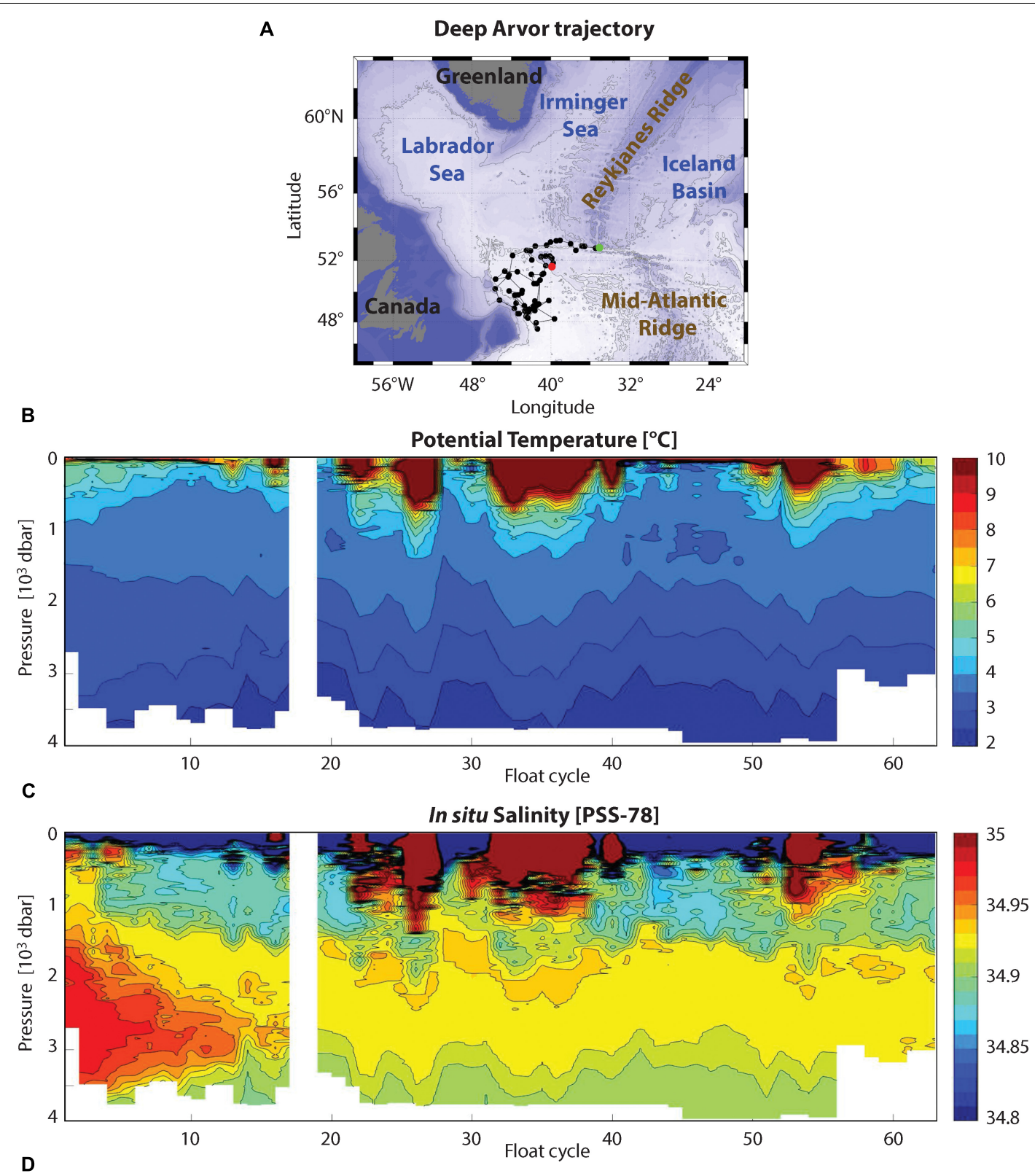

D

Dissolved Oxygen [ $\mu \mathrm{mol} \mathbf{k g}-1]$

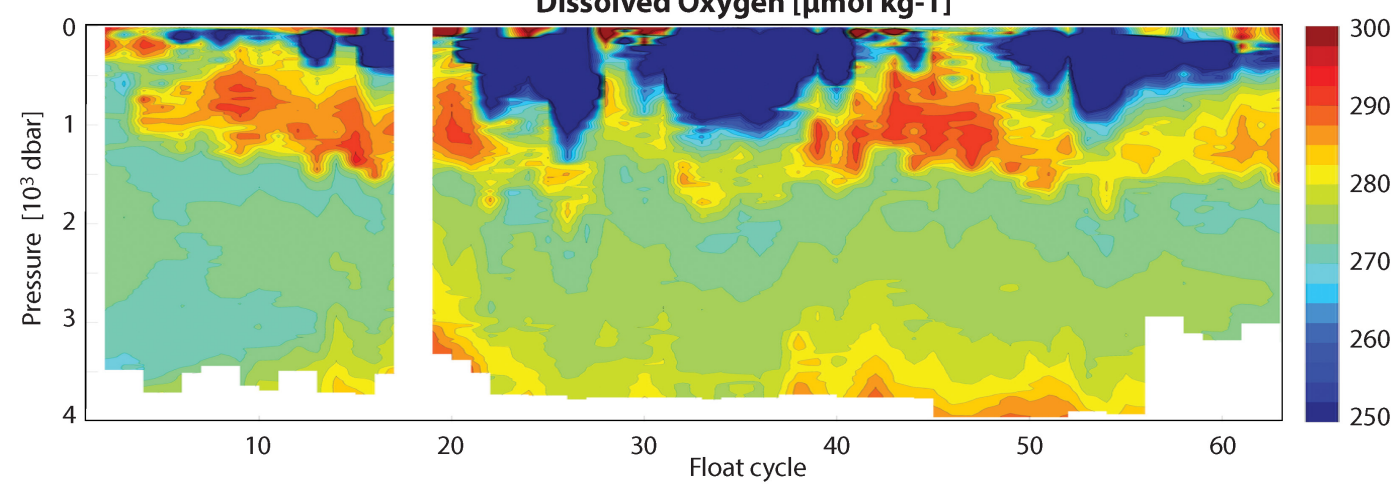

FIGURE 7 | (A) Trajectory of a Deep Arvor float (WMO ID 6901758) deployed in the North Atlantic Basin between July 2015 and March 2017. Locations of float deployment (rounded green symbol) and last observation (rounded red symbol) are indicated. (B) Potential temperature, (C) In situ salinity, and (D) Dissolved oxygen (DO) measurements from the Deep Arvor float between July 2015 and March 2017. 
Argo, the position uncertainty in Deep Argo float trajectories is highest in sea-ice covered areas, where estimated float positions are interpolated based on ice-free surface fixes that can be months apart.

Current challenges are to improve the accuracy and increase the long-term stability of the Deep Argo conductivity and pressure sensors. Target accuracies of Deep Argo P/T/S are \pm 3 dbar, $0.001^{\circ} \mathrm{C}$, and 0.002 PSS-78, similar to GO-SHIP standards (Hood et al., 2010). Comparisons of shipboard rosette-mounted SBE-61 CTDs with shipboard (SBE-911) CTD observations, show $\mathrm{P} / \mathrm{T} / \mathrm{S}$ uncertainties of $\pm 4.5 \mathrm{dbar}, \pm 0.001^{\circ} \mathrm{C}$, and \pm 0.005 PSS-78. Observations from SBE-61 CTDs mounted on Deep SOLO floats show a similar spread in salinity when compared with reference data (Figure 8). Efforts will continue to improve the performance of the Deep Argo CTD models to the accuracy levels envisioned by the Deep Argo community. Float recovery can be used to achieve CTD recalibration in regions with limited reference data.

Regional Deep Argo pilot arrays have been established in deep areas of the South Pacific, South Indian, and North Atlantic Ocean, with plans underway for deployments in the South Atlantic, to demonstrate the technology readiness and scientific potential of the Deep Argo Program. There were 69 Deep Argo floats active as of September 2018 (Figure 9).

\section{Argo Data Management}

The real time Argo data stream is used extensively by operational users who need the best quality data within $24 \mathrm{~h}$ of profile completion. To this end, Argo has transitioned from sending only TESAC ${ }^{4}$ ASCII messages to BUFR $^{5}$ onto the GTS which

\footnotetext{
${ }^{4}$ TEmperature, Salinity, and Current.

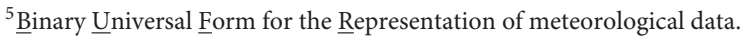

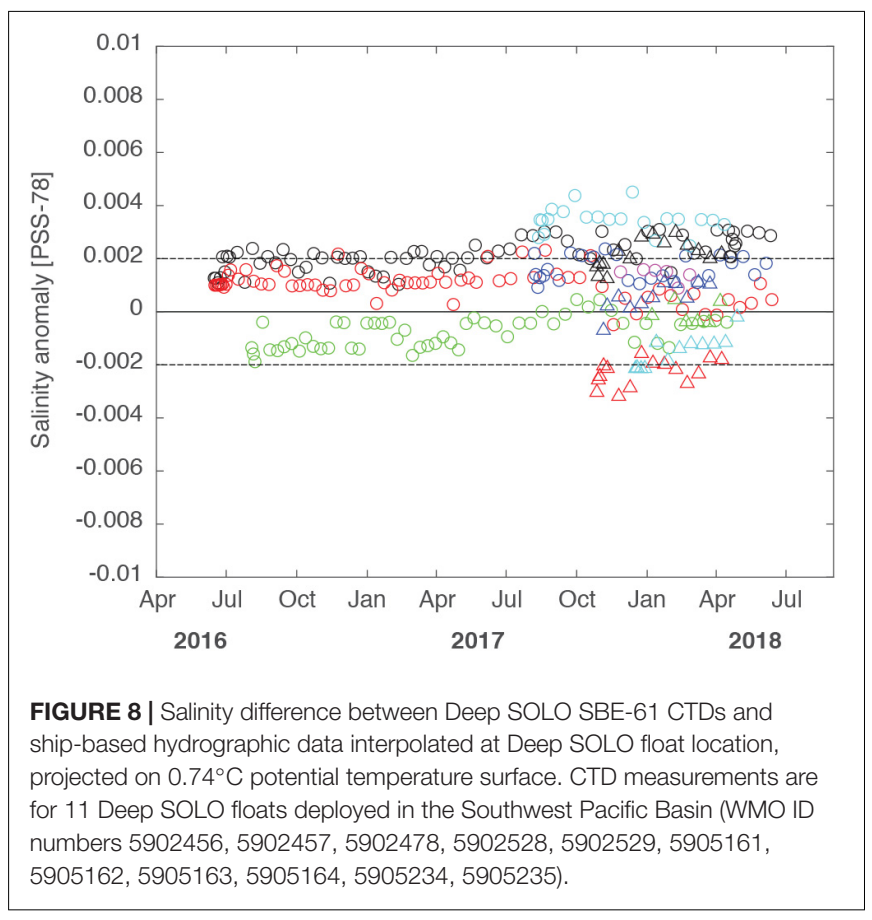

allows the inclusion of quality flags and additional profiles such as near-surface temperature and salinity as well as oxygen, and soon, other biogeochemical data. In 2017, Argo DACs delivered a monthly average of 12,328 TESAC messages, $93 \%$ of them within $24 \mathrm{~h}$. The NRT quality control tests have remained the same for many years and with the majority of floats being deployed with two-way communications and returning high resolution data, it is timely to re-consider these tests. Specifically, Argo is exploring a test against gradient climatologies for better detection of anomalous profiles and better spike removal. In addition, over the past several years, RTQC tests for BGC parameters have been developed, some being more mature than others. All BGC RTQC tests are documented ${ }^{6}$ and the documentation is updated as tests mature and additional sensors are deployed.

The Argo data system underwent a major format change when all Argo file formats were transitioned to version 3 and higher. This format change was needed to manage mission changes due to two way communications via Iridium, to better accommodate non-core Argo profiles, to cope with different pressure sampling schemes for some parameters and to store more float metadata. It has been a multi-year process to convert legacy Argo files to the version 3 formats, but the majority of files have been updated. The additional meta data facilitates tracking the health of the array and identifying floats with potentially bad sensors. A large effort was put into homogenizing the meta data and technical files to simplify them and to make cross float model comparisons easier. The ability to store multiple profiles within one cycle has allowed the addition of BGC profiles and other nonCore Argo parameters. To minimize the impact of this change on Core Argo users, the Core Argo profile files kept their name and basic structure and do not contain any BGC parameters. There may be additional profiles in a Core Argo profile file, such as shallow near-surface CTD profiles. B-files were added to the Argo data stream containing the BGC parameters and all the intermediate variables measured and used to convert to the ocean state BGC parameters. Finally, to accommodate users whose research requires all parameters in one file, $\mathrm{M}$-files are generated by the GDACs and contain both Core Argo and BGC profiles.

The transition into version 3 formats also allowed the development of GDAC File Checkers which check not only the format, but also the content of some variables. This has greatly increased the consistency and usability of Argo data. With all these changes, the GDACs today host over 2 million files comprising over $245 \mathrm{~GB}$.

Argo DMQC of Core Argo variables occurs about 12 months after observation. The Owens and Wong (2009) method is the Argo standard, with local modifications depending on profile location. An ongoing challenge is ensuring that Argo DMQC is performed consistently across the program, with the best possible quality reference database for comparison. To this end, Argo is beginning to study the Argo DM dataset in a more robust manner. The North Atlantic ARC has analyzed a variety of floats from different DMQC operators in the North Atlantic to see if DMQC software is being applied properly and consistently (Cabanes et al., 2016). DMQC workshops continue to be held as

\footnotetext{
${ }^{6}$ http://dx.doi.org/10.13155/40879
} 


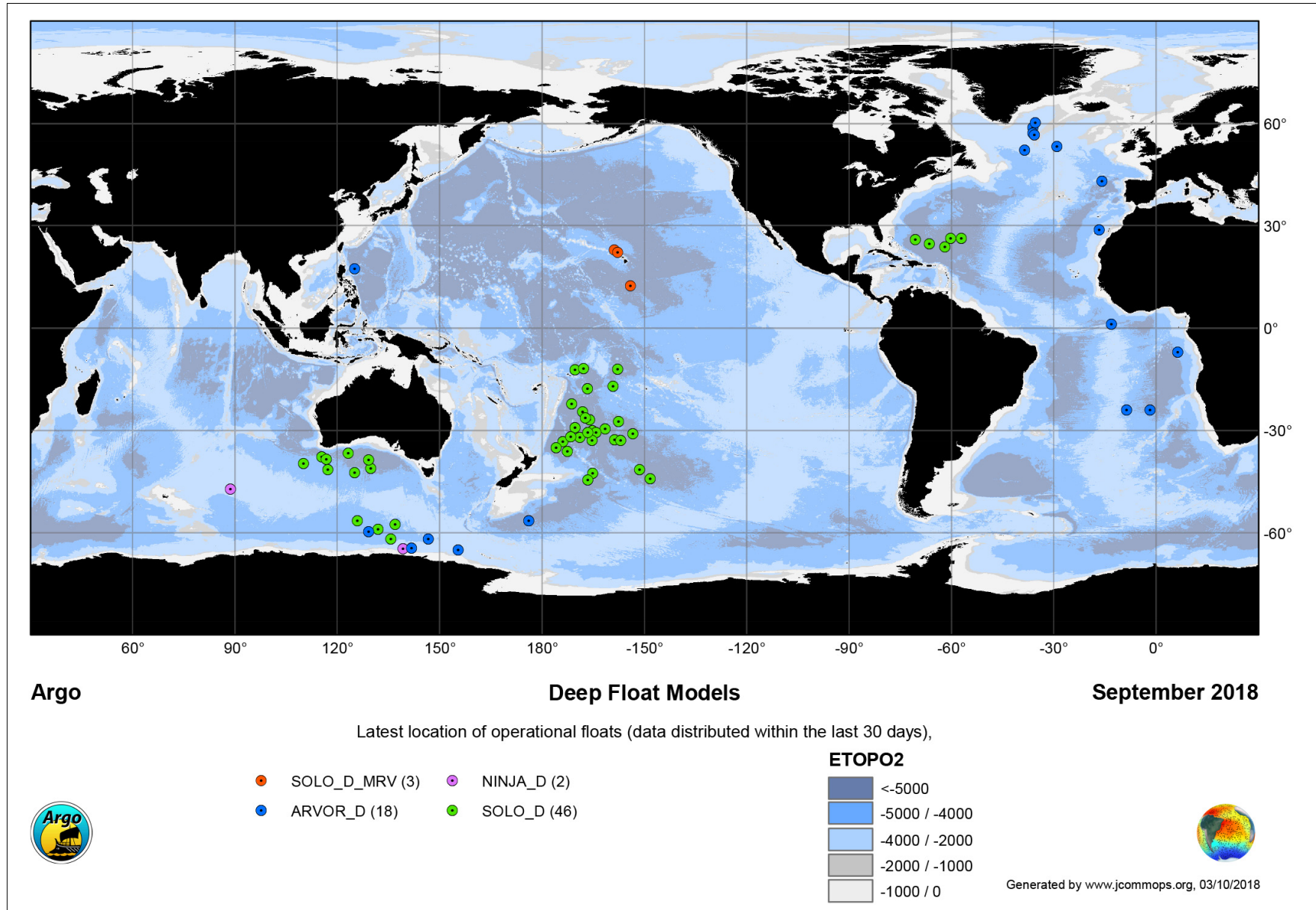

FIGURE 9 | Map of 69 operational Deep Argo floats in September 2018 (rounded symbols), including 46 Deep SOLOs, 3 MRV Deep SOLOs, 18 Deep Arvors, and 2 Deep NINJA. The underlying color indicates bottom depth shallower than 1000 m (white), depth between 1000-2000 m (light gray), 2000-4000 m (light blue), 4000-5000 m (dark blue), and greater than 5000 m (dark gray) (Source: JCOMMOPS).

needed to study the current status of DMQC data and to train new DMQC operators. To help with this, Argo is beginning to share source code more formally through Github, and with a Matlab version of the OW method that is publically available via https://github.com/ArgoDMQC/matlabow/.

The AIC has upgraded to an interactive dashboard interface that allows users to perform searches on different float samples, make plots, and display statistics of the float samples. This helps Argo PIs, users, and the public track Argo floats and monitor the health of the array on a real time basis.

Ensuring a timely and citable Argo dataset is a high priority. Over the past several years, the Argo GDACs have served the Argo data in a variety of ways. To facilitate research reproducibility, monthly snapshots of the GDACs are captured and are assigned digital object identifiers (DOIs) ${ }^{7}$. Through collaboration with the Research Data Alliance, Argo has moved ahead with issuing a DOI for its dataset with a monthly $\operatorname{tag}^{8}$

${ }^{7}$ http://doi.org/10.17882/42182

${ }^{8} \mathrm{http}: / /$ www.argodatamgt.org/Access-to-data/Argo-DOI-Digital-ObjectIdentifier that can be added to the end of the DOI string to indicate from which month Argo data was used in scientific papers. As work in this field progresses, if DOIs are further adapted to data sets that change over time, Argo will follow the recommended changes to adhere to the current DOI framework.

The Argo Data Management System has been acknowledged as a model for other observing systems and widely copied. Nevertheless, there are substantial challenges for sustaining this success. With additional Argo sensors comes increasing complexity, and the extra burden on human resources in the data management system is considerable to maintain consistent meta and technical data for all sensors and float types. Even the P/T/S elements of the Argo dataset increase in complexity as multiple vertical axes are accommodated, and new requirements for meta and technical data are implemented. Another strain on the human resources comes from producing high quality, consistent DM files in a timely manner for increasingly complex profile and trajectory files. Finally, integration of Argo and other elements of GOOS generates a need for improvement of interoperability across the GOOS data systems. 


\section{ArgoMix}

The quantification of fluxes through turbulence measurements and models based on turbulence theory (Osborn and Cox, 1972; Osborn, 1980) has developed over the past several decades. Robust testing constrained by physics (Figure 3 and tracer release experiments; e.g., Ledwell et al., 1993) demonstrates a mature technology. Mixing measurements consist of temperature microstructure measured from thermistors and/or shear measured from piezoelectric airfoil probes, measuring thermal and kinetic energy dissipation rates, $\chi$ and $\varepsilon$, respectively. Both types of measurements have been documented on profiling floats (Sherman and Davis, 1995; Nagai et al., 2015; Goto et al., 2016, 2018; Lucas et al., 2016; Shroyer et al., 2016) and also conducted but not published by groups at APL/UW and Scripps. These successful demonstrations show the possibilities of a more complete integration.

Shear probes and thermistors deployed on Argo floats would need to survive long immersion times ( $>5$ years) and 100 s of pressure cycles. Sensors on microstructure gliders (St. Laurent, Personal Communication) have lasted several 100 profiles over several weeks; it is not known whether reported failures originated from cycling or immersion. While we are not aware of any fundamental issues with fully-potted sensors remaining sensitive and waterproof for 100s of cycles over 5 years, their endurance and stability under Argo conditions will need to be demonstrated.

Typical power consumption of modern turbulence packages is $\sim 0.05 \mathrm{~W}$, or about $1 \mathrm{~kJ}$ for a $2000 \mathrm{~m}$ profile ( 6 and $12 \%$ of the energy for APEX and SOLO profiles, respectively) and $3 \mathrm{~kJ}$ for a $6000-\mathrm{m}$ profile (11\% of the energy for a deep SOLO float). Using best practices for data compression and onboard processing (Becherer and Moum, 2017), only two variables $(\varepsilon$ and $\chi$ ) need be telemetered, at similar data rates to Argo's normal T and S.

\section{PLANS FOR EVOLUTION, GROWTH, AND TECHNICAL ADVANCES}

\section{Core Argo}

Several enhancements to Argo's original sampling design are being piloted, refined and recommended for implementation. Besides the major expansions into the deep ocean and biogeochemical parameters, the changes to the Core Argo array design involve either spatial expansion or regional increases in float density. The combined plan for spatial enhancements, along with Deep and BGC Argo, is termed the Argo2020 design, which will transform Argo into a truly global array with improved resolution in critical regions.

\section{Seasonal Ice Zones}

Due to a dearth of historical data (particularly in winter) and the fast rates of change in the polar oceans and cryosphere, there is strong demand to extend Argo into the seasonal ice zones (Lee et al., 2019; Newman et al., 2019). Pilots have demonstrated good float reliability in the sea-ice zone with a remaining challenge being accurately estimating location for under-ice profiles. Experience with ice-avoidance in the Arctic is less mature than for the Antarctic, but pilots are underway. Access to remote ice-covered oceans for float deployment remains a logistical challenge that will require a dedicated effort.

\section{Marginal Seas}

Operating a float array in enclosed seas has been shown to be feasible, especially for Iridium-equipped floats, with successful arrays in the Mediterranean and Asian marginal seas. Many of these floats carry biogeochemical sensors (Figure 6). Only a modest number of floats are required to cover all marginal seas at double Argo density (Table 1). However, implementation requires active and strong logistical and political support from coastal states.

\section{Tropical Enhancements}

Tropical Oceans have a large influence on global climate variability and weather. The increasing challenges of maintaining large-scale tropical moored arrays (TMAs), combined with Argo's efficacy in measuring salinity and resolving narrow tropical thermoclines, have resulted in a recommendation to double the density of Argo sampling in the tropical Pacific. A similar increase is under consideration for the remaining basins (Foltz et al., 2019; Hermes et al., 2019; Smith et al., 2019).

\section{Western Boundary Current Enhancements}

With high levels of mesoscale variability, enhanced Argo sampling in WBC regions is needed to reduce noise in tracking the largescale temperature and salinity fields. A tentative design is to double the float density in regions of high eddy variability around western boundary currents.

The implementation of all of the above design changes would result in a 4600 float global array (Figure 10 and Table 1), roughly a $15 \%$ increase over present active float numbers, but also requiring a substantial redistribution of floats. Some of this might be achieved via efficiency, but full implementation will require additional resources and/or partners. Of the 4600 floats in the Argo2020 design, 1000 will be BGC-Argo (see section "BGC Argo" under the section "Plans for Evolution, Growth, and Technical Advances") and 1250 will be Deep Argo floats (see section "Deep Argo" under the section "Plans for Evolution, Growth, and Technical Advances").

Ongoing progress in extending float lifetimes (see section "Core Argo" under the section "The present status, problems, and strengths") will remain essential to Argo's future. Argo's ability to meet requirements for finer $(\sim 10 \mathrm{~cm})$ near-surface resolution is being piloted and may be extended across the Argo array. Longer term developments might also include using surface currents and/or a glide mode to keep floats apart, driving up the array sampling efficiency.

\section{BGC Argo}

The immediate goal for BGC-Argo is to obtain national commitments to the long-term implementation of BGC-Argo (contribution to the array and its data system) so that the 1000 -float array can begin full-scale deployment within the next 5 years. Successfully achieving long-term sustainability relies on clear long-term commitments from national agencies. Recently 


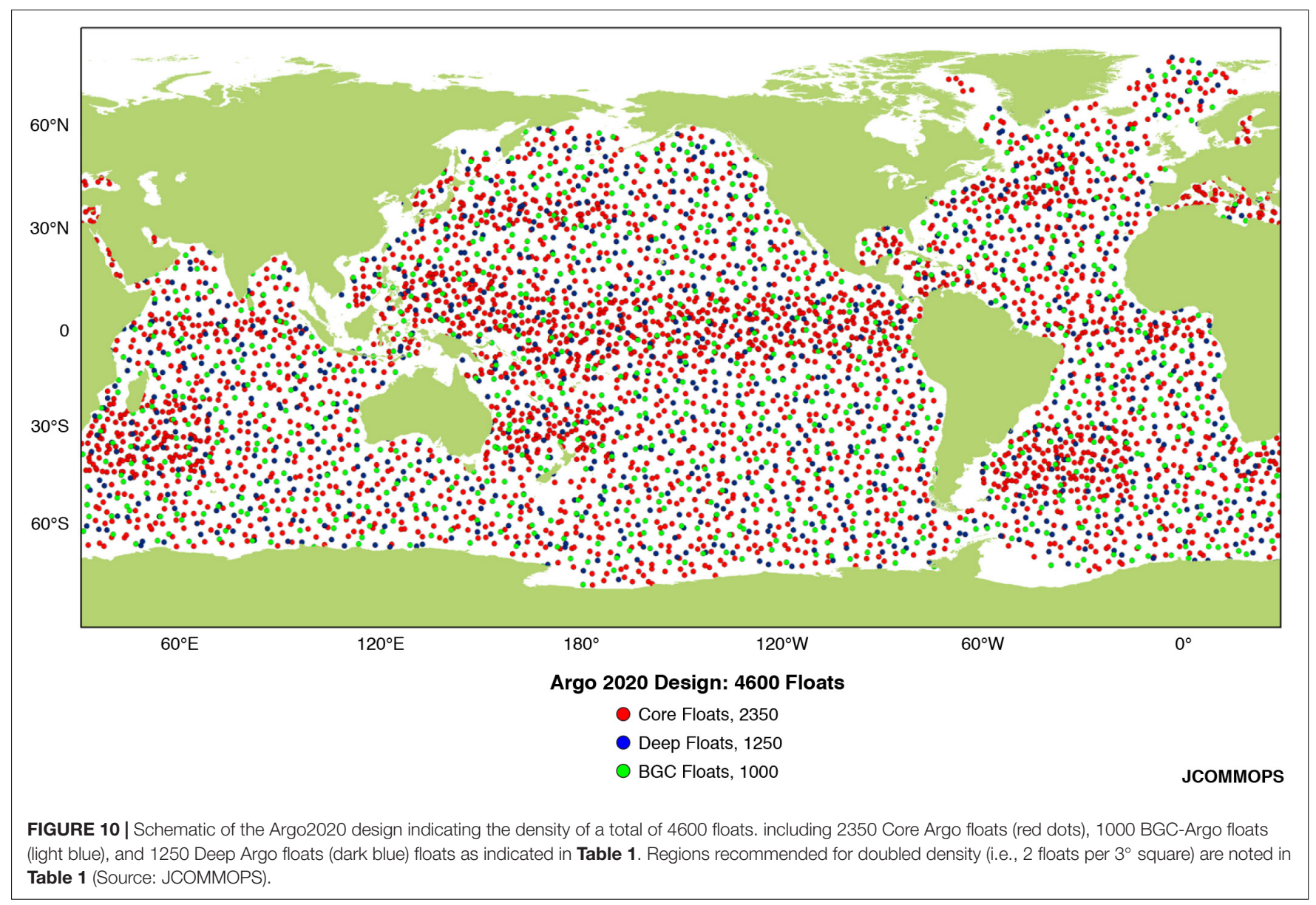

TABLE 1 | Summary of the Argo2020 design, including the required number of active floats and the present status of elements.

\begin{tabular}{lrl}
\hline $\begin{array}{l}\text { Design element 2x indicates } \\
\text { doubled density (i.e., 2 floats } \\
\text { per } \mathbf{3}^{\circ} \text { square) }\end{array}$ & $\begin{array}{r}\text { Active } \\
\text { floats }\end{array}$ & Status \\
\hline $\begin{array}{l}\text { Global - Original } \\
\text { Global - Antarctic }\end{array}$ & 3000 & $\begin{array}{l}\text { Implemented } \\
\text { Pilot completed; } \\
\text { implementation not resourced } \\
\text { Global - Arctic }\end{array}$ \\
$\begin{array}{l}\text { Global - Marginal Seas (2x) } \\
\text { Global - Total }\end{array}$ & 220 & $\begin{array}{l}\text { Implet underway } \\
\text { GOOS alliances are active }\end{array}$ \\
$\begin{array}{l}\text { Tropical Enhancement(2x) } \\
\text { Western Boundary Current }\end{array}$ & 5600 & $\begin{array}{l}\text { W. Pacific implementation } \\
\text { prioritized, but not resourced }\end{array}$ \\
Enhancement (2x) & 460 & $\begin{array}{l}\text { Kuroshio pilot completed. Final } \\
\text { design still in development. }\end{array}$ \\
Argo2020 Design & 4600 & \\
\hline
\end{tabular}

The needed number of deployments per year is equal to the number of active floats divided by the mean float lifetime, presently about 4.2 years. The number of floats is inclusive of Core Argo. Deep Argo, and BGC Argo floats, forming a single integrated Argo Program.

announced contributors to BGC-Argo include the government of Canada and the European Research Infrastructure consortium ERIC Euro-Argo (Euro-Argo-Eric, 2017).
Beside high level commitments at national or supranational levels, a key step toward increased accessibility for developing nations that wish to contribute to BGC-Argo is to increase costeffectiveness (Biogeochemical-Argo Planning Group, 2016). The price of BGC Argo floats could be lowered by developing a competitive market for biogeochemical sensors once there is a clear vision of the market size, with a view to decreasing sensor costs. Additionally, where feasible, float retrievals, and subsequent refitting (for $\sim 20 \%$ of their initial price) is a way to reduce the array cost.

Finally, long-term sustainability of BGC-Argo is dependent on data usage and satisfaction of the end-users, who are essential advocates for the BGC-Argo system. The continuous development of a large and diverse end-user community has to be a priority, in particular with the development of specific data products responding to user requirements. It will rely on the establishment of an evolving data management system that will combine (a) real-time data delivery for operational purposes, (b) delayed-mode quality-controlled data delivery for science purposes, and (c) new products supporting enduser requirements.

While the present BGC-Argo program target is to fulfill its objective of six core variables measured by 1000 operational floats, possible new extensions are being tested. These include passive acoustic listener (PAL) not only for wind and rain 
(Riser et al., 2008; Yang et al., 2015) but also for anthropogenic noise and mammals, miniaturized imaging sensors for resolving particle and plankton (size range from $100 \mu \mathrm{m}$ to few $\mathrm{mm}$ ), and hyperspectral radiometry (phytoplankton functional types). There are promising efforts to develop additional new chemical sensors, such as alkalinity (Briggs et al., 2017) and to adapt existing chemical sensors such as $\mathrm{pCO}_{2}$ (Fiedler et al., 2013).

\section{Deep Argo}

Plans for the next 10 years are to transition from the pilot phase to completed global implementation of the Deep Argo Program, including about 1250 Deep Argo floats at $5^{\circ} \times 5^{\circ}$ spacing (Johnson et al., 2015). Elements of the Deep Argo deployment strategy are as follows. Existing pilot arrays should be maintained to demonstrate the scientific value of Deep Argo, and to refine the design of the Deep Argo array as well as of its floats and data management system. The deployments of 4000 and 6000 dbar Deep Argo floats will be coordinated to achieve areal coverage of top-to-bottom $\mathrm{P} / \mathrm{T} / \mathrm{S}$ profiles. New pilot arrays will be implemented in regions believed to have substantial decadal changes, seasonal cycles, or other variability, and that are close to deep-water and bottom-water formation sites. The choices of globally-uniform or basin-specific parking depth, and of preferred cycle time are still under discussion. As occurred with Core Argo, the global implementation of an evenly distributed Deep Argo float array will follow from the expansion of the regional pilots.

Deep Argo's scientific objectives are to close the Earth's budgets of heat and freshwater, to assess the steric contribution to sea level rise, to improve our understanding of full-depth ocean circulation and water-mass transformation in the ocean interior, and to reduce uncertainties in coupled atmosphereocean models, reanalyses, and predictions. The deployment of new sensors for BGC or mixing parameters could enhance the value of Deep Argo, expand the scope of the Deep Argo dataset, and promote the integration of Deep Argo with other observing system elements. Power usage of additional sensors should be compatible with Deep Argo array refresh-times of 5 years or longer. Sensor accuracy should be sufficient to resolve interannual to decadal climate signals. Enhanced hydrographic sampling of the bottom boundary layer is recommended for studies of deep-ocean dynamics, including dense overflows, energy dissipation near topography, and geothermal heating (de Lavergne et al., 2016). Deep Arvor floats equipped with altimeter and bottom-looking ADCP, will be tested in the Weddell Sea as part of the Water Mass Transformation and Pathways in the Weddell Sea (WAPITI) project, to draw deep $\mathrm{P} / \mathrm{T} / \mathrm{S}$ and oxygen measurements closer to the topography, and to improve velocity estimates near the bottom. Deep SOLO floats already sample to within a few meters of the bottom, closer than GO-SHIP cruises, which stop at about $10 \mathrm{~m}$ off the bottom.

\section{Argo Data Management}

Argo encourages technological developments in floats and sensors, but the introduction of new floats and sensors places a burden on the Argo data stream. Therefore, the Argo Steering
Team (AST) and the ADMT have developed a framework for the definition of an Argo float, and have described a pathway for new sensors to become approved Argo sensors. Moving forward, there will be three categories of sensors: (1) accepted sensors that meet Argo's accuracy and performance standards, (2) sensors approved for pilot arrays by the AST and ADMT, and (3) experimental sensors on floats equipped with an approved Argo CTD where the experimental sensor data will be available in the Argo auxiliary data stream.

If pilot sensors are shown to meet Argo's accuracy and stability targets, the AST will accept these into the Argo data stream. This applies to Core Argo 2000 dbar CTDs, Deep Argo CTDs, and BGC-Argo sensors, and other prioritized parameters, and will involve coordination of the AST, ADMT, Deep Argo, BGC-Argo, and sensor manufacturers. The framework for incorporation into the Argo database explains what is required of float providers and articulates guidelines for the collection and distribution of all data collected by Argo floats. In summary, float providers must follow Argo governance rules for mandatory pre-deployment notification through the AIC and agree to timely data delivery of both NRT and DM data. In addition, float providers must have a plan for long term data ownership with a national Argo DAC. Floats that are to become part of the Core Argo Program should target the Core Argo profiling depth of 2000 dbar and the cycle time of 10 days.

Argo will also continue to develop more sophisticated code sharing among Argo DACs, PIs, and manufacturers to reduce the burden on DACs and PIs each time modifications are made to floats or sensors and to improve consistency across the Argo program. Code sharing may also help with turnover as scientists and DMQC operators within Argo retire and take some of their knowledge with them. Continuing to find interested and talented Argo data team members and train them appropriately in a timely manner will remain a challenge for the ADMT.

Quality control of Argo data involves a complex sequence of both automatic and manual tests to produce data of high scientific quality. With 400 profiles arriving daily, the burden on human resources dedicated to QC is large and Argo is considering whether machine learning can help ease the burden and improve the QC process.

Work still needs to be done on several BGC-Argo parameters to modify and develop DMQC procedures. In addition, the BGC Argo data community has developed BGC Argo products that colocate all parameters on the same pressure levels and that perform additional QC tests.

As Deep Argo continues to grow, there will be a need to extend the Core Argo DMQC processes to data deeper than $2000 \mathrm{dbar}$ and to understand how the CTDs, both the SBE-41 and the specially designed for Deep Argo SBE-61, behave over multi-year lifetimes.

Besides profile data, Argo also provides trajectory data which can be used to calculate the velocity of the ocean at the drift depth of 1000 dbar. To improve the accuracy of these velocity calculations, newer floats send back more timing information to populate additional timing variables in the new version 3 file format for trajectory files. Argo is developing a DM process for trajectory files. This will include correcting the $\mathrm{P} / \mathrm{T} / \mathrm{S}$ 
measurements made along the float's trajectory, and performing quality control on the float positions and on the timing variables.

\section{ArgoMix}

Some engineering challenges remain in designing and implementing the best (most reliable, lowest power, and least expensive) float-deployed microstructure system. The next step, already underway, is for turbulence and float groups to work together to develop design criteria and test procedures that will lead to experimental deployments of $\mathrm{O}(2-5)$ floats, carrying temperature and shear microstructure sensors and having Argo-like missions. Once success is demonstrated in experimental deployments, and data management is harmonized with Argo, a plan for regional pilot deployment of $\mathrm{O}(30-100)$ floats, to allow patterns of mixing to be mapped on regional scales, would be presented to the Argo Steering Team for consideration. In parallel, several next-generation sensors are being pursued such as pitot tubes, faster thermistors, and refractometers for resolution of salinity microstructure. In time, we expect the hardware to be simplified, and the endurance of the probes demonstrated, to the point that these measurements can be efficiently incorporated as an integral part of the global Argo Program.

\section{END USER ENGAGEMENT}

\section{Core Argo}

The Argo Program began with a strong "user pull" that informed decisions relating to the original Argo design (Argo Steering Team, 1998). Key beneficiaries of the Argo Program, along with the scientific community, include climate sensitive industries (e.g., agriculture, aquaculture, tourism, and fisheries), and industries with a significant maritime presence (e.g., oil and gas, shipping, and emergency response). In most cases, the Argo Community's end user engagement is maintained indirectly either through the forecasting community, or through the scientific community.

The forecasting community (e.g., CLIVAR GSOP, GODAE OceanView) has impact through the provision of forecasts that are underpinned by GOOS - primarily through Argo and satellite observations. Such forecasts depend on the routine delivery of NRT Argo data. Public availability of NRT data has remained a core principle of the Argo community since its beginning. Forecasts are regularly exploited by the Blue Economy (Willis, 2015; Wenhai et al., 2019) to inform tactical and strategic decision-making. Adding value to seasonal forecasts to inform the agricultural industry has proved beneficial (e.g., Hochman et al., 2009). For example, inclusion of SST forecasts in crop models improves farmers' ability to manage stocks and crops more efficiently. Short-range forecasts are often used to support emergency response to oil spills (e.g., Deepwater Horizon; Mariano et al., 2011), marine pollution (Potemra, 2012; Maximenko et al., 2019), and search and rescue (e.g., search for MH370; Griffin et al., 2017). The impact of Argo observations on forecast systems is well-documented (Davidson et al., 2019), and the complementary nature of information available from Argo observations and satellite observations (Ponte et al., 2019; Vinogradova et al., 2019), has been demonstrated many times (e.g., Oke and Schiller, 2007; Lea et al., 2014). An example of the impact of Argo data on FOAM - the $1 / 4^{\circ}$-resolution operational ocean forecast system run by the UKMet Office - is presented in Figure 11, showing the change of temperature at $110 \mathrm{~m}$ depth in the FOAM system after Argo data are withheld for just 1 month (Lea et al., 2014).

The delayed-mode Argo dataset underpins many efforts in global ocean reanalysis on climate-scales (e.g., de Boisséson et al., 2018) and eddy-scales (e.g., Oke et al., 2013), providing products that are the foundation for much scientific research. In return, the ocean reanalysis community, coordinated by the Global Ocean Data Assimilation Experiment (GODAE) OceanView Observing System Evaluation Task Team (Fujii et al., 2019) and the CLIVAR Global Synthesis and Observation Panel (GSOP), regularly articulate the ongoing need and continued benefit/impact the Core Argo program delivers to their stakeholders (e.g., Oke et al., 2009; Davidson et al., 2019; Fujii et al., 2019; Penny et al., 2019).

Impacts via the scientific community typically include the identification of potential opportunities and/or vulnerabilities that are "publicized" through popular media and/or the published scientific literature. Most of the relevant scientific studies are built on the publicly-available DM data - spanning the entire Argo mission (1999-present) - that have been subject to the highest standards of quality control (e.g., Wong et al., 2003; Kobayashi and Minato, 2005; Owens and Wong, 2009). The Argo dataset facilitates assessment of the variability and trends in ocean heat content (Figure 12, also see Wijffels et al., 2016), freshwater distribution, steric sea level rise, and intermediate depth ocean circulation. This fundamental monitoring program for the world climate facilitates understanding of climate variability and change with increased certainty and more precise attribution (e.g., Chen et al., 2017). Core Argo and BGC Argo also underpin the assessments of ecosystem health (Canonico et al., 2019) increasingly delivering metrics relevant to marine biodiversity and ocean acidification (e.g., Bender et al., 2016; Watanabe and Kawamiya, 2017; Cross et al., 2019).

A key Argo user community is Education, including K-12, undergraduate, and graduate levels. Gridded versions of the Argo dataset have been developed, as well as data viewers, to serve the needs of education, research, and government agency users having widely varying backgrounds and levels of technical expertise. Education applications of Argo have included teaching units tailored to curricula as well as "after school enrichment" web-based activities'. At the graduate level, about 300 Ph.D. theses have used Argo data. Despite substantial achievements in Education, there remains more to be done. All modern students should be aware of the Argo Program and able to explore the global ocean from their desktop.

It's important to the Argo Community that the Program remains relevant to the wider community and continues to deliver societal benefits. As new opportunities and vulnerabilities

\footnotetext{
${ }^{9} \mathrm{http} / / /$ www.argo.ucsd.edu/Educational_use.html
} 


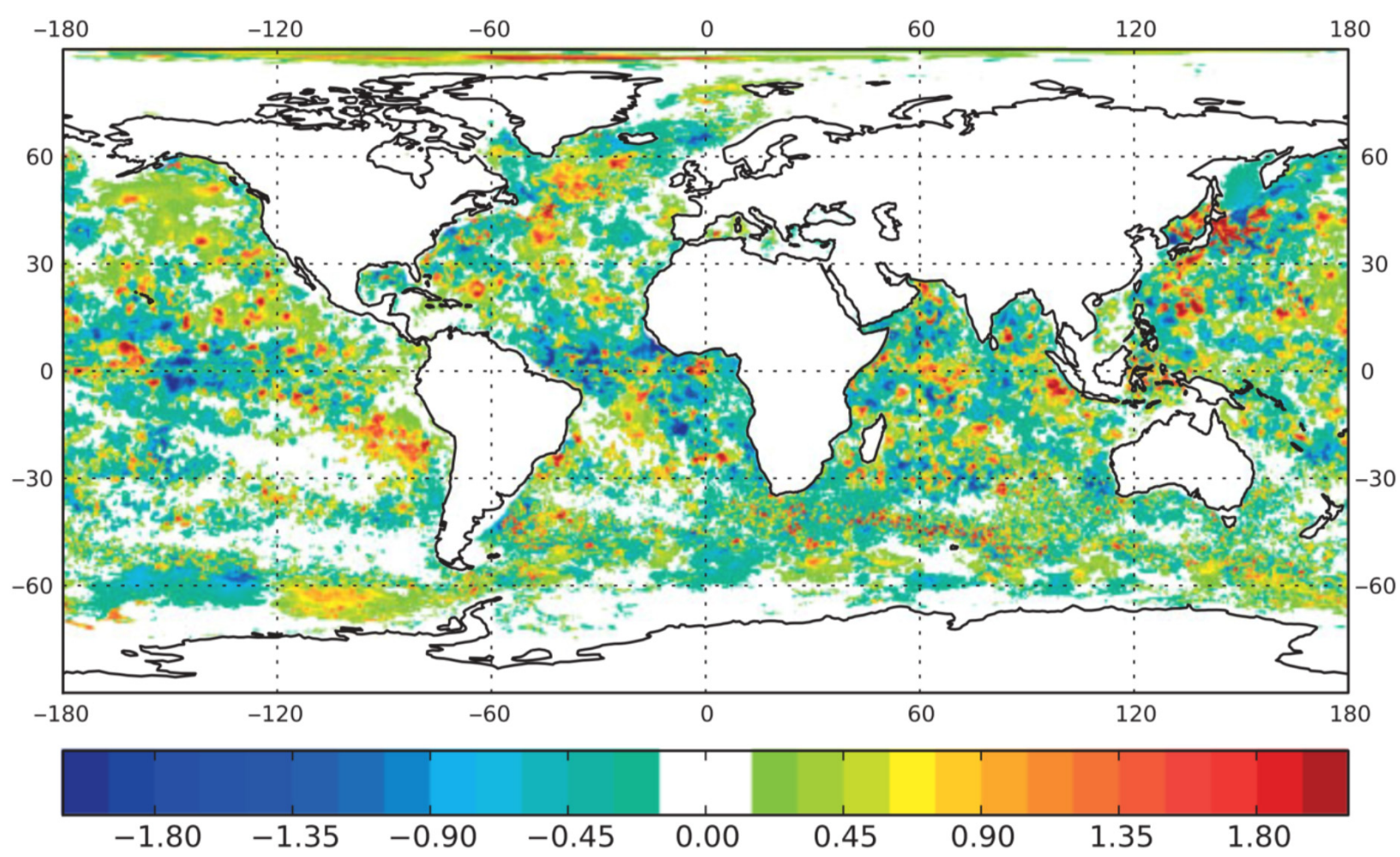

FIGURE 11 | Map of the temperature difference (operational minus 'no Argo'; ${ }^{\circ} \mathrm{C}$ ) at $110 \mathrm{~m}$ depth, calculated from daily average fields from the last day of an Argo Observing System Experiment (31 July, 2011) [Source: adapted from Lea et al. (2014), Copyright Royal Meteorological Society, printed with permission].

arise, and as requirements for ocean observing are better understood, the Argo design will continue to be refined to better meet ongoing societal needs (e.g., Jayne et al., 2017).

\section{BGC Argo}

Already supporting a growing community of scientific endusers, BGC-Argo has enormous potential to contribute to societal engagement through a wider group of stakeholders. In particular, these include end-users of ocean observation and modeling products and services that utilize BGC-Argo data. The BGCArgo science and implementation plan has notably identified two topics of societal relevance in the context of "climate variability and change" and "ocean ecosystem health."

With regard to the climate variability and change, better constraints on ocean carbon fluxes will reduce the present residual in global carbon budget estimates (Le Quéré et al., 2018) and improve quantification of air-sea $\mathrm{CO}_{2}$ fluxes (Gray et al., 2018). BGC-Argo floats measuring $\mathrm{O}_{2}$, salinity (to enable estimation of alkalinity) and $\mathrm{pH}$, have the potential to provide complete annual cycles of $\mathrm{pCO}_{2}$ throughout the ocean (Williams et al., 2017). Direct measurement of $\mathrm{pH}$ and $\mathrm{O}_{2}$ would contribute information on ocean acidification as well as deoxygenation.

Furthermore, the BGC-Argo network will provide an important underpinning for the observation of changes occurring at the base of the food web, for the attribution of changes in higher trophic levels to environmental stressors, and for the development of predictive capabilities for marine living resources. The network would, for the first time, enable global monitoring of environmental conditions such as $\mathrm{pH}$ and $\mathrm{O}_{2}$ concentrations, which directly affect the physiology and fitness of marine fauna. In addition, it would enable improved estimates of primary productivity, which supports the entire marine food web, through direct observation of nitrate concentrations and plankton biomass.

Realizing the potential of BGC-Argo for societal benefits will require provision of easily accessible products (Figure 13) through partnerships with the operational oceanographic community such as GODAE. Several national service providers are actively pursuing the development of biogeochemical forecasts and re-analyses but remain severely data limited. Currently the primary NRT data source for assimilation into numerical models is ocean color from satellites. BGC-Argo will fundamentally advance biogeochemical data assimilation by extending observations below the surface and significantly expanding the suite of observable parameters.

\section{Deep Argo}

Deep Argo will substantially reduce the uncertainty in the rate of deep ocean warming, helping to constrain future projections of ocean heat uptake and sea level rise. Sources of uncertainties in projections of the ocean heat uptake include changes in the deep-water and bottom-water mass formation rates, and in the strength of the deep meridional overturning circulation (Masuda et al., 2010; Marshall and Zanna, 2014). GODAE, and 


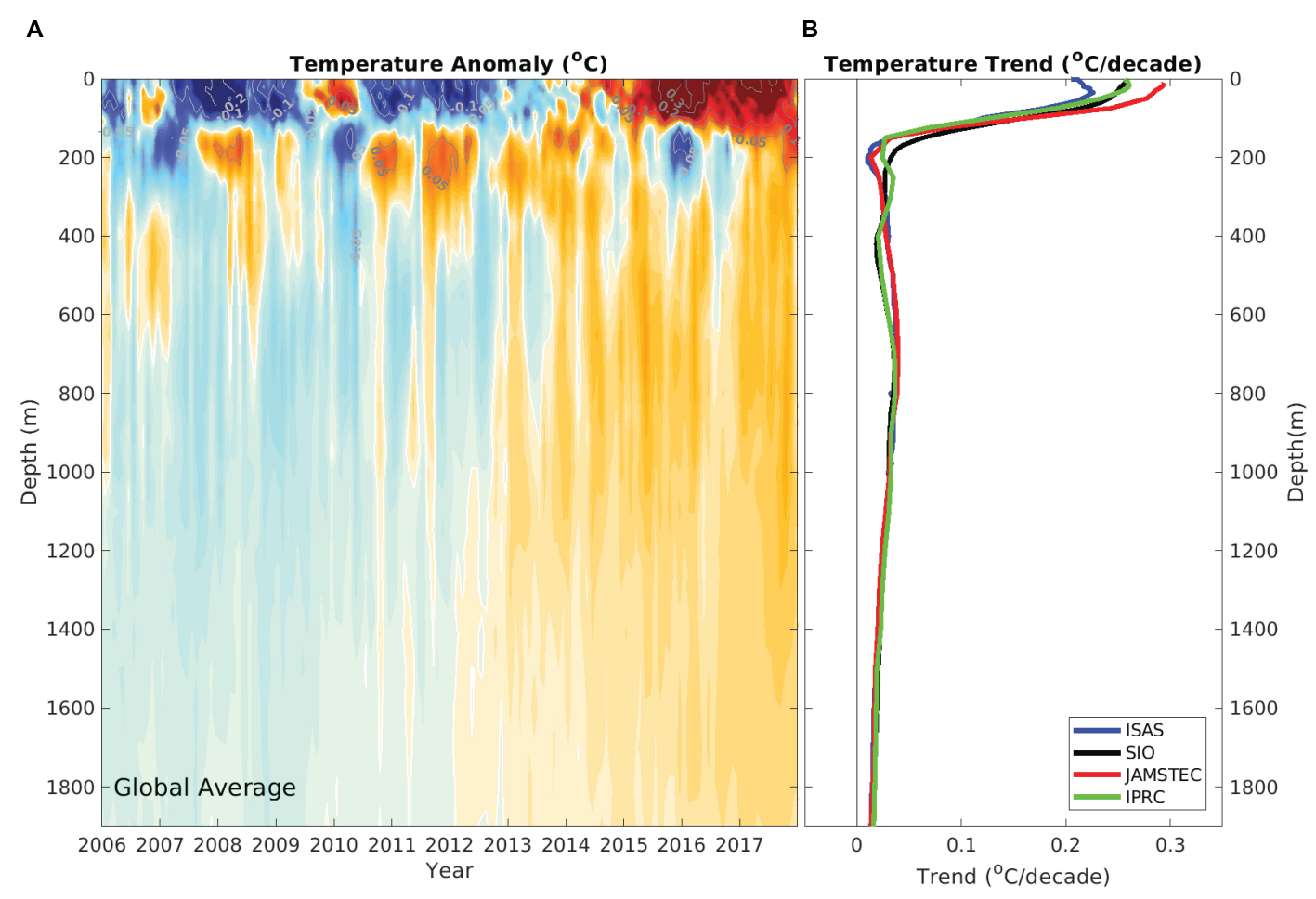

FIGURE 12 | (A) Global averaged temperature anomaly $\left({ }^{\circ} \mathrm{C}\right.$ - from SIO) and (B) trend $\left({ }^{\circ} \mathrm{C} /\right.$ decade, 2006-2018) versus depth, based on gridded Argo data. Gridded product sources are: ISAS (Gaillard et al., 2016), SIO (Roemmich and Gilson, 2009); IPRC- from the Asia-Pacific Data Research Center (http://apdrc.soest.hawaii.edu/projects/Argo/data/gridded/On_standard_levels/index-1.html); JAMSTEC (Hosoda et al., 2008).

BGC-Argo Network
1000 profiling floats with
$\mathrm{T} \& \mathrm{~S}$, oxygen, nitrate,
chl fluorescence, backscatter,
irradiance and $\mathrm{pH}$


its follow-on extensions have identified the need for deep ocean observations in data assimilation systems, to improve long-term predictions of atmosphere and ocean conditions (Bell et al., 2015). Deep Argo will help fill this need. A series of OSSEs has been performed to assess the impact of Deep Argo observations for ocean analysis and forecasting within the AtlantOS project (Visbeck et al., 2015; Gasparin et al., 2018). Early results show that the assimilation of full-depth temperature and salinity profiles using sampling density similar to the anticipated Deep Argo design $\left(5^{\circ} \times 5^{\circ} \times 15\right.$-day $)$, would reduce the root mean square errors of deep-ocean temperature and salinity by $20-40 \%$.

\section{Argo Data Management}

Argo has developed an efficient data system that is used as a model for other operational observing systems, as it (1) serves both operational and research users, (2) works to standardize information in its files (scientific, meta, and technical data), (3) acts as a key player in data citation methodology in real time data systems (see section "Core Argo" under the section "End User Engagement"). These attributes illustrate the importance of identifying end user communities and developing data management systems that serve their needs. Argo did this from the outset, developing links with CLIVAR and GODAE beginning in 1998. Argo continues today to evolve the data management system to provide greater value to communities engaged in basic research, climate assessment, operational reanalysis and forecasting, and education.

\section{ArgoMix}

Numerical experimentation over the last decade has shown that key aspects of Earth's climate depend on accurate representation of turbulent mixing in the ocean. In fact, the field has matured to a point where without better observational constraints on turbulent mixing it will be difficult to make further progress in understanding processes as diverse as abyssal circulation (Melet et al., 2016; MacKinnon et al., 2017), ice ages (Ferrari et al., 2016), tropical precipitation (Jochum et al., 2013), the sea-surface temperature cycle (Moum et al., 2013) or the extent of the oxygen minimum zones (Brandt et al., 2010). ArgoMix would provide the observational backbone needed to implement trustworthy parameterizations into climate models and achieve a deeper understanding of the Earth System.

\section{INTEGRATION WITH OTHER OBSERVING SYSTEM ELEMENTS}

\section{Core Argo}

From the outset, and explicitly with Argo's naming, there has been an emphasis on the complementarity of the Argo float array and the Jason satellite altimeter missions (Argo Steering Team, 1998). Argo captures the subsurface temperature, salinity and thus density structure, with sufficient coverage and resolution to constrain the steric component of the altimetric signal, enabling interpretation of altimetric sea surface height variability. On the other hand, the satellite altimetry provides spatial/temporal context, such as in terms of mesoscale variability, to each Argo profile. Even though the Argo/altimetry complementarity is remarkable, it is only one part of the synergy between Argo and many other in situ and satellite observing system elements (Roemmich and The Argo Steering Team, 2009; Freeland et al., 2010; Riser et al., 2016; Wuldera et al., 2016).

Argo, as a broad scale array with temperature and salinity measurements to $2000 \mathrm{~m}$, has remarkable synergies with repeat hydrography (Sloyan et al., 2019) and high resolution XBT (HRX) transects (Goni et al., 2019). These transect-oriented data provide estimates of geostrophic velocity and transport, and hence heat and freshwater transport into enclosed regions or across basinspanning transects. Combining estimated transports with Argo assessments of the storage of heat and fresh water in the enclosed regions provide a powerful tool for analyzing ocean heat and freshwater budgets along with air-sea flux products (Meyssignac et al., 2019). Argo also provides spatial/temporal context for HRX lines, which capture the mesoscale and hence the eddy flux for boundary current/basin scale heat flux measurements, and for the repeat hydrography program, whose decadal repeat cycle lacks the seasonal-to-interannual temporal resolution supplied by Argo. Also, high-quality repeat hydrography is also essential for quality control of Argo data.

While an accurate sea level record by satellite altimetry is key to monitoring and understanding sea level change, Argo serves as an essential integrant of the comprehensive observing system for sea level and its contributing components (Ponte et al., 2019). Argo provides an estimate of the steric component, resolving the temperature and salinity contributions and their depth distribution. Combining satellite altimetry and Argo, along with satellite gravimetry capturing ocean mass change, provide the capability to close local and global sea level budgets. The tide gauge network provided by GLOSS and coordinated global shipboard CTD observations provided by GO-SHIP are also essential parts of the observing system for sea level, by ensuring the accuracy of satellite altimetry and Argo data, respectively. Furthermore, variability in the globally integrated ocean heat content monitored by Argo provides constraints and validations of the satellite estimates of Earth's top-of-the-atmosphere radiative energy imbalance (Johnson et al., 2016).

Sea surface temperature and sea surface salinity (SSS) are provided by satellite remote sensing globally, but requires in situ measurements for calibration purposes and interpretation (Vinogradova et al., 2019). Argo plays an essential role in these requirements along with other in situ observing elements such as a voluntary observing ship (VOS) network, surface drifters and moorings. Argo's capability in this domain is being greatly improved by extension of profiles to include the nearsurface layer.

Since Argo is the only global subsurface network, it occupies a special role in the integrated GOOS consisting of satellite and in situ networks. Argo's interdependency and mutual value-adding relationships with other elements of the systems have been comprehensively discussed in the context of many regional observing systems such as the Tropical Pacific Observing System (TPOS 2020) (deYoung et al., 2019; Foltz et al., 2019; 
Hermes et al., 2019; Lee et al., 2019; Newman et al., 2019; Palazov et al., 2019; Smith et al., 2019; Todd et al., 2019).

\section{BGC Argo}

The BGC-Argo network has significant synergy with other ocean observing programs. Its global, year-around presence will fill observational gaps for key biogeochemical variables. In the coming years it is essential to strengthen the relations and interactions with other components of the ocean observation system.

\section{Argo}

The interaction with the Core Argo Program and its extensions (deep, marginal seas, polar seas) is a natural interaction. BGC-Argo floats also provide Core Argo measurements that are seamlessly integrated into the Argo data system. This interaction allows a more cost-effective and efficient usage of a multidisciplinary array. Additional synergies related to science have already been described (e.g., physical - biogeochemical coupling, radiometry, and heating rate of the upper ocean).

\section{OceanGliders}

As the target of Argo is the open ocean it is highly complementary to glider observations conducted over the shelf-open ocean interface. BGC-Argo and OceanGliders share biogeochemical sensors used with similar mode of operations.

\section{OceanSites}

Fixed point observatories integrate a strong biogeochemical component. Synergies between BGC Argo and Eulerian observatories will be beneficial from both scientific and technical perspectives.

\section{Ocean Color Remote Sensing}

Chla and particle backscatter are quantities that are inferred from remote sensing of ocean color. BGC-Argo floats provide the vertical dimension to the global and synoptic measurements of the satellites, with a high degree of consistency (Haëntjens et al., 2017). The integration of both observation systems has to target the development of merged 3D/4D products (already prototyped, e.g., Sauzède et al., 2016). Additionally building on float arrays dedicated to satellite calibration (IOCCG, 2011; Gerbi et al., 2016; Leymarie et al., 2018), the progressive densification of an in situ database, with the addition of radiometry, is a way to validate remote sensing products and eventually to refine algorithm retrieval, including at regional scales. BGC-Argo can be used to validate space-based products (e.g., Haëntjens et al., 2017), fill in gaps in cloudy regions, during polar night, and under ice.

\section{GO-SHIP/GLODAP}

GO-SHIP is an essential partner to BGC-Argo. Its cruises take calibrated observations of most of the biogeochemical variables measured by floats, providing an important source of reference data. The GO-SHIP data set and the merged internally consistent product produced by the GLODAP project (Olsen et al., 2016) are essential for construction of the reference data set used to detect errors and make adjustments in BGC sensors
(Johnson et al., 2017) in a similar manner to that used by Core Argo to detect salinity drift (Owens and Wong, 2009). Floats with chemical sensors $\left(\mathrm{O}_{2}, \mathrm{pH}, \mathrm{NO}_{3}\right)$ can then extend these accurate, but infrequent, GO-SHIP cruises in the time domain to seasonal and inter-annual scales over the entire ocean.

\section{SOCONET and SOCAT}

Floats with carbon system parameters, such as $\mathrm{pH}$, would extend the surface $\mathrm{pCO}_{2}$ observations collected by the SOCONET ship of opportunity and mooring network and served from the SOCAT database. SOCAT (Bakker et al., 2016) produces a collated dataset with annual updates of in situ surface $\mathrm{pCO}_{2}$ measurements. However, the observations are infrequent, at high latitude, and biased to summer months. Merged products of ship, mooring-based and float-based estimates have the potential to improve our understanding of seasonal and interannual variability of ocean acidification and air-sea $\mathrm{CO}_{2}$ fluxes.

\section{Deep Argo}

At present, the deep ocean is primarily monitored by temporallylimited repeat hydrographic sections provided by GO-SHIP, and fixed-point time-series at the OceanSITES moorings (e.g., TAO/TRITON, PIRATA, and RAMA arrays; Send et al., 2010). Deep Argo will complement existing deep-ocean observations by increasing the top-to-bottom $\mathrm{P} / \mathrm{T} / \mathrm{S}$ sampling in the ocean interior. As in Core Argo, Deep Argo's success will build upon collaboration and integration across elements of the GOOS. Comparison with highly accurate P/T/S and oxygen measurements from GO-SHIP is the basis for Deep Argo DMQC, to monitor CTD and oxygen sensor drift, and to provide scientific background for data analysis. The implementation of the Deep Argo array relies on partnerships with ship-based hydrography programs including GO-SHIP, the HRX network, Core Argo, and OceanSITES. Deep Argo sea-going operations will provide deployment opportunities for other observing system elements (e.g., the Global Drifter Program).

Deep Argo, will improve the integration of satellite remote sensing observations, including sea level, net top-of-the atmosphere energy flux, and ocean mass and land ice variability, with in situ ocean data. Changes below 2000 dbar account for about $8 \%$ of the steric sea level rise, and $10 \%$ of the ocean heat uptake since the 1990s (Purkey and Johnson, 2010; Desbruyères et al., 2016). Combining estimates of the deep-ocean thermal expansion derived from Deep Argo, with ocean mass observations from the Gravity Recovery and Climate Experiment (GRACE; Tapley et al., 2004), and sea surface height data from satellite altimetry, will advance current understanding of the partitioning of steric and mass contribution to sea level rise (Purkey et al., 2014). In addition, systematic observations of topto-bottom ocean temperature from Deep Argo will improve the monitoring of year-to-year fluctuations in the net accumulation of anthropogenic heat in the Earth system. Increased deep ocean $\mathrm{P} / \mathrm{T} / \mathrm{S}$ sampling will provide in situ validation for variability in the Earth's energy imbalance estimated from the satellitebased Clouds and the Earth's radiant Energy System (CERES; Johnson et al., 2016). 


\section{Argo Data Management}

Argo is already used by operational users and scientists together with other platform data and therefore enhancing the interoperability of Argo data with other data systems by implementing the FAIR ${ }^{10}$ principles is an important next step for Argo. Improving the FAIR-ness of the Argo data system may imply updates in data and metadata format as standards evolve or the addition of services to supplement existing services. As stated earlier, serving high quality data is a top priority for Argo and comparison with other observing systems will help each system with improving data quality and services (see section "Core Argo" under the section "Integration with Other Observing System Elements").

\section{ArgoMix}

ArgoMix would greatly expand the number of direct observations of ocean turbulence, enabling improvement of fine-scale parameterizations made from existing $\mathrm{P} / \mathrm{T} / \mathrm{S}$ measurements (Gregg, 1989; Polzin et al., 1995; Whalen et al., 2012, 2015). Turbulence measurements from ArgoMix can validate and improve the underlying assumptions applied to convert P/T/S measurements from Core Argo, Chi-pods, and ship based hydrography data to fine-scale parameterizations of turbulence. Additionally, ArgoMix would bridge the gap between geographically isolated moorings and process studies and the global scale of ocean mixing. Global spatial coverage will provide the necessary context to link moorings and process studies, which may be located in special regions of enhanced mixing, to the large scales of water mass properties and circulation, and their variability.

\section{CONCLUSION}

Argo's continuous, real-time, global coverage of $\mathrm{P} / \mathrm{T} / \mathrm{S}$ in the upper $2000 \mathrm{~m}$ of the ocean has revolutionized our ability to monitor, understand, and forecast ocean variability. Starting in the late 1990s, the oceanographic community recognized the need and opportunity for a major enhancement of subsurface measurements to complement recent advances in remote sensing and to better monitor the global oceans for climate variability and change. The community designed and implemented Argo as an autonomous profiling float array capable of sampling the interior global ocean in a temporally and spatially continuous way that revolutionized the existing GOOS (Argo Steering Team, 1998). Argo has been a tremendous success in terms of engineering advancements, data management, and data dissemination, accomplishing its initial goal of monitoring global change and its consequences, improving ocean forecasts, and providing data underpinning many scientific advances. By combining the successful groundwork of Core Argo with recent float and sensor technology developments, there is now the opportunity to expand Argo as a next-generation observational array with enhanced societal value. The expanded Argo array envisioned here is capable of documenting the regional and global heat

${ }^{10}$ FAIR: Findable Accessible Interoperable Re-Usable. and freshwater budgets, biogeochemistry, and circulation of the top-to-bottom and pole-to-pole ocean.

This report calls for the expansion of Argo by (1) supplementing Core Argo to cover marginal seas and seasonal ice zones, (2) increasing Core Argo float density in the tropics and western boundary current regions, (3) deploying a global BGCArgo float array capable of measuring chlorophyll fluorescence, particle backscatter, oxygen, nitrate, $\mathrm{pH}$, and irradiance, (4) deploying a global Deep Argo array to monitor ocean variability down to $6000 \mathrm{~m}$, (5) validating and deploying new sensors, for example those capable of measuring ocean turbulence, (6) maintaining and advancing data management to keep up with growing data inflow and new requirements, and (7) continuing to engage with the end-user community to best maximize accessibility and utility of Argo data for the scientific, Blue Economy, and educational communities alike. The expanded design of the Argo Program, termed Argo2020, includes all of the elements described above while continuing as a single unified ocean observing system sharing platforms, logistics, data management, scientific objectives, and international governance, and with powerful synergies across its components and with other satellite and in situ observing systems.

The expanded Argo array will increase end user engagement through enabling basic research, climate assessment, education, and industry, the latter by means of improved long and short term ocean reanalysis and forecasting. Argo will better monitor climate variability and ecosystem health to allow for improved parameterization in models, and thus improved climate forecasting enabling better societal adaptation. In addition, real time assimilation of ocean heat, freshwater, and biogeochemistry will improve short-term forecasts vital for climate sensitive industries such as agriculture, aquaculture, fisheries, oil and gas. Finally, full-depth monitoring of ocean heat content will allow for synthesis with satellite observations for better closure of the global sea level rise and energy budgets. The Argo2020 design (Figure 10 and Table 1) describes the next-generation Argo Program that includes enhancements to Core Argo as well as BGC-Argo and Deep Argo elements, all fully integrated in their spatial distributions and through the comprehensive Argo Data Management System.

In the face of increasing anthropogenic forcing and accelerating global and regional trends in sea level, heat content, ocean acidification and deoxygenation, there is urgent need to continuously monitor the physical and biogeochemical variability of the global ocean with sufficient spatial and temporal resolution. The Argo in situ profiling array is a crucial piece of GOOS, and essential for meeting this challenge. Here, we have outlined a practical and scientifically compelling plan to expand Argo into a global full-depth physical and biogeochemical observational array.

\section{AUTHOR CONTRIBUTIONS}

Lead authors, designated by the symbol " $\dagger$ " and listed first in the list of authors, had primary responsibility for writing entire sections of the manuscript as follows: Abstract - SPu and DR; 
Introduction - WBO and DR; Core Argo subsections - DR, BK, SWi, PO, and TS; BGC Argo subsections - KJ, HC, and EB; Deep Argo subsections - NZ; Argo Data Management subsections MS and SPo; ArgoMix subsections - MA and JM; Conclusion $\mathrm{SPu}$ and $\mathrm{DR}$; DR compiled the manuscript and edited it for consistency. Co-authors (DB, MBa, MBe, HB, EB, PC, FCa, TC, FCh, DC, FD, GD, DD, KF, IF, RF, GF, HF, TF, MG, BG, RH, TH, SH, SJ, MJ, GCJ, KK, NK, AK, TM, TN, JN, AG, AO, RP, SPr, SR, CSchmechtig, CSchmid, ES, AS, PS, LT, TTa, VT, ST, JTo, AT, TTr, JTu, PV, WW, HW, RW, AW, SWa, AWa, CW, AWo, JX, P-YT, Y-DL, GM, KM, and IY) wrote the passages, as coordinated and assembled by the lead authors, and edited the manuscript for completeness, clarity, and accuracy.

\section{FUNDING}

DR, MS, and NZ were supported by the US Argo Program through the NOAA Grant NA15OAR4320071 (CIMEC). WO, SJ, and SWi were supported by the US Argo Program through the NOAA Grant NA14OAR4320158 (CINAR). EuroArgo scientists were supported by the two grants: (1) AtlantOS funding by the European Union's Horizon 2020 Research and Innovation Programme under the Grant Agreement No. 633211 and (2) Monitoring the Oceans and Climate Change with Argo (MOCCA) Co-funded by the European Maritime and Fisheries Fund (EMFF) Project No. SI2.709624. This manuscript represents a contribution to the following research projects for $\mathrm{HC}, \mathrm{CaS}$, and FD: remOcean (funded by the European Research Council, grant 246777), NAOS (funded by the Agence Nationale de la Recherche in the frame of the French "Equipement d'avenir" program, grant ANR J11R107-F), AtlantOS (funded by the European Union's Horizon 2020 Research and Innovation Programme, grant 2014-633211), and the BGC-Argo project funded by the CNES. DB was funded by the EU RINGO project (730944 H2020-INFRADEV-2016-1). RF was supported by the AGS-1835576. GCJ was supported by the Global Ocean Monitoring and Observing Program, National Oceanic and

\section{REFERENCES}

Alford, M. H., Lukas, R., Howe, B., Pickering, A., and Santiago-Mandujano, F. (2011). Moored observations of episodic abyssal flow and mixing at station ALOHA. Geophys. Res. Lett. 38:L15606. doi: 10.1029/2011GL048075

Argo Steering Team (1998). On the Design and Implementation of Argo - an Initial Plan for the Global Array of Profiling Floats. The Hague: International CLIVAR Project Office, 32.

Bakker, D. C. E., Pfeil, B., Landa, C. S., Metzl, N., O’Brien, K. M., Olsen, A., et al. (2016). A multi-decade record of high-quality $\mathrm{fCO}_{2}$ data in version 3 of the Surface Ocean $\mathrm{CO}_{2}$ Atlas (SOCAT). Earth Syst. Sci. Data 8, 383-413. doi: 10.5194/essd-8-383-2016

Barnard, A. H., and Koegle, J. (2018). Comment on "Particulate concentration and seasonal dynamics in the mesopelagic ocean based on the backscattering coefficient measured with Biogeochemical-Argo floats" by Poteau, A., Boss, E., and H. Claustre. Geophys. Res. Lett. 44, 6933-6939. doi: 10.1002/2017g 1073949

Becherer, J., and Moum, J. N. (2017). An efficient scheme for onboard reduction of moored xpod data. J. Atmos. Ocean. Technol. 34, 2533-2546. doi: 10.1175/ JTECH-D-17-0118.1
Atmospheric Administration (NOAA), U.S., and the Department of Commerce and NOAA Research. LT was funded under the SOCCOM Grant No. NSF PLR-1425989. VT's contribution was supported by the French National Research Agency (ANR) through the EQUIPEX NAOS (Novel Argo Observing System) under the reference ANR-10-EQPX-40 and by the European H2020 Research and Innovation Programme through the AtlantOS project under the reference 633211. WW was supported by the Argo Poland program through the Ministry of Sciences and Higher Education Grant No. DIR/WK/2016/12. AmW was funded by the NSF-OCE1434722. K-RK is funded by the National Institute of Meteorological Sciences' Research and Development Program "Development of Marine Meteorology Monitoring and Next-generation Ocean Forecasting System" under the grant KMA2018-00421. CSchmid is funded by NOAA/AOML and the US Argo Program through NOAA/OOMD. $\mathrm{MBa}$ is funded by NOAA/AOML.

\section{ACKNOWLEDGMENTS}

Argo data were collected and made freely available by the International Argo Project and the national programs that contribute to it (http://www.argo.ucsd.edu, http://argo. jcommops.org, http://doi.org/10.17882/42182). The authors gratefully acknowledge support from their respective Argo National Programs and/or national agencies. This is the PMEL Contribution Number 4863. The Surface Ocean CO2 Atlas (SOCAT) is an international effort by numerous researchers and funding agencies to deliver a uniformly quality-controlled surface ocean CO2 database. BGC Argo data were collected and made freely available by the Southern Ocean Carbon and Climate Observations and Modeling (SOCCOM) Project funded by the NationalScience Foundation, Division of Polar Programs (NSF PLR -1425989), supplemented by both the NOAA and NASA. The authors would also like to thank the US Ocean Carbon and Biogeochemistry (US-OCB) for their support to BGC Argo in the United States.

Bell, M. J., Schiller, A., Le Traon, P. Y., Smith, N. R., Dombrowsky, E., and WilmerBecker, K. (2015). An introduction to GODAE OceanView. J. Operat. Oceanogr. 8, s2-s11. doi: 10.1080/1755876X.2015.1022041

Bender, M. L., Tilbrook, B., Cassar, N., Jonsson, B., Poisson, A., and Trull, T. W. (2016). Ocean productivity south of Australia during spring and summer. Deep Sea Res. Part I Oceanogr. Res. Pap. 112, 68-78. doi: 10.1016/j.dsr.2016. 02.018

Biogeochemical-Argo Planning Group (2016). "Biogeochemical-argo science and implementation plan," in The Scientific Rationale, Design and Implementation Plan for a Biogeochemical-Argo Float Array, eds K. Johnson and H. Claustre (France: IFREMER), 58. Available at: https://archimer.ifremer.fr/doc/00355/ 46601/46508.pdf (accessed November 03, 2016).

Bishop, J. K. B., Davis, R. E., and Sherman, J. T. (2002). Robotic observations of dust storm enhancement of carbon biomass in the north pacific. Science 298, 817-821. doi: 10.1126/science.1074961

Bittig, H. C., Steinhoff, T., Claustre, H., Fiedler, B., Williams, N. L., Sauzède, R., et al. (2018). An alternative to static climatologies: robust estimation of open ocean $\mathrm{CO}_{2}$ variables and nutrient concentrations from $\mathrm{T}, \mathrm{S}$, and $\mathrm{O}_{2}$ data using bayesian neural networks. Front. Mar. Sci. 5:328. doi: 10.3389/fmars.2018. 00328 
Boss, E., and Behrenfeld, M. (2010). In situ evaluation of the initiation of the North Atlantic phytoplankton bloom. Geophys. Res. Lett. 37:L18603. doi: 10. 1029/2010GL044174

Boss, E., Swift, D., Taylor, L., Brickley, P., Zaneveld, R., Riser, S., et al. (2008). Observations of pigment and particle distributions in the western North Atlantic from an autonomous float and ocean color satellite. Limnol. Oceanogr. 53, 2112-2122. doi: 10.4319/lo.2008.53.5_part_2.2112

Brandt, P., Hormann, V., Kortzinger, A., Visbeck, M., Krahmann, G., Stramma, L., et al. (2010). Changes in the ventilation of the oxygen minimum zone of the tropical North Atlantic. J. Phys. Oceanogr. 40, 1784-1801. doi: 10.1175/ 2010JPO4301.1

Briggs, E. M., Sandoval, S., Erten, A., Takeshita, Y., Kummel, A. C., and Martz, T. R. (2017). Solid state sensor for simultaneous measurement of total alkalinity and $\mathrm{pH}$ of seawater. ACS Sens. 2, 1302-1309. doi: 10.1021/acssensors.7b0 0305

Bryden, H. L., Longworth, H. R., and Cunningham, S. A. (2005). Slowing of the Atlantic meridional overturning circulation at $25^{\circ} \mathrm{N}$. Nature $438,655-657$. doi: 10.1038 /nature04385

Bushinsky, S. M., and Emerson, S. (2015). Marine biological production from in situ oxygen measurements on a profiling float in the subarctic Pacific Ocean. Glob. Biogeochem. Cycles 29, 2050-2060. doi: 10.1002/2015GB005251

Bushinsky, S. M., Gray, A. R., Johnson, K. S., and Sarmiento, J. L. (2017). Oxygen in the Southern Ocean from Argo floats: determination of processes driving air-sea fluxes. J. Geophys. Res. Oceans 122, 8661-8682. doi: 10.1002/2017JC012923

Cabanes, C., Thierry, V., and Lagadec, C. (2016). Improvement of bias detection in Argo float conductivity sensors and its application in the North Atlantic. Deep Sea Res. Part I Oceanogr. Res. Pap. 114, 128-136. doi: 10.1016/j.dsr.2016. 05.007

Canonico, G., Buttigieg, P. L., Montes, E., Stepien, C. A., Wright, D., Benson, A., et al. (2019). Global observational needs and resources for marine biodiversity. Front. Mar. Sci. (in press). doi: 10.3389/fmars.2019.00367

Carrassi, A., Guemas, V., Doblas-Reyes, F. J., Volpi, D., and Asif, M. (2016). Sources of skill in near-term climate prediction: generating initial conditions. Clim. Dyn. 47, 3693-3712. doi: 10.1007/s00382-016-3036-4

Chen, X., Zhang, X., Church, J. A., Watson, C. S., King, M. A., Monselesan, D., et al. (2017). The increasing rate of global mean sea-level rise during 1993-2014. Nat. Clim. Change 7, 492-495. doi: 10.1038/nclimate3325

Claustre, H., Bishop, J., Boss, E., Stewart, B., Berthon, F. J., Coatanoan, C., et al. (2010). "Bio-optical profiling floats as new observational tools for biogeochemical and ecosystem studies: potential synergies with ocean color remote sensing," in Proceedings of OceanObs'09: Sustained Ocean Observations and Information for Society, eds J. Hall, D. E. Harrison, and D. Stammer (Venice: ESA Publication). doi: 10.5270/OceanObs09.cwp.17

Cross, J. N., Turner, J. A., Cooley, S. R., Newton, J. A., Azetsu-Scott, K., Chambers, R. C., et al. (2019). Building the knowledge-to-action pipeline in North America: connecting ocean acidification research and actionable decision support. Front. Mar. Sci. 6:356. doi: 10.3389/fmars.2019. 00356

Dall'Olmo, G., Dingle, J., Polimene, L. R., Brewin, J. W., and Claustre, H. (2016). Substantial energy input to the mesopelagic ecosystem from the seasonal mixed-layer pump. Nat. Geosci. 9, 820-823. doi: 10.1038/ngeo 2818

Dall'Olmo, G., and Mork, K. A. (2014). Carbon export by small particles in the Norwegian Sea. Geophys. Res. Lett. 41, 2921-2927. doi: 10.1002/2014GL05 9244

Davidson, F. J., Chassignet, E., Vinayachandran, P. N., Lu, Y., Smith, G. C., Zhu, X., et al. (2019). Synergies in operational oceanography: the intrinsic need for sustained ocean observations. Front. Mar. Sci. (in press). doi: 10.3389/fmars.2019.00450

Davis, R. E., Sherman, J. T., and Dufour, J. (2001). Profiling ALACEs and other advances in autonomous subsurface floats. J. Atmos. Ocean. Technol. 18, 982993. doi: 10.1175/1520-0426(2001)018<0982:paaoai $>2.0 . c 0 ; 2$

de Boisséson, E., Balmaseda, M. A., and Mayer, M. (2018). Ocean heat content variability in an ensemble of twentieth century ocean reanalyses. Clim. Dyn. 50, 3783-3798. doi: 10.1007/s00382-017-3845-0

de Lavergne, C., Madec, G., Capet, X., Maze, G., and Roquet, F. (2016). Getting to the bottom of the ocean. Nat. Geosci. 9, 857-858. doi: 10.1038/ ngeo 2850
Desbruyères, D. G., Purkey, S. G., McDonagh, E. L., Johnson, G. C., and King, B. A. (2016). Deep and abyssal ocean warming from 35 years of repeat hydrography. Geophys. Res. Lett. 43, 10,356-10,365. doi: 10.1002/2016GL070413

deYoung, B., Visbeck, M., Araujo Filho, M. C., Baringer, M. O., Buch, E., Canonico, G., et al. (2019). Atlantic observing system. Front. Mar. Sci. (in press). doi: $10.3389 /$ fmars.2019.00428

D’Ortenzio, F., Lavigne, H., Besson, F., Claustre, H., Coppola, L., Garcia, N., et al. (2014). Observing mixed layer depth, nitrate and chlorophyll concentrations in the northwestern Mediterranean: a combined satellite and NO3 profiling floats experiment. Geophys. Res. Lett. 41, 6443-6451. doi: 10.1002/2014GL061020

Euro-Argo-Eric (2017). Strategy for Evolution of Argo in Europe, EA-2016-ERICSTRAT. V3.2. doi: 10.13155/48526 (accessed June 07, 2017).

Ferrari, R., Mashayek, A., McDougall, T. J., Nikurashin, M., and Campin, M. J. (2016). Turning ocean mixing upside down. J. Phys. Oceanogr. 46, 2239-2261. doi: 10.1175/JPO-D-15-0244.1

Ferrari, R., and Polzin, K. L. (2005). Finescale structure of the T-S relation in the eastern North Atlantic. J. Phys. Oceanogr. 35, 1437-1454. doi: 10.1175/JPO 2763.1

Fiedler, B., Fietzek, P., Vieira, N., Silva, P., Bittig, H. C., and Körtzinger, A. (2013). In situ $\mathrm{CO}_{2}$ and $\mathrm{O}_{2}$ measurements on a profiling float. J. Atmos. Ocean. Technol. 30, 112-126. doi: 10.1175/JTECH-D-12-00043.1

Foltz, G. R., Brandt, P., Richter, I., Rodríguez-Fonseca, B., Hernandez, F., Dengler, M., et al. (2019). The tropical atlantic observing system. Front. Mar. Sci. 6:206. doi: 10.3389/fmars.2019.00206

Forget, G., Ferreira, D., and Liang, X. (2015). On the observability of turbulent transport rates by Argo: supporting evidence from an inversion experiment. Ocean Sci. 11, 839-853. doi: 10.5194/os-11-839-2015

Freeland, H. J., Roemmich, D., Garzoli, S. L., Le Traon, P. Y., Ravichandran, M., Riser, S., et al. (2010). "Argo - a decade of progress," in Proceedings of OceanObs'09: Sustained Ocean Observations and Information for Society, eds J. Hall, D. E. Harrison, and D. Stammer (Mangere: ESA Publications). doi: 10.5270/OceanObs09.cwp.32

Fujii, Y., Remy, E., Zuo, H., Oke, P. R., Halliwell, G. R., Gasparin, F., et al. (2019). Observing system evaluation based on Ocean Data Assimilation and Prediction Systems: on-going challenges and future vision for designing/supporting ocean observational networks. Front. Mar. Sci. (in press). doi: 10.3389/fmars.2019. 00417

Gaillard, F., Reynaud, T., Thierry, V., Kolodziejczyk, N., and Von Schuckmann, K. (2016). In-situ based reanalysis of the global ocean temperature and salinity with ISAS: variability of the heat content and steric height. J. Clim. 29, 13051323. doi: $10.1175 /$ jcli-d-15-0028.1

Gasparin, F., Greiner, E., Lellouche, M. J., Legalloudec, O., Garric, G., Drillet, Y., et al. (2018). A large-scale view of oceanic variability from 2007 to 2015 in the global high resolution monitoring and forecasting system at Mercator Océan. J. Mar. Syst. 187, 260-276. doi: 10.1016/j.jmarsys.2018. 06.015

Gerbi, G. P., Boss, E., Werdell, P. J., Proctor, C. W., Haëntjens, N., Lewis, M. R., et al. (2016). Validation of ocean color remote sensing reflectance using autonomous floats. J. Atmos. Ocean. Technol. 33, 2331-2352. doi: 10.1175/JTECH-D-160067.1

Goni, G. J., Sprintall, J., Bringas, F., Cheng, L., Cirano, M., Dong, S., et al. (2019). More than 50 years of successful continuous temperature section measurements by the global eXpendable BathyThermograph (XBT) network, its integrability, future and impact on societal benefits, and future. Front. Mar. Sci. (in press). doi: 10.3389/fmars.2019.00452

Goto, Y., Yasuda, I., and Nagasawa, M. (2016). Turbulence estimation using fast-response thermistors attached to a free-fall vertical microstructure profiler. J. Atmos. Ocean. Technol. 33, 2065-2078. doi: 10.1175/JTECH-D-150220.1

Goto, Y., Yasuda, I., and Nagasawa, M. (2018). Comparison of turbulence intensity from CTD-attached and free-fall microstructure profilers. J. Atmos. Ocean. Technol. 35, 147-162. doi: 10.1175/JTECH-D-17-0069.1

Gray, A. R., Johnson, K. S., Bushinsky, S., Riser, S. C., Russell, J. L., Talley, L., et al. (2018). Autonomous biogeochemical floats detect significant carbon dioxide outgassing in the high-latitude Southern Ocean. Geophys. Res. Lett. 45, 9049-9057. doi: 10.1029/2018gl078013

Gregg, M. C. (1989). Scaling turbulent dissipation in the thermocline. J. Geophys. Res. Oceans 94, 9686-9698. doi: 10.1029/JC094iC07p09686 
Griffin, D. A., Oke, P. R., and Jones, E. (2017). The Search for MH370 and Ocean Surface Drift - Part II. CSIRO Report Number EP172633. doi: 10.4225/08/ 58fba83e73f2b (accessed April 13, 2017).

Gruber, N., Doney, S., Emerson, S., Gilbert, D., Kobayashi, T., Körtzinger, A., et al. (2007). The Argo-Oxygen Program: A White Paper to Promote the Addition of Oxygen Sensors to the International Argo Float Program. Available at: http: //www-argo.ucsd.edu/o2_white_paper_web.pdf (accessed April 4, 2007).

Gruber, N., Doney, S., Emerson, S. R., Gilbert, D., Kobayashi, T., Kortzinger, A., et al. (2010). "Addition oxygen to Argo: developing a global in situ observatory for ocean deoxygenation and biogeochemistry," in Proceedings of Oceanobs'09: Sustained Ocean Observations and Information for Society, eds J. Hall, D. E. Harrison, and D. Stammer (Venice: ESA Publication). doi: 10.5270/ OceanObs09.cwp.39

Haëntjens, N., Boss, E., and Talley, L. D. (2017). Revisiting Ocean Color algorithms for chlorophyll a and particulate organic carbon in the Southern Ocean using biogeochemical floats. J. Geophys. Res. Oceans 122, 6583-6593. doi: 10.1002/ 2017JC012844

Hennon, T. D., Riser, S. C., and Mecking, S. (2016). Profiling float-based observations of net respiration beneath the mixed layer. Glob. Biogeochem. Cycles 30, 920-932. doi: 10.1002/2016GB005380

Hermes, J. C., Masumoto, Y., Beal, L. M., Roxy, M. K., Vialard, J., Andres, M., et al. (2019). A sustained ocean observing system in the Indian Ocean for climate related scientific knowledge and societal needs. Front. Mar. Sci. 6:355. doi: 10.3389/fmars.2019.00355

Hochman, Z., van Rees, H., Carberry, P. S., Hunt, J. R., McCown, R. L., Gartmann, A., et al. (2009). Re-inventing model-based decision support with Australian dryland farmers. 4. Yield Prophet (R) helps farmers monitor and manage crops in a variable climate. Crop Pasture Sci. 60, 1057-1070. doi: 10.1071/CP 09020

Hood, E. M., Sabine, C. L., and Sloyan, B. M. (2010). The GO-SHIP Repeat Hydrography Manual: A Collection of Expert Reports and Guidelines. Available at: http://www.go-ship.org/HydroMan.html (accessed May 3, 2013).

Hosoda, S., Ohira, T., and Nakamura, T. (2008). A monthly mean dataset of global oceanic temperature and salinity derived from Argo float observations. JAMSTEC Rep. Res. Dev. 8, 47-59. doi: 10.5918/jamstecr.8.47

Intergovernmental Oceanographic Commission [IOC] (1999). Resolution XX-6, "Reports of Governing and Major Subsidiary Bodies, Twentieth Session of the Assembly”. Paris: Intergovernmental Oceanographic Commission.

IOCCG (2011). "Bio-optical sensors on Argo floats," in Reports of the International Ocean Colour Coordinating Group, $N^{\circ} 11$, ed. H. Claustre (Dartmouth: IOCCG).

Jayne, S. R., Roemmich, D., Zilberman, N. V., Riser, S. C., Johnson, K. S., Johnson, G. C., et al. (2017). The Argo program: present and future. Oceanography 30, 18-28. doi: 10.5670/oceanog.2017.213

Jochum, M., Briegleb, B. P., Danabasoglu, G., Large, W. G., Norton, N. J., Jayne, S. R., et al. (2013). The impact of oceanic near-inertial waves on climate. J. Clim. 26, 2833-2844. doi: 10.1175/JCLI-D-12-00181.1

Johnson, G. C. (2008). Quantifying antarctic bottom water and North Atlantic deep water volumes. J. Geophys. Res. Oceans 113:C05027. doi: 10.1029/ 2007JC004477

Johnson, G. C., and Birnbaum, A. N. (2017). As El Niño builds, Pacific Warm Pool expands, ocean gains more heat. Geophys. Res. Lett. 44, 438-445. doi: 10.1002/2016GL071767

Johnson, G. C., Lyman, J. M., and Loeb, N. G. (2016). Improving estimates of Earth's energy imbalance. Nat. Clim. Change 6, 639-640. doi: 10.1038/nclimate 3043

Johnson, G. C., Lyman, J. M., and Purkey, S. G. (2015). Informing deep Argo array design using Argo and full-depth hydrographic section data. J. Atmos. Ocean. Technol. 32, 2187-2198. doi: 10.1175/JTECH-D-150139.1

Johnson, K. S., Berelson, W. M., Boss, E. S., Chase, Z., Claustre, H., Emerson, S. R., et al. (2009). Observing biogeochemical cycles at global scales with profiling floats and gliders prospects for a global array. Oceanography 22, 216-225. doi: 10.5670/oceanog.2009.81

Johnson, K. S., Plant, J. N., Coletti, L. J., Jannasch, H. W., Sakamoto, C. M., Riser, S. C., et al. (2017). Biogeochemical sensor performance in the SOCCOM profiling float array. J. Geophys. Res. Oceans 122, 6416-6436. doi: 10.1002/ 2017JC012838
Johnson, K. S., Riser, S. C., and Karl, D. M. (2010). Nitrate supply from deep to near-surface waters of the North Pacific subtropical gyre. Nature 465, $1062-$ 1065. doi: 10.1038/nature09170

Kamenkovich, I., Haza, A., Gray, A. R., Dufour, C. O., and Garraffo, Z. (2017). Observing System Simulation Experiments for an array of autonomous biogeochemical profiling floats in the Southern Ocean. J. Geophys. Res. Oceans 122, 7595-7611. doi: 10.1002/2017JC012819

Klatt, O., Boebel, O., and Fahrbach, E. (2007). A profiling float's sense of ice. J. Atmos. Ocean. Technol. 24, 1301-1308. doi: 10.1175/JTECH2026.1

Kobayashi, T. (2013). Deep NINJA collects profiles down to 4,000 meters. Sea Technol. 54, 41-44.

Kobayashi, T., and Minato, S. (2005). Importance of reference dataset improvements for Argo delayed-mode quality control. J. Oceanogr. 61, 9951009. doi: 10.1007/s10872-006-0016-z

Körtzinger, A., Schimanski, J., Send, U., and Wallace, D. (2004). The ocean takes a deep breath. Science 306, 1337-1337. doi: 10.1126/science.1102557

Kouketsu, S., Doi, T., Kawano, T., Masuda, S., Sugiura, N., Sasaki, Y., et al. (2011). Deep ocean heat content changes estimated from observation and reanalysis product and their influence on sea level change. J. Geophys. Res. Oceans 116:C03012. doi: 10.1029/2010JC006464

Kouketsu, S., Inoue, R., and Suga, T. (2016). Western North Pacific integrated physical-biogeochemical ocean observation experiment (INBOX): part 3 . Mesoscale variability of dissolved oxygen concentrations observed by multiple floats during S1-INBOX. J. Mar. Res. 74, 101-131. doi: 10.1357/ 002224016819257326

Le Quéré, C., Andrew, R. M., Friedlingstein, P., Sitch, S., Pongratz, J., Manning, A. C., et al. (2018). Global carbon budget 2017. Earth Syst. Sci. Data 10, 405-448. doi: 10.5194/essd-10-405-2018

Le Reste, S., Dutreuil, V., André, X., Thierry, V., Renaut, C., Traon, P. Y. L., et al. (2016). "Deep-Arvor": a new profiling float to extend the Argo observations down to 4000-m depth. J. Atmos. Ocean. Technol. 33, 1039-1055. doi: 10.1175/ JTECH-D-15-0214.1

Lea, D. J., Martin, M. J., and Oke, P. R. (2014). Demonstrating the complementarity of observations in an operational ocean forecasting system. Q. J. R. Meteorol. Soc. 140, 2037-2049. doi: 10.1002/qj.2281

Ledwell, J. R., Watson, A. J., and Law, C. S. (1993). Evidence for slow mixing across the pycnocline from an open-ocean tracer-release experiment. Nature 364, 701-703. doi: 10.1038/364701a0

Lee, C. M., Starkweather, S., Eicken, H., Timmermans, M.-L., Wilkinson, J., Sandven, S., et al. (2019). A framework for the development, design and implementation of a sustained artic ocean observing system. Front. Mar. Sci. (in press). doi: 10.3389/fmars.2019.00451

Legler, D. M., Freeland, H. J., Lumpkin, R., Ball, G., McPhaden, M. J., North, S., et al. (2015). The current status of the real-time in situ Global Ocean Observing System for operational oceanography. J. Operat. Oceanogr. 8, s189-s200. doi: 10.1080/1755876X.2015.1049883

Leymarie, E., Penkerc'h, C., Vellucci, V., Lerebourg, C., Antoine, D., Boss, E., et al. (2018). ProVal: a new autonomous profiling float for high quality radiometric measurements. Front. Mar. Sci. 5:437. doi: 10.3389/fmars.2018.00437

Llort, J., Langlais, C., Matear, R., Moreau, S., Lenton, A., and Strutton, P. G. (2018). Evaluating southern ocean carbon eddy-pump from biogeochemicalArgo floats. J. Geophys. Res. Oceans 123, 971-984. doi: 10.1002/2017JC01 2861

Lozier, M. S., Bacon, S., Bower, A. S., Cunningham, S. A., Jong, M. F. D., Steur, L. D., et al. (2017). Overturning in the subpolar North Atlantic program: a new international ocean observing system. Bull. Am. Meteorol. Soc. 98, 737-752. doi: 10.1175/bams-d-16-0057.1

Lucas, A. J., Nash, J. D., Pinkel, R., MacKinnon, J., Tandon, A., Mahadevan, A., et al. (2016). Adrift upon a salinity-stratified sea: a view of upper-ocean processes in the bay of Bengal during the southwest monsoon. Oceanography 29, 134-145. doi: $10.5670 /$ oceanog. 2016.46

MacKinnon, J. A., Zhao, Z., Whalen, C. B., Waterhouse, A. F., Trossman, D. S., Sun, O. M., et al. (2017). Climate process team on internal wave-driven ocean mixing. Bull. Am. Meteorol. Soc. 98, 2429-2454. doi: 10.1175/BAMS-D-160030.1

Mariano, A. J., Kourafalou, V. H., Srinivasan, A., Kang, H., Halliwell, G. R., Ryan, E. H., et al. (2011). On the modeling of the 2010 Gulf of Mexico 
Oil Spill. Dyn. Atmos. Oceans 52, 322-340. doi: 10.1016/j.dynatmoce.2011. 06.001

Marshall, D. P., and Zanna, L. (2014). A conceptual model of ocean heat uptake under climate change. J. Clim. 27, 8444-8465. doi: 10.1175/JCLI-D-13-00344.1

Masuda, S., Awaji, T., Sugiura, N., Matthews, J. P., Toyoda, T., Kawai, Y., et al. (2010). Simulated rapid warming of abyssal north pacific waters. Science 329, 319-322. doi: 10.1126/science.118 8703

Maximenko, N., Corradi, P., Law, K. L., Van Sebille, E., Garaba, S. P., Lampitt, R. S., et al. (2019). Towards the integrated marine debris observing system. Front. Mar. Sci. (in press). doi: 10.3389/fmars.2019.00447

Meinen, C. S., Garzoli, S. L., Perez, R. C., Campos, E., Piola, A. R., Chidichimo, M. P., et al. (2017). Characteristics and causes of Deep Western Boundary Current transport variability at $34.5^{\circ} \mathrm{S}$ during 2009-2014. Ocean Sci. 13, 175-194. doi: 10.5194/os-13-175-2017

Melet, A., Legg, S., and Hallberg, R. (2016). Climatic impacts of parameterized local and remote tidal mixing. J. Clim. 29, 3473-3500. doi: 10.1175/JCLI-D-150153.1

Meyssignac, B., Boyer, T., Zhao, Z., Hakuba, M. Z., Landerer, F. W., Stammer, D., et al. (2019). Measuring global Ocean Heat Content to estimate the Earth Energy Imbalance. Front. Mar. Sci. (in press). doi: 10.3389/fmars.2019.00432

Mignot, A., Ferrari, R., and Claustre, H. (2018). Floats with bio-optical sensors reveal what processes trigger the North Atlantic bloom. Nat. Commun. 9:190. doi: 10.1038/s41467-017-02143-6

Mitchell, B. G. (2003). "Resolving spring bloom dynamics in the sea of Japan," in ALPS: Autonomous and Lagrangian Platforms and Sensors, Workshop Report, eds D. L. Rudnick and M. J. Perry (La Jolla, CA: ALPS), 26-27.

Moum, J. N., Lien, R. C., Perlin, A., Nash, J. D., Gregg, M. C., and Wiles, P. J. (2009). Sea surface cooling at the Equator by subsurface mixing in tropical instability waves. Nat. Geosci. 2, 761-765. doi: 10.1038/ngeo657

Moum, J. N., and Nash, J. D. (2009). Mixing measurements on an equatorial ocean mooring. J. Atmos. Ocean. Technol. 26, 317-336. doi: 10.1175/2008JTECHO 617.1

Moum, J. N., Perlin, A., Nash, J. D., and McPhaden, M. J. (2013). Seasonal sea surface cooling in the equatorial Pacific cold tongue controlled by ocean mixing. Nature 500, 64-67. doi: 10.1038/nature12363

Nagai, T., Inoue, R., Tandon, A., and Yamazaki, H. (2015). Evidence of enhanced double-diffusive convection below the main stream of the Kuroshio Extension. J. Geophys. Res. Oceans 120, 8402-8421. doi: 10.1002/2015JC011288

Naveira Garabato, A. C., Polzin, K. L., Ferrari, R., Zika, J. D., and Forryan, A. (2015). A microscale view of mixing and overturning across the Antarctic circumpolar current. J. Phys. Oceanogr. 46, 233-254. doi: 10.1175/JPO-D-150025.1

Newman, L., Heil, P., Trebilco, R., Katsumata, K., Constable, A. J., van Wijk, E., et al. (2019). Delivering sustained, coordinated and integrated observations of the Southern Ocean for global impact. Front. Mar. Sci. (in press). doi: $10.3389 /$ fmars.2019.00433

Oke, P. R., Balmaseda, M. A., Benkiran, M., Cummings, J. A., Dombrowsky, E., Fujii, Y., et al. (2009). Observing system evaluations using godae systems. Oceanography 22, 144-153. doi: 10.5670/oceanog.2009.72

Oke, P. R., Sakov, P., Cahill, M. L., Dunn, J. R., Fiedler, R., Griffin, D. A., et al. (2013). Towards a dynamically balanced eddy-resolving ocean reanalysis: BRAN3. Ocean Model. 67, 52-70. doi: 10.1016/j.ocemod.2013. 03.008

Oke, P. R., and Schiller, A. (2007). Impact of Argo, SST, and altimeter data on an eddy-resolving ocean reanalysis. Geophys. Res. Lett. 34:L19601. doi: 10.1029/ $2007 \mathrm{gl03} 1549$

Olbers, D., and Eden, C. (2013). A global model for the diapycnal diffusivity induced by internal gravity waves. J. Phys. Oceanogr. 43, 1759-1779. doi: 10. 1175/JPO-D-12-0207.1

Olsen, A., Key, R. M., van Heuven, S., Lauvset, S. K., Velo, A., Lin, X., et al. (2016). The global ocean data analysis project version 2 (GLODAPv2) - an internally consistent data product for the world ocean. Earth Syst. Sci. Data 8, 297-323. doi: 10.5194/essd-8-297-2016

Osborn, T. R. (1980). Estimates of the local rate of vertical diffusion from dissipation measurements. J. Phys. Oceanogr. 10, 83-89. doi: 10.1175/15200485(1980)010<0083:eotlro > 2.0.co;2
Osborn, T. R., and Cox, C. S. (1972). Oceanic fine structure. Geophys. Fluid Dyn. 3 , 321-345. doi: 10.1080/03091927208236085

Owens, W. B., and Wong, A. P. S. (2009). An improved calibration method for the drift of the conductivity sensor on autonomous CTD profiling floats by theta-S climatology. Deep Sea Res. Part I Oceanogr. Res. Pap. 56, 450-457. doi: 10.1016/j.dsr.2008.09.008

Palazov, A., Ciliberti, S., Peneva, E., Gregoire, M., Staneva, J., Lemieux-Dudon, B., et al. (2019). Black sea observing system. Front. Mar. Sci. 6:315. doi: 10.3389/ fmars.2019.00315

Pearlman, J., Bushnell, M., Coppola, L., Karstensen, J., Buttigieg, P. L., Pearlman, F., et al. (2019). Evolving and sustaining ocean best practices and standards for the next decade. Front. Mar. Sci. 6:277. doi: 10.3389/fmars.2019. 00277

Penny, S. G., Akella, S., Balmaseda, M. A., Browne, P., Carto, J. A., Chevallier, M., et al. (2019). Observational needs for improving ocean and coupled reanalysis, S2S prediction, and decadal prediction. Front. Mar. Sci. 6:391. doi: 10.3389/ fmars.2019.00391

Perlin, A., and Moum, J. N. (2012). Comparison of thermal variance dissipation rates from moored and profiling instruments at the equator. J. Atmos. Ocean. Technol. 29, 1347-1362. doi: 10.1175/JTECH-D-12-00019.1

Pinardi, N., Stander, J., Legler, D., O’Brien, K., Boyer, T., Cuff, T., et al. (2019). The Joint IOC (of UNESCO) and WMO collaborative effort for met-ocean services. Front. Mar. Sci. (in press). doi: 10.3389/fmars.2019.00410

Plant, J. N., Johnson, K. S., Sakamoto, C. M., Jannasch, H. W., Coletti, L. J., Riser, S. C., et al. (2016). Net community production at Ocean Station Papa observed with nitrate and oxygen sensors on profiling floats. Glob. Biogeochem. Cycles 30, 859-879. doi: 10.1002/2015GB005349

Pollman, F., Eden, C., and Olbers, D. (2017). Evaluating the global internal wave model IDEMIX using finestructure methods. J. Phys. Oceanogr. 47, 2267-2289. doi: 10.1175/JPO-D-16-0204.1

Polzin, K. L., Naveira Garabato, A. C., Huussen, T. N., Sloyan, B. M., and Waterman, S. (2014). Finescale parameterizations of turbulent dissipation. J. Geophys. Res. Oceans 119, 1383-1419. doi: 10.1002/2013JC008979

Polzin, K. L., Toole, J. M., and Schmitt, R. W. (1995). Finescale parameterizations of turbulent dissipation. J. Phys. Oceanogr. 25, 306-328. doi: 10.1038/s41598018-28554-z

Ponte, R. M., Carson, M., Cirano, M., Domingues, C., Jevrejeva, S., Marcos, M., et al. (2019). Towards comprehensive observing and modeling systems for monitoring and predicting regional to coastal sea level. Front. Mar. Sci. (in press). doi: 10.3389/fmars.2019.00437

Poteau, A., Boss, E., and Claustre, H. (2017). Particulate concentration and seasonal dynamics in the mesopelagic ocean based on the backscattering coefficient measured with Biogeochemical-Argo floats. Geophys. Res. Lett. 44, 6933-6939. doi: 10.1002/2017GL073949

Potemra, J. T. (2012). Numerical modeling with application to tracking marine debris. Mar. Pollut. Bull. 65, 42-50. doi: 10.1016/j.marpolbul.2011.06.026

Prakash, S. T., Balakrishnan Naira, T. M., daya Bhaskara, T. V. S. U., Prakasha, P., and Gilbert, D. (2012). Oxycline variability in the central Arabian Sea: an Argo-oxygen study. J. Sea Res. 71, 1-8. doi: 10.1016/j.seares.2012.03.003

Pujiana, K., Moum, J. N., and Smyth, W. D. (2018). The role of turbulence in redistributing upper-ocean heat, freshwater, and momentum in response to the MJO in the equatorial Indian ocean. J. Phys. Oceanogr. 48, 197-220. doi: 10.1175/JPO-D-17-0146.1

Purkey, S. G., and Johnson, G. C. (2010). Warming of global abyssal and deep southern ocean waters between the 1990s and 2000s: contributions to global heat and sea level rise budgets. J. Clim. 23, 6336-6351. doi: 10.1175/ 2010JCLI3682.1

Purkey, S. G., and Johnson, G. C. (2013). Antarctic bottom water warming and freshening: contributions to sea level rise, ocean freshwater budgets, and global heat gain. J. Clim. 26, 6105-6122. doi: 10.1175/JCLI-D-12-00834.1

Purkey, S. G., Johnson, G. C., and Chambers, D. P. (2014). Relative contributions of ocean mass and deep steric changes to sea level rise between 1993 and 2013. J. Geophys. Res. Oceans 119, 7509-7522. doi: 10.1002/2014JC01 0180

Riser, S. C., Freeland, H. J., Roemmich, D., Wijffels, S., Troisi, A., Belbeoch, M., et al. (2016). Fifteen years of ocean observations with the global Argo array. Nat. Clim. Change 6, 145-153. doi: 10.1038/nclimate2872 
Riser, S. C., and Johnson, K. S. (2008). Net production of oxygen in the subtropical ocean. Nature 451, 323-325. doi: 10.1038/nature06441

Riser, S. C., Nystuen, J., and Rogers, A. (2008). Monsoon effects in the Bay of Bengal inferred from profiling float-based measurements of wind speed and rainfall. Limnol. Oceanogr. 53, 2080-2093. doi: 10.4319/lo.2008.53.5_part_2.2080

Robson, J. I., Sutton, R. T., and Smith, D. M. (2012). Initialized decadal predictions of the rapid warming of the North Atlantic Ocean in the mid 1990s. Geophys. Res. Lett. 39:L19713. doi: 10.1029/2012GL053370

Roemmich, D., Boehme, L., Claustre, H., Freeland, H., Fukasawa, M., Goni, G., et al. (2010). "Integrating the ocean observing system: mobile platforms," in Proceedings of OceanObs'09: Sustained Ocean Observations and Information for Society, eds J. Hall, D. E. Harrison, and D. Stammer (Venice: ESA Publication). doi: 10.5270/OceanObs09.pp.33

Roemmich, D., and Gilson, J. (2009). The 2004-2008 mean and annual cycle of temperature, salinity, and steric height in the global ocean from the Argo Program. Prog. Oceanogr. 82, 81-100. doi: 10.1016/j.pocean.2009.03.004

Roemmich, D., and The Argo Steering Team (2009). Argo: the challenge of continuing 10 years of progress. Oceanography 22, 46-55. doi: 10.5670/oceanog. 2009.65

Roesler, C., Uitz, J., Claustre, H., Boss, E., Xing, X., Organelli, E., et al. (2017). Recommendations for obtaining unbiased chlorophyll estimates from in situ chlorophyll fluorometers: a global analysis of WET Labs ECO sensors. Limnol. Oceanogr. Methods 15, 572-585. doi: 10.1002/lom3. 10185

Rudnick, D. L., and Perry, M. J. (2003). ALPS: Autonomous and Lagrangian Platforms and Sensors Workshop Report. Available at: https://www.geo-prose. com/pdfs/alps_report.pdf (accessed August 25, 2014).

Sauzède, R., Claustre, H., Uitz, J., Jamet, C., Dall'Olmo, G., D’Ortenzio, F., et al. (2016). A neural network-based method for merging ocean color and Argo data to extend surface bio-optical properties to depth: retrieval of the particulate backscattering coefficient. J. Geophys. Res. Oceans 121, 2552-2571. doi: 10.1002/ 2015JC011408

Send, U., Weller, R. A., Wallace, D., Chavez, F., Lampitt, R., Dickey, T., et al. (2010). "OceanSITES," in Proceedings of the OceanObs'09: Sustained Ocean Observations and Information for Society, eds J. Hall, D. E. Harrison, and D. Stammer (Venice: ESA Publication). doi: 10.5270/OceanObs09.cwp.79

Sherman, J. T., and Davis, R. E. (1995). Observations of temperature microstructure in NATRE. J. Phys. Oceanogr. 25, 1913-1929. doi: 10.1175/ 1520-0485(1995)025<1913:ootmin >2.0.co;2

Shroyer, E., Rudnick, D. L., Farrar, J. T., Lim, B., Venayagamoorthy, K., St Laurent, L. C., et al. (2016). Modification of upper-ocean temperature structure by subsurface mixing in the presence of strong salinity stratification. Oceanography 29, 62-71. doi: 10.5670/oceanog.2016.39

Siedler, G., Church, J., and Gould, J. (eds). (2001). Ocean Circulation and Climate: Observing and Modeling the Global Ocean. London: Academic Press.

Sloyan, B. M., Wanninkhof, R., Kramp, M., Johnson, G. C., Talley, L., Tanhua, T., et al. (2019). The Global Ocean Ship-Base Hydrographic Investigations Program (GO-SHIP): a platform for integrated multidisciplinary ocean science. Front. Mar. Sci. (in press). doi: 10.3389/fmars.2019.00445

Smeed, D. A., Josey, S. A., Beaulieu, C., Johns, W. E., Moat, B. I., Frajka-Williams, E., et al. (2018). The North Atlantic ocean is in a state of reduced overturning. Geophys. Res. Lett. 45, 1527-1533. doi: 10.1002/2017GL076350

Smith, N., Kessler, W. S., Cravatte, S., Sprintall, J., Wijffels, S., Cronin, M. F., et al. (2019). Tropical pacific observing system. Front. Mar. Sci. 6:31. doi: 10.3389/ fmars.2019.00031

Stanev, E. V., Poulain, P. M., Grayek, S., Johnson, K. S., Claustre, H., and Murray, J. W. (2018). Understanding the dynamics of the oxic-anoxic interface in the black sea. Geophys. Res. Lett. 45, 864-871. doi: 10.1002/2017GL07 6206

Sukigara, C., Suga, T., Saino, T., Toyama, K., Yanagimoto, D., Hanawa, K., et al. (2011). Biogeochemical evidence of large diapycnal diffusivity associated with the subtropical mode water of the North Pacific. J. Oceanogr. 67, 77-85. doi: 10.1007/s10872-011-0008-5

Talley, L. D., Feely, R. A., Sloyan, B. M., Wanninkhof, R., Baringer, M. O., Bullister, J. L., et al. (2016). Changes in ocean heat, carbon content, and ventilation: a review of the first decade of GO-SHIP global repeat hydrography. Annu. Rev. Mar. Sci. 8, 185-215. doi: 10.1146/annurev-marine-052915100829
Tapley, B. D., Bettadpur, S., Watkins, M., and Reigber, C. (2004). The gravity recovery and climate experiment: mission overview and early results. Geophys. Res. Lett. 31:L09607. doi: 10.1029/2004GL019920

Todd, R. E., Chavez, F. P., Clayton, S., Cravatte, S. E., Goes, M. P., Graco, M. I., et al. (2019). Global perspectives on observing ocean boundary current systems. Front. Mar. Sci. (in press). doi: 10.3389/fmars.2019.00423

Verdy, A., and Mazloff, M. R. (2017). A data assimilating model for estimating Southern Ocean biogeochemistry. J. Geophys. Res. Oceans 122, 6968-6988. doi: 10.1002/2016JC012650

Vinogradova, N., Lee, T., Boutin, J., Drushka, K., Fournier, S., Sabia, R., et al. (2019). Satellite salinity observing system: recent discoveries and the way forward. Front. Mar. Sci. 6:243. doi: 10.3389/fmars.2019. 00243

Visbeck, M., Araujo, M., Boetius, A., Buch, E., Claustre, H., Dabrowski, T., et al. (2015). More integrated and more sustainable Atlantic ocean observing (AtlantOS). CLIVAR Exchanges 67, 18-20.

Watanabe, M., and Kawamiya, M. (2017). Remote effects of mixed layer development on ocean acidification in the subsurface layers of the North Pacific. J. Oceanogr. 73, 771-784. doi: 10.1007/s10872-017-0431-3

Waterhouse, A. F., MacKinnon, J. A., Nash, J. D., Alford, M. H., Kunze, E. Simmons, H. L., et al. (2014). Global patterns of diapycnal mixing from measurements of the turbulent dissipation rate. J. Phys. Oceanogr. 44, 18541872. doi: 10.1175/JPO-D-13-0104.1

Waterman, S., Polzin, K. L. A., Garabato, C. N., Sheen, K. L., and Forryan, A. (2014). Suppression of internal wave breaking in the Antarctic circumpolar current near topography. J. Phys. Oceanogr. 44, 1466-1492. doi: 10.1175/JPOD-12-0154.1

Wenhai, L., Cusack, C., Baker, M., Tao, W., Mingbao, C., Paige, K., et al. (2019). Successful blue economy examples with an emphasis on international perspectives. Front. Mar. Sci. 6:261. doi: 10.3389/fmars.2019.00261

Whalen, C. B., MacKinnon, J. A., Talley, L. D., and Waterhouse, A. F. (2015). Estimating the mean diapycnal mixing using a Finescale strain parameterization. J. Phys. Oceanogr. 45, 1174-1188. doi: 10.1175/JPO-D-140167.1

Whalen, C. B., Talley, L. D., and MacKinnon, J. A. (2012). Spatial and temporal variability of global ocean mixing inferred from Argo profiles. Geophys. Res. Lett. 39:L18612. doi: 10.1029/2012GL053196

Whitmire, A. L., Letelier, R. M., Villagrán, V., and Ulloa, O. (2009). Autonomous observations of in vivo fluorescence and particle backscattering in an oceanic oxygen minimum zone. Opt. Express 17, 21992-22004. doi: 10.1364/OE.17. 021992

Wijffels, S., Roemmich, D., Monselesan, D., Church, J., and Gilson, J. (2016). Ocean temperatures chronicle the ongoing warming of Earth. Nat. Clim. Change 6, 116-118. doi: 10.1038/nclimate2924

Williams, N. L., Juranek, L. W., Feely, R. A., Johnson, K. S., Sarmiento, J. L., Talley, L. D., et al. (2017). Calculating surface ocean $\mathrm{pCO}_{2}$ from biogeochemical Argo floats equipped with pH: an uncertainty analysis. Glob. Biogeochem. Cycles 31, 591-604. doi: 10.1002/2016GB005541

Willis, Z. (2015). "Chapter 2 - national ocean observing systems in a global context," in Coastal Ocean Observing Systems, eds Y. Liu, H. Kerkering, and R. H. Weisberg (Cambridge, MA: Academic Press), 11-25. doi: 10.1016/b978-0-12802022-7.00002-x

Wolf, M. K., Hamme, R. C., Gilbert, D., Yashayaev, I., and Thierry, V. (2018). Oxygen saturation surrounding deep water formation events in the Labrador sea from Argo- $\mathrm{O}_{2}$ data. Glob. Biogeochem. Cycles 32, 635-653. doi: 10.1002/ 2017GB005829

Wong, A. P. S., Johnson, G. C., and Owens, W. B. (2003). Delayed-mode calibration of autonomous CTD profiling float salinity data by theta-S climatology. J. Atmos. Ocean. Technol. 20, 308-318. doi: 10.1175/15200426(2003)020<0308:dmcoac $>2.0 . c 0 ; 2$

Wong, A. P. S., and Riser, S. C. (2011). Profiling float observations of the upper ocean under sea ice off the Wilkes land coast of Antarctica. J. Phys. Oceanogr. 41, 1102-1115. doi: 10.1175/2011JPO4516.1

Wuldera, M. A., White, J. C., Loveland, T. R., Woodcock, C. E., Belward, A., and Cohen, W. B. (2016). The Global Observing System for Climate: Implementation Needs. GCOS Implementation Plan 2016, GCOS-200 (GOOS214). Available at: https://unfccc.int/sites/default/files/gcos_ip_10oct2016.pdf doi: 10.1175/2011jpo4516.1 (accessed October 10, 2016). 
Wunsch, C., and Heimbach, P. (2014). Bidecadal thermal changes in the abyssal ocean. J. Phys. Oceanogr. 44, 2013-2030. doi: 10.1175/JPO-D-13-096.1

Yang, J., Riser, S. C., Nystuen, J. A., Asher, W. E., and Jessup, A. T. (2015). Regional rainfall measurements using the Passive Aquatic Listener during the SPURS field campaign. Oceanography 28, 124-133. doi: 10.5670/oceanog. 2015.10

Yeager, S., Karspeck, A., Danabasoglu, G., Tribbia, J., and Teng, H. (2012). A decadal prediction case study: late twentieth-century North Atlantic ocean heat content. J. Clim. 25, 5173-5189. doi: 10.1175/JCLI-D-1100595.1

Zilberman, N., and Maze, G. (2015). Report on the Deep Argo Implementation Workshop. Available at: http://www.argo.ucsd.edu/DAIW1report.pdf (accessed October 9, 2015).

Zilberman, N. V. (2017). Deep Argo: sampling the total ocean volume in state of the climate in 2016. Bull. Am. Meteorol. Soc. 98, S73-S74. doi: 10.1175/ 2017BAMSStateoftheClimate.1

Conflict of Interest Statement: The authors declare that the research was conducted in the absence of any commercial or financial relationships that could be construed as a potential conflict of interest.

Citation: Roemmich D, Alford MH, Claustre H, Johnson K, King B, Moum J, Oke P, Owens WB, Pouliquen S, Purkey S, Scanderbeg M, Suga T, Wijffels S, Zilberman N, Bakker D, Baringer M, Belbeoch M, Bittig HC, Boss E, Calil P, Carse F, Carval T, Chai F, Conchubhair DÓ, d'Ortenzio F, Dall'Olmo G, Desbruyeres D, Fennel K,
Fer I, Ferrari R, Forget G, Freeland H, Fujiki T, Gehlen M, Greenan B, Hallberg R, Hibiya T, Hosoda S, Jayne S, Jochum M, Johnson GC, Kang K, Kolodziejczyk N, Körtzinger A, Le Traon P-Y, Lenn Y-D, Maze G, Mork KA, Morris T, Nagai T, Nash J, Naveira Garabato A, Olsen A, Pattabhi RR, Prakash S, Riser S, Schmechtig C, Schmid C, Shroyer E, Sterl A, Sutton P, Talley L, Tanhua T, Thierry V, Thomalla S, Toole J, Troisi A, Trull TW, Turton J, Velez-Belchi PJ, Walczowski W, Wang H, Wanninkhof R, Waterhouse AF, Waterman S, Watson A, Wilson C, Wong APS, Xu J and Yasuda I (2019) On the Future of Argo: A Global, Full-Depth, MultiDisciplinary Array. Front. Mar. Sci. 6:439. doi: 10.3389/fmars.2019.00439

Copyright (ㄷ) 2019 Roemmich, Alford, Claustre, Johnson, King, Moum, Oke, Owens, Pouliquen, Purkey, Scanderbeg, Suga, Wijffels, Zilberman, Bakker, Baringer Belbeoch, Bittig, Boss, Calil, Carse, Carval, Chai, Conchubhair, d'Ortenzio, Dall'Olmo, Desbruyeres, Fennel, Fer, Ferrari, Forget, Freeland, Fujiki, Gehlen, Greenan, Hallberg, Hibiya, Hosoda, Jayne, Jochum, Johnson, Kang, Kolodziejczyk, Körtzinger, Le Traon, Lenn, Maze, Mork, Morris, Nagai, Nash, Naveira Garabato, Olsen, Pattabhi, Prakash, Riser, Schmechtig, Schmid, Shroyer, Sterl, Sutton, Talley, Tanhua, Thierry, Thomalla, Toole, Troisi, Trull, Turton, Velez-Belchi, Walczowski, Wang, Wanninkhof, Waterhouse, Waterman, Watson, Wilson, Wong, $\mathrm{Xu}$ and Yasuda. This is an open-access article distributed under the terms of the Creative Commons Attribution License (CC BY). The use, distribution or reproduction in other forums is permitted, provided the original author(s) and the copyright owner $(s)$ are credited and that the original publication in this journal is cited, in accordance with accepted academic practice. No use, distribution or reproduction is permitted which does not comply with these terms. 
OPEN ACCESS

Edited by:

Frank Edgar Muller-Karger, University of South Florida,

United States

Reviewed by:

Nicholas J. Bax,

Commonwealth Scientific and Industrial Research Organisation

(CSIRO), Australia

Vardis Maximilian Tsontos, NASA Jet Propulsion Laboratory

(JPL), United States

${ }^{*}$ Correspondence:

Molly Powers

mollyp@spc.int

Specialty section:

This article was submitted to

Ocean Observation

a section of the journal

Frontiers in Marine Science

Received: 30 October 2018

Accepted: 15 July 2019

Published: 02 August 2019

Citation:

Powers M, Begg Z, Smith G and

Miles E (2019) Lessons From the Pacific Ocean Portal: Building Pacific

Island Capacity to Interpret, Apply, and Communicate Ocean Information.

Front. Mar. Sci. 6:476

doi: 10.3389/fmars.2019.00476

\section{Lessons From the Pacific Ocean Portal: Building Pacific Island Capacity to Interpret, Apply, and Communicate Ocean Information}

\author{
Molly Powers ${ }^{1 *}$, Zulfikar Begg ${ }^{1}$, Grant Smith ${ }^{2}$ and Elaine Miles ${ }^{2}$ \\ ${ }^{1}$ Pacific Community (SPC), Suva, Fiji, ${ }^{2}$ Bureau of Meteorology, Melbourne, VIC, Australia
}

The need for improved access to ocean observations for Pacific Island countries (PICs) and territories has been increasingly recognized over the last decade, particularly in the face of a changing climate. Although more remote sensing and in situ data are available than ever before, however, oceanographic, and marine forecasting expertise in the region is limited. To support capacity building in these areas, the Climate and Oceans Support Program in the Pacific (COSPPac) has engaged with partners in the National Meteorological Services (NMS) and other relevant agencies in 14 Pacific Island nations, to identify priorities and to develop tools and training to address these needs. A key tool is the online Pacific Ocean Portal. With a focus on the Pacific Islands region, this website provides ocean data relevant to a range of sectors and applications such as tourism, fishing, shipping, coastal inundation, and environmental management. Via a user-friendly interface, the portal serves up data from a variety of sources including near real-time observations, historical information and forecast data. Training modules have been designed for portal users and delivery has gone hand-in-hand with in-country stakeholder engagement workshops, allowing sector users to make requests for ocean information products. Eight workshops have been delivered from November 2015 to June 2018, training a total of 97 NMS staff and 116 ocean sector stakeholders including port authorities, disaster management, tourism, fisheries, community leaders, and many more. As a result, five Pacific Island NMSs (Tonga, Tuvalu, Kiribati, Samoa, and Vanuatu) are now producing monthly Ocean Outlooks, guided by the needs of in-country stakeholders. Outlooks are tailored for each country and can include forecasts such as sea surface temperature, coral bleaching, and sea level, as well as information about current chlorophyll conditions, wind, and wave climate.

Keywords: Pacific Islands, capacity building, SIDS, portal, ocean outlook 


\section{INTRODUCTION}

Pacific Island countries (PICs) are custodians of $20 \%$ of global Exclusive Economic Zones (EEZs), where ocean space exceeds land area by an average factor of 300 to 1 . Pacific Island countries often referred to as small island developing states (SIDS), can be more accurately described as large ocean developing states due to the size of their EEZs, which are rich in biodiversity and natural resources (Appeltans et al., 2016). The majority of economic activities in these countries- from tourism to fishingrely upon the ocean, justifying the Pacific Island Forum's focus on the development of a sustainable Blue Pacific Economy (Pacific Islands Forum Pacific Islands Forum Secretariat, 2017).

The need to support SIDS in implementing the United Nations Sustainable Development Goal 14 was highlighted in the call for action from the 2017 Oceans Conference in New York. Ocean information has also been identified as a requirement for successful implementation of the United Nations-led initiative Global Framework for Climate Services (GFCS) in SIDS that are particularly vulnerable to climate variability and climate change (WMO, 2014).

The diffuse geography of PICs presents many challenges in disseminating ocean information to isolated communities. Datasets covering entire EEZs can be very large and this is especially true for data that extend below the ocean surface. Transmission of large datasets puts significant strain on internet bandwidth in locations that already have limited or unreliable capacity (Iwamoto et al., 2017). Datasets are becoming more sophisticated with improvements in spatial and temporal resolution, exacerbating transmission issues. Depending on the technical ability of the user, analysis of raw data may be too difficult and time consuming for practical application. Although many ocean data sources include visual representations, these may not always be ideally suited for the given application or target audience. Most ocean datasets only include a global or ocean basin view, which is not practical for small island nations.

Pacific Island National Meteorological Services (NMSs) are primarily responsible for weather observations, climate services, and seasonal climate outlooks. Many NMSs have expressed their aspiration to better engage the marine sector within their respective countries. For this to occur, NMSs require access to relevant ocean data that is frequently updated, low bandwidth, tailored for priority applications within their EEZs, and accompanied by a training program. It is essential that guidance is provided to NMSs and relevant stakeholders on how to access and interpret ocean data, which can then inform relevant decision-making (Bax et al., 2018).

The Climate and Oceans Support Program in the Pacific (COSPPac) was an AU\$36 million program funded by the Department of Foreign Affairs \& Trade (DFAT formerly AusAID) over 6 years (July 2012 to June 2018). It was recently extended for an additional 4 years and AU\$23 million until June 2022. The COSPPac program carries on the legacy from previous phases of support for NMSs in sea-level monitoring and climate prediction with the inclusion of capacity development and communication, information technology services, and ocean services (Commonwealth of Australia, 2012). The program objective is to enhance the capacity across NMSs and key stakeholders in 14 Pacific Island nations in climate and ocean variability and its applications. The countries participating in this program include the Cook Islands, Federated States of Micronesia, Fiji, Kiribati, Marshall Islands, Nauru, Niue, Palau, Papua New Guinea, Samoa, Solomon Islands, Tonga, Tuvalu and Vanuatu (illustrated in Supplementary Figure 1).

This paper offers a case study perspective on the development and roll-out of the COSPPac Pacific Ocean Portal (http:// oceanportal.spc.int), a web-based delivery system for ocean data in support of Pacific NMSs and in-country stakeholders. It also discusses lessons learnt, outcomes, and benefits to portal users, and explores future opportunities.

\section{OCEAN INFORMATION REQUIREMENTS FOR THE PACIFIC}

For the majority of Pacific Island countries, the ocean is the most significant natural asset available to support sustainable economic development. Pacific regional leaders have promoted numerous initiatives to secure the conservation and sustainable use of the ocean and its resources. These include, but are not limited to, the Western and Central Pacific Fisheries Convention of 2004, the Framework for Resilient Development in the Pacific (2017-2030), and the Framework for a Pacific Oceanscape of 2010. Building upon the "Blue Pacific" concept endorsed by Pacific Island Leaders in 2017, the 49th Pacific Islands Forum Meeting in 2018 highlighted oceans, fisheries, and climate change and disaster resilience among the priority issues confronting the region (Pacific Islands Forum Secretariat, 2018).

At the Pacific Meteorological Council in Nuku'alofa, Tonga in 2015, the region highlighted the critical need for improved ocean and marine services and established the Pacific Islands Marine and Ocean Services (PIMOS) Expert Panel, tasked to advise NMSs on how best to meet these needs. Subsequently, the mid-term review of the Pacific Islands Meteorological Strategy in 2017 resulted in the revision of Pacific Key Outcome (PKO) 2 to include: Improved marine weather and establishment of ocean services (Secretariat of the Pacific Regional Environment Programme, 2017).

Underscoring the regional effort are actions at national levels seeking a better understanding of ocean processes to support management and conservation. Despite growing political support in the Pacific, the ocean science capacity of Pacific governments is limited and oceanographic expertise is available almost exclusively at the regional level. Improved ocean science and analysis capacity is required, not only for informed and sustainable resource management, but also for robust early warning systems, sea-level rise monitoring, adaptation planning, climate-smart development, and growing the blue economy.

Feedback from representatives of 14 PICs captured at a consultation in April 2011 identified 65 references to ocean information products and services. There was significant focus on disaster mitigation, especially inundation events experienced from high tides, sea-level rise, high waves, and storm surge. Sea-level historical climatology, predictions, and long-term 
projections were of interest. Fisheries were identified as an important aspect of Pacific livelihoods requiring decisionmaking tools. Sea surface temperature relating to coral bleaching and the impact on local fisheries was also a key concern. Consequently, delivery of a set of PIC ocean products and services was planned under COSPPac (Commonwealth of Australia, 2012) to fulfill the outcome "Pacific Island NMSs and other relevant in-country agencies understand and use climate, ocean, and sea level products for the benefit of island communities and governments."

Based on requests and needs identified, it was determined that a Pacific Ocean services web portal would be useful for NMSs to deliver more comprehensive climate and ocean data services. Most of the data available in the portal can be accessed via other individual websites online, but the Pacific Ocean Portal seeks to make them available in a single location, organized by application, and visually consistent in their presentation, to facilitate interpretation.

A similar approach was successfully undertaken to synthesize climate change time series data into the Pacific Climate Change Data Portal under the Pacific Climate Change Science Program (McGree et al., 2014). In terms of a visual map-based portal, the most similar product for the Pacific is the PacIOOS Voyager tool (Iwamoto et al., 2017, Potemra et al., 2017). The Voyager website was developed for US-affiliated PICs and later extended to all PICs. It is comprehensive, including detailed historical, near-real time, and forecast data, resulting in a high bandwidth requirement. Feedback from Pacific users stated that it was difficult to ascertain the application of the different datasets when they were grouped by data sources or data type. User feedback and specialist reviews of these tools directly informed the development of the COSPPac Pacific Ocean Portal.

\section{PACIFIC OCEAN PORTAL DESIGN AND DATA}

The Pacific Ocean Portal is an interactive website for visualizing and accessing historical, real-time and predictive ocean data categorized by marine applications on an integrated and user-friendly platform. The intended primary users of the portal are scientists within NMSs and other government agencies, marine industry and conservation stakeholders, and the general public. It is a tool that aims to both obtain and communicate ocean information. A range of visual formats are employed to support different downstream applications (i.e., maps, time-series, statistical charts, profiles as illustrated in Supplementary Figure 2). Data sources include satellite observations, in situ observations, and numerical models (reanalysis and forecast).

From the outset, the portal aimed to provide regional-tocountry level information (Commonwealth of Australia, 2012). The intention was to make the portal as intuitive as possible through use of a point-and-click interface to access data, graphs, and tables. Early on in the project, an IT capacity mapping of 14 PIC NMSs was undertaken. Following the discovery that PIC internet speeds were all in the order of kilobytes per second, significant effort was made to ensure the portal minimized bandwidth use. The design needed support of all major browser platforms.

The architecture for the portal was informed by business requirements for the COSPPac project, user cases, and feedback from PIC and Australian climatologists. COSPPac prioritized the sustainability of products beyond the life of the project. With this in mind, only open-source components were selected so they could be used in the PICs without licensing fees. The tools and libraries selected are the most suitable for the purpose and also widely supported by developers and organizations around the world. Using familiar products ensures that it is easy to find experts capable of extending the tools and enables re-use of the components in future products. As will be discussed in section References, this design attribute has already proven valuable.

High-resolution datasets were chosen as the preferred option where possible to better resolve small islands, although there were limitations across many gridded datasets. The datasets and sources initially selected for integration into the portal are listed in Table 1, along with the potential applications of this data for PICs.

The portal's landing page, shown in Figure 1, is designed to direct specialist and non-specialist users alike toward the datasets or information that will be most relevant for their needs, based primarily on its application. All of the spatial datasets within the portal are accessible via an interactive mapping environment that permits exploration through zooming, scrolling, and data point extraction, allowing the user to create tailored imagery for communication purposes, and to screen capture data outputs for any location.

\section{EDUCATION, TRAINING, AND AWARENESS BUILDING}

As recommended, COSPPac sought to facilitate the uptake the Pacific Ocean Portal to PIC users (Commonwealth of Australia, 2012; Thompson et al., 2012). Sustainable capacity building is a frequent challenge in development initiatives, particularly those that introduce a new tool or technology. To address this challenge, an integrated approach was employed spanning portal design, tailored training, and training material development, collaboration with Pacific regional organizations and in-country partners, ongoing consultation, high-level endorsement, and ultimately, integration into existing regional products, services, courses and initiatives.

A series of "help" documents were created for each dataset that describes the dataset, background science behind the data, how to navigate the portal to obtain the information, suggested applications for the dataset in the Pacific, and technical details regarding the data sources, including references.

The portal was the key teaching tool introduced in a series of Ocean \& Tides Workshops delivered across the Pacific between 2015 and 2018. Sub-regional and national workshops were conducted in the Solomon Islands (November 2015), Fiji (September 2016), Cook Islands (November 2016), Marshall 
TABLE 1 | Datasets available within the Pacific Ocean Portal with data source and potential PIC applications.

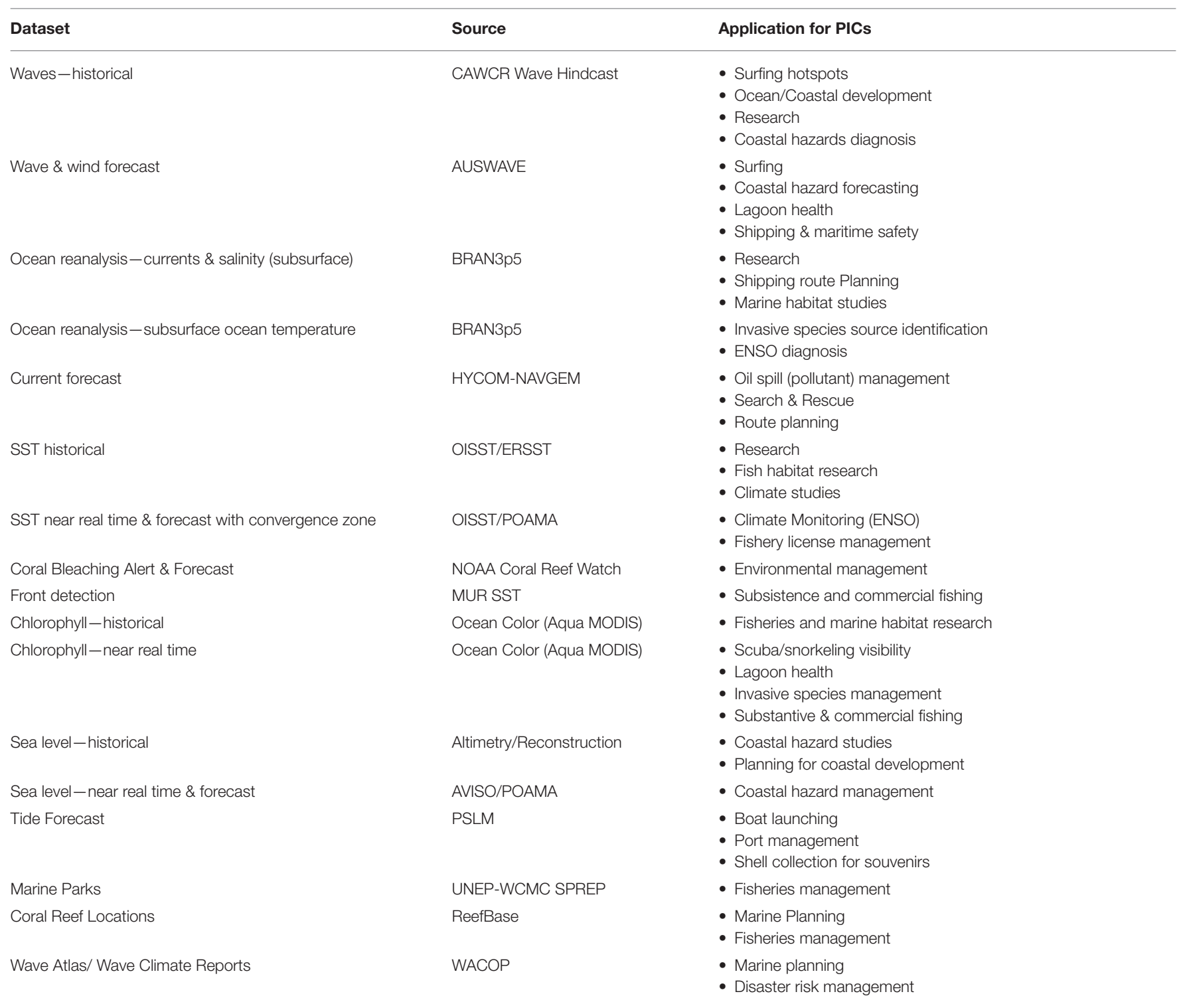

Islands (March 2017), Tuvalu (October 2017), Tonga (November 2017), Niue (March 2018), and Samoa (May 2018) with NMS and other ocean-related agencies and organizations in each country. A total of 97 NMS staff across 14 countries were trained, plus an additional 116 stakeholders. Of the 213 total Pacific Islanders trained, 41 of them were women (19\%).

The workshops aimed to provide background ocean science behind the portal, teach workshop participants how to navigate the portal, provide examples of marine applications, and support NMSs in initiating dialogue with in-country users of ocean data. Workshops were hosted by and developed in consultation with the local NMS, according to their needs. Typically, the program would include a few days of technical training for NMS staff, followed by a stakeholder training and consultation allowing sector users to make requests for ocean information products. Where practical, case studies, demonstrations, field trips, and presentations by local experts were incorporated to cater to different learning styles and reinforce the local relevance of science tools and training. At regional workshops, participants were encouraged to develop science posters using outputs from the portal and national workshops focused around the development of national ocean services and, where relevant, products using the portal as illustrated in Figure 1.

Nine training modules with explicit learning outcomes were designed to familiarize users with ocean science concepts, the portal, and other ocean data products. Each module includes a step-by-step guide, followed by a series of exercises to help self-directed learners explore. The modules were progressively tailored to ensure relevance to each country prior to the 


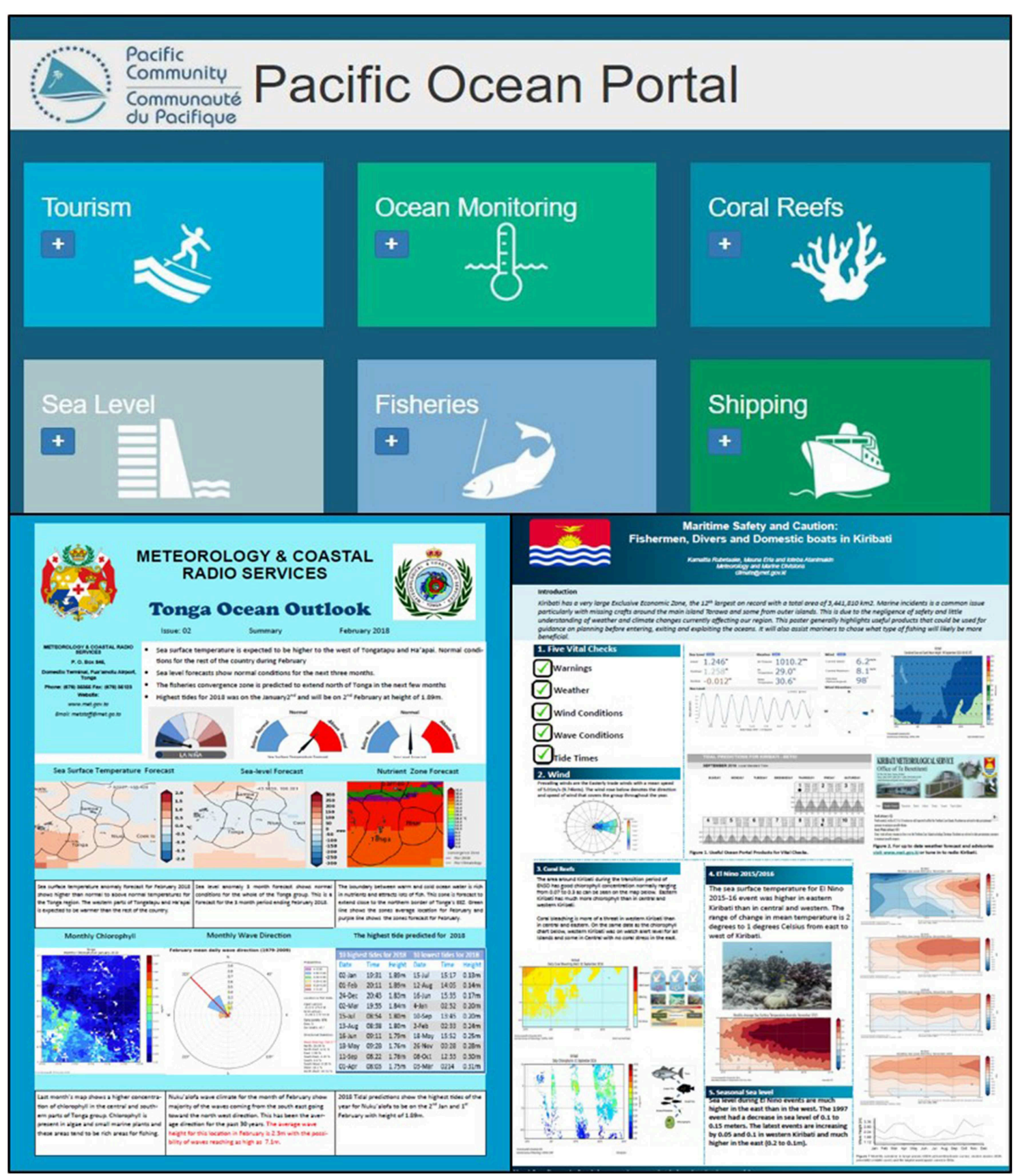

FIGURE 1 | (Top) landing page of the Pacific Ocean Portal http://oceanportal.spc.int (Bottom left) Monthly Ocean Outlook produced by Tonga Meteorological Service (Tonga Meteorological Service (TMS), 2018) and (Bottom right) an Ocean Science for Maritime Safety and Caution poster produced by Kiribati stakeholders at the Fiji Sub-regional Workshop in 2016 (Eria et al., 2016).

workshop, and later updated based upon participant and facilitator feedback.

The Pacific Ocean Portal was officially launched by the President of the Republic of Fiji, Jioji Konrote on World Oceans Day, 8th June 2018, in Suva, Fiji, as the opening event at the World Oceans Day Symposium. In the Pacific region, securing a visible and formal endorsement of such a tool is a unique opportunity to boost awareness and use among key stakeholders.
The 1-day event directly reached more than 200 participants, invited guests and visitors, and many more people through television, radio, and newspaper features and interviews. In addition, an introductory Know Your Ocean YouTube video demonstrating applications of the portal was produced for this event to reinforce the user-friendly nature of the portal, encourage sharing on social media, and promote ocean awareness and monitoring. 


\section{OUTCOMES, BENEFITS, AND FUTURE OPPORTUNITIES}

Since the soft-launch of the first portal version in 2015 until the official launch in June 2018, there have been a number of successful outcomes and significant benefits of this new tool to the Pacific Islands region. Notably, the portal has been integrated into routine early warning products and services of Pacific NMSs, regional organizations, and regional training courses, including the University of the South Pacific (USP)'s post-graduate Physical Oceanography course.

The open-source design of the portal has proven a prudent strategy, as hosting and management was successfully handedover from the Bureau of Meteorology (BoM) to the Pacific Community (SPC) based in Suva, Fiji in June 2018. This handover was intended to ensure the sustainability of the portal and to reinforce the regional ownership and relevance of the products. SPC is the Pacific Islands' regional technical organization, with an established Ocean \& Maritime Programme providing the region's hub of oceanographic expertise, and has led delivery of in-country portal training since 2016. The portal is also poised to contribute to the proposed Pacific Community Center for Ocean Science (PCCOS), an integrated facility to improve accessibility of ocean knowledge and facilitate interdisciplinary partnerships (Pacific Community, 2018a). As the regional coordinator for the UN Decade of Ocean Science for Sustainable Development 2021-2030, SPC is thus well-placed to ensure the continued investment in portal development and support for training.

Even with its low bandwidth design, the portal was observed to have difficulty loading larger maps and graphs in countries with lower bandwidth internet access. Since the portal was first designed, however, internet access across the region has improved significantly. Further upgrades are planned and therefore an associated improvement in portal performance is anticipated into the future. As a bandwidth comparison, accessing the portal web page, and producing the most recent SST anomaly required 1.9MB, compared to PacIOOS Voyager, which required 3.3MB. Since the transition, SPC has continued to add value to the portal and is currently integrating a new data management interface, updating wave climate reports, and adding new resources to the site. Regular maintenance is required as data sources are constantly changing and being upgraded. As new applied research and Pacific data become available, SPC can expand the portal to include these features if there is a clearly identified application and benefit for the additional data.

Since 2015, the website has received over 14,000 users worldwide with 3,500 separate users accessing the portal across Melanesia, Polynesia, and Micronesia. SPC counted over 2,500 hits in September 2018, and 285 users, 62\% of whom were returning users. The largest peaks in traffic occurred during cyclonic events in the Pacific, demonstrating that users have a strong interest in ocean information in the fields of risk management and disaster mitigation. There was a noticeable uptick in users in countries following COSPPac incountry workshops. Follow-up surveys conducted 6 months after workshops held in Niue and Samoa indicate that at least 25\% of workshop participants gained new skills, passed new skills onto another colleague, and have implemented changes to their work as a result the training.

Following in-country workshops and NMS staff attachment trainings at BoM and SPC, six Pacific Island NMS (Tonga, Tuvalu, Kiribati, Samoa, Fiji, and Vanuatu) are now producing monthly ocean outlooks, as in Figure 1. Ocean outlooks are tailored to individual country user needs and include forecasts such as SST, convergence zone location, coral bleaching alerts, and sea level, as well as information about current chlorophyll conditions, wind and wave climate. In Tonga, Tuvalu, Fiji, Vanuatu and Samoa, the outlooks were co-designed by users to include priority sector-specific information. With close collaboration between PICs and support from SPC, these outlooks offer other NMSs an example of what can be achieved using the portal. In another example, in March 2019, the Fiji Met Service used the portal to respond to requests from local media on marine heatwaves in the region, providing SST anomaly and bleaching risk maps, a textual interpretation of the data, and background science that was sourced from the included "help files" within the portal.

Targeted in-country training and stakeholder engagement on ocean data applications under COSPPac has helped to cement NMSs as focal agencies for ocean forecasting services while also providing tools and skills to other ocean-data users such as fisheries, shipping and maritime, environment, and disaster management. Ocean stakeholders have also reported beneficial outcomes. Following the Niue workshop in March 2018, the owner of Niue Tours and Commodore of the Niue Yacht Club reported, "After the workshop, we immediately started using the tide chart for our tour operations. I am also collating all the relevant information from the ocean portal to be included in the Niue Yacht Club's website for the coming season" (Pacific Community, 2018b).

SPC conducted a COSPPac Ocean Information Products usersurvey in September 2018. This is an inter-annual practice to assess the relevance of products, and to plan improvements and future products. Of 94 participants (out of 400 surveyed) from 15 different Pacific countries and territories, $65 \%$ indicated that it was a highly valuable tool for them or their work. Users reported that Ocean Monitoring (74.5\%) and Sea Level (71.3\%) were most useful datasets and functions of the portal. As knowledge brokers for climate and ocean science, NMSs were the most frequent users, integrating the portal into their daily operations, monitoring severe weather conditions, and producing monthly outlooks. In addition, other users reported using the portal as a reference for search and rescue operations, scheduling of recreational activities, fishing, and fieldwork, and even using outputs or information from helpfiles to create new information products or country reports. Survey results are reported in Supplementary Table 1.

Looking toward the future, SPC aims to continue to develop the portal in response to regional needs and requests. In coordination with the PMC, PIMOS Panel and WMO, SPC plans to develop an accredited online oceanographic competency module for NMSs that incorporates the portal and training materials. In addition, continued collaboration with regional 
educational institutions is proposed to explore the development of online courses and other options to formalize portal training and increase accessibility.

While satellite technology is revolutionizing ocean observations, the resolution of many large ocean datasets is still insufficient to resolve the small island states in the Pacific. Downscaled, local models are essential for understanding the impacts of increasing SSTs in atoll lagoons, sea-level rise, and wave transformation on small island coastlines, for example.

It is hoped that an increase in PIC oceanographic capacity will contribute to increased investment in ocean observations infrastructure and increased engagement with the global ocean observing community. The Pacific may be the world's largest ocean, but it is also the most data-sparse and PICs are significantly under-represented in global discussions on ocean observations (Secretariat of the Pacific Regional Environment Programme, 2017). The region also needs to engage in international discussions on improved data sharing standards so that systems can be upgraded easily and information shared.

The UN Decade of Ocean Science presents a timely opportunity to catalyze action on ocean monitoring, and the Pacific Islands region is poised to be a key player, as the stakes are high for Large Ocean States. Protecting ocean resources, ensuring economic security, understanding vulnerability to climate change and variability all rely on robust ocean monitoring systems. However, the importance of human capacity to interpret and act upon information available from these systems is equally vital. Facilitating the transformation of data to information, information to knowledge, and knowledge to policy and action is a critical challenge for ocean observations. This requires not only scientific expertise and technological advances, but an integrated

\section{REFERENCES}

Appeltans, W. Dujardin, F., Flavell, M., Miloslavich, P., and Webb, T. J. (2016). Biodiversity Baselines in the Global Ocean, The Open Ocean: States and Trends. Paris: UNESCO.

Bax, N. J., Appeltans, W., Brainard, R., Duffy, J. E., Dunstan, P., Hanich, Q., et al. (2018). Linking capacity development to GOOS monitoring networks to achieve sustained ocean observation. Front. Mar. Sci. 5:346. doi: $10.3389 /$ fmars.2018.00346

Commonwealth of Australia (2012). Climate and Ocean Support Program in the Pacific (COSPPac). Program Design Document, Melbourne.

Eria, M., Rubetaake, K., and Atanimakin, I. (2016). Maritime Safety and Caution: Fisherman, Divers, and Domestic Boats in Kiribati, Nadi: Fiji.

Iwamoto, M., Langenberger, F., and Ostrander, C. E. (2017). Ocean observing: serving stakeholders in the pacific islands, marine technology society. Journal 50:pp 47-54. doi: 10.4031/MTSJ. 50.3 .2

McGree, S., Whan, K., Jones, D., Alexander, L. V., Imielska, A., Diamond, H., et al. (2014). An updated assessment of trends and variability in total and extreme rainfall in the western Pacific. Int. J. Climatol. 34, 2775-2791. doi: $10.1002 /$ joc. 3874

Pacific Community (2018a). Pacific Community Centre for Ocean Science Implementation Strategy, Noumea, New Caledonia.

Pacific Community (2018b). Ocean and Tides Workshop Makes immediate Impact on Niue Communities, Suva, Fiji. 9 April 2018.

Pacific Islands Forum Secretariat (PIFS) (2017). State of Pacific Regionalism Report 2017, Suva, Fiji. approach informed by science communication, social science, user-centered design, and policy best-practice.

In conclusion, the Pacific Ocean Portal is an example of consultative design and, accompanied by outcome-driven training, it is already contributing significantly toward the development of ocean and marine services in the Pacific Islands. Embedded within SPC's Ocean and Maritime Programme and with the ongoing Australian Aid support through 2022, it is anticipated that the portal will continue to develop and play a key role in building the marine meteorological competency of Pacific NMSs to meet the needs of the Pacific marine community into the future.

\section{AUTHOR CONTRIBUTIONS}

MP: primary drafting, editing of the paper, circulated for feedback among other authors, Ocean Literacy \& Capacity Building Leader at SPC- led delivery of workshops. GS: drafted first three sections, shaped the content, edited, provided some references, led development of BoM Ocean Portal, curriculum development and helpfile drafting. ZB: edited, proof-read, and provided details on ocean portal data sets in Table 1, currently overseeing and maintaining Ocean Portal. EM: edited and suggested future-forward content, supported development of the Portal.

\section{SUPPLEMENTARY MATERIAL}

The Supplementary Material for this article can be found online at: https://www.frontiersin.org/articles/10.3389/fmars. 2019.00476/full\#supplementary-material
Pacific Islands Forum Secretariat (PIFS) (2018). Forty-Ninth Pacific Islands Forum Communique, Yaren, Nauru.

Potemra, J., Maurer, J., and Burns, E. S. (2017). "Providing Oceanogrpahic Data and Information to Pacific Island Communities," in Oceanographic and Marine Cross-Domain Data Management for Sustainable Development, eds P. Diviacco, A. Leadbetter, and H. Glaves (Hershey, PA: IGI Global), 253-281. doi: 10.4018/978-1-5225-0700-0.ch011

Secretariat of the Pacific Regional Environment Programme (SPREP) (2017). Pacific Islands Meteorological Strategy 2017-2026. Apia, Samoa.

Thompson, E., Howie, M., and Amjadali, A. (2012). Pacific Islands Climate Prediction Project Completion Report. Melbourne, Australia.

Tonga Meteorological Service (TMS) (2018). Tonga Ocean Outlook, Nuku'alofa, Tonga. Available online at: http://www.met.gov.to/

WMO (2014). Implementation Plan of the Global Framework for Climate Services, Geneva, Switzerland.

Conflict of Interest Statement: The authors declare that the research was conducted in the absence of any commercial or financial relationships that could be construed as a potential conflict of interest.

Copyright (C) 2019 Powers, Begg, Smith and Miles. This is an open-access article distributed under the terms of the Creative Commons Attribution License (CC BY). The use, distribution or reproduction in other forums is permitted, provided the original author(s) and the copyright owner(s) are credited and that the original publication in this journal is cited, in accordance with accepted academic practice. No use, distribution or reproduction is permitted which does not comply with these terms. 


\section{OPEN ACCESS}

Edited by:

Sanae Chiba

Japan Agency for Marine-Earth

Science and Technology, Japan

Reviewed by:

Alessandra Giorgetti,

Istituto Nazionale di Oceanografia e di

Geofisica Sperimentale (OGS), Italy

Hiroaki Saito,

The University of Tokyo, Japan

*Correspondence:

Nadia Pinardi

nadia.pinardi@unibo.it

Specialty section:

This article was submitted to

Ocean Observation,

a section of the journa

Frontiers in Marine Science

Received: 29 October 2018

Accepted: 03 July 2019

Published: 02 August 2019

Citation:

Pinardi N, Stander J, Legler DM, O'Brien K, Boyer T, Cuff T, Bahurel P,

Belbeoch M, Belov S, Brunner S, Burger E, Carval T, Chang-Seng D,

Charpentier E, Ciliberti S, Coppini G

Fischer A, Freeman E, Gallage C,

Garcia H, Gates L, Gong Z, Hermes J,

Heslop E, Grimes S, Hill K,

Horsburgh $K$, Iona A, Mancini S, Moodie N, Ouellet M, Pissierssens $P$,

Poli P, Proctor R, Smith N, Sun C, Swail V, Turton J and Xinyang $Y$ (2019) The Joint IOC (of UNESCO) and WMO

Collaborative Effort for Met-Ocean Services. Front. Mar. Sci. 6:410. doi: 10.3389/fmars.2019.00410

\section{The Joint IOC (of UNESCO) and WMO Collaborative Effort for Met-Ocean Services}

\author{
Nadia Pinardi ${ }^{1 *}$, Johan Stander ${ }^{2}$, David M. Legler ${ }^{3}, K_{\text {Kevin O'Brien }}^{4,5}$, Tim Boyer $^{6,7}$, \\ Tom Cuff $^{8}$, Pierre Bahurel ${ }^{9}$, Mathieu Belbeoch ${ }^{10}$, Sergey Belov ${ }^{11}$, Shelby Brunner ${ }^{3}$, \\ Eugene Burger ${ }^{4,5}$, Thierry Carval ${ }^{12}$, Denis Chang-Seng ${ }^{13}$, Etienne Charpentier ${ }^{14}$, \\ S. Ciliberti ${ }^{15}$, Giovanni Coppini ${ }^{15}$, Albert Fischer ${ }^{13}$, Eric Freeman ${ }^{7,16}$, Champika Gallage ${ }^{14}$, \\ Hernan Garcia ${ }^{6,7}$, Lydia Gates ${ }^{17}$, Zhiqiang Gong ${ }^{18}$, Juliet Hermes ${ }^{19}$, Emma Heslop ${ }^{13}$, \\ Sarah Grimes ${ }^{14}$, Katherine Hill ${ }^{14}$, Kevin Horsburgh ${ }^{20}$, Athanasia lona ${ }^{21}$, \\ Sebastien Mancini ${ }^{22}$, Neal Moodie ${ }^{23}$, Mathieu Ouellet ${ }^{24}$, Peter Pissierssens ${ }^{13}$, Paul Poli ${ }^{25}$, \\ Roger Proctor ${ }^{22}$, Neville Smith ${ }^{26}$, Charles Sun ${ }^{6,7}$, Val Swail ${ }^{27}$, Jonathan Turton ${ }^{28}$ and \\ Yue Xinyang ${ }^{29}$
}

${ }^{1}$ Department of Physics and Astronomy, University of Bologna, Bologna, Italy, ${ }^{2}$ South African Weather Service, Pretoria, South Africa, ${ }^{3}$ NOAA Climate Program Office, Silver Spring, MD, United States, ${ }^{4}$ NOAA, Pacific Marine

Environmental Laboratory, Seattle, WA, United States, ${ }^{5}$ Joint Institute for the Study of the Atmosphere and Ocean, University of Washington, Seattle, WA, United States, ${ }^{6}$ NOAA, National Centers for Environmental Information, Silver Spring, MD, United States, ${ }^{7}$ NOAA, National Centers for Environmental Information, Asheville, NC, United States, ${ }^{8}$ National Weather Service Organization, Silver Spring, MD, United States, ${ }^{9}$ Mercator Ocean, Toulouse, France, ${ }^{10}$ JCOMMOPS (WMO/IOC-UNESCO), Brest, France, " All Russian Research Institute of Hydrometeorological Information World Data Center, Obninsk, Russia, ${ }^{12}$ IFREMER, Brest, France, ${ }^{13}$ IOC of UNESCO, Paris, France, ${ }^{14}$ WMO, Geneva, Switzerland, ${ }^{15}$ CMCC Foundation, Lecce, Italy, ${ }^{16}$ Riverside Technology, Inc., Fort Collins, CO, United States, ${ }^{17}$ Deutscher Wetterdienst, Offenbach, Germany, ${ }^{18}$ China Meteorological Administration, Climate Division, Forecasting and Network Department, Beijing, China, ${ }^{19}$ South African Environmental Observation Network, Cape Town, South Africa, ${ }^{20}$ National Oceanography Center, Liverpool, United Kingdom, ${ }^{21}$ Hellenic Center for Marine Research, Athens, Greece, ${ }^{22}$ Australian Ocean Data Network, Integrated Marine Observing System, Hobart, TAS, Australia, ${ }^{23}$ Bureau of Meteorology, Melbourne, VIC, Australia, ${ }^{24}$ Fisheries and Oceans Canada, Ottawa, ON, Canada, ${ }^{25}$ Center de Météorologie Marine, Météo-France, Brest, France, ${ }^{26}$ GODAE Ocean Services, Canterbury, NSW, Australia, ${ }^{27}$ Climate Research Division, Environment and Climate Change Canada, Toronto, ON, Canada, ${ }^{28}$ UK Met Office, Exeter, United Kingdom, ${ }^{29}$ State Oceanic Administration, National Marine Data and Information Service, Tianjin, China

The WMO-IOC Joint Technical Commission for Oceanography and Marine Meteorology (JCOMM) has devised a coordination mechanism for the fit-for-purpose delivery of an end-to-end system, from ocean observations to met-ocean operational services. This paper offers a complete overview of the activities carried out by JCOMM and the status of the achievements up to 2018. The JCOMM stakeholders consist of the research and operational institutions of WMO members and the IOC member states, which mandated JCOMM to devise an international strategy to move toward the achievement of the United Nations Sustainable Development Goals. The three areas of activity are the Observation Program Area (OPA), the Data Management Program Area (DMPA) and the Services and Forecasting Services Program Area (SFSPA), and several expert teams have been established to contribute to the international coordination efforts. OPA is organized into observing networks connected by different observing technologies, DMPA organizes the overall near-real time and delayed mode data assembly, and the delivery methodology and architecture, and the SFSPA coordinates the met-ocean services 
resulting from the observations and data management. Future developments should enhance coordination in these three program areas by considering the inclusion of new and emergent observing technologies, the interoperability of met-ocean data assembly centers and the establishment of efficient research to operations protocols, in addition to better fit-for-purpose customized services in both the public and private sectors.

Keywords: marine meteorology and oceanography, global ocean observing networks, data management, ocean services, capacity development

\section{INTRODUCTION}

The World Meteorological Organization (WMO) and the Intergovernmental Oceanographic Commission of UNESCO (IOC) started the WMO-IOC Joint Technical Commission for Oceanography and Marine Meteorology (JCOMM) in 1999. JCOMM was mandated to enhance coordination between the meteorological and oceanographic communities to support the delivery of ocean forecasts and services, and the efficient design of global climate research programs, such as the Tropical Ocean Global Atmosphere (TOGA) program (McPhaden et al., 2010) and the Global Ocean Observing System (GOOS) (IOC Assembly Resolution XXVI-8, 2011; Task Team for the Integrated Framework for Sustained Ocean Observing, 2012).

The ocean economy is predicted to double by 2030 in terms of value-added products and employment (OECD, 2016) and population expansion and urbanization in coastal regions will increase people's vulnerability to climate change (McGranahan et al., 2007). To address this, JCOMM devised a coordination system that will help to standardize access to met-ocean data and services for the National Meteorological and Hydrological Services (NMHS) and for national oceanographic infrastructures. The system consists of the IOC International Oceanographic Data and Information Exchange (IODE), National Oceanographic Data Centers (NODCs) and the operational ocean forecasting services. JCOMM works at the interface between the WMO members, IOC member states and the research community (Figure 1), coordinating standardized research and operation transition, as required by environmental data services (Serafin et al., 2002; National Research Council, 2003; Shao et al., 2016). WMO members and IOC member states set the requirements of JCOMM activities, and the international community implements the solutions. JCOMM also facilitates the provision of observations to support other WMO and IOC applications, such as numerical weather prediction, climate monitoring, and other ocean services not solely governed via JCOMM.

In Internet terms, an end-to-end system is a network designed to connect nodes with maximum efficiency and quality. The application of such a principle to users and producers of meteorological and oceanographic information is a complex value chain structure, which is shown in Figure 2. JCOMM considers all the building blocks of the value-added chain, from the collection and distribution of observations in near-real time and delayed mode, the production of generic data products by modeling and data assembly centers, to the further customization of such products so they can be utilized by the final end-users to provide societal benefit. It should be noted that the value-adding chain shown in Figure 2 is not totally governed by JCOMM. The non-ocean and climate related components are governed by other international coordination entities that JCOMM must interface with.

The marine meteorology and ocean services generated by the meteorological and oceanographic (operational) centers and by research institutions provide information for a large number of users including governments, emergency responders, industry, navies and the public. JCOMM supports the National Meteorological and Hydrological Services (NHMS), the National Oceanographic Data Centers (NODC) and operational oceanographic services and strives to reach the UN's sustainable development goals (SDGs, Kates et al., 2005). The JCOMM beneficiaries are a complex network of research institutions, meteorological and oceanographic centers, navies, and industry that need international standards for exchanging observations and producing forecasts, to meet the challenges of climate change, coastal resilience, safe maritime transport and a productive and healthy ocean.

In this study we first provide an overview of the approach taken by the JCOMM in its coordination efforts (section The JCOMM Approach). We then examine the four pillars of JCOMM activities: the standardized collection of ocean

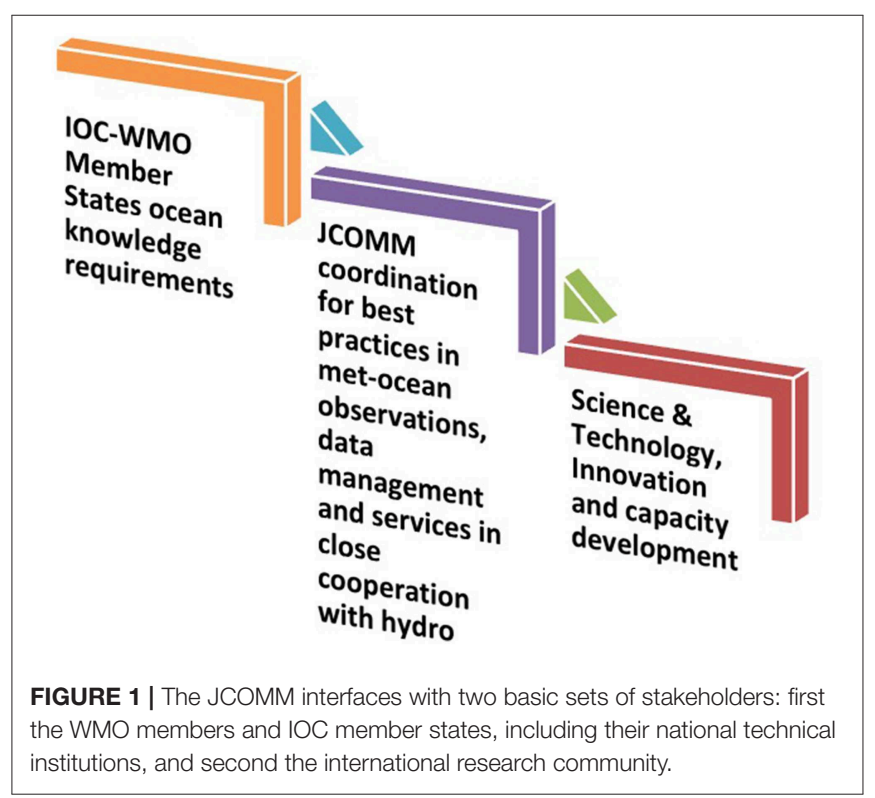




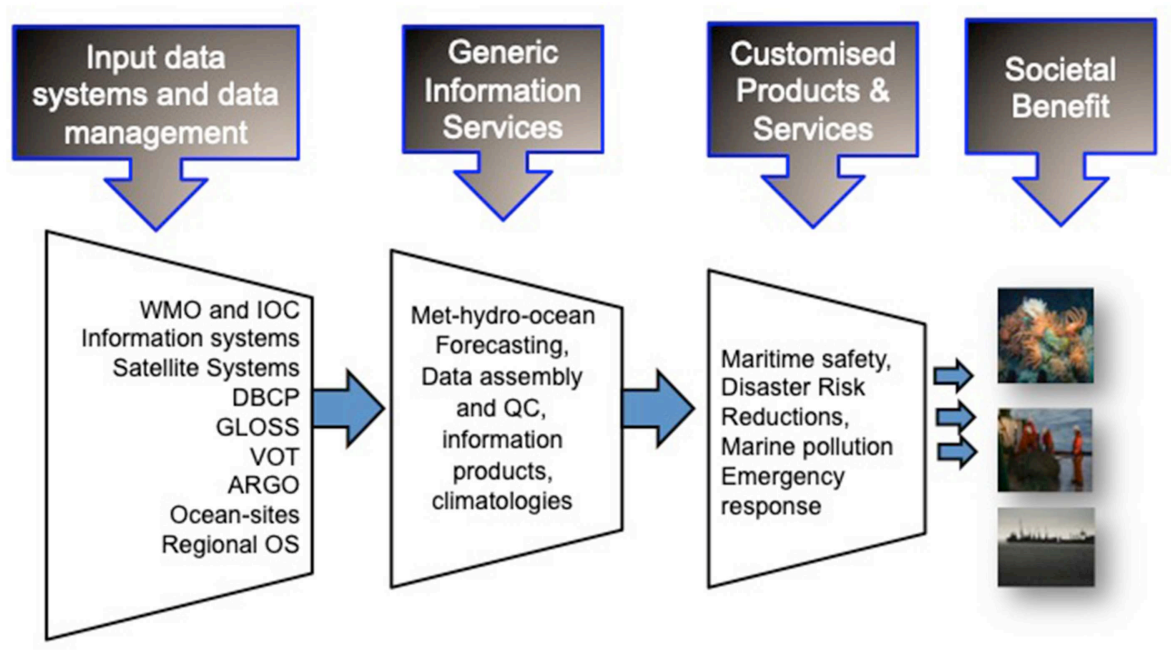

FIGURE 2 | The met-ocean end-to-end system at multiple time and space scales and for multiple users.

observations (section Operational Observing Networks and Systems), the networking of data management and data assembly centers (section Data Management Systems, From Near-Real Time to Delayed Mode), the ocean services (section From Monitoring to Services) and capacity building (section Capacity Development). A discussion and conclusions are provided in sections Discussion and Future Foci.

\section{THE JCOMM APPROACH}

In most nations of the world, NMHS, NODCs and oceanographic institutions are responsible for the delivery of meteorological and ocean marine products and services. They must consider the whole data information chain, from observations, to data assembly and predictions, to customized products for specific users (Figure 2). This delivery is the aim of the JCOMM international coordination activity, and to achieve it with the maximum interoperability, standardization, and sustainability.

The approach followed by JCOMM in building the international coordination for the met-ocean end-to-end system is summarized in Figure 3. The four building blocks are:

(1) The Member States met-ocean research and operational infrastructure, which is the basis of the WMO and IOC coordination responsibilities;

(2) The delivery of met-ocean products and services related to the WMO and IOC strategic goals, which require standardization and the transition from research to operations;

(3) Stakeholder and end-user assessments organized by JCOMM along with specific user consultation activities to evaluate the fitness for purpose of the service delivery;

(4) The scientific assessment of the status of the networks, which in principle should establish the fitness for use of the observations in the data product and service delivery,

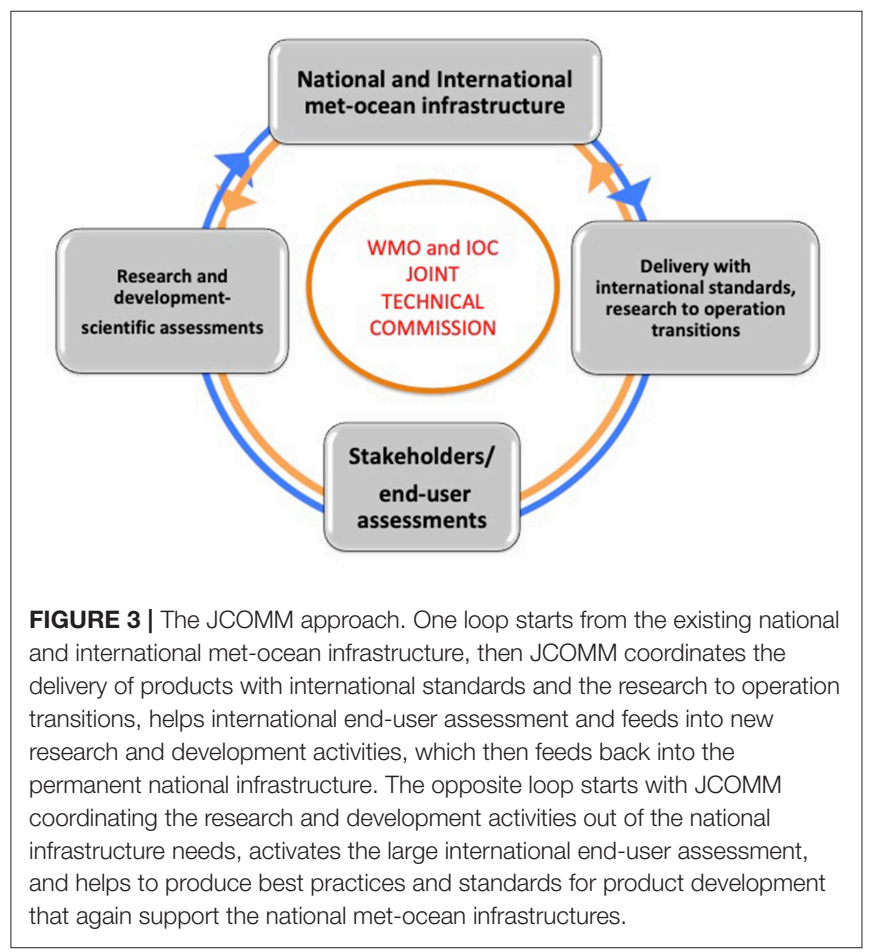

thus prompting new research and innovation activities to fill the gaps.

Since its inception 20 years ago, JCOMM has developed a coordinating structure for the delivery of met-ocean products and services. Over the past 5 years a scientific and stakeholder assessment of the existing coordinating structure has been conducted and will continue in the future.

To achieve its goals, JCOMM has been organized into four focal activity areas of technical expertise, as described in the next section. 


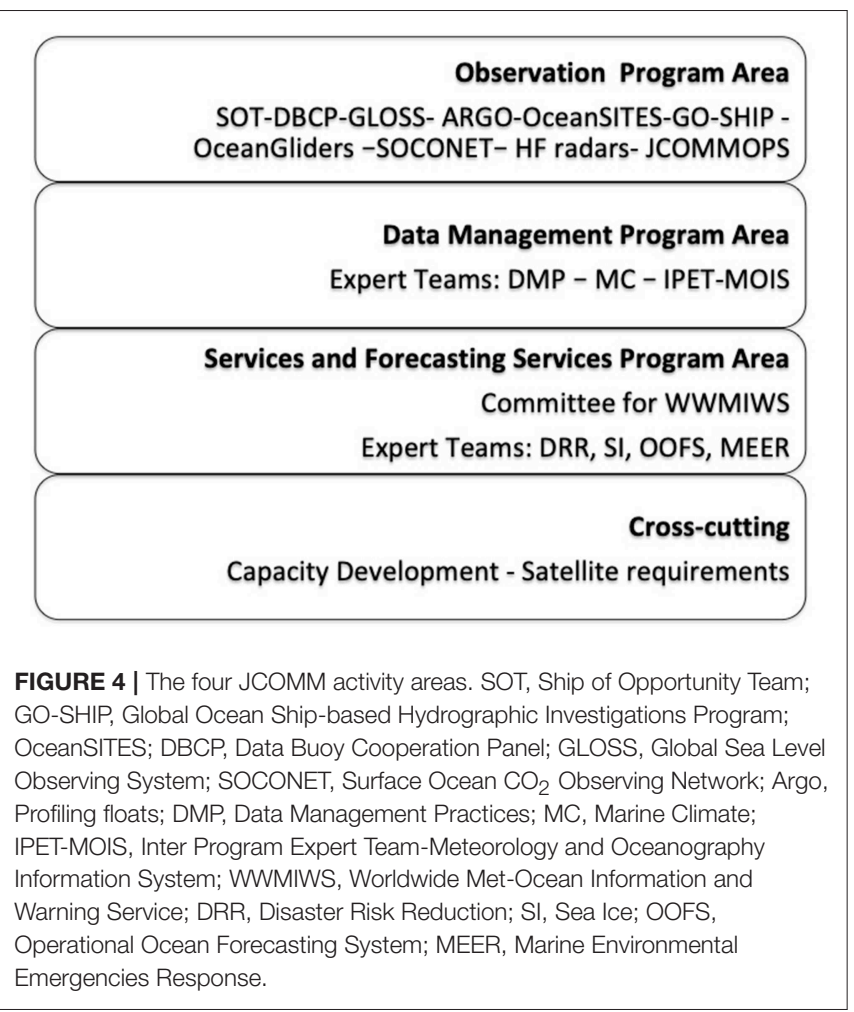

\section{The JCOMM Activity Areas}

In JCOMM, the end-to-end system (Figure 2) is sub-divided into three program areas and a cross-cutting activity, as shown in Figure 4. These are:

(1) The Observations Program Area (OPA), in which the Observations Coordination Group (OCG) is charged with reviewing, advising on, and coordinating the effective operation of the ocean and marine observing systems and related activities. This group also has the mandate to assess new and emerging technologies and enable their transition into operational networks.

(2) The Data Management Program Area (DMPA), in which the Data Management Coordination Group (DMCG) has the primary objective of reviewing, implementing and maintaining a fully integrated end-to-end data management system across the entire marine meteorology and oceanographic community. Recent work in this area includes metadata management and the implementation of the Marine Climate Data System (MCDS), which can be considered an interface between the WMO Information System (WIS, http://www.wmo.int/pages/prog/www/WIS/) and the future IOC Ocean Data and Information System (ODIS, https://www.iode.org/odis).

(3) The Services and Forecasting System Program Area (SFSPA), in which the Services Coordination Group (SCG) has the mandate to overview and coordinate state-of-the-art globally distributed and inter-connected marine services, including sea ice and emergency responses, natural- and humaninduced coastal hazards, and maritime safety.

(4) The cross-cutting area consists of two activities: Capacity Development (CD), whereby JCOMM has progressively implemented a training framework that considers the complete marine weather and oceanographic end-to-end system; and Satellite requirement activities, which focus on setting the requirements of WMO and IOC for the timely collection of remotely sensed met-ocean environmental data.

JCOMM activities have recently been evaluated, and selected conclusions are outlined in the next subsection.

\section{User-Based Surveys}

In April-May 2016 the IOC organized the first stakeholder survey on JCOMM performance. The survey was sent to the NMHS, NODCs and oceanographic institutions listed in the IOC and WMO JCOMM contacts and 90 responses were returned. The survey addressed the following areas: experience/familiarity with JCOMM (five major objectives/activities); impact/influence of JCOMM on Met-Ocean Institutions; importance/priority of JCOMM activities; management/coordination and capacity building of JCOMM; benefits of JCOMM; needs and new activities/approaches of JCOMM.

The results showed that $85 \%$ of respondents were involved in JCOMM activities for at least the past 5 years, but $40 \%$ were not aware of the breadth of topics in the program and the range of objectives. JCOMM was perceived to have a positive impact on NHMS and Oceanographic Institution activities by 35\% of respondents. Almost half of the answers confirmed that JCOMM was effective in coordinating the development of an integrated met-ocean system. However, the scores were low in terms of the training offered JCOMM for sharing met-ocean products and services, and it was suggested that JCOMM's role in the global development of tools/training for the local use of met-ocean products and services should be enhanced.

Recommended developments in JCOMM activities included the need for effective stakeholder consultation and less top-down governance, with enhanced inclusive and broader participation by countries and organizations. It was recommended that it should continue with its current activities, while improving its communication strategy and developing new partnerships with the private sector, and other programs.

The second survey was carried out by WMO to assess the perceptions of mariners on whether the Maritime Safety Information (MSI) services provided in the Global Maritime Distress and Safety (GMDSS) network met the user requirements. Overall, the survey demonstrated the importance of delivering wind, wave, sea ice, and visibility information to ships, but it also highlighted inadequate packaging of information, gaps in areas covered by observations, and the need for more wave information, such as swell, in addition to significant wave height and direction.

The outcomes of these initial surveys indicated that JCOMM was recognized as useful by the WMO and IOC stakeholders, but that much more work was needed to further raise awareness and update existing products and services. 


\section{Working Practices}

The primary governance structure of JCOMM is the management committee (MAN), which makes the strategic decisions regarding the programs and expert teams, evaluates the progress of the activities, and interacts with external groups. The JCOMM MAN consists of the two co-presidents, the IOC and WMO secretariats, the coordinators of the activity areas, and representatives from IODE, GOOS, and the GOOS Regional Alliances. External experts are invited as required, depending on the topic under discussion.

The expert teams (Figure 4) are the technical implementing bodies of the JCOMM strategy, and they can also directly feed back to the JCOMM management on problems and developments. This pyramidal structure, and thus the absence of a common "project-oriented" implementation strategy, was identified as a weakness of the system. In addition, the technical nature of the groups at all levels, from the JCOMM MAN to the expert teams, added to the complexity of making priorities and strategies for these joint activities, beyond their first decades. As discussed in the conclusions, a new structure is now envisaged for the future of JCOMM.

A more detailed analysis of the strengths and weaknesses of the JCOMM effort in general is presented in the discussion section, after we describe the work of the three program areas.

\section{OPERATIONAL OBSERVING NETWORKS AND SYSTEMS}

\section{Observations Program Area}

The JCOMM Observations Program Area (OPA) is appointed with reviewing and coordinating the effective operation of ocean and marine observing systems and related activities. Its focus is on developing synergies across the in situ observing networks, leading to a sustained global ocean observing system that meets research requirements and those of weather, ocean, and climate services, and maximizes opportunities for engaging with new technology and observing networks as they mature.

The Observations Coordination Group (OCG) leads the OPA. It is comprised of representatives of the major global networks involved in observational oceanography and marine meteorology. The OPA has defined the following attributes of a JCOMM ocean observing network, which are consistent with the Framework for Ocean Observing (Task Team for the Integrated Framework for Sustained Ocean Observing, 2012):

- Global in scale: Broader than regional, and if feasible, the intention to be global.

- Sustained observation: Sustained over multiple years, beyond the time-span of single research or experimental projects, by undertaking routine, systematic and essential observations.

- Community of practice: Has an identified community governance structure that provides a means of developing a multi-year strategy, implementation plans and targets, and standards and best practices.

- Delivers data that are free, open, and available in a timely manner: Has a defined data management infrastructure that delivers interoperable and inter-comparable data in real-time and/or with minimal delay after becoming available, through an internationally recognized data center/s and/or standard services, including the WMO Global Telecommunications System (GTS, real-time data), the MCDS (delayed-mode data) and web based services (real-time and delayed-mode data), and operates with a view of encompassing findable, accessible, interoperable and reusable (FAIR) principles (Wilkinson et al., 2016), with at least a minimum (as defined by JCOMMOPS) level of metadata.

- Observes one or more essential ocean variables or essential climate variables: Contributes to meeting requirements through observing one or more of the Global Ocean Observing System (GOOS) Essential Ocean Variables (EOVs) or Global Climate Observing System (GCOS) Essential Climate Variables (ECVs).

- Maintains network mission and targets: A role in the GOOS is defined and progress toward targets can be tracked and progress assessed.

- Develops, updates and follows standards and best practices: To ensure consistent delivery of observational data and encompassing measurement technique, deployment and sampling, reference materials and standards, calibration and validation, data retrieval and formatting, primary quality control and secondary quality control, as appropriate. Ideally these are documented, consistent with other OPA networks, and their use is encouraged.

The OCG works with the following global networks that have met these attributes (Figure 4): ship-based observations (managed by the Ship Observations Team-SOT), moored and drifting buoy observations (managed by the Data Buoy Cooperation Panel-DBCP), tide gauge observations (the Global Sea Level Observing System-GLOSS), Argo float observations (managed by the Argo Steering Team-Argo) and ocean reference stations (OceanSITES). Additional "emerging" networks are developing these attributes and are actively engaging in OPA activities and meetings: OceanGliders, HF Radar, SOCONET (Surface Ocean CO2 Observing Network), and Animal Marine Monitoring.

The OCG ensures all coordinated networks deliver data of known quality through appropriate data systems. Stakeholders include the research community, a range of weather, ocean, and marine service providers (many of whom are represented in JCOMM's parent organizations, the WMO and the IOC), and others who rely on ocean information.

\section{Ocean Observing System Requirements}

To communicate and advocate for the development of the ocean observing system, requirements are developed through a number of forums to ensure that the full range of stakeholders and implementers of ocean observations are engaged.

The JCOMM OPA works primarily with the GCOS-GOOSWCRP Ocean Observation Physics and Climate panel (OOPC) on setting the requirements for observations for climate applications. The OPA does not itself set requirements but contributes toward a requirement-setting iterative process, which considers the costs and feasibility of observations vs. their value or impact. To enable the consistent communication 
of requirements for observations, Essential Ocean Variable (EOV) specification sheets have been developed by the GOOS panels (OOPC, Biogeochemistry, and Biology and Ecosystem) to specify the requirements for the observation of a variable, its applications, the processes/phenomena that need to be captured, and how the observations of variables come together to meet these requirements. This was initiated by the OOPC for climate and operational services requirements and will enable the eventual evaluation of the observing system by each variable.

The OPA also engages with the WMO Rolling Review of Requirements (RRR) process, which compiles the requirements for the various WMO application areas by variable in terms of five criteria: horizontal resolution, vertical resolution, observing cycle, timeliness, and uncertainty. The WMO Observing Systems Capabilities Analysis and Review tool (OSCAR/Requirements) provides a record of observational user requirements formulated by WMO and various co-sponsored programs, such as GCOS, GOOS, and WCRP. The requirements are regularly reviewed by groups of experts nominated by these programs, although a systematic approach is still lacking.

\section{Coordination, Development, and Integration of Observing Systems}

The OPA assesses ocean observing enterprises and approaches and engages emerging and transitional observational networks that meet the aforementioned attributes of a JCOMM network as they mature and become ready for sustained operations. Developing guidance and monitoring the transitions of such capabilities into the sustained observing system will also become an important set of processes and products. The OPA also supports assessments/studies that consider the mix of platforms/technologies to best meet JCOMM requirements (e.g., SOCONET, Sabine et al., 2010). Changes in sensors and platforms, improved satellite capabilities and coverage, and shifts in the use of platforms (particularly from research ships, due to the high cost with respect to autonomous vehicles) are evolving due to technological advances and economies. This will require the OPA to coordinate strategic changes to the observing systems and manage transitions over time.

The OPA will also work to address new frontiers (e.g., Arctic, the deep ocean) that are presently poorly observed and bring about system improvements through regionally focused observing pilots (e.g., TPOS-2020; Cravatte et al., 2016). The OPA will also work with other JCOMM program areas to address the increased demand for information products and new services, such as for climate trends (Figure 5). It will work with service providers to encourage observing/service pilot activities to address ongoing and new needs.

\section{Observing System Reporting, Metrics, and JCOMMOPS}

The OPA is working with OOPC, GOOS, and the JCOMM networks to develop network specification sheets to document the role of components of the observing system, highlight interdependencies, missions, and to set targets for implementation. JCOMM networks are developing Key

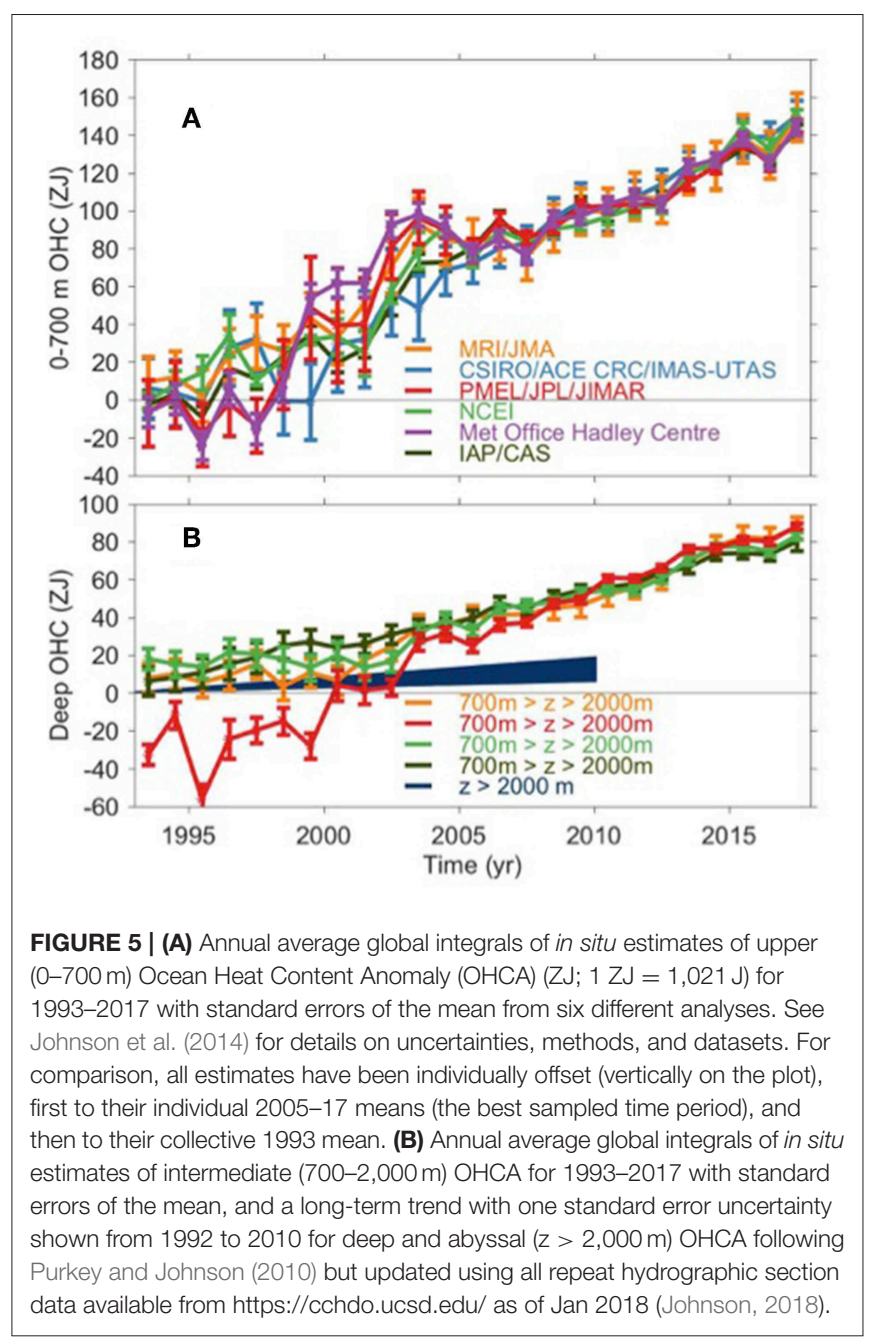

Performance Indicators (KPIs), in line with their mission targets to more easily track progress, such as identifying challenges/risks and reporting the state of the global ocean observing system to a broader set of stakeholders. System metrics beyond KPIs have demonstrated value in managing the observing system (e.g., coordinating deployment opportunities, identifying geographical observing gaps, and data flow). Additional metrics, to be aligned for future use by stakeholders (e.g., researchers, forecasters, and others), are also under consideration and development.

The JCOMM in situ Observations Program Support Center (JCOMMOPS—www.jcommops.org) was established in 2001 and it provides a focal point of technical coordination across the ocean observing networks, encourages timely reporting and the conveyance of data and metadata, and develops appropriate tools for monitoring the status and reporting of the observing system. JCOMMOPS functions are critical for the integrative development, operations, tracking, and monitoring of the global system.

In the recent years, JCOMMOPS has developed a new generation of Information System with an integrated metadata management and web applications. It has developed KPI to 
track network status and anticipated gaps. It gained enhanced responsibilities with JCOMM fifth session (e.g., allocation of unique identifiers for all met ocean platforms, production of WIGOS compliant metadata through WMO). In 2018 JCOMMOPS was reviewed through an independent team to identify strengths and challenges of the structure, and prepare its future through the development of a strategy plan.

A major JCOMMOPS deliverable, the JCOMM Ocean Observing System Report Card, takes full advantage of the development of new metrics. The first issue, distributed in 2017, was to inform major stakeholders and sponsors about the observing system. The 2018 version included more information on the status and projections of satellite systems, and an initial assessment of the health of the in situ observing networks. Future versions will provide an annual snapshot of system health, highlight developments and areas of concern, and could be expanded to include reports on regional networks and pilot projects (Figure 6).

\section{Observing System Standards and Best Practices}

Ocean Observation best practices are procedures and/or processes that have been shown by experience to produce reliable, efficient, accurate, and replicable results, and that are established or proposed as a reference technique or process suitable for widespread adoption. The OPA goal is to provide a standards and best practices framework to ensure the standardization of data collection protocols and the traceability of ocean data, so that the quality of the observing systems and resulting data are maintained at the highest possible levels.

Many JCOMM platforms are based on community practices, and have evolved to develop best practices. Although they may be widely accepted, they have not all undergone a documented review process. Data collection activities are often oriented around a range of instruments, hence measurements of the same variable are often made from different instruments with varying characteristics. Those, such as climatologists who use this data for products that involve all measurements find that many different programs must be consulted. One focus of the OPA Standards and Best Practices is to work toward consistency across the best practices around EOVs.

The OPA vision and strategy for observing system standards and best practices includes the following goals and activities:

- Identify, coordinate, and promote the development and use of standards and best practices across all areas of ocean observing and marine meteorological platforms of JCOMM, and all partner networks and activities;

- Develop a strategy to update these important resources;

- Create a publication or endorsement method through WMO regulatory and guidance materials (such as manuals and guides of GOS, CIMO, and WIGOS), IOC guides, and/or JCOMM technical reports, and peer-reviewed journal publications to communicate those best practices not currently disseminated;

- Support the development of best practices for new observing technologies, drawing from experienced networks;
- Make recommendations to JCOMM OPA for future actions to address these needs; and

- Work toward consistency across best practices for EOVs.

The OPA standards and best practices vision is already being addressed, with two initial foci: (1) a JCOMM network focus; and (2) the integration of JCOMM standards and best practices within a larger set of systems (e.g., IODE Ocean Best Practices, Pearlman et al., 2019) and improved organization of, and access to, this information (Hermes et al., 2018).

\section{Near-Real Time Ocean Data Acquisition and Distribution Importance of Real-Time Observations}

Much of the ocean information collected as part of the ocean observing system has a very high value in near realtime. These data, and their near real-time dissemination, are critical for ocean and weather prediction, disaster response and climate monitoring, detection, and mitigation, i.e., for the endto-end met-ocean system. In resource-limited environments, such as ocean research, it is essential to continually evaluate and implement workflows, which improve the efficiency and effectiveness of overall investments. One way to increase the impact of observations is to ensure that when possible, data have been at least minimally quality controlled and also are distributed in a timely fashion as possible.

Unfortunately, for many years ocean and marine meteorological data has faced barriers to near-real time distribution beyond the WMO system. The GTS (http:// www.wmo.int/pages/prog/www/TEM/GTS/index_en.html) has been the distribution mechanism of choice for global timecritical weather information, which also allows for near real-time distribution. WMO (2017c) defines the GTS as: "The coordinated global system of telecommunication facilities and arrangements for the rapid collection, exchange and distribution of observations and processed information within the framework of the World Weather Watch." The GTS has been an effective method of distributing both land-based and marine weather observations to all NMHS. However, the research community has faced difficulties in trying to mirror the weather world by utilizing the GTS for real-time data distribution. Limited connectivity to GTS endpoints, the use of standard WMO data formats (such as the Binary Uniform Form (BUFR) for the representation of meteorological data), and research-project technical maturity levels have limited the amount of global ocean data that is available in near-real time. Furthermore, for many years it has not been in the culture of research to make oceanographic data widely available in near real-time. Networks, such as Argo and drifting and moored buoys under DBCP have overcome this barrier because they incorporate real-time data transmission systems in their observing platforms. However, there remains a wealth of ocean data that is not being distributed in real-time, as only a small number of national met services have established links with their national oceanographic institutes to facilitate the delivery of their data to the GTS. For example, there are many tide gauges that are not reporting their data to the GTS. 


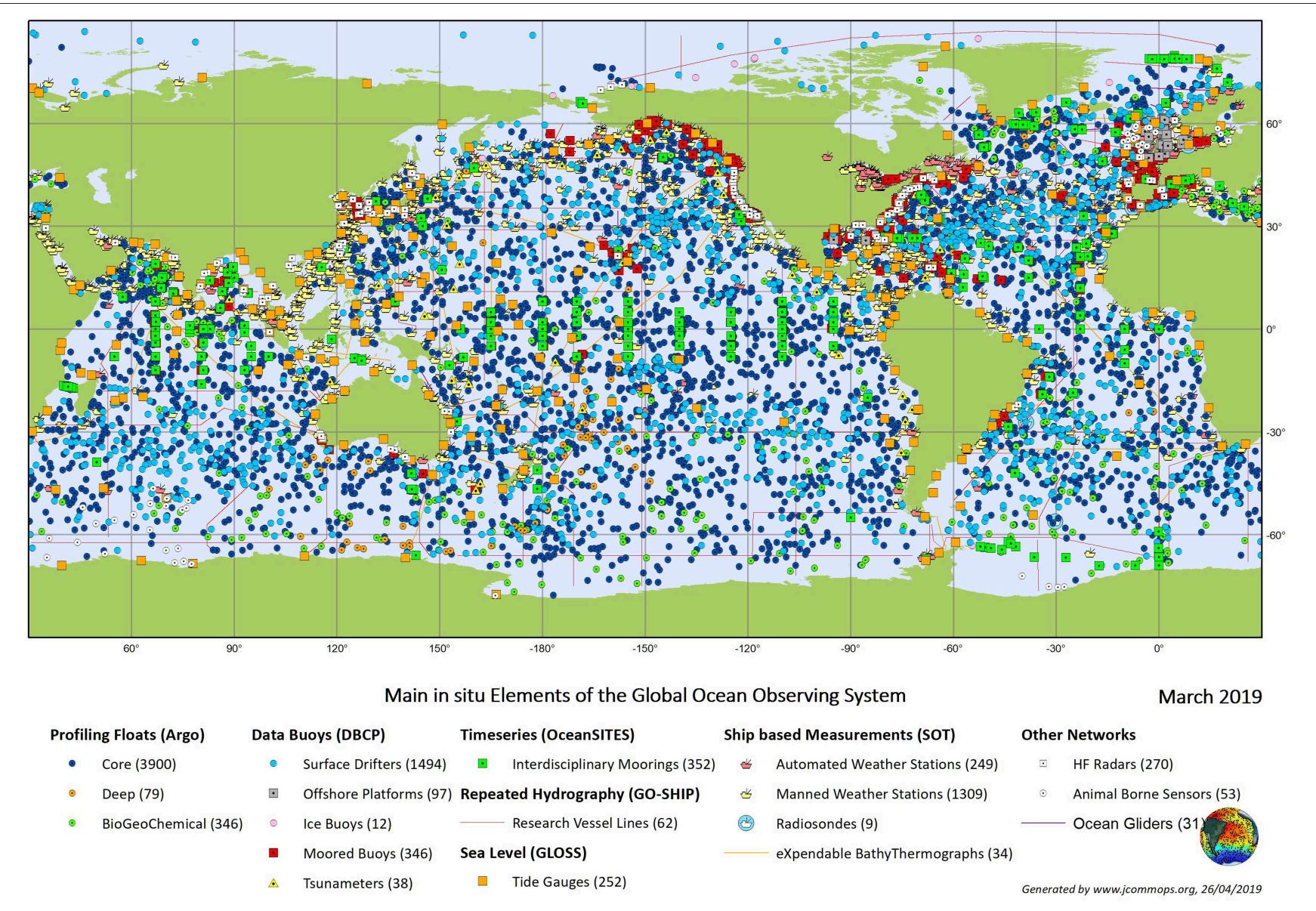

FIGURE 6 | Locations of JCOMM global ocean observing system platforms from the https://www.jcommops.org, where all the symbols are explained.

In an attempt to bridge this gap, the JCOMM OCG undertook a pilot project designed to make it easier for research institutions to distribute and access data using the GTS. The ocean data community has begun to embrace widely used standards and conventions, such as the climate and forecast $(\mathrm{CF})$ metadata conventions (http://cfconventions. org/), NetCDF binary file format (https://www.unidata.ucar.edu/ software/netcdf/), and distributed data service protocols, such as the Data Access Protocol (DAP) (https://cdn.earthdata.nasa.gov/ conduit/upload/512/ESE-RFC-004v1.1.pdf). By leveraging these services and conventions, it should be possible to create a simplified workflow that would provide data producers with an easier path for distributing their data to the real-time community.

The pilot project, aptly named "Open Access to GTS" (Figure 7), identified and utilized a dozen platforms whose data would not otherwise have been placed onto the GTS. These included well-known platforms, such as ocean moorings and sailing vessels, and new platforms, such as Saildrone (https:// www.saildrone.com/). In all cases we were able to leverage a simple workflow incorporating the widely used science data formats of ASCII and NetCDF, and the ERDDAP data platform (Simons, 2017). The use of ERDDAP allowed a National Data center in the US, the National Data Buoy Center (NDBC), to harvest the data collected from the various platforms. Once harvested through machine-to-machine services, the data was encoded by NDBC into BUFR, using the appropriate template, and distributed globally via the GTS. Among the many benefits of this workflow was the important advantage that the scientists and data providers were not required to understand the BUFR format, and only needed to make their data available through the ERDDAP distributed data service.

By leveraging such modern data services and conventions, it was also possible to provide access to this data beyond the distribution arc of the GTS. Although data submission to the GTS by the wider oceanographic community is strictly regulated, accessing GTS data for them is challenging. In the JCOMM OCG pilot the same data platform, ERDDAP, was used to provide access to the wider observations that are distributed on the GTS. This allows any user, regardless of their technical background, to access near-real time data from any of the $4,000+$ platforms currently reporting in the global oceans. This data is available for all without the burden of decoding BUFR, and users are able to use the data through any software client they are familiar with. There is no need to download, reformat, or chase down BUFR 


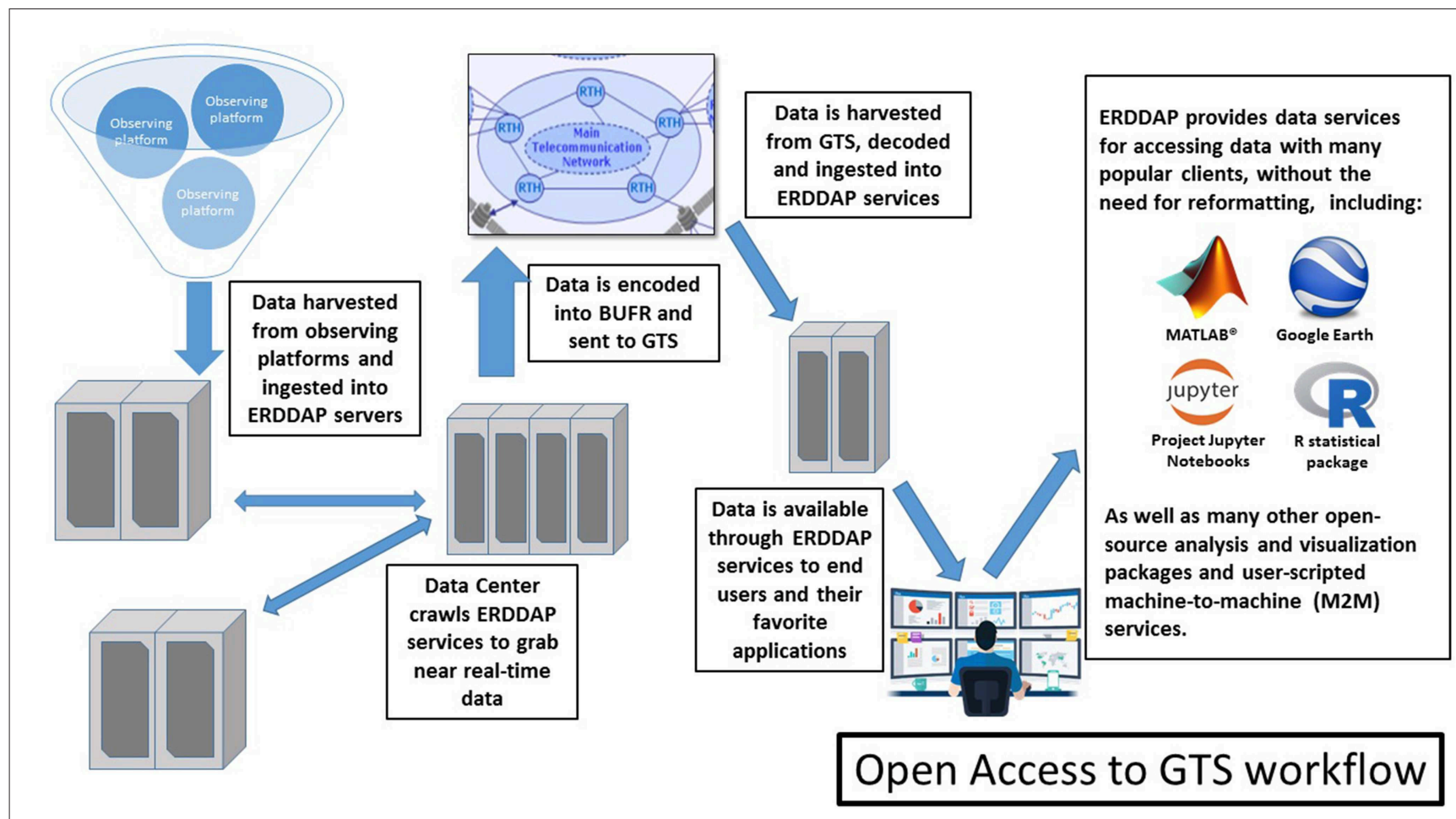

FIGURE 7 | The Open Access to GTS workflow developed for the JCOMM OCG Pilot Project. Symbols are defined in the Table of acronyms.

code tables. In addition, as the data is available through ERDDAP web services, it is much easier for traditional data systems to retrieve the data and ingest it into their products.

As the GTS distribution mechanism was established to support operational forecasting there are of course strict guidelines regarding what can be sent via the GTS and how. Although the JCOMM OCG pilot project was a demonstrable success, it is imperative to define a strategy for the wider implementation of this project. The JCOMM OCG has undertaken the task of developing an Open Access GTS strategy to ensure issues, such as data duplication and the national sensitivity of data are addressed. However, reducing the burden of requirements for GTS data distribution can clearly be accomplished using current technology and standards to provide an easier path for near real-time data distribution. The challenge is not a technical one but cultural, which must be addressed in the future to improve data availability.

This need to embrace modern data formats and distributed services is also reflected in the evolution of the WIS, as it develops to meet the current and future demands of data interoperability.

\section{Evolution of the WMO Information System}

As noted above, the WMO GTS has been successful in meeting its primary objective of the cost-effective dissemination of meteorological information in near-real time (https://wiswiki. wmo.int/tiki-index.php?page=ManualGTS). As the GTS was conceived, developed and implemented in a period where teletype communication was the norm some of the protocols, standards, and capabilities of the system evolved to meet the needs of the modern era. However, the full advantages of modern IT technologies have not always been allowed for in this evolution. While the GTS is well-managed, reliable and effective, it is also limited in capability and complexity and has restricted access.

To address the shortcomings of the GTS and to expand the data services offered by the WMO, the WIS offers three services areas (http://www.wmo.int/pages/prog/www/WIS/ overview.html):

1. A routine collection and dissemination service for timecritical and operation-critical data and products.

2. A data discovery, access, and retrieval service.

3. A timely delivery service for data and products.

While the first service area will incorporate the GTS, the third expands the GTS offering through the utilization of public networks, such as the Internet. It is this service area that has the potential to provide greater access to WMO data services.

The WIS does present a unique opportunity to increase the dissemination of marine observations and modeling data toward operational forecast use. The key to meeting this goal is to ensure the certification of oceanographic data centers as data providers to the WIS. The WIS should also adapt data transmission message standards to accommodate new platforms used in the oceanographic community.

The WIS has the potential to provide greater access to near real-time data without the procedural overhead now imposed by 
the GTS. The second service area listed above should address this need, and the WIS and its task teams must ensure the provision of adequate mechanisms for accessing these modern data services. The development of WIS 2.0 (the upgrade of WIS by 2030) will contribute to new and modern data access and dissemination services beyond GTS using cloud technologies.

\section{Improved Workflows for End-to-End Systems}

The early steps of a workflow (telecommunication, quality assurance, and communication) are usually the responsibility of the owner of the platform/instrument. JCOMM and other relevant bodies and initiatives, such as the OBP project, strive to standardize these steps and to facilitate efficient pathways from instrument/platform to databases. The workflow for ocean data is at its most intense during this period: many applications are run in real-time and demand rapid processing steps from the point of measurement to the point of use (assimilation, analysis, and monitoring).

The workflow must accommodate high-volume data streams, but often with repeated and similar characteristics (e.g., satellites), and at relatively low volume with more individual characteristics (e.g., surface data buoys). The users require a workflow that is efficient and timely, irrespective of the source, and standardized for ease of use.

Multiple concurrent usage, often in high-impact systems (e.g., weather prediction, safety, risk management, hazards), means the data value is at its peak. In contrast, the arrival of some data is delayed and is only used off-line, while yet other data may be subject to constraints (e.g., proprietary use) and may never move beyond a single user into the general data system. This is wasteful and inefficient.

The workflow for ocean data is open-ended. Reprocessing for reanalysis is now commonplace, to take advantage of knowledge that was not available in real-time, and/or to exploit improved techniques. Such reprocessing may occur multiple times. The added value of reprocessing can be compromised if the associated data processes do not provide clear details about how the data were re-processed, or how it is improved (or different) from earlier versions, and if they are not accompanied by adequate metadata/information to allow users to distinguish between differing versions.

A data system that contains different levels of data, from engineering units through to quality-controlled observations, presents significant challenges both to the workflow and to users: which is best for their application? How easy is it to decide and access the data? Does the user need to consider different formats and standards? The architecture of the system, its workflow, and its efficiency are all important factors.

Complexity does impact efficiency and, perhaps just as importantly, uptake on the provider side and utility on the user side. In reality there are multiple manifestations of such workflows, all aimed at similar outcomes but with slightly different assumptions and implementations, and often with different spatial-temporal windows and variables. As with ocean and climate models, such diversity can be a good thing, but it also comes with risks in terms of the efficiency and integrity of the database as a whole.
In summary, the explosion in observing technology over the last decade has exposed weaknesses in current global data management practices. The ever-increasing number of sensors and the volume of data they are producing demonstrates a need for improved data acquisition and distribution systems to meet the current and future needs of users, which equally applies to near-real time, delayed mode, and reprocessed data. However, technological advances and the embracing of standards and conventions by science communities provide opportunities to successfully meet these challenges.

\section{DATA MANAGEMENT SYSTEMS, FROM NEAR-REAL TIME TO DELAYED MODE The Met-Ocean Data Management System}

The JCOMM data management system is jointly organized by IOC's IODE (International Oceanographic Data and Information Exchange) program and the JCOMM Data Management Coordination Group (DMCG), and the existing marine meteorology data management programs in WMO are considered. The DMCG's mission is to maintain a data management plan that identifies, assesses, and specifies priorities of activities relevant to both near-real time and delayed mode marine meteorological and oceanographic data management. Expert teams and pilot projects review, assess, and coordinate appropriate new approaches, standards and best practices, methods, and tools.

Since 1961, IODE has coordinated a network of national oceanographic data centers (NODCs), and since 2013 associated data units (ADUs) and global data assembly centers (GDACs). These centers form the structural elements of IODE and JCOMM for data management, which provide access and stewardship for the national resources of oceanographic data. This requires the gathering, quality control, processing, summarizing, dissemination, exchange, and preservation of data generated by national and international agencies. National institutions (such as navies, universities, fishery agencies, government research bodies, and industry) and programs and projects collect oceanographic data. This wealth of data can be complementary to that collected and archived by the coordinated global and regional programs of JCOMM OPA described in the previous section. NODCs and ADUs often manage more data types than those of interest to JCOMM.

IODE structural elements are organized into networks and projects (ODIN) at regional and thematic levels. Examples of such network and projects are the pan-European Infrastructure for Ocean and Marine Data Management (SeaDataNet, https:// www.seadatanet.org/), the Ocean Data and Information Network for Africa (ODIN-Africa, http://www.odinafrica.org/), and the IODE Ocean Data Portal (ODP, http://www.oceandataportal. org/), which provide access to more than 100 IODE data centers worldwide.

Recently the IOC decided to undertake the development of its Ocean Data and Information System (ODIS), which will initially offer an inventory of existing online data and information sources (ODISCat: http://catalogue.odis.org). The system will 
considerably increase the discoverability of data and information, but more importantly, also of products and services, particularly those relevant to SDGs. The structural IODE units make available physical, chemical, and biological (including biogeographic) data and information that are the basis of at least SDG goals 13 and 14: NODCs are traditionally archiving facilities for climate trend assessment from in situ observations and ADU/GDACs collect and make available biogeochemical data that are fundamental to the sustainable use of marine resources. ODIS will make all these data assembling facilities more visible and discoverable.

Another very important action that is being promoted by IODE, GOOS, and JCOMM data management is the Ocean Best Practices System (OBP, http://www.oceanbestpractices.org) that aims to provide the ocean research, observing, and application communities with a mechanism to discover, review, agree upon, adopt, and support the widest possible dissemination of ocean best practices (see the paper by Pearlman et al., 2019, this issue). This is the most important activity for the JCOMM end-to-end system, because the capacity development of data access and dissemination for IOC member states and WMO members will be based on it.

While the real time data flow has been described in section Near-Real Time Ocean Data Acquisition and Distribution, here we describe the delayed mode data flow developed by JCOMM, so-called Marine Climate Data System. Both data flows, the near real time and the MCDS, are essential in the structure of ODIS. Near real time data should be converted into high quality climate data sets by enhanced quality control and they should contribute to the IODE structural elements as well as the MCDS. Furthermore, the near-real time data management system, as described in section Near-Real Time Ocean Data Acquisition and Distribution, should be the main link between DMPA and OPA network activities.

\section{The Marine Climate Data System Purpose and Structure of MCDS}

Delayed mode and quality-controlled ocean data are the cornerstone of earth climate monitoring, as they are the basis for the estimation of deep ocean trends. The air-sea and ice-seaatmosphere interface is a critical subcomponent of the earth's climate and it requires the assembly of observations at the marine surface that are collected by very different sensors. The MCDS has been developed to produce highly quality controlled marine meteorological and oceanographic data sets that can fulfill the IOC and WMO requirements for climate long-term series observations.

The purpose of the Marine Climate Data System (MCDS, http://www.iode.org/mcds) is essentially to enable the provision, on a free and unrestricted basis, of consistent, coherent, discoverable, and documented marine meteorological and oceanographic climate data sets of known quality to address the needs of WMO and IOC applications. Such applications include long-term climate monitoring (Global Climate Observing System-GCOS; https://public.wmo.int/en/programmes/globalclimate-observing-system), seasonal to inter-annual climate forecasts, and climate services. A critical function of the MCDS is to compile added-value and variable-based products,

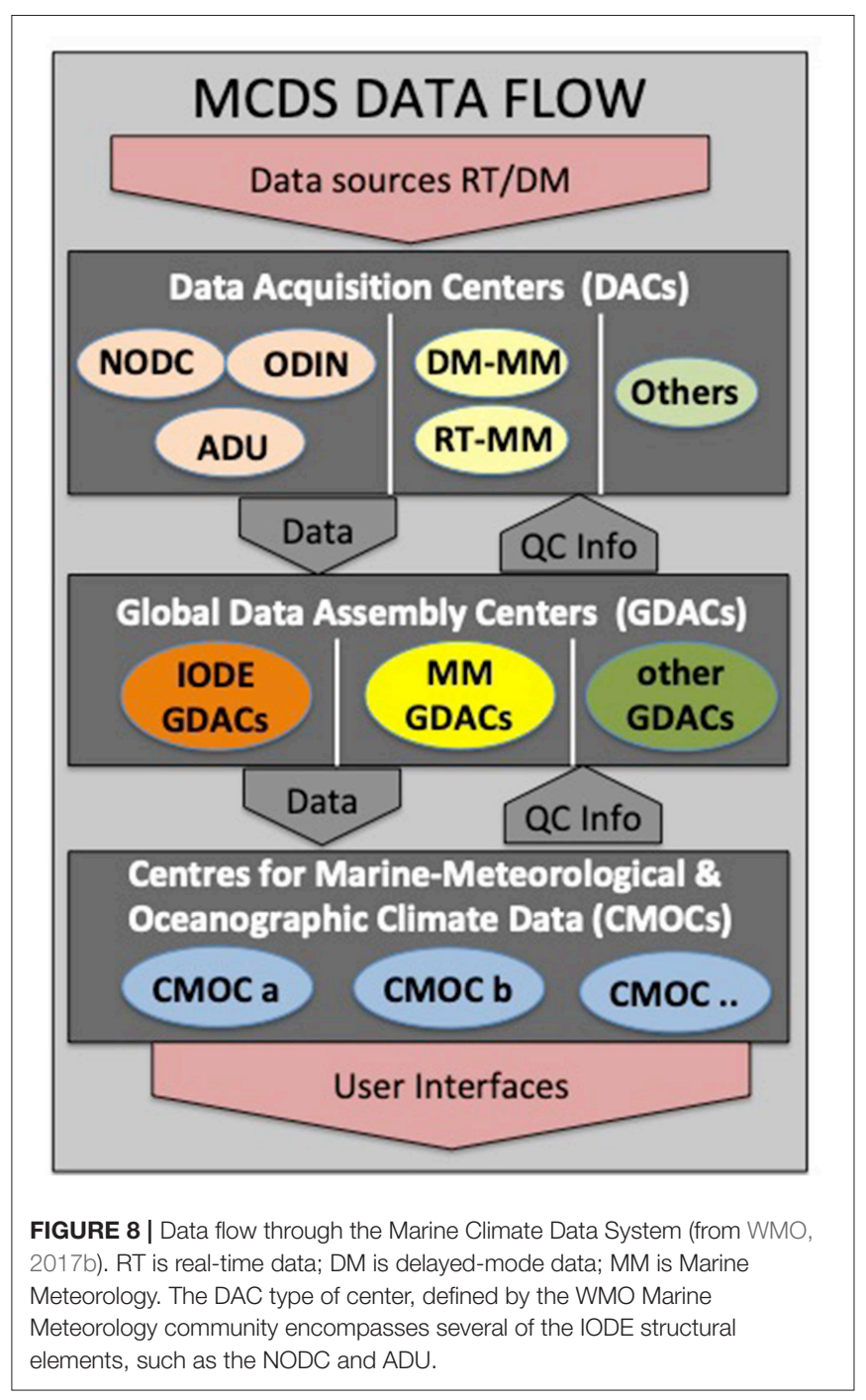

including essential ocean variables (EOVs; Task Team for the Integrated Framework for Sustained Ocean Observing, 2012) and relevant essential climate variables (ECVs; Bojinski et al., 2014). Other functions include the provision of added value bias-corrected data-sets.

This system is expected to improve the timescales for metocean climate data availability and facilitate the exchange of historical met-ocean climate data sets between countries, thereby increasing the amount of ocean observations eventually made available to the relevant end user applications. Furthermore, integrated data and metadata will be available that contains comprehensive dataset information, such as historic details on current and past data codes and formats.

The MCDS data flow is based on a three-tier structure (Figure 8) of Data Acquisition Centers (DAC), Global Data Assembly Centers (GDAC) and Centers for MarineMeteorological and Oceanographic Climate data (CMOC) that standardize data flows from operators to end users. The general structure coordinates data flow through different levels 
of the MCDS providing a traceable and robust flow of data of known quality:

- DAC is the basic level of the MCDS and receives data directly from the measurement source, applies basic quality/duplicate checks, and forwards the data to an associated GDAC. The definition of this type of center encompasses several of the IODE structural elements, including the NODC and ADU;

- GDACs combine the streams of associated DACs to produce a global aggregation for a specific observation system, apply further quality/duplicate checks, and provide the data to an associated CMOC;

- CMOCs collect data from GDACs, aggregate all the relevant data types for a specific set of environmental variables, and produce integrated datasets and products for end users.

Each center in the MCDS data flow is responsible for the completeness and consistency of the data they acquire and to provide it to other centers within the MCDS or to end users. This provides an efficient mechanism for routinely collecting, processing, and delivering high quality data with known quality control procedures and documented processing details of ocean data to various users. The terms of reference for the MCDS Centers is provided in the Manual WMO (2017a) and the Guide WMO (2017b) on Marine Meteorological Services. The harmonization of standards and nomenclature between MCDS and IODE structural elements has not yet been fully achieved, and is the goal for future collaborative work between IOC and WMO. Furthermore, the integration of the IODE networks in MCDS is still to be clarified.

\section{Present Status of the MCDS}

The aim of the MCDS is to implement the described structure for about ten ocean data areas and respective CMOCs. The two areas of surface drifters and ocean profile data are in the process of being implemented. A third, surface and marine meteorology data, is planned and will be built on existing legacy structures.

CMOC-China (http://www.cmoc-china.cn), operated by the National Marine Data and Information Service (NMDIS), was the first CMOC to be provisionally accepted into the MCDS in 2012, and then formally in 2015. While CMOC-China has wide-ranging activities, its contribution to the MCDS will focus on surface velocity products, essentially based on integrating data and metadata from Lagrangian drifters and constructing relevant data products. While the drifting buoy GDACs (see below) communicate well amongst themselves, coherent and well planned tools for disseminating and utilizing the surface velocity data will be of benefit, which will enhance the utility of the data for climate and environmental studies using the buoy trajectory information, and recorded environmental variables. CMOC China will also be involved in other activities, such as ocean observing platform metadata integration, and data rescue focusing on the Asia Pacific region.

Two GDACs for drifting buoys have been established, led by "Fisheries and Oceans Canada" and Coriolis (a French organization including Météo-France and Ifremer). Both GDACs also perform the function of DACs. The main data source consists of data circulated on the Global Telecommunication System
(GTS) of WMO. Both GDACs routinely compare GTS bulletin headings and the data volume received and are able to make data available to requesters (Canada: through an offline form request system, France: through the Copernicus Marine Environment Monitoring Service). A public FTP server distributing data and metadata using one NetCDF file per buoy is under construction.

The World Ocean Database (WOD; https://www.nodc.noaa. gov/OC5/WOD/pr_wod.html, Boyer et al., 2013, also available through http://wod.iode.org) was endorsed as a CMOC for ocean profile data in 2017. The WOD is a database operated from the U.S. National Centers for Environmental Information (NCEI) and is also a project of the IOC International Oceanographic Data and Information Exchange (IODE). The WOD aggregates historic and recent subsurface ocean profile EOVs. The WOD role as CMOC is to provide all available ocean profile data in a uniform way to the public through both machine-to-machine discovery and individual discovery and download. As a CMOC, the WOD can flexibly prepare metadata and discovery options, which feed into the WIS and ODIS systems for dissemination with other types of ocean and marine meteorological data.

Several ocean profile GDACs will in the future provide the infrastructure for collecting the most up-to-date and highest quality and resolution ocean profile data, integrate the near real-time data streams with historical (delayed-mode) data, and deliver the integrated data sets to relevant CMOCs for further products developments. Near real-time ocean profile data are those made available within $48 \mathrm{~h}$, often at less than full resolution and without full quality control/calibration. Delayedmode data are those made available after $48 \mathrm{~h}$, and often much after, usually with full quality control and calibration. Another key role for the ocean profile GDACs is to coordinate activities among all participating relevant DACs, to standardize quality control procedures (IOC, 1993). Ocean profile GDACs include, but are not limited to, Argo (http://www.argo.ucsd. edu/Argo_Project_Office.html; Jayne et al., 2017), CLIVAR and Carbon Hydrographic Data Office (CCHDO; https:// cchdo.ucsd.edu/), and the Global Temperature and Salinity Profile Program (GTSPP; https://www.nodc.noaa.gov/GTSPP/) for ocean temperature and salinity profiles.

\section{Building the MCDS}

The governance for defining the designation procedure, function, and evaluation process for MCDS centers (i.e., DACs, GDACs, and CMOCs) is recommended by JCOMM and adopted by both the WMO and IOC governing bodies. The host of a candidate MCDS center is required to produce a statement of compliance with requirements and commitments, and to list and demonstrate the capabilities of the proposed center, state the scope of the data and/or products managed, and formally commit to hosting the center in line with its specific terms of reference. A committee comprised of at least three members then evaluates the statement of compliance and a unanimous decision is required for approval.

The detailed designation and evaluation process for the DACs and GDACs is provided in WMO (2017a), while WMO (2017b) provides this for the CMOCs. 
Based on its terms of reference, and particularly the standards and procedures regarding the overall collection, management, exchanging, and archiving of high-quality marine meteorological and oceanographic data, information and products, JCOMM in collaboration with the IODE will further monitor the implementation status of MCDS through evaluating the efficiency of the network, including the operation of DACs, GDACs, and CMOCs. The CMOCs must provide the high level functions of the MCDS and make products available to end users, so it is essential that the flow of marine meteorological and other appropriate oceanographic data is ensured through the diversity of data sources, whether real-time or delayed mode, to the CMOCs. This data flow is ensured through oversight and regulation with the MCDS, provided by JCOMM and IODE. In addition, JCOMM will work to establish new centers to cover a wider spectrum of oceanographic and marine meteorological observing platform types. Currently, the JCOMM Expert Team on Marine Climatology (ETMC) and the JCOMM-IODE Expert Team on Data Management Practices (ETDMP) is responsible for the oversight, regulation, and additions to the MCDS. Active coordination between these teams and between the teams and the elements of the MCDS is essential for the maintenance and growth of the MCDS. More accessible documentation and outreach from the expert teams will also encourage the awareness of and participation in the MCDS.

One of the initial goals of the MCDS was to modernize and expand the existing Marine Climatological Summaries Scheme (MCSS) established in 1964 by WMO. Marine climatology then only consisted of data collected by the Ship Observation Team, which includes the traditional Voluntary Observing Ships (VOS) and Ships Of Opportunity Program (SOOP). The initial system was built around several VOS DACs responsible for collecting VOS data in delayed mode from recruited vessels, and for forwarding those data to two GDACs that applied minimum quality control standards and conducted duplicate removal. The GDACs apply additional reformatting where needed, along with additional metadata. The two VOS GDACs operate in parallel and provide mirroring services to avoid loss due to technological failures. The critical updates of the MCSS to the MCDS maintain data flow through the system and insert the global functionality of the CMOC. Currently there is no CMOC that can function as a repository for the VOS data, but the International Comprehensive Ocean-Atmosphere Data Set (ICOADS) is considering fulfilling a CMOC role and will collect the VOS data from the VOS GDACs together with other marine meteorological data sources.

\section{FROM MONITORING TO SERVICES}

The JCOMM value adding chain (Figure 2) requires international coordination for operational weather and oceanographic forecasting and the development of targeted applications, notably for maritime transport and fisheries, and for the coastal communities.

Improvements in numerical weather prediction through better data assimilation, improved algorithms, and increased spatial resolution, have been facilitated by advancements in supercomputing techniques and capacity (Bauer et al., 2015). Operational oceanographic services have also been developed, such as the Copernicus Marine Environment Monitoring Service (CMEMS) (Le Traon et al., 2019, this issue), producing high-resolution, short-term, and accurate forecasts of up to 10 days for global and regional wave and currents, temperature, sea level, and salinity, together with long term reconstructions of the ocean state over several decades. The broader operational use of ensembles and the transition to fully combined ocean, air, and cryosphere models will further improve numerical weather and ocean guidance. Hydrological forecasting is lagging behind, but this is soon to be addressed by new activities at WMO and IOC. As we develop and operationalize a total earth systems prediction capability in the coming decade we will bridge the gap between atmosphere and ocean weather and climate predictions, enabling us to predict across weather, sub-seasonal, seasonal, annual, and decadal time scales. To achieve these goals, JCOMM has collaborated with research networks, such as the Global Ocean Data Assimilation Experiment (GODAE, https://www.godaeoceanview.org/science/ocean-forecasting-systems/) and the WMO Global Data-Processing and Forecasting System (GDPFS, http://www.wmo.int/pages/prog/www/DPS/gdps.html).

To harmonize and strengthen the global network of weather and operational oceanographic services support for the maritime and coastal environments in terms of coastal populations and fisheries, the JCOMM Services and Forecasting Systems Program Area (hereafter referred to as JCOMM Services) provides standards and protocols to national authorities regarding the delivery of marine weather and ocean services, which protect lives and property at sea and in the sensitive coastal environment. While JCOMM services support a wide range of met-ocean activities, the primary focus is on promoting safe shipping, reducing the risk from disasters along the coasts, responding to marine emergencies, and providing the international coordination necessary for these services to be successful.

\section{The Maritime Safety Services}

Although the investments made in observations and forecasts described above have substantially improved predictability, ships still transit into hazardous conditions. Incidents of ships encountering extreme weather at sea in recent years highlight the challenges of exchanging information among ships, national marine forecasting centers, and commercial weather providers, which have adverse effects on the decision-making of mariners. The situation is further exacerbated by a maritime weather infrastructure that remains heavily reliant on decades-old technology for dissemination, such as radiofax, text, and brief voice broadcasts.

To ensure forecasts and hazardous weather warnings are provided to ships at sea, JCOMM has established a Worldwide Met-Ocean Information and Warning Service (WWMIWS) Committee. Aligned with the International Hydrographic Organization (IHO) Worldwide Navigation Warning Service (WWNWS) Sub-Committee, WWMIWS ensures that standards and procedures are developed for the preparation and issuance 
of marine weather support that are compatible with systems that disseminate navigation warnings to ships, and that these protocols are equitably implemented around the world. The basis for these services dates back to the initial international Convention on the Safety of Life at Sea (SOLAS), held in 1914 following the sinking of the RMS Titanic in 1912 by an iceberg in the waters of the North Atlantic Ocean. Subsequently adopted as the UN Convention on the SOLAS, the convention has been revised four times, most recently in 1974, with the International Maritime Organization (IMO) responsible for regulating under the technical guidance of the WMO, through JCOMM, and IHO.

IMO manages this maritime safety information through the Global Maritime Distress and Safety System (GMDSS). As shown in Figure 9, the world's oceans are divided into 21 zones called METAREAs. JCOMM WWMIWS guides the provision of services through METAREA coordinators, who are responsible for monitoring and reviewing the provision of meteorological maritime safety information within the GMDSS and also for other vessels not covered by the SOLAS Convention.

Sea ice is particularly dangerous to vessels. To address this specific navigational hazard, JCOMM's Expert Team on Sea Ice (ETSI) coordinates with and advises coastal states on products and services required by user communities in sea ice areas, to support navigation, coastal and offshore activities, and monitoring of the sea ice cover for both the northern and southern hemispheres. Such services are becoming more relevant as the polar seas warm and become less ice-bound during the summer months, resulting in an increase in shipping and other maritime activities in these regions. Recognizing this, the IMO adopted the International Code for Ships Operating in Polar Waters, and the "Polar Code" (MEPC, 2015) entered into force in 2017. ETSI will have the important role of providing guidance for national ice services to ensure the sea ice information they provide meets Polar Code requirements.

\section{The Disaster Risk Reduction Services}

Inundation in the coastal zone resulting from tsunamis, storm surge, increased river flow, and other weather-related causes kills thousands annually and hundreds of thousands more are displaced from their homes and communities. Although regional warning systems are increasingly available, these phenomena continue to wreak havoc due to the lack of preparedness and effective mitigation strategies. This led to the inclusion of a specific target in the Sendai Framework to "substantially increase the availability of and access to multi-hazard early warning systems and disaster risk information and assessments to people by $2030 . "$

Innovative solutions are required to minimize the impacts of these events, and since 2009 WMO and JCOMM have partnered to develop tools that can be used to improve prediction and lessen the effects of these disasters. The Coastal Inundation Forecasting Demonstration Project (CIFDP) is a cooperative effort by the WMO's Commission for Hydrology and JCOMM to pilot an initial capability in Bangladesh, Fiji, the island of Hispaniola encompassing both the Dominican Republic and Haiti, and Indonesia, with a focus on the development and implementation of multi-hazard early warning systems leading to long-term disaster risk reduction services in these coastal states (Swail et al., 2010). The project integrated observations of sea level, ocean waves, and meteorological variables including wind, pressure, and rainfall with hydrological information, such as river level and flow. This observational information was combined with the forecast modeling techniques used for waves, storm surge, tide, sea surface height anomaly and river flooding by organizations, such as the WMO's Regional Specialized Meteorological Center (RSMC) for Tropical Cyclones in Miami, USA, and the RSMC Tokyo's Typhoon Centre, along with national meteorological agencies where available, such as in Indonesia.

Based on the technical description of CIFDP, as given in Figure 10, the national forecast agencies can provide essential guidance on potential inundation hazards to national disaster management agencies according to their established protocols. The outcomes of these demonstration projects are being evaluated to help create an optimal path for the full operational implementation of a program to extend these multi-hazard forecast systems to other nations that are subject to disasters resulting from coastal inundation events.

To help provide coastal states with the technical expertise and tools needed to mitigate the impacts of these deadly coastal weather events, JCOMM Services established an Expert Team on Disaster Risk Reduction (ETDRR) in 2017. This ETDRR will be comprised of experts from both developed and developing coastal states, and is aimed at improving the links between observations and emergency management officials in states vulnerable to such disasters. In addition, to actively supporting the CIFDP, the expert team will help promote and develop regional coastal hazard warning solutions for all vulnerable nations, including capacity building activities. ETDRR will also promote trans-disciplinary connections between scientists, engineers, planners, and policy makers, to deliver the tools needed for effective coastal resilience.

\section{Marine Environmental Emergency Services}

Operational services that deliver marine pollution forecasts and hazard/risk mapping for marine environmental emergencies and disaster risk reduction will be co-ordinated. The initial framework involved the setting up of a network of response centers within the 21 WMO metareas (Figure 9) within the GMDSS, and is called the Marine Pollution Emergency Response Support System (MPERSS). This NMHS network liaises with oceanographic centers to provide environmental emergencies response information. These centers should provide basic meteorological and oceanographic forecasts and advectiondiffusion and transformation models capable of simulating and forecasting the movement and weathering of pollutants at the sea surface. The MPERSS system is being revised by the Expert Team on Marine Environmental Emergencies Response (ETMEER), which will facilitate the implementation and operation of GDPFS specialized centers for marine emergency management, and establish and maintain standards for information products, thus supporting members and member states in their responses to marine environmental emergencies. 

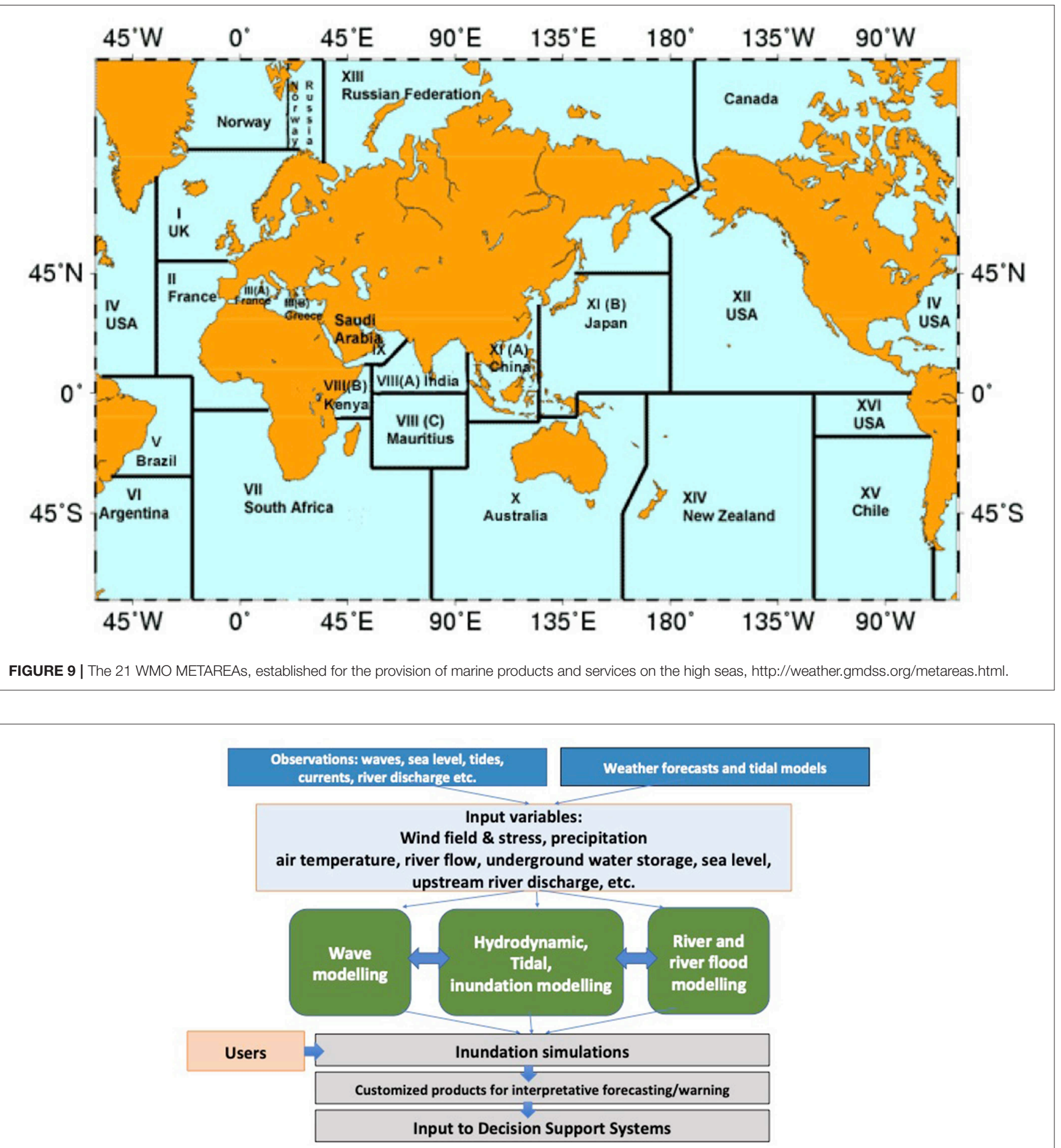

FIGURE 10 | Conceptual diagram of Coastal Inundation Forecasting Demonstration Project (CIFDP) forecast system.

This global system must liaise with the International Convention for the Prevention of Pollution from Ships (MARPOL), which is the main international convention addressing the prevention of pollution of the marine environment by ships from operational or accidental causes. It should also connect with regional conventions that develop contingency plans for marine pollution emergencies in the Mediterranean (REMPEC), the Baltic (HELCOM), and the 
North Sea (OSPAR). The International Maritime Organization (IMO) and International Atomic Energy Agency (IAEA) are already involved in JCOMM activities and provide guidance for ship pollution and radioactive accidents.

The complexity of such a network cannot be underestimated. Environmental emergencies range from radioactive material discharges, such as the Chernobyl nuclear power plant accident in 1986 (Margvelashvili et al., 1997; Staneva et al., 1999; Evangeliou et al., 2016), the Fukushima Daiichi nuclear power plant accident in 2011 (Dietze and Kriest, 2012; Estournel et al., 2012; Miyazawa et al., 2012; Tsumune et al., 2012; Draxler et al., 2015; Periáñez et al., 2015), and the ruthenium-106 release in 2017 (IRSN, 2017), to oil spills from operational release and/or ship or platform accidents (Price et al., 2003; Abascal et al., 2010; Olita et al., 2012; Fernandes et al., 2016; Liubartseva et al., 2016a; Sepp-Neves et al., 2016; Zodiatis et al., 2018), accidents related to objects/debris/people drift (Kawamura et al., 2014; Jansen et al., 2016; Trinanes et al., 2016; Maximenko et al., 2018), other hazardous and noxious substances (Neuparth et al., 2011; Legrand et al., 2016), harmful algal blooms (He et al., 2008; Li et al., 2009; Glibert et al., 2010; Hense, 2010; McGillicuddy, 2010; Anderson et al., 2012), and plastic pollution (Maximenko et al., 2012; Mansui et al., 2015; Van Sebille et al., 2015; Liubartseva et al., 2016b, 2018; Fossi et al., 2017; Politikos et al., 2017; Zambianchi et al., 2017). All of these accidents/events require specific information and competency, particularly if a global support system for mitigation and response has to be organized. Nothing is yet available for underwater pollution sources and hazard/risk mapping information, although severe accidents can result from underwater explosions and so risk/hazard mapping is extremely important for the few $10 \mathrm{~min}$ after the accident, to understand the potential environmental conditions that can affect pollutant dispersion.

\section{Ocean Forecasting From the Global to the Regional Scales}

Over the past 20 years oceanographic operational services have emerged and developed (Pinardi et al., 2017), and together with the sea state provide 10 days forecasts of the deep ocean state and long-term reanalyses. Ocean forecasting has been developed (Pinardi and Woods, 2002; Chassignet and Verron, 2006) under the scientific coordination and guidance of the Global Data Assimilation Experiment (Bell et al., 2009; Dombrowsky et al., 2009), which has now evolved into GODAE OceanPredict. This promotes the development and improvement of operational ocean analysis and forecasting systems worldwide and involves 15 different ocean forecasting systems from various countries and institutions. The most important aspects of this work relate to scientific advances in ocean modeling and data assimilation. The particular challenges for the future will be in terms of high-resolution physical modeling, downscaling, biogeochemical and ecosystem modeling, ocean/wave/atmosphere coupling, data assimilation and coupled data assimilation, error estimates, longterm reanalyses, and the use of new observations.

In 2012 JCOMM established an expert team to monitor and maintain an overview of the worldwide Operational Ocean
Forecasting Systems portfolio, including ocean physics and marine biogeochemistry, and to promote the adoption of international standards for ocean forecasting activities. Users of this new ocean forecasting are those within the socioeconomic sectors of maritime transport and safety, coastal and marine environment management and protection, marine resources and weather, and climate and seasonal forecasting. Tonani et al. (2015) offers an exhaustive overview of the global and regional operational forecasting systems across the world.

To achieve forecast accuracy, ocean operational systems must from the outset consider mesoscale resolution, from global to regional scales. For coastal forecasting, higher-resolution models are nested into global forecasts both as limited area models and as stand-alone systems that are initialized only once. Coastal models and forecasting introduce new challenges. The issue of big data management, in terms of the exchange of large amounts of data for initial and lateral boundary condition updates, a highresolution bathymetry-topography mapping to allow for coastal inundation, and the data assimilation of local observations into the nested models (De Mey et al., 2017). These problems will be partially tackled by JCOMM coordination to establish best practices in the NMHS and responsible oceanographic institutions, but they will remain a research, development, and innovation challenge.

\section{CAPACITY DEVELOPMENT}

JCOMM Capacity Development (CD) activities are carried out in each program area (Figure 2) using the existing IOC Capacity Development and WMO Education and Training structures, to leverage the $\mathrm{CD}$ expertise and experience in both organizations (i.e., WMO Global Campus and Regional Training Centres (RTC), the IOC-IODE Ocean Teacher Global Academy (OTGA) and its OTGA Regional Training Centres, IOC/WESTPAC Regional Training and Research Centres, etc.). JCOMM CD activities are also undertaken on the basis of the Partnership for New GEOSS Applications (PANGEA-https://www.jcomm. info/pangea-concept) using voluntary contributions from WMO members and IOC Member States. In the following we provide an overview of the main principles for CD activities in IOC and WMO.

The IOC Capacity Development Strategy (2015) Mission Statement states that "The IOC will undertake relevant actions to assist Member States with developing and sustaining the necessary capacity to undertake activities necessary to achieve the IOC vision at the national level as well as at the international cooperation level." It further suggests that the IOC Criteria and Guidelines on the Transfer of Marine Technology should be taken into account along with IOC's custodianship role for SDG 14 (ICSU, 2017), particularly under targets 14.3 and 14.a. The first target, 14.3 , is related to the minimization of ocean acidification including ocean scientific cooperation and the second, 14.a, is concerned with the increase of scientific knowledge and the development of research capacities in order to improve ocean health. 
Thus, member states have recognized the need to address the ocean scientific knowledge gaps in national infrastructures, NODCs, and operational ocean forecasting centers. In this context, IOC governing bodies have endorsed the proposal for an International (UN) Decade of Ocean Science for Sustainable Development to be established for the period 2021-2030, which will significantly contribute to $\mathrm{CD}$ across the end-to-end metocean system. From 2015 to 2021, the IOC CD strategy proposes a framework of outputs and activities, which are summarized in Table 1A.

The WMO CD mission is to foster stronger NMHS that can meet the need for further information on weather, climate, and water, for the safety and well-being of people throughout the world. CD should be conducted, particularly for developing countries, the least developed countries (LDCs), and small island development states (SIDSs), through advocacy, education and training, outreach, partnerships and resource mobilization, demonstration and pilot projects, service delivery, and research. The WMO Capacity Development Strategy identifies six strategic objectives and corresponding Strategic Approaches, which are summarized in Table 1B (https://www.wmo.int/pages/prog/dra/ documents/WMO_Capacity_Development_Strategy.doc).

The IOC and WMO CD strategies have several similar outputs and/or objectives, including increased education, research, human resources, visibility, awareness, strengthening of global, regional and sub-regional mechanisms, and sustained resource mobilization for $\mathrm{CD}$. Their differences are that the IOC CD strategy includes increased access to physical oceanographic infrastructures while the WMO CD strategy begins by explicitly defining the required capacities and deficiencies, emphasizing ownership and optimizing knowledge management.

\section{DISCUSSION}

Over the past 20 years JCOMM has worked to ensure coordination in the delivery of an integrated, global meteorological and ocean monitoring and forecasting system, for the benefit of WMO members and IOC member states. This coordination has been fruitful, and the efficient use of available resources has led to advancements in the overall management capacity of this complex system, from observations to generic services and customized products.

The demand for improved integration, coordination, and standards across global ocean observing and forecasting systems is increasing. The effective monitoring of the earth's climate requires a system that is continuously updated by new technologies, well-coordinated within the research community, and fully near-real time and operational. The four JCOMM activity areas only partially meet the expectations resulting from these new challenges. The JCOMM MAN has carried out a Strength and Weaknesses, Opportunities and Threats (SWOT) analysis (Table 2) to attempt to devise a better fit-for-purpose collaboration between IOC and WMO, in view of the changes that will involve JCOMM in 2020.

In general, the weaknesses outlined in Table 2 are due to the technical nature of the three program areas, which have not included any periodic review of stakeholder needs. A bottom-up approach has been taken in the program area activities, which has been adequate over their 20 years joint activity development period, but they now must be revisited.

In particular, there is a weakness in the connections between the observational program area and the ocean infrastructure of IODE and MCDS. Many real-time OPA networks have defined both their real-time and delayed mode data management strategies separately from the IODE structural elements described in section Data Management Systems, From Near-Real Time to Delayed Mode. Furthermore, there has been little cross-fertilization between the IODE system and the MCDS itself. The technical institutions represented by the WMO members and the IOC members states require international best practices across communities, so they can take advantage of past experiences in building a fit-for-purpose met-ocean data exchange system. JCOMM DMPA began to define a strategy for the met-ocean archiving system but failed because information about the working practices of IODE networks was not fully considered.

In an attempt to bridge the gap, IODE is developing an ODIS system that will initially be in the form of a "Catalogue of Sources" (http://catalogue.odis.org), aimed at being an online searchable catalogue of existing ocean-related web-based data and information sources/systems of as well as products and services. It will also provide information on products and visualize the landscape (entities and their connections) of ocean data and information sources. This may lead to a design for combining real-time and delayed mode data flows and the ongoing storage of the data. However, this process is far from being accomplished and will require future activities to focus on the strategic objectives. The future ODIS will be an eenvironment in which users can discover data, information, and associated products or services provided by member states, projects, and other partners associated with IOC and WMO. The system will aim to align itself with accepted community data management principles, such as the FAIR principles (Wilkinson et al., 2016) and, where appropriate, interoperate with existing data exchange solutions. ODIS will also allow additional metrics to be defined that record the data flow and if the data are securely archived within the IODE network and links with the satellite data centers.

To achieve this objective, JCOMM began defining standards for networking between IODE, OPA networks, and WIS (https://www.jcomm.info/index.php?option=com_oe\&task= viewDocumentRecord\&docID=20327). The Inter-Program Expert Team on Integrated Marine Meteorological and Oceanographic Services (IPET-MOIS) will continue this work (Figure 11). This group will define a formal procedure to interconnect all the relevant WIS to the IODE centers and eventually ODIS, using best practices with standards-based data and metadata exchange. With real-time data as its initial focus, IPET-MOIS will identify marine observing assets that have real-time value and facilitate the inclusion of these data on the GTS and WIS.

Another major weakness of the system is the lack of strong links between the three pillars of the met-ocean value chain 
TABLE 1 | (A) IOC CD Strategy; (B) WMO CD Strategy.

\section{(A) IOC (2015-2021)}

\section{Outputs}

1. Human resources developed

2. Access to physical infrastructure established or improved

3. Global, regional and sub-regional mechanisms strengthened

4. Development of ocean research policies in support of sustainable development objectives promoted

5. Visibility and awareness increased

6. Sustained (long-term) resource mobilization reinforced

\section{Activities}

1.1 Academic (higher) education

1.2 Continuous professional development

1.3 Sharing of knowledge and expertise/community building

1.4 Gender balance

2.1 Facilitating access to infrastructure (facilities, instruments, vessels)

3.1 Further strengthening and supporting secretariats of regional commissions 3.2 Enhance effective communication between regional sub-commission secretariats and global programs as well as other communities of practice (incl. other organizations)

4.1 Sharing of information on ocean research priorities

4.2 Developing national marine science management procedures and national policies

5.1 Public Information

5.2 Ocean Literacy

6.1 In-kind opportunities

6.2 Financial support by Member States to IOC activities

\section{(B) WMO}

\section{Objectives}

1. Define required capacities and identify deficiencies

2. Increase visibility and national ownership

3. Optimize knowledge management

4. Reinforce resource mobilization and project management

5. Strengthen global, regional and sub-regional mechanisms

6. Increase education and research opportunities

\section{Strategic approaches}

1.A: Emphasize compliance with WMO technical requirements to address priorities

1.B: Assist countries in identifying deficiencies of the NMHSs

1.C: Encourage development of services to address specific user needs

1.D: Establish modalities for partner and stakeholder engagement

2.A: Emphasize socio-economic benefits of services provided by NMHSs to decision makers 2.B: Assist NMHSs to incorporate requirements into national policy, legislative frameworks and national development plans

2.C: Enhance outreach to end users and decision makers

2.D: Develop leadership and management capacities

2.E: Reinforce national support to meet societal needs for weather, climate and hydrology services

3.A: Enhance mechanisms for collecting and sharing of up-to-date information relating to NMHSs development

3.B: Share best practices and success stories relating to the development of NMHSs

3.C: Enhance communities of practice dealing with the development of NMHSs

4.A: Enhance coordination and actively explore new funding opportunities and develop proposals through dialogue with stakeholders and development partners

4.B: Enhance capacity to develop, implement, monitor and evaluate projects

4.C. Encourage innovative voluntary and bilateral cooperation

0.1.1 5.A: Strengthen the work of global and regional centers

5.B: Strengthen global, regional and sub-regional mechanisms to provide support for weather, climate and hydrological services

0.1.2 6.A: Improve access to and provision of fellowships

6.B: Strengthen applications of research findings
(Figure 2). Neither the OPA networks nor the IODE/MCDS infrastructure get requirements from the forecasting and/or downstream services. As outlined in the user survey, it is clear that maritime safety services do not as yet benefit from the existing new observation data streams and the advanced metocean forecasts.

The activities carried out by JCOMM have begun to create strong and important links between the meteorological and the oceanographic communities, but the level of connections required surpasses the governance put together by JCOMM.

\section{FUTURE FOCI}

The areas identified as weaknesses and opportunities in Table 2 are considered the starting points for the focus of the new Joint IOC and WMO collaborative activities, which will begin in 2020.

The WMO reform advice was to abolish JCOMM and establish a new joint collaborative board between WMO and IOC that will take on all the activities of the present JCOMM but within a new governance structure and with new working practices. 
TABLE 2 | SWOT analysis for the Joint activities between WMO and IOC.

Strengths
Effective coordination between Meteorology and Oceanography (90\%
impact)
Global ocean observing system monitoring tool implemented and fully
operational
Increased number of Observing Networks connected to JCOMM
Near-real time data flow from oceanography to meteorology and vice
versa increased
Ocean Best Practices (OBP) System elevated to higher importance in
all PA

Opportunities

Strengthen connection between the PAs and GOOS and WMO reformed internal structures

Strengthen connections to Decade of the Ocean initiatives

Better research/innovation to operations coordination at all levels Strengthen JCOMMOPS/openGTS activities for new networks Put in place stronger connections with GRAs with RAs for "end-user" understanding

Strengthen OBP and CD activities

\section{Weaknesses}

Weak stakeholder analysis when setting the specific requirements for the Joint WMO and IOC activities

Weak across-program area activities

Capacity Development activities still weak in interfacing IOC and WMO specialized groups

Weak external and internal communication plan

Weak inter-program activities within WMO and IOC

Weak satellite-in situ observing connections

\section{Threats}

Many JCOMM processes still being in the phase of definition

Threat is to stop advancements for many years (services still in the phase of definition)

Design of met-ocean data assembly centers and interfaces with WIS halted

Loss of end-to-end system overview

Loss of impact at the level of NMHS and/or IODE national infrastructures

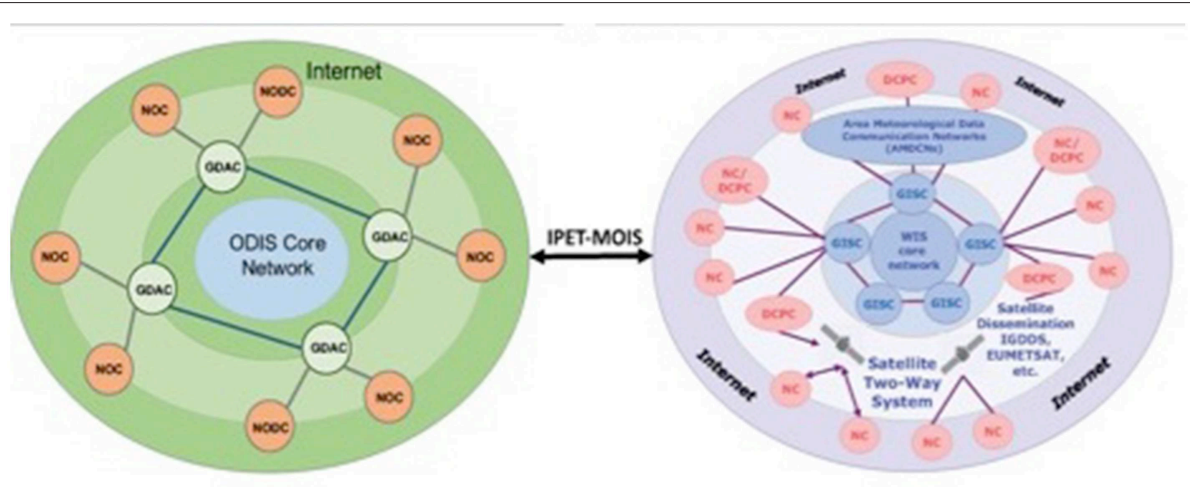

FIGURE 11 The high level structure of the ODIS components next to the WIS (http://www.wmo.int/pages/prog/www/WIS/). Symbols are defined in the Table of acronyms. The JCOMM IPET-MOIS team will facilitate the data interchange between existing oceanographic institutions part of ODIS and the WIS.

The new Joint Collaborative Board should develop and provide strategic advice, directly derived from the overall strategic plans of IOC and WMO, to achieve joint objectives with the mandate to propose decisions directly to IOC and WMO governing bodies.

It will also provide a forum for WMO and IOC bodies and external partners, to negotiate and fast-track projects and joint work plans, which include objectives that cut across meteorology and oceanography and WMO and IOC regional structures to enhance implementation and capacity development, and the value chain connecting observations, data management, forecasting systems, services, and research.

In this future structure, the expert teams will become involved in active IOC programs, such as GOOS, IODE, the IOC Tsunami Program, and the new two restructured commissions of the WMO (to be approved in June 2019). One of the major tasks of the new Joint Board will be to ensure the sustainability of the JCOMMOPS service, its connections with the IODE centers, and the full development of a fit-for-purpose data management system for the global met-ocean data and information system. The following goals form the backbone of the JCOMMOPS roadmap over the next $5-10$ years:

(i) Reach its metadata "gold" standard for all networks itemized in its initial Terms of Reference (Argo, OceanSITES, DBCP, SOT, GO-SHIP, GLOSS) and develop standard metadata services.

(ii) Expand its support and monitoring capacity; to new OCG networks (such as OceanGliders) and develop a regional pilot on e.g., Mediterranean Sea.

(iii) Enabling a responsive and truly global monitoring system. Over the long term, JCOMMOPS could expand its monitoring capacity to most of GOOS/WIGOS regions, and give visibility and monitoring capability to a cluster of coastal observing systems, with the cooperation of national focal points. 
The Joint Collaborative Board will oversee the work of the expert teams from an overall strategic point of view, and will organize the necessary exchanges with other programs, including GODAE OceanPredict, WCRP, GeoBluePlanet, etc. IMO and IHO should be invited to engage in the Joint Board, and particularly in the activities of maritime safety and bathymetric mapping, with an eye to the synergistic opportunities.

A major focus of the new Joint Board will be to organize projects that will advance the sharing of data and training on met-ocean products and information, including the new ocean forecasts, analyses and reanalyses, and the early warning systems, navigation safety services, and emergency response decision support systems.

In conclusion, we should again consider the success of the first 20 years of collaborative work between the IOC of UNESCO and WMO. Those in the fields of oceanography and meteorology have, at various levels and at the country level in particular, started to develop common plans and share expertise. These common activities are essential to gain knowledge and develop applications to fulfill the sustainable development goals.

\section{REFERENCES}

Abascal, A., Castanedo, S., Medina, R., and Liste, M. (2010). Analysis of the reliability of a statistical oil spill response model. Mar. Pollut. Bull. 60, 425-442. doi: 10.1016/j.marpolbul.2010.07.008

Anderson, D. M., Cembella, A. D., and Hallegraeff, G. M. (2012). Progress in understanding harmful algal blooms: paradigm shifts and new technologies for research, monitoring, and management. Annu. Rev. Mar. Sci. 4, 143-176. doi: 10.1146/annurev-marine-120308-081121

Bauer, P., Thorpe, A., and Gilbert, B. (2015). The quiet revolution of numerical weather prediction. Nature 525, 47-55. doi: 10.1038/nature 14956

Bell, M. J., Lefèbvre, M., Le Traon, P.-Y., Smith, N., and Wilmer-Becker, K. (2009). GODAE: the global ocean data assimilation experiment. Oceanography 22, 14-21. doi: 10.5670/oceanog.2009.62

Bojinski, S., Verstraete, M., Peterson, T. C., Richter, C., Simmons, A., and Zemp, M. (2014). The concept of essential climate variables in support of climate research, applications, and policy. Bull. Amer. Meteor. Soc. 95, 1431-1443. doi: 10.1175/BAMS-D-13-00047.1

Boyer, T. P., Antonov, J. I., Baranova, O. K., Coleman, C., Garcia, H. E., Grodsky, A., et al. (2013). World Ocean Database 2013, NOAA Atlas NESDIS, 72. Silver Spring, MD: NOAA Printing Office. Available online at: http://hdl.handle.net/ $11329 / 357$

Chassignet, E. P., and Verron, J. (2006). Ocean Weather Forecasting. An Integrated View of Oceanography. Dordrecht: Springer. doi: 10.1007/1-4020-4028-8

Cravatte, S., Kessler, W. S., Smith, N., Wijffels, S. E. (2016). First Report of TPOS 2020. GOOS-215, 200 pp. Available online at: http://tpos2020.org/first-report/

De Mey, P., Stanev, E., and Kourafalou, V. (2017). Science in support of coastal ocean forecasting - part 1. Ocean Dyn. 67:665. doi: 10.1007/s10236-017-1048-1

Dietze, H., and Kriest, I. (2012). ${ }^{137} \mathrm{Cs}$ off Fukushima Dai-ichi, Japanmodel based estimates of dilution and fate. Ocean Sci. 8, 319-332. doi: 10.5194/os-8-319-2012

Dombrowsky, E., Bertino, L., Brassington, G. B., Chassignet, E. P., Davidson, F., Hurlburt, H. E., et al. (2009). GODAE systems in operation. Oceanography 22, 80-95. doi: 10.5670/oceanog.2009.68

Draxler, R., Arnold, D., Masamichi, C., Galmarini, S., Hort, M., Jones, A., et al. (2015). World Meteorological Organization's model simulations of the radionuclide dispersion and deposition from the Fukushima Daiichi nuclear power plant accident. J. Environ. Radioact. 139, 172-184. doi: 10.1016/j.jenvrad.2013.09.014

Estournel, C., Bosc, E., Bocquet, M., Ulses, C., Marsaleix, P., Winiarek, V., et al. (2012). Assessment of the amount of cesium-137 released into the Pacific Ocean

\section{AUTHOR CONTRIBUTIONS}

NP suggested the contents of the paper and wrote the abstract, the whole introduction, part of section From Monitoring to Services, Capacity Development, and Discussion. DL together with JT, $\mathrm{JH}, \mathrm{EH}, \mathrm{SB}$, and others wrote the section Operational Observing Networks and Systems. KO'B wrote the section on real time data flow and TB the Marine Climate Data System with the help of PP. All the remaining authors corrected the different drafts.

\section{ACKNOWLEDGMENTS}

Several projects and national Institutions supported the authors of this paper. NP has received funding from the European Union's Horizon 2020 research and innovation program under grant agreement No. 633211 (AtlantOS). DL and SB acknowledge support from NOAA (FundRef 100007298). KO'B was partially funded by the Joint Institute for the Study of the Atmosphere and Ocean (JISAO) under NOAA Cooperative Agreement NA15OAR4320063, Contribution No. 2018-0176. after the Fukushima accident and analysis of its dispersion in Japanese coastal waters. J. Geophys. Res. 117:C11014. doi: 10.1029/2012JC007933

Evangeliou, N., Hamburger, T., Talerko, N., Zibtsev, S., Bondar, Y., Stohl, A., et al. (2016). Reconstructing the Chernobyl Nuclear Power Plant (CNPP) accident 30 years after. A unique database of air concentration and deposition measurements over Europe. Environ. Pollut. 216, 408-418. doi: 10.1016/j.envpol.2016.05.030

Fernandes, R., Braunschweig, F., Lourenço, F., and Neves, R. (2016). Combining operational models and data into a dynamic vessel risk assessment tool for coastal regions. Ocean Sci. 12, 285-317. doi: 10.5194/os-12285-2016

Fossi, M. C., Romeo, T., Baini, M., Panti, C., Marsili, L., Campan, T., et al. (2017). Plastic debris occurrence, convergence areas and fin whales feeding ground in the Mediterranean marine protected area Pelagos Sanctuary: a modeling approach. Front. Mar. Sci. 4:167. doi: 10.3389/fmars.2017. 00167

Glibert, P. M., Allen, J. I., Bouwman, A. F., Brown, C. W., Flynn, K. J., Lewitus, A. J., et al. (2010). Modeling of HABs and eutrophication: status, advances, challenges. J. Mar. Syst. 83, 262-275. doi: 10.1016/j.jmarsys.2010.05.004

He, R., McGillicuddy, D. J., Keafer, B. A., and Anderson, D. M. (2008). Historic 2005 toxic bloom of Alexandrium fundyense in the western Gulf of Maine: 2. Coupled biophysical numerical modeling. J. Geophys. Res. Oceans 113:C07040. doi: 10.1029/2007JC004602

Hense, I. (2010). Approaches to model the life cycle of harmful algae. J. Mar. Syst. 83, 108-114. doi: 10.1016/j.jmarsys.2010.02.014

Hermes, J., Pearlman, J., and Buttigieg, P. L. (2018). What's the best way to responsibly collect ocean data? Eos 99. doi: 10.1029/2018EO096533

ICSU (2017). A Guide to SDG Interactions: From Science to Implementation. Paris: International Council for Science.

IOC (1993). Manual of Quality Control Procedures for Validation of Oceanographic Data. Paris: UNESCO, 436pp. Intergovernmental Oceanographic Commission Manuals and Guides, 26. Available online at: http://hdl.handle.net/11329/167

IOC Assembly Resolution XXVI-8 (2011). Strengthening and Streamlining GOOS, IOC-XXVI/3. Available online at: ioc-unesco.org/ioc-26.

IRSN (2017). Detection of Ruthenium 106 in France and in Europe. Results of IRSN's Investigations. L'Institut de Radioprotection et de Sûreté Nucléaire. Available online at: https://www.irsn.fr/EN/newsroom/News/Documents/ IRSN_Information-Report_Ruthenium-106-in-europe_20171109.pdf

Jansen, E., Coppini, G., and Pinardi, N. (2016). Drift simulation of MH370 debris using superensemble techniques. Nat. Hazards Earth Syst. Sci. 16, 1623-1628. doi: 10.5194/nhess-16-1623-2016 
Jayne, S. R., Roemmich, D., Zilberman, N., Riser, S. C., Johnson, K. S., Johnson, G. C., et al. (2017). The argo program present and future. Oceanography 30, 18-28. doi: 10.5670/oceanog.2017.213

Johnson, G.C., Layman, J. M., Willis, J., Boyer, T., Antonov, J., Good, S. A., et al. (2014). Ocean heat content [in "State of the Climate in 2013"]. Bull. Am. Meteorol. Soc. 95, S54-S57. doi: 10.1175/2014BAMSStateoftheClimate.1

Johnson, G. C. (2018). Global oceans [in "State of the Climate in 2017"]. Bull. Am. Meteorol. Soc. 99, S143-S173. doi: 10.1175/2018BAMSStateoftheClimate.1

Kates, W. R., Parris, T. M., and Leiserowitz, A. A. (2005). What is sustainable development? Goals, indicators, values, and practice. Environ. Sci. Policy Sustain. Dev. 47, 8-21. doi: 10.1080/00139157.2005.10 524444

Kawamura, H., Kobayashi, T., Nishikawa, S., Ishikawa, Y., Usui, N., Kamachi, M., et al. (2014). Drift simulation of tsunami debris in the North Pacific. Glob. Environ. Res. 18, 81-96. Available online at: http://www.airies.or.jp/journal_182eng.html

Le Traon, P. Y., Reppucci, A., Fanjul, E. A., Aouf, L., Behrens, A., Belmonte, M., et al. (2019). From observation to information and users: the Copernicus Marine Service perspective. Front. Mar. Sci. 6:234. doi: 10.3389/fmars.2019. 00234

Legrand, S., Le Floch, S., Aprin, L., Parthenay, V., Donnay, E., Parmentier, K., et al. (2016). "Improving member states preparedness to face an HNS pollution of the marine system," in EGU General Assembly 2016, EGU2016, 17622. Available online at: https://meetingorganizer.copernicus.org/EGU2016/ EGU2016-17622.pdf

Li, Y., He, R., McGillicuddy, D.J., Anderson, D.M., and Keafer, B.A. (2009). Investigation of the 2006 Alexandrium fundyense bloom in the Gulf of Maine: in situ observations and numerical modeling. Cont. Shelf Res. 29, 2069-2082. doi: 10.1016/j.csr.2009.07.012

Liubartseva, S., Coppini, G., Lecci, R., and Clementi, E. (2018). Tracking plastics in the Mediterranean: 2D Lagrangian model. Mar. Pollut. Bull. 129, 151-162. doi: 10.1016/j.marpolbul.2018.02.019

Liubartseva, S., Coppini, G., Lecci, R., and Creti, S. (2016b). Regional approach to modeling the transport of floating plastic debris in the Adriatic Sea. Mar. Pollut. Bull. 103, 115-127. doi: 10.1016/j.marpolbul.2015. 12.031

Liubartseva, S., De Dominicis, M., Oddo, P., Coppini, G., Pinardi, N., and Greggio, N. (2016a). Oil spill hazard from dispersal of oil along shipping lanes in the Southern Adriatic and Northern Ionian Seas. Mar. Pollut. Bull. 90, 259-272. doi: 10.1016/j.marpolbul.2014.10.039

Mansui, J., Molcard, A., and Ourmieres, Y. (2015). Modelling the transport and accumulation of floating marine debris in the Mediterranean basin. Mar. Pollut. Bull. 91, 249-257. doi: 10.1016/j.marpolbul.2014.11.037

Margvelashvili, N., Maderich, V., and Zheleznyak, M. (1997). THREE-TOXcomputer code to simulate three-dimensional dispersion of radionuclides in stratified water bodies. Radiat. Protect. Dosimetry 73, 177-180. doi: 10.1093/oxfordjournals.rpd.a032128

Maximenko, N., Hafner, J., Kamachi, M., and MacFadyen, A. (2018). Numerical simulations of debris drift from the Great Japan Tsunami of 2011 and their verification with observational reports. Mar. Pollut. Bull. 132, 5-25. doi: 10.1016/j.marpolbul.2018.03.056

Maximenko, N., Hafner, N., and Niiler, J. (2012). Pathways of marine debris derived from trajectories of Lagrangian drifters. Mar. Pollut. Bull. 65, 151-162. doi: 10.1016/j.marpolbul.2011.04.016

McGillicuddy, D. J. (2010). Models of harmful algal blooms: conceptual, empirical, and numerical approaches. J. Mar. Syst. 83, 105-107. doi: 10.1016/j.jmarsys.2010.06.008

McGranahan, G., Balk, D., and Anderson, B. (2007). The rising tide: assessing the risks of climate change and human settlements in low elevation coastal zones. Environ. Urban. 19, 17-37. doi: 10.1177/0956247807076960

McPhaden, M.J., Busalacchi, A. J., and Anderson, D. L. T. (2010). A TOGA retrospective. Oceanography 23, 86-103. doi: 10.5670/oceanog.2010.26

MEPC (2015). Annex 10: International Code for Ships Operating in Polar Waters (Polar Code), MPEC 68/21. Marine Environment Protection Committee of International Maritime Organization.

Miyazawa, Y., Masumoto, Y., Varlamov, S. M., and Miyama, T. (2012). Transport simulation of the radionuclide from the shelf to open ocean around Fukushima. Cont. Shelf Res. 50-51, 16-29. doi: 10.1016/j.csr.2012.09.002
National Research Council (2003). Satellite Observations of the Earth's Environment: Accelerating the Transition of Research to Operations. Washington, DC: The National Academies Press.

Neuparth, T., Moreira, S., Santos, M. M., and Reis-Henriques, M. A. (2011). Hazardous and noxious substances (HNS) in the marine environment: prioritizing HNS that pose major risk in a european context. Mar. Pollut. Bull. 62, 21-28. doi: 10.1016/j.marpolbul.2010.09.016

OECD (2016). The Ocean Economy in 2030. Paris: OECD Publishing.

Olita, A., Cucco, A., Simeone, S., Ribotti, A., Fazioli, L., Sorgente, B., et al. (2012). Oil spill hazard and risk assessment for the shorelines of a Mediterranean coastal archipelago. Ocean Coast. Manage. 57, 44-52. doi: 10.1016/j.ocecoaman.2011.11.006

Pearlman, J., Bushnell, M., Coppola, L., Karstensen, J., Buttigieg, L., Pearlman, F., et al. (2019). Evolving and sustaining ocean best practices and standards for the next decade. Front. Mar. Sci. 6:277. doi: 10.3389/fmars.2019. 00277

Periáñez, R., Brovchenko, I., Duffa, C., Jung, K. T., Kobayashi, T., Lamego, F., et al. (2015). A new comparison of marine dispersion model performances for Fukushima Dai-ichi releases in the frame of IAEA MODARIA program. J. Environ. Radioact. 150, 247-269. doi: 10.1016/j.jenvrad.2015. 09.003

Pinardi, N., Cavaleri, L., Coppini, G., De Mey, P., Fratianni, C., Huthnance, J., et al. (2017). From weather to ocean predictions: an historical viewpoint. J. Mar. Res. 75, 103-159. doi: 10.1357/002224017821 836789

Pinardi, N., and Woods, J. (2002). Ocean Forecasting. Conceptual Basis and Applications. Berlin; Heidelberg: Springer-Verlag. doi: 10.1007/978-3-662-22648-3

Politikos, D. V., Ioakeimidis, C., Papatheodorou, G., and Tsiaras, K. (2017). Modeling the fate and distribution of floating litter particles in the Aegean Sea (E. Mediterranean). Front. Mar. Sci. 4:191. doi: 10.3389/fmars.2017. 00191

Price, J., Jonson, W., Marshall, C., Ji, Z., and Rainey, G. (2003). Overview of the oil spill risk analysis (OSRA) model for environmental impact assessment. Spill Sci. Technol. Bull. 8, 529-533. doi: 10.1016/S1353-2561(03)00003-3

Purkey, S. G., and Johnson, G. C. (2010). Warming of global abyssal and deep Southern Ocean waters between the 1990s and 2000s: contributions to global heat and sea level rise budgets. Clim. J. 23, 6336-6351. doi: 10.1175/2010JCLI3682.1

Sabine, C.L., Ducklow, H., and Hood, M. (2010). International carbon coordination: Roger Revelle's legacy in the Intergovernmental Oceanographic Commission. Oceanography 23, 48-61. doi: 10.5670/oceanog.2010.23

Sepp-Neves, A. A., Pinardi, N., and Martins, F. (2016). IT-OSRA: applying ensemble simulations to estimate the oil spill risk associated to operational and accidental oil spills. Clim. Dyn. 66, 939-954. doi: 10.1007/s10236-016-0960-0

Serafin, R. J., MacDonald, A. E., and Gall, R. L. (2002). Transition of weather research to operations. Bull. Am. Meteorol. Soc. 83, 377-392. doi: 10.1175/15200477(2002)083<0377:TOWRTO>2.3.CO;2

Shao, H., Derber, J., Huang, X., Hu, M., Newman, K., Stark, D., et al. (2016). Bridging research to operations transitions: status and plans of community GSI. Bull. Am. Meteorol. Soc. 97, 1427-1440. doi: 10.1175/BAMS-D-13-00245.1

Simons, R. A. (2017). ERDDAP. Monterey, CA: NOAA/NMFS/SWFSC/ERD. Available online at: https://coastwatch.pfeg.noaa.gov/erddap

Staneva, J. V., Buesseler, K. O., Stanev, E. V., and Livingston, H. D. (1999). The application of radiotracers to a study of Black Sea circulation: validation of numerical simulations against observed weapons testing and chernobyl ${ }^{137}$ Cs data. J. Geophys. Res. 104, 11099-11114. doi: 10.1029/1998JC 900121

Swail, V., Lee, B., Soares, A., Resio, D., Horsburgh, K., Murty, T., et al. (2010). "Storm surge," in Proceedings of OceanObs'09: Sustained Ocean Observations and Information for Society, Vol. 2, eds J. Hall, D. E. Harrison, and D. Stammer (Venice: ESA Publication WPP-306), 21-25 September 2009.

Task Team for the Integrated Framework for Sustained Ocean Observing (2012). A Framework for Ocean Observing (Revised in 2017). Paris: UNESCO, IOC/INF$1284 \mathrm{rev} .2$.

Tonani, M., Balmaseda, M., Bertino, L., Blockley, E., Brassington, G., Davidson, F., et al. (2015). Status and future of global and regional ocean prediction systems. J. Oper. Oceanogr. 8, 201-220. doi: 10.1080/1755876X.2015.1049892 
Trinanes, J. A., Olascoaga, M. J., Goni, G. J., Maximenko, N. A., Griffin, D. A., and Hafner, J. (2016). Analysis of potential MH370 debris trajectories using ocean observations and numerical model results. J. Oper. Oceanogr. 9, 126-138. doi: 10.1080/1755876X.2016.1248149

Tsumune, D., Tsubono, T., Aoyama, M., and Hirose, K. (2012). Distribution of oceanic $137 \mathrm{Cs}$ from the Fukushima Dai-ichi nuclear power plant simulated numerically by a regional ocean model. J. Environ. Radioact. 111, 100-108. doi: 10.1016/j.jenvrad.2011.10.007

Van Sebille, E., Wilcox, C., Lebreton, L., Maximenko, N. A., Hardesty, B. D., van Franeker, J. A., et al. (2015). A global inventory of small floating plastic debris. Environ. Res. Lett. 10:124006. doi: 10.1088/1748-9326/10/12/ 124006

Wilkinson, M. D., Dumontier, M., Aalbersberg, I. J., Appleton, G., Axton, M., Baak, A., et al. (2016). The FAIR Guiding Principles for scientific data management and stewardship. Sci. Data 3:160018. doi: 10.1038/sdata.2016.18

WMO (2017a). Guide to Marine Meteorological Services. WMO-471. 73 pp. Available online at: https://www.jcomm.info/index.php?option=com_oe\& task=viewDocumentRecord\&docID=19901

WMO (2017b). Manual on Marine Services. WMO-558. 62 pp. Available online at: https://www.jcomm.info/index.php?option=com_oe\&task= viewDocumentRecord\&docID $=19900$

WMO (2017c). Technical Regulations, Volume I: General Meteorological Standards and Recommended Practices. WMO-49. Available online at: https://library. wmo.int/index.php?lvl=notice_display\&id=14073
Zambianchi, E., Trani, M., and Falco, P. (2017). Lagrangian transport of marine litter in the Mediterranean Sea. Front. Mar. Sci. 5:5. doi: 10.3389/fenvs.2017.00005

Zodiatis, G., Liubartseva, S., Alves, T. M., Lardner, R., Kampanis, N., Spanoudaki, K., et al. (2018). "Monitoring and forecasting oil platforms leakages in the Mediterranean and Black Sea," in EGU General Assembly 2018, EGU2018, 550-1. Available online at: https://meetingorganizer.copernicus.org/EGU2018/ EGU2018-2550-1.pdf

Conflict of Interest Statement: The authors declare that the research was conducted in the absence of any commercial or financial relationships that could be construed as a potential conflict of interest.

At least a portion of this work is authored by David Legler, Kevin O'Brien, Tim Boyer, Tom Cuff, Eugene Burger, Shelby Brunner, Hernan Garcia, and Eric Freeman on behalf of the U.S. Government and, as regards Dr. Legler, Dr. O'Brien, Dr. Boyer, Dr. Cuff, Dr. Burger, Dr. Brunner, Dr. Garcia, Dr. Freeman, and the U.S. Government, is not subject to copyright protection in the United States. Foreign and other copyrights may apply. This is an open-access article distributed under the terms of the Creative Commons Attribution License (CC BY). The use, distribution or reproduction in other forums is permitted, provided the original author(s) and the copyright owner(s) are credited and that the original publication in this journal is cited, in accordance with accepted academic practice. No use, distribution or reproduction is permitted which does not comply with these terms. 


\section{TABLE OF ACRONYMS}

Argo

ADU

AIU

BUFR

$\mathrm{CCHDO}$

CD

CIFDP

CLIVAR

CMEMS

CMOC

DAC

DCPC

DMPA

EOV

ERDDAP

ETDMP

ETDRR

ETMEER

ETSI

FAIR

GCOS

GEO

GeoBluePlanet

GDAC

GFCS

GDPFS

GISC

GMDSS

GODAE

GOOS

GTSPP

HELCOM

IAEA

ICOADS

ICSU

$\mathrm{IHO}$

$\mathrm{IMO}$

IOC

IODE

IPET-MOIS

ISO

$\mathrm{KPI}$

JCOMM

JCOMMOPS

LDCs

MARPOL

MCDS

MCSS

MPERSS

$\mathrm{NC}$

NHMS

NMDIS

NOC

NODC
Global array of subsurface profiling floats

meteorological data.

Clivar \& Carbon Hydrographic Data Office

Capacity Development

Coastal Inundation Forecasting Demonstration Project

Climate and ocean-Variability, Predictability and Change

Copernicus Marine Environment Monitoring Service

WMO-IOC Centre for Marine. Meteorological and

Oceanographic Climate Data

Data Assembly Center

Data Collection or Production Center

Data Management Program Area (JCOMM)

Essential Ocean Variable

Guidelines for Data Distribution Systems (no longer an acronym)

Expert Team on Data Management Practices (JCOMM)

Expert Team on Disaster Risk Reduction (JCOMM)

Expert Team on Marine Environmental Emergencies

Response (JCOMM)

Expert Team on Sea Ice (JCOMM)

Findable, Accessible, Interoperable, Re-Usable

WMO Global Climate Observing System

Group on Earth Observations

Blue Planet Oceans and Society, a GEO program

Global Data Assembly Center

Global Framework for Climate Services

WMO Global Data-Processing and Forecasting System

Global Information System Center (WMO)

WMO Global Maritime Distress and Safety System

Global Ocean Data Assimilation Experiment

Global Ocean Observing System

Global Temperature and Salinity Profile Program

Baltic Marine Environment Protection Commission

International Atomic Energy Agency

International Comprehensive Ocean-Atmosphere Data Set

International Council for Science

International Hydrographic Organization

International Maritime Organization

Intergovernmental Oceanographic Commission of UNESCO

IOC International Oceanographic Data and Information

Exchange

Inter-Program Expert Team on Integrated Marine

Meteorological and Oceanographic Services

International Standards Organization

Key Performance Indicator

Joint Committee of Oceanography and Marine Meteorology

JCOMM in-situ Observing Platform Support Centre

Least Developed Countries (LDCs)

International Convention for the Prevention of Pollution from

Ships

Marine Climate Data System (JCOMM)

Marine Climatological Summaries Scheme

WMO Marine Pollution Emergency Response Support System

National Centers (Meteorology)

National Meteorological and Hydrological Services

National Marine Data and Information Service

National Oceanographic center

National Oceanographic Data Centre
OCG

ODIN

ODP

OECD

OPA

OSPAR

Observation Coordination Group (JCOMM)

IODE Ocean Data and Information Network

Ocean Data Portal

Organization for Economic Co-operation and Development

Observation Program Area (JCOMM)

The Convention for the Protection of the Marine Environment of the North-East Atlantic

OTGA

PANGEA

QC

IOC-IODE Ocean Teacher Global Academy

Partnership for New GEOSS Applications

Quality Control

Regional Marine Pollution Emergency Response Centre for the Mediterranean Sea

WMO Regional Specialized Meteorological Center

WMO Global Campus and Regional Training Centers

Sustainable Development Goal (s)

Simple Object Access Protocol

Ships Of Opportunity Program

Sea Surface Temperature

Tropical Ocean Global Atmosphere

Pan-European infrastructure for ocean \& marine data management

Services and Forecasting Services Program Area (JCOMM)

Small Island Development States

Safety of Life at Sea

Strength and Weaknesses, Opportunities and Threats

Task Team for Integrated Marine Meteorological and

Oceanographic Services

Voluntary Observing Ships

VOS

WIGOS.

WIS

WMO

WWMIWS

WWNWS
WMO Integrated Global Observing System (WIGOS)

WMO Information System

World Meteorological Organization

Worldwide Met-Ocean Information and Warning Service

IHO Worldwide Navigation Warning Service 


\section{OPEN ACCESS}

\section{Edited by: \\ Minhan Dai, \\ Xiamen University, China \\ Reviewed by: \\ Andrea Storto, \\ NATO Centre for Maritime Research and Experimentation, Italy \\ Sophie E. Cravatte, Institut de Recherche pour le Développement (IRD), France Wilhelm Petersen, Institute of Coastal Research, Helmholtz-Zentrum Geesthacht, \\ Germany \\ *Correspondence: \\ Shawn R. Smith \\ smith@coaps.fsu.edu \\ Specialty section: This article was submitted to \\ Ocean Observation, \\ a section of the journal \\ Frontiers in Marine Science \\ Received: 09 November 2018 \\ Accepted: 05 July 2019 \\ Published: 02 August 2019}

Citation:

Smith SR, Alory G, Andersson A,

Asher W, Baker A, Berry DI,

Drushka K, Figurskey D, Freeman E,

Holthus P, Jickells T, Kleta H, Kent EC,

Kolodziejczyk N, Kramp M, Loh Z,

Poli P, Schuster U, Steventon E,

Swart S, Tarasova O, de la Villéon LP

and Vinogradova-Shiffer N (2019)

Ship-Based Contributions to Global

Ocean, Weather, and Climate

Observing Systems.

Front. Mar. Sci. 6:434.

doi: 10.3389/fmars.2019.00434

\section{Ship-Based Contributions to Global Ocean, Weather, and Climate Observing Systems}

\author{
Shawn R. Smith ${ }^{1 *}$, Gaël Alory2 ${ }^{2}$ Axel Andersson ${ }^{3}$, William Asher ${ }^{4}$, Alex Baker ${ }^{5}$, \\ David I. Berry 6 , Kyla Drushka ${ }^{4}$, Darin Figurskey ${ }^{7}$, Eric Freeman ${ }^{8}$, Paul Holthus $^{9}$, \\ Tim Jickells ${ }^{5}$, Henry Kleta ${ }^{10}$, Elizabeth C. Kent ${ }^{6}$, Nicolas Kolodziejczyk ${ }^{11}$, Martin Kramp ${ }^{12}$, \\ Zoe Loh ${ }^{13}$, Paul Poli14, Ute Schuster ${ }^{15}$, Emma Steventon ${ }^{16}$, Sebastiaan Swart 17,18, \\ Oksana Tarasova ${ }^{19}$, Loic Petit de la Villéon ${ }^{20}$ and Nadya Vinogradova-Shiffer ${ }^{21}$
}

\begin{abstract}
${ }^{1}$ Center for Ocean-Atmospheric Prediction Studies, The Florida State University, Tallahassee, FL, United States, ${ }^{2}$ LEGOS, CNES/CNRS/IRD/UPS, Toulouse, France, ${ }^{3}$ Maritimes Datenzentrum, Deutscher Wetterdienst, Hamburg, Germany, ${ }^{4}$ Applied Physics Laboratory, University of Washington, Seattle, WA, United States, ${ }^{5}$ School of Environmental Sciences, University of East Anglia, Norwich, United Kingdom, ${ }^{6}$ National Oceanography Centre, Southampton, United Kingdom, ${ }^{7}$ Ocean Prediction Center, NOAA National Weather Service, College Park, MD, United States, ${ }^{8}$ ERT, Inc., National Centers for Environmental Information/CCOG, Asheville, NC, United States, ${ }^{9}$ World Ocean Council, Honolulu, HI, United States, ${ }^{10}$ Maritimes Messnetz, Deutscher Wetterdienst, Hamburg, Germany, ${ }^{11}$ Laboratory of Ocean Physics, University of Brest, Plouzané, France, ${ }^{12} \mathrm{JCOMMOPS}$, WMO/IOC-UNESCO, Brest, France, ${ }^{13}$ Oceans and Atmosphere, CSIRO, Aspendale, VIC, Australia, ${ }^{14}$ Centre de Météorologie Marine, Météo-France, Brest, France, ${ }^{15}$ College of Life and Environmental Sciences, Hatherly Laboratories, University of Exeter, Exeter, United Kingdom, ${ }^{16}$ Met Office, Exeter, United Kingdom, ${ }^{17}$ Department of Marine Sciences, University of Gothenburg, Gothenburg, Sweden, ${ }^{18}$ Department of Oceanography, University of Cape Town, Rondebosch, South Africa, ${ }^{19} \mathrm{Global}$ Atmosphere Watch Programme, World Meteorological Organization, Geneva, Switzerland, ${ }^{20}$ IFREMER/Sismer, Plouzané, France, ${ }^{21}$ Science Mission Directorate, NASA Headquarters, Washington, DC, United States
\end{abstract}

The role ships play in atmospheric, oceanic, and biogeochemical observations is described with a focus on measurements made near the ocean surface. Ships include merchant and research vessels; cruise liners and ferries; fishing vessels; coast guard, military, and other government-operated ships; yachts; and a growing fleet of automated surface vessels. The present capabilities of ships to measure essential climate/ocean variables and the requirements from a broad community to address operational, commercial, and scientific needs are described. The authors provide a vision to expand observations needed from ships to understand and forecast the exchanges across the ocean-atmosphere interface. The vision addresses (1) recruiting vessels to improve both spatial and temporal sampling, (2) conducting multivariate sampling on ships, (3) raising technology readiness levels of automated shipboard sensors and ship-to-shore data communications, (4) advancing quality evaluation of observations, and (5) developing a unified data management approach for observations and metadata that meet the needs of a diverse user community. Recommendations are made focusing on integrating private and autonomous vessels into the observing system, investing in sensor and communications technology development, developing an integrated data management structure that includes all types of ships, and moving toward a quality evaluation process that will result in a subset of ships being defined as mobile reference ships that will support climate studies. We envision a future where commercial, research, 
and privately owned vessels are making multivariate observations using a combination of automated and human-observed measurements. All data and metadata will be documented, tracked, evaluated, distributed, and archived to benefit users of marine data. This vision looks at ships as a holistic network, not a set of disparate commercial, research, and/or third-party activities working in isolation, to bring these communities together for the mutual benefit of all.

Keywords: ships, observations, meteorology, physical oceanography, biogeochemistry, data management, climatology

\section{INTRODUCTION}

Since the days when sailing vessels were the primary vehicle for commerce and exploration on the high seas, ships have observed the marine environment (Woodruff et al., 2005). With the exception of a few research voyages (e.g., HMS Beagle, Keynes, 2012; HMS Challenger, Corfield, 2003), these early observations of sea water temperature, winds, and atmospheric pressure were made to support day-to-day operations (e.g., Richardson, 1980). Today, all types of ships make routine weather and ocean observations that are shared internationally to support weather forecasting, safety at sea, and commercial ventures (e.g., energy, fisheries, and transportation), while dedicated oceanographic research vessels make a wide range of atmospheric, oceanographic, chemical, biological, and other observations to support research into the complex interactions between the marine atmosphere, hydrosphere, cryosphere, and biosphere. Research vessels provide an extremely versatile sampling platform from which highly sophisticated instrumentation can be deployed by national research facilities, navies, coast guards, universities, or private institutions. Many are designed to operate in remote and inhospitable waters, providing data from regions outside commercial shipping lanes; however, sampling from research vessels often suffers from a lack of regularity and repeat sampling at given locations and can be subject to large seasonal biases, with high latitude regions rarely visited during the winter months. In contrast, commercial ships tend to traverse the ocean along traditional and, with decreased Arctic ice cover in recent years, evolving shipping lanes, thus repeating observations in spatially limited regions of the ocean. Over long timescales (decades to centuries), meteorological and oceanographic observations made from pre-industrial sailing vessels, research vessels, and commercial ships and more recently autonomous surface vessels underpin our understanding of marine climate variability and change.

The focus herein is on the role ships presently play in atmospheric and oceanic observations and outlines a vision for the coming decade. The vision builds upon the recommendations from the OceanObs'09 meeting (Smith et al., 2010). Of the 14 recommendations made in Smith et al. (2010), successes include improving linkages between the physical, biological, and carbon communities [e.g., through the Joint Technical Commission for Oceanography and Marine Meteorology (JCOMM, see Appendix) Observation Coordination Group]; working toward standardized metadata [e.g., converging on World Meteorological Organization (WMO) Integrated Global
Observing System (WIGOS) metadata standards and developing unique identifiers for observing platforms]; identifying new ships to provide observations (e.g., recruiting racing yachts from the Volvo Ocean Race and research vessels to underway data programs); and outlining new approaches to recruit ships through non-traditional methods [e.g., JCOMM approval in 2018 of a new third-party class to recruit ships outside of the traditional national meteorological and hydrographic services (NMHS)]. There has also been success in developing automated precipitation systems for ships (e.g., Klepp, 2015) and including air-flow modeling in new ship design (e.g., specifically for the research vessels Sikuliaq, Sally Ride, and Neil Armstrong in the United States). One recent success in the area of addressing diplomatic obstacles was the workshop on enhancing ocean observations and research, and the free exchange of data, to foster services for the safety of life and property hosted by WMO in February 2019. The workshop recognized the importance of Observing System Simulation Experiments (OSSEs) and sensitivity analyses to be used to investigate the importance of data collected within nation's exclusive economic zones. Despite the successes since OceanObs'09, a number of the recommendations saw little progress in the past decade. There is still a need to advance instrument technology for autonomous sampling, particularly for cloud cover, cloud type, and sea state. Adequate resources have not been available to coordinate research vessel cruise data, to develop consolidated marine datasets, standardize data quality evaluation across multiple ship measurement programs, or conduct systematic comparisons of different sensor systems typically deployed on ships. The proposed vision includes some of the topics not addressed over the past decade.

Throughout this review, the term "ship" includes, but is not limited to, merchant and research vessels; cruise liners and ferries; fishing vessels; coast guard, military, and other governmentoperated ships; yachts and other private crafts; and a growing fleet of autonomous surface vessels. The primary focus will be on ships with a crew; however, autonomous surface vessels (e.g., Caccia et al., 2005; German et al., 2012) and large fixed or mobile platforms (e.g., drilling platforms and light towers) can provide similar observational capabilities. While recognizing the great importance of ships for deploying atmosphere- and oceanobserving technology (e.g., balloon soundings, McBean et al., 1986; expendable bathythermographs, Goni et al., 2019; Argo floats, Roemmich et al., 2009; drifters, Pazan and Niiler, 2004; moorings, McPhaden et al., 1998; Send et al., 2010; and gliders, Rudnick et al., 2004), the focus here is on measurements 
TABLE 1 | Parameters observed by ships near the ocean surface.

\begin{tabular}{|c|c|c|c|c|}
\hline Parameter & $\begin{array}{l}\text { Observation } \\
\text { method }\end{array}$ & Comments & Ship type & $\begin{array}{l}\text { First year } \\
\text { systematically } \\
\text { observed }\end{array}$ \\
\hline \multicolumn{5}{|l|}{ Essential climate/Ocean variables } \\
\hline Air Temperature & $A, M$ & & $\mathrm{C}, \mathrm{R}, \mathrm{P}, \mathrm{G}, \mathrm{Y}$ & 1784 \\
\hline Water Vapor & $A, M$ & A variety of humidity parameters are reported. & C, R, P, G & 1873 \\
\hline Atmospheric Pressure & $A, M$ & $\begin{array}{l}\text { Either pressure at measurement height or adjusted to } \\
\text { mean sea level can be reported. }\end{array}$ & $C, R, P, G, Y$ & 1785 \\
\hline Wind Direction and Speed & $A, M, V$ & $\begin{array}{l}\text { Visual estimates of winds derived from sea state (Beaufort } \\
\text { wind scale). Also manually recorded by crew reading } \\
\text { analog or digital display from anemometer. }\end{array}$ & $C, R, P, G, Y$ & 1750 \\
\hline Radiation & A & & $\mathrm{C}, \mathrm{R}, \mathrm{G}$ & 1970 \\
\hline Precipitation & A & & $C, R, P, G$ & 1970 \\
\hline Cloud Properties & M & & $C, R, P, G$ & 1852 \\
\hline Sea Water Temperature & $A, M$ & $\begin{array}{l}\text { Including water measurements at the ocean surface and } \\
\text { at depths within the scope of this paper }\end{array}$ & $C, R, P, G, Y$ & 1816 \\
\hline Salinity & $A, M$ & $\begin{array}{l}\text { Including salinity measurements at the ocean surface and } \\
\text { at depths within the scope of this paper }\end{array}$ & C, R, P, G, Y & 1873 \\
\hline Inorganic Carbon & $A, M$ & Including $\mathrm{pCO}_{2}$ measured in the atmosphere and ocean. & $C, R, P, G, Y$ & 1958 \\
\hline Dissolved Organic Carbon & A & & $R, G$ & 1990 \\
\hline Nutrients & A & & $R, G$ & 1921 \\
\hline Nitrous Oxide & A & & $\mathrm{R}$ & 2000 \\
\hline Oxygen & A & & $\mathrm{R}$ & 1900 \\
\hline Ocean Color & A & & $\mathrm{R}$ & 1954 \\
\hline Transient Tracers (e.g., CFC11) & M & & $\mathrm{R}$ & 1982 \\
\hline Aerosols & M & Semi-manual approach & $\mathrm{R}$ & 1995 \\
\hline Sea State & $A, M, V$ & $\begin{array}{l}\text { Visual estimates by crew, automated measurements via } \\
\text { wave radars and the Ship Bourne Wave Recorder }\end{array}$ & $\mathrm{C}, \mathrm{R}, \mathrm{G}$ & 1876 \\
\hline Surface and Subsurface Currents & A & $\begin{array}{l}\text { Measured directly by acoustic Doppler current profilers, } \\
\text { indirectly via ship drift calculations (using navigation data) }\end{array}$ & $\mathrm{C}, \mathrm{R}, \mathrm{G}, \mathrm{Y}$ & $\begin{array}{c}1920 \text { (surface), } \\
1985 \text { (subsurface) }\end{array}$ \\
\hline \multicolumn{5}{|c|}{ Additional shipboard measurements } \\
\hline Visibility & M & & $\mathrm{C}, \mathrm{G}$ & 1854 \\
\hline Sea lce & $\mathrm{A}, \mathrm{M}$ & Automated measurements by ice radars & $C, R, P, G$ & 1955 \\
\hline Chlorophyll Concentration & A & Supports ocean color and biomass EOVs & $\mathrm{R}, \mathrm{G}$ & 1954 \\
\hline Alkalinity/pH & A & & $R, G$ & 1972 \\
\hline
\end{tabular}

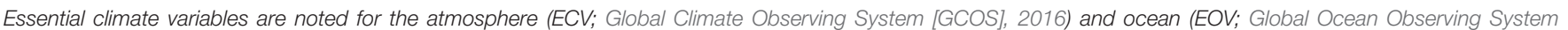

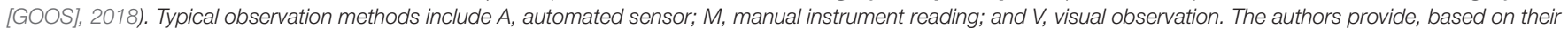

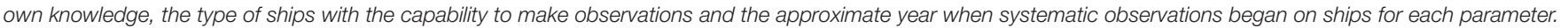

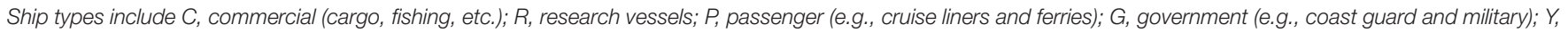
yachts and other private crafts; and $A$, autonomous surface vessels.

by automated instrumentation and both visual estimates and instrumental readings taken by observers on board ships. Ships provide a platform for simultaneous measurements of the physical and biogeochemical properties within the atmosphere and ocean through the use of fully automated instruments, manual observations, or a combination of the two. The suite of observations (Table 1) that can be made from ships includes essential climate variables (ECVs; Bojinski et al., 2014; Global Climate Observing System [GCOS], 2016) and essential ocean variables (EOVs; Global Ocean Observing System [GOOS], 2018), along with other parameters that address a wide range of applications. The instrumentation installed on ships (along with human observers) supports making measurements over a wide range of heights in the atmosphere and depths in the ocean. While we recognize that ship-based instruments can make atmospheric and ocean profiles (e.g., balloon sondes, rosette casts, and expendable bathythermographs) and include remote sensing systems (e.g., radar, sonar, and acoustic Doppler current profiler), the discussion herein focuses on measurements made near the ocean surface and typically within the physical dimensions of the ship. This limitation is motivated by community requirements (see Community Requirements) to observe those parameters near the ocean surface that are essential to (1) understand the processes that govern the energy, nutrient, and chemical exchanges at the ocean-atmosphere interface; (2) support operational weather, ocean, and climate forecasting; (3) provide observations to validate and evaluate space-based observations of the ocean's surface and numerical model analyses; (4) quantify biases in ship observations and derived products used in climate research and assessments; (5) examine and understand the variations in the near-surface marine climate system on 
timescales from hours to centuries; and (6) support ocean ecosystem management.

The decadal vision expands the multivariate observations needed from ships to understand and forecast the exchanges across the ocean-atmosphere interface. The vision addresses (1) recruiting additional vessels to improve both spatial and temporal sampling, (2) conducting multivariate sampling on ships, (3) raising technology readiness levels of automated shipboard sensors and ship-to-shore data communications, (4) advancing quality evaluation of observations, and (5) developing a unified data management approach for shipboard observations and metadata that meet the needs of a diverse user community. Leveraging existing programs and developing innovative methods will be a cost-effective approach to support the measurement of multiple physical and biogeochemical observations on individual ships, thereby maximizing ship contributions to GOOS. The authors envision a ship-based observational network that integrates across operational and research communities to deliver needed information.

\section{COMMUNITY REQUIREMENTS}

\section{Overview}

Marine data are used directly and indirectly by a broad community to address operational, commercial, and scientific needs. User requirements differ between the real-time versus delayed-mode (climate) communities resulting in various sampling and collection strategies, data transmission technologies, metadata and documentation, and data quality evaluation. A primary challenge is effectively managing resources provided by commercial, governmental, and private entities to meet these varied requirements. Most observations made to support operational marine forecasting are funded and managed by NMHS, while many other observations are supported by timelimited national or private sector research funding. A continuing challenge is how to sustain observations that are initiated within the research community, but where users see a need for longterm observations and data management. Equally challenging is ensuring that observations primarily funded and made available by the operational community, but used downstream for climate analyses and research, (a) are of sufficient quality and quantity, (b) are appropriately described by metadata, and (c) have suitable provision for sustained data management.

\section{Forecasting, Navigation, and Safety}

For centuries observations from ships have provided safetyrelated meteorological services for ships at sea and have been used for climatological purposes (e.g., Maury, 1854; Mallory, 1855; International Maritime Organization [IMO], 2018). The Safety of Life at Sea Convention (International Maritime Organization [IMO], 2002), Regulation 5, "Meteorological Forecasts and Warnings," specifies provisions whereby contracting governments are encouraged to arrange for a selection of ships to be equipped with tested marine meteorological instruments and to take, record, and transmit meteorological observations at the main standard times for surface synoptic observations. Contracting governments are also instructed to encourage other ships to make, record, and transmit observations in a modified form, particularly in areas with sparse data. In addition, ship observations have set an early open-access example: WMO (1995) Resolution 40 Annex 1 lists marine data among "data and products to be exchanged without charge and with no conditions on use." Presently, the Voluntary Observing Ships' (VOS) Scheme (Kent et al., 2010), a panel of the Ship Observations Team of the JCOMM of WMO and the Intergovernmental Oceanographic Commission (IOC), provides the governance by which ships are recruited by NMHSs for making and transmitting meteorological observations. For near-real-time applications, the data are delivered to users via the WMO Global Telecommunication System (GTS). VOS meteorological reports are a unique and invaluable contribution to operational meteorology and marine meteorological services (Fletcher, 2008).

Requirements for near-surface ship observations to support numerical weather prediction and operational forecasting include, but are not limited to, atmospheric pressure, wind speed and direction, air temperature, relative humidity, and sea surface temperature (SST), as well as wave height, direction, and period (Anderson, 2018). A major ongoing problem is the scarcity of in situ data from vast areas of the world's oceans. While the near global coverage from satellite-based remote sensing helps overcome this, data from ships remain essential. Ship-based observations provide parameters that satellites cannot observe (e.g., atmospheric pressure), data from regions with gaps in satellite coverage (Figure 1), and validation data that are relevant to forecast operations. Beyond their use in numerical weather prediction, data from ships are also used operationally in the preparation of forecasts and warnings, including those for the Global Maritime Distress and Safety System, and to support the routing of ships to avoid adverse weather and efficiently transport cargo (International Maritime Organization [IMO], 2018).

An example of ship observations assisting in the issuance of a warning for the high seas of the North Atlantic occurred July 29,2015 , when a ship observation on the near-west side of an extratropical cyclone reported hurricane-force sustained winds of 65 knots (Figure 2). The low-pressure center in the 6-h forecast of the National Centers for Environmental Prediction Environmental Modeling Center's Global Forecasting System model (Figure 3) was located too far west and was too weak. Using the ship observation, a forecaster at the National Weather Services Ocean Prediction Center upgraded high seas forecasts to include a hurricane-force wind warning for the cyclone. While the skill of global numerical weather prediction models is increasing (Bauer et al., 2015), human guidance still adds value to daily forecasts, watches, and warnings. This is especially true for high-impact events, specifically in the $12-48 \mathrm{~h}$ of the forecast period (e.g., Stern and Davidson, 2015).

Ship-based observations transmitted via the GTS are also essential to the growing field of operational oceanography, which provides a basis for our knowledge of the marine environment on timescales sufficient to support Blue Growth applications. Operational short-term ocean prediction systems (e.g., Bell et al., 2015) require both surface and subsurface observations of 


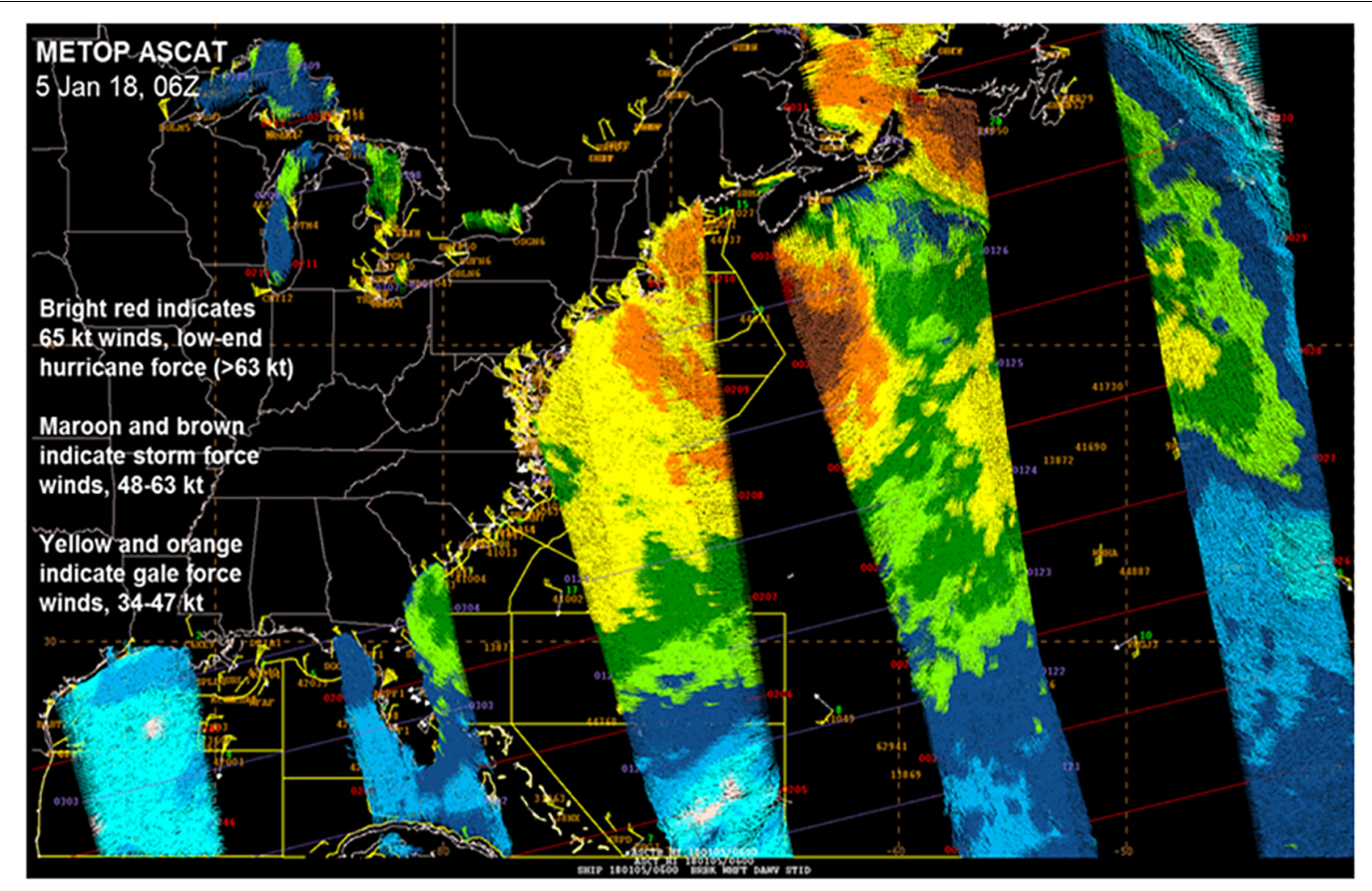

FIGURE 1 | EUMETSAT MetOp Advanced Scatterometer data (colored swaths), with available ship observations (using standard synoptic station notation), from 0600 UTC on 5 January 2018. Courtesy of National Oceanic and Atmospheric Administration, National Weather Service, Ocean Prediction Center.

sea water temperature, salinity, and ocean currents. Ships can provide these observations (Table 1), although SST is the most commonly measured parameter on ships, for direct assimilation or to support satellite product development (see Development and Evaluation of Models and Products) prior to assimilation of satellite products into the model.

\section{Climate Monitoring, Assessments, and Services}

Observations from ships are an essential component of the Global Climate Observing System (GCOS) and complement observations from other marine networks (e.g., Argo, drifting buoys, and moorings). The key challenge is that the surface marine climate record is mainly constructed from observations originally collected for other reasons, typically from weather logs that are part of normal ship activities or to support numerical weather prediction. Even among those observations collected for climate applications, the motivation was to establish normal conditions rather than to quantify variability and longterm change. Requirements for the construction of long-term surface marine records are detailed in Kent et al. (2019). Ship observations of ECVs and EOVs (Table 1) are far more valuable for the construction of climate records when they are a multivariate record described by extensive platform and observational metadata, including information related to quality assurance and quality control, uncertainty estimation, and bias adjustment. Routine weather reports from the VOS Scheme contribute to GCOS "comprehensive" global networks (Global Climate Observing System [GCOS], 2016), providing frequent sampling over much of the ocean to capture variability. Research vessel observations are often used in a similar way. Although research vessels are fewer in number, hence covering less of the ocean on any given day, they typically collect observations at higher temporal frequency (sampling rates of $1 \mathrm{~min}$ or higher) with sensors that are designed for research-quality observations; thus, they have the potential to be used for evaluation, quantification of uncertainty, and as "baseline" or "reference" observation stations.

Climate services provide climate information to assist decision-making. The sources of information used range from observational data, through model forecasts and hindcasts, to climate projections and socioeconomic data, with timescales ranging from a few days to decades and centuries. Marine climate services include applications in both coastal and open ocean environments, ranging from design criteria for vessels, offshore structures, and coastal defenses to seasonal prediction and forecasting seasonal energy production and demand. Examples of developing climate services can be found through the European Union Copernicus Climate Change Service and the Sectoral Information System (e.g., for global shipping, a demonstration project can be found at https://climate.copernicus.eu/globalshipping-project).

The variables required to develop climate services include, inter alia, the following: air and sea temperature and humidity (e.g., for human safety/comfort operating at sea and seasonal prediction); wind, waves, and pressure (e.g., to establish design criteria and wind/wave loading of structures, coastal inundation, and ship routing); and oceanographic parameters (for seasonal prediction, evaluating ecosystem health, and studying biogeochemical cycles). Historically, ship observations have been the primary source of many of these variables 


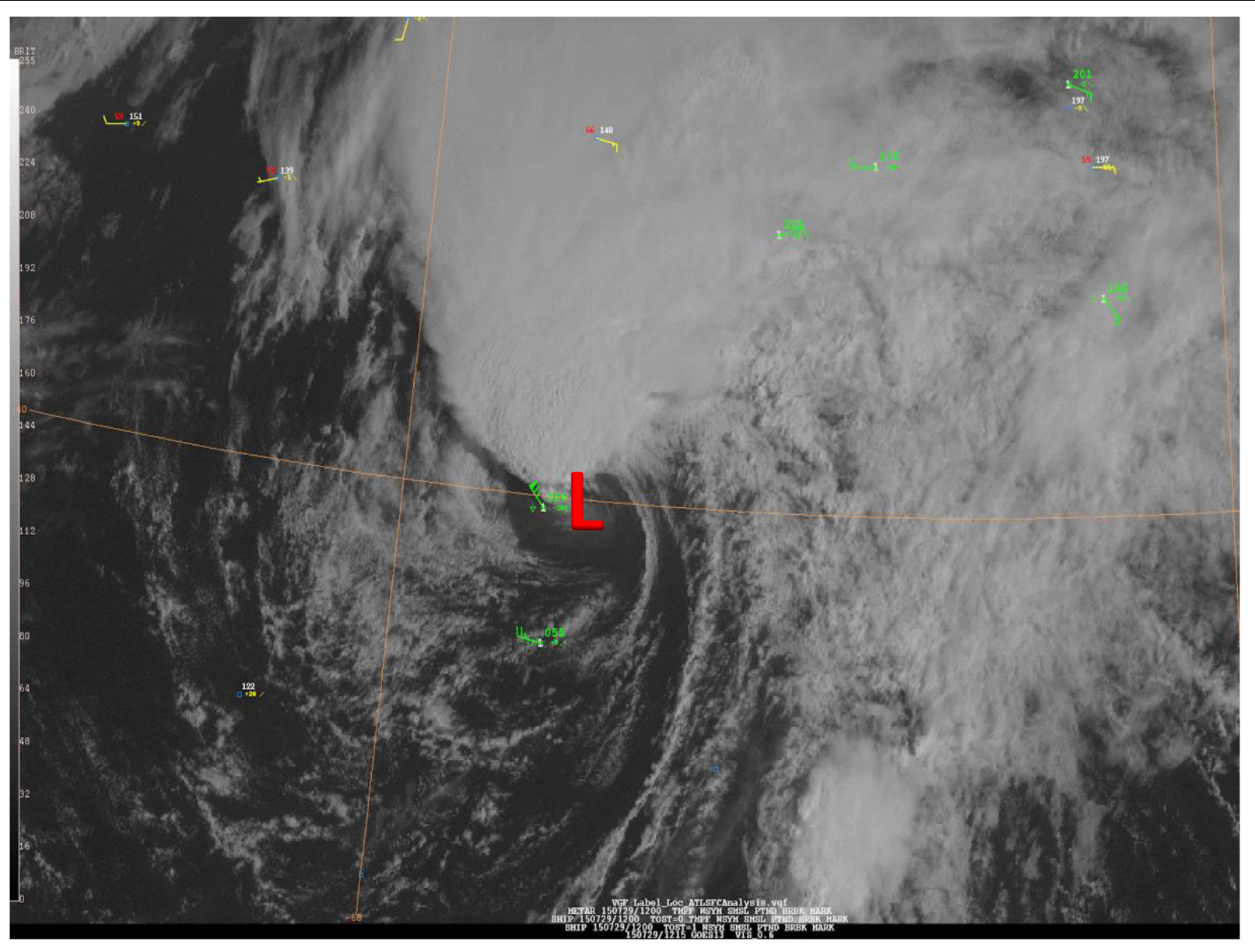

FIGURE 2 | Visible satellite image with available ship observations (overlain in green and yellow using standard synoptic station notation) in the central Atlantic Ocean (1200 UTC, 29 July 2015). Based on this image, the low pressure is centered near $40^{\circ} \mathrm{N}, 58^{\circ} \mathrm{W}$. Courtesy of National Oceanic and Atmospheric Administration, National Weather Service, Ocean Prediction Center.

(Table 1) and underpin many of the climate datasets used (e.g., Kent et al., 2019).

More recently, ship observations are used indirectly through assimilation into model forecasts, hindcasts, reanalyses, and resulting databases (e.g., Pilar et al., 2008; Geyer and Rockel, 2013; von Schuckmann et al., 2018) to support climate studies. Models provide a self-consistent and spatiotemporally complete representation of the atmosphere and oceans beyond what can be sampled directly. Ship observations, alongside other ocean, land-based, and satellite observations, are now regularly assimilated into global and regional climate analyses that depict the four-dimensional evolution of our environment. Atmospheric reanalyses (e.g., Gelaro et al., 2017), ocean reanalyses (e.g., Storto et al., 2016), coupled reanalyses (e.g., Saha et al., 2010), and ocean/sea-ice state estimates assimilate observations from various sources into domain models (ocean, land, atmosphere, and ice) used for a range of climate applications. New approaches combining components via coupling are emerging (Laloyaux et al., 2018), while traditional approaches continue to mature. Multivariate climate reanalysis products form the backbone of climate services, describing the past and present states of the climate.

The first use of ship data to describe the steady ocean circulation using different hydrographic datasets and inverse box applications was introduced in the 1970s (Wunsch, 1978). Since then, a great variety of integrated ship data products have been developed, demonstrating the essential role of ship data in producing ocean state estimates, including, but not limited to, circulation, ocean energetics, air-sea exchange, property fluxes, dynamical balances, and ventilation and mixing. For many applications, such gridded information forms the tip of the iceberg of Earth's environmental digital history, alongside geological and biological datasets (Keim, 2011).

Development of datasets to support climate studies began in 1963 when the international exchange of delayed-mode marine climatological data was put in place through establishment of the WMO Marine Climatological Summaries Scheme. The JCOMM Expert Team on Marine Climatology updated the summaries scheme to facilitate timely exchange, access, and longterm preservation of marine climate data (Pinardi et al., 2019). This new Marine Climate Data System (MCDS), endorsed in 


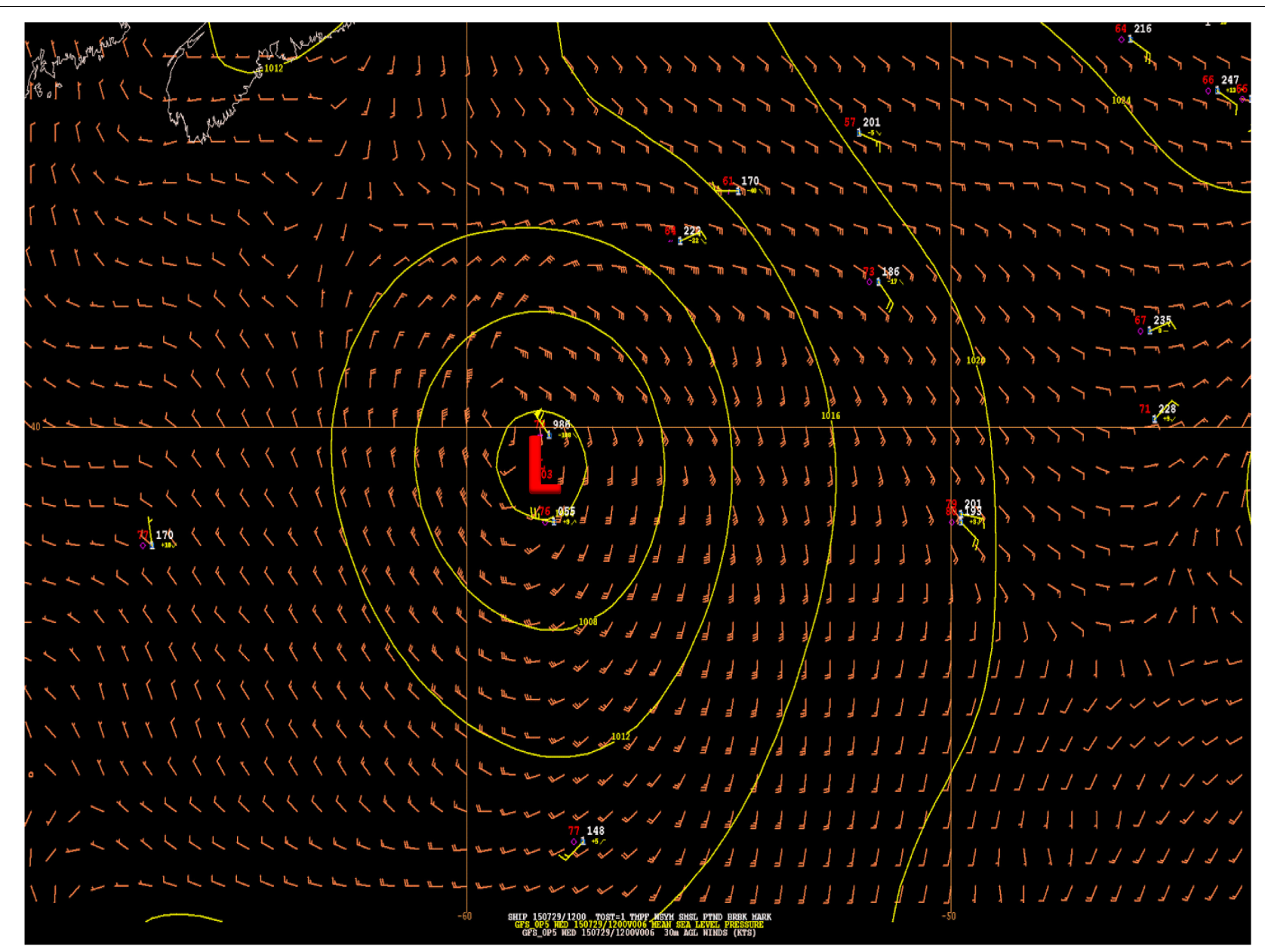

FIGURE 3 | GFS model 29 July 20150600 UTC 6-h forecast, valid 1200 UTC 29 July 2015, with available ship observations using standard synoptic station notation. Isobars are drawn with yellow lines, and the model wind field is indicated by wind barbs in orange. The low pressure center is located near $39^{\circ} \mathrm{N}, 58.5^{\circ} \mathrm{W}$. Courtesy of National Oceanic and Atmospheric Administration, National Weather Service, Ocean Prediction Center.

2012 by JCOMM, provides a JCOMM-wide unified approach to data management and higher-quality climate observations. The MCDS is now in the implementation phase and encompasses a data flow structure with defined roles and tasks to be applied to all data types across JCOMM. In 2016, JCOMM confirmed the establishment of the first MCDS Center for Marine Meteorological and Oceanographic Climate Data. Since then, additional centers have been established and the MCDS will be operational by 2020 .

Ship data, as in the International Comprehensive OceanAtmosphere Data Set (ICOADS; Freeman et al., 2017), are among the most important contributions to reanalyses (Buizza et al., 2018) and for climate studies. The surface marine observational record is almost entirely based on ship observations prior to the 1980s (Freeman et al., 2017) with records for some parameters dating back to approximately 1750 (Table 1). Efforts to extend and enhance the climate data record via data rescue (Brönnimann et al., 2018) are often motivated by the need to improve reanalyses (e.g., Allan et al., 2011). With the top few meters of the ocean containing as much heat as the atmosphere, global SST observations from ships are essential to develop longer reanalyses such as the 20th Century Reanalysis (Compo et al., 2011) or the ERA20C (Poli et al., 2016), which aim to estimate past climate and weather. These reanalyses also assimilate ship surface pressure observations (Figure 4) for a realistic timing of the synoptic events.

\section{Development and Evaluation of Models and Products}

Production of models or data products, for either climate research or operational needs, requires careful assessment of the underlying numerical system or data processing algorithms, evaluation of the model skill and realism, and definition of its application domain (i.e., scales and processes that are resolved by the model or analysis). Ship observations are routinely used in model development studies to demonstrate the system's stability, identify deficiencies in model physics, and derive new numerical schemes and parameterizations to enhance the system's overall performance. The majority of model evaluation studies (e.g., 


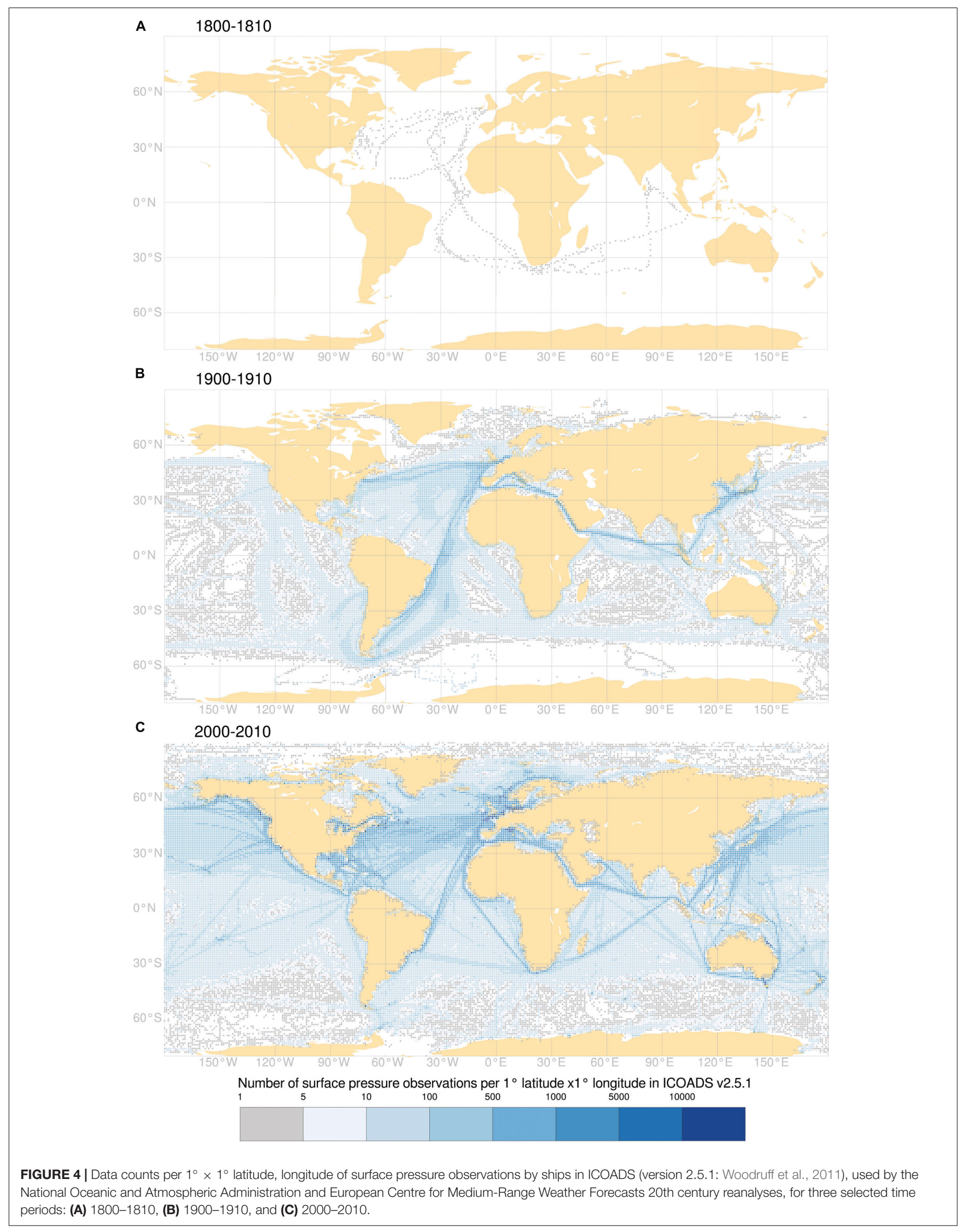


Smith et al., 2001, 2016a; Delcroix et al., 2011; Perlwitz et al., 2015; Jones et al., 2016) are completed using historical (delayed-mode) shipboard observations; however, real-time ship observations can be used to validate operational models and near-term forecasts. Another requirement for evaluation studies is for the shipboard observations to be independent of the observations assimilated into the model. For this reason, it is necessary to provide model feedback metadata (ship identifier, vessel type, data quality, etc.) that allow users to select observations that are not assimilated (frequently, these observations are from research vessels and other research platforms that are not routinely transmitted over the GTS). To evaluate fluxes and other derived fields within numerical models, it is essential to have multivariate observations (e.g., winds, SST, air temperature, pressure, and humidity) that are taken simultaneously (or as near as possible to the same time and location). This is one great advantage of ships as an observing platform-they are capable of carrying numerous instrument systems and making simultaneous observations.

Ships also provide observations of SST, waves, sea ice, salinity, chlorophyll concentration, humidity, air temperature, and winds (Table 1), each of which has corresponding remote sensing systems deployed on Earth-orbiting satellites or aircraft. Remote sensing communities rely on surface observations to develop and validate retrieval algorithms used to derive geophysical parameters from the measurements made by remote sensing systems. For example, satellites can measure a backscatter from the ocean surface that is then used to derive wind direction and speed (Naderi et al., 1991) or radiance, which is used to derive air temperature and humidity. Ship-based observations are used to develop and refine these retrieval algorithms (e.g., Benallal et al., 2016; Jackson and Wick, 2016). Satellitebased estimates of chlorophyll concentrations rely on in situ measurements of surface water optical properties to refine and calibrate retrieval algorithms (McClain, 2009; Brewin et al., 2016). In addition, in situ ship measurements are used to validate and evaluate products developed from one or more remote sensing systems (e.g., Bourassa et al., 2003; Liman et al., 2018). The remote sensing community is seeking observations taken at high temporal sampling rates (1 min or less) to support precise collocations with satellite measurements and from a wide range of ocean environments (e.g., tropics to polar regions and light winds to storm conditions) to help constrain the retrieval algorithms and ensure the measurements are accurate across all oceanic and atmospheric conditions. Ships can also measure the multiple, simultaneous parameters needed to adjust observations measured at various sensor heights to a common reference height or depth. The high horizontal resolution of ship measurements also resolves sub-footprint processes, helping to interpret and validate the satellite measurements (e.g., Kolodziejczyk et al., 2015b; Boutin et al., 2018).

\section{Monitoring and Process Studies}

Ship-based observations are used for a wide range of activities to monitor the marine environment and to conduct research into the fundamental processes that govern the interactions between the ocean and atmosphere. Observations of air/sea temperature, wind speed, relative humidity, radiation, and the concentration of dissolved gasses are critical in quantifying atmosphere-ocean fluxes of heat, freshwater, momentum, and gasses. Knowledge of these fluxes over the global ocean is important in understanding ocean dynamics, biogeochemical cycling, and the global water cycle. For example, SST, wind velocity, air temperature, and relative humidity are key factors in determining the magnitude and direction of the air-sea fluxes of momentum and latent/sensible heat (e.g., Smith et al., 2016b). Similarly, water temperature, wind speed, and the airsea difference in the partial pressure of carbon dioxide $\left(\Delta \mathrm{pCO}_{2}\right.$; which is a component of the inorganic carbon ECV) are important factors in determining the air-sea flux of carbon dioxide $\left(\mathrm{CO}_{2}\right.$; Watson et al., 2009). Ocean acidification is recognized as a major threat to the marine environment and methods for measuring ocean $\mathrm{pH}$ directly or indirectly via automated systems are just becoming available for use on ships (e.g., Shangguan et al., 2019). The water cycle drives tropical atmospheric circulation and is therefore a key component of climate and weather. Ship-based observations of salinity and temperature provide in situ data to monitor the water cycle response to increasing global mean temperature (e.g., Terray et al., 2012). On smaller scales, diurnal heating or freshwater input (from precipitation, rivers, or melting ice) can cause a stable density stratification with vertical gradients in temperature and/or salinity over the upper few meters of the ocean (e.g., Tomczak, 1995; Kawai and Wada, 2007). This stratification can affect the air-sea fluxes of heat and moisture, and a better understanding of its formation, evolution, spatiotemporal statistics, and effect on air-sea interaction and satellite remote sensing measurements is needed. The presence of near-surface temperature and salinity gradients (e.g., Reverdin et al., 2013; Anderson and Riser, 2014; Boutin et al., 2018) complicates the comparison between near-surface in situ and skin-layer satellite measurements, which are made at different depths. One way to address this issue is to make simultaneous measurements at multiple depths to characterize the near-surface vertical gradients and hence the in situ-satellite comparisons.

Atmospheric composition and processes over the oceans in general are poorly known. The flow of chemicals from land to ocean and changes in atmospheric composition (e.g., levels of pollution) have been greatly increased by human activity and have the potential to perturb ocean ecosystems. Working Group 38 of the United Nation's Joint Group of Experts on the Scientific Aspects of Marine Environmental Protection ${ }^{1}$ and others reveal that atmospheric deposition represents the major route by which nutrients, such as iron and nitrogen, and contaminants, such as cadmium, lead, and copper, reach the open ocean (Duce et al., 1991; Jickells et al., 2017). These substances are removed relatively quickly from the atmosphere, than are less reactive components (e.g., $\mathrm{CO}_{2}$ ), creating strong concentration gradients over the oceans. Accurate estimates of these atmospheric inputs, via deposition samples, are vital to improve estimates of atmospheric inputs into the ocean (e.g., Baker et al., 2010; Powell et al., 2015) and for prediction of their ecological impacts.

\footnotetext{
${ }^{1}$ http://www.gesamp.org/work/groups/38
} 
In several cases, especially for the trace metals, knowledge of the chemical form of the substance that is deposited is needed, since insoluble forms are typically unavailable to biota. Direct observation of atmospheric deposition chemical composition is therefore essential. Multi-year averaged observation- and model-based estimates of the inputs of these nutrients and contaminants are in good agreement at the ocean basin scale but are more difficult to evaluate at the higher resolutions relevant to ecosystem impacts (Baker et al., 2010, 2013). Improved model flux estimates require improved data on the atmospheric concentrations of these components to test and validate models. A step change in observations of nutrients and contaminants over and into the ocean, ideally using commercial ships, is required if we are to provide reliable estimates of atmospheric deposition at appropriate scales to quantify the impact of these atmospheric fluxes.

Building knowledge of atmospheric composition over the oceans requires a significantly greater number of direct observations. Several Global Atmosphere Watch (GAW) stations (e.g., Mauna Loa, Mace Head, Cape Verde, Cape Grim) ${ }^{2}$ are able to sample air that arrives from the open ocean, but these observatories are located on land and routinely measure terrestrial signals. Presently, the vast majority of sampling for aerosol composition over the ocean is done from research vessels, with a focus on the Southern Ocean. This allows researchers to develop a better understanding of the composition and chemistry of the marine atmosphere, which is important for climate science because understanding "pristine, pre-industriallike" environments is needed to reduce climate model uncertainty (Carslaw et al., 2013).

Supporting monitoring of the marine environment and process studies requires measuring or estimating the range of both surface ocean and atmospheric variables simultaneously. Only limited observational platforms are currently able to obtain these measurements, namely, ships (e.g., Berry and Kent, 2011) and surface moorings (e.g., Ogle et al., 2018). Ships also provide a platform to support sensors that require human intervention and monitoring. Additionally, routine underway observations from well-maintained and well-sited sensors on ships can provide information to quantify variability and co-variability of ECVs and EOVs. Improved quantification of short-term variability, including diurnal variations, would enable a more informed comparison of observations separated in space or time or that resolve different spatial or temporal scales.

\section{PRESENT CAPABILITIES AND CHALLENGES}

\section{Observations}

Weather, sea state, and surface ocean observations have been collected and disseminated on a systematic basis for over 200 years (Table 1). Observations from ships are now relayed to shore in real time, providing important information on meteorological conditions at sea, along with a long-term record

${ }^{2}$ https://gawsis.meteoswiss.ch/GAWSIS/index.html\# of observations important for climate applications. Traditionally, observations were made using human-read instrumentation supplemented by visual observations made by officers serving on ships operating worldwide. In recent decades, there has been a substantial shift toward shipboard automatic weather stations and a decline in the visual observations.

The VOS Scheme is the primary source of real-time meteorological observations from ships (Figure 5) and the main source of several ECVs, including air temperature and humidity. Unfortunately, the number of VOSs has declined over the last 30 years. This decline can be attributed to a number of factors, including, but not limited to, (1) successive changes in ships' ownership and widespread use of flag of convenience; (2) reduction in the number of crew members aboard vessels; (3) concerns of vessel owners and operators, for perceived or actual economic as well as legal reasons, not to provide any meteorological information that could reveal a vessel's geographical position; (4) ship companies claiming not to be able to take up meteorological tasks due to budgetary constraints; (5) perception that increased satellite and available buoy observations are sufficient and ship observations are not used; and (6) financial strain on NMHSs to recruit and retain vessels in national VOS fleets (JCOMM, 2002; Kent et al., 2010). But while the number of ships has declined, new technologies, such as automatic weather stations and electronic logbooks, have led to an increased number of reports per ship from a smaller VOS fleet, as well as some increase in the quality of observations (Ingleby, 2010). However, fewer ships (typically only 300-400 may be reporting at any given synoptic hour) mean less coverage of the oceans (e.g., Berry and Kent, 2017). The numerical weather prediction need for atmospheric pressure data has led to installation of pressure-only automatic weather stations, and this increase in pressure observations has been offset by a reduction in measurements of other ECVs (e.g., air temperature, humidity, clouds, weather, waves, sea ice, and sea state) required by the marine climate community.

Increasing satellite communications bandwidth continues to reduce the latency and to increase the volume of real-time VOS data transmissions. The recent introduction of table-driven codes (the Binary Universal Form for the Representation of meteorological data or BUFR) to replace traditional alphanumeric codes (i.e., FM-13 SHIP Code) will help to increase the availability of high-resolution data and more detailed metadata in the coming years. However, the transition to BUFR is ongoing, and challenges remain for real-time data systems relating to varying ship-to-shore data exchange formats, the shore-side conversion to BUFR, and ensuring that all required data and metadata make it from real-time observations into the long-term climate archives (Kent et al., 2019).

Meteorological observations on research vessels include both the standard weather observations made to support the VOS Scheme and also dedicated air-sea interaction experiments that directly measure turbulent exchanges (i.e., momentum, heat, water, gasses, and aerosols; Weller et al., 2008), radiative fluxes (typically in wavelength bands required to measure the upwelling/downwelling thermal infrared flux, solar radiation, and photosynthetically active radiation), precipitation 


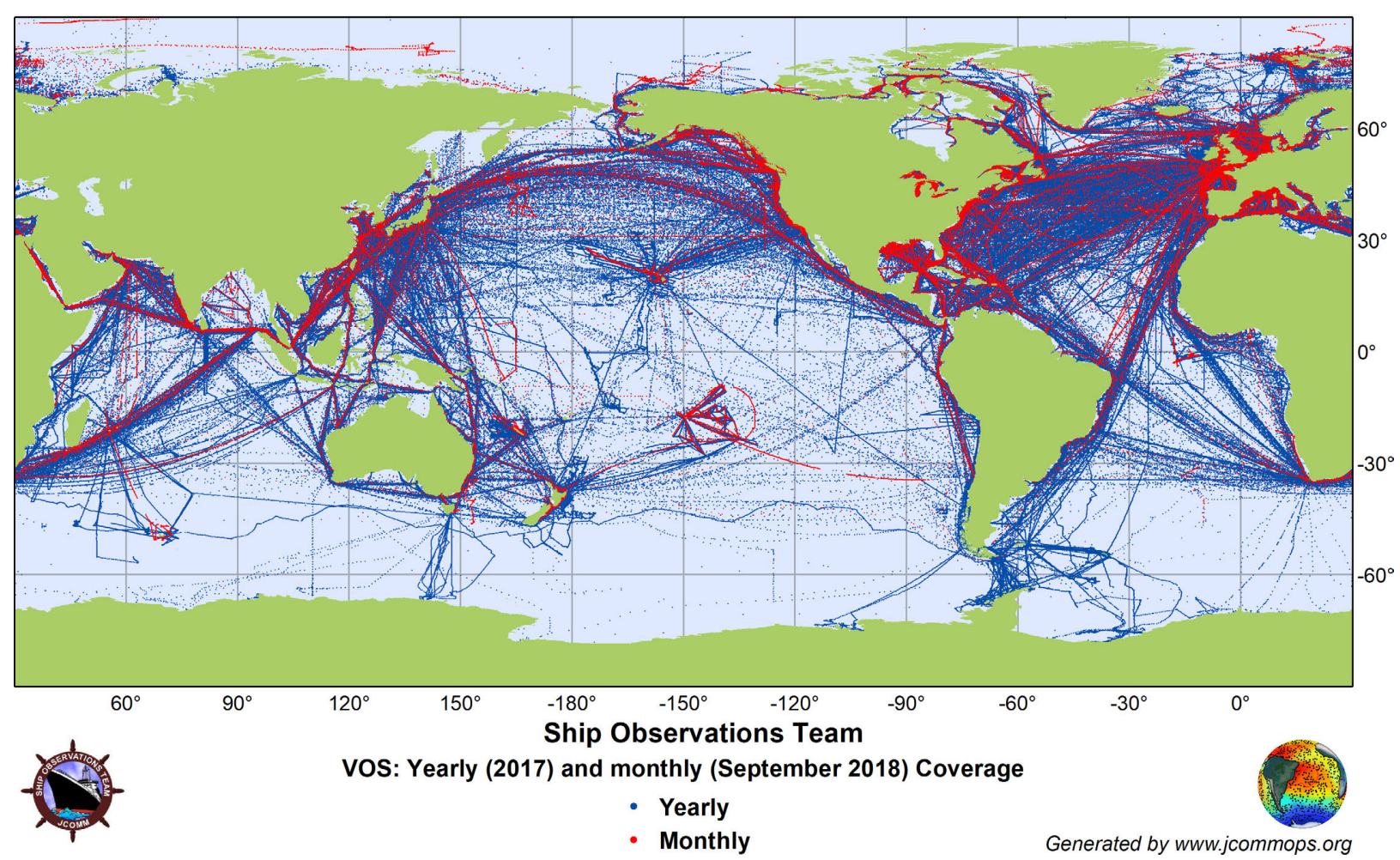

FIGURE 5 | Coverage of the ocean vessels participating in the VOS Scheme (including both character-based and BUFR reports) on monthly (red, September 2018) and yearly (blue, 2017) bases. While approximately 2,000 vessels contributed in total, more than $90 \%$ of the data are submitted by fewer than 600 ships.

(e.g., Galloway et al., 1983; Klepp, 2015), and atmospheric composition measurements (Baker et al., 2010, 2013). The longest observation-based estimates of surface heat and momentum fluxes are based on ICOADS (da Silva et al., 1994; Berry and Kent, 2011) and currently begin in the middle to late 20th century. Research vessels also measure variables necessary to interpret flux observations and develop parameterizations such as mean conditions (wind speed and direction, air and sea temperatures, humidity, pressure, $\mathrm{pCO}_{2}$, and salinity) and sea-state parameters (such as wave heights, periods, lengths, directions, spectra, and whitecap fraction). Although capability to make comprehensive flux measurements on other types of ships, moored buoys, or autonomous surface vessels is developing rapidly (Cronin et al., 2019; Swart et al., 2019), research vessels will be the primary platform used to evaluate data from these emerging technologies.

Research vessel observations are geolocated via the highprecision navigational systems typically deployed to support research vessel science operations. Research vessels often carry both standard weather instruments of the type supplied to vessels by NMHS to contribute to the VOS Scheme and sophisticated automatic weather stations, which are frequently custom designed and built by individual research vessel operators. Although research vessels may transmit a subset of their observations in real-time via the GTS, most observations are recorded and used in delayed mode, typically as part of an end of cruise dataset. There are no international standards for meteorological parameters to be sampled by research vessels, but since 2005, the U.S.-funded Shipboard Automated Meteorological and Oceanographic System (SAMOS) initiative (Smith et al., 2018) has provided guidance to the research vessel community. SAMOS has developed standard data formats for research vessel ship-to-shore data exchange, distribution, and archival along with training materials for marine technicians on research vessels with regard to sensor selection, siting and exposure, data processing, and quality evaluation.

Most research vessels, selected commercial vessels (e.g., Alory et al., 2015; Gaillard et al., 2015), and more recently racing yachts (e.g., Kramp et al., 2010) ${ }^{3}$ are equipped with flow-through sea water systems that measure sea temperature and salinity [using a thermosalinograph (TSG)], fluorescence, transmissivity, and other biogeochemical properties (e.g., oxygen, alkalinity, chlorophyll, and carbon). Flow-through water sampling systems on ships typically take water from a single port in the hull at a depth of a few meters. There is large uncertainty on this depth on commercial ships, as it can vary by several meters depending on the ship's load; moreover, the incoming water can be strongly mixed by the ship's wake. While these observations are invaluable for gaining information on the temporal variability in the ocean surface, they cannot provide information on vertical gradients of water properties or concentrations. To study vertical gradients in temperature, salinity, gas concentrations, and other properties,

\footnotetext{
${ }^{3}$ https://www.volvooceanrace.com/en/news/10225_What-is-the-ScienceProgramme.html
} 


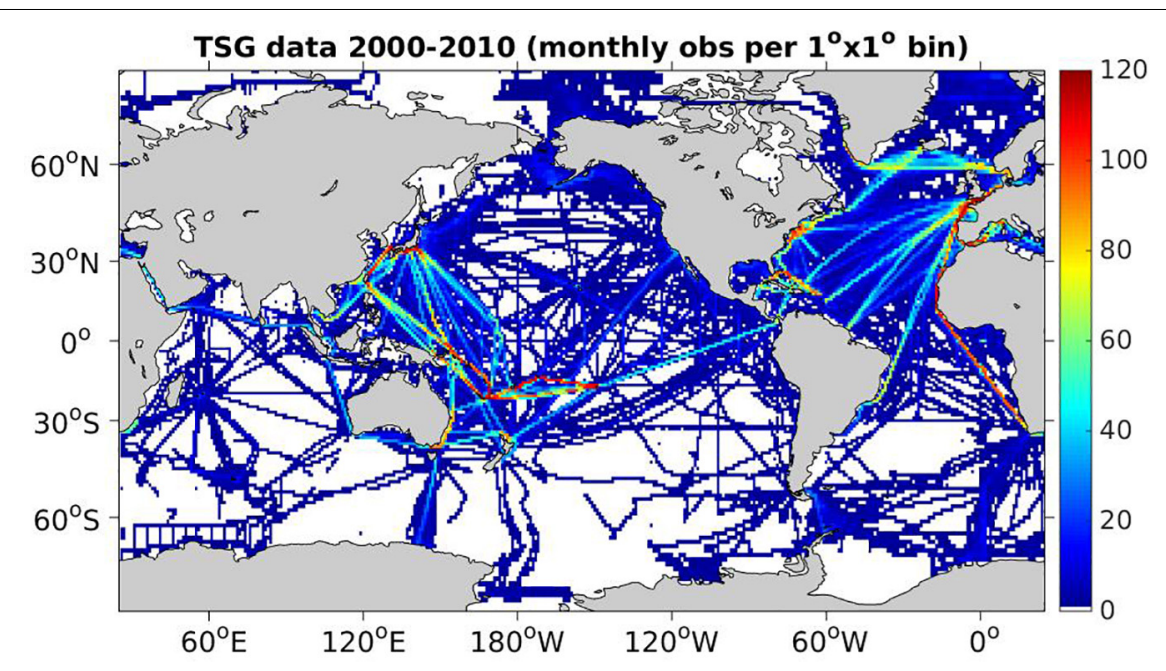

FIGURE 6 | Density map of global real-time and delayed-mode thermosalinograph observations covering 2000-2010 from multiple data sources, including the following: French Sea Surface Salinity Observation Service; GOSUD; SAMOS; National Oceanic and Atmospheric Administration Atlantic Oceanographic and Meteorological Laboratory, Pacific Marine Environmental Laboratory, and National Centers for Environmental Information; Japan Agency for Marine-Earth Science and Technology; Japan National Institute for Environmental Studies; VOS Nippon; Commonwealth Scientific and Industrial Research Organisation; Australian Antarctic Division; and the Alfred Wegener Institute for Polar and Marine Research.

the Applied Physics Laboratory at the University of Washington installed through-hull ports for sensors and water sampling at depths of 2 and $3 \mathrm{~m}$ (below mean water level) aboard three global-class research vessels $(R / V$ Thomas $G$. Thompson, $R / V$ Roger Revelle, and Ronald G. Brown; Jessup and Branch, 2008; Asher et al., 2014). When instrumented with salinity sensors (Asher et al., 2014), the data from the instruments in these ports combined with the ship's own TSG sampling at a depth of $5 \mathrm{~m}$ provide profiles of temperature and salinity in the upper ocean. These data have proven valuable in studying diurnal warm layers and rain-formed salinity gradients and could be readily extended to include underway measurement of vertical gradients in alkalinity, partial pressure of carbon dioxide, and biological properties of interest. Additionally, flow water systems can be used to pump water through filters to measure concentrations and conduct analyses of particulate, plankton, or microplastics.

The global network of ship-based TSG measurements (Figure 6) has enormously increased our ability to study variations in ocean salinity. Ongoing projects managing flowthrough water data include SAMOS, the U.S. Rolling Deck to Repository ${ }^{4}$, Global Ocean Surface Underway Data (GOSUD; Kolodziejczyk et al., 2015b), Ferryboxes (Petersen, 2014) ${ }^{5}$, and the Surface Ocean Carbon Observing NETwork (Wanninkhof, 2019). Although profiles of surface temperature and salinity are available at single points through Argo (Roemmich et al., 2009; Riser et al., 2016), conductivity-temperature-depth (CTD) casts (e.g., Talley et al., 2016), and moorings (McPhaden et al., 1998; Bourlès et al., 2008), only TSGs on ships, drifters, and autonomous surface vessels provide the capability to measure high-horizontal salinity variations in frontal and

${ }^{4}$ https://www.rvdata.us/

${ }^{5}$ www.ferrybox.org sub-mesoscale structures (e.g., Kolodziejczyk et al., 2015a). Repeat transects of ships instrumented with TSGs provide longterm salinity time series at relatively low cost (e.g., Alory et al., 2015). Additionally research vessels provide TSG (along with carbon) measurements in ocean regions outside of well-traveled commercial shipping lanes.

Regardless of the vessel, making continuous SSS measurements poses significant challenges. TSGs on research vessels can be serviced regularly, while those on commercial ships or autonomous surface vessels can only be inspected and serviced during harbor calls. Salinity measurements tend to drift due to sensor fouling in turbid waters or abruptly shift after calls in dirty harbors. Therefore, measurements often need to be calibrated with independent SSS data, from water samples collected onboard and later analyzed, from CTD casts done on the same ship, or from collocated Argo profiles. External temperature sensors cannot always be installed at the water intake, and the temperature measured by the TSG, often located in a hot engine room, is then biased warm. Systems without a flow meter can also have undetected data degradation when flow is insufficient.

The recent advancement of fairly small ( $\sim 2-7 \mathrm{~m}$ in length) and robust autonomous surface vessels have also provided opportunities for air-sea flux measurements. Miniaturized sensors, improved battery capacity, and energy harvesting techniques (currently limited to using wind, waves, and sunlight) have allowed these robotic platforms to support mainstream ocean observing approaches. Examples include Wave Gliders and Saildrones, but there is an ever-growing number of platforms emerging (e.g., Sailbuoy, Autonaut, Navocean's Nav2, and C-Enduro). Autonomous surface vessels are proven to work well in a variety of regions ranging from the tropics to the ice-free polar oceans and in extreme conditions such as enduring multi-month 
missions in the Southern Ocean (e.g., Monteiro et al., 2015; Schmidt et al., 2017; Thomson and Girton, 2017) or transecting through hurricanes (Lenain and Melville, 2014).

Several projects have shown over the last decade that sailing expedition vessels and ocean racing yachts can not only deploy autonomous instruments (drifters and floats) in sea areas with very limited or no other shipping (e.g., Arctic and Antarctic waters) but also directly gather ocean-atmosphere data with innovative underway instrumentation (Choquer, 2014). Such projects are often well covered by the media and thus help in reaching out to a broader public. The emerging data are of sufficient quality for many operational or research applications (Kramp et al., 2010), for example, the validation of SMOS remotely sensed salinity (Salat et al., 2013). The IOC has signed several cooperation agreements, including for the joint organization of the second international ocean research conference $^{6}$. The sailors are highly motivated and sometimes submit data gathered with their own instruments and at their own satellite cost (Kramp and Rusciano, 2016). Several roundthe-world races (Volvo Ocean Race, Barcelona World Race, and Vendée Globe) now comprise corresponding science projects and will thus create valuable time series from particularly the Southern Ocean.

Presently, RVs also provide the primary platform capable of measuring a wide range of chemical and biogeochemical properties and processes within the atmosphere and ocean, including atmospheric deposition of nutrients and contaminants (e.g., Powell et al., 2015) and characterization of atmospheric aerosols in the marine troposphere (Quinn and Bates, 2005). Examples include the collection of wet deposition samples onboard research vessels and the deployment of two dedicated atmospheric sampling laboratories on the research vessel Investigator $^{7}$, which measure major tropospheric long-lived greenhouse gasses along with carbon monoxide, ozone, radon, and a broad range of aerosol microphysical and chemical properties. The Investigator routinely transits the Southern Ocean, providing atmospheric and oceanic data in a region with minimal anthropogenic influence; however, reliance on research vessels and a limited number of coastal and island stations provides only limited measurements from a small fraction of the oceans. Aerosol sampling (e.g., Martino et al., 2014), sampling for climatologically important trace gasses (Nara et al., 2011, 2014; Yokouchi et al., 2012), and potentially deposition sampling from ships of opportunity is also possible but requires fairly high levels of support from the ship's crew and operators. Automation may simplify this sampling in the future, and further sampler development (e.g., automated switching of sampling cassettes in the case of aerosol collection) is required to decrease the impact of sampling on shipboard operations.

Although the focus of this paper is primarily on physical and chemical observations, a number of biologically relevant measurements can be made by ships. In addition to sensors in flow water systems that measure $\mathrm{pH}$, transmittance, fluorescence,

\footnotetext{
${ }^{6} \mathrm{http}: / /$ iocunesco-oneplanetoneocean.fnob.org/

${ }^{7}$ https://www.csiro.au/en/research/facilities/marine-national-facility/researchcase-studies
}

chlorophyll, oxygen, and inorganic carbon, we note that the Continuous Plankton Recorder (CPR) has been deployed regularly from a select group of commercial ships since the $1930 \mathrm{~s}^{8}$, with the initial aim of improving knowledge of zooplankton stocks for fisheries management purposes. The CPR has also delivered an immensely valuable long-term record of ocean biological variability and how marine species respond to climate change (e.g., Beaugrand and Kirby, 2018). However, the nature of the CPR operation is necessarily based on small numbers of ships with subsequent painstaking laboratory analyses of the collected samples and, hence, is rather different to the approach of using a wide range of research, commercial, and private ships discussed herein.

\section{Metadata}

Almost as important as the measurements themselves are the associated metadata that provide supporting information about host vessels, instrumentation, and the data. A sophisticated metadata catalog allows for detailed and standardized quality monitoring of individual vessels and instruments, as well as the health of the network of VOS, research vessels, and complementary private and autonomous vessels. WIGOS has developed metadata requirements (WMO, 2017) that are designed to make the observations (e.g., Table 1) useful to downstream users. Mapping community-developed metadata schemas (e.g., VOS, SAMOS, and GOSUD) to identify overlaps and gaps with respect to these requirements is an ongoing challenge.

Information on the ship's characteristics and meteorological instrument type and siting is also important for climate studies. Different observing methods have different error characteristics, with systematic errors leading to biases in derived climate products if not corrected. When the observing methods change over time, the mean biases also change, leading to artificial signals being introduced into the climate record. For example, the height at which air temperature observations have been made have changed by several tens of meters since the late 1800s. If unaccounted for, this would result in the recent climate warming being underestimated by $0.25^{\circ} \mathrm{C}$ over the past 100 years and by $0.1^{\circ} \mathrm{C}$ over the past 30 years (Kent et al., 2013). Conversely, if not accounted for, the change from SST measurements made using buckets to engine room intakes would result in an overestimation of the change over the last 100 years (Kent et al., 2017). To account for these changes, metadata are required, such as the height or depth of observation and the measurement method.

Metadata for ships participating in the VOS Scheme are recorded in the WMO Publication No. 47 (Pub 47; Kent et al., 2007). This framework was initially developed by the WMO but is presently managed by a task team of the JCOMM Ship Observation Team. In an effort to modernize and sustain the VOS metadata record and transition from Pub 47 to the WIGOS standard, a new VOS metadata database is being implemented and managed by the JCOMM Observations Programme Support Centre (JCOMMOPS). While Pub47 is an extensive metadata schema, there are only five mandatory metadata fields required to register a ship within the VOS Scheme: recruiting country,

\footnotetext{
${ }^{8}$ https://www.cprsurvey.org/
} 
callsign, VOS recruitment date, vessel type, and general observing practice. The present challenge is to map all the prior Pub47 metadata fields into the new WIGOS schema and define new metadata fields to support a broader user community while also working with VOS operators to collect and provide a larger number of mandatory metadata fields.

Another metadata challenge arose from an increased sensitivity of vessel owners and operators to the location and identification of their vessels (IDs) being available in near real time. There have been a number of different schemes [callsign masking, use of generic callsigns (e.g., SHIP), callsign encryption, etc.] implemented to accommodate the vessel operators and maintain their contribution to the VOS Scheme. However, these all come with limitations, for both the operational and climate communities. The loss of IDs makes quality control and blacklisting of low-quality data difficult for the operational users. The loss of callsigns makes associating the observations with vessel metadata (e.g., Pub 47) impossible, resulting in lower-quality climate monitoring products. Some attempts have been made to overcome this by expanding the range of metadata in the VOS real-time formats to include those required to fully understand and use the observations, but reporting of the additional metadata has been limited to date.

Parallel to these developments, advancements in Automated Information Systems (AIS) technology largely negate attempts to "hide" ships from unwanted interest. In light of this, JCOMMOPS is implementing a new approach for ship metadata that separates the "ship" metadata from the "observing station" metadata so that no detectable reference to the ship is included with the observation. A master list of all participating ships will be retained by JCOMMOPS to re-associate the ship with the instruments for authorized users. At present, not all countries operating VOS contribute their metadata to the international database. With the global decline in the number of manually observing VOS and the increasing number of automated systems, a fully representative metadata database containing records for all international VOS would improve the capabilities of operational monitoring and therefore maximize data availability for climate and research purposes.

Discovery metadata are required to enable users to find and retrieve the observations. This includes information such as the spatial and temporal coverage of the observations, information on the reported parameters, licensing and usage rights, and how to access and download the data. Historically, this information has been provided through project web pages, but there is an increasing requirement for the metadata to be machine readable and interoperable, driven by, inter alia, the following: the volume and distributed nature of the observations; ease of use and the increasing standardization of data delivery; and the development of web services. This is usually achieved through the publication of discovery metadata records for observation collections based on international standards (e.g., the ISO 19115 standard). Once published through online data catalogs, such as those provided by the IODE Ocean Data Portal ${ }^{9}$ and the ERDDAP software ${ }^{10}$,

\footnotetext{
${ }^{9}$ http://www.oceandataportal.org/

${ }^{10}$ https://coastwatch.pfeg.noaa.gov/erddap/
}

the observation collections are easily locatable and retrievable through a single or limited number of locations. In addition to providing access to observational collections, systems are being developed to provide bespoke access to individual observations (e.g., through the Copernicus Climate Data Store ${ }^{11}$, systems based on the OGC Web Feature Service interface).

\section{Quality Assurance and Control}

Well-documented data quality evaluation and adjustment procedures (e.g., post-deployment calibration) are essential to develop a wide range of products. Quality assurance is more of an end-to-end process where attention is given to all stages of data collection, transmission, processing, and distribution, while quality control focuses more on evaluating the observations relative to some standard of physical reality or plausibility. Quality control is typically done following the data collection and may or may not rely on or be an integral part of the quality assurance for an observing system. Additionally, the level of and approach to quality control depend on the particular purpose and use of the evaluated data. Most real-time quality control focuses on assigning flags to suspect data without removing these values. In contrast, quality control for data assimilation typically rejects suspect data or even blacklists entire data records, based on lack of agreement with the data assimilation assumptions. Finally, quality evaluation to create research data products, including the use of visual quality control, can result in a combination of value rejection and value flagging.

Real-time quality control is frequently done by NMHS and other centers to meet operational mission requirements and tends to be limited to basic quality tests (e.g., range checks, consistency, and validity of position data). The SAMOS initiative provides an example of a fully automated real-time quality control with the data processing being completed within a minute of data being received (Smith et al., 2018). Real-time quality control associated with the data assimilation process for numerical weather prediction or other operational modeling (e.g., Cummings, 2011) is basically a "buddy check" whereby the individual observations typically are compared to the first guess model fields and accepted or rejected according to tolerances set for the assimilation process.

Alternatively, a number of projects and centers [e.g., GOSUD; Kolodziejczyk et al., 2015b, SAMOS; Smith et al., 2018, Surface Ocean $\mathrm{CO}_{2}$ Atlas (SOCAT); Pfeil et al., 2013, Global Ocean Data Analysis Project (GLODAP); Olsen et al., 2016, MCDS, World Ocean Database ${ }^{12}$ ] focus on developing research products using delayed-mode quality evaluation, each using techniques that are specialized to their area of scientific expertise. For example, data from flow-through systems, for which fouling can induce sensor drift, typically undergo preliminary automatic quality tests but often require manual evaluation from science area experts to ensure the highest data quality. For time series adjustment, pre-calibration/post-calibration data can be used along with comparison with discrete samples or collocated data. The best practice for research product development is to employ

\footnotetext{
${ }^{11}$ https://cds.climate.copernicus.eu/

${ }^{12}$ https://www.nodc.noaa.gov/OC5/WOD/pr_wod.html
} 
a quality control system that only applies flags to data values and does not remove the values-decisions to accept or reject data failing quality control tests are left up to the data user. Furthermore, the best practice includes checking the consistency of the quality control and adjustment from different groups that produce delayed-mode ship data. Indeed, post-calibration or co-located measurements are not systematically available; thus, quality control and correction decisions need to be checked and discussed to provide the highest data quality for climate.

In performing observations, the traceability to the common standards also plays an important role. Such traceability especially for the measurement of the same qualities ensures that the biases are minimized and the data from different platforms can be used together (e.g., in the data assimilation systems). In the case of the physical measurements, the traceability is usually established to the international system of units through the initial calibration of equipment versus standards supported by the National Metrology Institutes. In the cases of the chemical or biological measurements, the primary standards and the measurement traceability are established by the relevant networks.

If data are accompanied by estimates of their uncertainty, this is extremely beneficial for users, allowing a simple assessment of whether the data quality meets the requirements for their application. In practice, the assignment of uncertainty estimates is challenging, and this is typically done by expert users rather than by data providers. In some cases, biases in the observations can be identified and quantified, adjustments applied, and the uncertainty in that adjustment estimated (Kent et al., 2019). The quality of the adjustment for bias and the estimation of uncertainty in observations are dependent on the availability of observational metadata (providing information on instruments, observing protocols, etc.), on information on data management practices (e.g., data precision and any conversions or calibrations applied), and, in some cases, on knowledge of the ambient environmental conditions (Kent et al., 2019).

An alternative to the provision of uncertainty estimates is an end-to-end quality assessment, which is much more challenging than quality control completed following data collection as described above. The GAW atmospheric composition monitoring program is one example of a quality assessment approach that ensures all its observing sites are comparable. This is essential to produce globally consistent datasets. GAW mandates the use of a primary standard for most parameters to define a measurement scale. The scale is maintained by a single institution and propagated to all other members of the program in a traceable manner. Clear data quality objectives have been articulated, and these are supported by measurement guidelines developed by the community of researchers. Detailed logbooks and metadata are required to be maintained, along with a commitment to submit data to the World Data Centers in a timely manner. Audits and inter-comparison programs are routine. Participating institutions are encouraged to deliver measurements with uncertainty within agreed limits.

The atmospheric laboratories on the research vessel Investigator have opted to adhere to the quality assessment guidelines of the GAW program, making it the first mobile
GAW station. Personnel for the research vessel Investigator have committed to the GAW quality assessment principles for a suite of ship-borne measurements. This includes regular maintenance of all instrumentation, routine calibration and quality control, annual delivery of data to the relevant World Data Centers (with a view to [near] real-time delivery in future), and provision of appropriate filter methods to remove local pollution (e.g., ship exhaust).

\section{Vision for the Next Decade}

An efficient shipboard observational network needs to include ships from commercial, private, and research fleets with sufficient operational flexibility to take advantage of continued advances in technology and communications. Many industries (e.g., cruise lines, ferries, fisheries, energy, and transportation) operate thousands of ships that could contribute to cost-effectively collecting data (Holthus, 2018). A comprehensive structure and process is needed to connect these industries with the scientific data collection community to expand the spatial and temporal extent of observations. Vessel owners and operators are often interested in the health of the oceans and seek opportunities to make measurements to support marine operational and research activities (e.g., Watson et al., 2009; Nara et al., 2011). These communities need a mechanism to register their instrumentation and provide observations to the community with both real-time or delayed-mode options. For research vessels, the challenges are less in making the observations but more focused on the coordination of observations between nations, standardizing observational methods and quality assessment/control, and ensuring consistent data management.

Over the next decade, the international community needs to focus on a unified program of marine observations from crewed ships and autonomous surface vessels. Challenges to be addressed include (1) recruiting additional vessels to improve both spatial and temporal sampling of the parameters outlined in Table 1, (2) conducting multivariate sampling on ships, (3) raising the technology readiness level of automated sensors for autonomous shipboard observation and ship-to-shore data communications, (4) advancing quality evaluation of observations, (5) and developing a unified data management approach for shipboard observations and metadata that meet the needs of a diverse user community. Given the diversity of operational and research applications (see Community Requirements), there is no ideal sampling scheme for near-surface marine observations from ships, but the need for additional sampling over the global oceans to increase measurements of parameters listed in Table $\mathbf{1}$ is recognized. More vessels are needed to increase sampling in remote oceans (e.g., polar seas, South Atlantic, and the Pacific), to increase the frequency of observations (more per day) even along well-traveled shipping lanes, and to measure multiple parameters simultaneously on a single ship.

\section{Vessel Recruitment}

The decline in ships contributing to the VOS Scheme and the impact this has on both operational forecasts/warnings and climate studies are described above. Recruiting additional ships to contribute to the VOS Schema and make other atmospheric 
and oceanic observations requires communication with the ocean industries that operate ships across the global ocean. The first recommendation to recruit additional vessels is for users of shipboard observations to create a list of the instruments they wish to see deployed on ships that will meet their needs to measure the parameters in Table 1. Once developed, this list can be used to approach owners/operators to determine what level of instrumentation they are willing to host on their ships. It is anticipated that partnerships will be needed between NMHS, World Ocean Council (WOC), the research community, and individual vessel owner/operators to deploy a combination of instrumentation on each vessel (see Coordinating Vessel Recruitment). Better coordination between the observational programs is essential for unified requests (i.e., not from multiple individual researchers or operational groups) to be made to the vessel owner/operators to ensure that requests do not adversely impact the core mission of the vessels. The WOC is one option to address these coordination needs.

Despite the diverse observing capabilities of research vessels, they are an underutilized component of the observing network. A concerted effort is needed to coordinate marine observations from research vessels. Firstly, we recommend all research vessels to be recruited into the VOS Scheme, have their metadata documented by JCOMMOPS, and be provided with tools to submit data via the GTS. Ensuring research vessel data acquisition systems are compatible with existing (e.g., TurboWin) or new VOS reporting software will increase research vessel capabilities to provide real-time observations. The authors further recommend engaging the research vessel operator community in a dialogue to (a) determine which research vessels routinely operate in high-priority ocean regions, (b) support installation of multiple sensor packages on research vessels in these regions, and (c) maximize data collection by ensuring that all sensor packages on each research vessel are operating on every voyage. The Arctic Research Icebreakers Consortium ${ }^{13}$ program provides an example of how countries are working to coordinate research vessels for data collection, and interaction with the commercial fleets via the WOC.

Additional ships are needed to collect observations to estimate air-sea fluxes by installing sensors and undertaking further automated approaches to collect the full suite of observations required. This is particularly true in regions where we currently have few observations and large uncertainties (e.g., the Southern Ocean, Swart et al., 2019; Western Boundary Currents, Bentamy et al., 2017) and yet where there is growing ship activity via research, tourist, or other ships. We recommend enhancing current capability for direct flux measurements from research vessels and ensuring that high temporal resolution underway observations from all research vessels and a subset of commercial ships are routinely collected and managed in a SAMOS-type data system, including real-time transmission if needs are identified. These enhancements will help identify biases and improve data quality. Direct flux measurements will always be sparse, so meeting required accuracy targets for regional and global fluxes (Cronin et al., 2019) requires expanding our knowledge of

\footnotetext{
${ }^{13}$ https://www.arice.eu/
}

near-surface atmospheric and oceanic states and improvements to parameterizations of air-sea exchange. Ship and potentially autonomous surface vessel observations can provide direct measurements of fluxes and the observations required to calculate fluxes via bulk methods to enable improvements to flux parameterizations and uncertainty characterization.

Since NMHS and the research community do not have adequate resources to deploy instrumentation on all the ships they may be able to recruit, it is essential for the marine community to develop a method for vessel operators to provide weather, ocean, and other measurements using instrumentation purchased and deployed by the owner/operator. Such "third party" data collection can be facilitated through a crowd-sourced data collection approach, which have been widely successful in other communities. Examples include the Community Collaborative Rain, Hail and Snow Network ${ }^{14}$, and Weather Underground's Personal Weather Station Network ${ }^{15}$. Several user communities (e.g., satellite product developers and numerical modelers) acknowledge the value of observations from thirdparty platforms, provided that the platform metadata are sufficient to allow these users to conduct quality evaluation necessary for their application. In some cases, this may result in rejection of these records based on comparison with model first guess fields or neighboring ship, mooring, or other observations with a known quality. The authors recommend establishing a pilot project, possibly in partnership with JCOMM, to develop a web portal to support third-party data collection. Such a portal would support registration of the vessel, collecting minimum vessel and instrumental metadata, and submission of their observations. Promotion of third-party ship observations may be achieved via international organizations (e.g., WOC and JCOMM), ship owners' associations, maritime exchanges, marine training institutes, mariners' or shipmasters' associations, and outreach to yacht and pleasure craft owners.

\section{Multivariate Shipboard Observations}

As platforms with ample space and power, along with the presence of human resources, ships are ideally suited for multivariate observations. Achieving the goals outlined by the broad community of shipboard data users requires collaboration between existing international research and operational activities to build a program to measure multiple parameters across a network of ships. Many users need co-located observations to address their operational and scientific goals. For example, the heights at which observations of the marine air temperature, humidity, and wind speed are made vary, which means that applications such as satellite calibration and evaluation require adjusting data to a common reference height. This requires co-located measurements of these parameters be combined with accurate metadata on observing heights. Furthermore, many users calculate fluxes of heat, moisture, momentum, carbon, and other quantities using shipboard observations (e.g., Smith et al., 2016b). Accurate flux estimation requires co-located measurements of winds, air and sea temperature,

\footnotetext{
${ }^{14}$ https://www.cocorahs.org/

${ }^{15}$ https://www.wunderground.com/weatherstation/overview.asp
} 
humidity, pressure, salinity, and other quantities (e.g., $\mathrm{pCO}_{2}$ and chemical properties). Temperature and salinity must always be measured on platforms where alkalinity and $\mathrm{pCO}_{2}$ are measured, as this allows improved empirical relationships for calculating the latter from the former that are more widely available (e.g., Lee et al., 2006; Bonou et al., 2016). To maximize efficiency by which observations are made, the authors recommend that multiple sensor systems be deployed on individual ships and every effort should be made to ensure all systems are operating and transmitting data on every voyage. As described in the "Quality Assurance and Control" section, the estimation of data uncertainty is often improved by the availability of co-located observations providing information on the ambient conditions.

\section{Technological Advancement}

Over the next decade, there is a need for additional investment to advance the technological readiness level of instrumentation and sensors, increase the capabilities of ship-to-shore communications, and enhance the design of new vessels to support marine observations.

Although autonomous sensors are becoming more common on all types of vessels, many technologies need improvement to expand autonomous marine measurements, as ships offer a cost-effective way to vastly increase data collection. Examples include automated atmospheric aerosol samplers (Sholkovitz and Sedwick, 2006) that could provide routine estimates of biogeochemical fluxes; sensors to measure cloud parameters, precipitation, and visibility; and automated sensors for carbon cycle parameters of high enough quality to be comparable with manual analytical instrumentation. Some land-based sensors for these measurements exist but are typically too costly and not sufficiently robust to be routinely deployed on ships. The authors recommend additional investment into autonomous sensors for shipboard use. We also recommend using research vessels as well-established labs for testing new sensors prior to their wider deployment on commercial and private ships. Any such effort will require partnerships with sensor manufacturers to leverage their design and engineering experience and also to allow scientists to inform the design to ensure that all essential data and metadata are correctly encoded into sensor data records. As a community, we should be moving away from the days when data and metadata (e.g., calibration factors and units) are provided separately from the data values-they should be provided simultaneously by "smart sensors" in any electronic data records using communitydeveloped methodology (e.g., SensorML ${ }^{16}$; Aloisio et al., 2006).

In addition to new sensor development, existing technologies should be further exploited. For example, the AIS may be used to increase the availability of observations globally. AIS is a maritime navigation safety communications system standardized by the International Telecommunication Union and adopted by the IMO that provides vessel information (i.e., identity, type, position, course, speed, navigational status, and other safetyrelated information) automatically to appropriately equipped shore stations, other ships, and aircraft. Its primary use is as a navigation tool for collision avoidance; however, a promising

\footnotetext{
${ }^{16} \mathrm{https} / / /$ www.opengeospatial.org/standards/sensorml
}

methodology has been recently developed to estimate surface currents from AIS navigation data (Guichoux et al., 2016), potentially in real time, as an updated version of historical ship drift data (e.g., Richardson and Walsh, 1986). Services like AIS can also be used to transmit Application Specific Messages $^{17}$. The Environmental Application Specific Messages can be incorporated into AIS messages and has great potential to transmit meteorological and oceanographic information from automated sensors. For example, pressure is variable from which other parameters could be derived to assist in improved weather forecasts and warnings over the high seas. From pressure, conceivably one could generate better model wind information that, in turn, could generate better model wave information. The potential of AIS technologies to increase ship observations can be estimated by viewing the hundreds of millions of ship locations from 2012 displayed at https://www.shipmap.org/.

As data volumes from autonomous sensor technology grow, continued development of ship-to-shore communications technology is needed. The obvious first step is to continue to increase the bandwidth of satellite broadband technology; however, collaboration with telecommunications companies is needed to develop reasonable cost models for these transmissions, including two-way communication. Much like space agencies communicate with satellites, shore-side personnel should be able to monitor shipboard sensor performance at sea, to provide updates to software/firmware, and to alter the sampling characteristic of the sensors as needed. To achieve further mutual benefit, the communities active in shipboard and autonomous surface vessel observations need to jointly develop and advance two-way communications.

Needs for marine observing should be built into new vessel design (Rossby, 2011), as this would simplify the installation of scientific instrumentation and ensure more consistent data quality when sensors are moved from vessel to vessel. For commercial ships, the focus should be on providing a dedicated water intake and location to install flow-through systems, together with cable and tubing routes (e.g., to the bridge level for satellite communication instrumentation and atmospheric gas sensors). On research vessels, designs should also include well-stabilized forward masts to support meteorological and air-sea flux sensors. Several examples of these designs have been implemented on recently constructed research vessels (e.g., Sikuliaq and RRS Sir David Attenborough). Whenever possible, all vessels should also undergo both atmospheric and oceanic flow modeling during the design phase, and these results should be made available to the user community. Finally, when vessel designs cannot be changed, the alternative is developing new sensor technology that can easily be fitted onto any vessel (e.g., magnetic sensor packages for deployment on ship hulls).

\section{Evaluating Data Quality}

All observations need to undergo timely, standardized quality control procedures established by the wider community of data managers to identify gross errors (e.g., check data within defined ranges, time and location, and climatological test).

\footnotetext{
${ }^{17}$ http://www.iala-aism.org/asm/weather-observation-report-ship/
} 
Near-real-time quality control should take advantage of collocated measurements from other marine networks (e.g., ships versus Argo) and be optimized by retrospective comparison with more refined delayed-mode processing (e.g., Alory et al., 2015). Coordination is needed between the different components of the marine observational community (e.g., meteorological, oceanic, and biogeochemical observations) working toward standard approaches to evaluate the data.

Ideally quality control activities are conducted by scientific experts using community-developed, accepted, and shared tools. Programs like SAMOS, GOSUD, ICOADS, SOCAT, GLODAP, Copernicus, and others have developed quality control software and tools specific to a type of ship (commercial versus research vessel) and various parameters. Many of these systems are highly customized, making them difficult to disseminate to the wider observational community, but these tools should be harmonized and open-source code made available. The authors recommend organizing an international working group to focus on developing and distributing quality control tools. Such an effort may be coordinated by the MCDS or JCOMM or via workshops to include the diverse research communities that conduct much of the not presently "operational" marine observing from ships.

A further challenge is to identify some shipboard observations to serve as "reference" stations similar to what is being done in the land-surface community (e.g., Thorne et al., 2018). Most likely, these would be research vessels, but they should adhere to the highest standard for quality assessment/control. Presently, no standards for shipboard reference stations exist, but lessons can be learned from GAW program or the definition of core voyages in GLODAP (Olsen et al., 2016). We recommend identifying a set of vessel operators willing to work toward developing and supporting a standard to be known as a mobile reference station. Ideally, these vessels would host as many data systems as possible, meeting the multivariate observing goal outlined in the "MultiVariate Shipboard Observations" section.

\section{Data Management}

Operational data need to be made available as soon as possible after transmission from the ship to shore. For synoptic meteorological data, procedures are well established to push observations onto the GTS; however, for a number of oceanographic and biogeochemical parameters, especially those made on research vessels, the procedures for real-time data dissemination are not as clearly defined. Over the next decade, there is a need for the meteorological, oceanographic, and biogeochemical observing communities to come together to establish protocols for real-time transmission of at least a subset of the parameters in Table 1. Further efforts will be needed to manage full-temporal resolution observations that are collected from vessels post voyage (in delayed mode).

It is important to recognize that data collected for operational purposes also serve a wide range of climate applications. For example, the National Centers for Environmental Information currently combines two GTS data streams to create monthly updates of ICOADS (Freeman et al., 2017). Creating such interim products supports climate assessment and monitoring.
Identifying duplicate and near-duplicate reports can be difficult, especially if ship identifiers have been masked. Data derived from the GTS are periodically combined with data from delayed-mode data systems in major ICOADS updates, again with challenges for identifying duplicate reports received from different data management systems.

For the climate record, it is sometimes the case that only a real-time observation exists, and there is no corresponding delayed-mode source. We recommend preserving all metadata included with BUFR or earlier fixed character-based messages. With the current transition to BUFR, more metadata can be made available alongside the observed data, which should eventually remove the need for the VOS delayed-mode data system. While metadata fields available in BUFR are much more expansive than those in past formats, encoding of metadata into the BUFR records may not be complete, depending on the network owner responsible for providing this information; so a central metadata repository, such as that provided by JCOMMOPS, is still required to maintain historical vessel/platform metadata and lineage.

GTS collections at different NMHS may vary in their contents (JCOMM, 2010), and in some cases, data are circulating on the GTS with a low level of metadata (a situation that will significantly improve when the BUFR format is fully adopted). To facilitate capturing the full range of observations on the GTS, the authors recommend establishing a real-time GTS data assembly center within the JCOMM MCDS to harvest and manage all ship observations circulating on the GTS. This center could accept data received from the GTS by multiple NMHS, conduct duplicate evaluation, and provide these observations to downstream users and product developers. The result would be a consolidated source for real-time GTS data with agreed quality control that could be aggregated to climate datasets such as ICOADS or the World Ocean Database, providing full provenance from instruments to archives/products as intended by the MCDS (Pinardi et al., 2019). Aggregating the data in the MCDS would provide a more complete collection and assure permanent preservation in international archives and repositories, guaranteeing their existence for future applications.

Beyond the real-time data transmitted via the GTS, there presently is no global repository dedicated to all data types (Table 1) collected onboard vessels. Most research vessel observations are neither available in near real time for operational applications nor processed in delayed mode to be submitted to global climate archives. There are examples of good stewardship of EOVs and ECVs, including the Rolling Deck to Repository, SAMOS, GOSUD, SOCAT, GLODAP, PANGAEA Data Publisher ${ }^{18}$, and the Australian Ocean Data Network, which provide quality assurance and dissemination and securely archive observations from research vessels (e.g., Gaillard et al., 2015). Much of the data are archived by different national data centers, and only some of these data are included in global marine climate databases (e.g., ICOADS and World Ocean Database). There are counter-examples where national research vessel operators do not always continuously log or manage data from underway systems. The authors recommend establishing

\footnotetext{
${ }^{18}$ www.Pangaea.de
} 
a distributed set of data assembly centers, possibly affiliated with the MCDS, to catalog, manage, and deliver underway data from research vessels in a manner that support efficient data exchange across different computer platforms. The first step is to develop a unified catalog of vessels collecting data in Table 1, including metadata on what parameters are being sampled, at what frequency, and who is responsible for managing the individual sensor systems on research vessels. Any global research vessel observation catalog will need to leverage ongoing vessel metadata efforts at JCOMMOPS and from individual operational and research projects. This could be achieved in the next few years by organizing cross network workshops to engage the diverse shipboard observing community with the goal being to unlock existing data collected by research vessels and to expand observing capabilities by coordinating deployment of existing and new autonomous technology.

\section{COMMUNITY ENGAGEMENT}

Coordination between the operational and research observational communities needs to be strengthened at national and international levels, drawing on expertise in making the observations, collecting and tracking comprehensive metadata, and ensuring the observations are distributed and available in a manner that is transparent to the user. Data user communities need to be involved to assess their requirements for observations (e.g., identifying regions to improve sampling and focusing on specific physical parameters). Coordination is needed in the recruitment of ships to make observations, in developing technology for autonomous use on ships, and in collecting, tracking, and archiving of both the observations and the metadata that are critical for real-time (e.g., forecasting and warnings, and safety of life at sea) and delayed-mode (e.g., reanalyses, process studies, satellite algorithm development and product evaluation, and model evaluation) applications of the observations.

\section{Coordinating Vessel Recruitment}

For vessel recruitment, additional coordination is needed between international governing bodies and panels that are central to specific ship communities. For example, additional interaction is needed between international research vessel operators, the passenger cruise industry (e.g., Cruise Lines International Association, International Association of Antarctic Tour Operators), and the panels of JCOMM to coordinate recruitment across these large fleets. The WOC Smart OceanSmart Industries program provides a potential platform/portal to facilitate communication between the scientific ocean observing community and marine industries. The program goal is to increase the number of companies from a range of ocean industries involved in the sustained collection and reporting of standardized data for input to operational and scientific programs that improve the safety and sustainability of commercial activities at sea and contribute to understanding the ocean. The WOC brings together representatives from (a) shipping, oil/gas, ferries, offshore wind, and fisheries industries; (b) marine technology, instrumentation, and IT/communications companies; (c) international and national oceanographic/meteorological organizations; and (d) existing voluntary observation programs.

One major issue in using commercial vessels as observing platforms is that ships are frequently on short-term charters due to changing markets in a globalized world, while climate monitoring requires repetitive long-term observations. The resulting instability of repeat ship lines makes climate monitoring difficult and costly since a ship recruited for one line can switch to another line, sometimes on the other side of the world, on very short notice when the contract ends, and the in-port window can be very short for retrieving instrument for installation on another ship. Advancing vessel design to support observations may help to reduce this problem (see Technological Advancement), but more direct coordination with shipping companies will be required.

Not all marine observations are made from or coordinated by vessels officially recruited by an NMHS. Where commercial operators have a requirement for meteorological data for their operations, these data can be purchased or shared with NMHSs as a supplement to official operated networks. An example of third-party data includes automatic weather stations situated on offshore rigs, platforms, and floating production units. The oil and gas industries have a requirement for highquality meteorological data (particularly wind) to carry out safe helicopter operations. Additional engagement with these third-party operators is essential to ensure valuable data are shared and made available, ideally free of cost, to the wider marine community.

Within the VOS Scheme, a dedicated "ZZ" category exists for recruited VOS ships that have no particular affiliation with a national VOS fleet. There are currently over 300 such ships registered within the VOS metadata database. This allows for the ad hoc recruitment of certain vessels where, for example, their voyage route looks to be beneficial (e.g., within a data sparse area), the voyage is only for a dedicated period of time, or there is limited resource to manage the data as part of a national fleet. Other benefits can be obtained by building relationships with private operators (e.g., yachts and racing teams) of vessels equipped with atmospheric or oceanic sensors, which may not be of the highest quality, but are occupying routes/regions not typically occupied by vessels recruited to VOS or research vessels (e.g., Southern Ocean). The advantage of doing so is that data supply is increased and the wider community is engaged with the making of ocean observations. As long as the platform metadata describe the source of the data sufficiently, data users can assess the usefulness for their operational or research goals. The recommendation to develop tools to collect third-party data using ships outside the traditional commercial and research vessel operators (see Vessel Recruitment) will engage new operators in the collection of the parameters in Table 1.

\section{Leveraging Technological Advances}

The authors recommend establishing routine communication between ship- and autonomous surface vessel-based science communities to develop and disseminate techniques between 
their domains, as an optimum ocean observational network is multi-platform. We expect autonomous surface vessels to play an ever-growing role in ocean observations for a variety of disciplines as new sensors become integrated (e.g., air-sea fluxes, ocean biogeochemistry, and fisheries; Swart et al., 2016; Centurioni et al., 2019). The role of the autonomous surface vessels to enhance and broaden existing and future shipbased observing needs to be defined. There is significant benefit to connect these communities, since the introduction of autonomous surface vessels creates the potential to scale up traditionally ship-limited observations and reduce the spacetime data gaps inherent with ship-based surveys (Greene et al., 2014; Swart et al., 2016), such as envisioning a quasi-permanent science platform presence and year-round monitoring in both local and remote ocean regions. Meanwhile, ships will continue to be a primary contributor to the marine observational network and will be required for calibration, validation, and technological testing of sensors deployed on autonomous surface vessels, as well as to launch/recover autonomous surface vessels.

As noted above, AIS is a promising option to transmit observations for AIS-equipped ships. Although AIS was not designed for satellite reception and it has very limited bandwidth, there has been substantial success in receiving AIS transmission from satellites, increasing its potential for collecting weather observations. Recently, the VHF Data Exchange System (VDES) is being developed with higher bandwidth and designed to include two-way satellite communication capabilities. VDES will augment AIS and holds promise to be well suited for collection of weather observations from ships. AIS and VDES are inexpensive, essentially free beyond equipment cost, and likely to be aboard many, including smaller, vessels. The authors recommend the establishment of pilot projects, to include industry, governments, and providers of hardware and software, which will transmit meteorological and oceanographic information via AIS messages. This is in keeping with the U.S. National Transportation Safety Board's recommendation to the National Oceanic and Atmospheric Administration in their report on the El Faro sinking $^{19}$ (United States Coast Guard [USCG], 2017).

\section{Data Users}

Within the marine climate research community, international workshops promote best practices: the JCOMM Workshop on Advances in Marine Climatology and the International Workshop on Advances in the Use of Historical Marine Climate Data. These workshops focus on data management aspects of marine climatology, the development and production of multidecadal to centennial climate products, and sharing improvements in bias adjustment and uncertainty estimation methodologies. Both series include dataset developers and users, but it would be beneficial and is recommended to expand the scope to include operational users of marine data, allowing connections to be made between the need to make observations to support immediate operational needs and also to foster understanding that reusing these data is essential to meet longerterm climate objectives.

\footnotetext{
${ }^{19}$ https://www.ntsb.gov/investigations/AccidentReports/Reports/MAR1701.pdf
}

Models, from simple to fully coupled atmosphere-ocean biogeochemical global circulation models, benefit from observations for assimilation and evaluation. A more coordinated, multivariate approach to the collection of marine data from ships, along with easing accessibility to and better documentation of these data, will increase the use of observations by the modeling community. For example, the annually published global carbon budget (Le Quéré et al., 2018) now routinely combines data from observations and models, which reduces uncertainties and increases the confidence level in contemporary analyses as well as future model predictions. Such efforts foster not only communicating result to non-specialists but also enabling information flow to policy makers, either direct or via other organizations (e.g., the Intergovernmental Panel on Climate Change). It is essential in the next decade that shipboard observation of biogeochemical parameters become routine, in addition to traditional meteorological and physical oceanographic measurements, and the authors recommend further investment in the personnel and technology required to advance shipboard biogeochemical observation.

The role of shipboard observations in retrieval algorithm development and the calibration/validation of remotely sensed marine parameters will continue throughout the next decade. Indefinitely, the role of these observations in transportation and safety will be very important. More advanced satellite and aircraft remote sensing system will be developed, each requiring in situ observations to evaluate sensors and systems. Continued collaboration between the shipboard and remote sensing communities, and even among these communities, NMHSs, and the private sector (e.g., AIS and instrumentation developers and manufacturers), is important, whether at joint workshops, meetings of opportunity, or, ideally, joint observing projects. The authors recommend that agencies supporting satellite and remote sensing systems development provide complimentary funding to enhance shipboard observations. In many cases, the cost will be minimal compared with the cost of deploying new remote sensing systems.

\section{SUMMARY AND RECOMMENDATIONS}

All types of ships have the capability to provide essential atmospheric, oceanic, and biogeochemical observations. We envision a future where commercial, research, and privately owned vessels are making multivariate observations using a combination of automated and human-observed measurements. All data and metadata will be documented, tracked, evaluated, distributed, and archived to benefit users of marine data. This vision looks at ships as a holistic network, not a set of disparate VOS, research, commercial, and/or third-party activities working in isolation. The idea is to bring these communities together for the mutual benefit of all.

Toward that end, the authors make the following summary recommendations to be discussed at OceanObs' $19^{20}$ and to be acted on over the coming decade. The authors note that the

\footnotetext{
${ }^{20}$ http://www.oceanobs19.net/
} 
level of capital investment, both financial and human, varies between these recommendations. Those marked with bold text are deemed by the authors to be feasible with lower investments that may be supported through the funding infrastructure of individual nations (or small collaboratives). The other recommendations, though necessary to advance the coordination of shipboard observing and data management, will require higher levels of international cooperation and capital investment.

- Expand recruitment of new ships to make observations of the parameters outlined in Table 1 to

○ include recruitment via traditional means, ensuring that all research vessels are contributing to operational and research data collection efforts, and seeking new collaborations with commercial and private ship owners/operators,

- increase and improve communications between the WOC, JCOMM, the research community, and relevant panels/communities in identifying additional ships to make observations, and

$\circ$ engage the user community to develop a list of sensors to be widely deployed on ships and to define high-priority regions of the ocean to target for increased sampling.

- The research vessel community should

o provide metadata regarding their observational capabilities to international metadata catalogs,

o expand capabilities to conduct direct flux measurements,

- host new technology to provide a testbed prior to installation on commercial or private ships,

o support installation of multiple sensor packages on research vessels, and

- ensure that all sensor packages on each research vessel operate on every voyage (to maximize data collection).

- Agencies should invest in technology development by, but not limited to

○ establish pilot projects; to include industry, governments, and equipment and software providers; and to develop methods to transmit meteorological and oceanographic information via AIS messages,

○ work with sensor manufacturers to develop costeffective, self-describing sensors that could deliver their metadata in a normalized format,

\section{REFERENCES}

Allan, R., Brohan, P., Compo, G. P., Stone, R., Luterbacher, J., and Brönnimann, S. (2011). The international atmospheric circulation reconstructions over the earth (ACRE) initiative. Bull. Amer. Meteor. Soc. 92, 1421-1425. doi: 10.1175/ 2011bams3218.1

Aloisio, G., Conte, D., Elefante, C., Marra, G. P., Mastrantonio, G., and Quarta, G. (2006). "Globus monitoring and discovery service and SensorML for grid sensor networks," in Proceedings of the 15th IEEE International Workshops on
○ develop automated atmospheric composition sampling systems that could be installed on ships of opportunity, and

$\circ$ develop a web portal and other recruitment tools to support third-party data collection.

- Improve data access and interoperability by

- agreeing internationally to a fully open policy for the exchange of and access to data and metadata from ships and

- establishing distributed, but interoperable, regional/ global data centers (affiliated with the MCDS), to catalog, evaluate, and distribute shipboard underway data, including VOS and research vessels, collected in real time and delayed mode. One center should focus on receiving, processing, and evaluating GTS data from multiple NMHS.

- Move ship observing networks toward standard quality control and assessment methodology by organizing an international working group to focus on developing and distributing tools and best practices.

\section{AUTHOR CONTRIBUTIONS}

All authors contributed to writing sections of the manuscript, and each read and approved the submitted version. DF, PP, MK, and GA developed and contributed the figures.

\section{FUNDING}

Preparation of this article by SRS and associated page charges are provided by a cooperative agreement (NA16OAR4320199) from the Climate Program Office, Ocean Observing and Monitoring Division of the National Oceanographic and Atmospheric Administration (FundRef\#100007298) via a subaward (191001.363513.01D) from the Northern Gulf of Mexico Cooperative Institute administered by the Mississippi State University. EK and DB were supported by the UK NERC under the CLASS program (NE/R015953/1). SS was supported by a Wallenberg Academy Fellowship (WAF 2015.0186). US was supported by the UK NERC projects ABC Fluxes project (NE/M005070/1), the SONATA (NE/P021417/1), and the EU Horizon 2020 projects RINGO (\#730944), and the AtlantOS (\#633211).

Enabling Technologies: Infrastructure for Collaborative Enterprises, Manchester, doi: 10.1109/WETICE.2006.44

Alory, G., Delcroix, T., Téchiné, P., Diverrès, D., Varillon, D., Cravatte, S., et al. (2015). The french contribution to the voluntary observing ships network of sea surface salinity. Deep Sea Res. 105, 1-18. doi: 10.1016/j.DSR.2015.08.005

Anderson, E. (2018). Statement of Guidance for Global Numerical Weather Prediction (NWP), WMO. Available at: https://www.wmo.int/pages/prog/www/ OSY/SOG/SoG-Global-NWP.pdf doi: 10.1016/j.dsr.2015.08.005 (accessed July 22, 2018). 
Anderson, J. E., and Riser, S. C. (2014). Near-surface variability of temperature and salinity in the near-tropical ocean: observations from profiling floats. J. Geophys. Res. 119, 7433-7448. doi: 10.1002/2014JC010112

Asher, W. E., Jessup, A. T., Branch, R., and Clark, D. (2014). Observations of rain-induced near-surface salinity anomalies. J. Geophys. Res. 119, 5483-5500. doi: $10.1002 / 2014$ jc009954

Baker, A. R., Adams, C., Bell, T. G., Jickells, T. D., and Ganzeveld, L. (2013). Estimation of atmospheric nutrient inputs to the Atlantic Ocean from $50^{\circ} \mathrm{N}$ to $50^{\circ} \mathrm{S}$ based on large-scale field sampling: iron and other dust-associated elements. Glob. Biogeochem. Cycles 27, 755-767. doi: 10.1002/gbc.20062

Baker, A. R., Lesworth, T., Adams, C., Jickells, T. D., and Ganzeveld, L. (2010), Estimation of atmospheric nutrient inputs to the atlantic ocean from $50^{\circ} \mathrm{N}$ to $50^{\circ} \mathrm{S}$ based on large-scale field sampling: fixed nitrogen and dry deposition of phosphorus. Glob. Biogeochem. Cycles 24:GB3006. doi: 10.1029/2009GB003634

Bauer, P., Thorpe, A., and Brunet, G. (2015). The quiet revolution of numerical weather prediction. Nature 525, 47-55. doi: 10.1038/nature14956

Beaugrand, G., and Kirby, R. R. (2018). How do marine pelagic species respond to climate change? Theories and observations. Annu. Rev. Mar. Sci. 10, 169-197. doi: 10.1146/annurev-marine-121916-063304

Bell, M. J., Schiller, A., Le Traon, P.-Y., Smith, N. R., Dombrowsky, E., and WilmerBecker, K. (2015). An introduction to GODAE OceanView. J. Oper. Oceanogr. 8, s2-s11. doi: 10.1080/1755876X.2015.1022041

Benallal, M., Moussa, H., Touratier, F., Goyet, C., and Poisson, A. (2016). Ocean salinity from satellite-derived temperature in the Antarctic Ocean. Antarct. Sci. 28, 127-134. doi: 10.1017/S0954102015000516

Bentamy, A., Piollé, J. F., Grouazel, A., Danielson, R., Gulev, S., Paul, F., et al. (2017). Review and assessment of latent and sensible heat flux accuracy over the global oceans. Remote Sens. Environ. 201, 196-218. doi: 10.1016/j.rse.2017. 08.016

Berry, D. I., and Kent, E. C. (2011). Air-sea fluxes from ICOADS: the construction of a new gridded dataset with uncertainty estimates. Int. J. Climatol. 31, 987-1001. doi: 10.1002/joc.2059

Berry, D. I., and Kent, E. C. (2017). Assessing the health of the global surface marine climate observing system. Int. J. Climatol. 37, 2248-2259. doi: 10.1002/joc.4914

Bojinski, S., Verstraete, M., Peterson, T. C., Richter, C., Simmons, A., and Zemp, M. (2014). The concept of essential climate variables in support of climate research, applications, and policy. Bull. Am. Meteor. Soc. 95, 1431-1443. doi: 10.1175/BAMS-D-13-00047.1

Bonou, F. K., Noriega, C., Lefèvre, N., and Araujo, M. (2016). Distribution of CO2 parameters in the Western Tropical Atlantic Ocean. Dyn. Atmos. Oceans 73, 47-60. doi: 10.1016/j.dynatmoce.2015.12.001

Bourassa, M. A., Legler, D. M., O'Brien, J. J., and Smith, S. R. (2003). SeaWinds validation with research vessels. J. Geophys. Res. 108:3019. doi: 10.1029/ 2001JC001028

Bourlès, B., Lumpkin, R., McPhaden, M. J., Hernandez, F., Nobre, P., Campos, E., et al. (2008). The PIRATA program: history, accomplishments, and future directions. Bull. Am. Meteor. Soc. 89, 1111-1126. doi: 10.1175/2008BAMS 2462.1

Boutin, J., Chao, Y., Asher, W. E., Delcroix, T., Drucker, R., Drushka, K., et al. (2016). Satellite and in situ salinity: understanding near-surface stratification and sub-footprint variability. Bull. Am. Meteor. Soc. 97, 1391-1407. doi: 10. 1175/BAMS-D-15-00032.1

Boutin, J., Vergely, J. L., Marchand, S., D’Amico, F., Hasson, A., Kolodziejczyk, N., et al. (2018). New SMOS sea surface salinity with reduced systematic errors and improved variability. Remote Sens. Environ. 214, 115-134. doi: 10.1016/j. rse.2018.05.022

Brewin, R. J. W., Dall'Olmo, G., Pardo, S., van Dongen-Vogels, V., and Boss, E. S. (2016). Underway spectrophotometry along the atlantic meridional transect reveals high performance in satellite chlorophyll retrievals. Remote Sens. Environ. 183, 82-97. doi: 10.1016/j.rse.2016.05.005

Brönnimann, S., Allan, R., Atkinson, C., Buizza, R., Bulygina, O., Dahlgren, P., et al. (2018). Observations for reanalyses. Bull. Am. Meteor. Soc. 99, 1851-1866. doi: 10.1175/BAMS-D-17-0229.1

Buizza, R., Poli, P., Rixen, M., Alonso-Balmaseda, M., Bosilovich, M. G., Brönnimann, S., et al. (2018). Advancing global and regional reanalyses. Bull. Am. Meteor. Soc. 99, ES139-ES144. doi: 10.1175/BAMS-D-17-0312.1

Caccia, M., Bono, R., Bruzzone, G., Bruzzone, G., Spirandelli, E., Veruggio, G., et al. (2005). "Design and exploitation of an autonomous surface vessel for the study of sea-air interactions," in Proceedings of the 2005 IEEE International Conference on Robotics and Automation, Barcelona, doi: 10.1109/ROBOT.2005. 1570665

Carslaw, K. S., Lee, L. A., Reddington, C. L., Pringle, K. J., Rap, A., Forster, P. M., et al. (2013). Large contribution of natural aerosols to uncertainty in indirect forcing. Nature 503, 67-71. doi: 10.1038/nature12674

Centurioni, L. R., Turton, J., Lumpkin, R., Braasch, L., Brassington, G., Chao, Y., et al. (2019). Global in-situ observations of essential climate and ocean variables at the air-sea interface. Front. Mar. Sci. doi: 10.3389/fmars.2019.00419

Choquer, M. (2014). Bark EUROPA: the OceanoScientific system onboard the three-master. Mariners Weather Log 58, 4-8.

Compo, G. P., Whitaker, J. S., Sardeshmukh, P. D., Matsui, N., Allan, R. J., Yin, X., et al. (2011). The twentieth century reanalysis project. Q. J. R. Meteorol. Soc. 137, 1-28. doi: 10.1002/qj.776

Corfield, R. (2003). The Silent Landscape: The Scientific Voyage of HMS Challenger. Washington, DC: Joseph Henry Press.

Cronin, M. F., Gentemann, C. L., Edson, J., Ueki, I., Bourassa, M., Brown, S., et al. (2019). Air-sea fluxes with a focus on heat and momentum. Front. Mar. Sci. 6:430. doi: $10.3389 /$ fmars. 2019.00430

Cummings, J. A. (2011). "Ocean data quality control," in Operational Oceanography in the 21st Century, eds A. Schiller and G. B. Brassington (Dordrecht: Springer), 91-121. doi: 10.1007/978-94-007-0332-2_4

da Silva, A. M., Young, C. C., and Levitus, S. (1994). Atlas of Surface Marine Data 1994, volume 1: Algorithms and Procedures. NOAA Atlas NESDIS 6. Silver Spring, MD: NESDIS.

Delcroix, T., Alory, G., Cravatte, S., Corrège, T., and McPhaden, M. (2011). A gridded sea surface salinity data set for the tropical Pacific with sample applications (1950-2008). Deep Sea Res. 58, 38-48. doi: 10.1016/j.dsr.2010. 11.002

Duce, R. A., Liss, P. S., Merrill, J. T., Atlas, E. L., Buat-Menard, P., Hicks, B. B., et al. (1991). The atmospheric input of trace species to the world ocean. Glob. Biogeochem. Cycles 5, 193-259. doi: 10.1029/91gb01778

Fletcher, J. (2008). Meteorological Observations from Ships. Seaways. 7-10. The Nautical Institute, Available at: http://sot.jcommops.org/vos/documents/ seaways-vos-200804.pdf (accessed July 22, 2019).

Freeman, E., Woodruff, S. D., Worley, S. J., Lubker, S. J., Kent, E. C., Angel, W. E., et al. (2017). ICOADS Release 3.0: a major update to the historical marine climate record. Int. J. Climatol. 37, 2211-2232. doi: 10.1002/joc.4775

Gaillard, F., Diverres, D., Jacquin, S., Gouriou, Y., Grelet, J., Le Menn, M., et al. (2015). Sea surface temperature and salinity from French research vessels, 2001-2013. Sci. Data 2:150054. doi: 10.1038/sdata.2015.54

Galloway, J. N., Knap, A. H., and Church, T. M. (1983). The composition of western Atlantic precipitation using shipboard collectors. J. Geophys. Res. 88, 10859-10864. doi: 10.1029/JC088iC15p10859

Gelaro, R., McCarty, W., Suárez, M. J., Todling, R., Molod, A., Takacs, L., et al. (2017). MERRA-2 Overview: the modern-era retrospective analysis for research and applications, version 2 (MERRA-2). J. Clim. 30, 5419-5454. doi: 10.1175/ JCLI-D-16-0758.1

German, C. R., Jukuba, M. V., Kinsey, J. C., Partan, J., Suman, S., Belani, A., et al. (2012). "A long term vision for long-range ship-free deep ocean operations: persistent presence through coordination of autonomous surface vehicles and autonomous underwater vehicles," in Proceedings of the 2012 IEEE/OES Autonomous Underwater Vehicles (AUV), Southampton, doi: 10.1109/AUV. 2012.6380753

Geyer, B., and Rockel, B. (2013). coastDat-2 COSMO-CLM Atmospheric Reconstruction. Hamburg: World Data Center for Climate, doi: 10.1594/ WDCC/coastDat-2_COSMO-CLM

Global Climate Observing System [GCOS] (2016). The Global Observing System for Climate: Implementation Needs. GCOS-200, GOOS-214, World Meteorological Organization. Available at: https://unfccc.int/sites/default/files/ gcos_ip_10oct2016.pdf (accessed July 22, 2018).

Global Ocean Observing System [GOOS] (2018). Essential Ocean Variables. Available at: http://www.goosocean.org/index.php?option=com_content\& view=article\&id $=14 \& I t e m i d=114$ (accessed 19 September 2018)

Goni, G., Sprintall, J., Bringas, F., Cheng, L., Cirano, M., Dong, S., et al. (2019). More than 50 years of successful continuous temperature section measurements by the global eXpendable BathyThermograph (XBT) network, its integrability, societal benefits, and future. Front. Mar. Sci. doi: 10.3389/fmars.2019.00452 
Greene, C. H., Meyer-Gutbrod, E. L., McGarry, L. P., Hufnagle, L. C., Chu, D., McClatchie, S., et al. (2014). A wave glider approach to fisheries acoustics: transforming how we monitor the nation's commercial fisheries in the 21st century. Oceanography 27, 168-174.

Guichoux, Y., Lennon, M., and Thomas, N. (2016). "Sea surface currents calculation using vessel tracking data," in Proceedings of the Maritime Knowledge Discovery and Anomaly Detection Workshop, (Ispra: Joint Research Centre),

Holthus, P. (2018). "The role of the world ocean council and the ocean business community in global ocean governance," in The IMLI Treatise on Global Ocean Governance: Volume I: UN and Global Ocean Governance, ed. D. Kritsiotis (London: International Maritime Organization).

Ingleby, B. (2010). Factors affecting ship and buoy data quality: a data assimilation perspective. J. Atmos. Ocean. Technol. 27, 1476-1489. doi: 10.1175/ 2010JTECHA1421.1

International Maritime Organization [IMO] (2002). Safety of Life at Sea, Chapter V. Available at: http://www.imo.org/en/OurWork/facilitation/documents/solas\% 20v\%20on\%20safety\%20of\%20navigation.pdf (accessed July 22, 2018).

International Maritime Organization [IMO] (2018). Participation in the WMO Voluntary Observing Ship Scheme, MSC.1 Circular 1293. Available at: http: //sot.jcommops.org/vos/documents/msc_1293.pdf (accessed July 22, 2018).

Jackson, D. L., and Wick, G. A. (2016). "Development of a 28-year (1987-2014) climatology of single and multi-sensor satellite-based retrievals of near-surface humidity and temperature," in Proceedings of the 20th Conference on air-sea Interactions, Madison, WI.

JCOMM (2002). JCOMM Ship Observation Team, First Session, National Reports. JCOMM Technical Report No. 17, WMO/TD-No. 1121. Available at: https:// library.wmo.int/pmb_ged/wmo-td_1121_en.pdf (accessed July 24, 2018).

JCOMM (2010). Third Session of the JCOMM Expert Team on Marine Climatology. JCOMM Meeting Report No. 70. Geneva: World Meteorological Organization.

Jessup, A. T., and Branch, R. (2008). Integrated ocean skin and bulk temperature measurements using the calibrated infrared in situ measurement system (CIRIMS) and through-hull ports. J. Atmos. Ocean Technol. 25, 579-597. doi: 10.1175/2007jtecho479.1

Jickells, T. D., Buitenhuis, E., Altieri, K., Baker, A. R., Capone, D., Duce, R. A., et al. (2017). A re-evaluation of the magnitude and impacts of anthropogenic nitrogen inputs on the ocean. Glob. Biogeochem. Cycles 31, 289-305. doi: 10. 1002/2016GB00558

Jones, R. W., Renfrew, I. A., Orr, A., Webber, B. G. M., Holland, D. M., and Lazzara, M. A. (2016). Evaluation of four global reanalysis products using in situ observations in the Amundsen Sea Embayment, Antarctica. J. Geophys. Res. Atmos. 121, 6240-6257. doi: 10.1002/2015JD024680

Kawai, Y., and Wada, A. (2007). Diurnal sea surface temperature variation and its impact on the atmosphere and ocean: a review. J. Oceanogr. 63, 721-744. doi: 10.1007/s10872-007-0063-0

Keim, B. (2011). Transcending Time: Great Long-Term Datasets. Wired.com 10.17.11. Available at: https://www.wired.com/2011/10/long-term-datasets/ (accessed July 31, 2018).

Kent, E. C., Ball, G., Berry, D. I., Fletcher, J., Hall, A., North, S., et al. (2010). “The Voluntary Observing Ship (VOS) Scheme," in Proceedings of the "OceanObs'09: Sustained ocean observations and information for society", Venice, eds J. Hall, D. E. Harrison, and D. Stammer (Venice: ESA Publication), 21-25. doi: 10.5270/ OceanObs09.cwp.48

Kent, E. C., Kennedy, J. J., Smith, T. M., Hirahara, S., Huang, B., Kaplan, A., et al. (2017). A call for new approaches to quantifying biases in observations of sea-surface temperature. BAMS 98, 1601-1616. doi: 10.1175/BAMS-D-1500251.1

Kent, E. C., Rayner, N. A., Berry, D. I., Eastman, R., Grigorieva, V. G., Huang, B., et al. (2019). Observing requirements for long-term climate records at the ocean surface. Front. Mar. Sci. 6:441. doi: 10.3389/fmars.2019.00441

Kent, E. C., Rayner, N. A., Berry, D. I., Saunby, M., Moat, B. I., Kennedy, J. J., et al. (2013). Global analysis of night marine air temperature and its uncertainty since 1880, the HadNMAT2 Dataset. J. Geophys. Res. Atmos. 118, 1281-1298. doi: $10.1002 /$ jgrd.50152

Kent, E. C., Woodruff, S. D., and Berry, D. I. (2007). Metadata from WMO Publication No. 47 and an assessment of voluntary observing ship observation heights in ICOADS. J. Atmos. Ocean Technol. 24, 214-234. doi: 10.1175/ JTECH1949.1
Keynes, R. D. (2012). The Beagle Record: Selections from the Original Pictorial Records and Written Accounts of the Voyage of HMS Beagle. Cambridge: Cambridge University Press.

Klepp, C. (2015). The oceanic shipboard precipitation measurement network for surface validation-OceanRAIN. Atmos. Res. 163, 74-90. doi: 10.1016/j. atmosres.2014.12.014

Kolodziejczyk, N., Diverres, D., Jacquin, S., Gouriou, Y., Grelet, J., Le Menn, M., et al. (2015a). Sea Surface Salinity from French RESearcH Vessels: Delayed Mode Dataset, Annual Release. France: SEANOE, doi: 10.17882/39475

Kolodziejczyk, N., Reverdin, G., Boutin, J., and Hernandez, O. (2015b). Observation of the surface horizontal thermohaline variability at meso- to submesoscales in the North-Eastern Subtropical Atlantic Ocean. J. Geophys. Res. Oceans 120, 2588-2600. doi: 10.1002/2014JC010455

Kramp, M., Gaillard, F., Blouch, P., and Fietzek, P. (2010). Scientific data acquisition by ocean-going sailing yachts: the OceanoScientific program. Revue de L'electricite et de L'electronique 10, 52-60.

Kramp, M., and Rusciano, E. (2016). Sailing and science: 27 days to Cape Horn with surface drifters and TurboWin. Mariners Weather Log 60, 4-7.

Laloyaux, P., de Boisseson, E., Balmaseda, M., Bidlot, J.-R., Broennimann, S., Buizza, R., et al. (2018). CERA-20C: a coupled reanalysis of the twentieth century. J. Adv. Model. Earth Syst. 10, 1172-1195. doi: 10.1029/2018MS001273

Le Quéré, C., Andrew, R. M., Friedlingstein, P., Sitch, S., Pongratz, J., Manning, A. C., et al. (2018). Global carbon budget 2017. Earth Syst. Sci. Data 10, 405-448. doi: 10.5194/essd-10-405-2018

Lee, K., Tong, L. T., Millero, F. J., Sabine, C. L., Dickson, A. G., Goyet, C., et al. (2006). Global relationships of total alkalinity with salinity and temperature in surface waters of the world's oceans. Geophys. Res. Lett. 33:L19605. doi: 10.1029/2006GL027207

Lenain, L., and Melville, W. K. (2014). Autonomous surface vehicle measurements of the ocean's response to tropical cyclone Freda. J. Atmos. Ocean. Technol. 31, 2169-2190. doi: 10.1175/JTECH-D-14-00012.1

Liman, J., Schröder, M., Fennig, K., Andersson, A., and Hollmann, R. (2018). Uncertainty characterization of HOAPS 3.3 latent heat-flux-related parameters. Atmos. Meas. Tech. 11, 1793-1815. doi: 10.5194/amt-11-1793-2018

Mallory, (1855). Origin of the Wind and Current Chart and Sailing Directions. Report, 32 Congress, The Senate of the United States, Rep. Com. No 443, 29 January 1855. Available at: https://books.google.co.uk/books?id= WacFAAAAQAAJ\&printsec $=$ frontcover\&source $=$ gbs_ge_summary_r\&cad $=$ $0 \# \mathrm{v}=$ onepage\&q\&f$=$ false (accessed July 22, 2018).

Martino, M., Hamilton, D., Baker, A. R., Jickells, T. D., Bromley, T., Nojiri, Y., et al. (2014). Western pacific atmospheric nutrient deposition fluxes, their impact on surface ocean productivity. Glob. Biogeochem. Cycles 28, 712-728. doi: 10.1002/2013gb004794

Maury, M. F. (1854). "Maritime conference held at brussels for devising a uniform system of meteorological observations at sea, August and September, 1853," in Explanations and Sailing Directions to Accompany the Wind and Current Charts, 6th Edn, eds E. C. and J. Biddle (Philadelphia, PA: Read Books Design), 54-96.

McBean, G. A., Phillips, D. J., and Mathieson, J. R. (1986). An intercomparison of two rawinsonde systems. Atmos. Ocean 24, 42-51. doi: 10.1080/07055900.1986. 9649239

McClain, C. R. (2009). A decade of satellite ocean color observations. Annu. Rev. Mar. Sci. 1, 19-42. doi: 10.1146/annurev.marine.010908.163650

McPhaden, M. J., Busalacchi, A. J., Cheney, R., Donguy, J. R., Gage, K. S., Halpern, D., et al. (1998). The tropical ocean-global atmosphere observing system: a decade of progress. J. Geophys. Res. 103, 14169-14240. doi: 10.1029/97JC02906

Monteiro, P. M. S., Gregor, L., Lévy, M., Maener, S., Sabine, C. L., and Swart, S. (2015). Seasonal-scale robotics experiment reveals the contribution made by fine scale dynamics to the uncertainties and biases in the mean seasonal CO2 flux in the Southern Ocean. Geophys. Res. Lett. 42, 8507-8514. doi: 10.1002/ 2015GL066009

Naderi, F., Freilich, M. H., and Long, D. G. (1991). Spaceborne radar measurement of wind velocity over the ocean: an overview of the NSCAT scatterometer system. Proc. IEEE 79, 850-866. doi: 10.1109/5.90163

Nara, H., Tanimoto, H., Nojiri, Y., Mukai, H., Machida, T., and Tohjima, Y. (2011). Onboard measurement system of atmospheric carbon monoxide in the Pacific by voluntary observing ships. Atmos. Meas. Tech. 4, 2495-2507. doi: 10.5194/amt-4-2495-2011 
Nara, H., Tanimoto, H., Tohjima, Y., Mukai, H., Nojiri, Y., and Machida, T. (2014). Emissions of methane from offshore oil and gas platforms in Southeast Asia. Sci. Rep. 4:6503. doi: 10.1038/srep06503

Ogle, S. E., Tamsitt, V., Josey, S. A., Gille, S. T., Cerovečki, I., Talley, L. D., et al. (2018). Episodic southern ocean heat loss and its mixed layer impacts revealed by the farthest south multiyear surface flux mooring. Geophys. Res. Lett. 45, 5002-5010. doi: 10.1029/2017GL076909

Olsen, A., Key, R. M., van Heuven, S., Lauvset, S. K., Velo, A., Lin, X., et al. (2016). The global ocean data analysis project version 2-an internally consistent data product for the world ocean. Earth Syst. Sci. Data 8, 297-323. doi: 10.5194/essd8-297-2016

Pazan, S. E., and Niiler, P. (2004). New global drifter data set available. EOS Trans. Am.Geophys. Union 85:17. doi: 10.1029/2004EO020007

Perlwitz, J. P., Perez Garcia-Pando, C., and Miller, R. L. (2015). Predicting the mineral composition of dust aerosols-part 2: model evaluation and identification of key processes with observations. Atmos. Chem. Phys. 15, 11629-11652. doi: 10.5194/acp-15-11629-2015

Petersen, W. (2014). FerryBox systems: state-of-the-art in Europe and future development. J. Mar. Syst. 140(Part A), 4-12. doi: 10.1016/j.jmarsys.2014. 07.003

Pfeil, B., Olsen, A., Bakker, D. C. E., Hankin, S., Koyuk, H., Kozyr, A., et al. (2013). A uniform, quality controlled Surface Ocean CO2 Atlas (SOCAT). Earth Syst. Sci. Data 5, 125-143. doi: 10.5194/essd-5-125-2013

Pilar, P., Guedes Soares, C., and Carretero, J. C. (2008). A 44-year wave hindcast for the North East Atlantic European coast. Coast. Eng. 55, 861-871. doi: 10.1016/ j.coastaleng.2008.02.027

Pinardi, N., Stander, J., Legler, D., O’Brien, K., Boyer, T., Cuff, T., et al. (2019). Marine monitoring to services: the IOC of UNESCO and WMO experience. Front. Mar. Sci. doi: 10.3389/fmars.2019.00410

Poli, P., Hersbach, H., Dee, D. P., Berrisford, P., Simmons, A. J., Vitart, F., et al. (2016). ERA-20C: an atmospheric reanalysis of the twentieth century. J. Clim. 29, 4083-4097. doi: 10.1175/JCLI-D-15-0556.1

Powell, C. F., Baker, A. R., Jickells, T. D., Bange, H. W., Chance, R. J., and Yodle, C. (2015). Estimation of the atmospheric flux of nutrients and trace metals to the eastern tropical North Atlantic Ocean. J. Atmos. Sci. 72, 4029-4045. doi: 10.1175/jas-d-15-0011.1

Quinn, P. K., and Bates, T. S. (2005). Regional aerosol properties: comparisons of boundary layer measurements from ACE 1, ACE 2, Aerosols99, INDOEX, ACE Asia, TARFOX, and NEAQS. J. Geophys. Res. 110:D14202. doi: 10.1029/ 2004JD004755

Reverdin, G., Morisset, S., Bellenger, H., Boutin, J., Martin, N., Blouch, P., et al. (2013). Near-sea surface temperature stratification from SVP drifters. J. Atmos. Ocean Technol. 30, 1867-1883. doi: 10.1175/JTECH-D-12-00182.1

Richardson, P. L. (1980). Benjamin franklin and timothy Folger's first printed chart of the gulf stream. Science 207, 643-645. doi: 10.1126/science.207.4431.643

Richardson, P. L., and Walsh, D. (1986). Mapping climatological seasonal variations of surface currents in the tropical Atlantic using ship drifts. J. Geophys. Res. 91, 10537-10550.

Riser, S. C., Freeland, H. J., Roemmich, D., Wijffels, S., Troisi, A., Belbeoch, M., et al. (2016). Fifteen years of ocean observations with the global Argo array. Nat. Clim. Chang. 6, 145-153. doi: 10.1038/nclimate2872

Roemmich, D., Johnson, G., Riser, S., Davis, R., Gilson, J., Owens, W., et al. (2009). The Argo program: observing the global ocean with profiling floats. Oceanography 22, 34-43. doi: 10.5670/oceanog.2009.36

Rossby, T. (2011). OceanScope: A Proposed Partnership Between the Maritime Industries and the Ocean Observing Community to Monitor the Global Ocean Water Column. Report of SCOR/IAPSO Working Group. Paris: SCOR.

Rudnick, D. L., Davis, R. E., Eriksen, C. C., Fratantoni, D. M., and Perry, M. J. (2004). Underwater gliders for ocean research. Mar. Technol. Soc. J. 38, 73-84.

Saha, S., Moorthi, S., Pan, H., Wu, X., Wang, J., Nadiga, S., et al. (2010). The NCEP climate forecast system reanalysis. Bull. Am. Meteor. Soc. 91, 1015-1058. doi: 10.1175/2010BAMS3001.1

Salat, J., Umbert, M., Ballabrera-Poy, J., Fernández, P., Salvador, K., and Martínez, J. (2013). The contribution of the Barcelona World Race to improved ocean surface information. A validation of the SMOS remotely sensed salinity. Contrib. Sci. 9, 89-100. doi: 10.2436/20.7010.01.167

Schmidt, K. M., Swart, S., Reason, C., and Nicholson, S. (2017). Evaluation of satellite and reanalysis wind products with in situ wave glider wind observations in the Southern Ocean. J. Atmos. Ocean. Technol. 34, 2551-2568. doi: 10.1175/ JTECH-D-17-0079.1

Send, U., Weller, R. A., Wallace, D., Chavez, F., Lampitt, R., Dickey, T., et al. (2010). "OceanSITES," in Proceedings of OceanObs'09: Sustained Ocean Observations and Information for Society, Vol. 2, eds J. Hall, D. E. Harrison, and D. Stammer (Noordwijk: European Space Agency), 913-922.

Shangguan, Q., Shu, H., Li, P., Lin, K., Byrne, R. H., Li, Q., et al. (2019). Automated spectrophotometric determination of carbonate ion concentration in seawater using a portable syringe pump based analyzer. Mar. Chem. 209, 120-127. doi: 10.1016/j.marchem.2019.01.007

Sholkovitz, E. R., and Sedwick, P. N. (2006). Open-ocean deployment of a buoymounted aerosol sampler on the Bermuda Testbed Mooring: aerosol iron and sea salt over the Sargasso Sea. Deep Sea Res. 53, 547-560. doi: 10.1016/j.dsr.2005. 12.002

Smith, S. R., Bourassa, M. A., Bradley, E. F., Cosca, C., Fairall, C. W., Goni, G. J., et al. (2010). "Automated underway oceanic and atmospheric measurements from ships," in Proceedings of the "OceanObs'09: Sustained Ocean Observations and Information for Society, Venice, eds J. Hall, D. E. Harrison, and D. Stammer (Venice: ESA Publication), 21-25. doi: 10.5270/OceanObs09.cwp.82

Smith, S. R., Briggs, K., Bourassa, M. A., Elya, J., and Paver, C. (2018). Shipboard automated meteorological and oceanographic system data archive: 2005-2017. Geosci. Data J. 5, 73-86. doi: 10.1002/gdj3.59

Smith, S. R., Briggs, K., Lopez, N., and Kourafalou, V. (2016a). Numerical model evaluation using automated underway ship observations. J. Atmos. Ocean. Technol. 33, 409-428. doi: 10.1175/JTECH-D-15-0052.1

Smith, S. R., Lopez, N., and Bourassa, M. A. (2016b). SAMOS air-sea fluxes: 2005-2014. Geosci. Data J. 3, 9-19. doi: 10.1002/gdj3.34

Smith, S. R., Legler, D. M., and Verzone, K. V. (2001). Quantifying uncertainties in NCEP reanalyses using high quality research vessel observations. J. Clim. 14, 4062-4072. doi: 10.1175/1520-0442(2001)014<4062:quinru>2.0.co;2

Stern, H., and Davidson, N. E. (2015). Trends in the skill of weather prediction at lead times of 1-14 days. Q. J. R. Meteorol. Soc. 141, 2726-2736. doi: 10.1002/qj. 2559

Storto, A., Masina, S., and Navarra, A. (2016). Evaluation of the CMCC eddypermitting global ocean physical reanalysis system (C-GLORS, 1982-2012) and its assimilation components. Q. J. R. Meteorol. Soc. 142, 738-758. doi: 10.1002/ qj. 2673

Swart, S., Gille, S. T., Delille, B., Josey, S., Mazloff, M., Newman, L., et al. (2019). Constraining southern ocean air-sea fluxes through enhanced observations. Front. Mar. Sci. 6:421. doi: 10.3389/fmars.2019.00421

Swart, S., Zietsman, J. J., Coetzee, J., Goslett, D. G., Hoek, A., Needham, D., et al. (2016). Ocean robotics in support of fisheries research and management. Afr. J. Mar. Sci. 38, 525-538. doi: 10.2989/1814232X.2016.1251971

Talley, L. D., Feely, R. A., Sloyan, B. M., Wanninkhof, R., Baringer, M. O., Bullister, J. L., et al. (2016). Changes in ocean heat, carbon content, and ventilation: a review of the first decade of GO-SHIP Global Repeat Hydrography. Annu. Rev. Mar. Sci. 8, 185-215. doi: 10.1146/annurev-marine-052915-100829

Terray, L., Corre, L., Cravatte, S., Delcroix, T., Reverdin, G., and Ribes, A. (2012). Near-surface salinity as nature's rain gauge to detect human influence on the tropical water cycle. J. Clim. 2, 958-977. doi: 10.1175/JCLI-D-10-05025.1

Thomson, J., and Girton, J. (2017). Sustained measurements of Southern Ocean air-sea coupling from a wave glider autonomous surface vehicle. Oceanography 30, 104-109. doi: 10.5670/oceanog.2017.228

Thorne, P. W., Diamond, H. J., Goodison, B., Harrigan, S., Hausfather, Z., Ingleby, N. B., et al. (2018). Towards a global land surface climate fiducial reference measurements network. Int. J. Climatol. 38, 2760-2774. doi: 10.1002/joc. 5458

Tomczak, M. (1995). Salinity variability in the surface layer of the tropical western Pacific Ocean. J. Geophys. Res. 100, 20499-20515. doi: 10.1029/95JC01544

United States Coast Guard [USCG] (2017). Marine Board's Report, Steam Ship El Faro (O.N. 561732), sinking and loss of the vessel with 33 persons missing and presumed deceased northeast of Acklins and Crooked Island, Bahamas on October 1, 2015. Available at: https://media.defense.gov/2017/Oct/01/ 2001820187/-1/-1/0/FINAL\%20PDF\%20ROI\%2024\%20SEP\%2017.PDF (accessed July 24, 2018).

von Schuckmann, K., Le Traon, P.-Y., Smith, N., Pascual, A., Brasseur, P., Fennel, K., et al. (2018). Copernicus marine service ocean state report. J. Oper. Oceanogr. 11, S1-S142. doi: 10.1080/1755876X.2018.1489208 
Wanninkhof, R., Pickers, P. A., Omar, A. M., Sutton, A. J., Murata, A., Olsen, A., et al. (2019). A surface ocean $\mathrm{CO}_{2}$ reference network, SOCONET and associated marine boundary layer $\mathrm{CO}_{2}$ measurements. Front. Mar. Sci. 6:400. doi: 10.3389/fmars.2019.00400

Watson, A. J., Schuster, U., Bakker, D. C. E., Bates, N. R., Corbiere, A. Gonzalez-Davila, M., et al. (2009). Tracking the variable North Atlantic sink for atmospheric CO2. Science 326, 1391-1393. doi: 10.1126/science.11 77394

Weller, R. A., Bradley, E. F., Edson, J. B., Fairall, C. W., Brooks, I., Yelland, M. J., et al. (2008). Sensors for physical fluxes at the sea surface: energy, heat, water, salt. Ocean Sci. 4, 247-263. doi: 10.5194/os-4-2472008

WMO (1995). "Resolution 40 (Cg-XII): WMO policy and practice for the exchange of meteorological and related data and products including guidelines on relationships in commercial activities," in Proceedings of the Twelfth World Meteorological Congress, (Geneva: WMO).

WMO (2017). WIGOS Metadata Standard. Geneva: WMO.

Woodruff, S. D., Diaz, H. F., Worley, S. J., Reynolds, R. W., and Lubker, S. J. (2005). Early ship observational data and ICOADS. Clim. Change 73, 169-194. doi: 10.1007/s10584-005-3456-3

Woodruff, S. D., Worley, S. J., Lubker, S. J., Ji, Z., Freeman, E. J., Berry, D. I., et al. (2011). ICOADS Release 2.5: extensions and enhancements to the surface marine meteorological archive. Int. J. Climatol. 31, 951-967. doi: 10.1002/joc. 2103
Wunsch, C. (1978). The North Atlantic general circulation west of $50^{\circ} \mathrm{W}$ determined by inverse methods. Rev. Geophys. 16, 583-620. doi: 10.1029/ RG016i004p00583

Yokouchi, Y., Nojiri, Y., Toom-Sauntry, D., Fraser, P., Inuzuka, Y., Tanimoto, H., et al. (2012). Long-term variation of atmospheric methyl iodide and its link to global environmental change. Geophys. Res. Lett. 39:L23805. doi: 10.1029/ 2012GL053695

Conflict of Interest Statement: EF was employed by ERT, Inc, as a contractor for the National Centers for Environmental Information.

The remaining authors declare that the research was conducted in the absence of any commercial or financial relationships that could be construed as a potential conflict of interest.

Copyright (C) 2019 Smith, Alory, Andersson, Asher, Baker, Berry, Drushka, Figurskey, Freeman, Holthus, Jickells, Kleta, Kent, Kolodziejczyk, Kramp, Loh, Poli, Schuster, Steventon, Swart, Tarasova, de la Villéon and Vinogradova-Shiffer. This is an openaccess article distributed under the terms of the Creative Commons Attribution License (CC BY). The use, distribution or reproduction in other forums is permitted, provided the original author(s) and the copyright owner(s) are credited and that the original publication in this journal is cited, in accordance with accepted academic practice. No use, distribution or reproduction is permitted which does not comply with these terms. 


\section{APPENDIX}

Discipline-specific terminology and a range of organizations, projects, and programs are identified in the manuscript using acronyms. Table A1 lists the acronyms and provides universal resource locaters (when available) to provide the reader with easy access to more information associated with each acronym.

TABLE A1 | Definitions and universal resource locators (when applicable) for acronyms used in this manuscript.

\begin{tabular}{|c|c|c|}
\hline Acronym & Full Name & Universal Resource Locator \\
\hline BUFR & $\begin{array}{l}\text { Binary Universal Form for the Representation of } \\
\text { meteorological data }\end{array}$ & https://www.wmo.int/pages/prog/www/WDM/Guides/Guide-binary-1A.html \\
\hline ECV & Essential Climate Variable & https://gcos.wmo.int/en/essential-climate-variables \\
\hline EOV & Essential Ocean Variable & http://www.goosocean.org/index.php?option=com_content\&view=article\&id=14\&ltemid=114 \\
\hline GAW & Global Atmosphere Watch & http://www.wmo.int/pages/prog/arep/gaw/gaw_home_en.html \\
\hline GCOS & Global Climate Observing System & https://gcos.wmo.int/en/home \\
\hline GLODAP & Global Ocean Data Analysis Project & https://www.glodap.info/ \\
\hline GOOS & Global Ocean Observing System & http://www.goosocean.org/ \\
\hline GOSUD & Global Ocean Surface Underway Data project & http://www.gosud.org/ \\
\hline GTS & Global Telecommunication System & https://public.wmo.int/en/programmes/global-telecommunication-system \\
\hline ICOADS & $\begin{array}{l}\text { International Comprehensive Ocean-Atmosphere } \\
\text { Data Set }\end{array}$ & https://icoads.noaa.gov/ \\
\hline $\mathrm{IMO}$ & International Maritime Organization & http://www.imo.org/en/Pages/Default.aspx \\
\hline IOC & Intergovernmental Oceanographic Commission & http://www.ioc-unesco.org/ \\
\hline JCOMM & $\begin{array}{l}\text { Joint Technical Commission for Oceanography and } \\
\text { Marine Meteorology }\end{array}$ & https://www.jcomm.info/ \\
\hline JCOMMOPS & JCOMM Observation Programme Support Centre & https://www.jcommops.org/ \\
\hline MCDS & Marine Climate Data System & https://www.iode.org/index.php?option=com_content\&view=article\&id=389\&ltemid=100140 \\
\hline NMHS & National meteorological and hydrographic services & Varies by nation \\
\hline SAMOS & $\begin{array}{l}\text { Shipboard Automated Meteorological and } \\
\text { Oceanographic System initiative }\end{array}$ & http://samos.coaps.fsu.edu/html/ \\
\hline SOCAT & Surface Ocean $\mathrm{CO}_{2}$ Atlas & https://www.socat.info/ \\
\hline VOS & Voluntary Observing Ships scheme & https://www.wmo.int/pages/prog/amp/mmop/JCOMM/OPA/SOT/vos.html \\
\hline WIGOS & WMO Integrated Global Observing System & http://www.wmo.int/pages/prog/www/wigos/index_en.html \\
\hline WMO & World Meteorological Organization & https://public.wmo.int/en \\
\hline WOC & World Ocean Council & https://www.oceancouncil.org/ \\
\hline
\end{tabular}




\title{
Ocean Reanalyses: Recent Advances and Unsolved Challenges
}

\author{
Andrea Storto1,2*, Aida Alvera-Azcárate ${ }^{3}$, Magdalena A. Balmaseda $^{4}$, Alexander Barth $^{3}$, \\ Matthieu Chevallier ${ }^{5}$, Francois Counillon ${ }^{6}$, Catia M. Domingues ${ }^{7}$, Marie Drevillon $^{8}$, \\ Yann Drillet ${ }^{8}$, Gaël Forget ${ }^{9}$, Gilles Garric ${ }^{8}$, Keith Haines ${ }^{10,11}$, Fabrice Hernandez ${ }^{8,12}$, \\ Doroteaciro lovino ${ }^{1}$, Laura C. Jackson ${ }^{13}$, Jean-Michel Lellouche ${ }^{8}$, Simona Masina ${ }^{1}$, \\ Michael Mayer ${ }^{4,14}$, Peter R. Oke ${ }^{15}$, Stephen G. Penny ${ }^{16}$, K. Andrew Peterson ${ }^{17}$, \\ Chunxue Yang $^{18}$ and Hao Zuo ${ }^{4}$
}

\begin{abstract}
${ }^{1}$ Euro-Mediterranean Center on Climate Change (CMCC) Foundation, Bologna, Italy, ${ }^{2}$ Centre for Maritime Research and Experimentation (CMRE), La Spezia, Italy, ${ }^{3}$ GHER, University of Liège, Liège, Belgium, ${ }^{4}$ European Centre for Medium-Range Weather Forecasts (ECMWF), Reading, United Kingdom, ${ }^{5}$ Météo-France, Toulouse, France, ${ }^{6}$ Nansen Environmental and Remote Sensing Center (NERSC), Bergen, Norway, ${ }^{7}$ ACE CRC, CLEX, IMAS, University of Tasmania (UTAS), Hobart, TAS, Australia, ${ }^{8}$ Mercator Ocean, Ramonville-Saint-Agne, France, ${ }^{9}$ Massachusetts Institute of Technology (MIT), Cambridge, MA, United States, ${ }^{10}$ Department of Meteorology, University of Reading, Reading, United Kingdom, ${ }^{11}$ National Centre for Earth Observation (NCEO), Reading, United Kingdom, ${ }^{12}$ LEGOS, Institut de Recherche pour le Développement (IRD), Toulouse, France, ${ }^{13}$ Met Office, Exeter, United Kingdom, ${ }^{14}$ Department of Meteorology and Geophysics, University of Vienna, Vienna, Austria, ${ }^{15}$ Commonwealth Scientific and Industrial Research Organisation (CSIRO), Hobart, TAS, Australia, ${ }^{16}$ Department of Atmospheric and Oceanic Science, University of Maryland, College Park, College Park, MD, United States, ${ }^{17}$ Environmental Numerical Research Section, Environment and Climate Change Canada, Dorval, QC, Canada, ${ }^{18}$ Institute of Marine Sciences, National Research Council of Italy, Rome, Italy
\end{abstract}

OPEN ACCESS

Edited by:

Laura Lorenzoni,

University of South Florida, Tampa,

United States

Reviewed by:

Nadya Vinogradova Shiffer, National Aeronautics and Space Administration (NASA), United States Joaquin A. Trinanes, University of Santiago de Compostela, Spain

*Correspondence: Andrea Storto andrea.storto@cmre.nato.int

Specialty section:

This article was submitted to Ocean Observation,

a section of the journal

Frontiers in Marine Science

Received: 30 October 2018 Accepted: 05 July 2019 Published: 31 July 2019

Citation:

Storto A, Alvera-Azcárate A Balmaseda MA, Barth A, Chevallier M,

Counillon F, Domingues CM

Drevillon $M$, Drillet $Y$, Forget $G$,

Garric G, Haines K, Hernandez F, lovino $D$, Jackson LC, Lellouche J-M,

Masina S, Mayer M, Oke PR,

Penny SG, Peterson KA, Yang C and Zuo H (2019) Ocean Reanalyses: Recent Advances and Unsolved Challenges. Front. Mar. Sci. 6:418. doi: 10.3389/fmars.2019.00418

Ocean reanalyses combine ocean models, atmospheric forcing fluxes, and observations using data assimilation to give a four-dimensional description of the ocean. Metrics assessing their reliability have improved over time, allowing reanalyses to become an important tool in climate services that provide a more complete picture of the changing ocean to end users. Besides climate monitoring and research, ocean reanalyses are used to initialize sub-seasonal to multi-annual predictions, to support observational network monitoring, and to evaluate climate model simulations. These applications demand robust uncertainty estimates and fit-for-purpose assessments, achievable through sustained advances in data assimilation and coordinated inter-comparison activities. Ocean reanalyses face specific challenges: (i) dealing with intermittent or discontinued observing networks, (ii) reproducing inter-annual variability and trends of integrated diagnostics for climate monitoring, (iii) accounting for drift and bias due, e.g., to air-sea flux or ocean mixing errors, and (iv) optimizing initialization and improving performances during periods and in regions with sparse data. Other challenges such as multi-scale data assimilation to reconcile mesoscale and large-scale variability and flowdependent error characterization for rapidly evolving processes, are amplified in longterm reanalyses. The demand to extend reanalyses backward in time requires tackling all these challenges, especially in the emerging context of earth system reanalyses and coupled data assimilation. This mini-review aims at documenting recent advances from the ocean reanalysis community, discussing unsolved challenges that require sustained activities for maximizing the utility of ocean observations, supporting data rescue and advancing specific research and development requirements for reanalyses.

Keywords: data assimilation, uncertainty, inter-comparisons, observational requirements, historical reanalyses 


\section{INTRODUCTION: THE USEFULNESS OF REANALYSES}

Ocean and sea-ice reanalyses (ORAs, or ocean syntheses) are reconstructions of the ocean and sea-ice states using an ocean model integration constrained by atmospheric surface forcing, and ocean observations via a data assimilation method (e.g., Storto and Masina, 2016). Over the last decade, performance of ocean reanalyses has improved thanks to (a) increased model resolution (e.g., Zuo et al., 2018); (b) improved physics (e.g., Breivik et al., 2015); (c) better forcing from new atmospheric reanalyses (e.g., Dee et al., 2011); (d) more and improved quality-controlled observation data sets (e.g., Legeais et al., 2018); and (e) advances in data assimilation methods (e.g., Sakov et al., 2012; Storto et al., 2018b). This is exemplified by several works showing the increase of verification skill scores between successive versions of reanalyses (e.g., Lellouche et al., 2013, 2018; Storto et al., 2016; Zuo et al., 2017). Balmaseda et al. (2015) gave a detailed overview of the recent development of ocean reanalyses, with focus on the uncertainty level of climate signals. Ocean reanalyses have thus become an established tool for a variety of climate services and science-driven studies.

For instance, on-line tools examining multiple ocean reanalyses (e.g., the CREATE$^{1}$ initiative, Potter et al., 2018) enable immediate evaluation of model simulations such as the Coupled Model Inter-comparison Project (CMIP5). Visualization tools for near real-time reanalyses permit the monitoring of climate-key diagnostics, such as heat and salinity budgets in the Tropics (Xue et al., 2017) ${ }^{2}$.

Reanalyses are superior to objective analyses since, by construction, they provide increased and multi-variate dynamical consistency (e.g., Forget, 2010; Forget et al., 2015a; Evans et al., 2017; Fukumori et al., 2018) as compared to objective analyses (i.e., statistical processing of observations without model constraints, e.g., Johnson G.C. et al., 2018). One major advantage of reanalyses is their potential usefulness as a tool for studying important climate diagnostics that are only partly or indirectly observed. Examples include deep ocean variability (Balmaseda et al., 2013b), ocean transports (Duan et al., 2016), Atlantic Meridional Overturning Circulation (AMOC, e.g., Jackson et al., 2018), sea-ice thickness and drift (Massonnet et al., 2013), deep convection, and many more.

Dynamical ocean reanalysis have shown better capabilities than their statistical counterparts to assess observables where short timescale variability, or a changing or irregular observing network have large influences. Toyoda et al. (2017) showed that an ensemble of ocean reanalysis could outperform monthly mean statistical gridded products when validating monthly means of (shallow, especially summer time) mixed layer depths derived directly from original profile data, likely due to the reanalysis ability to more accurately simulate the short temporal timescale fluctuations the profiles measured (Figure 1, top panel). Similarly, Storto et al. (2017) showed the enhanced capabilities of

\footnotetext{
${ }^{1}$ Available at: https://esgf.nccs.nasa.gov/search/create-ip/

${ }^{2}$ Real-Time Ocean Reanalysis Inter-comparison Project (RT ORA-IP), available at: https://www.cpc.ncep.noaa.gov/products/GODAS/multiora93_body.html
}

ocean reanalyses over gridded statistical products to capture the inter-annual variability of extra-tropical steric sea level changes, particularly in the southern ocean, where a sparse observational network combined with small temporal and spatial scale eddy activity would have a heavy influence (Figure 1, bottom panel).

Appropriateness of using reanalyses for ocean transport investigations is particularly relevant to understand large to regional scale climate changes (Zuo et al., 2011; Vidar et al., 2016). Transports are mostly unobserved, unavailable from objective analyses and poorly reproduced by free-model running simulations. Reanalyses have been shown to reproduce the AMOC variability seen in the RAPID data at $26^{\circ} \mathrm{N}$ (Jackson et al., 2016, 2018; Masina et al., 2017), although these data are not assimilated. Reanalyses extending back prior to the deployment of the RAPID array may indicate longer term variability, although the changing observational coverage from distinct periods may affect results. Heat and volume transports through the Indonesian Throughflow, which are not continuously observed and are relevant to ENSO monitoring, are another excellent example where ocean reanalyses successfully reproduce anomalous events (Mayer et al., 2018). Monitoring multiannual heat, mass and freshwater transports at key Arctic ocean gateways by means of reanalyses has also successfully emerged in several recent studies (Bricaud et al., 2018; Garric et al., 2018; Uotila et al., 2018).

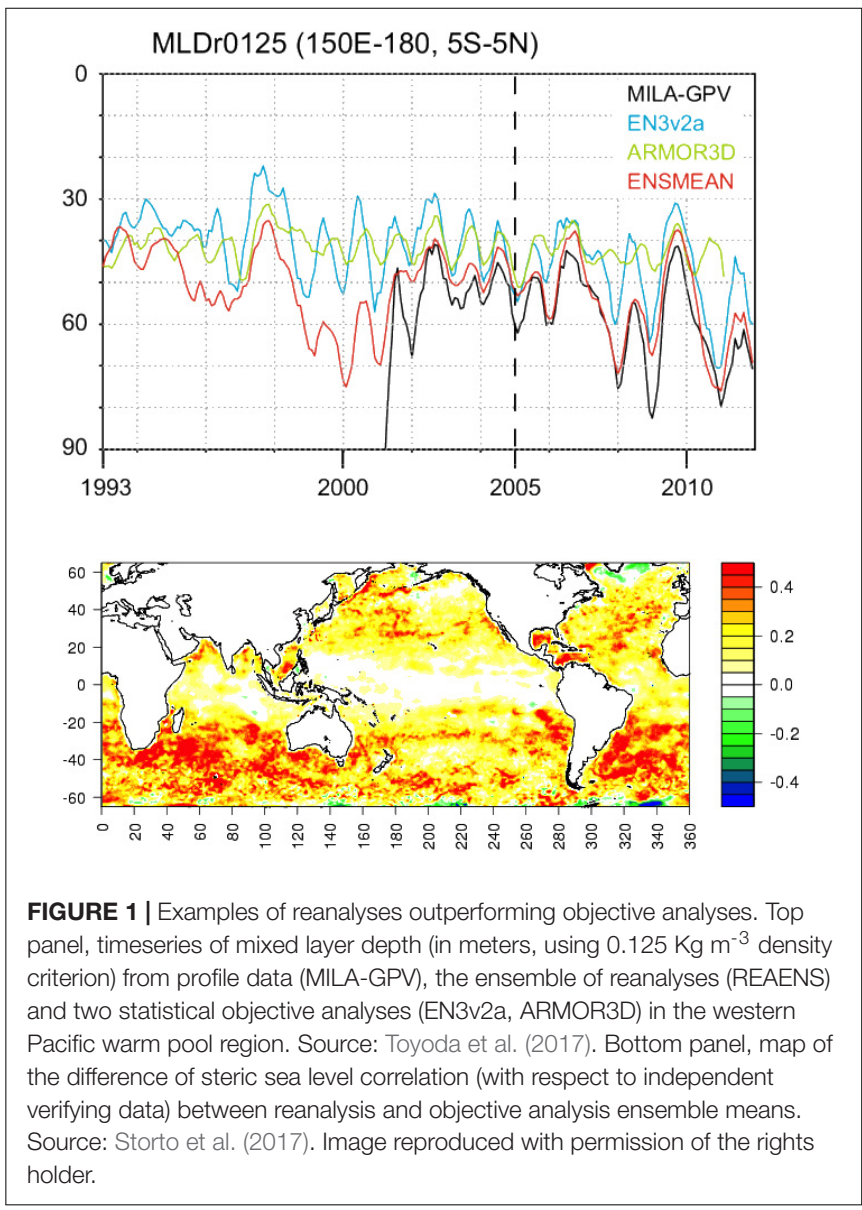


Reanalyses are also consistent integrators of the ocean dynamics, and there exist several studies that indicate the superiority of reanalyses with respect to model simulations and observation-only products, e.g., improving Mean Kinetic Energy at depth (1000 m) at low- and mid-latitudes (Storto et al., 2016); improving the near-surface Eddy Kinetic Energy (Masina et al., 2017), improving the statistics of the eddy population (Cipollone et al., 2017), and improving the dynamics of poorly observed regions (Koenig et al., 2017a).

Reanalyses that enforce strict budget closures such as ECCO (Estimating the Circulation and Climate of the Ocean) can be very informative for balance studies even though sometimes they may also underfit data (Forget and Ponte, 2015; Forget et al., 2015a). Reanalyses also avoid spurious high-frequency variability in global energy budgets, which is typically found in objective analyses (Trenberth et al., 2016).

In the cryosphere, sea-ice volume from the PIOMAS reanalysis fits well with independent validating data (Schweiger et al., 2011) although the PIOMAS data assimilation only ingests sea-ice concentration and sea surface temperature data. Melia et al. (2015) used the PIOMAS reanalysis to biascorrect climate model scenario predictions using both mean and interannual variability from the reanalysis, demonstrating a new use for such products.

One long-term application of ocean reanalyses has been for the initialization of ocean and sea ice components of seasonal (e.g., at Met Office, ECMWF, NCAR, GFDL, CMCC, MeteoFrance, etc.; e.g., Johnson S.J. et al., 2018), and more recently decadal, retrospective forecasts (e.g., Bellucci et al., 2011). The importance of the role of the ocean (e.g., Alessandri et al., 2010; Doblas-Reyes et al., 2013) and sea ice (e.g., Guemas et al., 2016) in initialization is indeed widely accepted. Despite the growing community interest, ocean reanalyses, and the ocean component of coupled reforecasts, are underutilized, largely due to the existing terrestrial emphasis of most of the derived products of these systems. Efforts are being made, for instance by the Copernicus Climate Change Service $\left(\mathrm{C}_{3} \mathrm{~S}^{3}\right)$ and Copernicus Marine Environment Monitoring Service $\left(\mathrm{CMEMS}^{4}\right)$, to release the ocean reanalysis component in a more timely manner, and to incorporate their information into climate monitoring reports (i.e., the Ocean State Report, von Schuckmann et al., 2018a,b, or the State of the Climate, Hartfield et al., 2018). A particular benefit of having the reanalysis of these systems readily available, is that this now leads to the ability to access error growth in the forecast components, leading to much better assessment of model forecast error and ultimately to bias correction and model improvement (e.g., Mulholland et al., 2016).

Global reanalyses also provide lateral boundary conditions for regional high-resolution simulations/reanalyses, which are found to significantly improve the local circulation compared to the use of, e.g., climatology (Barth et al., 2008). Additionally, reanalyses are extensively used to monitor the impact of past and present observing networks, thus helping the optimal maintenance and

\footnotetext{
${ }^{3}$ See product catalog at: https://climate.copernicus.eu/; https://cds.climate. copernicus.eu

${ }^{4}$ See product catalog at: http://marine.copernicus.eu/
}

the future design of the global ocean observation system (e.g., Fujii et al., 2019; Gasparin et al., 2019).

A relatively new application of reanalyses is the investigation of climate variability over centennial timescales by extending back throughout the 20th century and even further. Such historical ocean reanalyses (Giese et al., 2016; de Boisséson et al., 2017; Yang et al., 2017) can particularly provide initial conditions for more extensive decadal predictions. However, caution must be used with "20th century" ocean reanalyses because of the spurious variability resulting from the inhomogeneous observations, especially for ocean transport diagnostics (Yang et al., 2017). Historical ocean reanalyses rely on historical atmospheric reanalyses also extending back to the 19th century (e.g., 20CR, Compo et al., 2011), making the emerging field of historical coupled reanalyses (Laloyaux et al., 2018) doubly attractive to make use of the sparse observations.

\section{ASSESSING THE UNCERTAINTY OF OCEAN REANALYSES}

The quality of ocean reanalysis relies upon the accuracy of ocean models, forcing products, and observational data accuracy, namely both data and model errors contribute to the reanalysis uncertainty, as in any other data assimilation context. Model and forcing errors are reduced through assimilation of satellite and in situ data, and the availability of accurate observations, with reliable uncertainty estimates associated, is therefore crucial.

Unfortunately, the first satellite missions sensing the ocean surface (sea surface temperature and sea-ice concentration) are available only after 1979; high-precision altimetry missions sampling the sea surface height only began in 1992; in situ surface observations only increased when the Global Drifter Program became efficient, after the 1990s, and subsurface profile data from the Argo program achieved near-global coverage only around 2007 (Roemmich et al., 2019). This implies that ocean reanalyses are increasingly less reliable going further back in time. So, it is crucial to carefully assess their performance, particularly in light of the problems noted in the section "Introduction."

Estimating ocean reanalysis skill and uncertainty can be performed in several ways. First, validating the reanalyses against independent (non-assimilated) datasets, typically available from special campaigns at regional scale (e.g., Koenig et al., 2017b, for the Arctic Ocean), delayed time reprocessed data (e.g., drifterderived currents or cross-calibrated tide-gauge measurements) or by using multi-source data to evaluate complex frontal dynamics (Artana et al., 2018) and adopting popular skill score metrics (bias, root mean square error, etc.).

Second, probabilistic reanalyses based on ensemble data assimilation possibly maintained through perturbed forcing and physics, provide probability distribution functions of the ocean state. These also require that the dispersion of the limited-size ensemble also requires validation based on objective methods (Desroziers et al., 2005; Candille et al., 2015; Yan et al., 2015; Rodwell et al., 2016; Storto et al., 2018a).

Third, inter-comparison activities that assess reanalyses through evaluation of inter-system consistency provide valuable 
information. The spread of different reanalyses inform on the relative accuracy of the reanalyses for different ocean parameters. This approach has been performed by the CLIVAR/GSOP and GODAE communities, in particular during the recent ORAIP project (Balmaseda et al., 2015; and the Climate Dynamics Special Issue $\mathrm{e}^{5}$ ), followed by regional reanalysis inter-comparison assessments (e.g., the POLAR ORA-IP, Uotila et al., 2018).

An example is provided in Figure 2, which compiles findings from the ORA-IP project, showing the ensemble mean and spread of the reanalyses and reporting the Signal-to-Noise Ratio (SNR, see the figure caption for details) for several inter-compared parameters. The figure highlights how the net surface heat flux, the halosteric (hence salinity content) sea level, the thermosteric sea level below $700 \mathrm{~m}$ (hence heat content), and the sea-ice volume are much less constrained than sea surface temperature, $0-700 \mathrm{~m}$ thermosteric sea level and seaice concentration as indicated by the SNR values. CMEMS also provides multi-reanalysis assessments (e.g., Storto et al., 2018a) and a reanalysis ensemble mean product to end users, following a first attempt by Masina et al. (2017).

Inter-comparison exercises also provide detailed information on the deficiency of the current ocean observing network. For instance, Mignac et al. (2018) showed that the AMOC in the South Atlantic among several ocean reanalyses strongly disagree with each other, due to the discrepancies in the flow strength in the upper and deep western boundary currents. However, the interior gyre circulations showed much greater consistency between products. This shows the effectiveness of data assimilation in the basin interior but also highlights the difficulty of constraining the western boundary currents with the present observations of Argo and altimetry, and illustrates the requirement for a sustained coastal observing system (De Mey-Frémaux et al., 2019).

\section{CHALLENGES}

A number of specific challenges concern ocean reanalyses that are not necessarily shared with operational systems (Masina and Storto, 2017; Moore et al., 2019), because one of the fundamental goals of reanalyses is the reproduction of low-frequency (e.g., inter-annual) variability and trends. This is conceptually linked with the fact that most reanalyses implement sequential data assimilation tools that correct the state of the ocean at high frequencies (typically daily), thus introducing an intrinsic jump of spatial and temporal scales (Tardif et al., 2015).

Additionally, discontinuous observing networks often lead to inhomogeneous reanalysis time-series. The sampling of in situ profiles is always intrinsically irregular in space and time, but this is particularly so during periods of expansion of observing networks such as XBTs (1970s) and Argo floats (2000s). This is still true today with the inhomogeneous vertical sampling that can lead to large and unrealistic drift in unobserved layers below $2000 \mathrm{~m}$ depth (Lellouche et al., 2018).

\footnotetext{
${ }^{5}$ Climate Dynamics Special Issue on "Ocean estimation from an ensemble of global ocean reanalysis," Volume 49, Issue 3, August 2017, available at: https: //link.springer.com/journal/382/49/3/page/1
}

At the surface new observation types are set to provide more complete global coverage, for instance Surface Water and Ocean Topography (SWOT) altimetry will provide sea level measurements reaching the sub-mesoscale, extended HF-radar systems can provide high-resolution surface current observations in the coastal areas, and a strengthened constellation of oceansensing satellites (e.g., from the Copernicus program) should increase dramatically in the next decades. Reanalyses will need to adapt their capabilities (i.e., resolution, data assimilation schemes) to the new observation sampling and types in order to still provide consistent climatic metrics.

Accounting for drift and bias due, e.g., to air-sea flux errors, inadequate model resolution and parameterizations is another non-trivial problem (Zuo et al., 2017). Model bias correction through observation-based (Balmaseda et al., 2007b; Storto et al., 2016), analysis increments-based (Dee, 2005; Canter et al., 2016), ensemble (Zuo et al., 2018), or adaptive (Lea et al., 2008) bias estimates, assimilation of additional climatological observations (Lellouche et al., 2018), on-line correction of atmospheric forcing and mixing terms (Stammer et al., 2004; Forget and Ponte, 2015; Forget et al., 2015a), assimilating anomalies instead of full-field observations (Weber et al., 2015; Counillon et al., 2016), are all bias mitigation methods which have been used in the last decade.

In data-sparse periods, the initialization of ocean reanalyses remains challenging, due to our intrinsic lack of knowledge about the ocean state at earlier times, and is acute in ocean reanalyses before the 1980s (see, e.g., de Boisséson et al., 2017). Inclusion of stochastic initialization (Zuo et al., 2018), point-wise linear regression from objective analyses (Lellouche et al., 2018), or adjoint methods resolving the entire reanalysis period at once (Wunsch and Heimbach, 2007; Forget et al., 2015a) are all possible solution methods that generally require additional computational costs. Other problems such as spurious vertical velocity fields caused by dynamical imbalance after data assimilation, are particularly detrimental to downstream applications such as biogeochemical reanalyses, where these imbalances propagate onto unrealistic distributions of nutrients (Forget et al., 2015b; Park et al., 2018), revealing consistency problems otherwise neglected.

In the context of high-resolution ocean reanalyses that approach eddying scales, additional challenging topics, derived by the increase of ocean model resolution, include among others (i) the coupling with tides and waves; (ii) dealing with mesoscale eddies and sharp gradients in properties; (iii) two-way nested analysis schemes (e.g., Barth et al., 2007); (iv) discontinuities at choke points or in key coastal areas; (v) downscaling of atmospheric forcing, and (vi) accuracy of bathymetry. All these aspects call for a close collaboration between the ocean modeling and the reanalysis communities. Furthermore the increasing demand for temporal and spatial resolution by end-users, for instance for ocean downscaling applications, translate into a big data challenge for high-resolution reanalyses, making the dissemination of fullresolution datasets prohibitively expensive with the current data infrastructure paradigms. 


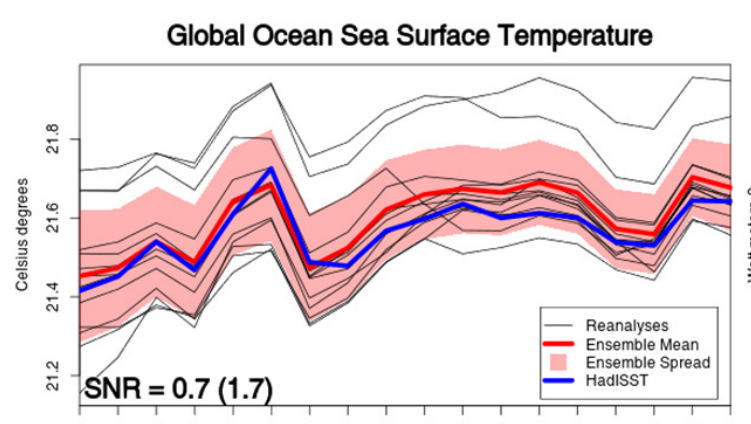

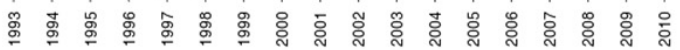

Global Ocean Thermosteric Sea Level (0-700m)

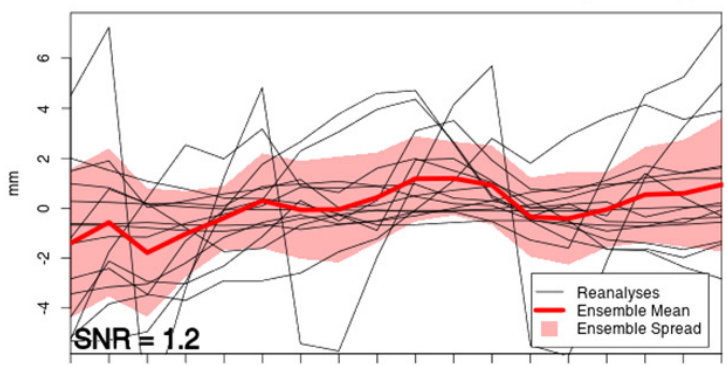

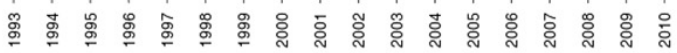

Global Ocean Thermosteric Sea Level (700-1500m)

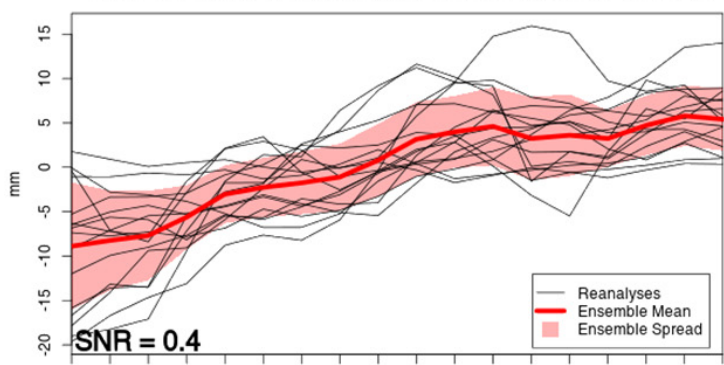

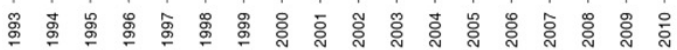

September Arctic Sea-ice Extent

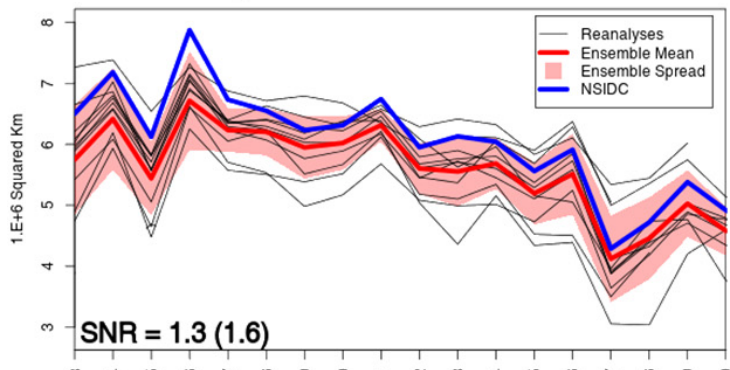

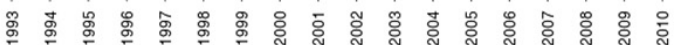

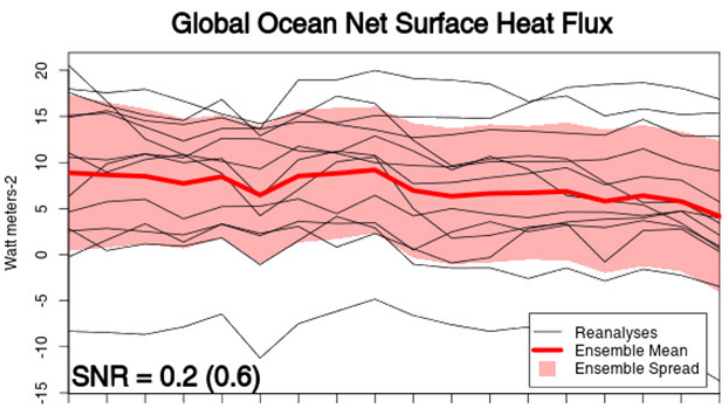

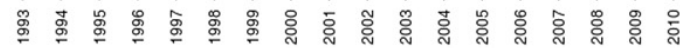

Global Ocean Halosteric Sea Level (0-700m)

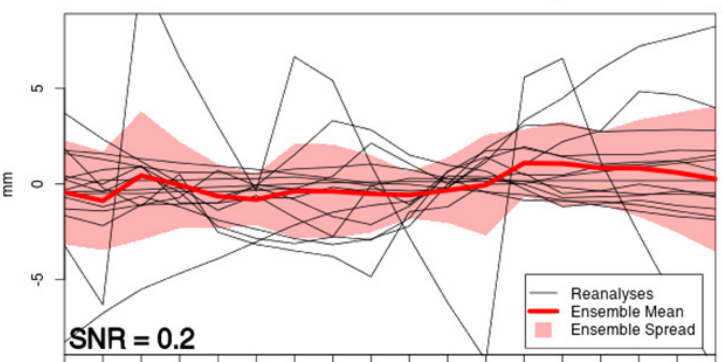

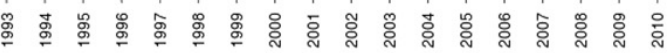

Global Ocean Halosteric Sea Level (700-1500m)

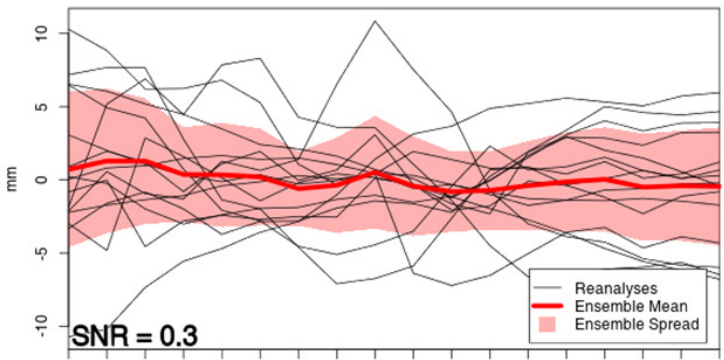

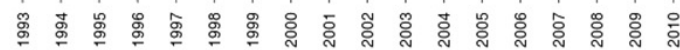

September Arctic Sea-ice Volume

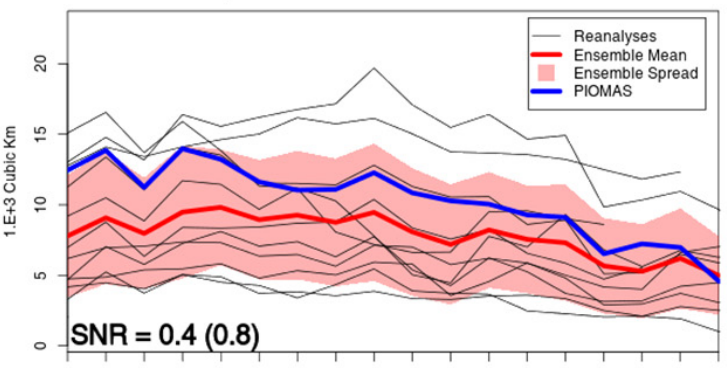

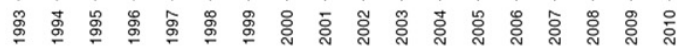

FIGURE 2 | Timeseries of global ocean sea surface temperature, net surface heat flux, thermo, and halo steric sea level (0-700 and 700-1500 m), September Arctic sea-ice extent and volume, from the ORA-IP inter-comparison project. Each plot shows the ensemble mean and standard deviation and the individual ocean reanalyses. Reference datasets are shown in blue for selected parameters. Each plot reports also the Signal-to-noise ratio (SNR), defined here for simplicity as the ratio between the temporal standard deviation of the ensemble mean divided by the temporal mean of the ensemble standard deviation. Numbers in parentheses refer to the SNR ratio computed from anomalies rather than full field data, except for thermo and halo steric sea level data that are already anomalies by construction. Adapted from Chevallier et al. (2017), Storto et al. (2017), and Valdivieso et al. (2017). Image reproduced with permission of the rights holder. 


\section{OBSERVATIONAL REQUIREMENTS}

The need for sustained, long-term global observations to support climate monitoring and forecast activities has long been recognized (Karl et al., 2010), as well as the complementary need for reliable and sophisticated pre-processing procedures (bias-correction, redundancy checks, quality control, etc.). The impact of different observing networks on the quality of ocean reanalyses and forecast systems has been assessed in several studies. Observational networks like Argo and altimetry satellite data are crucial to correctly resolve the vertical structure of the water column and the mesoscale ocean circulation, respectively (e.g., Oke et al., 2015; Shao et al., 2015; Zhang et al., 2017). Their availability therefore has a direct impact in the quality of ocean reanalyses (Balmaseda et al., 2007a, 2013). Tropical observing systems, like the TAO/TRITON, RAMA and PIRATA arrays, provide important information for the accuracy of ocean forecast systems (see Oke et al., 2015 and references therein). The recent degradation in the TAO/TRITON arrays had a significant detrimental impact on forecast skill in the tropical Pacific (Fujii et al., 2015), showing that it is critical to quickly address such degradations.

Although the number of oceanic observations has increased in recent decades, high-quality observations remain scarce in places, especially around and underneath the sea ice (Pellichero et al., 2017). While remotely sensed ice concentration has had good spatial coverage since 1979, the amount of in situ measurements of sea ice thickness (SIT) is very limited (Worby et al., 2008), and long-term and large-scale data sets do yet not exist. Nevertheless, improved SIT estimates are crucial to improve Arctic seasonal forecasts (Blockley and Peterson, 2018) and to reduce climate-model uncertainties (Lisæer et al., 2007; Mathiot et al., 2012; Melia et al., 2015). To improve model confidence in predicting polar sea ice conditions, satellite missions aiming at retrieving information on SIT (such as CryoSat2 and SMOS, and their combination, see Ricker et al., 2017) have been found to improve the performance of reanalyses in polar regions (Allard et al., 2018; Mu et al., 2018; Xie et al., 2018).

For global estimates of key climate parameters such as ocean heat content, the depths below $2000 \mathrm{~m}$ are currently unobserved, leading to obvious deficiencies of reanalyses in the representation of deep water characteristics and variability (Storto et al., 2017). Repeated observational transects such as GO-SHIP (Talley et al., 2016), as a follow-up to the WOCE sections (Macdonald, 1998), are fundamental for multi-decadal climate monitoring, but may be inadequate to constrain the deep ocean. For example, when numerous upper ocean observations are available, the use of unreliable vertical covariances in data assimilation may induce the wrong response at depth. There is hope that the developing deep Argo program (Zilberman, 2017) will gradually fill the gaps between the upper and deep ocean observing networks.

For both coupled and uncoupled reanalyses, observations of air-sea fluxes, at the moment available from a small number of dedicated ocean stations (e.g., Curry et al., 2004), appear crucial for understanding the coupled data assimilation paradigm and improving air-sea flux formulations. On top of all these observational requirements, independent research-quality validation datasets (e.g., research quality tide-gauge data, Caldwell et al., 2015) must be sustained and made available in standard format (e.g., ADCP data from vessel-mounted or fixed location instruments to validate currents from reanalyses) to ensure reliable estimation of reanalysis uncertainty. The same applies to the development of independent remote sensing capabilities, both from existing and new satellites, such as the use of Gravity Recovery and Climate Experiment (GRACE) data to verify steric sea level rise in ocean reanalyses (Storto et al., 2017), or the use of IceSat sea ice freeboard measurements to validate sea ice thickness in reanalyses (Chevallier et al., 2017; Uotila et al., 2018).

Finally, as important as the data availability itself and data rescue projects such as $\mathrm{GODAR}^{6}$, improvements to quality-control and bias adjustment procedures for reanalysis applications need to be maintained and supported. Efforts are on-going, testified by the recent release of the first interim version of the $\mathrm{IQuOD}^{7}$ temperature profile dataset (Domingues and Palmer, 2015) ${ }^{8}$.

\section{A FUTURE OUTLOOK}

Recent years have seen the pioneering production of Earth system reanalyses that simultaneously produce a dynamically consistent reconstruction of the atmosphere, ocean, land and seaice, although with varying complexity in the data assimilation coupling (e.g., Penny et al., 2017). These products are preliminary but provide promising results (e.g., Feng et al., 2018) and will likely be developed much further in the coming decade. Earth system reanalyses can potentially improve the representation of budgets (Mayer et al., 2017), although propagation and amplification of coupled biases and errors may occur (Zhang et al., 2013). The role of (uncoupled) ocean reanalyses is expected to focus on the added value of eddying resolution (tackling eddy energetics, including tides at global scale, and improving the representation and the impact of coastal and shallow water processes on the global ocean), as well as consolidating its role as a validation tool for assessing the ocean model component of Earth system simulations. Emerging multi-scale data assimilation schemes (e.g., Miyoshi and Kondo, 2013) may further reconcile the mesoscale and large-scale circulation variability, whose simultaneous correction is generally lacking in the current state-of-the-art reanalyses. The increase in resolution also poses the challenge of data infrastructures capable of handling these large datasets; cloud computing services, new format standards, extraction services, etc., should all be investigated to facilitate access to high-resolution reanalysis datasets.

\footnotetext{
${ }^{6}$ Global Oceanographic Data Archaeology and Rescue (GODAR), Information available at: https://www.nodc.noaa.gov/about/international_godar.html

${ }^{7}$ Data available at: https://data.nodc.noaa.gov/cgi-bin/iso?id=gov.noaa.nodc: IQuODv0.1

${ }^{8}$ www.iquod.org
} 
Flow-dependent error characterization for both rapidly evolving processes (marine weather) and low frequency processes (climate modes, changes in observational sampling, etc.) will likely become an important component of both long- and shortterm ocean reanalyses, inheriting the positive experience from atmospheric reanalyses (Poli et al., 2013). In particular, the importance of flow-dependent ensemble based data assimilation methods, compared to prescribed error implementations, has recently been demonstrated at global scales by Penny et al. (2015) and Storto et al. (2018b), and regionally for sub-polar gyre variability (Counillon et al., 2016), and also for the assimilation of sea ice (Sakov et al., 2012; Barth et al., 2015; Kimmritz et al., 2018).

Changes in the observing network expected over the next decade will offer reanalyses the possibility to integrate a larger number of heterogeneous observing networks. Deep Argo, surface salinity, high resolution altimetry, gravimetry, coastal observing networks, together with conventional profile data, may all be ingested to improve the representation of heat, mass and freshwater budgets in uncoupled and coupled reanalyses.

Finally data rescue programs also increase the appetite for the backward extension of ocean reanalyses, but need to be complemented with realistic error estimates and sophisticated bias- and drift- aware data assimilation methods that currently lack sufficient robustness. Enhancing the ocean observing network will also enable better bias estimates to be developed and used. This appears particularly relevant in light of new projects aiming at producing millennial timescale reanalyses from multi-proxy data (e.g., PAGES2k Consortium, 2017).

\section{REFERENCES}

Alessandri, A., Borrelli, A., Masina, S., Cherchi, A., Gualdi, S., Navarra, A., et al. (2010). The INGV-CMCC seasonal prediction system: improved ocean initial conditions. Mon. Wea. Rev. 138, 2930-2952. doi: 10.1175/2010MWR3178.1

Allard, R. A., Farrell, S. L., Hebert, D. A., Johnston, W. F., Li, L., Kurtz, N. T., et al. (2018). Utilizing CryoSat-2 sea ice thickness to initialize a coupled iceocean modeling system. Adv. Space Res. 62, 1265-1280. doi: 10.1016/j.asr.2017. 12.030

Artana, C., Lellouche, J.-M., Park, Y.-H., Garric, G., Koenig, Z., Sennéchael, N., et al. (2018). Fronts of the Malvinas current system: surface and subsurface expressions revealed by satellite altimetry, Argo floats, and Mercator operational model outputs. J. Geophys. Res. Oceans 123, 5261-5285. doi: 10. 1029/2018JC013887

Balmaseda, M. A., Dee, D., Vidard, A., and Anderson, D. L. (2007b). A multivariate treatment of bias for sequential data assimilation: application to the tropical oceans. Q.J. R. Meteorol. Soc. 133, 167-179. doi: 10.1002/qj.12

Balmaseda, M., Anderson, D., and Vidard, A. (2007a). Impact of Argo on analyses of the global ocean. Geophys. Res. Lett. 34:L16605. doi: 10.1029/2007GL030452

Balmaseda, M. A., Hernandez, A., Storto, M. D., Palmer, O., Alves, L., and Shi, G. C. (2015). The ocean reanalyses intercomparison project (ORA-IP). J. Operat. Oceanogr. 8(Suppl. 1), s80-s97. doi: 10.1080/1755876X.2015.1022329

Balmaseda, M. A., Trenberth, K. E., and Källén, E. (2013b). Distinctive climate signals in reanalysis of global ocean heat content. Geophys. Res. Lett. 40, 1754-1759. doi: 10.1002/grl.50382

Balmaseda, M. A., Mogensen, K., and Weaver, A. T. (2013). Evaluation of the ECMWF ocean reanalysis system ORAS4. Q. J. R. Meteorol. Soc. 139, 1132-1161. doi: 10.1002/qj.2063

\section{AUTHOR CONTRIBUTIONS}

AS coordinated the individual contributions and compiled them into the manuscript. All co-authors contributed to the text by proposing the text, points of discussion, and references, and provided the corrections to a preliminary version of the manuscript.

\section{FUNDING}

We are grateful to the "Evaluation of Ocean Synthesis (EOS)" COST Action ES1402, which provided support for visiting research periods and networking opportunities contributing to some of the cited ocean reanalysis inter-comparison works. CLIVAR through the Global Synthesis and Observations Panel (GSOP) has also provided support for the reanalysis intercomparison and assessment activities. CMD was funded by the Australian Research Council (FT130101532 and DP160103130); the Scientific Committee on Oceanic Research (SCOR) Working Group 148, funded by national SCOR committees and a grant to SCOR from the U.S. National Science Foundation (Grant OCE-1546580); and the Intergovernmental Oceanographic Commission of UNESCO/International Oceanographic Data and Information Exchange (IOC/IODE) IQuOD Steering Group.

\section{ACKNOWLEDGMENTS}

We thank the two reviewers for their valuable help in improving the quality of the manuscript.

Barth, A., Alvera-Azcárate, A., Beckers, J.-M., Rixen, M., and Vandenbulcke, L. (2007). Multigrid state vector for data assimilation in a two-way nested model of the Ligurian Sea. J. f Mar. Syst. 65, 41-59. doi: 10.1016/j.jmarsys.2005.07.006

Barth, A., Alvera-Azcárate, A., and Weisberg, R. H. (2008). Benefit of nesting a regional model into a large-scale ocean model instead of climatology. Application to the West Florida Shelf. Cont. ShelfRes. 28, 561-573. doi: 10.1016/ j.csr.2007.11.004

Barth, A., Canter, M., Van Schaeybroeck, B., Vannitsem, S., Massonnet, F., Zunz, V., et al. (2015). Assimilation of sea surface temperature, sea ice concentration and sea ice drift in a model of the Southern Ocean. Ocean Model. 93, 22-39. doi: 10.1016/j.ocemod.2015.07.011

Bellucci, A., Gualdi, S., Masina, S., Storto, A., Scoccimarro, E., Cagnazzo, C., et al. (2011). Decadal climate predictions with a coupled OAGCM initialized with oceanic reanalyses. Clim. Dyn. 40, 1483-1497. doi: 10.1007/s00382-0121468-z

Blockley, E. W., and Peterson, K. A. (2018). Improving Met Office seasonal predictions of Arctic sea ice using assimilation of CryoSat-2 thickness. Cryosphere 12, 3419-3438. doi: 10.5194/tc-12-3419-201

Breivik, $\varnothing$, Mogensen, K. J., Bidlot, R., Balmaseda, M. A., and Janssen, P. A. (2015). Surface wave effects in the NEMO ocean model: forced and coupled experiments. J. Geophys. Res. Oceans 120, 2973-2992. doi: 10.1002/ 2014JC010565

Bricaud, C., Garric, G., Drillet, Y., Zuo, H., Storto, A., Peterson, K. A., et al. (2018). The copernicus marine environment monitoring service ocean state report. J. Operat. Oceanogr. 11(Suppl. 1), S49-S53. doi: 10.1080/1755876X.2018.1489208

Caldwell, P. C., Merrifield, M. A., and Thompson, P. R. (2015). Sea Level Measured by Tide Gauges from Global Oceans - the Joint Archive for Sea Level holdings 
(NCEI Accession 0019568), Version 5.5. Silver Spring, MD: NOAA. doi: 10.7289/ V5V40S7W

Candille, G., Brankart, J.-M., and Brasseur, P. (2015). Assessment of an ensemble system that assimilates Jason-1/Envisat altimeter data in a probabilistic model of the North Atlantic ocean circulation. Ocean Sci. 11, 425-438. doi: 10.5194/ os-11-425-2015

Canter, M., Barth, A., and Beckers, J.-M. (2016). Correcting circulation biases in a lower-resolution global general circulation model with data assimilation. Ocean Dyn. 67, 1-18. doi: 10.1007/s10236-016-1022-3

Chevallier, M., Smith, G. C., Dupont, F., Lemieux, J.-F., Forget, G., and Fujii, Y. (2017). Intercomparison of the Arctic sea ice cover in global ocean-sea ice reanalyses from the ORA-IP project. Clim. Dyn. 49, 1107-1136. doi: 10.1007/ s00382-016-2985-y

Cipollone, A., Masina, S., Storto, A., and Iovino, D. (2017). Benchmarking the mesoscale variability in global ocean eddy-permitting numerical systems. Ocean Dyn. 67, 1313-1333. doi: 10.1007/s10236-017-1089-5

Compo, G. P., Whitaker, J. S., Sardeshmukh, D. P., Matsui, N., Allan, R. J., and Yin, X. (2011). The Twentieth Century reanalysis project. Q. J. R. Meteorol. Soc. 137, 1-28. doi: $10.1002 /$ qj.776

Counillon, F., Keenlyside, N., Bethke, I., Wang, Y., Billeau, S., Shen, M. L., et al. (2016). Flow-dependent assimilation of sea surface temperature in isopycnal coordinates with the Norwegian climate prediction model. Tellus A 68, 1-17. doi: 10.3402/tellusa.v68.32437

Curry, J. A., Bentamy, M. A., Bourassa, A., Bourras, D., Bradley, E. F., and Brunke, M. (2004). SEAFLUX. Bull. Am. Meteor. Soc. 85, 409-424. doi: 10.1175/BAMS85-3-409

de Boisséson, E., Balmaseda, M. A., and Mayer, M. (2017). Ocean heat content variability in an ensemble of twentieth century ocean reanalyses. Clim. Dyn. 50, 3783-3798. doi: 10.1007/s00382-017-3845-0

De Mey-Frémaux, P., Ayoub, N., Barth, A., Brewin, R., Charria, G., Campuzano, F., et al. (2019). Model-observations synergy in the coastal ocean. Front. Mar. Sci. 6:436. doi: 10.3389/fmars.2019.00436

Dee, D. P. (2005). Bias and data assimilation. Q. J. R. Meteorol. Soc. 131, 3323-3343. doi: 10.1256/qj.05.137

Dee, D. P., Uppala, S. M., Simmons, A. J., Berrisford, P., Poli, P., and Kobayashi, S. (2011). The ERA-Interim reanalysis: configuration and performance of the data assimilation system. Q. J. R. Meteorol. Soc. 137, 553-597. doi: 10.1002/ qj. 828

Desroziers, G., Berre, L., Chapnik, B., and Poli, P. (2005). Diagnosis of observation, background and analysis-error statistics in observation space. Q. J. R. Meteorol. Soc. 131, 3385-3396. doi: 10.1256/qj.05.108

Doblas-Reyes, F. J., García-Serrano, J., Lienert, F., Biescas, A. P., and Rodrigues, L. R. L. (2013). Seasonal climate predictability and forecasting: status and prospects. WIREs Clim. Change 4, 245-268. doi: 10.1002/wcc.217

Domingues, C., and Palmer, M. (2015). The IQuOD initiative: towards an international quality controlled ocean database. Clivar. Exchanges 19, 38-40.

Duan, Y., Liu, H., Yu, W., and Hou, Y. (2016). The mean properties and variations of the Southern Hemisphere subpolar gyres estimated by simple ocean data assimilation (SODA) products. Acta Oceanol. Sin. 3, 8-13. doi: 10.1007/s13131016-0901-2

Evans, D. G., Toole, J., Forget, G., Zika, J. D., Naveira Garabato, A. C., Nurser, A. G., et al. (2017). Recent wind-driven variability in atlantic water mass distribution and meridional overturning circulation. J. Phys. Oceanogr. 47, 633-647. doi: 10.1175/JPO-D-16-0089.1

Feng, X., Haines, K., Liu, C., de Boisséson, E., and Polo, I. (2018). Improved SST-precipitation intraseasonal relationships in the ECMWF coupled climate reanalysis. Geophys. Res. Lett. 45, 3664-3672. doi: 10.1029/2018GL0 77138

Forget, G. (2010). Mapping Ocean Observations in a dynamical framework: a 2004-06 Ocean Atlas. J. Phys. Oceanogr. 40, 1201-1221. doi: 10.1175/ 2009JPO4043.1

Forget, G., Campin, J.-M., Heimbach, P., Hill, C. N., Ponte, R. M., et al. (2015a). ECCO version 4: an integrated framework for non-linear inverse modeling and global ocean state estimation. Geosci. Model Dev. 8, 3071-3104. doi: 10.5194/ gmd-8-3071-2015

Forget, G., Ferreira, D., and Liang, X. (2015b). On the observability of turbulent transport rates by Argo: supporting evidence from an inversion experiment. Ocean Sci. 11, 839-853. doi: 10.5194/os-11-839-2015
Forget, G., and Ponte, R. M. (2015). The partition of regional sea level variability. Prog. Oceanogr. 137, 173-195. doi: 10.1016/ j.pocean.2015.06.002 doi: 10.1016/ j.pocean.2015.06.002

Fujii, Y., Cummings, J., Xue, Y., Schiller, A., Lee, T., and Balmaseda, M. A. (2015). Evaluation of the Tropical Pacific Observing System from the ocean data assimilation perspective. Q. J. R. Meteorol. Soc. 141, 2481-2496. doi: 10.1002/qj. 2579

Fujii, Y., Remy, E., Remy, H., Oke, P. R., Halliwell, G. R., Gasparin, F., et al. (2019). Observing system evaluation based on ocean data assimilation and prediction systems: on-going challenges and future vision for designing/supporting ocean observational networks. Front. Mar. Sci. 6:417. doi: 10.3389/fmars.2019.00417

Fukumori, I., Heimbach, P., Ponte, R. M., and Wunsch, C. (2018). A dynamically consistent, multi-variable ocean climatology. Bull. Amer. Meteor. Soc. 99, 21072128. doi: 10.1175/bams-d-17-0213.1

Garric, G., Hernandez, O., Bricaud, C., Storto, A., Peterson, K. A., and Zuo, H. (2018). Arctic Ocean freshwater content, in: the copernicus marine environment monitoring service ocean state report, issue 2. J. Operat. Oceanogr. 11(Suppl. 1), S70-S78. doi: 10.1080/1755876X.2018.1489208

Gasparin, F., Guinehut, S., Mao, C., Mirouze, I., Rémy, E., King, R. R., et al. (2019). Requirements for an Integrated in situ Atlantic ocean observing system from coordinated observing system simulation experiments. Front. Mar. Sci. 6:83. doi: $10.3389 /$ fmars.2019.00083

Giese, B. S., Seidel, H. F., Compo, G. P., and Sardeshmukh, P. D. (2016). An ensemble of ocean reanalyses for 1815-2013 with sparse observational input. J. Geophys. Res. Oceans 121, 6891-6910. doi: 10.1002/2016JC012079

Guemas, V., Blanchard-Wrigglesworth, E., Chevallier, M., Day, J. J., Déqué, M., Doblas-Reyes, F. J., et al. (2016). A review on Arctic sea-ice predictability and prediction on seasonal to decadal time-scales. Q. J. R. Meteorol. Soc. 142, 546-561. doi: 10.1002/qj.2401

Hartfield, G., Blunden, J., and Arndt, D. S. (2018). A Look at 2017: takeaway points from the State of the climate supplement. Bull. Am. Meteor. Soc. 99, 1527-1539. doi: 10.1175/BAMS-D-18-0173.1

Jackson, L., Dubois, C., Masina, S., Storto, A., and Zuo, H. (2018). Atlantic meridional overturning circulation. in copernicus marine service ocean state report, issue 2. J. Operat. Oceanogr. 11(Suppl.1), S65-S66. doi: 10.1080/ 1755876X.2018.1489208

Jackson, L. C., Peterson, K. A., Roberts, C. D., and Wood, R. A. (2016). Recent slowing of Atlantic overturning circulation as a recovery from earlier strengthening. Nat. Geosci. 9, 518-522. doi: 10.1038/ngeo2715

Johnson, G. C., Lyman, J. M., Boyer, T., Cheng, L., Domingues, C. M., Gilson, J., et al. (2018). Global oceans: ocean heat content. In state of the climate in 2017. Bull. Am. Meteorol. Soc. 99, S72-S77. doi: 10.1175/ 2018BAMSStateoftheClimate.1

Johnson, S. J., Stockdale, T. N., Ferranti, L., Balmaseda, M. A., Molteni, F., Magnusson, L., et al. (2018). SEAS5: the new ECMWF seasonal forecast system. Geosci. Model Dev. 12, 1087-1117. doi: 10.5194/gmd-2018-228

Karl, T. R., Diamond, H. J., Bojinski, S., Butler, J. H., Dolman, H., and Haeberli, W. (2010). Observation needs for climate information, prediction and application: capabilities of existing and future observing systems. Proc. Environ. Sci. 1, 192-205. doi: 10.1016/j.proenv.2010.09.013

Kimmritz, M., Counillon, F., Bitz, C. M., Massonnet, F., Bethke, I., and Gao, Y. (2018). Optimising assimilation of sea ice concentration in an Earth system model with a multicategory sea ice model. Tellus A 70, 1-23. doi: 10.1080/ 16000870.2018 .1435945

Koenig, Z., Provost, C., Sennéchael, N., Garric, G., and Gascard, J. C. (2017a). The Yermak pass branch: a major pathway for the Atlantic Water north of Svalbard? J. Geophys. Res. Oceans 122, 9332-9349. doi: 10.1002/2017JC013271

Koenig, Z., Provost, C., Villacieros-Robineau, N., Sennéchael, N., Meyer, A., Lellouche, J.-M., et al. (2017b). Atlantic Waters inflow north of Svalbard: insights from IAOOS observations and Mercator Ocean global operational system during N-ICE2015. JGR Oceans 122, 1254-1273. doi: 10.1002/ 2016JC012424

Laloyaux, P., de Boisseson, E., Balmaseda, M., Bidlot, J.-R., Broennimann, S., Buizza, R., et al. (2018). CERA-20C: a coupled reanalysis of the twentieth century. J. Adv. Model. Earth Syst. 10, 1172-1195. doi: 10.1029/2018MS001273

Lea, D. J., Drecourt, J., Haines, K., and Martin, M. J. (2008). Ocean altimeter assimilation with observational- and model-bias correction. Q. J. R. Meteorol. Soc. 134, 1761-1774. doi: 10.1002/qj.320 
Legeais, J. F., Ablain, M., Zawadzki, L., Zuo, H., Johannessen, J. A., and Scharffenberg, M. G. (2018). An improved and homogeneous altimeter sea level record from the ESA climate change initiative. Earth Syst. Sci. Data 10, 281-301. doi: 10.5194/essd-10-281-2018

Lellouche, J.-M., Drévillon, M., Régnier, C., Greiner, E., Garric, G., et al. (2013). Evaluation of global monitoring and forecasting systems at Mercator Océan. Ocean Sci. 9, 57-81. doi: 10.5194/os-9-57-2013

Lellouche, J.-M., Greiner, E., Le Galloudec, O., Garric, G., Regnier, C., Drevillon, M., et al. (2018). Recent updates on the copernicus marine service global ocean monitoring and forecasting real-time $1 / 12^{\circ}$ high resolution system. Ocean Sci. 14, 1093-1126. doi: 10.5194/os-14-1093-2018

Vidar, S. L., Solfrid, S. H., Skogen, M. D., Svendsen, E., Wehde, H., and Bertino, L. (2016). An Assessment of the added value from data assimilation on modelled Nordic Seas hydrography and ocean transports. Ocean Model. 99, 43-59. doi: 10.1016/j.ocemod.2015.12.010

Lisæter, K. A., Evensen, G., and Laxon, S. (2007). Assimilating synthetic CryoSat sea ice thickness in a coupled ice-ocean model. J. Geophys. Res. 112:C07023. doi: 10.1029/2006JC003786

Macdonald, A. M. (1998). The global ocean circulation: a hydrographic estimate and regional analysis. Prog. Oceanogr. 41, 281-382. doi: 10.1016/S00796611(98)00020-2

Masina, S., and Storto, A. (2017). Reconstructing the recent past ocean variability: status and perspective. J. Mar. Res. 75, 727-764. doi: 10.1357/ 002224017823523973

Masina, S., Storto, A., Ferry, N., Valdivieso, M., Haines, K., Balmaseda, M., et al. (2017). An ensemble of eddy-permitting global ocean reanalyses from the MyOcean project. Clim. Dyn. 49, 813-841. doi: 10.1007/s00382-015-2728-5

Massonnet, F., Mathiot, P., Fichefet, T., Goosse, H., König Beatty, C., Vancoppenolle, M., et al. (2013). A model reconstruction of the Antarctic sea ice thickness and volume changes over 1980-2008 using data assimilation. Ocean Model. 64, 67-75. doi: 10.1016/j.ocemod.2013.01.003

Mathiot, P., König Beatty, C., Fichefet, T., Goosse, H., Massonnet, F., and Vancoppenolle, M. (2012). Better constraints on the sea-ice state using global sea-ice data assimilation. Geosci. Model Dev. 5, 1501-1515. doi: 10.5194/gmd5-1501-2012

Mayer, M., Alonso Balmaseda, M., and Haimberger, L. (2018). Unprecedented 2015/2016 Indo-Pacific heat transfer speeds up tropical Pacific heat recharge. Geophys. Res. Lett. 45, 3274-3284. doi: 10.1002/2018GL077106

Mayer, M., Haimberger, L., Edwards, J. M., and Hyder, P. (2017). Toward consistent diagnostics of the coupled atmosphere and ocean energy budgets. J. Clim. 30, 9225-9246. doi: 10.1175/JCLI-D-17-0137.1

Melia, N., Haines, K., and Hawkins, E. (2015). Improved Arctic sea ice thickness projections using bias corrected CMIP5 simulations. Cryosphere 9, 2237-2251. doi: 10.5194/tc-9-2237-2015

Mignac, D., Ferreira, D., and Haines, K. (2018). South Atlantic meridional transports from NEMO-based simulations and reanalyses. Ocean Sci. 14, 53-68. doi: 10.5194/os-14-53-2018

Miyoshi, T., and Kondo, K. (2013). A multi-scale localization approach to an ensemble Kalman filter. SOLA 9, 170-173. doi: 10.2151/sola.2013-038

Moore, A. M., Martin, M. J., Akella, S., Arango, H. G., Balmaseda, M., Bertino, L., et al. (2019). Synthesis of ocean observations using data assimilation for operational, real-time and reanalysis systems: a more complete picture of the state of the ocean. Front. Mar. Sci. 6:90. doi: 10.3389/fmars.2019.00090

Mu, L., Yang, Q., Losch, M., Losa, S. N., Ricker, R., Nerger, L., et al. (2018). Improving sea ice thickness estimates by assimilating CryoSat-2 and SMOS sea ice thickness data simultaneously. Q. J. R. Meteorol. Soc. 144, 529-538. doi: $10.1002 /$ qj.3225

Mulholland, D. P., Haines, K., and Balmaseda, M. A. (2016). Improving seasonal forecasting through tropical ocean bias corrections. Q. J. R. Meteorol. Soc. 142, 2797-2807. doi: 10.1002/qj.2869

Oke, P. R., Larnicol, G., Fujii, Y., Smith, G. C., Lea, D. J., and Guinehut, S. (2015). Assessing the impact of observations on ocean forecasts and reanalyses: part 1, Global studies. J. Operat. Oceanogr. 8, s49-s62. doi: 10.1080/1755876X.2015. 1022067

PAGES2k Consortium (2017). A global multiproxy database for temperature reconstructions of the Common Era. Sci. Data 4:170088. doi: 10.1038/sdata. 2017.88
Park, J.-Y., Stock, C. A., Yang, X., Dunne, J. P., Rosati, A., John, J., et al. (2018). Modeling global ocean biogeochemistry with physical data assimilation: a pragmatic solution to the equatorial instability. J. Adv. Model. Earth Syst. 10, 891-906. doi: 10.1002/2017MS001223

Pellichero, V., Sallée, J. B., Schmidtko, S., Roquet, F., and Charrassin, J.B. (2017). The ocean mixed layer under Southern Ocean sea-ice: seasonal cycle and forcing. J. Geophys. Res. 122, 1608-1633. doi: 10.1002/2016jc0 11970

Penny, S. G., Akella, R., Sergey, F., Yosuke, F., Alicia, K., and Malaquias, P. (2017). Coupled Data Assimilation for Integrated Earth System Analysis and Prediction: Goals, Challenges, and Recommendations. World Weather Research Programme (WWRP) Report 2017-3. Geneva: World Meteorological Organization.

Penny, S. G., Behringer, W. D., Carton, J. A., and Kalnay, E. (2015). A hybrid global ocean data assimilation system at NCEP. Mon. Wea. Rev. 143, 4660-4677. doi: 10.1175/MWR-D-14-00376.1

Poli, P., Hersbach, H., Tan, D. G. H., Dee, D. P., Thépaut, J.-N., and Simmons, A. (2013). The data assimilation system and initial performance evaluation of the ECMWF pilot reanalysis of the 20th-century assimilating surface observations only (ERA-20C). ECMWF ERA Rep. 14:59.

Potter, G. L., Carriere, L., Hertz, J., Bosilovich, M., Duffy, D., Lee, T., et al. (2018). Enabling reanalysis research using the collaborative reanalysis technical environment (CREATE). Bull. Am. Meteor. Soc. 99, 677-687. doi: 10.1175/ BAMS-D-17-0174.1

Ricker, R., Hendricks, S., Kaleschke, L., Tian-Kunze, X., King, J., et al. (2017). A weekly Arctic sea-ice thickness data record from merged CryoSat-2 and SMOS satellite data. Cryosphere 11, 1607-1623. doi: 10.5194/tc-11-1607-2017

Rodwell, M. J., Lang, S. T. K., Ingleby, N. B., Bormann, N., Hólm, E., Rabier, F., et al. (2016). Reliability in ensemble data assimilation. Q. J. R. Meteorol. Soc. 142, 443-454. doi: 10.1002/qj.2663

Roemmich, D., Alford, M. H., Claustre, H., Johnson, K. S., King, B., Moum, J., et al. (2019). On the future of Argo: a global, full-depth, multi-disciplinary array. Front. Mar. Sci. (in press). doi: 10.3389/fmars.2019.00439

Sakov, P., Counillon, F., Bertino, L., Lister, K. A., Oke, P. R., and Korablev, A. (2012). TOPAZ4: an ocean-sea ice data assimilation system for the North Atlantic and Arctic. Ocean Sci. 8, 633-656. doi: 10.5194/os-8-6332012

Schweiger, A., Lindsay, R., Zhang, J., Steele, M., Stern, H., and Kwok, R. (2011). Uncertainty in modeled Arctic sea ice volume. J. Geophys. Res. 116:C00D06. doi: 10.1029/2011JC007084

Shao, C., Xuan, L., Cao, Y., Cui, X., and Gao, S. (2015). Impact of argo observation on the regional ocean reanalysis of China coastal waters and adjacent seas: a twin-experiment study. Adv. Meteorol. 2015:793825. doi: 10.1155/2015/793825

Stammer, D., Ueyoshi, K., Kohl, A., Large, W. G., Josey, S. A., and Wunsch, C. (2004). Estimating air-sea fluxes of heat, freshwater, and momentum through global ocean data assimilation. J. Geophys. Res. 109: C05023. doi: 10.1029/ 2003JC002082

Storto, A., and Masina, S. (2016). C-GLORSv5: an improved multipurpose global ocean eddy-permitting physical reanalysis. Earth Syst. Sci. Data 8, 679-696. doi: 10.5194/essd-8-679-2016

Storto, A., Masina, S., Balmaseda, M., Guinehut, S., Xue, Y., Szekely, T., et al. (2017). Steric sea level variability (1993-2010) in an ensemble of ocean reanalyses and objective analyses. Clim. Dyn. 49, 709-729. doi: 10.1007/s00382-015-2554-9

Storto, A., Masina, S., and Navarra, A. (2016). Evaluation of the CMCC eddypermitting global ocean physical reanalysis system (C-GLORS, 1982-2012) and its assimilation components. Q. J. R. Meteorol. Soc. 142, 738-758. doi: 10.1002/ qj. 2673

Storto, A., Oddo, P., Cipollone, A., Mirouze, I., and Lemieux-Dudon, B. (2018b). Extending an oceanographic variational scheme to allow for affordable hybrid and four-dimensional data assimilation. Ocean Model. 128, 67-86. doi: 10.1016/ j.ocemod.2018.06.005

Storto, A., Masina, S., Simoncelli, S., Iovino, D., Cipollone, A., Drevillon, M. et al. (2018a). The added value of the multi-system spread information for ocean heat content and steric sea level investigations in the CMEMS GREP ensemble reanalysis product. Clim. Dyn. 53, 287-312. doi: 10.1007/s00382-0184585-5

Talley, L. D., Feely, R. A., Sloyan, B. M., Wanninkhof, R., Baringer, M. O., and Bullister, J. L. (2016). Changes in ocean heat, carbon content, and ventilation: 
review of the first decade of global repeat hydrography (GO-SHIP). Ann. Rev. Mar. Sci. 8, 185-215. doi: 10.1146/annurev-marine-052915-100829

Tardif, R., Hakim, G. J., and Snyder, C. (2015). Coupled atmosphere-ocean data assimilation experiments with a low-order model and CMIP5 model data. Clim. Dyn. 45:1415. doi: 10.1007/s00382-014-2390-3

Toyoda, T., Fujii, Y., Kuragano, T., Masafumi, K., Yoichi, I., Shuhei, M., et al. (2017). Intercomparison and validation of the mixed layer depth fields of global ocean syntheses. Clim. Dyn. 49, 753-773. doi: 10.1007/s00382-0152637-7

Trenberth, K. E., Fasullo, J. T., von Schuckmann, K., and Cheng, L. (2016). Insights into Earth's Energy Imbalance from Multiple Sources. Clim. J. 29, 7495-7505. doi: 10.1175/JCLI-D-16-0339.1

Uotila, P., Goosse, H., Haines, K., Matthieu, C., Antoine, B., Clément, B., et al. (2018). An assessment of ten ocean reanalyses in the polar regions. Clim. Dyn. 52, 1613-1650. doi: 10.1007/s00382-018-4242-z

Valdivieso, M., Haines, K., Balmaseda, M., Chang, Y.-S., Drevillon, M., Ferry, N., et al. (2017). An assessment of air-sea heat fluxes from ocean and coupled reanalyses. Clim. Dyn. 49, 983-1008. doi: 10.1007/s00382-015$2843-3$

von Schuckmann, K., Le Traon, P.-Y., Smith, N., Pascual, A., Brasseur, P., and Fennel, K. (2018a). Copernicus marine service ocean state report. J. Operat. Oceanogr. 11, s1-s142. doi: 10.1080/1755876X.2018.148 9208

von Schuckmann, K., Storto, A., Simoncelli, S., Raj, R., Samuelsen, A., and de Pascual Collar, A. (2018b). Ocean heat content; in: copernicus marine service ocean state report, issue 2. J. Operat. Oceanogr. 11, s41-s45. doi: 10.1080/ 1755876X.2018.1489208

Weber, R. J., Carrassi, A., and Doblas-Reyes, F. J. (2015). Linking the anomaly initialization approach to the mapping paradigm: a proof-of-concept study. Mon. Wea. Rev. 143, 4695-4713. doi: 10.1175/MWR-D-14-00398.1

Worby, A. P., Geiger, C. A., Paget, M. J., Woert, M. L. V., Ackley, S. F., and DeLiberty, T. L. (2008). Thickness distribution of Antarctic sea ice. J. Geophys. Res. 113:C05S92. doi: 10/1029/2007JC004254

Wunsch, C., and Heimbach, P. (2007). Practical global oceanic state estimation. Physica D 230, 197-208. doi: 10.1016/j.physd.2006.09.040

Xie, J., Counillon, F., and Bertino, L. (2018). Impact of assimilating a merged sea ice thickness from CryoSat-2 and SMOS in the Arctic reanalysis. Cryosphere 12, 3671-3691. doi: 10.5194/tc-12-3671-2018

Xue, Y., Wen, C., Kumar, A., Balmaseda, M., Fujii, Y., Alves, O., et al. (2017). A real-time ocean reanalyses intercomparison project in the context of tropical pacific observing system and ENSO monitoring. Clim. Dyn. 49, 3647-3672. doi: $10.1007 / \mathrm{s} 00382-017-3535-\mathrm{y}$
Yan, Y., Barth, A., Beckers, J. M., Candille, G., Brankart, J. M., and Brasseur, P. (2015). Ensemble assimilation of ARGO temperature profile, sea surface temperature, and altimetric satellite data into an eddy permitting primitive equation model of the North Atlantic Ocean. J. Geophys. Res. Oceans 120, 5134-5157. doi: 10.1002/2014JC010349

Yang, C., Masina, S., and Storto, A. (2017). Historical ocean reanalyses (1900-2010) using different data assimilation strategies. Q. J. R. Meteorol. Soc. 143, 479-493. doi: 10.1002/qj.2936

Zhang, S., Chang, Y.-S., Yang, X., and Rosati, A. (2013). Balanced and coherent climate estimation by combining data with a biased coupled model. J. Clim. 27, 1302-1314. doi: 10.1175/JCLI-D-13-00260.1

Zhang, X., Sun, C., Liu, C., Zhang, L., Shao, C., Zhang, X., et al. (2017). Evaluation of the impact of argo data on ocean reanalysis in the Pacific Region. Adv. Meteorol. 2017, 7314106. doi: 10.1155/2017/ 7314106

Zilberman, N. V. (2017). Deep argo: sampling the total ocean volume in state of the climate in 2016. Bull. Am. Meteorol. Soc. 98, S73-S74. doi: 10.1175/ 2017BAMSStateoftheClimate.1

Zuo, H., Balmaseda, M. A., and Mogensen, K. (2017). The new eddypermitting ORAP5 ocean reanalysis: description, evaluation and uncertainties in climate signals. Clim. Dyn. 49, 791-811. doi: 10.1007/s00382-0152675-1

Zuo, H., Balmaseda, M. A., Mogensen, K., and Tietsche, S. (2018). OCEAN5: the ECMWF Ocean Reanalysis System and its Real-Time analysis component, ECMWF Tech Memo 823. Reading: ECMWF.

Zuo, H. R. I., Mugford, R. I., Haines, K., and Smith, G. C. (2011). Assimilation impacts on Arctic Ocean circulation, heat and freshwater. Ocean Model. 40, 147-163. doi: 10.1016/j.ocemod.2011.08.008

Conflict of Interest Statement: The authors declare that the research was conducted in the absence of any commercial or financial relationships that could be construed as a potential conflict of interest.

Copyright (๑) 2019 Storto, Alvera-Azcárate, Balmaseda, Barth, Chevallier, Counillon, Domingues, Drevillon, Drillet, Forget, Garric, Haines, Hernandez, Iovino, Jackson, Lellouche, Masina, Mayer, Oke, Penny, Peterson, Yang and Zuo. This is an openaccess article distributed under the terms of the Creative Commons Attribution License (CC BY). The use, distribution or reproduction in other forums is permitted, provided the original author(s) and the copyright owner(s) are credited and that the original publication in this journal is cited, in accordance with accepted academic practice. No use, distribution or reproduction is permitted which does not comply with these terms. 


\section{OPEN ACCESS}

Edited by:

Fei Chai,

State Oceanic Administration, China

Reviewed by:

Arthur J. Miller

University of California, San Diego,

United States

Lei Zhou,

Shanghai Jiao Tong University, China

*Correspondence. Detlef Stammer

detlef.stammer@uni-hamburg.de

Annalisa Bracco

abracco@gatech.edu

Specialty section:

This article was submitted to

Ocean Observation,

a section of the journal

Frontiers in Marine Science

Received: 18 November 2018

Accepted: 05 July 2019

Published: 31 July 2019

Citation:

Stammer D, Bracco A,

AchutaRao K, Beal L, Bindoff NL,

Braconnot $P$, Cai W, Chen D,

Collins M, Danabasoglu G, Dewitte B,

Farneti R, Fox-Kemper B, Fyfe J,

Griffies SM, Jayne SR, Lazar A,

Lengaigne $M$, Lin X, Marsland S,

Minobe $S$, Monteiro PMS,

Robinson W, Roxy MK,

Rykaczewski RR, Speich S, Smith IJ,

Solomon A, Storto A, Takahashi K,

Toniazzo T and Vialard J (2019)

Ocean Climate Observing

Requirements in Support of Climate

Research and Climate Information.

Front. Mar. Sci. 6:444.

doi: 10.3389/fmars.2019.00444

\section{Ocean Climate Observing Requirements in Support of Climate Research and Climate Information}

\author{
Detlef Stammer ${ }^{1 *}$, Annalisa Bracco ${ }^{2 *}$, Krishna AchutaRao $^{3}$, Lisa Beal ${ }^{4}$, \\ Nathaniel L. Bindoff5,6, Pascale Braconnot ${ }^{7}$, Wenju Cai ${ }^{8}$, Dake Chen ${ }^{9}$, Matthew Collins ${ }^{10}$, \\ Gokhan Danabasoglu'11, Boris Dewitte ${ }^{12,13,14,15}$, Riccardo Farneti ${ }^{16}$, Baylor Fox-Kemper ${ }^{17}$, \\ John Fyfe ${ }^{18}$, Stephen M. Griffies ${ }^{19}$, Steven R. Jayne ${ }^{20}$, Alban Lazar ${ }^{21}$, \\ Matthieu Lengaigne ${ }^{22}$, Xiaopei Lin ${ }^{23,24}$, Simon Marsland ${ }^{25}$, Shoshiro Minobe ${ }^{26}$, \\ Pedro M. S. Monteiro ${ }^{27}$, Walter Robinson ${ }^{28}$, Mathew Koll Roxy ${ }^{29}$, Ryan R. Rykaczewski ${ }^{30}$, \\ Sabrina Speich ${ }^{31}$, Inga J. Smith ${ }^{32}$, Amy Solomon ${ }^{33}$, Andrea Storto ${ }^{34}$, Ken Takahashis, \\ Thomas Toniazzo ${ }^{36}$ and Jerome Vialard ${ }^{37}$
}

\begin{abstract}
${ }^{1}$ Center für Erdsystemforschung und Nachhaltigkeit, Universität Hamburg, Hamburg, Germany, ${ }^{2}$ School of Earth and Atmospheric Sciences, Georgia Institute of Technology, Atlanta, GA, United States, ${ }^{3}$ Centre for Atmospheric Sciences, Indian Institute of Technology Delhi, New Delhi, India, ${ }^{4}$ Rosenstiel School of Marine and Atmospheric Science, University of Miami, Miami, FL, United States, ${ }^{5}$ Institute for Marine and Antarctic Studies, University of Tasmania, Hobart, TAS, Australia, ${ }^{6}$ CSIRO Oceans and Atmospheres, Hobart, TAS, Australia, ${ }^{7}$ Laboratoire des Sciences du Climat et de l'Environnement, Unité Mixte CEA-CNRS-UVSQ, Université Paris Saclay, Gif-sur-Yvette, France, ${ }^{8}$ Centre for Southern Hemisphere Oceans Research, CSIRO, Aspendale, VIC, Australia, ${ }^{9}$ State Key Laboratory of Satellite Ocean Environment Dynamics, Second Institute of Oceanography, State Oceanic Administration, Hangzhou, China, ${ }^{10}$ College of Engineering, Mathematics and Physical Sciences, University of Exeter, Exeter, United Kingdom, ${ }^{11}$ Climate and Global Dynamics Laboratory, National Center for Atmospheric Research, Boulder, CO, United States, ${ }^{12}$ Centro de Estudios Avanzados en Zonas Áridas, Coquimbo, Chile, ${ }^{13}$ Departamento de Biología, Facultad de Ciencias del Mar, Universidad Católica del Norte, Coquimbo, Chile, ${ }^{14}$ Millennium Nucleus for Ecology and Sustainable Management of Oceanic Islands (ESMOI), Coquimbo, Chile, ${ }^{15}$ LEGOS, Université de Toulouse, IRD, CNES, CNRS, UPS, Toulouse, France, ${ }^{16}$ Earth System Physics Section, Abdus Salam International Centre for Theoretical Physics, Trieste, Italy, ${ }^{17}$ Department of Earth, Environmental, and Planetary Sciences, Brown University, Providence, RI, United States, ${ }^{18}$ Canadian Centre of Climate Modelling and Analysis, Environment and Climate Change Canada, Victroia, BC, Canada, ${ }^{19}$ NOAA Geophysical Fluid Dynamics Laboratory, Princeton, NJ, United States, ${ }^{20}$ Department of Physical Oceanography, Woods Hole Oceanographic Institution, Woods Hole, MA, United States, ${ }^{21}$ CNRS, IRD, MNHN, Laboratoire d'Océanographie et du Climat: Expérimentations et Approches Numériques, Sorbonne Université, Paris, France, ${ }^{22}$ Laboratory of Oceanography and Climate: Experiments and Numerical Approaches, Institute of Research for Development, Paris, France, ${ }^{23}$ Physical Oceanography Laboratory, Institute for Advanced Ocean Studies, Ocean University of China, Qingdao, China, ${ }^{24}$ Qingdao National Laboratory for Marine Science and Technology, Qingdao, China, ${ }^{25}$ CSIRO Climate Science Centre, Canberra, ACT, Australia, ${ }^{26}$ Department of Earth and Planetary Sciences, Faculty of Science, Hokkaido University, Sapporo, Japan, ${ }^{27}$ Southern Ocean Carbon - Climate Observatory, CSIR, Cape Town, South Africa, ${ }^{28}$ Department of Marine, Earth, and Atmospheric Sciences, North Carolina State University, Raleigh, NC, United States, ${ }^{29}$ Centre for Climate Change Research, Indian Institute of Tropical Meteorology, Pune, India, ${ }^{30}$ School of the Earth, Ocean, and Environment, University of South Carolina, Columbia, SC, United States, ${ }^{31}$ UMR8539, IPSL, ENS - PSL, Laboratoire de Météorologie Dynamique, Paris, France, ${ }^{32}$ Department of Physics, University of Otago, Dunedin, New Zealand, ${ }^{33}$ Earth System Research Laboratory, National Oceanic and Atmospheric Administration, Cooperative Institute for Research in Environmental Sciences, University of Colorado, Boulder, Boulder, CO, United States, ${ }^{34}$ Centre for Maritime Research and Experimentation, La Spezia, Italy, ${ }^{35}$ Servicio Nacional de Meteorología e Hidrología del Perú, Instituto Geofisico del Peru, Lima, Peru, ${ }^{36}$ Bjerknes Centre for Climate Research, University of Bergen, Bergen, Norway, ${ }^{37}$ IRD, IPSL, CNRS, IRD, MNHN, LOCEAN Laboratory, Sorbonne University, Paris, France
\end{abstract}

Natural variability and change of the Earth's climate have significant global societal impacts. With its large heat and carbon capacity and relatively slow dynamics, the ocean plays an integral role in climate, and provides an important source of predictability at seasonal and longer timescales. In addition, the ocean provides the slowly evolving 
lower boundary to the atmosphere, driving, and modifying atmospheric weather. Understanding and monitoring ocean climate variability and change, to constrain and initialize models as well as identify model biases for improved climate hindcasting and prediction, requires a scale-sensitive, and long-term observing system. A climate observing system has requirements that significantly differ from, and sometimes are orthogonal to, those of other applications. In general terms, they can be summarized by the simultaneous need for both large spatial and long temporal coverage, and by the accuracy and stability required for detecting the local climate signals. This paper reviews the requirements of a climate observing system in terms of space and time scales, and revisits the question of which parameters such a system should encompass to meet future strategic goals of the World Climate Research Program (WCRP), with emphasis on ocean and sea-ice covered areas. It considers global as well as regional aspects that should be accounted for in designing observing systems in individual basins. Furthermore, the paper discusses which data-driven products are required to meet WCRP research and modeling needs, and ways to obtain them through data synthesis and assimilation approaches. Finally, it addresses the need for scientific capacity building and international collaboration in support of the collection of high-quality measurements over the large spatial scales and long time-scales required for climate research, bridging the scientific rational to the required resources for implementation.

Keywords: ocean observing system, ocean climate, earth observations, in situ measurements, satellite observations, ocean modeling, climate information

\section{INTRODUCTION}

The ocean is an integral part of the climate system: understanding how climate change and variability have shaped it and how climate will be influenced by future changes is a major societal challenge. Key to understanding the ocean's role in the Earth's climate system is monitoring changes in the ocean circulation, heat and carbon content, freshwater, biogeochemistry and sea level, as well as studying the interactions of the ocean with the atmosphere, cryosphere, land, and ecosystems. In addition, a firm knowledge of the changing physical environment is fundamental to understanding the ocean's biogeochemistry and ecosystem functioning, variability, and change. Obtaining high-quality ocean climate observations on a sustained basis is also key to attributing the observed changes of the ocean state and therefore obtaining reliable climate predictions for a range of applications and users (Clivar, 2018 Science $\operatorname{Plan}^{1}$ ).

Through OceanObs'99 (Koblinsky and Smith, 2001) and OceanObs'09 (Hall et al., 2010) and through a continuous discussion of the global climate observing system (GCOS) implementation strategy ${ }^{2}$, the design of the ocean observing system has emerged. It consisted, initially, in a definition of essential climate variables (ECVs) and their sampling characteristics in the ocean and elsewhere, and has evolved from a platform-centric perspective to an integrated cross-platform,

${ }^{1}$ http://www.clivar.org/news/clivar-released-second-generation-science-planand-implementation-strategy

${ }^{2}$ https://unfccc.int/sites/default/files/gcos_ip_10oct2016.pdf parameter-centric observing system. The challenge now is for the climate-oriented observing system to evolve further into one serving the increasing needs for ocean climate information at refined spatial and temporal scales, particularly in the face of advancing anthropogenic climate forcing.

In this whitepaper we review in section "Observing Requirements for Climate" the observing requirements for major processes of climate interest organized around global considerations and basins-specific aspects with an emphasis on the open ocean. Challenges and opportunities for the ocean margins are discussed in section "Observing Requirements for Bounday Currents and Continental Margins," while needs specific to climate modeling, forecasting and to synthesis and reanalysis efforts are presented in section "Ocean Observations for Climate Modeling and Climate Information," followed by concluding remarks in section "Summary and Final Remarks.”

\section{OBSERVING REQUIREMENTS FOR CLIMATE}

A key capability of the ocean is to store huge amounts of heat, freshwater, and carbon (Rhein et al., 2013). Without this capability, terrestrial anthropogenic heating would be more severe than it currently is. An excess of these quantities enters the ocean through its surface, eventually filling the deep ocean through ventilation in the high-latitude "windows." The heat, freshwater and carbon are then distributed through ocean 
currents and mixing, and feed back into the atmosphere and the cryosphere. Many processes and mechanisms contribute to this redistribution over a wide range of scales in space and time. Understanding and monitoring them is needed to quantify ocean climate variability and change, as well as to constrain and initialize climate models and remedy model biases for improved hindcasting and prediction.

An ocean observing system for climate needs to provide essential information on a sustained long-term basis for monitoring, understanding, and forecasting within all ocean basins (The National Academies of Sciences, Engineering, and Medicine [NASEM], 2017). Specifically, its components should cover climate-observing parameters concerned with changes of the ocean circulation and its budgets of heat, freshwater, carbon, and nutrients, and the exchanges of heat, momentum, freshwater, and gases between the ocean, atmosphere, land, and cryosphere. Parameters concerned with sea level as an integral indicator for climate change (see also Ponte et al., 2019) should also be included. Several of the observing requirements are general in nature and concern the global ocean domain; others concern regional specificities and are presented separately in each ocean basin.

\section{General Considerations Concerning the Global Ocean Domain}

General observing requirements involve sustained in situ and space-based approaches that sample essential climate parameters over a broad range of spatial and time scales, as outlined in the GCOS implementation plan. Space-based measurements cover globally the surface expression of a broad spectrum of ocean processes. In contrast, in situ measurements provide the full-depth resolution required to monitor the vertical structure of climate changes in the ocean. They are also essential to climate-quality satellite data for calibration and inter-calibration purposes. The combination of in situ and space measurements is indispensable in regional and global process studies and estimates of anthropogenic heat and carbon uptake, among others (e.g., Palmer et al., 2019; Wannikhov et al., 2019).

Space-based climate monitoring requirements involve sustained satellite measurements of sea surface temperature (SST) (O'Carroll et al., 2019), surface wind and wind stress (Cronin et al., 2019), sea surface height (SSH) from which surface geostrophic currents can be derived and gravity changes (mass changes in the climate system), surface fluxes of heat (Cronin et al., 2019) and freshwater including rainfall, sea surface salinity (SSS), surface waves (Villas Boas et al., 2019) and ocean color. They should be sampled at least twice daily, ideally four times a day, and at a spatial scale that resolves accurately the ocean mesoscale $[\mathrm{O}(1) \mathrm{km}]$. Technology developments, such as new sensors and satellite constellations, are key to improve and expand these measurements. One priority is pursuing the development of wide-swath altimeters to measure meso- and submeso-scale SSH variations in crucial energetic regions.

It is imperative to sustain these observations (satellite or in situ) over a long period with climate-quality stability and precision. This requirement is all the more important if the physical signal deviates from a Gaussian distribution, since in that case the statistical moment of the time series may not be stable in time, reflecting the occurrence of extreme events. Global budgets of ECVs, such as heat, freshwater, and carbon rely on top-to-bottom in situ measurements using various platforms. A global full-water column Argo float array (Claustre et al., 2019) and high-quality ship-based CTD sections are therefore mandatory, and should include measurements under sea ice. Equally important is the monitoring of boundary currents through systems that continuously measure their transport - a recommendation formulated during OceanObs'09 but never implemented.

In situ measurements of the above variables are currently coordinated by the global ocean observing system (GOOS) and collected through the global tropical moored buoy arrays, Argo and global drifter programs, high resolution XBT transects, and Go-SHIP surveys.

The surface drifter array provides a Lagrangian view of ocean circulation, as well as surface temperature, and a growing number of barometric pressure measurements essential to weather forecasting. Argo profiling floats sample temperature, salinity, and derived geostrophic currents more or less homogeneously throughout the ocean subsurface, except in the polar regions, and sparsely below $2000 \mathrm{~m}$ depth (Roemmich et al., 2015).

During OceanObs'09 the expansion to routinely include biogeochemical parameters, such as $\mathrm{O}_{2}$, carbon, $\mathrm{pH}$ and nutrients, was recommended. BioArgo floats, while still low in number, are being implemented, contributing oxygen profiles and, in some cases, additional biogeochemical variables. Improving globally the spatial and temporal coverage of subsurface measures of biogeochemical tracers collocated with physical measurements is even more critical today with accelerating acidification, oxygen depletion, and ecosystem change. New DeepArgo technology is also coming on line to increase observations below $2000 \mathrm{~m}$.

Surface and subsurface mooring arrays augment these observations, primarily along the equator and in critical boundary regions, with high temporal and/or spatial resolution observations of surface fluxes and subsurface T, S, currents, and at times oxygen, nutrients, and fluorescence. Moorings require routine maintenance and hence datasets are often short with respect to the climate signals of interest. Basinwide GO-SHIP transects are essential to provide full depth observations of climate variables, including a full suite of biogeochemical parameters that can only be measured through water collection. Paleoclimate proxy data, such as through ocean sedimentary cores and corals, provide critical extensions of the instrumental record.

Since changes in mass and heat content drive sea level changes, regionally and globally, their monitoring over the full water column and under sea ice is essential. These measurements, in combination with sustained altimetry and tide gauge data, form the basis for studies of sea level change, and its coastal impacts (a WCRP Grand Challenge).

Finally, water isotopes of seawater, rainwater, and water vapor are required to provide calibration datasets for satellite 
water isotope retrievals, as well as benchmarks for isotopeequipped climate model simulations of the global water cycle (also a WCRP Grand Challenge), as they intersect with clouds, circulation, and climate.

\section{The Atlantic Ocean \\ Background}

The Atlantic Ocean, through its Meridional Overturning Circulation (AMOC; Frajka-Williams et al., 2019), plays a unique role in the Earth's climate. Different from the vertical overturning circulation in the other basins, the AMOC carries warm saline water from the South Atlantic northward in the upper $1200 \mathrm{~m}$ (deemed as the "upper limb") that is then transformed into the North Atlantic deep water (NADW) in the subpolar North Atlantic and Nordic Seas, and returned southward in the "lower limb" (1200-4000 m). This circulation is far more complex than commonly portrayed and involves the whole water column, linking the upper, mode, intermediate, deep and bottom waters formed and transformed in this ocean, or imported from other basins. The AMOC influences the northward transport of heat (Bryden and Imawaki, 2001) and its release to the atmosphere, providing a significant fraction of the zonally integrated atmospheric energy flux northward of $30^{\circ} \mathrm{N}$ (Rhines et al., 2008). Additionally, it captures about $30 \%$ of the anthropogenic excess of heat (Häkkinen et al., 2015; Palmer et al., 2017) and carbon (Takahashi et al., 2009). It drives the inter-basin transport of freshwater, nutrients, carbon, oxygen and other biogeochemical quantities (Tagklis et al., 2017), impacts the marine ecosystem, and affects the global hydrological cycle, with a clear connection to predictability, from seasonal to decadal (Schlundt et al., 2014; Fu et al., 2018).

The AMOC is thought to be a driver of Atlantic multi-decadal variability (AMV), whose sub-polar center of action is linked to ocean dynamics (Kerr, 2000). The AMV, in turn, impacts Sahel rainfall, North American and European weather, the South and East Asian Monsoons, and Arctic sea ice (Sutton and Hodson, 2005). Decadal AMOC variability is positively correlated with northward energy transport in the ocean, but anticorrelated with transport in the tropical atmosphere; regional dynamical feedbacks, however, are unknown. Atmosphereocean interactions have been extensively studied on interannual time scales, but are less understood at decadal scales. The spatial dynamical scales involved in the variability and change of the Atlantic Ocean are small, as all current systems of interest are significantly affected by fronts and eddies at the mesoscales $(10-300 \mathrm{~km})$.

\section{Atlantic Observing Requirements}

The scientific community is moving toward a better rationalization of the Atlantic Observing System as a whole (AtlantOS, deYoung et al., 2019). Being the ocean that receives a large portion of the oceanic excess heat uptake, and that hosts major modes of climate variability, the Atlantic should be observed adequately to accompany the society in adaptation to sea level rise (Ponte et al., 2019), ocean warming (Palmer et al., 2019), ocean acidification (Tilbrook et al., 2019), and the increased intensity of extreme events, including hurricanes (Goni et al., 2019). It is necessary to improve process understanding (in particular, ocean interactions between the meso- and submeso-scales and the large-scale circulation, air-sea interactions at the meso- and submeso-scales, and heat, freshwater, oxygen and carbon uptake, transport and release to the atmosphere), monitor changes, and feed robustly model developments (Fox-Kemper et al., 2019; Subramanian et al., 2019) and predictions (Penny et al., 2019).

Because of the particular nature of the Atlantic Ocean circulation, observations of currents, salinity, and temperature fields are needed from the surface to the ocean floor. These variables are also mandatory to monitor climate change and enable robust numerical weather prediction, as well as intraseasonal to interdecadal forecasts. However, because of the different scales of variability, they could be sampled with reduced spatial and temporal resolution for depths greater than $2000 \mathrm{~m}$.

The current Atlantic basin observing system (schematized in Figure 1) is composed of AMOC (Frajka-Williams et al., 2019) and Tropical Atlantic PIRATA mooring arrays (Foltz et al., 2018). The AMOC array covers the full depth of the water column near the continental slope; the PIRATA array is limited to the upper ocean but includes heat and momentum flux observations. An assessment on the evolution of the tropical atlantic observing system (TAOS) to expand and rationalize observations in this region is ongoing (Johns et al., 2018; Foltz et al., 2019).

In the open-ocean upper 1500-2000 m, the core Argo program is well developed in the North Atlantic, but more sparse in the tropical, and even more so in the South Atlantic. In the North Atlantic, deployments of BGC Argo and Deep Argo have been

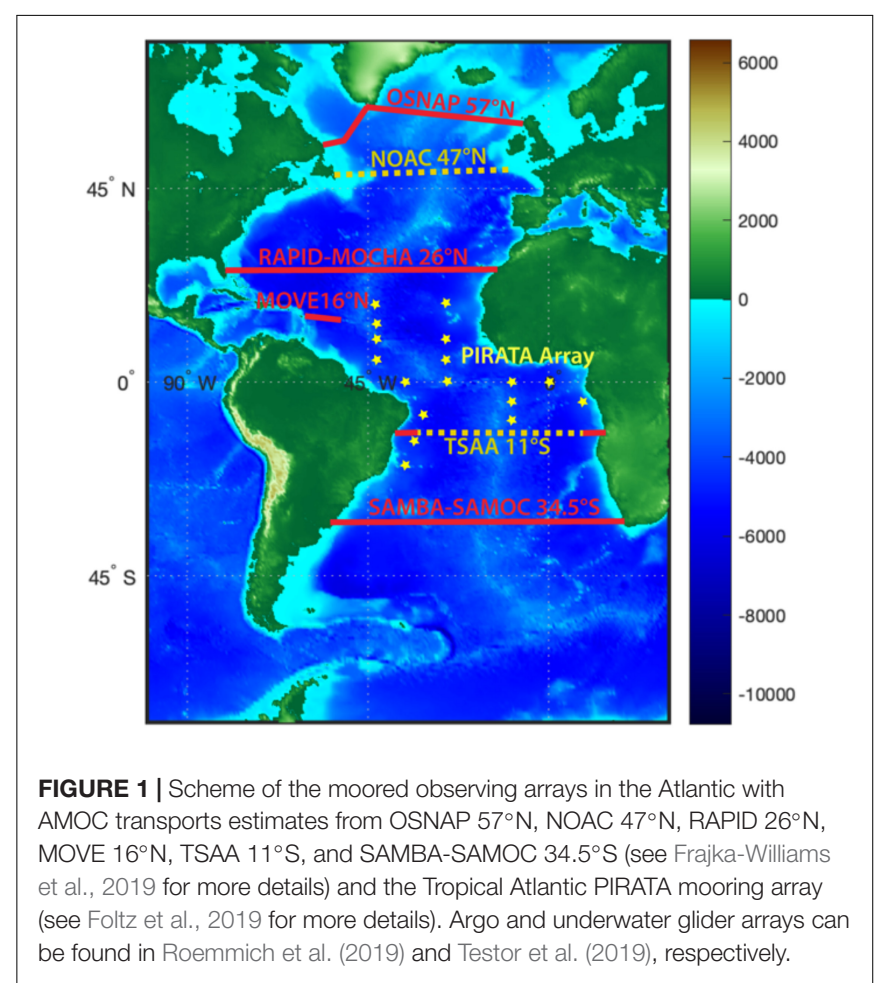


initiated but remain in an early development and coverage stage (Roemmich et al., 2019).

The Atlantic basin is regularly (on a 5-10 years base) surveyed by repeated GO-SHIP cruises (Sloyan et al., 2019), and other cruises servicing the various deep moorings and mooring arrays as well as oceanographic cruises organized by finite-time lifespan research projects. These cruises are essentials in providing regular sampling of physical and biogeochemical properties over the full ocean depth not attainable by the Argo core floats nor by the mooring arrays alone.

The ship of opportunity program (SOOP) acquires measurements from volunteer merchant ships that regularly undertake shipping routes crossing the basin. This program helps to increase the in situ sampling of the surface ocean (temperature and salinity via thermosalinographs instrument mounted close to the water intake of the vessel) and the upper $700 \mathrm{~m}$ of the ocean temperature (mainly) by a high resolution (every 10-35 km) deployment of expendable bathymographs (XBTs).

A dense array of buoys drogued at $15 \mathrm{~m}$ of depth that measure SST, surface currents and barometric pressure, via the global drifter program (GDP), is also maintained across the basin, mostly through the ship-volunteering program (Lumpkin et al., 2017).

Ocean underwater gliders deployment has been occurring along and near the North American continental slope within the global ocean gliders program (Testor et al., 2019). Typically, gliders profile from the surface to the bottom (over the continental shelf), or to 200-1000 m depth in the open ocean. However, their spatial coverage remains marginal compared to the extent of the Atlantic slope boundary. Indeed, sustained projects in the Atlantic include only observations in limited spots of the western, eastern and northern boundaries of the North Atlantic (Testor et al., 2019).

Observations should be enhanced in key regions related to the AMOC (the Labrador and Irminger Seas and the North Atlantic passages overflows, the South Atlantic that interconnects actively the North Atlantic with the rest of the world ocean) (FrajkaWilliams et al., 2019), in the Tropical Atlantic that plays an important control on weather extremes and climate modes (Foltz et al., 2019; Goni et al., 2019), and in the eastern and western boundary current systems (BCs) that are currently not sampled by the core Argo floats program, nor fully covered by the various Atlantic mooring arrays (Todd et al., 2019). Indeed, BCs not only regulate the meridional heat and fresh-water transports and cloud formation, but strongly influence atmosphere dynamics (Small et al., 2014; O'Reilly et al., 2017) and decadal variability (Gulev et al., 2013).

We recommend the continuation of all observing systems currently in place (core Argo and BGC Argo, AMOC, and PIRATA arrays, GO-SHIP cruises, surface drifters, VOS), and the enhancement of surface observations and observations in the atmosphere beyond current practices. In addition, we recommend enhancing subsurface ECV observations, as well as adding variables such as oxygen and $\mathrm{pH}$, by maintaining and expanding the sensors equipping mooring arrays and observations undertaken by ships, and by implementing Deep Argo and BGC Argo.

\section{The Pacific Ocean Background}

The Pacific Ocean is the largest of the world's oceans and hosts the strongest interannual tropical climate mode, the El Niño Southern oscillation (ENSO), an ocean-atmosphere coupled phenomenon (Timmermann et al., 2018). ENSO exhibits huge worldwide socio-economical and environmental impacts (McPhaden et al., 2006). The Pacific basin accounts for much of the interannual variability of precipitation and tropical cyclones, inducing an elevated risk of drought in the western part of the basin and of flooding on the eastern side that cause widespread ecosystems disruptions, including severe bleaching of corals. Because of its remote influence through atmospheric teleconnections, ENSO also impacts the Indian and Atlantic oceans (Klein et al., 1999), and is in turn impacted by both (Kucharski et al., 2011; Terray et al., 2016).

At decadal to multidecadal timescales, the interdecadal Pacific oscillation (IPO), driven partly by decadal ENSO modulations (Power et al., 1999), is a major source for low-frequency climate variations, worldwide. The IPO affects the weather and climate in many regions of the globe (Dong and Dai, 2015), drives important state transitions observed in marine ecosystems across the Pacific Ocean (Mantua et al., 1997), and has been hypothesized as major cause of the recent slowdown in global surface warming (Kosaka and Xie, 2013). The observed warming trend in the past century has a complicated structure in the Pacific, with 'hot spots' along the western boundary currents (Wu et al., 2012). There are large uncertainties about the nature of this trend, calling for the need of sustained and uninterrupted monitoring, also with respect to its likely impacts on phytoplankton growth (Marinov et al., 2010).

The eastern tropical Pacific Ocean hosts two of the four largest oxygen minimum zones (OMZ) in the world, located to the north and the south of the equator. Significant global deoxygenation together with expansion of the OMZ in the tropical Pacific has been observed over the past 50 years (Stramma et al., 2008). This expansion will result in the transition, adaptation, and/or extinction of different marine species in the tropical Pacific Ocean (Breitburg et al., 2018).

Understanding and projecting climate changes induced by anthropogenic forcing in this region, including changes in background state and ENSO characteristics (Cai et al., 2015), are critical for assessing future socioeconomic and environmental impacts, as well as planning adaptation strategies. The latter are especially important for the highly populated coastal regions, including the Pacific island communities that are vulnerable to climate change through sea level rise, ocean acidification, and changes in storms characteristics.

\section{Pacific Observing Requirements}

Several regions of the Pacific Ocean require enhanced climate observations on top and above what stated already in the general considerations. Among them are the tropical Pacific for its unique role in seasonal to decadal changes, the Kuroshio and its extensions into the North Pacific, the eastern boundaries for their role in the hemispheric communication and radiative heat budget, and the complex low-latitude western boundary 
systems, including the Indonesian throughflow (ITF) to the Indian Ocean. Because of the key role of the Pacific Ocean on global Earth's climate, it is also necessary to observe ocean surface wind stress and heat fluxes in the tropical Pacific at hourly intervals. Momentum and heat fluxes are essential for inter-calibrating satellite missions, driving ocean models and ocean reanalysis products, detecting ENSO precursors, and assessing and understanding tropical Pacific climate variations and climate change.

In the BCs of the Pacific heat and freshwater fluxes should be monitored through hourly observations of temperature, salinity, and velocity over the ocean's depth. These highly variables currents are indeed vital for predicting climate changes, for ENSO forecast and decadal prediction. Spatial and temporal coverage of subsurface measurements of oxygen, nutrients, chlorophyll, and $\mathrm{pH}$ should also be improved.

The parameters above could be observed by adding the necessary sensors to the existing Tropical Pacific Moored Array, which is a key component of the broader observing system. Eventual replacement of moorings with other observing technologies are being considered ${ }^{3}$ and should be done carefully, to ensure continuity of the climate records, e.g., with an overlap of the existing and new observing platforms over a sufficiently long time to allow a thorough examination of any consequences of the platform change. The existing Tropical Pacific Moored Array should be enhanced with additional direct surface flux measurements and biogeochemical and bio-optical sensors $\left(\mathrm{pCO}_{2}\right.$, chlorophyll concentration, particulate backscatter, oxygen, and nutrients). Furthermore, we recommend incorporating water isotope sampling/analysis on ships, including those servicing the Tropical Pacific Moored Array, as well as prioritizing island-based collection of rainwater, seawater, and vapor water isotopes wherever feasible, and deploying Argo floats with biogeochemical and bio-optical sensors, particularly in equatorial Pacific upwelling and OMZs regions. The current observing system, reviewed in this issue by Smith et al. (2019), would also benefit from an enhanced regional coordination in the far Eastern tropical Pacific where no mooring is currently available within the Tropical Pacific Moored Array albeit being the location of extreme El Nino development.

In general terms, we support developing a multi-platform approach for monitoring velocity, temperature, salinity, and biogeochemistry in the boundary currents of both the northern and southern hemispheres. This holds especially for the Pacific Ocean, building on the CLIVAR endorsed North Pacific Ocean circulation experiment (NPOCE) and the Southwest Pacific Ocean and climate experiment (SPICE). Finally, seasonal to interannual changes in the upper ITF that links the Pacific to the Indian Ocean should be monitored through direct observations and development of proxies for temperature, salinity, and velocity down to sill depths at hourly intervals. This can be achieved through enhancement of the IX1 XBT line with additional Argo floats and a pilot glider program, and maintaining multi-year mooring arrays to measure the full depth ITF of the major

${ }^{3}$ http://tpos2020.org/ inflow (e.g., Makassar, Maluku, and Halmahera) and outflow (e.g., Lombok, Ombai, and Timor) passages.

\section{The Indian Ocean Background}

Although the Indian Ocean is the smallest of the world's oceans, it has accounted for more than $50 \%$ of global ocean heat gain over the last decade (Vialard, 2015; Desbruyères et al., 2017) and harbors two of the most severe OMZs (Paulmier and RuizPino, 2009). Indian Ocean rim countries are home to almost one third of the global population. Many depend on fisheries and agriculture and have poor adaptive capacity, making them especially vulnerable to changes in ocean climate, including declines in fish catch, intensification of tropical cyclone intensity, increased drought (Roxy et al., 2015), and sea level rise. For instance, the Bay of Bengal accounts for $5 \%$ of tropical cyclones globally, but $80 \%$ of the associated fatalities (Paul, 2009).

The unique circulation and stratification of the Indian Ocean (Schott et al., 2009), driven by reversing monsoon winds, an influx of tropical waters from the Indonesian Seas, and massive run-off from the Asian continent, enhance important coupled modes of variability, such as the Madden-Julian oscillation (MJO) (Zhang, 2005), the monsoon intra-seasonal oscillation (MISO), and the Indian Ocean dipole (Webster et al., 1999), that have far-reaching impacts on rainfall, cyclogenesis, and ENSO. The recent rapid warming in the Indian Ocean has been attributed to an increasing trend in the ITF (Lee et al., 2015; Hu and Sprintall, 2017; Zhang et al., 2018) and an intensified Walker Circulation. Yet long-term variability in the ITF and in other dominant flux components of the basin-wide heat budget, e.g., the Agulhas Current (Bryden and Beal, 2001) or the upper-ocean overturning (Talley et al., 2003), remains poorly constrained. Modes of decadal variability also remain largely unknown in the Indian Ocean (Han et al., 2014), where the observing system dates back only 10 years.

The tropical Indian Ocean has a large warm pool (surface temperature $>27.5^{\circ} \mathrm{C}$ ), linked to the Pacific, that has a global reach, driving atmospheric convection, and the Walker Circulation. The warm pool, together with the thermocline ridge of the western tropical gyre, is strongly coupled with the atmosphere at intraseasonal timescales (MJO and MISO, Vialard et al., 2009; DeMott et al., 2015). Observing and simulating correctly these climate modes could improve monsoon forecasting and seasonal climate predictability throughout the tropics, yet measurements of upper ocean variability and surface fluxes have long been precluded in the western tropical Indian Ocean owing to piracy, while to the east, near the ITF exit, disagreements between flux products remain.

The OMZs in the northern Indian Ocean, together with changes in monsoon winds, are threatening marine ecosystems around the Asian subcontinent (Roxy et al., 2016). In the Arabian Sea, where hypoxia is well established, the OMZ has grown by $60 \%$ since the 1990s (Rixen et al., 2014), and may have already caused a dramatic shift in the ecosystem (Gomes et al., 2014). This OMZ could account for $20 \%$ of water column denitrification globally and may become a hotspot for outgassing of nitrous 
oxide, a greenhouse gas with almost 300 times the potency of $\mathrm{CO}_{2}$ (Naqvi et al., 2010). Observations of biogeochemical parameters to monitor subsurface expansion and impacts of these OMZs are lacking, and satellite ocean color proxies for tracking primary productivity have not been validated in this basin.

\section{Indian Ocean Observing Requirements}

The existing Indian Ocean sustainable observing system (IndOOS) is being reviewed under the leadership of the CLIVARIOC GOOS Indian Ocean Region $\mathrm{Panel}^{4}$. This review established that the current IndOOS system (see Figure 2 for its components) resolves reasonably the equatorial variability associated with the MJO and MISO, monsoon circulation and Indian Ocean Dipole. Key phenomena or regions that require enhanced observations are the heat and freshwater transport of the ITF, Agulhas and Leeuwin BCs, air-sea fluxes, and the Arabian Sea including biogeochemical aspects such as its OMZ. Variability in the ITF could be captured through enhancement of the IX1 XBT line with additional Argo floats, a pilot glider program, and exploration of the use of tomography for monitoring heat transport in the main passages of Lombok, Ombai, and Timor. Monitoring the subtropical boundary currents can be achieved with a mixture of tall moorings, CPIES, and glider programs. Pilot hydrographic end-point moorings as part of these arrays, in waters $2000 \mathrm{~m}$ deep or more, will allow a better handle on the basin-scale variations in oceanic heat content. In the tropics, the RAMA (Research Moored Array for African-Asian-Australian Monsoon Analysis and Prediction) tropical observing system should be expanded westward into the Arabian Sea (now that piracy is resolved) and enhanced with more direct flux measurements and a new surface flux reference site northwest of Australia. Finally, Argo floats with biogeochemical and bio-optical sensors should be deployed throughout the Indian Ocean, particularly in the Arabian Sea upwelling and OMZ regions and in the Bay of Bengal. An overview of the IO observing system requirements is given in Figure 2.

\section{Polar Oceans}

\section{Background}

The northern and southern polar oceans, present distinct, and at times shared, observational challenges and opportunities. The unique environment and the presence of sea ice complicate obtaining continuous, systematic observations, while promoting the development of new technology, and observational platforms. Polar oceans have experienced significant changes in heat/freshwater sources and fluxes from lower latitudes, sea ice melt, and river runoff. However, since the 1970s the decrease in Arctic sea ice cover has been widespread and dramatic (Vaughan et al., 2013), whereas Antarctic sea ice area changes have been strongly regional, with both increases and decreases (RichterMenge and Mathis, 2017; Stammerjohn and Scambos, 2017). More recently, polar amplification, where the polar region warms to higher temperatures and more rapidly than lower latitudes, has become of major concern in the northern hemisphere, but is not (yet) realized in the Southern Ocean (Lind et al., 2018).

\footnotetext{
${ }^{4}$ http://www.clivar.org/indoos-review-2006-2016
}

The Arctic Ocean plays a key role in the global overturning circulation and is a nexus of Atlantic Ocean variability, Pacific Ocean variability, and coastal environments, with run-off from ice sheets and large rivers being critical to ocean properties and circulation. A number of hypotheses for Arctic climate variability have been proposed but cannot be confirmed with current measurements. For example, how Atlantic water enters the Arctic, how it is redistributed within the Arctic interior, or the balance of forces that control the net outflow through the Canadian Arctic Archipelago. This complex system requires an integrated observational network to monitor and characterize sources and sinks of heat and salt into and out of the Arctic, and their changes. Direct observations of these sources are lacking, as in the case of fresh water inputs due to ice shelf basal melt and iceberg calving. However, in the broader region of the Arctic Mediterranean (Nordic Seas and Arctic Ocean), current meter arrays have been in operation at critical gateways or "choke points," such as the Bering Strait and across the GreenlandScotland Ridge, since the 1990s, providing monthly, if partly intermittent, time-series (e.g., Bringedal et al., 2018). Arrays operate also at key sections within the region, e.g., Svinøy Section, Barents Opening, and in the Fram Strait (Dickson et al., 2008), and in Davis Strait (Curry et al., 2014).

The Southern Ocean connects the Atlantic, Pacific, and Indian Oceans and, as explained in more detail in Newman et al., 2019, has a major impact on climate change signals. Recent estimates show that Southern Ocean circulation changes may account for $75 \%$ of the total excess heat linked to the airborne fraction of anthropogenic $\mathrm{CO}_{2}$ and $50 \%$ of the anthropogenic $\mathrm{CO}_{2}$ taken up by the ocean (Frölicher et al., 2015).

Decadal variability in heat and $\mathrm{CO}_{2}$ storage in the "upper" and "lower" loops of the MOC in the Southern Ocean may modulate both anthropogenic and natural $\mathrm{CO}_{2}$ reservoirs (Gruber et al., 2019). The Southern Ocean also plays a critical role in the ventilation of the low latitude ocean interior through midand deep-water masses. Freshening and volume decrease of the heaviest water mass in the world's ocean, the Antarctic bottom water (AABW), are the two strongest decadal signals ever observed in the deep oceans (Rintoul, 2018). AABW is a mixture of high salinity brine rejected in coastal polynyas during ice formation and surrounding circumpolar deep water, but the mixing process has not been observed directly, and links between surface forcing and AABW remain speculative. These factors, combined with the seasonal variability in sea ice, intense mesoand submesoscale features and extreme synoptic scale winds, pose a challenge to resolving the seasonal to decadal variability in the storage, and ventilations through sustained observations.

\section{High-Latitude Observing Requirements}

In the polar oceans, observations are needed to monitor and characterize the ice-ocean system, the coastal-open ocean system, and the freshwater/heat transports into and out of these systems. Satellite observations of the polar oceans have provided a multi-decadal record of climate variability, but only for a limited number of fields. Remote sensing allows for monitoring sea ice concentration, thickness, age, and drift. Furthermore, it provides SST, sea surface salinity, and ocean 


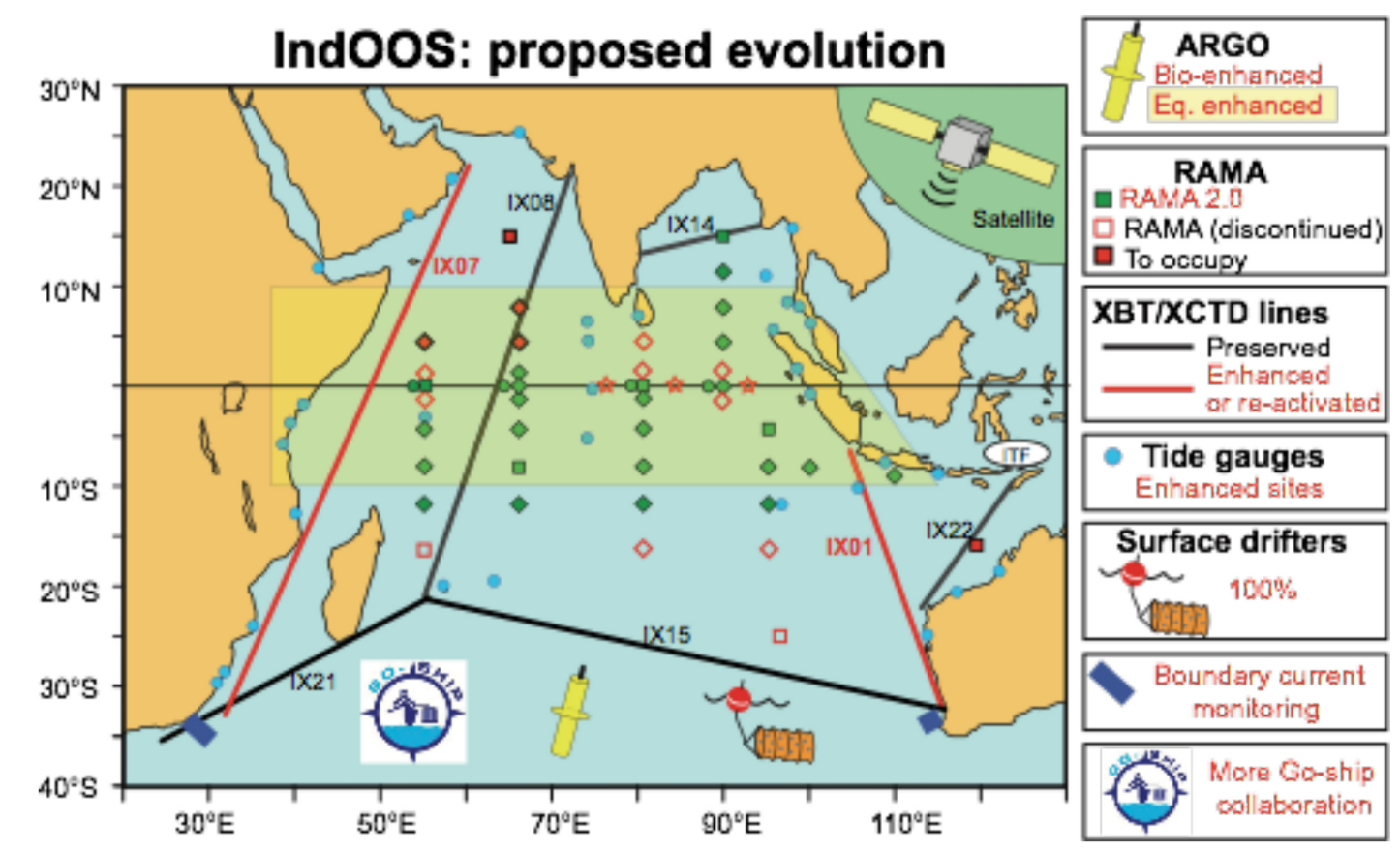

FIGURE 2 | IndOOS original design and current state. The original IndOOS design comprises the RAMA, Argo, XBT/XCTD, surface drifting buoys and tide gauges components.

bottom pressure. However, in situ measurements of these remote sensing products are rare, making calibration and validation of satellite algorithms challenging.

Free-drifting profiling floats present challenges in the polar oceans due to the likelihood of being trapped under ice and damaged. Technology developments allow the floats to sense the ice presence and store data for a number of years, but failure rates remain high. Efforts are underway to improve this problem. New technologies are being tested, such as the NOAA autonomous sailing drone that measures the near-surface ocean and atmosphere environments, and a system of moored sound sources maintained by the Alfred Wegener Institute in the Weddell Sea.

In the Arctic, well constrained transport estimates of volume, heat, and salt/freshwater in and out of the basin exist. It is imperative to maintain such gateway observations at a time when the climate system is in flux. However, key branches of the circulation and exchange, e.g., the Arctic circumpolar boundary current (ACBD), remain mostly unobserved. Significant work has been done to characterize freshwater storage within the Arctic Ocean (Rabe et al., 2014; Prowse et al., 2015), but additional observations are needed to characterize heat storage, transport, and mixing in this basin. Buoys to measure ice motion, surface pressure, and air temperature have been deployed and monitored by the International Arctic Buoy Program since $1978^{5}$, but measurements in the vicinity of sea ice remain limited.

Observing the Southern Ocean presents additional challenges due to the distance from population centers and higher costs

${ }^{5}$ http://iabp.apl.washington.edu to deploy ships and autonomous platforms. As in the Arctic, surface fluxes from the atmosphere and ice (both land-based and floating) need additional sustained observations. Among all climate variables, ocean-, and sea-ice surface flux estimates are simultaneously the most fundamental and uncertain (Bourassa et al., 2013). Their seasonal cycle is not well constrained and should be a focus for the next decade (Mongwe et al., 2018; Gruber et al., 2019). Seasonal biases in the observational record (Figure 3) pose significant challenges to constraining mean annual variability and trends (Fay et al., 2018). Significant gaps remain in observations of the ice-impacted oceans for nonsummer seasons (Newman et al., 2019).

The continuation of the high-resolution full depth CTD lines as part of GO-SHIP, particularly the 3 choke points (SR01, SR03, and A12), is essential on a 5 - 10-year repeat cycle to constrain not only storage rates but also their decadal variability. Greater focus should be given to the winter Mode Water and Intermediate Waters subduction areas at the boundary between the ACC and the sub-tropical systems. Floats and deep-diving gliders may contribute to a future integrated observatory of the Southern Ocean, complementing the seasonal biased ship-based data.

Although the Southern Ocean MOC cannot be directly quantified, it is possible to estimate the transport of mass, heat and other physical and biogeochemical tracers at the edge of it, usually defined to be at $30^{\circ} \mathrm{S}$. These transports are routinely computed in climate models, pointing to common biases such as the underestimation of bottom water and the overestimation of light water exports (Downes et al., 2015). Recent estimates of meridional transports of mass, heat and salt along the Atlantic sector at $32-35^{\circ} \mathrm{S}$ were obtained 


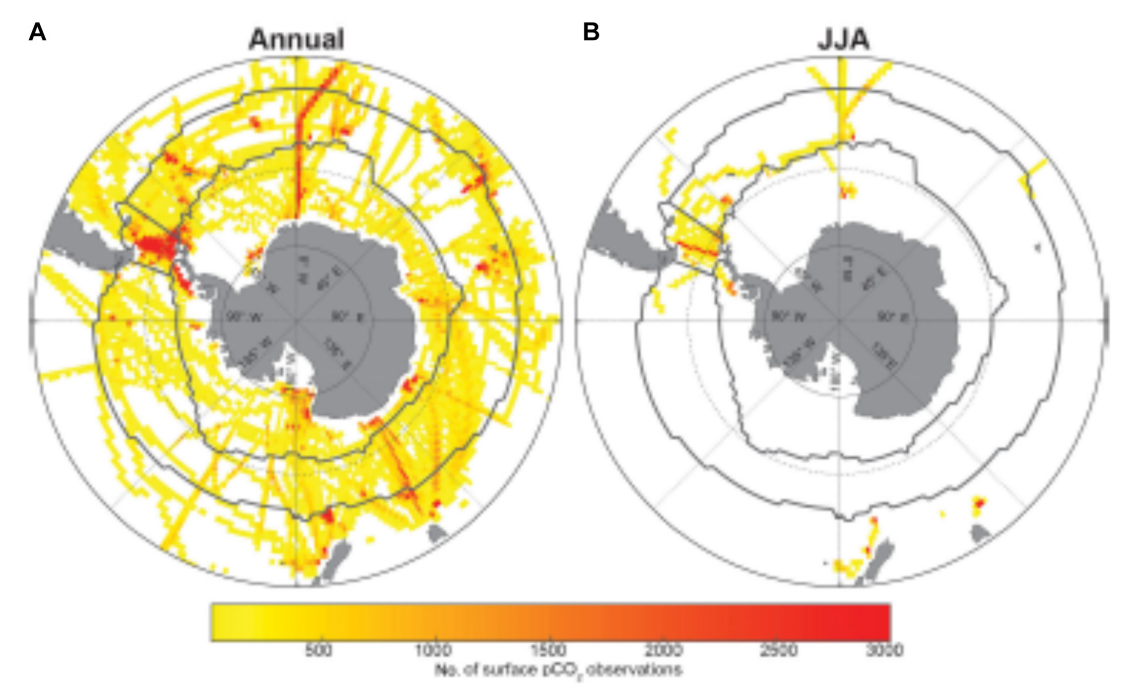

FIGURE 3 | Seasonal sampling bias for surface $\mathrm{pCO}_{2}$ observations in the Southern Ocean (Fay et al., 2018). The total annual observations (A) are dominated by summer observations whereas winter observations $\mathbf{( B )}$ are dominated by the Drake Passage and the more recent dedicated winter cruises by the South African SA Agulhas II.

through the SAMOC project ${ }^{6}$. Similarly, new observational estimates of ACC transport across Drake Passage reported values much larger than model estimates (Donohue et al., 2016). All these observations should be sustained and enhanced over the next decade. In this framework, we recommend establishing a Deep Ocean Observing System that would include instrumented sea floor cables, as routinely done in other regions.

Additional observations are needed to monitor the mixing and exchange of water masses between shelves and open ocean. Furthermore, sea-ice freshwater flux has been recognized as a key player in water mass formation and transformation in both data assimilating models (Abernathey et al., 2016) and a combination of observations (Pellichero et al., 2018). These unprecedented observations should be maintained and enhanced (Gray et al., 2018).

The paucity of Southern Ocean station data leads to relatively low accuracy in estimates of wind stress strengthening and poleward shift during the last decades, quantified as trends in the Southern Annular Mode (Swart and Fyfe, 2013; Swart et al., 2018). Reliable estimates of westerly winds and confidence in recent wind trends are fundamental. Accurate estimates of easterlies and katabatic coastal winds are also needed for understanding sea-ice extent and AABW production. Moreover, coastal winds may partially explain the subsurface warming to the west of the Antarctica Peninsula that is largely responsible for the glacial ice mass loss in the same region (Spence et al., 2017), and for significant changes in ACC transport (Zika et al., 2013).

Hydrographic observations since the 1950s in combination with earth system models indicate that the warming and freshening of the Southern Ocean over recent decades can be

${ }^{6}$ http://www.aoml.noaa.gov/phod/SAMOC_international/ attributed to a combination of greenhouse gas emissions and ozone depletion (Swart et al., 2018). It is critical that the Southern Ocean continues to be carefully monitored as the expectation is that warming and freshening will carry on into the foreseeable future with major implications for sea-ice, ice-sheets, as well heat and carbon uptake, in the region.

\section{OBSERVING REQUIREMENTS FOR BOUNDAY CURRENTS AND CONTINENTAL MARGINS}

\section{Background}

Tropical and subtropical coastal oceans host marine resources of great value to human societies and their sensitivity to anthropogenic change is amplified by proximity to growing population centers. The coastal oceans (e.g., areas within Large Marine Ecosystems; Sherman and Alexander, 1986) account for 95\% of the global fish catch (Stock et al., 2017) despite covering about 22\% of the ocean's surface area, and are exceptionally complex across a range of scales and steep gradients in physical and biogeochemical properties.

Over the past centuries interdisciplinary observations of the coastal oceans have offered unparalleled insight to the links among climate processes, fluid dynamics, ocean biogeochemistry, and marine fisheries (McCabe et al., 2016). Coastal upwelling, vertical mixing, and lateral and vertical transport of waters along and across coastal boundaries, combined with riverine delivery of allochthonous inorganic and organic matter (Liu et al., 2018), contribute to the biological production in the eastern BCs and broadly in tropical coastal systems. Canonical western BCs are characterized as oligotrophic 
marine environments, in which mesoscale processes, coastal bathymetry, and continental runoff can locally enhance primary production.

On both eastern and western margins of tropical and subtropical basins, interactions between topographic features, coastal atmospheric jets, coastally trapped and internal waves, Ekman transport and submesoscale and mesoscale circulations (Figure 4) further complicate our ability to represent coastal dynamics in climate models (Richter, 2015). These model biases affect seasonal prediction and climate change projections through nonlinear processes such as atmospheric deep convection (Belmadami et al., 2014), and cannot be easily corrected a posteriori.

Primary production and the fate of organic matter influence the capacity of the coastal oceans to capture atmospheric $\mathrm{CO}_{2}$ (Fiechter et al., 2014). Currently, the net effect of coastal productivity and $\mathrm{CO}_{2}$ flux on the global carbon cycle is highly uncertain. Closely coupled to $\mathrm{CO}_{2}$ atmosphere-ocean exchange is growing concern for acidification and deoxygenation of coastal waters (Ito et al., 2017). While empirical relationships between climate conditions and marine ecosystems have been documented, their stationarity over long time periods is uncertain, particularly as coastal systems are forced by physical climate conditions never observed in the recent observational record (Rykaczewski and Dunne, 2010).

The upwelling that occurs in eastern tropical-subtropical boundary currents is highly sensitive, depending on latitude, to the adjacent surface and subsurface water masses and/or to the coastal wind stress, the so-called coastal low-level jet (LLJ) (Fennel et al., 2012). This sensitivity is reflected in the biogeochemical consequences of the upwelling. The representation of both water masses and coastal wind stress are challenging in numerical models, and observations are lacking and/or are collected at national level with limited international coordination. LLJs are located at the atmospheric low-level inversion capping the planetary boundary layer (PBL) and separate moist, cool marine air-masses from warm, dry continental air-masses. They separate prevalent midtropospheric westerlies and lower-tropospheric easterlies, and are due to atmospheric subsidence associated with subtropical anticyclones (Takahashi and Battisti, 2007; Toniazzo et al., 2011). Currently almost no in situ data is available on some of the LLJs, such as that of the Benguela (Patricola and Chang, 2016) and scatterometer satellite data have limitations within the coastal fringe (Astudillo et al., 2017). Even where regular measurements exist (e.g., Chile-Peru and California coasts) stations are land-based and lateral gradient information is sparse.

Western BCs of the subtropical gyres, such as the Gulf Stream, the Kuroshio, the Brazil, the Agulhas, and the East Australian Currents play important roles in climate system. They export heat, salt, and nutrients from the tropics, release vast heat and moisture to the atmosphere, and play important roles in a number of atmospheric phenomena such as precipitation (Minobe et al., 2008), positioning of the storm track (Small et al., 2014), and blocking and positioning of the jet stream (O’Reilly et al., 2017). Alike eastern boundary upwellings, western boundary currents mediate the horizontal and vertical flux of water masses from the permanent thermocline to the mixed layer (Blanke et al., 2002). They can also have a strong influence on the uptake of carbon dioxide $\left(\mathrm{CO}_{2}\right)$ from the atmosphere (Takahashi et al., 2009), and thus be a key component of the carbon cycle. The ocean dynamics in the western boundary regions can control the local expression of large-scale sea-level rise on the eastern coasts of the continents (Minobe et al., 2017).

\section{Observing Requirements}

Major challenges in observing the BCs and the continental margins include elevated mesoscale and submesoscale variability, the heterogeneity across regions, and the complexity of processes affecting the dynamics and thermodynamics of the air-sea interface. Decades of consistent in situ observations are required to distinguish long-term anthropogenic change from changes associated with natural variability (Takahashi and Martinez, 2017). They are also necessary for better understanding extreme events, including marine heatwaves that often occur in the eastern boundary upwelling systems due to shallow thermocline associated with large-scale climate drivers and/or local air-sea interaction (Bond et al., 2015; Kataoka et al., 2018).

Multidecadal and interdisciplinary observations are available in some eastern BCs, such as the California (Goericke and Ohman, 2015) or Peru (Graco et al., 2017) Current systems and in all the western boundary currents. However, only baseline observations are available in most coastal oceans, particularly in the southern hemisphere. Given the coastal oceans' disproportionately large importance to human society and marine ecosystems, continued emphasis should be placed on increasing in-situ observations and sharing existing data collected within national programs.

Boundary current systems observing systems should be developed and implemented to cover the upper $1500 \mathrm{~m}$ continental slope in dynamical regions key to ocean-atmosphere interactions that are heavily undersampled (see Todd et al., 2019 for more insights). Enhanced the monitoring of both eastern and western boundary currents is required to more accurately characterize their variability and change, and campaigntype observational experiments are necessary to improve understanding of the mechanistic processes controlling them.

The accumulation of interdisciplinary oceanographic observations in boundary current regions make them central to CLIVAR's goals of understanding climate phenomena on a range of scales and informing adaptation and mitigation strategies regionally. Efforts, however, should be coordinated among countries, and data -for both ocean and atmosphere - made available to the scientific community. While the dissemination of open ocean observations has made exceptional steps in response to internationally coordinated programs, data collected in territorial waters remains subjected to observation protocols, and usage policies that differ widely between countries. They are often inaccessible to the international and even national community 


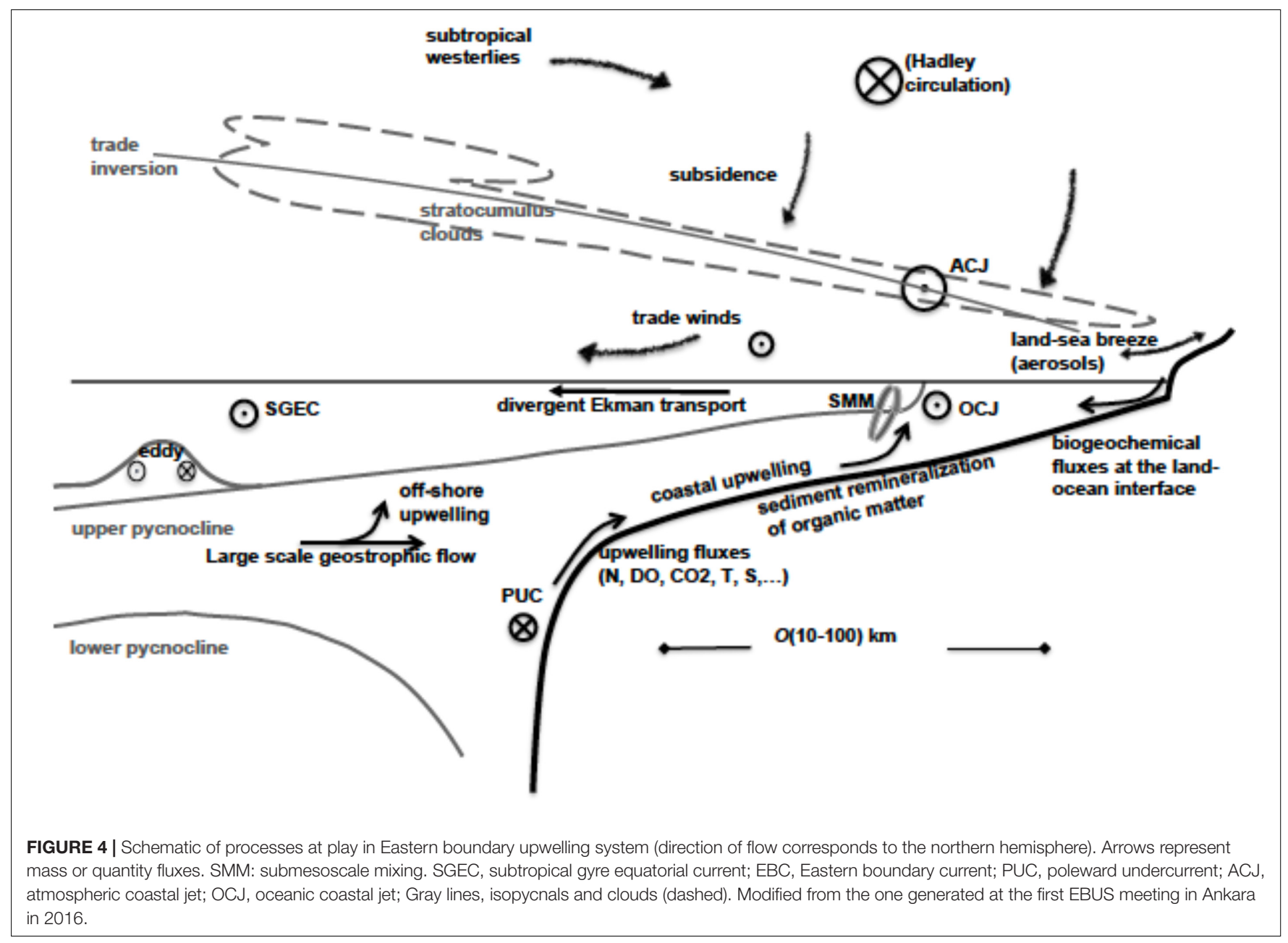

due to their sensitive nature in terms of economic (e.g., oil exploration and fish stocks), military and border enforcement considerations. Restrictions also involve sovereign airspace, limiting in situ observations of the atmospheric boundary layer, and which is a critical component of the coupled coastal upwelling system. Height-resolved atmospheric observations are particularly scarce and a major limitation for the understanding of coastal dynamics of upwelling regions.

We argue for fostering finer and more integrated physical and biogeochemical in situ sampling that would naturally involve $\mathrm{R} / \mathrm{V}$ surveys, autonomous sensors (drifters, profilers, and gliders), and moorings. Considering the general gap in coastal marine in situ data, a specific effort could target the ability of such communities to install and maintain long-term high-frequency ocean-atmosphere data-buoys at homogenous standards, especially in the tropics-subtropics. The ship time and sea-going capacity of various national laboratories for fishery assessment should also be better leveraged. Coordinated coastal process studies in different regions are required to document the relationship between coastal upwelling and meteorological conditions, and to improve understanding of the mechanical and thermal forcing of the near-surface ocean in coastal areas, of surface turbulent fluxes, air-sea exchanges, transport and mixing at scale of kilometers or less, and biogeochemical exchanges, interactions, and cycling.

\section{OCEAN OBSERVATIONS FOR CLIMATE MODELING AND CLIMATE INFORMATION}

\section{Background}

One of the key communities that use ocean observations are the participating governments through the inter-governmental panel on climate change (IPCC). Since the Fourth assessment report the IPCC assessment cycles includes a chapter on oceans. IPCC Working Group reports focus on climate change, frequently at global scales, over long periods of time, and have common sets of principles with consequences for the observing system. There is demand for multiple lines of evidence to support conclusions with estimates of uncertainty, and the scientific conclusions have to be traceable to the observations or simulations that underlie them. One of the fundamental questions is whether the observed changes can be attributed to anthropogenic forcing and alternative natural forcing, like variations in solar radiation, 
volcanic eruptions, and internal variability, can be excluded. There is also growing interest about ocean extremes and abrupt events, requiring the observational record to include time-scales of these extremes.

Since OceanObs'09, the role of ocean modeling and data assimilation in furthering understanding and enhancing prediction capabilities has increased to the point where today models and state estimations are integral to oceanography and climate science. In particular, models initialized from observed climate conditions through state estimation products are used in prediction or projection systems. State estimation involves a simultaneous analysis of the state of both ocean and atmosphere. These coupled state estimations will benefit from accurate and complete estimates of the exchange of heat and momentum at the ocean-atmosphere interface, noting the requirement for global balances.

Recent advances in the fidelity of models and their enhanced use arose through ongoing community efforts to improve the numerical and physical foundations of ocean models. These advances also concern the increased role of open source development: by embracing the input of any and all scientists and engineers, and offering a systematic and documented software framework to ingest that input, ocean model codes have been taken from the purview of a few experts circa 2009 into the light of an open and vigorous community circa 2019. Further details of the enhancements to numerics and physics are summarized in Fox-Kemper et al. (2019); here we focus on the role of observations for improving the skill of ocean models as part of assimilation/prediction systems.

From numerical schemes to parameterizations, ocean circulation models have improved over the last decade [see Griffies et al. (2010) for an assessment a decade ago]. New numerical approaches, including Arbitrary Lagrangian-Eulerian approaches to vertical coordinates and unstructured and nested horizontal grids, preserve large-scale budgets while allowing for regional specificity, and improved stability and accuracy. Coupled models - ocean-sea ice, ocean-atmosphere, and oceanice shelf - are evolving rapidly and reveal emergent phenomena that uncoupled models fail to capture. Consistent coupling requires numerical and parameterization advances that will lead to more robust estimates of oceanic and cryospheric changes, including sea level rise. Significant model biases have been reduced through improved parameterizations of mesoscales, submesoscales, boundary layers (Villas Boas et al., 2019), internal mixing, and other processes, in turn made possible by process-level understanding through high-resolution models and dedicated observational studies.

\section{Requirements for Climate Modeling and Forecasting}

As the community moves into higher-resolution and more accurate modeling, new observational challenges arise (FoxKemper et al., 2019). Continuous monitoring of global and process-level diagnostics is key to ensuring continued progress in model development. At the same time, coordinated sets of observing system simulation experiments (OSSEs) can help optimizing cost and scale effectiveness of future long-term observational systems (Mazloff et al., 2017).

Key diagnostics and their observational requirements include the annual cycle at various latitudes. In the polar oceans, for example, challenges in observing it within the seasonal ice zone and a bias toward summer values in the observations cause large model uncertainties in surface momentum and buoyancy flux that are, in turn, among the largest sources of model spread (Downes et al., 2015; Farneti et al., 2015). Sea ice thickness, sea ice volume, AABW and High Salinity Shelf Water formation rates and processes, ice shelf basal melt (including rates of changes), iceberg calving rates, and ocean heat fluxes are also among the data-driven products required to meet WCRP research and modeling needs, and reduce divergence in ocean reanalyses (Uotila et al., 2018).

In addition, many new applications of ocean and climate models, especially state estimation, monitoring, and decadal predictions, require observational data that is high quality in representativeness, accuracy, precision, and sampling. Realistic models benefit from these observations, and at the same time provide help to identify gaps in observational schemes.

\section{Observational Products for Climate Information}

Ocean reanalyses (or syntheses) are a fundamental tool for present-day climate monitoring because they provide a dynamically consistent, four-dimensional view of the oceans. Although the propagation of the observational content into unobserved regions or periods is non-trivial, the use of ocean reanalyses products increased significantly during the last decade (Stammer et al., 2016). Ocean reanalyses that combine an ocean general circulation model with available ocean observations use new, improved methods for comparing observations and modeled data. Reanalyses, if consistent mathematically and dynamically, can provide information for key climate diagnostics that are mostly unobserved, such as deep ocean heat content changes and their contributions to sea level rise (Karspeck et al., 2017; Palmer et al., 2017). Synthesis efforts are widely used in climate studies such as the annual State of the Climate report (Blunden et al., 2018), and applications ranging from downstream (biogeochemistry, fishery, and energy) modeling to downscaling (high-resolution regional simulations) deployments, depend upon their availability.

The development and production of ocean reanalyses calls for coordinated efforts in disseminating quality-controlled observational datasets, which may in turn benefit from delayed time quality checks e.g., relying on feedback information from assimilation systems and/or sophisticated buddy checks (e.g., Cabanes et al., 2013). Among these datasets, IQuOD is a recently produced collection of marine sub-surface observations, which aims at processing and disseminating unbiased climate data of the highest quality, consistency, and completeness (Domingues and Palmer, 2015 $)$. Among many existing datasets, in situ observations of biogeochemical parameters have been compiled and quality controlled by the global ocean data analysis project

\footnotetext{
${ }^{7}$ www.iquod.org
} 
(GLODAP), that includes over one million seawater samples (Olsen et al., 2016 ${ }^{8}$ ). Similar initiatives have been implemented by several space agencies for satellite parameters (e.g., the European Space agency climate change initiative (CCI), or the Making Earth System Data Records for Use in Research Environments (MEaSUREs) programs through the NASA Earth Science Data Systems). Finally, there is the need to sustain and maintain a large number of observational datasets (e.g., currents from drifters or ADCP, tide-gauge data, air-sea flux measurements, etc.) that are used for validation purposes and enable improvements of, and linkages among, ocean models, and assimilation systems (Balmaseda et al., 2015).

Investigating ocean heat content products, Abrams et al. (2013) concluded that a major source of error was in the interpolation-methods of the underlying time series. Cheng et al. (2017) identified the culprit in assuming in the interpolation that missing data can be treated as background climatological field. This assumption creates a low bias in the estimates, that for global heat content becomes a significant fraction of the trend (Figure 5). New estimates agree better with, and lie with the spread of, CMIP5 simulations of the historical period.

Controlling these biases demands high quality reference temperature dates sets with expendable measurements from Argo and XBT lines from ship of opportunities as done in

${ }^{8}$ www.glodap.info the IQuOD project. Additionally, the analysis and gridding of the observations should be monitored through systematic reviews and assessments. Finally, it is essential that the observed records are tested against those from climate models (Figure 5, lower panel).

Critical for reanalyses and objective analyses is the provision of uncertainties for each observational based product, ideally in form of error covariances. However, uncertainty information needs to be provided also for model results, and in either case should account for omissions, sampling and systematic errors.

Finally, an important downstream application is the initialization of coupled subseasonal to decadal prediction systems (Marotzke et al., 2016), where improved data assimilation schemes have led to increased predictability (Bellucci et al., 2011; Polkova et al., 2014; Liu et al., 2017). However, model-consistent coupled assimilation schemes will ultimately be required to optimize prediction skills and improve model biases.

\section{SUMMARY AND FINAL REMARKS}

Climate monitoring requires long, sustained and high-quality measurements of essential and process-oriented climate parameters in the ocean, including surface fluxes of heat, freshwater, and momentum. For climate purposes, the

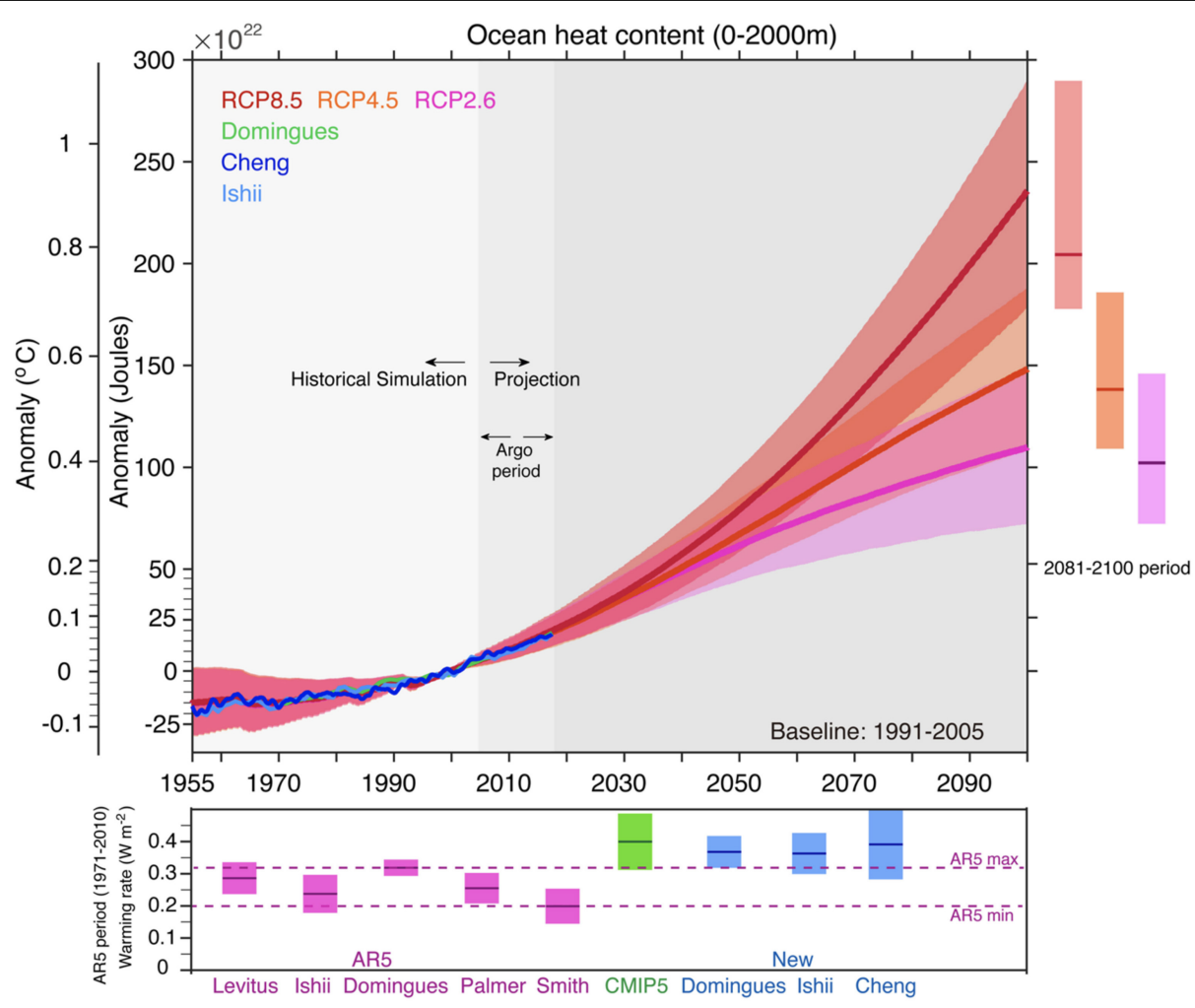

FIGURE 5 | Observational ocean heat content changes for the upper $2000 \mathrm{~m}$ from in situ observations in Domingues et al. (2008), Cheng et al. (2017), and Ishii et al. (2017), compared with the ensemble means of the CMIP5 models for historical simulations pre-2005 and projections after 2005. Model calculations performed for emissions projections RCP2.6, RCP4.5 and RCP8.5 (Taylor et al., 2012). All error bars are 90\% confidence interval. Credit: Lijing Cheng. 
length of uninterrupted timeseries, and the stability of the measurements are critical.

The climate observing system needs to include both satellite and in situ observations. A mix of platforms and technologies, in orbit and in situ, will greatly improve the chance to meet user requirements, regionally, and globally. Synergies between all components and platforms are fundamental also to insure the cross-calibration of parameters for quality purposes. Each satellite mission should have an in-situ counterpart that optimizes data calibration, stability and space/time sampling, and vice versa. Such a system is parameter oriented; to maximize its use and utility, the subsequent required step is the merging of observations into a parameter time series. Ocean and climate syntheses are therefore an integral part of this endeavor.

Subsurface measurements are critical to monitor and investigate the ocean role in the climate system. It is a priority to expand the Argo program to the full water column and under sea ice, and to augment high-quality sections and timeseries networks, especially in BCs. A global, continuous, in situ network of gliders would contribute effectively toward this objective. All existing elements should include $\mathrm{O}_{2}$ and $\mathrm{CO}_{2}$ sensors, as well as nutrient monitoring, a testimony of the increasingly interdisciplinary nature of climate science. Improving both quality and coverage of surface flux measurements of heat, carbon, freshwater, and momentum is mandatory. At the same time the existing satellite fleet should be sustained and expanded, to ensure that surface salinity, gravity, and winds etc are part of a long-term, stable monitoring system.

The climate observing system cannot be static, but needs to evolve scientifically and technically, considering new sampling needs, new sensors and technological advances. Reviewing the adequacy of present-day observing system and its future requirements will allow to focus research and innovation in areas where existing technologies do not meet expectations and needs.

Finally, building and sustaining a climate observing system in the ocean relies critically on capacity building, cooperation, and coordination among the international community. It is indispensable to motivate and support the scientific communities

\section{REFERENCES}

Abernathey, R. P., Cerovecki, I., Holland, P. R., Newsom, E., Mazloff, M., and Talley, L. D. (2016). Water-mass transformation by sea ice in the upper branch of the Southern Ocean overturning. Nat. Geosci. 9, 596-601. doi: 10.1038/ ngeo2749

Abrams, J. P., Baringer, M., Bindoff, N. L., Boyer, T., Cheng, L. J., Church, J. A., et al. (2013). A review of global ocean temperature observations: implications for ocean heat content estimates and climate change. Rev. Geophys. 51, 450-483. doi: 10.1002/rog.20022

Astudillo, O., Dewitte, B., Mallet, M., Frappart, F., Ruttlant, J., Ramos, M., et al. (2017). Surface winds off Peru-Chile: observing closer to the coast from radar altimetry. J. Rem. Sens. Environ. 191, 179-196. doi: 10.1016/j.rse.2017.01.010

Balmaseda, M. A., Hernandez, F., Storto, A., Palmer, M. D., Alves, O., Shi, L., et al. (2015). The ocean reanalyses intercomparison project (ORA-IP). J. Operat. Oceanogr. 8, s80-s97. doi: 10.1080/1755876X.2015.1022329

Bellucci, A., Gualdi, S., Masina, S., Storto, A., Scoccimarro, E., Cagnazzo, C., et al. (2011). Decadal climate predictions with a coupled OAGCM initialized with oceanic reanalyses. Clim. Dyn. 40, 1483-1497. doi: 10.1007/s00382-012-1468-z in emerging and developing economies to take ownership for long-term, quality-controlled climate observations, and to use them to produce actionable climate information. This last step is indispensable to the generation of regionally relevant climate information.

\section{AUTHOR CONTRIBUTIONS}

DS and $\mathrm{AB}$ created the outline of topics and wrote the proposal of the manuscript. All authors contributed to the writing and editing processes of the manuscript, and recommended the references and examples.

\section{FUNDING}

This work was partly supported by the DFG funded excellence center CliSAP of the Universituat Hamburg (DS). AB was supported by the National Science Foundation through award NSF-1658174 and by the NOAA through award NA16OAR4310173. SM was supported by the Earth Systems and Climate Change Hub of the Australian Government's National Environmental Science Program.

\section{ACKNOWLEDGMENTS}

Comments from the two reviewers are gratefully acknowledged. We thank all the CLIVAR panel members who contributed with helpful comments and suggestions to this work. The International Cooperation that CLIVAR enjoys would not be possible without the organizational leadership of the CLIVAR ICPO hosted by the First Oceanographic Institute (FIO) in Qingdao, China, the continuous support of the US-CLIVAR office, and the scientific leadership and support of the World Climate Research Programme. NCAR is sponsored by the US National Science Foundation. BD acknowledges supports from FONDECYT (project 1171861).

Belmadami, A., Echevin, V., Codron, F., Takahashi, K., and Junquas, C. (2014). What dynamics drive future wind scenarios for coastal upwelling off Peru and Chile? Clim. Dyn. 43, 1893-1914. doi: 10.1007/s00382-0132015-2

Blanke, B., Speich, S., Madec, G., and Maugé, R. (2002). A global diagnostic of interior ocean ventilation. Geophys. Res. Lett. 29, 108-1-108-4.

Blunden, J., Arndt, D. S., and Hartfield, G. (eds). (2018). State of the climate in 2017. Bull. Am. Meteorol. Soc. 99:Si-S310. doi: 10.1175/2018BAMSStateofthe Climate.1

Bond, N. A., Cronin, M. F., Freeland, H., and Mantua, N. (2015). Causes and impacts of the 2014 warm anomaly in the NE Pacific. Geoph. Res. Lett. 42, 3414-3420. doi: 10.1002/2015GL06330

Bourassa, M. A., Gille, S. T., Bitz, C., Carlson, D., Cerovecki, I., Clayson, C. A., et al. (2013). High latitude ocean and sea ice surface fluxes: challenges for climate research. Bull. Am. Meteorol. Soc. 94, 403-423. doi: 10.1175/BAMS-D11-00244.1

Breitburg, D., Levin, L. A., Oschlies, A., Grégoire, M., Chavez, F. P., Conley, D. J., et al. (2018). Declining oxygen in the global ocean and coastal waters. Science 359:eaam7240. doi: 10.1126/science.aam 7240 
Bringedal, C., Eldevik, T., Skagseth, Ø., Spall, M., and Østerhus, S. (2018). Structure and forcing of observed exchanges across the Greenland-Scotland Ridge. J. Clim. 31, 9881-9901. doi: 10.1175/JCLI-D-17-0889.1

Bryden, H. L., and Beal, L. M. (2001). Role of the agulhas current in indian ocean circulation and associated heat and freshwater fluxes. Deep Sea Res. Part I Oceanogr. Res. Papers 48, 1821-1845. doi: 10.1016/s0967-0637(00)00111-4

Bryden, H. L., and Imawaki, S. (2001). "Ocean heat transport" in Ocean Circulation and Climate: Observing and Modelling the Global Ocean, International Geophysics Series, eds G. Siedler, J. Church, and J. Gould, (Cambridge, MA: Academic Press) 455-474.

Cabanes, C., Grouazel, A., von Schuckmann, K., Hamon, M., Turpin, V., Coatanoan, C., et al. (2013). The CORA dataset: validation and diagnostics of in-situ ocean temperature and salinity measurements. Ocean Sci. 9, 1-18. doi: 10.5194/os-9-1-2013

Cai, W., Santoso, A., Wang, G., Yeh, S.-W., An, S.-I., Cobb, K., et al. (2015). ENSO and greenhouse warming. Nat. Clim. Change 5, 849-859. doi: 10.1038/ nclimate 2743

Cheng, L., Trenberth, K. E., Fasullo, J., Boyer, T., Abraham, J., and Zhu, J. (2017). Improved estimates of ocean heat content from 1960 to 2015. Sci. Adv. 3:e1601545. doi: 10.1126/sciadv.1601545

Claustre, H., Zilberman, N., Scanderbeg, M., Roemmich, D., and Alford, M.H. (2019). Argo technology and integration on the global ocean observing system. In Oceanobs19: an ocean of opportunity. Front. Mar. Sci. doi: 10.1126/sciadv. 1601545

Cronin, M. F., Ueki, I., Gentemann, C. L., Edson, J., Bentamy, A., Bourussa, M., et al. (2019). Air sea flux: heat and momentum. in oceanobs19: an ocean of opportunity. Front. Mar. Sci. doi: 10.3389/fmars.2019.00430

Curry, B., Lee, C. M., Petrie, B., Moritz, R. E., and Kwok, R. (2014). Multiyear volume, liquid freshwater, and sea ice transports through davis strait, 2004-10. J. Phys. Ocean. 44, 1244-1266. doi: 10.1175/JPO-D-13-0177.1

DeMott, C. A., Klingaman, N. P., and Woolnough, S. J. (2015). Atmosphereocean coupled processes in the Madden-Julian oscillation. Rev. Geophys. 53, 1099-1154. doi: 10.1002/2014RG000478

Desbruyères, D., McDonagh, E. L., King, B. A., and Thierry, V. (2017). Global and full-depth ocean temperature trends during the early twenty-first century from Argo and repeat hydrography. J. Clim. 30, 1985-1997. doi: 10.1175/JCLI-D-160396.1

deYoung, B., Visbeck, M., de Araujo Filho, M. C., Baringer, M. O., Black, C.-A., and Buch, E. et al. (2019). An integrated all-atlantic ocean observing system in 2030. Front. Mar. Sci. doi: 10.1175/jcli-d-16-0396.1

Dickson, B., Meincke, J., and Rhines, P. (eds) (2008). Arctic and Subarctic Ocean Fluxes: Defining the Role of The Northern Seas In Climate, Netherland: Springer.

Domingues, C. M., Church, J. A., White, N. J., Gleckler, P. J., Wijffels, S. E., Barker, P. M., et al. (2008). Improved estimates of upper-ocean warming and multi-decadal sea-level rise. Nature 453, 1090-1093. doi: 10.1038/nature 07080

Domingues, C. M., and Palmer, M. (2015). The IQuOD initiative: towards an international quality controlled ocean database. Clivar Exchanges 19, 38-40.

Dong, B., and Dai, A. (2015). The influence of the interdecadal pacific oscillationon temperature and precipitation over the globe. Clim. Dyn. 45, 2667-2681. doi: 10.1007/s00382-015-2500-x

Donohue, K. A., Tracey, K. L., Watts, D. R., Chidichimo, M. P., and Chereskin, T. K. (2016). Mean antarctic circumpolar current transport measured in drake passage. Geophys. Res. Lett. 43, 11,760-11,761. doi: 10.1002/2016GL0 70319

Downes, S., Farneti, R., Uotile, P., Griffies, S. M., Marsland, S. J., Bailet, D., et al. (2015). An assessment of Southern Ocean water masses and sea- ice during 1988-2007 in a suite of interannual CORE-II simulations. Ocean Modell. 94, 67-94. doi: 10.1016/j.ocemod.2015.07.022

Farneti, R., Downes, S. M., Griffies, S. M., Marsland, S. J., Behrens, E., Bentsen, M., et al. (2015). An assessment of antarctic circumpolar current and southern ocean meridional overturning circulation during 1958-2007 in a suite of interannual CORE-II simulations. Ocean Modell. 94, 84-120. doi: 10.1016/j. ocemod.2015.07.009

Fay, A. R., Lovenduski, N. S., McKinley, G. A., Munro, D. R., Sweeney, C., Gray, A. R., et al. (2018). Utilizing the drake passage time-series to understand variability and change in subpolar Southern Ocean pCO2. Biogeosciences 15, 3841-3855. doi: 10.5194/bg-15-3841-2018
Fennel, W., Junker, T., Schmidt, M., and Mohrholz, V. (2012). Response of the Benguela upwelling systems to spatial variations in the wind stress. Cont. Shelf Res. 45, 65-77. doi: 10.1016/j.csr.2012.06.004

Fiechter, J., Curchitser, E. N., Edwards, C. A., Chai, F., Goebel, N. L., and Chavez, F. P. (2014). Air-sea CO2 fluxes in the California current: impacts of model resolution and coastal topography. Glob. Biogeochem. Cycles 28, 371-385. doi: 10.1002/2013gb004683

Foltz, G. R., Brandt, P., Richter, I., Rodriguez-fonseca, B., Hernandez, F., Dengler, M., et al. (2019). The tropical atlantic observing system. Front. Mar. Sci. 6:206. doi: 10.3389/fmars.2019.00206

Foltz, G. R., Schmid, C., and Lumpkin, R. (2018). An enhanced PIRATA data set for tropical Atlantic ocean-atmosphere research. J. Clim. 31, 1499-1524. doi: 10.1175/JCLI-D-16-0816.1

Fox-Kemper, B., Adcroft, A., Boning, C. W., Chassignet, E. P., Curchitser, E., Danabasoglu, G., et al. (2019). Challenges and prospects in ocean circulation models. Front. Mar. Sci. 6:65. doi: 10.3389/fmars.2019.00065

Frajka-Williams, E., Ansorge, I. J., Baehr, J., Bryden, H. L., Chidichimo, M. P., Cunningham, S. A. et al. (2019). Atlantic meridional overturning circulation: observed transports and variability. Frontiers Mar. Sci. doi: 10.3389/fmars.2019. 00260

Frölicher, T. L., Sarmiento, J. L., Paynter, D. J., Dunne, J. P., Krasting, J. P., and Winton, M. (2015). Dominance of the Southern Ocean in anthropogenic carbon and heat uptake in CMIP5 models. J. Clim. 28, 862-886. doi: 10.1175/JCLI-D14-00117.1

Fu, Y., Karstensen, J., and Brandt, P. (2018). Atlantic meridional overturning circulation at $14.5^{\circ} \mathrm{N}$ in 1989 and 2013 and $24.5^{\circ} \mathrm{N}$ in 1992 and 2015: volume, heat, and freshwater transports. Ocean Sci. 14, 589-616. doi: 10.5194/os-14589-2018

Goericke, G., and Ohman, M. D. (2015). Introduction to CCE-LTER: responses of the California current ecosystem to climate forcing. Deep Sea Res. II 112, 1-5. doi: 10.1016/j.dsr2.2014.12.00

Gomes, H., Goes, J. I., Matondkar, S. G. P., Buskey, E. J., Basu, S., Parab, S., et al. (2014). Massive out-breaks of Noctiluca scintillans blooms in the Arabian Sea due to spread of hypoxia. Nat. Commun. 5:4862. doi: 10.1038/ncomms 5862

Goni, G. J., Sprintall, J., Bringas, F., Cheng, L., Cirano, M., Dong, S., et al. (2019). More than 50 years of successful continuous temperature section measurements by the global expendable bathythermograph network, its integrability, societal benefits, and future. Front. Mar. Sci. doi: 10.3389/fmars.2019. 00452

Graco, M., Purca, S., Dewitte, B., Castro, C. G., Moron, O., Ledesma, J., et al. (2017). The OMZ and nutrients features as a signature of interannual and low frequency variability off the Peruvian upwelling system. Biogeosciences 14, 4601-4617. doi: 10.5194/bg-14-4601-2017

Gray, A., Johnson, K. S., Bushinsky, S. M., Riser, S. C., Russell, J. L., Talley, L. D., et al. (2018). Autonomous biogeochemical floats detect significant carbon dioxide outgassing in the high-latitude Southern Ocean. Geophys. Res. Lett. 45, 9049-9057. doi: 10.1029/2018GL078013

Griffies, S. M., Adcroft, A. J., Banks, H., Boning, C. W., Chassignet, E. P., Danabasoglu, G., et al. (2010). Problems and prospects in large-scale ocean circulation models. in Proceedings of OceanObs'09: Sustained Ocean Observations and Information for Society, eds J. Hall, D.E. Harrison, and D. Stammer, (Noordwijk: European Space Agency), 437-458.

Gruber, N., Landscützer, P., and Lovenduski, N. (2019). The variable southern ocean carbon sink. Annu. Rev. Mar. Sci. 11, 159-186. doi: 10.1146/annurevmarine-121916-063407

Gulev, S. K., Latif, M., Keelyside, N., Park, W., and Koltermann, K. P. (2013). North Atlantic ocean control on surface heat flux on multidecadal timescales. Nature 499, 464-467. doi: 10.1038/nature12268

Häkkinen, S., Rhines, P. B., and Worthen, D. L. (2015). Heat content variability in the North Atlantic Ocean in ocean reanalyses. Geophys. Res. Lett. 42, 2901-2909. doi: 10.1002/2015GL063299

Hall, J., Harrison, D. E., and Stammer, D. (eds) (2010). Proceedings of OceanObs'09: "Sustained Ocean Observations and Information for Society. Venice: ESA Publication WPP-306.

Han, W., Vialard, J., McPhaden, M. J., Lee, T., Masumoto, Y., Feng, M., et al. (2014). Indian ocean decadal variability: a review. Bull. Am. Meteorol. Soc. 95, 1679-1703. doi: 10.1175/BAMS-D-13-00028.1 
Horwath, M., Hamlington, B., Cazenave, A., Kulaiappan, H., Marzeion, P., Paul, F., et al. (this issue). Observational needs: global sea level budget. in oceanobs19: an ocean of opportunity. Front. Mar. Sci.

$\mathrm{Hu}$, S., and Sprintall, J. (2017). Observed strengthening of interbasin exchange via the Indonesian seas due to rainfall intensification. Geophys. Res. Lett. 44, 1448-1456. doi: 10.1002/2016GL072494

Ishii, M., Fakuda, Y., Hirahara, S., Yasui, S., Suzuki, T., and Sato, K. (2017). Accuracy of global upper ocean heat content estimation expected from present observational data sets. SOLA 13, 163-167. doi: 10.2151/sola.2017-030

Ito, T., Minobe, S., Long, M., and Deutsch, C. (2017). The upper ocean oxygen trend: 1958-2015. Geoph. Res. Lett. 44, 4214-4223. doi: 10.1002/2017GL073613

Johns, B., Speich, S., Araujo, M., McPhaden, M., Visbeck, M., Tanhua, T., et al. (2018). TAOS Review, CLIVAR Report No. 09/2018. Available at: http://www.clivar.org/sites/default/files/documents/1st\%20TAOS\%20Review\% 20Workshop\%20Report_final.pdf (accessed July 5, 2019).

Karspeck, A. R., Stammer, D., Köhl, A., Danabasoglu, G., Balmaseda, M., Smith, D. M., et al. (2017). Comparison of the Atlantic Meridional overturning circulation between 1960 and 2007 in six ocean reanalysis products. Clim. Dyn. 49, 957-982. doi: 10.1007/s00382-015-2787-7

Kataoka, T., Masson, S., Izumo, T., Tozuka, T., and Yamagata, T. (2018). Can Ningaloo Niño/Niña develop without El Niño-Southern oscillation? Geoph. Res. Lett. 45, 7040-7048. doi: 10.1029/2018GL078188

Kerr, R. A. (2000). A North atlantic climate pacemaker for the centuries. Science 288, 1984-1986. doi: 10.1126/science.288.5473.1984

Klein, S. A., Soden, B. J., and Lau, N.-C. (1999). Remote sea surface temperature variations during enso: evidence for a tropical atmospheric bridge. J. Climate, 12, 917-932. doi: 10.1175/1520-0442(1999)012<0917:rsstvd>2.0.co;2

Koblinsky, C. J., and Smith, N. R. (eds) (2001). Observing the Oceans in the 21st Century. Melbourne: GODAE Project Office and Bureau of Meteorology. doi: 10.1175/1520-0442(1999)012<0917:rsstvd > 2.0.co;2

Kosaka, Y., and Xie, S.-P. (2013). Recent global-warming hiatus tied to equatorial Pacific surface cooling. Nature 501, 403-407. doi: 10.1038/nature12534

Kucharski, F., Kang, I.-S., Farneti, R., and Feudale, L. (2011). Tropical Pacific response to 20th century Atlantic warming. Geophys. Res. Lett. 38:L03702. doi: 10.1029/2010GL046248

Lee, S. K., Park, W., Baringer, M. O., Gordon, A. L., Huber, B., and Liu, Y. (2015). Pacific origin of the abrupt increase in Indian Ocean heat content during the warming hiatus. Nat. Geosci. 8, 445-449. doi: 10.1038/ngeo2438

Lind, S., Ingvaldsen, R. B., and Furevik, T. (2018). Arctic warming hotspot in the northern Barents Sea linked to declining sea-ice import. Nat. Clim. Change 8, 1-9. doi: 10.1038/s41558-018-0205-y

Liu, G., Bracco, A., and Passow, U. (2018). The influence of mesoscale and submesoscale circulation on sinking particles in the northern Gulf of Mexico. Elem. Sci. Anth. 6:36. doi: 10.1525/elementa.292

Liu, X., Köhl, A., and Stammer, D. (2017). Dynamical ocean response to projected changes of the global water cycle. J. Geophys. Res. Oceans 122, 6512-6532. doi: 10.1002/2017JC013061

Lumpkin, R., Özgökmen, T., and Centurioni, L. (2017). Advances in the application of surface drifters. Annu. Rev. Mar. Sci. 9, 59-81. doi: 10.1146/annurev-marine010816-060641

Mantua, N. J., Hare, S. R., Zhang, Y., Wallace, J. M., and Francis, R. C. (1997). A Pacific interdecadal climate oscillation with impacts on salmon production. Bull. Am. Met. Soc. 78, 1069-1079. doi: 10.1175/1520-0477(1997)078<1069: apicow $>2.0 . \operatorname{co} ; 2$

Marinov, I., Doney, S. C., and Lima, I. D. (2010). Response of ocean phytoplankton community structure to climate change over the 21st century: partitioning the effects of nutrients, temperature and light. Biogeosciences 7, 3941-3959. doi: 10.5194/bg-7-3941-2010

Marotzke, J., Müller, W. A., Vamborg, F., Becker, P., Cubasch, U., Feldmann, H., et al. (2016). MiKlip - a national research project on decadal climate prediction. Bull. Am. Meteorol. Soc. 97, 2379-2394. doi: 10.1175/BAMS-D-1500184.1

Mazloff, M. R., Sallée, J.-B., Menezes, V. V., Macdonald, A. M., Meredith, M. P., Newman, L., et al. (2017). "Southern Ocean" in: State of the Climate 2016, eds J. Blunden, and D. S. Arndt, (Boston, MA: American Meteorological Society), $166-167$.

McCabe, R. M., Hickey, B. M., Kudela, R. M., Lefebvre, K. A., Adams, N. G., Bill, B. D., et al. (2016). An unprecedented coastwide toxic algal bloom linked to anomalous ocean conditions. Geoph. Res. Lett. 43, 10366-10376. doi: 10.1002/ $2016 \mathrm{gl070023}$

McPhaden, M. J., Zebiak, S. E., and Glantz, M. H. (2006). ENSO as an integrating concept in earth science. Science 15, 1740-1745. doi: 10.1126/science.1132588

Minobe, S., Kuwano-Yoshida, A., Komori, N., Xie, S.-P., and Small, R. J. (2008). Influence of the gulf stream on the troposphere. Nature 452, 206-209. doi: 10.1038 /nature0669

Minobe, S., Terada, M., Qiu, B., and Schneder, N. (2017). Western boundary sea level: a theory, rule of thumb, and application to climate models. J. Phys. Oceanogr. 47, 957-977. doi: 10.1175/JPO-D-16-0144.1

Mongwe, N. P., Vichi, M., and Monteiro, P. M. S. (2018). The seasonal cycle of pCO2 and CO2 fluxes in the Southern Ocean: diagnosing anomalies in CMIP5 Earth system models. Biogeosciences 15, 2851-2872. doi: 10.5194/bg-15-2851-8

Naqvi, S. W. A., Bange, H. W., Farías, L., Monteiro, P. M. S., Scranton, M. I., and Zhang, J. (2010). Marine hypoxia/anoxia as a source of CH4 and N2O. Biogeosciences 7, 2159-2190. doi: 10.5194/bg-7-2159-2010

Newman, L., Heil, P., van Wijk, E., Constable, A. J., Trebilco, R., et al. (2019) Southern ocean observing system. In oceanobs19: an ocean of opportunity. Front. Mar. Sci. doi: 10.3389/fmars.2019.00433

Olsen, A., Key, R. M., van Heuven, S., Lauvset, S. K., Velo, A., Lin, X., et al. (2016). The global ocean data analysis project version 2 (GLODAPv2) - an internally consistent data product for the world ocean. Earth Syst. Sci. Data 8, 297-323. doi: 10.5194/essd-8-297-2016

O’Reilly, C. H., Minobe, S., Kuwano-Yoshida, A., and Woollings, T. (2017). The Gulf stream influence on wintertime North Atlantic jet variability. Q. J. R. Met. Soc. 143, 173-183. doi: 10.1002/qj.2907

Palmer, M. D., Roberts, C. D., Balmaseda, M. Chang, Y.-S., Chepurin, G., Ferry, N., et al. (2017). Ocean heat content variability and change in an ensemble of ocean reanalyses. Clim. Dyn. 49, 909-930. doi: 10.1007/s00382-015-2801-0

Palmer, M. D., Durack, P., Chidichimo, M. P., Church, J., Cravatte, S., Hill, K., et al. (2019) Adequacy of the ocean observing system for quantifying regional heat and freshwater storage and change. in Oceanobs19: an ocean of opportunity. Front. Mar. Sci.

Patricola, C. M., and Chang, P. (2016). Structure and dynamics of the Benguela low-level coastal jet. Clim. Dyn. 49, 2765-2788. doi: 10.1007/s00382-0163479-7

Paul, B. K. (2009). Why relatively fewer people died? The case of Bangladesh's Cyclone Sidr. Nat. Hazards 50, 289-304. doi: 10.1007/s11069-0089340-5

Paulmier, A., and Ruiz-Pino, D. (2009). Oxygen minimum zones (OMZs) in the modern ocean. Progr. Ocean. 80, 113-128. doi: 10.1016/j.pocean.2008.08.001

Pellichero, V., Sallée, J.-B., Chapman, C. C., and Downes, S. M. (2018). The southern ocean meridional overturning in the sea-ice sector is driven by freshwater fluxes. Nat. Commun. 9:1789. doi: 10.1038/s41467-018-04101-2

Penny, S. G., Akella, S., Balmaseda, M. A., Browne, P., Carton, J. A., Chevallier, M., et al. (2019). Observational needs for improving ocean and coupled reanalysis, S2S prediction, and decadal prediction. Front. Mar. Sci.

Polkova, I., Köhl, A., and Stammer, D. (2014). Impact of initialization procedures on the predictive skill of a coupled ocean - atmosphere model. Clim. Dyn. 42, 3151-3169. doi: 10.1007/s00382-013-1969-4

Ponte, R., Carson, M., Cirano, M., Domingues, C., Jevrejeva, S., Marcos, M., et al. (2019). Towards comprehensive observing and modeling systems for monitoring and predicting regional to coastal sea level. Front. Mar. Sci.

Power, S., Casey, T., Folland, C., Colman, A., and Mehta, V. (1999). Inter-decadal modulation of the impact of ENSO on Australia. Clim. Dyn. 15, 319-324. doi: $10.1007 / \mathrm{s} 003820050284$

Prowse, T., Bring, A., Mård, J., and Carmack, E. (2015). Arctic freshwater synthesis: introduction. J. Geophys. Res. Biogeosci. 120, 2121-2131. doi: 10.1002/ 2015JG003127

Rabe, B., Karcher, M., Kauker, F., Schauer, U., Toole, J. M., Krishfield, R. A., et al. (2014). Arctic Ocean basin liquid freshwater storage trend 1992-2012. Geoph. Res. Lett. 41, 961-968. doi: 10.1002/2013GL058121

snmRhein, M., Rintoul, S. R., Aoki, S., Campos, E., Chambers, D., Feely, R. A., et al. (2013). “Observations: Ocean." In Climate Change 2013: The Physical Science Basis. Contribution of Working Group I to the Fifth Assessment Report of the Intergovernmental Panel on Climate Change. eds T.F, Stocker, et al. (Cambridge: Cambridge University Press). 
Rhines, P., Häkkinen, S., and Josey, S. A. (2008). "Is oceanic heat transport significant in the climate system?" in Arctic-Subarctic Ocean Fluxes: Defining the Role of the Northern Seas in Climate, eds R. R. Dickson, J. Meincke, and P. Rhines, (Berlin: Springer), pp. 87-109 doi: 10.1007/978-1-4020-6774-7_5

Richter, I. (2015). Climate model biases in the eastern tropical oceans: causes, impacts and ways forward. WIREs Clim. Change, 6, 345-358. doi: 10.1002/ wcc. 33

Richter-Menge, J., and Mathis, J. T., (eds) (2017). “Arctic” in state of the climate in 2016. Bull. Am. Meteorol. Soc. 98, S129-S154.

Rintoul, S. R. (2018). The global influence of localized dynamics in the Southern Ocean. Nature 558, 209-218. doi: 10.1038/s41586-018-0182-3

Rixen, T., Baum, A., Gaye, B., and Nagel, B. (2014). Seasonal and interannual variations in the nitrogen 190 cycle in the Arabian Sea. Biogeoscience 11, 5733-5747. doi: 10.5194/bg-11-5733-2014

Roemmich, D., Alford, M. H., Claustre, H., Johnson, K. S., King, B., and Moum, J. (2019). On the future of Argo: an enhanced global array of physical and biogeochemical sensing floats. Front. Mar. Sci.

Roemmich, D., Church, J., Gilson, J., Monselesan, D., Sutton, P., and Wijffels, S. (2015). Unabated planetary warming and its ocean structure since 2006. Nat. Clim. Change. 5, 240-245. doi: 10.1038/nclimate2513

Roxy, M. K., Modi, A., Murtugudde, R., Valsala, V., Panickal, S., Kumar, S. P., et al. (2016). A reduction in marine primary productivity driven by rapid warming over the tropical Indian Ocean. Geophys. Res. Lett. 43, 826-833. doi: 10.1002/2015GL066979

Roxy, M. K., Ritika, K., Terray, P., Murtugudde, R., Ashok, K., and Goswami, B. N. (2015). Drying of indian subcontinent by rapid Indian ocean warming and a weakening land-sea thermal gradient. Nat. Commun. 6:7423. doi: 10.1038/ ncomms 8423

Rykaczewski, R. R., and Dunne, J. P. (2010). Enhanced nutrient supply to the California current ecosystem with global warming and increased stratification in an Earth system model. Geoph. Res. Lett. 37:L21606

Schlundt, M., Brandt, P., Dengler, M., Hummels, R., Fischer, T., Bumke, K., et al. (2014). Mixed layer heat and salinity budgets during the onset of the 2011 Atlantic cold tongue. J. Geophys. Res. Oceans 119, 7882-7910. doi: 10.1002/ 2014JC010021

Schott, F. A., Xie, S. P., and McCreary, J. P. (2009). Indian ocean circulation and climate variability. Rev. Geophys. 47:RG1002. doi: 10.1029/2007RG000245

Sherman, K., and Alexander, L. M. (eds). (1986). Variability and Management of Large Marine Ecosystems. AAAS Selected Symposium 99, (Boulder, CO: Westview Press).

Sloyan, B. M., Wanninkhof, R., Kramp, M., Johnson, G. C., Talley, L., Tanhua, T., et al. (2019). The global ocean ship-base hydrographic investigations program (GO-SHIP): a platform for integrated multidisciplinary ocean science. Front. Mar. Sci.

Small, R. J., Tomas, R. A., and Bryan, F. O. (2014). Storm track response to ocean fronts in a global high-resolution climate model. Clim. Dyn. 43, 805-828. doi: 10.1007/s00382-013-1980-9

Smith, N., Kessler, W. S., Cravatte, S., Sprintall, S. S., Cronin, M. F., Sutton, A. et al. (2019). Tropical Pacific Observing System. Front. Mar. Res. doi: 10.3389/fmars. 2019.00031

Spence, P., Holmes, R. M., Hogg, A. M., Griffies, S. M., Stewart, K. D., and England, M. H. (2017). Localized rapid warming of West Antarctic subsurface waters by remote winds. Nat. Clim. Change 7, 595-603. doi: 10.1038/nclimate 3335

Stammer, D., Balmaseda, M., Heimbach, P., Köhl, A., and Weaver, A. (2016). Ocean data assimilation in support of climate applications - status and perspectives. Ann. Rev. Mar. Sci. 8, 491-518. doi: 10.1146/annurev-marine-122414034113

Stammerjohn, S., and Scambos, T. A. (eds). (2017). Antarctica [in "(State)of the Climate in 2016"]. Bull. Am. Meteorol. Soc. 98, S155-S172.

Stock, C. A., John, J. G., Rykaczewski, R. R., Asch, R. G., Cheung, W. W. L., Dunne, J. P., et al. (2017). Reconciling fisheries catch and ocean productivity. Proc. Nat. Acad. Sci. 114, E1441-E1449. doi: 10.1073/pnas.1610238114

Stramma, L., Johnson, G. C., Sprintall, J., and Mohrholz, V. (2008). Expanding oxygen-minimum zones in the tropical oceans. Science 320, 655-658. doi: 10. $1126 /$ science. 1153847

Subramanian, A., Balmaseda, M. A., Chattopadhyay, R., Centurioni, L. R., Cornuelle, B. D., DeMott, C., et al. (2019). Ocean observations to improve our understanding, modeling, and forecasting of subseasonal-to-seasonal variability. Front. Mar. Sci. doi: 10.3389/fmars.2019.00427

O'Carroll, A. G., Armstrong, E. M., Beggs, H., Bouali, M., Casey, K. S., and Corlett, G. K. (2019). Observational needs of Sea Surface Temperature. Front. Mar. Sci. doi: $10.3389 /$ fmars.2019.00420

Sutton, R. T., and Hodson, D. L. (2005). Atlantic Ocean forcing of North American and European summer climate. Science 309, 115-118. doi: 10.1126/science. 1109496

Swart, N. C., and Fyfe, J. C. (2013). The influence of recent Antarctic ice sheet retreat on simulated sea ice area trends. Geophys. Res. Lett. 40, 4328-4332. doi: $10.1002 /$ grl.50820

Swart, N. C., Gille, S. T., Fyfe, J. C., and Gillett, N. (2018). Recent Southern Ocean warming and freshening driven by greenhouse gas emissions and ozone depletion. Nat. Geosci. 11, 836-841. doi: 10.1038/s41561-018-0226-1

Tagklis, F., Bracco, A., and Ito, T. (2017). Physically driven patchy O2 changes in the North Atlantic Ocean simulated by the CMIP5 Earth system models. Glob. Biogeochem. Cycles 31, 1218-1235. doi: 10.1002/2016GB005617

Takahashi, K., and Battisti, D. S. (2007). Processes controlling the mean tropical pacific precipitation pattern. Part I: the Andes and the Eastern Pacific ITCZ. J. Clim. 20, 3434-3451. doi: 10.1175/JCLI4198.1

Takahashi, K., and Martinez, A. G. (2017). The very strong coastal El Niño in 1925 in the far-eastern Pacific. Clim. Dyn. 52, 7389-7415. doi: 10.1007/s00382-0173702-1

Takahashi, T., Sutherland, S. C., Wanninkhof, R., Sweeney, C., Feely, R. A., Chipman, D. W., et al. (2009). Climatological mean and decadal change in surface ocean pCO2, and net sea-air CO2 flux over the global oceans. Deep Sea Res. II, 56, 554-577. doi: 10.1016/j.dsr2.2008.12.009

Talley, L. D., Reid, J. L., and Robbins, P. E. (2003). Data-based meridional overturning streamfunctions for the global ocean. J. Climate, 16, 3213-3226. doi: 10.1175/1520-0442(2003)016<3213:dmosft >2.0.co;2

Taylor, K. E., Stouffer, R. J., and Meehl, G. A. (2012). An overview of CMIP5 and the experiment design. Bull. Am. Meteorol. Soc. 93, 485-498. doi: 10.1175/BAMSD-11-00094.1

Terray, P., Masson, S., Prodhomme, C., Roxy, M. K., and Sooraj, K. P. (2016). Impacts of Indian and Atlantic oceans on ENSO in a comprehensive modeling framework. Clim. Dyn. 46, 2507-2533. doi: 10.1007/s00382-015-2715-x

Testor, P., DeYoung, B., Rudnick, D. L., Glenn, S., Hayes, D., and Lee, C., et al. (2019). OceanGliders: a component of the integrated GOOS. Front. Mar. Sci. doi: $10.3389 /$ fmars. 2019.00422

The National Academies of Sciences, Engineering, and Medicine [NASEM], (2017). Sustaining Ocean Observations To Understand Future Changes In Earth's Climate. (Washington, DC: The National Academics Press).

Tilbrook, B., Jewett, E. B., DeGrandpre, M. D., Hernandez-Ayon, J. M., Feely, R. A., Gledhill, D. K., et al. (2019). An enhanced ocean acidification observing network: from people to technology to data synthesis and information exchange. Front. Mar. Sci. 6:337. doi: 10.3389/fmars.2019.00337

Timmermann, A., An, S. I., Kug, J. S., Jin, F. F., Cai, W., Capotondi, A., et al. (2018). El Niño-Southern Oscillation complexity. Nature 559, 535-545. doi: 10.1038/s41586-018-0252-6

snmTodd, R. E., Chavez, F. P., Clayton, S., Cravette, S. E., Pereira Goes, M., Graco, N. I., et al. (2019). Global perspectives on observing ocean boundary current systems. Front. Mar. Sci. doi: 10.3389/fmars.2019.00423

Toniazzo, T., Abel, S. J., Wood, R., Mechoso, C. R., Allen, G., and Shaffrey, L. C. (2011). Large-scale and synoptic meteorology in the south-east Pacific during the observations campaign VOCALS-REx in austral Spring 2008. Atmos. Chem. Phys. 11, 4977-5009. doi: 10.5194/acp-11-4977-201

Uotila, P., Goosse, H., Haines, K., Chevallier, M., Barthelemy, A., Bricaut, C., et al. (2018). An assessment of ten ocean reanalyses in the polar regions. Clim. Dyn. doi: $10.1007 / \mathrm{s} 00382-018-4242-\mathrm{z}$

Vaughan, D. G., Comiso, J. C., Allison, I., Carrasco, J., Kaser, G., Kwok, R., et al. (2013). Observations: Cryosphere. in: Climate Change 2013: The Physical Science Basis. Contribution of Working Group I to the Fifth Assessment Report of the Intergovernmental Panel on Climate Change, eds Stocker, T. F., et al. (Cambridge: Cambridge University Press).

Vialard, J. (2015). Hiatus heat in the Indian Ocean, Nat. Geosci. 8, 423-424. doi: 10.1038 /ngeo2442

Vialard, J., Shenoi, S. S. C., McCreary, J. P., Shankar, D., Durand, F., Fernando, V., et al. (2009), Intraseasonal response of the northern Indian oceancoastal 
waveguide to the Madden-Julian oscillation. Geophys. Res. Lett. 36:L14606. doi: 10.1029/2009GL038450

Villas Boas, A. B., Ardhuin, F., Ayet, A., Bourassa, M. A., Brandt, P., Chapron, B., et al. (2019). Integrated observations and modeling of winds, currents, and waves: requirements and challenges for the next decade. Front. Mar. Sci. doi: 10.3389/fmars.2019.00425

Wannikhov, R. Pickers, P., Omar, A., Sutton, A. J., Murata, A., and Olsen, A. (2019). A surface ocean $\mathrm{CO}_{2}$ reference network, SOCONET and associated marine boundary layer $\mathrm{CO}_{2}$ measurements. Front. Mar. Sci. doi: 10.3389/fmars.2019. 00400

Webster, P. J., Moore, A. M., Loschnigg, J. P., and Leben, R. P. (1999). Coupled ocean-atmosphere dynamics in the Indian Ocean during 1997-98". Nature 401, 356-360. doi: 10.1038/43848

Wu, L., Cai, W., Zhang, L., Nakamura, H., Timmermann, A., Joyce, T. et al. (2012). Enhanced warming over the global subtropical western boundary currents. Nat. Clim. Change 2, 161-166. doi: 10.1038/nclimate1353

Zhang, C. (2005). Madden-Julian Oscillation. Rev. Geophys. 43:RG2003. doi: 10. 1029/2004RG000158

Zhang, Y., Feng, M., Du, Y., Phillips, H. E., Bindoff, N. L., and McPhaden, M. J. (2018). Strengthened indonesian throughflow drives decadal warming in the southern indian ocean. Geoph. Res. Lett. 45, 6167-6175.
Zika, J. D., Le Sommer, J., Dufour, C. O., Naveira-Garabato, A. C., and Blaker, A. T. (2013). Acceleration of the antarctic circumpolar current by wind stress along the coast of antarctica. J. Phys. Ocean. 12, 2772-2784. doi: 10.1175/JPO-D-13091.1

Conflict of Interest Statement: The authors declare that the research was conducted in the absence of any commercial or financial relationships that could be construed as a potential conflict of interest.

The handling Editor declared a shared affiliation, though no other collaboration, with one of the authors DC at the time of review.

Copyright $\odot 2019$ Stammer, Bracco, AchutaRao, Beal, Bindoff, Braconnot, Cai, Chen, Collins, Danabasoglu, Dewitte, Farneti, Fox-Kemper, Fyfe, Griffies, Jayne, Lazar, Lengaigne, Lin, Marsland, Minobe, Monteiro, Robinson, Roxy, Rykaczewski, Speich, Smith, Solomon, Storto, Takahashi, Toniazzo and Vialard. This is an open-access article distributed under the terms of the Creative Commons Attribution License (CC BY). The use, distribution or reproduction in other forums is permitted, provided the original author(s) and the copyright owner(s) are credited and that the original publication in this journal is cited, in accordance with accepted academic practice. No use, distribution or reproduction is permitted which does not comply with these terms. 


\section{Air-Sea Fluxes With a Focus on Heat and Momentum}

\author{
Meghan F. Cronin ${ }^{1 *}$, Chelle L. Gentemann², James Edson ${ }^{3}$, Iwao Ueki", \\ Mark Bourassa ${ }^{5,6}$, Shannon Brown ${ }^{7}$, Carol Anne Clayson ${ }^{3}$, Chris W. Fairall ${ }^{8}$, \\ J. Thomas Farrar ${ }^{3}$, Sarah T. Gille ${ }^{9}$, Sergey Gulev ${ }^{10}$, Simon A. Josey ${ }^{11}$, Seiji Kato ${ }^{12}$, \\ Masaki Katsumata ${ }^{4}$, Elizabeth Kent ${ }^{11}$, Marjolaine Krug ${ }^{13}$, Peter J. Minnett ${ }^{14}$, \\ Rhys Parfitt ${ }^{5,6}$, Rachel T. Pinker ${ }^{15}$, Paul W. Stackhouse Jr. 12, Sebastiaan Swart 16,17, \\ Hiroyuki Tomita ${ }^{18}$, Douglas Vandemark ${ }^{19}$, Robert A.Weller ${ }^{3}$, Kunio Yoneyama ${ }^{4}$, Lisan Yu ${ }^{3}$ \\ and Dongxiao Zhang ${ }^{20}$
}

\begin{abstract}
${ }^{1}$ Pacific Marine Environmental Laboratory, NOAA, Seattle, WA, United States, ${ }^{2}$ Earth and Space Research, Seattle, WA, United States, ${ }^{3}$ Woods Hole Oceanographic Institution, Woods Hole, MA, United States, ${ }^{4}$ Japan Agency for Marine-Earth Science and Technology, Yokosuka, Japan, ${ }^{5}$ Department of Earth, Ocean and Atmospheric Science, Florida State University, Tallahassee, FL, United States, ${ }^{6}$ Center for Ocean-Atmospheric Prediction Studies (COAPS), Florida State University, Tallahassee, FL, United States, ${ }^{7}$ Jet Propulsion Laboratory, California Institute of Technology, Pasadena, CA, United States, ${ }^{8}$ Earth System Research Laboratory, NOAA, Boulder, CO, United States, ${ }^{9}$ Scripps Institution of Oceanography, University of California, San Diego, La Jolla, CA, United States, ${ }^{10}$ P.P.Shirshov Institute of Oceanology, Moscow, Russia, " National Oceanography Centre, Southampton, United Kingdom, ${ }^{12}$ NASA Langley Research Center, Hampton, VA, United States, ${ }^{13}$ Council for Scientific and Industrial Research, Cape Town, South Africa, ${ }^{14}$ Rosensteil School of Marine and Atmospheric Science, University of Miami, Miami, FL, United States, ${ }^{15}$ University of Maryland, College Park, College Park, MD, United States, ${ }^{16}$ Department of Marine Sciences, University of Gothenburg, Gothenburg, Sweden, ${ }^{17}$ Department of Oceanography, University of Cape Town, Rondebosch, South Africa, ${ }^{18}$ Institute for Space-Earth Environmental Research, Nagoya University, Nagoya, Japan, ${ }^{19}$ College of Engineering and Physical Sciences, University of New Hampshire, Durham, $\mathrm{NH}$, United States, ${ }^{20}$ Joint Institute for the Study of the Atmosphere and Ocean, University of Washington, Seattle, WA, United States
\end{abstract}

Turbulent and radiative exchanges of heat between the ocean and atmosphere (hereafter heat fluxes), ocean surface wind stress, and state variables used to estimate them, are Essential Ocean Variables (EOVs) and Essential Climate Variables (ECVs) influencing weather and climate. This paper describes an observational strategy for producing 3-hourly, 25-km (and an aspirational goal of hourly at 10-km) heat flux and wind stress fields over the global, ice-free ocean with breakthrough 1-day random uncertainty of $15 \mathrm{~W} \mathrm{~m}^{-2}$ and a bias of less than $5 \mathrm{~W} \mathrm{~m}^{-2}$. At present this accuracy target is met only for OceanSITES reference station moorings and research vessels (RVs) that follow best practices. To meet these targets globally, in the next decade, satellite-based observations must be optimized for boundary layer measurements of air temperature, humidity, sea surface temperature, and ocean wind stress. In order to tune and validate these satellite measurements, a complementary global in situ flux array, built around an expanded OceanSITES network of time series reference station moorings, is also needed. The array would include 500-1000 measurement platforms, including autonomous surface vehicles, moored and drifting buoys, RVs, the existing OceanSITES network of 22 flux sites, and new OceanSITES expanded in 19 key regions. This array would be globally distributed, with 1-3 measurement platforms in each nominal $10^{\circ}$ by $10^{\circ}$ box. These improved moisture and temperature profiles and surface data, if assimilated into Numerical Weather Prediction (NWP) models, would lead to better representation of cloud formation processes, improving state variables and surface radiative and turbulent fluxes from these models. The in situ flux array provides globally distributed measurements and metrics for satellite algorithm development, 
product validation, and for improving satellite-based, NWP and blended flux products. In addition, some of these flux platforms will also measure direct turbulent fluxes, which can be used to improve algorithms for computation of air-sea exchange of heat and momentum in flux products and models. With these improved air-sea fluxes, the ocean's influence on the atmosphere will be better quantified and lead to improved longterm weather forecasts, seasonal-interannual-decadal climate predictions, and regional climate projections.

Keywords: air-sea heat flux, latent heat flux, surface radiation, ocean wind stress, autonomous surface vehicle, OceanSITES, ICOADS, satellite-based ocean monitoring system

\section{INTRODUCTION}

\section{Societal Importance of Air-Sea Fluxes}

The oceans impact weather and climate by heating (and cooling) the lower atmosphere. In particular, as seawater evaporates, the ocean surface cools; and when the moisture later condenses into cloud droplets, this heat is released, warming the atmosphere. This moistening, and then warming, makes the air buoyant, driving low-level baroclinicity and atmospheric convection, causing wind convergence at the surface and divergence aloft. At the equator, ocean heating of the atmosphere can result in towering convective clouds that reach the top of the troposphere. These disturbances in turn drive teleconnections in the atmosphere, affecting weather and climate remotely. Most dramatically, every 2-7 years, zonal shifts in the surface heating patterns along the equatorial Pacific, associated with El Niño Southern Oscillation (ENSO), lead to climate extremes across the world. Patterns of surface heat fluxes (Figures 1, 2) also affect large-scale atmospheric circulation patterns, with deep convection over the thermal equator forming the upward branch of the "Hadley Cell" that drives trade winds. Westerly jet streams in both hemispheres are likewise associated with vertical-meridional cells in the midlatitude and high latitudes. Again, their rising branches and storm tracks are aligned with the surface heating of the atmosphere associated with warm ocean western boundary currents that extend into the midlatitude ocean basins (Figures 1, 2). These surface wind patterns, e.g., westerly winds at high latitudes and easterly trades in the tropics, in turn drive the ocean general circulation. Western boundary currents associated with the wind-forced subtropical ocean gyres are particularly important as they carry warm water poleward, helping to transfer heat from the tropics (where there is greater heating of the earth's surface by solar radiation per area) to higher latitudes (where heat lost at the surface by latent and sensible heat flux and net infrared cooling is greater than that gained by solar radiation). As discussed in this paper, quantifying these air-sea fluxes, which represent the direct communication between the ocean and atmosphere, is challenging. Through the recommendations presented here, we believe that remaining large biases and uncertainties that result in differences in global fields (Figures 1C-F) could be reduced by up to an order of magnitude, enabling better resolution of phenomena on scales ranging from sub-diurnal and mesoscale to global and interannual.
Reducing inaccuracies (both biases and random uncertainty) in air-sea fluxes is important for improving long-term weather and climate predictions. Because the ocean's capacity to store heat is about 1000 times greater than that of the atmosphere, long-term weather and climate predictability has its origins in the oceans. Heat storage and release occurs on a range of time scales (Figure 2 and Supplementary Table S1) and can provide predictability out to 10-100 days (e.g., Madden-Julian Oscillation, Asian/Indian Monsoon), on seasonal-interannual time scales (e.g., ENSO), and out to decades (e.g., Pacific Decadal Oscillation, Atlantic Multidecadal Oscillation). Predictions of weather and climate on these time scales have great economic benefits for agriculture, water resource management, energy management, human and ecosystem health among others. Thus, to achieve useful predictions we must be able to quantify where, when and how much heat is released to the atmosphere. As a first step, here, we discuss strategies for improving our ability to quantify the amount of heat that at present is being exchanged between the ocean and atmosphere, regionally and globally. Because these air-sea heat exchanges are highly related to the surface dynamics and turbulent properties, we will also address quantification of wind stress.

Strong air-sea fluxes can occur on short time and space scales (Figure 2 and Supplementary Table S1), challenging both in situ (because of technical difficulties in extreme conditions and undersampling) and satellite observations (because of gridaveraging). The primary external time scales affecting air-sea fluxes are the diurnal cycle and the annual cycle associated orbital forcing. Internal dynamics lead to a range of other time and space scale variability in wind stress and air-sea heat fluxes. Sea-surface temperature (SST) fronts in the ocean are particularly critical to air-sea fluxes. The largest magnitude and temporal variability of air-sea fluxes is found in regions associated with SST fronts, specifically at western boundary currents such as the Gulf Stream, where intense poleward currents carry warm tropical water into the subtropics. In winter, large ocean heat loss is associated with cold air outbreaks, when cold and dry air blowing over much warmer water drive frequent episodic high flux events (e.g., Bond and Cronin, 2008; Shaman et al., 2010; Tilinina et al., 2018). Additionally, the intense SST gradients at ocean fronts result in strong heat flux gradients. These strong gradients in heat flux are known to be crucial for modulating both synoptic atmospheric variability and in turn the mean atmospheric state (Parfitt et al., 2016; Parfitt and Seo, 2018). Away from ocean fronts, whilst 

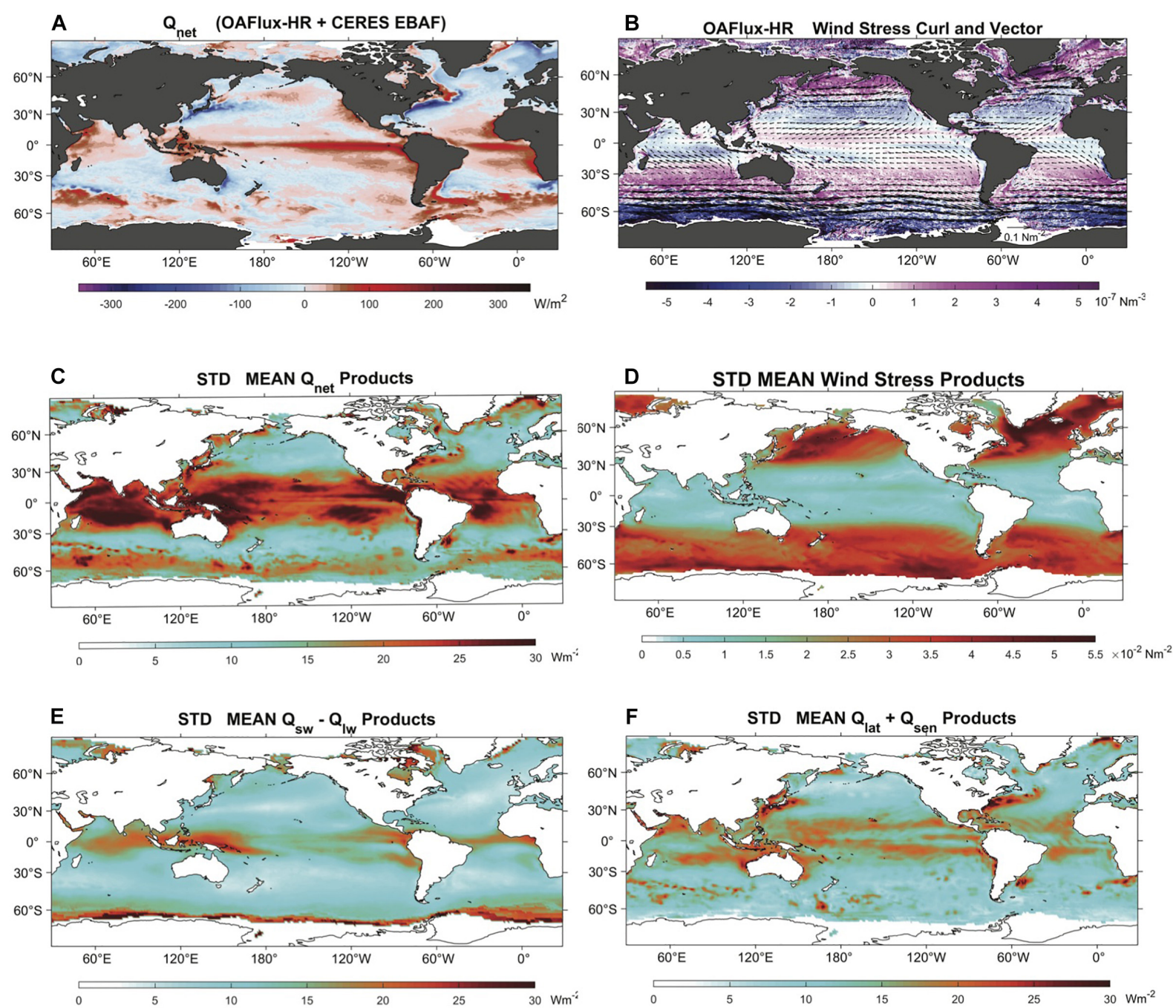

FIGURE 1 | (A) Annual mean net surface heat flux $\left(Q_{\text {net }}\right)$ for 2016 from the OAFlux-HR + CERES EBAFv4.0 product. (B) Annual mean wind stress curl and wind stress vectors for 2016 from the OAFlux-HR. (C) Standard deviation of annual-mean $Q_{n e t}$ from 12 products. (D) Standard deviation of annual mean of surface wind stress magnitude from 12 products. (E) Standard deviation of annual-mean surface shortwave and longwave $\left(Q_{S w}-Q_{I W}\right)$ from 10 products. (F) Standard deviation of annual-mean turbulent latent and sensible heat flux $\left(Q_{\text {lat }}+Q_{\text {sen }}\right)$ from 11 products. Based on Yu (2019).

turbulent mixing of colder and dryer air aloft generally results in a near surface air temperature cooler than the SST and relative humidity less than $100 \%$, the net surface heat loss from the ocean is much weaker. As will be discussed below, there are many challenges associated with resolving air-sea fluxes in regions of strong ocean fronts.

Because the specific heat capacity of water is considerably larger than that of land, air temperature is more variable over land than over the oceans, leading to a tendency for milder coastal climates than inland. Oceanic heat loss due to evaporation is associated with moisture fluxes that are an important source of water for agriculture and human consumption. Understanding and quantifying the exchange of heat and momentum between the ocean and atmosphere is therefore critically important for proper management of natural resources and reducing risks to vulnerable populations.

\section{Quantifying Air-Sea Exchanges of Heat and Momentum}

The net surface heat flux $\left(Q_{n e t}\right)$ comprises net shortwave (i.e., solar) $\left(Q_{S W}\right)$ and net longwave (i.e., infrared (IR) $\left(Q_{L W}\right)$ radiative fluxes, and surface turbulent (latent and sensible) heat fluxes:

$$
Q_{n e t}=Q_{s W}-Q_{L W}-Q_{l a t}-Q_{s e n}
$$

Surface latent heat flux, $Q_{\text {lat }}$, is the heat extracted from the ocean when seawater evaporates. This heat is released to the atmosphere when and where this vapor condenses, forming clouds. Likewise, sensible heat flux, $Q_{s e n}$, is the heat extracted from the ocean associated with an air-sea temperature difference. The sign convention used here enables each term to be expressed generally as a positive value (i.e., as a magnitude) for most applications. When averaged over the global oceans and a full year, there 


\section{Flux Accuracies and Processes}

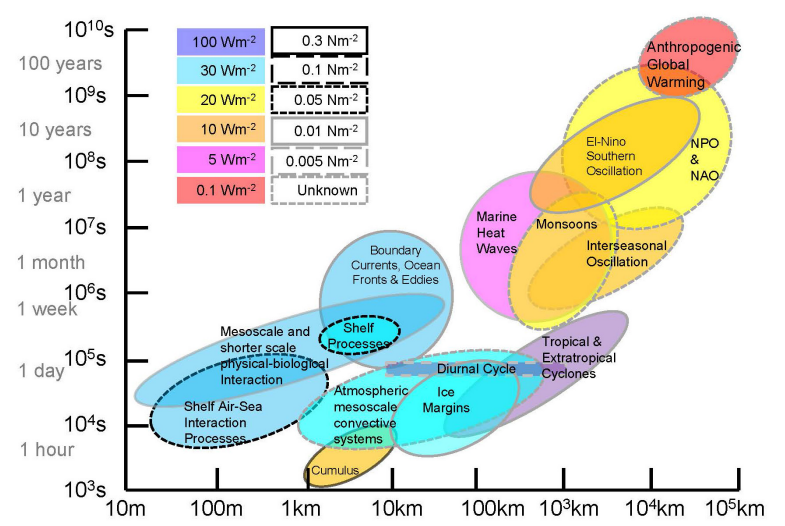

FIGURE 2 | Target flux accuracy and scales for different phenomena. The space and time scales are indicated by the extent of the bubble. The target accuracies, estimated at 10 to $20 \%$ of the observed variability, are indicated by the color for net heat flux, and by the bubble's outline for surface stress. The accuracy requirement for stress is a factor of 2 smaller for phenomena that are dependent on the curl of the stress rather than the stress magnitude. See Supplementary Table $\mathbf{S 1}$ for more detail. This figure is updated and adapted from Bourassa et al. (2013).

should be a near-balance between solar radiative heating of the ocean (reduced by net longwave radiative heat loss), latent heat loss due to evaporation and sensible heat loss induced by air-sea temperature and humidity differences. However, due to biases in flux estimates, existing products have difficulties closing the heat budget, as discussed in section "Current Capabilities for Gridded Flux Products."

The net shortwave radiation flux, $Q_{S W}$, is the net difference between the incoming (i.e., downwelling, $S W \downarrow$, and reflected outgoing shortwave radiations, $S W \uparrow$, and is commonly computed using a surface shortwave albedo, $\alpha=S W \uparrow / S W \downarrow$, estimate:

$$
Q_{S W}=S W \downarrow-S W \uparrow=S W \downarrow(1-\alpha) .
$$

Likewise, because the outgoing surface longwave radiation $L W \uparrow$ comprises both the IR radiation emitted by the surface of the ocean and the portion of atmospheric downwelling IR radiation $L W \downarrow$ that is not absorbed by the ocean surface, the net longwave radiation flux, $Q_{L W}$, can be expressed as:

$$
\begin{aligned}
Q_{L W}=L W \uparrow-L W \downarrow & =\epsilon \sigma_{S B} T_{s}^{4}+(1-\epsilon) L W \downarrow-L W \downarrow \\
& =\epsilon\left(\sigma_{S B} T_{s}^{4}-L W \downarrow\right)
\end{aligned}
$$

where $\epsilon$ is the IR surface emissivity $(\epsilon=1$ for black-body emission) and is taken to be equal to the absorptivity, $\sigma_{S B}$ is Stefan-Boltzmann constant, and $T_{s}$ is the surface (skin) temperature that is emitting the IR-radiation, in degrees Kelvin. The skin temperature of the ocean is generally cooler than the water beneath, as the ocean is nearly always and everywhere giving heat to the atmosphere (Fairall et al., 1996a). On the aqueous side of the interface, viscosity and the air-sea density difference prevent the turbulent transfer of heat from ocean to atmosphere and so the heat supplied to the interface to feed the latent and sensible heat fluxes and into the layer that emits infrared radiation to the atmosphere, is provided by molecular conduction, which requires a vertical temperature gradient. This temperature gradient is referred to as the thermal skin layer (Donlon et al., 2002; Minnett et al., 2011). As a result, the surface layer of the ocean is in nearly all cases cooler than at a depth of a millimeter or so. The thickness of the layer emitting infrared radiation that is subsequently measured by satellite radiometers to derive SST is comparable to the thermal skin layer $(\sim 0.1 \mathrm{~mm}$; Wong and Minnett, 2016, 2018), and so the derived temperature is referred to as the ocean skin temperature.

The latent and sensible heat fluxes are typically estimated from state variables, using a "bulk flux algorithm" (e.g., Fairall et al., 2003). As described in section "Turbulent Momentum and Heat Flux EOV and ECVs," the primary state variables for turbulent fluxes, including wind stress, are surface winds relative to surface currents, skin temperature, near-surface air temperature, and near-surface humidity. Because most in situ estimates of the oceanic near-surface properties are below the skin, as discussed in section "Parameterizations to Extrapolate Measurements to Air-Sea Interface," parameterizations must be used to extrapolate the bulk sea surface measurements to the airsea interface. Likewise, as described in section "Parameterizations to Extrapolate Measurements to Air-Sea Interface," flux EOV and ECV for the surface radiation include downward solar radiation, upward solar radiation (or surface albedo), downward longwave radiation, skin temperature, and longwave surface emissivity. We refer to the variables listed here as the "flux Essential Climate Variables (ECVs)" and "flux Essential Ocean Variables (EOVs)."

\section{Turbulent Momentum and Heat Flux EOV and ECVs}

The surface momentum (aka wind stress), sensible heat and latent heat fluxes provide surface boundary conditions for turbulent flux profiles in the lower atmosphere and upper ocean. These surface turbulent fluxes are most directly quantified by measuring the direct covariance (aka eddy correlation) between the fluctuating vertical velocity that drives the exchange with the fluctuating quantity of interest within the constant flux layer above the air-sea interface. For example, the directly measured latent heat flux is determined from

$$
Q_{\text {lat }}=\rho L_{v}\langle w q\rangle
$$

where $\rho$ is the density of air; $L_{v}$ is the latent heat of evaporation, $w$ and $q$ represent the fluctuating vertical velocity and specific humidity, respectively, and the brackets denote a temporal average of, generally, an hour or less. The turbulent fluxes, however, are difficult to measure at sea due to challenges that include platform motion contamination, flow distortion, high power requirements, rain and sea-spray contamination. Additionally, numerical forecast models do not resolve near surface turbulence, so surface fluxes must be parameterized.

These parameterizations are typically based on the assumption that the flux of some quantity is proportional to the vertical gradient of that quantity, e.g., the latent heat flux is proportional to the gradient in specific humidity. This approach, commonly referred to as the gradient or profile method, provides first-order 
closure in numerical models. The approach therefore requires vertical profiles of the observed or model-resolved non-turbulent state variables of temperature, specific humidity and velocity. The multiplicative factor that relates the flux to the gradient is known as the eddy viscosity for the momentum flux and the eddy diffusivity for the heat fluxes, e.g., the latent heat is determined from

$$
Q_{\text {lat }} \approx-\rho L_{v} K_{q} \frac{\partial Q}{\partial z}
$$

where $K_{q}$ is the eddy diffusivity for moisture, and $\partial Q / \partial z$ is the vertical gradient of the mean specific humidity. Commonly used parameterizations of the eddy viscosity and diffusivity in the surface (constant flux) layer assume that the efficiency of mixing by turbulent eddies scales with the height above the ocean surface. The efficiency of mixing is also a function of atmospheric stability, where mixing is suppressed under stable (thermally stratified) conditions and enhanced in unstable (convective) conditions. These two considerations predict semilogarithmic profiles that includes a function that accounts for atmospheric stability.

It is also difficult, however, to measure vertical profiles and implement the gradient method over the ocean due to many of the challenges given for the direct covariance method. Instead, the surface fluxes are generally estimated using sea-air differences in the mean "bulk" state variables measured (or modeled) at the surface and at some height within the surface layer. The bulk aerodynamic method links the turbulent fluxes to mean air-sea velocity, temperature and humidity difference using transfer coefficients:

$$
\begin{gathered}
\tau_{x}=-\rho\langle u w\rangle \approx \rho C_{D} S \Delta U, \quad \tau_{y}=-\rho\langle v w\rangle \approx \rho C_{D} S \Delta V \\
Q_{\text {lat }}=\rho L_{v}\langle w q\rangle \approx \rho L_{v} C_{E} S \Delta Q \\
Q_{\text {sen }}=\rho c_{p}\langle w \theta\rangle \approx \rho c_{p} C_{H} S \Delta \Theta
\end{gathered}
$$

where $c_{p}$ is specific heat at constant pressure; $u$ and $v$ are the fluctuating along-wind and cross-wind velocity components, respectively; and $\theta$ is the fluctuating potential temperature; $C_{D}, C_{E}$, and $C_{H}$ are the transfer coefficients (known as the drag coefficient) for momentum, latent heat and sensible heat, respectively; $S$ is the scalar wind speed relative to the ocean surface that includes gustiness; and $\Delta U, \Delta V, \Delta Q$, and $\Delta \Theta$ are the sea-air differences in the along-wind, crosswind, specific humidity and potential temperature, respectively.

At low winds, convective conditions, large-scale eddies drive gustiness that results in differences between the vector average wind components, $U$ and $V$, and the wind speed, $S$, that includes gustiness. Convective gustiness has been shown to drive surface fluxes even when the vector-averaged winds are close to zero (Beljaars, 1995; Fairall et al., 1996b). However, the wind speed is difficult to measure on a moving platform due to wave contamination of the anemometers (i.e., vector averaging is used to remove this contamination but at the expense of gustiness). Additionally, the momentum equations in numerical models generally predict the vector components. Therefore, a common solution is to add convective gustiness to the vector averaged winds such that $S^{2}=$
$U^{2}+V^{2}+U_{g}^{2}$ where $U_{g}$ represents the gustiness due to convection. This is the approach used in the COARE algorithm (Fairall et al., 1996b). However, gustiness parameterizations that provide a single value of $U_{g}$ regardless of height are physically inconsistent as convective gustiness is expected to vary with height within the boundary layer. More work is needed on this topic.

Although difficult, direct covariance estimates of the fluxes have been successfully measured from a variety of over-ocean platforms or a wide range of conditions as described in section "Current Capabilities." These fluxes provide direct estimates of the transfer coefficients after normalization of the appropriate bulk state variables as given by Eq. 1.6. The measured transfer coefficients are then used to develop parameterizations of these coefficients that take into account two principal effects: atmospheric stability and ocean surface roughness. For example, direct measurements of the momentum flux are used to parameterize the drag as

$$
C_{D}=\frac{-\langle u w\rangle}{S \Delta U}=\left(\frac{\kappa}{\ln \left(z / z_{0}\right)-\psi_{u}}\right)^{2}
$$

where the middle term is the measured drag coefficient and the last term is a parameterization that includes a roughness length, $z_{0}$, and a function that accounts for stability, $\psi_{u}$. This formulation is based on the assumption of a semi-logarithmic profile in the marine surface layer.

The impact of atmospheric stability is generally determined using Monin-Obukhov Similarity (MOS) scaling. MOS is used to develop functions (e.g., $\psi_{u}$ ) that account for the effects of stability in three overall stability classes: (1) neutral conditions, (2) unstable or convective conditions, and (3) stable or stratified conditions. Neutral surface layers are associated with high winds and little solar heating where turbulent mixing is driven by wind shear. The stability function equals zero under neutral conditions, i.e., $\psi_{u}($ neural $)=0$, in the absence of convection or stratification. Unstable surface layers range from situations where turbulent mixing is completely driven by convective processes (aka free convection) to more common situations where the mixing is driven by both buoyancy and wind shear (aka forced convection). Stable surface layers force the wind shear to do work against the stratification, thereby inhibiting mixing and turbulent exchange.

While the form of the stability functions can be guided by scaling arguments (e.g., in the free convective limit), the actual form of these functions must be determined empirically from direct measurements. Successful formulations are able to parameterize the entire range of stability classes. Reasonably consistent formulations have been determined through observations in a number of overland (e.g., Dyer and Hicks, 1970; Businger et al., 1971; Dyer and Bradley, 1982), over-ice (e.g., Grachev et al., 2007) and overwater (e.g., Edson and Fairall, 1998; Vickers and Mahrt, 1999; Edson et al., 2004, 2007, 2013) investigations, which have shown MOS scaling to be valid as long as the assumptions central to its application are not violated. These include a constant turbulent flux layer, stationarity and horizontal homogeneity.

Before developing parameterizations that account for the varying roughness of the underlying sea surface, the impact of 
atmospheric stability must first be removed (e.g., Fairall et al., 1996b). This is done using the MOS stability functions by adjusting the transfer coefficients to neutral conditions where, e.g., $\psi_{u}(0)=0$ and the neutral drag coefficient is defined as $C_{D N}=\left(\ln \left(z / z_{0}\right)\right)^{2}$. The roughness length is commonly parameterized using the roughness Reynolds number for smooth flow to provide the surface "roughness" at low winds (Smith, 1988). Other low wind studies have suggested the use of Weber number scaling to parameterize the roughness as a function of surface tension (Wu, 1994). The low-wind roughness is added to another roughness length that accounts for the increasing roughness of surface waves at increasingly higher wind speeds. This roughness length commonly relies on a relationship suggested by Charnock (1955). The Charnock relationship effectively models the surface roughness due to wind-waves as the ratio of surface forcing (i.e., the surface stress) to the restoring force of gravity. This ratio is multiplied by a variable of proportionality known as the Charnock parameter.

The Charnock parameter is expected to account for the widerange of physical processes that impact wind-wave interaction. Therefore, it should come as no surprise that there is a wide range of published parameterizations of the Charnock parameter. The transfer coefficients are known to have a wind speed dependence - strong for $C_{D}$ and weak for $C_{E}$ and $C_{H}$. This dependence has naturally led to the parameterization of the transfer coefficients as a function of wind speed (e.g., Large and Pond, 1981) or using a wind-speed dependent Charnock parameter (e.g., Fairall et al., 2003). Other bulk algorithms have explicit dependencies on sea state (e.g., significant wave height, wave period and wave steepness, wave-age, directional differences between the wind and wave fields), and fraction of ice cover. Such dependencies in measured fluxes are often modeled using Charnock's relationship where the Charnock parameter is parameterized in terms of the wind-speed, wave-slope, wave-age and ice fraction. However, understanding the relation between the roughness parameter and the sea state, and likewise the effect of sea state on the relative winds, remains an area of active research (e.g., Liu et al., 1979; Large and Pond, 1981; Donelan et al., 1993; Fairall et al., 1996b, 2003; Mahrt et al., 1996; Bourassa et al., 1999; Brunke et al., 2003; Drennan et al., 2005; Edson et al., 2013; Hristov and Ruiz-Plancarte, 2014).

The impact of waves on air sea-fluxes also extends to the sensible and latent heat fluxes. For example, many formulations of the transfer coefficients for sensible and latent heat include the drag coefficient to the one-half, $C_{D}^{1 / 2}$, times a scalar component for heat and moisture; e.g., the transfer coefficient for latent heat can be defined as $C_{E}=C_{D}^{1 / 2} C_{q}$ where $C_{q}$ is the scalar component of the moisture transfer. This formulation predicts that any waverelated dependencies in the drag coefficient will be included in these formulations in a somewhat muted form. Analogous to the aerodynamic roughness length used to define the drag coefficient, the scalar components are commonly defined using "thermal" roughness lengths. For example, the transfer coefficient for latent heat has been defined as

$$
C_{E}=C_{D}^{1 / 2} C_{q}=\left(\frac{\kappa}{\ln \left(z / z_{0}\right)-\psi_{u}}\right)\left(\frac{\kappa}{\ln \left(z / z_{0 q}\right)-\psi_{q}}\right)
$$

where $z_{0 q}$ is the thermal roughness length for moisture and $\psi_{q}$ is a MOS stability function for humidity. The thermal roughness lengths are often parameterized as a function of the roughness Reynolds number, which is defined using the aerodynamic roughness length (see Liu et al., 1979; Fairall et al., 1996b for details). As such, any wave-related parameterization of the aerodynamic roughness length will also be included in the thermal roughness lengths.

The more sophisticated algorithms account for the difference between a bulk water temperature and the interface temperature, wind gustiness, and surface currents, as discussed in section "Parameterizations to Extrapolate Measurements to Air-Sea Interface." However, there is a balance between increasing parameterization complexity and hence dependence on additional variables that may be uncertain or unknown, and improvements that may be gained by considering additional physics. The COARE algorithm transfer coefficients are claimed to be accurate on average to approximately $\pm 5 \%$ for $C_{D}$ and $C_{E}$ (set equal to $C_{H}$ ) for wind speeds from 2 to $20 \mathrm{~m} \mathrm{~s}^{-1}$. By this we mean that some $15,000 \mathrm{~h}$ of direct flux measurements, converted to transfer coefficients, and averaged in wind speed bins will be within $5 \%$ of the COARE transfer coefficients at the same wind speed. The scatter of individual 1-h measured values is about $25 \%$ and are generally wind speed and certainly platform dependent. This scatter is principally sampling error and is well-understood in terms of turbulence statistical theory (Blomquist et al., 2014). Research challenges that could lead to improved bulk flux parameterizations include:

- Development of wave-dependent surface flux parameterization that outperform wind-speed dependent parameterizations under a wide range of wind, wave and current conditions.

- Development of flux-profile relationships that account for both stratification and wave-induced perturbations on the wind profile though the wave boundary layer (WBL) and beyond under a wide range of wind, wave and current conditions.

- Development of surface flux and flux-profile relationships that account for directional differences between the wind and wave fields.

- Development of Geophysical Model Functions (GMF) to provide remotely sensed surface stress estimates that match or exceed the accuracy of bulk fluxes using in situ measurements.

- Development of convective gustiness parameterizations that are valid through the surface layer to the lowest grid point in high resolution forecast models.

- Development of gustiness parameterizations for coherent structures such as roll vortices in forced convection.

- Validation and continued development of models to simulate evaporating sea-spray and their impact on momentum, heat and mass fluxes under high to extreme wind conditions.

- Development of scale-dependent flux parameterizations for nested high-resolution models down to Large Eddy Simulation (LES) scale. 
- Development of coupled LES with sufficient accuracy to simulate wind-wave-current interaction near the ocean surface to provide output that can be considered data for parameterization and model development.

In summary, modern bulk algorithms need to better incorporate the impact of waves and currents on the magnitude and direction of the surface stress and their modulation of fluxes and mean profiles (e.g., Grachev et al., 2003; Hara and Belcher, 2004; Grare et al., 2013, 2018; Hristov and Ruiz-Plancarte, 2014; Buckley and Veron, 2016; Cifuentes-Lorenzen et al., 2018). The wavy boundary layer and shallow atmospheric boundary layers provide a number of additional challenges associated with seaspray, gustiness, severe stratification and extreme winds. The impact of boundary layer processes on surface fluxes above the surface layer are best studied with additional boundary layer measurement in combination with numerical models and simulations. Therefore, resolving the main issues with bulk algorithms will require a combination of field observations and specialized atmosphere-wave models. Because of the many dependencies, detailed research models such as advanced wave codes (e.g., Kukulka et al., 2007; Banner and Morison, 2010; Kudryavtsev et al., 2014) and wave-LES models (e.g., Sullivan et al., 2014, 2018; Hara and Sullivan, 2015), in combination with observations in many different regimes, can provide a rational way to explore the phase space of parameterization variables.

\section{Parameterizations to Extrapolate Measurements to Air-Sea Interface}

While the transfer coefficients used in bulk algorithm introduce some uncertainty into the estimation of the surface air-sea fluxes, perhaps a larger uncertainty comes from treatment of the flux state variables used to estimate the flux. In particular, for most state-of-the-art bulk algorithms, the sea surface temperature (SST) and specific humidity are assumed to be ocean skin values. If instead, a bulk sea temperature is used (i.e., sea temperature measured at depths ranging between 0.01 and $5 \mathrm{~m}$ or even deeper), then it should be adjusted to the surface using either parameterizations or models. Fairall et al. (1996a), for example, uses first estimates of the net surface heat flux and wind stress to force a one-dimensional mixed-layer model of the diurnal warm layer relative to the pre-dawn conditions. This is then used to account for stratification (i.e., the warm layer) above the depth of the bulk temperature measurement. Fairall et al. (1996a) also provides a "cool skin" model to account for the surface cooling from non-solar radiative components of the net surface heat flux to compute the SST or skin temperature. Other methods exist for making these extrapolations. For example, the Webster et al. (1996) method relies upon a specification of wind speed and peak solar flux to compute the diurnal warm SST variability at the surface. Parameterizations in terms of wind speed lead to a simple thermal skin effect correction (Donlon et al., 2002; Minnett et al., 2011). For tropical open ocean environments, the average warm layer correction leads to about a $5 \mathrm{~W} \mathrm{~m}^{-2}$ increase in $Q_{n e t}$, while the average cool skin adjustment is a decrease of about $10 \mathrm{~W} \mathrm{~m} \mathrm{~m}^{-2}$. However, corrections due to the warm layer can lead to substantially larger fluxes when the measurements are made at depth in the presence of large diurnal warming events.

Likewise, for these state-of-the-art bulk algorithms, surface current estimates are needed to compute the wind speed and vertical wind shear relative to ocean surface as given by $\Delta U$ and $\Delta V$ in Eq. 1.6. The resulting changes in stress are usually small compared to the stress except in regions of strong currents such as over western boundary currents. However, ignoring surface currents in the development of flux parameterization can lead to a systematic bias in the transfer coefficients (Edson et al., 2013) as the wind-and wave-driven currents are generally in the direction of the wind. As a result, the wind speed relative to water is generally smaller than the wind speed relative to earth. These differences are also large enough to cause substantial errors in the horizontal gradient of stress, which can have substantial impact on ocean circulation, upwelling, biology and biogeochemistry (Shi, 2017). Surface currents, however, are generally measured in situ at $10 \mathrm{~m}$ or deeper. There is growing appreciation that there can be non-negligible shear within the upper $10 \mathrm{~m}$ on timescales of the flux calculations. This can add to the errors in the relative wind, and potentially to errors in the flux parameterizations if the currents are not consistently adjusted to the surface. Brodeau et al. (2017) estimated the effect of surface currents on the wind stress to be on average within $\pm 0.005 \mathrm{~N} \mathrm{~m}^{-2}$ with the largest uncertainties amounting to $0.02-0.025 \pm 0.005 \mathrm{~N} \mathrm{~m}^{-2}$.

\section{Radiative Heat Flux EOV/ECV}

The net radiative component of the air-sea heat flux comprises a shortwave component that is emitted by the sun (SW $\downarrow$ ) and reflected from the ocean surface (SW $\uparrow$ ) in the spectral range of $0.3-4.0 \mu \mathrm{m}$, and a longwave component that is emitted by the atmosphere (LW $\downarrow$ ) and surface (LW $\uparrow$ ) in the spectral range of $4.0-100.0 \mu \mathrm{m}$ (Eqs 1.1-1.3). SW $\downarrow$ has a direct and a diffuse component that interacts differently with the underlying surface due to differences in their spectral composition and angularly dependent properties. About half of the solar radiation incident on the top of the atmosphere reaches the surface of the Earth after being transmitted through the atmosphere. Extinction of solar radiation in the atmosphere is mostly by ozone, water vapor, clouds, and aerosols. The vertical profiles of clouds, water vapor, and temperature largely determine the longwave emission by the atmosphere. Clouds play a major role in determining the net radiative balance at the surface, dependent on their amount and optical properties (e.g., optical depth, a general measure of the capacity of a cloud to control the amount of light that will reach the surface). Most atmospheric constituents (e.g., cloud, aerosols, and water vapor) can now be derived from satellite instruments. At the ground, $\mathrm{SW} \downarrow$ is measured with pyranometers (spectral range of 0.310 to $2.800 \mu \mathrm{m}$ ) and LW $\downarrow$ is measured with pyrgeometers (spectral range of 4.5 to $42 \mu \mathrm{m}$ ). The upward component of the surface solar radiation, SW $\uparrow$, depends upon SW $\downarrow$ spectral composition controlled by the solar zenith angle, atmospheric and cloud properties, as well as the surface optical properties, which depend upon the sea state (i.e., wind speed) and chlorophyll concentration in the upper ocean (Jin et al., 2004). In situ measurements of $S W \uparrow$ are very rare and thus it is typically estimated from the $S W \downarrow$ and surface albedo, $\alpha$ (Eq. 1.2). 
The strong albedo dependence upon solar zenith angle means that more reflection occurs at lower sun angles (during dawn and dusk, in winter and at higher latitudes). Payne (1972) used observations of both upwelling and downwelling shortwave radiation to develop a relation between albedo and solar zenith angle and atmospheric transmittance. At low solar zenith angles, the albedo is 0.03 to 0.06 , but at high solar zenith angles it can approach 0.3. Li et al. (2006) examined wind speed and zenith angle dependent models of the albedo from the perspective of upwelling shortwave at the top of the atmosphere. They found that differences among models were less than $10 \mathrm{~W} \mathrm{~m}^{-2}$ and the difference in the global mean was within $\pm 2.5 \mathrm{~W} \mathrm{~m}^{-2}$ compared with Clouds and the Earth's Radiant Energy System (CERES) data. In the TOGA COARE bulk formulae, Fairall et al. (1996a) use a fixed albedo of 0.055 . Errors in albedo can introduce errors in the net shortwave radiation and air-sea heat flux. Further work is needed to improve albedo parameterizations for use in state of the art bulk algorithms, Numerical Weather Prediction (NWP), and satellite radiation algorithms.

In situ observations of longwave radiation at sea have become more common only in the last 10-20 years. Prior to that, various bulk formulae for net longwave radiation at the sea surface were used that depended upon air- and sea-surface temperature, humidity, cloud cover (and type), and latitude (Fung et al., 1984). While in situ observations are used for local radiation budgets and for validations of computed radiative fluxes, only satellite observations can provide surface radiative fluxes at a global scale. To estimate radiative fluxes from satellite observations, we need to rely on radiative transfer models. Inputs for radiative transfer models include temperature and water vapor specific humidity vertical profiles, and cloud and aerosol properties. The accuracy of these properties largely influences the accuracy of surface radiative flux computations. Surface radiative fluxes are especially sensitive to near surface temperature and humidity profiles and boundary layer cloud properties. Consequently, improvements to these near surface properties are critical for reducing uncertainty in satellite-derived surface radiative fluxes.

\section{CURRENT CAPABILITIES}

Quantifying the air-sea fluxes over the global ice-free oceans requires a hierarchy of observations specifically targeted for (1) improving understanding of processes controlling air-sea exchange and their relationship to atmospheric and oceanic state variables, and specifically, for improving the "bulk algorithm" for computing these fluxes; (2) measuring flux EOVs and ECVs over the global ice-free ocean with sufficiently high spatial and temporal resolution, coverage, and accuracy to generate the global flux products; and (3) obtaining highquality long time series and regionally distributed measurements that can be used for validating and improving these flux products. Here we describe the current capabilities of the in situ networks for measuring air-sea heat and momentum fluxes, and remotely sensed capabilities. Each has its strengths and weaknesses and NWP models are commonly used to integrate these disparate observations into dynamically consistent fields.
We thus also describe current capabilities in NWP and hybrid NWP flux products.

\section{Current Capabilities for in situ Flux EOV/ECV Measurements ICOADS}

The International Comprehensive Ocean-Atmosphere Data Set (ICOADS, Freeman et al., 2017, 2019) collates surface marine data extending back three centuries. Before about 1970 almost all ICOADS observations are from ship voyages but as ocean technology has developed, data from more platforms (surface moorings, drifters, floats) have been incorporated into the archive. At present, nearly all near-real time surface marine data available through the Global Telecommunication System (GTS) are incorporated into the database and periodically data from delayed mode archives are incorporated. Flux ECV and EOVs available through ICOADS include: SST, air temperature, humidity, wind speed and direction, barometric pressure, visually observed characteristics of sea state, and coded weather information. ICOADS contains few radiation measurements, but does contain visually observed cloud observations (Eastman et al., 2011). Sampling in ICOADS is very heterogeneous with observations concentrated in the major shipping routes of the Northern Hemisphere. Sampling errors in surface turbulent fluxes computed from ICOADS reports may amount to more than $60 \mathrm{~W} \mathrm{~m}^{-2}$ in poorly sampled regions (e.g., Gulev et al., 2007). ICOADS observations can be challenging to use, but if handled with care, ICOADS provides data for in situ flux calculations and global surface flux products (Josey et al., 1999; Berry and Kent, 2009). ICOADS also provides a major input data source for reanalyses (e.g., ERA-Interim: Dee et al., 2011; 20th Century Reanalysis: Compo et al., 2011; and CFSR: Saha et al., 2010). ICOADS is also the main in situ data source for the construction of gridded analyses of surface marine ECVs and EOVs including those used as surface boundary conditions for atmospheric reanalyses (e.g., HadSST3: Kennedy et al., 2011; ERSSTv5: Huang et al., 2017; COBE-SST2: Hirahara et al., 2014; HadNMAT2: Kent et al., 2013). Additionally, ICOADS data are used for satellite data calibration and evaluation (e.g., Jackson and Wick, 2010; Liman et al., 2018; Tomita et al., 2018), as well as long-term regional reconstructions of surface fluxes (Gulev et al., 2013). ICOADS is an archive for observations but requires access to expertly managed data from each different network type. Presently there is no international system for the expert management and archival of surface meteorological observations from the GTS although several national weather services maintain their own collections.

\section{In situ Sensors for Measuring Fluxes}

A typical set up to estimate the momentum, sensible heat and latent heat flux on a moving platform includes a 3 -axis sonic anemometer/thermometer; a 3-axis motion package and an open path infrared hygrometer. Sonic anemometers/thermometers have become the tool of choice for marine research. Although they experience some dropouts and occasional spikes, particularly in rain, they are generally very reliable in the marine environment. Motion sensors are finding their way into 
many (non-marine) applications. As a result, researchers now have a number of small, low-power, reasonably inexpensive and sufficiently accurate motion packages to choose from. The infrared hygrometer is ideally deployed alongside a system that flushes the optics between rain events. A closed-path hygrometer avoids many issues associated with contamination of the optics. However, experience has shown that they suffer from uncorrectable attenuation of the signal due to the sticky nature of water vapor within sampling tubes in high humidity environments. The fluxes should be measured using the wind velocity relative to water, which requires a 2 -axis current meter. Investigation of wind-wave interaction requires surface wave information for the wave height, wave direction and wave period to compute variables such as wave-slope and wave-age from directional wave spectra. These estimates can be made from ships and surface moorings with some difficulty and limits in the frequency/wavenumber resolution due to the size of the platform. Instead, the latest generation of directional wave-buoys are recommended for these investigations. These buoys have onboard processing and near real-time data telemetry of key wave parameters. They are small enough to resolve a large fraction of the shorter wind-wave spectra in addition to the dominant waves. There is also a growing use of fast-response pressure sensors to investigate pressure-work and energy transfer primarily from fixed towers and specialized research platforms like the Floating Instrument Platform $(R / P$ FLIP). The latest generation of these sensors appear to measure absolute pressure to sufficient accuracy for these studies. The main challenge, however, is in the design of the sensor head required to remove dynamic pressure fluctuations to isolate the desired static pressure fluctuations.

The meteorology sensors are best deployed at a height above the wavy boundary layer (corresponding to the height of the dominant waves or more about mean sea level) due to issues that arise when attempting to measure the fluxes too close to the ocean surface. Specifically, since the size of the turbulent eddies supporting the fluxes scales with the height above the surface, ever higher frequency measurements are required to capture the flux as one nears the surface (Kaimal et al., 1972). Similarly, stronger winds shift energy toward higher frequencies. However, there is an inherent limitation to the size of the eddies that can be measured with sonic anemometers and infrared hygrometers. This stems from the $\sim 10 \mathrm{~cm}$ path length of these devices, which means that any fluctuations smaller than this length are path-averaged and unresolvable. This limits their ability to capture the flux and resolve the inertial subrange very near the ocean surface, particularly at high winds, no matter how fast the sampling is. High winds also produce sea spray that poses a number of measurement challenges (Andreas et al., 2008, 2015). Besides the challenge of actually measuring spray to investigate their modulation of the fluxes (e.g., Richter and Veron, 2016), the droplets are known to contaminate sensors used to measure the latent heat (moisture) flux. In fact, the direct measurement of latent heat flux under high wind and rainy conditions remains one of our greatest needs and challenges. Additionally, the measurement of surface fluxes under extreme wind conditions are complicated by additional considerations such as an increasing "pressure stress" term reported in the LES study by Hara and Sullivan (2015). However, a major challenge for air-sea interaction research is to observe momentum, heat and mass exchange within the wavy boundary layer. The observational challenge is to minimize these issues through the use of innovative platforms and new sensors specifically designed for the nearsurface environment.

\section{In situ Platforms for Observing Fluxes}

Historically, ships have been the primary platform for marine surface observations over the open ocean. Prior to 2000, nearly all direct covariance flux observations that have gone into developing the state of the art bulk flux algorithms were from ship-based observations (e.g., Fujitani, 1981, 1985; Donelan et al., 1997; Fairall et al., 1997); notable exceptions being fluxes measured from the R/P FLIP; (Fisher and Spiess, 1963) and the Air-Sea Interaction Spar (ASIS, Graber et al., 2000; Drennan et al., 2003, 2005). Care was taken in the development of these algorithms to account for issues associated with making surface marine observations from ships. For example, the large profile of the ship can cause flow distortion so that wind measured on the deck is not characteristic of the ambient wind (Yelland et al., 1998). Likewise, micro-climates on the ship decks can affect air temperature (Berry et al., 2004), humidity, and even barometric pressure measurements. Ideally, ship-based measurements are made on a well-exposed mast, forward of any obstructions.

The flow distortion in the measured mean winds can then be accounted for using empirically, modeled and/or wind tunnel derived corrections. Limiting relative wind directions and using aspirated radiation shields (or using naturally aspirated radiation shield when the relative winds are above some limit) reduce the errors due to heat island effects (i.e., ship micro-climate). The motion of the ship can also affect the surface turbulence measurements (e.g., Landwehr et al., 2015). In particular, wind and ocean currents must be transformed into Earth coordinates using high quality navigation data. In the past few years, the quality of inertial motion sensors has increased significantly, making it possible to now do motion correction routinely at $20 \mathrm{~Hz}$ resolution. Such technology, however, is not always available and even when available, as for example for Voluntary Observing Ships, motion correction is not always applied. For further discussion on best practices for surface marine data, see Bradley and Fairall (2006), which can be found in the Ocean Best Practice repository website for GO-SHIP measurements: https: //www.oceanbestpractices.net/handle/11329/386.

Many of these "field errors" are minimized in measurements taken from moored surface buoys. Progress has been made in both developing sensors suitable for unattended deployment at sea on surface buoys and in quantifying their uncertainties. As a consequence, in moderate conditions an accuracy of $8 \mathrm{~W} \mathrm{~m}^{-2}$ in net heat flux has been achieved over hours to days and longer (Tables 1, 2), and further improvements are possible. In wind speeds below $\sim 3 \mathrm{~m} \mathrm{~s}^{-1}$ active ventilation is needed of air temperature and humidity sensors and radiometers. New generation humidity sensors offering better stability and improved accuracy should be phased in. Platform tilts should be monitored as mean tilt is a source of error for incoming shortwave and longwave radiation observations. New sensors are 
TABLE 1 | Summary of flux EOV/ECV uncertainties based upon ASIMET sensor uncertainties stemming from laboratory calibration, sensor drift, and field impacts with estimates of total uncertainties in instantaneous, daily, and annual values (after Colbo and Weller, 2009).

\begin{tabular}{|c|c|c|c|c|c|c|}
\hline \multirow[b]{2}{*}{ Flux EOV/ECV } & \multirow[b]{2}{*}{ Lab Calibration } & \multirow[b]{2}{*}{ Drift } & \multirow[b]{2}{*}{ Field } & \multicolumn{3}{|c|}{ Sensor Error (and contribution to $Q_{n e t}$ error) } \\
\hline & & & & Instantaneous & Daily & Annual \\
\hline $\begin{array}{l}\text { Downwelling } \\
\text { Longwave } \\
\text { Radiation }\end{array}$ & $\begin{array}{l}\text { Coefficients of fit: } \\
1.5 \mathrm{~W} \mathrm{~m}^{-2} \\
\text { Noise: } 0.5 \mathrm{~W} \mathrm{~m}^{-2}\end{array}$ & $2 \mathrm{~W} \mathrm{~m}^{-2}$ & $\begin{array}{l}\text { Tilt: }<2 \mathrm{~W} \mathrm{~m}^{-2} \\
\text { Temperature gradients: } 4 \mathrm{~W} \mathrm{~m}^{-2} \\
\text { Salt spray: }<1 \mathrm{~W} \mathrm{~m}^{-2} \\
\text { Solar: }<1 \% \mathrm{SW}_{\text {in }}\end{array}$ & $7.5 \mathrm{~W} \mathrm{~m}^{-2}$ & $4 \mathrm{~W} \mathrm{~m}^{-2}$ & $4 \mathrm{~W} \mathrm{~m}^{-2}$ \\
\hline $\begin{array}{l}\text { Downwelling } \\
\text { Shortwave } \\
\text { Radiation }\end{array}$ & $2 \mathrm{~W} \mathrm{~m}^{-2}$ & $<2 \mathrm{~W} \mathrm{~m}^{-2}$ & $\begin{array}{l}\text { Tilt: }<2 \mathrm{~W} \mathrm{~m}^{-2} \\
\text { Temperature gradients: } 1-2 \mathrm{~W} \mathrm{~m}^{-2} \\
\text { Salt spray: }<1 \mathrm{~W} \mathrm{~m}^{-2}\end{array}$ & $\begin{array}{l}20 \mathrm{~W} \mathrm{~m}^{-2}, \text { more in } \\
\text { broken cloud }\end{array}$ & $6 \mathrm{~W} \mathrm{~m}^{-2}$ & $5 \mathrm{~W} \mathrm{~m}^{-2}$ \\
\hline Humidity & $\begin{array}{l}\text { Linear: } 0.16 \% \mathrm{RH} \\
\text { Cubic: } 0.1 \% \mathrm{RH}\end{array}$ & 0.9 & $\begin{array}{l}\text { Under } 95 \% \mathrm{RH}: \pm 1 \% \mathrm{RH} \\
\text { Heating in low winds: } 3 \% \mathrm{RH}\end{array}$ & $\begin{array}{l}1 \% \mathrm{RH}, 3.2 \mathrm{~W} \mathrm{~m}^{-2} \\
3 \% \mathrm{RH}, \text { low winds, } \\
10 \mathrm{~W} \mathrm{~m}^{-2}\end{array}$ & $\begin{array}{l}1 \% \mathrm{RH}, 3.2 \mathrm{~W} \mathrm{~m}^{-2} \\
3 \% \mathrm{RH}, 10 \mathrm{~W} \mathrm{~m}^{-2}\end{array}$ & $1 \% \mathrm{RH}, 3.2 \mathrm{~W} \mathrm{~m}^{-2}$ \\
\hline Air Temperature & $<0.03^{\circ} \mathrm{C}$ & $0.05^{\circ} \mathrm{C}$ & $\begin{array}{l}>1^{\circ} \mathrm{C}, \text { wind }<1 \mathrm{~m} \mathrm{~s}^{-1} \\
0.7^{\circ} \mathrm{C}, \text { wind }=2 \mathrm{~m} \mathrm{~s}^{-1} \\
0.4^{\circ} \mathrm{C}, \text { wind }=3 \mathrm{~m} \mathrm{~s}^{-1}\end{array}$ & $\begin{array}{l}0.2^{\circ} \mathrm{C}, \text { more in low } \\
\text { wind, } 3.5 \mathrm{~W} \mathrm{~m}^{-2}\end{array}$ & $0.1^{\circ} \mathrm{C}, 2.2 \mathrm{~W} \mathrm{~m}^{-2}$ & $0.1^{\circ} \mathrm{C}, 2.2 \mathrm{~W} \mathrm{~m}^{-2}$ \\
\hline $\begin{array}{l}\text { Barometric } \\
\text { Pressure }\end{array}$ & $0.06 \mathrm{hPa}$ & $\begin{array}{l}1.5 \mathrm{hPa}(\max ) \\
0.2 \mathrm{hPa}\end{array}$ & $\begin{array}{l}\text { Temperature: } 0.1 \mathrm{hPa} \\
\text { Wind: }<0.1 \mathrm{hPa} \\
\left(\text { wind }<10 \mathrm{~m} \mathrm{~s}^{-1} \text { ) }\right.\end{array}$ & $\begin{array}{l}0.3 \mathrm{hPa} \\
0.0 \mathrm{~W} \mathrm{~m}^{-2}\end{array}$ & $\begin{array}{l}0.2 \mathrm{hPa} \\
0.0 \mathrm{~W} \mathrm{~m}^{-2}\end{array}$ & $\begin{array}{l}0.2 \\
0.0 \mathrm{~W} \mathrm{~m}^{-2}\end{array}$ \\
\hline $\begin{array}{l}\text { Sea Surface } \\
\text { Temperature }\end{array}$ & $0.001^{\circ} \mathrm{C}$ & $0.05^{\circ} \mathrm{C}$ & $\begin{array}{l}\text { Low wind: } 0.1^{\circ} \mathrm{C} \\
\text { Cool skin: }<0.02^{\circ} \mathrm{C}\end{array}$ & $0.1^{\circ} \mathrm{C}, 4.4 \mathrm{~W} \mathrm{~m}^{-2}$ & $0.1^{\circ} \mathrm{C}, 4.4 \mathrm{~W} \mathrm{~m}^{-2}$ & $0.04^{\circ} \mathrm{C}, 1.7 \mathrm{~W} \mathrm{~m}^{-2}$ \\
\hline Wind Speed & $1 \%$ & $+0.1 \mathrm{~m} \mathrm{~s}^{-1}$ & $\begin{array}{l}\text { Tilt: }<0.3 \% \\
\text { Sea state: uncertain } \\
\text { Very low wind: } \pm 1 \mathrm{~m} \mathrm{~s}^{-1}\end{array}$ & $\begin{array}{l}\max \left(1.5 \%, 0.1 \mathrm{~m} \mathrm{~s}^{-1}\right) \\
\text { more in low wind } \\
1.7 \mathrm{~W} \mathrm{~m}^{-2}\end{array}$ & $\begin{array}{l}\max \left(1 \%, 0.1 \mathrm{~m} \mathrm{~s}^{-1}\right) \\
1.6 \mathrm{~W} \mathrm{~m}^{-2}\end{array}$ & $\begin{array}{l}\max \left(1 \%, 0.1 \mathrm{~m} \mathrm{~s}^{-1}\right) \\
1.6 \mathrm{~W} \mathrm{~m}^{-2}\end{array}$ \\
\hline Wind Direction & $\begin{array}{l}\text { Raw compass: } 1^{\circ} \\
\text { Buoy spin: } 4^{\circ}\end{array}$ & $2^{\circ}$ & $\begin{array}{l}\text { Low wind: } 1^{\circ} \\
\text { Flow distortion: }<5^{\circ}\end{array}$ & $\begin{array}{l}6^{\circ} \\
\text { (more in low wind) }\end{array}$ & $5^{\circ}$ & $5^{\circ}$ \\
\hline
\end{tabular}

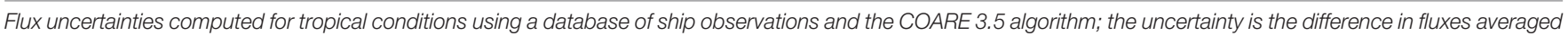

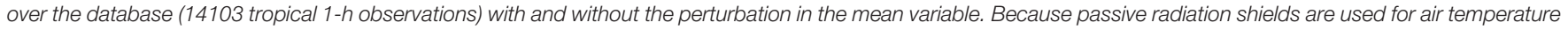
and humidity sensors, values are given for different ranges of wind speed; in low winds and high insolation, air temperature and humidity errors are larger.

TABLE 2 | Accuracy of long-term average of heat flux components, net heat flux, wind stress magnitude, and freshwater flux for an ASIMET system deployed in the subtropics, after Colbo and Weller (2009).

\begin{tabular}{|c|c|c|c|c|c|c|c|}
\hline & $Q_{L W}$ & $Q_{s w}$ & $Q_{\text {lat }}$ & $Q_{\text {sen }}$ & $Q_{\text {net }}$ & $|\tau|$ & E-P \\
\hline$\%$ Error (covariance flux \% Error) & 10 & 2.5 & $6(5)$ & $16(14)$ & $20(16)$ & $20(9)$ & $10 \mathrm{~cm}$ \\
\hline $\begin{array}{l}\text { Typical Error (covariance flux } \\
\text { typical error) }\end{array}$ & $3.9 \mathrm{~W} \mathrm{~m}^{-2}$ & $5 \mathrm{~W} \mathrm{~m}^{-2}$ & $\begin{array}{l}7 \mathrm{~W} \mathrm{~m}^{-2} \\
\left(5 \mathrm{~W} \mathrm{~m}^{-2}\right)\end{array}$ & $\begin{array}{c}2 \mathrm{~W} \mathrm{~m}^{-2} \\
\left(1.5 \mathrm{~W} \mathrm{~m}^{-2}\right)\end{array}$ & $\begin{array}{l}10 \mathrm{~W} \mathrm{~m}^{-2} \\
\left(8 \mathrm{~W} \mathrm{~m}^{-2}\right)\end{array}$ & $\begin{array}{l}0.007 \mathrm{~N} \mathrm{~m}^{-2} \\
\left(0.005 \mathrm{~N} \mathrm{~m}^{-2}\right)\end{array}$ & $10 \mathrm{~cm}$ \\
\hline
\end{tabular}

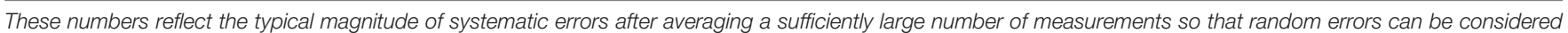

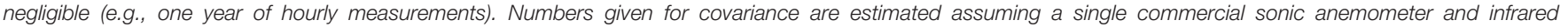

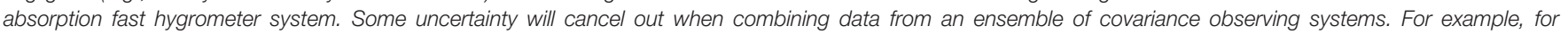
the PSD ship database containing data from 41 cruises residual statistical uncertainty in mean covariance stress (averaged in wind speed bins) is described by $\operatorname{sqr}\left[(0.0003)^{2}+(0.02|\tau|)^{2}\right]$.

being developed that can distinguish diffuse radiation, allowing the computation of the direct solar radiation component and its correction for effective changes in zenith angle associated with platform motion.

Researchers have recently begun collecting year-long time series of direct covariance flux measurements of momentum, heat and mass fluxes from surface moorings (Weller et al., 2012; Bigorre et al., 2013; Farrar et al., 2015; Ogle et al., 2018). The instrumentation on these moorings experiences less flow distortion than ships and measures a wider variety of conditions given their longer deployments. For example, fluxes were measured on a 3-m discus buoy for 15 months during the NSF CLIMODE field program. The buoy experienced wind conditions spanning $0-23 \mathrm{~m} \mathrm{~s}^{-1}$ over a wide range of stability and surface wave conditions. The fluxes measured under these conditions were used to develop the COARE 3.5 algorithm (Edson et al., 2013).

Similar flux packages have been deployed as part of NSF's Ocean Observatories Initiative (OOI), which have been measuring motion-corrected winds for over 5 years, which will enable direct stress and buoyancy flux measurements. Recently, fast response hygrometers were used in the NASA SPURS programs (Farrar et al., 2015) to directly measure the latent heat flux. Similar work is underway to develop buoy $\mathrm{CO}_{2}$ flux systems by drying the sample. Flux systems now exist that can compute and telemeter fluxes in near real-time to shore. In general, deployment duration on buoys is limited by battery power, although some sensors are subject to biofouling and 
other issues that affect the calibrations. Surface buoys are also exposed to weather, vandalism, waves, and sea birds. Redundant installation of meteorological sensors is often necessary to avoid data gaps due to sensor failures. This is particularly important for flux calculations since failure of any one of the primary state variables will result in a data gap in the air-sea flux. Even with these precautions, however, surface moorings must be refreshed at 12-18 month intervals, requiring a ship to transit to these distant locations and adding to their expense. On the other hand, these mooring cruises provide an opportunity to do repeat sections to key locations in the global climate system.

Computational fluid dynamic flow studies of the buoy tower and sensors are recommended to identify errors due to flow distortion and guide improved sensor placement and tower design. Protection from marine birds is recommended. In freezing conditions, heated sensors are required to prevent ice buildup; and heating of the buoy tower maybe required to prevent ice buildup leading to tipping or inversion of the surface buoy. Of course, adding heating and ventilation as well as additional sensors to measure the fluxes directly requires increased battery payloads. This has been done successfully using isolated battery packs to deliver power on a duty cycle. The buoys of the OOI provide continuous power using additional sources that include solar panels and wind generators with rechargeable storage batteries.

The success of buoy-based systems has led to the development and use of a wide variety of platforms for observing air-sea interactions, including Autonomous Surface Vehicles (ASVs), surface drifters and spar buoys (Figure 3). ASVs generally have propulsion powered by either waves (e.g., Wave Glider) or wind (e.g., Saildrone, Sailbuoy), and have electronics powered by solar energy and/or batteries. ASVs navigation can be controlled by setting corridor width and waypoints via satellite communication system (e.g., Iridium). With speeds of up to 2.5 knots for wave-propulsion ASV and 7 knots for wind-propulsion ASV (depending upon wind and ocean conditions), and endurances of 6 months to a year, ASVs can cover thousands of nautical miles. This gives ASVs the capability to either sample in a station-keeping mode, like moored buoys, to create a fixed time series, or in repeat section-mode, or adaptive sampling mode, to do surveys like a research vessel (RV). Recent examples include sampling through hurricanes/typhoons (Lenain and Melville, 2014; Mitarai and McWilliams, 2016) and in the harsh Southern Ocean (Monteiro et al., 2015; Schmidt et al., 2017; Thomson and Girton, 2017).

Nearly all components for calculating bulk EOV/ECV fluxes have been measured from ASVs, including wind speed and direction, air-temperature, humidity, solar and longwave radiation, bulk temperature, skin SST, and surface currents, although some of these measurements are less mature than others. While ASVs tend to have minimal flow distortion, their platform motion (pitch, roll, heading) must be removed when transforming their measurements into Earth coordinates. Improved Global Positioning Systems (GPS) enable corrections for platform motions at better than $10 \mathrm{~Hz}$. With sonic wind sensors measuring 3-dimension winds at $10 \mathrm{~Hz}$ or faster (particularly at high winds near the surface), field tests are underway to determine whether these platforms can be used to measure covariance flux wind stress directly in addition to the mean wind. The low height of sensors making atmospheric measurements on some of the ASVs remains a technical issue. The community also does not have a good handle on the effects of wave shadowing or distortion on the atmospheric boundary layer and its impact for example on the measured wind field (Schmidt et al., 2017) and further efforts are required to assess and address this. Quantification of the accuracy for measurements associated with air-sea heat and momentum fluxes are continuing.

ASV technology is new and currently at a pilot Technical Readiness Level (TRL) of 4 (“Trial") - 5 ("Verification") (Lindstrom et al., 2012). Before it can be expanded to a global array, the TRL needs to be increased to a mature TRL of 9 ("Sustained"). For this, all sensors and systems need to be validated against known standards under a wide range of field conditions on a routine basis. The platforms themselves must be understood with respect to flow distortion, height of the various instruments, and other complicating factors. Navigation needs to be automated in ways that maintain safety at sea, and enables coordinated work with other observational platforms, such as moorings, ships, and gliders (see Swart et al., 2019). Adaptive sampling of atmospheric (e.g., storms/hurricanes) or ocean (e.g., fronts and eddies) features require automatic identification and tracking by ASV. Such capability would enable optimal exploration of complex atmosphere-oceanic environments. Likewise, onboard data processing needs to be developed and tested, and sensor system, data, and metadata must be standardized. Finally, the ASV community must agree to common data delivery, archiving and best practice. An ASV governing body could help develop these standards and create an ASV network.

Drifting or Lagrangian platforms such as the ASIS (Graber et al., 2000) have been used to successfully measure the surface fluxes in field campaigns for decades. Drifting spar buoys generally require less motion correction, experience less flow-distortion and place sensors above the difficult-to-resolve processes within the wave-boundary layer (Hara and Sullivan, 2015); all of which results in accurate direct flux estimates (Edson et al., 2013; Drennan et al., 2014). Another advantage of a Lagrangian measurement of the air-sea fluxes in combination with oceanic temperature and salinity is that, to the extent the drifter follows the mean mixed layer currents, an ocean heat budget assessment can be simplified by reducing the advective flux divergence contribution to the budget (e.g., Silverthorne and Toole, 2013). Thus the surface fluxes measured by a drifter can be more directly constrained by changes in the upper ocean heat or salt content, and more directly compared to one-dimensional ocean models to evaluate the effects of surface forcing on the upper ocean (e.g., du Penhoat et al., 2002). Low-profile Lagrangian surface drifters provide reliable measurements of surface currents and waves over a wide range of conditions (e.g., Herbers et al., 2012). Recent advances in these platforms have included the ability to measure EOVs and subsurface turbulence with, e.g., SWIFT drifters (Thomson, 2012). Drifting versions of the traditional surface mooring are being developed at several institutions. These "minibuoys" provide flux measurements at 

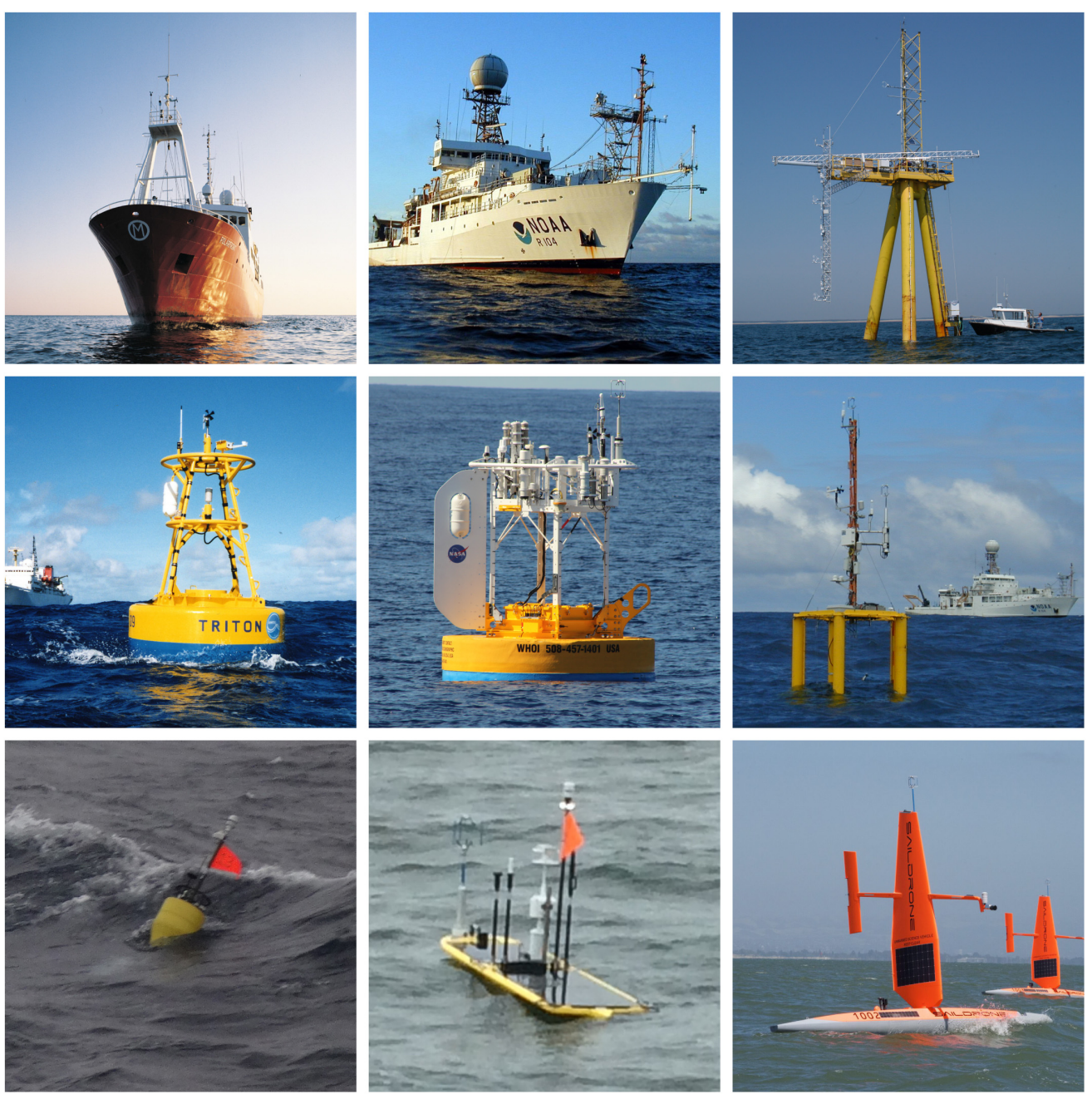

FIGURE 3 | Examples of different types of air-sea flux in situ platforms. Clockwise from upper left: Norwegian weathership Polarfront (image courtesy of Norwegian Meteorological Institute) (Yelland et al., 2009); NOAA ship Ron Brown (from www.noaa.gov); WHOI Air-Sea Interaction Tower (image courtesy Jayne Doucette Woods Hole Oceanographic Institution); RSMAS "ASIS" spar buoy (Graber et al., 2000); Saildrone, Inc., "Saildrone" ASV (image courtesy of Saildrone, Inc.; www.saildrone.com), Liquid Robotics "Wave Glider" ASV (from www.liquid-robotics.com with modifications by UW/APL), UW/APL "SWIFT" drifter (Thomson, 2012); JAMSTEC TRITON buoy (from JAMSTEC, www.jamstec.go.jp); and, in the center, the WHOI SPURS buoy (Farrar et al., 2015).

significantly lower cost for field programs and could also be used to significantly augment conventional operational networks such as NDBC and TAO. The community should be encouraged to continue its efforts to design innovative platforms and flux systems while observing and developing best practices that include assessment against accepted standards.

\section{OceanSITES Reference Time Series}

The accuracy of fluxes from moorings approaches and in some cases exceeds that required for monitoring many of the ocean air-sea interaction phenomena (Figure 2). Moorings thus can provide "reference time series" for tuning satellite measurements and assessing uncertainties in satellite and NWP fields. The purpose of the OceanSITES network ${ }^{1}$ is to collect, deliver and promote the use of high-quality multi-disciplinary data from

\footnotetext{
${ }^{1} \mathrm{http}: / /$ www.oceansites.org
}

long-term, high-frequency observations at fixed locations in the open ocean. These long time-series help to distinguish variations in EOVS due to temporal variability from that due to spatial variability. The large set of co-located EOVs at these sites (e.g., surface heat fluxes, ocean wind stress, subsurface temperature, salinity, velocity, surface mixed layer depth), allow many terms in the heat, momentum and salt equations to be evaluated and thus processes responsible for variability to be identified. Such analyses are critical for identifying causes of biases in NWP reanalyses and ultimately improving the model physics.

The OceanSITES network comprises moorings funded by individual principal investigators from oceanographic agencies in many different nations. Most sites were initiated through research process studies. For example, the Stratus mooring at $20^{\circ} \mathrm{S} 85^{\circ} \mathrm{W}$, was initiated during a cloud feedbacks study (Mechoso et al., 2014; Weller, 2015), while the Kuroshio Extension Observatory 
(KEO) was initiated during a field study of western boundary current physics (Cronin et al., 2013). Station Papa, a site of an ocean weather ship from 1940 to 1981 in the NE Pacific subpolar gyre, has been at the center of many oceanographic process studies (Freeland, 2007; Cronin et al., 2015). The NOAA surface mooring there was initiated during a process study of the carbon cycle. The WHOI Hawaii Ocean Timeseries (WHOT) mooring was initiated as an oceanic sentinel sister site to the Moana Loa "Keeling Curve." Monterey Bay Aquarium Research Institute (MBARI) maintains a station in the California Current system. Its primary purpose is for monitoring and understanding the ecosystem productivity and biogeochemical cycling in this upwelling zone. The OOI Irminger Sea station is part of the Overturning in the Subpolar North Atlantic Program (OSNAP). The Southern Ocean Flux Station (SOFS) south of Tasmania monitors the ventilation and mode water formation in the Subantarctic Zone (Schulz et al., 2012). SOFS and the OOI Southern Ocean $\left(50^{\circ} \mathrm{S}, 90^{\circ} \mathrm{W}\right)$ site west of Patagonia are the only two stations in the Southern Ocean. Both are subject to storms, waves, and strong currents. The Tropical Atmosphere and Ocean (TAO) mooring array in the Pacific was initiated to better understand, monitor and predict ENSO (McPhaden et al., 1998; Cronin et al., 2006), while the tropical array in the Atlantic was designed to monitor and predict both ENSO-like and meridional modes and the Indian Ocean tropical array was designed to also monitor monsoon variability (McPhaden et al., 2009). The commonality of these long time series sites is that they all are publicly available through the OceanSITES global data assembly center, in a common data format. Figure $\mathbf{4}$ shows a comparison of a satellite-based latent and sensible heat fluxes and OceanSITES moorings. Not all these moorings carry radiation sensors and therefore only a subset of these OceanSITES moorings monitor net surface heat flux.

The long time series provided by the sustained surface moorings of OceanSITES have proven to be of high value, and continuation of the sustained observing sites is recommended. Merged, quality-controlled time series are produced at a number of such sites and have been sought after by the modeling community, by the remote sensing community (Pinker et al., 2018), and by those evaluating new hybrid flux products (Valdivieso et al., 2017). Some of the time series are just now entering a third decade of observing, and these time series are capturing accurate records of decadal variability as well as of trends. Testing whether or not models and flux products replicate the broad range of time scales in the fluxes, out to decadal and beyond, is critical and requires sustained surface flux time series of high quality. Further, detection of long-term trends and separation of trends from decadal and multidecadal variability also requires ongoing long time series. These sustained time series sites also become foci for process studies that will improve understanding of air-sea interaction and fluxes and support further improvement of models.

Within the global ocean observing system, data from OceanSITES reference stations moorings are particularly important for validating gridded products of fluxes as they provide long records of high-quality flux EOV and ECV at high temporal resolution, co-located with other EOVs and ancillary ocean variables such as the surface ocean mixed layer. In this way, the sources of the biases can sometimes be determined, leading to improvements. The suite of sensors from OceanSITES flux moorings should include not only all flux EOV/ECV, but also, if possible, direct covariance flux estimates as well, although this may require technological development for the platforms. Likewise, sea state EOVs are being tested as flux EOVs and therefore should be included if possible. In addition, it is strongly encouraged to obtain additional environmental parameters which could help represent atmospheric and oceanic conditions that may affect the air-sea exchanges and their impacts. For example, Global Navigation Satellite System (GNSS) receiver, could provide precipitable water vapor, which has been shown to improve weather forecasts (Li et al., 2015).

Current EOV/ECV observations all suffer from different drawbacks. Comparison of point measurements from in situ instruments to satellite measurements, which inherently represent an average over some spatial footprint that is typically kilometers or more in extent, is made difficult by the differences in spatial and temporal sampling. These differences, caused by spatial variability on scales smaller than the satellite footprint, can be compensated somewhat by temporal averaging of the in situ data to effectively attenuate the small-scale variability (e.g., May and Bourassa, 2011; Lin et al., 2015), but the difference in time-space averaging in different observational approaches remains a fundamental difficulty. The in situ moored buoy data is accurate and has high temporal resolution, often for a long record, but these point measurements tend to be too sparsely distributed for mapping spatial patterns and understanding teleconnections. The moored buoys tend to be located along coastlines where they are easier to maintain, and in the three main tropical arrays. Furthermore, while the surface moorings that contribute to OceanSITES and coastal arrays and to research endeavors provide many flux EOV and $\mathrm{ECV}$, few measure all. In particular, only a small subset of these moorings measure solar radiation and not all of these sites measure downward longwave radiation. Likewise, surface current observations are available at only a small subset of surface mooring sites. There are also large gaps in the center of ocean basins and at high latitudes, especially in the Southern Hemisphere (see Swart et al., 2019). There are currently only 22 operating sites in this global network that measure net surface heat flux, with only 7 of those being in the Southern Hemisphere. This drastically undersamples important oceanatmospheric regimes that are known areas of high error for flux analyses. These long, high-quality time series are critical data for satellite algorithm developers, for model testing and development, and for analyzing critical processes in the climate system. These large gaps in coverage reduce the efficacy of the observation for the research and weather applications discussed in section "Introduction."

\section{Current Capabilities for Remotely Sensed Flux EOV/ECV Measurements}

The current capabilities for remotely sensed flux EOV/ECV are summarized in Table 3 and Figure 5. In particular, 
A $Q_{\text {lat }}$ bias

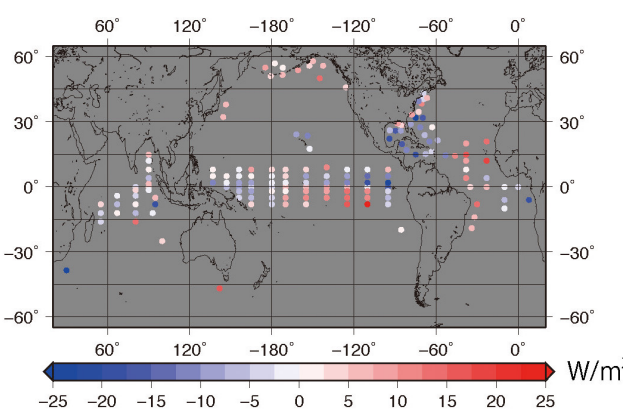

C $Q_{\text {sen }}$ bias

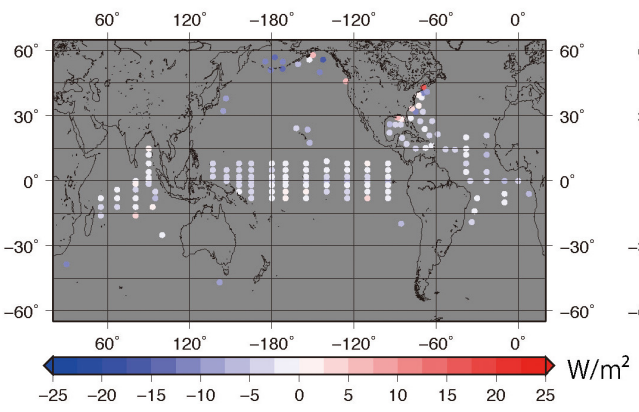

B $Q_{\text {lat }}$ RMSE

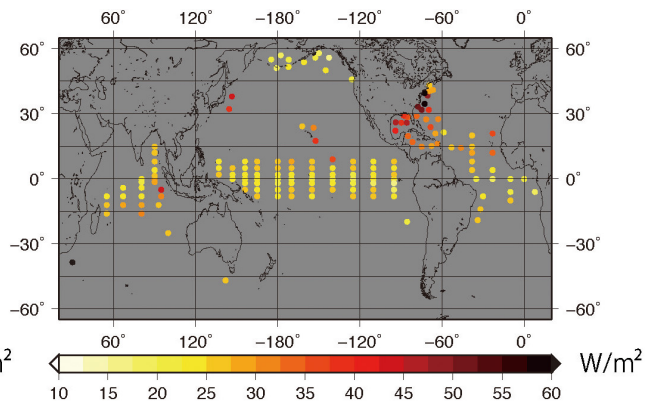

D $Q_{\text {sen }}$ RMSE

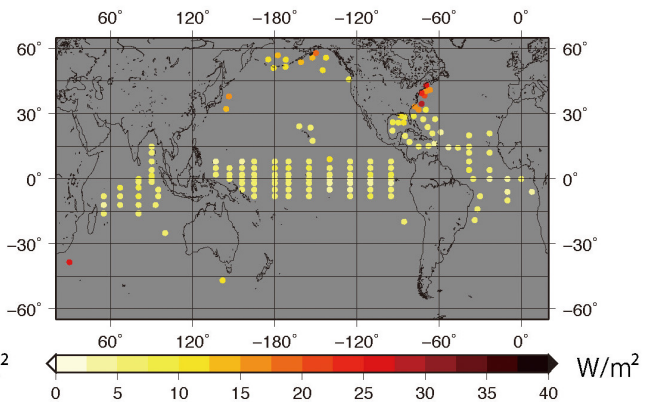

FIGURE 4 | Comparison of J-OFURO3 air-sea heat fluxes with daily-averaged buoys for the period 2002-2013, in units W m ${ }^{-2}$. (A) Latent heat flux (Qlat) bias (satellite minus buoy); (B) $Q_{\text {lat }}$ root-mean-square errors (RMSE); (C) Sensible heat flux $\left(Q_{\text {sen }}\right)$ bias; and (D) $Q_{\text {sen }}$ RMSE. From Figure 5 of Tomita et al. (2019).

typical uncertainty estimates for the highest resolution swath products as well as high resolution gridded products. These uncertainty estimates are presented along with their contribution to uncertainty in the net surface heat flux, estimated by linearizing Eq. 1.1 with respect to the EOV/ECV following Cronin et al. (2014). It should be noted that these uncertainties are based upon comparisons to buoys, which are primarily located in the tropics. Table 3 also describes the typical spatial and temporal resolution, and where technology developments are necessary. Figure 5 shows the status of the constellation for each EOV/ECV over the next decade, and actions needed for improvement. The status of each system is described briefly here.

The ocean surface roughness measured by satellite sensors is normally transformed into an ocean wind speed at $10 \mathrm{~m}$ height using algorithms developed through comparisons with ocean buoys and NWP products (Meissner and Wentz, 2012; Shibata, 2012; Meissner et al., 2014; Hirahara et al., 2015). In reality, the ocean surface roughness is related to the air-sea velocity difference, which is actually the variable of most interest for flux calculations. The measurement of ocean surface roughness from scatterometers (e.g., ASCAT, QuikSCAT, RapidSCAT) and passive microwave (MW) radiometers (e.g., SSMI, SSMI/S, WindSAT, AMSR-E, AMSR2) is already at a spatial resolution and accuracy sufficient for most global flux estimates. At NDBC, TAO, and PIRATA buoys, monthly mean satellite wind speeds are found to have average biases of $0.3 \mathrm{~m} \mathrm{~s}^{-1}$ and RMS of 0.73 and $0.81 \mathrm{~m} \mathrm{~s}^{-1}$ (QuikSCAT and SSMI, respectively) (Wallcraft et al., 2009). RMS is a bit larger for the daily mean wind speed; RMS of
$1.25 \mathrm{~m} \mathrm{~s}^{-1}$ at TAO buoy is reported (Hirahara et al., 2015). It is even larger in the Kuroshio Extension region; RMS is $1.6 \mathrm{~m} \mathrm{~s}^{-1}$ at KEO buoy for AMSR2 (Tomita et al., 2015).

The recent generation of satellite SST sensors (e.g., VIIRS, AATSR and its successor, SLSTR) are close to meeting the global uncertainty of $0.3 \mathrm{~K}$ for surface skin temperature measurements, but the uncertainty has regional non-random characteristics that may not always meet the uncertainty requirements (Petrenko et al., 2014). There have been efforts to generate a stable SST record (e.g., ESA Climate Change Initiative for SST, NOAA Pathfinder AVHRR, MODIS - VIIRS). In regions with persistent, seasonal cloud cover, observations are simply not possible from IR instruments, which hinders the accuracy of daily and monthly SST analyses (Liu and Minnett, 2016). Other sources of error, such as water vapor and atmospheric aerosols have regional and temporal characteristics that will impact the uncertainty (Luo et al., 2019). Passive microwave SSTs approximate to the subskin value, but with simultaneous observation of wind speeds, and further research into transformation of these observations into a skin value, they can provide essential observations in regions where the IR observations are simply not available due to cloud cover (see section "Systematic Uncertainties Near Fronts and Regions of Persistent Clouds"). Donlon et al. (2002) found the skin to subskin difference asymptotes to a value of $-0.14 \mathrm{~K}$ for wind speeds above approximately $6 \mathrm{~m} \mathrm{~s}^{-1}$. Since subsurface temperature measurements from buoys are widely used in IR atmospheric correction algorithm development and validation, an offset of $-0.17 \mathrm{~K}$ is used as an estimate of the global thermal 
TABLE 3 | Current capability in remotely sensed flux EOVs and corresponding error in net surface heat flux and wind stress.

\begin{tabular}{|c|c|c|c|c|c|}
\hline Observable & Sensor & $\begin{array}{l}\text { Horizontal } \\
\text { Temporal } \\
\text { Resolution }\end{array}$ & $\begin{array}{l}\text { Sensor accuracy of swath } \\
\text { (and contribution to } Q_{\text {net }} \\
\text { uncertainty) }\end{array}$ & $\begin{array}{l}\text { Uncertainty of gridded } \\
\text { product at available daily or } \\
\text { monthly resolution (and } \\
\text { contribution to } Q_{n e t} \\
\text { uncertainty) }\end{array}$ & References \\
\hline $\begin{array}{l}\text { Ocean surface wind } \\
\text { speed and direction }\end{array}$ & $\begin{array}{l}\text { Scatterometer and Passive } \\
\text { Microwave Radiometer }\end{array}$ & $25 \mathrm{~km} / 12 \mathrm{~h}$ & $0.6-1.6 \mathrm{~m} \mathrm{~s}^{-1}\left(13-26 \mathrm{~W} \mathrm{~m}^{-2}\right)$ & $0.6-1.6 \mathrm{~m} \mathrm{~s}^{-1}\left(9.6-26 \mathrm{~W} \mathrm{~m}^{-2}\right)$ & $\begin{array}{l}\text { Yu and Jin, 2012; Zhang } \\
\text { et al., } 2018\end{array}$ \\
\hline Skin SST & $\begin{array}{l}\text { Infrared Radiometer; Passive } \\
\text { Microwave Radiometer } \\
\text { (which measures an } \\
\text { approximation to the sub-skin } \\
\text { temperature) }\end{array}$ & $1 \mathrm{~km} / 12 \mathrm{~h}$ & $0.2-0.6 \mathrm{~K}^{\left(9-26 \mathrm{~W} \mathrm{~m}^{-2}\right)}$ & $0.2-0.6 \mathrm{~K}\left(9-26 \mathrm{~W} \mathrm{~m}^{-2}\right)$ & $\begin{array}{l}\text { Corlett et al., 2014; } \\
\text { Gentemann and Hilburn, } \\
\text { 2015; Kilpatrick et al., } \\
\text { 2015; Tu et al., 2015; } \\
\text { Bulgin et al., } 2016\end{array}$ \\
\hline $\begin{array}{l}\text { Near surface air } \\
\text { temp }\end{array}$ & $\begin{array}{l}\text { Technology advancements } \\
\text { needed }\end{array}$ & $25 \mathrm{~km} / 12 \mathrm{~h}$ & $1.3-1.55 \mathrm{~K}\left(18-22 \mathrm{~W} \mathrm{~m}^{-2}\right)$ & $\left.0.5-1.55 \mathrm{~K}^{(6-22} \mathrm{W} \mathrm{m}^{-2}\right)$ & $\begin{array}{l}\text { Jackson and Wick, 2010; } \\
\text { Roberts et al., 2010; Yu } \\
\text { and Jin, } 2018\end{array}$ \\
\hline $\begin{array}{l}\text { Near surface } \\
\text { specific air humidity }\end{array}$ & $\begin{array}{l}\text { Passive Microwave } \\
\text { Radiometer }\end{array}$ & $25 \mathrm{~km} / 12 \mathrm{~h}$ & $1-1.3 \mathrm{~g} / \mathrm{kg}\left(20-26 \mathrm{~W} \mathrm{~m}^{-2}\right)$ & $0.8-1 \mathrm{~g} / \mathrm{kg}\left(16-20 \mathrm{~W} \mathrm{~m}^{-2}\right)$ & $\begin{array}{l}\text { Roberts et al., 2010; } \\
\text { Tomita et al., 2018; Yu } \\
\text { and Jin, } 2018\end{array}$ \\
\hline $\begin{array}{l}\text { Surface solar } \\
\text { radiation }\end{array}$ & $\begin{array}{l}\text { Imagers (multi-channel), } \\
\text { CERES, ancillary }\end{array}$ & 100 km/3 h & $55 \mathrm{~W} \mathrm{~m}^{-2}\left(55 \mathrm{~W} \mathrm{~m}^{-2}\right)$ & $11 \mathrm{~W} \mathrm{~m}^{-2}\left(11 \mathrm{~W} \mathrm{~m}^{-2}\right)$ & $\begin{array}{l}\text { Rutan et al., 2015; Kato } \\
\text { et al., } 2018\end{array}$ \\
\hline $\begin{array}{l}\text { Surface longwave } \\
\text { radiation }\end{array}$ & $\begin{array}{l}\text { Imagers (multi-channel), } \\
\text { CERES, ancillary }\end{array}$ & $100 \mathrm{~km} / 3 \mathrm{~h}$ & $20 \mathrm{~W} \mathrm{~m}^{-2}\left(20 \mathrm{~W} \mathrm{~m}^{-2}\right)$ & $5 \mathrm{~W} \mathrm{~m}^{-2}\left(5 \mathrm{~W} \mathrm{~m}^{-2}\right)$ & $\begin{array}{l}\text { Rutan et al., 2015; Kato } \\
\text { et al., } 2018\end{array}$ \\
\hline
\end{tabular}

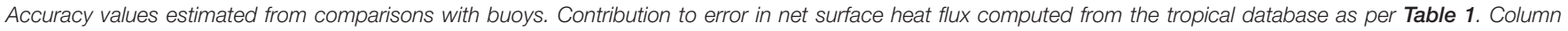

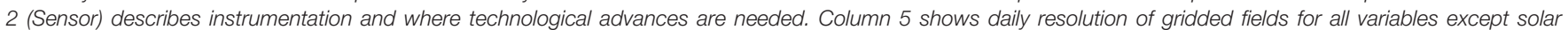

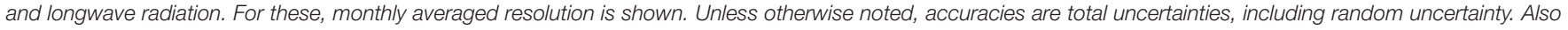

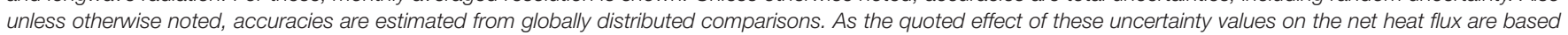

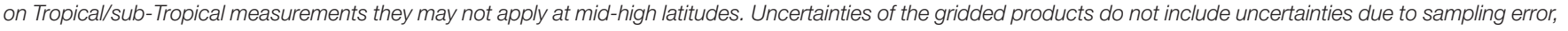
and therefore underestimate the true uncertainty by some unknown percentage.

skin effect, so the subsurface temperatures approximate to a skin SST (Kilpatrick et al., 2015).

Near-surface air temperature is an exceptionally difficult observation from satellite measurements, as existing instrumentation cannot adequately resolve the planetary boundary layer, which has thicknesses varying from $\sim 500 \mathrm{~m}$ to $3 \mathrm{~km}$ over the ocean (von Engeln and Teixeira, 2013). This observable is currently estimated using atmospheric sounders and hyperspectral sensors, both of which have drawbacks. The sounders have higher sensitivity to the upper, rather than lower, atmospheric temperatures and have low vertical resolution, making the measurement of near surface temperature exceptionally challenging. The hyperspectral instruments such as AIRS and IASI, have high spectral resolution and offer better vertical resolution, but still suffer from the fundamental physical problem that the vertical resolution of derived profiles is limited to $\sim 1 \mathrm{~km}$. The use of passive MW imagers to determine near-surface air temperature and humidity has been undertaken with some success, if a first-guess SST is used, with small $\left(<0.1^{\circ} \mathrm{C}\right)$ bias and roughly $1.5^{\circ} \mathrm{C}$ RMS (Roberts et al., 2010; Clayson and Brown, 2016).

Near-surface air humidity is very difficult to infer accurately from satellite radiometers, for the same reasons as for nearsurface air temperature. For both temperature and humidity, the weighting functions used for retrievals are dependent on the temperature and humidity profiles and, consequently, cannot be fixed for given wavelengths. Thus, there is a risk that the nearsurface variables are artificially correlated with the sea-surface temperature and the state of the atmosphere, and distinguishing the true physical correlations from those that are artifacts of the measurement is difficult. In addition, the retrieval algorithms for near-surface temperature and humidity are commonly trained with in situ buoy and/or ship measurements. The relationship between satellite measurements and near-surface variables is strongly regime dependent, displaying a step-like transition (or separation) from the warm/humid regime to the cold/dry regime (Yu and Jin, 2018). The evidence suggests that the skill of the retrieval algorithm is highly dependent of the vertical distribution of water vapor. Current remote-sensing algorithms to derive near-surface humidity using a satellite MW radiometer show RMS disagreements of $\sim 1.0 \mathrm{~g} \mathrm{~kg}^{-1}$ with smaller positive bias in mid-latitudes (Tomita et al., 2018). A recent regimedependent approach that treats the warm/humid and cold/dry regimes separately shows noted improvement with RMS of $0.8 \mathrm{~g} \mathrm{~kg}^{-1}$ for air specific humidity and $0.5^{\circ} \mathrm{C}$ for air temperature (Yu and Jin, 2018).

As discussed in section "Radiative Heat Flux EOV/ECV", surface radiative fluxes are computed using radiative transfer models with input provided by cloud properties retrieved from satellites combined with temperature and humidity profiles. Comparisons of in situ surface observations and satellite-derived irradiances are used to estimate the uncertainty in satellitederived irradiances; there are however, only a limited number of radiation measurements over the global ocean and most of these are in the tropics. Comparisons reported by Kato et al. (2018) show that surface monthly mean downward fluxes agree with observations to within a mean difference (RMS) of 5 (11) $\mathrm{W} \mathrm{m}^{-2}$, respectively for shortwave, and 2 (5) $\mathrm{W} \mathrm{m}^{-2}$ for longwave, when 


\begin{tabular}{|c|c|c|c|}
\hline Variable & $\begin{array}{l}\text { Instrument } \\
\text { type }\end{array}$ & Relevant Satellite Sensors - 2018-2030 & Status and Actions \\
\hline \multirow{5}{*}{$\begin{array}{l}\text { Ocean surface } \\
\text { wind speed/ } \\
\text { direction }\end{array}$} & $\begin{array}{l}\text { Radar } \\
\text { scatterometers }\end{array}$ & $\begin{array}{c}\text { ASCAT on MetOp-A, B, C; OSCAT on ScatSat-1 and OceanSat }-2,-3,-3 A \text {; } \\
\text { SCAT \& SWIM on CFOSAT, Follow-On mission from 2022; WindRAD on } \\
\text { FY-3E, 3H from 2019; SCAT on HY-2C, 2D, 2E, 2F from 2019; } \\
\text { SCA on MetOp SG from 2022. }\end{array}$ & $\begin{array}{l}\text { Technology and algorithms are mature, but } \\
\text { directional accuracy needs improvement. } \\
\text { Technology improvements to include } \\
\text { Doppler could give broad swath surface } \\
\text { currents. }\end{array}$ \\
\hline & $\begin{array}{l}\text { PWM } \\
\text { radiometers }\end{array}$ & $\begin{array}{l}\text { WindSat* on Coriolis; AMSR2* on GCOM-W1; SSMIS on DMSP F16, } \\
\text { F17, F18; GMI on GPM-Core Observatory. }\end{array}$ & $\begin{array}{l}\text { Technology and algorithms are mature. } \\
\text { Constellation is fragile. Sampling is poor. } \\
\text { Speed only in most cases. Directional } \\
\text { accuracy needs improvement. }\end{array}$ \\
\hline & SAR & $\begin{array}{l}\text { Sentinel } 1 \text { a \& b, followed by c \&d; TerraSar-X, TanDEM-X \& PAZ; } \\
\text { COSMO-SkyMed; RADARSAT-2; RADARSAT Constellation. }\end{array}$ & $\begin{array}{l}\text { Technology is mature, algorithm } \\
\text { improvement needed. High resolution, but } \\
\text { very poor global coverage. Directional } \\
\text { accuracy needs improvement. }\end{array}$ \\
\hline & $\begin{array}{l}\text { GNSS } \\
\text { reflectometry }\end{array}$ & CYGNSS.* & $\begin{array}{l}\text { Technology is quite mature, but GPS } \\
\text { satellites were not adequately } \\
\text { intercalibrated, algorithm improvement } \\
\text { needed. CYGNSS in tropics only. Launched } \\
2015 \text { for two-year mission. Speed only. } \\
\text { Long-term prospects unknown. }\end{array}$ \\
\hline & Doppler LIDAR & Atmospheric Dynamics Mission-Aeolus. & $\begin{array}{l}\text { Experimental. 2D wind profile in flight } \\
\text { direction, component in look direction. } \\
\text { Should reach into PBL. Launched in } 2018 \text { for } \\
\text { a 3-year mission. Large footprint, and poor } \\
\text { sampling }\end{array}$ \\
\hline \multirow[b]{2}{*}{$\begin{array}{l}\text { Surface } \\
\text { currents }\end{array}$} & SAR & $\begin{array}{l}\text { Sentinel } 1 \mathrm{a} \& \mathrm{~b} \text {, followed by } c \& d \text {. TerraSar-X, TanDEM-X \& PAZ; } \\
\text { COSMO-SkyMed; RADARSAT-2; RADARSAT Constellation. }\end{array}$ & $\begin{array}{l}\text { Experimental, narrow swath, poor } \\
\text { sampling, selected locations, current } \\
\text { component only in look direction. }\end{array}$ \\
\hline & Altimeters & $\begin{array}{c}\text { Sentinel } 3 \text { a \& b, followed by } c \text { \& d; JASON 3, followed by JASON-CS-A } \\
\text { and -B; SWOT in } 2022 .\end{array}$ & $\begin{array}{l}\text { Mature technology and algorithms. Derived } \\
\text { from surface topography along sub-satellite } \\
\text { track using geostrophy. SWOT is } \\
\text { experimental. Resolution is roughly } 200 \mathrm{~km} \\
\text { and } 5 \text { days. }\end{array}$ \\
\hline \multirow{3}{*}{$\begin{array}{l}\text { Sea-surface } \\
\text { temperature }\end{array}$} & $\begin{array}{l}\text { IR radiometers } \\
\text { - polar orbiters }\end{array}$ & $\begin{array}{l}\text { AVHRR on NOAA-19, MetOp-B, -C; MODIS* on Terra and Aqua; } \\
\text { VIIRS on S-NPP \& NOAA-20, JPSS-2, -3; SLSTR on Sentinel } 3 \mathrm{a} \& \mathrm{~b} \text {, } \\
\text { followed by } c \text { \& d; METimage on MetOp SG from } 2022 \text {. }\end{array}$ & $\begin{array}{l}\text { Technology is mature, algorithm } \\
\text { improvement needed to reach required } \\
\text { goals. }\end{array}$ \\
\hline & $\begin{array}{l}\text { IR radiometers } \\
\text { - geostationary }\end{array}$ & $\begin{array}{l}\text { Imager on GOES-15; SEVIRI on MSG-10; Imager on INSAT-3, -3DR, } \\
\text {-3DS; AHI on Himawari-8, -9; ABI on GOES-16 (IR bands on GOES-17 } \\
\text { have problems); AGRI on FY-4A, 4B, } 4 C \text {; GIIRS on FY-4A, } 4 B, 4 C, 4 D \text {, } \\
4 E ; A M I \text { on Geo-Kompsat-2A; FCI on MTG-I. }\end{array}$ & $\begin{array}{l}\text { Technology is mature, algorithm } \\
\text { improvement needed to reach required } \\
\text { goals. }\end{array}$ \\
\hline & $\begin{array}{l}\text { PWM } \\
\text { radiometers }\end{array}$ & WindSat* on Coriolis - current; AMSR2* on GCOM-W1. & $\begin{array}{l}\text { Technology is mature, algorithm } \\
\text { improvement needed to reach required } \\
\text { goals. Constellation is fragile. }\end{array}$ \\
\hline \multirow{3}{*}{$\begin{array}{l}\text { Near-surface } \\
\quad \text { air } \\
\text { temperature }\end{array}$} & $\begin{array}{l}\text { IR sounders } \\
\text { - polar orbiters }\end{array}$ & $\begin{array}{l}\text { AIRS* on Aqua; TES-N* on Aura; IASI on MetOp-B, -C; IASI-NG on } \\
\text { MetOp SG from 2022; CRIS on S-NPP \& NOAA-20; JPSS-2, -3. }\end{array}$ & \multirow{3}{*}{$\begin{array}{l}\text { Technology and algorithms mature for } \\
\text { coarse resolution sounding; new } \\
\text { algorithms, and probably new technology, } \\
\text { needed for PBL temperature. }\end{array}$} \\
\hline & $\begin{array}{l}\text { IR sounders } \\
\text {-geostationary }\end{array}$ & $\begin{array}{l}\text { Sounder on GOES-15; Sounder on INSAT-3, -3DR, -3DS; GIIRS on } \\
\text { FY-4A, } 4 B, 4 C, 4 D, 4 E ; \text {; IRS on MTG-S. }\end{array}$ & \\
\hline & $\begin{array}{l}\text { PMW } \\
\text { radiometers }\end{array}$ & $\begin{array}{l}\text { SSMIS on DMSP series; AMSU on MetOp-B, -C, and NOAA-19; ATMS } \\
\text { on S-NPP \& NOAA-20, JPSS-2, -3; MWS on MetOp SG from } 2022 .\end{array}$ & \\
\hline \multirow{3}{*}{$\begin{array}{l}\text { Near-surface } \\
\text { air humidity }\end{array}$} & $\begin{array}{l}\text { IR sounders - } \\
\text { polar orbiters }\end{array}$ & $\begin{array}{l}\text { AIRS* on Aqua; IASI on MetOp-B, -C; IASI-NG on MetOp SG from } \\
\qquad 2022 .\end{array}$ & \multirow{3}{*}{$\begin{array}{l}\text { Technology and algorithms mature for } \\
\text { coarse resolution sounding; new } \\
\text { algorithms, and probably new technology, } \\
\text { needed for PBL humidity. }\end{array}$} \\
\hline & $\begin{array}{l}\text { IR sounders - } \\
\text { geostationary }\end{array}$ & $\begin{array}{l}\text { Sounder on GOES-15; Sounder on INSAT-3,-3DR, -3DS; GIIRS on } \\
\qquad F Y-4 A, 4 B, 4 C, 4 D, 4 E \text {; IRS on MTG-S. }\end{array}$ & \\
\hline & PMW sounders & $\begin{array}{l}\text { AMSU on Aqua, MetOp-B, -C and NOAA-19; ATMS on S-NPP \& NOAA- } \\
20, \text { JPSS-2, -3; MWS on MetOp SG from } 2022 .\end{array}$ & \\
\hline $\begin{array}{l}\text { Surface } \\
\text { insolation }\end{array}$ & $\begin{array}{l}\text { VIS/IR } \\
\text { radiometers - } \\
\text { polar orbiters \& } \\
\text { geostationary }\end{array}$ & $\begin{array}{l}\text { CERES on Terra, Aqua, S-NPP, NOAA-20; RBI on JPSS-2 \& -3 from 2022; } \\
\text { Imager on GOES-15; SEVIRI on MSG-10; Imager on INSAT-3, -3DR, } \\
\text {-3DS, -3DS; AHI on Himawari-8, -9; ABI on GOES-16 (IR bands on } \\
\text { GOES-17 have problems); AGRI on FY-4A, 4B, } 4 C \text {; GIIRS on FY-4A, } 4 B \text {, } \\
\text { 4C, } 4 D, 4 E ; \text {; AMI on Geo-Kompsat-2A; FCI on MTG-I. }\end{array}$ & $\begin{array}{l}\text { Highly derived, needing frequent samples } \\
\text { ( } 1 \text { hr or better) of top-of-atmosphere } \\
\text { emitted radiance, 3D distributions of clouds, } \\
\text { aerosols, water vapor and some trace } \\
\text { gasses. Wind-speed dependent surface } \\
\text { albedo need to derive net insolation. } \\
\text { Accuracy of input data and algorithms need } \\
\text { improvement to reach goals. }\end{array}$ \\
\hline $\begin{array}{l}\text { Net surface } \\
\text { infrared } \\
\text { fluxes }\end{array}$ & $\begin{array}{l}\text { VIS/IR } \\
\text { radiometers - } \\
\text { polar orbiters \& } \\
\text { geostationary }\end{array}$ & $\begin{array}{l}\text { CERES on Terra, Aqua, S-NPP, NOAA-20; RBI on JPSS-2 \& -3 from 2022; } \\
\text { Imager on GOES-15; SEVIRI on MSG-10; Imager on INSAT-3, -3DR, } \\
\text {-3DS, -3DS; AHI on Himawari-8, -9; ABI on GOES-16 (IR bands on } \\
\text { GOES-17 have problems); AGRI on FY-4A, 4B, } 4 C \text {; GIIRS on FY-4A, } 4 B \text {, } \\
4 C, 4 D, 4 E ; \text { AMI on Geo-Kompsat-2A; FCI on MTG-I. }\end{array}$ & $\begin{array}{l}\text { Highly derived, needing frequent samples } \\
\text { (1 hr or better) of top-of-atmosphere } \\
\text { emitted radiance, 3D distributions of clouds, } \\
\text { aerosols, water vapor and some trace gases; } \\
\text { cloud base emissivity. Accurate skin SSTs } \\
\text { and surface emissivity needed to derive net } \\
\text { IR fluxes. Accuracy of input data and } \\
\text { algorithms need improvement to reach } \\
\text { goals. }\end{array}$ \\
\hline
\end{tabular}

FIGURE 5 | Satellite sensors that are producing data that can be used in deriving estimates of ocean surface fluxes. Normal text indicates current satellites and sensors, with * indicating those that are beyond their planned lifetime. Italic font shows missions that are expected to be launched in the next decade. Bold text shows areas needing attention in coming decade; red borders highlight where significant action and progress are needed. Not all derived variables from all sensors will reach the accuracies given in Table 3. 
the differences are averaged over 46 ocean sites. Rutan et al. (2015) using CERES Edition 3 3-hourly products found an RMS of 55 and $20 \mathrm{~W} \mathrm{~m}^{-2}$ for SW $\downarrow$ and LW $\downarrow$, respectively. These root-mean-square differences between observed and satellitederived 3-hourly and monthly mean irradiances are used for the uncertainty shown in Table 3. These are within the reported monthly averaged uncertainty of observed radiative fluxes at buoys of $\sim 5 \mathrm{~W} \mathrm{~m}^{-2}$ (Colbo and Weller, 2009). Comparison uncertainties are influenced by atmospheric, cloud and aerosol properties as well as temporal and spatial sampling issues. Ambient conditions, such as aerosol deposition, have also been shown to degrade buoy radiative flux measurements as well (Foltz et al., 2013). Although satellite derived surface radiative fluxes agree with observed radiative fluxes at buoys to within the uncertainty, most buoys are located in the tropics. To evaluate satellite derived radiative fluxes in a wide range of atmospheric conditions, observations in mid- and high-latitude regions are needed.

\section{Systematic Uncertainties Near Fronts and Regions of Persistent Clouds}

Because persistent clouds can form at fronts, IR satellite SST observations (e.g., AVHRR, MODIS, VIIRS ...) can be spatially patchy due to contamination by clouds. Conversely, IR data cloud screening algorithms can also mischaracterize actual IR-observed ocean SST variability near fronts as being cloud (Kilpatrick et al., 2019). A separate issue near fronts stems from the fact that the $25-50 \mathrm{~km}$ spatial footprint of microwave satellite SST retrievals often exceeds the frontal scale. Furthermore, these larger footprints and antenna side-lobes can allow land contamination to impact SST front detection in coastal regions. At present, many mapping products interpolate through these patches (e.g., Reynolds et al., 2013), leading to western boundary current fronts that are too smooth and that do not capture the mesoscale variability associated with the meandering of these fronts. Because the atmospheric response depends upon the sharpness of the SST gradient (Chelton et al., 2004; Minobe et al., 2010; Parfitt et al., 2016), this bias can result in a cascade of errors. Even multi-satellite merged data are unlikely to eliminate the gaps in surface variables completely in part because of land contamination in coastal microwave-based measurements, although a proposed higher resolution sensor could mitigate this problem (e.g., Bourassa et al., 2019; Rodríguez et al., 2019).

A strategy must be developed for handling patchy data in frontal and coastal regions, using alternative data sources. While drifters, ASVs and underway ship measurements may be able to fill these gaps at times, they are likely to be too sparse both in time and space to fill all gaps. Repeat sections from these platforms, however, may provide structure functions for sharp fronts, which when combined with information about the location of the front from altimeter and tidal data, could then help fill the gaps in the patchy data with properly located sharp fronts in flux EOV and ECVs fields. Ultimately, regional atmospheric modeling, preferably with non-hydrostatic model settings and high-resolution NWP products must be used to fill in remaining patches in data with dynamically consistent fields.

\section{Systematic Uncertainties Due to Inadequate Sampling of Diurnal Variability and Episodic Events}

Satellite instruments can provide global or near-global coverage, but the spatial coverage comes at the cost of reduced temporal resolution. Many of the satellites used for estimating surface fluxes are in sun-synchronous polar orbits. Polar orbits have the virtue of providing near global coverage, and sun-synchronous orbits have significant engineering advantages, as satellite observations are made within a narrow range of local times so that solar angles and sun glint are relatively consistent at all times. For some variables, such as SST, geostationary satellites are also useful, with the advantage of providing higher temporal sampling rates that can resolve the diurnal cycle of SST. SST can vary by more than 2-4 K over the course of a day in regions of high solar insolation and low winds. Resolving only nighttime SST can lead to biases of up to $5-10 \mathrm{~W} \mathrm{~m}^{-2}$ in the long-term mean heat fluxes (Clayson and Bogdanoff, 2013).

Resolving the diurnal cycle is particularly important since the diurnal cycle in solar radiation leads to an afternoon near-surface stratification that can trap surface forcing into a thin layer. As a consequence, rain-puddles formed during the daytime are fresher than ones formed at nighttime (Cronin and McPhaden, 1999). Likewise, "diurnal jets" can form, with anomalous wind-forced surface currents that are stronger in the afternoon than at nighttime (Cronin and Kessler, 2009, their Figure 5). The afternoon near-surface stratification also traps surface heat fluxes, making them more effective at causing SST change than at nighttime when the mixed layer depth is deeper. The diurnally varying mixed layer depth, in this way, causes rectification of diurnal variability into longer timescales (Bernie et al., 2005).

Satellite and NWP/reanalyses fluxes may also be biased due to poor representation of short-term extreme flux events associated with very strong winds and strong temperature and humidity gradients near the surface. In satellite-based fluxes these biases are likely due to poor sampling while in NWP and reanalyses they also might be associated with inability of atmospheric models to adequately simulate such conditions. Bentamy et al. (2017) using probability density function approach (Gulev and Belyaev, 2012) demonstrated that extreme fluxes derived from satellite products may deviate from flux extremes estimated from buoy measurements by tens of $\mathrm{W} \mathrm{m}^{-2}$, even if the mean flux estimates are comparable.

Except at high latitudes, a single polar orbiting satellite can provide a maximum of two measurements per day (e.g., at 10:30 am and 10:30 pm), which is inadequate for resolving atmospheric synoptic variability or for resolving the amplitude and phase of the diurnal cycle. The diurnal cycle can be resolved by either placing several satellites in different sun-synchronous orbit planes to sample several discrete local times or by a single satellite in a lower inclination orbit. In the latter case, the satellite will precess through the diurnal cycle for any location on the ground over a period of time (days to months) that is related to the orbit inclination. Placing several satellite in sunsynchronous orbits, while more costly, has the benefit of sampling the diurnal cycle daily at discrete local times and reduces the 
overall re-visit time for resolving rapidly evolving storms and short-lived extreme events.

\section{Current Capabilities for Gridded Flux Products}

To create the global flux products, the flux EOVs and ECVs must be observed or estimated with sufficient resolution and coverage to map their fields, or to constrain NWP model solutions and reanalyses to produce realistic flux fields. For flux products that extend back to the early-mid twentieth century, these gridded products based upon observations and NWP rely exclusively upon in situ data, primarily from underway ships. In the past decades, a satellite constellation has been building that can provide several of the gridded EOVs necessary for creating the flux products. However, because satellites are at present unable to provide some EOVs (e.g., surface air temperature and humidity) with acceptable accuracy, for some "blended" products such as the OAFlux product ( $\mathrm{Yu}$ and Weller, 2007), air temperature and humidity are instead based on bias-adjusted NWP outputs, while other flux EOVs and ECVs are derived from satellite data. Satellite measurements appear to provide sufficient signal-tonoise ratios to produce high-quality stress estimates; however, direct in situ measurements of stress are too sparse to provide adequate calibration. The common practice is to derive winds from satellite backscatter measurements by utilizing in situ wind measurements and compute wind stress from bulk flux parameterization.

Even NWP and blended flux products, however, have large biases and uncertainties (Cronin et al., 2006; Bentamy et al., 2017; Valdivieso et al., 2017; Tomita et al., 2019; Yu, 2019). Mean fields from 12 products show standard deviations of up to $30 \mathrm{~W} \mathrm{~m}^{-2}$ for net surface heat flux and $5 \times 10^{-2} \mathrm{~N} \mathrm{~m}^{-2}$ for wind stress (Figures 1C,D). It should be noted though that agreement between the different products does not mean that there are no biases; it may mean that all products are biased in the same way. In this case, the standard deviation around the multiproduct mean is not an appropriate estimate of the uncertainty. Moreover, the standard deviation could be overestimated due to outlying products. Thus, independent reference data are required to determine the accuracy of these products.

For the global average, ocean heat content estimates show the net surface heat flux into the ocean to be less than $1 \mathrm{~W} \mathrm{~m}^{-2}$ (e.g., Roemmich et al., 2015); for most products, the global average of the net air-sea heat flux is up to an order of magnitude larger than this (Figure 6A and Table 4). Comparisons of the state variables against moored reference station data show that a substantial part of this mean bias is due to deficiencies in the bulk algorithm used by the NWP (Jiang et al., 2005; Zhang et al., 2016). Bias can also be associated with the radiative heat flux components (Figure 6B), highlighting the need to improve satellite radiative observations and the presence of clouds in NWP. In order to understand the regional distribution of these errors and diagnose their causes, in situ reference stations are needed (Figure 4).

To improve NWP fluxes, marine boundary layer physics in NWP must be improved, including their algorithms for computing air-sea fluxes. In addition, more in situ and remotely sensed data with higher accuracy are needed to constrain the NWP. More accurate satellite-based moisture and temperature profiles and surface data would greatly improve remotely sensed estimates of surface radiation and latent and sensible heat fluxes, and if assimilated into NWP models, would likely lead to improved representation of cloud formation processes and thereby improved representation of the state variables and surface radiative and turbulent fluxes from these NWP models.

\section{ASPIRATIONAL SAMPLING REQUIREMENTS AND TARGET UNCERTAINTIES}

Air-sea fluxes are challenging to observe because high-frequency variability and gustiness rectifies into the longer time scales. To adequately represent the annual cycle of heat fluxes, synoptic variability associated with storms must be captured. Subdiurnal resolution is required to adequately represent fluxes associated with fast-moving storms or in regions with a large diurnal cycle in SST. Fast moving atmospheric synoptic transients are associated with surface flux extremes (e.g., Tilinina et al., 2018), which can only be resolved in high resolution data. A gridded air-sea flux product that has $25 \mathrm{~km}$ or better spatial resolution and at least 3-hourly temporal resolution with a random uncertainty of $15 \mathrm{~W} \mathrm{~m}^{-2}$ and a bias of less than $5 \mathrm{~W} \mathrm{~m}^{-2}$ would be able to capture most of the air-sea interaction phenomena shown in Figure 2. The corresponding wind stress product should have a random uncertainty of $\pm 0.01 \mathrm{~N} \mathrm{~m}^{-2}$ (or a $5 \%$ noise-tosignal ratio, whichever is larger) and a bias of $<0.005 \mathrm{~N} \mathrm{~m}^{-2}$. It is important though to realize that the native resolution of the source observations for this gridded product must be at least 2-4 times better - at least $10 \mathrm{~km}$ and hourly. While this represents an improved accuracy at this resolution for wind stress, for the heat flux product, achieving this accuracy would represent a breakthrough advancement. Table 5 summarizes the sampling requirements for each flux EOV/ECV needed to meet this aspirational target. Because SST is the primary ocean EOV that drives the air-sea flux, and can be controlled by ocean frontogenesis processes, SST must have the highest spatial resolution of all EOV/ECV. We set an aspirational target of $1 \mathrm{~km}$. This aspirational target, even now, is partially met for many parts of the global ocean.

\section{OPPORTUNITIES FOR IMPROVED FLUXES IN THE GLOBAL ICE-FREE OCEAN}

For global ice-free ocean estimates of turbulent heat and momentum fluxes, it is necessary to measure the flux EOVs and ECVs with sufficient accuracy, coverage and resolution to meet the targeted uncertainty and stability. As described in section "Current Capabilities," the primary state variables for computing turbulent air-sea fluxes of heat and momentum include near surface air temperature, ocean skin SST, near-surface humidity, and the surface wind relative to the ocean surface currents. 
A

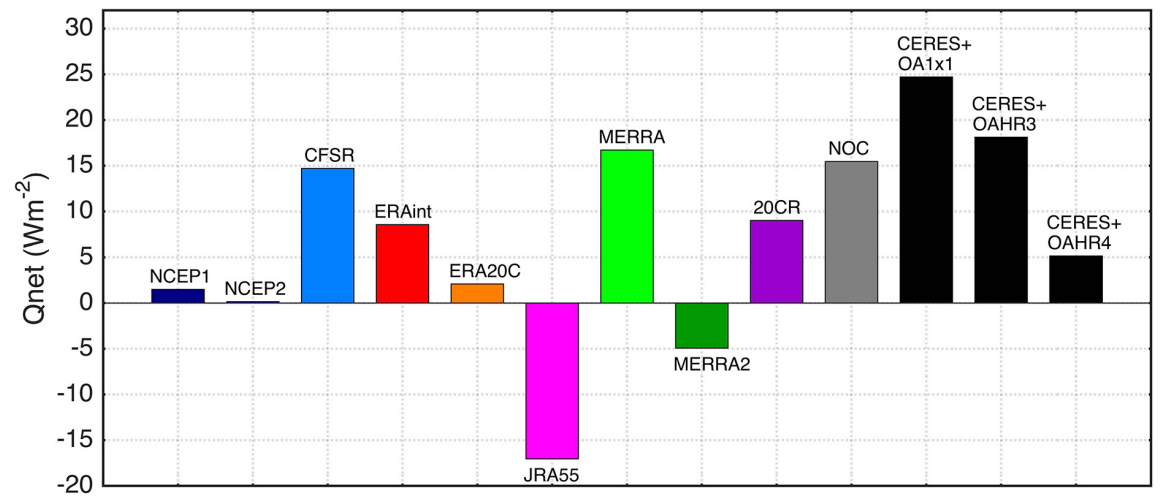

B

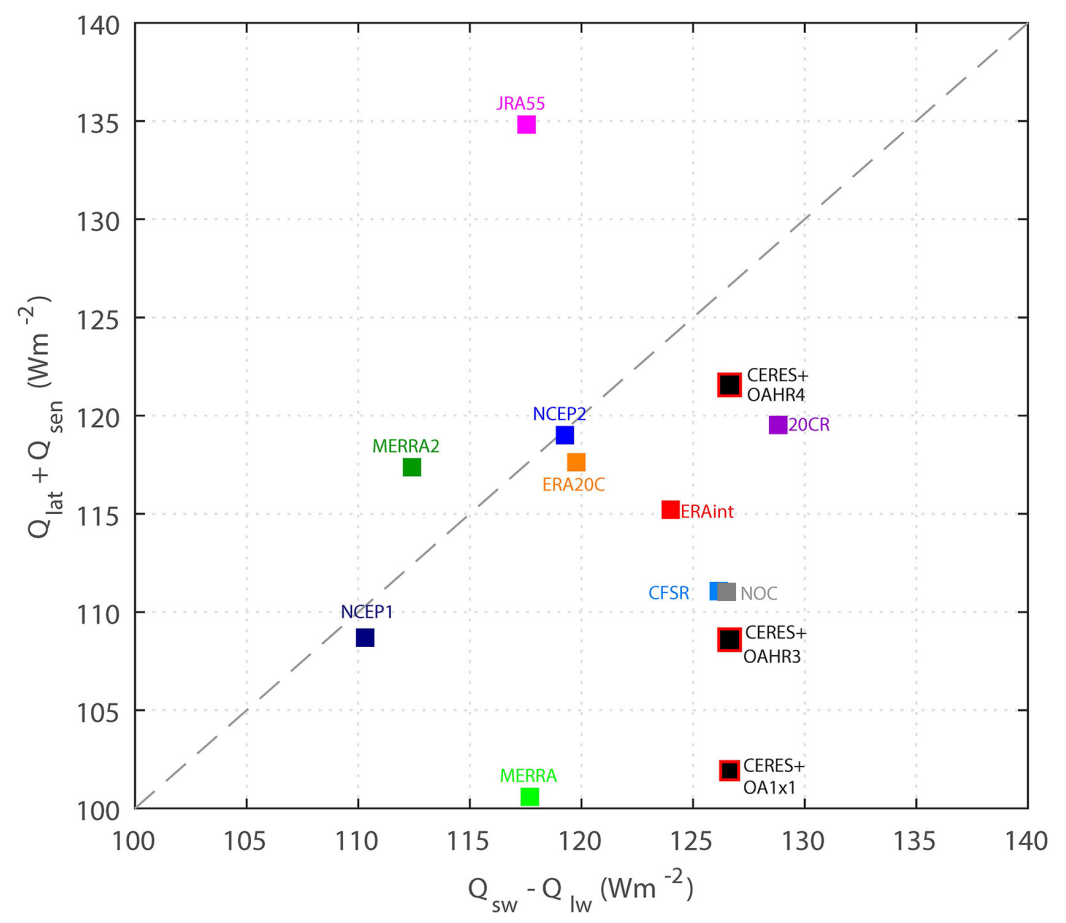

FIGURE 6 | (A) Global-ocean mean energy $\left(Q_{n e t}\right)$ budget. (B) The ratio of the mean averages of $Q_{S W}-Q_{L W}$ to $Q_{l a t}+Q_{s e n}$; the dashed line denotes that this ratio equals 1.0. Abbreviations: 20CR, Twentieth Century Reanalysis; CERES, Clouds and the Earth's Radiant Energy System-Energy Balanced and Filled; CFSR, Climate Forecast System Reanalysis; ERA-20C, European Centre for Medium-Range Weather Forecasts Twentieth Century; ERA-Interim, European Centre for Medium-Range Weather Forecasts Interim; GPCP, Global Precipitation Climatology Project; JRA-55, Japanese 55-Year Reanalysis; MERRA, Modern-Era Retrospective Analysis for Research and Applications; NCEP, National Center for Environmental Prediction; NOC, National Oceanography Centre; OAFlux-1 $\times 1$, $1^{\circ}$-gridded Objectively Analyzed Air-Sea Fluxes; OAFlux-HR3 and -HR4, high-resolution (0.25 ${ }^{\circ}$-gridded) Objectively Analyzed Air-Sea Fluxes analysis computed from Coupled Ocean-Atmosphere Response Experiment (COARE) version 3 and version 4, respectively. The 12 products used here are the same as used in Figure 1 from Yu (2019).

At present, humidity and air-temperature ECVs do not meet the requirements needed for global flux estimation (Tables 3, 5 and Figure 7). Our two major recommendations, described below, and our roadmap to flux improvement described in section "Roadmap for Improving Air-Sea Fluxes," will address these deficiencies and others, enabling adequate sampling for all primary flux EOV/ECV by 2030.

\section{Space-Based Sensor for Near Surface Moisture and Air-Temperature Retrievals}

A future remote-sensing measurement system designed for the boundary layer can address many of the issues that bedevil the present system. In particular, as described in section "Current Capabilities for Remotely Sensed Flux EOV/ECV Measurements," existing remote sensing 
TABLE 4 | Various flux products information (based on Bentamy et al., 2017; Valdivieso et al., 2017; Tomita et al., 2018; Yu, 2019), including temporal and horizontal (in degrees) resolution, period of availability, flux components [Shortwave $\left(Q_{S W}\right)$, Longwave $\left(Q_{L W}\right)$, Sensible $\left(Q_{s e n}\right)$, Latent $\left(Q_{l a t}\right)$, Net air-sea heat flux $\left(Q_{n e t}\right)$, Freshwater Flux $(F W)$, and Momentum flux $(\tau)]$, and global ocean mean value for $Q_{\text {net }}$ where available.

\begin{tabular}{|c|c|c|c|c|c|c|}
\hline Type & Product & Resolution & Period & Flux availability & $\begin{array}{l}\text { Global ocean mean } \\
\text { of } Q_{\text {net }}\left[\mathrm{W} \mathrm{m^{-2 } ]}\right.\end{array}$ & References \\
\hline \multirow[t]{6}{*}{ Satellite-based } & $\begin{array}{l}\text { CERES Surface } \\
\text { EBAF, SYN1Deg }\end{array}$ & $\begin{array}{l}\text { Hourly, daily } \\
\text { monthly, } 1^{\circ}\end{array}$ & $2000-$ & $Q_{S W}, Q_{L W}$ & - & $\begin{array}{l}\text { Rutan et al., 2015; Kato } \\
\text { et al., } 2018\end{array}$ \\
\hline & HOAPS3.2 & Daily, $0.5^{\circ}$ & 1987-2008 & $Q_{\text {lat }}, Q_{\text {sen }}, F W, \tau$ & - & Andersson et al., 2011 \\
\hline & IFREMER V4 & Daily, $0.25^{\circ}$ & 1992-June 2017 & $Q_{\text {lat }}, Q_{\text {sen }}$ & - & Bentamy et al., 2017 \\
\hline & J-OFURO3 & Daily, $0.25^{\circ}$ & 1988-2013 & $Q_{\text {lat }}, Q_{\text {sen }}, Q_{s W}, L W, Q_{\text {net }}, \tau, F W$ & 23 & Tomita et al., 2018 \\
\hline & OAFlux HR & Daily, $0.25^{\circ}$ & 1988- & $Q_{\text {lat }}, Q_{\text {sen }}, F W, \tau$ & $\begin{array}{l}5 \text { (when combined with } \\
\text { CERES EBAF) }\end{array}$ & Yu, 2019 \\
\hline & SeaFlux CDR & $3 \mathrm{~h}, 0.25^{\circ}$ & $1998-$ & $Q_{\text {lat }}, Q_{\text {sen }}$ & - & Clayson and Brown, 2016 \\
\hline \multirow{4}{*}{$\begin{array}{l}\text { Latest } \\
\text { atmospheric } \\
\text { reanalysis }\end{array}$} & CFSR & $1 \mathrm{~h}, \mathrm{~T} 382$ & $1979-$ & $Q_{\text {lat }}, Q_{\text {sen }}, Q_{S W}, Q_{L W}, Q_{\text {net }}, \tau, F W$ & 15 & Saha et al., 2010 \\
\hline & ERA-Interim & $6 \mathrm{~h}, \mathrm{~T} 255$ & $1979-$ & $Q_{\text {lat }}, Q_{\text {sen }}, Q_{S W}, Q_{L W}, Q_{\text {net }}, \tau, F W$ & 9 & Dee et al., 2011 \\
\hline & JRA-55 & 3 h, T319 & 1958- & $Q_{\text {lat }}, Q_{\text {sen }}, Q_{s W}, Q_{L W}, Q_{n e t}, \tau, F W$ & -15 & Kobayashi et al., 2015 \\
\hline & MERRA2 & $1 \mathrm{~h}, 0.5^{\circ}$ & 1979- & $Q_{\text {lat }}, Q_{\text {sen }}, Q_{S W}, Q_{L W}, Q_{\text {net }}, \tau, F W$ & -5 & Molod et al., 2015 \\
\hline \multirow[t]{3}{*}{ Blended } & CORE.2 & Monthly, $1^{\circ}$ & 1984-2006 & $Q_{\text {lat }}, Q_{s e n}, Q_{s W}, Q_{L W}, Q_{n e t}, \tau, F W$ & $\begin{array}{l}30 \text { (unadjusted) } 2 \\
\text { (adjusted) }\end{array}$ & $\begin{array}{l}\text { Large and Yeager, 2009; } \\
\text { Josey et al., } 2013\end{array}$ \\
\hline & JRA-55-do & $3 \mathrm{~h}, 1.25^{\circ}$ & & $Q_{\text {lat }}, Q_{\text {sen }}, Q_{s w}, Q_{L W}, Q_{n e t}, \tau, F W$ & 1.8 & Tsujino et al., 2018 \\
\hline & OAFlux & $\begin{array}{l}\text { Daily, } 1^{\circ} \\
\text { Monthly, } 1^{\circ}\end{array}$ & 1983- 1958- & $Q_{\text {lat }}, Q_{\text {sen }}, Q_{s W}, Q_{L W}, Q_{n e t}, \tau$ & $\begin{array}{l}25 \text { (when combined } \\
\text { with ISCCP) }\end{array}$ & Yu and Weller, 2007 \\
\hline Ship-based & NOC 2 & Monthly, $1^{\circ}$ & $1973-$ & $Q_{\text {lat }}, Q_{\text {sen }}, Q_{s W}, Q_{L W}, Q_{\text {net }}, \tau, F W$ & 24 & Berry and Kent, 2011 \\
\hline
\end{tabular}

TABLE 5 | Sampling and uncertainty targets for producing a 3-hourly 25-km (aspirational goal of hourly at 10-km) gridded product of $Q_{\text {net }}$ with a 1-day random uncertainty of $15 \mathrm{~W} \mathrm{~m}^{-2}$ and a bias of less than $5 \mathrm{~W} \mathrm{~m}^{-2}$.

\begin{tabular}{|c|c|c|c|c|}
\hline Flux EOV/ECV & Comment & $\begin{array}{l}\text { Native Spatial } \\
\text { Resolution }\end{array}$ & $\begin{array}{c}\text { Temporal } \\
\text { Resolution }\end{array}$ & Uncertainty Target (1-day) \\
\hline Net surface heat flux & $\begin{array}{l}\text { Technology advancements/improvement of } \\
\text { bulk flux parameterization needed }\end{array}$ & $\leq 10 \mathrm{~km}$ & Hourly & Random: $15 \mathrm{~W} \mathrm{~m}^{-2}$ Bias: $<5 \mathrm{~W} \mathrm{~m}^{-2}$ \\
\hline Wind stress & Technology advancements & $\leq 10 \mathrm{~km}$ & Hourly & Random: $0.01 \mathrm{~N} \mathrm{~m}^{-2}$ or $4 \%$ Bias: $0.005 \mathrm{~N} \mathrm{~m}^{-2}$ \\
\hline Ocean surface wind speed/direction & Technology advancements for resolution & $\leq 10 \mathrm{~km}$ & Hourly & Random: $0.4 \mathrm{~m} \mathrm{~s}^{-1}$ or $4 \%$ Bias: $0.24 \mathrm{~m} \mathrm{~s}^{-1}$ \\
\hline SST & Algorithm improvement needed & $1 \mathrm{~km}$ & Hourly & Random: $0.2 \mathrm{~K}$ Bias: $0.1 \mathrm{~K}$ \\
\hline Near surface air temp & $\begin{array}{l}\text { Technology advancements/algorithm } \\
\text { improvements needed }\end{array}$ & $\leq 10 \mathrm{~km}$ & Hourly & Random: $0.5 \mathrm{~K}$ Bias: $0.2 \mathrm{~K}$ \\
\hline Near surface specific air humidity & $\begin{array}{l}\text { Technology advancements/algorithm } \\
\text { improvements needed }\end{array}$ & $\leq 10 \mathrm{~km}$ & Hourly & Random: $0.5 \mathrm{~g} \mathrm{k}^{-1}$ Bias: $0.3 \mathrm{~g} \mathrm{k}^{-1}$ \\
\hline Sea surface current & Technology advancement needed & $\leq 10 \mathrm{~km}$ & 3 hourly & $0.3 \mathrm{~m} \mathrm{~s}^{-1}$ \\
\hline $\begin{array}{l}\text { Upwelling/downwelling surface solar } \\
\text { radiation }\end{array}$ & $\begin{array}{l}\text { Technology advancements/algorithm } \\
\text { improvements }\end{array}$ & $\leq 10 \mathrm{~km}$ & Hourly & Random: $5 \mathrm{~W} \mathrm{~m}^{-2}$ Bias: $3 \mathrm{~W} \mathrm{~m}^{-2}$ \\
\hline $\begin{array}{l}\text { Upwelling/downwelling surface } \\
\text { longwave radiation }\end{array}$ & $\begin{array}{l}\text { Technology advancements/algorithm } \\
\text { improvements }\end{array}$ & $\leq 10 \mathrm{~km}$ & Hourly & Random: $5 \mathrm{~W} \mathrm{~m}^{-2}$ Bias: $3 \mathrm{~W} \mathrm{~m}^{-2}$ \\
\hline
\end{tabular}

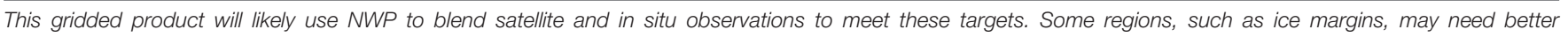
resolution than these.

satellites are able to measure near surface wind speed and surface skin temperature with sufficient accuracy using either microwave or IR instruments, although further algorithm development is needed since these measurements are often tuned to bulk (rather than skin) SST and wind speed (rather than wind stress). Current atmospheric sounders are able to measure vertical profiles of temperature and water vapor, and these observations are the basis for deriving satellite-based near-surface air temperature and humidity.
Three major challenges need to be addressed to improve remotely sensed estimates of heat fluxes: improvements to retrievals, improvements to time coincidence of EOVs/ECVs, and improvements to algorithms. The first challenge is to improve the sensitivity and vertical resolution of microwave sounders in the boundary layer. Existing microwave sounders typically only have a few channels giving sensitivity at less than 10 evenly spaced levels from the surface to $20 \mathrm{~km}$ for temperature and to $10 \mathrm{~km}$ for water vapor. These channels are also fixed in frequency and often introduce biases due to the 


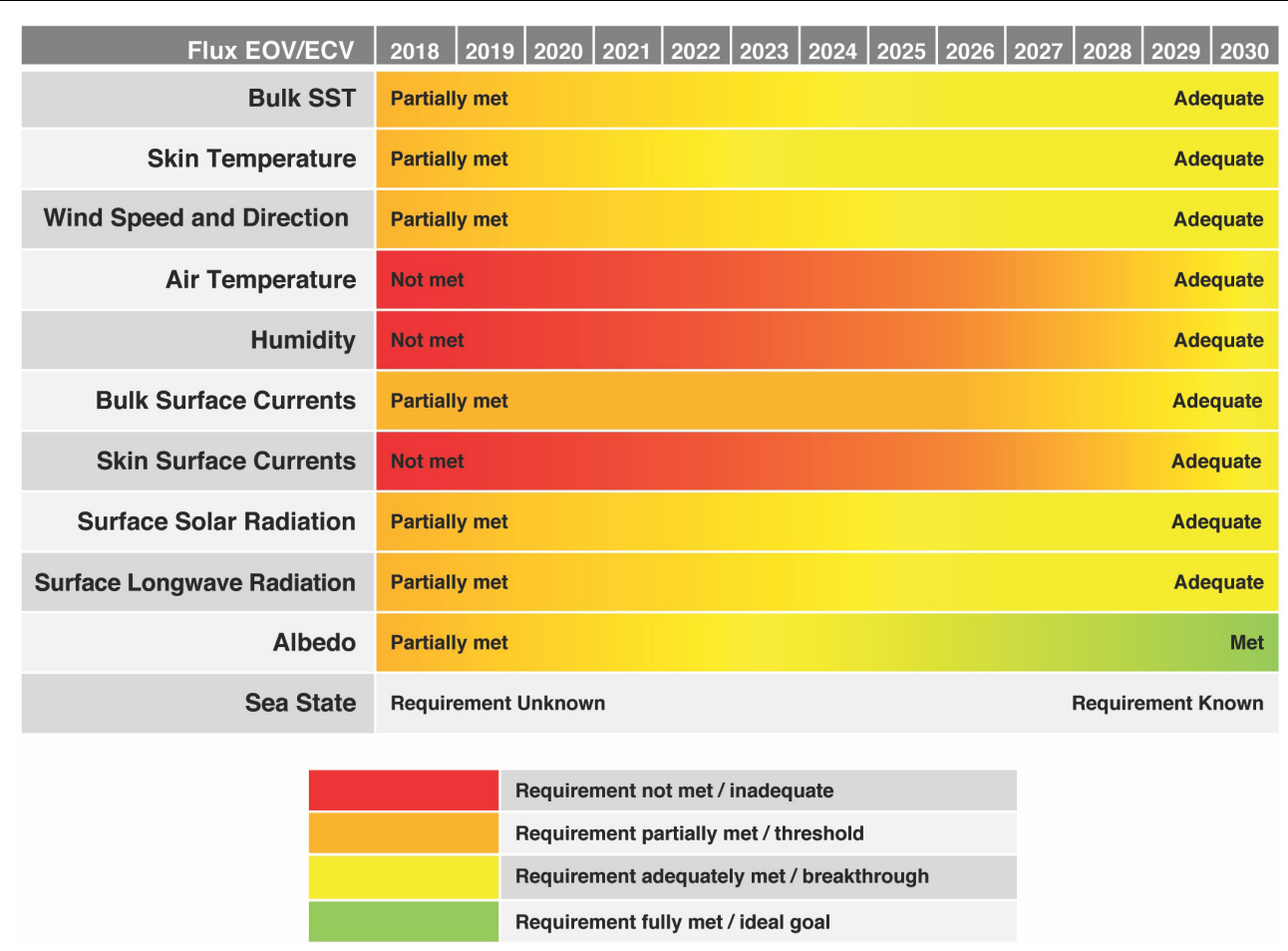

FIGURE 7 | Overall status of flux EOV/ECV, assuming recommendations made here are followed.

shift of the weighting functions (see section "Current Capabilities for Remotely Sensed Flux EOV/ECV Measurements"). This may be overcome by adding additional channels, such as hyperspectral microwave sounding spectrometers at 50 and $183 \mathrm{GHz}$. Simulations of a notional hyperspectral sensor with a neural network retrieval algorithm predict uncertainties of $0.6^{\circ} \mathrm{C}$ for near-surface air temperature and $7 \%$ error for nearsurface specific humidity, effectively doubling the accuracy of existing retrievals, for $1 \mathrm{~km}$ layers from the surface to 10 $20 \mathrm{~km}$ altitude (Blackwell, 2005; Blackwell et al., 2011; Boukabara and Garrett, 2011). Assimilation of these better-resolved profiles into NWP should lead to additional improvements to the representation of near-surface conditions and thus airsea fluxes.

The second challenge is to improve the time coincidence of the remotely sensed flux EOV/ECV measurements, which are typically made by independent systems and therefore usually not coincident in time, introducing de-correlation errors when computing fluxes from merged data. Time coincidence could be achieved by combining instruments on the same platform, or by using instruments on separate platforms flying in formation, which would most likely require a set of small satellite sensors to be cost effective. Alternatively, a passive microwave radiometer that combined low-frequency window channels used to measure SST and wind speed with atmospheric temperature and water vapor sounding channels would address the co-incidence issue with a single instrument. Radiometer instrument technology has made rapid strides in the past several years to miniaturize and lower the cost of the sensors envisioned for this constellation, coupled with equal advancements in lowcost CubeSat and small satellites. The US Air Force plans to demonstrate a low-cost conical microwave imager (Brown et al., 2017) and NASA has two missions to demonstrate CubeSat microwave imager/sounders (Reising et al., 2016; Blackwell et al., 2018). In addition, launch costs have decreased through rideshare opportunities.

The third challenge is to improve the retrieval algorithms for near-surface air temperature and humidity. While SST and wind speed accuracies are typically better than $0.3-0.5^{\circ} \mathrm{C}$ (Gentemann, 2014; Kilpatrick et al., 2015) and $0.5-1.4 \mathrm{~ms}^{-1}$ (Zhang et al., 2018), direct retrievals of near-surface air temperature and humidity a few meters above the sea surface remains challenging. Remote sensing retrievals provide average values for the lower $1 \mathrm{~km}$. The relationship between this average and the air temperature and specific humidity at the sea surface requires an understanding of the vertical distribution of moisture within the atmospheric boundary layer and how this varies regionally and temporally. The suggested roadmap therefore includes a holistic program to improve and tune the retrieval algorithms and our understanding of their uncertainties, which will require improved remote sensing observations and a global array of in situ observation.

\section{Globally Distributed in situ Network of Flux EOV and ECV}

As discussed in section "Current Capabilities for Remotely Sensed Flux EOV/ECV Measurements," the sparsity of the in situ network of flux EOV/ECV observations, particularly in large 
regions of the Southern Hemisphere, stands as a major obstacle to progress. Lack of in situ data in these regions and other regimes lead to large uncertainties in satellite air humidity and temperature retrievals because retrieval algorithms are not sufficiently trained to represent various near-surface boundary conditions ( $\mathrm{Yu}, 2019)$. In regions of low winds, where SST can have a large diurnal cycle and wind gustiness contributes significantly to the wind variance, in situ surface observations may capture the temporal variability of air-sea fluxes better than polar-orbiting satellite. Likewise, in situ observations may be better at capturing temporal variability of fast moving storms in the northern and southern hemisphere storm tracks. These tend to be regions with convective rainfall, which also can contaminate satellite IR scatterometer measurements. Finally, while satellite observations have a spatial resolution that a global in situ array could never meet, as discussed in section "Systematic Uncertainties Near Fronts and Regions of Persistent Clouds," satellite can have data gaps due to persistent clouds in frontal regions precisely where air-sea fluxes are expected to be large. Together, these strengths and weaknesses of remotely sensed vs. in situ observations argue for developing a global in situ flux-observing array to complement the global remote-sensing constellation.

Mazloff et al. (2018, their Table 1) provide estimates for heat and carbon flux decorrelation scales in the Southern Ocean, sorted into low-pass and high-pass scales. The lowpass requirements need very sparse sampling $\left(90^{\circ}\right.$ by $10^{\circ} ; 11$ measurements), but high-pass sampling is more substantial: 156 measurements in the $70^{\circ}$ to $35^{\circ}$ latitude range, or roughly one measurement per $4^{\circ}$ by $4^{\circ}$ box. Further work will need to be done to determine the optimal design of this regionally distributed plan. But at its center might be pairs or clusters of flux platforms within a regular grid (e.g., $10^{\circ}$ by $10^{\circ}$ ) box. For reference, nodes of a $10^{\circ}$ grid are shown in Figure 8, with 368 nodes in the global ocean. We envision a global network of flux observing platforms made up of ASVs, drifting and moored buoys, and R/Vs, with the exact choice of platform dependent upon conditions and scientific needs. This large-scale network of high-resolution sampling would ideally capture frontal structures that are critical to the generation of large exchanges of heat between the ocean and atmosphere.

These flux platforms could carry other sensor suites too, such as air-sea carbon dioxide flux packages and ocean acidification sensors. In this way, the network could be multi-functional and serve a range of communities and stakeholders beyond those described here. In the future, as true coupled data assimilation becomes standard for NWP, we would expect that this network of co-located surface ocean and atmospheric in situ observations would be particularly valuable for assimilations.

This globally distributed array should be built around an expanded OceanSITES network. At present, the OceanSITES surface flux array is too sparse, with 22 sites that measure net surface heat flux. Nineteen key regions needing OceanSITES time series stations are shown in Figure 8. These regions include eleven discussed in section "In situ Platforms for Observing Fluxes," as well as regions in the western equatorial Pacific and western North Atlantic, and eight additional high priority regions in the Southern Hemisphere. These regions have no OceanSITES flux reference station. As discussed in section "In situ Platforms for Observing Fluxes," an OceanSITES station not only monitors the air-sea fluxes, but also the local response of the ocean, atmosphere, and ecosystem to these air - sea exchanges. Tozuka et al. (2018) have shown that the contribution of surface heat fluxes to surface frontogenesis/frontolysis depends not just on the flux gradients, but also on the distribution of mixed layer depth, which controls the effective heat capacity of the upper ocean. The OceanSITES flux network provides these co-located air-sea flux, mixed layer depth observations, and other EOVs so that budget analyses can be performed to determine causes for surface variability.

In some regions, such as the tropical Pacific, the number of OceanSITES flux reference stations could be increased simply by adding a few sensors to existing OceanSITES surface moorings. In other regions, new mooring stations are needed. The Tropical Pacific Observing System (TPOS)-2020 project is working to optimize flux measurements in the tropical Pacific, including the eastern and western boundary regions (Smith et al., 2019). Likewise, the Southern Ocean Flux working group is in the process of developing a deployment strategy to optimize the positioning of a sparse internationally supported array of flux moorings (Swart et al., 2019). Regional groups, like these, have the scientific expertise and local knowledge that are critical for making these long-term stations successful. Nevertheless, the following list of new sites are considered to be a high priority as they are all in regions that do not at present have any flux reference station:

New Flux Reference Sites for 8 Key Regions with no Flux Station at Present

(1) Tropical Western Pacific $\left(9^{\circ} \mathrm{N}, 150^{\circ} \mathrm{E}\right.$; equatorial region west of $165^{\circ} \mathrm{E}$ ): Typhoon corridor, monsoon, intraseasonal oscillations, and ENSO variability.

(2) Southern Ocean (order $60^{\circ} \mathrm{S}$, any longitude): Ice-edge conditions. Southern Hemisphere storm track.

(3) Southern Ocean $\left(40 \mathrm{~S}^{\circ}-60^{\circ} \mathrm{S}\right.$, any longitude): Southern Hemisphere storm track.

(4) Agulhas $\left(40^{\circ} \mathrm{S}, 36^{\circ} \mathrm{E}\right)$ : Intense heat loss region associated with S. Indian Ocean western boundary current (WBC), extratropical storms, interbasin eddy heat exchanges, mixed layer depth role in front gradient.

(5) Southeast Indian Ocean $\left(25^{\circ} \mathrm{S}, 110^{\circ} \mathrm{E}\right)$ : Strongest ocean evaporation region (globally) in trade wind return flow, subject to "Ningaloo" mid-latitude marine heat waves. Subtropical mode water formation site.

(6) Gulf Stream $\left(35^{\circ} \mathrm{N}, 60^{\circ} \mathrm{W}\right)$ : Intense heat loss associated with N. Atlantic WBC, eddy rich region, tropicalextratropical cyclones and interactions, cold-air outbreaks.

(7) East Australian Current $\left(35^{\circ} \mathrm{S}, 160^{\circ} \mathrm{E}\right)$ : S. Pacific WBC, Tasman Front, tropical-extratropical interactions.

(8) Brazil-Malvinas Confluence $\left(42^{\circ} \mathrm{S}, 42^{\circ} \mathrm{W}\right)$ : S. Atlantic $\mathrm{WBC}$, eddy rich region, tropical-extratropical interactions.

These regions listed here are high priority because they at present have no OceanSITES flux reference station in them. Our 


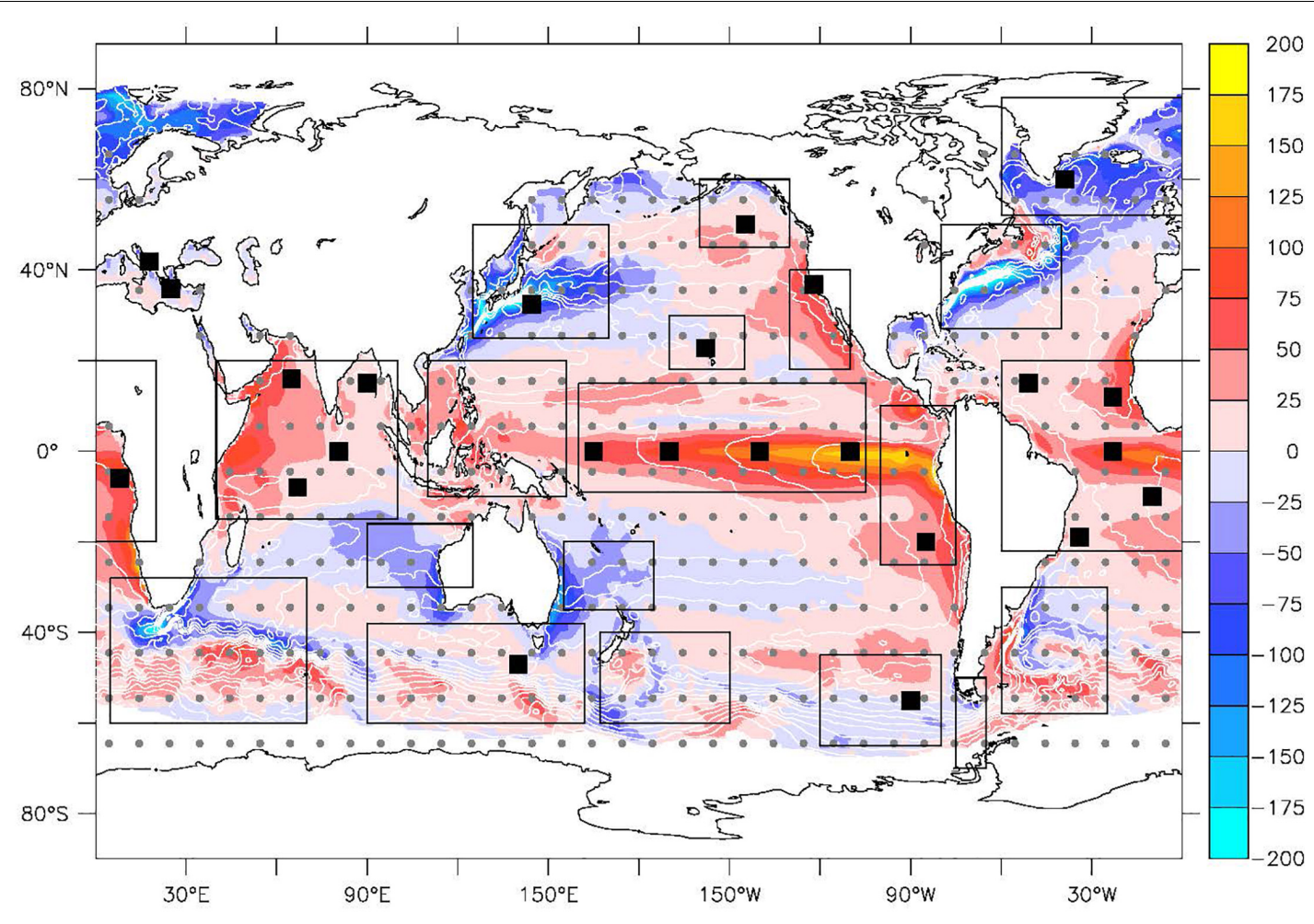

Mean Net Surface Heat Flux $\left(\mathrm{Wm}^{-2}\right)$

FIGURE 8 | Existing OceanSITES network of surface heat fluxes (filled black squares) with priority regions for new OceanSITES stations indicated by black boxes. The gray dots indicate nodes of a $10^{\circ}$ by $10^{\circ}$ grid, with 368 grid boxes in the global oceans. The background mean net surface heat flux is constructed from OAFlux-HR (Yu, 2019) and CERES EBAF (Kato et al., 2018) for the period of 2001-2015.

boxed regions, however, are large, covering major quadrants of each basin. Each have multiple regimes, sometimes separated by major fronts. For many regions, such as the tropics, multiple stations are justified. Careful array design studies by regional experts is recommended.

A subset of the long-term reference stations should be enhanced for process studies and to provide high resolution turbulent flux and mean profiles that extend 20 to $100 \mathrm{~m}$ into the marine atmosphere. These "super sites" would provide critical observations to validate and guide model physics and bulk flux and satellite retrieval algorithm development. Such a system, with multiple levels of fast-response sensors to directly measure momentum, heat and mass exchange, could be based on fixed towers, large moored spar buoys or other floating platforms, which are being developed and used by the offshore wind industry. Remote sensing systems deployed on the platforms and from nearby moorings (e.g., Lidar buoys) could provide further characterization of the coupled boundary layer, including cloud information, boundary layer height and mixed layer depth. Other key variables to be observed at these sites could include wave information, radiative fluxes (including both upwelling and downwelling fluxes), skin SST, precipitation, high-resolution upper ocean currents, fast static pressure, and a suite of oceanic boundary measurements, among others. These supersites and their associated infrastructure would also serve as testbeds for validation of innovative platforms and systems, such as lowpower sensors and remote sensing systems for deployment on buoys and mobile platforms. In addition, it is recommended that some of the ships servicing the moorings and supersites be enhanced to monitor the coupled boundary layer, and host unmanned aircraft systems for observing along-wind and cross-wind variations, obtaining data to improve albedo parameterizations, and for monitoring a wide-range of coupled boundary layer processes. The breakthroughs highlighted in Figure 7 depend not only upon improved remote sensing and expanded in situ observations, but also upon improved models, algorithms and parameterizations. This ambitious goal is predicated upon vigorous research fed by these coupled boundary layer observations described here.

\section{ROADMAP FOR IMPROVING AIR-SEA FLUXES}

Quantifying air-sea fluxes is very challenging as multiple co-located flux EOV/ECV must be measured at high temporal and spatial resolution, with high accuracy. At 
present, the global ocean observing system does not meet the necessary requirements (Figure 7), mainly due to the lack of global coverage of surface humidity and air temperature. Our two major recommendations rectify this and other deficiencies:

\section{Major Recommendation \#1}

Create a remote-sensing retrieval system designed for accurate boundary layer measurement of air temperature, humidity, SST, and surface stress. This would involve a holistic approach to improve resolution of satellite retrievals, time coincidence of remotely sensed surface flux EOVs/ECVs, and algorithms that relate the retrievals to near-surface conditions.

\section{Major Recommendation \#2}

Create a global in situ array of flux observing platforms, built around an expanded OceanSITES network of time series reference station moorings. The global array would include 500-1000 platforms including ASVs, moored and drifting buoys, and RVs, with $1-3$ platforms in nominal $10^{\circ}$ by $10^{\circ}$ boxes. The OceanSITES network of 22 flux sites must be maintained and expanded in up to 19 key regions. In addition, the in situ array should include a few super sites with enhanced observations of the coupled boundary layer.

These major recommendations depend upon a number of steps taking place, as shown in the Roadmap (Figure 9):

- The TRL for ASV and other new flux platforms must be increased before being used to form a large global network. These platforms must be continually evaluated against satellite and proven technology, including RVs, flux towers with vertical extent, and OceanSITES and OOI moorings. It is recommended that an international ASV expert group form to coordinate ASV data stream, evaluate data, and develop best practices and standardizations.

- Array designs for the in situ flux network must be studied. It is likely that a flux network would have some similarities to an Argo array in being globally distributed, but also have some distinct differences due to the importance of fronts and rapid variations in flux EOV/ECV. Likewise, in contrast to Argo, adaptive sampling capabilities of ASV could allow the globally distributed array to target certain phenomena and to sample in combination with other fixed platforms. Determining the optimal array design will require both model studies and pilot field studies.

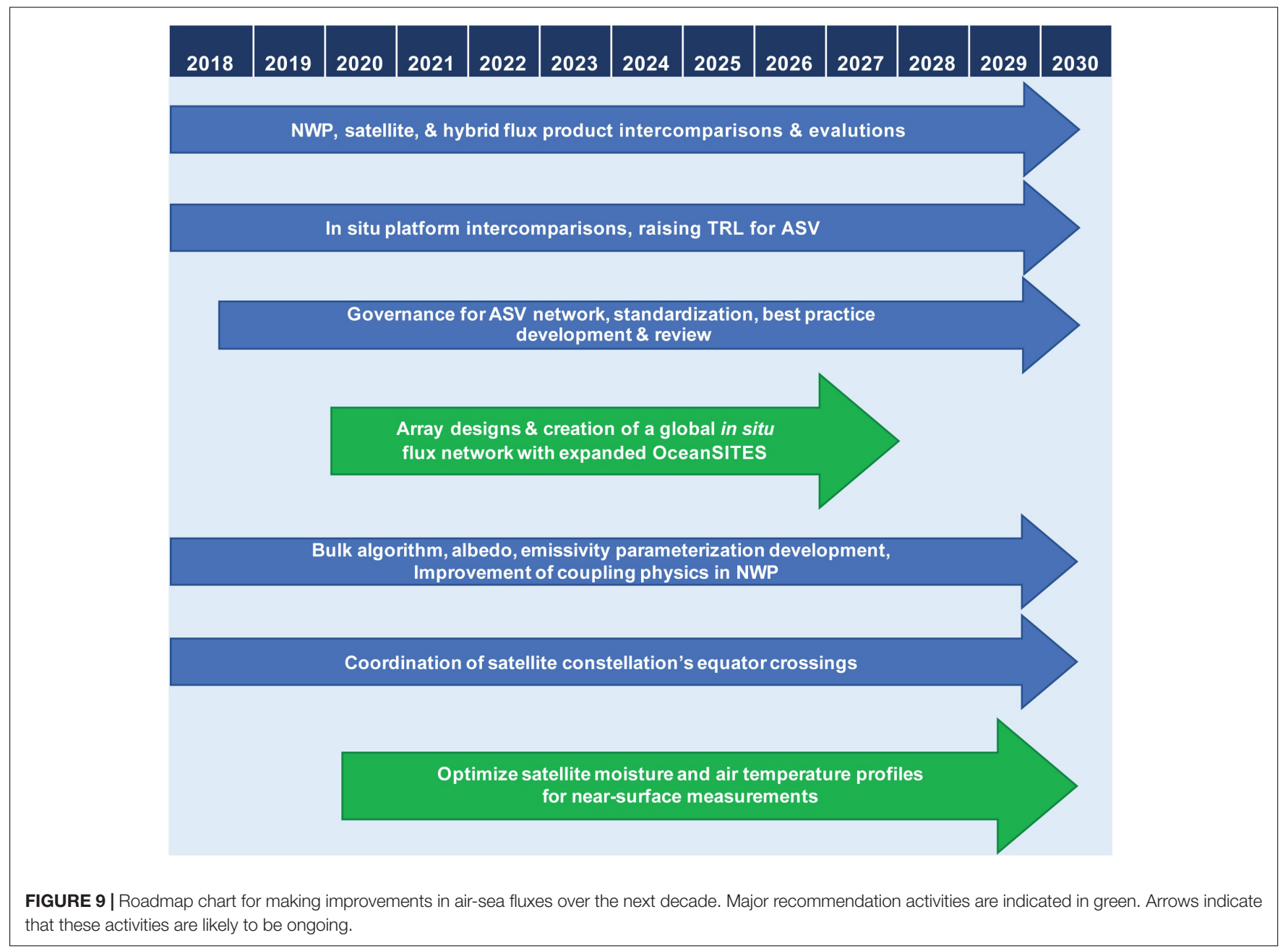


- International coordination of data management and archival must be extended to flux-related data streams, including for all underway RV observations, ASVs, drifting and other flux platforms, and for observations transmitted in near real time in support of NWP on the Global Telecommunications System (GTS).

- With the expanded in situ array of platforms that measure all flux EOV/ECV including direct covariance flux estimates of the turbulent fluxes, the state of the art bulk aerodynamic flux algorithm can be improved, leading to reduced uncertainties. It is critical that the bulk algorithm in NWP be improved.

- Parameterizations for transforming bulk EOV/ECV into bulk algorithm state variables must be improved. These include (but are not limited to) extrapolation of bulk sea surface state variables to the air-sea interface, and parameterizations of albedo and emissivity.

- Cross-platform and cross-product intercomparisons must take place and differences must be reconciled. Sensors across all in situ platforms should have laboratory calibrations traceable to international standards, and field intercomparisons to verify consistency. Identification of field errors should lead to changes in best practices. Identification of model errors should lead to recommendations for improved physics and parameterizations.

\section{CONCLUSION}

In this paper, we lay out an observational strategy for producing 3 -hourly at $25-\mathrm{km}$ (and an aspirational goal of hourly at $10-\mathrm{km}$ ) gridded heat fluxes and wind stress fields over the global, icefree ocean that have a breakthrough improvement in accuracy for heat flux and, for these scales, an improved accuracy in wind stress. The target for the net heat flux product is 1day random uncertainty of $15 \mathrm{~W} \mathrm{~m}^{-2}$ (or $5 \%$ error for each radiative component and the sum of the turbulent heat fluxes) and a bias of less than $5 \mathrm{~W} \mathrm{~m}^{-2}$. For the wind stress product the target is 1-day random uncertainty of $\pm 0.01 \mathrm{~N} \mathrm{~m}^{-2}$ (or a $5 \%$ noise-to-signal ratio, whichever is higher) and a bias of $0.005 \mathrm{~N} \mathrm{~m}^{-2}$. At present the heat flux uncertainty target is met only at OceanSITES reference station moorings and RVs that follow best practices. To meet these targets in the next decade, we make two major recommendations: (1) Create a remote-sensing measurement system designed for accurate boundary layer measurements of air temperature, humidity, SST, and surface stress; and (2) Create a complementary global in situ array of flux observing platforms, built around an expanded OceanSITES network of time series reference station moorings. The global array would include $\sim 500-1000$ platforms including ASV, moored and drifting buoys, and RVs, with $1-3$ platforms in nominal $10^{\circ}$ by $10^{\circ}$ boxes. The OceanSITES network of 22 flux sites must be maintained and expanded in up to 19 key regions. The in situ flux array would provide globally distributed measurements and metrics for satellite algorithm development and product validation. In addition, in situ flux EOV and ECV observations that also include direct covariance flux observations could be used to improve "bulk aerodynamic algorithm" for computation of airsea exchange of heat and momentum. With improved coupling of the ocean and atmosphere in NWP, and constrained by the improved moisture and air temperature profiles, these NWP are expected to have improved representation of cloud formation processes and radiative properties. Together, these will lead to a more accurate satellite-based (and blended) flux product. This better quantification of the ocean's influence on the atmosphere will lead to improved long-term weather forecasts, seasonal-interannual-decadal climate predictions, and regional climate projections.

\section{AUTHOR CONTRIBUTIONS}

MFC, CLG, JE, and IU were first authors for abstracts that were merged into the present version. MFC wrote the first draft of the manuscript. CLG, JE, SB, IU, EK, RAW, SAJ, RTP, SK, CWF, HT, PJM, JTF, SS contributed to text sections. LY, MB, PJM, and MFC contributed to figures. All authors contributed to manuscript revision, and read and approved the submitted version.

\section{FUNDING}

EK was funded by the NERC CLASS Program (NE/R015953/1). CLG was funded by NASA grant 80 NSSC18K0837. SG was funded by MEGAGRANT P220 program (\#14.W03.31.0006).

\section{ACKNOWLEDGMENTS}

The three reviewers provided insightful and detailed comments that significantly improved the manuscript. The authors gratefully acknowledge the contributions from Drs. Kentaro Ando, Tatsuya Fukuda, Yasuhisa Ishihara, and Makito Yokota (all from JAMSTEC, Japan), Dr. Aderrahim Bentamy (IFREMER, France), Mr. Shawn Smith (FSU, United States), Dr. Scott Miller (Albany University, United States), and Sandra Bigley (NOAA PMEL). This work benefited from discussions within the OOPC, AOPC, WRCP Data Advisory Council (WDAC) Surface Flux Task Team, National Academy of Science Workshop "The Future of Atmospheric Boundary Layer Observing, Understanding, and Modeling," Southern Ocean Flux Working Group (SOFLUX), and Tropical Pacific Observing System (TPOS)-2020 Planetary Boundary Layer Task Team. This is PMEL publication 4842.

\section{SUPPLEMENTARY MATERIAL}

The Supplementary Material for this article can be found online at: https://www.frontiersin.org/articles/10.3389/fmars.2019. 00430/full\#supplementary-material 


\section{REFERENCES}

Andersson, A., Klepp, C., Fennig, K., Bakan, S., Grassl, H., and Schulz, J. (2011). Evaluation of HOAPS-3 ocean surface freshwater flux components. J. Appl. Meteor. Climatol. 50, 379-398. doi: 10.1175/2010JAMC 2341.1

Andreas, E. L., Mahrt, L., and Vickers, D. (2015). An improved bulk air-sea surface flux algorithm, including spray-mediated transfer. Q. J. R. Meteorol. Soc. 141, 642-654. doi: 10.1002/qj.2424

Andreas, E. L., Persson, P. O. G., and Hare, J. E. (2008). A bulk turbulent air-sea flux algorithm for high-wind, spray conditions. J. Phys. Oceanogr. 38, 1581-1596. doi: 10.1175/2007JPO3813.1

Banner, M. L., and Morison, R. P. (2010). Refined source terms in wind wave models with explicit wave breaking forecasts. Part I: model framework and validation against field data. Ocean Modell. 33, 177-189. doi: 10.1016/j.ocemod. 2010.01.002

Beljaars, A. C. M. (1995). The parametrization of surface fluxes in large-scale models under free convection. Q. J. R. Meteorol. Soc. 121, 255-270. doi: 10. 1256/smsqj.52202

Bentamy, A., Piollé, J. F., Grouazel, A., Danielson, R., Gulev, S., Paul, F., et al. (2017). Review and assessment of latent and sensible heat flux accuracy over the global oceans. Remote Sens. Environ. 201, 196-218. doi: 10.1016/j.rse.2017. 08.016

Bernie, D. J., Woolnough, S. J., Slingo, J. M., and Guilyardi, E. (2005). Modeling diurnal and intraseasonal variability of the ocean mixed layer. J. Clim. 18, 1190-1202. doi: 10.1175/JCLI3319.1

Berry, D. I., and Kent, E. C. (2009). A new air-sea interaction gridded dataset from ICOADS with uncertainty estimates. Bull. Am. Meteor. Soc. 90, 645-656. doi: 10.1175/2008bams2639.1

Berry, D. I., and Kent, E. C. (2011). Air-sea fluxes from ICOADS: the construction of a new gridded dataset with uncertainty estimates. Int. J. Climatol. 31, 987-1001. doi: 10.1002/joc.2059

Berry, D. I., Kent, E. C., and Taylor, P. K. (2004). An analytical model of heating errors in marine air temperatures from ships. J. Atmos. Oceanic Technol. 21, 1198-1215. doi: 10.1175/1520-04262004021<1198:AAMOHE<2.0.CO;2

Bigorre, S., Weller, R. A., Lord, J., Edson, J. B., and Ware, J. D. (2013). A surface mooring for air-sea interaction research in the Gulf Stream. Part 2: analysis of the observations and their accuracies. J. Atmos. Ocean. Tech. 30, 450-469. doi: 10.1175/jtech-d-12-00078.1

Bishop, S. P., Small, R. J., Bryan, F. O., and Tomas, R. A. (2017). Scale dependence of midlatitude air-sea interaction. J. Clim. 30, 8207-8221. doi: 10.1175/JCLI-D17-0159.1

Blackwell, W. J. (2005). A neural-network technique for the retrieval of atmospheric temperature and moisture profiles from high spectral resolution sounding data. IEEE Trans. Geosci. Remote Sens. 43, 2535-2546. doi: 10.1109/ tgrs.2005.855071

Blackwell, W. J., Bickmeier, L. J., Leslie, R. V., Pieper, M. L., Samra, J. E., Surussavadee, C., et al. (2011). Hyperspectral microwave atmospheric sounding. IEEE Trans. Geosci. Remote Sens. 49, 128-142.

Blackwell, W. J., Braun, S., Bennartz, R., Velden, C., DeMaria, M., Atlas, R., et al. (2018). An overview of the TROPICS NASA Earth Venture Mission. Q. J. R. Meteorol. Soc. 144, 16-26. doi: 10.1002/qj.3290

Blomquist, B. W., Huebert, B. J., Fairall, C. W., Bariteau, L., Edson, J. B., Hare, J. E., et al. (2014). Advances in air-sea CO2 flux measurement by eddy correlation. Bound. Layer Meteorol. 152, 245-276. doi: 10.1007/s10546-014-9926-2

Bond, N. A., and Cronin, M. F. (2008). Regional weather patterns during anomalous air-sea fluxes at the kuroshio extension observatory (KEO). J. Clim. 21, 1680-1697. doi: 10.1175/2007JCLI1797.1

Bond, N. A., Cronin, M. F., Freeland, H., and Mantua, N. (2015). Causes and impacts of the 2014 warm anomaly in the NE Pacific. Geophys. Res. Lett. 42, 3414-3420. doi: 10.1002/2015gl063306

Boukabara, S. A., and Garrett, K. (2011). "Benefits of a hyperspectral microwave sensor," in Proceedings of the 2011 IEEE Conference on Sensors, (Limerick), 1881-1884. doi: 10.1109/ICSENS.2011.6127357

Bourassa, M., Gille, S., Bitz, C., Carlson, D., Cerovecki, I., Cronin, M., et al. (2013). High-latitude ocean and sea ice surface fluxes: requirements and challenges for climate research. Bull. Am. Meteorol. Soc. 94, 403-423. doi: 10.1175/BAMS-D11-00244.1
Bourassa, M. A., Meissner, T., Cerovecki, I., Chang, P., Xiaolong, D., De Chiara, G., et al., (2019). Remotely sensed winds and wind stresses for marine forecasting and ocean modeling. Front. Mar. Sci. doi: 10.3389/fmars.2019.00443

Bourassa, M. A., Vincent, D. G., and Wood, W. L. (1999). A flux parameterization including the effects of capillary waves and sea state. J. Atmos. Sci. 56, 11231139. doi: 10.1175/1520-0469(1999)056<1123:afpite > 2.0.co;2

Bradley, F., and Fairall, C. (2006). A Guide to Making Climate Quality Meteorological and Flux Measurements at Sea, NOAA Technical Memorandum OAR PSD-311. Boulder, CO: Earth System Research Laboratory, Physical Sciences Division.

Brodeau, L., Barnier, B., Gulev, S. K., and Woods, C. (2017). Climatologically significant effects of some approximations in the bulk parameterizations of turbulent air-sea fluxes. J. Phys. Oceanogr. 47, 5-28. doi: 10.1175/JPO-D-160169.1

Brown, S., Focardi, P., Kitiyakara, A., Maiwald, F., Milligan, L., Montes, O., et al. (2017). "The COWVR Mission: Demonstrating the capability of a new generation of small satellite weather sensors," in Proceedings of the 2017 IEEE Aerospace Conference, (Big Sky, MT), 1-7. doi: 10.1109/AERO.2017.7943884

Brunke, M. A., Fairall, C. W., Zeng, X., Eymard, L., and Curry, J. A. (2003). Which bulk aerodynamic algorithms are least problematic in computing ocean surface turbulent fluxes? J. Clim. 16, 619-635. doi: 10.1175/1520-04422003016<0619: WBAAAL $<2.0 . \mathrm{CO} ; 2$

Buckley, M. P., and Veron, F. (2016). Structure of the airflow above surface waves. J. Phys. Oceanogr. 46, 1377-1397. doi: 10.1175/jpo-d-15-0135.1

Bulgin, C. E., Embury, O., Corlett, G., and Merchant, C. J. (2016). Independent uncertainty estimates for coefficient based sea surface temperature retrieval from the along-track scanning radiometer instruments. Remote Sens. Environ. 178, 213-222. doi: 10.1016/j.rse.2016.02.022

Businger, J. A., Wyngaard, J. C., Izumi, Y., and Bradley, E. F. (1971). Flux-profile relationships in the atmospheric surface layer. J. Atmos. Sci. 28, 181-189. doi: 10.1175/1520-04691971028<0181:FPRITA<2.0.CO;2

Charnock, H. (1955). Wind stress on a water surface. Q. J. R. Meteor. Soc. 81, 639-640. doi: 10.1002/qj.49708135027

Chelton, D. B., Schlax, M. G., Freilich, M. H., and Milliff, R. F. (2004). Satellite measurements reveal persistent small-scale features in ocean winds. Science 303, 978-983. doi: 10.1126/science.1091901

Chuda, T., Niino, H., Yoneyama, K., Katsumata, M., Ushiyama, T., and Tsukamoto, O. (2008). A statistical analysis of surface turbulent heat flux enhancements due to precipitating clouds observed in the tropical Western Pacific. J. Meteorol. Soc. Jpn. 86, 439-457. doi: 10.2151/jmsj.86.439

Cifuentes-Lorenzen, A., Edson, J. B., and Zappa, C. J. (2018). Air-sea interaction in the Southern Ocean: exploring the height of the wave boundary layer at the airsea interface. Bound Layer Meteorol. 169, 461-482. doi: 10.1007/s10546-0180376-0

Clayson, C. A., and Bogdanoff, A. (2013). The effect of diurnal sea surface temperature warming on climatological air-sea fluxes. J. Clim. 26, 2546-2556. doi: $10.1175 /$ jcli-d-12-00062.1

Clayson, C. A., and Brown, J. (2016). NOAA Climate Data Record Ocean Surface Bundle (OSB) Climate Data Record (CDR) of Ocean Heat Fluxes, Version 2. Climate Algorithm Theoretical Basis Document (C-ATBD). Asheville, NC: NOAA National Center for Environmental Information, doi: 10.7289/ V59K4885

Colbo, K., and Weller, R. A. (2009). Accuracy of the IMET sensor package in the subtropics. J. Atmos. Ocean. Tech. 26, 1867-1890. doi: 10.1175/2009jtecho667.1

Compo, G. P., Whitaker, J. S., Sardeshmukh, P. D., Matsui, N., Allan, R. J., Yin, X., et al. (2011). The Twentieth Century Reanalysis Project. Q. J. R. Meteor. Soc. 137, 1-28. doi: 10.1002/qj.776

Corlett, G. K., Merchant, C. J., Minnett, P. J., and Donlon, C. J. (2014). “Assessment of long-term satellite derived sea surface temperature records," in Experimental Methods in the Physical Sciences, Vol 47, Optical Radiometry for Ocean Climate Measurements, eds G. Zibordi, C. J. Donlon, and A. C. Parr (Cambridge, MA: Academic Press), 639-677. doi: 10.1016/B978-0-12-417011-7.00021-0

Cronin, M. F., Bond, N. A., Farrar, J. T., Ichikawa, H., Jayne, S. R., Kawai, Y., et al. (2013). Formation and erosion of the seasonal thermocline in the Kuroshio Extension Recirculation Gyre. Deep Sea Res. Part II 85, 62-74. doi: 10.1016/j. dsr2.2012.07.018

Cronin, M. F., Bourassa, M., Clayson, C. A., Edson, J., Fairall, C., Feely, D., et al. (2014). "White Paper \#11-Wind stress and air sea fluxes observations: status, 
implementation and gaps," in Proceedings of the Tropical Pacific Observing System 2020 Workshop, A Future Sustained Tropical Pacific Ocean Observing System for Research and Forecasting, (La Jolla, CA).

Cronin, M. F., Fairall, C. W., and McPhaden, M. J. (2006). An assessment of buoyderived and numerical weather prediction surface heat fluxes in the tropical Pacific. J. Geophys. Res. 111:C06038. doi: 10.1029/2005JC003324

Cronin, M. F., and Kessler, W. S. (2009). Near-surface shear flow in the tropical Pacific cold tongue front. J. Phys. Oceanogr. 39, 1200-1215. doi: 10.1175/ 2008JPO4064.1

Cronin, M. F., and McPhaden, M. J. (1999). Diurnal cycle of rainfall and surface salinity in the western Pacific warm pool. Geophys. Res. Lett. 26, 3465-3467.

Cronin, M. F., Pelland, N. A., Emerson, S. R., and Crawford, W. R. (2015). Estimating diffusivity from the mixed layer heat and salt balances in the North Pacific. J. Geophys. Res. Oceans 120, 7346-7362. doi: 10.1002/2015JC011010

Dee, D., Uppala, S. M., Simmons, A. J., Berrisford, P., Poli, P., Kobayashi, S., et al. (2011). The ERA-Interim reanalysis: configuration and performance of the data assimilation system. Q. J. R. Meteorol. Soc. 137, 553-597. doi: 10.1002/ qj. 828

Donelan, M. A., Dobson, F. W., Smith, S. D., and Anderson, R. J. (1993). On the dependence of sea surface roughness on wave development. J. Phys. Oceanogr. 23, 2143-2149. doi: 10.1175/1520-0485(1993)023<2143:otdoss>2.0.co;2

Donelan, M. A., Drennan, W. M., and Katsaros, K. B. (1997). The air-sea momentum flux in conditions of wind sea and swell. J. Phys. Oceanogr. 27, 2087-2099. doi: 10.1175/1520-04851997027<2087:Tasmfi<2.0.Co;2

Donlon, C. J., Minnett, P. J., Gentemann, C., Nightingale, T. J., Barton, I. J., Ward, B., et al. (2002). Toward improved validation of satellite sea surface skin temperature measurements for climate research. J. Clim. 15, 353-369. doi: 10.1175/1520-04422002015<0353:TIVOSS<2.0.CO;2

Drennan, W. M., Graber, H. C., Collins, C. O. III, Herrera, A., Potter, H., Ramos, R. J., et al. (2014). EASI: an air-sea interaction buoy for high winds. J. Atmos. Ocean. Tech. 31, 1397-1409. doi: 10.1175/jtech-d-13-00201.1

Drennan, W. M., Graber, H. C., Hauser, D., and Quentin, C. (2003). On the wave age dependence of wind stress over pure wind seas. J. Geophys. Res. 108:8062. doi: 10.1029/2000JC000715

Drennan, W. M., Taylor, P. K., and Yelland, M. J. (2005). Parameterizing the sea surface roughness. J. Phys. Oceanogr. 35, 835-848. doi: 10.1175/jpo2704.1

du Penhoat, Y., Reverdin, G., and Caniaux, G. (2002). A Lagrangian investigation of vertical turbulent heat fluxes in the upper ocean during tropical oceanglobal atmosphere/coupled ocean-atmosphere response experiment (TOGACOARE). J. Geophys. Res. 107, 7-1-7-14. doi: 10.1029/2001JC000926

Dyer, A. J., and Bradley, E. F. (1982). An alternative analysis of flux-gradient relationships at the 1976 ITCE. Bound. Layer Meteorol. 22, 3-19. doi: 10.1007/ bf00128053

Dyer, A. J., and Hicks, B. B. (1970). Flux-gradient relationships in the constant flux layer. Q. J. R. Meteor. Soc. 96, 715-721. doi: 10.1002/qj.49709641012

Eastman, R., Warren, S. G., and Hahn, C. J. (2011). Variations in cloud cover and cloud types over the ocean from surface observations, 1954-2008. J. Clim. 24, 5914-5934. doi: 10.1175/2011jcli3972.1

Edson, J., Crawford, T., Crescenti, J., Farrar, T., Frew, N., Gerbi, G., et al. (2007). The coupled boundary layers and air-sea transfer experiment in low winds. Bull. Am. Meteorol. Soc. 88, 341-356. doi: 10.1175/BAMS-88-3-341

Edson, J. B., and Fairall, C. W. (1998). Similarity relationships in the marine surface layer. J. Atmos. Sci. 55, 2311-2328. doi: 10.1111/j.1462-2920.2010.02154.x

Edson, J. B., Jampana, V., Weller, R. A., Bigorre, S., Plueddemann, A. J., Fairall, C. W., et al. (2013). On the exchange of momentum over the open ocean. J. Phys. Oceanogr. 43, 1589-1610. doi: 10.1175/jpo-d-12-0173.1

Edson, J. B., Zappa, C. J., Ware, J. A., McGillis, W. R., and Hare, J. E. (2004). Scalar flux profile relationships over the open ocean. J. Geophys. Res. 109:C08S09. doi: 10.1029/2003JC001960

Fairall, C. W., Bradley, E. F., Godfrey, J. S., Wick, G. A., Edson, J. B., and Young, G. S. (1996a). Cool skin and warm layer effects on the sea surface temperature. J. Geophys. Res. 101, 1295-1308. doi: 10.1029/95jc03190

Fairall, C. W., Bradley, E. F., Rogers, D. P., Edson, J. B., and Young, G. S. (1996b). Bulk parameterization of air-sea fluxes or the tropical ocean coupled-ocean atmosphere response experiment. J. Geophys. Res. 101, 3747-3764. doi: 10.1029/ 95JC03205

Fairall, C. W., Bradley, E. F., Hare, J. E., Grachev, A. A., and Edson, J. B. (2003). Bulk parameterization of air-sea fluxes: updates and verification for the COARE algorithm. J. Clim. 16, 571-591. doi: 10.1175/1520-04422003016<0571: BPOASF $<2.0 . \mathrm{CO} ; 2$

Fairall, C. W., White, A. B., Edson, J. B., and Hare, J. E. (1997). Integrated shipboard measurements of the marine boundary layer. J. Atmos. Ocean. Tech. 14, 338-359. doi: 10.1175/1520-0426(1997)014<0338:ismotm>2.0.co;2

Farrar, J. T., Rainville, L., Plueddemann, A. J., Kessler, W. S., Lee, C., Hodges, B. A., et al. (2015). Salinity and temperature balances at the SPURS Central Mooring during fall and winter. Oceanography 28, 56-65. doi: 10.5670/oceanog.2015.06

Fisher, F. H., and Spiess, F. N. (1963). Flip-floating instrument platform. J. Acoust. Soc. Am. 35, 1633-1644. doi: 10.1121/1.1918772

Foltz, G. R., Evan, A. T., Freitag, H. P., Brown, S., and McPhaden, M. J. (2013). Dust accumulation biases in PIRATA shortwave radiation records. J. Atmos. Ocean. Tech. 30, 1414-1432. doi: 10.1175/jtech-d-12-00169.1

Freeland, H. (2007). A short history of Ocean Station Papa and Line P. Prog. Oceanogr. 75, 120-125. doi: 10.1016/j.pocean.2007.08.005

Freeman, E., Kent, E. C., Brohan, P., Cram, T., Gates, L., Huang, B., et al. (2019). The international comprehensive ocean-atmosphere data set - meeting users needs and future priorities. Front. Mar. Sci. doi: 10.3389/fmars.2019.00435

Freeman, E., Woodruff, S. D., Worley, S. J., Lubker, S. J., Kent, E. C., Angel, W. E., et al. (2017). ICOADS Release 3.0: a major update to the historical marine climate record. Int. J. Climatol. 37, 2211-2232. doi: 10.1002/joc.4775

$\mathrm{Fu}, \mathrm{X}$., and Wang, B. (2004). The boreal summer intraseasonal oscillations simulated in a hybrid coupled atmosphere-ocean model. Mon. Wea. Rev. 132, 2628-2649. doi: $10.1175 / \mathrm{mwr} 2811.1$

Fujitani, T. (1981). Direct measurement of turbulent fluxes over the sea during AMTEX. Pap. Meteorol. Geophys. 32, 119-134. doi: 10.1016/j.dib.2018.09.108

Fujitani, T. (1985). Method of turbulent flux measurement on a ship by using a stable platform system. Pap. Meteorol. Geophys. 36, 157-170. doi: 10.2467/ mripapers. 36.157

Fung, I. Y., Harrison, D. E., and Lacis, A. A. (1984). On the variability of the net longwave radiation at the sea surface. Rev. Geophys. Space Phys. 22, 177-193.

Gentemann, C. L. (2014). Three way validation of MODIS and AMSR-E sea surface temperatures. J. Geophys. Res. Oceans 119, 2583-2598. doi: 10.1002/ 2013JC009716

Gentemann, C. L., and Hilburn, K. A. (2015). In situ validation of sea surface temperatures from the GCOM-W1 AMSR2 RSS calibrated brightness temperatures. J. Geophys. Res. 120, 3567-3585. doi: 10.1002/2014JC010574

Graber, H. C., Terray, E., Donelan, M. A., Drennan, W. M., Van Leer, J. C., and Peters, D. B. (2000). ASIS-a new air-sea interaction Spar Buoy: design and performance at sea. J. Atmos. Ocean. Tech. 17, 708-720. doi: 10.1175/15200426(2000)017<0708:aanasi>2.0.co;2

Grachev, A. A., Andreas, E. L., Fairall, C. W., Guest, P. S., and Persson, P. O. G. (2007). SHEBA flux-profile relationships in the stable atmospheric boundary layer. Bound. Layer Meteorol. 124, 315-333. doi: 10.1007/s10546-007-9177-6

Grachev, A. A., Fairall, C. W., Hare, J. E., Edson, J. B., and Miller, S. D. (2003). Wind stress vector over ocean waves. J. Phys. Oceanogr. 33, 2408-2429. doi: 10.1175/1520-0485(2003)033<2408:wsvoow>2.0.co;2

Grare, L., Lenain, L., and Melville, W. K. (2013). Wave-coherent airflow and critical layers over ocean waves. J. Phys. Oceanogr. 43, 2156-2172. doi: 10.1175/jpo-d13-056.1

Grare, L., Lenain, L., and Melville, W. K. (2018). Vertical profiles of the waveinduced airflow above ocean surface waves. J. Phys. Oceanogr. 48, 2901-2922. doi: 10.1175/jpo-d-18-0121.1

Grossman, R. L., and Betts, A. K. (1990). Air-sea interaction during an extreme cold air outbreak from the eastern coast of the United States. Mon. Wea. Rev. 118, 324-342. doi: 10.1175/1520-0493(1990)118<0324:aidaec>2.0.co;2

Gulev, S. K., and Belyaev, K. P. (2012). Probability distribution characteristics for surface air-sea turbulent heat fluxes over the global ocean. J. Clim. 25, 184-206. doi: 10.1175/2011JCLI4211.1

Gulev, S. K., Jung, T., and Ruprecht, E. (2007). Estimation of the impact of sampling errors in the VOS observations on air-sea fluxes. Part I. Uncertainties in climate means. J. Clim. 20, 279-301. doi: 10.1175/jcli4010.1

Gulev, S. K., Latif, M., Keenlyside, N., Park, W., and Koltermann, K. P. (2013). North Atlantic Ocean control on surface heat flux on multidecadal timescales. Nature 499, 464-467. doi: 10.1038/nature12268

Hara, T., and Belcher, S. E. (2004). Wind profile and drag coefficient over mature ocean surface wave spectra. J. Phys. Oceanogr. 34, 2345-2358. doi: 10.1175/ jpo2633.1 
Hara, T., and Sullivan, P. P. (2015). Wave boundary layer turbulence over surface waves in a strongly forced condition. J. Phys. Oceanogr. 45, 868-883. doi: 10.1175/jpo-d-14-0116.1

Herbers, T. H. C., Jessen, P. F., Janssen, T. T., Colbert, D. B., and MacMahan, J. H. (2012). Observing ocean surface waves with GPS-tracked buoys. J. Atmos. Ocean Tech. 29, 944-959. doi: 10.1175/jtech-d-11-00128.1

Hirahara, S., Ishii, M., and Fukuda, Y. (2014). Centennial-scale sea surface temperature analysis and its uncertainty. J. Clim. 27, 57-75. doi: 10.1175/JCLID-12-00837.1

Hihara, T., Kubota, M., and Okuro, A. (2015). Evaluation of sea surface temperature and wind speed observed by GCOM-W1/AMSR2 using in situ data and global products. Remote Sens. Environ. 164, 170-178. doi: 10.1016/j. rse.2015.04.005

Hobday, A. J., Oliver, E. C. J., Sen Gupta, A., Benthuysen, J. A., Burrows, M. T., Donat, M. G., et al. (2018). Categorizing and naming marine heatwaves. Oceanography 31, 162-173. doi: 10.5670/oceanog.2018.205

Hristov, T., and Ruiz-Plancarte, J. (2014). Dynamic balances in a wavy boundary layer. J. Phys. Oceanogr. 44, 3185-3194. doi: 10.1175/JPO-D-13-0209.1

Huang, B., Thorne, P. W., Banzon, V. F., Boyer, T., Chepurin, G., Lawrimore, J. H., et al. (2017). Extended reconstructed sea surface temperature, version 5 (ERSSTv5): upgrades, validations, and intercomparisons. J. Clim. 30, 81798205. doi: 10.1175/JCLI-D-16-0836.1

Jackson, D. L., and Wick, G. A. (2010). Near-surface air temperature retrieval derived from AMSU-A and sea surface temperature observations. J. Atmos. Ocean Technol. 27, 1769-1776. doi: 10.1175/2010JTECHA1414.1

Jacob, S. D., Shay, L. K., and Mariano, A. J. (2000). The 3D oceanic mixed layer response to Hurricane Gilbert. J. Phys. Oceanogr. 30, 1407-1429. doi: 10.1175/ 1520-04852000030<1407:TOMLRT<2.0.CO;2

Jiang, C., Cronin, M. F., Kelly, K. A., and Thompson, L. (2005). Evaluation of a hybrid satellite- and NWP-based turbulent heat flux product using Tropical Atmosphere-Ocean (TAO) buoys. J. Geophys. Res. Oceans 110:C09007. doi: 10.1029/2004JC002824

Jin, Z., Charlock, T. P., Smith, W. L., and Rutledge, K. (2004). A parameterization of ocean surface albedo. Geophys. Res. Lett. 31:L22301. doi: 10.1029/ 2004GL021180

Josey, S. A., Gulev, S., and Yu, L. (2013). "Exchanges through the ocean surface," in Ocean Circulation and Climate: A 21st Century Perspective, 2nd Edn, eds G. Siedler, S. Griffies, J. Gould, and J. Church (Oxford: Academic Press), 115-140. doi: 10.1016/b978-0-12-391851-2.00005-2

Josey, S. A., Kent, E. C., and Taylor, P. K. (1999). New insights into the ocean heat budget closure problem from analysis of the SOC air-sea flux climatology. J. Clim. 12, 2856-2880. doi: 10.1175/1520-0442(1999)012<2856:niitoh> 2.0.co;2

Kaimal, J. C., Wyngaard, J. C., Izumi, Y., and Coté, O. R. (1972). Spectral characteristics of surface-layer turbulence. Q. J. R. Meteor. Soc. 98, 563-589. doi: 10.1002/qj.49709841707

Kato, S., Rose, F. G., Rutan, D. A., Thorsen, T. J., Loeb, N. G., Doelling, D. R., et al. (2018). Surface irradiances of Edition 4.0 Clouds and the Earth's Radiant Energy System (CERES) Energy Balanced and Filled (EBAF) data product. J. Clim. 31, 4501-4527. doi: 10.1175/JCLI-D-17-0523.1

Kawamura, R., Fukuta, Y., Ueda, H., Matsuura, T., and Iizuka, S. (2002). A mechanism of the onset of the Australian summer monsoon. J. Geophys. Res. Atmos. 107, ACL5-1-ACL5-15.

Kennedy, J. J., Rayner, N. A., Smith, R. O., Parker, D. E., and Saunby, M. (2011). Reassessing biases and other uncertainties in sea surface temperature observations measured in situ since 1850: 1. Measurement and sampling uncertainties. J. Geophys. Res. 116:D14103. doi: 10.1029/2010JD015218

Kent, E. C., Rayner, N. A., Berry, D. I., Saunby, M., Moat, B. I., Kennedy, J. J., et al. (2013). Global analysis of night marine air temperature and its uncertainty since 1880: the HadNMAT2 data set. J. Geophys. Res. Atmos. 118, 1281-1298. doi: 10.1002 /jgrd. 50152

Kilpatrick, K. A., Podestá, G., Walsh, S., Williams, E., Halliwell, V., Szczodrak, M., et al. (2015). A decade of sea surface temperature from MODIS. Remote Sens. Environ. 165, 27-41. doi: 10.1016/j.rse.2015.04.023

Kilpatrick, K. A., Podestá, G., Williams, E., Walsh, S., and Minnett, P. J. (2019). Alternating decision trees for cloud masking MODIS and VIIRS NASA SST products. J. Atmos. Ocean Tech. 36, 387-407. doi: 10.1175/JTECH-D-180103.1
Kobayashi, S., Ota, Y., Harada, Y., Ebita, A., Moriya, M., Onoda, H., et al. (2015). The JRA-55 reanalysis: general specifications and basic characteristics. J. Meteorol. Soc. Jpn. 93, 5-48. doi: 10.2151/jmsj.2015-001

Kudryavtsev, V., Chapron, B., and Makin, V. (2014). Impact of wind waves on the air-sea fluxes: a coupled model. J. Geophys. Res. Oceans 119, 1217-1236. doi: 10.1002/2013JC009412

Kukulka, T., Hara, T., and Belcher, S. E. (2007). A model of the air-sea momentum flux and breaking-wave distribution for strongly forced wind waves. J. Phys. Oceanogr. 37, 1811-1828. doi: 10.1175/JPO3084.1

Landwehr, S., O'Sullivan, N., and Ward, B. (2015). Direct flux measurements from mobile platforms at sea: motion and airflow distortion corrections revisited. J. Atmos. Oceanic Tech. 32, 1163-1178. doi: 10.1175/jtech-d-14-00137.1

Large, W. G., and Pond, S. (1981). Open ocean momentum flux measurements in moderate to strong winds. J. Phys. Oceanogr. 11, 324-336. doi: 10.1175/152004851981011<0324:OOMFMI<2.0.CO;2

Large, W. G., and Yeager, S. G. (2009). The global climatology of an interannually varying air-sea flux data set. Clim. Dyn. 33, 341-364. doi: 10.1007/s00382-0080441-3

Lenain, L., and Melville, W. K. (2014). Autonomous surface vehicle measurements of the ocean's response to Tropical Cyclone Freda. J. Atmos. Oceanic Tech. 31, 2169-2190. doi: 10.1175/jtech-d-14-00012.1

Levitus, S., Antonov, J. I., Boyer, T. P., Baranova, O. K., Garcia, H. E., Locarnini, R. A., et al. (2012). World ocean heat content and thermosteric sea level change (0-2000 m), 1955-2010. Geophys. Res. Lett. 39:L10603. doi: 10.1029/ 2012GL051106

Li, J., Scinocca, J., Lazare, M., McFarlane, N., von Salzen, K., and Solheim, L. (2006). Ocean surface albedo and its impact on radiation balance in climate models. J. Clim. 19, 6314-6333. doi: 10.1175/jcli3973.1

Li, X., Dick, G., Lu, C., Ge, M., Nilsson, T., Ning, T., et al. (2015). Multi-GNSS meteorology: real-time retrieving of atmospheric water vapor from BeiDou, Galileo, GLONASS, and GPS observations. IEEE Trans. Geosci. Remote Sens. 53, 6385-6393. doi: 10.1109/tgrs.2015.2438395

Liman, J., Schröder, M., Fennig, K., Andersson, A., and Hollmann, R. (2018). Uncertainty characterization of HOAPS 3.3 latent heat-flux-related parameters. Atmos. Meas. Tech. 11, 1793-1815. doi: 10.5194/amt-11-1793-2018

Lin, W., Portabella, M., Stoffelen, A., Verhoef, A., and Turiel, A. (2015). ASCAT wind quality control near rain. IEEE Trans. Geosci. Remote Sens. 53, 4165-4177. doi: 10.1109/TGRS.2015.2392372

Lindstrom, E. J., Gunn, A., Fischer, A., and McCurdy, L. K. (2012). A Framework for Ocean Observing. By the Task Team for an Integrated Framework for Sustained Ocean Observing. UNESCO 2012, IOC/INF-1284 rev. Paris: UNESCO.

Liu, W. T., Katsaros, K., and Businger, J. (1979). Bulk parameterization of airsea exchanges of heat and water vapor including the molecular constraints at the interface. J. Atmos. Sci. 36, 1722-1735. doi: 10.1175/1520-0469(1979) $036<1722$ :bpoase $>2.0 .00 ; 2$

Liu, Y., and Minnett, P. J. (2016). Sampling errors in satellite-derived infrared seasurface temperatures. Part I: global and regional MODIS fields. Remote Sens. Environ. 177, 48-64. doi: 10.1016/j.rse.2016.02.026

Luo, B., Minnett, P. J., Gentemann, C., and Szczodrak, G. (2019). Improving satellite retrieved night-time infrared sea surface temperatures in aerosol contaminated regions. Remote Sens. Environ. 223, 8-20. doi: 10.1016/j.rse.2019. 01.009

Mahrt, L., Vickers, D., Howell, J., Højstrup, J., Wilczak, J. M., Edson, J. B., et al. (1996). Sea surface drag coefficients in RASEX. J. Geophys. Res. 101 327-314.

May, J., and Bourassa, M. A. (2011). Quantifying variance due to temporal and spatial difference between ship and satellite winds. J. Geophys. Res. 116:C08013. doi: 10.1029/2010JC006931

Mazloff, M. R., Cornuelle, B. D., Gille, S. T., and Verdy, A. (2018). Correlation lengths for constraining the large-scale carbon and heat content of the Southern Ocean. J. Geophys. Res. Oceans 123, 883-901. doi: 10.1002/2017JC0 13408

McPhaden, M. J., Busalacchi, A. J., Cheney, R., Donguy, J.-R., Gage, K. S., Halpern, D., et al. (1998). The Tropical Ocean Global Atmosphere observing system: a decade of progress. J. Geophys. Res. 103:240. doi: 10.1029/97JC02906

McPhaden, M. J., Meyers, G., Ando, K., Masumoto, Y., Murty, V. S. N., Ravichandran, M., et al. (2009). RAMA: the research moored array for AfricanAsian-Australian monsoon analysis and prediction. Bull. Am. Meteor. Soc. 90, 459-480. doi: 10.1175/2008bams2608.1 
Mechoso, C. R., Wood, R., Weller, R., Bretherton, C. S., Clarke, A. D., Coe, H., et al. (2014). Ocean-cloud-atmosphere-land interactions in the southeastern Pacific: the VOCALS program. Bull. Amer. Meteor. Soc. 95, 357-375. doi: 10. 1175/BAMS-D-11-00246.1

Meissner, T., and Wentz, F. J. (2012). The emissivity of the ocean surface between 6 and $90 \mathrm{GHz}$ over a large range of wind speeds and earth incidence angles. IEEE Trans. Geosci. Remote Sens. 50, 3004-3026. doi: 10.1109/tgrs.2011.217 9662

Meissner, T., Wentz, F. J., and Ricciardulli, L. (2014). The emission and scattering of L-band microwave radiation from rough ocean surfaces and wind speed measurements from the Aquarius sensor. J. Geophys. Res. Oceans 119, 64996522. doi: $10.1002 / 2014$ jc009837

Minnett, P. J., Smith, M., and Ward, B. (2011). Measurements of the oceanic thermal skin effect. Deep-Sea Res. Part II-Top. Stud. Oceanogr. 58, 861-868. doi: $10.1016 /$ j.dsr2.2010.10.024

Minobe, S., Miyashita, M., Kuwano-Yoshida, A., Tokinaga, H., and Xie, S. P. (2010). Atmospheric response to the Gulf Stream: seasonal variations. J. Climate 23, 3699-3719. doi: 10.1175/2010jcli3359.1

Mitarai, S., and McWilliams, J. C. (2016). Wave glider observations of surface winds and currents in the core of Typhoon Danas. Geophys. Res. Lett. 43 312-311. doi: 10.1002/2016GL071115

Molod, A., Takacs, L., Suarez, M., and Bacmeister, J. (2015). Development of the GEOS-5 atmospheric general circulation model: evolution from MERRA to MERRA2. Geosci. Model Dev. 8, 1339-1356. doi: 10.5194/gmd-8-1339-2015

Monteiro, P. M. S., Gregor, L., Lévy, M., Maenner, S., Sabine, C. L., and Swart, S. (2015). Intraseasonal variability linked to sampling alias in air-sea CO2 fluxes in the Southern Ocean. Geophys. Res. Lett. 42, 8507-8514. doi: 10.1002/ 2015GL066009

Morey, S. L., Dukhovskoy, D. S., and Bourassa, M. A. (2009). Connectivity of the Apalachicola River flow variability and the physical and bio-optical oceanic properties of the northern West Florida Shelf. Cont. Shelf Res. 29, 1264-1275. doi: 10.1016/j.csr.2009.02.003

Ogle, S. E., Tamsitt, V., Josey, S. A., Gille, S. T., Cerovečki, I., Talley, L. D., et al. (2018). Episodic Southern Ocean heat loss and its mixed layer impacts revealed by the farthest south multiyear surface flux mooring. Geophys. Res. Lett. 45, 5002-5010. doi: 10.1029/2017GL076909

Parfitt, R., Czaja, A., Minobe, S., and Kuwano-Yoshida, A. (2016). The atmospheric frontal response to SST perturbations in the Gulf Stream region. Geophys. Res. Lett. 43, 2299-2306. doi: 10.1002/2016gl067723

Parfitt, R., and Seo, H. (2018). A new framework for near-surface wind convergence over the Kuroshio Extension and Gulf Stream in wintertime: the role of atmospheric fronts. Geophys. Res. Lett. 45, 9909-9918. doi: 10.1029/ 2018gl080135

Payne, R. E. (1972). Albedo of the sea surface. J. Atmos. Sci. 29, 959-970. doi: 10.1175/1520-0469(1972)029<0959:aotss >2.0.co;2

Petrenko, B., Ignatov, A., Kihai, Y., Stroup, J., and Dash, P. (2014). Evaluation and selection of SST regression algorithms for JPSS VIIRS. J. Geophys. Res. Atmos. 119, 4580-4599. doi: 10.1002/2013JD020637

Pinker, R. T., Zhang, B. Z., Weller, R. A., and Chen, W. (2018). Evaluating surface radiation fluxes observed from satellites in the southeastern Pacific Ocean. Geophys. Res. Lett. 45, 2404-2412. doi: 10.1002/2017GL076805

Potter, H., Graber, H. C., Williams, N. J., Collins, C. O., Ramos, R. J., and Drennan, W. M. (2014). In situ measurements of momentum fluxes in typhoons. J. Atmos. Sci. 72, 104-118. doi: 10.1175/JAS-D-14-0025.1

Reising, S. C., Gaier, T. C., Kummerow, C. D., Padmanabhan, S., Lim, B. H., Brown, S. T., et al. (2016). “Temporal experiment for storms and tropical systems technology demonstration (TEMPEST-D): reducing risk for $6 \mathrm{U}$-Class nanosatellite constellations," in Proceedings of the 2016 IEEE International Geoscience and Remote Sensing Symposium (IGARSS), (Beijing), 5559-5560.

Renfrew, I. A., and Moore, G. W. K. (1999). An extreme cold-air outbreak over the Labrador Sea: roll vortices and air-sea interaction. Mon. Wea. Rev. 127, 2379-2394. doi: 10.1175/1520-0493(1999)127<2379:aecaoo>2.0.co;2

Reynolds, R. W., Chelton, D. B., Roberts-Jones, J., Martin, M. J., Menemenlis, D., and Merchant, C. J. (2013). Objective determination of feature resolution in two sea surface temperature analyses. J. Clim. 26, 2514-2533. doi: 10.1175/JCLI-D12-00787.1

Richter, D. H., and Veron, F. (2016). Ocean spray: an outsized influence on weather and climate. Phys. Today 66, 34-39. doi: 10.1063/pt.3.3363
Roberts, J. B., Clayson, C. A., Robertson, F. R., and Jackson, D. (2010). Predicting near-surface characteristics from SSM/I using neural networks with a first guess approach. J. Geophys. Res. 115:D19113. doi: 10.1029/200d9JD01 3099

Rodríguez, E., Bourassa, M., Chelton, D., Farrar, J. T., Long, D., PerkovicMartin, D., et al. (2018). The winds and currents mission concept. Front. Mar. Sci. doi: 10.3389/fmars.2019.00438

Roemmich, D., Church, J., Gilson, J., Monselesan, D., Sutton, P., and Wijffels, S. (2015). Unabated planetary warming and its ocean structure since 2006. Nat. Clim. Change 5, 240-245. doi: 10.1038/NCLIMATE2513

Rutan, D. A., Kato, S., Doelling, D. R., Rose, F. G., Nguyen, L. T., Caldwell, T. E., et al. (2015). CERES Synoptic product: methodology and validation of surface radiant flux. J. Atmos. Oceanic Technol. 32, 1121-1143. doi: 10.1175/JTECH-D14-00165.1

Saha, S., Moorthi, S., Pan, H.-L., Wu, X., Wang, J., Nadiga, S., et al. (2010). The NCEP Climate forecast system reanalysis. Bull. Am. Meteor. Soc. 91, 1015-1057. doi: 10.1175/2010BAMS3001.1

Schmidt, K. M., Swart, S., Reason, C., and Nicholson, S.-A. (2017). Evaluation of satellite and reanalysis wind products with in situ wave glider wind observations in the Southern Ocean. J. Atmos. Ocean Tech. 34, 2551-2568. doi: 10.1175/jtechd-17-0079.1

Schulz, E. W., Josey, S. A., and Verein, R. (2012). First air-sea flux mooring measurements in the Southern Ocean. Geophys. Res. Lett. 39:L16606. doi: 10. 1029/2012GL052290

Shaman, J., Samelson, R. M., and Skyllingstad, E. (2010). Air-sea fluxes over the Gulf Stream region: atmospheric controls and trends. J. Clim. 23, 2651-2670. doi: $10.1175 / 2010$ jcli3269.1

Shi, Q. (2017). Coupling Ocean Currents and Waves with Wind Stress Over the Gulf Stream. Ph.D. dissertation. Florida State University: Tallahassee, FL.

Shibata, A. (2012). Ocean wind speed retrieval algorithm using the frequency $36 \mathrm{GHz}$ vertical/horizontal and $6 \mathrm{GHz}$ horizontal data of the Advanced Microwave Scanning Radiometer (AMSR). Eur. J. Remote Sens. 45, 133-140. doi: 10.5721/EuJRS20124513

Shinoda, T., Hendon, H., and Glick, J. (1998). Intraseasonal variability of surface fluxes and sea surface temperature in the tropical western Pacific and Indian Oceans. J. Clim. 11, 1685-1702. doi: 10.1175/1520-0442(1998)011<1685: ivosfa $>2.0 . \operatorname{co} ; 2$

Silverthorne, K. E., and Toole, J. M. (2013). Quasi-Lagrangian observations of the upper ocean response to wintertime forcing in the Gulf Stream. Deep Sea Res. Part II 91, 25-34. doi: 10.1016/j.dsr2.2013.02.021

Smith, N., Kessler, W. S., Cravatte, S., Sprintall, J., Wijffels, S., Cronin, M. F., et al. (2019). Tropical pacific observing system. Front. Mar. Sci. 6:31. doi: 10.3389/ fmars.2019.00031

Smith, S. D. (1988). Coefficients for sea surface wind stress, heat flux, and wind profiles as a function of wind speed and temperature. J. Geophys. Res. 93, 467-415. doi: 10.1029/JC093iC12p15467

Sullivan, P. P., Banner, M. L., Morison, R. P., and Peirson, W. L. (2018). Turbulent flow over steep steady and unsteady waves under strong wind forcing. J. Phys. Oceanogr. 48, 3-27. doi: 10.1175/jpo-d-17-0118.1

Sullivan, P. P., McWilliams, J. C., and Patton, E. G. (2014). Large-eddy simulation of marine atmospheric boundary layers above a spectrum of moving waves. J. Atmos. Sci. 71, 4001-4027. doi: 10.1175/jas-d-14-0095.1

Swart, S., Gille, S. T., Delille, B., Josey, S., Mazloff, M., Newman, L., et al. (2019). Constraining Southern Ocean air-sea-ice fluxes through enhanced observations. Front. Mar. Sci. 6:421. doi: 10.3389/fmars.2019.00421

Thomson, J. (2012). Wave breaking dissipation observed with "SWIFT" drifters. J. Atmos. Ocean. Tech. 29, 1866-1882. doi: 10.1175/jtech-d-1200018.1

Thomson, J., and Girton, J. (2017). Sustained measurements of Southern Ocean airsea coupling from a Wave Glider autonomous surface vehicle. Oceanography 30, 104-109. doi: 10.5670/oceanog.2017.228

Tilinina, N., Gavrikov, A., and Gulev, S. (2018). Association of the North Atlantic surface turbulent heat fluxes with midlatitude cyclones. Mon. Wea. Rev. 146, 3691-3715. doi: 10.1175/MWR-D-17-0291.1

Tomita, H., Hihara, T., Kako, S., Kubota, M., and Kutsuwada, K. (2019). An introduction to J-OFURO3, a third-generation Japanese ocean flux data set using remote-sensing observations. J. Oceanogr. 75, 171-194. doi: 10.1007/ s10872-018-0493-x 
Tomita, H., Hihara, T., and Kubota, M. (2018). Improved satellite estimation of near-surface humidity using vertical water vapor profile information. Geophys. Res. Lett. 45, 899-906. doi: 10.1002/2017GL076384

Tomita, H., Kawai, Y., Cronin, M. F., Hihara, T., and Kubota, M. (2015). Validation of AMSR2 sea surface wind and temperature over the Kuroshio Extension region. SOLA 11, 43-47. doi: 10.2151/sola.2015-010

Tozuka, T., Ohishi, S., and Cronin, M. F. (2018). A metric for surface heat flux effect on horizontal sea surface temperature gradients. Clim. Dyn. 51, 547-561. doi: 10.1007/s00382-017-3940-2

Tsujino, H., Urakawa, S., Nakano, H., Small, R. J., Kim, W. M., Yeager, S. G., et al. (2018). JRA-55 based surface dataset for driving ocean-sea-ice models (JRA55-do). Ocean Model. 130, 79-139. doi: 10.1016/j.ocemod.2018.07.002

Tu, Q., Pan, D., and Hao, Z. (2015). Validation of S-NPP VIIRS sea surface temperature retrieved from NAVO. Remote Sens. 17:245.

Valdivieso, M., Haines, K., Balmaseda, M., Chang, Y.-S., Drevillon, M., Ferry, N., et al. (2017). An assessment of air-sea heat fluxes from ocean and coupled reanalyses. Clim. Dyn. 49, 983-1008. doi: 10.1007/s00382-015-2843-3

Vickers, D., and Mahrt, L. (1999). Observations of non-dimensional wind shear in the coastal zone. Q. J. R. Meteor. Soc. 125, 2685-2702. doi: 10.1002/qj. 49712555917

Villas Bôas, A. B., Sato, O. T., Chaigneau, A., and Castelão, G. P. (2015). The signature of mesoscale eddies on the air-sea turbulent heat fluxes in the South Atlantic Ocean. Geophys. Res. Lett. 42, 1856-1862. doi: 10.1002/2015gl06 3105

von Engeln, A., and Teixeira, J. (2013). A planetary boundary layer height climatology derived from ECMWF reanalysis data. J. Clim. 26, 6575-6590. doi: 10.1175/JCLI-D-12-00385.1

Wallcraft, A. J., Kara, A. B., Barron, C. N., Metzger, E. J., Pauley, R. L., and Bourassa, M. A. (2009). Comparisons of monthly mean $10 \mathrm{~m}$ wind speeds from satellites and NWP products over the global ocean. J. Geophys. Res. Atmos. 114:D16109. doi: 10.1029/2008JD011696

Wang, W., and McPhaden, M. J. (2000). The surface-layer heat balance in the equatorial Pacific Ocean. Part II: interannual variability. J. Phys. Oceanogr. 30, 2989-3008. doi: 10.1175/1520-0485(2001)031<2989:tslhbi>2.0.co;2

Webster, P. J., Clayson, C. A., and Curry, J. A. (1996). Clouds, radiation, and the diurnal cycle of sea surface temperature in the tropical western Pacific. J. Clim. 9, 1712-1730. doi: 10.1175/1520-0442(1996)009<1712:cratdc $>2.0 . c 0 ; 2$

Weller, R. A. (2015). Variability and trends in surface meteorology and air-sea fluxes at a site off northern Chile. J. Clim. 28, 3004-3023. doi: 10.1175/JCLID-14-00591.1

Weller, R. A., Bigorre, S. P., Lord, J., Ware, J. D., and Edson, J. B. (2012). A surface mooring for air-sea interaction research in the Gulf Stream. Part 1: mooring design and instrumentation. J. Atmos. Ocean. Tech. 29, 1363-1376. doi: $10.1175 /$ jtech-d-12-00060.1

Wong, E. W., and Minnett, P. J. (2016). Retrieval of the ocean skin temperature profiles from measurements of infrared hyperspectral radiometers-Part I: derivation of an algorithm*. IEEE Trans. Geosci. Remote Sens. 54, 1879-1890. doi: 10.1109/TGRS.2015.2483746

Wong, E. W., and Minnett, P. J. (2018). The response of the ocean thermal skin layer to variations in incident infrared radiation. J. Geophys. Res. Oceans 123, 2475-2493. doi: 10.1002/2017JC013351

$\mathrm{Wu}$, J. (1994). The sea surface is aerodynamically rough even under light winds. Bound. Layer Meteorol. 69, 149-158. doi: 10.1007/bf00713300

Yelland, M. J., Moat, B. I., Taylor, P. K., Pascal, R. W., Hutchings, J., and Cornell, V. C. (1998). Wind stress measurements from the open ocean corrected for airflow distortion by the ship. J. Phys. Oceanogr. 28, 1511-1526. doi: 10.1175/ 1520-04851998028<1511:WSMFTO<2.0.CO;2
Yelland, M. J., Pascal, R. W., Taylor, P. K., and Moat, B. I. (2009). AutoFlux: an autonomous system for the direct measurement of the air-sea fluxes of CO2, heat and momentum. J. Oper. Oceanogr. 2, 15-23. doi: 10.1080/1755876X.2009. 11020105

Yokoi, S., Katsumata, M., and Yoneyama, K. (2014). Variability in surface meteorology and air-sea fluxes due to cumulus convective systems observed during CINDY/DYNAMO. J. Geophys. Res. Atmos. 119, 2064-2078. doi: 10. 1002/2013JD020621

Yu, L. (2019). Global air-sea fluxes of heat, fresh water, and momentum: energy budget closure and unanswered questions. Annu. Rev. Mar. Sci. 11, 227-248. doi: 10.1146/annurev-marine-010816-060704

Yu, L., and Jin, X. (2012). Buoy perspective of a high-resolution global ocean vector wind analysis using passive radiometers and active scatterometers from 1987 to the present. J. Geophys. Res. 117:C11013. doi: 10.1029/2012JC0 08069

Yu, L., and Jin, X. (2018). A regime-dependent retrieval algorithm for near-surface air temperature and specific humidity from multi-microwave sensors. Remote Sens. Environ. 215, 199-216. doi: 10.1016/j.rse.2018.06.001

Yu, L., Jin, X., Schulz, E. W., and Josey, S. A. (2017). Air-sea interaction regimes in the sub-Antarctic Southern Ocean and Antarctic marginal ice zone revealed by icebreaker measurements. J. Geophys. Res. Oceans 122, 6547-6564. doi: 10. 1002/2016JC012281

Yu, L., and Weller, R. A. (2007). Objectively analyzed air-sea heat fluxes for the global ice-free oceans (1981-2005). Bull. Am. Meteor. Soc. 88, 527-539.

Zhang, D., Cronin, M. F., Wen, C., Xue, Y., and Kumar, A. (2016). Assessing surface heat fluxes in atmospheric reanalyses with a decade of data from the NOAA Kuroshio Extension Observatory. J. Geophys. Res. 121, 6874-6890. doi: 10.1002/2016JC011905

Zhang, L., Shi, H., Wang, Z., Yu, H., Yin, X., and Liao, Q. (2018). Comparison of wind speeds from spaceborne microwave radiometers with in situ observations and ECMWF data over the global ocean. Remote Sens. 10:425. doi: 10.3390/ rs10030425

Conflict of Interest Statement: NOAA PMEL has had a Cooperative Research and Development Agreement (CRADA) with Saildrone, Inc., since 2014 that covers work with Saildrone ASV by MFC. This has involved in kind contributions from Saildrone, Inc., toward NOAA ASV missions. It is expected that this work will benefit NOAA's mission and the entire ASV industry. CLG had a contract for 6 weeks of her time from Saildrone, Inc. The contract was to coordinate a 2-month Saildrone cruise that occurred in Spring 2018. DV had a contract for 2 weeks of his time from Saildrone Inc., in 2018 to advise on the data collection and processing of turbulent flux measurements using their platform.

The remaining authors declare that the research was conducted in the absence of any commercial or financial relationships that could be construed as a potential conflict of interest.

Copyright (c) 2019 Cronin, Gentemann, Edson, Ueki, Bourassa, Brown, Clayson, Fairall, Farrar, Gille, Gulev, Josey, Kato, Katsumata, Kent, Krug, Minnett, Parfitt, Pinker, Stackhouse, Swart, Tomita, Vandemark, Weller, Yoneyama, Yu and Zhang. This is an open-access article distributed under the terms of the Creative Commons Attribution License (CC BY). The use, distribution or reproduction in other forums is permitted, provided the original author(s) and the copyright owner(s) are credited and that the original publication in this journal is cited, in accordance with accepted academic practice. No use, distribution or reproduction is permitted which does not comply with these terms. 


\section{OPEN ACCESS}

Edited by:

Sabrina Speich,

École Normale Supérieure, France

Reviewed by:

Brent Else,

University of Calgary, Canada

Tong Lee,

NASA Jet Propulsion Laboratory

(JPL), United States

*Correspondence:

Sebastiaan Swart

sebastiaan.swart@marine.gu.se

Specialty section:

This article was submitted to

Ocean Observation,

a section of the journal

Frontiers in Marine Science

Received: 31 October 2018 Accepted: 05 July 2019

Published: 31 July 2019

Citation:

Swart S, Gille ST, Delille B,

Josey $S$, Mazloff M, Newman $L$, Thompson AF, Thomson J, Ward B, du Plessis MD, Kent EC, Girton J, Gregor L, Heil P, Hyder P, Pezzi LP, de Souza RB, Tamsitt V, Weller RA and Zappa CJ (2019) Constraining Southern Ocean Air-Sea-Ice Fluxes

Through Enhanced Observations.

Front. Mar. Sci. 6:421.

doi: 10.3389/fmars.2019.00421

\section{Constraining Southern Ocean Air-Sea-Ice Fluxes Through Enhanced Observations}

\author{
Sebastiaan Swart ${ }^{1,2 *}$, Sarah T. Gille ${ }^{3}$, Bruno Delille ${ }^{4}$, Simon Josey ${ }^{5}$, Matthew Mazloff ${ }^{3}$, \\ Louise Newman', Andrew F. Thompson ${ }^{7}$, Jim Thomson ${ }^{8}$, Brian Ward ${ }^{9}$, \\ Marcel D. du Plessis ${ }^{2,10}$, Elizabeth C. Kent ${ }^{11}$, James Girton ${ }^{8}$, Luke Gregor ${ }^{10,12,}$ \\ Petra Heil ${ }^{13}$, Patrick Hyder ${ }^{14}$, Luciano Ponzi Pezzi ${ }^{15}$, Ronald Buss de Souza ${ }^{16}$, \\ Veronica Tamsitt ${ }^{17,18}$, Robert A. Weller ${ }^{19}$ and Christopher J. Zappa ${ }^{20}$
}

\begin{abstract}
'Department of Marine Sciences, University of Gothenburg, Gothenburg, Sweden, ${ }^{2}$ Department of Oceanography, University of Cape Town, Cape Town, South Africa, ${ }^{3}$ Scripps Institution of Oceanography, University of California, San Diego, San Diego, CA, United States, ${ }^{4}$ Chemical Oceanography Unit, University of Liège, Liège, Belgium, ${ }^{5}$ National Oceanography Centre, Southampton, Southampton, United Kingdom, ${ }^{6}$ Southern Ocean Observing System International Project Office, University of Tasmania, Hobart, TAS, Australia, ${ }^{7}$ Division of Geological and Planetary Sciences, Department of Environmental Science and Engineering, California Institute of Technology, Pasadena, CA, United States, ${ }^{8}$ Applied Physics Laboratory, University of Washington, Seattle, WA, United States, ${ }^{9}$ AirSea Laboratory, School of Physics and Ryan Institute, National University of Ireland Galway, Galway, Ireland, ${ }^{10}$ Southern Ocean Carbon and Climate Observatory, Council for Scientific and Industrial Research, Pretoria, South Africa, " National Oceanography Centre, Southampton, Southampton, United Kingdom, ${ }^{12}$ Environmental Physics, Institute of Biogeochemistry and Pollutant Dynamics, ETH Zürich, Zurich, Switzerland, ${ }^{13}$ Australian Antarctic Division and Antarctic Climate and Ecosystem CRC, University of Tasmania, Hobart, TAS, Australia, ${ }^{14}$ Met Office Hadley Centre, Exeter, United Kingdom, ${ }^{15}$ Earth Observation General Coordination, National Institute for Space Research, São Paulo, Brazil, ${ }^{16}$ Center for Weather Forecasting and Climate Studies, National Institute for Space Research, São Paulo, Brazil, ${ }^{17}$ Climate Change Research Centre, University of New South Wales, Sydney, NSW, Australia, ${ }^{18}$ Centre for Southern Hemisphere Oceans Research, CSIRO Oceans and Atmosphere, Hobart, TAS, Australia, ${ }^{19}$ Woods Hole Oceanographic Institution, Woods Hole, MA, United States, ${ }^{20}$ Lamont-Doherty Earth Observatory, Columbia University, Palisades, NY, United States
\end{abstract}

Air-sea and air-sea-ice fluxes in the Southern Ocean play a critical role in global climate through their impact on the overturning circulation and oceanic heat and carbon uptake. The challenging conditions in the Southern Ocean have led to sparse spatial and temporal coverage of observations. This has led to a "knowledge gap" that increases uncertainty in atmosphere and ocean dynamics and boundary-layer thermodynamic processes, impeding improvements in weather and climate models. Improvements will require both process-based research to understand the mechanisms governing air-sea exchange and a significant expansion of the observing system. This will improve flux parameterizations and reduce uncertainty associated with bulk formulae and satellite observations. Improved estimates spanning the full Southern Ocean will need to take advantage of ships, surface moorings, and the growing capabilities of autonomous platforms with robust and miniaturized sensors. A key challenge is to identify observing system sampling requirements. This requires models, Observing System Simulation Experiments (OSSEs), and assessments of the specific spatialtemporal accuracy and resolution required for priority science and assessment of observational uncertainties of the mean state and direct flux measurements. Yearround, high-quality, quasi-continuous in situ flux measurements and observations of extreme events are needed to validate, improve and characterize uncertainties in 
blended reanalysis products and satellite data as well as to improve parameterizations. Building a robust observing system will require community consensus on observational methodologies, observational priorities, and effective strategies for data management and discovery.

Keywords: air-sea/air-sea-ice fluxes, Southern Ocean, ocean-atmosphere interaction, climate, ocean-ice interaction

\section{INTRODUCTION}

Air-sea fluxes determine how properties, such as momentum, heat, freshwater, and gasses, exchange between the atmosphere and the ocean. They are essential to understanding the global energy budget (Trenberth and Fasullo, 2010), evaluating and improving weather prediction and climate models, and understanding oceanic and atmospheric processes that depend on the upper ocean [ocean heat uptake, mixed-layer temperature, Sea Surface Temperature (SST) variability] (Dong et al., 2007). This is exemplified in the Southern Ocean (SO), where extreme winds and sea states, strong temperature gradients, and strong seasonal changes (e.g., sea-ice cover) enhance air-sea exchanges in a region that is key to global climate (e.g., Sabine et al., 2004; Frölicher et al., 2015; Rintoul, 2018) and highly responsive to changing climate conditions (e.g., Andrews et al., 2015).

In situ observations of variables needed to estimate fluxes, in particular, meteorological measurements, are sparse in the SO (Figure 1), with almost no observations from autumn or winter months or at any time in the regions impacted by permanent or seasonal sea-ice cover. This results in large errors in SO reanalysis products (see section "Status of Flux Reanalyses and Requirements to Reduce Uncertainty," Figure 2) and poor representation in climate models, with significant inter-related biases in SSTs, ocean thermal structure, winds and clouds, due to its strongly coupled dynamics (e.g., Thompson et al., 2011; Bodas-Salcedo et al., 2014, 2016; Hermanson et al., 2018; Hyder et al., 2018). The lack of in situ data also limits our ability to improve remote sensing products and optimize blending of model and remote sensing inputs (e.g., Objectively Analyzed Flux - Yu and Weller, 2007), and increases uncertainty in atmosphere and ocean dynamics and boundarylayer thermodynamic processes. The consequent uncertainties in climate projections further hinder our ability to develop climate mitigation and adaptation measures. To assess and improve flux estimates and understand key processes in the SO there is an urgent requirement for accurate, direct airsea-ice flux observations, and the meteorological variables on which they depend.

This paper builds on the high-latitude flux review of Bourassa et al. (2013). The objectives of this paper are to highlight the Southern Ocean-specific observational gaps, scales, and challenges in estimating air-sea-ice fluxes, to summarize key focus areas for future flux research, and to offer recommendations for improving estimates of fluxes. In particular, recent technological progress, such as surface flux moorings and autonomous surface vehicles, provide new mechanisms to improve high-latitude flux observations and research in the coming decade. For details on broad-scale heat and momentum fluxes for the global ice-free ocean the paper by Cronin et al. (2019) should also be referred to. This paper articulates a charge for the SO air-sea flux community to come together to plan, collect and share air-sea-ice flux information, experience and knowledge. It is a product of the Southern Ocean Observing System (SOOS) international working group on SO air-sea fluxes (SOFLUX; Gille et al., 2016), which coordinates observing-system capabilities and requirements for SO air-sea-ice fluxes.

\section{KEY SPATIAL AND TEMPORAL SCALES OF FLUXES}

The short time- and length-scale variability of SO air-sea fluxes makes quantifying exchanges challenging (Lenton et al., 2006; Monteiro et al., 2015). This variability is predominantly driven by the high-frequency structure of winds (Yuan et al., 2009) and high-wavenumber structure of the ocean state (Chelton et al., 1998). However, one may exploit the low-frequency variability of ocean properties and low-wavenumber structure of the atmospheric properties to constrain the fluxes. For example, Observing System Simulation Experiments (OSSEs) show that the Argo float array is able to constrain the upperocean climate signals of heat, carbon, and freshwater content on seasonal timescales (Majkut et al., 2014; Kamenkovich et al., 2017; Mazloff et al., 2018). Spatial and temporal integrals of oceanic heat content change provide information on net heat fluxes, which can be used to assess biases in the bulk formulae derived from point measurements. Yet large-scale budgets do not provide information about the mechanisms driving smallscale air-sea fluxes, which are vital to understanding the system and can be revealed by in situ time-series observations (Ogle et al., 2018).

SOFLUX has prioritized the collection of high-quality in situ point measurements necessary to understand true small-scale flux variability, while also measuring the large-scale properties that reveal integrated flux values. High-frequency measurements suggest that Subantarctic air-sea $\mathrm{CO}_{2}$ fluxes should be measured at a 3-day frequency to provide an unbiased constraint, particularly during the more stratified summer period. Sampling at lower temporal frequency would potentially alias the more rapid response of mixing and entrainment to storm events (Monteiro et al., 2015). Alternatively, Mazloff et al. (2018) suggest that maintaining platforms every $20^{\circ}$ longitude by $6^{\circ}$ latitude would constrain the climate-scale heat and carbon inventory, and validate integrated air-sea fluxes. While fluxes 


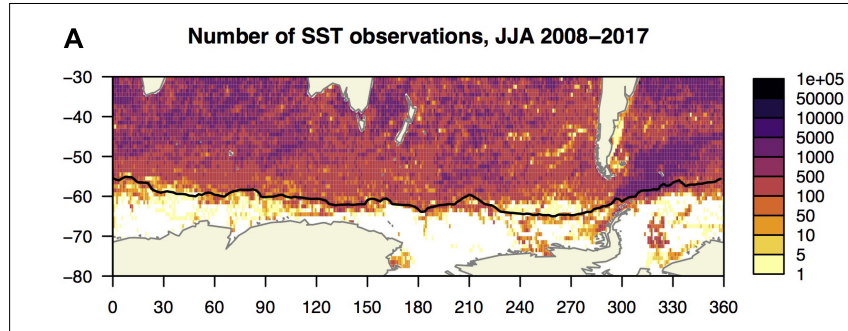

B Number of air temperature observations, JJA 2008-2017

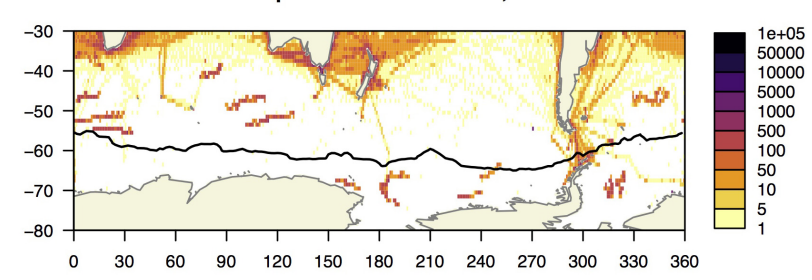

C Number of humidity observations, JJA 2008-2017

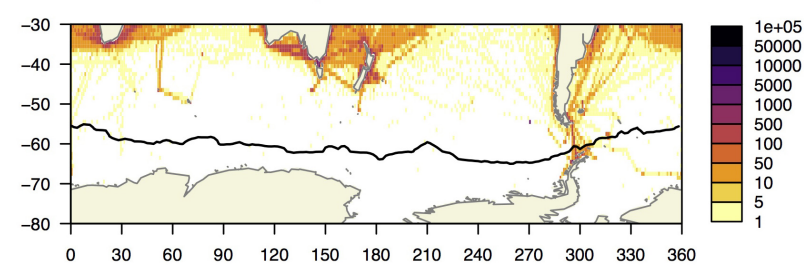

D Number of wind speed observations, JJA 2008-2017

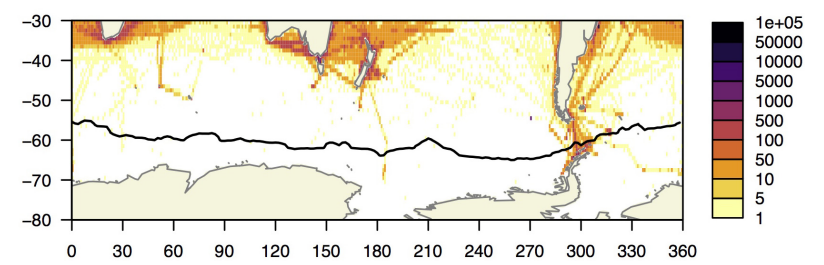

E

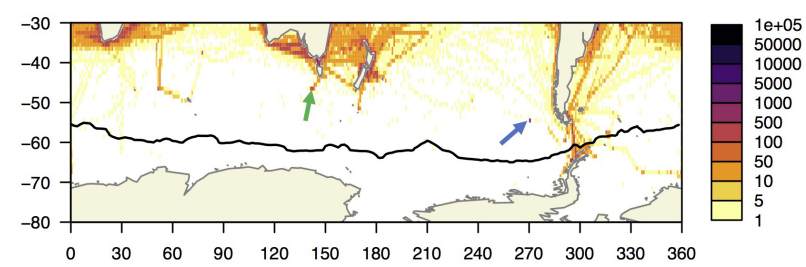

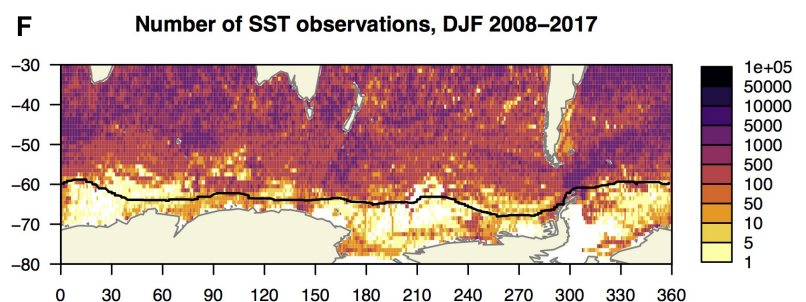

G Number of air temperature observations, DJF 2008-2017

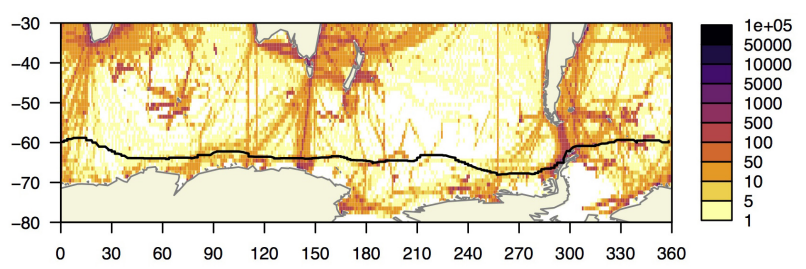

H Number of humidity observations, DJF 2008-2017

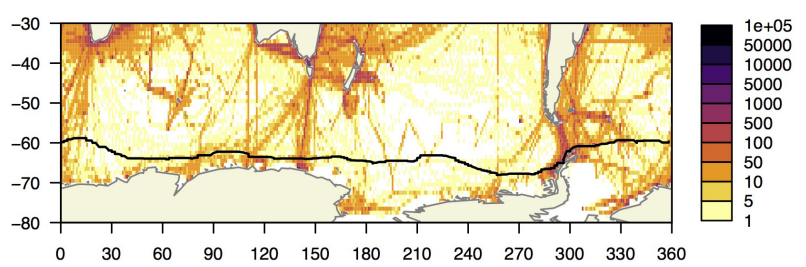

I Number of wind speed observations, DJF 2008-2017

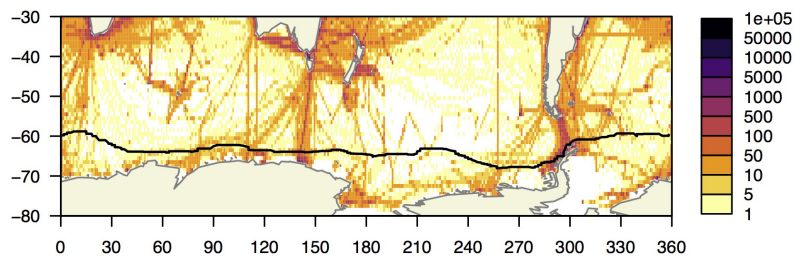

J Number of observations for LHF, DJF 2008-2017

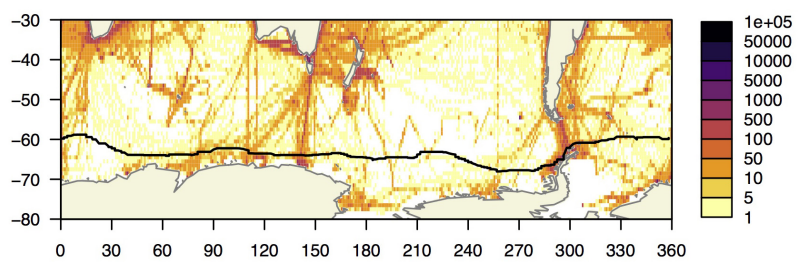

FIGURE 1 | Observation count for critical air-sea flux ocean and atmospheric parameters for austral winter (June-August) on the left versus summer (December-February) on the right, for 10 years (2008-2017): (A,F) SST, (B,G) air temperature, (C,H) humidity, (D,I) wind speed, and (E,J) latent heat flux (LHF). Humidity observations include dew point temperature and relative humidity. LHF observations include co-located measurements of SST, air temperature, humidity and wind speed. Data from International Comprehensive Ocean-Atmosphere Data Set (ICOADS), as described by Freeman et al. (2017). The two existing surface mooring sites, $\mathrm{OOI}$ and SOFS, are indicated in panel (E) using a blue and green arrow, respectively. Black contour denotes the 10-year mean of the 15\% sea ice concentration from NCEP/DOE AMIP-II Reanalysis, representing the sea-ice edge in winter (left panels) and summer (right panels).

themselves have significant high-frequency variability, the lowfrequency component has large spatial scales of approximately $90^{\circ}$ longitude by $10^{\circ}$ latitude. Climate-scale fluxes for the ocean between $35^{\circ} \mathrm{S}$ and $70^{\circ} \mathrm{S}$, could therefore be determined with 100 optimally spaced platforms. The current Argo array of $>600$ floats results in non-negligible uncertainty in estimating climate signals, with crucial gaps in the sea-ice impacted regions (Mazloff et al., 2018). Nevertheless, the results imply that reducing 

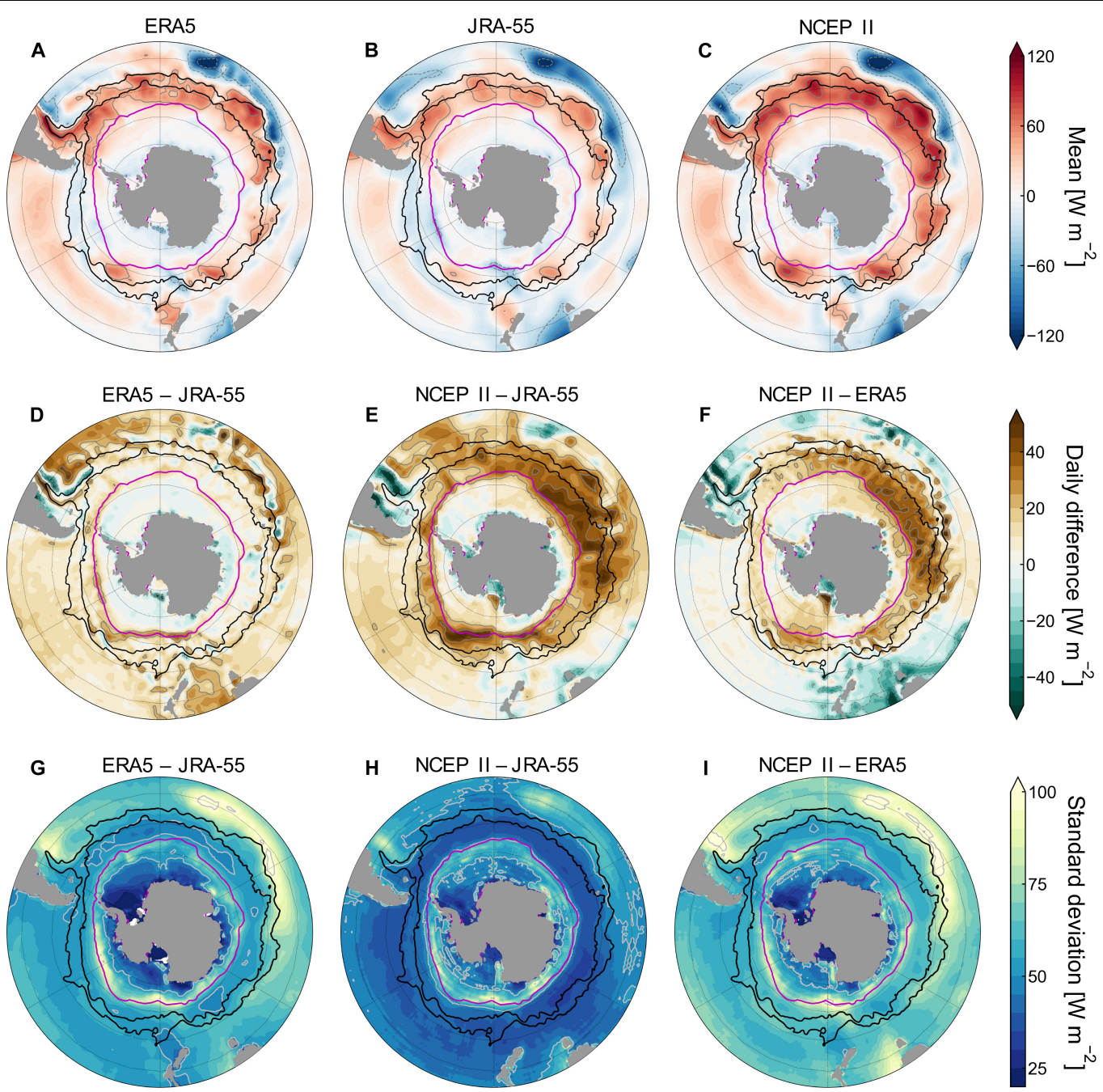

FIGURE 2 | (A-C) The 10-year (2008-2017) mean net heat flux $\left(\mathrm{W} \mathrm{m}^{-2}\right.$ ) for three reanalysis products: ERA5, JRA-55 and NCEP-II. (D-F) The 10-year mean difference in daily net heat flux (in $\mathrm{W} \mathrm{m}^{-2}$ ) between the products. (G-I) Standard deviation of the daily differences (in $\mathrm{W} \mathrm{m}^{-2}$ ) shown in panel (D-F). Positive (negative) values denote a heat flux into (out of) the ocean. Magenta line denotes the 10-year mean of the 15\% sea ice concentration from NCEP/DOE AMIP-II Reanalysis representing the maximum sea-ice edge. Gray lines contour the mean (-120 to $120 \mathrm{~W} \mathrm{~m}^{-2}$ intervals of $\left.40 \mathrm{~W} \mathrm{~m}^{-2}\right)$, the daily difference $\left(-40\right.$ to $40 \mathrm{~W} \mathrm{~m}^{-2}$ intervals of $20 \mathrm{~W} \mathrm{~m}^{-2}$ ), and standard deviation (50 and $100 \mathrm{~W} \mathrm{~m}^{-2}$ ). Solid (dashed) contours represent positive (negative) values. Black contours indicate the 10-year mean position of the Sub-Antarctic Front (north) and Antarctic Polar Front (south) from the AVISO Absolute Dynamic Topography (as in Swart et al., 2010).

uncertainty in fluxes and their parameterizations, is feasible. Statistical significance of observed trends will improve as fixedpoint time series are sustained. Constraining the marginal ice zone (MIZ), however, remains a priority (Figures 1A,F).

\section{STATUS OF FLUX REANALYSES AND REQUIREMENTS TO REDUCE UNCERTAINTY}

Gridded satellite and reanalysis datasets in the SO suffer from a large inter-product spread in estimates of the net air-sea heat flux (e.g., Liu et al., 2011), stemming from insufficient observations, particularly in winter (Figures 1A-E), and the need to determine the net exchange as the sum of four individual components (latent - Figures 1E,J, sensible, shortwave, longwave; Bentamy et al., 2017). Evaluation of the spread in net heat flux between atmospheric reanalysis products has revealed regional differences up to $60 \mathrm{Wm}^{-2}$ (Liu et al., 2011). These differences persist when zonally averaged and show shipbased and reanalysis products differing by $30-40 \mathrm{Wm}^{-2}$ at a given latitude (e.g., Josey et al., 2013).

To further demonstrate first-order uncertainties around SO air-sea fluxes, a comparison is made between three commonly used reanalysis products of net heat flux (NCEP-II, ERA5, and JRA-55; Figure 2). Large mean state (10 year) differences (up to $50 \mathrm{~W} \mathrm{~m}^{-2}$; Figures $2 \mathrm{~A}-\mathbf{C}$ ) and mean daily differences (reaching up to $40 \mathrm{~W} \mathrm{~m}^{-2}$; Figures 2D-F) exist between products for large swaths of the SO (e.g., in Figure 2E: mean daily differences $>20 \mathrm{~W} \mathrm{~m}^{-2}$ for $35 \%$ of area south of $35^{\circ} \mathrm{S}$ ), 
particularly in the NCEP-II comparisons. These differences are highest in the central Antarctic Circumpolar Current (ACC) domains, at the boundaries to strong subtropical originating currents (Malvinas Confluence and Agulhas Return Current), and around the southern ACC to the edge of the winter sea-ice extent (especially in the Pacific and Indian sectors). The standard deviation of these daily differences (Figures 2G-I) emphasizes the larger range of differences (reaching $>100 \mathrm{~W} \mathrm{~m}^{-2}$ ) found in both the northern ACC domain and at the winter sea-ice edge and extending into the MIZ. The larger uncertainty associated with the edge or within sea-ice regions suggests complexities in constraining fluxes impacted by ice-ocean processes and/or the limitation of satellites/reanalysis products to estimate fluxes in ice-impacted domains (Yu et al., 2017). Strong ocean gradients at the boundary to the subtropical gyres could be leading to enhanced heat flux differences (Figures 2D-I; see section "Ocean Impact on Fluxes") and these regions should be targeted for enhanced observational coverage to reduce uncertainty (see section "Specific Recommendations for the Coming Decade").

The momentum (wind stress) flux is better determined in a climatological sense than the heat flux, as it is a function of fewer, more easily determined variables that are reasonably estimated from satellite observations. However, significant wind speed differences still exist between gridded products and in situ data (Schmidt et al., 2017) with biases of up to $7.5 \mathrm{~m} \mathrm{~s}^{-1}$ depending on the wind speed magnitude (higher wind speeds are subject to considerably higher errors). Consequently, there is only medium confidence that SO wind-stress forcing has strengthened on decadal timescales (Swart and Fyfe, 2012; Rhein et al., 2013) as is expected from coupled model-based studies (Russell et al., 2018).

Given the uncertainties noted above, improvement in SO heat and momentum flux accuracies is vital. This has long been recognized (e.g., OceanObs' 99 and '09 - Fairall et al., 2010) and the deployment of two surface flux moorings ${ }^{1}$ is progressing towards this goal. The time-series are currently insufficient to evaluate whether reanalysis products accurately represent interannual and decadal variability, thus sustaining these timeseries is key to reducing these uncertainties. Recently, analysis of ship data across the open ocean to the MIZ has enabled better understanding of air-sea interaction in this key transition region (Yu et al., 2017), although winter measurements remain lacking (Figure 1).

\section{OCEAN IMPACT ON FLUXES}

The exchange of tracers and energy across the air-sea interface is modulated by a complex upper-ocean "mixed layer" structure (Kilbourne and Girton, 2015). Instruments that quantify upperocean turbulence (e.g., Air-Sea Interaction Profiler; Ward et al., 2014) provide direct estimates of the mixing and mixed-layer depths from measurements of the dissipation rate of turbulent kinetic energy $(\varepsilon)$ and density $(\rho)$, respectively. Combined

\footnotetext{
${ }^{1}$ Integrated Marine Observing System (IMOS) SO Flux Station (SOFS) south of Tasmania (Schulz et al., 2012) and the Ocean Observatories Initiative (OOI) mooring upstream of Drake Passage at $55^{\circ} \mathrm{S}, 90^{\circ} \mathrm{W}$ (Ogle et al., 2018).
}

with velocity scales associated with wind, wave, and buoyancy (e.g., after Belcher et al., 2012), improved parameterizations of mixing in the SO can be determined. It is anticipated turbulence from wave breaking and Langmuir circulation will play a dominant role in the turbulence generation and scaling (Esters et al., 2018). As ocean turbulence is a major driver for air-sea fluxes, such studies will lead to improved parameterizations in the SO.

Additionally, small-scale turbulence in the mixed-layer is influenced by oceanic motions spanning a few hundred meters (submesoscale) to hundreds of kilometers (mesoscale). For example, Ardhuin et al. (2017) show a strong covariance of mesoscale currents and surface-wave energy, especially in the Drake Passage. This implies a change in mesoscale surface roughness and thus effective surface-ocean stress (Small et al., 2008; O'Neill et al., 2012). This dependence impacts gas exchange via surface wave breaking (Brumer et al., 2017), although the magnitude remains to be directly measured. Furthermore, remote-sensing products indicate up to $20 \%$ of South Atlantic turbulent heat flux variance is explained by mesoscale eddies (Villas Bôas et al., 2015; Figure 2). Autonomous surface vehicles (ASVs) are key for augmenting in situ measurements of surface ocean variability, which is dominated by smallscale, submesoscale gradients (Thomson and Girton, 2017). Strong coupling exists between both waves and surface currents as well as between oceanic and atmospheric temperatures (Villas Bôas et al., 2015). This supports earlier work showing that fronts and eddies (and associated SST gradients) can substantially impact the air-sea exchange and near-surface atmospheric conditions in vigorous SO boundary-current regions (Pezzi et al., 2005, 2009; Messager et al., 2012; de Camargo et al., 2013; Rouault et al., 2016).

Submesoscale $(0.1-20 \mathrm{~km})$ lateral density gradients in the upper ocean can also significantly modulate the depth of the mixed-layer on sub-seasonal time scales (Rosso et al., 2015; du Plessis et al., 2017, 2019), with implications for heat and carbon uptake, water mass modification due to surface buoyancy fluxes, and the timing of biological processes (Swart et al., 2015). The intensity of these features is strongly localized by the circulation of the ACC and its interaction with topographic features (Viglione et al., 2018). The MIZ may also be a site of enhanced vertical velocities due to the coupling between thin sea ice and near-surface oceanic flows (Manucharyan and Thompson, 2017). This can lead to enhanced lateral and vertical heat fluxes toward sea-ice, as well as modifying air-sea fluxes through, for example, changes in sea-ice concentration and floe size distributions (Horvat et al., 2016).

\section{OBSERVING FLUXES THROUGH ICE}

Sea-ice presence and concentration dramatically affect physical, gas, and aerosol fluxes (Bourassa et al., 2013), which in turn, provide a significant feedback to sea-ice state, extent, and thickness (e.g., Perovich et al., 2008). As sea-ice parameters change in a warming climate, a significant adjustment in the balance of the high-latitudes air-sea-ice fluxes is likely. 
Ice-covered regions of the SO are acutely under sampled, especially in autumn and winter - with the hypothesis that open water in autumn before freeze-up is exposed to enhanced wind stress and mixing, amplifying air-sea exchange. Fluxes within open waters surrounded by sea ice (MIZ, leads and polynyas) can be estimated by classical approaches, but must account for (i) logistical constraints and (ii) ice-specific processes, such as reduced fetch area, wave attenuation and scattering, shear in the ice-ocean boundary layer, buoyant convection or stratification, short period gravity waves, and ice-flow interactions.

Air-sea-ice fluxes are 'in this sense' dramatically different from air-sea fluxes and often require separate methodology. For instance, heat flux budgets require assessment of sea-ice growth using ice mass-balance buoys, which provide spatially restricted point-measurements and require consolidated ice for deployment (i.e., not on unconsolidated ice formed in polynyas). While sea-ice processes drive gas fluxes at the air-sea-ice interface, assessment of these fluxes must take into account the unique gas dynamics and transport within sea ice, which are derived from techniques used for terrestrial fluxes. In addition, snow load and structure affect energy and gas transfer in different ways and are poorly constrained.

Finally, budgeting fluxes in ice-covered regions requires understanding of spatial and temporal variability of the sea-ice cover (Schröder et al., 2003; Matear et al., 2015), for which remote sensing is critical. However, deriving sea-ice volume, growth, type, temperature, snow load and structure, and momentum absorption from remote sensing is challenging. These properties are not measured directly and require models that are prone to errors. Further field process studies (see section "Specific Recommendations for the Coming Decade") are needed in sea ice to constrain these uncertainties so as to improve the accuracy of fluxes that are reliant on satellite-derived information about sea ice characteristics.

\section{TECHNOLOGICAL REQUIREMENTS FOR THE COMING DECADE}

SO flux observations are almost exclusively collected by ships, with valuable time-series observations provided by two moored surface buoys (Figure 1). Expansion of these observing systems are necessary (see recommendation 1 below). Yet, ships suffer superstructure flow distortion effects on measurement accuracy (Yelland et al., 2002) and collect data for relatively short periods (days to weeks), while moorings provide point-location estimates and have significant maintenance costs. Detailed flow distortion calculations for research vessels operating in the SO would help improve the analysis of ship-based flux observations (e.g., Moat et al., 2006). Studies of the flow distortion around the towers of surface moorings shows minimal impact on wind velocity, although buoy position should be tracked to allow the wind observations relative to the buoy to be corrected to absolute wind velocity. More significant challenges involve coping with icing, surface waves and sea birds and so improvements to current surface buoy designs are recommended. These include phasing-in heating of key sensors to prevent ice and frost build up, including anemometers and radiometers, adding cameras to document icing (being implemented on Irminger Sea Buoy) and actively ventilating air temperature and humidity sensor enclosures. In addition, incorporation of turbulent flux sensors (3-D sonic anemometers, fast response humidity, and temperature) and platform attitude and motion sensors can be done now to allow computation of the turbulent fluxes by the Direct Covariance Flux methods (Edson et al., 1998).

Recent advances in relatively cheap, small (2-7 m) and robust ASV with associated miniaturized sensors, improved battery capacity and energy harvesting, are demonstrating the capability to collect multi-month time-series of high-resolution air-sea flux and meteorology observations. The latest generation of ASV, such as Wave Gliders and Saildrone, have been deployed successfully in the SO (Monteiro et al., 2015; Schmidt et al., 2017; Thomson and Girton, 2017). Key technical issues for ASV flux measurements center on the low height of the atmospheric measurements and the effect of platforms tilts on observations, such as incoming shortwave and longwave radiation. Bulk algorithms for estimating fluxes rely on the gradients beyond the ocean-atmosphere interface, which increase (and therefore are more reliably measured) with height. Very close to the surface, these measurements may have an insufficient signal-to-noise ratio. Further, measurements within the wave-affected portion of the atmospheric boundary layer may not be appropriate for comparison to the larger scale fluxes used in circulation models (e.g., Schmidt et al., 2017). These issues require careful calibration and validation over the full dynamic range of conditions.

The question now becomes how best to coordinate the distribution of these assets to ensure collection of high-quality data for end users, including agreement on data standards and management. To date, ASV deployments have been shortterm project-based missions. A sustained and coordinated effort to deploy multiple ASVs (and Lagrangian platforms SWIFT/drifting buoys) in the SO, potentially centered around key mooring sites, will require international resources and effort (see Newman et al., 2019). This type of system will address the data gaps between sparse vessel-based observations and achieve year-round presence in harsh/remote regions. In order to achieve this vision, recommended enhancements include; extending deployment duration (harness alternate forms of energy from current or wind; improved battery capability), autonomous piloting techniques (on-board route decision making), ocean/atmosphere feature tracking and automated coordination between platforms, and upper ocean profiling (e.g., automated hydrographic casts). Additionally, year-round flux observations in the seasonal ice-covered regions of the SO are required. Given that current ASV technology is limited to the ice-free ocean, new technologies need to be developed for the unique characteristics of Antarctic sea ice, which largely melts and reforms on an annual basis thereby not allowing for multiseason installations of platforms that are proven capabilities in the Arctic (e.g., Ice Tethered Profilers, Arctic Ocean Flux Buoys, and meteorological stations). One approach could be surface floating platforms that are able to withstand being frozen into the ice pack and then continue to operate once the sea ice melts and 
releases the platform into the ocean until the next freezing period (e.g., UpTempO buoys).

Unmanned aerial vehicles (UAV) are a new platform supplementing flux sensing capabilities from existing platforms, with duration of fixed-wing missions of up to $24 \mathrm{~h}$. UAVs, like satellites and aircraft, can provide a synoptic view of a relatively large area, while providing unprecedented ground resolutions (as fine as $1 \mathrm{~cm}$ ) due to their slower speed, making for an exciting approach for process studies in particular. Significant progress has been made in developing cutting-edge UAV payloads for air-sea turbulent fluxes, radiative fluxes, surface albedo, SST variability, ocean surface waves, and ocean biogeochemistry (e.g., Reineman et al., 2016). Applications for UAVs in air-sea interaction have been demonstrated in polar seas, such as from polynyas (Cassano et al., 2010) and the MIZ.

\section{SPECIFIC RECOMMENDATIONS FOR THE COMING DECADE}

\section{Targeted Field Observations}

Enhanced flux observations are required almost everywhere (Figure 1), however, we particularly require them in (i) varying conditions (e.g., transient events/storms, different types of seaice, and sea state); (ii) regions with higher uncertainty and variability in reanalyses (marked on Figure 2, see section "Status of Flux Reanalyses and Requirements to Reduce Uncertainty"), which are predominantly in the boundary regions of the SO (e.g., Agulhas Return Current, Malvinas Current region), regions prone to higher energetics/eddy kinetic energy (e.g., downstream of Campbell Plateau, Drake Passage), and in the seasonal ice zone, especially in the region of the winter maximum sea ice extent. Further, SOFLUX recommends efforts towards achieving year-round time-series (i.e., mooring sites supplemented with new technologies, such as ASVs that can provide a spatial length-scale component to the time-series observations). Seasonally, the autumn and winter are priority periods to obtain even the most basic flux-related observations. Given that almost no flux-related observations exist in the seasonal and permanent sea-ice covered regions (approximately the entire SO south of $60^{\circ} \mathrm{S}$ ), the community should see this as one of the highest priorities with potentially the most important priority being areas prone to abrupt flux events driven by spurious gaps in the sea ice (from coastal and open ocean polynyas down to the scale of leads in the pack ice). The SOCCOM float array is starting to address this, especially in the seasonal ice zone, but higher "carbon capable" $(\mathrm{pH})$ float density is needed (further guidelines for density in Mazloff et al., 2018). OSSEs are valuable for optimizing the quantity and locations of time-series measurements, and flow distortion studies would enable better interpretation of vessel flux observations.

\section{Dedicated Studies to Understand Air-Sea-Ice Flux Processes}

Process studies are critical to improve flux and model parameterizations. Experiments from both poles would deliver process information on fluxes under various conditions. Studies should leverage existing flux time-series provided by mooring sites to enhance understanding of annual and inter-annual flux variability. More specifically, observations able to resolve intraseasonal processes and variability that impact fluxes are needed, such as those associated with oceanic eddies and fronts or the passage of atmospheric storms. This would provide insight into the temporal-spatial scales over which the atmosphere and ocean respond to changes imposed by the other. This justifies targeted process studies aimed at measuring flux differences over the scale of the first baroclinic Rossby radius (50 km or less) and over strong oceanic (temperature) gradients. Further detail can also be found in Newman et al. (2019).

\section{Improve Satellite Flux Capabilities}

Satellite retrievals of air-sea-ice fluxes pose a series of challenges, many of which are addressed by Bourassa et al. (2013). The challenges vary depending on the flux: (1) Momentum fluxes are well measured by scatterometers, but the global network of scatterometers does not provide the spatio-temporal coverage that is desired for tracking rapidly evolving and otherwise poorly sampled SO storms. The proposed SKIM (waves and currents) and $\mathrm{WaCM}$ (winds and currents) missions would be particularly valuable for assessing the direct transfer of momentum from atmosphere to wave and current fields. (2) Turbulent heat fluxes are typically inferred using bulk formulae from ship or buoy measurements. Satellites do not provide measurements of temperature and humidity in the planetary boundary layer that are necessary to apply bulk formulae, but they can measure properties of the ocean surface. Therefore, further work to develop reliable retrievals of turbulent heat fluxes would be valuable, particularly for the high wind, high sea spray conditions typical of the SO. The small Rossby radius in the SO means narrow frontal features (ACC fronts and eddies) have the potential to influence fluxes significantly, but are not necessarily well resolved in existing retrievals - high spatial resolution would be of value. (3) Freshwater fluxes depend on precipitation and evaporation. The Global Precipitation Mission estimates precipitation, but only in the latitude range from $65^{\circ} \mathrm{S}$ to $65^{\circ} \mathrm{N}$ (missing snow and rain within the Antarctic circle). Future satellite missions providing full coverage of the high latitudes would be valuable towards constraining air-sea-ice freshwater fluxes.

\section{Accessibility of Data}

Flux and meteorological data must be made more accessible, with uniformity of data processing and quality control. Polarspecific data discovery may be a way to address this (e.g., through SOOSmap). Likewise, the efforts of the Shipboard Automated Meteorological and Oceanographic System (SAMOS), which collects flux-related data from vessels and provides data collection standardization information and targets, needs to be supported/expanded (see Smith et al., 2015, 2019). Furthermore, increased field and vessels access to conduct flux measurements (e.g., available ship berth space or sensor installations) should be encouraged. The SOOS tool "DueSouth: Database of Upcoming Expeditions to the SO" provides support towards this end. 


\section{AUTHOR CONTRIBUTIONS}

SS and SG conceptualized the manuscript with inputs from the SOFLUX working group and wrote the first draft of the manuscript. AT, BdS, JT, SJ, MM, and BW led the sections of the manuscript. SS, EK, MdP, and MM conceptualized and developed the figures. All authors contributed to the writing of the manuscript and read and approved its final version.

\section{FUNDING}

SS was funded by a Wallenberg Academy Fellowship (WAF 2015.0186). EK was funded by the NERC ORCHESTRA Project (NE/N018095/1). LP was funded by the Advanced Studies in Oceanography of Medium and High Latitudes (CAPES 23038.004304/2014-28) and the Research Productivity Program (CNPq 304009/2016-4). BdS was a research associate at the F.R.S-FNRS. PeH was supported by the Australian Antarctic Science Projects 4301 and 4390, and the Australian Government's Cooperative Research Centres Programme through the Antarctic Climate and Ecosystems Cooperative Research Centre and the International Space Science Institute Project 406. SG and

\section{REFERENCES}

Andrews, T., Gregory, J. M., and Webb, M. J. (2015). The dependence of radiative forcing and feedback on evolving patterns of surface temperature change in climate models. J. Clim. 28, 1630-1648. doi: 10.1175/jcli-d-14-00545.1

Ardhuin, F., Gille, S. T., Menemenlis, D., Rocha, C. B., Rascle, N., Chapron, B., et al. (2017). Small-scale open ocean currents have large effects on wind wave heights. J. Geophys. Res. Oceans 122, 4500-4517. doi: 10.1002/2016JC012413

Belcher, S. E., Grant, A. L. M., Hanley, K. E., Fox-Kemper, B., Van Roekel, L., Sullivan, P. P., et al. (2012). A global perspective on Langmuir turbulence in the ocean surface boundary layer. Geophys. Res. Lett. 39:L18605. doi: 10.1029/ 2012GL052932

Bentamy, A., Piollé, J. F., Grouazel, A., Danielson, R., Gulev, S., and Paul, F. (2017). Review and assessment of latent and sensible heat flux accuracy over the global oceans. Remote Sens. Environ. 201, 196-218. doi: 10.1016/j.rse.2017.08.016

Bodas-Salcedo, A., Hill, P. G., Furtado, K., Williams, K. D., Field, P. R., Manners, J. C., et al. (2016). Large contribution of supercooled liquid clouds to the solar radiation budget of the Southern Ocean. J. Clim. 29, 4213-4228. doi: 10.1175/ jcli-d-15-0564.1

Bodas-Salcedo, A., Williams, K. D., Ringer, M. A., Beau, I., Cole, J. N. S., Dufresne, J. L., et al. (2014). Origins of the solar radiation biases over the Southern Ocean in CFMIP2 models. J. Clim. 27, 41-56. doi: 10.1175/jcli-d-13-00169.1

Bourassa, M. A., Gille, S. T., Bitz, C., Carlson, D., Clayson, C. A., and Cerovecki, I. (2013). High-latitude ocean and sea ice surface fluxes: challenges for climate research. Bull. Am. Met. Soc. 94, 403-423. doi: 10.1175/bams-d-1100244.1

Brumer, S. E., Zappa, C. J., Blomquist, B. W., Fairall, C. W., Cifuentes-Lorenzen, A., Edson, J. B., et al. (2017). Wave-related reynolds number parameterizations of CO2 and DMS transfer velocities. Geophys. Res. Lett. 44, 9865-9875. doi: 10.1002/2017GL074979

Cassano, J. J., Maslanik, J. A., Zappa, C. J., Gordon, A. L., Cullather, R. I., and Knuth, S. L. (2010). Observations of antarctic polynya with unmanned aircraft systems. Eos Trans. Am. Geophys. Union 91, 245-246. doi: 10.1029/ 2010EO280001

Chelton, D. B., deSzoeke, R. A., Schlax, M. G., El Naggar, K., and Siwertz, N. (1998), Geographical variability of the first baroclinic rossby radius of deformation. J. Phys. Oceanogr. 28, 433-460. doi: 10.1175/1520-04851998028<0433: GVOTFB $<2.0 . \mathrm{CO} ; 2$
MM were funded by National Science Foundation awards OCE-1658001 and PLR-1425989. AT was supported by NASA (NNX15AG42G) and NSF (OCE-1756956).

\section{ACKNOWLEDGMENTS}

This manuscript is a contribution to the Southern Ocean Air-Sea Flux (SOFLUX) capability working group of the SCAR-SCOR Southern Ocean Observing System (SOOS). SOFLUX thanks the SCAR and SCOR for financial support in order to host meetings and support travel. The ICOADS data was acquired at the National Center for Atmospheric Research, Computational and Information Systems Laboratory, Boulder, CO, United States (available online at https://doi.org/10.5065/D6ZS2TR3). The three satellite-reanalysis products used include: (1) NCEP_ Reanalysis 2 data provided by the NOAA/OAR/ESRL PSD, Boulder, CO, United States, available at www.esrl.noaa. gov/psd/; (2) ERA5 is generated using Copernicus Climate Change Service Information, available at www.ecmwf.int/en/ forecasts/datasets/archive-datasets/reanalysis-datasets/era5; and (3) JRA-55: Japanese 55-year Reanalysis, available at: https: //doi.org/10.5065/D6HH6H41.

Cronin, M., Gentemann, C. L., Edson, J., Ueki, I., Bourassa, M., Brown, S., et al. (2019). Air-sea fluxes with a focus on heat and momentum. Front. Mar. Sci. doi: 10.3389/fmars.2019.00430

de Camargo, R., Todesco, E., Pezzi, L. P., and de Souza, R. B. (2013). Modulation mechanisms of marine atmospheric boundary layer at the Brazil-Malvinas confluence region. J. Geophys. Res. Atmos 118, 6266-6280. doi: 10.1002/jgrd. 50492

Dong, S., Gille, S. T., and Sprintall, J. (2007). An assessment of the Southern Ocean mixed layer heat budget. J. Clim. 20, 4425-4442. doi: 10.1175/JCLI4259.1

du Plessis, M., Swart, S., Ansorge, I. J., and Mahadevan, A. (2017). Submesoscale processes promote seasonal restratification in the Subantarctic Ocean. J. Geophys. Res. 122, 2960-2975. doi: 10.1002/2016jc012494

du Plessis, M. D., Swart, S., Ansorge, I. J., Mahadevan, A., and Thompson, A. F. (2019). Southern Ocean seasonal restratification delayed by submesoscale windfront interactions. J. Phys. Ocean. 49, 1035-1053. doi: 10.1175/JPO-D-180136.1

Edson, J. B., Hinton, A. A., Prada, K. E., Hare, J. E., and Fairall, C. W. (1998). Direct covariance flux estimates from mobile platforms at sea. J. Atmos. Ocean. Technol. 15, 547-562. doi: 10.1175/1520-0426015<0547:DCFEFM<2.0.CO;2

Esters, L., Breivik, Ø, Landwehr, S., ten Doeschate, A., Sutherland, G., Christensen, K. H., et al. (2018). Turbulence scaling comparisons in the ocean surface boundary layer. J. Geophys. Res. 123, 2172-2191. doi: 10.1002/2017JC013525

Fairall, C. W., Barnier, B., Berry, D. I., Bourassa, M. A., Bradley, E. F., and Clayson, C. A. (2010). "Observations to quantify air-sea fluxes and their role in climate variability and predictability," in Proceedings of OceanObs'09: Sustained Ocean Observations and Information for Society, 2, eds J. Hall, D. E. Harrison, and D. Stammer (Paris: European Space Agency), 299-313.

Freeman, E., Woodruff, S. D., Worley, S. J., Lubker, S. J., Kent, E. C., Angel, W. E., et al. (2017). ICOADS release 3.0: a major update to the historical marine climate record. Int. J. Climatol. 37, 2211-2232. doi: 10.1002/joc.4775

Frölicher, T. L., Sarmiento, J. L., Paynter, D. J., Dunne, J. P., Krasting, J. P., Winton, M., et al. (2015). Dominance of the Southern Ocean in anthropogenic carbon and heat uptake in CMIP5 models. J. Clim. 28, 862-886. doi: 10.1175/jcli-d14-00117.1

Gille, S., Josey, S., and Swart, S. (2016). New approaches for air-sea fluxes in the Southern Ocean. Eos 97. doi: 10.1029/2016EO052243

Hermanson, L., Ren, H. L., Vellinga, M., Dunstone, N. D., Hyder, P., Ineson, S., et al. (2018). Different types of drifts in two seasonal forecast systems and their 
dependence on ENSO. Clim. Dyn. 51, 1411-1426. doi: 10.1007/s00382-0173962-9

Horvat, C., Tziperman, E., and Campin, J. M. (2016). Interaction of sea ice floe size, ocean eddies, and sea ice melting. Geophys. Res. Lett. 43, 8083-8090. doi: $10.1002 / 2016$ gl069742

Hyder, P., Edwards, J. M., Allan, R. P., Hewitt, H. T., Bracegirdle, T. J., Gregory, J. M., et al. (2018). Critical Southern Ocean climate model biases traced to atmospheric model cloud errors. Nat. Commun. 9:3625. doi: 10.1038/s41467018-05634-2

Josey, S. A., Gulev, S., and Yu, L. (2013). "Exchanges through the ocean surface," in Ocean Circulation and Climate: A 21st Century Perspective, eds G. Siedler, J. Church, W. J. Gould, and S. Griffies (Cambridge, MA: Academic Press), 115-140. doi: 10.1016/b978-0-12-391851-2.00005-2

Kamenkovich, I., Haza, A., Gray, A. R., Dufour, C. O., and Garraffo, Z. (2017). Observing system simulation experiments for an array of autonomous biogeochemical profiling floats in the Southern Ocean. J. Geophys. Res. Oceans 122, 7595-7611. doi: 10.1002/2017JC012819

Kilbourne, B. F., and Girton, J. B. (2015). Surface boundary layer evolution and near-inertial wind power input. J. Geophys. Res. 120:75067520.

Lenton, A., Matear, R. J., and Tilbrook, B. (2006). Design of an observational strategy for quantifying the Southern Ocean uptake of CO2. Glob. Biogeochem. Cycl. 20:GB4010.

Liu, J., Tingyin, X., and Chen, L. (2011). Intercomparisons of air-sea heat fluxes over the Southern Ocean. J. Clim. 24, 1198-1211. doi: 10.1175/2010jcli 3699.1

Majkut, J. D., Carter, B. R., Frölicher, T. L., Dufour, C. O., Rodgers, K. B., and Sarmiento, J. L. (2014). An observing system simulation for Southern Ocean carbon dioxide uptake. Philos. Trans. R. Soc. 372:20130046. doi: 10.1098/rsta. 2013.0046

Manucharyan, G. E., and Thompson, A. F. (2017). Submesoscale sea ice-ocean interactions in marginal ice zones. J. Geophys. Res 122, 9455-9475. doi: 10.1002/ $2017 \mathrm{jc} 012895$

Matear, R. J., O’Kane, T. J., Risbey, J. S., and Chamberlain, M. (2015). Sources of heterogeneous variability and trends in Antarctic sea-ice. Nat. Commun. 6:8656. doi: $10.1038 /$ ncomms 9656

Mazloff, M. R., Cornuelle, B. D., Gille, S. T., and Verdy, A. (2018). Correlation lengths for estimating the large-scale carbon and heat content of the Southern Ocean. J. Geophys. Res. Oceans 123, 883-901. doi: 10.1002/2017jc 013408

Messager, C., Speich, S., and Key, E. (2012). Marine atmospheric boundary layer over some Southern Ocean fronts during the IPY BGH cruise. Ocean Sci. 8, 1001-1023. doi: 10.5194/os-8-1001-2012

Moat, B. I., Yelland, M. J., Pascal, R. W., and Molland, A. F. (2006). Quantifying the airflow distortion over merchant ships. Part I: validation of a CFD Model. J. Atmos. Ocean. Technol. 23, 341-350. doi: 10.1175/JTECH 1858.1

Monteiro, P. M. S., Gregor, L., Lévy, M., Maenner, S., Sabine, C. L., and Swart, S. (2015). Intraseasonal variability linked to sampling alias in air-sea CO2 fluxes in the Southern Ocean. Geophys. Res. Lett. 42, 8507-8514. doi: 10.1002/ 2015GL066009

Newman, L., Heil, P., Trebilco, R., Katsumata, K., Constable, A. J., van Wijk, E., et al. (2019). Delivering sustained, coordinated and integrated observations of the Southern Ocean for global impact. Front. Mar. Sci. doi: 10.3389/fmars.2019. 00433

Ogle, S. E., Tamsitt, V., Josey, S. A., Gille, S. T., Ceroveèki, I., Talley, L. D., et al. (2018). Episodic Southern Ocean heat loss and its mixed layer impacts revealed by the furthest south multi-year surface flux mooring. Geophys. Res. Lett. 45, 5002-5010. doi: 10.1029/2017gl076909

O'Neill, L. W., Chelton, D. B., and Esbensen, S. K. (2012). Covariability of surface wind and stress responses to sea surface temperature fronts. J. Clim. 25, 5916-5942. doi: 10.1175/jcli-d-11-00230.1

Perovich, D. K., Richter-Menge, J. A., Jones, K. F., and Light, B. (2008). Sunlight, water, and ice: extreme Arctic sea ice melt during the summer of 2007. Geophys. Res. Lett. 35:L11501.

Pezzi, L. P., Souza, R. B., Acevedo, O., Wainer, I., Mata, M. M., Garcia, C. A. E., et al. (2009). Multiyear measurements of the oceanic and atmospheric boundary layers at the Brazil-Malvinas confluence region. J. Geophys. Res. 114:D19103. doi: $10.1029 / 2008$ JD011379
Pezzi, L. P., Souza, R. B., Dourado, M. S., Garcia, C. A. E., Mata, M. M., and Silva-Dias, M. A. F. (2005). Ocean-atmosphere in situ observations at the Brazil-Malvinas Confluence region. Geophys. Res. Lett. 32:L22603.

Reineman, B. D., Luc, L., and Melville, W. K. (2016). The use of ship- launched fixed-wing UAVs for measuring the marine atmospheric boundary layer and ocean surface processes. J. Atmos. Ocean. Technol. 33, 2029-2052. doi: 10.1175/ jtech-d-15-0019.1

Rhein, M., Rintoul, S. R., Aoki, S., Campos, E., Chambers, D., Feely, R. A., et al. (eds) (2013). “Observations: ocean," in Climate Change 2013: The Physical Science Basis. Contribution of Working Group I to the Fifth Assessment Report of the Intergovernmental Panel on Climate Change, ed T. F. Stocker, (Cambridge: Cambridge University Press).

Rintoul, S. R. (2018). The global influence of localised dynamics in the Southern Ocean. Nature 558, 209-218. doi: 10.1038/s41586-018-0182-3

Rosso, I., McC. Hogg, A., Kiss, A. E., and Gayen, B. (2015). Topographic influence on submesoscale dynamics in the Southern Ocean. Geophys. Res. Lett. 42, 1139-1147. doi: 10.1002/2014gl062720

Rouault, M., Verley, P., and Backeberg, B. (2016). Wind changes above warm agulhas current eddies. Ocean Sci. 12, 495-506. doi: 10.5194/os-12-495-2016

Russell, J. L., Kamenkovich, I., Bitz, C., Ferrari, R., Gille, S. T., Goodman, P. J., et al. (2018). Metrics for the evaluation of the Southern Ocean in coupled climate models and earth system models. J. Geophys. Res. Oceans 123, 3120-3143. doi: 10.1002/2017JC013461

Sabine, C. L., Feely, R. A., Gruber, N., Key, R. M., Lee, K., Bullister, J. L., et al. (2004). The Oceanic Sink for Anthropogenic CO2. Science 305, 367-371. doi: 10.1126/science. 1097403

Schmidt, K. M., Swart, S., Reason, C., and Nicholson, S.-A. (2017). Evaluation of satellite and reanalysis wind products with in situ Wave Glider wind observations in the Southern Ocean. J. Atmos. Ocean. Tech 34. doi: 10.1175/ JTECH-D-17-0079.1

Schröder, D., Vihma, T., Kerber, A., and Brümmer, B. (2003). On the parameterization of turbulent surface fluxes over heterogeneous sea ice surfaces. J. Geophys. Res. 108:3195.

Schulz, E. W., Josey, S. A., and Verein, R. (2012). First air-sea flux mooring measurements in the Southern Ocean. Geophys. Res. Lett. 39:L16606. doi: 10.1029/2012GL052290

Small, R. J., DeSzoeke, S. P., Xie, S. P., O’Neill, L., Seo, H., and Song, Q. (2008). Airsea interaction over ocean fronts and eddies. Dyn. Atmos. Oceans 45, 274-319. doi: 10.1016/j.dynatmoce.2008.01.001

Smith, S., Gaël, A., Axel, A., William, A., Alex, B., David, I. B., et al. (2019). Shipbased contributions to global ocean, weather, and climate observing systems. Front. Mar. Sci. doi: 10.3389/fmars.2019.00434

Smith, S. R., Lopez, N., and Bourassa, M. A. (2015). Shipboard Automated Meteorological and Oceanographic System (SAMOS) Air-Sea Fluxes. Boulder, CO: National Center for Atmospheric Research, doi: 10.5065/D6930R70

Swart, N. C., and Fyfe, J. C. (2012). Observed and simulated changes in the Southern Hemisphere surface westerly wind-stress. Geophys. Res. Lett. 39:L16711. doi: 10.1029/2012GL052810

Swart, S., Speich, S., Ansorge, I. J., and Lutjeharms, J. R. E. (2010). An altimetrybased gravest empirical mode south of Africa: 1. Development and validation. J. Geophys. Res. 115:C03002. doi: 10.1029/2009JC005299

Swart, S., Thomalla, S. J., and Monteiro, P. M. S. (2015). The seasonal cycle of mixed layer dynamics and phytoplankton biomass in the Sub-Antarctic Zone: a highresolution glider experiment. J. Mar. Syst. 147, 103-115. doi: 10.1016/j.jmarsys. 2014.06.002

Thompson, D. W. J., Solomon, S., Kushner, P. J., England, M. H., Grise, K. M., Karoly, D. J., et al. (2011). Signatures of the Antarctic ozone hole in Southern Hemisphere surface climate change. Nat. Geosci. 4, 741-749. doi: 10.1038/ ngeo1296

Thomson, J., and Girton, J. (2017). Sustained measurements of Southern Ocean airsea coupling from a Wave Glider autonomous surface vehicle. Oceanography 30, 104-109. doi: 10.5670/oceanog.2017.228

Trenberth, K. E., and Fasullo, J. T. (2010). Simulation of present day and twenty first century energy budgets of the Southern Oceans. J. Clim. 23, 440-454. doi: 10.1175/2009jcli3152.1

Viglione, G. A., Thompson, A. F., Flexas, M. M., Sprintall, J., and Swart, S. (2018). Abrupt transitions in submesoscale structure in southern Drake Passage: glider 
observations and GCM results. J. Phys. Oceanogr. 48, 2011-2027. doi: 10.1175/ jpo-d-17-0192.1

Villas Bôas, A. B., Sato, O. T., Chaigneau, A., and Castelão, G. P. (2015). The signature of mesoscale eddies on the air-sea turbulent heat fluxes in the South Atlantic Ocean. Geophys. Res. Lett. 42, 1856-1862. doi: 10.1002/2015GL0 63105

Ward, B., Fristedt, T., Callaghan, A. H., Sutherland, G., Sanchez, X., Vialard, J., et al. (2014). The Air-Sea Interaction Profiler (ASIP): an autonomous upwardly-rising profiler for microstructure measurements in the upper ocean. J. Atmos. Ocean. Technol. 31, 2246-2267. doi: 10.1175/jtech-d-1400010.1

Yelland, M. J., Moat, B. I., Pascal, R. W., and Berry, D. I. (2002). CFD Model Estimates of the airflow distortion over research ships and the impact on momentum flux measurements. J. Atmos. Ocean. Technol. 19, 1477-1499. doi: 10.1175/1520-04262002019<1477:CMEOTA<2.0.CO;2

Yu, L., Jin, X., Schulz, E. W., and Josey, S. (2017). Air-sea regimes in the sub-Antarctic Southern Ocean and Antarctic marginal ice zone revealed by icebreaker measurements. J. Geophys. Res. Oceans 122, 6547-6564. doi: 10.1002/ 2016JC012281
Yu, L., and Weller, R. A. (2007). Objectively analyzed air-sea heat Fluxes (OAFlux) for the global ice-free oceans. Bull. Am. Meteorol. Soc. 88, 527-533. doi: 10.1175/ BAMS-88-4-527

Yuan, X., Patoux, J., and Li, C. (2009). Satellite-based midlatitude cyclone statistics over the Southern Ocean: 2. Tracks and surface fluxes. J. Geophys. Res. Atmos. 114:D04106. doi: 10.1029/2008JD010874

Conflict of Interest Statement: The authors declare that the research was conducted in the absence of any commercial or financial relationships that could be construed as a potential conflict of interest.

Copyright (c) 2019 Swart, Gille, Delille, Josey, Mazloff, Newman, Thompson, Thomson, Ward, du Plessis, Kent, Girton, Gregor, Heil, Hyder, Pezzi, de Souza, Tamsitt, Weller and Zappa. This is an open-access article distributed under the terms of the Creative Commons Attribution License (CC BY). The use, distribution or reproduction in other forums is permitted, provided the original author(s) and the copyright owner(s) are credited and that the original publication in this journal is cited, in accordance with accepted academic practice. No use, distribution or reproduction is permitted which does not comply with these terms. 


\section{OPEN ACCESS}

Edited by:

Carlos M. Duarte,

King Abdullah University of Science and Technology, Saudi Arabia

Reviewed by:

Kentaro Ando,

Japan Agency for Marine-Earth Science and Technology, Japan Martin Ostrowski, University of Technology Sydney,

Australia

${ }^{*}$ Correspondence:

Vladimir Ryabinin

v.ryabinin@unesco.org

Specialty section:

This article was submitted to

Ocean Observation,

a section of the journal

Frontiers in Marine Science

Received: 30 March 2019

Accepted: 12 July 2019

Published: 31 July 2019

Citation:

Ryabinin $V$, Barbière J, Haugan $P$, Kullenberg G, Smith N, McLean C, Troisi A, Fischer A, Aricò S, Aarup T,

Pissierssens $P$, Visbeck $M$,

Enevoldsen HO and Rigaud J (2019)

The UN Decade of Ocean Science

for Sustainable Development.

Front. Mar. Sci. 6:470.

doi: 10.3389/fmars.2019.00470

\section{The UN Decade of Ocean Science for Sustainable Development}

\author{
Vladimir Ryabinin ${ }^{1 *}$, Julian Barbière1, Peter Haugan², Gunnar Kullenberg1, \\ Neville Smith ${ }^{3}$, Craig McLean ${ }^{4}$, Ariel Troisi ${ }^{5}$, Albert Fischer ${ }^{1}$, Salvatore Aricò', \\ Thorkild Aarup ${ }^{1}$, Peter Pissierssens ${ }^{1}$, Martin Visbeck ${ }^{6}$, Henrik Oksfeldt Enevoldsen ${ }^{1}$ and \\ Julie Rigaud ${ }^{1}$ \\ ${ }^{1}$ Intergovernmental Oceanographic Commission of UNESCO, Paris, France, ${ }^{2}$ Geophysical Institute of the University of \\ Bergen and the Institute of Marine Research, Bergen, Norway, ${ }^{3}$ GODAE Ocean Services, Canterbury, NSW, Australia, \\ ${ }^{4}$ National Oceanic and Atmospheric Administration, Silver Spring, MD, United States, ${ }^{5}$ Servício de Hídrografia Naval, \\ Buenos Aires, Argentina, ${ }^{6}$ GEOMAR, Helmholtz-Zentrum für Ozeanforschung, Kiel Marine Biogeochemistry, Kiel, Germany
}

Our civilization needs a clean, resilient, productive, safe, well-observed, documented and predicted ocean. "The ocean we need for the future we want" was the motto of the Intergovernmental Oceanographic Commission proposal to the United Nations to consider the merit of an Ocean Science Decade. By proclaiming the Decade, the UN General Assembly offered the oceanographic community a unique, once in a life-time, opportunity to change the way we do things, make oceanography fit for purpose of effectively supporting sustainable development, and energize the ocean sciences for future generations. The Decade is the chance to put in place a more complete and sustainable observing system and feed the resulting data into a science-based informed decision-making system allowing increased reliance of our civilization on the ocean, its ecosystem services and, at the same time, preserving ocean health. Strong and proactive engagement of the oceanographic community in the design of the Decade and its observing component and subsequent energetic implementation of the ideas are sought. Participants in OceanObs'19 are invited to consider the additional possibilities and requirements associated with the Decade in their contributions to and brainstorming at the Conference. It is essential to use collective wisdom of OceanObs'19 to help developing an ambitious and also realistic implementation plan for the Decade, with a strong observational component.

Keywords: ocean observing, science, observations, decade, sustainable development, societal, data management

\section{INTRODUCTION}

The Intergovernmental Oceanographic Commission (IOC) of UNESCO, a specialized organization of the United Nations system for ocean observations, data, services and related capacity development, proposed to the United Nations to proclaim in 2021-2030 an international Decade of Ocean Science for Sustainable Development. The idea of an ocean decade was directly linked to the 2030 Agenda adopted by the United Nations in 2015. The Decade can mobilize the ocean community behind the ideas of sustainable development and serve to focus the research and technological development in oceanography on existentially important issues of protection 
and sustainable use of the ocean. This will be a decisive contribution by the ocean community to the implementation of the Sustainable Development Goals (SDGs) - not only the $14^{\text {th }}$ ("Ocean") SDG, but many others as well. Ocean observations are at the heart of this initiative.

The IOC proposal to the United Nations was successful and the UN Decade of Ocean Science for Sustainable Development was proclaimed by the United Nations General Assembly to start on 1 January, 2021. This decision potentially opens a new era in oceanography, implying a major change in the way the ocean community works and how it is organized and supported, and how it can contribute to the future of our civilization.

This article presents motivations for the Decade, gives background information and describes goals, anticipated societal outcomes, main areas of research and development, and the approach to use them in achieving societal outcomes. The IOC must present an Implementation Plan for the Decade to the UN General Assembly of 2020. This work is in progress. The plan will be a living document and will be adjusted in the future to accommodate new needs and undertakings, and, importantly, partners. Engagement of the ocean community, including ocean observation specialists, in designing the Decade and its subsequent implementation is strongly encouraged.

\section{MOTIVATIONS FOR THE UN DECADE OF OCEAN SCIENCE FOR SUSTAINABLE DEVELOPMENT}

The ocean, the largest ecosystem on our planet, provides our civilization with a range of existentially important services (Stocker, 2015; Visbeck, 2018). To continue benefitting from them, a globally shared information and knowledge system is needed that would inform, on one side, actions for the restoration and maintenance of the ocean's health (Duarte et al., 2018), and on the other side, use of the ocean space and resources to achieve global sustainable development. At present, such a consolidated system is not in place. However, many elements that could be connected in a more synergistic way to build such a system either exist, are being designed, or being worked on. These seeds of growth reside in the ocean observing systems, research and development activities, advances in the domains of technology and oceanographic information services, scientific assessments, national and international legislation and policies, best practices, standards, capacities, and resources-including human and economic resources available to industries,-and the private sector.

There are numerous international agreements aimed at preserving the ocean, either in the form of treaties or frameworks, which have various degrees of being legally binding. The 2030 Agenda with its 17 Sustainable Development Goals (SDGs), including the SDG 14 on the Ocean and its 10 targets, was unanimously endorsed in 2015 by the UN General Assembly ${ }^{1}$. The Paris Agreement of 2015 under the UN Framework

${ }^{1}$ https://www.un.org/sustainabledevelopment/blog/2015/09/historic-newsustainable-development-agenda-unanimously-adopted-by-193-un-members
Convention on Climate Change refers to "the importance of ensuring the integrity of all ecosystems, including the oceans. $^{2 "}$ The Sendai Framework for Disaster Risk Reduction of 2015 intends to address, inter alia, the risks to countries' coastlines. The UN Convention on Biological Diversity of 1992, with its Protocols and Implementation Plan components ${ }^{3}-$ such as Aichi Targets-refers to the need to manage marine and other aquatic ecosystems scientifically. The 1995 Code of Conduct for Responsible Fisheries ${ }^{4}$ and the Agreement on Port State Measures to Prevent, Deter and Eliminate Illegal, Unreported and Unregulated Fishing of $2009^{5}$ are key global instruments under the UN Food and Agricultural Organization to make fisheries sustainable. The Regional Seas Conventions of the UN Environment Programme and regional fisheries management organizations provide regional framework for conserving the ocean health and its living marine resources. The International Maritime Organization monitors the implementation the UN Convention for Safety of Life at Sea (SOLAS Convention of 1974) ${ }^{6}$ and a series of international conventions against marine pollution. The UN Convention on the Law of the Sea (UNCLOS) of 1982 http://www.un.org/Depts/ los/convention_agreements/texts/unclos/unclos_e.pdf provides overall regulatory framework for the conservation and sustainable use of ocean and its resources.

An United Nations Intergovernmental Conference is being held in the years 2019-2020 to agree an international legally binding instrument under UNCLOS on the conservation and sustainable use of marine biological diversity of areas beyond national jurisdiction. A number of important targets in relation to the sustainable use of the ocean are set in the Small Island Developing States (SIDS) Accelerated Modalities Of Action [S.A.M.O.A.] Pathway ${ }^{7}$, adopted at the Third International Conference on Small Island Developing States in Samoa in 2014. Since 1995, the Global Programme of Action for the Protection of the Marine Environment from Land-based Activities ${ }^{8}$ has been working to identify the sources of land-based pollution or harmful activities and prepare measures to reduce them. It is even more relevant now as we know about the huge amount of plastics transported to the ocean from the shore. Taken together, all of these and other frameworks provide building blocks for the potential ocean information and knowledge system aimed at supporting policies and actions for ocean health and sustainable use of the ocean.

At present, there is tremendous goodwill in the global community to protect the ocean. It strongly manifested itself at the first UN Conference to Support the Implementation of Sustainable Development Goal 14 that took place at UN

\footnotetext{
${ }^{2}$ https://unfccc.int/sites/default/files/english_paris_agreement.pdf

${ }^{3}$ https://www.cbd.int

${ }^{4}$ http://www.fao.org/3/a-v9878e.htm

${ }^{5}$ http://www.fao.org/fileadmin/user_upload/legal/docs/037t-e.pdf

${ }^{6} \mathrm{http} / /$ www.imo.org/en/about/conventions/listofconventions/pages/ international-convention-for-the-safety-of-life-at-sea-(solas),-1974.aspx

${ }^{7}$ http://www.sids2014.org/index.php?menu=1537

${ }^{8}$ https://www.unenvironment.org/nairobiconvention/unep-global-programmeaction-unepgpa
} 
Headquarters in June of 2017 (the Ocean Conference ${ }^{9}$ ). More than 1,500 various commitments to that effect were received by the organizing committee of the Conference. Nine Communities of Ocean Action are following up on the implementation of those commitments. Another venue for seeking commitments is the series of Our Ocean annual conferences organized by national governments after the founding conference in 2014 called by the U.S. Ex-Secretary of State John Kerry. New networks and groups have been and will be created to conserve ocean health and promote sustainable means of using ocean resources. Engagement of the private sector holds a major promise in that respect and is stepping up.

The mobilization of the communities is the result of growing awareness and concern about the decline in ocean health. According to the First World Ocean Assessment conducted by $\mathrm{UN}^{10}$, due to the impact of multiple negative stressors, our civilization is running out of time to start managing the ocean sustainably. Symptoms of the decline in ocean health include warming of the ocean water at all depths, including submarine heat waves (Stocker, 2015), ocean acidification and its multiple consequences (Hoegh-Guldberg et al., 2014), reduced generation or depletion of oxygen, the massive scale of various types of ocean pollution including plastics and accidental pollution, dire consequences of the illegal, unreported and unregulated fishing and of fishing subsidies, ocean habitat destruction, harmful impacts of invasive species, and some other negative effects. The World Ocean Assessment lists some other impacts on the society: the sea-level rise, growing occurrences of more intense tropical (and, potentially extratropical) cyclones, harmful algal blooms, fish and human poisoning, inundation of the coasts by the Sargasso and some other factors. Migration of the population to the coast along with the continuing population growth exacerbate the risks to lives and property in the coastal zone, and earthquakes and tsunamis continue to represent a major threat.

According to the first Global Ocean Science Report, prepared by the IOC of UNESCO in $2017^{11}$, the investment into ocean science is less than $4 \%$ of the global funding of natural sciences, with very large variations among countries. The current knowledge of the ocean, in terms of its physics and dynamics, might be close to sufficient for alerting the society and governments about many of the existing or emerging threats. However, there is a strong need for more specific knowledge and its systematic delivery and application to enable effective and efficient actions toward sustainability, at the global and regional levels. Mainstreaming oceanography and targeting it and ocean related components of natural and social sciences on practically assisting the sustainable development agenda were the main motivations for the IOC to develop the proposal to the UN to organize a Decade of Ocean Science for Sustainable Development (hereafter - the Decade ${ }^{12}$ ) in 2021-2030.

\footnotetext{
${ }^{9}$ https://oceanconference.un.org

${ }^{10} \mathrm{http}: / /$ www.worldoceanassessment.org/

${ }^{11}$ https://en.unesco.org/gosr

${ }^{12}$ https://en.unesco.org/ocean-decade
}

\section{BACKGROUND AND MAIN GOALS OF THE DECADE}

The proposal of the Decade came from a brainstorming meeting of the IOC Chair, Vice-Chairs and senior secretariat staff held in Gilleleje, Denmark in January 2016. Dr. Gunnar Kullenberg, a former IOC Executive Secretary, made significant contributions resulting in the meeting's success. During 2016 and first half of 2017, IOC Member States and many other interested parties were consulted on the concept and potential value of a Decade of Ocean Science. A proposal for the Decade was drafted for consideration by IOC Member States (IOC/INF1341, 2 February, 2017) and a revised version of the proposal (IOC/INF-1341 REV) was presented to the twenty-ninth session of the IOC Assembly and subsequently endorsed through a Resolution (IOC Resolution XXIX-1, IOC-Unesco, 2017). In addition, recognizing the cross-cutting role of ocean science in SDG 14 of the 2030 Agenda, the Decade proposal was registered by the IOC as a voluntary commitment to the first UN Ocean Conference that took place in June of 2017 in New York ${ }^{9}$. In the middle of 2017, the IOC submitted the proposal for consideration of the $72^{\text {nd }}$ Session of the UN General Assembly (UNGA).

It was proposed that the Decade start exactly fifty years after the International Decade of Ocean Exploration (IDOE) that took place in 1971-1980 (Holland and Pugh, 2010). IDOE was coordinated by the IOC and successfully stimulated oceanographic research and exploration, creating additional interest and support to the discipline. The, IDOE was followed by several seminal developments in ocean management and sciences, including the preparation and signature of the UN Convention on the Law of the Sea (1982), the World Ocean Circulation Experiment (1990-2002) and several other major international undertakings in the domain of the ocean.

On 5 December 2017, the UNGA, within Part XI of the Omnibus Resolution for Oceans and the law of the sea (RES 72/73) relating to "Marine science," decided (United Nations General Assembly, 2017, paragraph 292) to proclaim

the United Nations Decade of Ocean Science for Sustainable Development for the 10-year period beginning on 1 January 2021, within existing structures and available resources.

\section{The UNGA:}

called upon the Intergovernmental Oceanographic Commission to prepare an implementation plan for the Decade in consultation with Member States, specialized agencies, funds, programmes and bodies of the United Nations, as well as other intergovernmental organizations, non-governmental organizations and relevant stakeholders.

\section{Further, the UNGA:}

- Requested that the Intergovernmental Oceanographic Commission provide information on the development of the implementation plan and regularly consult with, and report to, Member States on the United Nations Decade of Ocean Science and its implementation;

- Invited the Secretary-General to inform the General Assembly about the implementation of the United Nations Decade of 


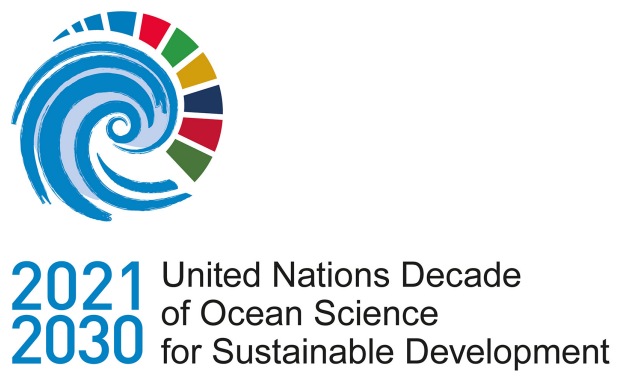

FIGURE 1 | Decade of Ocean Science for Sustainable Development logo. Guidelines to request the logo and obtain permission to use it are available on the Decade website (https://en.unesco.org/ocean-decade).

Ocean Science through his report on oceans and the law of the sea, on the basis of information to be provided by the Intergovernmental Oceanographic Commission;

- Invited UN-Oceans and its participants to collaborate with the Intergovernmental Oceanographic Commission on the United Nations Decade of Ocean Science.

The proclamation of the Decade by the UN General Assembly is an acknowledgment by UN Member States and their Governments of the importance, need for and role of ocean science, data and information exchange for sustainable development, and that science can play an important role in helping the ocean support the 2030 Agenda. The Decade requires response and delivery from the IOC and other UN bodies, the scientific community as a whole, working in close contact with governments, industry and business, as well as with the civil society.

The idea of the Decade is to achieve a major change in the knowledge and management of the ocean. It is reflected in the following two over-arching goals that provide the high-level motivation for the Decade:

Goal 1: To generate the scientific knowledge and underpinning infrastructure and partnerships needed for sustainable development of the ocean.

Goal 2: To provide ocean science, data and information to inform policies for a well-functioning ocean in support of all Sustainable Development Goals of the 2030 Agenda.

The design of the Decade should address both deep disciplinary understanding of ocean processes and solutionoriented research to generate the knowledge needed for reducing pressures on the ocean, preserving and restoring ocean ecosystems and safeguarding ocean-related prosperity for future generations. The Decade should turn the scientific knowledge and understanding into effective actions supporting improved ocean stewardship for sustainable development. The generation of societal outcomes of the Decade would trigger positive environmental and societal changes and would support peaceful cooperation of nations. The logo of the Decade (Figure 1) aims to demonstrate the role of ocean in all aspects of sustainable development.

\section{SOCIETAL OUTCOMES}

The Decade will aim to achieve considerable progress in a number of research and technology development areas with a view of generating the following six societal outcomes.

A clean ocean, whereby sources of pollution are identified, quantified and reduced and pollutants removed from the ocean in an efficient manner. Integrated research will assess the human and environmental shorter-term and long-term risks from ongoing and future types of ocean pollution and generate new ideas on how to reduce ocean pressures by recycling, improved waste management, and strengthening the governance regimes that encourage more sustainable production and consumption.

A healthy and resilient ocean, whereby marine ecosystems are mapped and protected, multiple impacts on them, including climate change, are quantified and, where possible, reduced and provision of ocean ecosystem services is maintained. The Decade will promote research aimed at elucidating impacts of cumulative stressors on the ocean, its seas, ecosystems and resources, hence providing required information to enable actions, which can reverse the ocean ecosystem degradation. Improved evaluation and appreciation of the economic and societal value of the ocean and its ecosystems will also be instrumental to stimulate marine spatial planning, marine protected areas, coastal zone management and other ecosystembased management approaches.

A predicted ocean, whereby society has the capacity to understand current and predict future ocean conditions and their impact on human well-being and livelihoods. Under the Decade, sustained and systematic ocean observations would be expanded to all ocean basins and depths to document ocean change, initialize coupled models and facilitate improved ocean understanding. Knowledge of present and future conditions is a pre-requisite to the development of sustainable ocean economic policies and ecosystem-based management. More detailed and complete accounts of ocean processes can help to improve climate prediction in a significant way. The Decade will also build on advances in ocean robotics and the combination of remote and in situ ocean observations, which offer new opportunities and reduce operational costs; it will also promote multi-stakeholder contributions by governments, the private sector and citizens.

A safe ocean, whereby human communities are much better protected from ocean hazards and where the safety of operations at sea and on the coast is ensured. The Decade will promote research aimed at minimizing impacts of various changes and risk reduction through adaptation and mitigation. It will also support the development of integrated multi-hazard warning systems (MHWSs) in all basins, hence, contributing to enhanced preparedness and awareness of society with regard to ocean risks. Community resilience and adaptive capacity, with elevated education and awareness concerning the use of observations and data, will also contribute to reduced impacts and improved efficiency of early warning systems for natural and man-made hazards. This area of research will be of great interest for the insurance and reinsurance industries.

A sustainably harvested and productive ocean, ensuring the provision of food supply and alternative livelihoods. The Decade 
should create a better understanding of the interactions and interdependencies of the ocean ecosystem and environmental conditions and processes, the use of resources and the economy. A major task in context of the development of the ocean economy will be in documenting the potential impacts from environmental changes on the established and emerging maritime industries and their ability to generate growth, especially for least developed countries and SIDS. Defining safe and sustainable thresholds for economic operations in the ocean will help policy-makers and stakeholders in implementing a truly sustainable blue economy (see for example, Smith-Godfrey, 2016; Visbeck, 2018). New research should develop and flesh out sustainable blue-green growth agendas and link it to efforts in ecosystem protection.

A “transparent and accessible" ocean, whereby all nations, stakeholders and citizens have access to ocean data and information technologies and the capacities to inform their decisions. The enormous need for more ocean information in the scientific, governmental, private and public sectors demands a step change in ocean education at all levels. New technologies and the digital revolution are transforming the ocean sciences; these will be harnessed to deliver data and information to all stakeholders. Science-policy interface for the ocean should be enhanced as well. Open access to ocean information, increased interactions between the academic and societal actor communities, and ocean literacy for all should capacitate all citizens and stakeholders to have a more responsible and informed behavior toward the ocean and its resources. Innovative capacity development schemes between south-south and northsouth ocean actors as well as courses for ocean professionals will be key in raising ocean awareness and promoting better solutions.

The six societal outcomes of the Decade are holistic. In order to be achieved, most of them require actions by the society, governments, or by key stakeholders. However, there is no causal link to achieving them that would be entirely scientific. Nevertheless, progress in several thematic areas of ocean science is either necessary or very useful to achieve them. Scientific papers should not be the sole measure of success of the Decade. Impact to society, appropriately measured against clear objectives, should also be a measure of success. Such initial priority areas of research and technology development (R\&D) are outlined below. There is no priority order among them. They are interconnected but allow focused design and planning. Progress in these areas is necessary to facilitate protection and the sustainable use of the ocean, on global and more localized scale.

\section{R\&D Priority Area 1: Comprehensive Map (Georeferenced Digital Atlas) of the Ocean}

This R\&D Area includes and goes beyond the domain of mapping the ocean bottom topography, and its importance can be illustrated by the deficiency of current global ocean depth maps. Current, largely satellite-based mapping of the global ocean renders a horizontal resolution between 2 and $5 \mathrm{~km}$. At this resolution, many features are not detectable and result in only a coarse understanding of the ocean bottom. Technologies such as multibeam echo-sounders can deliver much higher resolution, either as hull mounted on surface ships or on undersea vehicles that can be positioned closer to the seabed. At present the multibeam echo-sounder coverage of the world ocean is scarcely over $5 \%$, leaving much work to be done and only a sketch of the true detail that exists under the ocean. The current map of the ocean does not represent therefore many important underwater features. If a parallel to the land surface is evoked, the equivalent resolution of terrestrial maps would level out almost all prominent topographic features. With the current level of knowledge, the recent searchers for missing aircraft simply did not know the true initial depth of water they were supposed to operate in.

Current efforts under the International Hydrographic Organization (IHO) and the IOC, particularly the IHO/IOC Project "General Bathymetric Chart of the Ocean" $\left(\mathrm{GEBCO}^{13}\right)$ and its flagship Project Seabed 2030, sponsored by the Nippon Foundation, address the formidable task of mapping the ocean depth for most of the global ocean.

The task of mapping the ocean depth is being currently addressed through coordinated assembly of depth measurements made using various platforms, such as satellites, ship and autonomous underwater vehicle (gliders) echo sounding. It may be promising to review other sources of data, e.g., seismic information that is available from various seismic networks to enrich the volume of data, particularly for poorly covered regions. The feasibility of using new data and approaches needs to be studied.

A comprehensive map of the ocean should include much more information than just ocean depth. It requires other variables describing the physical, biological, chemical, and geological environments, ecosystems, cultural objects, boundaries, resources, etc. The shipping and transport community, weather and ocean forecasters, fishing industries and authorities, marine resource managers, and coastal cities and communities vulnerable to sea level rise, tsunami, and tropical cyclones are all dependent upon accurate ocean maps. Maritime spatial planning is a way of optimally putting many interdependent maritime activities on a single map. Establishment of marine protected areas is also based on mapping. Similar requirements exist in the coastal zone management. A common digital atlas of ocean information is necessary in order to address the prevailing and future societal pressures on the ocean. Advanced prototypes of such an atlas already exist in many regions of the world, sometimes encompassing high resolution maps.

Designing a digital georeferenced atlas of the ocean that would satisfy many of the future uses of the ocean and its protection is therefore a necessary and innovative scientific undertaking that requires consolidation of existing knowledge, review of requirements, new $\mathrm{R} \& \mathrm{D}$, and comprehensive assemblage on regional and local scales.

\section{R\&D Priority Area 2: A Comprehensive Ocean Observing System}

One cannot manage what one cannot measure. Ocean observations are the key to understanding weather, climate

\footnotetext{
${ }^{13}$ https://www.gebco.net
} 
and the future state of marine ecosystems and resources. The Global Ocean Observing System $\left(\mathrm{GOOS}^{14}\right)$ domain of competence is physical state variables in the upper $2 \mathrm{~km}$ of the water column and at the surface. It is expanding to include additional measurements in the deeper ocean and in the domains of biogeochemistry, biology, and ecosystems. The approach based on Essential Ocean Variables (Lindstrom et al., 2012) is effective and new variables should be taken on board, making the scope of observations capable to help monitoring and management of large marine ecosystems (LMEs) and coastal and offshore areas.

Global Ocean Observing System is a shared undertaking. All nations, even landlocked, benefit from the associated data products and services. United Nations Member States need to engage further to construct and sustain a global ocean observing system that covers all the world's major ocean basins and also addresses coastal and local interests. This system should be routinely maintained, with observations collected to a uniform and common standard of foundational or basic data, with information gathered made openly available to all and should be readily adaptive to both emerging circumstances and priorities as well as to local or regional needs, in addition to basic parameters. All the societal outcomes listed above would benefit from additional observations and parameters, and in particular, in the southern ocean and the Arctic that are currently under-observed. Efforts should also focus on expanding observations to the deep ocean in all ocean basins to characterize its physical state, biogeochemistry and ecosystems, and detect changes.

The Decade should produce a regime of international cooperation that fully monitors the major ocean basins of the world, at all depths, blending inputs into the GOOS, and maintained by the nations of the basin region, among others. It should synergistically use in situ and remotely sensed observations and strongly benefit from emerging observing technologies. The Decade should create conditions for involvement of downstream beneficiaries, such as the private sector, of the ocean observations (for example, as has been done for the tropical Pacific; Smith et al., 2019). Their input would provide very useful feedback on the quality and quantity of the observations information provided, and their interest in obtaining the data should create a "pull" and a better resource base for expanding and sustaining the ocean observations. It should be noted that such beneficiaries often operate on local scales and require information of high resolution.

\section{R\&D Priority Area 3: A Quantitative Understanding of Ocean Ecosystems and Their Functioning as the Basis for Their Management and Adaptation}

The Census of Marine Life produced an inventory of species in the ocean, enhancing the scientific knowledge on what lived, lives, and will live in the ocean. This work is successfully continuing under the IOC Ocean Biogeographic Information System

\footnotetext{
${ }^{14}$ http://www.goosocean.org
}

(OBIS). The biological component of GOOS is approaching the pilot phase. Emerging technologies are becoming more mature and available, such as Environmental DNA sampling (eDNA; Ausubel et al., 2019). These new approaches will enrich more mature molecular methods and, combined, create a capacity for imaging life in the ocean in near real time. From viruses and plasmids to whales, and their interaction with each other, the new state of molecular and genetic science will reveal much of what has not been able to be measured or understood in the past. This knowledge will be instrumental to better understand the ecosystem services, including, for example, nitrogen fixation, primary production, nutrient cycling, and surface colonization (with thanks to the manuscript reviewer).

Increasingly, tools are available to replace the laboratory bound analysis with deployable laboratories that produce the resulting information, rather than simply collect the sample. A combination of methods exists to produce an ongoing measurement of micro- to the macro- and mega-sized components of the marine ecosystem inventory and assess its health. The new technologies will be able to help the researchers to better understand the functioning of deep-sea ecosystems, measure the cumulative impacts of ocean stressors and define the carrying capacity of ocean ecosystem to sustain human impacts and economic development. For the first time in history, this opens a possibility to meaningfully predict the evolution of ocean ecosystems, using biogeochemical and ecosystem models that are forced by modern climate prediction or projection models and are verified against a bulk of standardized in situ eDNA and other observations.

Availability of environmental predictions also offers potential to guide the adaptation to the new climatic and environmental conditions of certain valuable ecosystems (not only individual species), e.g., some coral reef ecosystems. The approach may consist of selective breeding of ecosystem components in a controlled basin of water with environmental conditions corresponding to the predicted regime of temperature (including estimates of its future variability and emergence of heat waves), salinity, water $\mathrm{pH}$, oxygen, turbidity and, potentially, some other stressors. World aquariums possess significant potential to conduct such experiments.

Ecosystem monitoring with quantitative criteria of ecosystem health, enhancing knowledge of management solutions and availability of best practices and emerging predictive capacities hold promise to create a foundation for the science-based management of LMEs, with engagement of all key stakeholders, such as environmentalists and fisheries.

\section{R\&D Priority Area 4: Data and Information System}

The IOC International Ocean Data and Information Exchange Programme $\left(\mathrm{IODE}^{15}\right)$ focuses on the discovery, exchange of, and access to marine data and information including metadata, products and information. It sets international standards in that domain. The system, composed of an approximately 100 Data

\footnotetext{
${ }^{15}$ https://www.iode.org
} 
Centers and associated data units, is capable of providing for long-term archival, preservation and documentation of marine data and information products, and facilitate related capacity development and the use of best practices.

Under the Decade, the oceanographic information system needs to be significantly expanded, upgraded and truly opened to the whole world to ensure flow of data and products between providers and users at different levels. The core of the future oceanographic data and information system is currently seen as a portal of ocean data that acts as a link between demand and supply and has a capacity to facilitate matching requirements for data and products. This engine is expensive to design and build, but it can be gradually created based on existing elements and advances in data processing and cataloging of data and product sources. Big data and cloud data processing and computing approaches offer additional perspectives to implement the portal. The initial steps toward creating the portal will likely consist of upgrading the current ocean data and information system while seeking resources and involvement of partners from IT industry and private sector to develop the future system in orderly manner, respecting the requirements of interoperability, the standards with acknowledgment of data originators. A Decade data policy will need to be developed and proposed. Compliance with the IOC data policy of free, unrestricted and open access to data should be considerably strengthened in the course of the Decade. A major international project on ocean data and information system development may be required, and, potentially, a global conference of the scale of OceanObs'19 to bring together known and new partners in the ocean data discipline, going beyond classical oceanographic data and starting to work with economic and social data.

\section{R\&D Priority Area 5: Ocean Dimension in an Integrated Multi-Hazard Warning System}

Currently, there are a number of unconnected warning systems for ocean-related hazards. Some of them are operational, e.g., for tsunami generated by earthquakes, some are incomplete, e.g., for storm surges, and some emerging, e.g., for harmful algal blooms. Widely recognized and reflected in the Sendai Framework for Disaster Risk Reduction is the need to strengthen and harmonize the warning systems. An effective warning system has to be based on the knowledge of risks and corresponding emergency planning and warnings. The potentially affected communities need to be prepared to act appropriately on a warning. The hazard has to be detected, followed, and/or forecast. The warning then needs to be generated and timely, fully, and correctly transmitted, received, and acted upon.

This combination of actions and responsibilities necessarily involves more than one responsible agency. Experience shows a strong advantage of developing MHWSs able to act on more than one type of risk. During the Decade, a concerted effort should be made to incorporate ocean components into emerging or existing MHWSs and, as well, to more effectively use the ocean information for warnings of ocean-related hazards at a variety of time scales, from immediate threats, such as tropical storms, to long-term, high-impact events like droughts, heat waves, forest fires, floods, etc. Until now, humankind has largely reacted too late to such hazards. There is significant progress in saving lives, but economic and infrastructural losses from natural disasters remain overwhelming and often strongly affect the capacity of a country or a region to recover after a natural hazard event.

The ocean-related warning systems, especially the one for tsunami, still require major methodological development, e.g., to embrace other sources of tsunami such as submarine landslides and volcanoes. Most importantly, the system needs to strengthen and upscale the preparedness of communities at risk. This quality of the system needs to be addressed during the Decade through involvement of appropriate governmental authorities and international mechanisms. The problem of "last mile" needs to be addressed so that the warning reaches the local community, which is prepared to act correctly, and community does so.

\section{R\&D Priority Area 6: The Ocean in an Earth-System Observation, Research, and Prediction}

Ocean science is a part of the Earth system science. GOOS is a contributor to the Global Climate Observing System and, more widely, a part of the planet observing system of systems. To study and predict the future state of the ocean, one needs to incorporate it into various types of Earth system models. Industries, behavioral and societal changes, and economy all need to be eventually included in the observations and predictive modeling scope. As the time range of the prediction lengthens, more complexity is usually required to exploit the predictability of the interacting components of the Earth system. The longer the prediction range, the higher generally is the role of ocean processes in the prediction.

In addition, there exists an untapped potential in oceanographic observation and modeling that can help to improve a number of important services to humanity. This area of societal benefit includes many types and ranges of weather predictions, climate information services, various thematic assessments, and ocean space and resources management. Activities in the coastal zones and operations at sea are becoming more and more interdependent and need to be regulated accordingly. Activities require real-time decision making and anticipatory planning. Ocean modeling and prediction capacity that could meet such emerging requirements does not currently exist. Therefore, the Decade might engage at present unconnected modeling groups and industries into a design of a future multi-scale ocean observation and prediction system. Development of ethical principles of human interactions with the ocean, and short- and long-term economic analyses of ocean sustainability and the role of ocean science in it are urgently required. Quantitative assessments of tangible benefits and appreciation of intangible assets related to the ocean are needed for decisions to act on urgent issues including land-based sources of pollution, unsustainable ways of fishing and aquaculture and for ensuring protection of underwater cultural heritage. 


\section{R\&D Priority Area 7: Capacity Building and Accelerated Technology Transfer, Training and Education, Ocean Literacy}

All above R\&D areas will move the cutting-edge of ocean science forward. They will augment the ocean science capacity and make it fit for the purpose of informing and even guiding sustainable development. The enabling elements for the progress are human potential, infrastructure, cooperation, resources and adequate social conditions of successful research and development. The IOC has started and will keep assessing the capacity of ocean science through the Global Ocean Science Report process. At present, the oceanographic capacities are highly uneven in the world, not only in terms of ability to contribute to the research but also in terms of ability to benefit from the scientific knowledge and technology, which is defined as also including ocean data and information. Based on ethical considerations and on the principle of common but differentiated responsibilities, all countries and communities should be able to benefit from the ocean life-supporting services, which requires capacity to act in a scientifically sound way. The Decade should strengthen the existing capacity development, training and education work and set in motion the transfer of marine technology (TMT) mechanisms. Infrastructure, training and teaching materials exist in many organizations. Their potential will be better sustained and potentially multiplied once the TMT Clearing House is put in place, linking supply and demand in capacity development, training and education.

One outcome of the Decade should be a major step forward in ocean literacy for various categories of people. The motivation for the Decade itself comes the realization of the role of ocean for people and planet and from the understanding that the ocean is largely underexplored. A vigorous ocean literacy program of activities has to be designed. The major target audiences have to be students, which requires including ocean literacy in the school curriculum, decision makers-including governmental authorities-and the public at large (Santoro et al., 2017).

\section{STRATEGIC APPROACH OF THE DECADE: TWO-WAY INTERACTION BETWEEN RESEARCH AND PRACTICAL APPLICATIONS}

It is anticipated that progress in $\mathrm{R} \& \mathrm{D}$ will create new, more beneficial conditions for practical applications of new knowledge and technology. It is essential that ambitions of the private sector, governments and involved managers grow along with the progress in research. For that, active, intensive, and efficient communication of advances on ocean sciences will be a key enabling factor. The seven $\mathrm{R} \& \mathrm{D}$ priority areas listed above (or additional) will trigger accelerated development of the following means of using and protecting the ocean.

Protecting the ocean should employ the ecosystem-based approach, supported by observations and knowledge, with adequate regulation, and efficient and implemented policies including knowledge based establishment of marine protected areas in key locations.
The anticipation for the culmination year of the Sustainable Development Agenda, i.e., 2030, would be to have approximately $30 \%$ of the total area of the world Exclusive Economic Zones covered by scientifically-managed and governmentally approved maritime spatial plans ${ }^{16}$. This will bring multiple advantages for ocean "blue" economy and will facilitate management of ocean ecosystems and preserve ocean health. Similar progress is expected in the coastal zone management. It is essential that new MSP and coastal zone management approaches take into account not only the current state of the ocean and activities in it but are forward-looking, taking into account scenarios of future activities and appropriate environmental predictions.

Management of fisheries and aquaculture under the conditions of growing population, changing climate and the need to abate marine pollution, needs to fully capitalize on the multitude of ocean observations and on predictions of climate and ocean health and productivity. Observations and science can help increase transparency in managing the living marine resources and compliance with existing regulations, as well as stimulate countries to adopt progressive policies in the domain of fisheries and aquaculture.

Based on the analysis in the Intergovernmental Panel on Climate Change Special Report on the Ocean and Cryosphere in Changing Climate and gaps of knowledge and capacity to be identified in it, systematic work on adaptation solutions and search for climate change mitigation actions will be intensified.

\section{WAY FORWARD, COMMUNITY AND UN ENGAGEMENT, RESOURCES}

The Intergovernmental Oceanographic Commission was mandated by the United Nations General Assembly (UNGA) to lead the preparations of the Decade and develop its Implementation Plan in consultation with Member States, UN partners as well as other relevant stakeholders. The Implementation Plan must be submitted to the UN General Assembly in the second half of 2020. In the first part of 2018, a roadmap (IOC-Unesco, 2018b) was developed as an evolving document to guide the preparatory phase of the Decade by defining preliminary objectives, societal outcomes, governance and engagement processes.

In 2018, the $51^{\text {st }}$ session of the IOC Executive Council welcomed the roadmap and decided to establish an Executive Planning Group (EPG) consisting of high-level experts to advise IOC governing bodies. The IOC convened the $1^{\text {st }}$ meeting of the EPG from 17 to 19 December 2018 to brainstorm on scientific, governance, communications and engagement elements of the Decade. Beyond the scientific process itself, wide consensus was achieved within the EPG that the Decade must transform the way nations around the world invest in ocean science, both from the public and private sectors and how decision-makers use the available scientific knowledge to inform policies for ocean management. Experts also sought to lay out the basis for an effective strategy to communicate the objectives of the

\footnotetext{
${ }^{16}$ https://en.unesco.org/mspglobal
} 
Decade, engage stakeholders, and ultimately influence behaviors and perceptions of scientists, decision-makers and the public around the value of ocean science for sustainable development. They stressed that capacity development and the TMT should be a key crosscutting priority, embedded deeply into the Decade planning process.

Executive Planning Group also agreed that early involvement of stakeholders in the planning phase would be key to achieving the objectives in an efficient manner and to secure broad and consistent implementation of the Decade. Stakeholders will be invited to contribute to the co-design of the Decade Implementation Plan through a consultation process that will be organized around Global Planning Meetings and a series of regional workshops foreseen in 2019 and early 2020 that will channel stakeholders' inputs into the preparatory process. Further to these consultative meetings, a Stakeholder Forum will be established and open to a broad range of communities (science, technology, ocean management, private sector, civil society) that are interested in contributing to the Decade.

UN partners with a focus on ocean are also associated to this consultation process. UN bodies were invited to contribute to the development of the Implementation Plan during a UNOceans' meeting (the UN inter-agency mechanism on ocean affairs) at UNESCO Headquarters in March of 2018. A dedicated task group was established at the UN-Oceans meeting held at the World Meteorological Organization in February 2019 to structure and reinforce its members' contributions to the Decade. The collaboration of the different UN bodies will also be encouraged through the development of both new and renewed partnerships with the IOC. It is essential to ensure that the UN agencies exercise leadership on aspects of the Decade that are relevant for their mandate. Communication and outreach efforts have intensified to present the objectives of the Decade and engage all these stakeholders.

The UNGA tasked the IOC with preparing the Decade Implementation Plan "within existing structures and available resources." Following the IOC Executive Council Decision, at its $51^{\text {st }}$ session (IOC-Unesco, 2018a), urging Member States to provide voluntary contributions for the preparation of the Decade, the IOC issued a Call for expression of interest in June of 2018. UN agencies and partners' organizations expressed strong support for the Decade and several Member States offered to host regional, global or thematic workshops scheduled during the Preparatory Phase of the Decade. More tangible support is still needed for the communication and outreach activities that will be developed for these consultative and planning meetings. Additional resources will be sought through alternative funding mechanisms such as public/private partnerships, as well as contributions of the philanthropic sector.

\section{REFERENCES}

Ausubel, J. H., Stoeckle, M. Y., and Gaffney, P. (2019). Final Report of the 1st US National Conference on Marine Environmental DNA (eDNA). Available at: https://phe.rockefeller.edu/eDNAmarine2018/docs/MURU_ eDNA_Conference_final_report.pdf; https://undocs.org/en/A/RES/72/73 (accessed December 5, 2017).

\section{CONCLUSION: DECADE AND OCEANOBS'19 COMMUNITY}

The Decade is a major chance for oceanography to put in place a more complete and sustainable observing system and feed the resulting data into a science-based ocean management system satisfying the increasing reliance of our civilization on the ocean, its ecosystem services, and, at the same time, preserving ocean health. The OceanObs conferences are also decadal but periodic events intended to give momentum and direction to developing ocean observing systems. The Decade responds well to the main goal of this OceanObs'19, which is to establish the value chain from ocean observations through research to longterm societal benefits and key stakeholders. It is hoped therefore that the OceanObs'19 community will see the Decade as a key opportunity to propose ideas for making ocean observations even more societally relevant and, hence, better supported, and will actively participate and strongly contribute to preparatory phase of UN Decade. In summer 2020 IOC will need to submit the Implementation Plan to the $75^{\text {th }}$ UN General Assembly of United Nations. The opportunities to contribute are multiple. The proceedings of OceanObs'19 will be carefully studied in crafting the Plan. Regional and global meetings will be organized to further develop ideas and digest them into the plan. The Decade stakeholder forum is a platform to collect ideas. The link to it is at https://en.unesco.org/ocean-decade/get-involved.

\section{AUTHOR CONTRIBUTIONS}

VR wrote the first draft of the manuscript taking into account various documents, to which JB, GK, NS, and some other authors made an input. PP and JR wrote some individual sections of the manuscript. All authors contributed to the manuscript revision, and read and approved the submitted version of the manuscript.

\section{FUNDING}

Preparation of this manuscript was supported by IOC/UNESCO.

\section{ACKNOWLEDGMENTS}

We thank many people who contributed to preparation of the proposal to proclaim 2021-2030 an international Decade of Ocean Science for Sustainable Development and those who continue to work on the planning and support of it that is currently underway.

Duarte, C. M., Poiner, I., and Gunn, J. (2018). Perspectives on a global observing system to assess ocean health. Front. Mar. Sci. 5:265. doi: 10.3389/fmars.2018. 00265

Hoegh-Guldberg, O., Cai, R., Poloczanska, E. S., Brewer, P. G., Sundby, S., Hilmi, K., et al. (2014). “The ocean," in Climate Change 2014: Impacts, Adaptation, and Vulnerability. Part B: Regional Aspects. Contribution of Working Group II to the Fifth Assessment Report of the Intergovernmental Panel on Climate Change, eds 
V. R. Barros, C. B. Field, D. J. Dokken, M. D. Mastrandrea, K. J. Mach, T. E. Bilir, et al. (Cambridge: Cambridge University Press), 1655-1731.

Holland, G., and Pugh, D. (2010). Troubled Waters: Ocean Science and Governance. Geoff Holland and David Pugh. Cambridge: Cambridge University Press.

IOC-Unesco (2017). Assembly Resolution XXIX-1. Paris: UNESCO.

IOC-Unesco (2018a). Executive Council Resolution EC-LI.1. Paris: UNESCO.

IOC-Unesco (2018b). Roadmap for the UN Decade of Ocean Science for Sustainable Development, Version 2.0. Paris: UNESCO.

Lindstrom, E. J., Gunn, A., Fischer, A., McCurdy, A., and Glover, L. K. (2012). A Framework for Ocean Observing. By the Task Team for an Integrated Framework for Sustained Ocean Observing. Paris: UNESCO.

Santoro, F., Santin, S., Scowcroft, G., Fauville, G., and Tuddenham, P. (2017). Ocean Literacy for All: a Toolkit. Paris: UNESCO.

Smith, N., Kessler, W. S., Cravatte, S., Sprintall, J., Wijffels, S., Cronin, M. F., et al. (2019). Tropical pacific observing system. Front. Mar. Sci. 6:31. doi: 10.3389/ fmars.2019.00031

Smith-Godfrey, S. (2016). Defining the blue economy. Marit. Aff. 12, 58-64. doi: $10.1080 / 09733159.2016 .1175131$

Stocker, T. F. (2015). The silent services of the world ocean. Science 350, 764-765. doi: $10.1126 /$ science.aac8720
United Nations General Assembly. (2017). Resolution A/RES/72/73, Part XI of the Omnibus Resolution for Oceans and the law of the sea. Available at: https://undocs.org/en/a/res/72/73 (accessed December 5, 2017).

Visbeck, M. (2018). Ocean science research is key for a sustainable future. Nat Commun. 9:690. doi: 10.1038/s41467-01803158-3

Conflict of Interest Statement: The authors declare that the research was conducted in the absence of any commercial or financial relationships that could be construed as a potential conflict of interest.

Copyright () 2019 Ryabinin, Barbière, Haugan, Kullenberg, Smith, McLean, Troisi, Fischer, Aricò, Aarup, Pissierssens, Visbeck, Enevoldsen and Rigaud. This is an openaccess article distributed under the terms of the Creative Commons Attribution License (CC BY). The use, distribution or reproduction in other forums is permitted, provided the original author(s) and the copyright owner(s) are credited and that the original publication in this journal is cited, in accordance with accepted academic practice. No use, distribution or reproduction is permitted which does not comply with these terms. 


\section{OPEN ACCESS}

Edited by:

Amos Tiereyangn Kabo-Bah,

University of Energy and Natural

Resources, Ghana

Reviewed by:

Zeke J. Hausfather,

University of California, Berkeley,

United States

Matthew Mayernik,

National Center for Atmospheric Research (UCAR), United States

*Correspondence: Elizabeth C. Kent eck@noc.ac.uk

Specialty section: This article was submitted to

Ocean Observation,

a section of the journal Frontiers in Marine Science

Received: 15 November 2018 Accepted: 05 July 2019

Published: 30 July 2019

Citation:

Kent EC, Rayner NA, Berry DI, Eastman R, Grigorieva VG, Huang B, Kennedy JJ, Smith SR and Willett KM

(2019) Observing Requirements for Long-Term Climate Records

at the Ocean Surface.

Front. Mar. Sci. 6:441.

doi: 10.3389/fmars.2019.00441

\section{Observing Requirements for Long-Term Climate Records at the Ocean Surface}

\author{
Elizabeth C. Kent ${ }^{1 *}$, Nick A. Rayner ${ }^{2}$, David I. Berry ${ }^{1}$, Ryan Eastman ${ }^{3}$, \\ Vika G. Grigorieva ${ }^{4}$, Boyin Huang ${ }^{5}$, John J. Kennedy ${ }^{2}$, Shawn R. Smith ${ }^{6}$ and \\ Kate M. Willett ${ }^{2}$
}

\begin{abstract}
${ }^{1}$ National Oceanography Centre, Southampton, United Kingdom, ${ }^{2}$ Met Office Hadley Centre, Exeter, United Kingdom, ${ }^{3}$ Department of Atmospheric Sciences, University of Washington, Seattle, WA, United States, ${ }^{4}$ Shirshov Institute of Oceanology, Russian Academy of Sciences, Moscow, Russia, ${ }^{5}$ National Oceanic and Atmospheric Administration, National Centers for Environmental Information, Asheville, NC, United States, ${ }^{6}$ Center for Ocean-Atmospheric Prediction Studies, Florida State University, Tallahassee, FL, United States
\end{abstract}

Observations of conditions at the ocean surface have been made for centuries, contributing to some of the longest instrumental records of climate change. Most prominent is the climate data record (CDR) of sea surface temperature (SST), which is itself essential to the majority of activities in climate science and climate service provision. A much wider range of surface marine observations is available however, providing a rich source of data on past climate. We present a general error model describing the characteristics of observations used for the construction of climate records, illustrating the importance of multi-variate records with rich metadata for reducing uncertainty in CDRs. We describe the data and metadata requirements for the construction of stable, multi-century marine CDRs for variables important for describing the changing climate: SST, mean sea level pressure, air temperature, humidity, winds, clouds, and waves. Available sources of surface marine data are reviewed in the context of the error model. We outline the need for a range of complementary observations, including very high quality observations at a limited number of locations and also observations that sample more broadly but with greater uncertainty. We describe how high-resolution modern records, particularly those of high-quality, can help to improve the quality of observations throughout the historical record. We recommend the extension of internationally-coordinated data management and curation to observation types that do not have a primary focus of the construction of climate records. Also recommended is reprocessing the existing surface marine climate archive to improve and quantify data and metadata quality and homogeneity. We also recommend the expansion of observations from research vessels and high quality moorings, routine observations from ships and from data and metadata rescue. Other priorities include: field evaluation of sensors; resources for the process of establishing user requirements and determining whether requirements are being met; and research to estimate uncertainty, quantify 
biases and to improve methods of construction of CDRs. The requirements developed in this paper encompass specific actions involving a variety of stakeholders, including funding agencies, scientists, data managers, observing network operators, satellite agencies, and international co-ordination bodies.

Keywords: marine, climate, measurement, uncertainty, ocean, atmosphere, ship, buoy

\section{INTRODUCTION}

Observations of environmental conditions near the ocean surface have been made from ships for centuries, and more recently from a wider range of observing platforms, including satellites. This paper will introduce the different types of measurements that have been made and describe the methods used to assemble the observations to generate records that can be used to characterize the changing conditions over the oceans. The most well-known long term marine climate record is that of sea surface temperature (SST), but there are also observations of air temperature, pressure, humidity, wind, clouds, waves, and weather conditions that have been used to generate climate records.

Most in situ surface marine climate records are based on the International Comprehensive Ocean-Atmosphere Data Set (ICOADS, Freeman et al., 2019), presently on Release 3.0 (Freeman et al., 2017). ICOADS is the most complete archive of its kind presently available and forms the basis for our discussion.

The requirements for data and metadata to construct long term climate records are hard to summarize in a simple form. Requirements for climate monitoring are collated by the Global Climate Observing System (GCOS) as part of the Observing Systems Capability Analysis and Review Tool (OSCAR) requirements database. The World Meteorological Organization (WMO) operate a "Rolling Review of Requirements (RRR)" in which user requirements for observations are compared with the capabilities of present and planned observing systems. For climate monitoring the outcomes of this review are published as reports on observing system status (GCOS, 2015) and requirements (GCOS, 2016). OSCAR considers a range of different application areas, other areas related to climate include climate science, applications and services, but climate monitoring is the most relevant to the construction of long-term climate records. The OSCAR/RRR process presents requirements for each variable as a desired accuracy at a chosen space and time resolution, plus a temporal stability. This is well-suited to measurements derived from satellites, but problematic for marine in situ measurements from mobile platforms (Berry and Kent, 2017). This paper therefore discusses the wide range of considerations underlying the development of accurate and stable long-term records in the context of an error model. Examples of the types of information that feed into the error model and therefore have an impact on the observing requirements are the availability of metadata describing measurement methods and protocols, and requirements for ancillary observations, for example for bias estimation or height adjustment. There are a range of other organizations concerned with the development of user requirements for surface marine data, for example the Copernicus Climate Change Service, the European Space Agency
Climate Change Initiative, and groups focused on particular variables such as SST (the Group for High Resolution SST) and winds (the International Ocean Vector Winds Science Team). Where relevant to climate monitoring and the development of long-term climate records requirements from such groups feed into the WMO RRR.

The GCOS defines three different types of observation networks: reference, baseline, and global (GCOS, 2016). These give a hierarchy of high quality traceable measurements at limited locations (reference network), through good quality measurements made more widely (baseline), to less accurate but widespread measurements to capture the important scales of variability (global). Such a suite of measurements can be combined to give the quality and coverage to provide stable, long term, climate records. The task is to assess the accuracy and sampling required for the different networks and how well the available historical observations map onto the requirements. Only then will it be possible to specify requirements for the continuation of long term records with the future observing system, and for prioritization of data and metadata recovery to improve the historical record.

\section{Scope and Terminology}

Here we consider the construction of climate data records (CDRs) of physical parameters observed near the ocean surface. These include the GCOS Essential Climate Variables (ECVs, Bojinski et al., 2014; GCOS, 2016) and Global Ocean Observing System (GCOS, 2018) Essential Ocean Variables (EOVs). Hereafter, we will refer to this combination of ECVs and EOVs simply as ECVs. Specifically we consider SST, marine air temperature (MAT), humidity, wind speed and direction, atmospheric sea level pressure (SLP) and also sea state and cloud parameters. Our criterion in this paper for considering a CDR to be long is greater than about 50 years, longer than the recent period with extensive satellite observations, and ideally centennial or longer.

This scope presently excludes the consideration of records derived from satellite data only as these have a maximum record length of about 40 years and are the subject of other papers in this issue (e.g., Ardhuin et al., 2019; Bourassa et al., 2019; O’Carroll et al., 2019). We note that many of the themes contained in this paper are relevant to both in situ and satellite data. It is critically important that high-quality satellite ECV records are maintained, evaluated and characterized with uncertainty estimates to create stand-alone CDRs, to enable the construction of long records jointly with in situ observations, to provide estimates of ECV variability and also data for evaluation. The Committee for Earth Observation Satellites (CEOSs) and the 
Coordination Group for Meteorological Satellite (CGMS) Joint Working Group on Climate provide coordination for activities related to the construction of CDR from satellites.

It is helpful to define some terminology for this paper.

Long term: about 50 years or ideally longer.

Large-scale: ranges from several degrees latitude/longitude to global scale.

Data: a collection of individual observations or measurements including information on date, time, and location.

Platform: any type of structure from which observations are made. Platforms providing records longer than 50 years include various types of ship, and fixed platforms such as rigs or coastal stations. Platforms providing shorter records include moored and drifting buoys, satellites, autonomous profilers, and surface vehicles.

Metadata: information describing characteristics of the platform, instrument, environmental conditions, observing protocols or data management that are helpful for interpreting the observations and estimating their uncertainty.

Climate data record: a time series of measurements of sufficient length, consistency, and continuity to determine climate variability and change and ideally accompanied by estimates of uncertainty and its correlation structure.

Gridded analysis: a timeseries of fields on a regular spatial grid, sometimes in-filled, typically at monthly or daily resolution, constructed from CDRs and ideally accompanied by estimates of uncertainty in the gridded values and the correlation structure of the uncertainty.

Data product: usually a gridded analysis, CDR or collection of data enhanced with derived variables or metadata.

\section{Introduction to the Generation of Climate Data Records (CDRs) and Data Products}

Figure 1 shows in schematic form the process involved in constructing a long-term dataset. In summary this consists of the following steps:

Step 1 - understand what is required by different end users, prioritize

Step 2 - gather together available data and metadata, or digitize new data

Step 3 - determine the structure of the error model for each observation type and source and estimate contributing uncertainties and their dependencies

Step 4 - clean up and bias adjust observations, propagating uncertainties and assessing uncertainty in the adjustments

Step 5- compare measurements from different components of the observing system in the context of their estimated uncertainties and user requirements, revisit steps 2-5 (and possibly 1 ) if needed

Step 6 - produce data products tailored for particular applications

Step 7 - evaluate products by comparison with withheld observations or independent reference data if available, and with other similar data products if available

Step 8 - disseminate products with appropriate metadata, uncertainty estimates and documentation.
As shown in Figure 1 this should be an iterative process, as changes to any part of the system will affect successive parts, and all can be refined by iteration.

\section{Structure of This Paper}

A general error model is first introduced along with an overview of approaches to uncertainty estimation and the generation of gridded and gap-filled data products (Section "An Error Model and Its Application"). The section "Overview of Available Data and Observational Metadata" describes the main types of platforms providing data and ancillary information relevant for the construction of surface marine CDRs, how each contributes to CDR construction and how the available observations might be improved and extended. The next section considers "User Needs for Data and Data Products" followed by an overview of approaches to the construction of CDRs, including summaries of specific issues for each ECV (Section "Considerations When Creating Internally-Consistent Records for Marine Surface ECVs"). The final sections look to the future and provide reflections and recommendations.

\section{AN ERROR MODEL AND ITS APPLICATION}

\section{Introduction to an Error Model for Individual Observations}

The observed value of measurand $(O)$ at location co-ordinates $(x$, $y)$, date and time $(t)$ and observing height or depth $(h)$, hereafter location $(x, y, t, h)$ is an approximation to the true value $(T)$ at a desired nearby location $\left(x^{\prime}, y^{\prime}, t^{\prime}, h^{\prime}\right)$. The definition of "nearby" will depend on the application, for example on the size of output grid or distance from a reference observation. The observed value will contain systematic and random errors (e.g., JCGM, 2008), and is an approximation for $T$. It may be possible to estimate the component due to systematic errors $(B)$. Similarly, it may be possible to estimate the component due to differences in location or spatiotemporal representativeness $(L)$. In each case we wish to correct the observations for bias or location, leaving residual errors $\varepsilon_{b}$ and $\varepsilon_{l}$ respectively. The local random errors, after any adjustment, can be represented by $\varepsilon_{o}$.

The systematic errors ( $B$, hereafter bias) may depend on location, the general measurement method $(m)$, the specific instrument used $(i)$, the platform $(p)$, the ambient environmental conditions at the time of the observation $(a)$, and how the data were recorded, transmitted, exchanged, stored and archived $(d)$ or other factors. The differences due to location $(L)$ will depend on the gradients in the field of interest and on the distance. Examples of such location adjustments are to adjust observations to a common reference level (such as the adjustment of marine air temperature measurements to a common reference level, Kent et al., 2013), or time (such as the adjustment of satellite observations of SST to a common reference time to remove inhomogeneity in retrievals due to changing overpass times relative to local time of day, Merchant et al., 2014). Following correction for location differences and systematic observational 


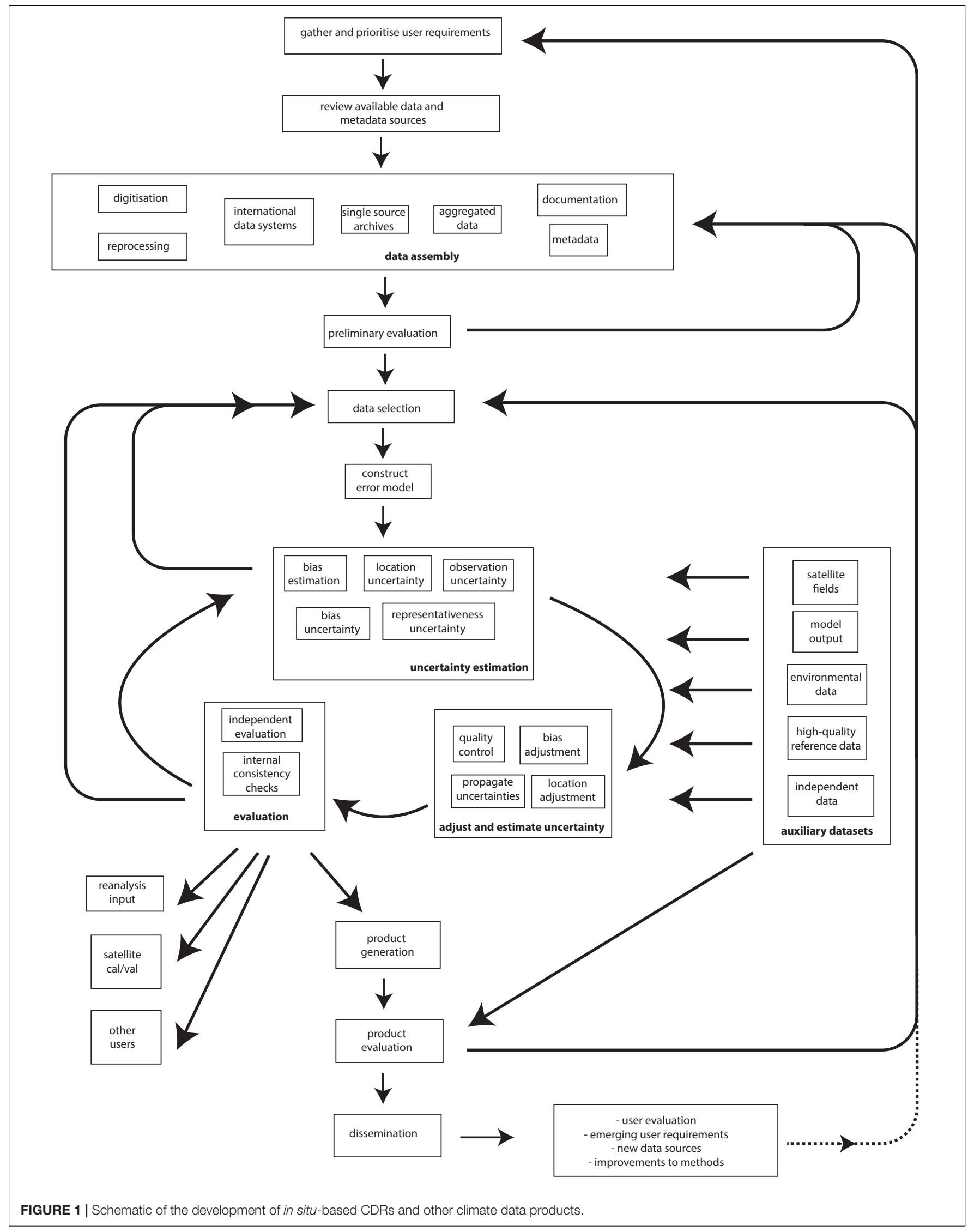


errors our best estimate is that all error terms $\left(\varepsilon_{o}, \varepsilon_{b}, \varepsilon_{l}\right)$ will have zero mean across a large number of observations, but there may be structural relationships between subsets of errors that might be, for example, correlated across $x, y, t, m, i, p, a$, or $d$.

This error model can be written:

$$
\begin{aligned}
& O(x, y, t, h)=T\left(x^{\prime}, y^{\prime}, t^{\prime}, h^{\prime}\right)+B(x, y, t, h, m, i, p, a, d, \ldots) \\
& +L\left(\frac{\partial T}{\partial x}, \frac{\partial T}{\partial y}, \frac{\partial T}{\partial t}, \frac{\partial T}{\partial h}, \Delta x, \Delta y, \Delta t, \Delta h\right)+\varepsilon_{0}+\varepsilon_{b}+\varepsilon_{l}
\end{aligned}
$$

With the residual difference between our observed and approximated value given by:

$$
\begin{gathered}
O(x, y, t, h)-\bar{B}(x, y, t, h, m, i, p, a, d, \ldots)-\bar{L}\left(\frac{\partial T}{\partial x}\right. \\
\left.\frac{\partial T}{\partial y}, \frac{\partial T}{\partial t}, \frac{\partial T}{\partial h}, \Delta x, \Delta y, \Delta t, \Delta h\right)-T\left(x^{\prime}, y^{\prime}, t^{\prime}, h^{\prime}\right)=\varepsilon_{0}+\varepsilon_{b}+\varepsilon_{l}
\end{gathered}
$$

Where the overbar indicates an estimated value of the systematic $(\bar{B})$ and location errors $(\bar{L})$. Estimates of $T$ and gradients in $T$ will have further dependencies, for example on $x, y, t, h$, or $a$.

Typically we also need estimates of the expected error variances $\left(\left\langle\varepsilon_{0}^{2}\right\rangle+\left\langle\varepsilon_{b}^{2}\right\rangle+\left\langle\varepsilon_{l}^{2}\right\rangle\right)$ and their covariance structure. The random errors are usually considered independent and the covariance structure between observations takes the form of a diagonal covariance matrix. The covariance structures for the other error terms are more complicated. For example, the residual bias and location errors may be considered to be fully correlated across observations sharing particular characteristics (e.g., same $m, i, p, a$ or $d$ or nearby) and uncorrelated otherwise (JCGM, 2008).

The general error model informs data requirements. The most obvious requirement is for observations $(O)$ of the parameter of interest at known dates, times, and locations. Next is for information on the methods and instruments used in order to accurately estimate any bias correction $(\bar{B})$ and any auxiliary information required to calculate the location adjustment $(\bar{L})$, for example atmospheric stability for the air temperature height adjustment. The number of observations required will depend on the objective (e.g., location or region, spatiotemporal resolution, and desired accuracy), and on the uncertainty in the observations (and its correlation structure). These requirements will be discussed further for specific variables in Section "User Needs for Data and Data Products." In addition to estimates of the adjustments required for biases and location differences we also need estimates of the expected variance of the residual errors, these are addressed in the next section.

\section{Approach to Uncertainty Estimation}

Historical data vary in quality and have a variety of errors. Understanding these errors, the degree to which they can be corrected, and the residual uncertainty associated with the adjusted measurements is essential for making use of the data. Quantification of uncertainties is likely to reveal complex dependencies of $\left\langle\varepsilon_{0}^{2}\right\rangle+\left\langle\varepsilon_{b}^{2}\right\rangle+\left\langle\varepsilon_{l}^{2}\right\rangle$ (e.g., on $\left.x, y, t, h, m, i, d, p, a\right)$.
There are two primary ways of estimating the uncertainty in the observations, either through the direct observation and estimation of the probability density functions for the different errors (Type A) or through the use of assumed probability density functions based on other evidence (Type B) (JCGM, 2008). Instruments calibrated to international (and national or community) standards will conform with the Type A approach prior to deployment. However, even if international standards are followed and an instrument installed on a ship is accurate, siting of the instrument and misreporting can still lead to significant errors (e.g., Beggs et al., 2012). Drifting buoys are rarely recovered, so their calibration chain is effectively broken upon deployment. Moored buoys, such as those in the TAO array, are periodically recalibrated, but detailed calibration information is not presently delivered alongside the observations.

Typically, other solutions following the Type B approach are required. For example, one solution for detecting drifts in the calibration of sensors on drifting buoys is to fit each drifter with multiple sensors (Reverdin et al., 2010; Poli et al., 2018). Another solution is to compare coincident or nearly coincident measurements from different instruments such as using variograms (Kent and Berry, 2005). Triple co-locations (e.g., Stoffelen, 1998; O'Carroll et al., 2008) can be used in situations where errors can be considered to be independent. Application of such techniques requires redundancy in observing systems and consideration of the correlation structure of the errors. Comparisons between nearly coincident measurements are also important for quality control and bias estimation.

Quality control systems such as the in situ SST quality monitor (iQuam) Atkinson et al. (2013) and Xu and Ignatov (2016) use comparisons between in situ measurements and a reference field derived from a satellite-based analysis to quantify biases and standard deviations for individual platforms, which are effectively estimates of uncertainties. Others (for example Kent et al., 1993; Stoffelen, 1998; Ingleby, 2010) have used weather forecast fields as a basis for estimating measurement errors and uncertainties. More modern statistical infilling techniques can also be used to estimate and assess errors and uncertainties (see, e.g., Kent et al., 2017) but it is challenging to implement methods with complex dependencies globally and with high data volumes, for example, as associated with satellite data.

\section{The Construction of Gridded and Gap-Filled Records}

One of the main requirements for long-term CDRs is the creation of datasets containing summary statistics, such as the mean, on a regular grid. These gridded products tend to be more usable than the individual observations for many applications and are widely used. The simplest gridded products are based on the arithmetic mean of all observations within a grid box passing quality control. Examples include the ICOADS summaries (Freeman et al., 2017) and HadSST3 (Kennedy et al., 2011a,b). Such products are typically easy to use and maybe relatively easy to produce, but suffer from a variety of problems such as unresolved biases, incomplete coverage and inhomogeneous variance in time and space owing to the vastly different sampling density in 
different times and places. Gridbox uncertainty estimation should account for all important components of the appropriate error model for the observation types used, and will typically require observational and platform metadata to correctly represent the expected error covariances. Kennedy et al. (2011a) showed large increases in the uncertainty in the global average SST associated with measurement error when covarying errors were taken into account.

Simple gridded products often form the basis of more sophisticated data treatments. A number of data sets interpolate or fill in gaps in the data, mostly for SST (e.g., ERSSTv5, Huang et al., 2017; COBE-SST2, Hirahara et al., 2014; HadISST, Rayner et al., 2003; Kaplan SST, Kaplan et al., 1998), but also for pressure (HadSLP2, Allan and Ansell, 2006). For filling of large-scale gaps as is required particularly in the 19th century (Figure 2A), these reconstruction methods typically use patterns (e.g., principal components or empirical orthogonal functions) derived from modern well-sampled data to reconstruct sparsely observed past climate states. The advantage of having globally or regionally complete fields is obvious and such analyses are widely used. A number of generic difficulties are expected and seen in such analyses including loss of variance, under-estimation of trends, over-fitting, and under-estimation of uncertainty. This highlights the difficulty of representing the broad spectrum of possible climate states from the relatively small and noisy sample of observed historical variability. In addition, these methods do not typically take into account the full range of error structures seen in the data and typically assume that errors are uncorrelated. Smaller-scale gaps and smoothing of data can also use techniques based on describing the expected local structure of variability such as optimal interpolation (e.g., Reynolds et al., 2002) or using a statistical model of the mid-scale variability (Karspeck et al., 2012).

One aspect of gap-filling that has not yet been widely used is exploitation of co-variability between different variables (except in the context of dynamical reanalysis production which exploits physical relationships between variables via assimilation into a weather forecasting model). Examples where this is likely to be useful include: joint analysis of winds and pressure; pressure or atmospheric circulation and temperature, humidity or cloud cover; and of SST, MAT, and humidity.

\section{OVERVIEW OF AVAILABLE DATA AND OBSERVATIONAL METADATA}

\section{The Different Types of Observing Platforms}

Figure 2 summarizes data availability based on the ICOADS Release 3.0 from each of the in situ observation types considered in Section "The Different Types of Observing Platforms." Figure 2A shows sampling by ECV for 1800 to 1899, a period dominated by ship observations (Figure 3). Reports typically contain several ECVs with humidity and sea state reported less frequently than SST, MAT, SLP or winds. Numbers of observations and coverage generally increase over time, with peaks associated with the start of international coordination (Maury, 1854) and the ingest of a large collection of US logbooks. Observation numbers and coverage both increase over 1900 to 1969 (Figure 2B) with decreases associated with the two world wars. There is an increasing contribution from meteorological observations associated with oceanographic measurements (Boyer et al., 2013). Humidity and waves remain less well-sampled than the other variables, their coverage depending on the sources of data in this period. Since 1970 the range of platform types diversifies (Figure 3) but while the number of observations increases, the coverage is lower by 2017 than in 1970 for all ECVs (Figures 2C, 3). This is due to a declining contribution by ships since the 1990s, partially compensated for SST and SLP by an increase in coverage from drifting buoys (noting that ICOADS is currently missing some drifting buoy observations from 2016 onwards).

\section{Non-specialist Observing Ships}

The creation of long records depends on combining many different types of observations. Many ship observations were originally made for ship operations or navigation, or in support of numerical weather prediction (NWP). Some observations arise from programs that did aim to understand marine climate variability, but typically to chart long term mean conditions (climatology) such as Maury (1854) or the WMO Marine Climatological Summaries Scheme (MCSS, WMO, 2012). The amount of information we have for such vessels varies dramatically with the data source. For observations digitized recently the value of multi-variate records with rich metadata is recognized and typically an attempt is made to recover all relevant information. However, in the past data and metadata are often lost through the many format conversions that observations may have been subjected to.

The earliest observations come from digitization of observations from individual voyages of discovery (Woodruff et al., 2005). The earliest systematic observations come from logbooks of the East India Company covering the period 1789 to 1834 (Freeman et al., 2017) and then from international co-ordination of marine observing by Maury (1854). This international co-ordination is now under the WMO Voluntary Observing Ships (VOSs) scheme (WMO, 2012). Presently data from VOS are collected in near real time (NRT) in support of NWP, via the WMO Global Telecommunications System (GTS). Archives of GTS-derived reports are kept by several National Weather Services (NWSs) but there is no systematic international system that ensures completeness and quality of these records that become the mainstay of the climate observing system.

Some VOS observations are also made available in delayed mode with additional parameters and improved quality control through dedicated WMO Global Assembly Centers (GDACs) in the United Kingdom and Germany. Over the years ICOADS has acquired major collections of surface marine observations from prior to the VOS scheme that were not collected by Maury, notable examples are from the United States, Japan (Kobe collection), Norway, and Australia (Manabe, 1999; Worley et al., 2005). 


\section{A (i) Number of SST observations 1800 to 1899}

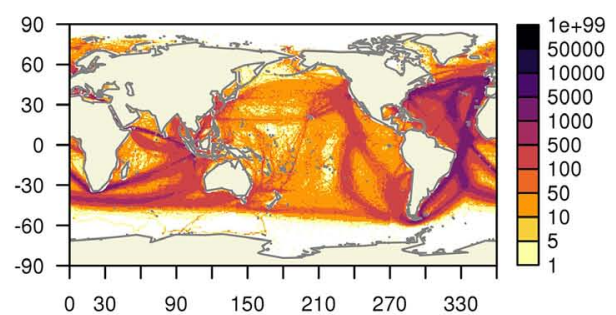

(iii) Number of SLP observations 1800 to 1899

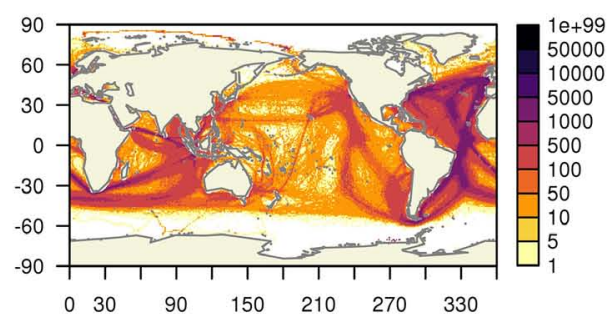

(v) Number of wind speed observations 1800 to 1899

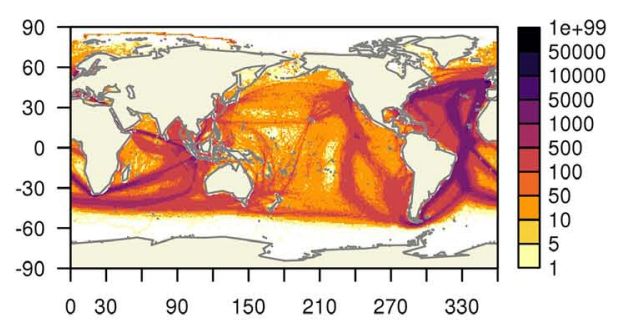

(vii) Number of wave observations 1800 to 1899

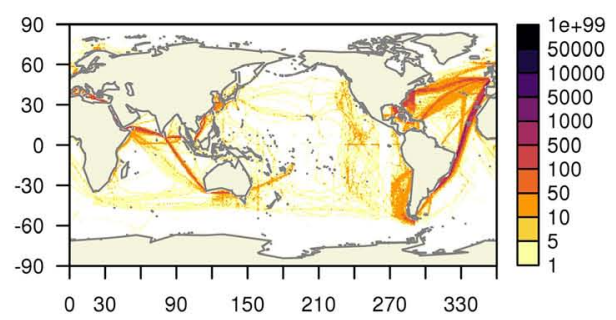

(ix) Number of observations 1800 to 1899

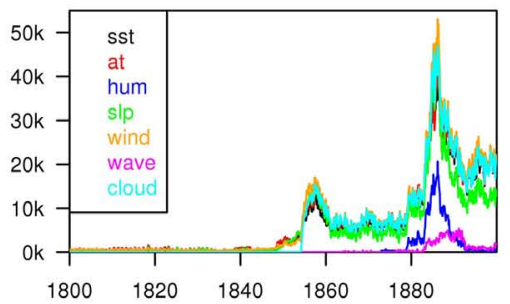

(ii) Number of AT observations 1800 to 1899

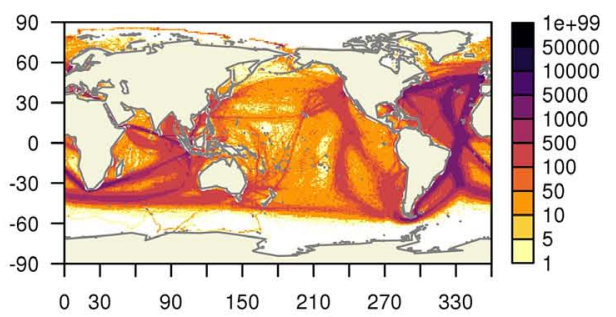

(iv) Number of humidity observations 1800 to 1899

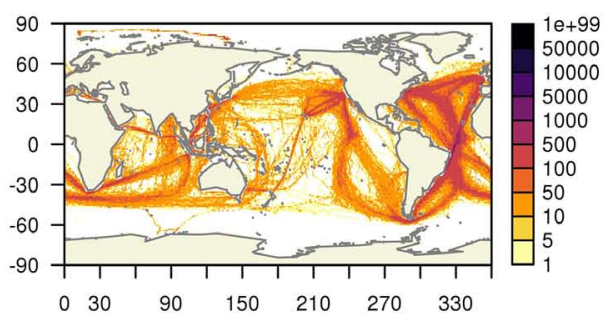

(vi) Number of wind direction observations 1800 to 1899

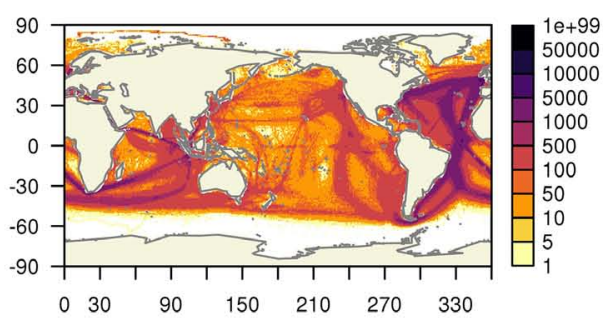

(viii) Number of cloud observations 1800 to 1899

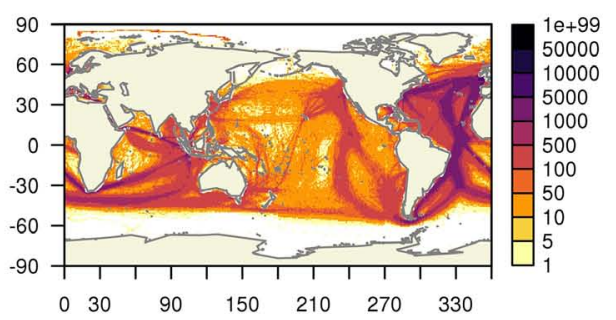

(x) Coverage at $1^{\star} 1^{*}$ monthly 1800 to 1899

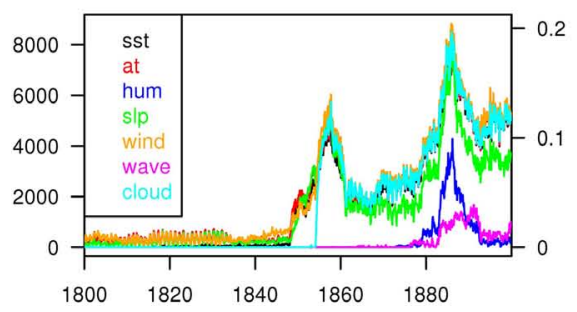

FIGURE 2 | Continued 
B (i) Number of SST observations 1900 to 1969

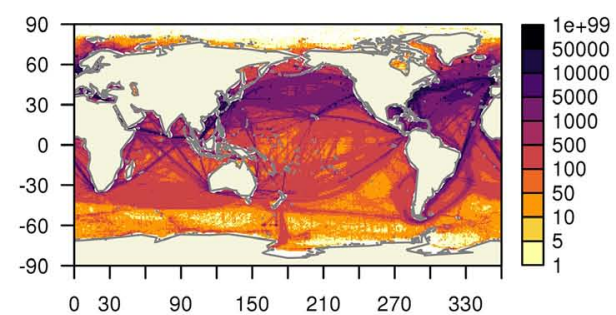

(iii) Number of SLP observations 1900 to 1969

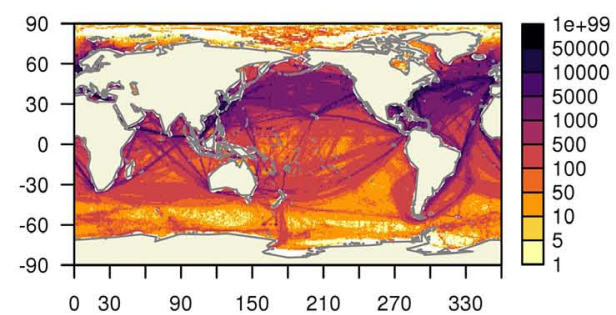

(v) Number of wind speed observations 1900 to 1969

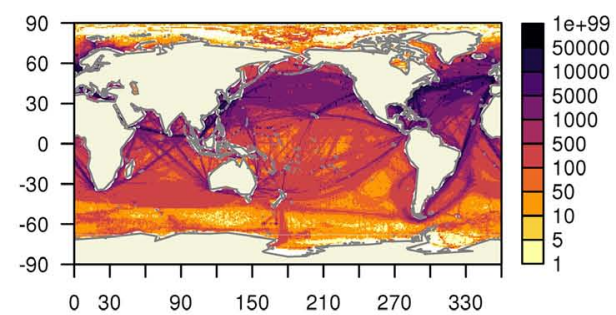

(vii) Number of wave observations 1900 to 1969

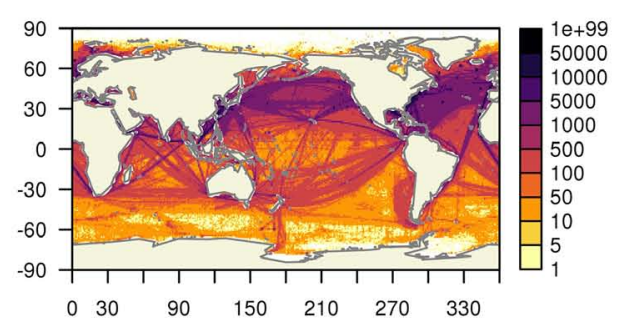

(ix) Number of observations 1900 to 1969

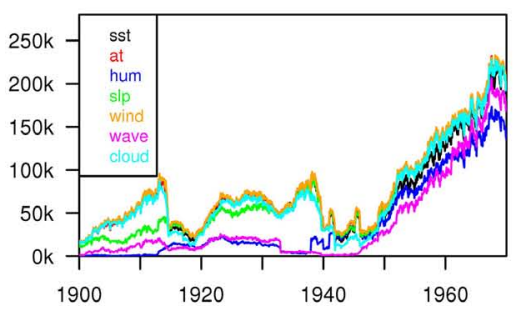

(ii) Number of AT observations 1900 to 1969

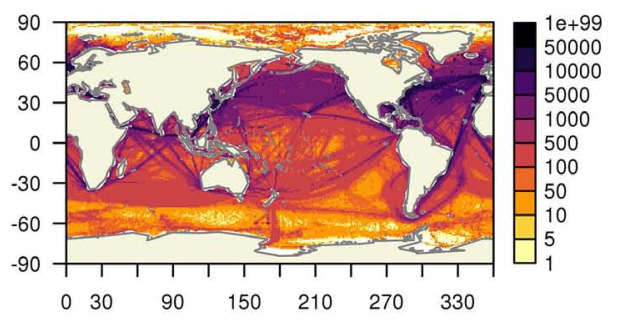

(iv) Number of humidity observations 1900 to 1969

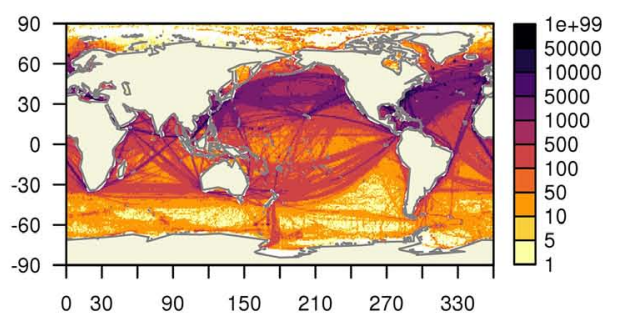

(vi) Number of wind direction observations 1900 to 1969

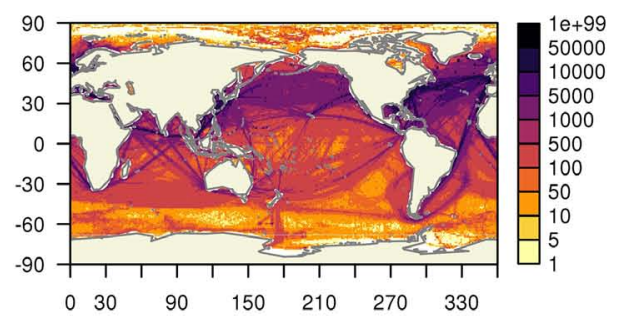

(viii) Number of cloud observations 1900 to 1969

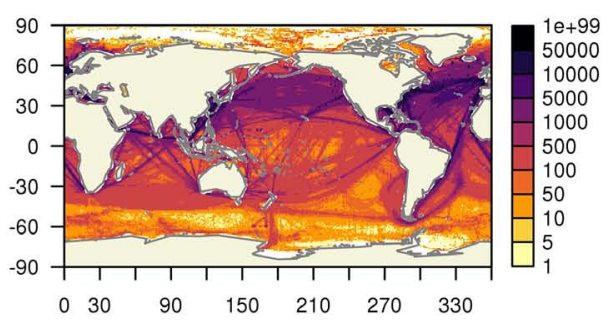

(x) Coverage at $1^{*} 1^{*}$ monthly 1900 to 1969

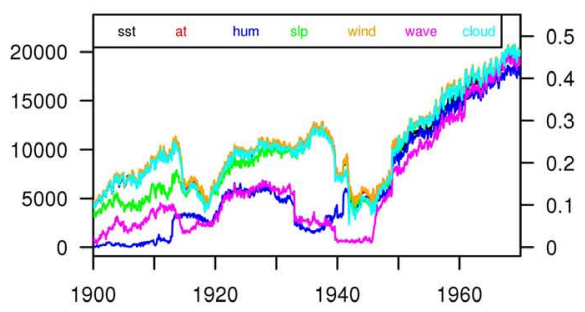

FIGURE 2 | Continued 


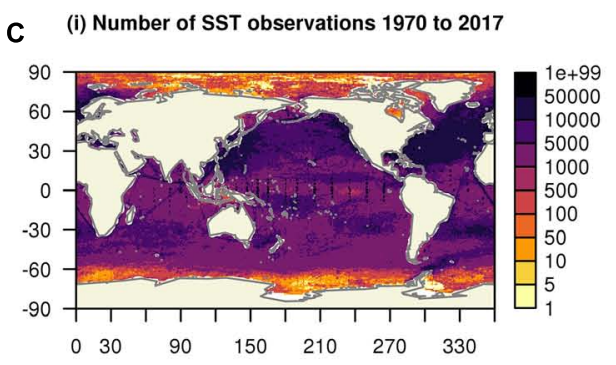

(iii) Number of SLP observations 1970 to 2017

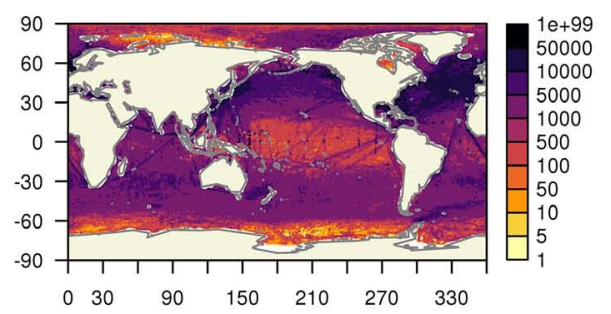

(v) Number of wind speed observations 1970 to 2017

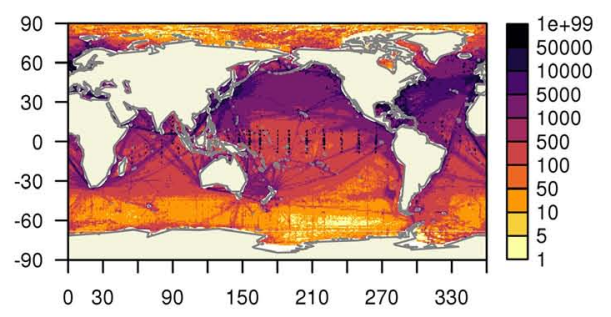

(vii) Number of wave observations 1970 to 2017

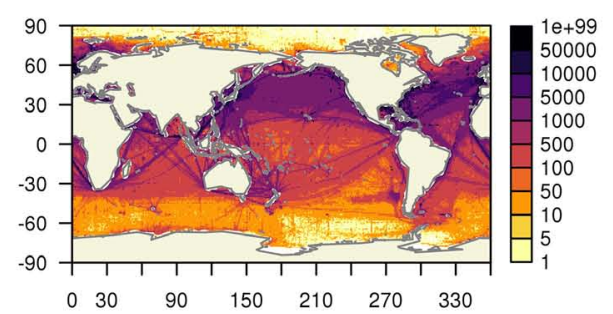

(ix) Number of observations 1970 to 2017

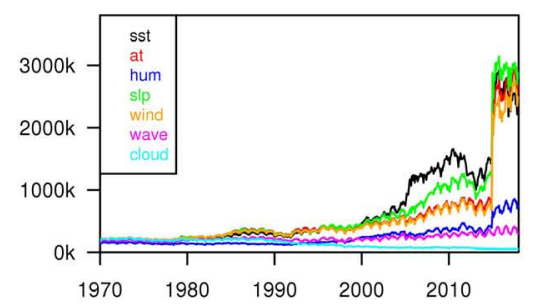

(ii) Number of AT observations 1970 to 2017

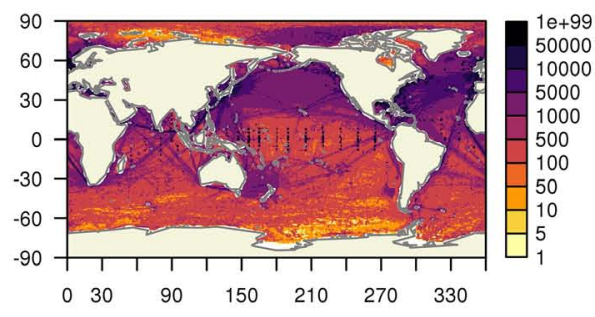

(iv) Number of humidity observations 1970 to 2017

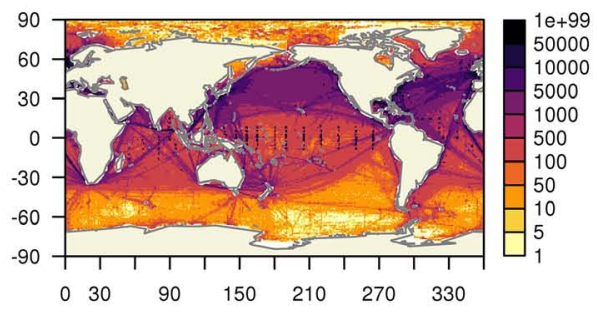

(vi) Number of wind direction observations 1970 to 2017

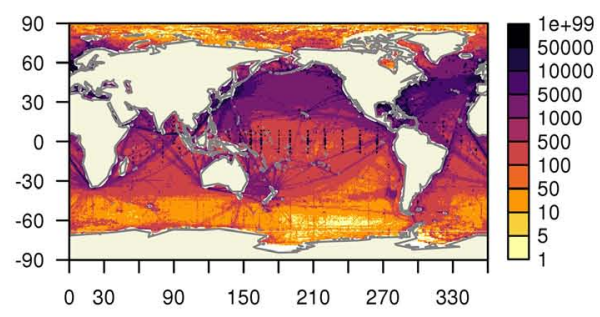

(viii) Number of cloud observations 1970 to 2017

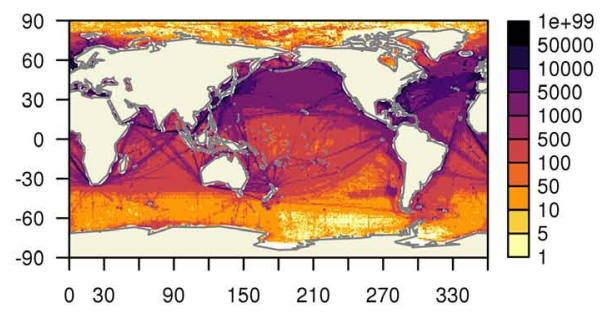

(x) Coverage at $1^{*} 1^{*}$ monthly 1970 to 2017

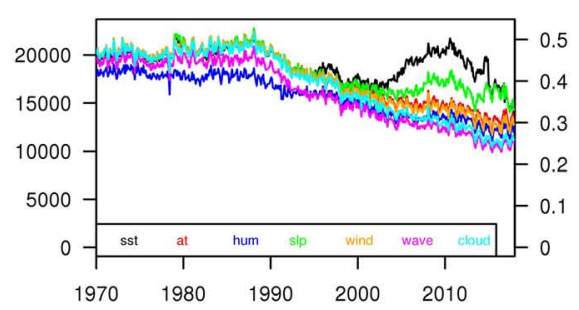

FIGURE 2 | (A) Total number of observations on a $1^{\circ}$ latitude-longitude grid for selected ECVs from ICOADS Release 3.0 over the period, $1800-1899$. The lower panels show (ix) the total number of monthly observations and (x) the number of $1^{\circ}$ monthly areas sampled (left axis) and fraction of ocean $1^{\circ}$ areas (right axis). No QC or other data selection has been applied. (B) As 2a but for 1900-1969. (C) As 2a but for 1970-2017. 


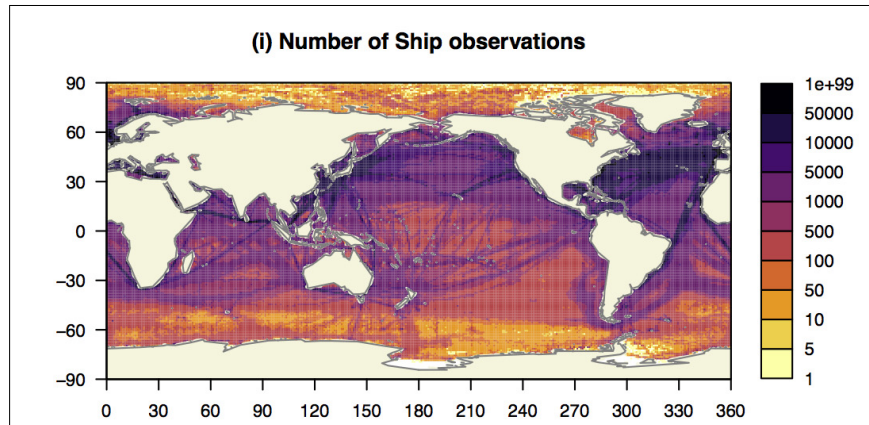

(iii) Number of drifting buoy observations

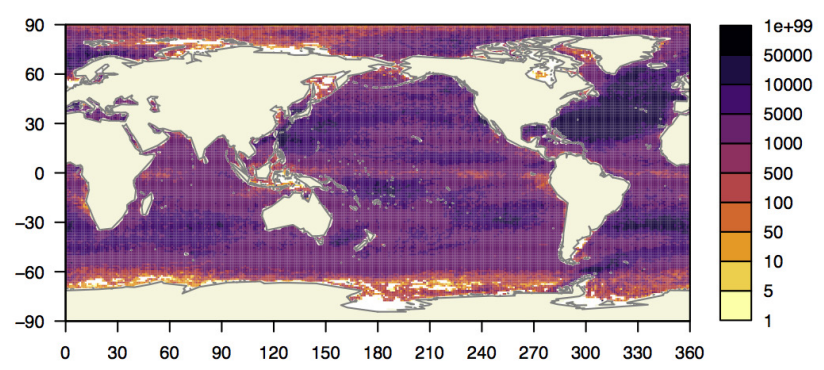

(v) Number of observations 1850 to 2017

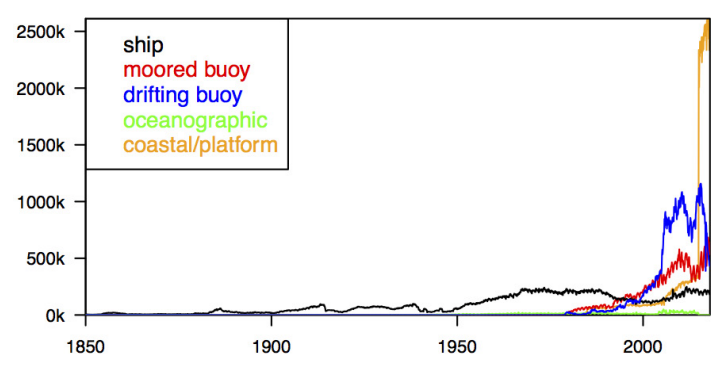

(vii) Number of observations 1970 to 2017

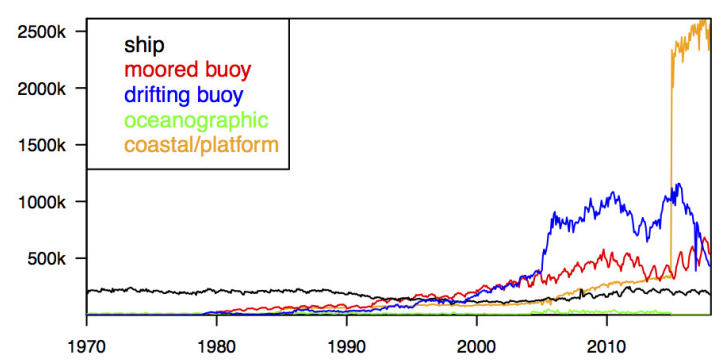

(ii) Number of moored buoy observations

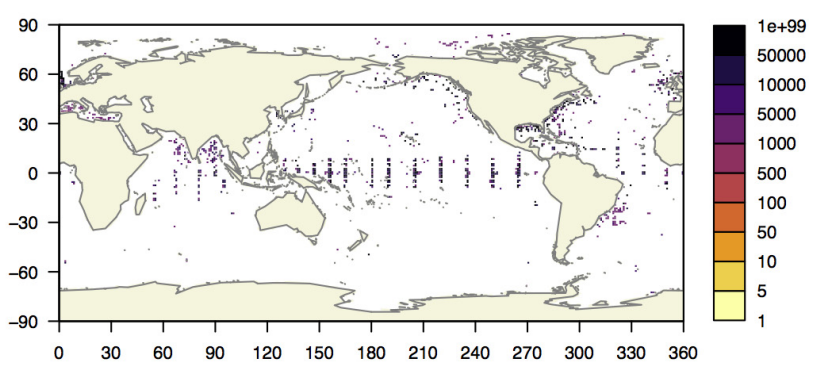

(iv) Number of oceanographic observations

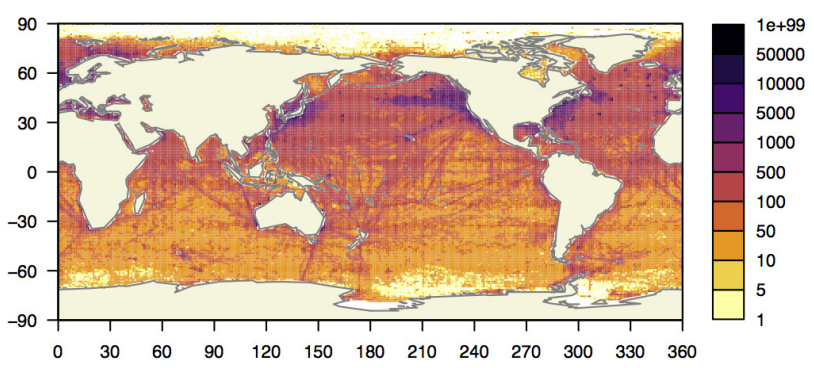

(vi) Coverage at $1^{*} 1^{*}$ monthly 1850 to 2017

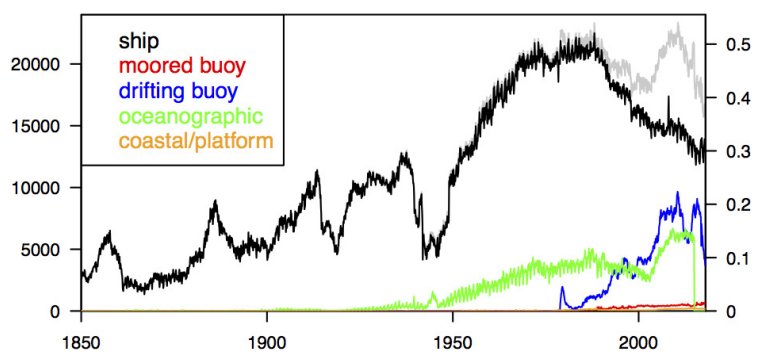

(viii) Coverage at $1^{\star} 1^{\star}$ monthly 1970 to 2017

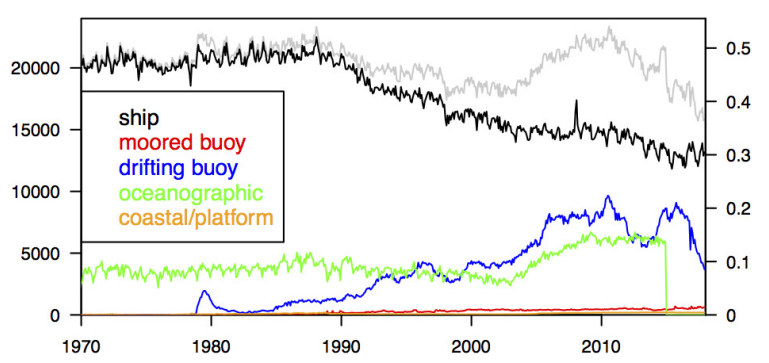

FIGURE 3 | Number of observations between 1850 and 2017 (as Figure 2) but by ICOADS platform type (PT): ships (PT 0-5); moored buoys (PT 6), drifting buoys (PT 7), oceanographic observations (PT 10-12, 19-21), and coastal and other platforms (9, 13-16). Note that some missing PT have been estimated based on data sources. The gray line in panels vi and viii shows the coverage across all platforms. Panels vii and viii show the same information as $v$ and vi but starting in 1970. Oceanographic data are ingested into ICOADS in delayed mode, data for 2015 onwards will be ingested in the next ICOADS release.

\section{Specialist Observing Ships}

Observations from specialist vessels obviously have the potential for providing higher quality observations than non-specialist ships. However, in order for such observations to be most useful for the construction of long term records the data must be clearly identifiable and accompanied by extensive platform and observational metadata. ICOADS contains observations from research vessels (RVs), ocean weather ships (OWSs), and light vessels (LVs) that have the potential to be used in a variety of ways either as a source of observations in sometimes 
sparse ocean regions, or as high quality data for evaluation (e.g., Smith et al., 2001).

International Comprehensive Ocean-Atmosphere Data Set mainly contains observations from RVs operating as VOS and using a separate set of instruments from those used for research applications. In some cases, particularly in the US, VOS observations from RVs may come from the research instruments. Additionally, some RV data in ICOADS are from delayed-mode data sources that are focused on collection and quality evaluation of measurements from research instrumentation (e.g., Smith et al., 2018). There is the potential for some confusion as to the source of any observations identified as coming from RVs by their identifiers (callsign or ship name, hereafter IDs). In some cases, the same RV may provide VOS and delayed mode observations from two different instrument systems. A similar situation exists for OWS, which can be found in ICOADS in several different source data collections. Some OWS observations are clearly identified as such, either through being identified with a specialist source, or through the ICOADS "platform type" identifier (PT). However some ICOADS data sources contain mixtures of OWS and other data types, not always clearly identified, some duplicating observations in dedicated OWS sources likely to be of higher quality and completeness, and some that may be unique.

Data from RVs are typically managed at the national level with no dedicated international data management or archival system (Smith et al., 2019). Some nations do have dedicated data management systems for RVs including the US (Smith et al., 2018) and Australia [Integrated Marine Observing System (IMOS)], full international integration of such national RV data systems would enable the construction of high quality datasets for evaluation of a wide range of data and data products.

Data from specialist observing ships could have huge value for the development of long-term datasets, but the lack of an integrated international management system for RV data means that observations are not well-utilized for uncertainty estimation and quality evaluation.

\section{Moored Buoys}

Moored buoy observations have contributed to the global observing system since the 1970s (Figures 2C, 3) and are found in ICOADS in data sources deriving from both NRT and delayed mode archives. The two largest sources of moored buoy observations are the Global Tropical Moored Buoy Array (GTMBA) providing measurements in the Pacific, Atlantic and Indian Oceans (McPhaden et al., 1998) and the coastal network of buoys that make observations in support of NWP (DBCP, 2016). GTMBA data are curated by the NOAA Pacific Marine Environmental Laboratory (PMEL) who provide consolidated access to data from the full tropical array ${ }^{1}$ (accessed 26 March 2019). ICOADS ingested the GTMBA archive as of February 2016 for Release 3.0 which included data collected up to the end of 2014 (Freeman et al., 2017). The PMEL archive is updated when improved information such as from post-calibration becomes

\footnotetext{
${ }^{1}$ https://www.pmel.noaa.gov/tao/drupal/disdel/
}

available, but updates and changes to the archive are not prominently publicized.

Observations from national coastal networks have not been a priority for historical curation, and no definitive archive for the data and metadata exist. Whilst potentially valuable, a combination of mixed data quality with many moorings sited in highly-variable coastal locations has meant that these moorings have not been widely exploited for climate applications (e.g., Wentz et al., 2017).

The OceanSITES aim is to collect, deliver and promote the use of high-quality data from long-term, high-frequency observations at fixed locations in the open ocean. Its scope is wider than considered here, including also biogeochemical and subsurface observations. OceanSITES funds a Technical Coordinator and IT staff who are based at the JCOMMOPS Project Office (OceanSITES, 2016). OceanSITES collates timeseries data from many different providers, and makes it available in a consistent format (OceanSITES, 2015) via two Data Assembly Centres. Several of the GTMBA moorings are designated as OceanSITES as are long-term moorings from the Woods Hole Oceanographic Institution.

\section{Other Moored and Fixed Platforms}

Observations made in coastal regions and from fixed platforms such as oil rigs can also contribute to the marine climate record (Figure 3). Land-based coastal observations are often excluded from CDRs and other data products as unrepresentative of open ocean conditions, but have a role to play for example for evaluation of datasets (see Hanawa et al., 2000; Kent et al., 2017; Cowtan et al., 2018 in the context of SST).

\section{Surface Drifters}

Surface drifters predominantly report SST and SLP (Figures 2C, 3), providing critical data for NWP and reanalysis (Centurioni, 2018). The development of the surface drifter observing system has been facilitated by clear user requirements (e.g., Zhang et al., 2006) and assessment of the impact of the data (e.g., Ingleby and Isaksen, 2018). However the reliance on surface drifters has led to a decline in observations of important ECVs such as MAT, humidity, wind, and cloud (Kent et al., 2006, also Figure 2C). Some drifting buoys have reported MAT, but their quality has not yet been evaluated. There is the potential for correlated sampling uncertainty as drifters may get trapped in eddies and will follow currents. They cannot provide good sampling in regions of divergence or areas of upwelling. Lack of multivariate sampling can be problematic for analysis, for example Morak-Bozzo et al. (2016) associated model output with drifting buoy measurements to characterize the dependencies of their diurnal cycles.

\section{Oceanographic or Profile Measurements for Temperature}

Historically, typically from 1950 to 2000s, ocean temperature was measured by reversing thermometers (attached to hydrographic bottles), conductivity-temperature-depth (CTD), mechanical bathythermographs (MBTs), and expendable bathythermographs (XBTs). Globally, there were 5000-20,000 measurements within $5 \mathrm{~m}$ of the ocean surface each month from 1950 to $2000 \mathrm{~s}$ 

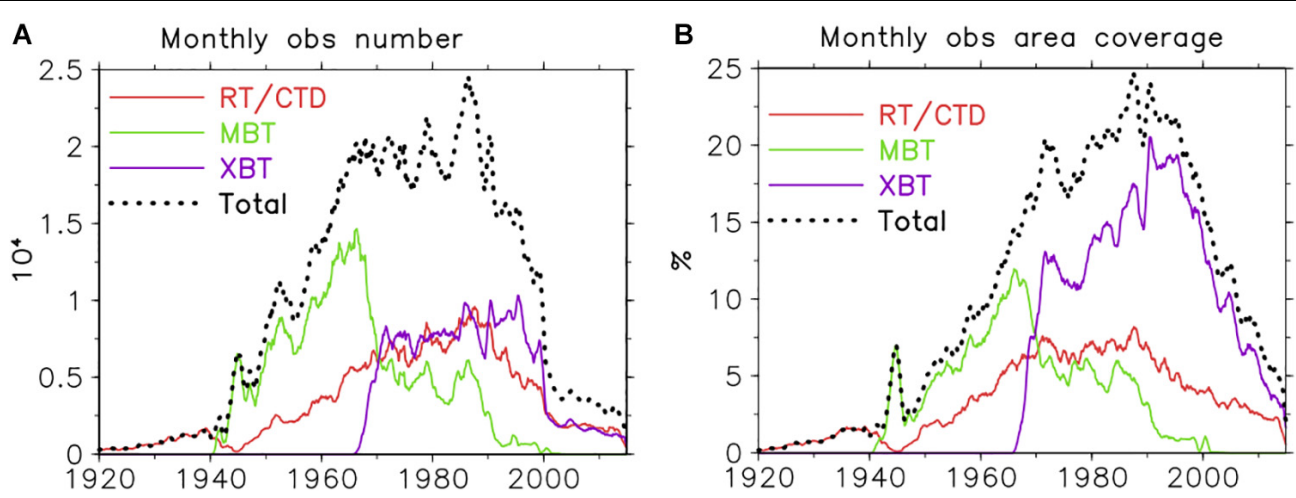

FIGURE 4 | (A) Number and (B) area coverage of monthly measurements from RT/CTD (solid red), MBT (solid green), XBT (solid purple), and total (dotted black) in the global oceans.

(Figure 4A), which covers $5-20 \%$ area of the ocean on $2^{\circ} \times 2^{\circ}$ grids (Figure 4B). Argo floats provide highly accurate temperature measurements but observations are sparse due to the typical 10 day sampling cycle. Argo data are only available for regions where the ocean is deeper than $\sim 2 \mathrm{~km}$.

These measurements were commonly used to estimate ocean heat content (e.g., Levitus et al., 2009), but XBT are problematic for SST analysis due to biases caused by the shock of entry into the water. Recent studies have however (e.g., Gouretski et al., 2012; Hausfather et al., 2017; Berry et al., 2018; Huang et al., 2018) have used these measurements to evaluate SST data products.

\section{Satellites}

Data from satellites have become an important resource for climate. Their record length is presently not yet sufficient on their own to meet our definition of a long term climate record. Increasingly CDRs will be constructed from satellite observations without blending with in situ observations, and the same considerations for stability and uncertainty will apply. In situ data are also required for calibration and validation of satellite climate records (e.g., Belmonte Rivas et al., 2007; Berry et al., 2018). As with in situ networks, satellite missions have not always been designed with climate applications foremost, so typically substantial work has to go into constructing stable records (Hollmann et al., 2013; Verhoef et al., 2017). However there are now efforts to establish and maintain traceability of global fiducial reference measurements, including for satellitederived surface temperature (Snook, 2016).

Sea surface temperature is one ECV where satellite data has been most used for the construction of CDR, notably in the HadISST dataset (Rayner et al., 2003). Substantial effort has been put into the construction of stable and accurate SST records (Merchant et al., 2012, 2014). Other variables where satellites will play an important part in the construction of long-term CDR are for clouds and radiation (e.g., Loeb et al., 2012), winds (e.g., Verhoef et al., 2017), and waves (e.g., Young et al., 2011; Ardhuin et al., 2019). However, to date only SST has extended the satellite record back in time using in situ observations. Air temperature and humidity are hard to derive from space (e.g., Andersson et al., 2011; Prytherch et al., 2015) but doing so would be valuable for estimation of air-sea exchanges (Weller, 2018; Cronin et al., 2019).

The satellite community are important users of in situmeasured surface ECVs (e.g., Belmonte Rivas et al., 2007; Stoffelen et al., 2015; Jackson and Wick, 2016; Kinzel et al., 2016; Berry et al., 2018; Liman et al., 2018; Thorne et al., 2018), and recommendations that aim to improve the quantity, quality, and consistency of in situ data will be of huge benefit for satellite calibration and evaluation.

\section{Selection of Data Sources \\ Selection of in situ Observations for the CDR}

The construction of long records inevitably means that a range of data sources needs to be considered and that there will be compromises on data quality to increase sampling. Most modern in situ-based surface marine climate records are built using the ICOADS (Freeman et al., 2017). ICOADS collates surface marine data from a range of different observing platforms, keeping the majority of available parameters and metadata together. Obvious requirements are measurements or visual estimates of the parameter of interest, along with information about the date and time of the observation and its location. Observations in ICOADS may have substantial uncertainty in their locations, dates, and times. Some data sources have positions recorded to the nearest degree, and some positions, dates and times have gross errors (Carella et al., 2017). Increasingly information on the identity of the measurement platform is used, for quality control, but also as part of bias adjustment and the construction of error covariances in gridded analyses. Observational metadata giving measurement methods, heights or depths of sensors is also valuable, but can be estimated (e.g., Kennedy et al., 2011a; Kent et al., 2013; Carella et al., 2018). It is also becoming more common to use information on the ambient environmental conditions as input to bias adjustment schemes and uncertainty estimation, which may further require estimates of ship speed and course, winds, cloud, temperature, humidity, pressure, wave conditions, and coded weather information. The availability of a full suite of estimates of environmental conditions also permits multivariate quality control. 
Despite ICOADS providing all the information in a common format, the content and quality of records from different sources varies markedly. Much of the data provided in ICOADS (then COADS) Release 1 came from data that had been initially recorded in ships logbooks, then stored on punchcards and later transitioned to reel-to-reel tapes (Woodruff et al., 1987). Each format change and re-archival inevitably results in some degree of lost information, and the introduction of transcription errors. The present ICOADS format [International Marine Meteorological Archive (IMMA) format version 1] (Smith et al., 2016) allows the preservation of the entire record, but at cost of considerable complexity. Recently ingested data, such as that from the GTS or data recovery (Allan et al., 2011) therefore can retain a more complete record of the original data and observational metadata.

The amount of information we have for such vessels varies dramatically with the data source, summarized in ICOADS by the DCK (derived from a "deck" of punch cards) and SID (source identifier) indicators. Presently the information required to select observations from ICOADS based on an objective quantification of the quality of the data and metadata needed to construct CDRs is not available. A reprocessing of ICOADS to improve its compatibility with WMO Integrated Global Observing System (WIGOS) data and metadata standards would substantially improve this deficit.

\section{Observations for Bias and Location Adjustment and Uncertainty Estimation}

Methods for the estimation of data uncertainty such as the calculation of variograms (e.g., Kent and Berry, 2005) are based only on the variability of observations themselves, although these may be calculated for subsets of data, for example by measurement method. Bias estimation may use measures of internal consistency between subsets of measurements made using different methods (e.g., Folland and Parker, 1995; Kent and Kaplan, 2006; Kennedy et al., 2011a; Hirahara et al., 2014; Carella et al., 2018), between different platforms, or based on other characteristics (Chan et al., 2019), requiring metadata to identify appropriate subsets.

High quality observations are important for the detection and evaluation of biases (Hausfather et al., 2017; Berry et al., 2018). Specially designed datasets are also useful, often containing enhanced metadata (Kent et al., 1993; Berry and Kent, 2005), and/or co-located observations from different methods (James and Fox, 1972; Berry et al., 2004). Comparison of data products of similar ECVs that are likely to have largely independent errors (for example SST and MAT) can be used for bias adjustment (Huang et al., 2017) or evaluation (Cowtan et al., 2018).

Nearby observations are needed for some types of QC (see section "Quality Control") but can also be used to diagnose relative data biases (e.g., Thomas et al., 2005; Chan and Huybers, 2019).

Observations of ambient environmental conditions which may be based on measurements or climatology are needed for estimation of $B, L$ and their uncertainties, and may also feed into estimation of other components of uncertainty. Coded weather information is particularly useful for identifying ambient conditions, for example whether or not it is raining.

\section{Observations for Variability}

Estimates of local ECV variability and gradients are needed for quantification of location uncertainty, as input to QC and for implementation of local gap-filling and smoothing algorithms. Estimates of large-scale variability are needed to provide patterns of expected modes of variability for reconstructions. Estimates of temporal variability are often based on in situ observations, moored buoys are particularly good for quantification of high-frequency temporal variability, and for understanding covariability among different ECVs. Many ECVs show diurnal variability, both real and spurious. Real diurnal variations are often not considered, but ideally variations in local sampling time should be assessed as part of $L$. Satellite observations can be valuable for the estimation of spatial variability, but for variables such as MAT and humidity can only be assessed from VOS observations at present (Figures 2C, 3).

\section{Observations for Evaluation}

Ideally evaluation would use high-quality independent data widely distributed and spanning the entire period of record. These data would also be independent of those used for evaluation of biases and uncertainty. Examples where such evaluation has been possible are largely limited to SST using observations from Argo or other near surface measurements from oceanographic profiles (e.g., Hausfather et al., 2017; Huang et al., 2018), drifting buoys (e.g., Berry et al., 2018), moored buoys (e.g., Merchant et al., 2012). Evaluation for marine surface CDRs often relies on co-evaluation of different data products, which can provide important information on structural uncertainty (Kennedy, 2014; Kennedy et al., 2019) and be used to evaluate uncertainty estimates (Kent et al., 2017). Examples include: evaluation of SST using MAT from ships (Kent et al., 2013); coastal SST (Hanawa et al., 2000); or coastal air temperatures (Cowtan et al., 2018). Folland (2005) used a climate model to evaluate SST bias adjustments. Triple colocation (Stoffelen, 1998) is often used for evaluation of satellite winds, but has typically not been applied to historical in situ observations due to lack of collocated observations with independent errors.

An alternative, is to define subsets of data for evaluation that are excluded from the construction of data products. Near surface temperatures from Argo have often been excluded from analyses for use in validation (e.g., Martin et al., 2012) and the evaluation of air temperatures over land has also taken this approach by creating a separate version withholding a high quality subset (Hausfather et al., 2016).

When comparing different types of observation, or different gridded products, it is important to account for mismatches in spatiotemporal scales, known as representativeness. An example is when comparing in situ temporal average measurements at a single point with satellite measurements that are an almost instantaneous average across a spatial footprint. In order to estimate the expected uncertainty due to representativeness it is necessary to quantify the differences in scales and the variability of the ECV field across those scales. 
Lack of independent high-quality observations is a barrier to the evaluation of long CDRs. As record lengths increase for timeseries stations such as OceanSITES and the GTMBA, and for satellite CDRs it will be possible to extend evaluation of in situ CDRs to other variables. The continuation and extension of OceanSITES will enable much evaluation of a wider range of ECVs with more independent data, at least for the modern period. RV observations are underutilized for evaluation, mainly because their data management is not internationally coordinated into quality-evaluated archives (Smith et al., 2019). Some potentially high-quality sources of data for evaluation, including from RVs, OWS and coastal stations, may require data rescue (see section "Data and Metadata Rescue") or reprocessing (see section "Reprocessing of Existing Archives").

\section{Enhancing the Observational Archive Quality Control}

Initial checking can identify reports with incorrect values for date, time and position, unphysical values for elements or incorrectly coded parameters or metadata. If these errors are systematic, it may be possible to re-translate the available observation source, or to provide feedback to the data provider and obtain a revised version. More often than not such problems are discovered too late for such remedial action (e.g., the original data may have been lost, or staff may no longer be in post) and the data source may be excluded from the analysis, but some values might be correctable.

Basic checks can identify unusable data or impossible values such as non-existent dates or locations, or observations made over land. Any data failing checks should be flagged rather than discarded as changes to tests may be required in the future, and analysis of reasons for data failing checks can be used in the refinement of QC. Simple tests for each variable include ensuring there is a measured value which is reasonable given typical conditions, usually tested against a monthly or daily climatology with allowances for a combination of the expected real variability in the parameter and observational uncertainty. Further checks may be possible, for example, cloud and weather codes can be checked for consistency (Hahn et al., 1988) and relative humidity should be close to saturation when it is raining or foggy.

More sophisticated checks may then be implemented such as evaluation of the tracks of individual ships or drifting buoys. Tests applied by Rayner et al. (2006) include limits on the inferred speed of the ship, consistency between actual and reported heading, consistency between interpolated locations and reported locations. Other tests that can be performed on individual ships include: checks for repeated values, repeated super-saturation, and counts of observations from the platform (ships which make very small numbers of reports are typically less reliable). More extensive checks can also be performed at this level (e.g., Atkinson et al., 2013; Xu and Ignatov, 2016) by comparing measurements from an individual ship or buoy to a reference field and making decisions based on metrics such as the average bias or standard deviation relative to the reference data set.

A further step may be comparison of nearby observations (Rayner et al., 2006). Typically, a single observation is a compared to a mean of its neighbors. These "buddy checks" are of varying degrees of sophistication and effectiveness depending on the expected density and quality of the neighbors.
At each stage in the process, data which pass or fail individual checks are flagged and an overall decision is made based on some combination of these checks.

Following quality evaluation and flagging particular data sources or platforms that are identified as being of consistently low quality can be explicitly excluded from further analysis. Depending on the volume and characteristics of the observations that are flagged as erroneous or suspect, it may be advantageous to reevaluate parameters and limits within the QC process (Figure 1).

\section{Enhancing Metadata}

The amount of metadata that appears in ICOADS varies dramatically with data source (DCK and SID). Some sources have no platform IDs or observational metadata, some sources have IDs and rich metadata, particularly those that have been more recently digitized or processed. Most data sources have associated documentation, and a systematic review of this information is likely to substantially enhance ICOADS metadata availability. Examples of untapped metadata include mappings of ship numbers to ship names for some Japanese and Australian sources and documentation describing the transcription of data for punchcards. External documentary sources have also been used to infer metadata such as measurement methods (Kennedy et al., 2011b). Metadata from a WMO catalog has been associated with individual observations (Kent et al., 2007) based on IDs, and also used indirectly to infer metadata based on the recruiting country (Kent et al., 2010; Kennedy et al., 2011b). However to take full advantage of new information an improved data system and more flexible data models will be required.

Availability of IDs makes track checking possible (see section "Quality Control") which can identify mispositioned observations. Recent masking of ship callsigns (Woodruff et al., 2011) in response to ship operators concerns about security and commercial interests, has led to a degradation of data in ICOADS and other archives. If no ID information is available then it is not possible to fully apply QC, to easily identify mispositioned or duplicate reports, to appropriately propagate uncertainty and to associate external metadata (Kent et al., 2007). Coded IDs have been adopted by some operators, which avoid some of these issues, but it may still not be possible to associate existing external metadata, and may preclude association of information that becomes available in the future.

It is possible to extend metadata using the characteristics of the data themselves. Examples include: the clustering of reports likely to be made on the same platform by ship tracking (Carella et al., 2017); the inference of data units or reporting precision from the distribution of reported values (Rhines et al., 2015); or the assignment of observing methods based on the data characteristics (Carella et al., 2018). Ideally such indirect methods of deducing metadata should be supported by full descriptions including observing instructions, information on instruments, their locations and installation and also documenting each stage of report coding and recoding.

There is useful information available from a wide range of other sources that have not yet been systematically integrated with marine observations. Where ship names are available it 
is often possible to determine a wealth of information about the ship, including pictures and quantitative information on size and tonnage from the internet. It may also be possible to infer ship names for reports with a numerical ID based on ports of departure and arrival from online sources. Many other sources of information exist that might provide relevant metadata, for example databases held by Lloyds Register of Shipping or metadata transmitted with Automatic Identification System (AIS) vessel tracking.

\section{Merging Different Sources and Archives}

If data from different sources are to be combined and analyzed together then clear metadata is required to indicate the data sources and typically effort will be needed to harmonize data formats, particularly metadata and data flags. Documentation of all steps is essential to avoid misinterpretation.

The identification of any reports that may derive from the same original observation may be required. Examples include where observations may become available in delayed mode in enhanced formats or with additional checks. In this case, any NRT version of the same report should be identified and is usually flagged as inferior (although in some cases inadequacies in the archival process may have degraded the data). This may be straightforward, but in some cases, especially where corruption has occurred, different versions of the same observation can only be identified with uncertainty. For example, prior to ICOADS Release 2.5 (Woodruff et al., 2011) reports identified as inferior duplicates are only available in depreciated data formats, so it is not easy to test the efficacy of the duplicate identification procedures applied. Another issue arises where newly-digitized data sources should replace older versions, but it is not always possible to clearly identify previous digitizations of the same source material.

Ideally the identification of data likely to be derived from the same original observations should allow for a specified tolerance for the permissible degree of difference between candidate reports. This should be based where possible on known recording, conversion, and data management practices (ideally from metadata flags). Where this is not possible the classification of expected differences can be built up from a comparison of the data sources themselves. This classification of expected differences should feed into uncertainty estimation and be used when selecting the preferred version of observations thought to be duplicates.

Data for ICOADS Releases prior to 2.5 (Woodruff et al., 2011) for sources that have been given low priority for selection in the ICOADS “dupelim" duplicate elimination processing and are not available in the present archive should be prioritized for recovery and reprocessing. This will provide improved information on likely differences between data sources and also information on typical errors/miscoding.

\section{The Need for Expert Data System Design and Management}

The modern observing system is diverse and each data type requires careful management, documentation, evaluation and quality control. Observations collected primarily for applications other than climate monitoring, typically for NWP, form the majority of the surface marine climate observing system. It is therefore critical that the needs of climate monitoring are considered in observing system design, and that observations needed for climate monitoring have dedicated data management systems and centers, whatever their origin. Whilst single variable repositories are used for some applications, observations made on the same platform should primarily be managed together and with adequate resources to allow expert evaluation, QC, and bias estimation.

\section{USER NEEDS FOR DATA AND DATA PRODUCTS}

\section{Understanding the Needs of Different Applications}

Needs for observational climate records have in recent years been discussed in a number of contexts, for example under the ESA Climate Change Initiative, the Copernicus Climate Change Service and the 5th International Conference on Reanalyses.

In general, these experiences yield a requirement for:

(1) More data, i.e., existing observing arrays should be maintained, and sparsely observed regions should be better monitored and the potential of past measurements needs to be unlocked via digitisation and wider data sharing;

(2) More research to be undertaken into creating consistent records so that multi-decadal records can be used for the evaluation of decadal re-forecasts, for example;

(3) More research to be undertaken into quantifying uncertainty components and their covariance structures; and

(4) Better statistical modeling techniques to create analyses and allow better representation of the full data distribution to allow, for example, provision of observational constraints on future climate projections.

As the need for higher resolution information (in space and time) grows, there needs to be continued development of long-term products from in situ and satellite which are consistent to support the optimal combination of these different data types. This growing requirement for higher resolution information places high demands on the historical and current observing systems.

To support services with frequent updates, such as seasonal forecasting, and in short delay, such as short-delay event attribution, short-delay updates to monitoring and SST and sea ice boundary forcing data sets (and their underpinning data bases, like ICOADS) need to be developed which are consistent with the long-term record (e.g., Schwab et al., 2015). ECV products need to be developed with consistent coverage through time (in-filled) and good uncertainty estimates.

The adequacy of observing systems for variables that are not yet associated with requirements for operational services has declined in recent years (Figure 2C, e.g., Kent et al., 2006; Berry and Kent, 2017). An example is air temperature 
where a need has been identified for long term records for comparison with climate model output (Richardson et al., 2018). Observations of air temperature from VOS are required to extend coverage to enable the continued production of global air temperature analyses. To resolve this sampling deficit it will be necessary to relate observation requirements for gridded datasets to requirements for numbers and sampling strategies for VOS and other observing networks.

In the following, we explore two specific experiences: gathering of user requirements for sea-surface temperature and the needs for observations of dynamical reanalysis development.

\section{Gathering User Requirements for Marine Climate Data}

The development of user requirements covering a broad range of applications is increasingly important, and it is necessary to ensure that a narrow specification of requirements for a particular application does not lead to a degradation for other applications. An example of such a broad approach to gathering user requirements is that of the European Space Agency (ESA) Climate Change Initiative (CCI) SST project which aims to improve SST satellite data records to meet the requirements of the climate research community. A User Requirements exercise undertaken by the project (Good and Rayner, 2010) and then repeated 5 years later (Rayner, 2017) gathered the needs of the climate research community for observed SST information in general (not just for satellite SST products) via six methods:

(1) a literature review of relevant documents from bodies such as the GCOS;

(2) review of lessons learned information provided by other projects;

(3) a questionnaire, which asked about;

(a) currently available SST data; and

(b) future needs for SST data, 5 years from now;

(4) discussion sessions;

(5) review of user requirements found in other related projects; and

(6) a user workshop on uncertainties ${ }^{2}$.

Current and future users of SST data were invited to enter their requirements into an online questionnaire and over 100 people responded on each occasion from all over the world. Respondents' work spanned the full range of climate applications, together with air quality modeling, fisheries, atmospheric chemistry, agricultural research, etc.

In general, different applications require different levels of data and the SST CCI User Requirements Document (URD) supports this conclusion. At least half of the users of SST information surveyed each time stated a need for information which has been made complete using a statistical infilling technique. However, it is also important to provide both individual observations and gridded, but not infilled, information for the other types of application such as data assimilation and climate change detection and attribution

\footnotetext{
${ }^{2}$ http://www.esa-sst-cci.org/PUG/pdf/CombinedSSTUserWorkshopReport.pdf
}

respectively. Furthermore, data from different instruments should be combined where this will allow weaknesses in individual datasets to be overcome. For example, there is a requirement for SSTs retrieved from infrared and microwave satellite instruments to be combined to reduce biases and data gaps in particular regions. By making available singlesensor records, sensor-series datasets, and multiple-sensor analyses and extending records back before the satellite era using in situ measurements, the needs of different users can be met.

A large majority of survey respondents needs global information, but different applications require different resolutions of information, both spatially and temporally. The full range of options from $<1 \mathrm{~km}$ to $>1$ degree latitude-longitude and $<3$-hourly to monthly were required, which indicates that the climate observing system needs to be able to support some very exacting requirements for some climate applications and with a sizeable minority needing information about diurnal variability. The most common response in terms of length of record needed was $>30$ years, but as an objective $>100$ years would be ideal for many applications with compatibility between satellite and in situ data being extremely important. Analysis of decadal variability and the study of climate extremes, detection of long-term trends and the study of long-term changes associated with coral systems, fish growth or genetic changes all need long records. These potential users want to be able to use data before the satellite era but also want to take advantage of satellite-derived products, so it is important that the two are consistent.

The concept of interim CDRs, i.e., short-delay updates to CDRs consistent with the long-term record, is relevant to many users. For some, a delay of less than a day is needed until data receipt. Some climate monitoring applications ideally need data within just a few days, but some can tolerate longer delays. This continuing need for climate quality data can be addressed by ensuring that the data record is extendable in the future when new instrumentation is available, but also requires conversion of data processing systems from research to operational, supported by the convergence of climate and operational requirements.

Typically, biases in SST data are not tolerated above about $0.1^{\circ} \mathrm{C}$ and precision is required to be $0.1^{\circ} \mathrm{C}$ or better. Strict stability requirements of better than $0.05^{\circ} \mathrm{C} /$ decade are stated. Users require these statistics to be demonstrated over spatial scales of $\sim 100 \mathrm{~km}$, which is not typically possible everywhere and certainly not unless large high-quality data subsets are reserved for evaluation.

The SST CCI User Workshop on Uncertainty ${ }^{3}$ identified a significant interest within the user community of the provision of uncertainty information via an ensemble (a set of plausible realizations of each SST field which span the uncertainties in the data). Respondents to a second questionnaire were asked if SST uncertainty information were to be represented by an ensemble, how many ensemble members would they need for their application? Over half of respondents indicated they would require more than 10 ensemble members, and a similar number also felt they would benefit from the provision of uncertainty

\footnotetext{
${ }^{3}$ http://www.esa-sst-cci.org/PUG/workshop.htm
} 
information via a parameterized error covariance matrix. These responses indicate a movement amongst the SST user community toward much more consideration of observational uncertainties.

Users also felt that confidence in uncertainty estimates needs to be stated and that uncertainty characteristics should be verified by comparison against independent observations. As with the evaluation of bias, precision and drift over time, this places strict requirements on the availability of independent, high quality reference data.

\section{Observational Needs for Reanalysis}

Many climate users access information about the climate system from dynamical reanalyses. Historical marine observations are key data sources for the production of climate-quality reanalyses. At the Fifth International Conference on Reanalysis (Buizza et al., 2018), the session on observations discussed the needs for observations of reanalysis production and the processing required to ensure a climate-quality outcome. These processing steps (data assembly, data rescue, quality control, bias correction, and data assimilation feedback analysis) and stated needs echo, in many cases, the processing and the SST needs discussed above, and demonstrate that they are generalizable to other variables.

Work on observations for reanalyses requires a sustained, well-supported effort involving cooperation with reanalysis producers; operational services are inadequate to support this work alone. Operationally-produced reanalyses are susceptible to changes in observing practice or transmission practice, such as the recent general move to the use of BUFR format for message transmission. Operationally-sustained production of underlying key data sets, consistent with the climate record, is essential to avoid discontinuities that affect downstream services.

Ongoing data rescue and recalibration, both of satellite and in situ data sources are very important for improving the quality of reanalyses and extending their quality back in time. In particular, data rescue is crucial for preserving data currently on fragile tape or paper. Reprocessing and making observational [both ECV and parameters used to generate Fundamental Climate Data Records (FCDRs) such as radiance (GCOS, 2011)] series more consistent allows more of the information to be included in a reanalysis and allows our reanalyses to better reflect the observations. This requires a sustained program (Brönnimann et al., 2018a,b).

The fundamental observational record needs to be carefully preserved for future reanalyses and for every other activity we may need it for now and in the future. This needs ongoing, adequate support. Previous experience demonstrates that once observational holdings become fragmented, it requires a great deal of effort to correctly reconstruct them.

Development of future assimilation systems needs to consider how best to use observations and information on their uncertainties and this effort needs to be supported. End-to-end propagation of uncertainties through observation processing is required to enable this.

The reanalysis community need to clearly state their ongoing requirements for the observing system, considering also the known downstream needs of applications. For example: a subset of the observations used are particularly key and reduce uncertainty so much that they could be considered akin to reference series - these should be defined and particularly cared for; resolution of boundary/constraining SST fields is key to reproducing precipitation over frontal zones and reducing tropical biases - microwave SST data should be continued and its resolution increased; and funding for new satellite missions should be continued, since that provides for future innovation.

\section{How Do User Needs Impact on the Construction of CDRs?}

Producers of CDR and other climate data products have responded to user requests for more frequent updates, particularly important for climate monitoring. There is inevitably a tension between the speed with which products are made available and their quality. Observations may not be available in time for cut-off deadlines, improvements in data quality from delayed mode processing are not available, and assumptions must be made that data quality is similar to that seen previously. For those products aiming to meet the needs of users with requirements for fast data delivery, updates are typically made a few days after the end of every month. Except where particular problems are apparent, revision of these fast delivery products is usually every few years. This is a compromise between having relatively stable versions, and ensuring the highest quality.

Other trade-offs that may occur between different user needs are between quality, resolution and completeness. Infilled datasets are needed for some applications but uncertainty is bound to increase in unsampled regions. Need for increased coverage, or higher resolution, may require that lower quality data sources are utilized, perhaps without the desired metadata. In all these cases careful consideration of the terms in the error model will guide data selection, and provide estimates of data uncertainty that can guide user choice.

\section{CONSIDERATIONS WHEN CREATING INTERNALLY-CONSISTENT RECORDS FOR MARINE SURFACE ECVs}

\section{Air Temperature}

Observations of MAT have been made on ships since about 1750 , but observation numbers increased with availability of measurements from the East India Company in the late 18th Century (Freeman et al., 2017) (Figure 2A). Early in the record measurements of MAT are more common than SST, which only become consistently observed following Maury (1854). Measurements are made with thermometers with a transition between mercury thermometers to electronic sensors over time (Kent et al., 2007). The most important factors for MAT data quality are thought to be the location, ventilation, and degree of sheltering from the sun (Kent et al., 1993).

Marine air temperature observations need to be adjusted to a common reference height (Kent et al., 2013), requiring information on the actual observing height $(h)$ and an estimate 
of the stability of near surface atmosphere which depends on ambient conditions ( $a) . h$ may be available from Publication 47 (Kent et al., 2007), but only from around the 1970s, prior to this some rather broad generalizations must be made (Kent et al., 2013). A further source of uncertainty is the difference in measurement heights and depths that occurs with a change in ship loading. Elements of $a$ required are wind speed, airsea temperature difference, and ideally the humidity. Present adjustments applied to ICOADS use climatological estimates of $a$ and its uncertainty. For high-quality data sources it is likely that using observed values would be an improvement, but this will require further research to quantify uncertainties for each data source and their impact on height adjustment. Improvements are likely to be possible for MAT without high-quality a through, for example, further constraining the likely climatological distribution of atmospheric stability with additional information such as wind direction.

The most prevalent source of bias $(B)$ in MAT is due to the daytime influence of spurious heating by the ship infrastructure (Berry et al., 2004). This effect will be larger for sensors that are installed close to the deck as is required for manual reading of thermometers installed in Stevenson Screens (Berry and Kent, 2005). The move to remote reading electronic thermometers enables sensors to be installed in well-exposed locations should reduce the magnitude of $B$, but this has not yet been evaluated across ICOADS. Additional metadata, for measurement method $(m)$, instrument $(i)$, ship size and type and height above deck may in the future provide valuable information for more refined estimates of $B$ and $\varepsilon_{b}$ that may depend on proximity to the heat source and the ventilation of the sensor (for example an estimate of the ambient air flow and $i, m$ ). An estimate of $\bar{B}$ (Berry et al., 2004) has been implemented with ICOADS from 1973 (Berry and Kent, 2011), but existing longer records minimize $B$ by selecting night-MAT only (Rayner et al., 2003; Kent et al., 2013), restricting the record to start after 1850 . Other $B$ specific to particular data sources, periods or regions are treated ad hoc (Rayner et al., 2003; Kent et al., 2013).

\section{Humidity}

Humidity observation records exist intermittently from the late 1800 s, reaching reasonable monthly coverage $(\sim 30-40 \%$ ocean area) from around 1960 onwards (Figure 2). Likely, many more observations exist but these have not typically been prioritized in data rescue efforts. Ships are the most prolific, and arguably most reliable, platform providing humidity observations given that they are manned and sensors are located further from sea level, and therefore from sea spray contamination, than moored buoys. However, since the 1990s moored buoys have steadily increased in number, rapidly from around 2010 to now almost double the number of ship observations (Figures 2C, 3).

Humidity has been typically measured using paired wet-bulb and dry-bulb thermometers (psychrometers) in either a hand held sling or within a ventilated screen. Humidity is then often converted and reported as dew point temperature, any such conversions should be documented $(d)$. Since the 1990s, available metadata (40-60\% of ships) shows that capacitance and electric sensors have steadily increased in number to approximately $40 \%$ of the ships with metadata combined. These typically measure relative humidity which is then converted and reported as dew point temperature.

Sources of error for humidity observations depend on $m$. For wet-bulb thermometers the wick surrounding the thermometer can dry out or become contaminated with sea spray. Relative humidity sensors are also prone to contamination and can drift relatively rapidly, particularly in humid environments (Ingleby, 2010). While psychrometers tend to have better accuracy in moist environments, relative humidity sensors perform better in drier environments. Field evaluations of relative humidity sensors in the marine environment, extending the Ingleby et al. (2013) study of the United Kingdom land station network, are urgently required. If not well-ventilated, through active whirling or artificial ventilation, sensors will be biased humid. Adjustments have been applied for naturally ventilated screens (Berry and Kent, 2009, 2011), but require knowledge of $m$ or $i$. Errors in MAT due to daytime heating of ship structures may not directly lead to biased humidity measurements (Kent and Taylor, 1996), but both sources of bias are likely to be larger for poorly exposed or ventilated sensors. As for MAT, humidity observations need to be adjusted to a common reference level requiring $h$ and $a$. Sources of $B$ for humidity largely follow those for MAT but are more complex owing to the derived and non-linear nature of humidity.

\section{Sea-Surface Temperature}

Sea surface temperature provides the marine component of global surface temperature, combined with air temperatures measured over land (Hartmann et al., 2013) and is the marine variable that receives the most attention because many activities in climate science and service provision rely upon it. Its uncertainties and bias adjustment have been recently reviewed (Kennedy, 2014; Kent et al., 2017). A wide range of different types of SST data are available, from satellites (e.g., Merchant et al., 2014), and in situ from ships, drifting buoys, moored buoys, platforms and coastal stations and the upper parts of temperature profiles from XBTs, CTDs, and Argo floats. There are several long-term global SST data sets (HadSST3, Kennedy et al., 2011a,b; HadISST, Rayner et al., 2003; ERSSTv5, Huang et al., 2017; COBE-SST2, Hirahara et al., 2014). This redundancy enables an estimation of structural uncertainty (Kennedy, 2014), demonstration that differences in $B$ for different datasets exceeds their joint uncertainty (Kent et al., 2017) and that significant biases likely remain at the scale of individual ocean basins (Davis et al., 2018).

There is a need to reconcile near-surface and sub-surface measurements of temperature. The near surface layer is complicated (Kawai and Wada, 2007) and sampling just below the surface is worse than that close to the surface (Figures 2, 4). Despite this upper measurements from temperature profiles have been used to evaluate SST products (e.g., Gouretski et al., 2012; Hausfather et al., 2017; Berry et al., 2018; Huang et al., 2018).

Estimation of $B$ for SST is relatively mature (Kent et al., 2017) and ideally requires knowledge of $p, m, d$, and $a$. The need for improved estimates of $m$ and $p$ for SST has driven improvements in metadata estimation (see section "Enhancing Metadata"). Improved estimates of $B$ will need further improvements to $m$ 
and $p$, better documentation of past data management $(d)$ and ambient conditions ( $a$ ), facilitated by a reprocessing of ICOADS to improve duplicate identification and uncertainty estimation. Measurements from sensors attached to the hulls of ships are yet to be evaluated for uncertainty.

Availability of data for evaluation is better than for most ECVs, but improvements through data rescue and reprocessing would still be valuable (Kent et al., 2017).

\section{Wind Speed and Direction}

Wind speed observations based on the Beaufort scale and derived either from descriptions on the sails carried, or of the sea state, are available from the 18th century (Figure 2A). The transition from these visual observations to measurements by anemometers (Kent and Taylor, 1997) resulted in a spurious trend in mean wind speeds (Cardone et al., 1990; Thomas et al., 2008). This is compounded by an overall increase in $h$ over time, and biases due the disturbance of air flow around ships (Moat et al., 2006). True wind speed and direction (relative to the earth or ocean surface rather than to the frame of reference of the ship) needs to be calculated from measured or estimated relative wind speed and direction and the ship motion. The procedure is different for measured and visually estimated winds and is not always performed correctly (Smith et al., 1999). There are also stronger diurnal variations in visual wind estimates from ships than in those measured with anemometers, probably due to the difficulty of observing sea state when it is dark (Thomas et al., 2008). To minimize inhomogeneity Tokinaga and Xie (2011) generated a long-term wind product based only on visual wind estimates and anemometers with known heights.

Measurements from buoys also contain uncertainties: any averaging applied needs to be documented, including whether scalar or vector averaging was used (Thomas et al., 2005), and measurements made close to the sea surface may be affected by waves. The moored buoy network provides calibration for satellite winds (Wentz et al., 2017) and helps to anchor wind products such as the Cross-Calibrated Multi-Platform (CCMP) winds (Atlas et al., 2011). Comparisons between in situ satellite winds should account for geophysical differences between the records such as the effects of surface currents (Plagge et al., 2012; Rodriguez et al., 2019).

\section{Atmospheric Pressure}

An extensive review of historical atmospheric pressure observations was conducted by Allan and Ansell (2006) and Ansell et al. (2006). Pressure observations need adjustment to sea level $(L)$ requiring information on $h$ and temperature. Mercury barometers need adjustment for local gravity and thermal expansion (requiring $m, x, y$ and temperature) and also information on whether these adjustments have already been applied and if so how $(d)$. Diurnal variations are significant (Ansell et al., 2006).

Lack of clarity on past data management remains a substantial problem for the use of pressure observations, with examples of adjustments having been applied twice, incorrectly, or not at all. A comprehensive review of available documentation, reprocessing of ICOADS original data sources and comparison between those sources would be particularly beneficial for the historical pressure record, including for reanalysis.

\section{Waves and Sea State}

Visual observations of sea state have been made for centuries (Figure 2), observing practice was defined in the 1850s (Maury, 1854) and constitute the longest record of wind waves. Visual observations can identify several wave systems when a welltrained observer estimates a number of sea state characteristics along with atmospheric parameters. The observer's estimation is dependent on surrounding parameters (weather conditions, place of observation, type of vessel, stationary point, etc.). Even though the approach is essentially qualitative, it is possible to integrate all of these factors into a comprehensive observation. VOS data provide basic wave characteristics, such as wind sea, first and second swell heights, periods, and the directions of propagation of those wave components. Reported parameters enable the estimation of significant wave height, dominant period, wave age, steepness and wavelength (Gulev et al., 2003; Grigorieva et al., 2017) and have been used in extreme waves analysis (Grigorieva and Gulev, 2008), and for the assessment of long-term tendencies in wave parameters (Gulev and Grigorieva, 2004, 2006).

Observational practice has not changed, however, significant changes to the coding system occurred in 1950 and it is important to account for this when developing consistent climate records (Gulev et al., 2003; Grigorieva and Badulin, 2016; Grigorieva et al., 2017). Evaluation of the internal consistency of the wave record over time is critical for the identification of coding changes and their impact. Fitting of Weibull distributions to available data can produce more reliable gridbox values. Using these methods a century long record of wave parameters has been developed, that has not yet been fully exploited for applications such as comparison with satellite-based wave records or with model output.

\section{Cloud Type, Cloud Cover, and Coded Weather Information}

Visual cloud observations from human surface observers (Figure 2) contain a great deal of information about the atmosphere, interacting with radiation and modifying weather. The structure, height, and shape of clouds, which can be quantified by weather observers at the surface, can be used to assess the atmospheric state (Norris, 1998). The cloud record has been used to study how cloud cover interacts with the SST and changing meteorological variables such as lower tropospheric stability as well as to examine long-term trends in ocean cloudiness whilst revealing some spurious long-term variations (Eastman et al., 2011).

Visual cloud observations board ships throughout the oceans have been recorded using the same methodology and format, since the 1950s, 20 years before satellites. The Extended Edited Cloud Reports Archive (EECRA, Hahn et al., 1988; Hahn and Warren, 2009) contains millions of visual cloud reports from 1954 through 2008, based on observations in the synoptic code of the WMO that have been assessed for internal consistency 
and QCd. The EECRA provides a measure of lowest-level cloud cover and total cloud cover along with a cloud type at three levels: high, middle, and low. At least 10 cloud types are defined at each level and selected meteorological information is also included in the record. EECRA methodology has been applied to ICOADS reports from 1950 to 2014 to provide an enhanced cloud record, but this has not yet been used to derive gridded estimates. VOS reports contain coded weather information that can be used to provide context of the environmental conditions that may be used in uncertainty or bias estimation. Precipitation data products have been produced using such codes (Petty, 1995; Josey et al., 1999).

\section{FUTURE IMPROVEMENTS}

\section{Ensuring the Future Record Reference Observations}

OceanSITES provides the most consistent source of surface marine data presently available with unified access and common data formats. Whilst it does not strictly conform to the definition of a reference network used for observations over land (Thorne et al., 2017b) the maintenance of a high quality array of moored observations is critical for the maintenance of long-term records. The number of OceanSITES currently providing most of the ECVs in the scope of this paper is about 20, but an extension of similar size has been recommended in support of air-sea fluxes (Cronin et al., 2019), sampling regions thought to be particularly important for understanding the mechanisms of air-sea interaction and providing validation observations for satellites. Such an extension to approximately 40 sites would be extremely valuable for the construction of CDRs.

Select RVs have the potential, based on their level of technical personnel onboard and typically research-quality instrumentation, to act as mobile reference stations, provided a high-level of quality assessment can be achieved (Smith et al., 2019). This would be extremely valuable to link up fixed point high-quality OceanSITES, particularly if the same RVs are used to service OceanSites, and provide more distributed data.

Argo provides a reference network for SST, but suffers from large sampling uncertainty due to the relatively short time spent at the surface. Surface drifters with higher quality sensors have been deployed to quantify sensor drift (Reverdin et al., 2010; Poli et al., 2018) and if deployed systematically in the future might provide a reference network for SST. Satellite missions such as the ATSR-series (Merchant et al., 2012) that are designed with stable orbits and sampling strategies such as dual view to enable the removal of artifacts such as aerosol contamination should be maintained and extended to provide stable longterm global records.

\section{Baseline Observations}

Baseline observations should be of good quality, widespread, and provide observations to link the reference and global networks. RVs that do not meet the criteria for reference observations are an obvious choice, but presently their data and metadata are not collected and managed systematically, and there is no globally integrated data management system (Smith et al., 2019). Moored buoys other than OceanSITES are also candidates for contribution to baseline networks, but as for RVs there is no integrated data and metadata management system. Enhanced VOS, once evaluated, may also provide baseline observations.

\section{Global Observations}

Global networks are those data types that may not reach accuracy requirements for baseline networks but provide widespread measurements to capture the important scales of variability. Standard VOS form such a network, and data from AIS may in the future add to the global network of ship observations. Autonomous observations from Argo and drifting buoys contribute to the reference and baseline networks, but data from emerging autonomous vehicles will initially contribute to a global network. Observations from satellites have the potential to extend sampling to data sparse regions and can provide observations to quantify variability. Long term records blending satellite and in situ data to extend records prior to the satellite era should be developed for ECVs beyond the presently-available SST. New technology, especially from autonomous vehicles will also provide valuable observations for long term climate records. Such data can improve our knowledge of ECV variability over the oceans which will contribute to improved CDRs throughout the record and allow the extension of reconstruction methods to a wider range of ECVs. It also needs to be recognized that single variable observations are not as useful as multivariate ones which typically provide more of the ancillary information needed to quantify $B, L$ and quantify uncertainty and its correlation structure.

Construction of long term records means that new observing technologies need to be continually incorporated into the record. This is made substantially easier when the changes are well-managed, there is co-located overlap between old and new types of observations, and all observations are described by appropriate metadata. It is critical that we retain a network of high quality observations, and evaluations of existing and new technologies are designed to exploit these high quality data.

\section{Data and Metadata Rescue}

The digitization of marine observations has dramatically extended the climate record (e.g., Freeman et al., 2017). Most data and metadata rescue activities are presently overseen by the Atmospheric Circulation Reconstructions over the Earth (ACRE) initiative (Allan et al., 2011). International coordination of data rescue is needed for several reasons, most obviously to avoid duplication of effort but also because those countries or organizations with the resources or requirements for data digitization may not be the same as those with holdings of undigitized data. The first step is the identification and cataloging of suitable material, typically ships logbooks, observing instructions, or other documentation. This may involve visits to libraries, archives or other institutions. If resources are identified then the material will be imaged, the 
resulting images digitized, the data or metadata evaluated, QC applied and data made available for ingestion into climate archives such as ICOADS. The prioritization of resources for imaging will depend on the likely quality and quantity of data, the parameters available, the period and region covered, the match to user requirements and the level of risk for deterioration of the original material. Best practice is that as much information as possible should be digitized from each image along with all of the relevant metadata and ancillary data.

In the past all data digitization was performed by manual keying. Often the output data format was severely restricted, such as that for punchcards. In these cases it is particularly valuable to have documentation describing the procedures used. Sometimes the more complete original sources were then destroyed, but there may be examples where it may be possible to recover data from original sources. Automatic techniques for digitization do not yet work well with most data sources, but this likely to improve in the future, for example with tailored post-processing of output from standard optical character recognition algorithms. Recently crowdsourcing has been used with good success (Brohan et al., 2009; Burt and Hawkins, 2019). Crowdsourcing can be very effective, but requires substantial preparation of material so is most suitable for large volumes of data in similar formats. It is also necessary to maintain the enthusiasm of volunteers, and to demonstrate the value of their contributions.

Much recent digitization has been driven by requirements for reanalysis (e.g., Allan et al., 2011; Brönnimann et al., 2018a,b), and records containing pressure observations are most valuable for this application, especially in data sparse regions or periods. However there are many other applications that would also benefit from data recovery. Of particular value for the construction of long-term records are high-quality independent validation data, especially those that are expected to be consistent through periods of rapid changes in the observing system, for example during the World Wars.

Much marine data rescue has focused on old ships logbooks and metadata such as observing instructions, but there are other data sources that need to be integrated into international archives. Some RV operators have data archives on archaic media or in proprietary formats that could be lost and should be cataloged and prioritized for rescue. Many national services hold collections of GTS data that might provide additional observations, or if duplicating existing archives, information about uncertainty. Early satellite data also exist that need painstaking rescue from at-risk media.

\section{Reprocessing of Existing Archives}

The surface marine climate community has long benefited from the ICOADS integrated archive, the value of which is widely recognized (Thorne et al., 2017a). However compromises made during past data management are now reducing the potential for application of advanced methods for estimation of bias and location adjustments, thereby degrading gridded analyses and limiting further improvement. ICOADS has retained original data sources and the available documentation, so it is possible to re-ingest these data, without constraints on formats or data volumes and in a way more compatible with international standards (see section "An Integrated Data System").

Advantages of reprocessing selected ICOADS original data sources include:

- Recovery of observations thought to be duplicates and excluded from further analysis. This will allow the development of improved approaches to duplicate identification, and the quantification of differences between versions of the same measurements due to past data management.

- Improved identification of data from sources likely to be useful for evaluation, such as OWS or RVs.

- Recovery of data and metadata that were not retained in past data formats.

- Extension of metadata through a comprehensive review of documentation.

A further advantage would come from a reprocessing of GTS accessions to better identify reports that should be replaced with higher quality data from delayed mode sources.

\section{An Integrated Data System}

As noted above, the observing system is made up of a diverse range of platforms, observing systems and data streams, each with their own issues. In order to efficiently use the observations in the generation of CDRs a number of basic requirements need to be met:

- Observations from identifiable sources at the platform level

- Instrument metadata (methods, error characteristics, etc.) associated with the observations

- Open data sources for reproducibility

- Consistent conventions and format used to represent the observations (and metadata)

- Timely access to the observations (and metadata)

- Redundancy of NRT data streams and archive access.

These requirements are beginning to be addressed for surface ocean data through a number of initiatives. For example, within JCOMM a database containing the instrumental and platform metadata for all observing platforms contributing to the JCOMM programs (e.g., VOS, DBCP, etc.) has been developed and populated. Similarly, a Marine Climate Data System is under development to improve the timely flow of delayed mode and real time data as well as promoting best practices and standards. For the VOS the JCOMM metadata database has been built based on the WMO Publication 47 metadata and, in part, been driven by development of the WIGOS and metadata requirements from the WIGOS Metadata Standard. Integrated data management and archival systems are needed for all components of the GOOS and GCOS providing ECVs for the construction of CDRs. WIGOS, if used fully, provides an appropriate framework for the provision of data and metadata together. Whilst the developments of the JCOMM and WMO systems has focused on operational data flows other systems are being developed for climate data. An 
exemplar is the C3S Climate Data Store part of the European Union funded Copernicus Climate Change Service.

\section{REFLECTIONS AND RECOMMENDATIONS}

\section{Reflections}

The construction of CDRs for all ECVs has been made more difficult by the lack of availability of good platform and observational metadata. This has in the past been compounded by a focus on rather narrow user requirements, for example for NWP, reanalysis or satellite bias adjustment. This has led to a narrowing of the range of ECVs that are typically measured together, and in some cases to truncated data formats and lack of metadata. Each set of observations has a non-trivial error model and this is linked with everything done to the data. Understanding, documenting, and encoding in metadata everything relevant about the platform, instruments, observing protocols, coding and recoding helps to reduce the uncertainty in CDRs and enables reconciliation between different measurement types (GCOS, 2016).

New data systems under development, for example at JCOMM and for the C3S Climate Data Store, should enable the relevant information to be captured and provided to users. But having the ability to record metadata is only part of the story. Data providers need also to be diligent in ensuring that metadata are reported, and archival formats need to preserve it.

Compromises are inevitable when the needs of CDR construction interact with the needs of real users, particularly in the area of timeliness vs. quality. The impact of any compromise will be minimized if the data system understands these potentially conflicting needs from the start.
The decline in the number of VOS reports has had a severe and detrimental effect on the sampling for several ECVs, in particular MAT, humidity, winds, clouds, and waves. The resulting decline in the number of multivariate records also has an impact on ability to bias adjust and quantify uncertainty those ECVs that are reported.

Rescue through digitization of data and metadata are key to the extension and improvements of CDRs. In many cases the priorities for CDR construction are similar to those for applications such as reanalysis which target data sparse regions and periods. But other requirements for CDRs include recovery of metadata and documentation, high quality and consistent timeseries, data with measurements of the same ECV made by different methods, and might prioritize reports that do not contain pressure measurements.

User requirements for GCOS are typically expressed in terms of a required accuracy at particular spatiotemporal resolutions ${ }^{4}$. Requirements for CDRs are often described in terms of quite strict stability of anomalies over large space and time scales. An example is a need for SST data sets which are global and demonstrably stable to much better than $0.1^{\circ} \mathrm{C}$ decade $^{-1}$, ideally with deviations less than $0.01^{\circ} \mathrm{C}$ decade $^{-1}$. This has been achieved for satellite SST (Merchant et al., 2012) but requires complementary and independent data for evaluation. SST and winds are the only record for which the community is able to demonstrate compliance with stringent user requirements, and typically only for relatively short periods and limited regions (e.g., Verhoef et al., 2017; Berry et al., 2018). SST is the only ECV where there are multiple long term data products with uncertainty estimates which

${ }^{4}$ http://www.wmo-sat.info/oscar/observingrequirements

\section{Recommendations}

\begin{tabular}{|c|c|c|c|c|}
\hline & Recommendation & Outcome & To whom directed & Supporting information section \\
\hline 1 & Extensions to observations & & & \\
\hline 1.1 & $\begin{array}{l}\text { The OceanSITES network of high } \\
\text { quality time series data should be } \\
\text { maintained as an ocean reference } \\
\text { network and expanded to } \\
\text { approximately double its existing } \\
\text { number of sites. }\end{array}$ & $\begin{array}{l}\text { Provision of adequate reference } \\
\text { observations for a wide range of } \\
\text { ECVs }\end{array}$ & $\begin{array}{l}\text { Network managers } \\
\text { Funding agencies }\end{array}$ & $\begin{array}{l}\text { Reference observations } \\
\text { Observations for evaluation }\end{array}$ \\
\hline 1.2 & $\begin{array}{l}\text { RVs evaluated as providing high quality } \\
\text { measurements of ECVs should be } \\
\text { managed as a reference network and } \\
\text { field comparisons with OceanSITES } \\
\text { data regularly performed. }\end{array}$ & $\begin{array}{l}\text { Increased availability of high quality } \\
\text { data for a wide range of ECVs }\end{array}$ & RV operators & $\begin{array}{l}\text { Reference observations } \\
\text { Observations for evaluation }\end{array}$ \\
\hline 1.3 & $\begin{array}{l}\text { All RVs should aim to contribute to the } \\
\text { provision of ECVs for climate archives, } \\
\text { keeping sensors running at all times } \\
\text { whilst at sea. Data should be evaluated } \\
\text { and RVs designated as providing data } \\
\text { suitable for reference, baseline and } \\
\text { global networks identified and } \\
\text { managed appropriately. }\end{array}$ & $\begin{array}{l}\text { Increased availability of high quality } \\
\text { data for a wide range of ECVs }\end{array}$ & RV operators & $\begin{array}{l}\text { Reference observations } \\
\text { Observations for evaluation }\end{array}$ \\
\hline
\end{tabular}




\begin{tabular}{ll}
\hline \multicolumn{3}{c}{ Recommendation } \\
\hline $1.4 \quad \begin{array}{l}\text { The coverage of ECV observations from } \\
\text { VOS needs to be expanded, as } \\
\text { coverage of observations of MAT, } \\
\text { humidity, clouds, waves has } \\
\text { substantially declined since the 1990s. }\end{array}$ \\
$\begin{array}{l}\text { Data rescue needs to be continued, } \\
\text { designed to meet a wide range of user } \\
\text { requirements. }\end{array}$ \\
Continuation of a dual-view reference \\
satellite sensor series (e.g., in the family \\
of ATSR/SLSTR) in a stable orbit with \\
fixed Local Equator Crossing Time. \\
Enhancement to data management \\
and QC systems
\end{tabular}

2.1 Distributed but interoperable data centers, affiliated with JCOMM should be established building on national activities, for the archival, quality evaluation and distribution of surface marine data from RVs. All RVs should run measurement systems continuously whilst at sea and contribute their data and metadata

2.2 Distributed but interoperable data centers, affiliated with JCOMM should be established, collecting and archiving GTS data for all surface marine ECVs and EOVs. One center should have responsibility for integrating and evaluating GTS data across data centers for each platform type (e.g., ships, moored buoys, drifting buoys).

2.3 Ensure that data from new technologies (e.g., autonomous vehicles) and systems (e.g., AIS) have appropriate data management systems in place, recognizing the needs of downstream users including climate.

2.4 Review and enhance data and metadata management for networks established in support of NWP to ensure that observations of ECV meet the requirements for climate applications.

2.5 Recognize the value in terms of enhanced data quality and traceability of the management of delayed mode archives by those with expertise with particular types of measurements and systems and ensure that adequate funding is provided for this.

\subsection{Multi-variate reports should be} managed together, and data subsequently pushed to single variable repositories if required. It needs to be easier for users to select and access data subsets.

\section{Outcome}

Adequate records of MAT,

humidity, cloud cover, etc. for use in climate science

Increased information content of long-term surface marine records to support higher resolution applications Stable, accurate SSTs, particularly in the presence of dust or other high aerosol loading

Establishment of a WMO/IOC Global Data Assembly Centre (GDAC) for RV surface marine observations Increased availability of RV observations

Establishment of Data Assembly Centres (DACs) and Global Data Assembly Centres (GDACs) for real time data, contributing to the JCOMM Marine Climate Data system. More consistency in GTS holdings

Avoidance of the need for future reorganization work Reduced uncertainty

Avoidance of the need for future reorganization work Reduced uncertainty

Reduced uncertainty

Improved access to data for users

\section{To whom directed}

JCOMM

VOS network managers GCOS

GCOS

Holders of relevant archive material Funding agencies

Satellite agencies

Funding agencies GCOS

Funding agencies GCOS

Funding agencies

Funding agencies JCOMM

Funders of Global Data Assembly Centres

Network data managers

\section{Supporting information section}

Understanding the needs of different applications Considerations when creating internally-consistent records for marine surface ECVs Global observations Data and metadata rescue

Reference observations

Reference observations Observations for evaluation

Merging different sources and archives Reprocessing of existing archives

Introduction to an error model for individual observations Enhancing the observational archive

Introduction to an error model for individual observations Enhancing the observational archive

The need for expert data system design and management

The need for expert data system design and management 


\begin{tabular}{|c|c|c|c|c|}
\hline & Recommendation & Outcome & To whom directed & Supporting information section \\
\hline 2.7 & $\begin{array}{l}\text { Approaches to QC should be shared and } \\
\text { open source code and systems for } \\
\text { implementation developed according to } \\
\text { best practice. }\end{array}$ & Reduction in uncertainty & QC developers & Quality control \\
\hline 3 & Field evaluation of sensors & & & \\
\hline 3.1 & $\begin{array}{l}\text { The uncertainty of surface ECV data from } \\
\text { all observing systems and instrument } \\
\text { types should be quantified in the field, } \\
\text { and periodically re-evaluated. Examples } \\
\text { where such field evaluations are urgently } \\
\text { required are: humidity from relative } \\
\text { humidity sensors SST from hull sensors } \\
\text { MAT sensors with remote reading }\end{array}$ & Better quantified uncertainty & Network managers & $\begin{array}{l}\text { Observations for evaluation } \\
\text { Humidity } \\
\text { Sea-surface temperature } \\
\text { Wind speed and direction }\end{array}$ \\
\hline 4 & $\begin{array}{l}\text { Improvements to ICOADS and the } \\
\text { surface marine archive }\end{array}$ & & & \\
\hline 4.1 & $\begin{array}{l}\text { The data format used by ICOADS } \\
\text { predates WIGOS standards, and needs } \\
\text { updating (Freeman et al., 2019). }\end{array}$ & $\begin{array}{l}\text { Better communication of } \\
\text { metadata }\end{array}$ & ICOADS & Enhancing metadata \\
\hline 4.2 & $\begin{array}{l}\text { A reprocessing of ICOADS sources from } \\
\text { the most original sources available. This } \\
\text { will enable better identification of } \\
\text { duplicate observations, vessel tracking, } \\
\text { uncertainty estimation and the better } \\
\text { identification of data from different types } \\
\text { of observing system. }\end{array}$ & Reduction of uncertainty & $\begin{array}{l}\text { ICOADS } \\
\text { Funding agencies }\end{array}$ & $\begin{array}{l}\text { Merging different sources and } \\
\text { archives } \\
\text { Atmospheric pressure } \\
\text { Reprocessing of existing archives }\end{array}$ \\
\hline 4.3 & $\begin{array}{l}\text { A systematic review of documentation } \\
\text { and coding practice for the accession of } \\
\text { data into ICOADS should be undertaken } \\
\text { alongside an evaluation of data and } \\
\text { metadata content, to ensure that } \\
\text { documentation, data and metadata are } \\
\text { consistent. }\end{array}$ & $\begin{array}{l}\text { Greater consistency in } \\
\text { information } \\
\text { Reduced uncertainty }\end{array}$ & $\begin{array}{l}\text { ICOADS } \\
\text { Funding agencies }\end{array}$ & $\begin{array}{l}\text { Specialist observing ships } \\
\text { Merging different sources and } \\
\text { archives }\end{array}$ \\
\hline 5 & User requirements & & & \\
\hline 5.1 & $\begin{array}{l}\text { Engage with user groups to establish } \\
\text { their requirements, recognizing that } \\
\text { resources are required both to gather } \\
\text { those requirements and to establish } \\
\text { whether or not they are being met. }\end{array}$ & $\begin{array}{l}\text { Products better suit the needs } \\
\text { of users } \\
\text { Adequate observing system }\end{array}$ & $\begin{array}{l}\text { Network operators } \\
\text { Funding agencies } \\
\text { WMO/GCOS/JCOMM }\end{array}$ & $\begin{array}{l}\text { User needs for data and data } \\
\text { products }\end{array}$ \\
\hline 5.2 & $\begin{array}{l}\text { Develop observing requirement } \\
\text { statements in terms of network design } \\
\text { including for observation accuracy, } \\
\text { sampling, coverage, and accompanying } \\
\text { metadata. }\end{array}$ & $\begin{array}{l}\text { Improved information for } \\
\text { observing network design and } \\
\text { monitoring } \\
\text { Adequate observing system }\end{array}$ & $\begin{array}{l}\text { GCOS } \\
\text { Funding agencies }\end{array}$ & $\begin{array}{l}\text { User needs for data and data } \\
\text { products }\end{array}$ \\
\hline 6 & $\begin{array}{l}\text { Research to improve quantification of } \\
\text { bias and uncertainty in ECVs }\end{array}$ & & & \\
\hline 6.1 & $\begin{array}{l}\text { Research to quantify biases and } \\
\text { uncertainty in surface marine ECVs and } \\
\text { EOVs and to construct improved CDRs } \\
\text { and other data products needs to be } \\
\text { adequately funded. }\end{array}$ & $\begin{array}{l}\text { Better quantified uncertainty } \\
\text { Improved products }\end{array}$ & Funding agencies & Observational needs for reanalysis \\
\hline
\end{tabular}

enables us to also evaluate uncertainty estimates and state that uncertainties are underestimated (Kent et al., 2017; Davis et al., 2018).

\section{AUTHOR CONTRIBUTIONS}

EK and NR wrote the first draft of the manuscript. All authors provided text and comments and approved the final draft.

\section{FUNDING}

EK and $\mathrm{DB}$ were supported by the NERC under the grants NE/R015953/1 and NE/J020788/1. NR, JK, and KW were supported by the Met Office Hadley Centre Climate Programme funded by the BEIS and Defra. VG was supported by the Russian Ministry of Science and Education under the Project 14.W03.31.0006. 


\section{REFERENCES}

Allan, R., and Ansell, T. (2006). A new globally complete monthly historical gridded mean sea level pressure dataset (HadSLP2): 1850-2004. J. Clim. 19, 5816-5842. doi: 10.1175/JCLI3937.1

Allan, R., Brohan, P., Compo, G. P., Stone, R., Luterbacher, J., and Brönnimann, S. (2011). The international atmospheric circulation reconstructions over the earth (ACRE) initiative. Bull. Am. Meteorol. Soc. 92, 1421-1425. doi: 10.1175/ 2011BAMS3218.1

Andersson, A., Klepp, C., Fennig, K., Bakan, S., Grassl, H., and Schulz, J. (2011). Evaluation of HOAPS-3 ocean surface freshwater flux components. J. Appl. Meteorol. Climatol. 50, 379-398. doi: 10.1175/2010JAMC2341.1

Ansell, T. J., Jones, P. D., Allan, R. J., Lister, D., Parker, D. E., Brunet, M., et al (2006). Daily mean sea level pressure reconstructions for the european-north atlantic region for the period 1850-2003. J. Clim. 19, 2717-2742. doi: 10.1175/ JCLI3775.1

Ardhuin, F., Stopa, J. E., Chapron, B., Collard, F., Jensen, R. E., Johannessen, J., et al. (2019). Observing sea states. Front. Mar. Sci. 6:124. doi: 10.3389/fmars. 2019.00124

Atkinson, C. P., Rayner, N. A., Roberts-Jones, J., and Smith, R. O. (2013). Assessing the quality of sea surface temperature observations from drifting buoys and ships on a platform-by-platform basis: assessing buoy and ship SST observations. J. Geophys. Res. Oceans 118, 3507-3529. doi: 10.1002/jgrc. 20257

Atlas, R., Hoffman, R. N., Ardizzone, J., Leidner, S. M., Jusem, J. C., Smith, D. K., et al. (2011). A cross-calibrated, multiplatform ocean surface wind velocity product for meteorological and oceanographic applications. Bull. Am. Meteorol. Soc. 92, 157-174. doi: 10.1175/2010BAMS2946.1

Beggs, H. M., Verein, R., Paltoglou, G., Kippo, H., and Underwood, M. (2012) Enhancing ship of opportunity sea surface temperature observations in the Australian region. J. Oper. Oceanogr. 5, 59-73. doi: 10.1080/1755876X.2012. 11020132

Belmonte Rivas, M., Stoffelen, A., Verspeek, J., Verhoef, A., Neyt, X., and Anderson, C. (2007). Cone metrics: a new tool for the intercomparison of scatterometer records. IEEE J. Sel. Top. Appl. Earth Obs. Remote Sens. 10, 2195-2204. doi: $10.1109 /$ jstars.2017.2647842

Berry, D., Corlett, G., Embury, O., and Merchant, C. (2018). Stability assessment of the (A)ATSR sea surface temperature climate dataset from the european space agency climate change initiative. Remote Sens. 10:126. doi: 10.3390/rs100 10126

Berry, D. I., and Kent, E. C. (2005). The effect of instrument exposure on marine air temperatures: an assessment using VOSClim data. Inter. J. Clim. 25, 1007-1022. doi: 10.1002/joc.1178

Berry, D. I., and Kent, E. C. (2009). A new air-sea interaction gridded dataset from ICOADS with uncertainty estimates. Bull. Am. Meteorol. Soc. 90, 645-656. doi: 10.1175/2008BAMS2639.1

Berry, D. I., and Kent, E. C. (2011). Air-Sea fluxes from ICOADS: the construction of a new gridded dataset with uncertainty estimates. Inter. J. Clim. 31, 987-1001. doi: 10.1002/joc.2059

Berry, D. I., and Kent, E. C. (2017). Assessing the health of the in situ global surface marine climate observing system. Inter. J. Clim. 37, 2248-2259. doi: 10.1002/joc.4914

Berry, D. I., Kent, E. C., and Taylor, P. K. (2004). An analytical model of heating errors in marine air temperatures from ships. J. Atmos. Ocean. Technol. 21, 1198-1215. doi: 10.1175/1520-0426(2004)021<1198:aamohe>2.0.co;2

Bojinski, S., Verstraete, M., Peterson, T. C., Richter, C., Simmons, A., and Zemp, M. (2014). The concept of essential climate variables in support of climate research, applications, and policy. Bull. Am. Meteorol. Soc. 95, 1431-1443. doi: 10.1175/BAMS-D-13-00047.1

Bourassa, M. A., Meissner, T., Cerovecki, I., Chang, P., Xiaolong, D., De Chiara, G., et al. (2019). Remotely sensed winds and wind stresses for marine forecasting and ocean modeling. Front. Mar. Sci. submitted.

Boyer, T. P., Antonov, J. I, Baranova, O. K., Coleman, C., Garcia, H. E., Grodsky, A., et al. (2013). World Ocean Database 2013. Silver Spring, MD: NOAA Printing Officce.

Brohan, P., Allan, R., Freeman, J. E., Waple, A. M., Wheeler, D., Wilkinson, C., et al. (2009). Marine observations of old weather. Bull. Am. Meteorol. Soc. 90, 219-230. doi: 10.1175/2008BAMS2522.1
Brönnimann, S., Allan, R., Atkinson, C., Buizza, R., Bulygina, O., Dahlgren, P., et al. (2018a). Observations for reanalyses. Bull. Am. Meteorol. Soc. 99, 1851-1866. doi: 10.1175/BAMS-D-17-0229.1

Brönnimann, S., Brugnara, Y., Allan, R. J., Brunet, M., Compo, G. P., Crouthamel, R. I., et al. (2018b). A roadmap to climate data rescue services. Geosci. Data J. 5, 28-39. doi: 10.1002/gdj3.56

Buizza, R., Poli, P., Rixen, M., Alonso-Balmaseda, M., Bosilovich, M. G., Brönnimann, S., et al. (2018). Advancing global and regional reanalyses. Bull. Am. Meteor. Soc. 99, ES139-ES144. doi: 10.1175/BAMS-D-17-0312.1

Burt, S., and Hawkins, E. (2019). Near-zero humidities on Ben Nevis, Scotland, revealed by pioneering nineteenth-century observers and modern volunteers. Inter. J. Climatol. doi: 10.1002/joc.6084 [Epub ahead of print].

Cardone, V. J., Greenwood, J. G., and Cane, M. A. (1990). On trends in historical marine wind data. J. Clim. 3, 113-127. doi: 10.1175/1520-0442(1990)003<0113. otihmw $>2.0 . \operatorname{co} ; 2$

Carella, G., Kennedy, J. J., Berry, D. I., Hirahara, S., Merchant, C. J., Morak-Bozzo, S., et al. (2018). Estimating sea surface temperature measurement methods using characteristic differences in the diurnal cycle. Geophys. Res. Lett. 45, 363-371. doi: 10.1002/2017GL076475

Carella, G., Kent, E. C., and Berry, D. I. (2017). A probabilistic approach to ship voyage reconstruction in ICOADS. Inter. J. Climatol. 37, 2233-2247. doi: 10.1002 /joc.4492

Centurioni, L. R. (2018). "Drifter Technology and Impacts for Sea Surface Temperature, Sea-Level Pressure, and Ocean Circulation Studies," in Observing the Oceans in Real Time, eds R. Venkatesan, A. Tandon, E. D'Asaro, and M. A Atmanand (Cham: Springer International Publishing), 37-57. doi: 10.1007/ 978-3-319-66493-4 3

Chan, D., and Huybers, P. (2019). Systematic differences in bucket sea surface temperature measurements amongst nations identified using a linear-mixed-effect method. J. Clim. 32, 2569-2589. doi: 10.1175/JCLI-D-180562.1

Chan, D., Kent, E. C., Berry, D. I., and Huybers, P. (2019). Correcting datasets leads to more homogeneous early-twentieth-century sea surface warming. Nature 571, 393-397. doi: 10.1038/s41586-019-1349-2

Cowtan, K., Rohde, R., and Hausfather, Z. (2018). Evaluating biases in sea surface temperature records using coastal weather stations. Q. J. R. Meteorol. Soc. 144, 670-681. doi: 10.1002/qj.3235

Cronin, M. F., Gentemann, C. L., Edson, J. B., Ueki, I., Bourassa, M., Brown, S et al. (2019). Air-sea fluxes with a focus on heat and momentum. Front. Mar. Sci. doi: 10.3389/fmars.2019.00430

Davis, L. L. B., Thompson, D. W. J., Kennedy, J. J., and Kent, E. C. (2018) The importance of unresolved biases in 20th century sea-surface temperature observations. Bull. Am. Meteorol. Soc. 100, 621-629. doi: 10.1175/BAMS-D-180104.1

DBCP (2016). Data Buoy Co-Operation Panel, A Retropective, 2016'. Available at: https:/www.wmo.int/pages/prog/amp/mmop/documents/dbcp/Dbcp56/ DBCP\%20TD\%20No\%2056.pdf (accessed November 2015).

Eastman, R., Warren, S. G., and Hahn, C. J. (2011). Variations in cloud cover and cloud types over the ocean from surface observations, 1954-2008. J. Clim. 24 5914-5934. doi: 10.1175/2011JCLI3972.1

Folland, C. (2005). Assessing bias corrections in historical sea surface temperature using a climate model. Inter. J. Climatol. 25, 895-911. doi: 10.1002/joc.1171

Folland, C. K., and Parker, D. E. (1995). Correction of instrumental biases in historical sea surface temperature data. Q. J. R. Meteorol. Soc. 121, 319-367. doi: 10.1002/qj.49712152206

Freeman, E., Kent, E. C., Brohan, P., Cram, T., Gates, L., Huang, B., et al. (2019). The international comprehensive ocean-atmosphere data set - meeting users needs and future priorities. Front. Mar. Sci. doi: 10.3389/fmars.2019. 00435

Freeman, E., Woodruff, S. D., Worley, S. J., Lubker, S. J., Kent, E. C., and Angel, W. E. (2017). ICOADS Release 3.0: a major update to the historical marine climate record. Inter. J. Climatol. 37, 2211-2232. doi: 10.1002/joc. 4775

GCOS (2011). 'Systematic Observation Requirements for Satellite-Based Data Products for Climate. Available at: https://library.wmo.int/doc_num.php? explnum_id=3710 (accessed December 2011)

GCOS (2015). Status of the Global Observing System for Climate. Available at: https://library.wmo.int/pmb_ged/gcos_195_en.pdf (accessed October 2015). 
GCOS (2016). The Global Observing System for Climate: Implementation Needs. Available at: https://unfccc.int/sites/default/files/gcos_ip_10oct2016.pdf (accessed July 2019).

GCOS (2018). Essential Ocean Variables. Available at: http://www.goosocean.org/ index.php?option=com_content\&view=article\&id=14\&Itemid=114 (accessed July 2019)

Good, S., and Rayner, N. A. (2010). User Requirements Document, CCI Phase 1 (SST), Document Ref SST_CCI-URD-UKMO-001. Available at: http://www.esa-sst-cci.org/sites/default/files/Documents/public/SST_cci\% 20URD\%20Issue\%202\%20(2010\%2011\%2030) (accessed July 12, 2019).

Gouretski, V., Kennedy, J., Boyer, T., and Köhl, A. (2012). Consistent near-surface ocean warming since 1900 in two largely independent observing networks. Geophys. Res. Lett. 39:L19606. doi: 10.1029/2012GL052975

Grigorieva, V. G., and Badulin, S. I. (2016). Wind wave characteristics based on visual Observations and satellite altimetry. Oceanology 56, 19-24. doi: 10.1134/ S0001437016010045

Grigorieva, V., and Gulev, S. (2008). "Extreme waves in visual wave observations by VOS," in Proceedings of the Rogue Waves 2008 Workshop, October 1315, Brest, 41-49. Available at: http://www.ifremer.fr/web-com/stw2008/rw/full/ Grigorieva.pdf (accessed July 12, 2019).

Grigorieva, V. G., Gulev, S. K., and Gavrikov, A. V. (2017). Global historical archive of wind waves based on voluntary observing ship data. Oceanology 57, 229-231. doi: 10.1134/S0001437017020060

Gulev, S. K., and Grigorieva, V. (2004). Last century changes in ocean wind wave height from global visual wave data. Geophys. Res. Lett. 31:L24302. doi: 10.1029/ 2004GL021040

Gulev, S. K., and Grigorieva, V. (2006). Variability of the winter wind waves and swell in the north atlantic and north pacific as revealed by the voluntary observing ship data. J. Clim. 19, 5667-5685. doi: 10.1175/JCLI3936.1

Gulev, S. K., Grigorieva, V., Sterl, A., and Woolf, D. (2003). Assessment of the reliability of wave observations from voluntary observing ships: insights from the validation of a global wind wave climatology based on voluntary observing ship data. J. Geophys. Res. 108, 3236-3257. doi: 10.1029/2002JC001437

Hahn, C. J., and Warren, S. G. (2009). Extended Edited Cloud Reports from Ships and Land Stations over the Globe, 1952-1996 (2009 Update)'. Carbon Dioxide Information Analysis Center Numerical Data Package NDP-026C. Available at: https://cdiac.ess-dive.lbl.gov/epubs/ndp/ndp026c/ndp026c.html (accessed April, 2009).

Hahn, C. J., Warren, S. G., London, J., Jenne, R. L., and Chervin, R. M. (1988). Climatological Data for Clouds Over the Globe from Surface Observations (1988) (NDP-026). Carbon Dioxide Information Analysis Center (CDIAC), Oak Ridge National Laboratory (ORNL), Oak Ridge, TN (United States). doi: 10.3334/ CDIAC/CLI.NDP026

Hanawa, K., Yasunaka, S., Manabe, T., and Iwasaka, N. (2000). Examination of correction to historical sst data using long-term coastal sst data taken around Japan. J. Meteorol. Soc. Japan Ser. II 78, 187-195. doi: 10.2151/jmsj1965. 78.2_187

Hartmann, D. L., Klein Tank, A. M. G., Rusticucci, M., Alexander, L. V., Brönnimann, S., Charabi, Y., et al. (2013). "Observations: atmosphere and surface," in Climate Change 2013: The Physical Science Basis. Contribution of Working Group I to the Fifth Assessment Report of the Intergovernmental Panel on Climate Change, eds T. F. Stocker, D. Qin, G.-K. Plattner, M. Tignor, S. K. Allen, J. Boschung, et al. (Cambridge: Cambridge University Press).

Hausfather, Z., Cowtan, K., Clarke, D. C., Jacobs, P., Richardson, M., and Rohde, R. (2017). Assessing recent warming using instrumentally homogeneous sea surface temperature records. Sci. Adv. 3:e1601207. doi: 10.1126/sciadv.16 01207

Hausfather, Z., Cowtan, K., Menne, M. J., and Williams, C. N. (2016). Evaluating the impact of U.S. historical climatology network homogenization using the U.S. climate reference network. Geophys. Res. Lett. 43, 1695-1701. doi: 10.1002/ 2015GL067640

Hirahara, S., Ishii, M., and Fukuda, Y. (2014). Centennial-Scale sea surface temperature analysis and its uncertainty. J. Clim. 27, 57-75. doi: 10.1175/JCLID- $12-00837.1$

Hollmann, R., Merchant, C. J., Saunders, R., Downy, C., Buchwitz, M., and Cazenave, A. (2013). The ESA climate change initiative: satellite data records for essential climate variables. Bull. Am. Meteorol. Soc. 94, 1541-1552. doi: 10.1175/BAMS-D-11-00254.1
Huang, B., Angel, W., Boyer, T., Cheng, L., Chepurin, G., Freeman, E., et al. (2018). Evaluating SST analyses with independent ocean profile observations. J. Clim. 31, 5015-5030. doi: 10.1175/JCLI-D-17-0824.1

Huang, B., Thorne, P. W., Banzon, V. F., Boyer, T., Chepurin, G., Lawrimore, J. H., et al. (2017). Extended reconstructed sea surface temperature, version 5 (ersstv5): upgrades, validations, and intercomparisons. J. Clim. 30, 8179-8205. doi: 10.1175/JCLI-D-16-0836.1

Ingleby, B. (2010). Factors affecting ship and buoy data quality: a data assimilation perspective. J. Atmos. Ocean. Technol. 27, 1476-1489. doi: 10.1175/ 2010JTECHA1421.1

Ingleby, B., and Isaksen, L. (2018). Drifting buoy pressures: impact on NWP. Atmos. Sci. Lett. 19:e822. doi: 10.1002/asl.822

Ingleby, B., Moore, D., Sloan, C., and Dunn, R. (2013). Evolution and accuracy of surface humidity reports. J. Atmos. Ocean. Technol. 30, 2025-2043. doi: 10.1175/JTECH-D-12-00232.1

Jackson, D. L., and Wick, G. A. (2016). "Development of a 28-year (1987-2014) climatology of single and multi-sensor satellite-based retrievals of near-surface humidity and temperature," in Proceedings of the 20th Conference on Air-Sea Interactions, (Madison, WI: American Meteorological Society).

James, R. W., and Fox, P. T. (1972). "Comparative sea surface temperature measurements," in World Meteorological Organization, Reports on Marine Science Affairs, Report No. 5. (Geneva: World Meteorological Organization No. 336)

JCGM (2008). Evaluation of Measurement Data - Guide to the Expression of Uncertainty in Measurement (GUM)'. Available at: http://www.bipm.org/ utils/common/documents/jcgm/JCGM_100_2008_E.pdf (accessed September 2008).

Josey, S. A., Kent, E. C., and Taylor, P. K. (1999). New insights into the ocean heat budget closure problem from analysis of the SOC air-sea flux climatology. J. Clim. 12, 2856-2880. doi: 10.1175/1520-0442(1999)012<2856:niitoh> 2.0.co;2

Kaplan, A., Cane, M. A., Kushnir, Y., Clement, A. C., Blumenthal, M. B., and Rajagopalan, B. (1998). Analyses of global sea surface temperature 1856-1991. J. Geophys. Res. Oceans 103, 18567-18589. doi: 10.1029/97JC 01736

Karspeck, A. R., Kaplan, A., and Sain, S. R. (2012). Bayesian modelling and ensemble reconstruction of mid-scale spatial variability in North Atlantic seasurface temperatures for 1850-2008. Q. J. R. Meteorol. Soc. 138, 234-248. doi: 10.1002/qj.900

Kawai, Y., and Wada, A. (2007). Diurnal sea surface temperature variation and its impact on the atmosphere and ocean: a review. J. Oceanogr. 63, 721-744. doi: 10.1007/s10872-007-0063-0

Kennedy, J. J. (2014). A review of uncertainty in in situ measurements and data sets of sea surface temperature. Rev. Geophys. 52, 1-32. doi: 10.1002/2013RG0 00434

Kennedy, J. J., Rayner, N. A., Atkinson, C. P., and Killick, R. E. (2019). An ensemble data set of sea-surface temperature change from 1850: the Met Office Hadley Centre HadSST.4.0.0.0 data set. J. Geophys. Res. Atmos. 124. doi: 10.1029/ 2018JD029867

Kennedy, J. J., Rayner, N. A., Smith, R. O., Parker, D. E., and Saunby, M. (2011a). Reassessing biases and other uncertainties in sea surface temperature observations measured in situ since 1850: 1. measurement and sampling uncertainties. J. Geophys. Res. 116:D14103. doi: 10.1029/2010JD0 15218

Kennedy, J. J., Rayner, N. A., Smith, R. O., Parker, D. E., and Saunby, M. (2011b). Reassessing biases and other uncertainties in sea surface temperature observations measured in situ since 1850: 2. biases and homogenization. J. Geophys. Res. 116:D14104. doi: 10.1029/2010JD015220

Kent, E. C., and Berry, D. I. (2005). Quantifying random measurement errors in Voluntary Observing Ships' meteorological observations. Inter. J. Climatol. 25, 843-856. doi: 10.1002/joc.1167

Kent, E. C., Berry, D. I., Woodruff, S. D., and Taylor, P. K. (2006). Voluntary Observing Ships: a Vital Observing System in Decline. CLIVAR Exchanges 11(3): 20-21. Available at: http://www.clivar.org/sites/default/files/documents/ Exchanges38.pdf (accessed 25 March 2019).

Kent, E. C., and Kaplan, A. (2006). Toward estimating climatic trends in SST. Part III: systematic biases. J. Atmos. Ocean. Technol. 23, 487-500. doi: 10.1175/ JTECH1845.1 
Kent, E. C., Kennedy, J. J., Berry, D. I., and Smith, R. O. (2010). Effects of instrumentation changes on sea surface temperature measured in situ. Wiley Interdiscip. Rev. Clim. Change 1, 718-728. doi: 10.1002/wcc.55

Kent, E. C., Kennedy, J. J., Smith, T. M., Hirahara, S., Huang, B., and Kaplan, A. (2017). A call for new approaches to quantifying biases in observations of sea surface temperature. Bull. Am. Meteorol. Soc. 98, 1601-1616. doi: 10.1175/ BAMS-D-15-00251.1

Kent, E. C., Rayner, N. A., Berry, D. I., Saunby, M., Moat, B. I., Kennedy, J. J., et al. (2013). Global analysis of night marine air temperature and its uncertainty since 1880: the HadNMAT2 data set. J. Geophys. Res. Atmos. 118, 1281-1298. doi: 10.1002 /jgrd.50152

Kent, E. C., and Taylor, P. K. (1996). Accuracy of humidity measurement on ships: consideration of solar radiation effects. J. Atmos. Ocean. Technol. 13, 1317-1321. doi: 10.1175/1520-0426(1996)013<1317:aohmos>2.0.co;2

Kent, E. C., and Taylor, P. K. (1997). Choice of a beaufort equivalent scale. J. Atmos. Ocean. Technol. 14, 228-242. doi: 10.1175/1520-0426(1997)014<0228:coabes> 2.0.co; 2

Kent, E. C., Taylor, P. K., Truscott, B. S., and Hopkins, J. S. (1993). The accuracy of voluntary observing ships' meteorological observations-results of the VSOPNA. J. Atmos. Ocean. Technol. 10, 591-608. doi: 10.1175/1520-0426(1993) 010<0591:taovos $>2.0 . c 0 ; 2$

Kent, E. C., Woodruff, S. D., and Berry, D. I. (2007). Metadata from WMO publication no. 47 and an assessment of voluntary observing ship observation heights in ICOADS. J. Atmos. Ocean. Technol. 24, 214-234. doi: 10.1175/ JTECH1949.1

Kinzel, J., Fennig, K., Schröder, M., Andersson, A., Bumke, K., and Hollmann, R. (2016). Decomposition of random errors inherent to HOAPS-3.2 near-surface humidity estimates using multiple triple collocation analysis. J. Atmos. Ocean. Technol. 33, 1455-1471. doi: 10.1175/JTECH-D-150122.1

Levitus, S., Antonov, J. I., Boyer, T. P., Locarnini, R. A., Garcia, H. E., and Mishonov, A. V. (2009). Global ocean heat content 1955-2008 in light of recently revealed instrumentation problems. Geophys. Res. Lett. 36:L07608. doi: 10.1029/2008GL037155

Liman, J., Schröder, M., Fennig, K., Andersson, A., and Hollmann, R. (2018). Uncertainty characterization of HOAPS 3.3 latent heat-flux-related parameters. Atmos. Meas. Tech. 11, 1793-1815. doi: 10.5194/amt-11-17932018

Loeb, N. G., Lyman, J. M., Johnson, G. C., Allan, R. P., Doelling, D. R., Wong, T., et al. (2012). Observed changes in top-of-the-atmosphere radiation and upper-ocean heating consistent within uncertainty. Nat. Geosci. 5:110. doi: 10.1038/ngeo1375

Manabe, T. (1999). The digitized kobe collection, phase I: historical surface marine meteorological observationsin the archive of the japan meteorological agency. Bull. Am. Meteorol. Soc. 80, 2703-2715. doi: 10.1175/1520-0477(1999) $080<2703:$ tdkcpi $>2.0 . c 0 ; 2$

Martin, M., Dash, P., Ignatov, A., Banzon, V., Beggs, H., and Brasnett, B. (2012). Group for high resolution sea surface temperature (GHRSST) analysis fields inter-comparisons. Part 1: a GHRSST multi-product ensemble (GMPE). Deep Sea Res. Part II Top. Stud. Oceanogr. 7, 21-30. doi: 10.1016/j.dsr2.2012. 04.013

Maury, M. F. (1854). "Maritime Conference held at Brussels for devising a uniform system of meteorological observations at sea, August and September, 1853," in Proceedind of the Maritime Conference Explanations and Sailing Directions to Accompany the Wind and Current Charts, 6th Edn, (Philadelphia, PA: Biddle), 54-96.

McPhaden, M. J., Busalacchi, A. J., Cheney, R., Donguy, J. R., Gage, K. S., Halpern, D., et al. (1998). The tropical ocean-global atmosphere observing system: a decade of progress. J. Geophys. Res.: Oceans 103, 14169-14240. doi: 10.1029/ 97JC02906

Merchant, C. J., Embury, O., Rayner, N. A., Berry, D. I., Corlett, G. K., Lean, K., et al. (2012). A 20 year independent record of sea surface temperature for climate from Along-Track scanning radiometers. J. Geophys. Res. Oceans 117:C12013. doi: 10.1029/2012JC008400

Merchant, C. J., Embury, O., Roberts-Jones, J., Fiedler, E., Bulgin, C. E., Corlett, G. K., et al. (2014). Sea surface temperature datasets for climate applications from Phase 1 of the European Space Agency Climate Change Initiative (SST CCI). Geosci. Data J. 1, 179-191. doi: 10.1002/gdj3.20
Moat, B. I., Yelland, M. J., and Molland, A. F. (2006). Quantifying the airflow distortion over merchant ships. Part II: application of the model results. J. Atmos. Ocean. Technol. 23, 351-360. doi: 10.1175/JTECH1859.1

Morak-Bozzo, S., Merchant, C. J., Kent, E. C., Berry, D. I., and Carella, G. (2016). Climatological diurnal variability in sea surface temperature characterized from drifting buoy data. Geosci. Data J. 3, 20-28. doi: 10.1002/gdj3.35

Norris, J. R. (1998). Low cloud type over the ocean from surface observations. part I: relationship to surface meteorology and the vertical distribution of temperature and moisture. J. Clim. 11, 369-382. doi: 10.1175/1520-0442(1998) $011<0369:$ lctoto $>2.0$. co; 2

O'Carroll, A. G., Armstrong, E. M., Beggs, H. M., Bouali, M., Casey, K. S., Corlett, G. K., et al. (2019). Observational needs of sea surface temperature. Front. Mar. Sci. doi: $10.3389 /$ fmars. 2019.00420

O'Carroll, A. G., Eyre, J. R., and Saunders, R. W. (2008). Three Way error analysis between AATSR, AMSR-E, and in situ sea surface temperature observations. J. Atmos. Ocean. Technol. 25, 1197-1207. doi: 10.1175/2007JTECHO 542.1

OceanSITES (2015). OceanSITES Data Format Reference Manual, NetCDF Conventions and Reference Tables Version 1.3. Available at: http: //www.oceansites.org/docs/oceansites_data_format_reference_manual.pdf (accessed July, 2017).

OceanSITES (2016). Organization and Governance. Available at: http://www. oceansites.org/documents/Organization_and_Governance_17Mar2016.pdf (accessed Mar, 2016).

Petty, G. W. (1995). Frequencies and characteristics of global oceanic precipitation from shipboard present-weather reports. Bull. Am. Meteorol. Soc. 76, 1593-1616. doi: 10.1175/1520-0477(1995)076<1593:facogo >2.0.co;2

Plagge, A. M., Vandemark, D., and Chapron, B. (2012). Examining the impact of surface currents on satellite scatterometer and altimeter ocean winds. J. Atmos. Ocean. Technol. 29, 1776-1793. doi: 10.1175/JTECH-D-12-00017.1

Poli, P., Lucas, M., O’Carroll, A., Le Menn, M., David, A., Corlett, G. K., et al. (2018). The copernicus surface velocity platform drifter with barometer and reference sensor for temperature (SVP-BRST): genesis, design, and initial results. Ocean Sci. Discuss. 15, 199-214. doi: 10.5194/os-2018-109

Prytherch, J., Kent, E. C., Fangohr, S., and Berry, D. I. (2015). A comparison of SSM/I-derived global marine surface-specific humidity datasets. Inter. J. Climatol. 35, 2359-2381. doi: 10.1002/joc.4150

Rayner, N. A. (2017). User Requirements Document, CCI Phase 1 (SST), Document ref SST_CCI-URD-UKMO-201. Available at: http://www.esa-sstcci.org/PUG/pdf/SST_CCI-URD-UKMO-201-Issue_2.1-signed.pdf (accessed July 12, 2019).

Rayner, N. A., Brohan, P., Parker, D. E., Folland, C. K., Kennedy, J. J., Vanicek, M., et al. (2006). Improved analyses of changes and uncertainties in sea surface temperature measured in situ since the mid-nineteenth century: the HadSST2 dzataset. J. Clim. 19, 446-469. doi: 10.1175/JCLI3637.1

Rayner, N. A., Parker, D. E., Horton, E. B., Folland, C. K., Alexander, L. V., Rowell, D. P., et al. (2003). Global analyses of sea surface temperature, sea ice, and night marine air temperature since the late nineteenth century. J. Geophys. Res. 108, 4407-4437. doi: 10.1029/2002JD002670

Reverdin, G., Boutin, J., Martin, N., Lourenco, A., Bouruet-Aubertot, P., Lavin, A., et al. (2010). Temperature measurements from surface drifters. J. Atmos. Ocean. Technol. 27, 1403-1409. doi: 10.1175/2010JTECHO741.1

Reynolds, R. W., Rayner, N. A., Smith, T. M., Stokes, D. C., and Wang, W. (2002). An improved in situ and satellite SST analysis for climate. J. Clim. 15, 1609-1625. doi: 10.1175/1520-0442(2002)015<1609:aiisas >2.0.co;2

Rhines, A., Tingley, M. P., McKinnon, K. A., and Huybers, P. (2015). Decoding the precision of historical temperature observations: decoding observational precision. Q. J. R. Meteorol. Soc. 141, 2923-2933. doi: 10.1002/qj. 2612

Richardson, M., Cowtan, K., and Millar, R. J. (2018). Global temperature definition affects achievement of long-term climate goals. Environ. Res. Lett. 13:054004. doi: 10.1088/1748-9326/aab305

Rodriguez, R., Bourassa, M. A., Chelton, D., Farrar, J. T., Long, D., PerkovicMartin, D., et al. (2019). The winds and currents mission concept. Front. Mar. Sci. doi: 10.3389/fmars.2019.00438

Schwab, M., Meinke, I., Vanderlinden, J.-P., and Touili, N. (2015). Empirical Working Paper: Analysing Stakeholders' Needs and Understanding, European Climate and Weather Events: Interpretation and Attribution Deliverable 
D4.2. Available at: https://eucleia.eu/wp-content/uploads/2018/07/d4-2_final. pdf (accessed July 12, 2019).

Smith, S. R., Briggs, K., Bourassa, M. A., Elya, J., and Paver, C. R. (2018). Shipboard automated meteorological and oceanographic system data archive: 2005-2017. Geosci. Data J. 5, 73-86. doi: 10.1002/gdj3.59

Smith, S. R., Alory, G., Andersson, A., Asher, W., Baker, A., Berry, D. I., et al. (2019). Ship-based contributions to global ocean, weather, and climate observing systems. Front. Mar. Sci. doi: 10.3389/fmars.2019.00434

Smith, S. R., Bourassa, M. A., and Sharp, R. J. (1999). Establishing more truth in true winds. J. Atmos. Ocean. Technol. 16, 939-952. doi: 10.1175/15200426(1999)016<0939:emtitw>2.0.co;2

Smith, S. R., Freeman, J. E., Lubker, S. J., Woodruff, S. D., Worley, S. J., and Angel, W. E. (2016). The International Maritime Meteorological Archive (IMMA) Format. Available at: http://icoads.noaa.gov/e-doc/imma/R3. 0-imma1.pdf (accessed July 2016).

Smith, S. R., Legler, D. M., and Verzone, K. V. (2001). Quantifying uncertainties in ncep reanalyses using high-quality research vessel observations. J. Clim. 14, 4062-4072. doi: 10.1175/1520-0442(2001)014<4062:quinru> 2.0.co;2

Snook, I. B. (2016). Fiducial Reference Measurements for Validation of Surface Temperature from Satellites (FRM4STS), Project Brochure'. OFE-D-30-V1-Iss-1. Available at: http://www.frm4sts.org/wp-content/uploads/sites/3/2017/12/ D30-FRM4STS-project-brochure-with-cover-16Nov16-signed.pdf (accessed October 2016).

Stoffelen, A. (1998). Toward the true near-surface wind speed: error modeling and calibration using triple collocation. J. Geophys. Res. Oceans 103, 7755-7766. doi: 10.1029/97JC03180

Stoffelen, A., Jur Vogelzang, J., and Wenming, L. (2015). On Buoys, Scatterometers and Reanalyses for Globally Representative Winds. Available at: https://www. nwpsaf.eu/publications/tech_reports/nwpsaf-kn-tr-024.pdf (accessed August 2015).

Thomas, B. R., Kent, E. C., and Swail, V. R. (2005). Methods to homogenize wind speeds from ships and buoys. Inter. J. Climatol. 25, 979-995. doi: 10.1002/joc. 1176

Thomas, B. R., Kent, E. C., Swail, V. R., and Berry, D. I. (2008). Trends in ship wind speeds adjusted for observation method and height. Inter. J. Climatol. 28 , 747-763. doi: 10.1002/joc.1570

Thorne, P. W., Allan, R. J., Ashcroft, L., Brohan, P., Dunn, R. J. H., and Menne, M. J. (2017a). Toward an integrated set of surface meteorological observations for climate science and applications. Bull. Am. Meteorol. Soc. 98, 2689-2702. doi: 10.1175/BAMS-D-16-0165.1

Thorne, P. W., Diamond, H. J., Goodison, B., Harrigan, S., Hausfather, Z., and Ingleby, N. B. (2018). Towards a global land surface climate fiducial reference measurements network. Inter. J. Climatol. 38, 2760-2774. doi: 10.1002/joc. 5458

Thorne, P. W., Madonna, F., Schulz, J., Oakley, T., Ingleby, B., Rosoldi, M., et al. (2017b). Making better sense of the mosaic of environmental measurement networks: a system-of-systems approach and quantitative assessment. Geosci. Instrum. Method. Data Sys. 6, 453-472. doi: 10.5194/gi-6-453-2017
Tokinaga, H., and Xie, S.-P. (2011). Wave and anemometer-based sea surface wind (WASWind) for climate change analysis. J. Clim. 24, 267-285. doi: 10.1175/ 2010JCLI3789.1

Verhoef, A., Vogelzang, J., Verspeek, J., and Stoffelen, A. (2017). Long term scatterometer wind climate data records. IEEE J. Sel. Top. Appl. Earth Obs. Remote Sens. 10, 2186-2194. doi: 10.1109/JSTARS.2016.2615873

Weller, R. A. (2018). “Observing Surface Meteorology and Air-Sea Fluxes," in ., eds R. Venkatesan, A. Tandon, E. D’Asaro, and M. A. Atmanand (Cham: Springer International Publishing), 17-35. doi: 10.1007/978-3-319-66493-4_2

Wentz, F. J., Ricciardulli, L., Rodriguez, E., Stiles, B. W., Bourassa, M. A., and Long, D. G. (2017). Evaluating and extending the ocean wind climate data record. IEEE J. Sel. Top. Appl. Earth Obs. Remote Sens. 10, 2165-2185. doi: 10.1109/JSTARS.2016.2643641

WMO (2012). Manual on Marine Meteorological Services, Volume I - Global Aspects'. Available at: https://library.wmo.int/pmb_ged/wmo_558_en-v1.pdf (accessed July 12, 2019).

Woodruff, S. D., Diaz, H. F., Worley, S. J., Reynolds, R. W., and Lubker, S. J. (2005). Early ship observational data and icoads. Clim. Change 73, 169-194. doi: 10.1007/s10584-005-3456-3

Woodruff, S. D., Slutz, R. J., Jenne, R. L., and Steurer, P. M. (1987). A comprehensive ocean-atmosphere data set. Bull. Am. Meteorol. Soc. 68, 1239-1250. doi: 10.1175/1520-0477(1987)068<1239:acoads $>2.0$. co;2

Woodruff, S. D., Worley, S. J., Lubker, S. J., Ji, Z., Eric Freeman, J., Berry, D. I., et al. (2011). ICOADS Release 2.5: extensions and enhancements to the surface marine meteorological archive. Inter. J. Climatol. 31, 951-967. doi: 10.1002/joc. 2103

Worley, S. J., Woodruff, S. D., Reynolds, R. W., Lubker, S. J., and Lott, N. (2005). ICOADS release 2.1 data and products. Inter. J. Climatol. 25, 823-842. doi: 10.1002/joc.1166

Xu, F., and Ignatov, A. (2016). Error characterization in I Quam SSTs using triple collocations with satellite measurements. Geophys. Res. Lett. 43:834. doi: 10.1002/2016GL070287

Young, I. R., Zieger, S., and Babanin, A. V. (2011). Global trends in wind speed and wave height. Science 332, 451-455. doi: 10.1126/science.1197219

Zhang, H. M., Reynolds, R. W., and Smith, T. M. (2006). Adequacy of the in situ observing system in the satellite era for climate SST. J. Atmos. Ocean. Technol. 23, 107-120. doi: 10.1175/JTECH1828.1

Conflict of Interest Statement: The authors declare that the research was conducted in the absence of any commercial or financial relationships that could be construed as a potential conflict of interest.

Copyright (C) 2019 Kent, Rayner, Berry, Eastman, Grigorieva, Huang, Kennedy, Smith and Willett. This is an open-access article distributed under the terms of the Creative Commons Attribution License (CC BY). The use, distribution or reproduction in other forums is permitted, provided the original author(s) and the copyright owner(s) are credited and that the original publication in this journal is cited, in accordance with accepted academic practice. No use, distribution or reproduction is permitted which does not comply with these terms. 


\section{OPEN ACCESS}

Edited by:

John Siddorn,

Met Office, United Kingdom

Reviewed by:

Girishkumar Madathil

Sivasankaran,

Indian National Centre for Ocean

Information Services, India

Saša Gaberšek,

Naval Research Laboratory Marine Meteorology Division, United States

*Correspondence.

Ricardo Domingues

Ricardo.Domingues@noaa.gov

Specialty section:

This article was submitted to

Ocean Observation,

a section of the journal

Frontiers in Marine Science

Received: 20 November 2018

Accepted: 05 July 2019

Published: 30 July 2019

Citation:

Domingues $R$,

Kuwano-Yoshida A,

Chardon-Maldonado P, Todd RE,

Halliwell G, Kim H-S, Lin I-I, Sato K,

Narazaki T, Shay LK, Miles T, Glenn S,

Zhang JA, Jayne SR, Centurioni L,

Le Hénaff M, Foltz GR, Bringas F,

Ali MM, DiMarco SF, Hosoda S,

Fukuoka T, LaCour B, Mehra A,

Sanabia ER, Gyakum JR, Dong J,

Knaff JA and Goni G (2019) Ocean Observations in Support of Studies and Forecasts of Tropical

and Extratropical Cyclones.

Front. Mar. Sci. 6:446.

doi: 10.3389/fmars.2019.00446

\section{Ocean Observations in Support of Studies and Forecasts of Tropical and Extratropical Cyclones}

Ricardo Domingues ${ }^{1,2 *}$, Akira Kuwano-Yoshida ${ }^{3}$, Patricia Chardon-Maldonado ${ }^{4,5}$, Robert E. Todd ${ }^{6}$, George Halliwell ${ }^{2}$, Hyun-Sook Kim ${ }^{2,7}$, I.-I. Lin ${ }^{8}$, Katsufumi Sato' Tomoko Narazaki ${ }^{9}$, Lynn K. Shay ${ }^{10}$, Travis Miles ${ }^{11}$, Scott Glenn ${ }^{11}$, Jun A. Zhang ${ }^{1,2}$, Steven R. Jayne ${ }^{6}$, Luca Centurioni ${ }^{12}$, Matthieu Le Hénaff ${ }^{1,2}$, Gregory R. Foltz ${ }^{2}$, Francis Bringas ${ }^{2}$, M. M. Ali ${ }^{13}$, Steven F. DiMarco ${ }^{14}$, Shigeki Hosoda ${ }^{15}$, Takuya Fukuoka ${ }^{9}$, Benjamin LaCour', Avichal Mehra², Elizabeth R. Sanabia ${ }^{16}$, John R. Gyakum ${ }^{17}$, Jili Dong'2, John A. Knaff² and Gustavo Goni

${ }^{1}$ Cooperative Institute for Marine and Atmospheric Studies, University of Miami, Miami, FL, United States, ${ }^{2}$ Atlantic Oceanographic and Meteorological Laboratory, National Oceanic and Atmospheric Administration, Miami, FL, United States, ${ }^{3}$ Shirahama Oceanographic Observatory Shionomisaki Wind Effect Laboratory, Disaster Prevention Research Institute, Kyoto University, Kyoto, Japan, ${ }^{4}$ Caribbean Coastal Ocean Observing System, Mayagüez, Puerto Rico, ${ }^{5}$ University of Puerto Rico at Mayagüez, Mayagüez, Puerto Rico, ${ }^{6}$ Woods Hole Oceanographic Institution, Woods Hole, MA, United States, ${ }^{7}$ I.M. Systems Group, Inc., Rockville, MD, United States, ${ }^{8}$ Department of Atmospheric Sciences, National Taiwan University, Taipei, Taiwan, ${ }^{9}$ Atmosphere and Ocean Research Institute, University of Tokyo, Kashiwa, Japan, ${ }^{10}$ Rosenstiel School of Marine and Atmospheric Science, University of Miami, Miami, FL, United States, ${ }^{11}$ Department of Ocean Sciences, Rosenstiel School of Marine and Atmospheric Science, University of Miami, Coral Gables, FL, United States, ${ }^{12}$ Scripps Institution of Oceanography, La Jolla, CA, United States, ${ }^{13}$ Center for Ocean-Atmospheric Prediction Studies, Florida State University, Tallahassee, FL, United States, ${ }^{14}$ Texas A\&M University, College Station, TX. United States, ${ }^{15}$ Japan Agency for Marine-Earth Science and Technology, Yokosuka, Japan, ${ }^{16}$ United States Naval Academy, Annapolis, MD, United States, ${ }_{17}$ U.S. Integrated Ocean Observing System, National Oceanic and Atmospheric Administration, Miami, FL, United States

Over the past decade, measurements from the climate-oriented ocean observing system have been key to advancing the understanding of extreme weather events that originate and intensify over the ocean, such as tropical cyclones (TCs) and extratropical bomb cyclones (ECs). In order to foster further advancements to predict and better understand these extreme weather events, a need for a dedicated observing system component specifically to support studies and forecasts of TCs and ECs has been identified, but such a system has not yet been implemented. New technologies, pilot networks, targeted deployments of instruments, and state-of-the art coupled numerical models have enabled advances in research and forecast capabilities and illustrate a potential framework for future development. Here, applications and key results made possible by the different ocean observing efforts in support of studies and forecasts of TCs and ECs, as well as recent advances in observing technologies and strategies are reviewed. Then a vision and specific recommendations for the next decade are discussed.

Keywords: tropical cyclones, extratropical bomb cyclones, upper-ocean temperature, ocean heat content, global ocean observing system, weather extremes, natural hazards, coupled ocean-atmosphere forecasts 


\section{INTRODUCTION}

Extreme weather events are natural hazards that affect marine and terrestrial areas around the world and are associated with different temporal and spatial scales (Elsner et al., 2008; Menkes et al., 2016; Zhou et al., 2018). Tropical cyclones (TCs) and their extratropical counterparts, often referred to as extratropical "bomb" cyclones (ECs), are deep low pressure systems that produce and sustain high intensity winds. TCs develop exclusively over the ocean (e.g., Knapp et al., 2010), while ECs predominantly form in the land-ocean margin, in the vicinity of western boundary currents (e.g., Sanders and Gyakum, 1980), and can also occasionally develop over land (e.g., Hakim et al., 1995). TCs and ECs are among the deadliest and most destructive types of extreme weather, often causing widespread damage due to strong winds, storm surge, and heavy precipitation. Understanding their dynamical mechanisms and having the ability to accurately forecast them is a critical societal need but remains a challenge.

The understanding of upper-ocean processes leading to extreme weather events has largely benefited from the climate-oriented sustained ocean observing system (e.g., Legler et al., 2015) and observational process studies. Clearly, ocean observations are vital in ECs and TCs research and forecasts, since they enable examining the details of air-sea interaction processes that can lead to the formation and intensification of these systems (e.g., Leipper and Volgenau, 1972; Sanders and Gyakum, 1980; Mainelli et al., 2008; Kuwano-Yoshida and Minobe, 2017). In addition, ocean observations have been increasingly acknowledged by the forecast community as a critical piece to improve extreme weather forecasts (e.g., Dong et al., 2017). Their important role will likely continue to increase in light of improvements in coupled model capabilities (see below), and future extreme weather projections, which expects intense weather systems to become more frequent (e.g., Colle et al., 2015; Bacmeister et al., 2018). In fact, more and more occurrences of record-breaking "Cat-6" types of TCs were observed over the last decade, with three Cat- 6 storms ( $>=165 \mathrm{kts}$ ) forming during 2008-2018 (Haiyan, November, 2013; Patricia, October, 2015; and Meranti, September, 2016), and no Cat-6 TCs recorded globally in the earlier decade during 1999-2008. These recent changes and projections in occurrence of intense TCs and ECs emphasize the need for a sustained observing system in support of extreme weather studies and forecasts.

Recent advances in research and ocean observing efforts during the past decade have largely addressed specific recommendations identified by the scientific community during OceanObs' 09 (e.g., Goni et al., 2010) about key areas to focus in support of TC studies and forecasts. Since then, new technologies, pilot networks, and targeted deployments have further expanded the reach of the global observing system and have been used in weather analyses, outlooks, and improved ocean-atmosphere numerical forecasts models. For instance, (i) extensive synergies between the scientific and operational communities continue to facilitate transitioning of research results into operations (e.g., Shay et al., 2014); (ii) networks specifically designed in support of TC studies and forecasts have been implemented (e.g., Domingues et al., 2015; Miles et al., 2015); (iii) targeted airborne profiling observations ahead of forecasted TCs have been extensively implemented (e.g., D’Asaro et al., 2014; Meyers et al., 2015; Jaimes et al., 2016; Zhang et al., 2018); (iv) substantial progress has been made toward understanding the role of upper ocean salinity (e.g., Balaguru et al., 2012a,b; Domingues et al., 2015) and temperature stratification (Price, 2009; Lin et al., 2013a,b; Balaguru et al., 2015, 2018; Glenn et al., 2016) in controlling TC intensity and development; (v) real-time assimilation of ocean observations into coupled weather forecast systems continues to provide critical information for improved ocean representation (e.g., Chen et al., 2017; Dong et al., 2017); and (vi) new, state-of-theart, coupled numerical weather models have evolved and are now being used in experimental and operational forecasting modes (e.g., Kim et al., 2014).

On a broader context, recent advances in ocean observations in support of extreme weather were accompanied by an overall improvement of global coverage based on in situ and satellite ocean observations, and in their analysis. For example: improved sensor technology and satellite coverage enabled substantial advances in satellite altimetry (Verrier et al., 2018); satellite-derived salinity measurements (Meissner et al., 2018), now available with the launch of the Aquarius and the Soil-Moisture Active Passive (SMAP) missions, have produced unprecedented spatial coverage of sea surface salinity observations (Meissner et al., 2018); advances in in situ observing systems and networks (e.g., Foltz et al., 2019; Goni et al., 2019; Roemmich et al., 2019; Todd et al., 2019) have also enabled many groundbreaking research of the global oceans and climate system. In addition, community-wide efforts aimed at advancing data availability, and data assimilation within high-resolution numerical models through the US GODAE (Chassignet et al., 2009), the GODAE/Mercator-Ocean forecast system (Drévillon et al., 2008), and other similar efforts, have also provided significant advances in oceanic forecasting capabilities. Currently, there are also ongoing efforts from the Environmental Modeling Center (EMC) within the National Oceanic and Atmospheric Administration (NOAA) to implement a global data assimilation scheme into the Real-Time Ocean Forecast System (RTOFS), and a similar scheme more specifically in support of hurricane modeling systems. Some examples of such systems include the regional scale coupled HYbrid Coordinate Ocean Model - Hurricane Weather Research and Forecast (HYCOM-HWRF), and the HYCOM - Hurricanes in a Multi-scale Ocean-coupled Nonhydrostatic model (HYCOM-HMON). Additional details of such hurricane models are explored further in Section "Impact of Ocean Data in Tropical Cyclone Intensity Forecasts" of this manuscript.

In this community white paper, we describe some of the most important advances in the observational efforts in support of studies and forecasts of TCs and ECs since OceanObs' 09 , and present and discuss some potential enhancements to ocean observing strategies for the upcoming future, as well as provide specific recommendations. This manuscript is organized 
as follows: Sections "Tropical Cyclones" and "Extratropical Bomb Cyclones" provide detailed descriptions of TCs and ECs, respectively, and discuss the role of the ocean in their genesis and intensification; in Section "Ocean Observations and Parameters in Support of Extreme Weather Studies and Forecasts," key components of the ocean observing system, products, and pilot networks in support of TC and/or ECs studies and forecasts are described; Section "Highlighted Applications and Results" presents selected applications of ocean observations in support of research into TCs and ECs; in Section "Impact of Ocean Data in Tropical Cyclone Intensity Forecasts," the impact of ocean observations in the forecast of TC is specifically addressed; Section "Data Management" describes recent advances and needs concerning data management; and in Section "The Vision for the Next 10 years," the recommendations from the scientific community and the vision for the upcoming decade are provided.

\section{Tropical Cyclones}

A TC is a fast-rotating storm system that is characterized by a low pressure center that forms in the tropics. TCs are observed in seven ocean basins worldwide (Figure 1), namely the: North Atlantic, Northeast Pacific, Northwest Pacific, Southwest Indian, North Indian, Southeast Indian, and South Pacific. In the North Atlantic Ocean basin, for example, 11-12 named TCs, 6 hurricanes, and 2-3 major hurricanes develop between June and November in a typical year (e.g., Landsea, 1993). TC development is associated with unstable atmospheric conditions, which are primarily linked with boundary layer temperature [i.e., sea surface temperatures (SST) under the eyewall], and to a lesser extent to the upper-level temperatures (see discussion in Balaguru et al., 2015). In addition, favorable atmospheric conditions for TC genesis and intensification, in order of importance, include: (i) relatively little variation in the vertical profile of the environmental winds (low vertical wind shear); (ii) an atmosphere characterized by lower-level convergence and upper-level divergence; (iii) sufficient environmental midand upper-level moisture; and (iv) relatively slow (less than $8 \mathrm{~m} \mathrm{~s}^{-1}$ ) deep layer steering. Favorable environmental conditions for TC intensification have been thoroughly documented in many studies (e.g., DeMaria and Kaplan, 1994; Knaff et al., 2005, 2018; Kaplan et al., 2010; DeMaria et al., 2014).

Under favorable atmospheric conditions, SSTs above $26^{\circ} \mathrm{C}$ are needed for development and maintenance of TCs (e.g., Leipper and Volgenau, 1972; Dare and McBride, 2012). The vertical structure of the upper ocean plays a key role in the intensification and weakening of TCs with both thermal structure (e.g., Leipper and Volgenau, 1972; Shay et al., 2000; Mainelli et al., 2008; Goni et al., 2009; Shay and Brewster, 2010) and stratification (e.g., Lin et al., 2008; Price, 2009; Balaguru et al., 2012a,b, 2015, 2018; Domingues et al., 2015; Emanuel, 2015; Huang et al., 2015; Seroka et al., 2016; Rudzin et al., 2017, 2018) being important. The upper ocean heat content $(\mathrm{OHC})$ is an indicator of how much energy is potentially available for TC intensification, while the stratification can act as a barrier to TC-induced mixing, suppressing upper ocean cooling, and helping maintain enthalpy fluxes from the ocean into the TC. Intense upper ocean mixing events caused by the strong hurricane winds can quickly erode the thermal signature of subsurface warm or cold features (Pickard and Emery, 1990), leading to SST misrepresenting the ocean thermal energy, and potential TC intensity. Several studies (e.g., Mao et al., 2000; Shay et al., 2000; Ali et al., 2007, 2013; Mainelli et al., 2008; Lin et al., 2013b) demonstrated the importance of ocean thermal energy, represented by warm ocean features.

Tropical cyclone intensification involves the interaction of very complex mechanisms at a range of scales, such as internal TC dynamics, upper ocean interaction, and atmospheric circulation. Rapid intensification is often associated with TCs moving over warm ocean features (i.e., upper-OHC values larger than $60 \mathrm{~kJ} \mathrm{~cm}^{-2}$, Figure 2), which maintain warmer SSTs (due to suppression of TC-induced sea surface cooling) near the convective center of the TC (Shay et al., 2000; Lin et al., 2005, 2009). The thermal energy across the sea surface is central to the enthalpy fluxes that transport heat and moisture from the ocean to the atmosphere, fueling the TC. For this reason, $\mathrm{OHC}$ estimates from a variety of datasets and methodologies are routinely used to provide operational guidance and carry out studies for intensity change and rapid intensification (e.g., Goni and Trinanes, 2003; DeMaria et al., 2005; Kaplan et al., 2010; Shay and Brewster, 2010; Meyers et al., 2014; Rogers et al., 2017; Knaff et al., 2018; Yamaguchi et al., 2018).

Despite these advances in understanding the role that the upper ocean plays in TC intensification, officially-issued shortterm intensity forecast errors have not been significantly reduced over the past two decades in any basin (e.g., Figure 3; DeMaria et al., 2007, 2014). One of the factors contributing to the lag in improvement of TC intensity forecasts relative to TC track forecasts may be the lack of a dedicated ocean observing system with sustained and targeted ocean observations to correctly represent the ocean component in ocean-atmosphere coupled intensity forecast models. Nonetheless, operational intensity forecast tools and models have been improving and are starting to reduce official intensity errors (DeMaria et al., 2014), which may, in part, be due to improved atmospheric and ocean data assimilation (DA).

\section{Extratropical Bomb Cyclones}

An EC is an extratropical cyclone that undergoes rapid deepening of its low pressure by $24 \mathrm{hPa}$ or more in a period of $24 \mathrm{~h}$ (i.e., 'explosive cyclogenesis'; Sanders and Gyakum, 1980). This process is predominantly maritime, with seldom occurrences over continental land masses. Though they are typically winter events, and their genesis involves processes distinct from those associated with TC development, ECs produce winds as strong as hurricanes and are often associated with large rainfall and storm surges. Explosive cyclogenesis is mainly observed within the four basins, namely the Northwest Pacific, the North Atlantic, the Southwest Pacific, and the South Atlantic (Black and Pezza, 2013). Though ECs in the North Atlantic basin occur preferentially along the east coast of North America, in the vicinity of the Gulf Stream current (Sanders and Gyakum, 1980), several cases of ECs have occurred offshore of western Europe (e.g., Young et al., 1987; Burt and Mansfield, 1988). Additionally, ECs do occasionally occur over continents. 


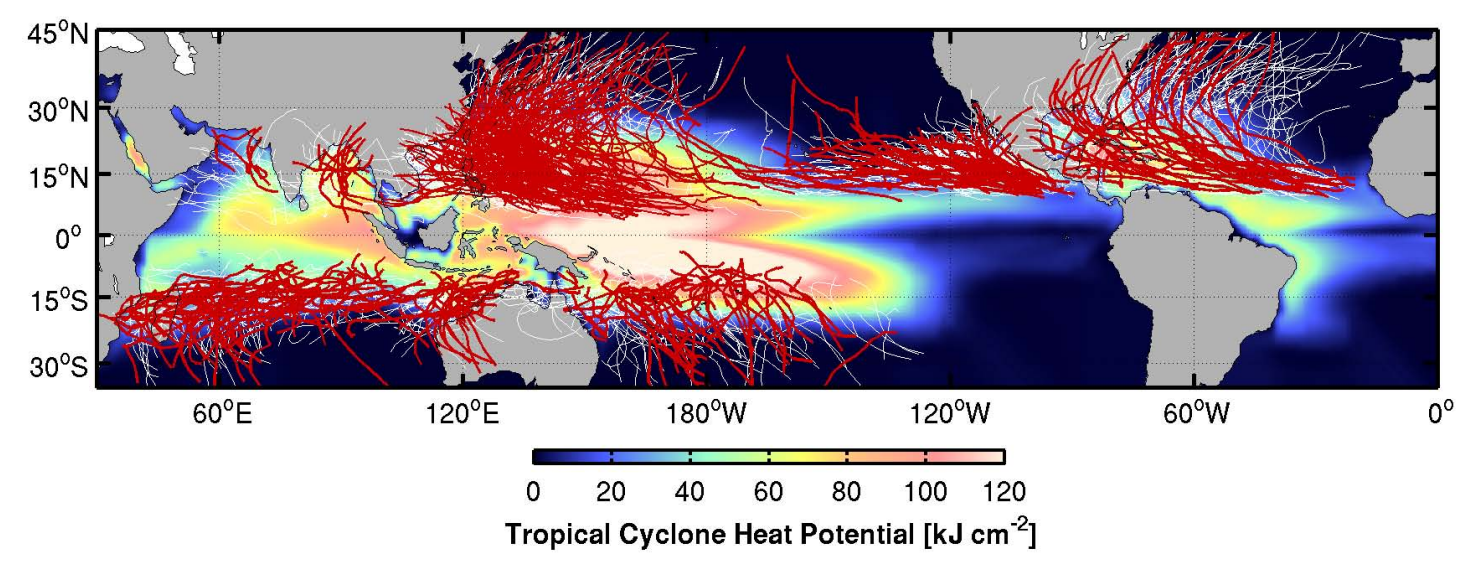

FIGURE 1 | Global TC tracks during the time period of 1993-2011, overlaid on the average hurricane season satellite-derived tropical cyclone heat potential (TCHP) (upper-ocean heat content $(\mathrm{OHC})$ above $26^{\circ} \mathrm{C}$ isotherm) for each hemisphere computed for the same time period. Thin white lines show the tracks of TCs with Cat-2 and below according to the Saffir-Simpson TC wind scale, and red lines show tracks of major TCs with Cat-3 and above.

Examples include the "Cleveland Superbomb" in January 1978 (Hakim et al., 1995) and the more recent Colorado EC of March 2019.

Atmospheric baroclinic instabilities, upper-level vorticity coupling, and diabatic processes have been acknowledged as the main atmospheric mechanisms causing ECs genesis (Shapiro et al., 1999; Yoshida and Asuma, 2004; Kuwano-Yoshida and Asuma, 2008). Over the North Atlantic ocean, the number of explosive cyclogenesis events is modulated by the North Atlantic Oscillation (NAO), with positive NAO favoring a larger number of ECs due to stronger atmospheric baroclinicity (Pinto et al., 2009). Large-scale heat convergence linked with the Atlantic Meridional Overturning Circulation is also thought to influence atmospheric baroclinicity and modulate EC activity (Gómara et al., 2016). While these atmospheric and ocean conditions are mostly linked with the number of ECs developing, the strength, intensification and trajectory of the ECs are known for being directly influenced by upper ocean conditions (Kuwano-Yoshida and Minobe, 2017), with their genesis often associated with oceanic frontal systems. For example, the maximum frequency of explosive cyclogenesis and deepening is found to occur near the Kuroshio or the Gulf Stream; these oceanic western boundary currents are associated with large heat fluxes from the ocean to the atmosphere (Sanders and Gyakum, 1980; Ulbrich et al., 2009; Hirata et al., 2015, 2016, 2018; KuwanoYoshida and Minobe, 2017). Future projections suggest that strong ECs will tend to increase, while the total number of ECs is expected to decrease (Colle et al., 2015; Chang, 2017). Some of these climate model projections, however, cannot fully resolve sharp gradients linked with the Kuroshio and the Gulf Stream currents, and may lack in the accurate representation of the number of ECs forming (Haarsma et al., 2016). Further studies based on observations, or on highresolution numerical simulations will likely be needed to confirm these trends.

In addition, the intensities of ECs that develop over warmer ocean regions are usually underestimated when compared to those that develop over cooler ocean regions (Kuwano-Yoshida and Enomoto, 2013). This is because cloud condensation associated with latent heat release over warm ocean areas is more important for rapid development than the upper-level vorticity forcing (Catto et al., 2010). For example, recent analyses using satellite-based high-resolution SST measurements suggest that ECs are affected by SST fluctuations associated with fronts and mesoscale eddies around western boundary currents (Booth et al., 2012; Hirata et al., 2015, 2016, 2018; Kuwano-Yoshida and Minobe, 2017). Considering that the western boundary currents such as the Gulf Stream and the Kuroshio are warming more rapidly than the global average rate (Wu et al., 2012), the ocean's effect on ECs generation is likely to increase in the future. With respect to ocean response to ECs winds, recent ocean simulations suggest that ECs can induce surface horizontal divergence and upwelling reaching depths of $6,000 \mathrm{~m}$, which can impact the deep ocean circulation and ecosystems through mixing and biogeochemical transport (Kuwano-Yoshida et al., 2017).

\section{OCEAN OBSERVATIONS AND PARAMETERS IN SUPPORT OF EXTREME WEATHER STUDIES AND FORECASTS}

The Global Ocean Observing System (GOOS) provides measurements from a diverse suite of observing platforms that enable studies of the complex dynamics of extreme weather systems and help improve the overall skill of extreme weather forecast models. Accurate TC and EC forecasts based on coupled models, for example, require a correct representation of the upper-OHC, vertical density structure, and the mesoscale eddy field. This in turn requires upper ocean observations with high spatial and temporal resolution.

Analyses of ocean observations in the vicinity of TCs and ECs have led to improved understanding of their development and 


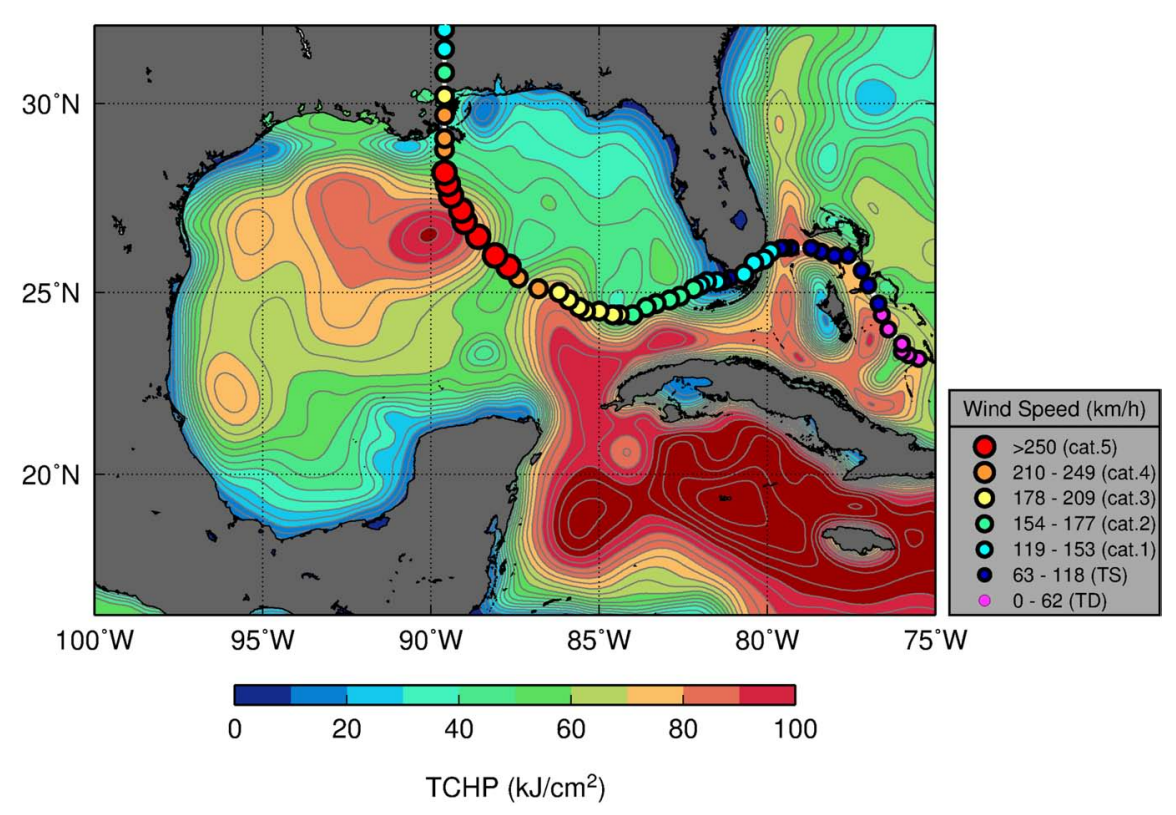

FIGURE 2 | Track of Hurricane Katrina (2005) overlaid on tropical cyclone heat potential (TCHP, upper-OHC) conditions in the Gulf of Mexico on 08/20/2005 (prior to the passage of Katrina). Gray contours are displayed every $5 \mathrm{~kJ} \mathrm{~cm}^{-2}$ units.

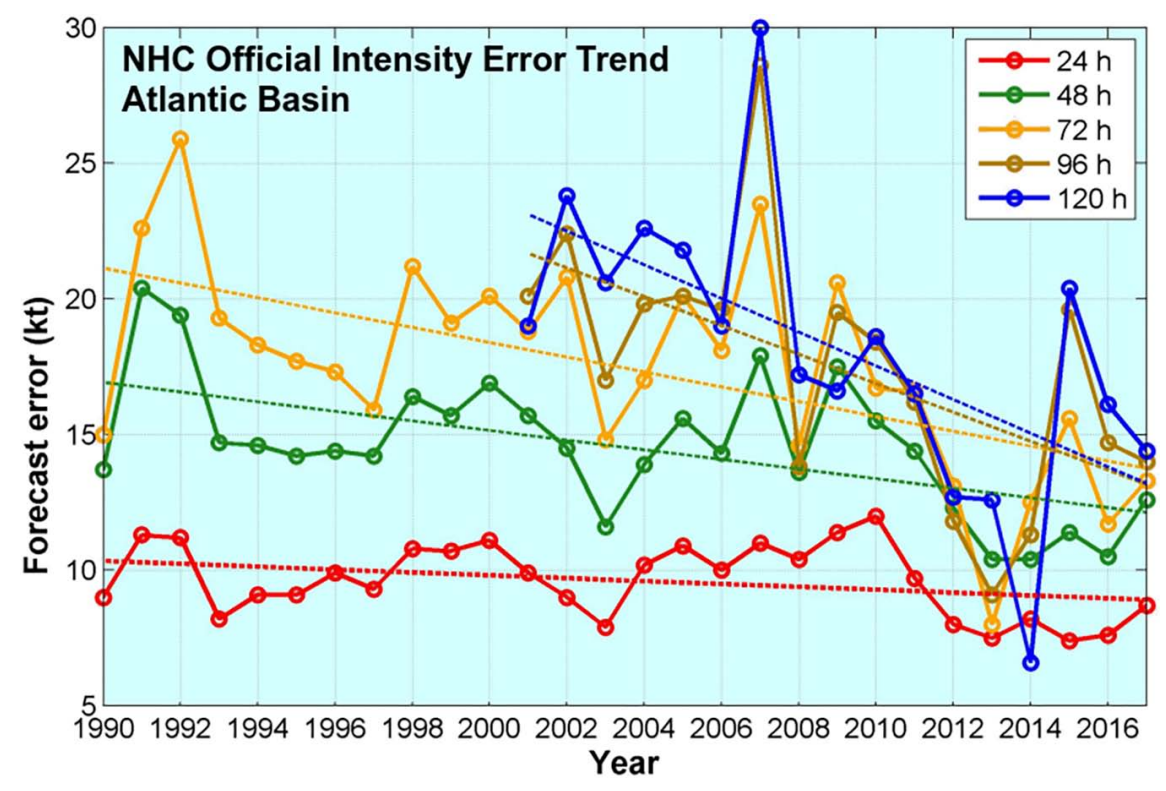

FIGURE 3 | Official Atlantic hurricane intensity forecast error for the Atlantic basin reported by NOAA's National Hurricane Center. Source: https://www.nhc.noaa.gov/ verification/verify5.shtml.

intensification, which occur over distinct geographic domains and during different seasons. The GOOS includes several multi-national ocean observing efforts that support studies and forecasts of both TCs and ECs. While the observational requirements and needs for TCs and ECs are different, some of the observing platforms are used in support of studies on both types of storms, and some are specifically used to provide observations for TCs or ECs.
Here we discuss the importance of the integrated ocean observing system and of targeted ocean observations, focusing on their application to TCs and ECs. We also provide an overview of these various components based on several successful examples, which illustrate applications that are helping understand the dynamics of these extreme weather systems, and are also helping to improve the overall skill of their forecast. To be most effective for operational forecasting, observing 
platforms should transmit their data in real-time via the Global Telecommunications System (GTS).

\section{Ocean Observations Satellites}

Satellite-derived fields of SST ${ }^{1}$ and sea surface height (SSH) ${ }^{2}$ are used to estimate the upper-OHC (Leipper and Volgenau, 1972), which is sometimes also referred to as Tropical Cyclone Heat Potential ${ }^{3}$ (TCHP; Goni and Trinanes, 2003). The TCHP is defined as the excess heat content in the water column from the sea surface to the depth of the $26^{\circ} \mathrm{C}$ isotherm. TCHP fields can provide key qualitative and quantitative spatial information about areas where TCs may intensify, mainly by identifying the location and thermal characteristics of the oceanic upper layer, including warm eddies and current frontal regions (Meyers et al., 2014). For example, high values of TCHP (larger than approximately $50 \mathrm{~kJ} \mathrm{~cm}^{-2}$ ) have been shown to be linked to intensification of Atlantic hurricanes (e.g., Mainelli et al., 2008). It should be acknowledged, however, that TCHP from satellite-derived SST and SSHA can sometimes result in inaccurate estimates in regions that are strongly influenced by freshwater sources. In the Bay of Bengal, for example, where river water can persist in the near surface layer, salinity can have a dominant role in determining the subsurface density structure and the SSH (e.g., Yu and McPhaden, 2011); this can have a detrimental effect on the TCHP derived from satellite observations. Nevertheless, comparison of TCHP values derived from satellites and in situ observations in the Bay of Bengal has shown that satellite-derived estimates are generally unbiased, and estimates with a precision better than $20 \mathrm{~kJ} \mathrm{~cm}^{-2}$ are often obtained (Nagamani et al., 2012).

Fields of SST and TCHP are routinely used by the NOAA National Hurricane Center (NHC) and the Joint Typhoon Warning Center for their subjective TC intensity forecasts and quantitatively in the Statistical Hurricane Intensity Prediction Scheme (SHIPS; DeMaria and Kaplan, 1994; DeMaria et al., 2005) and rapid intensification aids (Kaplan et al., 2010; Knaff et al., 2018). Notable examples of use of satellite fields to assess links between the ocean and hurricane intensification include Hurricane Opal (Shay et al., 2000), super-typhoon Maemi (Lin et al., 2005), Hurricane Katrina (Figure 2; Goni et al., 2009), and "killer cyclone" Nargis (Lin et al., 2009).

\section{Underwater Gliders}

Autonomous underwater gliders (Rudnick, 2016) provide measurements of temperature and salinity in the upper several $100 \mathrm{~m}$ and are becoming key components of the ocean observing system (Liblik et al., 2016; Testor et al., 2019). In addition to the standard measurements of temperature, salinity, and depth-average currents, gliders can be equipped to measure

\footnotetext{
${ }^{1}$ NOAA High-resolution Blended Analysis of Daily SST available at: https://www. esrl.noaa.gov/psd/data/gridded/data.noaa.oisst.v2.highres.html.

${ }^{2}$ Satellite altimetry products made available by the Copernicus Marine Environment Monitoring Service: http://marine.copernicus.eu/.

${ }^{3}$ TCHP fields made available by the NOAA Atlantic Oceanographic and Meteorological Laboratory (NOAA/AOML) at: http://www.aoml.noaa.gov/phod/ cyclone/data/.
}

current profiles (e.g., Todd et al., 2017), bio-optical properties, dissolved oxygen, and turbulent microstructure. Because of their adaptability and versatility, gliders fill important observational gaps in the ocean observing system (Liblik et al., 2016), particularly with respect to TC intensity forecasts. Glider observations are, for example, frequently assimilated into oceanatmosphere coupled models and used for hurricane intensity forecasts. Sustained glider deployments monitor upper ocean conditions in areas frequently impacted by TCs (e.g., Domingues et al., 2015; Miles et al., 2015; Todd et al., 2018) and are part of the NOAA Hurricane Field Program ${ }^{4}$. Gliders also have particular utility measuring ocean processes on continental shelves before and during landfall of TCs, where alternative ocean observations are scarce (e.g., Glenn et al., 2016; Miles et al., 2017). In addition to measuring physical variables, gliders can carry specialized sensor payloads; sensors for key biogeochemical variables offer the promise of advancing our understanding of the role of TCs and ECs on ecosystems. For instance, dissolved oxygen measurements could help to characterize the stormdriven ventilation of subsurface waters in areas with oxygen minimum zones (e.g., the Arabian Sea; Morrison et al., 1999).

Since gliders move slowly (about $25 \mathrm{~km} /$ day) compared to most atmospheric cyclones $O(300 \mathrm{~km} /$ day $)$, actively piloting them into the paths of storms is generally not feasible due to the short lead times of forecasts. Thus, sustained deployment of gliders at locations prone to TCs or ECs (e.g., Domingues et al., 2015; Glenn et al., 2016; Perry et al., 2017) or along oceanic boundaries as part of boundary current observing systems (Todd et al., 2018, 2019; Testor et al., 2019) is preferable. Compared to rapid response deployments (e.g., Miles et al., 2015; Goni et al., 2017), sustained glider surveillance has the distinct advantage of providing critical high-resolution observations in the open ocean and over the continental shelf prior to storm arrival; these observations have been shown to improve the representation of the ocean in operational coupled forecast models of hurricane intensity (e.g., Dong et al., 2017). Most underwater glider data collected in support of Atlantic Hurricane studies and forecasts are transmitted in real-time to the GTS and U.S. Integrated Ocean Observing System (IOOS) underwater glider data assembly center $^{5}$, and made available for immediate use by operational forecast centers.

\section{Surface Drifters}

Different types of drifters provide observations of ocean current velocities, SST, and sea level pressure (SLP) that are also used in support of weather forecasts, including TCs and ECs. Sustained global observations from drifters are used for constraining satellite SST errors and biases (e.g., Zhang et al., 2009) and have had a positive impact on global weather forecast throughout the troposphere thanks to assimilation of in situ SLP observations (Centurioni et al., 2017; Horányi et al., 2017; Ingleby and Isaksen, 2018). Horányi et al. (2017) showed that, in the case of intense cyclogenesis, SLP observations from drifters made

${ }^{4}$ NOAA Hurricane Field Program: https://www.aoml.noaa.gov/hrd/programs sub/HFP.html.

${ }^{5}$ U.S. Glider Data Assembly Center: https://gliders.ioos.us/data/. 
possible large reduction in forecast errors, sometimes the largest among all the other assimilated observations. Furthermore, targeted deployments of drifters are sometimes carried out in front of TCs. During the Atlantic hurricane season, for example, Surface Velocity Program (SVP), Surface Velocity Program Barometer (SVPB) and Autonomous Drifting Ocean Station drifters (Centurioni, 2010; see also Centurioni, 2018, for a complete description of the drifter technology) are often airdeployed in front of TCs that may impact the US mainland. New drifters capable of measuring the directional wave spectra of surface gravity waves, termed Directional Wave Spectra Drifters (Centurioni et al., 2016, 2019) have also been deployed ahead of TCs. Successful deployments of various drifters have been carried out in the Atlantic Ocean during the following TCs: Fabian (2003), Frances (2004), Rita (2005), Dean (2007), Gustav (2008), Ike (2008), Isaac (2012) and Michael (2018), and in the western Pacific Ocean during Hagupit (2008), Jangmi (2008), Fanapi (2010), and Malakas (2010). In addition, drifters that are part of the Global Drifter Program ${ }^{6}$ array, often come close to TCs and provide valuable SST and SLP observations. In the 20132018 period a total of 116 SVP and SVPB drifters were located within $55 \mathrm{~km}$ of the tracks of systems that eventually developed into hurricanes (Centurioni et al., 2019). However, the effect of SLP drifter observation in improving TC track forecast has yet to be studied.

\section{Air Deployed Profiling Instruments}

Airborne profiling instruments are often deployed in targeted sampling mode in front of TCs in the Atlantic and Pacific basins. In the Atlantic, deployments of Airborne eXpendables Bathythermographs (AXBTs), Airborne eXpendable Conductivity Temperature and Depth (AXCTDs), and Airborne eXpendable Current Profilers (AXCPs) are generally conducted to sample ocean conditions ahead and under TCs as part of the NOAA Hurricane Field Program (Meyers et al., 2015; Jaimes et al., 2016; Zhang et al., 2018). Paired deployments of AXBTs and dropsondes, for example, provide collocated measurements of SST, air temperature, humidity and TC wind speed that allow for the estimation of bulk air-sea fluxes. Exchange coefficients used in such computations are based on direct flux data (Zhang et al., 2008). These data provide valuable information that is used to evaluate and improve TC model physics such as boundary-layer parameterizations (e.g., Zhang et al., 2015). In recent years, the following Atlantic TCs were sampled: Edouard (2014), Harvey (2017), Irma (2017), Maria (2017), Nate (2017), and Michael (2018). In the Pacific, paired deployments of AXBTs and dropsondes were also carried out during the 2010 Impact of Typhoon on Pacific (ITOP) international field experiment (D'Asaro et al., 2014, see section “The 2010 ITOP Field Campaign").

More recently, the Air-Launched Autonomous MicroObserver (ALAMO) (Jayne and Bogue, 2017) profiling float was developed to be deployed from an aircraft Sonobuoy-A size tube, similarly to AXBTs (Sanabia et al., 2013). A key distinction

${ }^{6}$ The Global Drifter Program: http://www.aoml.noaa.gov/phod/gdp/index.php and http://gdp.ucsd.edu. between ALAMO floats and other airborne expendable profiling instruments is that these floats are capable of sampling 100s of profiles continuously before, during, and after the passage of TCs for up to 6 months, depending on instrument configurations. During the 2014-2018 period, a total of 60 ALAMO floats were deployed in support of the NOAA Hurricane Field Program in both the Atlantic and eastern North Pacific basins.

\section{Profiling Floats}

Profiling floats (e.g., Riser et al., 2016; Roemmich et al., 2019) offer the advantage of providing a sustained long-term and largescale record of most of global oceans. Temperature and salinity observations from Argo floats ${ }^{7}$ are routinely assimilated into operational ocean models (e.g., Chassignet et al., 2009) that are used to initialize the ocean component of hurricane forecast models. In addition, operational Argo floats have been found to be very important in regions where routine and/or opportunistic airborne AXBTs observations are lacking, e.g., in the case of "Cat-6" supertyphoon Haiyan which devastated the Philippines in 2013 (Lin et al., 2014).

Profiling floats also offer valuable information of upper ocean processes contributing to ECs formation and intensification. The typical observation interval for individual Argo floats, however, is 10 days, which is generally too long to capture the rapid air-sea interactions associated with enthalpy fluxes and exchanges. High-frequency and adaptive fine scale profiling float sampling are generally needed to fully capture mesoscale ocean features usually associated with storm intensification, and also to characterize the storm-induced upper ocean response in detail. Argo floats under developing ECs were used to obtain 73 high-frequency profiles of the upper $700 \mathrm{~m}$ at 6 -h intervals during the 2015/2016 and 2016/2017 winters. These data were critical to understanding ocean changes under ECs in the Northwestern Pacific (Kuwano-Yoshida et al., 2018) and emphasize the advantage of adaptive profiling float sampling using two-way communication systems. Observation interval and depth of modern Argo floats can be controlled using twoway satellite communication. Interactive operation of the floats with satellite and assimilated data enables high-frequency and high-resolution observation at fronts, i.e., the floats observe short interval if satellite and assimilated data suggest that the floats are located near the SST fronts. These in situ observations will complement satellite observations, increase temporal sampling, and enable resolving the fine structure associated with SST fronts that may help improve EC and TC forecasts.

In addition, expanding coverage of Biogeochemical Argo float observations also offer opportunities for evaluating phytoplankton response to mixing forced by TCs and ECs (e.g., Chacko, 2017) and studying the role that these extreme weather events play in ventilating subsurface waters in oxygen minimum zones. These new applications of profiling floats will enable detailed investigations of the upper ocean processes involved in EC intensification and the role that these extreme weather events play in the ocean biogeochemistry within their main formation basins, such as the Northwest Pacific and North Atlantic ocean.

\footnotetext{
${ }^{7}$ Argo Global Data Assembly Center at: http://www.usgodae.org/argo/argo.html.
} 


\section{EM-APEX (Electromagnetic Autonomous Profiling Explorer) Floats}

EM-APEX were developed to measure profiles of upper ocean temperature, salinity and currents. Velocity estimates are based on measuring the voltage induced by seawater moving through the earth's magnetic field as first pioneered with expendable current profilers (Sanford et al., 1987; Shay et al., 1992) and later added to standard profiling floats (Sanford et al., 2007, 2011). EM-APEX floats were successfully air-deployed in front of Hurricane Frances (2004), during the ITOP missions in the western Pacific (2010), and more recently during Hurricane Michael (2018). The EM-APEX floats can profile to depths of $2000 \mathrm{~m}$ or over a specific depth region such as the mixed layer through the seasonal thermocline. Profiling configurations (i.e., sampling rate, and depth) can be changed via two-way Iridium communications, allowing for significant flexibility in an adaptive sampling before, during, and after storm passage, capturing air-sea interactions, and the oceanic response for several weeks following passage. For example, during Hurricane Michael (2018), a EM-APEX float sampled from 30 to $300 \mathrm{~m}$ during the storm at $1 \mathrm{~h}$ intervals to assess the role of current shear in vertical mixing processes to evaluate model parameterizations. With the high-resolution measurements, the evolution of the Richardson numbers could be determined at vertical resolution of 2-4 $\mathrm{m}$ in an active entrainment zone. In addition, the momentum flux from the surface wind stress into the surface mixed layer provides a method to back out the surface drag coefficient that are needed in examining the complex air-sea interactions that occur during TC passage (e.g., Shay and Brewster, 2010).

\section{Biologging}

It has also been used to collect in situ meteorological and physical oceanographic observations. Marine mammals (Campagna et al., 2000; Boehlert et al., 2001; Boyd et al., 2001), seabirds (Koudil et al., 2000; Watanuki et al., 2001; Charrassin et al., 2002; Wilson et al., 2002), sea turtles (Fukuoka et al., 2015; Narazaki et al., 2015) and fish (Block et al., 2001) have been adopted as autonomous samplers of oceanographic parameters such as temperature, conductivity and depth (Koudil et al., 2000; Block et al., 2001; Watanuki et al., 2001; Charrassin et al., 2002; Wilson et al., 2002; Fedak, 2013). These animals often live around western boundary currents and frontal systems where TCs and ECs are often observed (Figure 4). For example, biologging by sea turtles observed daily temperature profiles in surface layers in the northwestern Pacific (Fukuoka et al., 2015; Narazaki et al., 2015). The profiles were collected during 77 EC events in 6 consecutive winters. Both Argo floats and biologging captured rapid temperature changes under ECs. These ocean observations are crucial to identify near-surface baroclinic zones and oceanatmosphere fluxes of heat and moisture. Such processes are crucial to the successful predictions and simulations of ECs.

\section{Ocean Metrics for TC/EC Intensification Studies}

Various parameters derived from in situ and remotely sensed observations that describe energy available in the upper ocean have been used to estimate the potential for TC and EC development and intensification. The TCHP is one example, but it has dimensions that differ from SST and so cannot be used in place of SST in numerical models. Moreover, Price (2009) demonstrated that depth-averaged temperature is a more robust metric of hurricane-ocean interaction than is TCHP. Ali et al. (2015) then used satellite-derived TCHP and the depth of the $26^{\circ} \mathrm{C}$ isotherm (D26) to estimate ocean mean temperature (OMT) with a few assumptions. This OMT estimate was a better predictor for Indian monsoon rainfall than SST (Ali et al., 2015; Venugopal et al., 2018). Use of OMT in place of SST in numerical models offers potential for improvement in cyclone forecasting.

Thermodynamically, the subsurface ocean affects TCs through its control of TC-induced cold SST wakes. When the cold wake is weak (less than about $0.5^{\circ} \mathrm{C}$ ), TCHP is a very good predictor of TC intensification, exceeding the skill of other predictors such as SST and vertically-averaged temperature (Figure 5; Balaguru et al., 2018). However, when the cold wake is strong $\left(>0.5^{\circ} \mathrm{C}\right.$ ) (e.g., when SST is very warm and temperature stratification is shallow) ocean dynamic temperature (Tdy) performs significantly better (Figure 5; Balaguru et al., 2018). Tdy is defined as the ocean temperature averaged from the surface to the post-storm mixed layer depth, which depends on the upper-ocean stratification as well as the TC intensity and translation speed (Balaguru et al., 2015). An additional limitation of temperature-based metrics such as TCHP and OMT is that they do not account for salinity, which has been shown to be important in all TC basins (Wang et al., 2011; Balaguru et al., 2012b, 2016; Grodsky et al., 2012; Neetu et al., 2012; Domingues et al., 2015; Foltz and Balaguru, 2016). Satellite-based sea surface salinity measurements, when combined with subsurface in situ observations, can provide further information about the salinity stratification. This additional metric, when incorporated into Tdy may result in further improvements to statistical TC prediction schemes and enable more meaningful validation of operational ocean analyses and forecasts.

\section{HIGHLIGHTED APPLICATIONS AND RESULTS}

In this section, key results, sampling strategies, and applications of ocean observations in support of studies and forecasts of TCs and ECs are described. These case studies provide additional information on some of the successful examples of employing data derived from the GOOS, new pilot networks, and targeted deployments to enhance our understanding of the ocean-atmosphere interaction processes that can lead to TC and EC intensification.

\section{The 2011 and 2012 Atlantic Hurricane Seasons: Hurricanes Irene (2011) and Sandy (2012)}

Over the broad continental shelf of the Middle Atlantic Bight along the US East Coast, research carried out with gliders observations has shown that cool subsurface waters (i.e., the "Cold Pool"; Houghton et al., 1982) can be mixed with the 

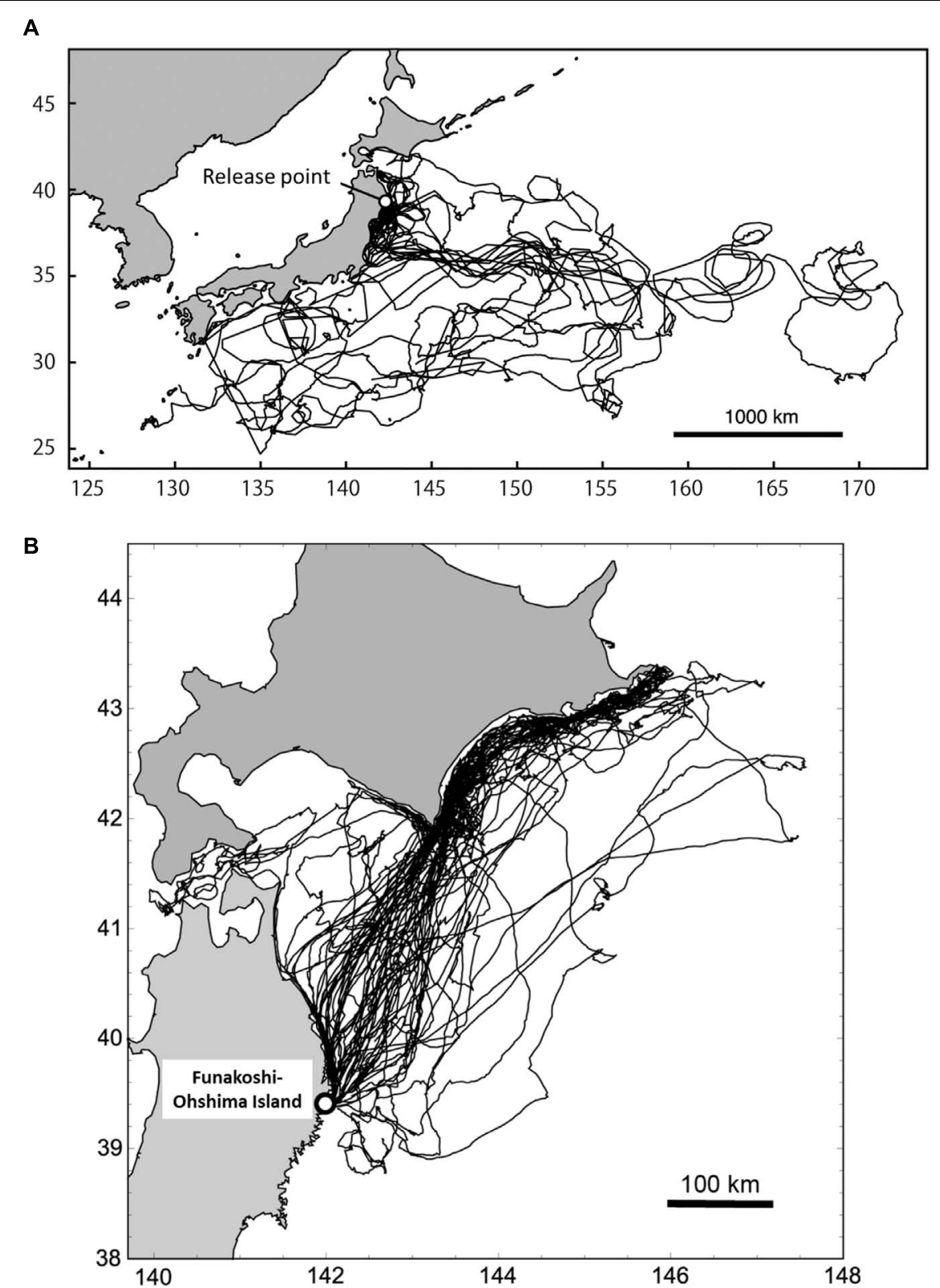

FIGURE 4 | (A) Satellite tracks of 15 loggerhead turtles released from Sanriku coast, Japan (open circle) between 2010 and 2014. (B) Tracks of 33 streaked shearwaters from a breeding colony in Funakoshi-Ohshima Island, Japan (open circle) between August and September in 2013 and 2014.

surface waters under intense wind conditions, thereby impacting storm intensity. Since the Cold Pool is obscured from the view of satellites, in situ observations, such as those obtained by gliders, are needed to capture its properties and impact on cyclone intensity (Glenn et al., 2016; Seroka et al., 2016). For example, a glider deployed ahead of Hurricane Irene (2011) observed larger than usual ahead-of-eye-center cooling of over $6^{\circ} \mathrm{C}$ (Seroka et al., 2016) caused by intense mixing of surface waters with cold subsurface waters forced by the hurricane winds. Subsequent ocean and atmosphere model sensitivity studies identified this process as the missing component necessary to capture Irene's rapid weakening just prior to landfall. In contrast, glider observations collected during Hurricane Sandy (2012) showed that the storm winds were downwelling favorable and led to offshore advection of the subsurface Cold Pool waters, which prevented upper ocean cooling and favored the sustained intensity of Sandy (Miles et al., 2015, 2017).

\section{The 2014 Atlantic Hurricane Season: Hurricanes Gonzalo (2014) and Fay (2014)}

Studies carried out using all ocean observations, including those from underwater gliders, in the western tropical Atlantic and Caribbean Sea, were used to assess the pre- and post-storm 

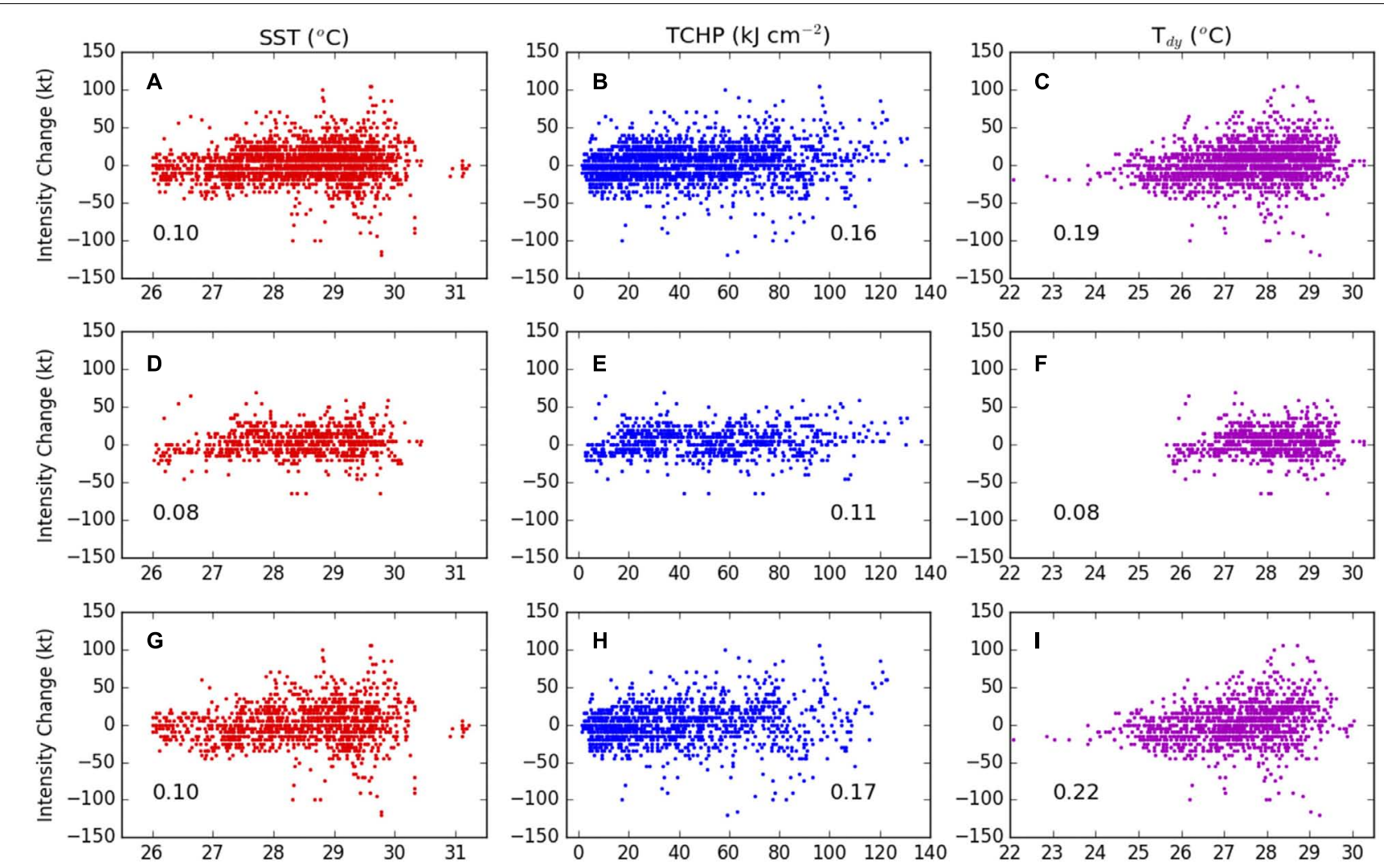

FIGURE 5 | Scatter between 36-h intensity changes of Atlantic TCs and SST (red), TCHP (blue), and Tdy (magenta) for the 10-year period 2005-2014. (A-C) All storm locations, (D-F) cases where the magnitude of the hurricane-induced SST cooling is below $0.5^{\circ} \mathrm{C}$, and (G-I) cases where the cold wake magnitude is greater than $0.5^{\circ} \mathrm{C}$. Correlation coefficients are also indicated in each panel. Reproduced from Balaguru et al. (2018).

ocean conditions associated with Hurricanes Fay and Gonzalo (2014). When Hurricane Gonzalo passed north of Puerto Rico, the general background ocean conditions were provided by Argo floats and satellite-derived SST and SSH fields. In addition, there was one glider surveying upper ocean temperature and salinity structure in the vicinity of the projected path of Gonzalo (Figure 6A). This glider was the only observing platform to capture the presence of a 20-m-thick barrier layer (Domingues et al., 2015), a salinity stratified layer (e.g., Sprintall and Tomczak, 1992) within the deeper isothermal layer. This layer inhibited vertical mixing and limited surface cooling forced by Gonzalo's winds to only $0.4^{\circ} \mathrm{C}$, allowing the storm to intensify to Cat- 4 (Domingues et al., 2015). When Gonzalo subsequently crossed the path of Fay near Bermuda (Figure 6A), it weakened from Cat3 to Cat-2 due to the upper ocean cooling of approximately $4^{\circ} \mathrm{C}$ observed in the wake of Fay (Goni et al., 2017).

\section{The 2017 Atlantic Hurricane Season: Hurricanes Harvey, Irma, Jose, Maria, and Nate}

The 2017 Atlantic hurricane season was one of the most active in recent history with 17 named storms, and six major hurricanes. Underwater gliders, profiling floats, XBTs, airborne observations, and other observing platforms collected crucial ocean data to assess upper ocean conditions and changes before, during, and after the passage of multiple hurricanes. Here we describe ocean observations and key results from Hurricanes Harvey, Irma, Maria, Jose, and Nate. Data from the ocean observing system were used in support of operational hurricane intensity forecasts.

In August, Hurricane Harvey developed in the tropical Atlantic and passed through the Caribbean Sea south of Puerto Rico. In this area, observations from one underwater glider showed that a relatively shallow mixed layer favored cooling of the upper ocean. Together with the moderate wind shear, this contributed to Harvey's lack of intensification within the Caribbean. Once Harvey reached the Gulf of Mexico, where TCHP derived from Argo floats was at a record level and SST exceeded $30^{\circ} \mathrm{C}$ (Trenberth et al., 2018), it intensified from a tropical depression (16 $\mathrm{m} \mathrm{s}^{-1} / 56 \mathrm{~km} \mathrm{~h}^{-1}$ sustained winds) into a Cat-4 hurricane (59 $\mathrm{m} \mathrm{s}^{-1} / 212 \mathrm{~km} \mathrm{~h}^{-1}$ sustained winds) in less than $48 \mathrm{~h}$ before making landfall along the Texas coast with devastating effects.

In September, SST values of $\sim 30^{\circ} \mathrm{C}$ were observed across the western Atlantic and Caribbean (Figure 7), which, along with low wind shear, helped sustain the development and intensification of Hurricanes Irma, Maria and Jose (Camp et al., 2018). Hurricane Irma, the strongest TC globally in 2017, reached its maximum intensity (Cat-5) on September 6, while traveling over waters north of Puerto Rico and Hispaniola. 

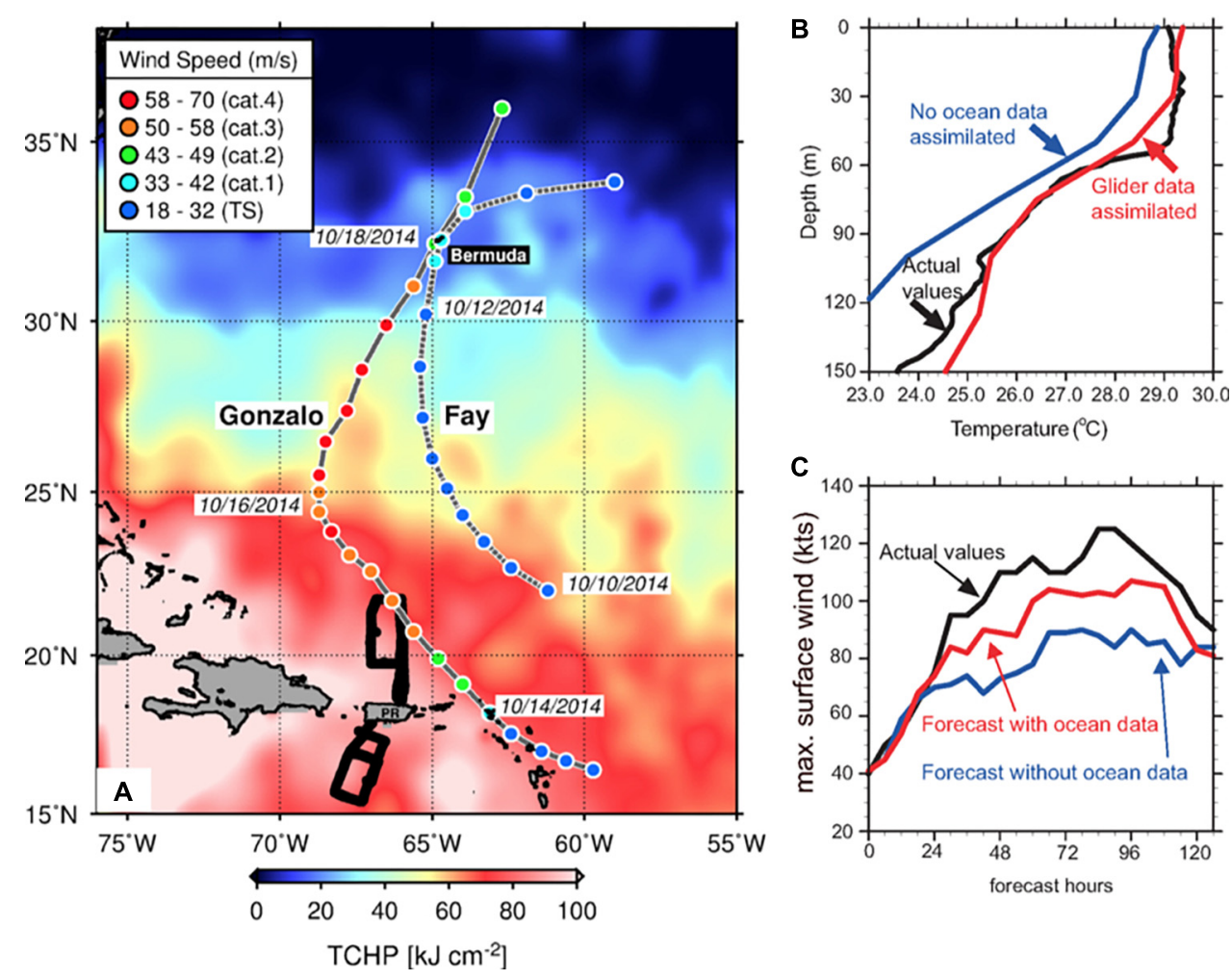

C

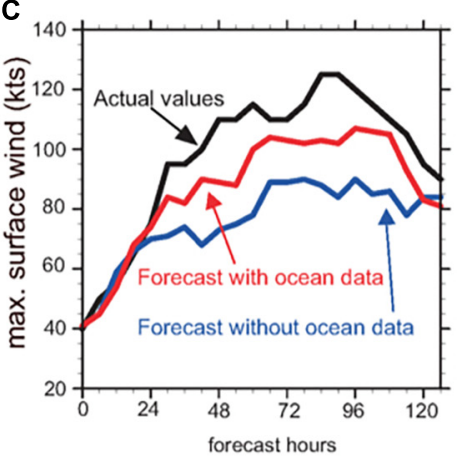

FIGURE 6 | (A) Tracks of Hurricanes Gonzalo (2014) and Fay (2014) superimposed on the altimetry-derived tropical cyclone heat potential (TCHP) during October 2014. (B) Impact of glider temperature profiles on the initialization of HYCOM-HWRF. (C) Impact of glider and other ocean data to reduce errors in TC intensity (maximum wind speed) during the forecast of Gonzalo tested on October 13, 2014. Figure adapted from Goni et al. (2017).

Observations from underwater gliders showed that a fresh water barrier layer $\sim 15 \mathrm{~m}$ thick (Figure 8A) inhibited mixing between the upper ocean and colder underlying waters, similar to Hurricane Gonzalo (2014; Domingues et al., 2015; Dong et al., 2017). These observations also revealed that the upper $50 \mathrm{~m}$ of the ocean cooled by approximately $1^{\circ} \mathrm{C}$ as a result of storm-induced mixing. A few days after Irma, Hurricane Jose passed 2-3 degrees of latitude to the north of Irma's track. Jose's trajectory coincided with the cold wake left by Hurricane Irma, so it interacted with a relatively cooler and well-mixed upper-ocean as observed by underwater glider data. These cooler ocean conditions may have contributed to its weakening from Cat-4 to Cat-3. Later in the month, Hurricane Maria passed through the Caribbean Sea and then the same area as Irma in the tropical North Atlantic. Following a landfall in Dominica, Maria reached peak intensity on September 20 with maximum sustained winds of $78 \mathrm{~m} \mathrm{~s}^{-1}\left(280 \mathrm{~km} \mathrm{~h}^{-1}\right)$. Underwater glider observations revealed the existence of a very stable barrier layer that was approximately $30 \mathrm{~m}$ deep along the path of Maria (Figure 8A), providing favorable ocean conditions for intensification. On September 20, Maria made landfall in Puerto Rico as an intense Cat- 4 hurricane. By the end of September, positive SST anomalies recorded before the passage of these hurricanes had dissipated due to the intense mixing caused by these major storms, and SSTs closer to neutral conditions were observed (Figure 7A). Farther north, Todd et al. (2018) used glider observations and volume transport measurements in the Florida Straits to show that the Gulf Stream exhibited a large freshwater anomaly that was attributable to rains from Irma and also a transient reduction in volume transport that was attributable to wind forcing associated with the passing storm; further studies with numerical simulations are needed to better understand the dynamics of the storm impacts on the western boundary current.

In October, Hurricane Nate developed and steadily gained strength over warm waters of the northwestern Caribbean Sea. Once Nate reached the Gulf of Mexico, EM-APEX floats located near the projected track (Figure 9) were reprogrammed to profile every 2 to $4 \mathrm{~h}$, returning vertical profiles of temperature, salinity, currents, dissolved oxygen, chlorophyll fluorescence, backscatter as a proxy of particle concentration, and chromophoric dissolved organic carbon. In addition, 140 AXCPs and AXCTDs were deployed from the NOAA WP-3D aircraft prior to, during and after Nate (Figure 9). These observations showed an upper ocean velocity response with magnitude of $0.5-0.75 \mathrm{~m} \mathrm{~s}^{-1}$ and rotation of the current vectors with increasing depth that led to strong current shear at depths of 40-60 m. The development of strong shear favored the deepening of the oceanic mixed layer under Nate by 10 to $15 \mathrm{~m}$ and mixed layer cooling of $1.5-2^{\circ} \mathrm{C}$. The observed response was predominantly near-inertial in character, and likely impacted the air-sea fluxes and the intensity and structure of the storm (e.g., Jaimes et al., 2016). 

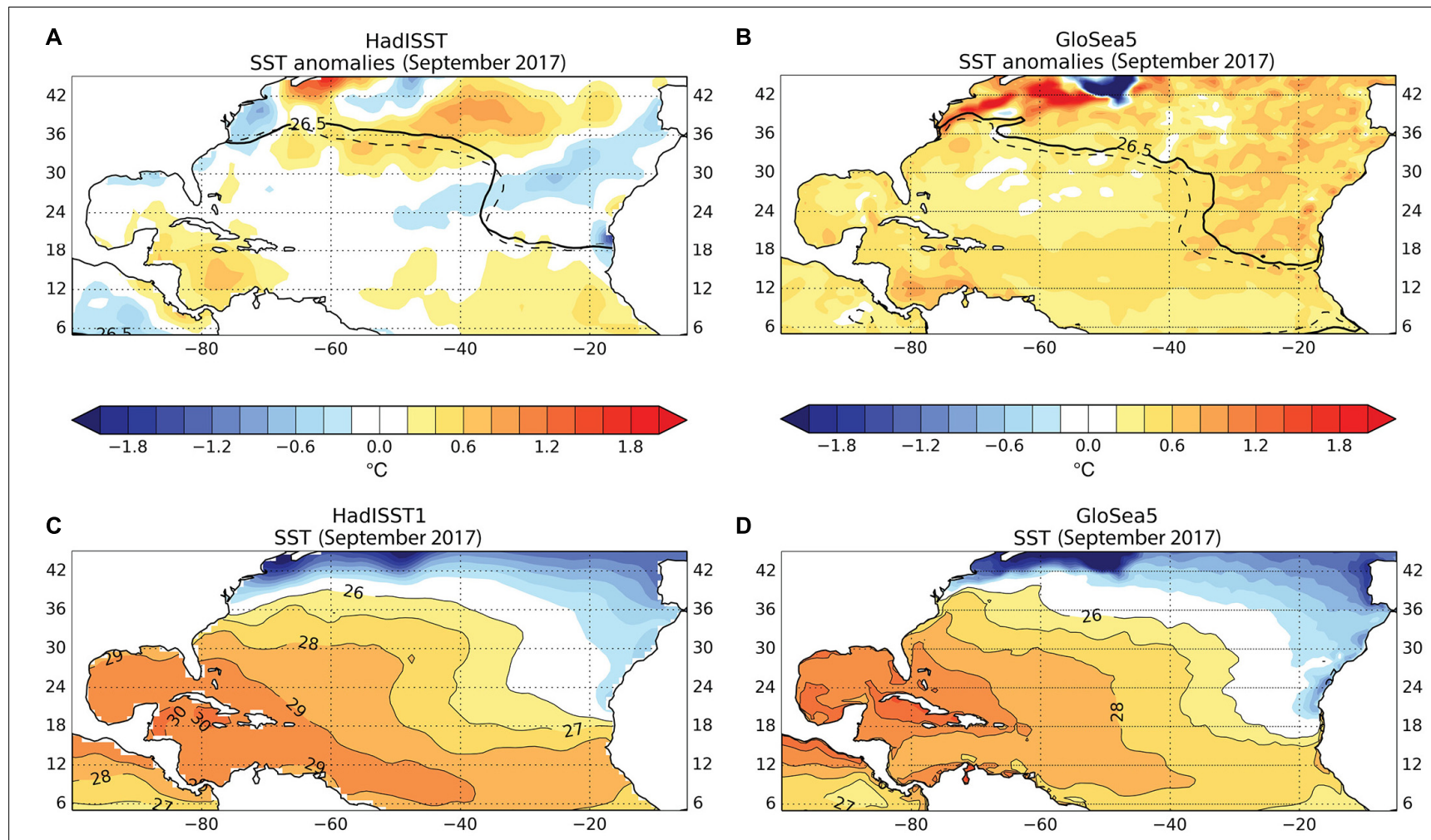

D
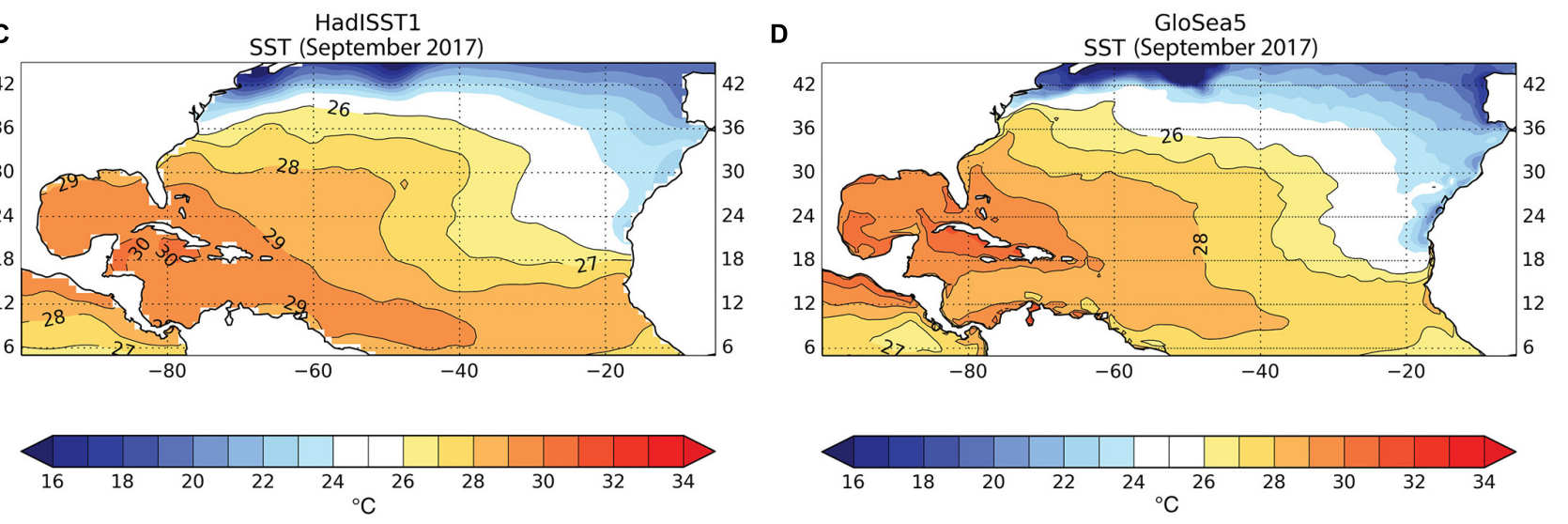

FIGURE 7 | North Atlantic sea surface temperature forecasts: (A) observed and (B) forecast mean Atlantic SST anomalies during September 2017. The location of the $26.5^{\circ} \mathrm{C}$ isotherm during September 2017 (solid black line) relative to average September conditions during $1993-2015$ (dashed line) are also shown.

(C) Observed and (D) forecast mean Atlantic SSTs during September 2017. Anomalies relative to September 1993-2015. Adapted from Camp et al. (2018).

\section{Impact of Riverine Outflows on Tropical Cyclones}

Areas in the Caribbean Sea and Tropical North Atlantic, where hurricanes commonly intensify, are sensitive to different freshwater sources, including major rivers such as the Amazon and Orinoco (e.g., Kelly et al., 2000; Balaguru et al., 2012a; Johns et al., 2014), and the Mississippi River (Goni and Domingues, 2019), which can contribute to the formation of barrier layers. In regions under the influence of strong fresh water sources, such as in the Bay of Bengal, low salinity conditions at the surface may sustain thermal inversions in the upper layer, which may further help suppress the TC-induced SST cooling (e.g., Sengupta et al., 2008). Barrier layers can be tens of meters thick, and have been indicated as a potential contributor to the rapid intensification of several TCs worldwide (e.g., Balaguru et al., 2012b).

Several major Atlantic hurricanes in 2017 encountered pre-existing barrier layer conditions along their trajectories (see section "The 2017 Atlantic Hurricane Season: Hurricanes Harvey, Irma, Jose, Maria, and Nate," Figure 8A). Analysis of satellite-derived chlorophyll data ${ }^{8}$ for August 2017 (Figure 8B)

${ }^{8}$ NASA Ocean Color website: https://oceancolor.gsfc.nasa.gov/. indicates that freshwater plumes from the Amazon and Orinoco rivers were advected into the Caribbean basin, contributing to barrier layer formation. Comparison with historical chlorophyll data for the tropical North Atlantic Ocean and Caribbean Sea (Figures 8C,D) suggests that entrainment of freshwater plumes from these rivers into the basinscale circulation in 2017 may have caused unusually strong freshwater transport into these areas. While investigation is still ongoing to assess the potential impact of these freshwater conditions on the 2017 hurricanes, these results emphasize that the correct representation of salinity conditions within coupled TC forecast models can be key to produce accurate hurricane predictions. This may be especially true for areas that are particularly sensitive to large freshwater sources, such as the Caribbean Sea, Gulf of Mexico, and tropical North Atlantic Ocean.

\section{Development of Biologging as an Ocean Observation Platform for ECs}

Flight and drift paths of sea birds soaring and floating over the ocean surface enable measurement of fine-scale winds and currents. Yonehara et al. (2016) and Goto et al. (2017) found 


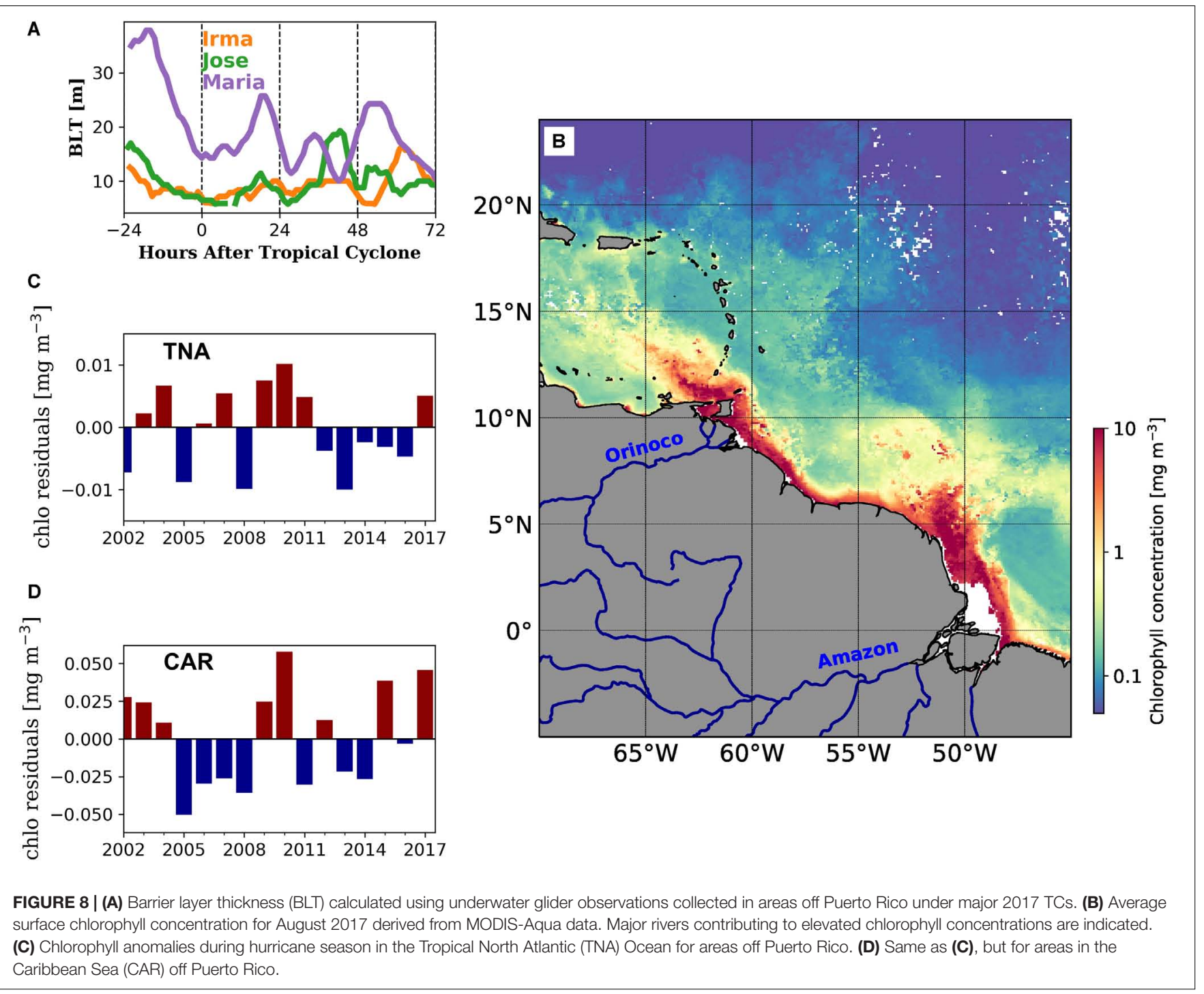

out that fine-scale flight trajectories by recording one position per second and minute provide 5 -min to 1 -h interval surface wind direction and speed along the trajectories. The birdestimated wind directions showed good agreement with those from satellite, although wind speeds were slower than satellite winds because sea birds flew at lower altitudes than $10 \mathrm{~m}$ at which satellite winds were calibrated. Only the wind estimates from three birds had meaningful impact on data assimilation when severe rainfall occurred in Japan associated with two typhoons using regional numerical forecast system (Wada et al., 2017). Yoda et al. (2014) developed a new method for obtaining in situ ocean current measurements by using sea birds with GPS/GNSS loggers floating at the surface as Lagrangian current sensors akin to drifting buoys. The sea birds forage boundary areas between two oceanic mesoscale eddies where primary productivity and prey density are thought to be high. The current data from sea birds improved reproducibility of eddies through data assimilation into an operational ocean nowcast/forecast system (Miyazawa et al., 2015).
Biologging of temperature and salinity measurements derived from turtles also has the potential for improving numerical simulations in support of EC forecasts. Loggerhead turtles, for example, favor waters warmer than $15^{\circ} \mathrm{C}$, which corresponds to the northern edge of the Kuroshio and its extension near the surface in winter. A feasibility study for data assimilation of temperature measurements by the turtles suggests that the turtle measurements captured the warm core rings separating from the Kuroshio Extension better than the Oyashio intrusion branches (Miyazawa et al., 2019). The improved ocean representation of such features may allow for better EC forecast through a more accurate simulation of air-sea interaction fluxes associated with these warm ocean rings and meanders.

\section{The 2010 ITOP Field Campaign}

The ITOP international field campaign in the western North Pacific Ocean is an important example for future field observation strategy and planning (Figure 10; D'Asaro et al., 2014). The western North Pacific was chosen because this basin is where 

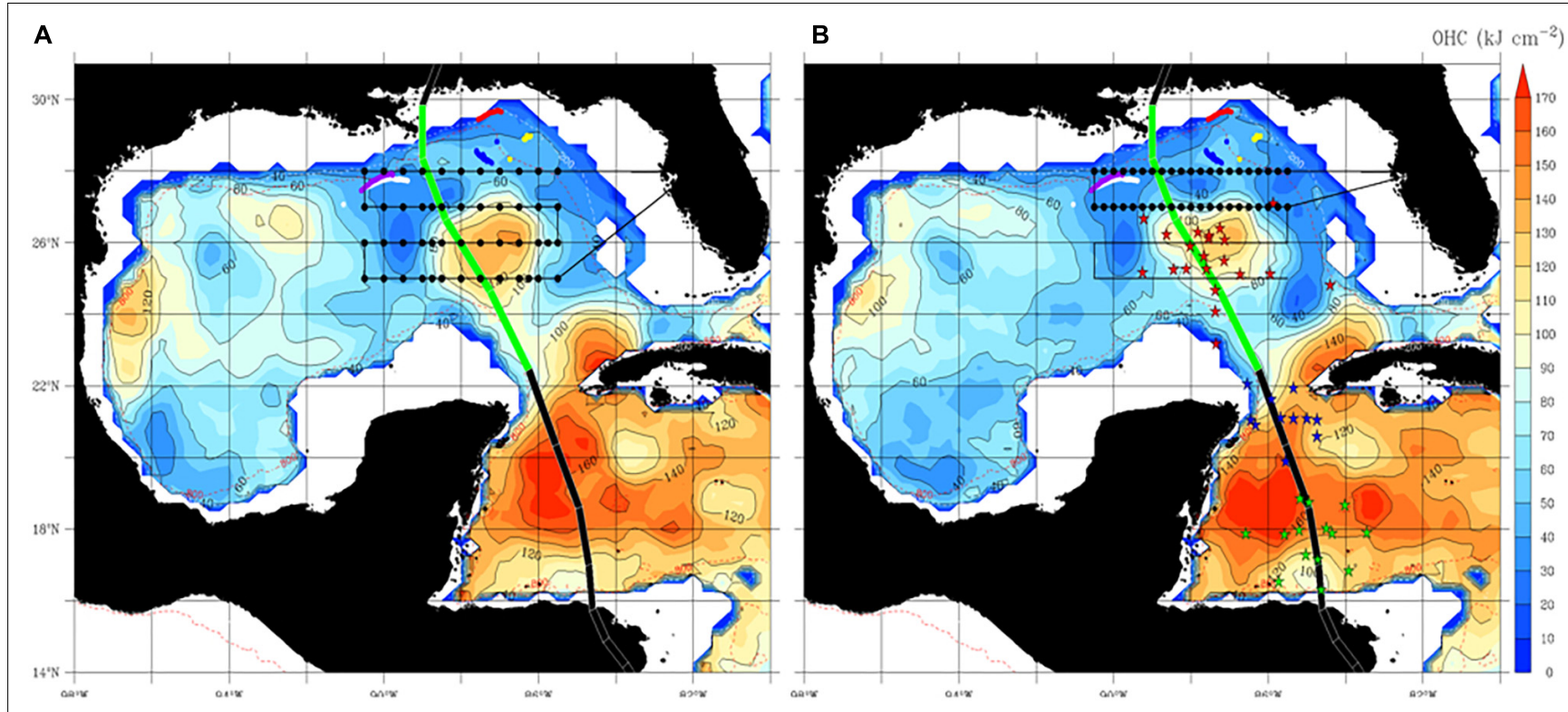

FIGURE 9 | The separation of a LC warm core eddy during the passage of Hurricane Nate of 2017 . The color scale is for $\mathrm{OHC}$ relative to the $26^{\circ} \mathrm{C}$ isotherm, from the satellite fields (Meyers et al., 2014). APEX-EM floats (purple, white, red, and yellow dots) that were active during Nate's passage of Nate over the Gulf.

(A) Pre-storm OHC structure on 5 October, 2017. (B) Post-storm OHC structure on 10 October, 2017. Black dots in (A,B) depict airborne ocean profilers deployed from NOAA WP-3D research aircraft; green, blue, and red stars in (B) represent in-storm oceanographic and atmospheric airborne profilers.

the largest number and the most intense TCs are usually recorded (Figure 1). In the summer of 2010, the ITOP field campaign used targeted aircraft AXBT observations to collect the pre-storm temperature profiles ahead of three TCs of distinct intensity: Megi, Fanapi, and Malakas. Supertyphoon Megi (with peak intensity $82 \mathrm{~m} \mathrm{~s}^{-1} / 296 \mathrm{~km} / \mathrm{h}$, Cat-5) was the most intense TC recorded globally until 2010, while Fanapi was a Cat-3 moderate TC and Malakas was a Cat- 2 TC. The pre-TC ocean conditions were different for these three TCs (Figure 11A). Among the three, Megi intensified over warm ocean temperatures, characterized by TCHP values larger than $140 \mathrm{~kJ} \mathrm{~cm}^{-2}$, and D26 of $120 \mathrm{~m}$. In contrast, both Fanapi and Malakas traveled over waters with shallower D26 and TCHP values lower than $100 \mathrm{~kJ} \mathrm{~cm}^{-2}$. Analysis of the available ocean observations revealed that these large differences in upper-OHC played a key role in the intensification of these TCs (Lin et al., 2013a; D’Asaro et al., 2014).

In addition to assessing the pre-TC ocean conditions using AXBT profiles, the paired ocean-atmosphere observations during TC intensification were also collected. These observations were used to evaluate the SST and air temperature at the TC core (Figure 11B), and their derived air-sea sensible and latent heat fluxes. The correct representation of these fluxes is needed to obtain accurate TC intensification forecasts. Direct observations of air-sea fluxes were obtained deploying co-incident/colocated atmospheric dropsoundes and ocean AXBTs during TC-penetration flights (see Figure 11C). With these unique observations obtained during ITOP, accurate air-sea sensible and latent fluxes were obtained (Lin et al., 2013a; D'Asaro et al., 2014), revealing that enthalpy fluxes were substantially larger during Supertyphoon Megi as it reached Cat-2 (Figure 11C) and then continued to intensify into a Cat-5 Supertyphoon. Results from ITOP emphasize the value of paired, co-located, ocean-atmosphere observations to improve model prediction performance and for improving our understanding on the role that different types of ocean conditions can play in the TC intensification processes.

\section{IMPACT OF OCEAN DATA IN TROPICAL CYCLONE INTENSITY FORECASTS}

A variety of observations collected near TCs in recent years have impacted the fidelity of TCs forecasts, typically by reducing errors and biases in analyses used to initialize the ocean component of coupled prediction models. For example, Halliwell et al. (2011) analyzed the impact of multiple factors toward reducing errors in HYCOM ocean analyses in the Gulf of Mexico prior to Hurricane Isaac (2005). They determined that assimilation of ocean observations is a leading-order factor in reducing initialization errors in comparison to ocean model attributes such as vertical mixing and surface flux parameterizations, along with model resolution.

More recently, Dong et al. (2017) conducted observing system experiments (OSEs) focused on the influence of conventional ocean observing systems plus underwater glider data on prediction of Gonzalo's intensity. A twin experiment was performed comparing an analysis that assimilated underwater glider data from July 15 to October 13 along with other in situ and satellite observations to an analysis produced by an unconstrained (ocean observations not assimilated) model simulation. These two analyses were then used to initialize the 


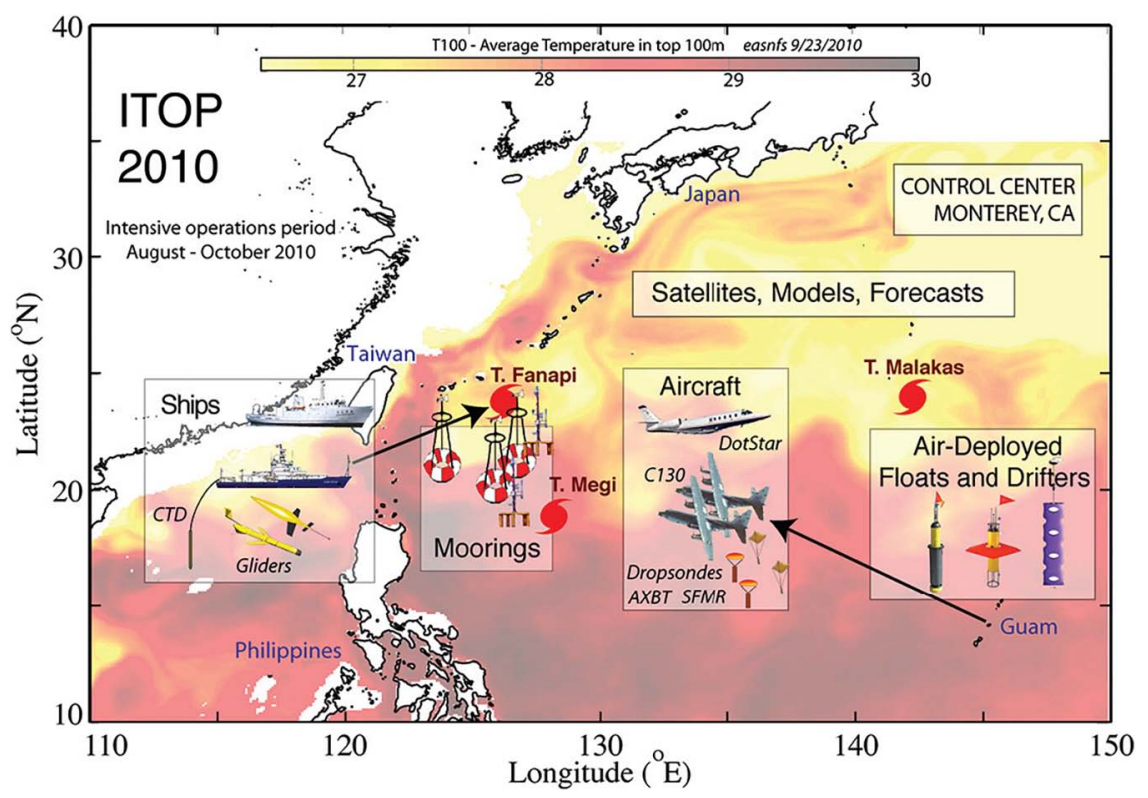

FIGURE 10 | Background color map of average temperature for the upper $100 \mathrm{~m}$ (T100) from the East Asia Seas Nowcast/Forecast System on 23 September, 2010. Overlaid are graphical representations of the ITOP operations area, experimental tools, and strategy. Locations of the three major ITOP storms at the time of maximum sampling are shown by storm symbols. Figure originally from D’Asaro et al. (2014), ${ }^{\circ}$ American Meteorological Society. Used with permission.
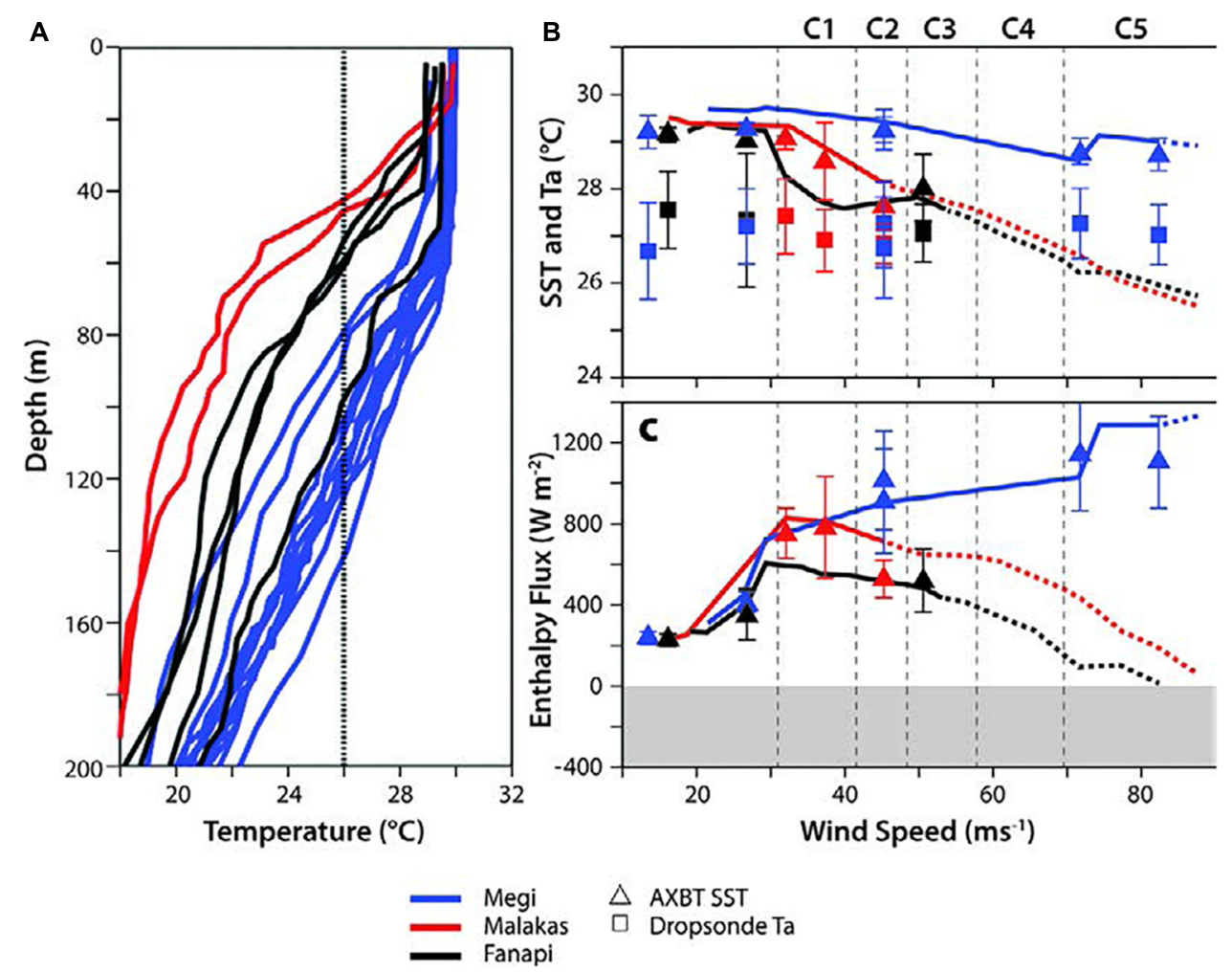

FIGURE 11 | Evolution of temperature and air-sea fluxes for three ITOP TCs (Megi, Fanapi, and Malakas). (A) Pre-TC temperature profiles from ARGO floats.

(B) Symbols: SST and air temperature at the core of each TC as measured by dropsonde/AXBT pairs. Lines: results of an ocean model (Price et al., 1994) driven by the observed TC (solid) and extrapolated to higher wind speeds (dashed). (C) As in (B), but for estimated total enthalpy flux (after Lin et al., $2013 a)$. 
high-resolution HWRF-HYCOM coupled forecast system (Dong et al., 2017). Assimilation of subsurface observations from gliders improved the representation of pre-storm vertical structure of both temperature (Figure 6B) and salinity, capturing the barrier layer previously observed in the region (Domingues et al., 2015). Consequently, forecast intensity errors (e.g., Figure 6C) were reduced by approximately $50 \%$ as a result of assimilating all available observations, enabling a substantially improved forecast for Hurricane Gonzalo.

Observing system experiments are now being conducted for the 2017 North Atlantic hurricane season. The fields of mean TCHP and D26 presented in Figure 12 demonstrate the impact of assimilating all ocean profilers (Argo and Alamo floats plus underwater gliders). Comparing fields produced by an unconstrained model simulation (Figures 12C,D) to observation-based estimates provided by the NOAA/AOML TCHP analysis product (Figures 12A,B), the unconstrained model produces TCHP that is too small and an upper-ocean warm layer that is too thin across the entire North Atlantic hurricane development region. Assimilation of all available ocean profiles (Figures 12E,F) substantially corrects these large-scale biases. The planned next step in this analysis will be to assess the impact on intensity prediction by using these fields to initialize the HYCOM-HWRF forecasting model.

Observing System Simulation Experiments (OSSEs) have also been performed over the North Atlantic hurricane region. Given that the Nature Run, a validated, unconstrained, and realistic ocean simulation by a state-of-the-art ocean model, is known, it is possible to evaluate new observing systems and alternate deployment strategies for existing systems. Previous OSSEs have quantitatively assessed the positive impacts of existing observing systems and different deployment strategies for systems, such as underwater gliders and picket-fence deployments of thermistor chains (Halliwell G. R. H. et al., 2017), and also for prestorm airborne ocean profiler surveys (Halliwell G. R. et al., 2017). More recently, OSSEs were performed to demonstrate the advantages of collecting ocean profiles from moving platforms such as gliders compared to collecting profiles from stationary platforms. These results are summarized by Fujii et al. (2019). Moving forward, OSSEs will continue to be an important tool

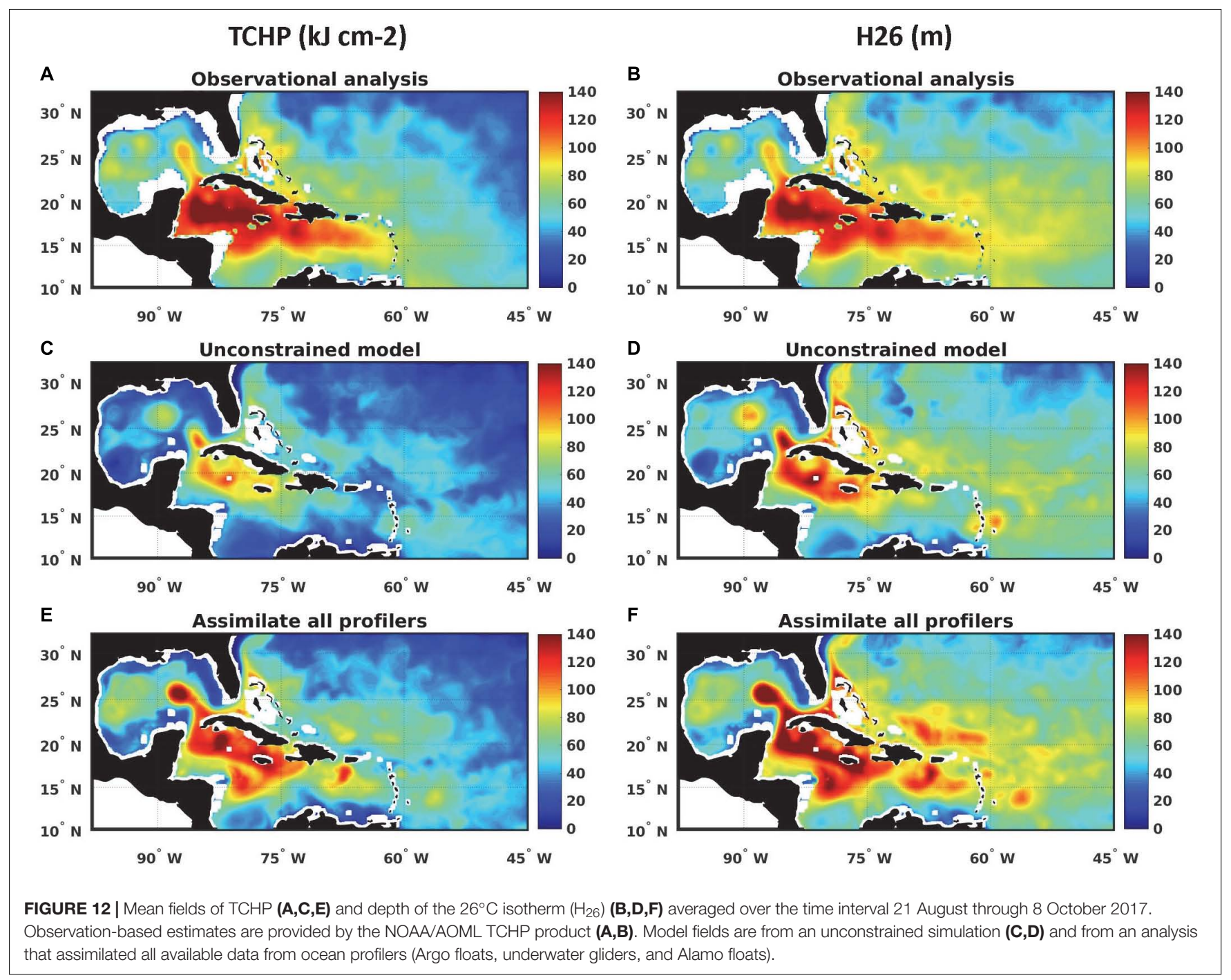


for the design and implementation of optimized ocean sampling strategies in support of both TCs and ECs forecasts, while OSEs will also continue providing further quantitative information on the impacts of different components of the existing ocean observing system.

\section{DATA MANAGEMENT}

Efficient data management, including data transmission is critical for ensuring observations are available in real-time or nearreal-time for assimilation into forecast models. Latency in data availability can have unwanted downstream effects on the use of observations for operational purposes.

For weather forecasting, it is critical that Data Assembly Centers (DACs) and operators transmit data in real time to systems such as the GTS to ensure data availability for forecasters and to validate models. In order to make the data available for assimilation into forecast numerical models, most of the data obtained by the different observational platforms considered here need to be transmitted in real-time or nearreal time through different satellite networks. After reception on land, the data typically undergo platform-dependent quality assurance (QA) and quality control (QC) procedures that are designed to identify possible inaccuracies in the observations. For most platforms these QA/QC procedures include tests designed to identify data gaps or missing values, spikes or unrealistic gradients in the data, and invalid dates or locations, among other error sources. The data are normally not modified during QA/QC, but individual records are flagged according to the results of the tests applied, or the data from a malfunctioning platform may be blacklisted and removed altogether from GTS distribution. The data are then encoded into different traditional alphanumeric formats (e.g., FM 63-XI Ext. BATHY for XBTs and AXBTs, FM 64-XI Ext. TESAC for Argo floats and underwater gliders; World Meteorological Organization (WMO), 2015a Part A), or into binary universal form for the representation of meteorological data (FM 94-XIV BUFR, World Meteorological Organization (WMO), 2015a - Part B). For example, the data format TM315009 is used by the Lagrangian Drifter Laboratory at the Scripps Institution of Oceanography, by MeteoFrance, and by the UK Met Office for their contribution to the Global Surface Drifter Array, and for submission into the GTS (World Meteorological Organization (WMO), 2015b) for near realtime distribution and numerical model assimilation. Other data centers includes the Global Temperature and Salinity Profile Program (GTSPP; XBT, Argo floats, underwater glider), the U.S. IOOS Glider Data Assembly Center (GDAC), and NOAA/NCEI (XBT, Argo, underwater glider) as part of long term archival and for distribution for other delayed-mode scientific applications. At this step the data may be submitted to delayed-mode QC that may result in flags for individual records or in modifications to the data set to ensure the highest possible data quality for all applications.

For research and retrospective analysis, data management is important to ensuring collected observations from various platforms, operated by diverse organizations, is easily available,
QA/QCed, and compatible with relevant standards. DACs can be leveraged to provide a diverse observation platform community a single place to store, share, archive, and quality control their data. In addition to providing standardized, easy to access, QA/QCed ocean observations critical for extreme weather events.

\section{THE VISION FOR THE NEXT 10 YEARS}

\section{Ocean Observations in Support of Tropical Cyclones Studies and Forecasts}

An integrated multiplatform ocean observing system for studies and forecasts of TCs is not currently in place. Analysis of ocean observations from the largely climate-focused ocean observing system often provides valuable information on the mechanisms and processes associated with these extreme weather conditions. Ocean data in support of extreme weather events need to focus on resolving upper ocean features such as barrier layers, spatial variability of warm currents, mesoscale OHC changes, and surface waves (Centurioni et al., 2019) prior to and during the season in each basin where TCs occur, with distribution of data in real-time. However, the scientific and operational requirements of observing platforms, such as profiling floats (Roemmich et al., 2019), moorings (Foltz et al., 2019; Masumoto et al., 2019; Smith et al., 2019), and expendable probes (Goni et al., 2019), do not explicitly target these needs. Sustained and targeted high-resolution ocean observations provide a means to better understand the processes responsible for the rapid evolution of the ocean and its feedback on the atmosphere during these extreme weather conditions. These concerns have been presented and discussed in workshops on TCs from a global perspective, as for example where WMO Recommendations focused on structure and intensity of TCs (Shay et al., 2014).

Pilot networks of sustained multi-platform observations and targeted observations in the tropical Atlantic during hurricane season have proven to provide key upper ocean observations to initialize numerical ocean-atmosphere coupled forecast models in areas where TC intensification and weakening may occur. The assimilation of ocean observations allows for a better representation of ocean conditions within coupled TC forecast models, which in turn provides a more realistic simulation of air-sea interactions and flux exchanges, generally resulting in an improved TC intensity forecast (e.g., Chen et al., 2017; Dong et al., 2017). OSEs (e.g., Dong et al., 2017) need to be extended to more storms in order to provide a more robust estimate of the benefit of various types of observations. These experiments should ideally be performed using operational models so as to quantify the benefit of ocean observations in operational conditions. Furthermore, the OSSE approach dedicated to hurricanes should continue to be followed in order to optimize the deployment of dedicated TC ocean observations, typically gliders and air-deployed profilers. Carrying out OSSEs and OSEs to design, implement, and assess the impact of new sustained components within the ocean observing system (e.g., underwater gliders, profiling floats, drifters, etc.) will be key to continued improvement of TC intensity forecasts, since significant errors still remain in data-assimilative ocean analyses due to existing 
observations being scarce in space and time. Improvements in spatial and temporal coverage of ocean observations should improve the ocean representation within coupled TC forecast models, which in turn will allow for better forecasts. Targeted ocean sampling, when appropriate, also has the potential to help improve TC predictions (e.g., Chen et al., 2017). In addition, improvements in data availability for the forecast community are also essential for ensuring that ocean observations reach operational forecast centers in real-time. In one effort to help with this requirement, the EMC and the National Data Buoy Center (NDBC) within NOAA are working to increase the frequency of data transfer to the GTS.

For the next decade, coupled model systems will extend to multi-way dynamic coupling. In recent years, NOAA/EMC has demonstrated three-way dynamic coupling with HYCOMWaveWatchIII-HWRF model. This would allow for revisiting air-sea interaction dynamics in greater detail, and also exploring observational measurements to support research and simulations. The importance of air-sea flux exchanges to TC development is widely known, yet simulations are still based on bulk parameterizations. To support evolving modeling efforts, observational efforts should accordingly extend to collecting data on waves, sea spray, roughness, turbulence, and relative humidity over the ocean. For example, measurements derived from turbulent microstructure sensors, such as those based on underwater gliders (e.g., St. Laurent and Merrifield, 2017) and moorings (e.g., Warner et al., 2016) will help obtain direct measurements of diapycnal heat flux and temperature diffusivity that can be used to develop, assess, and validate turbulent mixing schemes employed in coupled forecast models.

Expansion of sustained and targeted upper ocean observations in locations where TCs often intensify is one of the best strategies to support hurricane studies and forecasts. Underwater gliders and other autonomous vehicles offer one option for carrying out sustained surveillance in support of TCs studies and forecasts, given that these vehicles can be remotely operated along predetermined routes, they can provide observations in real-time continuously for several months, they withstand hurricane-force winds, and they can be refurbished and serviced for multiyear applications. Targeted and rapid-response observations also provide critical information that instruments surveying in sustained mode cannot. For instance, air-deployed instruments are particularly useful since they are deployed from aircraft already tasked with storm surveillance, and they are logistically easier to position along the forecast track ahead of a TC. Flexible deployments of in situ marine and airborne platforms also allows for co-located measurements with other air/ocean observing systems that are key for advancing our understanding air-sea fluxes across the oceanic surface.

Further advances in satellite remote sensing are also expected to improve the representation of features that impact storm development. For instance, the advent of wide-swath, highresolution satellite altimetry (e.g., Fu and Ubelmann, 2014) will enable the evaluation of air-sea interaction processes during highwind events in detail, such as, for example, the generation of internal waves in the wake of TCs that help drive upper-ocean mixing. Satellite measurements of surface salinity also have potential for improving our understanding of the oceanic factors and processes that lead to TC intensification, especially in the western Atlantic and the Bay of Bengal, where there is persistent shallow salinity stratification. It is important that these measurements continue, along with satellite SST, sea level, and winds.

Considering the positive impacts of upper-ocean observations from pilot networks, and targeted deployments, the following key recommendations have been identified to continue and enhance ocean observations in support of TCs:

- Maintain the elements of the observing system that have proven valuable for Tropical Cyclone ocean research and operational intensity forecast.

- Utilize numerical Observing System Experiments to quantify the impact of the current ocean observing platforms in Tropical Cyclone forecasts.

- Evaluate optimal ocean observational strategies in support of Tropical Cyclone studies and forecasts using numerical Observing System Simulation Experiments.

- Implement sustained and targeted ocean observations (gliders, profiling floats, drifters, etc.) dedicated to improving Tropical Cyclone intensity forecasts; and foster co-incident, co-located air-deployed profile observations (AXBTs, AXCTDs, floats, thermistor chains, etc.) of ocean temperature, salinity, and currents.

- Foster additional sustained measurements of sea level pressure (e.g., from drifters and moorings), and of waves, sea spray, and mixed-layer turbulence (e.g., from gliders) to help develop, evaluate, and validate boundary layer parameterizations.

- Use upper ocean metrics (e.g., Tropical Cyclone Heat Potential, ocean mean temperature, barrier layer thickness, etc.) derived from profile and satellite ocean observations in the operational evaluation and validation of numerical forecast models.

- Continue with efforts focused on improving coupled oceanatmospheric numerical weather models, especially those relating to enhancing ocean data assimilation techniques and mixed layer parametrizations.

- Create an ocean database easily accessible to the scientific community to facilitate research in support of assessments of the role of the ocean in Tropical Cyclones studies.

- Enhance data management efforts to transmit and QA/QC data in real-time for assimilation in operational forecast models.

\section{Ocean Observations in Support of Extratropical Bomb Cyclones Studies}

Recommendations to improve the understanding of oceanatmosphere interactions during EC events are:

- Increase efforts to implement and improve coverage of high-frequency and high-resolution observations using profiling instruments and biologging to detect oceanic fronts associated with western boundary currents in winter. 
- Enhance efforts dedicated to observing surface wind, and waves, using surface drifters, and floating seabirds equipped with weather, GNSS, and motion sensors, respectively, to estimate air-sea flux exchanges under Extratropical Bomb Cyclones.

- Foster additional efforts aimed at observing ocean turbulent mixing induced by Extratropical Bomb Cyclones using, profiling floats and other platforms (e.g., gliders, moorings, floats, etc.).

- Incorporate real-time meteo-ocean observations, including ocean bottom pressure, in moorings from the Tsunami monitoring network in support of Extratropical Bomb Cyclones studies and forecasts.

Air-sea interactions under ECs are poorly understood because of the sparseness of in situ observations and lack of satellite observations caused by thick clouds and heavy rain. Seabirds are often observed to fly and float under ECs to forage (Yoda et al., 2014; Yonehara et al., 2016; Goto et al., 2017), providing an additional potential source of environmental data. Estimation of surface winds and waves using biologging, GNSS and motion sensor can provide useful information about air-sea interaction processes under ECs as well as their temperature and pressure measurements. In addition, the development of profiling floats equipped with motion sensors can also help to provide metrics to evaluate ocean mixing near the sea surface.

To monitor Tsunami, several real-time observation networks of ocean bottom pressure have been established (Kaneda, 2010; Bernard and Meinig, 2011; Lawson et al., 2011; Mochizuki et al., 2017). Most of the sites in the networks are located under the area where ECs frequently develop. Recently, seismic stations on land can catch microseisms induced by ECs (Nishida and Takagi, 2016). The real-time monitoring networks will provide oceanic responses to ECs and informations of winds and waves which may contribute to forecast improvement of ECs.

\section{SUMMARY}

In this community white paper, we provide a summary of current ocean observing efforts, and recent research findings in support of studies and forecasts of TCs and ECs. Substantial progress has been made over the past decade in terms of ocean observations, improving our understanding of the role that the ocean plays in the evolution of TCs and ECs, and on transitioning state-of-the art coupled forecast models to operational mode. These advances have largely addressed recommendations made

\section{REFERENCES}

Ali, M. M., Jagadeesh, P. S. V., and Jain, S. (2007). Effects of eddies on Bay of Bengal cyclone intensity. EOS Trans. Am. Geophys. Union 88, 93-95. doi: 10.1029/2007EO080001

Ali, M. M., Kashyap, T., and Nagamani, P. V. (2013). Use of sea surface temperature for cyclone intensity prediction needs a relook. EOS Trans. Am. Geophys. Union 94, 177-177. doi: 10.1002/2013EO190005

Ali, M. M., Nagamani, P. V., Sharma, N., Gopal, R. T. V., Rajeevan, M., Goni, G. J., et al. (2015). Relationship between ocean mean temperatures and Indian summer monsoon rainfall. Atmos. Sci. Lett. 16, 408-413. doi: 10.1002/asl2.576 by the scientific community during OceanObs'09 (e.g., Goni et al., 2010) and emphasize the critical value of sustained and targeted ocean observations, real-time data transmission, and multi-platform efforts.

With recent advances in ocean modeling and coupled atmosphere-ocean modeling, operational forecasts increasingly rely on assimilating real-time ocean measurements to produce accurate ocean, weather and extreme weather forecasts. For example, assimilation of ocean observations can dramatically improve hurricane intensity forecasts (e.g., Dong et al., 2017). OSEs can assist in quantifying the impact of upper ocean observations on TC and ECs forecasts. Similarly, OSSEs can also be applied to various regions to design optimal and costeffective deployment strategies for both targeted and sustained observations in support of ECs and TCs.

Given the large benefits provided by the ocean observing system in support of extreme weather studies and forecasts, it is critical that current components are maintained and possibly expanded over the next decade. In addition, new technologies, pilot networks, and targeted deployments are greatly expanding the observation capabilities; incorporating these components into the sustained observing system will likely greatly benefit studies and forecasts of TCs and ECs. Finally, considering the large number of countries whose coastal areas are often impacted by TCs and ECs, results and advances presented here emphasize the critical value of carrying out a coordinated international effort in the design, implementation, maintenance, and data management of key aspects of ocean observations that will ensure the feasibility of logistical, operational, and research activities.

\section{AUTHOR CONTRIBUTIONS}

$\mathrm{RD}, \mathrm{AK}-\mathrm{Y}, \mathrm{PC}-\mathrm{M}$, and GG elaborated the initial outline, and wrote the first draft of the manuscript. All authors of this community white paper have provided input in the form of language, figures, and recommendations for the future of the ocean observing system in support of studies and forecasts of Tropical and Extratropical Cyclones.

\section{FUNDING}

This study was supported by the National Oceanic and Atmospheric Administration and JSPS KAKENHI (Grant Numbers: JP17K19093, JP16K12591, and JP16H01846).

Bacmeister, J. T., Reed, K. A., Hannay, C., Lawrence, P., Bates, S., Truesdale, J. E., et al. (2018). Projected changes in tropical cyclone activity under future warming scenarios using a high-resolution climate model. Climatic Change 146, 547-560. doi: 10.1007/s10584-016-1750-x

Balaguru, K., Chang, P., Saravanan, R., and Jang, C. J. (2012a). The barrier layer of the Atlantic warm pool: formation mechanism and influence on the mean climate. Tellus Dyn. Meteorol. Oceanogr. 64:18162. doi: 10.3402/tellusa.v64i0. 18162

Balaguru, K., Chang, P., Saravanan, R., Leung, L. R., Xu, Z., Li, M., et al. (2012b). Ocean barrier layers' effect on tropical cyclone intensification. Proc. Natl. Acad. Sci. U.S.A. 109, 14343-14347. doi: 10.1073/pnas.1201364109 
Balaguru, K., Foltz, G. R., Leung, L. R., Asaro, E. D., Emanuel, K. A., Liu, H., et al. (2015). Dynamic potential intensity: an improved representation of the ocean's impact on tropical cyclones. Geophys. Res. Lett. 42, 6739-6746. doi: 10.1002/2015GL064822

Balaguru, K., Foltz, G. R., Leung, L. R., and Emanuel, K. A. (2016). Global warminginduced upper-ocean freshening and the intensification of super typhoons. Nat. Commun. 7:13670. doi: 10.1038/ncomms13670

Balaguru, K., Foltz, G. R., Leung, L. R., Hagos, S. M., and Judi, D. R. (2018). On the use of ocean dynamic temperature for hurricane intensity forecasting. Weather Forecast. 33, 411-418. doi: 10.1175/WAF-D-17-0143.1

Bernard, E. N., and Meinig, C. (2011). "History and future of deep-ocean tsunami measurements," in Proceedings of the OCEANS' 11 MTS/IEEE, Kona, (Waikoloa, HI: IEEE)

Black, M. T., and Pezza, A. B. (2013). A universal, broad-environment energy conversion signature of explosive cyclones. Geophys. Res. Lett. 40, 452-457. doi: $10.1002 /$ grl.50114

Block, B. A., Dewar, H., Blackwell, S. B., Williams, T. D., Prince, E. D., Farwell, C. J., et al. (2001). Migratory movements, depth preferences, and thermal biology of Atlantic bluefin tuna. Science 293, 1310-1314. doi: 10.1126/science.1061197

Boehlert, G. W., Costa, D. P., Crocker, D. E., Green, P., O’Brien, T., Levitus, S., et al. (2001). Autonomous pinniped environmental samplers: using instrumented animals as oceanographic data collectors. J. Atmospheric Ocean. Technol. 18, 1882-1893. doi: 10.1175/1520-0426(2001)018<1882:apesui $>2.0 . c 0 ; 2$

Booth, J. F., Thompson, L., Patoux, J., and Kelly, K. A. (2012). Sensitivity of midlatitude storm intensification to perturbations in the sea surface temperature near the Gulf Stream. Mon. Weather Rev. 140, 1241-1256. doi: 10.1175/MWR-D-11-00195.1

Boyd, I. L., Hawker, E. J., Brandon, M. A., and Staniland, I. J. (2001). Measurement of ocean temperatures using instruments carried by Antarctic fur seals. J. Mar. Syst. 27, 277-288. doi: 10.1016/s0924-7963(00)00073-7

Burt, S. D., and Mansfield, D. A. (1988). The great storm of 15-16 October 1987. Weather 43, 90-110. doi: 10.1002/j.1477-8696.1988.tb03885.x

Camp, J., Scaife, A. A., and Heming, J. (2018). Predictability of the 2017 North Atlantic hurricane season. Atmos. Sci. Lett. 19:e813. doi: 10.1002/asl.813

Campagna, C., Rivas, A. L., and Marin, M. R. (2000). Temperature and depth profiles recorded during dives of elephant seals reflect distinct ocean environments. J. Mar. Syst. 24, 299-312. doi: 10.1016/s0924-7963(99)00091-3

Catto, J. L., Shaffrey, L. C., and Hodges, K. I. (2010). Can climate models capture the structure of extratropical cyclones? J. Clim. 23, 1621-1635. doi: 10.1175/ 2009JCLI3318.1

Centurioni, L. R. (2018) "Drifter technology and impacts for sea surface temperature, sea-level pressure, and ocean circulation studies," in Observing the Oceans in Real Time, eds R. Venkatesan, A. Tandon, E. D'Asaro, and M. Atmanand. (Cham: Springer), 37-57.

Centurioni, L., Braasch, L., Lauro, E. D., Contestabile, P., Leo, F. D., Casotti, R., et al. (2016). A new strategic wave measurement station off Naples port main breakwater. Coas. Eng. Proc. 1:36. doi: 10.9753/icce.v35.waves.36

Centurioni, L., Horányi, A., Cardinali, C., Charpentier, E., and Lumpkin, R. (2017). A global ocean observing system for measuring sea level atmospheric pressure: effects and impacts on numerical weather prediction. Bull. Am. Meteorol. Soc. 98, 231-238. doi: 10.1175/bams-d-15-00080.1

Centurioni, L. R. (2010). Observations of large-amplitude nonlinear internal waves from a drifting array: instruments and methods. J. Atmospheric Ocean. Technol. 27, 1711-1731. doi: 10.1175/2010jtecho774.1

Centurioni, L. R., Turton, J. D., Lumpkin, R., Braasch, L., Brassington, G., Chao, Y., et al. (2019). Multidisciplinary global in-situ observations of essential climate and ocean variables at the air-sea interface. Front. Mar. Sci. 6:419. doi: 10.3389/ fmars.2019.00419

Chacko, N. (2017). Chlorophyll bloom in response to tropical cyclone Hudhud in the Bay of Bengal: Bio-Argo subsurface observations. Deep Sea Res. Part Oceanogr. Res. Pap. 124, 66-72. doi: 10.1016/j.dsr.2017.04.010

Chang, E. K. (2017). Projected significant increase in the number of extreme extratropical cyclones in the Southern Hemisphere. J. Clim. 30, 4915-4935. doi: 10.1175/jcli-d-16-0553.1

Charrassin, J.-B., Park, Y.-H., Maho, Y. L., and Bost, C.-A. (2002). Penguins as oceanographers unravel hidden mechanisms of marine productivity. Ecol. Lett. 5, 317-319. doi: 10.1046/j.1461-0248.2002.00341.x

Chassignet, E. P., Hurlburt, H. E., Metzger, E. J., Smedstad, O. M., Cummings, J. A., Halliwell, G. R., et al. (2009). US GODAE: global ocean prediction with the HYbrid Coordinate Ocean Model (HYCOM). Oceanography 22, 64-75. doi: 10.5670/oceanog.2009.39

Chen, S., Cumming, J. A., Schmidt, J. M., Sanabia, E. R., and Jayne, S. R. (2017). Targeted ocean sampling guidance for tropical cyclones. J. G. R Oceans 122, 3505-3518. doi: 10.1002/2017JC01272

Colle, B. A., Booth, J. F., and Chang, E. K. (2015). A review of historical and future changes of extratropical cyclones and associated impacts along the US East Coast. Curr. Clim. Change Rep. 1, 125-143. doi: 10.1007/s40641-0150013-7

Dare, R. A., and McBride, J. L. (2012). Sea surface temperature response to tropical cyclones. Mon. Wea. Rev. 139, 3798-3808. doi: 10.1175/mwr-d-10-05019.1

D’Asaro, E. A., Black, P. G., Centurioni, L. R., Chang, Y.-T., Chen, S. S., Foster, R. C., et al. (2014). Impact of typhoons on the ocean in the Pacific. Bull. Am. Meteorol. Soc. 95, 1405-1418.

DeMaria, M., and Kaplan, J. (1994). A statistical hurricane intensity prediction scheme (SHIPS) for the Atlantic basin. Weather Forecast. 9, 209-220. doi: 10.1175/1520-0434(1994)009<0209:aships>2.0.co;2

DeMaria, M., Knaff, J. A., and Sampson, C. (2007). Evaluation of long-term trends in tropical cyclone intensity forecasts. Meteorol. Atmospheric Phys. 97, 19-28. doi: 10.1007/s00703-006-0241-4

DeMaria, M., Mainelli, M., Shay, L. K., Knaff, J. A., and Kaplan, J. (2005). Further improvements to the statistical hurricane intensity prediction scheme (SHIPS). Weather Forecast. 20, 531-543. doi: 10.1175/waf862.1

DeMaria, M., Sampson, C. R., Knaff, J. A., and Musgrave, K. D. (2014). Is tropical cyclone intensity guidance improving? Bull. Am. Meteorol. Soc. 95, 387-398. doi: 10.1175/bams-d-12-00240.1

Domingues, R., Goni, G., Bringas, F., Lee, S.-K., Kim, H.-S., Halliwell, G., et al. (2015). Upper ocean response to Hurricane Gonzalo (2014): salinity effects revealed by targeted and sustained underwater glider observations. Geophys. Res. Lett. 42, 7131-7138. doi: 10.1002/2015gl065378

Dong, J., Domingues, R., Goni, G., Halliwell, G., Kim, H.-S., Lee, S.-K., et al. (2017). Impact of assimilating underwater glider data on Hurricane Gonzalo (2014) forecasts. Weather Forecast. 32, 1143-1159. doi: 10.1175/waf-d-16-0182.1

Drévillon, M., Bourdallé-Badie, R., Derval, C., Lellouche, J. M., Rémy, E., Tranchant, B., et al. (2008). The GODAE/Mercator-Ocean global ocean forecasting system: results, applications and prospects. J. Oper. Oceanogr. 1, 51-57. doi: 10.1080/1755876x.2008.11020095

Elsner, J. B., Kossin, J. P., and Jagger, T. H. (2008). The increasing intensity of the strongest tropical cyclones. Nature 455, 92-95. doi: 10.1038/nature07234

Emanuel, K. (2015). Effect of upper-ocean evolution on projected trends in tropical cyclone activity. J. Clim. 28, 8165-8170. doi: 10.1175/jcli-d-15-0401.1

Fedak, M. A. (2013). The impact of animal platforms on polar ocean observation. Deep Sea Res. Part Top. Stud. Oceanogr. 8, 7-13. doi: 10.1016/j.dsr2.2012.07.007

Foltz, G. R., and Balaguru, K. (2016). Prolonged El Niño conditions in 2014-2015 and the rapid intensification of Hurricane Patricia in the eastern Pacific: El Niño and Hurricane Patricia. Geophys. Res. Lett. 43:355. doi: 10.1002/2016GL070274

Foltz, G. R., Brandt, P., Rodriguez-Fonseca, M., Hernandez, F., Dengler, M., Rodrigues, R., et al. (2019). The Tropical Atlantic observing system. Front. Mar. Sci. 6:36. doi: 10.3389/fmars.2019.00206

Fu, L.-L., and Ubelmann, C. (2014). On the transition from profile altimeter to swath altimeter for observing global ocean surface topography. J. Atmospheric Ocean. Technol. 31, 560-568. doi: 10.1175/JTECH-D-13-00109.1

Fujii, Y., Remy, E., Zuo, H., Oke, P. R., Halliwell, G. R., Garparin, F., et al. (2019). Observing system evaluation based on ocean data assimilation and prediction systems: on-going challenges and future vision for designing/supporting ocean observational networks. Front. Mar. Sci. 6:417. doi: 10.3389/fmars.2019.00417

Fukuoka, T., Narazaki, T., and Sato, K. (2015). Summer-restricted migration of green turtles Chelonia mydas to a temperate habitat of the northwest Pacific Ocean. Endanger. Species Res. 28, 1-10. doi: 10.3354/esr00671

Glenn, S. M., Miles, T. N., Seroka, G. N., Xu, Y., Forney, R. K., Yu, F., et al. (2016). Stratified coastal ocean interactions with tropical cyclones. Nat. Commun. 7:10887. doi: $10.1038 /$ ncomms 10887

Gómara, I., Rodríguez-Fonseca, B., Zurita-Gotor, P., Ulbrich, S., and Pinto, J. G. (2016). Abrupt transitions in the NAO control of explosive North Atlantic cyclone development. Clim. Dyn. 47, 3091-3111. doi: 10.1007/s00382-0163015-9

Goni, G., DeMaria, M., Knaff, J., Sampson, C., Ginis, I., Bringas, F., et al. (2009). Applications of satellite-derived ocean measurements to tropical cyclone intensity forecasting. Oceanography 22, 190-197. doi: 10.5670/oceanog.2009.78 
Goni, G., DeMaria, M., Knaff, J., Sampson, C., Price, J., Mehra, A., et al. (2010). "The ocean observing system for tropical cyclone intensification forecasts and studies," in Proceedings of the OceanObs' 09 Conference, (Venice).

Goni, G., and Domingues, R. (2019). "The particular upper ocean conditions in the gulf of mexico during hurricane michael (2018)," in State of the Climate in 2018, eds J. Blunden, D. S. Arndt, and G. Hartfield (Massachusetts: Bulletin of the American Meteorological Society).

Goni, G. J., Sprintall, J., Bringas, F., Cheng, L., Cirano, M., Dong, S., et al. (2019). More than 50 years of successful continuous temperature section measurements by the global expendable bathythermograph network, its integrability, societal benefits, and future. Front. Mar. Sci. 6:452. doi: 10.3389/fmars.2019.00452

Goni, G. J., Todd, R. E., Jayne, S. R., Halliwell, G., Glenn, S., Dong, J., et al. (2017). Autonomous and Lagrangian ocean observations for Atlantic tropical cyclone studies and forecasts. Oceanography 30, 92-103. doi: 10.5670/oceanog.2017.227

Goni, G. J., and Trinanes, J. A. (2003). Ocean thermal structure monitoring could aid in the intensity forecast of tropical cyclones. EOS Trans. Am. Geophys. Union 84, 573-578.

Goto, Y., Yoda, K., and Sato, K. (2017). Asymmetry hidden in birds' tracks reveals wind, heading, and orientation ability over the ocean. Sci. Adv. 3:e1700097. doi: 10.1126/sciadv.1700097

Grodsky, S. A., Reul, N., Lagerloef, G., Reverdin, G., Carton, J. A., Chapron, B., et al. (2012). Haline hurricane wake in the Amazon/Orinoco plume: AQUARIUS/SACD and SMOS observations. Geophys. Res. Lett. 39:L20603. doi: 10.1029/2012GL053335

Haarsma, R. J., Roberts, M. J., Vidale, P. L., Senior, C. A., Bellucci, A., Bao, Q., et al. (2016). High Resolution Model Intercomparison Project (HighResMIP v1.0) for CMIP6. Geosci. Model Dev. 9, 4185-4208. doi: 10.5194/gmd-9-4185-2016

Hakim, G. J., Bosart, L. F., and Keyser, D. (1995). The Ohio Valley wave merger cyclogenesis event of 25-26 January 1978. Part 1: multiscale case study. Mon. Wea. Rev. 123, 2663-2692. doi: 10.1175/1520-0493(1995)123<2663:tovwmc > 2.0.co;2

Halliwell, G. R., Shay, L. K., Brewster, J. K., and Teague, W. J. (2011). Evaluation and sensitivity analysis of an ocean model response to Hurricane Ivan. Mon. Weather Rev. 139, 921-945. doi: 10.1175/2010MWR3104.1

Halliwell, G. R. H., Mehari, M. F., Hénaff, M. L., Kourafalou, V. H., Androulidakis, I. S., Kang, H. S., et al. (2017). North Atlantic Ocean OSSE system: evaluation of operational ocean observing system components and supplemental seasonal observations for potentially improving tropical cyclone prediction in coupled systems. J. Oper. Oceanogr. 10, 154-175. doi: 10.1080/1755876X.2017.1322770

Halliwell, G. R., Mehari, M., Shay, L. K., Kourafalou, V. H., Kang, H., Kim, H.-S., et al. (2017). OSSE quantitative assessment of rapid-response prestorm ocean surveys to improve coupled tropical cyclone prediction. J. Geophys. Res. Oceans 122, 5729-5748. doi: 10.1002/2017JC012760

Hirata, H., Kawamura, R., Kato, M., and Shinoda, T. (2015). Influential role of moisture supply from the Kuroshio/Kuroshio extension in the rapid development of an extratropical cyclone. Mon. Weather Rev. 143, 4126-4144. doi: 10.1175/MWR-D-15-0016.1

Hirata, H., Kawamura, R., Kato, M., and Shinoda, T. (2016). Response of rapidly developing extratropical cyclones to sea surface temperature variations over the western Kuroshio-Oyashio confluence region. J. Geophys. Res. Atmos. 121, 3843-3858. doi: 10.1002/2015JD024391

Hirata, H., Kawamura, R., Kato, M., and Shinoda, T. (2018). A positive feedback process related to the rapid development of an extratropical cyclone over the Kuroshio/Kuroshio extension. Mon. Weather Rev. 146, 417-433. doi: 10.1175/ MWR-D-17-0063.1

Horányi, A., Cardinali, C., and Centurioni, L. (2017). The global numerical weather prediction impact of mean-sea-level pressure observations from drifting buoys. Q. J. R. Meteorol. Soc. 143, 974-985. doi: 10.1002/qj.2981

Houghton, R. W., Schlitz, R., Beardsley, R. C., Butman, B., and Chamberlin, J. L. (1982). The middle Atlantic bight cold pool: evolution of the temperature structure during summer 1979. J. Phys. Oceanogr. 12, 1019-1029. doi: 10.1175/ 1520-0485(1982)012<1019:tmabcp > 2.0.co;2

Huang, P., Lin, I.-I., Chou, C., and Huang, R.-H. (2015). Change in ocean subsurface environment to suppress tropical cyclone intensification under global warming. Nat. Commun. 6:7188. doi: 10.1038/ncomms 8188

Ingleby, B., and Isaksen, L. (2018). Drifting buoy pressures: impact on NWP. Atmospheric Sci. Lett. 19:e822. doi: 10.1002/asl.822
Jaimes, B., Shay, L. K., and Brewster, J. K. (2016). Observed air-sea interactions in tropical cyclone Isaac over Loop Current mesoscale eddy features. Dyn. Atmospheres Oceans 76, 306-324. doi: 10.1016/j.dynatmoce.2016. 03.001

Jayne, S. R., and Bogue, N. M. (2017). Air-deployable profiling floats. Oceanography 30, 29-31. doi: 10.5670/oceanog.2017.214

Johns, E. M., Muhling, B. A., Perez, R. C., Müller-Karger, F. E., Melo, N., Smith, R. H., et al. (2014). Amazon river water in the northeastern Caribbean Sea and its effect on larval reef fish assemblages during April 2009. Fish. Oceanogr. 23, 472-494. doi: 10.1111/fog. 12082

Kaneda, Y. (2010). "The advanced ocean floor real time monitoring system for megathrust earthquakes and tsunamis-application of DONET and DONET2 data to seismological research and disaster mitigation," in Proceedings of the OCEANS 2010 MTS/IEEE, (Seattle), 1-6. doi: 10.1109/OCEANS.2010.5664309

Kaplan, J., DeMaria, M., and Knaff, J. A. (2010). A revised tropical cyclone rapid intensification index for the Atlantic and eastern North Pacific basins. Weather Forecast. 25, 220-241. doi: 10.1175/2009WAF2222280.1

Kelly, P. S., Lwiza, K. M. M., Cowen, R. K., and Goni, G. J. (2000). Lowsalinity pools at Barbados, West Indies: their origin, frequency, and variability. J. Geophys. Res. Oceans 105, 19699-19708. doi: 10.1029/1999JC900328

Kim, H.-S., Lozano, C., Tallapragada, V., Iredell, D., Sheinin, D., Tolman, H. L., et al. (2014). Performance of Ocean coupled HWRF-HYCOM model. J. Atmospheric Ocean. Technol. 31, 545-559. doi: 10.1175/JTECH-D-1300013.1

Knaff, J. A., Sampson, C. R., and DeMaria, M. (2005). An operational statistical typhoon intensity prediction scheme for the Western North Pacific.Wea. Forecasting 20, 688-699. doi: 10.1175/waf863.1

Knaff, J. A., Sampson, C. R., and Musgrave, K. D. (2018). An operational rapid intensification prediction aid for the western North Pacific. Weather Forecast. 33, 799-811. doi: 10.1175/WAF-D-18-0012.1

Knapp, K. R., Kruk, M. C., Levinson, D. H., Diamond, H. J., and Neumann, C. J. (2010). The international best track archive for climate stewardship (IBTrACS) unifying tropical cyclone data. Bull. Am. Meteorol. Soc. 91, 363-376. doi: 10.1175/2009bams2755.1

Koudil, M., Charrassin, J.-B., Le Maho, Y., and Bost, C.-A. (2000). Seabirds as monitors of upper-ocean thermal structure. king penguins at the Antarctic polar front, east of Kerguelen sector. Comptes Rendus Académie Sci. Ser. III Sci. Vie 323, 377-384. doi: 10.1016/S0764-4469(00)00144-X

Kuwano-Yoshida, A., and Asuma, Y. (2008). Numerical study of explosively developing extratropical cyclones in the northwestern Pacific region. Mon. Weather Rev. 136, 712-740. doi: 10.1175/2007MWR2111.1

Kuwano-Yoshida, A., and Enomoto, T. (2013). Predictability of explosive cyclogenesis over the northwestern Pacific region using ensemble reanalysis. Mon. Weather Rev. 141, 3769-3785. doi: 10.1175/MWR-D-12-00161.1

Kuwano-Yoshida, A., and Minobe, S. (2017). Storm-track response to SST fronts in the northwestern Pacific region in an AGCM. J. Clim. 30, 1081-1102. doi: 10.1175/JCLI-D-16-0331.1

Kuwano-Yoshida, A., Sasaki, H., Sasai, Y., and Hosoda, S. (2018). "Impact of explosive cyclones on the deep ocean in the North Pacific: simulations and observations," in Proceedings of the OCEANS' 18 MTS/IEE Kobe/Techno-Ocean 2018, (Kobe).

Kuwano-Yoshida, A., Sasaki, H., and Sasai, Y. (2017). Impact of explosive cyclones on the deep ocean in the North Pacific using an eddy-resolving ocean general circulation model. Geophys. Res. Lett. 44, 320-329. doi: 10.1002/2016GL071367

Landsea, C. W. (1993). A climatology of intense (or major) Atlantic hurricanes. Mon. Weather Rev. 121, 1703-1713. doi: 10.1175/1520-0493(1993)121<1703: acoima $>2.0 . \operatorname{co} ; 2$

Lawson, R. A., Graham, D., Stalin, S., Meinig, C., Tagawa, D., LawrenceSlavas, N., et al. (2011). "From research to commercial operations: the next generation Easy-to-Deploy (ETD) tsunami assessment buoy," in Proceedings of the OCEANS 2011 IEEE, (Spain).

Legler, D. M., Freeland, H. J., Lumpkin, R., Ball, G., McPhaden, M. J., North, S., et al. (2015). The current status of the real-time in-situ Global Ocean Observing System for operational oceanography. J. Oper. Oceanogr. 8, s189-s200. doi: 10.1080/1755876X.2015.1049883

Leipper, D. F., and Volgenau, D. (1972). Hurricane heat potential of the Gulf of Mexico. J. Phys. Oceanogr. 2, 218-224. doi: 10.1175/1520-0485(1972) $002<0218$ :hhpotg $>2.0$. co; 2 
Liblik, T., Karstensen, J., Testor, P., Alenius, P., Hayes, D., Ruiz, S., et al. (2016). Potential for an underwater glider component as part of the Global Ocean observing system. Methods Oceanogr. 17, 50-82. doi: 10.1016/j.mio.2016. 05.001

Lin, I.-I., Black, P., Price, J. F., Yang, C.-Y., Chen, S. S., Lien, C.-C., et al. (2013a). An ocean coupling potential intensity index for tropical cyclones. Geophys. Res. Lett. 40, 1878-1882. doi: 10.1002/grl.50091

Lin, I.-I., Goni, G. J., Knaff, J. A., Forbes, C., and Ali, M. M. (2013b). Ocean heat content for tropical cyclone intensity forecasting and its impact on storm surge. Nat. Hazards 66, 1481-1500. doi: 10.1007/s11069-012-0214-5

Lin, I.-I., Chen, C.-H., Pun, I.-F., Liu, W. T., and Wu, C.-C. (2009). Warm ocean anomaly, air sea fluxes, and the rapid intensification of tropical cyclone Nargis (2008). Geophys. Res. Lett. 36:L03817. doi: 10.1029/2008GL03 5815

Lin, I.-I., Pun, I.-F., and Lien, C.-C. (2014). “Category-6” supertyphoon Haiyan in global warming hiatus: contribution from subsurface ocean warming. Geophys. Res. Lett. 41, 8547-8553. doi: 10.1002/2014GL061281

Lin, I.-I., Wu, C.-C., Emanuel, K. A., Lee, I.-H., Wu, C.-R., and Pun, I.-F. (2005). The interaction of supertyphoon Maemi (2003) with a warm ocean eddy. Mon. Weather Rev. 133, 2635-2649. doi: 10.1175/MWR3005.1

Lin, I.-I., Wu, C.-C., Pun, I.-F., and Ko, D.-S. (2008). Upper-ocean thermal structure and the western North Pacific category 5 typhoons. Part I: ocean features and the category 5 typhoons'. Intensification. Mon. Weather Rev. 136, 3288-3306. doi: 10.1175/2008MWR2277.1

Mainelli, M., DeMaria, M., Shay, L. K., and Goni, G. (2008). Application of oceanic heat content estimation to operational forecasting of recent Atlantic category 5 hurricanes. Weather Forecast. 23, 3-16. doi: 10.1175/2007WAF200 6111.1

Mao, Q., Chang, S. W., and Pfeffer, R. L. (2000). Influence of large-scale initial oceanic mixed layer depth on tropical cyclones. Mon. Weather Rev. 128, 4058-4070. doi: 10.1175/1520-0493(2000)129<4058:iolsio>2.0.co;2

Masumoto, Y., Hermes, J. C., Beal, L. M., Roxy, M. K., Vialard, J., Andres, M., et al. (2019). Sustained Indian Ocean observing system. Front. Mar. Sci. 6:355. doi: 10.3389/fmars.2019.00355

Meissner, T., Wentz, F., and Le Vine, D. (2018). The salinity retrieval algorithms for the NASA Aquarius version 5 and SMAP version 3 releases. Remote Sens. 10:1121. doi: 10.3390/rs10071121

Menkes, C. E., Lengaigne, M., Lévy, M., Ethé, C., Bopp, L., Aumont, O., et al. (2016). Global impact of tropical cyclones on primary production. Glob. Biogeochem. Cycles 30, 767-786. doi: 10.1002/2015GB005214

Meyers, P. C., Shay, L. K., and Brewster, J. K. (2014). Development and analysis of the systematically merged Atlantic regional temperature and salinity climatology for oceanic heat content estimates. J. Atmospheric Ocean. Technol. 31, 131-149. doi: 10.1175/JTECH-D-13-00100.1

Meyers, P. C., Shay, L. K., Brewster, J. K., and Jaimes, B. (2015). Observed ocean thermal response to Hurricanes Gustav and Ike. J. Geophys. Res. Oceans 121, 162-179. doi: 10.1002/2015JC010912

Miles, T., Seroka, G., and Glenn, S. (2017). Coastal ocean circulation during Hurricane Sandy. J. Geophys. Res. Oceans 122, 7095-7114. doi: 10.1002/ 2017JC013031

Miles, T., Seroka, G., Kohut, J., Schofield, O., and Glenn, S. (2015). Glider observations and modeling of sediment transport in Hurricane Sandy. J. Geophys. Res. Oceans 120, 1771-1791. doi: 10.1002/2014JC010474

Miyazawa, Y., Guo, X., Varlamov, S. M., Miyama, T., Yoda, K., Sato, K., et al. (2015). Assimilation of the seabird and ship drift data in the north-eastern sea of Japan into an operational ocean nowcast/forecast system. Sci. Rep. 5:17672. doi: 10.1038/srep 17672

Miyazawa, Y., Kuwano-Yoshida, A., Doi, T., Nishikawa, H., Narazaki, T., Fukuoka, T., et al. (2019). Temperature profiling measurements by sea turtles improve ocean state estimation in the Kuroshio-Oyashio Confluence region. Ocean Dyn. 69, 267-282. doi: 10.1007/s10236-018-1238-5

Mochizuki, M., Kanazawa, T., Uehira, K., Shimbo, T., Shiomi, K., Kunugi, T., et al. (2017). "S-net project: construction of large scale seafloor observatory network for tsunamis and earthquakes in Japan," in Proceedings of the AGU Fall Meeting 2017, (New Orleans).

Morrison, J. M., Codispoti, L. A., Smith, S. L., Wishner, K., Flagg, C., Gardner, W. D., et al. (1999). The oxygen minimum zone in the Arabian Sea during 1995. Deep Sea Res. Part Top. Stud. Oceanogr. 46, 1903-1931.
Nagamani, P. V., Ali, M. M., Goni, G. J., Pedro, D. N., Pezzullo, J. C., Udaya Bhaskar, T. V., et al. (2012). Validation of satellite-derived tropical cyclone heat potential with in situ observations in the North Indian Ocean. Remote Sens. Lett. 3, 615-620. doi: 10.1080/01431161.2011.640959

Narazaki, T., Sato, K., and Miyazaki, N. (2015). Summer migration to temperate foraging habitats and active winter diving of juvenile loggerhead turtles Caretta caretta in the western North Pacific. Mar. Biol. 162, 1251-1263. doi: 10.1007/ s00227-015-2666-0

Neetu, S., Lengaigne, M., Vincent, E. M., Vialard, J., Madec, G., Samson, G., et al. (2012). Influence of upper-ocean stratification on tropical cyclone-induced surface cooling in the Bay of Bengal. J. Geophys. Res. Oceans 117:C12020. doi: 10.1029/2012JC008433

Nishida, K., and Takagi, R. (2016). Teleseismic S wave microseisms. Science 353, 919-921. doi: 10.1126/science.aaf7573

Perry, R. L., Leung, P. T., Bouchard, R., Petraitis, D., Smith, W. C., Sharma, N., et al. (2017). "The continuation of an ocean observing collaboration to improve hurricane and loop current forecasting and modeling in the Gulf of Mexico," in Proceedings of the OCEANS 2017, (Anchorage), 1-7.

Pickard, G. L., and Emery, W. J. (1990). Descriptive Physical Oceanography. New York, NY: Academic Press.

Pinto, J. G., Zacharias, S., Fink, A. H., Leckebusch, G. C., and Ulbrich, U. (2009). Factors contributing to the development of extreme North Atlantic cyclones and their relationship with the NAO. Climate Dyn. 32, 711-737. doi: 10.1007/ s00382-008-0396-4

Price, J. F. (2009). Metrics of hurricane-ocean interaction: vertically-integrated or vertically-averaged ocean temperature? Ocean Sci. 5, 351-368. doi: 10.5194/os5-351-2009

Price, J. F., Sanford, T. B., and Forristall, G. Z. (1994). Forced stage response to a moving hurricane. J. Phys. Oceanogr. 24, 233-260. doi: 10.1175/15200485(1994)024<0233:FSRTAM>2.0.CO;2

Riser, S. C., Freeland, H. J., Roemmich, D., Wijffels, S., Troisi, A., Belbéoch, M., et al. (2016). Fifteen years of ocean observations with the global Argo array. Nat. Clim. Change 6, 145-153. doi: 10.1038/nclimate2872

Roemmich, D., Alford, M. H., Claustre, H., Johnson, K. S., King, B., Moum, J., et al. (2019). On the future of Argo: a global, full-depth, multi-disciplinary array. Front. Mar. Sci. 6:439. doi: 10.3389/fmars.2019.00439

Rogers, R. F., Aberson, S., Bell, M. M., Cecil, D. J., Doyle, J. D., Kimberlain, T. B., et al. (2017). Rewriting the tropical record books: the extraordinary intensification of Hurricane Patricia (2015). Bull. Am. Meteorol. Soc. 98, 20912112. doi: 10.1175/BAMS-D-16-0039.1

Rudnick, D. L. (2016). Ocean Research Enabled by Underwater Gliders. Annu. Rev. Mar. Sci. 8, 519-541. doi: 10.1146/annurev-marine-122414-033913

Rudzin, J. E., Shay, L. K., Jaimes, B., and Brewster, J. K. (2017). Upper ocean observations in eastern Caribbean Sea reveal barrier layer within a warm core eddy. J. Geophys. Res. Oceans 122, 1057-1071. doi: 10.1002/2016JC01 2339

Rudzin, J. E., Shay, L. K., and Johns, W. E. (2018). The influence of the barrier layer on SST response during tropical cyclone wind forcing using idealized experiments. J. Phys. Oceanogr. 48, 1471-1478. doi: 10.1175/JPO-D-17-0279.1

Sanabia, E. R., Barrett, B. S., Black, P. G., Chen, S., and Cummings, J. A. (2013). Real-time upper-ocean temperature observations from aircraft during operational hurricane reconnaissance missions: AXBT demonstration project year one results. Weather Forecast. 28, 1404-1422. doi: 10.1175/WAF-D-1200107.1

Sanders, F., and Gyakum, J. R. (1980). Synoptic-dynamic climatology of the "bomb. 108, 1589-1606. doi: 10.1175/1520-0493(1980)108<1589:sdcot $>2.0 . c 0 ; 2$

Sanford, T. B., Black, P. G., Haustein, J. R., Feeney, J. W., Forristall, G. Z., and Price, J. F. (1987). Ocean response to a hurricane. Part I: observations. J. Phys. Oceanogr. 17, 2065-2083. doi: 10.1371/journal.pone.0153735

Sanford, T. B., Price, J. F., and Girton, J. B. (2011). Upper-ocean response to Hurricane Frances (2004) observed by profiling EM-APEX floats. J. Phys. Oceanogr. 41, 1041-1056. doi: 10.1175/2010JPO4313.1

Sanford, T. B., Price, J. F., Girton, J. B., and Webb, D. C. (2007). Highly resolved observations and simulations of the ocean response to a hurricane. Geophys. Res. Lett. 34:L13604. doi: 10.1029/2007GL029679

Sengupta, D., Goddalehundi, B. R., and Anitha, D. S. (2008). Cyclone-induced mixing does not cool SST in the post-monsoon North Bay of Bengal. Atmos. Sci. Lett. 9, 1-6. doi: 10.1002/asl.162 
Seroka, G., Miles, T., Xu, Y., Kohut, J., Schofield, O., and Glenn, S. (2016). Hurricane Irene sensitivity to stratified coastal ocean cooling. Mon. Weather Rev. 144, 3507-3530. doi: 10.1175/MWR-D-15-0452.1

Shapiro, M., Wernli, H., Bao, J.-W., Methven, J., Zou, X., Doyle, J., et al. (1999). "A planetary-scale to mesoscale perspective of the life cycles of extratropical cyclones: the bridge between theory and observations," in The Life Cycles of Extratropical Cyclones, eds M. A. Shapiro and S. Grønås (Boston, MA: American Meteorological Society), 139-185. doi: 10.1007/978-1-935704-09-6_14

Shay, L. K., Ali, M. M., Chen, S., Ginis, I., Halliwell, G., Kim, H., et al. (2014). Air-sea Interface and Oceanic Influences (Topic 4.4)", Jeju, Republic of Korea. Geneva: World Meteorological Organization.

Shay, L. K., Black, P. G., Mariano, A. J., Hawkins, J. D., and Elsberry, R. L. (1992). Upper ocean response to Hurricane Gilbert. J. Geophys. Res. Oceans 97, 20227-20248. doi: 10.1029/92JC01586

Shay, L. K., and Brewster, J. K. (2010). Oceanic heat content variability in the eastern Pacific Ocean for hurricane intensity forecasting. Mon. Weather Rev. 138, 2110-2131. doi: 10.1175/2010MWR3189.1

Shay, L. K., Goni, G. J., and Black, P. G. (2000). Effects of a warm oceanic feature on Hurricane Opal. Mon. Weather Rev. 128, 1366-1383. doi: 10.1175/15200493(2000) $128<1366$ :eoawof $>2.0$. co; 2

Smith, E. R., Kessel, W., Cravatte, S., Sprintall, S., Cronin, S., Sutton, M., et al. (2019). Tropical Pacific observing system. Front. Mar. Sci. 6:31. doi: 10.3389/ fmars.2019.00031

Sprintall, J., and Tomczak, M. (1992). Evidence of the barrier layer in the surface layer of the tropics. J. Geophys. Res. Oceans 97, 7305-7316. doi: 10.1029/ 92JC00407

St Laurent, L., and Merrifield, S. (2017). Measurements of near-surface turbulence and mixing from autonomous ocean gliders. Oceanography 30, 116-125. doi: 10.5670/oceanog.2017.231

Testor, P., DeYoung, B., Rudnick, D. L., Glenn, S., Hayes, D., Lee, C., et al. (2019). OceanGliders: a component of the integrated GOOS. Front. Mar. Sci. 6:422. doi: $10.3389 /$ fmars.2019.00422

Todd, R. E., Asher, T. G., Heiderich, J., Bane, J. M., and Luettich, R. A. (2018). Transient response of the Gulf Stream to multiple hurricanes in 2017. Geophys. Res. Lett. 10:519. doi: 10.1029/2018GL079180

Todd, R. E., Chavez, F. P., Clayton, S., CRAVATTE, S. E., Goes, M. P., Graco, M. I., et al. (2019). Global Perspectives on Observing Ocean Boundary Current Systems. Front. Mar. Sci. 6:423. doi: 10.3389/fmars.2019.00423

Todd, R. E., Rudnick, D. L., Sherman, J. T., Owens, W. B., and George, L. (2017). Absolute velocity estimates from autonomous underwater gliders equipped with Doppler current profilers. J. Atmospheric Ocean. Technol. 34, 309-333. doi: 10.1175/JTECH-D-16-0156.1

Trenberth, K. E., Cheng, L., Jacobs, P., Zhang, Y., and Fasullo, J. (2018). Hurricane harvey links to ocean heat content and climate change adaptation. Earths Future 6, 730-744. doi: 10.1029/2018EF000825

Ulbrich, U., Leckebusch, G. C., and Pinto, J. G. (2009). Extra-tropical cyclones in the present and future climate: a review. Theor. Appl. Climatol. 96, 117-131. doi: 10.1007/s00704-008-0083-8

Venugopal, T., Ali, M. M., Bourassa, M. A., Zheng, Y., Goni, G. J., Foltz, G. R., et al. (2018). Statistical evidence for the role of southwestern Indian Ocean heat content in the Indian summer monsoon rainfall. Sci. Rep. 8:12092. doi: 10.1038/s41598-018-30552-0

Verrier, S., Le Traon, P. Y., Remy, E., and Lellouche, J. M. (2018). Assessing the impact of SAR altimetry for global ocean analysis and forecasting. J. Oper. Oceanogr. 11, 82-86. doi: 10.1080/1755876x.2018.1505028

Wada, A., Kunii, M., Yonehara, Y., and Sato, K. (2017). Impacts on local heavy rainfalls of surface winds measurement by seabirds. CAS/JSC WGNE Res. Activities Atm. And. Oceanic Model. 47, 1-25.

Wang, X., Han, G., Qi, Y., and Li, W. (2011). Impact of barrier layer on typhooninduced sea surface cooling. Dyn. Atmospheres Oceans 52, 367-385. doi: 10. 1016/j.dynatmoce.2011.05.002

Warner, S. J., Becherer, J., Pujiana, K., Shroyer, E. L., Ravichandran, M., Thangaprakash, V. P., et al. (2016). Monsoon mixing cycles in the Bay of Bengal: a year-long subsurface mixing record. Oceanography 29, 158-169. doi: 10.5670/oceanog. 2016.48

Watanuki, Y., Mehlum, F., and Takahashi, A. (2001). Water temperature sampling by foraging Brünnich's Guillemots with bird-borne data loggers. J. Avian Biol. 32, 189-193. doi: 10.1034/j.1600-048X.2001.320214.x
Wilson, R. P., Grémillet, D., Syder, J., Kierspel, M. A. M., Garthe, S., Weimerskirch, H., et al. (2002). Remote-sensing systems and seabirds: their use, abuse and potential for measuring marine environmental variables. Mar. Ecol. Prog. Ser. 228, 241-261. doi: 10.3354/meps228241

World Meteorological Organization (WMO) (2015a). Manual on Codes (WMO 306). Geneva: World Meteorological Organization.

World Meteorological Organization (WMO) (2015b). Manual on the Global Telecommunication System (WMO 386). Geneva: World Meteorological Organization.

Wu, L., Cai, W., Zhang, L., Nakamura, H., Timmermann, A., Joyce, T., et al. (2012). Enhanced warming over the global subtropical western boundary currents. Nat. Clim. Change 2, 161-166. doi: 10.1038/nclimate1353

Yamaguchi, M., Owada, H., Shimada, U., Sawada, M., Iriguchi, T., Musgrave, K. D., et al. (2018). Tropical cyclone intensity prediction in the western North Pacific basin using SHIPS and JMA/GSM. SOLA 14, 138-143. doi: 10.2151/sola. 2018-024

Yoda, K., Shiomi, K., and Sato, K. (2014). Foraging spots of streaked shearwaters in relation to ocean surface currents as identified using their drift movements. Prog. Oceanogr. 122, 54-64. doi: 10.1016/j.pocean.2013.12.002

Yonehara, Y., Goto, Y., Yoda, K., Watanuki, Y., Young, L. C., Weimerskirch, H., et al. (2016). Flight paths of seabirds soaring over the ocean surface enable measurement of fine-scale wind speed and direction. Proc. Natl. Acad. Sci. U.S.A. 113, 9039-9044. doi: 10.1073/pnas.1523853113

Yoshida, A., and Asuma, Y. (2004). Structures and environment of explosively developing extratropical cyclones in the northwestern Pacific region. Mon. Weather Rev. 132, 1121-1142. doi: 10.1175/1520-0493(2004)132<1121: saeoed $>2.0$. co; 2

Young, M. V., Monk, G. A., and Browning, K. A. (1987). Interpretation of satellite imagery of a rapidly deepening cyclone. Q. J. R. Meteorol. Soc. 113, 1089-1115. doi: $10.1002 /$ qj. 49711347803

Yu, L., and McPhaden, M. J. (2011). Ocean preconditioning of Cyclone Nargis in the Bay of Bengal: interaction between Rossby waves, surface fresh waters, and sea surface temperatures. J. Phys. Oceanogr. 41, 1741-1755. doi: 10.1175/ 2011jpo4437.1

Zhang, H.-M., Reynolds, R. W., Lumpkin, R., Molinari, R., Arzayus, K., Johnson, M., et al. (2009). An integrated global observing system for sea surface temperature using satellites and in-situ data: research to operations. Bull. Am. Meteorol. Soc. 90, 31-38. doi: 10.1175/2008BAMS2577.1

Zhang, J. A., Atlas, R., Emmitt, G. D., Bucci, L., and Ryan, K. (2018). Airborne Doppler wind lidar observations of the tropical cyclone boundary layer. Remote Sens. 10:825. doi: 10.3390/rs10060825

Zhang, J. A., Black, P. G., French, J. R., and Drennan, W. M. (2008). First direct measurements of enthalpy flux in the hurricane boundary layer: the CBLAST results. Geophys. Res. Lett. 35:L14813. doi: 10.1029/2008GL034374

Zhang, J. A., Nolan, D. S., Rogers, R. F., and Tallapragada, V. (2015). Evaluating the impact of improvements in the boundary layer parameterization on hurricane intensity and structure forecasts in HWRF. Mon. Weather Rev. 143, 3136-3155. doi: 10.1175/MWR-D-14-00339.1

Zhou, L., Chen, D., Karnauskas, K. B., Wang, C., Lei, X., Wang, W., et al. (2018). Introduction to special section on oceanic responses and feedbacks to tropical cyclones. J. Geophys. Res. Oceans 123, 742-745. doi: 10.1002/2018JC013809

Conflict of Interest Statement: H-SK was employed by company I.M. Systems Group, Inc.

The remaining authors declare that the research was conducted in the absence of any commercial or financial relationships that could be construed as a potential conflict of interest.

Copyright (C) 2019 Domingues, Kuwano-Yoshida, Chardon-Maldonado, Todd, Halliwell, Kim, Lin, Sato, Narazaki, Shay, Miles, Glenn, Zhang, Jayne, Centurioni, Le Hénaff, Foltz, Bringas, Ali, DiMarco, Hosoda, Fukuoka, LaCour, Mehra, Sanabia, Gyakum, Dong, Knaff and Goni. This is an open-access article distributed under the terms of the Creative Commons Attribution License (CC BY). The use, distribution or reproduction in other forums is permitted, provided the original author(s) and the copyright owner(s) are credited and that the original publication in this journal is cited, in accordance with accepted academic practice. No use, distribution or reproduction is permitted which does not comply with these terms. 


\section{Observing System Evaluation Based on Ocean Data Assimilation and Prediction Systems: On-Going Challenges and a Future Vision for Designing and Supporting Ocean Observational Networks}

OPEN ACCESS

Edited by:

Laura Lorenzoni,

University of South Florida,

United States

Reviewed by:

Zhijin Li,

NASA Jet Propulsion Laboratory

(JPL), United States

Nadya Vinogradova Shiffer,

National Aeronautics and Space Administration (NASA), United States

*Correspondence: Yosuke Fuji

yfujii@mri-jma.go.jp

Specialty section:

This article was submitted to

Ocean Observation,

a section of the journal

Frontiers in Marine Science

Received: 09 November 2018

Accepted: 05 July 2019

Published: 29 July 2019

Citation:

Fujii Y, Rémy E, Zuo H, Oke P, Halliwell G, Gasparin F, Benkiran M, Loose N, Cummings J, Xie J, Xue Y, Masuda S, Smith GC, Balmaseda M, Germineaud C, Lea DJ, Larnicol G, Bertino L, Bonaduce A, Brasseur $P$

Donlon C, Heimbach P, Kim Y, Kourafalou V, Le Traon P-Y, Martin M,

Paturi S, Tranchant B and Usui N (2019) Observing System Evaluation Based on Ocean Data Assimilation and Prediction Systems: On-Going Challenges and a Future Vision for Designing and Supporting Ocean

Observational Networks.

Front. Mar. Sci. 6:417.

doi: 10.3389/fmars.2019.00417
Yosuke Fujii ${ }^{1 *}$, Elisabeth Rémy ${ }^{2}$, Hao Zuo ${ }^{3}$, Peter Oke ${ }^{4}$, George Halliwell ${ }^{5}$, Florent Gasparin ${ }^{2}$, Mounir Benkiran ${ }^{2}$, Nora Loose ${ }^{6,7}$, James Cummings ${ }^{8}$, Jiping Xie ${ }^{9}$, Yan Xue ${ }^{10}$, Shuhei Masuda ${ }^{11}$, Gregory C. Smith ${ }^{12}$, Magdalena Balmaseda ${ }^{3}$, Cyril Germineaud ${ }^{5,13}$, Daniel J. Lea ${ }^{14}$, Gilles Larnicol ${ }^{15}$, Laurent Bertino ${ }^{9}$, Antonio Bonaduce ${ }^{16}$, Pierre Brasseur ${ }^{17}$, Craig Donlon ${ }^{18}$, Patrick Heimbach ${ }^{6}$, YoungHo Kim ${ }^{19}$, Villy Kourafalou ${ }^{20}$, Pierre-Yves Le Traon ${ }^{2}$, Matthew Martin ${ }^{14}$, Shastri Paturi ${ }^{8}$, Benoit Tranchant ${ }^{15}$ and Norihisa Usui ${ }^{1}$

${ }^{1}$ Meteorological Research Institute, Japan Meteorological Agency (JMA), Tsukuba, Japan, ${ }^{2}$ Mercator Ocean International, Ramonville-Saint-Agne, France, ${ }^{3}$ European Centre for Medium-Range Weather Forecasts (ECMWF), Reading,

United Kingdom, ${ }^{4}$ Commonwealth Scientific and Industrial Research Organisation Oceans and Atmosphere, Hobart, TAS, Australia, ${ }^{5}$ National Oceanic and Atmospheric Administration, Atlantic Oceanographic and Meteorological Laboratory, Physical Oceanography Division, Miami, FL, United States, ${ }^{6}$ Oden Institute for Computational Engineering and Sciences, Jackson School of Geosciences, The University of Texas at Austin, Austin, TX. United States, ${ }^{7}$ Department of Earth Science, University of Bergen, Bergen, Norway, ${ }^{8}$ I.M Systems Group, NCEP Environmental Modeling Center, College Park, MD, United States, ${ }^{9}$ Nansen Environmental and Remote Sensing Center (NERSC), Bergen, Norway, ${ }^{10}$ Climate Prediction Center, National Oceanic and Atmospheric Administration, National Weather Service, National Centers for Environmental Prediction, College Park, MD, United States, ${ }^{11}$ Research Institute for Global Change, Japan Agency for Marine-Earth Science and Technology (JAMSTEC), Yokosuka, Japan, ${ }^{12}$ Environmental Numerical Prediction Research Section, Meteorological Research Division, Environment and Climate Change Canada, Dorval, QC, Canada, ${ }^{13}$ Cooperative Institute for Marine and Atmospheric Studies, University of Miami, Miami, FL, United States, ${ }^{14}$ Met Office, Exeter, United Kingdom, ${ }^{15}$ Collecte Localisation Satellites (CLS), Ramonville-St-Agne, France, ${ }^{16} \mathrm{Helmholtz-Zentrum} \mathrm{Geesthacht} \mathrm{(HZG),} \mathrm{Centre} \mathrm{for} \mathrm{Materials} \mathrm{and}$ Coastal Research, Geesthacht, Germany, ${ }^{17}$ Université Grenoble Alpes, CNRS, IRD, Grenoble INP, IGE, Grenoble, France,

${ }^{18}$ European Space Agency (ESA), European Space Research and Technology Centre, Noordwijk, Netherlands, ${ }^{19}$ Ocean Circulation and Climate Research Center, Korea Institute of Ocean Science and Technology, Busan, South Korea,

${ }^{20}$ Department of Ocean Sciences, University of Miami, Miami, FL, United States

This paper summarizes recent efforts on Observing System Evaluation (OS-Eval) by the Ocean Data Assimilation and Prediction (ODAP) communities such as GODAE OceanView and CLIVAR-GSOP. It provides some examples of existing OS-Eval methodologies, and attempts to discuss the potential and limitation of the existing approaches. Observing System Experiment (OSE) studies illustrate the impacts of the severe decrease in the number of TAO buoys during 2012-2014 and TRITON buoys since 2013 on ODAP system performance. Multi-system evaluation of the impacts of assimilating satellite sea surface salinity data based on OSEs has been performed to demonstrate the need to continue and enhance satellite salinity missions. Impacts of underwater gliders have been assessed using Observing System Simulation Experiments (OSSEs) to provide guidance on the effective coordination of the western North Atlantic observing system elements. OSSEs are also being performed under H2O2O AtlantOS 
project with the goal to enhance and optimize the Atlantic in-situ networks. Potential of future satellite missions of wide-swath altimetry and surface ocean currents monitoring is explored through OSSEs and evaluation of Degrees of Freedom for Signal (DFS). Forecast Sensitivity Observation Impacts (FSOI) are routinely evaluated for monitoring the ocean observation impacts in the US Navy's ODAP system. Perspectives on the extension of OS-Eval to coastal regions, the deep ocean, polar regions, coupled data assimilation, and biogeochemical applications are also presented. Based on the examples above, we identify the limitations of OS-Eval, indicating that the most significant limitation is reduction of robustness and reliability of the results due to their system-dependency. The difficulty of performing evaluation in near real time is also critical. A strategy to mitigate the limitation and to strengthen the impact of evaluations is discussed. In particular, we emphasize the importance of collaboration within the ODAP community for multi-system evaluation and of communication with ocean observational communities on the design of OS-Eval, required resources, and effective distribution of the results. Finally, we recommend further developing OS-Eval activities at international level with the support of the international ODAP (e.g., OceanPredict and CLIVAR-GSOP) and observational communities.

Keywords: observing system evaluation, ocean data assimilation, ocean prediction, OSSE (observing system simulation experiment), OSE (observing system experiment), GODAE OceanView, OceanPredict, CLIVAR-GSOP

\section{INTRODUCTION}

Ocean Data Assimilation and Prediction (ODAP; see Table 1 for summary of essential acronyms) systems, which include ocean reanalysis systems for seasonal forecasting and longterm ocean state estimation systems, are used in a large range of oceanic applications and weather and climate forecasting services as an essential tool for integrating ocean observations and numerical forecasting models (e.g., Davidson et al., 2009; Brassington et al., 2015; Le Traon et al., 2017). The Global Ocean Observing System (GOOS) and Regional Ocean Observing Systems (ROOS) largely depend on ODAP systems for delivering analysis products used to promote safety (e.g., search and rescue or identifying periods of high risk to oil and gas operators) and efficiency (e.g., seasonal prediction to support agriculture or optimal ship-routing) at sea, and societal well-being (e.g., monitoring of our changing climate and protection of marine resources). Therefore, evaluating impacts of ocean observation networks on ODAP products in a scientific way provides important feedback to agencies responsible for maintaining and enhancing the GOOS/ROOS. Observing System Evaluation (OS-Eval) is invaluable to assess the effectiveness in ODAP systems of a novel observation type and to determine optimal network design when planning a new observing system or reorganizing existing observing networks (e.g., Fujii et al., 2015a; Oke et al., 2015a,b). In this context, several efforts of evaluating the GOOS/ROOS using ODAP systems have been made and some are ongoing under the support of ODAP communities such as GODAE OceanView (GOV; Bell et al., 2015) and CLIVAR Global Synthesis and Observations Panel (CLIVAR-GSOP, Caltabiano et al., 2015). In particular, GOV formed the OS-Eval Task Team (TT) in order to promote international collaboration and information sharing on these efforts.

There are several methods of OS-Eval based on ODAP systems that are proposed or have been used before. In the Observing System Simulation Experiment (OSSE) framework, synthetic observation data, intended to mimic observations from the proposed observing system, are generated from a freerunning simulation run of an ocean model that is intended to represent the "true" ocean (and is thus called the "Nature Run"), with observation errors added based on information from the observing community (e.g., Hoffman and Atlas, 2015). The synthetic data are assimilated into a different data-assimilative simulation run, called an OSSE, in which different initial/forcing conditions are used. It is also preferable to change the model sufficiently from the Nature Run, either due to reduced resolution or differences in physical parameterizations. The impact of the synthetic data on forecast improvement is assessed from the error reduction in OSSEs when assimilating the new data. In this framework, the errors on the full model space can be directly estimated by taking difference of variables between the Nature Run and OSSE fields. An important aspect of OSSEs is that they can be used for evaluating both existing and future observation data types (e.g., Halliwell et al., 2017).

To evaluate existing observations, Observing System Experiments (OSEs) may be performed. An OSE is a data assimilative run in which a certain observation-type is withheld from, or added to, the regularly assimilated data. The impact of these withheld/added data are assessed by comparing the OSE with the control simulation in which only the regular data are assimilated. Although the methodology of OSEs is simple, it is widely applied due to its applicability to any ODAP system including highly non-linear or stochastic systems, and ability to 
TABLE 1 | Essential acronyms used in this article.

\begin{tabular}{ll}
\hline ACC & Anomaly Correlation Coefficient \\
BGC & Biogeochemical \\
CLIVAR-GSOP & CLIVAR Global Synthesis and observations Panel \\
DFS & Degree of Freedom for Signal \\
ECCO & Estimating the Circulation and Climate of the Ocean \\
EnKF & Ensemble Kalman Filter \\
FSOI & Forecast Sensitivity Observation Impacts \\
GOOS & Global Ocean Observing System \\
GOV & GODAE OceanView \\
HYCOM & HYbrid Coordinate Ocean Model \\
MJO & Madden-Julian Oscillation \\
NRT-OSE & Near-Real-Time OSE \\
ODAP & Ocean Data Assimilation and Prediction \\
OGCM & Ocean General Circulation Model \\
OIS & Observation Impact Statement \\
OSE & Observing System Experiment \\
OS-Eval & Observing System Evaluation \\
OSSE & Observing System Simulation Experiment \\
RMS & Root Mean Square \\
ROOS & Regional Ocean Observing Systems \\
SSH & Sea Surface Height \\
SSS & Sea Surface Salinity \\
SST & Sea Surface Temperature \\
S2S & Subseasonal-to-Seasonal \\
TPOS & Tropical Pacific Observing System \\
TT & Task Team \\
3DVAR & Three-Dimensional Variational \\
$4 D V A R$ & Four-Dimensional Variational \\
&
\end{tabular}

directly give the differences caused by assimilation of targeted data (e.g., Oke and Schiller, 2007; Turpin et al., 2016; Xue et al., 2017b). In the OSE framework, reduction of errors cannot be estimated accurately because the true state is unknown. Instead, errors are evaluated using reference data, which are preferably independent from the data assimilation process performed in advance in the OSEs in order to perform fair evaluation (Fujii et al., 2015b).

Adjoint based sensitivity methods are also very applicable for OS-Eval. In particular, Langland and Baker (2004) proposed a technique to compute the variation in forecast error due to the assimilated data using an adjoint model, which is often called Forecast Sensitivity Observation Impact (FSOI) and widely used for monitoring observation impacts in weather centers. Meanwhile, Degrees of Freedom for Signal (DFS) are used to indicate how effectively signals which can potentially improve analysis accuracy are observed (e.g., Cardinali et al., 2004). In an Ensemble Kalman Filter (EnKF) system, FSOI, and DFS are evaluated based on statistical information of ensemble members. In addition, the analysis uncertainty (i.e., error variance) in an ODAP system can also be exploited for OS-Eval because the impact of assimilating specific observations can be measured by the uncertainty reduction. The uncertainty is estimated from the inverse Hessian matrix of the cost function in a 4-dimensional Variational (4DVAR) system (Thacker, 1989), while it is approximated by the variance among ensemble members in an EnKF system (Evensen, 2003). These methods are very powerful and allow the impact of all observations to be assessed at once. The main downside of the methods is that make the assumption of linearity. Effective application of these methodologies to oceanic OS-Eval is one of the on-going challenges in ODAP communities.

This paper introduces recent OS-Eval results shared in GOV OS-Eval TT and CLIVAR-GSOP. We then discuss the value of current OS-Eval methods and the future strategy toward more comprehensive and reliable evaluation. The effective use of OS-Eval in support of observing system review and design is also discussed.

\section{OBSERVING SYSTEM EXPERIMENTS (OSEs)}

In OSEs the impact of an existing observing system or observations is tested by adding or removing it from a data assimilating system. See section Introduction for more details.

\section{OSE Studies for Evaluation of TAO/TRITON Array}

The TAO/TRITON array provides subsurface temperature and salinity data in the equatorial Pacific, which was vital for an ocean data assimilation system to provide oceanic initial condition for coupled atmosphere-ocean models in ENSO forecasts before the Argo era (McPhaden et al., 1998). However, the rapid increase in the number of Argo floats in 2000s was presumed to reduce the importance of the TAO/TRITON array as the primary provider of tropical Pacific subsurface data, which in view of the costs of maintaining the array make it crucial to reassess the importance of TAO/TRITON data in seasonal predictions.

ECMWF $^{1}$ evaluated the impacts of TAO/TRITON, as well as Argo and satellite altimetry data, in their operational seasonal forecasting system through OSEs, and find complementarity among those data in late 2000s (Balmaseda et al., 2007; Balmaseda and Anderson, 2009). Fujii et al. (2011) carried out a similar OSE study to evaluate the impacts of TAO/TRITON data on the ENSO forecasts using the JMA ${ }^{2}$ 's seasonal forecasting system at the request of JAMSTEC ${ }^{3}$, the agency deploying TRITON buoys as a part of the array west of $165^{\circ} \mathrm{E}$. These results were shared in the GOV OS-Eval TT (Oke et al., 2009), and presented in OceanObs'09 (Balmaseda et al., 2010).

In spite of these efforts, the data received from the TAO buoys deployed by $\mathrm{NOAA}^{4}$ as a part of the TAO/TRITON array east of $165^{\circ} \mathrm{E}$ was severely reduced in the period of 2012-2014 (Tollefson, 2014). In addition, the number of the TRITON buoys has also started to reduce since 2013. The influence of the reduction of subsurface temperature and salinity data in the equatorial Pacific was discussed in the Tropical

\footnotetext{
${ }^{1}$ European Centre for Medium-Range Weather Forecasts.

${ }^{2}$ Japan Meteorological Agency.

${ }^{3}$ Japan Agency for Marine-Earth Science and Technology.

${ }^{4}$ National Oceanic and Atmospheric Administration.
} 
Pacific Observing System (TPOS) workshop in 2014 [hereafter TPOS2014 workshop; Ocean Observations Panel for Climate (OOPC) (2014)]. OSE studies in ECMWF and JMA introduced above and new OSE studies in $\mathrm{NCEP}^{5}$ (Xue et al., 2017b) were summarized in order to highlight the importance of the array for seasonal forecasting (Fujii et al., 2015a). Consequently, the workshop recommended the scientists to make a proposal of reorganizing the TPOS, which includes buoys, Argo, other insitu measurements and satellite data, in a more efficient and sustainable way by launching the TPOS2020 project (Smith G. C. et al., 2019).

During the TPOS2014 workshop, inconsistency among the impacts of the subsurface data was recognized in OSE results. For example, the results in JMA (Figure 1) indicates that removal of only near-equatorial buoys causes larger decrease of Anomaly Correlation Coefficients (ACCs) compared to the removal of all TAO/TRITON buoys in the forecast Sea Surface Temperature (SST) averaged in the NINO3 region for 9-12 months leadtime and in the NINO4 region for 5-12 months lead-time although Argo and TAO/TRITON data have positive impacts in all cases. The participants considered that these inconsistencies were mostly caused by large systematic errors, or biases, of coupled models, which prevented the systems from extracting the full potential impact of the observation data. The dependency on systematic errors is recognized as a general limitation of OSE studies.

Another problem found in the workshop was that OSEs do not fully evaluate the value of all observation data. It cannot evaluate the value of data which are not assimilated in the system. Therefore, near-surface atmospheric data observed by moorings are not evaluated although they are vital for an ODAP system for seasonal predictions because those data can effectively constrain the atmospheric forcing which is crucial to determine the oceanic interior state. Even the value of temperature and salinity data are not fully evaluated because these data are not only assimilated, but also used for the determination of prescribed error statistics and biases which are indispensable for data assimilation.

In addition, our inability to evaluate the impacts of temporal changes of the observing system in a timely way was also revealed in the workshop (see section Near-Real-Time Evaluation). This motivates work to start routine near real time intercomparison of operational ODAP products (section Use of Multi-system Ensemble Spread).

\section{OSEs for Evaluating Satellite SSS Data Impacts During the El Nino 2015 Event}

Monitoring Sea Surface Salinity (SSS) is crucial for understanding and forecasting the ocean circulation, water cycle and the changing of the climate. The recent satellite missions of $\mathrm{ESA}^{6}$ 's SMOS, NASA ${ }^{7}$ 's Aquarius, and SMAP missions have made it possible for the first time to measure SSS from space, and it is important to assess the impact of these measurements in order to justify follow-on missions. However, the assimilation

\footnotetext{
${ }^{5}$ National Centers for Environmental Prediction.

${ }^{6}$ European Space Agency.

${ }^{7}$ National Aeronautics and Space Administration.
}

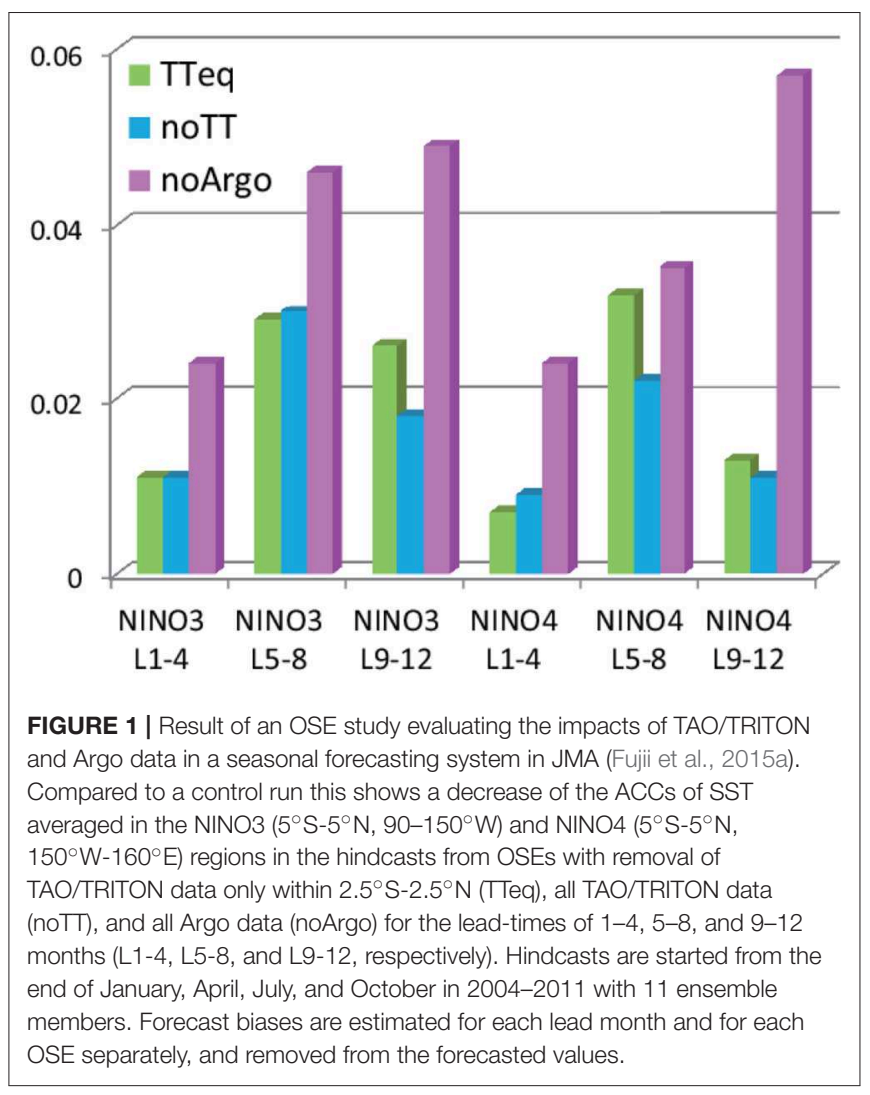

of satellite SSS observations is still challenging because of the various and complex biases that affect them. The difference between the forecast and the satellite SSS can be 5 times larger than the misfit between the forecast and near surface Argo salinity. Nevertheless, several studies (Lee et al., 2012; Reul et al., 2013; Toyoda et al., 2015) show that SSS measured from space can bring new information.

In the framework of the SMOS-Nino15 ESA project ${ }^{8}$, OSEs were designed to explore the potential impact of SMOS, Aquarius and SMAP SSS data assimilation using the ODAP systems of $\mathrm{UKMO}^{9}$ and Mercator Ocean. The project was expected to produce an Observation Impact Statement (OIS; see section Community Collaborations) based on the OSE activities for the GOV OS-Eval TT. The 2015/16 ENSO event has been chosen as a case study for this project. The ODAP systems are based on the same Ocean General Circulation Model (OGCM) (NEMO; Madec, 2016) but with different settings and atmospheric forcing fields. The data assimilation techniques are also quite different; Mercator Ocean uses a reduced order, localized Kalman Filter approach with a 1-week assimilation window and UKMO uses a 3-dimensional Variational (3DVAR) approach with a 1day assimilation window. Assimilated data sets in the control simulation are along-track satellite Sea Surface Height (SSH), SST, and in-situ temperature and salinity profiles. The SSS data ${ }^{8}$ See https://www.godae-oceanview.org/projects/smos-nino15
${ }^{9}$ UK Met Office. 
assimilation is restricted to the $40^{\circ} \mathrm{S}-40^{\circ} \mathrm{N}$ area, due to increasing uncertainty in the salinity observations at higher latitudes. For both systems, specific bias correction schemes have been adapted to estimate the bias for the different SSS datasets. Performing OSEs with different ODAP systems and data sources allow for more robust conclusions to be made on the sensitivity of ocean estimates to SSS data assimilation.

The success of assimilating SSS data was confirmed by the reduction seen of the Root Mean Square (RMS) differences between SSS observations and model forecast (Figure 2). In both systems, the differences are around or $>0.2$ PSU when assimilating SSS data (Martin et al., 2018; Tranchant et al., 2019). Changes due to SSS data assimilation are mainly confined to the first $50 \mathrm{~m}$ of the water column. Other variables, such as the temperature, velocity and sea level fields are also modified. Looking at the innovation (observation-minus-background differences) statistics for in-situ and altimetry confirms that information brought by the assimilation of SSS data is consistent with that from already assimilated data sets.

These results demonstrate that the ocean forecasting systems are ready to assimilate SSS data, without introducing incoherent information compared to the other assimilated observations. Note to achieve this result an observation bias correction scheme is still needed within the assimilation process, even if a "debiased" SSS data product is used. The RMS differences between model forecasts and observations are, in the Tropical Pacific region, around 0.2 PSU, which gives a quite stringent request on the accuracy of any new measurements.

\section{OSE Studies for Ocean Reanalysis and Seasonal Forecasting}

Adding new observation data in an ocean reanalysis should in principle never degrade the quality of analysis or forecast, assuming that the data are assimilated in a consistent way. However, in practice the exploitation of observations is challenging in ocean reanalysis and seasonal forecasts. The forecasts need balanced initial conditions consistent with the model dynamics and physics, so they are able to retain the observational information for longer than the typical assimilation window. In reanalysis, because it is important to retain temporal consistency, systems should be stable to changes in the observing system. OSEs are a practical way to validate consistency of a data assimilation system as well as a method for evaluating impacts of observation data.

For this reason, OSEs are routinely conducted at ECMWF. For example, Balmaseda et al. (2007) reported the impact of the Argo data in the climate of the ocean reanalysis simulated by a previous operational ocean reanalysis system (Balmaseda et al., 2008), showing the large impact in the estimation of salinity, especially in the Southern Ocean. An ensemble of OSEs withholding Argo data was exploited to verify that the increased heat uptake during the recent warming hiatus found in ocean reanalysis was robust to the changes in the observing system (Balmaseda et al., 2013b). OSEs were also conducted to assess the impact of XBT bias corrections and different SST products on the climate variability (Balmaseda et al., 2013a). Assessment of ocean observation impacts on seasonal forecasts is firstly conducted by Alves et al. (2004), which showed that assimilating observations improved the skill of the first operational seasonal forecasting system in ECMWF. Balmaseda and Anderson (2009) reported the impact of different observing systems in a later seasonal forecasting system. They reported that the individual observing systems were shown to improve the seasonal forecasts in most ocean basins, except in the tropical Atlantic, probably because of problems with the data assimilation system in that area.

A series of OSEs has been carried out using a low-resolution version of the latest ECMWF operational ocean reanalysis system OCEAN5 (Zuo et al., 2018). Since sea-level anomaly data were also assimilated in these OSEs, they are different from the OSEs carried out by Zuo et al. (2019). Maps of temperature RMS difference for the upper $700 \mathrm{~m}$ between the OSEs and the control experiment (Figure 3) suggest that removal of mooring data from TAO/TRITON, PIRATA, and RAMA arrays (NoMooring) mostly affects the tropical regions alone. Ship-based observations (XBT/MBT and CTD) show important contributions especially in the high-latitude where Argo floats are not often available (e.g., in the Arctic basin). Removal of Argo floats (NoArgo) degrades the ocean state almost everywhere except for the tropical Pacific and Indian Oceans, where the ocean state is well constrained by the assimilation of satellite SSH data (Zuo et al., 2017). Removal of all ocean in-situ observations (NoInsitu) gives an estimation about the total impact of the global in-situ data, which is not a simple linear combination of individual observation types. Note that in the Southern Ocean the RMS difference is sometimes larger in NoArgo than in NoInsitu, which indicates some inadequacy of the data assimilation process. Overall, the weak impact of removal of observations in the Indian Ocean is possibly related to the comparatively sparse observing system in that region. And the tropical Atlantic seems to be generally more sensitive to the removal of in-situ observations than the other tropical ocean basins. It would be useful to assess the robustness of these results by doing the same experiment with different ODAP systems in a multi-system approach (see section Multi-system Evaluation).

OSEs for assessing ocean observation impacts in seasonal forecasts are now being carried out with the current operational ocean reanalysis and coupled prediction systems in ECMWF. This time, the OSEs are planned to be also used for assessing impacts in sub-seasonal predictions (see section Medium-Range and S2S Coupled Prediction and Coupled Data Assimilation).

\section{OSE Study for Evaluation of Coastal Gliders in a High-Resolution Coastal Model}

OSEs are also used to evaluate impacts of coastal observation data/platforms on a high-resolution ocean model (e.g., Oke and Schiller, 2007; Aydogdu et al., 2016). An example of the impact of the assimilation of ocean glider data is presented in Figure 4. The ocean model used is configured based on the Sparse Hydrodynamic Ocean Code (Herzfeld, 2006) for south-eastern Tasmania (Figures 4D,E), and run for a period during April 2009. The model grid spacing ranges from $250 \mathrm{~m}$ in the estuary to $2.5 \mathrm{~km}$ near the open boundaries (Figure $4 \mathbf{F}$ ). In the control 


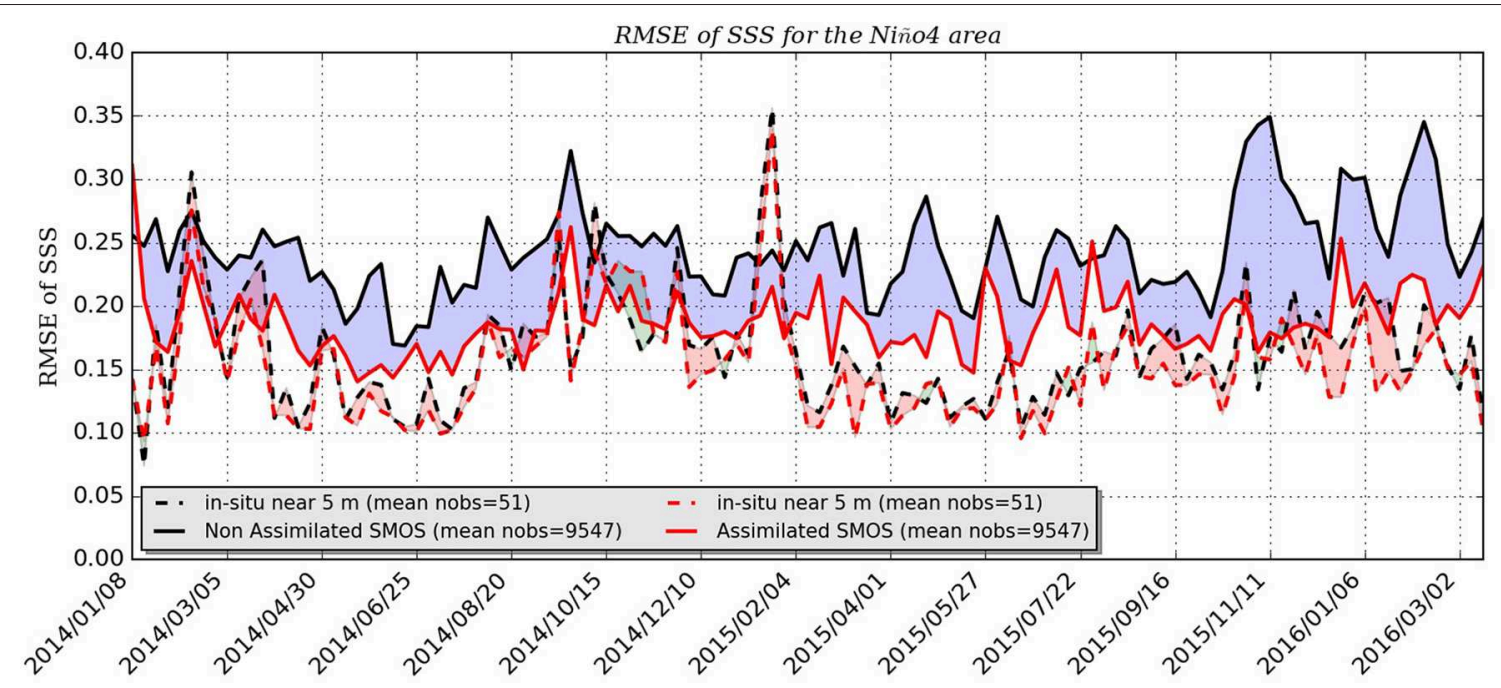

FIGURE 2 | RMS of innovation for SMOS (thick lines) and near surface (5 m depth) in-situ salinity observations (dashed lines) averaged over the NINO4 region: in black the control experiment, in red the OSE with assimilating SMOS data by the Mercator Ocean ODAP system.

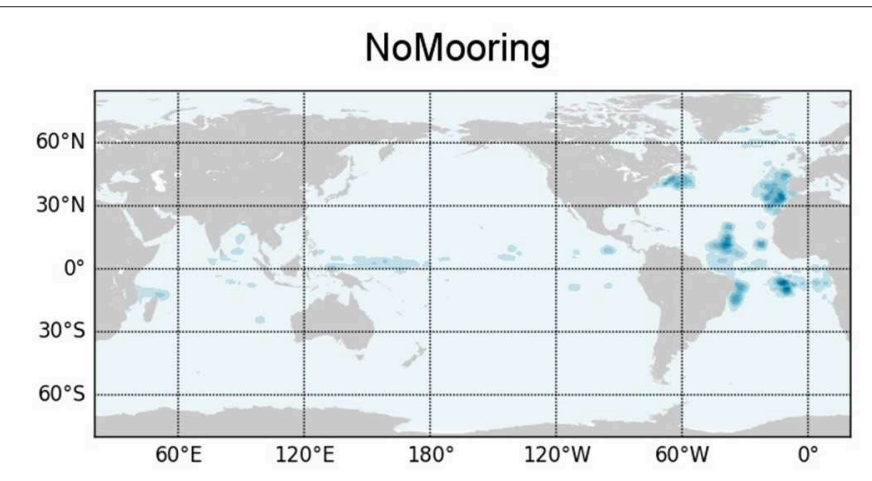

NoArgo

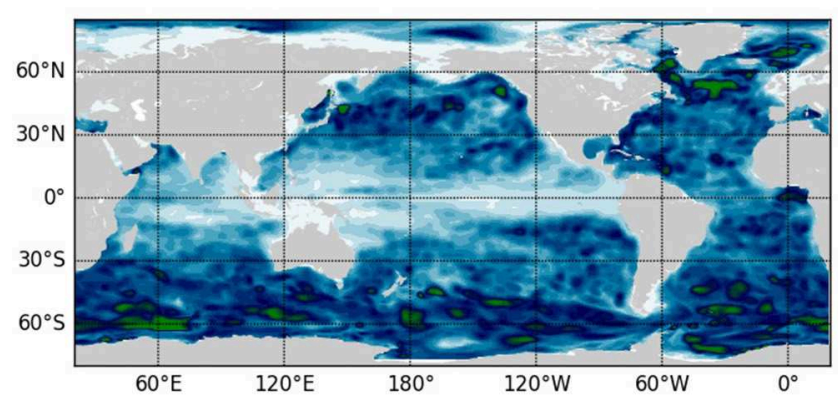

NoShip

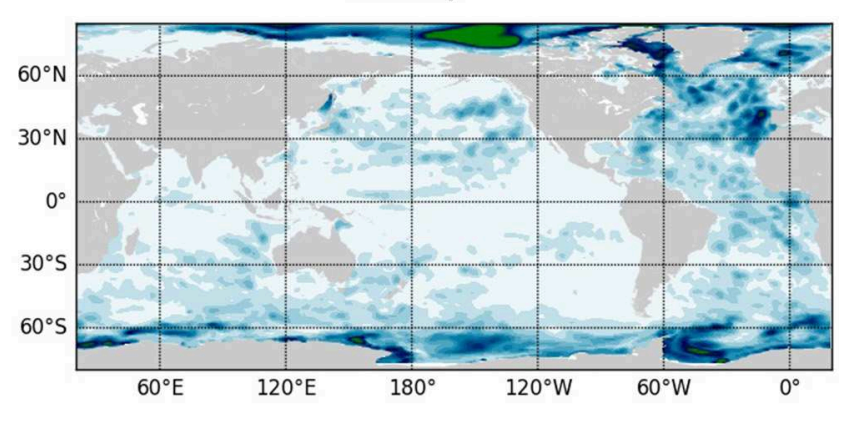

Nolnsitu

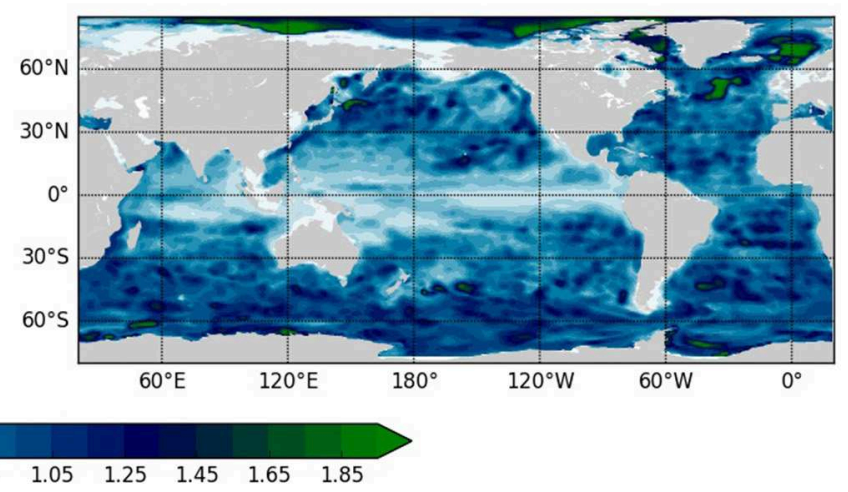

FIGURE 3 | Impacts of ocean observation data evaluated by OSE studies using the latest operational ocean reanalysis system in ECMWF. Plots of normalized RMS differences of upper 700-m column-averaged temperature between the control experiment, in which all in-situ observations are assimilated, and OSEs with removal of mooring data (NoMooring), XBT, MBT, and CTD data (NoShip), Argo data (NoArgo), and all in-situ observations (Nolnsitu). Statistics are computed using monthly-mean anomaly data over the 2008-2014 period after removal of the seasonal cycle information, then normalized against the temporal standard deviation of temperature over the same period in the control experiment. 
run without data assimilation, the model mis-represents both the potential temperature (Figures 4A,B,G) and salinity (not shown) fields with temperature errors in excess of $2^{\circ} \mathrm{C}$ at depth and over $1.4^{\circ} \mathrm{C}$ at the surface. This error is mostly due to uncertainty in fluxes from the nearby rivers. Then, in the OSE run in this study, glider data are assimilated once a day using the ensemble optimal interpolation (Oke et al., 2002, 2010), with a 91-member ensemble of weekly anomalies. For a full description of the model, assimilation, and experiments performed, the reader is referred to Jones et al. (2012). For this example, the model-observation misfit is reduced by up to $90 \%$ with the OSE run reproducing mixing (e.g., 10 April) and re-stratification (e.g., 15 April) events.

The impact of glider data on this high-resolution coastal model is further demonstrated by comparison with unassimilated SST data (Figures 4G,H). The differences between simulated and observed SST are reduced from $0.4-1.4^{\circ} \mathrm{C}$ (Figure 4G) when the model is run with no data assimilation (the control run) to $>0.3^{\circ} \mathrm{C}$ in the OSE run with data assimilation (Figure $4 \mathbf{H}$ ). For this example, Jones et al. (2012) report that assimilation of glider data not only helped reproduce the realistic variability of the properties in the coastal ocean, but it also reduced the bias of temperature from 1 to $>0.2^{\circ} \mathrm{C}$.

\section{OBSERVING SYSTEM SIMULATION EXPERIMENTS (OSSES)}

In OSSEs potential new observations or observing systems are tested by assimilating synthetic observations. See section Introduction for more details.

\section{OSSEs for Evaluating an Underwater Glider Array in Western North Atlantic}

A research ocean OSSE system has been developed through collaboration between the NOAA/AOML ${ }^{10}$ and the University of Miami. The system uses two substantially different configurations of the HYbrid Coordinate Ocean Model (HYCOM) for the free-running Nature Run and the dataassimilative OSSEs. The design and evaluation procedures follow strict guidelines developed for atmospheric OSSE systems to ensure that credible impact assessments are obtained (Atlas, 1997; Hoffman and Atlas, 2015). The system was initially set up and evaluated in the Gulf of Mexico (Halliwell et al., 2014), and then was expanded into a larger Atlantic domain $\left(5^{\circ} \mathrm{S}\right.$ to $45^{\circ}$ $\mathrm{N}$, extending east to $20^{\circ} \mathrm{W}$ ). Rigorous validation of the Atlantic system is presented in Kourafalou et al. (2016), Androulidakis et al. (2016), and Halliwell et al. (2017).

OS-Eval with this OSSE system is used to assess the impact of deploying arrays of underwater gliders on initialization errors in ocean prediction models. Particular focus was given to document the additional positive impact that is realized by using moving platforms, such as gliders, compared to using stationary platforms, such as moorings. Maps of RMS errors with respect to the truth represented by the Nature Run calculated over the time interval July to October 2014 are presented for dynamic height

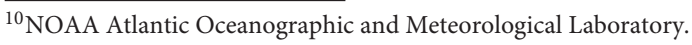

at the surface relative to $1,000 \mathrm{~m}$ (D1000, Figure 5). For profiles collected from stationary platforms, large error reduction is concentrated around each measurement location. Observations collected at fixed locations therefore have a limited radius of influence. By contrast, the ability of each moving glider to map a sub-region of the analysis domain extends the radius of influence and roughly equalizes error reduction across the domain.

\section{Evaluation of the Observing System Network in the Atlantic}

The H2020 AtlantOS project aims at building a multinational framework for a better-coordinated ocean observing system for the Atlantic by involving the main stakeholders from science and society (Visbeck et al., 2015), and leads coordinated efforts by European forecasting centers to provide quantitative information of potential impacts of further evolution of the in-situ networks on global ODAP systems. The main objectives are (i) to help defining and testing future observing systems from an integrated system perspective involving satellite and in-situ observations and numerical models, and (ii) to optimize the use of observation information in the analysis step and to improve the assimilation component. This initiative includes a set of physical OSSEs, using four global eddy-permitting $\left(1 / 4^{\circ}\right)$ systems, i.e., three ODAP systems (Mercator Ocean, CMCC ${ }^{11}$, UKMO) and one modelindependent analysis system $\left(\mathrm{CLS}^{12}\right)$. It is important to note that these experiments take fully into account the complementarity between satellite and in-situ observations. Impact of a given in-situ network is not analyzed in isolation and its value is assessed taking into account the information already provided by satellites.

The originality of this study lies in the assimilation in the four global systems of exactly the same synthetic data sets, which are derived from the Nature Run produced with the Mercator Ocean global $1 / 12^{\circ}$ unconstrained model (Gasparin et al., 2018). Potential future evolutions of the in-situ observing system, including Argo floats, drifting buoys, and mooring arrays, are defined based on strong interactions between observation agencies and forecasting centers. To our knowledge, this is the first time that such a coordinated effort is made using OSSEs. The multi-model and multi-approach feature is a critical point to ensure the robustness of the results (see section Multi-System Evaluation).

OSSEs are conducted for five potential future observing system designs as well as for the reference design which includes the satellite component (three altimeters and SST), moorings, $\mathrm{XBT}$, and Argo floats ( 1 float in every $3^{\circ} \times 3^{\circ}$ square). For each design, at least two groups conducted OSSEs and assessed the impacts of the integrated observing system. The doubling of Argo floats in the western boundary current regions and along the equator demonstrates an improvement of both temperature and salinity representation for the entire Atlantic of 5-10\% compared with the reference design. Stronger improvements are found in the western boundary current regions and along the equator. These results are consistent with Oke et al. (2015b) and Turpin

\footnotetext{
${ }^{11}$ Centro Euro-Mediterraneo per i Cambiamenti Climatici.

${ }^{12}$ Collecte Localisation Satellites.
} 


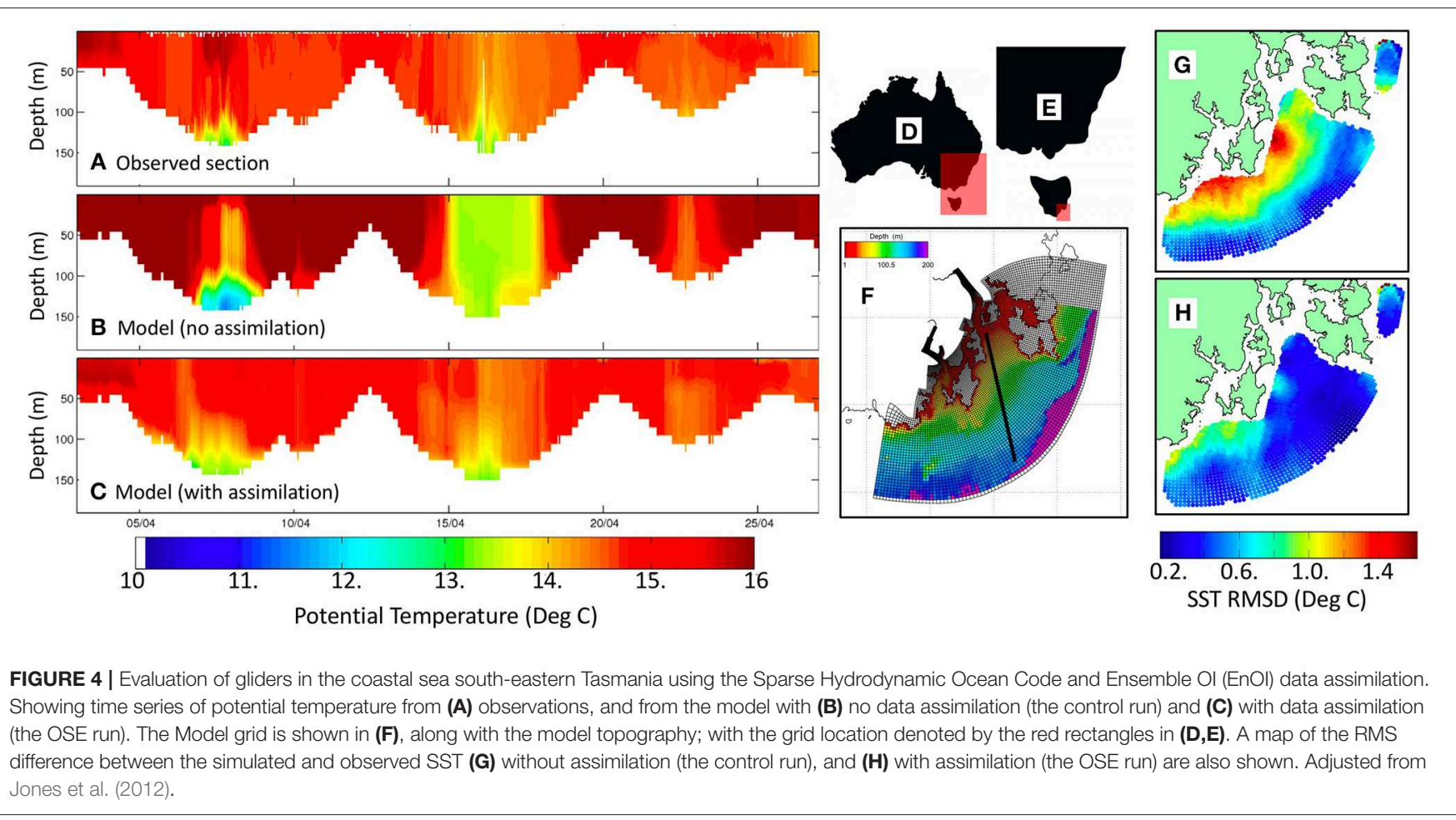

et al. (2016), who have investigated the impacts of removing half of the existing Argo floats. The implementation of a deep Argo array ( 1 float in every $5^{\circ} \times 5^{\circ}$ square, monthly), which samples to $4,000 \mathrm{~m}$ or to the bottom, shows a significant impact in reducing the bias in the temperature and salinity fields in the deep ocean. The ensemble mean of the four systems shows large error reduction of temperature and salinity up to $40 \%$, but the associated standard deviation indicates that there is quantitative difference among the systems (Figure 6). The implementation of a global drifter array equipped with a thermistor chain to $150 \mathrm{~m}$, which is an optimistic perspective on future evolution of the observing system, shows significant improvement of the temperature and salinity representation in the surface layer (10$20 \%$ of error reduction). The impact of removing the current mooring array is localized near the moorings, and does not affect significantly the large-scale structures, consistent with the findings of Fujii et al. (2015a).

Overall, this original study has demonstrated a positive impact of the different simulated observation networks, and is further detailed in a synthesis publication (Gasparin et al., 2019a).

\section{Evaluation of Impact of Wide-Swath Altimetry Missions}

The impact of forthcoming wide-swath altimetry missions was investigated at Mercator Ocean with OSSEs using a $1 / 12^{\circ}$ resolution regional ODAP system of the Iberian-Biscay-Ireland (IBI) region (Lellouche et al., 2013). The synthetic observations are derived from the Nature Run simulated by the same OGCM at $1 / 36^{\circ}$ resolution over the same region (Bonaduce et al., 2018). All the experiments assimilated the same synthetic observations of SST and temperature and salinity profiles. The synthetic data for conventional altimeters were derived from the sampling of the Nature Run over the theoretical tracks of the satellite missions Jason2, Jason1 on a geodetic orbit (Jason1g), and Envisat, with a sampling frequency of $1 \mathrm{~Hz}(\sim 7 \mathrm{~km})$. An observational white noise of $1 \mathrm{~cm}$ RMS was added to these pseudo-observations. The JPL simulator (Gaultier et al., 2016) was used to produce synthetic SWOT data. The wide-swath data were derived considering a 20.9-day repeat orbit at a spatial resolution of $7 \mathrm{~km}$ along and across the swath. Figure $7 \mathbf{A}$ shows examples of SSH fields in the Nature Run and the spatial coverage of simulated satellite altimetry data for conventional nadir and SWOT altimeter missions. Only the Ka-Band Radar Interferometer (Karin) noise in accordance with the error budget of SWOT mission concept (e.g., Durand et al., 2010; Rodriguez and Esteban-Fernandez, 2010). With $7 \times 7 \mathrm{~km}$ spatial resolution, the noise ranges from $0.2 \mathrm{~cm}$ RMS in the inner part of the swath to about $0.35 \mathrm{~cm}$ on the outer edges of the swath.

Four experiments were run OSSE0, OSSE1, OSSE2, and OSSE3. A free run experiment without assimilation (OSSE0) is used as a reference to quantify the contribution of the various data sets. SST and in-situ profiles are assimilated in all other experiments. In addition, synthetic SSH data along the tracks of conventional altimeters were assimilated in OSSE1. Only the synthetic data of SWOT were assimilated in OSSE2. Note that they include the nadir altimeter in the middle of the swaths of the SWOT satellite. Synthetic SSH data of both the nadir altimeters and SWOT were assimilated in OSSE3. All experiments are performed with the same initial conditions and forcing for 1 year January to December 2009. 

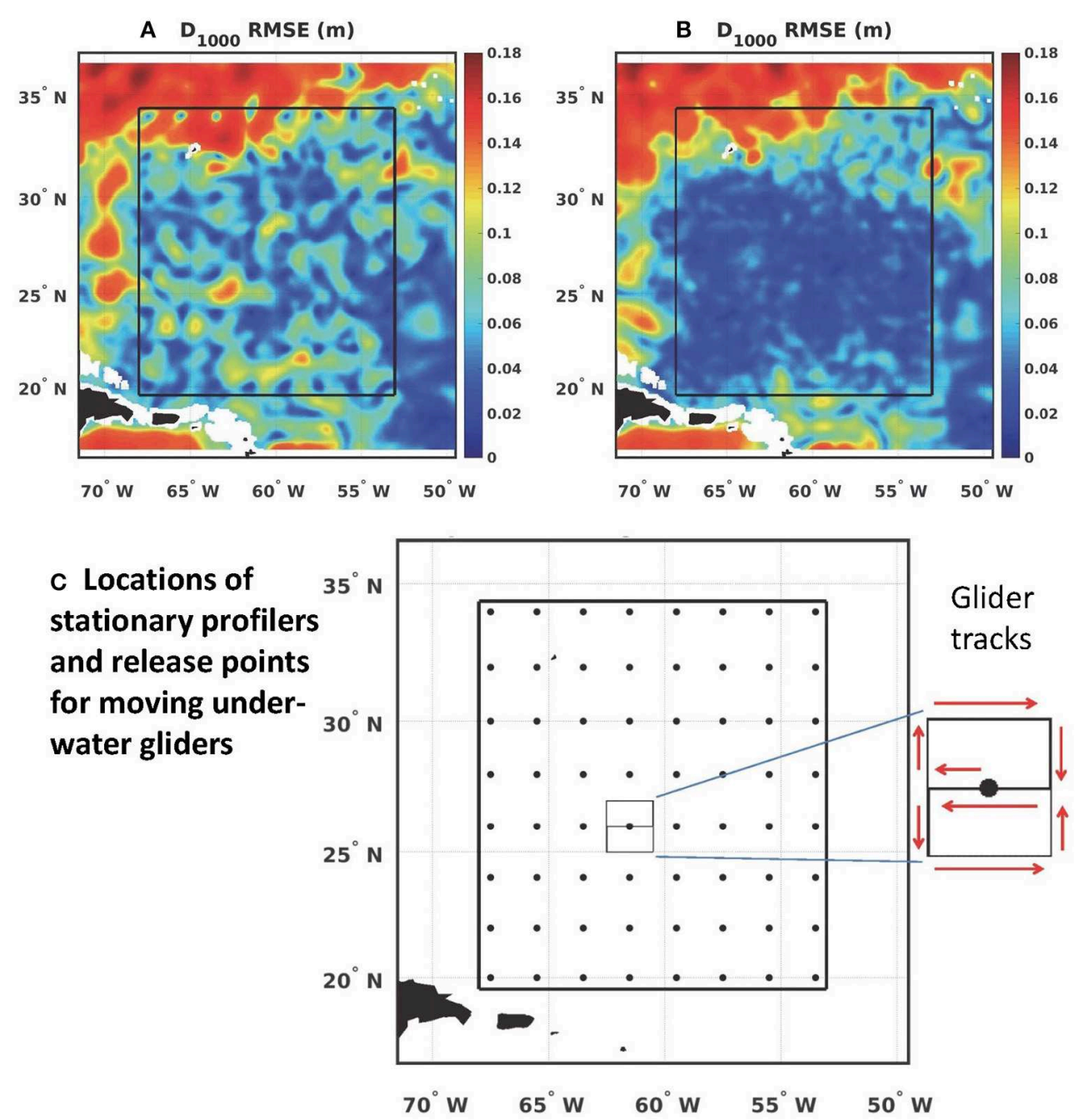

FIGURE 5 | Maps of RMS error in dynamic height at the surface relative to $1,000 \mathrm{~m}$ for (A) the stationary profiler experiment, and (B) the moving glider experiment, calculated over the time interval from July to October 2014. Stationary profilers and gliders were released at longitude-latitude grid points shown in (C) with separation distances of $2.0^{\circ}$. All gliders released at these same points executed a reverse figure eight pattern over a track spanning $2^{\circ}$ longitude and latitude while traveling at a speed of $0.25 \mathrm{~ms}^{-2}$. Both stationary and moving platforms sample temperature and salinity profiles to $1,000 \mathrm{~m}$ depth. The experiments are conducted with the research ocean OSSE system developed by the NOAA/AOML and the University of Miami.

Figure 7B shows the impact of assimilating synthetic SSH data evaluated by comparing the SSH errors in each OSSE. OSSE1 shows a clear reduction of the error by the assimilation of the nadir altimeter data compared to the free model (OSSE0). The best solution is the OSSE3 with assimilating SWOT and nadir altimeter data together. A constellation of SWOT and nadir altimeters shows a significant reduction of the error over the entire domain. The error variance does not exceed $9 \mathrm{~cm}^{2}$ except at the North Atlantic drift (between $50^{\circ} \mathrm{N}$ and $53^{\circ} \mathrm{N}$ ) and close to the open boundaries which are unchanged in these experiments. A coherency analysis (Thomson and Emery, 2014) was done to investigate the reliability of the SSH signal in the OSSEs at the different spatial scales (Figure 7C). If 0.6 is chosen as a threshold for reasonable performance, the figure implies that assimilation of nadir and SWOT altimeter data performs well for wavelengths down to $110 \mathrm{~km}$ in OSSE3, while OSSE1 performs well-down to $180 \mathrm{~km}$. This indicates that smaller scale features in the SSH fields are better captured when assimilating wide-swath altimeter data compared to nadir altimeter data.

\section{ADJOINT AND ENSEMBLE-BASED APPROACHES}

\section{Use of Adjoint Models and Uncertainty Quantification}

An ODAP system based on a 4DVAR method employs the adjoint of a forecast model in order to minimize the cost function which, in part, represents the model-data misfit. An adjoint model calculates the sensitivity of a scalar function 


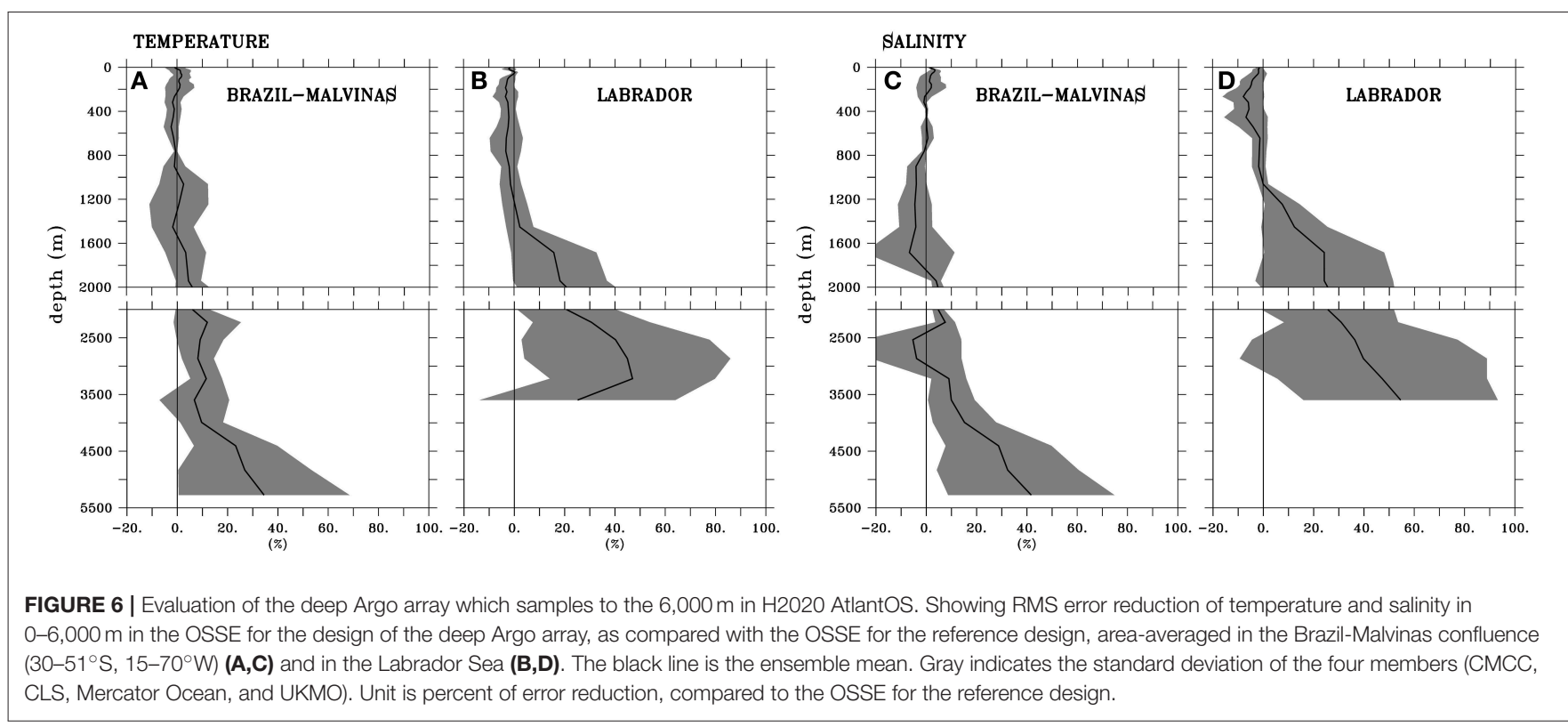

based on the model state at any time during a model integration (not limited to the 4DVAR cost function) to model state variables, boundary conditions, and parameters applied at previous times. Adjoint sensitivity experiments are, thus, applicable for OS-Eval studies. For example, an adjoint sensitivity study by Masuda et al. (2010) indicated that the temperature of the bottom water in the North Pacific is sensitive to the air-sea heat flux and temperature of the whole water column off the Adélie Coast of Antarctica multiple decades back in time. This result highlights the importance of temperature observations in that area, and motivated JAMSTEC to enhance ocean observation in that area through deep Argo floats. Meanwhile, singular vector analysis (e.g., Fujii et al., 2008; Zanna et al., 2011) and its non-linear extension, conditional non-linear optimal perturbation analysis (e.g., Wang et al., 2013) also employ adjoint models and provide insight into where observations are needed. Fujii et al. (2008) identified the rapidly developing perturbation which affects the Kuroshio large meandering, and indicated the importance of observations southeast of Kyushu in Japan where the perturbation is originated.

Hessian-based uncertainty quantification is an extension of adjoint sensitivity studies, and is performed within the framework of ocean state and parameter estimation. This framework uses the 4DVAR method, where the assimilation window is chosen equal to the entire estimation period, covering multiple years to decades. The long assimilation window guarantees a data-constrained solution for the time-evolving ocean state that is entirely dynamically and kinematically consistent, a desirable property for studying the ocean's climate and variability (e.g., Wunsch and Heimbach, 2013; Stammer et al., 2016). Uncertainty quantification is an appropriate tool to evaluate and design observing systems that are targeted at climate monitoring.
An example is measuring ocean connectivity via the mooring array deployed by the Overturning in the Subpolar North Atlantic Program (OSNAP; Lozier et al., 2016). The eigenvectors of the local Hessian of the cost function represent the state directions that are informed by the observations. They can be interpreted as prior-weighted adjoint sensitivities of the observed quantities to all control variables, as highlighted by Loose (2019). For instance, Figure 8A shows the state direction (truncated to the control variable meridional wind stress) that is informed by heat transport measurements across the OSNAPEast leg (MHT-OSNAPe), computed within the Estimating the Circulation and Climate of the Ocean (ECCO) framework. It is given by the prior-weighted adjoint sensitivity of MHTOSNAPe to meridional wind stress. Figure $\mathbf{8 B}$ shows the priorweighted meridional wind stress sensitivity of an unobserved quantity, namely subsurface temperature close to the Southeast Greenlandic coast (T-EG). Strong projection of the two patterns in Figure 8 reflects a high potential for the observed quantity MHT-OSNAPe to inform the unobserved quantity T-EG. How much of this information potential can actually be extracted is determined by a signal-to-noise ratio, which incorporates magnitudes of prior and observation uncertainties.

Extracting the leading eigenvectors of the Hessian for large observing systems replaces performing thousands to millions of adjoint sensitivity experiments (where each observed quantity would be the objective function of a new experiment). Importantly, uncertainty quantification evaluates observation uncertainties and prior information. Moreover, the orthogonal Hessian eigenvectors represent data-informed state directions with data redundancy removed. Unlike conventional adjoint sensitivities, they inform about data redundancy and complementarity of new observing systems in the context of existing observations, e.g., Argo profiling floats. Although full Hessian-based uncertainty quantification has not yet been 
A

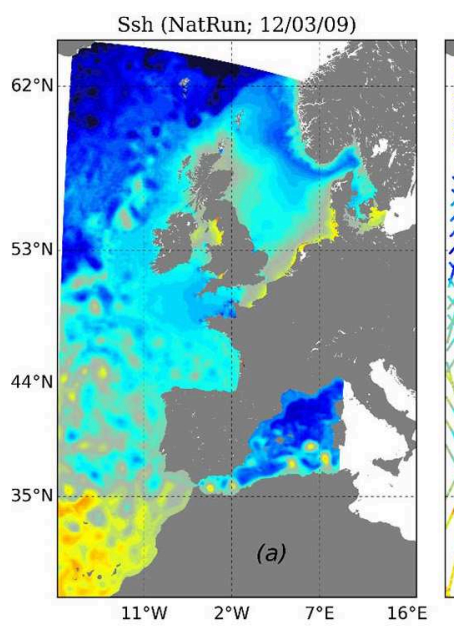

Ssh (J2+J1g+En; 09-14/03/09)

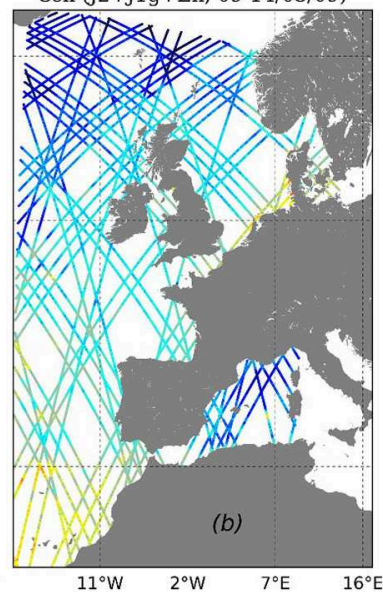

Ssh (Swot; 09-14/03/09)

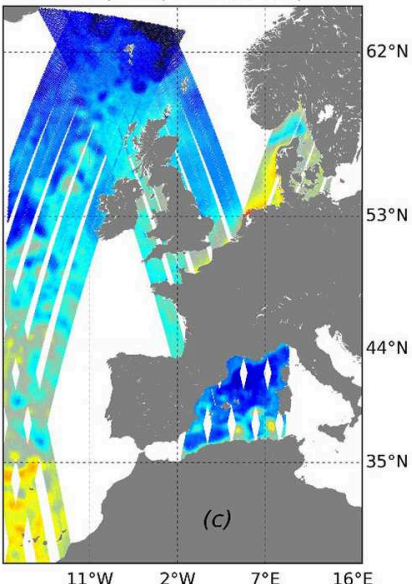

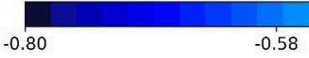

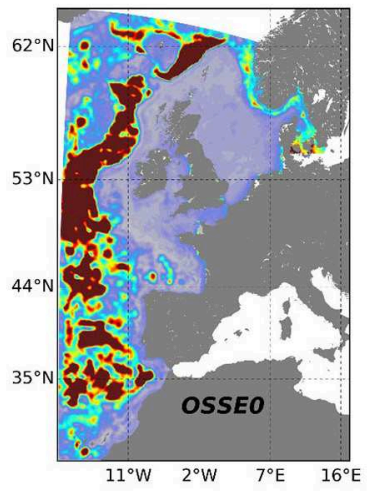

Control:Free Model

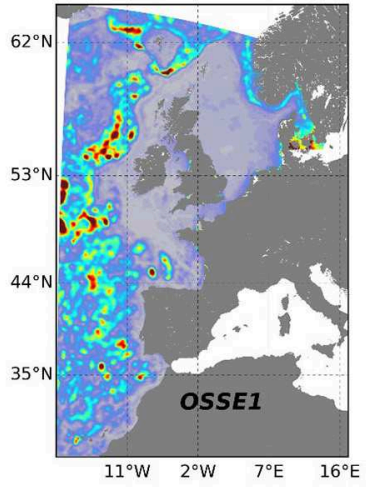

Assim: J2+ J1g+ En

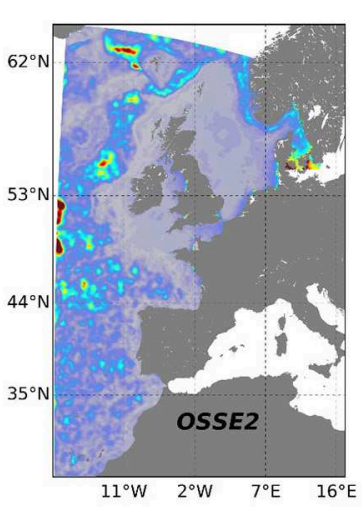

Assim: Swot

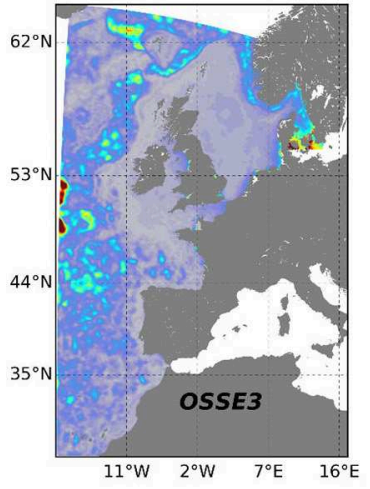

Assim: $5 w o t+J 2+J 1 g+E n$

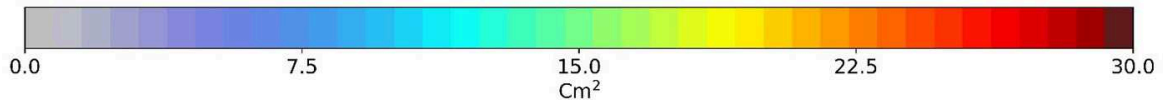

C

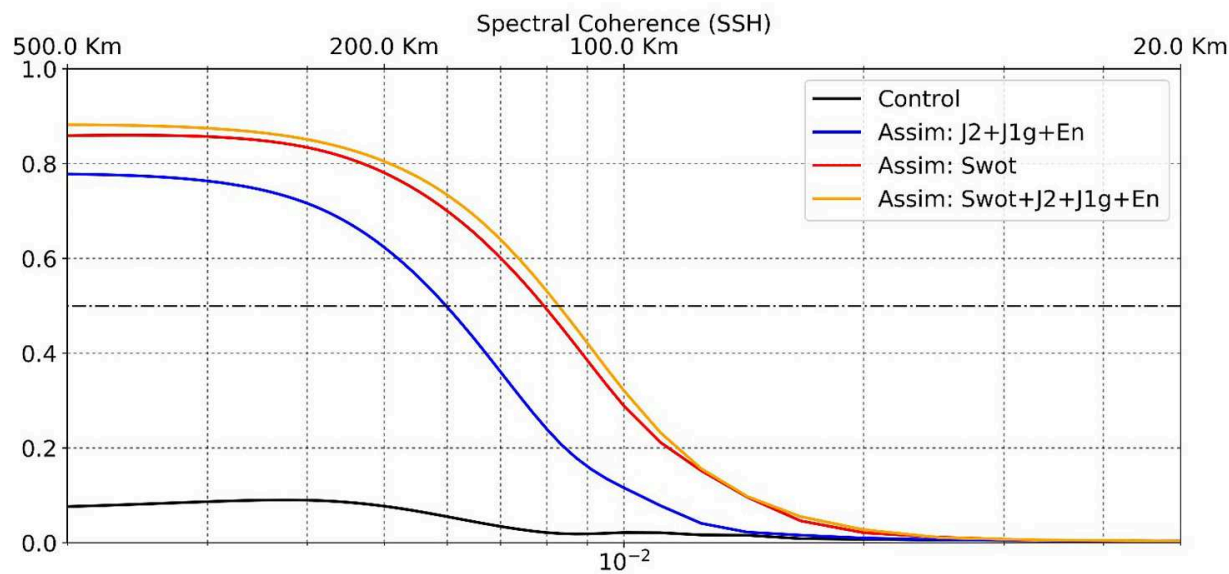

FIGURE 7 | Evaluation of a constellation of nadir and wide-swath altimeters in Mercator. (A) SSH field simulated in the Nature Run on March 122009 (left), and synthetic SSH data for Jason2, Jason1g and ENVISAT (middle), and SWOT synthetic data (right) in a 5-day assimilation cycle from March 12 2009. (B) Maps of the variance of SSH errors in the ocean analysis of OSSEO, OSSE1, OSSE2, and OSSE3 (from the left to right) over the IBI domain during 2009. Units in $\mathrm{cm}^{2}$. (C) Spectral coherence in OSSEO (black line), OSSE1 (blue line), OSSE2 (red lines), OSSE3 (orange lines) with respect to the Nature Run. 

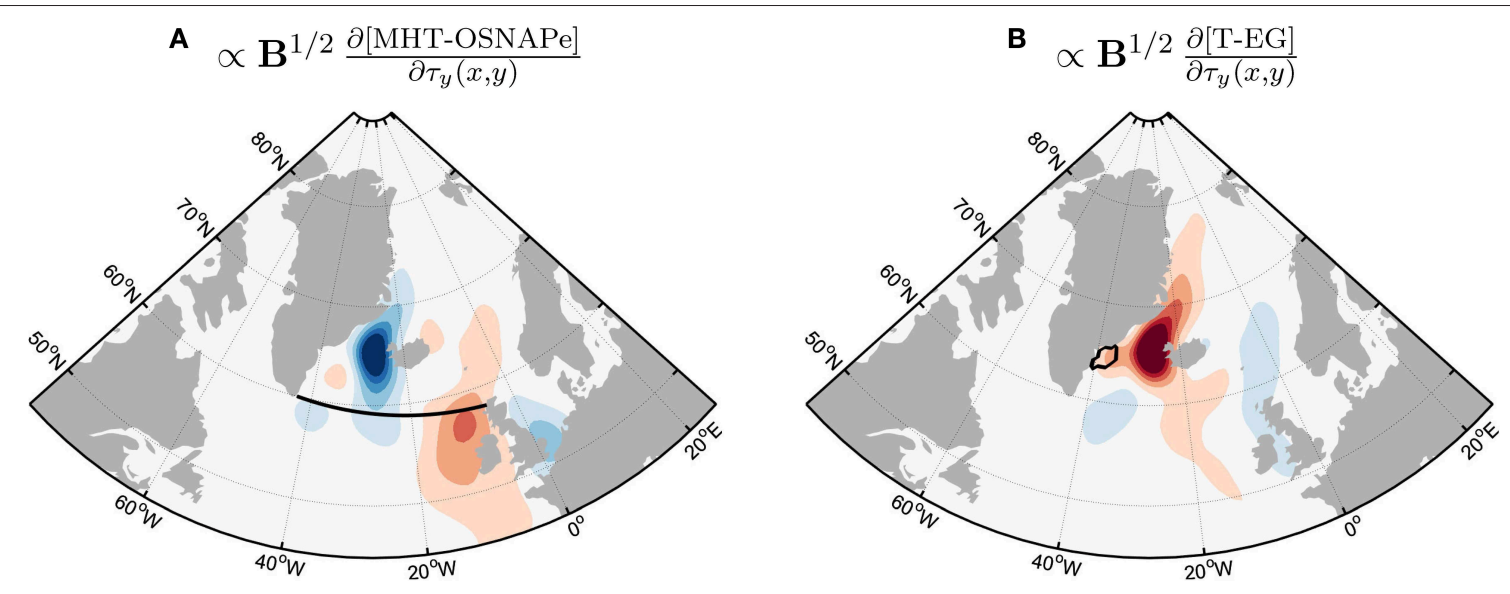

FIGURE 8 | Linear sensitivity of (A) meridional heat transport across the OSNAP-East section (MHT-OSNAPe) and (B) subsurface ocean temperature (150-550 m) close to the Southeast Greenlandic coast (T-EG) to meridional wind stress $\tau_{y}$, derived with the ECCO adjoint model, weighted by the square root of the prior error covariance matrix $\mathbf{B}$, and normalized. The solid black contour delineates the (A) OSNAP-East transect and (B) horizontal area for the respective calculations of (A) heat transport and (B) mean temperature. Sensitivities are accumulated over 5 years. Red (blue) colors indicate that an increase in the northward wind stress would lead to a subsequent increase (decrease) in (A) MHT-OSNAPe and (B) T-EG on a 5 year timescale. (A) An observed state direction (truncated to the control $\tau_{\mathrm{y}}$ ), informed by MHT-OSNAPe measurements. (B) The target direction (truncated to the control $\tau_{\mathrm{y}}$ ) for the unobserved quantity T-EG. The good projection of (A,B) reflects a high potential for the observed quantity MHT-OSNAPe to inform the unobserved quantity T-EG.

realized for global state estimation, substantial progress has been made within ECCO (Kalmikov and Heimbach, 2014, 2018). The coming decade will bring to bear the full potential of uncertainty quantification and optimal design of observing systems.

\section{Use of FSOI for Monitoring Ocean Observation Impacts in an ODAP System}

FSOI measures the variation in forecast error due to the assimilated data, and its evaluation is another promising application of an adjoint model for OS-Eval. Evaluation of FSOI has recently been applied to the ODAP system in operation at the US Navy and NOAA centers (Cummings and Smedstad, 2014). The system uses the global HYCOM and 3DVAR schemes, and measures the impact of all observations assimilated on reducing 48-h forecast temperature and salinity forecast error every day. Calculation of FSOI is formulated such that a negative value indicates a beneficial observation in that assimilation of that observation reduced forecast error. A positive value indicates a non-beneficial observation in that forecast error actually increased from assimilation of the observation. Non-beneficial impacts are not expected but if they occur, and they are persistent, then that may indicate problems with data quality or model performance. Thus, FSOI can be used as an effective observation monitoring tool with feedback to data providers on potential issues with their data.

To illustrate the routine application of FSOI, Figure 9A shows the geographic variation of the impacts of assimilating Argo temperature and salinity profiles from July 30 through August 18, 2018 in the Atlantic basin. In general, assimilating Argo data has beneficial impacts on reducing forecast error across the basin, although slightly non-beneficial impacts can be seen in some Argo temperature profiles. FSOI can also be partitioned by observing system. Figure 9B shows histograms of FSOI averaged within observing system in the Atlantic basin for the same time period. Here, impacts are normalized by the number of observations in an observing system such that the results are presented on a per observation basis. The results show that the most important temperature observing system is animal borne sensors, while for salinity the most important observing system is fixed moorings. When the per observation impacts are calculated without normalization, satellite altimeters, and satellite SST have the greatest impacts simply from the overwhelming number of observations generated by those observing systems. It is important to note that all ocean observing systems assimilated have beneficial impacts on reducing forecast error.

Evaluation of FSOI provides an all at once approach to estimating observation impacts. The method automatically adjusts to changes in the observation suite assimilated as new observing systems are introduced and to changes in the forecast model as model resolution increases or new physics are introduced. It is now possible to efficiently and routinely evaluate the entire global set of oceanographic observations assimilated in the HYCOM system, determining which data are most valuable and which data are redundant or do not add significant value. Like other OS-Eval methods, FSOI strongly depends on the ODAP system. Thus, the data impact results thorough evaluation of FSOI cannot be generalized to all ODAP systems.

\section{Use of DFS for Evaluating the Impact of the SKIM Mission}

In contrast to FSOI, DFS represent impacts of observations on the analysis fields. The DFS can be conveniently computed in an EnKF setting as a by-product of the calculation of the Kalman Gain without additional computing costs (Sakov et al., 2012). It is particularly relevant in the planning phase of a new observing system when the actual data are not yet available, 

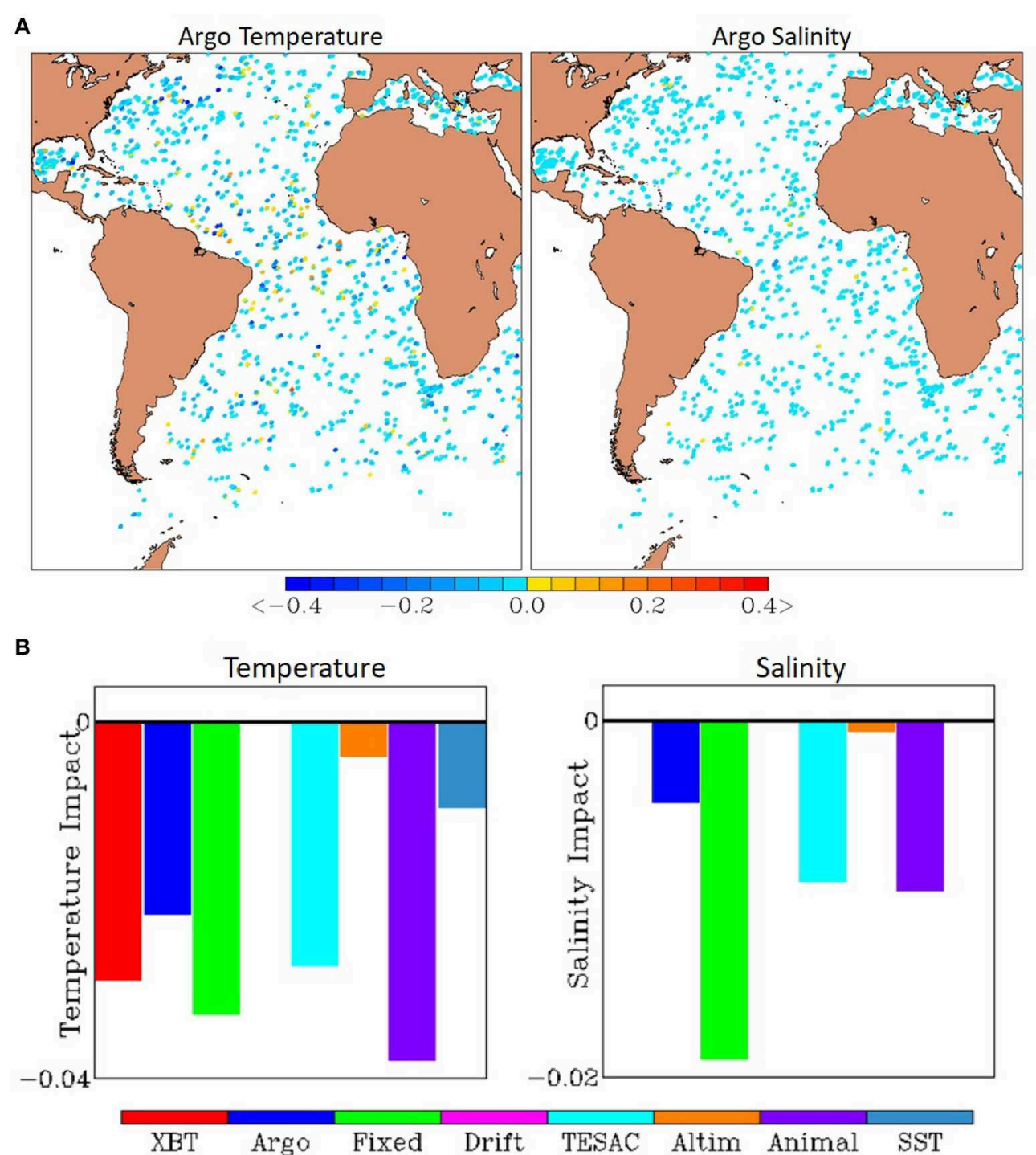

FIGURE 9 | FSOI evaluated for the ODAP system based on the global HYCOM. (A) Impacts of Argo profiles on reducing 48-h forecast error in the Atlantic basin from 30 July through 18 August 2018. Cool colors indicate beneficial impacts; warm colors indicate non-beneficial impacts. (B) Per observation impacts for temperature (left) and salinity (right) observing systems in the Atlantic for 30 July through 18 August, 2018 . Temperature units are ${ }^{\circ} \mathrm{C}$ and salinity units are PSU.

but some knowledge of the locations of observations and their uncertainties are sufficient to measure their potential impact on a given modeling system assimilating observations from a pre-existing network.

An initial evaluation of the impact of surface current data from ESA Earth Explorer 9 candidate mission SKIM ${ }^{13}$ (Ardhuin et al., 2018) has been performed using the TOPAZ ODAP system (Sakov et al., 2012) based on DFS. SKIM is designed with rotating altimeter and SAR Doppler technology at two incidence angles $\left(6^{\circ}\right.$ and $\left.12^{\circ}\right)$. SKIM is the first satellite mission designed to measure the ocean surface current directly, and also designed to measure wave spectral parameters and sea ice drift.

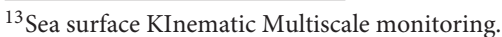

In this study, the surface currents from both the $6^{\circ}$ and $12^{\circ}$ beam angles have been considered with observation errors of 0.23 and $0.19 \mathrm{~ms}^{-1}$, respectively (Lucile Gaultier, personal communication). Considering that the TOPAZ system is only eddy-permitting and the SKIM currents are likely sensitive to submesoscale processes, high representativeness errors have been accounted, although very crudely, by applying a factor of 5 on the above standard deviations. Since the absolute values of DFS have little physical meaning on their own, the relative fraction of each observation type to the total DFS are presented.

The restart files from a TOPAZ ensemble have been extracted in the end of a 25-years reanalysis (Xie et al., 2017) in May 2017 which assimilated SST, along-track SSH, sea ice concentrations, in-situ temperature and salinity profiles. We compare the relative 
impact of SKIM surface currents to that from the along-track SSH which also informs surface currents (Figure 10). In view of the above simplifying assumptions on the observation errors and the fact that the simulated SKIM data does not cover the TOPAZ area completely, the quantitative share of DFS (22.6\% for SSH against $10.2 \%$ for SKIM) are only indications that the SKIM dataset is potentially valuable even in the presence of a good observing network as of May 2017. The patterns of the relative impacts in Figure 10 show strong benefits in the equatorial band where the SSH data has no information content and the area close to the ice edge. These improvements are likely to carry over to other ODAP systems. The high impacts seen in the Gulf Stream area may on the contrary be system-dependent and may diminish if the observation representativeness errors are increased to account for the intensity of ocean submesoscale activity.

\section{Use of Multi-System Ensemble Spread}

The spread of a multi-system ensemble can be used for evaluating the uncertainty of ODAP products. The International Real-Time Ocean Reanalysis Intercomparison project (Xue et al., 2017a), provides beneficial information for OS-Eval as the ensemble spread can be monitored as the observing network changes in time. The project was started in 2014 following to the recommendation of the TPOS2014 workshop (see section OSE Studies for Evaluation of TAO/TRITON Array). In the project an ensemble of nine ODAP products has been routinely collected at NCEP, and the temporal and spatial variations of the ensemble spread of total temperature and anomalous temperature have been monitored and used to discern the influences of the TPOS data on the uncertainty of the ODAP products in near real time.

Figure 11 shows the temporal variations of the number of daily temperature profiles and the ensemble spread of temperature in the upper $300 \mathrm{~m}$ for the TAO and TRITON regions, respectively. For the TAO array region, the number of temperature profiles dropped rapidly in early 2012 and then recovered to the historical level after 2015. In responding to the TAO decline in 2012-2013, the international Argo community significantly increased the deployment of Argo floats in the equatorial Pacific in early 2014. The ensemble spread of total and anomalous temperature is closely related to the number of temperature profiles: it had decreased gradually since early 2000s due to the increase of the Argo data, increased rapidly in 2012-2013 due to the TAO decline, and then decreased in 2014-2015 due to the increase of temperature profiles. This clearly demonstrated that ocean observations played a critical role in reducing the uncertainties among the ODAP products.

Another urgent issue is the decline of the TRITON array since 2013. Figure 11C shows that the spread of both total and temperature anomaly increased rapidly since 2014 due to the TRITON decline. The spread of total and anomalous temperature became comparable after 2016. Therefore, we need to address the data decline in the region, and also be aware of the influences of the time evolution of ocean observations on model climatology and anomalies.

\section{NEW CHALLENGES FOLLOWING EVOLUTION OF ODAP SYSTEMS}

\section{Coastal Regions}

Many coastal applications based on ODAP systems have been developed, and are being used as essential tools for improving coastal predictions. Coastal observation data are indispensable to improve the performance of the systems and to ensure that socioeconomic benefits can be obtained. Therefore, needs of OSEval studies for designing, developing, and sustaining coastal observing systems are now increasing. Relevant OSE and OSSE studies have been conducted as introduced in sections OSE Study for Evaluation of Coastal Gliders in a High-Resolution Coastal Model, and OSSEs for Evaluating an Underwater Glider Array in Western North Atlantic. Adjoint-based evaluations have also been applied for coastal regions (e.g., Moore et al., 2011). These studies have showcased the importance of various observation platforms in different coastal regions (e.g., HF radar, ocean gliders, etc.). Coastal regions have diverse characteristics and it is challenging to conduct OS-Eval studies that can be compared and contrasted. Due to the unique nature of the coastal regions a coordinated effort is needed from the international community of coastal ODAP systems.

\section{Deep Ocean}

The deep ocean, in particular below 2,000 $\mathrm{m}$ depth, is a new frontier for ocean observations in contrast to the upper ocean which is now well-sampled by standard Argo floats. Several deep cast cruises have explored the changes in the deep ocean (e.g., Johnson et al., 2008; Purkey and Johnson, 2010; Kouketsu et al., 2011; Desbruyères et al., 2016) following Fukasawa et al. (2004)'s finding of bottom-water warming in the abyssal North Pacific Ocean. Due to the huge volume of ocean involved, subtle thermal changes in the deep ocean are known to be an important signal for the trends of world climate (e.g., Severinghaus et al., 1998; IPCC, 2014). Recently, the success of the monitoring network of Argo for the upper ocean (e.g., Riser et al., 2016) motivates the construction of a similar global monitoring network for the deep ocean (e.g., Roemmich et al., 2019). Within this background, a deep ocean state estimation and prediction is greatly anticipated. Studies on deep ocean state estimation is, however, at an early stage. Some examples of work on this are found in Kouketsu et al. (2011), Balmaseda et al. (2013b), and Osafune et al. (2015).

As mentioned in section Use of Adjoint Models and Uncertainty Quantification, the adjoint sensitivity experiments by Masuda et al. (2010) motivate deployment of deep floats. Masuda et al. (2018) try examine the impact of available deep float data on deep ocean state estimation by comparing two data sets from a set of comparative experiments with and without the deep-float data. The results demonstrate that the available float data enables corrections of the modeled ocean state locally for each float. The impact of the deep float data on a small basin $\left(50^{\circ}\right.$ latitude and $10^{\circ}$ longitude) deep ocean state estimation is quantitatively estimated to show its dependence on the float density. In addition, the H2020 AtlantOS project (section Evaluation of the Observing System Network in the Atlantic) and other recent studies (e.g., Chang et al., 2018; Gasparin et al., 

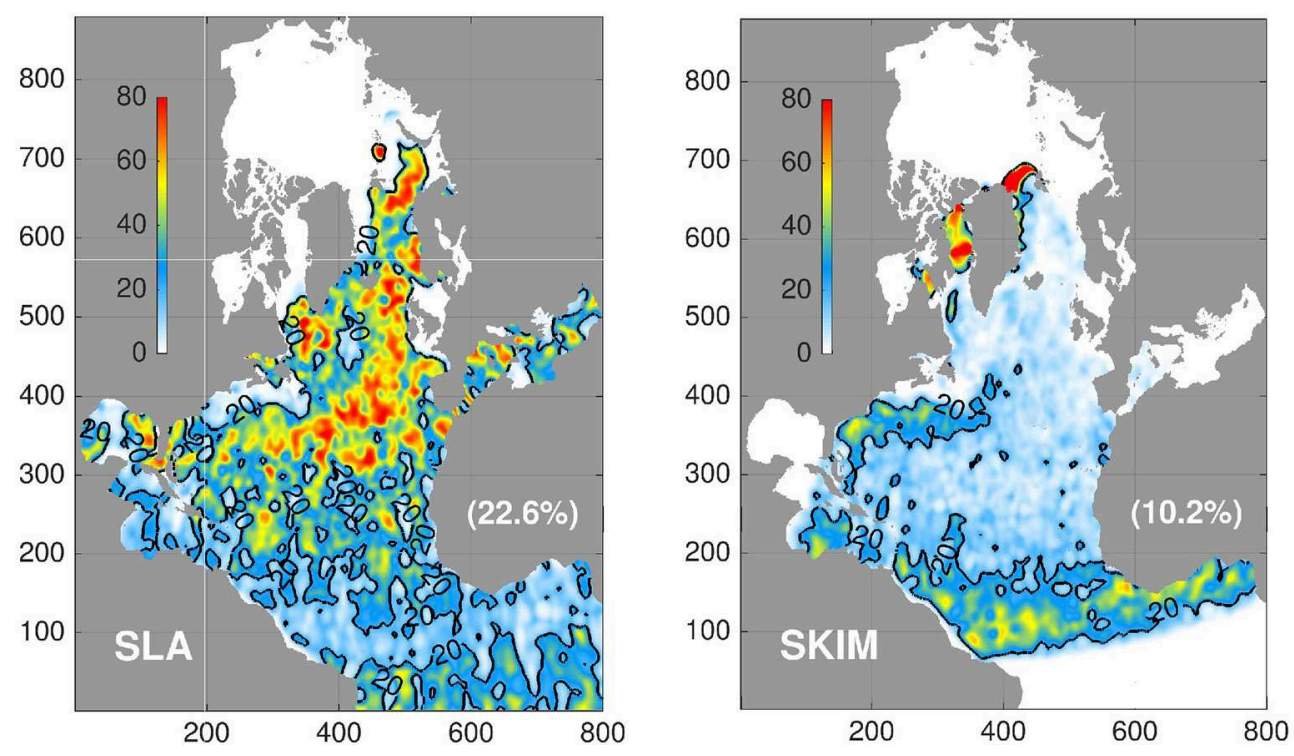

FIGURE 10 | Relative DFS impact factor projected in model coordinates in the TOPAZ system. The area-averaged impact fraction is indicated in parenthesis. The chart is presented in model native coordinates and shows the whole model domain.
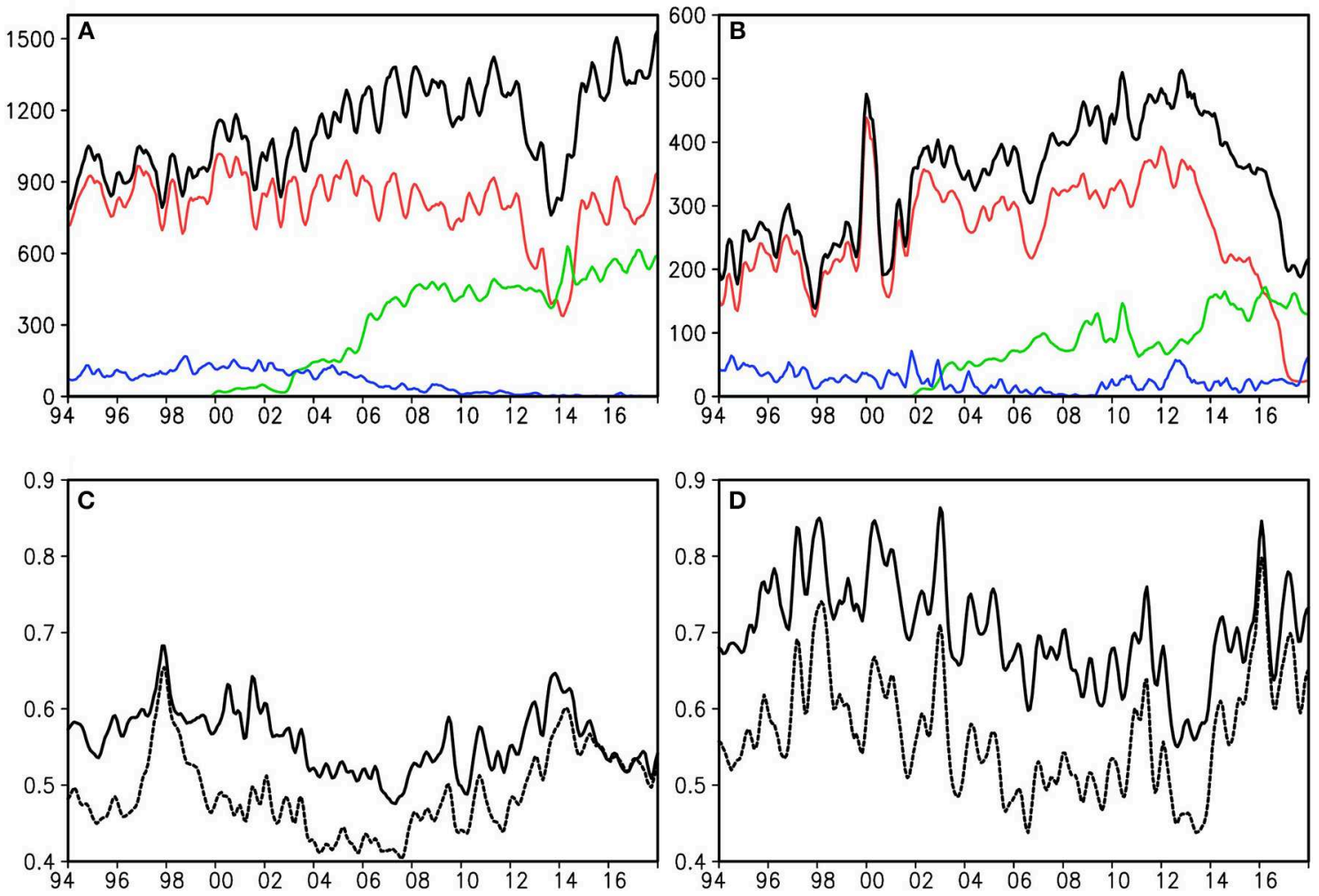

FIGURE 11 | Time variations of the number of daily temperature profiles per month accumulated in the (A) TAO and (C) TRITON array region from the moorings (red line), Argo (green line), XBT (blue line), and all together (black line) from January 1994 to 2018. Time evolution of the multi-system ensemble spread of total temperature (solid lines) and anomalous temperature (dash lines) calculated in the upper $300 \mathrm{~m}$ for the (B) TAO and (D) TRITON array region from January 1994 to 2018, evaluated in the Real-Time Ocean Ranalysis Intercomparison Project. 
2019b) have investigated the impact on assimilating a deep Argo array. However, the limited number of studies does not allow for firm statements to be made about the optimal spatial and temporal resolution of the deep Argo array. Further investigation is required to better understand the impact of the deep Argo array in the assimilation systems at long time scale.

\section{Arctic Ocean}

The relative remoteness and harsh environmental conditions over polar regions hinder efforts to provide adequate observations for ODAP systems. Improvements in observing technology and capabilities provide new avenues for sustained observations in polar regions suitable for ODAP systems (Smith N. et al., 2019). While these technologies make a more comprehensive polar ocean observing system, including sea-ice observations, feasible, the question remains, is it worth the cost?

A partial answer to the cost-benefit question may come from an ongoing international activity led by the World Weather Research Programme's Polar Prediction Project (PPP Steering Group, 2013, 2014). In particular, a Year of Polar Prediction (YOPP) for 2017-2019 aims to help address this question by coordinating international observing activities and fostering efforts to assess the impact of additional observations on environmental prediction skill, including impacts on downstream users and products (Jung et al., 2016). This effort will include OSEs to assess the benefit to forecast skill and product quality afforded by the additional observations, and evaluation of sea-ice observations will be an important target. The YOPP core period will be followed by a consolidation phase during which these experiments will be performed and results synthesized. This effort culminates in a YOPP summit, planned for 2022, which should provide an ideal opportunity for funding and implementation agencies to benefit from this community effort.

\section{Medium-Range and S2S Coupled Prediction and Coupled Data Assimilation}

The evaluation of the ocean observing system is gaining increasing importance in the context of medium-range and Subseasonal-to-Seasonal (S2S) coupled prediction and coupled data assimilation. Coupled atmosphere-ocean-land-sea-ice models are adopted in current operational medium-range weather forecasts at ECMWF (Buizza et al., 2018) and the CCMEP $^{14}$ (Smith et al., 2018), and other several operational weather centers developed their systems in this direction. Operational centers also examine feasibility of sub-seasonal predictions with a coupled model, and started development of coupled data assimilation for further improvement of prediction skills (e.g., Lea et al., 2015; Laloyaux et al., 2016).

Although the impact of the ocean in medium-range and sub-seasonal predictions is not as dominant as in seasonal, those predictions are still sensitive to air-sea interaction in the tropics, mixed layer physics, and sharp SST fronts. For example, the impact of ocean heat content on tropical cyclones is welldemonstrated in previous studies (e.g., Mogensen et al., 2017). The idea that ocean fields affect Madden-Julian Oscillation

${ }^{14}$ Canadian Centre for Meteorological and Environmental Prediction.
(MJO) is also widely accepted. Therefore, ocean observations are considered to have potential to increase the skill of those predictions. Thus, it is expected we now assess what is an effective ocean observing network for coupled predictions. In particular, the assessment of the adequacy of the current ocean observing system for the sub-seasonal range will be a focus activity of the WWRP/WCRP S2S prediction project [World Weather Research Program (WWRP) (2017)] in the next few years. An example of such work is that of Subramanian et al. (2019) who found that prediction skill of tropical cyclones, in the operational coupled prediction system at ECMWF, is increased with the assimilation of SSH satellite data.

A coupled data assimilation system can propagate impact of observation data across the sea surface and thus exploit the data more effectively. In addition, it enables us to assimilate newtypes of observation data around the sea surface in a more physically consistent manner. For example, Laloyaux et al. (2017) showed that satellite scatterometer sea surface wind data are more effectively assimilated giving improved oceanic mixed layer temperature in a coupled data assimilation system. Akella et al. (2017) reported on effective assimilation of satellite skin SST data in an atmospheric data assimilation system coupled with a skin SST model.

Applying regular OS-Eval techniques to a coupled data assimilation system usually requires some new developments. OSSEs with a coupled model requires a Nature Run based on a simulation of a coupled model (see section Nature Run and Calibration of OSSEs). Adjoint codes of a coupled model are required for calculating the FSOI of a coupled data assimilation system.

\section{Evaluation of BGC Argos}

Observing and forecasting systems for ocean biogeochemistry are not yet as mature as for the physical systems considered in the previous sections. Ocean color satellites routinely provide global observations of optical properties and chlorophyll concentration for over two decades. This has proved an invaluable tool for reanalysis and forecasting (Gehlen et al., 2015), but the coverage is restricted to the near-surface and cloud-free conditions, and limited information can be obtained about other variables such as nutrient concentrations. The in-situ observing network should develop significantly over the next few years with increase in the Biogeochemical (BGC) Argo network (Biogeochemical-Argo Planning Group, 2016). The deployment of a sustained global array of $\sim 1,000$ BGC Argo floats is foreseen, with a capacity to measure a variety of new variables (e.g., oxygen concentration, nitrate concentration, $\mathrm{pH}$, etc.).

In addition to OSSE experiments reported in section Evaluation of the Observing System Network in the Atlantic, H2020 AtlantOS have conducted observation impact studies to help in the design of the future BGC Argo network in the Atlantic basin, and assess the complementarity with existing satellite ocean color observations. For monitoring and forecasting purposes, the effect of uncertainties due to various BGC model imperfections (e.g., simplified biology, unresolved biological diversity, and unresolved scales) has to be properly simulated as it should play a key role in estimating the dynamical behavior 
of ocean ecosystems. To better represent model uncertainties, Brankart et al. (2015) and Garnier et al. (2016) investigated the use of an ensemble Monte Carlo approach based on the inclusion of stochastic processes in the NEMO-PISCES modeling framework. This study showed the potential of such an approach by explicitly simulating the joint effects of uncertain biological parameters and unresolved scales using a stochastic model to simulate an ensemble of 60 members in a $1 / 4^{\circ}$ resolution North Atlantic configuration.

Verification tools used in conventional OSSEs, such as RMS error metrics, need to be adapted for evaluating ensemble-based experiments, requiring a probabilistic approach. Thus, this BGC Argo OSSE study set up an integrated ensemble-based probability score methodology based on a set of probabilistic verification tools which have been mostly developed within the numerical weather prediction community to assess ensemble forecasts, and include a modified version of the entropy skill score introduced by Roulston and Smith (2002). For the sake of brevity, only the results associated with entropy are shown here.

This new methodology was applied to a single date, $15 / 04 / 2005$ to compare four basic deployment scenarios while keeping numerical costs tractable. In the scenarios of BGCArgo array deployment without satellite ocean color data (Figures 12A,B), the entropy of the updated ensemble related to chlorophyll distributions at $24 \mathrm{~m}$ depth shows a significant gain of information at the positions of the assimilated BGC floats, meaning that the prior uncertainty is only reduced locally. In the other two scenarios in which both BGC-Argo arrays and ocean color data are included (Figures 12C,D), the prior uncertainty is mostly reduced within a zonal band across the North Atlantic Basin at around $30^{\circ} \mathrm{N}$, matching with the best coverage of satellite ocean color tracks assimilated at the surface, while BGC-Argo data (especially on nominal array) adds significant information gain in the equatorial region and at high latitudes in the northern basin. Thus, inclusion of BGC sensors on roughly one quarter of the current Argo array (around 1,000 floats) is expected to provide major improvements. There is also some evidence that a higher density network of BGC floats would add further value.

\section{LIMITATIONS AND EFFORTS TOWARD IMPROVED OBSERVING SYSTEM EVALUATION}

\section{Limitations of OS-Eval and System Dependency}

Although OS-Eval gives practical feedback to observation communities, it has several limitations which should be noted in interpreting the results. In order to use the OS-Eval results effectively, we should recognize the limitations, and interpret the results appropriately based on this knowledge. Here, the limitations are summarized as follows:

(i) OS-Eval results inevitably depend on the properties of the ODAP system employed for the evaluation, including systematic errors, or biases, of the forecast model, and the characteristics of the data assimilation scheme. They are thus "system dependent."

(ii) OS-Eval also depends on the forecast/monitoring target (e.g., ENSO, variation of the Kuroshio axis, coastal phenomena, rapid tides, etc.), forecast leadtime, and the period for the evaluation. They are thus "question dependent."

(iii) OS-Eval generally evaluates only the value of observation data as those to be assimilated, and the value for other purposes (e.g., as forcing data, as reference data in system validation or for the determination of prescribed error statistics and biases) is not accounted for.

(iv) OS-Eval is usually unable to evaluate impacts of changes within observing networks in near real time.

(v) OS-Eval often requires heavy computer and human resources.

Among the limitation above, the most influential one is probably (i) the system dependency. In particular, this limitation was regarded as the main reason of the inconsistency among evaluations in the TPOS2014 workshop (section OSE Studies for Evaluation of TAO/TRITON Array). In this workshop it was concluded that large systematic errors of coupled atmosphereocean models severely reduce the reliability of the OS-Evals. Similarly, reasonable evaluation of coastal observing systems may be limited by insufficient representation of small-scale coastal features by the current generation of ocean models. An essential way to mitigate this limitation is by reducing the systematic errors by improving the forecast model.

The effectiveness of a data assimilation scheme in using observation information can vary which will affect OS-Eval results. Figure 13 clearly demonstrates this. This figure depicts impacts of in-situ temperature and salinity profiles, mainly profiles of Argo floats, on 100-m-depth temperature fields produced by 3DVAR and 4DVAR versions of the same ODAP system in JMA (Usui et al., 2015). Although a common large impact can be seen in the subarctic region and inside of the subtropical gyre, the large impacts around the Kuroshio Extension found in the evaluation with the 3DVAR system disappears in the 4DVAR evaluation due to high ability of 4DVAR to reproduce the thermal front from satellite altimetry data alone.

Because of the system-dependency, the impacts of observing systems in practical operation continuously change following to evolution of prediction models and data assimilation technologies. Meanwhile, we can only perform OS-Eval based on current ODAP systems. We thus need to consider future evolution of models and data assimilation in order to assess the true potential of an observing system. OS-Eval results also depend on how the system used for the evaluation is tuned. ODAP systems are usually tuned for the currently existing observation network. An OS-Eval study may, therefore, underestimate the impact of a future observing network as the system may need to be retuned to obtain the full benefit of it.

\section{Multi-System Evaluation}

In spite of continuous progress of model and data assimilation technologies, it is not likely to be feasible to reduce the influence 

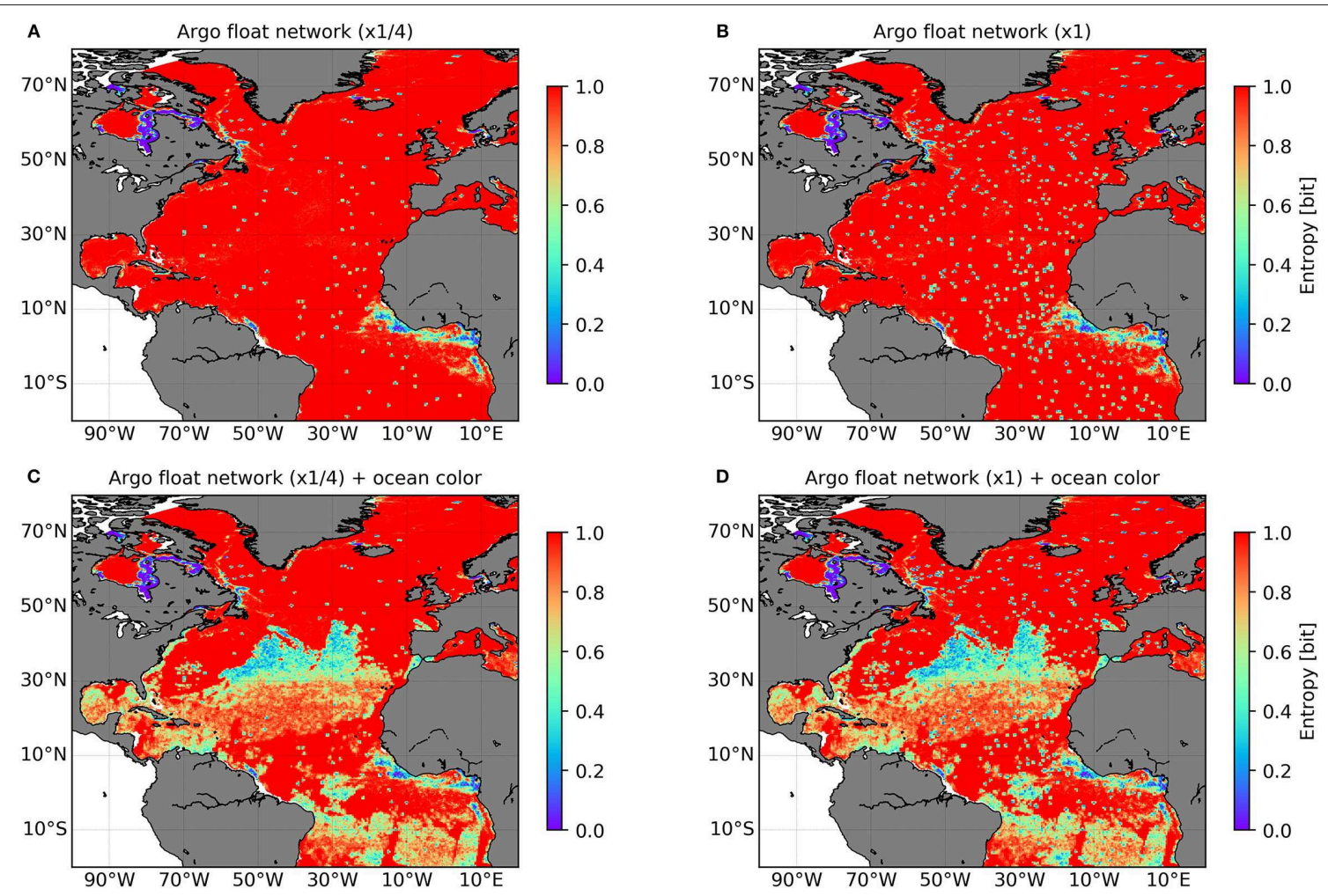

FIGURE 12 | Result of the OSSE studies for the future deployment scenarios of BGC Argo floats in H2020 AtlantOS. Showing entropy skill score related to the chlorophyll distribution at $24 \mathrm{~m}$ in the scenarios of (A) BGC-Argo sensors on 1/4 of the nominal Argo array, (B) BGC-Argo sensors on the full nominal Argo array, (C) daily satellite ocean color data and BGC-Argo on 1/4 of the nominal array, and (D) daily satellite ocean color data and BGC-Argo on nominal array. Note that reducing prior uncertainty of the system is having entropy values lower than 1 (red) and $>0$ (blue), with respect to a set of probabilistic events that are relevant to compare the different scenarios.
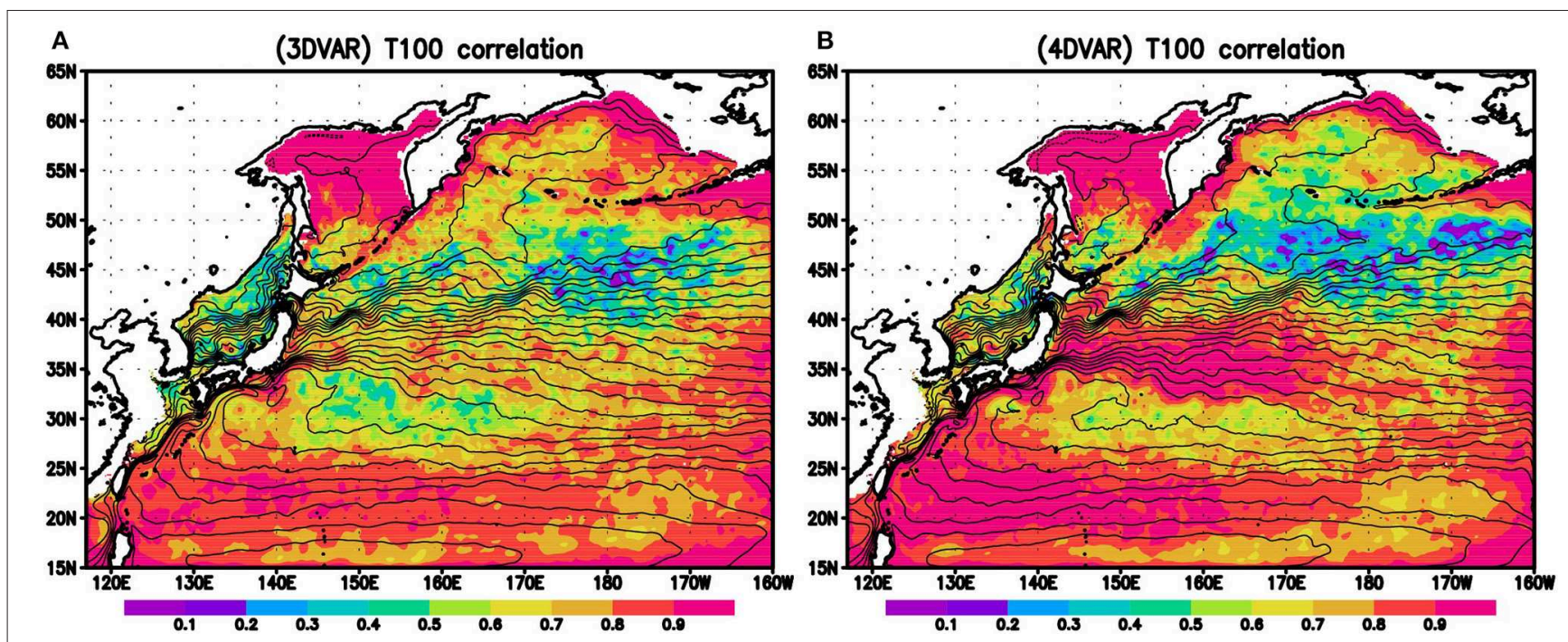

FIGURE 13 | Impacts of in-situ temperature and salinity profiles in (A) 3DVAR and (B) 4DVAR versions of JMA's operational western North Pacific ODAP system. Panels show correlation coefficients of 100-m-depth temperature anomaly with respect to the monthly climatology between assimilation runs with and without assimilating in-situ temperature and salinity profiles. The correlation is calculated for 2005-2011. Low correlation indicates high impact. 
of the system-dependency to a negligible level. But using multiple systems in OS-Eval is a potential way to reduce influence of dependency on a single system because the systematic errors of each system may be independent, and will be canceled out, to some extent, when the results from several systems are averaged or summarized. An ideal way to use multiple systems for evaluation is to conduct OSEs/OSSEs simultaneously using several different systems based on a common protocol. However, this requires coordinating the common protocol among operational/research centers. In addition, OSE/OSSE requires large computer resources as pointed out above. A singlesystem OSE/OSSE study typically requires one OSE/OSSE by each observation type to be evaluated and one control run. The period of the OSEs/OSSEs must be sufficiently long in order to obtain statistically robust results. Forecasts from the results of OSEs/OSSEs and control experiments for sufficient cases are also required if impacts on the forecast skill are evaluated. Ensemble forecasts are required if the system is highly stochastic. Furthermore, single-OSEs/OSSEs must be conducted simultaneously in several centers for a multi-system OSE.

Actually, the GOV OS-Eval TT has investigated the possibility of conducting multi-system OSEs in order to assess the tropical arrays, and the TPOS2014 workshop recommended the proposal. Although it has not been fully implemented yet an initial intercomparison of OSE results is presented in Fujii et al. (2015a). A main reason of the effort did not fully succeed is probably the lack of financial support, needed to perform such a resourceconsuming experiment at the same time in different groups. Nevertheless, multi-system OSEs for the evaluation of satellite salinity data have been successfully conducted using the UKMO and Mercator Ocean ODAP systems with financial support from ESA for the SMOS-Nino15 project (section OSEs for Evaluating Satellite SSS Data Impacts During the El Nino 2015 Event). In addition, a multi-system OSSEs are also conducted for evaluation and design of the Atlantic in-situ observation network within the H2020 AtlantOS project (section Evaluation of the Observing System Network in the Atlantic). This activity is feasible due to the close relationship among European countries and financial support from the European Union.

Although some European centers do successfully conduct multi-system activities, the number of the participating systems is still not sufficiently large (two for SMOS-Nino15, and four for H2020 AtlantOS). In order to perform more significant and more robust evaluation, it is desirable to conduct multisystem OSEs/OSSEs in a broader community. The growing understanding of the benefits of this work and the increasing requests to assess the merits of observing systems improves the chance of obtaining effective support for multi-system OSEval from operational and research centers and/or observational agencies. In fact, a common protocol on the method of OS-Eval may not be needed. It would still be beneficial to compare various evaluations of a specific observation types. This is more feasible, and may indeed offer more reliable and useful results because they do not depend on a single evaluation method. This type of collaboration is also ongoing in the community (see section Community Collaborations).

\section{Nature Run and Calibration of OSSEs}

OSSEs are widely used for the evaluation of future observing systems and network designs as introduced in section OSSEs. A clear advantage of OSSEs is that wide range of observation data, even data that do not exist in reality, can be evaluated by synthesizing the data from a simulated true state. Another advantage of OSSEs is that estimation errors in each experiment are easily calculated using the Nature Run as the true state. However, several limitations are identified through the H2020 AtlantOS project (see section Evaluation of the Observing System Network in the Atlantic). In particular, it should be noted that OSSEs operate under the following two limitations: the dependency on the performance of the Nature Run, and the difficulty in synthesizing observation data with realistic error properties.

In preliminary OSSE studies, the model used in the data assimilative experiment is also used as is, or with some changes of settings to generate the true state. However, the adequacy of OSSEs strongly depends on how appropriately the true state is simulated. The true state should favorably include a wide-range of phenomena that exist in the real world and are considered to affect the quality of data assimilation. Therefore, the true state is preferably simulated by a state-of-the-art model with an especially high resolution and no data assimilation. Such a very high performance free-running simulation generated for OSSE studies is often called the "Nature Run." The community often collaborate to generate a Nature Run because it requires very large computer resources (e.g., Gasparin et al., 2018).

For an OSSE to give a realistic real world idea of the impact of the tested observing system the Nature Run needs to be sufficiently different from the forecast model (which is known to have systematic errors). Ideally the Nature run would be as close as possible to the real world. The OSSE studies introduced in section OSSEs all give special attention to the generation of good quality Nature Run. Thorough validation of the Nature Run is a critical part of this work (Kourafalou et al., 2016). Even so the Nature Run will still inevitably have systematic errors and will lack some real world physics processes which can degrade the applicability of the OSSEs.

It is also not straightforward to generate synthesis observations from the Nature Run because adequate observation errors must be added to the model equivalent values calculated from the Nature Run. Observation errors include measurement and representativeness errors, and the errors due to inaccuracies of the observation operator. It is quite difficult to estimate those errors appropriately. Thus, modern OSSEs require the calibration of the error setting such that the OSSEs can provide impact assessments similar to OSEs with the same ODAP systems, as for example conducted in Halliwell et al. (2014), in order to guarantee that a similar result will be obtained even if the real observations are assimilated. The requirements of modern OSSEs are summarized in Hoffman and Atlas (2015).

Evolution of observation technology and ODAP systems makes further requirements for OSSE preparation. For example, some new-type observations may reflect both the ocean 
and atmospheric state. In order to evaluate the impacts of assimilating those data in a coupled data assimilation system, the true state must include both the atmosphere and ocean fields, and therefore the Nature Run must be generated by a coupled model. Considering the fact that state-of-the-art coupled models still have significant systematic errors, this may still be a big challenge. In addition, we need to design a new observation operator and its systematic errors carefully for the new-type observations, as well as its measurement and representativeness errors.

\section{Near-Real-Time Evaluation}

One of major limitation of current OS-Eval is inability to assess the effectiveness of the current observing network in near real time because the status of observing systems continuously change due to a break of an instrument for example. GOV OS-Eval TT recognized this limitation since the TT was formed, and had recommended to conduct routine OSEs in near real time in order to overcome this limitation.

According to the recommendation, a series of the NearReal-Time OSEs (NRT-OSEs) with a period of a month were performed with FOAM, the UKMO operational ODAP system, in 2011 (Lea et al., 2014). The method was to run a parallel version of the operational system identical to it except that a particular observation type is excluded. The results of the NRT-OSEs demonstrated the importance of all the observation types excluded and showed a good amount of complementarity between observations. Particularly notable is that excluding Argo data results in an increase in surface height errors as well as temperature and salinity. Even though all the altimeter data were assimilated it is necessary to have a good T-S structure in the model in order to obtain the full benefit of altimeter data assimilation.

The trial in UKMO, thus, demonstrated the potential of NRT-OSEs to evaluate observing systems in near real time. However, it is important to note that these results may not be robust due to the system-dependency as discussed in section Limitations of OS-Eval and System Dependency. The hope was that NRT-OSEs would become routine and would be run by other operational centers which would have given a better picture of the benefits of the components of the observing system in near real time. As of yet this has not happened and this remains the only example of NRT-OSEs in the ocean. The main barrier to wider implementation is the expense in terms of computer and human resources of running an additional version of the operational suite.

The importance of near real time evaluation was also highlighted in the TPOS2014 preliminary workshop. Although the influence of the reduction of the number of TAO data on seasonal predictions and other operational ocean services had to be evaluated promptly, there were few ways to examine the impacts at that time. Finally, the spread of the equatorial Pacific temperature anomaly fields among ocean reanalyses of operational centers are examined (Fujii et al., 2015a). The spread increased after the reduction of TAO data, implying it caused the increased uncertainty. This experience demonstrates that near-real-time intercomparison of ODAP products has a potential value for real time assessment of observing system status. Thus, the Real-Time Ocean Reanalysis Intercomparison project (Xue et al., 2017a) started after the workshop (section Use of Multi-system Ensemble Spread). We can quickly see the impact of changes in the observing system status by monitoring the spread among ocean reanalysis fields. For example, sudden break of observations by a specific platform may be identified from a spread increase in near real time. In contrast to the NRT-OSEs this uses existing runs and so does not require large computer resources in addition to operational calculation of ODAP systems. Thus, this strategy is very efficient and effective for monitoring observing system impacts.

The FSOI and DFS methods are also suitable for examining the impacts of observation data in near real time because these methods made evaluation in each analysis-prediction cycle, and are, therefore, widely used in operational weather forcasting systems. FSOI and DFS are also used in the US Navy's ODAP system (section Use of FSOI for Monitoring Ocean Observation Impacts in an ODAP System), and the TOPAZ ODAP system (section Use of DFS for Evaluating the Impact of the SKIM Mission). Since evaluation at each analysisprediction cycle is not robust, averaging for a sufficiently long period is necessary to obtain a reliable conclusion. Additional developments are required to calculate FSOI and DFS. For example, FSOI requires an adjoint model or results of ensemble predictions. But computational cost is relatively small as these methods simultaneously estimate impacts of all observation data assimilated on a particular metric. The results can be partitioned for any subset of the observations (e.g., instrument type, geographic region, etc.). Therefore, use of these methods for routine evaluation of observing systems in operational centers is expected to be increased in the future. There is still a role for NRT-OSEs as although they requires more computational resources. They can provide fully non-linear sensitivity, which can be used for calibration of FSOI and DFS which relies on the linear assumption.

\section{Community Collaborations}

Close community collaboration within the ODAP community is indispensable to integrate OS-Eval results in order to mitigate influence of dependency on the systems and methodology and to increase the reliability as discussed in section Multisystem Evaluation. This collaboration can also provide a forum for the exchange of ideas on OS-Eval studies and to review the latest achievements and ongoing activities. Such forum is essential to share our experience with newcomers. Awareness of the importance of this collaboration, GOV established the OS-Eval TT, which is continued in its followon program, "OceanPredict." CLIVAR-GSOP has also made significant support to OS-Eval efforts. Effective collaboration with the ocean modeling community will also be essential to increase the reliability of OS-Eval by reducing the model systematic errors.

We also emphasize the importance of communication between the ODAP and the ocean observational communities. While ocean observations provide indispensable information for 
ODAP systems, OS-Eval results can give valuable feedback to the observation community by providing scientific information on the value of ocean observations and their effective design, which supports development, maintenance, and improvement of the ocean observing system. For example, OS-Eval activities in near real time may help to target areas where Argo and drifting buoys need to be deployed for effective sampling at any given time. This is extremely useful for organizations such as the JCOMM Drifting Buoy Cooperation Panel tasked with overseeing the drifting buoy array. In order to perform a meaningful OS-Eval, requests of observational agencies/groups on the future observing networks and potential of emerging observation technologies should be considered in designing OS-Eval studies. The communication is necessary to build up the understanding on the potential of ODAP systems in the observational community to help in securing financial and human resources and to set up an adequate infrastructure in operational/research centers for OS-Eval activities. As a tool of the communication, the GOV/OceanPredict OS-Eval TT now plans to publish the Observing Impacts Statement (OIS; Lea, 2012) ideally once a year for a specific observing platform based on integration of OS-Eval results within the TT. The SMOS-Nino15 work (section OSEs for Evaluating Satellite SSS Data Impacts During the El Nino 2015 Event) highlights the interest of space agencies in developing OIS to justify follow-on missions for SSS. OISs, thus, support observational agencies to secure resources for sustaining and improving ocean observing systems.

The evolving nature of the ocean observing system (emerging technologies, possible obsolescence of existing approaches), as well as the ever changing societal needs (new applications and services, new science questions), implies that assessment of how fit-for-purpose of the ocean observing system requires continuous evaluation. Thus, the initiative on the OS-Eval by the GOV/OceanPredict OS-Eval TT and CLIVAR-GSOP should continue in the future. They are essential as an objective and authoritative source of advice on the relevance of the observing system design.

\section{CONCLUSION}

We have reviewed the methodologies of OS-Eval based on the ODAP systems, highlighting recent achievements and ongoing challenges in the evaluation of various ocean observation systems. We discussed the need to, and the strategy to, increase robustness and reliability of OS-Eval results. We showed the future vision of increasing the value of this work for designing and supporting ocean observing networks. Consequently, we make the following recommendations:

- Improvement of data assimilation methods in ODAP systems is required.

- Increased sophistication of OS-Eval methodology is needed.

- There should be clearer interpretation of the results, considering OS-Eval limitations to help the observational community.

- We should work toward performing OS-Eval in near real time.
- We should continue the efforts toward new frontiers of the ODAP systems, such as coastal regions, the deep ocean, polar regions, coupled data assimilation, and BGC applications, and to contribute the observing systems that underpin those frontiers.

- There should be closer collaboration between the ODAP and ocean modeling communities to reduce model systematic errors.

- Multi-system evaluation is needed under international coordination to improve the robustness of the results with moderating system-dependency.

- Enhanced communication is required between the ODAP and observational communities to better understand the respective needs and issues of each group. Specifically, the ODAP community must understand the key needs, opportunities, and issues of those managing the observing system; and the observational community should understand the potential of ODAP systems, as well as their strengths and weaknesses.

- Provision of human and financial resources and infrastructure is required for the OS-Eval activities under the collaboration between the ODAP and observational communities.

- Implementation of standard reporting in the form of OISs is needed to support decision-making and to provide quantitative demonstrations of data impacts that may strengthen the case for funding and improvements to ocean observing systems.

Finally, we recommend the continued development of OSEval activities at international level with the support of the international ODAP (e.g., OceanPredict and CLIVARGSOP) and observational communities. This is mandatory to guide the development of the observing system and to monitor its impact.

\section{AUTHOR CONTRIBUTIONS}

YF: decided the structure of the manuscript and coordinated the writing team. Each subsection is mainly contributed by following people: YF and PO: Introduction. YF: OSE studies for evaluation of TAO/TRITON array. ER, BT, MM, and CD: OSEs for evaluating satellite SSS data impacts during the El Nino 2015 event. HZ and MBa: OSE studies for ocean reanalysis and seasonal forecasting. PO: OSE study for evaluation of coastal gliders in a high-resolution coastal model. GH and VK: OSSEs for evaluating an underwater glider array in Western North Atlantic. FG, P-YL, and ER: Evaluation of the observing system network in the Atlantic. MBe, $\mathrm{AB}$, and ER: Evaluation of impact of wide-swath altimetry missions. NL, PH, YF, and SM: Use of adjoint models and uncertainty quantification. JC: Use of FSOI for monitoring ocean observation impacts in an ODAP system. JX and LB: Use of DFS for evaluating the impact of the SKIM mission. YX: Use of multi-system ensemble spread. YF and VK: Coastal regions. SM and FG: Deep ocean. GS: Arctic ocean. MBa and YF: Medium-range and S2S coupled prediction and coupled data assimilation. CG and PB: Evaluation of BGC Argos. YF 
and NU: Limitations of OS-eval and system dependency. YF and ER: Multi-system evaluation. YF: Nature run and calibration of OSSEs. DL and YF: Near-real-time evaluation. GL, YF, and CD: Community collaborations. YF, P-YL, and PO: Conclusion. The manuscript is edited by YF with the support of YK, SP, CD, VK, MM, ER, PO, and DL.

\section{FUNDING}

SSS OSE studies at CLS, Mercator Ocean, and UKMO were funded through the ESA SMOS-Nino15 project. OSE studies in ECMWF have been partially funded by the EU-H2020 program through the AtlantOS project (633211). OSSE studies on in-situ observations in Mercator Ocean and CNRS/IGE have been funded by the EU-H2020 program through the

\section{REFERENCES}

Akella, S., Todling, R., and Suarez, M. (2017). Assimilation for skin SST in the NASA GEOS atmospheric data assimilation system. Q. J. R. Meteorol. Soc. 143, 1032-1046. doi: 10.1002/qj.2988

Alves, O., Balmaseda, M., Anderson, D., and Stockdale, T. (2004). Sensitivity of dynamical seasonal forecasts to ocean initial conditions. Q. J. R. Meteorol. Soc. 130, 647-668. doi: 10.1256/qj.03.25

Androulidakis, Y., Kourafalou, V., Halliwell, G., Le Hénaff, M., Kang, H., Mehari, M., et al. (2016). Hurricane interaction with the upper ocean in the Amazon-Orinoco plum region. Ocean Dyn. 66, 1559-1588. doi: 10.1007/s10236-016-0997-0

Ardhuin, F., Aksenov, Y., Benetazzo, A., Bertino, L., Brandt, P., Caubet, E., et al. (2018). Measuring currents, ice drift, and waves from space: the Sea surface KInematics Multiscale monitoring (SKIM) concept. Ocean Sci. 14, 337-354. doi: 10.5194/os-14-337-2018

Atlas, R. (1997). Atmospheric observations and experiments to assess their usefulness in data assimilation. J. Meteor. Soc. Jap. 75, 111-130. doi: 10.2151/jmsj1965.75.1B_111

Aydogdu, A., Pinardi, N., Pistoi, J., Martinelli, M., Belardinelli, A., and Sparnocchia, S. (2016). Assimilation experiments for the Fishery Observing System in the Adriatic Sea. J. Mar. Syst 162, 126-136. doi: 10.1016/j.jmarsys.2016.03.002

Balmaseda, M., and Anderson, D. (2009). Impact of initialization strategies and observations on seasonal forecast skill. Geophys. Res. Lett. 36:L01701. doi: 10.1029/2008GL035561

Balmaseda, M. A., Anderson, D., and Vidard, A. (2007). Impact of Argo on analyses of the global ocean. Geophys. Res. Lett. 34, L16605. doi: 10.1029/2007GL0 30452

Balmaseda, M. A., Fujii, Y., Alves, O., Awaji, T., Behringer, D., Ferry, N., et al. (2010). "Initialization for seasonal and decadal forecasts," in Proceedings of OceanObs'09: Sustained Ocean Observations and Information for Society, Vol. 2, eds J. Hall, D. E. Harrison and D. Stammer (Venice: ESA Publication WPP-306), 19-26. doi: 10.5270/OceanObs09.cwp.02

Balmaseda, M. A., Mogensen, K., and Weaver, A. T. (2013a). Evaluation of the ECMWF ocean reanalysis system ORAS4. Q. J. R. Meteorol. Soc. 139, 1132-1161. doi: 10.1002/qj.2063

Balmaseda, M. A., Trenberth, K. E., and Källén, E. (2013b). Distinctive climate signals in reanalysis of global ocean heat content, Geophys. Res. Lett. 40, 1754-1759. doi: 10.1002/grl.50382

Balmaseda, M. A., Vidard, A., and Anderson, D. L. T. (2008). The ECMWF ocean analysis system: ORA-S3. Mon. Wea. Rev. 136, 3018-3034. doi: 10.1175/2008MWR2433.1

Bell, M., Schiller, A., Le Traon, P. Y., Smith, N., Dombrowsky, E., and Wilmer-Becker, K. (2015). An introduction to GODAE OCEANVIEW. J. Oper. Oceanogr. 8, s2-s11. doi: 10.1080/1755876X.2015.10 22041
AtlantOS project (633211). YF and SM are supported by JSPS KAKENHI Grant Number JP15H03727. YF is also supported by JPS KAKENHI grant Number JP17H00728. GH and VK are supported by the NOAA Hurricane Sandy Disaster Relief Act [OAR-M8R2WHSP01 and NA13OAR4830224] and from the NOAA Quantitative Observing System Assessment Program [QOSAP; OARP8R2W02PQF and NA15OAR4320064]. The publication fee is provided by JMA/MRI.

\section{ACKNOWLEDGMENTS}

This synthesis work was conducted as a part of the OS-Eval TT activity and underpinned by a number of studies within GOV/OceanPredict. CLIVAR-GSOP also supports this work.

Biogeochemical-Argo Planning Group (2016). The Scientific Rationale, Design, and Implementation Plan for a Biogeochemical-Argo Float Array. BiogeochemicalArgo Planning Group, 58.

Bonaduce, A., Benkiran, M., Rémy, E., Le Traon, P. Y., and Garric, G. (2018). Contribution of future wide swath altimetry missions to ocean analysis and forecasting. Ocean Sci. 14, 1405-1421. doi: 10.5194/os-14-1405-2018

Brankart, J. M., Candille, G., Garnier, F., Calone, C., Melet, A., Bouttier, P. A., et al. (2015). A generic approach to explicit simulation of uncertainty in the NEMO ocean model. Geosci. Model Develop. 8, 1285-1297. doi: 10.5194/gmd-8-1285-2015

Brassington, G. B., Martin, M. J., Tolman, H. L., Akella, S., Balmeseda, M., Chambers, C. R. S., et al. (2015). Progress and challenges in shortto medium-range coupled prediction. J. Oper. Oceanogr. 8, s239-s258. doi: 10.1080/1755876X.2015.1049875

Buizza, R., Balsamo, G., and Haiden, T. (2018). IFS Upgrade Brings More Seamless Coupled Forecasts. ECMWF Newsletter 156, 18-22. Available online at: https:// www.ecmwf.int/en/newsletter/156/meteorology/ifs-upgrade-brings-moreseamless-coupled-forecasts (accessed July 9, 2019).

Caltabiano, N., Lee, T., Haines, K., and Palmer, M. D. (2015). The CLIVAR Global Synthesis and Observations Panel (GSOP): Coordination Partnerships on Ocean Observations and Synthesis. CLIVAR Exchanges, 19, 52-54. Available online at: http://www.clivar.org/sites/default/files/documents/Exchanges_OceanObs_ No67_0.pdf

Cardinali, C., Pezzulli, S., and Andersson, E. (2004). Influence-matrix diagnostic of a data assimilation system. Q. J. R. Meteorol. Soc. 130, 2767-2786. doi: $10.1256 /$ qj. 03.205

Chang, Y. S., Zhang, S., Rosati, A., and Vecchi, G. A., Yang, X. (2018). An OSSE study for deep argo array using the GFDL ensemble coupled data assimilation system. Ocean Sci. J. 53, 179-189. doi: 10.1007/s12601-018-0007-1

Cummings, J. A., and Smedstad, O. M. (2014). Ocean data impacts in global HYCOM. J. Atmos. Ocean. Technol 31, 1771-1791. doi: 10.1175/JTECH-D-14-00011.1

Davidson, F. J. M., Allen, A., Brassington, G. B., Breivik, Ø., Daniel, P., Kamachi, M., et al. (2009). Applications of GODAE ocean current forecasts to search and rescue and ship routing. Oceanography. 22, 176-181. doi: $10.5670 /$ oceanog.2009.76

Desbruyères, D. G., Purkey, S. G., McDonagh, E. L., Johnson, G. C., and King, B. A. (2016). Deep and abyssal ocean warming from 35 years of repeat hydrography. Geophys. Res. Lett. 43, 10356-10365. doi: 10.1002/2016GL070413

Durand, M., Fu, L. L., Lettenmaier, D. P., Alsdorf, D. E., Rodriguez, E., and Esteban-Fernandez, D. (2010). The surface water and ocean topography mission: observing terrestrial surface water and oceanic submesoscale eddies. Proc. IEEE 98, 766-779. doi: 10.1109/JPROC.2010.20 43031

Evensen, G. (2003). The Ensemble Kalman Filter: theoretical formulation and practical implementation. Ocean Dyn. 53, 343-367. doi: $10.1007 / \mathrm{s} 10236-003-0036-9$ 
Fujii, Y., Cummings, J., Xue, Y., Schiller, A., Lee, T., Balmaseda, M. A., et al. (2015a). Evaluation of the tropical pacific observing system from the ocean data assimilation perspective. Q. J. R. Meteorol. Soc. 141, 2481-2496. doi: 10.1002/qj.2579

Fujii, Y., Kamachi, M., Nakaegawa, T., Yasuda, T., Yamanaka, G., Toyoda, T., et al. (2011). "Assimilating ocean observation data for ENSO monitoring and forecasting," in Climate Variability - Some Aspects, Challenges and Prospects, ed A. Hannachi (Rijeka: InTech Open), 75-98. doi: 10.5772/30330

Fujii, Y., Ogawa, K., Brassington, G. B., Ando, K., Yasuda, T., and Kuragano, T. (2015b). Evaluating the impacts of the tropical Pacific observing system on the ocean analysis fields in the global ocean data assimilation system for operational seasonal forecasts in JMA. J. Oper. Oceanogr. 8, 25-39. doi: 10.1080/1755876X.2015.1014640

Fujii, Y., Tsujino, H., Usui, N., Nakano, H., and Kamachi, M. (2008). Application of singular vector analysis to the Kuroshio large meander. J. Geophys. Res. 113:C07026. doi: 10.1029/2007JC004476

Fukasawa, M., Freeland, H., Perkin, R., Watanabe, T., Uchida, H., and Nishina, A. (2004). Bottom water warming in the North Pacific Ocean. Nature 427, 825-827. doi: 10.1038/nature02337

Garnier, F., Brankart, J.-M., Brasseur, P., and Cosme, E. (2016). Stochastic parameterizations of biogeochemical uncertainties in a $1 / 4^{\circ} \mathrm{NEMO} / \mathrm{PISCES}$ model for probabilistic comparisons with ocean color data. J. Mar. Syst. 155(Suppl. C), 59-72. doi: 10.1016/j.jmarsys.2015.10.012

Gasparin, F., Greiner, E., Lellouche, J. M., Legalloudec, O., Garric, G., Drillet, Y., et al. (2018). A large-scale view of oceanic variability from 2007 to 2015 in the global high resolution monitoring and forecasting system at Mercator Océan. J. Mar. Syst. 187, 260-276. doi: 10.1016/j.jmarsys.2018. 06.015

Gasparin, F., Guinehut, S., Mao, C., Mirouze, I., Remy, E., King, R., et al. (2019a). Requirements for an integrated in situ atlantic ocean observing system from coordinated observing system simulation experiments. Front. Mar. Sci. 6:83. doi: 10.3389/fmars.2019.00083

Gasparin, F., Hamon, M., Remy, E., and Le Traon, P. Y. (2019b). How deep Argo will improve the deep ocean in an ocean reanalysis. J. Clim. [Epub ahead of print].

Gaultier, L., Ubelmann, C., and Fu, L. L. (2016). The challenge of using future SWOT data for oceanic field reconstruction. J. Atmos. Ocean. Technol. 33, 119-126. doi: 10.1175/JTECH-D-15-0160.1

Gehlen, M., Barciela, R., Bertino, L., Brasseur, P., Butenschön, M., Chai, F., et al. (2015). Building the capacity for forecasting marine biogeochemistry and ecosystems: recent advances and future developments. J. Oper. Oceanogr. 8, s168-s187. doi: 10.1080/1755876X.2015.10 22350

Halliwell, G. R., Mehari, M. F., Le Henaff, M., Kourafalou, V. H., Androulidakis, I. S., Kang, H. S., et al. (2017). North Atlantic ocean OSSE system: evaluation of operational ocean observing system components and supplemental seasonal observations for potentially improving tropical cyclone prediction in coupled systems. J. Oper. Oceanogr. 10, 154-175. doi: 10.1080/1755876X.2017.13 22770

Halliwell, G. R. Jr., Srinivasan, A., Kourafalou, V., Yang, H., Willey, D., and Atlas, R. (2014). Rigorous evaluation of a fraternal twin ocean OSSE system for the open Gulf of Mexico. J. Atmos. Oceanic Technol. 31, 105-130. doi: 10.1175/JTECH-D-13-00011.1

Herzfeld, M. (2006). An alternative coordinate system for solving finite difference ocean models. Ocean Model. 14, 174-196. doi: 10.1016/j.ocemod.2006.04.002

Hoffman, R. N., and Atlas, R. (2015). Future observing system simulation experiments. Bull. Amer. Meteorol. Soc. 96, 1601-1616. doi: 10.1175/BAMS-D-15-00200.1

IPCC (2014). "Climate change 2014: synthesis report," in Contribution of Working Groups I, II and III to the Fifth Assessment Report of the Intergovernmental Panel on Climate Change, eds Core Writing Team, R.K. Pachauri and L.A. Meyer (Geneva: IPCC), 151.

Johnson, G. C., Purkey, S. G., and Bullister, J. L. (2008). Warming and freshening in the abyssal southeastern Indian Ocean. J. Clim. 21, 5351-5363. doi: 10.1175/2008JCLI2384.1

Jones, E. M., Oke, P. R., Rizwi, F., and Murray, L. (2012). Assimilation of glider and mooring data into a coastal ocean model. Ocean Model. 47, 1-13. doi: 10.1016/j.ocemod.2011.12.009
Jung, T., Gordon, N. D., Bauer, P., Bromwich, D. H., Chevallier, M., Day, J., et al. (2016). Advancing polar prediction capabilities on daily to seasonal time scales, Bull. Amer. Meteorol. Soc 97, 1631-1647. doi: 10.1175/BAMS-D-14-00246.1

Kalmikov, A. G., and Heimbach, P. (2014). A hessian-based method for uncertainty quantification in global ocean state estimation. SIAM J. Sci. Comput. 36, S267-S295. doi: 10.1137/130925311

Kalmikov, A. G., and Heimbach, P. (2018). On barotropic mechanisms of uncertainty propagation in estimation of drake passage transport. Eprint arXiv:1804.06033v2. Available online at: https://arxiv.org/abs/1804.06033v2 (accessed July 11, 2019).

Kouketsu, S., Doi, T., Kawano, T., Masuda, S., Sugiura, N., Toyoda, T., et al. (2011). Deep ocean heat content changes estimated from observation and reanalysis product and their influence on sea level change. J. Geophys. Res. 116:C03012. doi: 10.1029/2010JC006464

Kourafalou, V., Androulikakis, Y., Halliwell, G. R. Jr., Kang, H., Mehari, M. et al. (2016). North Atlantic Ocean OSSE system development: nature run evaluation and application to hurricane interaction within the Gulf Stream. Prog. Oceanogr. 148, 1-25. doi: 10.1016/j.pocean.2016.09.001

Laloyaux, P., Balmaseda, M., Dee, D., Mogensen, K., and Janssen, P. (2016). A coupled data assimilation system for climate reanalysis. Q. J. R. Meteorol. Soc. 142, 65-78. doi: 10.1002/qj.2629

Laloyaux, P., Thépaut, J. N., and Dee, D. (2017). Impact of scatterometer surface wind data in the ECMWF coupled assimilation system. Mon. Wea. Rev 144, 1203-1217. doi: 10.1175/MWR-D-15-0084.1

Langland, R. H., and Baker, N. L. (2004). Estimation of observation impact using the NRL atmospheric variational data assimilation adjoint system. Tellus 56A, 189-201. doi: 10.1111/j.1600-0870.2004.00056.x

Le Traon, P. Y., Dibarboure, G., Jacobs, G., Martin, G., Remy, E., and Schiller, A. (2017). "Use of satellite altimetry for operational oceanography," in Satellite Altimetry Over Oceans and Land Surfaces, eds D. Stammer and A. Cazenave, (Boca Raton: CRC Press). doi: 10.1201/9781315151779-18

Lea, D. J. (2012). Observation Impact Statements for Operational Ocean Forecasting Weather Science Technical Report, 568, Exeter: Met Office. Available online at: https://digital.nmla.metoffice.gov.uk/download/file/digitalFile_a747fc880535-495c-885b-66855fd67766 (accessed July 9, 2017).

Lea, D. J., Martin, M. J., and Oke, P. R. (2014). Demonstrating the complementarity of observations in an operational ocean forecasting system. Q. J. R. Meteorol. Soc. 140, 2037-2049. doi: 10.1002/qj.2281

Lea, D. J., Mirouze, I., Martin, M. J., King, R. R., Hines, A., Walters, D., et al. (2015). Assessing a new coupled data assimilation system based on the Met Office Coupled Atmosphere-Land-Ocean-Sea ice model. Mon. Wea. Rev. 143, 4678-4694. doi: 10.1175/MWR-D-15-0174.1

Lee, T., Lagerloef, G., Gierach, M. M., Kao, H.-Y., Yueh, S., and Dohan, K. (2012). Tropical instability waves linked to sea surface salinity anomalies. Geophys. Res. Lett. 39:L12610. doi: 10.1029/2012GL052232

Lellouche, J. M., Le Galloudec, O., Drévillon, M., Régnier, C., Greiner, E., Garric, G., et al. (2013). Evaluation of global monitoring and forecasting systems at Mercator Océan. Ocean Sci. 9, 57-81. doi: 10.5194/os-9-57-2013

Loose, N. (2019). Adjoint modeling and observing system design in the subpolar North Atlantic (Ph.D dissertation). University of Bergen, Bergen, Norway.

Lozier, M. S., Bacon, S., Bower, A. S., Cunningham, S. A., de Jong, M. F., de Steur, L., et al. (2016). Overturning in the subpolar North Atlantic program: a new international ocean observing system. Bull. Amer. Meteorol. Soc. 98, 737-752. doi: 10.1175/BAMS-D-16-0057.1

Madec, G. (2016). NEMO Ocean Engine. Available online at: http://www.nemoocean.eu/About-NEMO/Reference-manuals/NEMO_book_3.6_STABLE (accessed March 15, 2018).

Martin, M. J., King, R. R., While, J., and Martin, A. A. (2018). Assimilating satellite sea surface salinity data from SMOS, Aquarius and SMAP into a global ocean forecasting system. Q. J. R. Meteorol. Soc. 145, 705-726. doi: 10.1002/qj.3461

Masuda, S., Awaji, T., Sugiura, N., Matthews, J. P., Toyoda, T., Kawai, Y., et al. (2010). Simulated rapid warming of abyssal north Pacific waters. Science 329, 319-322. doi: 10.1126/science.1188703

Masuda, S., Osafune, S., and Hemmi, T. (2018). Deep-float salinity data synthesis for deep ocean state estimation: method and impact. Progr. Earth Planet. Sci. 5:89. doi: 10.1186/s40645-018-0247-9

McPhaden, M. J., Busalacchi, A. J., Cheney, R., Donguy, J., Gage, K. S., Halpern, D., et al. (1998). The tropical Ocean-Global Atmosphere observing system, 
a decade of progress. J. Geophys. Res. 103, 14169-14240. doi: 10.1029/97JC 02906

Mogensen, K. S., Magnusson, L., and Bidlot, J. R. (2017). Tropical cyclone sensitivity to ocean coupling in the ECMWF coupled model. Q. J. R. Meteorol. Soc. 122, 4392-4412. doi: 10.1002/2017JC012753

Moore, A. M., Arango, H. G., Broquet, G., Edwards, C., Veneziani, M., Powell, B., et al. (2011). The Regional Ocean Modeling System (ROMS) 4-dimensional variational data assimilation systems: part III - Observation impact and observation sensitivity in the California Current System. Prog. Oceangr. 91, 74-94. doi: 10.1016/j.pocean.2011.05.005

Ocean Observations Panel for Climate (OOPC), (2014). "Tropical Pacific observing system," in 2020 Workshop (TPOS 2020) Vol. 1: Workshop Report and Recommendations GCOS-184/GOOS-205/WCRP-6/2014, 66. Available online at: http://tpos2020.org/wp-content/uploads/TPOS-2020-WorkshopReport-FINAL-300114.pdf

Oke, P. R., Allen, J. S., Miller, R. N., Egbert, G. D., and Kosro, P. M. (2002). Assimilation of surface velocity data into a primitive equation coastal ocean model. J. Geophys. Res. 107, 3122-3147. doi: 10.1029/2000JC000511

Oke, P. R., Balmaseda, M. A., Benkiran, M., Cummings, J. A., Dombrowsky, E., Fujii, Y., et al. (2009). Observing system evaluations using GODAE systems. Oceanography 22, 144-153. doi: 10.5670/oceanog.2009.72

Oke, P. R., Brassington, G. B., Griffin, D. A., and Schiller, A. (2010). Ocean data assimilation: a case for ensemble optimal interpolation. Aust. Meteorol. Oceanogr. J. 59, 67-76. doi: 10.22499/2.5901.008

Oke, P. R., Larnicol, G., Fujii, Y., Smith, G. C., Lea, D. J., Guinehut, S., et al. (2015a). Assessing the impact of observations on ocean forecasts and reanalyses: part 1, Global studies. J. Oper. Oceanogr. 8, s49-s62. doi: 10.1080/1755876X.2015.1022067

Oke, P. R., Larnicol, G., Jones, E. M., Kourafalou, V. H., Sperrevik, A. K., Carse, F., et al. (2015b). Assessing the impact of observations on ocean forecasts and reanalyses: part 2: regional applications. J. Oper. Oceanogr. 8, S63-S79. doi: 10.1080/1755876X.2015.1022080

Oke, P. R., and Schiller, A. (2007). Impact of Argo, SST, and altimeter data on an eddy-resolving ocean reanalysis. Geophys. Res. Lett. 34:L19601. doi: 10.1029/2007GL031549

Osafune, S., Masuda, S., Sugiura, N., and Doi, T. (2015). Evaluation of the applicability of the Estimated Ocean State for Climate Research (ESTOC) dataset. Geophys. Res. Lett. 42, 4903-4911. doi: 10.1002/2015GL064538

PPP Steering Group (2013). WWRP Polar Prediction Project Implementation Plan. WMO Report. WWRP/PPP 2-2013, 72. Available at: https://www. polarprediction.net/fileadmin/user_upload/www.polarprediction.net/Home/ Documents/WWRP-PPP_IP_Final_12Jan2013_v1_2.pdf (accessed October 29, 2018).

PPP Steering Group (2014). WWRP Polar Prediction Project Implementation Plan for the Year of Polar Prediction (YOPP). WMO Report. WWRP/PPP 3-2014, 53. Available at: https://www.polarprediction.net/fileadmin/user_upload/www. polarprediction.net/Home/Documents/WWRP-PPP_YOPP_Plan_2014_v1_ 1.pdf (accessed October 29, 2018)

Purkey, S. G., and Johnson, G. C. (2010). Warming of global abyssal and deep Southern Ocean waters between the 1990s and the 2000s: contributions to global heat and sea level rise budgets. J. Clim. 23, 6336-6351. doi: 10.1175/2010JCLI3682.1

Reul, N., Fournier, S., Boutin, J., Hernandez, O., Maes, C., Chapron, B., et al. (2013). Sea surface salinity observations from space with the SMOS Satellite: a new means to monitor the marine branch of the water cycle. Surv. Geophys. 35, 681-772. doi: 10.1007/s10712-013-9244-0

Riser, S. C., Freeland, H. J., Roemmich, D., Wijffels, S., Troisi, A., Belbéoch, M., et al. (2016). Fifteen years of ocean observations with the global Argo array. Nat. Clim. Change 6, 145-153. doi: 10.1038/nclimate2872

Rodriguez, E., and Esteban-Fernandez, D. (2010). “The Surface Water and Ocean Topography Mission (SWOT): the Ka-band Radar Interferometer (KaRIn) for water level measurements at all scales." in Proceedings of the SPIE Remote Sensing Conference, Vol. 7826, Sensors, Systems, and Next-Generation Satellites $X I V$, eds R. Meynart, S. P. Neeck, and H. Shimoda (Toulouse), 782614. doi: $10.1117 / 12.868525$

Roemmich, D., Alford, M.H., Claustre, H., Johnson, K., King, B., Moum, J., et al. (2019). On the future of Argo: an enhanced global array of physical and biogeochemical sensing floats. Front. Mar. Sci.
Roulston, M. S., and Smith, L. A. (2002). Evaluating probabilistic forecasts using information theory. Mon. Wea. Rev. 130, 1653-1660. doi: 10.1175/1520-0493(2002)130<1653:EPFUIT>2.0.CO;2

Sakov, P., Counillon, F., Bertino, L., Lisæter, K. A., Oke, P. R., and Korablev, A. (2012). TOPAZ4: an ocean-sea ice data assimilation system for the North Atlantic and Arctic. Ocean Sci. 8, 633-656. doi: 10.5194/os-8-633-2012

Severinghaus, J. P., Sowers, T., Brook, E. J., Alley, R. B., and Bender, M. L. (1998). Timing of abrupt climate change at the end of the Younger Dryas interval from thermally fractionated gases in polar ice. Nature 391, 141-146. doi: $10.1038 / 34346$

Smith, G. C., Allard, R., Babin, M., Bertino, L., Chevallier, M., Corlett, G. K., et al. (2019). Polar ocean observations: a critical gap in the observing system and its role in affecting polar environmental prediction. Front. Mar. Sci.

Smith, G. C., Bélanger, J. M., Roy, F., Pellerin, P., Ritchie, H., and Onu, K. (2018). Impact of Coupling with an ice-ocean model on global medium-range NWP forecast skill. Mon. Wea. Rev. 146, 1157-1180. doi: 10.1175/MWR-D-17-0157.1

Smith, N., Kessler, W. S., Cravatte, S., Sprintall, J., Wijffels, S., Cronin, M. F., et al. (2019). Tropical Pacific observing system. Front. Mar. Sci. 6:31. doi: 10.3389/fmars.2019.00031

Stammer, D., Balmaseda, M., Heimbach, P., Köhl, A., and Weaver, A. (2016). Ocean data assimilation in support of climate applications: status and perspectives. Ann. Rev. Mar. Sci 8, 491-518. doi: 10.1146/annurev-marine-122414-034113

Subramanian, A., Balmaseda, M. A., Chattopadhyay, R., Centurioni, L. C., Cornuelle, B. C., DeMott, C. A., et al. (2019). Ocean observations to improve our understanding, modeling, and forecasting of subseasonal-to-seasonal variability. Front. Mar. Sci.

Thacker, W. C. (1989). The role of the Hessian matrix in fitting models to measurements. J. Geophys. Res. 94, 6177-6196. doi: 10.1029/JC094iC05p06177

Thomson, R. E., and Emery, W. J. (2014). "Chapter 5: time series analysis methods," in Data Analysis Methods in Physical Oceanography, 3rd Edn, eds R. E. Thomson and W. J. Emery (Boston, MA: Elsevier), 425-591. doi: 10.1016/B978-0-12-387782-6.00005-3

Tollefson, J. (2014). El Niño tests forecasters. Nature 508, 20-21. doi: $10.1038 / 508020$ a

Toyoda, T., Fujii, Y., Kuragano, T., Matthews, J. P., Abe, H., Ebuchi, N., et al. (2015). Improvements to a global ocean data assimilation system through the incorporation of Aquarius surface salinity data. Q. J. R. Meteorol. Soc. 141, 2750-2759. doi: 10.1002/qj.2561

Tranchant, B., Remy, E., Greiner, E., and Legalloudec, O. (2019). Data assimilation of Soil Moisture and Ocean Salinity (SMOS) observations into the Mercator Ocean operational system: focus on the EL Nino 2015 event. Ocean Sci. 15, 543-563. doi: 10.5194/os-15-543-2019

Turpin, V., Rémy, E., and Le Traon, P. Y. (2016). How essential are Argo observations to constrain a global ocean data assimilation system. Ocean Sci. 12, 257-274. doi: 10.5194/os-12-257-2016

Usui, N., Fujii, Y., Sakamoto, K., and Kamachi, M. (2015). Development of a four-dimensional variational assimilation system toward coastal data assimilation around Japan. Mon. Wea. Rev. 143, 3874-3892. doi: 10.1175/MWR-D-14-00326.1

Visbeck, M., Araujo, M., Boetius, A., Buch, E., Claustre, H., Dabrowski, T., et al. (2015). More Integrated and More Sustainable Atlantic Ocean Observing System (AtlantOS). CLIVAR Exchanges 67, 18-20.

Wang, Q., Mu, M., and Dijkstra, H. A. (2013). Effects of nonlinear physical processes on optimal error growth in predictability experiments of the Kuroshio Large Meander. J. Geophys. Res. 118, 6425-6438. doi: 10.1002/2013JC009276

World Weather Research Program (WWRP) (2017). Subseasonal-to-Seasonal Prediction Project. Driving Innovation Together; The World Weather Research Program. WMO-No. 1191, 13-16. Available online at: http://s2sprediction.net/ file/documents_reports/1191_WWRP_Driving_Innovation_JN17380(2).pdf

Wunsch, C., and Heimbach, P. (2013). "Dynamically and kinematically consistent global ocean circulation and ice state estimates," in Ocean Circulation and Climate: A 21st Century Perspective, 2nd Edn, Vol. 103 (Amsterdam: Elsevier Ltd.), 553-579. doi: 10.1016/B978-0-12-391851-2.00021-0

Xie, J., Bertino, L., Counillon, F., Lisæter, K. A., and Sakov, P. (2017). Quality assessment of the TOPAZ4 reanalysis in the Arctic over the period 1991-2013. Ocean Sci. 13, 123-144. doi: 10.5194/os-13-123-2017 
Xue, Y., Wen, C., Kumar, A., Balmaseda, M., Fujii, Y., Alves, O., et al. (2017a). A real-time ocean reanalyses intercomparison project in the context of tropical Pacific observing system and ENSO monitoring. Clim. Dyn. 49, 3647-3672. doi: 10.1007/s00382-017-3535-y

Xue, Y., Wen, C., Yang, X., Behringer, D., Kumar, A., Vecchi, G., et al. (2017b). Evaluation of tropical Pacific observing system using NCEP and GFDL ocean data assimilation systems. Clim. Dyn. 49, 843-868. doi: 10.1007/s00382-015-2743-6

Zanna, L., Heimbach, P., Moore, A. M., and Tziperman, E. (2011). Optimal excitation of interannual Atlantic meridional overturning circulation variability. J. Clim. 24, 413-427. doi: 10.1175/2010JCLI3610.1

Zuo, H., Balmaseda, M. A., and Mogensen, K. (2017). The new eddypermitting ORAP5 ocean reanalysis: description, evaluation and uncertainties in climate signals. Clim. Dyn. 49, 791-811. doi: 10.1007/s00382-0152675-1

Zuo, H., Balmaseda, M. A., Mogensen, K., and Tietsche, S. (2018). OCEAN5: The ECMWF Ocean Reanalysis System and Its Real-Time Analysis Component. ECMWF Technical Memo 823, 44.

Zuo, H., Balmaseda, M. A., Tietsche, S., Mogensen, K., and Mayer, M. (2019). The ECMWF operational ensemble reanalysis-analysis system for ocean and sea-ice: a description of the system and assessment. Ocean Sci. 15, 779-808. doi: 10.5194/os-15-779-2019

Conflict of Interest Statement: JC and SP were employed by company I.M Systems Group (IMSG). GL and BT were employed by company Collecte Localisation Satellites (CLS).

The remaining authors declare that the research was conducted in the absence of any commercial or financial relationships that could be construed as a potential conflict of interest.

Copyright (c) 2019 Fujii, Rémy, Zuo, Oke, Halliwell, Gasparin, Benkiran, Loose, Cummings, Xie, Xue, Masuda, Smith, Balmaseda, Germineaud, Lea, Larnicol, Bertino, Bonaduce, Brasseur, Donlon, Heimbach, Kim, Kourafalou, Le Traon, Martin, Paturi, Tranchant and Usui. This is an open-access article distributed under the terms of the Creative Commons Attribution License (CC BY). The use, distribution or reproduction in other forums is permitted, provided the original author(s) and the copyright owner(s) are credited and that the original publication in this journal is cited, in accordance with accepted academic practice. No use, distribution or reproduction is permitted which does not comply with these terms. 


\section{OPEN ACCESS}

Edited by:

Sabrina Speich,

École Normale Supérieure, France

Reviewed by:

Tong Lee,

NASA Jet Propulsion Laboratory

(JPL), United States

Katherine Louise Hill,

World Meteorological Organization,

Switzerland

${ }^{*}$ Correspondence:

Brad deYoung

bdeyoung@mun.ca

Specialty section:

This article was submitted to

Ocean Observation,

a section of the journal

Frontiers in Marine Science

Received: 02 November 2018 Accepted: 05 July 2019 Published: 26 July 2019

Citation:

deYoung $B$, Visbeck $M$, de Araujo Filho MC, Baringer MO, Black CA,

Buch E, Canonico G, Coelho P,

Duha JT, Edwards M, Fischer AS,

Fritz J-S, Ketelhake S, Muelbert JH, Monteiro P, Nolan G, O'Rourke E,

Ott M, Le Traon PY, Pouliquen S,

Sousa-Pinto I, Tanhua T, Velho F and

Willis Z (2019) An Integrated

All-Atlantic Ocean Observing System in 2030. Front. Mar. Sci. 6:428.

doi: 10.3389/fmars.2019.00428

\section{An Integrated All-Atlantic Ocean Observing System in 2030}

\author{
Brad deYoung ${ }^{1 *}$, Martin Visbeck ${ }^{2}$, Moacyr Cunha de Araujo Filho ${ }^{3}$, \\ Molly O'Neil Baringer ${ }^{4}$, CarolAnne Black ${ }^{5}$, Erik Buch ${ }^{6}$, Gabrielle Canonico', \\ Paulo Coelho ${ }^{8}$, Janice T. Duha ${ }^{9}$, Martin Edwards ${ }^{10}$, Albert Fischer ${ }^{11}$, Jan-Stefan Fritz ${ }^{12}$, \\ Sandra Ketelhake ${ }^{13}$, Jose-Henrique Muelbert ${ }^{14}$, Pedro Monteiro ${ }^{15}$, Glenn Nolan ${ }^{6}$, \\ Eleanor O'Rourke ${ }^{16}$, Michael Ott'11, Pierre Y. Le Traon ${ }^{17}$, Sylvie Pouliquen ${ }^{18}$, \\ Isabel Sousa-Pinto ${ }^{19}$, Toste Tanhua ${ }^{13}$, Filomena V. Velho ${ }^{8}$ and Zdenka Willis ${ }^{20}$
}

\begin{abstract}
1 Physics and Physical Oceanography, Memorial University, St. John's, NL, Canada, ${ }^{2}$ GEOMAR Helmholtz Centre for Ocean Research Kiel, Kiel University, Kiel, Germany, ${ }^{3}$ Laboratório de Oceanografia Fisica Estuarine e Costeria, Departamento de Oceanografia da Universidad Federal de Pernambuco, Recife, Brazil, ${ }^{4}$ Atlantic Oceanographic and Meteorological Laboratory, National Oceanic and Atmospheric Administration, Miami, FL, United States, ${ }^{5}$ Independent Researcher, Ottawa, ON, Canada, ${ }^{6}$ EuroGOOS, Brussels, Belgium, ${ }^{7}$ US IOOS, National Oceanic and Atmospheric Administration, Silver Spring, MD, United States, ${ }^{8}$ National Fisheries Research Institute, Luanda, Angola, ${ }^{9}$ Advisor of Ocean Affairs of the Secretary of STI of the Brazilian Navy, Rio de Janeiro, Brazil, ${ }^{10}$ Marine Biological Association, Plymouth, United Kingdom, ${ }^{11}$ Intergovernmental Oceanographic Commission of UNESCO, Paris, France, ${ }^{12}$ German Marine Research Consortium, Berlin, Germany, ${ }^{13}$ GEOMAR Helmholtz Centre for Ocean Research, Kiel, Germany, ${ }^{14}$ Federal University of Rio Grande, Rio Grande, Brazil, ${ }^{15}$ Council for Scientific and Industrial Research, Cape Town, South Africa, ${ }^{16}$ Marine Institute, Galway, Ireland, ${ }^{17}$ Ifremer and Mercator Ocean, Brest, France, ${ }^{18}$ Ifremer and Coriolis, Brest, France, ${ }^{19}$ Climar, University of Porto and Euromarine Network, Porto, Portugal, ${ }^{20}$ Veraison Consulting LLC, Great Falls, VA, United States
\end{abstract}

The ocean plays a vital role in the global climate system and biosphere, providing crucial resources for humanity including water, food, energy, and raw materials. There is a compelling need to develop an integrated basin-scale ocean observing system to support of ocean management. We articulate a vision for basin-scale ocean observing A comprehensive All-Atlantic Ocean Observing Systems that benefits all of us living, working and relying on the ocean. Until now, basin-scale ocean observation has been conducted through loosely-aligned arrangements of national and international efforts. The All-Atlantic Ocean Observing System (AtlantOS) is an integrated concept for a forward-looking framework and basin-scale partnership to establish a comprehensive ocean observing system for the Atlantic Ocean as a whole. The system will be sustainable, multi-disciplinary, multi-thematic, efficient, and fit-for-purpose. Platforms, networks, and systems do already exist that operate at various maturity levels. AtlantOS will go beyond the status quo by bringing together the observing communities and countries of the Atlantic basin, providing the opportunity to join and support the system. AtlantOS will build upon the coordinated work of the Global Ocean Observing System (GOOS) and the Group on Earth Observations (GEO), two international bodies that support and coordinate global ocean observing. AtlantOS will complement those efforts and offers a new approach to organizing ocean observing at the basin-scale. AtlantOS will focus not only on the physics but also the biology, ecology and biogeochemistry of the ocean and seafloor and will enhance new partnerships among governments, science, civil society and the private sector.

Keywords: ocean observation, Atlantic Ocean, ocean observing system, ocean governance, basin-scale, global ocean observing system, framework for ocean observing 


\section{VISION FOR AN ALL-ATLANTIC OCEAN OBSERVING SYSTEM IN 2030}

\section{A Comprehensive All-Atlantic Ocean Observing System That Benefits All of Us Living, Working and Relying on the Ocean}

The All-Atlantic Ocean Observing System (AtlantOS) is a concept for a forward-looking framework and basin-scale partnership to establish a comprehensive ocean observing system for the Atlantic Ocean as a whole (Figure 1). The system will be sustainable, multi-disciplinary, efficient, and fit-for-purpose. AtlantOS recognizes that platforms, networks, and systems already exist that operate at various maturity levels. AtlantOS will go beyond the status quo by bringing together the countries of the Atlantic basin providing the opportunity to join and support the system. AtlantOS, the All-Atlantic Ocean Observing System, grows out of the European Union's (EU) Horizons 2020 project AtlantOS into a program that will build upon the coordinated work of the Global Ocean Observing System (GOOS) and the Group on Earth Observations (GEO), two international bodies that support and coordinate global ocean observing. AtlantOS will complement those efforts and offers a new approach to organize ocean observing. AtlantOS will focus not only on the physics but also the biology, ecology and biogeochemistry of the ocean and seafloor.

Ocean observations touch our lives every day from the food we eat, to the clothes we wear, to how we spend our leisure time. The ocean is estimated to be the seventh largest economy in the world. Goods and services from coastal and marine environments have been estimated at US $\$ 2.5$ trillion each year worldwide (Hoegh-Guldberg et al., 2015).

In building AtlantOS it is important that we define and follow clear and transparent principles. The implementation of this vision will lead to a sustainable comprehensive observing system in the Atlantic basin from which societies, governments, and industries around the Atlantic will benefit (Figure 2). To guide this development, this paper lays out the vision and shows why the community should work together toward and invest in ocean observing. It defines the principles of the system, how partners could contribute, and presents the benefits that will be derived from an effective ocean observing system. This vision then needs to be complemented by the development of an Implementation Plan, that offers a more detailed review that points to the operational challenges and approaches and offers guidance on implementation of the ocean observing system and the roles of different partners.

This high-level strategy and its vision were developed to provide a structural framework for guiding the development of the Atlantic Ocean Observing System. We will outline a baseline of core platforms, networks and systems that make up AtlantOS and a path: from initial capability at the beginning of the United Nations (UN) Decade of Ocean Science for Sustainable Development in 2021, to full operating capability at the end of the decade in 2030. The program will build upon past development of ocean observing. Ocean Observations are critical to public services, facilitate commercial activity, support scientific research, foster well-being, and protect lives and property. While much has been accomplished, there remain gaps in fully meeting user needs at the basin-scale.

By 2030, we foresee that AtlantOS will have the following attributes:

- AtlantOS is the All-Atlantic part of the Global Ocean Observing System,

- All designated AtlantOS data providers are coordinated, integrated and cooperating to efficiently make accessible all relevant Essential Ocean Variables (EOVs) as part of the Global Ocean Observing System and the Group on Earth Observations Blue Planet programme,

- User needs are met by keeping connected with users and understanding their evolving requirements,

- A fully functioning governance framework is in place and provides a forum for coordination, resource mobilization, review and decision making, and

- Long-term sustainability has been achieved through national and stakeholder commitments with a goal of reaching $75 \%$ of sustained resources, in line with present meteorological observational support.

Full operating capability does not imply the end of development but an objective that defines the initial establishment of a fully functional AtlantOS. AtlantOS will be an agile system that will evolve as user needs change, new technologies are developed and new frontiers in ocean and earth system research are discovered.

AtlantOS will support many users including governmental and intergovernmental, commercial, non-governmental organizations, and scientific sectors to advance the use of ocean observations in the delivery of public services, sustained economic benefits and earth systems research. We envisage a basin-scale system that is compatible with global aspirations and serves as a model for other ocean basins, to realize the potential of well-integrated ocean information in support of a wide range of benefits including sustainable ocean economy with strong ocean stewardship. AtlantOS will serve a multi-dimensional range of interests relevant to the broader social and economic concerns of society.

Key examples of where AtlantOS ocean observing and information enables benefits:

- Disaster Resilience: Storm surge, hurricane and tsunami warnings are provided with enough advanced notice and precision to support successful emergency response.

- Blue Economy: Supporting sustainable prosperity from the ocean through advanced seafloor ecosystem mapping and habitat characterization, pollution tracking, identification of renewable energy options, carbon sequestration mechanisms, and other of ecosystem services.

- Food Security: Helping to achieve global food security, maximizing the sustainable food benefit that we can extract from the ocean now and in the future, by supporting sustainable fisheries and mariculture operations and management. 


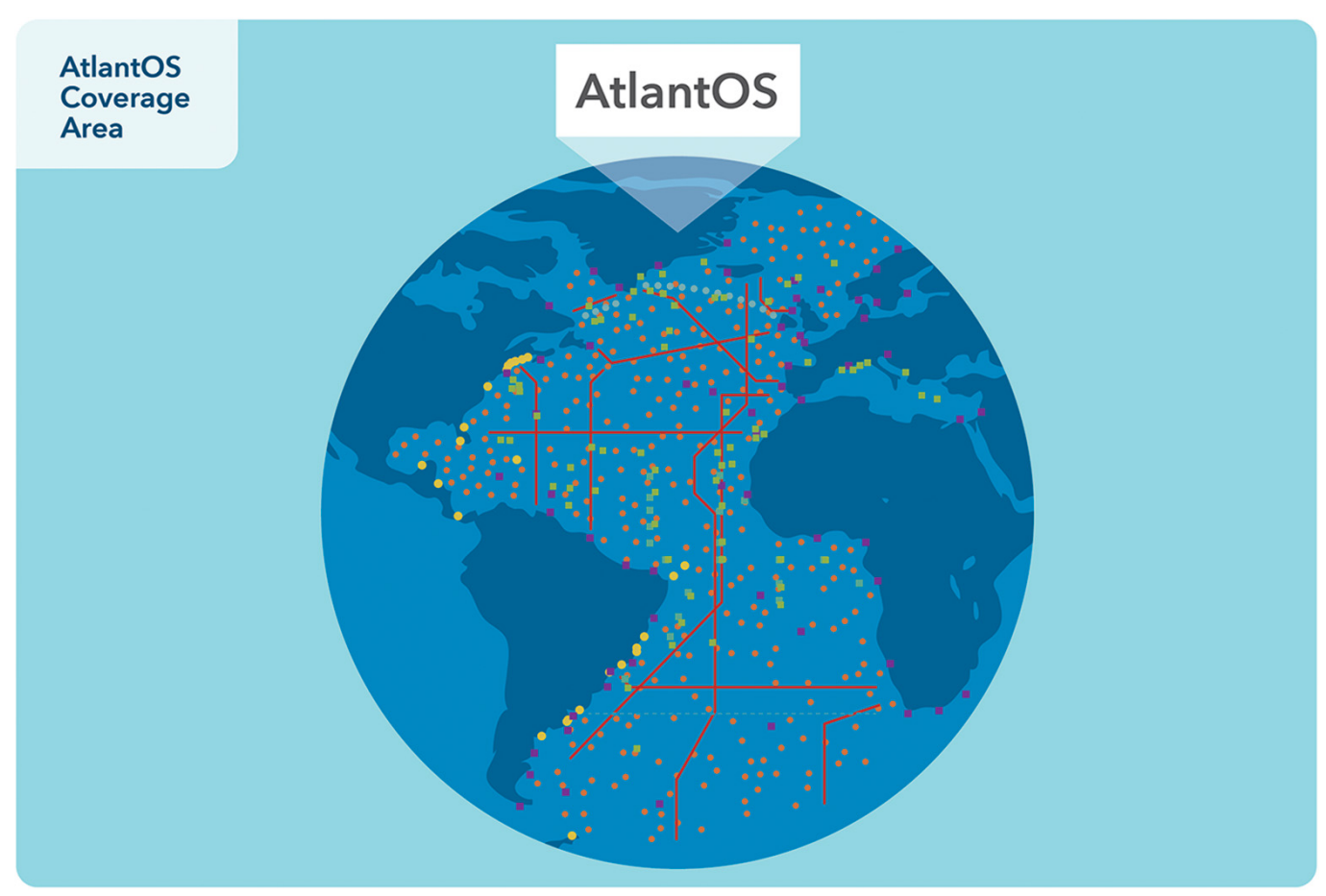

FIGURE 1 | AtlantOS coverage area. The Atlantic Ocean, depicting the focus area of ocean observing for AtlantOS. Dots and lines conceptually indicate current ocean observing activities by major networks, such as Argo, GO-SHIP, PIRATA, and OSNAP.

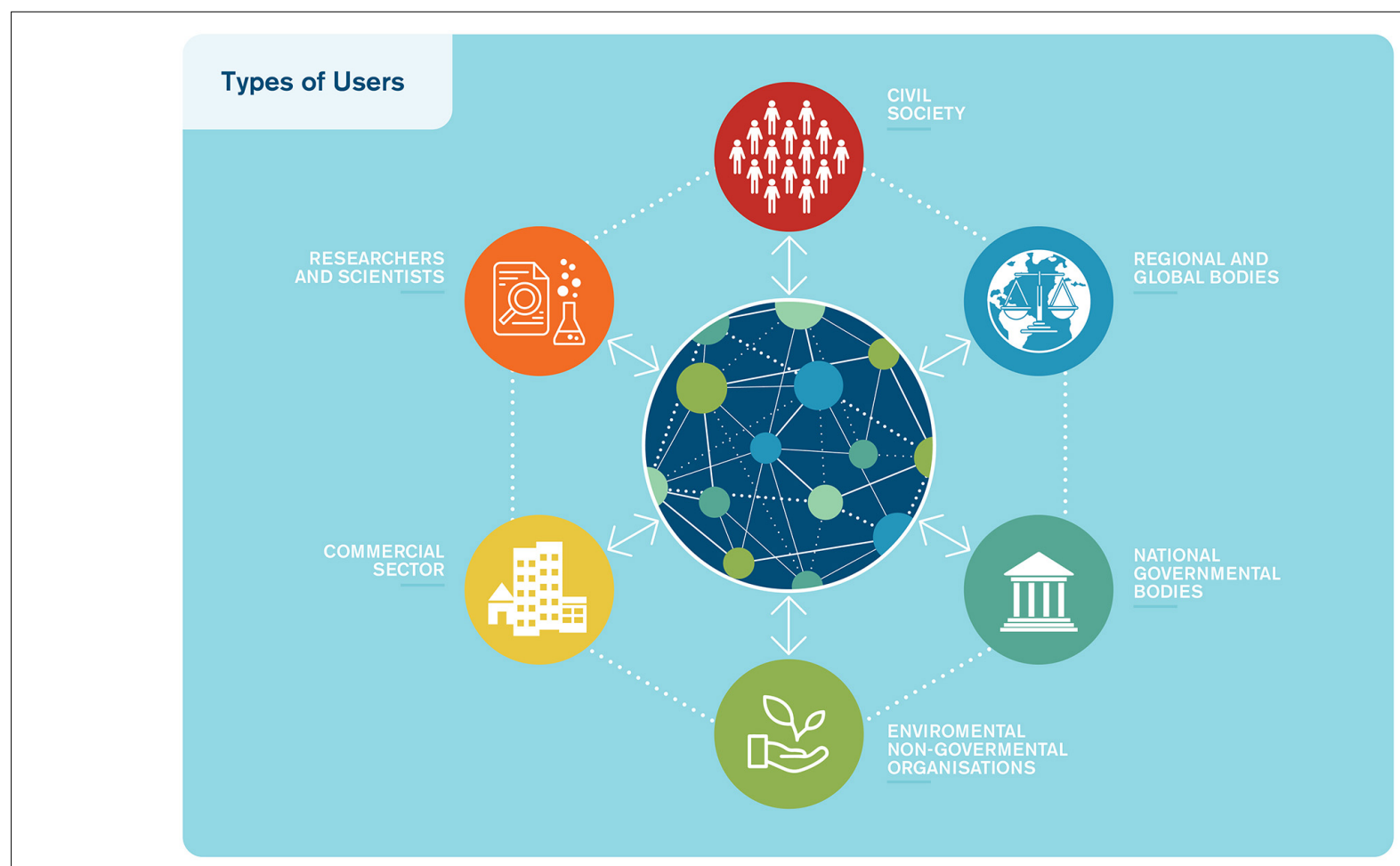

FIGURE 2 | Types of users. The data and data products provided by AtlantOS will support many user groups, ranging from industry to private citizens, from government to non-governmental organizations. 


\section{- Biodiversity and Ecosystem Sustainability:} Understanding changes in biodiversity and ecosystems to determine impacts on natural capital and ecosystem services and ensure our ocean resources can support human nutritional, recreational, and health needs. This includes monitoring the present abundance and distribution of organisms and improved forecasting of events such as harmful algal blooms (HABs), marine life mortality, or coral bleaching, which impact recreational and commercial use of the ocean.

- Marine Transportation: Forecasts of extreme wave events, hurricanes and tropical storm tracks, forecasts of ocean currents, sea-ice monitoring and iceberg tracking, oil spill and pollutant dispersals, and the locations of whales and other protected species, with advisories to modify ship routes or decrease speeds to avoid collisions in the North Atlantic with the busiest shipping lanes on the planet.

- Climate Change: Research that includes climate change indicators, such as the Atlantic Meridional Overturning Circulation, including measurements of ocean heat and circulation providing regional sea level monitoring, ocean circulation changes and climate feedbacks, and changes that affect ocean life, such as regional $\mathrm{pH}$ and oxygen levels.

- Ocean System Science: Research that allows discovery of new processes and phenomena of the ocean system, supports embedded process observations, enables the development and validation of ocean system models and supports innovation in ocean state assessment and prediction.

As an example of the type of service that could be offered at the basin-scale, the operational ecosystem model SEAPODYM (Spatial Ecosystem And Population Dynamics Model) is used to simulate the mid-trophic levels of the ocean, i.e., the micronekton, and then the spatial dynamics of tuna and their fisheries. The optimal sampling conditions of epipelagic and mesopelagic biomass have been identified with modeling to support the calibration of this ecosystem variables now included in the CMEMS catalog. Then, a new management and monitoring system is proposed for tuna fisheries (Dragon et al., 2015) and illustrated with the Atlantic Albacore tuna. This pre-operational forecast system (Figure 3) can bring value by providing increased information about changes in catch rates due to environmental variability, e.g., changes in abundance over time and space by age class from larvae to adults. It also permits distinction between fishing impact and natural variability of the stock and supports real-time monitoring of fishing activity and stock assessment analyses.

We must see ocean observation as a fundamental requirement for a global society. Over the past century, humanity has come to see roads, electricity, policing and many other services as fundamental requirements of today's society. So too must we shift our perspective about ocean observation in the twentyfirst century. We have a responsibility to act as stewards of our ocean - in our regions and globally, the international community has identified a range of goals and drivers that require systematic ocean observations (e.g., Miloslavich et al., 2018; Tanhua et al., 2019).

The ocean provides crucial resources for humanity and plays a vital role in the global biosphere and climate system. Ocean resources include food, energy, raw materials, recreational use, global trade and cultural services. According to a study by the Organization for Economic Cooperation and Development (OECD), by 2030 the ocean could more than double its contribution to the global economy, reaching over US\$ 3 trillion. To conserve and protect our ocean and the vast resources it provides, we must observe and understand ocean regions and how they interact - through transfer of heat, freshwater, nutrients, oxygen, carbon dioxide and marine life.

Improved and sustained observation of our ocean is necessary for informed decision-making to ensure human-ocean interactions are sustainable (Weller et al., 2019). We need coordination of observations, bringing together the data from many sources and countries: surface buoys measuring wind and waves; robots documenting the ocean's interior, ships and other methods for surveying fish stocks; and satellites providing the view from above. We should share information widely, opening data to many uses and the greatest value. We will save time, money, and energy by working together.

The ocean observing community has produced a Framework for Ocean Observing (Task Team for an Integrated Framework for Sustained Ocean Observing, 2012 - FOO), a guide to help us decide what to measure in our ocean and why. The FOO has been a useful guide in the writing this visionary strategy and will be helpful as we shift focus to the Implementation Plan.

\section{Why Now?}

Coordinated ocean observations have been in practice since the creation of GOOS in 1991. GOOS initially focussed on physics and climate issues. As a result of the OceanObs series of conferences $(1999,2009)$, GOOS was expanded to include ocean chemistry and biology as well as a focus on coastal observing. Indeed, these two meetings laid the foundations for the ocean observing systems that have developed since and documents such as the FOO, which arose from the OceanObs'09 meeting, guide much of the ocean observing community. New demands and expectations, and the upcoming OceanObs'19 meeting, offer opportunities to assess how we are conducting ocean observing and consider fresh approaches.

Our capabilities to observe the ocean, and our understanding of it, have been arisen from key collaborative science programs such as the World Ocean Circulation Experiment (WOCE), the Climate Variability and Predictability Programme (CLIVAR), the Joint Global Ocean Flux Study (JGOFS) and the Global Ocean Ecosystem Dynamics (GLOBEC) programs. These multi-disciplinary, large scale programs stimulated multinational collaborations and stimulated technological developments critical to our present ocean observing systems.

In 2015, the United Nations agreed to the 2030 Agenda for Sustainable Development and its 17 Goals (SDGs), and 195 countries agreed on the Paris Accord. In 2017, a call for action was adopted by member states at the high-level UN Ocean Conference supporting the implementation of SDG 14 

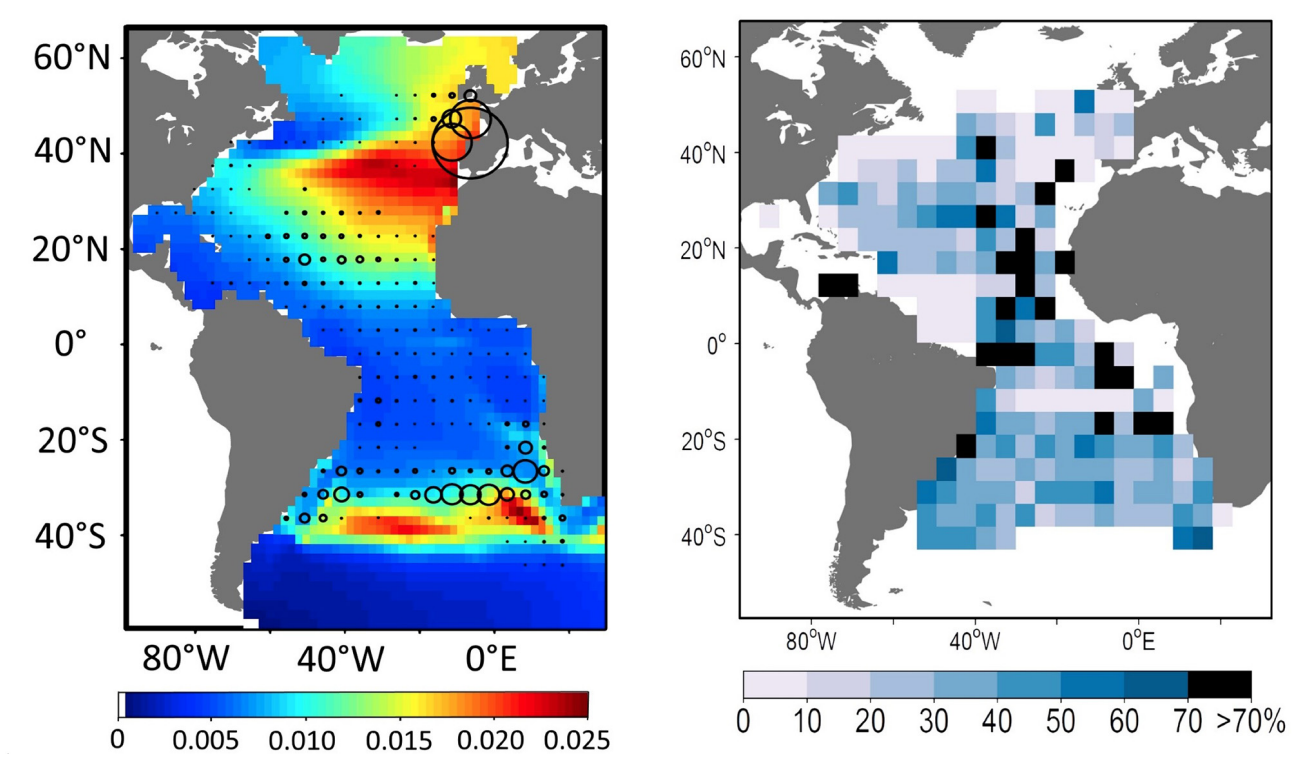

FIGURE 3 | The SEAPODYM (spatial ecosystem and population dynamics model) operational system supports near real-time prediction of albacore tuna stocks in the Atlantic. Left Density of adult fish $\left(\mathrm{kg} / \mathrm{km}^{2}\right)$ and observed catch (circles) in July. Right Error in predicted total catch.

to "conserve and sustainably use the oceans, seas and marine resources for sustainable development." G7 leaders have also shown an interest in the importance of ocean science since 2016, most recently with the 2018 publication, Charlevoix Blueprint for Healthy Oceans, Seas and Resilient Communities.

Two international Atlantic cooperative agreements for marine science provide an incentive and context for a focus on ocean observing with a particular emphasis on the Atlantic. These are: the Galway Statement on Atlantic Ocean Cooperation, signed by the European Union, Canada and the United States of America (U.S.) in 2013; and the Belém Statement on Atlantic Research and Innovation Cooperation, signed by the European Union, South Africa and Brazil in 2017 with additional EU-Argentina and EU-Cabo Verde agreements and the ambition to include other countries around the Atlantic Basin.

Another opportunity for working together and growing ocean observing and information systems is the United Nations Decade of Ocean Science for Sustainable Development (20212030) which was declared in late 2017. We must seize these opportunities, to focus our efforts on developing an ocean observing system that fulfills our Atlantic and global stewardship responsibilities.

\section{MEETING USER NEEDS}

Meeting user needs begins with assessing the societal benefits of sustained ocean observations, and then understanding how an observing system can provide those benefits (Weller et al., 2019). Growing coastal populations and use of the ocean's resources are creating increased demand for ocean information. Limited funds mean the observing system must be efficient, cost-effective, and responsive to user needs. We must make the best use of the diverse range of ocean observations available. Much of the work in designing an integrated ocean observing system for the Atlantic Ocean involves ensuring that existing programs are integrated coherently into a system that is fit-for-purpose, i.e., responsive to the needs of stakeholders.

The process of identifying and refining components of the value chain, as discussed in the FOO, from instruments measuring the ocean to information in users' hands, should be ongoing and iterative. We must pay attention to evolving technologies, changes in the ocean, societal needs, political alignment and leverage, and our ability to use observations to benefit society. Activities at each step in the value chain should be justified by the needs of one or more user communities. Different approaches are possible and all should be employed bottom up, working from the data, or top-down working from users' needs. Reviews of the system should be conducted on a regular basis.

GOOS has developed an open-access tool we can use to follow the links in the value chain. We can trace scientific questions, phenomena, Essential Ocean Variables, and the observing networks required to satisfy a societal benefit. Alternatively, we can determine the phenomena and societal benefits that rely on an EOV or on observing networks.

A key challenge is to distinguish what ocean variables are essential (and feasible) from those that are only desirable. The ocean is vast and there are many possible variables to measure, and at many scales. We must focus to ensure that our observations create the greatest benefit. The needs of the users, which are diverse and complex, should be the main consideration for the development of products and services.

The aim of a multiplatform, multidisciplinary, multisector basin-wide system is to optimize observations at basin-scale 
and maximize the benefits of the data collected. Data from observing platforms should be used for many different observing objectives in real-time and over and over again in delayed mode. The capacity and gap analysis should be done on the full value chain, for both societal benefit and improved scientific understanding.

\section{Challenges in Meeting User Needs}

An effective observing network or system needs clear standards for components and careful consideration of the societal benefits. The FOO document identifies lessons learned from the successes of existing observing efforts, and provides an internationally accepted common language and guidance for expanded collaboration in sustained ocean observations. The FOO uses EOVs as a framework to determine the key observations that are required to achieve the goals of the observing system. EOVs are negotiated based on feasibility and impact. Essential means that these observations are the minimum subset required; they are not replaceable by other variables. This framework, while challenging to implement, has been accepted by the user community as a guide to determine the key or essential measurements that should be made.

The review process should involve formal and analytical physical, biogeochemical, biological/ecological, and socioeconomic metrics, as well as qualitative approaches. Many aspects of our present observing system, such as some historical coastal stations, were selected based on pragmatic considerations. Expert panels have often played a key role. For example, the observing system for climate was originally developed through international panels such as the Ocean Observing System Development Panel (OOSDP, 1995) and the following Ocean Observing Panel on Climate (OOPC) which supported early developments of sustained ocean observing systems. Today, the advancement of computer models, data assimilation systems and prediction systems have enabled more quantitative, purposeful specifications for ocean observing (Moore et al., 2019). Computer model-based experiments, or Observing System Experiments (OSEs), enable testing of existing observation networks and systems, and Observing System Simulation Experiments (OSSEs), "simulate" testing of future observing networks and systems.

Several international efforts are working to coordinate systematic observing system and modeling system evaluations through programs, e.g., CLIVAR - Global Synthesis and Observations Panel (CLIVAR-GSOP) and the Global Ocean Data Assimilation Experiment (GODAE) Ocean View (now OceanPredict) are two such programs, with a focus on physical oceanography. OceanPredict is also dealing with biogeochemical modeling and data assimilation. The EU Horizon 2020 project AtlantOS includes a focus on the Atlantic Ocean, using state-of-the-art models to refine methods used in computer models (OSSEs, OSEs) to provide consistent, calibrated results. The improvement of observing system technologies must develop hand-in-hand with improvements in modeling and data assimilation systems (see Smith et al., 2019, for some thoughts on approaches to consider). Given our capabilities, we should rely on evidence-based approaches for the design of AtlantOS, the All-Atlantic Ocean Observing System (Ostrander et al., 2019).

\section{PRESENT OCEAN OBSERVING IN THE ATLANTIC}

AtlantOS is not a new system but rather a new approach to provide a comprehensive, state of the art observing system for the Atlantic Basin that can be fully realized and sustained for the long term. AtlantOS will build upon existing observing assets. Like other ocean observing systems, AtlantOS proposes to take measurements of ocean properties and use those measurements to understand how the ocean system functions, how it affects us, and how we affect the ocean. The system must encompass many types of platforms and sensors and needs to fully take into account the synergies and complementarities with present and future satellite observations. We learn a lot about the ocean from satellites including sea level and geostrophic ocean currents, water temperature, ocean color, sea ice, sea surface salinity, waves and winds (Le Traon et al., 2015). Satellites play a critical role in providing real time, global high space and time resolution observations of key ocean variables that are essential to constraining ocean models and for operational use (Le Traon et al., 2019). New capabilities (SWOT, SKIM) are also developed to observe the ocean dynamics at very high resolution (Morrow et al., 2019) or to directly observe surface ocean currents from space. Satellite observations are, however, largely restricted to the ocean surface. To fully understand, describe and forecast the 3D ocean, in situ observations (measurements taken in and out of the water) are mandatory. In situ observations are also essential to calibrate and validate satellite observations.

We still need direct measurements in the ocean - which can be from instruments left at a fixed spot in the ocean, from a ship or from drifting or mobile platforms (e.g., gliders or tagged animals). When we put these different data streams together, plus measurements of the atmosphere, we can see how the ocean and atmosphere work together, giving us a powerful tool to predict what is happening in our ocean and how this supports many societal benefit areas. Using evidence derived from ocean observations, we can keep people safe, support robust communities, and foster sustainable use while enabling the stewardship required to adapt and manage human-ocean interactions in changing ocean ecosystems.

Figure 4 would suggest that the whole ocean is fully covered and begs the question as to why there is concern about the state of ocean observation. Our response is that our observations are constantly improving, but still inadequate to respond to rapidly growing societal needs, and that the coordination and integration of the existing observations is less than it should be. We can get more value out of the effort we presently put into ocean observation. To do this, we need to understand what observing is happening and what user needs those activities meet.

To begin to realize a fully operational AtlantOS, we have proposed a methodology for determining what elements 


\section{JCOMMOPS \\ AtlantOS Status Map}

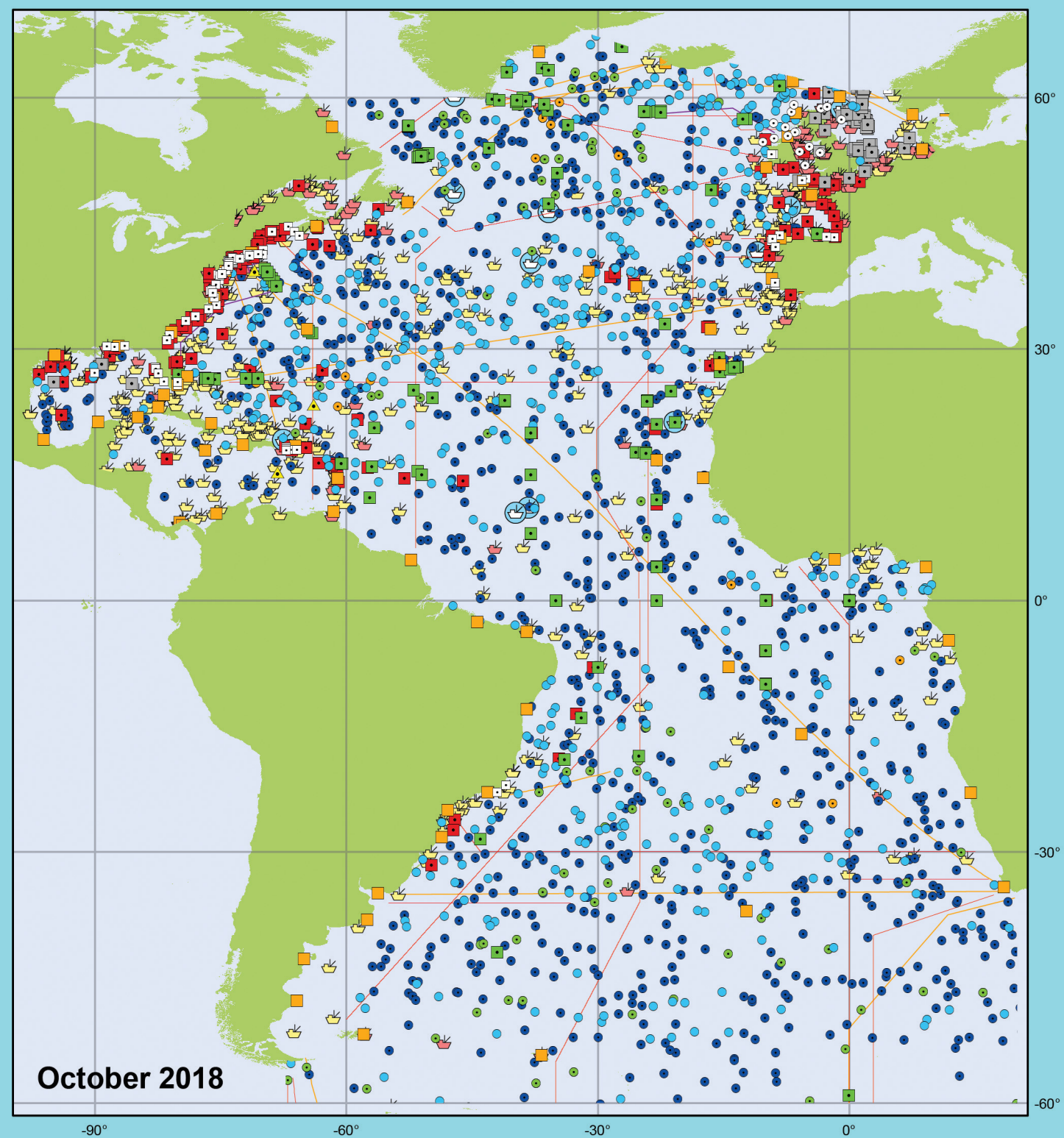

FIGURE 4 | Global ocean observing system (GOOS) status map for September 2018 (Source: JCOMMOPS). Map of the Atlantic Ocean showing the location of ocean platforms (drifters, moorings, buoys, etc.) with onboard instruments collecting ocean observations. Coverage map is of observing activities by GOOS network members. 


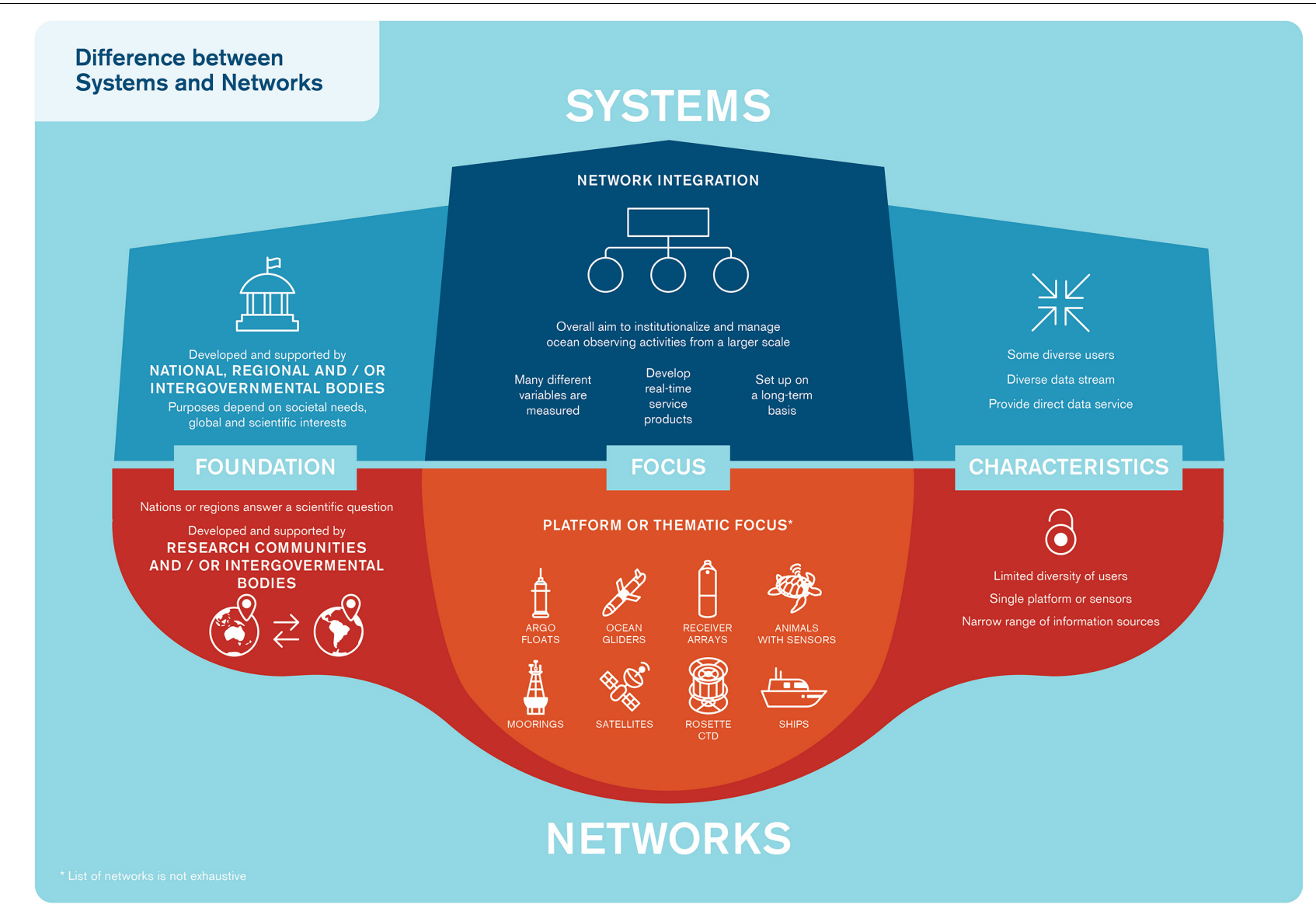

FIGURE 5 | Difference between systems and networks. Major ocean observing activities can be organized into two categories: Systems and Networks. AtlantOS, as a system, will coordinate networks conducting ocean observing in the Atlantic.

represent the initial core network and which systems would be included. Existing observing activities can be described in two main forms (Figure 5). Often the terms network and system are used interchangeably, however, networks and systems are different:

- Ocean observing networks are typically platform- or theme-focused activities - where platforms can be ships, moorings, drifters, ocean wave gliders and more, to which instruments can be mounted - based on common technology and data types, and often cater to focused ocean issues; themes include topics such as biodiversity and acidification. Networks are typically founded via international collaborations between research institutes and countries (like GO-SHIP, MBON, OceanSITES, SOOP, and Argo) with the aim to observe the ocean together (Duffy et al., 2013). They have common practices and standards, shared scientific infrastructure within their own network but with policies to share data openly. Global networks, such as Argo, are often built around a particular instrument platform. As an example, the growing High Frequency (HF) Radar network is built around coastal radar networks that provide measurements of coastal current and waves. The focus of networks is generally narrower, technology focussed and more specific than is true for observing systems.

- Ocean observing systems are large-scale national, regional or international programs founded and funded by governments and stakeholders that build on networks within a region. Three clear examples (presented later) are GOOS, EuroGOOS/EOOS and the U.S. Integrated Ocean Observing System (U.S. IOOS $\left.{ }^{\circledR}\right)$. Many different variables are measured in an ocean observing system, such as temperature, sea state, ocean currents, salinity, ocean acidity, dissolved gas concentration, biodiversity or seafloor properties. The motivation is to coordinate ocean observing activities carried out by research institutes within a geographic region. Their purposes depend on societal needs and governmental interests, as well as scientific interests. Observing systems typically have some forms of legal bases. In general, the aim is to institutionalize and manage ocean observing activities on a larger scale. Ocean observing systems promote open access to data and cooperate with their members to develop products for users. Observing systems are set up on a long-term basis. Ocean observing networks are members of ocean 
observing systems and are supported by them, e.g., via financial contributions.

For the All-Atlantic Ocean Observing System to succeed and provide benefit, ocean observing governance needs to evolve and recognize the basin scale as governing scale. There are key challenges with the coordination and governance of ongoing observation of the Atlantic Ocean:

- Today, not all of the networks and systems needed for AtlantOS are affiliated with GOOS and adequately governed or coordinated Some systems, such as U.S. IOOS and EuroGOOS do identify as GOOS Regional Alliances (GRAs) and parts of the global system. Other networks, such as the Marine Biodiversity Observation Network $(\mathrm{MBON})$, reside under the Group on Earth Observations Biodiversity Observation Network (GEO BON) and operate in explicit partnership with GOOS and the IOC. Others are national or regional in scope and governance - often established by treaty or other agreements or to satisfy national reporting requirements; these often exist and operate outside of GOOS or any other global body.

- There are differences in how systems are defined. For example, US IOOS is an adaptive, federated network of ocean observation, data management and communications, and modeling and analysis capabilities. EuroGOOS is a non-governmental organization (NGO) that coordinates national observing systems and funds coordinating activities.

These diverse groups, with different structures and responsibilities, present coordination challenges and gaps and there is widespread recognition that those gaps must be addressed if we are to embrace a true basin-scale observing approach.

Ocean observing systems exist also at the sub-basin scale. Two examples are presented here: EuroGOOS is a regional observing system with 42 members from 20 European countries. Members join EuroGOOS to have a single common voice, e.g., at the European Commission level. Since February 2013, EuroGOOS has been established as an international non-profit organization it can enter into contracts and have direct managerial control over the secretariat. The system promotes an open data policy and funding includes membership contributions, external funding via research projects and tender contracts. At present an European Ocean Observation System (EOOS) is being developed that would integrate EuroGOOS and increase the range of activities from the European system. US IOOS is a nationally-funded program that aims to gather observing data and develop tracking and predictive tools to benefit the economy, the environment, and public safety across their nations and around the globe.

A few key examples of current Atlantic and global ocean observing networks and systems are:

- Argo is an international ocean observing network of nearly 4,000 freely profiling floats that measure temperature and salinity in the upper 2,000 meters of the global ocean and are also extending to deeper ocean and bio-geochemistry EOVs. Since 2000, when the network formed, Argo has revolutionized oceanography, providing publicly-accessible data in near real-time. Argo data are vital for understanding ocean temperature, salinity, and current variations and their effects on weather, climate and ecosystems. Argo is a voluntary network, with the implication that longterm funding is not guaranteed. Argo's primary users are the World Meteorological Organization (WMO), the operational oceanographic services and the research community - easily identified groups with which Argo has close connections. The tight link between users and networks suggests that support from users in systems, such as AtlantOS, may be more challenging as the path from the data collection to the users is harder to identify.

- In the Atlantic there are three regional scale observing networks (OSNAP, RAPID, and SAMOC) all measuring the Atlantic Meridional Overturning Circulation (AMOC) that is a major process for moderating climate change, transferring heat and carbon dioxide from the surface to the deep ocean and from the tropics to the subpolar regions. These three networks have simple governance models, taking the governance approach of major international scientific programs, mainly because they have voluntary contribution models and indeed most of the funding comes from scientific funding councils of the contributing nation states.

- The Marine Biodiversity Observation Network works in partnership with GOOS, the Ocean Biogeographic Information System (OBIS), and a broad range of global partners to coordinate and make available the marine biological and ecosystem observations needed to ensure living marine resources are sustainably conserved and managed and can support essential human needs. MBON is refining methods for environmental DNA applications in an operational ocean observing context and has developed advanced methods to evaluate marine biogeographic provinces (seascapes) using multidisciplinary satellite remote sensing data. The MBON Pole to Pole initiative is building a community of practice that helps nations to improve conservation planning and environmental impact mitigation, serves the scientific community, and satisfies commitments to the Intergovernmental SciencePolicy Platform on Biodiversity and Ecosystem Services (IPBES), Aichi Targets of the Convention of Biological Diversity (CBD), and the UN 2030 Agenda for Sustainable Development Goals, as well as supporting efforts to produce and international, legally binding instrument under UNCLOS (United Nation Convention on the Law of the Sea) on the conservation and sustainable use of marine biological diversity of areas beyond national jurisdiction. MBON Pole to Pole is achieving this goal through: (1) Enhanced coordination of coastal and ocean observations and data collection among nations, (2) improved collection of harmonized data, and furthering data standards and methodologies, (3) integration of physical and biogeochemical observations with biodiversity information to characterize status and trends, and (4) generation of products needed for informed management of the ocean. 
- PIRATA is a regional-scale ocean observing system in the Tropical Atlantic founded in 1995 and has recently been renamed to "Prediction and Research Moored Array in the Tropical Atlantic." It is recognized as the reference network of oceanic and atmospheric observations in the Tropical Atlantic, as well as for climate-dedicated research and for operational climate and ocean prediction. The network was designed for studying ocean-atmosphere interactions in the tropical Atlantic that affect regional weather and climate variability on seasonal, interannual and longer time scales. Ocean-atmosphere interactions in this region influence the development of droughts, floods, severe tropical storms and hurricanes, with impacts felt by millions of people in America and Africa. This system is supported mainly by three member countries - the U.S., France and Brazil and collaborates with others such as Germany. The governance structure is more "formal" because the member countries work through a Memorandum of Understanding that is renewed every four to five years.

- The IOC-WMO Joint Technical Commission for Oceanography and Marine Meteorology (JCOMMOPS, 2017) provides organizational support through the JCOMM Ocean Coordination Group (OCG) and status reviews and metrics through the JCOMM Operational Program Support (OPS). Most of the programs supported by JCOMM operate as networks and not as systems. Many global networks supported by JCOMM are wellestablished (e.g., ARGO, GO-SHIP, OceanSITES) but there are new emerging networks such as HF Radar and Ocean Gliders, which are also JCOMM networks. All JCOMM networks support free access to open data and quality control processes.

\section{Challenges for Existing Ocean Observation Networks and Systems}

Ideally these existing networks would be integrated into coordinated systems at the global level. That is true for example of the AtlantOS components of Argo, OceanSITES and GOSHIP. But overall there is a lack of coordination linking the national/regional to the basin-scale (i.e., AtlantOS) and from the basin scale to the global scale. It is therefore the right time to articulate how we can adjust or integrate these component networks to form a desired fit-for-purpose, basin-scale system. While many may agree on the goal for a clearer more integrated system, the steps to reach or achieve this goal are actively debated by the ocean observing community.

Several key issues, or challenges can be identified:

\section{Multiple Sensors and Platforms}

First, the existing observing landscape includes many sensors and platforms. Many states conduct monitoring activities within their Exclusive Economic Zone (EEZ) that do not link systematically with the GOOS networks [e.g., Continuous Plankton Recorder (CPR) or fish stock monitoring that respond to EU habitat requirements]. Such activities use platforms and methodologies that meet local/national needs and are managed and archived at the national level. For a sustainable ocean observation system, it will be important to incorporate all these networks into a more connected governance structure. This might be more effectively coordinated at the basin-scale level. The coastal networks should be aligned so that vital ocean information products like storm surge, hurricane, oil spills or harmful algal bloom forecasts as well as changes in distributions of fish or other organisms could be predicted earlier and more reliably.

\section{Focus Too Narrow}

Many existing observing networks are too focussed on the physical Essential Ocean Variables articulated by the GOOS physics panel, e.g., ocean temperature, salinity, currents, waves, and sea level. There is increasing interest from users, and the scientific community to incorporate biogeochemistry, biology and ecosystem EOVs; GOOS panels are developing specification sheets and implementation plans to guide that effort, which includes newer activities such as animal tracking. One challenge as we widen the scope of our interest is how to provide guidance (e.g., using the FOO) for practitioners to select from an ever growing set of measurement expectations and requirements.

\section{Independent Operation}

Ocean observing networks and systems tend to work and be comfortable within their own structures. Collaborations between the different networks and systems, like the Argo-GOSHIP cooperation, where the two networks collect overlapping data types and can validate and build on one another, hold valuable potential and are only partly exploited. Increased collaborations will lead to a faster-evolving, more integrated and effective observing in the Atlantic Ocean where time, money, and knowledge would be used in an optimized and sustainable way.

\section{System Evaluation and User Feedback}

Ocean observing networks and systems are largely thematically or issue focussed and often started with a scientific need for information. AtlantOS has the ambition to connect existing systems, facilitate platform and information sharing and improve overall system efficiency. However, a system evaluation and feedback mechanism needs to be established to ensure the AtlantOS system is meeting multiple user needs. Thematic reviews, user consultations and discussions and agreements between resource providers are essential.

\section{Lack of Long-Term Funding}

Most observing networks lack long-term, sustainable funding. They depend on voluntary contributions from members or national governments, which often change from year to year. Much of the present funding structure is based on research funding and may not be sustainable. Without adequate and reliable sustainable funding, user needs cannot be met and innovation and capacity building will weaken. Governments must recognize the societal and economic benefits of open data policies and equipment and resource sharing. The governance structures of ocean observing systems should be oriented toward reliable, long-term strategies, akin to what we have for weather 
observations. The funding structures should be similarly aligned. With the development of AtlantOS, we can grow and coordinate collaborations between ocean observing systems, to improve data sharing and build a common data policy, and promote an effective voluntary funding structure and support capacity building activities.

\section{MOVING FROM THE PRESENT TO ATLANTOS}

In considering how to develop AtlantOS, it is clear that we will not start from nothing. There is a wide array of infrastructure in place. Many global networks presently operate well as do many coastal and regional systems. How do we move forward from this present patchwork of activity to the more coordinated and comprehensive system that we envisage?

A first step is to determine which present networks and systems could form the core of the developing AtlantOS system, which could be emerging networks, and which might best be partners to AtlantOS. In considering the wide array of existing networks and systems, the first step is to define the criteria by which to assess the state and status of a network and system. We suggest following: The general approach to this problem has been addressed practically by the JCOMM OCG (Legler et al., 2016) and as considered in the FOO. Adapting this approach, we suggest four key criteria to consider in determining how to organize existing activities into the basinscale AtlantOS system:

1. Location and scale - it must take place in the Atlantic. This includes both coastal and open ocean areas but likely not the regional seas such as the Baltic and Mediterranean and the northern and southern boundaries are purposely vague.

2. There should be an intention for operation over multiple years. There is no expectation that funding is guaranteed but there should be a plan for ongoing operations.

3. There must be some formal governance structure including a management team, defined standards and plans and a multi-year strategy for operations.

4. There should be a policy for free and open data that is available in a timely manner.

We reviewed key existing networks and systems in the Atlantic, North and South, and present the following as examples (see Bourlès et al., 2008; Rayner, 2010; Roemmich et al., 2010; Cooke et al., 2011; Duffy et al., 2013; Legler et al., 2015, 2016; McQuatters-Gollop et al., 2015; Talley et al., 2016; Mayer et al., 2018) to demonstrate the types of networks and systems that could be included and to demonstrate that there are many existing activities that could contribute to the developing core of AtlantOS:

\section{Persistent Core Components Networks \\ - $\operatorname{Argo}$ \\ - GO-SHIP}

- Ocean Tracking Network (OTN)

- OceanSITES (including OSNAP, RAPID, SAMOC)

- PIRATA

- Ship Observations Team (SOT)/Ship-of-Opportunity Programme (SOOP)

- Global Ocean Acidification Observing Network (GOA-ON)

- Continuous Plankton Recorder

- Global Sea Level Observing System (GLOSS)

- Marine Biodiversity Observation Network

- SeaBed 2030 (GEBCO)

\section{Systems}

- U.S. Integrated Ocean Observing System

- EuroGOOS

- South African Environmental Observation Network (SAEON)

- Sistema de Monitoramento da Costa Brasileira (SiMCosta) Brazilian Coastal Monitoring System

\section{Partners}

- Voluntary Observing Ship (VOS)

- Satellite remote sensing (e.g., CEOS)

- Tsunameter Networks

- Private Sector Installations (Oil-Riggs, Wind Farms, Offshore Mariculture)

- NGOs and Civil Society observing

\section{Examples of Emerging Components}

- OceanGliders

- HF Radar

- Surface Ocean $\mathrm{CO}_{2}$ Observing Network (SOCONET)

- Atlantic Seabed Mapping International Working Group (ASMIWG)

- Global Omics Observatory Network (GLOMICON)

- Canadian Integrated Ocean Observing System (C-IOOS)

- IOCARIBE

- ocEATLAN

- Global Coral Reef Monitoring Network

The first steps in developing AtlantOS could begin with coordination and integration of those well-established networks that see the benefit of collaboration with an Atlantic focus. The demonstrated benefits of coordination would encourage other groups to join and stimulate further developed of ocean observing in the Atlantic. These benefits should be defined by AtlantOS and their assessment would further support sustainability of these networks and systems and expansion of new ocean observing activity. The two immediate benefits to existing networks and systems would be expanded access and use of data being collected, by reaching wider communities of users, and improved demonstration of the societal benefit derived from the observational activity stimulating improved financial support and long-term sustainability.

AtlantOS will adopt an inclusive operating environment with well established and transparent ways by which new networks and systems can developed, accelerated and adopted as part of the basin-scale system. 


\section{GETTING DATA AND INFORMATION TO USERS}

Observations have no meaning without open data policies and effective data management. They ensure observations are retained and accessible for current and future users. Data management that enables access, use and interpretation of data and products is essential and must be based on collaboration across activities, such as quality assurance and control (QA/QC), which enable local and interoperable discovery and access, and secure archiving.

The present data management landscape is complex and hard to navigate. There are many specific data management infrastructures for specific date types, which can cause delayed and duplicate data receipts, missing data and metadata, and undocumented data processing procedures. There are also missing data management structures making it hard to retrieve some specific data types. Streamlined, modern data management structures are required: they can simplify, automate, and make more efficient the flow of data. New data systems should be interoperable. This would support routine data exchanges within and between observation networks, and user-friendly tools for data/products discovery, viewing and access. Community standards are the foundation for interoperability (Figure 6).

We need better coordination of the national and international efforts for data management and communication. Our goal: to harmonize data processing and distribution. It is also important to set up internet portals to make it easy to access a network's data. Such data portals and access are particularly well-developed in the meteorological community: for example, they support operational meteorology through the World Meteorological Organization. Using and expanding the system of portals that are either EOV-based (primarily for delayed mode data) or platform based (for near real-time data but also scientifically assessed data) supports a rigorous and effective quality assessment service for data providers.

Ocean observations must be available to users on a free and unrestricted basis. There is global recognition that open access encourages wide use of data, and the development of new data products. A key component of data management is close monitoring and quality control of data streams. Communication between the observing system operators and data managers is important. Data centers should give priority to using modern information and communication technology. Data and associated metadata have to be discoverable through portals, catalogs and search engines (Figure 7). To assist discoverability and to ensure correct citation of all data, each dataset requires a unique permanent reference for identification.

\section{Principles and Approaches to Data}

The FAIR principles must guide the Atlantic Ocean data management system and should be central to the AtlantOS approach (e.g., Tanhua et al., 2019). There are four FAIR principles. First, data should be Findable - this goal requires globally unique and eternally persistent identifiers for each dataset, rich standardized metadata attached to each dataset, and that data be searchable through standardized discovery services. Second, data should be Accessible - this goal will be achieved by establishing free and unrestricted access to data, ensuring full and open exchange of data, metadata and products, all with minimum delays. Third, data should be Interoperable and understandable - observations should always be provided with at least a minimum set of metadata, to enable traceability. Fourth, data should be Re-usable there should be comparability built from agreement on quality control procedures, there should be clear recognition and citation to assist discoverability and data should be preserved so that there accessibility will remain indefinitely (Wilkinson et al., 2016).

The study of the many interrelationships that exist in the ocean requires the integration of multidisciplinary approaches and different investigative skills and methods. The ocean observing community therefore has a responsibility to provide the best scientific knowledge, based on international collaboration, to enable wise global strategic decisions. We need to adopt ethical behaviors such as highlighted in the Cape Town Statement on Geoethics: (a) ethical values regarding behavior, honesty, integrity, courtesy and equity; (b) social values calling for sustainability, prevention and education; and (c) cultural values to strengthen community relations (Peppoloni and Di Capua, 2017). The basis and criterion of geo-(marine)ethics is responsibility. Ethics is a sum of all elements that will enable a sustainable research/monitoring endeavor, and we include elements drawn from the philosophical, social, and natural scientific dimensions.

The ocean observation community is responsible to respect for and minimize impacts from research and monitoring on ocean ecosystems, to respect and engage local people in research activities from multiple culture and diverse sector, to work with the goal of global benefit based on reciprocal relation, transparency and responsibility, to maximize efficiency and quality of observations in research activities, engage the society, communicate to and advise policymakers, encourage learning, and share data - acquire once, use multiple times (Buck et al., 2019).

\section{Basin-Scale Computer Models}

Coordinated basin-scale modeling will:

inform the blue economy and infrastructure planning, support ecosystem-based management, and predict and help mitigate the impacts of human actions and climate change.

Cyberinfrastructure requirements for a coordinated approach to developing basin-scale models will include both highperformance and cloud-based computing. A basin-scale approach requires seamless tools for integrating models, remote sensing observations (e.g., from satellites) and in situ observations, all at the basin and large regional scales. Computer models should be developed using a sequenced approach, 


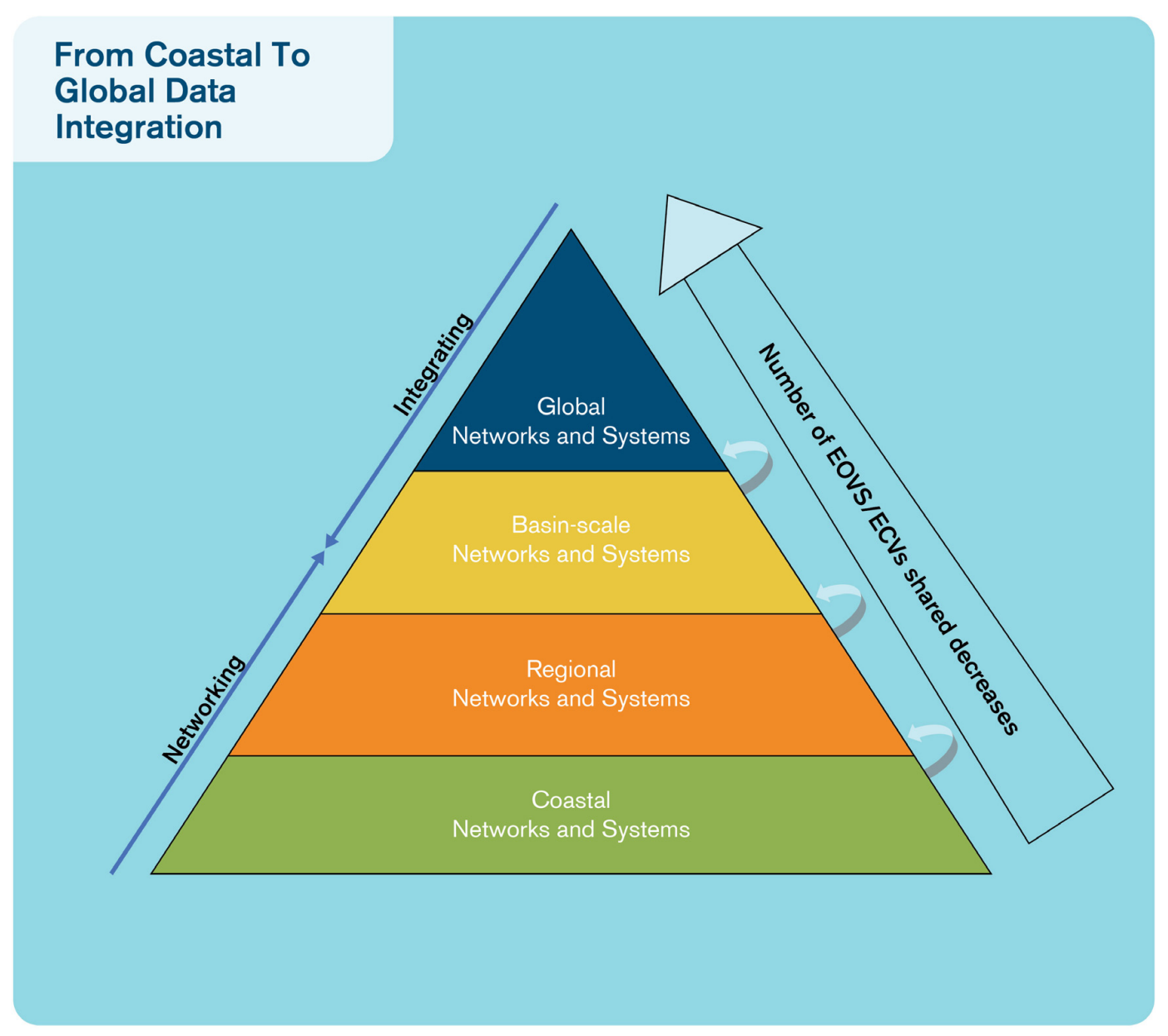

FIGURE 6 | From coastal to global data integration. For data to be made most accessible and used most efficiently, all data management systems must be interoperable, and data must be made available from central data repositories.

building on existing models. A framework for the seamless integration of models, remote sensing and in situ observations (Figure 8) will take time to develop and should address how basin-scale modeling can be seamless. Basin-scale models should be seamless:

- In space: basin - continental shelf - coastal - estuary;

- With regard to processes: land - coastal - ocean atmosphere;

- In time: from seconds (e.g., waves) to hundreds of years (e.g., meridional overturning circulation); and

- In variables: referring back to Essential Ocean Variables for physics, biogeochemistry, biology and ecosystems.

\section{INNOVATION, GOOD PRACTICE AND CAPACITY BUILDING IN OCEAN OBSERVING}

Innovation is key to ocean observing - it means creating developments or ideas that substantially enhance how we operate.
Many different areas of ocean observing have been enhanced by innovation including data communication, integration and sharing; the development and deployment of new sensors and platforms; ocean model guided observing system design, and new cooperative models for observing system governance. Innovation benefits all aspects of ocean observing (Figure 9).

A key innovation with big impact on ocean observing is citizen science. Citizen science programs are taking off. More and more people are getting involved in science - they're getting their hands wet! The development and expansion of citizen science programs is well timed. Consumer electronics are becoming part of the scientific arsenal and scientific instruments are increasingly userfriendly. Citizen scientists can now easily and quickly share their data, and the results of their efforts are just as easily shared with the wider community. Advances such as smart sensors, autonomous vehicles, and web-based data systems will enable important roles for citizens in ocean observation. In addition, citizen scientists often have access to areas that are not as often reached by scientists, such as school groups heading to rocky shores, and divers collecting data in shallow ecosystems like kelp forests and seagrass beds. 


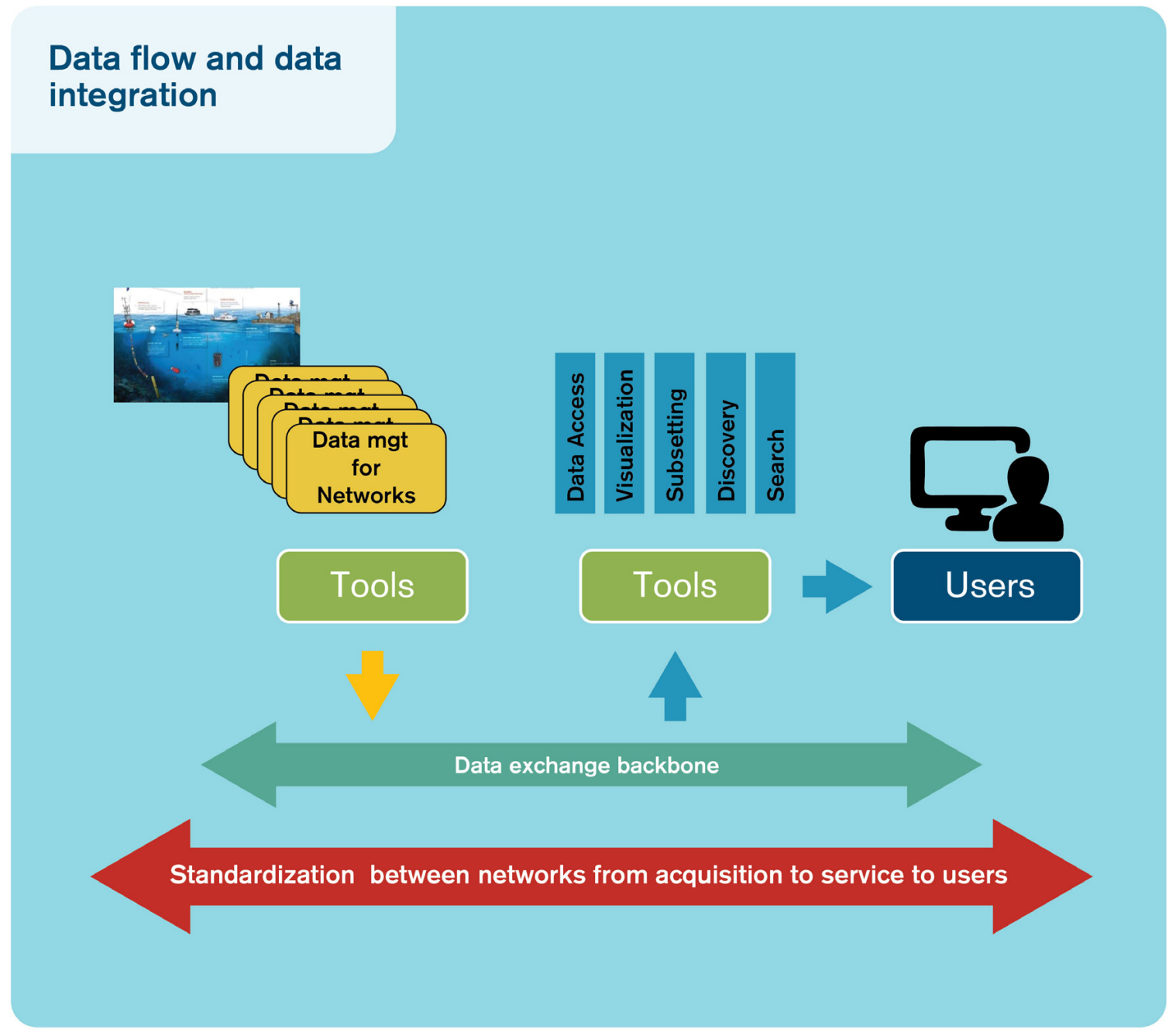

FIGURE 7 | Data flow and data integration. Schematics of the target ocean data flow from observations to users (in yellow: observing network data systems; in blue: integrators; in green and red: harmonization and integration elements). Source: AtlantOS.

Even in more extreme and inaccessible environments, citizen scientists are collecting data - from images taken by Remotely Operated Vehicles (ROVs) and Autonomous Underwater Vehicles (AUVs), they are identifying species from the deep sea ecosystems or kelp patches in collections of satellite pictures that span decades. This work is time consuming and would not be possible solely with professional scientists. Citizen scientists are more than ever critical to science, and they offer an opportunity to extend our reach into the ocean and to expand on the partners who will contribute to ocean observing systems. We should encourage further development of citizen science activities and share in the development of best practices for their creation and innovation.

One important aspect of improving ocean observation methods and technology is through sharing technology (Figure 10) and best practices. Partnerships between countries and across ocean sectors permit new opportunities for collaboration and innovation that result in improved ocean observing and downstream information for a large range of societal benefits. The ocean observing community can innovate by developing strategies for the use of new technology.

Capacity building activities benefit the ocean observation community. The biggest challenges to better education and technical skills lie in the disparate opportunities between countries - geographical, political, financial, economic and technical. AtlantOS should include formal structures to support capacity building and innovation, and to share best practices (Figure 11). We must do better to address issues of gender equity and diversity. The voices of those who are poorly represented must be better heard. We must raise our expectations and encourage wider participation in ocean observing to achieve greater participation and inclusion.

Each actor has particular skills and capabilities that should be shared widely across the community. Getting all Atlantic rim countries involved will enable a sense of community and ownership in each. It will also support synergies between 


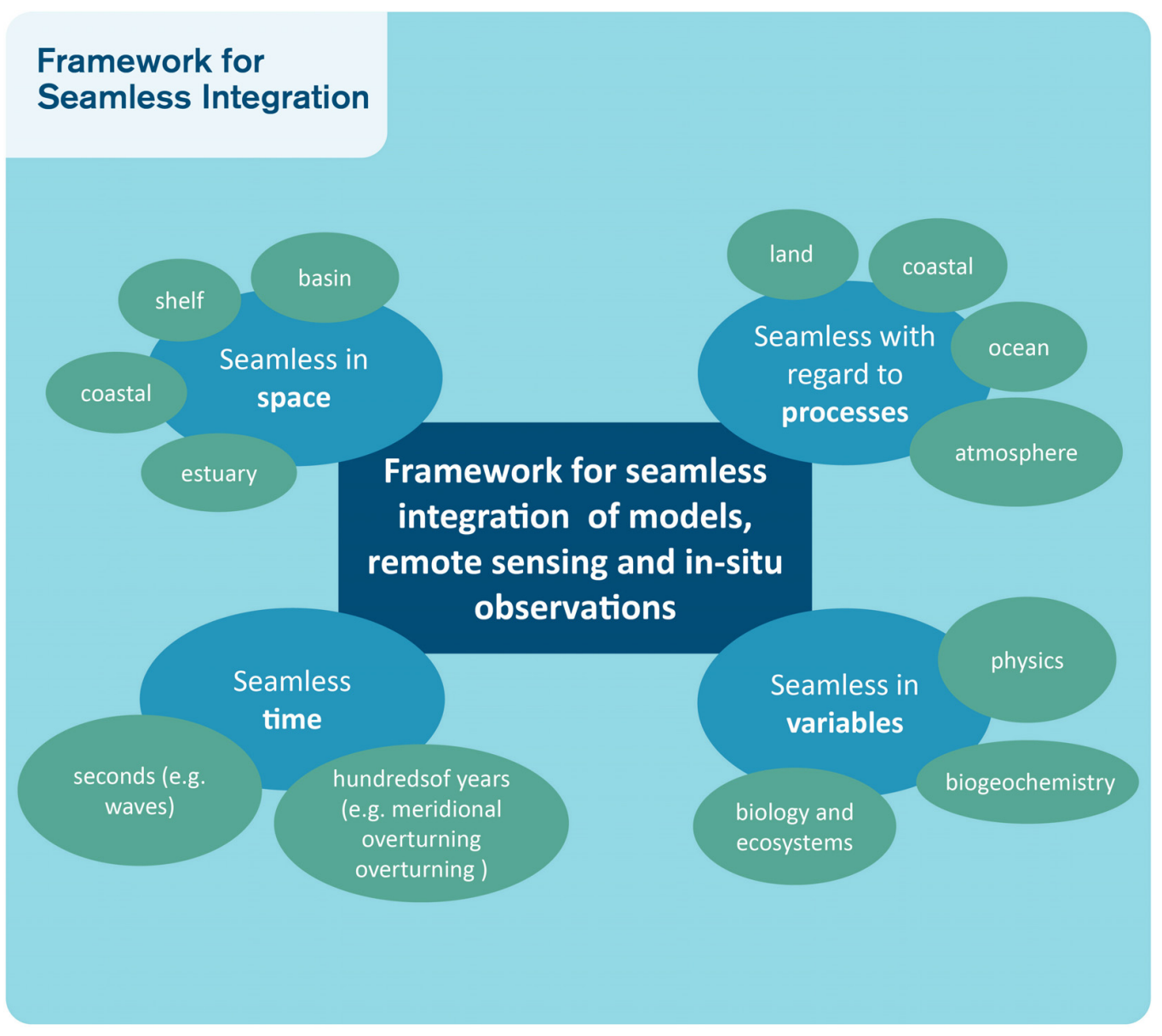

FIGURE 8 | Framework for seamless integration. Content of a framework for the seamless integration of models, remote sensing (satellites) and in situ (in-water) observations.

infrastructure investments and reduce barriers to multinational efforts. Capacity building should include fostering cooperation between Atlantic rim countries. Cooperation can be fostered by exchanges of capacity, shared platform deployment opportunities, joint scientific expert teams to ensure high quality and diffusion of data, and focus on new measurements, including biological and bio-geo-chemical and seafloor variables (Barth et al., 2019).

\section{GOVERNANCE AND PARTNERSHIPS AT THE ATLANTIC BASIN-SCALE}

Existing regional and national observation systems have both strengths and weaknesses. Their strengths lie in the transparency of the approaches used by different networks. Their key weakness is a lack of or loose coordination. A clear governance structure requires defined responsibilities and tasks for different actors. In general terms, a governance structure is a collection of functions and activities that support AtlantOS' development and management of policy, plans, guidance, resources, processes, tools and infrastructure. The degree of governance control varies from the local to the basin-scale systems like AtlantOS. At a local level, governance is straightforward, and funding is often from a single source and is sustained as long as there is a user demand. At the national level, the governance structure becomes more complicated because it often involves more than one agency. For a national level system such as U.S. IOOS, the funding is complicated because different sectors (public, private, and academia) are involved and the governance model is more one of consensus, so funding has become more complex but is still within a single nation. For AtlantOS, governance will be based on the willingness and sense of purpose of individuals, governments and entities. International funding is often complex and tied to the political climate. For AtlantOS to be successful, the management framework must be built upon clearly expressed and transparent principles.

AtlantOS proposes six guiding principles of governance to be adopted and reviewed as the system evolves (Figure 12):

- Engaging Stakeholders: The diverse range of stakeholders must be identified, to address user needs for new and review existing ocean information products. 


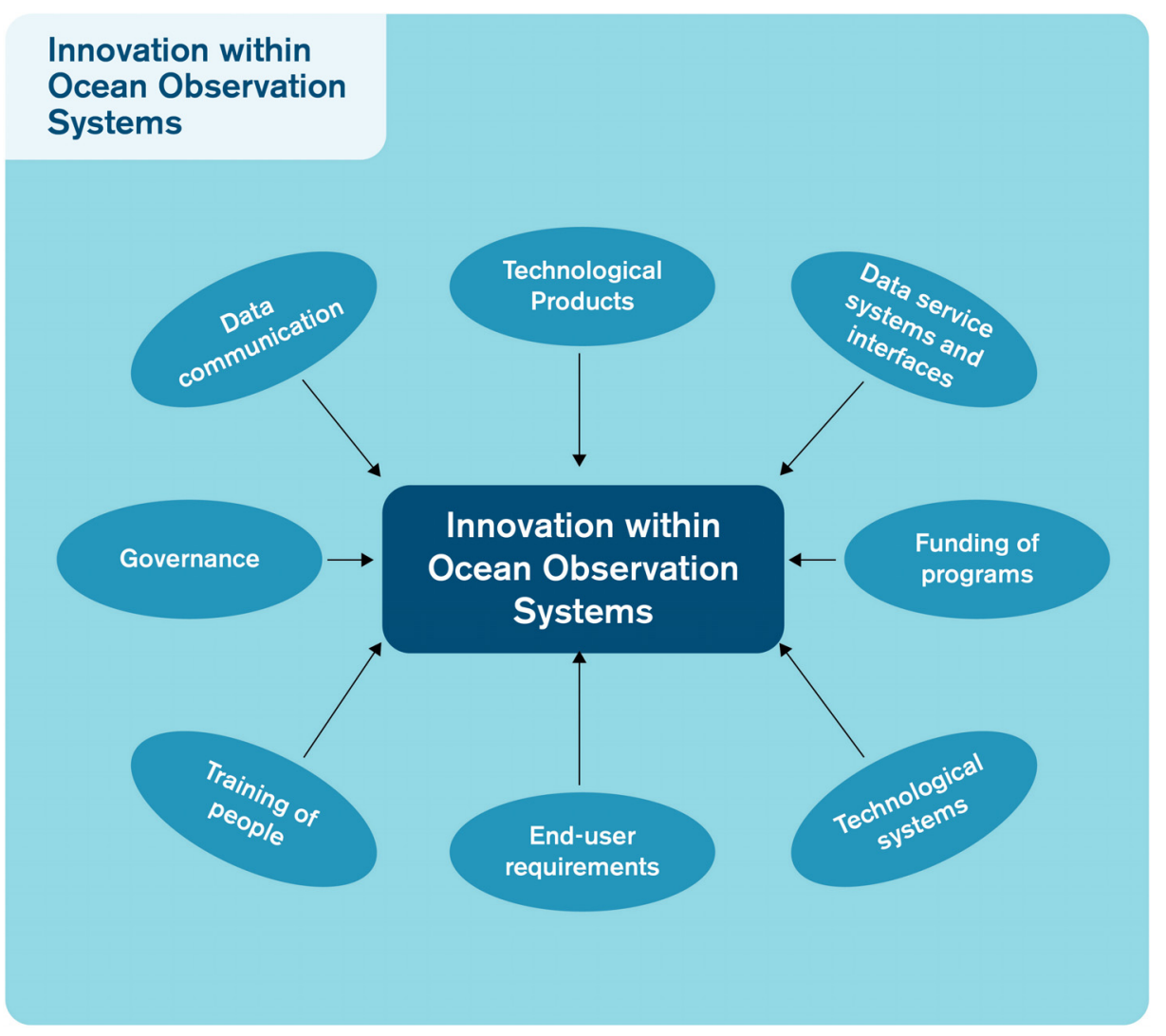

FIGURE 9 | Innovation within ocean observation systems. Innovation in ocean observing can take many forms. For sustainable ocean observing, all forms of innovating, including reviewing and updating, will need to be integrated into AtlantOS.

- Collaborative and coordinated: The stakeholder groups should form partnerships to define the roles and responsibilities of the partners and to extend and enhance the observational capability of the network or system.

- Integrated and transparent: The network or system should bring together all the observing components, partners and users to ensure that all of their interests are best met and that the maximum value is derived from each component. All partners must recognize and agree to their participation, and understand their expected contributions to and benefits from the observing network or system.

- Flexible and responsive: The observation network or system should be adaptive and responsive and have the ability to change in response to a shifting ocean environment and to changes in societal needs and structures.

- Open with free access to data and information: The ocean observation network or system should support and promote the open exchange of data to ensure that the maximum benefit is derived from the data collected.

- Reliably resourced: An effective ocean observation network or system requires reliable funding to ensure that it is sustainable.

Ocean observing is largely publicly-funded, and therefore public support is required. Present and future networks and systems should build on voluntary participation from governments, research communities, and other stakeholders. Voluntary participation helps to ensure that the partners are actively engaged in supporting and sharing the success of the system and its sustainable nature.

\section{Recommendations for Effective Governance of AtlantOS Coordination Mechanisms That Span Three Dimensions: The Observing System, Resource Engagement, and Ocean Information Delivery}

A single supervisory body is needed to coordinate the three dimensions with their respective tasks and responsibilities. A clear coordination structure of the different subgroups covering the three dimensions should exist, where tasks and responsibilities are distributed between all observation system contributors. As a result, overlapping and loosely coordinated structures that exist now could be streamlined.

\section{An AtlantOS Executive Board}

Governance and coordination of the AtlantOS system is important to better connect to the observing systems and networks operating in the Atlantic, and to the global system (GOOS). 


\section{Technological Ocean Observation Options / Development}

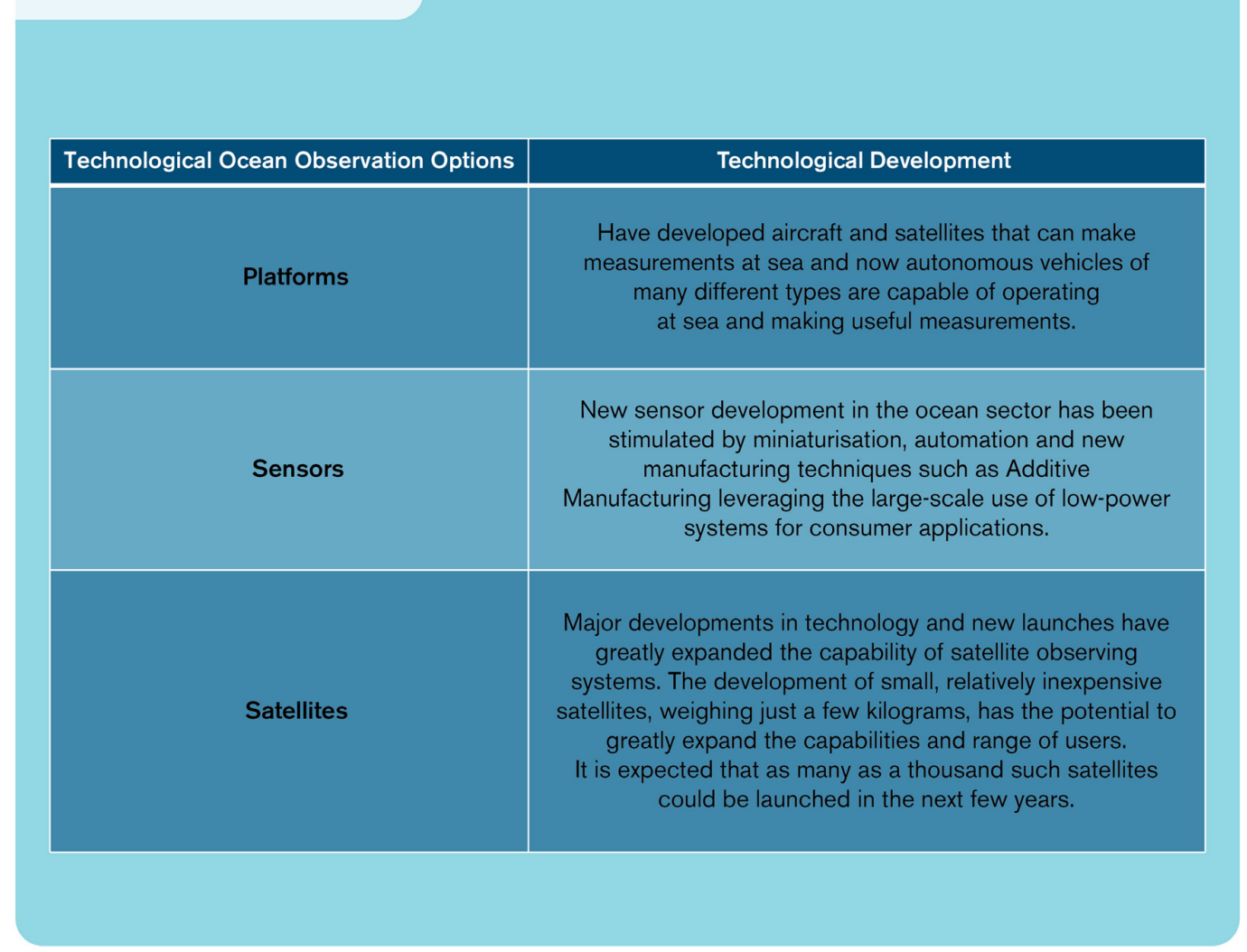

FIGURE 10 | Technological ocean observation options/development. Aspects of technological development leading to innovations in ocean observing.

\section{A Resource Board to Promote Long-Term Funding}

To promote long-term funding, the AtlantOS community should establish a Resource Board on a voluntary basis within this governance system. It should be arranged in a "resource engagement" dimension.

\section{Implementation of a Formalized, Dynamic, and Flexible Ocean Partnership Panel (OPP)}

A mechanism to inform the ocean observing community about the role of ocean data and products as well as their societal benefits, with representatives from within each stakeholder group.

\section{A Review Process}

Regularly held to determine the adequacy of ocean observing activities and programs, as well as the system's requirements. A review process would ensure long-term support and sustainability of the system. For this process, countries should be asked for information regarding the adequacy of the system, as well as ways to gain more resources and plans for future activities. The proposed timeline is every 5 years.

\section{Countries Should Establish an Ocean Focal Point or Ocean Ministry}

At the national level, responsibilities for ocean activities are often distributed across multiple sectorial actors, such as ministries or agencies (like transportation, finance, or environment). Several nations have already started an internal process to connect its actors and identified an Ocean Observing focal point. This mechanism can develop engagement opportunities, capabilities and national knowledge to more effectively contribute to govern their contributions and engagement with ocean observing and information generation. At the highest level of ambition, it could be beneficial if nations or regions create an Ocean Ministry or Ocean Focal Point Mechanism to combine and coordinate ocean information gathering and to provide advice to their governments and stakeholders on ocean issues (Degnarian and Stone, 2017).

In approaching governance at the basin-scale, spanning the Atlantic, there are already many programs with some form of governance. Many of these existing systems and networks exhibit one or more of the principles outlined, but few of them fully realize these principles. It is therefore a pragmatic challenge as to how to move forward building 


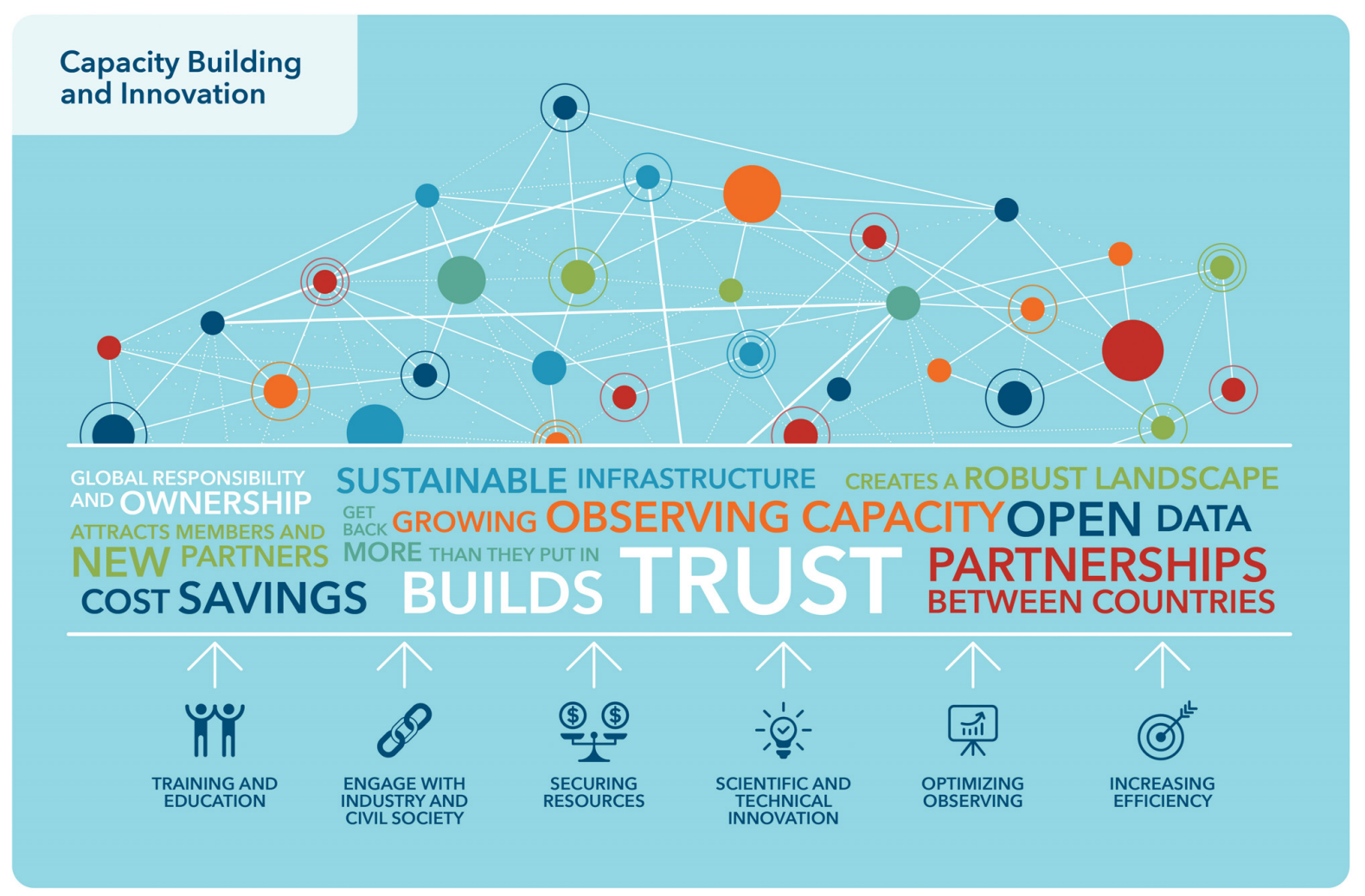

FIGURE 11 | Capacity building and innovation. Activities related to capacity building and innovation should take place across the system and should support and encourage collaboration between the system partners.

upon existing structures so that we do not waste effort by working to "reinvent the wheel" but at the same time ensure that we follow the key principles needed to ensure sustainability.

It may be too late to develop a fully new governance structure, as there are too many already in place, from the local to the regional to the global scale. It is also important to recognize that funding for the existing systems and networks has been allocated based upon previous agreements with particular expectations, all of which may be difficult to change. We should avoid harming good governance systems in search of a new perfect governance model. Therefore, care must be taken to build a basin-scale system that can integrate all the relevant existing structures but may evolve to attain a fully integrated governance structure following the principles we laid out.

\section{0 - REALIZING THE VISION}

In the coming decade, we will likely see many new changes in the ocean. A recent report stated that in the future:

scientific and technological advances are expected to play a crucial role both in addressing many of the ocean-related environmental challenges...and in the further development of ocean-based economic activities (OECD, 2016, p. 13).
Ocean observatories are expected to contribute data and information to these environmental and economic developments. We must shift our perspective as to why we make ocean observations and why we invest in ocean observing systems. Such activities should be seen as fundamental to a global society.

Ocean observing is a necessary service to fulfill our collective stewardship responsibility. To fully realize this service, we must change our view of the value of environmental data. Environmental data should be seen as contributing to the global public good and should be freely available to all. Thus, environmental data collected by fishermen should be openly shared, as too should data on seabed features collected in support of deep-sea mining, alongside scientific data. Commercial interests can be protected while at the same time ensuring that the environmental value of these data is not lost. Providing environmental data for the societal good must be seen as fundamental to a twenty-first century society.

When looking at existing sustainable observing infrastructures and systems that have been funded and implemented over the longer-term, e.g., U.S. IOOS and EuroGOOS, most share the following four characteristics:

- Meeting defined user needs;

- Inclusiveness such that partners or clients find their interests are served; 


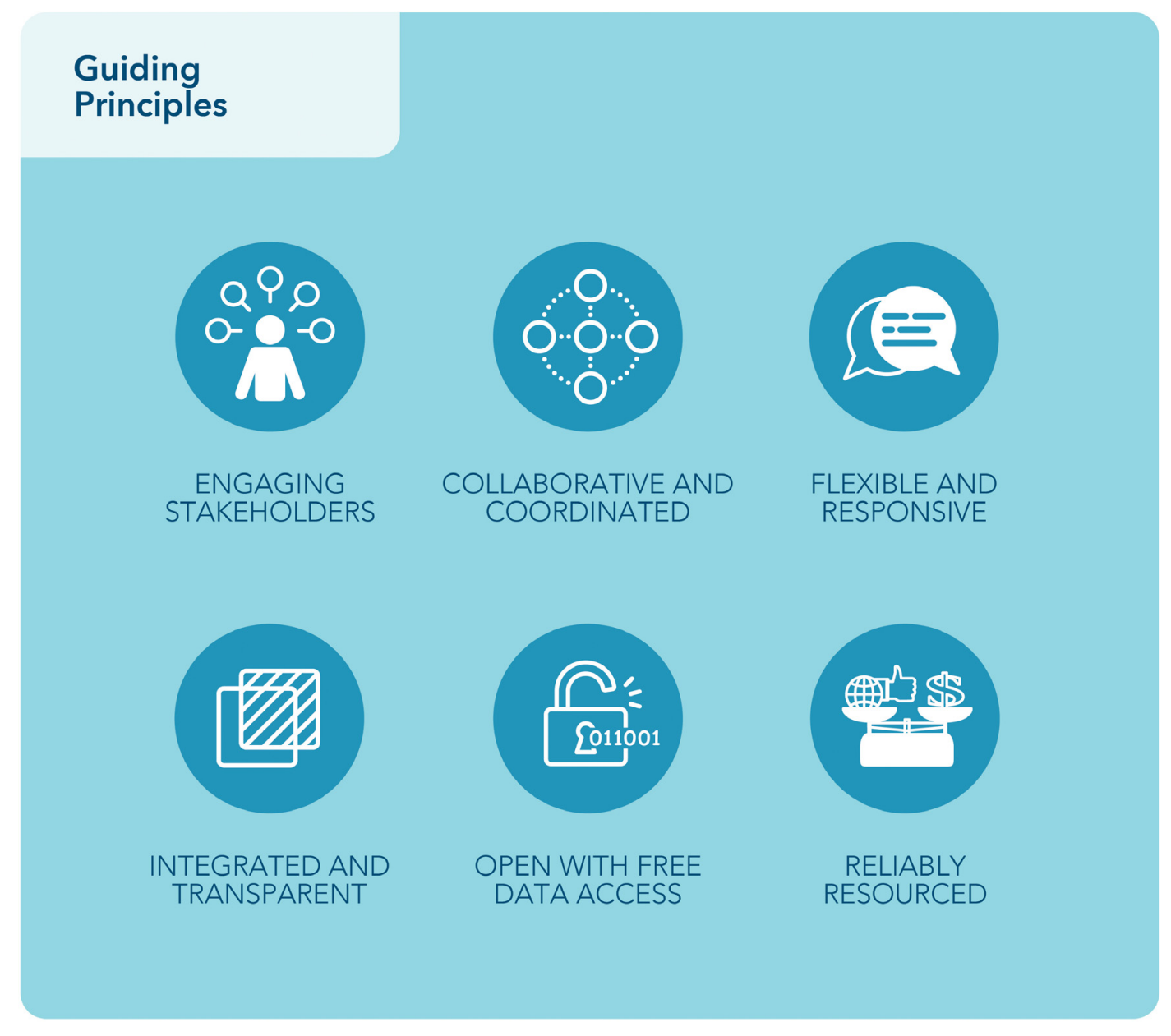

FIGURE 12 | Guiding principles. Six guiding principles of governance have been identified as necessary for creating a sustainable, effectively-governed system.

- Promoting open sharing and exchange of information; and

- Promoting partnerships between the private and public sectors, the academic community and users.

For a sustainable AtlantOS to benefit us in the long term, the system needs three key elements (discussed in more detail in the previous sections):

\section{Governance Sustainability}

Given that ocean observing is largely publicly-funded, basin-scale, ocean observing must have state support. At the international level, such support should be expressed through a governance framework that provides political and legal guidance, within a functional institutional setting. The governance framework encompasses the policies and rules that give any initiative a legal and political raison d'être. A governance framework also lays down the principles by which the observing system should function. Open data entails that data and information collected within the framework of an observing system should be freely available to all collaborators and of course the general public. Free and open exchange of data and information are essential to support collaboration and encourage cooperation and capacity building. To date, ocean observing has been seen as a scientific-technical rather than socio-political challenge. In the future, an intergovernmental ocean observing system should play a special role in communicating the condition of the ocean to the world (Fritz, 2016). Ocean data governance must ensure that the principle of "measure once, use multiple times" is realized.

\section{Institutional and Operational Sustainability}

A coordinating body should be identified to provide a forum for coordinating in situ ocean observing networks. GOOS provides a good example for such a body but must be reviewed and strengthened to balance top-down strategy development amongst the owners/operators/funders of observing stations and intergovernmental bodies with the bottom-up inclusion of the scientific, commercial and societal communities. To date, strategies tend to be priority lists and supply-sided plans for the future. In future, more detailed analyses and interactive foresight 
processes should be initiated to promote critical and reflective assessments of the current state and future of ocean observing.

\section{Financial Sustainability}

The diversity and number of ocean observing initiatives means that financial sustainability will not be equal in all initiatives. At best, financial sustainability of an observing system composed of ocean observing networks will require that, while each effort remains financially independent, together they be embedded in long value chains. From data collection to end use, ocean observing should be driven by providing societal or commercial benefits. To this end, efforts to secure more funding must consider capital vs. operational expenditures, so that technology is acquired and operated over the longer-term. A rigorous and continuous economic assessment of ocean observing is necessary, beginning with an assessment of the value added by investing in ocean observing for funders and stakeholders of an Atlantic Ocean observing system.

\section{CONCLUSION}

Looking forward, we see AtlantOS delivering comprehensive ocean observations that truly address existing and emerging needs of all those living by, working on and impacted by our Atlantic Ocean. We do not need to start from scratch -several successful networks and systems are effective in meeting user needs and can be integrated into this newly formed system. However, several key developments are required to enable the creation of a basin-scale system. New technologies present a wealth of opportunity - for making ocean observations, particularly in biology and biogeochemistry, and for facilitating the open and free access to ocean data to make sure the data we are already collecting can be used by all.

While some new funding is likely necessary to meet our needs, it is not just about spending more money. It is about working together more effectively, being driven by user needs, transparent sharing of data, and minimizing duplication of activities. Most importantly, it is about working together, coordinating our activities, and learning from each other so we can address some of our greatest challenges.

The AtlantOS All-Atlantic Ocean Observing System must be sustainable, offering the best value for the collective community investment. Only through such a basin-scale system can we truly understand our shared ocean, for the benefit of all.

\section{ABBREVIATIONS}

AMOC, Atlantic Meridional Overturning Circulation (Argo Broad-scale global array of temperature/salinity profiling floats that is a major component of the ocean observing system and exemplifies international collaboration, with 31 nations contributing floats (2017) and a data management system that delivers consistent quality controlled data streams.); ASMIWG, Atlantic Seabed Mapping International Working Group (AtlantOS Atlantic Ocean Observing System [and
European Union Horizon 2020 funded project is called 'AtlantOS - Optimizing and Enhancing the Integrated Atlantic Ocean Observing Systems (2015-2019)]); AUV, Autonomous Underwater Vehicle; BON, Biodiversity Observation Network; CBD, Convention of Biological Diversity; C-IOOS, Canadian Integrated Ocean Observing System; CLIVAR, Climate and Ocean: Variability, Predictability and Change; CLIVAR-GSOP CLIVAR, Global Synthesis and Observations Panel; CPR Continuous Plankton Record; DOI, Data Object Identifier; EEZ, Exclusive Economic Zone; EOOS, European Ocean Observing System; EOV, Essential Ocean Variable (Essential Ocean Variables are a set of quantities derived from observational data that have a high scientific value, and measuring them is technologically feasible. EOVs are suited to providing routine estimates of the ocean state (physical, biogeochemical, ecosystem); EU, European Union; EuroGOOS, European Global Ocean Observing System; FAIR, principles Findable, Accessible, Interoperable and Reusable principles; FOO, Framework for Ocean Observing; G7, Group of 7 nations; GEBCO, General Bathymetric Chart of the Oceans; GEO, Group of Earth Observation; GLOBEC, Global Ocean Ecosystem Dynamics; GLOMICON, Global Omics Observatory Network; GLOSS, Global Sea Level Observing System; GODAE, Global Ocean Data Assimilation Experiment (now OceanPredict); GOOS, Global Ocean Observing System; GOA-ON, Global Ocean Acidification Observing Network; GO-SHIP, Global Ocean Shipbased Hydrographic Investigations Program (GO-SHIP is a globally coordinated network of sustained hydrographic sections that aims to bring together scientists with interests in physical oceanography, the carbon cycle, marine biogeochemistry and ecosystems, and other users and collectors of hydrographic data.); GRA, GOOS Regional Alliance; HABs, Harmful Algal Blooms; HF, High Frequency; IOC, International Oceanographic Commission (IOCARIBE GOOS Regional Alliance for the Caribbean and adjacent regions - responsible for the promotion, development and coordination of IOC marine scientific research programs, the ocean services, and related activities); IPBES, Intergovernmental Science-Policy Platform on Biodiversity and Ecosystem Services; JCOMM, Joint Technical Commission for Oceanography and Marine Meteorology; JCOMM OPS, Joint Technical Commission for Oceanography and Marine Meteorology in situ Observing Programs Support Center; JGOFS, Joint Global Ocean Flux Study; MBON, Marine Biodiversity Observation Network; OBIS, Ocean Biogeographic Information System; OceanSITES, The mission of OceanSITES is to collect, deliver and promote the use of high-quality data from long-term, high-frequency observations at fixed locations in the open ocean. OceanSITES typically aim to collect physical, biogeochemical, and biology/ecosystem data worldwide using open-ocean, full-depth water column as well as the overlying atmosphere; OCEATLAN GOOS, Regional Alliance for the Upper Southwest and Tropical Atlantic represents the regional efforts of institutions from Argentina, Brazil, and Uruguay to jointly develop and implement an operational oceanographic system to monitor and investigate the oceanic processes in the Upper Southwest and Tropical Atlantic; OCG, Observations Coordination Group; OECD, 
Organization for Economic Cooperation and Development; OOPC, Ocean Observing Panel on Climate; OOSDP, Ocean Observing System Development Panel; OPP, Ocean Partnership Panel; OSE, Observing System Experiment; OSNAP, Overturning in the Subpolar North Atlantic Program; OSSE, Observation System Simulation Experiment; OTN, Ocean Tracking Network; PIRATA, Prediction and Research Moored Array in the Tropical Atlantic; QA/QC, Quality assurance and quality control; RAPID, Array across the Atlantic at $26^{\circ} \mathrm{N}$ as well as a project with three phases (2001-2007; 2008-2014 and 2014-2020) to observe different changes in this array that could be responsible for a rapid climate change; ROV, Remotely Operated Vehicle; SAEON, South African Environmental Observation Network; SAMOC, South Atlantic Meridional Overturning Circulation; SEAPODYM, Spatial Ecosystem And Population Dynamics Model; SDG, Sustainable Development Goal; SiMCosta, Sistema de Monitoramento da Costa Brasileira - Brazilian Coastal Monitoring System; SKIM, Sea surface KInematics Multiscale; SOCONET, Surface Ocean CO2 Observing Network; SOOP, Ship-of-Opportunity Programme; SOT, Ship Observations Team; SWOT, Surface Water and Ocean Topography; UN, United Nations; UNCLOS, United Nations Convention on the Law of the Sea; UNESCO, United Nations Educational, Scientific and Cultural Organization; U.S., United States of America; U.S. IOOS, United States Integrated Ocean Observing System; VOS, Voluntary Observing Ship (Programm); WMO, World Meteorological Organization; WOCE, World Ocean Circulation Experiment; XBT XBT, (eXpendable BathyThermograph) is a probe that is dropped from a ship and measures the temperature

\section{REFERENCES}

Barth, J. A., Allen, S. E., Dever, E. P., Dewey, R. K., Evans, W., Feely, R. A., et al. (2019). Better regional ocean observing through cross-national cooperation: a case study from the Northeast Pacific. Front. Mar. Sci. 6:93.

Bourlès, B., Lumpkin, R., McPhaden, M. J., Hernandez, F., Nobre, P., Campos, E., et al. (2008). The PIRATA program: history, accomplishments, and future directions. Bul. Am. Meteorol. Soc. 89, 1111-1126. doi: 10.1175/2008bams 2462.1

Buck, J. J. H., Bainbridge, S. J., Burger, E. F., Kraberg, A. C., Casari, M., Casey, K. S., et al. (2019). Ocean data product integration through innovation-The next level of data interoperability. Front. Mar. Sci. 6:32.

Cooke, S. J., Iverson, S. J., Stokesbury, M. A. W., Hinch, S. G., Fisk, A. T., and Vander Zwaag, D. L. (2011). Ocean tracking network canada: a network approach to addressing critical issues in fisheries and resource management with implications for ocean governance. Fisheries 36, 583-592. doi: 10.1080/ 03632415.2011 .633464

Degnarian, N., and Stone, G. S. (2017). 83 Countries are More Ocean Than Land, World. Available at: https://www.weforum.org/agenda/ 2017/10/global-ocean-governance-all-at-sea (accessed October 30, 2017).

Dragon, A. C., Senina, I., Conchon, A., Titaud, O., Arrizabalaga, H., and Lehodey, P. (2015). Modeling spatial population dynamics of North Atlantic Albacore tuna under the influence of both fishing and climate variability. Can. J. Fish. Aquatic Sci. 72, 864-878.

Duffy, J. E., Amaral-Zettler, L. A., Fautin, D. G., Paulay, G., Rynearson, T. A., Sosik, H. M., et al. (2013). Envisioning a marine biodiversity observation network. Bioscience 63, 350-361. doi: 10.1525/bio. 2013.63.5.8 as it falls through the water. In a joint effort between research and government institutions and the private industry, XBTs are usually launched from cargo, research, or cruise ships.

\section{AUTHOR CONTRIBUTIONS}

BdeY led the process and was the primary author with all coauthors substantially contributing to the document.

\section{ACKNOWLEDGMENTS}

This manuscript is the output of the "BluePrint for an Integrated Atlantic Ocean Observing System" team that has received funding and support from the European Union's Horizon 2020 Research and Innovation Programme Project AtlantOS (Grant Agreement No. 633211). We thank P. Lehodey who provided the operational tuna example shown in Figure 3. The team has also received support from the EuroGOOS, the pan-European network that is part of the Global Ocean Observing System (GOOS) of the Intergovernmental Oceanographic Commission (IOC) of the UNESCO and from the Marine Environmental Observation Prediction and Response (MEOPAR) program funded through the Networks of Centers of Excellence Program in Canada and from the Integrated Oceanography and Multiple Uses of the Continental Shelf and the Adjacent Ocean Integrated Center of Oceanography (INCT-Mar COI, CNPq, Proc. 56062/2010-7).

Fritz, J. S. (2016). Observations, Diplomacy, and the Future of Ocean Governance in Science \& Diplomacy. Available at: http://www.sciencediplomacy.org/ article/2016/ observations-diplomacy-and-future-ocean-governance (accessed October 30, 2017).

Hoegh-Guldberg, O., Tanzer, J., Gamblin, P., and Burgener, V. (2015). Reviving the Ocean Economy: the Case for Action. Gland: WWF International.

Le Traon, P.-Y., Antoine, D., Bentamy, A., Bonekamp, H., Breivik, L. A., Chapron, B., et al. (2015). Use of satellite observations for operational oceanography: recent achievements and future prospects. J. Operat. Oceanogr. 8(Suppl.1.), s12-s27. doi: 10.1080/1755876x.2015.102 2050

Le Traon, P.-Y., Reppucci, A., Alvarez Fanjul, E., Aouf, L., Behrens, A., Belmonte, M., et al. (2019). From observation to information and users: The Copernicus Marine service perspective. Front. Mar. Sci. 6:234. doi: 10.3389/fmars.2019. 00234

Legler, D. M., Freeland, H. J., Lumpkin, R., Ball, G., McPhaden, M. J., North, S., et al. (2015). The current status of the real-time in situ global ocean observing system for operational oceanography. J. Operat. Oceanogr. 8(Suppl. 2), s189-s200. doi: 10.1080/1755876x.2015.1049883

Legler, D. M., Meldrum, D. T., Hill, K. L., and Charpentier, E. (2016). Coordination and Integration of Global Ocean Observing Through JCOMM. Washington, D.C: American Geophysical Union.

Mayer, L., Jakobsson, M., Allen, G. L., Dorschel, B., Falconer, R., and Ferrini, V. (2018). The nippon foundation-GEBCO seabed 2030 project: the quest to see the world's oceans completely mapped by 2030. Geosciences 8, 63. doi: $10.3390 /$ geosciences 8020063

McQuatters-Gollop, A., Edwards, M., Helaouët, P., Johns, D. G., Owens, N. J. P., and Raitsos, D. E. (2015). The continuous plankton recorder survey: how can long-term phytoplankton datasets contribute to the assessment of good 
environmental status? Estuar., Coast. Shelf Sci. 162, 88-97. doi: 10.1016/j.ecss. 2015.05.010

Miloslavich, P., Bax, N. J., Simmons, S. E., Klein, E., Appeltans, W., AburtoOropeza, O., et al. (2018). Essential ocean variables for global sustained observations of biodiversity and ecosystem changes. Glob. Chang. Biol. 24, 2416-2433. doi: 10.1111/gcb.14108

Moore, A. M., Martin, M. J., Akella, S., Arango, H. A., Balmaseda, M., Bertino, L., et al. (2019). Synthesis of ocean observations using data assimilation for operational, real-time and reanalysis systems: a more complete picture of the state of the Ocean. Front. Mar. Sci. 6:90.

Morrow, R., Fu, L. L., Ardhuin, F., Benkiran, M., Chapron, B., Cosme, E., et al. (2019). Global observations of fine-scale ocean surface topography with the surface water and ocean topography (SWOT) mission. Front. Mar. Sci. 6:232

OECD (2016). The Ocean Economy in 2030. Paris: OECD Publishing.

OOSDP (1995). The Scientific Design for the Common Module of the Global Climate Observing System and the Global Ocean Observing System, Report of the Ocean Observing System Development Panel. College Station, TX: Texas A\&M University.

JCOMMOPS (2017). Monthly Map September 2017. Available at: http://www. jcommops.org/board? contacts $=($ accessed October 30, 2017).

Ostrander, C. E., Melissa, M. I., and Langenberger, F. (2019). An innovative approach to design and evaluate a regional coastal ocean observing system. Front. Mar. Sci. 6:111. doi: 10.3389/fmars.2019.00111

Peppoloni, S., and Di Capua, G. (2017). "Ethics," in Encyclopedia of Engineering Geology, Encyclopedia of Earth Sciences Series, eds P. T. Bobrowsky and B. Marker (New York, NY: Springer International Publishing), 1-5.

Rayner, R. (2010). The US integrated ocean observing system in a global context. Mar. Technol. Soc. J. 44, 26-31. doi: 10.4031/mtsj.44.6.1

Roemmich, D., Boehme, L., Claustre, H., Freeland, H., Fukasawa, M., Goni, G., et al. (2010). Integrating the ocean observing system: mobile platforms. Proc. OceanObs 9, 33.

Smith, N., Kessler, W. S., Cravatte, S., Sprintall, J., Wijffels, S., Cronin, M. F., et al. (2019). Tropical Pacific Observing System. Front. Mar. Sci. $6: 31$.
Talley, L. D., Feely, R. A., Sloyan, B. M., Wanninkhof, R., Baringer, M. O., Bullister, J. L., et al. (2016). Changes in ocean heat, carbon content, and ventilation: a review of the first decade of GO-SHIP global repeat hydrography. Ann. Rev. Mar. Sci. 8, 185-215. doi: 10.1146/annurev-marine-052915-10 0829

Tanhua, T., Pouliquen, S., Hausman, J., O’brien, K., Bricher, P., Bruin, T. D., et al. (2019). Ocean FAIR data services. Front. Mar. Sci. doi: 10.3389/fmars.2019. 00440

Task Team for an Integrated Framework for Sustained Ocean Observing (2012). A Framework for Ocean Observing, UNESCO, IOC/INF-1284. Available at: http://www.eoos-ocean.eu/download/GOOSFrameworkOceanObserving.pdf (accessed July 11, 2019).

Weller, R. A., Baker, D. J., Glackin, M. M., Roberts, S. J., Schmitt, R. W., Twigg, E. S., et al. (2019). The challenge of sustaining ocean observations. Front. Mar. Sci. 6:105

Wilkinson, M. D., Dumontier, M., Aalbersberg, I. J., Appleton, G., Axton, M., Baak, A., et al. (2016). The FAIR guiding principles for scientific data management and stewardship. Sci Data. 3:160018. doi: 10.1038/sdata.2016.18

Conflict of Interest Statement: ZW was employed by the company Veraison Consulting LLC.

The remaining authors declare that the research was conducted in the absence of any commercial or financial relationships that could be construed as a potential conflict of interest.

Copyright (c) 2019 deYoung, Visbeck, de Araujo Filho, Baringer, Black, Buch, Canonico, Coelho, Duha, Edwards, Fischer, Fritz, Ketelhake, Muelbert, Monteiro, Nolan, O'Rourke, Ott, Le Traon, Pouliquen, Sousa-Pinto, Tanhua, Velho and Willis. This is an open-access article distributed under the terms of the Creative Commons Attribution License (CC BY). The use, distribution or reproduction in other forums is permitted, provided the original author(s) and the copyright owner(s) are credited and that the original publication in this journal is cited, in accordance with accepted academic practice. No use, distribution or reproduction is permitted which does not comply with these terms. 


\title{
Observing the Oceans Acoustically
}

\author{
Bruce M. Howe ${ }^{1 *}$, Jennifer Miksis-Olds², Eric Rehm³ ${ }^{3}$ Hanne Sagen ${ }^{4}$, \\ Peter F. Worcester ${ }^{5}$ and Georgios Haralabus ${ }^{6}$
}

\begin{abstract}
${ }^{1}$ Department of Ocean and Resources Engineering, University of Hawai'i at Mānoa, Honolulu, HI, United States, ${ }^{2}$ School of Marine Science and Ocean Engineering, University of New Hampshire, Durham, NH, United States, ${ }^{3}$ Takuvik Joint International Laboratory, Université Laval, Quebec City, QC, Canada, ${ }^{4}$ Nansen Environmental and Remote Sensing Center, Bergen, Norway, ${ }^{5}$ Scripps Institution of Oceanography, University of California, San Diego, La Jolla, CA, United States,

${ }^{6}$ Hydro-Acoustics, Engineering and Development, IMS, CTBTO, Vienna, Austria
\end{abstract}

\section{OPEN ACCESS}

Edited by:

Tong Lee,

NASA Jet Propulsion Laboratory

(JPL), United States

Reviewed by:

Dimitris Menemenlis,

NASA Jet Propulsion Laboratory

(JPL), United States

Carl Wunsch,

Massachusetts Institute

of Technology, United States

*Correspondence:

Bruce M. Howe

bhowe@hawaii.edu

Specialty section:

This article was submitted to

Ocean Observation,

a section of the journal

Frontiers in Marine Science

Received: 01 November 2018

Accepted: 05 July 2019

Published: 26 July 2019

Citation:

Howe BM, Miksis-Olds J,

Rehm E, Sagen H, Worcester PF and Haralabus G (2019) Observing

the Oceans Acoustically.

Front. Mar. Sci. 6:426.

doi: 10.3389/fmars.2019.00426
Acoustics play a central role in humankind's interactions with the ocean and the life within. Passive listening to ocean "soundscapes" informs us about the physical and bio-acoustic environment from earthquakes to communication between fish. Active acoustic probing of the environment informs us about ocean topography, currents and temperature, and abundance and type of marine life vital to fisheries and biodiversity related interests. The two together in a multi-purpose network can lead to discovery and improve understanding of ocean ecosystem health and biodiversity, climate variability and change, and marine hazards and maritime safety. Passive acoustic monitoring (PAM) of sound generated and utilized by marine life as well as other natural (wind, rain, ice, seismics) and anthropogenic (shipping, surveys) sources, has dramatically increased worldwide to enhance understanding of ecological processes. Characterizing ocean soundscapes (the levels and frequency of sound over time and space, and the sources contributing to the sound field), temporal trends in ocean sound at different frequencies, distribution and abundance of marine species that vocalize, and distribution and amount of human activities that generate sound in the sea, all require passive acoustic systems. Acoustic receivers are now routinely acquiring data on a global scale, e.g., Comprehensive Nuclear-Test-Ban Treaty Organization International Monitoring System hydroacoustic arrays, various regional integrated ocean observing systems, and some profiling floats. Judiciously placed low-frequency acoustic sources transmitting to globally distributed PAM and other systems provide: (1) high temporal resolution measurements of large-scale ocean temperature/heat content variability, taking advantage of the inherent integrating nature of acoustic travel-time data using tomography; and (2) acoustic positioning ("underwater GPS") and communication services enabling basin-scale undersea navigation and management of floats, gliders, and AUVs. This will be especially valuable in polar regions with ice cover. Routine deployment of sources during repeat global-scale hydrographic ship surveys would provide high spatial coverage snapshots of ocean temperatures. To fully exploit the PAM systems, precise timing and positioning need to be broadly implemented. Ocean sound is now a mature Global Ocean Observing System (GOOS) "essential ocean variable," which is one crucial step toward providing a fully integrated global multi-purpose ocean acoustic observing system.

Keywords: acoustical oceanography, passive acoustic monitoring, ocean acoustic tomography, acoustic positioning, multi-purpose acoustic networks, marine bio-acoustics, soundscape, essential ocean variable 


\section{INTRODUCTION}

The mesoscale revolution in oceanography was enabled by the acoustic tracking of floats. In the late 1950s, John Swallow and his colleagues deployed floats off Bermuda, returning a few weeks later to locate them acoustically. They expected them to have drifted only kilometers-but the floats had disappeared. When deploying the next set, they stayed and tracked them. Rather than moving slowly in one direction, as originally expected, they moved at relatively high speeds in random directions. It was from this study that mesoscale eddies were discovered (Crease, 1962; Swallow, 1971).

Marine mammals depend on acoustics in very fundamental ways, but this was not known early on. In the 1950s, classified navy surveillance systems heard many signals of unknown origin, including $20 \mathrm{~Hz}$ pulses or BLIPs (from the paper strip chart recorders) that were observed to be highly repetitive, point sources traveling at 1-4 knots. Various interpretations ranged from mechanical and geophysical sources to the breathing or heartbeat of a whale. Finally, in 1963, they were attributed to finback whale courtship displays (Schevill et al., 1964; Tavolga, 2012). This was but one step in revealing the rich babel of life in the ocean.

Since then float tracking and marine mammal studies have been major applications of acoustics in oceanography, with improvements along the way. As Watkins (1981) said, "The sounds from finback whales (Schevill et al., 1964) provided the stimulus for much of the early progress in design of equipment and techniques for the acoustic observations at sea." Ever more acoustic tools were developed, including longbaseline positioning $(\sim 10 \mathrm{~km}$, originally to support drillship dynamic positioning), inverted and bio-acoustic echosounders, and acoustic doppler profilers. Early autonomous underwater vehicles (AUVs), and mobile platforms in general in the 1990s, motivated more work on combined positioning and communication and miniaturized sensors. A mix of acoustic and non-acoustic tools were developed to directly measure the ocean and also to provide necessary support infrastructure.

Ocean acoustic tomography as proposed by Munk and Wunsch (1982) catalyzed new directions. Many process experiments have amply demonstrated the concepts [measuring sound speed (temperature) and currents, $N^{2}$ growth of information with number of instruments], technology (broadband low frequency sources, precise timing and positioning, and coherent signal processing enabling precise travel time determination), and utility for measuring coastal, meso- and larger scale ocean variability (heat content changes, vorticity, baroclinic tides, deep ocean convection, to name a few). A defining event was the Acoustic Thermometry of Ocean Climate (ATOC) project (1996-2006), that demonstrated and served as a pilot for a basin scale ocean heat content observing system (with cabled instrumentation), and catalyzed new efforts in ocean bio-acoustics and passive acoustic monitoring (PAM). The latter includes more than bio-acoustics, with wind, rain, ice, earthquakes, volcanoes, and anthropogenic sound. Throughout, ocean data assimilation techniques struggle to accommodate the new data types and large amount of data generated.
The hydroacoustic monitoring component of the global Comprehensive Nuclear-Test-Ban Treaty Organization International Monitoring System (CTBTO IMS) has been in operation in the Indian Ocean since 2000. The original and primary purpose of the CTBTO IMS is to monitor for signals related to unsanctioned nuclear activity, but the scientific value of the recordings has been demonstrated in numerous studies related to ocean ambient sound, marine mammal behavior, glacial/iceberg noise, human ocean use, tsunami warning, and search and rescue.

These developments-ocean tomography and attendant technology, PAM, mobile platforms, and data assimilationmotivated ideas about regional (and together global) tomography systems (for the OceanObs99 conference; Dushaw et al., 2001), integrated acoustic systems for ocean observing (Howe and Miller, 2004), global ocean acoustic observing network (for OceanObs09 conference; Dushaw et al., 2009), and multipurpose acoustic networks in the integrated Arctic Ocean observing system (Mikhalevsky et al., 2015).

The vision we present in this paper is of a multi-purpose acoustic ocean observing system where the sum is greater than the individual parts. It is the network formed by the parts that yield the gains, both in terms of raw number of data but also the ocean information gain. The individual parts or components-sources, receivers, platforms-can all be shared among the various applications, including PAM, undersea and under-ice mobile platform positioning, navigation and communication, tomography, and more, including cost (Figures 1, 2). Each individual dataset then increases in value within a global context. Because acoustic instrumentation is in general robust and scalable, it is well suited for sustained, long term, multi-scale deployment as part of the Global Ocean Observing System (GOOS).

The goal of this OceanObs19 community white paper is to provide evidence to the readers and conference attendees that such a multi-purpose acoustic ocean observing system is an achievable component of GOOS that will bring many benefits.

A major outcome of the OceanObs09 conference was the Framework for Ocean Observing (Lindstrom et al., 2014). This document outlined the overall goals, objectives, structures, and processes of the GOOS. These include requirements that GOOS must: (1) serve multiple users/requirements with the same observing system; (2) make sustained observations over time with global-scale coverage; (3) be driven by end-user requirements; (4) incorporate continual evaluation, feedback and re-evaluation of system; (5) execute a phased implementation of components (concept/pilot/mature) based on technical readiness levels, and essential ocean variables with high feasibility and impact.

"Ocean Sound" has just been accepted by the Biology and Ecosystem Panel of GOOS as a mature Essential Ocean Variable (EOV; see Ocean Sound EOV, 2018). This is an important milestone primarily addressing PAM. This recognizes the technical readiness, feasibility, high impacts, and operational status of systems already in place. The latter is especially important and is reflected in the longestablished operational global array of hydroacoustic stations as part of the CTBTO IMS (Figure 3). Further, there 


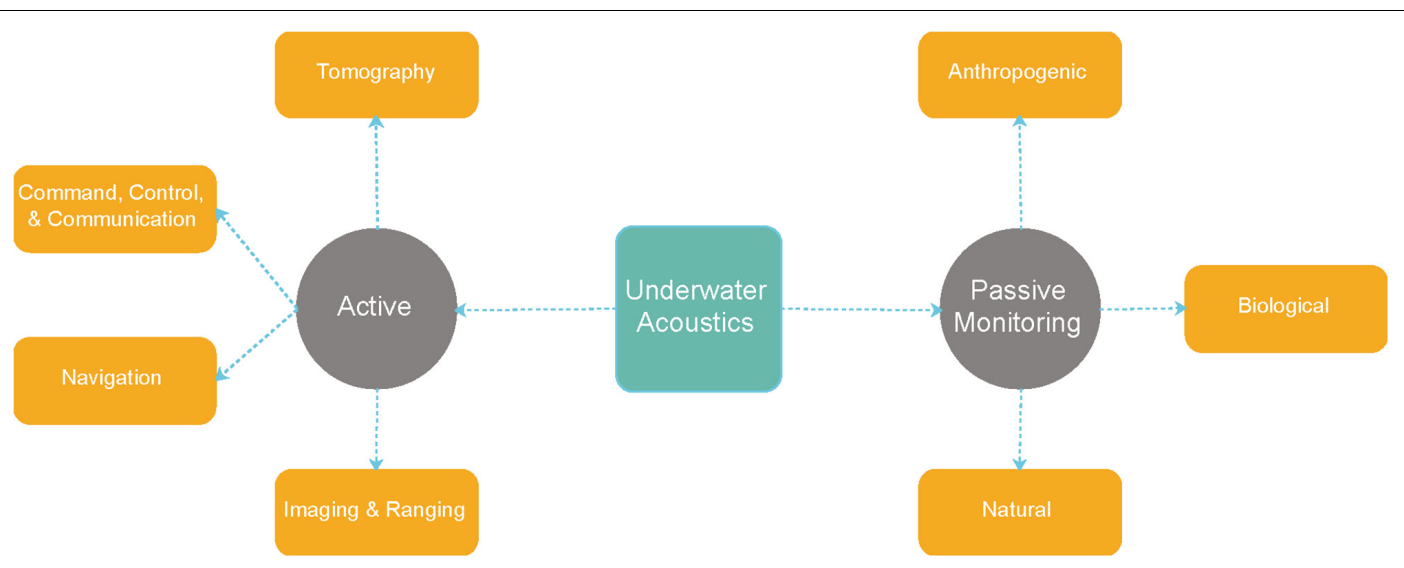

FIGURE 1 | Key core elements of a multipurpose acoustic system for ocean observing (Figure provided by E. Rehm, used with permission).

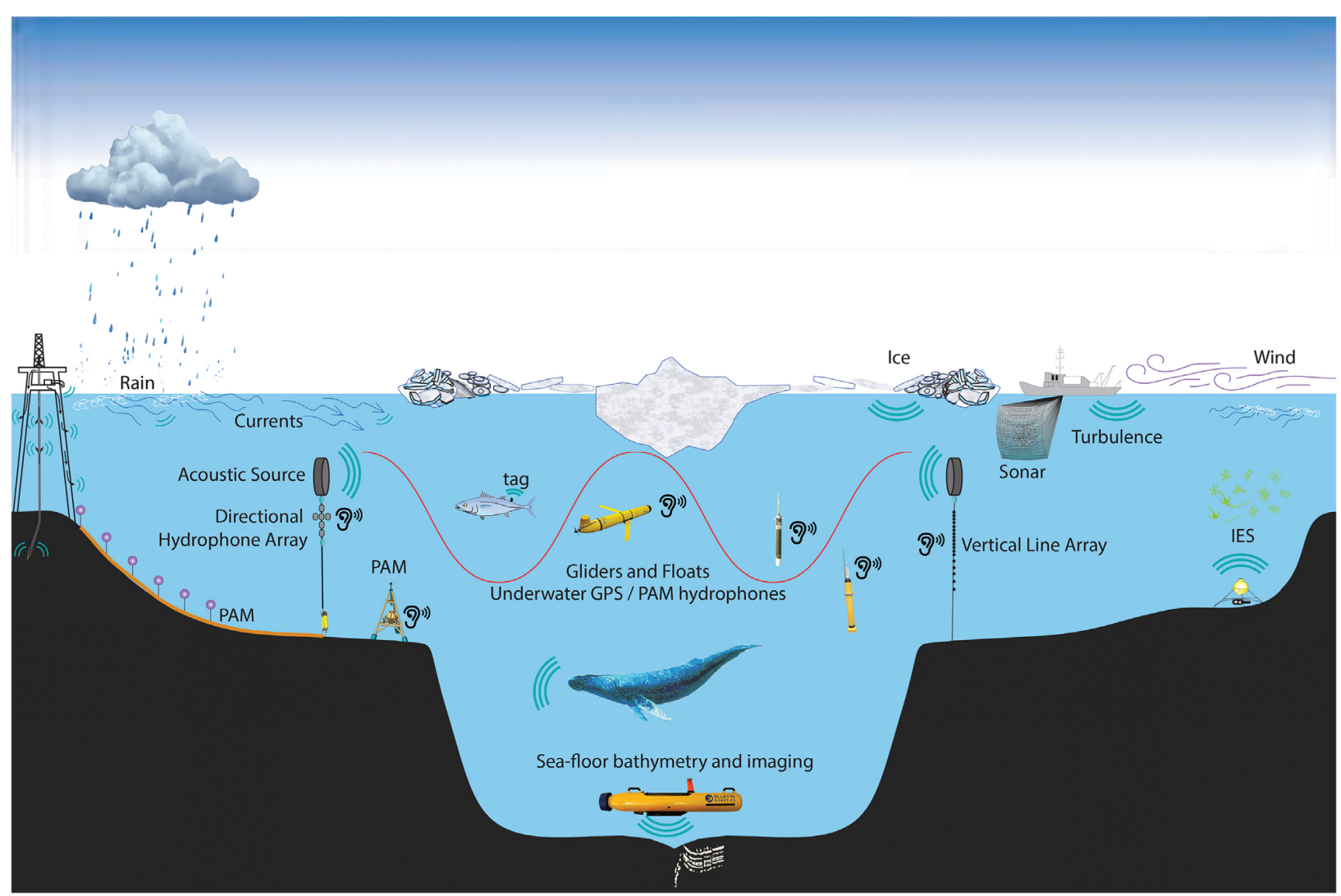

FIGURE 2 | A multipurpose acoustic observing system provides a range of capabilities: two-way transceivers provide support for low-frequency acoustic tomography (heat content, averaged currents), navigation, and communications as well as broadband passive acoustic listening posts. Bottom-mounted passive acoustic monitoring (PAM) stations provide additional coverage for observing soundscapes of natural (wind, rain, ice), biological (plankton, fish, marine mammals), and anthropogenic (ships, sonar, drilling platform) sources. The shifting paths of ocean currents and zooplankton populations can be detected using inverted echo sounders (IES). Autonomous platforms (floats, glides) take advantage of acoustic sources providing "underwater GPS" services and can also provide PAM services. (Figure provided by E. Rehm, used with permission).

are many additional ongoing regional programs, including the ALOHA Cabled Observatory (ACO), NEPTUNE in the northeast Pacific, Perennial Acoustic Observatory in the Antarctic Ocean (PALAOA), and NOAA Ocean Noise Reference
Station Network. There are a number of challenges, though, including: sustained funding for acoustic data management and network or system components; making ocean sound measurement ubiquitous on "integrated instrument packages" 

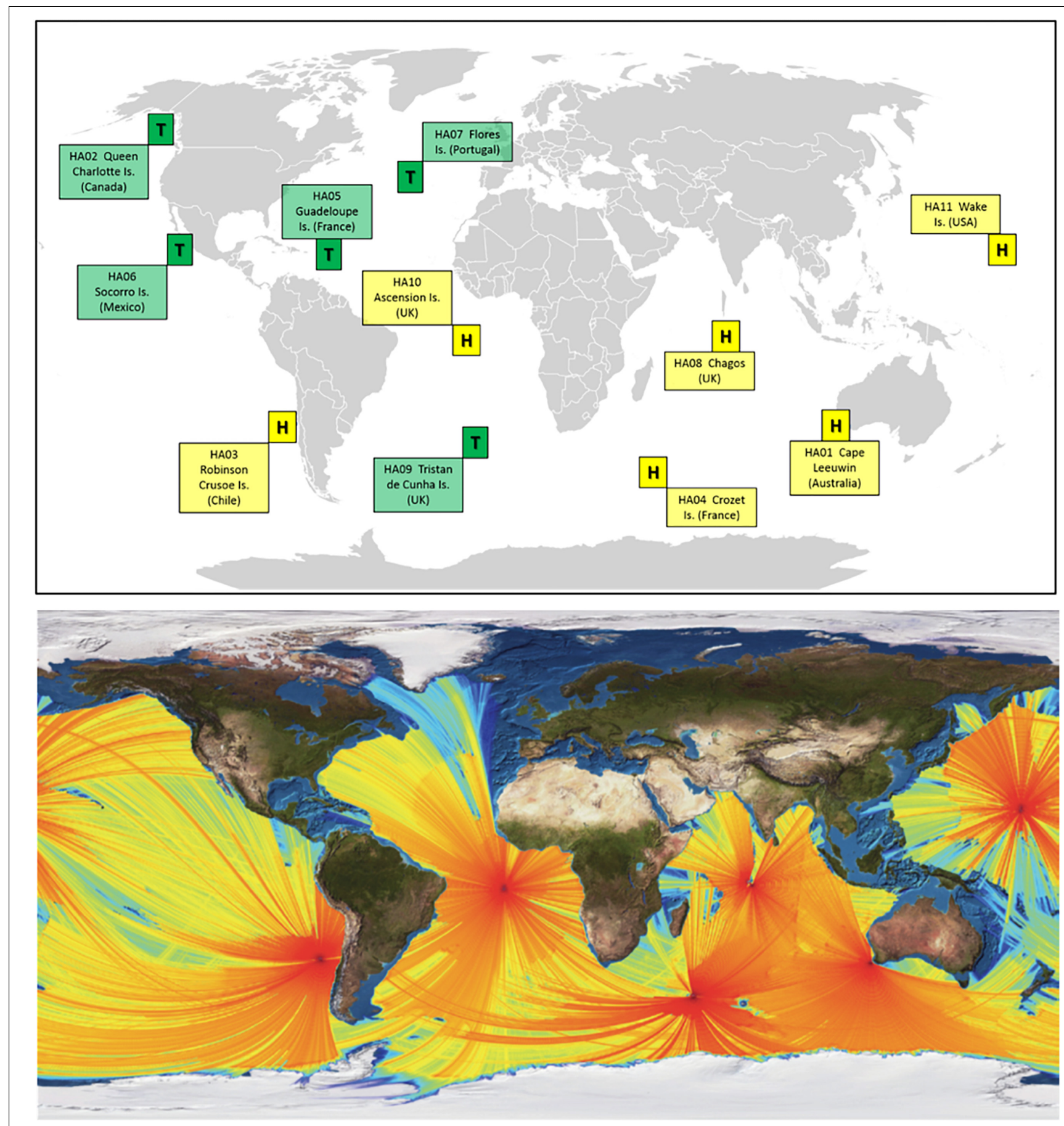

FIGURE 3 | The CTBTO as a global acoustic receiving array, sensitive to many anthropogenic sounds. (Top) A world map showing the Comprehensive Nuclear-Test-Ban Treaty Organization International Monitoring System (CTBTO IMS) hydroacoustic component. Cabled hydrophone stations (1-100 Hz) are represented by the letter "H", while T-stations (seismometers) are represented by the letter "T" (Haralabus et al., 2017). (Bottom) Modeled low frequency (<100 Hz) acoustic coverage of the CTBTO IMS hydrophone H-stations (Heaney, 2015, used with permission).

meant for broad deployment; integrated data management (easy access to any and all data - a "big data" problem); and broadening the scope to more explicitly include geophysical variables such as wind, rain, T-phase/earthquake/tsunami detections. For the purposes of a multi-purpose acoustic observing system, with positioning/tomography integral components thereof, it is important to require that receivers have adequate timing and positioning accuracy $(1 \mathrm{~ms}$ and $1 \mathrm{~m})$.

Other existing EOVs will directly benefit from measurements from multi-purpose acoustic systems. These include: EOV 
Subsurface Temperature from acoustic tomography; EOV Subsurface Velocity from acoustic tomography and mobile platform tracking; EOV Sea State from ocean sound/wind; EOV Marine Turtles, Birds, Mammals Abundance and Distribution from fixed and autonomous PAM sensors; EOV Zooplankton Biomass and Diversity in high-latitude marginal ice zones from mobile platform (float, glider) tracking.

In the balance of the paper, we begin with a review of acoustic technology and its capabilities, emphasizing the aspects that are important to the multipurpose nature of the acoustic observing system. To address the OceanObs19 Observing Needs themes, we have sections on discovery, ecosystems, climate and variability, and hazards and maritime safety. This is followed by a discussion that synthesizes the preceding sections. Concluding remarks and recommendations are given in the final section.

\section{OBSERVING TECHNOLOGY AND NETWORK}

\section{Multipurpose Acoustic Systems}

Multipurpose acoustic networks directly provide unique observations while supporting and complementing other in situ observations - all are needed for a complete GOOS. Acoustic tomography provides observations of large-scale temperature and current. PAM records sounds generated by marine life, ice, earthquakes, volcanoes, wind (e.g., Vagle et al., 1990), rain (e.g., Nystuen, 2001), and other natural sources, as well as by anthropogenic sources such as ships and seismic air gun arrays. In a multipurpose acoustic system, PAM instruments will also record the signals from acoustic sources used for acoustic tomography and undersea positioning and navigation. Acoustic networks facilitate underwater and under-ice geo-positioning of floats, gliders, and other autonomous vehicles. Figure 4 illustrates the various applications (an elaboration of Figure 2).

It is important to appreciate the network aspects that typically are involved in acoustics systems. In many cases, multiple receivers will record multiple sources, both natural and anthropogenic. As an example, data from a number of widely spaced PAM receivers could be used to simultaneously track a pod of blue whales, monitor volcanic activity along a mid-ocean ridge, and record transmissions from several acoustic sources to measure the temperature field. If desired, AUVs from a cabled docking station could be diverted from their routine mapping to monitor the ridge activity, using the acoustic positioning signals for locating themselves and navigating in real time (Figure 2).

Receiver and source technologies are mature, available, and ready for integration into an ocean observing system (e.g., Worcester et al., 2009; Morozov et al., 2016). The technology provides the required accuracy for tomography and underwater geo-positioning systems, as well as recordings of ambient sound and acoustic backscatter from the water column. Passive and active acoustic sensors are available for different frequency bands and different observing platforms, e.g., moorings, floats, bottom landers, and cabled systems. Passive systems can be quite broadband. The High-frequency Acoustic Recording Package (HARP), for example, has a bandwidth of $10-100,000 \mathrm{~Hz}$
(Wiggins and Hildebrand, 2007). Acoustic sources with center frequencies ranging from $20 \mathrm{~Hz}$ (Mikhalevsky and Gavrilov, 2001) to several $\mathrm{kHz}$ (e.g., Send et al., 2002) have been used in acoustic tomography experiments, depending on the range and application.

\section{Tomographic Instrumentation and Signal Processing}

The low-frequency acoustic source technology used to make large-scale tomographic measurements has advanced significantly in recent years. The bottom-mounted, cableconnected, 75- Hz ATOC sources deployed during the 1990s in the mid-latitude North Pacific Ocean were large and heavy $(\sim 5000 \mathrm{~kg})$ (ATOC Instrumentation Group, 1994). The autonomous acoustic sources used to generate the $\sim 20 \mathrm{~Hz}$ signals needed to propagate for long ranges under the ice in the Arctic Ocean for the Trans-Arctic Propagation (TAP) experiment in 1994 and the Acoustic Climate Observations using Underwater Sound (ACOUS) project beginning in 1999 were one-of-a-kind, large devices (Mikhalevsky and Gavrilov, 2001). With the dramatic decrease in multiyear ice (e.g., Meier et al., 2014) and the associated deep ice keels, it is now feasible to effectively transmit higher acoustic frequencies over long ranges in the Arctic. Sources that operate at $32 \mathrm{~Hz}$ and are of manageable size and weight have recently become commercially available (Figure 5). These require pressure compensation to keep the inner gas pressure equal to the external water pressure, using a (largely) passive bladder system with $20 \mathrm{~m}$ dynamic range for operation at $100 \mathrm{~m}$ depth on a mooring in under-ice Arctic conditions. Slightly modified versions of the same source and pressure compensation technologies can be used at sound channel depths in mid-latitudes $(\sim 1000 \mathrm{~m})$ at the ATOC frequency of $75 \mathrm{~Hz}$. In this case, the same pressure compensation unit would have a dynamic range of $200 \mathrm{~m}$ to accommodate stronger currents and mooring pull down. Furthermore, these sources can be coupled together in small arrays that broaden the bandwidth and provide more power and some vertical directionality. (A vertical array would reduce vertically propagating energy in favor of more horizontal energy in the sound channel).

Acoustic receiver technology has also improved. Sub-basinscale ocean acoustic tomography experiments have taken advantage of the flexibility of autonomous, moored systems. The expected magnitude of travel-time perturbations associated with the ocean mesoscale is roughly $100 \mathrm{~ms}$. The differential travel-time signal from reciprocal transmissions is an order of magnitude smaller. The requirements to keep time with millisecond accuracy in submerged instruments for a period of a year or more, measure the motion of the moored sources and receivers to within a meter or so in order to correct for travel-time perturbations due to changes in range, and record the large amounts of data generated by the acoustic receptions made for early instruments that were sufficiently complex to be accessible only to highly specialized research groups. [Munk et al. (1995) provides a detailed discussion of the observational methods used in acoustic tomography]. Modern developments 


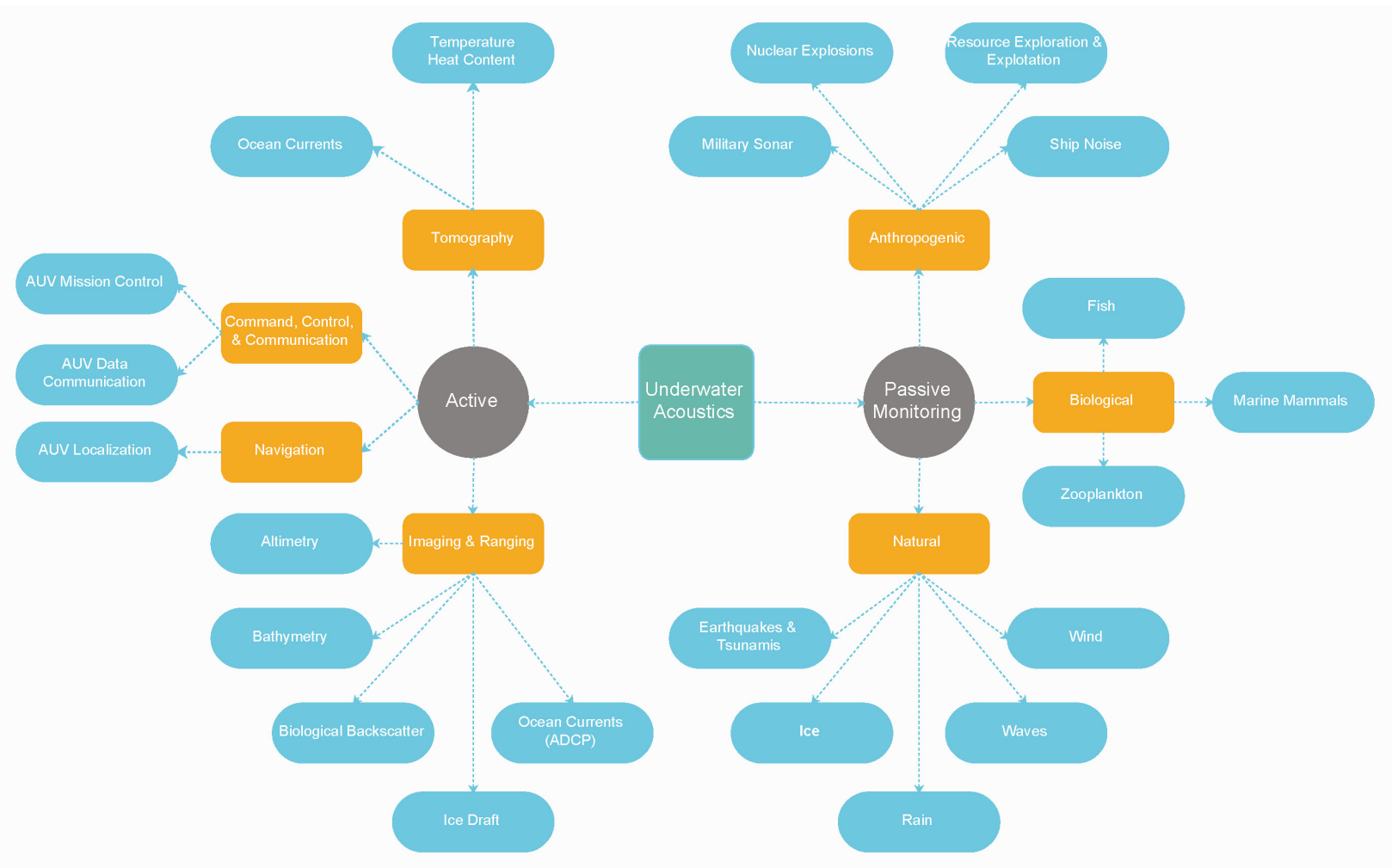

FIGURE 4 | Ocean observing applications of a multipurpose acoustic system, building on the core elements. (Figure provided by E. Rehm, used with permission).

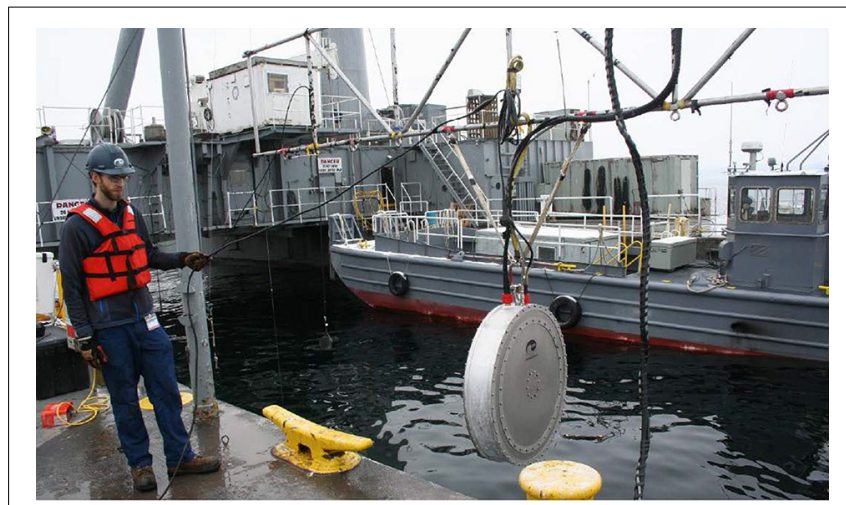

FIGURE 5 | The Geospectrum, Inc. low frequency source (LFS) $\left(f_{0}=32 \mathrm{~Hz}\right.$, $\Delta f=10 \mathrm{~Hz}, 1 \mathrm{~m}$ diameter, $0.2 \mathrm{~m}$ thick, $270 \mathrm{~kg}$ ). (Figure provided by Geospectrum, used with permission).

in data acquisition systems and data storage have now made the required instrumentation much more user-friendly. For climate-oriented, basin-scale experiments with requirements for high power and long duration, however, cabled systems are preferred. (Hybrid systems are of course possible). Mooring motion and accurate timing are not problems in systems mounted on the seafloor and cable-connected to shore. The ATOC project relied on cabled systems, for example, augmented by a few autonomous vertical receiving arrays deployed for year-long periods to better characterize the propagation (ATOC Instrumentation Group, 1994).

The processing of tomographic data to obtain time series of travel times has become much more routine in recent years. For both moored and cabled systems, the signal processing and generation of time series of travel times for resolved acoustic ray paths has been labor intensive and subjective. After matched filtering and beamforming of the received signals, the procedure is to pick the peaks in the processed receptions. The peaks must then be associated with predicted ray arrivals. This procedure must be repeated for each reception for every source-receiver pair. However, internalwave-induced scattering of the received signals impairs the ability to associate a measured peak with a computed ray path. Two procedures were developed to address these issues (Dzieciuch, 2014). First, an estimator-correlator that explicitly accounts for scattering is used to smooth the arrival pulse and improve the signal-to-noise ratio. Second, after defining an error metric that accounts for peak amplitude, travel time, and vertical arrival angle, the Viterbi algorithm has been successfully adapted to the task of automated peak tracking, eliminating the need to manually select appropriate peaks in each reception.

In the past, stochastic inverse methods have most commonly been used to interpret acoustic travel times to obtain ocean temperatures and currents (e.g., Munk et al., 1995). With recent improvements in the vertical resolution and realism of ocean 
circulation models, acoustic travel times are now being used to directly constrain the models, taking advantage of the models to combine acoustic (and other data) from different times and locations in a way that is consistent with ocean dynamics (e.g., Lebedev et al., 2003). Time-evolving ocean circulation models implicitly supply a large amount of information about the ocean by enforcing the conservation of mass, momentum, and other properties. The practice of combining data with models, referred to as state estimation or assimilation, simultaneously tests and constrains the models. A variety of approaches are available to solve this problem, e.g., variational data assimilation (4DVAR) and ensemble Kalman filtering (EnKF) (e.g., Munk et al., 1995; Gopalakrishnan et al., 2019).

Combining integral tomographic data with time-evolving models does not differ in any fundamental way from using other data types, but tomographic data do differ from most other oceanographic data because their path-integral sampling and information content tend to be localized in spectral space rather than in physical space. It is therefore important to use methods that preserve the integral information contained in the acoustic travel times. Approximate data assimilation methods optimized for measurements localized in physical space are generally inappropriate because they do not preserve the non-local tomographic information (Cornuelle and Worcester, 1996). The data types used to constrain the models are largely transparent to the user of the resulting ocean state estimates, placing the rather unfamiliar acoustic data on the same footing as more familiar data types.

Although state estimation employing general ocean circulation models in ice-free regions has received the most attention, state estimation employing coupled ocean-sea ice models has received increased attention in the past decade (Fenty and Heimbach, 2013; Stammer et al., 2016; Nguyen et al., 2017). Present ice-ocean coupled models can assimilate sea ice parameters from remote sensing and profiles from floats and drifting ice buoys. The sea ice forecasts are improved through the assimilation of sea ice parameters. However, there is a significant gap in under-ice observations to evaluate or constrain the ice-ocean models. The ice-ocean models are therefore unconstrained and their performance is more or less unknown. The effect of repeating assimilation only on the surface and keeping the ocean under the ice unconstrained with data is unknown. Acoustic methods can be used to provide data to test the large-scale behavior of the ocean-sea ice models and, eventually, to constrain them.

\section{Underwater Acoustic Positioning and Navigation}

Positioning, Navigation and Timing (PNT) are vital infrastructure services of our modern technical society. PNT needs to be extended throughout the subsea environment. PNT is a combination of three distinct, constituent capabilities:

1. Positioning is the ability to accurately and precisely determine one's location referenced to a standard geodetic system;
2. Navigation is the ability to determine current and desired position (relative or absolute) and apply corrections to course, orientation, and speed to attain a desired position anywhere in the domain of concern; and

3. Timing is the ability to acquire and maintain accurate and precise time anywhere in the domain of interest within user-defined timeliness parameters; it also includes time transfer.

Navigation is real time and necessarily depends on positioning, and positioning depends on timing.

A key aspect of the multi-purpose concept is to transfer the precise measurement of travel time between a source and receiver, as done in tomography, to the long-range underwater positioning and navigation community as exemplified by RAFOS float positioning and AUV navigation. Providing an underwater navigation capability is particularly important in ice-covered regions, where floats, gliders, and AUVs have difficulty surfacing to obtain their positions. Multipurpose acoustic networks allow economy in source signaling: the same acoustic signals are used for both tomography and positioning. The Global Navigation Satellite System (GNSS), of which the US Global Positioning System (GPS) is part, has enabled ionospheric (electron density) and atmospheric (temperature, precipitable water vapor) tomography, while providing its core PNT service. The relatively expensive satellites provide signals to $10-100$ s of low earth orbiting satellites (radio occultation) and 1000s of ground stations to obtain dense, global coverage over land and ocean. For the ocean case, acoustic sources can transmit to both mobile vehicles and fixed receivers, solving for position and sound speed (temperature) in a joint estimation problem.

The difference between RAFOS and what has been called RAFOS-2 is comparable to the difference between old long-range navigation (LORAN) and Transit positioning and GPS today. The increased bandwidth of RAFOS-2/tomographic signals yields better positioning accuracy (Duda et al., 2006; Van Uffelen et al., 2016). Figure 6 shows simulated arrival patterns for a narrow band $1.6-\mathrm{Hz}$ RAFOS signal, which is about $1 \mathrm{~s}$ in duration, equivalent to $1600 \mathrm{~m}$ position uncertainty, and for a RAFOS-2/tomography signal with a $50-\mathrm{Hz}$ bandwidth, where individual arrival peaks are $20 \mathrm{~ms}$ wide, giving roughly $30 \mathrm{~m}$ range uncertainty (assuming $1500 \mathrm{~m} / \mathrm{s}$ sound speed).

Precision glider positioning was in fact demonstrated in the 2010-2011 North Pacific Acoustic Laboratory (NPAL) Philippine Sea Experiment (PhiiSea10) (Van Uffelen et al., 2016). Six broadband, 250-Hz tomography sources were deployed in a pentagonal array $660 \mathrm{~km}$ in diameter. Four Seagliders, each equipped with an acoustic receiver and the ability to keep precise time (syncing to GPS time while the Seagliders were at the surface), recorded receptions. Subsequent analysis showed that estimated underwater position errors were reduced from $900 \mathrm{~m}$ RMS using standard glider positioning to about $80 \mathrm{~m}$ RMS when incorporating measured travel times, over $700 \mathrm{~km}$ ranges (Figure 7).

In ice-covered regions, work has been progressing along several lines. Using conventional narrow bandwidth RAFOS sources, Argo floats have been positioned (after the fact) to 

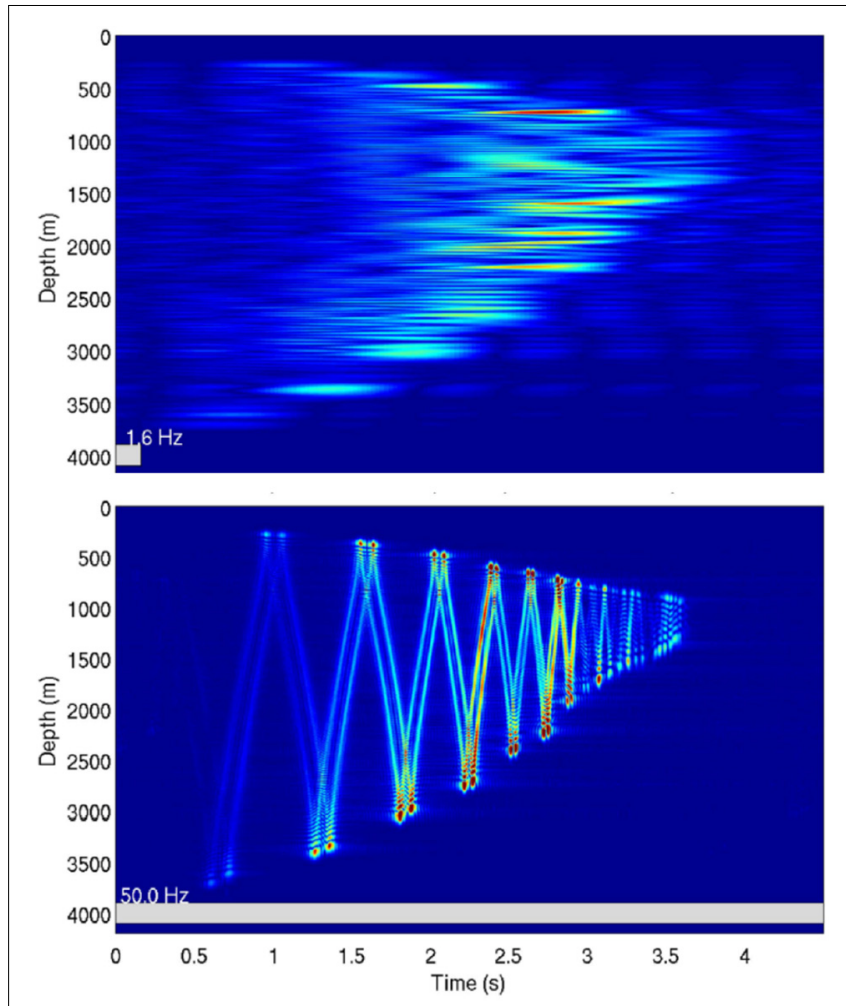

FIGURE 6 | Arrival patterns for a 1145-km transmission path in the North Atlantic Ocean for systems with 80 -s linear FM signals of $1.6-\mathrm{Hz}$ (upper, RAFOS) and 50- $\mathrm{Hz}$ bandwidth (lower, RAFOS-2/tomography) and $260-\mathrm{Hz}$ center frequency (source at $700 \mathrm{~m}$ depth, receiver at $2000 \mathrm{~m}$ depth). The time scale is reduced time, which removes the gross transmission delay between the source and receiver. Positioning uncertainty for the first case is (sound speed/bandwidth) 1000 m, while for the second it is $30 \mathrm{~m}$ (Duda et al., 2006, used with permission).

$400 \mathrm{~km}$ range (Klatt et al., 2007). Gliders have navigated under ice in Davis Strait (Webster et al., 2014) and Fram Strait (Sandven et al., 2011) with position uncertainties on the order of kilometers or more. More recently in the Arctic, higher frequency $(900 \mathrm{~Hz})$ instrumentation has been used in an integrated system with ice-tethered GPS synchronized navigation transceivers (i.e., the "satellites") talking to gliders. As with GPS, low data rate source position information is transmitted to enable the gliders to perform absolute geo-positioning and navigation. Additional information can also be transmitted, in this case new surfacing target waypoints. This system has been tested; performance is a function of range, the particular acoustic conditions present and the vertical position of the glider relative to structure in the sound channel (Freitag et al., 2015; Webster et al., 2015; Lee et al., 2017). These examples show that no one solution fits all, but that significant progress is being made toward multipurpose acoustic systems; especially in the last case all the elements are there.

The incremental cost of using a broadband tomography source relative to a narrowband RAFOS source is small compared to mooring and ship time costs. This simultaneous improvement in positioning and associated navigation accuracy coupled with obtaining tomographic temperature is a major motivator for the multi-purpose concept. To obtain subsea positioning accuracy of $10 \mathrm{~s}$ of meters, one will need to account for the 4-D variation in sound-speed (e.g., mesoscale variability). For realtime subsea navigation this is clearly challenging; for delayed mode positioning, this would likely be done in the context of a large joint ocean-position estimation procedure.

\section{Passive Acoustic Monitoring}

Passive acoustic monitoring recording devices are now key to regional strategic plans for the successful monitoring of natural, anthropogenic, and animal sound. Audio data receivers support multiple hydrophones and associated recording channels and contain massive amounts of data storage, thanks to advances in non-volatile memory storage capacity and low-power digital signal processors. An autonomous directional PAM acoustic receiver array on a bottom lander is shown in Figure 8 . Some acoustic recording systems contain sufficient on-board processing power to support real-time data reduction in the form of spectrogram generation, detection and classification of specific sounds, and localization of sounds from hydrophone arrays. For example, for ocean gliders equipped with acoustic receivers with on-board whale call classification software, only short data packets need be transmitted via Iridium satellite data connections to then allow real-time whale positions to be added to the automated identification system (AIS) used on ships and by vessel traffic services (Davis et al., 2016) to track vessel locations.

Passive acoustic monitoring receivers that are part of multipurpose acoustic observing systems need to have accurate and synchronized time bases. Timing is absolutely fundamental to undersea positioning, navigation and tomography, which use precision travel-time data, necessitating synchronized time bases across all platforms. Clock drift between acoustic sources and a receiver on an autonomous platform is the primary source of positioning uncertainty (Webster et al., 2015). Achieving synchronous time is straightforward for systems that have regular access to GPS signals. It is more difficult for autonomous subsea systems, but the technology to do so has been demonstrated, both via use of chip-scale atomic clocks and using time transfer methods, via acoustic communications.

\section{Network Technology}

One of the technologies that will be key to the vision of a multipurpose acoustic network as part of ocean observing systems is flexible low-power software-defined underwater signal processing systems. To support underwater sensor networks, next-generation Underwater Acoustic Networks (UANs) must be able to adapt their communication and networking protocols in real-time based on the environmental and application conditions. UAN developers initially found that commercially available acoustic modems, their embedded software systems being largely closed and proprietary, were not flexible enough to satisfy the requirements. This has led to a modular, evolving software-defined radio (SDR) framework for both research and commercial UAN devices supporting all layers of a network protocol stack, including the ability to flexibly encode/decode, in software, acoustic signal modulation at the physical layer (Dol et al., 2017). Such SDR technology enables, for example, 
an AUV to flexibly decode and process a multiplicity of source signal types: narrow-band RAFOS signals; wide-band frequencymodulated (FM) or phase-shift-keyed tomography/navigation (RAFOS-2) signals; or simple phase-shift keyed data packets. This allows a variety of acoustic source types to co-exist and evolve on multipurpose acoustic networks. Similarly, software algorithms for specific signal classification (e.g., commands, whale call identification, ship noise, estimates of rainfall, wind, or specific ice deformation events) can co-exist on board such an AUV or tomography SDR. Eventually, these decoded characteristics can be relayed to shore as compact metadata rather than voluminous raw acoustic data. An example of such SDR flexibility was demonstrated by the WHOI Micro-Modem 2 (Gallimore et al., 2010), deployed in 2014 on Seagliders in the marginal ice zone of the Chukchi Sea, which decoded RAFOS-like signals for traveltime estimates (linear FM sweep, $900-\mathrm{Hz}$ center frequency, $25-\mathrm{Hz}$ bandwidth, per above), as well as a phase-modulated signal that encoded a 72-bit data packet containing an ice-tethered (moving) acoustic source location (high resolution latitude and longitude) and a new target glider waypoint (low resolution latitude and longitude) (Freitag et al., 2015; Webster et al., 2015).

\section{ACOUSTICS ADDRESSING OCEAN OBSERVING NEEDS}

\section{Discovery}

Innovative tools that lead to new discoveries, which ultimately lead to hypothesis-driven science and finally sustained application, enable forward momentum in science. Because sound travels farther and faster than any other signal underwater, acoustics is the primary mode of probing the ocean volume, a form of remote sensing, whether it be fish navigating by the sound of surf or AUVs mapping the mid-ocean ridges, whales communicating and socializing, or measuring ocean basin heat content and currents.

Using the new tools to pick the "low-hanging fruit" catalyzes discovery. New tools or techniques in one field can be repurposed for ocean use, or a simple upgrade to an existing tool can make it much more versatile. Here we briefly explore the vision of what acoustics might bring to ocean observing on time scales of decades and longer, based on the technologies and networking described above.

There are direct analogies between acoustic underwater positioning and the global navigation system of systems (GNSS) that provide precise and accurate time and positioning. Our science and our society are now totally dependent on the latter and take it as an essential critical infrastructure service. We envision that multi-scale acoustic navigation systems will enable the same incredible development of applications that GNSS has created. It will be a major tool in helping us transition from a rather incoherent and chaotic view of the ocean, to a focused, evolving, coherent image. It will enable humankind (using mostly robotic avatars) to "see" the ocean volume.

With precise navigation one can envision using longrange/endurance AUVs to map the ocean floor (Woelfl et al., 2019). They will transit between a rather sparse network of

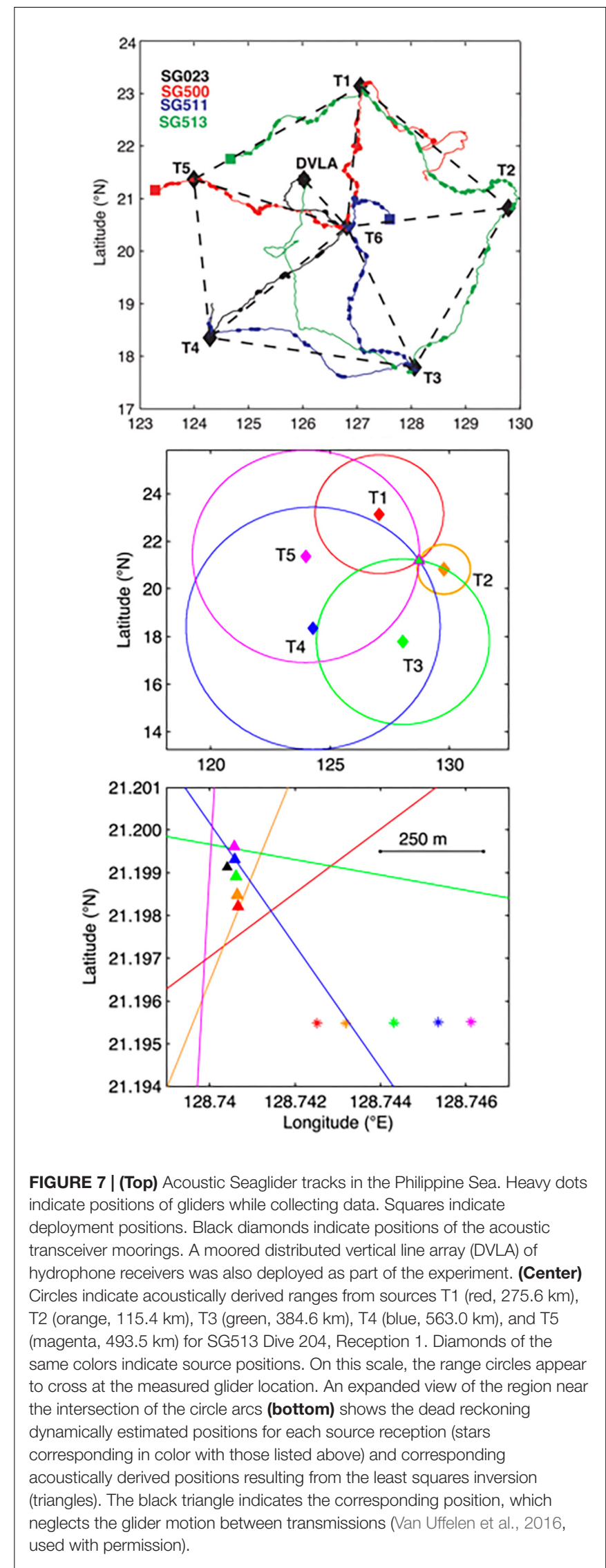




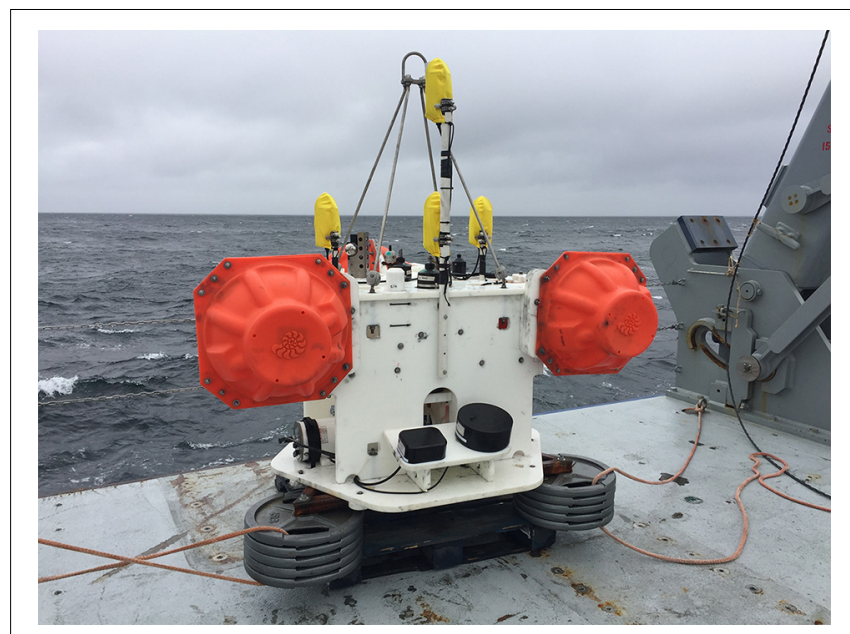

FIGURE 8 | Atlantic Deepwater Ecosystem Observatory Network (https://adeon.unh.edu) acoustic lander. A tetrahedral hydrophone array comprising four M36 hydrophones within an Autonomous Multichannel Acoustic Recorder (AMAR; JASCO Applied Sciences) located under the yellow protection covers. Two sets of transducers are shown mounted on the bottom of the lander looking upwards as part of a 4-frequency Acoustic Zooplankton-Fish Profiler (AZFP, ASL Environmental Sciences) echosounder system for capturing water column backscatter. (Photo by J. Miksis-Olds, used with permission).

cabled docking stations, which will also host the acoustic sources of the multipurpose system. There is no question that they will discover numerous volcanoes, hot and cold vent systems, mineral resources, and new life forms within these extreme environments. The latter is directly relevant to the conservation and sustainable use of marine biological diversity of areas beyond national jurisdiction (BBJN). With precise navigation, AUVs can fly over repeat paths to absolute locations with certainty to observe changes, enabling and servicing autonomous platforms, and serving as energy "tankers" and communications gateways. These platforms (as well as the AUVs) will be sampling a myriad of ocean and geophysical variables, including, for instance, heat and material fluxes from the seafloor (Deep Ocean Observing Strategy [DOOS], 2016, 2018; Levin et al., 2019).

Very importantly, such navigation is essential to sampling under ice in polar regions (Arctic and Antarctic), both for the physics as well as the ecosystems in such an extreme and ephemeral environment, e.g., massive springtime plankton blooms under Arctic sea ice that control the timing of water column primary production (Arrigo et al., 2012). While summer ice may disappear in the near future, ice during the rest of the year will likely persist for decades. Support for underice navigation would apply to AUVs as well as gliders and floats (ANCHOR Working Group, 2008; Mikhalevsky et al., 2015; Smith et al., 2019). In this case, not only would basic navigation be enabled, precise geolocation would allow results between the various disparate sampling platforms to be combined coherently within the framework of ocean data assimilation models (running the joint positioning/ocean state estimation). This applies equally to non-polar regions as well. High resolution in time and space tracking will open up a new domain of sampling space that includes smaller and faster ocean processes that are lost in crude RAFOS tracking $(2 \mathrm{~km}$, a few times per day).

We know there are sharp fronts in the ocean, but they are often difficult to sample. If they are sampled with mobile platforms, accurate georeferencing is important so that multiple sources of data can be coherently combined. Long-range acoustic propagation paths used in tomography are also sensitive to such features. One-second changes in travel time are observed as a result of Gulf Stream or Kuroshio meanders, for example (Spiesberger et al., 1983; Lebedev et al., 2003).

The use of "noise" sources of opportunity in a tomographic context is tantalizing, with sources being diffuse (wind, waves) to near points (ships, whales, earthquakes, glaciers). By cross-correlating noise signals received by spatially separated receivers, one can, in principle, infer acoustic arrival patterns for the intervening medium as if they were transceivers. This is well proven and utilized in seismology and many other fields. The critical factor in the ever-changing ocean, however, is the duration over which one can compute the cross-correlation before changes in the ocean medium cause the time delays between the receivers to vary (Kuperman, 2018). Some results have been obtained, for example using Antarctic ice noise as received on distant CTBTO arrays, and using vertical arrays listening to ships over short range. It may be that with the ever-increasing number of receivers being deployed with coherent signal processing, "passive tomography" will come to fruition (see Kuperman, 2018, for a recent review).

Extending this further, acoustic tagging of pelagic marine life is well underway (e.g., marine mammals, sharks) to address questions related to communication, foraging ecology, and impacts related to sound exposure (Johnson et al., 2009). With tags that include acoustic positioning capability, one can envision coherently processing the acoustic data in the context of other environmental data collected on other platforms (assuming the tag is eventually recovered). Information from animal tags have already been integrated into the Australian Ocean Data Network (AODN) (Hidas et al., 2018) and serve as a model for what is possible on a global scale.

Using acoustics to listen to marine life, from very small to very large, whether remotely or with a tag on an animal, will improve our understanding of communication and behavior. With accurate positioning, we can obtain finer and finer views of what the animals are doing, and why. With the improved ocean state estimates obtained by including acoustic tomography data, we will be able to more directly link animal behavior with ocean physics and determine how marine mammals function and adapt to the constantly changing conditions of a dynamic ocean.

Finally, the combined use of active and passive acoustics, as appropriate, to simultaneously observe the physics and biology will greatly aid the interpretation of the data. New insights will result when multi-frequency sonars and "acoustic vision" are coupled with observations of the physical flow throughout the water column, from the photic zone with the deep scattering layer, to the deepest hadal reaches. 


\section{Ecosystem Health and Biodiversity}

Following closely on the heels of initial discovery is the quest to gain a full understanding of the environment surrounding targeted findings. The combination of active and passive acoustic technology is a powerful tool in building a comprehensive concept of ocean dynamics shaping the local or regional ecosystem. Ocean processes, marine life dynamics, and human ocean use are each inherently three-dimensional and timedependent, and each occurs at many spatial and temporal scales. The versatility of acoustic observations provides the opportunity to obtain information over wide temporal and spatial scales. The value of ocean acoustic observations for understanding biology and ecosystems has been internationally recognized through the acceptance of the proposal by the International Quiet Ocean Experiment program to the GOOS Biology and Ecosystems Panel for the consideration of Ocean Sound as an Essential Ocean Variable (Miksis-Olds et al., 2018) and by the convening body of the Deep Ocean Observing Strategy (Deep Ocean Observing Strategy [DOOS], 2016, 2018). Additionally, the application of passive acoustic recordings in constructing underwater soundscapes to better understand ocean ecosystem dynamics has gained community traction. Clear evidence of the value and application of underwater soundscapes is demonstrated by the effort of the International Organization for Standardization (ISO) in developing ISO standard 18405 on Underwater Acoustics-Terminology to help ensure research measurements are repeatable and comparable across projects (International Organization for Standardization [IOS], 2017). ISO 18405 defines the underwater soundscape for the first time and illustrates the growing maturity of this ocean variable.

Ocean sound is a national and international focus because it crosses borders unimpeded. Acoustic signals, as opposed to visual and chemical signals, can propagate long distances in the ocean and provide a means for marine life and humans to gain information about the environment and for marine animals to exchange critical information. Passive acoustic technology allows us to eavesdrop on marine life interactions and physical processes that create the local soundscape. A soundscape is the characterization of the ambient sound in terms of its spatial, temporal and frequency attributes, and the types of sources contributing to the sound field (International Organization for Standardization [IOS], 2017). Passive acoustic recordings are used non-invasively to assess environmental sound levels, surface conditions, human activity, and the distribution and biodiversity of vocalizing marine life. Active acoustic technology provides a high-resolution (in both time and space) measure of biological (zooplankton and fish abundance and distribution) and physical oceanographic processes (internal waves, micro-turbulence, and frontal systems) through time series of acoustic backscatter measurements (Lavery et al., 2009).

A great deal of information related to ocean ecosystems can be gained simply by assessing the ambient sound. The concept of using information in ambient sound as cues to direct movement or identify appropriate habitats has recently been identified as a new field of study referred to as soundscape orientation, and the concept is also included within the broader field of soundscape ecology in the scientific literature (Slabbekoorn and Bouton, 2008; Pijanowski et al., 2011). A large number of aquatic species use sound cues contained in local soundscapes to navigate, forage, select habitat, detect predators, and communicate information related to critical life functions (e.g., migration, breeding, etc.). Consequently, marine animals ranging from the smallest larvae to the largest whales have evolved mechanisms for both producing and receiving acoustic signals. Information contained in underwater soundscapes provide a means to better understand the influence of environmental parameters on local acoustic processes (McWilliam and Hawkins, 2013; Miksis-Olds et al., 2013; Staaterman et al., 2014), assess habitat quality and health (Parks et al., 2014; Staaterman et al., 2014), and better understand the impacts and risks of human contributions to the soundscape on marine life.

Underwater soundscapes are dynamic, varying in space and time within and between habitats. Underwater soundscapes are highly influenced by local and regional conditions but, unlike most terrestrial soundscapes, distant sources are also significant contributors because sound propagates such great distances underwater. The underwater soundscape may be composed of contributions from human activity (e.g., shipping, seismic air gun surveys), natural abiotic processes (e.g., wind, rain, ice), non-acoustic biotic factors (e.g., animal movement), and acoustic contributions from sound producing, biological sources (e.g., marine mammals, fish, and crustaceans). The soundscape can be selectively decomposed and visualized to gain a greater understanding of the sources and environmental dynamics contributing to and shaping the temporal, spatial, and spectral patterns of the acoustic environment. In the early 1990s, the ATOC program began collecting ambient sound in order to begin to better understand soundscapes and answer such basic questions such as how often does the sound level exceed a certain value, and can the constituents of ambient sound be discriminated (Curtis et al., 1999). Figure 9 shows recent examples addressing these two questions.

Indicators of habitat quality and biodiversity that were developed for terrestrial applications are now being applied to marine habitats and soundscapes (Denes et al., 2014; Parks et al., 2014; Staaterman et al., 2014). Acoustic analysis of a habitat's soundscape using high level indicators such as the acoustic complexity index (ACI), acoustic entropy index, or acoustic similarity/dissimilarity indices provide a quantitative way to assess biodiversity and compare/contrast soundscapes of different habitats (Parks et al., 2014). Compared to in situ measurements of marine habitats by human divers, acoustic biodiversity indicators are less costly and less labor intensive. Acoustic systems also provide the added benefit of high temporal resolution resulting from long-term deployments of remote passive acoustic sensors. Acoustic diversity indicators are estimated by mathematically assessing the ratio of energy at different spectrum frequencies to make inferences about local community biodiversity. The larger the frequency bandwidth of recordings, the more information is available to accurately capture species and habitat diversity (Parks et al., 2014). 


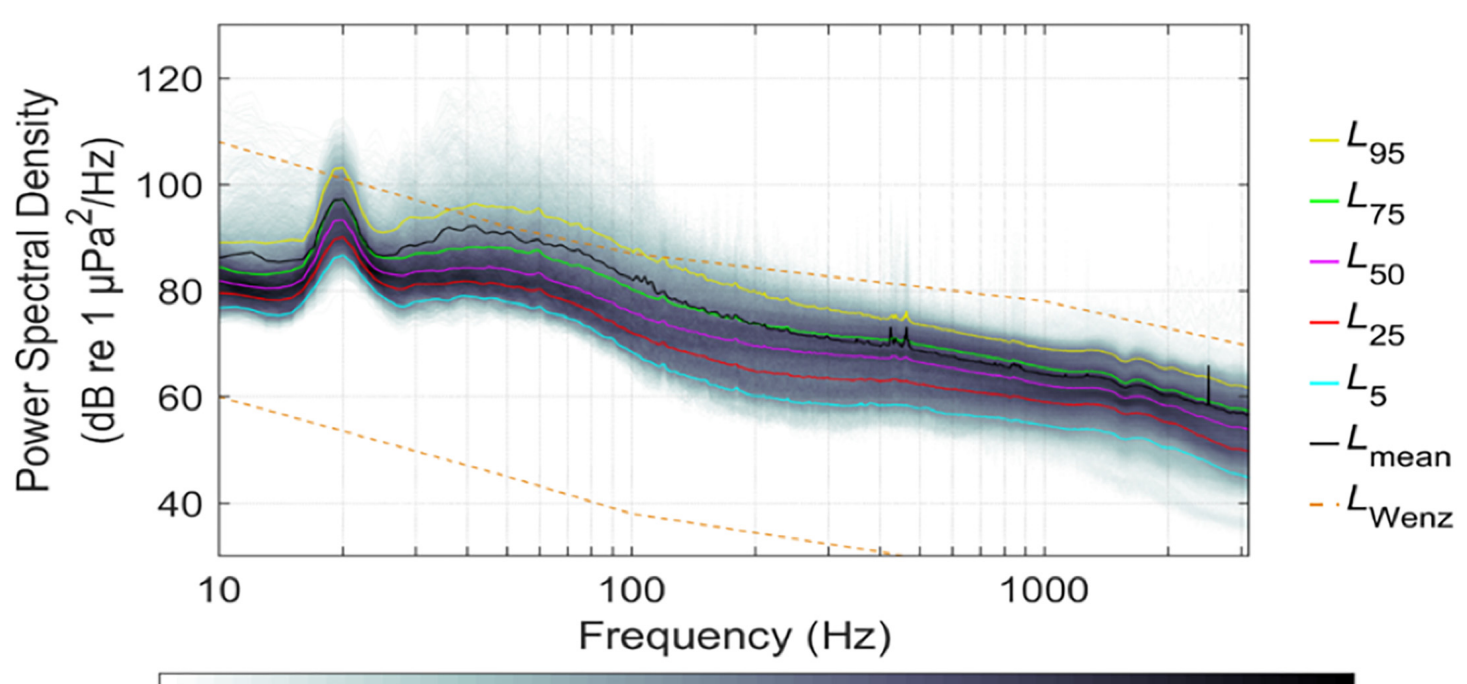

Relative Spectral Probability Density

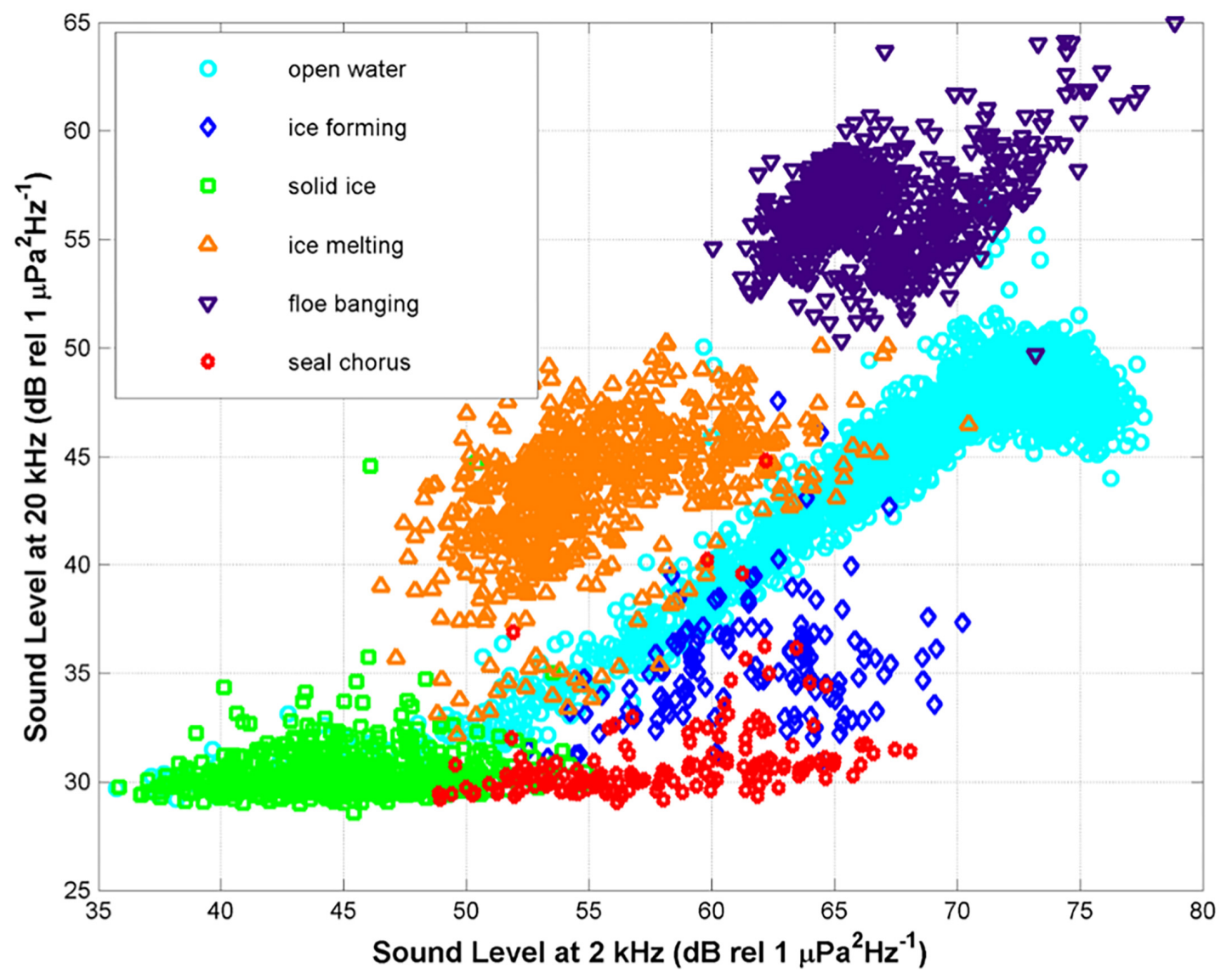

FIGURE 9 | Two examples of how soundscape data is visualized. (Top) Summary of sound levels by an ADEON lander (Figure 8) along the Outer Continental Shelf east of Virginia based on 1-min, $1 \mathrm{~Hz}$ resolution analysis of data sampled at $375 \mathrm{kHz}$. Percentiles and relative spectral probability density for the $1 \mathrm{~Hz}$ spectra (Figure provided by Bruce Martin, JASCO Applied Science, used with permission). (Bottom) Soundscape recorded in the winter/spring of 2009 in the central region of the Bering Sea Shelf. Each point on the image represents the ratio of sound pressure level between 2 and $20 \mathrm{kHz}$ at a specific point in time. The acoustic environment changed based on the presence of vocalizing ice seals and state of sea ice at the surface. Sources are color-coded based on their stereotyped source characteristics. [Figure was produced by Jeffrey Nystuen (APL-UW) and is a reproduction from Figure 5 in Van Opzeeland and Miksis-Olds, 2012, used with permission]. 
Successful examples of linking biodiversity and ocean sound come from studies on coral reefs and kelp beds. Healthy coral reefs and kelp habitats support high levels of biodiversity and produce an overall soundscape rich in temporal and spectral signatures created by the cacophony of vocalizing animals ranging from low-frequency fish calls to high-frequency, broadband sounds of snapping shrimp (Radford et al., 2008a; Staaterman et al., 2013). Kelp forests of New Zealand have shown diel, lunar, and seasonal trends in sound production with the most intense sounds occurring at dawn, dusk, and during the summer months when the abundance of sea urchins, snapping shrimp, and noise-emitting fish are highest (Radford et al., 2008b, 2010). In this system, there was good agreement between measures of acoustic and in situ diver collected biodiversity, illustrating the potential value of acoustic metrics for monitoring and assessing biodiversity of kelp habitats (Harris et al., 2016).

As powerful as passive acoustics can be in detecting, localizing, and providing information about local soundscape sources, passive acoustic technology fails when objects or phenomena are not producing sound. Active echosounder technology provides a time series of acoustic backscatter information that not only provides critical information on biology but also physical components of the water column (Lavery et al., 2009; BenoitBird and Lawson, 2016). The integration of multi-frequency echosounders in cabled and remotely deployed observation systems have contributed invaluable knowledge on marine life community structure, distribution and size of marine organisms, oceanic microstructure, and suspended sediments. By recording acoustic backscatter from at least two frequencies, the differences in backscatter between the two frequencies can be used to distinguish between different scatterers in the water column (Watkins and Brierley, 2002; Warren et al., 2003). Successful incorporation of upward looking, single beam echosounders in moorings at Ocean Station Papa (Trevorrow et al., 2005) and in the Bering Sea (Miksis-Olds et al., 2013; Miksis-Olds and Madden, 2014; Stauffer et al., 2015) demonstrate the maturity of this technology in providing time series of acoustic backscatter used to investigate the abundance and behavior of zooplankton and fish, predator-prey relationships, and community structure.

Successful use of multiple single-frequency echosounders to study ecosystem dynamics has led to the evolution of broadband systems (e.g., Lavery et al., 2010; Stanton et al., 2010; Benoit-Bird and Lawson, 2016), which are now being used in both cabled and moored configurations for inferring species composition. Broadband data have the advantage of improved spatial resolution, allowing better target isolation and noise suppression through the use of pulse compression techniques (Chu and Stanton, 1998; Ross et al., 2013). Broadband measurements and theoretical physics-based approaches for classifying zooplankton were successfully combined to classify biological scattering layers from the Victoria Experimental Network Under the Sea (VENUS) mooring in Saanich Inlet, British Columbia (Ross et al., 2013). Two years of broadband data $(85-155 \mathrm{kHz})$ were collected from the VENUS system. Data processing classified scattering layers based on their assemblages into four animal groups: (1) diel migrating euphausiids; (2) chaetognaths; (3) fish; and (4) a mix of pteropods and bottom-to-oxycline migrating amphipods. Data generated from active acoustic systems provide biological information on trophic levels containing fish and zooplankton. When combined with the information obtained from passive acoustic systems related to physical conditions (e.g., surface conditions, ice cover, etc.), upper trophic level dynamics of marine mammals and other top predators, and even human use factors, underwater acoustics becomes a valuable tool in monitoring ecosystems in terms of overall function, biodiversity, and health.

\section{Climate Variability and Change}

Nearly four decades ago Munk and Wunsch (1982) proposed the establishment of a system for "Observing the ocean in the 1990s." The hypothetical system that they described had two major observational components: ocean acoustic tomography and satellite measurements of sea surface topography (altimetry) and wind stress (scatterometry). (They also mentioned drifting floats but floats capable of profiling the upper $1000 \mathrm{~m}$ or so of the ocean did not exist at that time.) They expected that these complementary observations would "be assimilated into numerical modeling of the ocean circulation." They listed the

\footnotetext{
"advantages and disadvantages of tomography as a tool for providing large-scale (and hence climatological) data...
}

\begin{abstract}
Advantages
Large-scale spatial integration; information increases geometrically with the number of moorings; unattended recording over a year or more (1/3 year demonstrated so far); submerged instrumentation (of especial interest in regions of seasonal ice formation); remote sensing (of potential use in regions of strong currents); good vertical resolution.
\end{abstract}

\section{Disadvantages}

Sound speed is not a unique measure of temperature or density. However, in regions with stable temperature-salinity relations the separate temperature and salinity fields (and hence the density field) can be inferred to adequate precision..."

The notion of "unattended recording over a year or more" is now so routine that it seems quaint. Nonetheless, the basic advantages and disadvantages of the application of acoustic methods for studying climate variability are unchanged. The key advantage of measurements of climatological variability is that the spatial integration inherently provided by long-range acoustic transmissions suppresses the effects of mesoscale and smaller scale variability. The advantages of acoustic remote sensing for observing ocean climate variability were reiterated at the OceanObs'99 (Dushaw et al., 2001) and OceanObs'09 (Dushaw et al., 2010) conferences. These advantages still exist today. Obtaining the large-scale spatial averages needed for measuring climate change from profiles in which the majority of the variance is due to mesoscale and smaller scale variability is still very much a challenge. Satellite measurements of sea-surface properties, high-resolution vertical profiles of temperature and salinity (e.g., Argo), and large-scale average temperature and heat content from acoustic tomography are complementary for adequately sampling an ocean that varies on all time and space scales. As 
Munk and Wunsch (1982) noted, "A condition underlying any ocean observing system is that the ocean is transparent to sound, but opaque to electromagnetic radiation."

What makes the case for the application of acoustic methods for measuring ocean climate variability more compelling now than in past decades? Munk and Wunsch (1982) were clearly ahead of their time. The first three-dimensional test of ocean acoustic tomography, the 1981 Tomography Demonstration Experiment, which lasted only 4 months, had just been completed (Behringer et al., 1982). The numerical ocean models available at the time (and in the 1990s, for that matter) did not have the vertical resolution needed for acoustic calculations. The situation is now dramatically different.

\section{Long-Range Ocean Acoustic Thermometry}

Transmissions over ranges of $1000 \mathrm{~km}$ or more have been used to measure large-scale ocean temperature and heat content, beginning with recording of the transmissions during the 1981 Tomography Demonstration Experiment in the Northwest Atlantic Ocean on bottom-mounted receivers at ranges of 1000-2000 km (Spiesberger et al., 1983). The application of long-range transmissions to measure temperature is often referred to as "acoustic thermometry," usually in the context of transmissions for which there are few or no crossing acoustic paths. (Thermometry is a subset of acoustic tomography). Longrange measurements have also been made in the North Pacific Ocean, Mediterranean Sea, and Arctic Ocean. Transmissions over global scales were made during the 1991 Heard Island Feasibility Test (HIFT), but these very long ranges are not optimal from the perspective of measuring climate variability because the transmissions average across distinct climatic provinces (Munk et al., 1994).

Acoustic transmissions from sources located near the soundchannel axis to vertical receiving arrays over gyre scales $(1000 \mathrm{~km})$ (Worcester et al., 1994) and basin scales $(3250 \mathrm{~km})$ (Worcester et al., 1999) established conclusively that acoustic methods can measure range- and depth-averaged temperatures at gyre- and basin-scale ranges with a precision of a few millidegrees C (Figure 10). The early portions of the acoustic arrival patterns at long ranges consist of ray-like wave fronts that are resolvable, identifiable, and stable. The later parts of the arrival patterns (finale) do not contain identifiable ray-like arrivals due to scattering from internal-wave-induced sound-speed fluctuations. Nonetheless, the time at which the near-axial acoustic reception ends can be used as a surrogate for the group delay of the lowest acoustic normal mode, providing information on near-axial temperatures. These experiments were unique in combining long vertical receiving arrays, extensive concurrent environmental measurements, and broadband signals designed to measure acoustic travel times with millisecond precision.

Although these results were favorable, at the time it was not understood how the early time fronts could be stable in the presence of internal-wave-induced scattering of the acoustic signals. In the (non-linear) geometric optics approximation, rays were expected to become chaotic at long ranges. Subsequent analyses showed that scattering tends to occur along wave fronts, rather than across them, giving resolvable, stable wave fronts (Godin, 2007). Further, wave-theoretic modeling, using normal mode and parabolic equation methods, has been applied to obtain travel-time sensitivity kernels (TSKs) without making the geometric optics approximation describing how travel times are affected by localized sound-speed perturbations anywhere in the medium (Skarsoulis and Cornuelle, 2004). Investigations of the structure and stability of the TSK in the presence of small-scale oceanographic variability that scatters acoustic signals show that ray-based travel-time inversions are valid even in this case (Dzieciuch et al., 2013). There is now a firm theoretical basis for the application of ray inversion methods.

\section{Long-Range Transmissions and Ocean Models}

Early attempts to use gyre- and basin-scale travel times to constrain ocean models were made by Menemenlis et al. (1997) and the Acoustic Thermometry of Ocean Climate (ATOC) Consortium (1998). At the time, primitive equation ocean general circulation models (OGCMs) did not have the vertical resolution needed to characterize ocean acoustic propagation and permit the accurate calculation of travel times. Statistical inverse methods were therefore used to convert the travel times to range-averaged ocean temperatures, which were then used as data to constrain primitive equation models.

The ATOC project continued over the decade 1996-2006, using sources installed off central California (1996-1999) and north of Kauai (1997-1999, 2002-2006) that transmitted to bottom-mounted and moored receivers in the North Pacific. These measurements were subsequently used to test more modern OGCMs (Dushaw et al., 2009, 2013). When attempting to compare the travel-time variability observed at bottommounted receivers for the duration of the ATOC project with model estimates, Dushaw et al. (2009) found that calculations based on the climatology in the World Ocean Atlas 2005 (WOA05) were able to reproduce the observed acoustic arrival patterns. The OGCM estimates available at the time proved incapable of doing so, however. The critical parameter for acoustic propagation calculations is the vertical sound-speed gradient, which was sufficiently unrealistic in the OGCM estimates to make the acoustic calculations fail. In order to proceed, the time means of the model temperature and salinity fields were removed and replaced with the annual mean fields from WOA05, making the assumption that the variability in the model estimates was realistic even though the mean fields were not. The differences between the observed travel time variability and that calculated from the models were sometimes substantial. Dushaw et al. (2013) subsequently used receptions on three vertical hydrophone arrays that were installed for about a year each in 1996 and 1998 to test the time-mean properties of the OGCM estimates. The observed acoustic arrival patterns were found to be in relatively good agreement with those computed for state estimates made by the Estimating the Circulation and Climate of the Ocean, Phase II (ECCO2) project, indicating that the numerical ocean models had reached a level of maturity by the time of Dushaw et al. (2013) such that the acoustic data could 

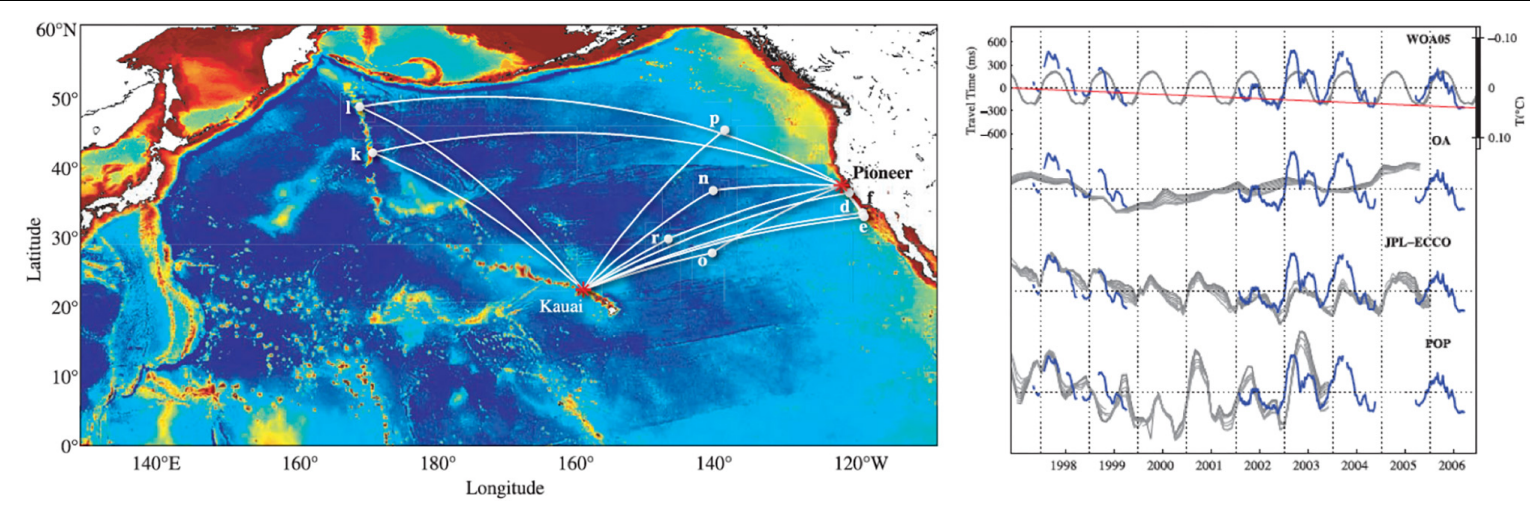

FIGURE 10 | (Left) Acoustic propagation paths from the Kauai and Pioneer Seamount sources to receivers in the Pacific for the Acoustic Thermometry of Ocean Climate (ATOC) program. (Right) Comparison of travel times (blue) for acoustic paths with ocean climate models (gray). Jet Propulsion Laboratory "Estimating the Circulation and Climate of the Ocean" (JPL-ECCO) = ocean circulation model + data assimilation; OA, objective analysis; POP, Parallel Ocean Program model; WOA05, World Ocean Atlas 2005 (Dushaw et al., 2009, used with permission).

be used to provide useful additional constraints for ocean state estimation.

\section{Ice-Covered Seas}

The case for using acoustic methods to study climate variability is especially compelling in ice-covered regions, where long-term, large-scale, continuous observations of the ocean interior are difficult to obtain using other approaches. Most attention to date has focused on the rapidly changing Arctic (e.g., Jeffries et al., 2013), but acoustic methods are equally applicable in the seasonally ice-covered Southern Ocean around Antarctica.

Satellite images show a large reduction of sea ice in the Arctic (e.g., Meier et al., 2014). The satellites cannot observe the interior of the ocean underneath the sea ice, and less is therefore known about what is occurring in the ocean under the ice. Just below the sea ice there is a cold, fresh water layer that protects the ice from the warmer, saltier waters deeper in the ocean. Point measurements in some areas of the Arctic indicate that this layer is disappearing (Lique, 2015; Polyakov et al., 2017). This change can accelerate the melting of the sea ice. Additionally, it is not known how much heat is stored in the water masses under the protective water layer.

Previous basin-scale acoustic measurements made in the 1990s showed that the Atlantic Intermediate Water (Atlantic Layer) was warming. During the 1994 Transarctic Acoustic Propagation (TAP) experiment, ultralow-frequency $(19.6 \mathrm{~Hz})$ acoustic transmissions propagated across the entire Arctic basin from a source located north of Svalbard to a receiving array located in the Beaufort Sea at a range of about $2630 \mathrm{~km}$ (Mikhalevsky and Gavrilov, 2001). Modal travel-time measurements yielded the surprising result that the Atlantic Layer was about $0.4^{\circ} \mathrm{C}$ warmer than expected from historical data. Acoustic data collected on a similar path during April 1999 as part of the Acoustic Climate Observations Using Underwater Sound (ACOUS) project indicated further warming of about $0.5^{\circ} \mathrm{C}$. These results were subsequently confirmed by direct measurements made from icebreakers and submarines (Mikhalevsky and Gavrilov, 2001).
Mikhalevsky et al. (2015) advocated the application of multipurpose acoustic networks in an integrated Arctic observing system (Figure 11). Several year-long experiments in Fram Strait (Sagen et al., 2016, 2017) and the Canada Basin have demonstrated the technology at a regional scale. It is now 20 years since the TAP and ACOUS measurements were made. The Coordinated Arctic Acoustic Thermometry Experiment (CAATEX), jointly funded by the United States, Norway, and Canada, will repeat these basin-scale measurements during 2019-2020. In addition to acoustic remote sensing (tomography), an integrated acoustic system would provide passive monitoring of ambient sound (ice, seismic, biologic, and anthropogenic) and under-ice navigation for drifting floats, gliders, and AUVs. Given the rapid rate at which the Arctic is changing, implementation of a sustained integrated observing system is urgent.

\section{Straits and Climate Choke Points}

Geographically constrained regions of the ocean function as gateways for important exchanges of heat, salt, nutrients, and marine life itself, with implications for climate change and variability. For example, water mass exchanges between the Arctic Ocean and the Atlantic and Pacific Oceans occur through the Fram Strait, Bering Strait, Davis Strait, and the Canadian Archipelago. Long-term acoustic tomography measurements can help determine heat and salt fluxes through these straits, taking advantage of the integrating property of acoustic data.

For example, models show a series of complex interactions within Baffin Bay in which melting Greenland ice sheets lead to decreased southward transport of cold Arctic water via the Canadian Archipelago and increased northward transport of fresh Atlantic water via Davis Strait due to a strengthening of the gyre circulation in Baffin Bay. A positive feedback cycle develops as warmer water enters Greenland fjords, further enhancing the melting of marine-terminating glaciers (de la Guardia et al., 2015). Clearly, monitoring the long-term changes in heat content, salinity, and mean circulation are paramount to evaluating the importance of this feedback cycle. 


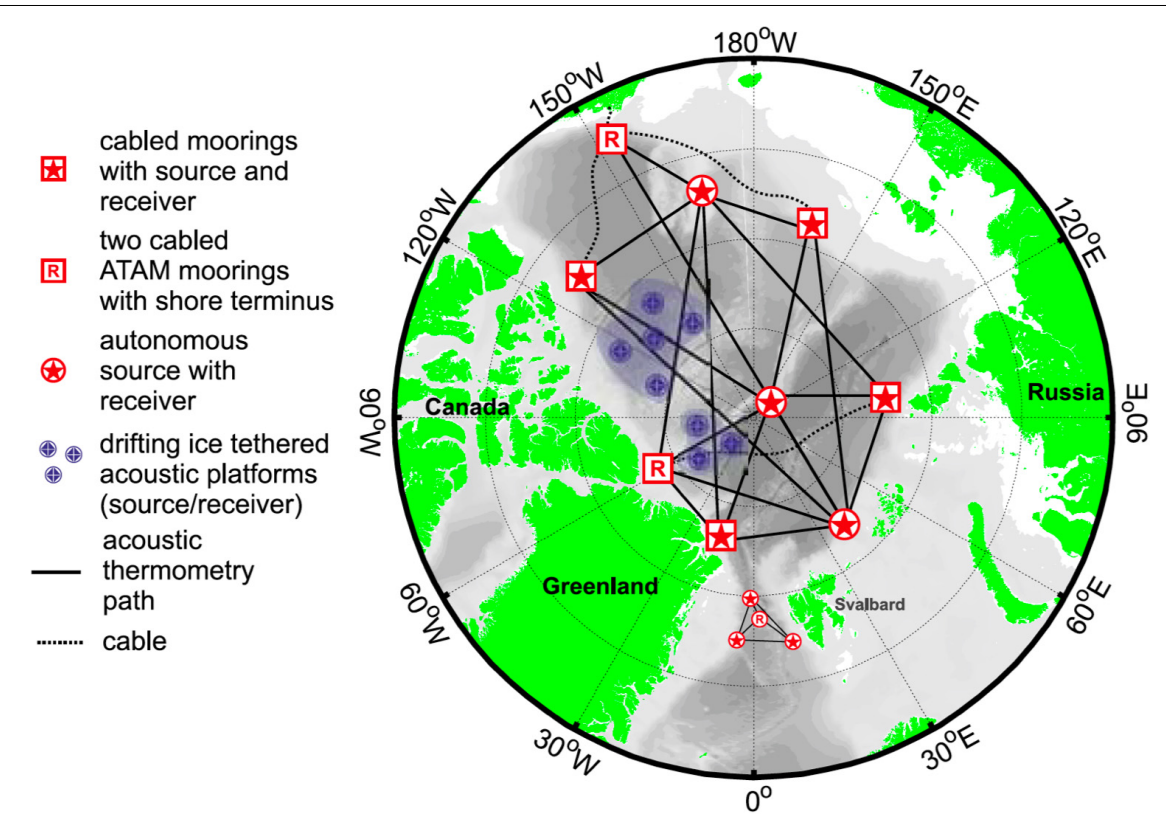

FIGURE 11 | The notional basin-wide Arctic mooring network for acoustic tomography, oceanography, and underwater "GPS" system for navigation of and low rate communications with floats, gliders, and UUVs. The Acoustic Thermometry and Multipurpose Mooring (ATAM) applies to all the moorings shown (Mikhalevsky et al., 2015, used with permission).

In another example, Pacific Waters (PW) represent one-third of all Arctic freshwater and supply and $10-20 \%$ of the oceanic heat to the Arctic Ocean. The large Arctic sea-ice retreat of 2007 was likely caused by extreme oceanic flux of PW. Remotely sensed sea surface temperature (SST) is insufficient for quantifying the variability in estimating the heat content in the Bering Strait region (Woodgate et al., 2012), which suggests an opportunity for temperature estimates from measurements of acoustic travel time to contribute to estimates of heat content in that region.

A study in Fram Strait showed that while point measurements from moorings and integrated measurements from acoustic tomography lead to similar uncertainties in sound speed (from which salt water properties are retrieved), objective estimates using combined mooring and tomographic measurement lead to a threefold reduction in uncertainties. Further, unlike a 2-D mooring array, adding tomographic measurements offers the opportunity to capture the 3-D variability necessary to fully understand transports through Fram Strait (Dushaw and Sagen, 2016).

Tomographic measurements have been made in other Straits as well, in locations of climatic importance. For 3 months in 1996, $2 \mathrm{kHz}$ transceivers were deployed in the Strait of Gibraltar. Reciprocal travel-time measurements diagonally across the Strait performed best for determining path-averaged velocity, while sum travel times provided good temperature measurements (Send et al., 2002). For the last decade, investigators led by A. Kaneko conducted many measurements in coastal seas. Most recently, the focus has been on measuring in the straits associated with the Indonesian Through Flow (ITF). Measurements in the Bali Strait resolved a five-layer vertical structure of flow as well as strong non-linear tides (Syamsudin et al., 2017). Future plans are to sustain these measurements and extend them to the Lombok and other straits. In this application, the remote sensing capability of the acoustics comes into play as nearshore (e.g., pier-mounted) equipment has a much higher probability of surviving than do instruments in the strait itself.

\section{Hazards and Maritime Safety}

The Comprehensive Nuclear-Test Ban Treaty Organization (CTBTO) is supporting the scientific use of International Monitoring System (IMS) data for disaster warning, marine hazard prevention and overall promotion of human welfare. The IMS functions as a Global Alarm System designed to detect not only nuclear explosions but also earthquakes able to produce tsunamis (CTBTO website: Disaster Warning and Science ${ }^{1}$ ).

The verification regime of the Comprehensive Nuclear-TestBan Treaty (CTBT) regime relies on the IMS, which consists of 337 facilities worldwide and provides global coverage for signs of nuclear explosions. Of the 337 facilities, 11 are hydroacoustic stations responsible for covering the oceans. The hydroacoustic stations include five T-stations, which use on-shore seismometers to detect waterborne signals coupled into the Earth's crust, and six cabled hydrophone stations with two triplets of moored hydrophones in a horizontal triangular configuration with a separation of $2 \mathrm{~km}$ (with the exception of one station in Australia which has a single triplet). At present, 100 IMS stations, both hydroacoustic and seismic, provide near real-time data to tsunami warning centers in 14 countries to enhance their capability to issue timely and precise warnings (Figure 12).

\footnotetext{
${ }^{1}$ https://www.ctbto.org/verification-regime/spin-offs-for-disaster-warning-and-
} science/ 


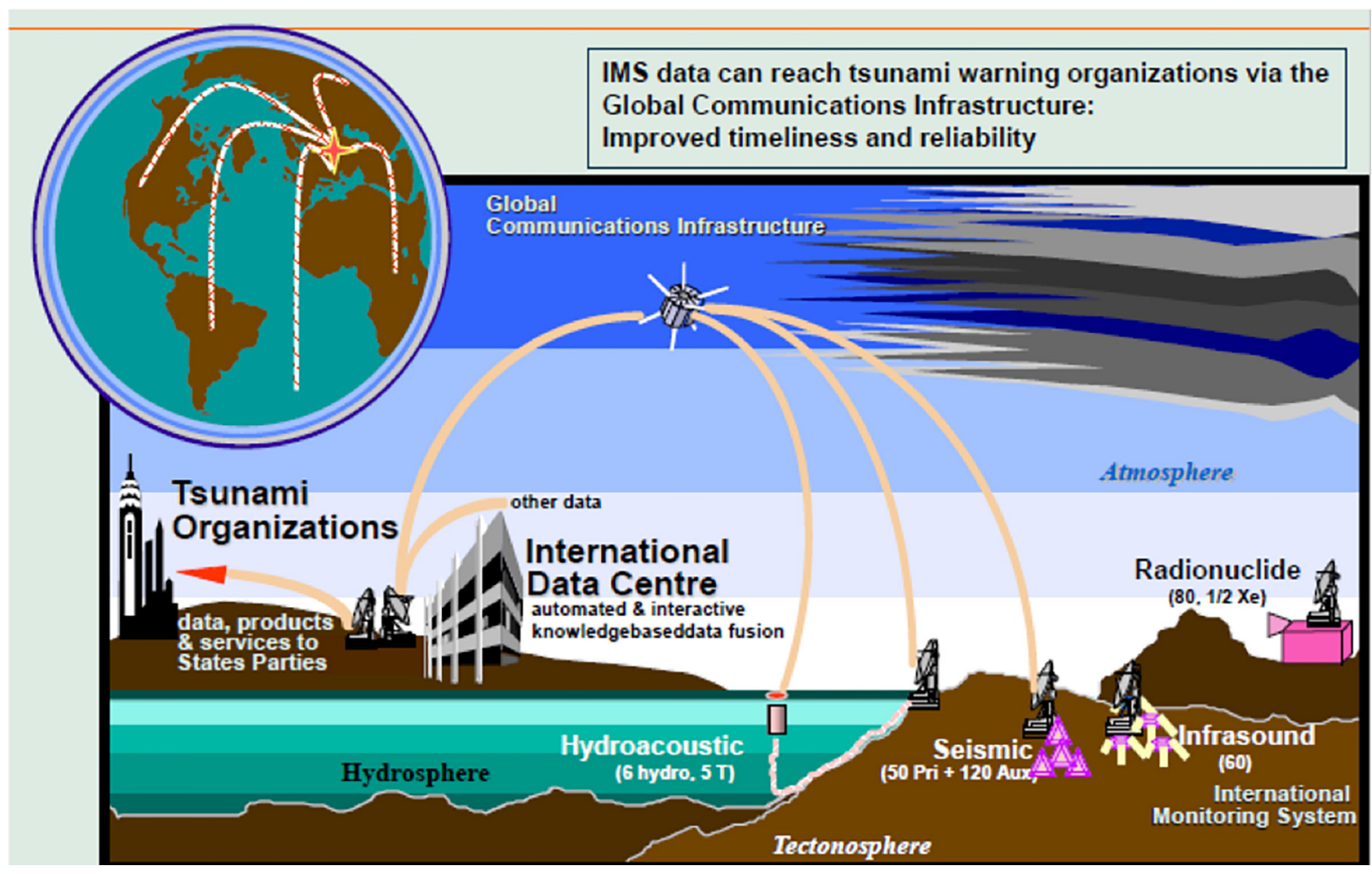

FIGURE 12 | Data flow within the CTBTO IMS, with appropriate data and products forwarding to tsunami warning centers in real time (Figure provided by G. Haralabus, used with permission).
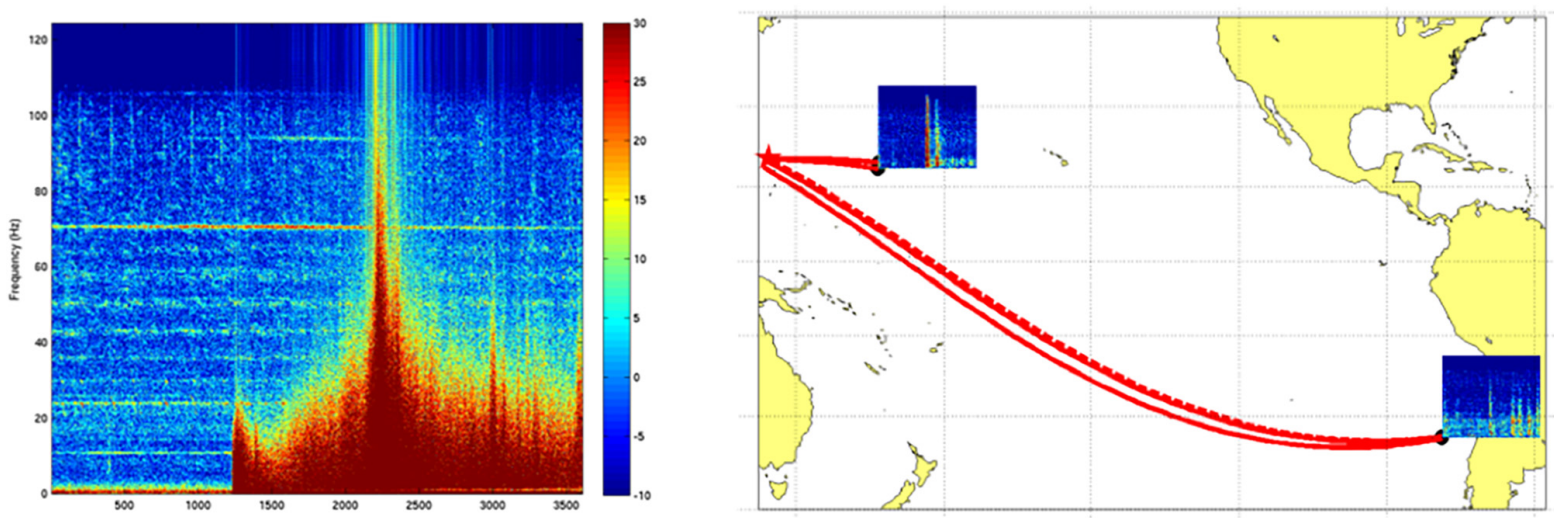

FIGURE 13 | (Left) Frequency content of the 1 April 2014 northern Chile earthquake signal on an HA03 hydrophone (Juan Fernandez, Chile) versus time (in seconds) on the horizontal axis. The color scale is in decibels (dB), with red denoting higher energy content. Early arrivals are attributed to seismic waves traveling through the ocean crust and leaking acoustic energy into the water, however, most of the acoustic energy arrived later in the form of T-phase propagation in the SOFAR. (Right) Hydrophone recordings at HA11 (Wake Island, United States) and HA03 (15,000 km away) pertaining to bursting underwater gas bubbles emitted by an undersea volcano near the Mariana Islands (Figure provided by G. Haralabus, used with permission).

Hydroacoustic station warnings of underwater volcanic eruptions or undersea earthquakes could bring significant benefits to maritime traffic. For example, during the Tohoku earthquake and the subsequently induced tsunami that struck Japan on 11 March 2011, the HA11 Wake Island (United States) hydrophone station helped track the wave as it propagated across the Pacific Ocean. Another example of the multitude of natural signals recorded at the IMS hydroacoustic stations is the magnitude 8.2 earthquake that occurred on 1 April 2014 in Northern Chile and was recorded at the HA03 Juan Fernandez (Chile) hydrophone station (Figure 13).

One of the greatest advantages offered by hydroacoustic stations is that they cover exceptionally large areas, compared to 
other technologies, due to the efficient way sound propagates in the water (Figure 3). In April 2014, underwater explosion-like signals emitted by an undersea volcano near the Mariana Islands in the North Pacific Ocean were received at HA03, more than 15,000 km away (Figure 13).

Hydroacoustic data from the IMS network provided assistance in the search for the missing Argentine submarine ARA San Juan (S-42). On 15 November 2017, two CTBTO hydroacoustic stations, namely HA10 (Ascension Island) and HA04 (Crozet), detected an unusual signal in the vicinity of the last known position of the submarine. The signal had the characteristics of an underwater impulsive event and occurred at 13:51 GMT on 15 November. Details and data were made available to the Argentinian authorities to support the ongoing search operations. The mutually beneficial collaboration between Argentinian researchers and hydroacoustic experts at CTBTO continues with further analysis of this unusual acoustic event (СТВTO, 2017a,b).

Passive acoustic monitoring is also currently being applied at a local and regional level to alert the shipping industry to the presence of hazards in the form of large whales. Off the east coast of the US, the endangered North Atlantic right whale migrates up and down the coast annually. Their surface behavior related to foraging puts them at high risk for ship collisions (Parks et al., 2014), which is costly to both the animal and the ship. The Right Whale Listening Network ${ }^{2}$ implements a smart acoustic buoy system to continually listen for whale calls. If a right whale call is detected any time in the last $24 \mathrm{~h}$, it is reflected on the website, and this information is also broadcast to mariners to reduce vessel speeds below 10 knots in an effort to reduce ship strikes. This technology has expanded in the form of a Whale Alert app $p^{3}$, which aims to reduce lethal whale ship strikes worldwide and across all large whale species.

\section{DISCUSSION}

The implementation of a global multipurpose acoustic ocean observing system is beginning. The establishment of ocean sound as an Essential Ocean Variable is an important milestone in this process. Ocean sound is uniquely suited to observing ocean ecology and biology, both in passive and active forms. The infrastructure for positioning, navigation, timing (PNT) and communication, which is so critical to effective operations of all observing platforms in the ocean, directly leads to tomographic observations. With these active and passive components, the multipurpose system can be realized.

The elements to accomplish this have sufficient technical readiness and feasibility to implement the system. Passive receivers, often with arrays, are now commonplace; accurate timing and geo-referencing are required for them to be coherent elements of the larger system. Active sources suited to long-range acoustic propagation necessary for PNT and tomography are now off-the-shelf. Similarly, autonomous echosounder systems

${ }^{2} \mathrm{http}: / /$ www.listenforwhales.org

${ }^{3}$ www.whalealert.org for short-range monitoring of water column biology are now commercially available. Acoustic signal processing is up-tothe-task, though the "big data" aspects are already somewhat daunting. The assimilation of tomographic travel time data into ocean models is understood but needs to be made routine with sustained data streams from operational systems. The same applies to acoustically determined high-resolution float and vehicle tracks; this needs to be done as a joint estimation problem.

A large proportion of PAM research programs and applications focus on questions pertaining to coastal waters due to the relatively easy access compared to outer continental shelves, concentration of human activities along the coasts, and regulatory/management concerns under national, regional, and local jurisdictions. PAM networks, like the CTBTO, demonstrate the value of global coverage, but have historically been limited to low frequency recordings due to constraints in power, sensor data storage capacity, required post-processing resources, and data management services. As discussed in this work, many of these obstacles have been overcome or are presently being addressed through advances in technology or repurposing methodologies from other fields. Consequently, PAM is a mature tool for ocean monitoring that is expanding in bandwidth, geographical scope, and application.

Future innovation related to PAM technology and applications is likely to come in the form of combining PAM with other nonacoustic sensing methods. For example, over the past 2-3 years, environmental DNA (eDNA) techniques, which have been used more in freshwater ecosystems, are now being explored in marine environments (Andruszkiewicz et al., 2017; Bakker et al., 2017; Gargan et al., 2017). The eDNA from targeted marine mammal species is detectable and comparable to both visual and acoustic surveys $2 \mathrm{~h}$ after an animal has swum through a volume of water (Baker et al., 2018). As eDNA technology and methodologies continue to develop in association with marine ecosystems, it is possible to envision an ocean observation system systematically addressing questions of ecosystem connectivity from estuarine habitats to deep ocean environments far offshore (Levin et al., 2019). Combining PAM with eDNA or genomic sensing is only one of the non-acoustic techniques that hold future promise in enhancing our ability to autonomously observe the ocean, and the likelihood of future generations developing completely new methods that are presently inconceivable is high.

To be used effectively, multipurpose acoustic observing systems should be deployed where they can uniquely make the largest contributions, filling gaps in the present observing system. Polar regions, near and under the ice in the Arctic and Antarctic are in desperate need of observations and sustained monitoring that only acoustics can provide. Such systems would simultaneously observe the changing heat content and effects on melting ice using tomography while at the same time providing positioning and navigation capability for under-ice floats and vehicles. It is especially important that broadband acoustic sources be used in conjunction with accurate clocks.

At the same time, the multitude of widely distributed PAM receivers in non-polar regions (e.g., CTBTO) can motivate multipurpose systems on global scale, perhaps with a longer time constant to implement. Judiciously 
locating cabled sources and using sources deployed from GO-SHIP vessels (providing sustained repeat hydrographic sections on decadal repeat timescales) can quickly provide wide-scale coherent tomographic coverage and derived heat content estimates, supplementing and complementing other, often incoherently sampled, observing elements. SMART telecommunication cables with integrated sensors will play a role as well (Howe et al., 2019).

Observing the ocean acoustically creates strong synergies with other observing programs, as reflected in the white papers in this Special Issue for OceanObs'19. Closing the sealevel budget (Leuliette, 2015; WCRP Global Sea Level Budget Group, 2018) requires accurate knowledge of ocean bathymetry (Woelfl et al., 2019), ocean sea level (Ponte et al., 2019), barystatic changes (mass of freshwater added or removed; Palmer et al., 2019), and thermostatic changes (e.g., changes in heat content; Meyssignac et al., 2019). Multipurpose acoustic observing systems will contribute to improved monitoring of volume, freshwater, and long-term heat fluxes, as well as monitoring long-term heat content changes at basin-scale, as well as in specific regions of global importance (e.g., Indonesian throughflow; Ravichandran, 2011; Sprintall et al., 2019). Multipurpose acoustic systems can provide ground-truth for ecosystem modeling and forecasting. Acoustic in situ rainfall and wind speed estimates can improve our observations of air-sea fluxes (Centurioni et al., 2019), particularly in sparely sampled regions such as the Southern Ocean (Swart et al., 2019) and polar regions (Smith et al., 2019) as well as improve our ability to monitor wind/current/wave interactions (Villas Bôas et al., 2019). The direct estimates of ocean state variables from acoustic tomography and acoustically located and navigated autonomous platforms operating in undersampled high-latitude regions will improve ocean state estimation (Heimbach et al., 2019). The rich applications of hydroacoustic monitoring from the CTBTO IMS demonstrate that additional observations from fixed acoustic transceiver nodes, coupled with soundscape maps provided by PAM, are important components of Eulerian observational systems, including tsunami warning (Angove et al., 2019).

\section{SUMMARY AND RECOMMENDATIONS}

We have outlined the current status of multi-purpose acoustic systems, noted the contributions they are making now and can make in the future, and discussed what gaps they can fill in the current ocean observing system. Based on this, we suggest that the following recommendations be included in the outcomes from the OceanObs19 conference.

Recommendation 1: OceanObs'19 recognizes the utility, both in terms of observing system capability as well as reduced effort and cost, of multipurpose acoustic observing systems, and recommends implementation in GOOS.

Recommendation 2: GOOS must develop a community capability to evaluate the present observing system and proposed additions thereto and apply the same to evaluating the benefits of precision acoustic navigation (of mobile platforms) and tomography. This could be accomplished by transitioning GODAE Ocean View to operational status as part of GOOS.

Recommendation 3: OceanObs'19 encourages implementation steps related to the ocean sound EOV, e.g., the incorporation of suitable acoustic receivers in general purpose instrument packages with necessary timing and geo-positioning capability and improved data access.

Recommendation 4: OceanObs'19 recognizes the extreme urgency of observing and monitoring the polar oceans, made difficult by ice cover, and recommends that multipurpose acoustic observing systems, along with other complementary systems including cabled ones, should be brought to operational status.

\section{DEDICATION}

This article is dedicated to Walter Munk, Oceanographer and Geophysicist, 1917-2019.

\section{AUTHOR CONTRIBUTIONS}

$\mathrm{BH}$ led the effort and wrote the Introduction, Discovery, Discussion, and Summary. BH and ER wrote the Ocean Technology section. JM-O wrote the Ecosystem section. PW wrote the Climate Variability section. GH and JM-O wrote the Hazards and Maritime Safety section. All authors contributed to overall editing.

\section{FUNDING}

The authors were supported by their home institutions.

\section{ACKNOWLEDGMENTS}

As part of the OceanObs'19 Conference white paper process, the Program Committee selected four related abstracts to combine into one for this Special Issue article:

Global Passive Monitoring of Sound to Understand Trends in Ocean Sound and Marine Life

Multipurpose Acoustic Networks in Ocean Observing Systems in Ice-Covered Regions

The Roles of Underwater Acoustics in Observing the Global Ocean

Baffin Bay Acoustic Navigation and Communication System: A Feasibility Study

This manuscript is the result. We gratefully acknowledge the contributions of the co-authors of these four abstracts, as well as others:

K. Atakan, W. Au, J. Ausubel, M. Babin, A. BeszczynskaMöller, O. Boebel, R. Brainard, S. Carr, B. Chakraborty, C. Clark, B. Cornuelle, C. de Jong, L. di Iorio, T. Duda, B. Dushaw, 
M. Dzieciuch, D. Fissel, L. Freitag, A. Gavrilov, P. Gorringe, C. Gourcuff, T. Hamre, K. Heaney, G. Heard, S. Jayne, B. Klein, M. Lammers, K. Latarius, D. Lecchini, C. Lee, H. Lin, R. McCauley, D. Menemenlis, P. Mikhalevsky, J. Miller, A. Mooney, S. Moore, A. Morozov, W. Munk, S. Nedelec, J. Orcutt, M. Parsons, C. Radford, S. Rintoul, S. Riser, M. Rixen, S. Sandven, S. Seeyave, S. Simpson,

\section{REFERENCES}

ANCHOR Working Group (2008). "Acoustic navigation and communications for high-latitude ocean research," in Proceedings of the ANCHOR Workshop 2006 (Seattle, WA: University of Washington Applied Physics Laboratory).

Andruszkiewicz, E. A., Starks, H. A., Chavez, F. P., Sassoubre, L. M., Block, B. A., and Boehm, A. B. (2017). Biomonitoring of marine vertebrates in monterey bay using eDNA metabarcoding. PLoS One 12:e0176343. doi: 10.1371/journal.pone. 0176343

Angove, M., Arcas, D., Bailey, R., Carrasco P., Coetzee, D., Fry, B., et al. (2019). Ocean observations required to minimize uncertainty in global tsunami forecasts, warnings, and emergency response. Front. Mar. Sci. 6:350. doi: 10. 3389/fmars.2019.00350

Arrigo, K. R., Perovich, D. K., Pickart, R. S., Brown, Z. W., Van Dijken, G. L., Lowry, K. E., et al. (2012). Massive phytoplankton blooms under arctic sea ice. Science 336:1408. doi: 10.1126/science. 1215065

Baker, C. S., Steel, D., Nieukirk, S., and Klinck, H. (2018). Environmental DNA (eDNA) from the wake of the whales: droplet digital PCR for detection and species identification. Fron. Mar. Sci. 5:133. doi: 10.3389/fmars.2018.00133

Bakker, J., Wangensteen, O. S., Chapman, D. D., Boussarie, G., Buddo, D., Guttridge, T. L., et al. (2017). environmental DNA reveals tropical shark diversity in contrasting levels of anthropogenic impact. Sci. Rep. 7:16886. doi: 10.1038/s41598-017-17150-2

Behringer, D., Birdsall, T., Brown, M., Cornuelle, B., Heinmiller, R., Knox, R., et al. (1982). A demonstration of ocean acoustic tomography. Nature 299, 121-125. doi: 10.1038/299121a0

Benoit-Bird, K. J., and Lawson, G. L. (2016). Ecological insights from pelagic habitats acquired using active acoustic techniques. Ann. Rev. Mar. Sci. 8, 463-490. doi: 10.1146/annurev-marine-122414-034001

Centurioni, L. R., Turton, J. D., Lumpkin, R., Braasch, L., Brassington, G., Chao, Y., et al. (2019). Global in-situ observations of essential climate and ocean variables at the air-sea interface. Front. Mar. Sci. 6:419. doi: 10.3389/fmars.2019.00419

Chu, D., and Stanton, T. K. (1998). Application of pulse compression techniques to broadband acoustic scattering by live individual zooplankton. J. Acoust. Soc. Am. 104, 39-55. doi: 10.1121/1.424056

Cornuelle, B. D., and Worcester, P. F. (1996). "Ocean acoustic tomography: integral data and ocean models," in Modern Approaches to Data Assimilation in Ocean Modeling, ed. P. Malanotte-Rizzoli (Amsterdam: Elsevier Science BV), 97-115.

Crease, J. (1962). Velocity measurements in the deep water of the western North Atlantic: summary. J. Geophys. Res. 67, 3173-3176. doi: 10.1029/ JZ067i008p03173

CTBTO (2017a). Available at: https://www.ctbto.org/press-centre/mediaadvisories/2017/media-advisory-ctbto-hydroacoustic-data-to-aid-in-searchfor-missing-sub-san-juan/ (accessed November 23, 2017).

CTBTO (2017b). Available at: https://www.ctbto.org/press-centre/highlights/ 2017/ctbto-hydroacoustic-data-used-to-aid-search-for-missing-submarineara-san-juan/ (accessed November 24, 2017).

Curtis, K. R., Howe, B. M., and Mercer, J. A. (1999). Low frequency ambient sound in the north pacific: long time series observations. J. Acoust. Soc. Am. 106, 3189-3200. doi: $10.1121 / 1.428173$

Davis, R., Baumgartner, M., Comeau, A., Cunningham, D., Davies, K., Furlong, A., et al. (2016), "Tracking whales on the Scotian Shelf using passive acoustic monitoring on ocean gliders," in Proceedings of the OCEANS 2016 MTS/IEEE (Monterey: IEEE), 1-4.

de la Guardia, L. C., Hu, X., and Myers, P. G. (2015). Potential positive feedback between greenland ice sheet melt and Baffin Bay heat content on the west greenland shelf. Geophys. Res. Lett. 42, 4922-4930. doi: 10.1002/2015GL064626

Denes, S. L., Miksis-Olds, J. L., Mellinger, D. K., and Nystuen, J. A. (2014). Assessing the cross-platform performance of marine mammal indicators
E. Skarsoulis, H. Søiland, M. Sørensen, B. Southall, K. Stafford, J. Stanley, R. Stephen, D. Tollefsen, J. Tougaard, P. Tyack, E. Urban, L. Van Uffelen, A. Vedenev, K. Vigness-Raposa, and C. Wunsch. We would like to thank CTBTO for its contribution and for promoting civil and scientific uses of data acquired by its international monitoring system. SOEST Contribution 10746.

between two collocated acoustic recorders. Ecol. Inform. 21, 74-80. doi: 10. 1016/j.ecoinf.2013.10.005

Dol, H. S., Casari, P., Van Der Zwan, T., and Otnes, R. (2017). Software-defined underwater acoustic modems: historical review and the NILUS approach. IEEE J. Oceanic Eng. 42, 722-737. doi: 10.1109/joe.2016.2598412

Deep Ocean Observing Strategy [DOOS] (2016). DOOS 2016 Workshop Proceedings, Available at: http://www.deepoceanobserving.org/wpcontent/uploads/2018/07/DOOS-2016-Workshop-Report-2017-04-13-V2.pdf (accessed July 11, 2019).

Deep Ocean Observing Strategy [DOOS] (2018). Science and Implementation Guide, Available at: http://www.deepoceanobserving.org/wp-content/uploads/ 2018/07/DOOS-SIG_CF_July2018_V2.pdf (accessed July 11, 2019).

Duda, T., Morrozov, A. K., Howe, B. M., Brown, M. G., et al. (2006). "Evaluation of a long-range joint acoustic navigation / thermometry system", in Proceedings of the Oceans 2006 Conference (Boston, MA: IEEE).

Dushaw, B. D., and Sagen, H. (2016). A comparative study of moored/point and acoustic tomography/integral observations of sound speed in fram strait using objective mapping techniques. J. Atmos. Ocean. Technol. 33, 2079-2093. doi: 10.1175/jtech-d-15-0251.1

Dushaw, B. D., Au, W. W. L., Beszczynska-Möller, A., Brainard, R. E., Cornuelle, B. D., Duda, T. F., et al. (2010). "A global ocean acoustic observing network," in Proceedings of OceanObs'09: Sustained Ocean Observations and Information for Society, eds J. Hall, D. E. Harrison and D. Stammer, Vol. 2 (Venice: ESA Publication WPP-306). doi: 10.5270/OceanObs09.cwp.25

Dushaw, B. D., Bold, G., Chiu, C.-S., Colosi, J. A., Cornuelle, B. D., Desaubies, Y., et al. (2001). "Observing the ocean in the 2000's: a strategy for the role of acoustic tomography in ocean climate observation," in Observing the Oceans in the 21st Century eds C. J. Koblinsky and N. R. Smith (Melbourne, VIC: Bureau of Meteorology), 391-418.

Dushaw, B. D., Worcester, P. F., Dzieciuch, M. A., and Menemenlis, D. (2013). On the time-mean state of ocean models and the properties of long-range acoustic propagation, J. Geophys. Res. Oceans 118, 4346-4362. doi: 10.1002/jgrc.20325

Dushaw, B. D., Worcester, P. F., Munk, W. H., Spindel, R. C., Mercer, J. A., Howe, B. M., et al. (2009), A decade of acoustic thermometry in the North Pacific Ocean. J. Geophys. Res. Oceans 114, 1-24. doi: 10.1029/2008JC005124

Dzieciuch, M. A. (2014). Signal processing and tracking of arrivals in ocean acoustic tomography. J. Acoust. Soc. Am. 136, 2512-2522. doi: 10.1121/1. 4897404

Dzieciuch, M. A., Cornuelle, B. D., and Skarsoulis, E. K. (2013). Structure and stability of wave-theoretic kernels in the ocean. J. Acoust. Soc. Am. 134, 3318-3331. doi: 10.1121/1.4818846

Fenty, I., and Heimbach, P. (2013). Coupled sea ice-ocean-state estimation in the labrador sea and baffin bay. J. Phys. Oceanogr. 43, 884-904. doi: 10.1175/JPOD- $12-065.1$

Freitag, L., Ball, K., Partan, J., Koski, P., and Singh, S. (2015). "Long range acoustic communications and navigation in the Arctic," in Proceeding of the OCEANS 2015 - MTS/IEEE Washington (Washington, DC: IEEE).

Gallimore, E., Partan, J., Vaughn, I., Singh, S., Shusta, J., and Freitag, L. (2010). “The WHOI micromodem-2: a scalable system for acoustic communications and networking," in Proceeding of the Oceans 2010 MTS/IEE (Seattle, WA: IEEE).

Gargan, L. M., Morato, T., Pham, C. K., Finarelli, J. A., Carlsson, J. E. L., and Carlsson, J. (2017). Development of a sensitive detection method to survey pelagic biodiversity using eDNA and quantitative PCR: a case study of devil ray at seamounts. Mar. Biol. 164:112. doi: 10.1007/s00227-017-3141-x

Godin, O. A. (2007). Restless rays, steady wave fronts. J. Acoust. Soc. Am. 122, 3353-3363. doi: 10.1121/1.2799479

Gopalakrishnan, G., Hoteit, I., Cornuelle, B. D., and Rudnick, D. L. (2019). Comparison of 4DVAR and EnKF state estimates and forecasts in the 
Gulf of Mexico, Q. J. R. Meteorol. Soc.145, 1354-1376. doi: 10.1002/qj. 3493

Haralabus, G., Zampolli, M., Grenard, P., Prior, M., and Pautet, L. (2017). "Underwater acoustics in nuclear-test-ban treaty monitoring", in Applied Underwater Acoustics: Leif Bjørnø, eds T. Neighbors and D. Bradley (Chicago: Elsevier Science).

Harris, S. A., Shears, N. T., and Radford, C. A. (2016). Ecoacoustic indices as proxies for biodiversity on temperate reefs. Methods Ecol. Evol. 7, 713-724. doi: 10.1111/2041-210x.12527

Heaney, K. D. (2015). "Advanced computation of three-dimensional long-range acoustic propagation for improved localisation methods," in Proceedings of the International Hydroacoustics Workshop (Vienna: CTBTO).

Heimbach, P., Fukumori, I., Hill, C. N., Ponte, R. M., Stammer D., Wunsch, C., et al. (2019). Putting it all together: adding value to the global ocean and climate observing systems with complete self-consistent ocean state and parameter estimates. Front. Mar. Sci. 6:55. doi: 10.3389/fmars.2019.00055

Hidas, M., Proctor, R., Mancini, S., Blain, P., Gordon, L., Scheibner, A., et al. (2018). "Automated data ingestion for the australian ocean data network," in Proceedings of the 20th EGU General Assembly Conference Abstracts, Vienna.

Howe, B. M., Arbic, B. K., Aucan, J., Barnes, C., Bayliff, N., Becker, N., et al. (2019). SMART cables for observing the global ocean: science and implementation. Front. Mar. Sci. 6424. doi: 10.3389/fmars.2019.00424

Howe, B. M., and Miller, J. H. (2004). Acoustic sensing for ocean research. J. Mar. Tech. Soc. 38, 144-154. doi: 10.4031/002533204787522811

International Organization for Standardization [IOS] (2017). Underwater acoustics-Terminology. ISO/DIS 18405.2:2017. Available at: https: //www.iso.org/standard/62406.html (accessed July 11, 2019).

Jeffries, M. O., Overland, J. E., and Perovich, D. K. (2013), The Arctic shifts to a new normal. Phys. Today 66, 35-40. doi: 10.1063/PT.3.2147

Johnson, M., de Soto, N. A., and Madsen, P. T. (2009). Studying the behaviour and sensory ecology of marine mammals using acoustic recording tags: a review. Mar. Ecol. Prog. Ser. 395, 55-73. doi: 10.3354/meps08255

Klatt, O., Boebel, O., and Fahrbach, E. (2007). A profiling float's sense of ice. J. Atmos. Ocean. Technol. 24, 1301-1308. doi: 10.1175/JTECH2026.1

Kuperman, W. A. (2018). Using noise to image the ocean. Bridge 48, 44-53.

Lavery A. C., Chu, D. Z., and Moum J. N. (2009). Measurements of acoustic scattering from zooplankton and oceanic microstructure using a broadband echosounder. ICES J. Mar. Sci. 67, 379-394. doi: 10.1093/icesjms/fsp242

Lavery, A. C., Chu, D. Z., and Moum, J. N. (2010). Observations of broadband acoustic backscattering from nonlinear internal waves: assessing the contribution from microstructure. IEEE J. Ocean. Eng. 35, 695-709. doi: 10.1109/joe.2010.2047814

Lebedev, K. V., Yaremchuk, M., Mitsudera, H., Nakano, I., and Yuan, G. (2003). Monitoring the kuroshio extension with dynamically constrained synthesis of the acoustic tomography, satellite altimeter and in situ data. J. Oceangr. 59, 751-763. doi: 10.1023/B:JOCE.0000009568.06949.c5

Lee, C. M., Thomson, J, The Marginal Ice Zone, and Arctic Sea State Teams. (2017). An autonomous approach to observing the seasonal ice zone in the western Arctic. Oceanography 30, 56-68. doi: 10.5670/oceanog.2017.222

Leuliette, E. W. (2015). The balancing of the sea-level budget. Curr. Clim. Change Rep. 1, 185-191. doi: 10.1007/s40641-015-0012-8

Levin, L., Betts, B. J., Gates, A. R., Heimbach, P., Howe, B. M., Janssen, F., et al. (2019). Global observing needs in the deep ocean. Front. Mar. Sci. 6241. doi: 10.3389/fmars.2019.00241

Lindstrom, E., Gunn, J., Fischer, A., McCurdy, A., and Glover, L. K. et al. (2014). A framework for Ocean Observing. (Paris: UNESCO).

Lique, C. (2015). Arctic sea ice heated from below. Nat. Geosci. 8, 172-173. doi: $10.1038 /$ ngeo2357.

McWilliam, J. N., and Hawkins, A. D. (2013). A comparison of inshore marine soundscapes. J. Exper. Mar. Biol. Ecol. 446, 166-176. doi: 10.1016/j.jembe.2013. 05.012

Meier, W. N., Hovelsrud, G. K., van Oort, B. E. H., Key, J. R., and Kovacs, K. M., (2014), Arctic sea ice in transformation: a review of recent observed changes and impacts on biology and human activity. Rev. Geophys. 52, 185-217. doi: 10.1002/2013RG000431

Menemenlis, D., Webb, T., Wunsch, C., Send, U., and Hill, C. (1997). Basinscale ocean circulation from combined altimetric, tomographic and model data. Nature 385, 618-621. doi: 10.1038/385618a0

Meyssignac, B., Boyer, T., Zhao, Z., Hakuba, M. Z., Landerer, F. W., Stammer, D., et al. (2019). Measuring global ocean heat content to estimate the earth energy imbalance. Front. Mar. Sci. 6432. doi: 10.3389/fmars.2019. 00432

Mikhalevsky, P. N., and Gavrilov, A. N. (2001). Acoustic thermometry in the arctic ocean. Polar Res. 20, 185-192. doi: 10.1111/j.1751-8369.2001.tb00055.x

Mikhalevsky, P. N., Sagen, H., Worcester, P. F., Baggeroer, A. B., Orcutt, J., Moore, S. E., et al. (2015). Multipurpose acoustic networks in the integrated arctic ocean observing system. Arctic 68, 11-27. doi: 10.14430/arctic4449

Miksis-Olds, J. L., and Madden, L. E. (2014). Environmental predictors of ice seal presence in the Bering Sea. PLoS One 9:e106998. doi: 10.1371/journal.pone. 0106998

Miksis-Olds, J. L., Martin, B., and Tyack, P. L. (2018). Exploring the ocean through soundscapes. Acoust. Today 14, 26-34.

Miksis-Olds, J. L., Stabeno, P. J., Napp, J. M., Pinchuk, A. I., Nystuen, J. A., Warren, J. D., et al. (2013). Ecosystem response to a temporary sea ice retreat in the Bering Sea. Prog. Oceanogr. 111, 38-51. doi: 10.1016/j.pocean.2012.10.010

Morozov, A. K., Webb, D. C., Chiu, C. -S., Worcester, P. F., Dzieciuch, M. A., Sagen, H., et al. (2016). High-efficient tunable sound sources for ocean and bottom tomography, 15 years of operating history. Paper Presented at OCEANS 2016 MTS/IEEE, Monterey.

Munk, W. H., and Wunsch, C. (1982). Observing the ocean in the 1990s. Philos. Trans. R. Soc. Lond. A 307, 439-464. doi: 10.1098/rsta.1982.0120

Munk, W. H., Worcester, P. F., and Wunsch, C. (1995). Ocean Acoustic Tomography (Cambridge: Cambridge University Press).

Munk, W. H., Spindel, R. C., Baggeroer, A. B., and Birdsall, T. G. (1994). The heard island feasibility test. J. Acoust. Soc. Am. 96, 2330-2342. doi: 10.1121/1.410105

Nguyen, A. T., Ocaña, V., Garg, V., Heimbach, P., Toole, J. M., Krishfield, R. A., et al. (2017). On the benefit of current and future ALPS data for improving Arctic coupled ocean-sea ice state estimation. Oceanography 30, 69-73. doi: 10.5670/oceanog.2017.223

Nystuen, J. A. (2001). Listening to raindrops from underwater: an acoustic disdrometer. J. Atmos. Ocean. Technol. 18, 1640-1657. doi: 10.1175/152004262001018<1640:LTRFUA<2.0.CO

Ocean Sound EOV (2018). Available at: http://www.goosocean.org/index.php? option=com_oe\&task=viewDocumentRecord\&docID=22567 (accessed July 11, 2019).

Palmer, M., Durack, P., Chidichimo, M. P., Church, J., Cravatte, S. E., Hill, K. L., et al. (2019). Adequacy of the ocean observation system for quantifying regional heat and freshwater storage and change. Front. Mar. Sci. 6416. doi: 10.3389/ fmars.2019.00416

Parks, S. E., Miksis-Olds, J. L., and Denes, S. L. (2014). Assessing marine ecosystem acoustic diversity across ocean basins. Ecol. Inform. 21, 81-88. doi: 10.1016/j. ecoinf.2013.11.003

Pijanowski, B. C., Villanueva-Rivera, L. J., Dumyahn, S. L., Farina, A., Krause, B. L., Napoletano, B. M., et al. (2011). Soundscape ecology the science of sound in the landscape. BioScience 61, 203-216.

Polyakov, I. V., Pnyushkov, A. V., Alkire, M. B., Ashik, I. M., Baumann, T. M., Carmack, E. C., et al. (2017). Greater role for atlantic inflows on sea-ice loss in the eurasian basin of the arctic ocean, Science 356, 285-291. doi: 10.1126/ science.aai8204

Ponte, R. M., Carson, M., Cirano, M., Domingues, C., Jevrejeva, S., Marcos, M., et al. (2019). Towards comprehensive observing and modeling systems for monitoring and predicting regional to coastal sea level. Front. Mar. Sci. 6437. doi: 10.3389/fmars.2019.00437

Radford, C., Jeffs, A. G., Tindle, C. T., and Montgomery, J. C. (2008b). Temporal patterns in ambient noise of biological origin from a shallow water temperate reef. Oecologia 156, 921-929. doi: 10.1007/s00442-008-1041-y

Radford, C., Jeffs, A., Tindle, C., and Montgomery, J. C. (2008a). Resonating sea urchin skeletons create coastal choruses. Mar. Ecol. Prog. Ser. 362, 37-43. doi: 10.3354/meps07444

Radford, C., Stanley, J. A., Tindle, C. T., Montgomery, J. C., and Jeffs, A. G. (2010). Localised coastal habitats have distinct underwater sound signatures. Mar. Ecol. Prog. Ser. 401, 21-29. doi: 10.3354/meps08451

Ravichandran, M. (2011). "In-situ ocean observing system," in Operational Oceanography in the 21 st Century, eds A. Schiller and G. Brassington (Springer: Dordrecht), 55-90.

Ross, T., Keister, J. E., and Lara-Lopez, A. (2013). On the use of highfrequency broadband sonar to classify biological scattering layers from a cabled observatory in saanich inlet, british columbia. Methods Oceanogr. 5, 19-38. doi: 10.1016/j.mio.2013.05.001 
Sagen, H., Dushaw, B. D., Skarsoulis, E. K., Dumont, D., Dzieciuch, M. A., and Beszczynska-Möller, A. (2016). Time series of temperature in fram strait determined from the 2008-2009 DAMOCLES acoustic tomography measurements and an ocean model, J. Geophys. Res. 121, 4601-4617. doi: 10. 1002/2015JC011591

Sagen, H., Worcester, P. F., Dzieciuch, M. A., Geyer, F., Sandven, S., Babiker, M., et al. (2017). Resolution, identification, and stability of broadband acoustic arrivals in fram strait. J. Acoust. Soc. Am. 141:2055. doi: 10.1121/1.4978780

Sandven, S., Sagen, H., Bertino, L., Beszczynska-Möller, A., Fahrbach, E., Worcester, P. F., et al. (2011). "The Fram Strait integrated ocean observing and modelling system," in Proceedings of the Sixth International Conference on EuroGOOS. Sustainable Operational Oceanography (Sopot: EuroGOOS), 50-58.

Schevill, W. E., Watkins, W. A., and Backus, R. H. (1964). "The 20-cycle signals and Balaenoptera physalus (fin whales)," in Marine Bio-Acoustics, ed. W. N. Tavolga (New York, NY: Pergamon Press).

Send, U., Worcester, P. F., Cornuelle, B. D., Tiemann, C. O., and Baschek, B. (2002). Integral measurements of mass transport and heat content in the strait of gibraltar from acoustic transmissions. Deep Sea Res. 49, 4069-4095. doi: 10.1016/S0967-0645(02)00143-141

Skarsoulis, E. K., and Cornuelle, B. D. (2004). Travel-time sensitivity kernels in ocean acoustic tomography. J. Acoust. Soc. Am. 116, 227-238. doi: 10.1121/1. 1753292

Slabbekoorn, H., and Bouton, N. (2008). Soundscape orientation: a new field in need of sound investigation. Anim. Behav. 76, e5-e8. doi: 10.1016/j.anbehav. 2008.06.010

Smith, G. C., Allard, R., Babin, M., Bertino, L., Chevallier, M., Corlett, G. K., et al. (2019). Polar ocean observations: a critical gap in the observing system and its effect on environmental predictions from hours to a season Front. Mar. Sci. 6429. doi: $10.3389 /$ fmars.2019.00429

Spiesberger, J. L., Birdsall, T. G., Metzger, K., Knox, R. A., Spofford, C. W., and Spindel, R. C. (1983). Measurements of gulf stream meandering and evidence of seasonal thermocline development using long-range acoustic transmissions. J. Phys. Oceanogr. 13, 1836-1846. doi: 10.1175/1520-04851983013<1836: MOGSMA<2.0.CO;2

Sprintall, J., Gordon, A. L., Wijffels, S. E., Feng, M., Hu, S., Koch-Larrouy, A., et al. (2019). Detecting change in the Indonesian Seas. Front. Mar. Sci. 6257. doi: $10.3389 /$ fmars.2019.00257

Staaterman, E., Rice, A. N., Mann, D. A., and Paris, C. B. (2013). Soundscapes from a tropical eastern pacific reef and a caribbean sea reef. Coral Reefs 32, 553-557. doi: 10.1007/s00338-012-1007-8

Staaterman, E., Paris, C. B., DeFerrari, H. A., Mann, D. A., Rice, A. N., and D’Alessandro, E. K. (2014). Celestial patterns in marine soundscapes. Mar. Ecol. Prog. Ser. 508, 17-32. doi: 10.3354/meps10911

Stammer, D., Balmaseda, M., Heimbach, P., Köhl, A., and Weaver, A. (2016). Ocean data assimilation in support of climate applications: status and perspectives. Ann. Rev. Mar. Sci. 8, 491-518. doi: 10.1146/annurev-marine-122414-034113

Stanton, T. K., Chu, D. Z., Jech, J. M., and Irish, J. D. (2010). New broadband methods for resonance classification and high-resolution imagery of fish with swim bladders using a modified commercial broadband echosounder. ICES J. Mar. Sci. 67, 365-378. doi: 10.1093/icesjms/fsp262

Stauffer, B. A., Miksis-Olds, J. L., and Goes, J. I. (2015). Cold regime interannual variability of community composition of primary and secondary producers in the southeastern Bering Sea. PLoS One 10:e0131246. doi: 10.1371/journal.pone. 0131246

Swallow, J. C. (1971). The Aries current measurements in the Western North Atlantic. Phil. Trans. Roy. Soc. London A 270, 451-460. doi: 10.1098/rsta.1971. 0084

Swart, S., Gille, S. T., Delille, B., Josey, S., Mazloff, M., Newman, L., et al. (2019). Constraining southern ocean air-sea-ice fluxes through enhanced observations. Front. Mar. Sci. 6421. doi: 10.3389/fmars.2019.00421

Syamsudin, F., Chen, M., Kaneko, A., Adityawarman, Y., Zheng, H., Mutsuda, H., et al. (2017). Profiling measurement of internal tides in bali strait by reciprocal sound transmission. Acoust. Sci. Technol. 38, 246-253. doi: 10.1250/ast.38.246

Tavolga, W. N. (2012). "Listening backward: early days of marine bioacoustics," in The Effects of Noise on Aquatic Life, eds A.N. Popper and A. Hawkins (Berlin: Springer).

Trevorrow, M. V., Mackas, D. L., and Benfield, M. C. (2005). Comparison of multifrequency and in situ measurements of zooplankton abundances in knight inlet, British Columbia. J. Acoust. Soc. Am. 117, 3574-3588. doi: 10.1121/1.1920087
Vagle, S., Large, W. G., and Farmer, D. M. (1990), An evaluation of the WOTAN technique of inferring oceanic winds from underwater ambient sound, J. Atmos. Oceanic Technol. 7, 576-595. doi: 10.1175/1520-04261990007<0576: AEOTWT $<2.0 . \mathrm{CO} ; 2$

Van Opzeeland, I.C., and Miksis-Olds, J.L. (2012). "Acoustic ecology of pinnipeds in polar habitats," in Aquatic Animals: Biology, Habitats, and Threats, ed. D.L. Eder (Hauppauge, NY: Nova Science Publishers).

Van Uffelen, L. J., Howe, B. M., Nosal, E. M., Carter, G. S., Worcester, P. F., and Dzieciuch, M. A. (2016). Localization and subsurface position error estimation of gliders using broadband acoustic signals at long range. IEEE J. Ocean. Eng. 41, 501-508. doi: 10.1109/JOE.2015.2479016

Villas Bôas, A. B., Ardhuin, F., Ayet, A., Bourassa, M. A., Chapron, B., Brandt, P., et al. (2019). Integrated observations and modeling of global winds, currents, and waves: requirements and challenges for the next decade. Front. Mar. Sci. 6425. doi: 10.3389/fmars.2019.00425

Warren J. D., Stanton, T. K., Wiebe, P. H., and Seim, H. E. (2003). Inference of biological and physical parameters in an internal wave using multiplefrequency, acoustic-scattering data. ICES J. Mar. Sci. 60, 1033-1046. doi: 10. 1016/s1054-3139(03)00121-8

Watkins, W. A. (1981). Activities and underwater sounds of fin whales. Sci. Rep. Whales Res. Inst. 33, 83-117.

Watkins, J. L., and Brierley, A. S. (2002). Verification of acoustic techniques used to identify and size antarctic krill. ICES J. Mar. Sci. 59, 1326-1336. doi: 10.1006/ jmsc.2002.1309

WCRP Global Sea Level Budget Group (2018). Global sea-level budget 1993-present. Earth Syst. Sci. Data 10, 1551-1590. doi: 10.5194/essd-10-15512018

Webster, S. E., Lee, C. M., and Gobat, J. I. (2014). "Preliminary results in under-ice acoustic navigation for seagliders in davis strait," in Proceedings of the IEEE/MTS OCEANS (St. John's: IEEE).

Webster, S. E., Freitag, L. E., Lee, C. M., and Gobat, J. I. (2015). "Towards real-time under-ice acoustic navigation at mesoscale ranges," in Proceedings of the IEEE International Conference on Robotics and Automation (Seattle: Washington).

Wiggins, S. M., and Hildebrand, J. A. (2007). "High-frequency Acoustic Recording Package (HARP) for broad-band, long-term marine mammal monitoring", in Proceedings of the 2007 Symposium on Underwater Technology and Workshop on Scientific Use of Submarine Cables and Related Technologies. (Tokyo: IEEE).

Woelfl, A.-C., Snaith, H., Amirebrahimi, S., Devey, C., Dorschel, B., Ferrini, V., et al. (2019). Seafloor mapping - the challenge of a truly global ocean bathymetry. Front. Mar. Sci. 6283. doi: 10.3389/fmars.2019.00283

Woodgate, R. A., Weingartner, T. J., and Lindsay, R. (2012). Observed increases in Bering Strait oceanic fluxes from the Pacific to the Arctic from 2001 to 2011 and their impacts on the Arctic Ocean water column. Geophys. Res. Lett. 39:L24603. doi: 10.1029/2012GL054092

Worcester, P. F., Cornuelle, B. D., Hildebrand, J. A., Hodgkiss, W. S., Duda, T. F., Boyd, J., et al. (1994). A comparison of measured and predicted broadband acoustic arrival patterns in travel time-depth coordinates at $1000-\mathrm{km}$ range. J. Acoust. Soc. Am. 95, 3118-3128. doi: 10.1121/1.409977

Worcester, P. F., Cornuelle, B. D., Dzieciuch, M. A., Munk, W. H., Howe, B. M., Mercer, J. A., et al. (1999). A test of basin-scale acoustic thermometry using a large-aperture vertical array at $3250-\mathrm{km}$ range in the eastern North Pacific Ocean. J. Acoust. Soc. Am. 105, 3185-3201. doi: 10.1121/1.424649

Worcester, P. F., Carey, S., Dzieciuch, M. A., Green, L. L., Horwitt, D., Lemire, J. C., et al. (2009). Distributed Vertical Line Array (DVLA) acoustic receiver. Paper presented at Proceedings of the 3rd International Conference on Underwater Acoustic Measurements: Technologies and Results, Foundation for Research and Technology, Heraklion.

Conflict of Interest Statement: The authors declare that the research was conducted in the absence of any commercial or financial relationships that could be construed as a potential conflict of interest.

Copyright $\odot 2019$ Howe, Miksis-Olds, Rehm, Sagen, Worcester and Haralabus. This is an open-access article distributed under the terms of the Creative Commons Attribution License (CC BY). The use, distribution or reproduction in other forums is permitted, provided the original author(s) and the copyright owner(s) are credited and that the original publication in this journal is cited, in accordance with accepted academic practice. No use, distribution or reproduction is permitted which does not comply with these terms. 
OPEN ACCESS

Edited by:

Justin Manley,

Just Innovation, Inc., United States

Reviewed by:

Jan Robert Van Smirren,

Ocean Sierra LLC, United States

Jay S. Pearlman,

Institute of Electrical and Electronics

Engineers (France), France

${ }^{*}$ Correspondence:

Derrick Snowden

derrick.snowden@noaa.gov

Specialty section:

This article was submitted to

Ocean Observation,

a section of the journal

Frontiers in Marine Science

Received: 15 November 2018

Accepted: 05 July 2019

Published: 26 July 2019

Citation:

Snowden D, Tsontos VM, Handegard NO, Zarate M, O' Brien K,

Casey KS, Smith N, Sagen H,

Bailey K, Lewis MN and Arms SC (2019) Data Interoperability Between

Elements of the Global Ocean

Observing System.

Front. Mar. Sci. 6:442

doi: 10.3389/fmars.2019.00442

\section{Data Interoperability Between Elements of the Global Ocean Observing System}

\author{
Derrick Snowden ${ }^{*}$, Vardis M. Tsontos ${ }^{2}$, Nils Olav Handegard ${ }^{3}$, Marcos Zarate, \\ Kevin O' Brien ${ }^{5}$, Kenneth S. Casey ${ }^{6}$, Neville Smith $^{7}$, Helge Sagen $^{8}$, Kathleen Bailey', \\ Mirtha N. Lewis ${ }^{4}$ and Sean C. Arms ${ }^{9}$
}

\begin{abstract}
1 US Integrated Ocean Observing System Program, National Oceanic and Atmospheric Administration/National Ocean Service, Silver Spring, MD, United States, ${ }^{2}$ PO.DAAC, NASA Jet Propulsion Laboratory, Pasadena, CA, United States, ${ }^{3}$ Institute of Marine Research, Bergen, Norway, ${ }^{4}$ Centre for the Study of Marine Systems, Patagonian National Research Centre, National Scientific and Technical Research Council, Puerto Madryn, Argentina, ${ }^{5}$ Joint Institute for the Study of the Atmosphere and Ocean, University of Washington, Seattle, WA, United States, ${ }^{6}$ National Centers for Environmental Information, National Oceanic and Atmospheric Administration, Silver Spring, MD, United States, ${ }^{7}$ GODAE Ocean Services, Melbourne, VIC, Australia, ${ }^{8}$ Institute of Marine Research, Norwegian Marine Data Centre, Bergen, Norway, ${ }^{9}$ University Corporation for Atmospheric Research/Unidata, Boulder, CO, United States
\end{abstract}

The data management landscape associated with the Global Ocean Observing System is distributed, complex, and only loosely coordinated. Yet interoperability across this distributed landscape is essential to enable data to be reused, preserved, and integrated and to minimize costs in the process. A building block for a distributed system in which component systems can exchange and understand information is standardization of data formats, distribution protocols, and metadata. By reviewing several data management use cases we attempt to characterize the current state of ocean data interoperability and make suggestions for continued evolution of the interoperability standards underpinning the data system. We reaffirm the technical data standard recommendations from previous OceanObs conferences and suggest incremental improvements to them that can help the GOOS data system address the significant challenges that remain in order to develop a truly multidisciplinary data system.

Keywords: interoperability, data management, data lifecycle, data preservation, standards, metadata

\section{INTRODUCTION}

Ocean observing programs of varying geographic or disciplinary scope have been coordinating globally for decades in an effort to develop an efficient, sustainable, and complete Global Ocean Observing System (GOOS) of systems. Many efforts focus on national or regional priorities that are often limited in geographical scope. Others are globally focused but constrained by sampling methodologies such as through the use of profiling floats [e.g., Argo (Riser et al., 2016)] or by sampling geometry such as time series measurement at one location [e.g., OceanSITES (Send et al., 2010)]. These programs are independently governed and funded and can serve different stakeholders, though they often have commonalities. Addressing the needs of the individual stakeholders influences how the observing programs design the information systems that manage and distribute the observations. This individuality of the stakeholders leads to individuality of the information systems, which contributes to a lack of interoperability across systems. 
The Framework for Ocean Observation (FOO) (Lindstrom et al., 2012) defines a set of key processes to guide the ocean observing community toward the establishment of an integrated, sustained ocean observing system with fit-for-purpose data/information streams for societal and scientific benefit. It provides a set of overarching principles and conceptual structures useful in guiding the coherent development of ocean observing systems and coordinating their supporting data infrastructures in a manner that mitigates the aforementioned structural issues. The FOO is based on observing system success stories and best practices, a collaboration-focused governance structure, and the concept of Essential Ocean Variables (EOVs). Global progress is measured through maturity/readiness levels which are central organizing principles for the establishment of coherent requirements for observing system elements consistent with systems engineering approaches. The FOO also highlights the critical role of data management and data interoperability standards in addressing the enormous challenge of open access to harmonized, integrated data across a very diverse ocean observing "system of systems," comprised of multi-scale, multiplatform/sensor observations supporting various applications and science domains. Given the associated highly heterogeneous data landscapes and data management infrastructures, "the desire to 'measure once and use many times' requires that standards be developed and adopted by observing components." Data interoperability across the data lifecycle and information value chain, from raw observational data through modeled synthesis products, is seen as a foundational element of ocean observing systems that are efficient and fit for purpose. Efficient, fit for purpose, observing systems provide useful inputs to science-based, data-driven decision support processes of societal importance, relevant to ecosystem management, food security, maritime safety, energy, climate monitoring, and other emerging areas of the Blue economy. The FOO additionally recognizes the role of international entities such as the WMO-IOC Joint Technical Commission for Oceanography and Marine Meteorology (JCOMM), and the International Oceanographic Data and Information Exchange (IODE) in coordinating such data management efforts for physical, geological, chemical, and biological observing system elements of the GOOS, leveraging also work done by Earth science data standards authorities. Finally, the Framework identifies the importance of education, outreach, and capacity building, including in the area of data interoperability standards and best practices for oceanographic data management.

While the Framework acknowledges the importance of data interoperability and provides a structure for global collaboration toward better interoperability, it does not describe specific steps, tools, or actions to be taken. In this paper we focus on data interoperability across the global ocean observing community. We first describe this global community and define interoperability between community members. We then examine several use cases that demonstrate various levels of interoperability in an effort to distill best practices that can be widely adopted. The use cases also help demonstrate limitations of our current understanding of data interoperability that we suggest can be opportunities for future work or investment.
Finally, we close with listing recommendations for evolving the information management systems supporting the GOOS.

\section{SIGNIFICANCE OF DATA INTEROPERABILITY FOR OCEAN OBSERVING}

\section{Defining the GOOS Data System}

The GOOS ${ }^{1}$ is a global system for sustained, interdisciplinary observations of the ocean comprising the oceanographic component of the Global Earth Observing System of Systems $(\text { GEOSS })^{2}$. It is a coordinated but highly decentralized system and organizational network overseeing the planning and implementation of observations for the world's oceans, aimed ultimately at delivering data, related services, and information products in support of research and applications. By necessity and design, it leverages heavily from regional and national programs and infrastructures but also from community partnerships that are related to specific observing system elements under the umbrella of JCOMM. Data is managed in a federated manner and accessible via regional observing system nodes, JCOMM partnership program data assembly centers (DACs), space agency satellite data centers, integrated DACs and designated IODE/International Council for Science (ICSU) World Data Centers (WDC) (Figure 1). Data offerings are diverse in type, coverage, and extent. They include multi-variate observational data from in situ and remote sensing platforms that are sustained, and often available in near real-time, and data from research field campaigns that are more ephemeral in nature. Data from numerical models are also an important data type that differ from observational in some important ways (e.g., model data are less diverse in structure but typically much higher volume). The ability to integrate across such a distributed and complex, multi-agency data management landscape, in support of GOOS, hinges critically on the widespread adoption of data interoperability standards.

Through national efforts such as the U.S. Integrated Ocean Observing System, or through GOOS Regional Alliances like EuroGOOS, system are being built that integrate data from across numerous observing system elements in an attempt to provide integrated products to regional or local customers. These efforts frequently result in more customer focused relationships and products. However, integrating across different platforms taxes the data interoperability standards which are often not general enough to span use cases across platforms and across disciplines. Maintaining a strong standards foundation while still providing flexible enough tools to tailor products to local customer needs is an ongoing challenge for the global ocean observations community.

\section{Why Is Interoperability Important?}

While we are unaware of any single effort to design and construct a monolithic GOOS Data System, in this paper we use this term

\footnotetext{
${ }^{1}$ http://www.goosocean.org/

${ }^{2}$ https://www.earthobservations.org/geoss.php
} 


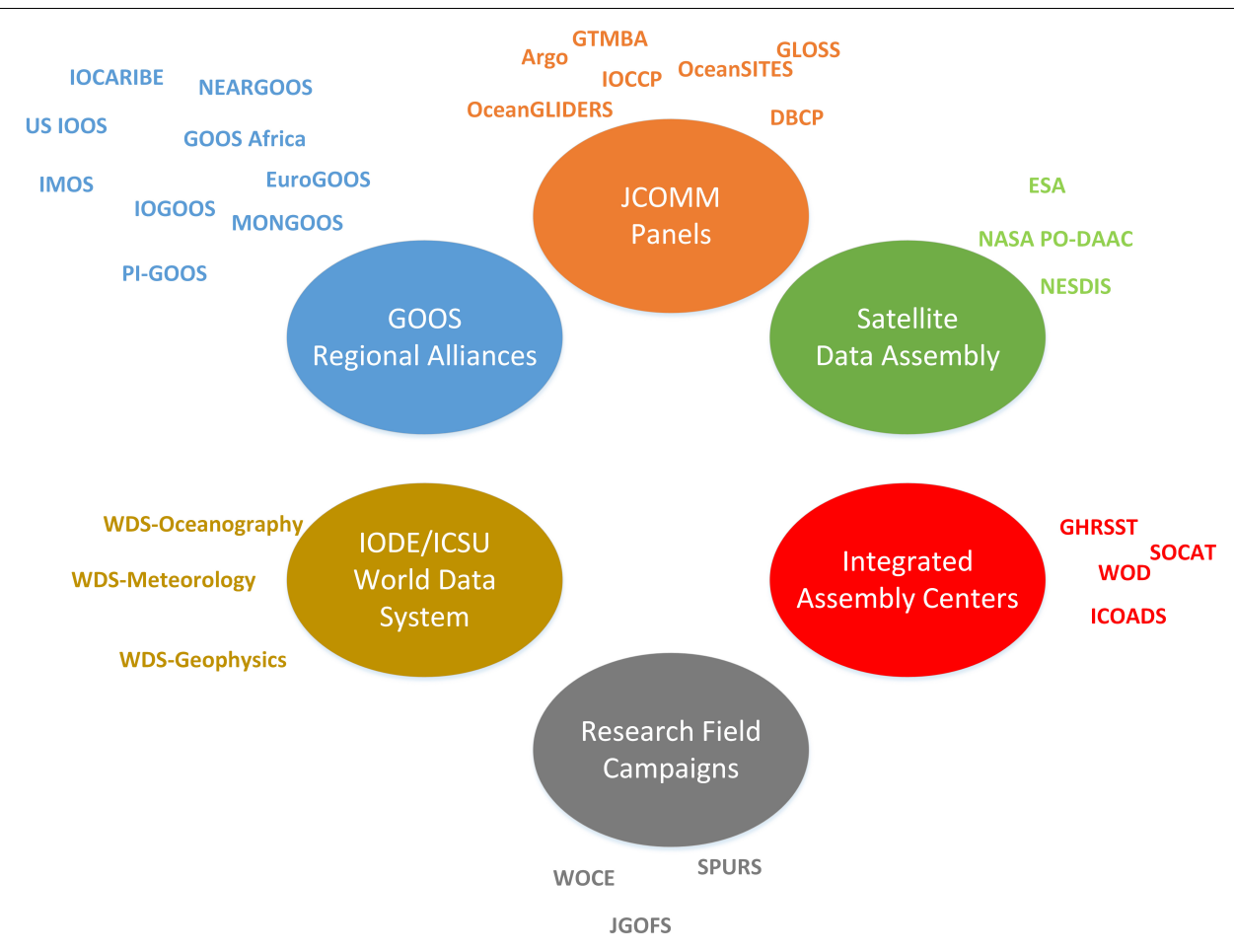

FIGURE 1 | The GOOS Data System comprised of many efforts that are funded and coordinated nationally and internationally. To ensure the successful information sharing between the component systems is enhanced through (i) data interoperability standards and protocols for measurements, data exchange and data management, (ii) ensure effective and efficient linkage among elements (measurements, data management and communications, data analysis), (iii) formulate rules of engagement for establishing the operational elements of the global ocean.

to collectively and generically refer to the independent but often coordinated efforts across all of the organizations in Figure 1. It is a distributed data system with responsibilities for different stages of the data management lifecycle spread across myriad organizations. We make a few assumptions about the principles that each of the component efforts share. If these assumptions are true, they serve as design principles for the GOOS Data System. Enhancing data interoperability then becomes a means to achieve these design principles. There are efforts that will not ascribe to these principles and for those efforts, we must exclude them from consideration in the GOOS Data System for pragmatic reasons. However, we believe that the trend toward embracing these principles will increase as policy and technology evolve.

\section{Data Reuse}

Data reuse is one of the Guiding Principles for the FOOs (see section "Web Service Based Data Exchange: Data Access Services," Lindstrom et al., 2012). The measure once/use many times philosophy underpins the entire FOO and is increasingly driving policy at federal levels. For example, the United States Government has committed to an Open Data policy ${ }^{3}$ predicated on the notion that open accessible data will spur innovation and lead to efficiencies. Examining the global policy landscape for data sharing is beyond the scope of this paper but we note that increased data sharing may be motivated by recognition that

${ }^{3}$ https://www.data.gov/developers/open-data-executive-order/ reusing data has potential economic benefits and encourage open data sharing across the GOOS Data System.

\section{Data Preservation}

A further, often unappreciated benefit stemming from Earth science data standards adoption is that long-term preservation of archive quality data with associated metadata and provenance information is facilitated. This ensures protection not only of significant public investments in costly data collection but also preservation of observations that are unique in time and space and thus irreplaceable. Understanding and alleviating underlying challenges and constraints to widespread adoption of applicable data standards, whether technical, capacity or resource related, will be central to ensuring sustained contributions to and effective usage of a vibrant and integrated ocean observing system data commons.

\section{Data Integration}

Many data management efforts are organized around an observing platform such as research vessels, profiling floats $\left(\mathrm{Argo}^{4}\right)$ or particular satellites (e.g., AVHRR Pathfinder ${ }^{5}$ ). This organization is sensible because in the early stages of the data lifecycle, there are efficiencies to be gained by managing all data from a single platform in a single place. However, for

\footnotetext{
${ }^{4}$ http://www.argodatamgt.org/

${ }^{5}$ https://podaac.jpl.nasa.gov/datasetlist?ids=\&values=\&search $=$ Pathfinder
} 
scientific or operational applications, stakeholders are often more interested in obtaining data organized around a sampling method (e.g., all ocean profile data in World Ocean Database ${ }^{6}$ ) or by EOV (e.g., Sea Surface Temperature data from the Group for High Resolution Sea Surface Temperature, GHRSST ${ }^{7}$ ). Creating these integrated data sets is made easier if all of the source data sets are available in interoperable standards compliant formats. It is worth noting that Argo, World Ocean Database (WOD), and GHRSST have all adopted common conventions for representing data and metadata in file formats that enhance system wide interoperability.

\section{Minimizing Lifecycle Costs}

The importance of data interoperability for the development of an operationally sustainable GOOS lies in the significant cost saving and scalability that automated data discovery, access and processing pipelines provide. Data conforming to established Earth science data interoperability standards have the necessary structural, syntactic and semantic characteristics rendering them searchable, more easily integrated within software systems and amongst themselves, and generally more usable. Standards compliance increases the likelihood that custom, unmaintainable and invariably costly to implement one-off solutions for handling of data are averted.

Software development is an essential and expensive part of the data management lifecycle. Widespread adoption of data standards encourages the development of generic rather than single purpose software tools. Generic tools, especially when developed using Open Source Software principles and methods, can attract more developers because they can focus their time as a team working on a common tool rather than individually on their particular application. This often results in higher quality, better documented, and better tested software which has implications for the efficiency of the global community. Open Source software policies should accompany Open Data Sharing Policies in the GOOS governance framework. This recommendation should not be adopted without consideration of long term implications. For example, the legal framework for software licensing is complex and potentially conflicts with some institutional policies. Further, open source software may not have dedicated technical support. In sum, the authors believe that Open Source Software is a net positive for the community but acknowledge that some caution is warranted.

\section{FAIR Principles}

These considerations are effectively embodied by the FAIR guiding principles for the improved data management, stewardship and accessibility of science that have recently been advanced (Wilkinson et al., 2016) and that are gaining increased traction. FAIR emphasizes: (1) Findable data, with machine-readable metadata essential for automated discovery and utilization of data and metadata by software services. (2) Access to data and persistent metadata records using open/free, standards-based protocols that support authentication. (3)

${ }^{6} \mathrm{https} / / /$ www.nodc.noaa.gov/OC5/WOD/pr_wod.html

${ }^{7}$ https://www.ghrsst.org/
Interoperable such that data are machine interpretable and can be automatically combined with other data, leveraging standard vocabularies and ontologies for knowledge representation accessible via semantic Web technologies. (4) Reusable data: well-characterized, rich community metadata enabling traceable, reproducible, and easily integrated data in support of research and applications into the future. FAIR provides a high-level conceptual framework useful to the design of contemporary information systems in support of ocean observation, the more detailed technical underpinnings of which are based on widely applied Earth science data interoperability standards that we now describe. While the focus of this paper is on data interoperability, it is difficult to decouple Interoperability from the other elements of FAIRness when describing the GOOS Data System current and future states (Tanhua et al., 2019).

\section{DEFINING INTEROPERABILITY FOR OCEAN DATA STAKEHOLDERS}

Interoperability in a general sense can be defined as the "degree to which two or more systems, products or components can exchange information and use the information that has been exchanged" (ISO/IEC/IEEE, 2017). It is the ability by which coupled software systems can communicate and exchange data via common formats and protocols and also meaningfully interpret and reproducibly act on exchanged data. This definition prompts an important recognition: interoperability is a characteristic of a relationship between two or more systems. It is not a characteristic of a single data file or data set. The two (or more) systems in this relationships are both stakeholders in the exchange of information. Generically, these stakeholders can be classified as Data Producers/Providers or Data Consumers.

Data Producers/Providers are responsible for generating data, typically through observation or simulation and make it available to a consumer. The scope of these activities can be local to an individual Principal Investigator conducting a lone experiment, or globally coordinated efforts like the World Ocean Circulation Experiment of the 1990s or the Argo program of today. These stakeholders have common objectives, to plan and design their experimental or sampling scheme so that the data addresses the scientific or operational objective. As noted above, we also assume that these Producers/Providers subscribe to the principles listed above. They wish to see their data maximally reused, preserved for the future, combined with other data into integrated products, and they want to minimize costs in the process. If Data Producers/Providers do not subscribe to these common principles, then the significant overhead of adopting and using global data interoperability standards is a resource drain that is often un- or under-funded. It is a requirement for national and international governance groups and funding agencies to encourage adherence to these common goals and to resource the work adequately if the GOOS Data System is to become more interoperable. However, it is also critical for the scientific Information Management community to help bridge the gap between data producers and the relevant metadata and data standards by providing tools that can improve conformance 
of their data to well-established data interoperability standards. This will facilitate integration of data in a range of software applications and Web services including data distribution, processing, modeling and visualization capabilities, thus enabling more widespread usage of data.

Data Consumers require data to solve problems or make decisions. They could be interested in obtaining data for scientific study, for assimilation into a numerical model, or to create a web based visualization. A scientist likely requires all available data and extensive metadata to ensure that they understand as much as possible about how the data was generated. They are interested in ingesting the data into their analysis tool of choice and may be willing to wait to download enormous file collections. A web developer on the other hand may only require a small subset of a data set but they need it on demand with minimal latency. For the scientist, downloading a large collection of files from an FTP server may be an acceptable access method while the web developer requires a web based Application Programming Interface (API) with flexible query methods and extremely fast response. By observing and documenting different types of Provider - Consumer relationships that are successful, we can generalize strategies that enhance the likelihood that future relationships are successful - i.e., interoperable. The following sections describe principles or design patterns that have underscored successful interoperable Provider - Consumer relationships and offer background for the Use Cases that follow.

\section{Common Elements of Data Interoperability}

Typical Provider-Consumer exchanges occur when a file subset or a file are transferred from one system to another. The ability of the consumer system to understand and use the information received has both syntactic and semantic elements. Syntactic elements describe the Consumers ability to decode the electronic data file and accurately access the objects within the file. Semantic elements describe the Consumer's ability to understand the data objects. Simple semantics include understanding parameter names and units while more complex semantics allow for translating between colloquial names for species types and standardized registries of species names like the World Register of Marine Species (WoRMS). Interoperability standards for Earth science data are comprised of three core elements (Figure 2):

- File standards, based on self-describing scientific data file formats;

- Common data and metadata models;

- Controlled vocabularies and ontologies that define terms, concepts and their relationships for a given science domain.

Much of the emphasis on the data file stems historically from its ubiquitous usage as the storage and exchange medium for science instrument data, including from ocean observing systems. Widely used scientific data file formats such as $\mathrm{HDF}^{8}$ and netCDF ${ }^{9}$ provide compact, binary formats optimized for efficient storage and access of large, complex datasets. They

${ }^{8}$ https://www.hdfgroup.org/solutions/hdf5/

${ }^{9}$ https://doi.org/10.5065/D6H70CW6 include features such as internal compression, and support hierarchical structuring of data within files. Significantly, from a data interoperability and data preservation perspective, they are, or can be made to be, self-describing. A self-describing file includes metadata that describes both the data and data structures comprising a file. Further, self-describing files minimize or eliminate the need for external sources of information (e.g., quality control code tables) allowing the file stand alone and be understood by a consumer. Common data models (CDMs) are important enablers of interoperability because they allow generic software to predict the structure of these self-describing files and access the encoded data as the author intended. Interoperability has been further facilitated by the parallel community development of metadata models supporting a broad range of geospatial Earth science data that have been built around or are compatible with the CDM. These include the Climate Forecast (CF) conventions ${ }^{10}$, the Attribute Conventions for Data Discovery (ACDD) $)^{11}$, and the ISO $19115^{12}$ standard metadata schema for geographic information and services. These conventions provide a standard set of attributes and technical framework for the encoding of metadata and data in selfdescribing data files. Such common data and metadata models have in turn promoted the development of an ecosystem of broadly used, open source software libraries, APIs, web service standards and Web server technologies. Hankin et al. (2010) describe the important role that the Climate and Forecast Conventions and the netCDF file format fill in our global data management and dissemination framework. In the decade since OceanObs '09, CF/netCDF has been further cemented as the de facto standard for file based storage and exchange of in situ, remotely sensed, and model generated data. Further progress since OceanObs '09 is nicely summarized in Tanhua et al. (2019).

The third ingredient for data to be interoperable relates to the semantic interpretability of the metadata that qualify or describe the geophysical data values themselves via the use of controlled vocabularies. Of particular importance is the application of a standard term for the observable parameter and its associated units, but also the use of standard vocabularies for metadata attributes relating to the sampling platform, sensor, and other categorical keyword descriptors. Application of standard terms is vital from a data interoperability perspective because it ensures valid interpretation of values by human users and enables correct aggregation and computation on integrated sets of data to be performed. Examples of actively maintained and widely used vocabularies include the CF standard names ${ }^{13}$ and the UDUnits library ${ }^{14}$ and the NASA Global Change Master Directory (GCMD) keywords. There are also specific science domain ontologies that are being developed by particular expert communities that once integrated provide a refined set of terms applicable to broader types of ocean science data. Vocabulary

\footnotetext{
${ }^{10}$ The CF Conventions are a set of guidelines for creating netCDF files with good interoperability characteristics (http://cfconventions.org/).

${ }^{11}$ http://wiki.esipfed.org/index.php/Attribute_Convention_for_Data_Discovery

${ }^{12}$ https://www.iso.org/standard/53798.html

${ }^{13} \mathrm{http} / /$ cfconventions.org/Data/cf-standard-names/60/build/cf-standard-nametable.html

${ }^{14}$ https://doi.org/10.5065/D6KD1WN0
} 


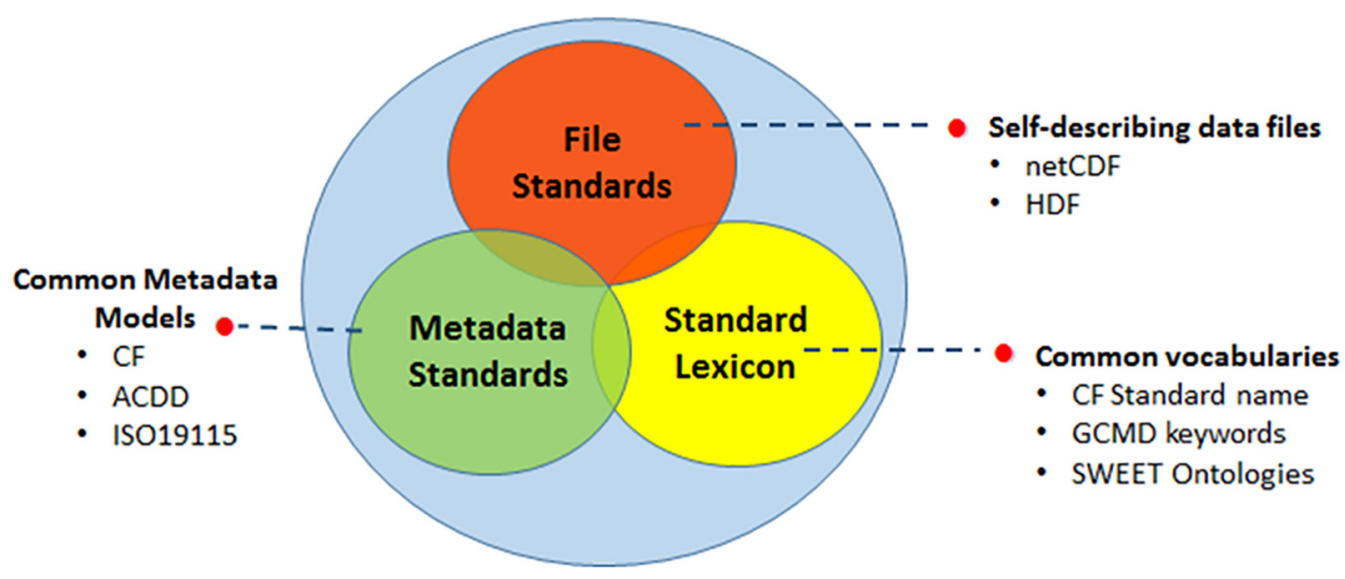

FIGURE 2 | The three main categories of data interoperability standards: file standards, metadata standards and standard lexicon, allow integration of data in a range of software applications and Web services including data distribution, processing, modeling and visualization capabilities, thus enabling more widespread usage of data.

servers, such as those maintained by the British Oceanographic Data Center (BODC) $)^{15}$ and the Marine Metadata Interoperability project $(\mathrm{MMI})^{16}$, aggregate and systematically organize such curated lists of standardized terms covering a broad spectrum of oceanographic disciplines that are machine query able and can help resolve metadata definitions or reconcile ambiguity in terms being applied.

In describing the key elements underlying Earth science data interoperability standards appropriate to the ocean observing domain, it is important to understand that there a couple of levels at which these are applicable and between which there are some differences: the dataset and granule levels. A dataset or collection is an aggregation comprised of data files or granules of a common type. While the definition of granule can be somewhat arbitrary, it is simply a convenient building block for the complete data set. For example, the Aquarius L3 Sea Surface Salinity V5.0 dataset ${ }^{17}$ is a collection of the entire series of data files ("granules") over the course of the Aquarius/SAC-D satellite mission spanning 2011-Aug-25 to 2015-Jun-07. The individual granules in this dataset represent 7-day averaged snapshots covering the entire globe. Similarly for in situ data, there is an analogous collection of over 2000 profiles (granules) from an underwater glider that are aggregated together into a complete dataset that represents a glider deployment between November 11 and $15,2015^{18}$. Metadata characterizing the complete dataset is different in composition and detail to that of the constituent data files comprising the collection, although both will ideally conform to the same ISO19115 geospatial metadata standard framework that enables interoperability. The use of files as a basic element of designing an information system is based on their use in common data management systems today, and because their ubiquity makes it easier to describe some of the conceptual

\footnotetext{
${ }^{15}$ https://www.bodc.ac.uk/resources/products/web_services/vocab/

${ }^{16}$ https://mmisw.org/ont/\#/

${ }^{17}$ http://dx.doi.org/10.5067/AQR50-3S7CS

${ }^{18}$ https://data.ioos.us/dataset/cp_387-20151014t011988915
}

elements of interoperability. However, this is not meant to preclude the use of other Information Technology staples such as Relational Database Management Systems, sometimes referred to as geodatabases. Geodatabases can be equally well-suited to interoperable data systems.

\section{Web Service Based Data Exchange: Data Access Services}

Data exchange between the nodes of the distributed system shown in Figure 1 requires APIs that are designed for Web protocols. Downloading collections of files published as hyperlinks on web pages or on FTP sites cannot scale to address the types of exchanges needed for distributed global science and operational needs. Machine to machine APIs, or Web Services, enable querying, subsetting, and other advantages over bulk download of files.

Mature, well-supported software to enable web based APIs for data and metadata access exists for use by the entire GOOS Data System. Hankin et al. (2010) describe the utility of the OPeNDAP protocol for serving data. OPeNDAP ${ }^{19}$ has become ubiquitous in the earth science community and several software packages implement this protocol. For example, THREDDS ${ }^{20}$, Hyrax $^{19}$, and ERDDAP $^{21}$ are three data servers that ingest data files in various formats and publish data to the web using the OPeNDAP protocol.

The THREDDS Data Server, developed by Unidata, is an implementation of the OPeNDAP protocol which enables aggregations of WMO GriB files and netCDF files to be aggregated and served as a single data resource. The APIs implemented by THREDDS allow subsetting in space and time. THREDDS is particularly well-suited to gridded data collections and is currently being enhanced to serve unstructured and

\footnotetext{
${ }^{19}$ https://www.opendap.org/

${ }^{20}$ https://doi.org/10.5065/D6N014KG

${ }^{21}$ https://upwell.pfeg.noaa.gov/erddap/information.html
} 
staggered grids common in modern ocean models (see section "Operational and Research Modeling").

Another example is the ERDDAP data platform that was developed by NOAA's Southwest Fisheries Environmental Research Division ${ }^{22}$. As an open source data platform, one of the functions ERDDAP performs is to serve data to users through web-based services. Similar to the THREDDS Data Server, ERDDAP supports the OPeNDAP protocol, allowing for remote accessing of data. To the data consumer, ERDDAP provides a uniform, RESTful service for accessing data that allows for machine-to-machine exchange. Many interesting tools have been built to take advantage of the services that ERDDAP provides ${ }^{23}$ and ERDDAP is a key element of the TPOS 2020 strategy (see section "TPOS 2020").

Both THREDDS and ERDDAP are developed as open source software projects that encourage outside developers to contribute feature enhancements and bug fixes.

\section{Web Service Based Metadata Exchange: Data Discovery Services}

While the focus of this article is on interoperability, the need to support data discovery is also an important driver for developing and adhering to interoperability standards. Well-structured and well-populated granule and dataset metadata makes discovery possible. Tools exist that extract information from data files to create discovery metadata records that can be indexed into queryable metadata catalogs.

Support for common metadata standards at both file and dataset levels, allow geospatial metadata services to make data discoverable through efficiently queryable metadata repositories. Furthermore, support for common metadata standards across repositories facilitates integration and unified search horizontally, agency-wide or even across inter-agency repositories. NASA's Common Metadata Repository $(\mathrm{CMR})^{24}$ and NOAA's OneStop ${ }^{25}$ system are examples of distributed metadata systems that integrate information on holdings across distributed data archives conforming to the aforementioned enterprise architecture and interoperability standards that enables unified search and access to science data enterprise-wide. Taking this approach to an even broader level are schema.org-based approaches like the new Google Dataset Search ${ }^{26}$ utility. While schema.org limits the amount of metadata to a relatively narrow set of searchable fields, that simplification makes it easier for many distributed groups to ensure their holdings are available to the big commercial search engines and other interoperable data systems. It also makes it possible for data access services that support schema.org markup, to be discoverable from Google searches.

Support for common metadata standards in turn enables integration of distinct agency metadata repository systems for discovery and access to data by users centrally with much greater

\footnotetext{
${ }^{22}$ https://upwell.pfeg.noaa.gov/erddap/information.html

${ }^{23} \mathrm{https} / /$ github.com/IrishMarineInstitute/awesome-erddap

${ }^{24} \mathrm{https} / /$ earthdata.nasa.gov/cmr

${ }^{25}$ https://data.noaa.gov/onestop

${ }^{26}$ https://toolbox.google.com/datasetsearch
}

efficiency across agency, national, or other jurisdictions. An example of cross-jurisdictional discovery is the Committee on Earth Observation Systems (CEOS) Common Data Assets (CDA) infrastructure, which facilitates federated search of interagency data holdings, including, NASA, NOAA, ESA, and other space agencies. This general architecture and approach, fundamentally enabled by data interoperability across systems that stems from the harmonized use of International Organization for Standardization (ISO) geospatial metadata standards, serves as a scalable implementation model for GEOSS, of which GOOS is a part. Improved integration of distributed ocean observing systems should leverage such existing development models, data system architectures and their associated data interoperability standard frameworks. Currently, these appear to implemented more at the regional and national level by responsible entities such as $\operatorname{IOOS}^{27}$ for the US, the Integrated Marine Observing System (IMOS) $)^{28}$ for Australia and the European Marine Observation and Data Network (EMODnet) ${ }^{29}$ for Europe, or for particular observing system elements, such as the JCOMMOPS ${ }^{30}$ asset monitoring system.

\section{USE CASES}

\section{Long Term Preservation at US National Centers for Environmental Information}

The FOO places high importance on data preservation, so those data can be reused in the future. Long term preservation is the mandate of archive centers such as the US National Centers for Environmental Information (NCEI). In this use case, NCEI is the Data Consumer, receives data from the Data Producers and assuming the responsibility for data stewardship. This transfer of stewardship responsibility places a heavy burden on the archive centers, because they must ensure future users, who will undoubtedly use different tools than today's users, can find, access, and understand the data. The archives cannot rely on personal connections to Data Producers, who won't be around decades into the future to explain their methods. Instead, the archives must ensure that the necessary information is captured today and encoded, preferably in machine readable data systems, so it is usable for solving tomorrow's problems.

Long term preservation requires managing data through information technology evolutions. Over the next 50 years the storage system will evolve from spinning disks to some yet to be envisioned technology. Similarly the software libraries enabling the use of scientific data formats will evolve. For example, the netCDF libraries in use today are primarily version 3.0 and 4.0. As hardware architectures evolve it is reasonable to assume that netCDF 3.0 will no longer be supported at some point and the archive center will need to undertake a mass migration of millions of data sets encoded in netCDF 3.0 to netCDF 5, 6 , or 7 , or some other representation suitable for cloud-based

\footnotetext{
${ }^{27}$ https://ioos.noaa.gov/

${ }^{28}$ http://imos.org.au/

${ }^{29}$ http://www.emodnet.eu/

${ }^{30}$ https://www.jcommops.org/
} 
processing, for example. The scale of a task like that underscores the need for the archive data holdings to be as interoperable as possible to allow for more automated data migration. For highly heterogeneous holdings, data migrations involving substantial individual analysis and human interventions will be prohibitively expensive.

To address these concerns, and to facilitate the broader adoption of these Earth science data interoperability standards components within the oceans community, NOAA/NCEI developed the netCDF templates ${ }^{31}$. These templates, along with documentation and examples, serve as a practical roadmap for the implementation of existing CF and ACDD standards to the range of spatial feature types characteristic of ocean and other environmental data: point, profile, trajectory, time series, and combinations of these discrete geometry types. These templates are being leveraged by other agency data centers such as NASA/PODAAC to ensure that oceanographic field campaign datasets submitted are archive quality and interoperable, such that they can be readily assimilated and disseminated via standards-aware tools/services and consumed by remote software applications. Regional data management efforts such as IOOS and IMOS have also adopted these templates as the de facto standard for data formats, supporting both current dissemination and long term preservation strategies. Global adoption of the NCEI templates would greatly enhance GOOS Data System interoperability.

\section{TPOS 2020}

The tropical Pacific Ocean has hosted some of most innovative ocean data and information management initiatives over the last 30 years (McPhaden et al., 1998). Smith and Hankin (2014) examined user requirements for the 2014 Tropical Pacific Observing System (TPOS) 2020 review and Smith (2018) undertook a similar task for the review of the Tropical Atlantic Observing System. One common finding was that neither observing system had significant gaps or issues at a technical level that were peculiar to that region; globally implemented systems such as those overseen by WMO and IOC data and information systems and including those under JCOMM oversight (Pinardi et al., 2019), were the best route for improvement, innovation and enhancements. Routine ocean and climate productions systems (data assembly, analyses, forecasts) and associated downstream users drove real-time data and information system requirements, but in both cases, research remained an important pathway for impact.

There are no systems or components that are TPOS specific. Rather TPOS information is managed and delivered by the information systems that support the platforms comprising the tropical Pacific observing system: Argo, tropical moorings, the Voluntary Observing Ship Program (VOS), the Ship-ofOpportunity Program (SOOP) (Goni et al., 2010), and surface drifters (Elipot et al., 2016). Independently, these data are collected, subjected to automated Quality Control and submitted to the Global Telecommunication System. For moorings, the infrastructure developed for TAO/TRITON (and for PIRATA

\footnotetext{
${ }^{31}$ https://www.nodc.noaa.gov/data/formats/netcdf/v2.0/
}

in the Atlantic) continue to be supported, for delayed-mode QC and for reprocessing, among other things. Standards have been developed so that tropical observations are intercomparable across the basins, but interoperability across platforms is more problematic. Knowledge of the climate of these basins is needed for this process. The arrays are at different levels of maturity, and involve multi-national efforts, so basin-centric coordination is needed. As well, the various components that comprise the TPOS are at different levels of maturity in terms of meeting the FAIR guidelines. Data systems, such as the aforementioned Argo and tropical mooring systems, are rather mature and therefore have higher levels of conformity to those guidelines. Interoperability among the various networks, however, is an issue that needs improvement and that the JCOMM community is working to improve (Pinardi et al., 2019). The most successful data systems, such as Argo, tend to be those systems that are also widely used by the community for which they were built. The Argo community uses the Argo data system, and therefore has provided feedback on the completeness of the data and metadata, and on the utility of the data system. This feedback, and the enhancements it provides, benefits the global community of users as well, and therefore improves overall interoperability - both of the data and the data system.

Remote sensing data are generally global in coverage and are provided without any distinction between basins. For more experimental data streams, e.g., research vessel measurements of pCO2, BioGeoChemical-Argo, there is a transition from PI-based to regional and then global data systems.

Opportunities for improved efficiency, robustness and effectiveness were identified in both cases. "We want it now" was a common theme among users which impacts consideration of timeliness, efficiency, and simplicity. Systems that deliver services through multiple channels, and with different offerings in terms of integration and quality, were seen as a priority (for example, ERDDAP Pinardi et al. (2019), Tanhua et al. (2019), and Harscoat and Pouliquen for AtlantOS, personal communication). A significant finding was that complexity was a barrier to stakeholder engagement, either as a provider or as a user.

That complexity arose as a barrier to engagement should not be surprising. The tropical Pacific Ocean is home to a variety of observing elements, particularly when compared to the design of the original mooring array. As other systems, such as unmanned autonomous surface vehicles, become more common, the complexity of integrating data streams from these heterogeneous platforms will increase. Though TPOS 2020 recommends the use of CF compliant netCDF formatted data files, as previously noted, in some cases this can provide a barrier to stakeholder engagement. Typically, the barrier is in the creation of data files that conform to a standard, such as CF, by a data producer that doesn't typically use those types of files, either in their own work or within their community. However, there is clearly a big advantage to having metadata attached to the data file, as it is in netCDF. In order to successfully engage all data providers, it is advantageous to allow providers to work in the data formats they are comfortable with, while still providing the data to the global community via data standards, conventions and web services. This is possible with brokering tools such as 
ERDDAP (see section "Recommendation for Addressing Some Gaps"). It is important to note, however, that such tools are less able to broker metadata between communities, such as physical oceanography and ecology communities. This is a role in which linked semantic data concepts, as discussed later in this paper, are very relevant.

National and institutional data policy also remains an issue despite successive OceanObs conferences highlighting the value of a data sharing paradigm being adopted across all systems. In the tropical Pacific it is mainly an institutional/research issue, while for developing countries in both basins it has both technical capability and historical roots. Because of this, it will be important for TPOS 2020 to embrace a distributed data landscape.

Smith (2018) noted that the FAIR Principles do provide a basis for defining a set of essential characteristics for data and information system. Such principles, and the best practice efforts, might provide a more effective pathway for improved harmonization and performance; maturity levels are useful for individual technical elements but are very uneven across the data system. Finally, both papers highlight the need for improved knowledge and use of systems architecture. Improvements over the next decade will be difficult without this.

\section{Fisheries Data From Trawl Surveys}

Surveys for fish, plankton and zooplankton have a long history, and some time series are more than 100 years old. Examples include the Norwegian beach seine survey from 1921, the Sir Alister Hardy Foundation for Ocean Science (SAHFOS) continuous plankton recorder survey from 1931, the Norwegian spring spawning herring series and the northeast arctic cod series, both from 1900. The series serve as input to fisheries stock assessments (Gulland, 1988; Beamish et al., 2009), and are important for studies addressing fish and nekton responses to climate change, ecological regime shifts etc. (Cury et al., 2008). The series are typically tracking regional populations of a species (fish stocks), and is typically regional in extent.

Several data centers are hosting the data from these surveys, including international bodies like the International Council for Exploration of the Sea and national institutions like NOAA Fisheries, CSIRO Australia, and Institute of Marine Research, Norway. The data sets typically consist both of sample data at a station or transect level, and integrated time series that tracks the abundance of a fish stock used as input to assessment or other models. Discoverability metadata is implemented to a varying degree, for example at the International Council for the Exploration of the Sea (ICES) data center.

Data Access solutions for fisheries data can vary significantly. The sample data from the joint Norwegian-Russian winter survey underpinning the advice for the North East Arctic Cod are only partially available due to a strict Russian data policy. The SAHFOS data is freely available, but only upon request. The data from most European trawl surveys are typically available through the ICES data center, and the data is accessible for automated downloads via web services. There are also standard vocabularies available, and fields like platform or vessel, species and gear types are in some cases available through web services, e.g., from the ICES data center.

Since most of these surveys are regional, the emphasis has been to ensure that data time series are consistent rather than trying to harmonize between the various interoperability standards. There is, however, a push from data managers to employ metadata standards to facilitate better discoverability and interoperability. Physical oceanography has been leading this field and advanced much further than the biological component in this respect. However, when moving forward with the biological data, employing existing standards that were developed for data from other disciplines may pose some challenges and impart additional costs. This can be in terms of costs in developing mechanisms to host the information, adding additional labor costs during data collection, or costs for make historical data compliant to the new standard. This may be less of a problem if the sensors can supply this information directly, but any manual labor is costly.

The other challenge is that it may create a false conception of interoperability in cases where a given metadata standard is used to accommodate data types that it was not designed for. An example may be trawl survey data, where the information from each trawl station is available (the primary sampling unit), but where no standard exists for the other key parameters, like survey design, survey area, stratification, data filtering parameters etc. Without this information and in the absence of suitable accompanying documentation, the data cannot be correctly used even if the metadata on the individual trawl station, from a data center point of view, conforms to the FAIR principles.

What would be the best way forward to obtain more complete interoperability for such complex datasets? Trawl surveys have one great strength: the data is tightly linked to management decision. This allows us to map out, machine to machine, if necessary, the pipeline from raw data to the data product and ensure that the information crucial to derive the desired data product, being indices of abundance for fisheries assessments or biodiversity indices for ecosystem state studies, are in place. At IMR the process of making open source software for the processing, e.g., the StoX program (Johnsen et al., 2019), relied on this approach, and it offered an approach to prioritize what was critically important for the process. It does not necessarily mean that other metadata fields are not important, but it offered a method to prioritize what was needed to obtain interoperability.

The next step would be to review the process and define a best practice guide for coding trawl survey data that is based on the actual processing pipeline. Rather than adopting a standard that was fit for another field and data type, we argue that this process would be more efficient when moving trawl surveys toward true interoperability. It may turn out that there are large overlaps with existing standards, but that should not be the prior assumption.

\section{Cross-Disciplinary Research Cruises}

The Ministry of Science, Technology and Productive Innovation (MINCyT Argentina) established a national initiative to promote improved scientific understanding of the Argentine Sea as a scientific basis for defining a national policy of biodiversity conservation. The initiative, known as Pampa Azul, was officially launched in 2014 to link interdisciplinary oceanographic cruise 
datasets and develop repositories capable of disseminating Marine data $\left(\mathrm{SNDM}^{32}\right)$ and biodiversity data $\left(\mathrm{SNDB}^{33}\right)$ from National Data Systems. These systems were created to integrate historical, current, and future information consistent with national policy and international programs. For oceanographic data, SNDM uses the Ocean Data Portal $^{34}$ with BODC vocabulary servers and for biodiversity data SNDB uses the Darwin Core standard.

In this policy and technical framework of stakeholders, the principal investigators began by planning cross-disciplinary oceanographic cruises. The implementation of interoperability concepts from the beginning had different levels of maturity in each discipline. In the case of physical oceanography, the maturity is high because principal investigators are familiarized with interoperability concepts and have history of participation in global projects (e.g., SAMOC, ATLANTOS, IPCC). Chemical oceanography adopted the BODC vocabulary (I1 and I2) for laboratory analysis and the IODE developed training courses to highlight best practices, but there is no data currently being uploaded to the systems (low maturity). Fisheries biology data has some mature elements, but is not currently widely accessible and is administered by the National Fisheries Agency (acronym INIDEP). They apply acoustic methods, perform validations of abundance and biomass data, and have developed protocols defining processes from the acquisition to the analysis of importance commercial species.

In this attempt to integrate all data types into a single system, it is important that new cross disciplinary oceanographic cruises, recognize the interoperability challenge and plan in advance, where possible, to comply with FAIR principles.

The steering committee of the Pampa Azul adopted the IMBER recommendations which elaborated protocols for crossdisciplinary cruises and explained, in detail, the processes for each stage of a cruise [see IMBER Cookbook (Pollard et al., 2011)]. The IMBER recommendations were the starting point for the design of the data acquisition system which was shared between the members of the cruise. The acquisition system managed the various types of data collected: (1) continuous shipboard data; (2) observation data collected by principal investigators; (3) the analysis of samples in the laboratory; and (4) the derived products. Ideally metadata describing transect design, data collection activities, the instruments to be used and the different surveys at each station should be integrated in a single platform. However, this can be a challenge because often the surveys are conducted from different vessels and equipment across vessels can vary. Therefore, the presence of the data manager, starting in the early stages of planning project, is helpful to coordinate and simplify the collection of data and metadata.

One of the first post-cruise responsibilities is to submit the report to the co-participants. It is at this stage where a nonintegrated system reveals its limitations. Due the heterogeneity of the observational data, as well as the laboratory analyzed

\footnotetext{
${ }^{32}$ http://www.datosdelmar.mincyt.gob.ar/

${ }^{33}$ https://datos.sndb.mincyt.gob.ar/ala-hub/search

${ }^{34}$ http://www.oceandataportal.org/
}

data, unless the metadata has clearly documented the processes, modeling groups that use the data may struggle in understanding it. Often, this disruption of the data life cycle can be traced back to the origin of the data. If, at that time, the data was not documented properly using standards, there is little hope for improvement as the data moves toward access and archival.

At the system level, the SNDM and SNDB work as two separate worlds and due to these drawbacks we propose improving the interoperability with the use of Linked Data (LD) (Janowicz et al., 2014).

Linked Data as a paradigm describes how to break up data silos $^{35}$ and support the publication, retrieval, reuse, and interlinkage of data on the Web. Together with other Semantic Web (SW) technologies, Linked Data shows promise to address many challenges that have affected semantic interoperability between repositories and services within and across domains that are highly heterogeneous in nature (Berners-Lee et al., 2001).

We develop an oceanographic linked dataset following the life cycle proposed by Villazón-Terrazas et al. (2011) with information provided by national cruises. For this we use the controlled vocabularies NERC ${ }^{36}$, ISO19115 standard to represent metadata records in conjunction with the geospatial standard for the SW GeoSPARQL ${ }^{37}$ and the reuse of the ontology design pattern (ODP) for oceanographic cruises (Krisnadhi et al., 2014). Publishing the vocabularies and metadata in standard RDF XML and exposing SPARQL endpoints renders them five-star Linked Data repositories.

In addition to enabling FAIR principles for these datasets, the benefits of this approach include: greater interoperability between the metadata created by cross-disciplinary projects; improved data discovery and newly developed tools can be used to explore the data. Here we have shown that the linked data provide a framework for better discovery and access to data, and it is possible to provide the highest standard of linked oceanographic data, and some of the benefits of the approach. Particularly when the results of the research have multiple stakeholders or are used by non-experts for manage and conservation purposes.

The interaction of biological, physical, geological, and chemical data in a single platform leads to the loss of information in the generalization of some parameters. From the technical point of view, each discipline has international formats and, as in the case of biological ones, they have extensions that avoid the loss of complementary information. With the use of linked data it is possible to keep the distributed system and solve the problem of the combination of different disciplines and sources of information.

There is no doubt, from a technical perspective, that solutions exist. However, often the problem lies in the fact that policies are required for actual implementation of those solutions. For example the permanent position of data manager in government agencies facilitate curation of data. For collecting new data, it is

\footnotetext{
${ }^{35}$ An information silo, or a group of such silos, is an insular management system in which one information system or subsystem is incapable of reciprocal operation with others that are, or should be, related.

${ }^{36}$ https://www.bodc.ac.uk/resources/vocabularies/vocabulary_search/P01/

${ }^{37} \mathrm{http}: / /$ www.opengeospatial.org/standards/geosparql
} 
necessary to promote traceability throughout the whole data life cycle and ensure the availability of essential and observational metadata collected during cruise surveys.

As a final recommendation, international coordination entities should encourage national agencies to implement FAIR and ISO standards in their data systems as a requirement for project funding.

\section{Operational and Research Modeling}

Operational and research numerical modeling efforts form a critical source of information to complement ocean observations. Numerical models are consumers of observational data and they are providers of data sets generated through simulation. They require observational data for model validation and verification and they increasingly ingest observational through data assimilation methods during the model integration. As such, they provide a use case for testing the efficacy of data interoperability standards for both ingesting observations and publishing model results.

Operational modeling centers typically access observational data through private networks governed by the World Meteorological Organization (WMO) standards, like the Global Telecommunications System (GTS) (Blanc et al., 2010; Hankin et al., 2010). While these networks are built upon different standards than are described here they serve their intended purpose for a very specific, and important, group of stakeholders. The broader research community does not have easy access to the GTS and therefore requires an alternative access pathway. Wilkin et al. (2017) describe a framework that would advance coastal modeling in the US and advocate for an open access portal that serves quality controlled near real time observations for ingestion into ocean models. Ideally these portals also include deep archives of long time series observations in addition to near real time data. These comprehensive access portals are a challenge to design because they involve integrating data from many individual data sources with their own data stewardship lifecycles. Nevertheless, providing a reliable source of quality observations to both operational and research modelers will accelerate the transition of modeling advances from the research community to the operational community. Ensuring both communities are served should be a requirement for the GOOS Data System. The JCOMM Open Access to GTS project is taking steps to address this inefficiency by simplifying the process of getting data onto and off of the GTS for the research community (Pinardi et al., 2019). NOAA's Observing System Monitoring Center (OSMC) ${ }^{38}$ provides access to near real-time ocean observations through the ERDDAP data platform. The interoperable web services that ERDDAP provides allows consumers of varied technical levels to access and use the real time data stream from the GTS through the software clients with which they are most familiar. Capabilities like these become ever important as the WMO community continues to require complicated, binary, table-based data formats for distribution on the GTS.

\footnotetext{
${ }^{38}$ http://www.osmc.noaa.gov
}

Signell and Snowden (2014), describe a framework for model data dissemination built on $\mathrm{CF} /$ netCDF/THREDDS that provides standards compliant data through a THREDDS Data Server. The THREDDS data service provides access to data through, among others, the OPeNDAP protocol which has been a popular tool for providing access to model data on a regular horizontal grid (Hankin et al., 2010). Recent developments in standardizing the encoding of unstructured grids (UGRID) ${ }^{39}$ and staggered grids (SGRID) ${ }^{40}$ have led to proposed improvements in the CF conventions. These improvements are at the Concept phase of maturity but are prompting debate within the standards governance community and development of software libraries to further test the concepts. Further development of software tools, along with adoption of these standards by the community modeling developers, is necessary to advance UGRID and SGRID to the Mature phase.

Standards for model data, and their inclusion in web service tools is critical due to the high volumes of data models provide. Increasingly it is infeasible to download and entire simulation for scientific or operational application. Therefore it is essential to support development of robust flexible server side subsetting tools if data consumers are to fully exploit the high volumes of information created by the modeling community. A promising area of development is in server side processing, especially when combined with cloud computing architectures (Vance et al., 2019).

\section{LIMITATIONS OF CURRENT SYSTEMS}

\section{Challenges to Data Interoperability Adoption}

There are several constraints, conceptual and practical, to the broader adoption of the kinds of established data interoperability standards described to projects involved in ocean observation, which in turn impacts the usability and accessibility of the data, and the advancement of GOOS more generally.

\section{Importance of Standards Not Understood}

First, the importance of data standards often are not fully understood, and the broader value of publicly sponsored data collection efforts beyond the specific science purpose for which they may have been collected and as part of an important data commons may not be adequately appreciated. This plus the implementation of data management practices that ensure the preservation and usability of data assets long-term must motivate and be promoted amongst stakeholders at all levels and further reinforced both by engineering requirements and governing program policies.

\section{Data Standards Are Hard to Understand and Use}

A second significant constraint is the lack of understanding of the applicable technical data interoperability standards that we have previously described (e.g., TPOS 2020) and which are

\footnotetext{
${ }^{39} \mathrm{http}$ //ugrid-conventions.github.io/ugrid-conventions/

${ }^{40}$ http://sgrid.github.io/sgrid/
} 
integral to ocean observing data management best practices. For non-experts, just understanding Earth science/geospatial data standards such as CF, ACDD, Darwin Core, ISO19115, and then how to practically apply them in the context of one's own particular datasets is a significant effort. Therefore, implementation of these standards is non-trivial. Our experience in working with oceanographic field campaigns and other in situ data producers is that even with resources such as the NCEI netCDF templates, implementing the necessary custom software routines to correctly undertake the necessary conversions and validate outputs for individual datasets is often involved and an iterative process. Even with the availability of compliance checker utilities online ${ }^{41}$, the process often requires multiple consultations with experts at data archives to resolve issues. The effort and resources necessary to do this is typically underestimated, and often included in project data management plans as an afterthought. Increasing the focus on data management and stewardship to ensure adequate technical skills and funding, at the proposal stage is a strong recommendation of this article.

\section{Recommendation for Addressing Some Gaps}

Addressing these issues will require a multifaceted approach, including further outreach, education, resources and practical tools, promoting improved data interoperability best practices. As has been emphasized, tackling interoperability issues as early on in the data lifecycle and as close as possible to the time of production is important. An area that can be improved upon and can have significant impact is greater engagement and partnership with in situ instrument and platform manufacturers to facilitate production of standards compliant data file outputs natively at source as an option in their processing software. Manufacturers are responsive to market demands, and so should be receptive to user, project and program sponsor requests in this space given the necessary awareness. There are examples where this has been achieved and produced the desired outcomes. Invariably, however, it is a process to secure the necessary buy-in to affect change if the business case and incentive for doing so is unclear.

\section{Tools facilitate file creation and translation}

While well-structured, complete, self-describing data files that comply with accepted metadata content models are a building block of a more interoperable data system, the complexity of these files is a barrier to adoption for many data providers. Tools are needed to enable conversion from commonly used formats like Comma Separated Value into netCDF and to augment the converted files with rich metadata complying with modern data content standards. ROSETTA ${ }^{42}$ and ERDDAP are two examples of software that facilitate file creation.

ROSETTA is a Web-based data format transformation service developed by Unidata and available Open Source ${ }^{43}$. It provides an easy, wizard-based interface ${ }^{44}$ and service for data providers to interactively transform their ASCII output instrument data into

\footnotetext{
${ }^{41}$ https://compliance.ioos.us/

${ }^{42}$ https://doi.org/10.5065/D6N878N2

${ }^{43}$ https://github.com/Unidata/rosetta

${ }^{44}$ https://youtu.be/2IrSDTUfeNU
}

Climate and Forecast (CF) compliant netCDF files. ROSETTA also provides a RESTful web service interface (API) for bulk data conversion $^{45}$. In addition to CF, ROSETTA supports also ACDD, the NCEI templates, a metadata standard profile developed for the biologging community, and is readily extensible to support metadata profiles for other science domains. ROSETTA provides full support for all spatial feature types associated with the range of discrete sampling geometries characteristic of in situ data consistent with the CF standard and NCEI templates. ROSETTA is built upon the netCDF-Java library, which is an implementation of the CDM, and which underlies widely used data access technologies such as THREDDS. It also employs commonly used web-based technologies such as Spring and JavaScript for the Web-front end.

ERDDAP takes a slightly different approach to creating standards compliant netCDF files. In addition to functioning as a data access service to publish data on the web, ERDDAP acts as a data broker that can convert between dozens of scientific file formats, including CF compliant netCDF files. For example, ERDDAP can ingest a collection of CSV files and serve the same information to users as a CF/netCDF file. The dataset can be augmented with additional metadata through a markup language that is part of the server configuration file. ERDDAP also provides automatic generation of ISO 19115 metadata, and can create BagIt ${ }^{46}$ archive packages which can automate submission to national archive data centers.

\section{Limitations of Existing Technical Standards}

Earth science data interoperability standards that govern the production of archive-quality data files support a broad range of oceanographic data types and are integral to ocean observing system data management infrastructures. However, as has been illustrated in some of the use cases above, there are some limitations to current standards such as CF/ACDD and the associated oceanographic NCEI netCDF template implementations that constrain their more universal application.

One issue is that these standards focus primarily on geospatial characteristics of data and their metadata representation. There is a need to extend standards specifications so as to additionally support richer sets of metadata that may be specific to certain science domains and to package such augmented metadata in a non-ad hoc, machine-readable manner within self-describing science data files (nc, HDF). Such metadata attributes would document more fully for example critical information on aspects of instrument deployment, sampling and other protocols necessary to properly and reproducibly interpret the associated file data. This is important because dataset level metadata used to catalog collections of data files invariably do not capture this necessary information with sufficient granularity for in situ datasets that will differ in descriptive content between files. This is also likely to be particularly important for certain classes of data, such as biological datasets. The ability to package richer metadata in a machine-readable

\footnotetext{
${ }^{45}$ https://youtu.be/_4jIDvrqiZo

${ }^{46}$ https://tools.ietf.org/html/draft-kunze-bagit- 17
} 
manner will inevitably have broader applicability and facilitate improved, more granular data search. It can also enable the integration of the existing SensorML metadata framework to better describe instrument characteristics with associated data and then potentially expose that information via Open Geospatial Consortium (OGC) Sensor Web Enablement ${ }^{47}$ (SWE) standards. There are already examples of satellite missions such as SMAP utilizing Group structures in HDF5 data files to organize hierarchically related sets of metadata attributes and encode these consistent with ISO standards. An analogous approach has also been implemented to support community metadata specifications for animal telemetry data involving 130 attributes organized thematically within 10 categorical Groups and dispositioned as required/recommended/optional. The biodiversity community uses the Darwin Core metadata standard to encode several categories of attributes related to the discrete sampling events, their geospatial characteristics, taxonomic composition and associated quantitative metrics for which the aforementioned framework will also be applicable for. A further example comes from the marine bio-acoustics community in which the international MESOPP ${ }^{48}$ (Mesopelagic Southern Ocean Prey and Predators) project has delivered acoustic backscatter and related modeled data products as netCDF files with CF metadata supplemented with ICES metadata conventions for active acoustic systems ${ }^{49}$.

A further interoperability challenge relates to frequently observed partial overlap of certain categories of attributes used by different science domain metadata frameworks. Several schemas share certain categories of attributes (e.g., Geospatial) that may conflict. There are also examples where even closely related communities (Biodiversity and Ecological sciences) may be using different metadata models (Darwin Core and EML) to represent even the exact same types of data. There is also considerable overlap in geospatial attributes across domain metadata conventions and Earth science such as CF/ACDD. This highlights the importance of establishing mappings across these schemas to facilitate semantic reconciliation of attributes between datasets produced by different communities, and to be able to do so in an automated service-based manner so as to enable also improved granule level search.

Improved support for provenance information is another important area for extensions to existing Earth Science data standards. This is becoming increasingly important as greater integration of data occurs and there is a proliferation of derived products. Currently CF foresees only a single History attribute to capture provenance and processing information, invariably implemented in ad hoc ways that are generally also undocumented. $\mathrm{PROV}^{50}$ is a W3C standard for the representation of provenance information that could be leveraged here, and implemented using groups in a manner consistent with the aforementioned suggested approach to

\footnotetext{
${ }^{47}$ http://www.opengeospatial.org/ogc/markets-technologies/swe

${ }^{48}$ http://www.mesopp.eu/

${ }^{49}$ http://www.ices.dk/sites/pub/Publication\%20Reports/ICES\%20Survey\% 20Protocols\%20(SISP)/SISP-4\%20A\%20metadata\%20 convention $\% 20$ for $\%$ 20processed $\% 20$ acoustic $\% 20$ data $\% 20$ from $\% 20$ active $\% 20$ acoustic $\% 20$ systems.pdf

${ }^{50}$ https://www.w3.org/TR/prov-overview/
}

support richer metadata more generally. Tracking of provenance in the evolution of metadata standards themselves and their inter-relationships is another area that can be improved upon to facilitate improved granule level search across data series where a blend of metadata profiles and versions may have been employed over time.

There is considerable interest in the inclusion of measurement uncertainty and data quality information in data files. CF standards provide a framework for quality flagging of observational data at a very granular level within variables of self-describing data files. Furthermore, certain communities (e.g., GHRSST) have worked to develop a standardized approach to the representation of uncertainty in geophysical data. However, there is a need to extend these approaches to also represent error in geolocation variables given that positional determination (and in some cases estimation) may be a particular issue for in situ datasets and may take the form of a qualitative category code or quantitative error estimate. The Argo profiling float data format standard makes extensive use of category codes, including beyond geolocation variables.

The trawl survey use case discussed in the previous section highlights the broader issue of how to better support complex datasets in a manner that better captures inter-relationships between recorded data elements when Earth Science data interoperability standards and typical system architectures focus on simple collections of discrete data files. Even with support for hierarchical structured data in the nc4/HDF5 data models, the ability to comprehensively represent complex event-based datasets such as cruise data in a single data file is an unrealistic expectation. Instead there may be more promise in developing a framework in which time course and functional relationships between data files are represented in a standard manner so as to better define a given dataset or file collection.

\section{SUMMARY AND RECOMMENDATIONS}

The use cases in the previous section lead to several key considerations for charting the course ahead for the next 10 years of ocean data management, specifically in enhancing interoperability across components of the GOOS Data System. These issues impact stewardship, discoverability and access to ocean data. In general, we recommend adherence to the FAIR data principles, though the details of what precisely that entails may differ between communities, and specific definitions of what FAIR compliance means within a community are still under discussion (Tanhua et al., 2019). The coming decade should do much to provide more specific implementation details for FAIR compliance.

Hankin et al. (2010) asserted that the combination of the netCDF file format, the Climate and Forecast Conventions, and the OPeNDAP network protocol formed the basis of a data management strategy for GOOS. Although at that time, the strength of this combination was gridded data. In the last 10 years the importance of netCDF/CF/OPeNDAP has grown and the capabilities now extend to in situ data types (points, profiles, time series etc.). The standards and 
the tools have evolved methodically and deliberately to address important data management problems. We conclude that a netCDF/CF/OPeNDAP remain the building blocks for ocean data management and that by focusing on incremental enhancements to mature technologies will be a sustainable path for the global community. The NCEI templates are the gold standard examples for many of the common data types encountered in GOOS and should be the starting point for any data management effort.

This collection of standards is complex and incomplete. The complexity requires strategies to simplify adoption through education and tool development. Communities of practice help spread knowledge and focus development efforts on tools of general utility, especially when combined with Open Source development practices. The incompleteness requires evolving the family of standards and finding ways to cross-reference and link to data that is managed according to other discipline specific but mature technologies. The issues surrounding multidisciplinary observations in general (see section "Cross-disciplinary Research Cruises") and trawl surveys in particular (see section "Fisheries Data from Trawl Surveys"), shed light on the differences between community data standards across science disciplines and suggest that pragmatic ways to bridge those standards is more likely to garner adoption than attempting to develop one standard for all data types. Further, the balance between global interoperability and local project priorities is a factor that should be considered in the funding and policy framework nationally and internationally. Linked Open Data strategies show promise in bridging discipline specific communities but those capabilities are nascent and at a relatively low maturity level.

Standards provide a framework to record and share essential metadata, but without concerted effort to populate the files with metadata, interoperability is still lacking. Strategies to incorporate metadata into the data stream automatically and without human

\section{REFERENCES}

Beamish, R. J., Rothschild, B. J., and American Institute of Fishery Research, Biologists. (2009). The Future of Fisheries Science in North America. Dordrecht: Springer.

Berners-Lee, T. I. M., Hendler, J., and Lassila, O. R. A. (2001). The semantic web. Sci. Am. 284, 34-43.

Blanc, F., Baralle, V., Blower, J. D., Bronner, E., Cornillon, P., deLaBeaujardiere, J., et al. (2010). "Evolution in Data and Product Management for Serving Operational Oceanography, a GODAE Feedback," in Proceedings of OceanObs'09: Sustained Ocean Observations and Information for Society, (Paris: ESA), 40-46.

Cury, P. M., Shin, Y.-J., Planque, B., Durant, J. M., Fromentin, J.-M., Kramer-Schadt, S., et al. (2008). Ecosystem oceanography for global change in fisheries. Trends Ecol. Evol. 23, 338-346. doi: 10.1016/j.tree.2008. 02.005

Elipot, S., Lumpkin, R., Perez, R. C., Lilly, J. M., Early, J. J., and Sykulski, A. M. (2016). A global surface drifter data set at hourly resolution. J. Geophys. Res. Oceans 121, 2937-2966. doi: 10.1002/2016JC011716

Goni, G., Roemmich, D., Molinari, R., Meyers, G., Sun, C., Boyer, T., et al. (2010). "The ship of opportunity program," in Proceedings of OceanObs'09: Sustained Ocean Observations and Information for Society, Vol. 2, eds J. Hall, D. E. Harrison, and D. Stammer (Venice: ESA Publication WPP-306). doi: 10.5270/ OceanObs09.cwp.35 intervention will be critical to automating work flows and dealing with growing volumes of data. Engaging the sensor and platform manufacturers and applying market pressure is an essential step.

Finally, the information management and data science skills needed to develop a truly global and interoperable system require an influx of talent from outside traditional marine disciplines. Incorporating data science and system engineering perspectives into the global policy framework can help identify areas for collaboration in the future. Balancing an operational perspective based on mature technologies (e.g., CF/netCDF/OPeNDAP) against the need for research into new technologies (e.g., the semantic web and Linked Open Data) to bridge communities will be essential.

\section{AUTHOR CONTRIBUTIONS}

DS, VT, NH, MZ, KO'B, and KC helped to conceive the manuscript, coordinate author contributions, wrote text, and edited and contributed figures. NS, HS, KB, ML, and SA contributed manuscript ideas and text.

\section{FUNDING}

This work was partially funded by the Joint Institute for the Study of the Atmosphere and Ocean (JISAO) under the NOAA Cooperative Agreement NA15OAR4320063, Contribution No. 2018-0178.

\section{ACKNOWLEDGMENTS}

We acknowledge the contributions of specific colleagues, institutions, or agencies who aided the efforts of the authors.

Gulland, J. A. (ed.) (1988). Fish Population Dynamics: Implications for Management. New York, NY: John Wiley and Sons.

Hankin, S. C., Blower, J. D., Carval, T., Casey, K. S., Donlon, C., Lauret, O., et al. (2010). "NetCDF-CF-OPeNDAP: Standards for Ocean Data Interoperability and Object Lessons for Community Data Standards Processes," in Proceedings of OceanObs'09, (Paris: European Space Agency), $450-458$.

ISO/IEC/IEEE. (2017). 24765:2017 Systems and Software Engineering - Vocabulary. Geneva: ISO.

Janowicz, K., Hitzler, P., Adams, B., Kolas, D., and Charles Vardeman, I. (2014). Five stars of Linked Data vocabulary use. Semant. Web 5, 173-176.

Johnsen, E., Totland, A., Skålevik, Å., Holmin, A. J., Dingsør, G. E., Fuglebakk, E., et al. (2019). StoX - an open source software for marine survey analyses. Methods Ecol. Evol. (in press). doi: 10.1111/2041-210x. 13250

Krisnadhi, A., Arko, R., Carbotte, S., Chandler, C., Cheatham, M., Finin, T., et al. (2014). An Ontology Pattern for Oceanographic Cruises: Towards an Oceanographer's Dream of Integrated Knowledge Discovery. Available at: https: //corescholar.libraries.wright.edu/cse/167/

Lindstrom, E., Gunn, J., Fischer, A., McCurdy, A., and Glover, L. K. (2012). A Framework for Ocean Observing. Available at: http://www.oceanobs09.net/foo/ FOO_Report.pdf

McPhaden, M. J., Busalacchi, A. J., Cheney, R., Donguy, J.-R., Gage, K. S., Halpern, D., et al. (1998). The tropical ocean-global atmosphere observing system: a 
decade of progress. J. Geophys. Res. Oceans 103, 14169-14240. doi: 10.1029/ 97JC02906

Pinardi, N., Stander, J., Legler, D., O’Brien, K., Boyer, T., Cuff, T., et al. (2019). The joint IOC (of UNESCO) and WMO collaborative effort for met-ocean services. Front. Mar. Sci. 6:410. doi: 10.3389/fmars.2019.00410

Pollard, R., Moncoiffé, G., and O'Brien, T. D. (2011). The IMBER Data Management Cookbook - A Project Guide to good Data practices. Plouzane: Institut Universitaire European de la Mer (IUEM).

Riser, S. C., Freeland, H. J., Roemmich, D., Wijffels, S., Troisi, A., Belbéoch, M., et al. (2016). Fifteen years of ocean observations with the global Argo array. Nat. Clim. Chang. 6, 145-153.

Send, U., Weller, R. A., Wallace, D., Chavez, F., Lampitt, R. L., Dickey, T., et al. (2010). "OceanSITES," in Proceedings of OceanObs'09: Sustained Ocean Observations and Information for Society, Vol. 2, eds J. Hall, D. E. Harrison, and D. Stammer (Venice: ESA Publication WPP-306). doi: 10.5270/OceanObs09. cwp.79

Signell, R., and Snowden, D. (2014). Advances in a Distributed Approach for Ocean Model Data Interoperability. J. Mar. Sci. Eng. 2, 194-208. doi: 10.3390/ jmse2010194

Smith, N. (2018). Data Flow and Information Products. Available at: http://www. clivar.org/sites/default/files/3.1_Data\%20Flow\%20and\%20Information\%20Pro ducts_Neville\%20Smith_0.pdf

Smith, N., and Hankin, S. (2014). "White Paper \#13 - Data and information delivery: communication, assembly and uptake," in Proceedings of the Tropical Pacific Observing System 2020 Workshop, (La Jolla CA: Ocean Observations Panel for Climate).

Tanhua, T., Pouliquen, S., Hausman, J., O’Brien, K., Bricher, P., Bruin, T. D., et al. (2019). Ocean FAIR data services. Front. Mar. Sci. (in press). doi: 10.3389/fmars. 2019.00440
Vance, T. C., Wengren, M., Burger, E., Hernandez, D., Kearns, T., Medina-Lopez, E., et al. (2019). From the oceans to the cloud: opportunities and challenges for data, models, computation and workflows. Front. Mar. Sci. 6:211. doi: 10.3389/ fmars.2019.00211

Villazón-Terrazas, B., Vilches-Blázquez, L. M., Corcho, O., and Gómez-Pérez, A. (2011). "Methodological Guidelines for Publishing Government Linked Data, in Linking Government Data, ed. D. Wood (New York, NY: Springer), 27-49. doi: 10.1007/978-1-4614-1767-5_2

Wilkin, J., Rosenfeld, L., Allen, A., Baltes, R., Baptista, A., He, R., et al. (2017). Advancing coastal ocean modelling, analysis, and prediction for the US integrated ocean observing system. J. Operat. Oceanogr. 10, 1-12. doi: 10.1080/ 1755876X.2017.1322026

Wilkinson, M. D., Dumontier, M., Aalbersberg, I. J., Appleton, G., Axton, M., Baak, A., et al. (2016). The FAIR Guiding Principles for scientific data management and stewardship. Sci. Data 3:160018. doi: 10.1038/sdata.20 16.18

Conflict of Interest Statement: The authors declare that the research was conducted in the absence of any commercial or financial relationships that could be construed as a potential conflict of interest.

Copyright (C) 2019 Snowden, Tsontos, Handegard, Zarate, O’ Brien, Casey, Smith, Sagen, Bailey, Lewis and Arms. This is an open-access article distributed under the terms of the Creative Commons Attribution License (CC BY). The use, distribution or reproduction in other forums is permitted, provided the original author(s) and the copyright owner(s) are credited and that the original publication in this journal is cited, in accordance with accepted academic practice. No use, distribution or reproduction is permitted which does not comply with these terms. 


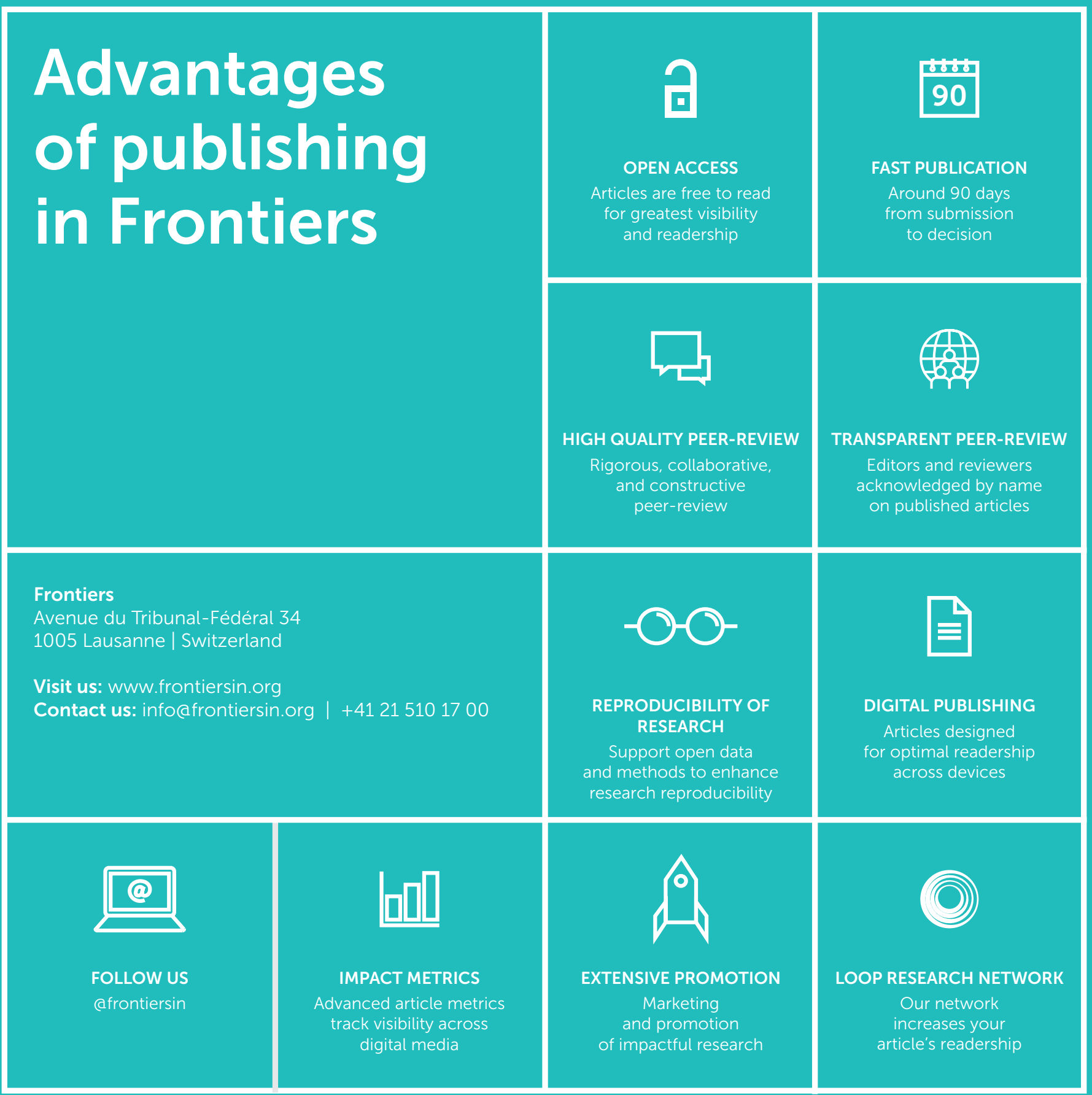

